\title{
AGC-2 Graphite Preirradiation Data Analysis Report
}

\author{
William E. Windes \\ W. David Swank \\ David T. Rohrbaugh \\ Joseph R. Lord
}

April 2013

The INL is a

U.S. Department of Energy

National Laboratory

operated by

Battelle Energy Alliance

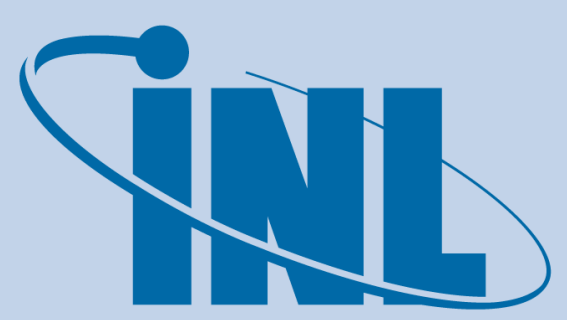

Idaho National Laboratory

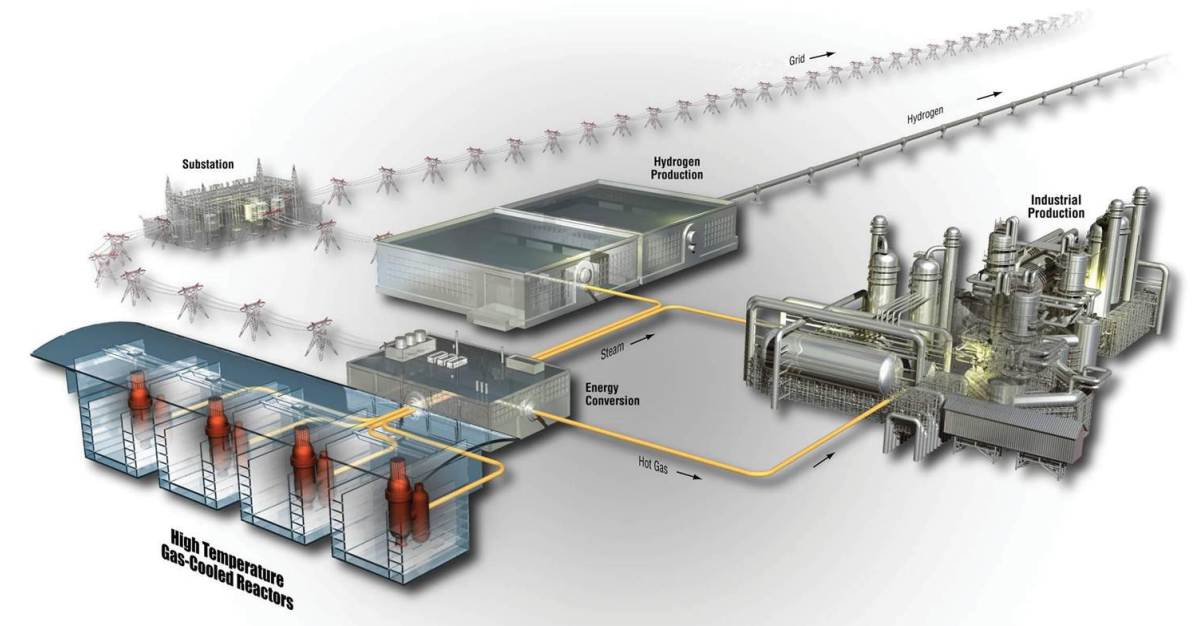




\section{DISCLAIMER}

This information was prepared as an account of work sponsored by an agency of the U.S. Government. Neither the U.S. Government nor any agency thereof, nor any of their employees, makes any warranty, expressed or implied, or assumes any legal liability or responsibility for the accuracy, completeness, or usefulness, of any information, apparatus, product, or process disclosed, or represents that its use would not infringe privately owned rights. References herein to any specific commercial product, process, or service by trade name, trade mark, manufacturer, or otherwise, does not necessarily constitute or imply its endorsement, recommendation, or favoring by the U.S. Government or any agency thereof. The views and opinions of authors expressed herein do not necessarily state or reflect those of the U.S. Government or any agency thereof. 
INL/EXT-13-28612

Revision 0

\title{
AGC-2 Graphite Preirradiation Data Analysis Report
}

\author{
William E. Windes \\ W. David Swank \\ David T. Rohrbaugh \\ Joseph R. Lord
}

April 2013

\begin{abstract}
Idaho National Laboratory
VHTR Program

Idaho Falls, Idaho 83415
\end{abstract}

http://www.inl.gov

Prepared for the

U.S. Department of Energy

Office of Nuclear Energy

Under DOE Idaho Operations Office

Contract DE-AC07-05ID14517 



\section{VHTR Program}

\section{AGC-2 Graphite Preirradiation Data Analysis Report}

\section{INL/EXT-13-28612}

Revision 0

April 2013

Approved by:

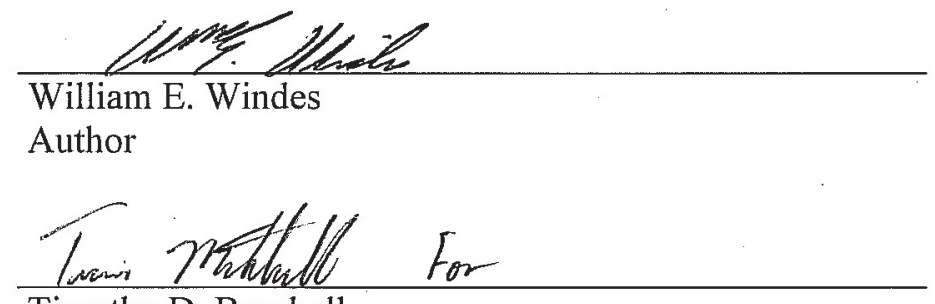

Timothy D. Burchell

ORNL Graphite Technical Peer Reviewer

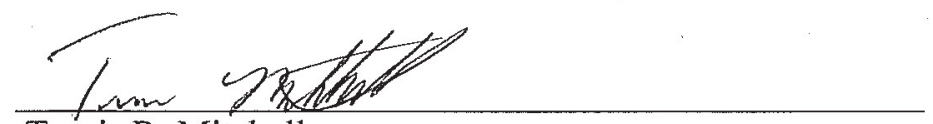

Travis R. Mitchell

VHTR TDO Project Manager
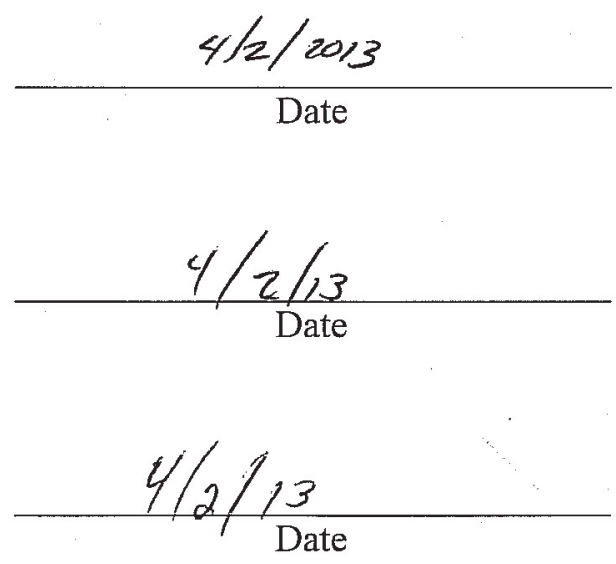



\section{SUMMARY}

This report describes the specimen-loading order and documents all preirradiation examination material property measurement data for graphite specimens contained within the Second Advanced Graphite Capsule (AGC-2) irradiation capsule. The AGC-2 capsule is the second in six planned irradiation capsules comprising the Advanced Graphite Creep (AGC) test series. The AGC test series is used to irradiate graphite specimens in order to garner quantitative data necessary for predicting the irradiation behavior and operating performance of new nuclear-grade graphites. This testing will ascertain the in-service behavior of the graphite for pebble-bed and prismatic very high temperature reactor designs. Similar to the First Advanced Graphite Capsule (AGC-1) preirradiation examination report, material property tests were conducted on specimens from 18 nuclear-grade graphite types. However, AGC-2 tested an increased number of specimens (i.e., 512) prior to loading them into the AGC-2 irradiation assembly. All AGC-2 specimen testing was conducted at Idaho National Laboratory (INL) from July 2009 to August 2010.

This report also details the specimen-loading methodology for graphite specimens inside the AGC-2 irradiation capsule. The AGC-2 capsule design requires "matched pair" creep specimens that have similar dose levels above and below the neutron flux profile mid-plane. This provides similar specimens with and without an applied load. Analysis in this document utilizes the neutron flux profile calculated for the AGC-2 capsule design, the capsule dimensions, and the size (i.e., length) of the selected graphite specimens to create a stacking order that produces "matched pairs" of graphite specimens above and below the AGC-2 capsule elevation mid-point, thus providing specimens with similar neutron dose levels. 


\section{CONTENTS}

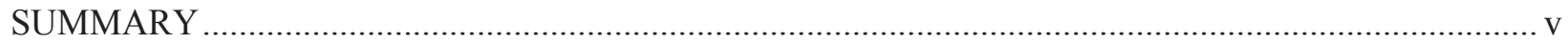

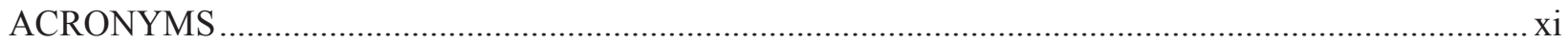

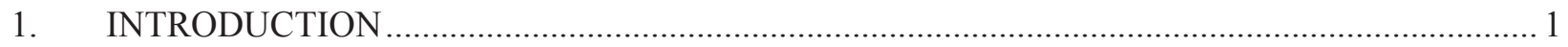

2. Description of the Advanced Graphite Creep Experiment ...........................................................2

2.1 Background Information for the Advanced Graphite Creep Experiment .............................. 2

2.2 Description of AGC-2 Test Series ............................................................................... 4

2.2.1 Establishing the Capsule's Physical Centerline to the Core Neutron Flux

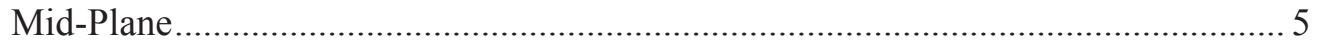

2.2.2 Establishing the Dose Levels as a Function of Position within the Capsule............... 6

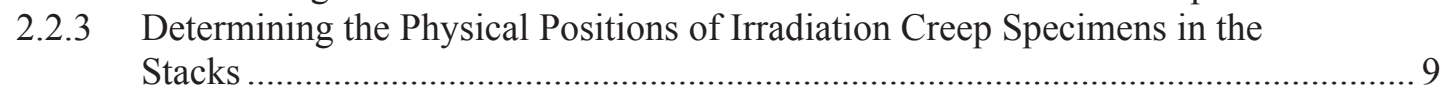

2.2.4 Determining the Physical Positions of Piggyback Specimens in the Central

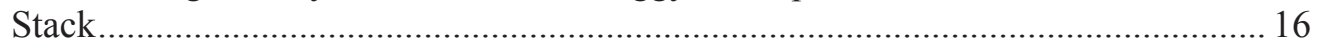

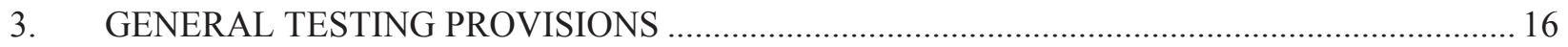

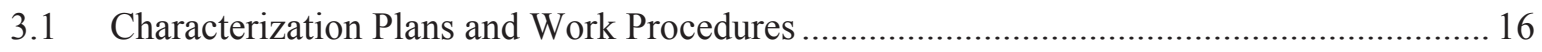

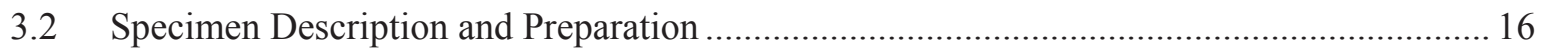

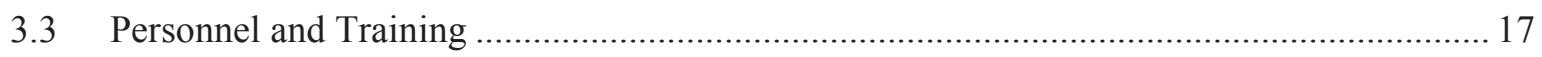

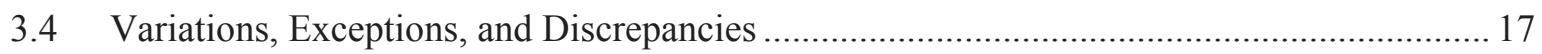

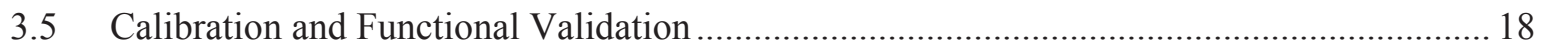

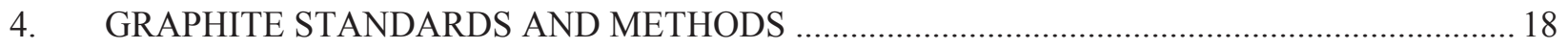

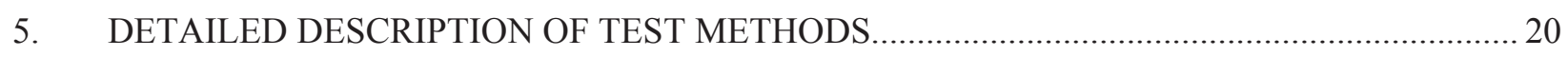

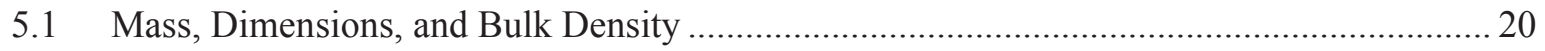

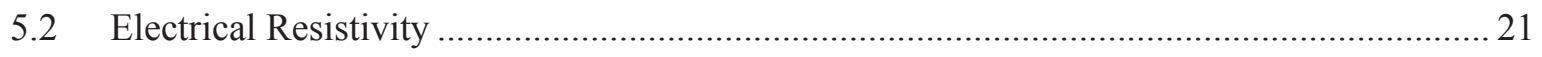

5.3 Approximation of Elastic Modulus from the Measurement of Sonic Velocity .................... 22

5.4 Modulus of Elasticity by Measurement of Fundamental Frequency ................................... 24

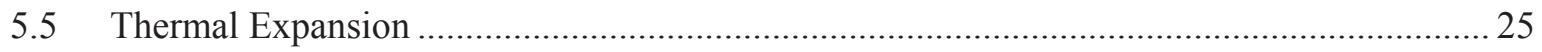

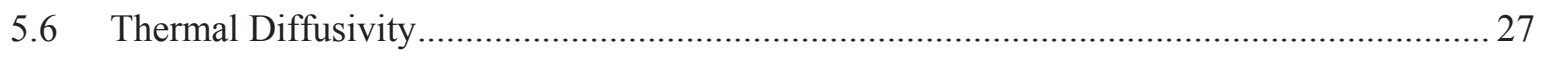

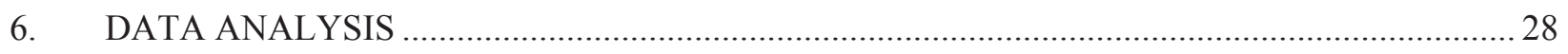

6.1 Analysis of Mass, Dimensions, and Density Data ............................................................ 29

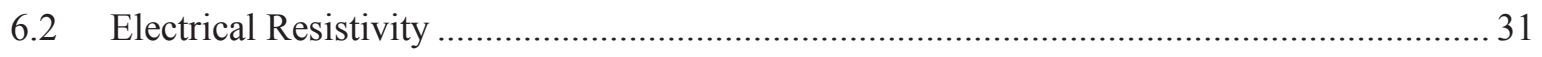

6.3 Approximation of Elastic Modulus from the Measurement of Sonic Velocity ..................... 32

6.4 Modulus of Elasticity by Measurement of Fundamental Frequency ................................... 34

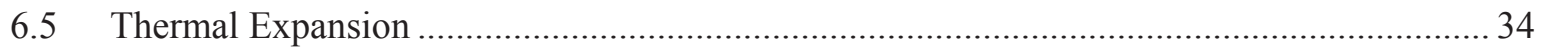

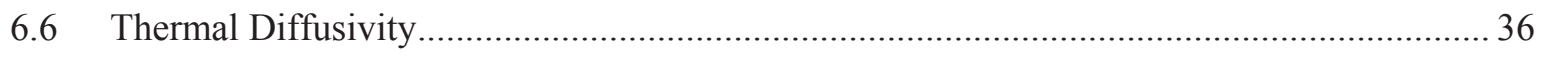

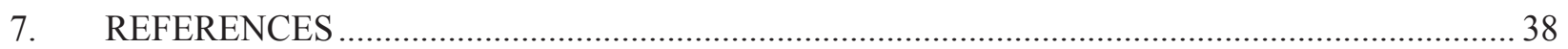




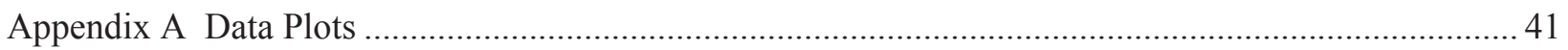

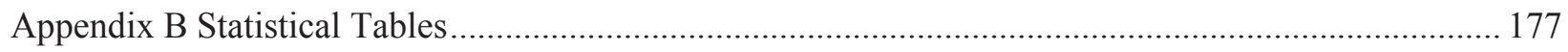

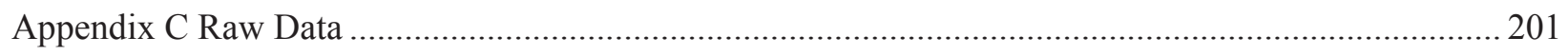

\section{FIGURES}

Figure 1. Irradiation dose and temperature parameters of the AGC test series. 3

Figure 2. Revised irradiation dose and temperature parameters of the AGC test series. Note the gray area between AGC-1 and AGC-2 is the expected dose overlap between capsule irradiation.

Figure 3. Elevation sketch of the Advanced Graphite Creep capsule.................................................. 6

Figure 4. Calculated dose levels for AGC-2 after 140 effective full-power days...................................... 7

Figure 5. Advanced Test Reactor flux profile, as a function of core mid-plane position........................... 8

Figure 6. Typical AGC-2 dose profile for creep-graphite specimens utilizing similar applied stress levels in matched stacks. 10

Figure 7. Estimated creep-specimen dose profiles for each major graphite grade. .................................. 11

Figure 7. Estimated creep-specimen dose profiles for each major graphite grade. .................................. 12

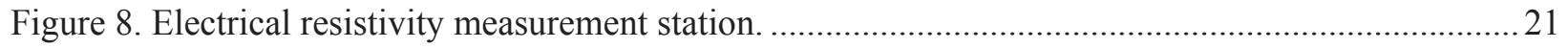

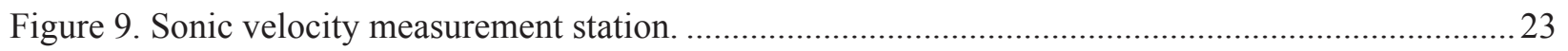

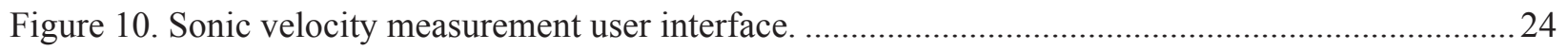

Figure 11. Fundamental frequency measurement station. ............................................................... 25

Figure 12. Commercial push rod dilatometer for measuring the coefficient of thermal expansion............26

Figure 13. Laser flash apparatus measurement station for determining thermal diffusivity......................27

Figure 14. Schematic showing location and dimensional details of NBG-17 billet density

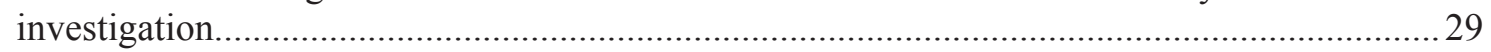

Figure 15. Density of NBG-18 as a function of depth in the billet......................................................... 30

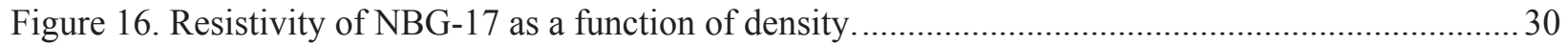

Figure 17. Electrical resistivity for several nuclear-grade graphite types. The anisotropy ratio is

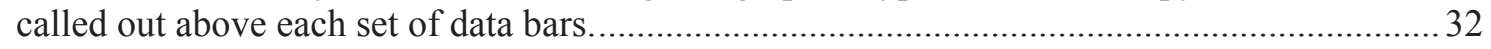

Figure 18. Measured longitudinal velocity as a function of density for NBG-17 creep specimens. .......... 33

Figure 19. Mean coefficient of thermal expansion for several types of nuclear-grade graphite as a function of temperature. Error bars represent \pm 1 standard deviation in the data used to obtain the averages plotted.

Figure 20. Coefficient of thermal expansion anisotropy ratio for several types of nuclear-grade graphite as a function of temperature.

Figure 21. Thermal diffusivity for various with-grain graphite types. Error bars are 1 standard deviation in the data and in some cases smaller than the data symbol. The percent difference between H451 and IG-110 is plotted on the right hand scale. 
Figure 22. Thermal diffusivity anisotropy ratio for several types of nuclear-grade graphite as a function of temperature.

\section{TABLES}

Table 1. Typical dose differences between matched pairs in the AGC-1 test-series capsule.....................9

Table 2. Total number of irradiated creep specimens in the AGC-2 test-series capsule. .......................... 10

Table 3. Final loading configuration for AGC-2 creep specimens in outer stacks. ................................ 14

Table 4. NGNP Graphite specimen measurement and test equipment. .................................................. 19

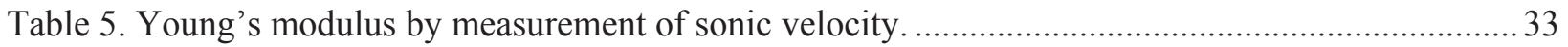

Table 6. Young's modulus by measurement of fundamental frequency.................................................. 34 


\section{ACRONYMS}

AGC Advanced Graphite Creep

AGC-1 First Advanced Graphite Capsule

AGC-2 Second Advanced Graphite Capsule

ASTM American Society for Testing and Materials

ATR Advanced Test Reactor

CCL Carbon Characterization Laboratory

$\mathrm{COV}$ coefficient of variation

CTE coefficient of thermal expansion

dpa Displacements per atom

EFPD effective full-power days

HTGR high-temperature gas-cooled reactor

INL Idaho National Laboratory

IQR interquartile range

LFA Laser Flash Apparatus

NDMAS NGNP Data Management and Analysis System

NGNP Next Generation Nuclear Plant

MCNP Monte Carlo N-Particle Transport Code

PB Piggy-Back specimen

PIE post-irradiation examination

R\&D research and development

TC Thermocouple

TDP Technology Development Plan

VHTR very high temperature reactor 


\section{INTRODUCTION}

The Next Generation Nuclear Plant (NGNP) will be a helium-cooled, very high temperature reactor (VHTR) with a large graphite core. In past applications, graphite has been used effectively as a structural and moderator material in both research and commercial high temperature gas-cooled reactor (HTGR) designs. ${ }^{[1,2]}$ Nuclear graphite H-451, used previously in the United States for nuclear reactor graphite components, is no longer available. New nuclear graphites have been developed and are considered suitable candidates for the new NGNP reactor design. To support the design and licensing of NGNP core components within a commercial reactor, a complete properties database must be developed for these current grades of graphite. Quantitative data on in-service material performance is required for the physical, mechanical, and thermal properties of each graphite grade with a specific emphasis on data accounting for the life-limiting effects of irradiation creep on key physical properties of the NGNP candidate graphites. Further details on the research and development activities and associated rationale required to qualify nuclear-grade graphite for use within the NGNP are documented in the NGNP graphite technology research and development plan. ${ }^{[3,4]}$

Based on experience with previous graphite-core components, the phenomenon of irradiation-induced creep within the graphite has been shown to be critical to the total useful lifetime of graphite components. Irradiation-induced creep occurs under the simultaneous application of high temperatures, neutron irradiation, and applied stresses within the graphite components. Significant internal stresses within the graphite components can result from a second phenomenon-irradiation-induced dimensional changewhere the graphite physically changes (i.e., first shrinking and then expanding with increasing neutron dose). This disparity in material-volume change can induce significant internal stresses within graphite components. Irradiation-induced creep relaxes these large internal stresses, thus reducing the risk of crack formation and component failure. Obviously, higher irradiation-creep levels tend to relieve more internal stress, thus allowing the components longer useful lifetimes within the core. Determining the irradiation-creep rates of nuclear-grade graphites is critical for determining the useful lifetime of graphite components and is a major component of the Advanced Graphite Creep (AGC) experiment.

The AGC experiment is currently underway to determine the in-service behavior of these new graphites for both pebble-bed and prismatic reactor designs. This experiment will examine properties and behavior of nuclear-grade graphites over a large spectrum of temperatures, irradiation fluencies, and applied stress levels that are expected to induce irradiation creep strains within a VHTR graphite component. Irradiation data are provided through the AGC test series, which comprises six planned capsules irradiated in the Advanced Test Reactor (ATR) in a large flux trap ${ }^{[3]}$ at the Idaho National Laboratory (INL) Site. This test series exposes the selected graphite specimens to temperatures and a range of doses that are expected within a VHTR design. Each irradiation capsule consists of over 400 graphite specimens that are characterized before and after irradiation.

The First Advanced Graphite Capsule (AGC-1) of the AGC test series was initially characterized, loaded into ATR, irradiated, and is currently undergoing post-irradiation examination (PIE). ${ }^{[5,6,7]}$ Characterization of the graphite specimens for the Second Advanced Graphite Capsule (AGC-2) was recently completed, and the measurements are reported in this document. Data gathered for the characterization of $\mathrm{AGC}-2$ specimens is contained in Appendixes A, $\mathrm{B}$, and $\mathrm{C}$ of this report. The original design called for AGC-1 and -2 capsule irradiations to be as similar as possible-similar graphites, irradiation temperatures, and loads applied to the creep specimens with the only difference being total received dose to the specimens. Consequently, material property tests were conducted on specimens of similar graphites as tested in the AGC-1 capsule, but on an increased number of specimens (i.e., 512) due to a change in the stacking order of the specimens within the capsule. All AGC-2 specimen testing was conducted from July 2009 to August 2010. ${ }^{[8]}$ 
To achieve the proper irradiation conditions and applied loads to the creep specimens, an exact specimen loading order is critical. Because irradiation creep is usually determined by the difference in dimensional change occurring within specimens that have an applied load and those that do not, these "matched pair" specimens are assumed to have the same irradiation dose and irradiation temperature values. To achieve these similar irradiation conditions for "matched pairs," a careful map of where each specimen resides within the irradiation capsule is required. A detailed analysis of the reactor flux profile is required to ascertain the dose levels for each specimen, as well as the designed loading configurations within the capsule, in order to guarantee that the matched pairs experience the same temperature and dose levels. This document discusses details of the specimen loading order, the capsule loading design, the flux profile within ATR, and the resulting estimated dose profiles for each graphite specimen for the AGC-2 irradiation capsule.

\section{Description of the Advanced Graphite Creep Experiment}

The AGC experiment is designed to establish the data necessary to determine the safe operating envelope of graphite core components for a VHTR by measuring the irradiated material property changes and behavior of several new nuclear grade graphites over a large range of temperatures, neutron fluencies, and mechanical compressive loads. The experiment consists of three interrelated stages; pre-irradiation characterization of the graphite specimens, the irradiation test series (designated as six separate irradiation capsules), and post-irradiation examination (PIE) of the graphite specimens after irradiation. Separate reports for each distinct stage are prepared after the activity is completed. The pre-irradiation examination reports detail the total number of graphites and specimens, the specimen loading configuration to expose all specimens to the entire range of irradiation conditions, and the pre-irradiation material property testing results. The irradiation test series reports detail the irradiation history of each capsule while in reactor, noting any changes from the technical and functional specifications for each specific test series capsule, and identifying the possible improvements to the next test series capsule design. The PIE reports detail the changes in the specimen material property measurements, compare the results to the pre-IE material property measurements, and analyze the data to assist in determining a credible safe operating envelope for graphite core components in a VHTR design and licensing application.

\subsection{Background Information for the Advanced Graphite Creep Experiment}

The AGC experiments will provide data on irradiated material properties for current graphites available for use within a VHTR design. Due to volume limitations within typical material test reactors (i.e. ATR), only a limited number of specimens can be irradiated, far fewer than can be used in an accurate statistical specimen population analysis. Therefore, the AGC only measures the changes in irradiated material properties and the behavior of relatively few specimens of new nuclear-grade graphites over the anticipated range of operating temperatures, neutron fluence, and mechanical loads. The experiment does generate quantitative material property change data (and limited irradiation creep data), which will be used in conjunction with the as-fabricated material property to predict the in-service behavior and operating performance of these new nuclear-grade graphites for pebble-bed and prismatic reactor designs. Changes to key thermal, physical, and mechanical material properties are determined by comparing the material properties of each specimen before and after irradiation. Differences measured from the irradiation conditions will provide irradiation-behavior data for graphite, with a specific emphasis on those data that account for the life-limiting effects of irradiation creep on key physical properties of several candidate graphites for use in NGNP.

The critical component of the AGC experiment is the irradiation test series, which irradiates the graphite specimens after preirradiation-examination characterization has been completed. The AGC test series comprises six planned irradiation capsules, which are irradiated in ATR in a large flux trap, as 
described in the Graphite Technology Development Plan. ${ }^{[3]}$ The test series exposes test specimens of the selected nuclear-grade graphites to temperatures and the range of doses that are expected within an VHTR design. Specifically, graphite specimens will be exposed to fast neutron dose ranging from 1 to $7 \mathrm{dpa}$ and temperatures of 600,900 , and $1200^{\circ} \mathrm{C}$, as shown in Figure 1. Similar to the AGC-1 irradiation capsule, AGC-2 was designed to be irradiated within the ATR's South Flux Trap. ${ }^{[9]}$ Generally, irradiations within the South Flux Trap require approximately 175 effective full-power days to provide a nominal

fast-neutron-dose range (in graphite) of 1-3.5 dpa. For those capsules requiring a 3.5-7.0-dpa dose range, the irradiation capsule (containing the graphite specimens) is irradiated for twice as long inside the ATR, for approximately 350 effective full-power days.

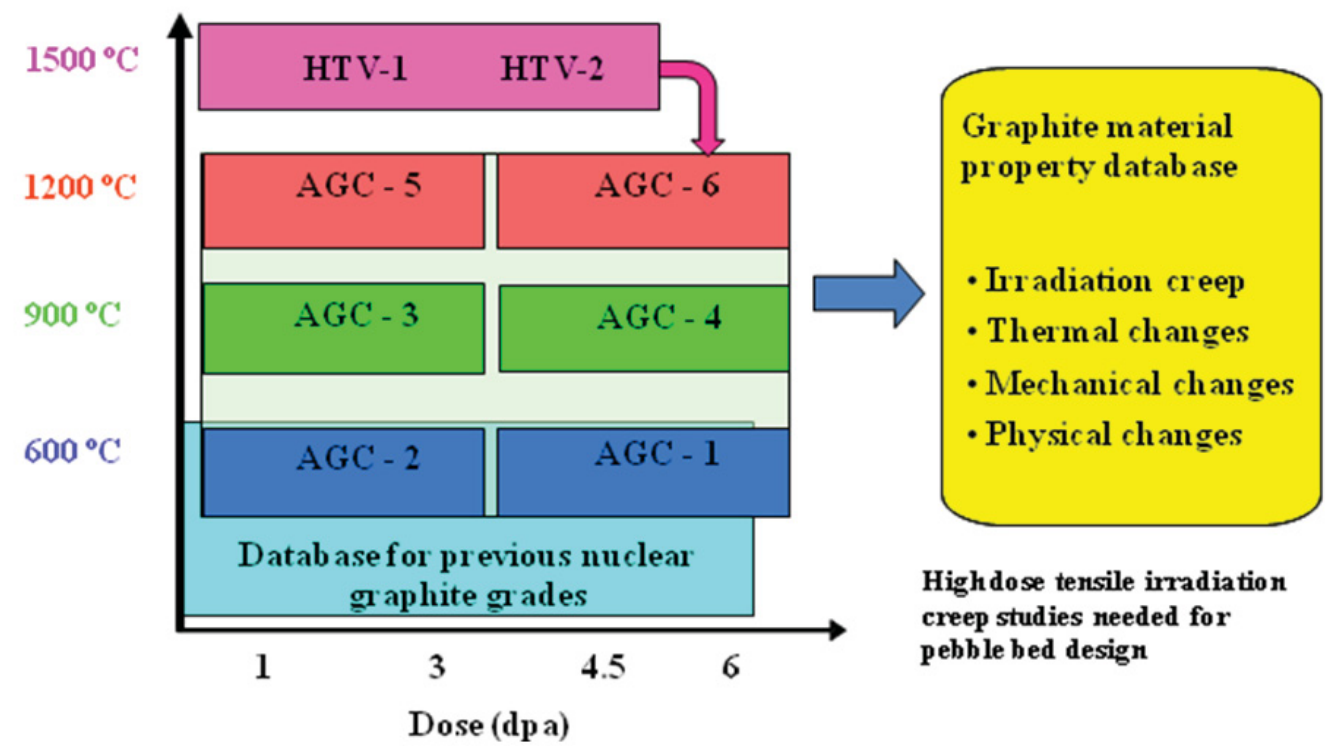

Figure 1. Irradiation dose and temperature parameters of the AGC test series.

The AGC test series dedicates a significant amount of scope to determining rates of irradiation-induced creep for different nuclear-grade graphite. The traditional method for measuring irradiation-induced creep is to apply a significant load to half the specimens during irradiation while leaving the remaining half of the specimens unloaded. The resulting difference in dimensional change between the loaded and unloaded specimens (assuming that temperature and dose levels are the same) provides the amount of irradiation-induced strain for each "matched pair" of graphite specimens. From this strain level, a creep rate for each graphite grade can be calculated as a function of dose if both specimens were irradiated at the same constant temperature. Thus, each capsule is designed to be irradiated at a constant temperature, allowing only the dose and applied mechanical load to vary within the test train of each test-series capsule. With all graphite specimens at a constant temperature, only the applied load and dose will affect the calculated creep rate of each graphite grade within a test series capsule.

To ascertain the temperature dependency of irradiation-induced material property changes, the creep rates of similar graphite specimens, at similar dose and load levels, must be compared between capsules. This implies that similar graphite grades must be in the same locations in every capsule to receive similar dose and load levels at different temperatures.

To provide all necessary material property tests in the AGC experiments, each test-series capsule contains two primary specimens: (1) creep specimens, providing irradiation creep-rate values as well as mechanical properties, and (2) "piggyback" specimens, providing thermal material property changes to 
the graphite. Generally, the creep specimens are larger $(25.4 \mathrm{~mm}$ tall) and are irradiated in the mechanically loaded outer stack positions of the capsule body where an applied load can be imposed upon half of the specimens. Piggyback specimens are short (i.e., $6 \mathrm{~mm}$ tall) button specimens that reside in the axial spine of the capsule or the lower half of the outer stack positions - receiving no applied load and subjected only to neutron irradiation at high operating temperatures - to assess the effects of a reactor environment on the specific graphite grade. Together, both types of specimens provide the changes in material properties for stressed and unstressed graphite grades. The physical dimensions for both "Creep" and "Piggy-back" specimens are shown in INL Drawing 600786, Rev. 2. ${ }^{[10]}$

\subsection{Description of AGC-2 Test Series}

AGC-2 was originally designed to be the longer duration irradiation capsule providing specimens irradiated at the 3.5-7.0-dpa dose level (see Figure 1) at the $600^{\circ} \mathrm{C}$ temperature conditions. However, due to issues with temperature control within the first AGC-1 capsule, ${ }^{[6]}$ it was decided during AGC-1 irradiation to hold the capsule inside ATR as long as possible to ascertain the viability of the capsule design at high dose levels. Therefore, the irradiation dose levels for AGC-1 were changed to the longer duration irradiation (i.e., $3.5-7.0 \mathrm{dpa}$ ) at the $600^{\circ} \mathrm{C}$ temperature conditions.

Because the AGC-1 specimen temperature limits exceeded the technical specifications, determining material property changes and creep rates at a constant temperature proved to be difficult. Therefore, an intermediate, $1.5-5$-dpa dose level, irradiated at $600^{\circ} \mathrm{C}$, was selected as the final irradiation condition for the AGC-2 test series to bridge between the two original $600^{\circ} \mathrm{C}$ test-series capsules, Figure 2 . It was assumed that some of the specimens from AGC-1 could be utilized in conjunction with the AGC-2 graphite specimens to produce more reliable material property changes at the constant $600^{\circ} \mathrm{C}$-temperature condition. While not optimal, it was determined that providing graphite specimens, irradiated over this overlapping dose level at a more consistent temperature condition, would be sufficient to ascertain the irradiation creep and material property changes for the selected graphites.

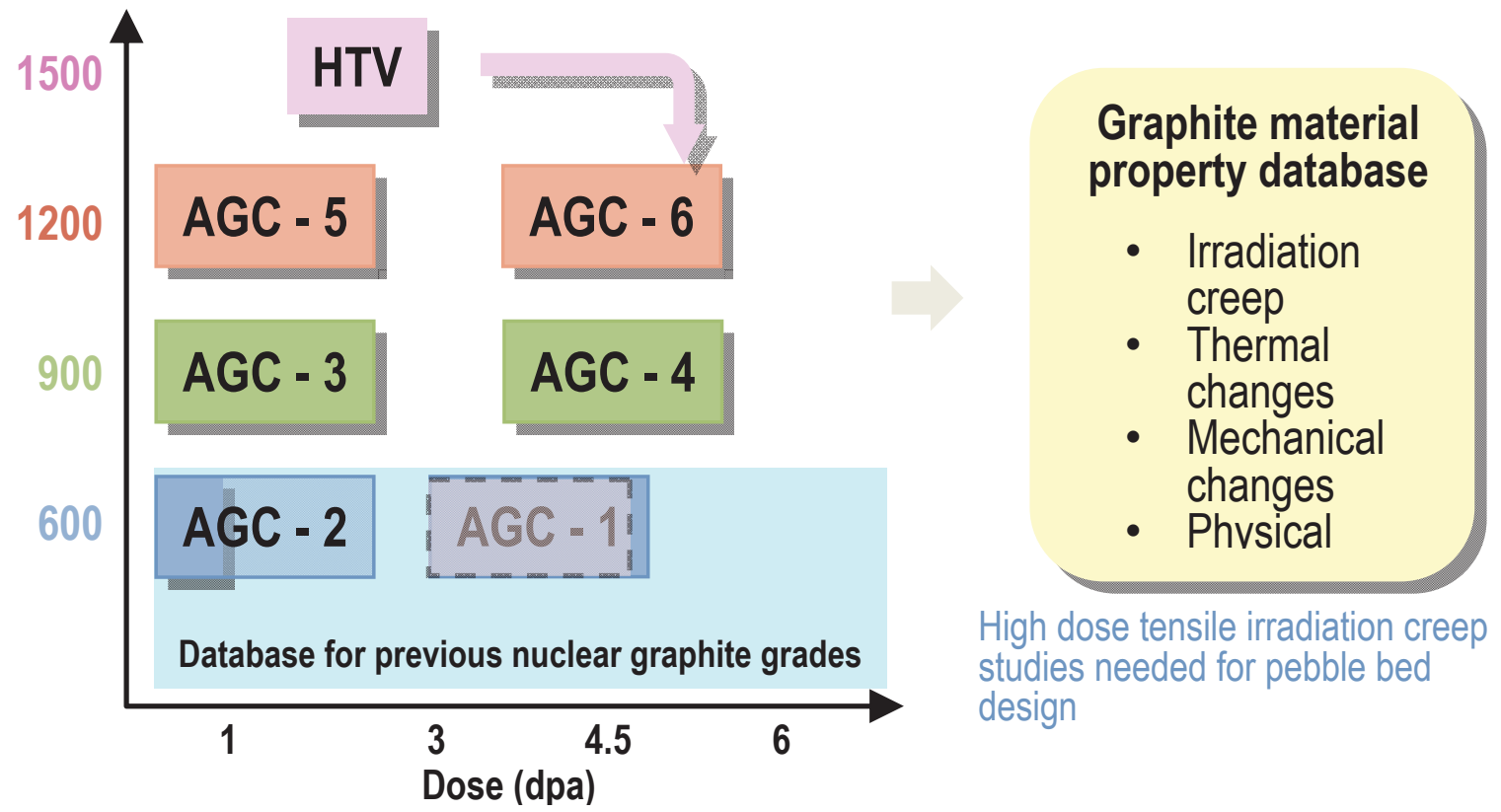

Figure 2. Revised irradiation dose and temperature parameters of the AGC test series. Note the gray area between AGC-1 and AGC-2 is the expected dose overlap between capsule irradiation. 
Additionally, one of the graphite grades was changed by request of the graphite vendor (Mersen, USA). Piggy-back specimens of graphite grade 2114 were directly substituted for graphite grade 2020 and irradiated within the AGC-2 central stack (axial spine of capsule). All other graphites irradiated within AGC-2 were similar to the graphites in AGC-1. To duplicate the specimen stacking order as in the AGC-1 test series capsule, the AGC-2 capsule contained the following major graphite grades NBG-17, NBG-18, PCEA, IG-430, H-451, and IG-110. The minor grades of graphite (i.e., "piggyback" specimens) included NBG-25, PCIB, PPEA, NBG-10, BAN, HLM, PGX, 2114, HOPG, and A3 Matrix.

\subsubsection{Establishing the Capsule's Physical Centerline to the Core Neutron Flux Mid-Plane}

The capsule elevation sketch provided in Figure 3 was generated from a number of AGC-2 capsule and ATR core drawings and used to determine the position of each specimen in the capsule as a function of height above and below the mid-plane of the core neutron flux profile. ${ }^{[11,12]}$ Other considerations included the size of each creep specimen, the need for periodically placed spacers containing flux wires, and the space requirements in the top of the stacks for the push rods. The core flux mid-plane, in relation to the capsule arrangement, was established so that the reactor neutron flux field could be correlated to the physical elevations and positions in the capsule. 


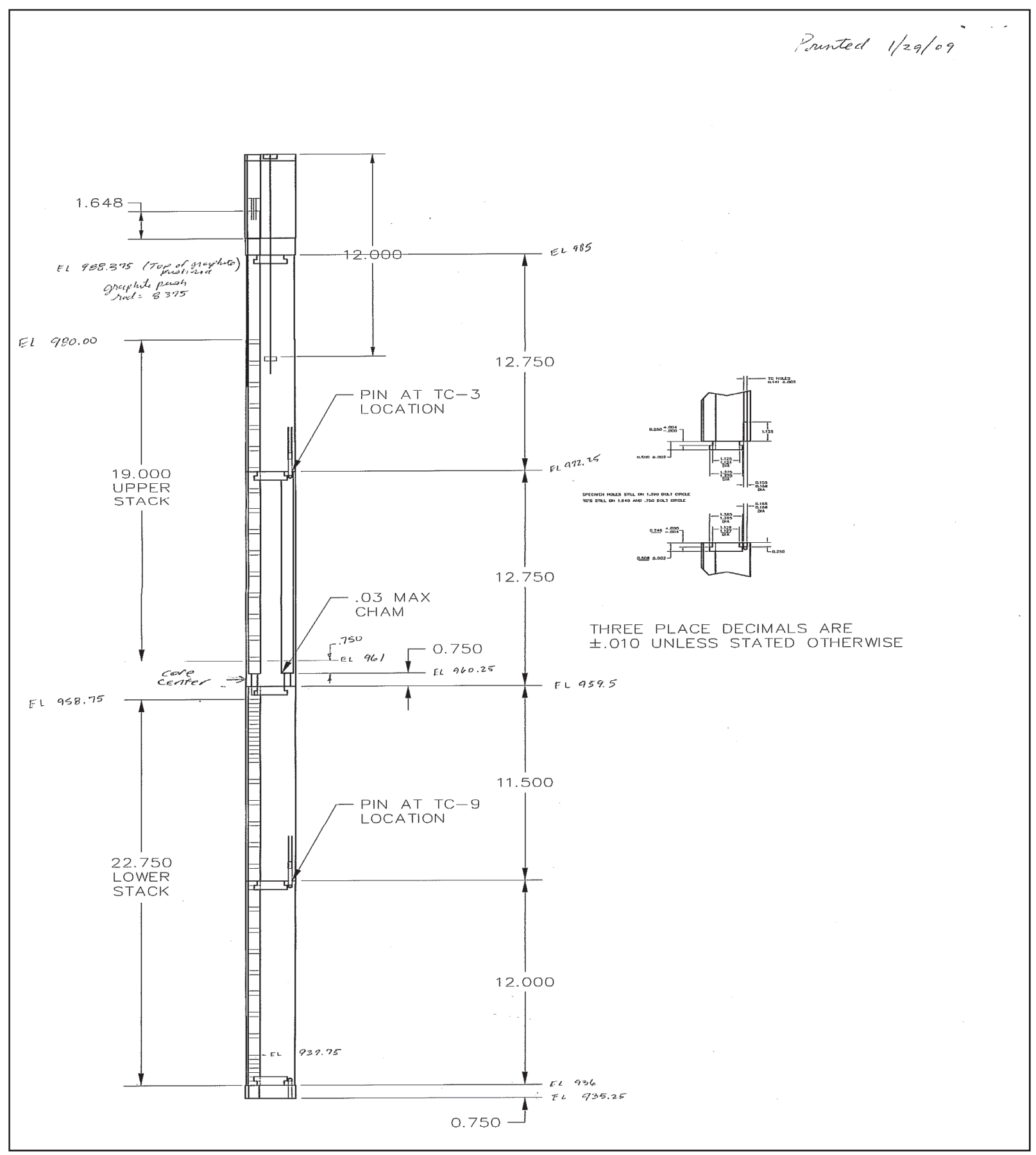

Figure 3. Elevation sketch of the Advanced Graphite Creep capsule.

\subsubsection{Establishing the Dose Levels as a Function of Position within the Capsule}

Once the physical elevation dimensions were established and correlated to the reactor core centerline, the dose as a function of distance from the core centerline needed to be determined. The estimated AGC- 2 experiment fluence profiles were calculated by Reactor Engineering utilizing a Monte Carlo Neutral Particle (transport code) model based on the known neutron flux profile of ATR, the position of the 
experiment in the South Flux Trap, and the number of effective full-power days planned for the test-series capsule.

Irradiation dose values, as a function of distance from the reactor core centerline, were calculated from the total estimated fluence, using standard conversion factors for carbon in a fast neutron irradiation field $(\mathrm{E}>0.1 \mathrm{MeV}) .{ }^{[13]}$ There is a neutron flux gradient across the capsule thickness requiring the capsule to be rotated 180 degrees at the irradiation mid-point. This rotation results in a uniform neutron-fluence profile for all stacks, regardless of their position within the capsule, as shown in Figure 4. Because all stacks within the capsule are estimated to have similar dose profiles after complete irradiation, the dose profile for the center stack within the capsule was used to determine the proper stacking order and specimen offset position in the lower half of the irradiation capsule.

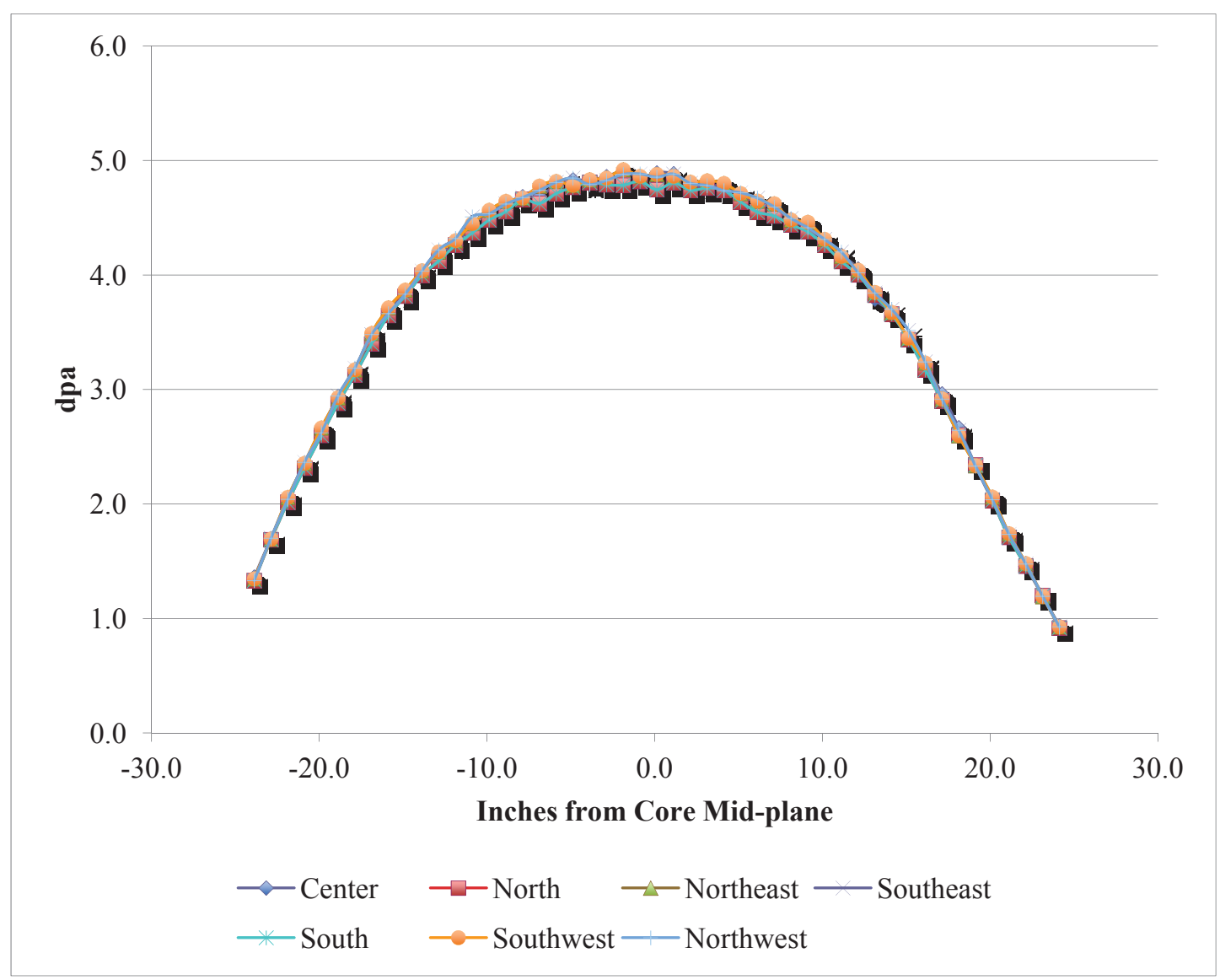

Figure 4. Calculated dose levels for AGC-2 after 140 effective full-power days.

As described in Section 1, "Introduction," of this report and as shown in Figure 4, the ATR neutron flux profile is not completely symmetrical along the vertical axis. Thus, to produce matched-pair specimens that have similar dose profiles both above and below the core mid-plane, an offset from the mid-plane is required. This offset adjusts the specimen positions and to matches the dose levels for "matched pair" specimen. For AGC-2, the offset spacing was determined using the accumulated dose levels from the central stack profile, both below and above the core mid-plane. Average dose values at 0.25-in. increments (i.e. height of a piggyback specimen) were calculated along both top and bottom curves allowing for direct comparison between matched positions within each stack. 
The dose profiles for the lower and upper core were superimposed upon each other, as shown in Figure 5. To determine the length of the offset space, the dose values above and below the core mid-plane were compared, and the specimens in the bottom half of the AGC capsule were moved farther away from the mid-plane, Figure 5. An offset of 1.25 inches from the core mid-plane for the bottom creep specimens produced the closest dose matches between specimens.

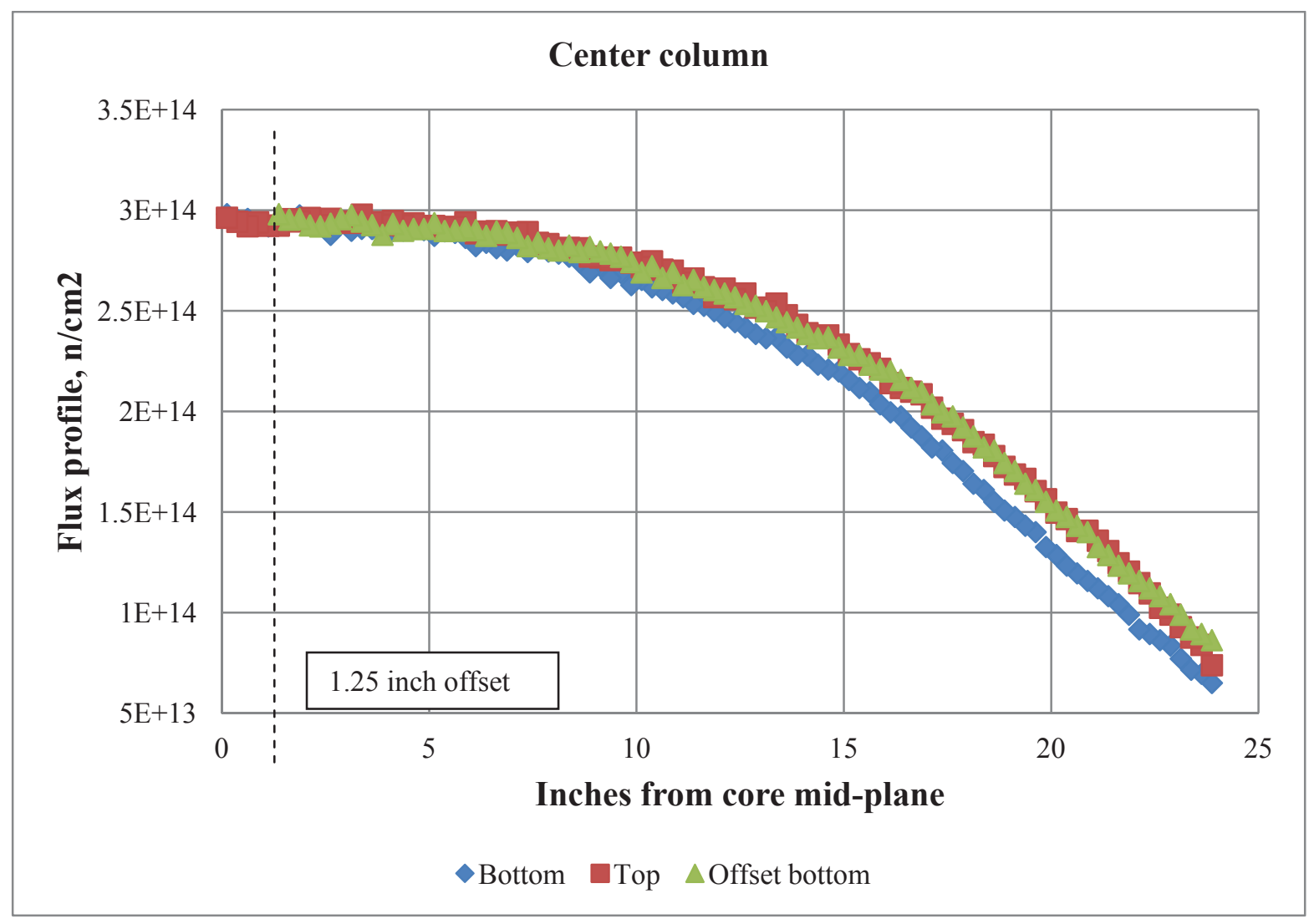

Figure 5. Advanced Test Reactor flux profile, as a function of core mid-plane position.

While it was impossible to exactly match both the upper and lower specimens, the dose levels for each specimen pair were fairly close, ranging from $0-11 \%$. In addition, over time, the specimens in the top stacks should dimensionally shrink more than the bottom specimens (during irradiation) due to the compressive load applied. This effectively moves the top specimens closer to the centerline, thus creating a closer dose match to the specimens in the bottom stack. While this irradiation-induced shrinkage of the top stack of specimens is anticipated to be relatively small at the end of irradiation (i.e., $<6 \mathrm{~mm}$ for the total stack of specimens), the dose levels do slightly converge rather than diverge.

It should be noted here that the specimen offset distance/position in the lower half of the AGC-1 capsule is significantly different from these offset calculations for the AGC-2 capsule. ${ }^{[14,15]}$ AGC-1 used an older ATR flux profile that did not accurately reflect the current flux profiles within the South Flux Trap. Consequently, the offset was approximately 3.75 in. (i.e., 15 piggyback specimen heights), leading to slightly larger differences between the upper and lower matched-specimen pairs than expected for AGC-2, as shown in Table 1. This will need to be addressed in detail in the AGC-1 PIE report to be issued after PIE is complete. 
Table 1. Typical dose differences between matched pairs in the AGC-1 test-series capsule.

\begin{tabular}{|c|c|}
\hline \multicolumn{2}{|c|}{$\begin{array}{c}\text { Representative Stack } \\
\text { (Stack No. 1) }\end{array}$} \\
\hline Matched Pairs & $\begin{array}{c}\text { AGC-1 Estimated Dose } \\
\text { Difference (\%) }\end{array}$ \\
\hline 1S1/1U1 & 0.34 \\
\hline 1S2/1U2 & 0.69 \\
\hline 1S3/1U3 & 2.11 \\
\hline 1S4/1U4 & 1.81 \\
\hline 1S5/1U5 & 2.57 \\
\hline 1S6/1U6 & 3.01 \\
\hline 1S7/1U7 & 1.54 \\
\hline 1S8/1U8 & 5.76 \\
\hline 1S9/1U9 & 6.55 \\
\hline 1S10/1U10 & 9.43 \\
\hline 1S11/1U11 & 13.40 \\
\hline 1S12/1U11 & 3.09 \\
\hline 1S13/1U12 & 5.23 \\
\hline 1S14/1U13 & 3.33 \\
\hline 1S15/1U14 & 1.53 \\
\hline
\end{tabular}

\subsubsection{Determining the Physical Positions of Irradiation Creep Specimens in the Stacks}

Once the specimen-position offset was established for the bottom half of the specimens, the number of total creep specimens for each grade of graphite was determined. To increase the number of creep specimens in the AGC-2 test-series capsule, the 0.25-in.-tall NBG-25 graphite spacers between creep specimens were eliminated. This decision to eliminate the spacers increased the total number of specimens by over 20 in the entire capsule. This allowed more specimens per graphite grade to be irradiated within the AGC-2 capsule.

A further decision was made to increase the creep specimen number population for the newer graphite grades because little-to-no irradiation data are available on these grades. Specifically, more specimens of graphite grades NBG-18 and PCEA were chosen to be irradiated than the IG-110, IG-430, and NBG-17 graphite grades. NBG-18 and PCEA were determined to have 16 specimens per applied stress level, for a total of 48 specimens within AGC-2. Graphite grades IG-110 and IG-430 were represented by only 12 specimens per applied stress level, for a total of 36 specimens within AGC-2. Table 2 shows the total number of specimens irradiated per graphite grade. 
Table 2. Total number of irradiated creep specimens in the AGC-2 test-series capsule.

\begin{tabular}{|l|c|}
\hline Graphite Grade & $\begin{array}{c}\text { Total Number of } \\
\text { Creep Specimens }\end{array}$ \\
\hline PCEA & 48 \\
\hline NBG-18 & 48 \\
\hline IG-110 & 36 \\
\hline IG-430 & 36 \\
\hline NBG-17 & 24 \\
\hline H-451 & 24 \\
\hline
\end{tabular}

Other factors had to be considered before the stacking order for each stack could be finalized. First, three load levels are applied to the specimens in the loaded upper parts of the stacks (i.e., loads of 13.8, 17.2, and 20.7 MPa). The six outer stacks in the capsule allow the specimens in two of the stacks to be loaded at $13.8 \mathrm{MPa}$, while the other two pairs of stacks are loaded at 17.2 and $20.7 \mathrm{MPa}$, respectively. Because two stacks are at similar applied stress levels, the specimen loading order can be shifted between the two stacks, allowing the same grade of graphite loaded at the specimen stress levels to be exposed over a broader neutron dose range, as shown in Figure 6. Assuming that both stacks will have the same applied stress level, receive similar dose levels per position, and have a constant temperature allows for this shifting of the specimens, and consequently more uniform, smoother dose profile for each graphite grade.

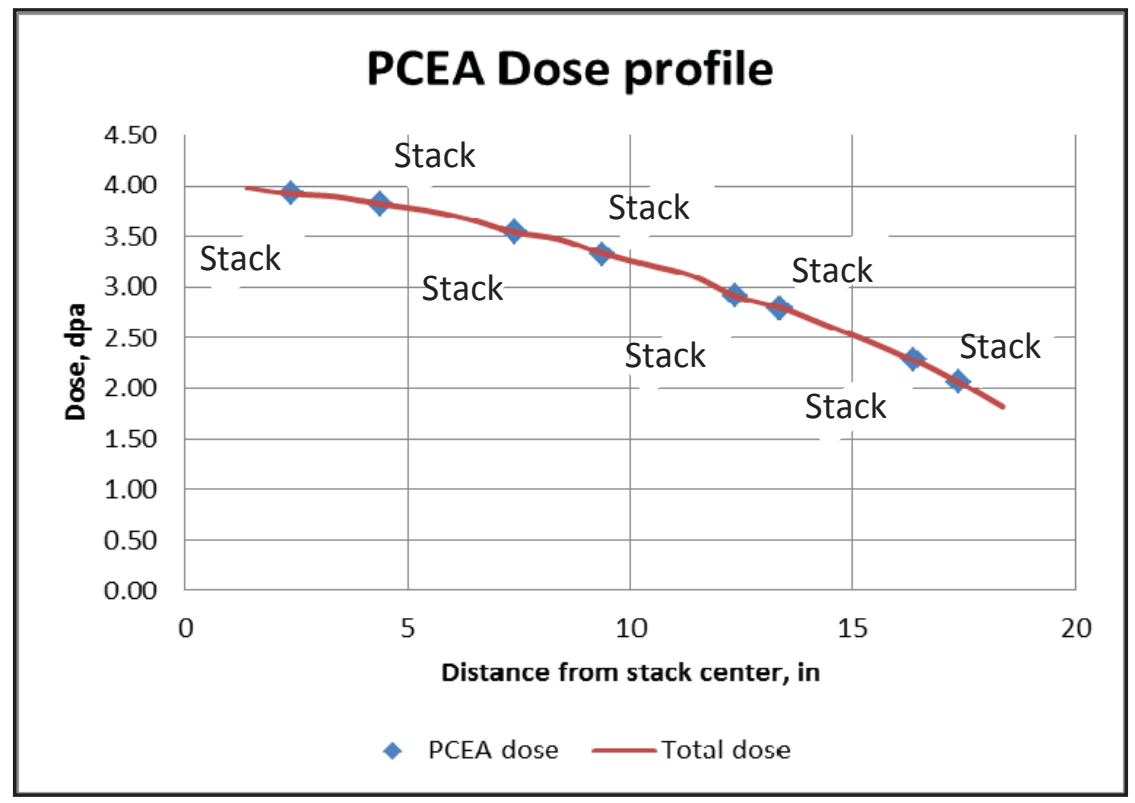

Figure 6. Typical AGC-2 dose profile for creep-graphite specimens utilizing similar applied stress levels in matched stacks.

A further consideration is the grain orientation of the specimens. A decision was made to have approximately $75 \%$ of the specimens be orientated in the "with-grain" (WG) direction and $25 \%$ of the specimens be "against-grain" (AG). However, in the case of the vibration-molded graphites (i.e., NBG-17 and -18), there are actually two with-grain directions and one against-grain direction as a consequence of the fabrication process. As such, it was logical to split the with-grain and against-grain specimens evenly (i.e., 50/50 ratio) rather than following the 75/25 ratio established for the other specimens. 
Once these considerations were accounted for, the dose-level profiles were determined for each graphite grade and within each stack. The estimated creep specimen dose profiles for each graphite grade for each stress level are illustrated in Figure 7.
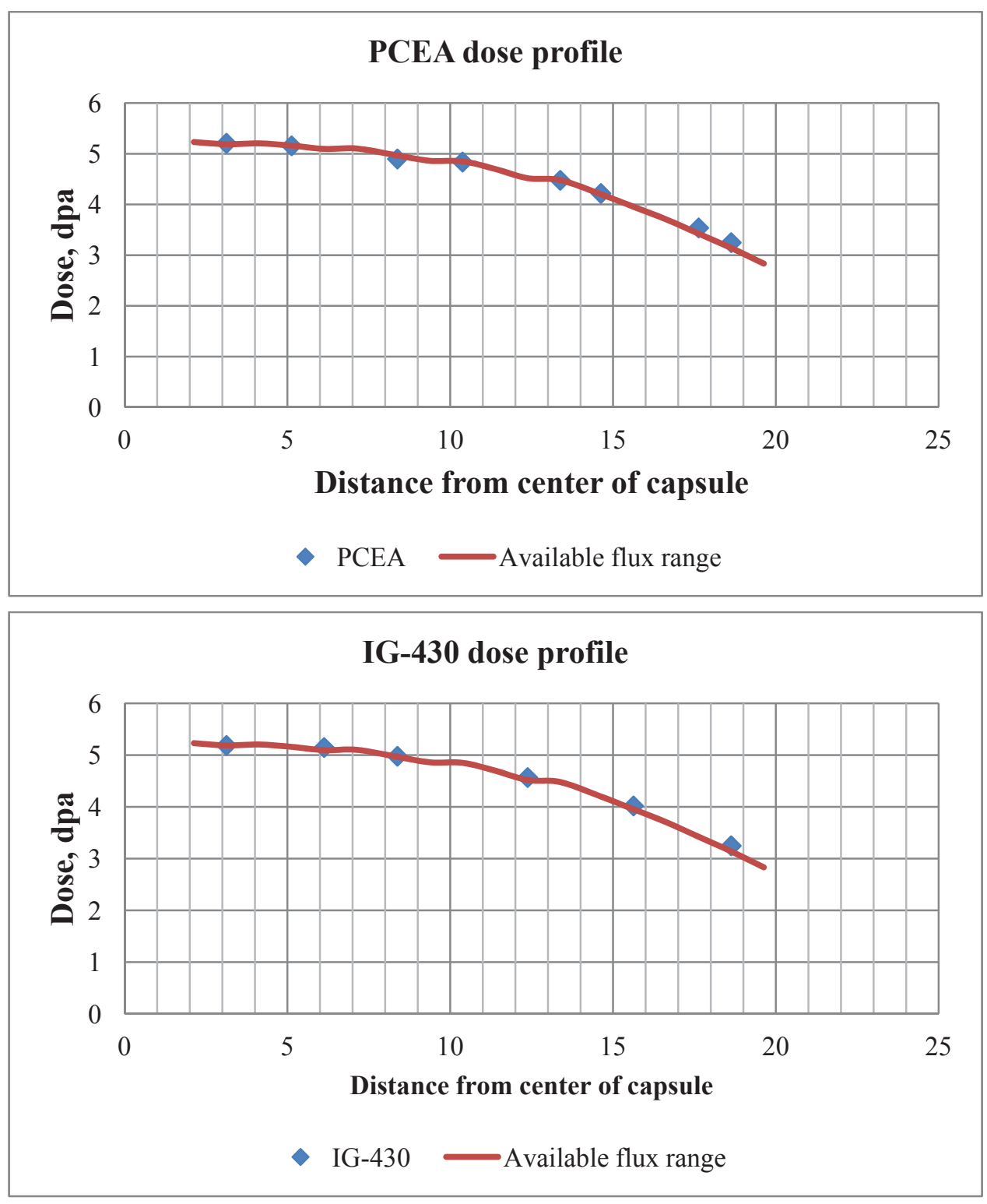

Figure 7. Estimated creep-specimen dose profiles for each major graphite grade. 

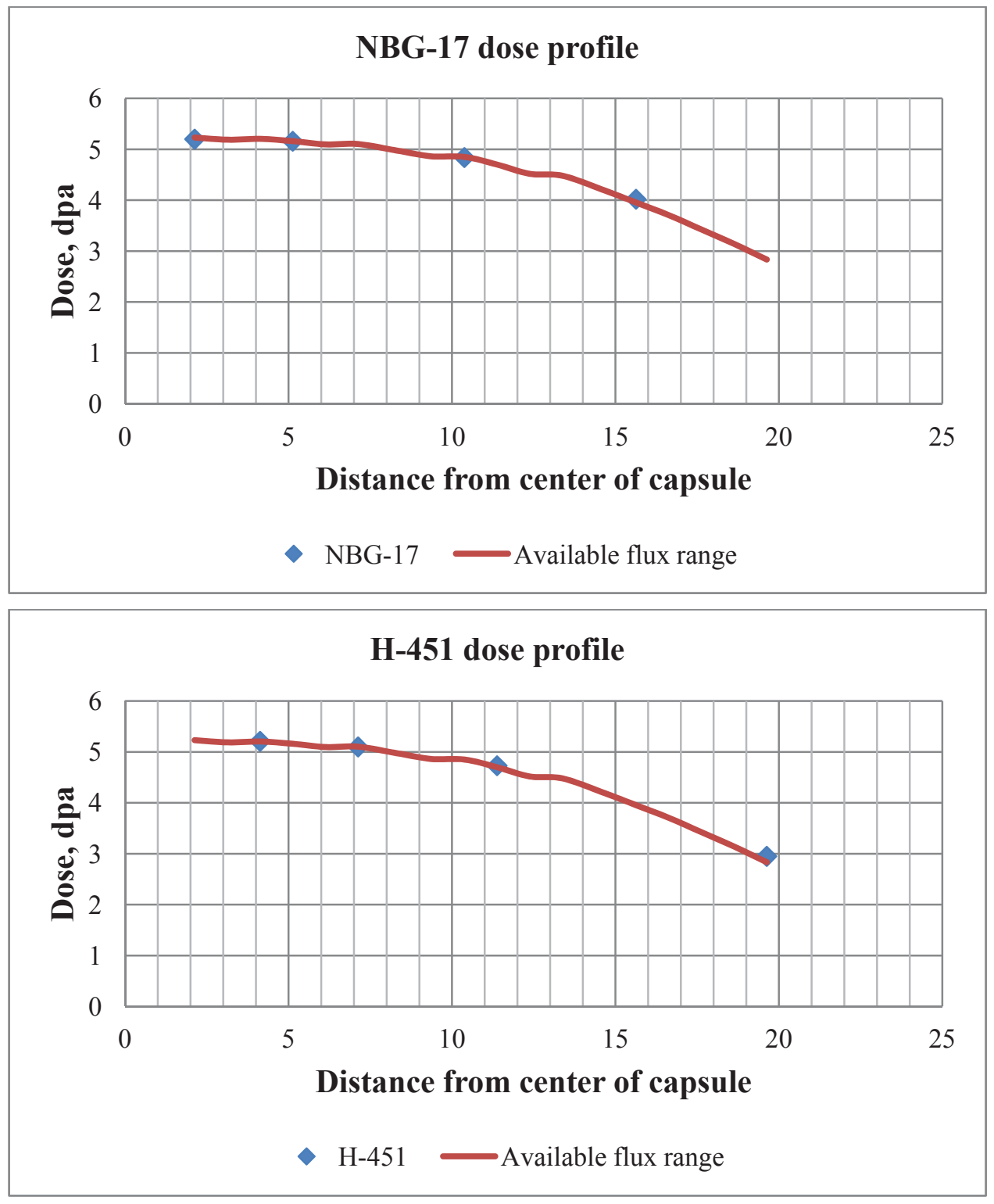

Figure 7. Estimated creep-specimen dose profiles for each major graphite grade. 

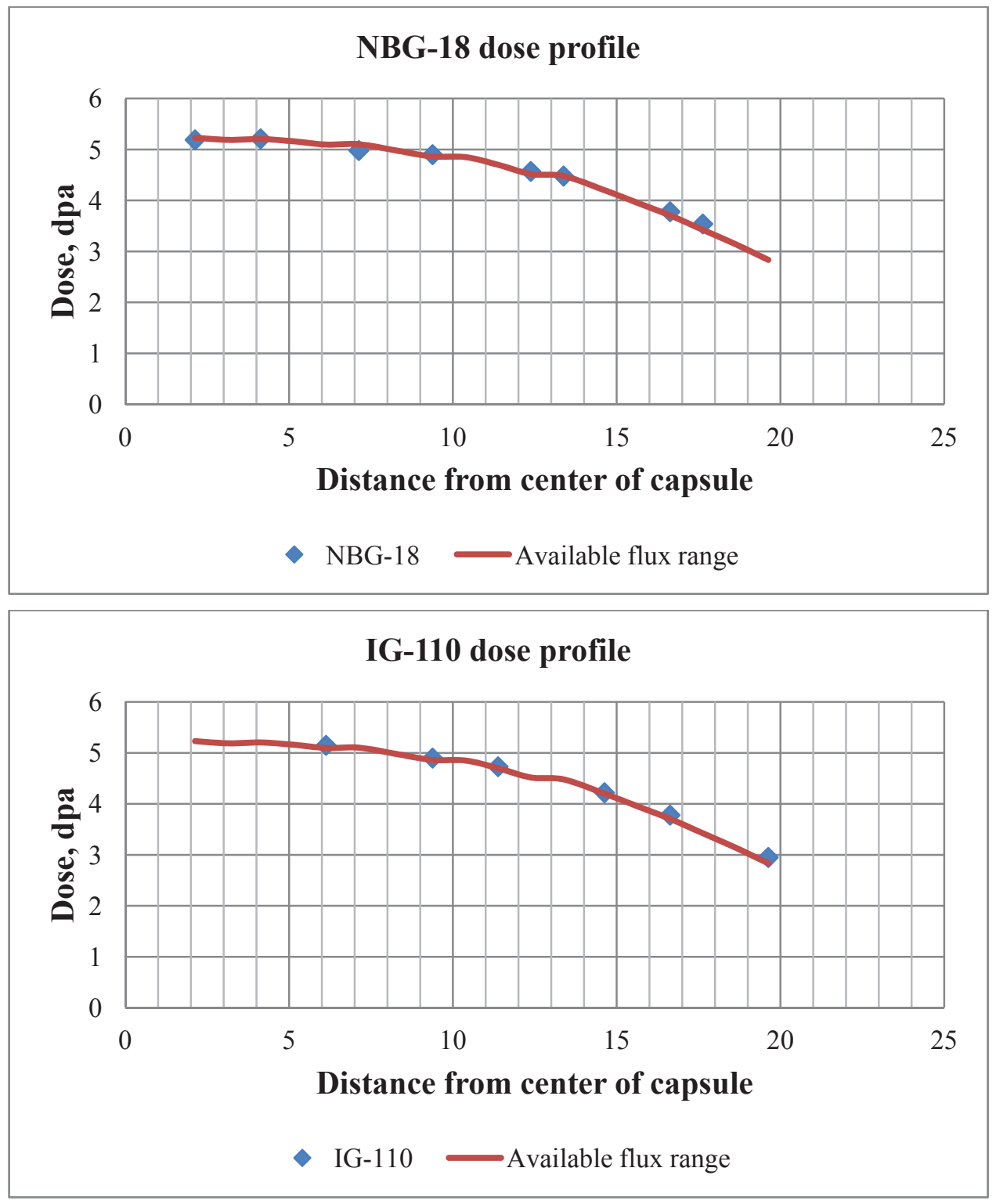

Figure 7. Estimated creep-specimen dose profiles for each major graphite type.

The final loading configuration for the outer stacks was established once a smooth dose profile was achieved for each graphite grade, Figure 7. The lower stack offset, the flux wire spacers, creep specimens, and specimen symmetry above and below the capsule mid-plane were mapped for each graphite specimen in Table 3. 


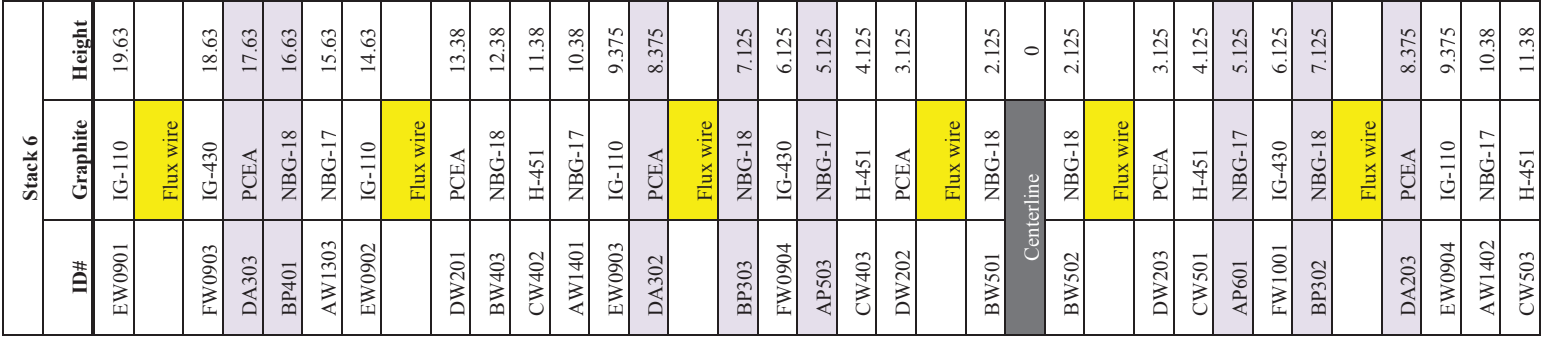

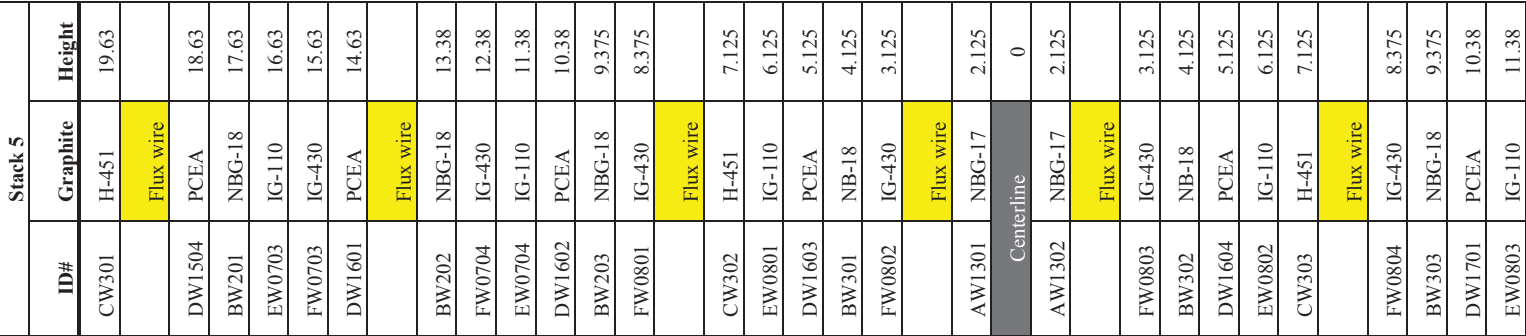

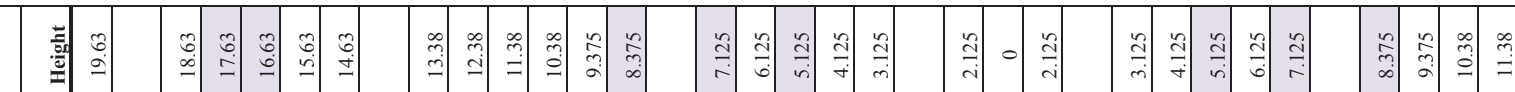

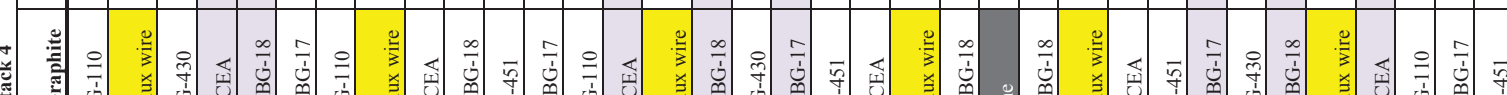

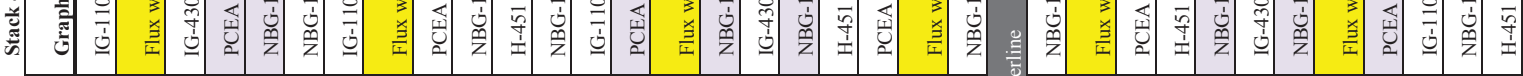

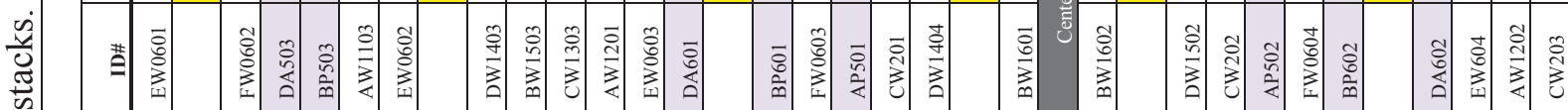

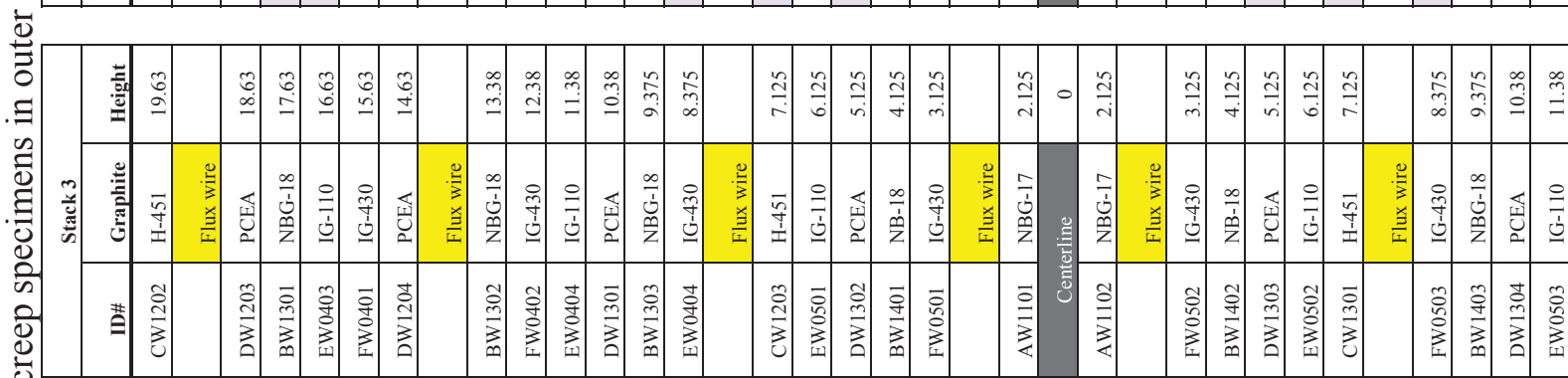

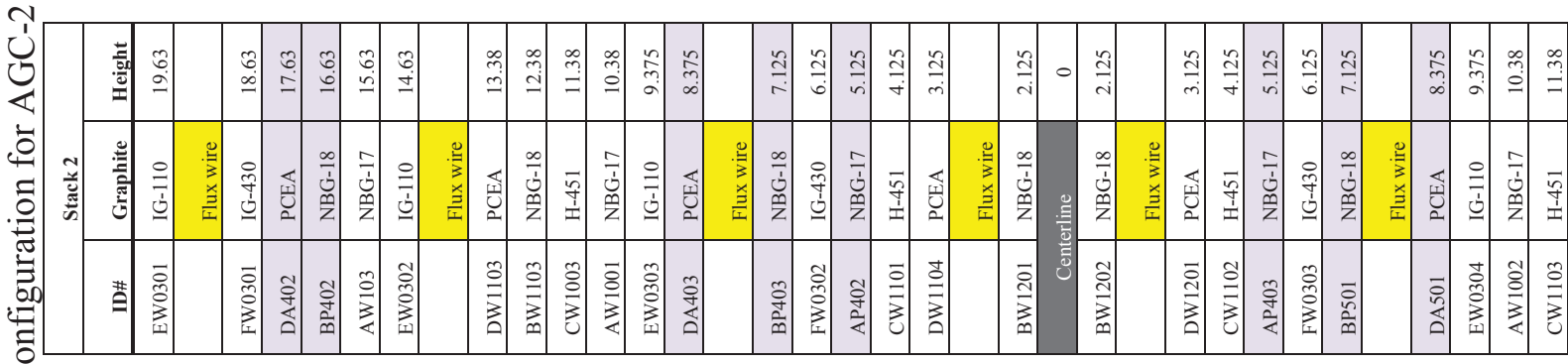

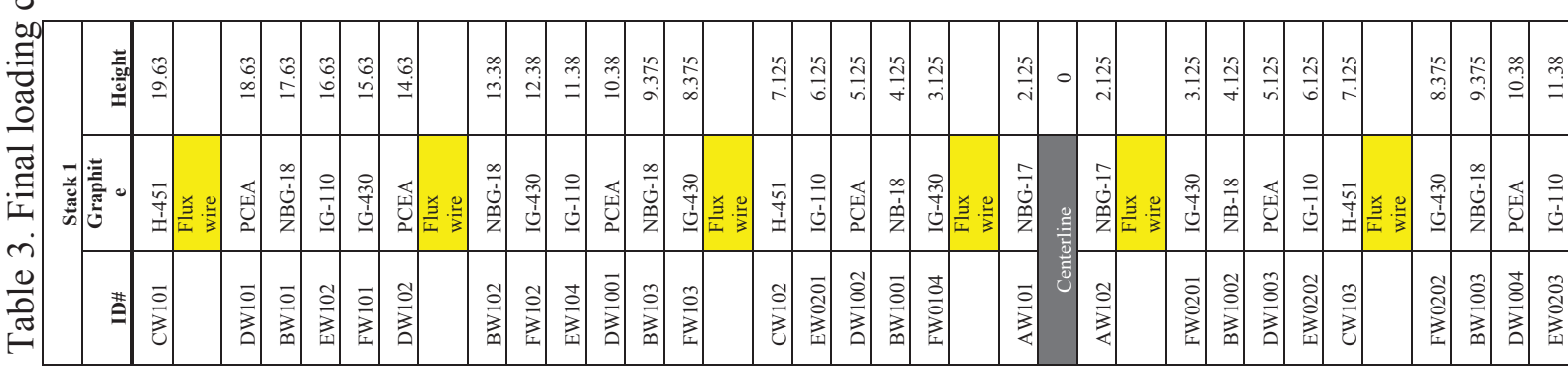




\begin{tabular}{|c|c|c|c|c|c|c|c|c|c|}
\hline & 檯 & 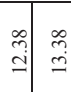 & & 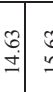 & & \begin{tabular}{c}
$:$ \\
\hdashline \\
\hdashline
\end{tabular} & $\begin{array}{l}\infty \\
\infty \\
\infty\end{array}$ & & \begin{tabular}{|l|l} 
\\
\hdashline
\end{tabular} \\
\hline 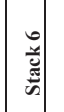 & 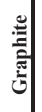 & \begin{tabular}{l|l}
$\frac{\infty}{b}$ \\
$\stackrel{\hat{b}}{2}$
\end{tabular} & 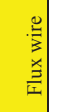 & & & 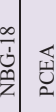 & 疍 & & 蒠 \\
\hline & 搨 & 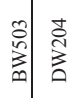 & & 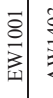 & 全 & 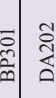 & $\frac{8}{3}$ & & $\frac{\tilde{8}}{3}$ \\
\hline
\end{tabular}

\begin{tabular}{|c|c|c|c|c|c|c|c|c|c|}
\hline & 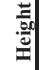 & 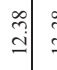 & & & 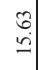 & | & & & 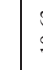 \\
\hline & 氧 & & & & $\stackrel{\dot{b}}{0}$ & & & & 童 \\
\hline & 頨| & 章 & & 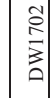 & $\begin{array}{l}\text { : } \\
\vdots \\
\vdots\end{array}$ & 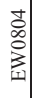 & & & \\
\hline
\end{tabular}

\begin{tabular}{|c|c|c|c|c|c|c|c|c|c|}
\hline & 衰离 & 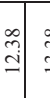 & & $\begin{array}{l}6 \\
\pm \\
\end{array}$ & $\underline{v}$ & & & - & 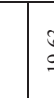 \\
\hline & 氛 & $\bar{z}$ & & $\stackrel{\circ}{3}$ & 尊 & 资 & & 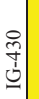 & 部 \\
\hline & 龍 & & & 是 & & & & 吝 & \\
\hline
\end{tabular}

\begin{tabular}{|c|c|c|c|c|c|c|c|c|c|}
\hline & 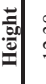 & 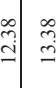 & & & & & \begin{tabular}{|c}
0 \\
$\stackrel{0}{0}$
\end{tabular} & & 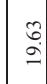 \\
\hline & 善 & 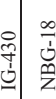 & 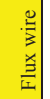 & & & & 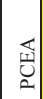 & & $\frac{\vec{q}}{q}$ \\
\hline & 蓼 & 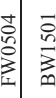 & & & 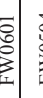 & & 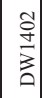 & & \\
\hline
\end{tabular}

\begin{tabular}{|c|c|c|c|c|c|c|c|c|c|}
\hline & 言 & 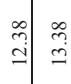 & & & & \begin{tabular}{ll}
8 \\
\hdashline
\end{tabular} & $\begin{array}{l}\delta \\
\infty \\
\varnothing\end{array}$ & & 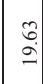 \\
\hline 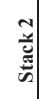 & 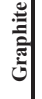 & 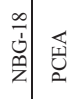 & 旁 & & 禽 & 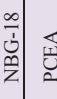 & 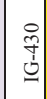 & & 它 \\
\hline & 㹃 & 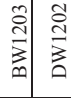 & & 预 & 络 & ڤั) & 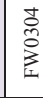 & & 萣 \\
\hline
\end{tabular}

韭

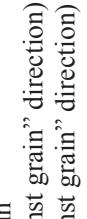

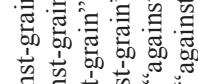

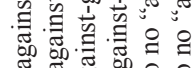

s

茟茟

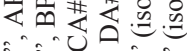

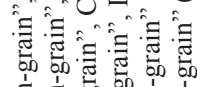

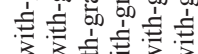
उ 30,3$\}$

\begin{tabular}{|c|c|c|c|c|c|c|c|}
\hline & $\mid \begin{array}{c}\infty \\
\stackrel{2}{\mathrm{i}} \\
\end{array}$ & $\stackrel{\infty}{0}$ & $\mid \begin{array}{l}3 \\
\pm \\
\pm\end{array}$ & & : & $\mid \begin{array}{l}0 \\
\stackrel{\infty}{\infty} \\
-\infty\end{array}$ & : \\
\hline 4 & 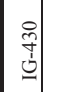 & & 离 & & 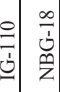 & 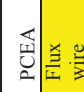 & 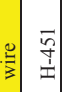 \\
\hline & 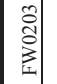 & 司 & & 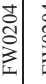 & 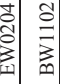 & 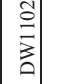 & $\begin{array}{l}\text { 总 } \\
3 \\
3\end{array}$ \\
\hline
\end{tabular}

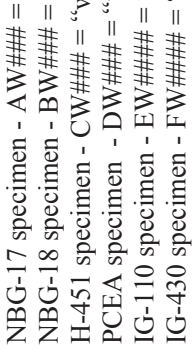




\subsubsection{Determining the Physical Positions of Piggyback Specimens in the Central Stack}

Similar to the AGC-1 design, the AGC-2 piggyback specimens were contained within the central stack and did not have an applied stress imposed. Because AGC-2 has the same number of major and minor graphite types as the AGC-1 design, the same piggyback stacking order was used. ${ }^{[14,15]}$

One major change to AGC-2 piggy-back specimens was the elimination of a central hole through each graphite type specimen. AGC-1 piggyback had a central hole to allow a series of SiC temperature monitors to be placed down the central spine of the experiment to provide independent temperature measurements from the capsule thermocouples (TC). Unfortunately, this central hole in the specimens precluded thermal diffusivity testing on the specimens above room temperature.

A decision was made to not machine a central hole in the AGC-2 piggyback specimens and allow each to be tested for high temperature thermal diffusivity. This eliminated the independent temperature monitoring capability for the AGC-2 capsule but it was determined that being able to measure the changes to the thermal diffusivity at high temperatures was of more importance.

\section{GENERAL TESTING PROVISIONS}

A significant level of preparation was needed to meet Nuclear Quality Assurance Level 2 quality requirements prior to actual material property testing. An approved characterization plan was developed that was dependent upon the two graphite specimens geometry and on the material properties to be measured. In general, all testing was performed through ASTM approved standards however due to small size of the graphite specimens some methods required modifications and variations of the testing standards. Details of these testing standard variations along with equipment calibration, personnel training on testing methodology, and data acquisition are provided.

\subsection{Characterization Plans and Work Procedures}

The AGC-2 specimens have been characterized in accordance with PLN-3267 "AGC-2 Characterization Plan." ${ }^{[16]}$ This plan describes thermal, physical, and mechanical measurement methods used to characterize the graphite specimens and is intended to meet the requirements of MCP-1380, "Research and Development Test Control." ${ }^{[17]}$ Described within the plan are the instruments, fixtures, and methods used for preirradiation material-property measurements of bulk density, thermal diffusivity, coefficient of thermal expansion (CTE), elastic modulus, and electrical resistivity.

All work was performed in accordance with LWP-21220, "Work Management." ${ }^{,[18]}$ All records designated in implementing documents as Quality Assurance records were controlled in accordance with PLN-3319, "Records Management Plan for the VHTR Technology Development Office Program."

Data resulting from the preirradiation characterization are plotted in Appendix A and listed in Appendix C. Statistical evaluation has been performed using an inner quartile range analysis to identify levels of uncertainty and outliers in the data. The measured properties and characteristics of different graphite types will be compared along with the effect of grain orientation.

\subsection{Specimen Description and Preparation}

The major grades of the nuclear graphite to be tested in AGC-2 are NBG17, NBG-18, PCEA, IG-430, H-451, 2114, and IG-110. Minor grades of graphite include NBG-25, PCIB, PPEA, NBG-10, BAN, HLM, PGX, 2114, HOPG, and A3 Matrix. All major grades have been characterized fully in accordance with PLN-3267, and the minor grades have only had dimensional, density, and thermal diffusivity measurements performed on them. The two primary specimen types in the AGC experiments are creep specimens and piggyback specimens. "Creep" specimens from major grade graphite types are shown in INL Drawing 600786, Rev. 1, “ATR Advanced Graphite Capsule (AGC-2) Graphite Specimen 
Machining Details," ${ }^{[20]}$ and will be subjected to a mechanical load during irradiation to induce irradiation creep within the specimens. "Piggyback" specimens from both major and minor grade graphite types are shown in INL Drawing 600786, Rev. 1. They are not subjected to a mechanical load and are subjected only to neutron irradiation at high operating temperatures to assess the effects of a reactor environment on the specific graphite grade.

All specimens are $12.7 \mathrm{~mm}$ in diameter, with the creep specimens being $25.4 \mathrm{~mm}$ long and the piggyback specimens being $6 \mathrm{~mm}$ long (INL Drawing 600786, Rev. 2) ${ }^{[10]}$ Details of how specimens were cut from the graphite blocks are contained in INL Drawing 600787, Rev. 3. ${ }^{[21]}$

Immediately after being machined, each specimen is placed in an individual container that is bar coded with a unique identification number, in accordance with INL Drawing 600787, Rev. 3. Each graphite specimen is then laser-engraved with that same unique identification number around the circumference at one end. Prior to any material property measurement, each specific specimen is identified by its unique identification number, and the data are recorded/stored under this identification number. After the specimens have been laser-engraved, they are ultrasonically cleaned, as follows:

1. Handle the specimens only while wearing cotton or powder-free nitrile gloves

2. Remove all dust and debris using an aerosol pressurized dust-off product

3. Ultrasonically clean specimens for 20 minutes in deionized water

4. Rinse specimens in ethyl alcohol to help displace water

5. Allow to air dry

6. Place specimens in a laboratory oven at $130^{\circ} \mathrm{C}$ for 2 hours.

Allow specimens to cool in a desiccator and retain there in storage until resistivity or bulk density measurements are taken.

It should be noted that irradiated specimens are not washed again prior to characterization measurements. However, for measurements of density and resistivity, Steps 6 and 7 above are followed (i.e., irradiated specimens are dried in a laboratory oven at $130^{\circ} \mathrm{C}$ for 2 hours and allowed to cool in a desiccators, where they are retained until resistivity and/or bulk density measurements are performed).

\subsection{Personnel and Training}

Personnel who perform the measurements identified in this report are qualified in accordance with MCP-3052, "VHTR TDO Personnel Qualification and Certification." ${ }^{, 22]}$ Their ability to adequately perform measurements described in this report is demonstrated by instrument manufacturers' training and certification and/or performance of an instrument/measurement operational validation. Personnel qualifications are reviewed by the NGNP Graphite Research and Development Technical Lead and documented in the laboratory notebooks.

\subsection{Variations, Exceptions, and Discrepancies}

Several variations, exceptions, and discrepancies may occur. The first is a known departure from the applicable American Society for Testing and Materials (ASTM) standard. These departures are typically related to geometrical constraints. All currently known departures or exceptions taken to the ASTM standard are described in detail in Section 3 of this plan. Any departure not captured in this plan will be recorded in laboratory notebooks associated with the measurement. In most cases, the effects of the exception or departure from the ASTM method/standard on the measured value are not well understood. When possible, sensitivity studies will be performed and documented in laboratory notebooks to understand the impact of these exceptions and departures. 
It is likely that the ASTM standards and/or test methods will be revised and improved during the $10+$ year AGC experiment cycle. Each revision or development will be evaluated for how it could impact future measurements and their consistency with measurements made under previous revisions or techniques. A programmatic determination will be made whether to continue with the current version of the ASTM method or use the updated version. This determination will be documented in laboratory notebooks associated with the affected measurement.

While measurements are being made, it is possible that something out of the ordinary may occur. Any unusual event that occurs during a measurement will be documented in the laboratory notebook associated with that specific measurement and duly noted within the database associated with the data generated for this program. The principal investigator will be notified of the event and will determine what impact it has on the data. The significance of the result will be documented in the laboratory notebook by the principal investigator.

\subsection{Calibration and Functional Validation}

The measurement protocol consists of calibration, functional validation, and data acquisition. Functional validations established for each measurement, in collaboration with the instrument manufacturer, will be performed periodically to ensure that accurate and consistent data are acquired. All validations will be performed on traceable standards and documented in retrievable laboratory notebooks associated with each measurement. In the event that an instrument functional validation fails, the reason for the failure will be investigated and resolved prior to that measurement being used for further characterization. Upon resolution, a determination will be made as to the impact the failure might have had on data taken prior to the failure and back to the last valid measurement. If it is determined the data captured during this interval are suspect, the impacted data will be evaluated for accuracy.

MCP-3066, "VHTR TDO Control of Measuring and Test Equipment,"[23] will be followed for calibration standards, methods, and frequencies that have been established for each measurement. Where it is not possible to use the INL Standards and Calibration Laboratory, calibration by user procedures will be established, based on ASTM standards and manufacturers' instructions and performed against international standards. These procedures will be documented in laboratory notebooks associated with each measurement.

\section{GRAPHITE STANDARDS AND METHODS}

A brief summary of the AGC specimen testing methods is included in Table 4 and includes precise dimensional measurements (before and after heating to $1000^{\circ} \mathrm{C}$ in the dilatometer) and nondestructive characterization of the physical properties. A detailed description of each preirradiation testing method is provided in Section 5, Detailed Description of Test Methods. 
Table 4. NGNP Graphite specimen measurement and test equipment.

\begin{tabular}{|c|c|c|c|c|}
\hline Measurement & Standard & Instrumentation & Calibration Method & Result \\
\hline \begin{tabular}{|l|} 
Physical \\
dimensions and \\
mass
\end{tabular} & $\begin{array}{l}\text { ASTM C559-90 } \\
\text { (reapproved 2010) }\end{array}$ & $\begin{array}{l}\text { Mitutoyo Micrometer } \\
\text { 121-155 } \\
\text { INL ID: } 725884 \\
\text { INL ID: } 727312 \\
\text { Mitutoyo Caliper CD-6" } \\
\text { CSX } \\
\text { INL ID: } 725813 \\
\text { INL ID: } 726607 \\
\text { INL ID: } 727194 \\
\text { Sartorius Scale ME235P } \\
\text { INL ID: } 412642 \\
\text { INL ID: } 415907\end{array}$ & $\begin{array}{l}\text { INL Standards and } \\
\text { Calibration Laboratory }\end{array}$ & Bulk density \\
\hline $\begin{array}{l}\text { Fundamental } \\
\text { frequency }\end{array}$ & $\begin{array}{l}\text { ASTM C747-93 } \\
\text { (reapproved 2010) } \\
\text { ASTM } \\
\text { C1259-08 } \\
\end{array}$ & $\begin{array}{l}\text { J. W. Lemmens } \\
\text { Grindosonic } \\
\text { INL ID: } 412850\end{array}$ & $\begin{array}{l}\text { No calibration required } \\
\text { per instrument } \\
\text { manufacturer }\end{array}$ & $\begin{array}{l}\text { Elastic } \\
\text { modulus }\end{array}$ \\
\hline Sonic velocity & ASTM C769-09 $9^{[26]}$ & $\begin{array}{l}\text { Olympus NDT Sq. Wave } \\
\text { Pulser/Receiver 5077PR } \\
\text { INL ID: } 728024 \\
\text { National Instruments } \\
\text { Digitizer USB 5133 } \\
\text { INL ID: } 726725 \\
\text { INL ID: } 415868 \\
\end{array}$ & $\begin{array}{l}\text { INL Standards and } \\
\text { Calibration Laboratory }\end{array}$ & $\begin{array}{l}\text { Young's } \\
\text { modulus, Shear } \\
\text { modulus, } \\
\text { Poisson ratio }\end{array}$ \\
\hline $\begin{array}{l}\text { Four-point } \\
\text { electrical } \\
\text { resistivity }\end{array}$ & $\begin{array}{l}\text { ASTM C611-98 } \\
\text { (reapproved 2010) }\end{array}$ & $\begin{array}{l}\text { Kiethly } 6220 \text { Precision } \\
\text { Current Source } \\
\text { INL ID: } 725865 \\
\text { INL ID: } 727290 \\
\text { Kiethly } 2182 \text { A Nano } \\
\text { Voltmeter } \\
\text { INL ID: } 725866 \\
\text { INL ID: } 727289\end{array}$ & $\begin{array}{l}\text { INL Standards and } \\
\text { Calibration Laboratory }\end{array}$ & $\begin{array}{l}\text { Electrical } \\
\text { resistivity }\end{array}$ \\
\hline $\begin{array}{l}\text { Laser flash } \\
\text { diffusivity }\end{array}$ & ASTM E1461-07 & $\begin{array}{l}\text { Netzsch LFA } 4572 \text { ea. } \\
\text { INL ID: } 412855 \\
\text { INL ID: } 412864\end{array}$ & $\begin{array}{l}\text { Calibration by user per } \\
\text { manufacturer's } \\
\text { instructions }\end{array}$ & $\begin{array}{l}\text { Thermal } \\
\text { diffusivity }\end{array}$ \\
\hline $\begin{array}{l}\text { Push rod } \\
\text { dilatometry }\end{array}$ & ASTM E228-06 & $\begin{array}{l}\text { Netzsch DIL } 402 \text { C } 2 \text { ea. } \\
\text { INL ID: } 412860 \\
\text { INL ID: } 412861\end{array}$ & $\begin{array}{l}\text { Calibration by user per } \\
\text { manufacturer's } \\
\text { instructions }\end{array}$ & $\begin{array}{l}\text { Coefficient of } \\
\text { thermal } \\
\text { expansion }\end{array}$ \\
\hline $\begin{array}{l}\text { Environmental } \\
\text { monitoring }\end{array}$ & All & $\begin{array}{l}\text { Visala Pressure, Humidity } \\
\text { and Temperature PTU301 } \\
\text { INL ID: } 726912 \\
\text { INL ID: } 727884 \\
\text { INL ID: } 727502\end{array}$ & $\begin{array}{l}\text { INL Standards and } \\
\text { Calibration Laboratory }\end{array}$ & $\begin{array}{l}\text { Laboratory } \\
\text { environmental } \\
\text { conditions }\end{array}$ \\
\hline
\end{tabular}

The equipment necessary for the measurements listed in the above Table 4 are segregated into individual stations. Each station has a bar scanner for reading unique specimen identification bar codes, a computer for automated data acquisition, and the test equipment. The bar code of the individual specimen container is read, and the file for that specimen is automatically opened for data input prior to each measurement. Associated with each measurement type/station is a unique laboratory notebook maintained in accordance with MCP-2875, "Maintaining Laboratory Notebooks,",[27] and PLN-2690, "VHTR Technology Development Office Quality Assurance Program Plan, ${ }^{,[28]}$ paragraph 3.3. Accepted data are 
stored in the NGNP Data Management and Analysis System, a satellite file location for NGNP. Data are transmitted in standardized Excel file format to the NGNP Data Management and Analysis System using Form 435.78, "VHTR Technology Development Office Information Input Sheet,"[29] in accordance with PLN-3319.

In addition to data stored in laboratory notebooks, the specific measuring instruments are networked to a server computer where the measurement data are automatically stored. This has been implemented in the INL Carbon Characterization Laboratory where custom LabVIEW software was written to facilitate automated data acquisition. This software comprises five main programs: Manufacturers Data, Physical and Dimensional Measurements, Electrical Resistivity Measurements, Sonic Resonance (Fundamental Frequency) Measurements, and Sonic Velocity Measurements. These five programs acquire data from instrumentation or user input and record the results in an Excel spreadsheet located on a server computer. In the case of thermal expansion and thermal diffusivity measurements, two other LabVIEW programs have also been written to parse vendor software-acquired data into Excel spreadsheets. MCP-3058, "VHTR TDO Software Quality Assurance,",[30] and FRM-959, "VHTR Software Management Plan,",[31] are currently used to govern the development, accuracy, and configuration control of this software.

Measurements are made in the following sequence:

1. Wash and dry - all specimens

2. Mass and dimensions - all specimens

3. Thermal diffusivity - piggyback specimens

4. Elastic modulus by sonic resonance - creep specimens

5. Electrical resistivity - creep specimens

6. Elastic modulus by measurement of sonic velocity - creep specimens

7. Wash and dry to remove couplant - creep specimens

8. Coefficient of thermal expansion - creep specimens

9. Postcharacterization of mass and dimensions - all specimens.

\section{DETAILED DESCRIPTION OF TEST METHODS}

Before any measurements are made, specimen numbers and basic information about each type of graphite will be entered into the manufacturer's data program. Once basic information about the graphite type has been recorded, it will be automatically saved to an Excel spreadsheet file, and the individual specimen numbers will be entered using a bar code reader. Following the initial input of general information, individual material property measurements will be made, starting with mass and dimensional measurements for determining bulk density.

\subsection{Mass, Dimensions, and Bulk Density}

Dimensional change is one of the key parameters affecting the performance of graphite in a neutron environment. Determining volumetric and linear dimension as a function of temperature and radiological dose is necessary to understand critical performance measures (e.g., dimensional change turnaround, irradiation creep, and internal stresses imposed upon graphite components). Dimensional and mass measurements will be performed to ASTM Standard C559-90, "Standard Test Method for Bulk Density by Physical Measurements of Manufactured Carbon and Graphite Articles" (reapproved 2010), ${ }^{[32]}$ which describes in detail the procedure for making dimensional measurements and calculating bulk density.

The accuracy of the dial micrometers used here is stated by the manufacture to be $2 \mu \mathrm{m}$. For the larger graphite creep specimens with a $25.4 \mathrm{~mm}$ length measurement this corresponds to a $0.008 \%$ accuracy for this technique. However, when evaluating the uncertainty of the density determination other 
factors must be considered, such as, the hardness of the material and the force with which the micrometer blade is engaged with the material, specimen temperature variation, technician skill, etc. These and other factors were considered in a propagation of error analysis to arrive at an uncertainty of $0.08 \%$ with the measurement of the specimen diameter being the largest contributor to the error.

\subsection{Electrical Resistivity}

Electrical resistivity is used as a rapid, simple means to determine grain orientation, structure, and crystallinity of graphite. In conjunction with optical microscopy, it can be used to determine the microstructural texture of graphite components without much specimen preparation work. Resistivity is measured following ASTM C611-98, "Standard Test Method for Electricity Resistivity of Manufactured Carbon and Graphite Articles at Room Temperature" (reapproved 2010). ${ }^{[33]}$ The measurement technique is commonly referred to as "four-point probe." It consists of passing a known current through the specimen and measuring the voltage across the specimen at known locations. Based on Ohms law, the resistance is determined, and the resistivity is calculated from Equation (1):

$$
\rho=R \cdot A / L
$$

where

$$
\begin{gathered}
\rho=\text { Electrical resistivity } \\
\mathrm{R}=\text { Measured resistance } \\
\mathrm{A}=\text { Cross sectional area } \\
\mathrm{L}=\text { Length over which the voltage is measured. }
\end{gathered}
$$

Figure 8 shows a test fixture fabricated at INL that allows a specimen to be rotated for multiple measurements of voltage around its periphery.

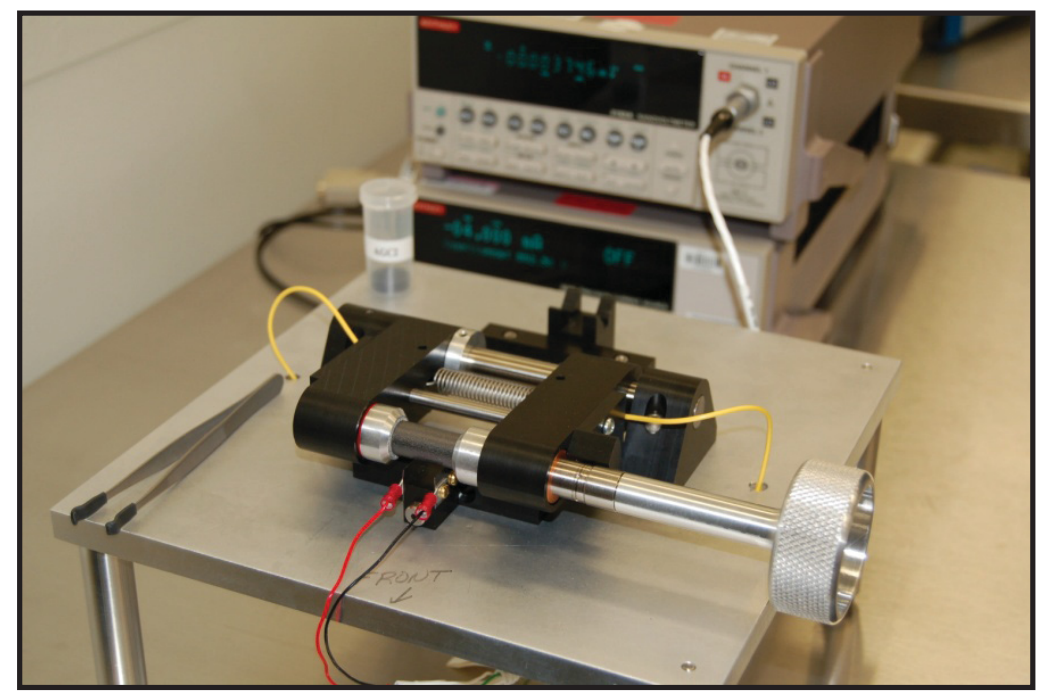

Figure 8. Electrical resistivity measurement station.

Uncertainty in the resistivity measurement is mainly comprised of the contact resistance between the specimen and the contacting blades for the voltage measurement. Specimen temperature and the temperature of other bimetal junctions in the voltage measuring leads are also significant factors. These effects are minimized by passing the current through the sample in two directions and averaging the measured voltage for each direction. In this way any thermoelectric or small differences in junction resistances will cancel. A round robin test series reported in ASTM C 611 precision and bias section 
states a lab to lab variability of $2.5 \%$. A round robin test series such as this would take into account the variables discussed above and is considered a good estimate of the measurement uncertainty.

\subsection{Approximation of Elastic Modulus from the Measurement of Sonic Velocity}

The mechanical properties of graphite are necessary to determine the structural integrity of graphite components. These properties are vital to determining the viability of the structural strength and integrity of the reactor core. The as-received and irradiated values are needed for whole-core models, which will be used for the graphite design code. This test is carried out in accordance with ASTM C 769-09, "Standard Test Method for Sonic Velocity in Manufactured Carbon and Graphite Materials for Use in Obtaining Young's Modulus." In this measurement, the transmitting piezoelectric transducer sends a 2.25-MHz sound wave through the specimen. At the opposite end of the specimen, the acoustic wave is received by another piezoelectric transducer. The sonic velocity of the specimen is the ratio of specimen length to the signal time lapse between transducers. An approximate value for Young's modulus, E, can be obtained from Equation (2):

$$
E=\rho V^{2}
$$

where

$$
\begin{array}{ccc}
\mathrm{E} & =\text { Young's modulus } \\
\rho & =\text { Specimen density } \\
\mathrm{V} & =\text { Sonic velocity. }
\end{array}
$$

Figure 9 shows the sonic velocity measurement station. In the foreground are fixtures for clamping the specimen between the transducer and receiver. These fixtures have unique features that improve measurement accuracy, precision, and efficiency. Specimens are easily and rapidly loaded into the fixture using the cam-operated clamp. Measurement precision is improved because the spring-loaded clamp applies consistent pressure between the transducers and specimen, resulting in repeatable couplant thickness.

As specified in paragraphs 8.1 and 8.5.1 of ASTM C 769-09, a suitable coupling medium should be used and reported with the data. Here, "Shear Gel," manufactured by Sonotech Inc., is used for a shear wave couplant, and "Ultra Gel II," also manufactured by Sonotech Inc., is used for the transverse wave couplant. 


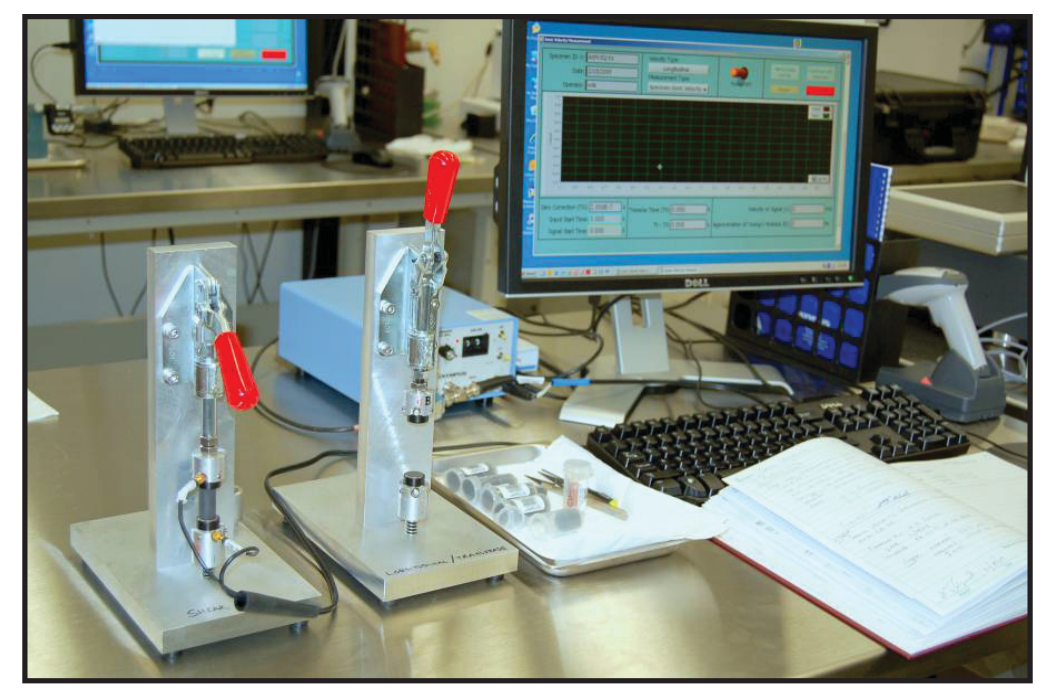

Figure 9. Sonic velocity measurement station.

Figure 9 shows the LabVIEW software user interface display for sonic velocity measurements after scanning the bar code of the specimen to be tested. This screen is used to acquire sonic velocity measurements of a specimen in both the longitudinal and shear directions. Operating much like an oscilloscope, the cursors automatically mark the time between the transmitted wave and the received wave. Also shown in Figure 9 are two examples of the shear wave and transverse wave timing locations for properly coupled specimens. The specimen length divided by this transit time yields the sonic velocity.

The uncertainty in determining of elastic moduli from the measurement of sonic velocity comes from several sources. First there is the effect of material and geometry related dispersion of the transmitted wave. ASTM C-769 provides guidance on how to minimize this problem by choosing the correct frequency. This technique also assumes linear elastic behavior and graphite is not completely linearly elastic. And finally, the operator's judgment on the positions of the timing cursors is somewhat objective. Clean wave forms to base these judgments on are highly dependent on the quality of the transducer-material coupling. These sources of error are difficult to quantify and therefore difficult to combine in a propagation of error analysis. However, ASTM C769 describes in some detail a round robin test series between different labs. Using round robin test data to determine a coefficient of variation $(\mathrm{COV})$ is a good means of estimating the measurement uncertainty. With caution, the COV of $3.8 \%$ (reported in C769) is taken here to be representative of the uncertainty for these measurements. When considering a single material and making comparisons between the pre and post irradiation signal values the precision of these measurements is good enough to consider differences greater than $4 \%$ significant. However, one is cautioned to refrain from using the values here as absolute or better than $\pm 10 \%$ accurate.

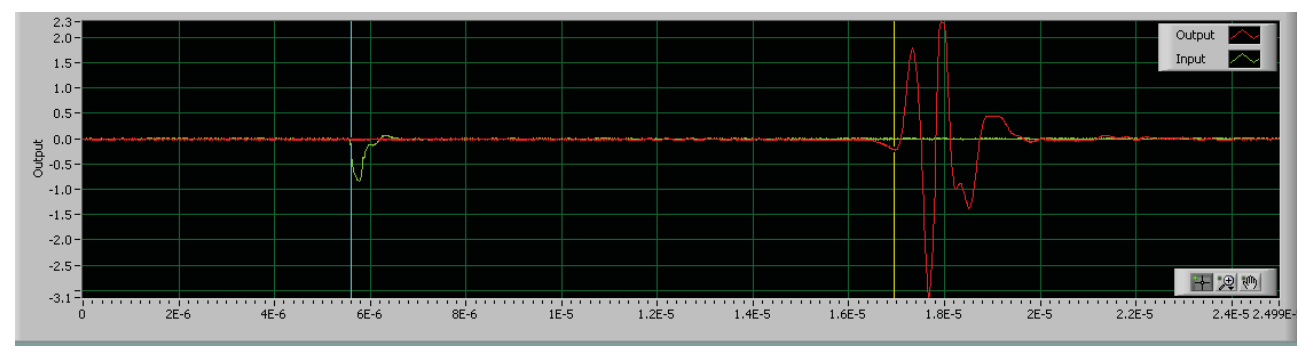

Shear wave timing 


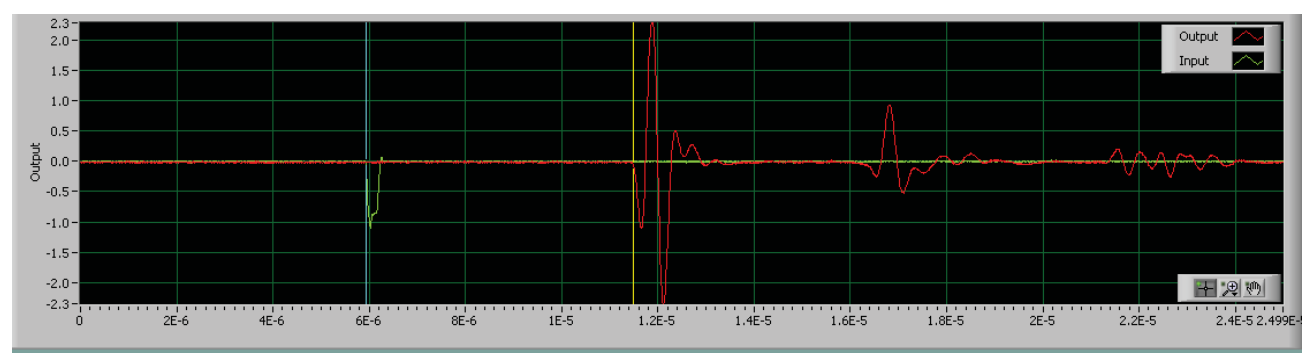

Transverse wave timing

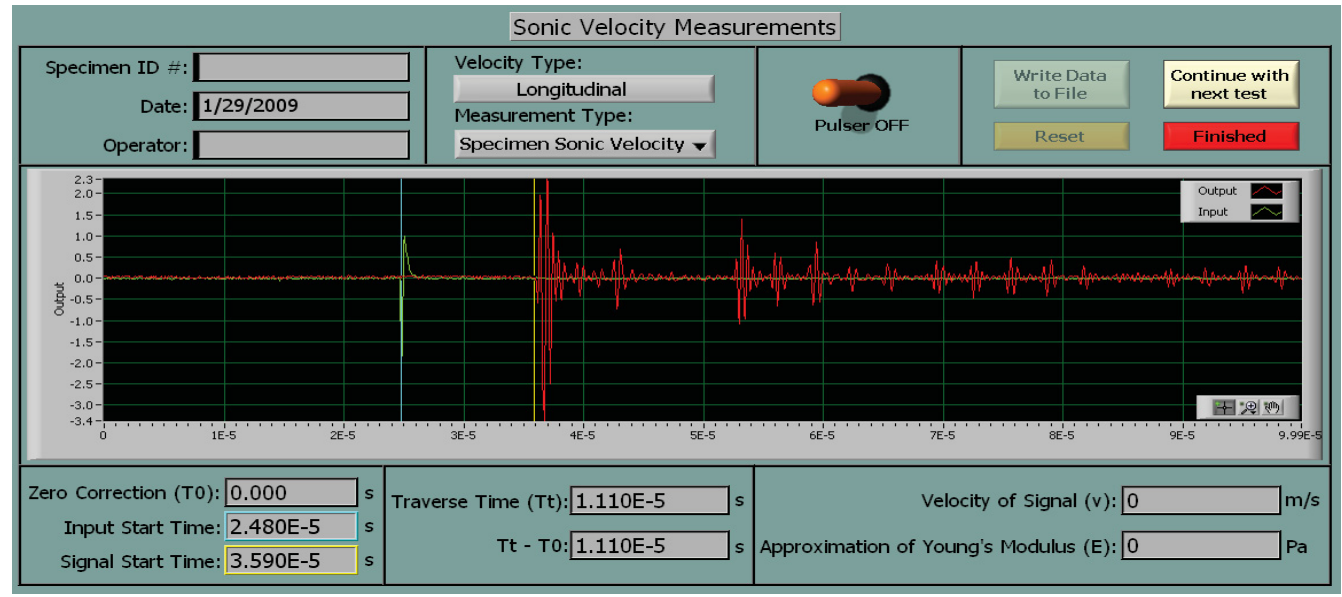

Figure 10. Sonic velocity measurement user interface.

After the longitudinal and shear modulus values have been determined, each specimen Poisson's Ratio can be calculated from these results through equation (3):

$$
v=\left[\frac{E}{2 G}\right]-1
$$

Where:

$$
\begin{gathered}
v=\text { Poisson's ratio } \\
\mathrm{E}=\text { Elastic Modulus } \\
\mathrm{G}=\text { Shear Modulus }
\end{gathered}
$$

\subsection{Modulus of Elasticity by Measurement of Fundamental Frequency}

Understanding the mechanical properties of graphite is necessary to determine the structural integrity of graphitic components. These properties are vital to determining the viability of the structural strength and integrity of the reactor core. This test method measures the fundamental resonant frequency of test specimens of suitable geometry by exciting them mechanically with a singular elastic strike. Specimen supports, impulse locations, and signal pick-up points are selected to induce and measure specific modes of the transient vibration of the specimen. The transient signals are analyzed, and the fundamental resonant frequency is isolated and measured by the signal analyzer. The measured fundamental resonant frequency, specimen dimensions, and mass are used to calculate the dynamic Young's modulus, shear modulus, and Poisson's ratio in accordance with ASTM C747-93, "Standard Test Method for Moduli of 
Elasticity and Fundamental Frequencies of Carbon and Graphite Materials by Sonic Resonance," (reapproved 2010) in combination with apparatus and calculations described in ASTM C1259-08, "Standard Test Method for Dynamic Young's Modulus, Shear Modulus, and Poisson's Ratio for Advanced Ceramics by Impulse Excitation of Vibration." The fundamental frequency measurement station is shown in Figure 11.

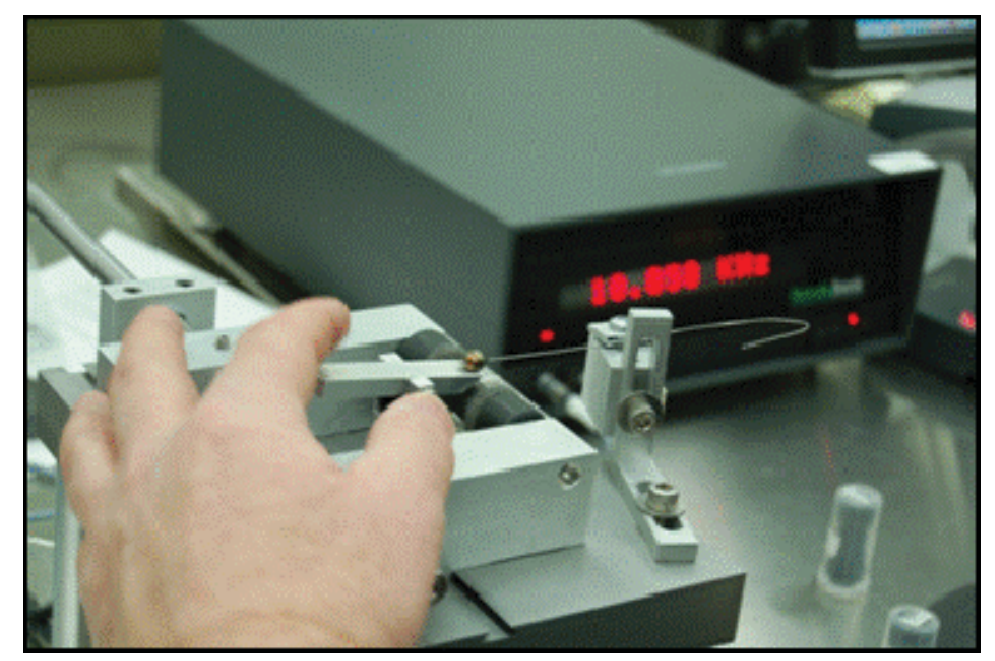

Figure 11. Fundamental frequency measurement station.

After placing a specimen in the test fixture, the user excites it by lightly tapping it with a small mechanical impulse. A consistent impulse is achieved by placing the ball hammer onto a lever that rotates out from under the hammer as it is raised. The specimen is supported in such a way that it vibrates at its natural frequency. A microphone placed underneath one end of the specimen, in combination with the Grindosonic electronics, measures this frequency, which is recorded and displayed by the computer. The modulus of elasticity is calculated and displayed next to the newly acquired frequency. If the results are satisfactory, the user can press the "Save $1^{\text {st }}$ Frequency" button and go on to the next measurement. Following the recommendations of ASTM C-1259-08, 10 readings of the fundamental frequency are measured before the results of the test are written to the applicable Excel spreadsheet.

ASTM C-1259 describes in detail a round robin test series using ceramic materials along with an analysis of the propagation of errors in the calculation of moduli from the measurement of resonant frequency, geometry and mass of the sample. This error analysis shows the major sources of experimental variation are the measurement of the fundamental frequency and the smallest dimension (diameter) of the specimens due to their higher exponent in the modulus calculations. Both the propagation of error analysis and round robin data indicated an uncertainty of less than $2 \%$. However, the creep specimens tested here do not meet the geometry requirements of C-1259. With the current specimen length-to-diameter ratio of only 2 (rather than 5 as specified) the AGC-2 graphite specimens are, at times, difficult to excite consistently and in a single mode of vibration. After significant training, an experienced operator was able to consistently obtain a resonant frequency within $2 \%$ uncertainty for the flexural mode of vibration within the specimens.

\subsection{Thermal Expansion}

Understanding the CTE for graphite components is critical for determining the dimensional changes that occur as a result of temperature cycles. Localized external stresses can be imposed upon mechanically interlocked graphite-core components because the individual pieces suffer differential thermal expansion. Internal stresses can occur within larger graphite components if there is a temperature gradient causing differential expansion within the piece (i.e., one side has a higher temperature than the 
other). Finally, the thermal expansion is highly dependent upon the graphite microstructure (e.g., orientation/anisotropy, pore size and distribution, and crystallinity). Irradiation damage can significantly alter graphite microstructure and thus CTE values. Determining the extent of the changes as a function of irradiation dose and temperature will be a key parameter for reliable calculation of stress states within graphite components, volumetric changes, and irradiation creep rates.

The CTE measured here follows ASTM E228-06, "Standard Test Method for Linear Thermal Expansion of Solid Materials with a Push-Rod Dialometer." ${ }^{\text {[34] }}$ This test method uses a push-rod type dilatometer to determine the change in length of a graphite specimen relative to that of the holder as a function of temperature from the specified reference temperature. The temperature is varied over the desired range at a slow, constant heating or cooling rate. The linear thermal expansion and mean CTE, $\alpha$, are calculated from the recorded data using Equation (4):

$$
\alpha=\frac{1}{L_{0}} \frac{\Delta L}{\Delta T}
$$

where

$$
\begin{aligned}
\alpha= & \text { Mean CTE } \\
\mathrm{L}_{0}= & \text { Specimen initial length } \\
\Delta \mathrm{L}= & \text { Change in length } \\
\Delta \mathrm{T}= & \text { Temperature difference between a specified reference } \\
& \text { temperature and the temperature at which the change in } \\
& \text { length was measured. }
\end{aligned}
$$

The Netzsch DIL 402 C commercial system, shown in Figure 12, currently used at INL does not have the capability to cool the specimen below ambient temperature. Therefore, the initial length at $20^{\circ} \mathrm{C}$ is linearly extrapolated from expansion data between 100 and $150^{\circ} \mathrm{C}$, and the mean CTE is calculated from a $20^{\circ} \mathrm{C}$ reference temperature.

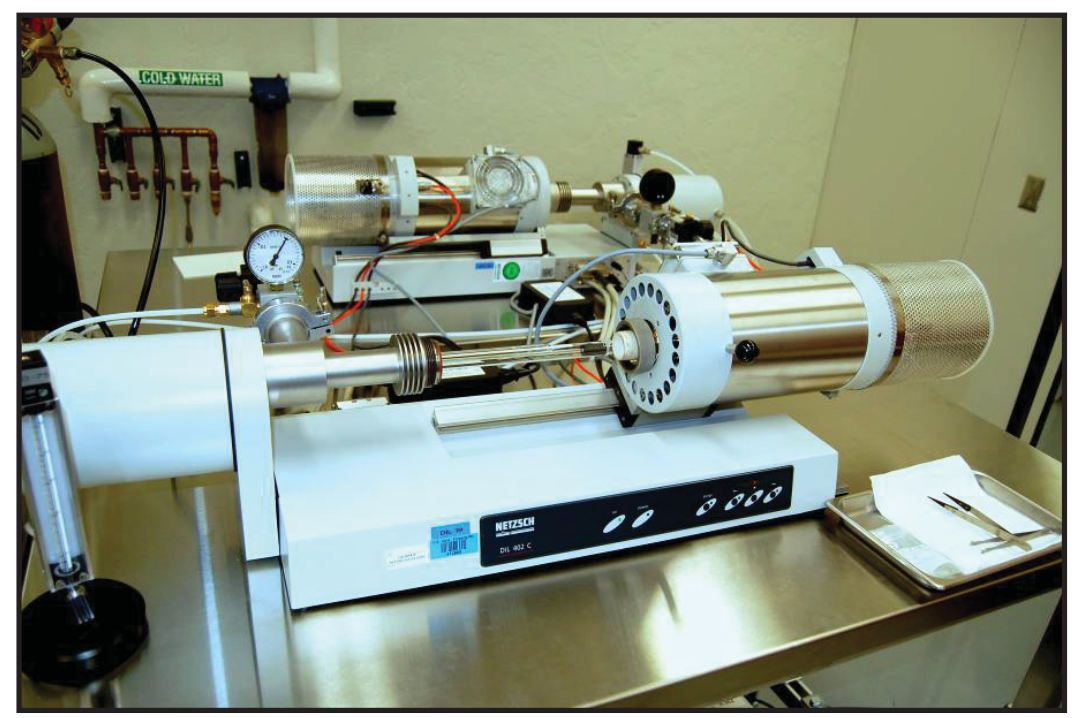

Figure 12. Commercial push rod dilatometer for measuring the coefficient of thermal expansion.

The greatest source of experimental error in the dilatometry method described here is the correction made for the expansion of the specimen holder and push rod/LVDT mechanism. This differential between the specimen and the apparatus must be accounted for in order to isolate the specimen expansion only. 
Studies reported in the precision and bias section of ASTM E228 have indicated that this type of dilatometry can be accurate to $4 \%$ when calibrations are performed carefully.

\subsection{Thermal Diffusivity}

The ability to conduct heat through the graphite core is critical to the passive removal of decay heat. Reduction of the thermal conductivity within graphite can have a significant effect on the passive heat-removal rate and thus the peak temperature that the core and, subsequently, the fuel particles will experience during off-normal events. Determining changes to the conductivity as a function of irradiation dose and temperature is important for the HTGR safety analysis. Here, ASTM E1461-07, "Standard Test Method for Thermal Diffusivity by the Flash Method," ${ }^{[35]}$ is followed for calculating thermal diffusivity and conductivity. Thermal diffusivity $(\delta)$ is measured and defined as the ratio of thermal conductivity to volumetric heat capacity, as shown in Equation (5):

$$
\delta=\frac{k}{\rho C_{P}}
$$

Where

$$
\begin{gathered}
\delta=\text { Thermal diffusivity } \\
\mathrm{k}=\text { Thermal conductivity } \\
\rho=\text { Density } \\
\mathrm{C}_{\mathrm{P}}=\text { Specific heat. }
\end{gathered}
$$

The measurement is performed on small, thin, disk-shaped specimens. A pulsed laser is used to subject one surface of the specimen to a high-intensity, short-duration energy pulse. The energy of this pulse is absorbed on the front surface of the specimen, and the resulting rise in rear-face temperature is recorded. The thermal diffusivity is calculated from the specimen thickness and the time required for the rear-face temperature to reach $50 \%$ of its maximum value. A commercially available laser flash apparatus, complete with vendor-developed software for instrument control and data acquisition, is used, as shown in Figure 13.

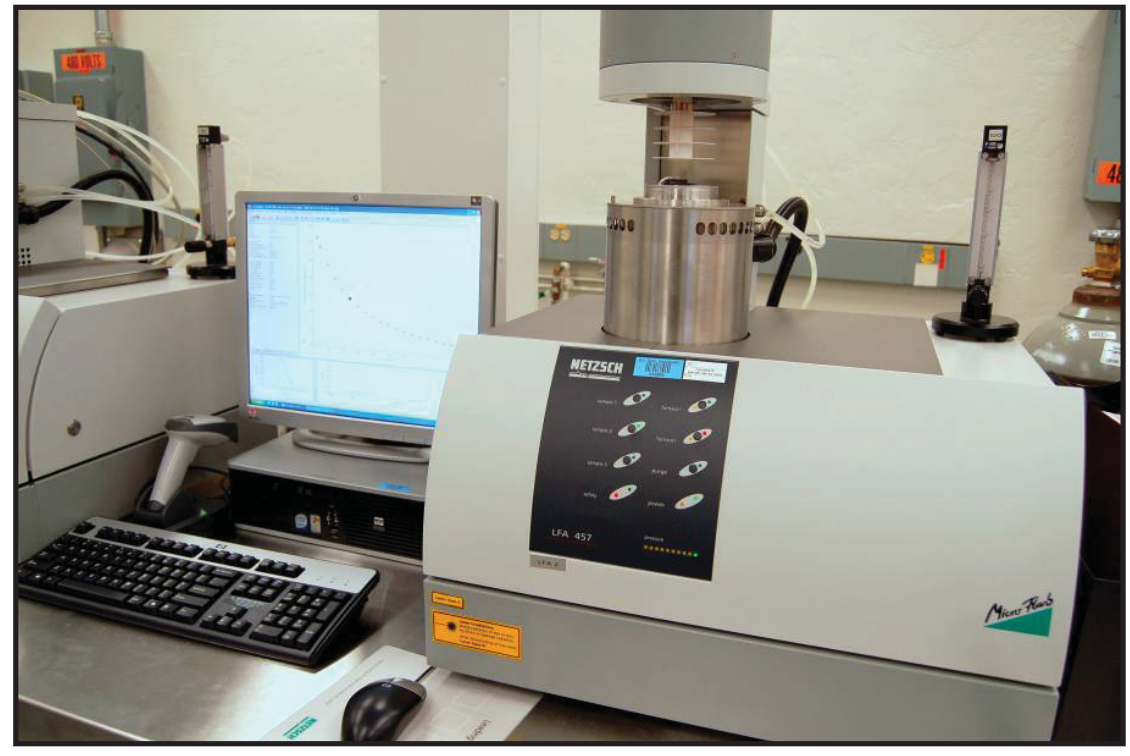

Figure 13. Laser flash apparatus measurement station for determining thermal diffusivity. 
Uncertainty in the measurement of thermal diffusivity occurs from specimen heat loss and temperature measurement. Specimen temperature measurement is performed with a calibrated type $\mathrm{S}$ thermocouple in the near vicinity of the specimen. Being a relatively straight forward, the specimen temperature measurement is typically a small contribution to the overall measurement error or uncertainty.

The main contributor to the measurement uncertainty is heat loss from the specimen. Because this measurement technique depends on the assumption of one-dimensional heat transfer from the flat face receiving the laser pulse to the flat face radiating to the detector, heat loss errors are mainly attributed to radiative heat loss from the circumference of the specimen at temperatures above $300^{\circ} \mathrm{C}$. Typically provided with the instrument software are several correction models to account for this heat loss. As the specimen diameter to thickness ratio decreases the heat loss increases to the point that the correction models no longer can account for the error. A study was performed to gain a fuller understanding of the limits of the models made available with the NETZSCH LFA and the dependence of the diameter to thickness ratio on measurement error. In this study the heat loss models were applied to data taken on specimens with various diameter to thickness ratios and at specimen temperatures between $25^{\circ} \mathrm{C}$ and $1000^{\circ} \mathrm{C}$. It was determined that the Cowan ${ }^{[36]}$ model along with diameter to thickness ratios greater than or equal to 2 resulted in determination of the diffusivity within ASTM E1461 and the manufactures specified uncertainties of $4 \%$ and $3 \%$ respectively. This was further verified by instrument functional tests performed monthly on a pure iron validation sample for which the diffusivity was determined to be within $3 \%$ of the Touloukian ${ }^{[37]}$ values between $100^{\circ} \mathrm{C}$ and $700^{\circ} \mathrm{C}$.

\section{DATA ANALYSIS}

Data gathered for the characterization of AGC-2 specimens is contained in the appendixes of this report. Appendix A contains plots of the individual data points for each specimen. Shown by the dashed lines in each plot are the upper and lower limits of the interquartile range (IQR). These limits are established by the lesser of either the least or greatest value in the data or by multiplying the interquartile range by 1.5 and adding or subtracting this value from the third and first quartile. Any datum value outside of these limits is a suspected outlier of the established pattern. However, it is important to note that these outlying values are not only subject to measurement variability but also material variability and, therefore, cannot necessarily be discarded. These outlying values are examined in the context of the entire data set and will be evaluated further following irradiation. Other statistical parameters are calculated and presented in the tables of Appendix B. The mean, standard deviation and coefficient of variance are all calculated for the different measurement data sets and graphite types. Upper and lower limits called out in the tables of Appendix B are the IQR limits described above. Appendix C contains all of the raw data, including parameters specified by the applicable ASTM standard (e.g., dates, performer identifier, and room conditions).

There are many ways to combine and compare the data presented here. In doing so, the validity of the data is exercised and scrutinized. First, the data sets are considered independently with the statistical analysis described above. Additionally, a limited comparison of the absolute property values is performed between different graphite types and grades. The degree of isotropy is also evaluated for a limited number of grades by calculating the anisotropy ratio, as shown in Equation (6).

$$
\text { Anisotropyratio }=\frac{\text { Value of the propertyin the against }- \text { grain direction }}{\text { Value of the propertyin the with }- \text { grain direction }}
$$

Note that in the case of isostatically molded graphite "with-grain" and "against-grain" indicate specimens taken from orthogonal planes in the billet. 


\subsection{Analysis of Mass, Dimensions, and Density Data}

Plots of the measured mass, dimensions, and density for all AGC-2 specimens are shown in Figures A-1 through A-88 (see Appendix A). Beginning with graphite grade H-451, the calculated density of with-grain specimens has a higher degree of scatter as compared to the against-grain specimens (see Figure A-1). Both length and mass measurements for the with-grain specimens have relatively high COV (see Figures A-23 and A-67, respectively), but do not compensate for each other in the distribution of density. The higher scatter in density is most likely a result of material variation in the area of the billet from which these specimens were machined; however, it is difficult to completely rule out measurement inaccuracies. Therefore, the with-grain specimen length and mass measurements should be treated with caution. Comparison to the postirradiation measurements should provide more information on this observation.

Similar variations in density were observed in graphite grade NBG-17. In this case, a bimodal distribution in density was observed for both with- and against-grain specimens (see Figure A-4). Dimensional measurements were consistent and well-behaved for all specimens, but the bimodal distribution was observed in the mass measurements. To understand this unusual distribution, a study was completed to investigate the density as a function of depth in the NBG-17 billet. Twenty-three specimens $(12 \times 12 \times 5.5 \mathrm{~mm}$ thick) were cut from the top of the billet to a depth of $\sim 125 \mathrm{~mm}$ (see Figure 14). Figure 15 shows the density of specimens as a function of depth from the surface. In just $25 \mathrm{~mm}$, the density increases $3 \%$. This low-density area or area of inconsistent properties at the outer surface of the billet is often referred to as the skin effect of the billet.

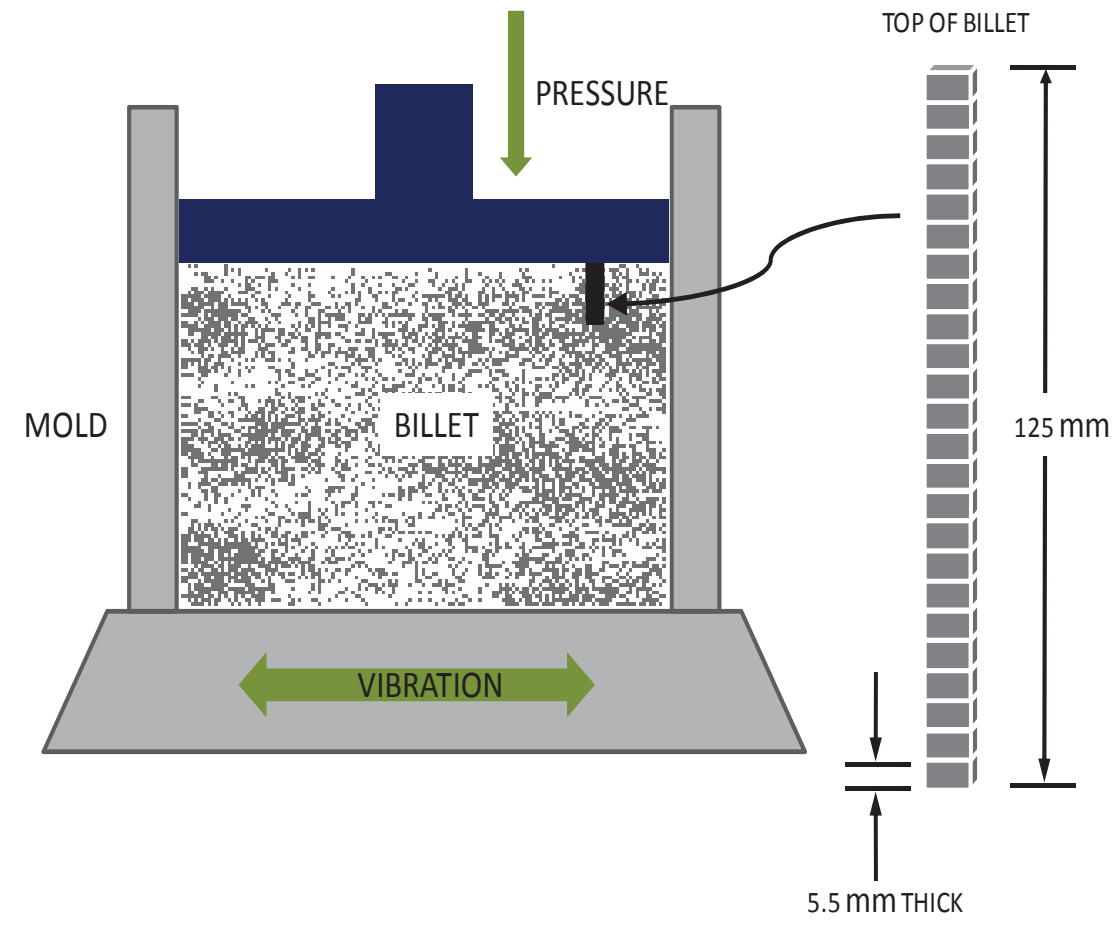

Figure 14. Schematic showing location and dimensional details of NBG-17 billet density investigation. 


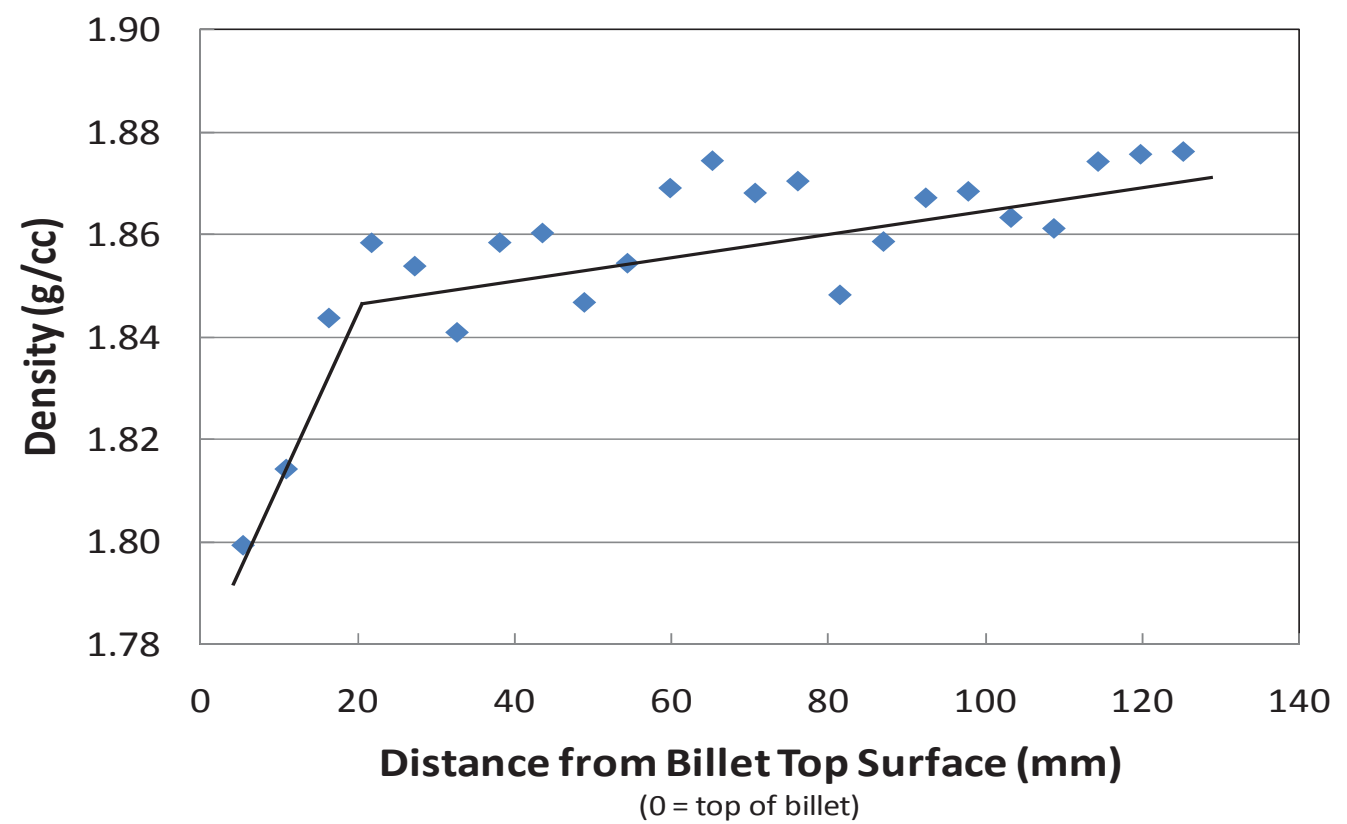

Figure 15. Density of NBG-18 as a function of depth in the billet.

Initially, it appeared unfortunate that specimens were taken from the skin of the billet, but in hindsight, this provided a variation in a significant property. With specimens of the same graphite type (i.e., coke, binder, and particle size) other material variables are held constant. This makes it possible to correlate the effect of density on other intrinsic properties (e.g., resistivity, elastic modulus, and diffusivity). Figure 16 shows an example of this for the resistivity measurements of the NBG-17 specimens. The expected difference in resistivity due to grain orientation is shown for the specimens at a similar density of $\sim 1.864 \mathrm{~g} / \mathrm{cc}$. The higher resistivity for low-density with-grain specimens compared to the higher density specimens is most likely a result of higher porosity or micro-crack density inhibiting the flow of electrons. Further discussion of the resistivity data is provided in Section 3.2 of this report.

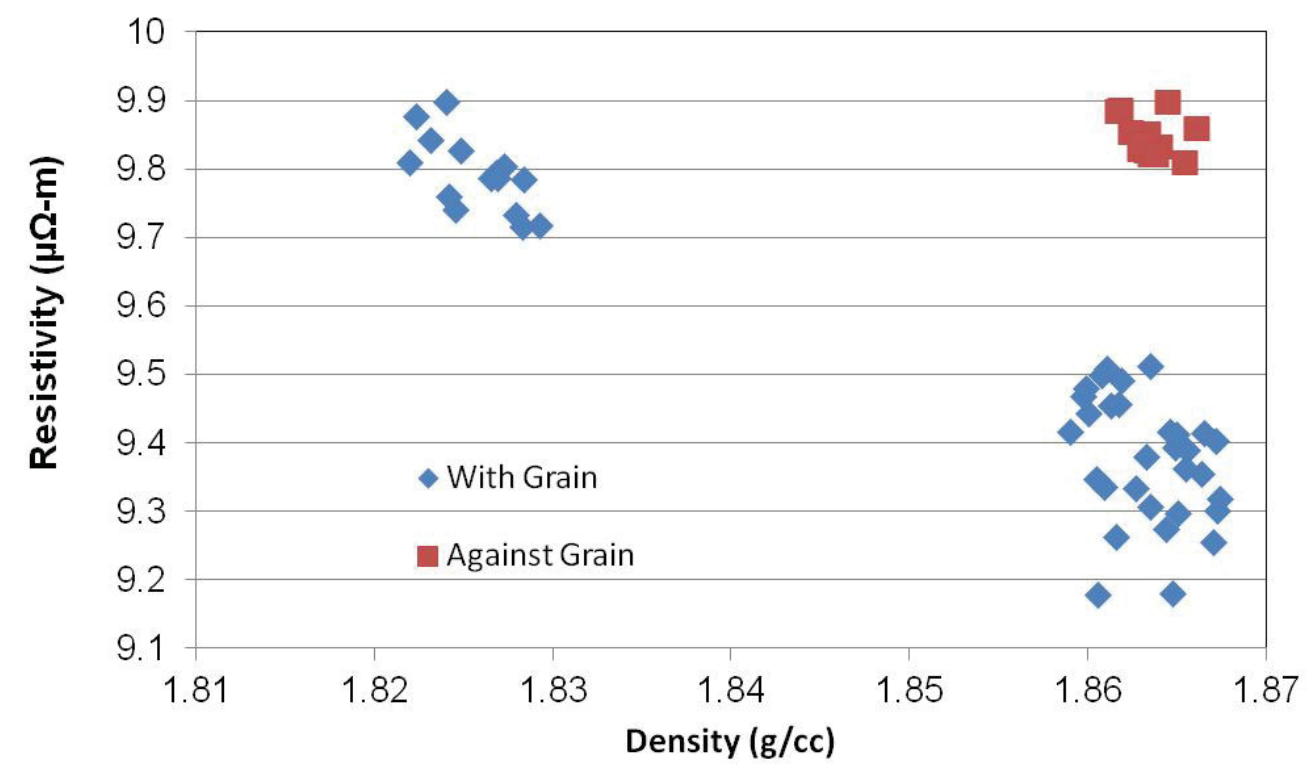

Figure 16. Resistivity of NBG-17 as a function of density. 
Occasions arise when the determination of bulk density can be used to evaluate outliers or data that are near the IQR limits in either the dimensional or mass measurements. For example, Figures A-24, A-27, and A-28 show several data values for IG-110, NBG-18 and PCEA dimensional measurements that are near or outside the IQR limits. Evaluation of the corresponding density plots, Figures A-5 and A-16, show that all values fall within the quartile limits, with relatively low COVs. From this, it can be concluded that the dimensional outliers are simply specimens that were machined with different dimensions, resulting in them being outside or near the limit of the quartile analysis.

The density data of all other graphite grades (i.e., 2114, IG-110, NBG-18, NBG-25, BAN, HLM, IG-430, NBG-10, PCIB, PPEA, A3 Matrix, and New Matrix) is well-behaved and consistent with what was expected. The fact that these density data are so well-behaved indicates any outliers found in the associated dimensional or mass data are simply a result of machining tolerances and are valid.

Additionally, the dimensions and mass were measured following the thermal measurement of the CTE and diffusivity. These measurements were performed up to $1000^{\circ} \mathrm{C}$, and concerns existed that the specimens maintained some residual stress due to the machining operations and that this stress would relax during the thermal cycle to $1000^{\circ} \mathrm{C}$. Figures A-137 through A-202 contain plots of a comparison between the pre and post thermal treatment dimensions and mass. The comparison plots of specimen length show a slight trend of shrinkage of approximately $0.03 \%$. For a $25-\mathrm{mm}$ specimen, the accuracy of the dial micrometers used is $0.01 \%$. Uncertainty in the measurement comprises not only accuracy of the dial micrometer but also other variables (e.g., room temperature, micrometer clutch wear, micrometer blade design, and velocity of blade approach) relative to the specimen hardness. Although there is a definite trend to the decrease in length measurements after the thermal measurements, it is difficult to draw concrete conclusions, considering that the length change is probably on the order of the measurement uncertainty. Furthermore, the annealing of residual stress would tend to increase the size of the specimen, not decrease it. However, oxidation could decrease the size of the specimen during the thermal measurements if the furnace is not purged of all air; and indeed, the post mass measurements trend to lower values by $\sim 0.03 \%$ on average, indicating that a very small amount of oxidation was occurring. Several specimens of graphite type 2114 (specimen numbers TP19 and TP21 were used as filler piggybacks in capsules 2 and 4, respectively, and TP21 was not used in the irradiation experiment) and NBG-18 (specimen number BP502 was not used in the irradiation experiment) displayed mass loss in excess of $0.5 \%$ (see Figures A-185 and A-187). This suspected oxidation is corroborated in the relatively large reduction in length and diameter of these specimens. In the analysis of postirradiation dimensional change, these specimens will require careful evaluation, using the post thermal measurement dimensions, if they are used for any calculation of irradiation creep.

\subsection{Electrical Resistivity}

Plots of electrical resistivity are shown in Figures A-119 through A-124 for graphite grades of PCEA, NBG-18, H-451, IG-110, IG-430, and NBG-17. All of the resistivity measurements were performed on the creep specimens only. Several noteworthy observations resulted from the statistical analysis, and all are related to billet density variations discussed in Section 3.1. Looking at Figure A-122, resistivity measurements for NBG-17 were only performed on the against-grain specimens taken from central locations in the billet where the density is consistent (refer to INL Drawing 600787, Rev. 3). In this case, the resistivity values are extremely consistent, with a coefficient of variance of $0.3 \%$, which is well below the $2.5 \%$ uncertainty reported in a round robin test series of ASTM C611-98. The COV of with-grain specimens is much higher than the against-grain specimens at $2.2 \%$. Although this value is relatively high, analysis of the data clearly shows a correlation of specimen density to resistivity (see Figure 15), and therefore, the high COV is a result of material density variation. A similar conclusion can be drawn for the spread of resistivity shown in Figure A-124 for the against-grain specimens of PCEA.

An example of a simple comparison between graphite grades and grain orientation is shown in Figure 17. Here, the resistivity values of several primary grades of graphite are plotted for both grain 
orientations, and the anisotropy ratio is displayed above the bars. It is interesting to note that all current grades of graphite have improved resistivity isotropy. This plot also shows that there is a $30 \%$ difference in resistivity for the current graphite types of interest.

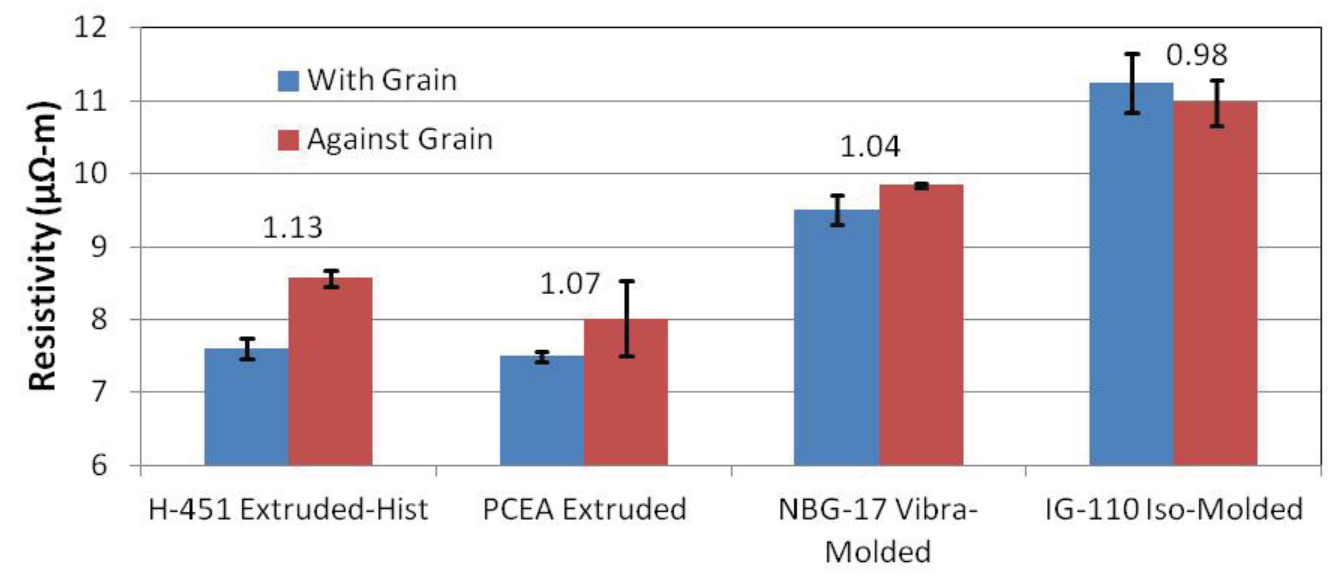

Figure 17. Electrical resistivity for several nuclear-grade graphite types. The anisotropy ratio is called out above each set of data bars.

\subsection{Approximation of Elastic Modulus from the Measurement of Sonic Velocity}

Figures A-125 through A-136 are plots of Young's and shear moduli determined from the measurement of sonic velocity. Statistical parameters are shown in Tables C-10 and C-11 for Young's and shear moduli, respectively. The IQR analysis does not reveal any inconsistency or outlier problems. As with resistivity, the moduli are easily correlated with the density of the specimens. Figure 18 shows that the sonic velocity increases with density for NBG-17. Therefore, in the calculation of the elastic modulus (i.e., density $\times$ the velocity squared), the density effect is compounded. This known behavior results from the fact that material containing more porosity and cracks (lower density) is more compliant and, therefore, will have a lower modulus of elasticity. The bimodal distribution of moduli resulting from density variations in the NBG-17 and PCEA specimens is clearly seen in Figures A-128, A-130, and A-134. 


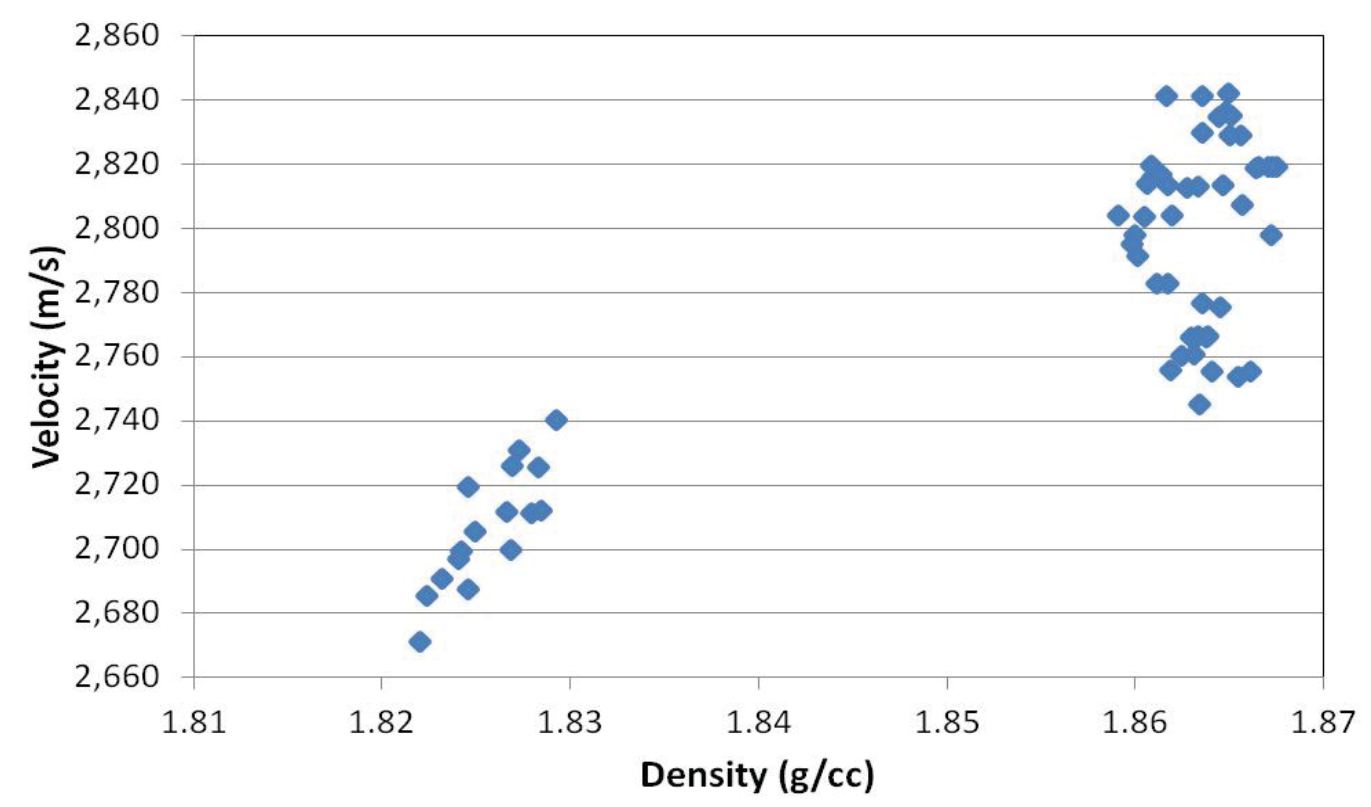

Figure 18. Measured longitudinal velocity as a function of density for NBG-17 creep specimens.

Table 5 shows Young's modulus measured using the sonic velocity technique. Although the COV includes potential material variability, these values compare favorably to a COV of $3.8 \%$ reported in the precision and bias section of ASTM C769. Consistent with the discussion of the effects of density here, it is observed that the highest-density graphite (i.e., NBG-17 and -18) produce the highest moduli. With the exception of IG-430, the modulus isotropy of the relevant graphite grades characterized here is better than the historical grade of $\mathrm{H}-451$.

Table 5. Young's modulus by measurement of sonic velocity.

\begin{tabular}{|l|c|c|c|c|c|}
\hline $\begin{array}{c}\text { Graphite } \\
\text { Type and } \\
\text { Process } \\
\text { Method }\end{array}$ & $\begin{array}{c}\text { Young's } \\
\text { Modulus } \\
\text { Against-Grain } \\
\text { Mean } \\
\text { (GPA) }\end{array}$ & $\begin{array}{c}\text { Against-Grain } \\
\text { COV } \\
(\%)\end{array}$ & $\begin{array}{c}\text { Young's } \\
\text { Modulus } \\
\text { With-Grain } \\
\text { Mean } \\
(\text { GPA }\end{array}$ & $\begin{array}{c}\text { With-Grain } \\
\text { COV } \\
(\%)\end{array}$ & $\begin{array}{c}\text { Young's Modulus } \\
\text { Anisotropy Ratio }\end{array}$ \\
\hline $\begin{array}{l}\text { H451 } \\
\text { extruded-hist }\end{array}$ & 10.29 & 0.94 & 12.06 & 2.94 & 0.85 \\
\hline $\begin{array}{l}\text { IG-110 } \\
\text { iso-molded }\end{array}$ & 11.20 & 3.97 & 10.81 & 3.08 & 1.04 \\
\hline $\begin{array}{l}\text { IG-430 } \\
\text { iso-molded }\end{array}$ & 12.01 & 1.94 & 10.46 & 2.54 & 1.15 \\
\hline $\begin{array}{l}\text { NBG-17 } \\
\text { vibra-molded }\end{array}$ & 14.23 & 0.72 & 14.31 & 4.86 & 0.99 \\
\hline $\begin{array}{l}\text { NBG-18 } \\
\text { vibra-molded }\end{array}$ & 16.11 & 1.08 & 15.46 & 1.70 & 1.04 \\
\hline $\begin{array}{l}\text { PCEA } \\
\text { extruded }\end{array}$ & 11.76 & 0.97 & 12.47 & 1.40 & 0.94 \\
\hline $\begin{array}{l}\text { COV = coefficient of variation } \\
\text { GPA = grade point average }\end{array}$ & & & \\
\hline
\end{tabular}




\subsection{Modulus of Elasticity by Measurement of Fundamental Frequency}

Young's modulus, determined by measurement of fundamental frequency, is plotted in Figures A-113 through A-118, and the statistical data are contained in Table B-8. Statistically, these data are well-behaved, with the IQR analysis showing no problems with outliers. Again, a bimodal effect of density is shown for the with-grain specimens of NBG-17 (see Figure A-116).

Table 5 shows a comparison of Young's modulus from the measurement of fundamental frequency for the primary grades of graphite. The COVs for all except the against-grain specimens of PCEA compare favorably with $\sim 3 \%$ uncertainty in the measurement technique. The scatter in modulus values for the against-grain specimens can be seen in Figure A-118. It was noted throughout the resonance testing of PCEA that achieving consistent values for the resonant frequency was difficult. More than 100 strikes were necessary to obtain 10 consistent values, and the average standard deviation of the frequencies measured was an order of magnitude higher than that of the other graphite grades. This can only be explained by the relatively large elongated porosity that existed in the PCEA graphite billet tested. This porosity is termed "wiggler porosity," and based on the testing in this program, the manufacturer has since corrected this problem in their manufacturing process. These elongated pores appear as cracks that run parallel to the grain and extrusion direction. In against-grain specimens, the size of these cracks or flaws can be on the order of the diameter of the specimen and run perpendicular to the direction of the flexural vibration mode, causing very inconsistent damping of the vibrational energy.

Also shown in Table 6 is the consistent fact that the new grades of graphite being tested exhibit better isotropy.

Table 6. Young's modulus by measurement of fundamental frequency.

\begin{tabular}{|l|c|c|c|c|c|}
\hline $\begin{array}{c}\text { Graphite } \\
\text { Type and } \\
\text { Process } \\
\text { Method }\end{array}$ & $\begin{array}{c}\text { Young's } \\
\text { Modulus } \\
\text { Against-Grain } \\
\text { Mean } \\
\text { (GPA) }\end{array}$ & $\begin{array}{c}\text { Against-Grain } \\
\text { COV } \\
(\%)\end{array}$ & $\begin{array}{c}\text { Young's } \\
\text { Modulus } \\
\text { With-Grain } \\
\text { Mean } \\
\text { (GPA) }\end{array}$ & $\begin{array}{c}\text { With-Grain } \\
\text { COV } \\
(\%)\end{array}$ & $\begin{array}{c}\text { Young's Modulus } \\
\text { Anisotropy Ratio }\end{array}$ \\
\hline $\begin{array}{l}\text { H451 } \\
\text { extruded-hist }\end{array}$ & 8.01 & 1.64 & 9.94 & 3.07 & 0.80 \\
\hline $\begin{array}{l}\text { IG-110 } \\
\text { iso-molded }\end{array}$ & 9.99 & 2.92 & 9.65 & 3.16 & 1.04 \\
\hline $\begin{array}{l}\text { IG-430 } \\
\text { iso-molded }\end{array}$ & 10.64 & 2.07 & 9.39 & 2.43 & 1.13 \\
\hline $\begin{array}{l}\text { NBG-17 } \\
\text { vibra-molded }\end{array}$ & 11.16 & 0.29 & 11.28 & 4.51 & 0.99 \\
\hline $\begin{array}{l}\text { NBG-18 } \\
\text { vibra-molded }\end{array}$ & 12.42 & 0.84 & 11.99 & 1.59 & 1.04 \\
\hline $\begin{array}{l}\text { PCEA } \\
\text { extruded }\end{array}$ & 8.73 & 23.35 & 9.92 & 2.38 & 0.87 \\
\hline $\begin{array}{l}\text { COV = coefficient of variation } \\
\text { GPA = grade point average }\end{array}$ & & & \\
\hline
\end{tabular}

\subsection{Thermal Expansion}

Mean CTE data are plotted in Figures A-89 through A-112. A statistical evaluation of the CTE data was performed at three discrete temperatures (i.e., 100,500 , and $900^{\circ} \mathrm{C}$ ) for each graphite type. Again, the dashed lines in these plots indicate the upper and lower IQR limits, and there are no outliers to consider. Tables B-5 through B-7 (see Appendix B) contain the mean, standard deviation, and COV values for data evaluated at the discrete temperatures. With the exception of PCEA, all COVs are below or on the order 
of the $\pm 3 \%$ measurement uncertainty when calculated for the with- and against-grain specimen groups individually.

Figure 19 shows the average of all specimens for several of the primary grades of graphite, with error bars indicating \pm 1 standard deviation. All increase with temperature in a near-linear fashion between 100 and $1000^{\circ} \mathrm{C}$. As an example of how these data may be used in future reactor designs, engineers will need to account for the fact that NBG-18 has a mean CTE that is $\sim 50 \%$ higher than the historical grade $\mathrm{H}-451$ if that grade is chosen for the core material.

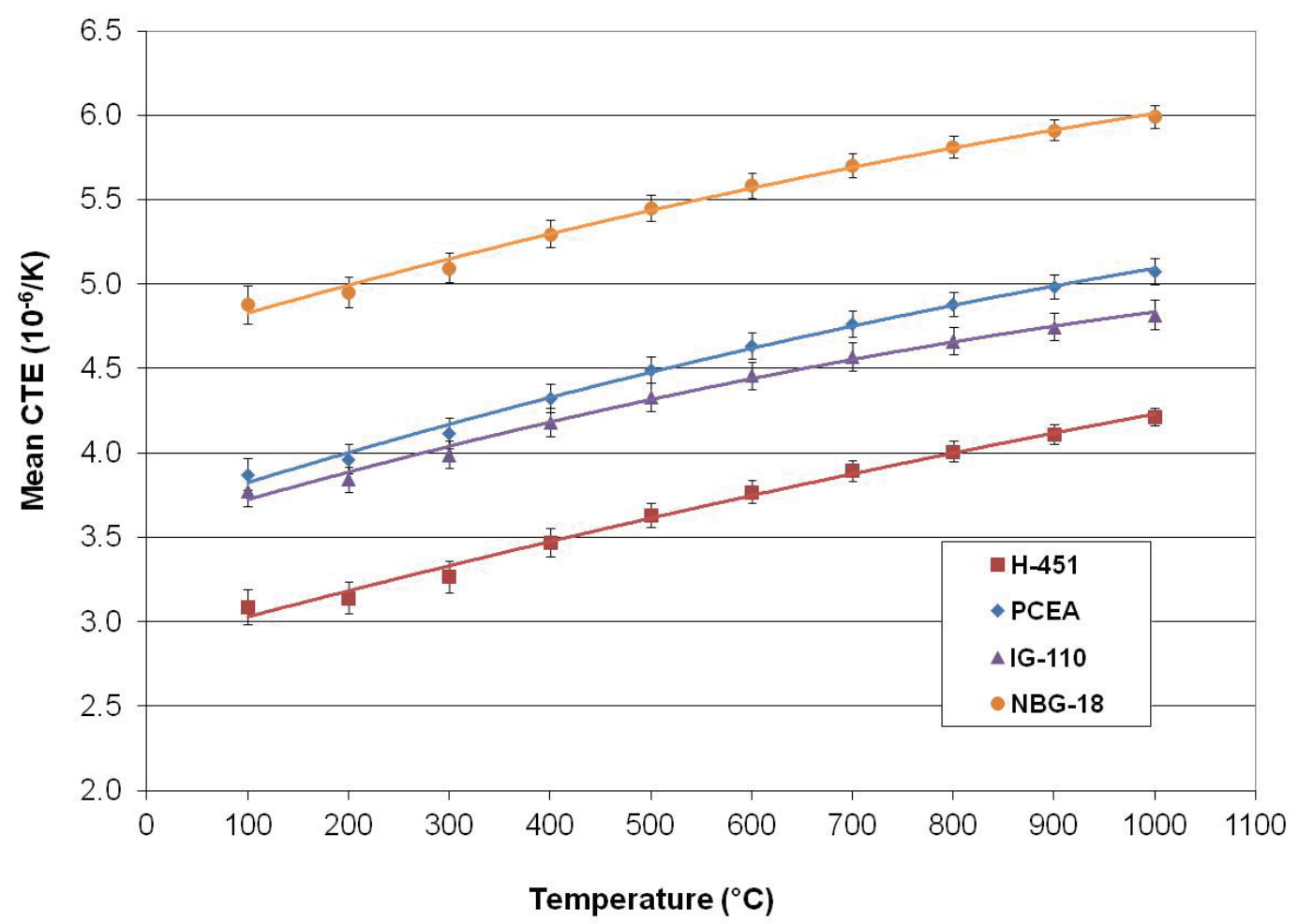

Figure 19. Mean coefficient of thermal expansion for several types of nuclear-grade graphite as a function of temperature. Error bars represent \pm 1 standard deviation in the data used to obtain the averages plotted.

Measurements of CTE were performed on both with-grain and against-grain specimens. Figure 20 shows the CTE anisotropy ratio for the same primary grades of graphite as a function of temperature. All are relatively constant with temperature. As in other properties, the new grades are more isotropic. 


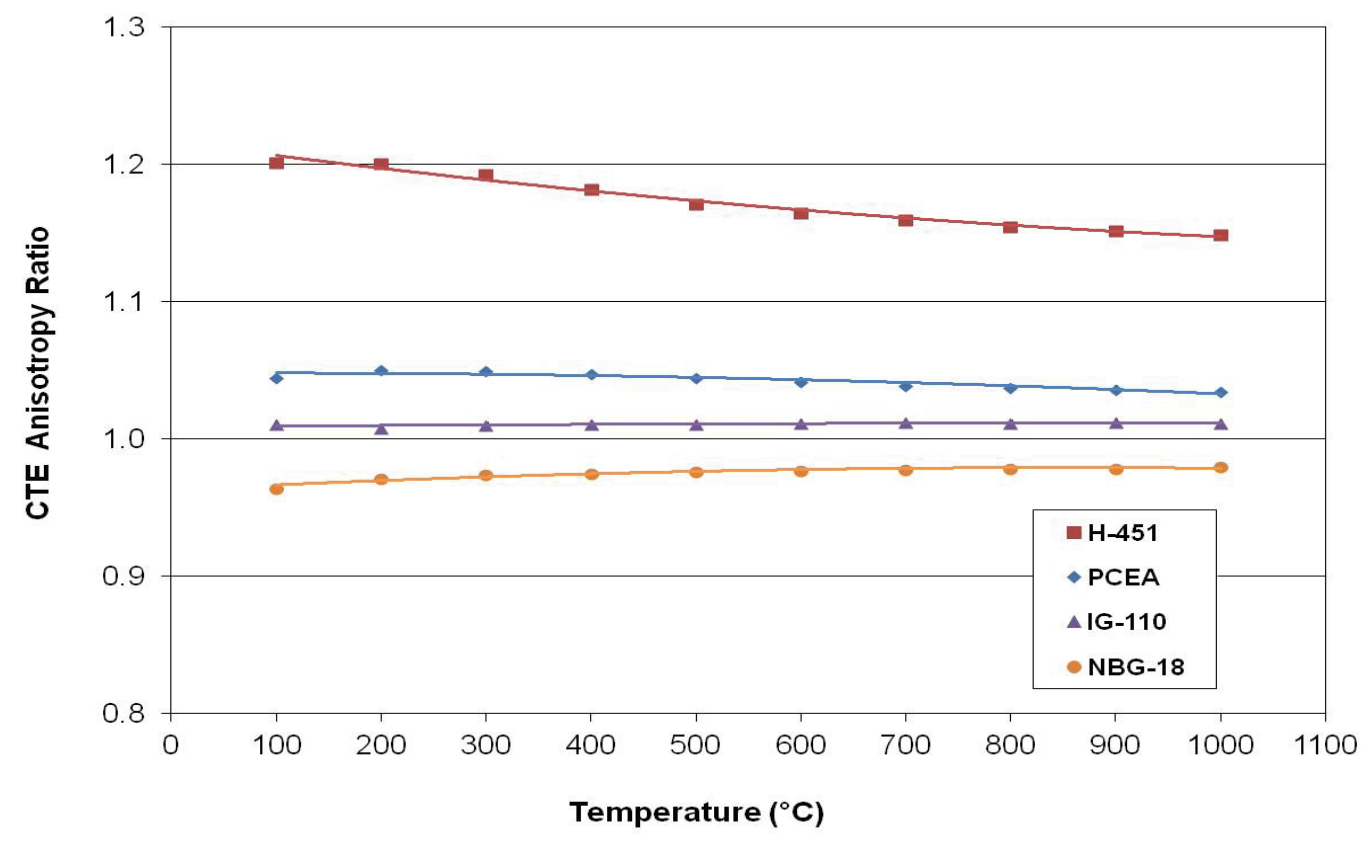

Figure 20. Coefficient of thermal expansion anisotropy ratio for several types of nuclear-grade graphite as a function of temperature.

\subsection{Thermal Diffusivity}

Plots of thermal diffusivity are shown in Figures A-203 through A-266. As with the CTE data, discrete temperatures of 100,500 , and $1000^{\circ} \mathrm{C}$ were statistically evaluated. Table B-16a through B-18c contain values of the mean, standard deviation, and COV. Four graphite types show COVs that are higher than the measurement uncertainty of $\pm 3 \%$. Graphite types A3 Matrix and New Matrix are both extremely small-batch graphite specimens, mixed and pressed individually with intentional variations in their composition. Therefore, it is not surprising that these specimens vary in their thermal diffusivity. The variation in diffusivity of PCEA is most likely due to the material variable called wiggler porosity discussed in Section 3.1, and it is speculated that the variation in H-451 is also due to material variability in the billet. For the most part, the diffusivity data are well-behaved, with only a few values that somewhat exceed the IQR limits and again are most likely a result of slight material variability.

Figure 21 shows the average thermal diffusivity for four of the primary graphites of interest. Error bars are \pm 1 standard deviation and, in most cases, are smaller than the plotted symbol. Also shown on the alternate ordinate is the percent difference between the historical grade of graphite $\mathrm{H}-451$ and the relatively new grade, IG-110. The current grades of graphite all have a lower diffusivity than the historical grade, H-451, with IG-110 being $20-33 \%$ lower, depending on the temperature. $\mathrm{H}-451$ is an extruded grade graphite made with relatively large filler coke particle size $(0.5 \mathrm{~mm})$. IG-110 is an isomolded graphite and has a filler particle size of 20 microns. This is why $\mathrm{H}-451$ has a high diffusivity in the with-grain orientation. As seen in the CTE measurements, these differences in diffusivity are significant and will need to be considered in future reactor designs.

Figure 22 shows the anisotropy ratio for the same graphite grades. The anisotropy for all grades of graphite are constant with temperature, and as with the other properties, the current grades of graphite show much-improved isotropy of thermal diffusivity. 


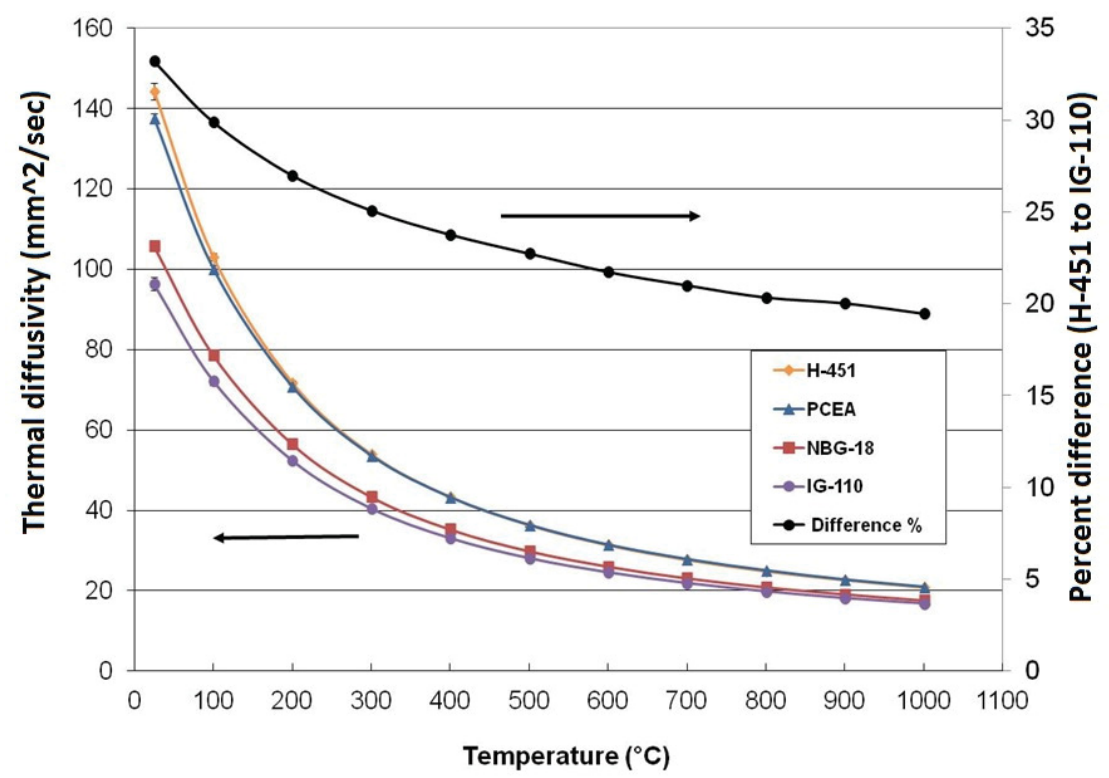

Figure 21. Thermal diffusivity for various with-grain graphite types. Error bars are 1 standard deviation in the data and in some cases smaller than the data symbol. The percent difference between H451 and IG-110 is plotted on the right hand scale.

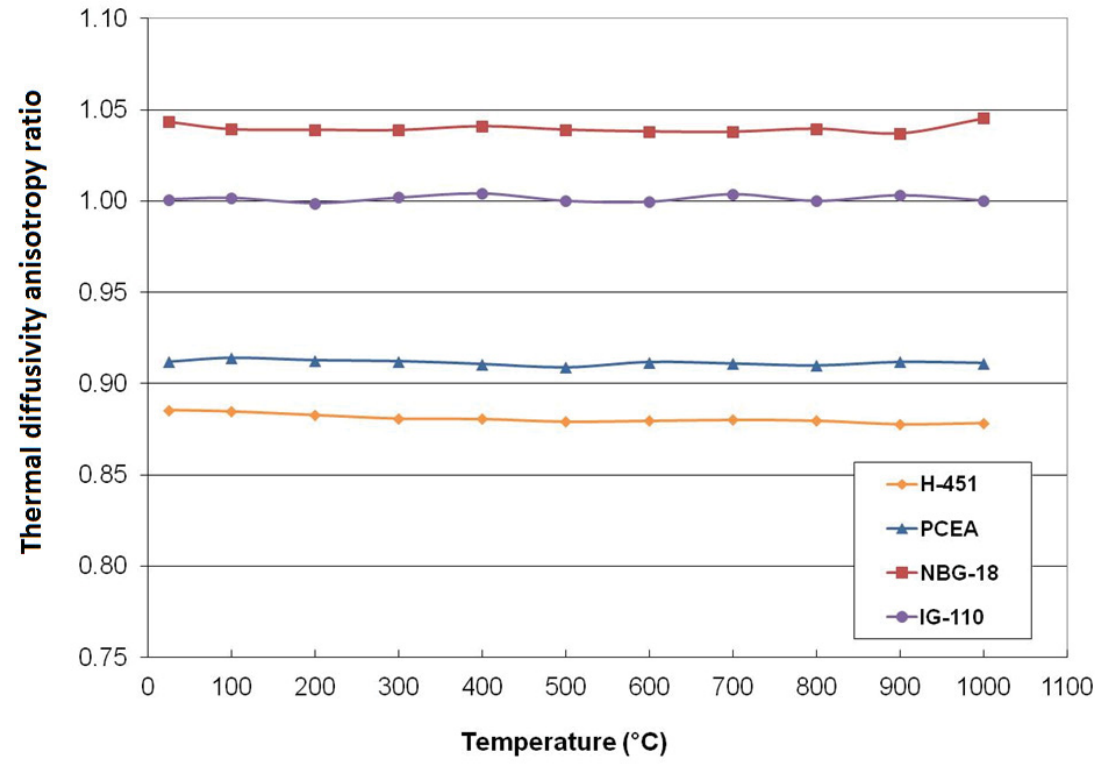

Figure 22. Thermal diffusivity anisotropy ratio for several types of nuclear-grade graphite as a function of temperature. 


\section{REFERENCES}

1 T. Burchell, R. Bratton, W. Windes, NGNP Graphite Selection and Acquisition Strategy, ORNL/TM-2007/153, September 2007.

2. R. L. Bratton and T. D. Burchell, 2005, NGNP Graphite Testing and Qualification Specimen Selection Strategy, INL/EXT-05-00269, Idaho National Laboratory, May 2005.

3. INL/EXT-07-13165, "Graphite Technology Development Plan," Rev. 0, Idaho National Laboratory, September 2007.

4. PLN-2497, 2010, “Graphite Technology Development Plan,” Rev. 1, Idaho National Laboratory, October 2010.

5. T. Burchell, J. Strizak, and M. Williams, 2011, AGC-1 Specimen Preirradiation Data Report, ORNL/TM-2010/285, Oak Ridge National Laboratory, August 2011.

6. T. Reed, 2012, "AGC-1 Individual Fluence, Temperature, and Load Calculation and Tabulation," ECAR-1943, Idaho National Laboratory, September 2012

7. T. Reed, 2012, “AGC-1 As Run Thermal Results," ECAR-1944, Idaho National Laboratory, September 2012.

8. D. Swank, 2010, AGC-2 Graphite Preirradiation Data Package, INL/EXT-10-19588, Rev. 0, Idaho National Laboratory, August 2010.

9. TFR-645, 2010, Advanced Graphite Capsule AGC-2 Experiment Test Train, Rev. 0, Idaho National Laboratory, July 2010.

10 INL Drawing 600786, 2012, “ATR Advanced Graphite Capsule (AGC-2) Graphite Specimen Machining Details," Rev. 2, Idaho National Laboratory, July 2012.

11. DWG 601266, 2012, “ATR Advanced Graphite Capsule Number 2 (AGC-2) Capsule Facility Assemblies," Rev. 2, Idaho National Laboratory, July 2012.

12. DWG 601258, 2012, “ATR Advanced Graphite Capsule 2 (AGC-2) Graphite Specimen Holder Assemblies and Details," Rev. 1, Idaho National Laboratory, July 2012.

13. J. R. Parry, 2010, "Engineering Calculations and Analysis Report: Reactor Physics Projections for the AGC-2 Experiment Irradiated in the ATR South Flux Trap," ECAR-1050, Rev. 0, Idaho National Laboratory, October 13, 2010.

14. T. Burchell and R. Bratton, 2005, Graphite Irradiation Creep Capsule AGC-1 Experimental Plan, ORNL/TM-2005/505, Oak Ridge National Laboratory, May 2005.

15. T. Burchell, 2009, A Revised AGC-1 Creep Capsule Layout, ORNL/TM-2009/009, Oak Ridge National Laboratory, January 2009.

16. PLN-3267, 2010, “AGC-2 Characterization Plan,” Rev. 0, Idaho National Laboratory, March 19, 2010.

17. MCP-1380, 2012, "Research and Development Test Control," Rev. 3, Idaho National Laboratory, March 2012.

18. LWP-21220, 2012, “Work Management,” Rev. 10, Idaho National Laboratory, October 2012. 
19. PLN-3319, 2012, "Records Management Plan for the VHTR Technology Development Office Program,” Rev. 2, Idaho National Laboratory, May 2012.

20. INL Drawing 600786, 2009, “ATR Advanced Graphite Capsule (AGC-2) Graphite Specimen Machining Details,” Rev. 1, Idaho National Laboratory, March 2009.

21. Drawing 600787, 2010, “ATR Advanced Graphite Capsule (AGC-2) Experiment Graphite Specimen Cut-Out Diagrams,” Rev. 3, Idaho National Laboratory, July 2010.

22. MCP-3052, 2012, "VHTR TDO Personnel Qualification and Certification," Rev. 1, Idaho National Laboratory, May 2012.

23. MCP-3066, 2012, "VHTR TDO Control of Measuring and Test Equipment," Rev. 2, Idaho National Laboratory, May 2012.

24. ASTM C747-93, 2010, "Standard Test Method for Moduli of Elasticity and Fundamental Frequencies of Carbon and Graphite Materials by Sonic Resonance," American Society for Testing and Materials, 2010.

25. ASTM C1259-08, 2008, "Standard Test Method for Dynamic Young's Modulus, Shear Modulus, and Poisson's Ratio for Advanced Ceramics by Impulse Excitation of Vibration," American Society for Testing and Materials, 2008.

26. ASTM C769-09, 2009, "Standard Test Method for Sonic Velocity in Manufactured Carbon and Graphite Materials for Use in Obtaining Young's Modulus," American Society for Testing and Materials, 2009.

27. MCP-2875, 2012, “Maintaining Laboratory Notebooks,” Rev. 11, Idaho National Laboratory, March 2012.

28. PLN-2690, 2013, “VHTR Technology Development Office Quality Assurance Program Plan,” Rev. 10, Idaho National Laboratory, January 2013.

29. Form 435.78, 2012, "VHTR Technology Development Office Information Input Sheet," Rev. 2, April 2012.

30. MCP-3058, 2012, "VHTR TDO Software Quality Assurance,” Rev. 2, Idaho National Laboratory, July 2012.

31. FRM-959, 2012, "VHTR Software Management Plan,” Rev. 1, Idaho National Laboratory, 2012.

32. ASTM Standard C559-90, "Standard Test Method for Bulk Density by Physical Measurements of Manufactured Carbon and Graphite Articles," American Society for Testing and Materials, 2010.

33. ASTM C611-98, 2010, "Standard Test Method for Electricity Resistivity of Manufactured Carbon and Graphite Articles at Room Temperature," American Society for Testing and Materials, 2010.

34. ASTM E228-06, 2006, "Standard Test Method for Linear Thermal Expansion of Solid Materials with a Push-Rod Dialometer," American Society for Testing and Materials, 2006.

35. ASTM E1461-07, 2007, "Standard Test Method for Thermal Diffusivity by the Flash Method," American Society for Testing and Materials, 2007.

36. R.D. Cowan (1963). "Pulse Method of Measuring Thermal Diffusivity at High Temperatures". Journal of Applied Physics 34 (4): 926. 
37. Y.S. Touloukian "Thermophysical Properties of Matter - Thermal Diffusivity", John Wiley \& Sons Ltd (May 6, 1973) 
Appendix A

\section{Data Plots}




\section{Appendix A Data Plots}

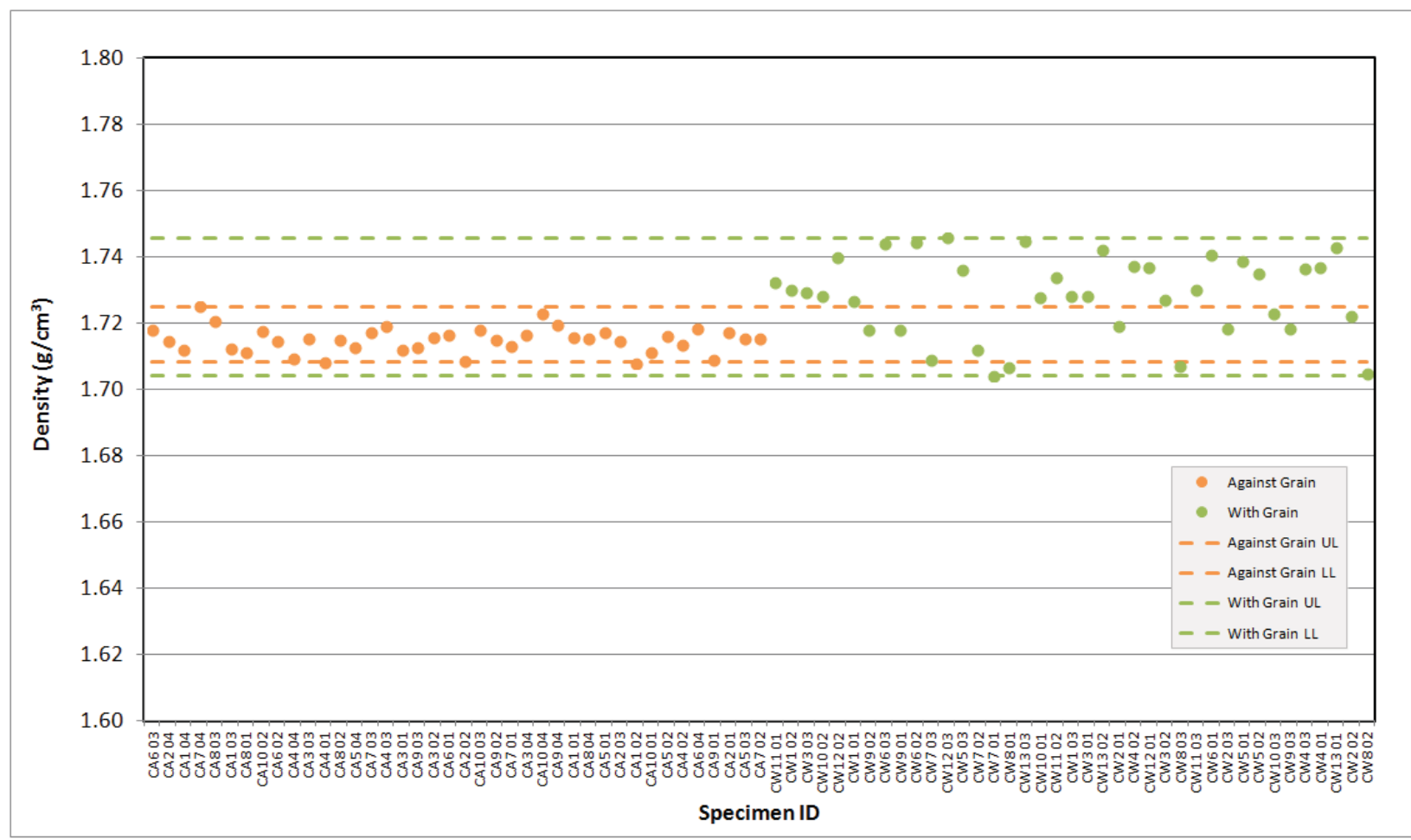

Figure A-1. H-451 Creep Pre Thermal Measurement Density.

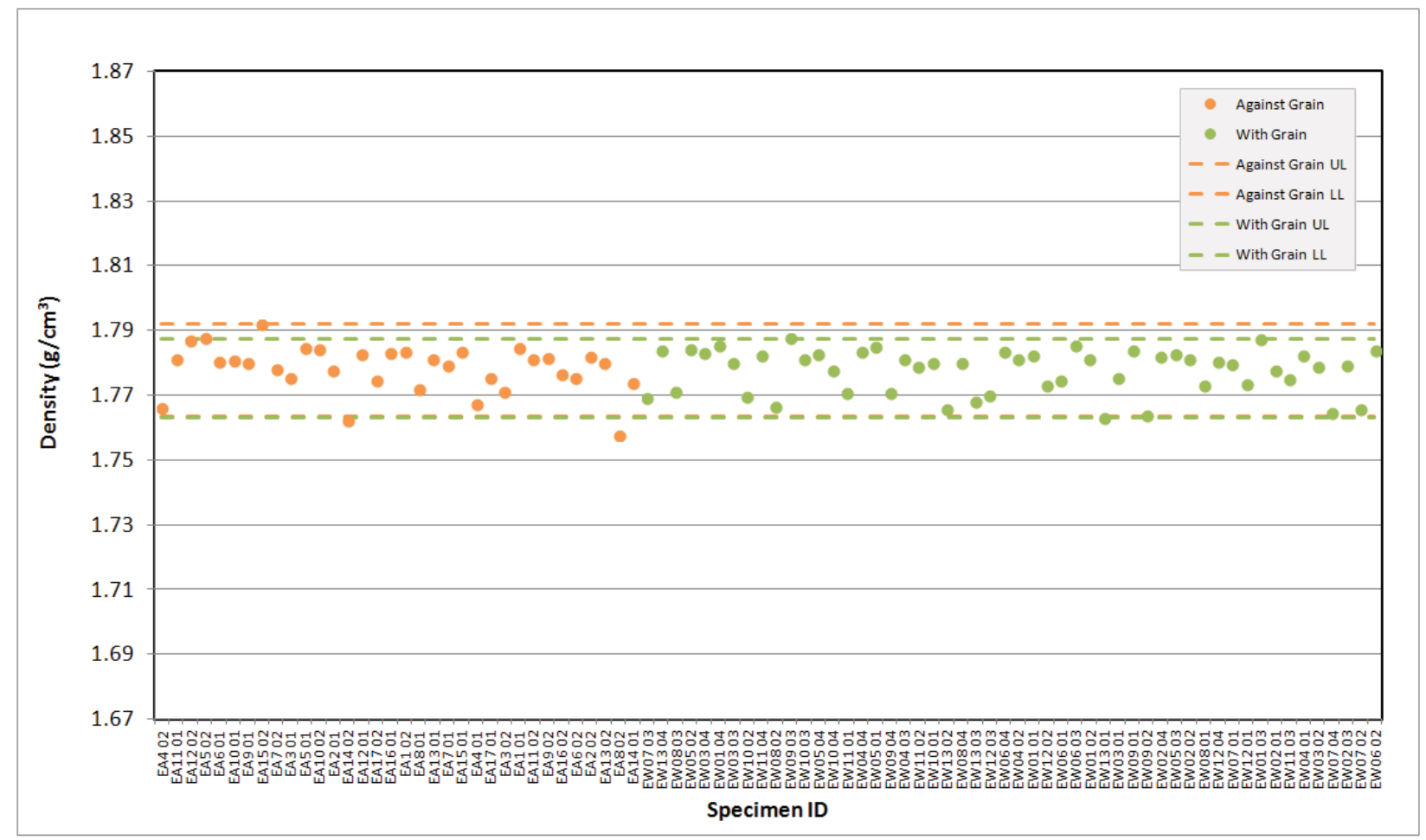

Figure A-2. IG-110 Creep Pre Thermal Measurement Density. 


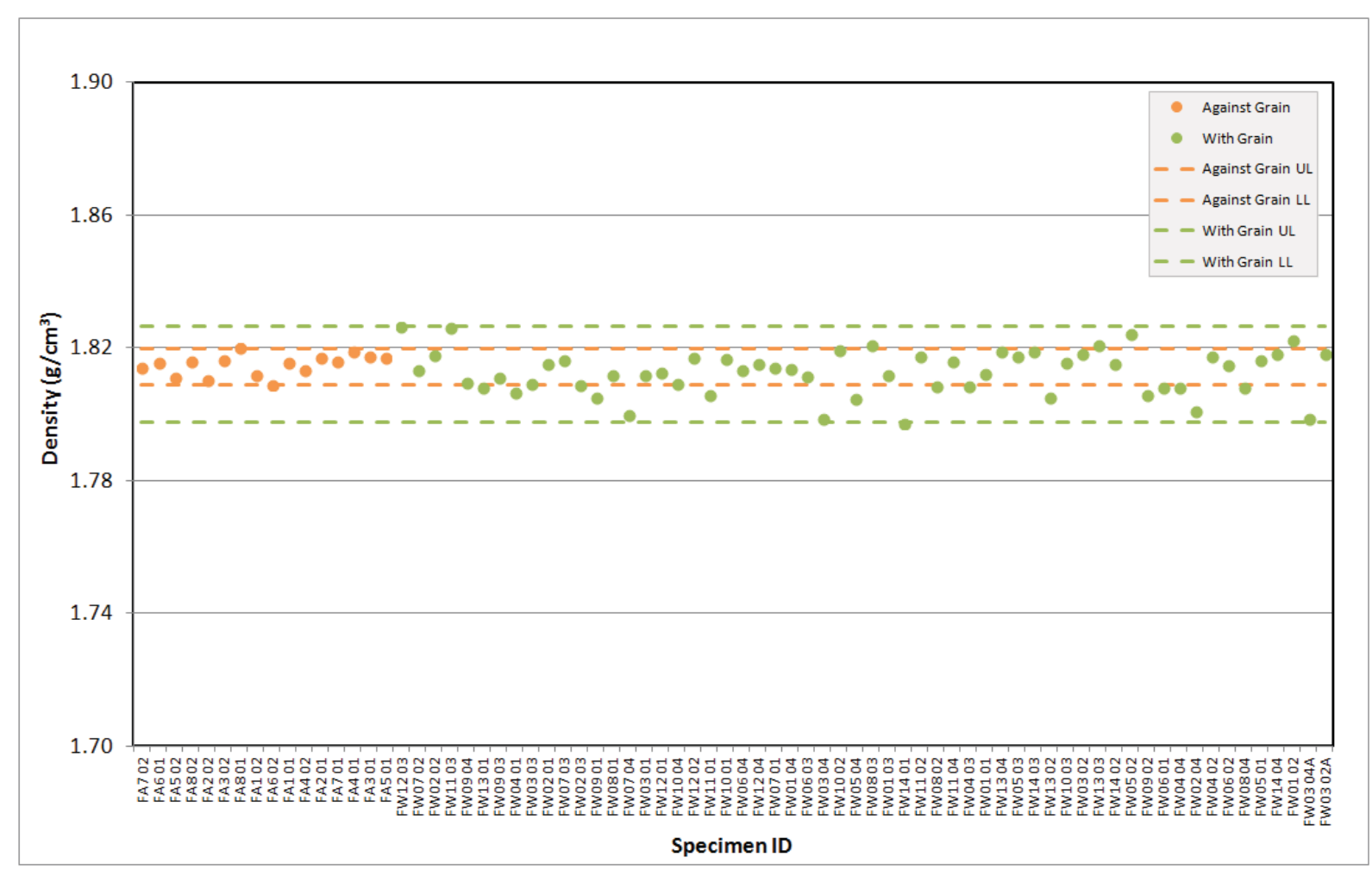

Figure A-3. IG-430 Creep Pre Thermal Measurement Density.

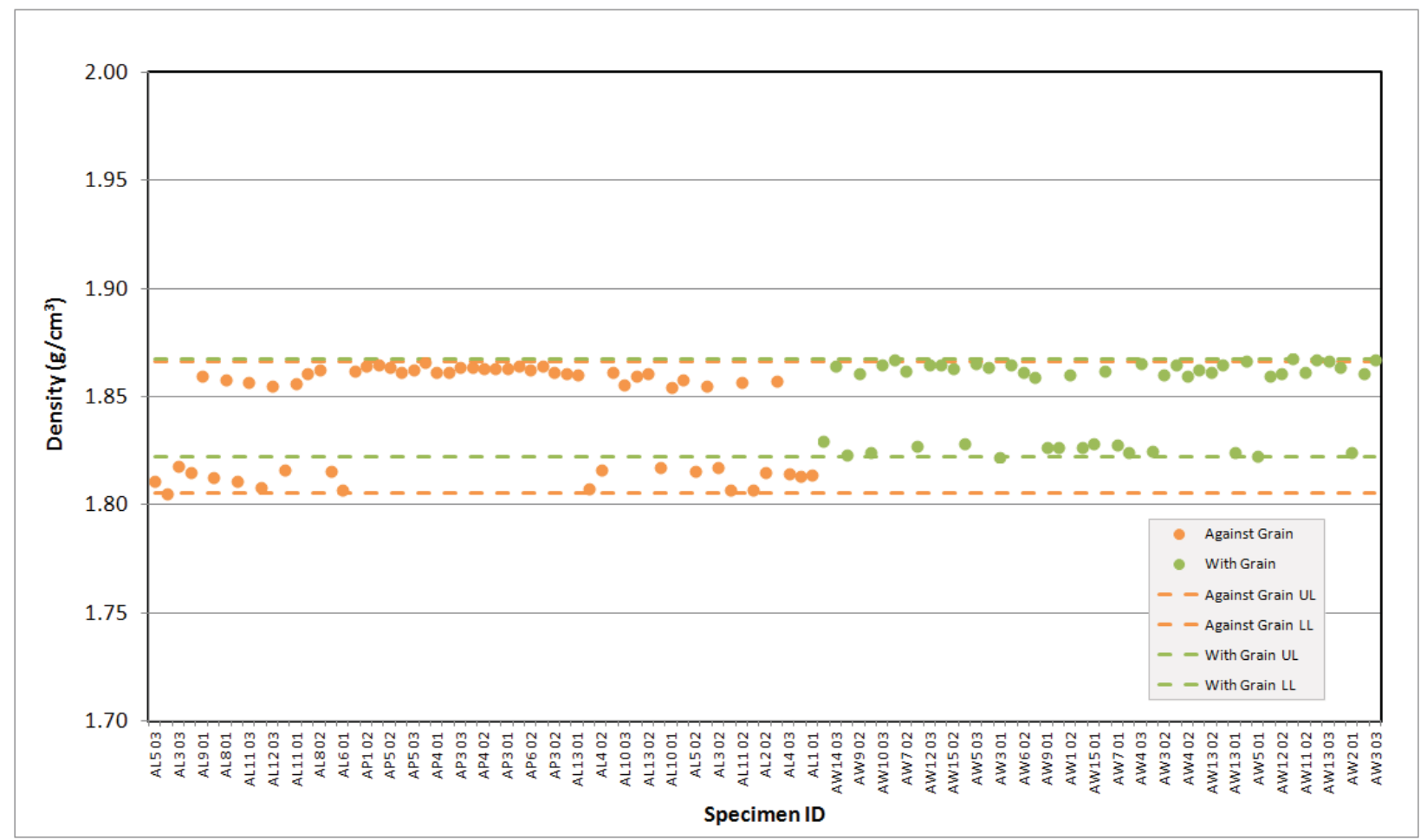

Figure A-4. NBG-17 Creep Pre Thermal Measurement Density. 


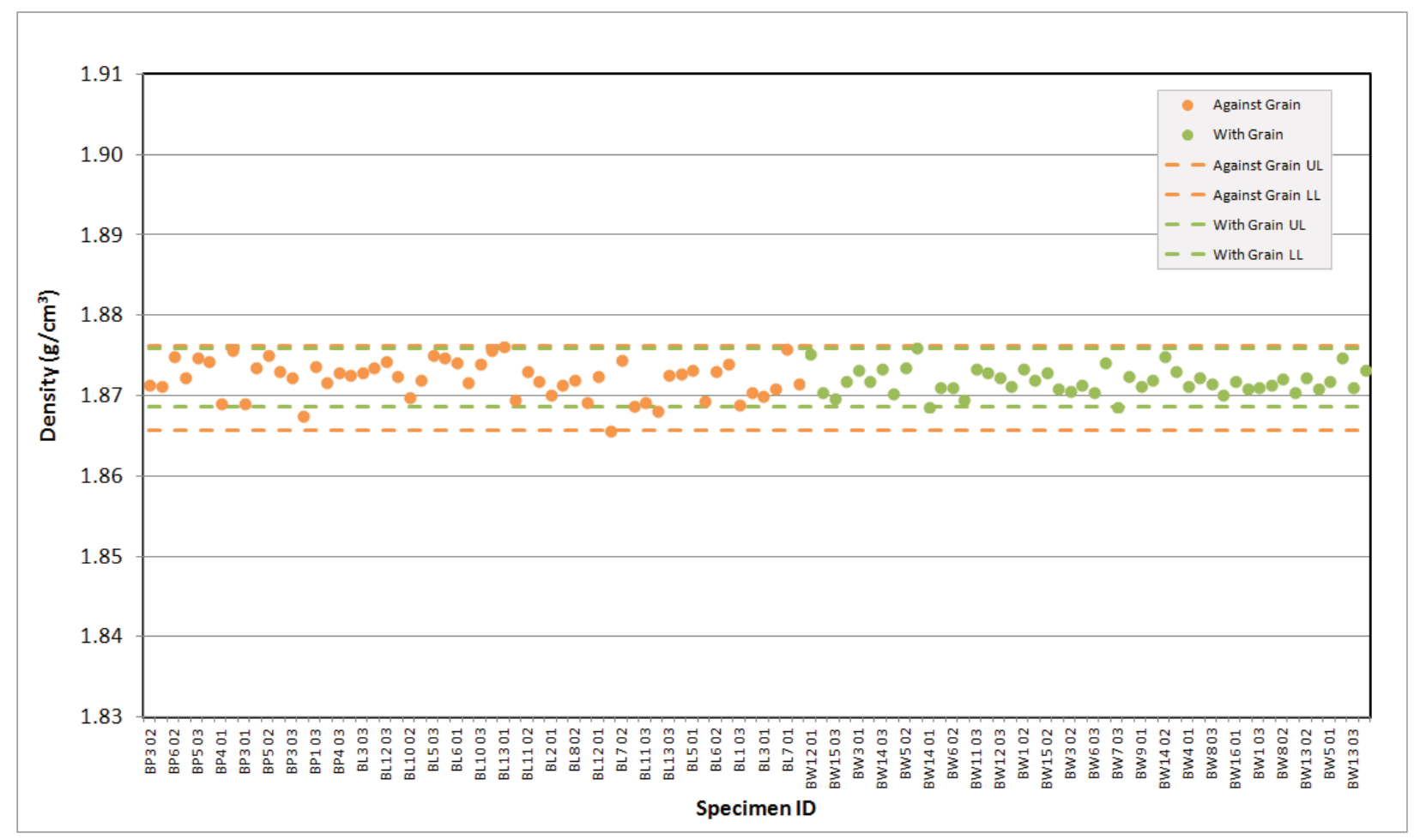

Figure A-5. NBG-18 Creep Pre Thermal Measurement Density.

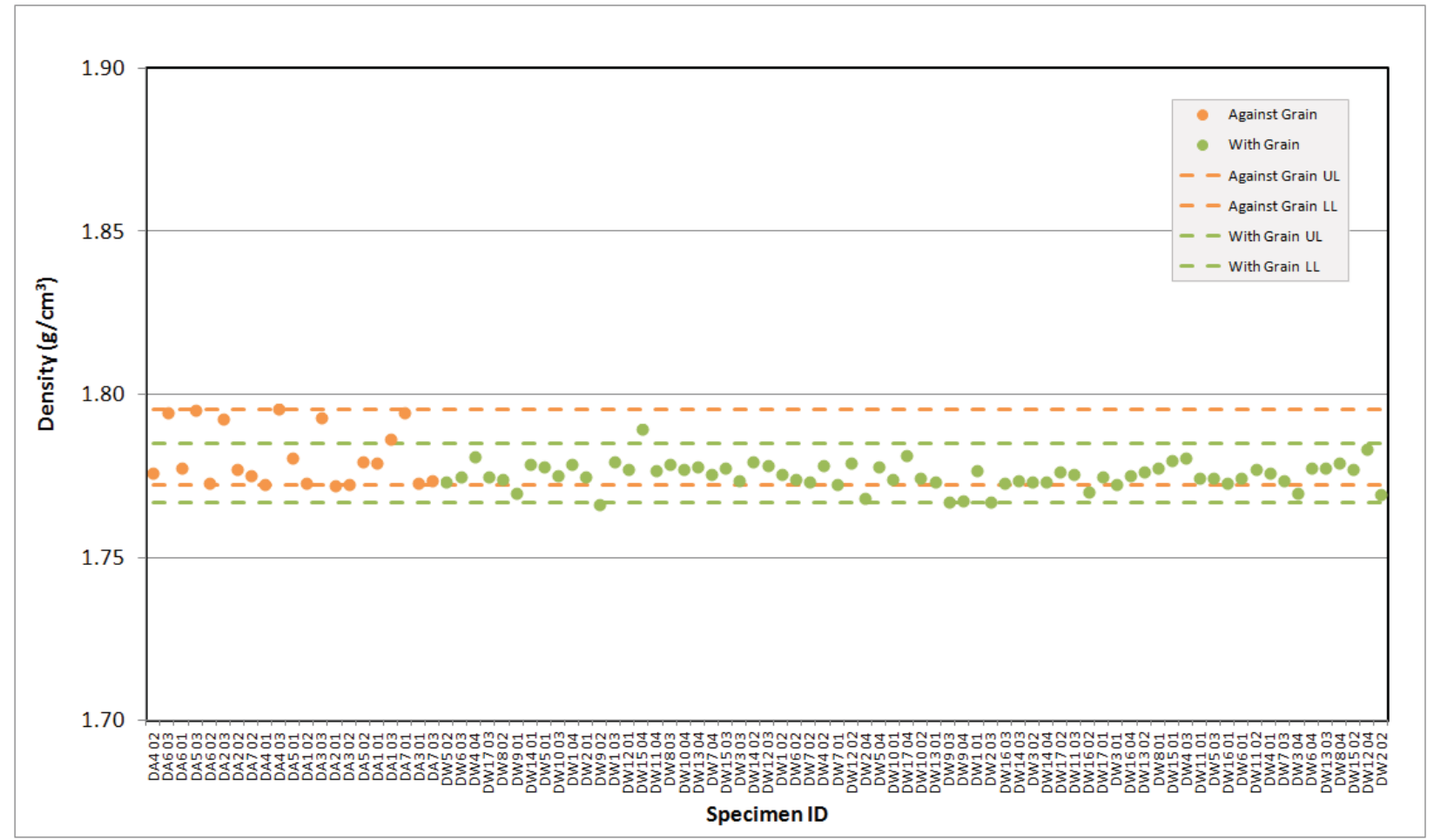

Figure A-6. PCEA Creep Pre Thermal Measurement Density. 


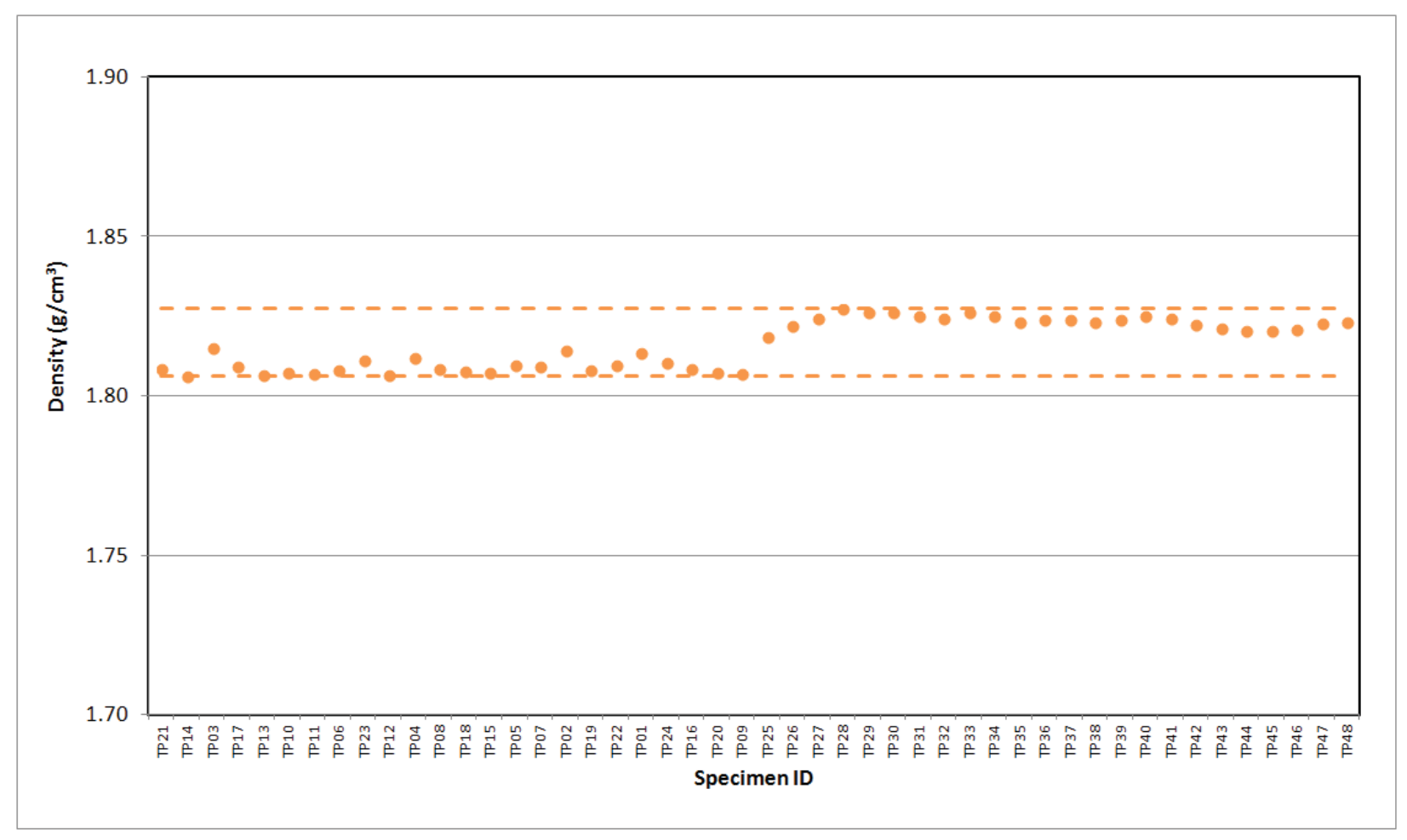

Figure A-7. 2114 Piggyback Pre Thermal Measurement Density.

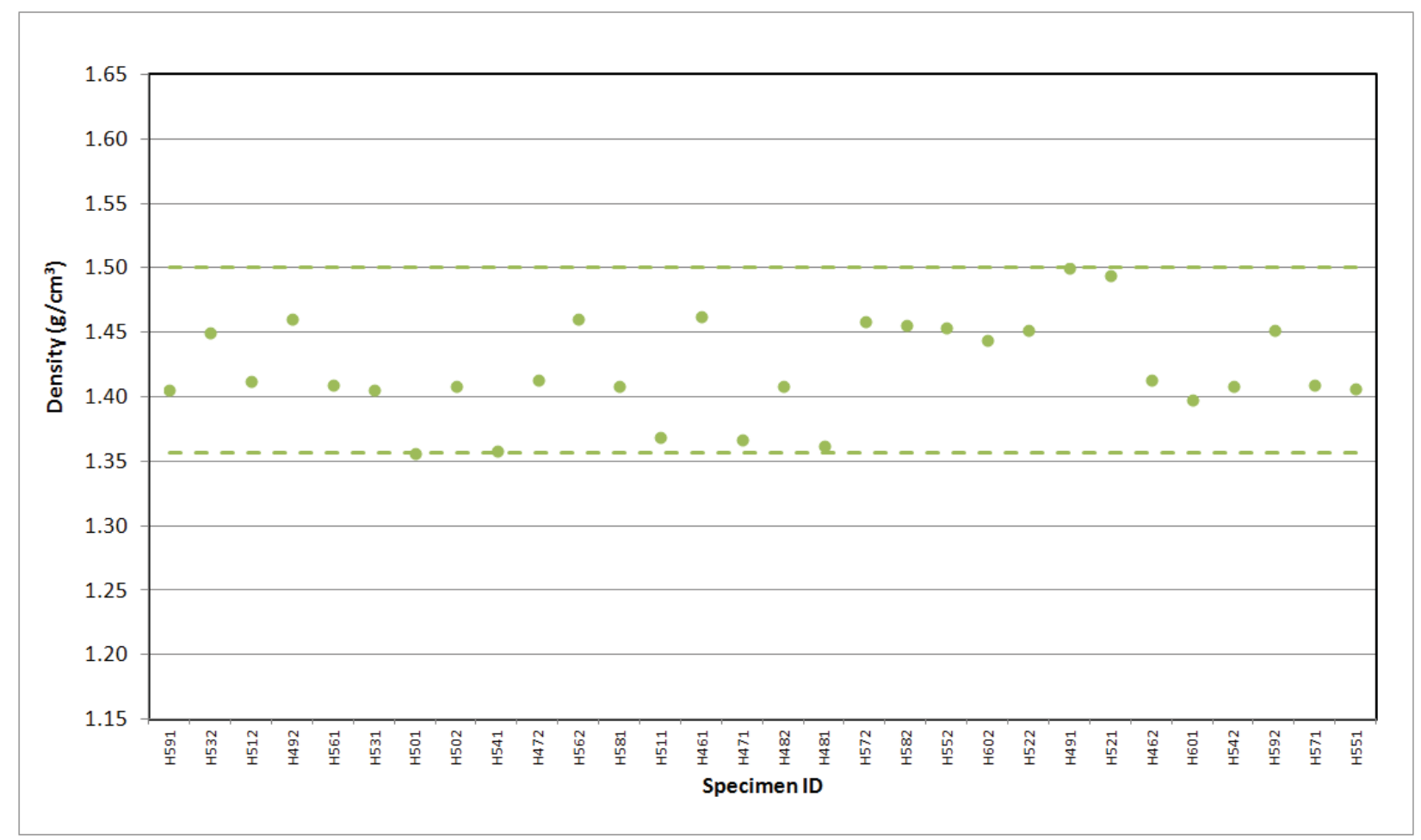

Figure A-8. A3 Matrix Piggyback Pre Thermal Measurement Density. 


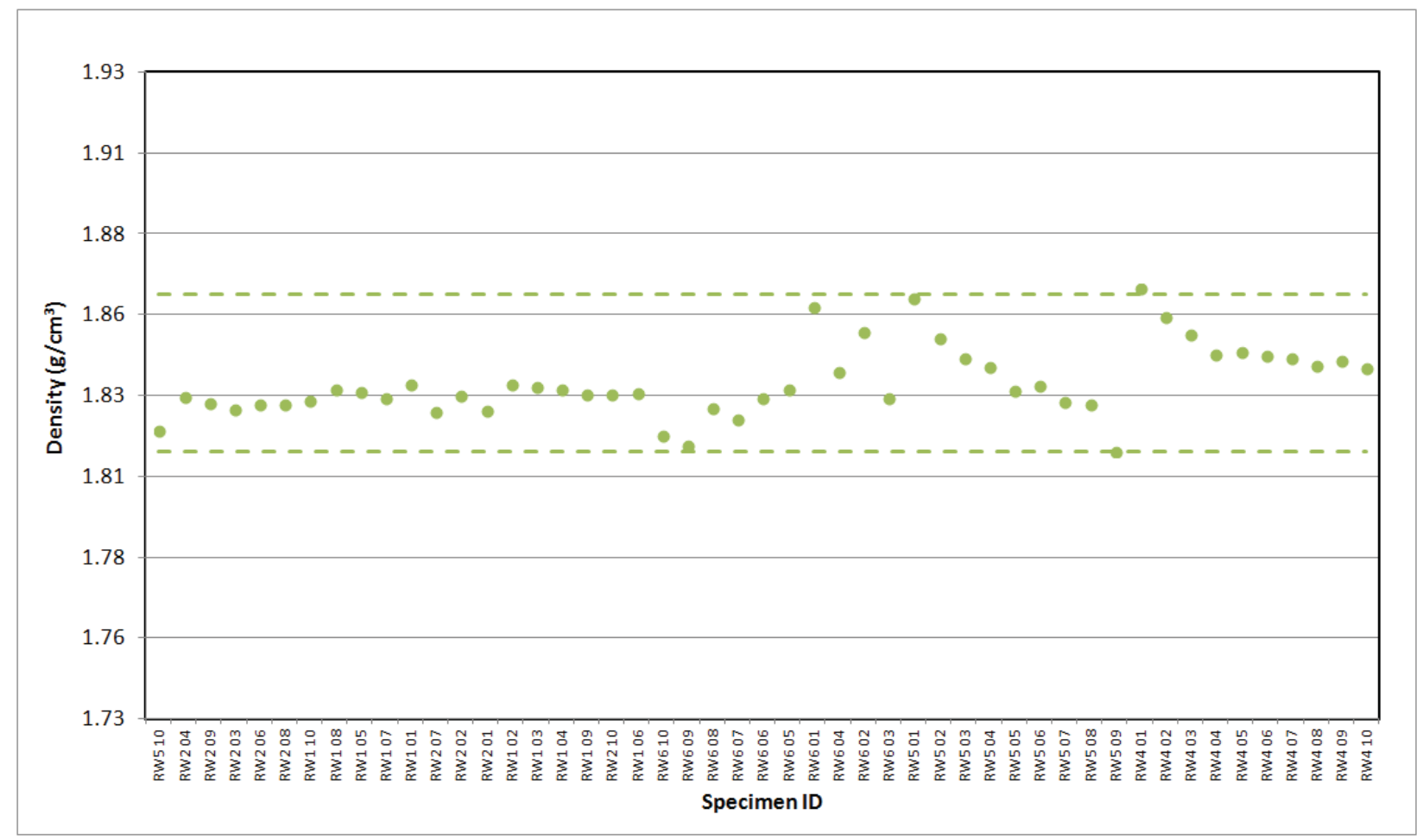

Figure A-9. BAN Piggyback Pre Thermal Measurement Density.

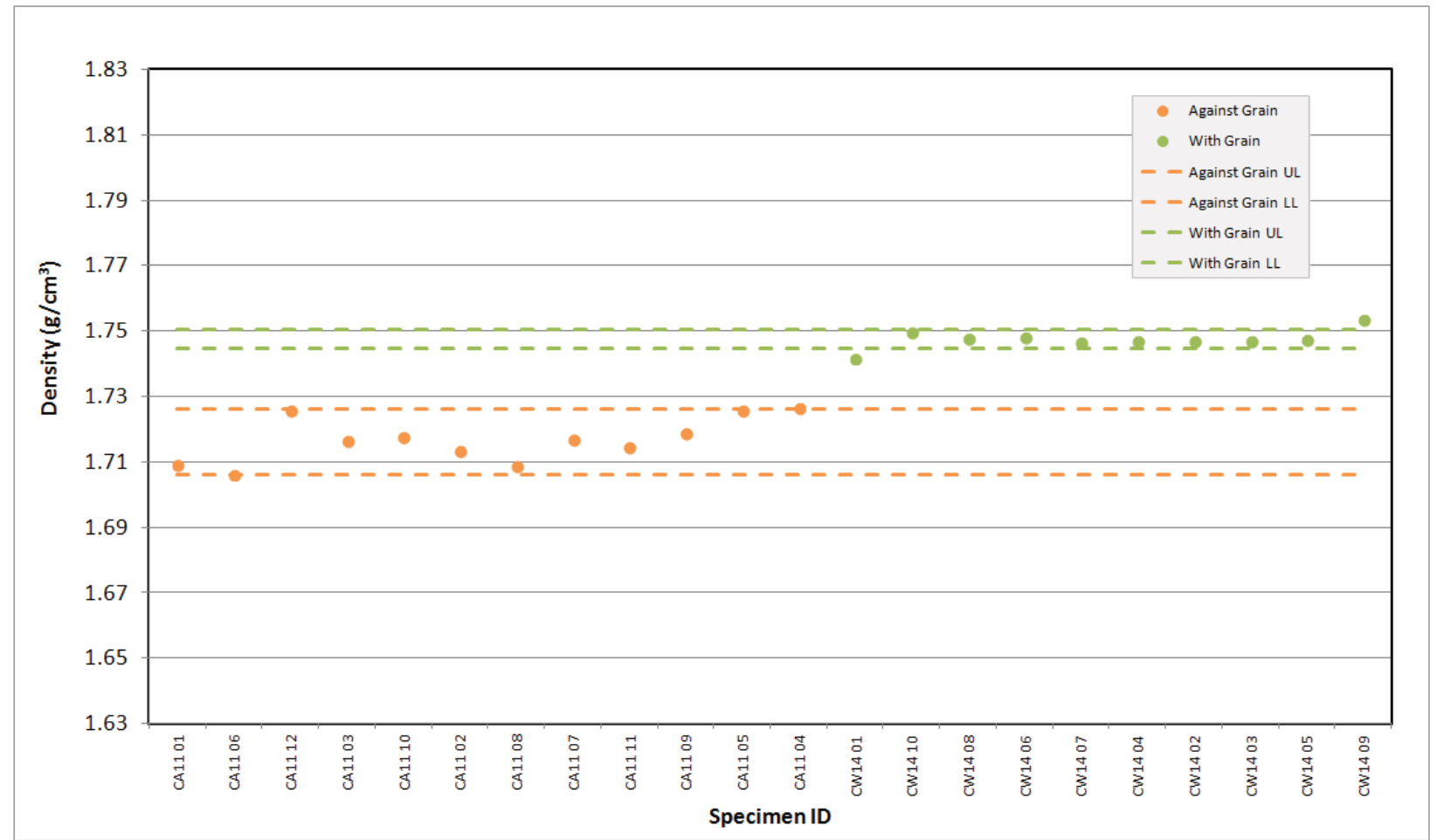

Figure A-10. H-451 Piggyback Pre Thermal Measurement Density. 


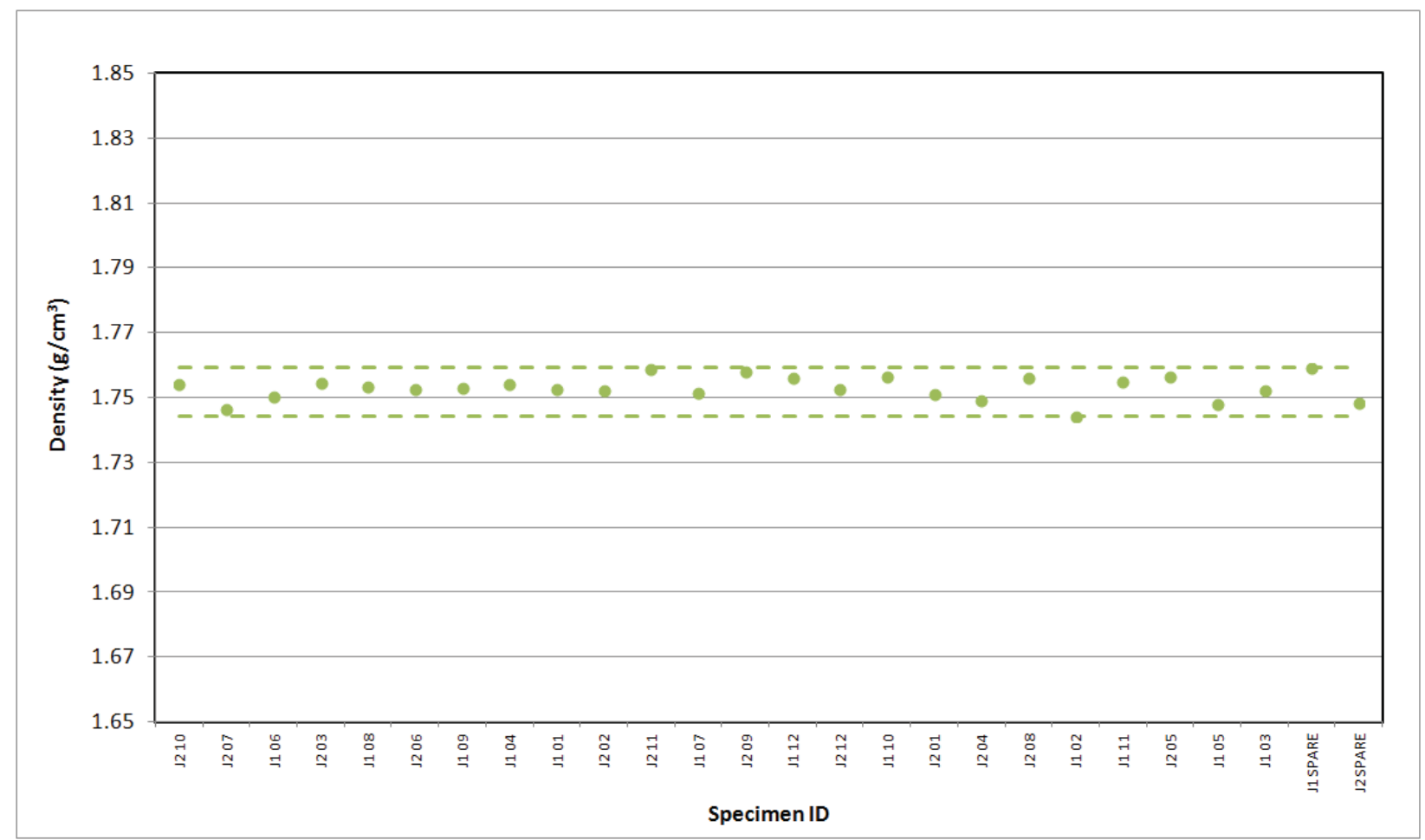

Figure A-11. HLM Piggyback Pre Thermal Measurement Density.

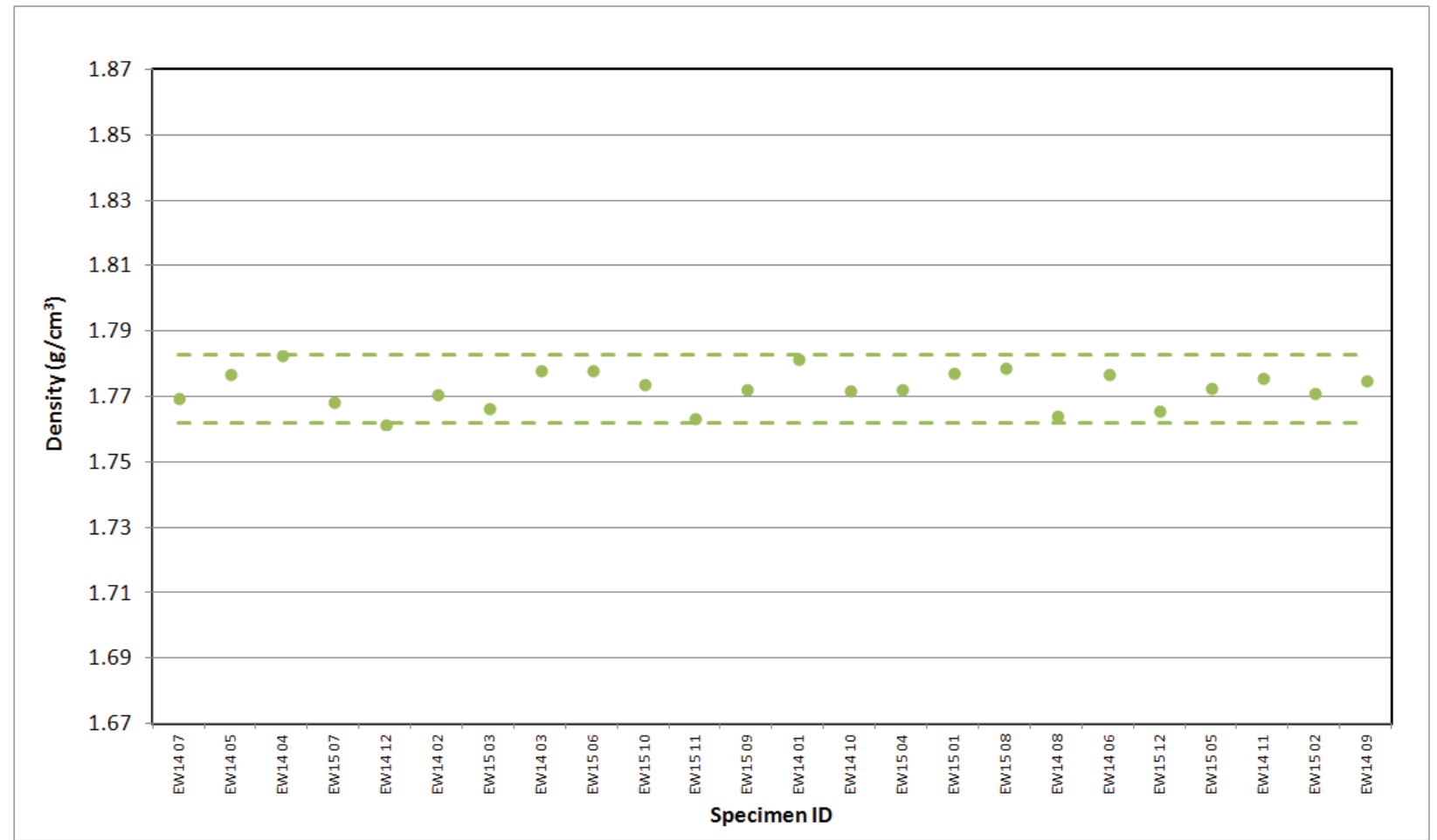

Figure A-12. IG-110 Piggyback Pre Thermal Measurement Density. 


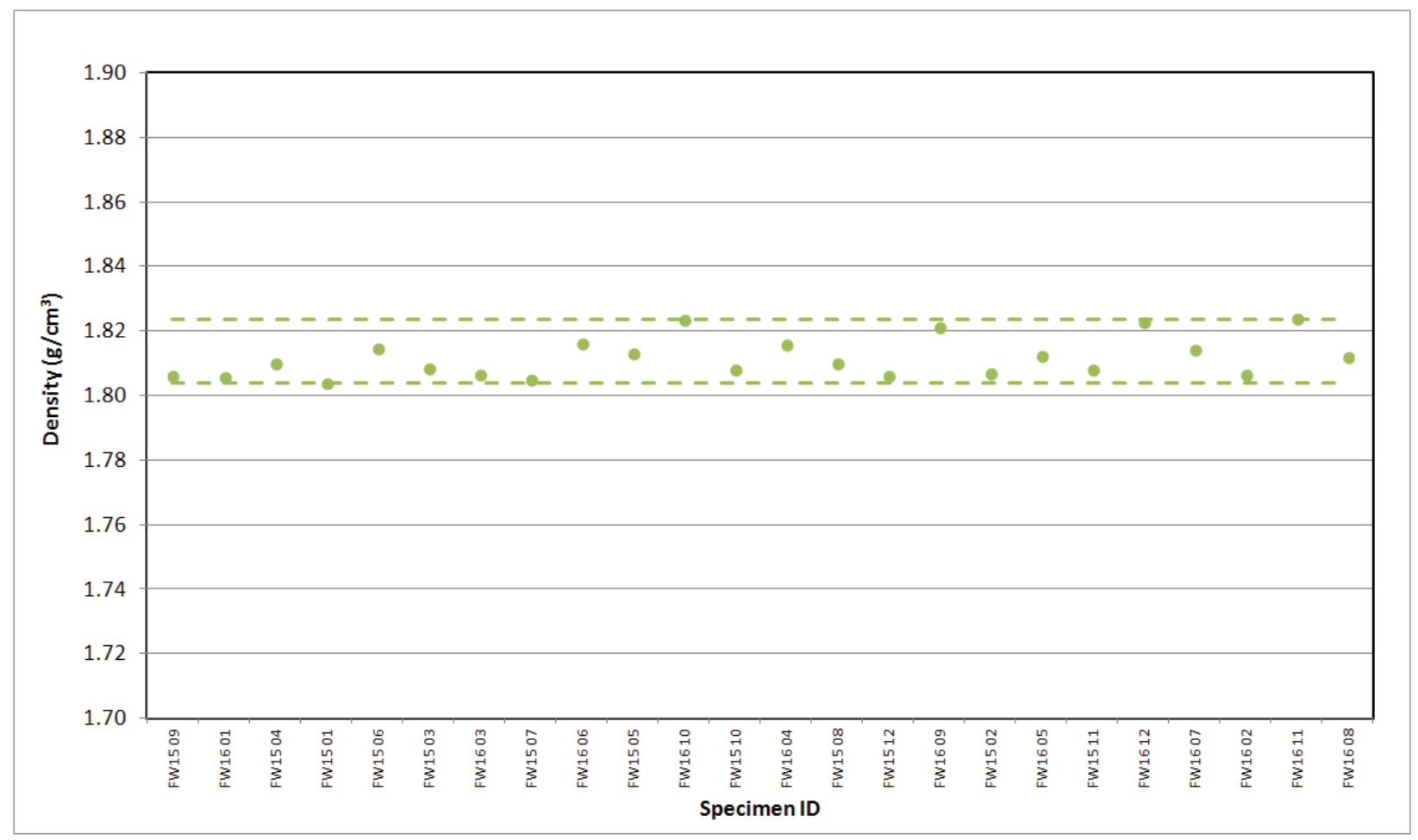

Figure A-13. IG-430 Piggyback Pre Thermal Measurement Density.

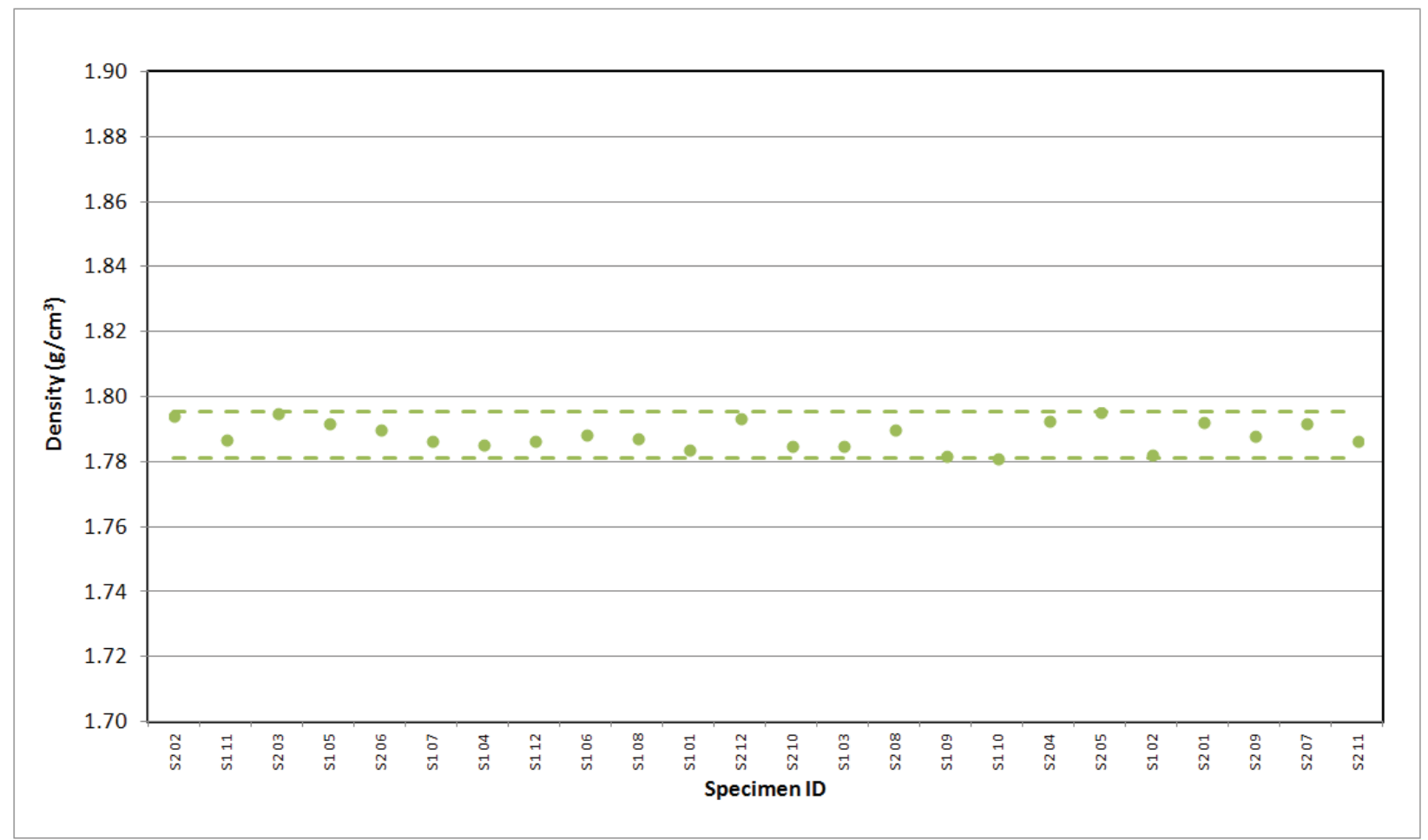

Figure A-14. NBG-10 Piggyback Pre Thermal Measurement Density. 


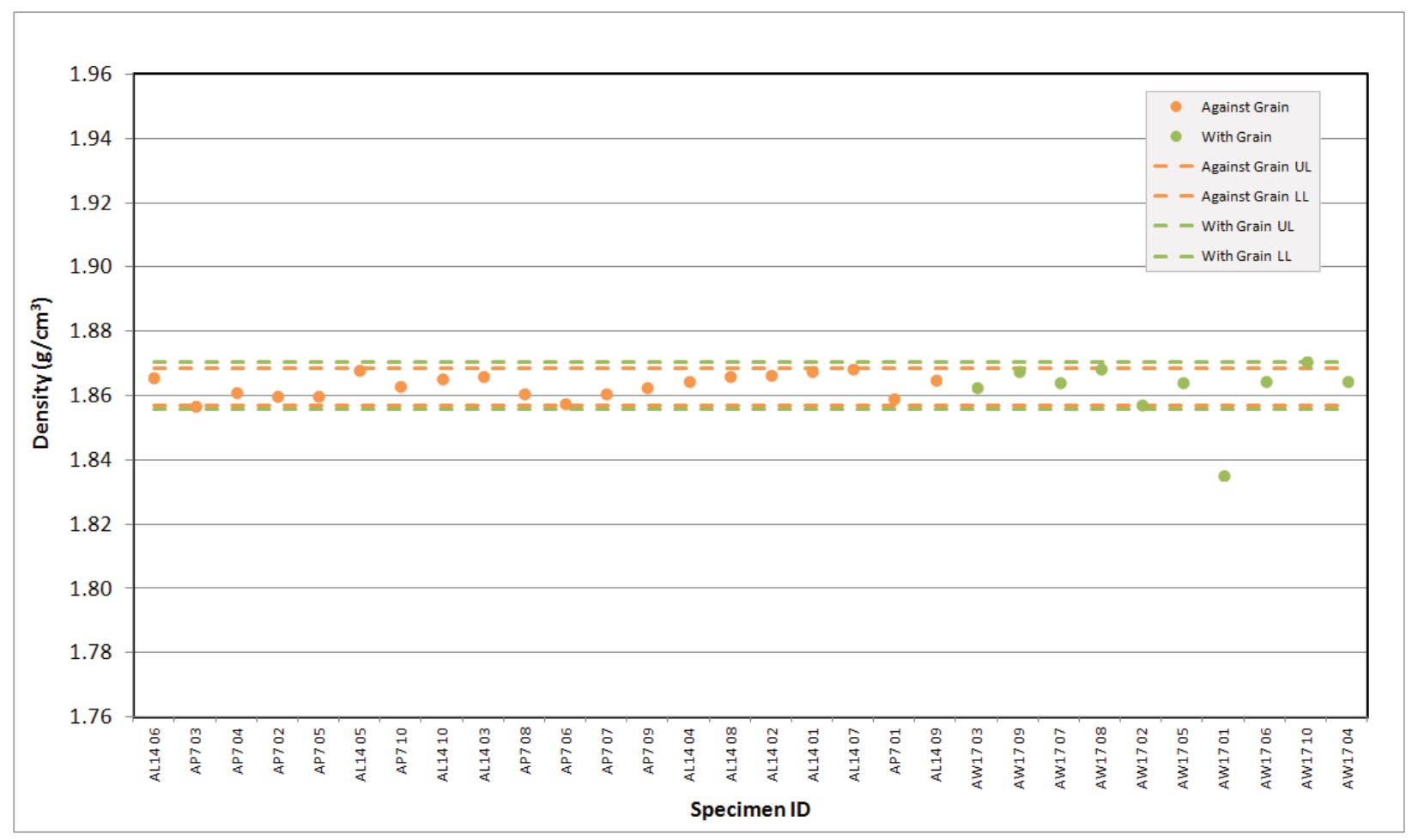

Figure A-15. NBG-17 Piggyback Pre Thermal Measurement Density.

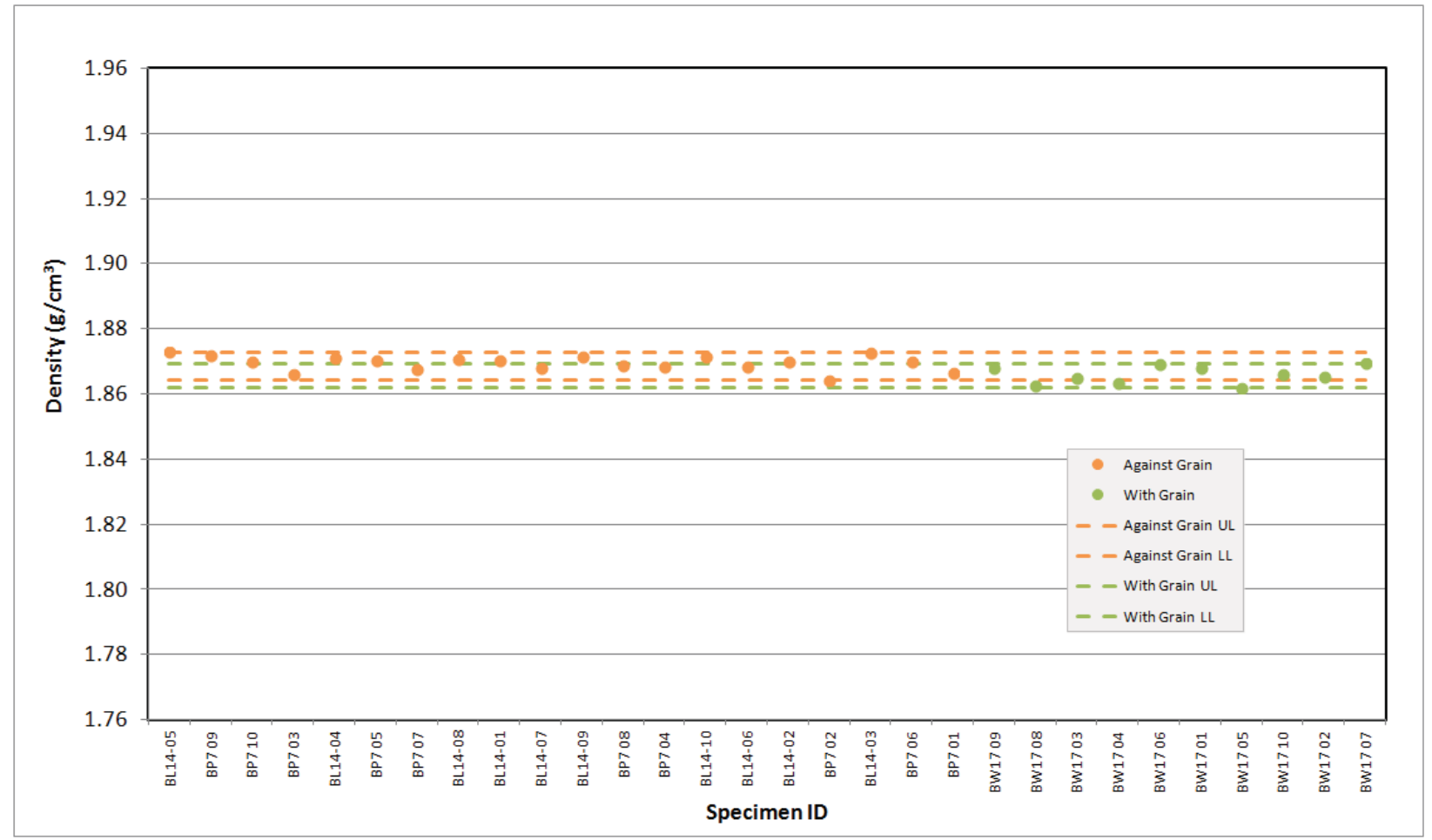

Figure A-16. NBG-18 Piggyback Pre Thermal Measurement Density. 


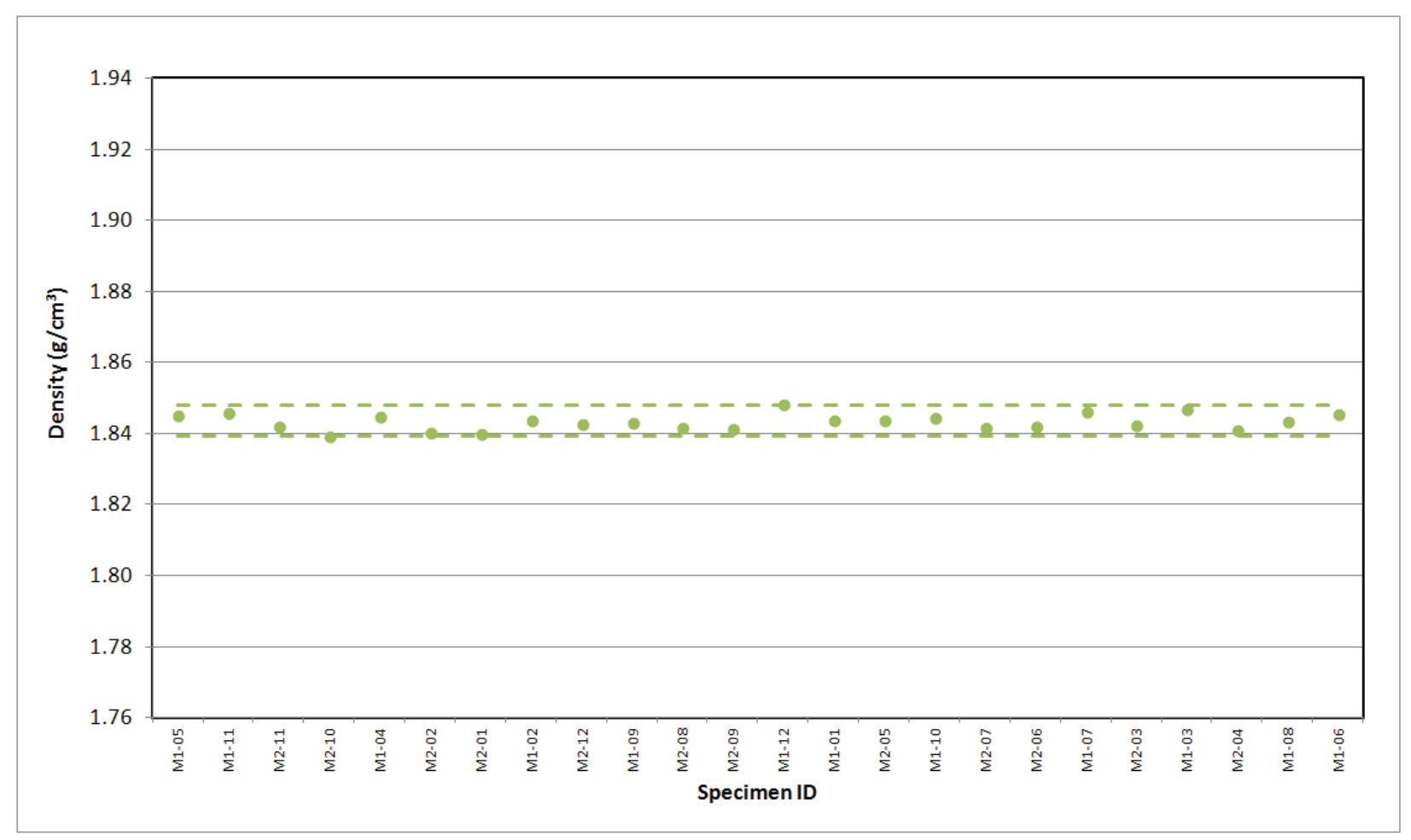

Figure A-17. NBG-25 Piggyback Pre Thermal Measurement Density.

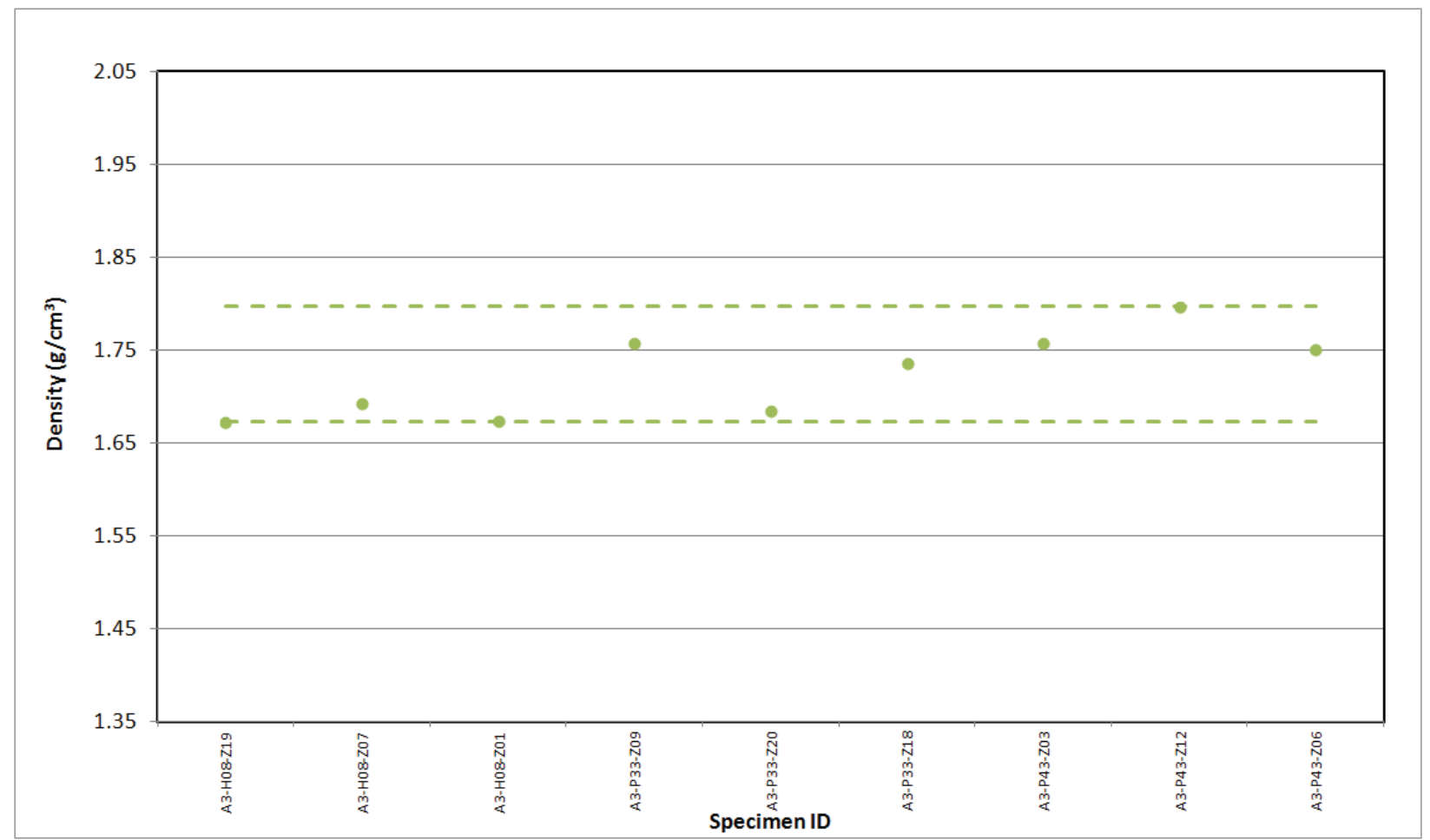

Figure A-18. New Matrix Piggyback Pre Thermal Measurement Density. 


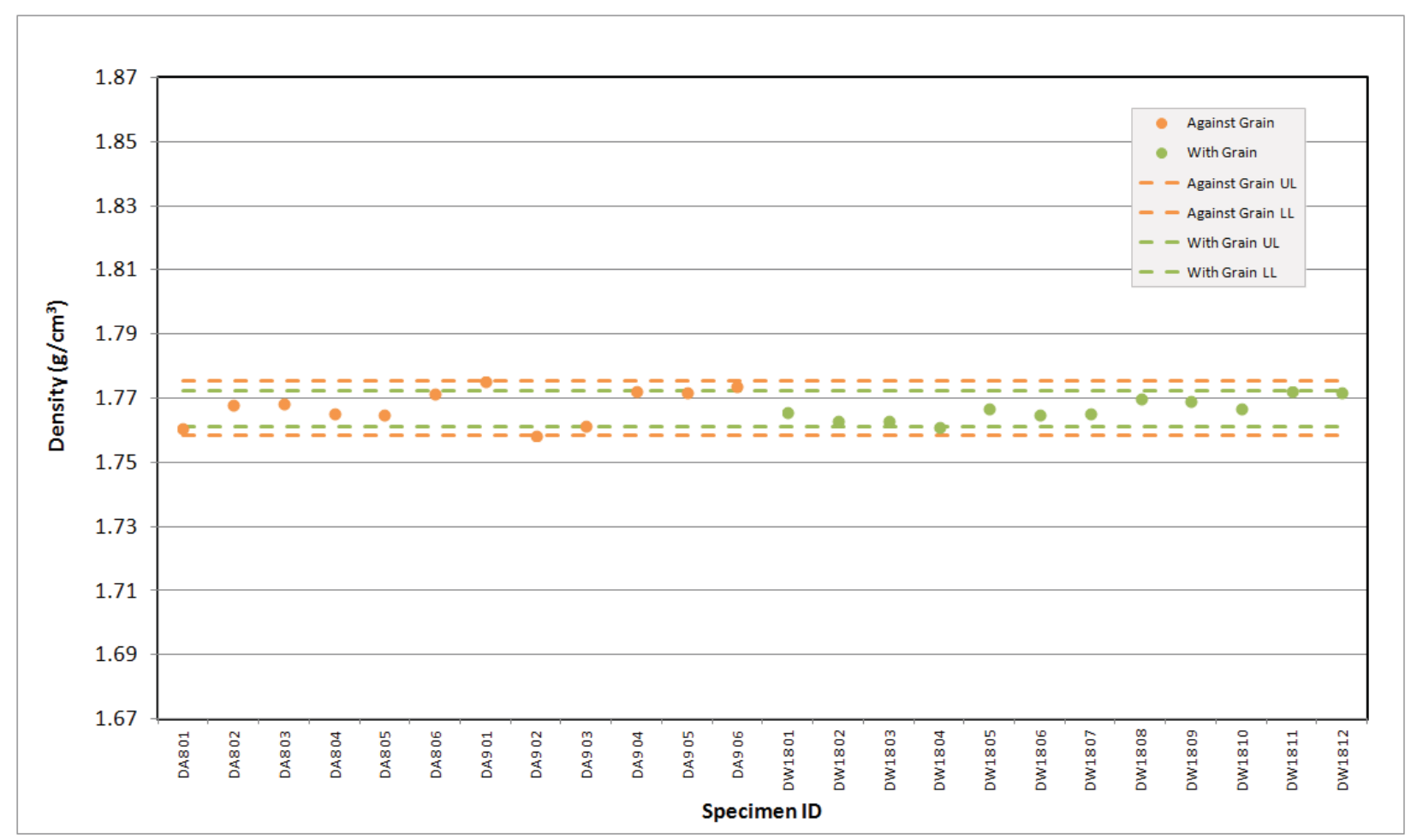

Figure A-19. PCEA Piggyback Pre Thermal Measurement Density.

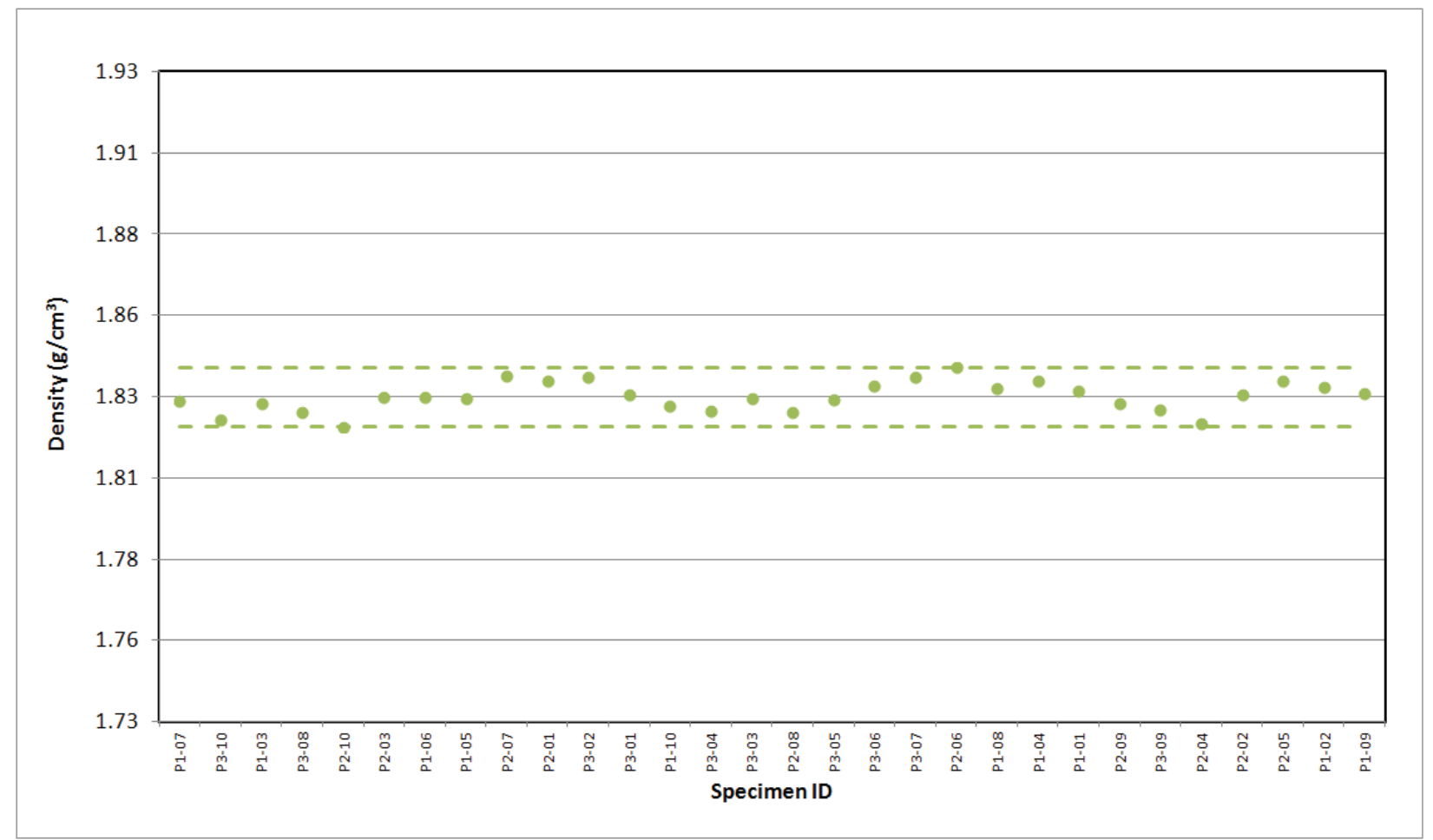

Figure A-20. PCIB Piggyback Pre Thermal Measurement Density. 


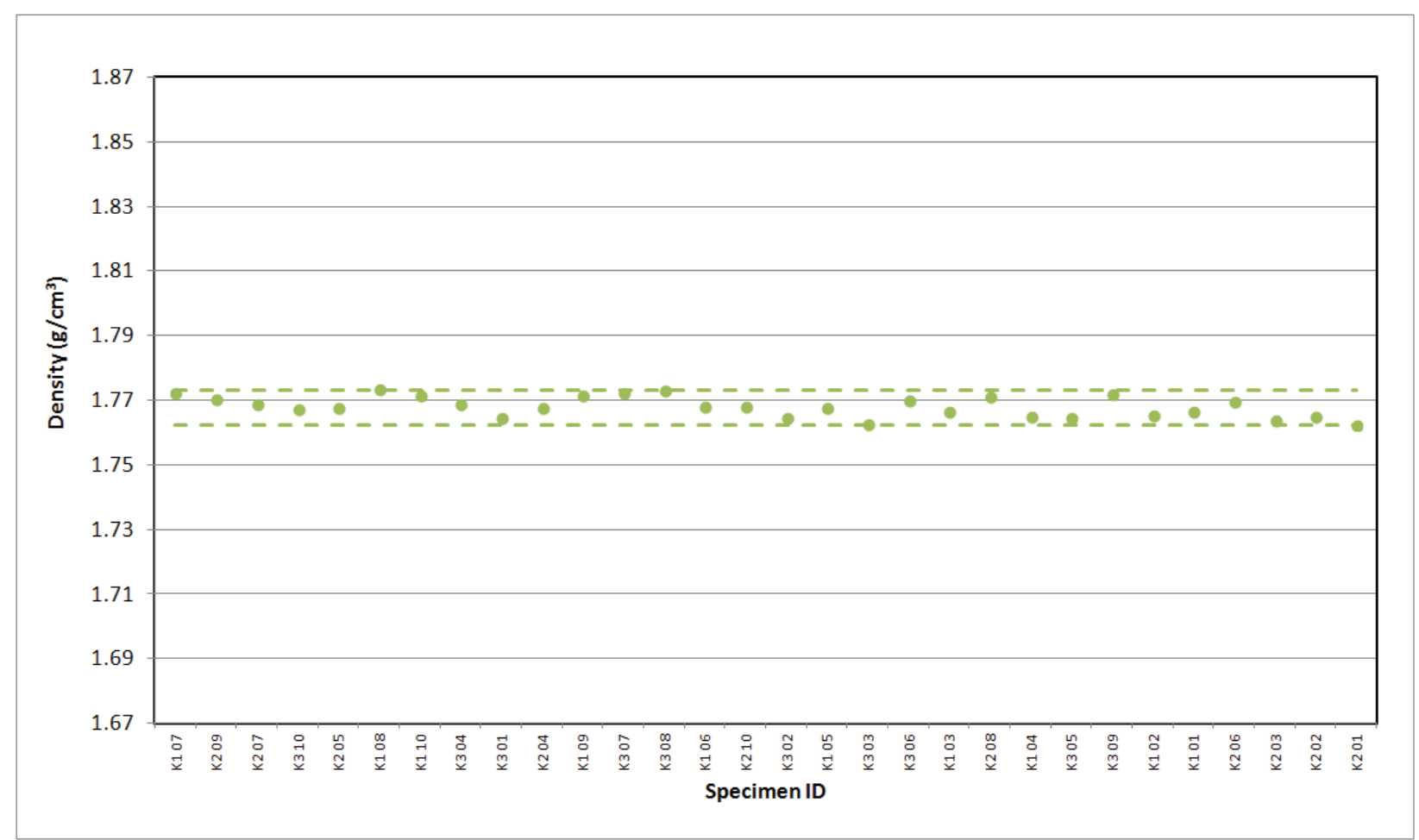

Figure A-21. PGX Piggyback Pre Thermal Measurement Density.

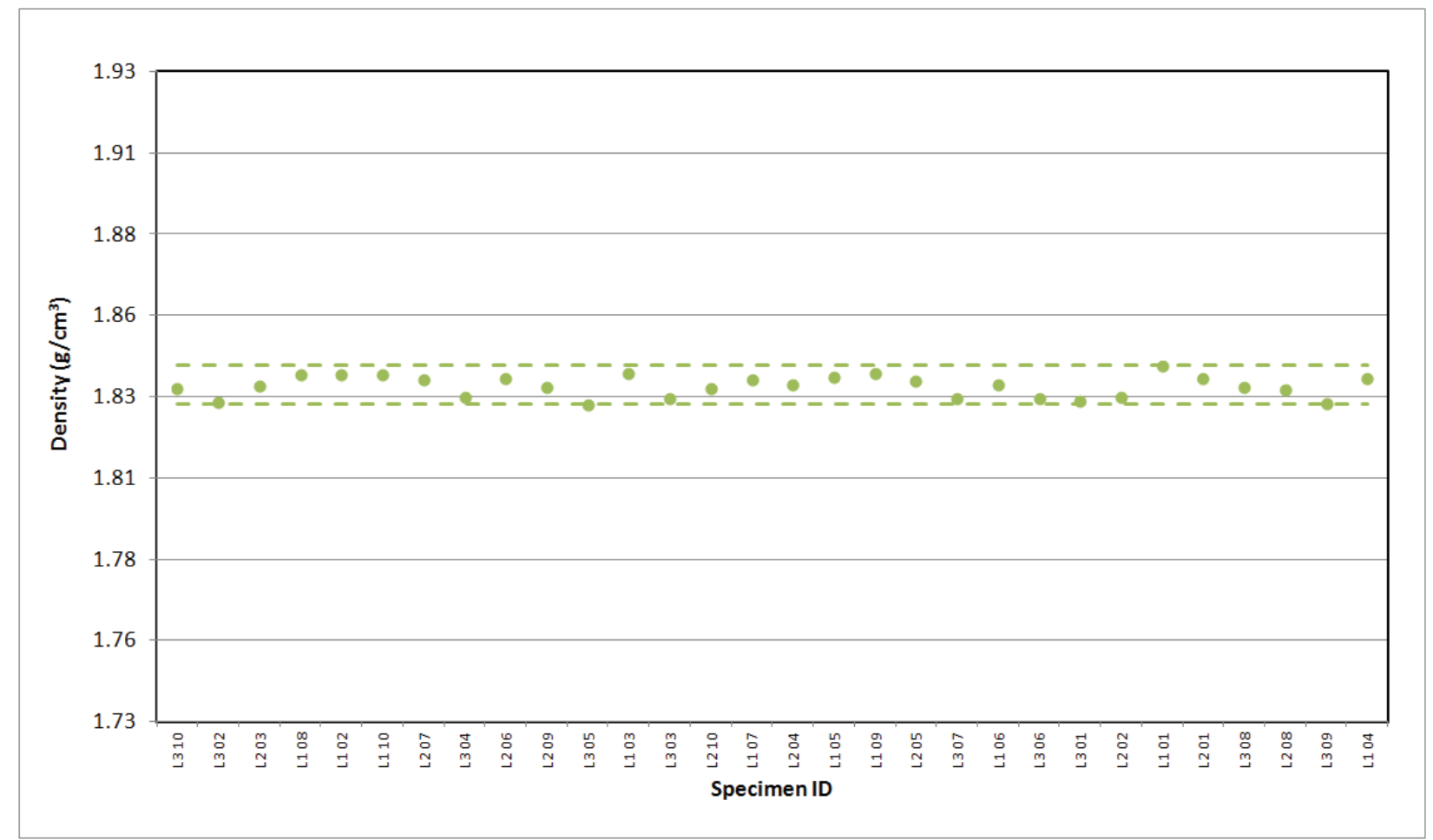

Figure A-22. PPEA Piggyback Pre Thermal Measurement Density. 


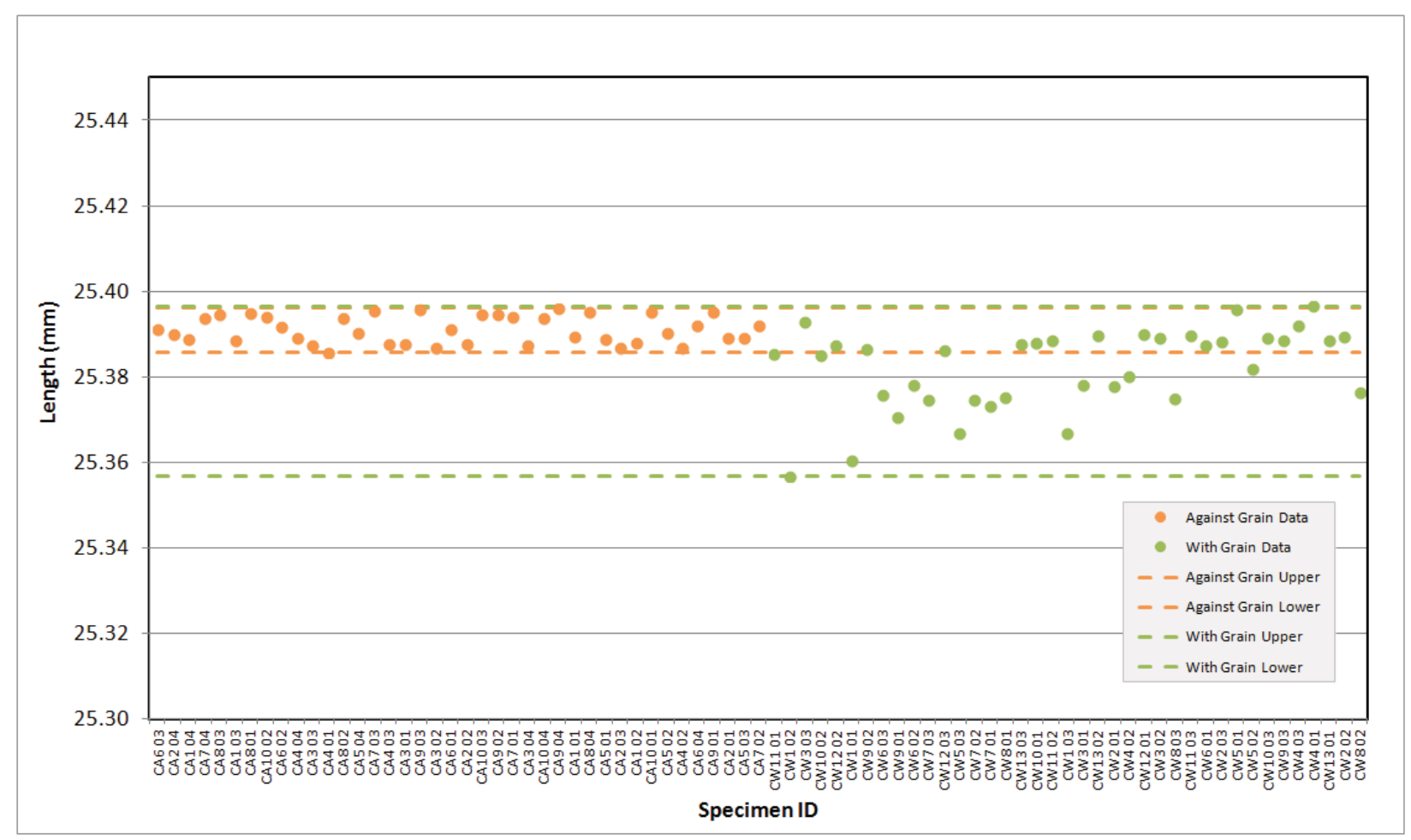

Figure A-23. H-451 Creep Pre Thermal Measurement Length.

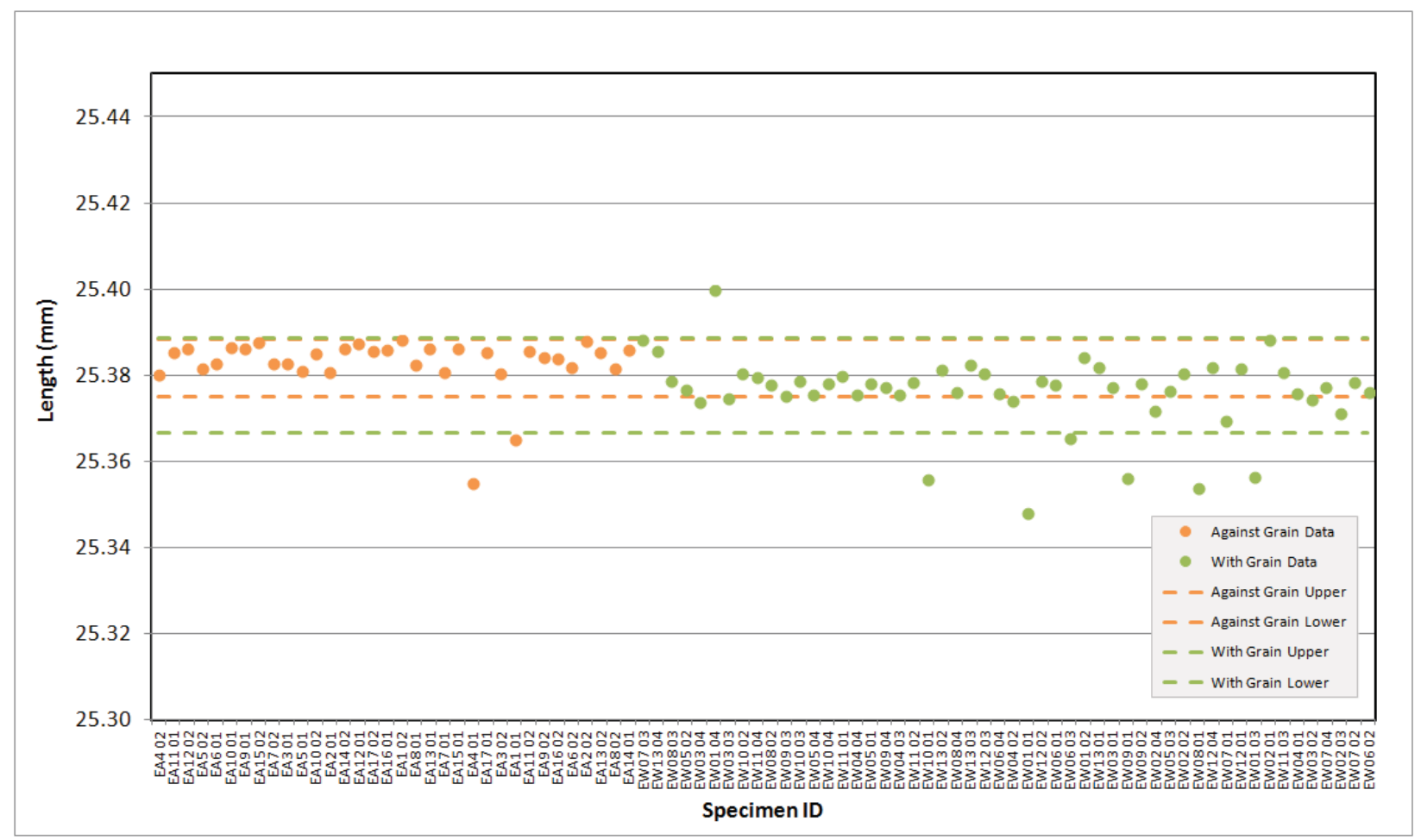

Figure A-24. IG-110 Creep Pre Thermal Measurement Length. 


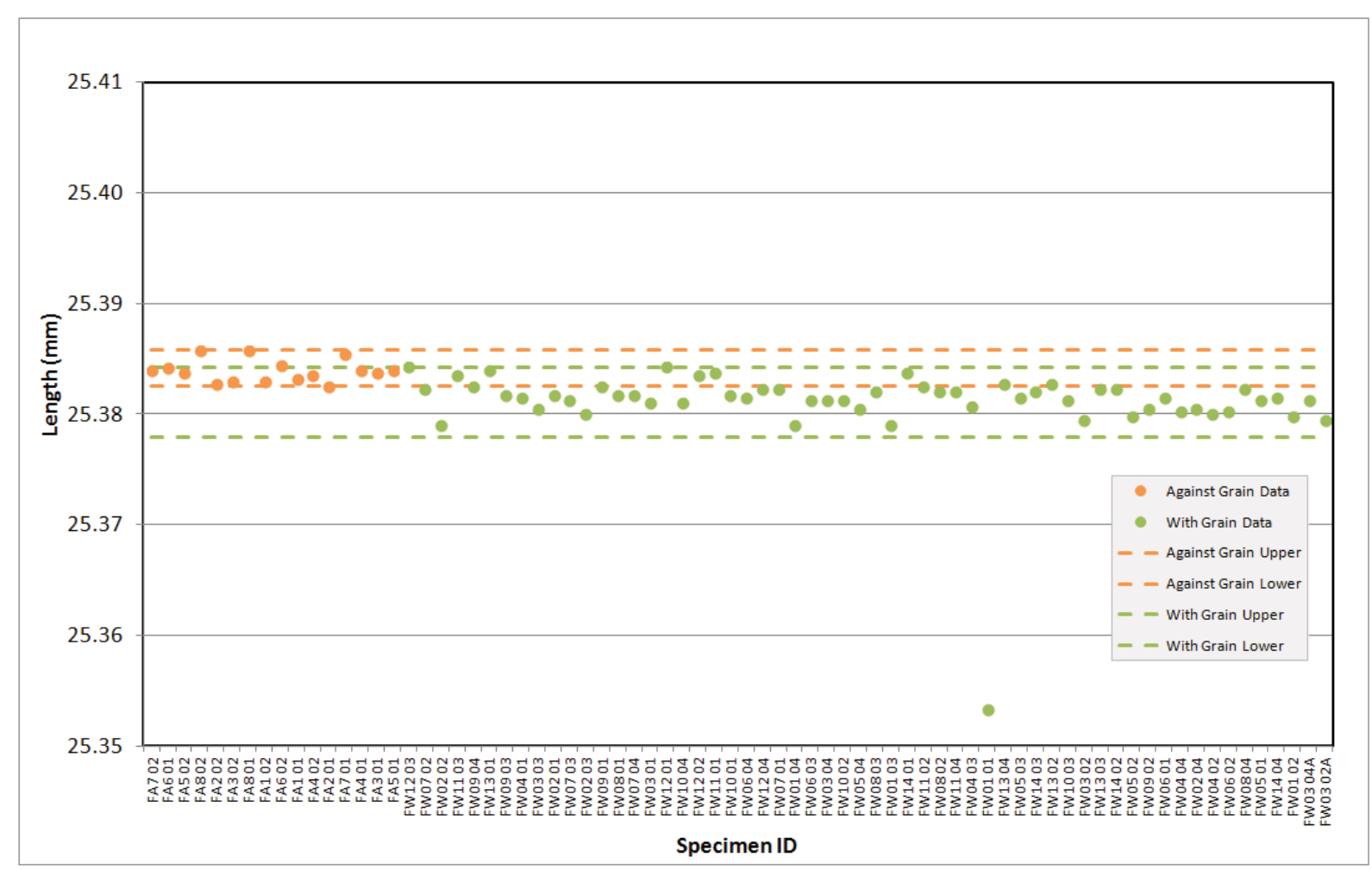

Figure A-25. IG-430 Creep Pre Thermal Measurement Length.

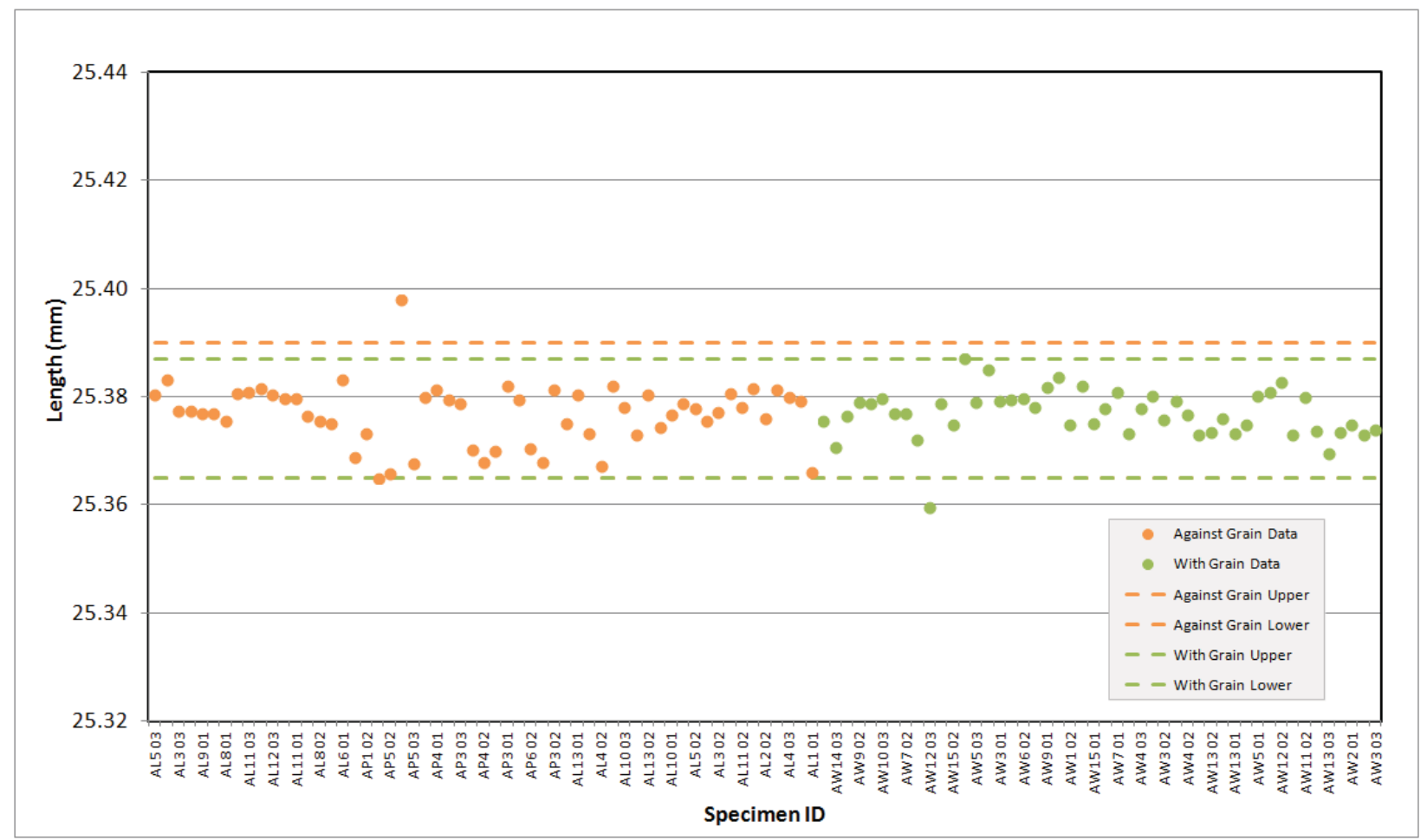

Figure A-26. NBG-17 Creep Pre Thermal Measurement Length. 


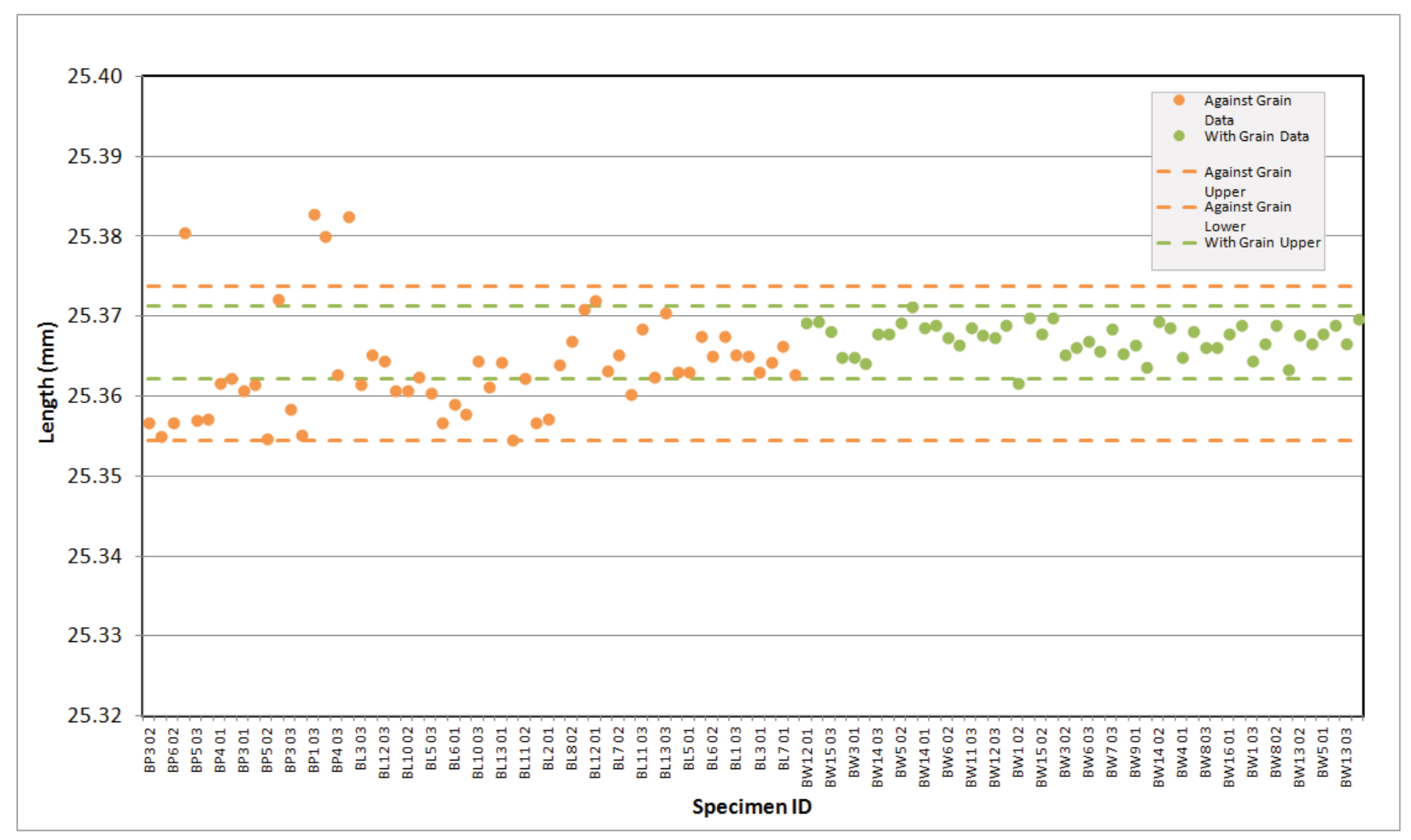

Figure A-27. NBG-18 Creep Pre Thermal Measurement Length.

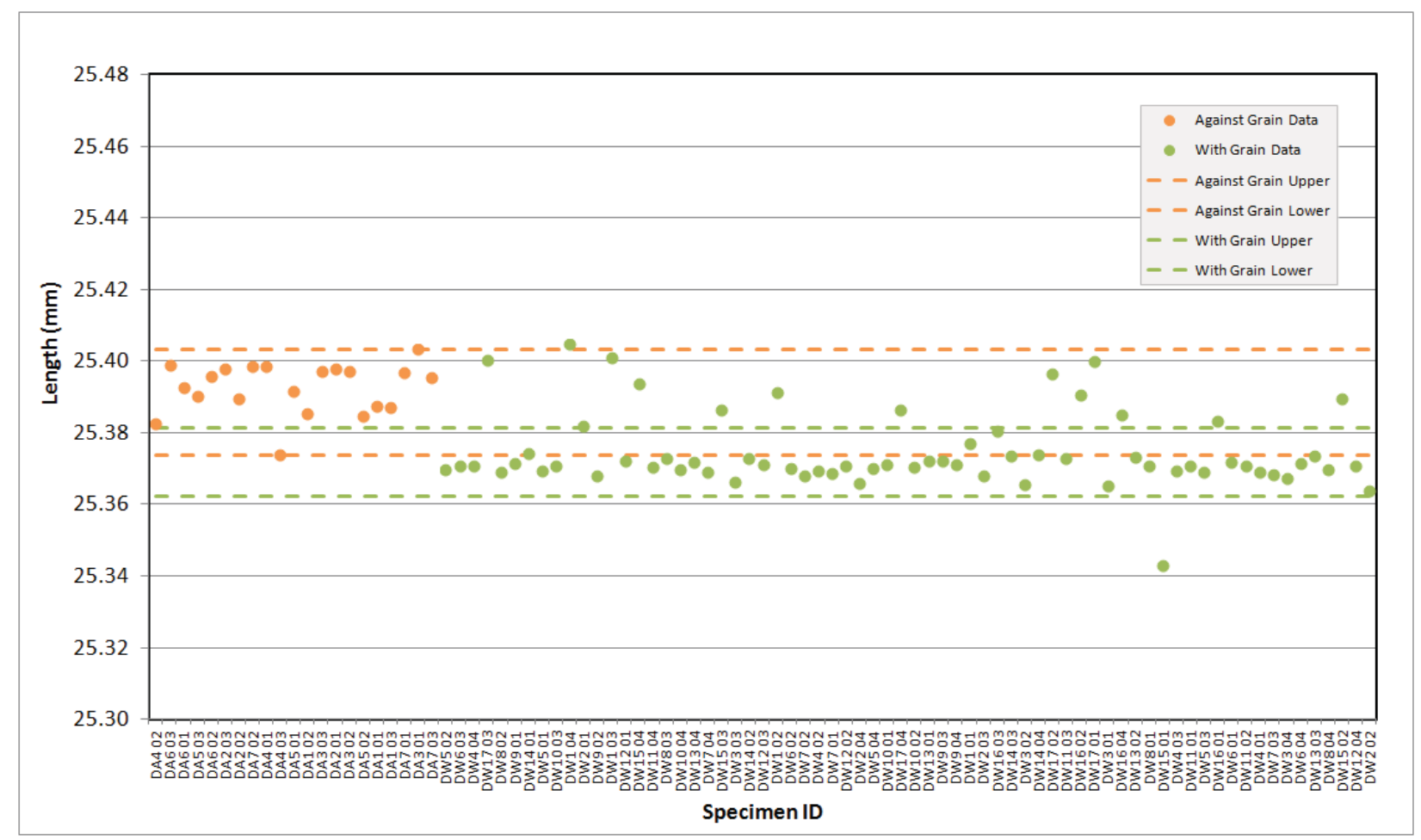

Figure A-28. PCEA Creep Pre Thermal Measurement Length. 


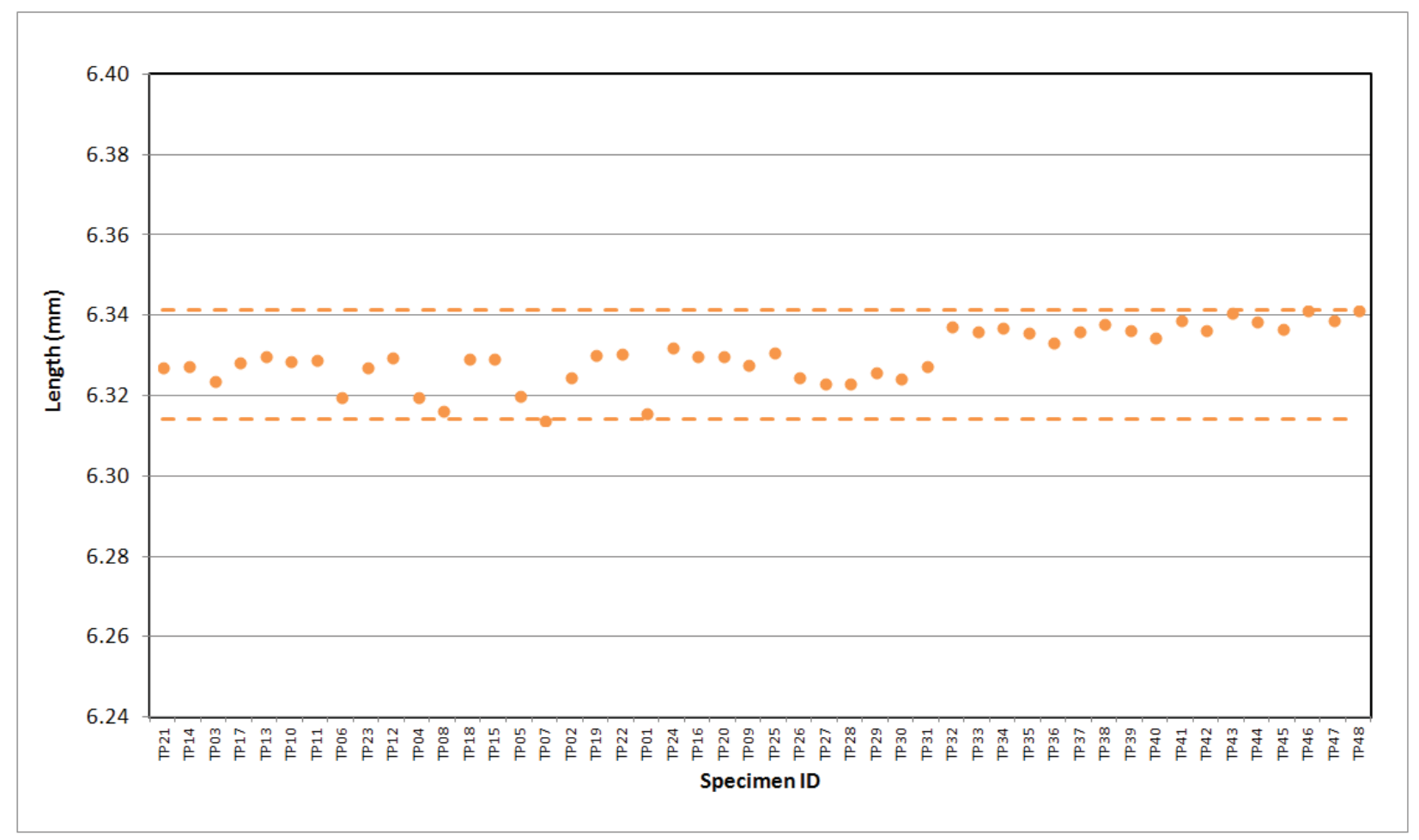

Figure A-29. 2114 Piggyback Pre Thermal Measurement Length.

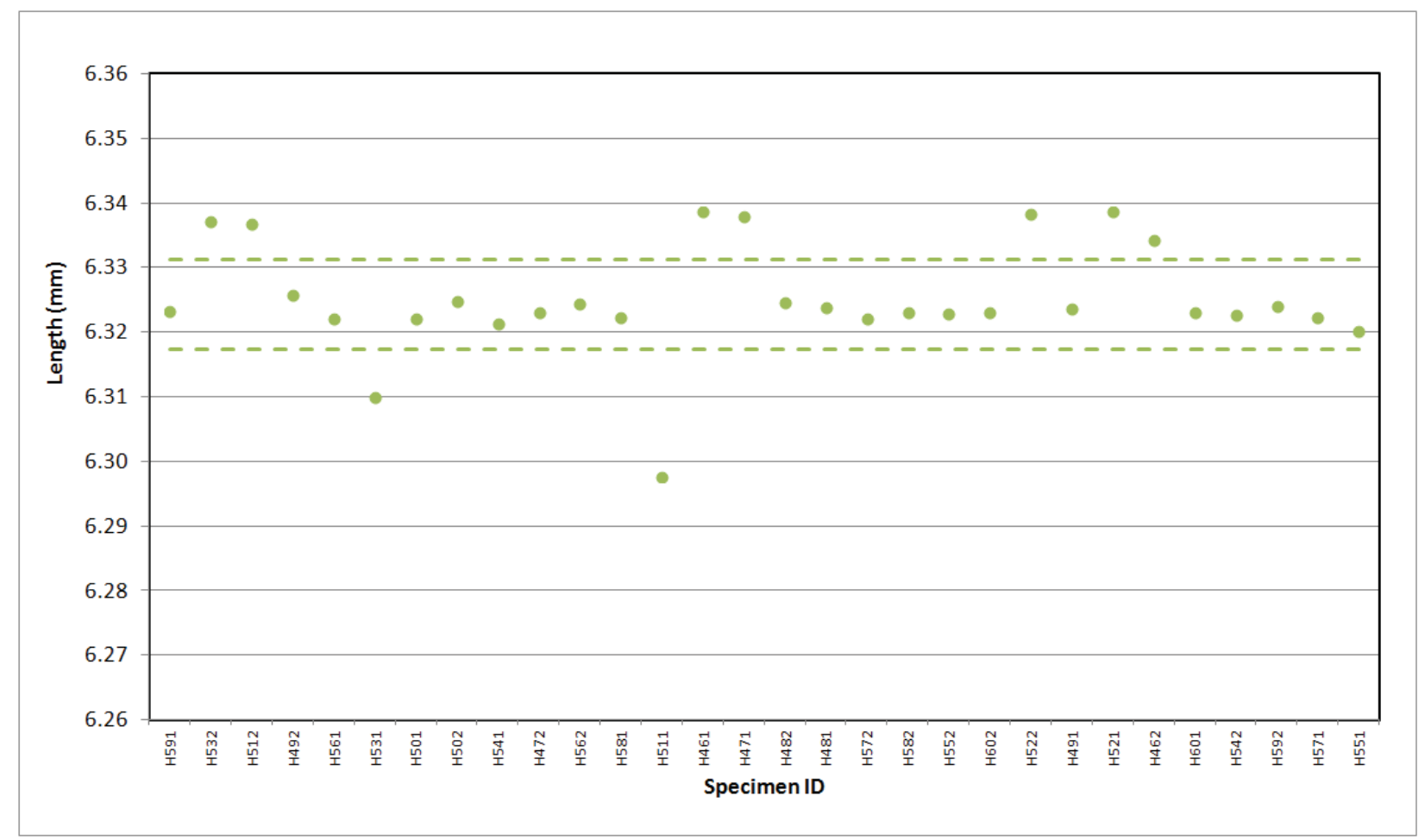

Figure A-30. A3 Matrix Piggyback Pre Thermal Measurement Length. 


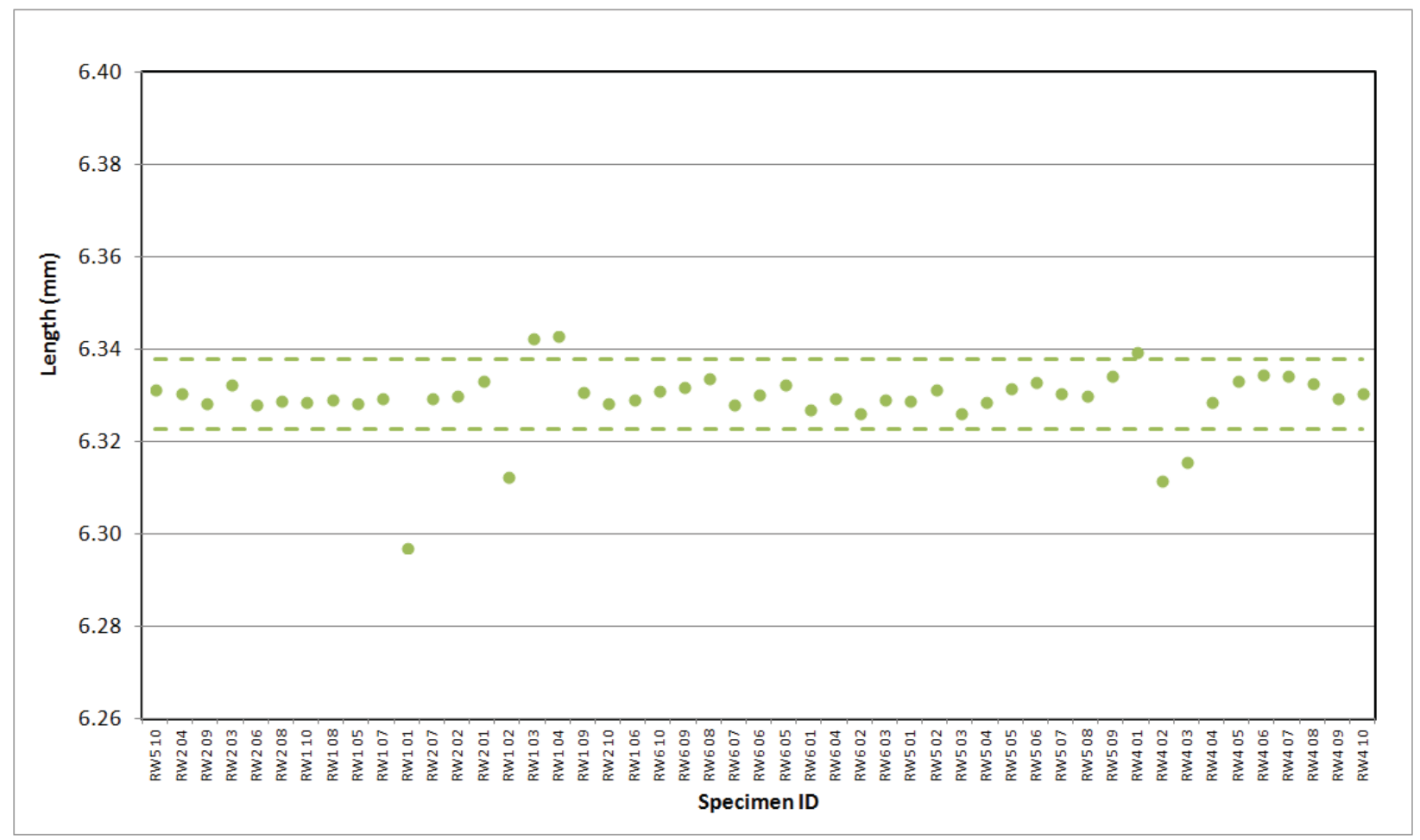

Figure A-31. BAN Piggyback Pre Thermal Measurement Length.

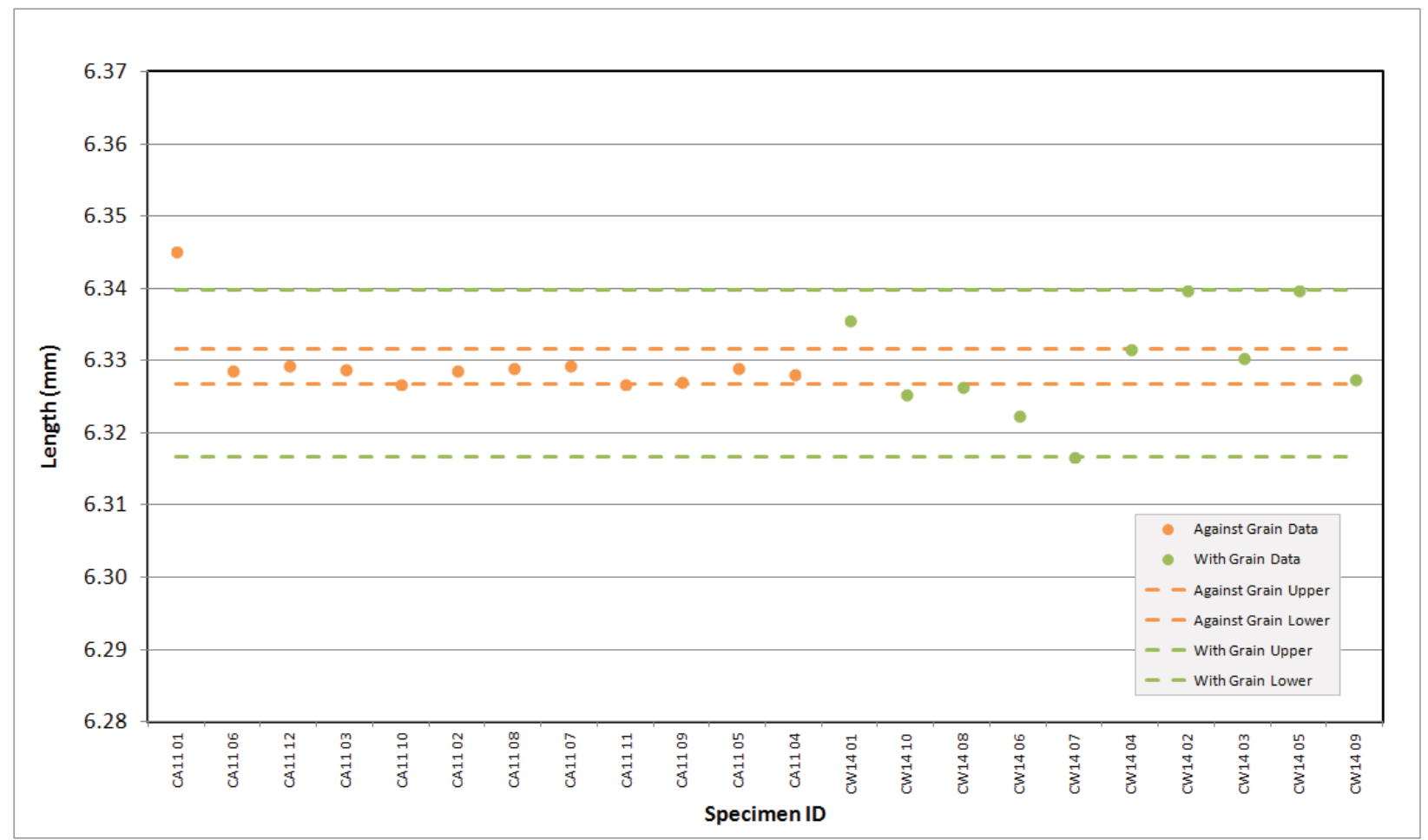

Figure A-32. H-451 Piggyback Pre Thermal Measurement Length. 


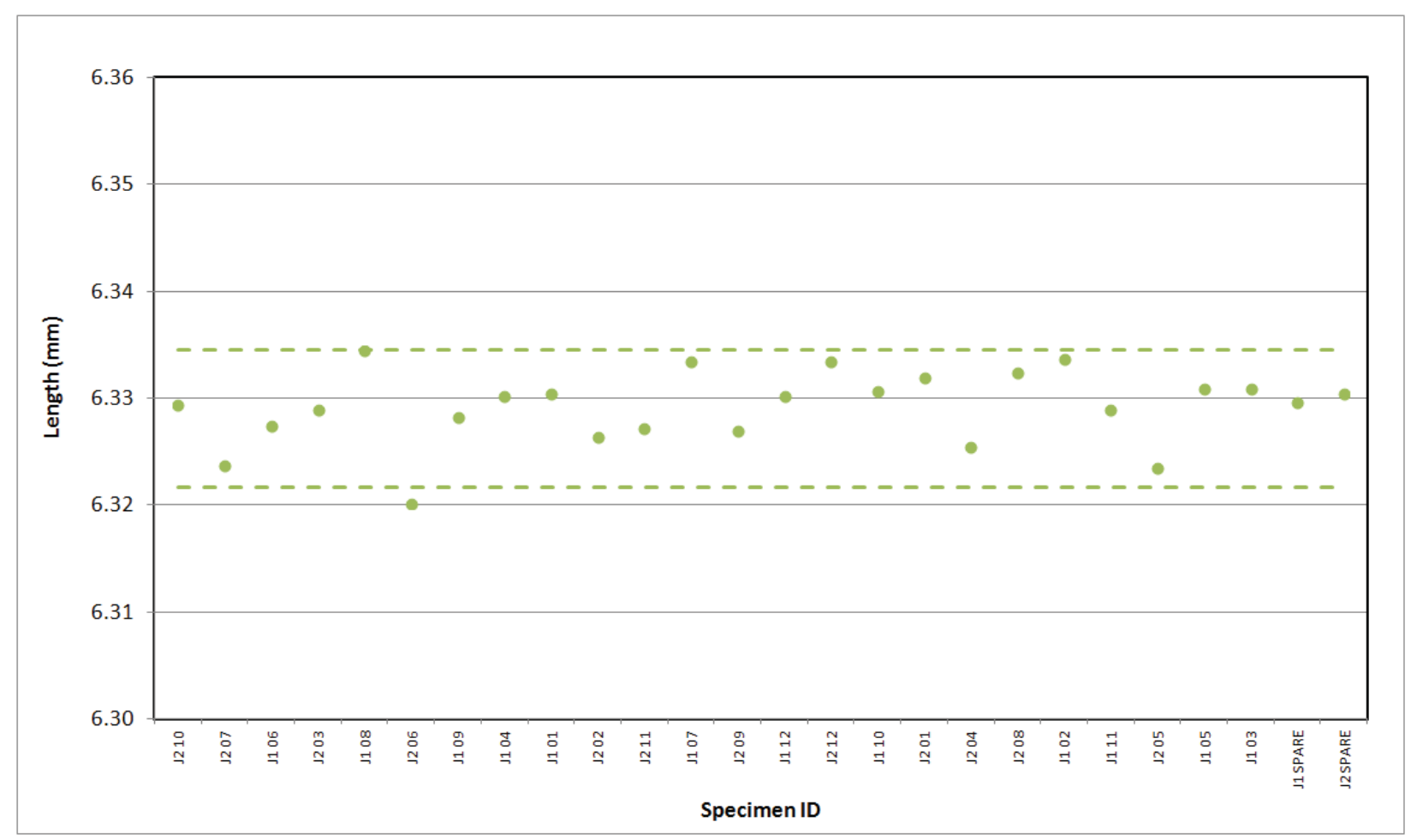

Figure A-33. HLM Piggyback Pre Thermal Measurement Length.

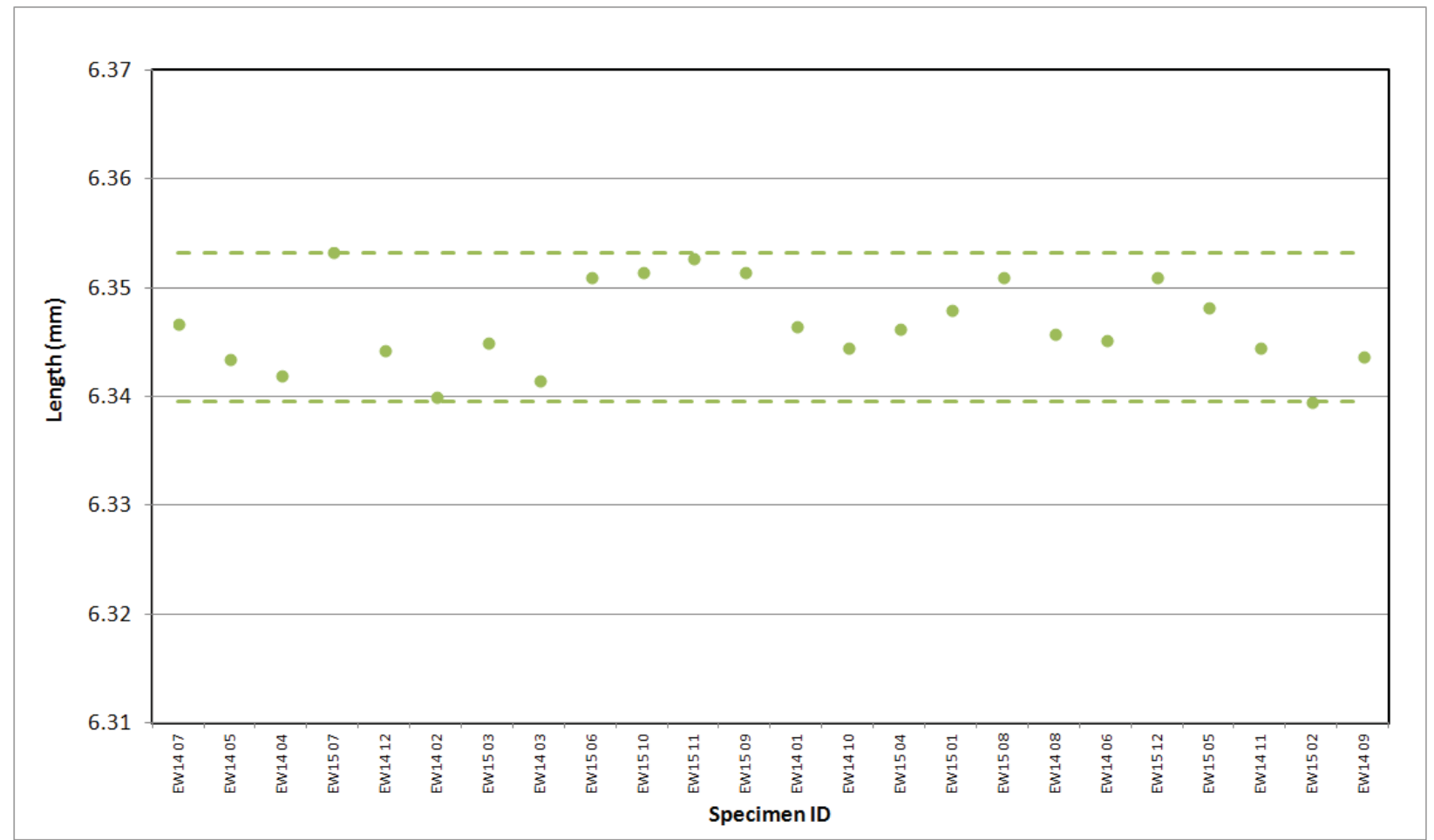

Figure A-34. IG-110 Piggyback Pre Thermal Measurement Length. 


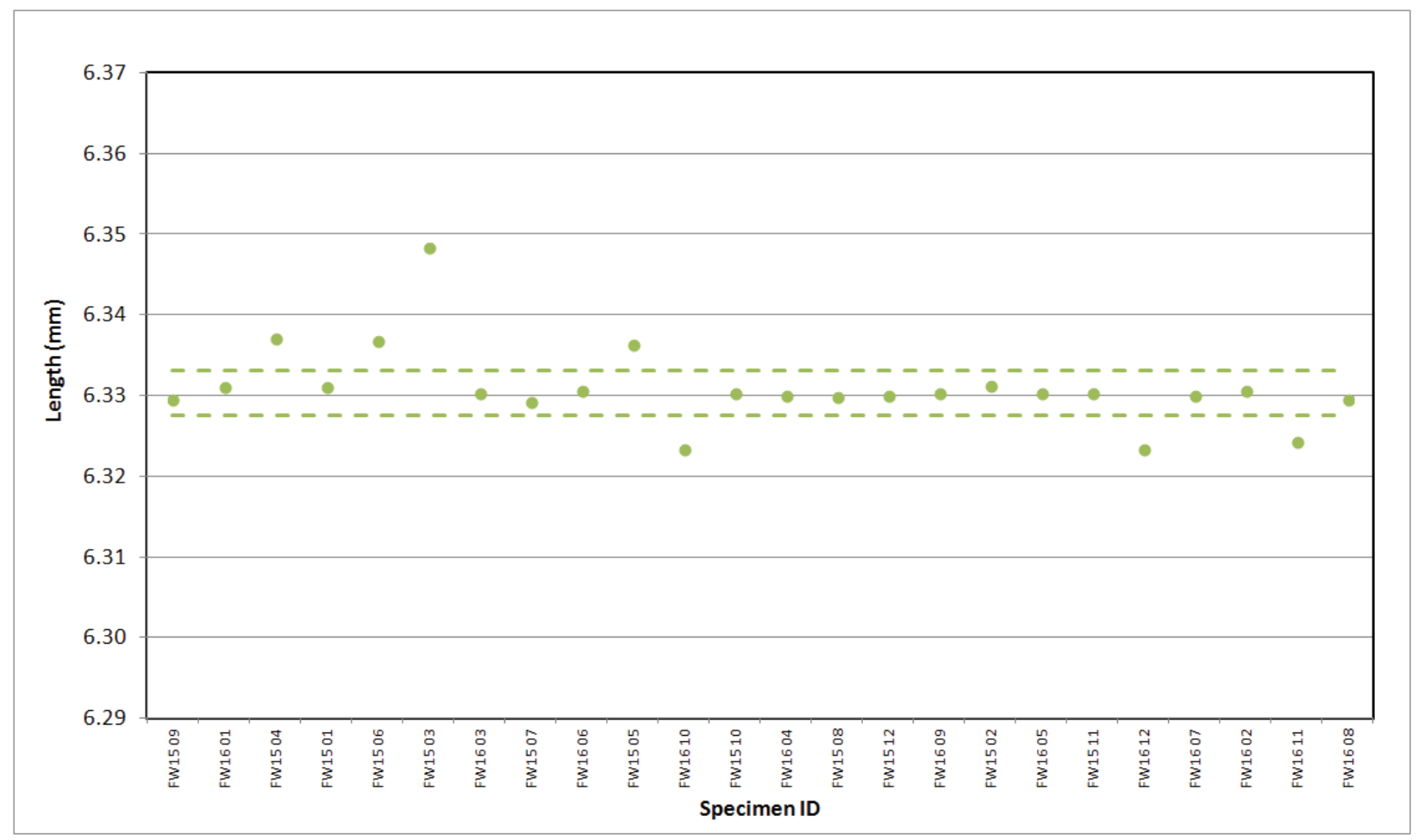

Figure A-35. IG-430 Piggyback Pre Thermal Measurement Length.

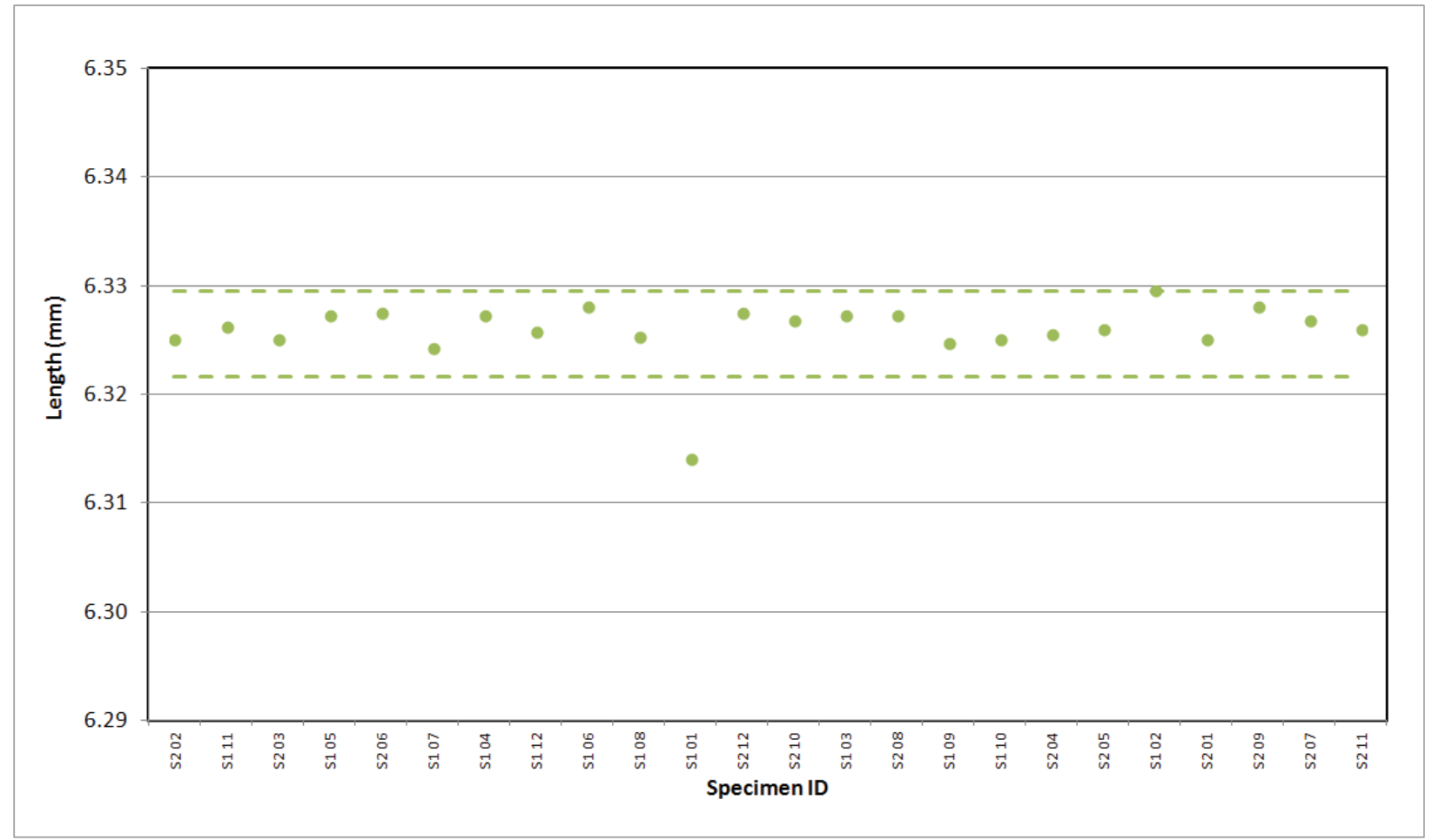

Figure A-36. NBG-10 Piggyback Pre Thermal Measurement Length. 


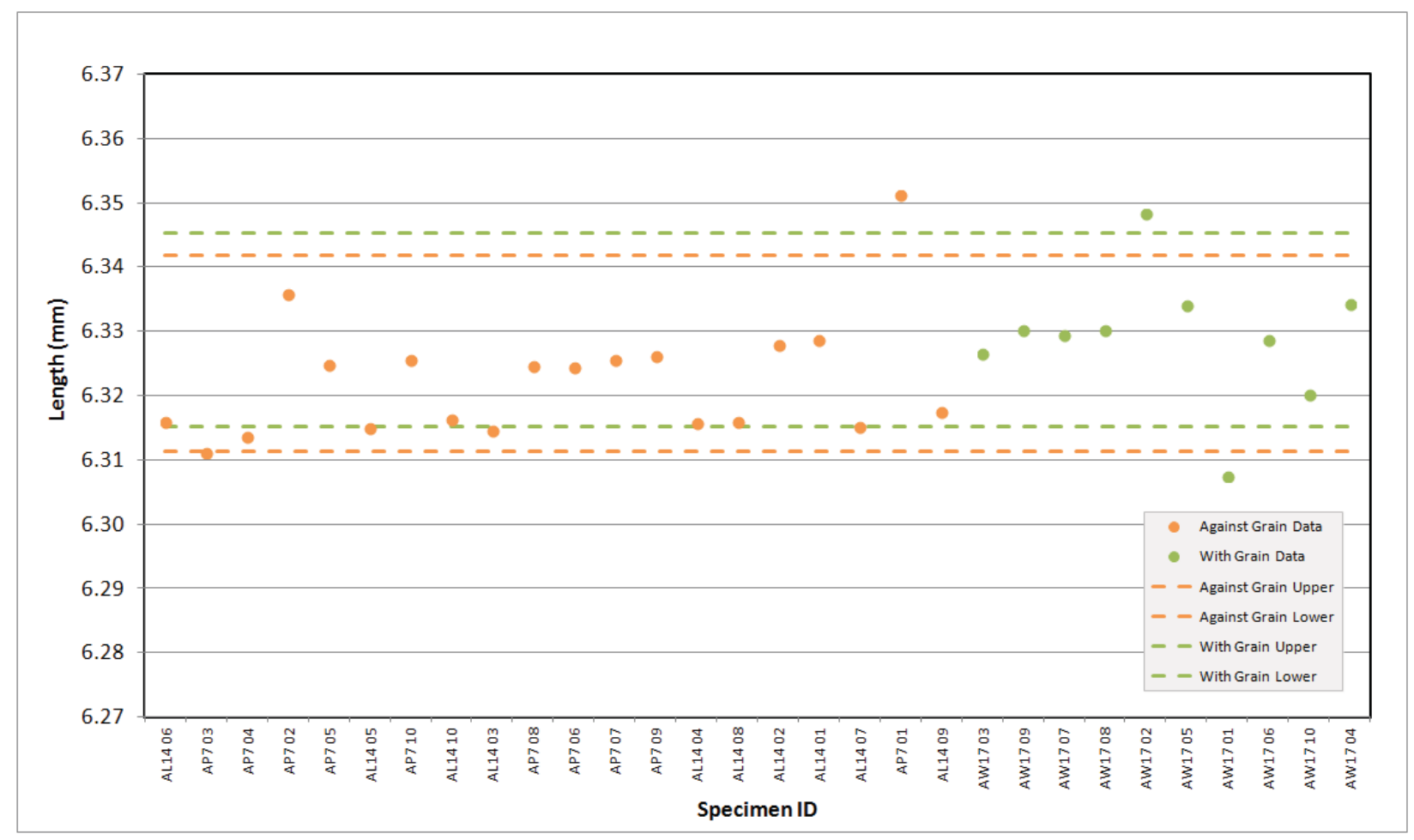

Figure A-37. NBG-17 Piggyback Pre Thermal Measurement Length.

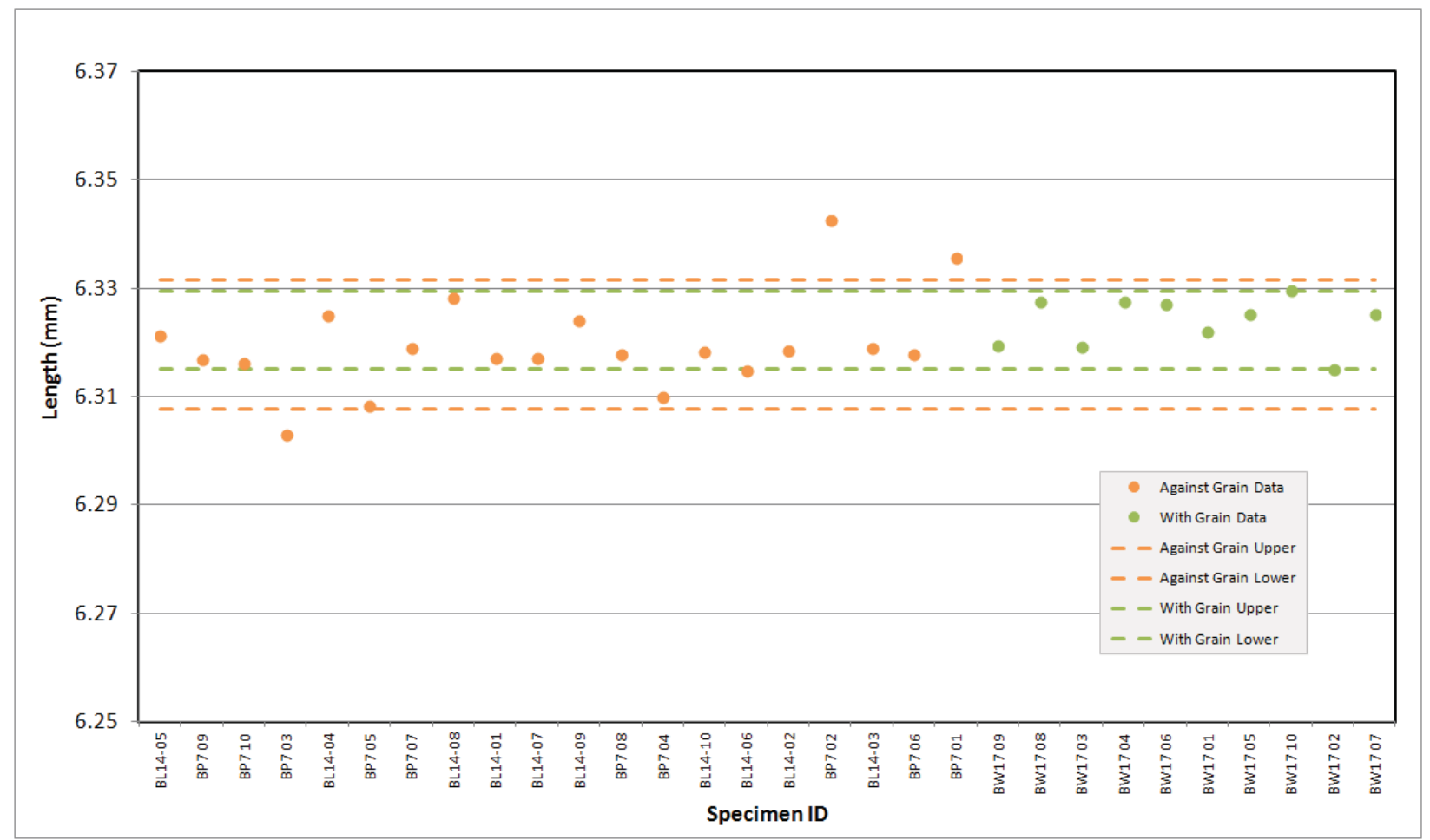

Figure A-38. NBG-18 Piggyback Pre Thermal Measurement Length. 


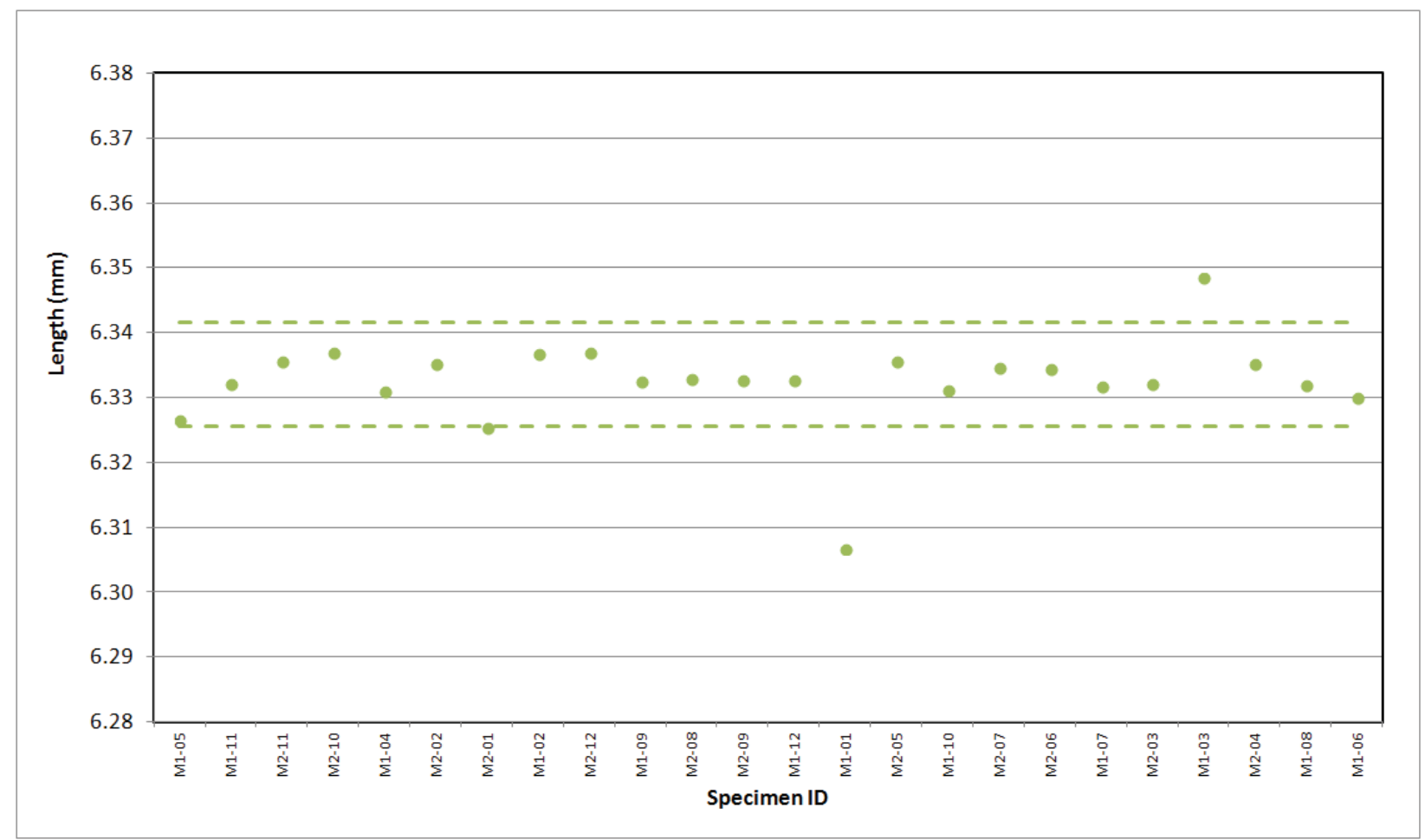

Figure A-39. NBG-25 Piggyback Pre Thermal Measurement Length.

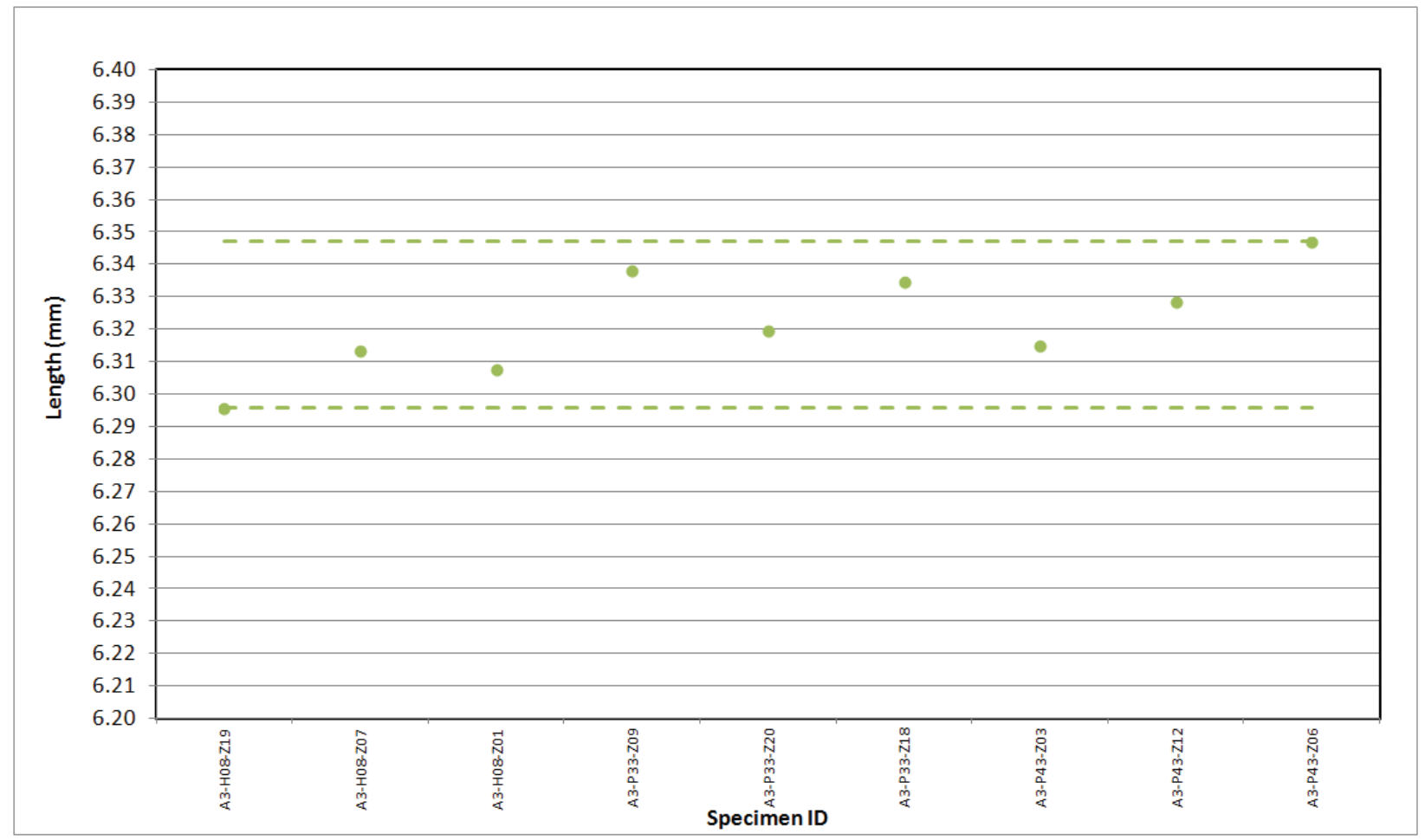

Figure A-40. New Matrix Piggyback Pre Thermal Measurement Length. 


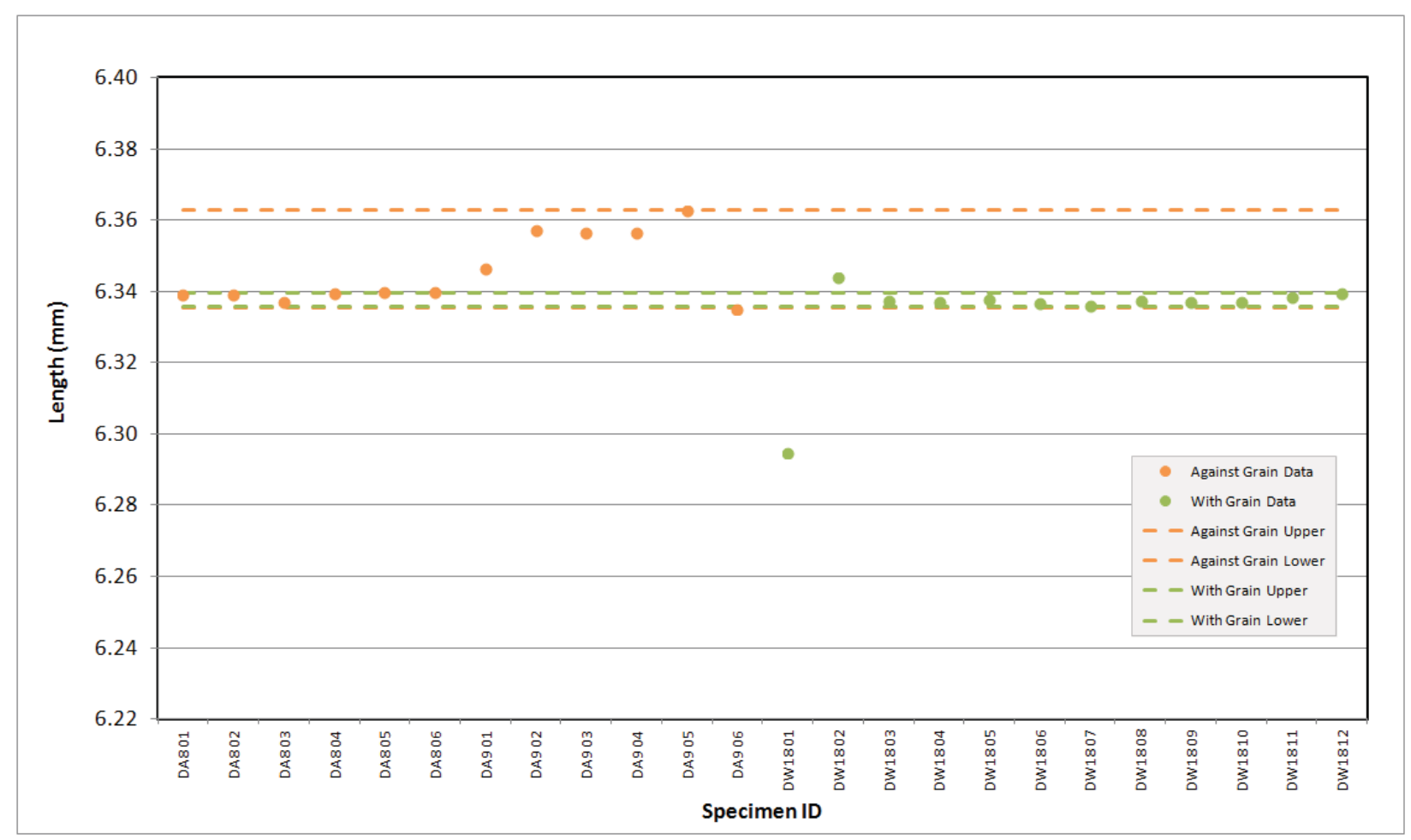

Figure A-41. PCEA Piggyback Pre Thermal Measurement Length.

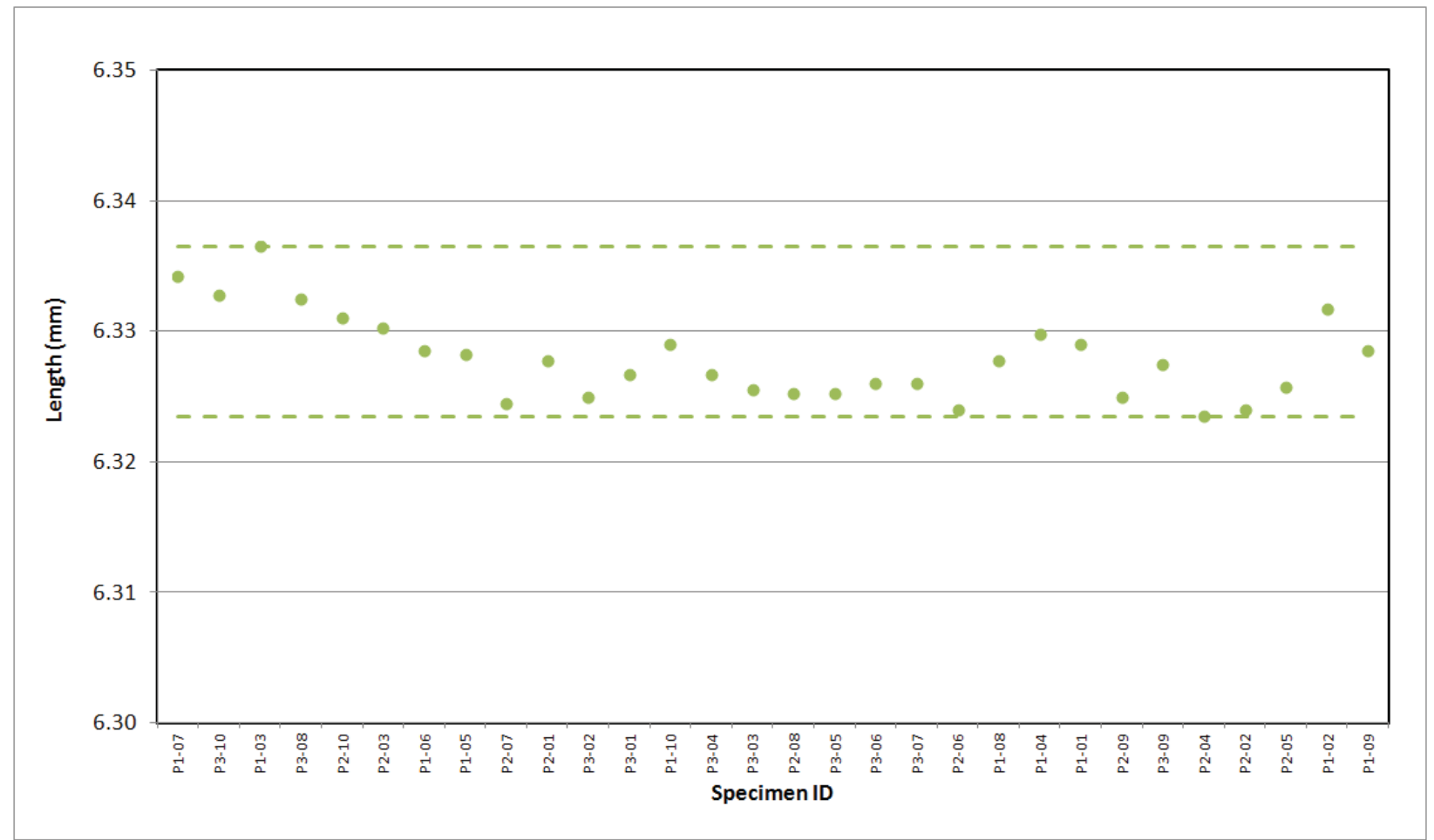

Figure A-42. PCIB Piggyback Pre Thermal Measurement Length. 


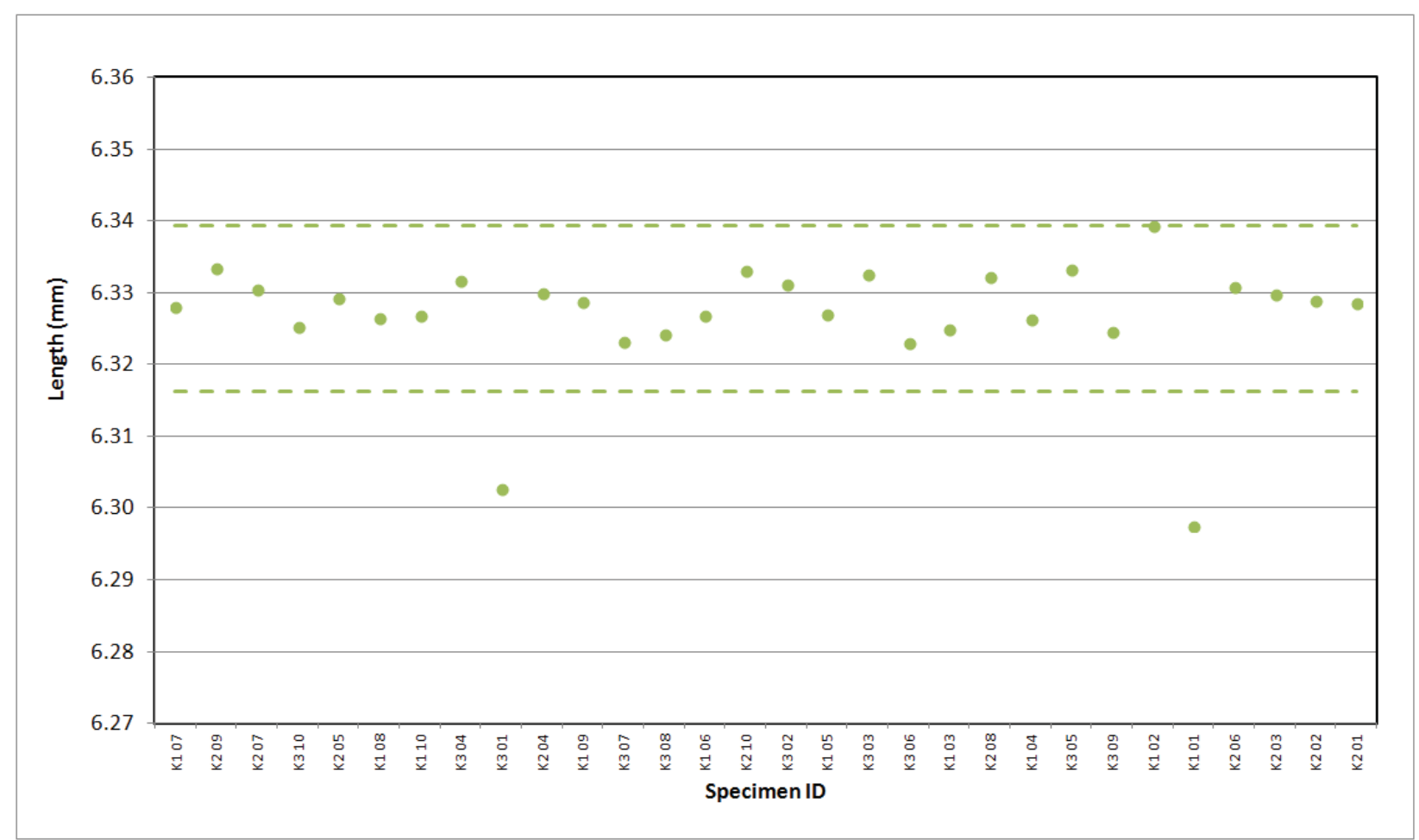

Figure A-43. PGX Piggyback Pre Thermal Measurement Length.

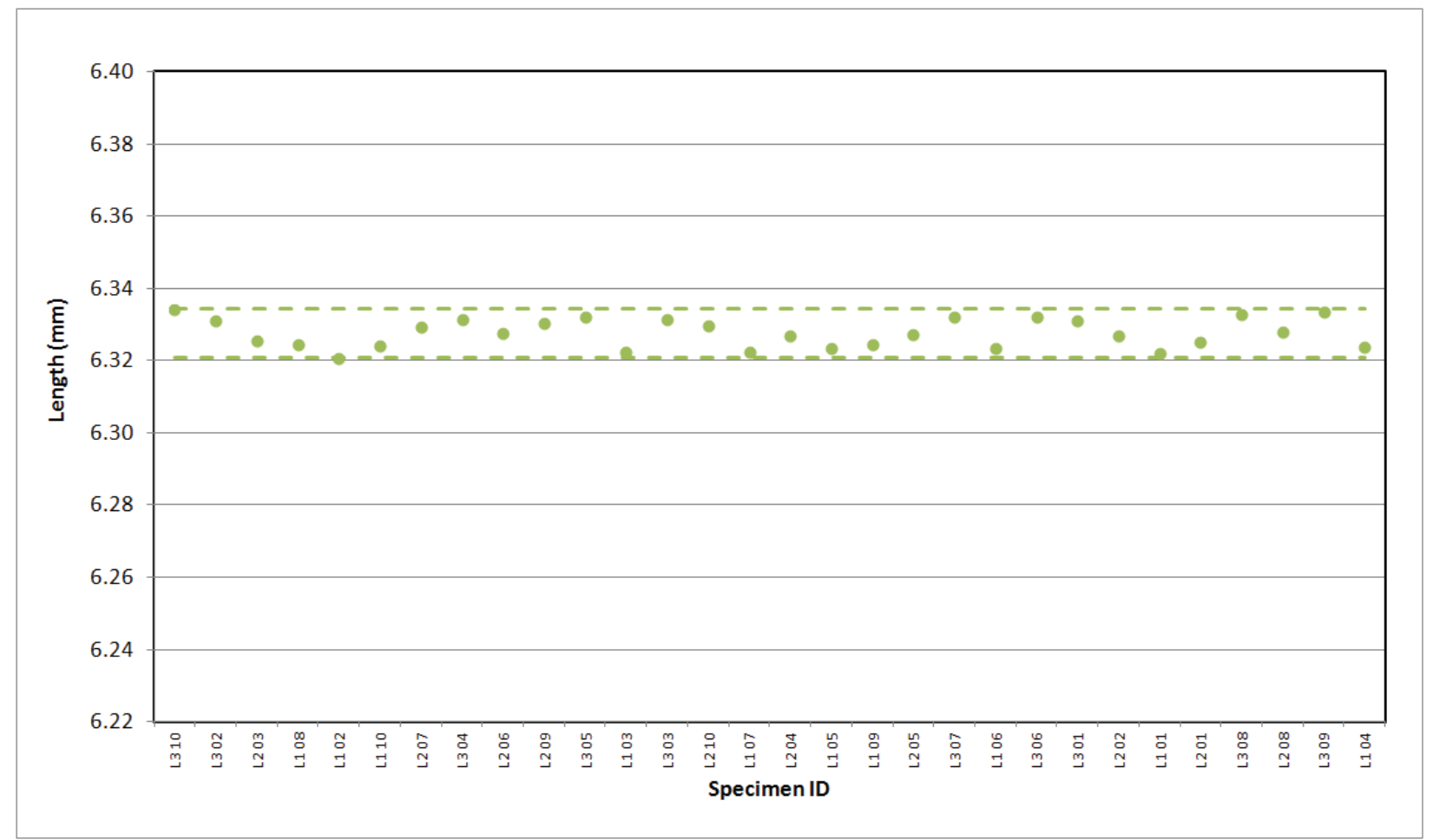

Figure A-44. PPEA Piggyback Pre Thermal Measurement Length. 


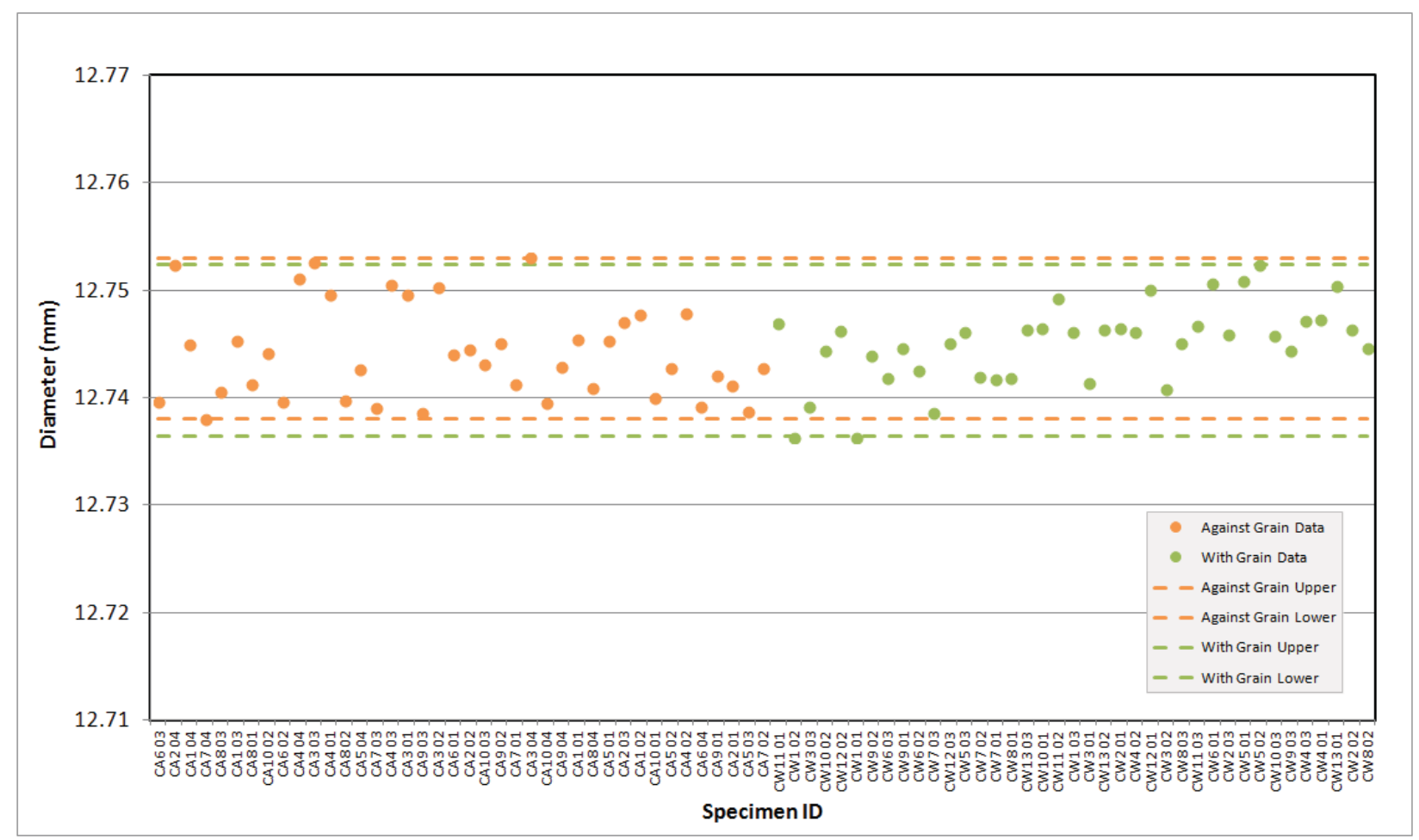

Figure A-45. H-451 Creep Pre Thermal Measurement Diameter.

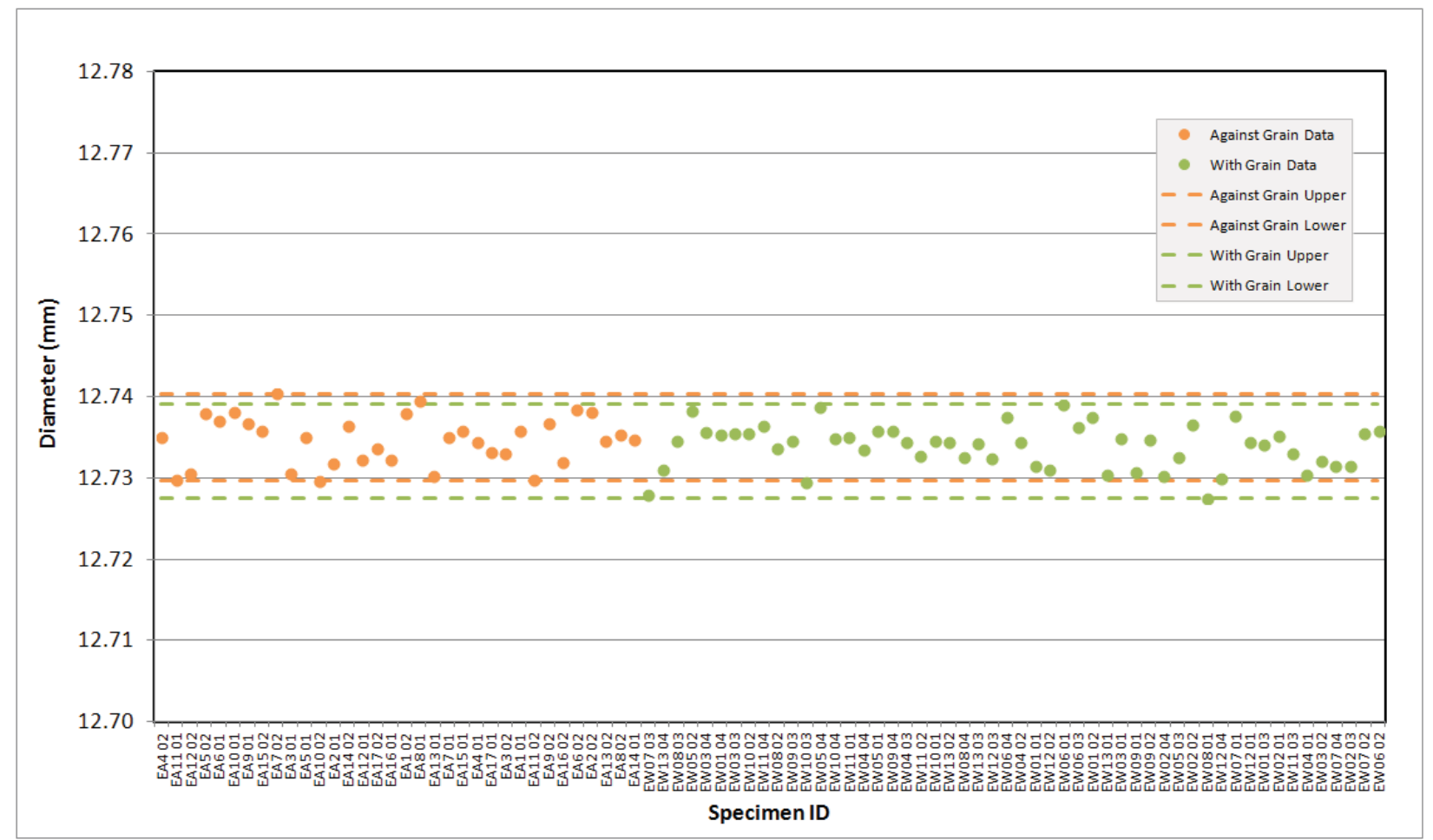

Figure A-46. IG-110 Creep Pre Thermal Measurement Diameter. 


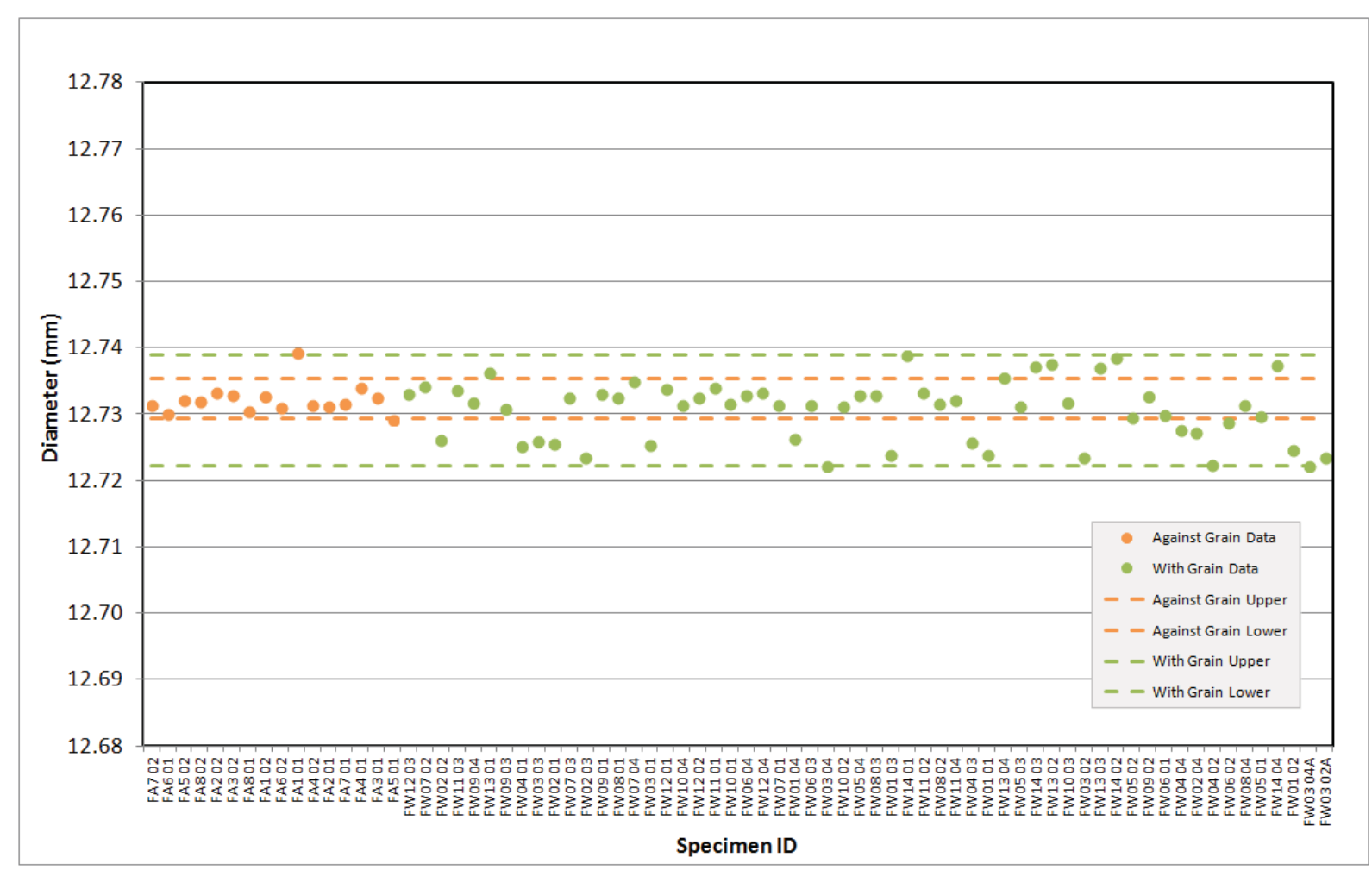

Figure A-47. IG-430 Creep Pre Thermal Measurement Diameter.

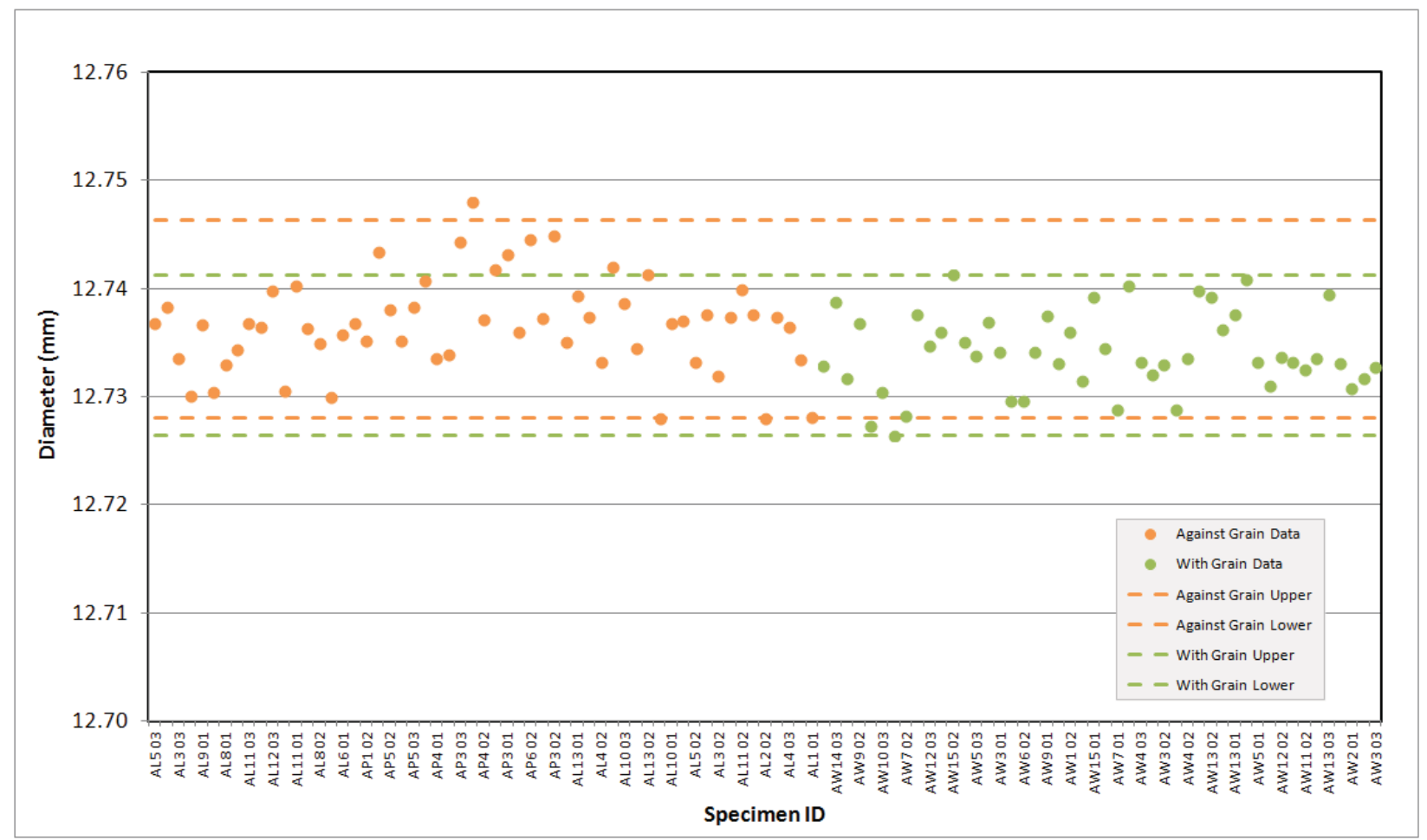

Figure A-48. NBG-17 Creep Pre Thermal Measurement Diameter. 


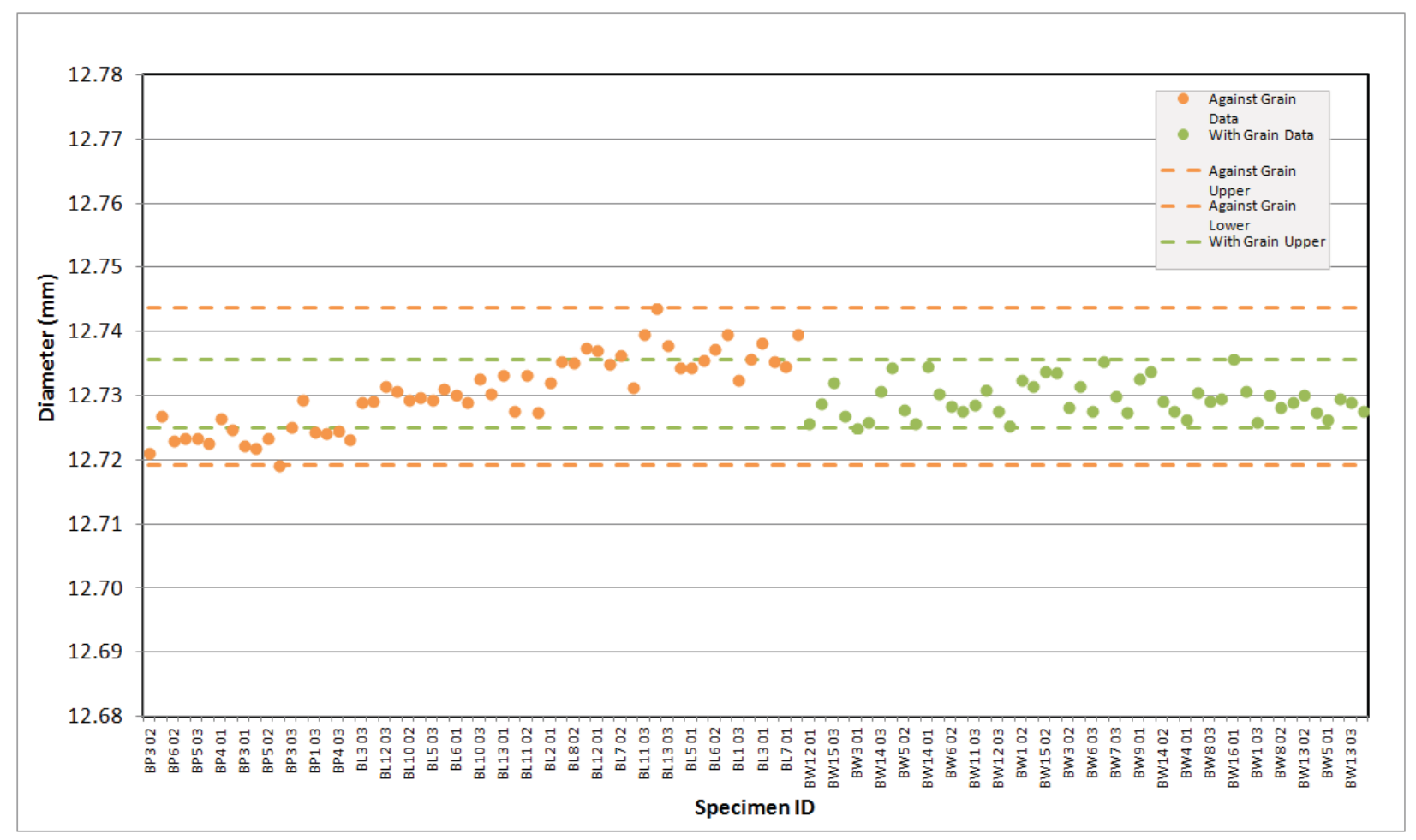

Figure A-49. NBG-18 Creep Pre Thermal Measurement Diameter.

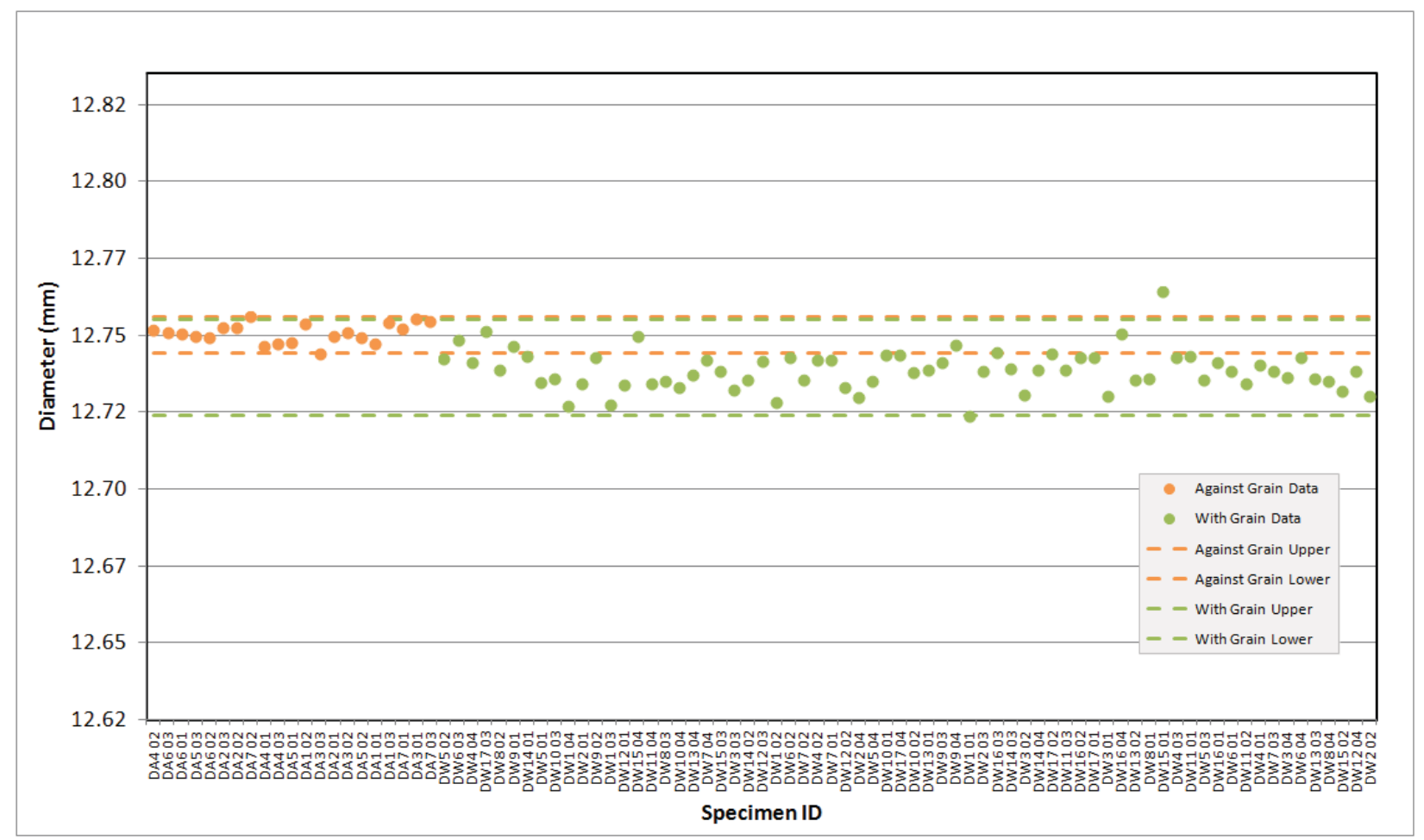

Figure A-50. PCEA Creep Pre Thermal Measurement Diameter. 


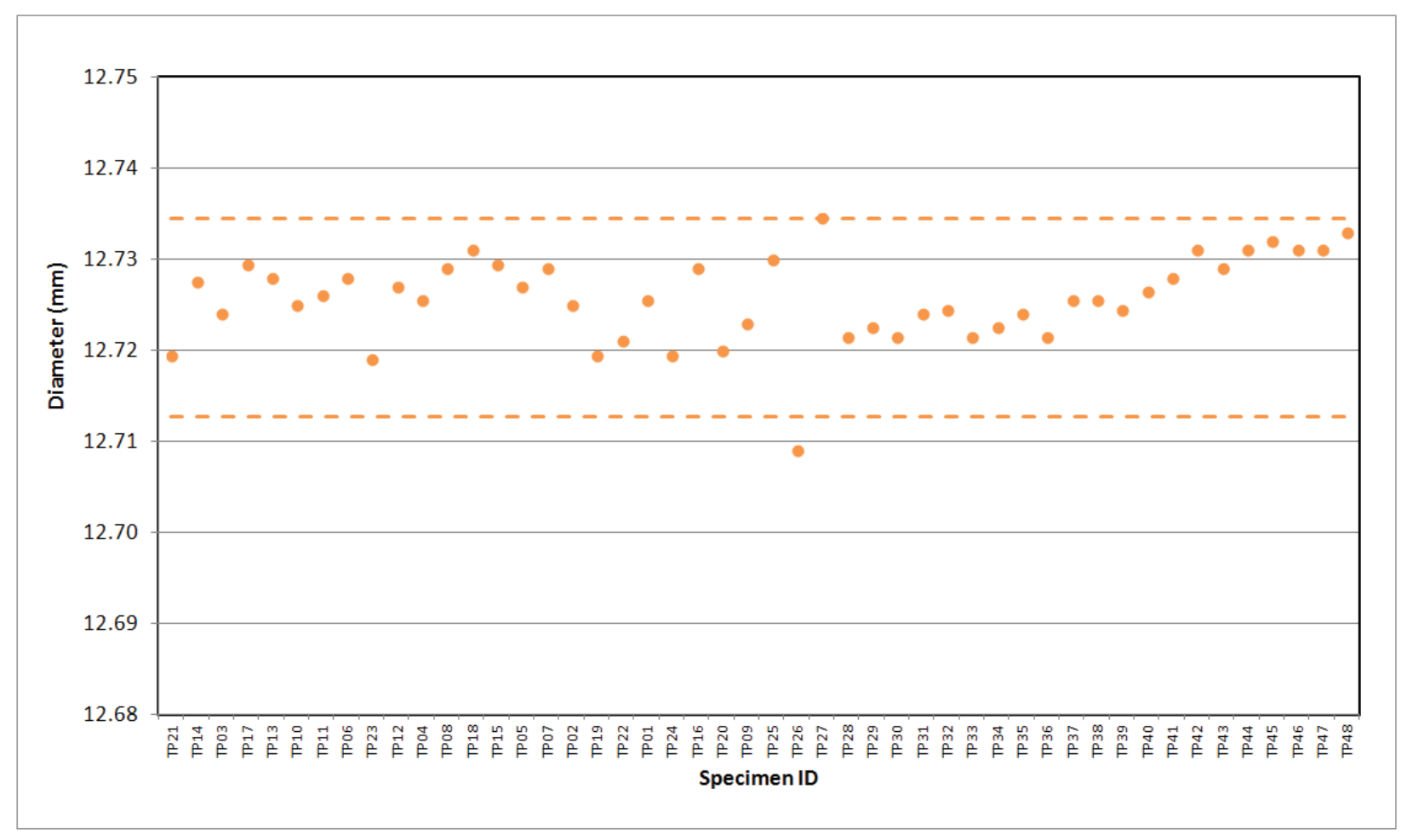

Figure A-51.2114 Piggyback Pre Thermal Measurement Diameter.

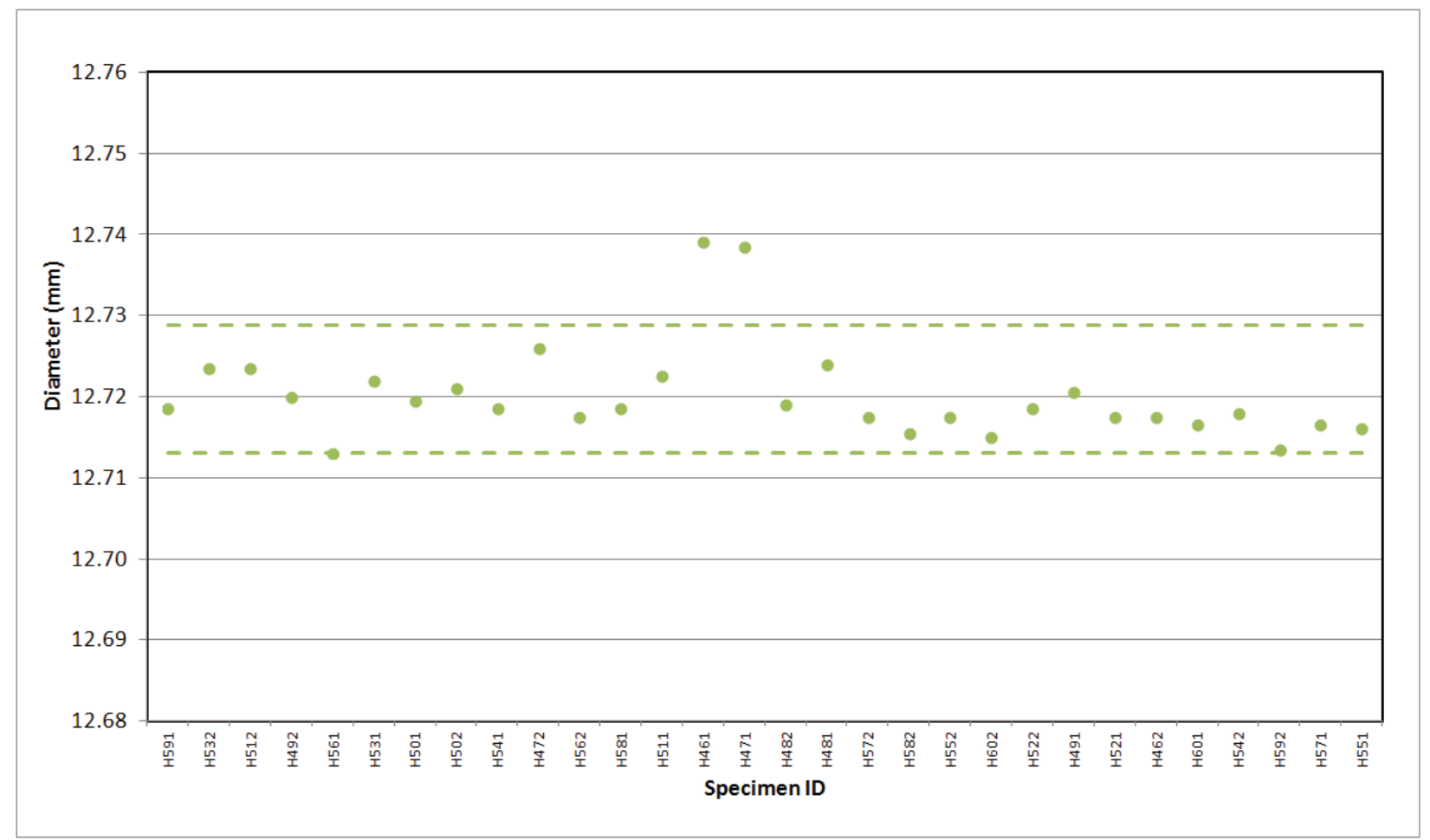

Figure A-52. A3 Matrix Piggyback Pre Thermal Measurement Diameter. 


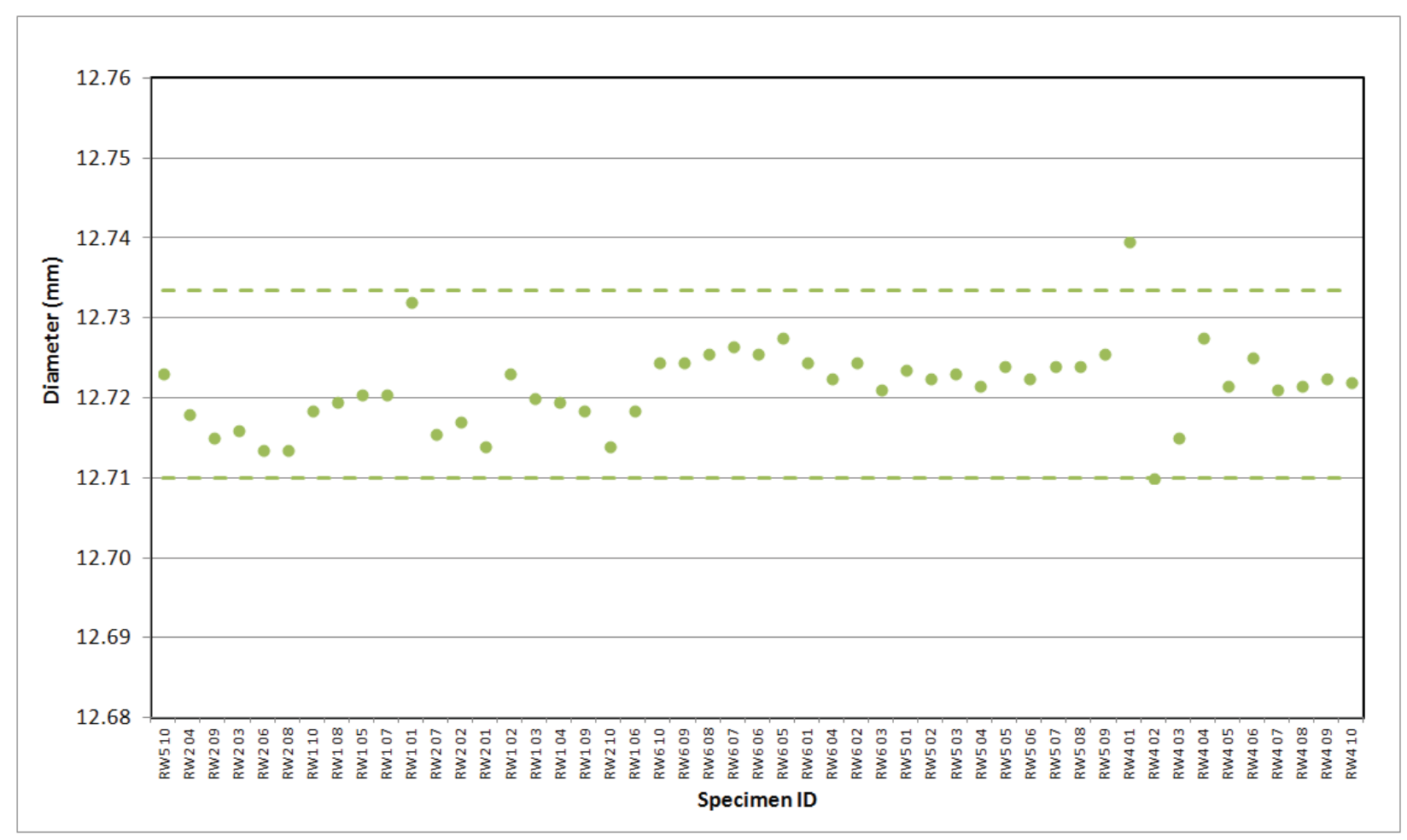

Figure A-53. BAN Piggyback Pre Thermal Measurement Diameter.

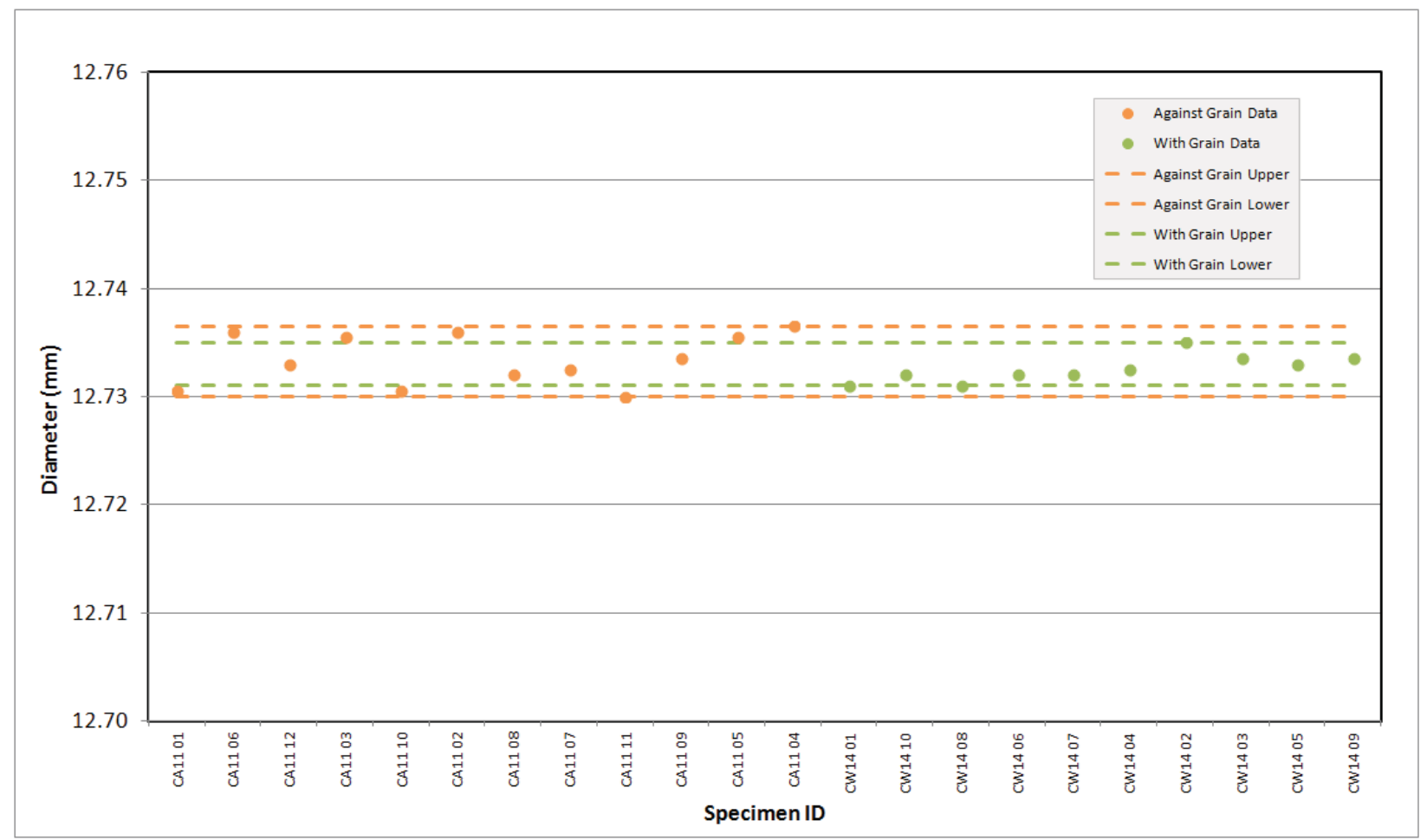

Figure A-54. H-451 Piggyback Pre Thermal Measurement Diameter. 


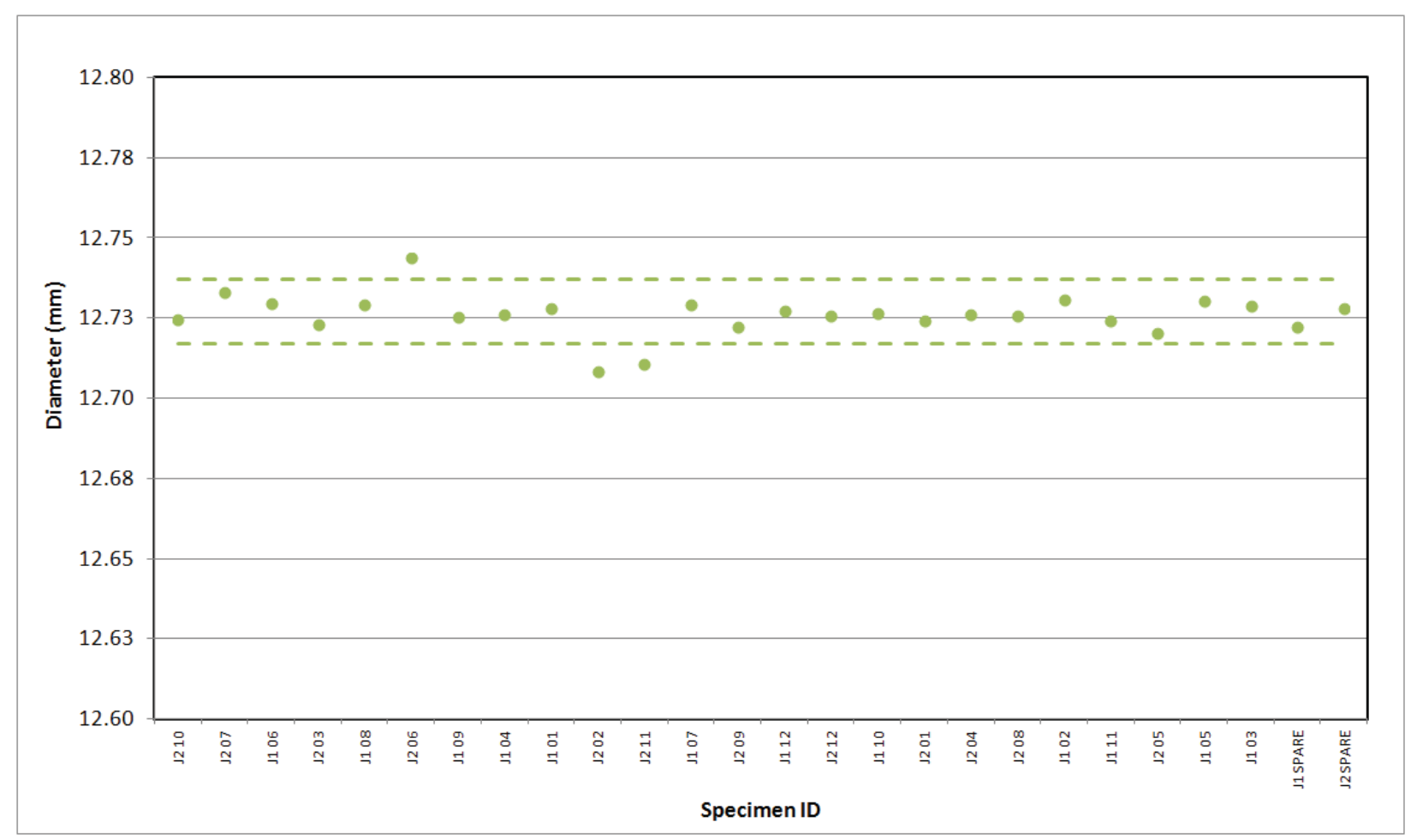

Figure A-55. HLM Piggyback Pre Thermal Measurement Diameter.

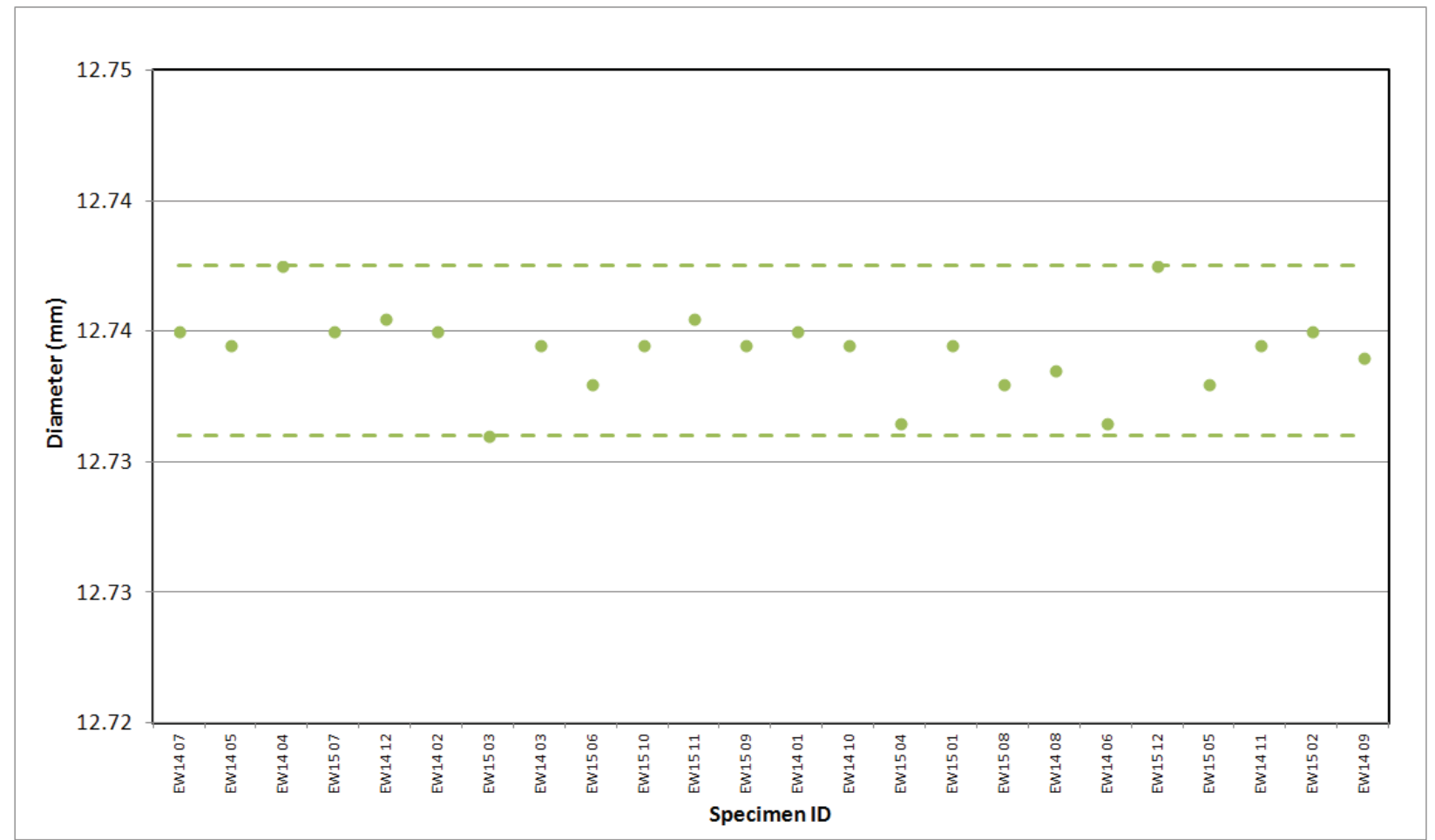

Figure A-56. IG-110 Piggyback Pre Thermal Measurement Diameter. 


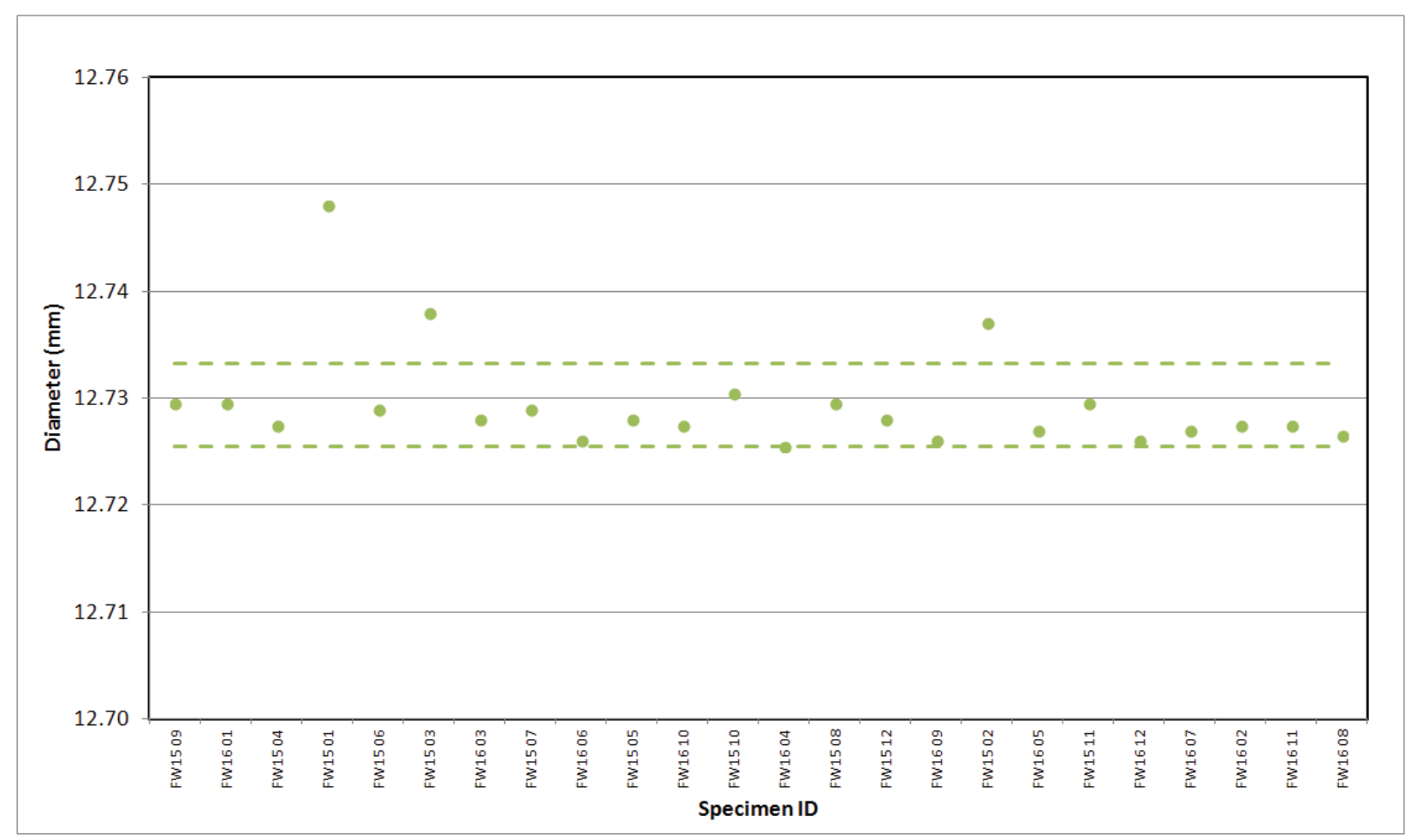

Figure A-57. IG-430 Piggyback Pre Thermal Measurement Diameter.

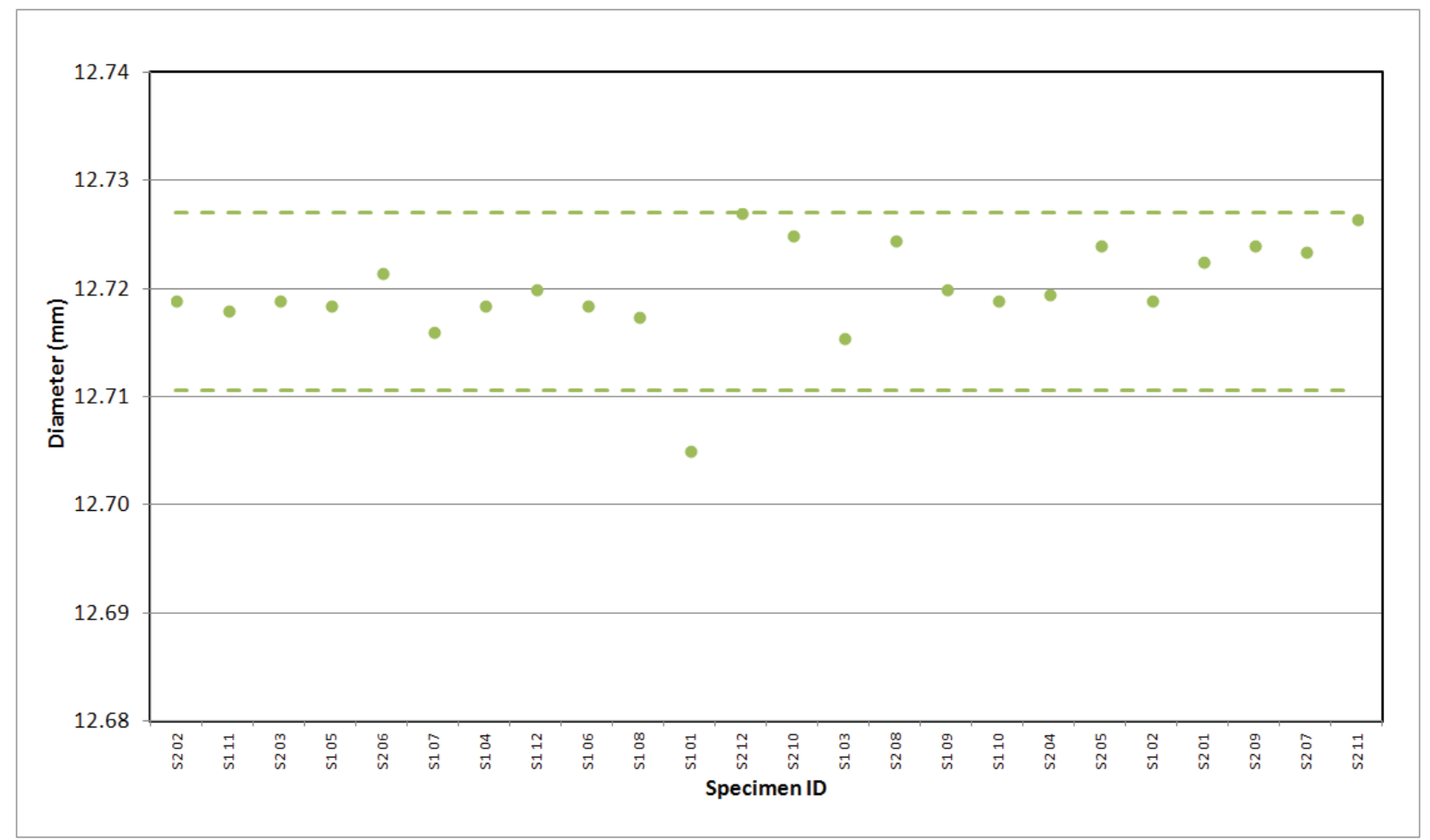

Figure A-58. NBG-10 Piggyback Pre Thermal Measurement Diameter. 


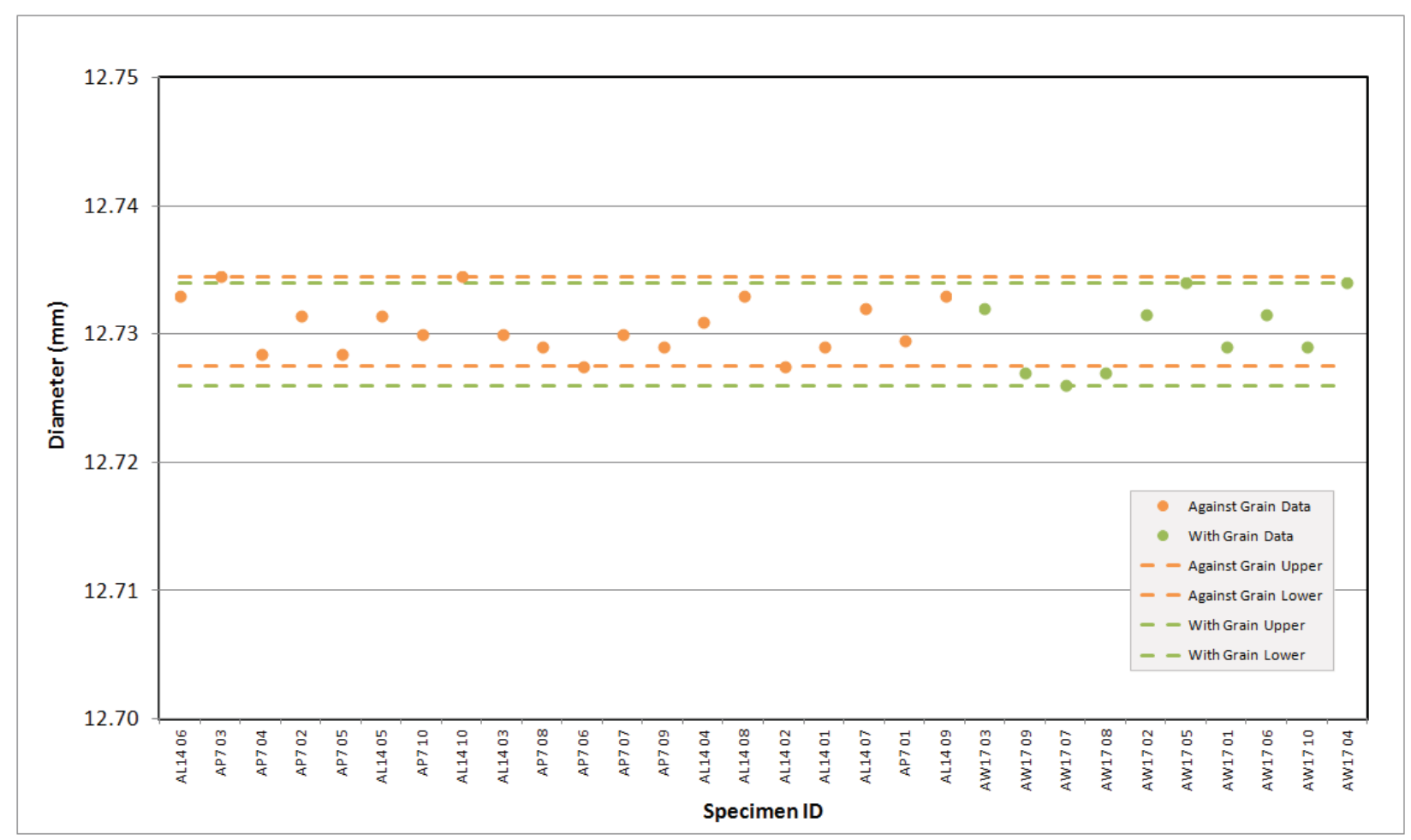

Figure A-59. NBG-17 Piggyback Pre Thermal Measurement Diameter.

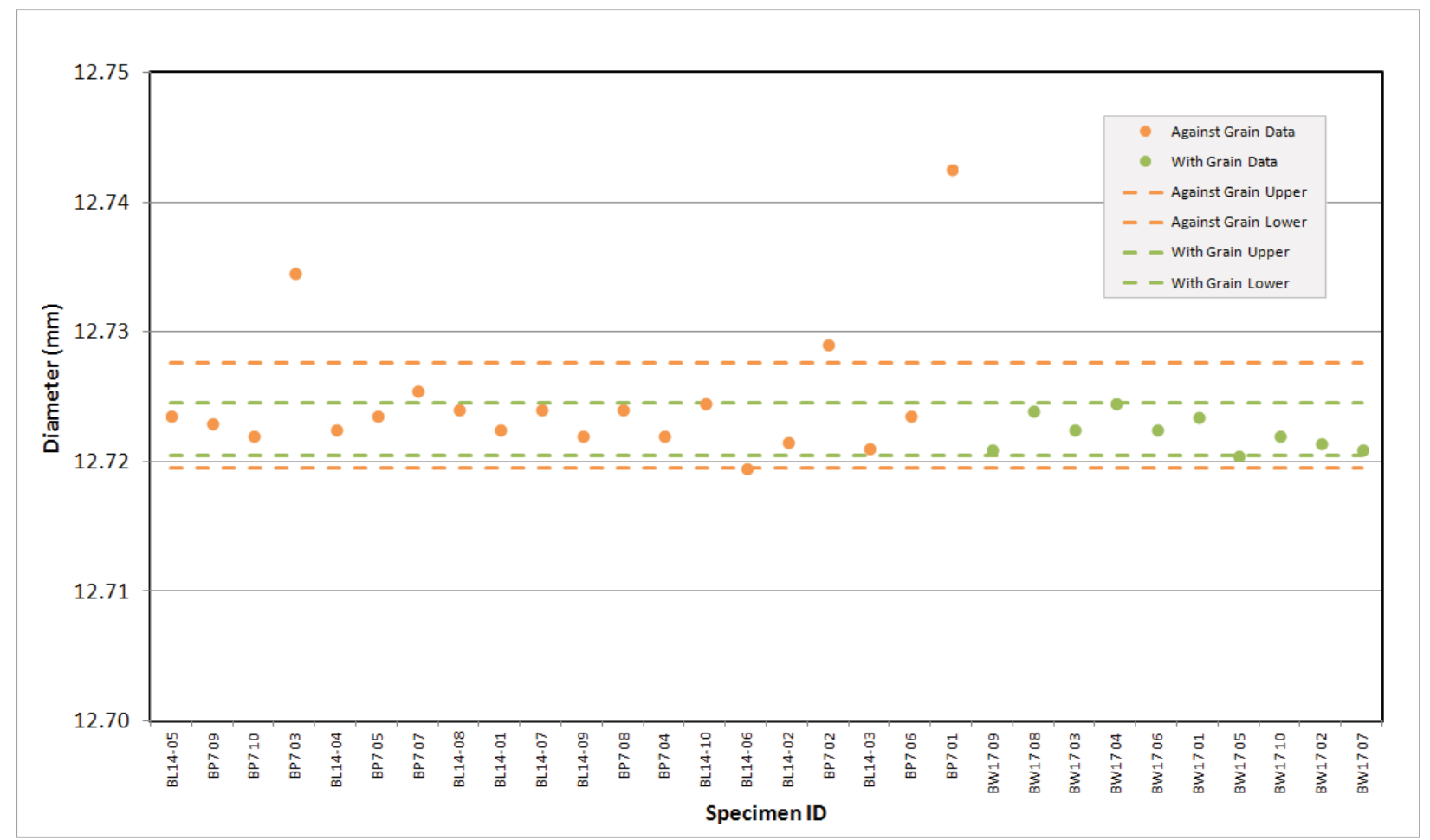

Figure A-60. NBG-18 Piggyback Pre Thermal Measurement Diameter. 


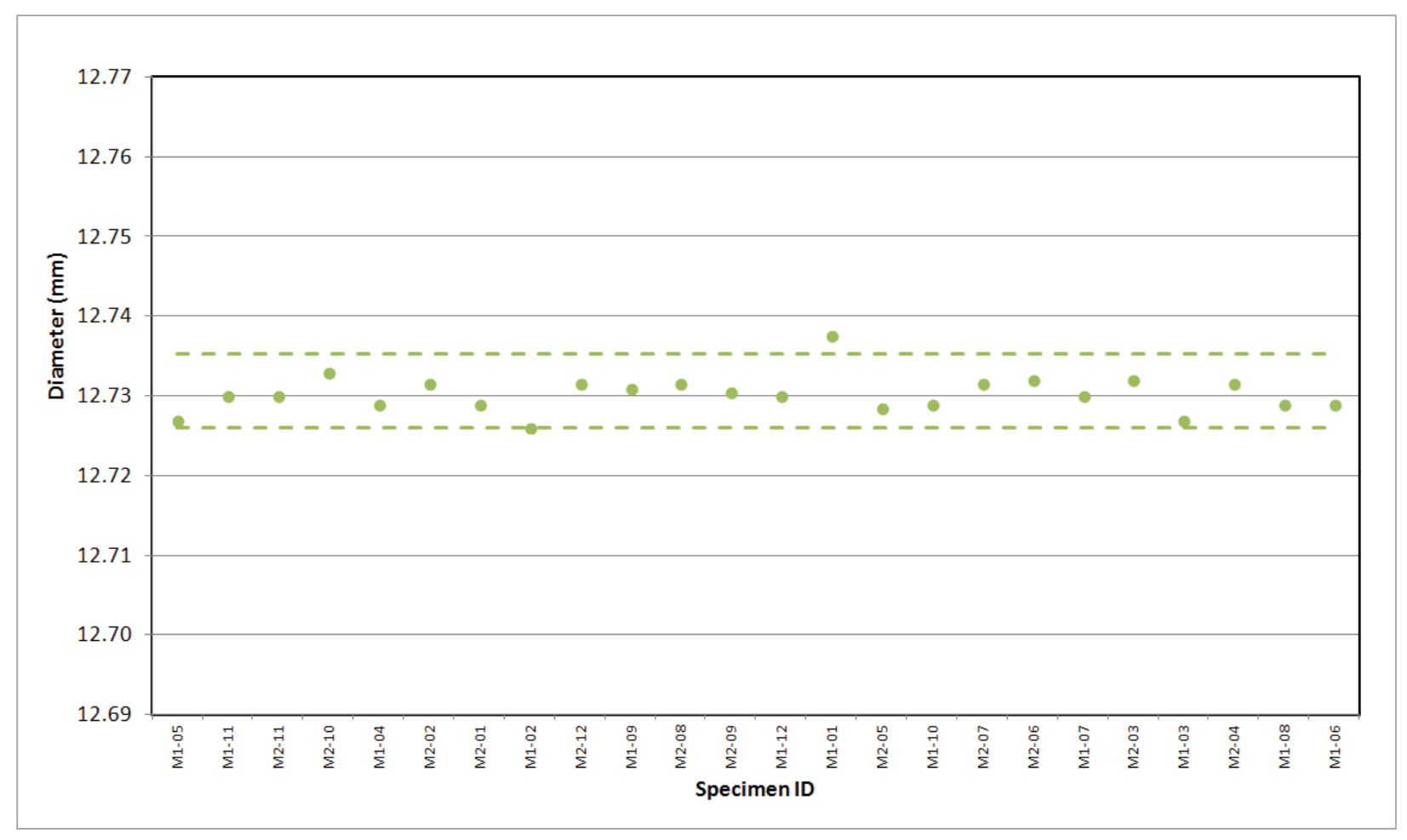

Figure A-61. NBG-25 Piggyback Pre Thermal Measurement Diameter.

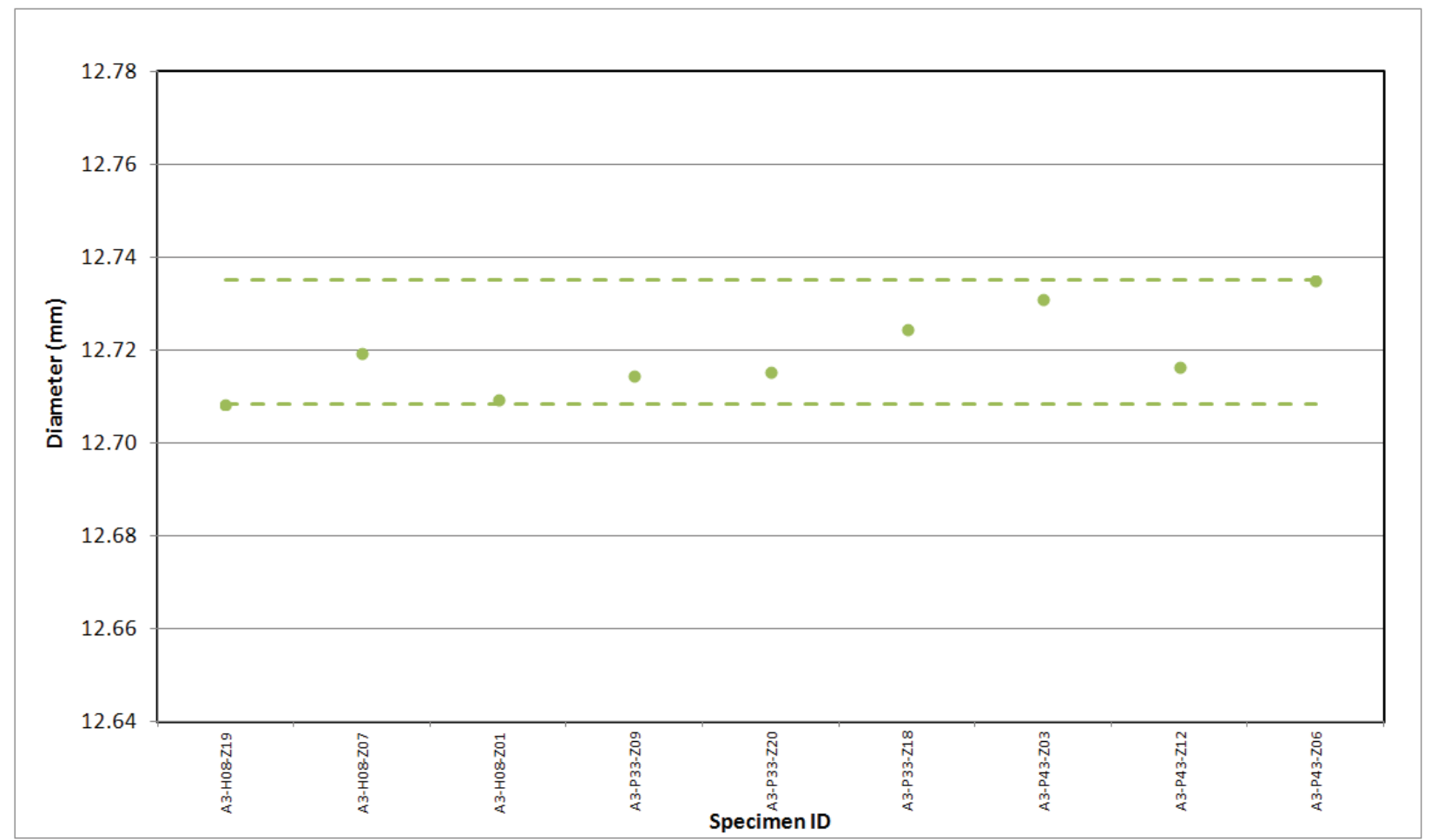

Figure A-62. New Matrix Piggyback Pre Thermal Measurement Diameter. 


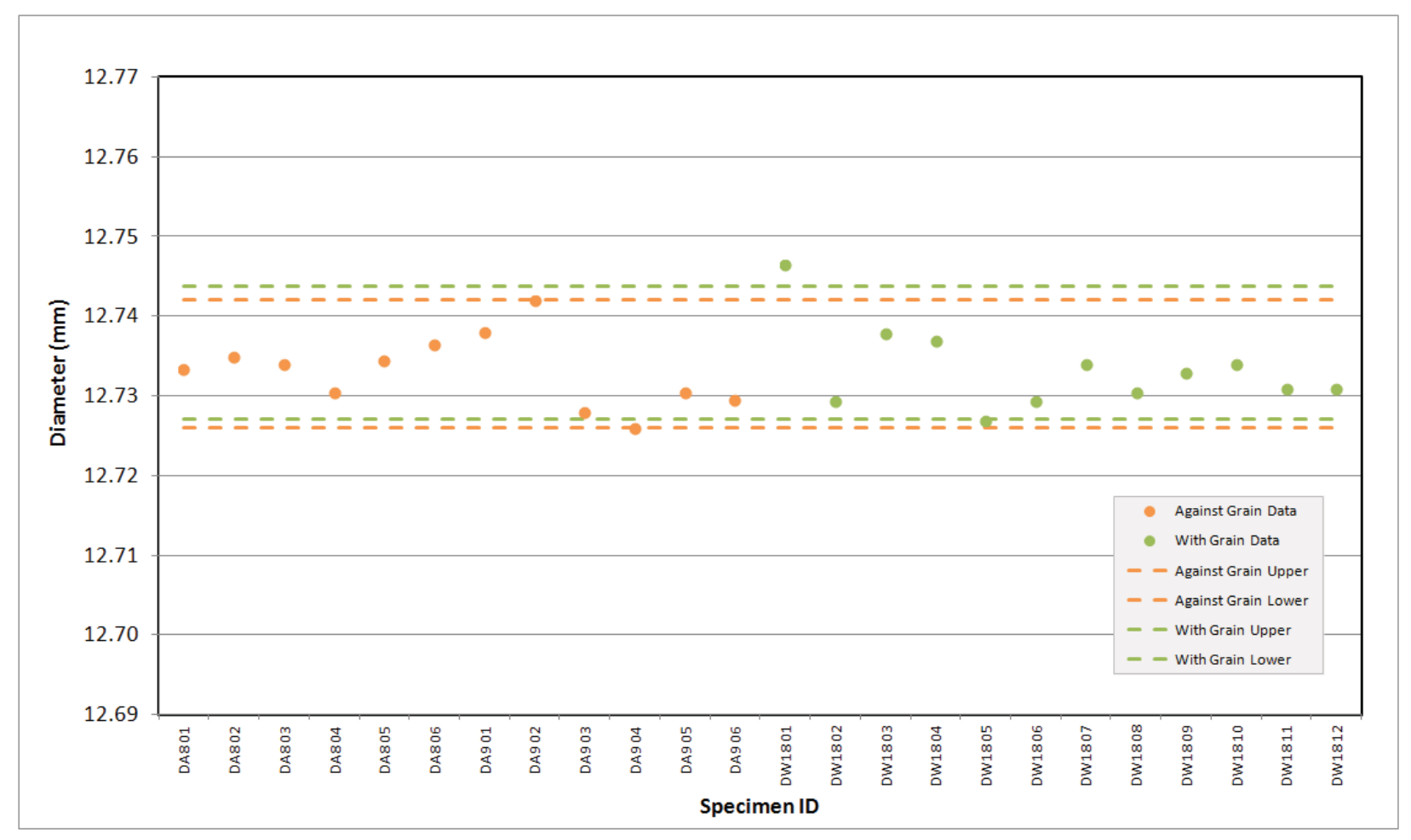

Figure A-63. PCEA Piggyback Pre Thermal Measurement Diameter.

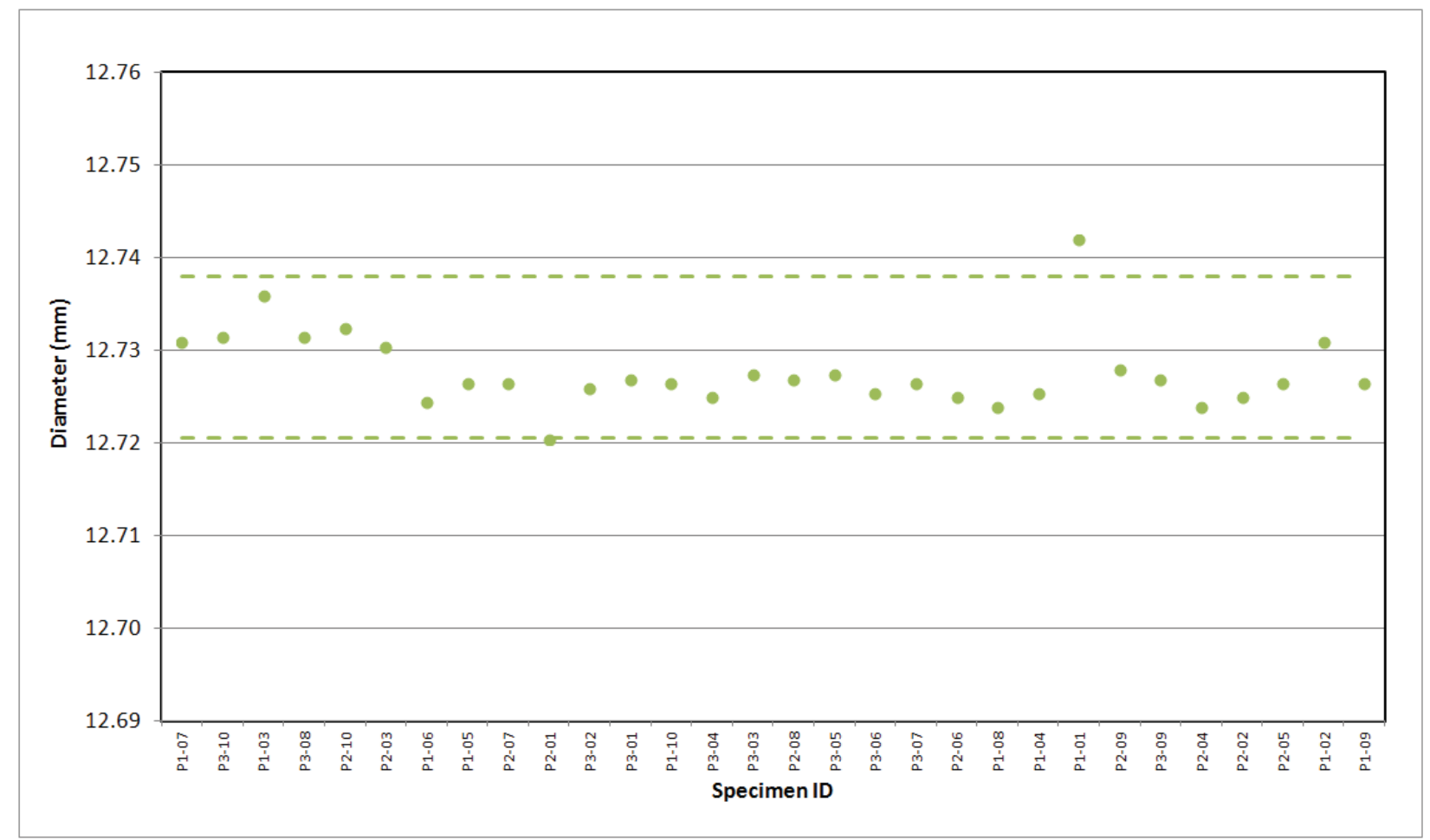

Figure A-64. PCIB Piggyback Pre Thermal Measurement Diameter. 


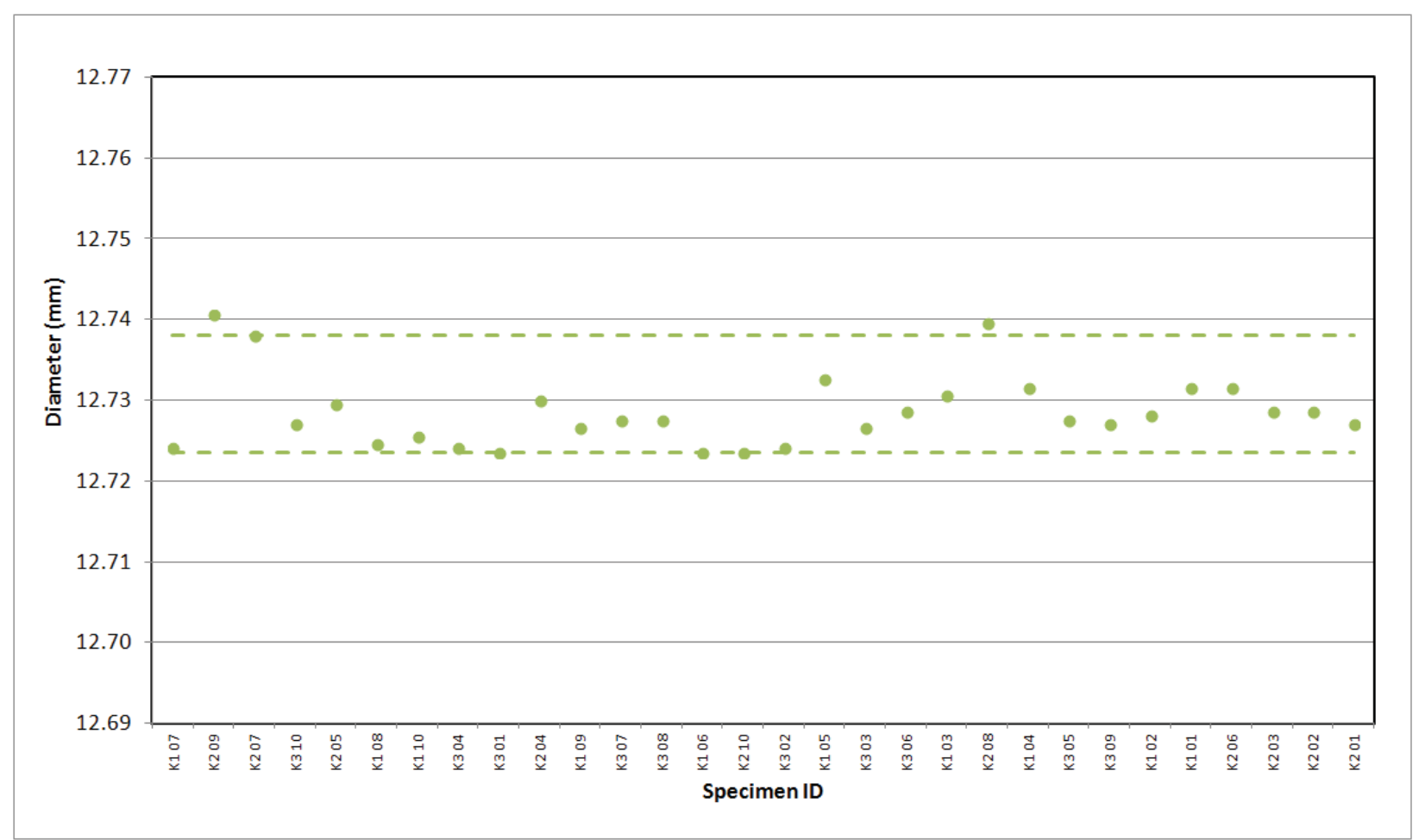

Figure A-65. PGX Piggyback Pre Thermal Measurement Diameter.

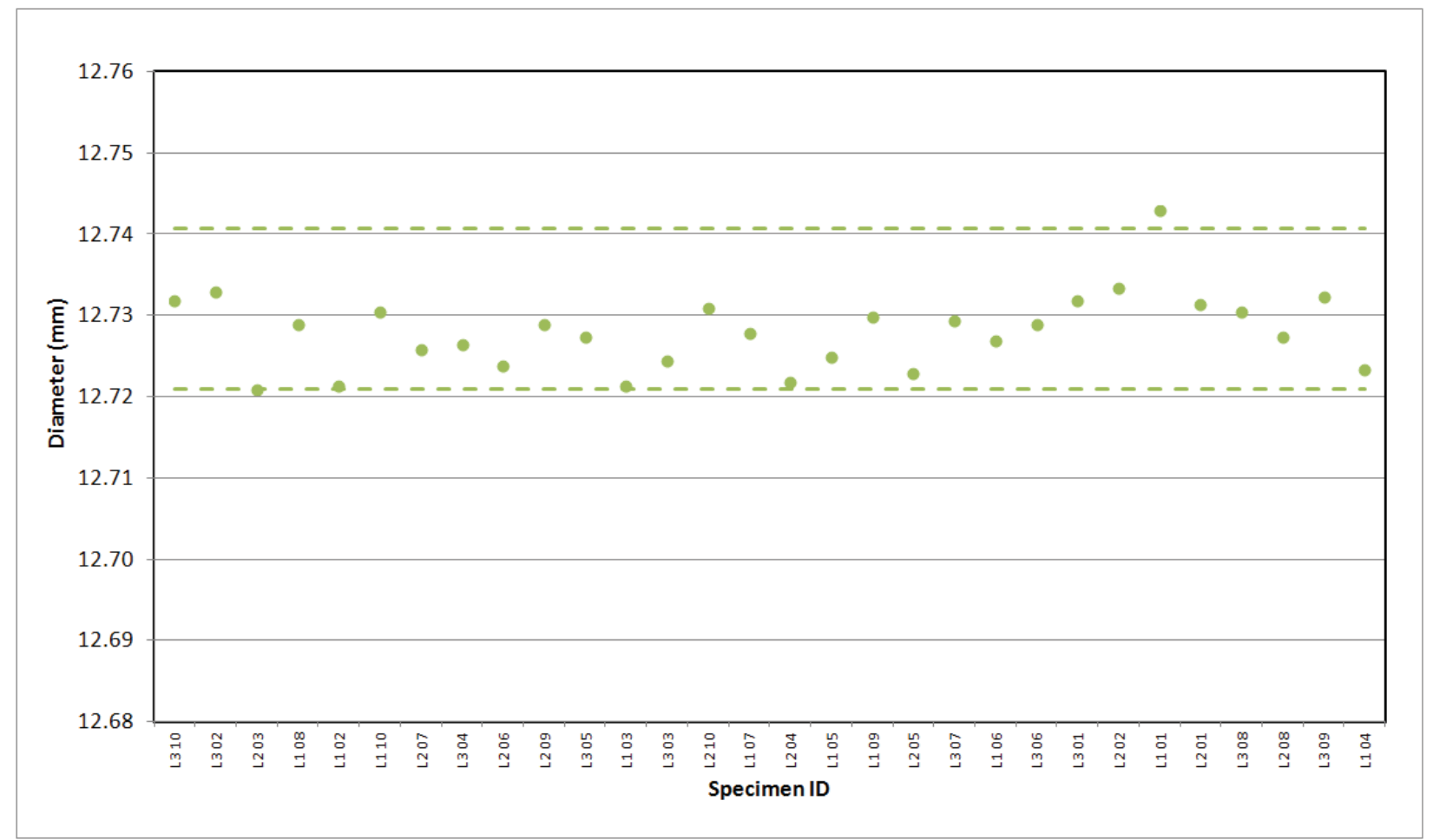

Figure A-66. PPEA Piggyback Pre Thermal Measurement Diameter. 


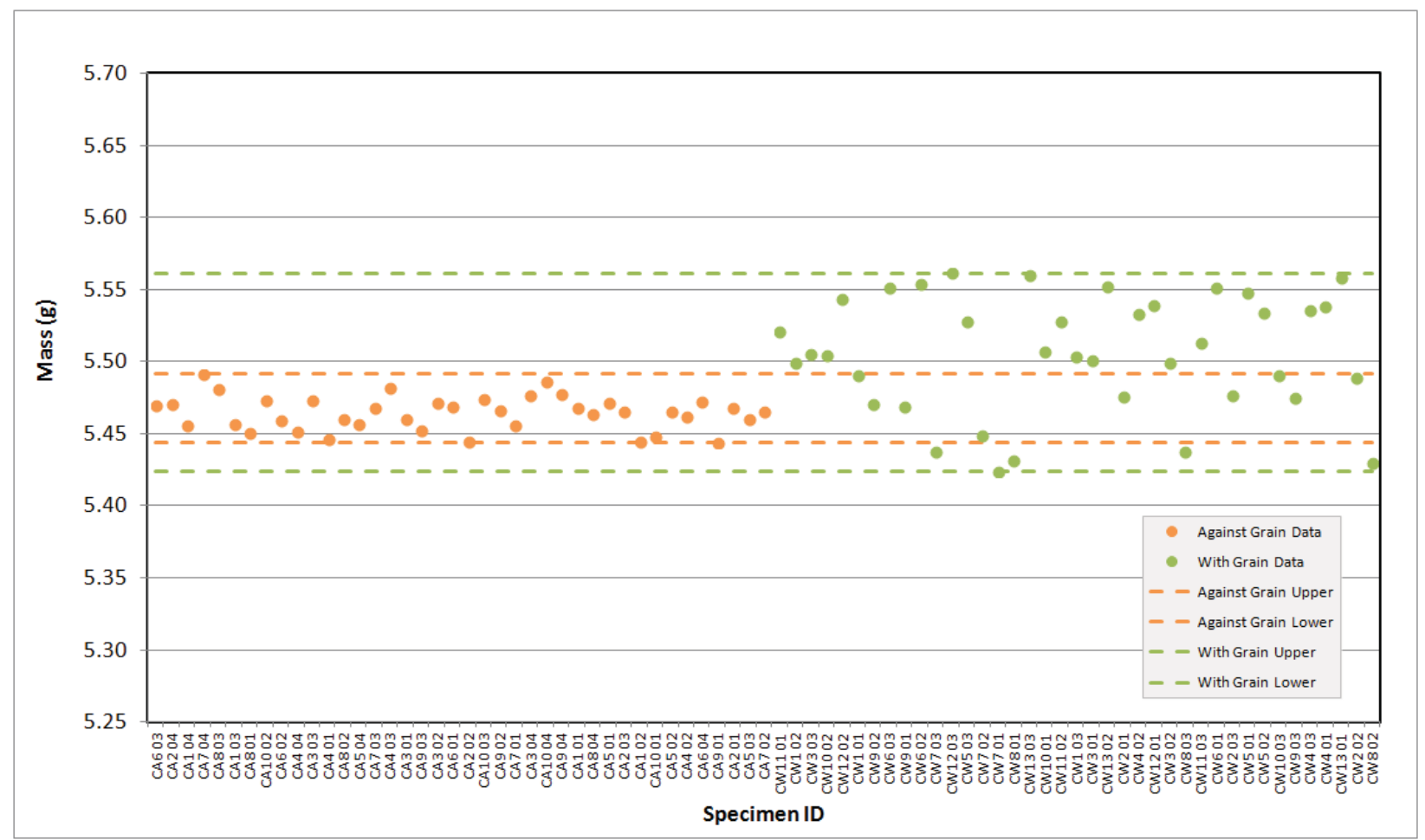

Figure A-67. H-451 Creep Pre Thermal Measurement Mass.

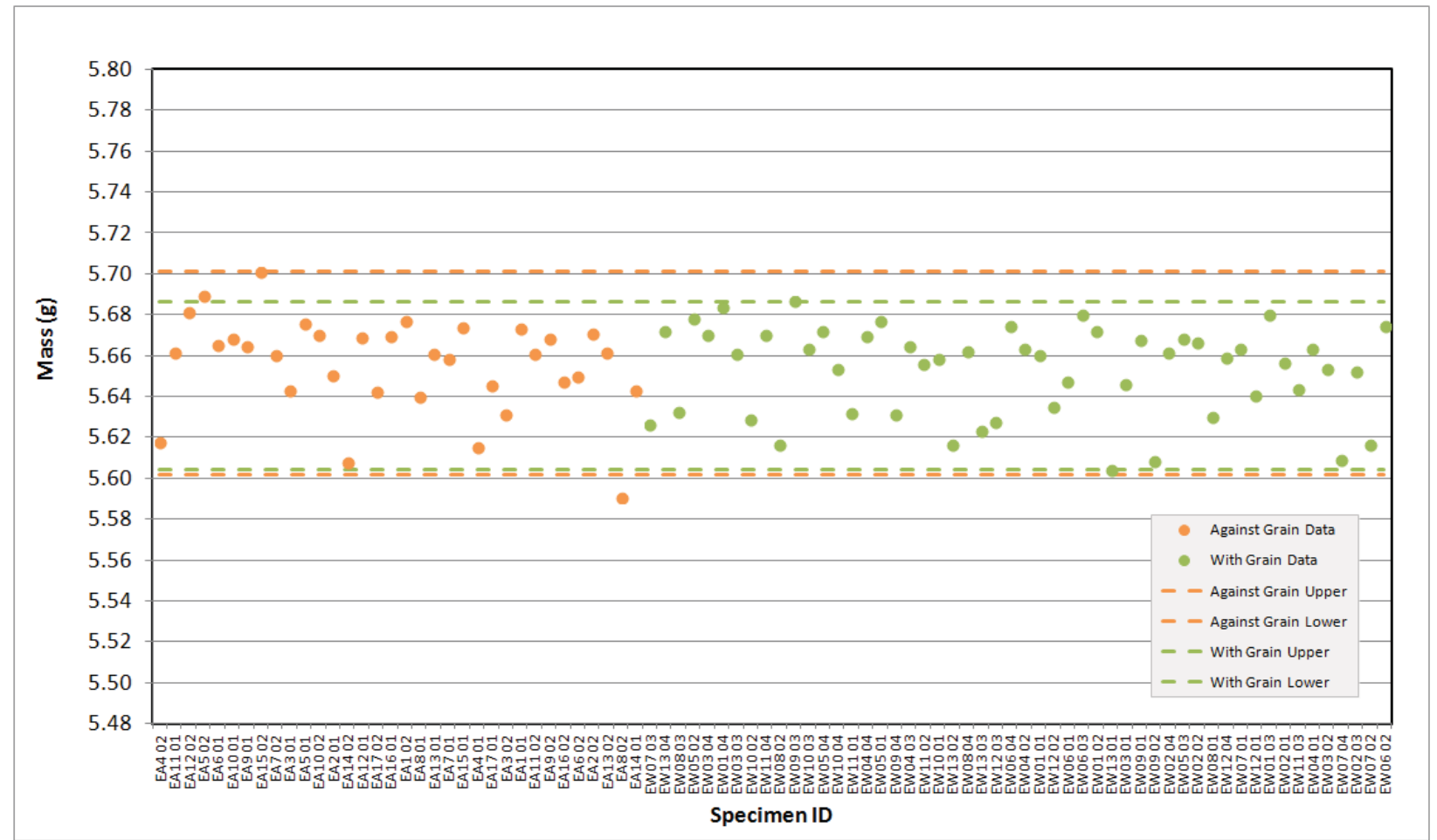

Figure A-68. IG-110 Creep Pre Thermal Measurement Mass. 


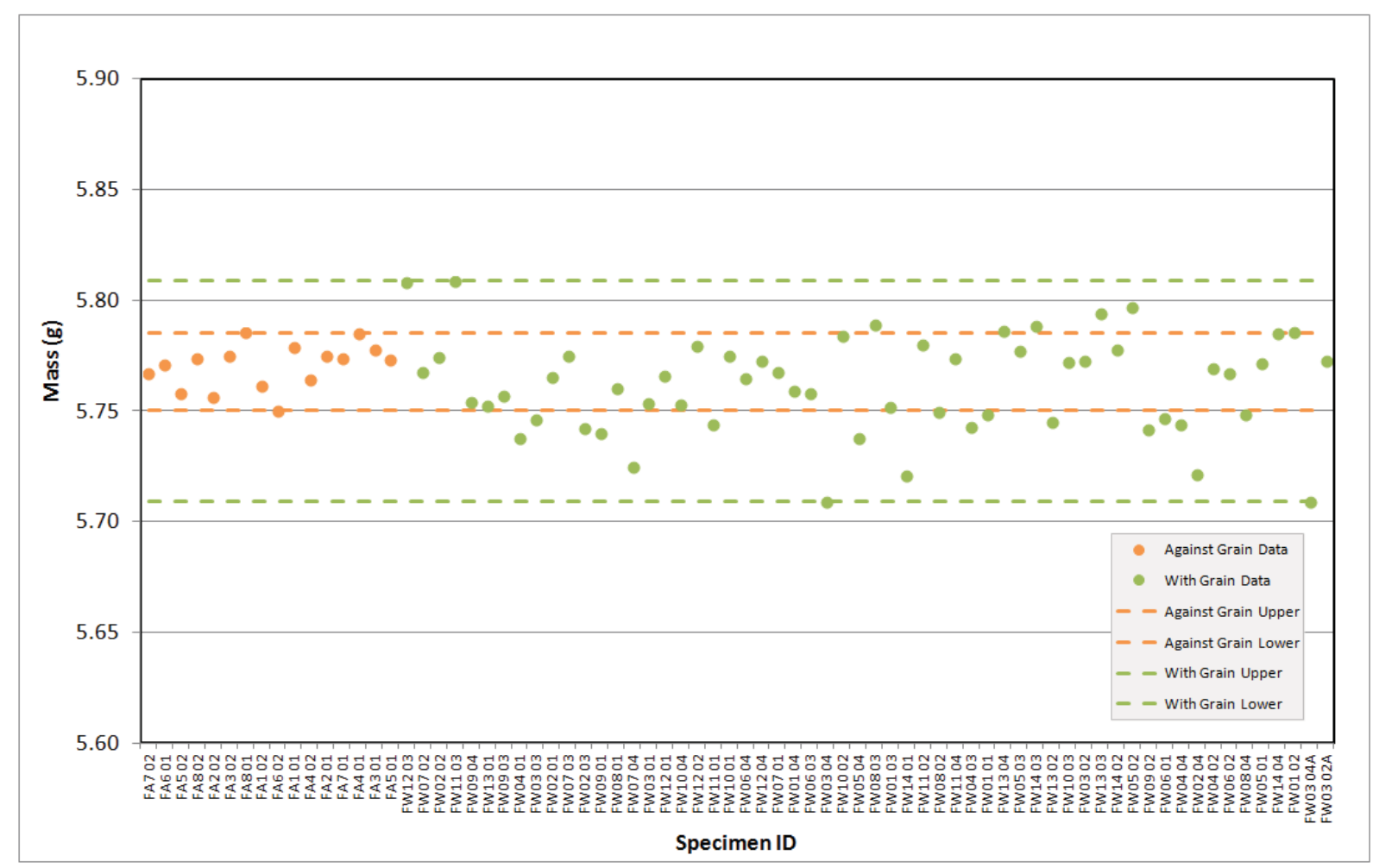

Figure A-69. IG-430 Creep Pre Thermal Measurement Mass.

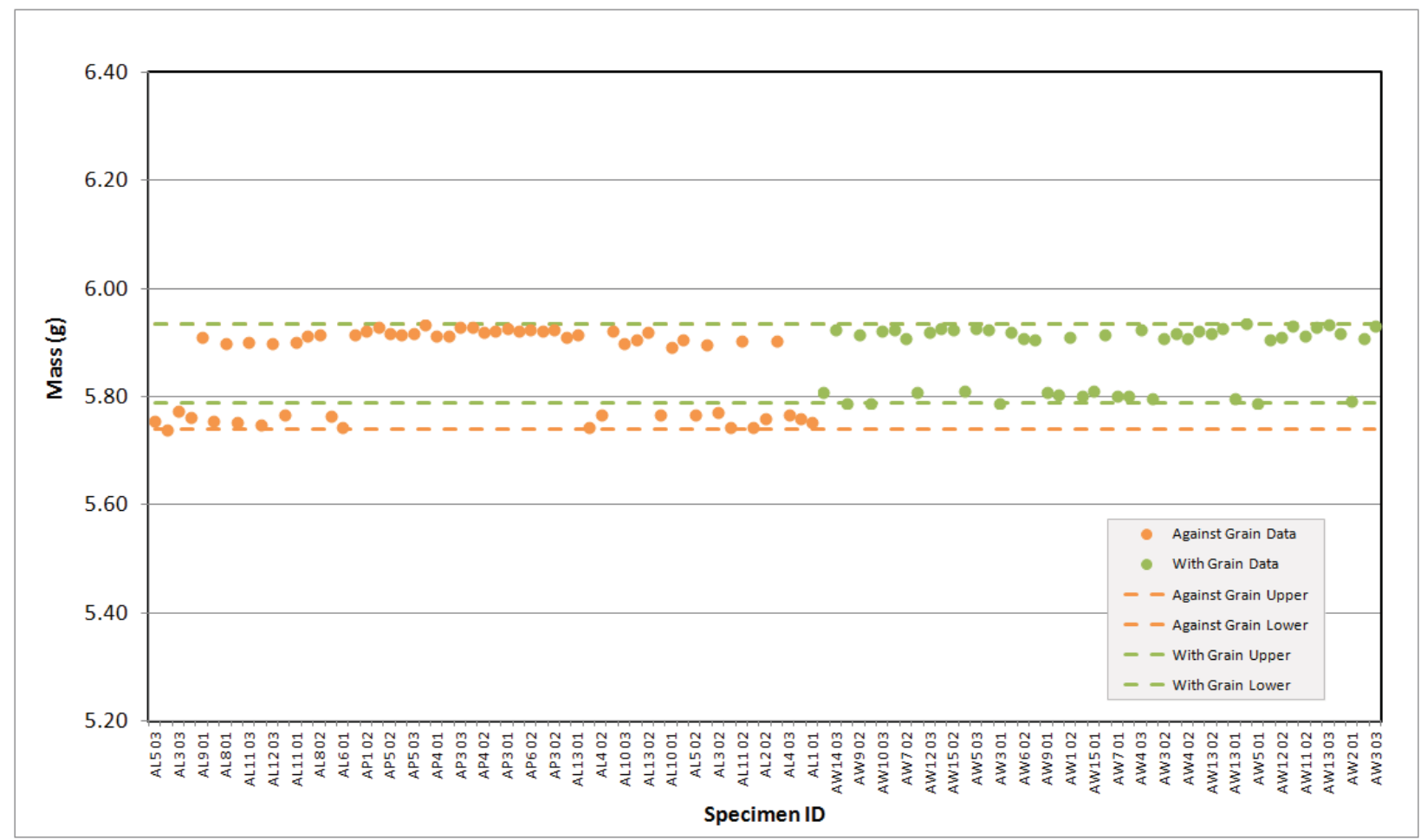

Figure A-70. NBG-17 Creep Pre Thermal Measurement Mass. 


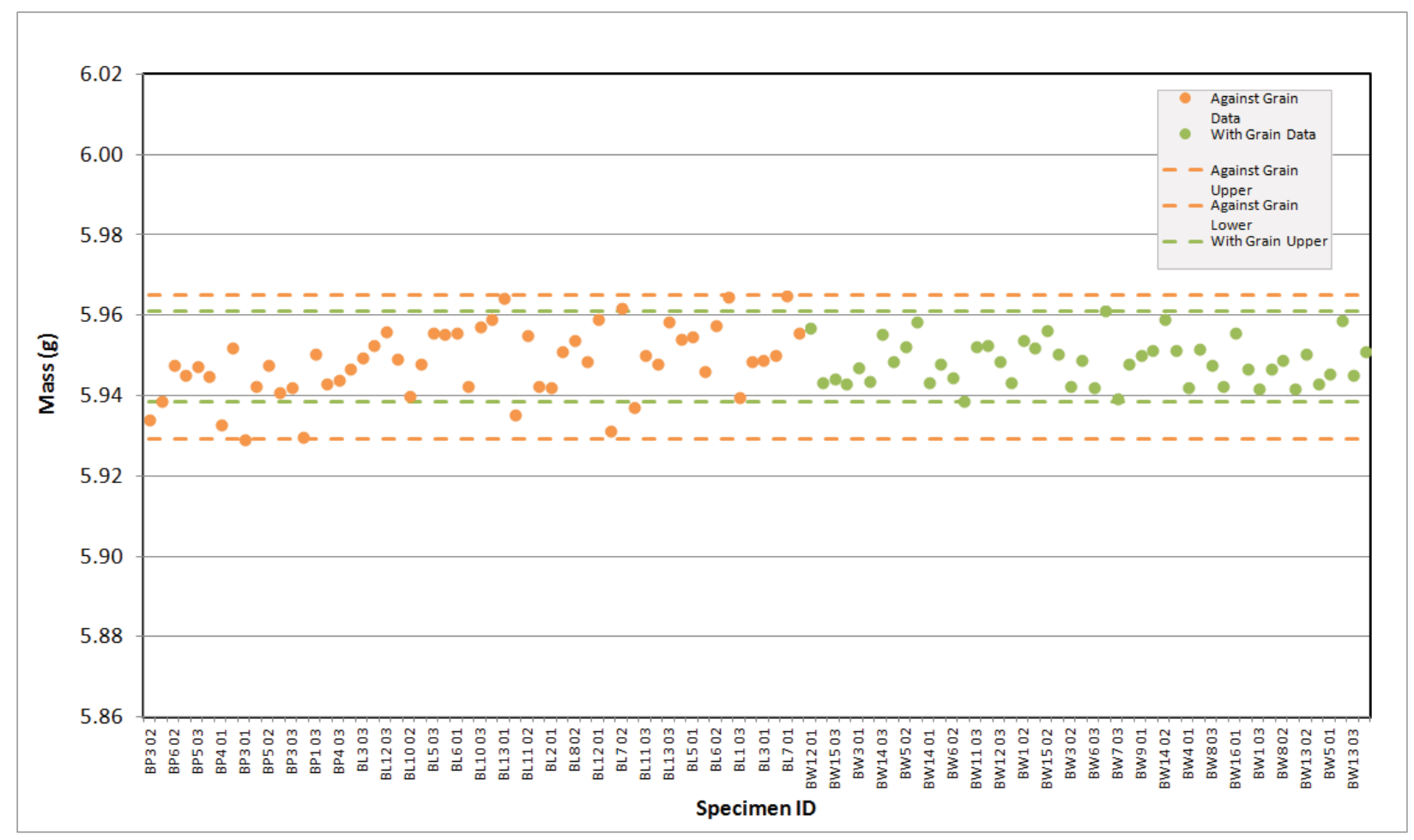

Figure A-71. NBG-18 Creep Pre Thermal Measurement Mass.

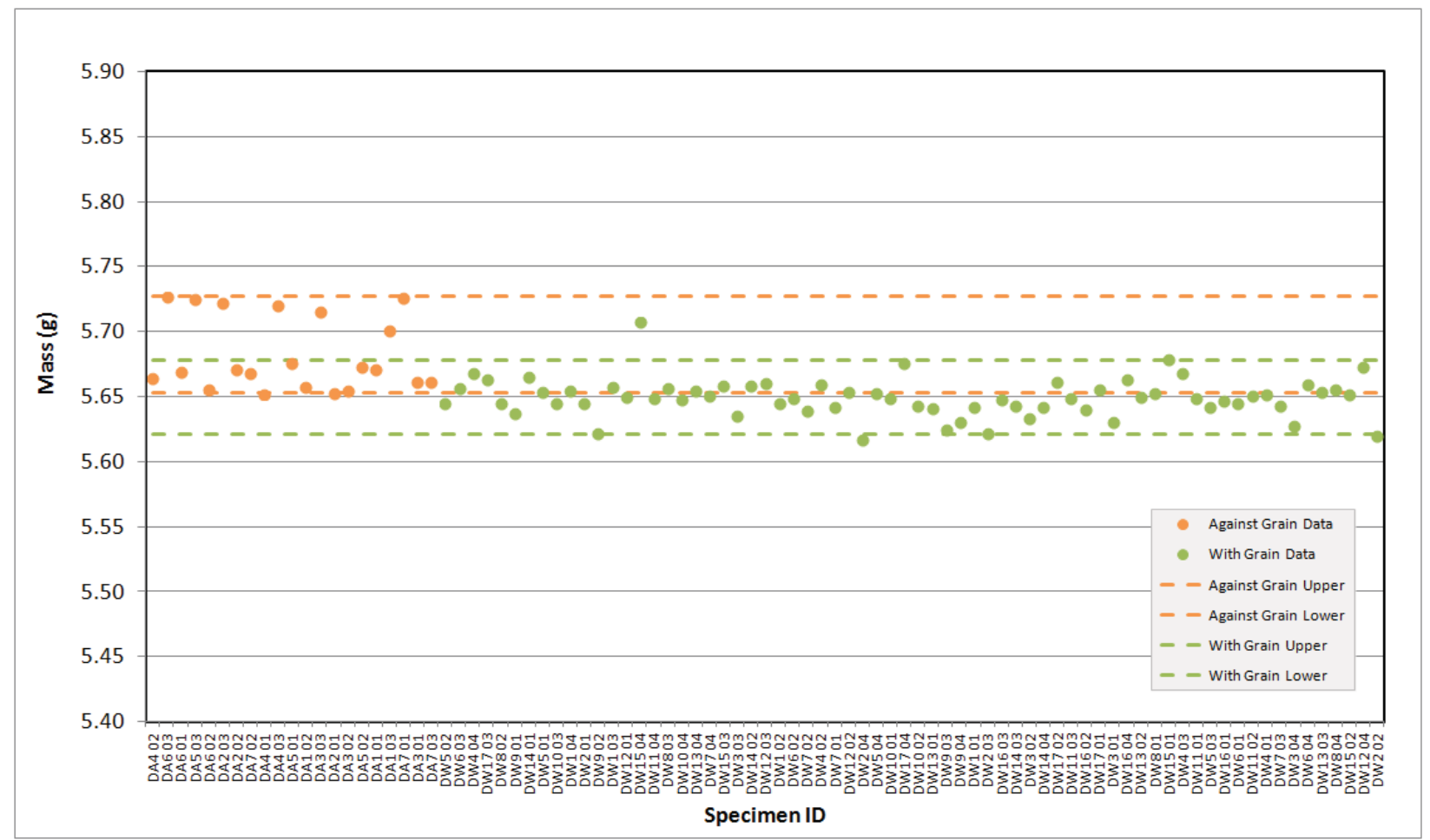

Figure A-72. PCEA Creep Pre Thermal Measurement Mass. 


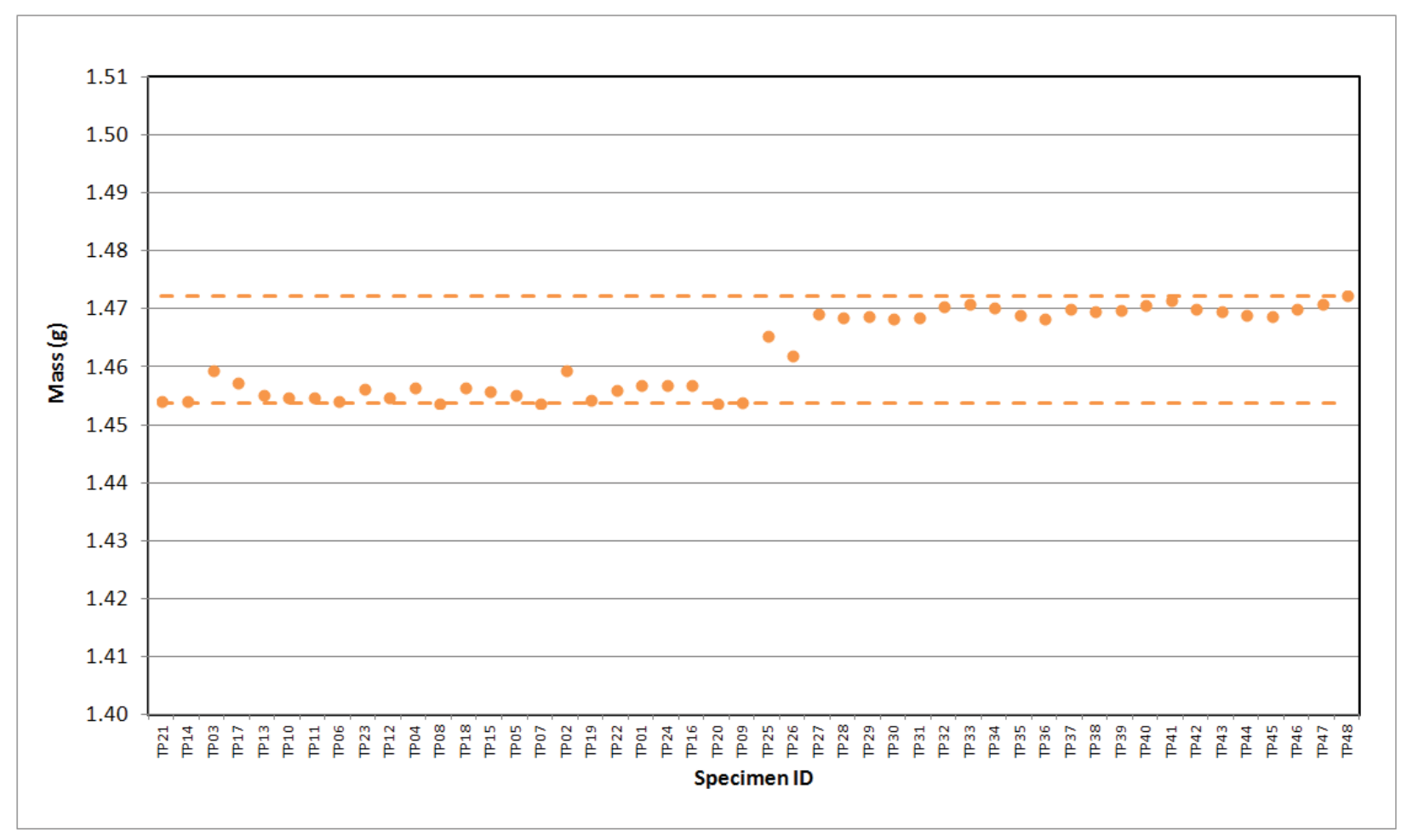

Figure A-73. 2114 Piggyback Pre Thermal Measurement Mass.

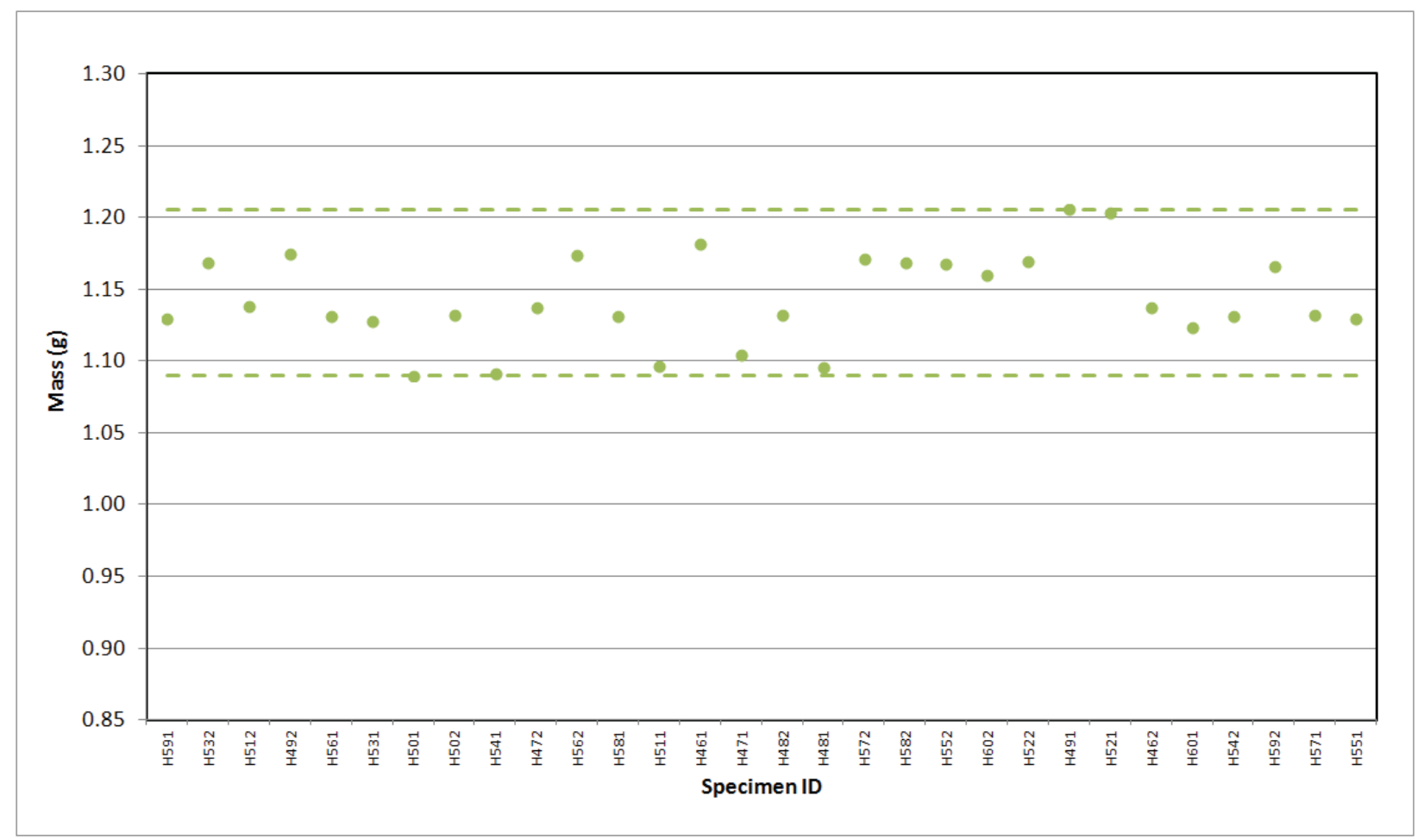

Figure A-74. A3 Matrix Piggyback Pre Thermal Measurement Mass. 


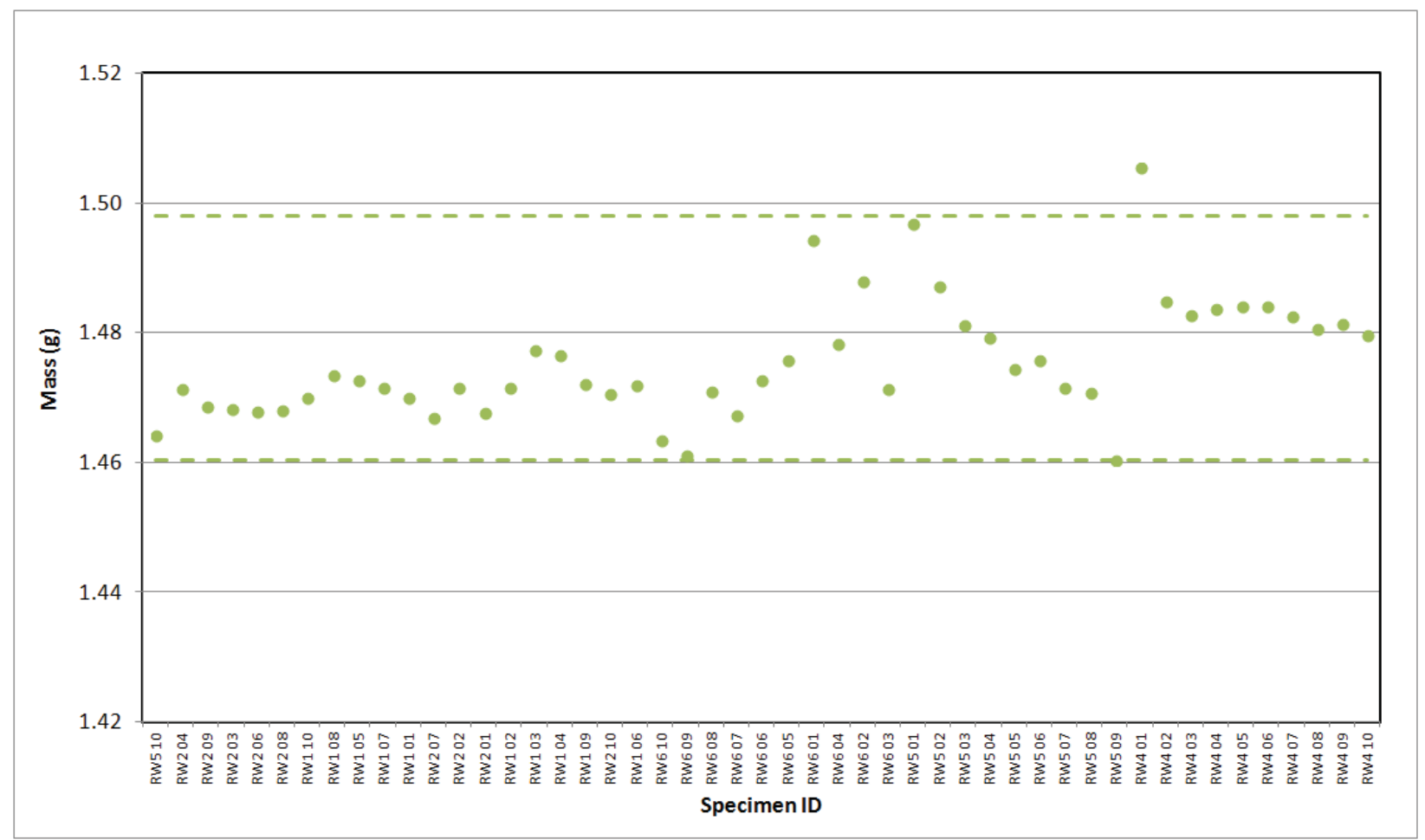

Figure A-75. BAN Piggyback Pre Thermal Measurement Mass.

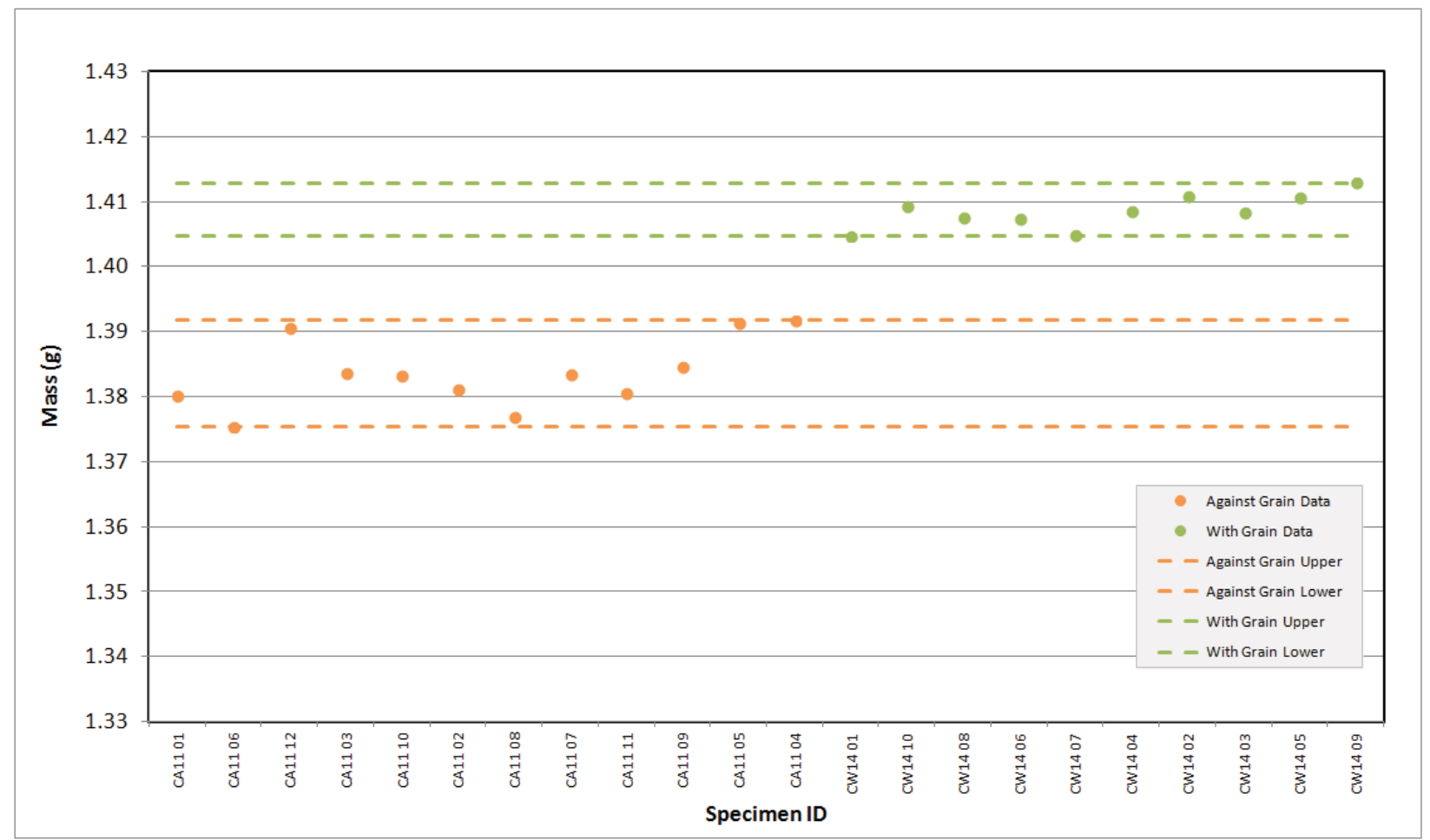

Figure A-76. H-451 Piggyback Pre Thermal Measurement Mass. 


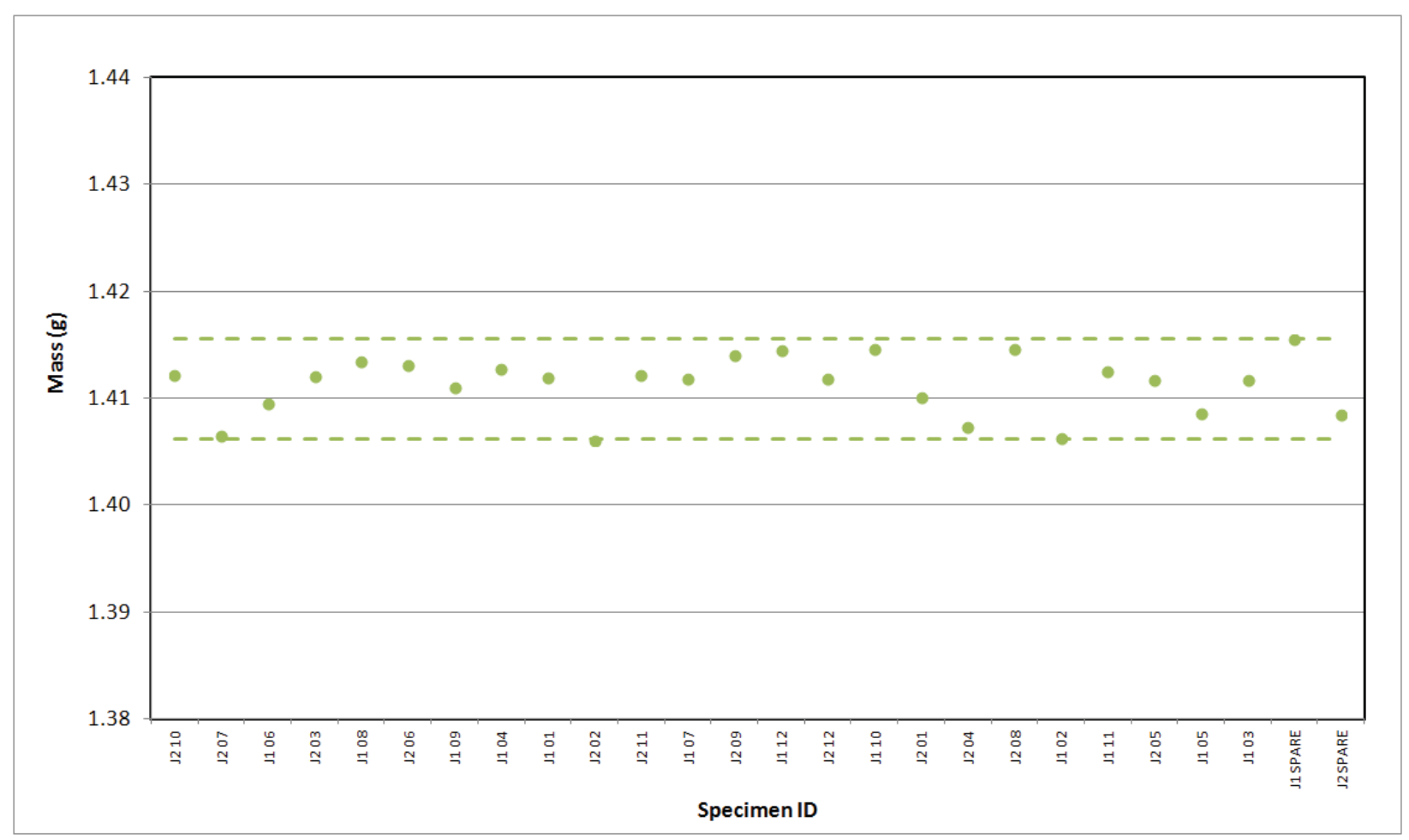

Figure A-77. HLM Piggyback Pre Thermal Measurement Mass.

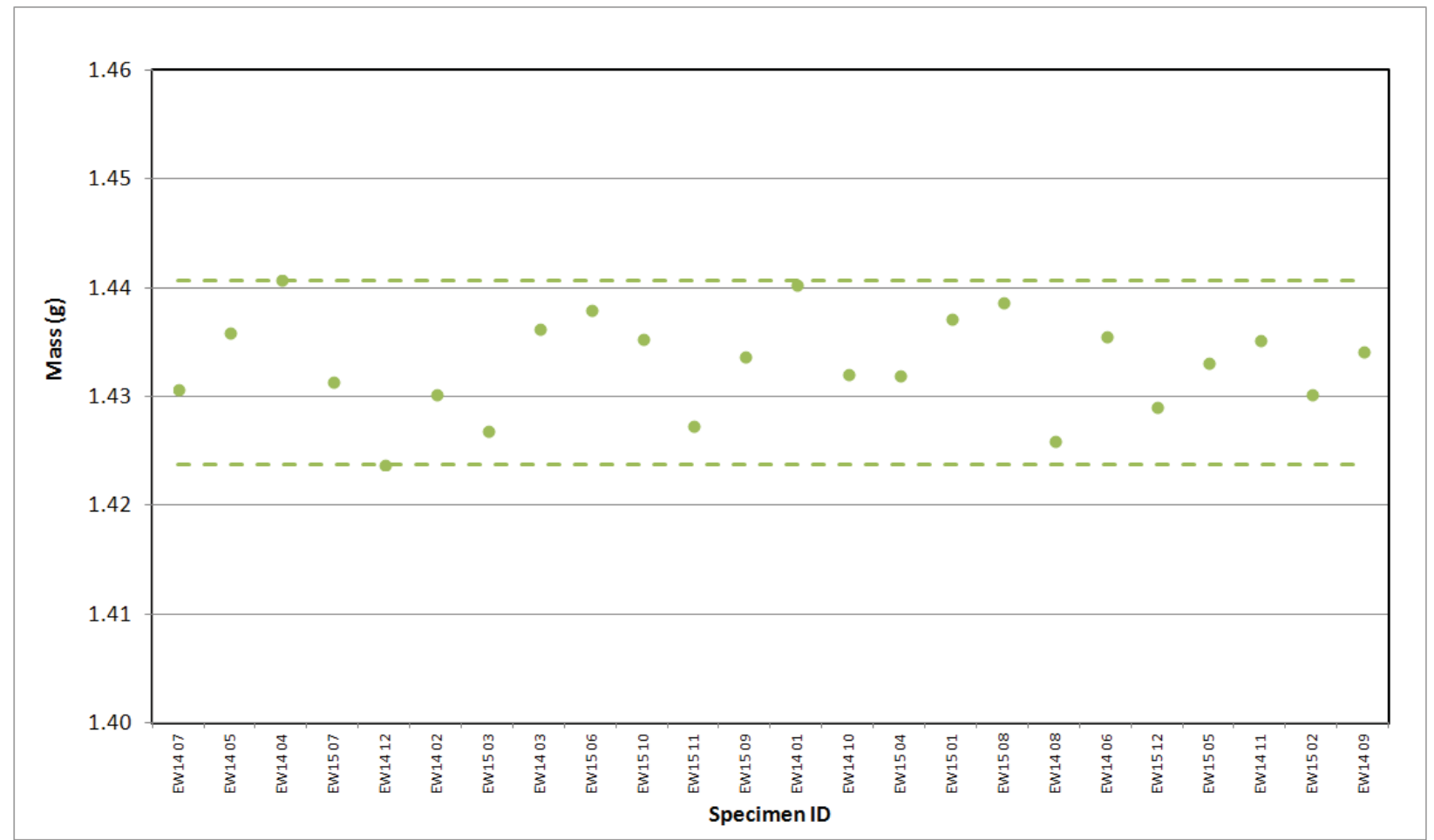

Figure A-78. IG-110 Piggyback Pre Thermal Measurement Mass. 


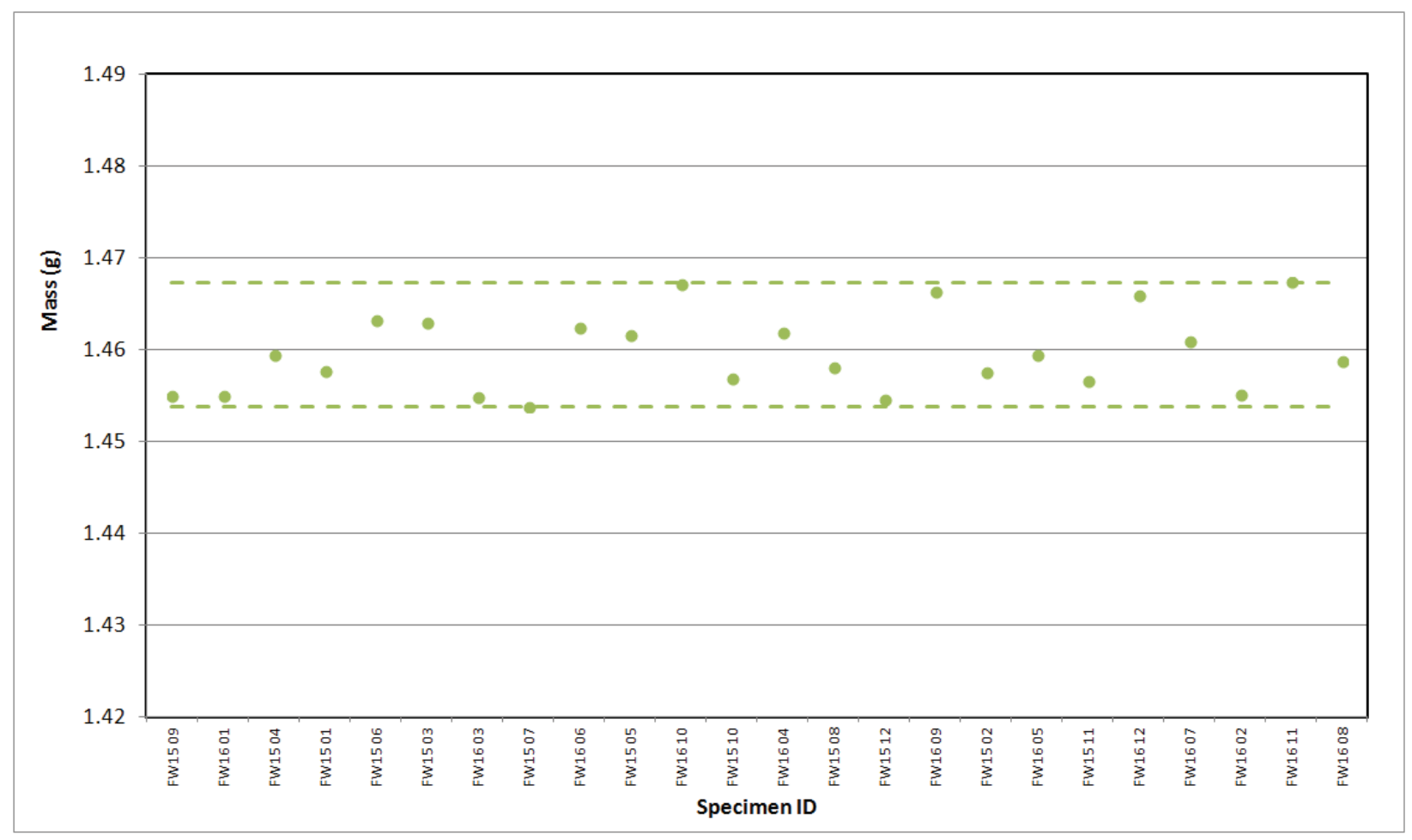

Figure A-79. IG-430 Piggyback Pre Thermal Measurement Mass.

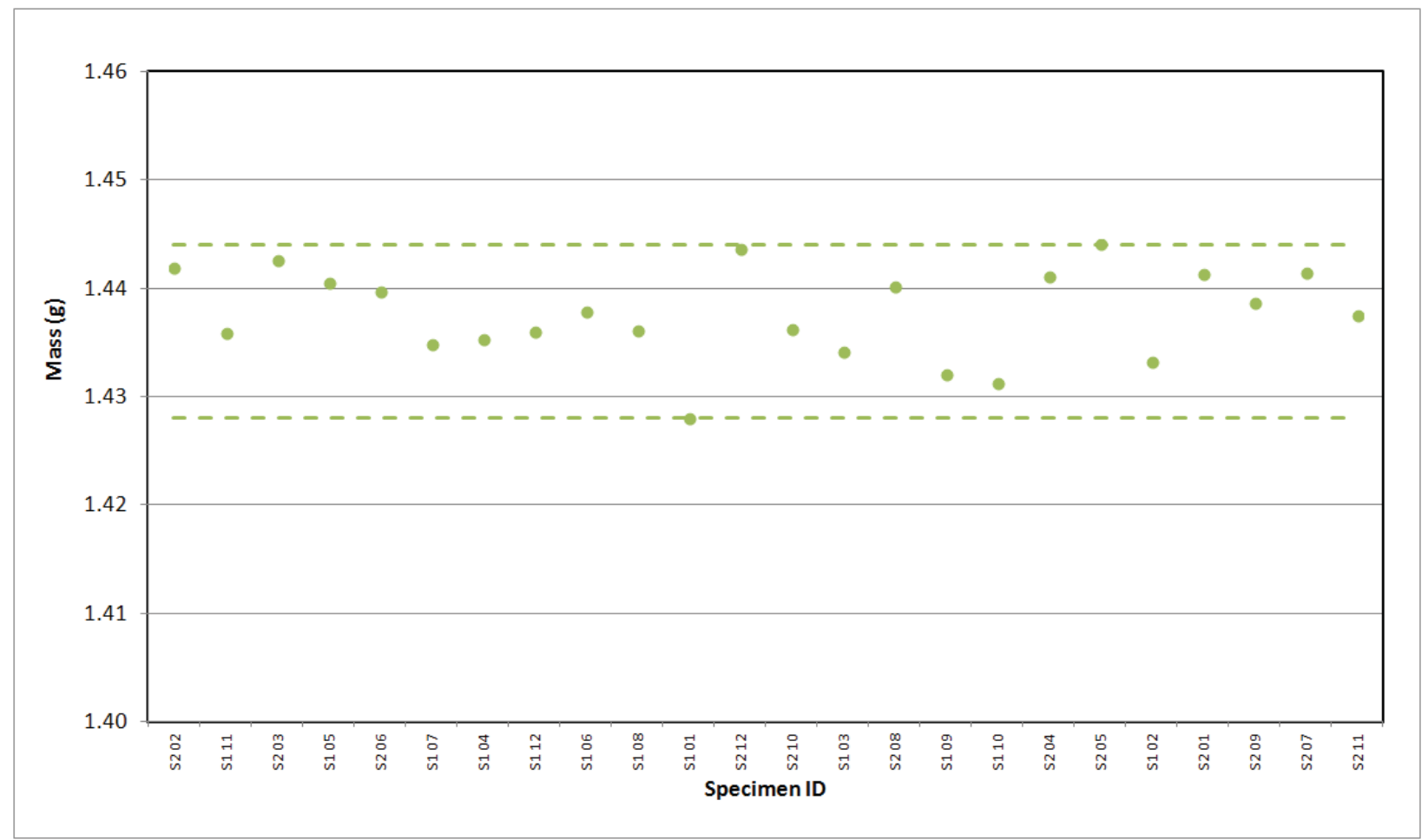

Figure A-80. NBG-10 Piggyback Pre Thermal Measurement Mass. 


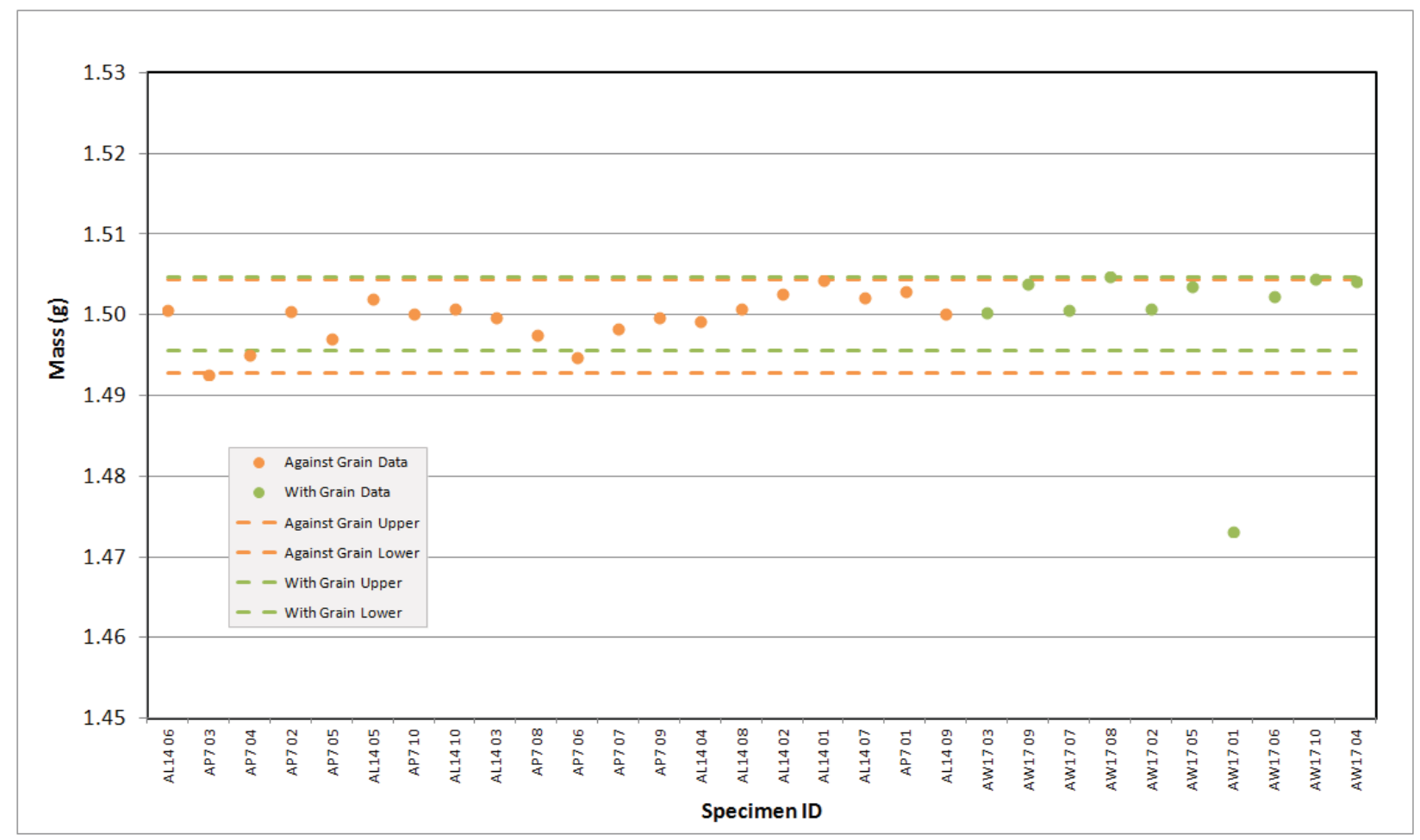

Figure A-81. NBG-17 Piggyback Pre Thermal Measurement Mass.

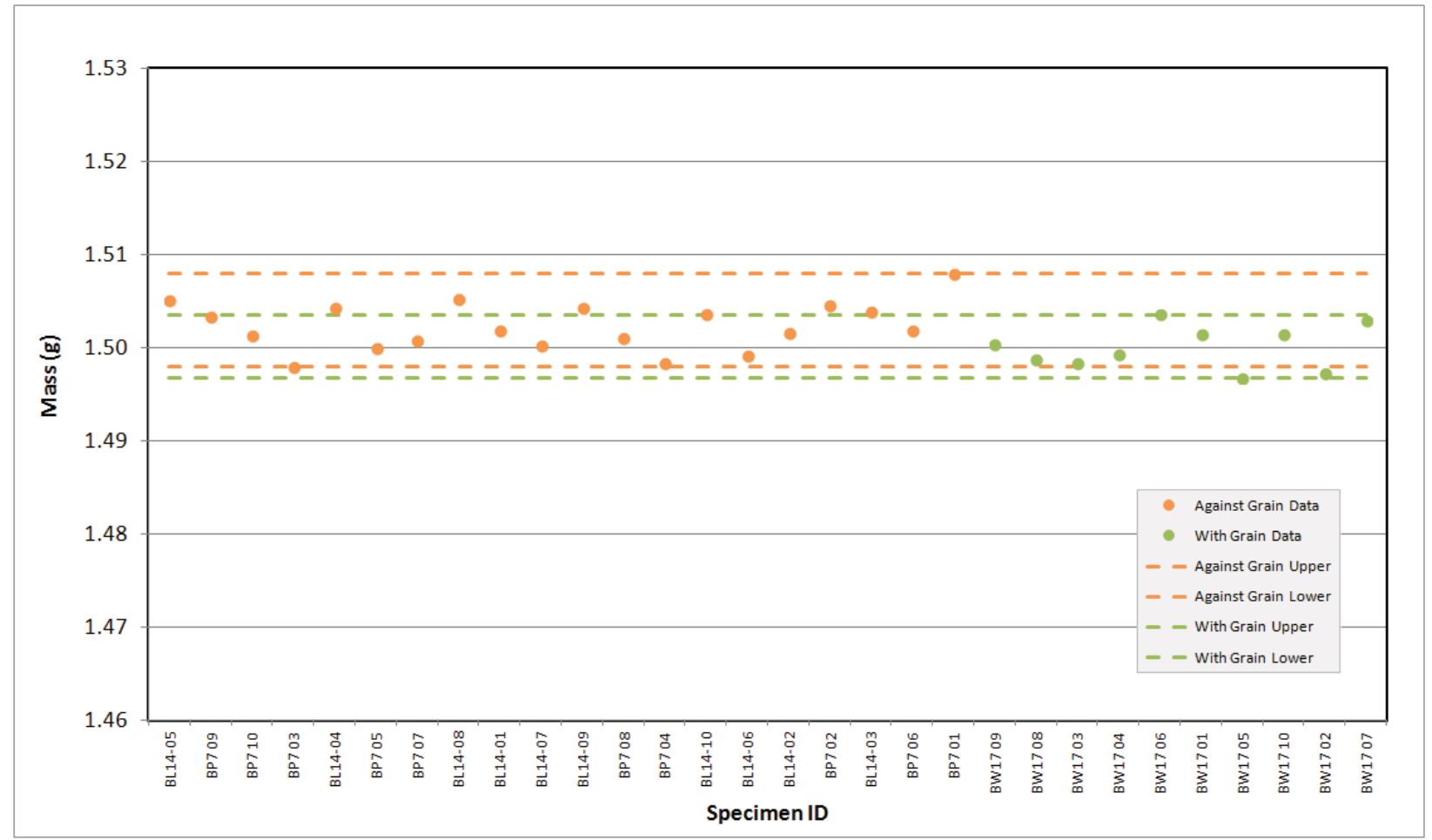

Figure A-82. NBG-18 Piggyback Pre Thermal Measurement Mass. 


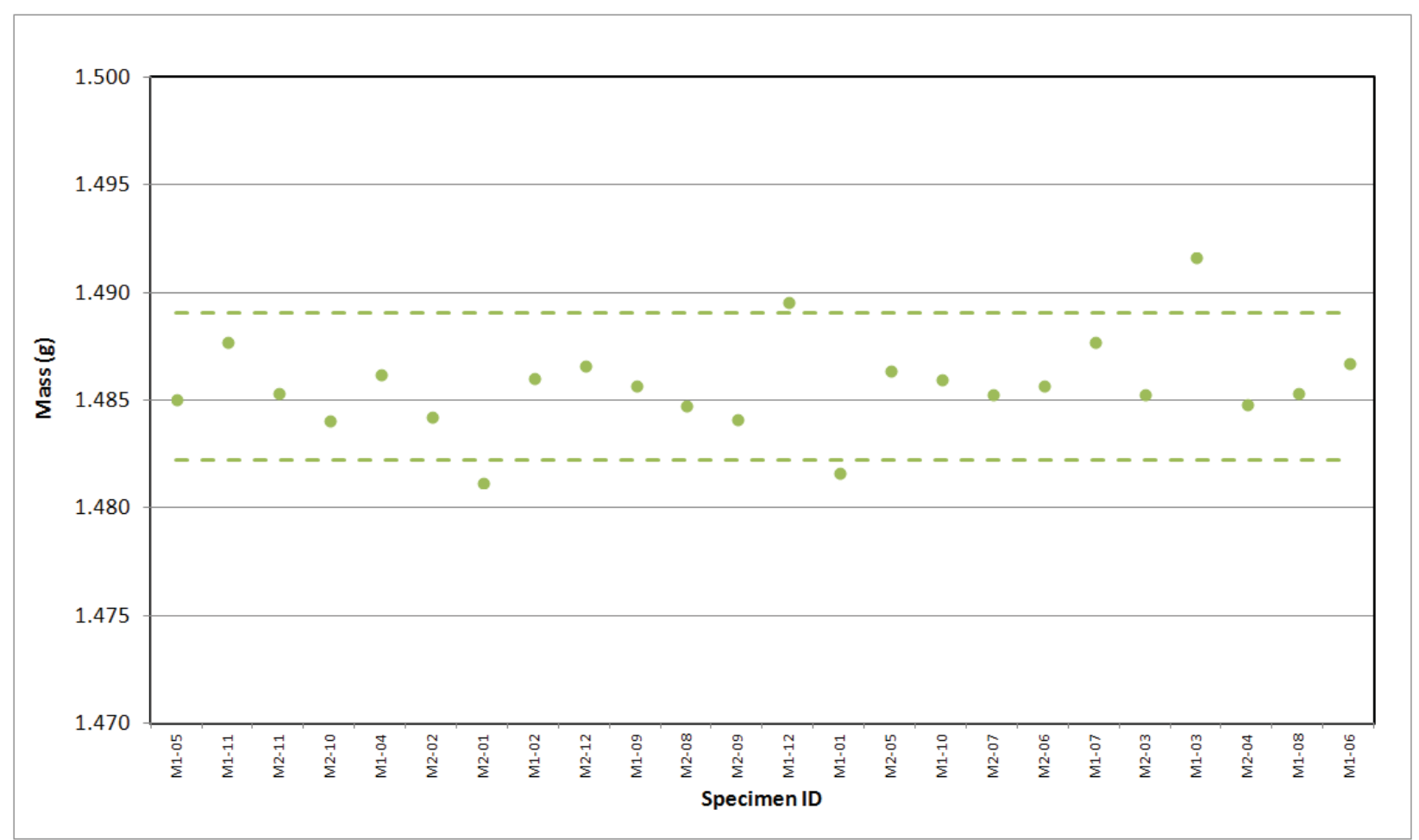

Figure A-83. NBG-25 Piggyback Pre Thermal Measurement Mass.

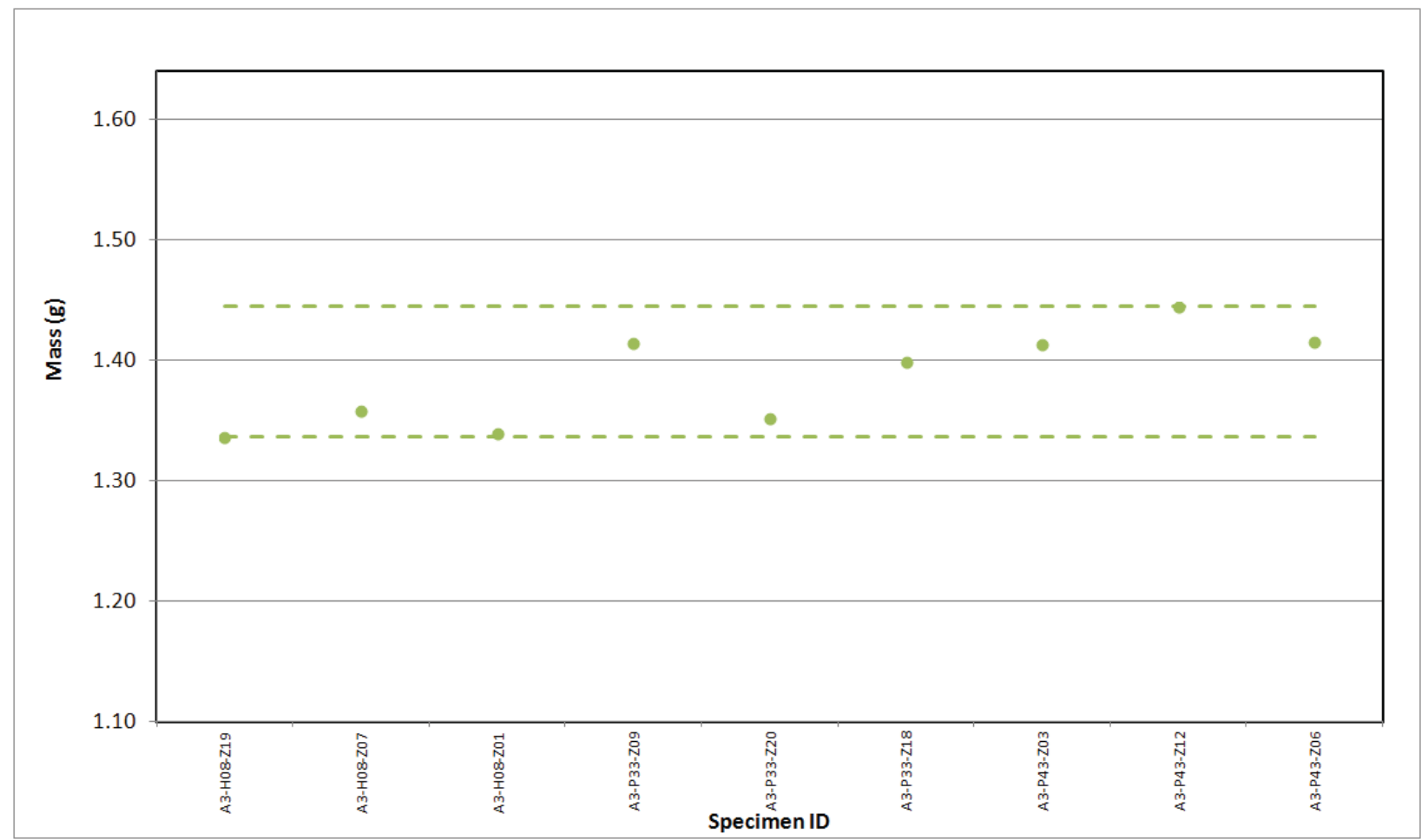

Figure A-84. New Matrix Piggyback Pre Thermal Measurement Mass. 


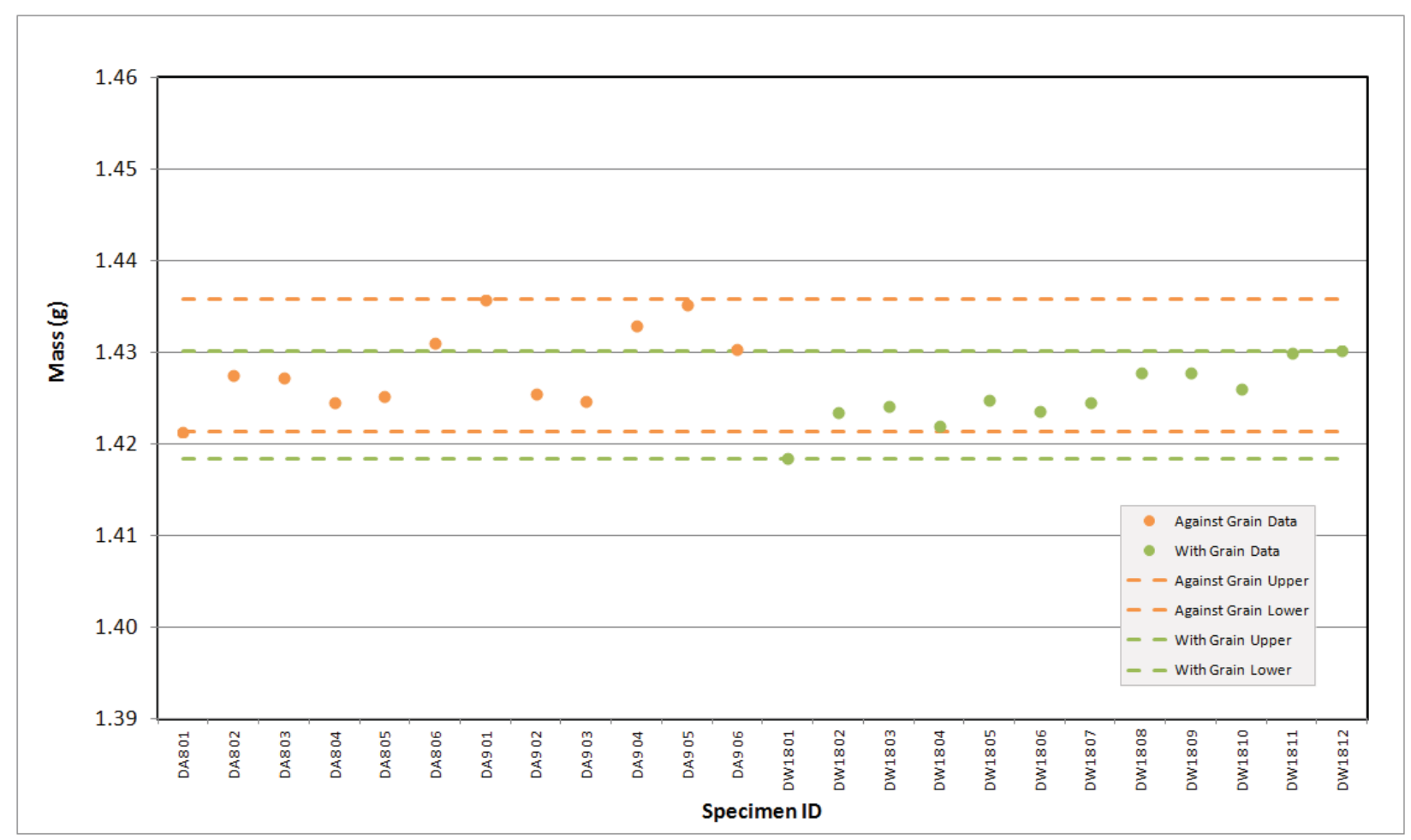

Figure A-85. PCEA Piggyback Pre Thermal Measurement Mass.

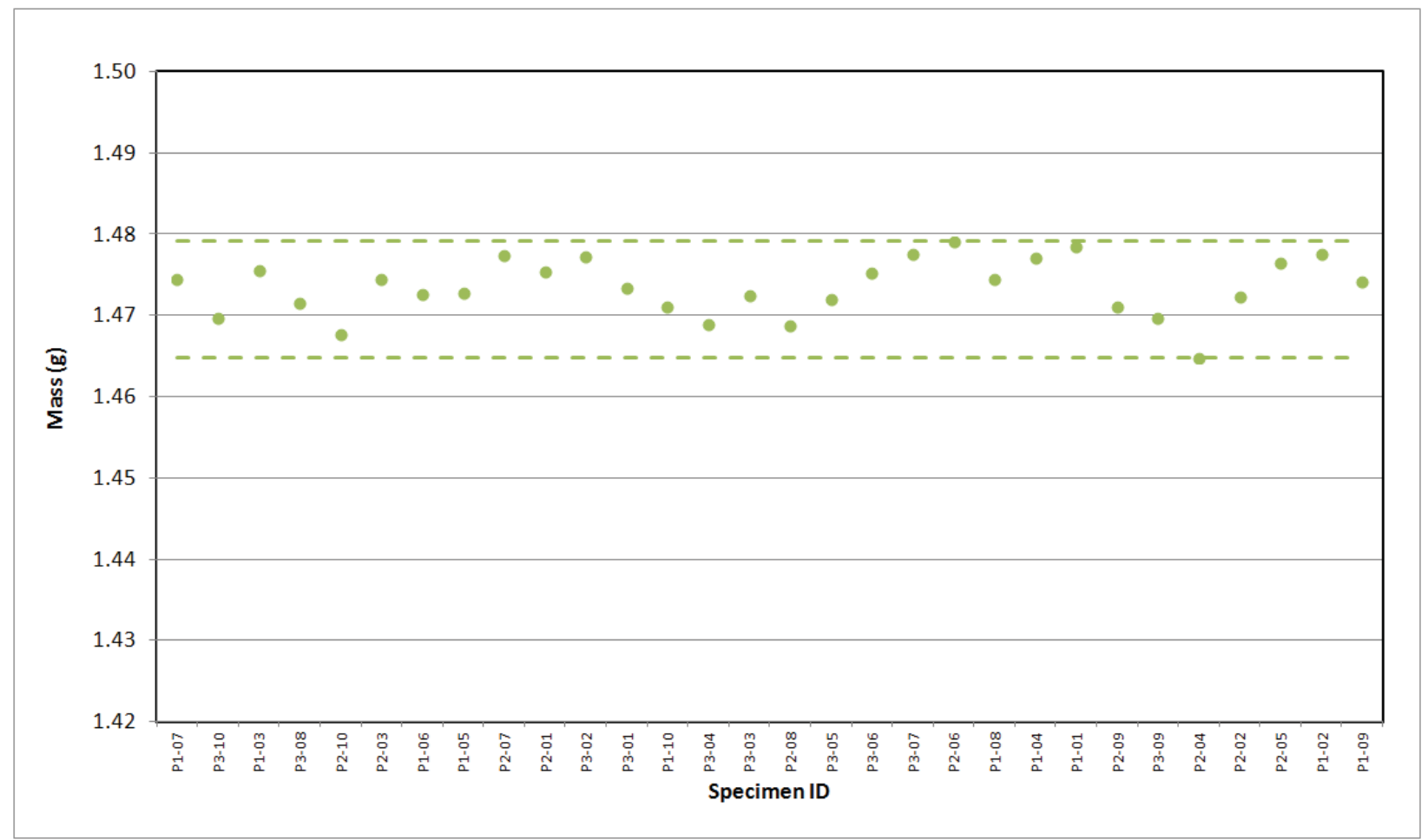

Figure A-86. PCIB Piggyback Pre Thermal Measurement Mass. 


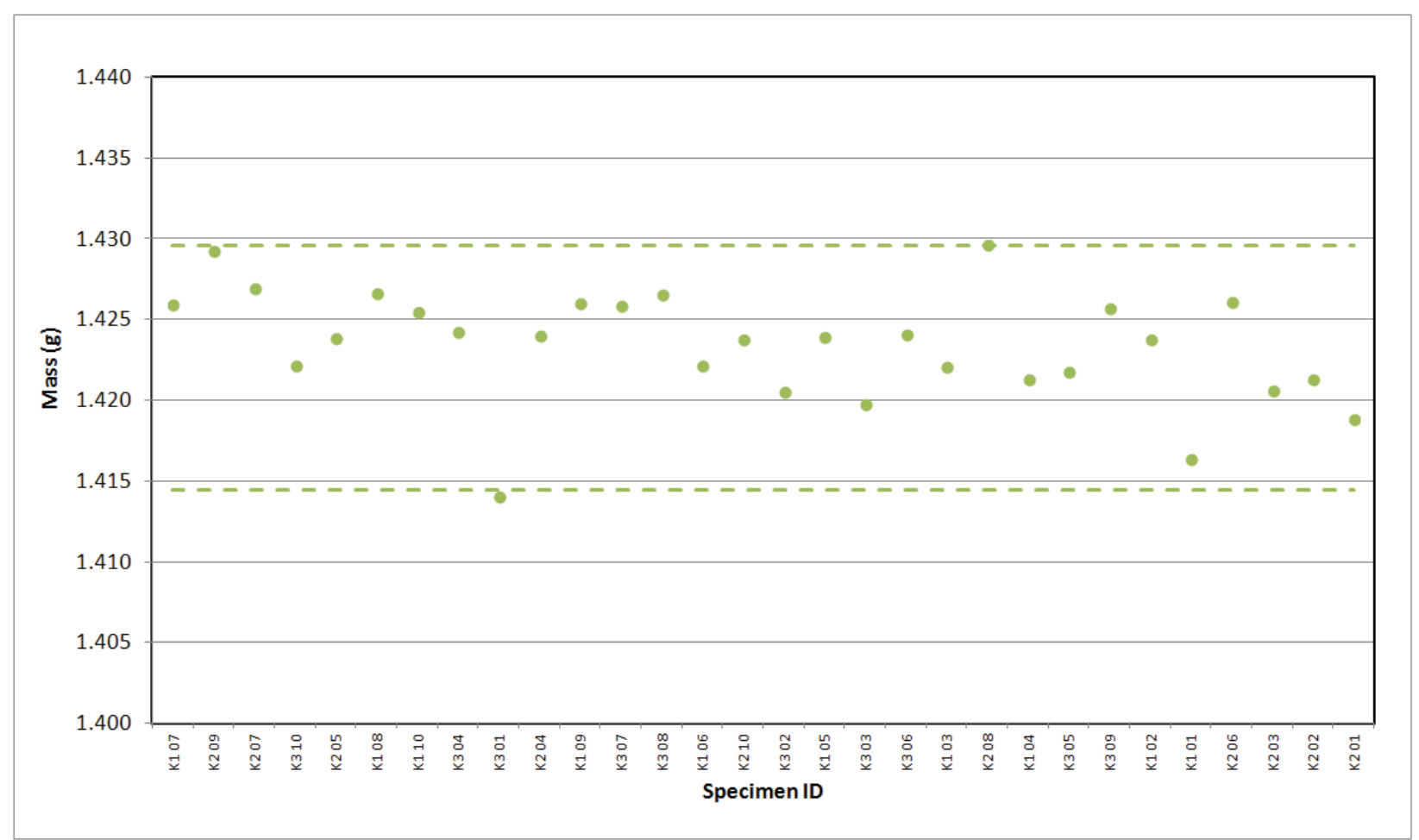

Figure A-87. PGX Piggyback Pre Thermal Measurement Mass.

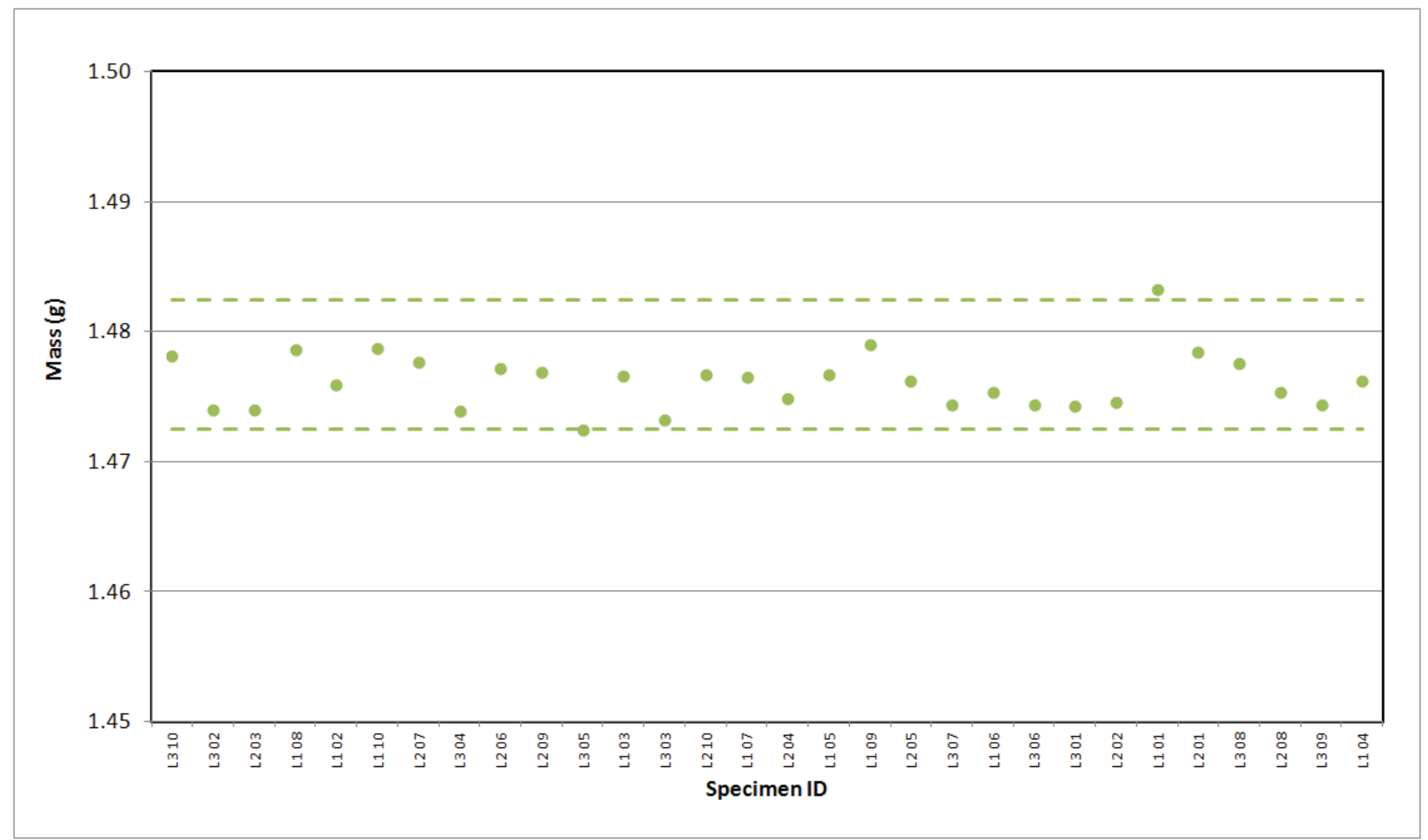

Figure A-88. PPEA Piggyback Pre Thermal Measurement Mass. 


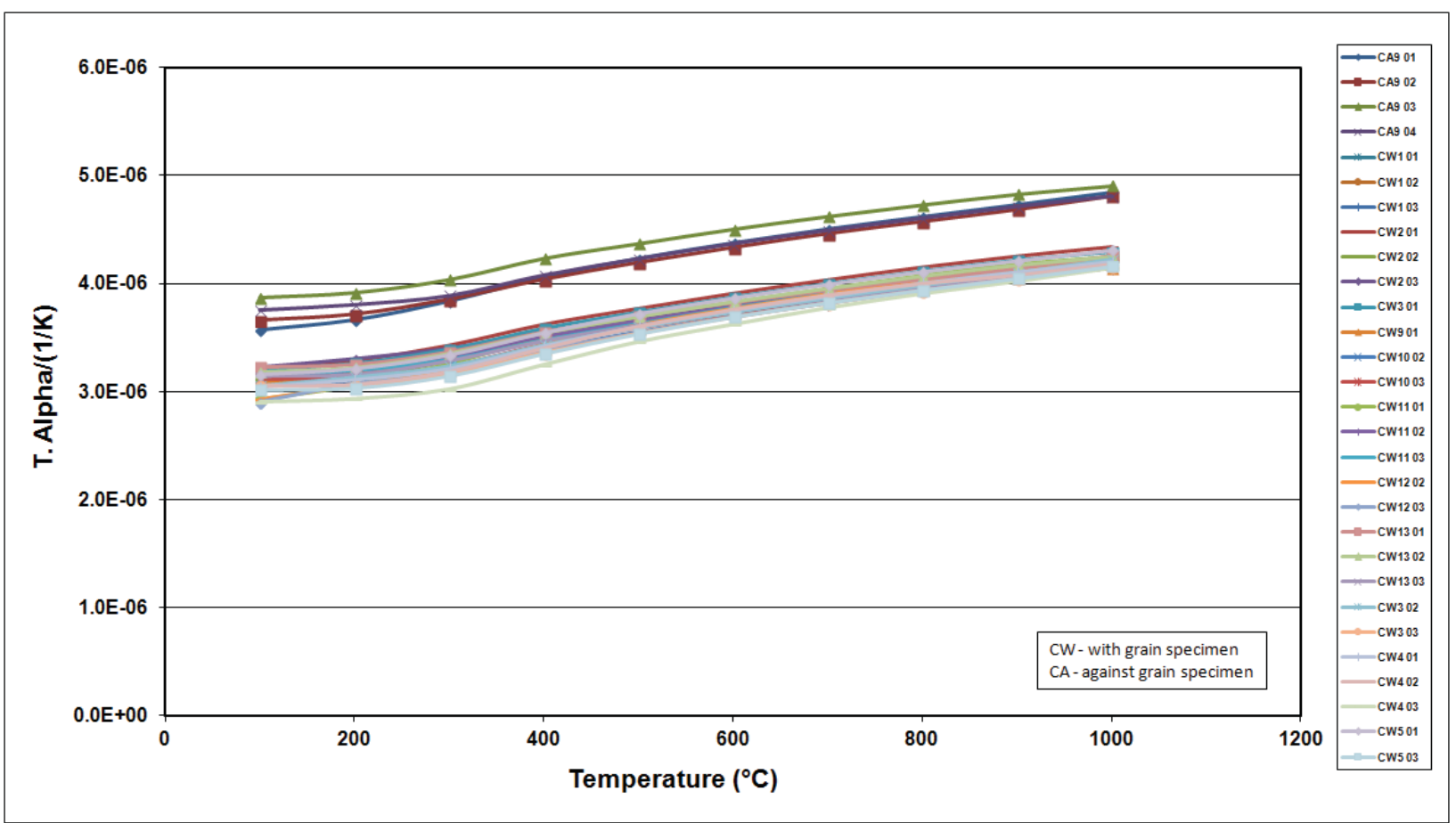

Figure A-89. H-451 Creep Coefficient of Thermal Expansion.

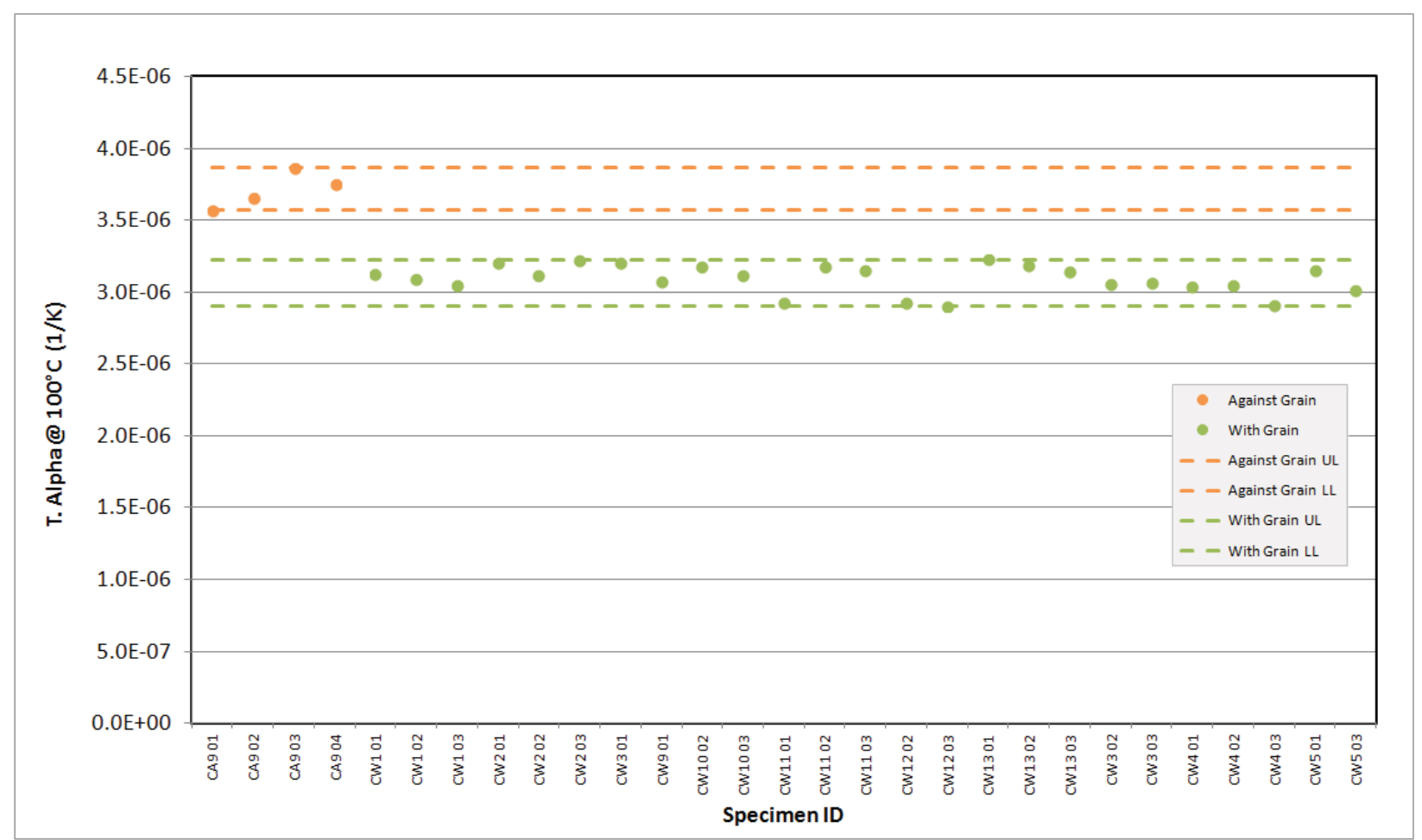

Figure A-90. H-451 Creep Coefficient of Thermal Expansion@ $100^{\circ} \mathrm{C}$. 


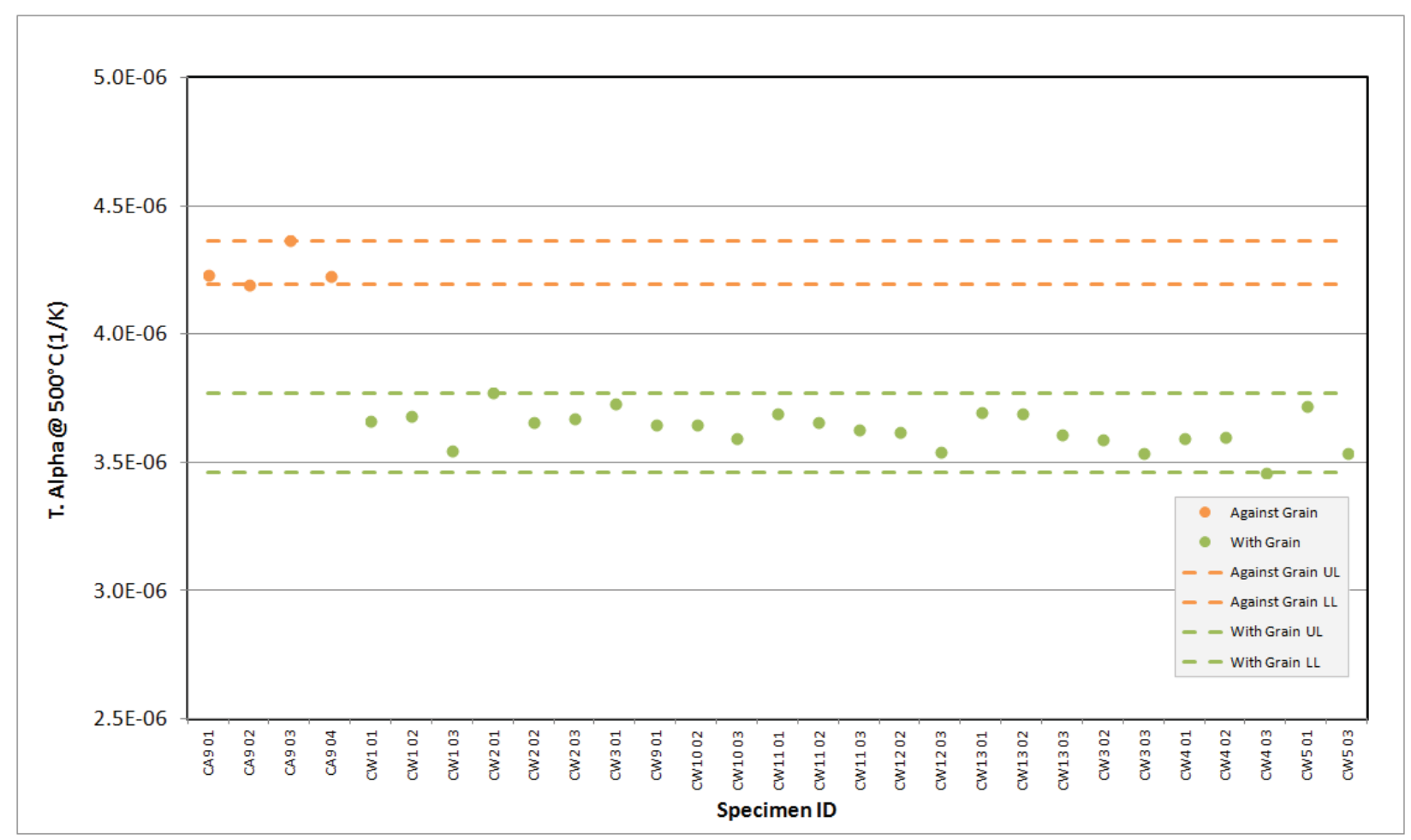

Figure A-91. H-451 Creep Coefficient of Thermal Expansion@ @ 500 $\mathrm{C}$.

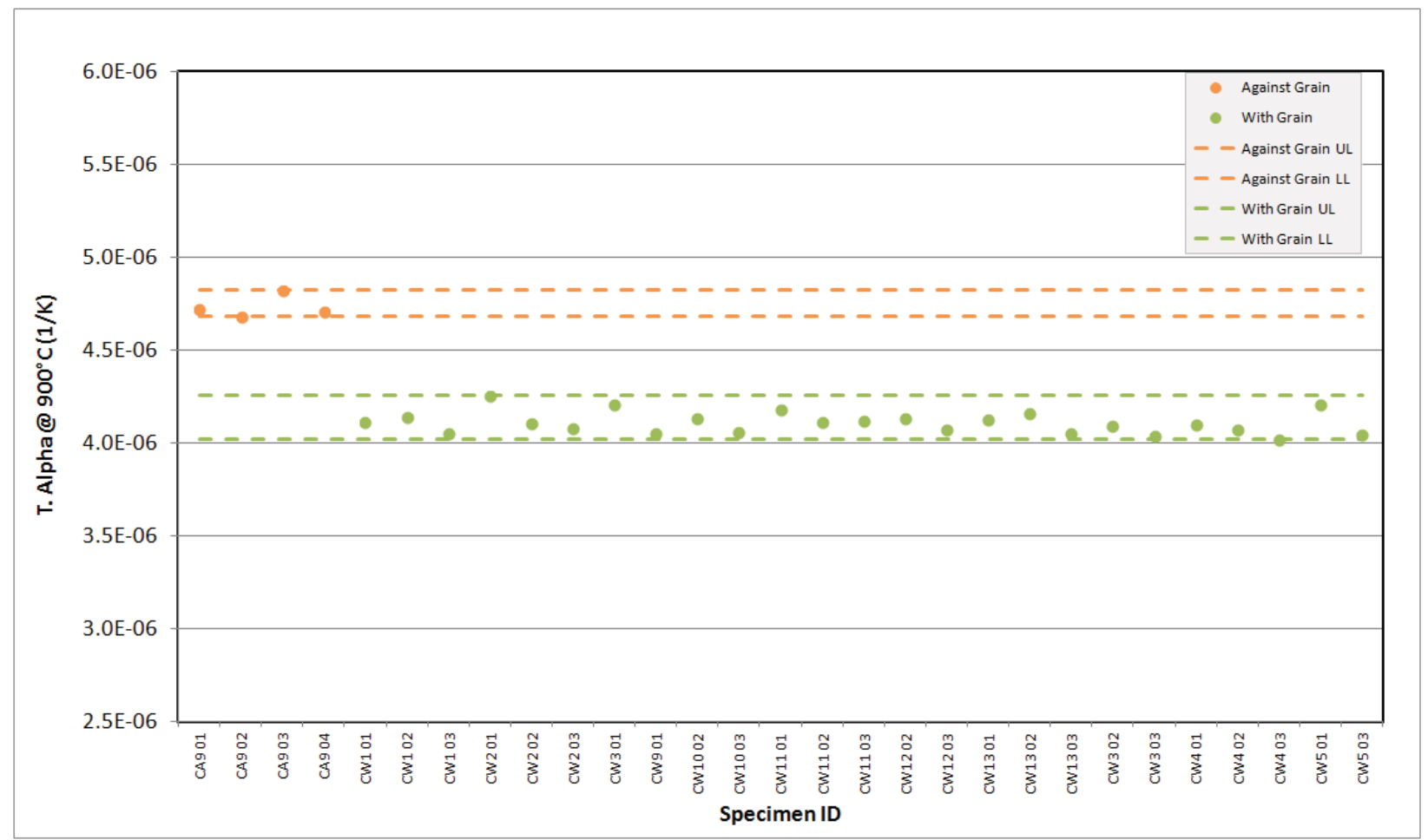

Figure A-92. H-451 Creep Coefficient of Thermal Expansion @ 900 $\mathrm{C}$. 


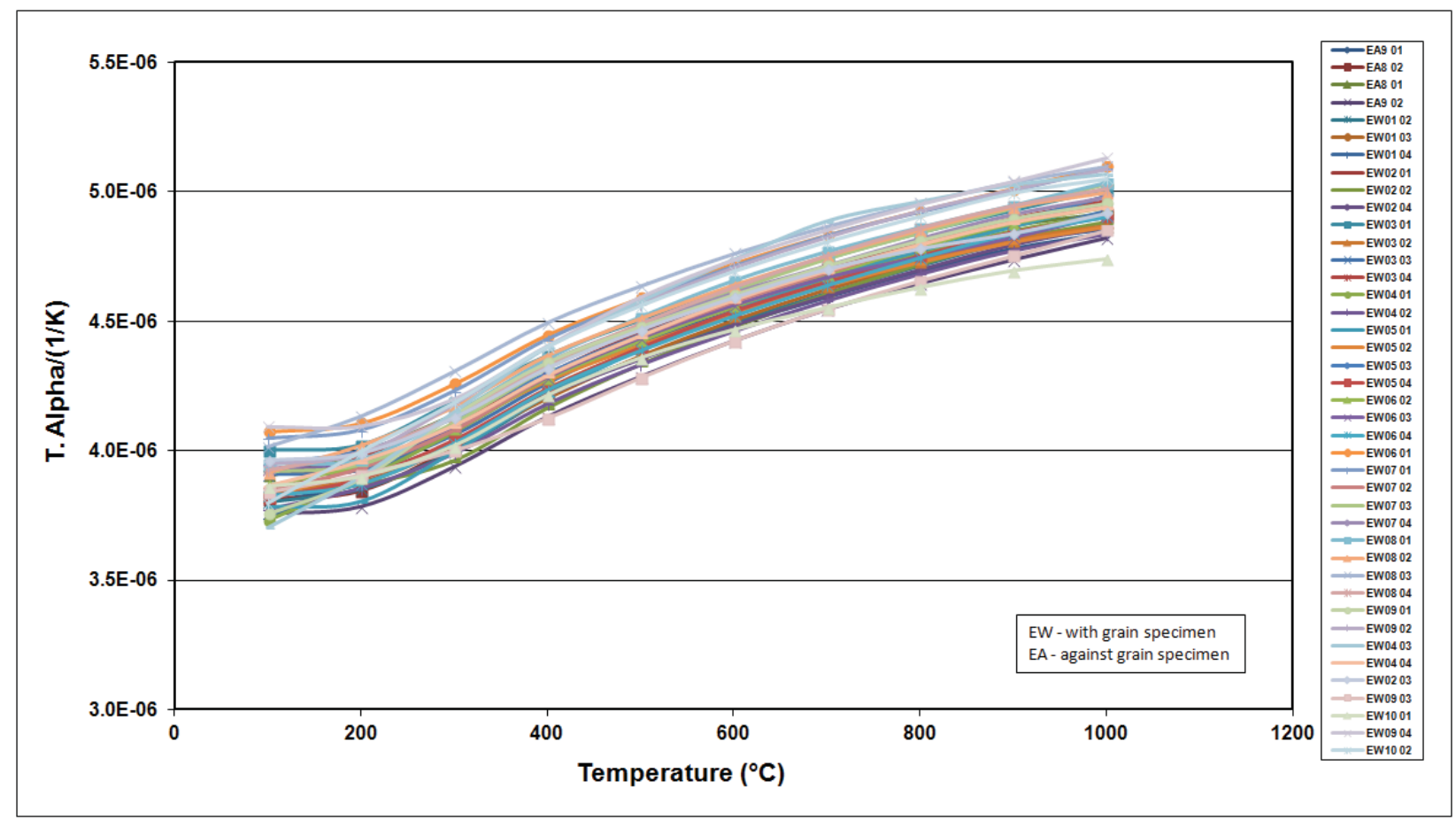

Figure A-93. IG-110 Creep Coefficient of Thermal Expansion.

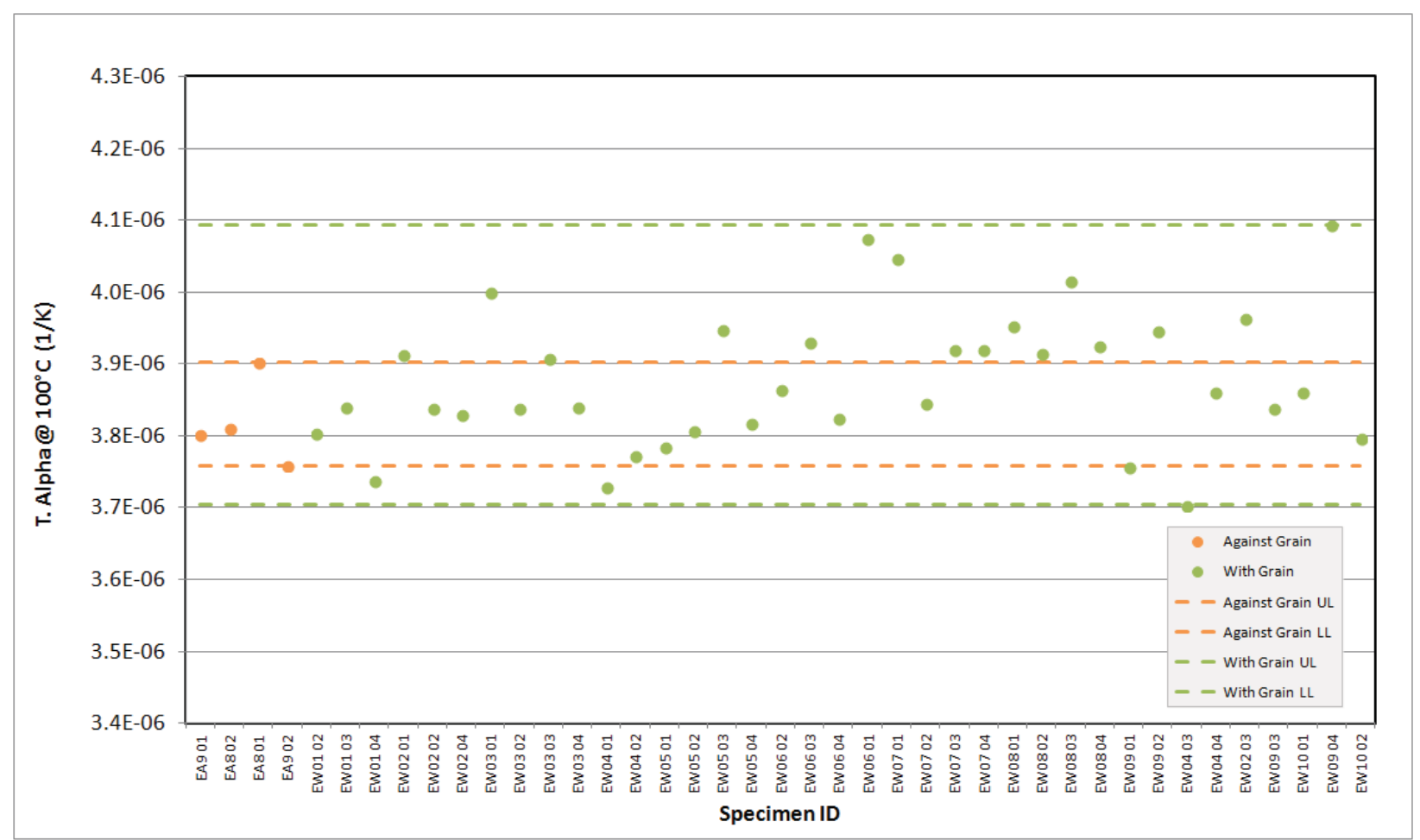

Figure A-94. IG-110 Creep Coefficient of Thermal Expansion@ $100^{\circ} \mathrm{C}$. 


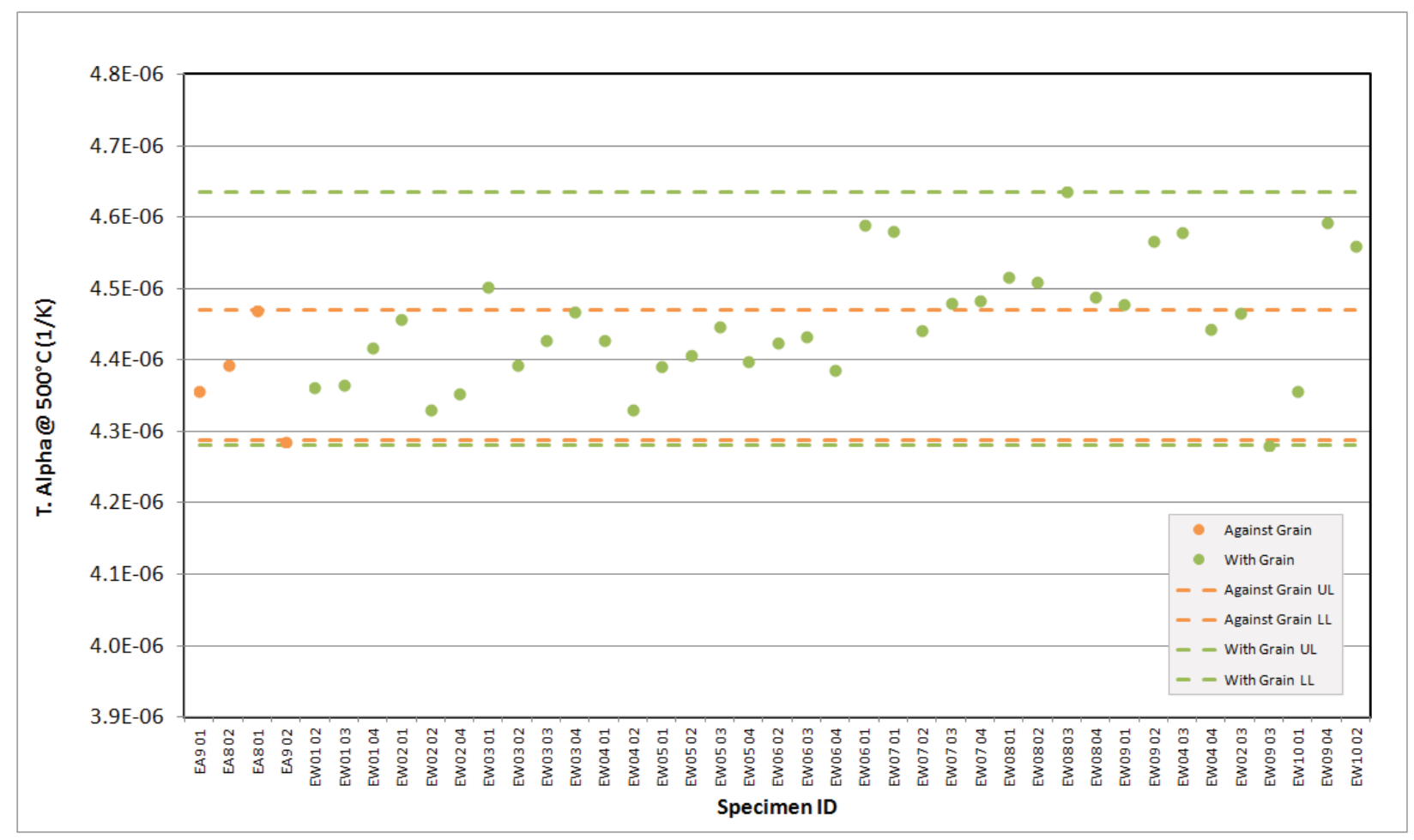

Figure A-95. IG-110 Creep Coefficient of Thermal Expansion @ 500 $\mathrm{C}$.

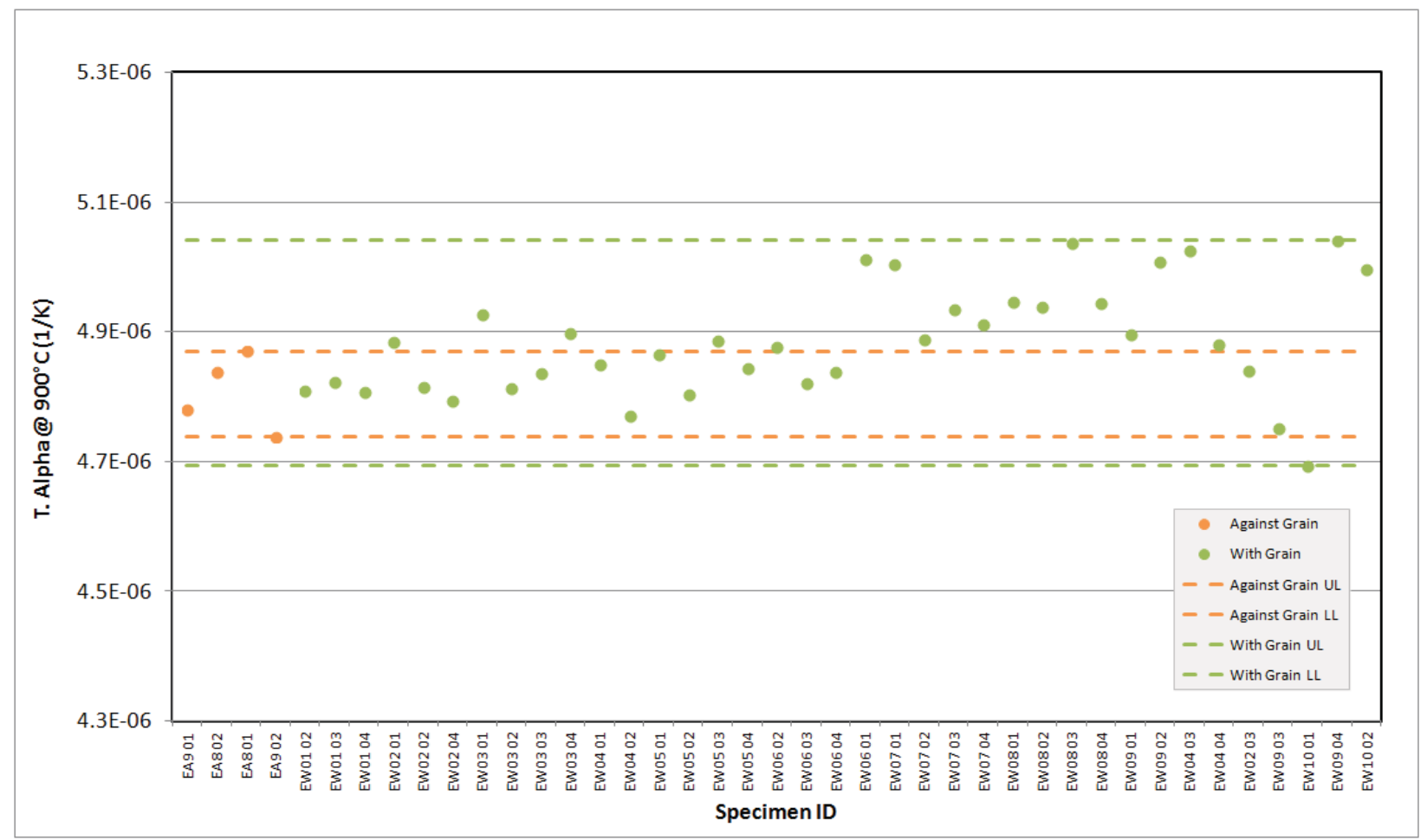

Figure A-96. IG-110 Creep Coefficient of Thermal Expansion @ $900^{\circ} \mathrm{C}$. 


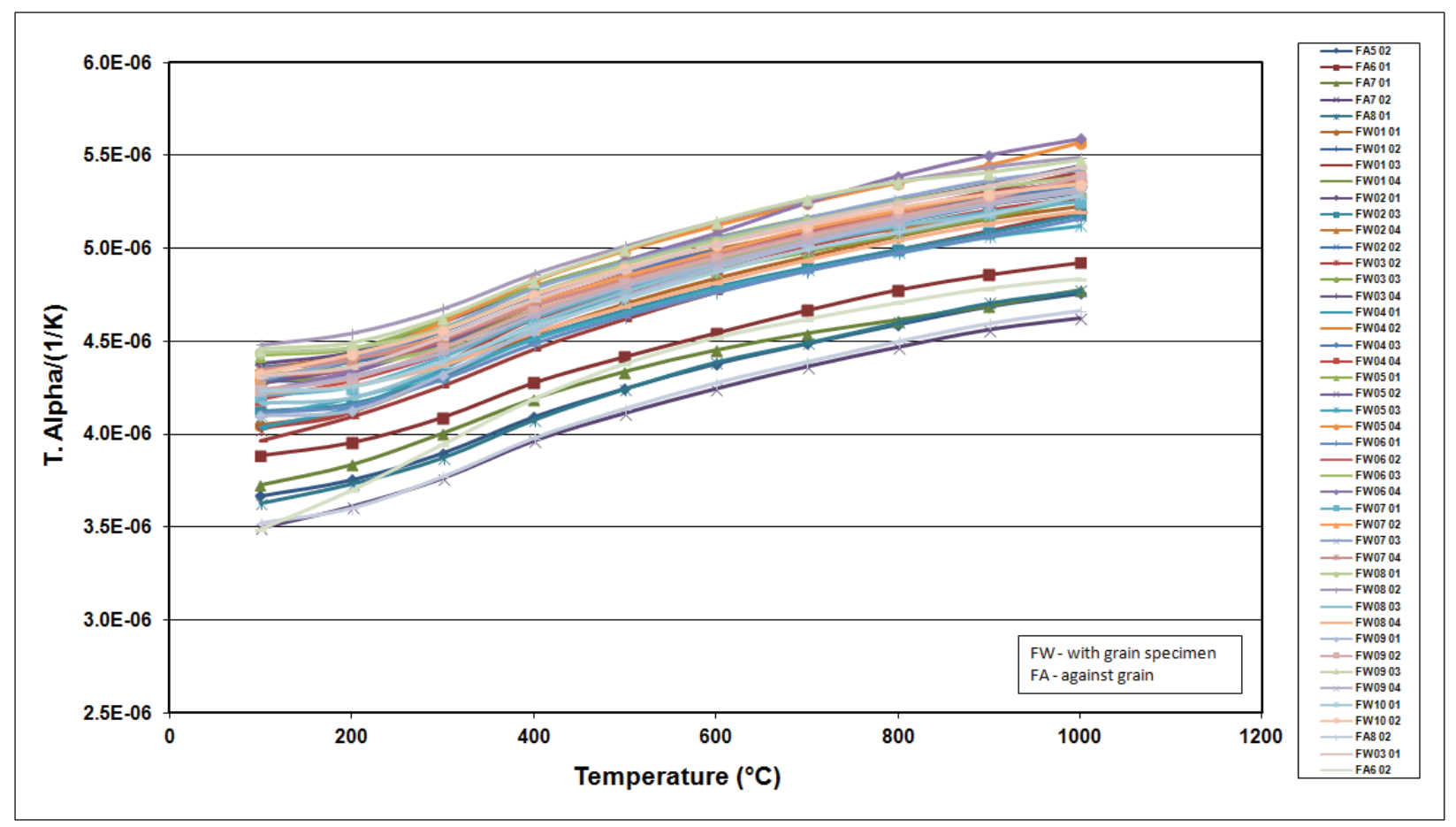

Figure A-97. IG-430 Creep Coefficient of Thermal Expansion.

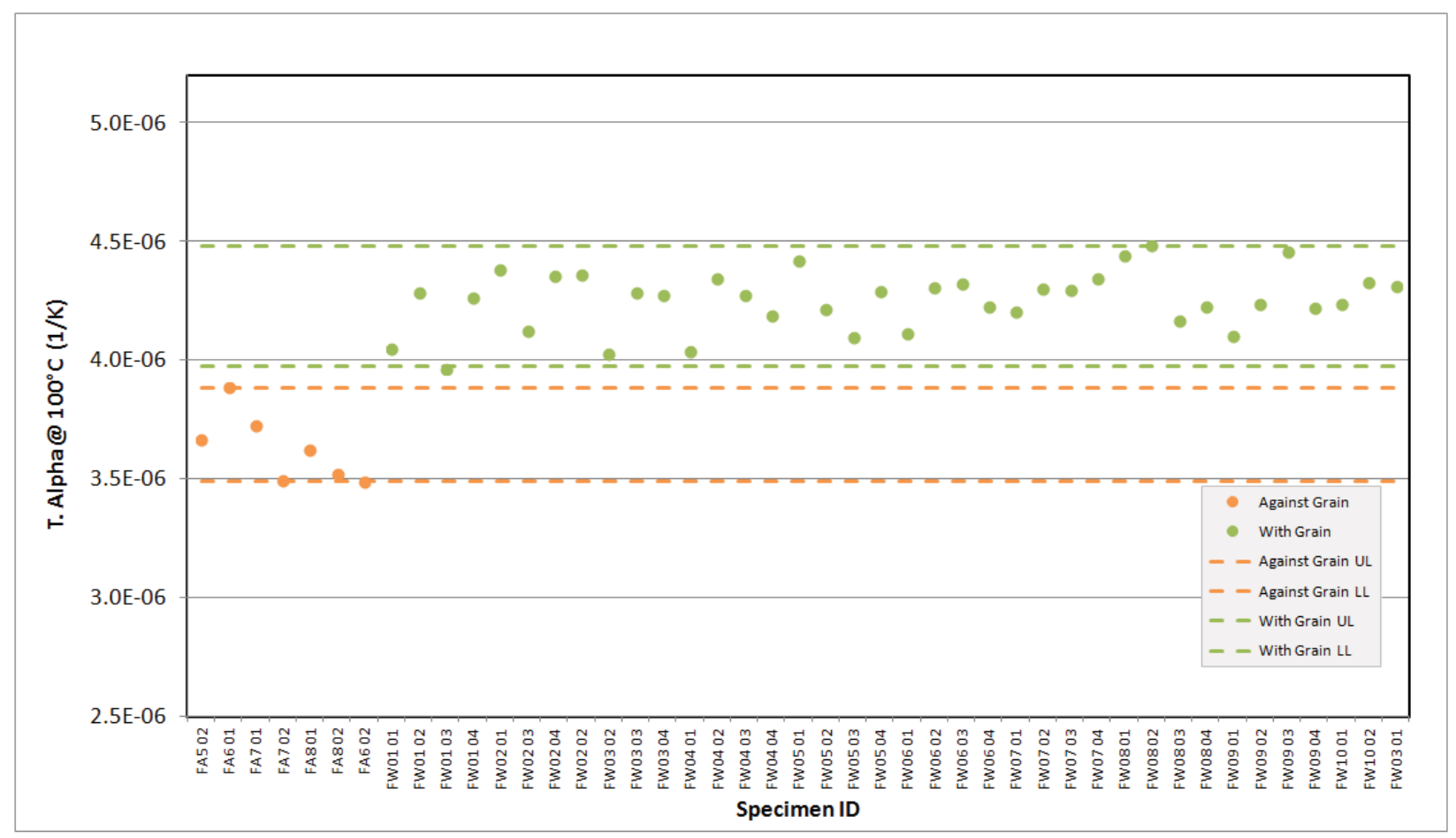

Figure A-98. IG-430 Creep Coefficient of Thermal Expansion @ $100^{\circ} \mathrm{C}$. 


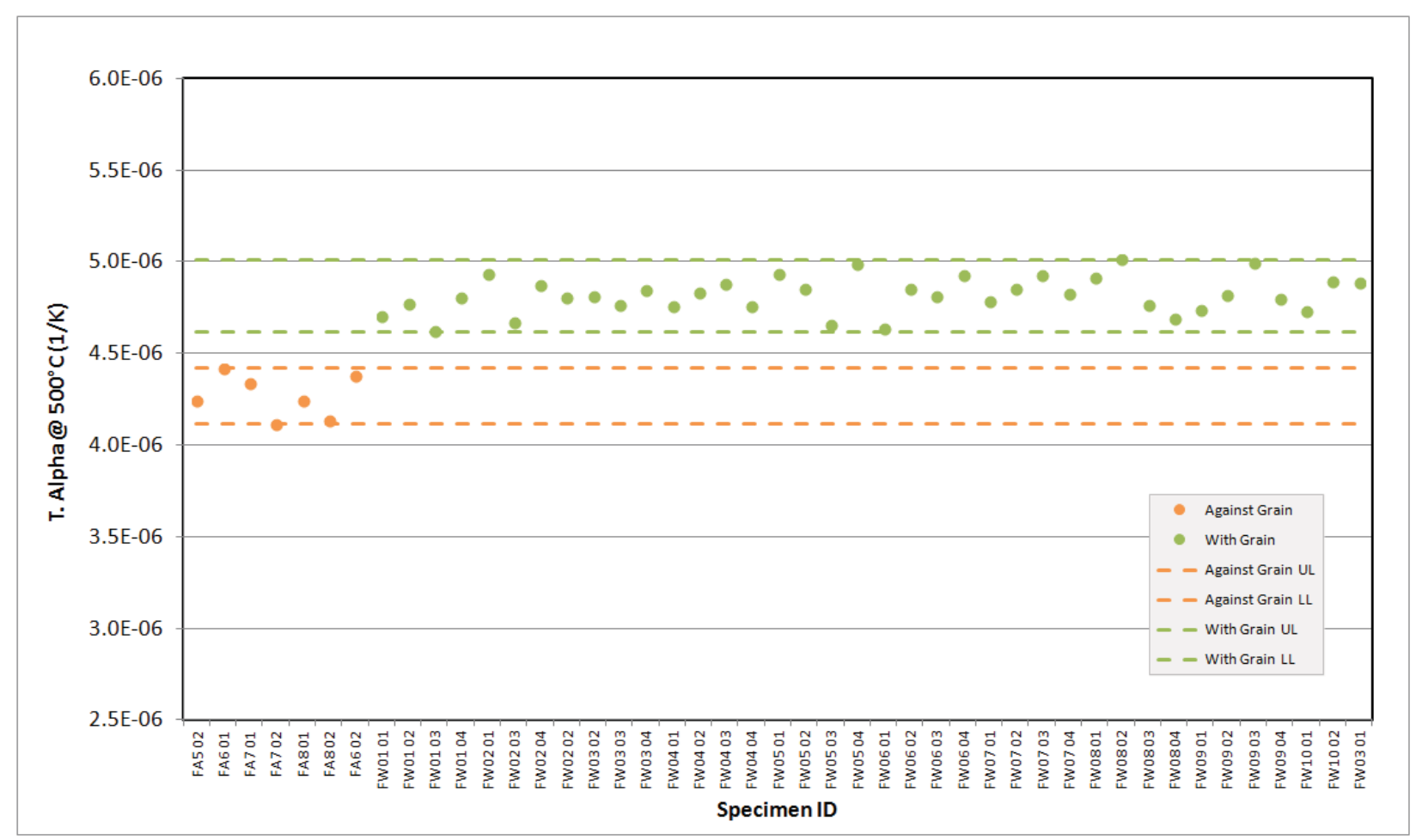

Figure A-99. IG-430 Creep Coefficient of Thermal Expansion @ $500^{\circ} \mathrm{C}$.

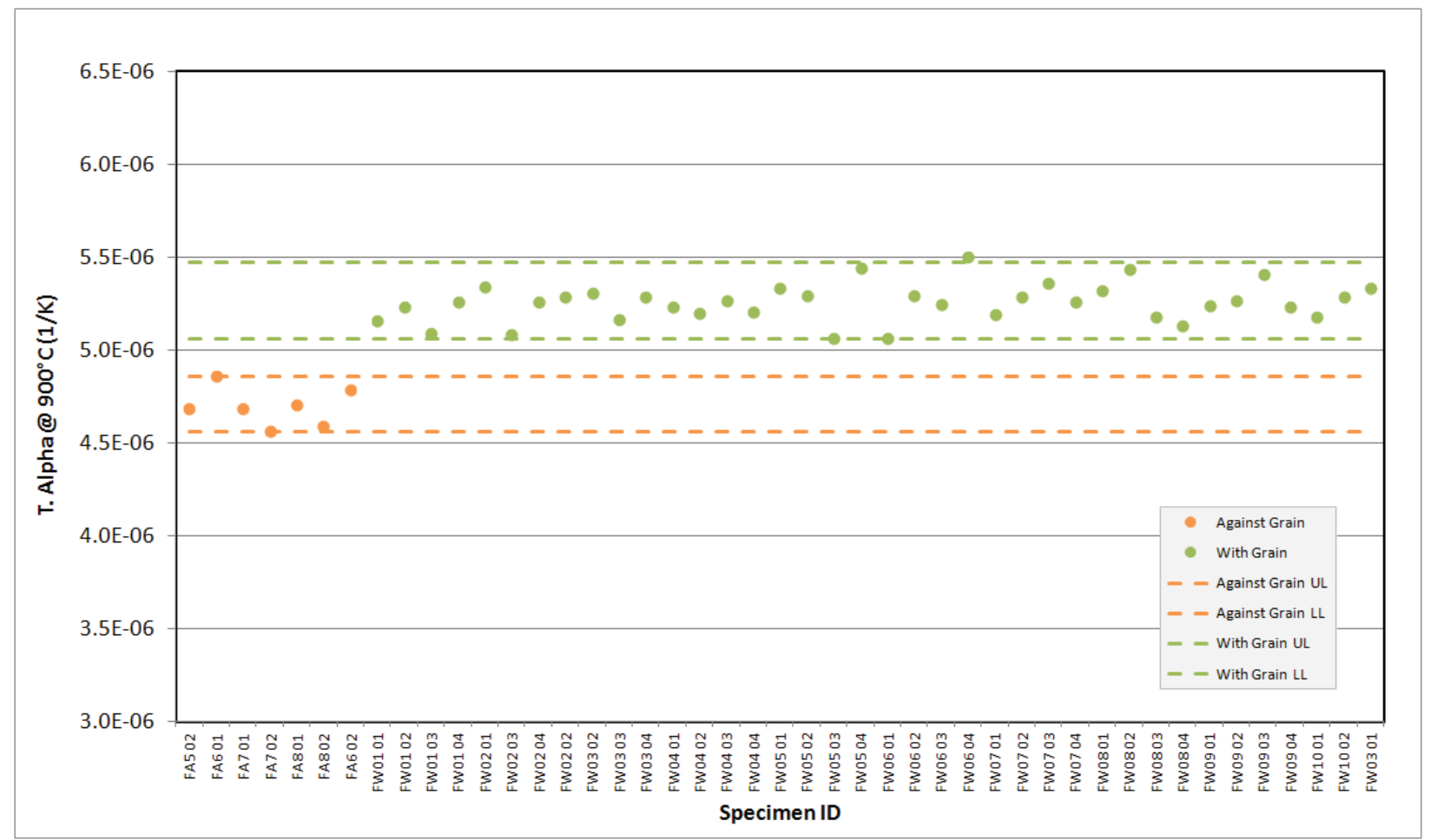

Figure A-100. IG-430 Creep Coefficient of Thermal Expansion @ $900^{\circ} \mathrm{C}$. 


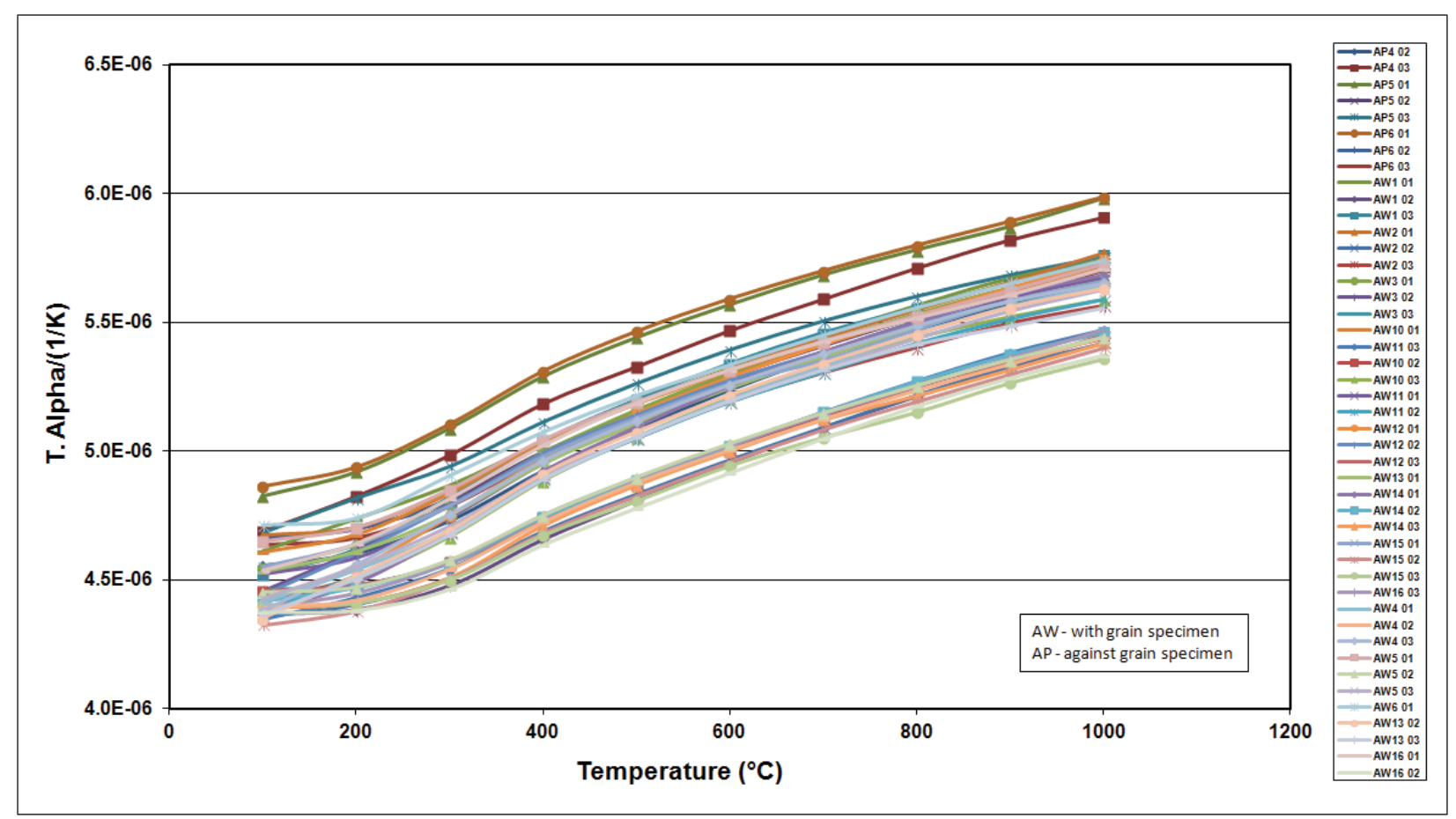

Figure A-101. NBG-17 Creep Coefficient of Thermal Expansion.

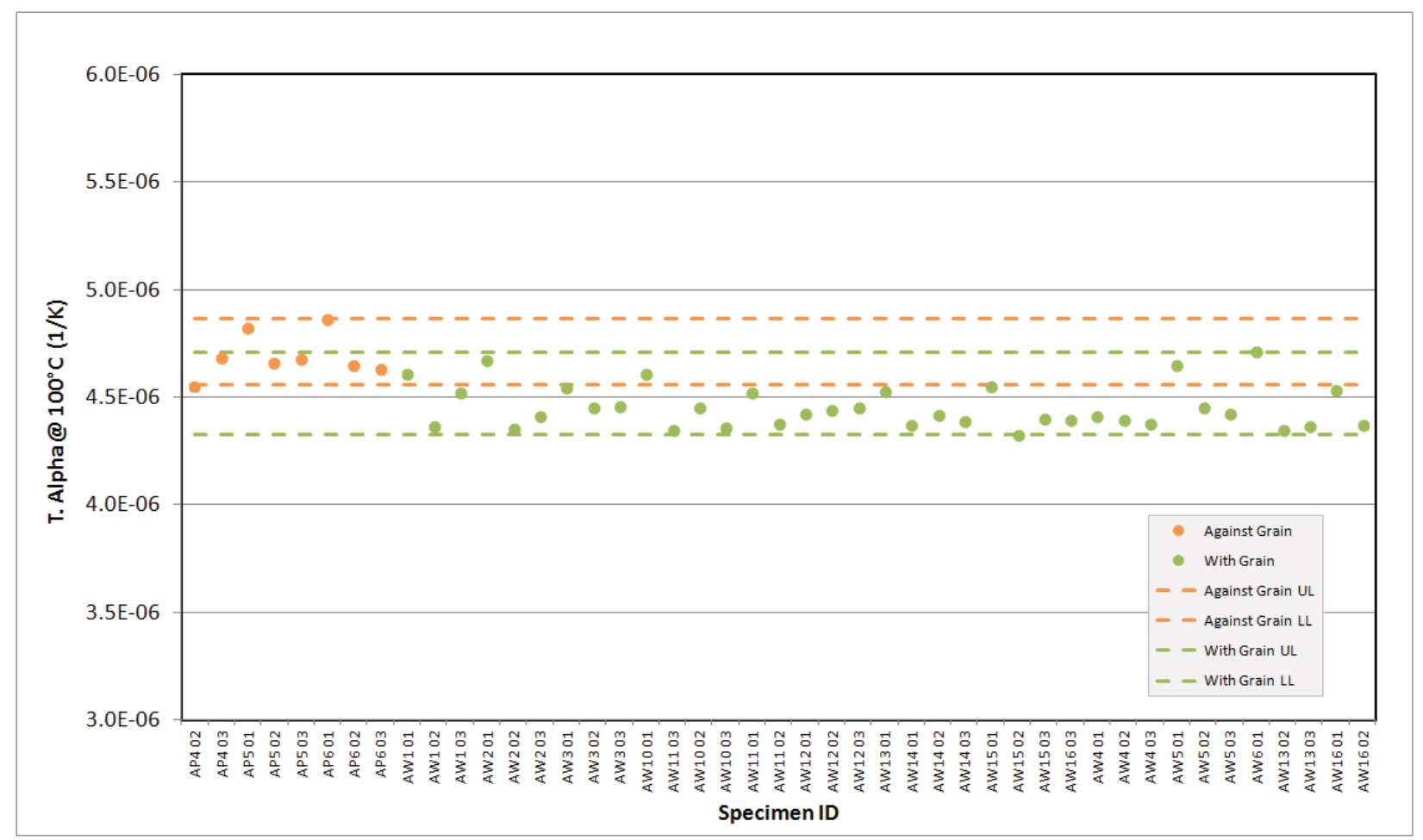

Figure A-102. NBG-17 Creep Coefficient of Thermal Expansion @ 100 C. 


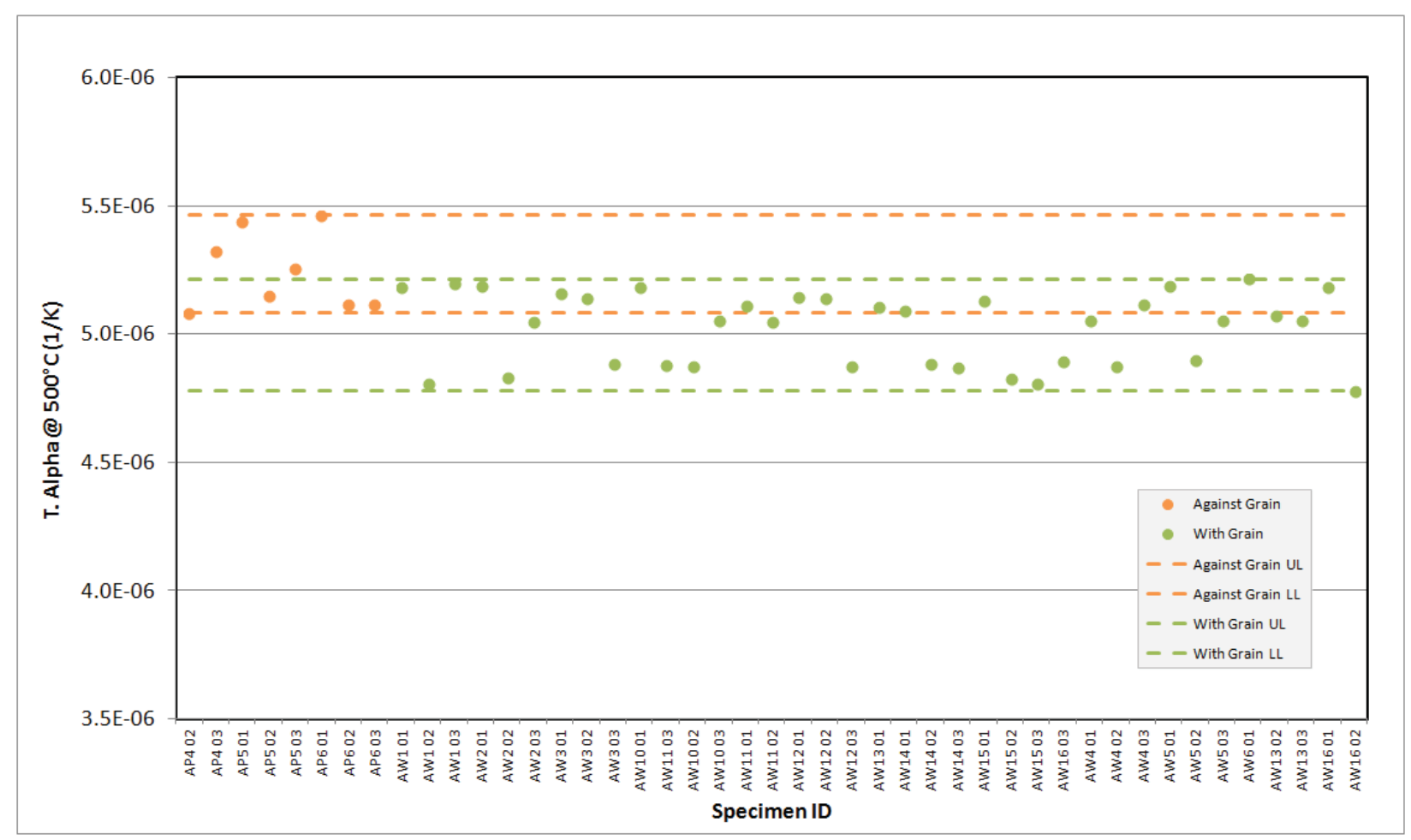

Figure A-103. NBG-17 Creep Coefficient of Thermal Expansion @ 500 $\mathrm{C}$.

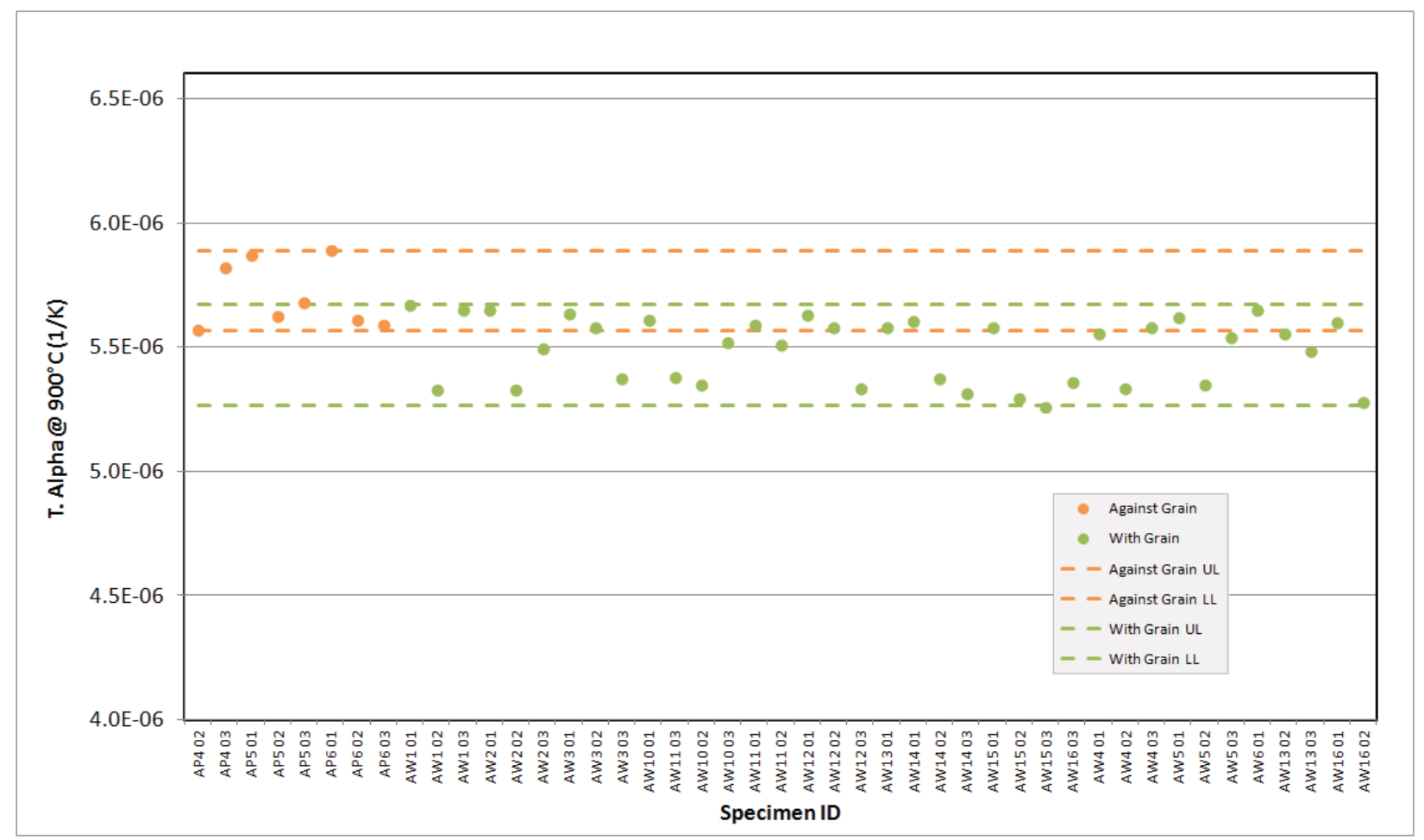

Figure A-104. NBG-17 Creep Coefficient of Thermal Expansion @ 900 ${ }^{\circ}$. 


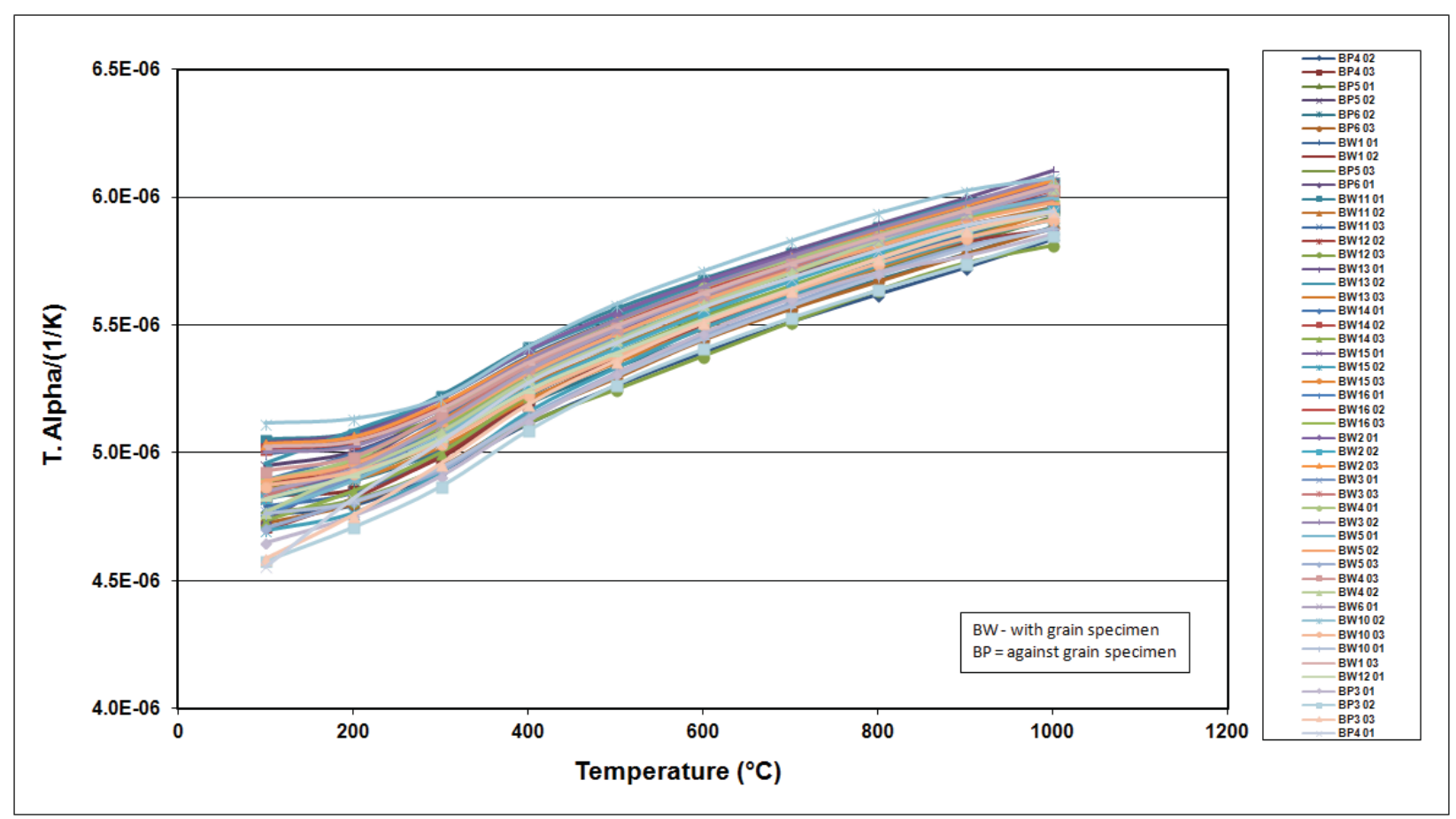

Figure A-105. NBG-18 Creep Coefficient of Thermal Expansion.

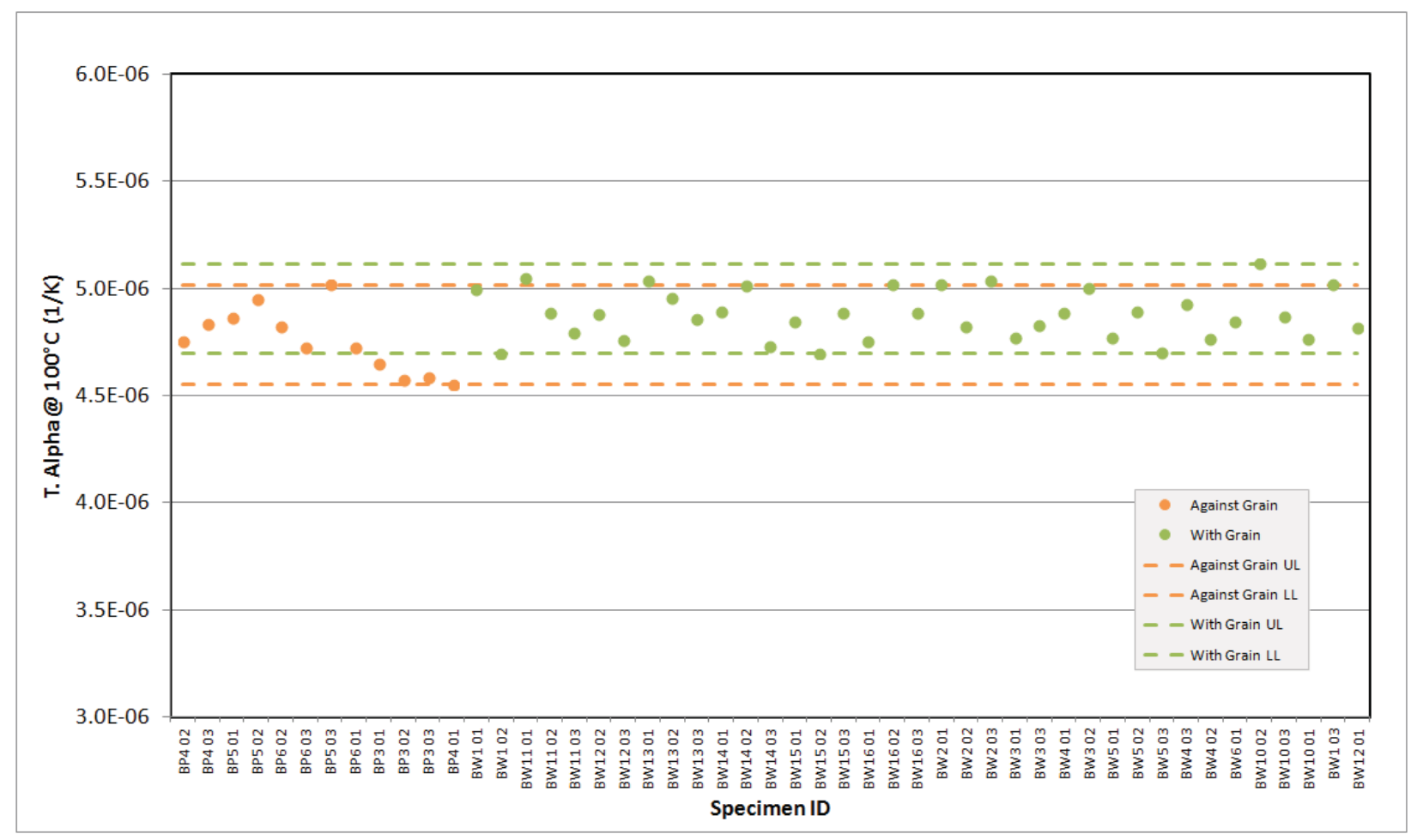

Figure A-106. NBG-18 Creep Coefficient of Thermal Expansion @ 100 C. 


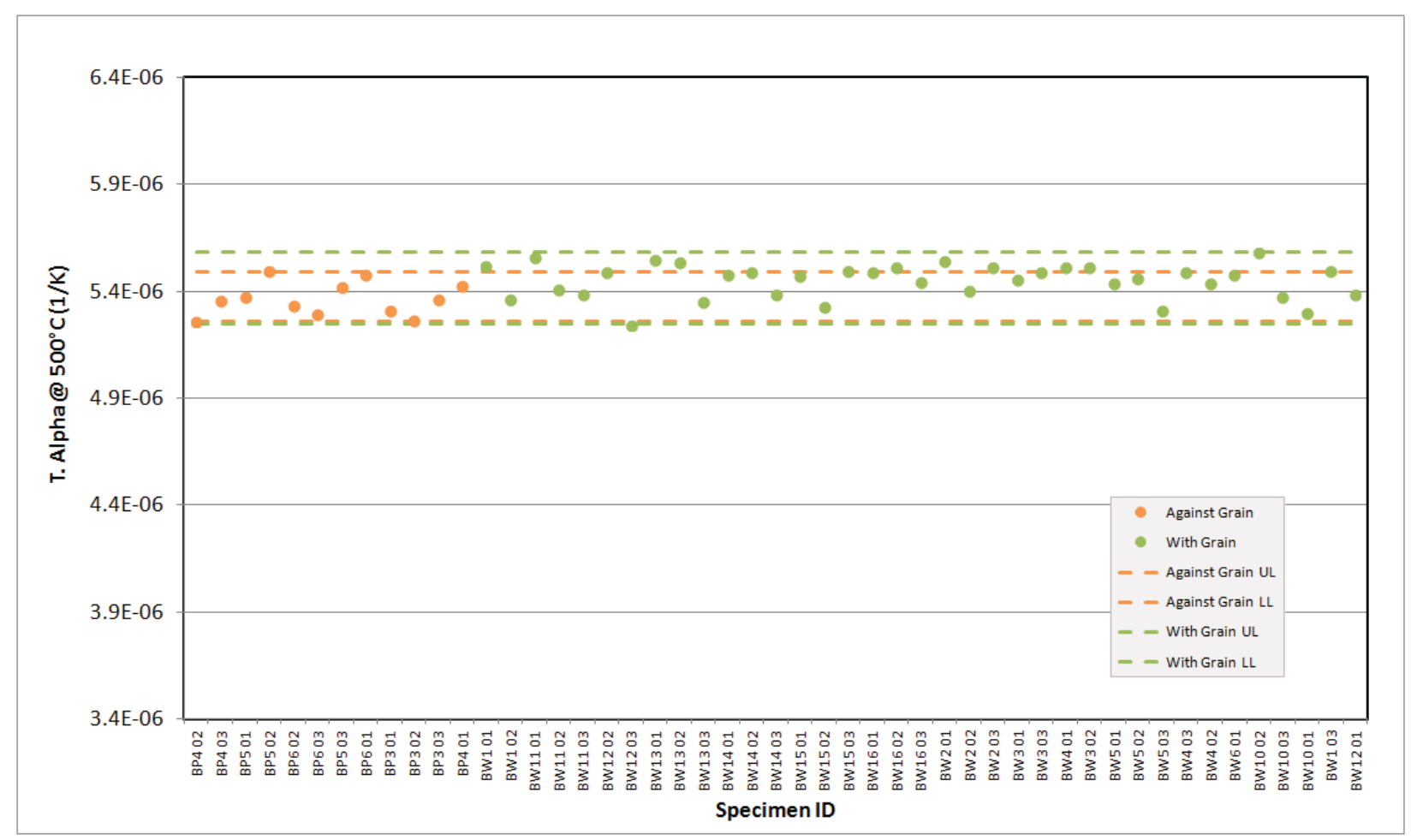

Figure A-107. NBG-18 Creep Coefficient of Thermal Expansion @ 500 $\mathrm{C}$.

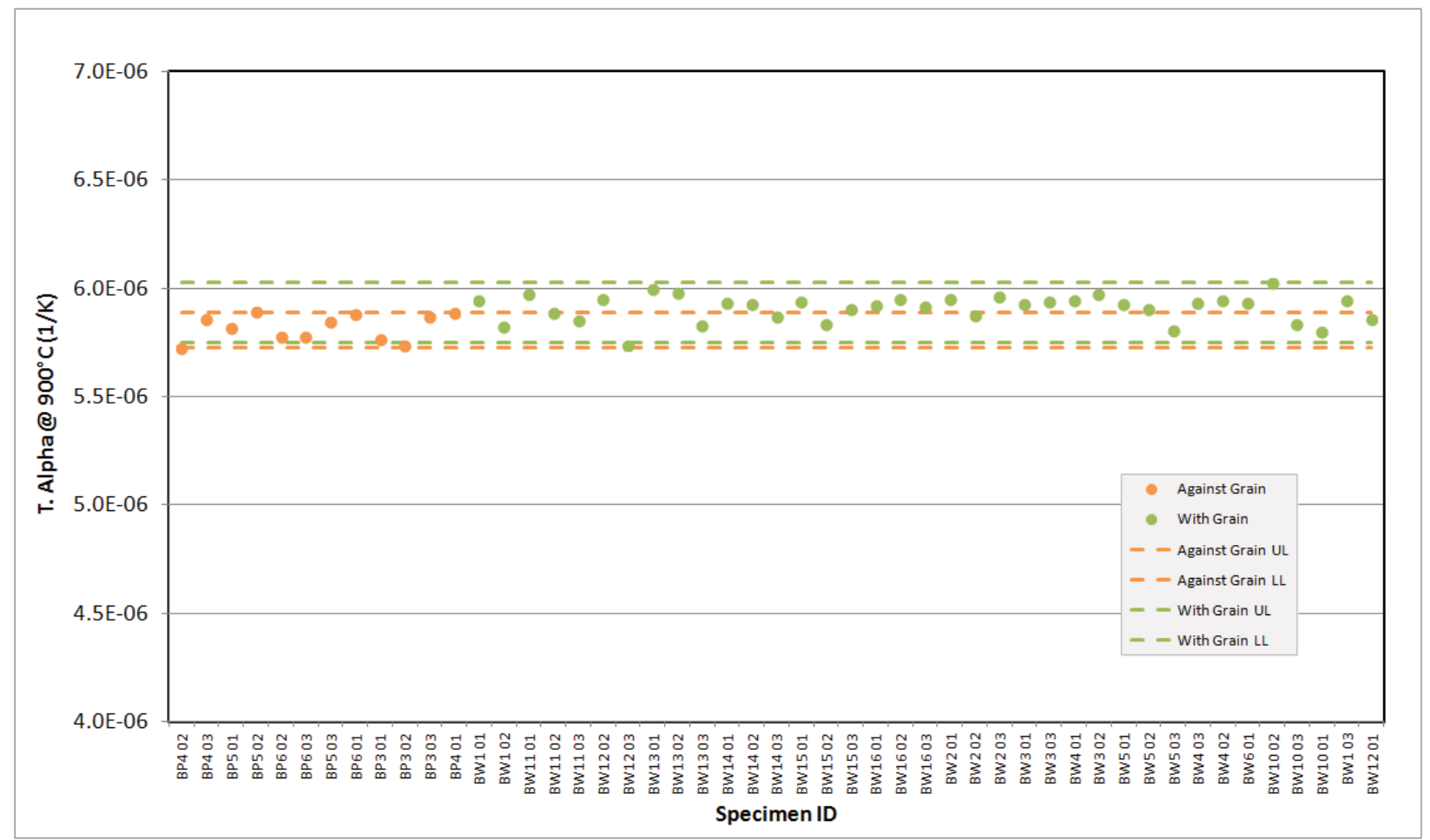

Figure A-108. NBG-18 Creep Coefficient of Thermal Expansion @ 900 ${ }^{\circ}$. 


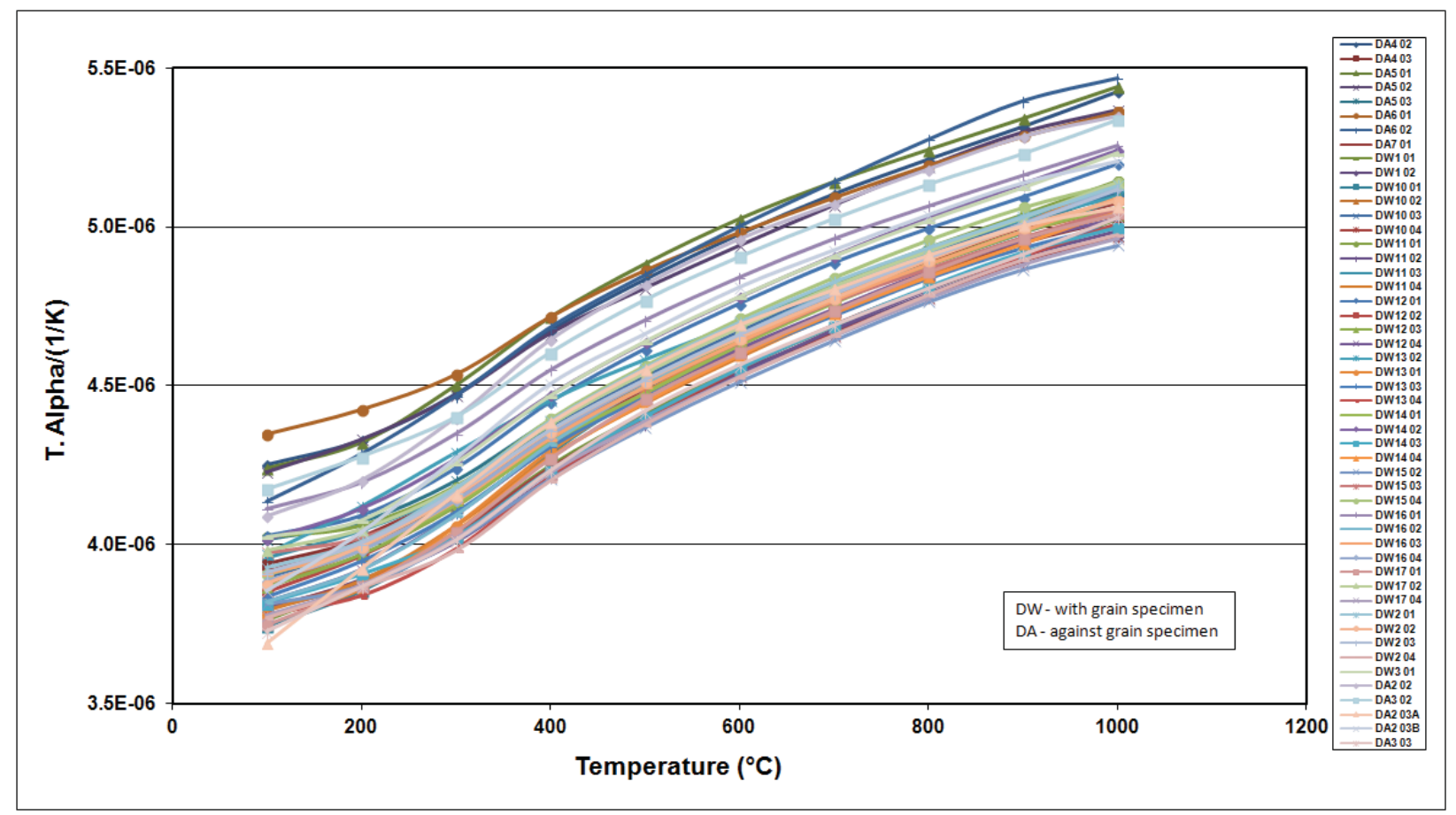

Figure A-109. PCEA Creep Coefficient of Thermal Expansion.

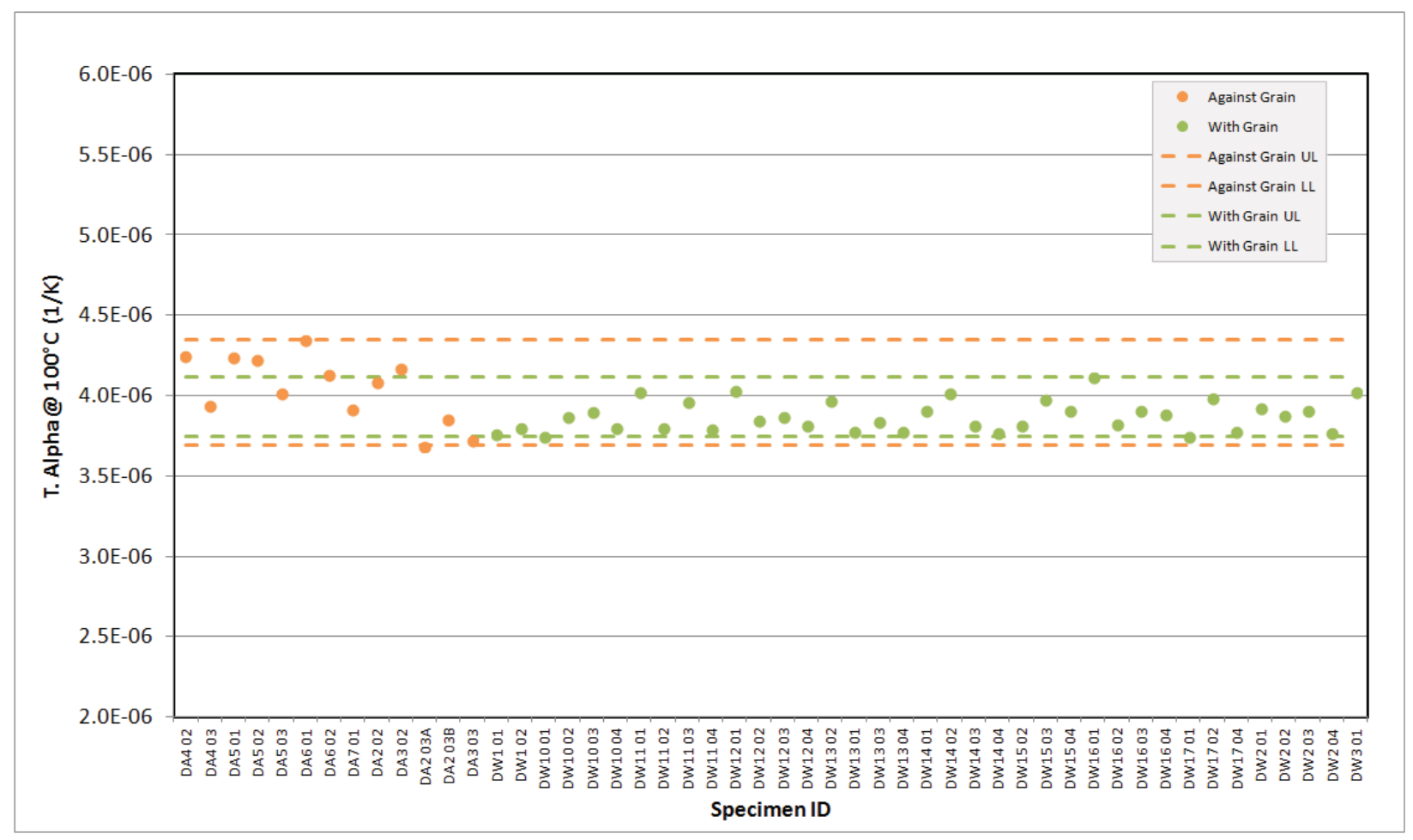

Figure A-110. PCEA Creep Coefficient of Thermal Expansion @ $100^{\circ} \mathrm{C}$. 


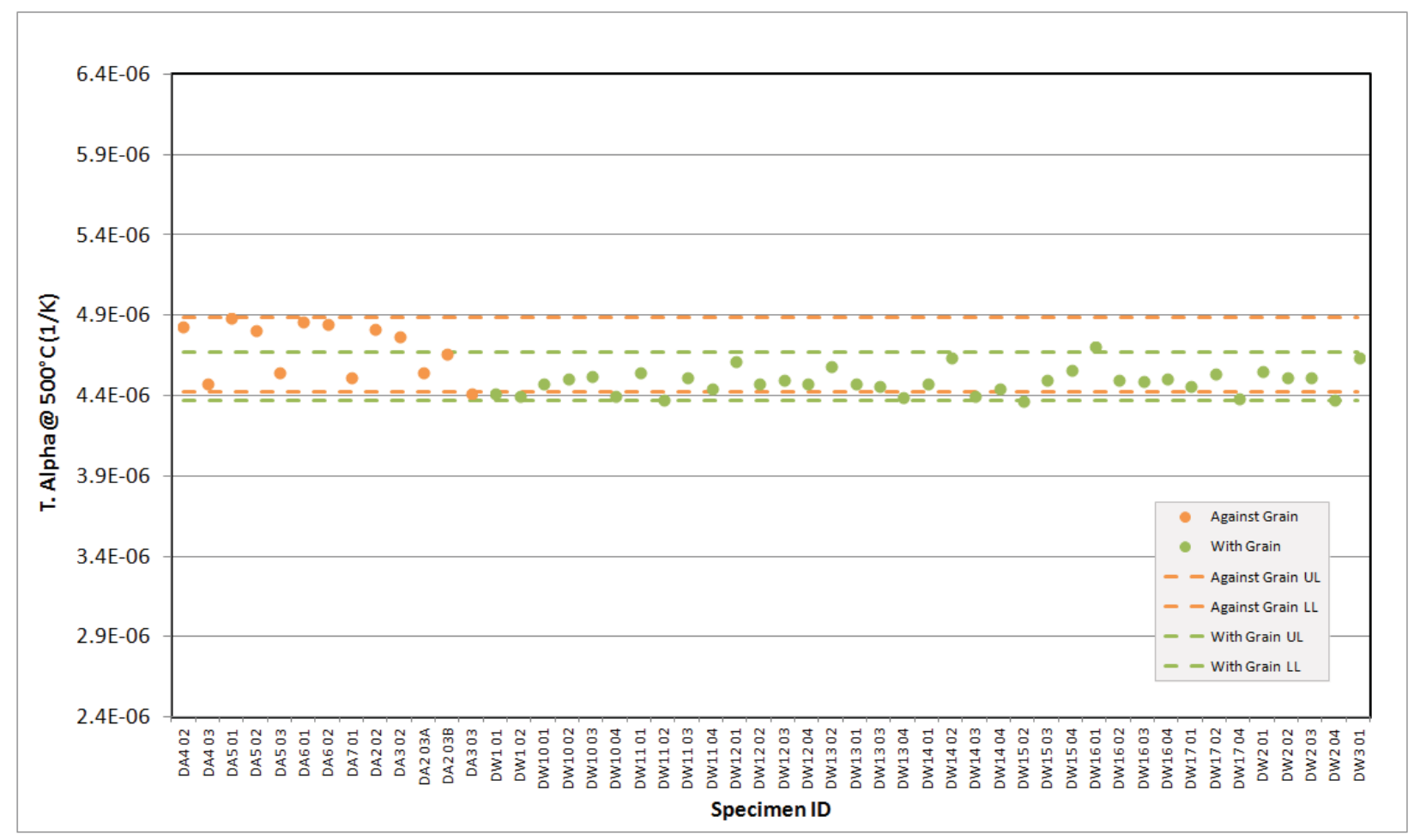

Figure A-111. PCEA Creep Coefficient of Thermal Expansion@ @ 500 C.

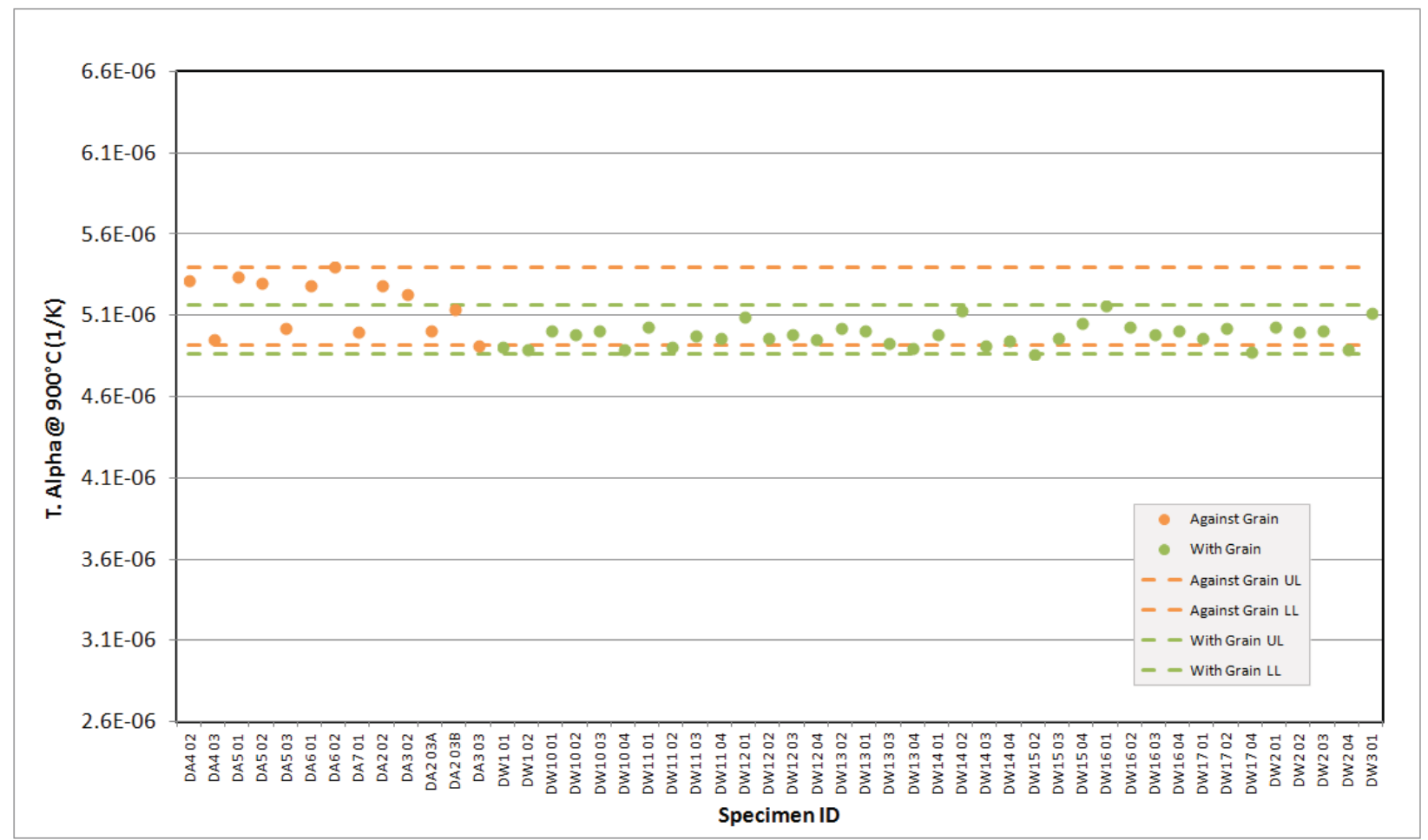

Figure A-112. PCEA Creep Coefficient of Thermal Expansion @ $900^{\circ} \mathrm{C}$. 


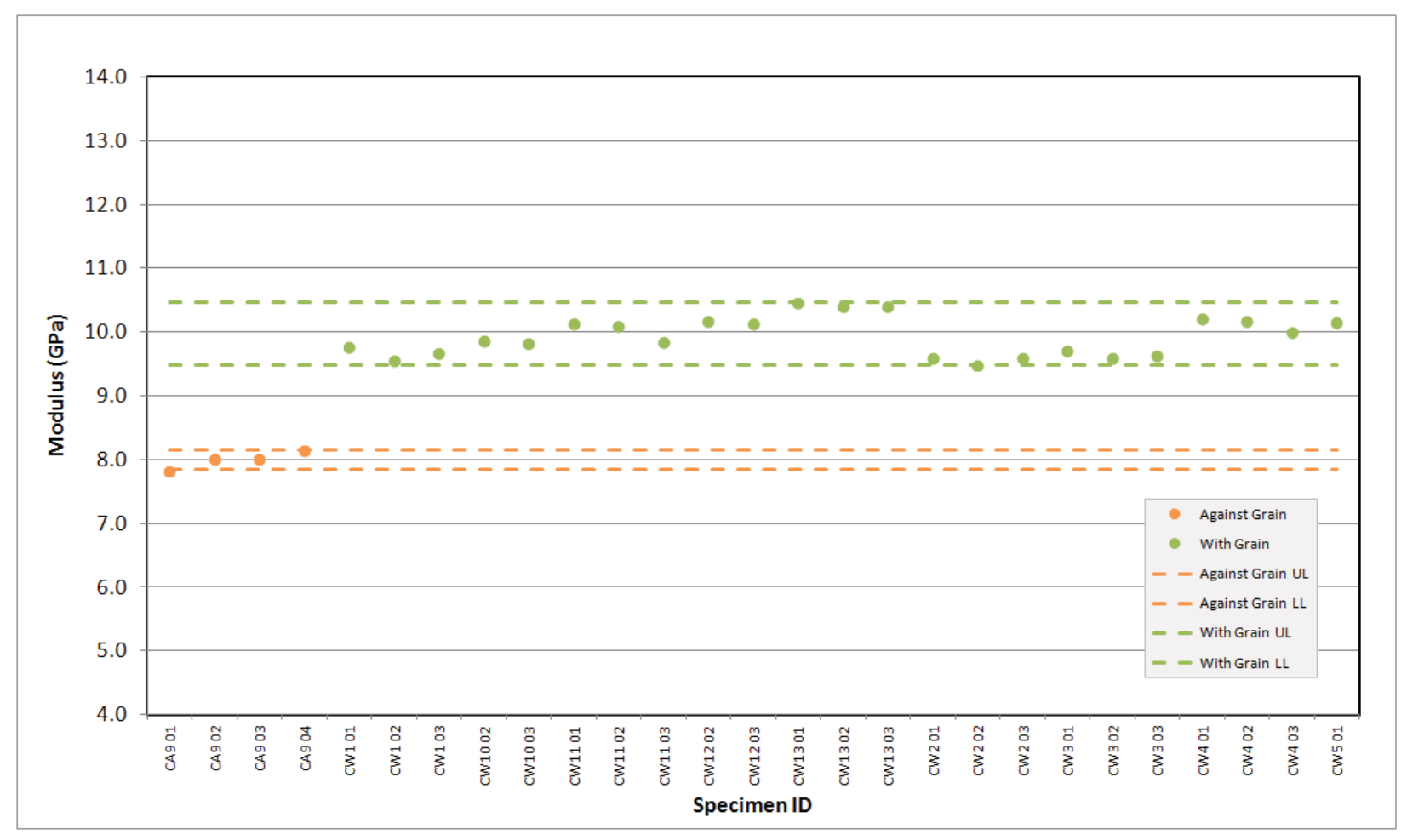

Figure A-113. H-451 Creep Modulus by Sonic Resonance.

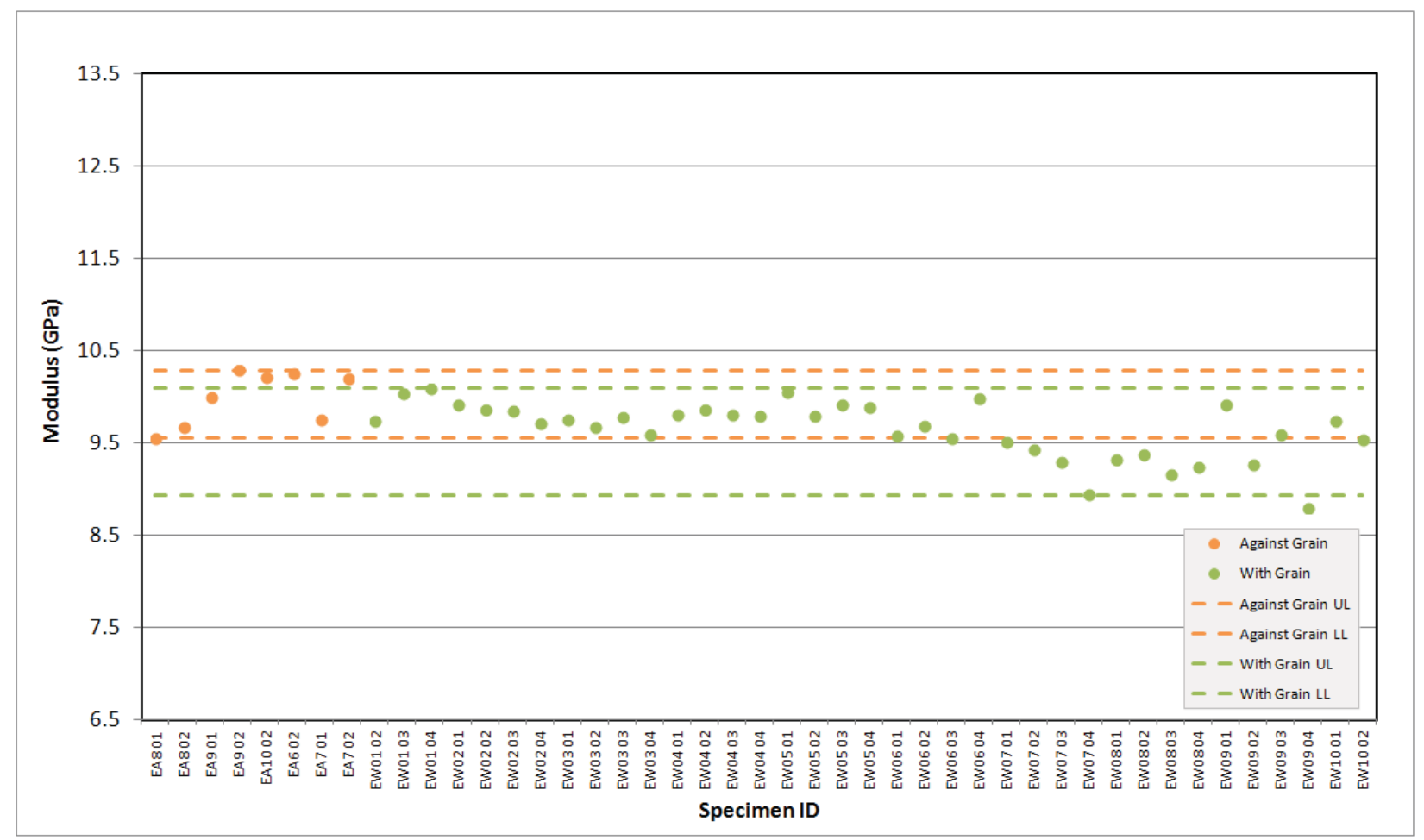

Figure A-114. IG-110 Creep Modulus by Sonic Resonance. 


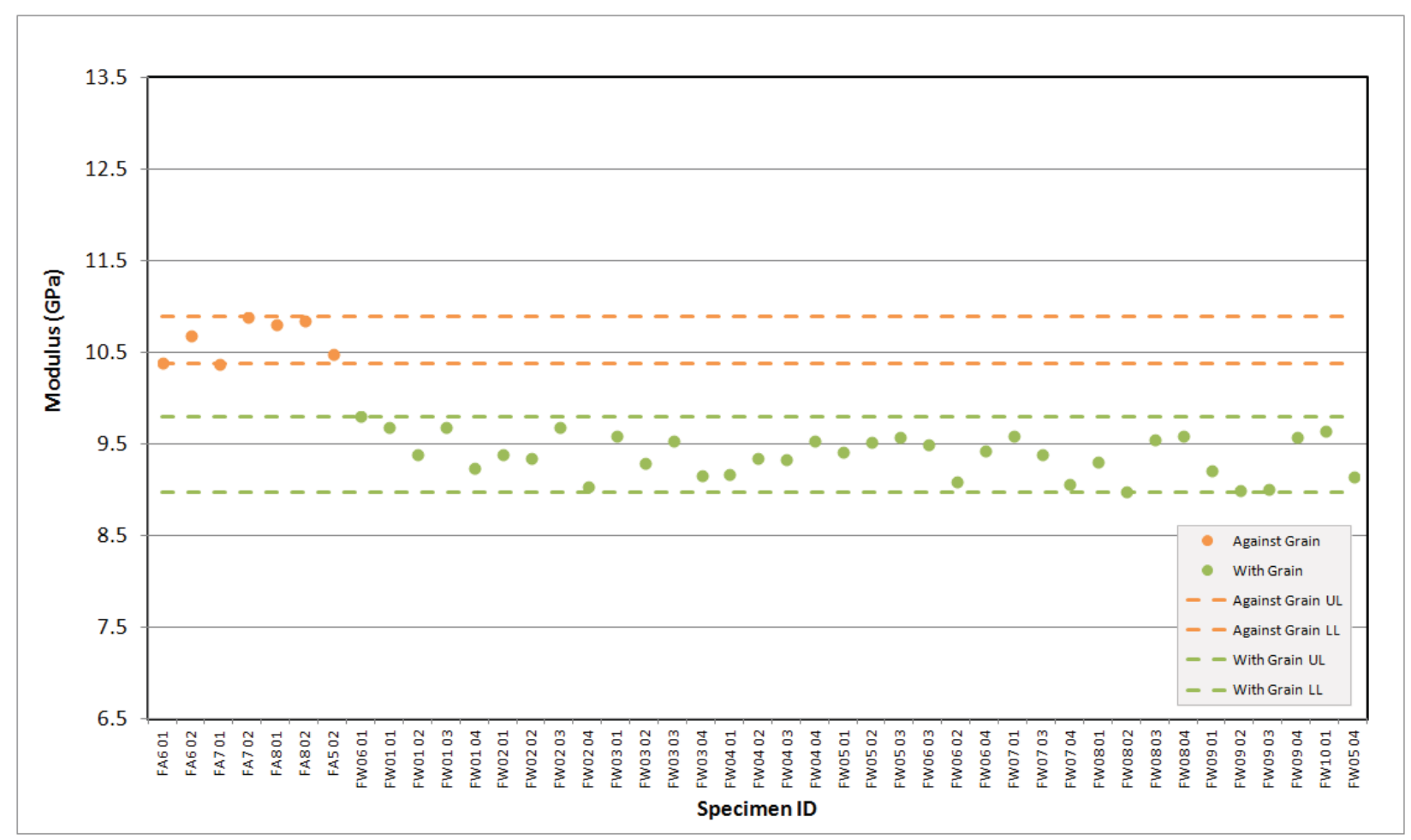

Figure A-115. IG-430 Creep Modulus by Sonic Resonance.

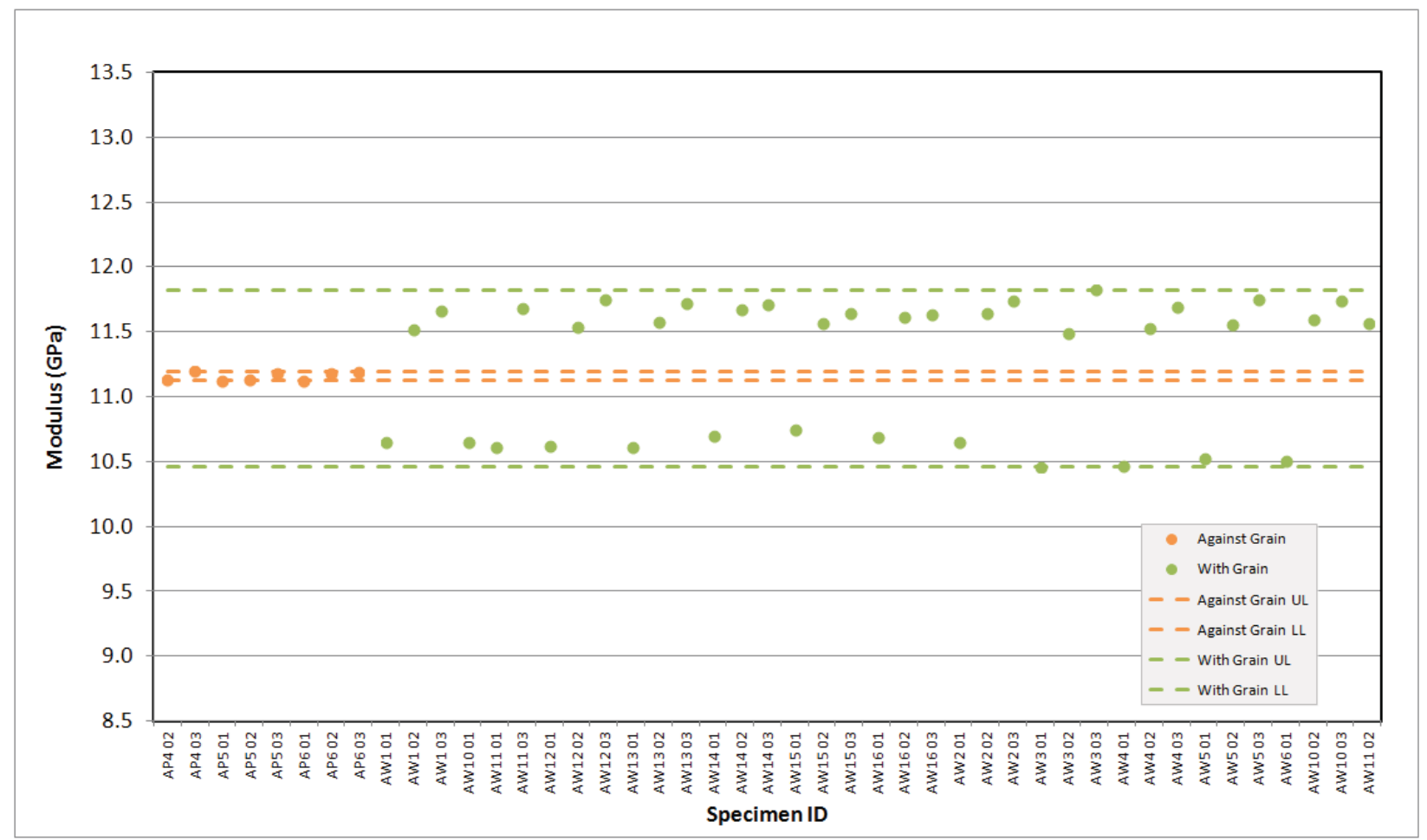

Figure A-116. NBG-17 Creep Modulus by Sonic Resonance. 


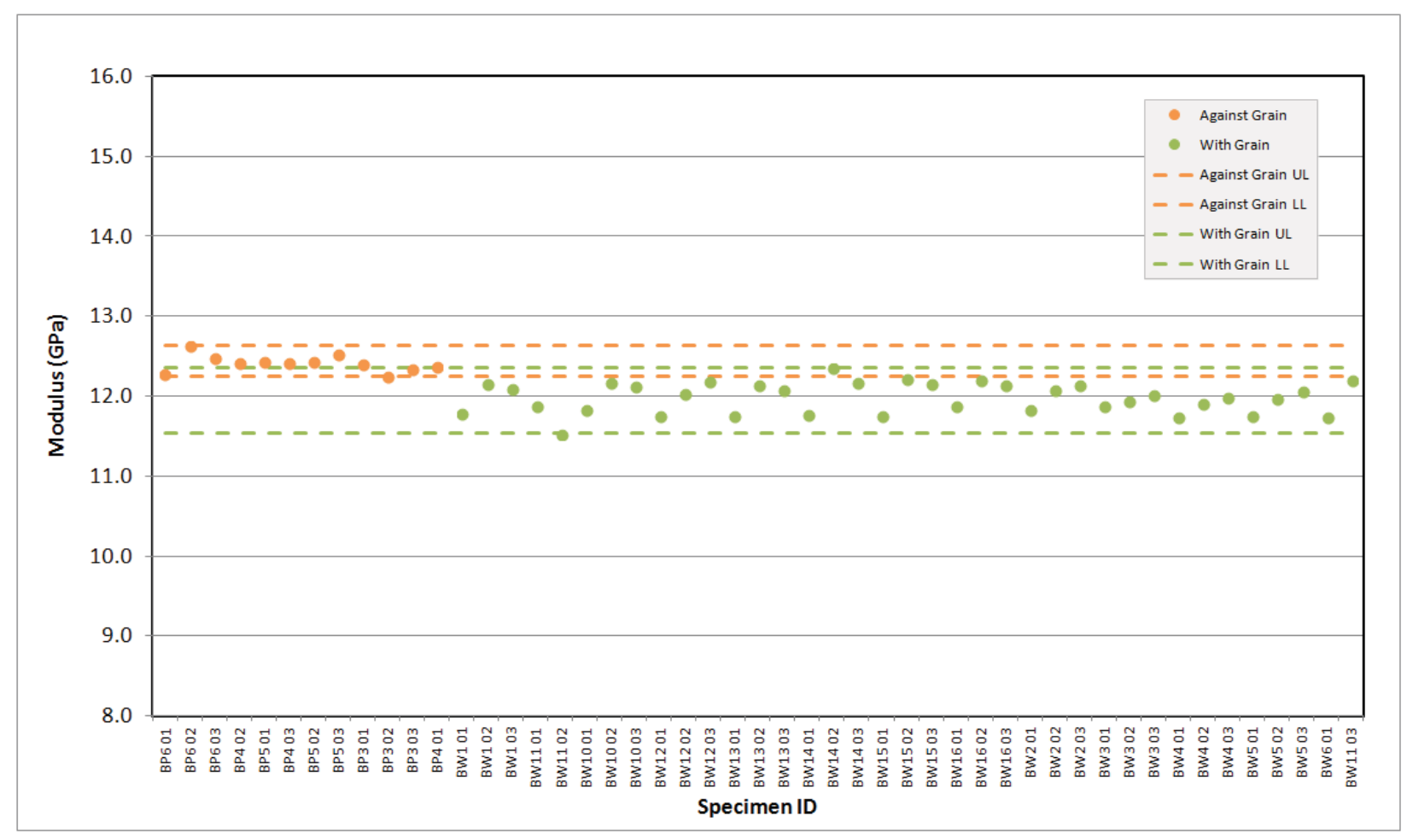

Figure A-117. NBG-18 Creep Modulus by Sonic Resonance.

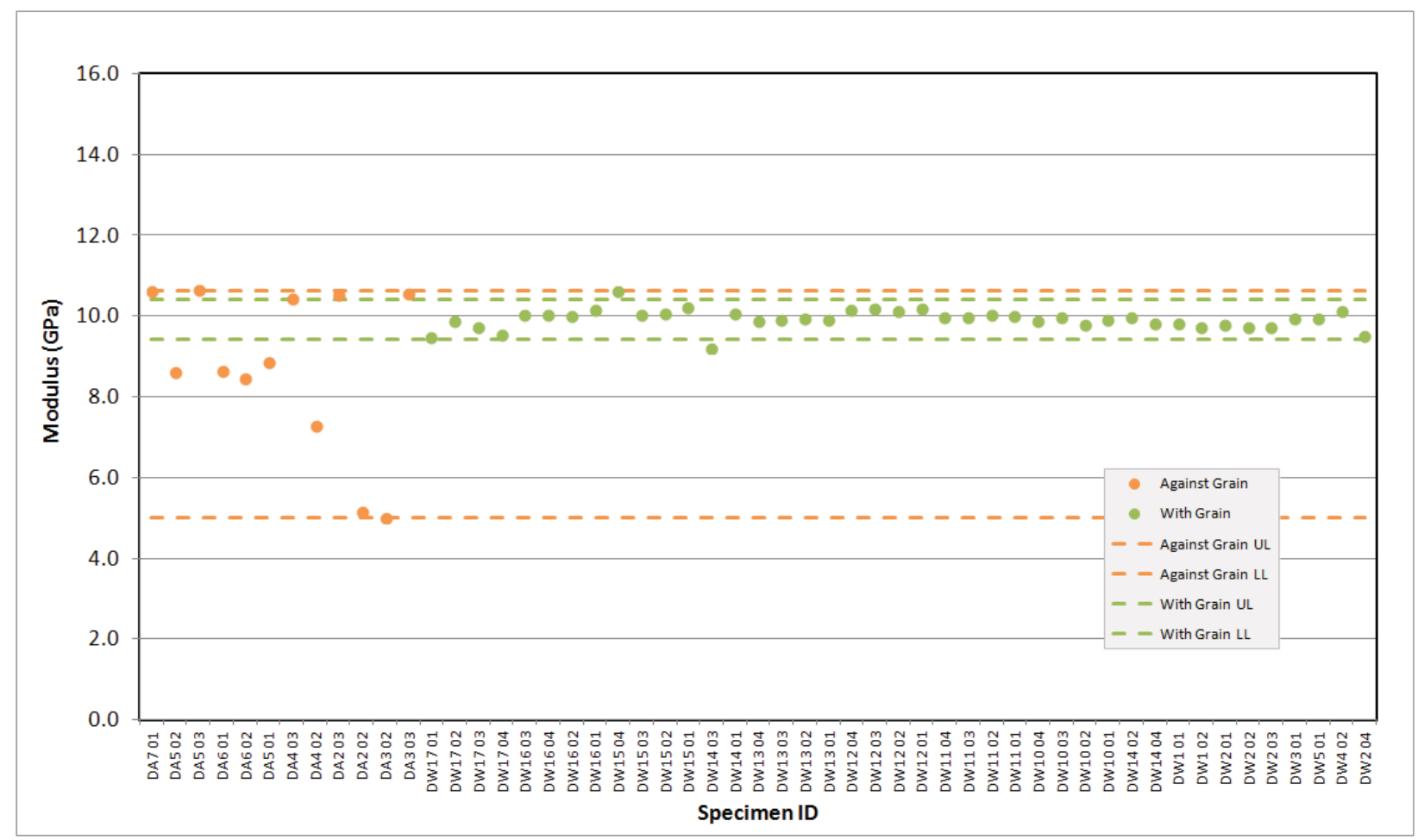

Figure A-118. PCEA Creep Modulus by Sonic Resonance. 


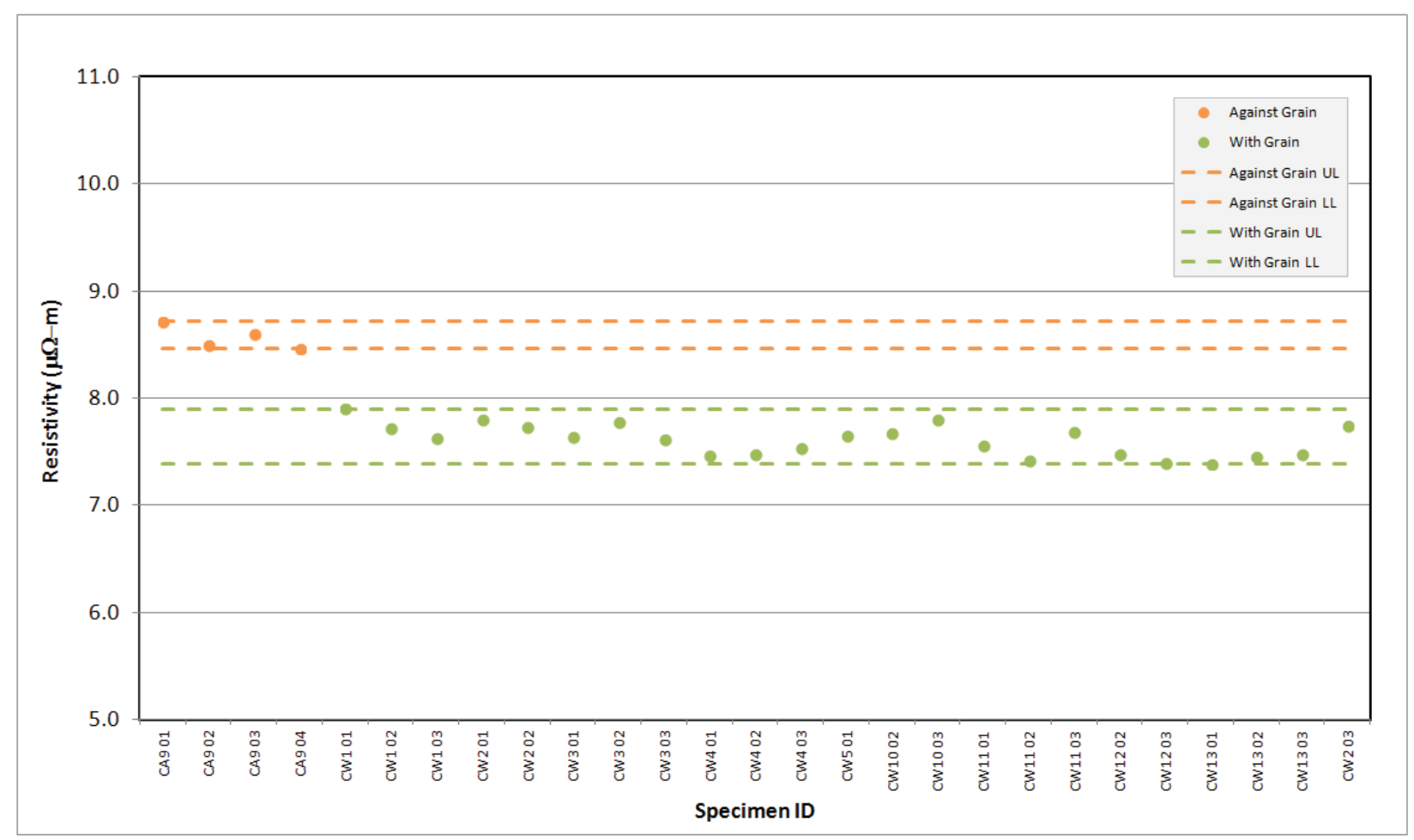

Figure A-119. H-451 Creep Resistivity.

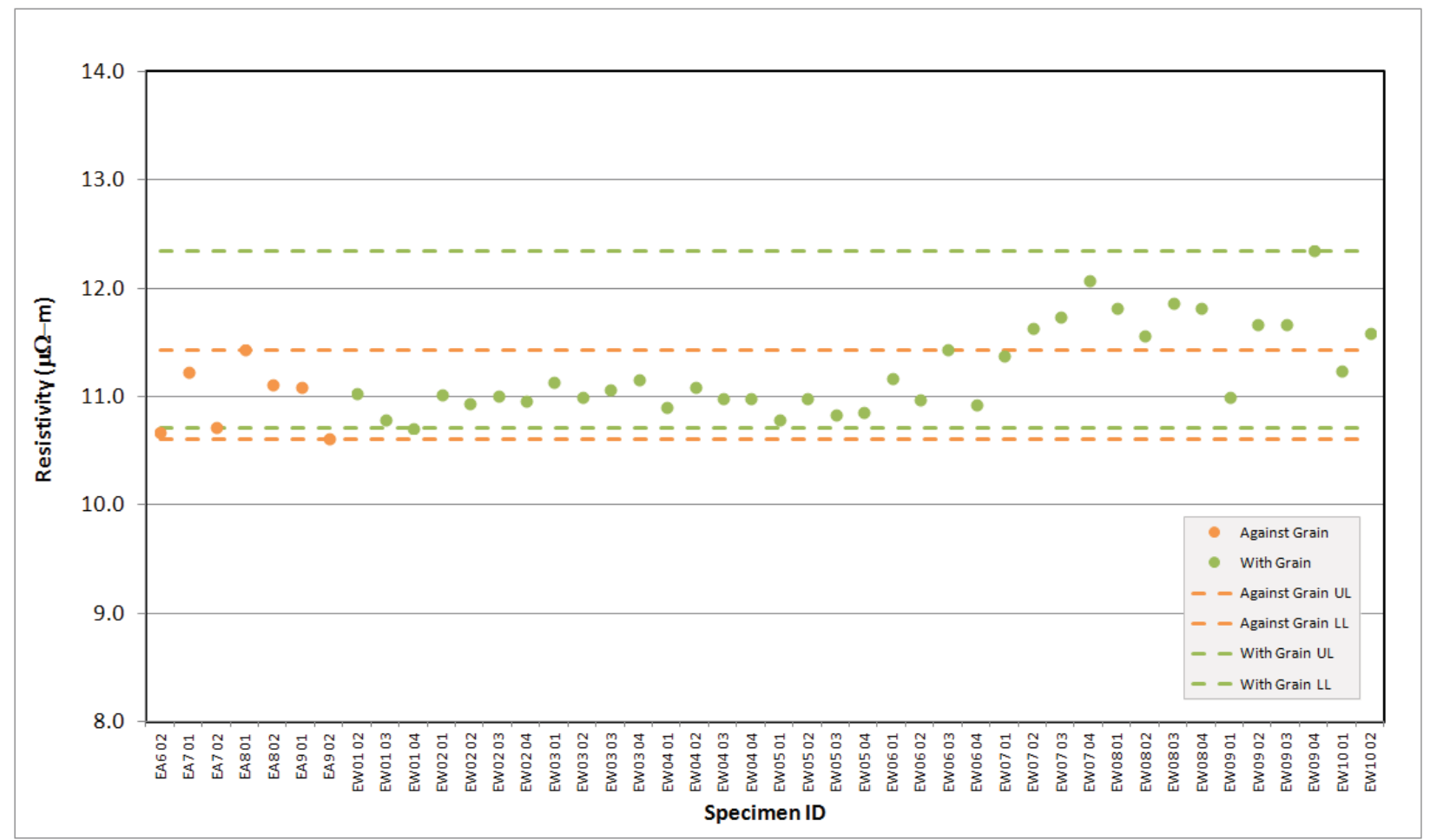

Figure A-120. IG-110 Creep Resistivity. 


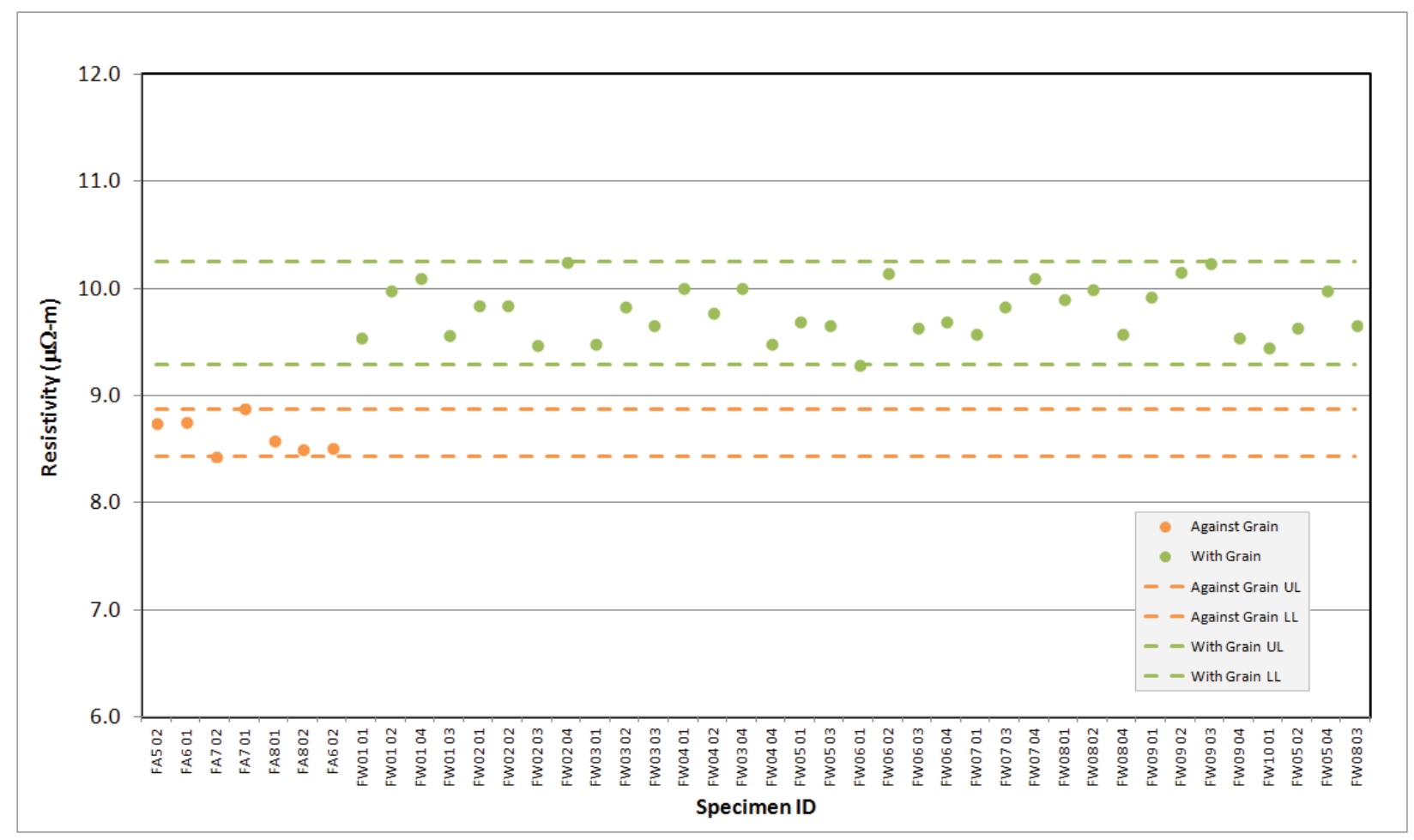

Figure A-121. IG-430 Creep Resistivity.

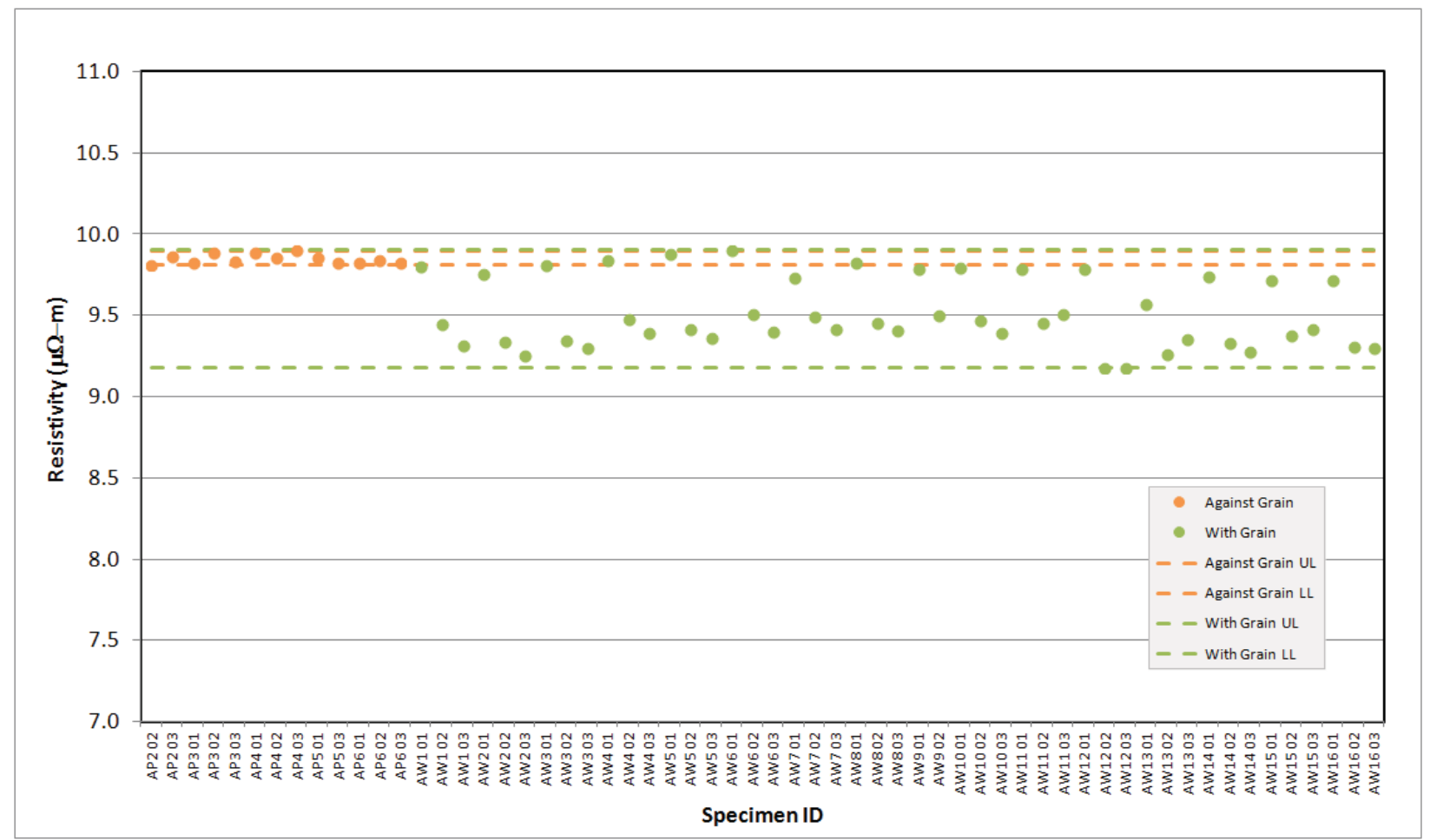

Figure A-122. NBG-17 Creep Resistivity. 


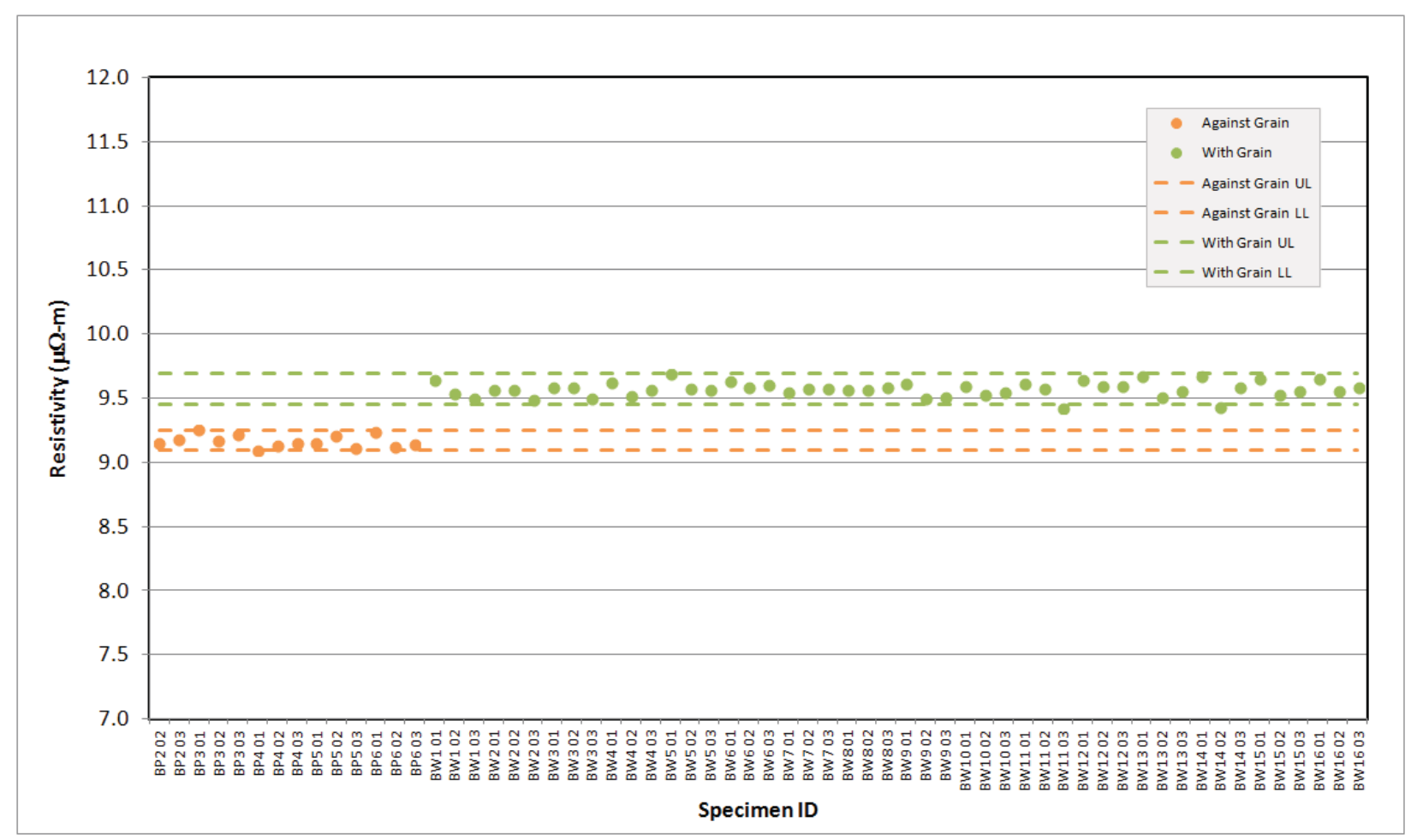

Figure A-123. NBG-18 Creep Resistivity.

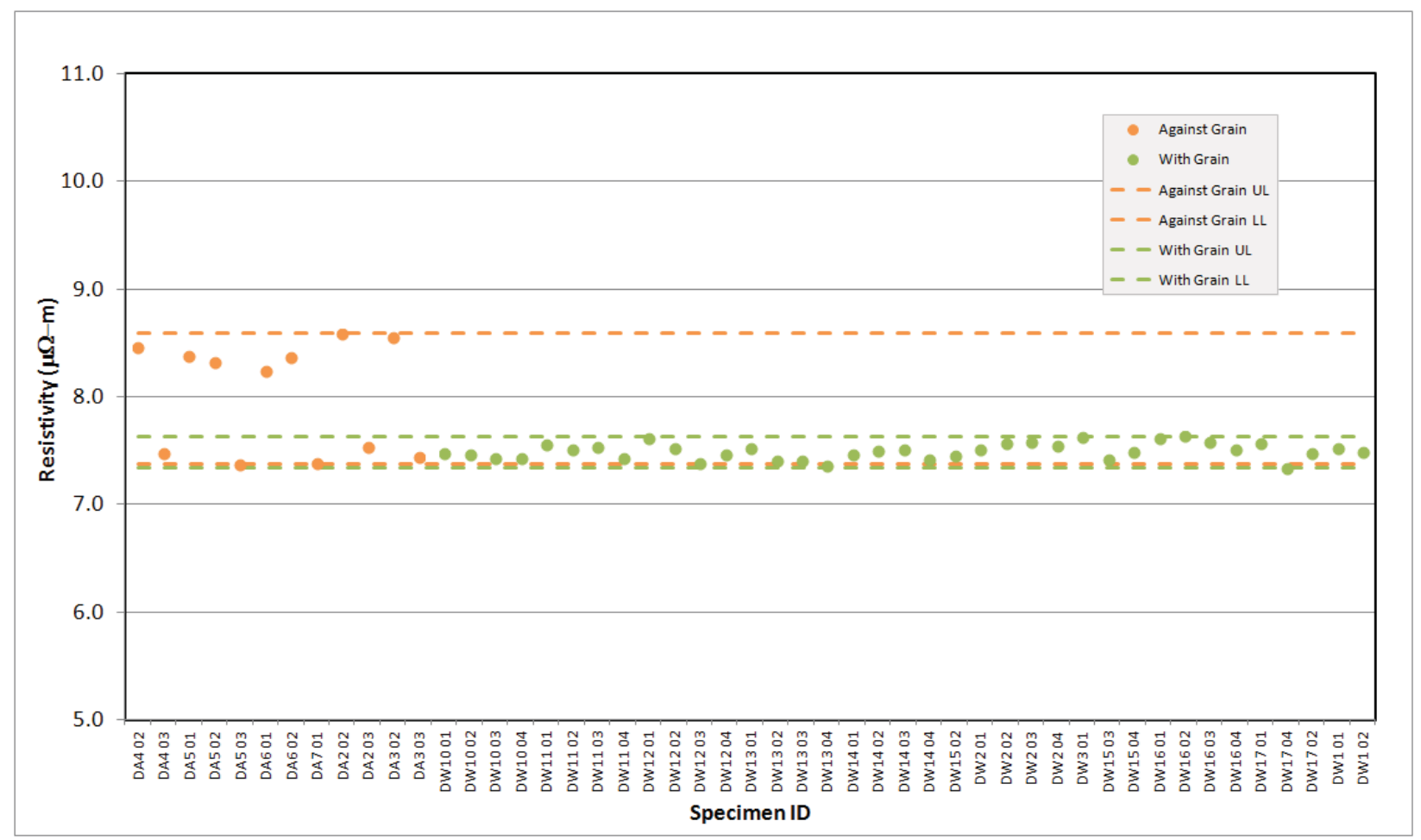

Figure A-124. PCEA Creep Resistivity. 


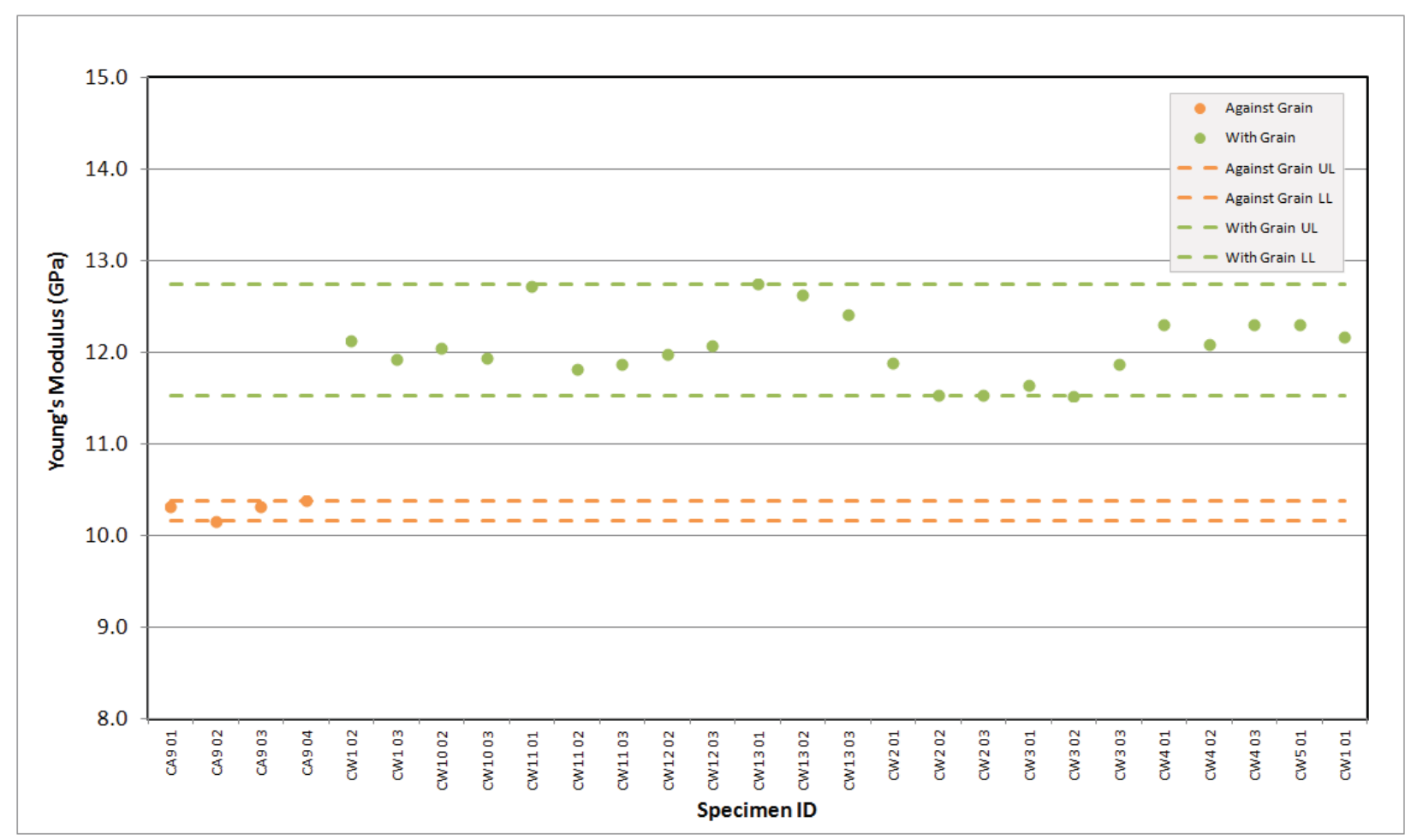

Figure A-125. H-451 Creep Young's Modulus by Sonic Velocity.

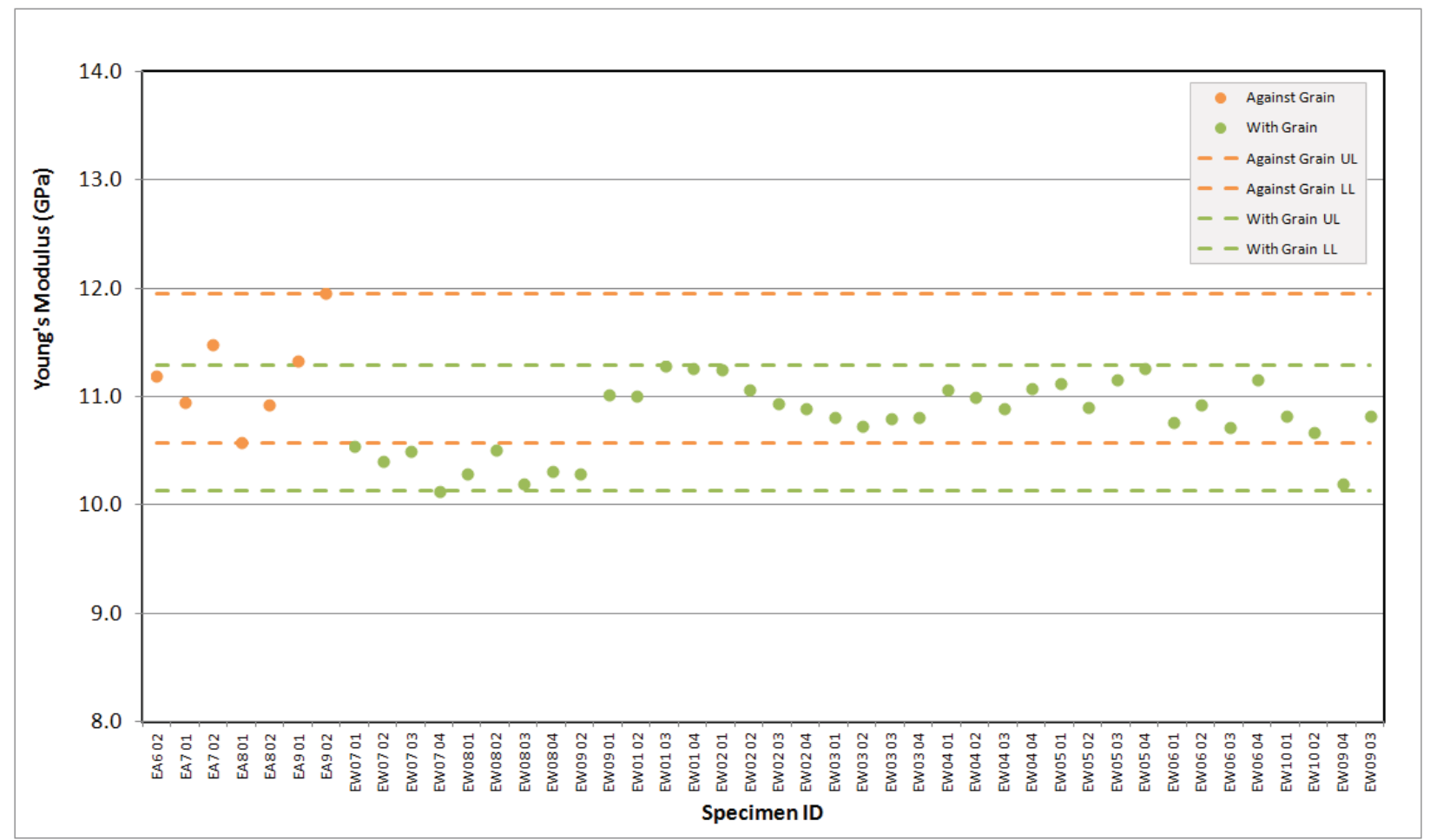

Figure A-126. IG-110 Creep Young's Modulus by Sonic Velocity. 


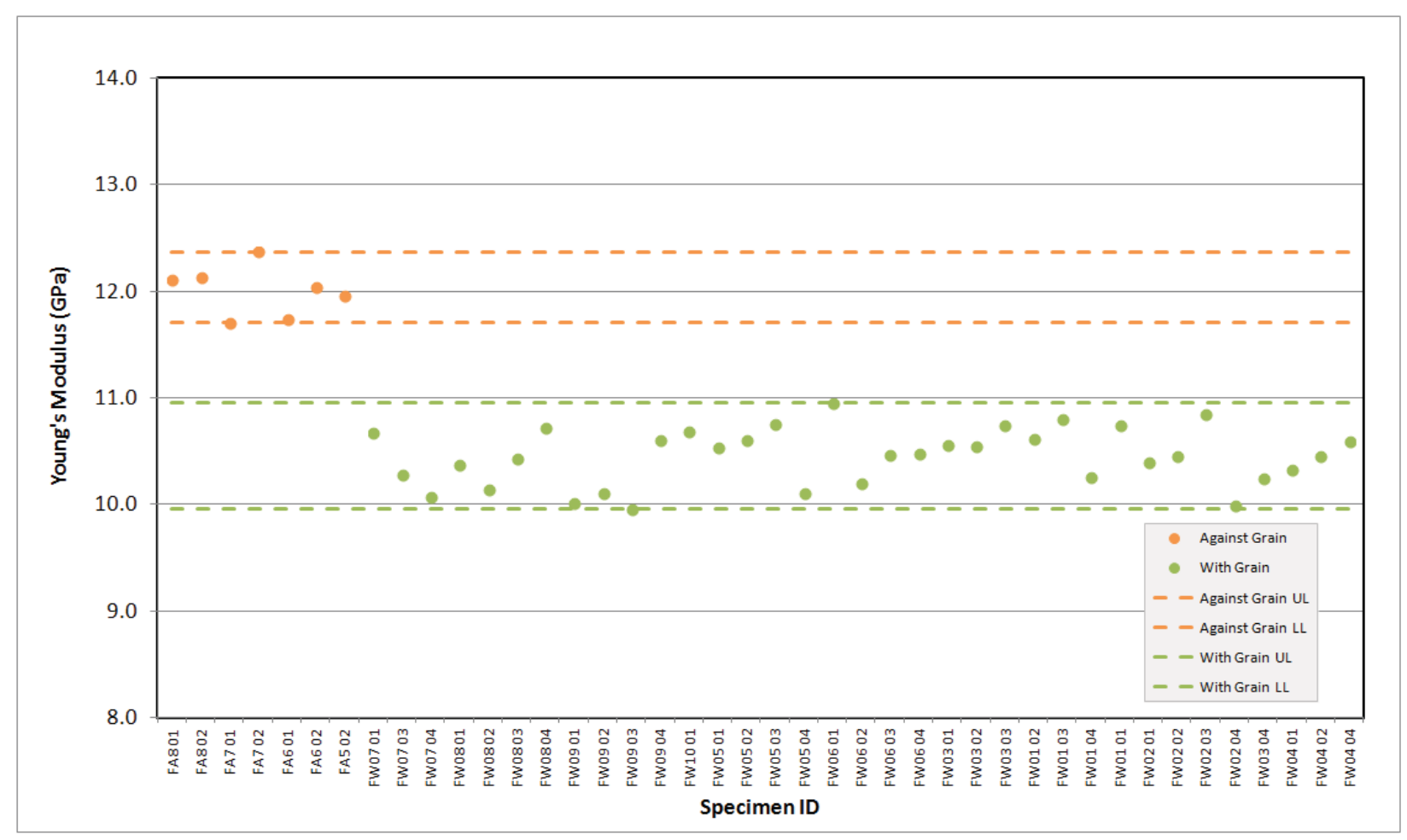

Figure A-127. IG-430 Creep Young's Modulus by Sonic Velocity.

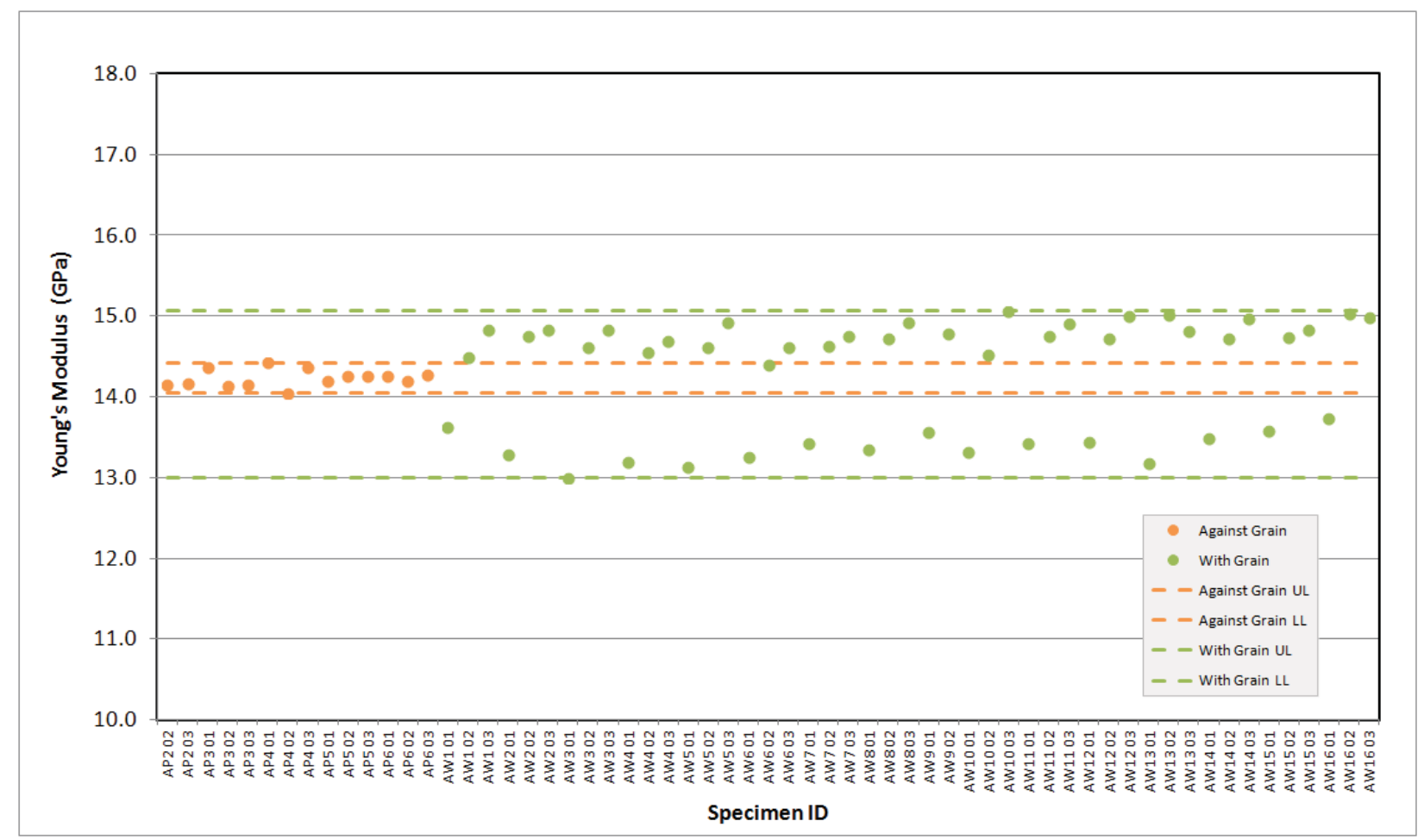

Figure A-128. NBG-17 Creep Young's Modulus by Sonic Velocity. 


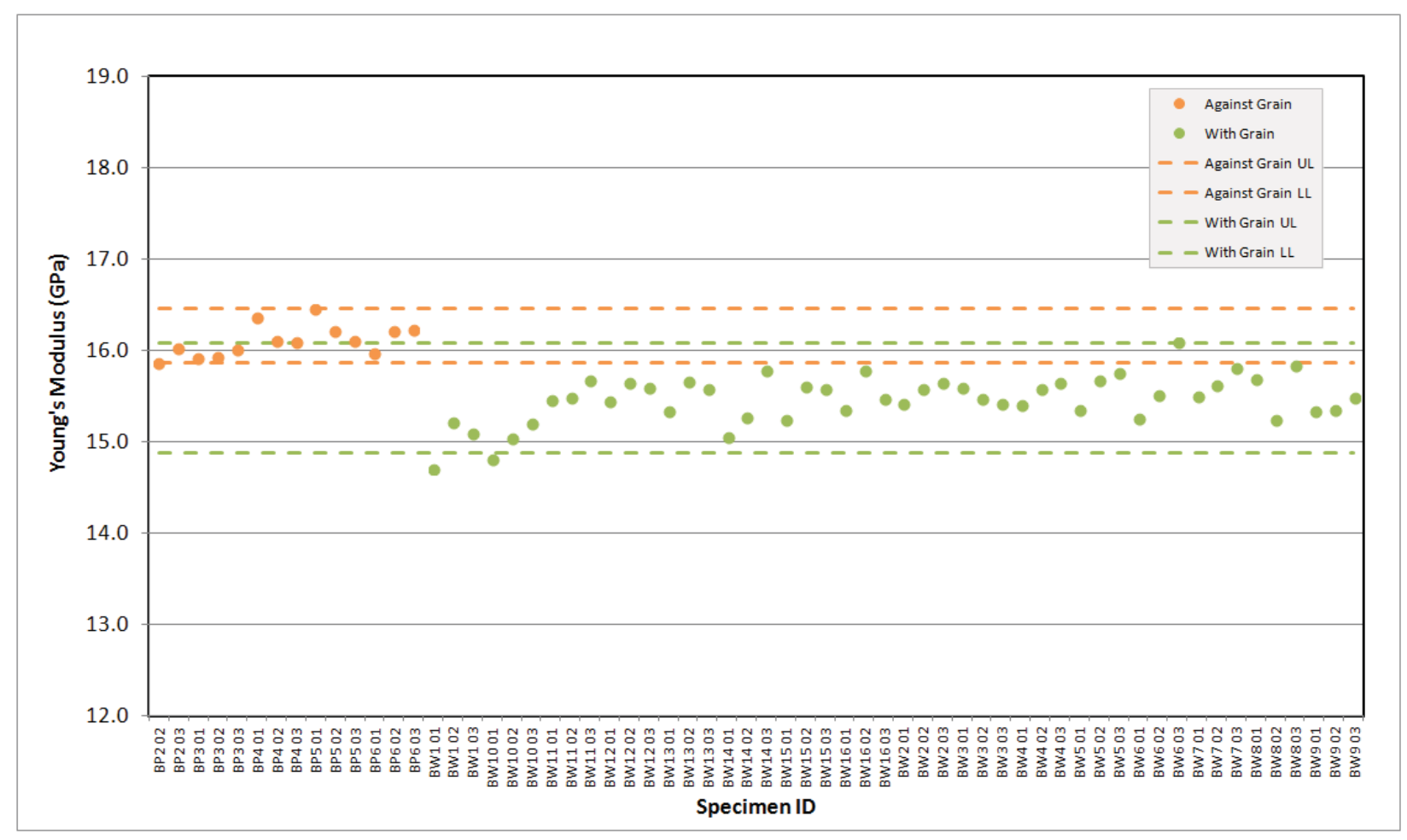

Figure A-129. NBG-18 Creep Young's Modulus by Sonic Velocity.

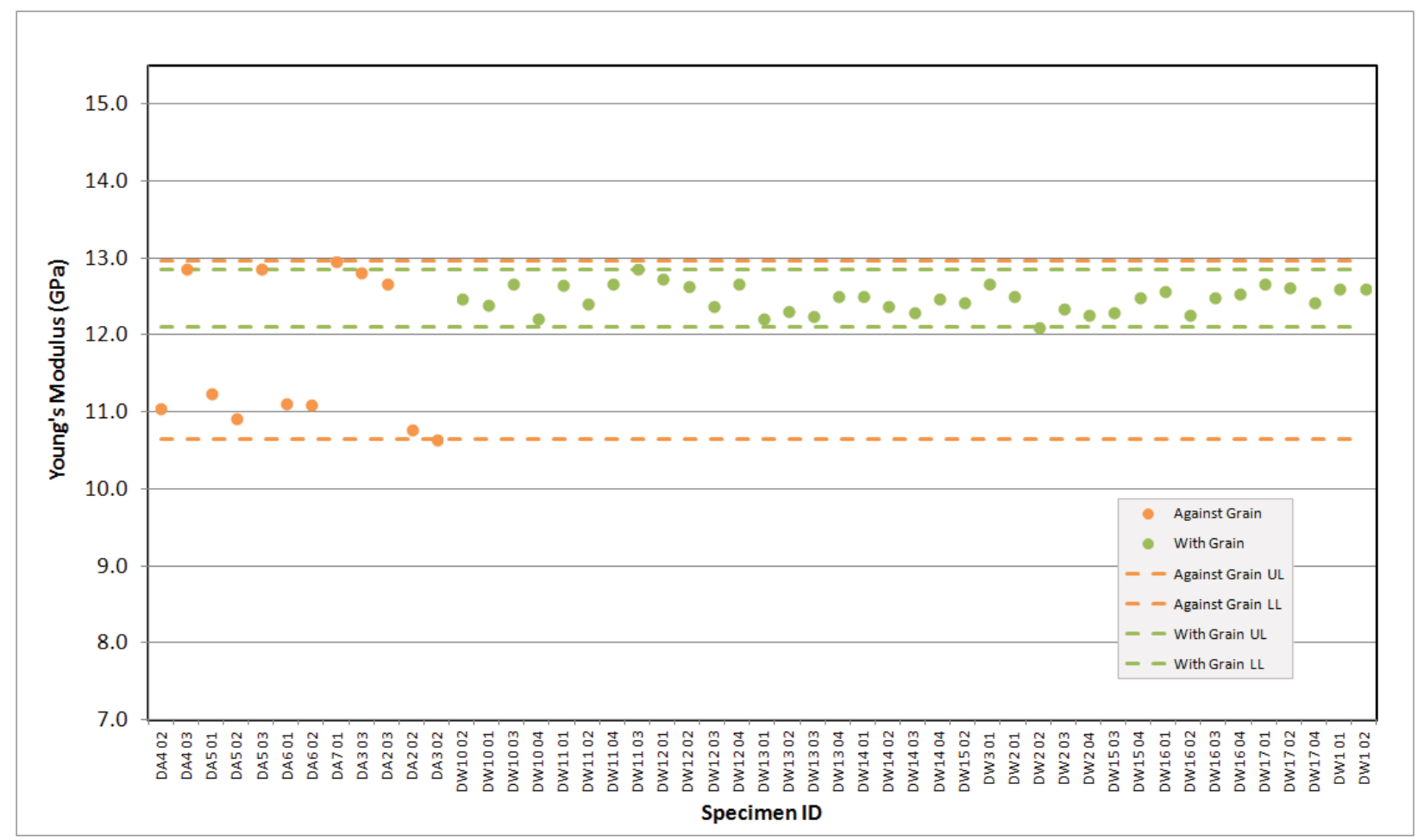

Figure A-130. PCEA Creep Young's Modulus by Sonic Velocity. 


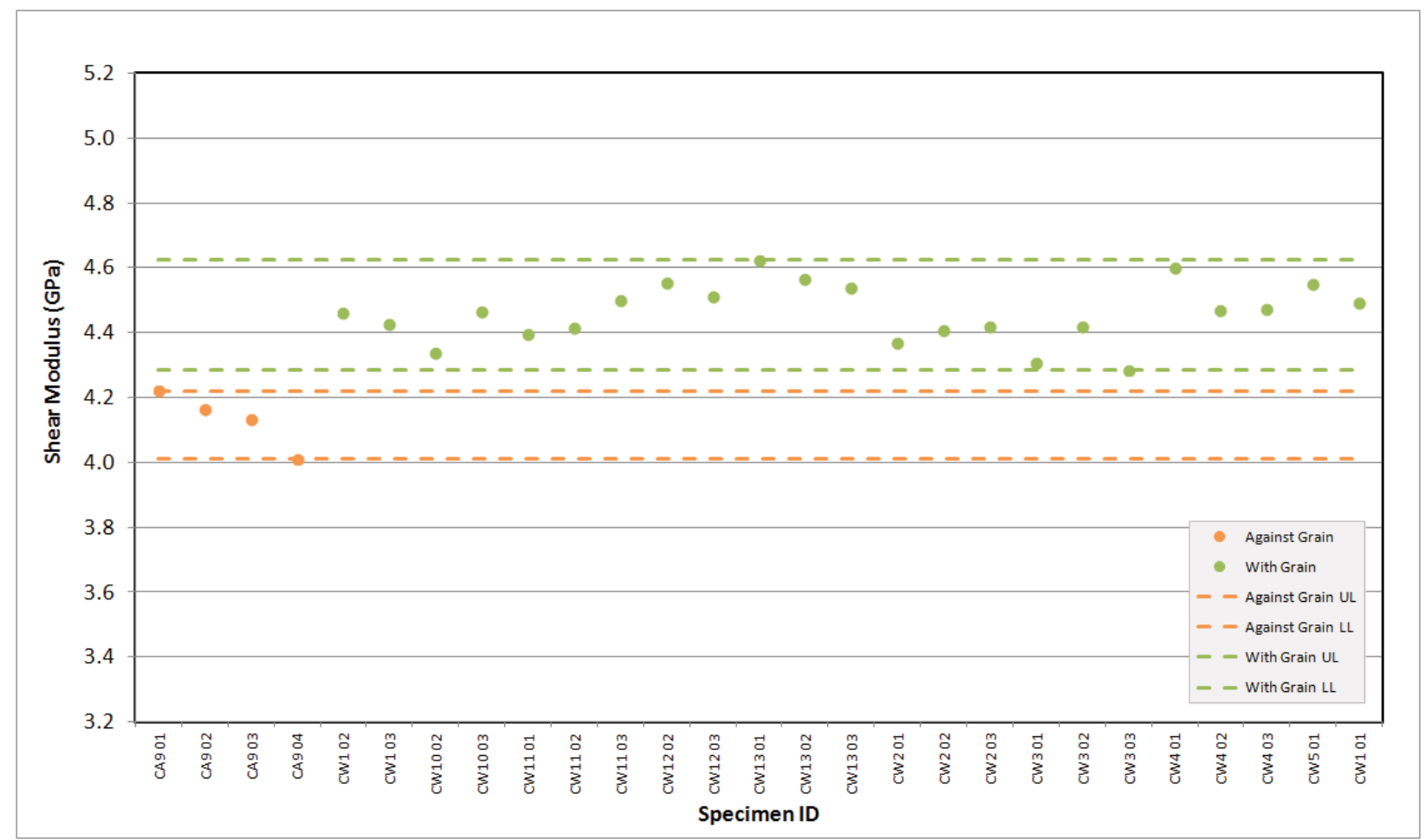

Figure A-131. H-451 Creep Shear Modulus by Sonic Velocity.

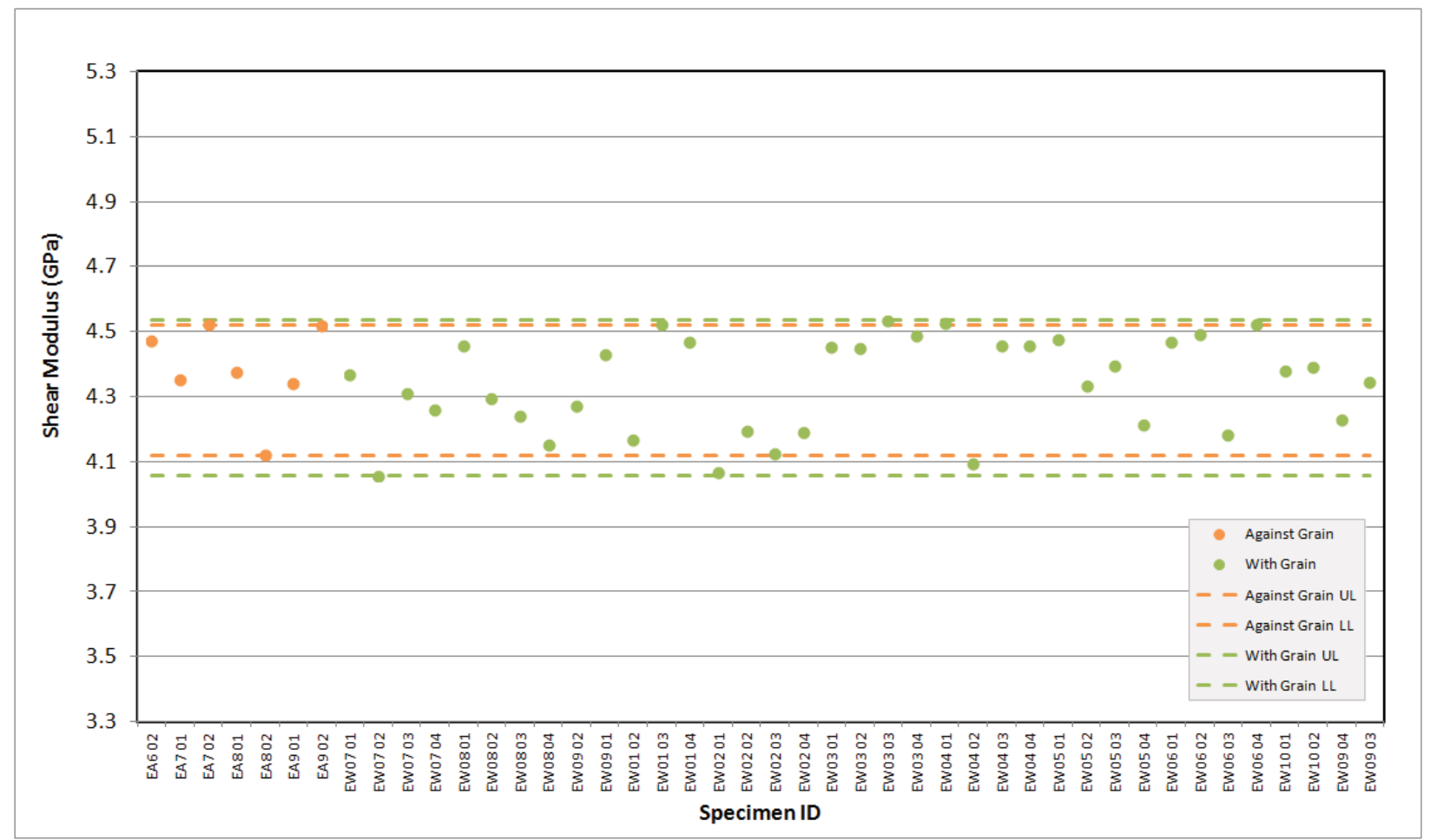

Figure A-132. IG-110 Creep Shear Modulus by Sonic Velocity. 


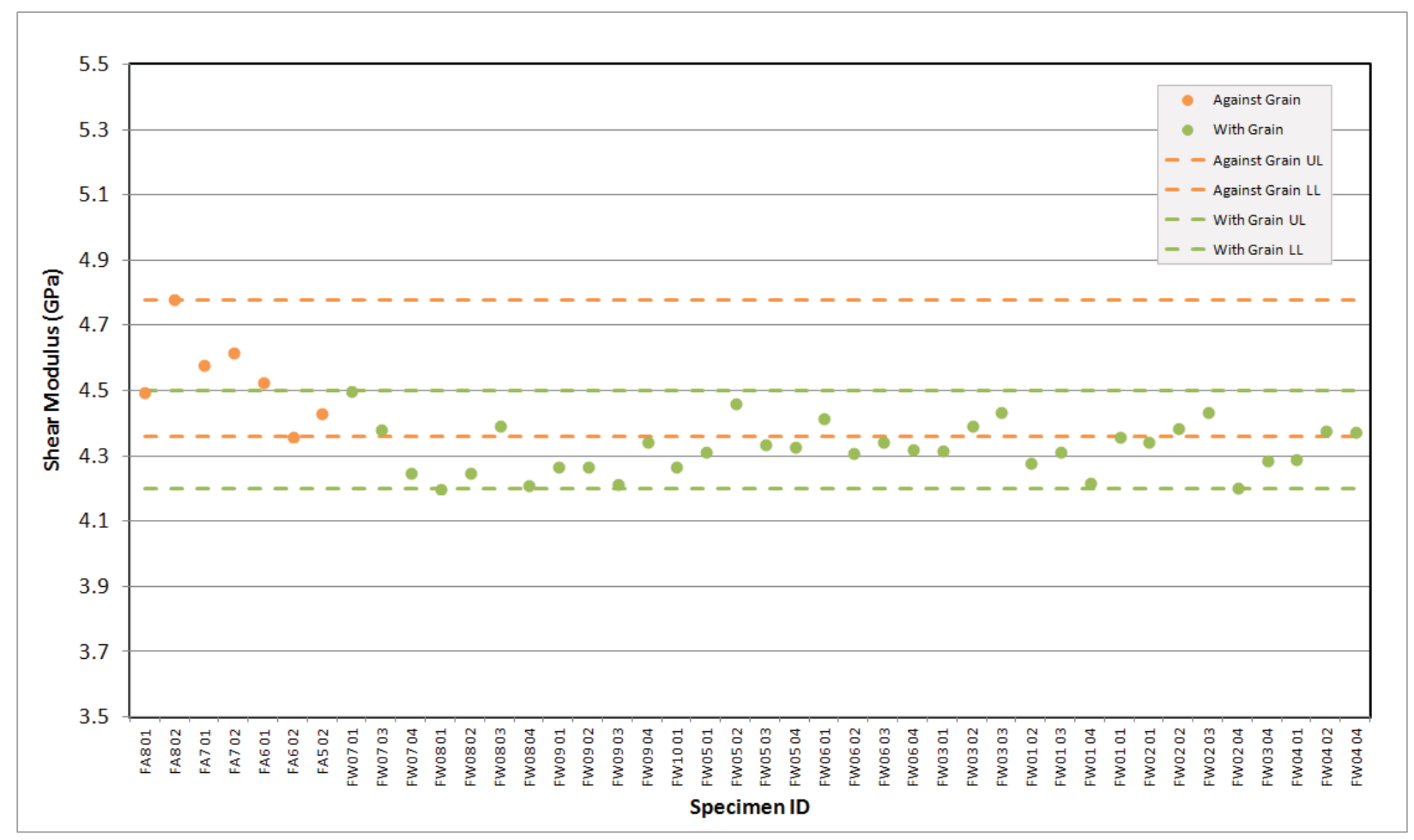

Figure A-133. IG-430 Creep Shear Modulus by Sonic Velocity.

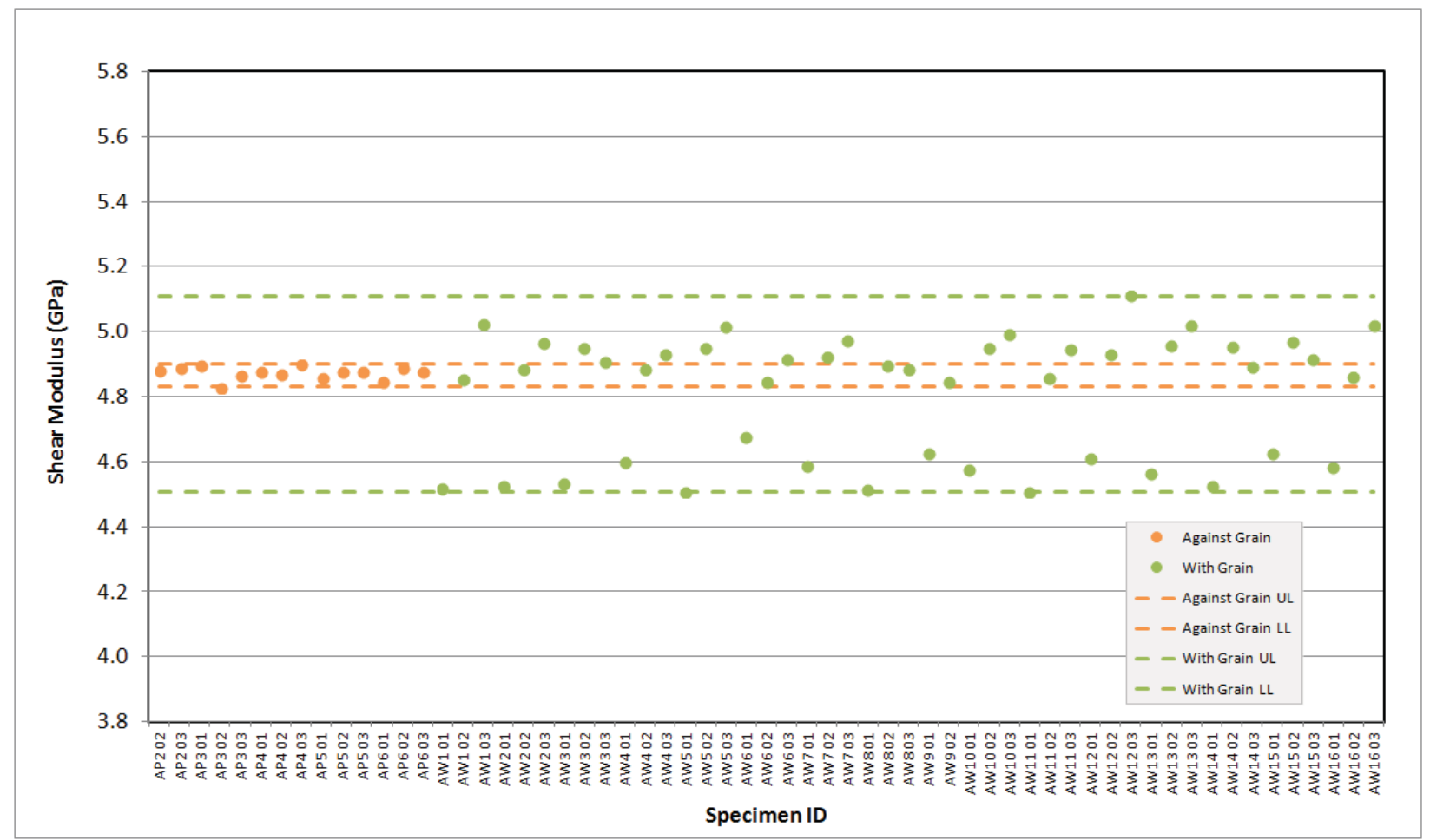

Figure A-134. NBG-17 Creep Shear Modulus by Sonic Velocity. 


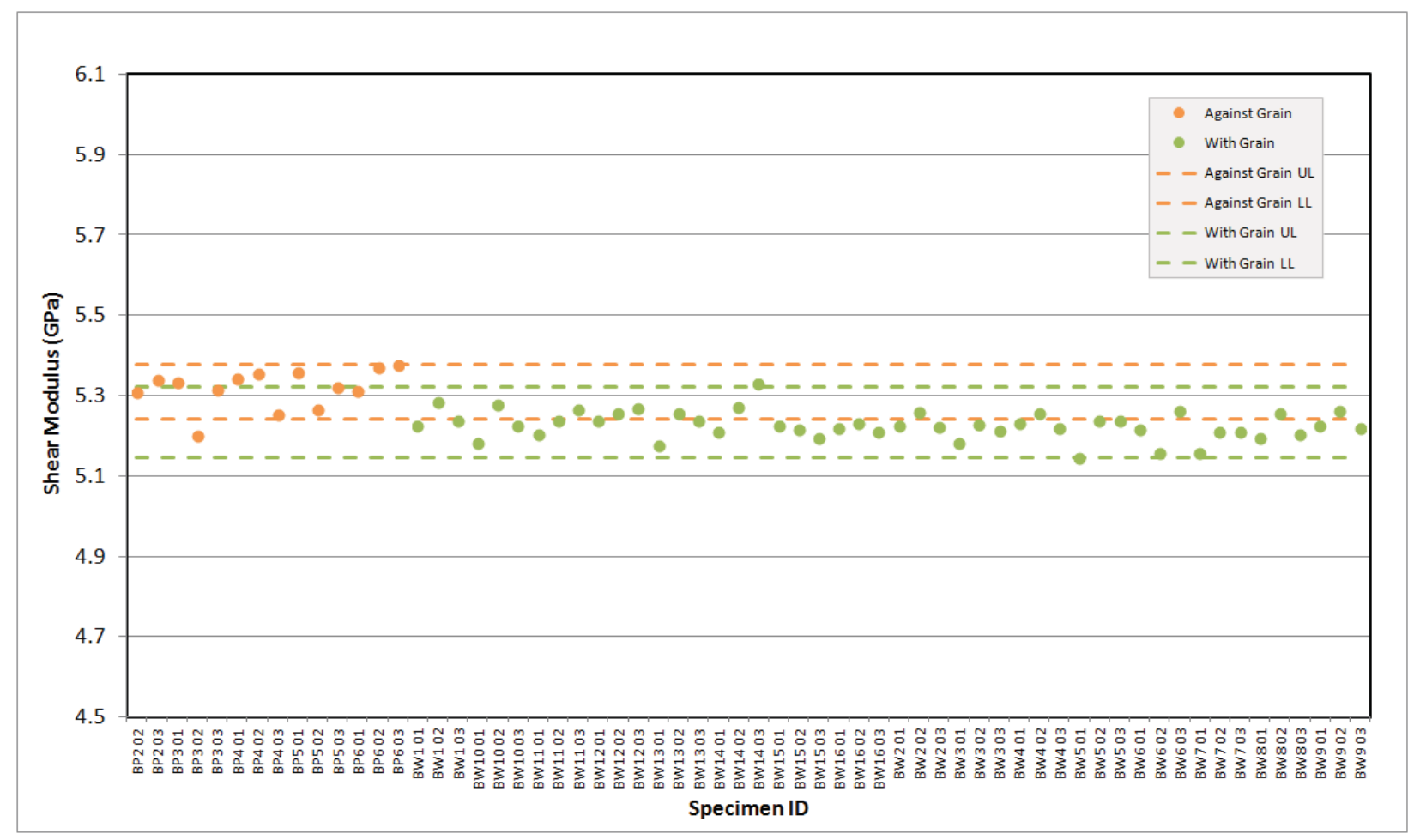

Figure A-135. NBG-18 Creep Shear Modulus by Sonic Velocity.

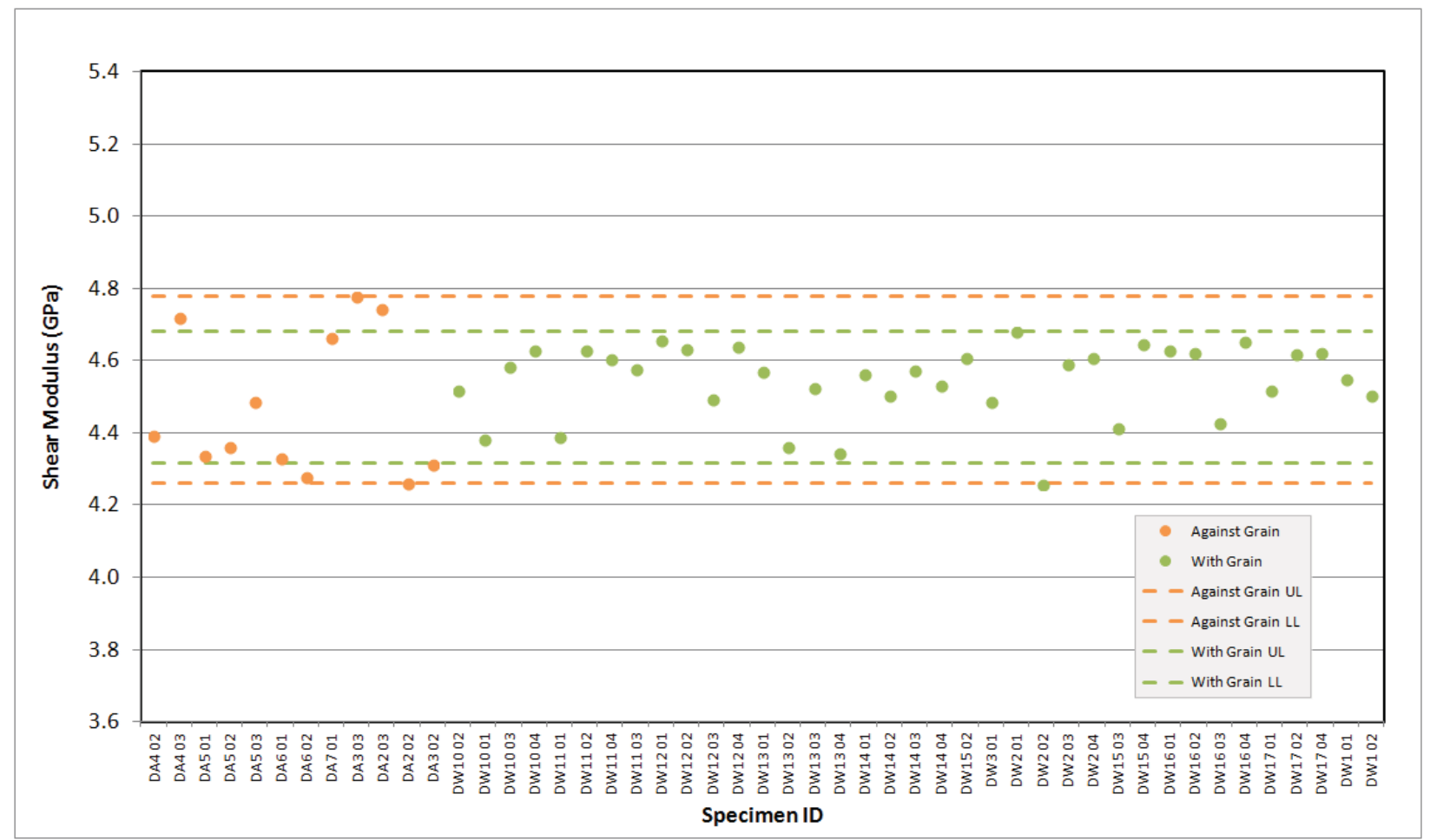

Figure A-136. PCEA Creep Shear Modulus by Sonic Velocity. 


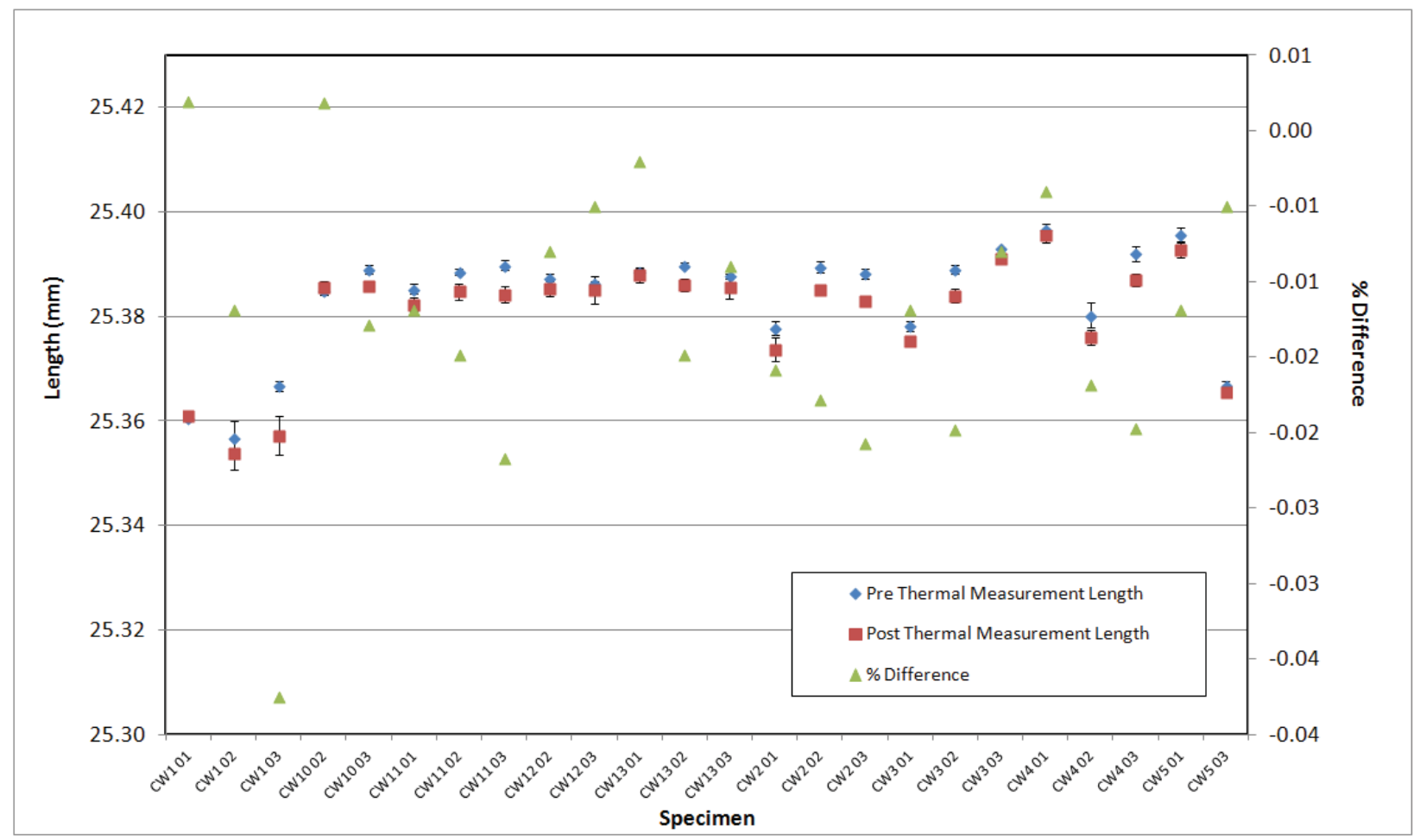

Figure A-137. H-451 Creep Pre vs. Post Thermal Measurement Length Comparison.

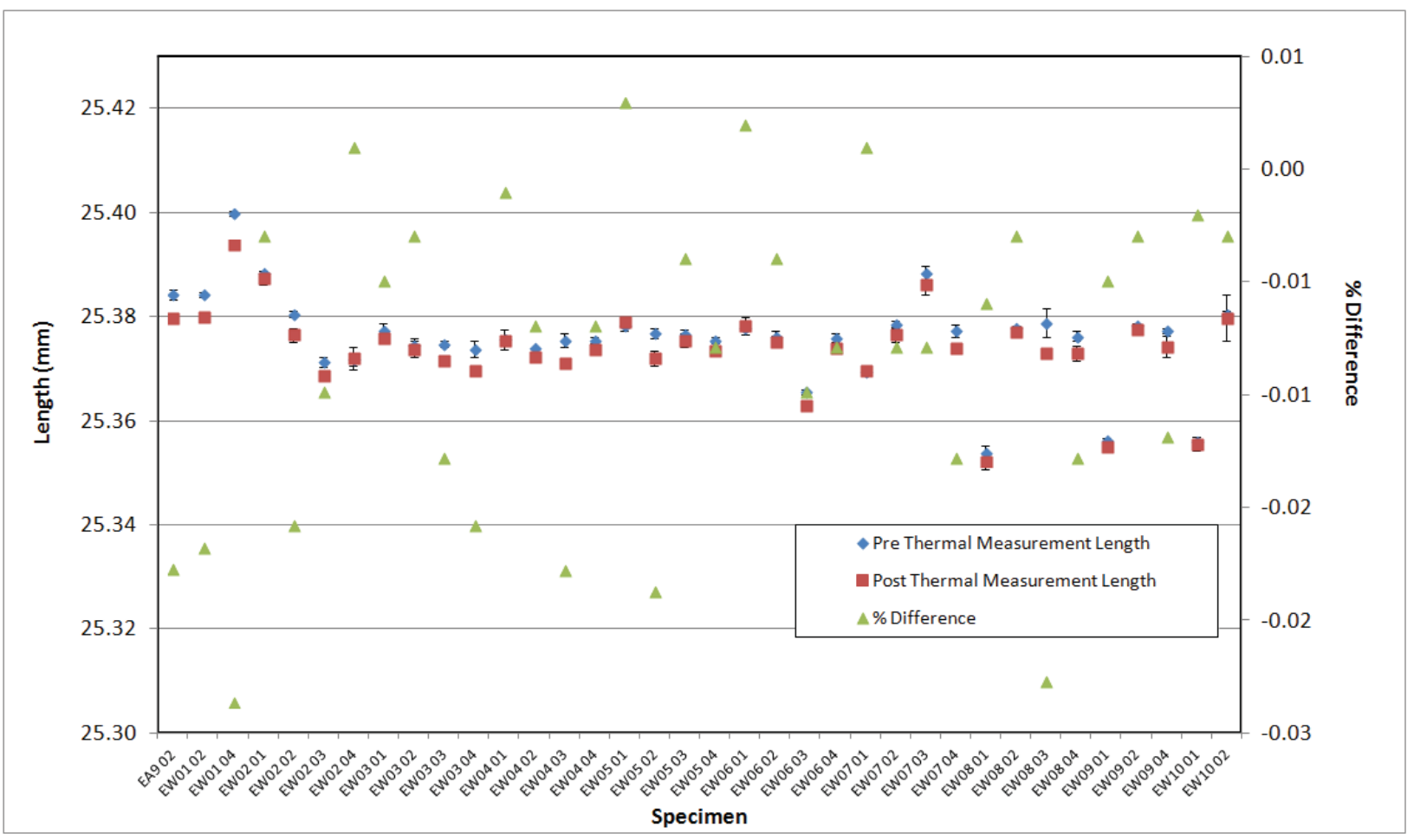

Figure A-138. IG-110 Creep Pre vs. Post Thermal Measurement Length Comparison. 


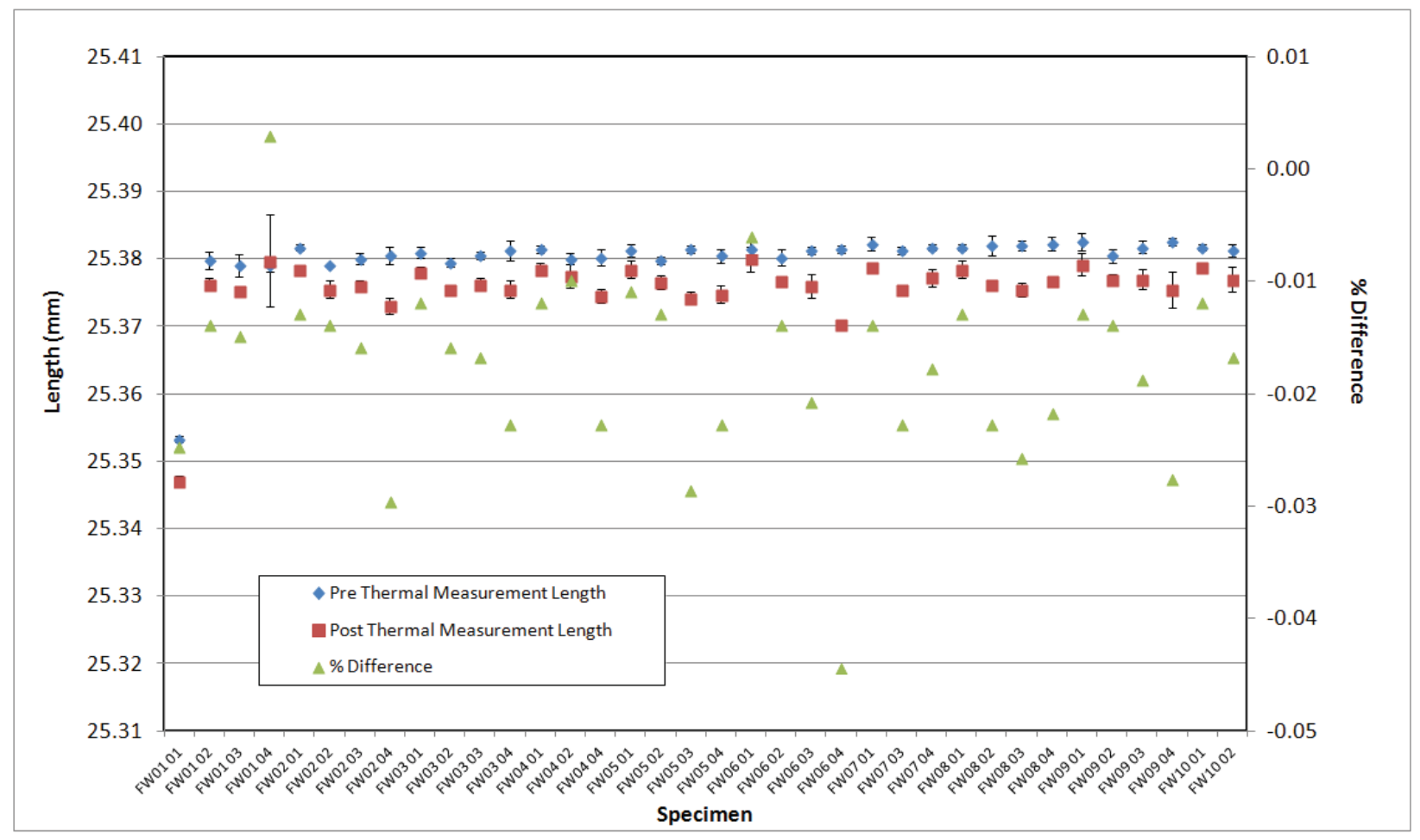

Figure A-139. IG-430 Creep Pre vs. Post Thermal Measurement Length Comparison.

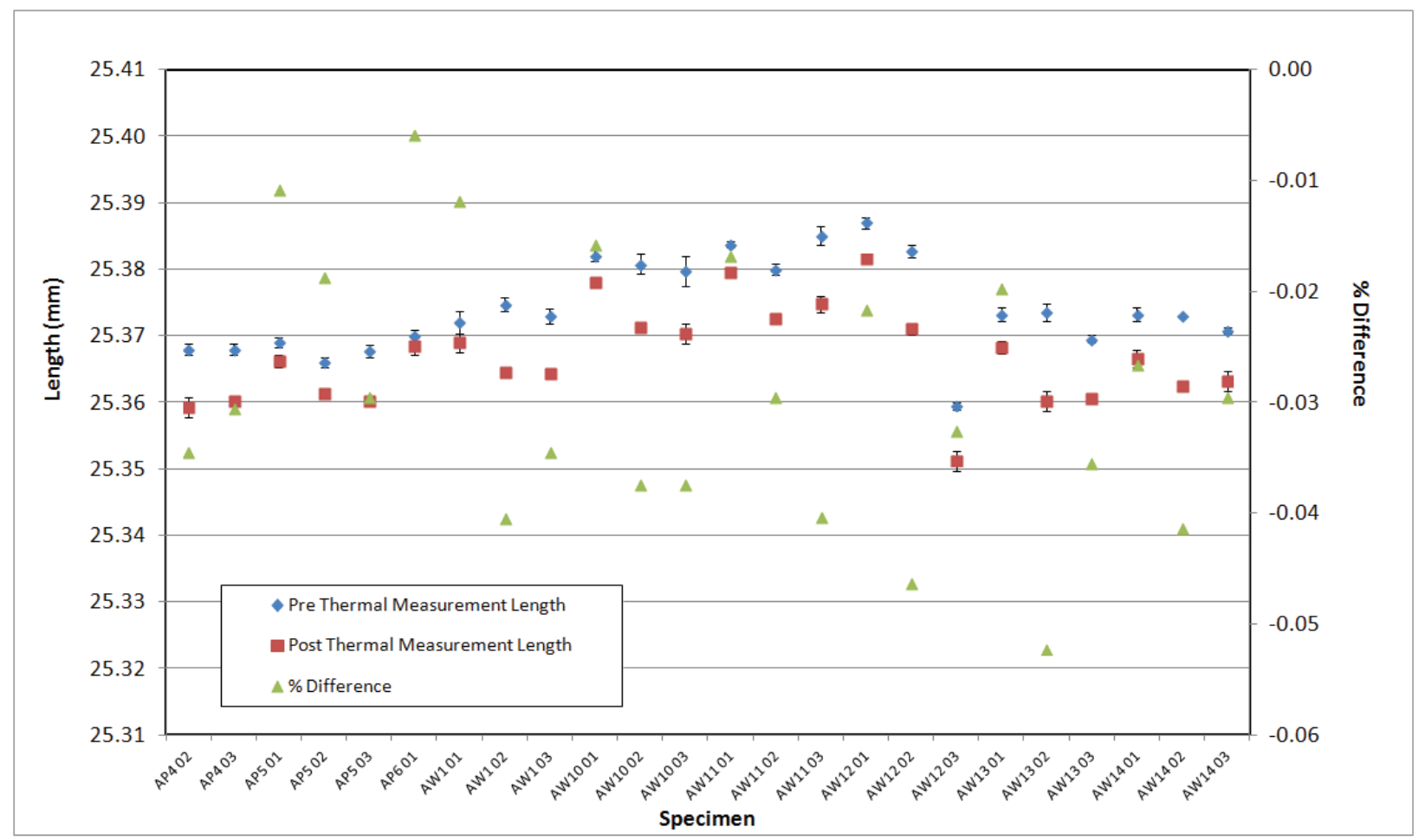

Figure A-140. NBG-17 Creep Pre vs. Post Thermal Measurement Length Comparison. 


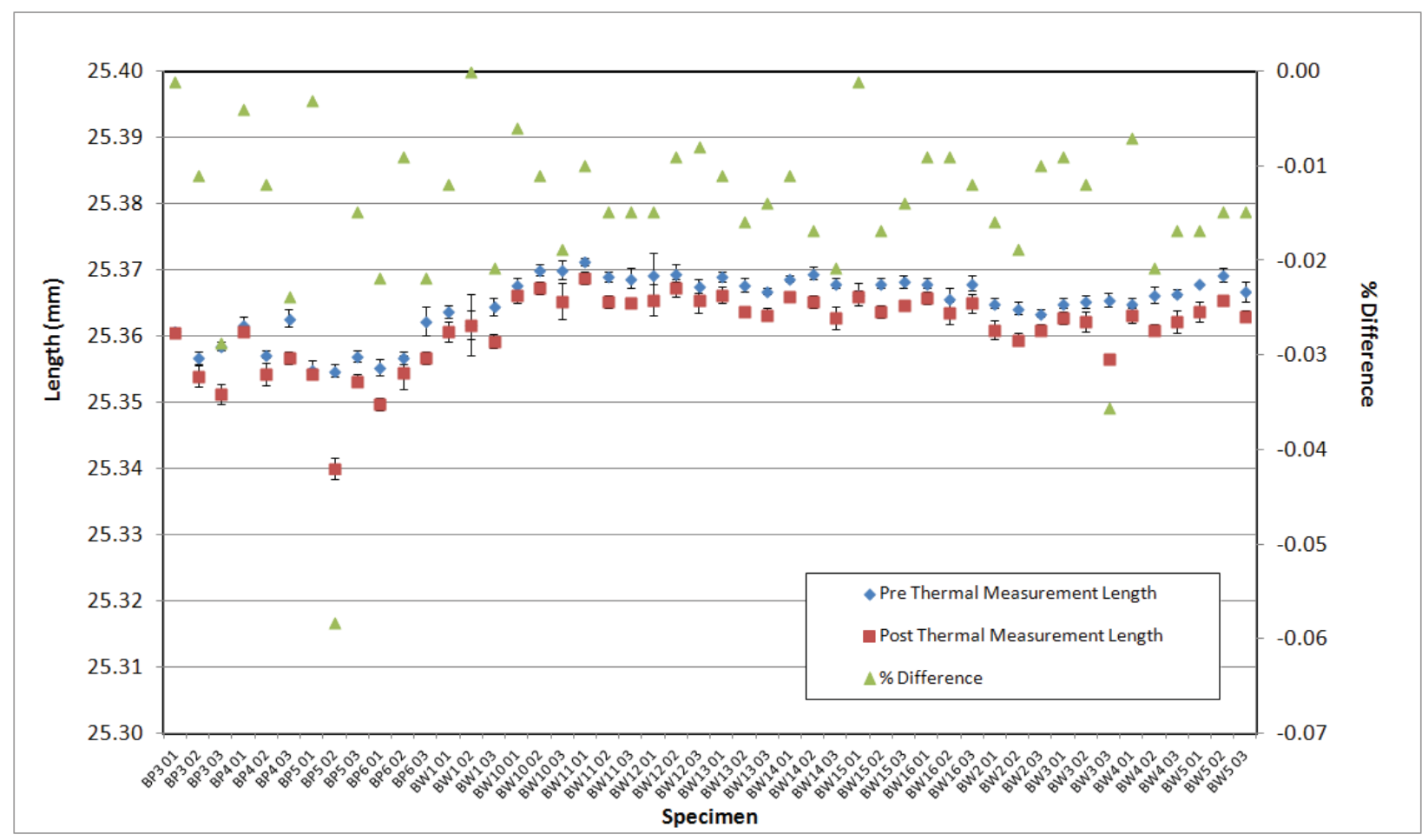

Figure A-141. NBG-18 Creep Pre vs. Post Thermal Measurement Length Comparison.

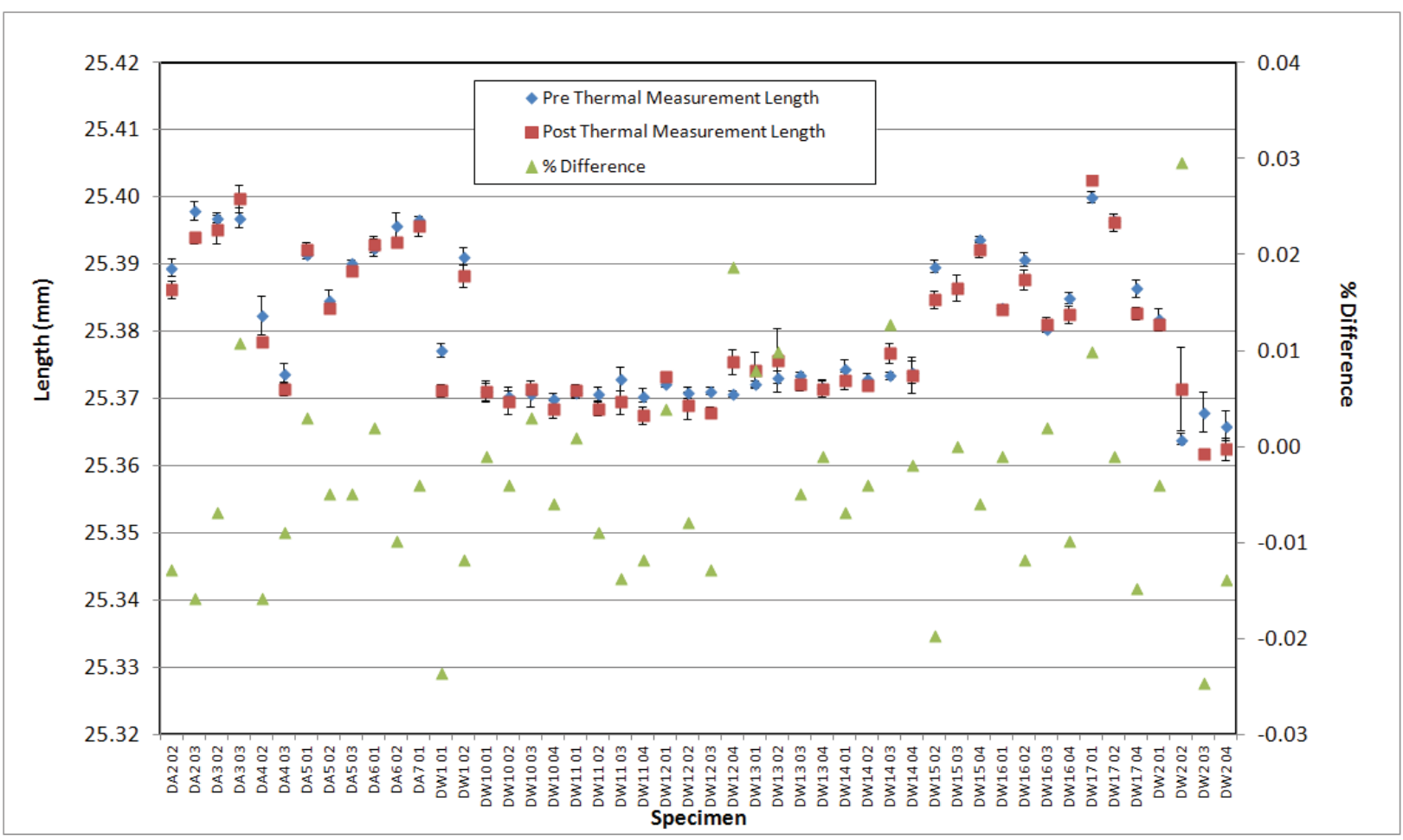

Figure A-142. PCEA Creep Pre vs. Post Thermal Measurement Length Comparison. 


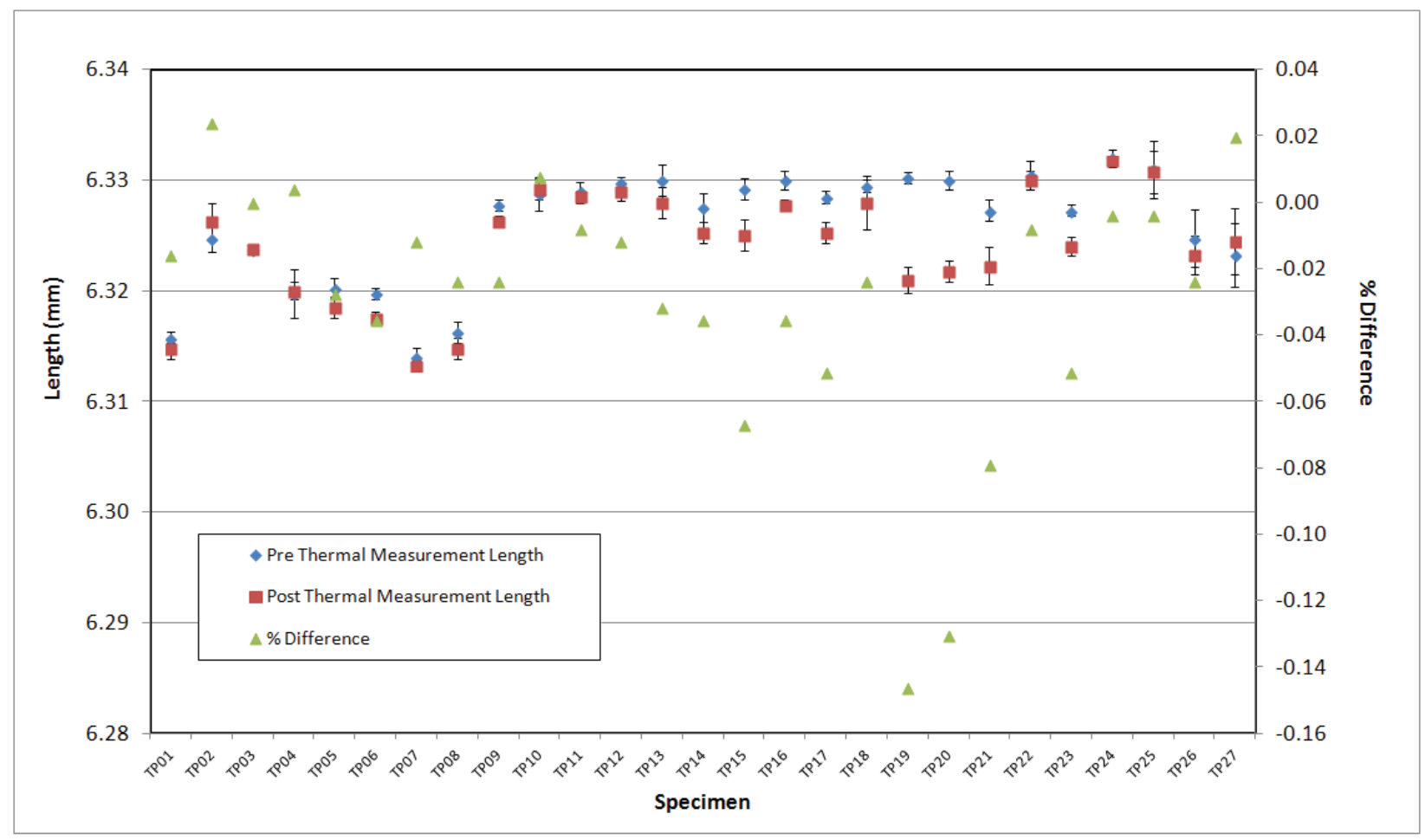

Figure A-143. 2114 Piggyback Pre vs. Post Thermal Measurement Length Comparison.

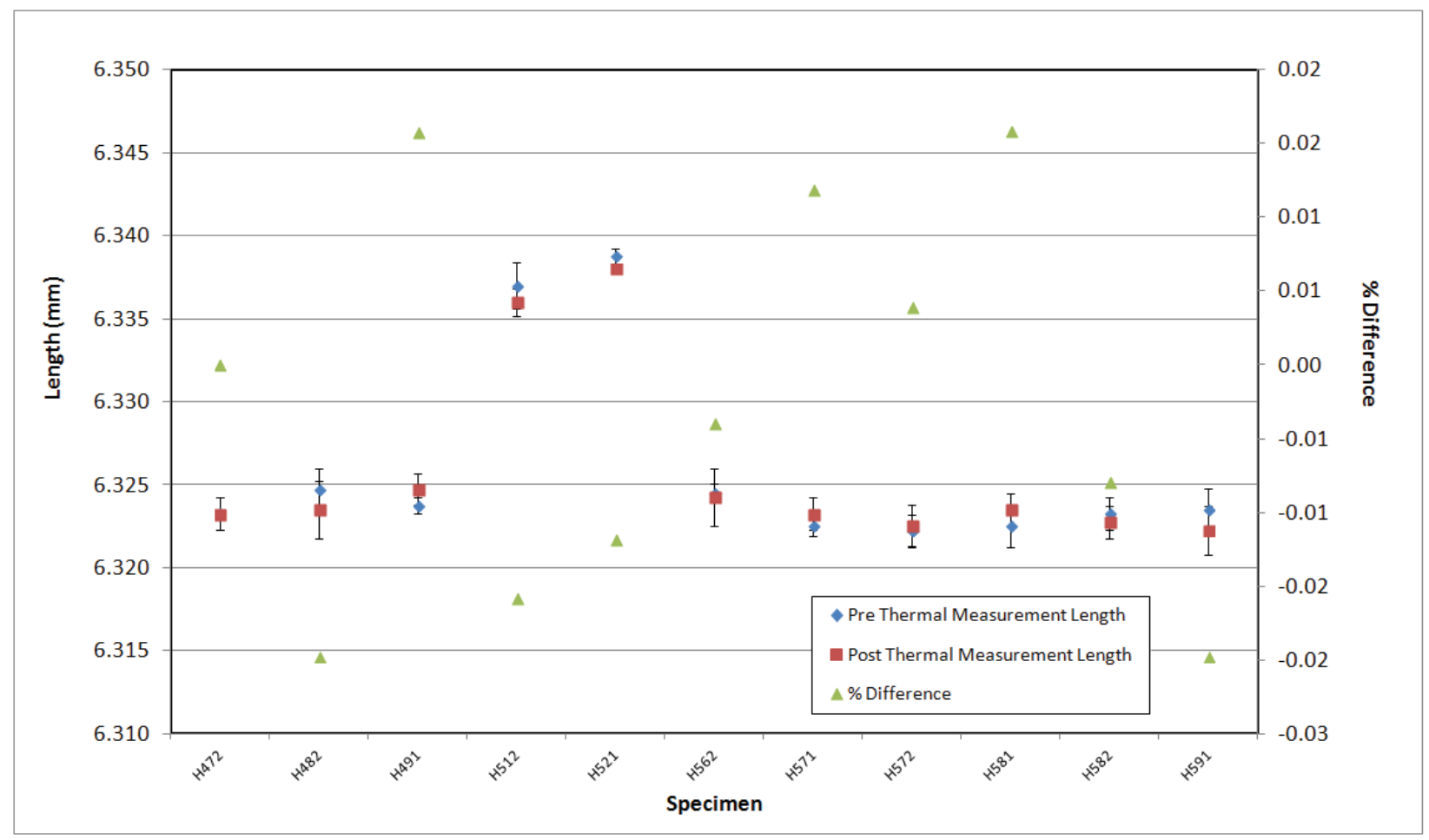

Figure A-144. A3 Matrix Piggyback Pre vs. Post Thermal Measurement Length Comparison. 


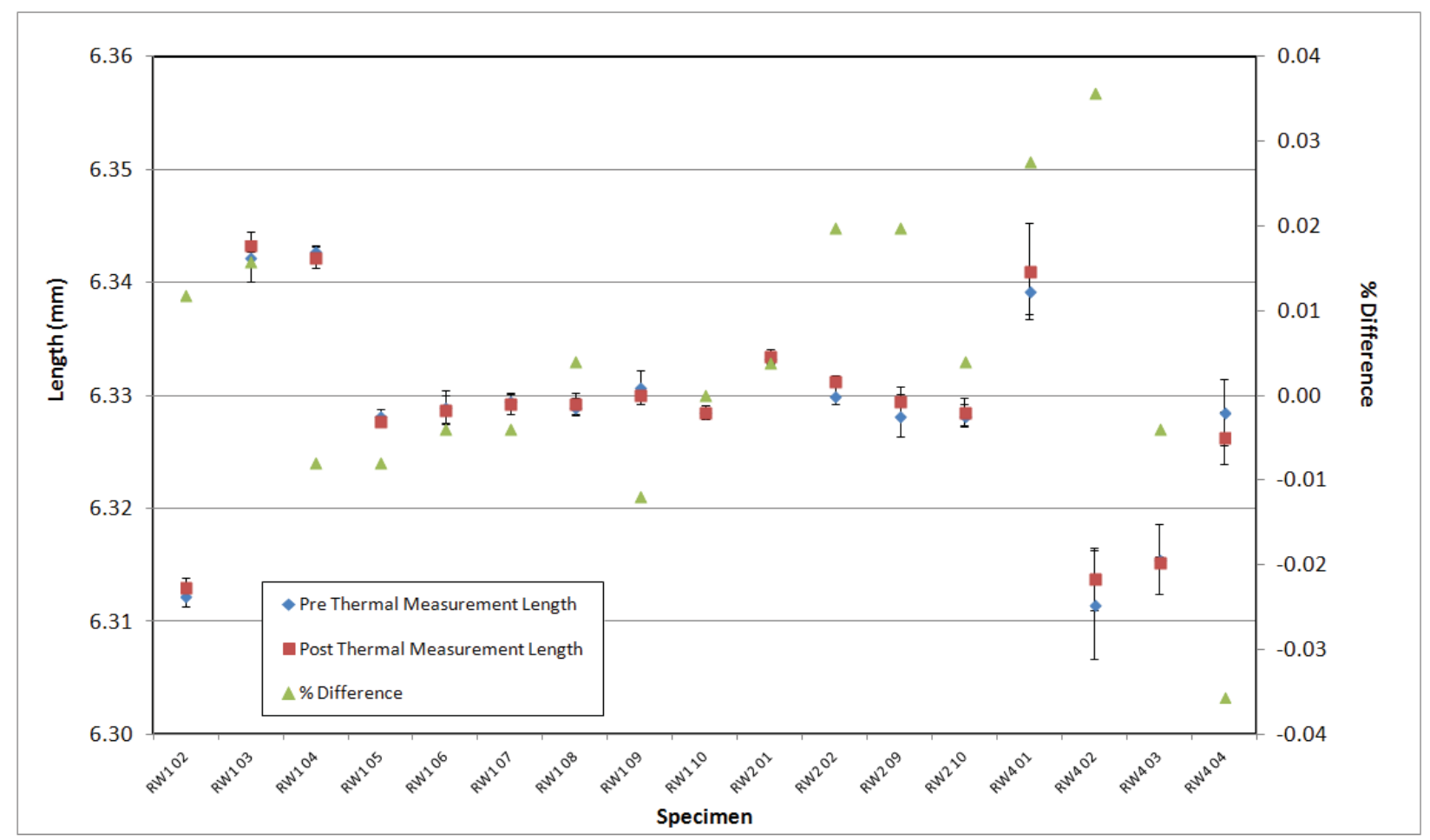

Figure A-145. BAN Piggyback Pre vs. Post Thermal Measurement Length Comparison.

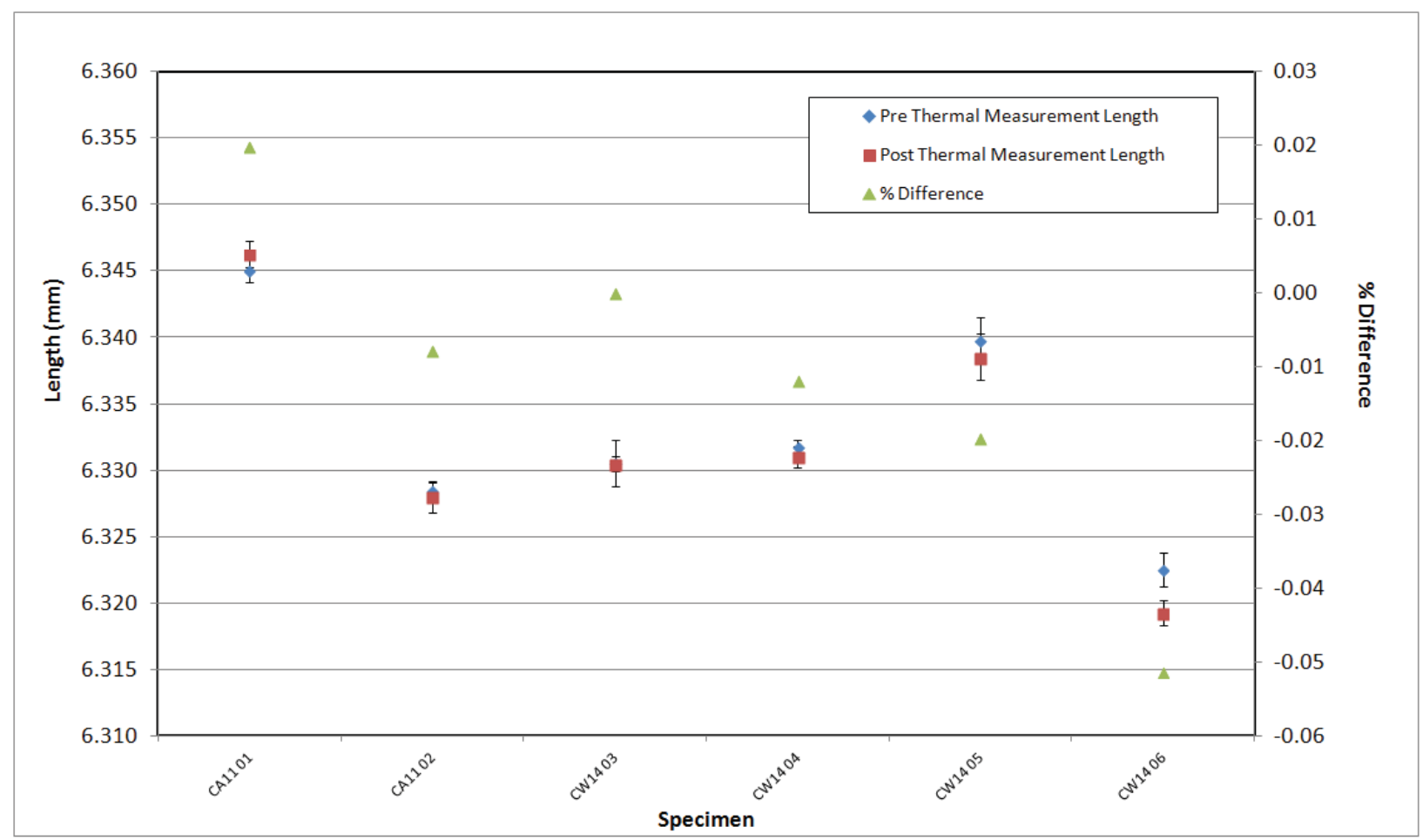

Figure A-146. H-451 Piggyback Pre vs. Post Thermal Measurement Length Comparison. 


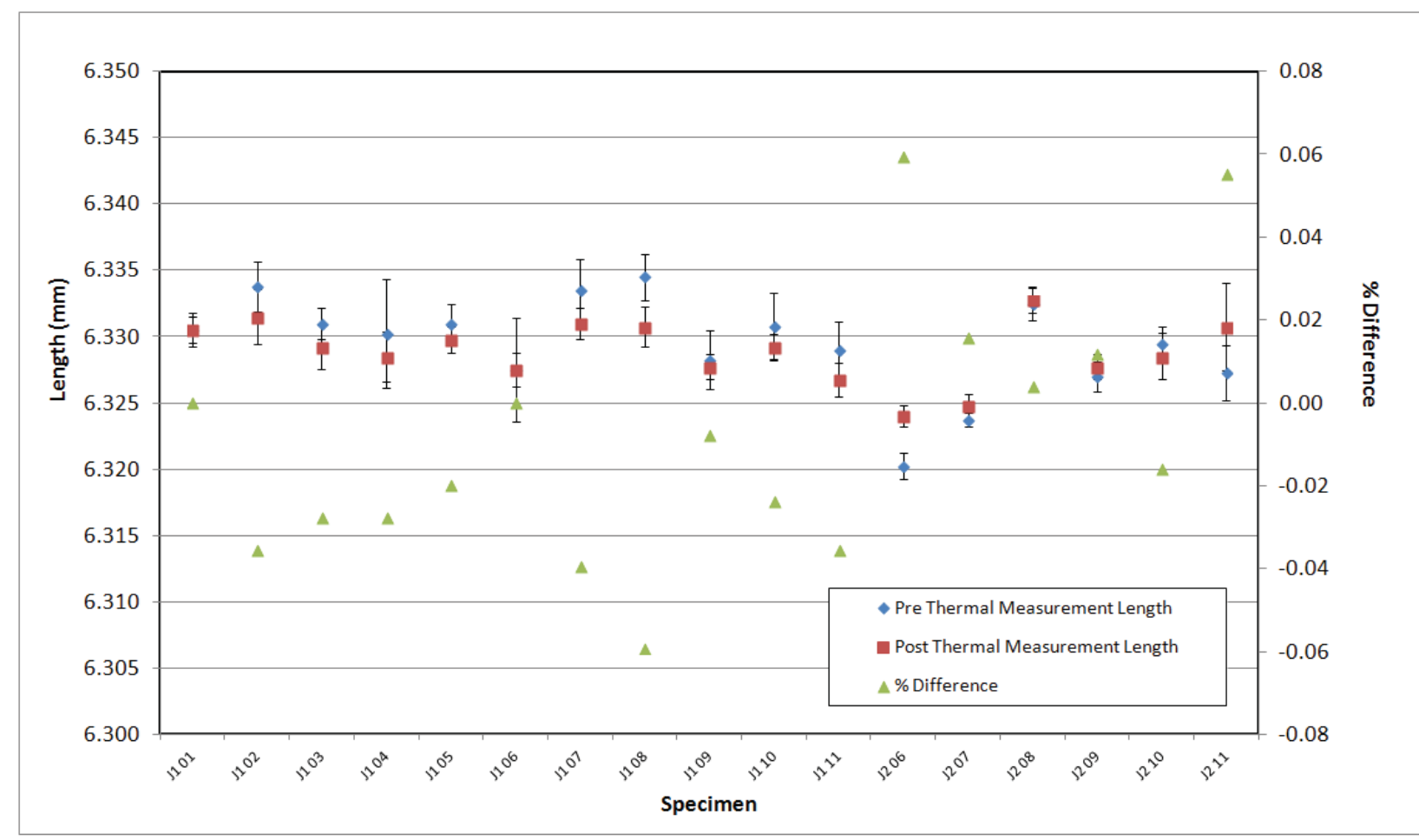

Figure A-147. HLM Piggyback Pre vs. Post Thermal Measurement Length Comparison.

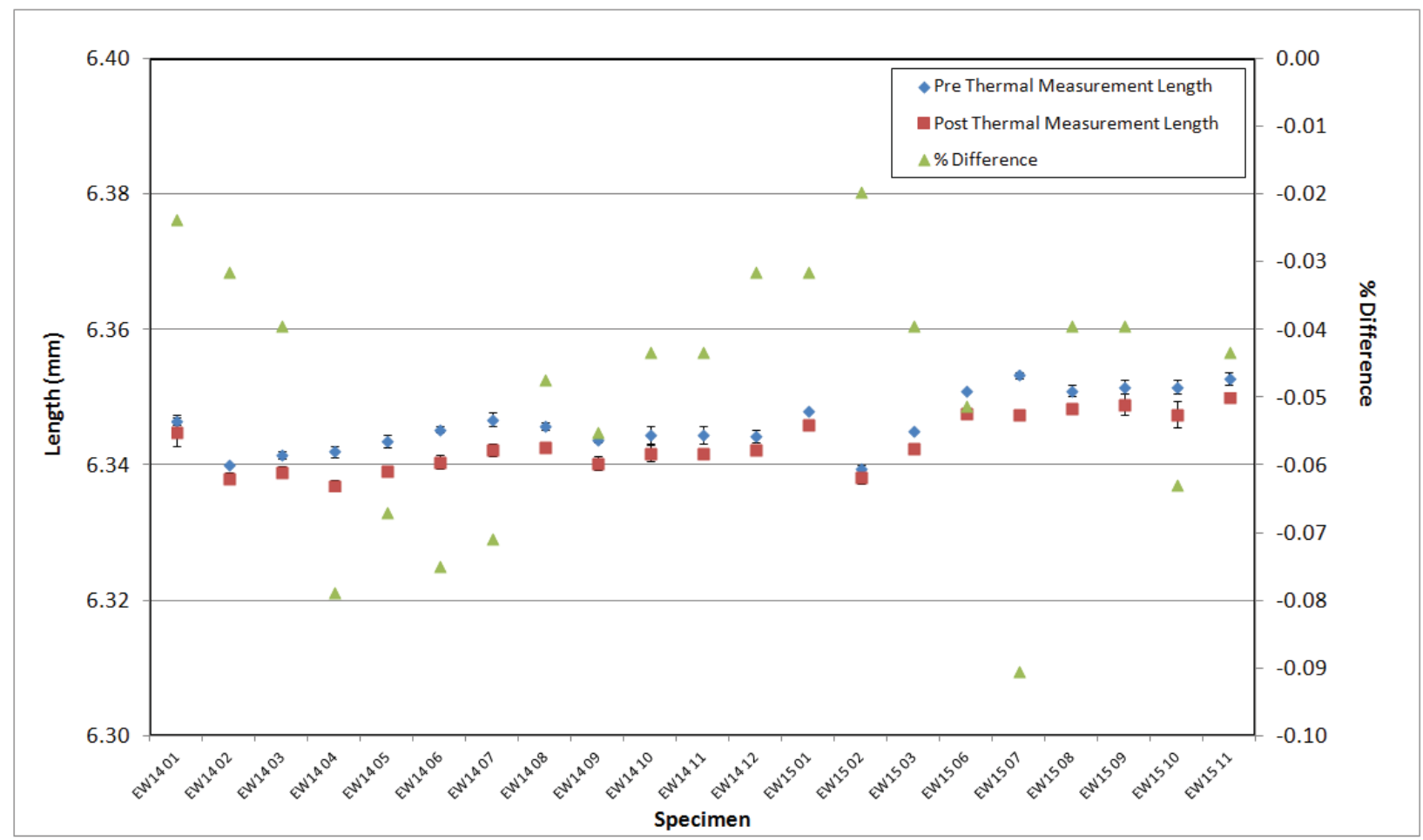

Figure A-148. IG-110 Piggyback Pre vs. Post Thermal Measurement Length Comparison. 


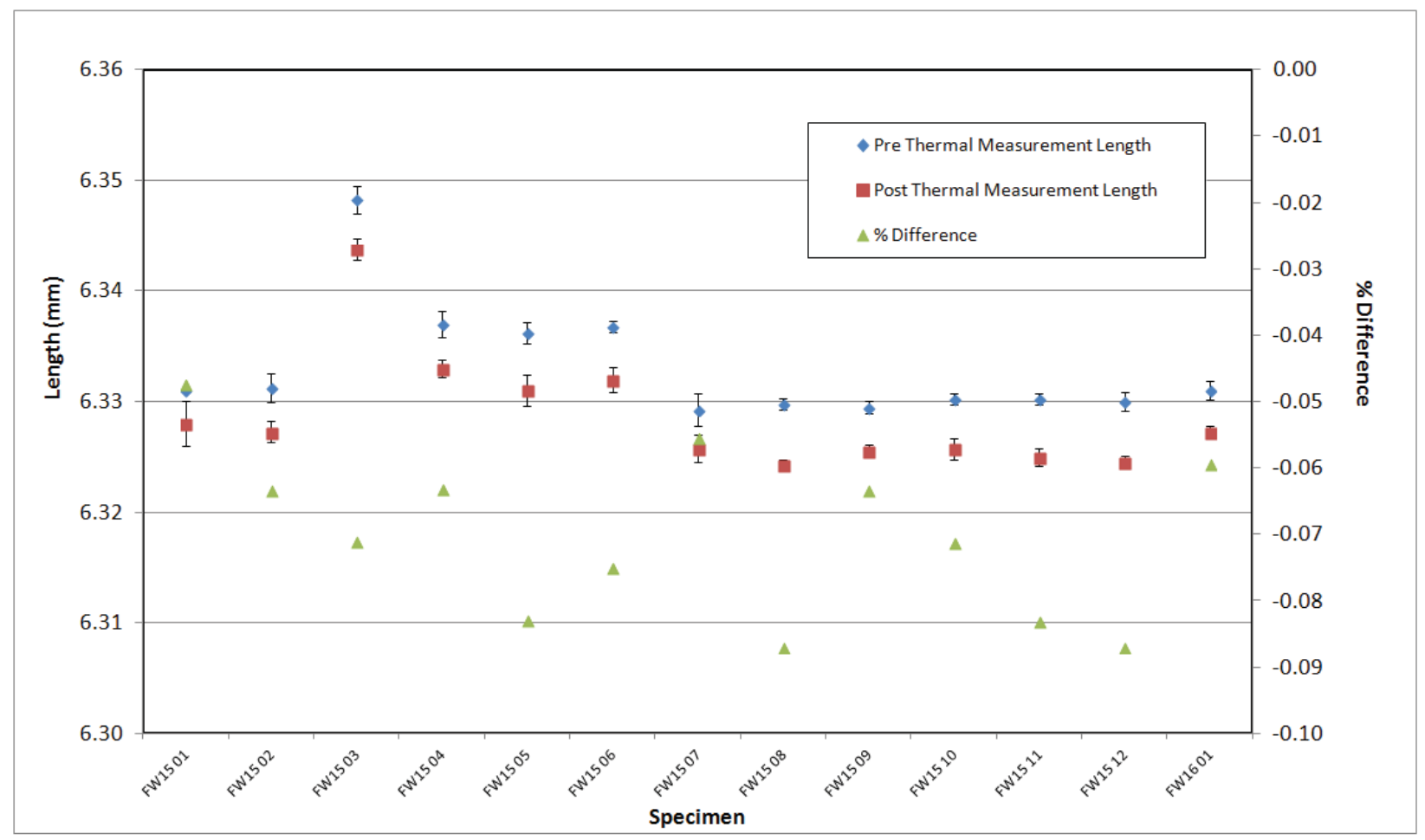

Figure A-149. IG-430 Piggyback Pre vs. Post Thermal Measurement Length Comparison.

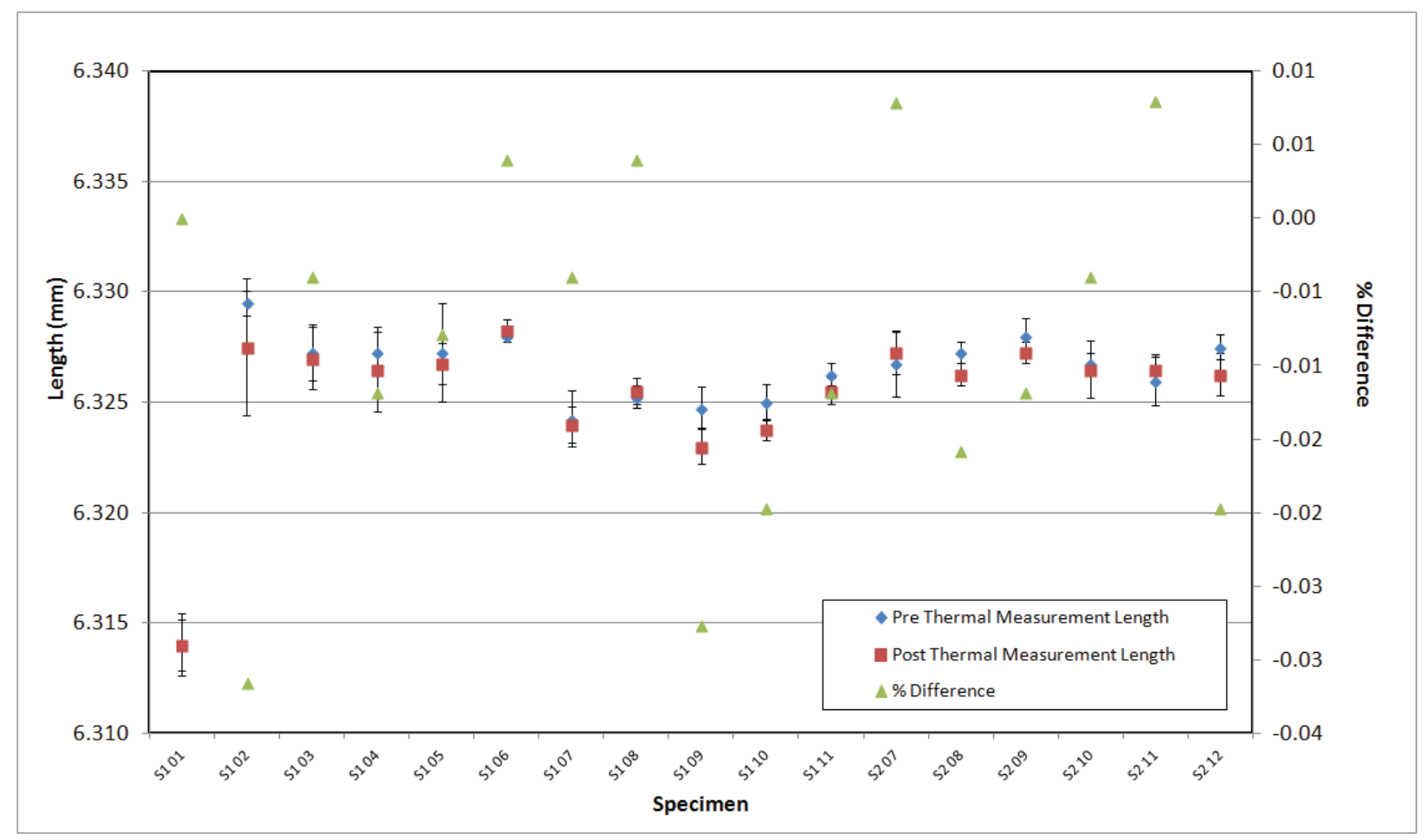

Figure A-150. NBG-10 Piggyback Pre vs. Post Thermal Measurement Length Comparison. 


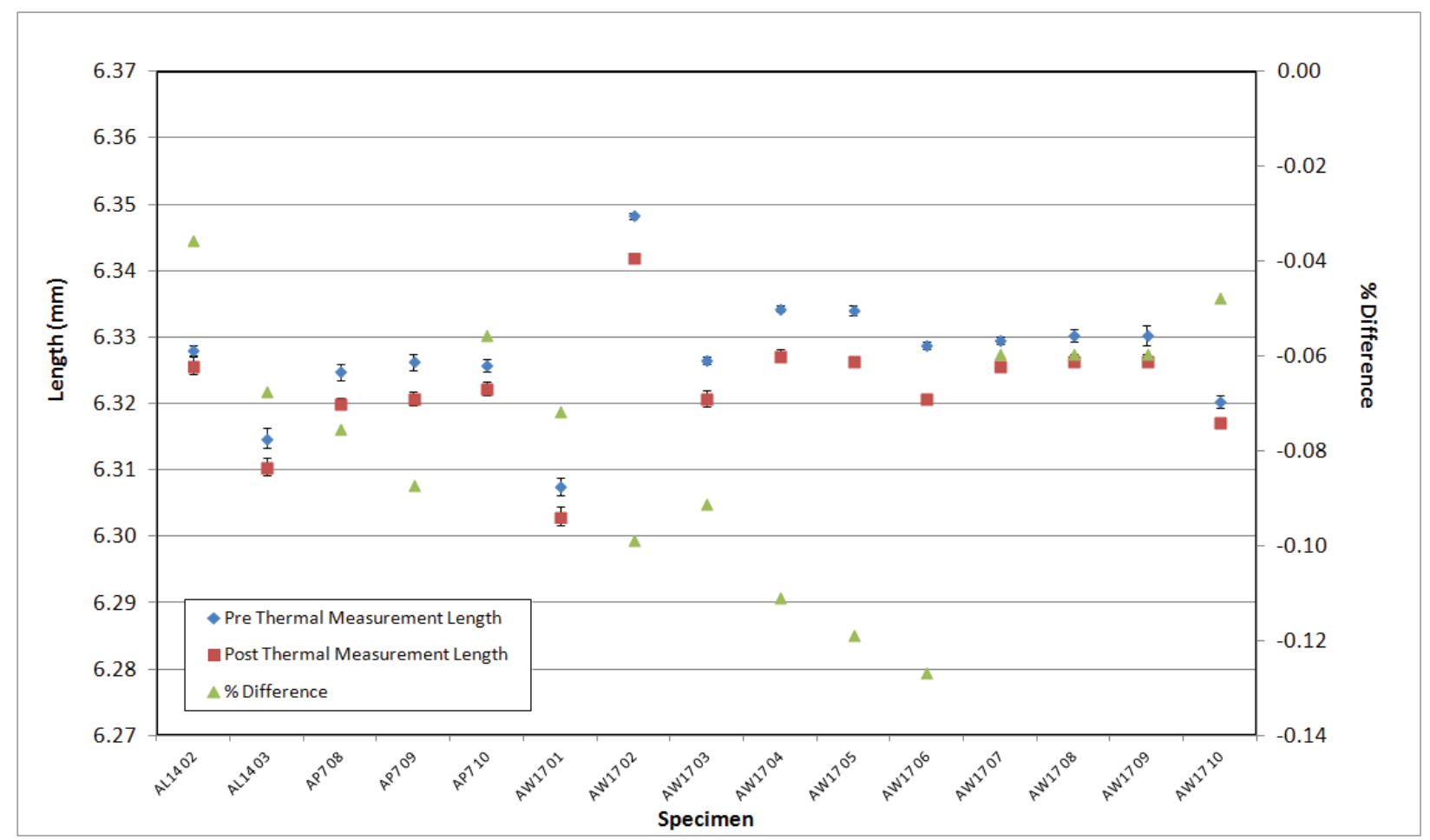

Figure A-151. NBG-17 Piggyback Pre vs. Post Thermal Measurement Length Comparison.

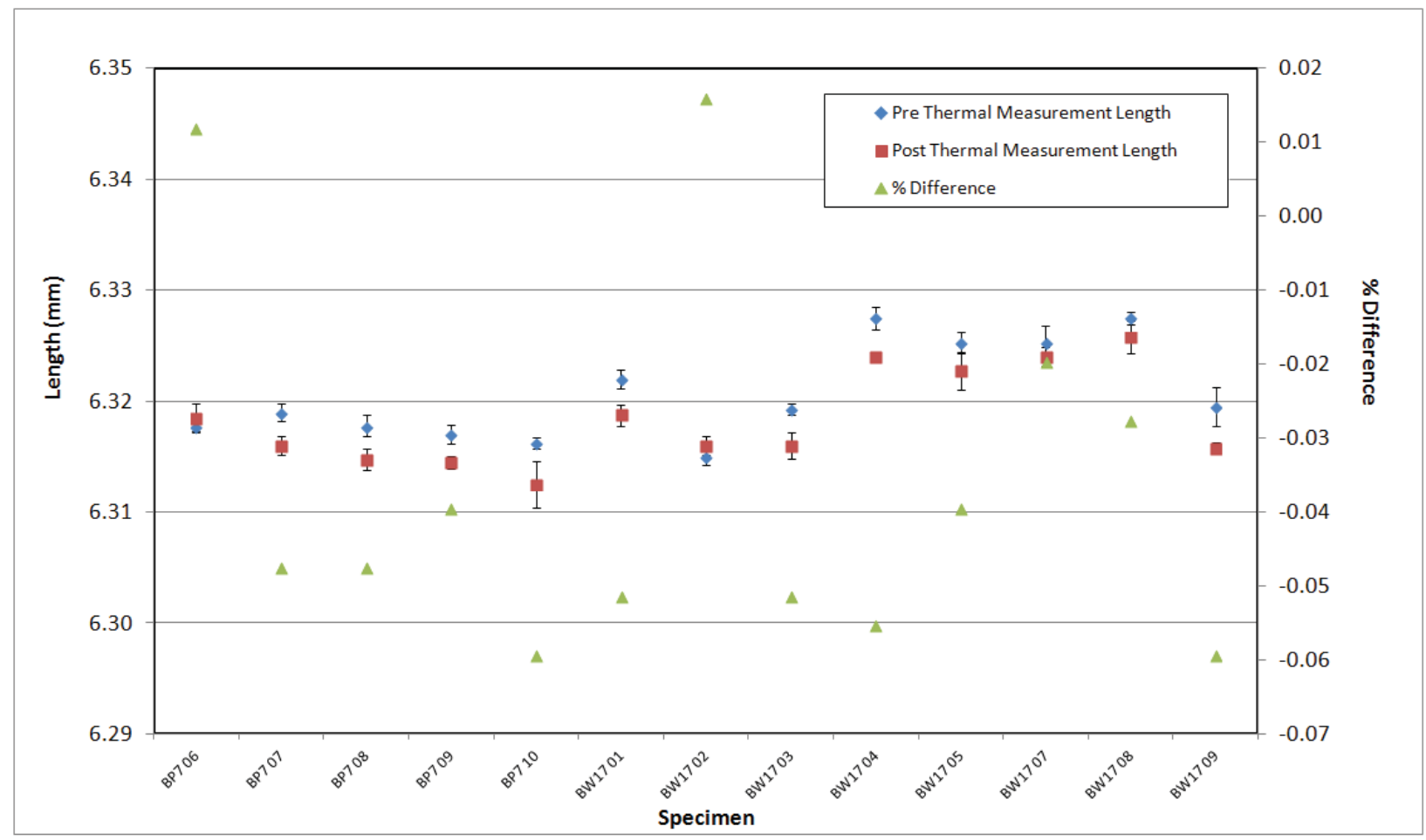

Figure A-152. NBG-18 Piggyback Pre vs. Post Thermal Measurement Length Comparison. 


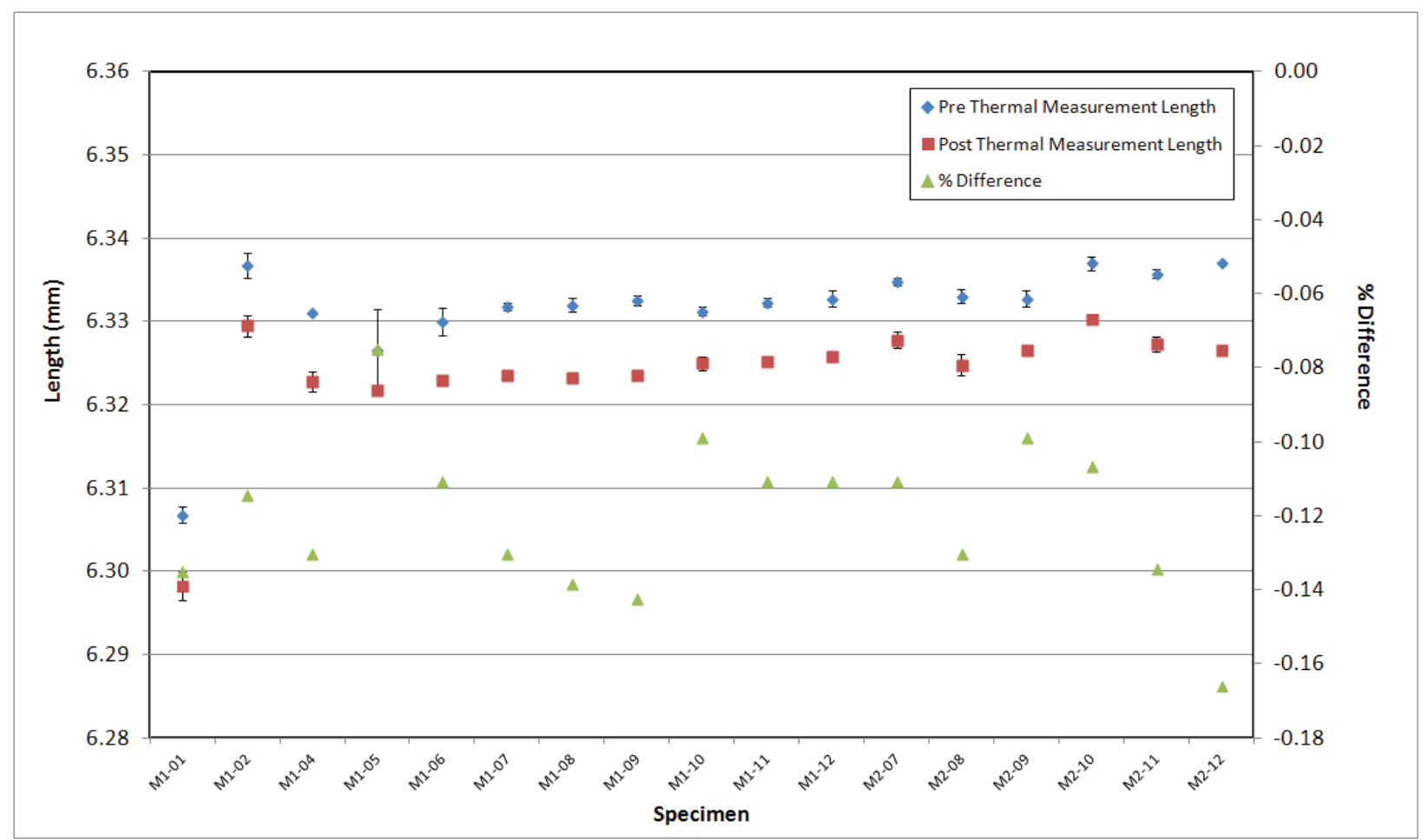

Figure A-153. NBG-25 Piggyback Pre vs. Post Thermal Measurement Length Comparison.

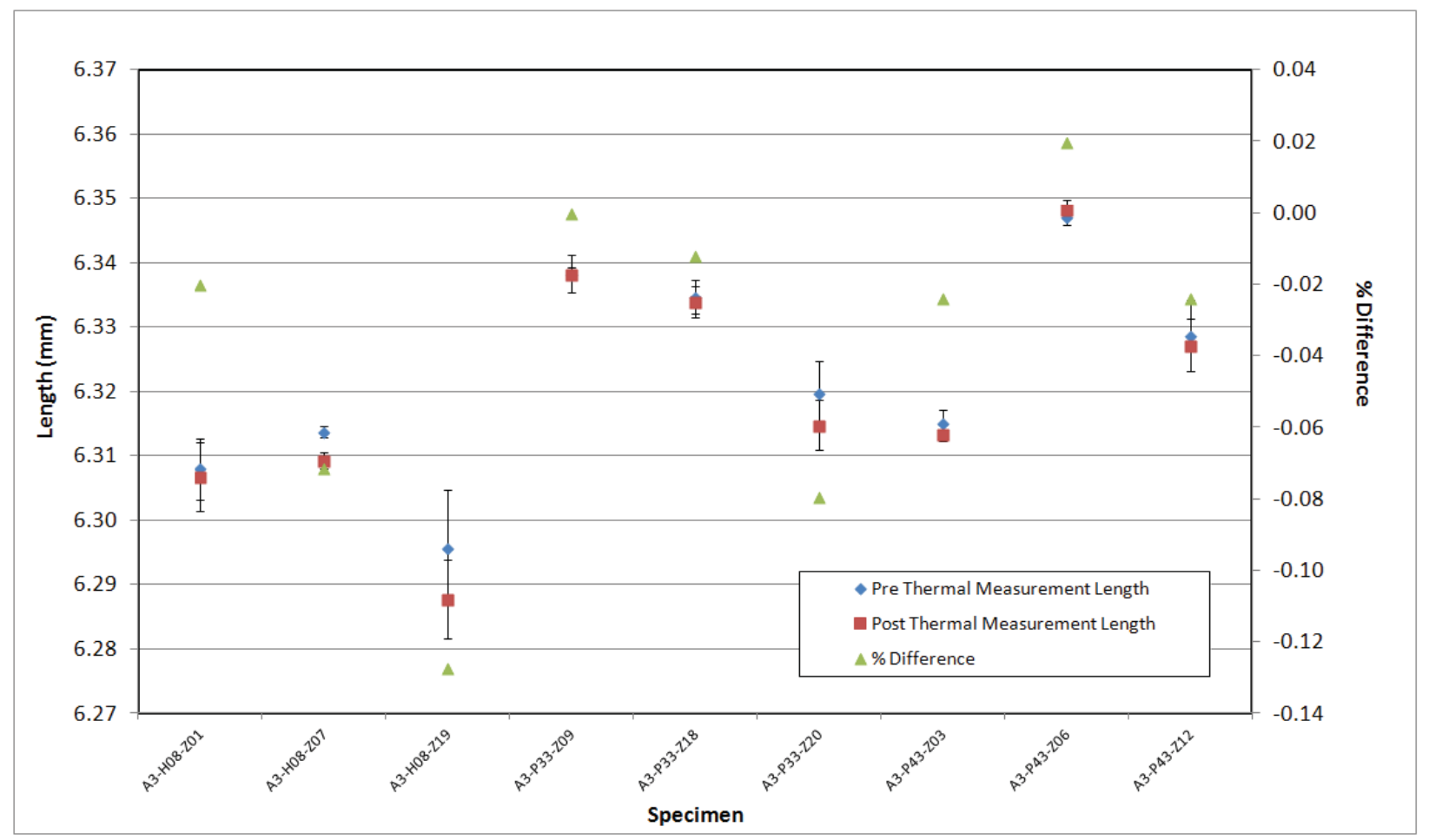

Figure A-154. New Matrix Piggyback Pre vs. Post Thermal Measurement Length Comparison. 


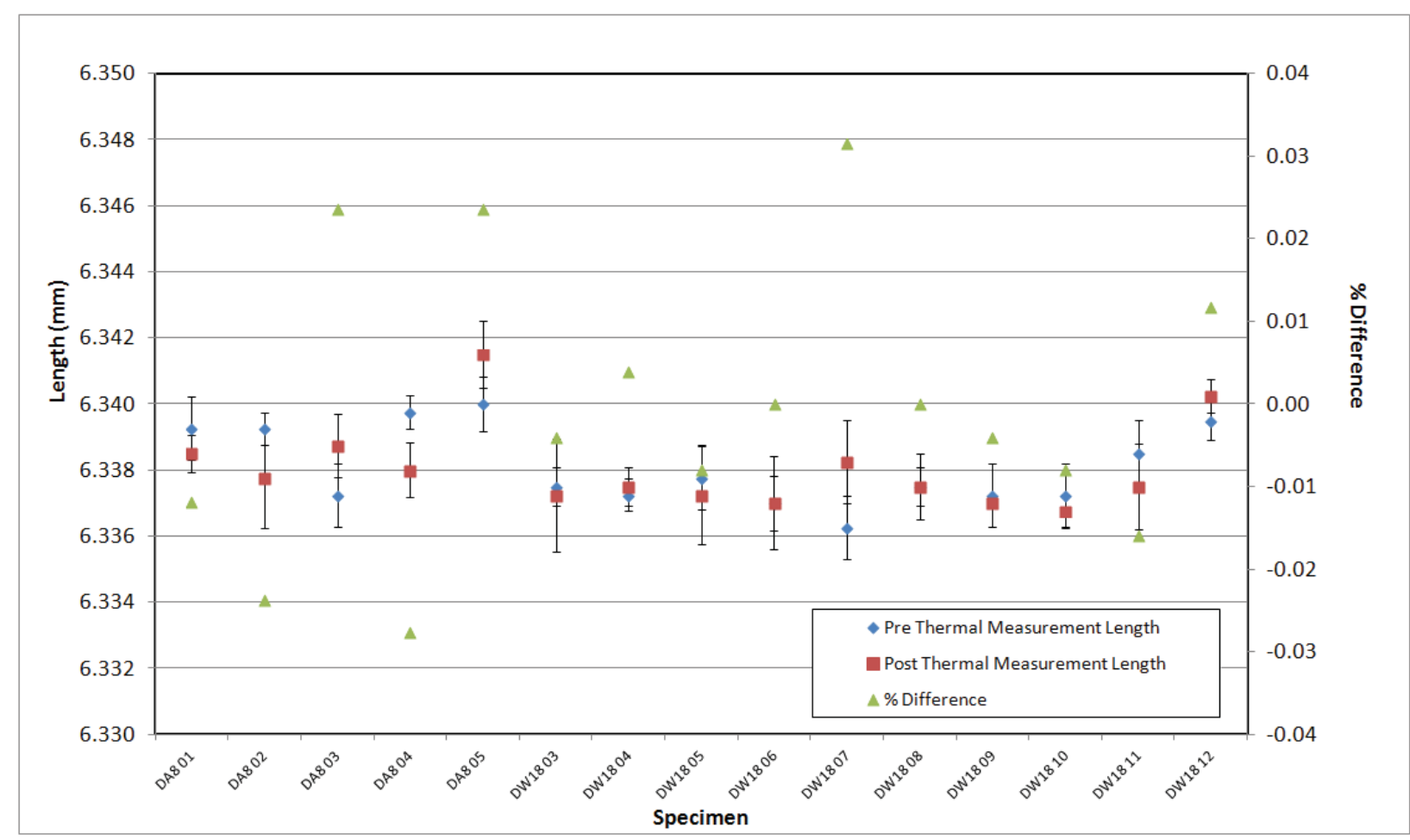

Figure A-155. PCEA Piggyback Pre vs. Post Thermal Measurement Length Comparison.

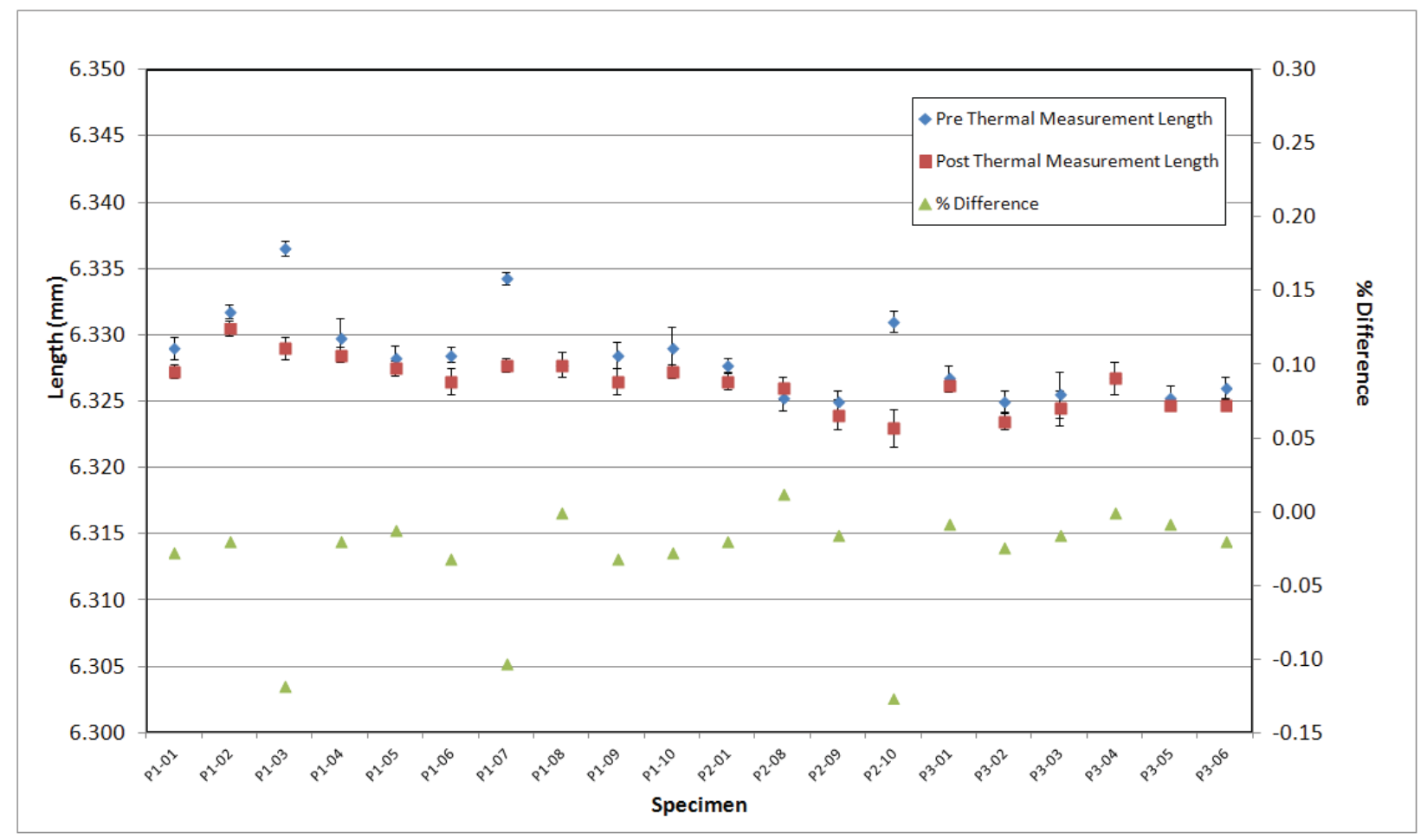

Figure A-156. PCIB Piggyback Pre vs. Post Thermal Measurement Length Comparison. 


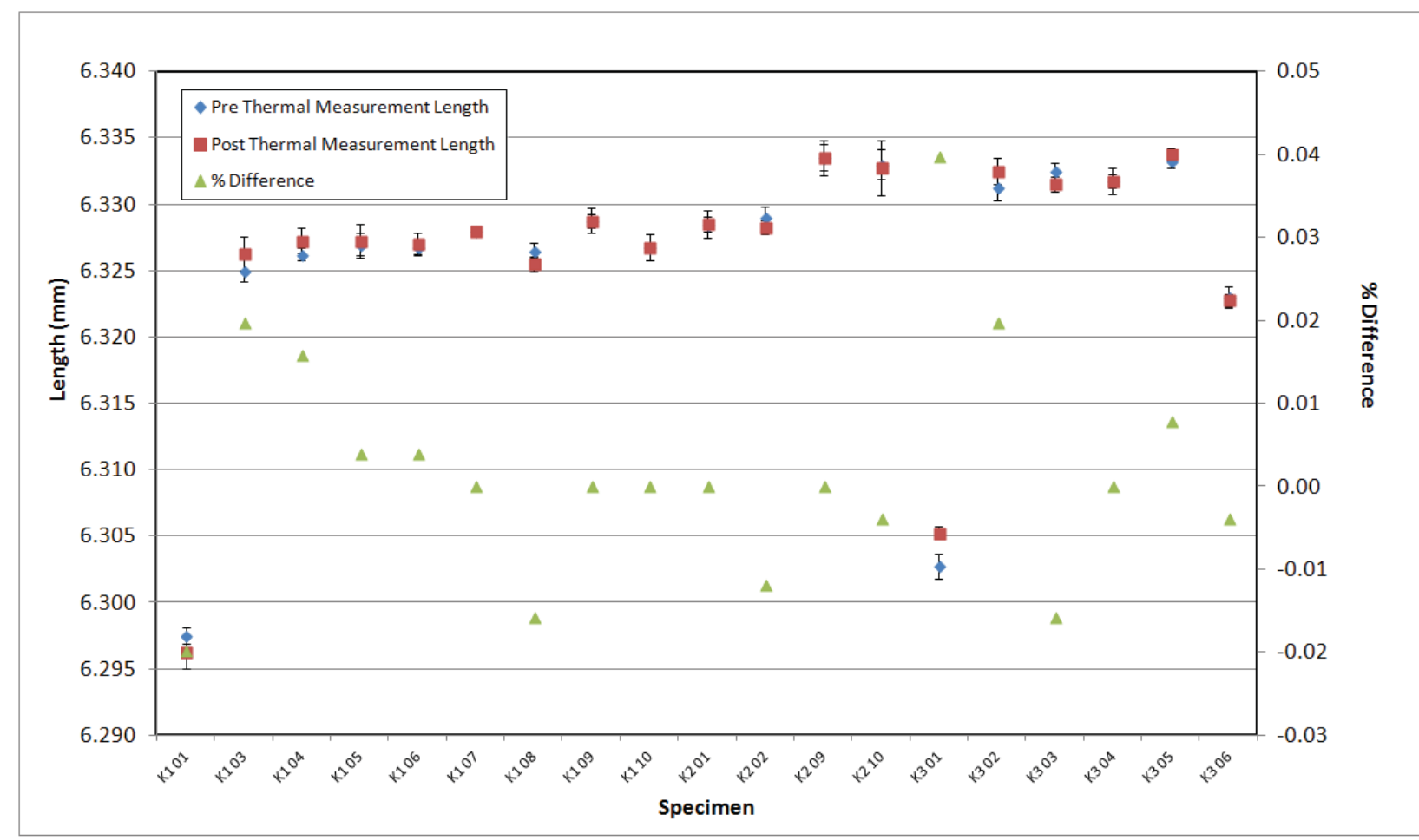

Figure A-157. PGX Piggyback Pre vs. Post Thermal Measurement Length Comparison.

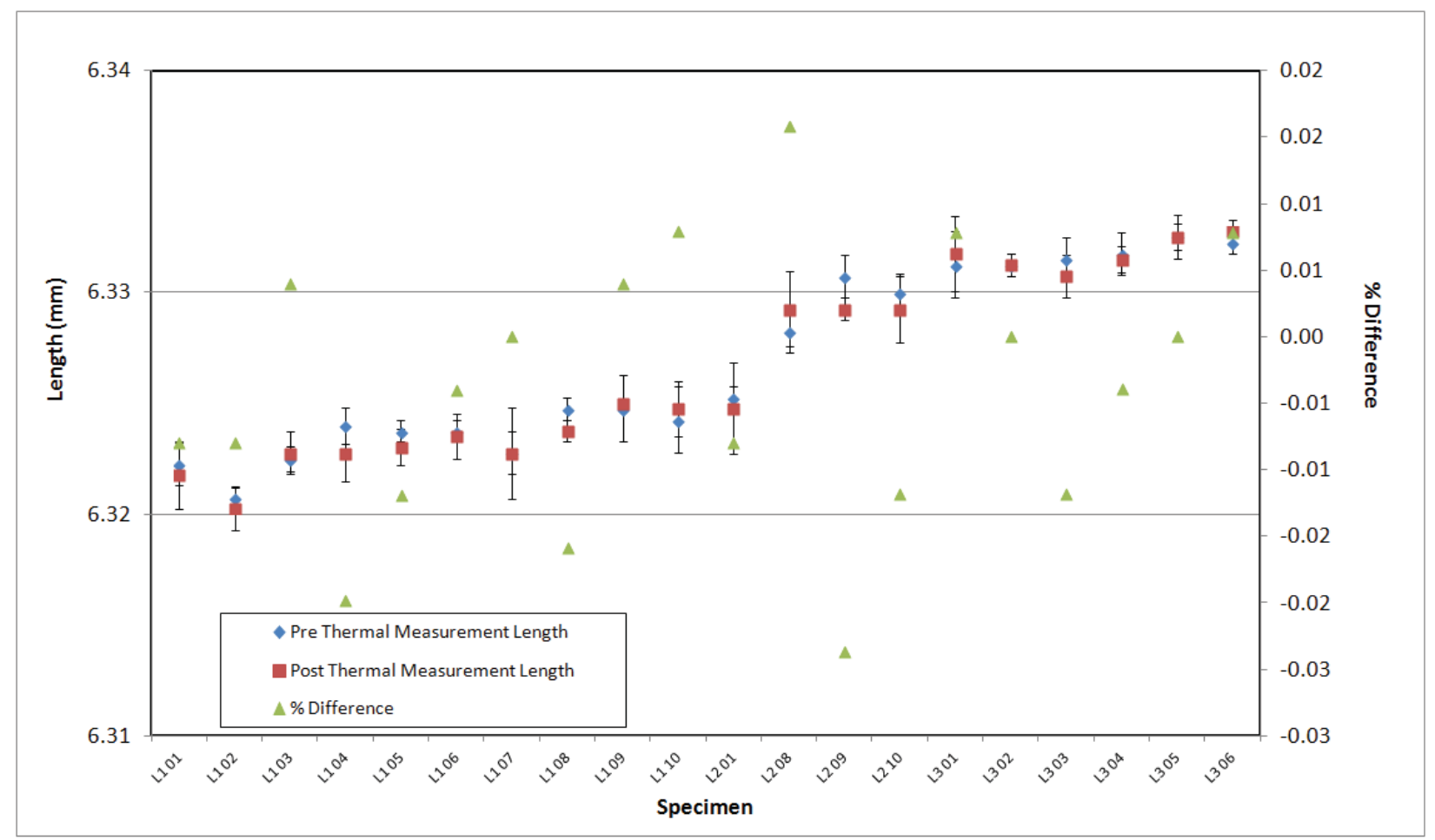

Figure A-158. PPEA Piggyback Pre vs. Post Thermal Measurement Length Comparison. 


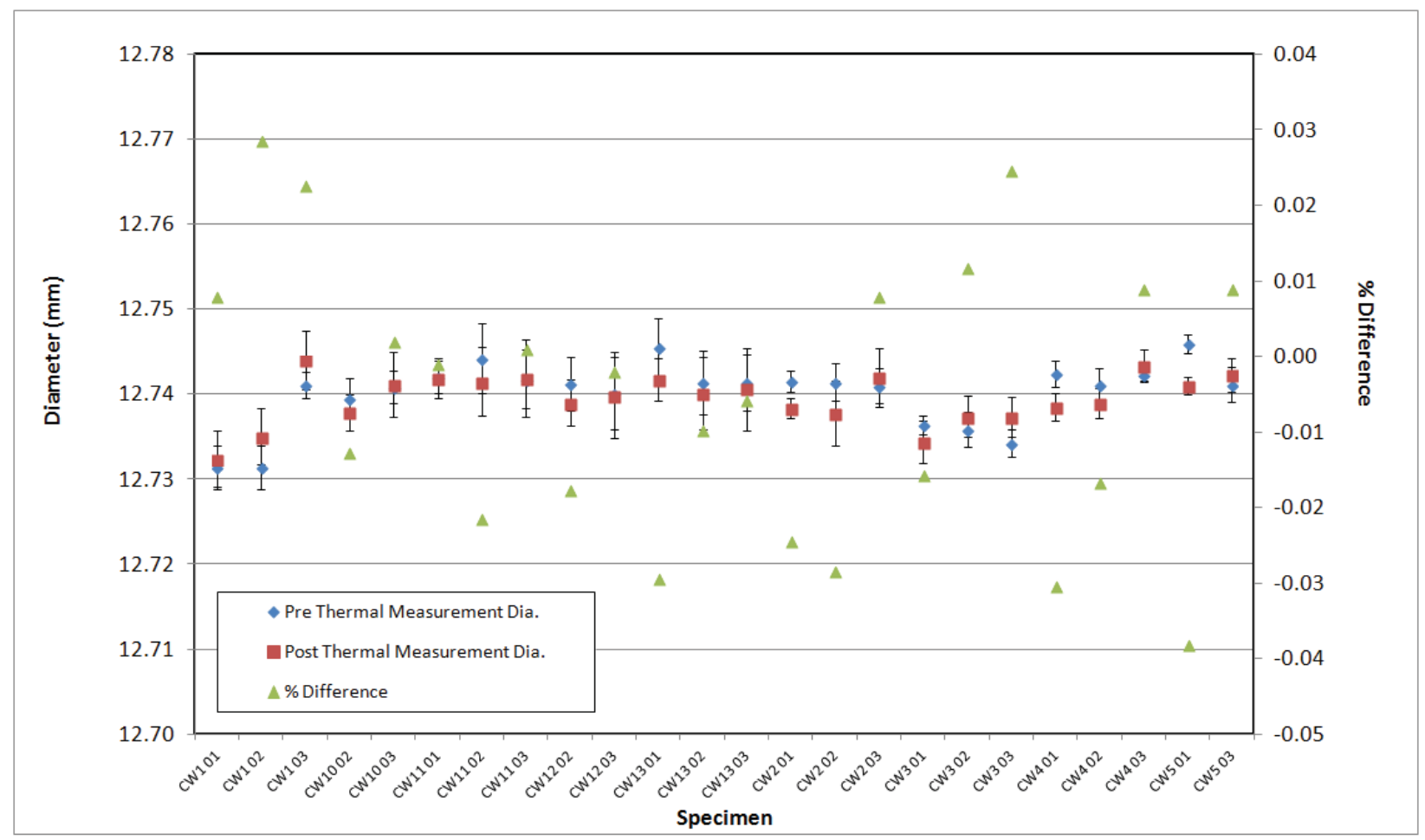

Figure A-159. H-451 Creep Pre vs. Post Thermal Measurement Diameter Comparison.

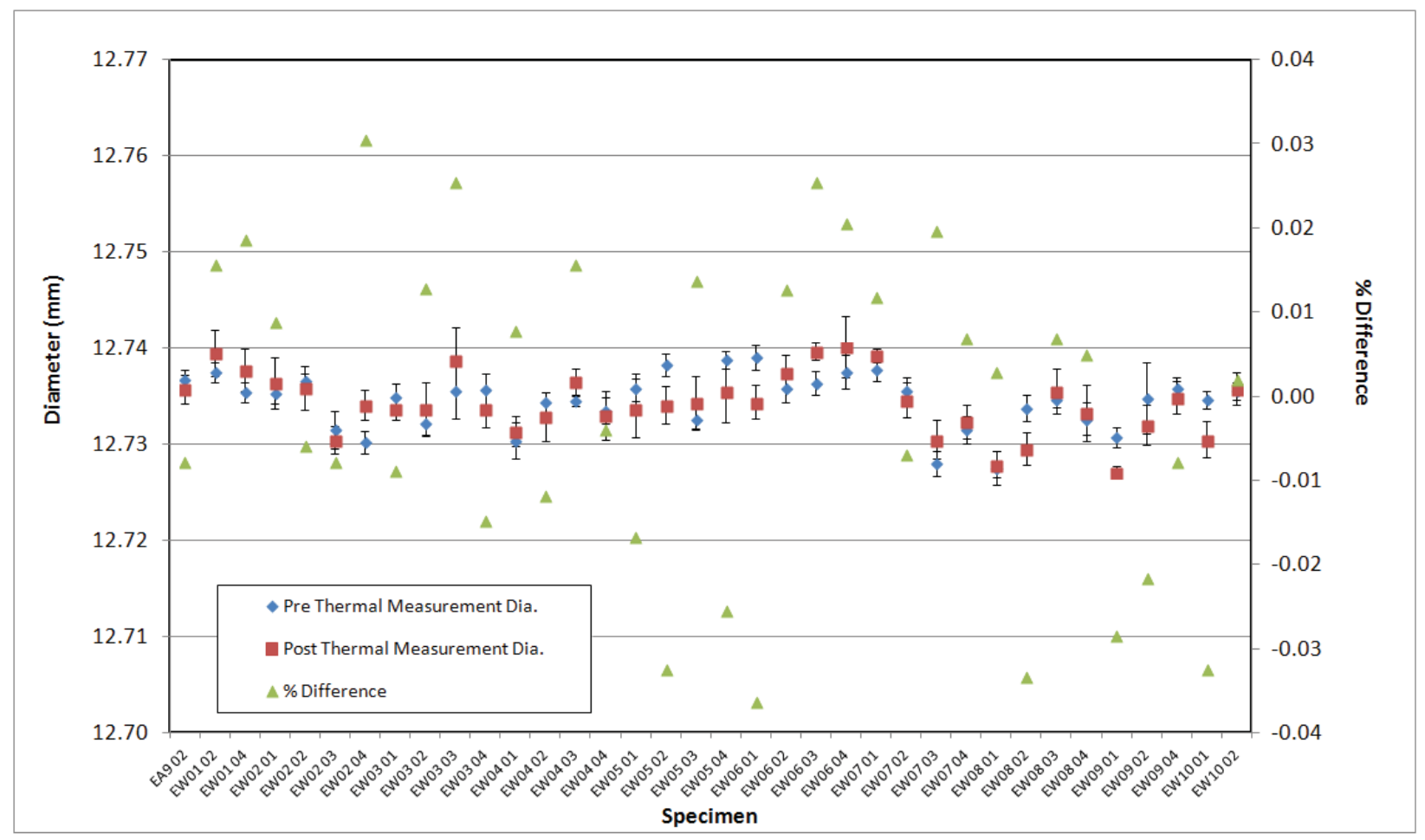

Figure A-160. IG-110 Creep Pre vs. Post Thermal Measurement Diameter Comparison. 


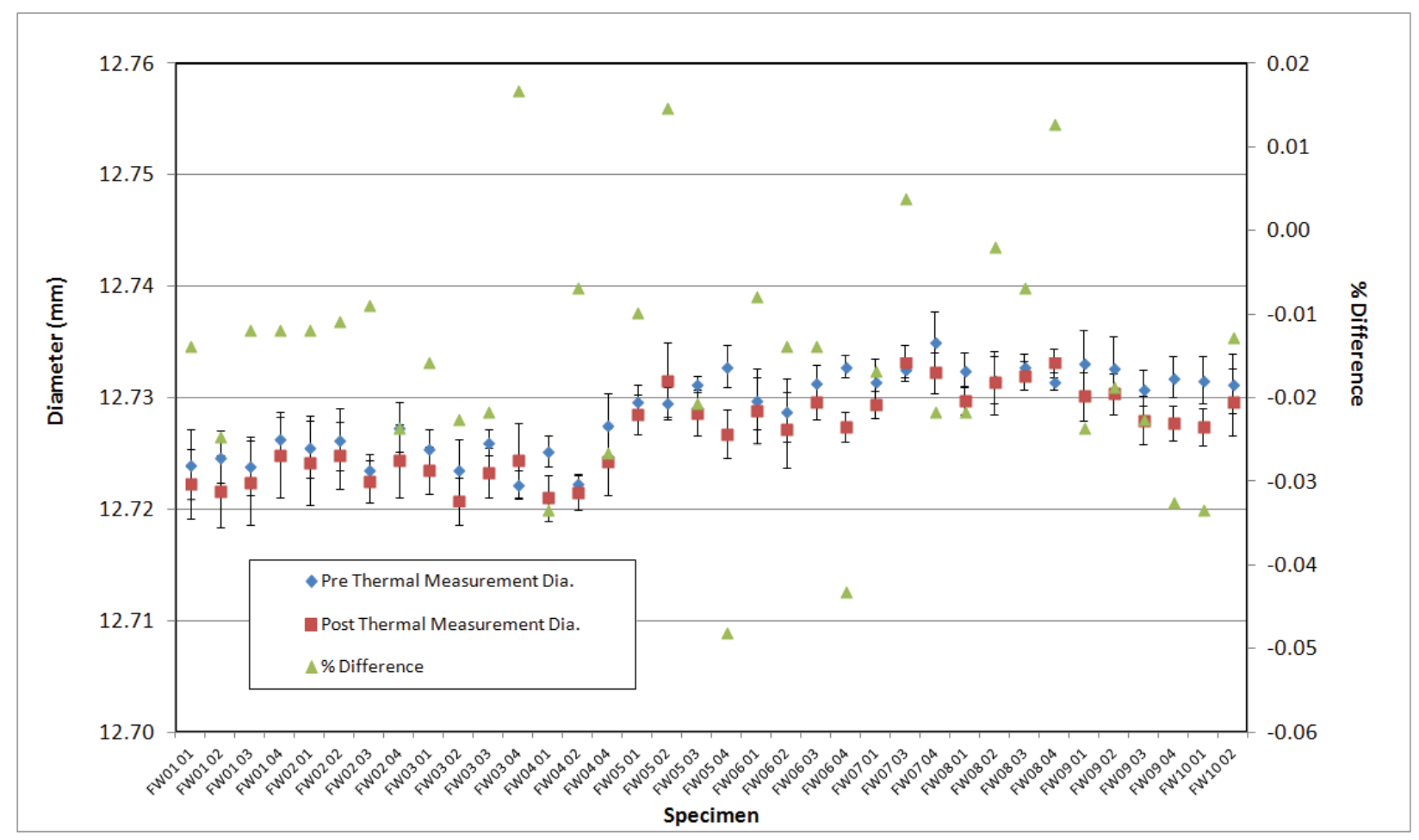

Figure A-161. IG-430 Creep Pre vs. Post Thermal Measurement Diameter Comparison.

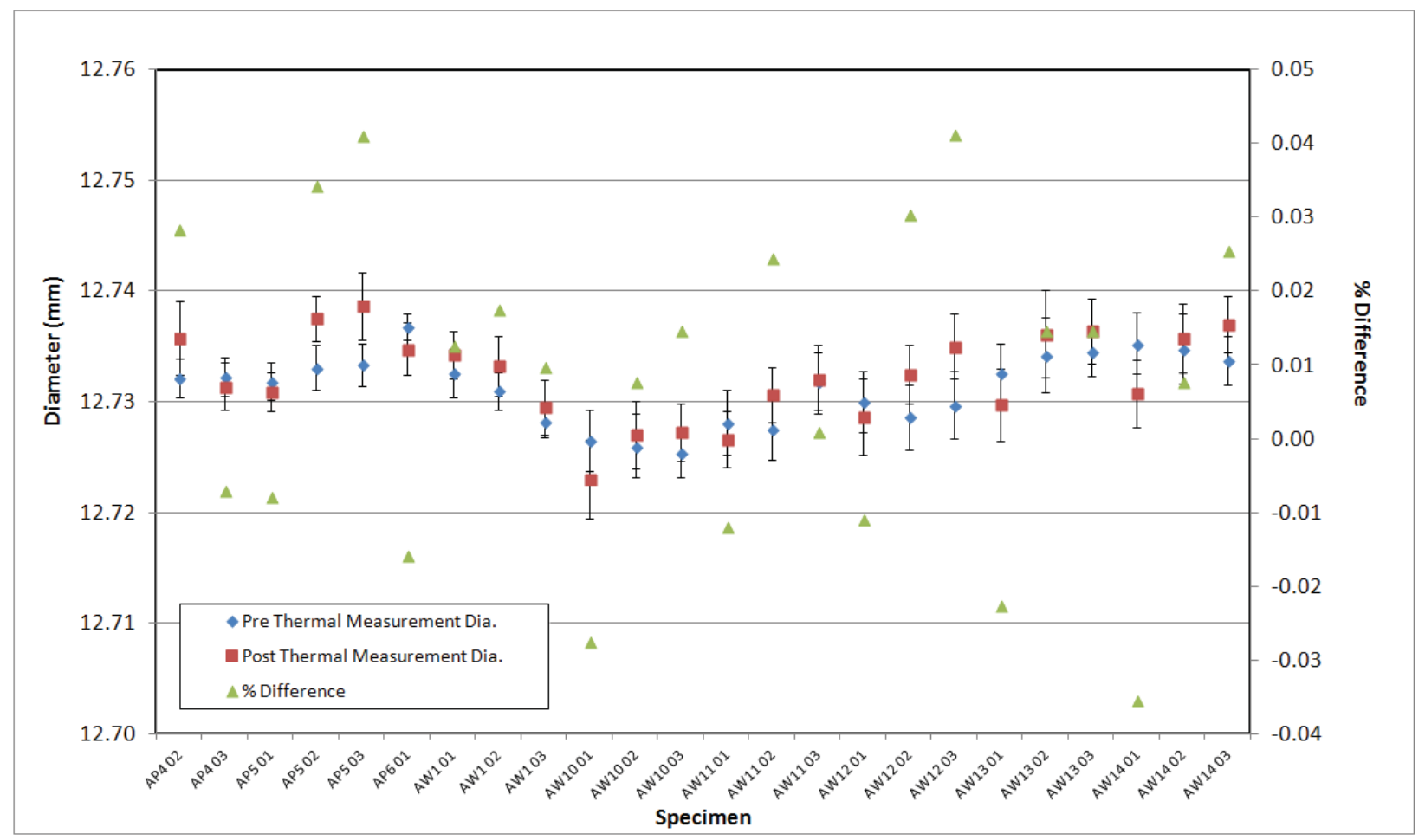

Figure A-162. NBG-17 Creep Pre vs. Post Thermal Measurement Diameter Comparison. 


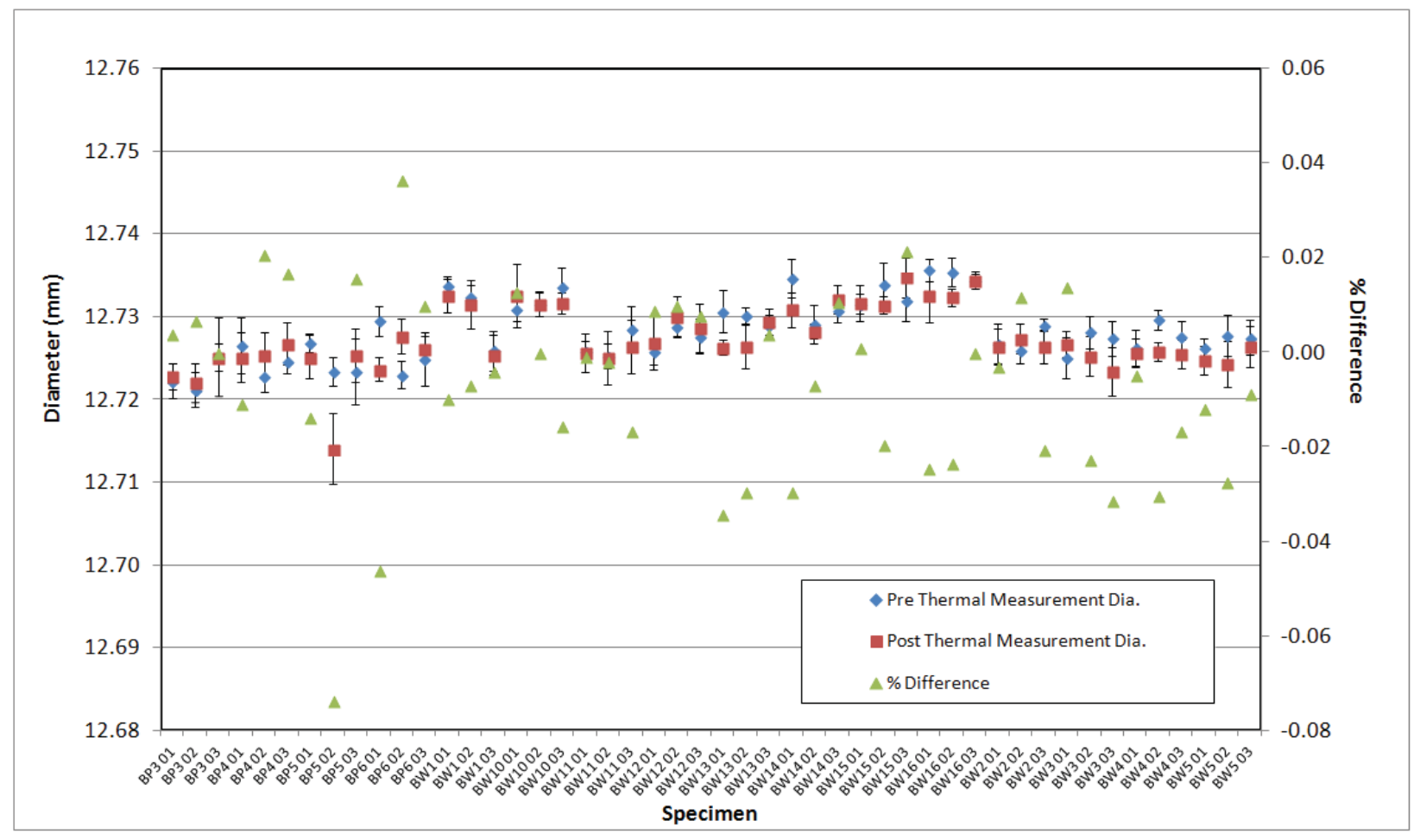

Figure A-163. NBG-18 Creep Pre vs. Post Thermal Measurement Diameter Comparison.

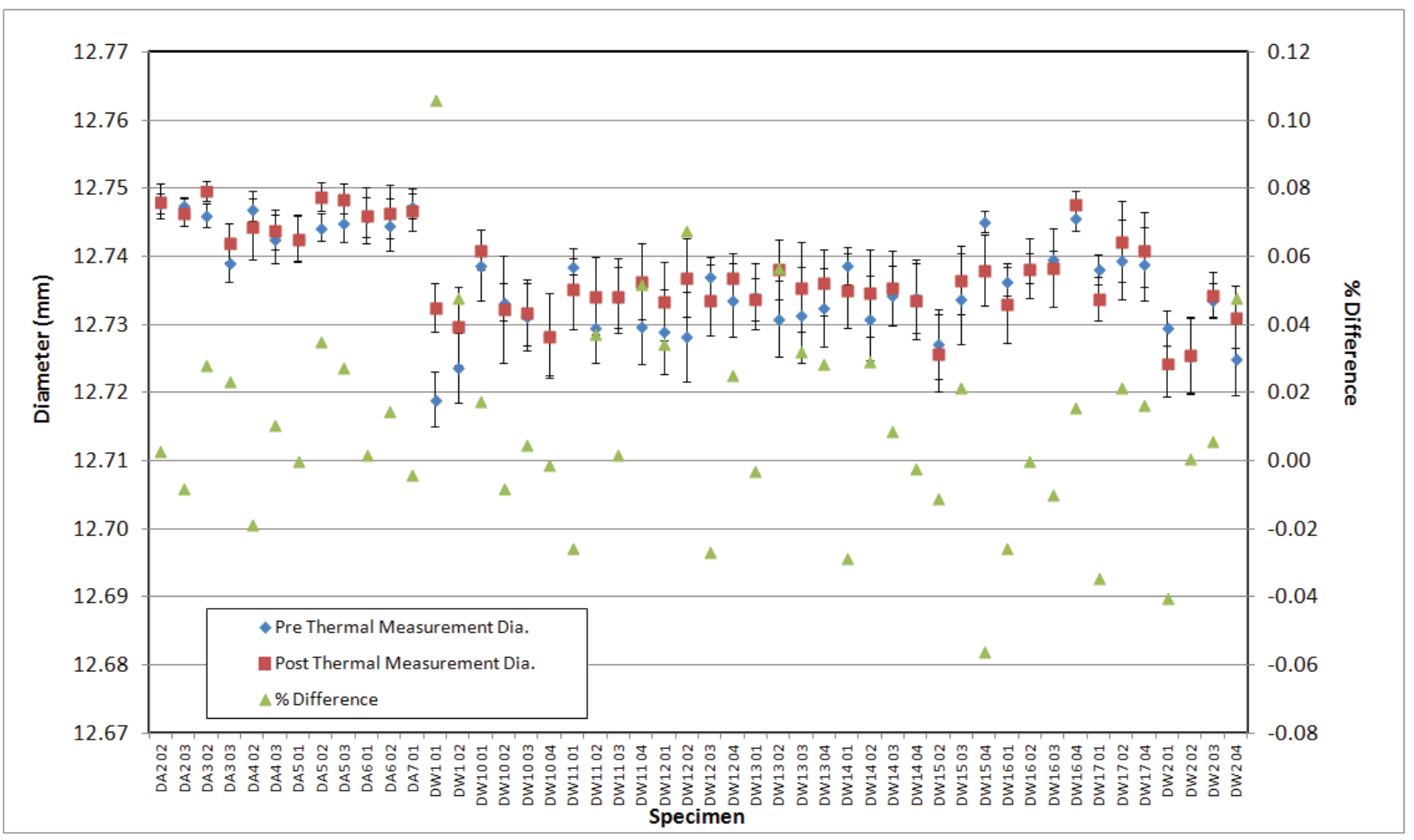

Figure A-164. PCEA Creep Pre vs. Post Thermal Measurement Diameter Comparison. 


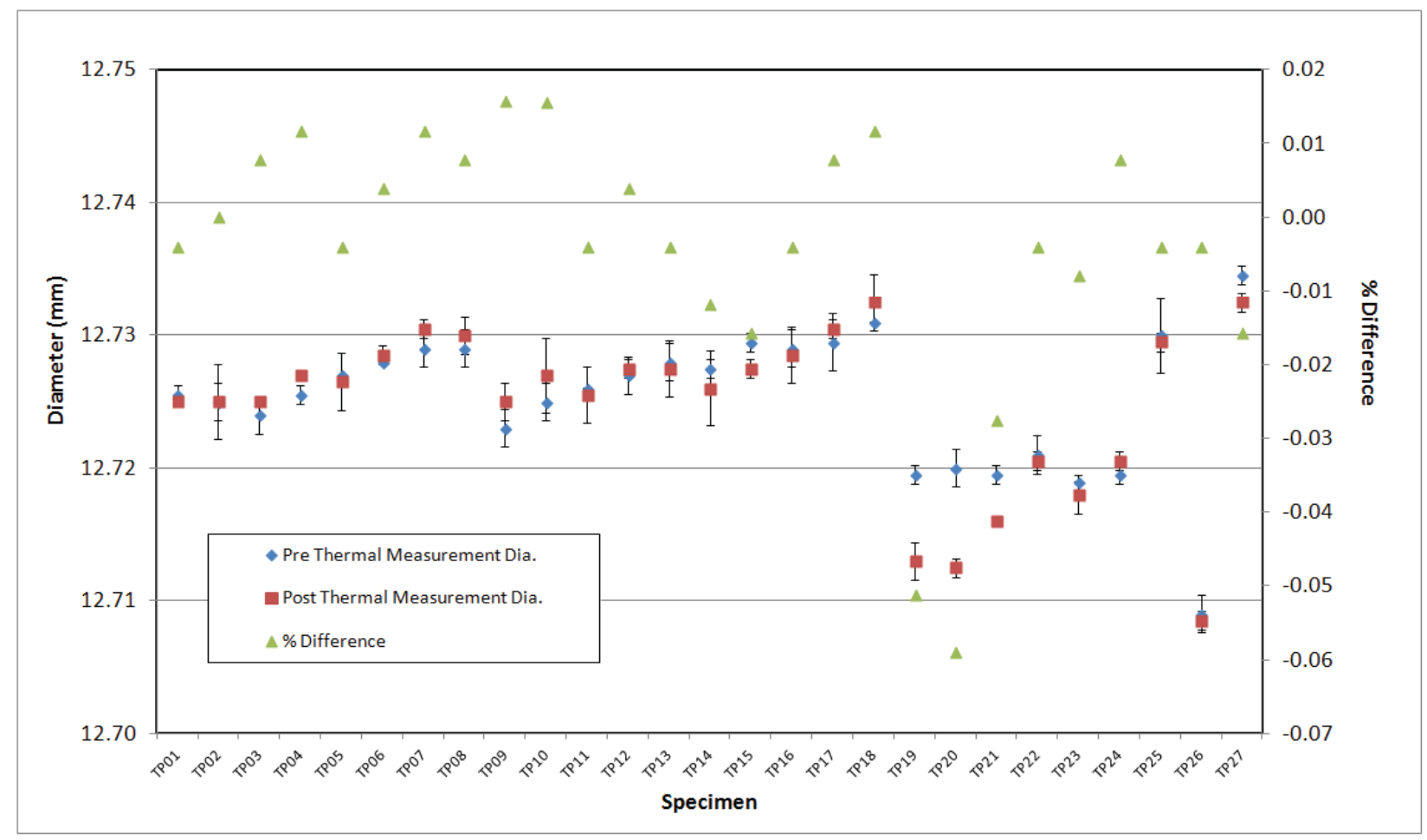

Figure A-165. 2114 Piggyback Pre vs. Post Thermal Measurement Diameter Comparison.

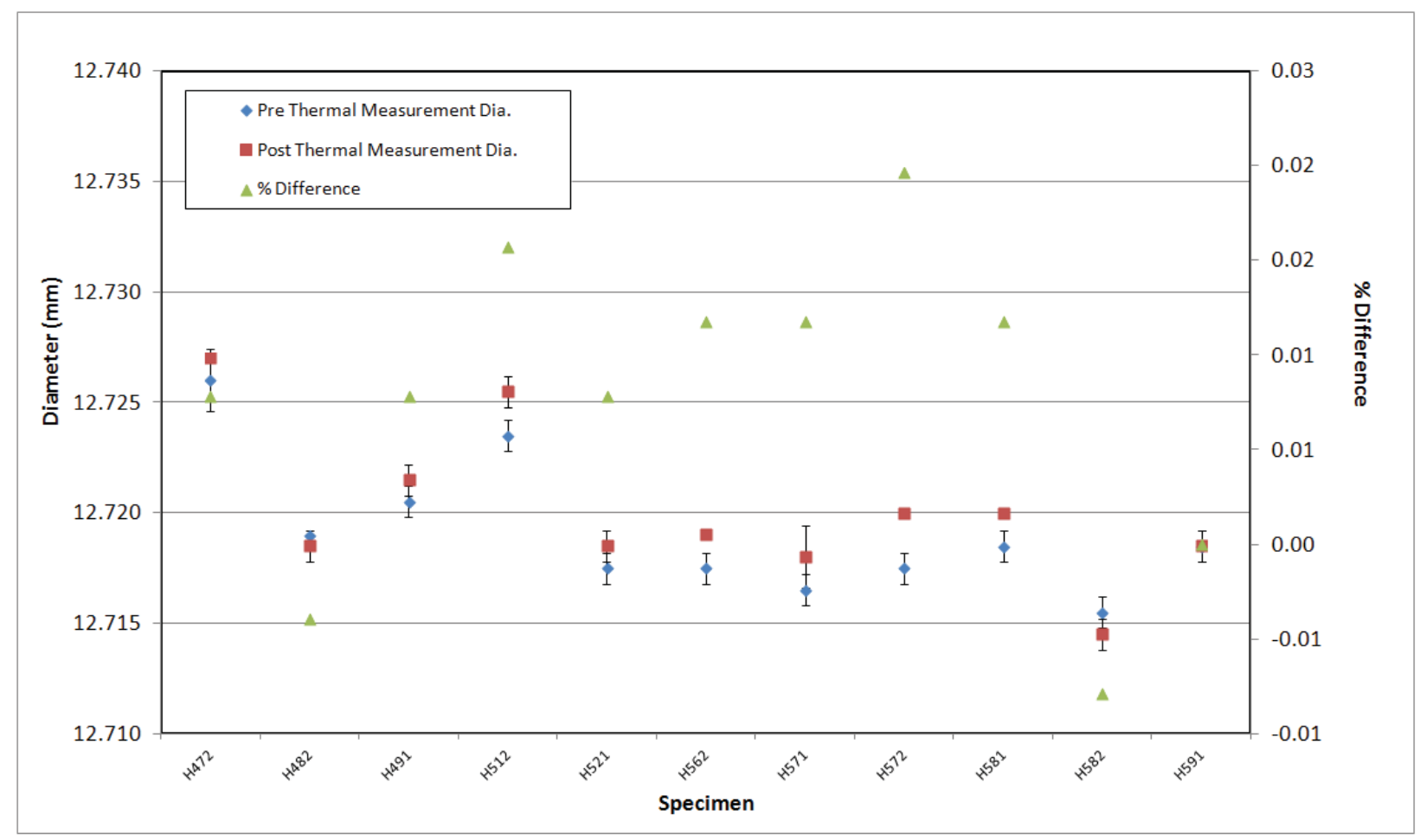

Figure A-166. A3 Matrix Piggyback Pre vs. Post Thermal Measurement Diameter Comparison. 


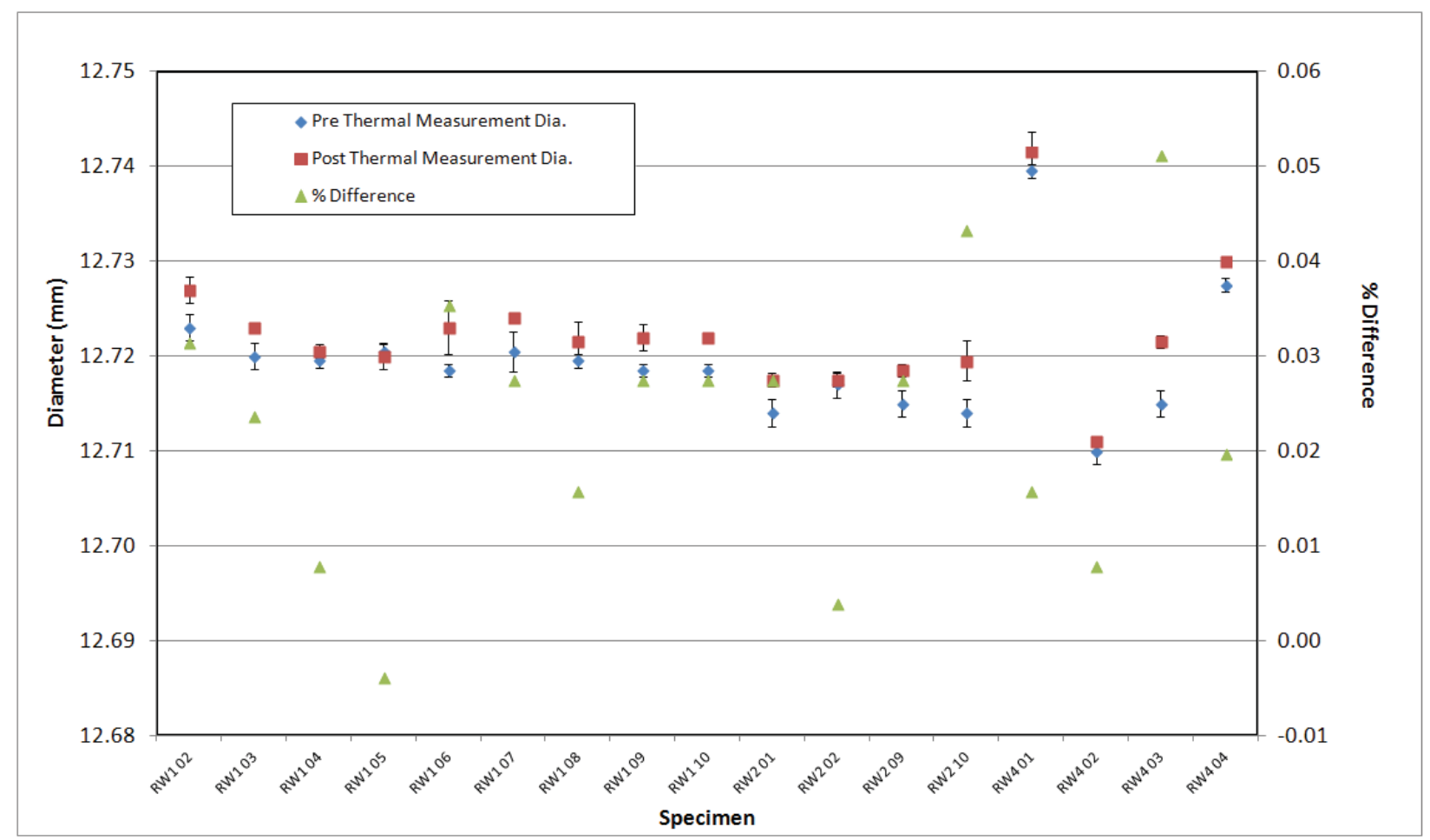

Figure A-167. BAN Piggyback Pre vs. Post Thermal Measurement Diameter Comparison.

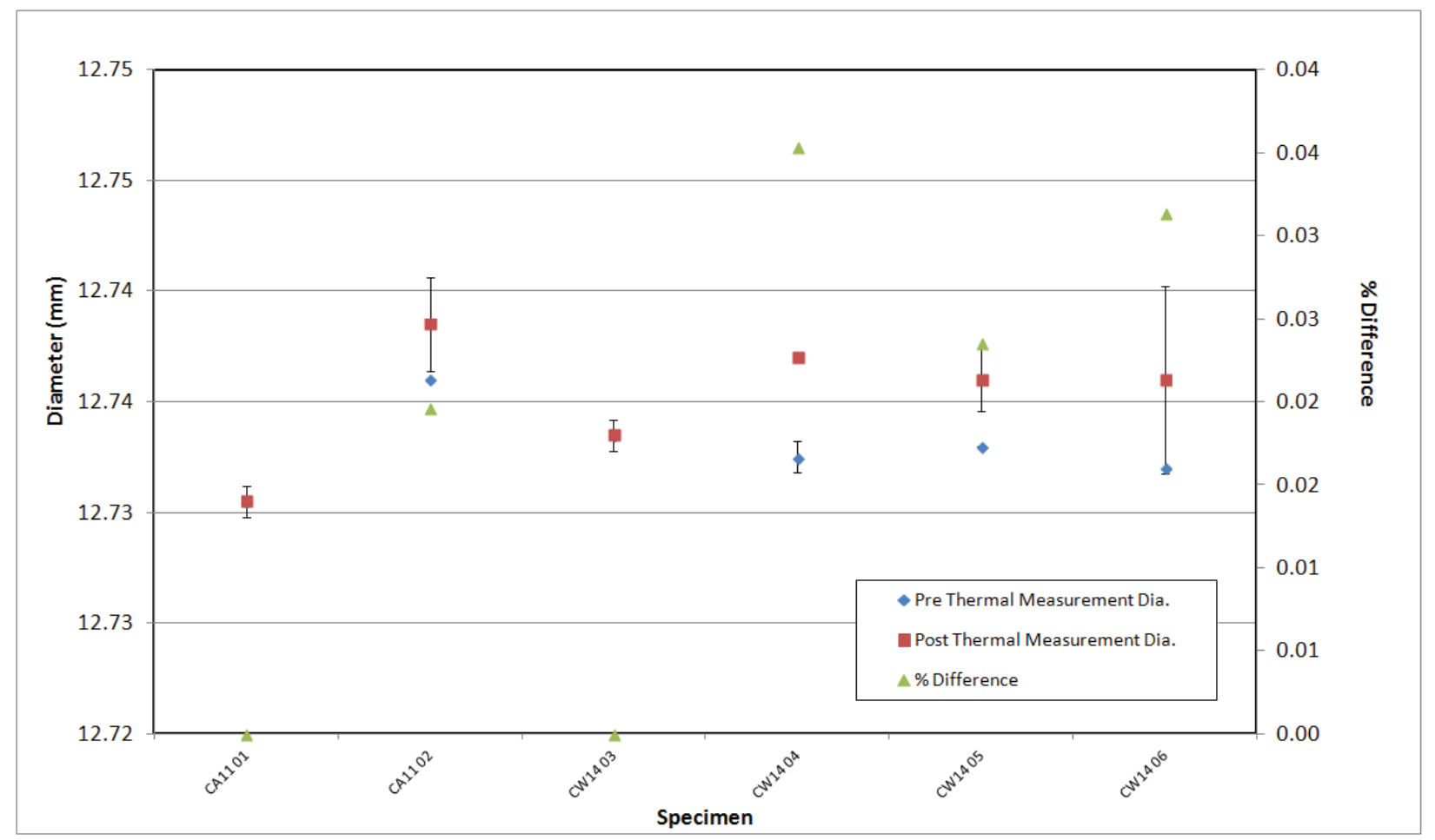

Figure A-168. H-451 Piggyback Pre vs. Post Thermal Measurement Diameter Comparison. 


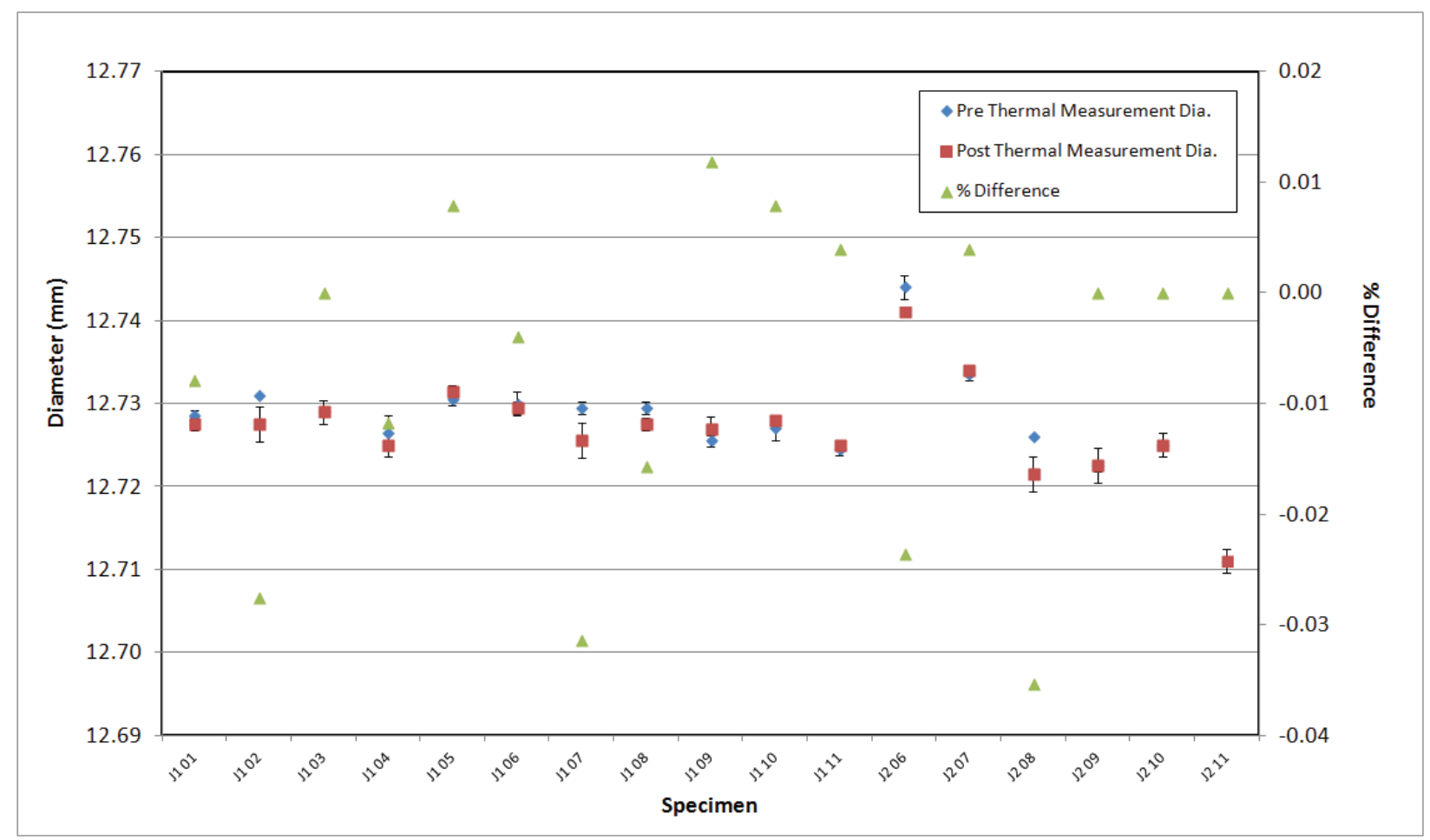

Figure A-169. HLM Piggyback Pre vs. Post Thermal Measurement Diameter Comparison.

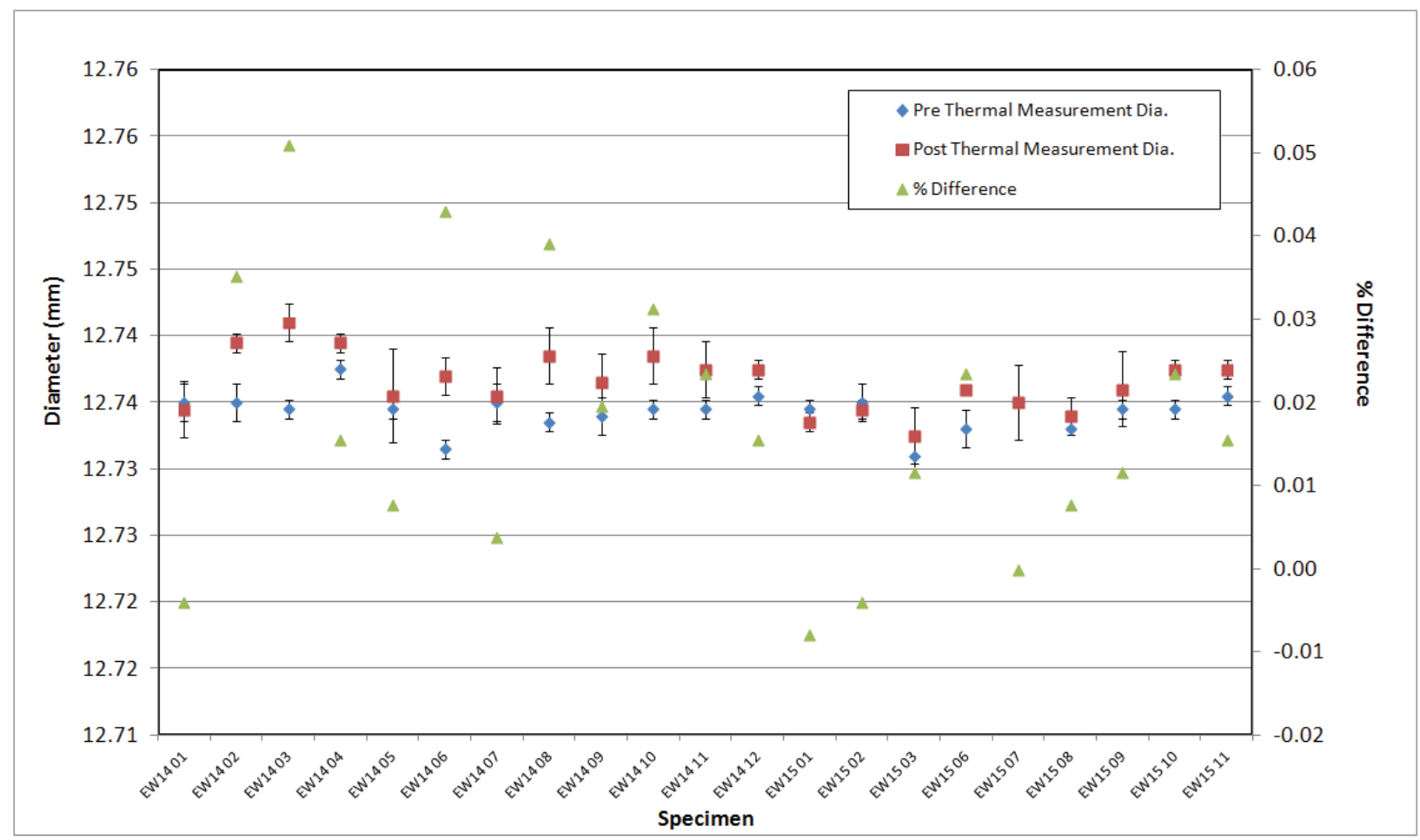

Figure A-170. IG-110 Piggyback Pre vs. Post Thermal Measurement Diameter Comparison. 


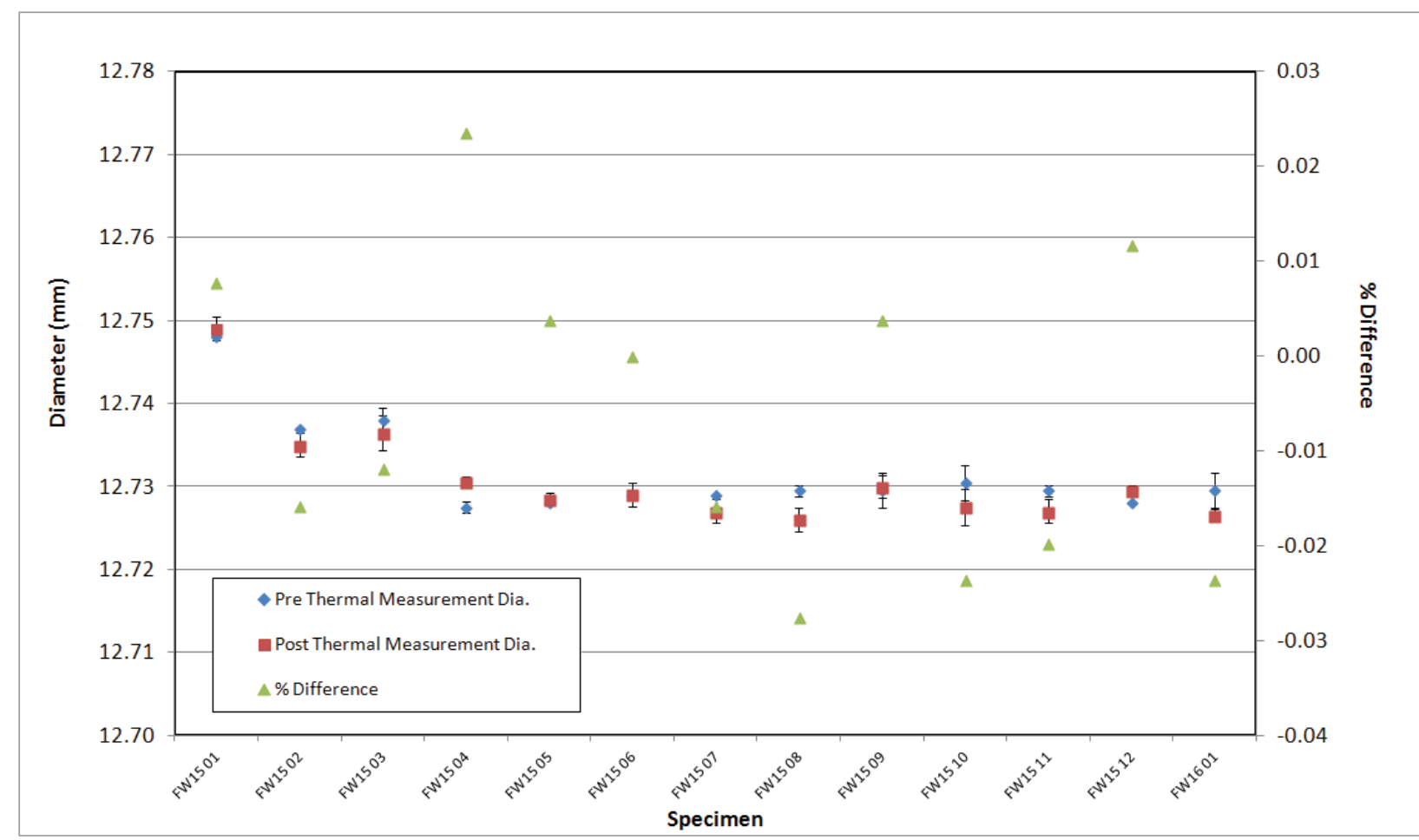

Figure A-171. IG-430 Piggyback Pre vs. Post Thermal Measurement Diameter Comparison.

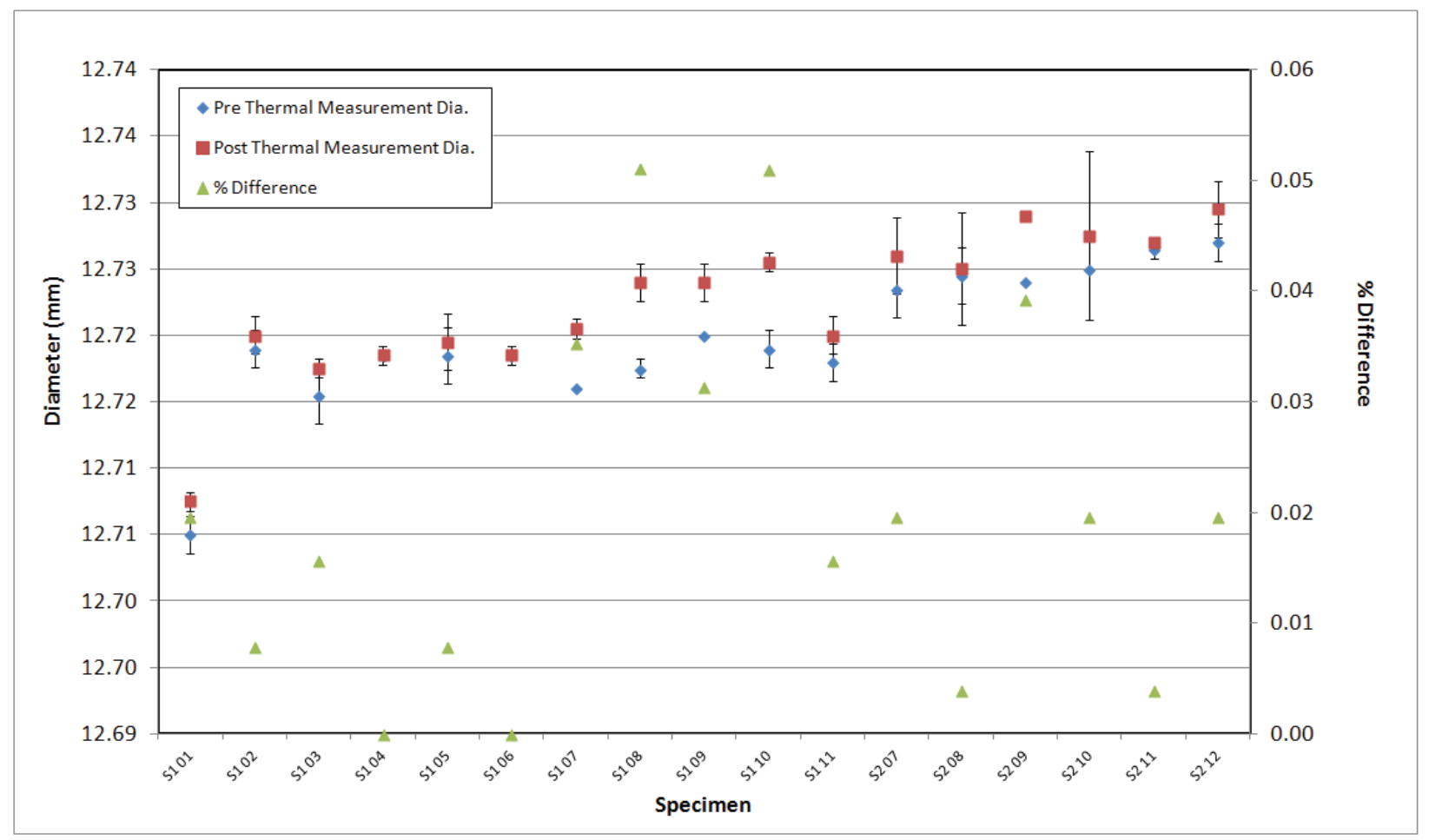

Figure A-172. NBG-10 Piggyback Pre vs. Post Thermal Measurement Diameter Comparison. 


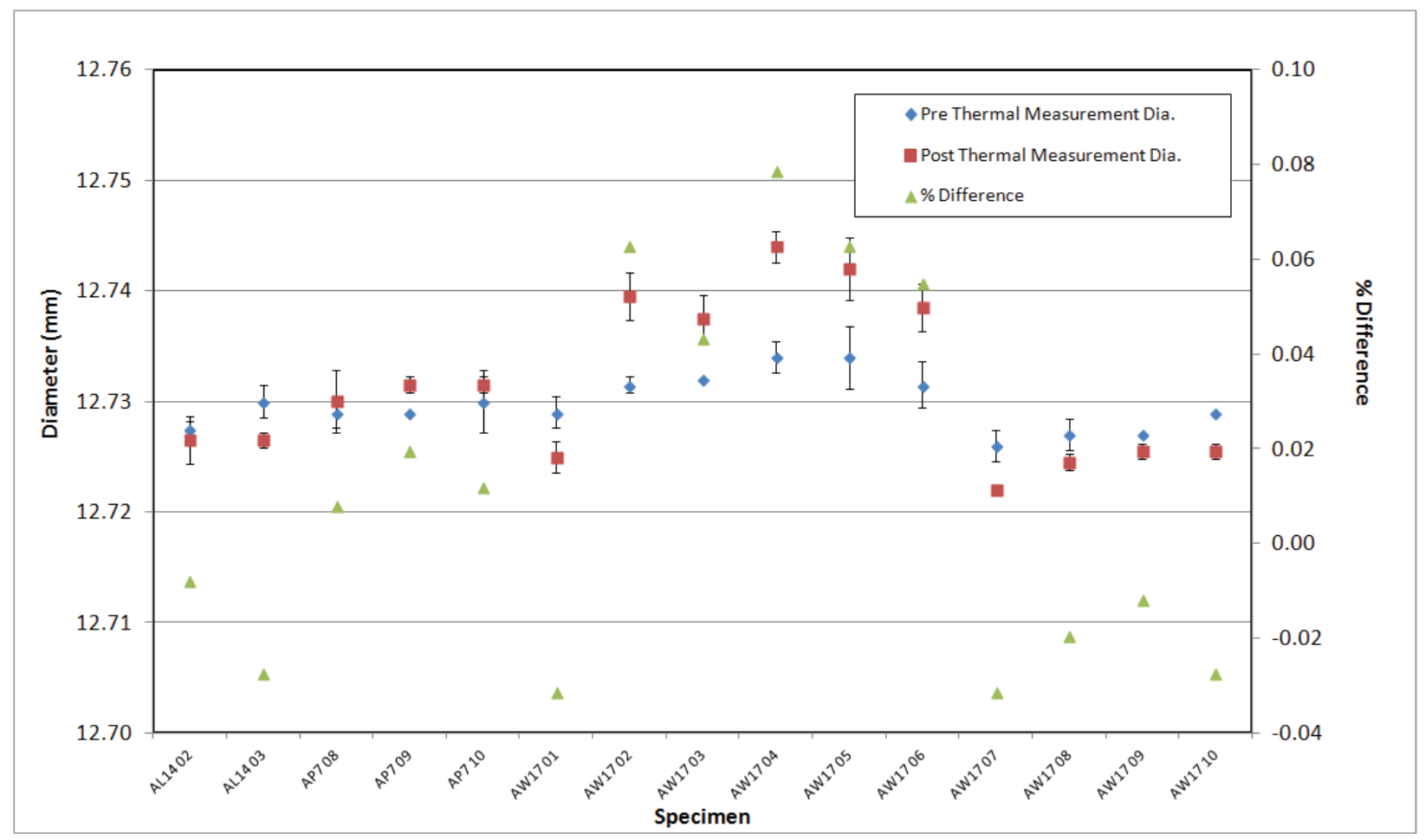

Figure A-173. NBG-17 Piggyback Pre vs. Post Thermal Measurement Diameter Comparison.

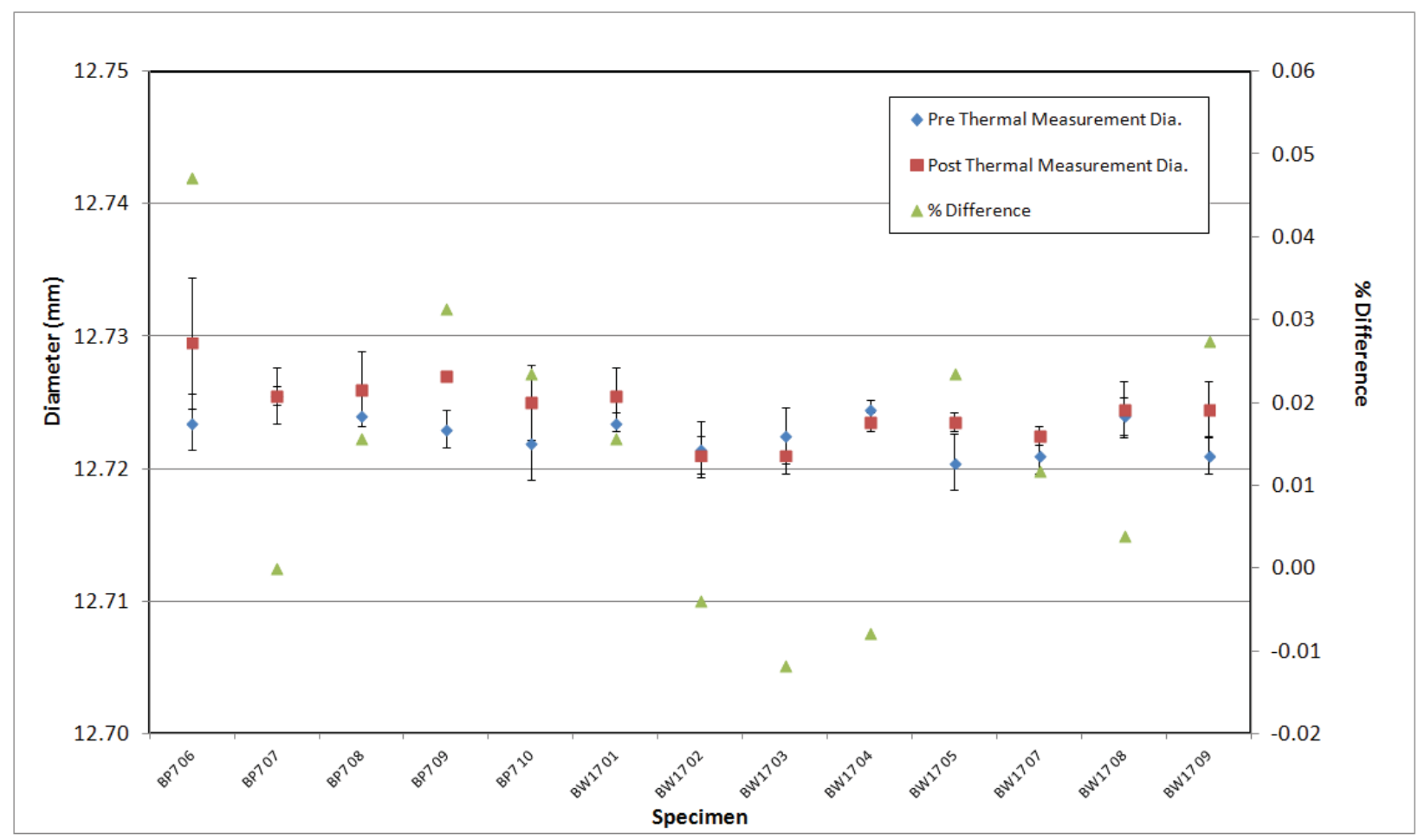

Figure A-174. NBG-18 Piggyback Pre vs. Post Thermal Measurement Diameter Comparison. 


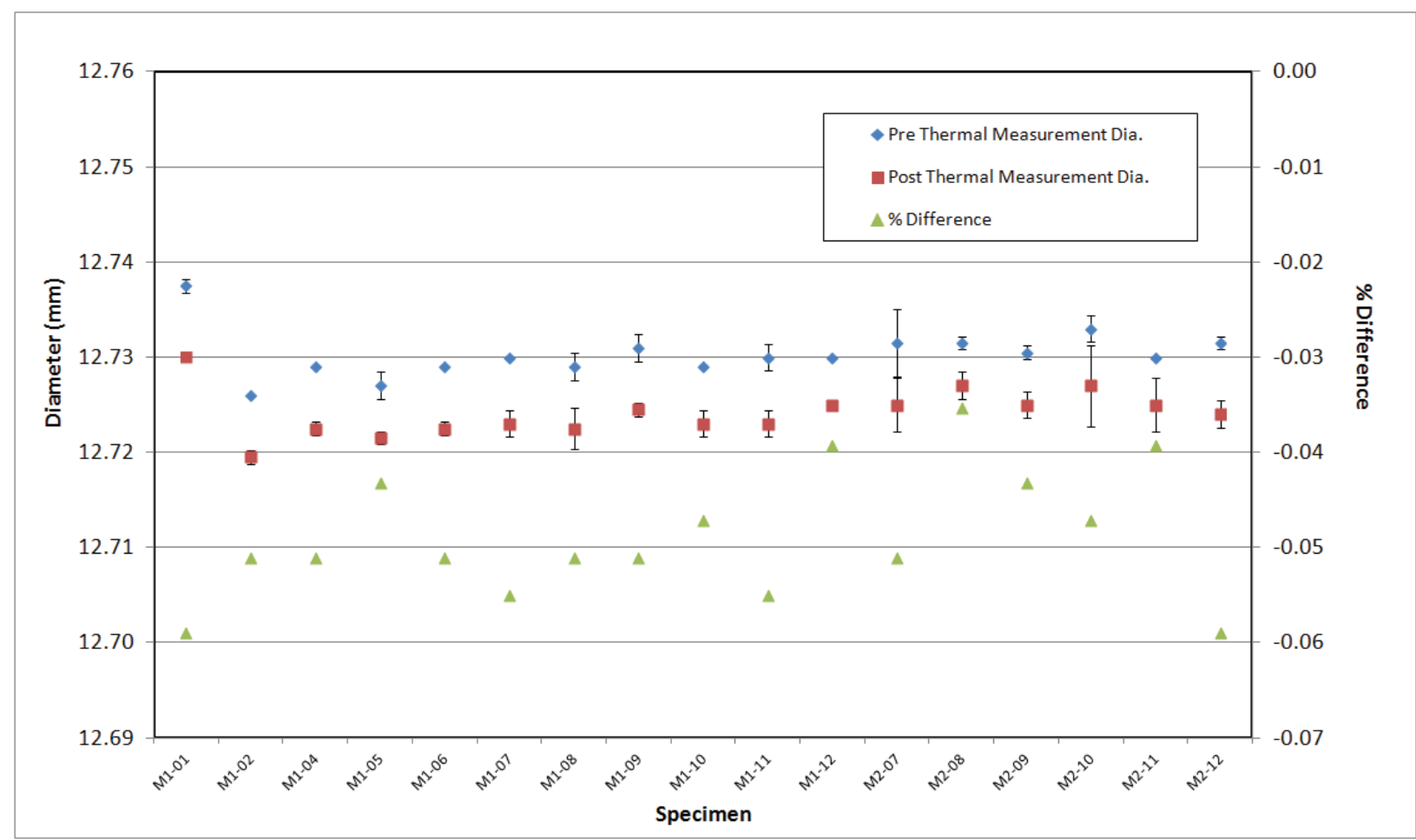

Figure A-175. NBG-25 Piggyback Pre vs. Post Thermal Measurement Diameter Comparison.

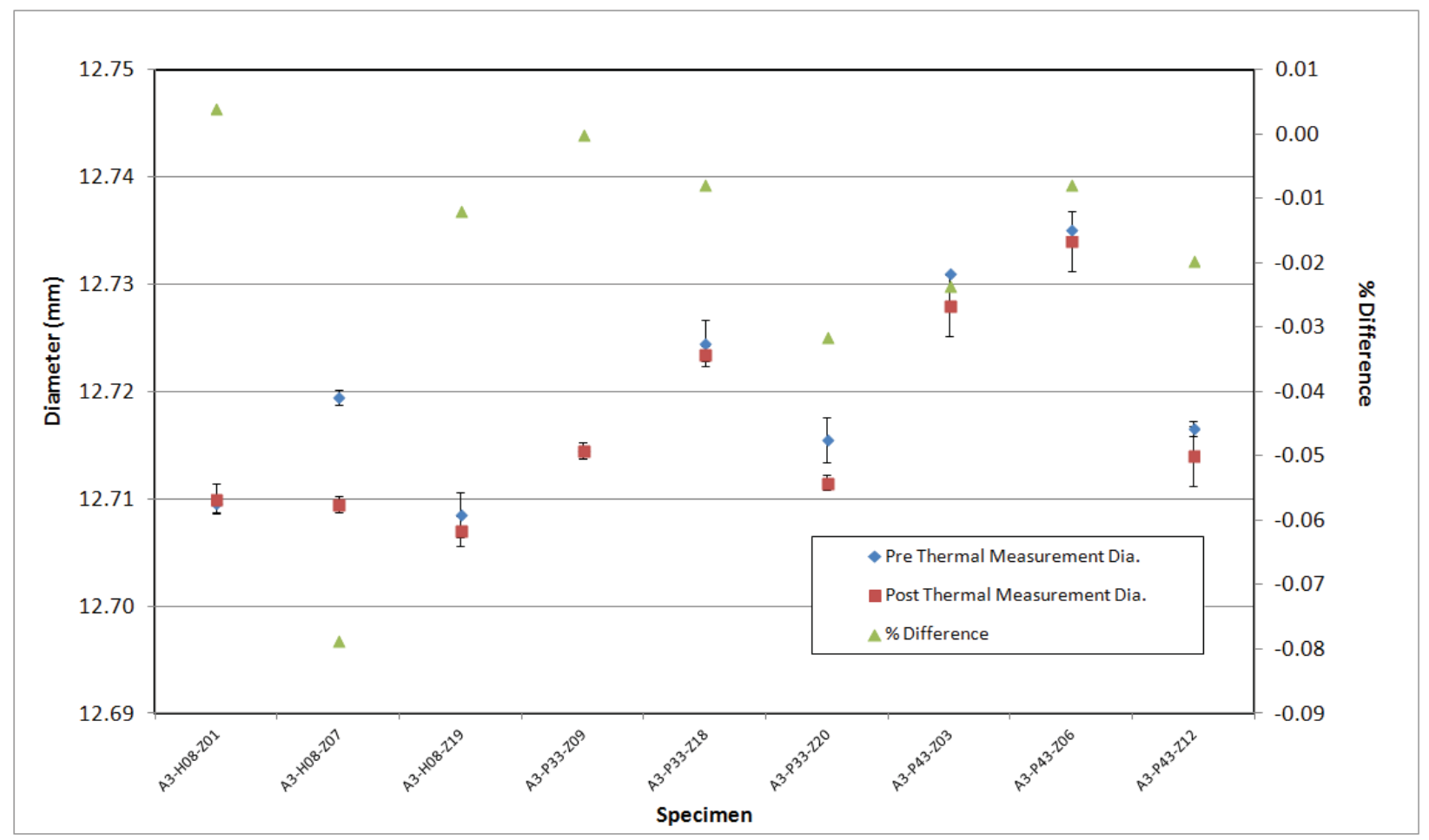

Figure A-176. New Matrix Piggyback Pre vs. Post Thermal Measurement Diameter Comparison. 


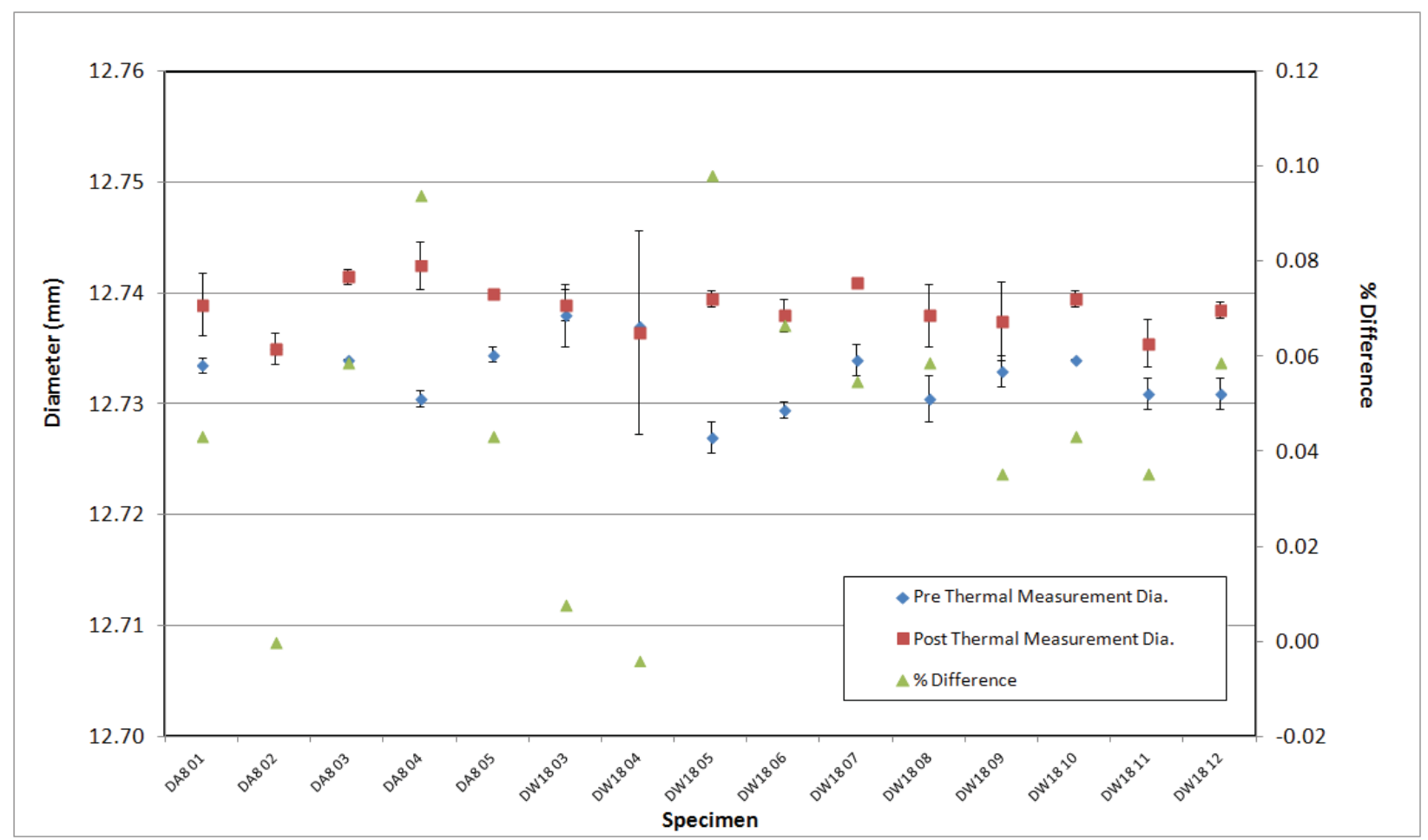

Figure A-177. PCEA Piggyback Pre vs. Post Thermal Measurement Diameter Comparison.

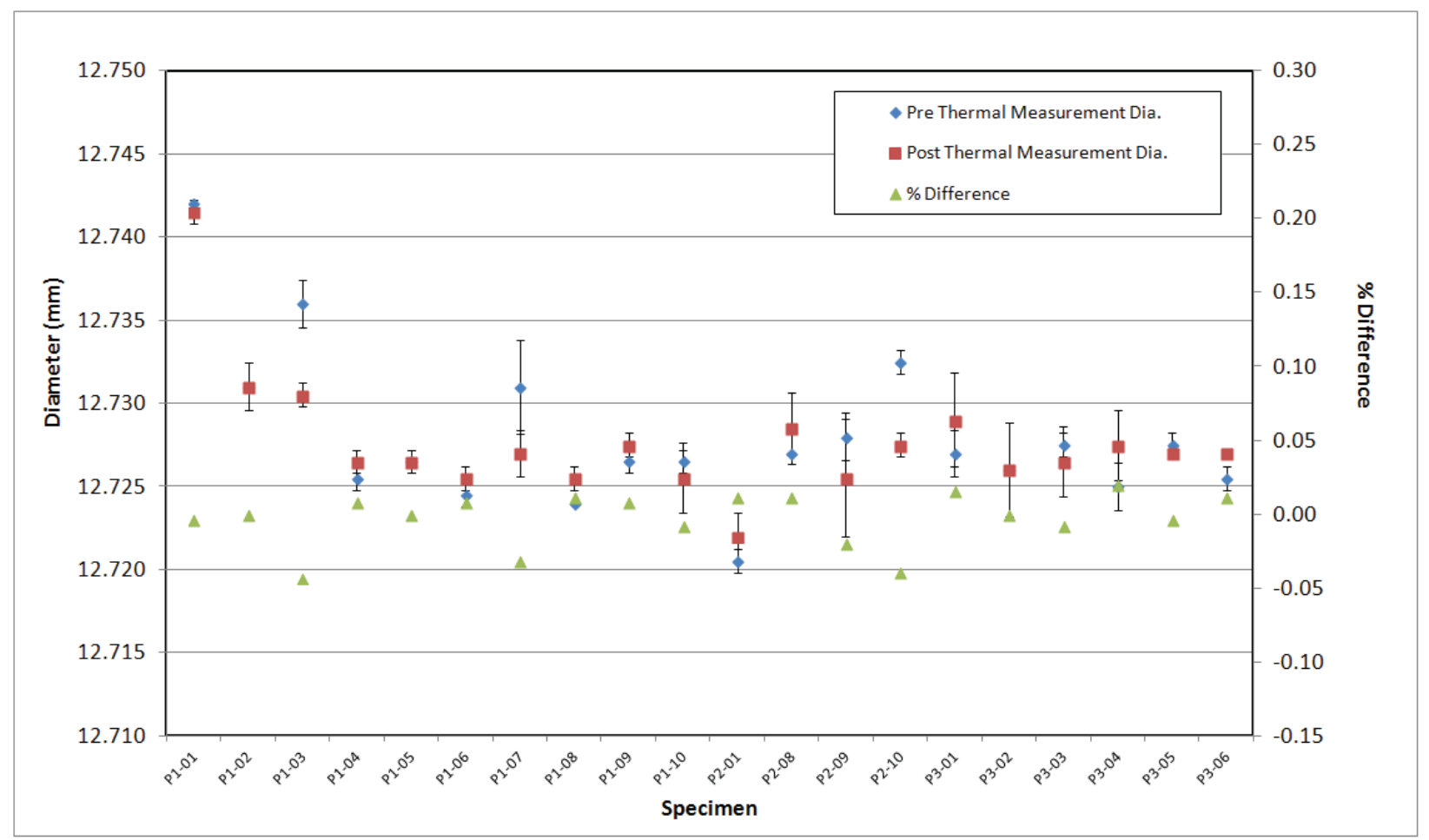

Figure A-178. PCIB Piggyback Pre vs. Post Thermal Measurement Diameter Comparison. 


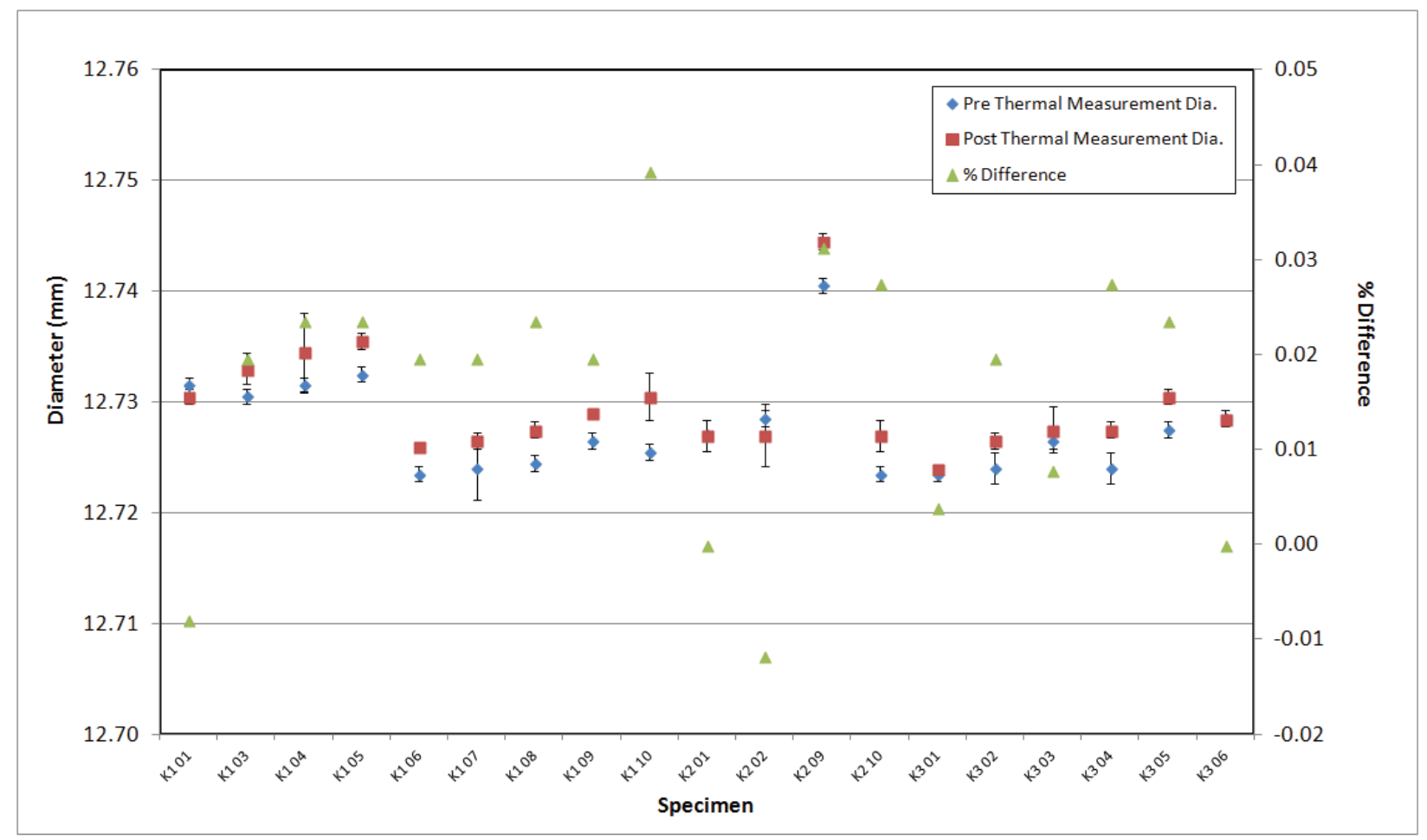

Figure A-179. PGX Piggyback Pre vs. Post Thermal Measurement Diameter Comparison.

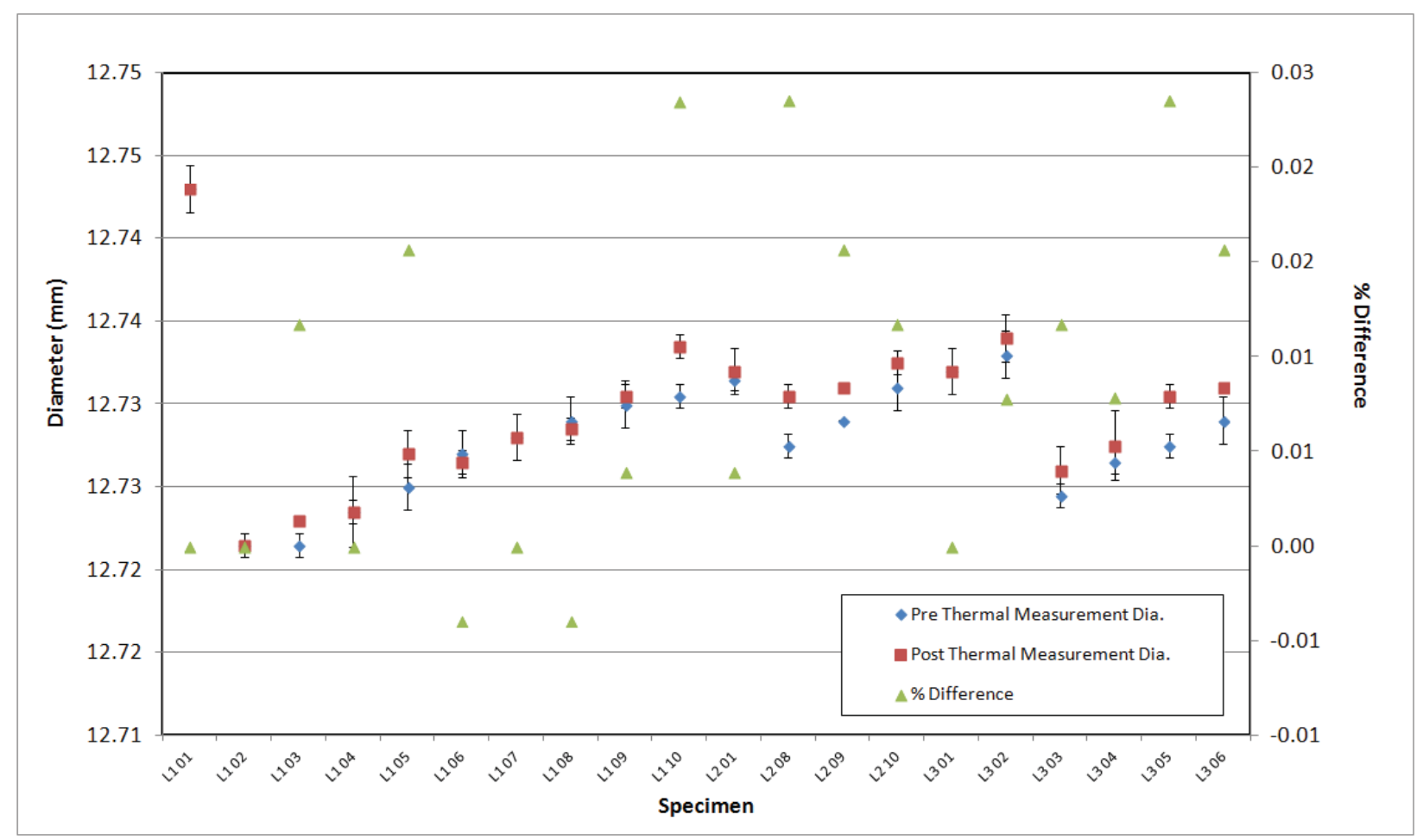

Figure A-180. PPEA Piggyback Pre vs. Post Thermal Measurement Diameter Comparison. 


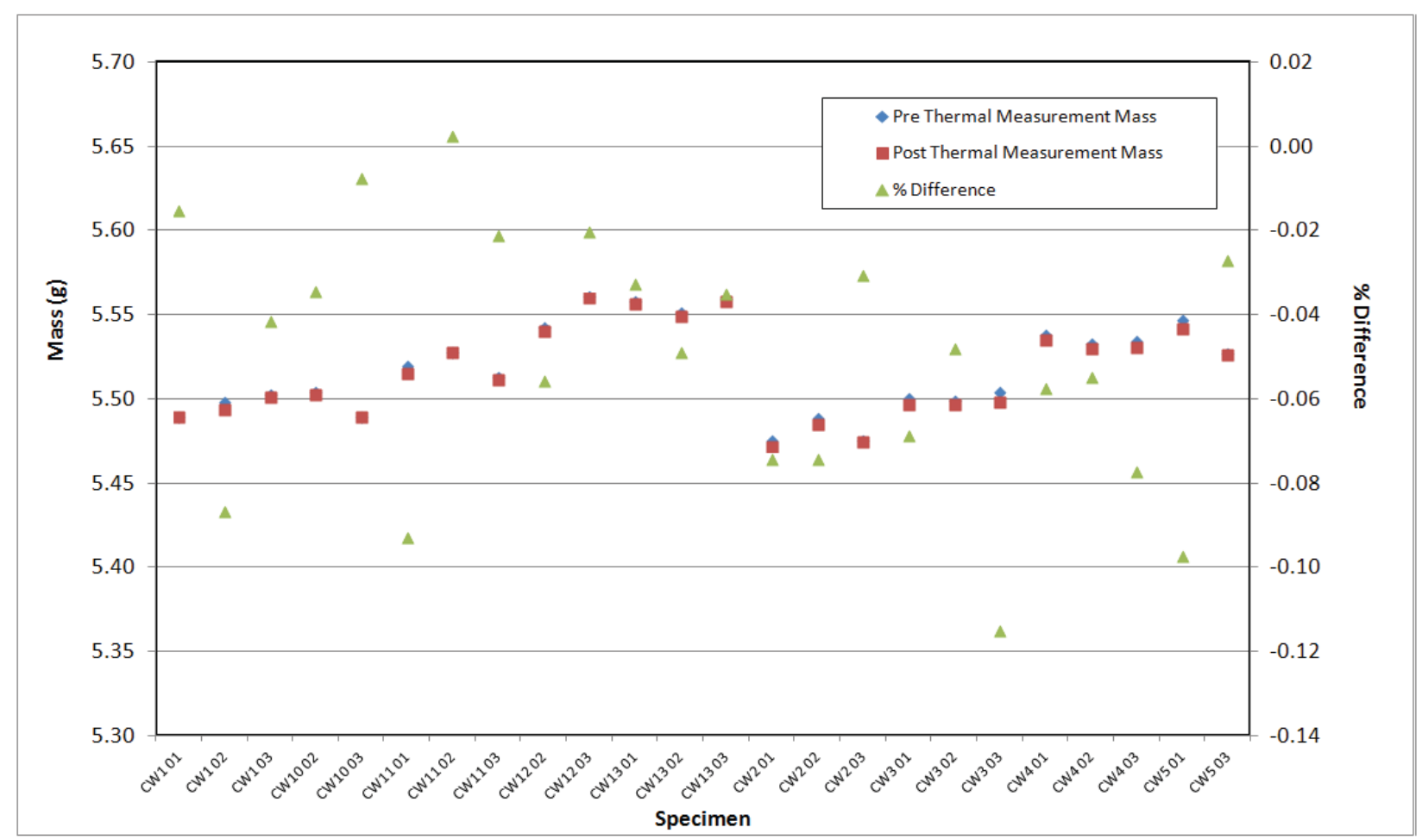

Figure A-181. H-451 Creep Pre vs. Post Thermal Measurement Mass Comparison.

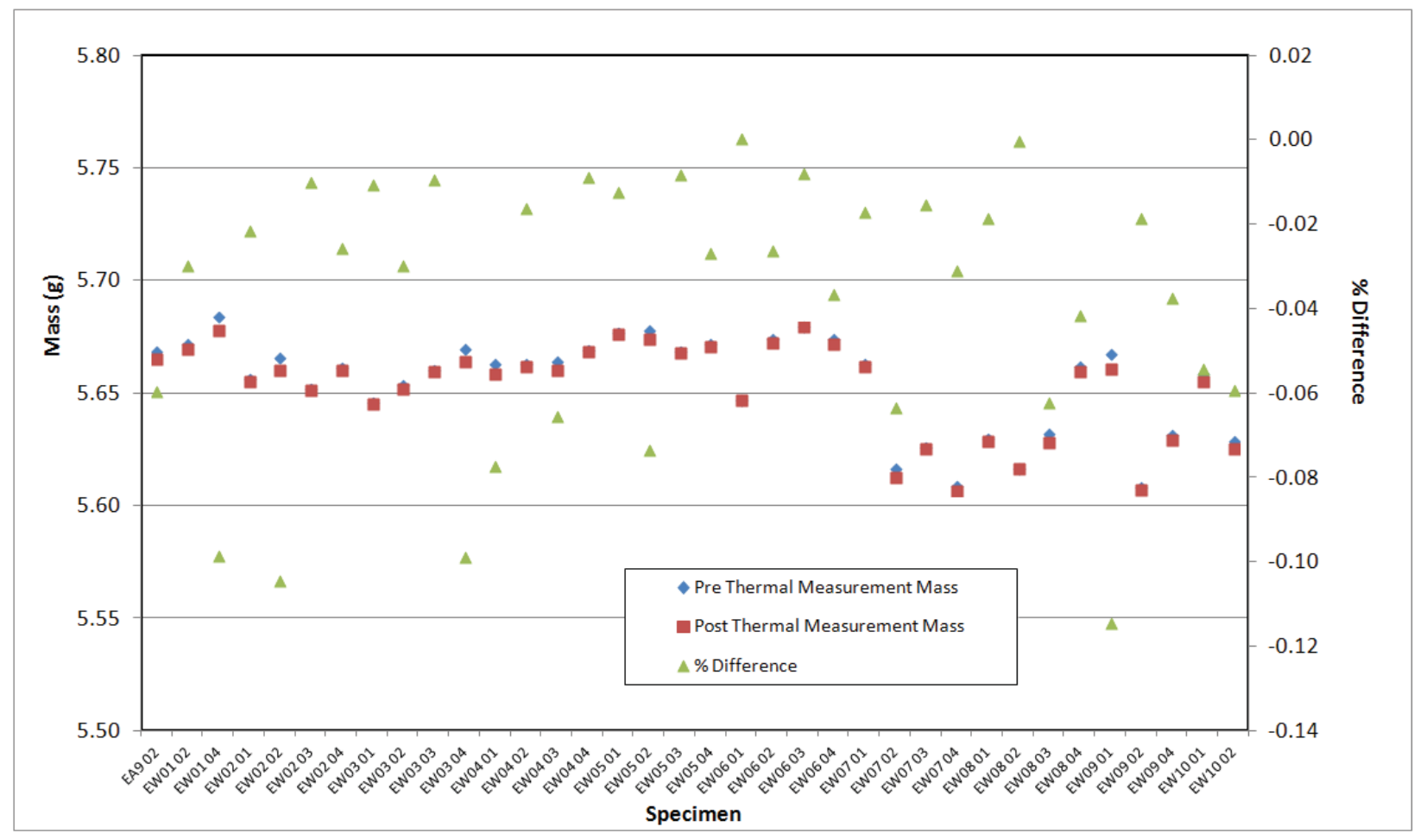

Figure A-182. IG-110 Creep Pre vs. Post Thermal Measurement Mass Comparison. 


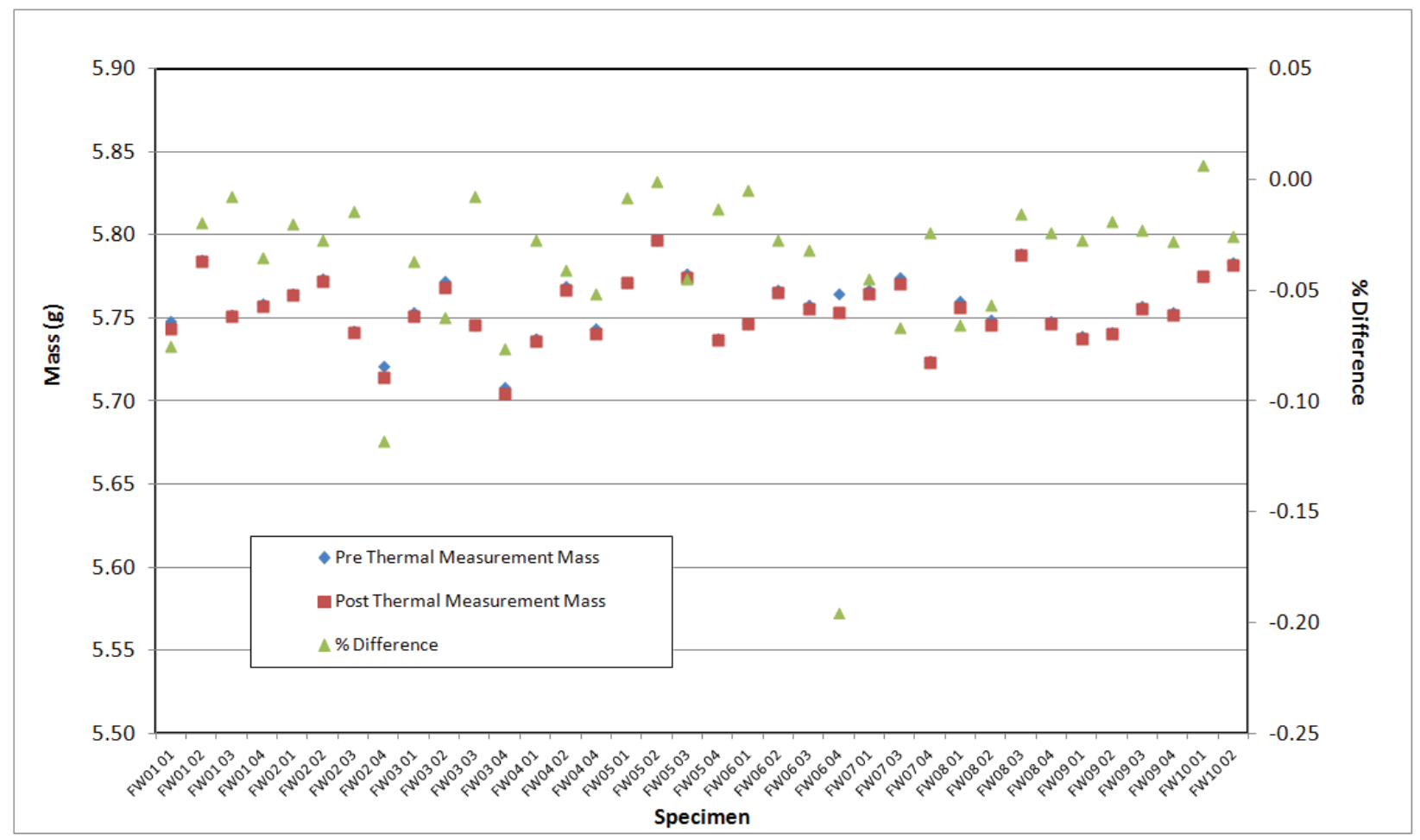

Figure A-183. IG-430 Creep Pre vs. Post Thermal Measurement Mass Comparison.

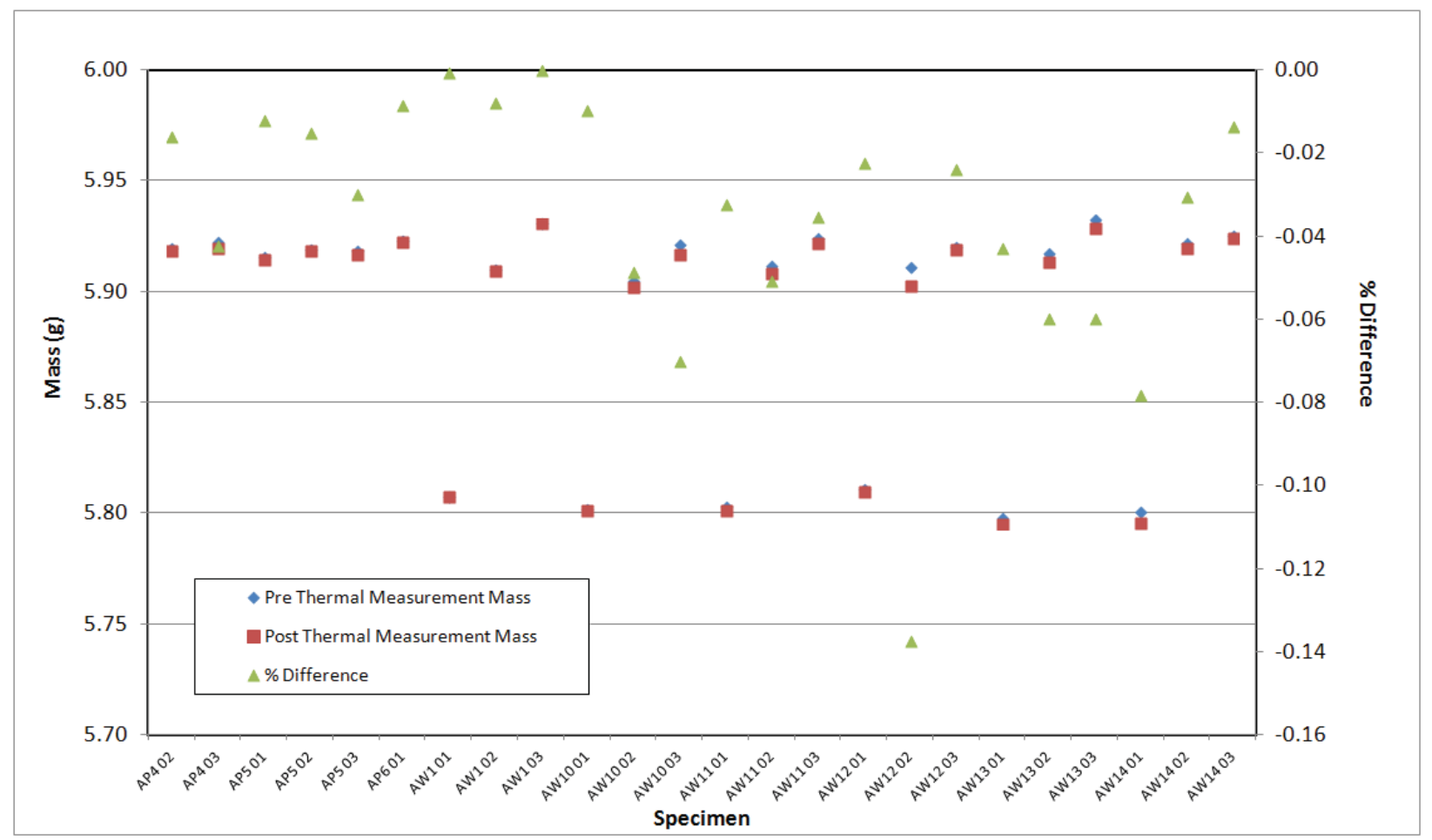

Figure A-184. NBG-17 Creep Pre vs. Post Thermal Measurement Mass Comparison. 


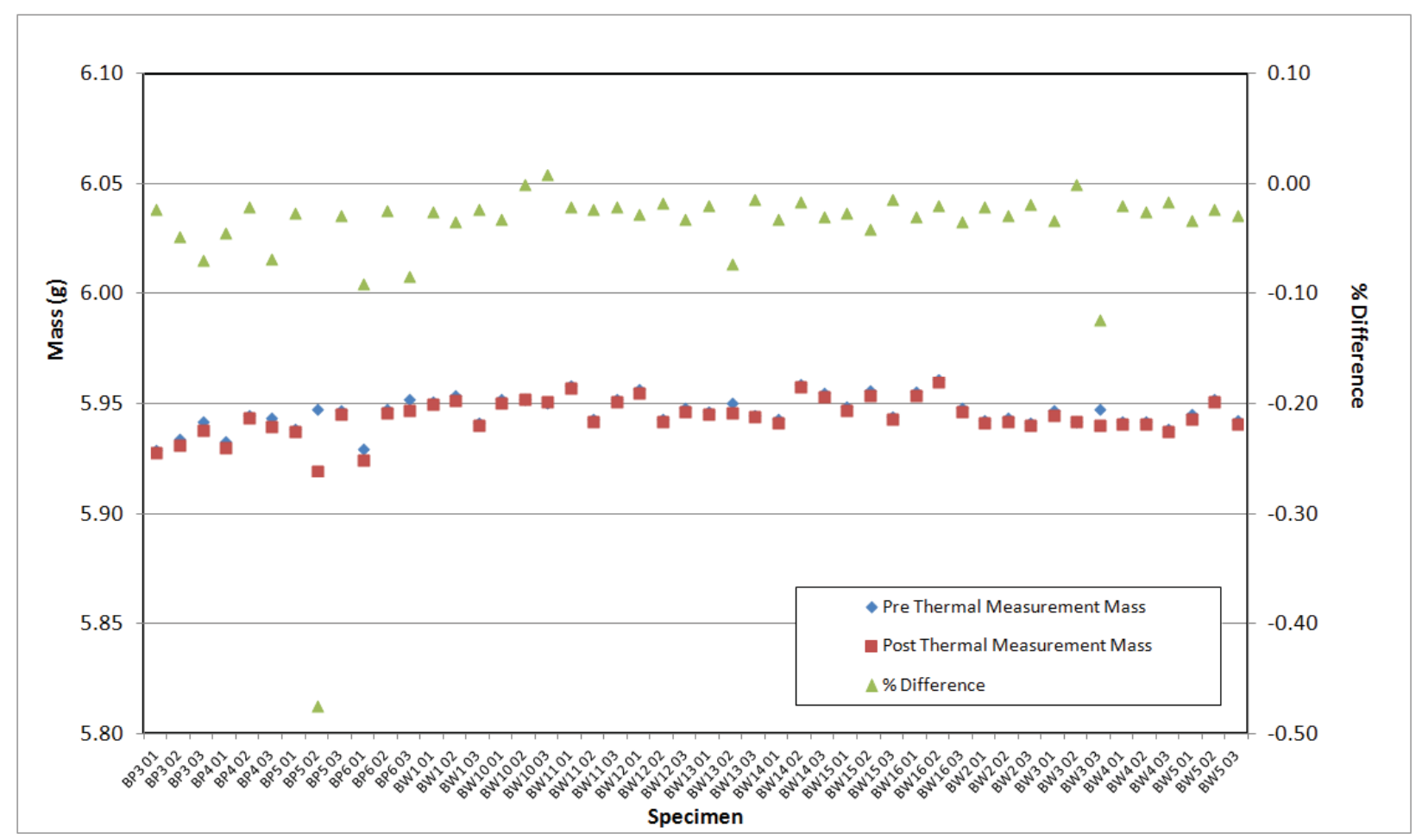

Figure A-185. NBG-18 Creep Pre vs. Post Thermal Measurement Mass Comparison.

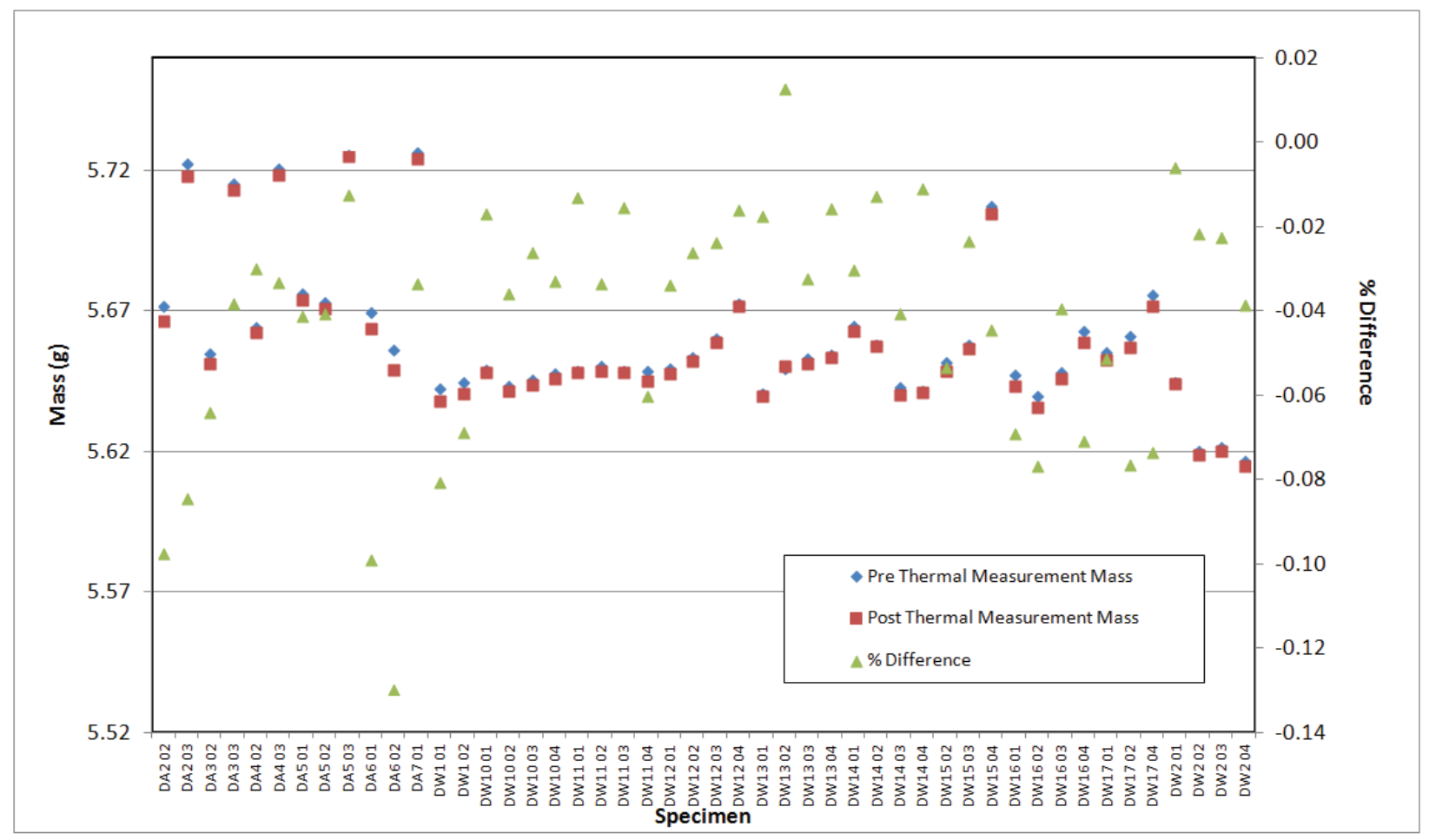

Figure A-186. PCEA Creep Pre vs. Post Thermal Measurement Mass Comparison. 


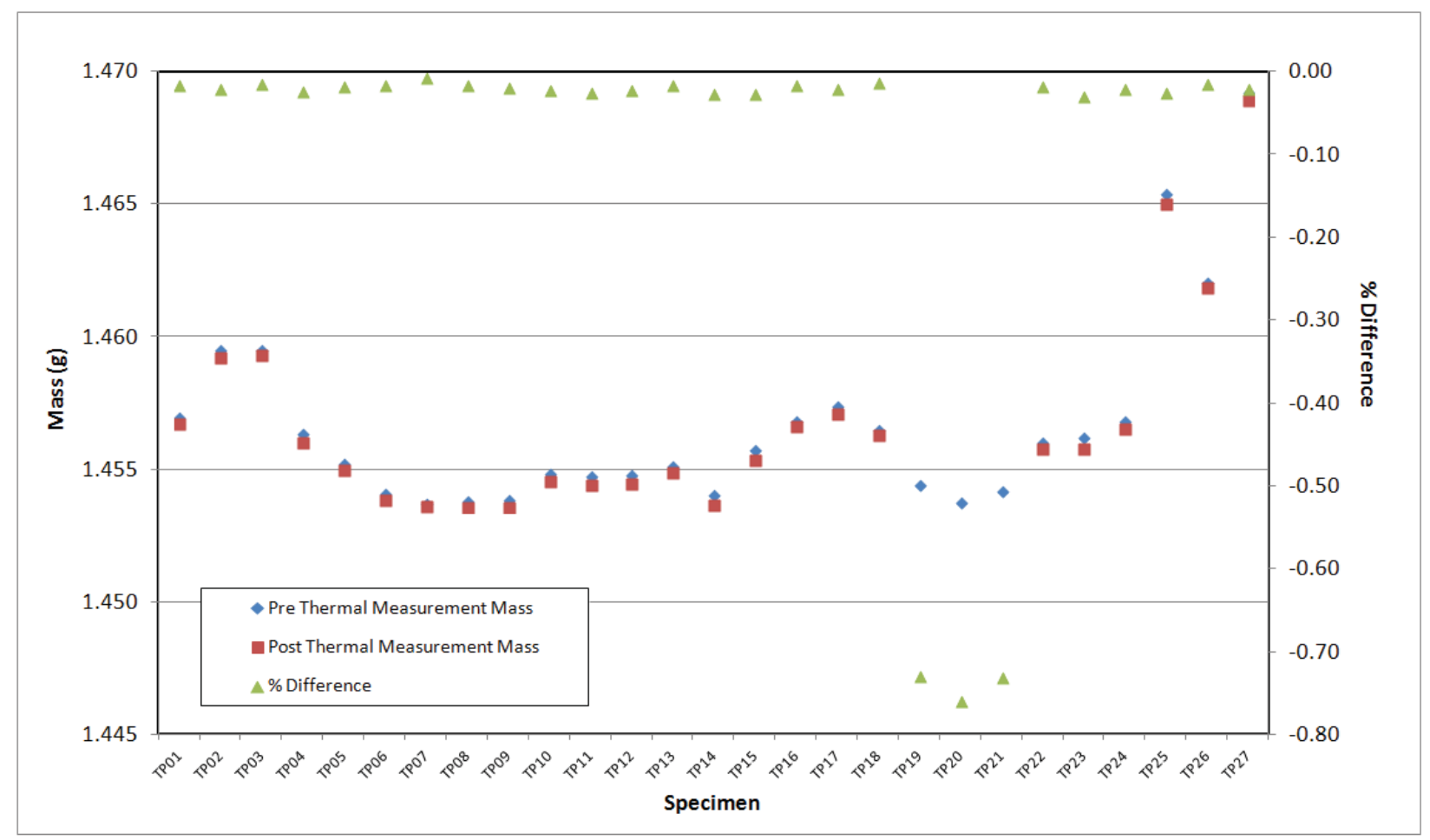

Figure A-187. 2114 Piggyback Pre vs. Post Thermal Measurement Mass Comparison.

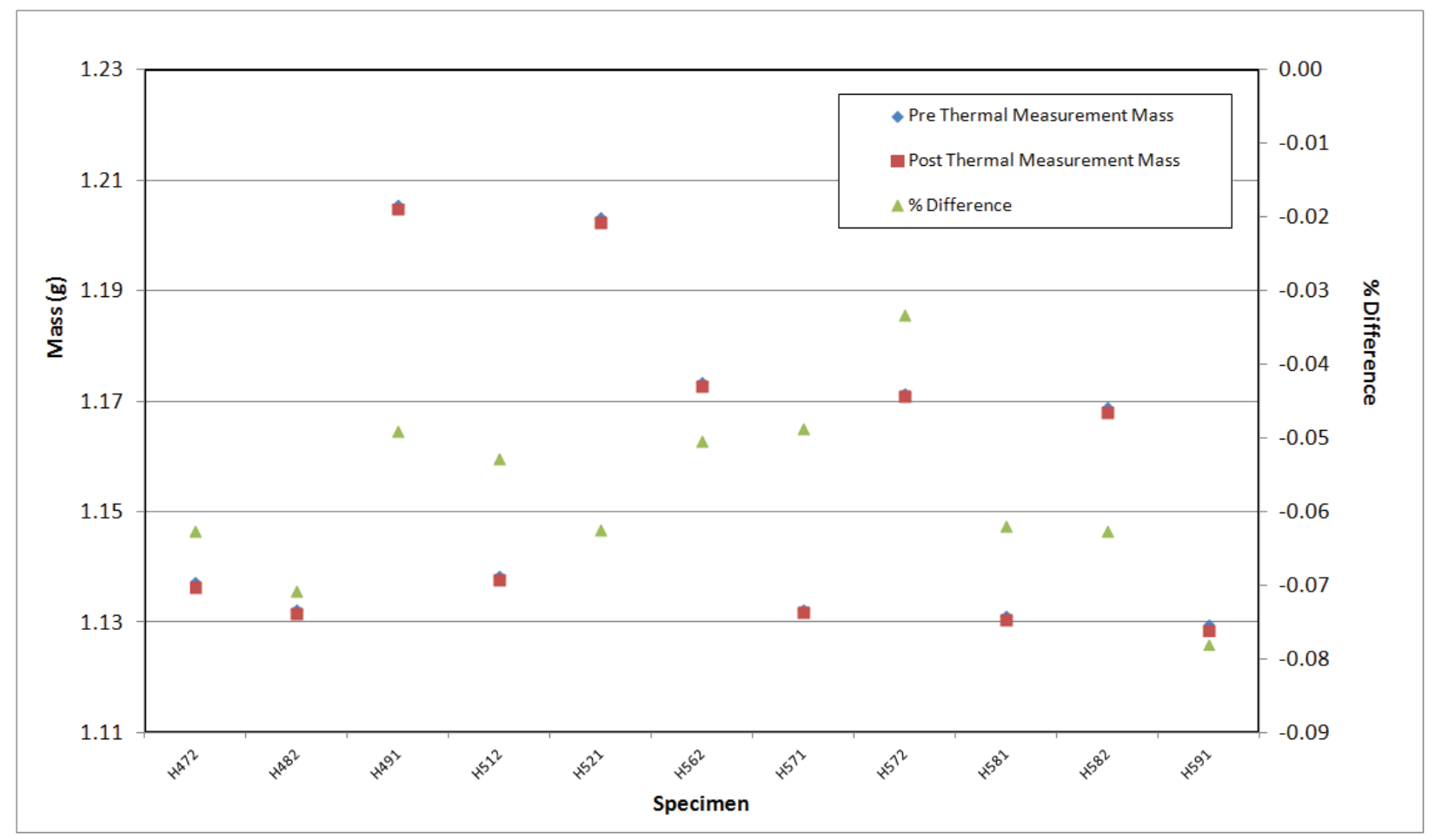

Figure A-188. A3 Matrix Piggyback Pre vs. Post Thermal Measurement Mass Comparison. 


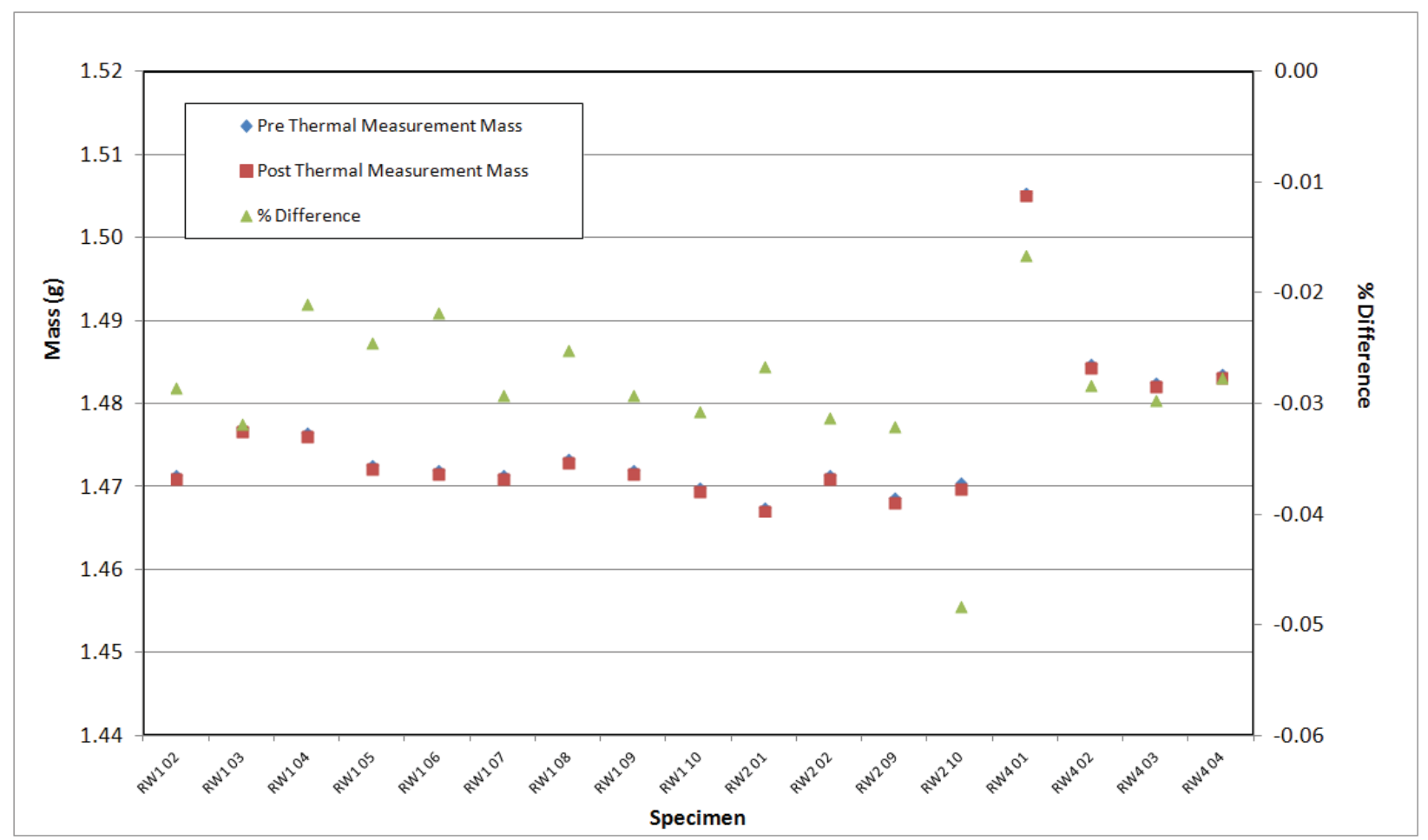

Figure A-189. BAN Piggyback Pre vs. Post Thermal Measurement Mass Comparison.

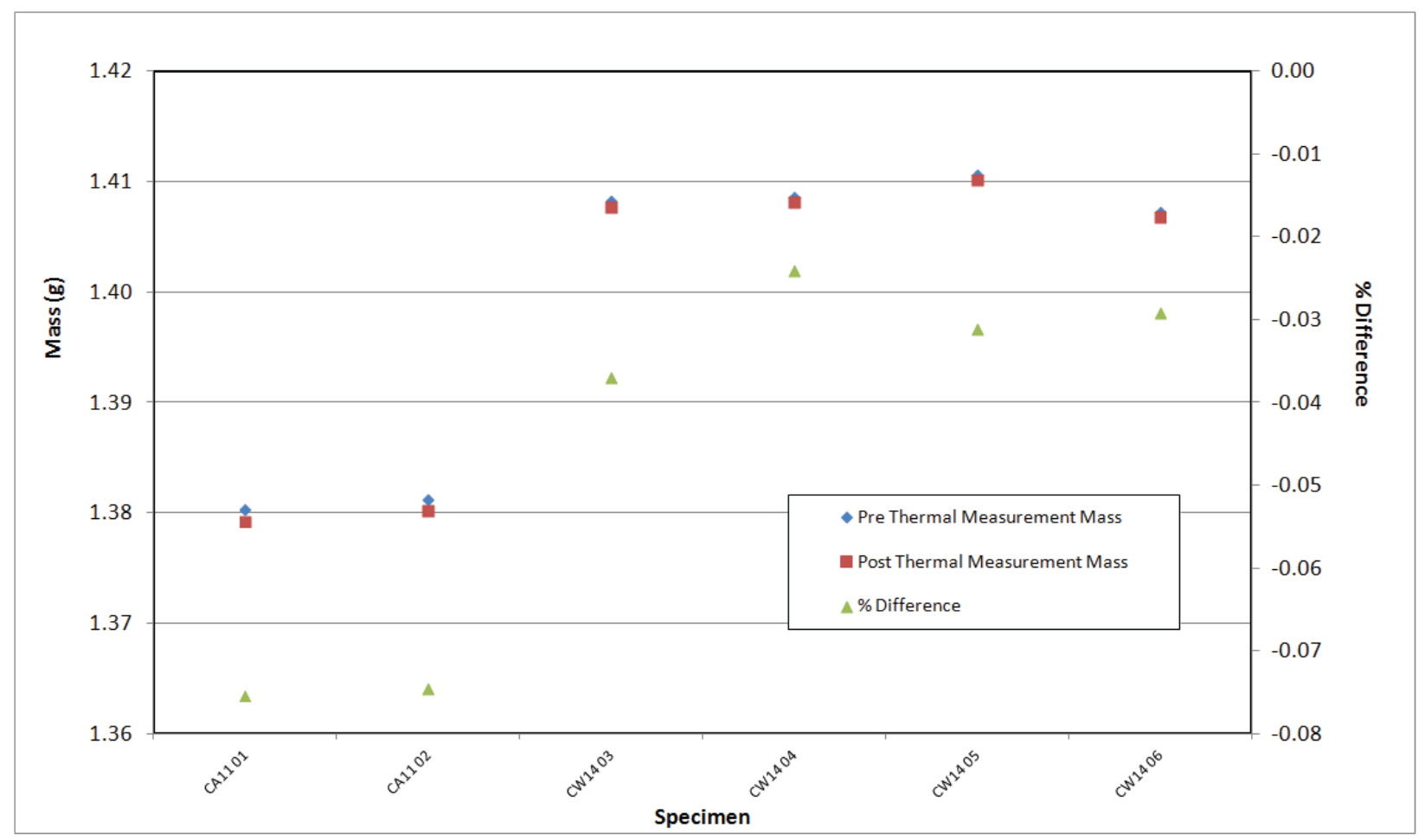

Figure A-190. H-451 Piggyback Pre vs. Post Thermal Measurement Mass Comparison. 


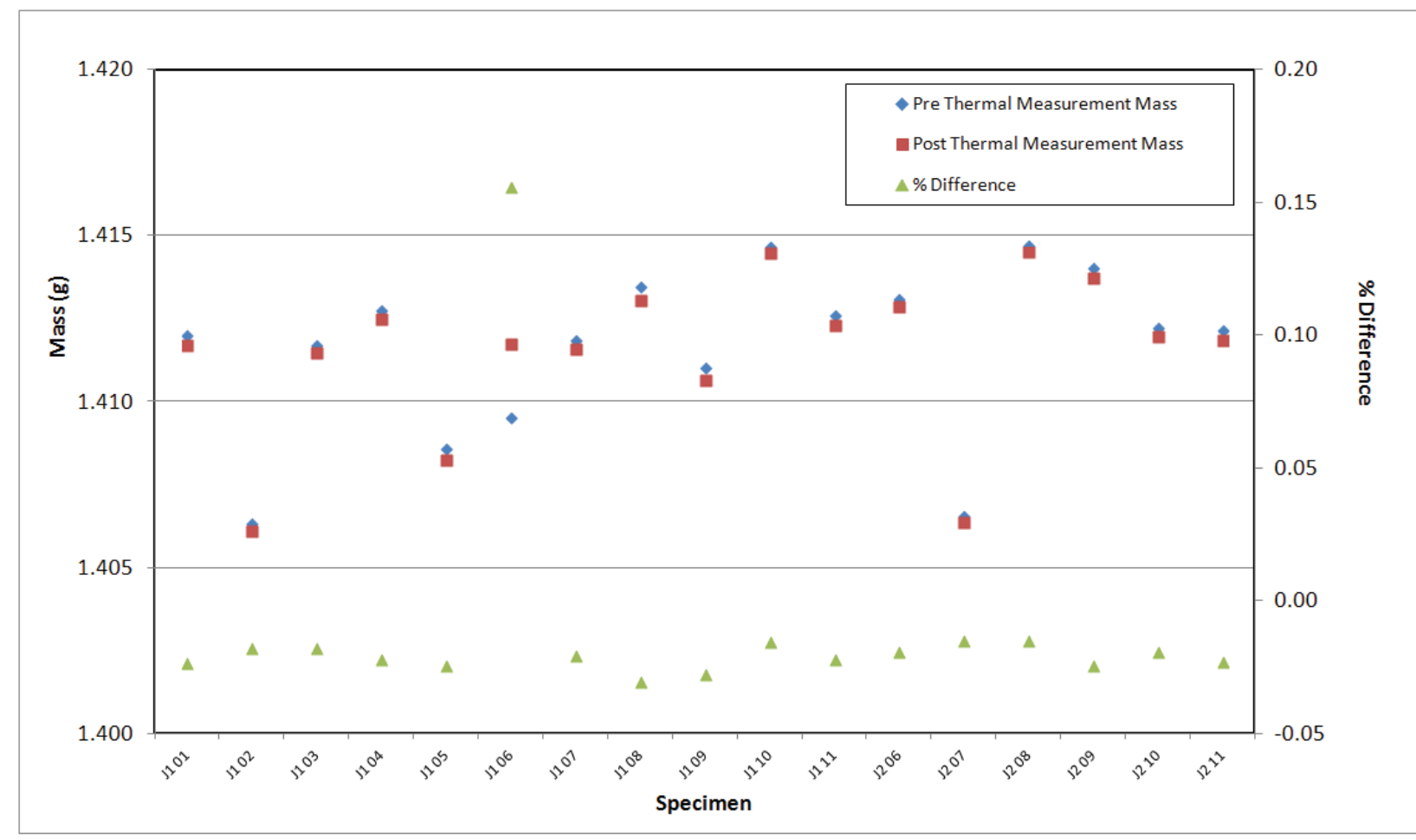

Figure A-191. HLM Piggyback Pre vs. Post Thermal Measurement Mass Comparison.

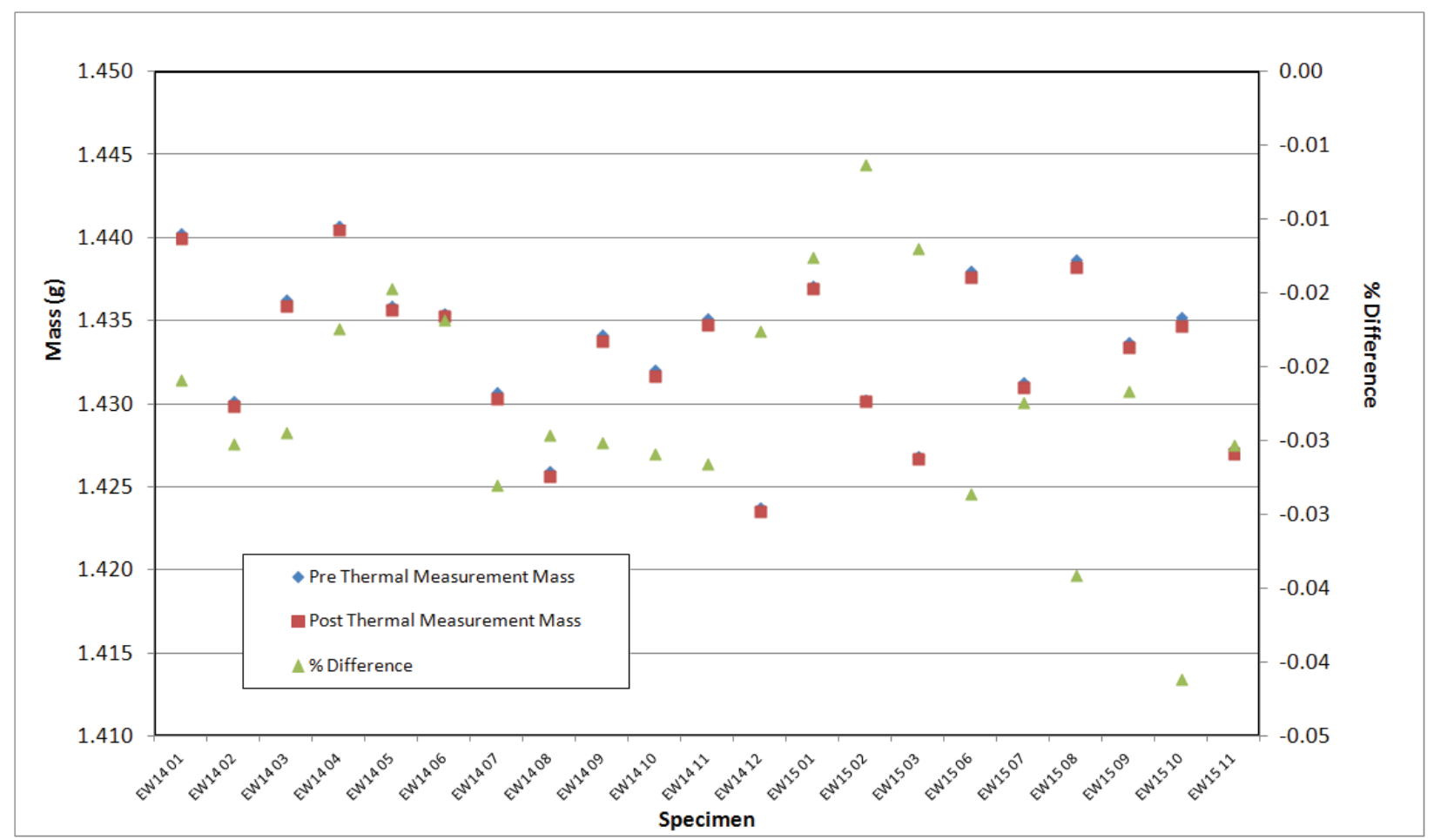

Figure A-192. IG-110 Piggyback Pre vs. Post Thermal Measurement Mass Comparison. 


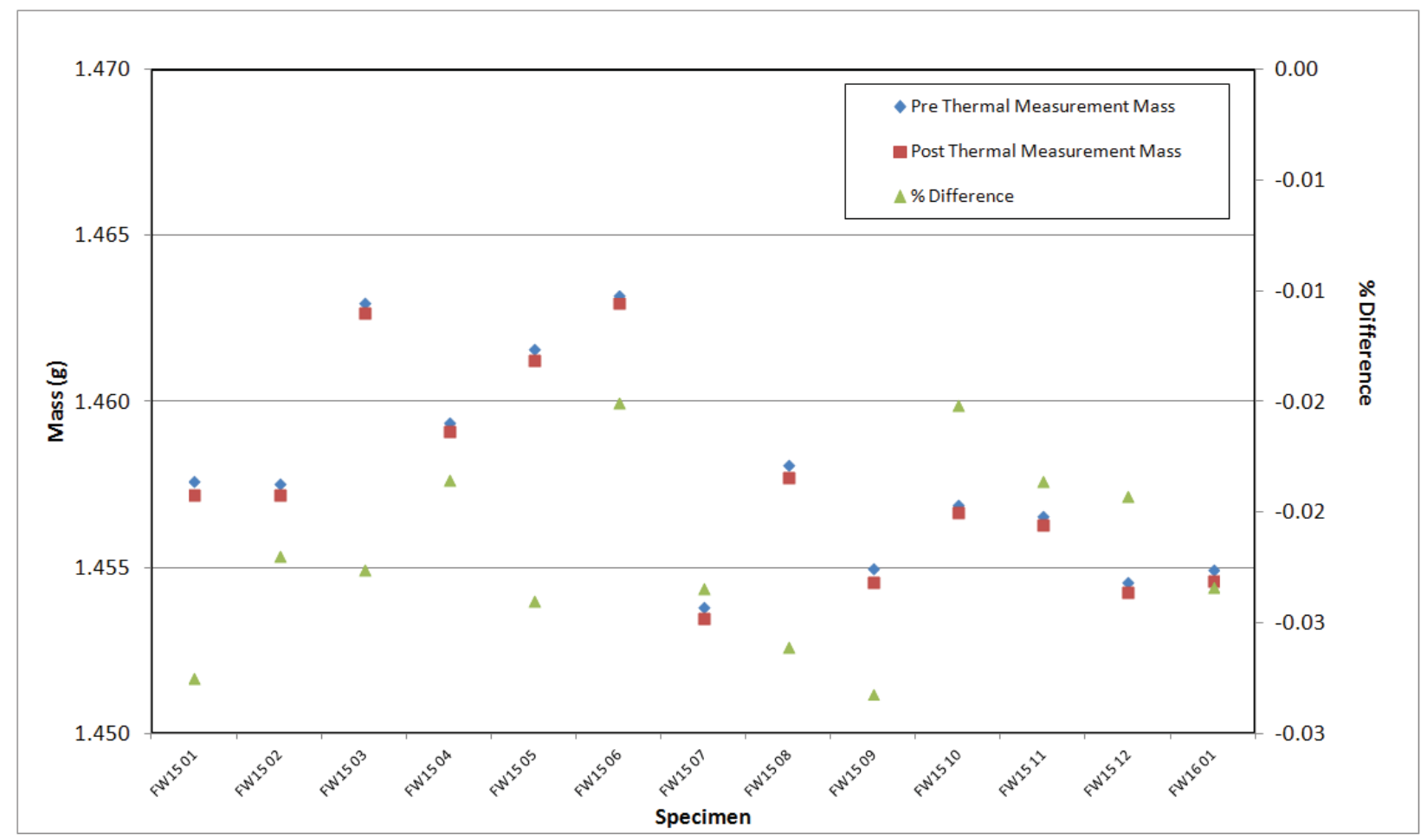

Figure A-193. IG-430 Piggyback Pre vs. Post Thermal Measurement Mass Comparison.

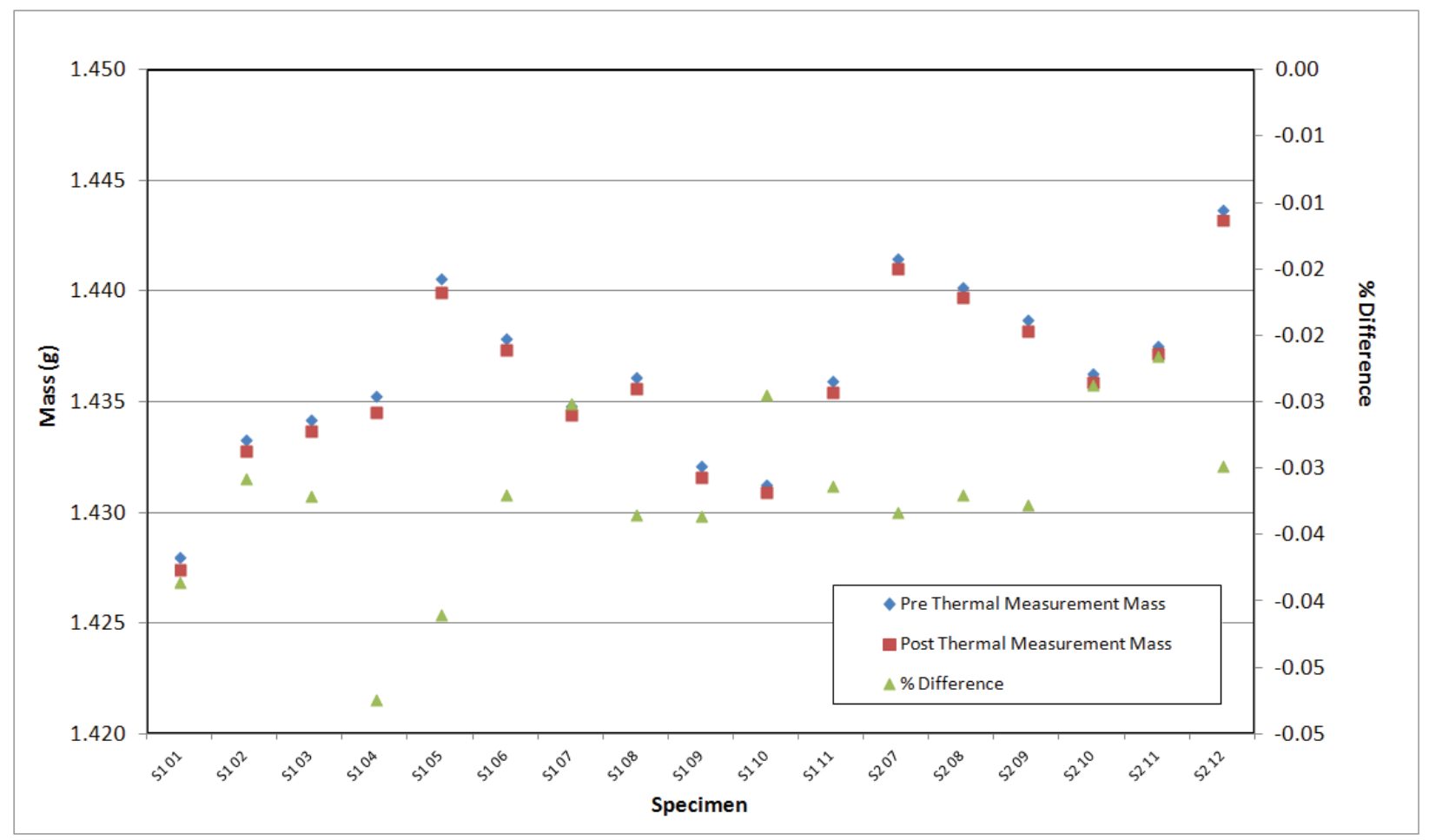

Figure A-194. NBG-10 Piggyback Pre vs. Post Thermal Measurement Mass Comparison. 


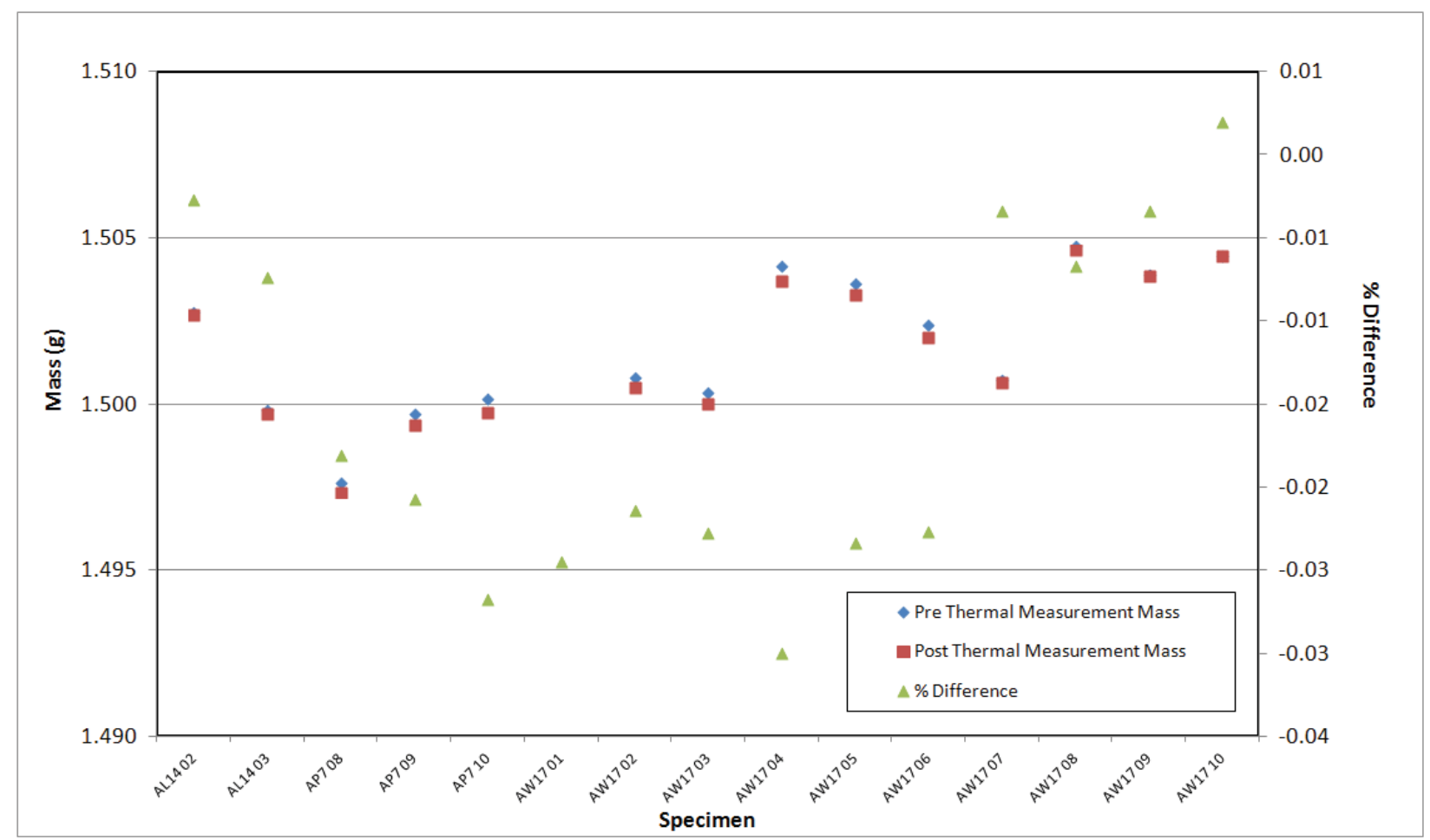

Figure A-195. NBG-17 Piggyback Pre vs. Post Thermal Measurement Mass Comparison.

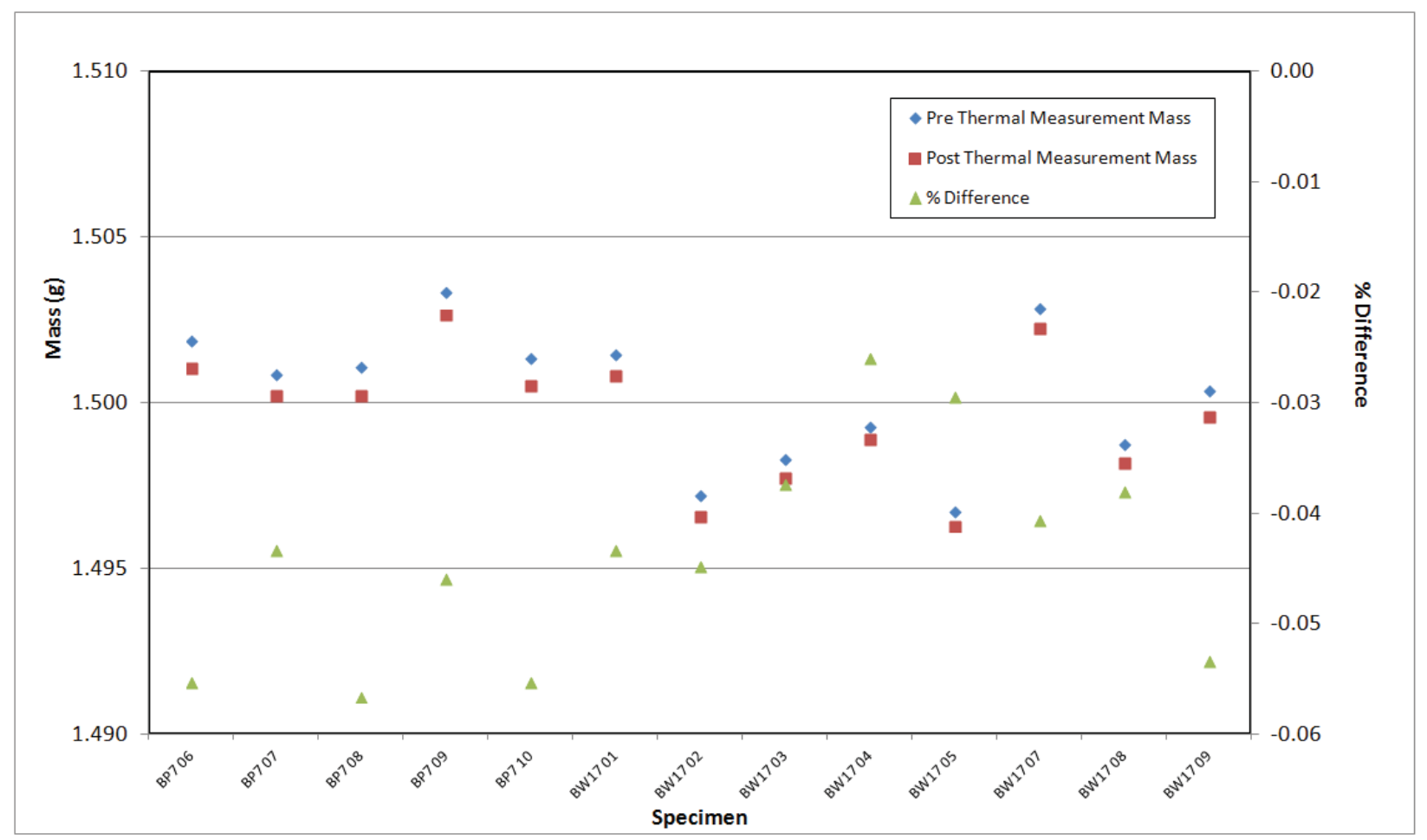

Figure A-196. NBG-18 Piggyback Pre vs. Post Thermal Measurement Mass Comparison. 


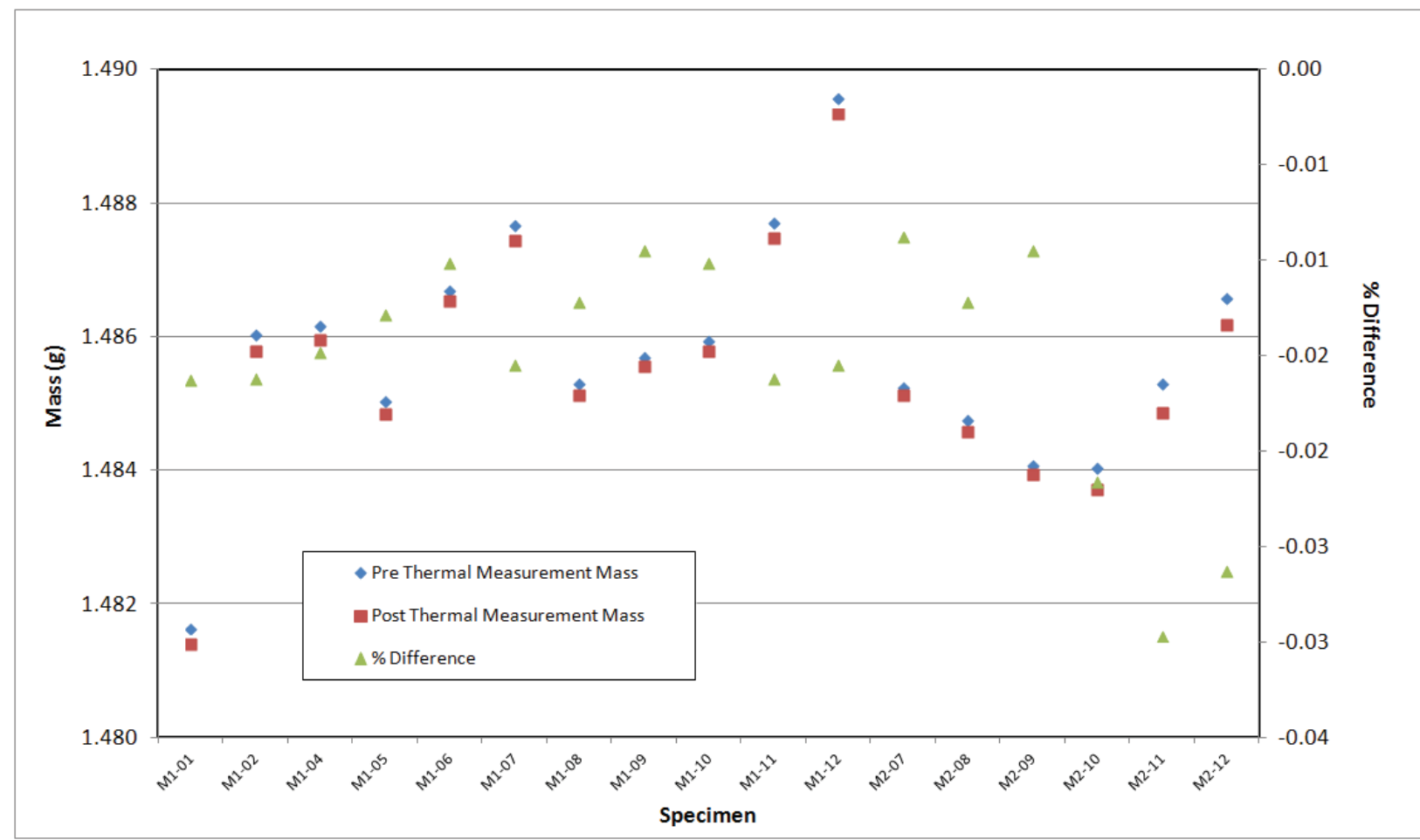

Figure A-197. NBG-25 Piggyback Pre vs. Post Thermal Measurement Mass Comparison.

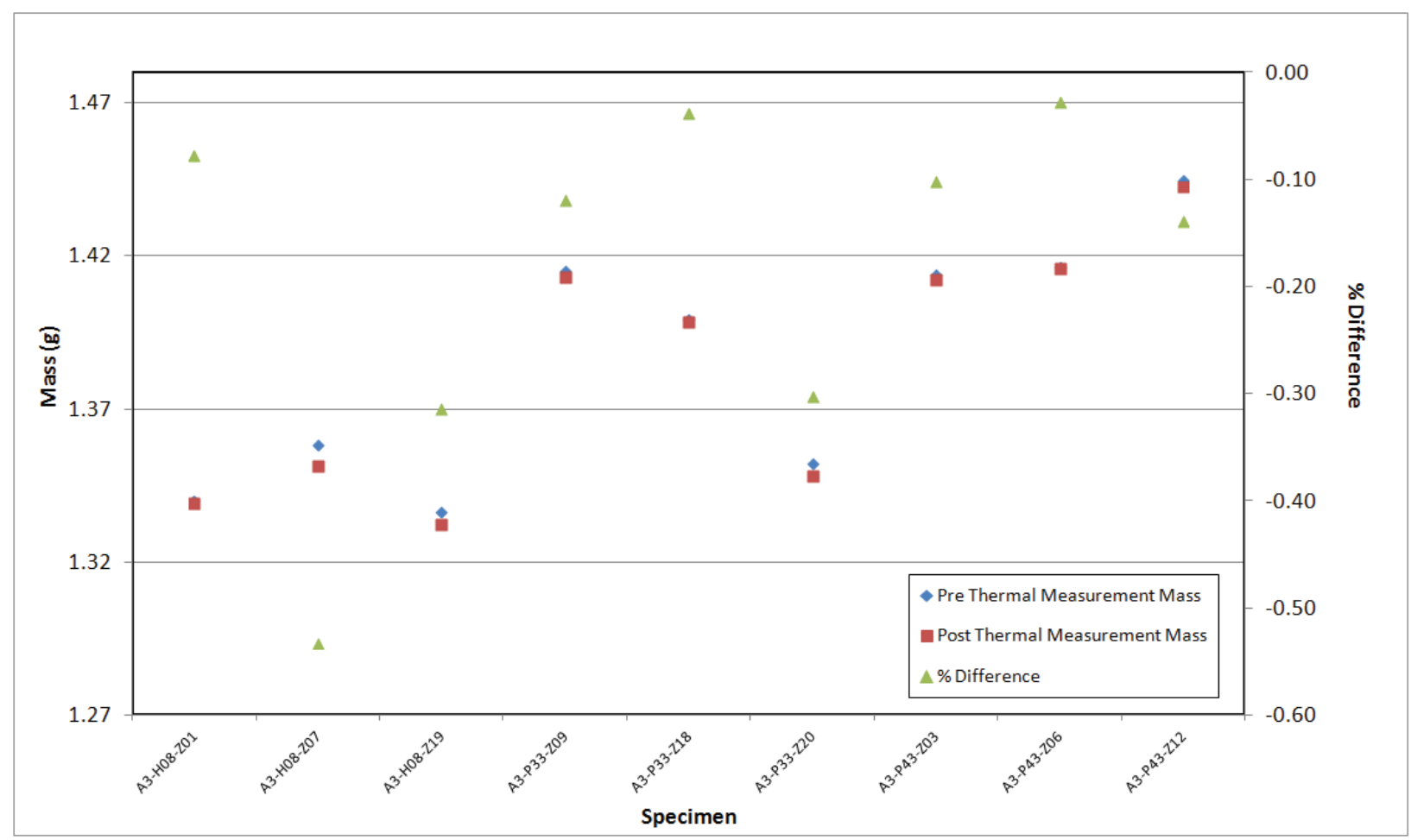

Figure A-198. New Matrix Piggyback Pre vs. Post Thermal Measurement Mass Comparison. 


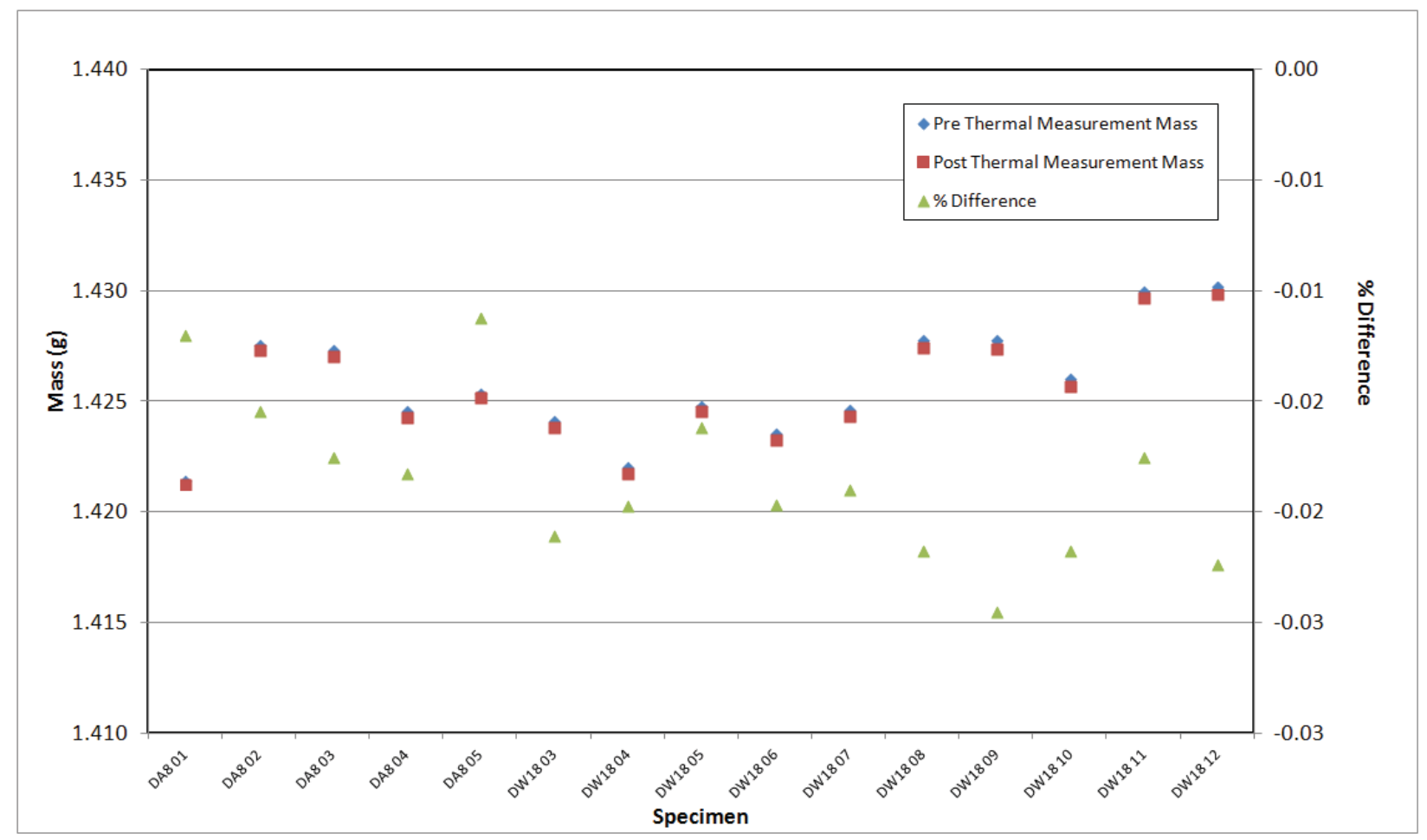

Figure A-199. PCEA Piggyback Pre vs. Post Thermal Measurement Mass Comparison.

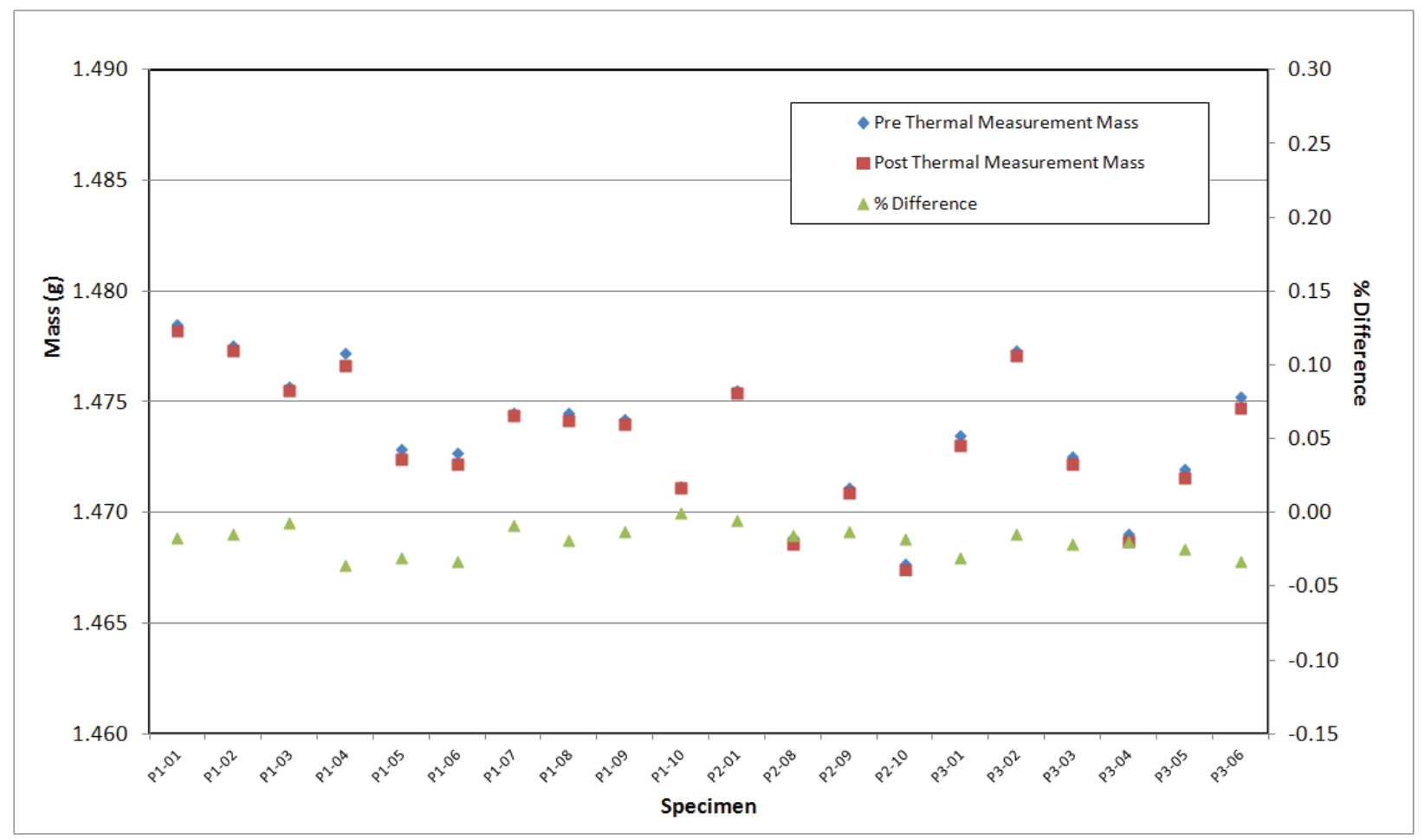

Figure A-200. PCIB Piggyback Pre vs. Post Thermal Measurement Mass Comparison. 


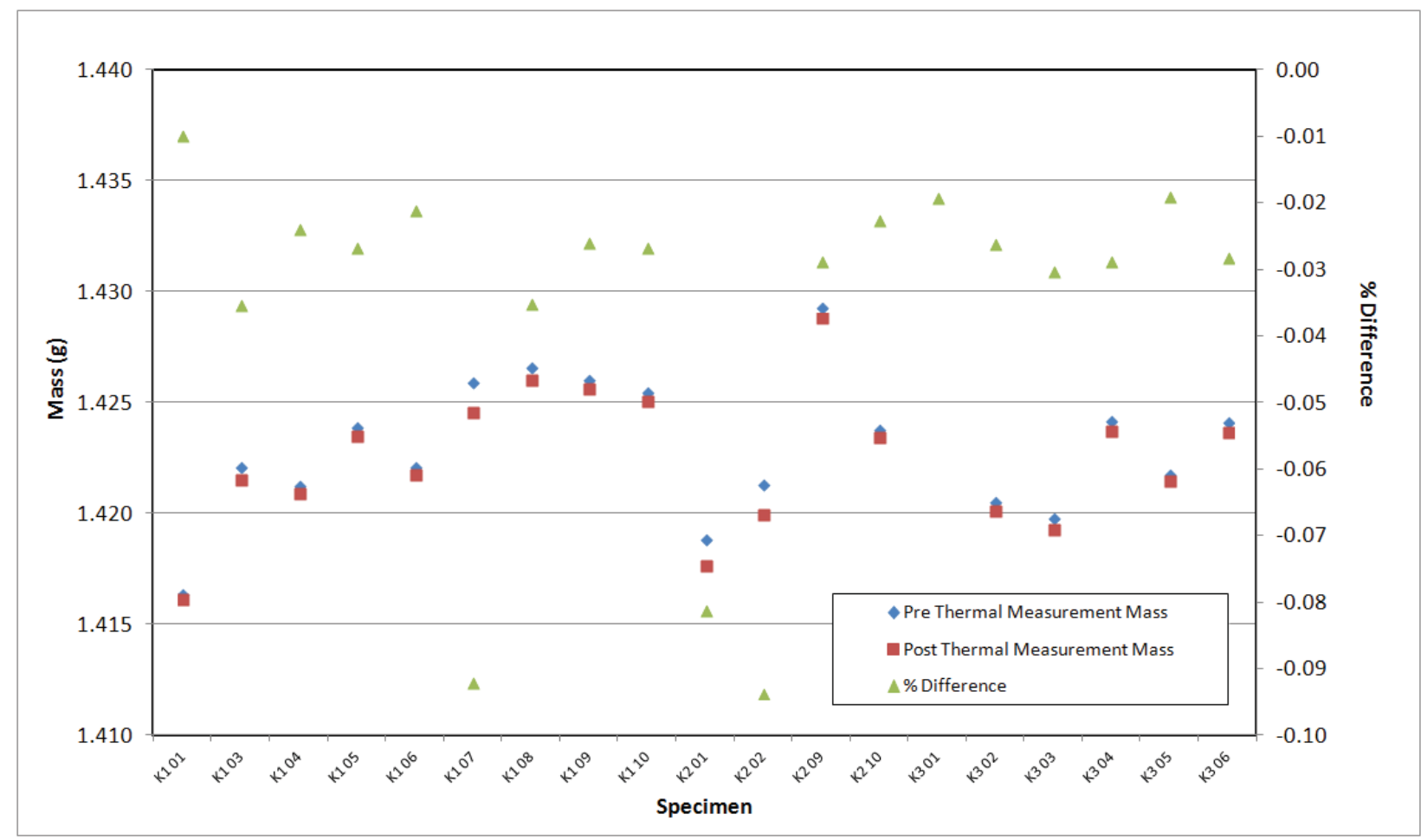

Figure A-201. PGX Piggyback Pre vs. Post Thermal Measurement Mass Comparison.

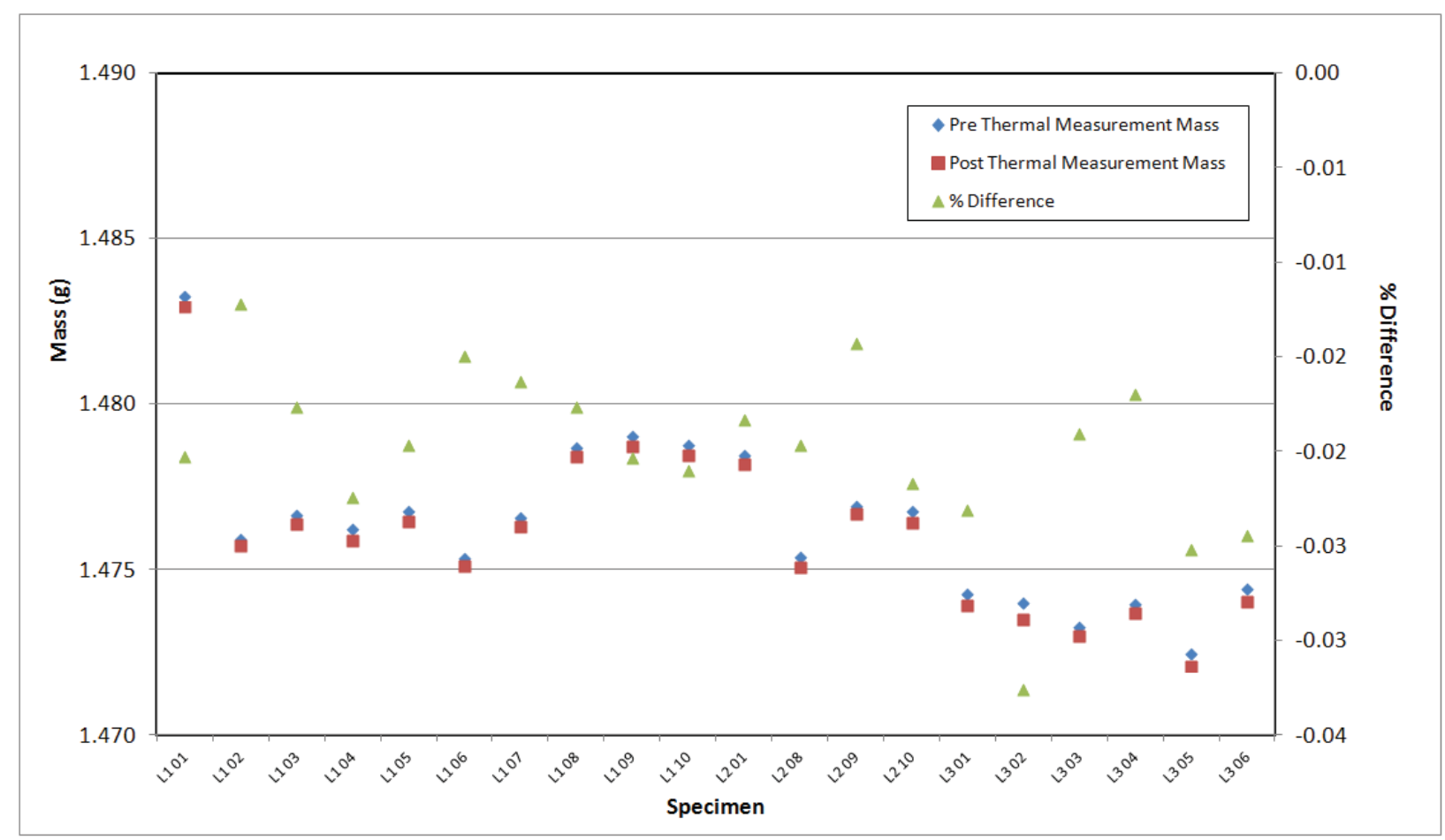

Figure A-202. PPEA Piggyback Pre vs. Post Thermal Measurement Mass Comparison. 


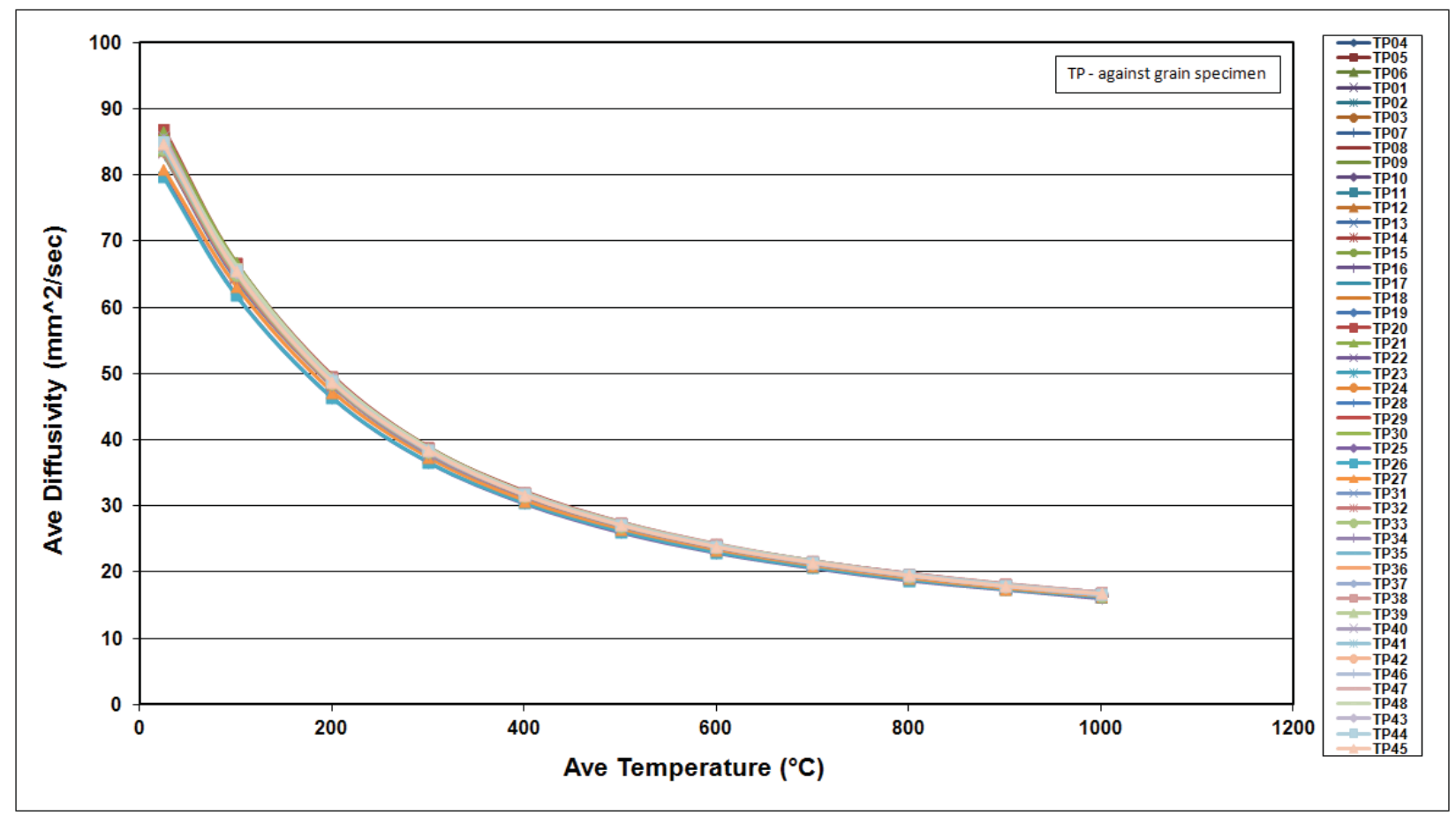

Figure A-203. 2114 Piggyback Diffusivity.

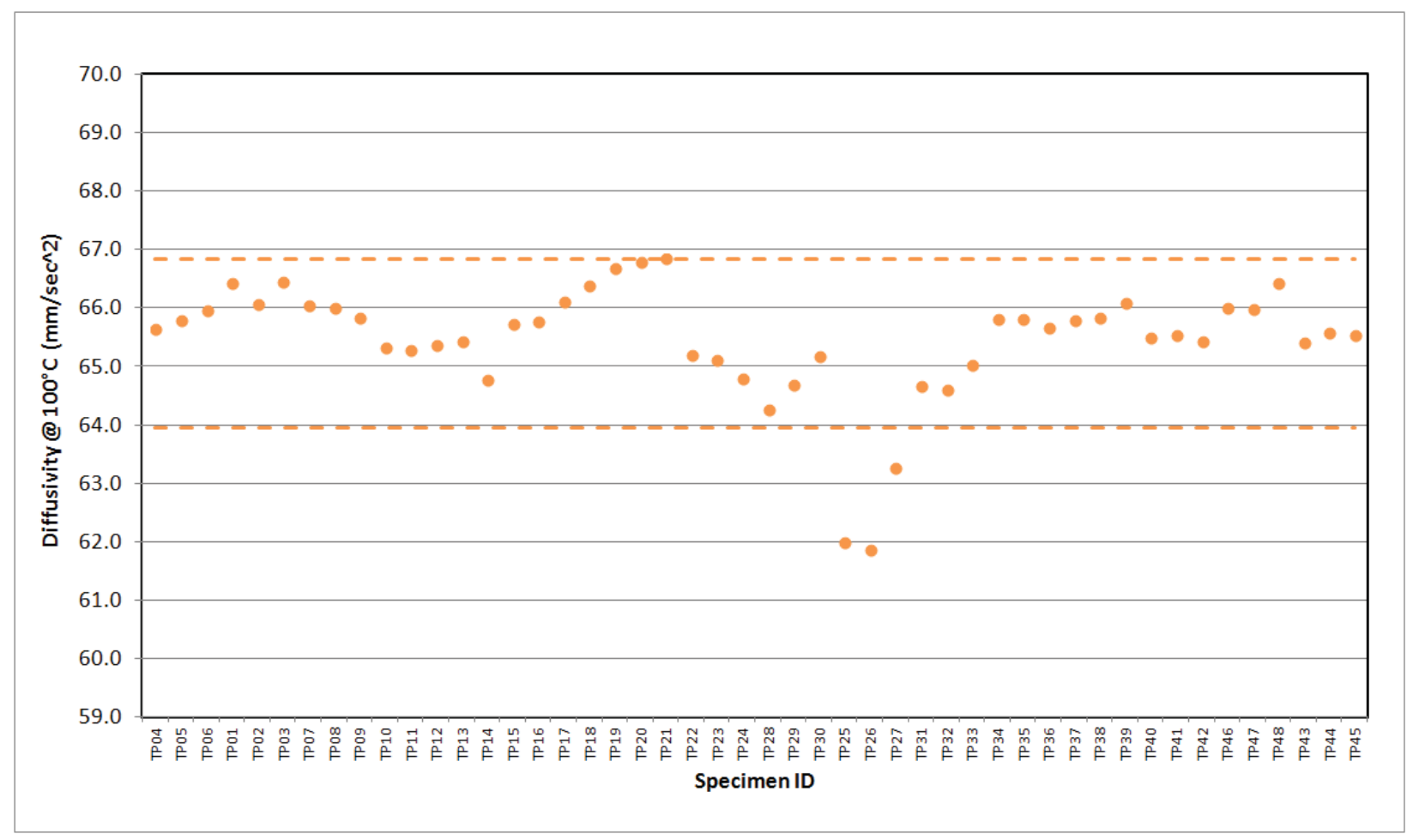

Figure A-204.2114 Piggyback Diffusivity @ 100․ 


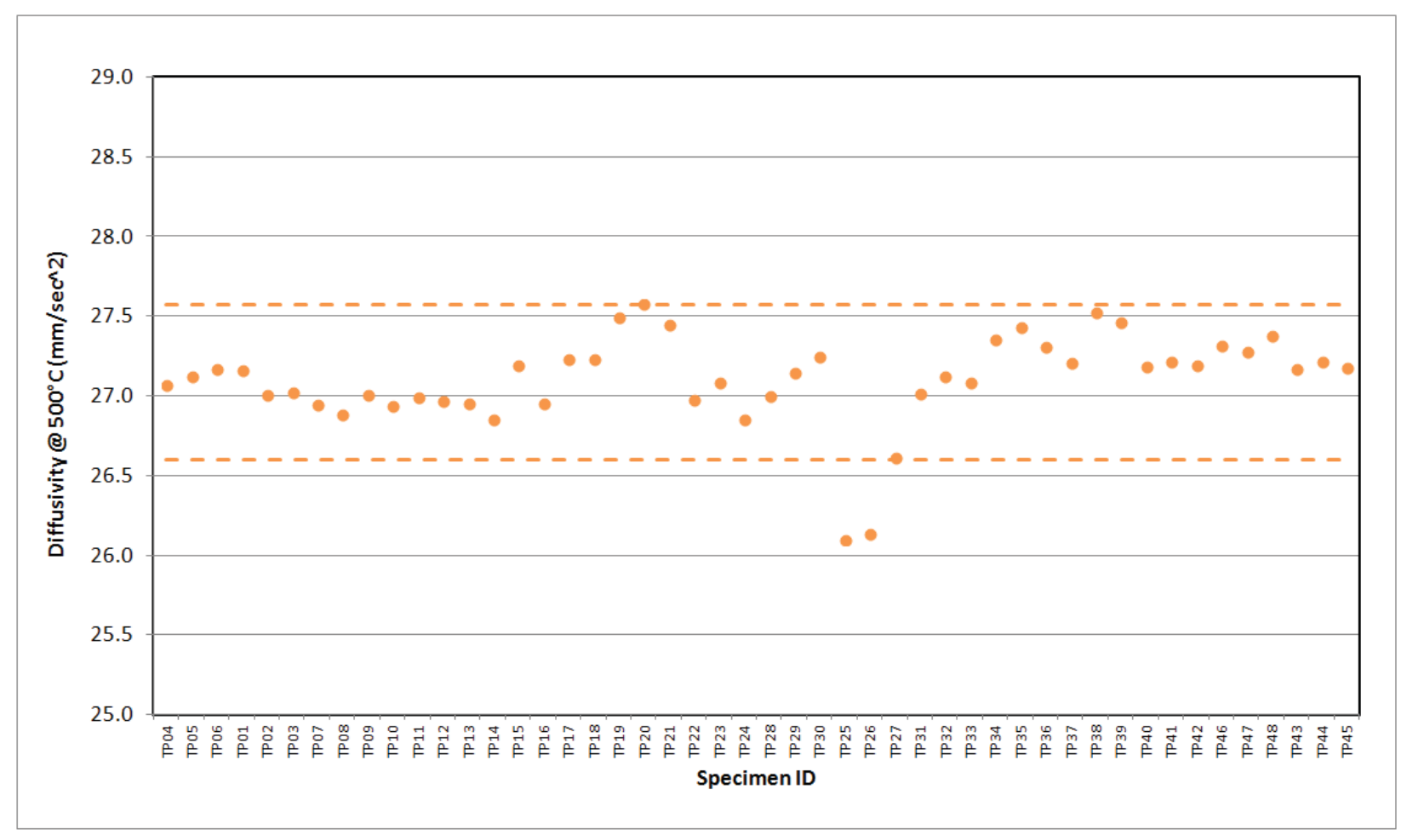

Figure A-205. 2114 Piggyback Diffusivity @ 500․

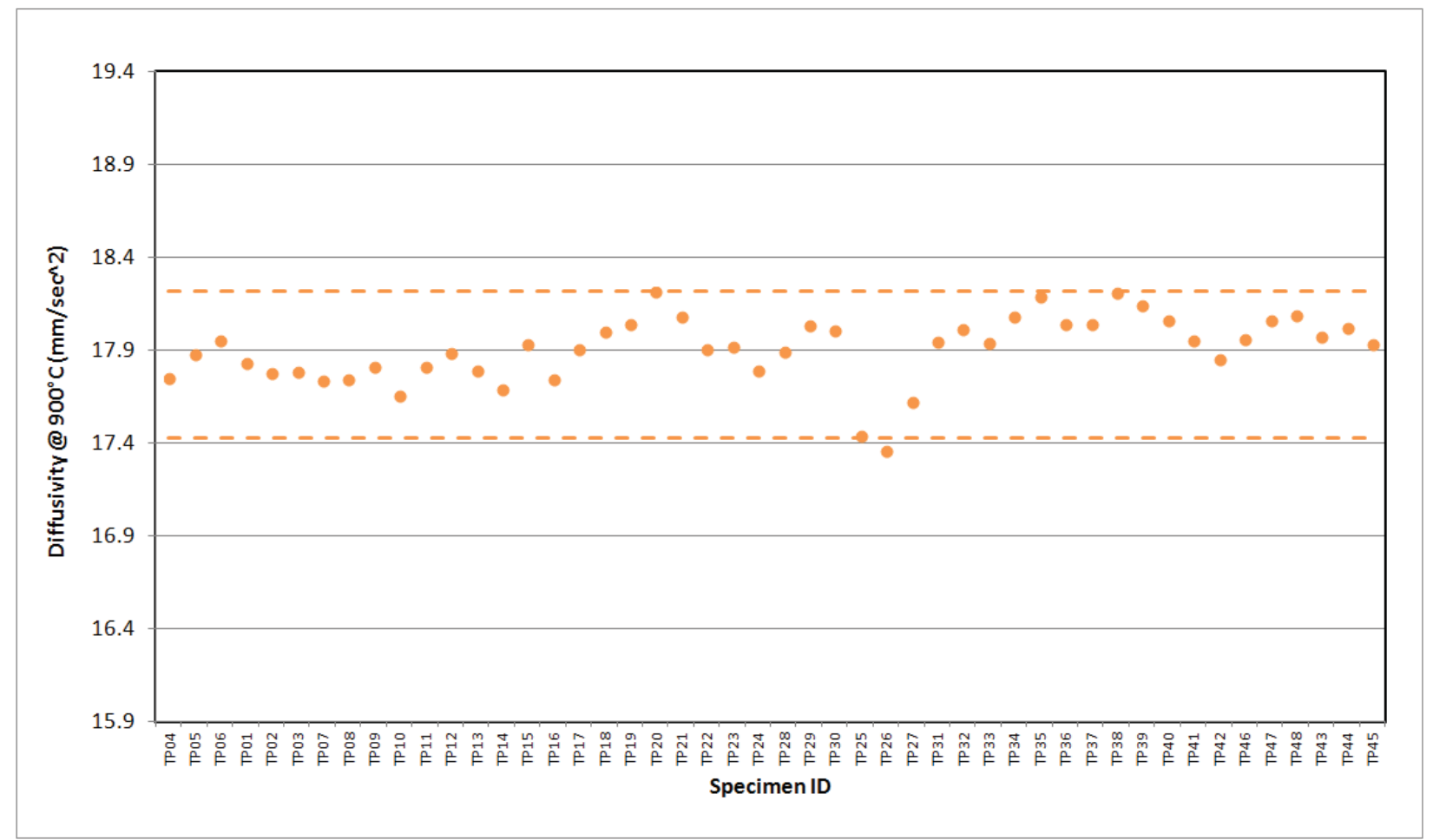

Figure A-206. 2114 Piggyback Diffusivity@900C. 


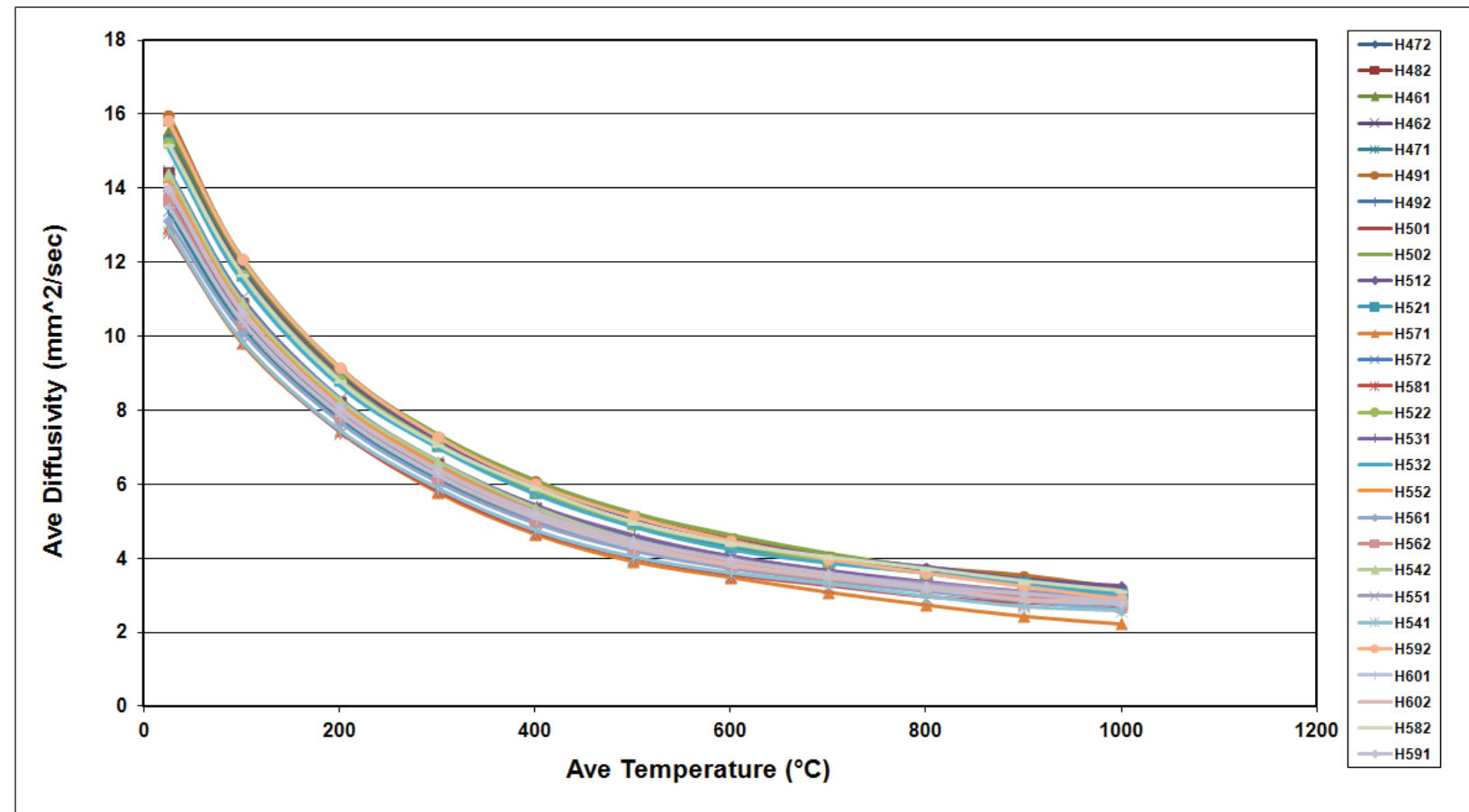

Figure A-207. A3 Matrix Piggyback Diffusivity.

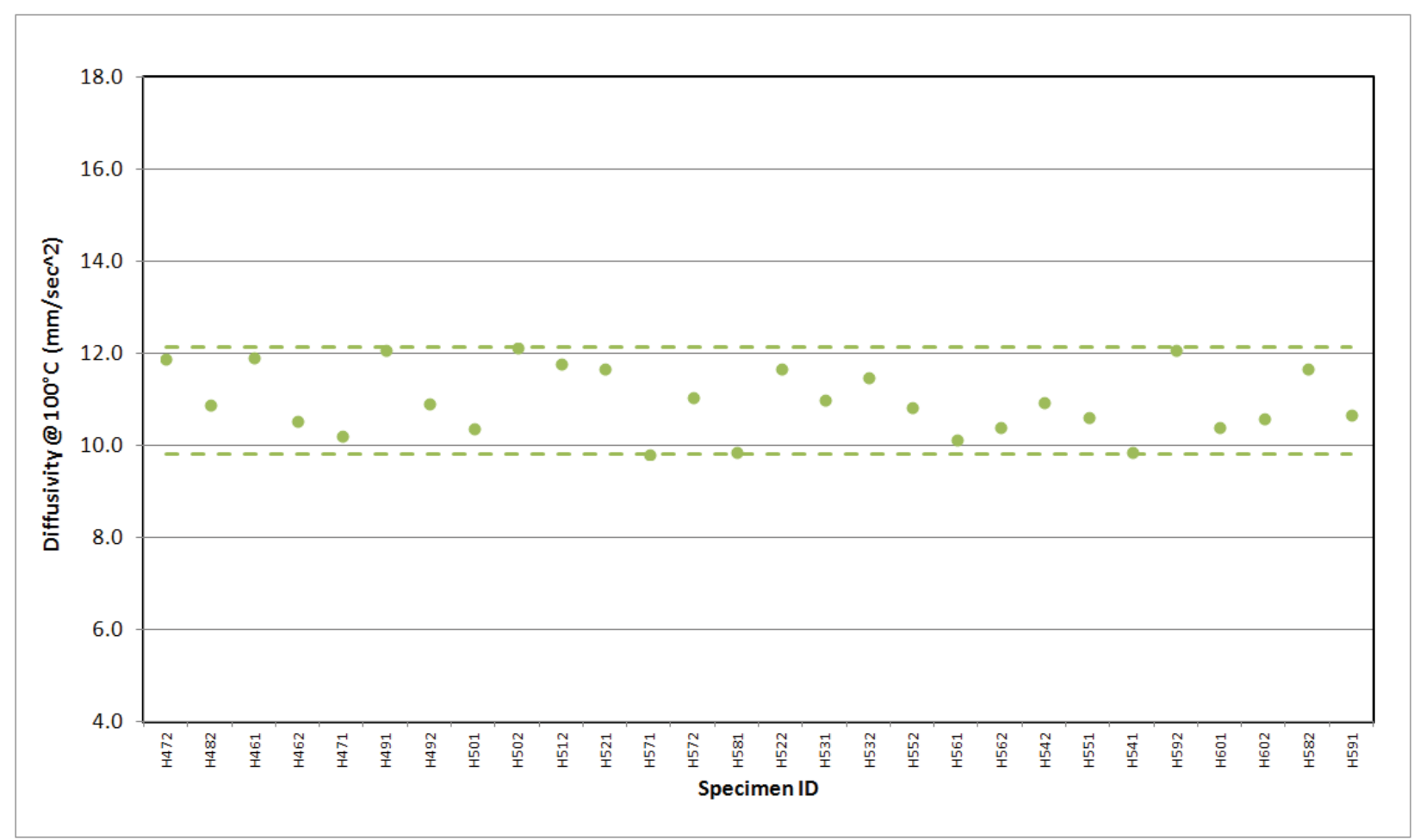

Figure A-208. A3 Matrix Piggyback Diffusivity @ $100^{\circ} \mathrm{C}$. 


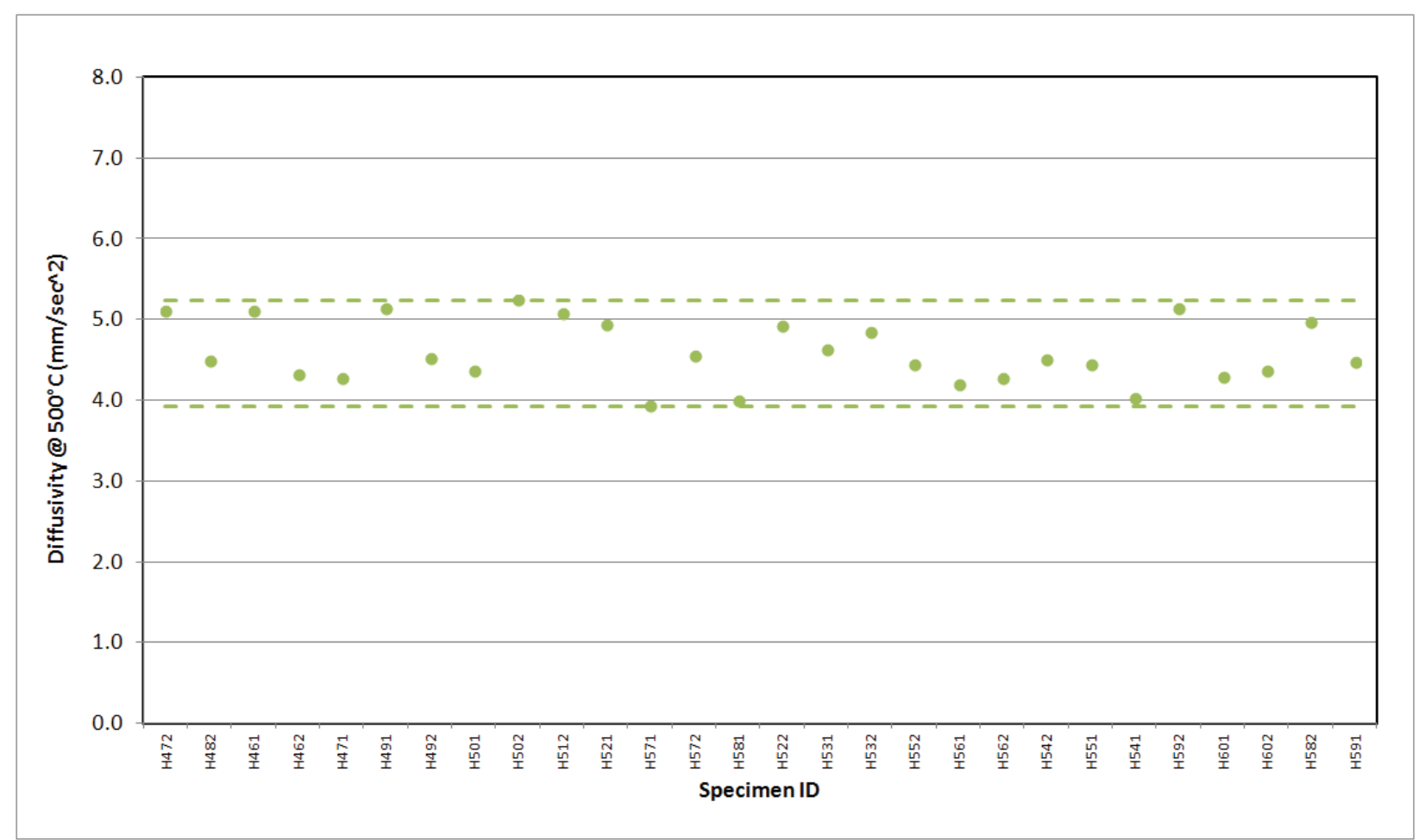

Figure A-209. A3 Matrix Piggyback Diffusivity @ 500C.

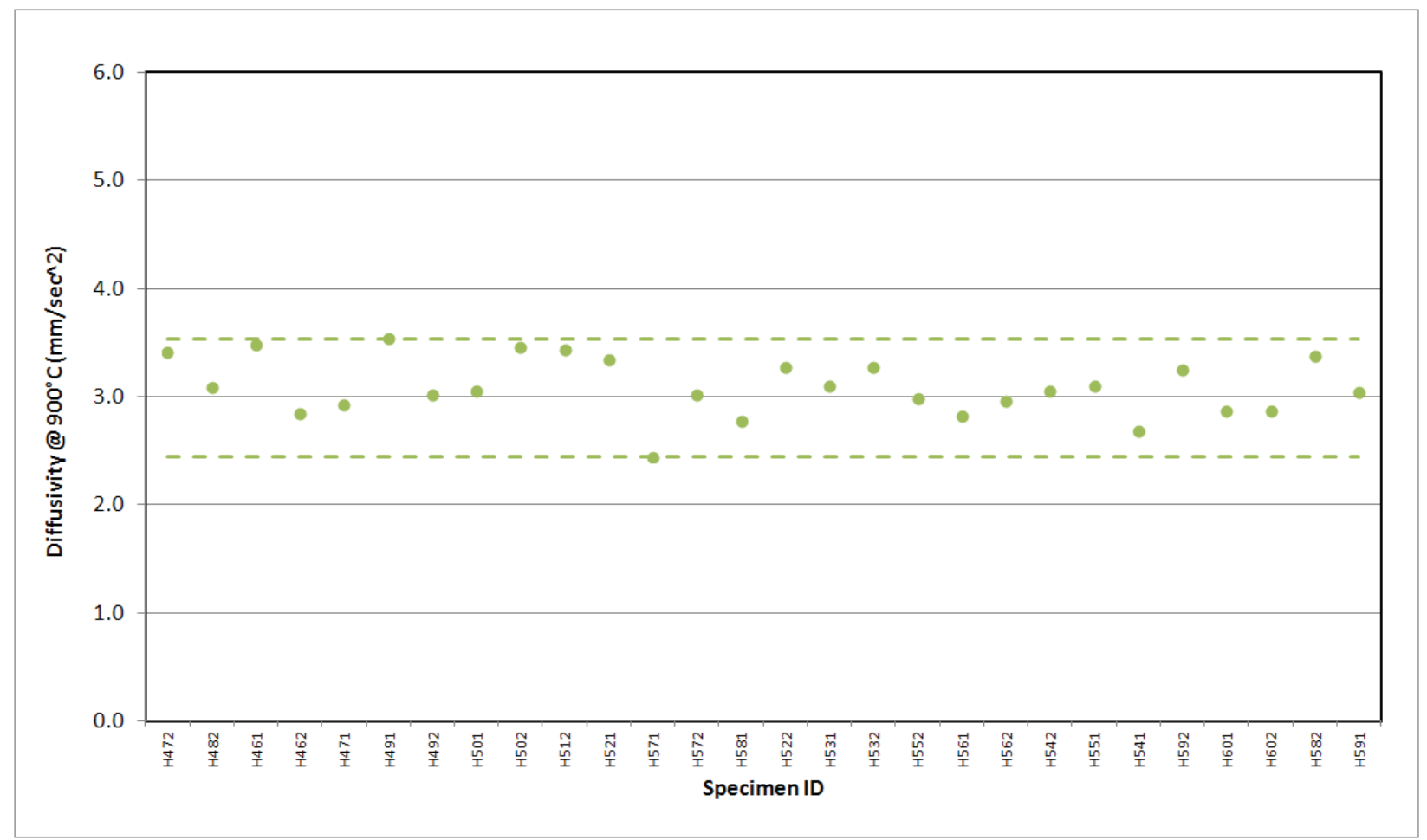

Figure A-210. A3 Matrix Piggyback Diffusivity@900C. 


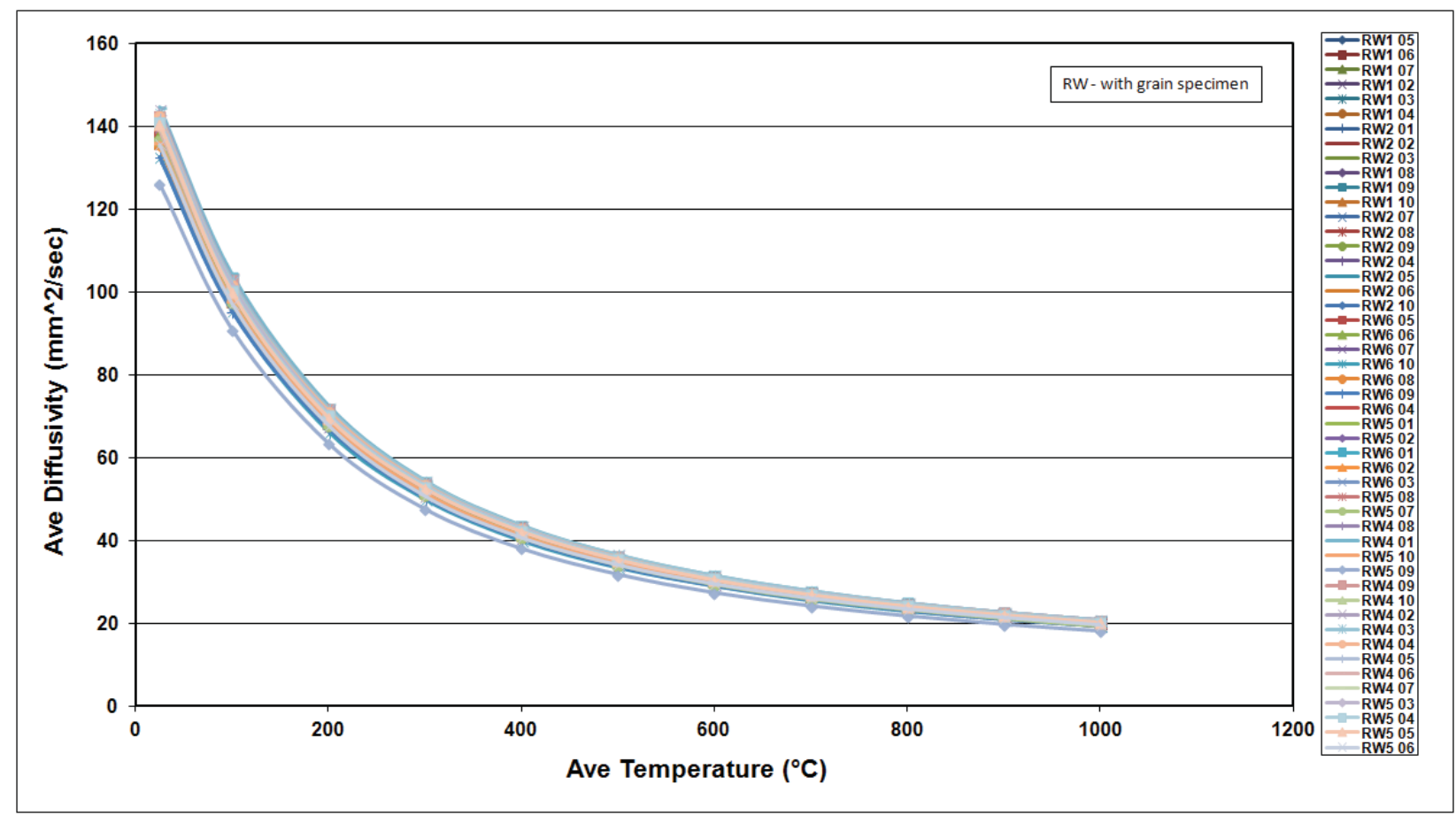

Figure A-211. BAN Piggyback Diffusivity.

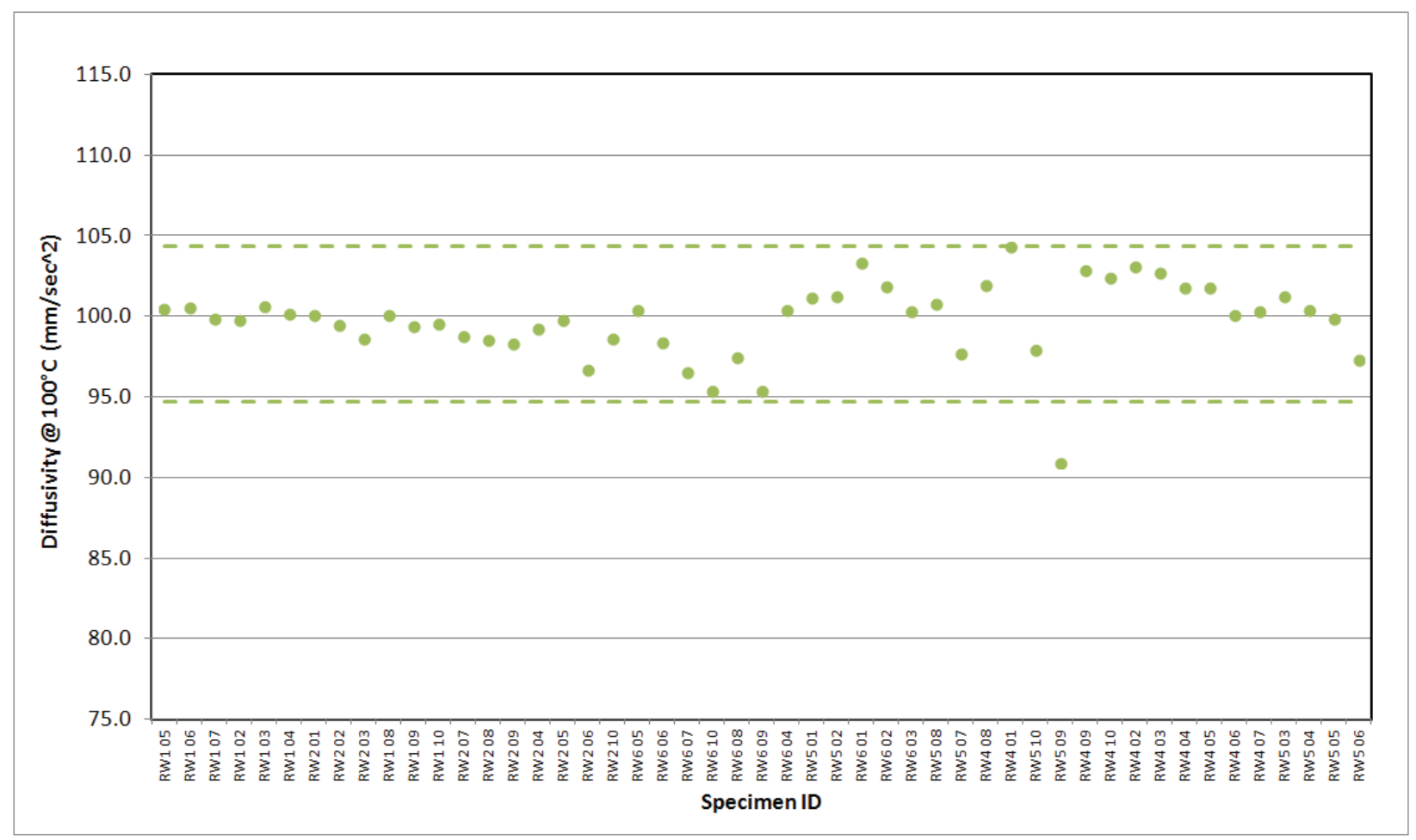

Figure A-212. BAN Piggyback Diffusivity@100². 


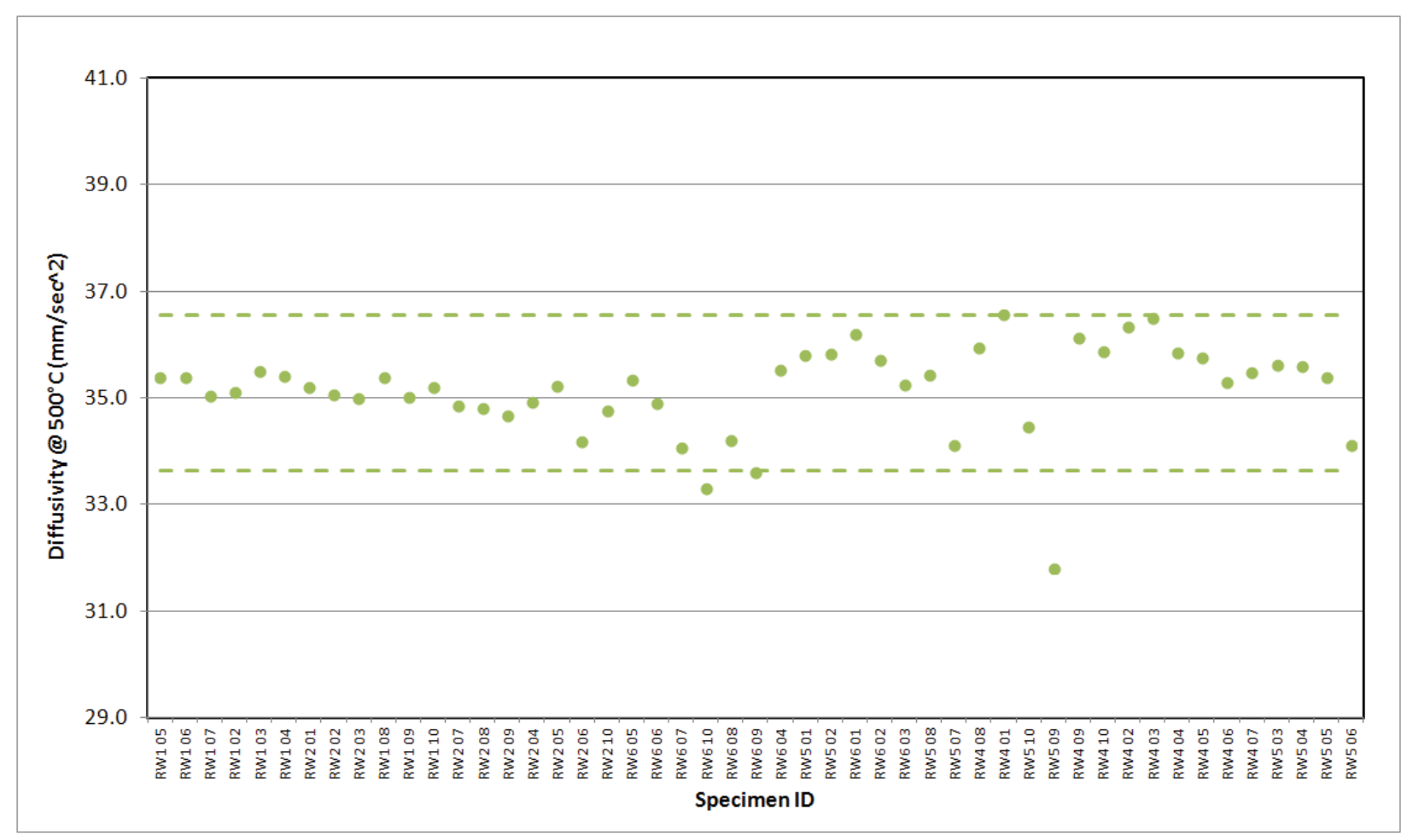

Figure A-213. BAN Piggyback Diffusivity @ 500․

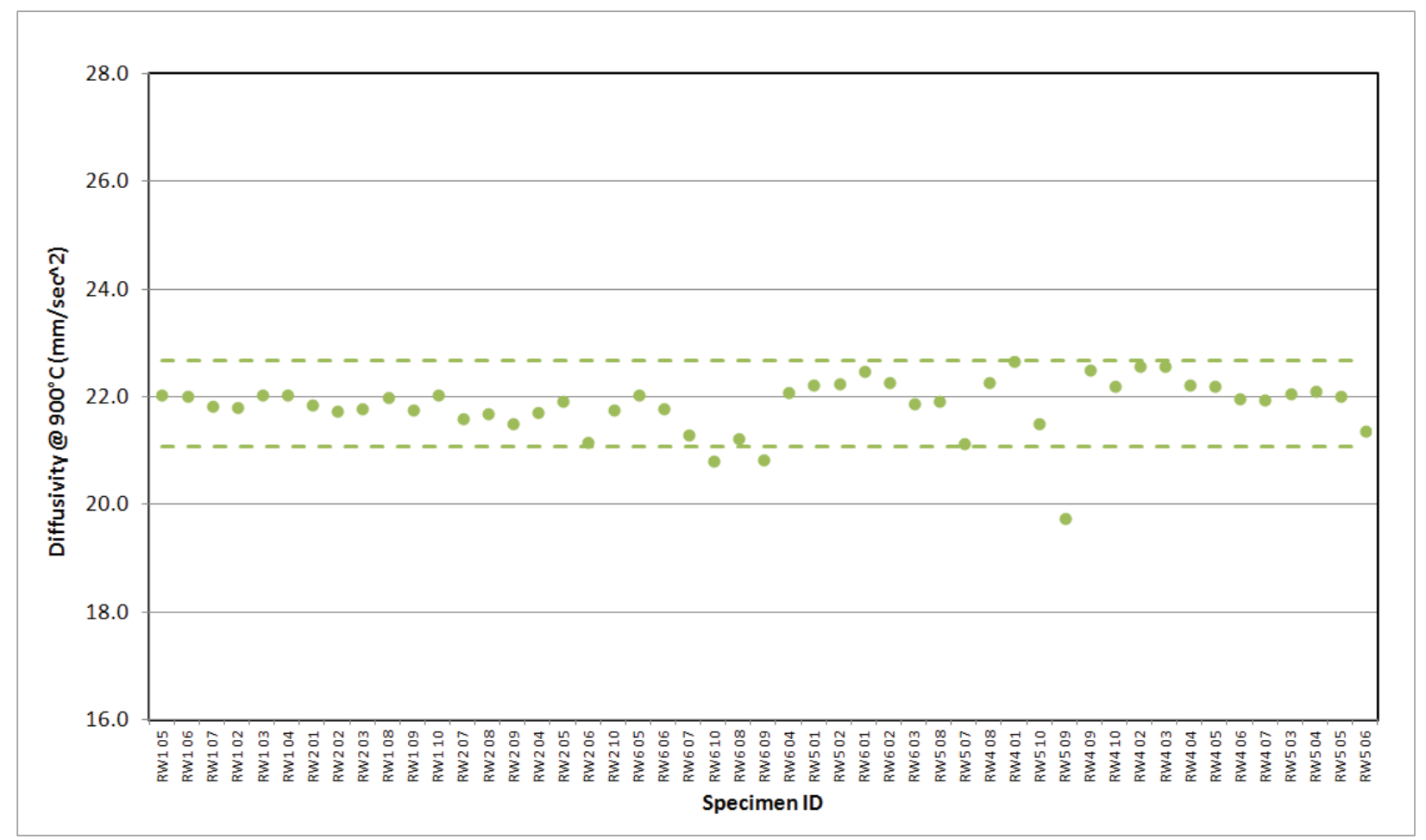

Figure A-214. BAN Piggyback Diffusivity @ 900C. 


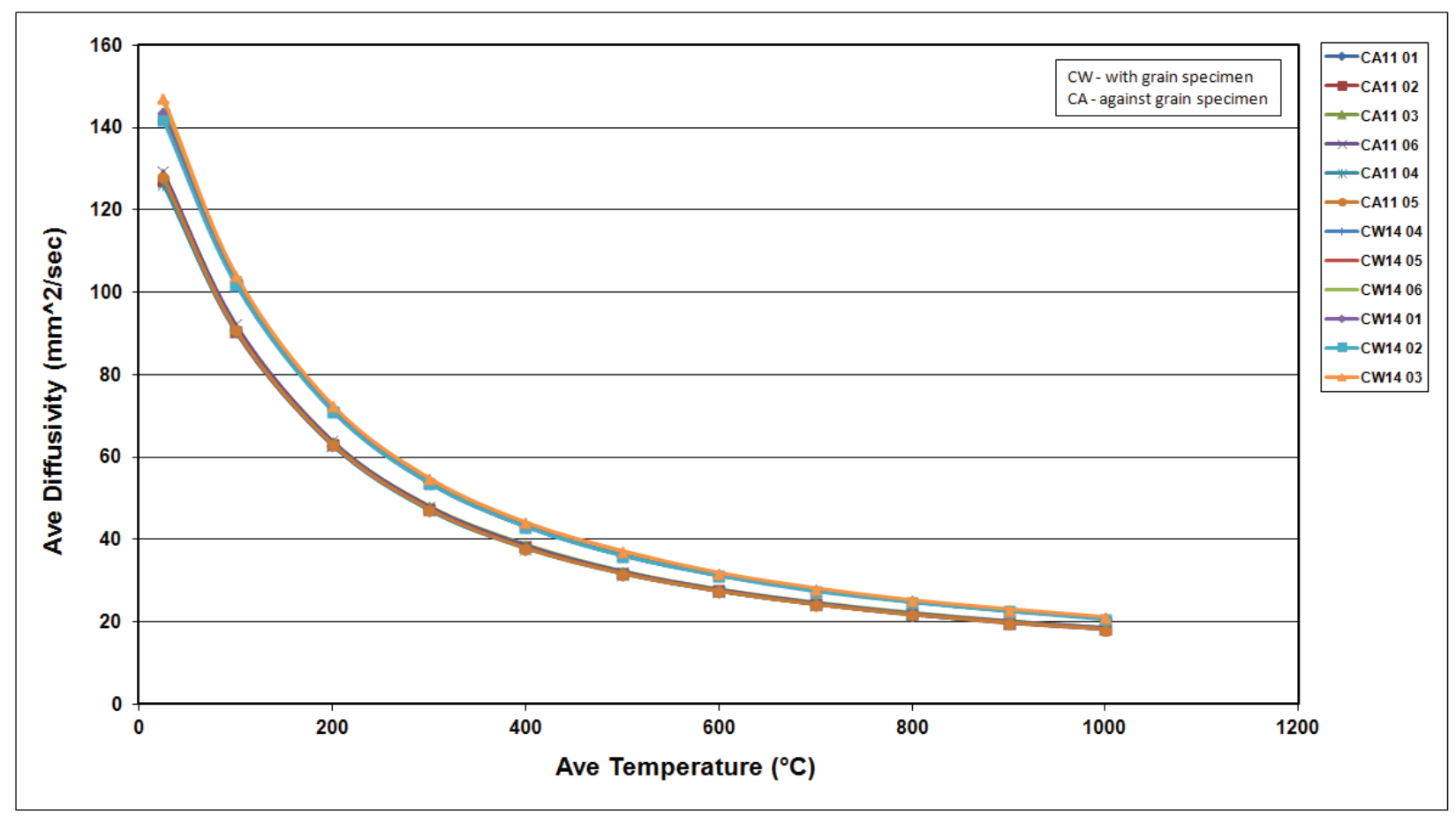

Figure A-215. H-451 Piggyback Diffusivity.

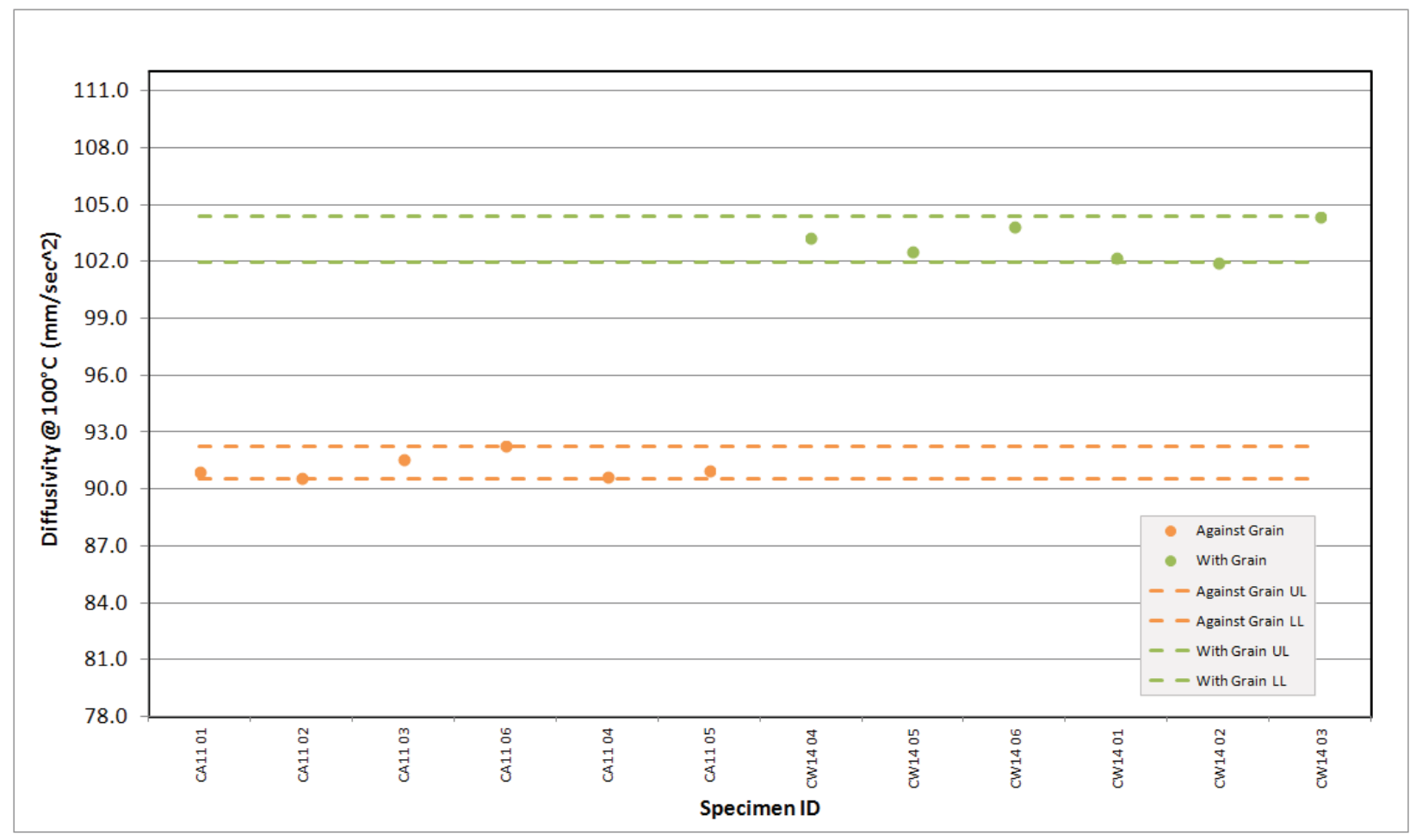

Figure A-216. H-451 Piggyback Diffusivity @ $100^{\circ} \mathrm{C}$. 


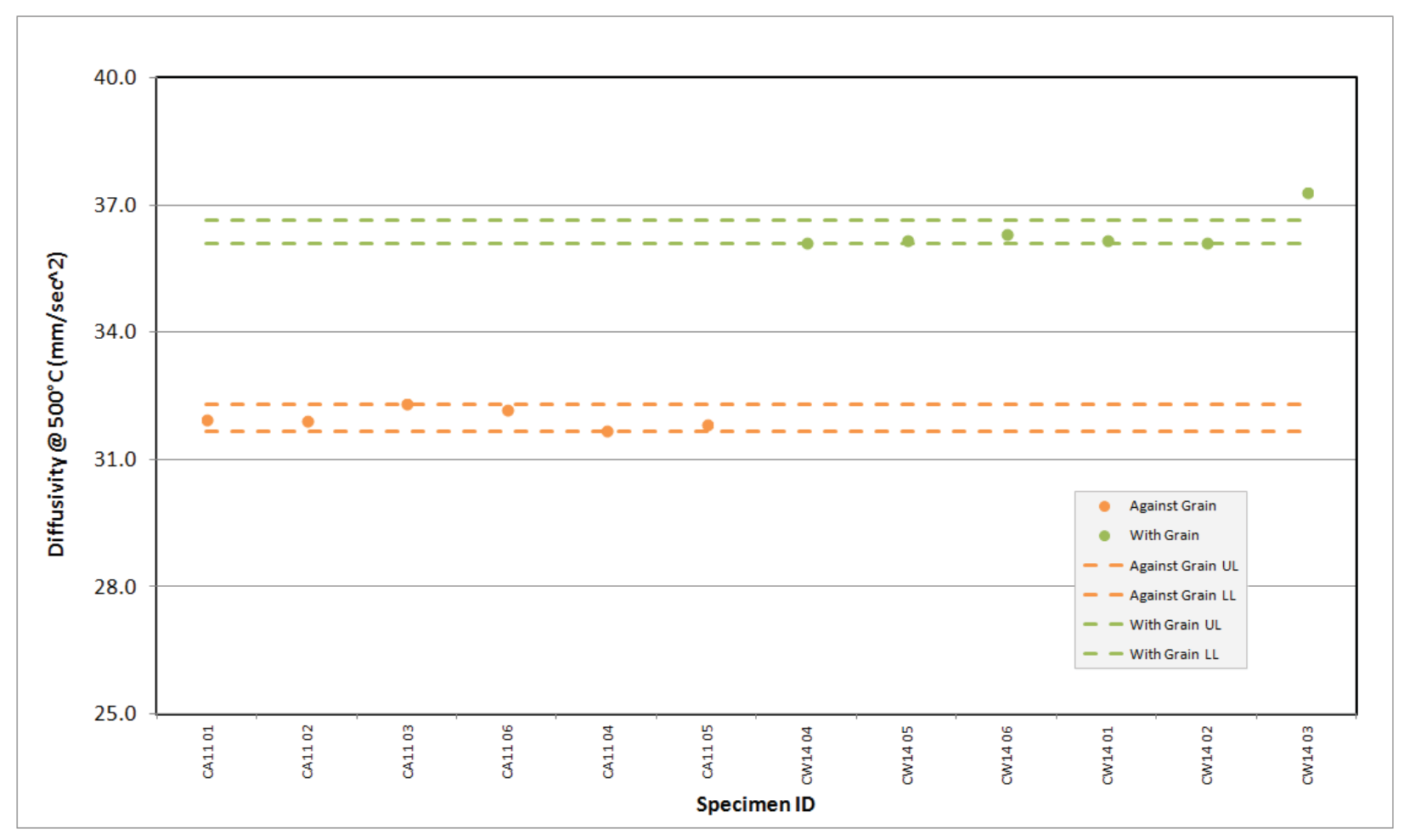

Figure A-217. H-451 Piggyback Diffusivity @ 500 C.

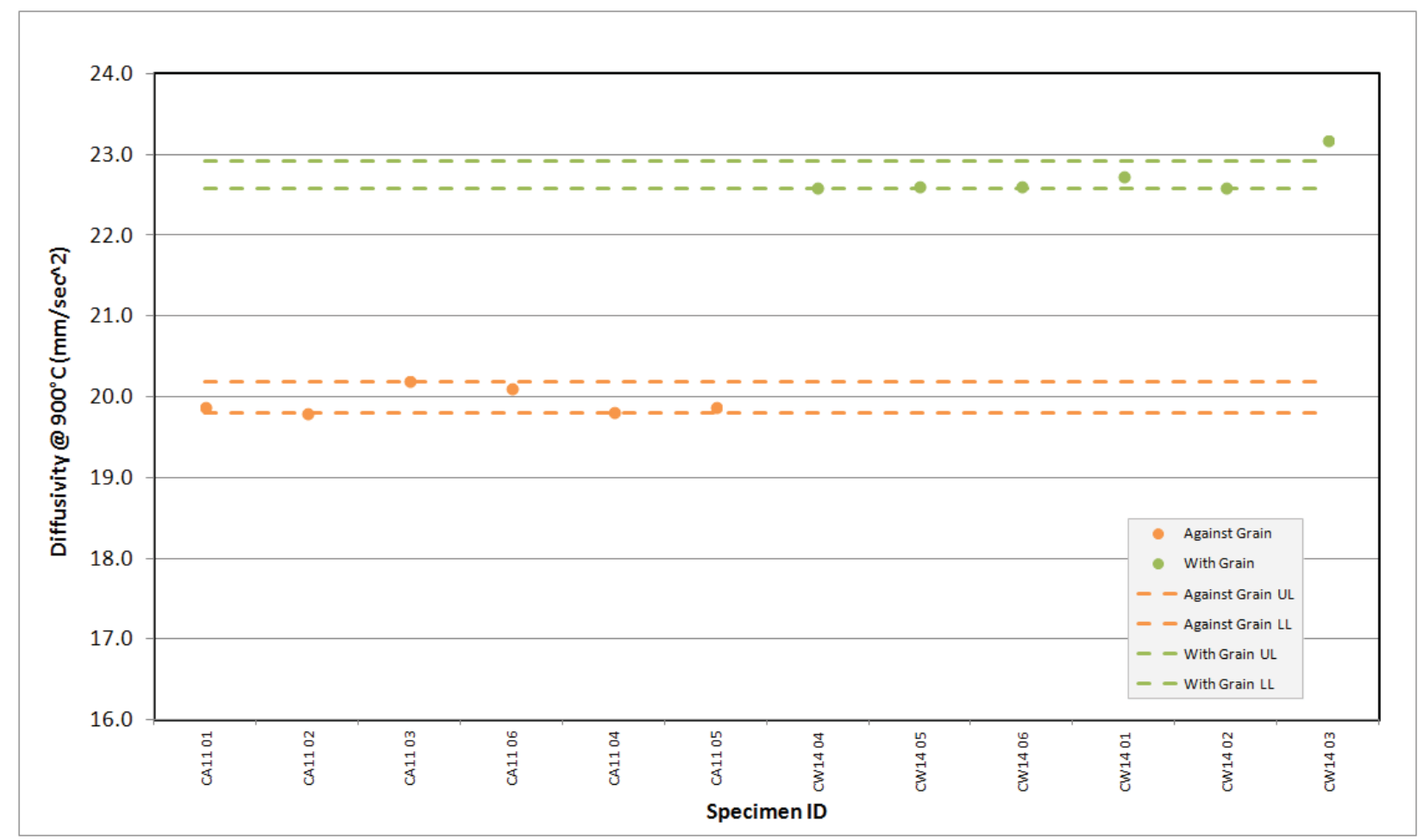

Figure A-218. H-451 Piggyback Diffusivity@900C. 


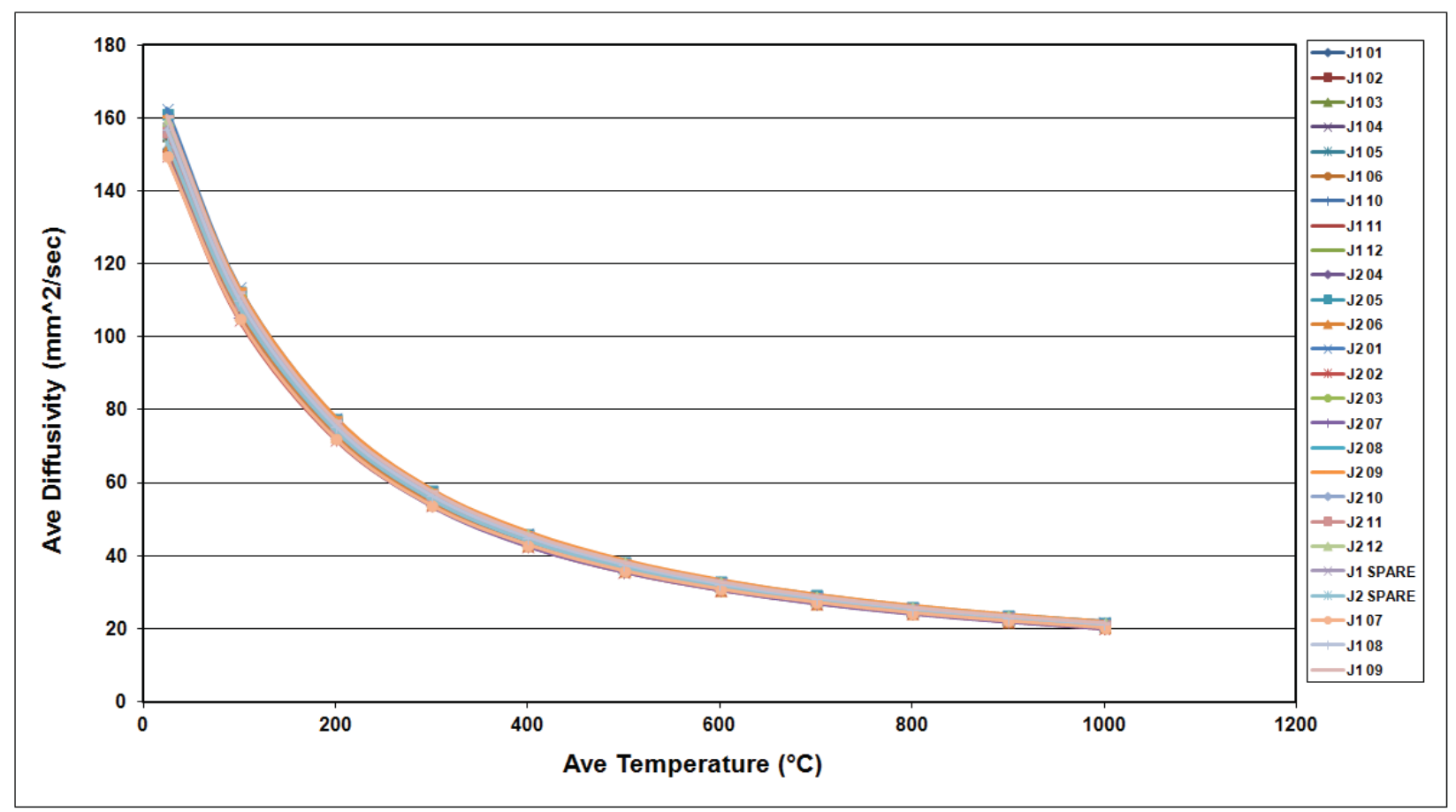

Figure A-219. HLM Piggyback Diffusivity.

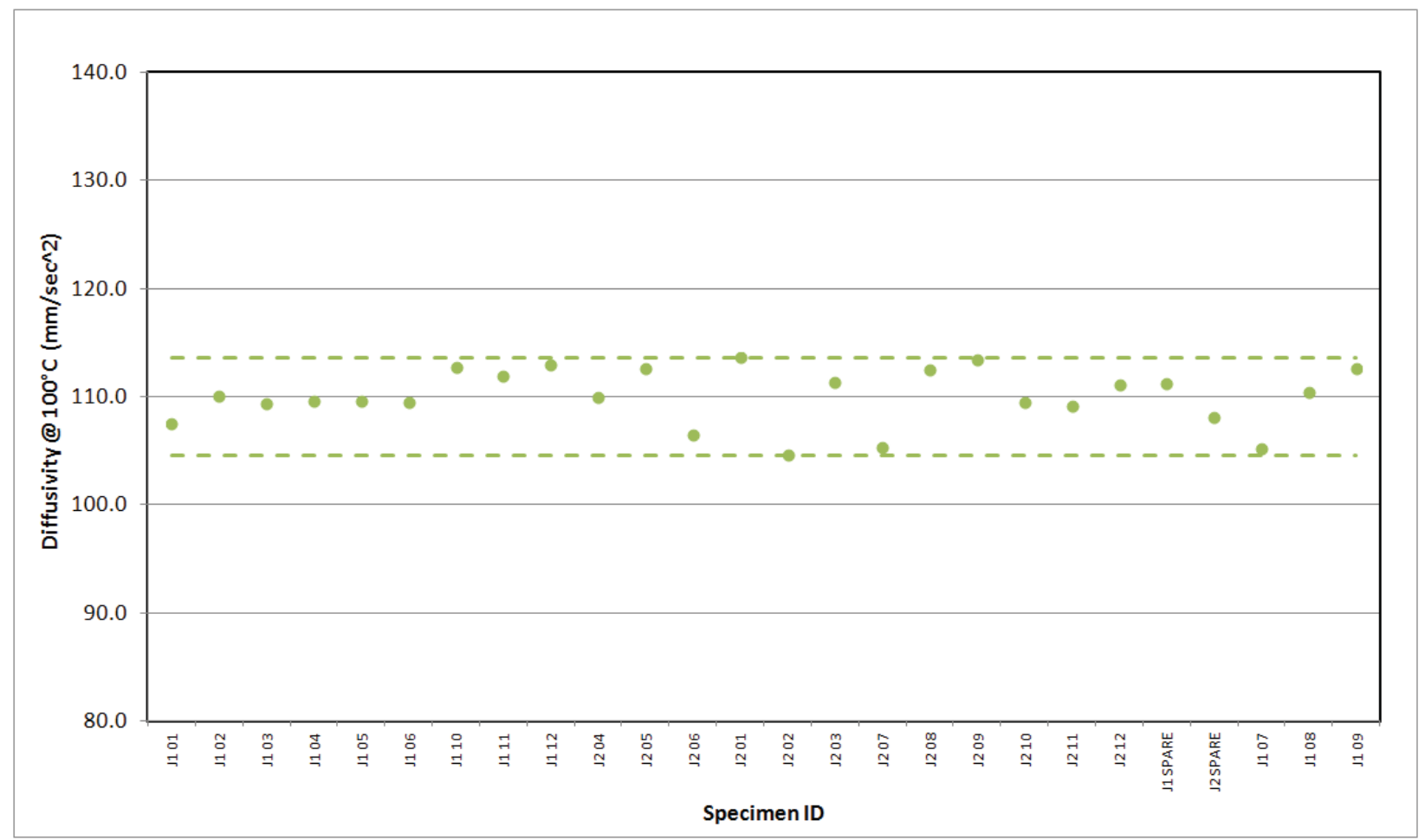

Figure A-220. HLM Piggyback Diffusivity@100C. 


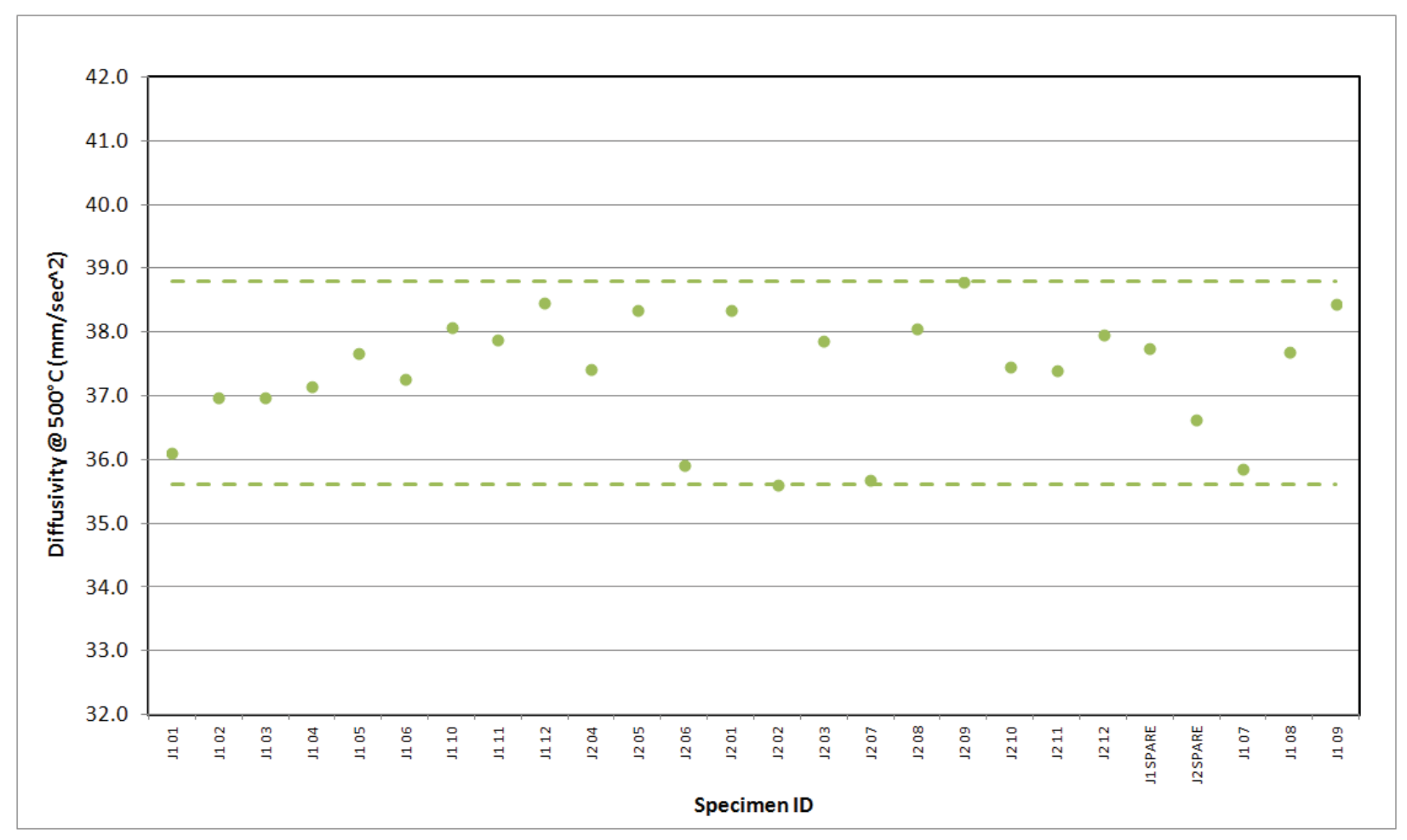

Figure A-221. HLM Piggyback Diffusivity @ 500․

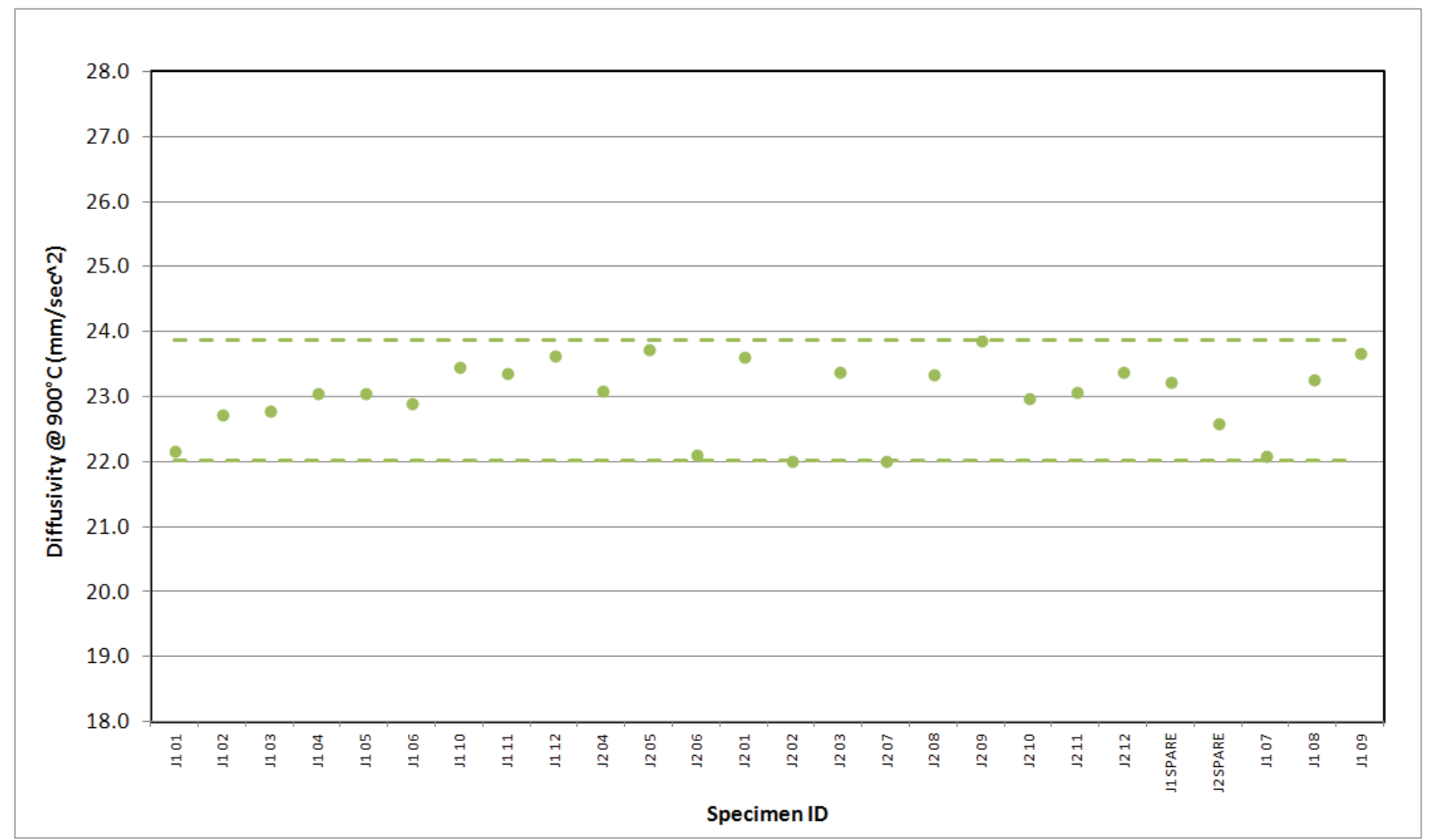

Figure A-222. HLM Piggyback Diffusivity @ 900․ 


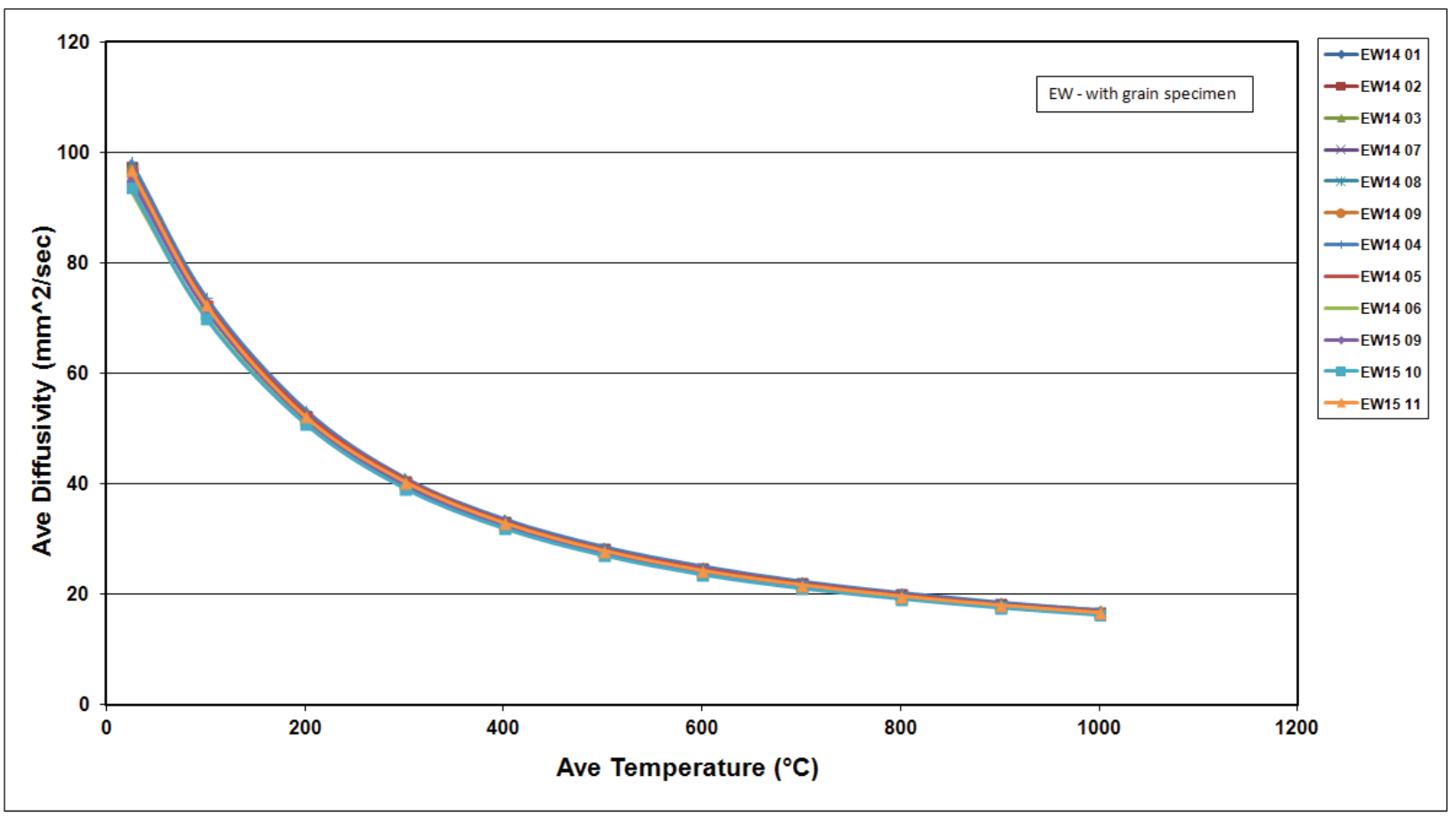

Figure A-223. IG-110 Piggyback Diffusivity.

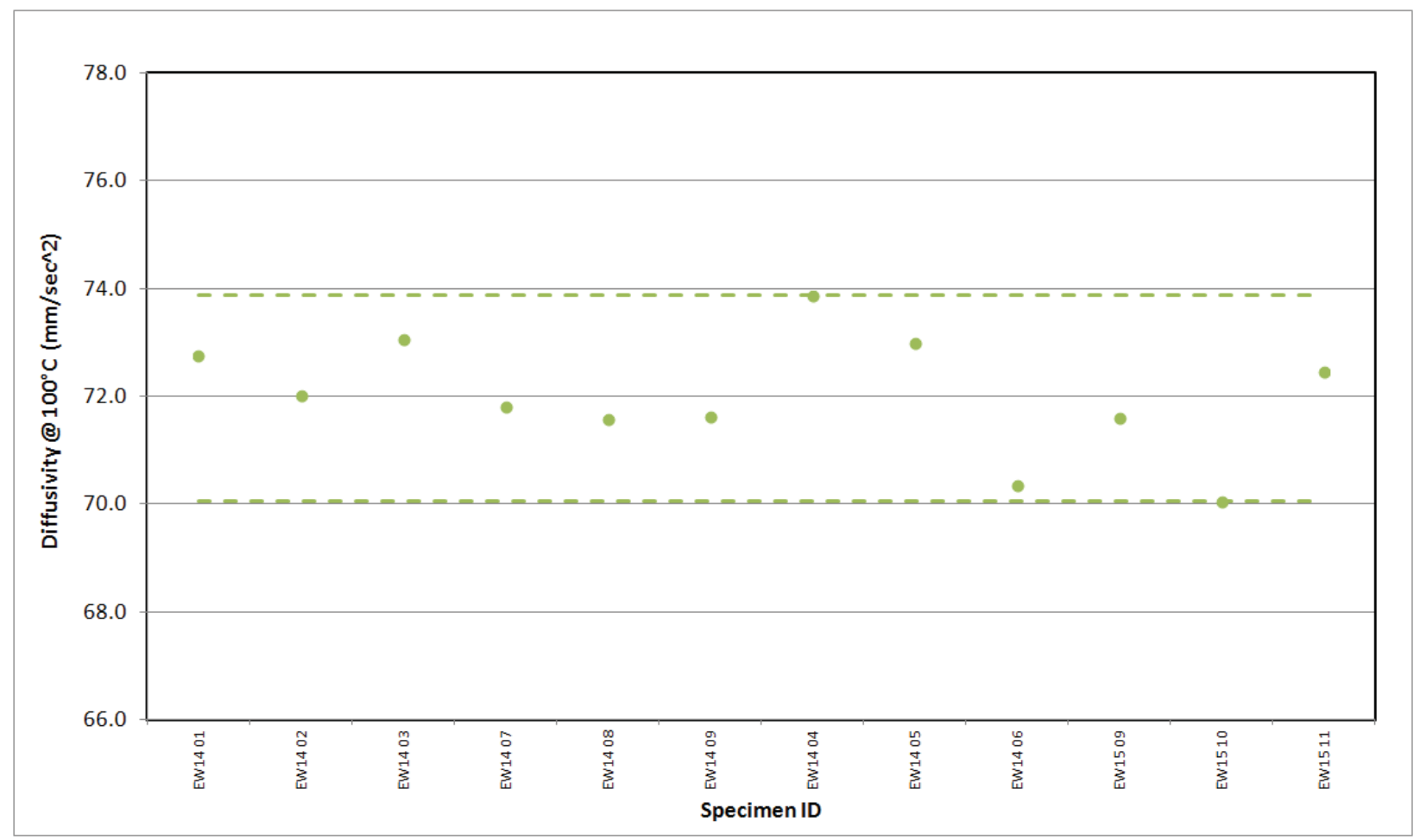

Figure A-224. IG-110 Piggyback Diffusivity @ $100^{\circ} \mathrm{C}$. 


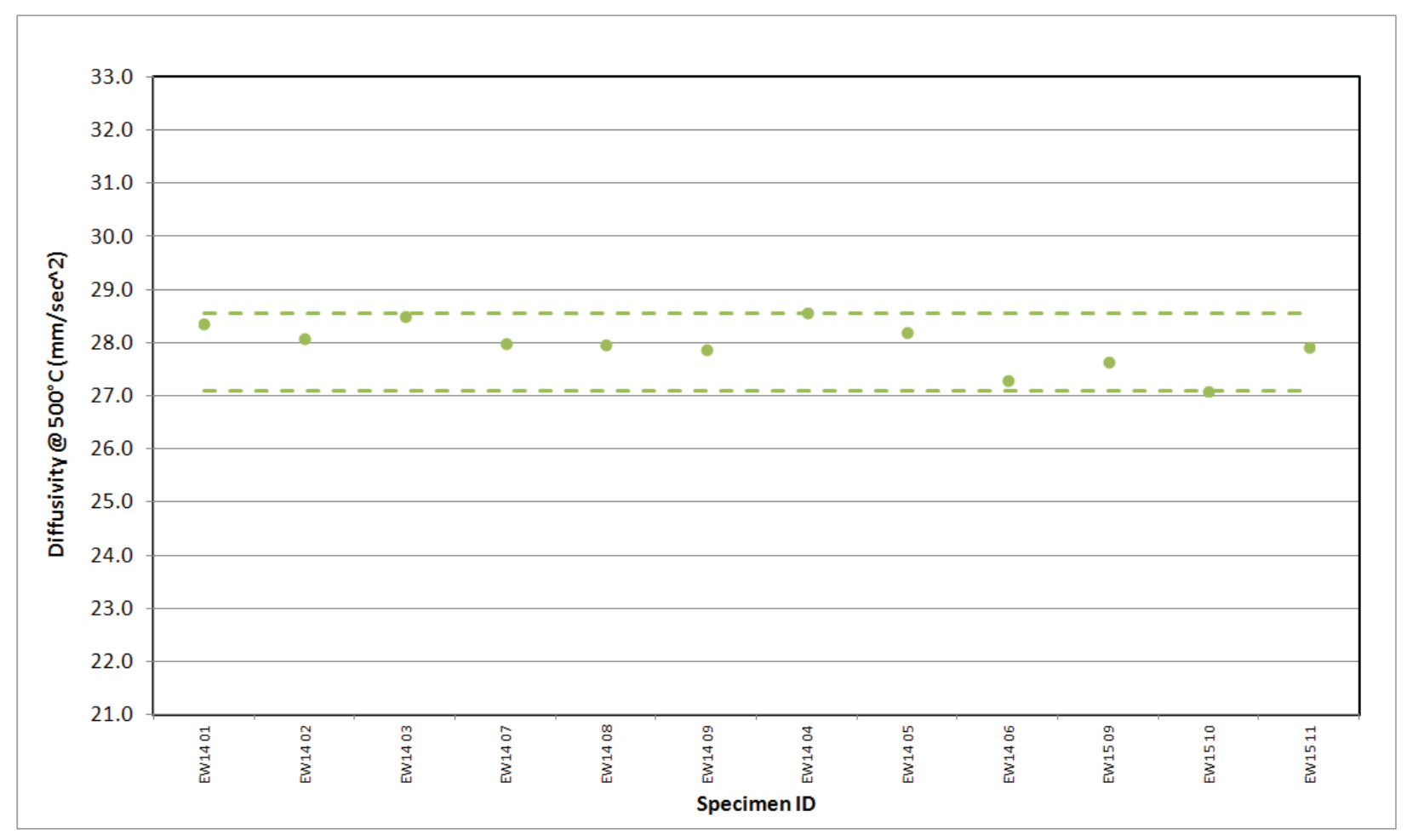

Figure A-225. IG-110 Piggyback Diffusivity @ 500 ${ }^{\circ} \mathrm{C}$.

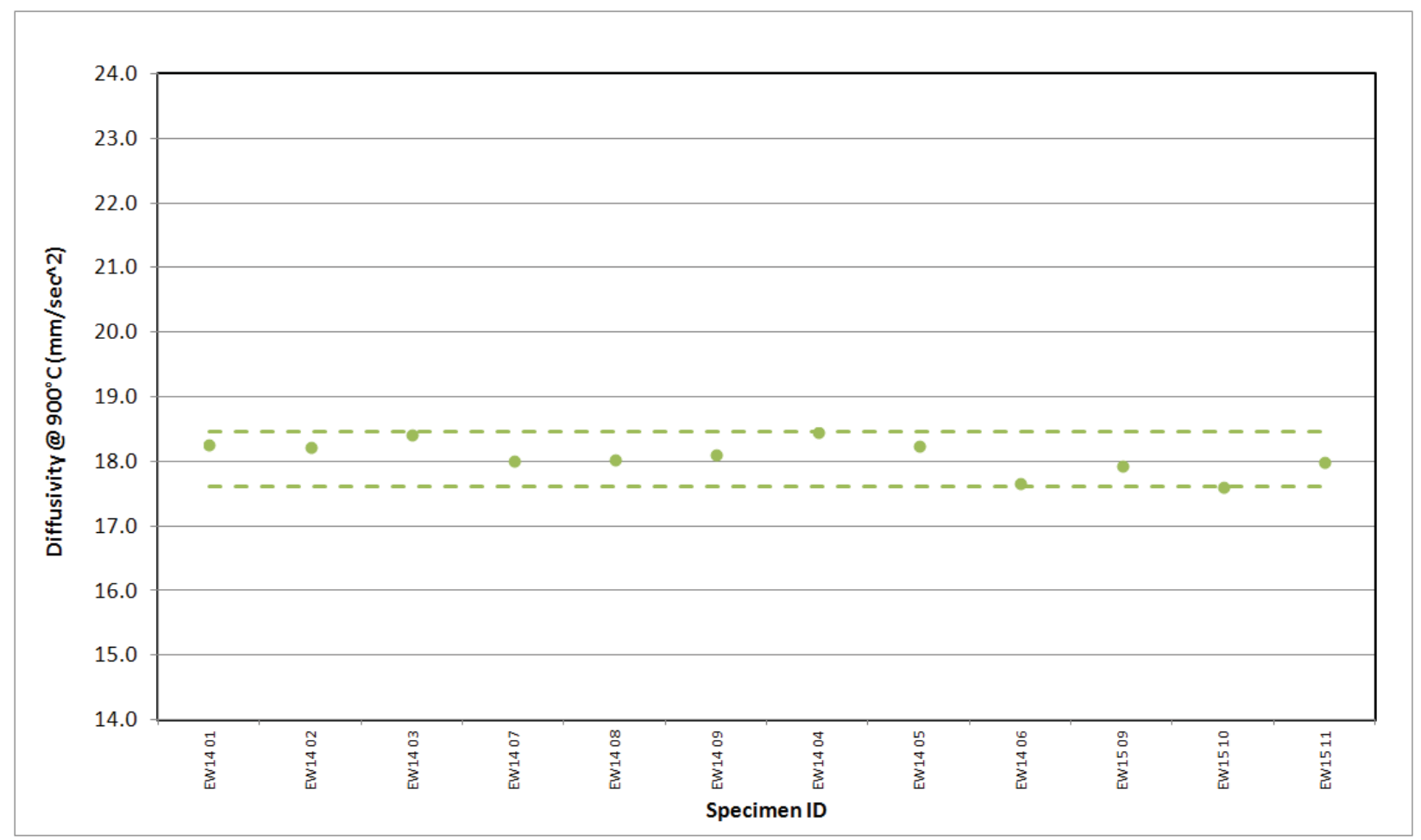

Figure A-226. IG-110 Piggyback Diffusivity@900C. 


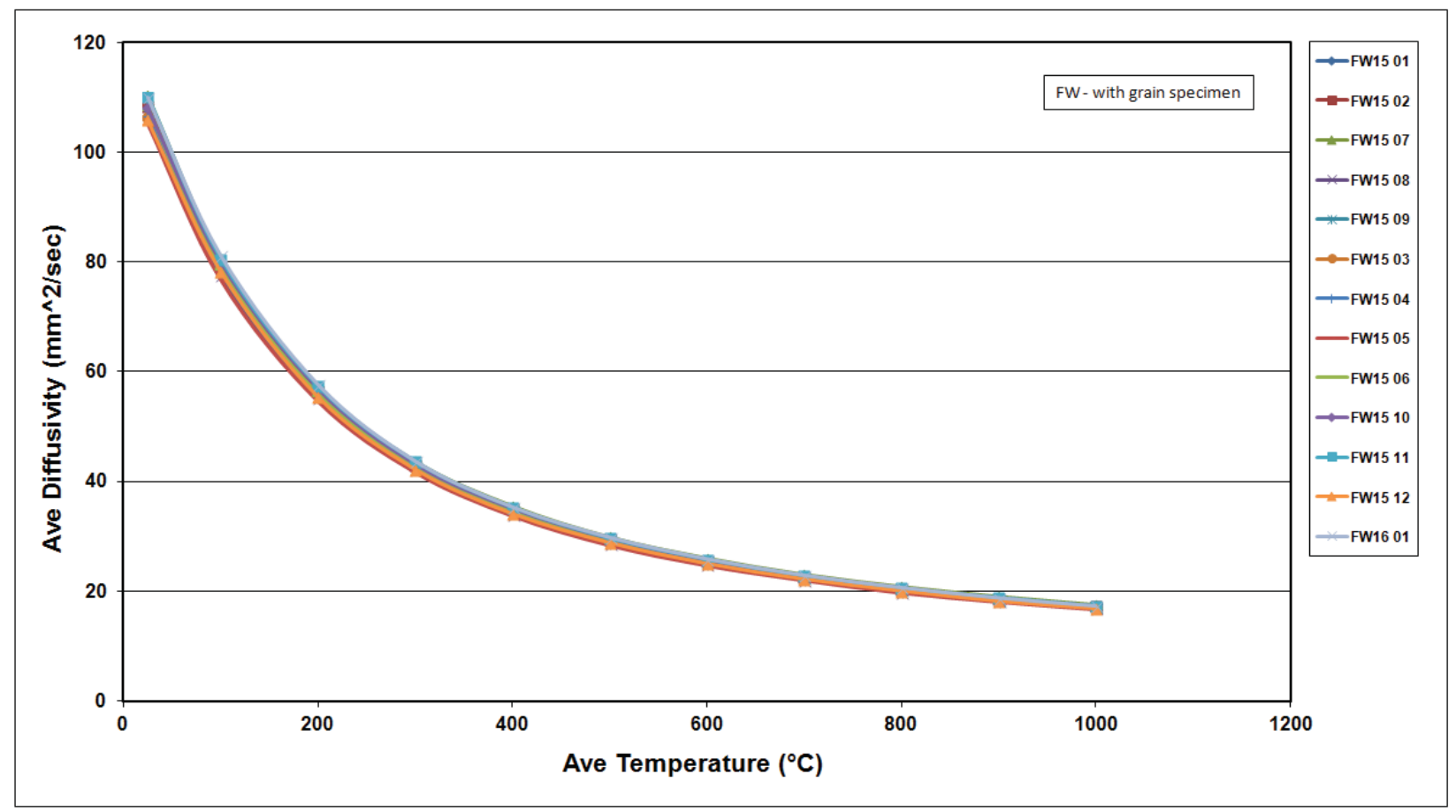

Figure A-227. IG-430 Piggyback Diffusivity.

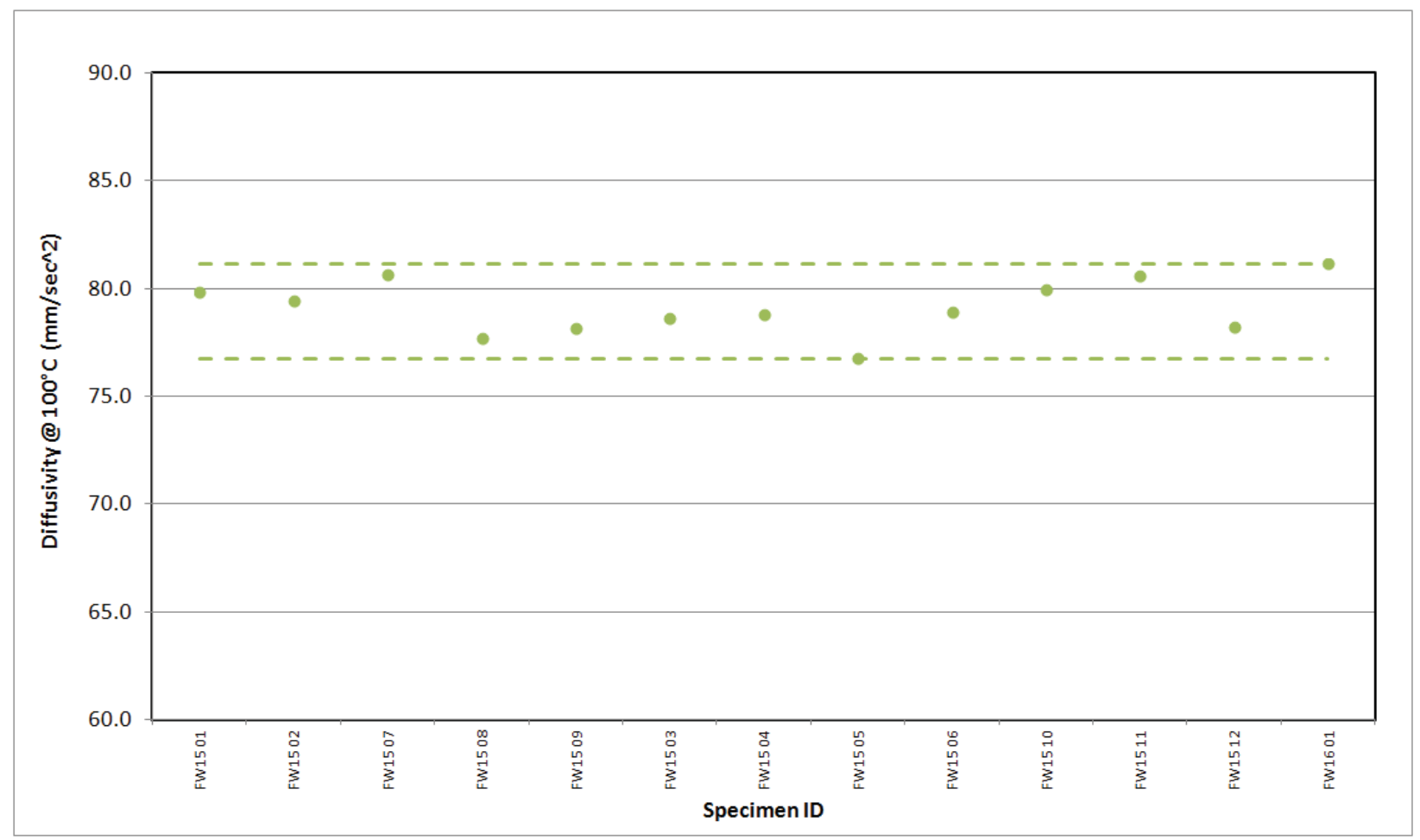

Figure A-228. IG-430 Piggyback Diffusivity @ $100^{\circ} \mathrm{C}$. 


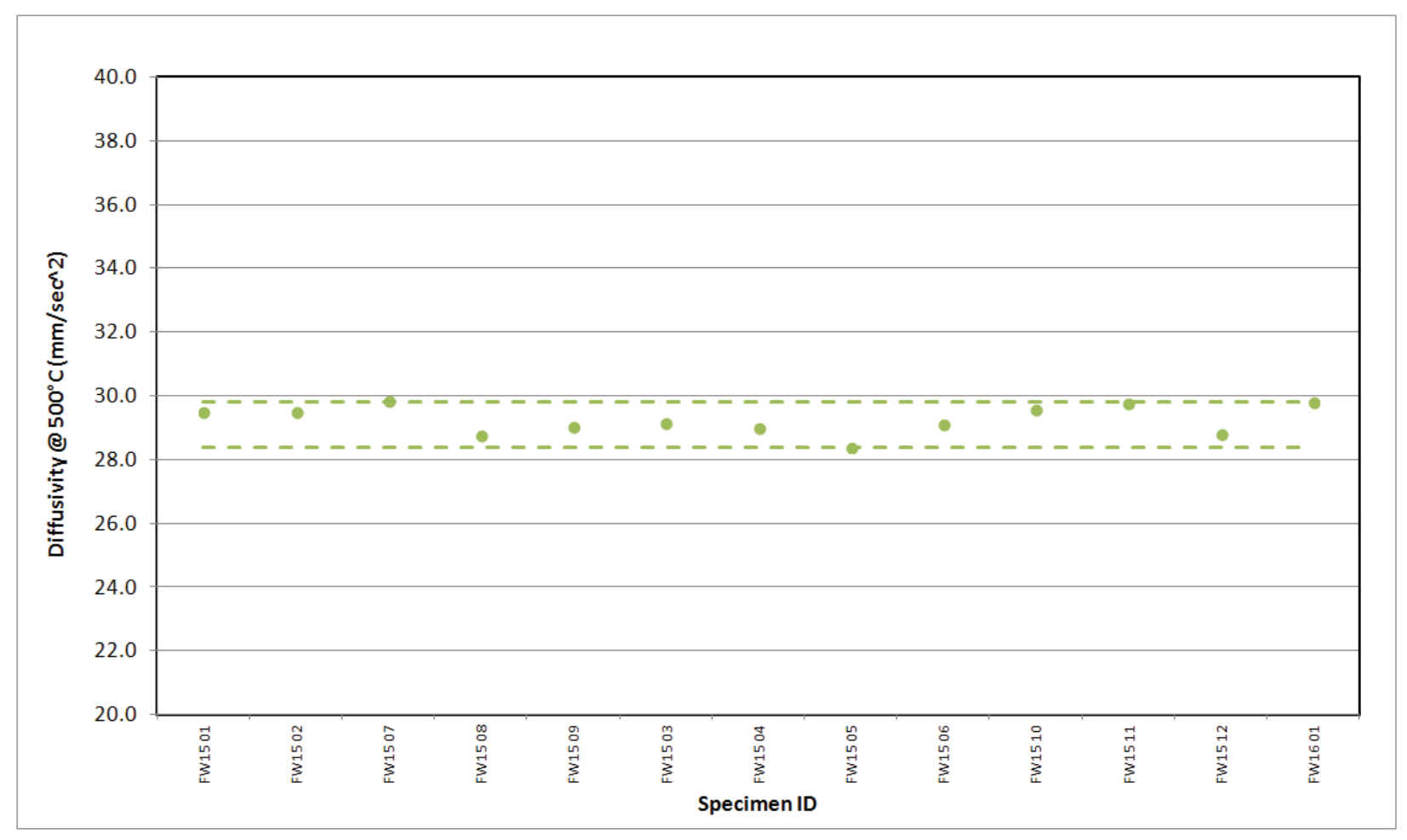

Figure A-229. IG-430 Piggyback Diffusivity @ 500C.

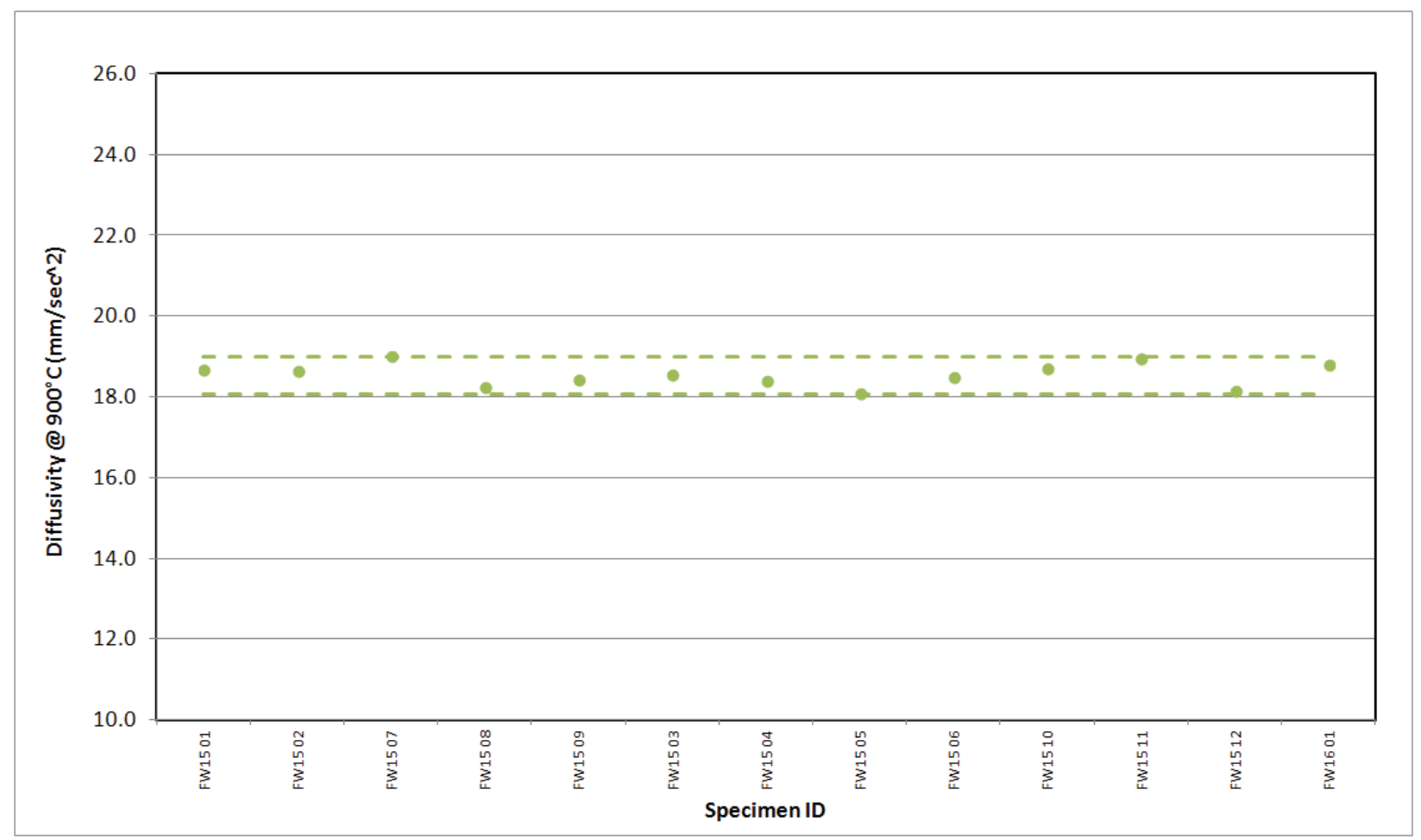

Figure A-230. IG-430 Piggyback Diffusivity@900C. 


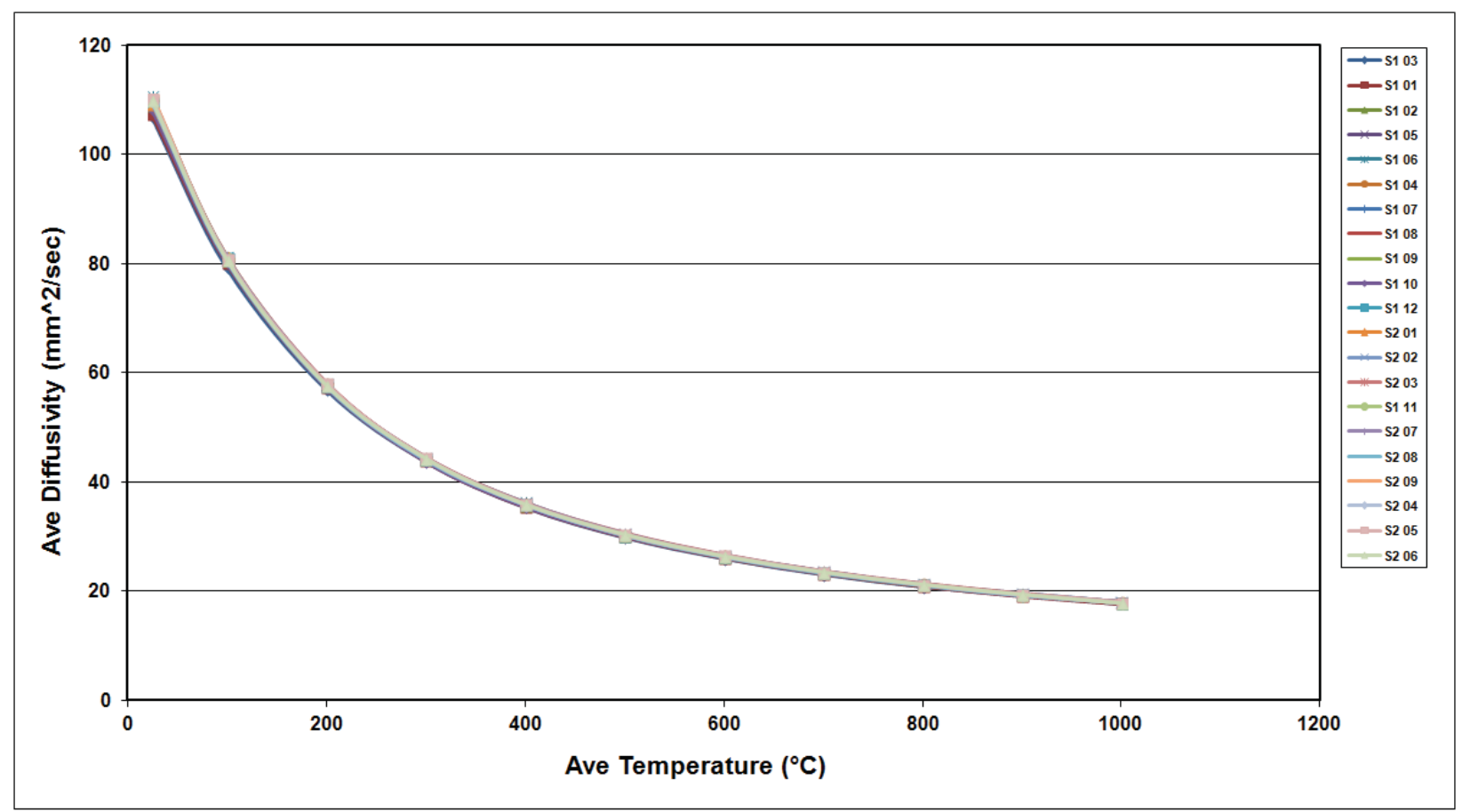

Figure A-231. NBG-10 Piggyback Diffusivity.

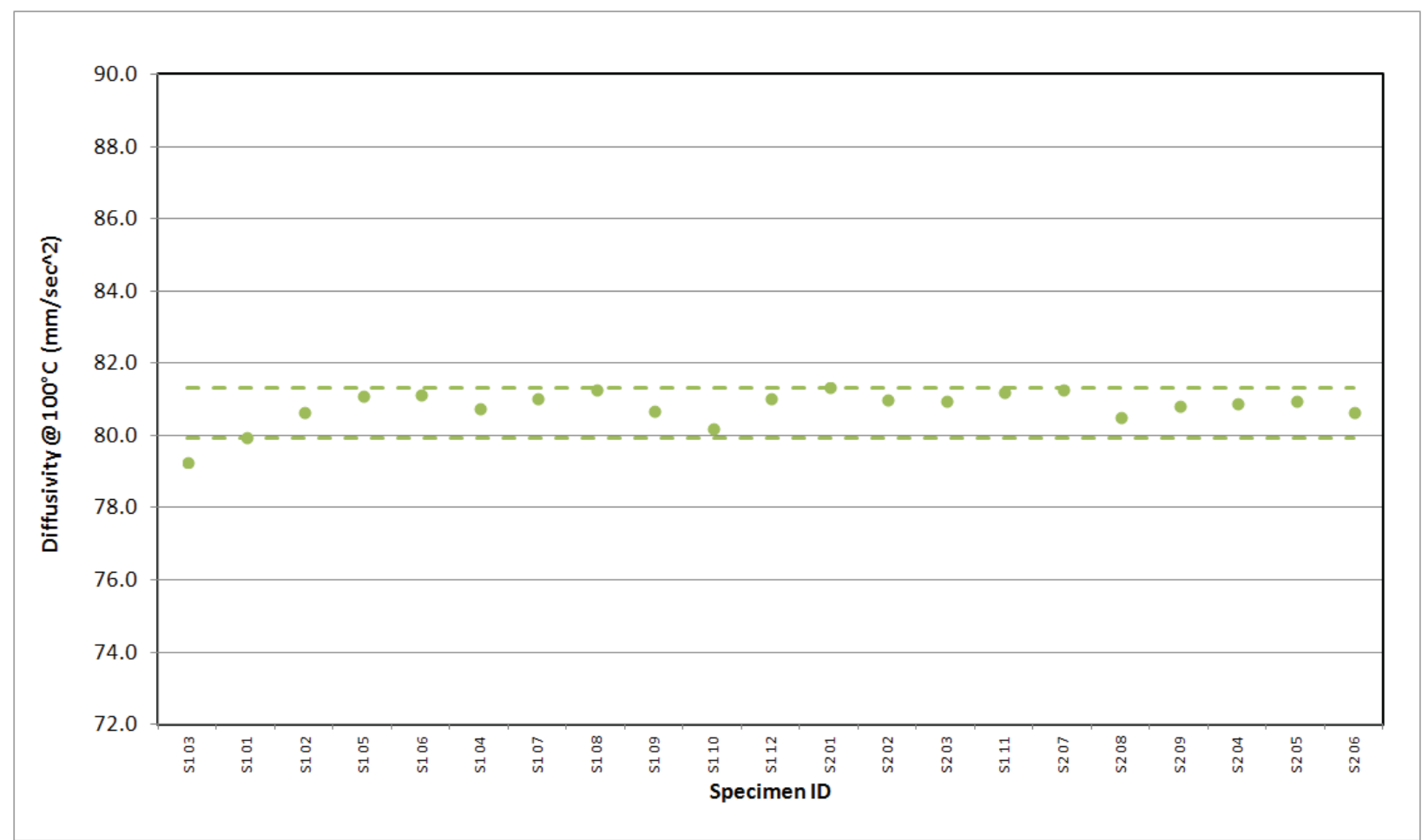

Figure A-232. NBG-10 Piggyback Diffusivity @ 100². 


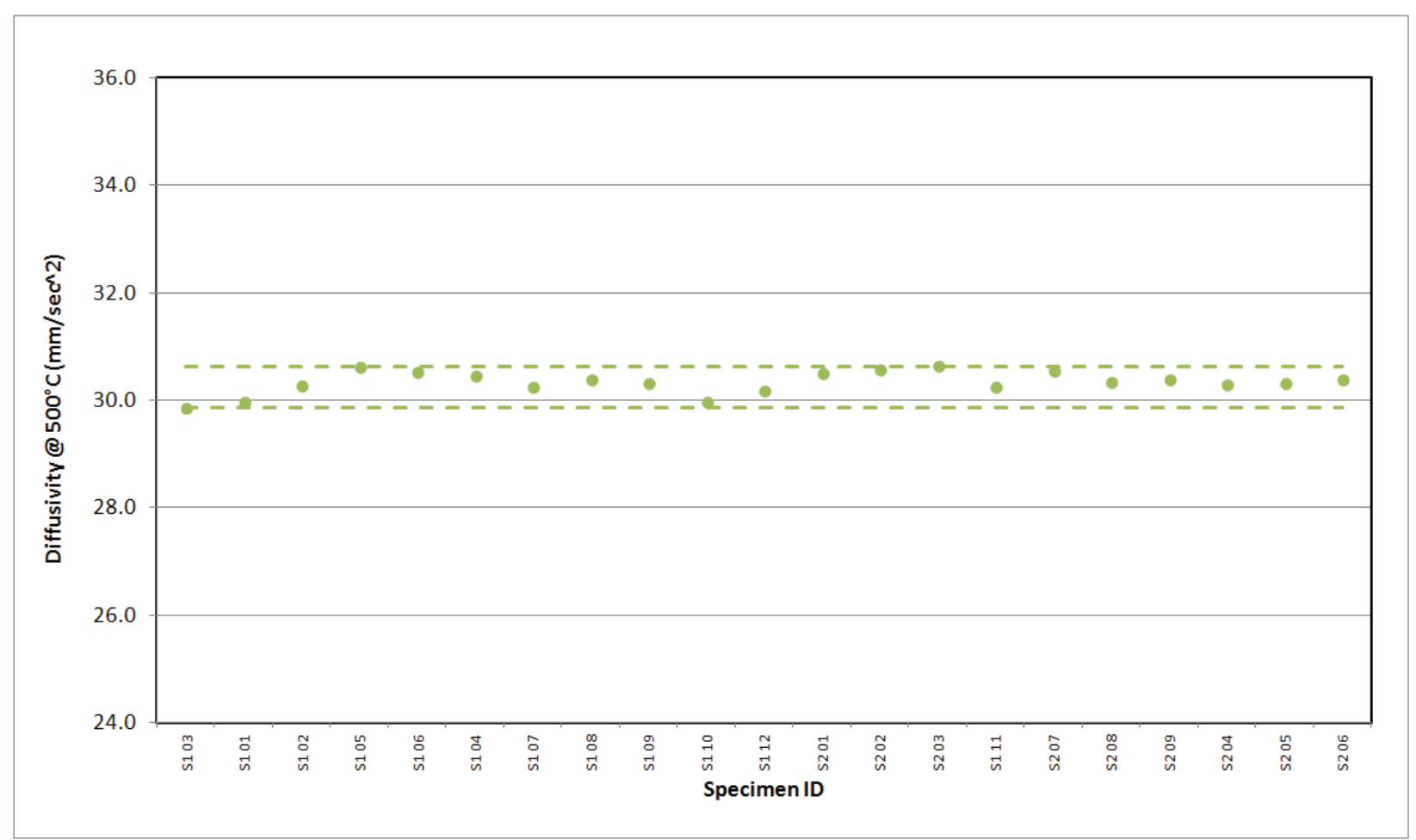

Figure A-233. NBG-10 Piggyback Diffusivity@500C.

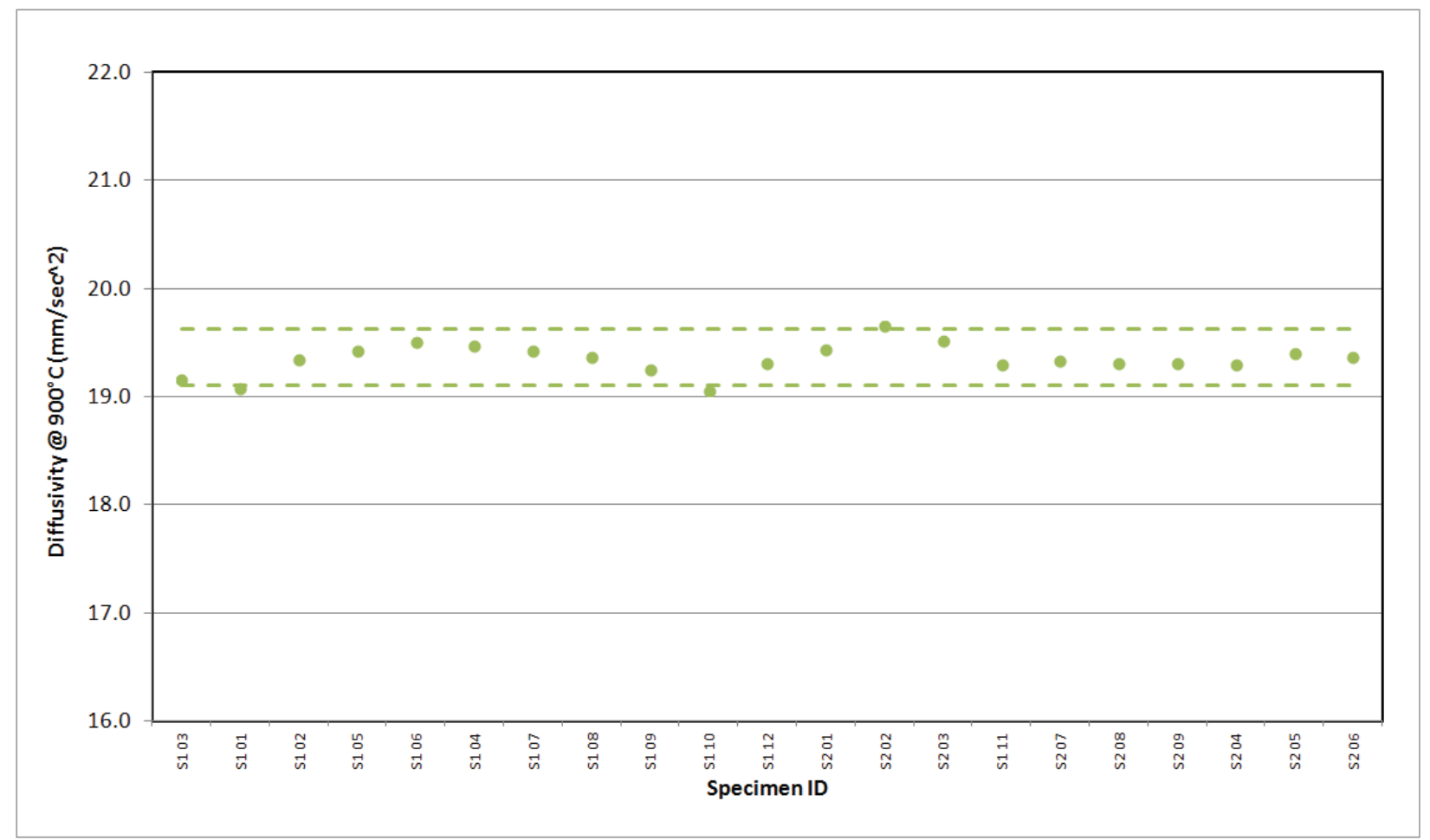

Figure A-234. NBG-10 Piggyback Diffusivity @ $900^{\circ} \mathrm{C}$. 


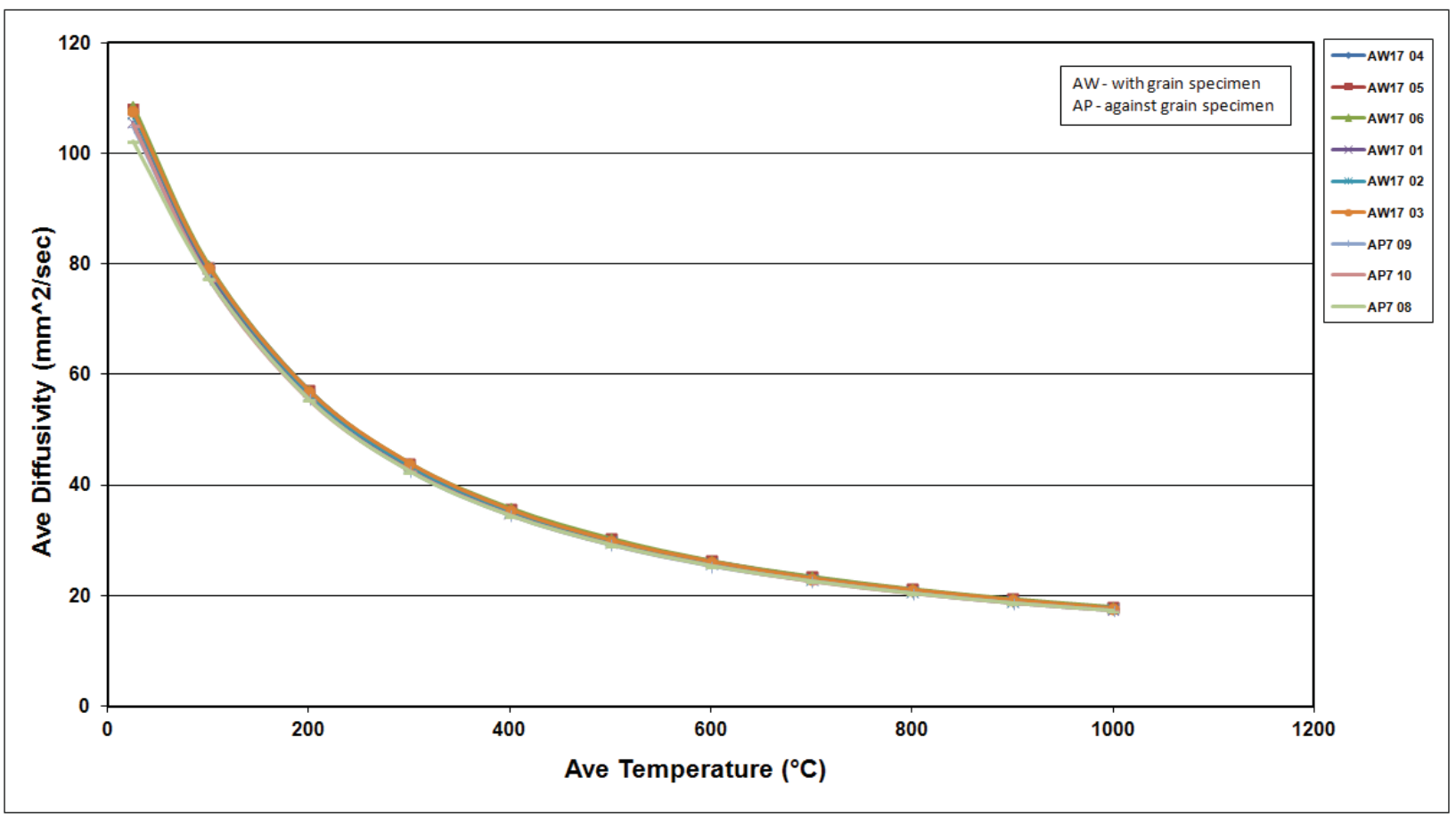

Figure A-235. NBG-17 Piggyback Diffusivity.

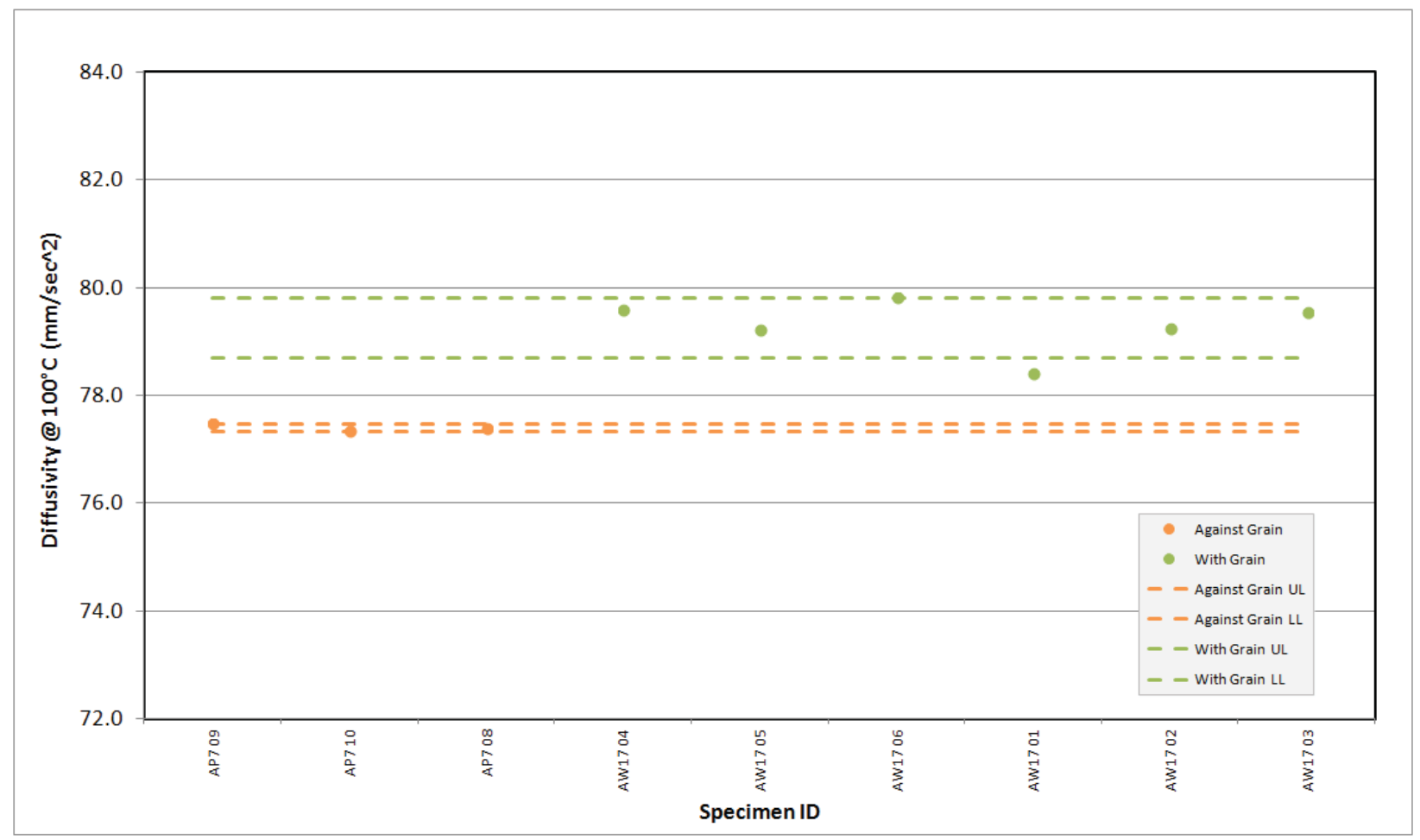

Figure A-236. NBG-17 Piggyback Diffusivity@100². 


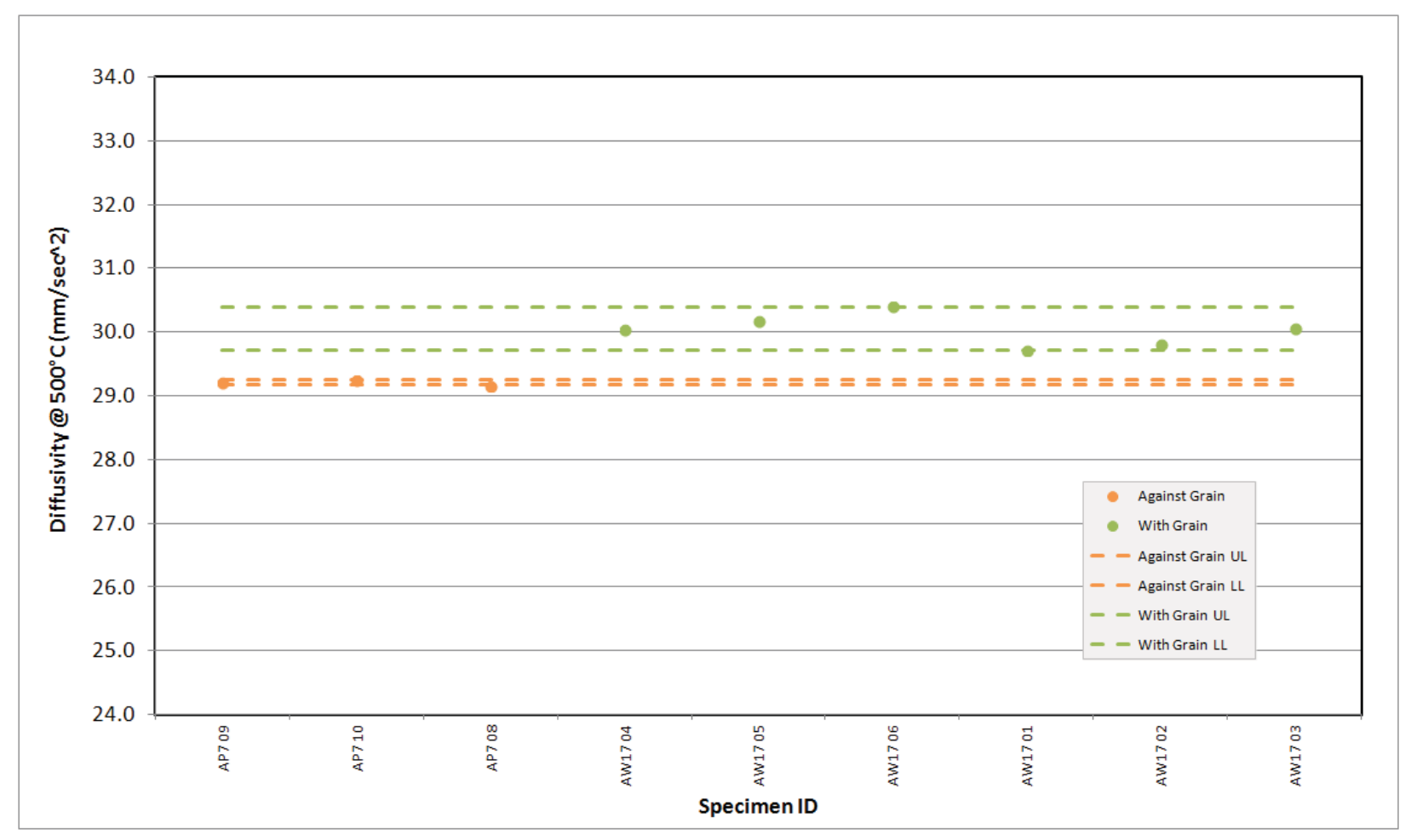

Figure A-237. NBG-17 Piggyback Diffusivity@500C.

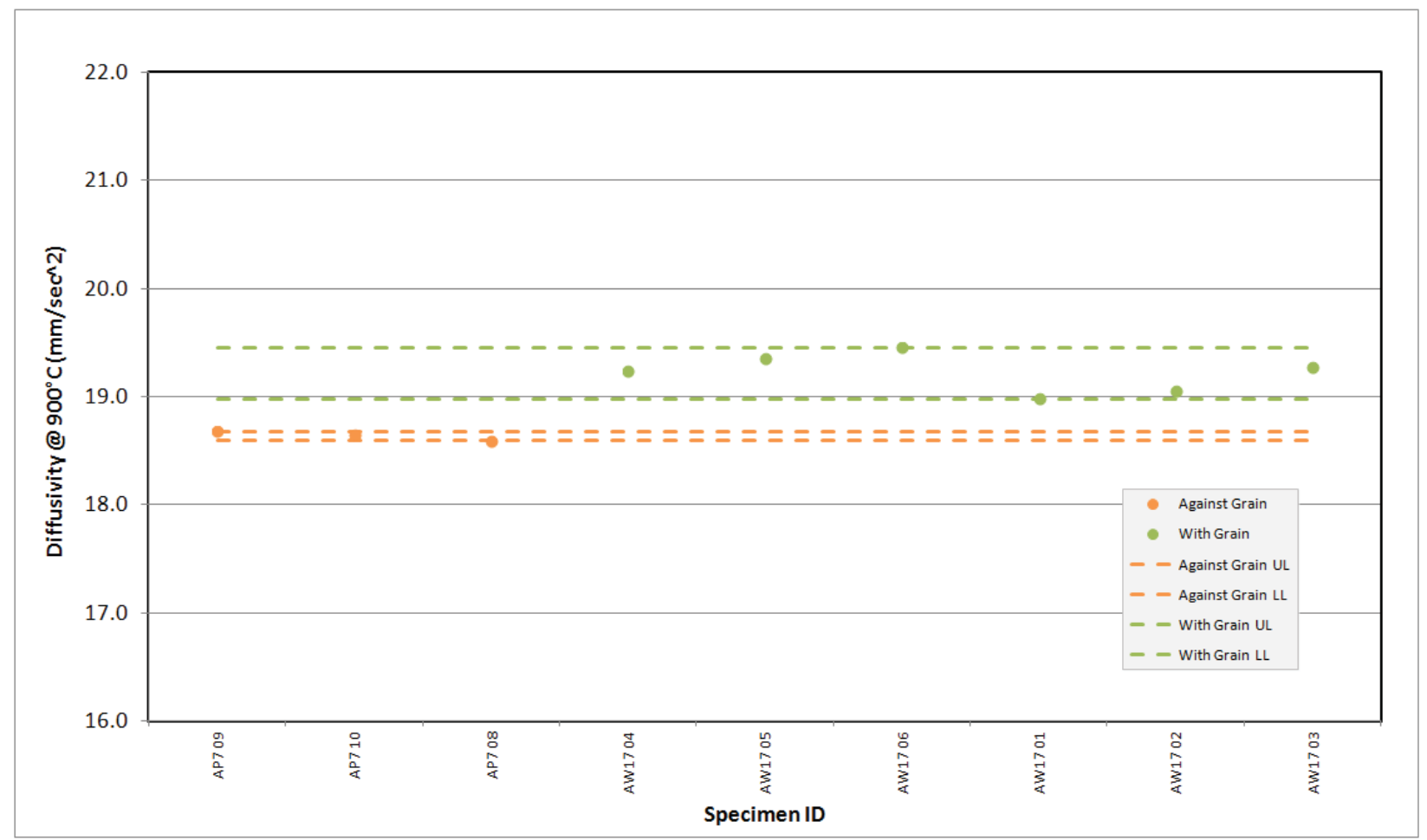

Figure A-238. NBG-17 Piggyback Diffusivity @ $900^{\circ} \mathrm{C}$. 


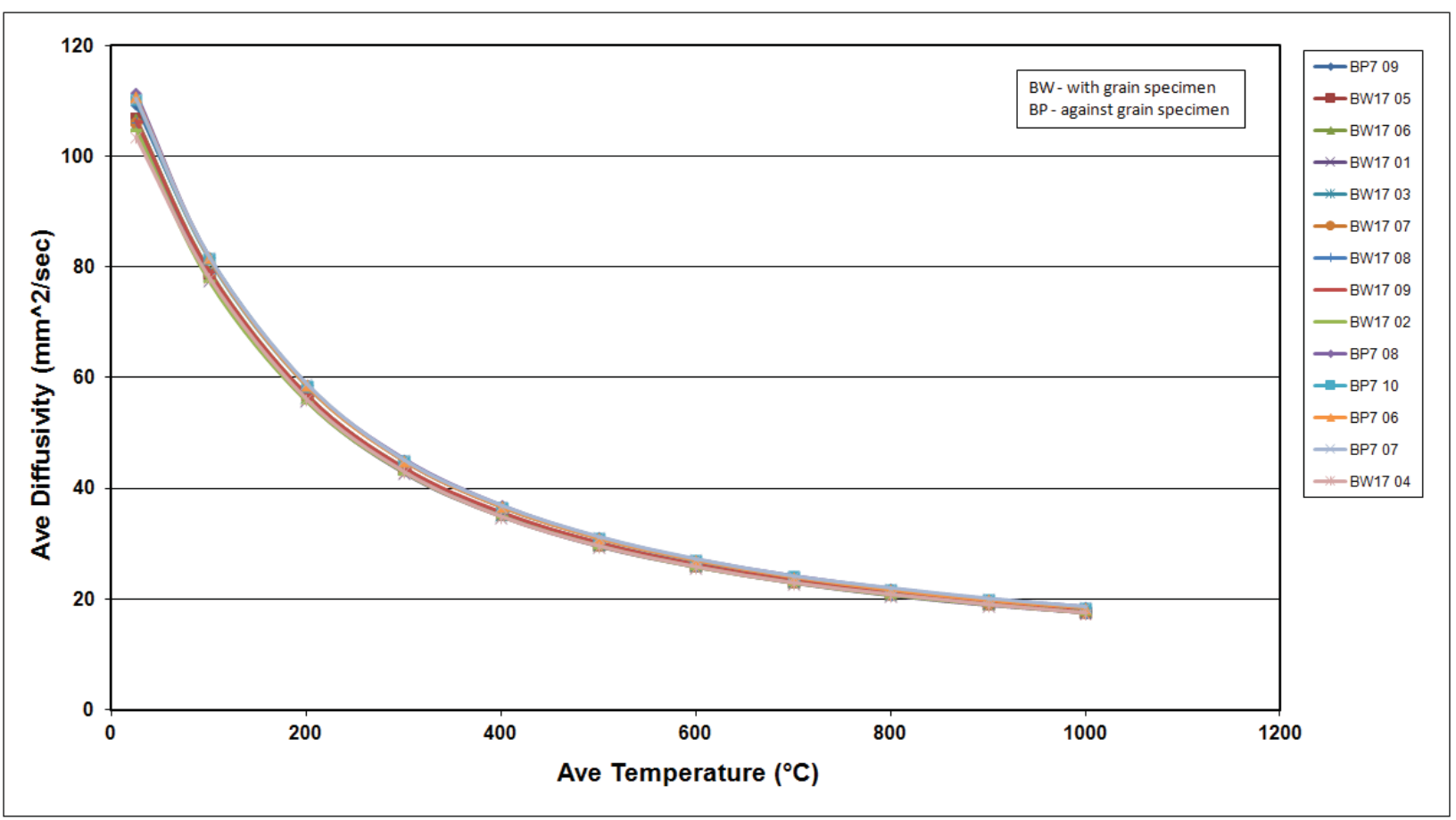

Figure A-239. NBG-18 Piggyback Diffusivity.

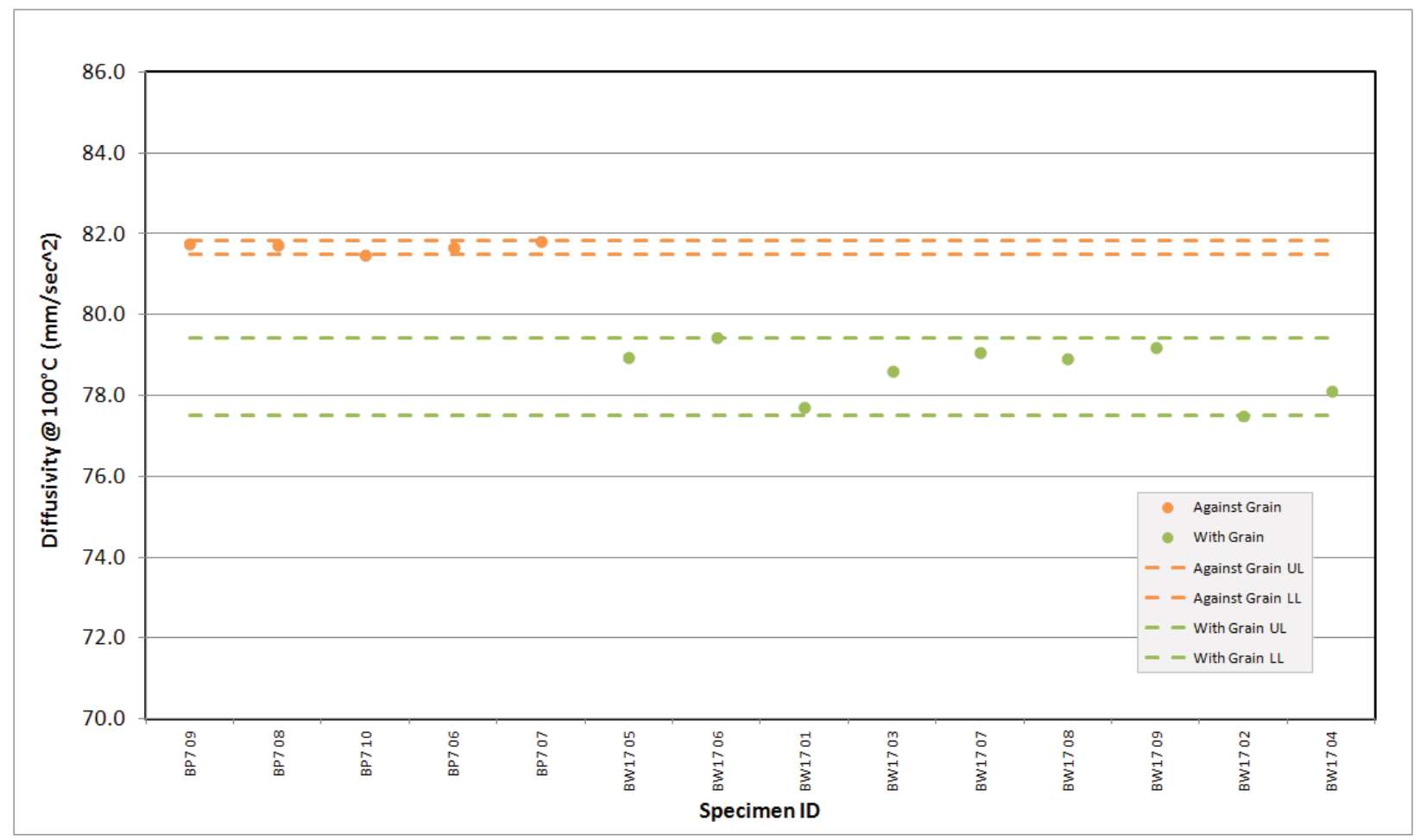

Figure A-240. NBG-18 Piggyback Diffusivity @100². 


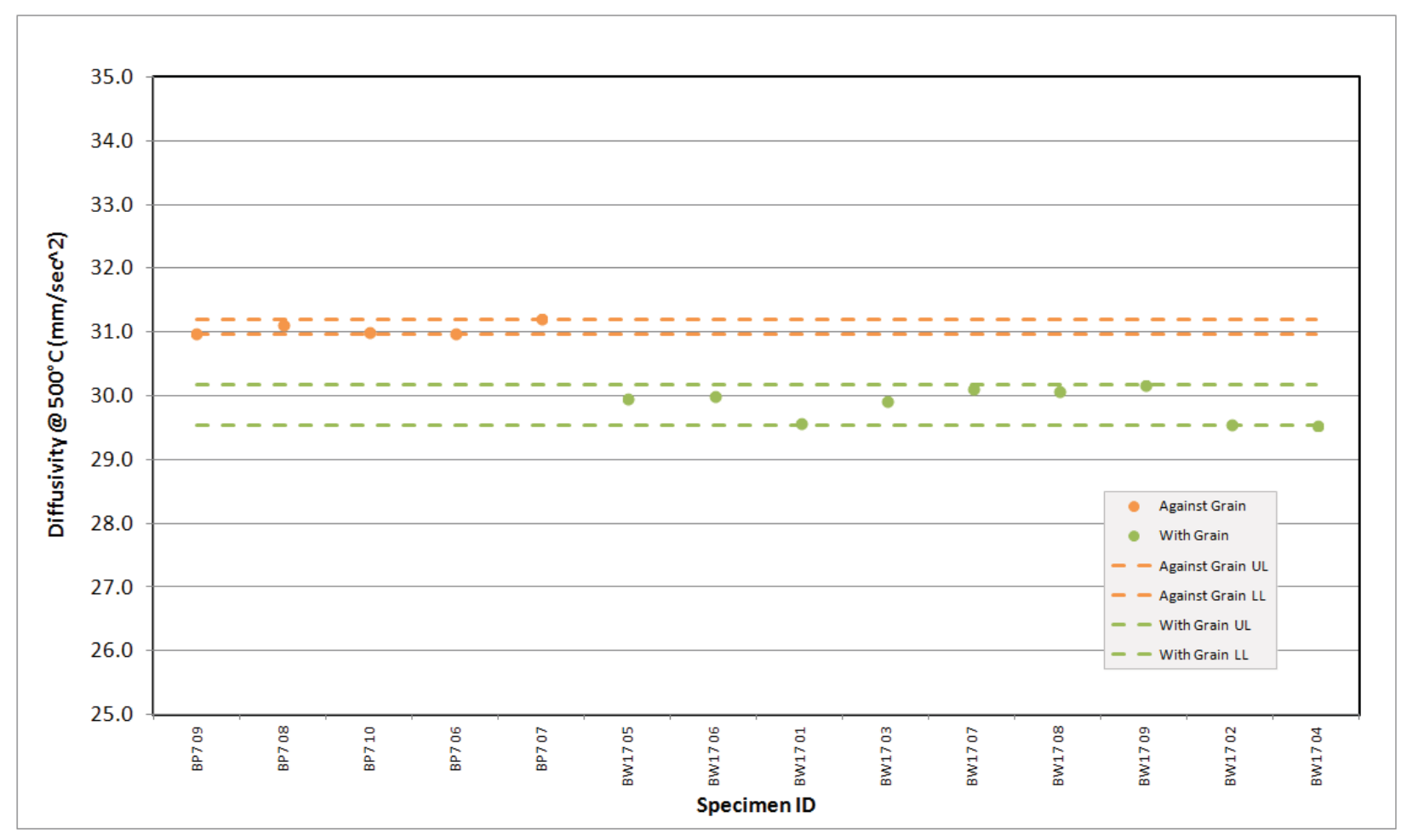

Figure A-241. NBG-18 Piggyback Diffusivity@500C.

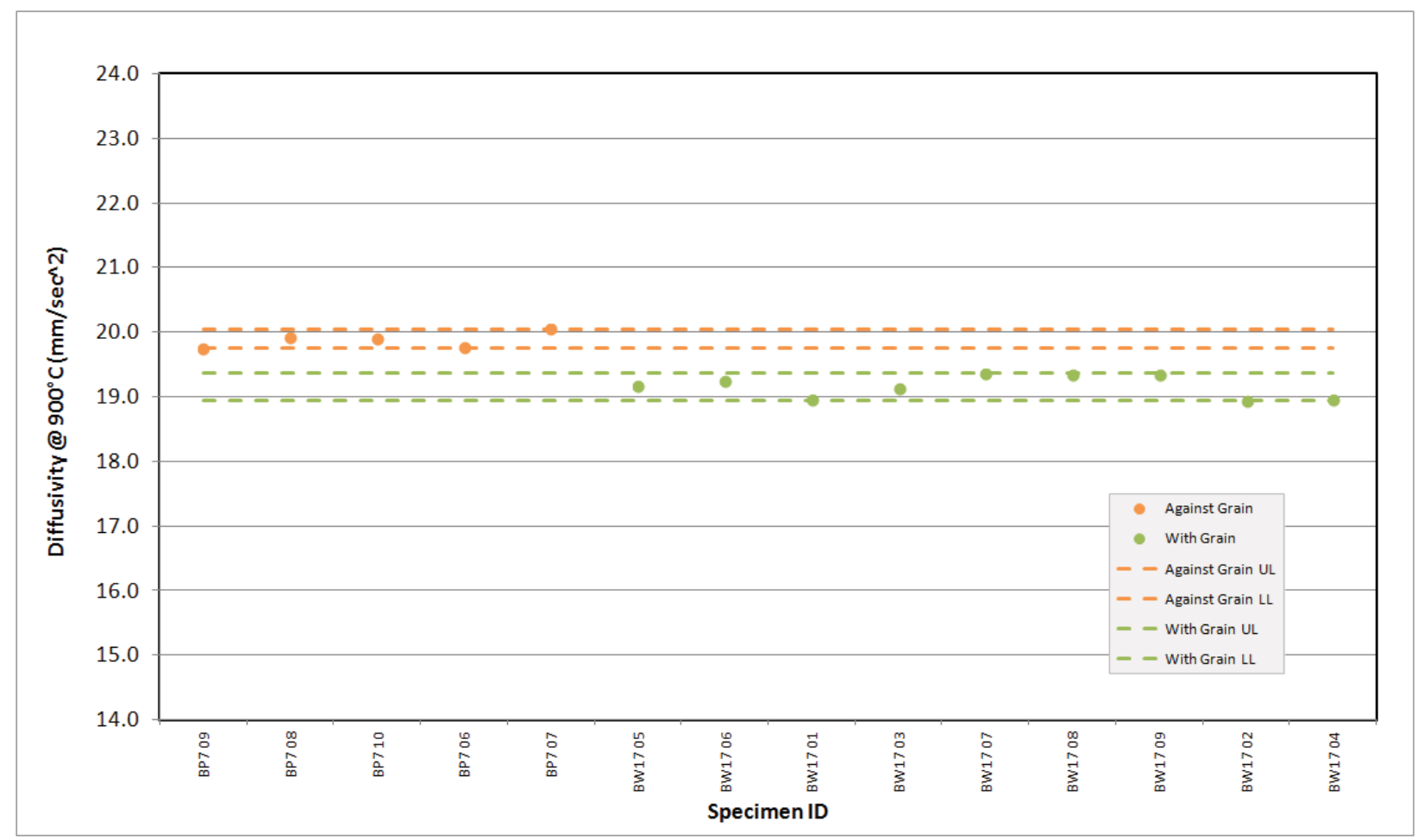

Figure A-242. NBG-18 Diffusivity @ 900 C. 


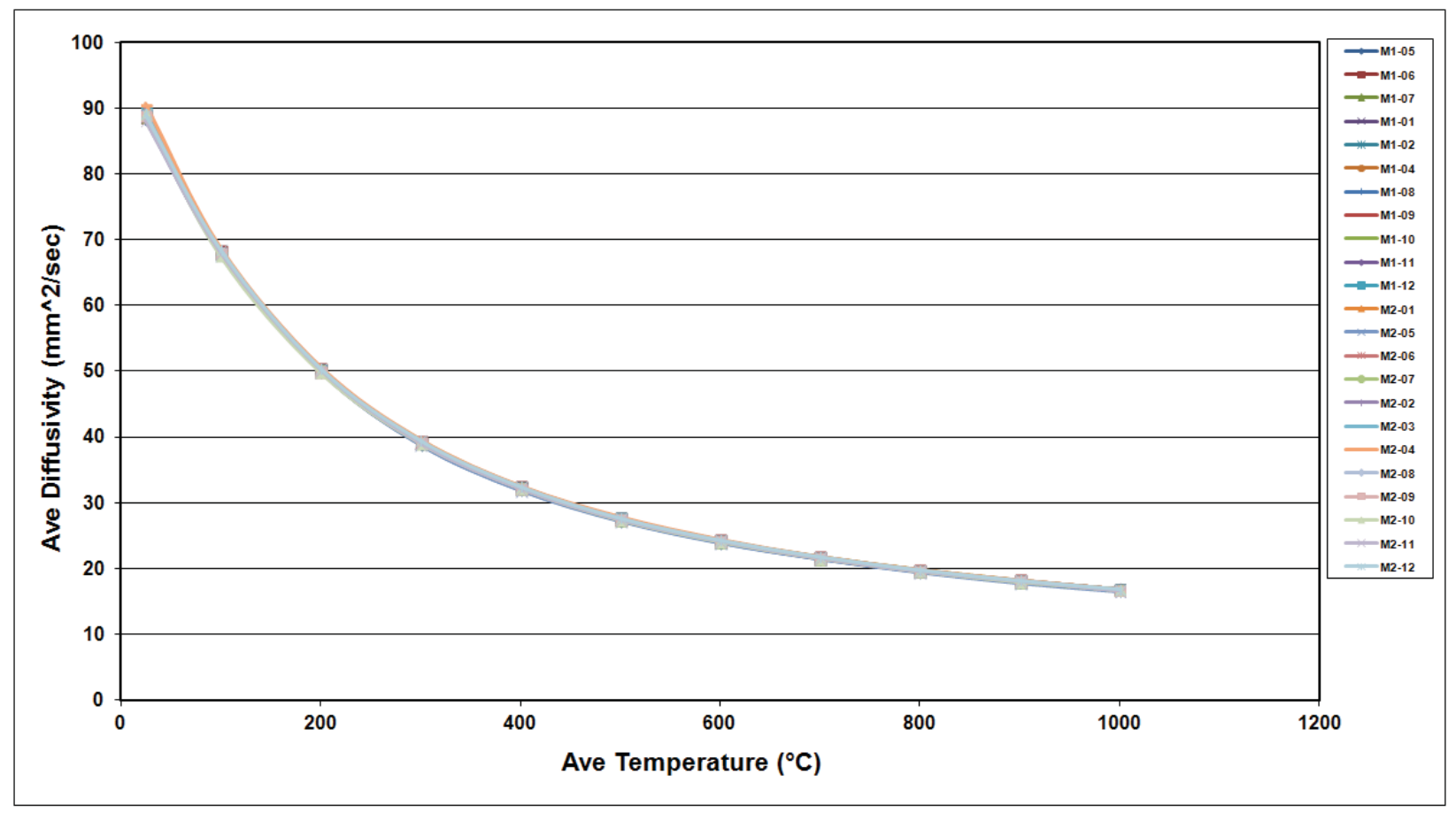

Figure A-243. NBG-25 Piggyback Diffusivity.

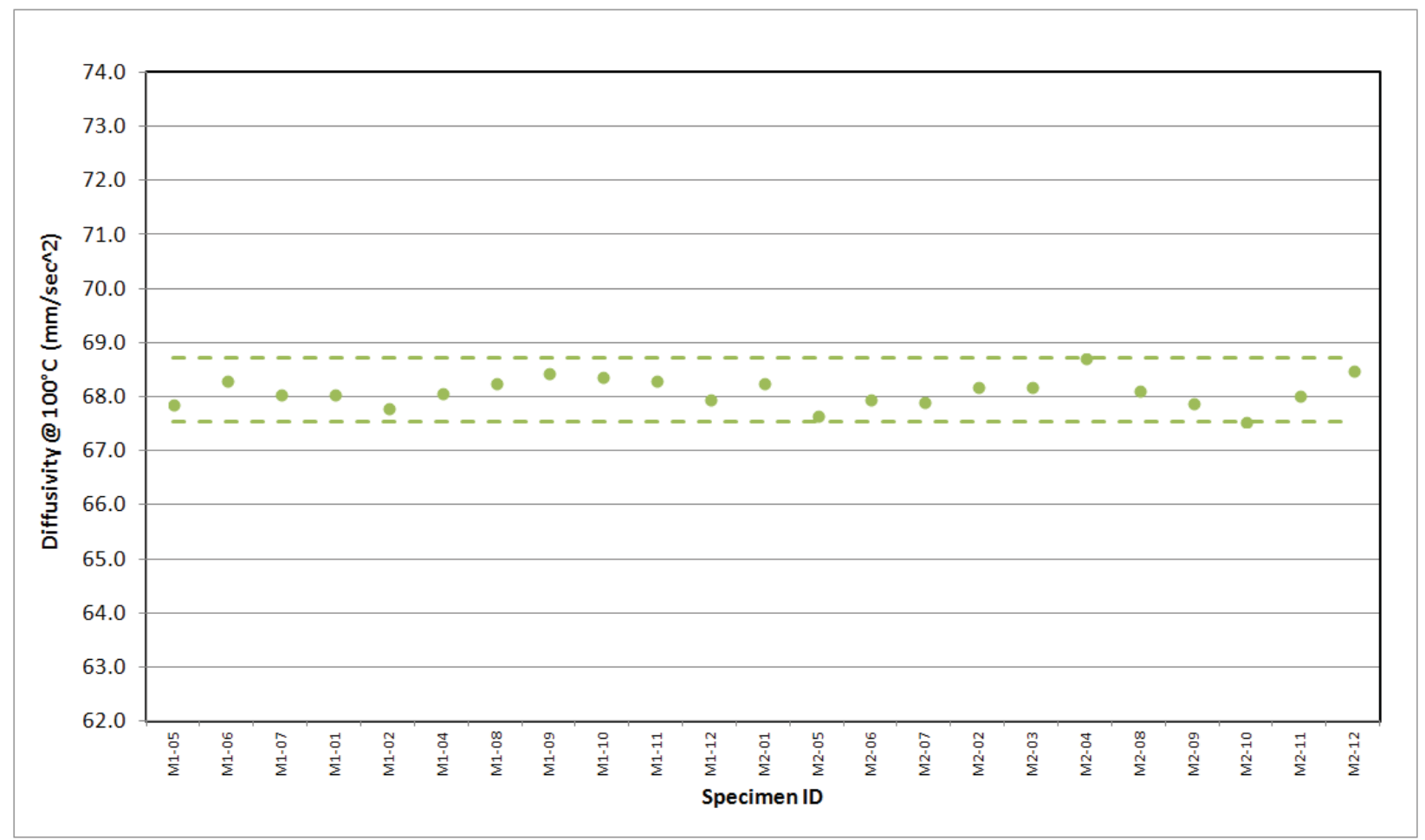

Figure A-244. NBG-25 Piggyback Diffusivity@100C. 


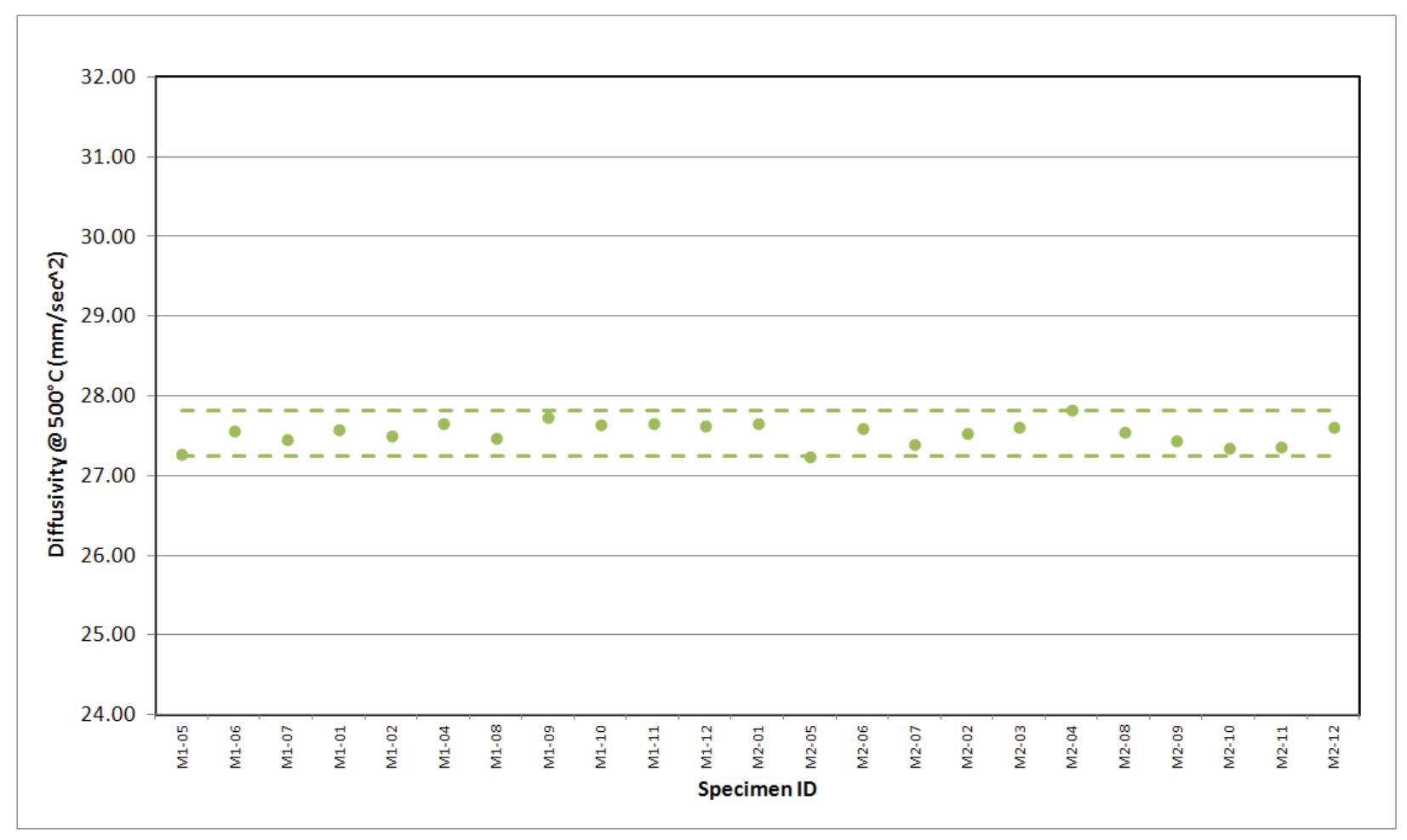

Figure A-245. NBG-25 Piggyback Diffusivity@500C.

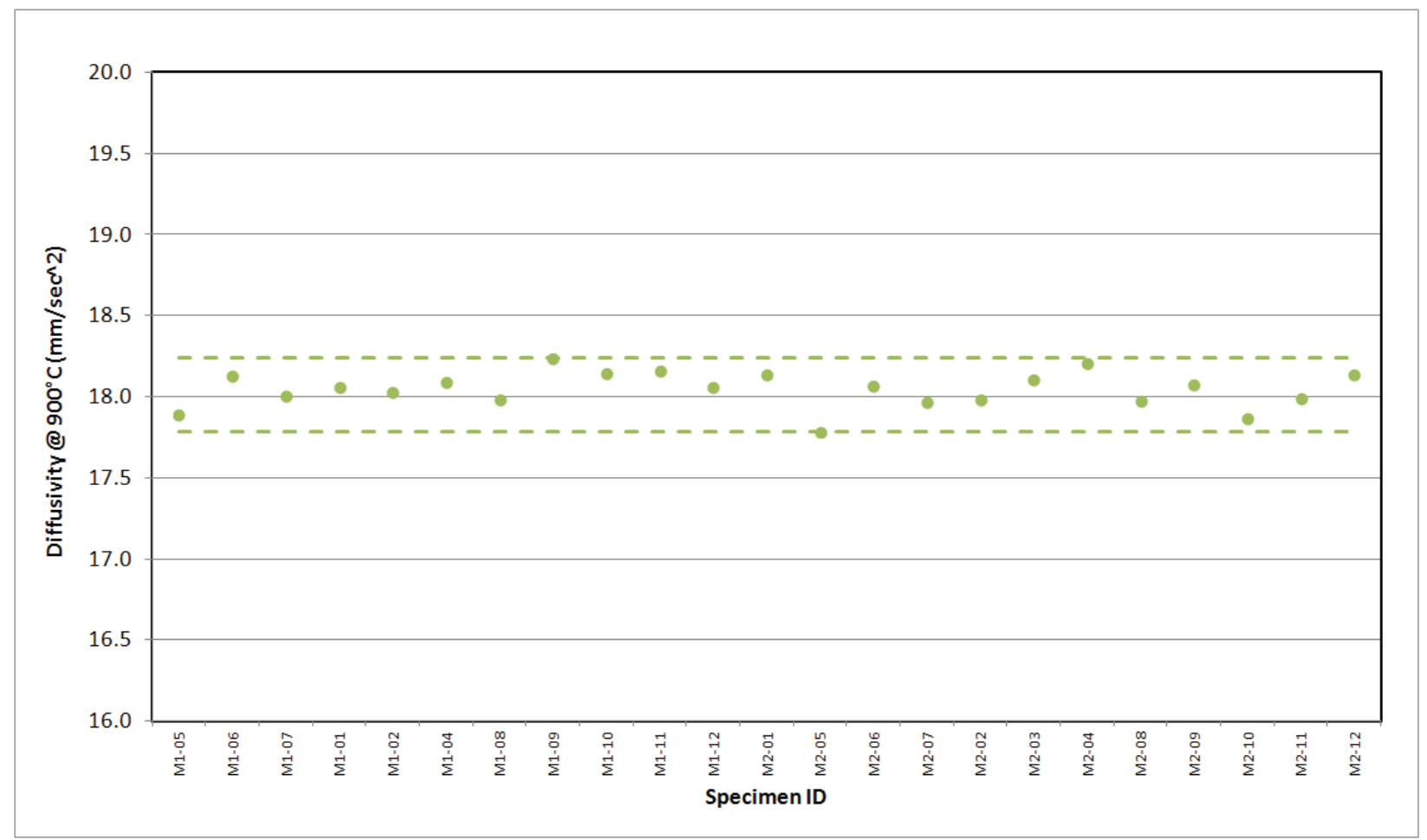

Figure A-246. NBG-25 Piggyback Diffusivity @ $900^{\circ} \mathrm{C}$. 


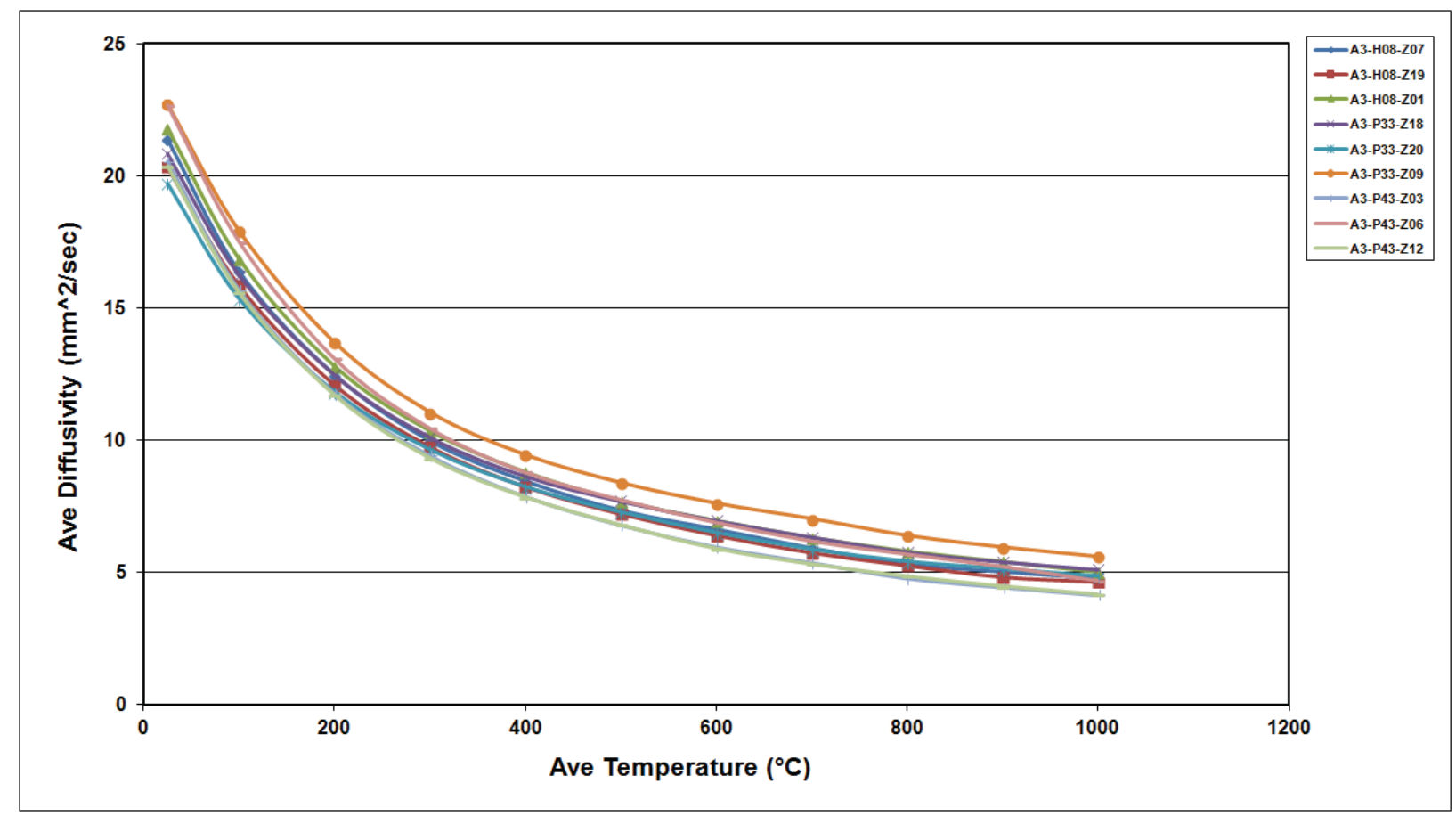

Figure A-247. New Matrix Piggyback Diffusivity.

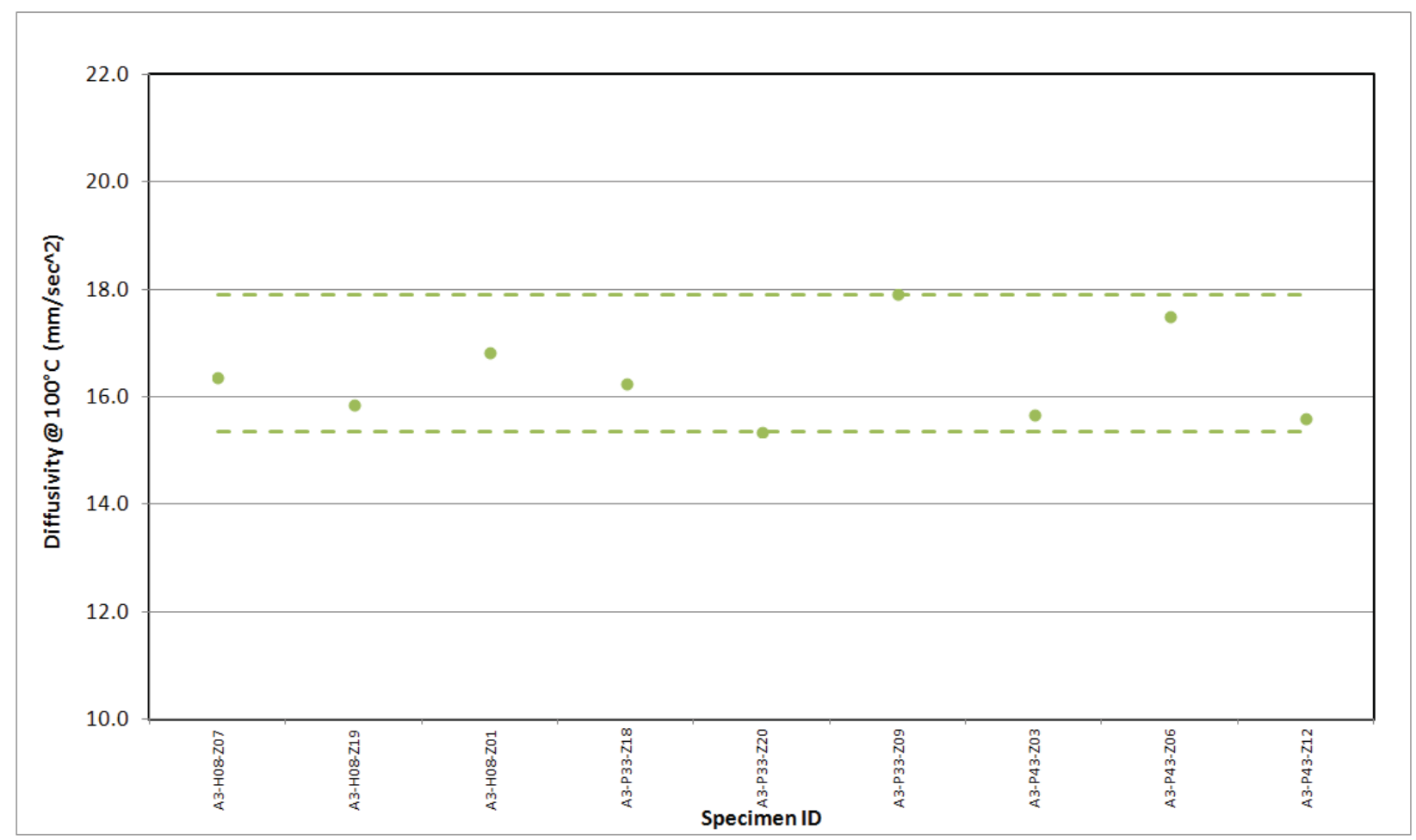

Figure A-248. New Matrix Piggyback Diffusivity @ $100^{\circ} \mathrm{C}$. 


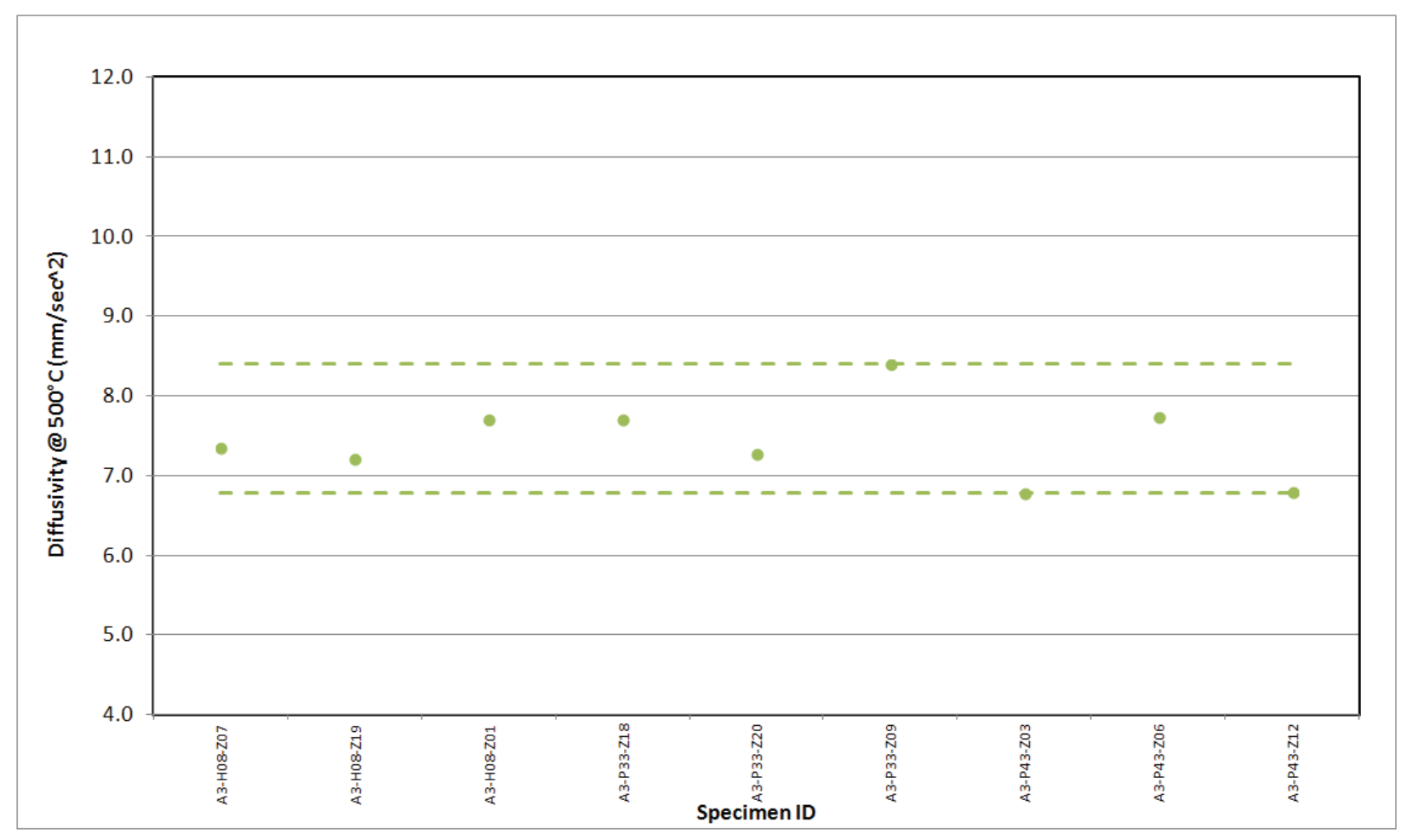

Figure A-249. New Matrix Piggyback Diffusivity @ 500․

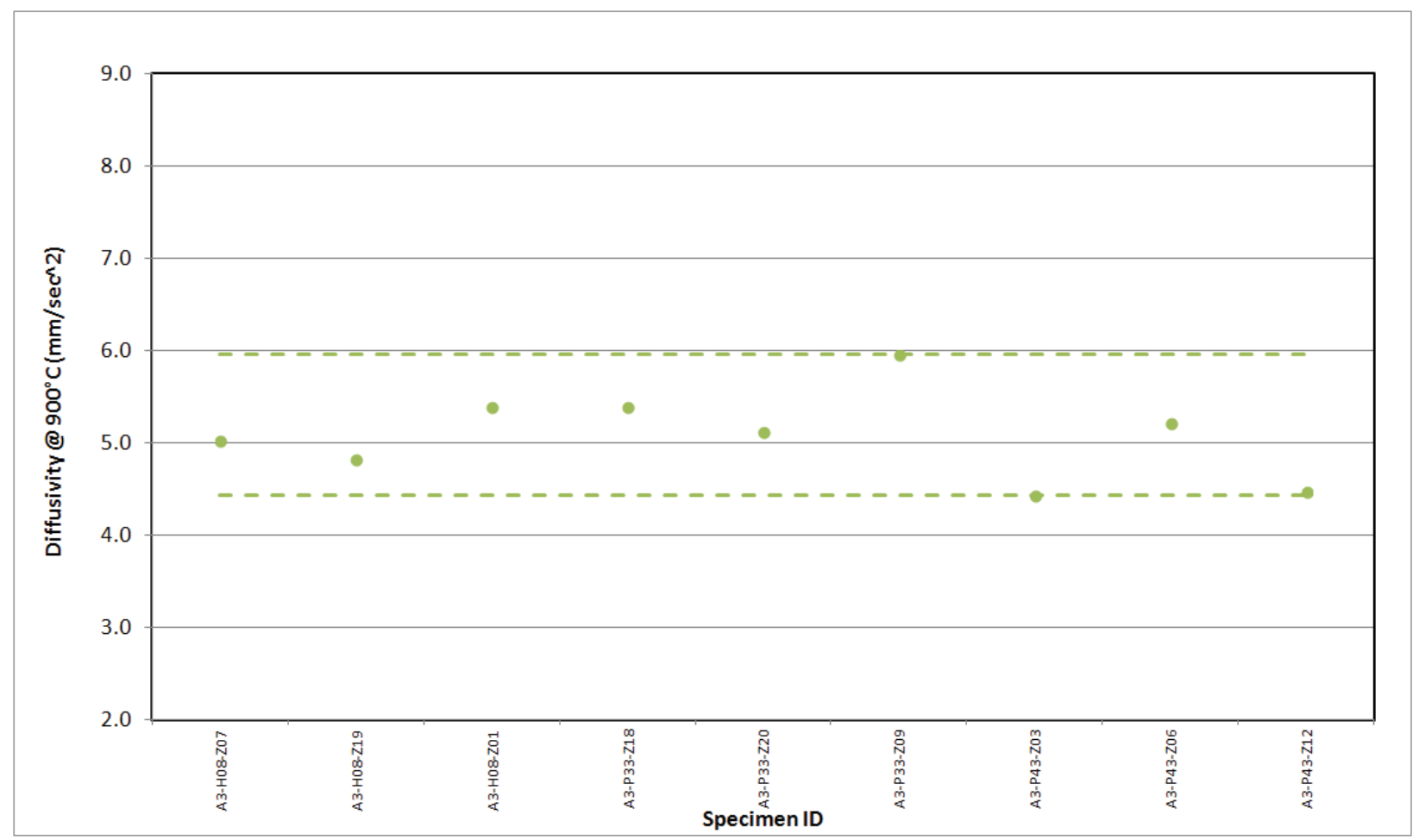

Figure A-250. New Matrix Piggyback Diffusivity @ 900C. 


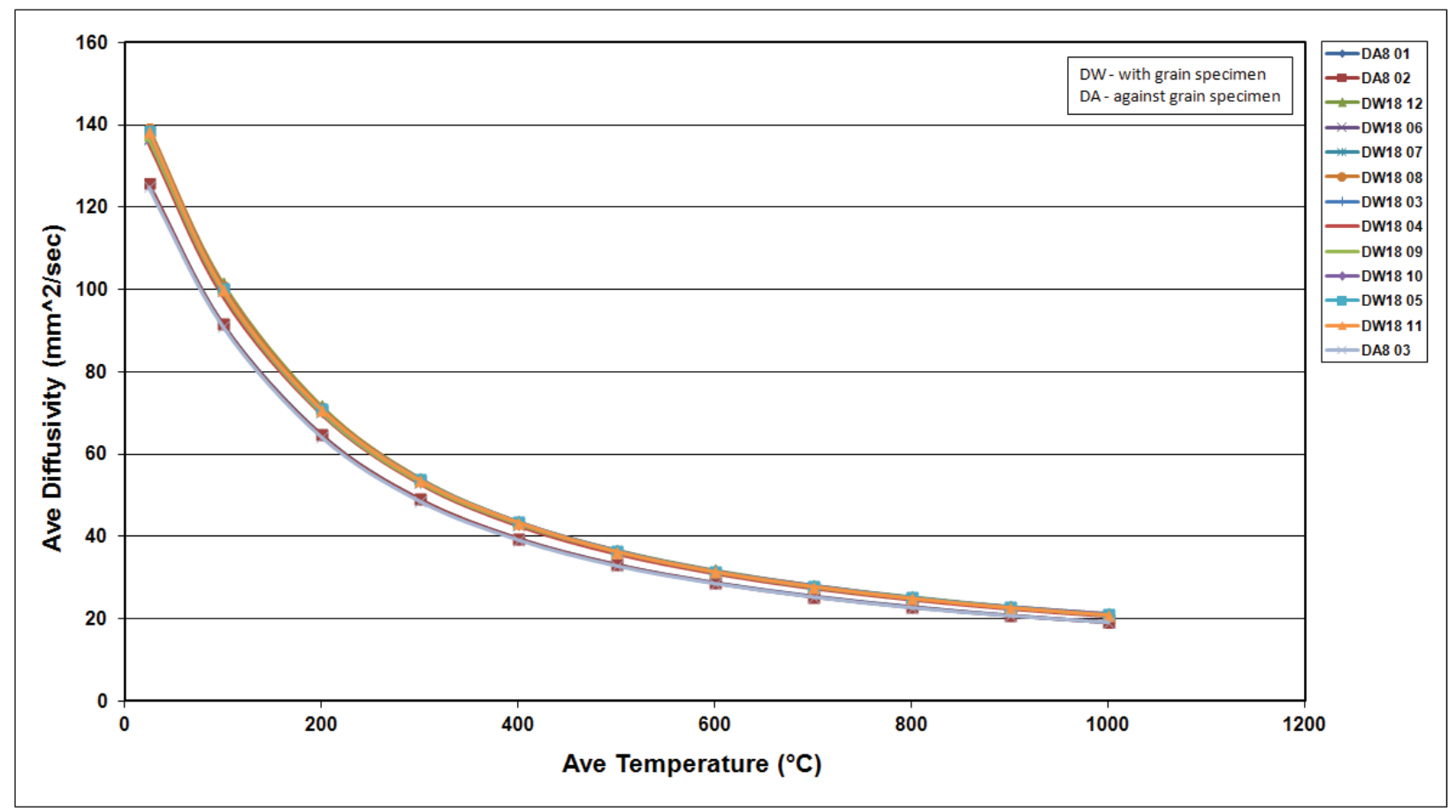

Figure A-251. PCEA Piggyback Diffusivity.

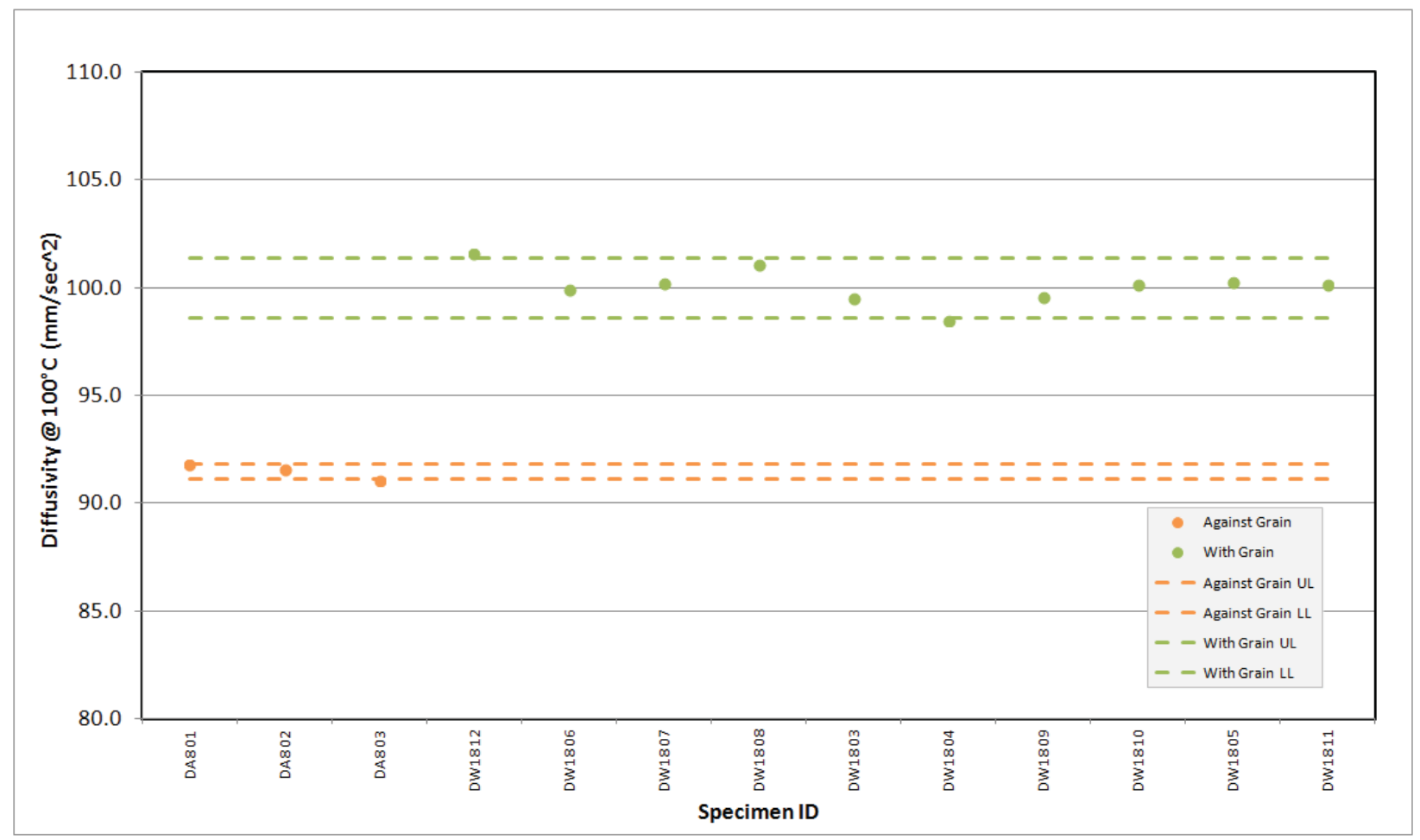

Figure A-252. PCEA Piggyback Diffusivity @ $100^{\circ} \mathrm{C}$. 


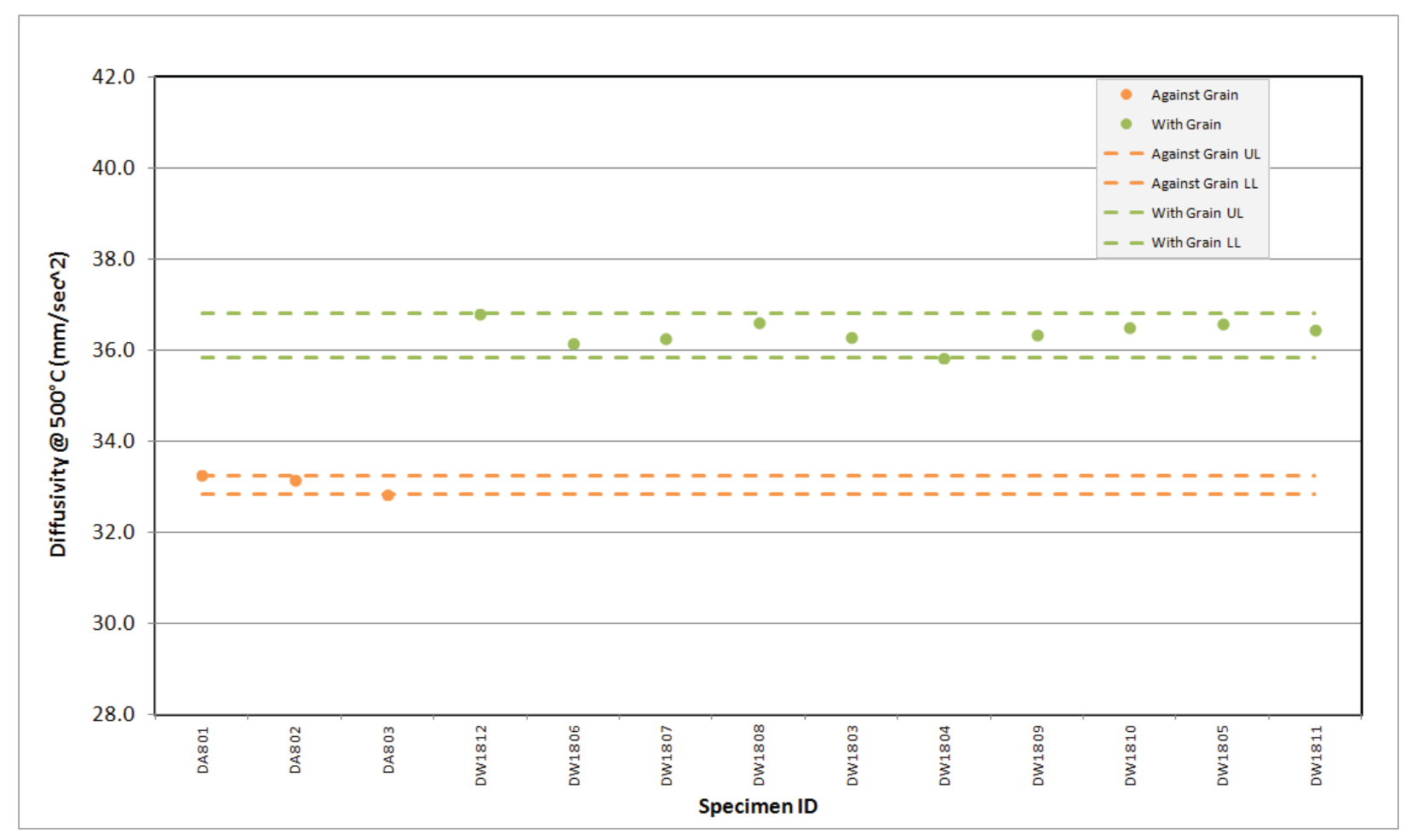

Figure A-253. PCEA Piggyback Diffusivity @ 500 ${ }^{\circ}$.

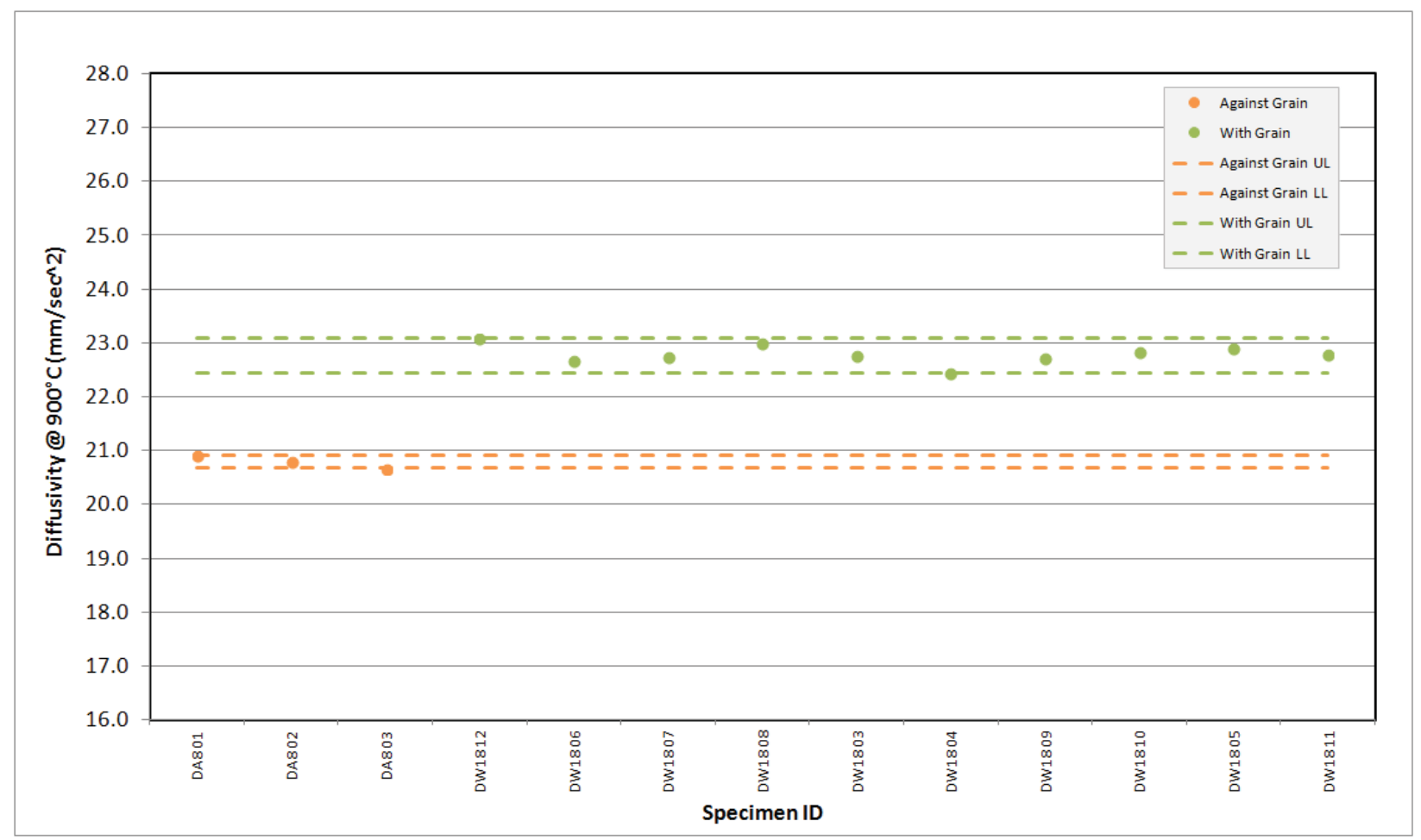

Figure A-254. PCEA Piggyback Diffusivity@900². 


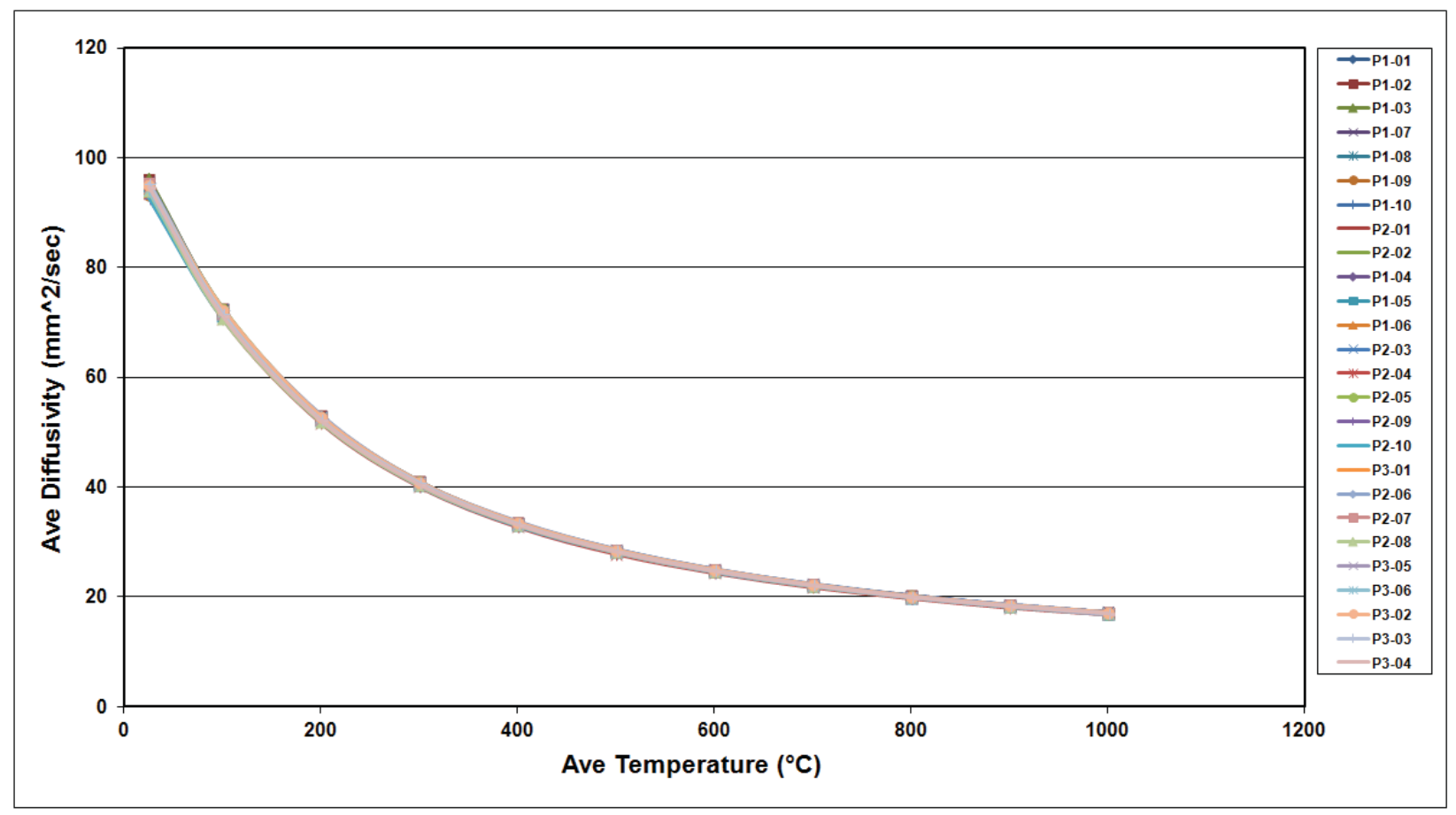

Figure A-255. PCIB Piggyback Diffusivity.

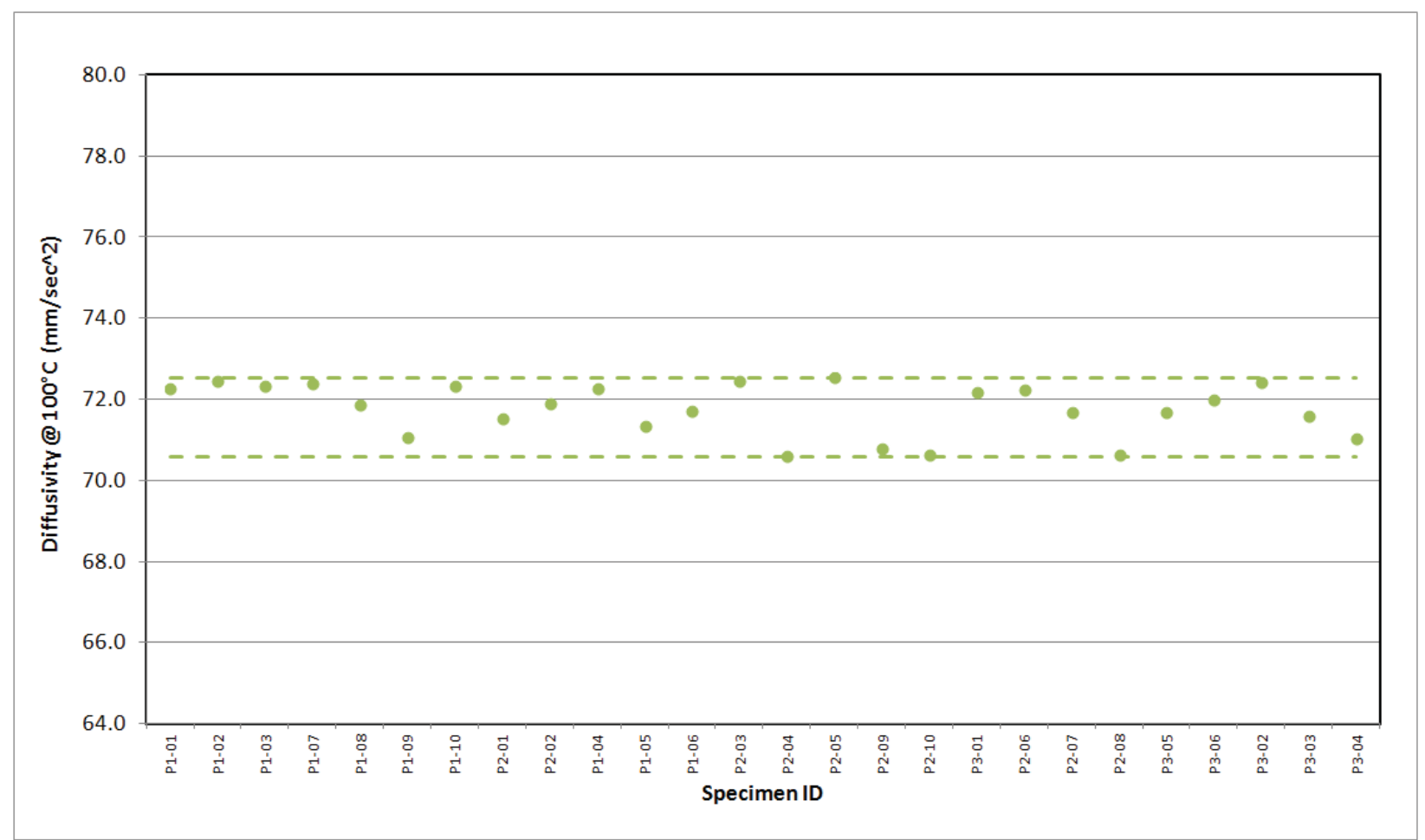

Figure A-256. PCIB Piggyback Diffusivity @ $100^{\circ} \mathrm{C}$. 


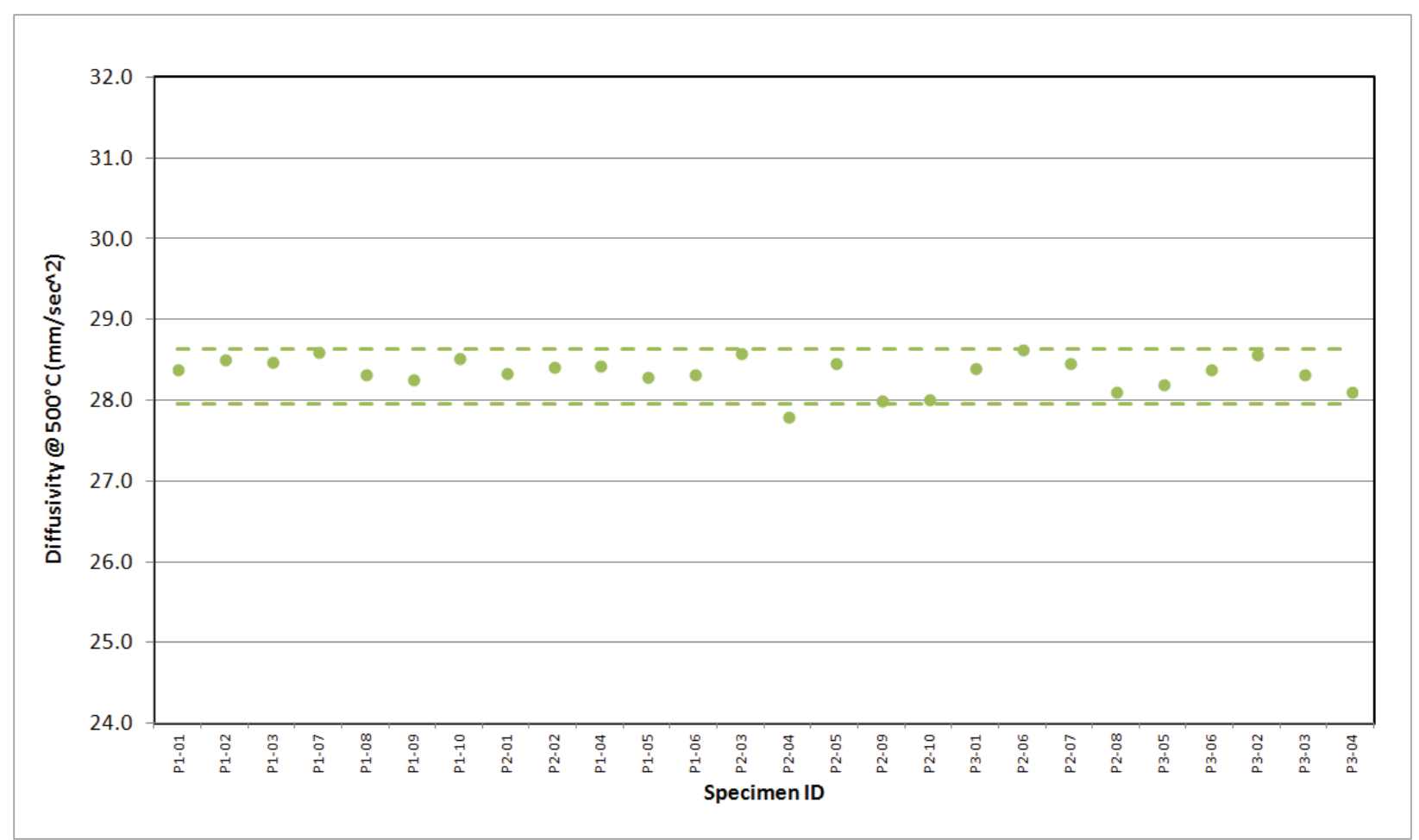

Figure A-257. PCIB Piggyback Diffusivity @ 500․

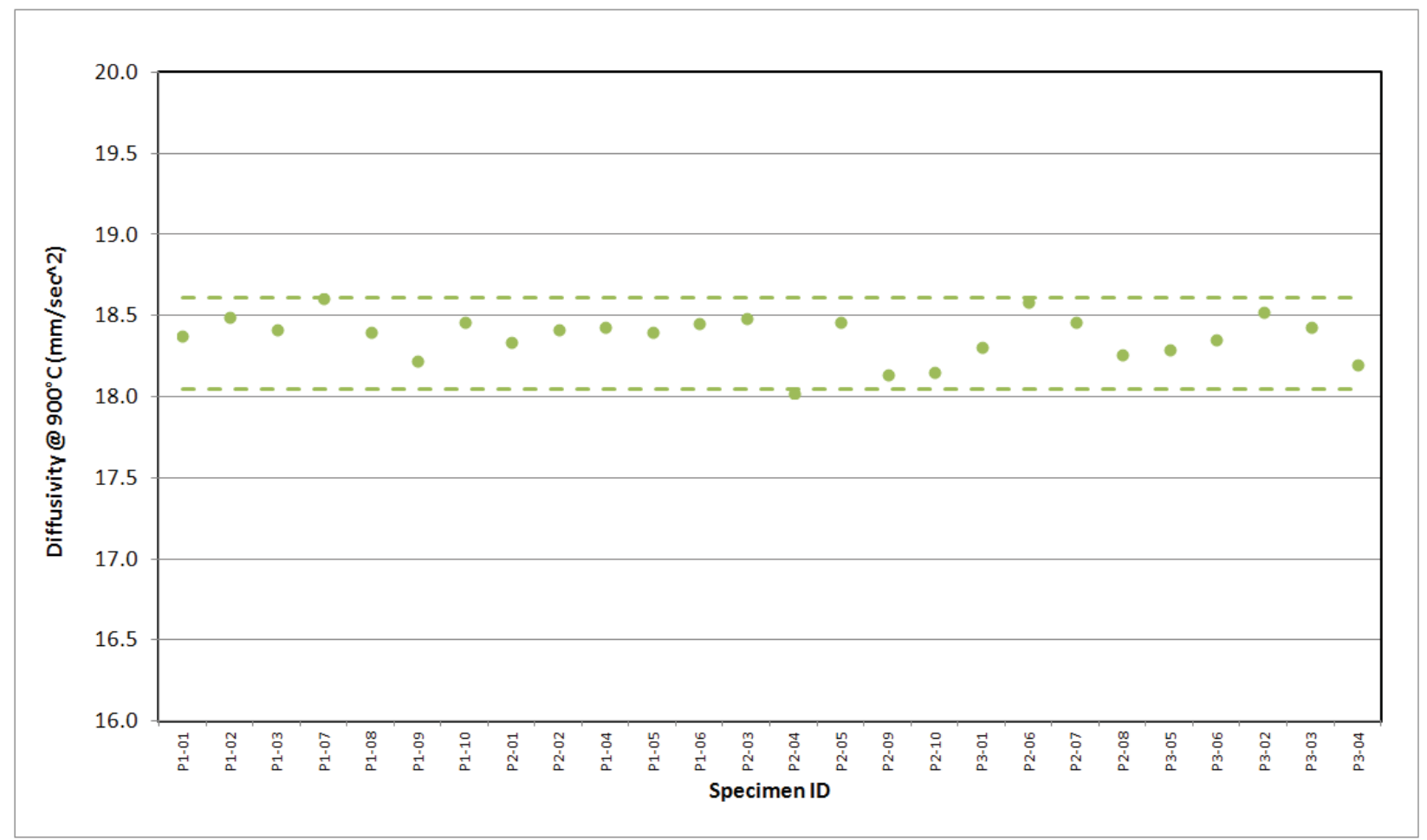

Figure A-258. PCIB Piggyback Diffusivity @ 900․ 


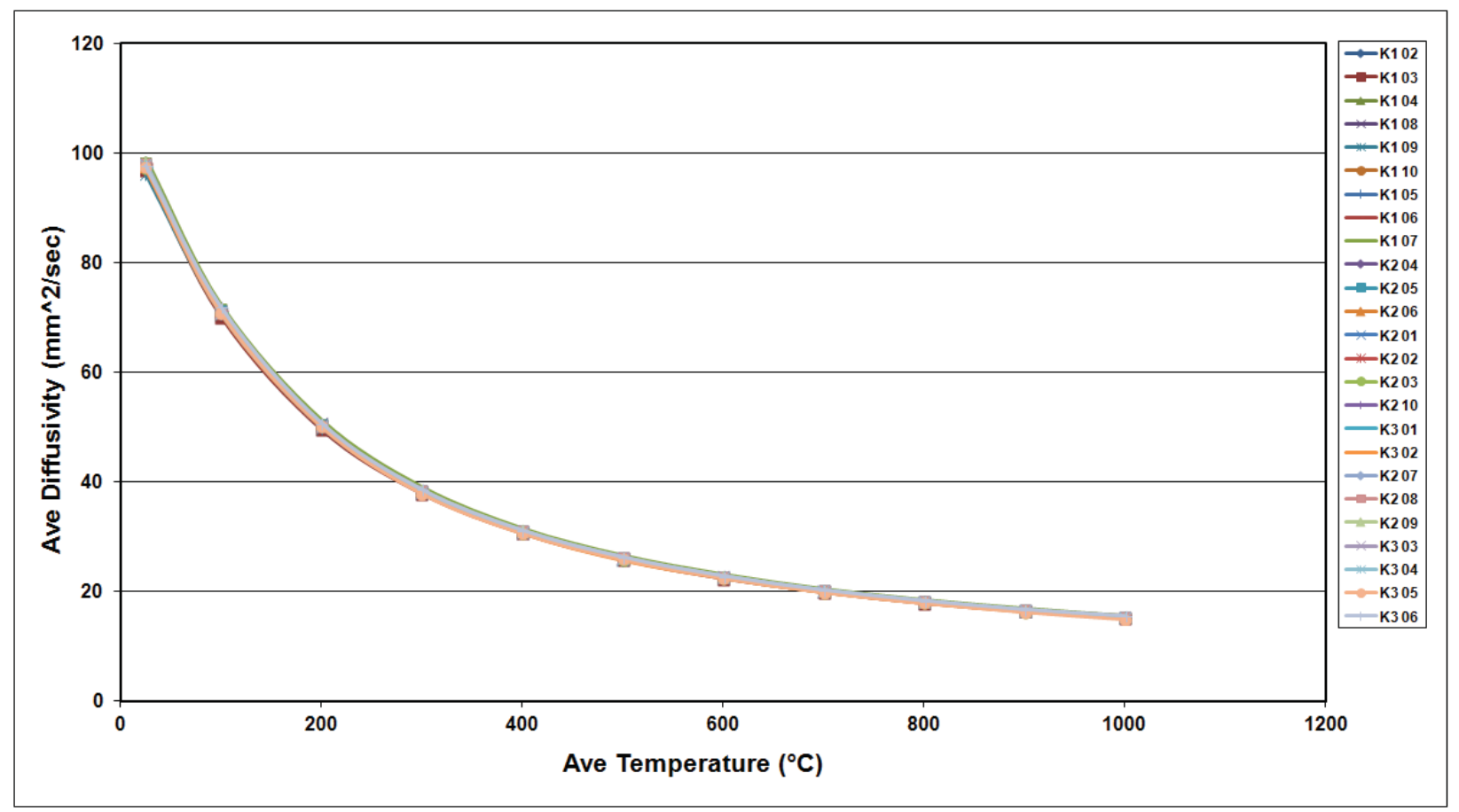

Figure A-259. PGX Piggyback Diffusivity.

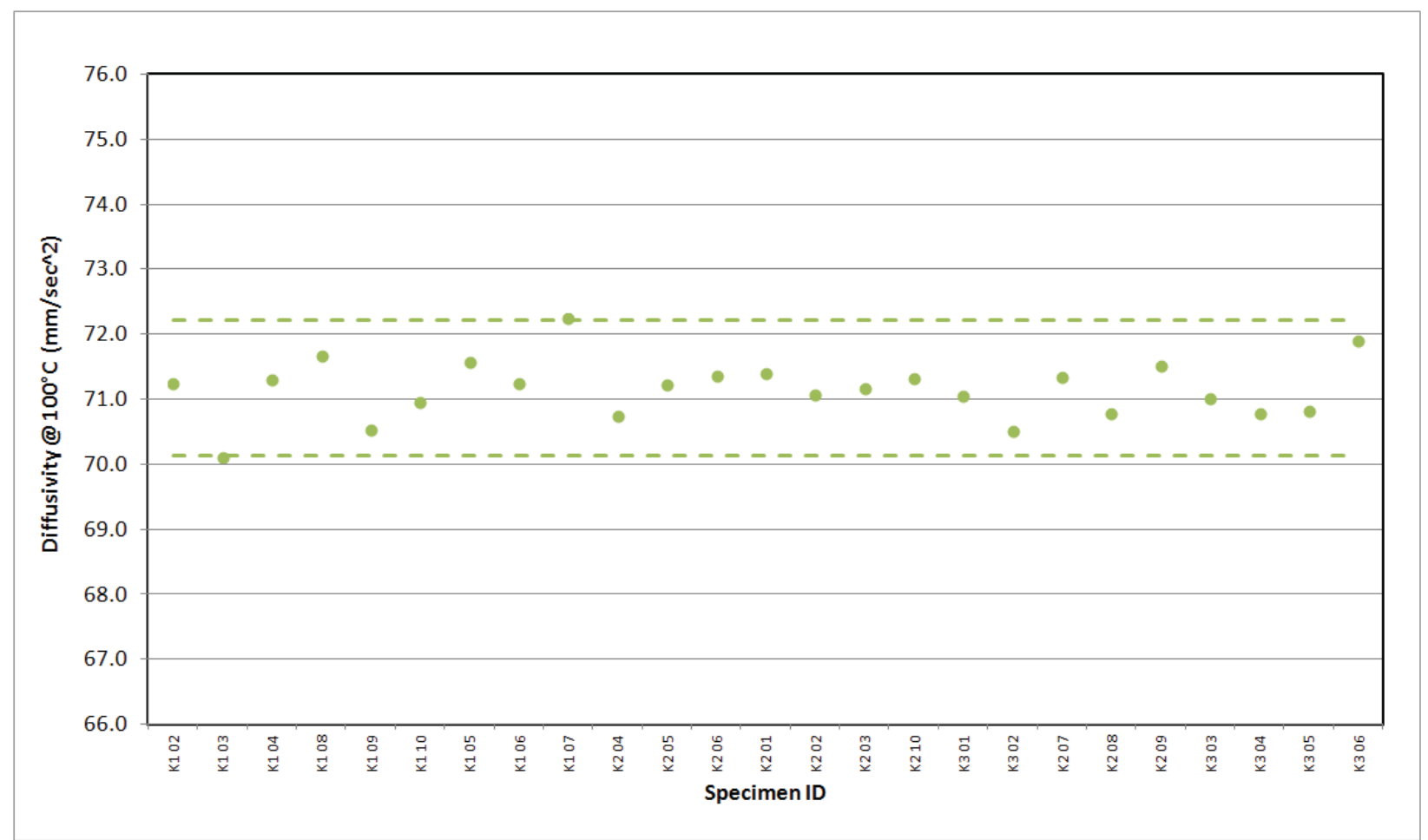

Figure A-260. PGX Piggyback Diffusivity @ $100^{\circ} \mathrm{C}$. 


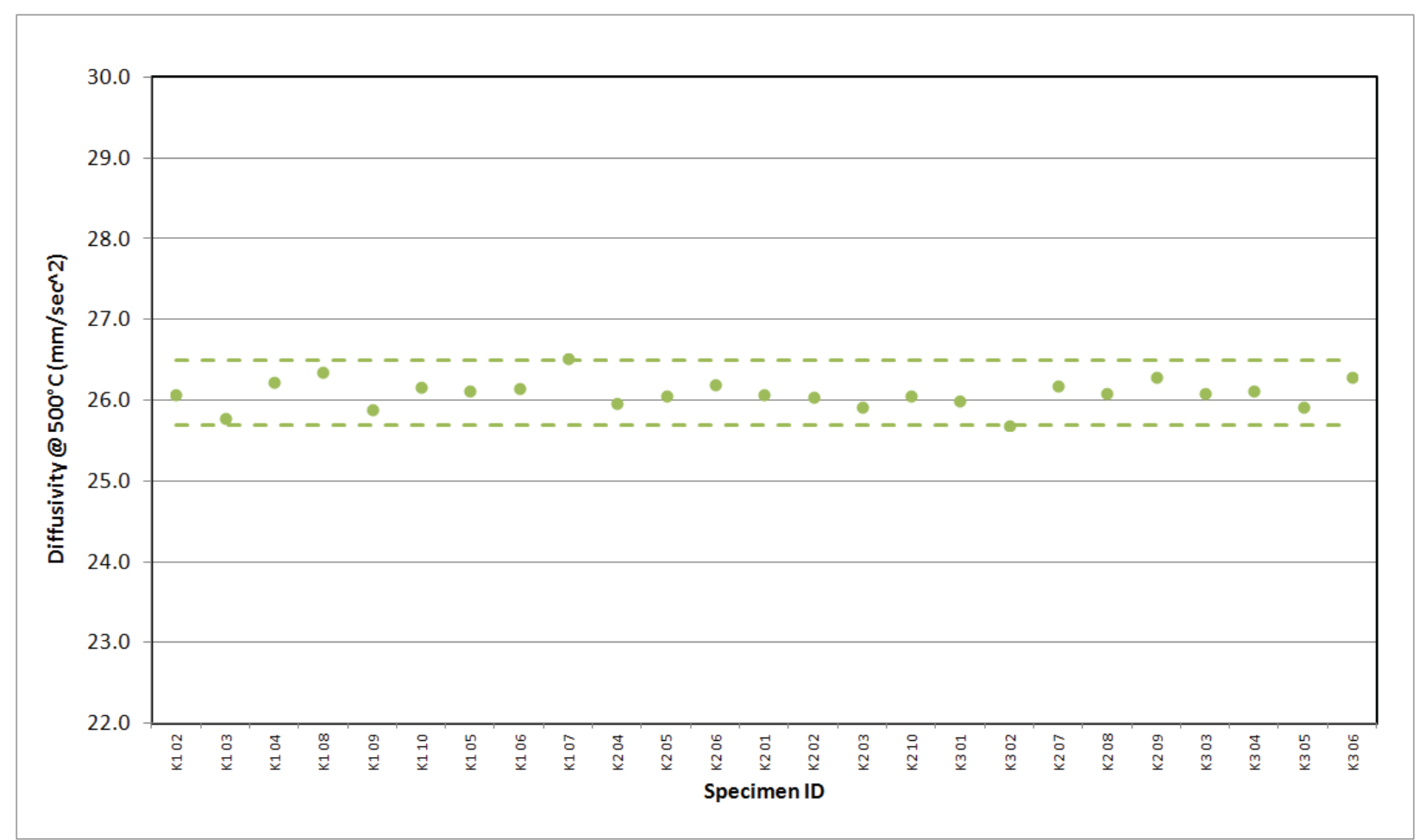

Figure A-261. PGX Piggyback Diffusivity @ 500․

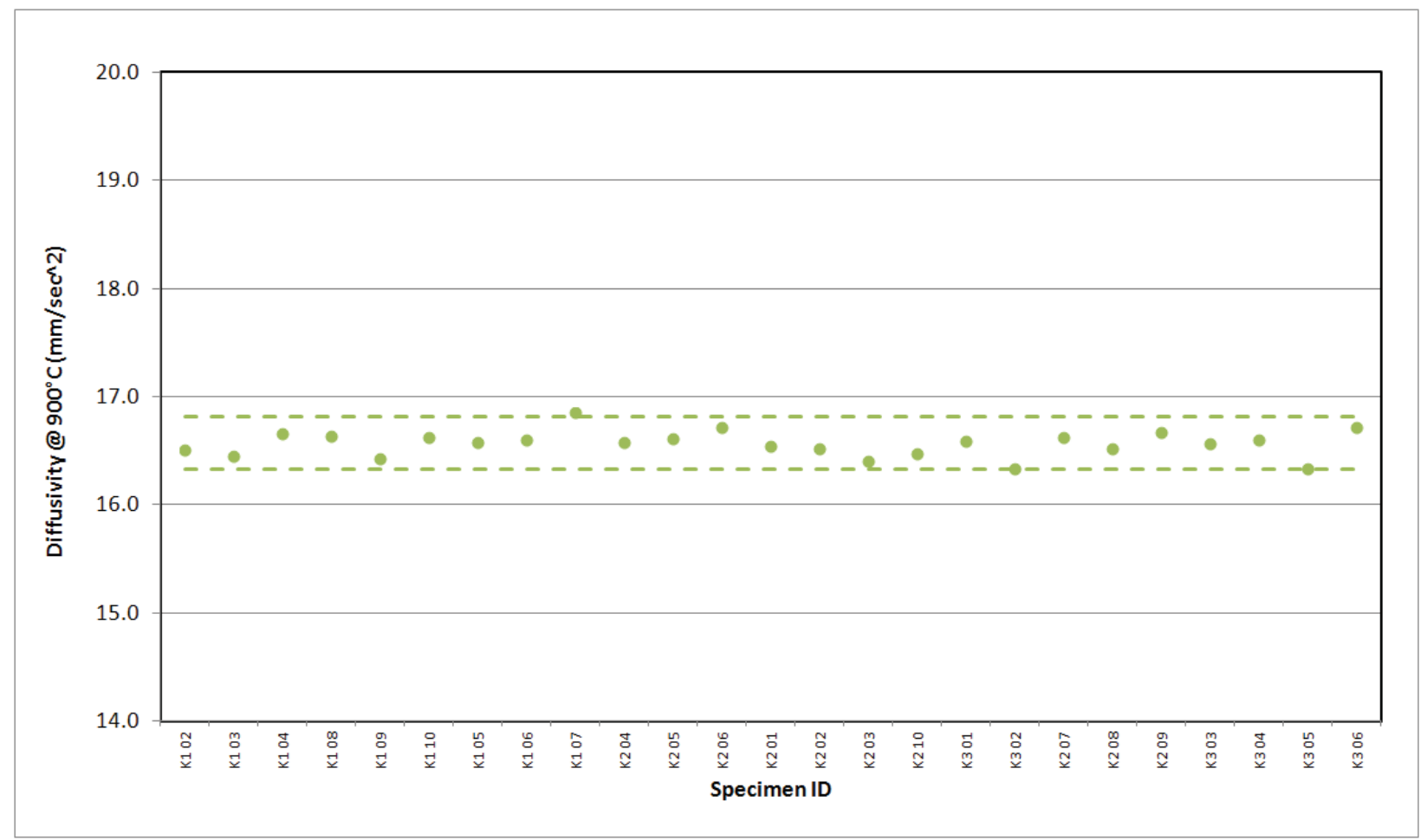

Figure A-262. PGX Piggyback Diffusivity@900 C. 


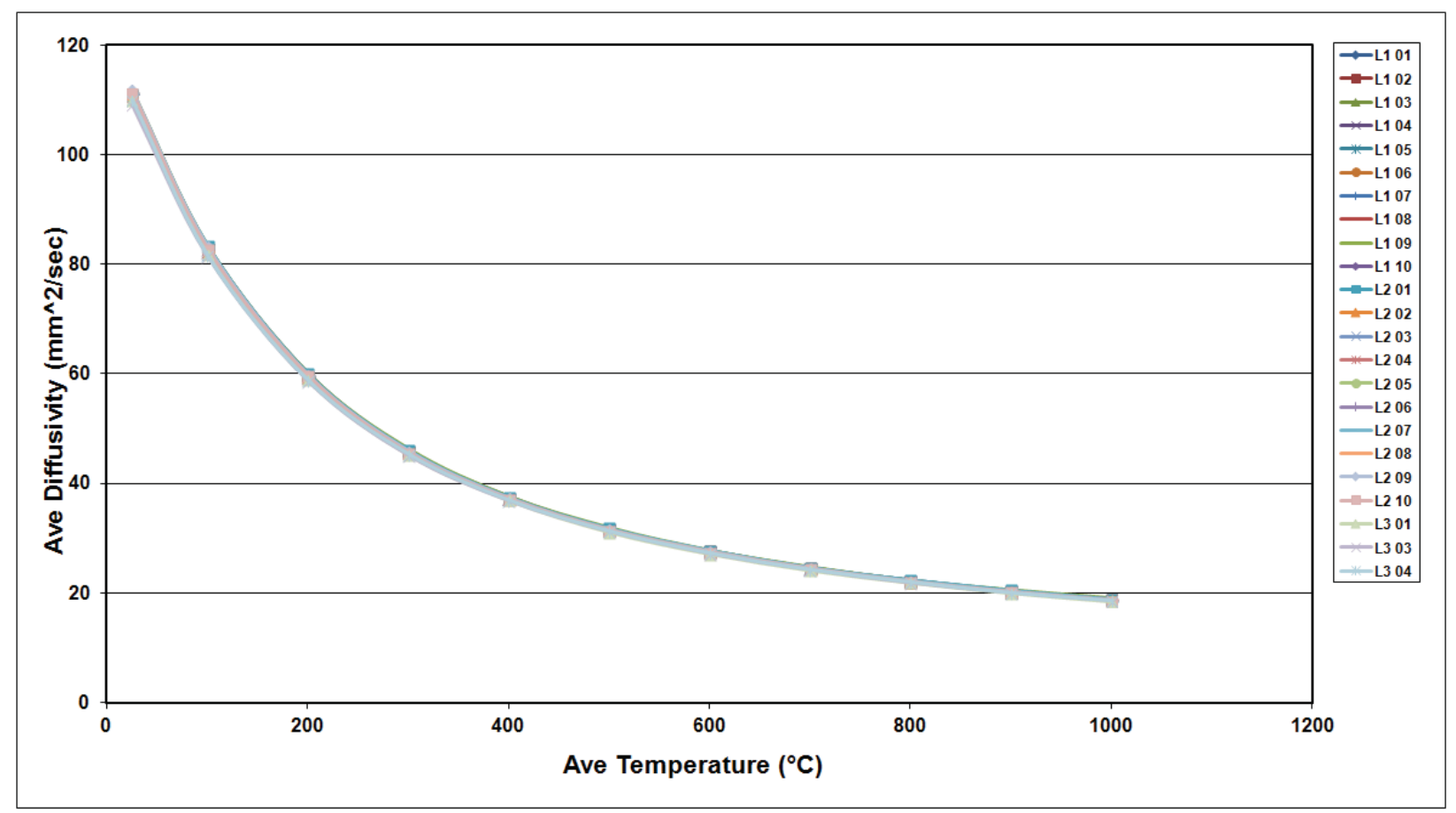

Figure A-263. PPEA Piggyback Diffusivity.

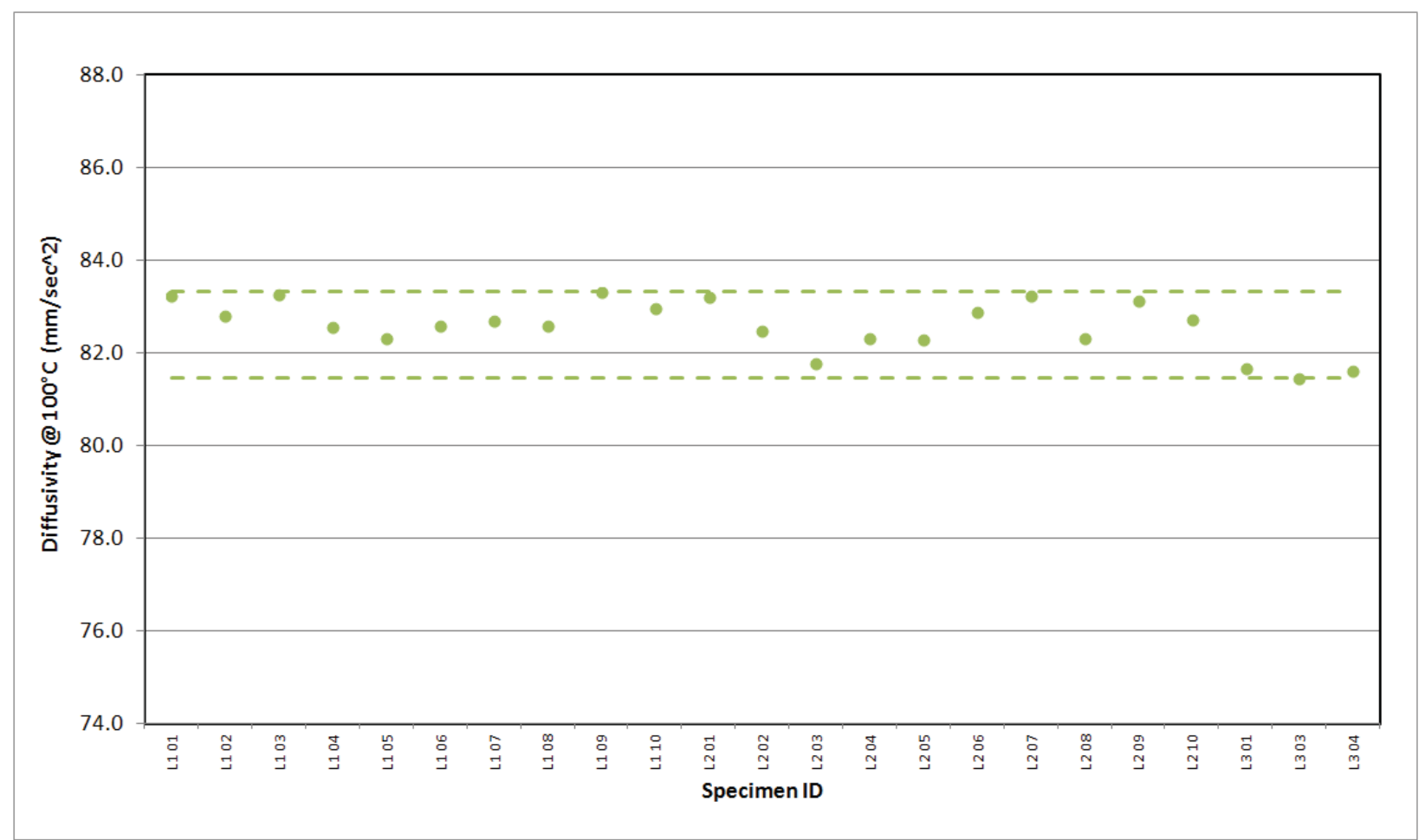

Figure A-264. PPEA Piggyback Diffusivity@100². 


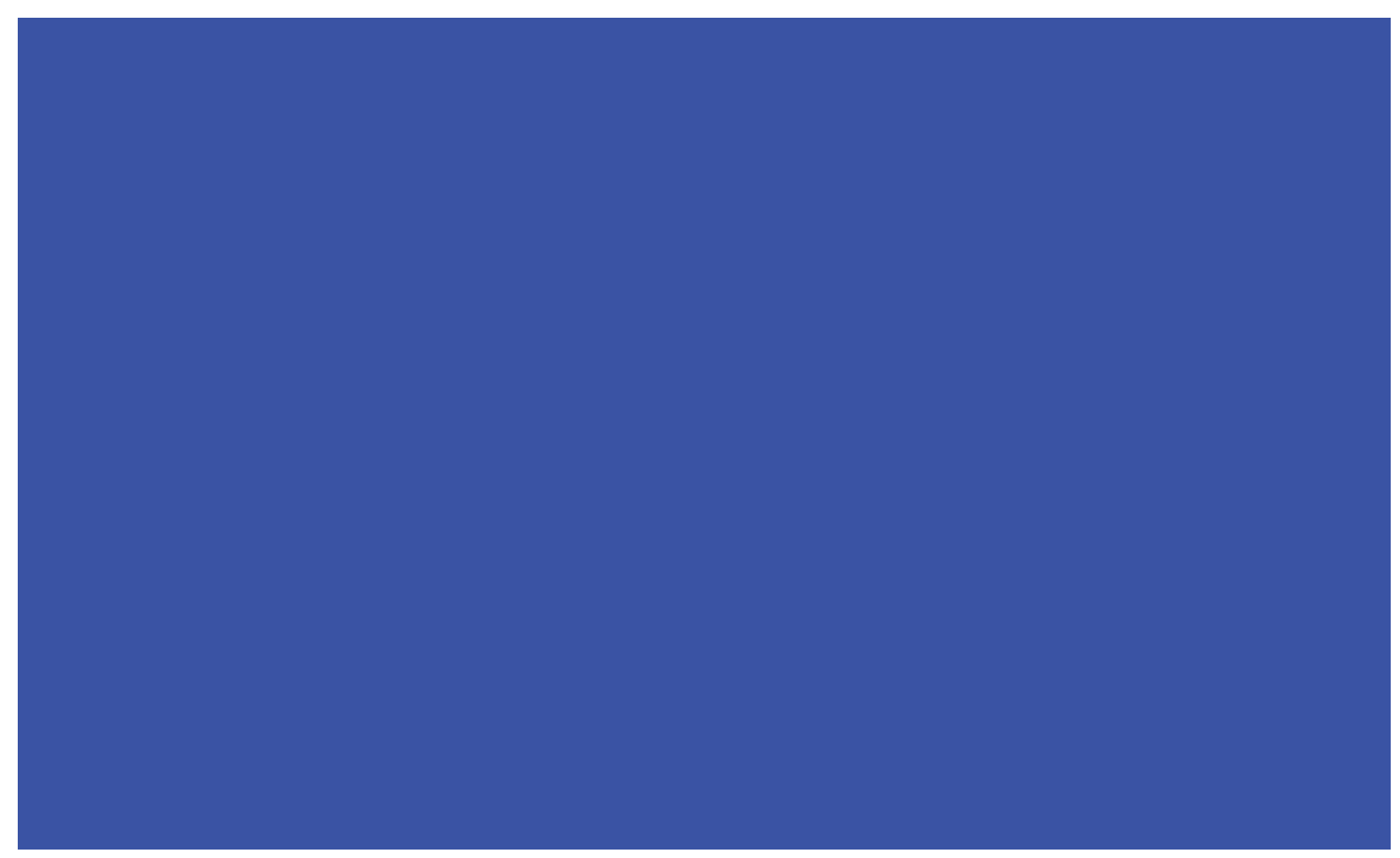

Figure A-265. PPEA Piggyback Diffusivity@500 C.

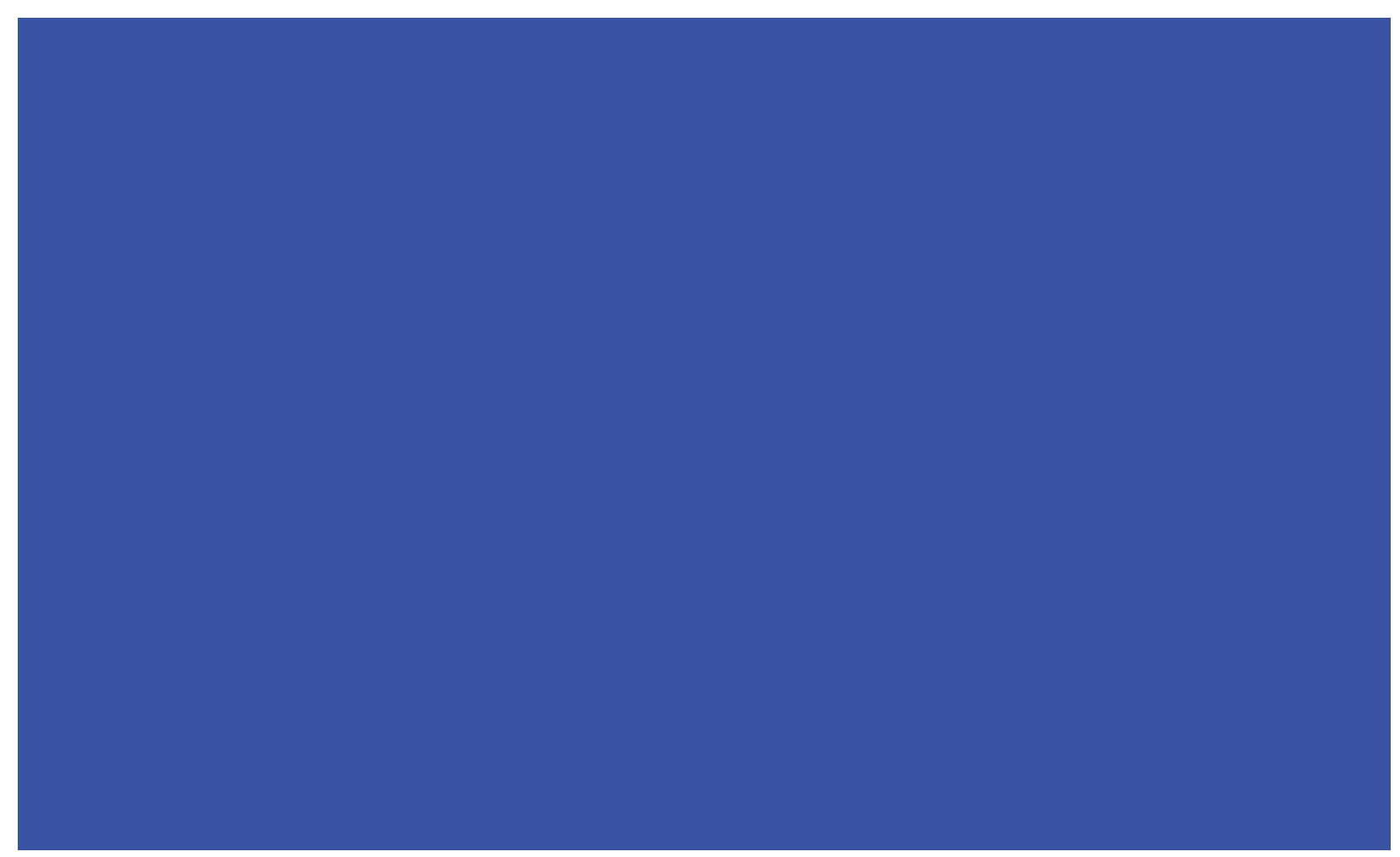

Figure A-266. PPEA Piggyback Diffusivity@900 . 
Appendix B

\section{Statistical Tables}




\section{Appendix B Statistical Tables}

Table B-1. Creep Pre Thermal Measurement Length (mm) Summary Statistics.

\begin{tabular}{|c|c|c|c|c|c|c|}
\hline $\begin{array}{l}\text { Combined } \\
\text { Specimens } \\
\end{array}$ & Mean & Std Dev & $\operatorname{CoV}(\%)$ & Median & Upper Limit & Lower Limit \\
\hline H451 & 25.387 & 0.0083 & 0.03 & 25.389 & 25.397 & 25.377 \\
\hline IG-110 & 25.379 & 0.0087 & 0.03 & 25.381 & 25.399 & 25.363 \\
\hline IG-430 & 25.382 & 0.0037 & 0.01 & 25.382 & 25.386 & 25.378 \\
\hline NBG-17 & 25.377 & 0.0052 & 0.02 & 25.378 & 25.390 & 25.364 \\
\hline NBG-18 & 25.365 & 0.0053 & 0.02 & 25.365 & 25.377 & 25.355 \\
\hline PCEA & 25.379 & 0.0124 & 0.05 & 25.373 & 25.405 & 25.343 \\
\hline $\begin{array}{c}\text { Against } \\
\text { Grain } \\
\text { Specimens } \\
\end{array}$ & Mean & Std Dev & $\operatorname{CoV}(\%)$ & Median & Upper Limit & Lower Limit \\
\hline H451 & 25.391 & 0.0032 & 0.01 & 25.391 & 25.396 & 25.386 \\
\hline IG-110 & 25.383 & 0.0064 & 0.03 & 25.385 & 25.388 & 25.375 \\
\hline IG-430 & 25.384 & 0.0010 & 0.00 & 25.384 & 25.386 & 25.383 \\
\hline NBG-17 & 25.377 & 0.0057 & 0.02 & 25.378 & 25.390 & 25.365 \\
\hline NBG-18 & 25.364 & 0.0066 & 0.03 & 25.363 & 25.374 & 25.355 \\
\hline PCEA & 25.392 & 0.0070 & 0.03 & 25.396 & 25.403 & 25.374 \\
\hline $\begin{array}{l}\text { With Grain } \\
\text { Specimens }\end{array}$ & Mean & Std Dev & CoV $(\%)$ & Median & Upper Limit & Lower Limit \\
\hline H451 & 25.382 & 0.0095 & 0.04 & 25.386 & 25.397 & 25.357 \\
\hline IG-110 & 25.376 & 0.0089 & 0.04 & 25.378 & 25.389 & 25.367 \\
\hline IG-430 & 25.381 & 0.0039 & 0.02 & 25.382 & 25.384 & 25.378 \\
\hline NBG-17 & 25.377 & 0.0045 & 0.02 & 25.377 & 25.387 & 25.365 \\
\hline NBG-18 & 25.367 & 0.0020 & 0.01 & 25.368 & 25.371 & 25.362 \\
\hline PCEA & 25.375 & 0.0105 & 0.04 & 25.371 & 25.381 & 25.362 \\
\hline
\end{tabular}


Table B-2. Creep Pre Thermal Measurement Diameter ( $\mathrm{mm}$ ) Summary Statistics.

\begin{tabular}{|c|c|c|c|c|c|c|}
\hline $\begin{array}{l}\text { Combined } \\
\text { Specimens }\end{array}$ & Mean & Std Dev & $\operatorname{CoV}(\%)$ & Median & Upper Limit & Lower Limit \\
\hline H451 & 12.740 & 0.0041 & 0.03 & 12.740 & 12.748 & 12.731 \\
\hline IG-110 & 12.734 & 0.0028 & 0.02 & 12.735 & 12.740 & 12.728 \\
\hline IG-430 & 12.731 & 0.0042 & 0.03 & 12.732 & 12.739 & 12.723 \\
\hline NBG-17 & 12.731 & 0.0043 & 0.03 & 12.730 & 12.740 & 12.721 \\
\hline NBG-18 & 12.730 & 0.0047 & 0.04 & 12.730 & 12.743 & 12.719 \\
\hline PCEA & 12.737 & 0.0078 & 0.06 & 12.737 & 12.759 & 12.719 \\
\hline $\begin{array}{c}\text { Against } \\
\text { Grain } \\
\text { Specimens } \\
\end{array}$ & Mean & Std Dev & $\operatorname{CoV}(\%)$ & Median & Upper Limit & Lower Limit \\
\hline H451 & 12.739 & 0.0044 & 0.03 & 12.738 & 12.748 & 12.733 \\
\hline IG-110 & 12.735 & 0.0030 & 0.02 & 12.735 & 12.740 & 12.730 \\
\hline IG-430 & 12.732 & 0.0022 & 0.02 & 12.732 & 12.735 & 12.729 \\
\hline NBG-17 & 12.732 & 0.0043 & 0.03 & 12.732 & 12.741 & 12.723 \\
\hline NBG-18 & 12.731 & 0.0058 & 0.05 & 12.731 & 12.744 & 12.719 \\
\hline PCEA & 12.746 & 0.0031 & 0.02 & 12.746 & 12.751 & 12.739 \\
\hline $\begin{array}{l}\text { With Grain } \\
\text { Specimens }\end{array}$ & Mean & Std Dev & $\operatorname{CoV}(\%)$ & Median & Upper Limit & Lower Limit \\
\hline H451 & 12.740 & 0.0037 & 0.03 & 12.741 & 12.747 & 12.731 \\
\hline IG-110 & 12.734 & 0.0027 & 0.02 & 12.734 & 12.739 & 12.728 \\
\hline IG-430 & 12.731 & 0.0045 & 0.04 & 12.732 & 12.739 & 12.722 \\
\hline NBG-17 & 12.729 & 0.0037 & 0.03 & 12.729 & 12.736 & 12.721 \\
\hline NBG-18 & 12.729 & 0.0028 & 0.02 & 12.729 & 12.736 & 12.725 \\
\hline PCEA & 12.734 & 0.0065 & 0.05 & 12.734 & 12.750 & 12.719 \\
\hline
\end{tabular}


Table B-3.Creep Pre Thermal Measurement Mass (g) Summary Statistics.

\begin{tabular}{|c|c|c|c|c|c|c|}
\hline $\begin{array}{l}\text { Combined } \\
\text { Specimens }\end{array}$ & Mean & Std Dev & $\operatorname{CoV}(\%)$ & Median & Upper Limit & Lower Limit \\
\hline H451 & 5.485 & 0.0360 & 0.66 & 5.473 & 5.561 & 5.424 \\
\hline IG-110 & 5.654 & 0.0225 & 0.40 & 5.661 & 5.701 & 5.597 \\
\hline IG-430 & 5.764 & 0.0205 & 0.36 & 5.767 & 5.809 & 5.711 \\
\hline NBG-17 & 5.867 & 0.0694 & 1.18 & 5.909 & 5.935 & 5.740 \\
\hline NBG-18 & 5.948 & 0.0075 & 0.13 & 5.948 & 5.965 & 5.929 \\
\hline PCEA & 5.657 & 0.0234 & 0.41 & 5.653 & 5.691 & 5.617 \\
\hline $\begin{array}{c}\text { Against } \\
\text { Grain } \\
\text { Specimens } \\
\end{array}$ & Mean & Std Dev & $\operatorname{CoV}(\%)$ & Median & Upper Limit & Lower Limit \\
\hline H451 & 5.465 & 0.0117 & 0.21 & 5.466 & 5.491 & 5.444 \\
\hline IG-110 & 5.656 & 0.0234 & 0.41 & 5.662 & 5.701 & 5.602 \\
\hline IG-430 & 5.770 & 0.0101 & 0.17 & 5.773 & 5.785 & 5.750 \\
\hline NBG-17 & 5.857 & 0.0771 & 1.32 & 5.904 & 5.934 & 5.740 \\
\hline NBG-18 & 5.948 & 0.0088 & 0.15 & 5.949 & 5.965 & 5.929 \\
\hline PCEA & 5.683 & 0.0282 & 0.50 & 5.671 & 5.727 & 5.652 \\
\hline $\begin{array}{c}\text { With Grain } \\
\text { Specimens }\end{array}$ & Mean & Std Dev & $\operatorname{CoV}(\%)$ & Median & Upper Limit & Lower Limit \\
\hline H451 & 5.505 & 0.0408 & 0.74 & 5.505 & 5.561 & 5.424 \\
\hline IG-110 & 5.652 & 0.0220 & 0.39 & 5.659 & 5.686 & 5.604 \\
\hline IG-430 & 5.762 & 0.0222 & 0.39 & 5.765 & 5.809 & 5.709 \\
\hline NBG-17 & 5.879 & 0.0575 & 0.98 & 5.911 & 5.935 & 5.788 \\
\hline NBG-18 & 5.949 & 0.0056 & 0.09 & 5.948 & 5.961 & 5.939 \\
\hline PCEA & 5.649 & 0.0147 & 0.26 & 5.649 & 5.678 & 5.621 \\
\hline
\end{tabular}


Table B-4. Creep Pre Thermal Measurement Density $\left(\mathrm{g} / \mathrm{cm}^{3}\right)$ Summary Statistics.

\begin{tabular}{|c|c|c|c|c|c|c|}
\hline $\begin{array}{l}\text { Combined } \\
\text { Specimens }\end{array}$ & Mean & Std Dev & $\operatorname{CoV}(\%)$ & Median & Upper Limit & Lower Limit \\
\hline H451 & 1.7217 & 0.0110 & 0.64 & 1.7180 & 1.7457 & 1.7041 \\
\hline IG-110 & 1.7779 & 0.0069 & 0.39 & 1.7802 & 1.7918 & 1.7591 \\
\hline IG-430 & 1.8132 & 0.0062 & 0.34 & 1.8141 & 1.8264 & 1.7974 \\
\hline NBG-17 & 1.8468 & 0.0216 & 1.17 & 1.8601 & 1.8675 & 1.8053 \\
\hline NBG-18 & 1.8720 & 0.0020 & 0.11 & 1.8720 & 1.8761 & 1.8673 \\
\hline PCEA & 1.7769 & 0.0060 & 0.34 & 1.7758 & 1.7869 & 1.7665 \\
\hline $\begin{array}{c}\text { Against } \\
\text { Grain } \\
\text { Specimens }\end{array}$ & Mean & Std Dev & $\operatorname{CoV}(\%)$ & Median & Upper Limit & Lower Limit \\
\hline H451 & 1.7153 & 0.0038 & 0.22 & 1.7156 & 1.7248 & 1.7082 \\
\hline IG-110 & 1.7784 & 0.0072 & 0.41 & 1.7804 & 1.7918 & 1.7633 \\
\hline IG-430 & 1.8148 & 0.0031 & 0.17 & 1.8157 & 1.8197 & 1.8087 \\
\hline NBG-17 & 1.8433 & 0.0237 & 1.28 & 1.8579 & 1.8661 & 1.8053 \\
\hline NBG-18 & 1.8722 & 0.0024 & 0.13 & 1.8725 & 1.8761 & 1.8656 \\
\hline PCEA & 1.7812 & 0.0091 & 0.51 & 1.7773 & 1.7954 & 1.7720 \\
\hline $\begin{array}{l}\text { With Grain } \\
\text { Specimens }\end{array}$ & Mean & Std Dev & $\operatorname{CoV}(\%)$ & Median & Upper Limit & Lower Limit \\
\hline H451 & 1.7283 & 0.0121 & 0.70 & 1.7292 & 1.7457 & 1.7041 \\
\hline IG-110 & 1.7775 & 0.0068 & 0.38 & 1.7801 & 1.7875 & 1.7629 \\
\hline IG-430 & 1.8127 & 0.0067 & 0.37 & 1.8133 & 1.8264 & 1.7974 \\
\hline NBG-17 & 1.8509 & 0.0181 & 0.98 & 1.8610 & 1.8675 & 1.8220 \\
\hline NBG-18 & 1.8719 & 0.0016 & 0.09 & 1.8718 & 1.8759 & 1.8686 \\
\hline PCEA & 1.7756 & 0.0040 & 0.22 & 1.7755 & 1.7848 & 1.7669 \\
\hline
\end{tabular}


Table B-5. Creep Coefficient of Thermal Expansion $(1 / \mathrm{K})$ at $100^{\circ} \mathrm{C}$ Summary Statistics.

\begin{tabular}{|c|c|c|c|c|c|c|}
\hline $\begin{array}{l}\text { Combined } \\
\text { Specimens }\end{array}$ & Mean & Std Dev & CoV (\%) & Median & Upper Limit & Lower Limit \\
\hline H451 & $3.18 \mathrm{E}-06$ & $2.40 \mathrm{E}-07$ & 7.54 & $3.12 \mathrm{E}-06$ & $3.44 \mathrm{E}-06$ & $2.90 \mathrm{E}-06$ \\
\hline IG-110 & $3.87 \mathrm{E}-06$ & 9.32E-08 & 2.41 & $3.84 \mathrm{E}-06$ & 4.09E-06 & $3.70 \mathrm{E}-06$ \\
\hline IG-430 & $4.16 \mathrm{E}-06$ & $2.60 \mathrm{E}-07$ & 6.26 & 4.24E-06 & $4.48 \mathrm{E}-06$ & $3.74 \mathrm{E}-06$ \\
\hline NBG-17 & $4.50 \mathrm{E}-06$ & $1.36 \mathrm{E}-07$ & 3.02 & $4.45 \mathrm{E}-06$ & 4.86E-06 & 4.32E-06 \\
\hline NBG-18 & $4.85 \mathrm{E}-06$ & $1.33 \mathrm{E}-07$ & 2.75 & $4.85 \mathrm{E}-06$ & $5.12 \mathrm{E}-06$ & $4.55 \mathrm{E}-06$ \\
\hline PCEA & $3.92 \mathrm{E}-06$ & $1.53 \mathrm{E}-07$ & 3.89 & $3.89 \mathrm{E}-06$ & 4.34E-06 & $3.69 \mathrm{E}-06$ \\
\hline $\begin{array}{c}\text { Against } \\
\text { Grain } \\
\text { Specimens } \\
\end{array}$ & Mean & Std Dev & $\operatorname{CoV}(\%)$ & Median & Upper Limit & Lower Limit \\
\hline H451 & $3.71 \mathrm{E}-06$ & $1.26 \mathrm{E}-07$ & 3.41 & $3.71 \mathrm{E}-06$ & $3.86 \mathrm{E}-06$ & $3.57 \mathrm{E}-06$ \\
\hline IG-110 & $3.82 \mathrm{E}-06$ & $6.01 \mathrm{E}-08$ & 1.57 & $3.81 \mathrm{E}-06$ & $3.90 \mathrm{E}-06$ & $3.76 \mathrm{E}-06$ \\
\hline IG-430 & $3.63 \mathrm{E}-06$ & $1.45 \mathrm{E}-07$ & 4.00 & 3.63E-06 & $3.89 \mathrm{E}-06$ & $3.49 \mathrm{E}-06$ \\
\hline NBG-17 & $4.70 \mathrm{E}-06$ & $1.01 \mathrm{E}-07$ & 2.15 & 4.67E-06 & $4.86 \mathrm{E}-06$ & $4.56 \mathrm{E}-06$ \\
\hline NBG-18 & $4.76 \mathrm{E}-06$ & $1.49 \mathrm{E}-07$ & 3.12 & 4.74E-06 & $5.02 \mathrm{E}-06$ & $4.55 \mathrm{E}-06$ \\
\hline PCEA & $4.05 \mathrm{E}-06$ & $2.09 \mathrm{E}-07$ & 5.17 & 4.09E-06 & 4.35E-06 & $3.69 \mathrm{E}-06$ \\
\hline $\begin{array}{l}\text { With Grain } \\
\text { Specimens }\end{array}$ & Mean & Std Dev & $\operatorname{CoV}(\%)$ & Median & Upper Limit & Lower Limit \\
\hline H451 & $3.09 \mathrm{E}-06$ & $9.90 \mathrm{E}-08$ & 3.20 & $3.12 \mathrm{E}-06$ & $3.22 \mathrm{E}-06$ & $2.90 \mathrm{E}-06$ \\
\hline IG-110 & $3.88 \mathrm{E}-06$ & $9.49 \mathrm{E}-08$ & 2.45 & $3.86 \mathrm{E}-06$ & 4.09E-06 & $3.70 \mathrm{E}-06$ \\
\hline IG-430 & $4.25 \mathrm{E}-06$ & $1.23 \mathrm{E}-07$ & 2.90 & $4.28 \mathrm{E}-06$ & $4.48 \mathrm{E}-06$ & $3.97 \mathrm{E}-06$ \\
\hline NBG-17 & $4.45 \mathrm{E}-06$ & $9.95 \mathrm{E}-08$ & 2.23 & 4.42E-06 & 4.71E-06 & 4.32E-06 \\
\hline NBG-18 & $4.88 \mathrm{E}-06$ & $1.14 \mathrm{E}-07$ & 2.35 & $4.88 \mathrm{E}-06$ & $5.12 \mathrm{E}-06$ & $4.70 \mathrm{E}-06$ \\
\hline PCEA & $3.87 \mathrm{E}-06$ & $9.52 \mathrm{E}-08$ & 2.46 & $3.87 \mathrm{E}-06$ & 4.11E-06 & $3.74 \mathrm{E}-06$ \\
\hline
\end{tabular}


Table B-6. Creep Coefficient of Thermal Expansion $(1 / \mathrm{K})$ at $500^{\circ} \mathrm{C}$ Summary Statistics.

\begin{tabular}{|c|c|c|c|c|c|c|}
\hline $\begin{array}{l}\text { Combined } \\
\text { Specimens }\end{array}$ & Mean & Std Dev & CoV (\%) & Median & Upper Limit & Lower Limit \\
\hline H451 & $3.72 \mathrm{E}-06$ & $2.30 \mathrm{E}-07$ & 6.17 & $3.66 \mathrm{E}-06$ & $3.86 \mathrm{E}-06$ & $3.46 \mathrm{E}-06$ \\
\hline IG-110 & $4.45 \mathrm{E}-06$ & 8.61E-08 & 1.94 & 4.44E-06 & 4.64E-06 & $4.28 \mathrm{E}-06$ \\
\hline IG-430 & 4.73E-06 & $2.25 \mathrm{E}-07$ & 4.76 & $4.80 \mathrm{E}-06$ & 5.01E-06 & $4.39 \mathrm{E}-06$ \\
\hline NBG-17 & $5.06 \mathrm{E}-06$ & $1.66 \mathrm{E}-07$ & 3.27 & 5.09E-06 & $5.46 \mathrm{E}-06$ & 4.78E-06 \\
\hline NBG-18 & $5.43 \mathrm{E}-06$ & 8.72E-08 & 1.61 & $5.45 \mathrm{E}-06$ & $5.58 \mathrm{E}-06$ & $5.25 \mathrm{E}-06$ \\
\hline PCEA & 4.54E-06 & $1.39 \mathrm{E}-07$ & 3.06 & $4.51 \mathrm{E}-06$ & 4.77E-06 & $4.37 \mathrm{E}-06$ \\
\hline $\begin{array}{c}\text { Against } \\
\text { Grain } \\
\text { Specimens } \\
\end{array}$ & Mean & Std Dev & $\operatorname{CoV}(\%)$ & Median & Upper Limit & Lower Limit \\
\hline H451 & 4.25E-06 & 7.61E-08 & 1.79 & $4.23 \mathrm{E}-06$ & $4.36 \mathrm{E}-06$ & 4.19E-06 \\
\hline IG-110 & $4.38 \mathrm{E}-06$ & 7.61E-08 & 1.74 & $4.38 \mathrm{E}-06$ & 4.47E-06 & 4.29E-06 \\
\hline IG-430 & 4.27E-06 & $1.17 \mathrm{E}-07$ & 2.74 & $4.25 \mathrm{E}-06$ & 4.42E-06 & 4.11E-06 \\
\hline NBG-17 & $5.25 \mathrm{E}-06$ & $1.51 \mathrm{E}-07$ & 2.88 & $5.21 \mathrm{E}-06$ & $5.46 \mathrm{E}-06$ & $5.08 \mathrm{E}-06$ \\
\hline NBG-18 & $5.36 \mathrm{E}-06$ & $7.79 \mathrm{E}-08$ & 1.45 & $5.36 \mathrm{E}-06$ & $5.49 \mathrm{E}-06$ & $5.26 \mathrm{E}-06$ \\
\hline PCEA & 4.69E-06 & $1.68 \mathrm{E}-07$ & 3.57 & 4.77E-06 & $4.88 \mathrm{E}-06$ & 4.42E-06 \\
\hline $\begin{array}{l}\text { With Grain } \\
\text { Specimens }\end{array}$ & Mean & Std Dev & $\operatorname{CoV}(\%)$ & Median & Upper Limit & Lower Limit \\
\hline H451 & $3.63 \mathrm{E}-06$ & $7.18 \mathrm{E}-08$ & 1.98 & $3.65 \mathrm{E}-06$ & $3.77 \mathrm{E}-06$ & $3.46 \mathrm{E}-06$ \\
\hline IG-110 & $4.45 \mathrm{E}-06$ & $8.46 \mathrm{E}-08$ & 1.90 & $4.44 \mathrm{E}-06$ & 4.64E-06 & $4.28 \mathrm{E}-06$ \\
\hline IG-430 & 4.82E-06 & $9.88 \mathrm{E}-08$ & 2.05 & $4.81 \mathrm{E}-06$ & $5.01 \mathrm{E}-06$ & 4.62E-06 \\
\hline NBG-17 & $5.02 \mathrm{E}-06$ & $1.41 \mathrm{E}-07$ & 2.81 & $5.05 \mathrm{E}-06$ & $5.21 \mathrm{E}-06$ & $4.78 \mathrm{E}-06$ \\
\hline NBG-18 & $5.45 \mathrm{E}-06$ & 7.97E-08 & 1.46 & $5.48 \mathrm{E}-06$ & $5.58 \mathrm{E}-06$ & $5.25 \mathrm{E}-06$ \\
\hline PCEA & $4.49 \mathrm{E}-06$ & 7.97E-08 & 1.77 & $4.50 \mathrm{E}-06$ & 4.67E-06 & 4.37E-06 \\
\hline
\end{tabular}


Table B-7. Creep Coefficient of Thermal Expansion $(1 / \mathrm{K})$ at $900^{\circ} \mathrm{C}$ Summary Statistics.

\begin{tabular}{|c|c|c|c|c|c|c|}
\hline $\begin{array}{l}\text { Combined } \\
\text { Specimens } \\
\end{array}$ & Mean & Std Dev & $\operatorname{CoV}(\%)$ & Median & Upper Limit & Lower Limit \\
\hline H451 & $4.20 \mathrm{E}-06$ & $2.27 \mathrm{E}-07$ & 5.40 & 4.12E-06 & 4.37E-06 & 4.02E-06 \\
\hline IG-110 & $4.88 \mathrm{E}-06$ & $8.59 \mathrm{E}-08$ & 1.76 & $4.87 \mathrm{E}-06$ & $5.04 \mathrm{E}-06$ & 4.69E-06 \\
\hline IG-430 & $5.17 \mathrm{E}-06$ & $2.28 \mathrm{E}-07$ & 4.42 & $5.24 \mathrm{E}-06$ & $5.50 \mathrm{E}-06$ & $4.86 \mathrm{E}-06$ \\
\hline NBG-17 & $5.53 \mathrm{E}-06$ & $1.55 \mathrm{E}-07$ & 2.80 & $5.58 \mathrm{E}-06$ & 5.89E-06 & $5.26 \mathrm{E}-06$ \\
\hline NBG-18 & $5.89 \mathrm{E}-06$ & $7.29 \mathrm{E}-08$ & 1.24 & $5.91 \mathrm{E}-06$ & $6.03 \mathrm{E}-06$ & $5.72 \mathrm{E}-06$ \\
\hline PCEA & $5.03 \mathrm{E}-06$ & $1.31 \mathrm{E}-07$ & 2.60 & $5.00 \mathrm{E}-06$ & $5.23 \mathrm{E}-06$ & $4.87 \mathrm{E}-06$ \\
\hline $\begin{array}{c}\text { Against } \\
\text { Grain } \\
\text { Specimens } \\
\end{array}$ & Mean & Std Dev & $\operatorname{CoV}(\%)$ & Median & Upper Limit & Lower Limit \\
\hline H451 & $4.74 \mathrm{E}-06$ & $6.13 \mathrm{E}-08$ & 1.29 & 4.72E-06 & 4.82E-06 & $4.68 \mathrm{E}-06$ \\
\hline IG-110 & 4.81E-06 & $5.88 \mathrm{E}-08$ & 1.22 & 4.81E-06 & $4.87 \mathrm{E}-06$ & $4.74 \mathrm{E}-06$ \\
\hline IG-430 & $4.70 \mathrm{E}-06$ & $1.03 \mathrm{E}-07$ & 2.18 & $4.69 \mathrm{E}-06$ & $4.86 \mathrm{E}-06$ & $4.56 \mathrm{E}-06$ \\
\hline NBG-17 & $5.71 \mathrm{E}-06$ & $1.32 \mathrm{E}-07$ & 2.32 & $5.65 \mathrm{E}-06$ & $5.89 \mathrm{E}-06$ & $5.57 \mathrm{E}-06$ \\
\hline NBG-18 & $5.82 \mathrm{E}-06$ & $6.06 \mathrm{E}-08$ & 1.04 & $5.83 \mathrm{E}-06$ & $5.89 \mathrm{E}-06$ & $5.72 \mathrm{E}-06$ \\
\hline PCEA & $5.17 \mathrm{E}-06$ & $1.68 \mathrm{E}-07$ & 3.26 & $5.23 \mathrm{E}-06$ & $5.40 \mathrm{E}-06$ & 4.91E-06 \\
\hline $\begin{array}{l}\text { With Grain } \\
\text { Specimens }\end{array}$ & Mean & Std Dev & $\mathrm{CoV}(\%)$ & Median & Upper Limit & Lower Limit \\
\hline H451 & 4.11E-06 & $5.95 \mathrm{E}-08$ & 1.45 & 4.11E-06 & $4.26 \mathrm{E}-06$ & 4.02E-06 \\
\hline IG-110 & $4.88 \mathrm{E}-06$ & $8.54 \mathrm{E}-08$ & 1.75 & $4.88 \mathrm{E}-06$ & $5.04 \mathrm{E}-06$ & $4.69 \mathrm{E}-06$ \\
\hline IG-430 & $5.26 \mathrm{E}-06$ & $1.02 \mathrm{E}-07$ & 1.93 & $5.26 \mathrm{E}-06$ & $5.47 \mathrm{E}-06$ & $5.06 \mathrm{E}-06$ \\
\hline NBG-17 & $5.49 \mathrm{E}-06$ & $1.32 \mathrm{E}-07$ & 2.41 & $5.54 \mathrm{E}-06$ & $5.67 \mathrm{E}-06$ & $5.26 \mathrm{E}-06$ \\
\hline NBG-18 & $5.91 \mathrm{E}-06$ & $6.16 \mathrm{E}-08$ & 1.04 & $5.93 \mathrm{E}-06$ & $6.03 \mathrm{E}-06$ & $5.75 \mathrm{E}-06$ \\
\hline PCEA & 4.99E-06 & $7.08 \mathrm{E}-08$ & 1.42 & $4.98 \mathrm{E}-06$ & $5.16 \mathrm{E}-06$ & $4.87 \mathrm{E}-06$ \\
\hline
\end{tabular}


Table B-8. Creep Modulus (GPa) by Sonic Resonance Summary Statistics.

\begin{tabular}{|c|c|c|c|c|c|c|}
\hline $\begin{array}{l}\text { Combined } \\
\text { Specimens }\end{array}$ & Mean & Std Dev & $\operatorname{CoV}(\%)$ & Median & Upper Limit & Lower Limit \\
\hline H451 & 9.66 & 0.76 & 7.83 & 9.83 & 10.46 & 8.74 \\
\hline IG-110 & 9.71 & 0.33 & 3.38 & 9.75 & 10.29 & 9.00 \\
\hline IG-430 & 9.59 & 0.52 & 5.43 & 9.50 & 10.31 & 8.98 \\
\hline NBG-17 & 11.26 & 0.46 & 4.11 & 11.52 & 11.82 & 10.46 \\
\hline NBG-18 & 12.09 & 0.25 & 2.10 & 12.13 & 12.63 & 11.53 \\
\hline PCEA & 9.65 & 1.08 & 11.23 & 9.93 & 10.62 & 9.19 \\
\hline $\begin{array}{c}\text { Against } \\
\text { Grain } \\
\text { Specimens } \\
\end{array}$ & Mean & Std Dev & $\operatorname{CoV}(\%)$ & Median & Upper Limit & Lower Limit \\
\hline H451 & 8.01 & 0.13 & 1.64 & 8.03 & 8.15 & 7.83 \\
\hline IG-110 & 9.99 & 0.29 & 2.92 & 10.10 & 10.29 & 9.55 \\
\hline IG-430 & 10.64 & 0.22 & 2.07 & 10.69 & 10.89 & 10.38 \\
\hline NBG-17 & 11.16 & 0.03 & 0.29 & 11.16 & 11.20 & 11.12 \\
\hline NBG-18 & 12.42 & 0.10 & 0.84 & 12.43 & 12.63 & 12.25 \\
\hline PCEA & 8.73 & 2.04 & 23.35 & 8.76 & 10.63 & 5.00 \\
\hline $\begin{array}{l}\text { With Grain } \\
\text { Specimens }\end{array}$ & Mean & Std Dev & $\operatorname{CoV}(\%)$ & Median & Upper Limit & Lower Limit \\
\hline H451 & 9.94 & 0.30 & 3.07 & 9.87 & 10.46 & 9.49 \\
\hline IG-110 & 9.65 & 0.30 & 3.16 & 9.74 & 10.09 & 8.93 \\
\hline IG-430 & 9.39 & 0.23 & 2.43 & 9.39 & 9.80 & 8.98 \\
\hline NBG-17 & 11.28 & 0.51 & 4.51 & 11.56 & 11.82 & 10.46 \\
\hline NBG-18 & 11.99 & 0.19 & 1.59 & 12.02 & 12.35 & 11.53 \\
\hline PCEA & 9.92 & 0.24 & 2.38 & 9.94 & 10.41 & 9.43 \\
\hline
\end{tabular}


Table B-9. Creep Resistivity $(\mu \Omega-m)$ Summary Statistics.

\begin{tabular}{|c|c|c|c|c|c|c|}
\hline $\begin{array}{l}\text { Combined } \\
\text { Specimens }\end{array}$ & Mean & Std Dev & $\operatorname{CoV}(\%)$ & Median & Upper Limit & Lower Limit \\
\hline H451 & 7.75 & 0.38 & 4.86 & 7.65 & 8.27 & 7.39 \\
\hline IG-110 & 11.21 & 0.40 & 3.60 & 11.08 & 12.33 & 10.61 \\
\hline IG-430 & 9.60 & 0.50 & 5.19 & 9.66 & 10.25 & 8.74 \\
\hline NBG-17 & 9.59 & 0.23 & 2.43 & 9.51 & 9.90 & 9.18 \\
\hline NBG-18 & 9.48 & 0.18 & 1.90 & 9.56 & 9.69 & 9.21 \\
\hline PCEA & 7.62 & 0.34 & 4.45 & 7.51 & 7.79 & 7.34 \\
\hline $\begin{array}{c}\text { Against } \\
\text { Grain } \\
\text { Specimens } \\
\end{array}$ & Mean & Std Dev & $\operatorname{CoV}(\%)$ & Median & Upper Limit & Lower Limit \\
\hline H451 & 8.57 & 0.11 & 1.30 & 8.56 & 8.71 & 8.47 \\
\hline IG-110 & 10.98 & 0.32 & 2.88 & 11.09 & 11.43 & 10.61 \\
\hline IG-430 & 8.63 & 0.17 & 1.92 & 8.58 & 8.87 & 8.43 \\
\hline NBG-17 & 9.85 & 0.03 & 0.28 & 9.84 & 9.90 & 9.81 \\
\hline NBG-18 & 9.17 & 0.05 & 0.53 & 9.15 & 9.25 & 9.10 \\
\hline PCEA & 8.02 & 0.51 & 6.39 & 8.29 & 8.59 & 7.38 \\
\hline $\begin{array}{l}\text { With Grain } \\
\text { Specimens }\end{array}$ & Mean & Std Dev & $\operatorname{CoV}(\%)$ & Median & Upper Limit & Lower Limit \\
\hline H451 & 7.61 & 0.15 & 1.94 & 7.62 & 7.90 & 7.39 \\
\hline IG-110 & 11.25 & 0.41 & 3.63 & 11.07 & 12.35 & 10.71 \\
\hline IG-430 & 9.79 & 0.25 & 2.56 & 9.78 & 10.25 & 9.29 \\
\hline NBG-17 & 9.51 & 0.21 & 2.22 & 9.46 & 9.90 & 9.18 \\
\hline NBG-18 & 9.58 & 0.06 & 0.60 & 9.58 & 9.69 & 9.45 \\
\hline PCEA & 7.50 & 0.07 & 1.00 & 7.51 & 7.64 & 7.34 \\
\hline
\end{tabular}


Table B-10. Creep Young's Modulus (GPa) by Sonic Velocity.

\begin{tabular}{|c|c|c|c|c|c|c|}
\hline $\begin{array}{l}\text { Combined } \\
\text { Specimens }\end{array}$ & Mean & Std Dev & $\operatorname{CoV}(\%)$ & Median & Upper Limit & Lower Limit \\
\hline H451 & 11.80 & 0.72 & 6.10 & 11.94 & 12.74 & 10.50 \\
\hline IG-110 & 10.87 & 0.38 & 3.46 & 10.91 & 11.83 & 10.13 \\
\hline IG-430 & 10.72 & 0.64 & 5.97 & 10.56 & 11.46 & 9.96 \\
\hline NBG-17 & 14.29 & 0.61 & 4.28 & 14.42 & 15.06 & 13.00 \\
\hline NBG-18 & 15.61 & 0.37 & 2.34 & 15.59 & 16.46 & 14.70 \\
\hline PCEA & 12.30 & 0.58 & 4.70 & 12.47 & 12.96 & 11.68 \\
\hline $\begin{array}{c}\text { Against } \\
\text { Grain } \\
\text { Specimens }\end{array}$ & Mean & Std Dev & $\operatorname{CoV}(\%)$ & Median & Upper Limit & Lower Limit \\
\hline H451 & 10.29 & 0.10 & 0.94 & 10.32 & 10.38 & 10.16 \\
\hline IG-110 & 11.20 & 0.45 & 3.97 & 11.19 & 11.95 & 10.58 \\
\hline IG-430 & 12.01 & 0.23 & 1.94 & 12.04 & 12.37 & 11.70 \\
\hline NBG-17 & 14.23 & 0.10 & 0.72 & 14.23 & 14.42 & 14.05 \\
\hline NBG-18 & 16.11 & 0.17 & 1.08 & 16.10 & 16.46 & 15.86 \\
\hline PCEA & 11.76 & 0.97 & 8.24 & 11.19 & 12.96 & 10.65 \\
\hline $\begin{array}{l}\text { With Grain } \\
\text { Specimens }\end{array}$ & Mean & Std Dev & $\operatorname{CoV}(\%)$ & Median & Upper Limit & Lower Limit \\
\hline H451 & 12.06 & 0.36 & 2.94 & 12.04 & 12.74 & 11.52 \\
\hline IG-110 & 10.81 & 0.33 & 3.08 & 10.83 & 11.29 & 10.13 \\
\hline IG-430 & 10.46 & 0.27 & 2.54 & 10.48 & 10.95 & 9.96 \\
\hline NBG-17 & 14.31 & 0.70 & 4.86 & 14.64 & 15.06 & 13.00 \\
\hline NBG-18 & 15.46 & 0.26 & 1.70 & 15.48 & 16.09 & 14.88 \\
\hline PCEA & 12.47 & 0.17 & 1.40 & 12.50 & 12.85 & 12.10 \\
\hline
\end{tabular}


Table B-11. Creep Shear Modulus (GPa) by Sonic Velocity.

\begin{tabular}{|c|c|c|c|c|c|c|}
\hline $\begin{array}{l}\text { Combined } \\
\text { Specimens }\end{array}$ & Mean & Std Dev & CoV (\%) & Median & Upper Limit & Lower Limit \\
\hline H451 & 4.41 & 0.15 & 3.36 & 4.43 & 4.62 & 4.10 \\
\hline IG-110 & 4.35 & 0.14 & 3.32 & 4.37 & 4.53 & 4.06 \\
\hline IG-430 & 4.36 & 0.12 & 2.72 & 4.34 & 4.62 & 4.20 \\
\hline NBG-17 & 4.83 & 0.17 & 3.43 & 4.88 & 5.11 & 4.51 \\
\hline NBG-18 & 5.25 & 0.05 & 1.03 & 5.24 & 5.35 & 5.15 \\
\hline PCEA & 4.53 & 0.13 & 2.95 & 4.57 & 4.78 & 4.26 \\
\hline $\begin{array}{c}\text { Against } \\
\text { Grain } \\
\text { Specimens } \\
\end{array}$ & Mean & Std Dev & $\operatorname{CoV}(\%)$ & Median & Upper Limit & Lower Limit \\
\hline H451 & 4.13 & 0.09 & 2.14 & 4.15 & 4.22 & 4.01 \\
\hline IG-110 & 4.39 & 0.14 & 3.19 & 4.38 & 4.52 & 4.12 \\
\hline IG-430 & 4.54 & 0.14 & 2.99 & 4.52 & 4.78 & 4.36 \\
\hline NBG-17 & 4.87 & 0.02 & 0.40 & 4.88 & 4.90 & 4.83 \\
\hline NBG-18 & 5.32 & 0.05 & 0.92 & 5.33 & 5.38 & 5.24 \\
\hline PCEA & 4.47 & 0.20 & 4.43 & 4.38 & 4.78 & 4.26 \\
\hline $\begin{array}{l}\text { With Grain } \\
\text { Specimens }\end{array}$ & Mean & Std Dev & CoV (\%) & Median & Upper Limit & Lower Limit \\
\hline H451 & 4.46 & 0.09 & 2.01 & 4.47 & 4.62 & 4.29 \\
\hline IG-110 & 4.34 & 0.15 & 3.35 & 4.37 & 4.53 & 4.06 \\
\hline IG-430 & 4.33 & 0.08 & 1.76 & 4.32 & 4.50 & 4.20 \\
\hline NBG-17 & 4.81 & 0.19 & 3.87 & 4.89 & 5.11 & 4.51 \\
\hline NBG-18 & 5.23 & 0.03 & 0.67 & 5.23 & 5.32 & 5.15 \\
\hline PCEA & 4.55 & 0.10 & 2.24 & 4.57 & 4.68 & 4.31 \\
\hline
\end{tabular}


Table B-12a. Piggyback Pre Thermal Measurement Length ( $\mathrm{mm}$ ) Summary Statistics.

\begin{tabular}{|l|c|c|c|c|c|c|}
\hline $\begin{array}{c}\text { Combined } \\
\text { Specimens }\end{array}$ & Mean & Std Dev & CoV (\%) & Median & Upper Limit & Lower Limit \\
\hline 2114 & 6.330 & 0.007 & 0.11 & 6.330 & 6.341 & 6.314 \\
\hline A3 Matrix & 6.325 & 0.009 & 0.14 & 6.323 & 6.331 & 6.317 \\
\hline BAN & 6.329 & 0.007 & 0.11 & 6.330 & 6.338 & 6.323 \\
\hline H451 & 6.330 & 0.006 & 0.10 & 6.329 & 6.336 & 6.321 \\
\hline HLM & 6.329 & 0.003 & 0.05 & 6.330 & 6.335 & 6.322 \\
\hline IG-110 & 6.347 & 0.004 & 0.06 & 6.346 & 6.353 & 6.340 \\
\hline IG-430 & 6.331 & 0.005 & 0.08 & 6.330 & 6.333 & 6.328 \\
\hline NBG-10 & 6.326 & 0.003 & 0.04 & 6.326 & 6.330 & 6.322 \\
\hline NBG-17 & 6.325 & 0.010 & 0.16 & 6.325 & 6.350 & 6.308 \\
\hline NBG-18 & 6.321 & 0.008 & 0.12 & 6.319 & 6.337 & 6.305 \\
\hline NBG-25 & 6.333 & 0.007 & 0.11 & 6.333 & 6.342 & 6.326 \\
\hline New Matrix & 6.322 & 0.016 & 0.26 & 6.320 & 6.347 & 6.296 \\
\hline PCEA & 6.340 & 0.012 & 0.20 & 6.339 & 6.349 & 6.330 \\
\hline PCIB & 6.328 & 0.003 & 0.05 & 6.328 & 6.337 & 6.324 \\
\hline PGX & 6.327 & 0.008 & 0.13 & 6.329 & 6.339 & 6.316 \\
\hline PPEA & 6.328 & 0.004 & 0.06 & 6.328 & 6.334 & 6.321 \\
\hline
\end{tabular}

Table B-12b. Piggyback Pre Thermal Measurement Length ( $\mathrm{mm}$ ) Summary Statistics.

\begin{tabular}{|c|c|c|c|c|c|c|}
\hline $\begin{array}{c}\text { Against } \\
\text { Grain } \\
\text { Specimens }\end{array}$ & Mean & Std Dev & $\operatorname{CoV}(\%)$ & Median & Upper Limit & Lower Limit \\
\hline 2114 & 6.330 & 0.007 & 0.11 & 6.330 & 6.341 & 6.314 \\
\hline \multicolumn{7}{|l|}{ A3 Matrix } \\
\hline \multicolumn{7}{|l|}{ BAN } \\
\hline H451 & 6.330 & 0.005 & 0.08 & 6.329 & 6.332 & 6.327 \\
\hline \multicolumn{7}{|l|}{ HLM } \\
\hline \multicolumn{7}{|l|}{ IG-110 } \\
\hline \multicolumn{7}{|l|}{ IG-430 } \\
\hline \multicolumn{7}{|l|}{ NBG-10 } \\
\hline NBG-17 & 6.322 & 0.009 & 0.15 & 6.321 & 6.342 & 6.311 \\
\hline NBG-18 & 6.320 & 0.009 & 0.14 & 6.318 & 6.332 & 6.308 \\
\hline \multicolumn{7}{|l|}{ NBG-25 } \\
\hline \multicolumn{7}{|l|}{ New Matrix } \\
\hline PCEA & 6.346 & 0.010 & 0.15 & 6.340 & 6.363 & 6.335 \\
\hline \multicolumn{7}{|l|}{ PCIB } \\
\hline \multicolumn{7}{|l|}{ PGX } \\
\hline PPEA & & & & & & \\
\hline
\end{tabular}


Table B-12c. Piggyback Pre Thermal Measurement Length ( $\mathrm{mm}$ ) Summary Statistics.

\begin{tabular}{|l|c|c|c|c|c|c|}
\hline $\begin{array}{l}\text { With Grain } \\
\text { Specimens }\end{array}$ & Mean & Std Dev & CoV (\%) & Median & Upper Limit & Lower Limit \\
\hline $\mathbf{2 1 1 4}$ & & & & & & \\
\hline A3 Matrix & & & & & & \\
\hline BAN & 6.329 & 0.007 & 0.11 & 6.330 & 6.338 & 6.323 \\
\hline H451 & 6.330 & 0.007 & 0.12 & 6.329 & 6.340 & 6.317 \\
\hline HLM & & & & & & \\
\hline IG-110 & 6.347 & 0.004 & 0.06 & 6.346 & 6.353 & 6.340 \\
\hline IG-430 & 6.331 & 0.005 & 0.08 & 6.330 & 6.333 & 6.328 \\
\hline NBG-10 & & & & & & 6.345 \\
\hline NBG-17 & 6.329 & 0.010 & 0.16 & 6.330 & 6.325 & \\
\hline NBG-18 & 6.324 & 0.005 & 0.07 & 6.325 & 6.330 & 6.315 \\
\hline NBG-25 & & & & & & \\
\hline New Matrix & & & & & & \\
\hline PCEA & 6.335 & 0.013 & 0.20 & 6.337 & 6.340 & 6.336 \\
\hline PCIB & & & & & & \\
\hline PGX & 6.327 & 0.008 & 0.13 & 6.329 & 6.339 & \\
\hline PPEA & & & & & & \\
\hline
\end{tabular}

Table B-13a. Piggyback Pre Thermal Measurement Diameter ( $\mathrm{mm}$ ) Summary Statistics.

\begin{tabular}{|l|c|c|c|c|c|c|}
\hline $\begin{array}{c}\text { Combined } \\
\text { Specimens }\end{array}$ & Mean & Std Dev & CoV (\%) & Median & Upper Limit & Lower Limit \\
\hline $\mathbf{2 1 1 4}$ & 12.726 & 0.005 & 0.04 & 12.726 & 12.735 & 12.713 \\
\hline A3 Matrix & 12.720 & 0.006 & 0.05 & 12.719 & 12.729 & 12.713 \\
\hline BAN & 12.721 & 0.005 & 0.04 & 12.722 & 12.734 & 12.710 \\
\hline H451 & 12.733 & 0.002 & 0.02 & 12.733 & 12.737 & 12.730 \\
\hline HLM & 12.726 & 0.007 & 0.05 & 12.727 & 12.737 & 12.717 \\
\hline IG-110 & 12.734 & 0.002 & 0.01 & 12.735 & 12.738 & 12.731 \\
\hline IG-430 & 12.729 & 0.005 & 0.04 & 12.728 & 12.733 & 12.726 \\
\hline NBG-10 & 12.720 & 0.005 & 0.04 & 12.719 & 12.727 & 12.711 \\
\hline NBG-17 & 12.730 & 0.002 & 0.02 & 12.730 & 12.735 & 12.726 \\
\hline NBG-18 & 12.724 & 0.004 & 0.04 & 12.723 & 12.727 & 12.720 \\
\hline NBG-25 & 12.730 & 0.002 & 0.02 & 12.730 & 12.735 & 12.726 \\
\hline New Matrix & 12.719 & 0.009 & 0.07 & 12.717 & 12.735 & 12.709 \\
\hline PCEA & 12.733 & 0.005 & 0.04 & 12.733 & 12.744 & 12.726 \\
\hline PCIB & 12.728 & 0.004 & 0.03 & 12.727 & 12.738 & 12.721 \\
\hline PGX & 12.729 & 0.004 & 0.04 & 12.728 & 12.738 & 12.724 \\
\hline PPEA & 12.728 & 0.005 & 0.04 & 12.729 & 12.741 & 12.721 \\
\hline
\end{tabular}


Table B-13b. Piggyback Pre Thermal Measurement Diameter ( $\mathrm{mm})$ Summary Statistics.

\begin{tabular}{|c|c|c|c|c|c|c|}
\hline $\begin{array}{c}\text { Against } \\
\text { Grain } \\
\text { Specimens }\end{array}$ & Mean & Std Dev & CoV (\%) & Median & Upper Limit & Lower Limit \\
\hline 2114 & 12.726 & 0.005 & 0.04 & 12.726 & 12.735 & 12.713 \\
\hline \multicolumn{7}{|l|}{ A3 Matrix } \\
\hline \multicolumn{7}{|l|}{ BAN } \\
\hline H451 & 12.733 & 0.002 & 0.02 & 12.733 & 12.737 & 12.730 \\
\hline \multicolumn{7}{|l|}{ HLM } \\
\hline \multicolumn{7}{|l|}{ IG-110 } \\
\hline \multicolumn{7}{|l|}{ IG-430 } \\
\hline \multicolumn{7}{|l|}{ NBG-10 } \\
\hline NBG-17 & 12.731 & 0.002 & 0.02 & 12.730 & 12.735 & 12.728 \\
\hline NBG-18 & 12.725 & 0.005 & 0.04 & 12.724 & 12.728 & 12.720 \\
\hline \multicolumn{7}{|l|}{ NBG-25 } \\
\hline \multicolumn{7}{|l|}{ New Matrix } \\
\hline PCEA & 12.733 & 0.005 & 0.04 & 12.734 & 12.742 & 12.726 \\
\hline \multicolumn{7}{|l|}{ PCIB } \\
\hline \multicolumn{7}{|l|}{ PGX } \\
\hline PPEA & & & & & & \\
\hline
\end{tabular}

Table B-13c. Piggyback Pre Thermal Measurement Diameter (mm) Summary Statistics.

\begin{tabular}{|l|c|c|c|c|c|c|}
\hline $\begin{array}{c}\text { With Grain } \\
\text { Specimens }\end{array}$ & Mean & Std Dev & CoV (\%) & Median & Upper Limit & Lower Limit \\
\hline $\mathbf{2 1 1 4}$ & & & & & & \\
\hline A3 Matrix & & & & & & \\
\hline BAN & 12.721 & 0.005 & 0.04 & 12.722 & 12.734 & 12.710 \\
\hline H451 & 12.733 & 0.001 & 0.01 & 12.732 & 12.735 & 12.731 \\
\hline HLM & & & & & & \\
\hline IG-110 & 12.734 & 0.002 & 0.01 & 12.735 & 12.738 & 12.731 \\
\hline IG-430 & 12.729 & 0.005 & 0.04 & 12.728 & 12.733 & 12.726 \\
\hline NBG-10 & & & & & & 12.734 \\
\hline NBG-17 & 12.730 & 0.003 & 0.02 & 12.730 & 12.726 \\
\hline NBG-18 & 12.722 & 0.001 & 0.01 & 12.722 & 12.725 & 12.721 \\
\hline NBG-25 & & & & & & \\
\hline New Matrix & & & & & & 12.744 \\
\hline PCEA & 12.733 & 0.005 & 0.04 & 12.732 & 12.738 & 12.724 \\
\hline PCIB & & & & & & \\
\hline PGX & 12.729 & 0.004 & 0.04 & 12.728 & & \\
\hline PPEA & & & & & & \\
\hline
\end{tabular}


Table B-14a. Piggyback Pre Thermal Measurement Mass (g) Summary Statistics.

\begin{tabular}{|l|c|c|c|c|c|c|}
\hline $\begin{array}{c}\text { Combined } \\
\text { Specimens }\end{array}$ & Mean & Std Dev & CoV (\%) & Median & Upper Limit & Lower Limit \\
\hline $\mathbf{2 1 1 4}$ & 1.462 & 0.01 & 0.49 & 1.461 & 1.472 & 1.454 \\
\hline A3 Matrix & 1.143 & 0.03 & 2.74 & 1.135 & 1.205 & 1.090 \\
\hline BAN & 1.475 & 0.01 & 0.61 & 1.473 & 1.498 & 1.460 \\
\hline H451 & 1.395 & 0.01 & 0.96 & 1.392 & 1.413 & 1.375 \\
\hline HLM & 1.411 & 0.00 & 0.19 & 1.412 & 1.416 & 1.406 \\
\hline IG-110 & 1.433 & 0.00 & 0.32 & 1.433 & 1.441 & 1.424 \\
\hline IG-430 & 1.460 & 0.00 & 0.29 & 1.459 & 1.467 & 1.454 \\
\hline NBG-10 & 1.438 & 0.00 & 0.29 & 1.438 & 1.444 & 1.428 \\
\hline NBG-17 & 1.500 & 0.01 & 0.39 & 1.501 & 1.505 & 1.494 \\
\hline NBG-18 & 1.502 & 0.00 & 0.18 & 1.501 & 1.508 & 1.497 \\
\hline NBG-25 & 1.486 & 0.00 & 0.15 & 1.485 & 1.489 & 1.482 \\
\hline New Matrix & 1.386 & 0.04 & 2.86 & 1.399 & 1.445 & 1.337 \\
\hline PCEA & 1.427 & 0.00 & 0.30 & 1.426 & 1.436 & 1.418 \\
\hline PCIB & 1.474 & 0.00 & 0.24 & 1.474 & 1.479 & 1.465 \\
\hline PGX & 1.423 & 0.00 & 0.24 & 1.424 & 1.430 & 1.414 \\
\hline PPEA & 1.476 & 0.00 & 0.15 & 1.476 & 1.482 & 1.472 \\
\hline
\end{tabular}

Table B-14b. Piggyback Pre Thermal Measurement Mass (g) Summary Statistics.

\begin{tabular}{|c|c|c|c|c|c|c|}
\hline $\begin{array}{c}\text { Against } \\
\text { Grain } \\
\text { Specimens } \\
\end{array}$ & Mean & Std Dev & $\operatorname{CoV}(\%)$ & Median & Upper Limit & Lower Limit \\
\hline 2114 & 1.462 & 0.01 & 0.49 & 1.461 & 1.472 & 1.454 \\
\hline \multicolumn{7}{|l|}{ A3 Matrix } \\
\hline \multicolumn{7}{|l|}{ BAN } \\
\hline H451 & 1.384 & 0.01 & 0.39 & 1.383 & 1.392 & 1.375 \\
\hline \multicolumn{7}{|l|}{ HLM } \\
\hline \multicolumn{7}{|l|}{ IG-110 } \\
\hline \multicolumn{7}{|l|}{ IG-430 } \\
\hline \multicolumn{7}{|l|}{ NBG-10 } \\
\hline NBG-17 & 1.500 & 0.00 & 0.20 & 1.500 & 1.504 & 1.493 \\
\hline NBG-18 & 1.502 & 0.00 & 0.17 & 1.502 & 1.508 & 1.498 \\
\hline \multicolumn{7}{|l|}{ NBG-25 } \\
\hline \multicolumn{7}{|l|}{ New Matrix } \\
\hline PCEA & 1.428 & 0.00 & 0.32 & 1.427 & 1.436 & 1.421 \\
\hline \multicolumn{7}{|l|}{ PCIB } \\
\hline \multicolumn{7}{|l|}{ PGX } \\
\hline PPEA & & & & & & \\
\hline
\end{tabular}


Table B-14c. Piggyback Pre Thermal Measurement Mass (g) Summary Statistics.

\begin{tabular}{|c|c|c|c|c|c|c|}
\hline $\begin{array}{c}\text { With Grain } \\
\text { Specimens }\end{array}$ & Mean & Std Dev & $\operatorname{CoV}(\%)$ & Median & Upper Limit & Lower Limit \\
\hline \multicolumn{7}{|l|}{2114} \\
\hline \multicolumn{7}{|l|}{ A3 Matrix } \\
\hline BAN & 1.475 & 0.01 & 0.61 & 1.473 & 1.498 & 1.460 \\
\hline H451 & 1.408 & 0.00 & 0.18 & 1.408 & 1.413 & 1.405 \\
\hline \multicolumn{7}{|l|}{ HLM } \\
\hline IG-110 & 1.433 & 0.00 & 0.32 & 1.433 & 1.441 & 1.424 \\
\hline IG-430 & 1.460 & 0.00 & 0.29 & 1.459 & 1.467 & 1.454 \\
\hline \multicolumn{7}{|l|}{ NBG-10 } \\
\hline NBG-17 & 1.500 & 0.01 & 0.63 & 1.503 & 1.505 & 1.496 \\
\hline NBG-18 & 1.500 & 0.00 & 0.15 & 1.500 & 1.504 & 1.497 \\
\hline \multicolumn{7}{|l|}{ NBG-25 } \\
\hline \multicolumn{7}{|l|}{ New Matrix } \\
\hline PCEA & 1.425 & 0.00 & 0.24 & 1.425 & 1.430 & 1.418 \\
\hline \multicolumn{7}{|l|}{ PCIB } \\
\hline PGX & 1.423 & 0.00 & 0.24 & 1.424 & 1.430 & 1.414 \\
\hline PPEA & & & & & & \\
\hline
\end{tabular}

Table B-15a. Piggyback Pre Thermal Measurement Density $\left(\mathrm{g} / \mathrm{cm}^{3}\right)$ Summary Statistics.

\begin{tabular}{|l|c|c|c|c|c|c|}
\hline $\begin{array}{c}\text { Combined } \\
\text { Specimens }\end{array}$ & Mean & Std Dev & CoV (\%) & Median & Upper Limit & Lower Limit \\
\hline 2114 & 1.8164 & 0.0076 & 0.42 & 1.8168 & 1.8273 & 1.8062 \\
\hline A3 Matrix & 1.4224 & 0.0384 & 2.70 & 1.4115 & 1.5000 & 1.3567 \\
\hline BAN & 1.8341 & 0.0110 & 0.60 & 1.8313 & 1.8612 & 1.8127 \\
\hline H451 & 1.7307 & 0.0167 & 0.96 & 1.7260 & 1.7534 & 1.7060 \\
\hline HLM & 1.7531 & 0.0037 & 0.21 & 1.7530 & 1.7591 & 1.7443 \\
\hline IG-110 & 1.7729 & 0.0057 & 0.32 & 1.7726 & 1.7827 & 1.7617 \\
\hline IG-430 & 1.8117 & 0.0062 & 0.34 & 1.8101 & 1.8237 & 1.8038 \\
\hline NBG-10 & 1.7884 & 0.0043 & 0.24 & 1.7877 & 1.7952 & 1.7810 \\
\hline NBG-17 & 1.8629 & 0.0063 & 0.34 & 1.8643 & 1.8705 & 1.8527 \\
\hline NBG-18 & 1.8683 & 0.0029 & 0.16 & 1.8685 & 1.8728 & 1.8619 \\
\hline NBG-25 & 1.8433 & 0.0023 & 0.12 & 1.8432 & 1.8481 & 1.8391 \\
\hline New Matrix & 1.7255 & 0.0449 & 2.60 & 1.7370 & 1.7972 & 1.6736 \\
\hline PCEA & 1.7673 & 0.0046 & 0.26 & 1.7670 & 1.7752 & 1.7584 \\
\hline PCIB & 1.8302 & 0.0046 & 0.25 & 1.8300 & 1.8390 & 1.8207 \\
\hline PGX & 1.7680 & 0.0032 & 0.18 & 1.7678 & 1.7732 & 1.7623 \\
\hline PPEA & 1.8335 & 0.0033 & 0.18 & 1.8336 & 1.8395 & 1.8277 \\
\hline
\end{tabular}


Table B-15b. Piggyback Pre Thermal Measurement Density $\left(\mathrm{g} / \mathrm{cm}^{3}\right)$ Summary Statistics.

\begin{tabular}{|c|c|c|c|c|c|c|}
\hline $\begin{array}{c}\text { Against } \\
\text { Grain } \\
\text { Specimens } \\
\end{array}$ & Mean & Std Dev & CoV (\%) & Median & Upper Limit & Lower Limit \\
\hline 2114 & 1.8164 & 0.0076 & 0.42 & 1.8168 & 1.8273 & 1.8062 \\
\hline \multicolumn{7}{|l|}{ A3 Matrix } \\
\hline \multicolumn{7}{|l|}{ BAN } \\
\hline H451 & 1.7165 & 0.0068 & 0.40 & 1.7166 & 1.7262 & 1.7060 \\
\hline \multicolumn{7}{|l|}{ HLM } \\
\hline \multicolumn{7}{|l|}{ IG-110 } \\
\hline \multicolumn{7}{|l|}{ IG-430 } \\
\hline \multicolumn{7}{|l|}{ NBG-10 } \\
\hline NBG-17 & 1.8634 & 0.0035 & 0.19 & 1.8641 & 1.8684 & 1.8569 \\
\hline NBG-18 & 1.8694 & 0.0023 & 0.12 & 1.8699 & 1.8728 & 1.8641 \\
\hline \multicolumn{7}{|l|}{ NBG-25 } \\
\hline \multicolumn{7}{|l|}{ New Matrix } \\
\hline PCEA & 1.7678 & 0.0055 & 0.31 & 1.7682 & 1.7752 & 1.7584 \\
\hline \multicolumn{7}{|l|}{ PCIB } \\
\hline \multicolumn{7}{|l|}{ PGX } \\
\hline PPEA & & & & & & \\
\hline
\end{tabular}

Table B-15c. Piggyback Pre Thermal Measurement Density $\left(\mathrm{g} / \mathrm{cm}^{3}\right)$ Summary Statistics.

\begin{tabular}{|l|c|c|c|c|c|c|}
\hline $\begin{array}{c}\text { With Grain } \\
\text { Specimens }\end{array}$ & Mean & Std Dev & CoV (\%) & Median & Upper Limit & Lower Limit \\
\hline $\mathbf{2 1 1 4}$ & & & & & & \\
\hline A3 Matrix & & & & & & \\
\hline BAN & 1.8341 & 0.0110 & 0.60 & 1.8313 & 1.8612 & 1.8127 \\
\hline H451 & 1.7476 & 0.0029 & 0.17 & 1.7472 & 1.7504 & 1.7447 \\
\hline HLM & & & & & & \\
\hline IG-110 & 1.7729 & 0.0057 & 0.32 & 1.7726 & 1.7827 & 1.7617 \\
\hline IG-430 & 1.8117 & 0.0062 & 0.34 & 1.8101 & 1.8237 & 1.8038 \\
\hline NBG-10 & & & & & & 1.8556 \\
\hline NBG-17 & 1.8619 & 0.0100 & 0.54 & 1.8643 & 1.8705 & 1.8619 \\
\hline NBG-18 & 1.8659 & 0.0027 & 0.14 & 1.8657 & & \\
\hline NBG-25 & & & & & & \\
\hline New Matrix & & & & & & \\
\hline PCEA & 1.7668 & 0.0036 & 0.20 & 1.7663 & 1.7722 & 1.7611 \\
\hline PCIB & & & & & & \\
\hline PGX & 1.7680 & 0.0032 & 0.18 & 1.7678 & 1.7732 & 1.7623 \\
\hline PPEA & & & & & & \\
\hline
\end{tabular}


Table B-16a. Piggyback Diffusivity $\left(\mathrm{mm}^{2} / \mathrm{sec}\right)$ at $100^{\circ} \mathrm{C}$ Summary Statistics.

\begin{tabular}{|l|c|c|c|c|c|c|}
\hline $\begin{array}{c}\text { Combined } \\
\text { Specimens }\end{array}$ & Mean & Std Dev & CoV (\%) & Median & Upper Limit & Lower Limit \\
\hline 2114 & 65.45 & 1.00 & 1.53 & 65.65 & 66.84 & 63.95 \\
\hline A3 Matrix & 10.99 & 0.73 & 6.68 & 10.91 & 12.13 & 9.82 \\
\hline BAN & 99.77 & 2.35 & 2.35 & 100.09 & 104.32 & 94.70 \\
\hline H451 & 97.10 & 6.25 & 6.44 & 97.10 & 104.36 & 90.57 \\
\hline HLM & 110.02 & 2.60 & 2.36 & 110.01 & 113.60 & 104.61 \\
\hline IG-110 & 72.02 & 1.10 & 1.53 & 71.93 & 73.87 & 70.06 \\
\hline IG-430 & 79.15 & 1.28 & 1.62 & 78.93 & 81.15 & 76.75 \\
\hline NBG-10 & 80.79 & 0.50 & 0.61 & 80.95 & 81.32 & 79.94 \\
\hline NBG-17 & 78.67 & 1.03 & 1.31 & 79.23 & 79.81 & 77.33 \\
\hline NBG-18 & 79.72 & 1.63 & 2.04 & 79.13 & 81.82 & 77.50 \\
\hline NBG-25 & 68.11 & 0.28 & 0.40 & 68.08 & 68.71 & 67.54 \\
\hline New Matrix & 16.37 & 0.89 & 5.41 & 16.25 & 17.91 & 15.36 \\
\hline PCEA & 98.13 & 3.85 & 3.92 & 99.92 & 101.60 & 91.75 \\
\hline PCIB & 71.77 & 0.64 & 0.89 & 71.88 & 72.53 & 70.59 \\
\hline PGX & 71.17 & 0.46 & 0.64 & 71.25 & 72.22 & 70.13 \\
\hline PPEA & 82.60 & 0.56 & 0.68 & 82.62 & 83.32 & 81.46 \\
\hline
\end{tabular}

Table B-16b. Piggyback Diffusivity $\left(\mathrm{mm}^{2} / \mathrm{sec}\right)$ at $100^{\circ} \mathrm{C}$ Summary Statistics.

\begin{tabular}{|c|c|c|c|c|c|c|}
\hline $\begin{array}{c}\text { Against } \\
\text { Grain } \\
\text { Specimens } \\
\end{array}$ & Mean & Std Dev & $\operatorname{CoV}(\%)$ & Median & Upper Limit & Lower Limit \\
\hline 2114 & 65.45 & 1.00 & 1.53 & 65.65 & 66.84 & 63.95 \\
\hline \multicolumn{7}{|l|}{ A3 Matrix } \\
\hline \multicolumn{7}{|l|}{ BAN } \\
\hline H451 & 91.17 & 0.65 & 0.71 & 90.95 & 92.26 & 90.57 \\
\hline \multicolumn{7}{|l|}{ HLM } \\
\hline \multicolumn{7}{|l|}{ IG-110 } \\
\hline \multicolumn{7}{|l|}{ IG-430 } \\
\hline \multicolumn{7}{|l|}{ NBG-10 } \\
\hline NBG-17 & 77.40 & 0.07 & 0.09 & 77.39 & 77.47 & 77.33 \\
\hline NBG-18 & 81.70 & 0.13 & 0.16 & 81.73 & 81.82 & 81.49 \\
\hline \multicolumn{7}{|l|}{ NBG-25 } \\
\hline \multicolumn{7}{|l|}{ New Matrix } \\
\hline PCEA & 91.51 & 0.36 & 0.39 & 91.60 & 91.81 & 91.11 \\
\hline \multicolumn{7}{|l|}{ PCIB } \\
\hline \multicolumn{7}{|l|}{ PGX } \\
\hline PPEA & & & & & & \\
\hline
\end{tabular}


Table B-16c. Piggyback Diffusivity $\left(\mathrm{mm}^{2} / \mathrm{sec}\right)$ at $100^{\circ} \mathrm{C}$ Summary Statistics.

\begin{tabular}{|l|c|c|c|c|c|c|}
\hline $\begin{array}{c}\text { With Grain } \\
\text { Specimens }\end{array}$ & Mean & Std Dev & CoV (\%) & Median & Upper Limit & Lower Limit \\
\hline $\mathbf{2 1 1 4}$ & & & & & & \\
\hline A3 Matrix & & & & & & \\
\hline BAN & 99.77 & 2.35 & 2.35 & 100.09 & 104.32 & 94.70 \\
\hline H451 & 103.04 & 0.96 & 0.94 & 102.91 & 104.36 & 101.94 \\
\hline HLM & & & & & & \\
\hline IG-110 & 72.02 & 1.10 & 1.53 & 71.93 & 73.87 & 70.06 \\
\hline IG-430 & 79.15 & 1.28 & 1.62 & 78.93 & 81.15 & 76.75 \\
\hline NBG-10 & & & & & & 79.81 \\
\hline NBG-17 & 79.30 & 0.49 & 0.62 & 79.39 & 79.69 \\
\hline NBG-18 & 78.61 & 0.68 & 0.87 & 78.93 & 79.43 & 77.50 \\
\hline NBG-25 & & & & & & \\
\hline New Matrix & & & & & & \\
\hline PCEA & 100.12 & 0.84 & 0.84 & 100.16 & 101.36 & 98.58 \\
\hline PCIB & & & & & & \\
\hline PGX & 71.17 & 0.46 & 0.64 & 71.25 & 72.22 & 70.13 \\
\hline PPEA & & & & & & \\
\hline
\end{tabular}

Table B-17a. Piggyback Diffusivity $\left(\mathrm{mm}^{2} / \mathrm{sec}\right)$ at $500^{\circ} \mathrm{C}$ Summary Statistics.

\begin{tabular}{|l|c|c|c|c|c|c|}
\hline $\begin{array}{c}\text { Combined } \\
\text { Specimens }\end{array}$ & Mean & Std Dev & CoV (\%) & Median & Upper Limit & Lower Limit \\
\hline 2114 & 27.10 & 0.29 & 1.06 & 27.15 & 27.57 & 26.60 \\
\hline A3 Matrix & 4.59 & 0.39 & 8.44 & 4.50 & 5.24 & 3.93 \\
\hline BAN & 35.15 & 0.85 & 2.43 & 35.29 & 36.56 & 33.64 \\
\hline H451 & 34.16 & 2.32 & 6.79 & 34.20 & 37.28 & 31.68 \\
\hline HLM & 37.39 & 0.92 & 2.47 & 37.57 & 38.79 & 35.61 \\
\hline IG-110 & 27.96 & 0.44 & 1.58 & 27.97 & 28.56 & 27.10 \\
\hline IG-430 & 29.25 & 0.45 & 1.55 & 29.16 & 29.83 & 28.39 \\
\hline NBG-10 & 30.33 & 0.21 & 0.70 & 30.34 & 30.63 & 29.86 \\
\hline NBG-17 & 29.76 & 0.46 & 1.54 & 29.81 & 30.40 & 29.17 \\
\hline NBG-18 & 30.30 & 0.62 & 2.03 & 30.11 & 31.20 & 29.54 \\
\hline NBG-25 & 27.54 & 0.15 & 0.53 & 27.58 & 27.82 & 27.24 \\
\hline New Matrix & 7.44 & 0.51 & 6.84 & 7.36 & 8.40 & 6.78 \\
\hline PCEA & 35.63 & 1.48 & 4.14 & 36.31 & 36.80 & 33.18 \\
\hline PCIB & 28.35 & 0.20 & 0.72 & 28.39 & 28.63 & 27.95 \\
\hline PGX & 26.09 & 0.18 & 0.68 & 26.08 & 26.49 & 25.69 \\
\hline PPEA & 31.53 & 0.18 & 0.58 & 31.56 & 31.87 & 31.19 \\
\hline
\end{tabular}


Table B-17b. Piggyback Diffusivity $\left(\mathrm{mm}^{2} / \mathrm{sec}\right)$ at $500^{\circ} \mathrm{C}$ Summary Statistics.

\begin{tabular}{|c|c|c|c|c|c|c|}
\hline $\begin{array}{c}\text { Against } \\
\text { Grain } \\
\text { Specimens } \\
\end{array}$ & Mean & Std Dev & CoV (\%) & Median & Upper Limit & Lower Limit \\
\hline 2114 & 27.10 & 0.29 & 1.06 & 27.15 & 27.57 & 26.60 \\
\hline \multicolumn{7}{|l|}{ A3 Matrix } \\
\hline \multicolumn{7}{|l|}{ BAN } \\
\hline H451 & 31.97 & 0.23 & 0.72 & 31.92 & 32.30 & 31.68 \\
\hline \multicolumn{7}{|l|}{ HLM } \\
\hline \multicolumn{7}{|l|}{ IG-110 } \\
\hline \multicolumn{7}{|l|}{ IG-430 } \\
\hline \multicolumn{7}{|l|}{ NBG-10 } \\
\hline NBG-17 & 29.21 & 0.04 & 0.14 & 29.22 & 29.25 & 29.17 \\
\hline NBG-18 & 31.05 & 0.10 & 0.33 & 31.00 & 31.20 & 30.97 \\
\hline \multicolumn{7}{|l|}{ NBG-25 } \\
\hline \multicolumn{7}{|l|}{ New Matrix } \\
\hline PCEA & 33.08 & 0.22 & 0.66 & 33.15 & 33.25 & 32.84 \\
\hline \multicolumn{7}{|l|}{ PCIB } \\
\hline \multicolumn{7}{|l|}{ PGX } \\
\hline PPEA & & & & & & \\
\hline
\end{tabular}

Table B-17c. Piggyback Diffusivity $\left(\mathrm{mm}^{2} / \mathrm{sec}\right)$ at $500^{\circ} \mathrm{C}$ Summary Statistics.

\begin{tabular}{|l|c|c|c|c|c|c|}
\hline $\begin{array}{c}\text { With Grain } \\
\text { Specimens }\end{array}$ & Mean & Std Dev & CoV (\%) & Median & Upper Limit & Lower Limit \\
\hline $\mathbf{2 1 1 4}$ & & & & & & \\
\hline A3 Matrix & & & & & & \\
\hline BAN & 35.15 & 0.85 & 2.43 & 35.29 & 36.56 & 33.64 \\
\hline H451 & 36.36 & 0.46 & 1.26 & 36.17 & 36.63 & 36.10 \\
\hline HLM & & & & & & \\
\hline IG-110 & 27.96 & 0.44 & 1.58 & 27.97 & 28.56 & 27.10 \\
\hline IG-430 & 29.25 & 0.45 & 1.55 & 29.16 & 29.83 & 28.39 \\
\hline NBG-10 & & & & & & 29.72 \\
\hline NBG-17 & 30.04 & 0.25 & 0.82 & 30.06 & 30.40 & 29.54 \\
\hline NBG-18 & 29.88 & 0.26 & 0.85 & 29.97 & 30.17 & \\
\hline NBG-25 & & & & & & \\
\hline New Matrix & & & & & & \\
\hline PCEA & 36.40 & 0.27 & 0.74 & 36.41 & 36.80 & 35.84 \\
\hline PCIB & & & & & & \\
\hline PGX & 26.09 & 0.18 & 0.68 & 26.08 & 26.49 & 25.69 \\
\hline PPEA & & & & & & \\
\hline
\end{tabular}


Table B-18a. Piggyback Diffusivity $\left(\mathrm{mm}^{2} / \mathrm{sec}\right)$ at $900^{\circ} \mathrm{C}$ Summary Statistics.

\begin{tabular}{|l|c|c|c|c|c|c|}
\hline $\begin{array}{c}\text { Combined } \\
\text { Specimens }\end{array}$ & Mean & Std Dev & CoV (\%) & Median & Upper Limit & Lower Limit \\
\hline $\mathbf{2 1 1 4}$ & 17.91 & 0.18 & 1.00 & 17.93 & 18.22 & 17.43 \\
\hline A3 Matrix & 3.09 & 0.27 & 8.63 & 3.06 & 3.53 & 2.45 \\
\hline BAN & 21.86 & 0.52 & 2.37 & 21.97 & 22.67 & 21.07 \\
\hline H451 & 21.33 & 1.46 & 6.85 & 21.39 & 23.17 & 19.79 \\
\hline HLM & 23.03 & 0.56 & 2.45 & 23.09 & 23.86 & 22.02 \\
\hline IG-110 & 18.08 & 0.26 & 1.44 & 18.08 & 18.45 & 17.61 \\
\hline IG-430 & 18.54 & 0.29 & 1.55 & 18.54 & 18.99 & 18.08 \\
\hline NBG-10 & 19.35 & 0.14 & 0.74 & 19.35 & 19.63 & 19.11 \\
\hline NBG-17 & 19.03 & 0.33 & 1.72 & 19.05 & 19.46 & 18.59 \\
\hline NBG-18 & 19.42 & 0.38 & 1.98 & 19.36 & 20.05 & 18.95 \\
\hline NBG-25 & 18.05 & 0.11 & 0.60 & 18.07 & 18.24 & 17.79 \\
\hline New Matrix & 5.10 & 0.48 & 9.40 & 5.13 & 5.96 & 4.44 \\
\hline PCEA & 22.34 & 0.90 & 4.01 & 22.75 & 23.09 & 20.86 \\
\hline PCIB & 18.38 & 0.14 & 0.76 & 18.41 & 18.61 & 18.05 \\
\hline PGX & 16.57 & 0.12 & 0.72 & 16.58 & 16.82 & 16.33 \\
\hline PPEA & 20.24 & 0.14 & 0.71 & 20.25 & 20.50 & 19.96 \\
\hline
\end{tabular}

Table B-18b. Piggyback Diffusivity $\left(\mathrm{mm}^{2} / \mathrm{sec}\right)$ at $900^{\circ} \mathrm{C}$ Summary Statistics.

\begin{tabular}{|c|c|c|c|c|c|c|}
\hline $\begin{array}{c}\text { Against } \\
\text { Grain } \\
\text { Specimens } \\
\end{array}$ & Mean & Std Dev & $\operatorname{CoV}(\%)$ & Median & Upper Limit & Lower Limit \\
\hline 2114 & 17.91 & 0.18 & 1.00 & 17.93 & 18.22 & 17.43 \\
\hline \multicolumn{7}{|l|}{ A3 Matrix } \\
\hline \multicolumn{7}{|l|}{ BAN } \\
\hline H451 & 19.94 & 0.16 & 0.82 & 19.87 & 20.19 & 19.79 \\
\hline \multicolumn{7}{|l|}{ HLM } \\
\hline \multicolumn{7}{|l|}{ IG-110 } \\
\hline \multicolumn{7}{|l|}{ IG-430 } \\
\hline \multicolumn{7}{|l|}{ NBG-10 } \\
\hline NBG-17 & 18.64 & 0.05 & 0.24 & 18.65 & 18.68 & 18.59 \\
\hline NBG-18 & 19.88 & 0.12 & 0.62 & 19.92 & 20.05 & 19.75 \\
\hline \multicolumn{7}{|l|}{ NBG-25 } \\
\hline \multicolumn{7}{|l|}{ New Matrix } \\
\hline PCEA & 20.79 & 0.12 & 0.56 & 20.80 & 20.91 & 20.68 \\
\hline \multicolumn{7}{|l|}{ PCIB } \\
\hline \multicolumn{7}{|l|}{ PGX } \\
\hline PPEA & & & & & & \\
\hline
\end{tabular}


Table B-18c. Piggyback Diffusivity $\left(\mathrm{mm}^{2} / \mathrm{sec}\right)$ at $900^{\circ} \mathrm{C}$ Summary Statistics.

\begin{tabular}{|c|c|c|c|c|c|c|}
\hline $\begin{array}{l}\text { With Grain } \\
\text { Specimens }\end{array}$ & Mean & Std Dev & $\operatorname{CoV}(\%)$ & Median & Upper Limit & Lower Limit \\
\hline \multicolumn{7}{|l|}{2114} \\
\hline \multicolumn{7}{|l|}{ A3 Matrix } \\
\hline BAN & 21.86 & 0.52 & 2.37 & 21.97 & 22.67 & 21.07 \\
\hline H451 & 22.71 & 0.23 & 1.01 & 22.61 & 22.91 & 22.58 \\
\hline \multicolumn{7}{|l|}{ HLM } \\
\hline IG-110 & 18.08 & 0.26 & 1.44 & 18.08 & 18.45 & 17.61 \\
\hline IG-430 & 18.54 & 0.29 & 1.55 & 18.54 & 18.99 & 18.08 \\
\hline \multicolumn{7}{|l|}{ NBG-10 } \\
\hline NBG-17 & 19.23 & 0.18 & 0.94 & 19.26 & 19.46 & 18.98 \\
\hline NBG-18 & 19.17 & 0.18 & 0.92 & 19.18 & 19.37 & 18.95 \\
\hline \multicolumn{7}{|l|}{ NBG-25 } \\
\hline \multicolumn{7}{|l|}{ New Matrix } \\
\hline PCEA & 22.80 & 0.18 & 0.79 & 22.79 & 23.09 & 22.44 \\
\hline \multicolumn{7}{|l|}{ PCIB } \\
\hline PGX & 16.57 & 0.12 & 0.72 & 16.58 & 16.82 & 16.33 \\
\hline PPEA & & & & & & \\
\hline
\end{tabular}


Appendix C

\section{Raw Data}


Graphite Grade:

Graphite Manufacturer:

Forming Process:

Coke Particle Size:

Coke Type:

ASTM Class:

Specimen Geometry:
$\mathrm{H}-451$

SGL Carbon Company

Extruded

Medium grain

Petroleum coke

ENHP

Cylinder

Specimen ID \#'s:

CW1 01

CW1 02

CW1 03

CW2 01

CW2 02

CW2 03

CW3 01

CW3 02

CW3 03

CW4 01

CW4 02

CW4 03

CW5 01

CW5 02

CW5 03

CW6 01

CW6 02

CW6 03

CW7 01

CW7 02

CW7 03

CW8 01

CW8 02

CW8 03

CW9 01

CW9 02

CW9 03

CW10 01

CW10 02

CW10 03

CW11 01

CW11 02

CW11 03

CW12 01

CW12 02

CW12 03

CW13 01

CW13 02

CW13 03

CA1 01

CA1 02

CA1 03 
Specimen ID \#'s:

CA1 04

CA2 01

CA2 02

CA2 03

CA2 04

CA2 01

CA2 02

CA2 03

CA2 04

CA3 01

CA3 02

CA3 03

CA3 04

CA4 01

CA4 02

CA4 03

CA4 04

CA5 01

CA5 02

CA5 03

CA5 04

CA6 01

CA6 02

CA6 03

CA6 04

CA7 01

CA7 02

CA7 03

CA7 04

CA8 01

CA8 02

CA8 03

CA8 04

CA9 01

CA9 02

CA9 03

CA9 04

CA10 01

CA10 02

CA10 03

CA10 04 


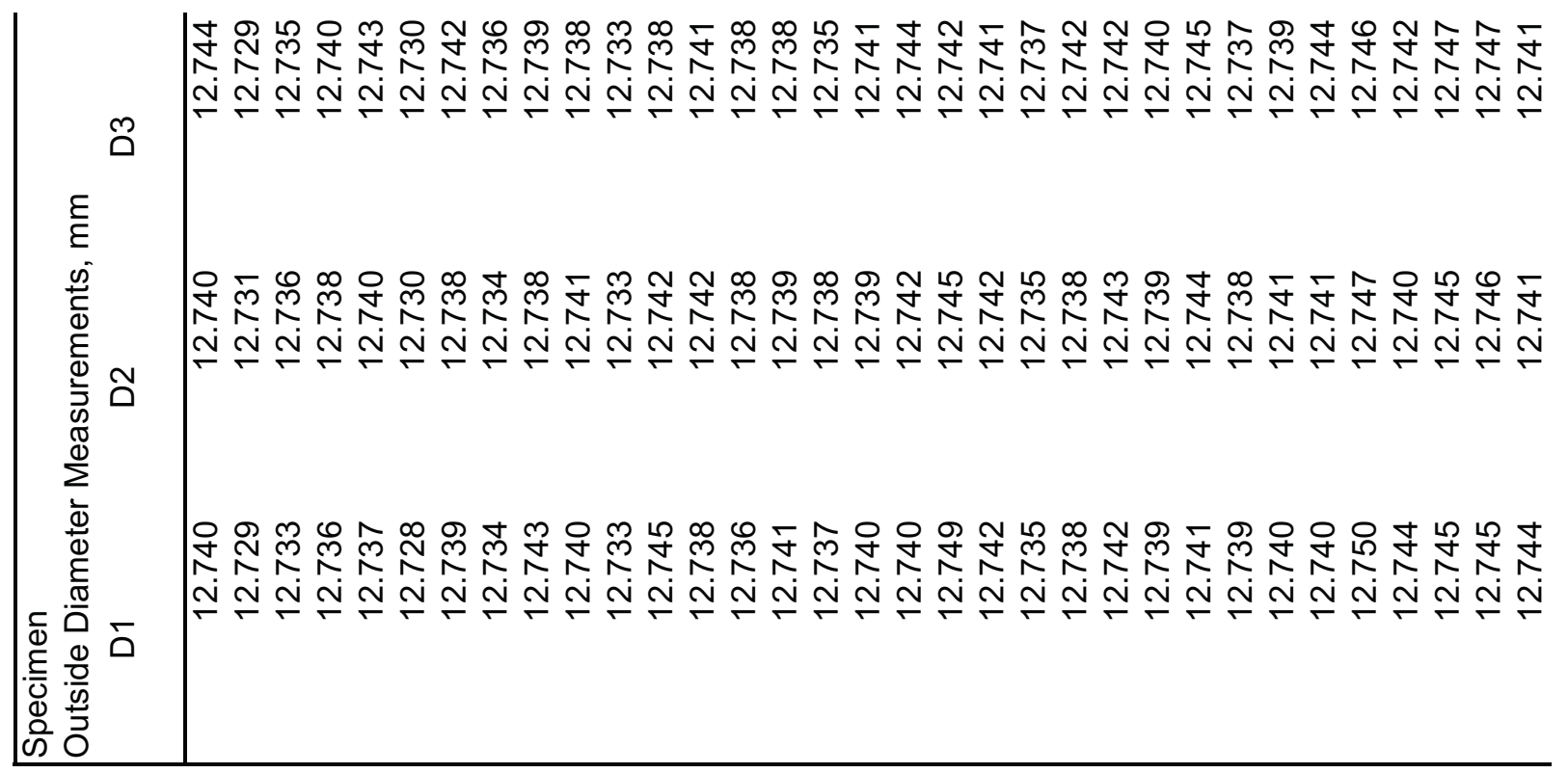

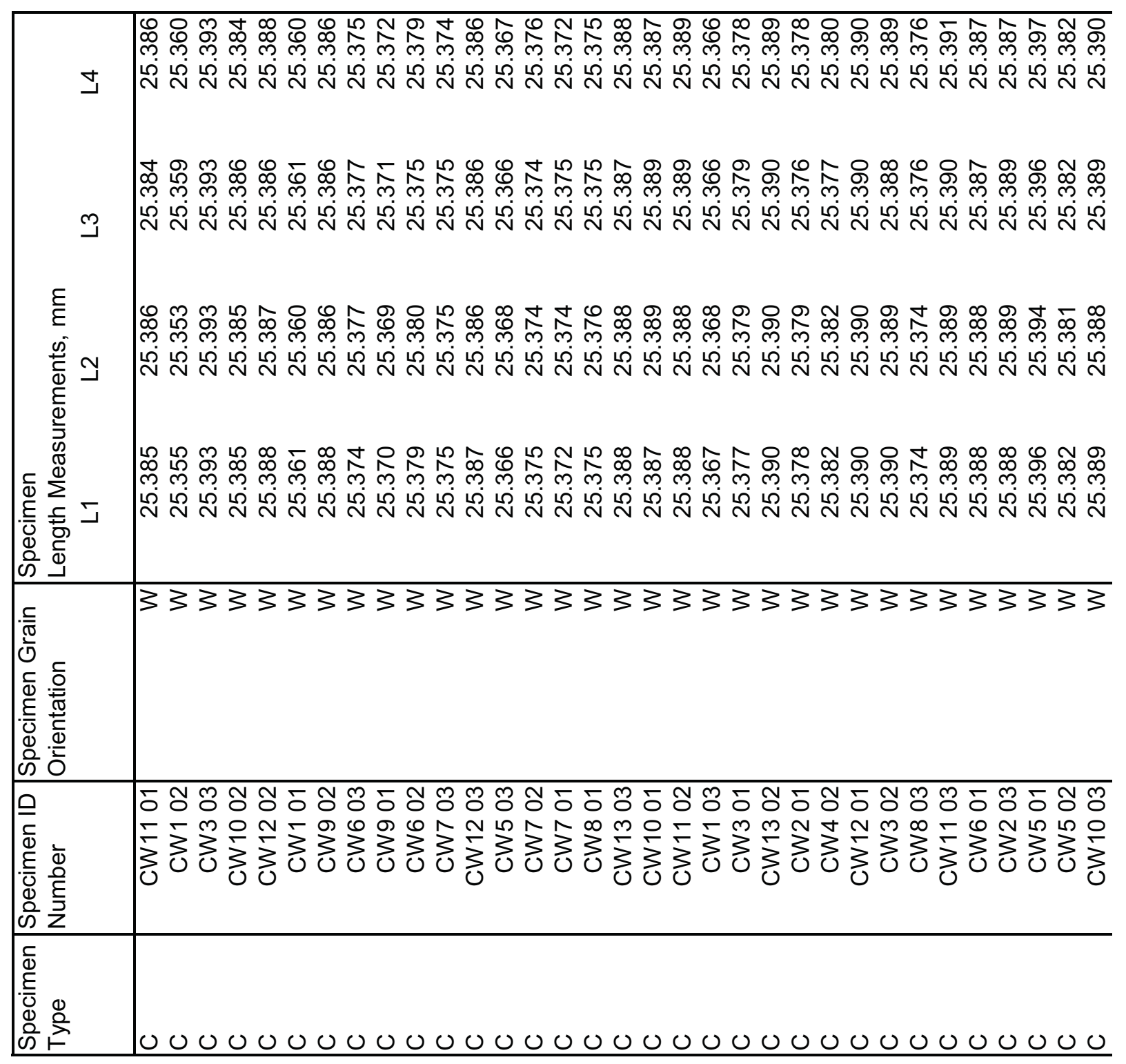




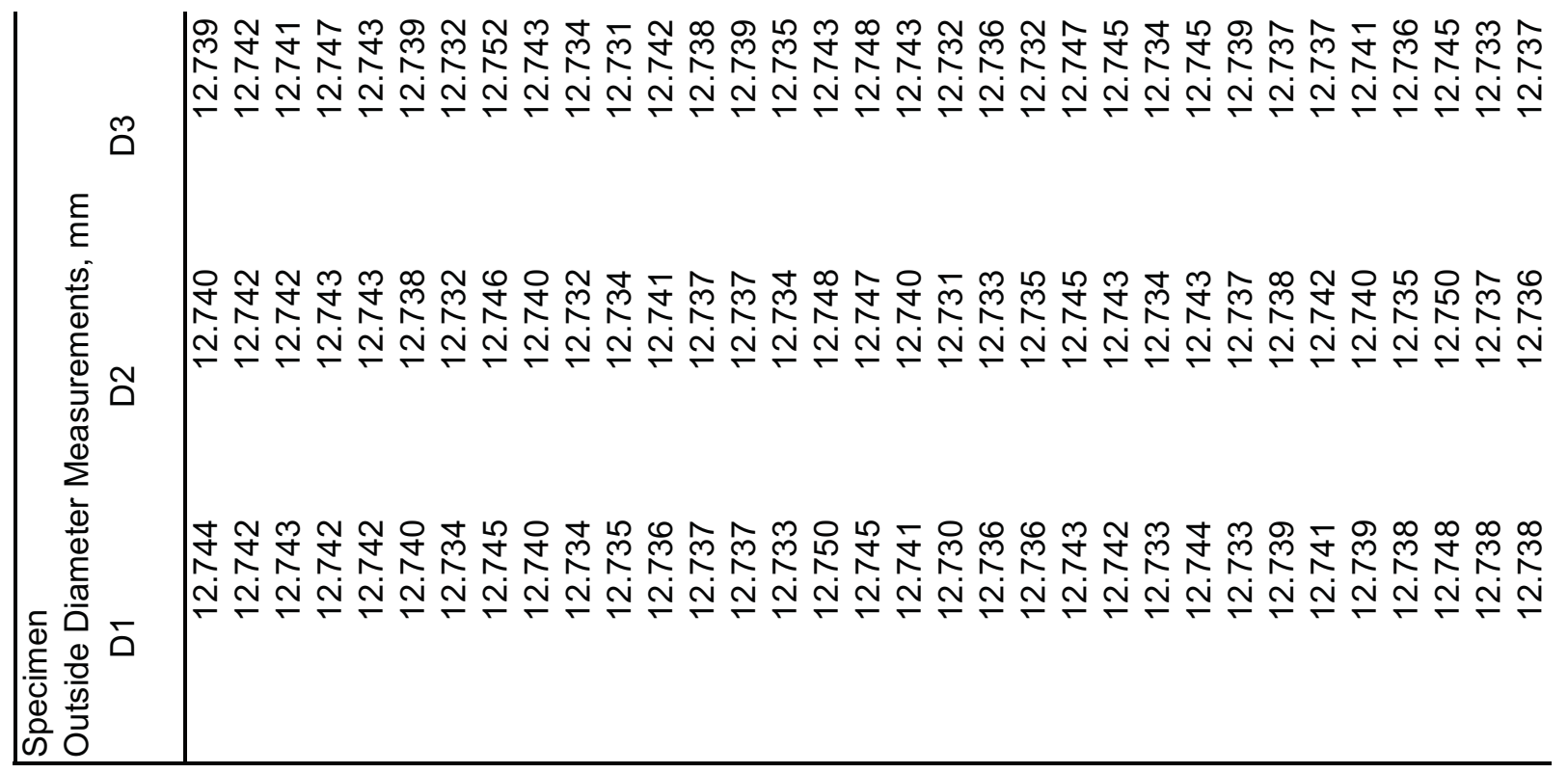

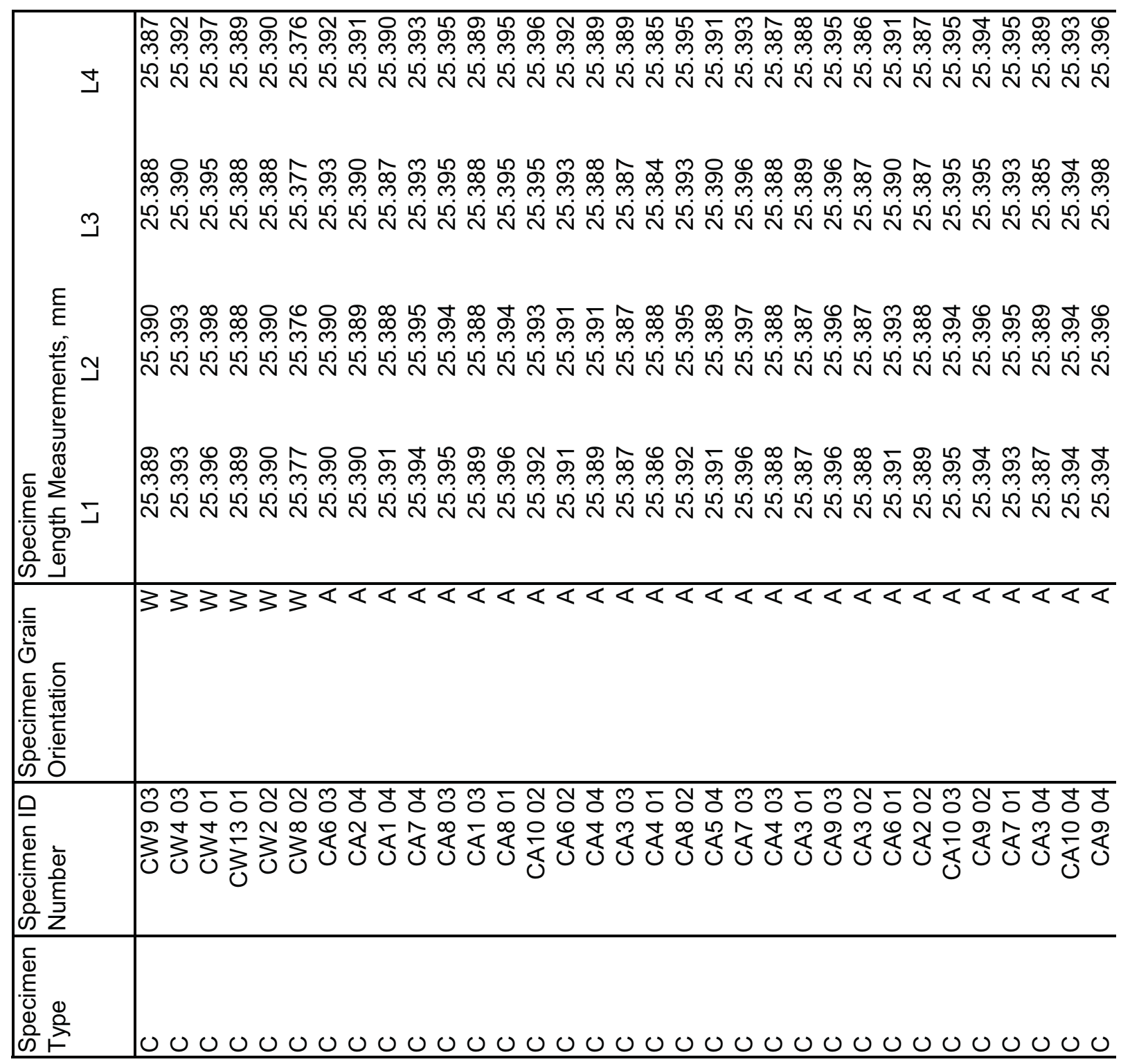



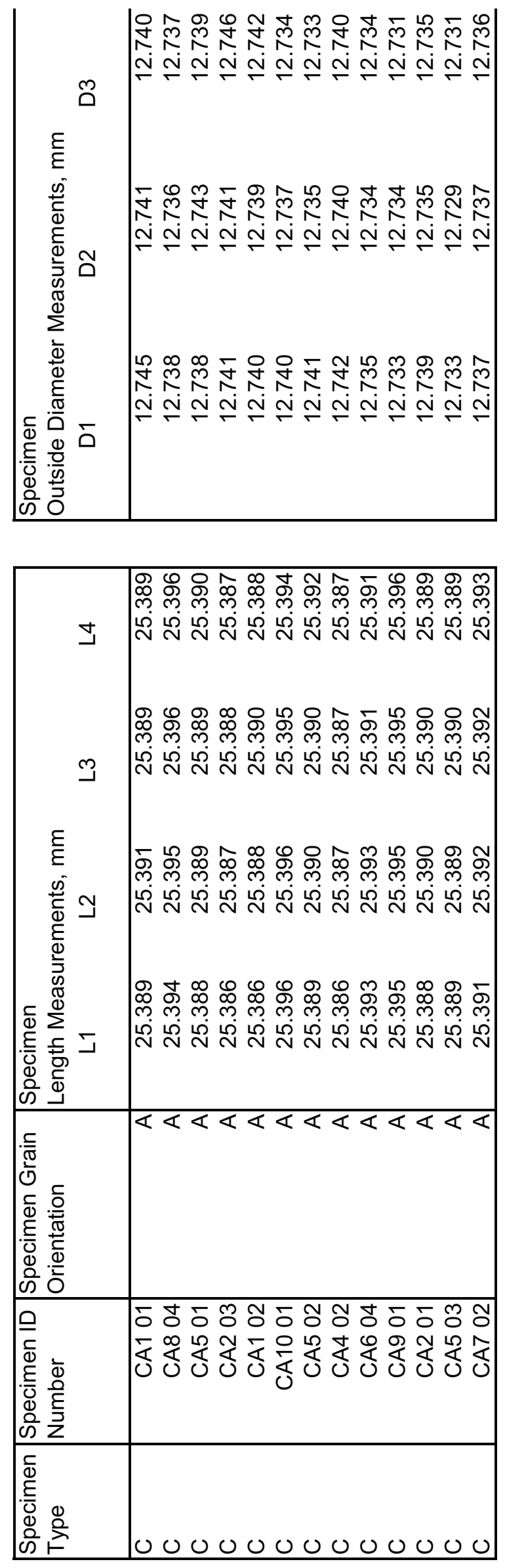


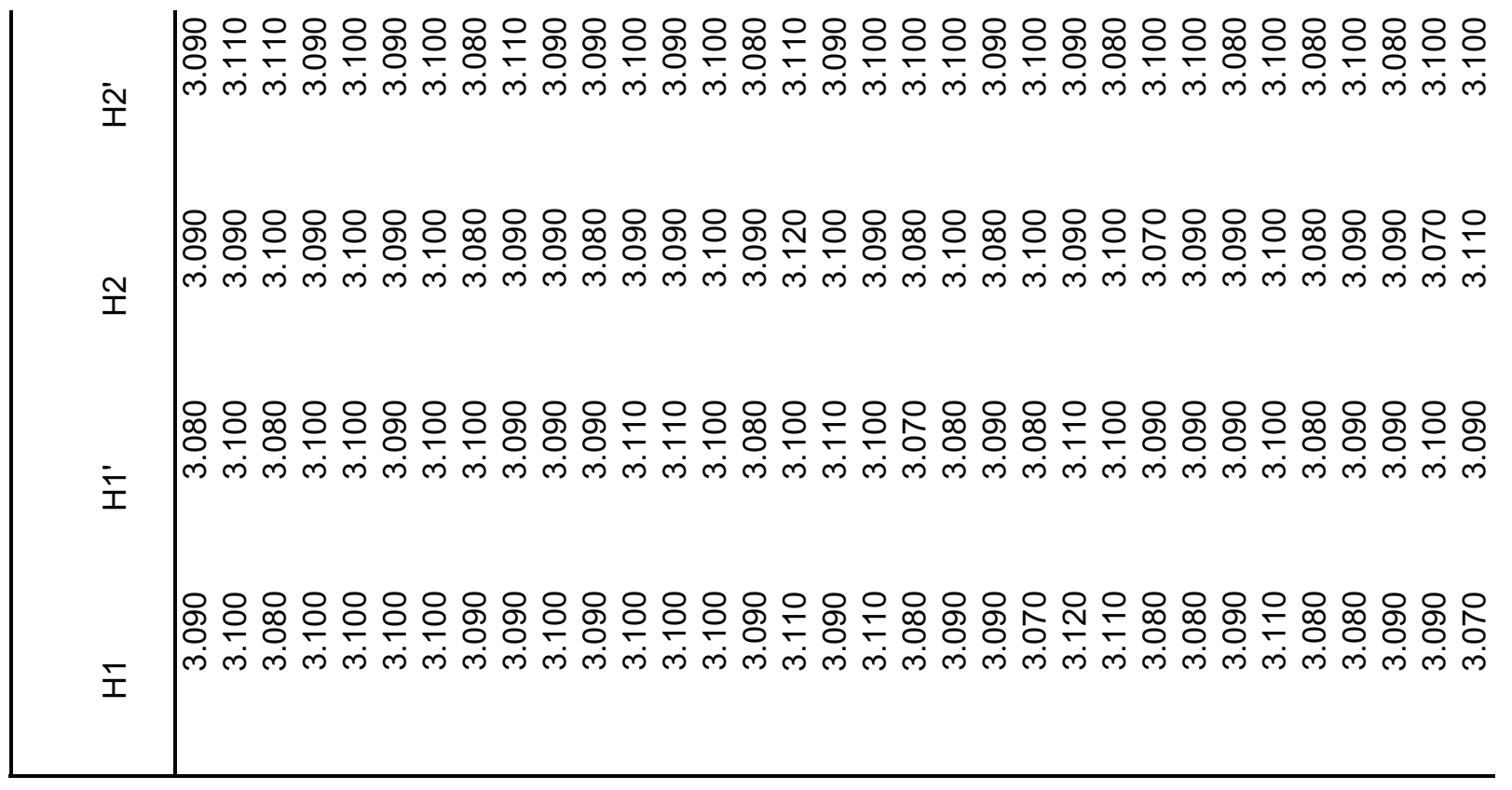

\begin{tabular}{|c|c|}
\hline & 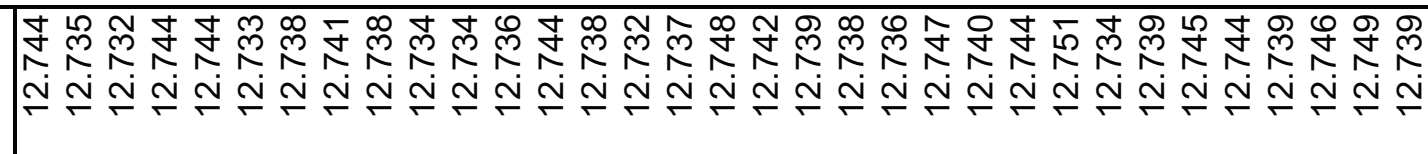 \\
\hline & 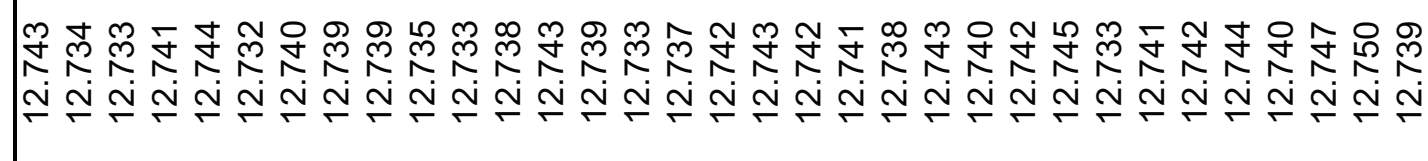 \\
\hline & 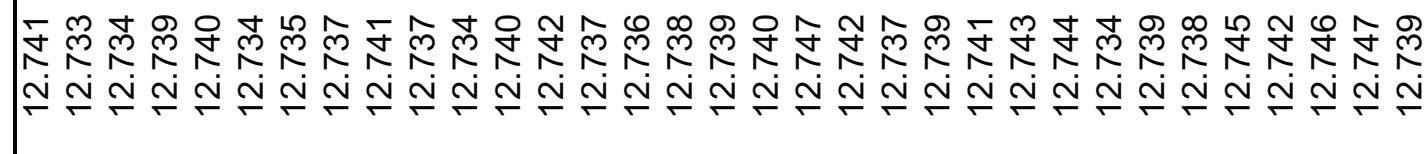 \\
\hline & 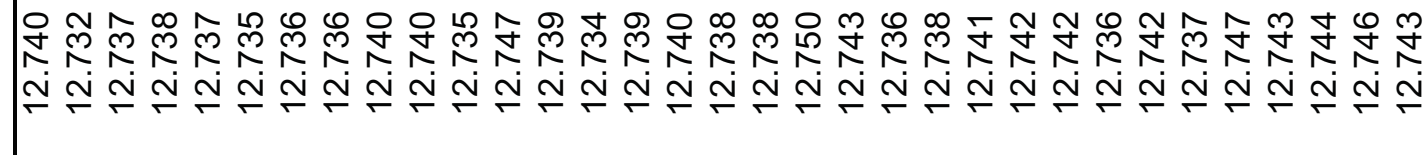 \\
\hline & 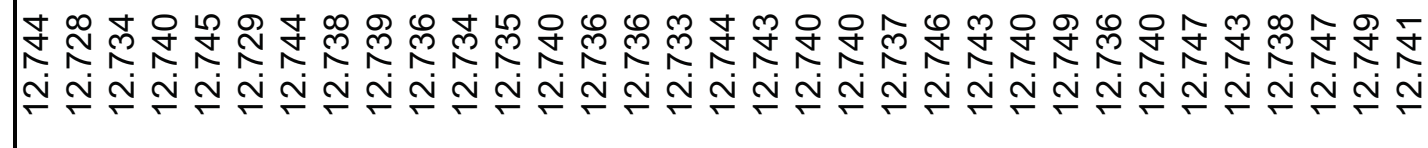 \\
\hline 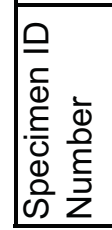 & 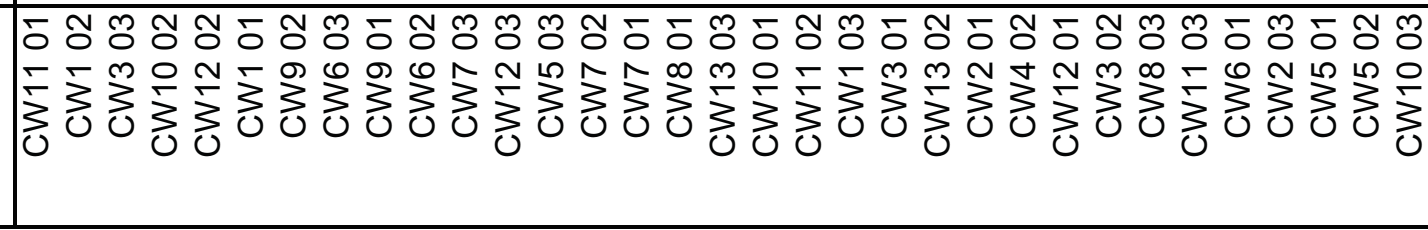 \\
\hline
\end{tabular}




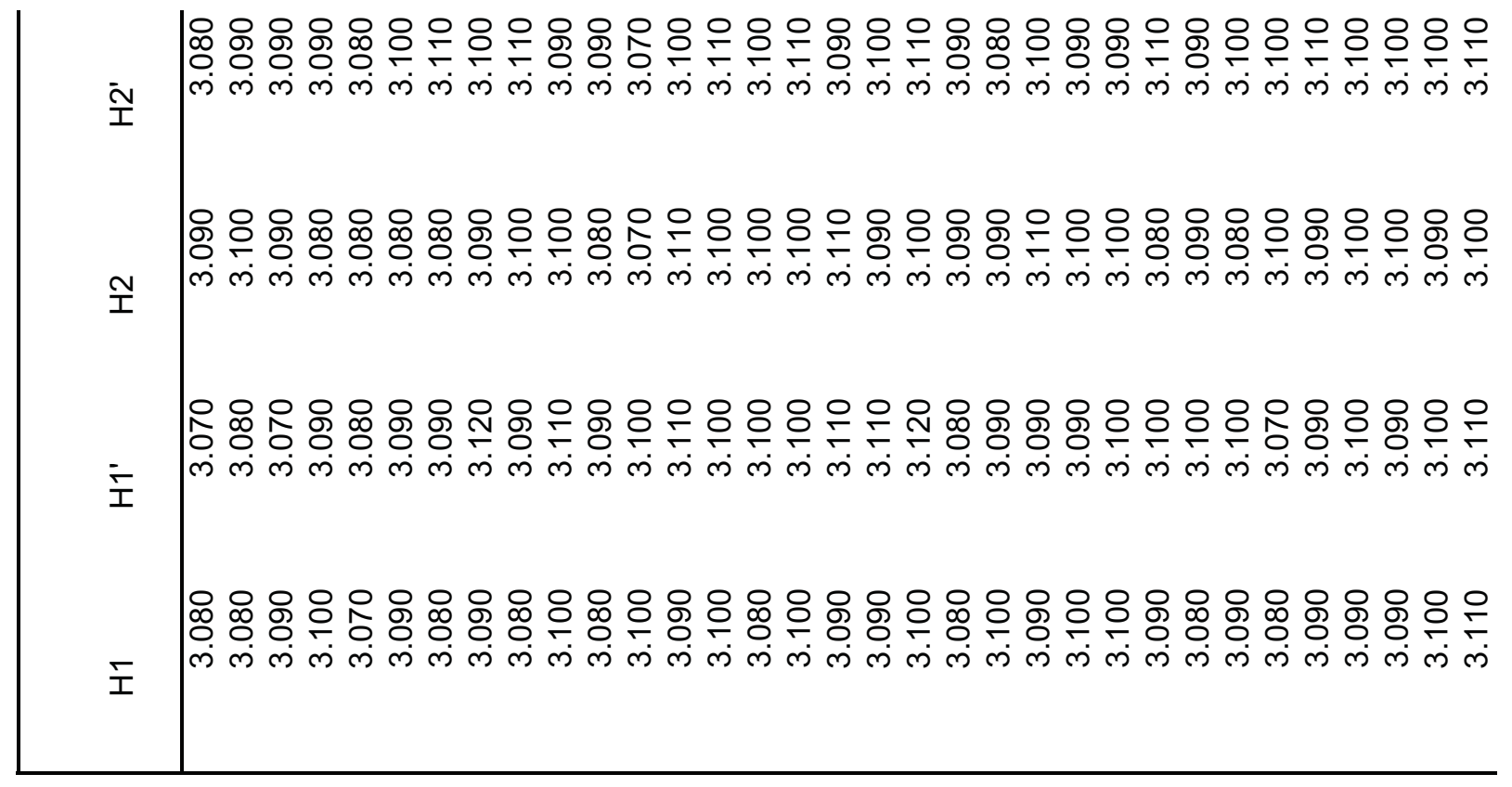

\begin{tabular}{|c|c|}
\hline & 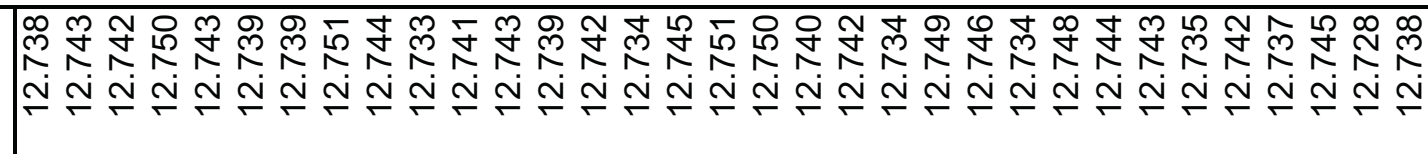 \\
\hline & 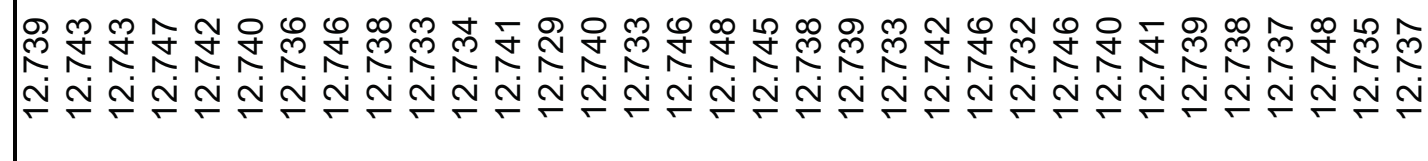 \\
\hline & 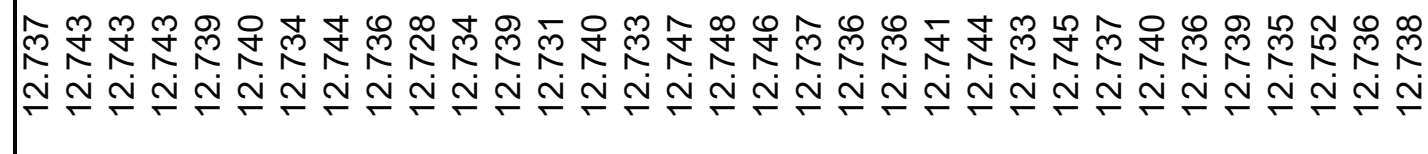 \\
\hline & 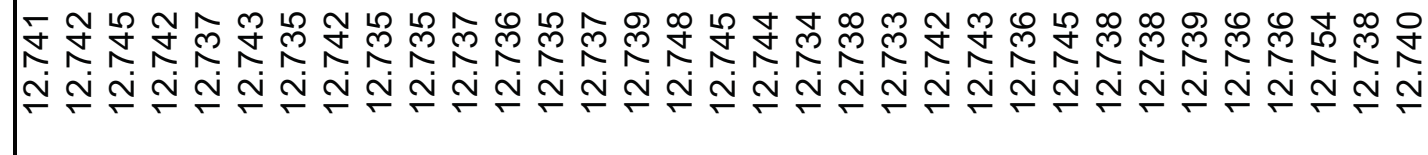 \\
\hline & 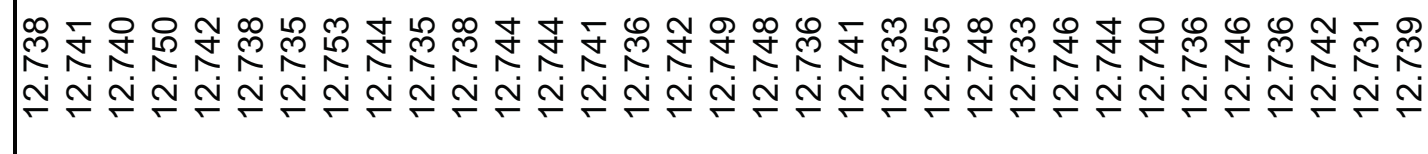 \\
\hline 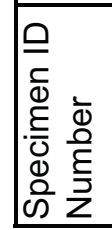 & 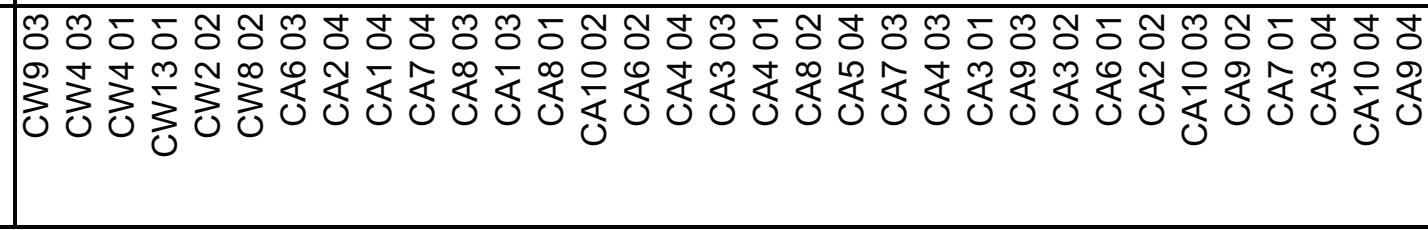 \\
\hline
\end{tabular}




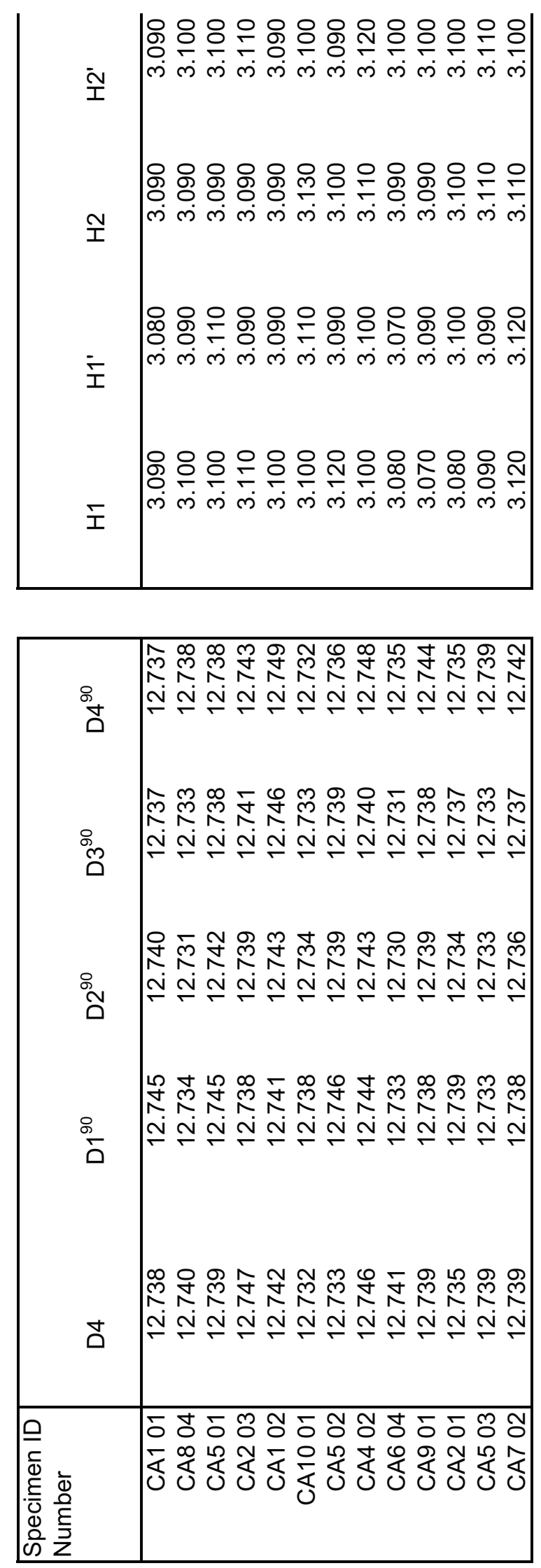




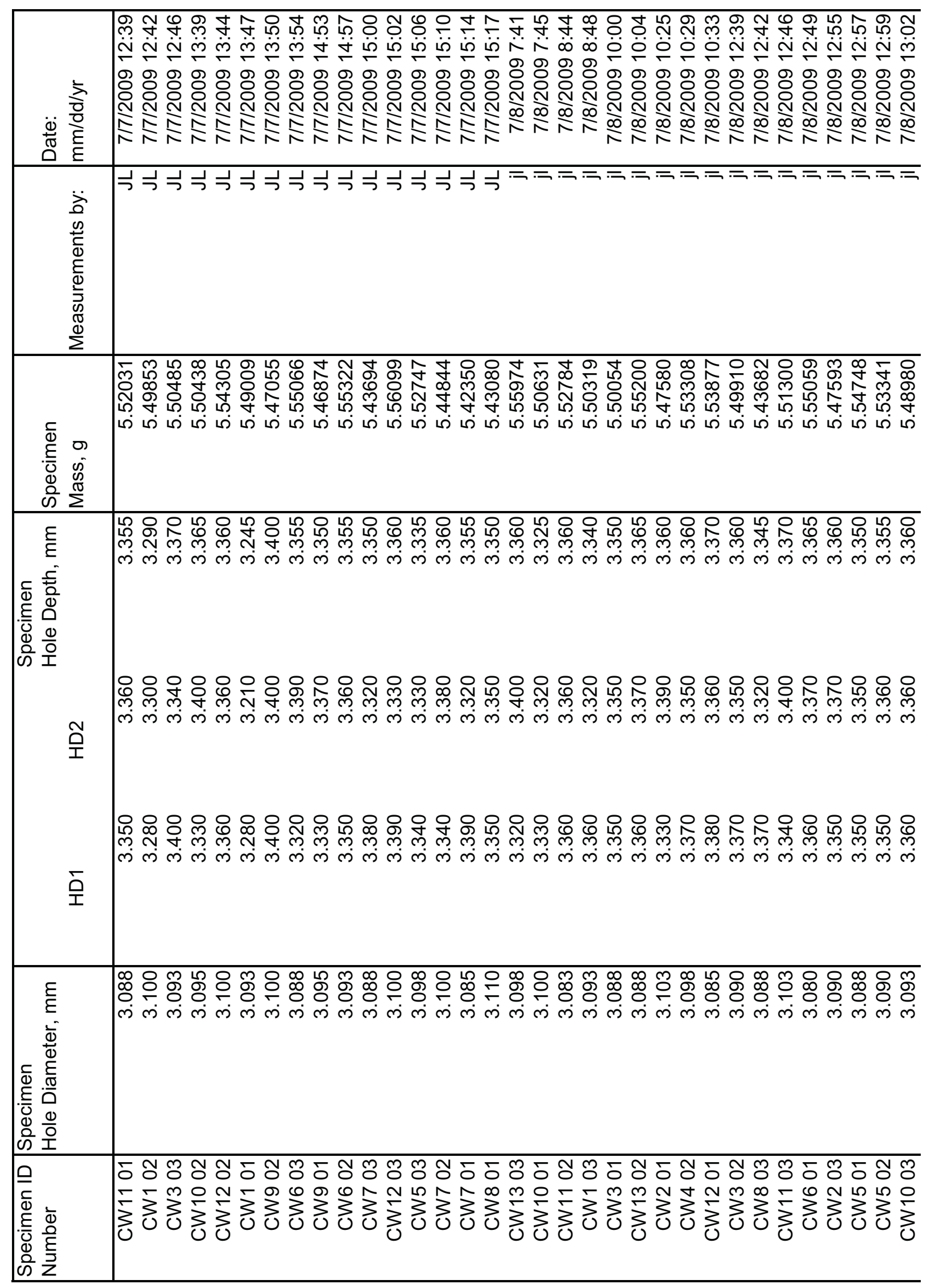




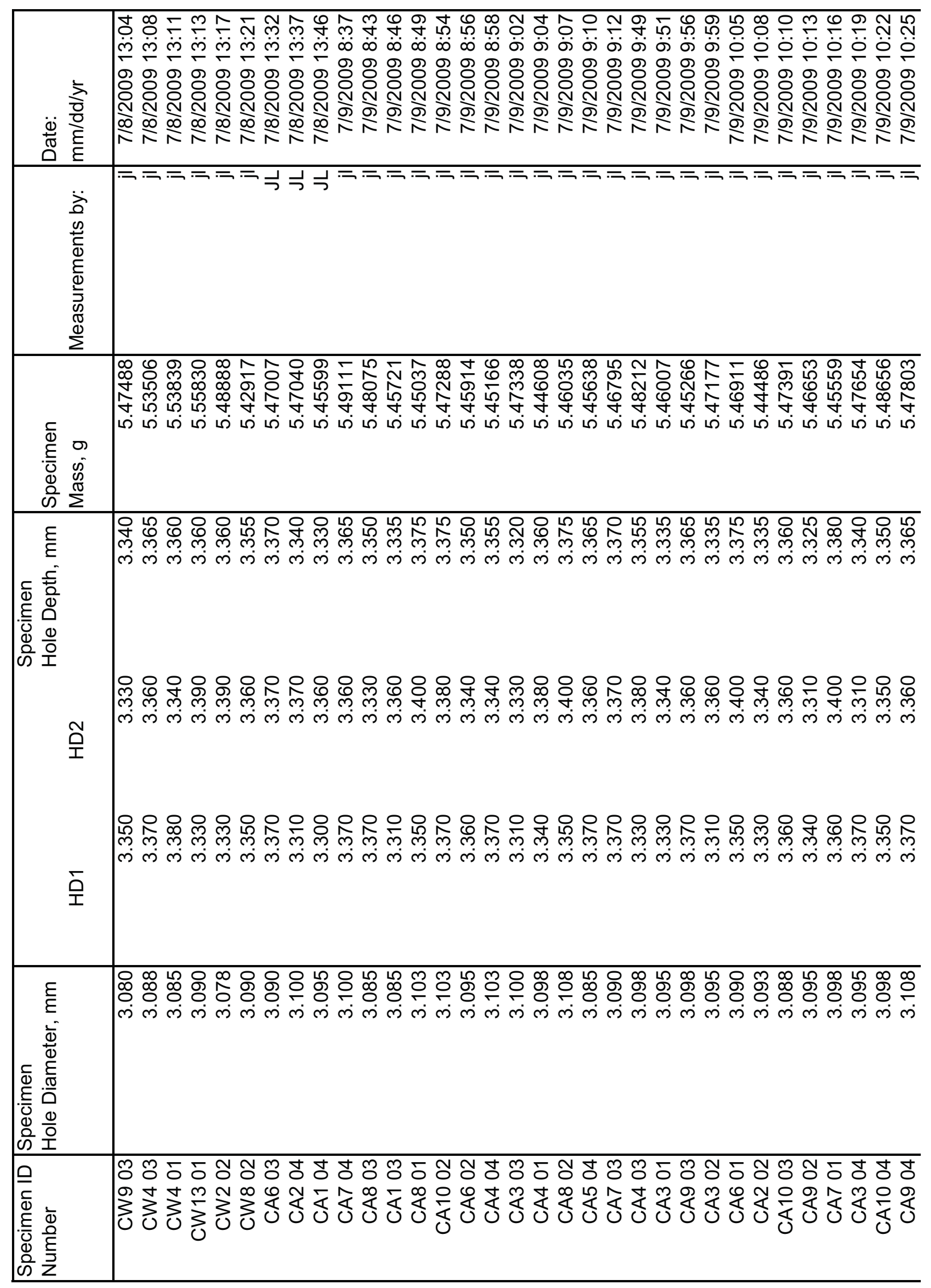




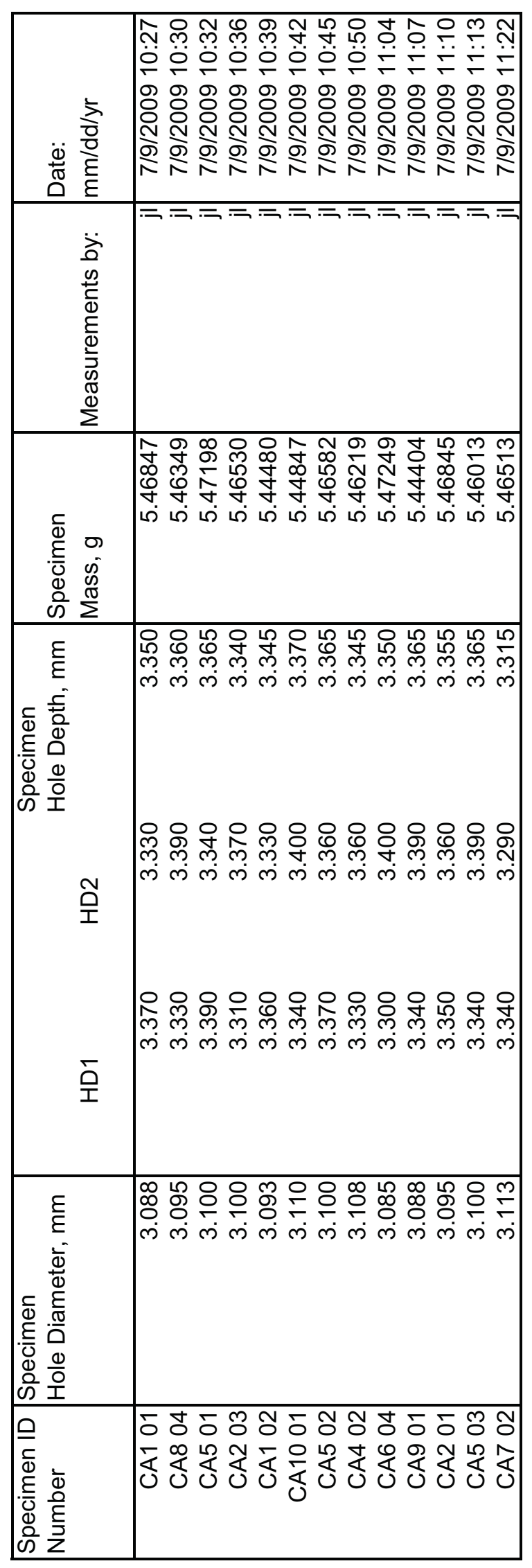

$\stackrel{n}{\sim}$ 


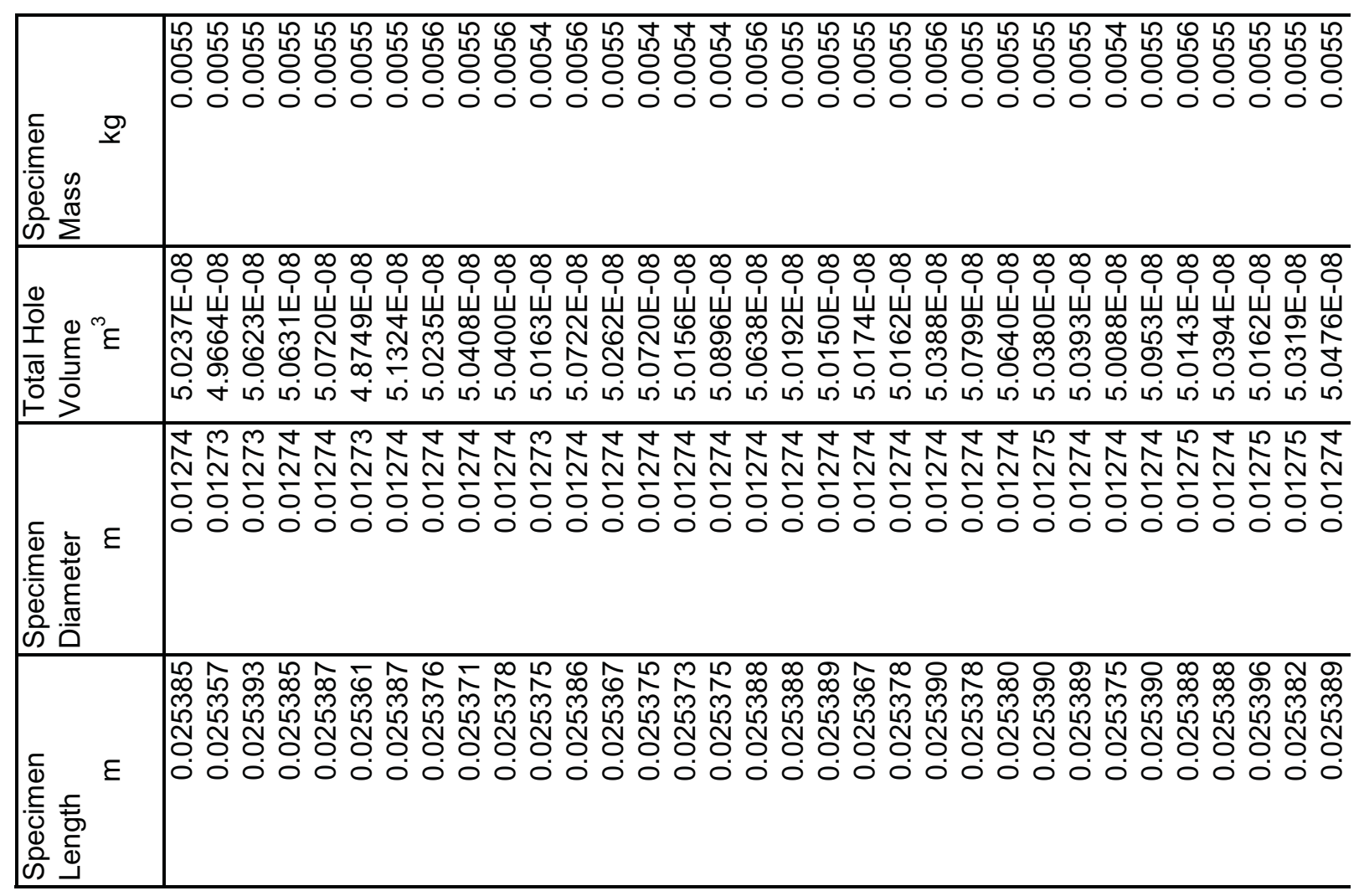

\begin{tabular}{|c|c|}
\hline 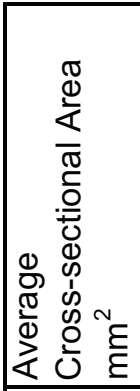 & 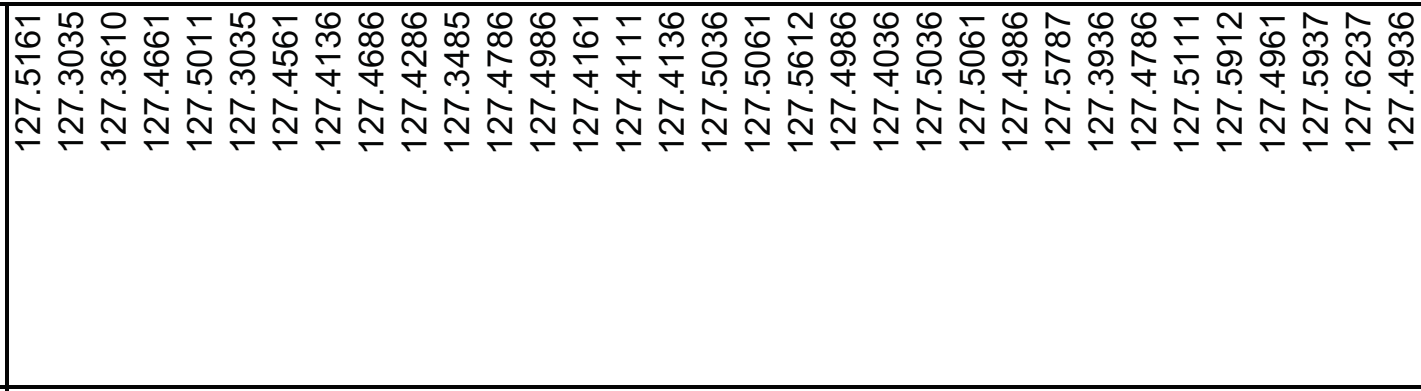 \\
\hline 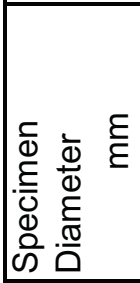 & 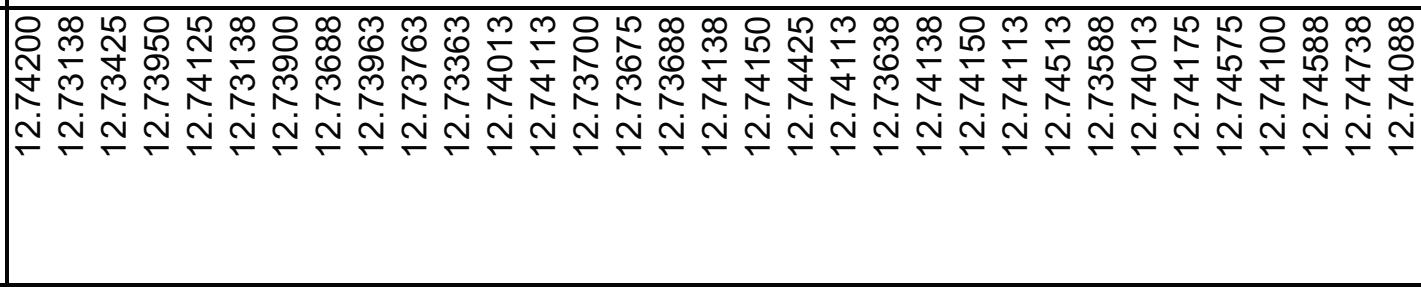 \\
\hline 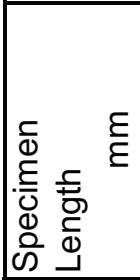 & 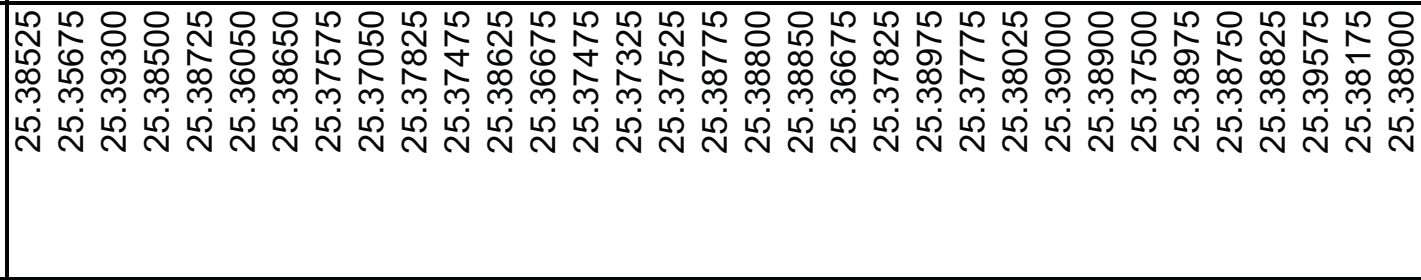 \\
\hline 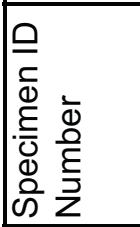 & 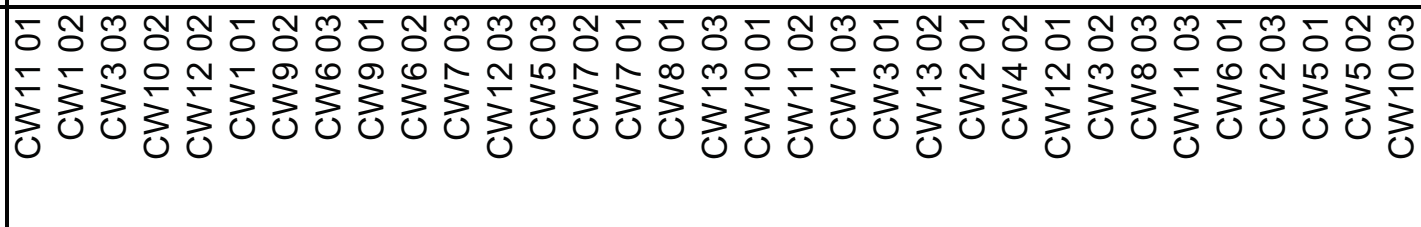 \\
\hline
\end{tabular}




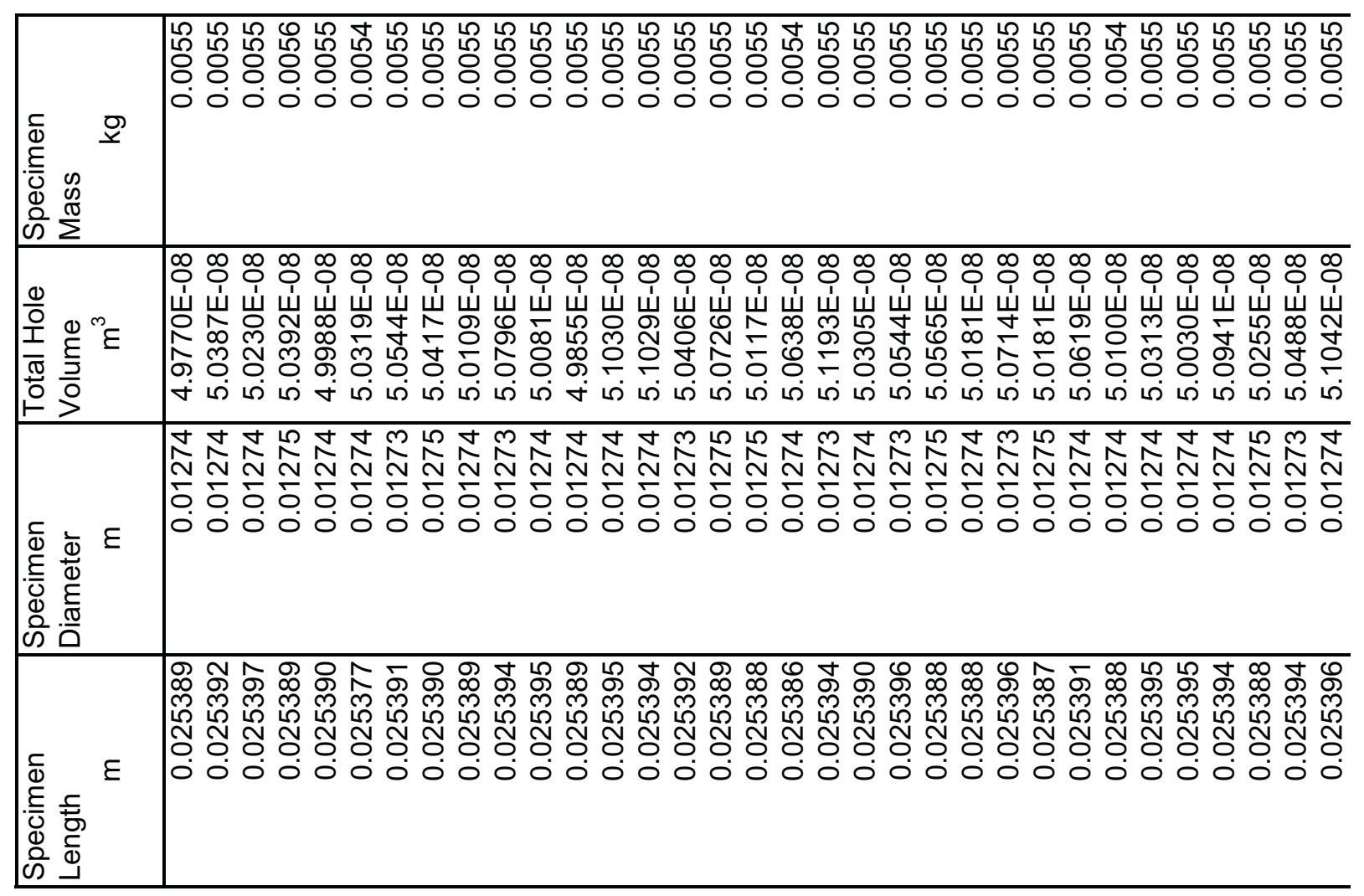

\begin{tabular}{|c|c|}
\hline 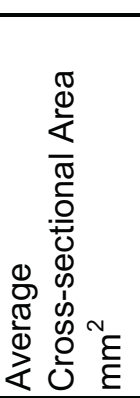 & 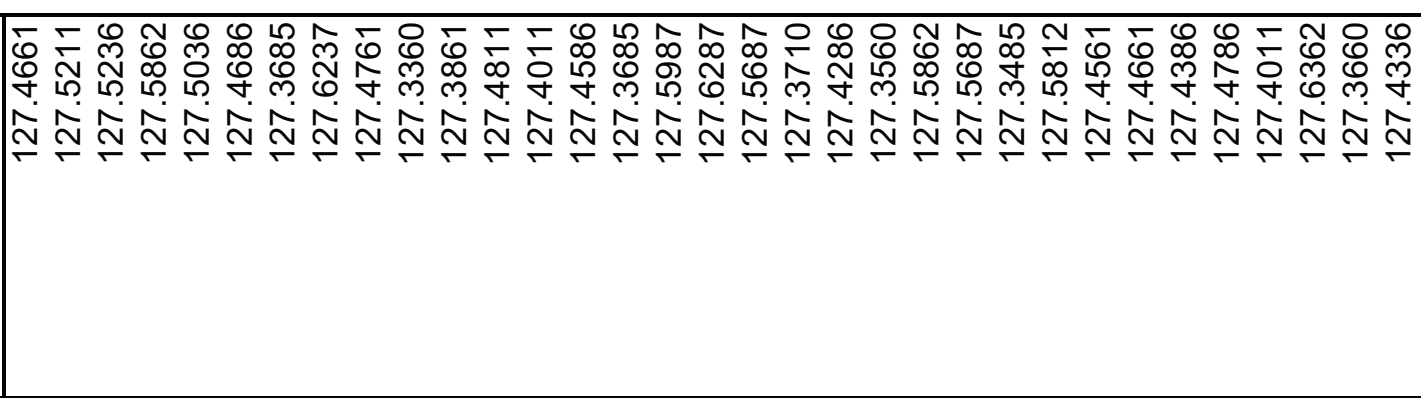 \\
\hline 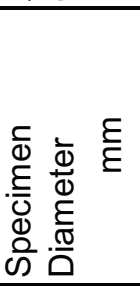 & 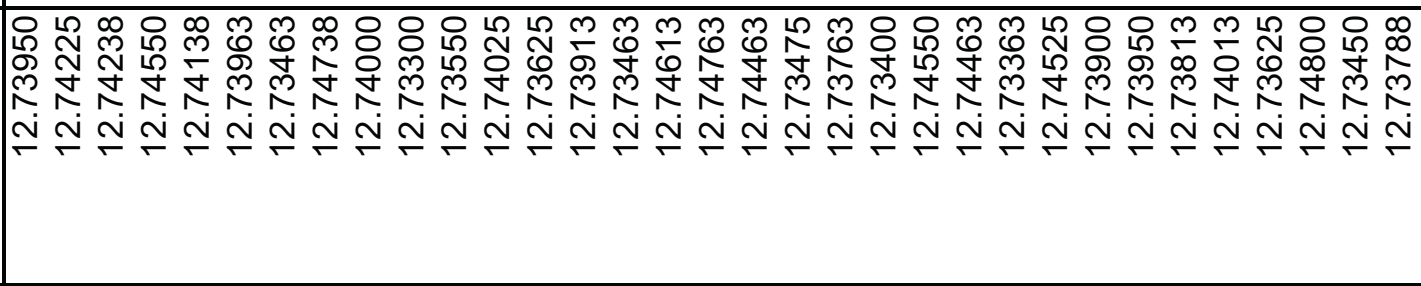 \\
\hline 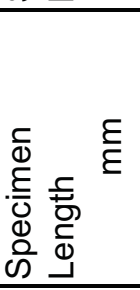 & 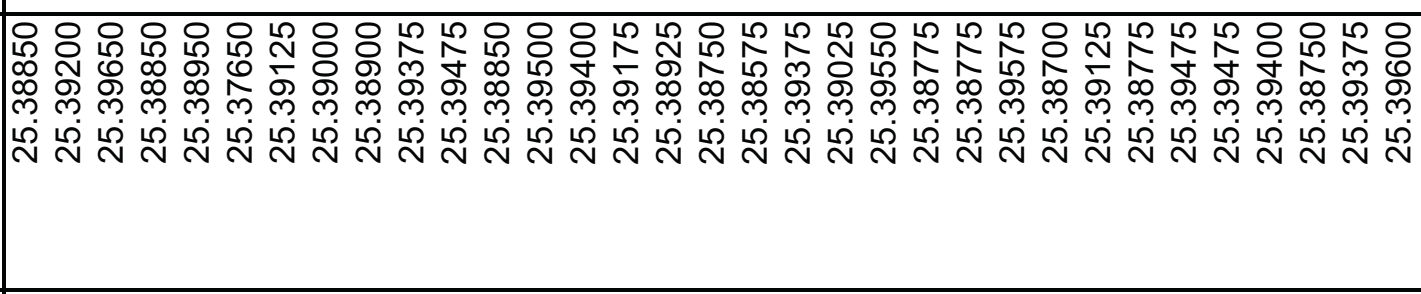 \\
\hline 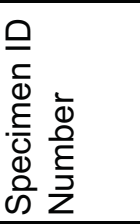 & 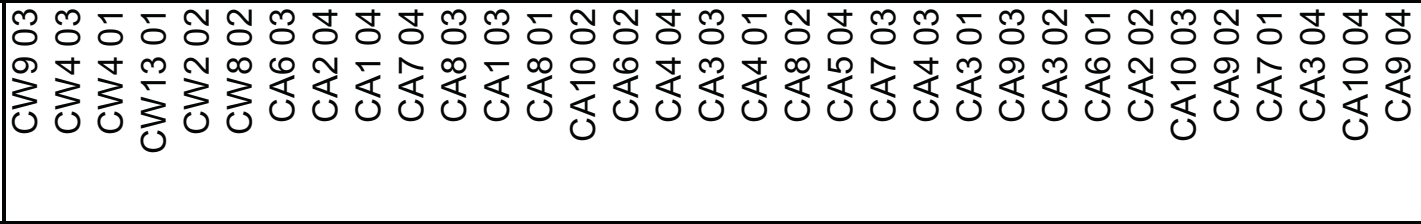 \\
\hline
\end{tabular}



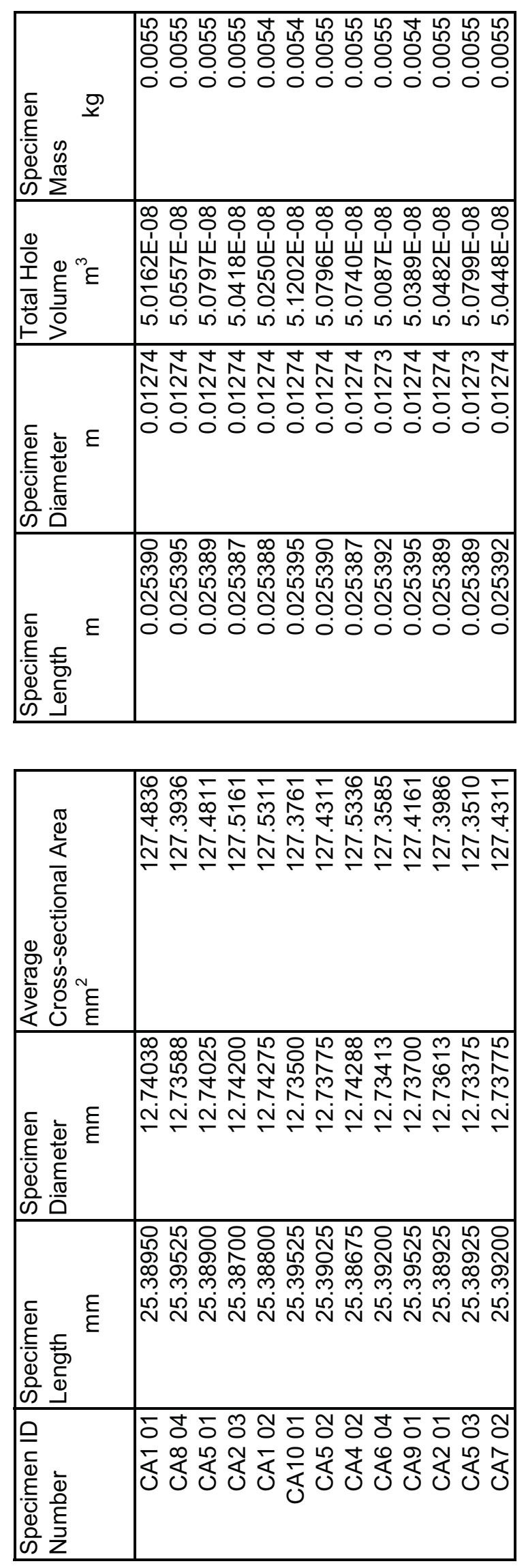


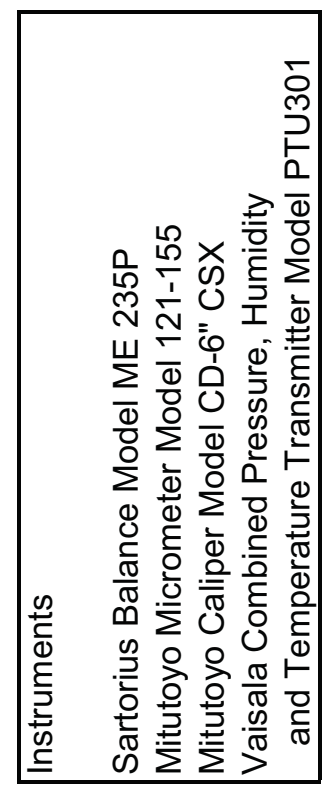

\begin{tabular}{|c|c|}
\hline 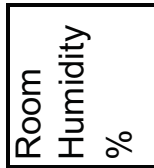 & 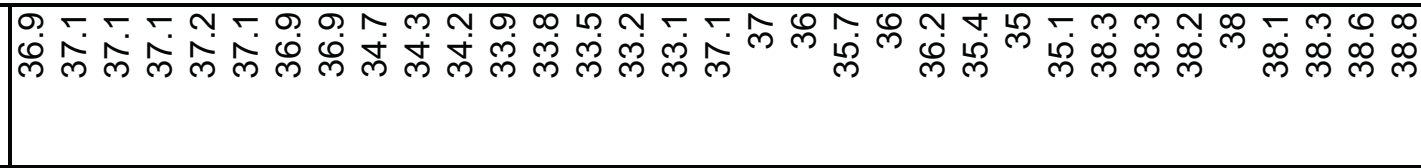 \\
\hline 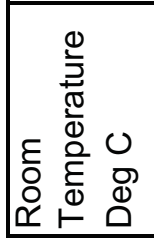 & 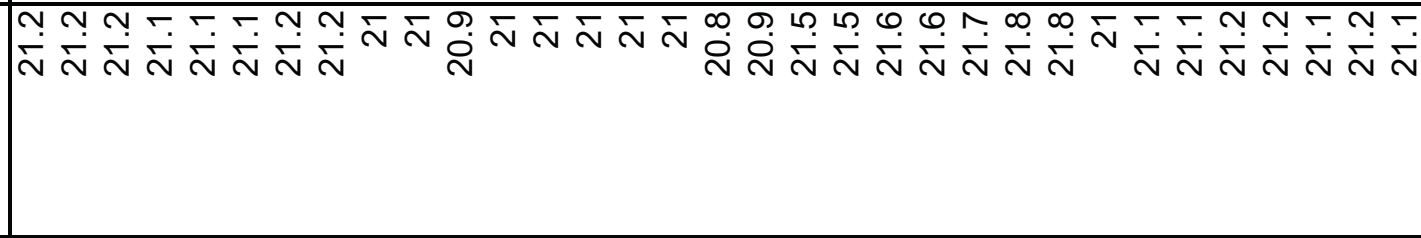 \\
\hline 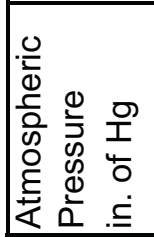 & 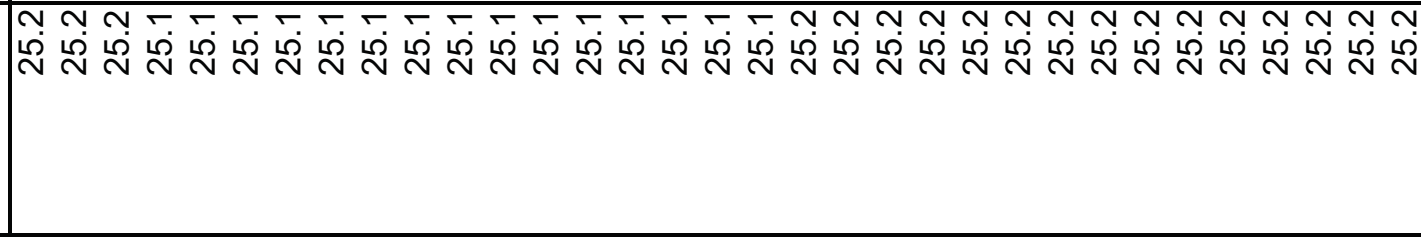 \\
\hline
\end{tabular}

\begin{tabular}{|c|c|}
\hline 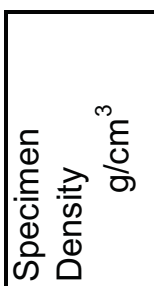 & 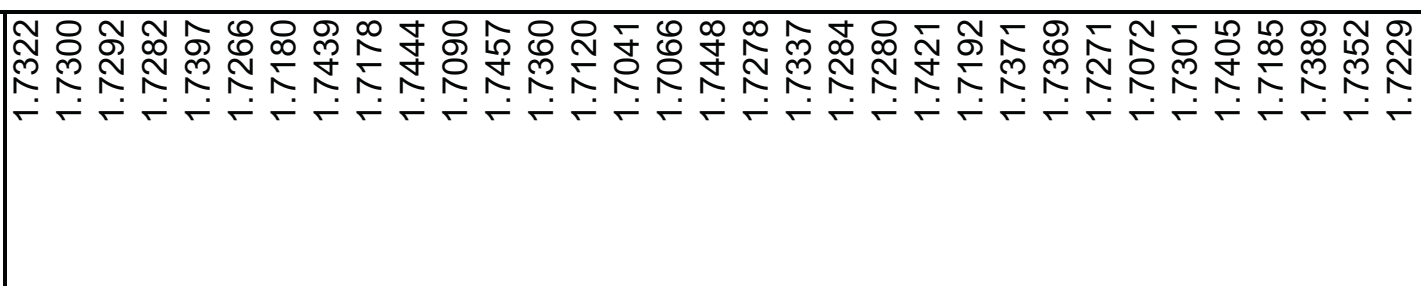 \\
\hline 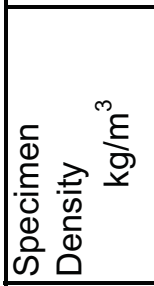 & 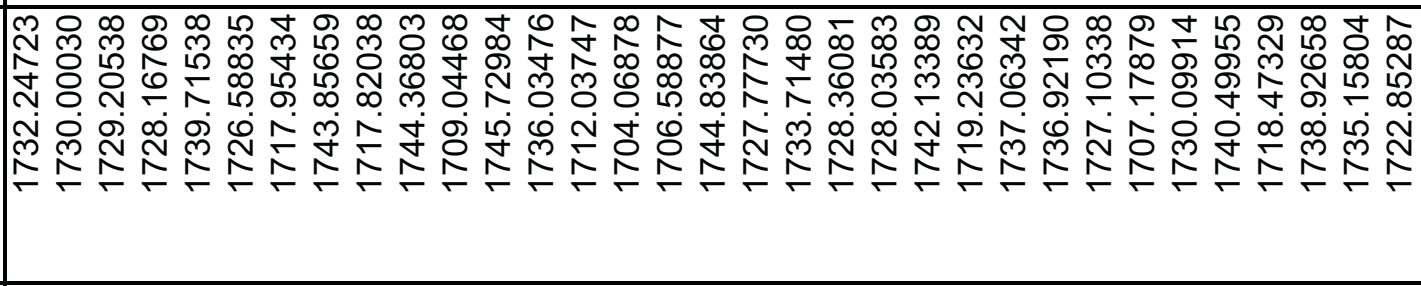 \\
\hline 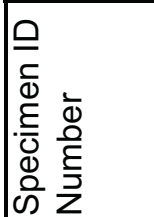 & 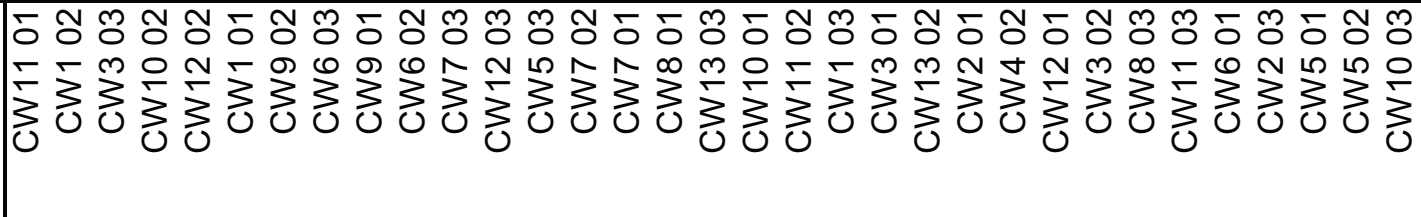 \\
\hline
\end{tabular}




\begin{tabular}{|c|c|}
\hline E & 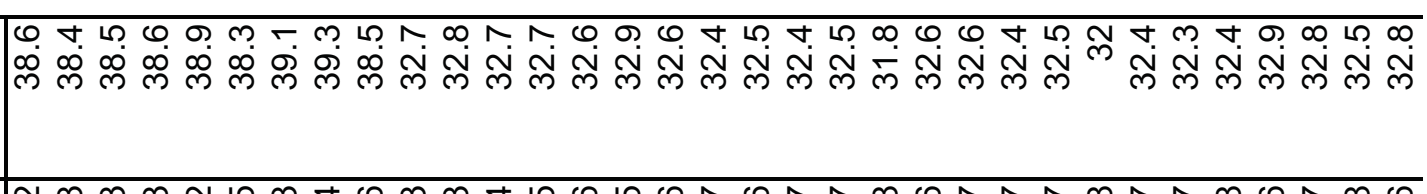 \\
\hline م. & 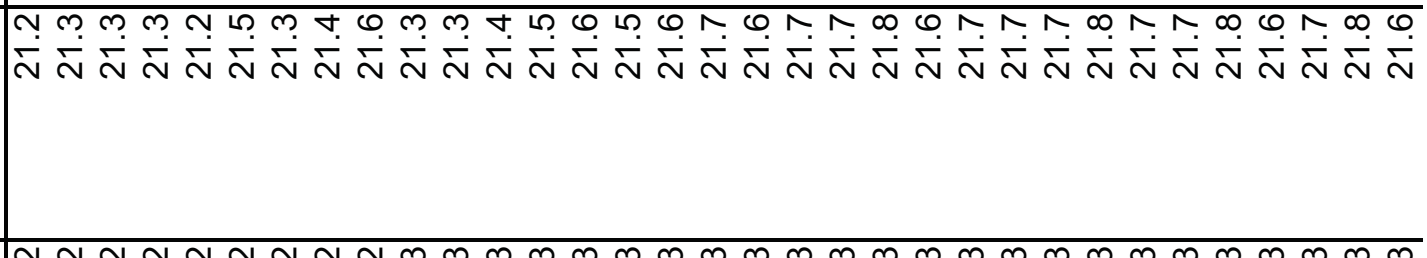 \\
\hline 罢 & 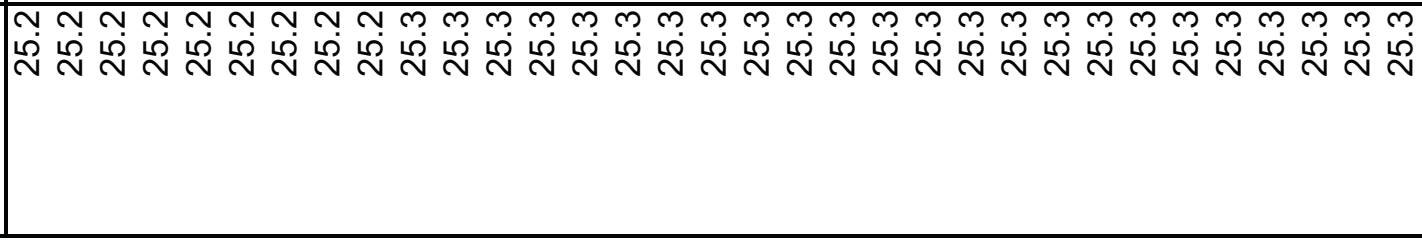 \\
\hline & 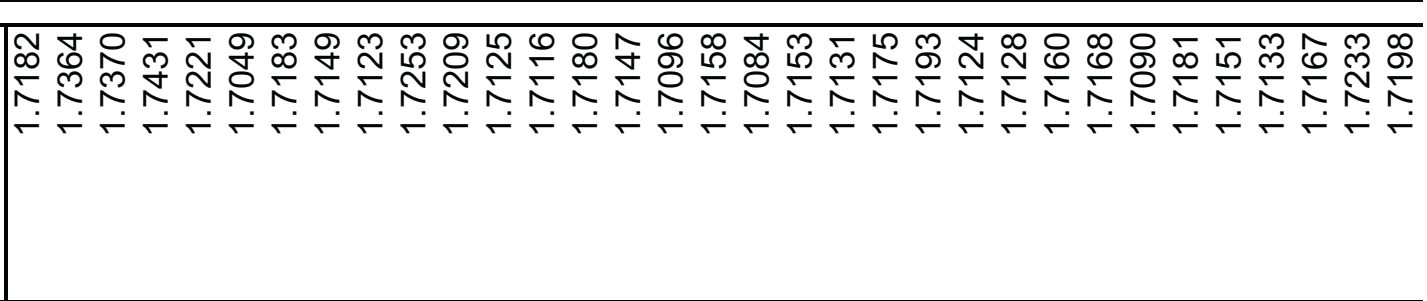 \\
\hline 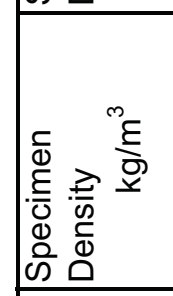 & 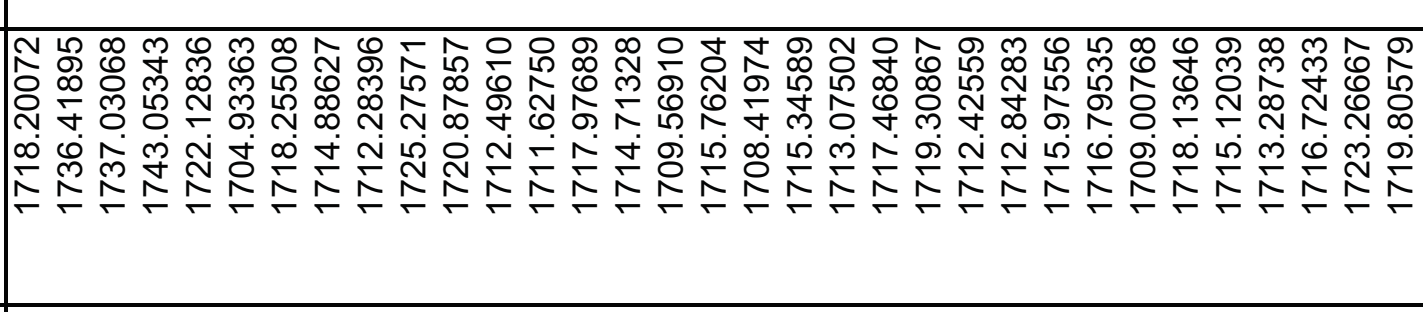 \\
\hline D & 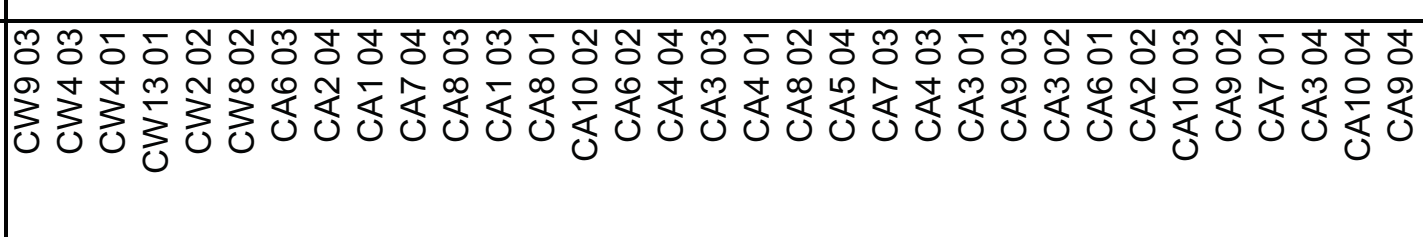 \\
\hline
\end{tabular}




\begin{tabular}{|c|c|}
\hline 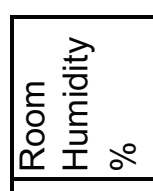 & 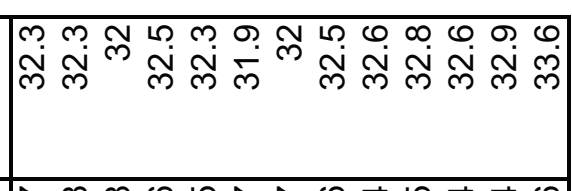 \\
\hline 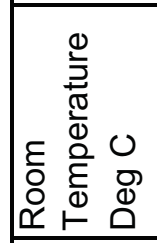 & 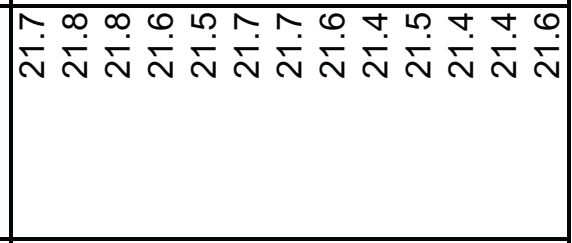 \\
\hline 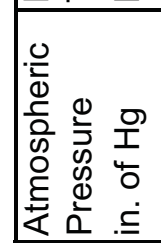 & m m m m m m m m m m m m \\
\hline
\end{tabular}

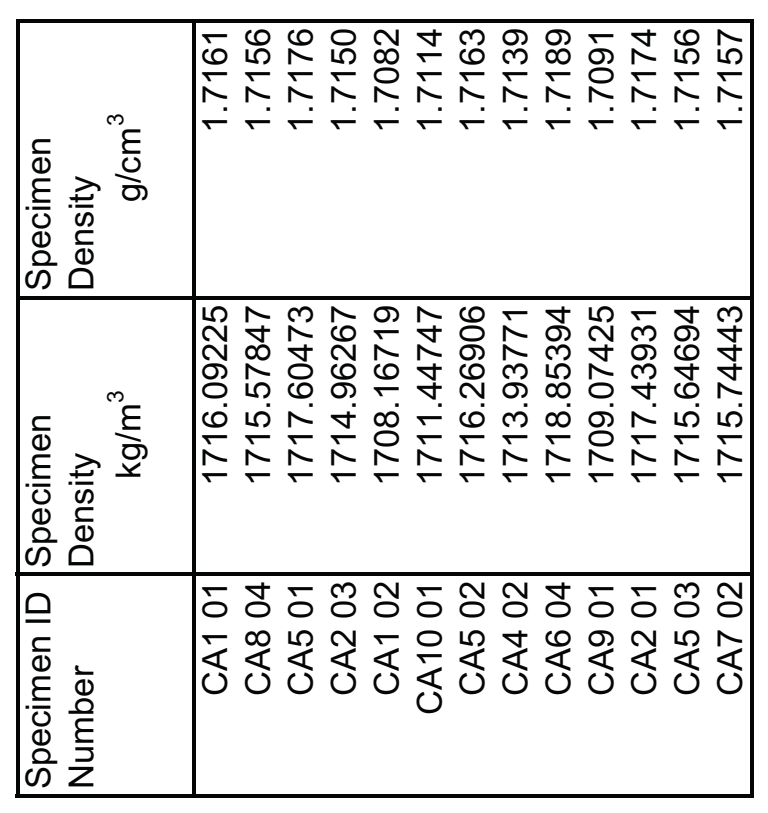




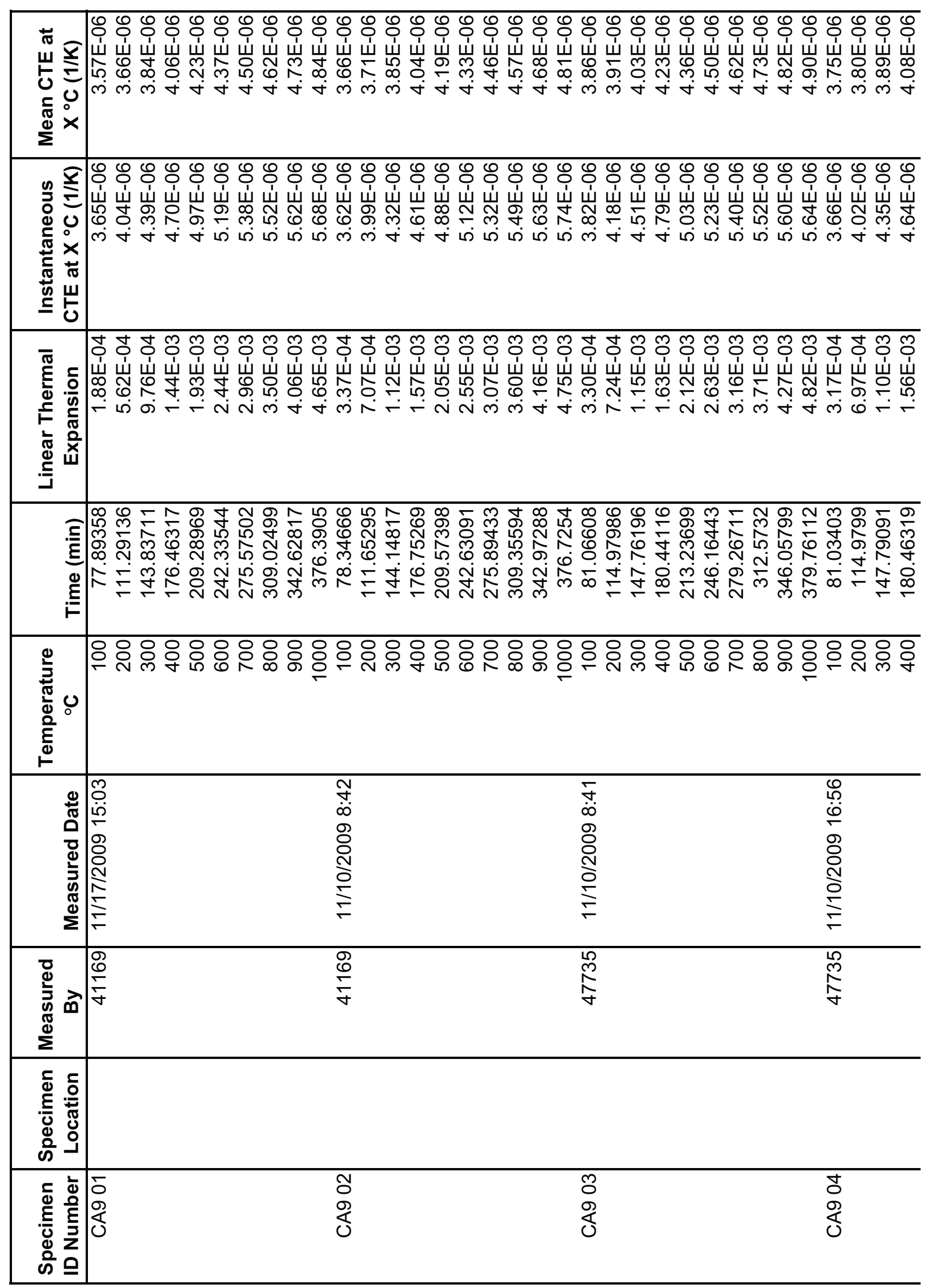




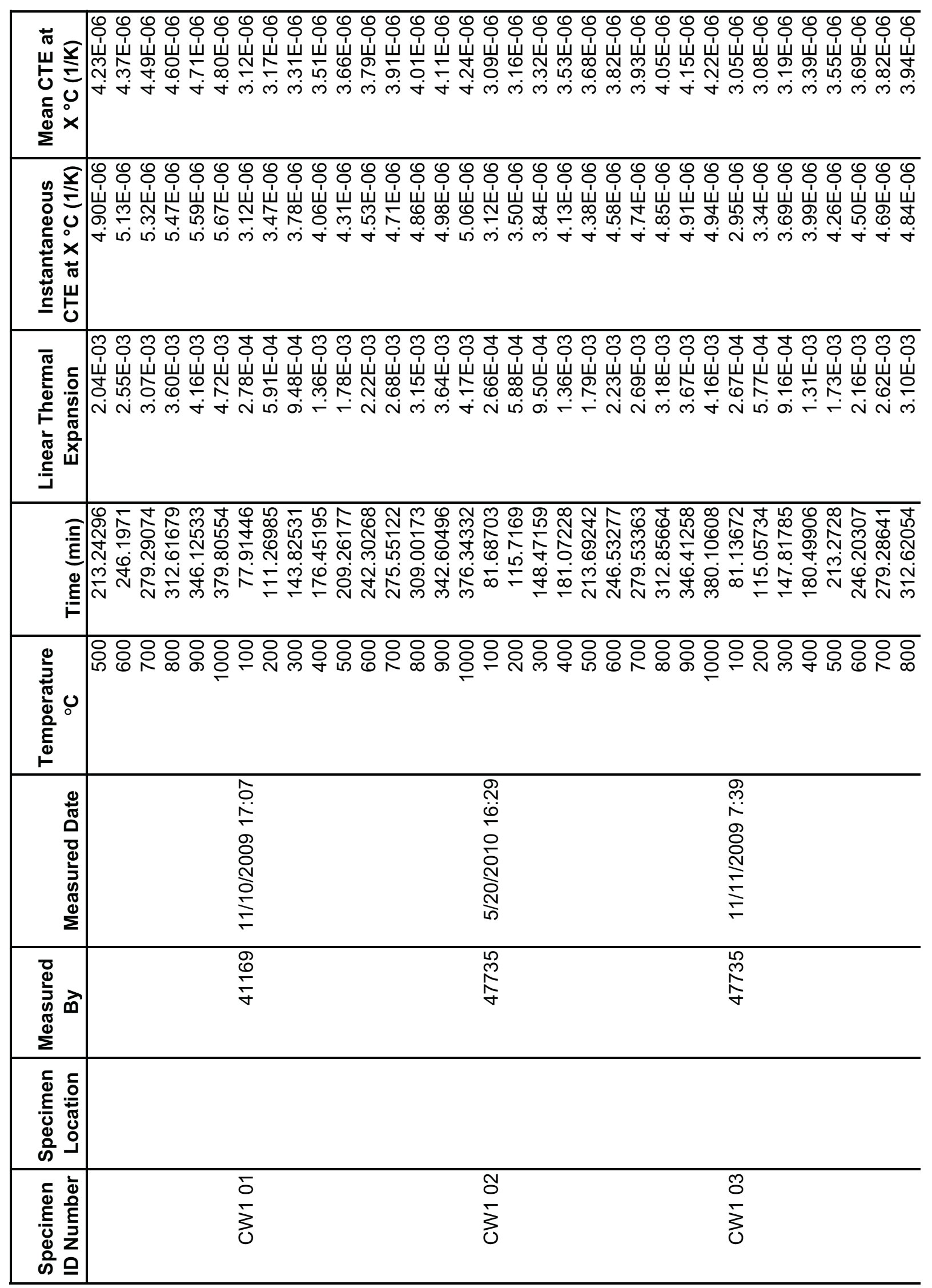




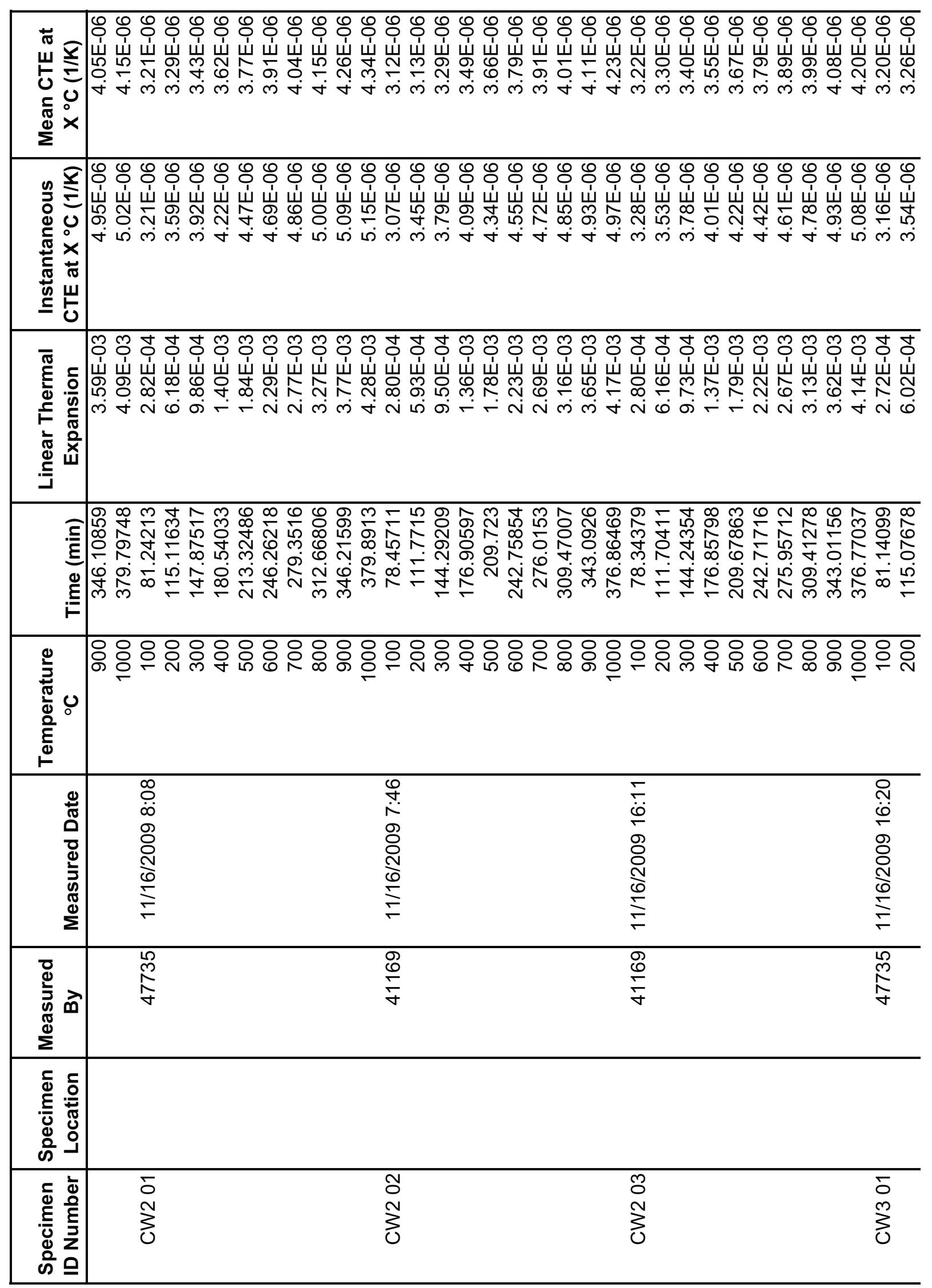




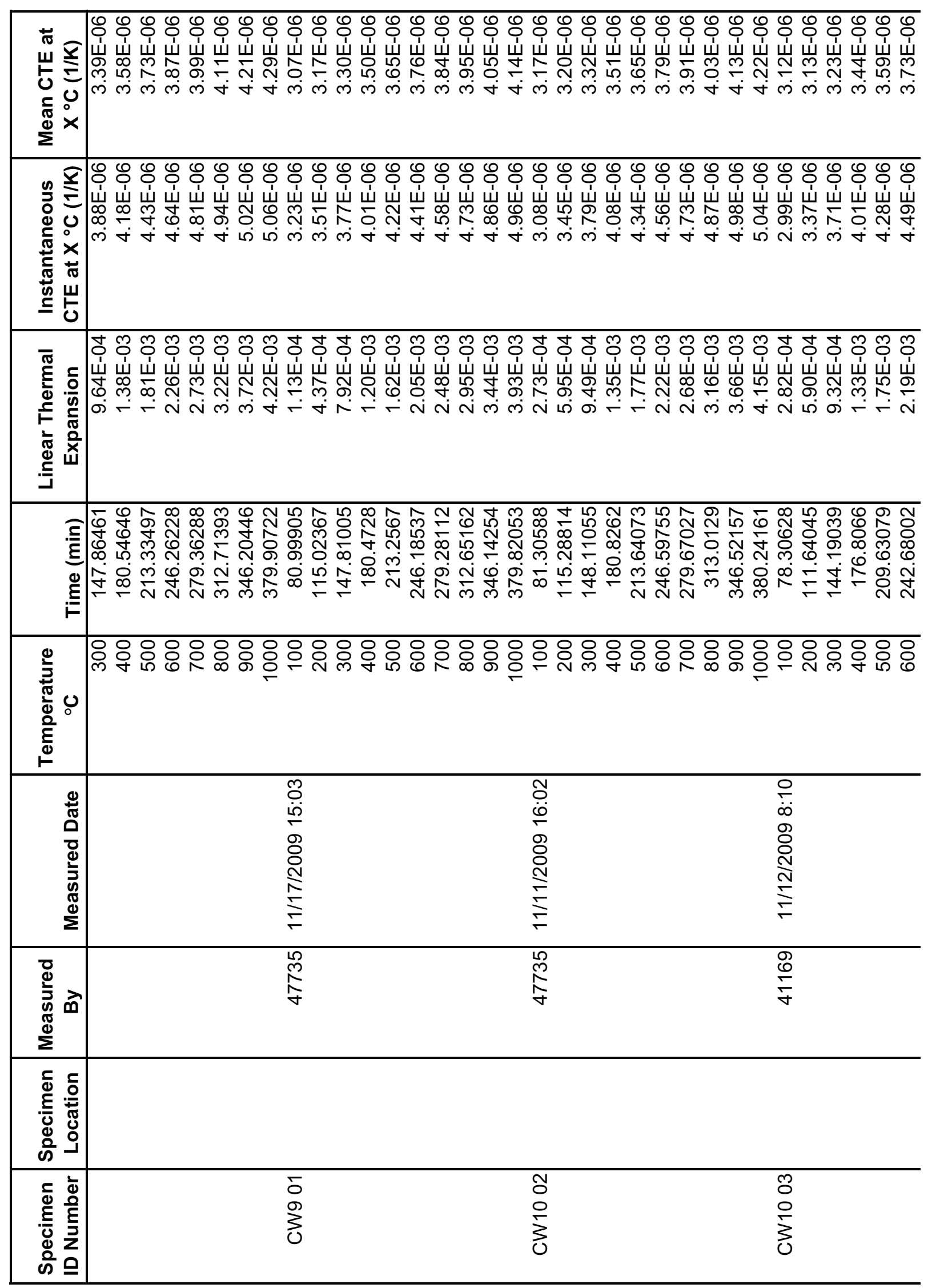




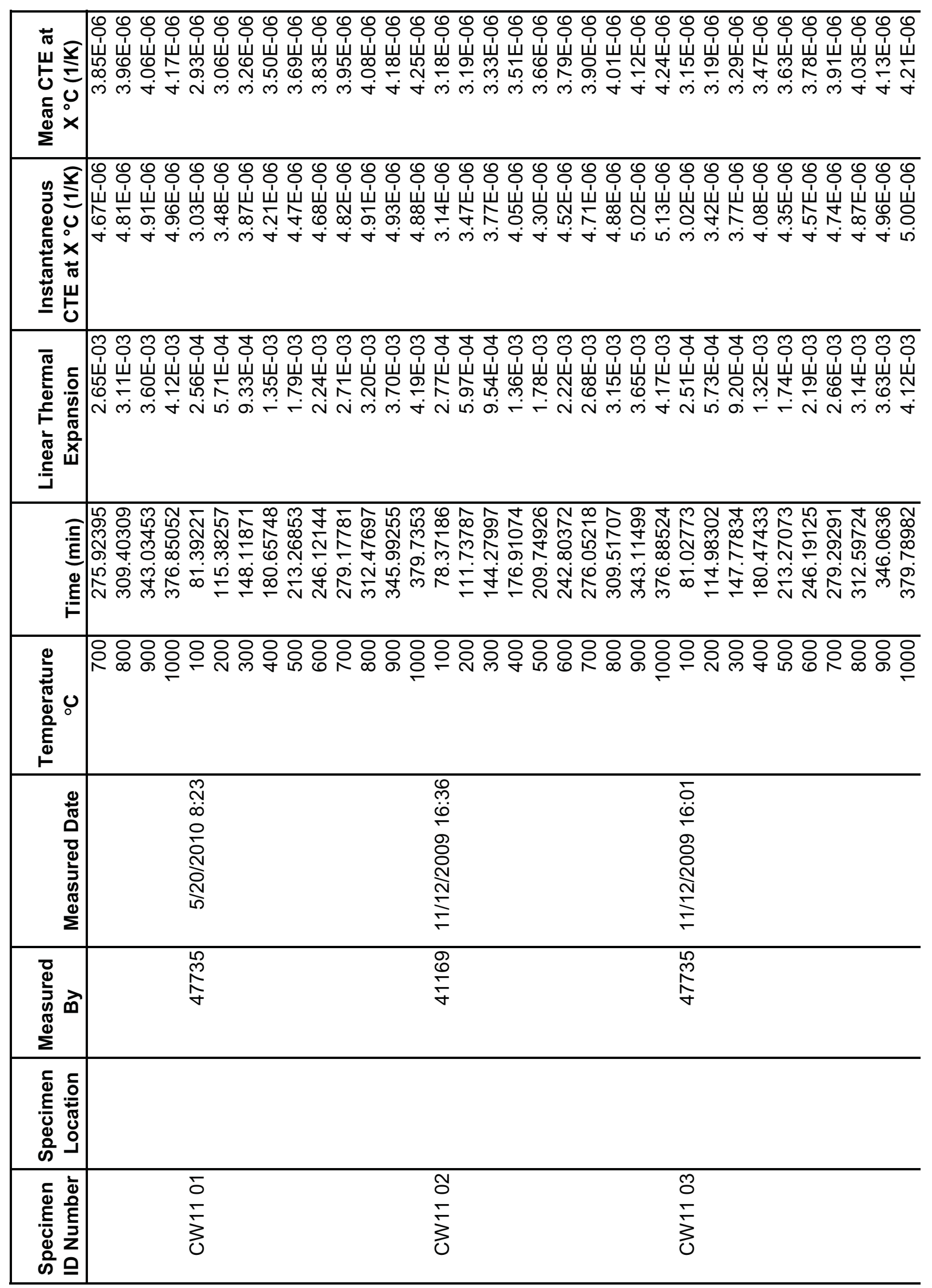




\begin{tabular}{|c|c|c|c|}
\hline 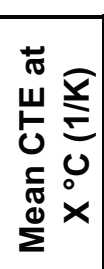 & \multirow{2}{*}{\multicolumn{3}{|c|}{ 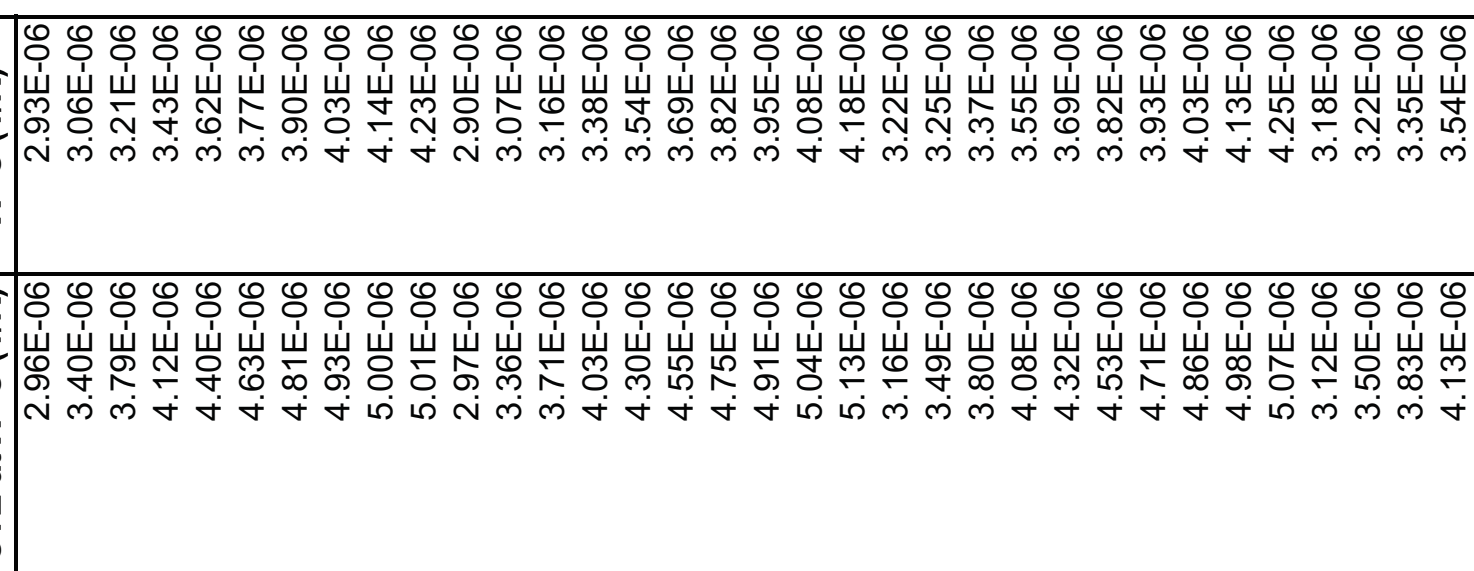 }} \\
\hline 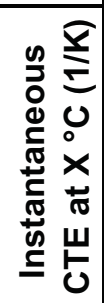 & & & \\
\hline 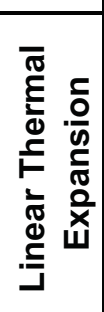 & 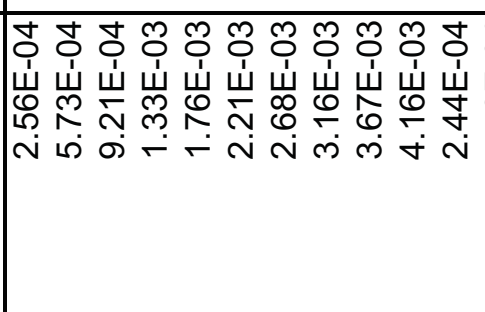 & 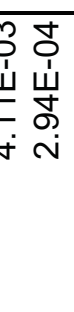 & 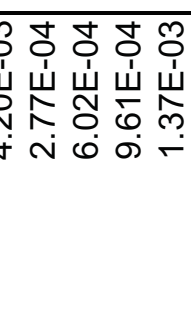 \\
\hline $\begin{array}{l}\widehat{\underline{\underline{\varepsilon}}} \\
\underline{\underline{\xi}} \\
\stackrel{0}{\xi} \\
\underline{\underline{\xi}}\end{array}$ & 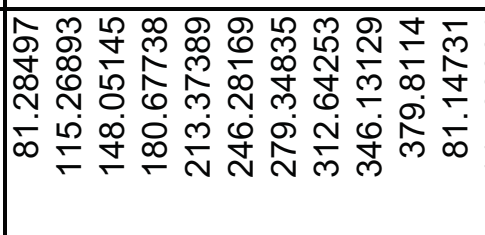 & & 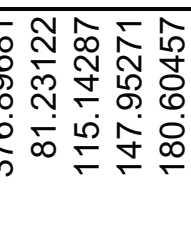 \\
\hline 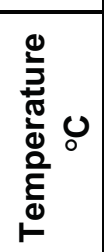 & 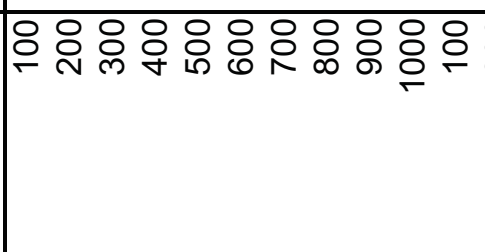 & 음음 & క్రం유융ㅇㅁ \\
\hline 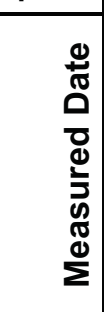 & 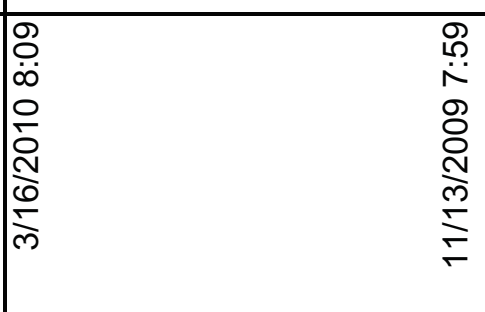 & 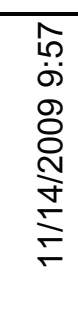 & 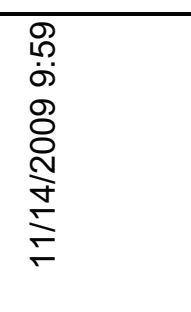 \\
\hline 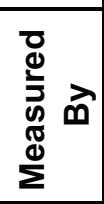 & 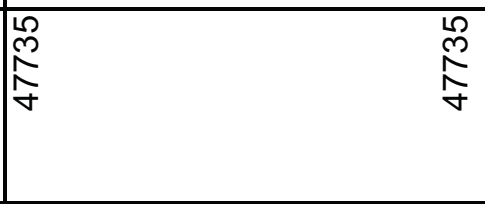 & $\frac{8}{\frac{8}{6}}$ & 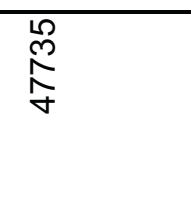 \\
\hline 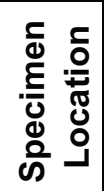 & & & \\
\hline 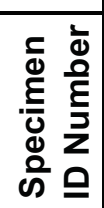 & 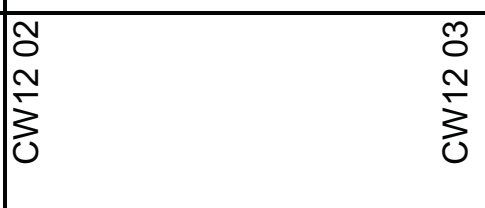 & $\sum_{0}^{\infty}$ & 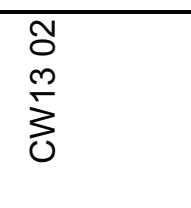 \\
\hline
\end{tabular}




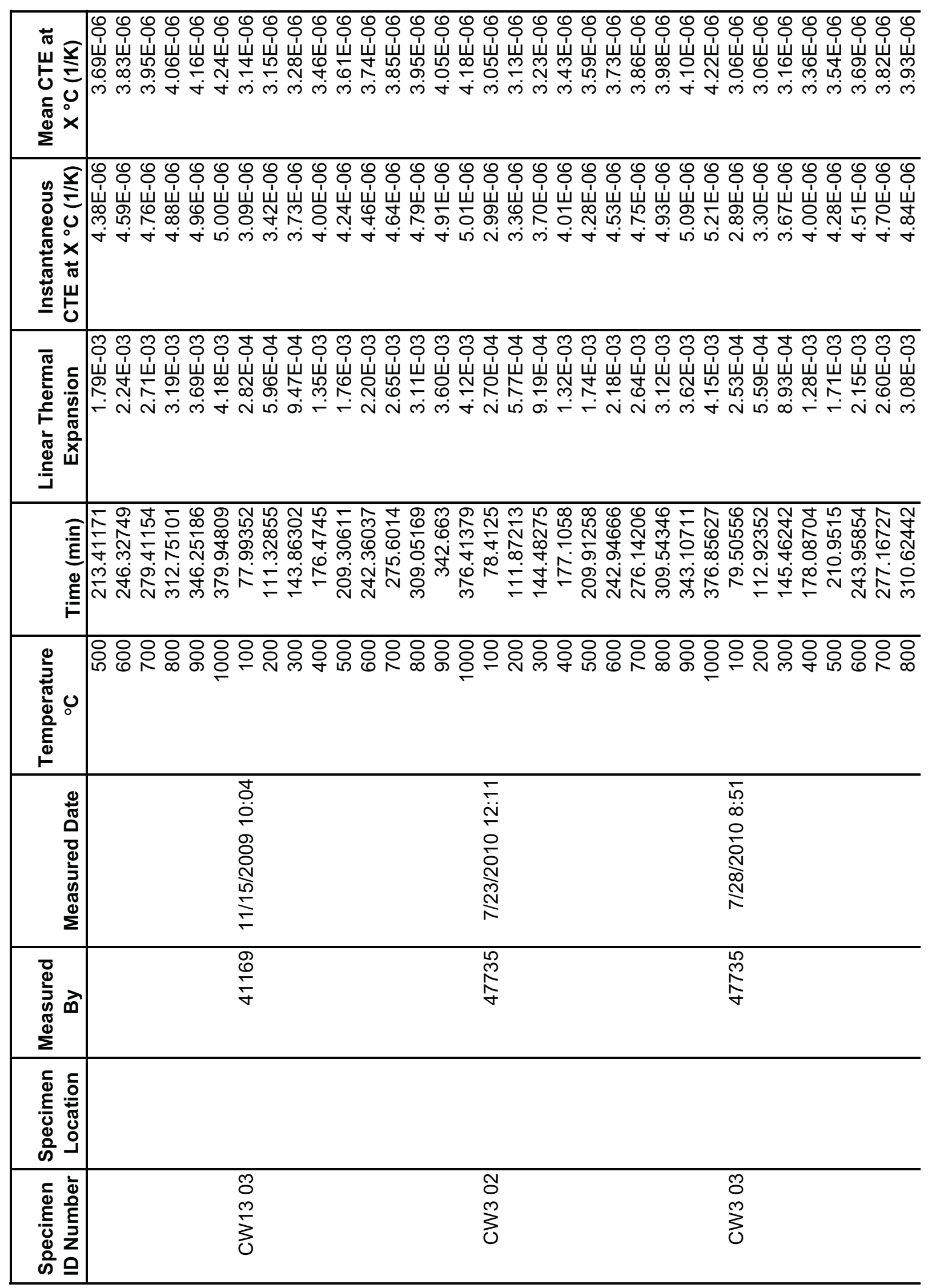




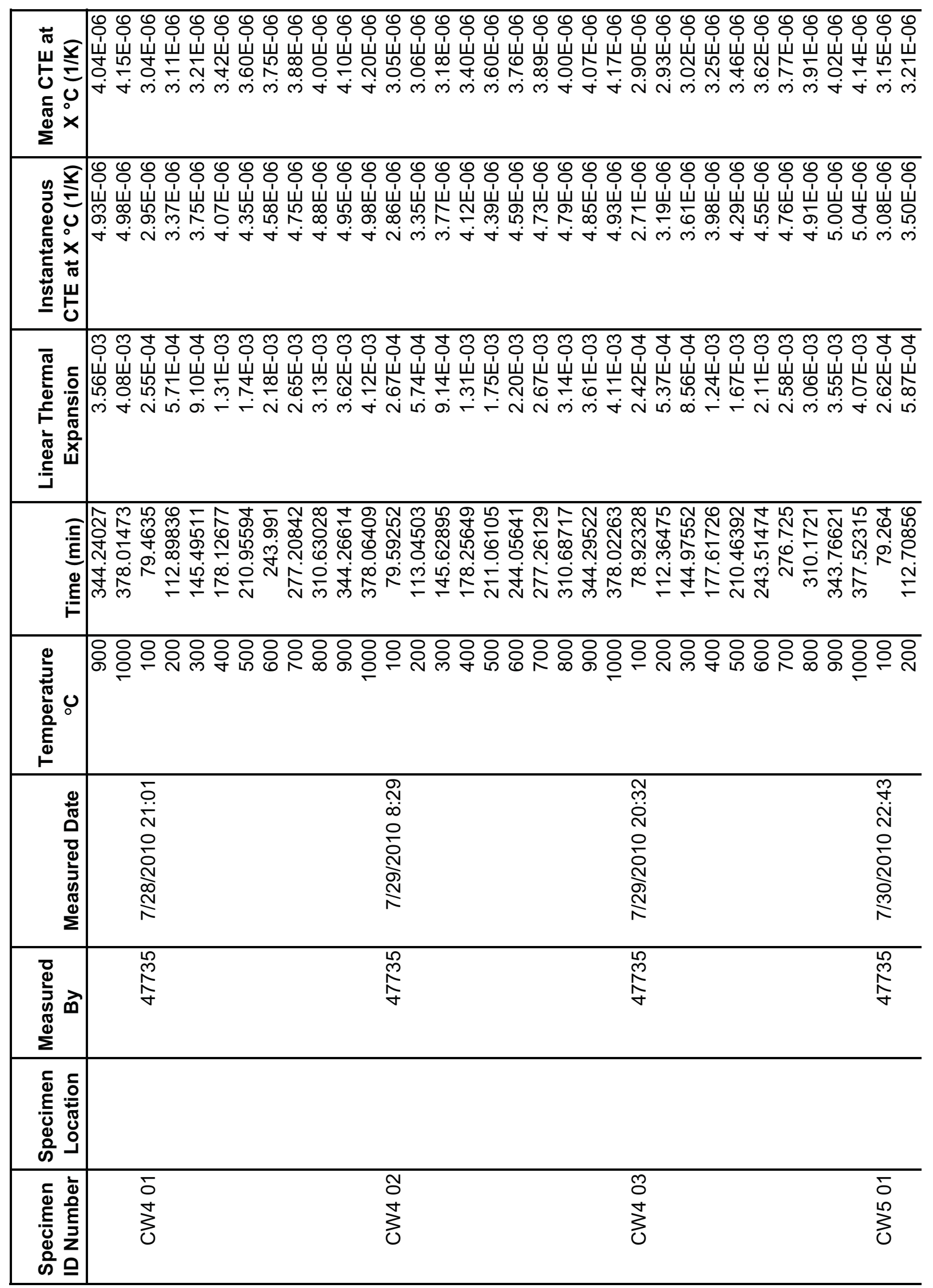




\begin{tabular}{|c|c|}
\hline 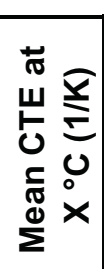 & 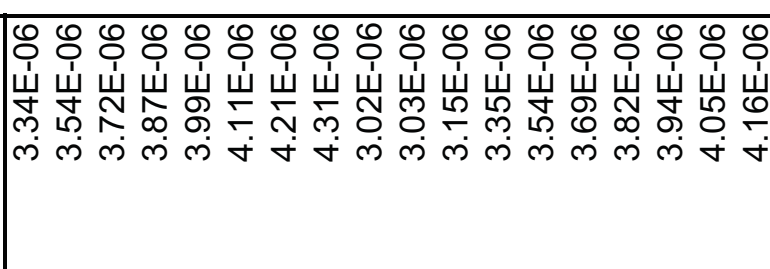 \\
\hline 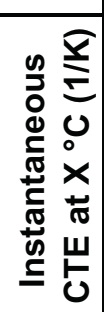 & 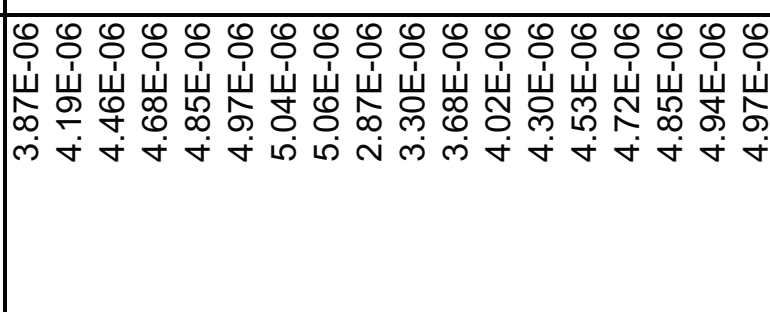 \\
\hline 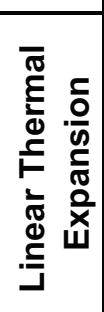 & 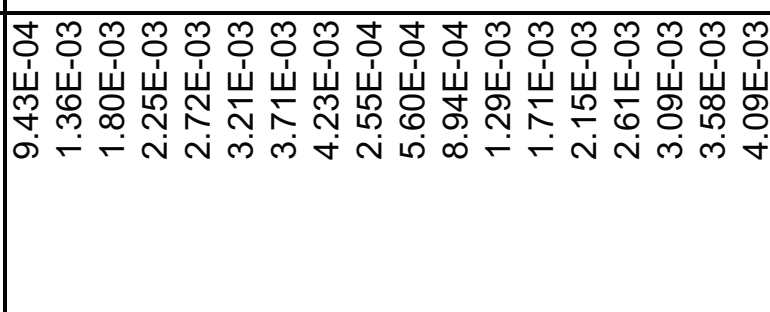 \\
\hline 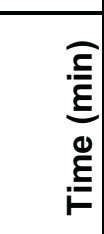 & 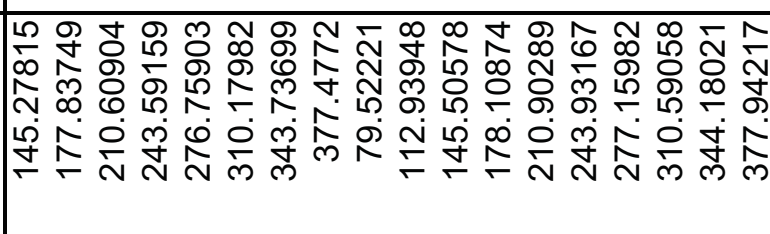 \\
\hline 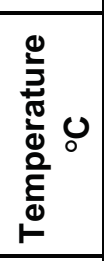 & 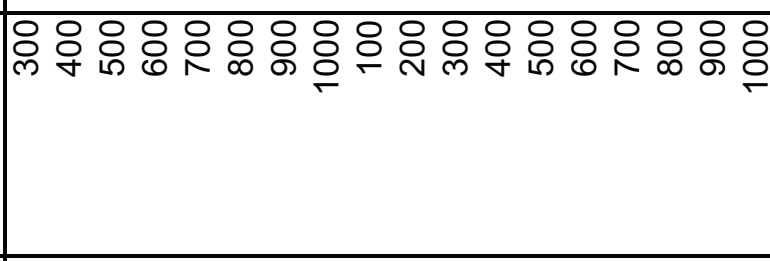 \\
\hline 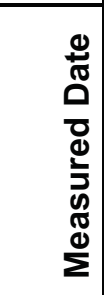 & 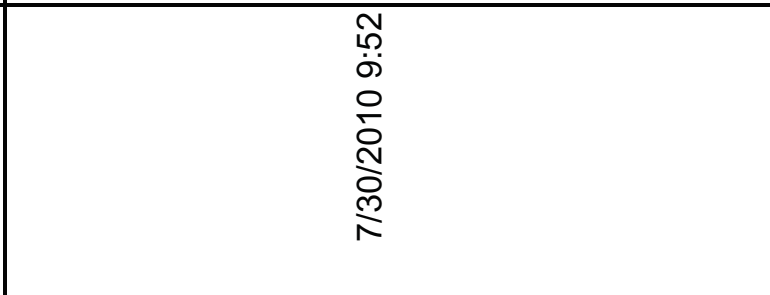 \\
\hline 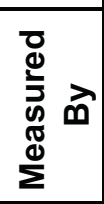 & 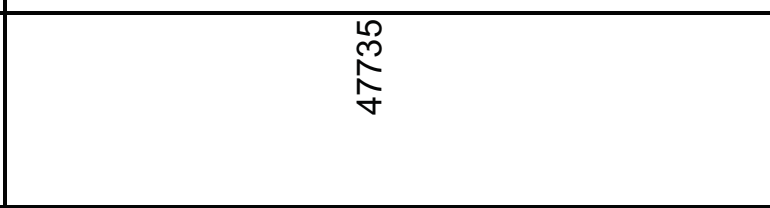 \\
\hline 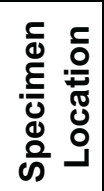 & \\
\hline 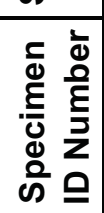 & 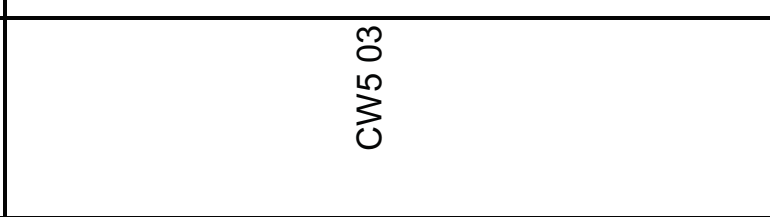 \\
\hline
\end{tabular}




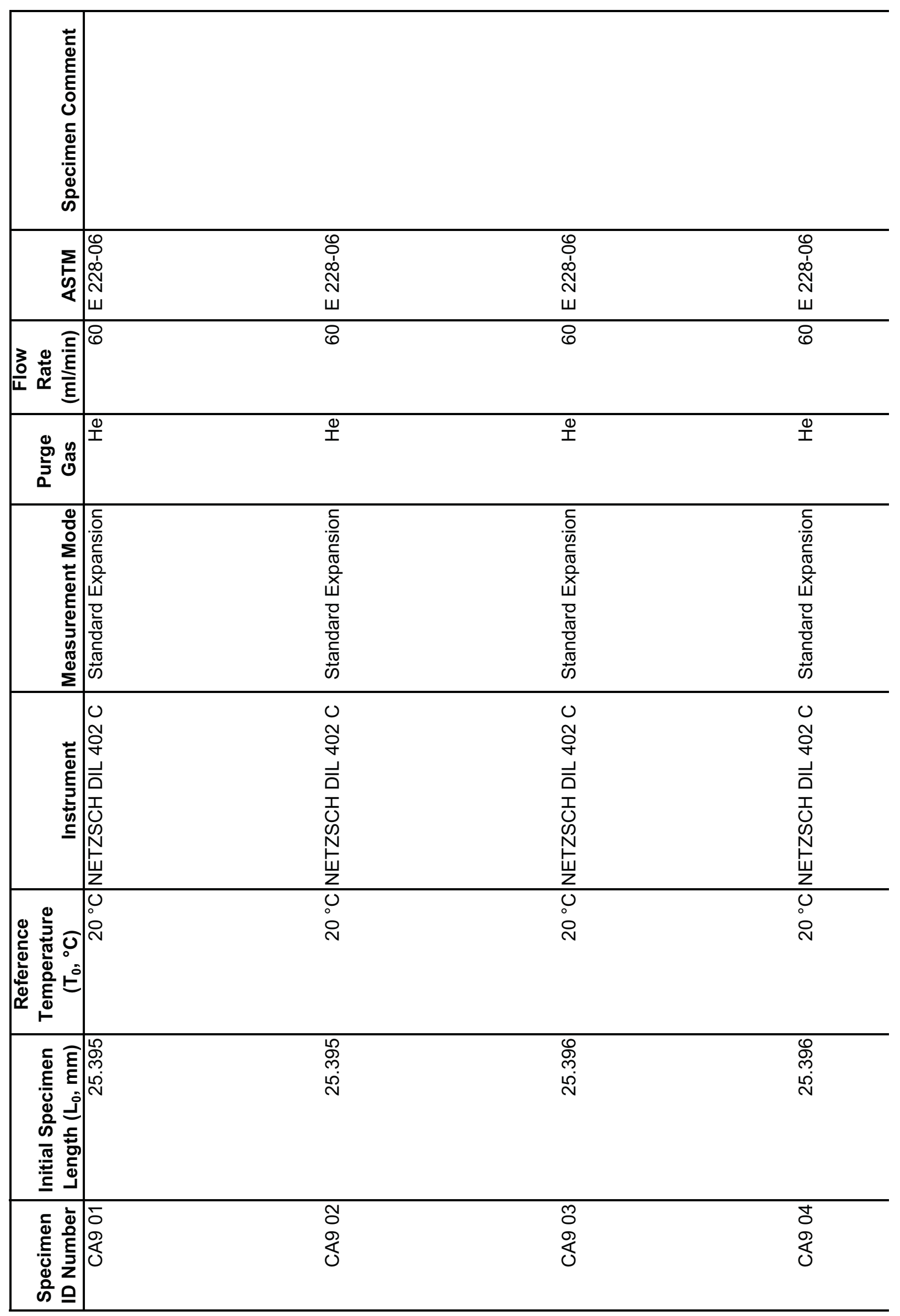




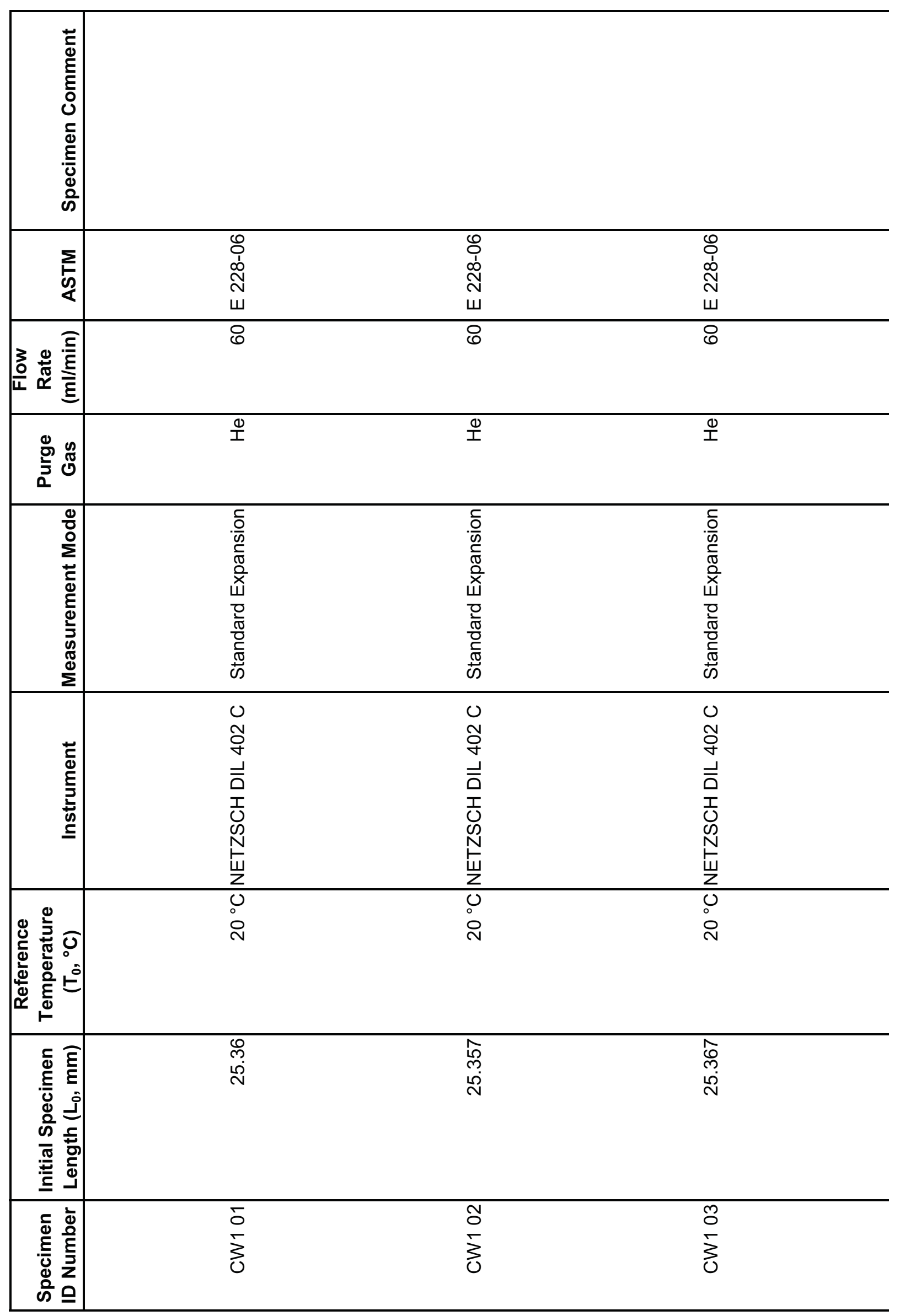




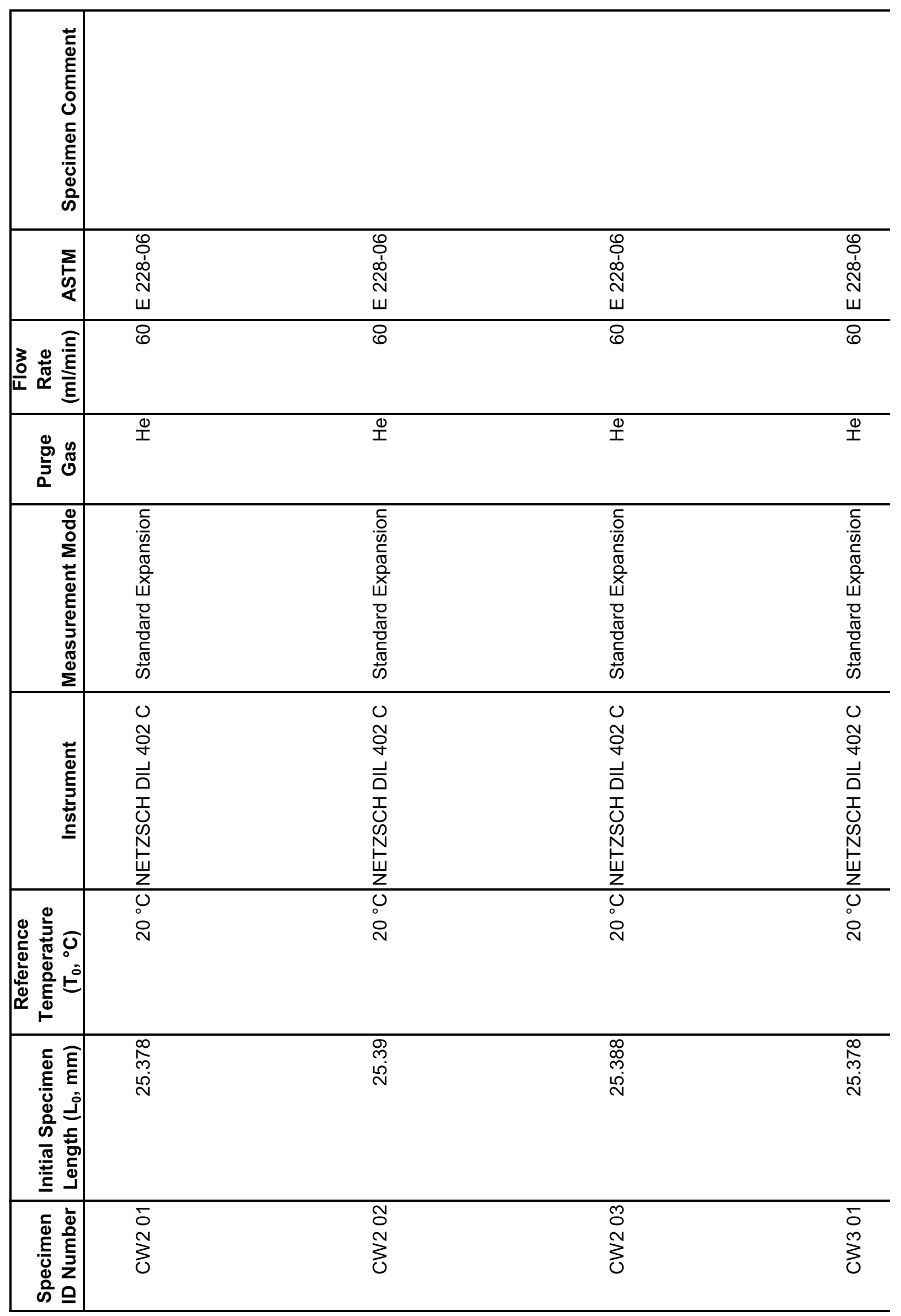

$\sqrt{n}$ 


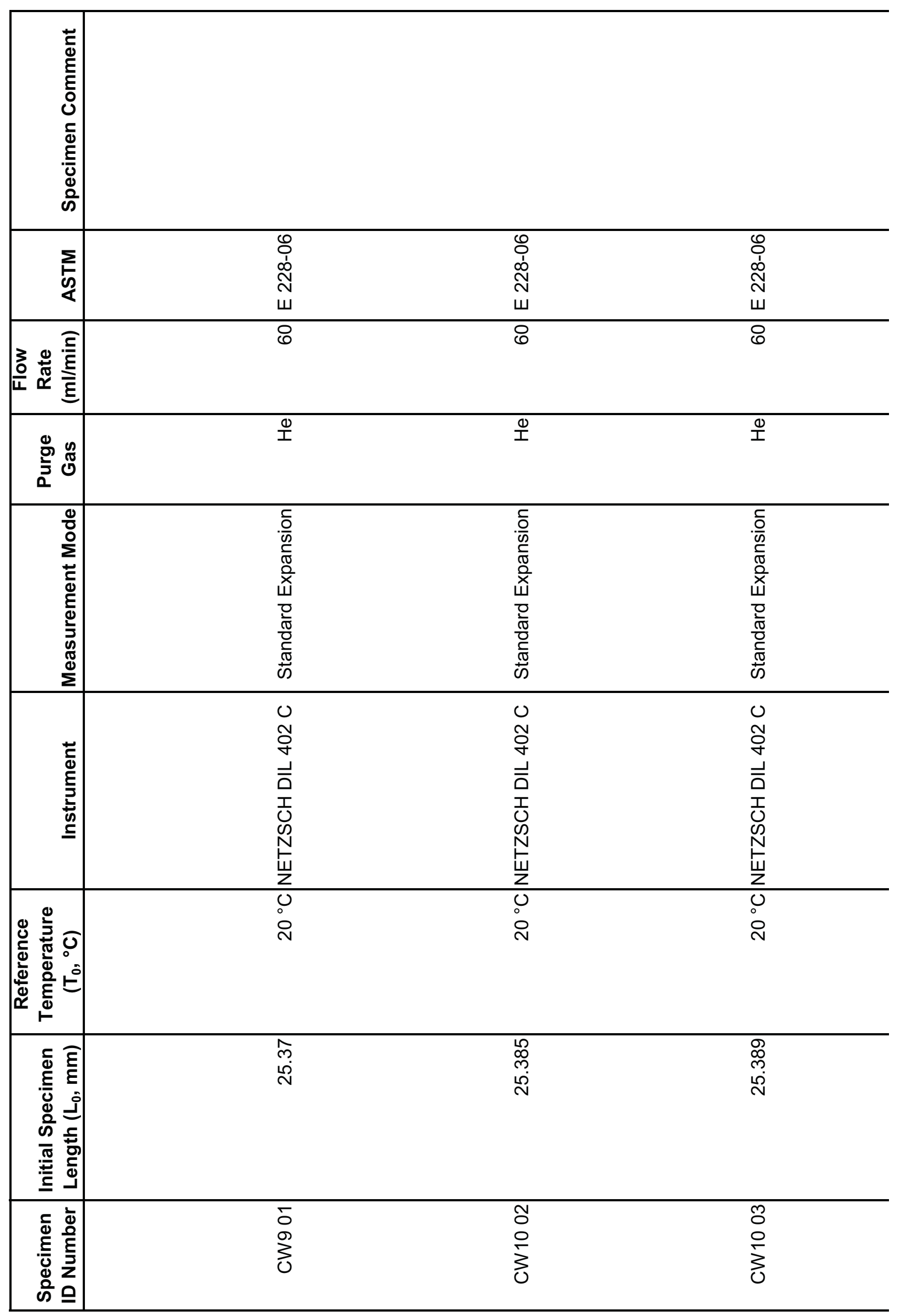




\begin{tabular}{|c|c|c|c|}
\hline 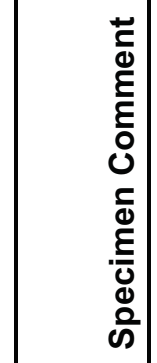 & & & \\
\hline$\underset{\substack{\mathbf{g} \\
\mathbf{Q}}}{\mathbf{\Sigma}}$ & $\begin{array}{l}0 \\
0 \\
0 \\
\mathbb{N} \\
\text { W } \\
\text { w }\end{array}$ & $\begin{array}{l}\text { O } \\
\text { Dे } \\
\text { N̦ } \\
\text { w }\end{array}$ & 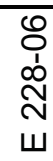 \\
\hline 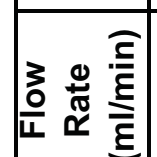 & 8 & 8 & 8 \\
\hline 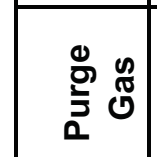 & $\stackrel{\oplus}{I}$ & $\frac{\Phi}{I}$ & $\stackrel{\oplus}{I}$ \\
\hline 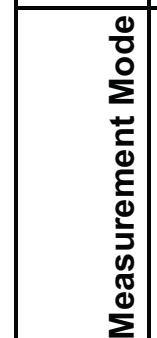 & 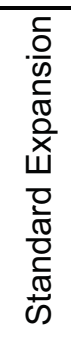 & 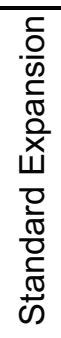 & 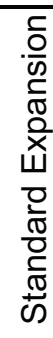 \\
\hline 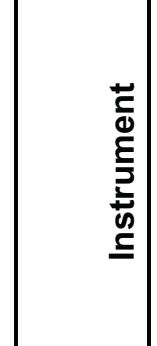 & 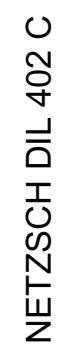 & 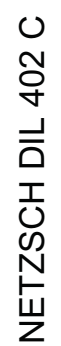 & 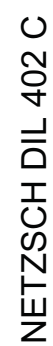 \\
\hline 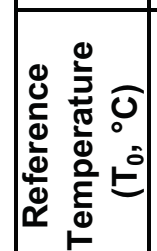 & $\begin{array}{l}0 \\
0 \\
\text { Oे }\end{array}$ & $\begin{array}{l}0 \\
0 \\
\text { N }\end{array}$ & $\begin{array}{l}\text { O } \\
\text { O }\end{array}$ \\
\hline 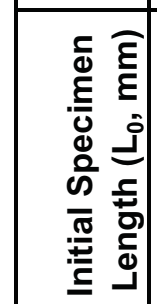 & $\begin{array}{l}\infty \\
\infty \\
\infty \\
\stackrel{\infty}{N} \\
\stackrel{N}{N}\end{array}$ & 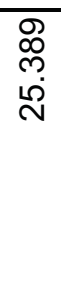 & 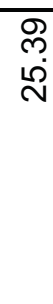 \\
\hline 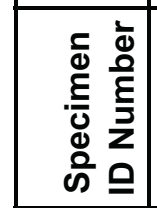 & $\begin{array}{l}\bar{\delta} \\
\frac{5}{3} \\
0\end{array}$ & $\begin{array}{l}\text { N } \\
\sum \\
\sum\end{array}$ & $\sum_{0}^{\frac{0}{0}}$ \\
\hline
\end{tabular}




\begin{tabular}{|c|c|c|c|c|}
\hline 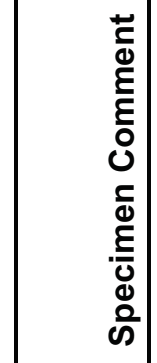 & & & & \\
\hline 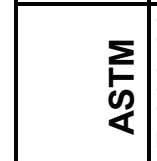 & 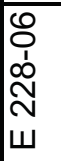 & $\begin{array}{l}0 \\
0 \\
0 \\
\text { N } \\
\text { w }\end{array}$ & 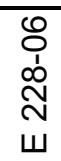 & 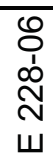 \\
\hline 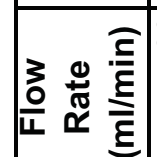 & 8 & 8 & 8 & 8 \\
\hline 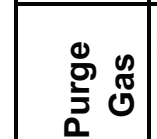 & $\frac{\Phi}{I}$ & $\underset{I}{\Psi}$ & $\frac{\Phi}{I}$ & $\frac{\otimes}{I}$ \\
\hline 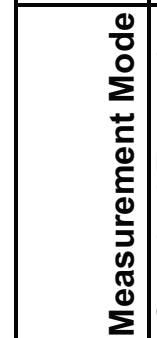 & 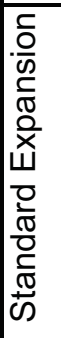 & 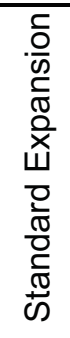 & 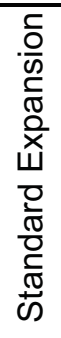 & 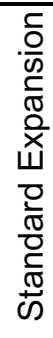 \\
\hline 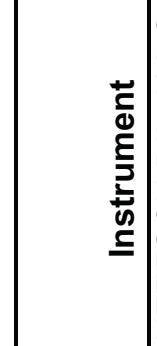 & 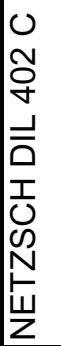 & 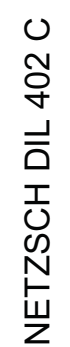 & 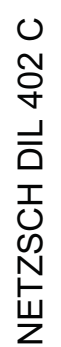 & 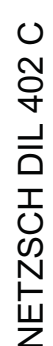 \\
\hline 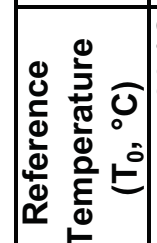 & $\begin{array}{l}0 \\
0 \\
i\end{array}$ & $\begin{array}{l}0 \\
0 \\
\text { Oे }\end{array}$ & $\begin{array}{l}0 \\
0 \\
i\end{array}$ & $\begin{array}{l}0 \\
0 \\
D\end{array}$ \\
\hline 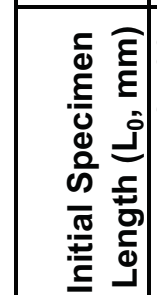 & 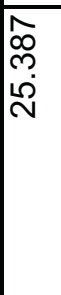 & 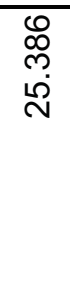 & $\begin{array}{l}\text { \& } \\
\text { m. } \\
\stackrel{\rho}{N}\end{array}$ & 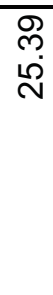 \\
\hline 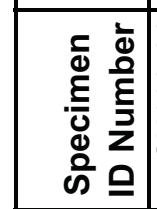 & 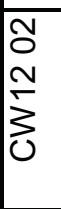 & $\begin{array}{l}\text { OO } \\
\stackrel{N}{N} \\
\sum_{0}\end{array}$ & $\begin{array}{l}\overline{0} \\
\sum_{0}^{m}\end{array}$ & $\begin{array}{l}\text { N } \\
\text { m } \\
\sum_{0}\end{array}$ \\
\hline
\end{tabular}




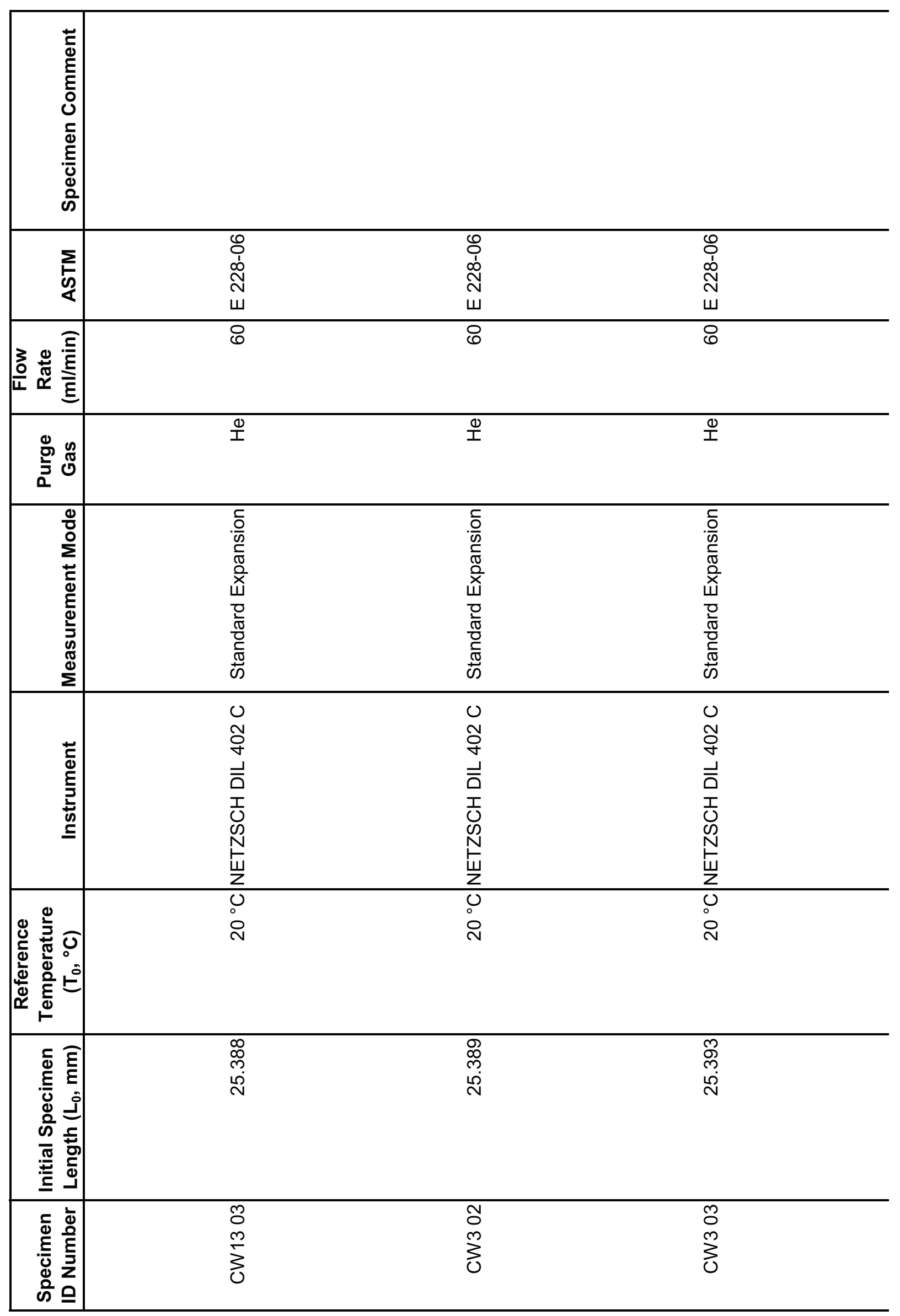




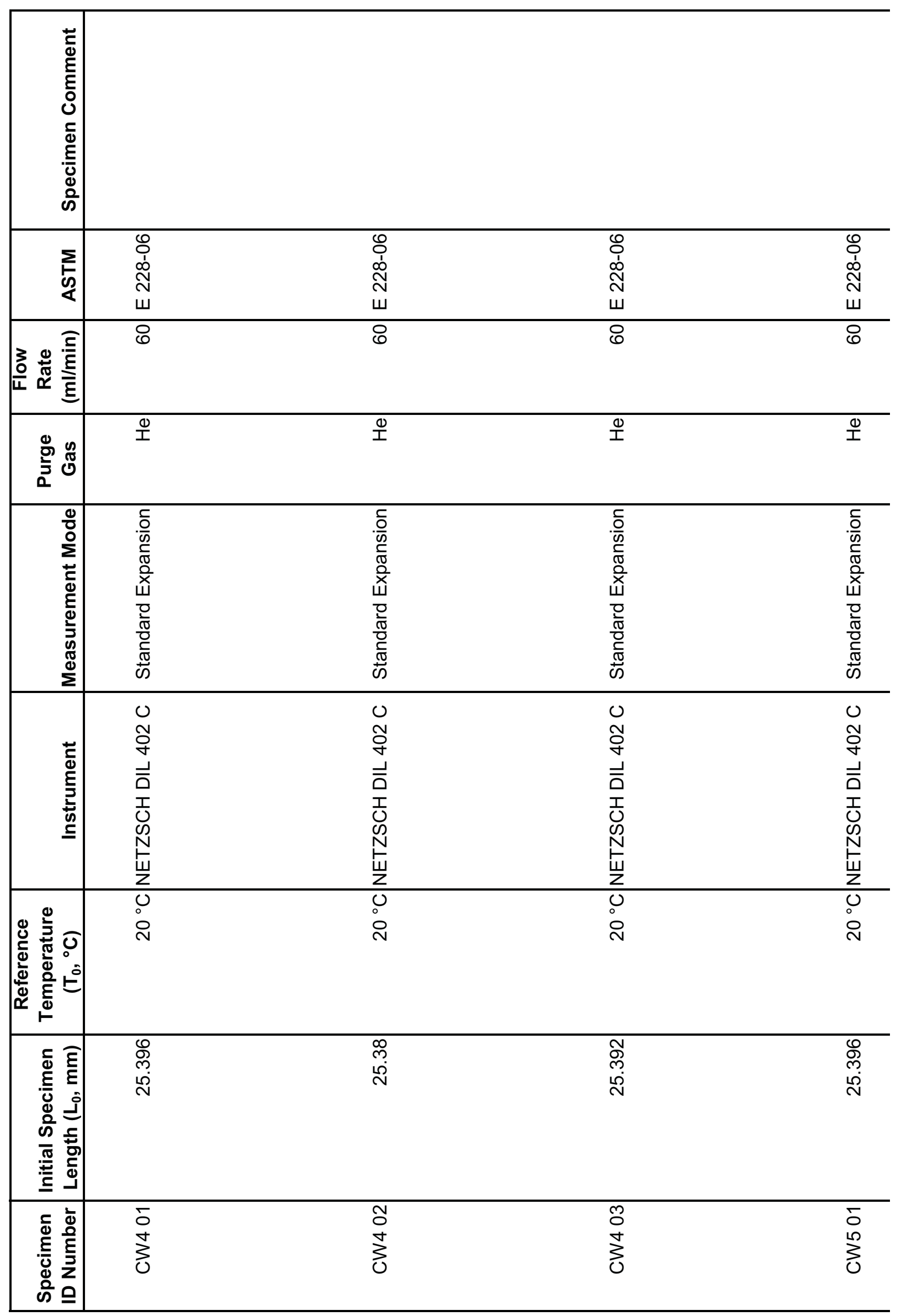




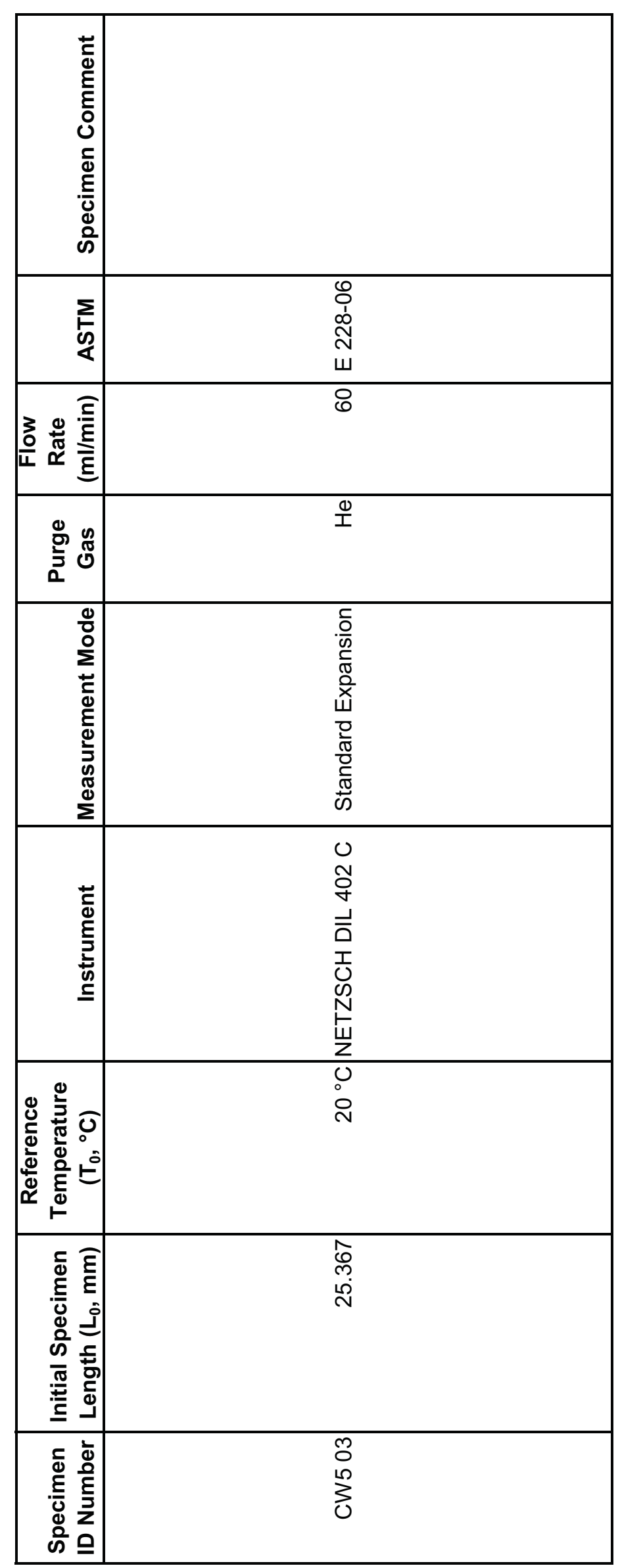

๙ิ 


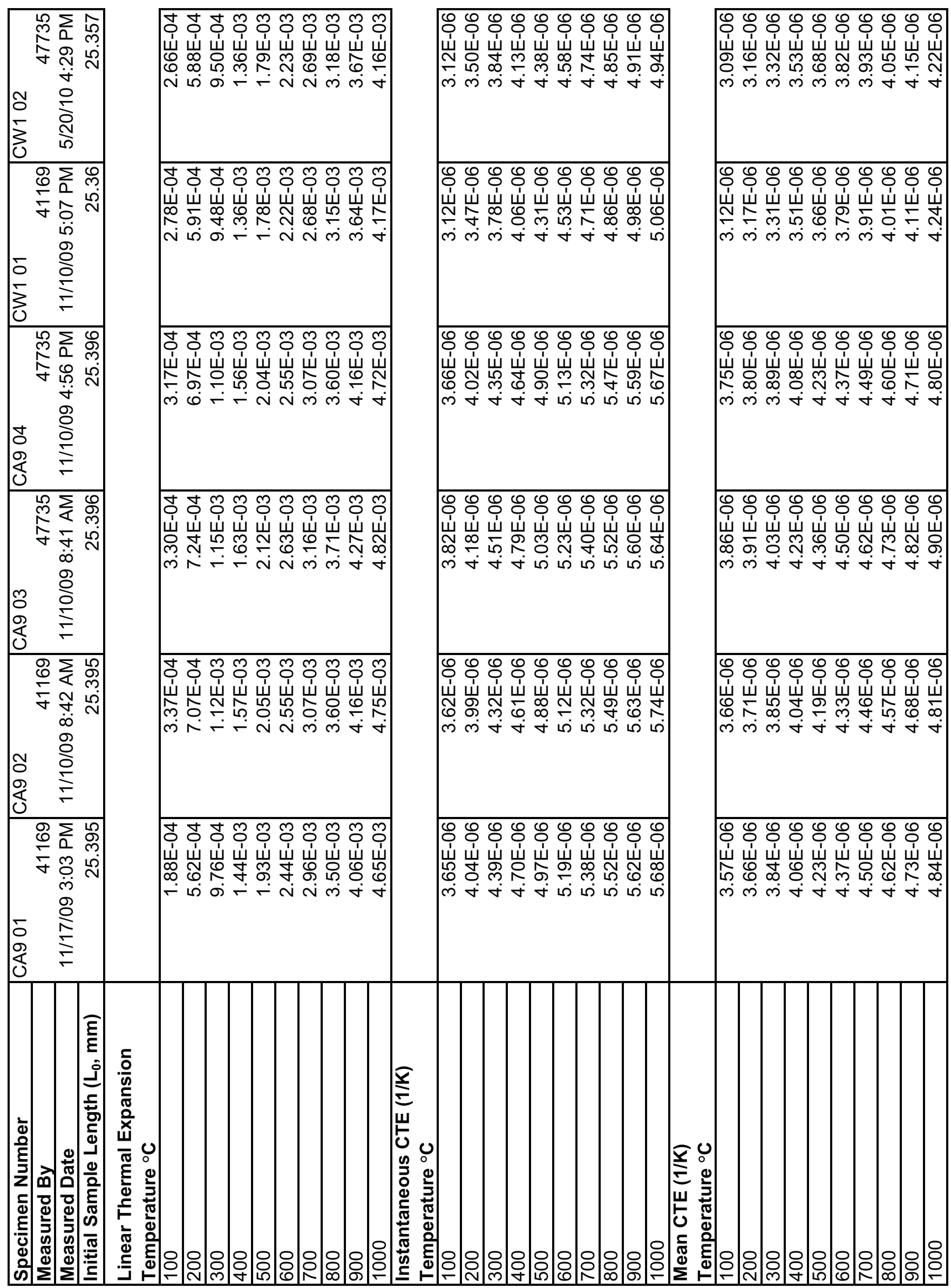




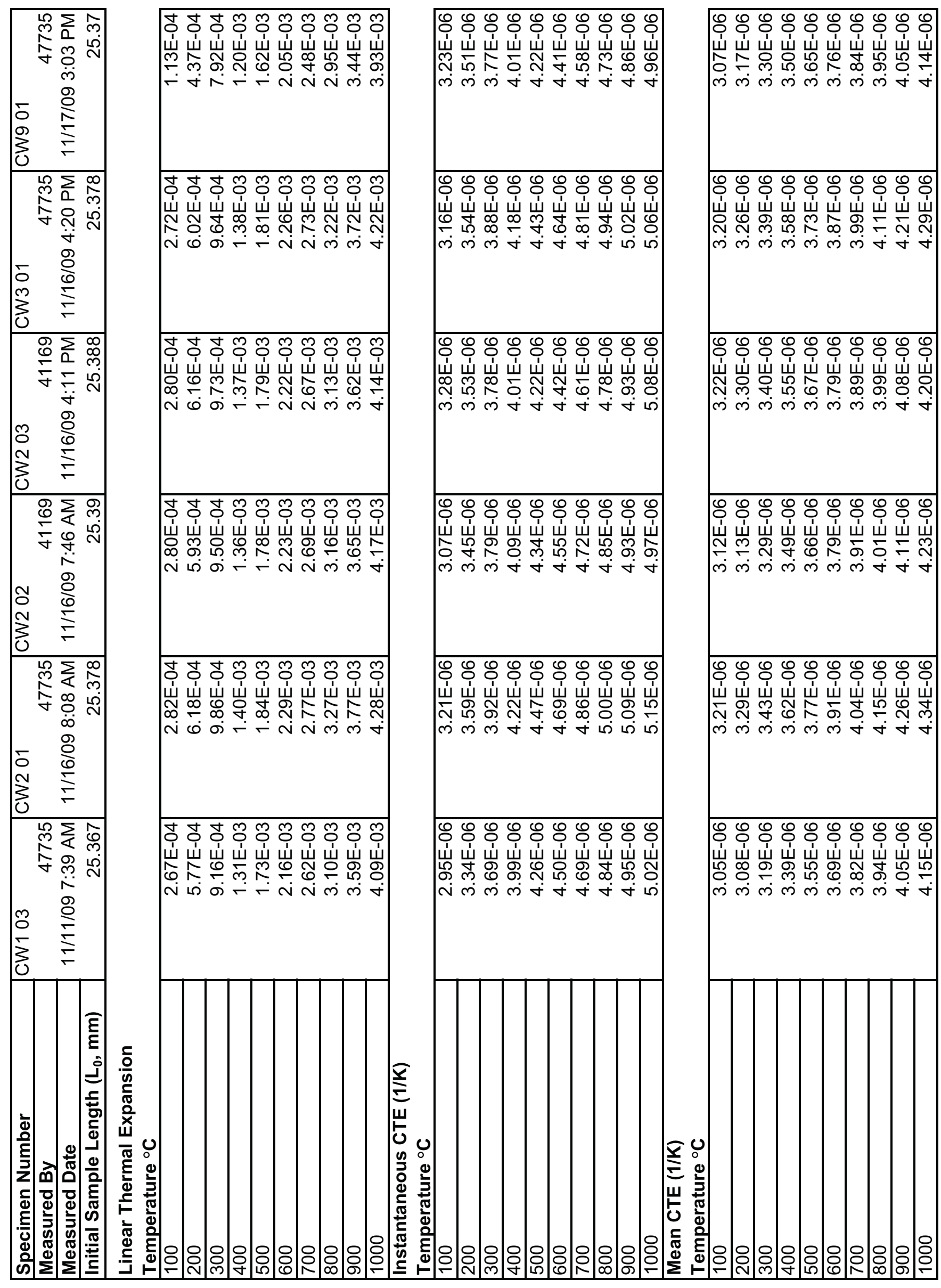




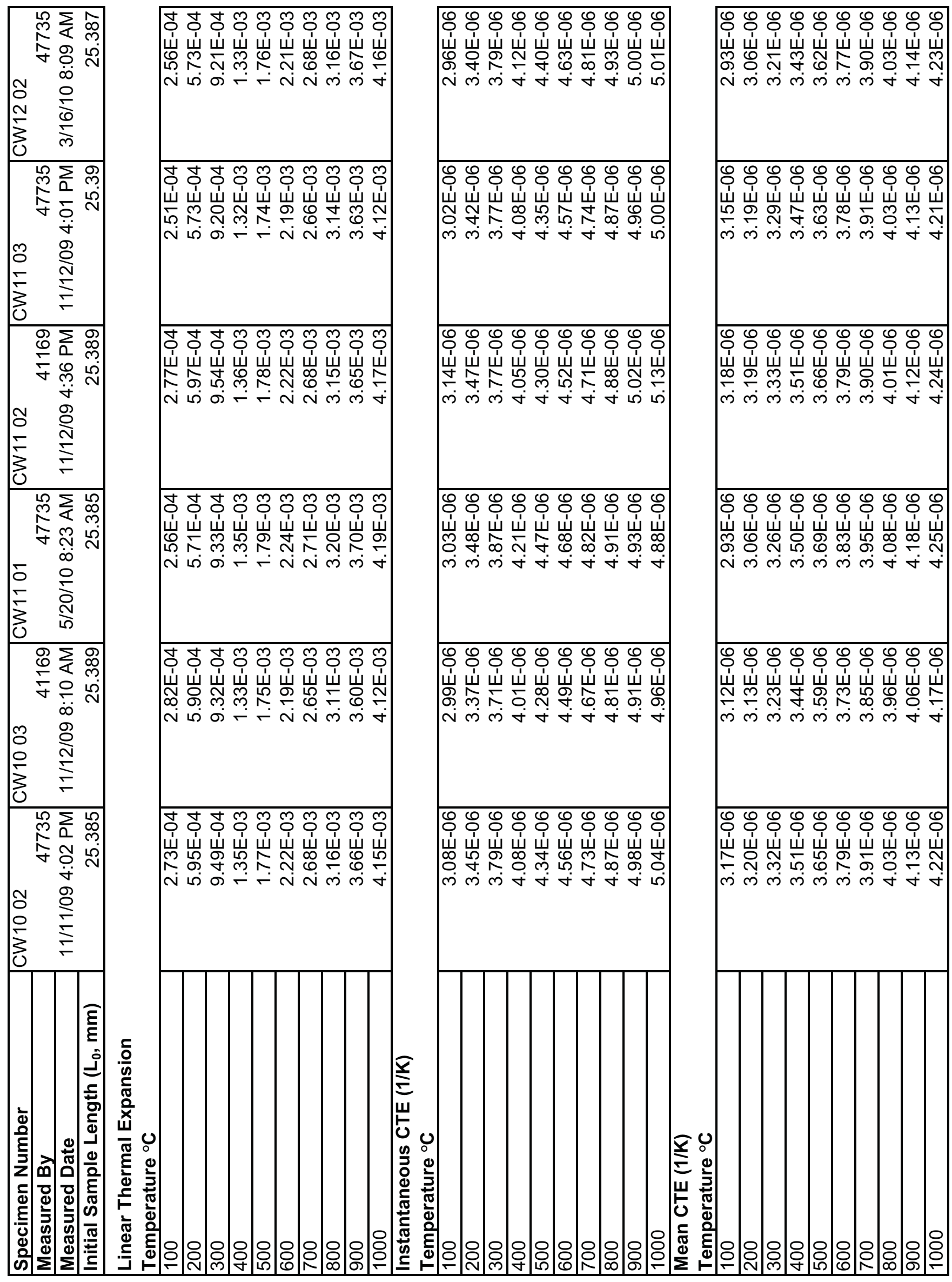




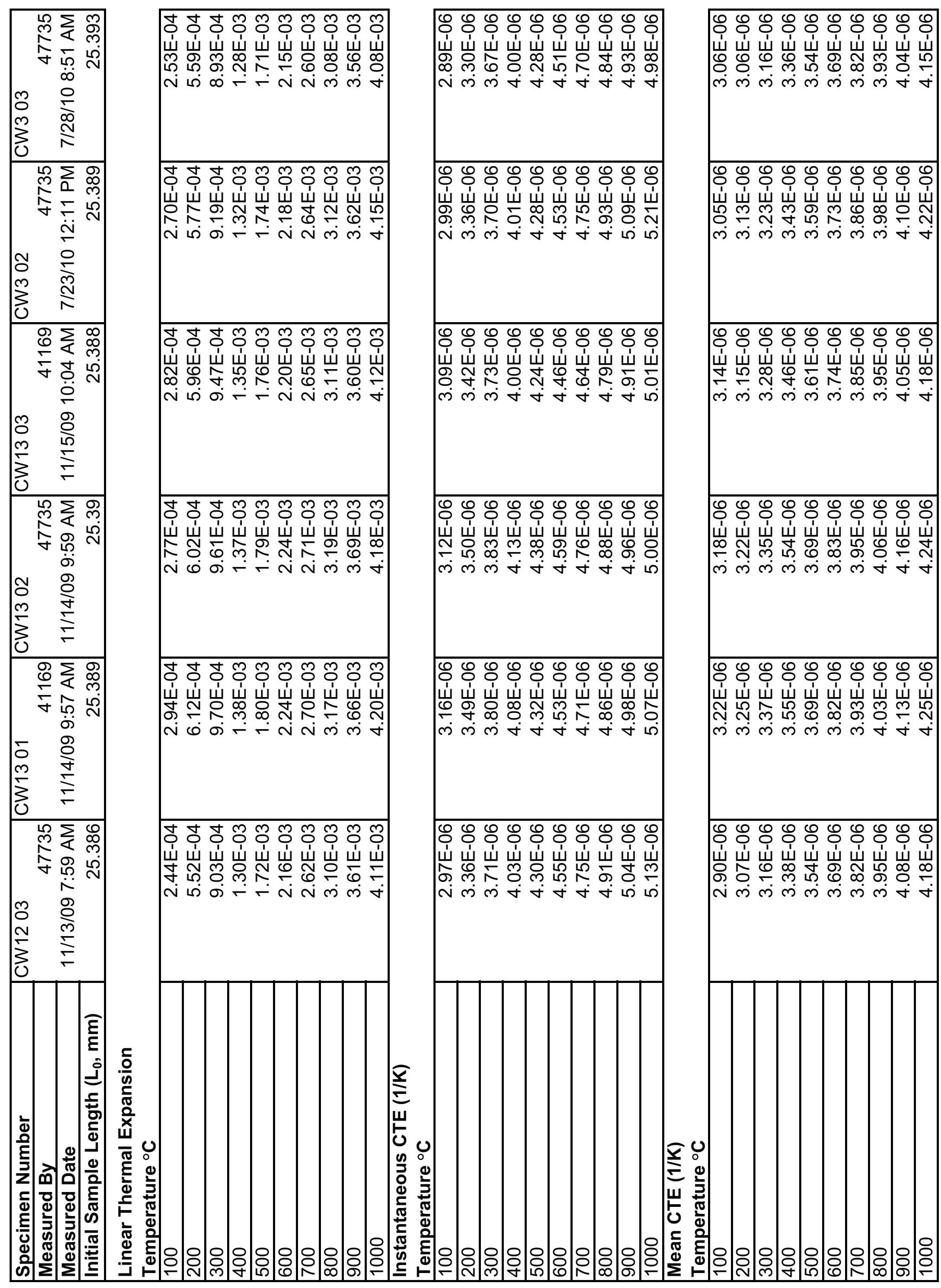




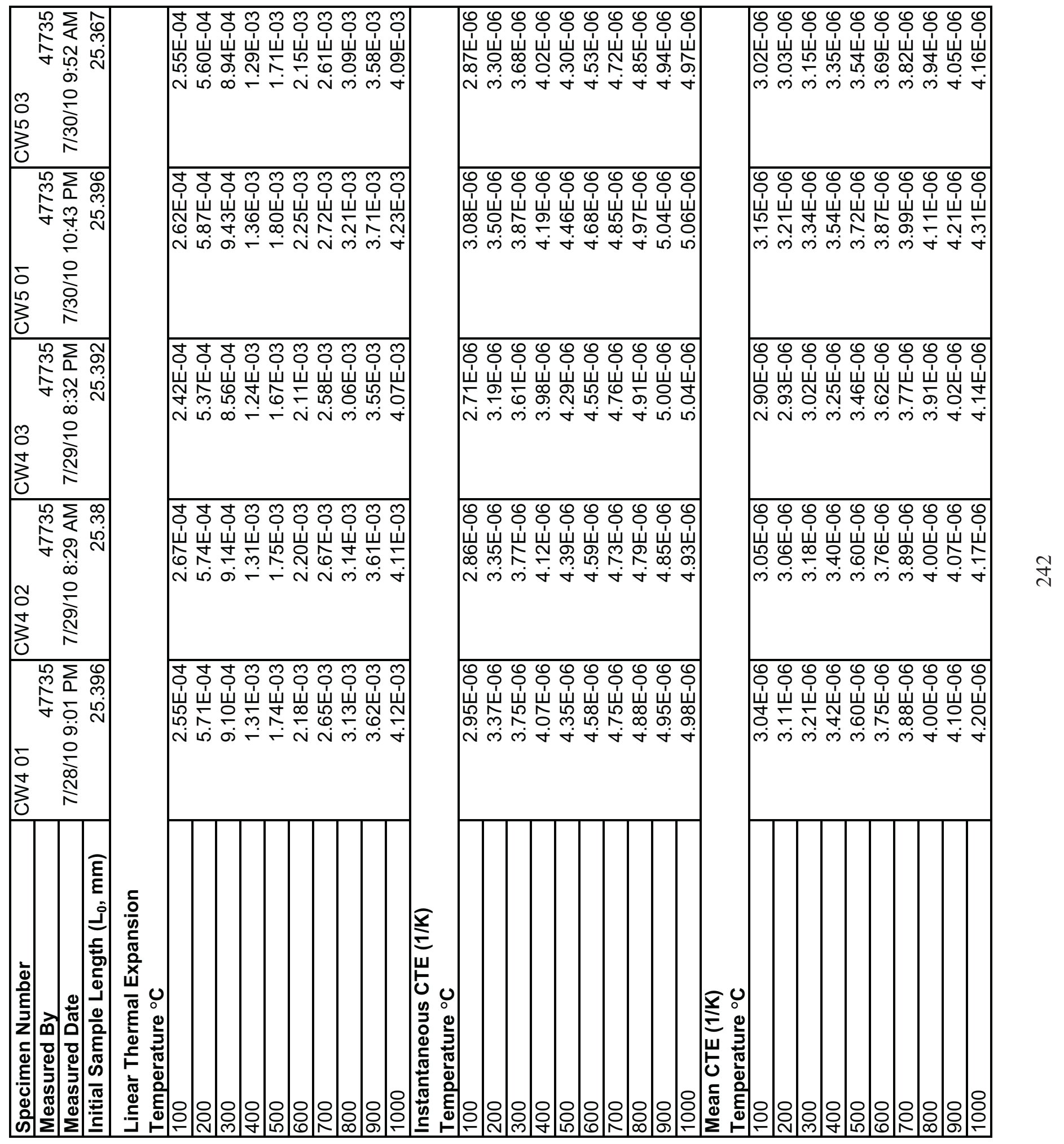




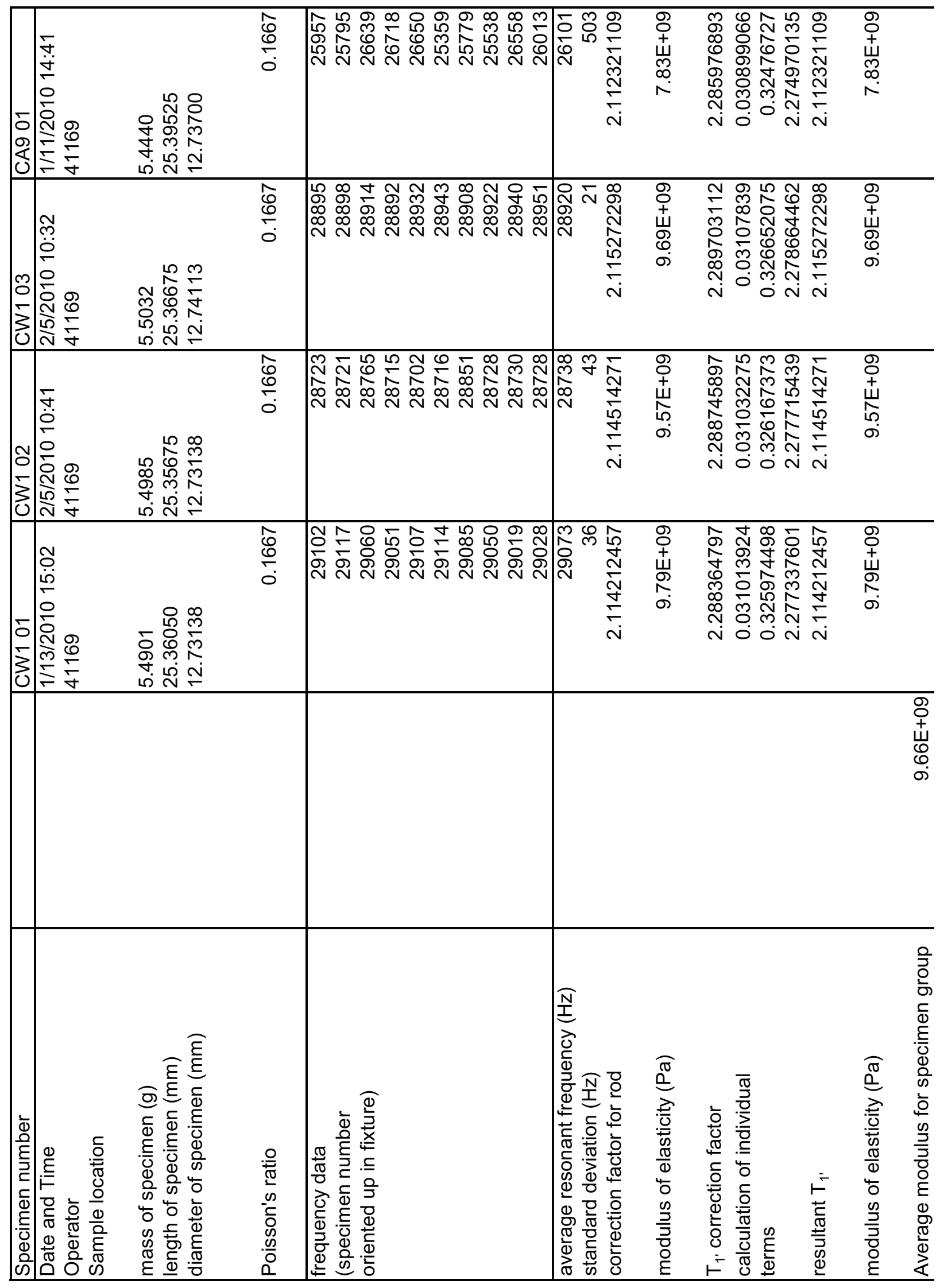




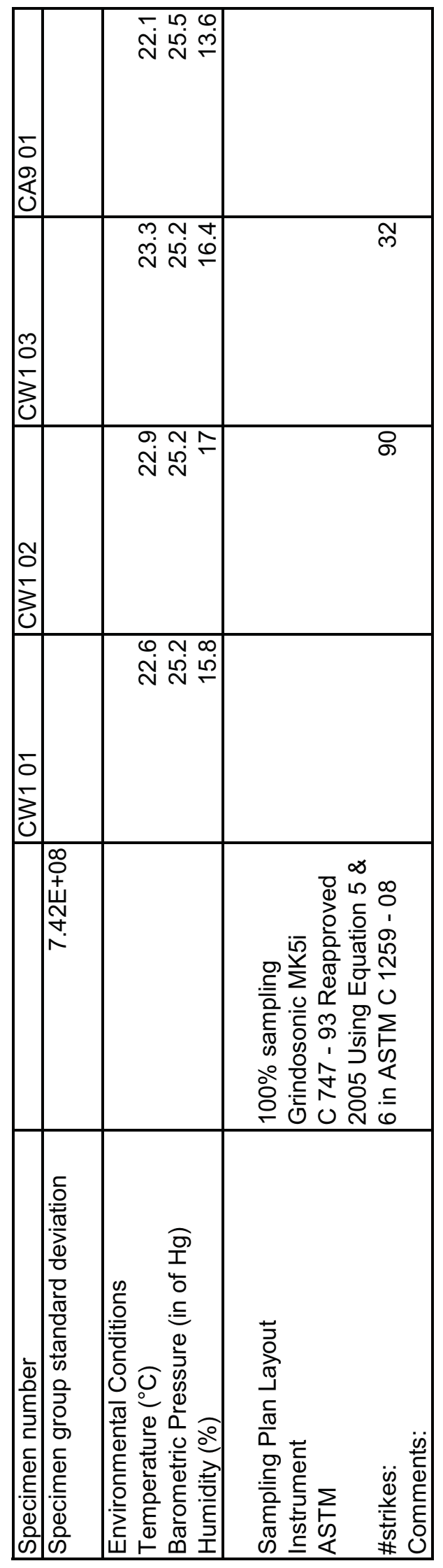




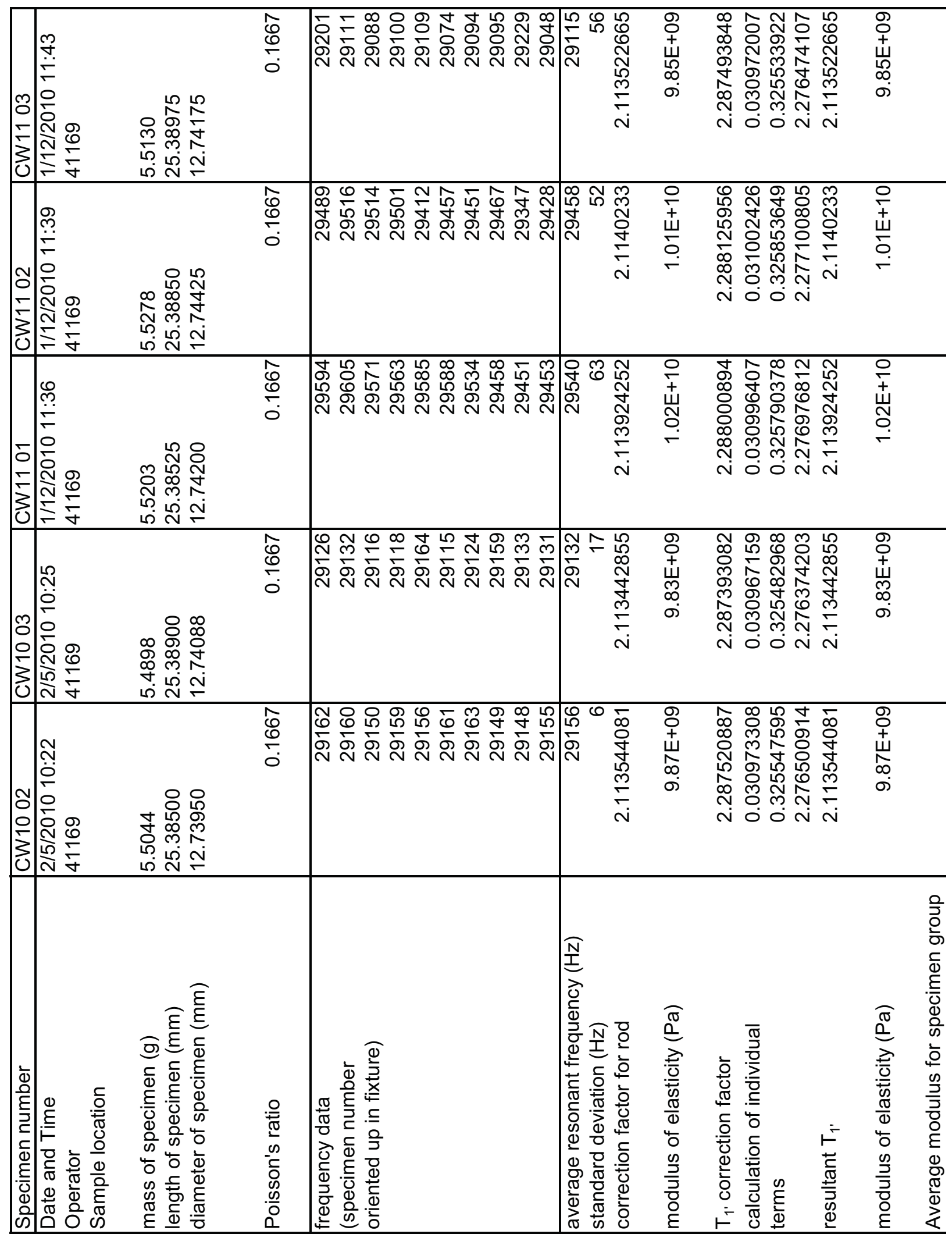




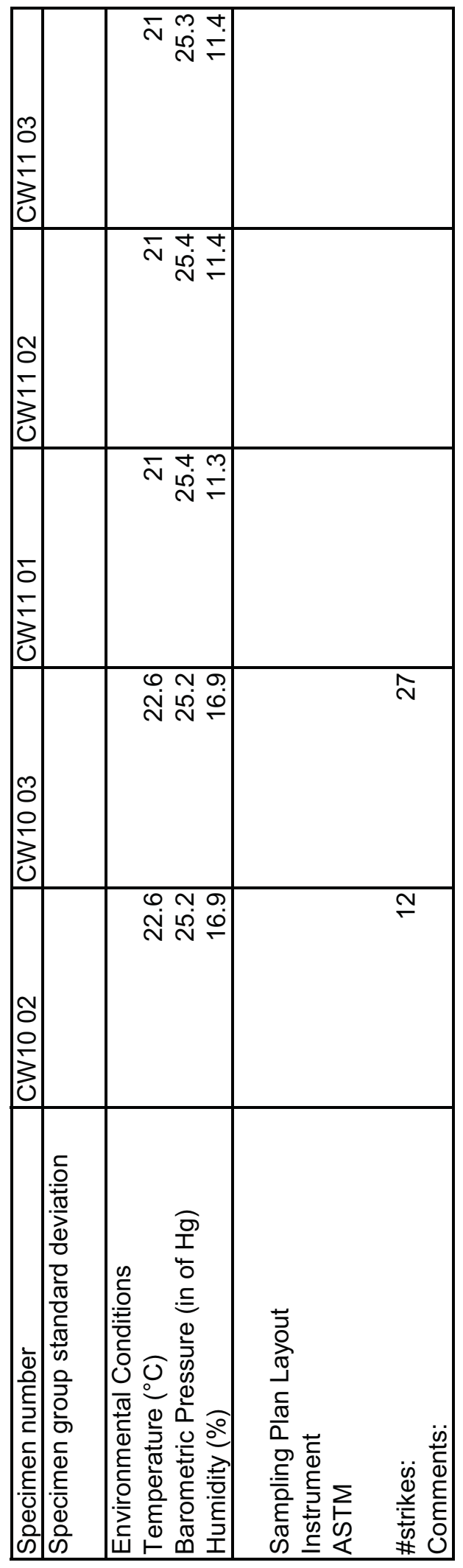




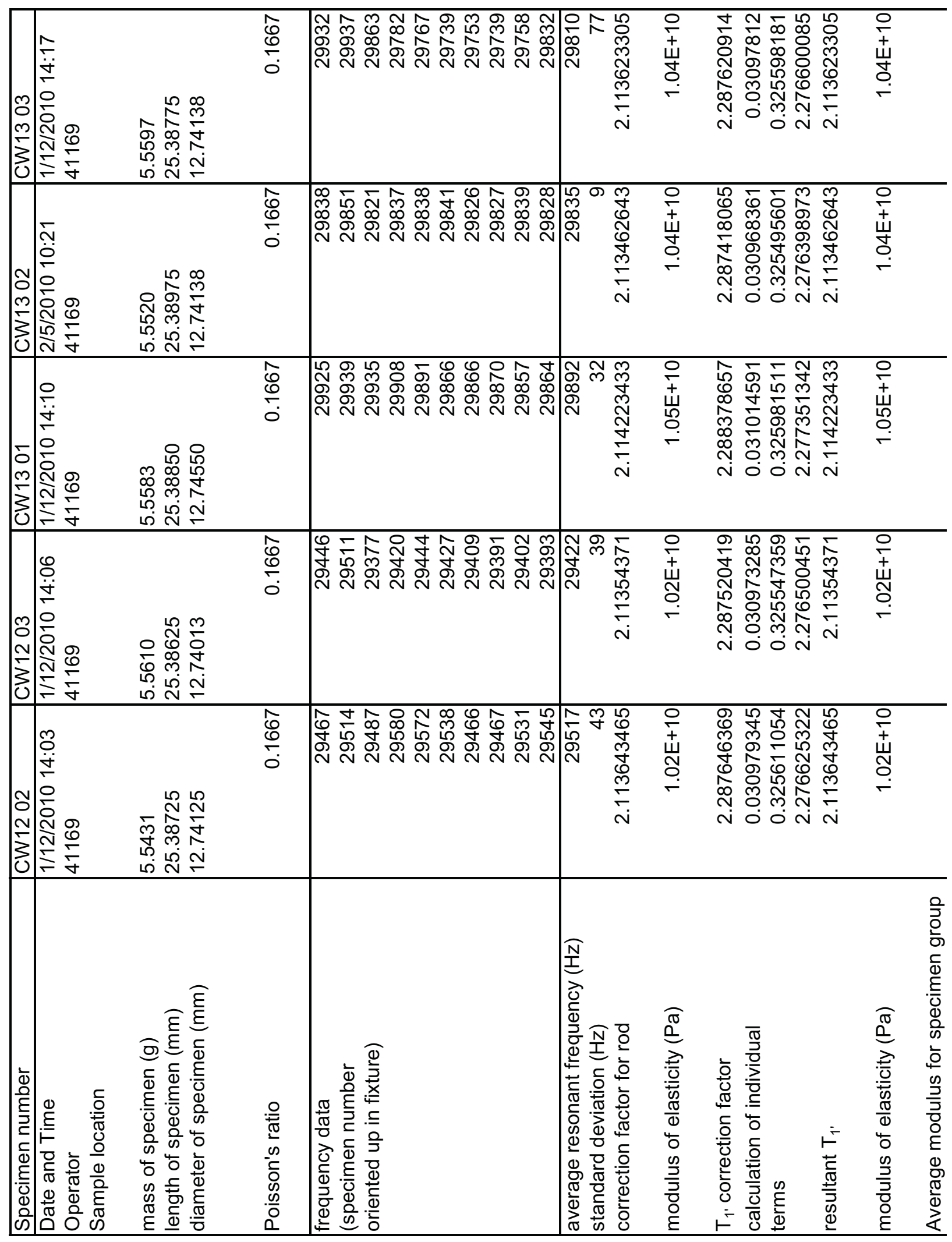




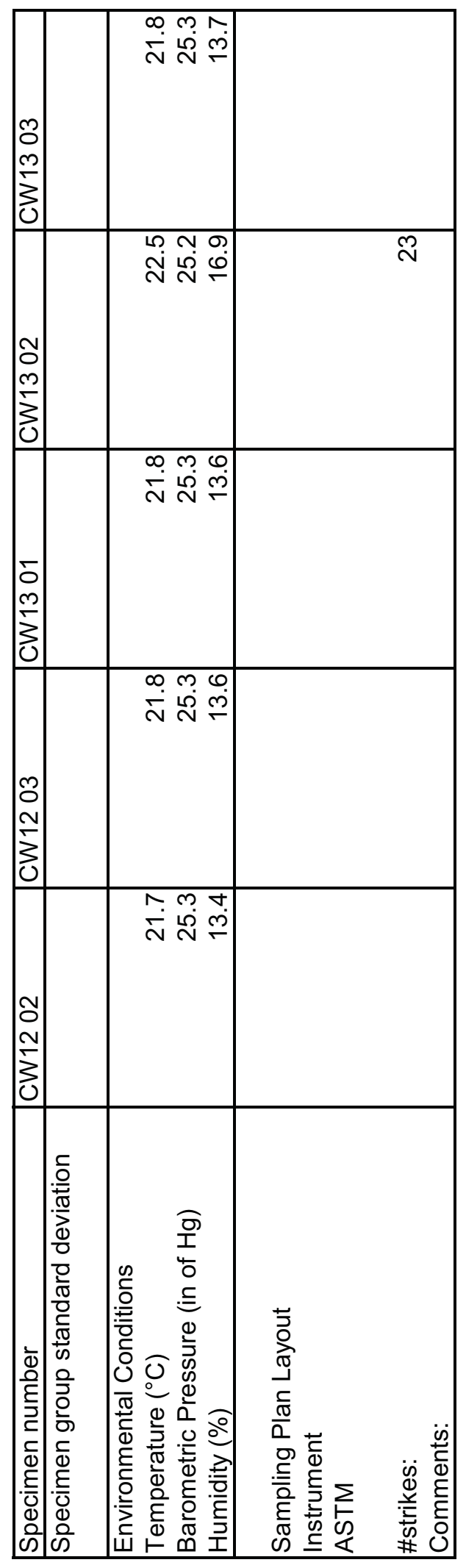




\begin{tabular}{|c|c|c|c|c|c|c|c|c|c|}
\hline 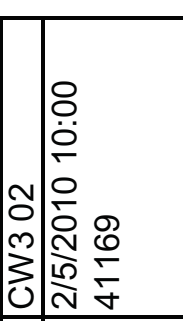 & 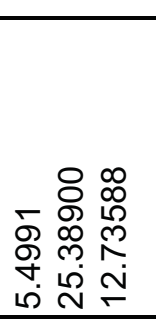 & $\begin{array}{l}\stackrel{0}{0} \\
\stackrel{0}{0}\end{array}$ & 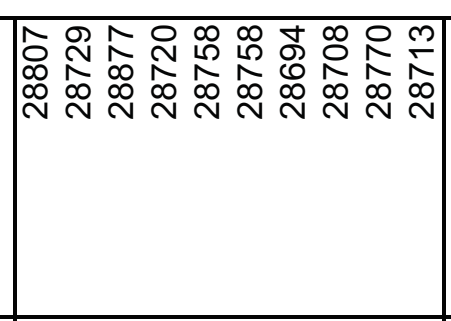 & 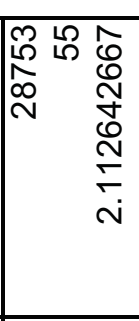 & $\begin{array}{l}0 \\
0 \\
+ \\
\stackrel{+}{0} \\
0 \\
0\end{array}$ & 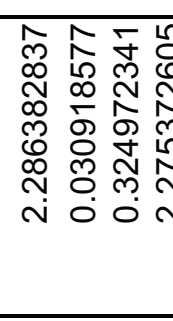 & 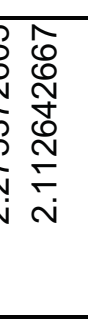 & $\begin{array}{l}\text { O } \\
+ \\
+ \\
\dot{+} \\
0 \\
0\end{array}$ & \\
\hline 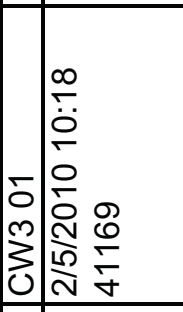 & 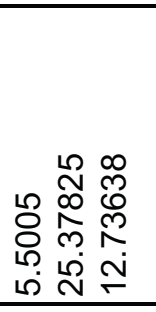 & $\begin{array}{l}\overline{0} \\
\stackrel{\emptyset}{0} \\
0\end{array}$ & 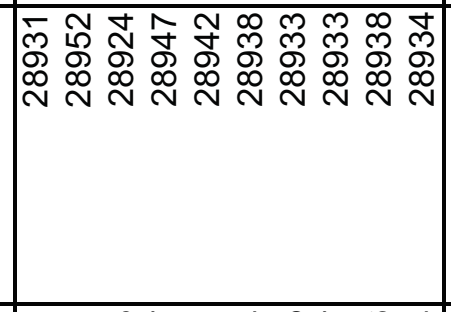 & 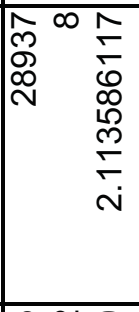 & $\begin{array}{l}\stackrel{8}{0} \\
\stackrel{+}{N} \\
\infty \\
\infty\end{array}$ & 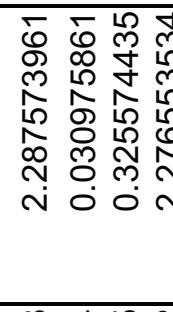 & 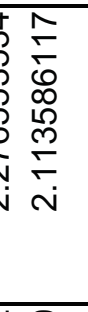 & 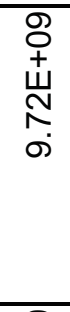 & \\
\hline 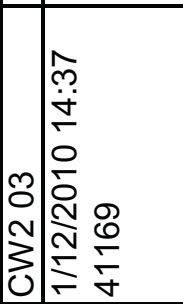 & 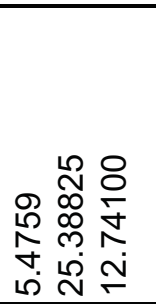 & $\begin{array}{l}\hat{0} \\
\stackrel{0}{0} \\
\dot{0}\end{array}$ & 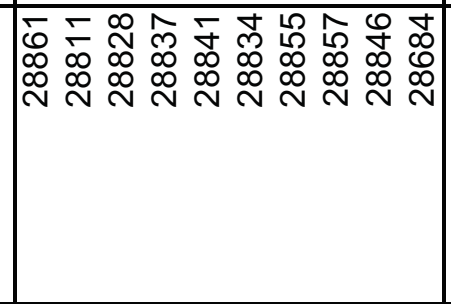 & 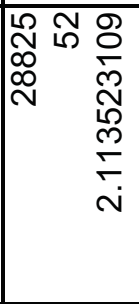 & 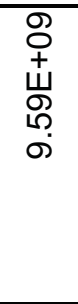 & 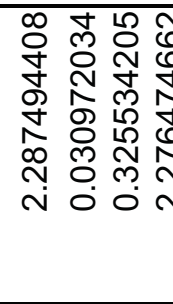 & 至 & $\begin{array}{l}\text { o } \\
+ \\
\dot{0} \\
0 \\
0\end{array}$ & \\
\hline 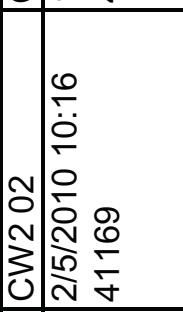 & 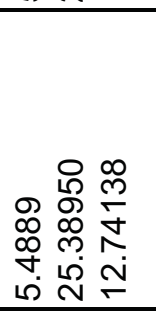 & $\begin{array}{l}\hat{0} \\
\stackrel{0}{0} \\
\vdots\end{array}$ & 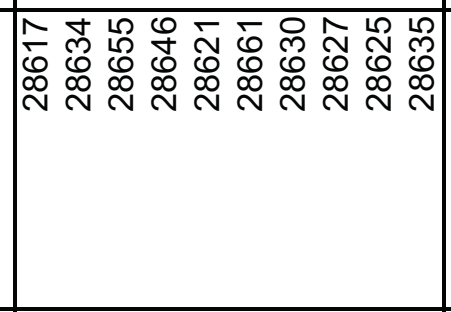 & 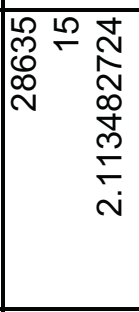 & 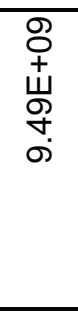 & 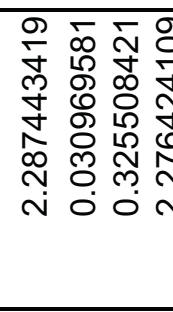 & 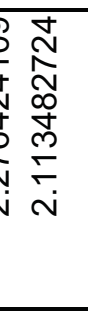 & 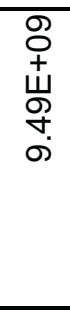 & \\
\hline 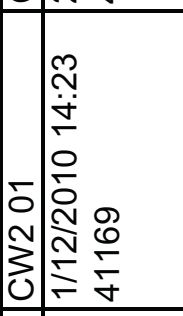 & 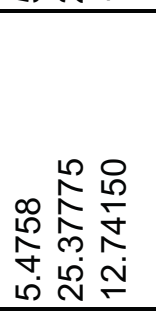 & $\begin{array}{l}\hat{\ddot{\theta}} \\
\stackrel{0}{0}\end{array}$ & 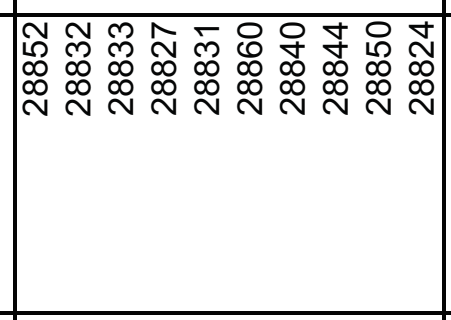 & 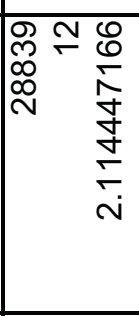 & 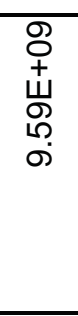 & 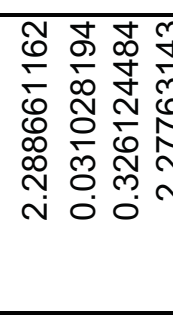 & 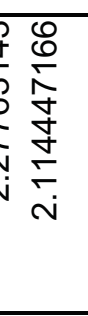 & 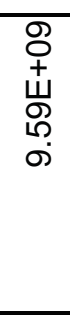 & \\
\hline 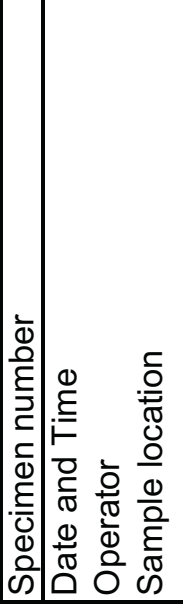 & 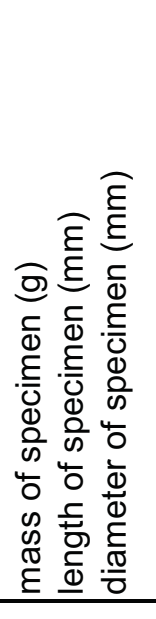 & 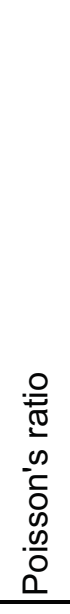 & 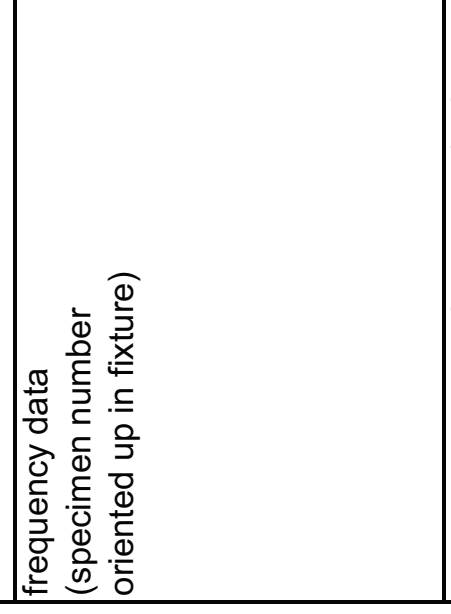 & 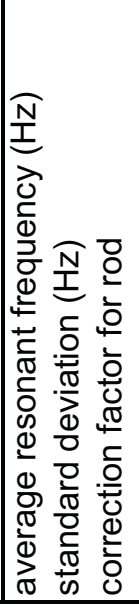 & 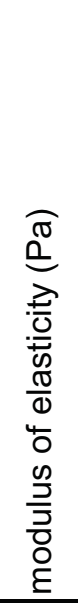 & 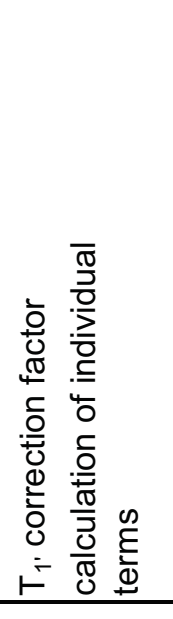 & 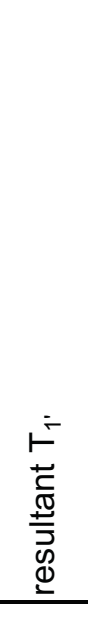 & 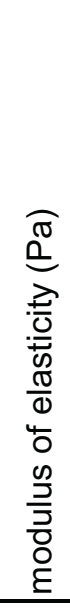 & 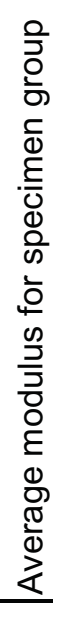 \\
\hline
\end{tabular}




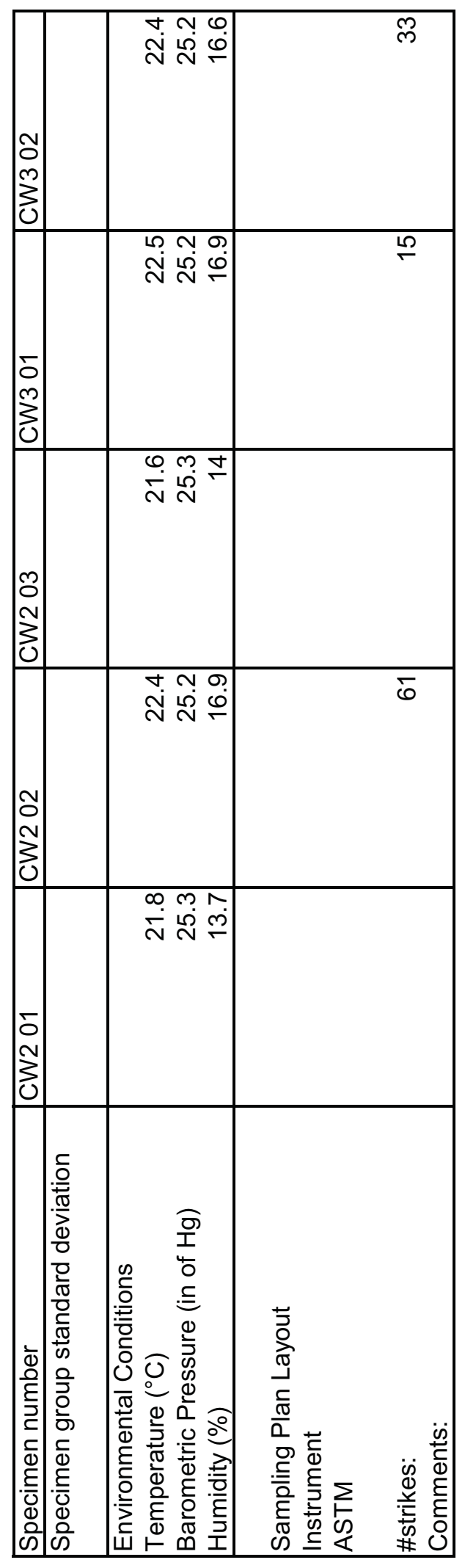




\begin{tabular}{|c|c|c|c|c|c|c|c|}
\hline 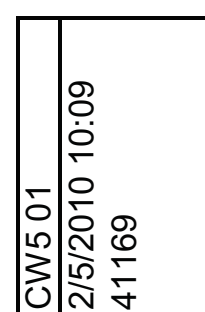 & 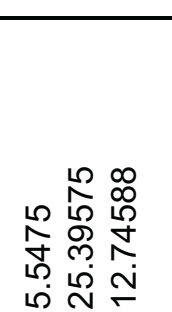 & 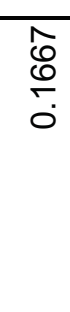 & 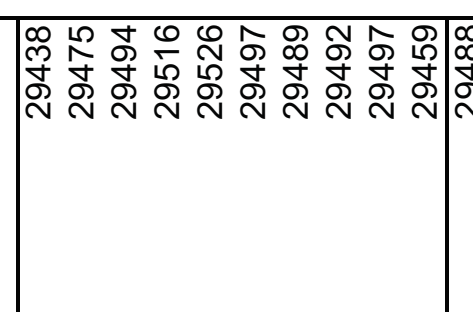 & 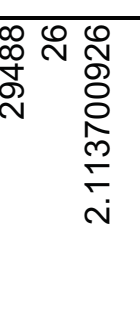 & 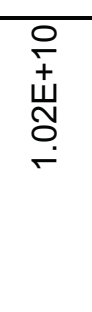 & 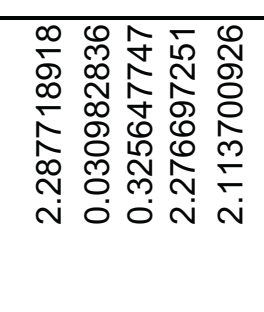 & 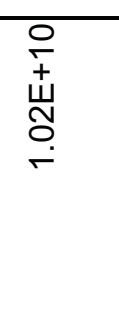 \\
\hline 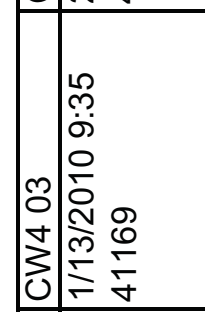 & 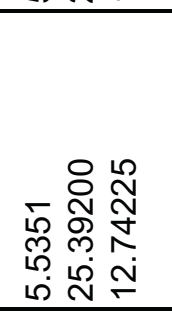 & 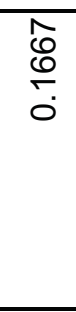 & 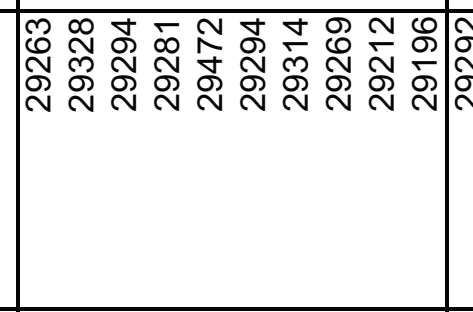 & ר) & $\begin{array}{l}\stackrel{\circ}{+} \\
\stackrel{+}{\dot{O}} \\
\stackrel{+}{+}\end{array}$ & 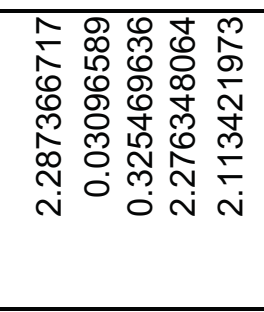 & 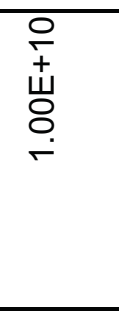 \\
\hline 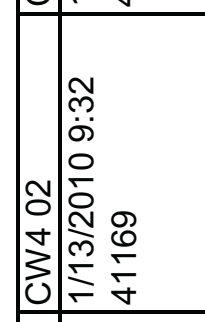 & 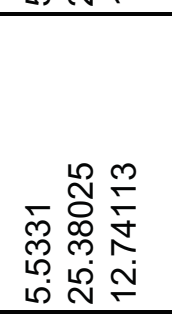 & $\begin{array}{l}\frac{\pi}{0} \\
\vdots \\
0\end{array}$ & 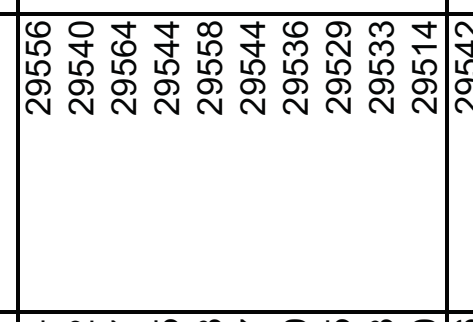 & 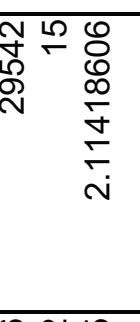 & 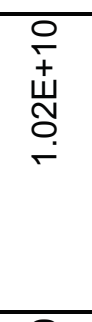 & 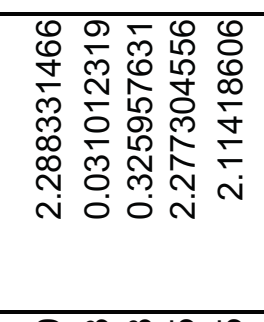 & 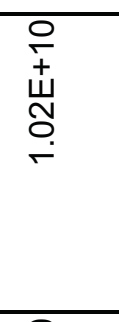 \\
\hline 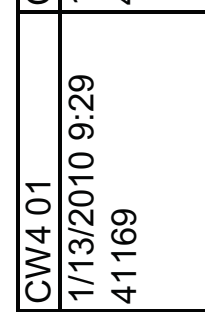 & 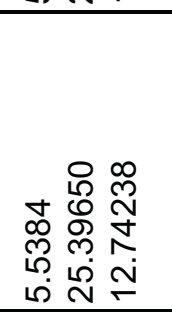 & $\begin{array}{l}\frac{\pi}{0} \\
\vdots \\
0\end{array}$ & 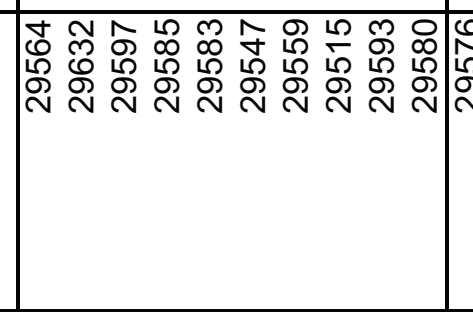 & 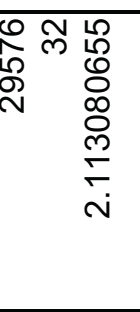 & 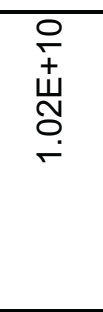 & 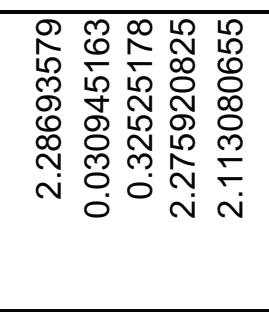 & 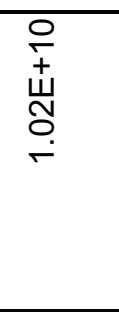 \\
\hline 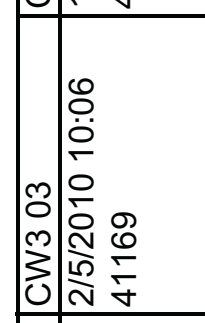 & 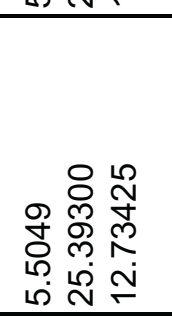 & $\begin{array}{l}\overline{0} \\
\stackrel{0}{0} \\
0\end{array}$ & 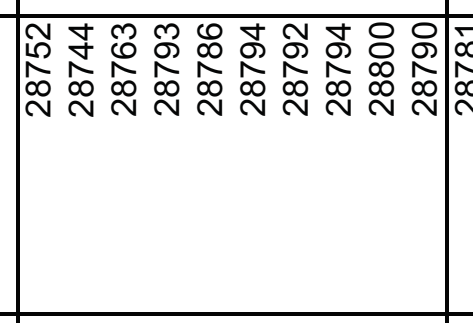 & 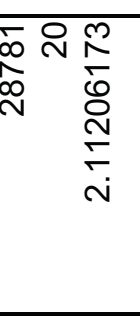 & 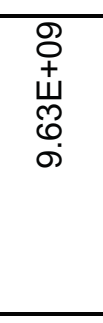 & 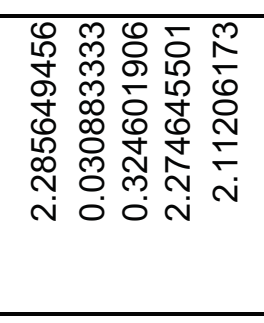 & 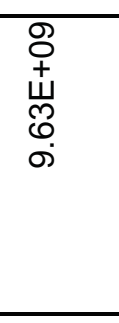 \\
\hline 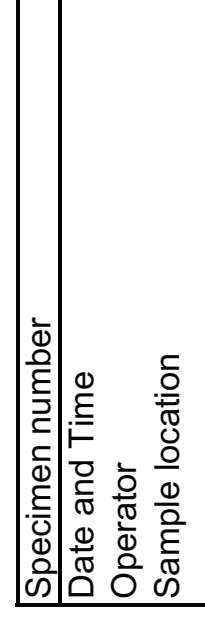 & 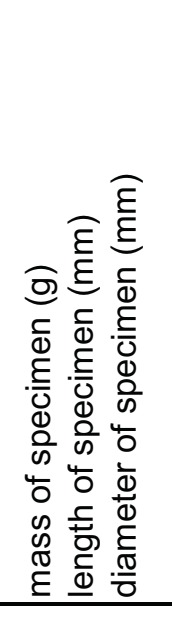 & 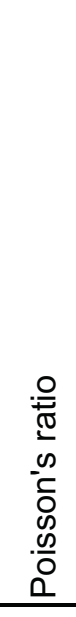 & \begin{tabular}{|l} 
\\
\\
\end{tabular} & 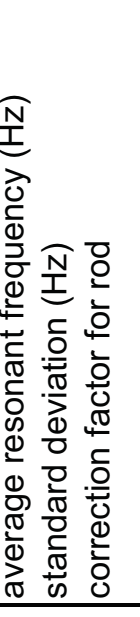 & 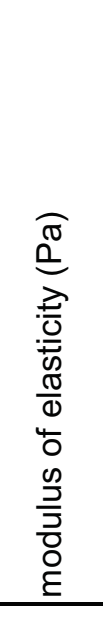 & 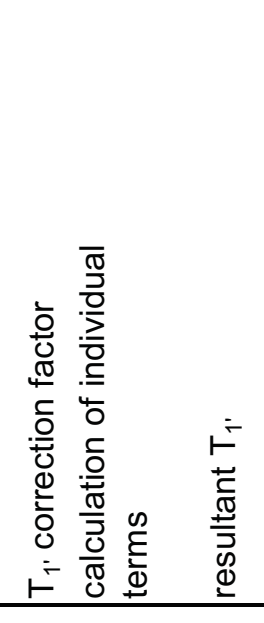 & 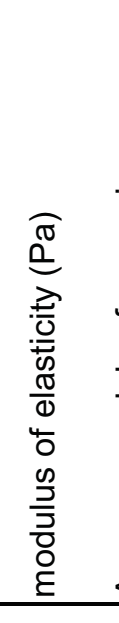 \\
\hline
\end{tabular}




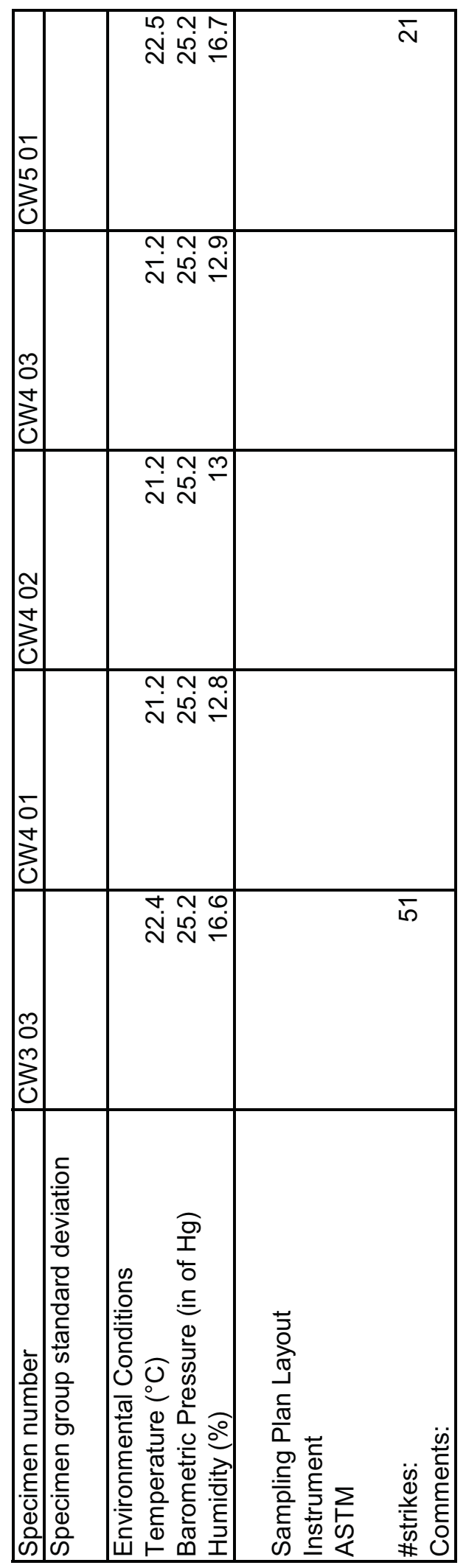




\begin{tabular}{|c|c|c|c|c|c|c|c|}
\hline 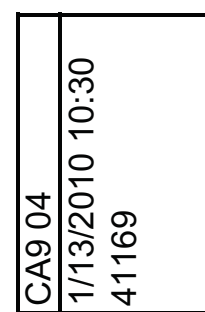 & 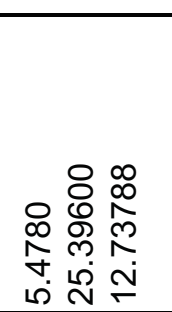 & 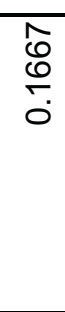 & 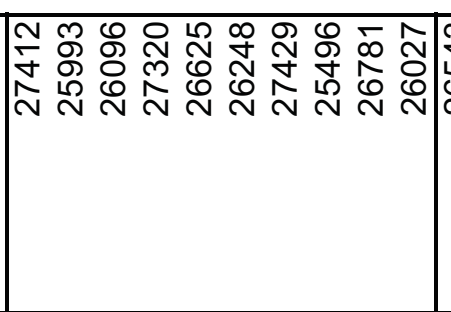 & 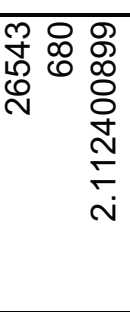 & 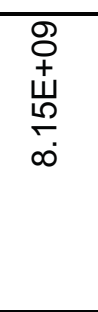 & 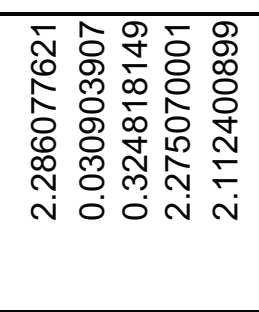 & 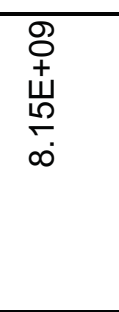 \\
\hline 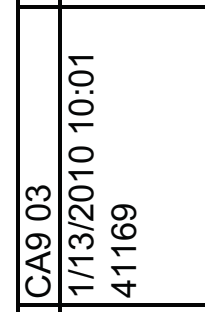 & 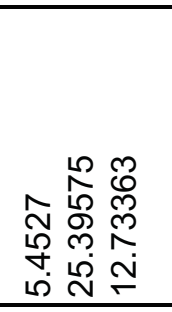 & $\begin{array}{l}\frac{\pi}{0} \\
\stackrel{0}{0}\end{array}$ & 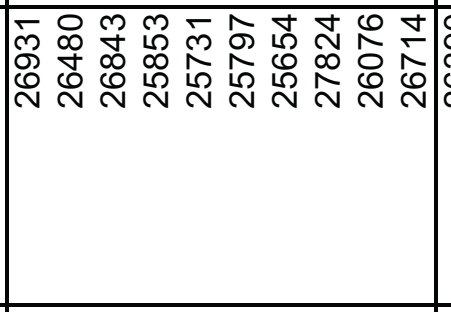 & 尊 & 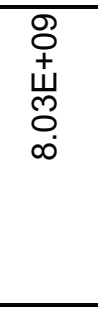 & 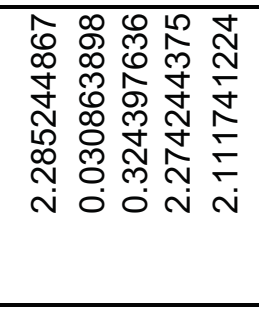 & 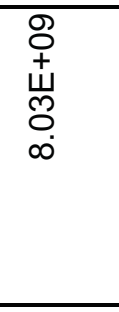 \\
\hline 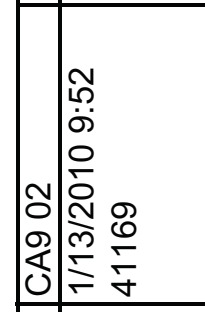 & 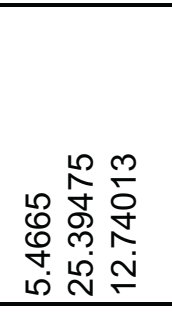 & 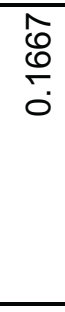 & 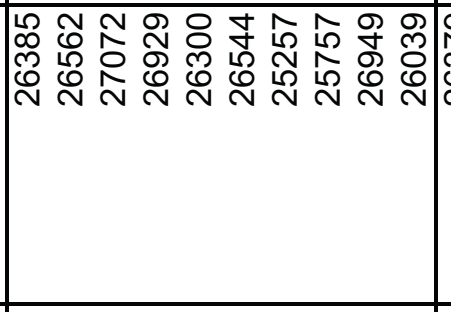 & 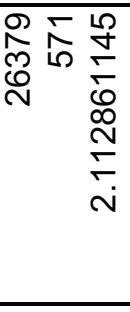 & 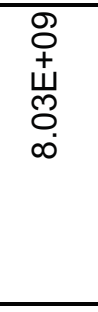 & 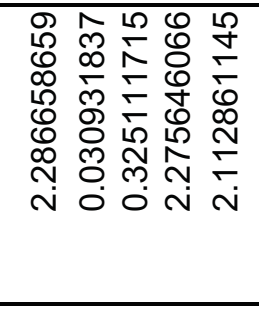 & 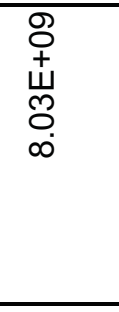 \\
\hline 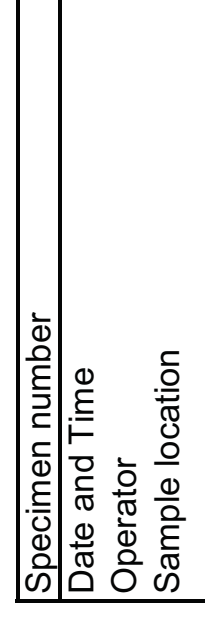 & 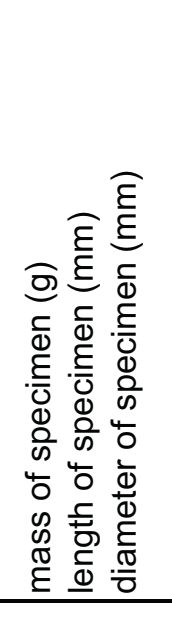 & 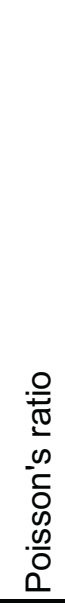 & 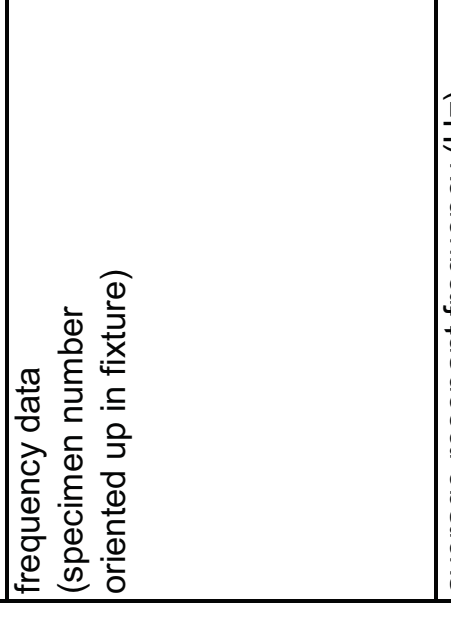 & 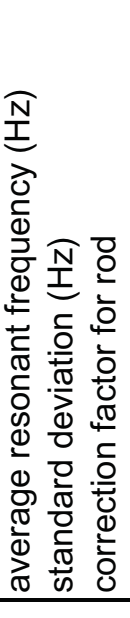 & 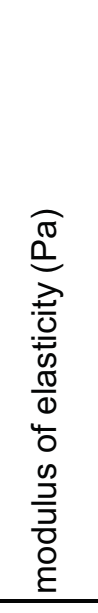 & 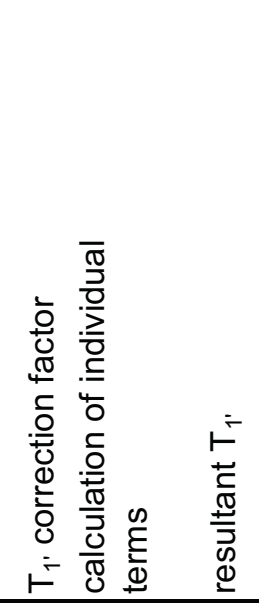 & 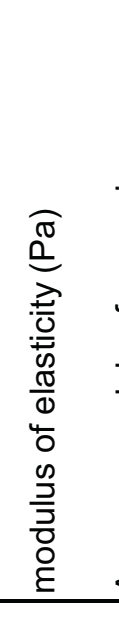 \\
\hline
\end{tabular}




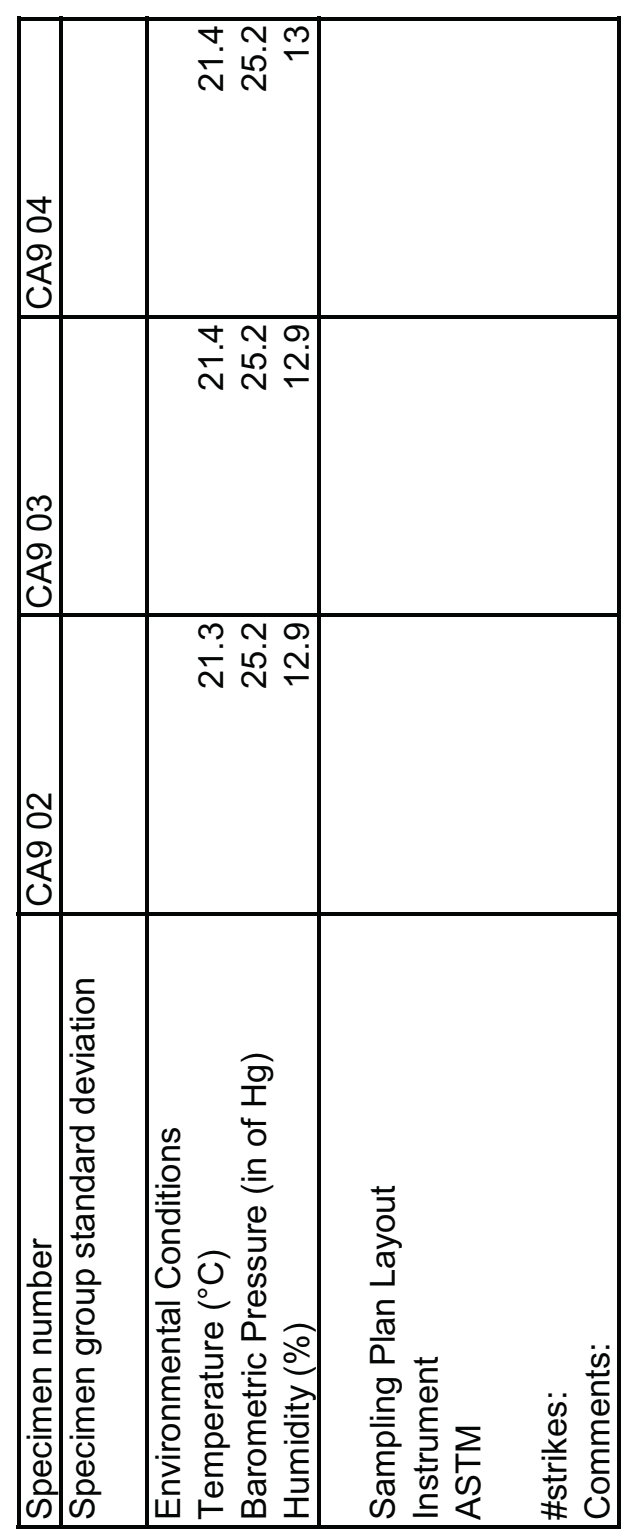




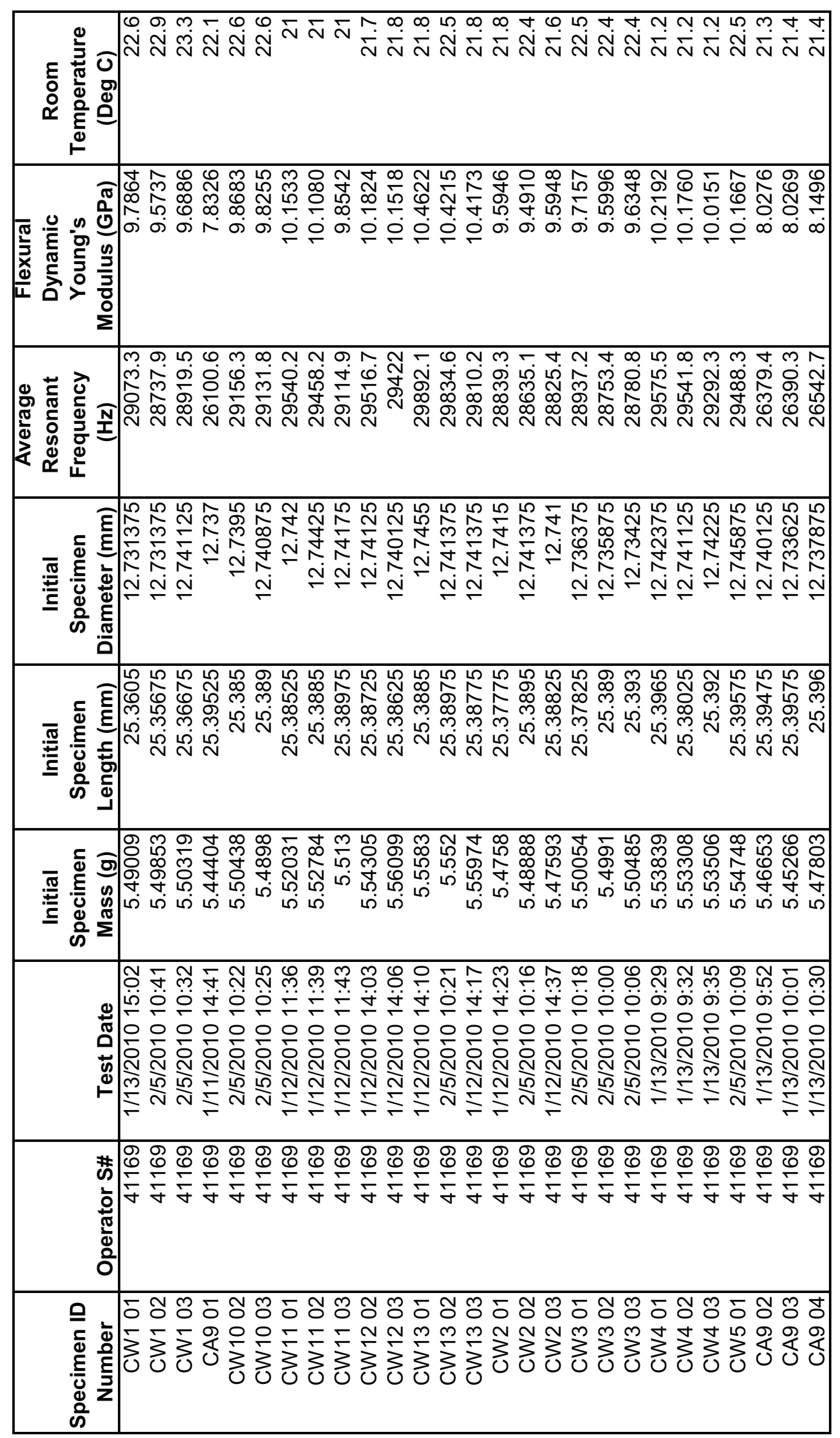




\begin{tabular}{|c|c|}
\hline 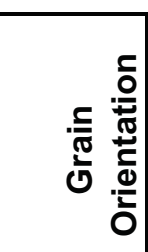 & 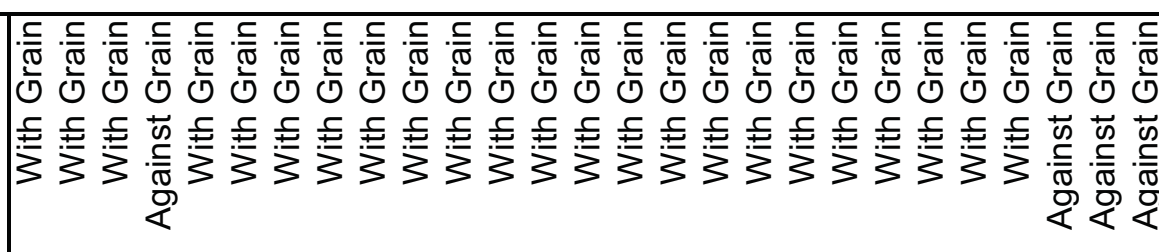 \\
\hline 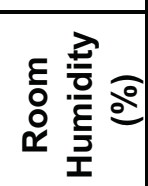 & 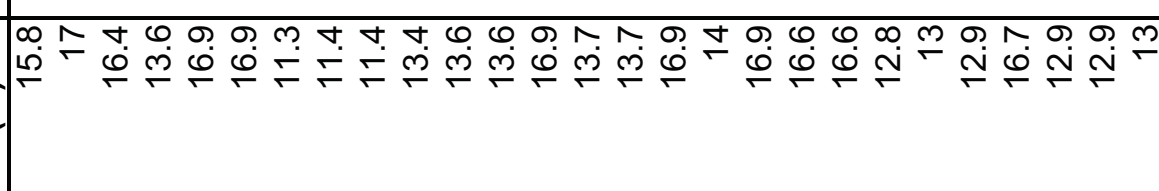 \\
\hline 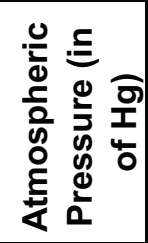 & 赵 N N \\
\hline 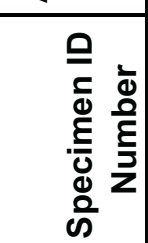 & 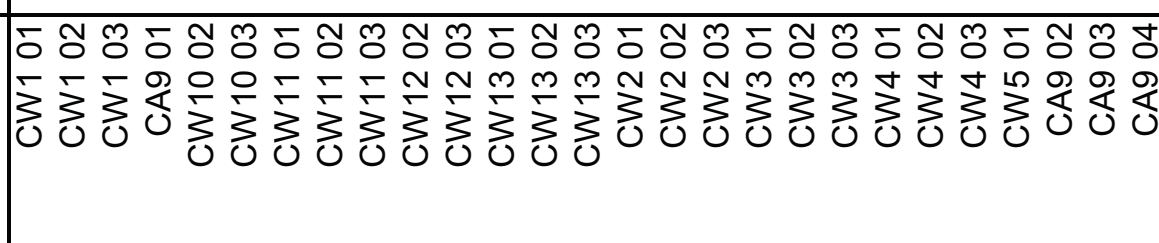 \\
\hline
\end{tabular}




\begin{tabular}{|c|c|c|c|c|c|c|c|c|}
\hline 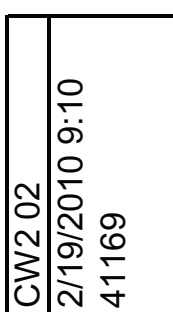 & 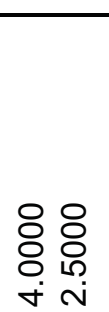 & & 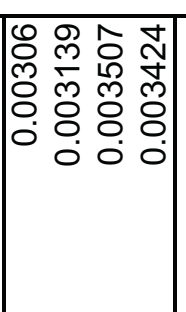 & 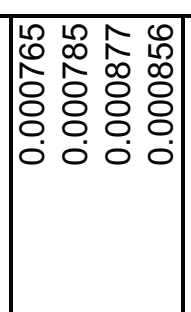 & 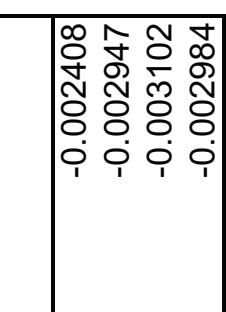 & 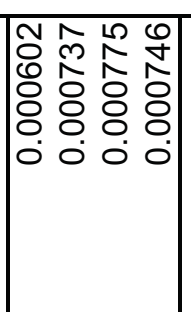 & & 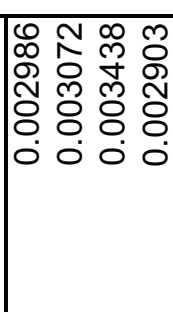 \\
\hline 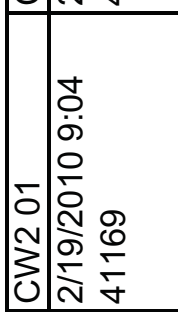 & 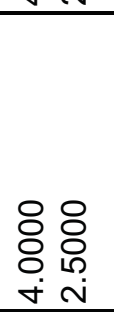 & & 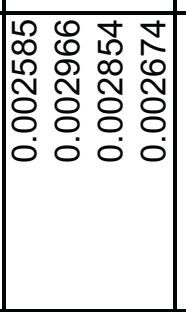 & 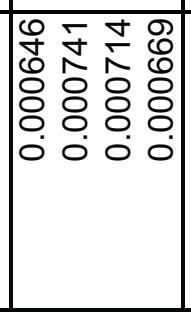 & 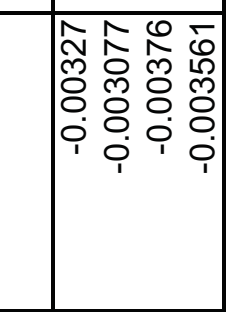 & 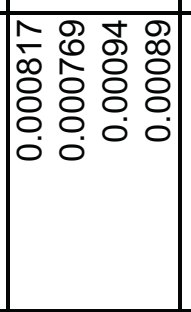 & & 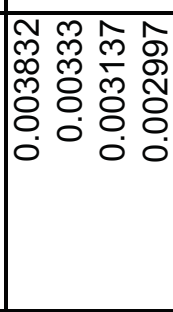 \\
\hline 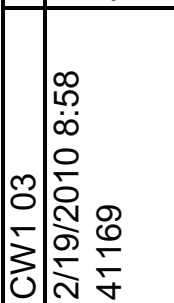 & 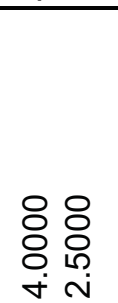 & & 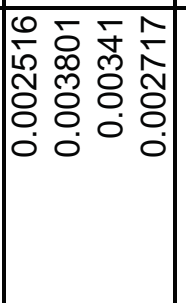 & 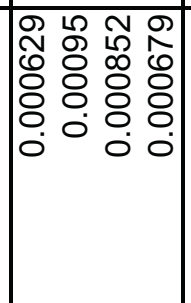 & 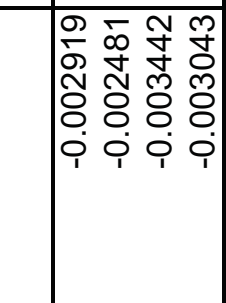 & 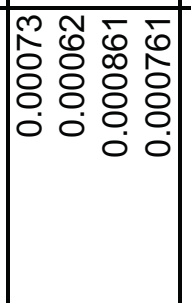 & & 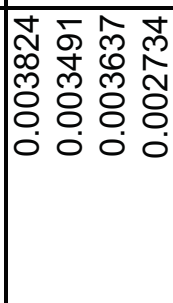 \\
\hline 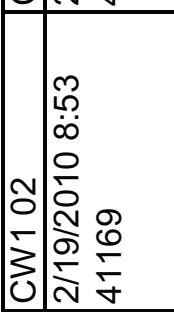 & 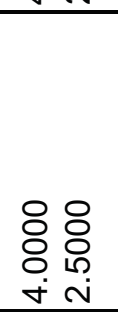 & & 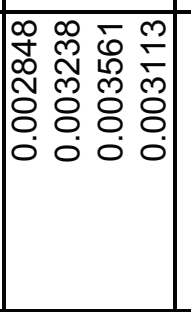 & 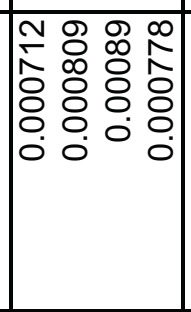 & 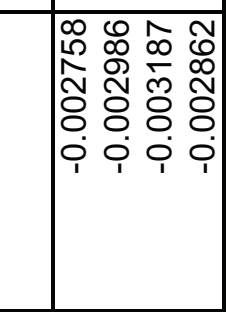 & 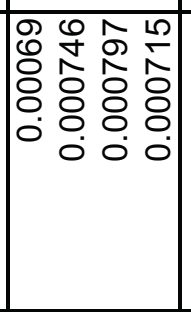 & & 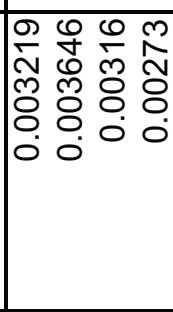 \\
\hline 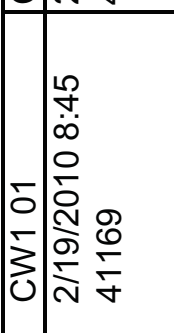 & 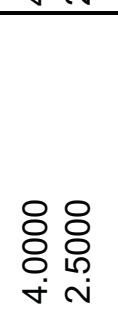 & & 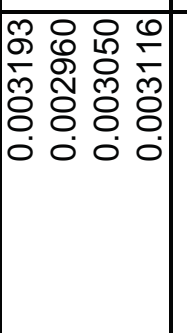 & 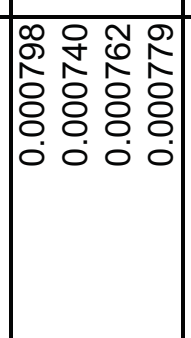 & 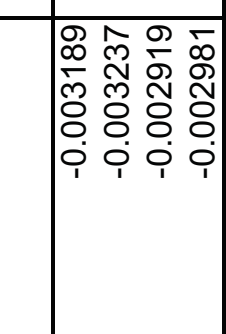 & 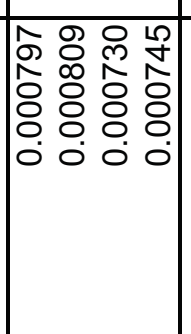 & & 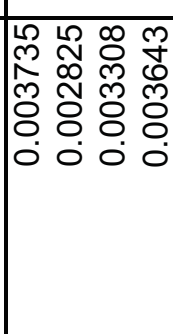 \\
\hline 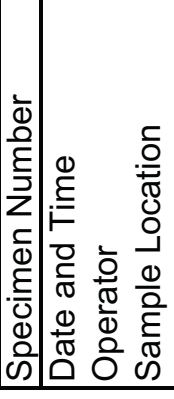 & 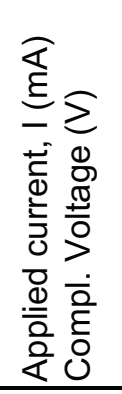 & 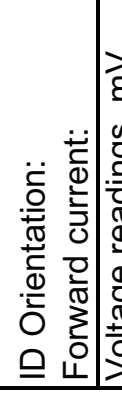 & 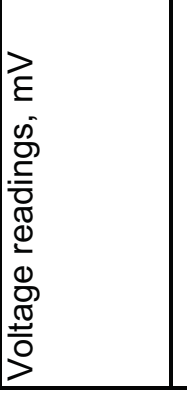 & 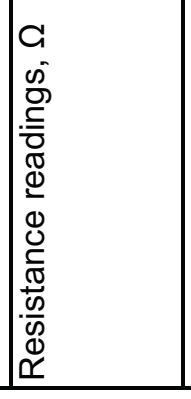 & 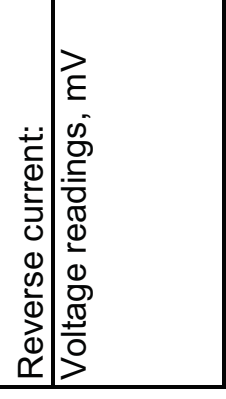 & 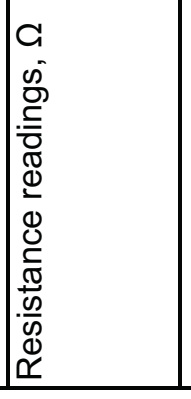 & 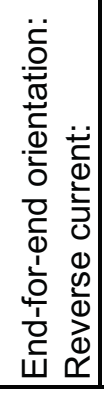 & 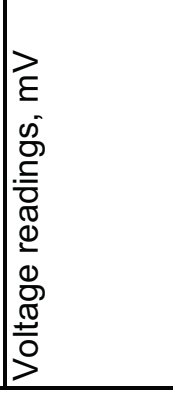 \\
\hline
\end{tabular}




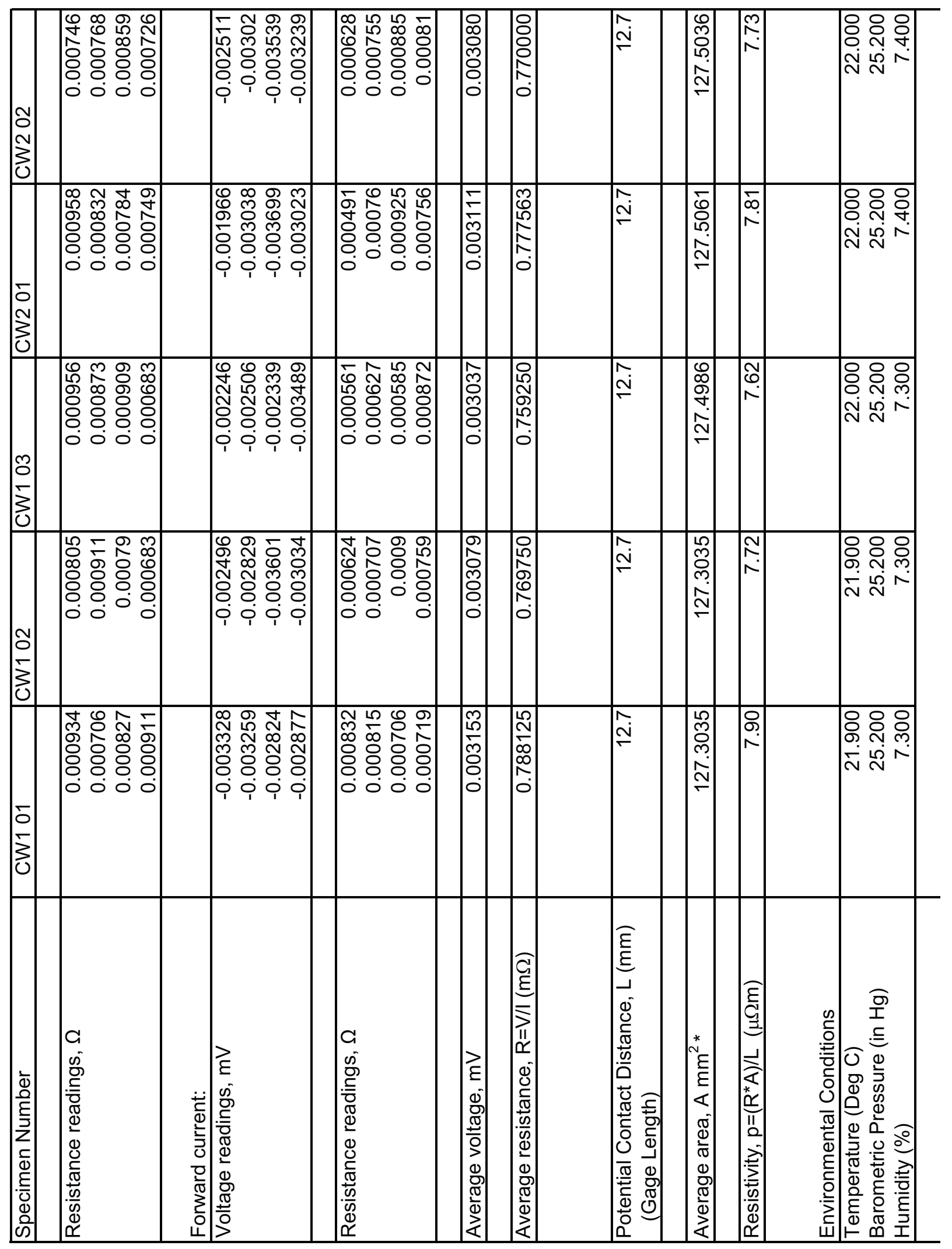




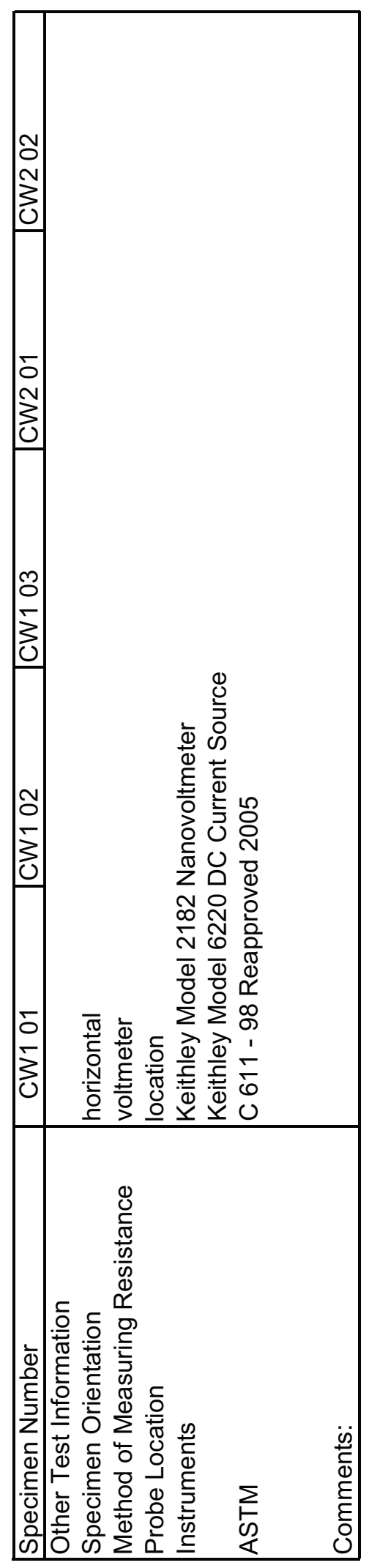




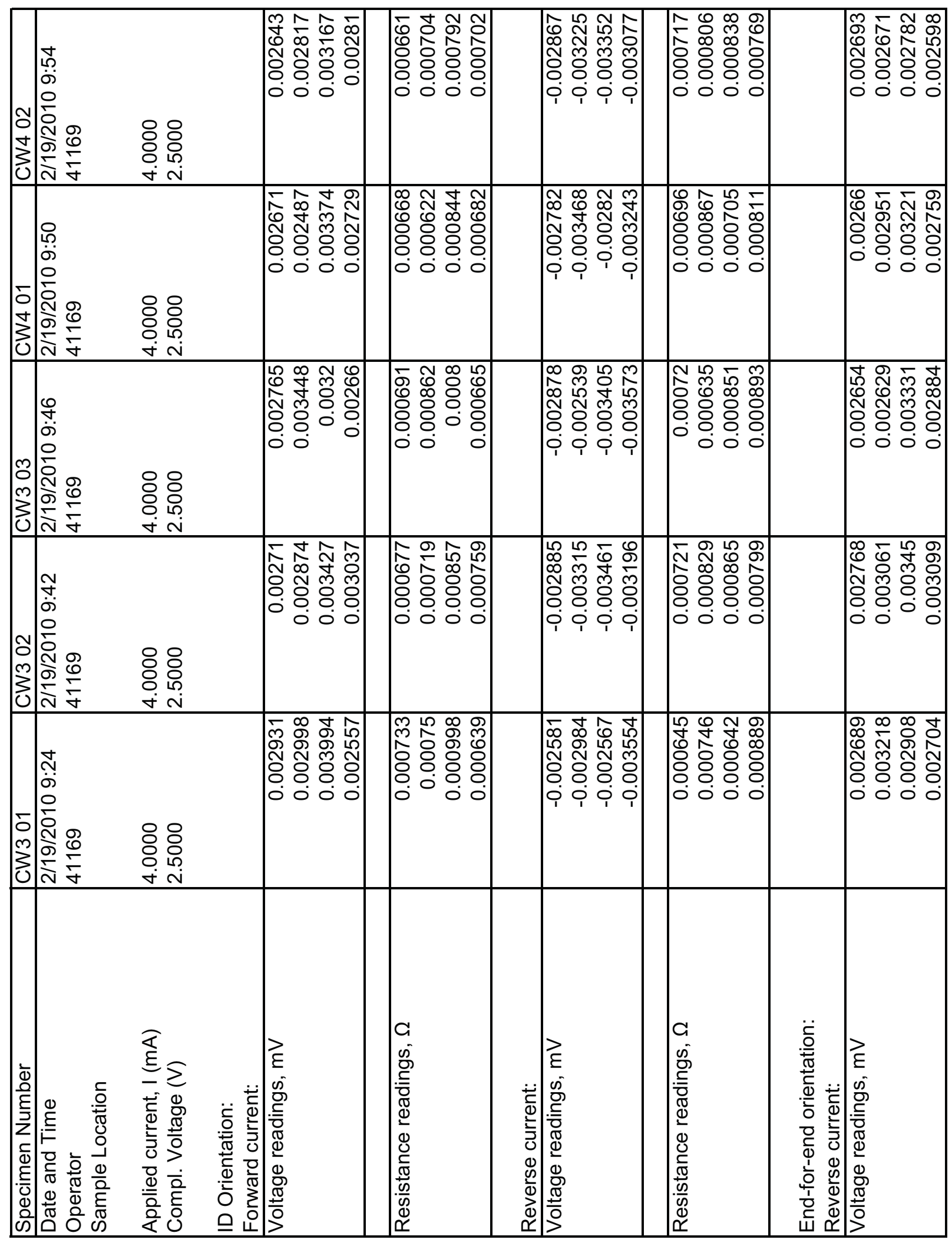




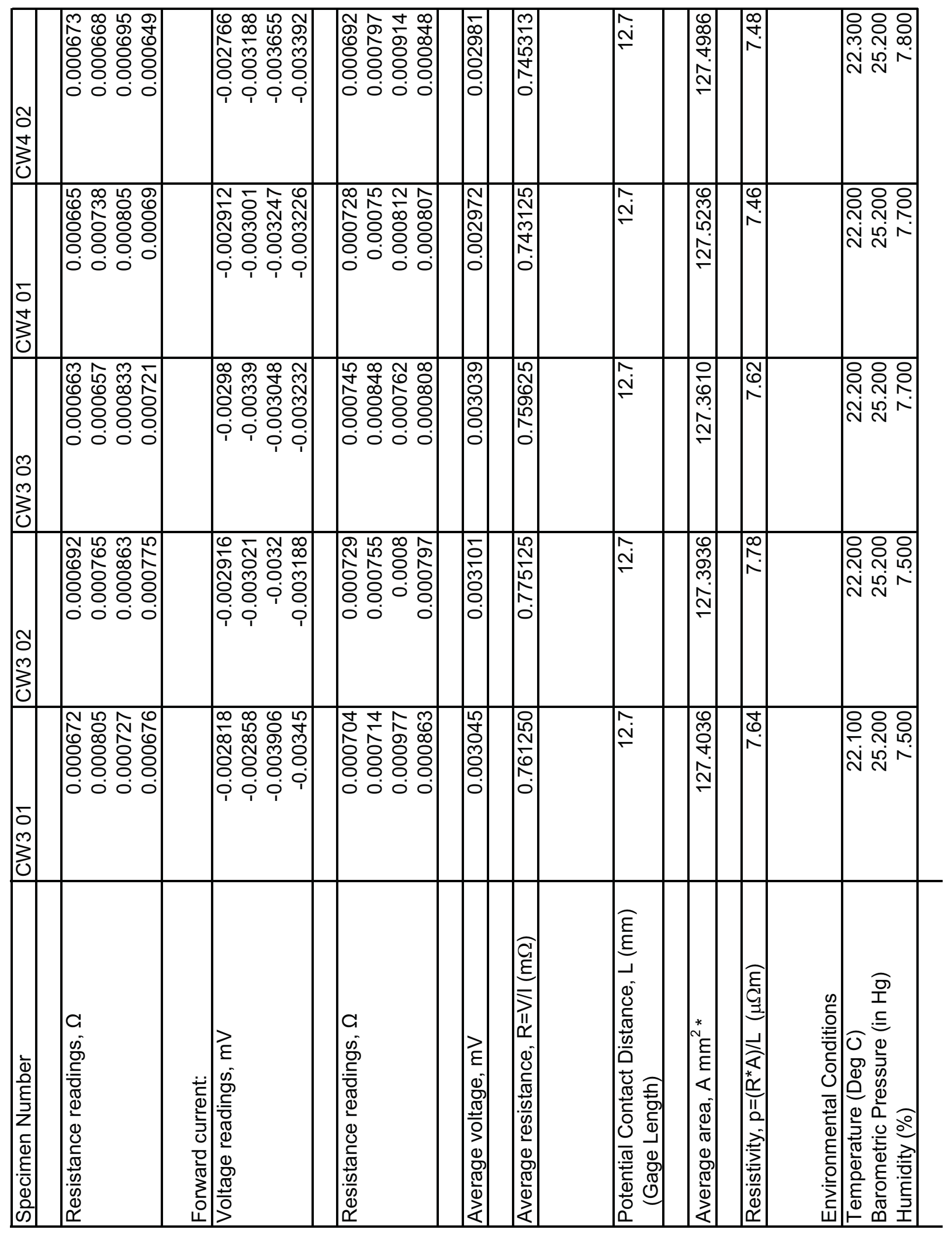




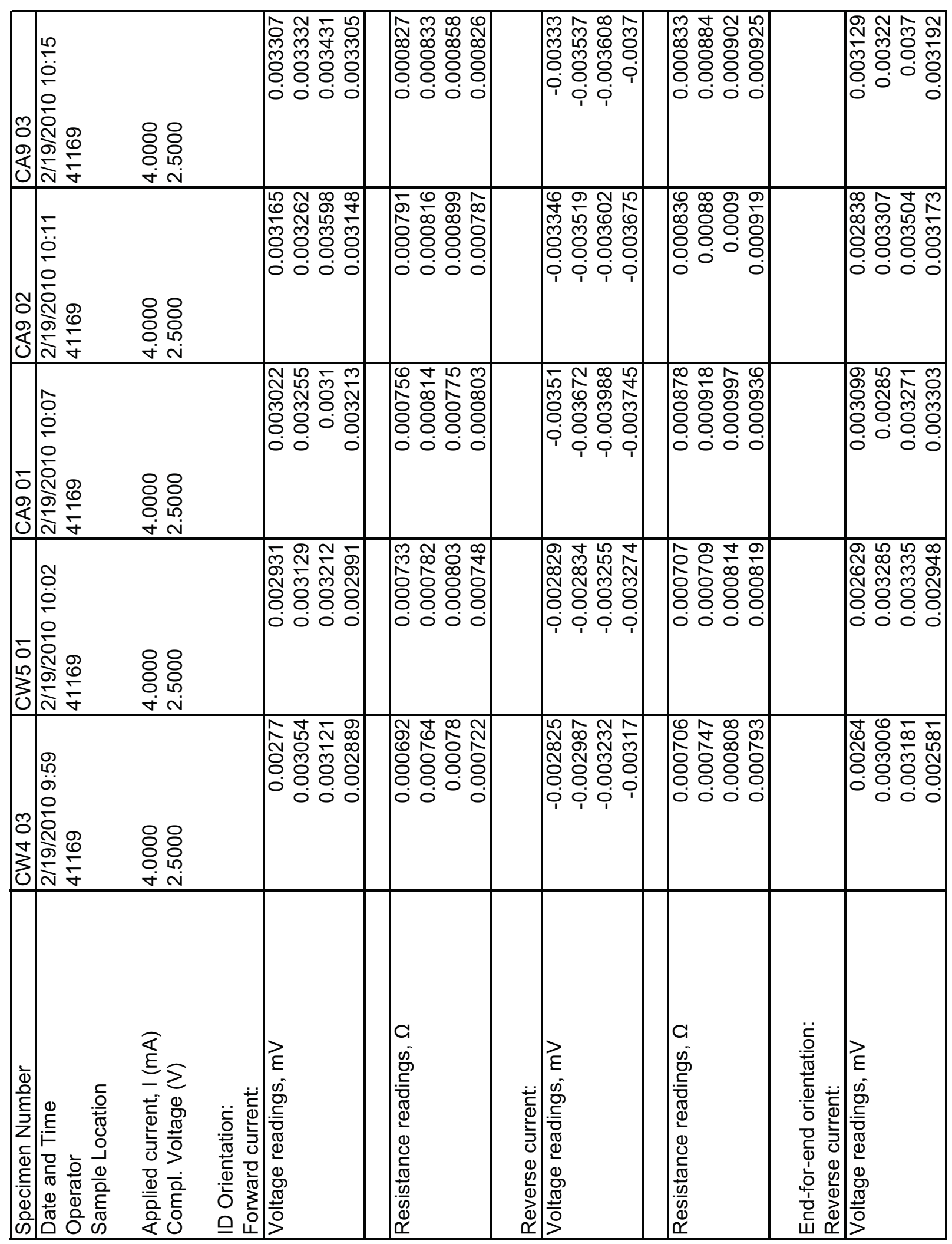




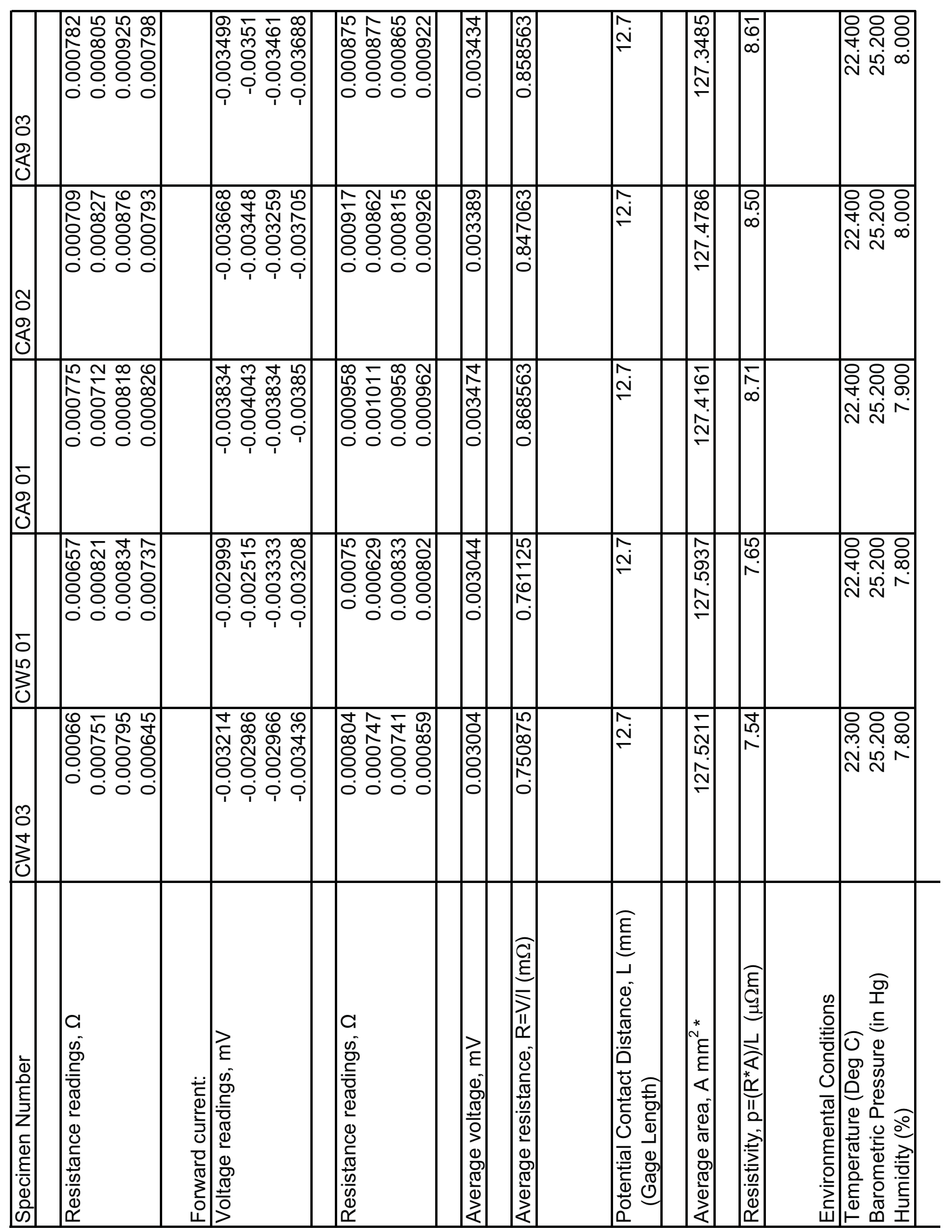




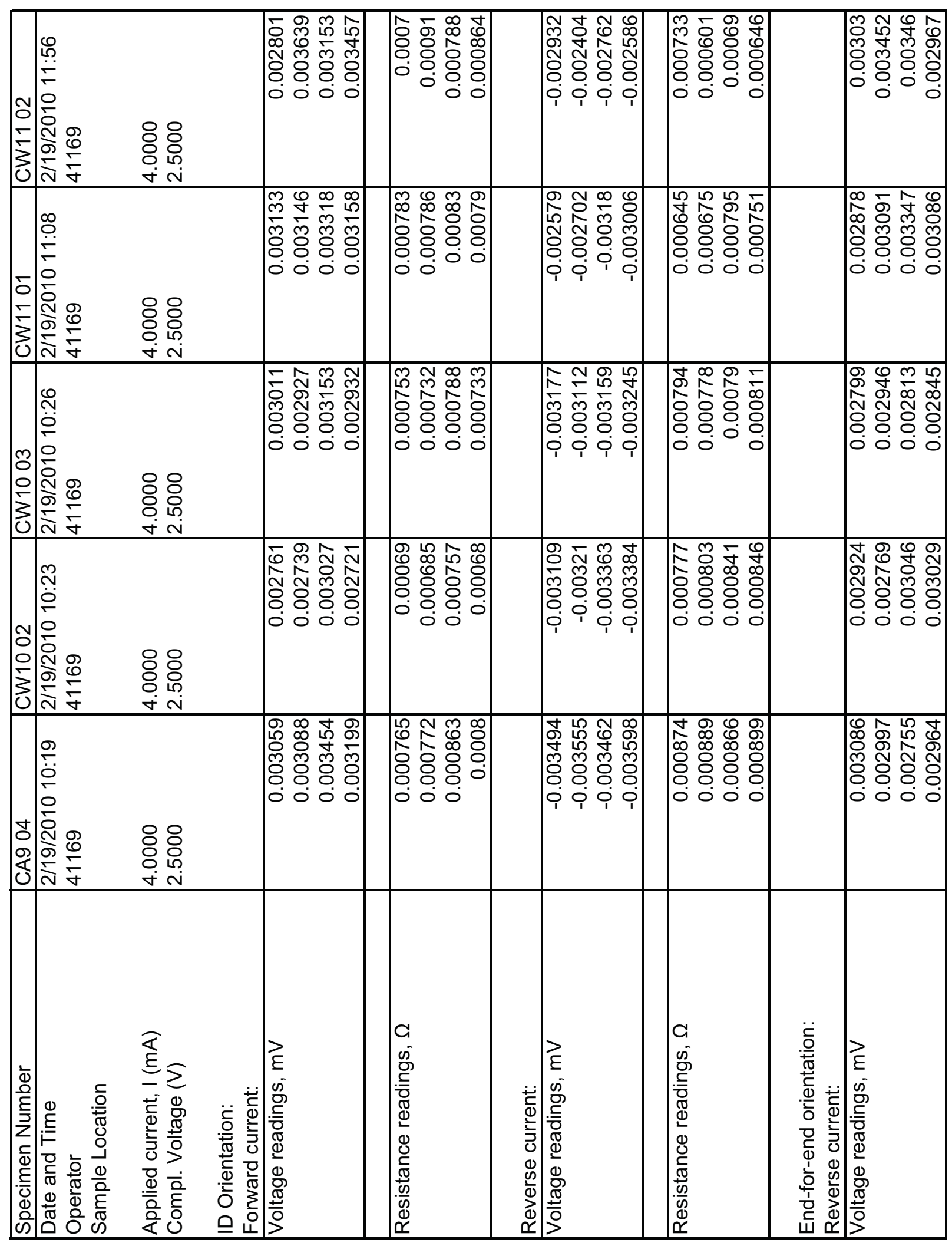




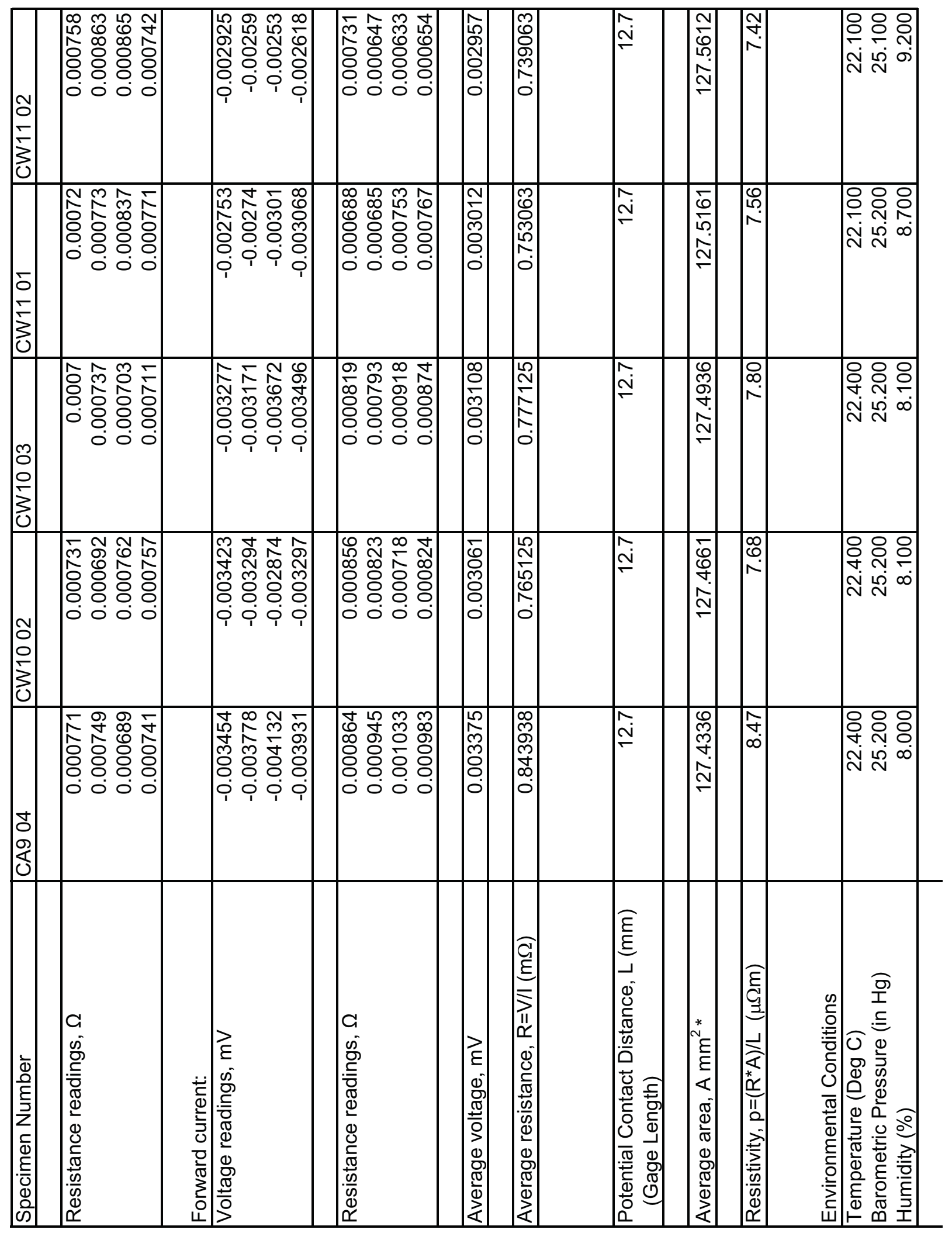




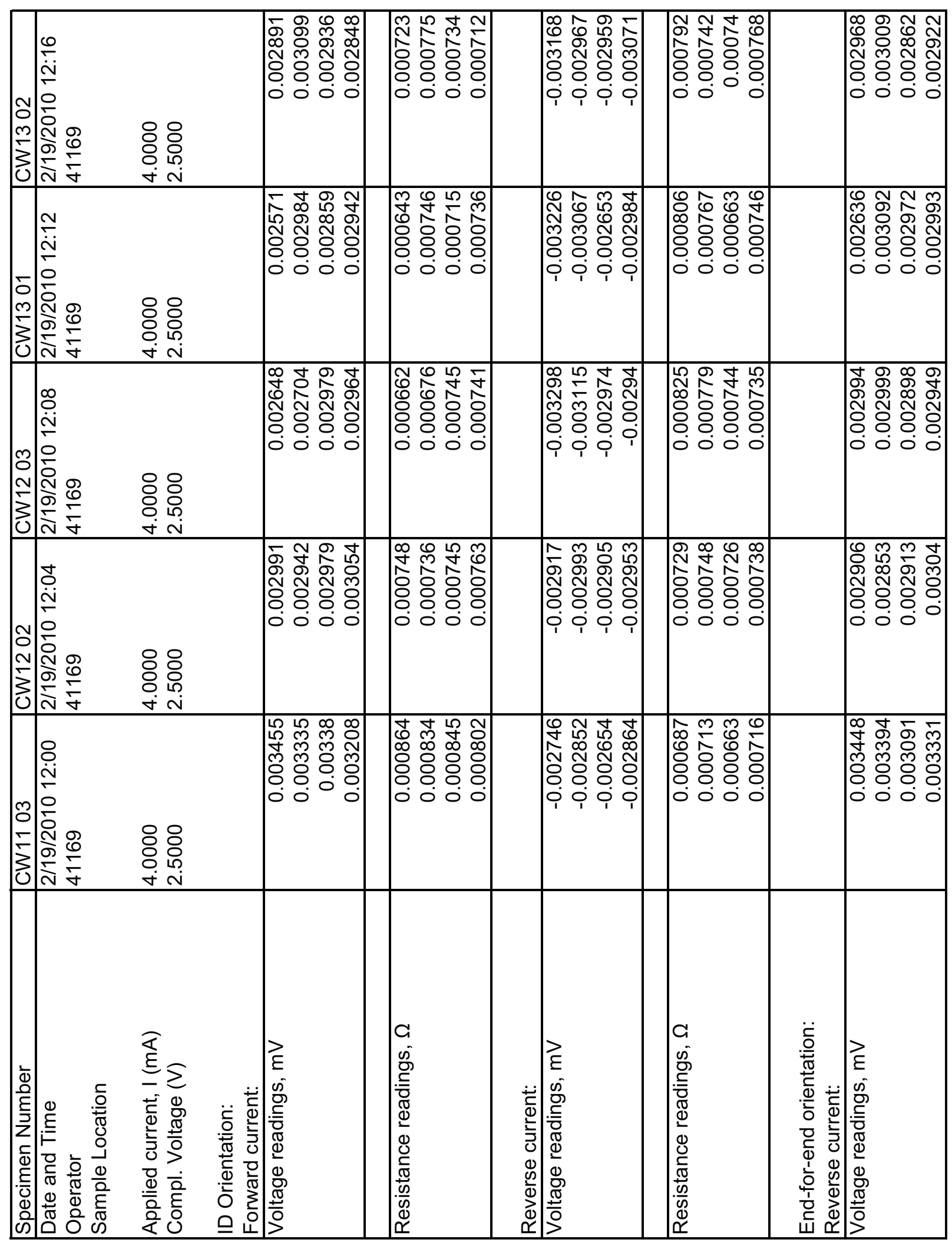




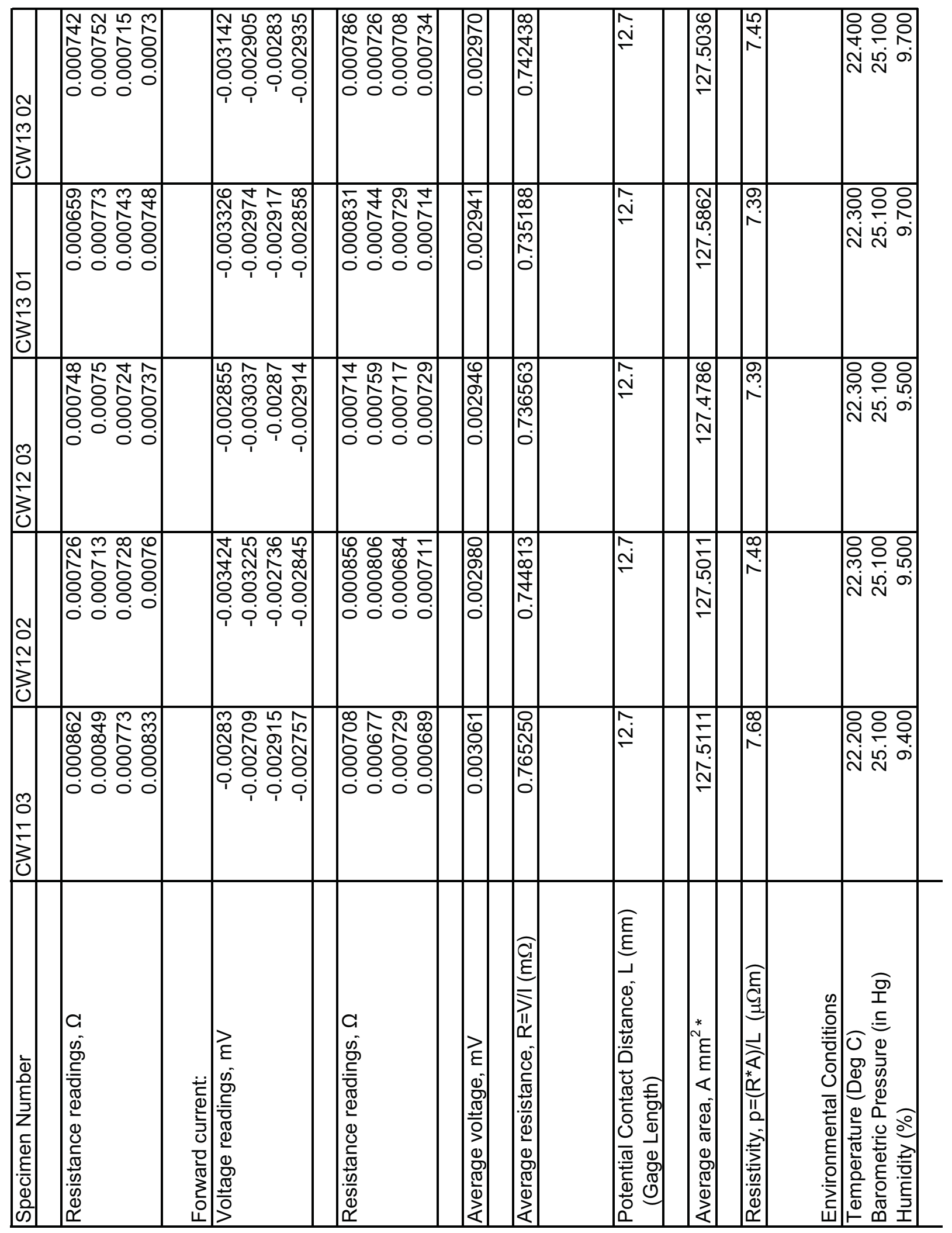




\begin{tabular}{|c|c|c|c|c|c|c|c|c|}
\hline 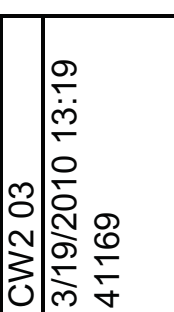 & 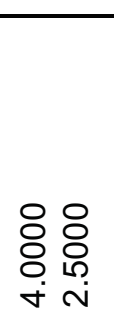 & & 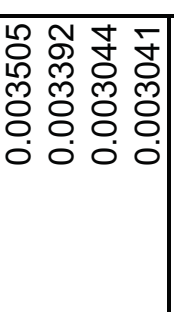 & 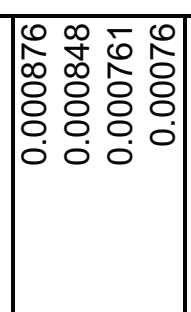 & 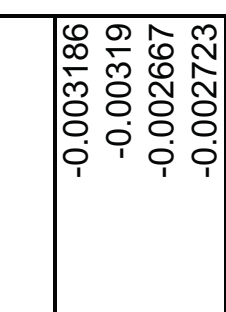 & 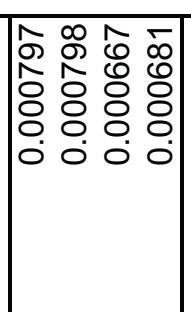 & & 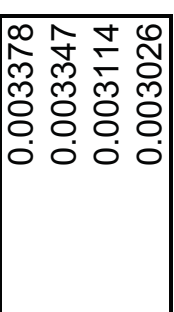 \\
\hline 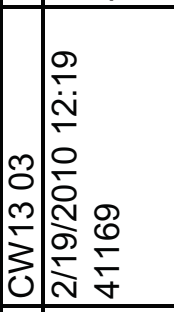 & 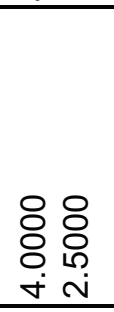 & & 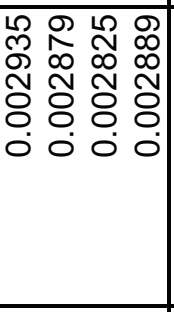 & 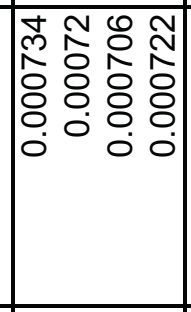 & 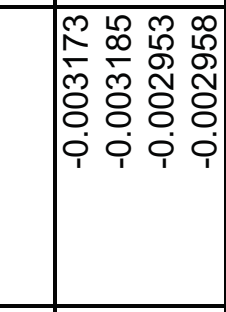 & 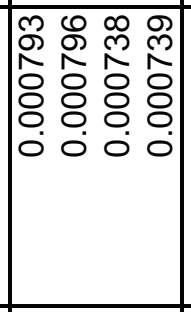 & & 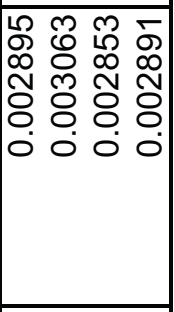 \\
\hline 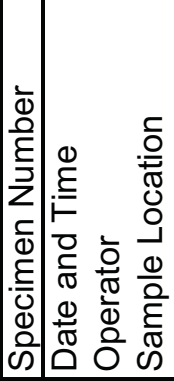 & 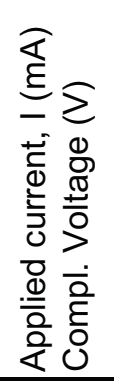 & 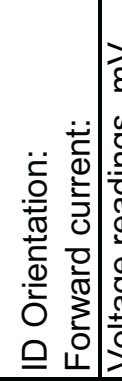 & 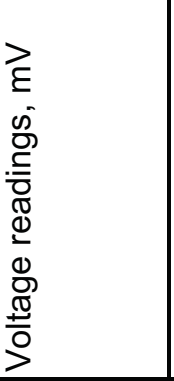 & 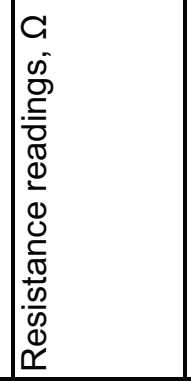 & 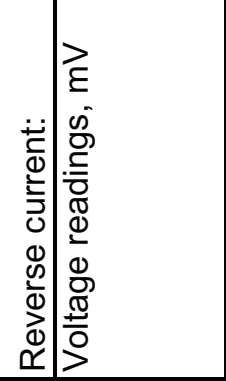 & 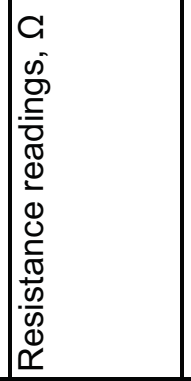 & 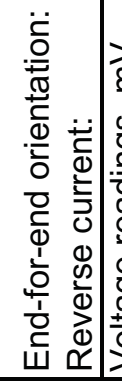 & 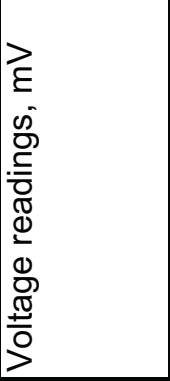 \\
\hline
\end{tabular}




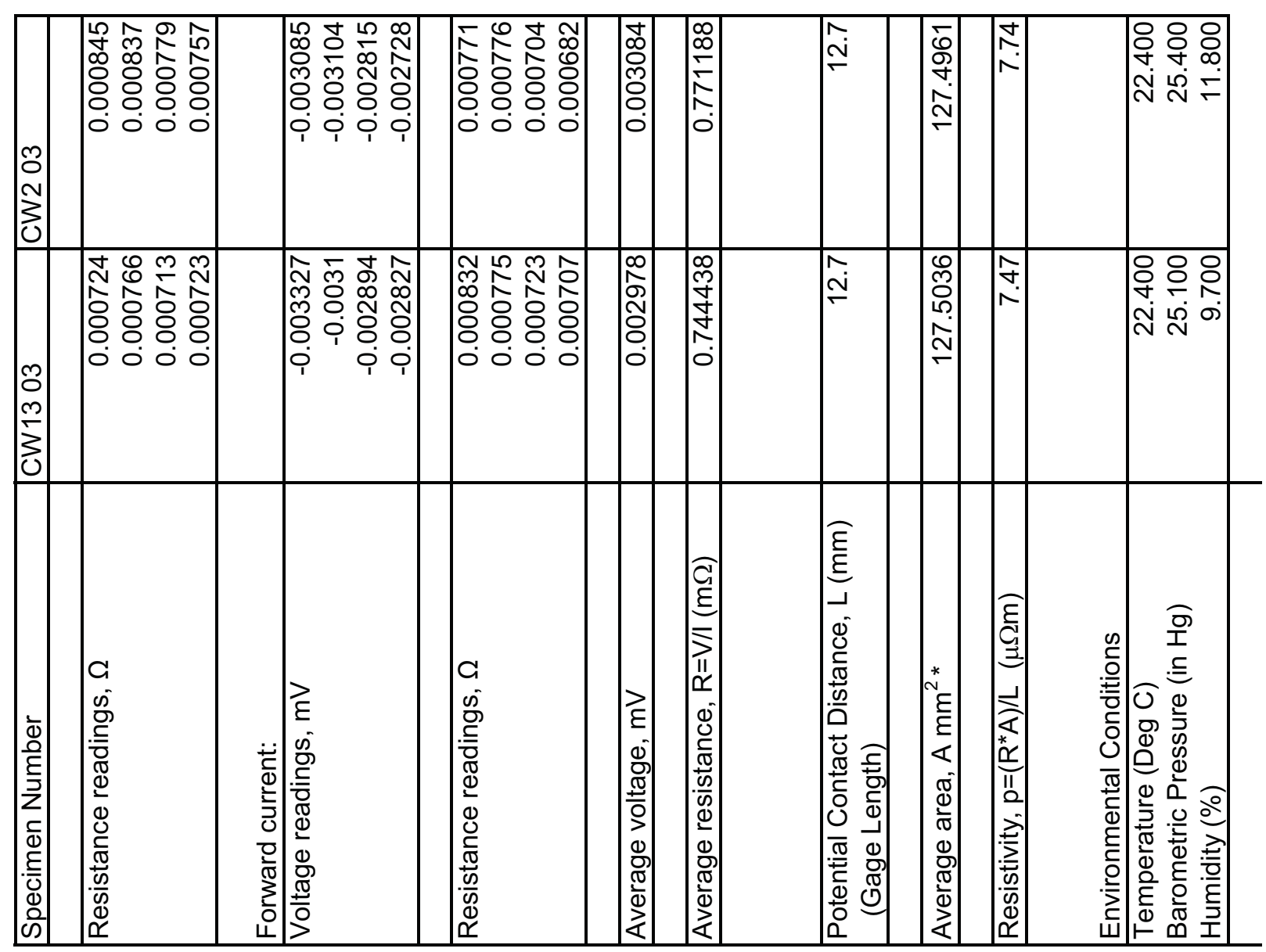




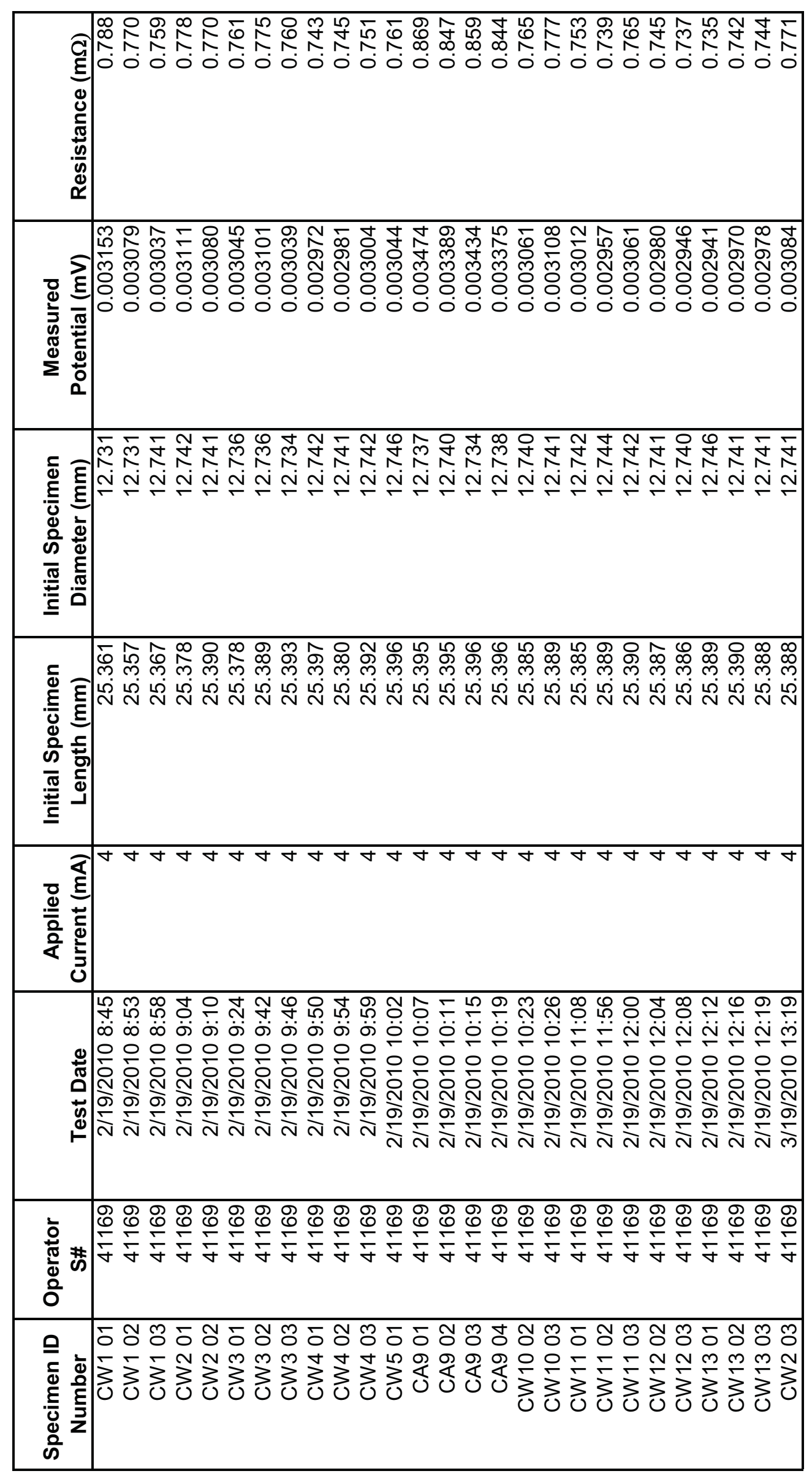




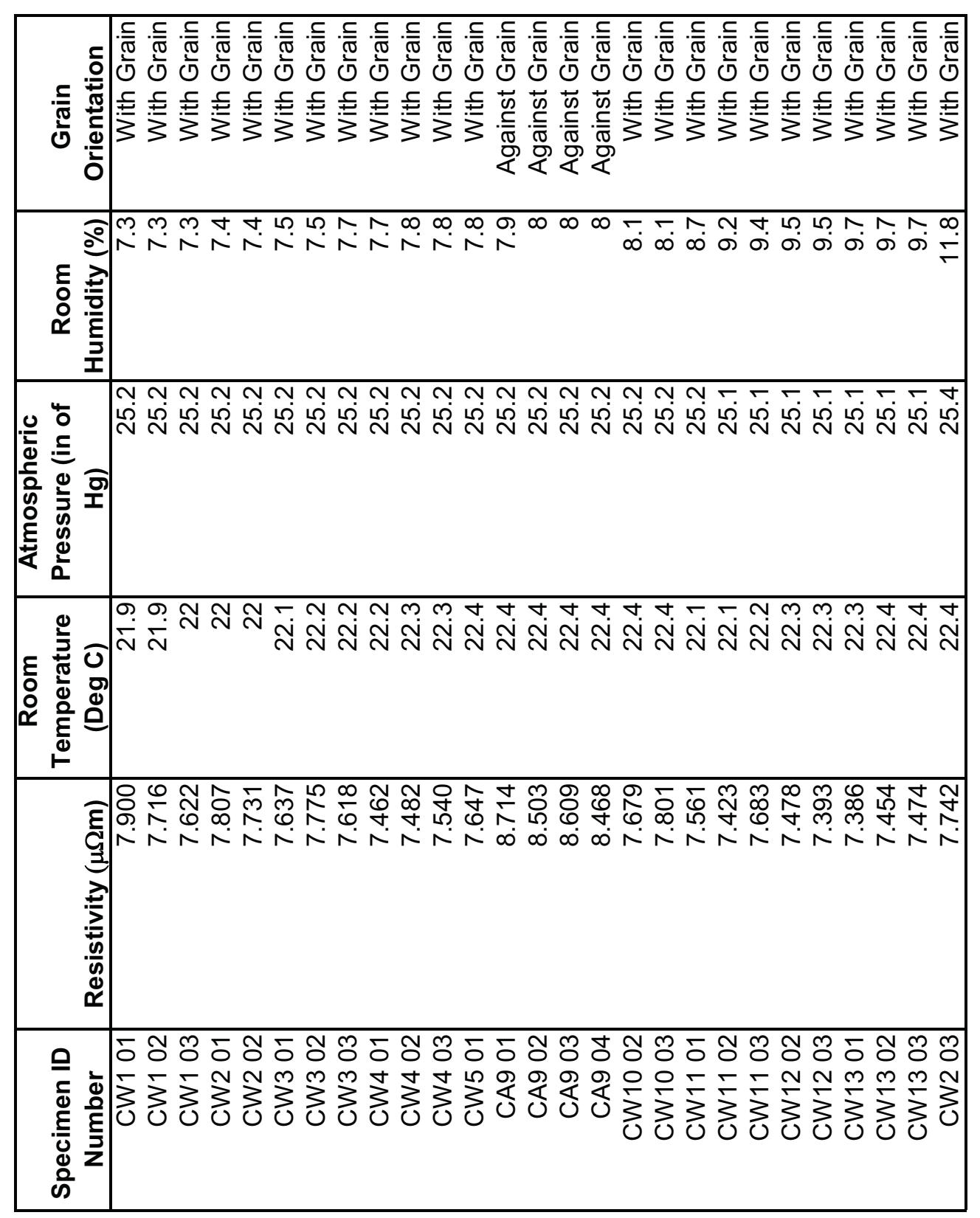




\begin{tabular}{|c|c|}
\hline 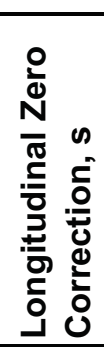 & 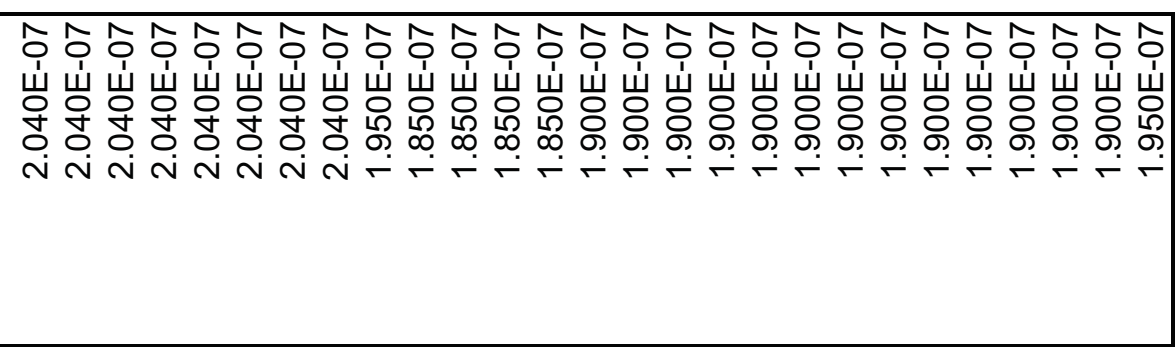 \\
\hline 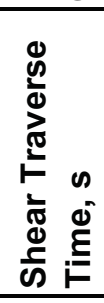 & 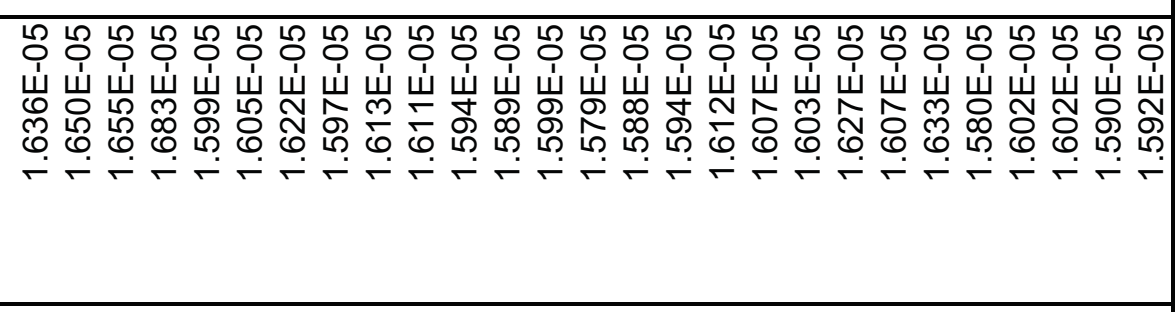 \\
\hline 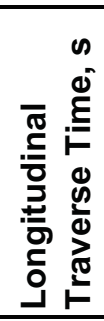 & 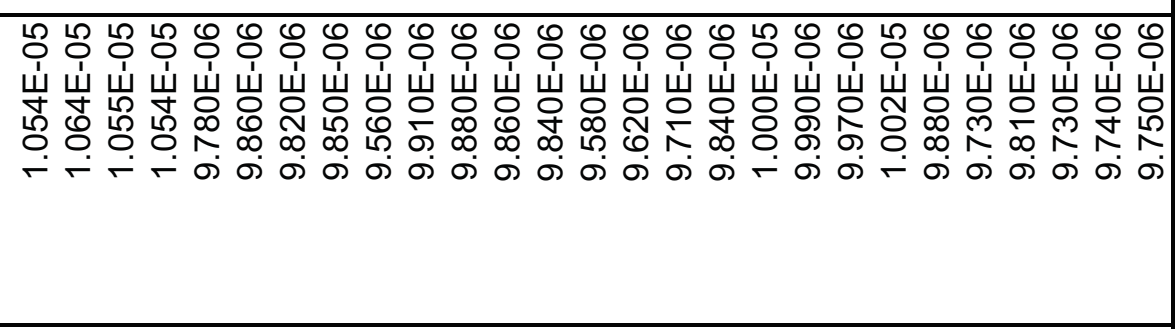 \\
\hline 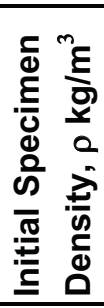 & 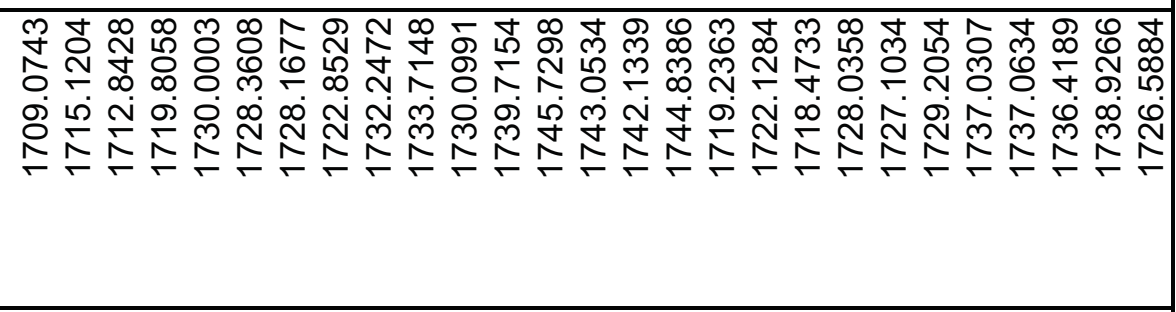 \\
\hline 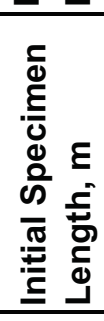 & 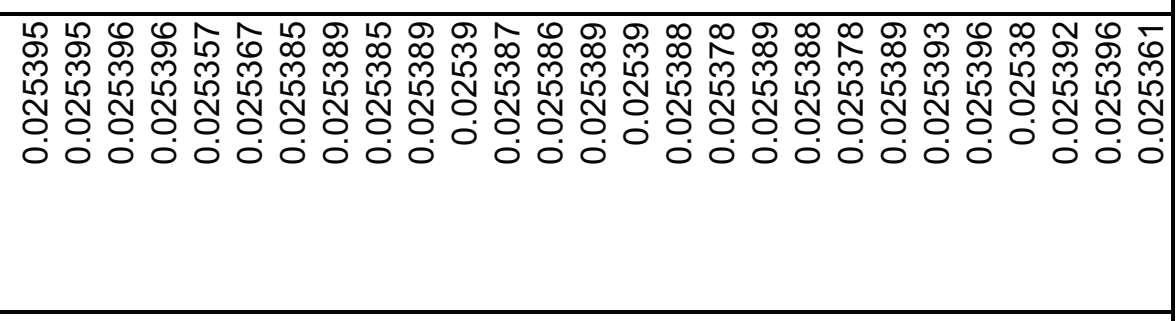 \\
\hline 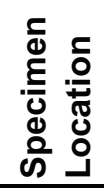 & \\
\hline 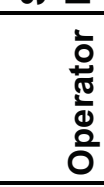 & 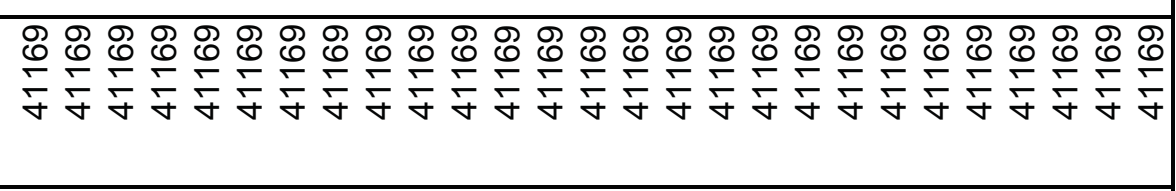 \\
\hline 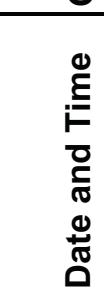 & 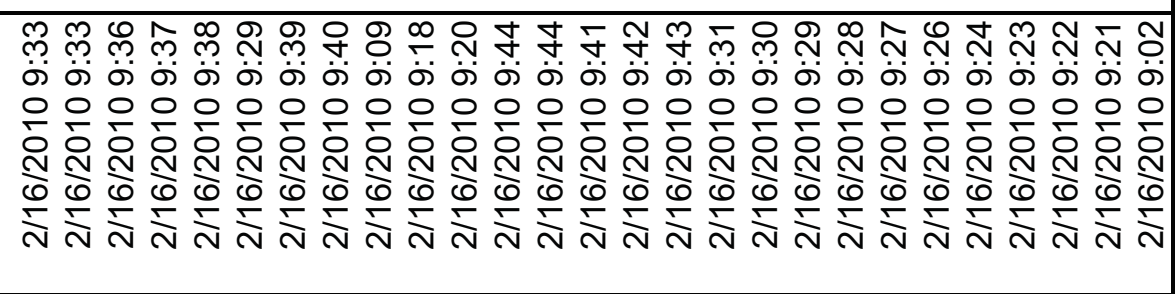 \\
\hline 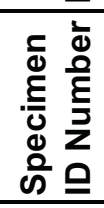 & 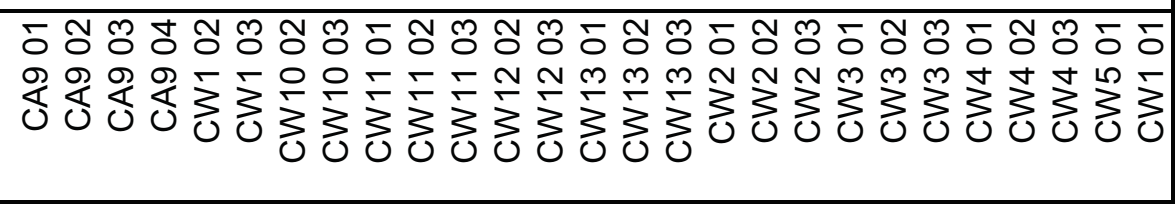 \\
\hline
\end{tabular}




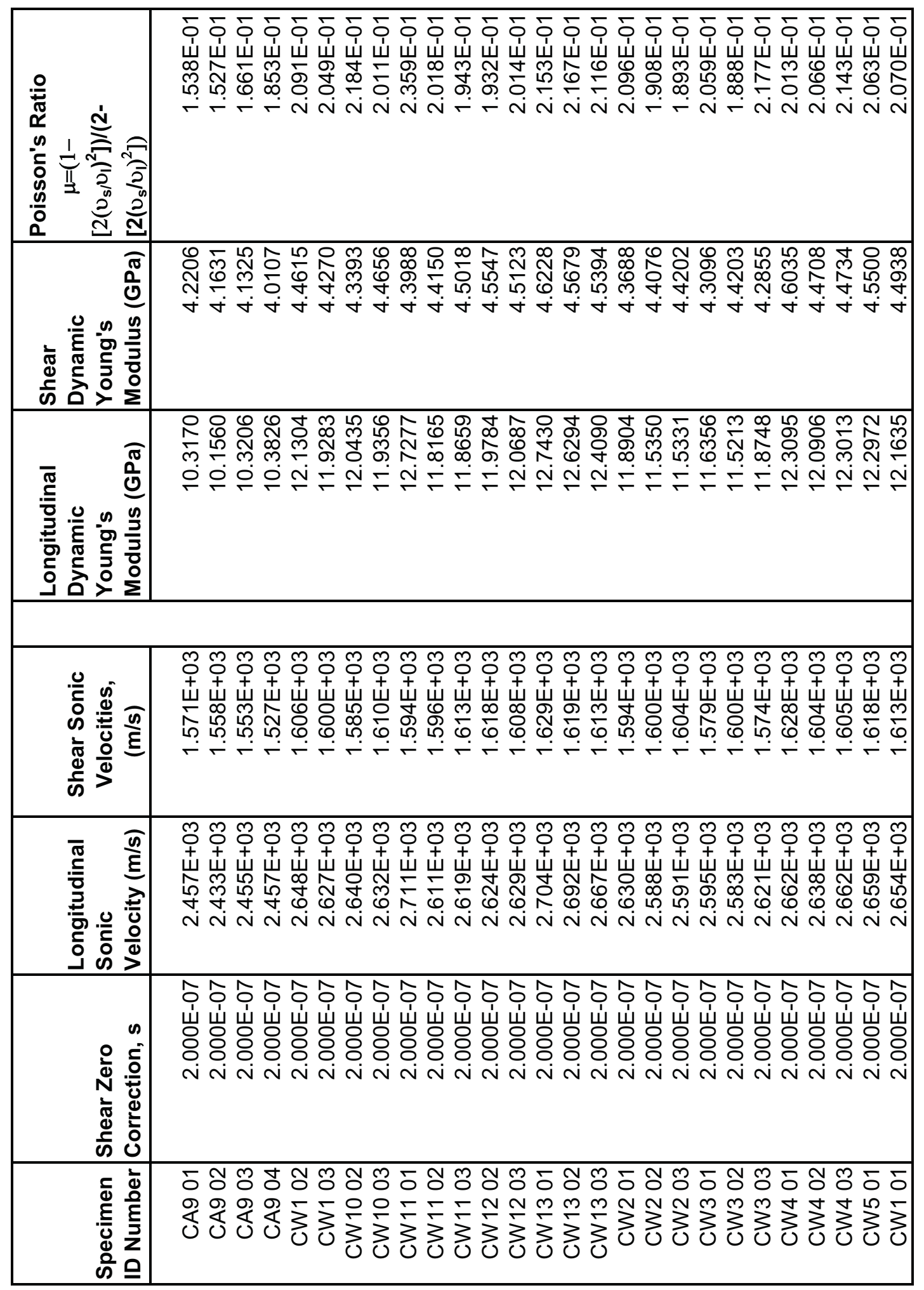




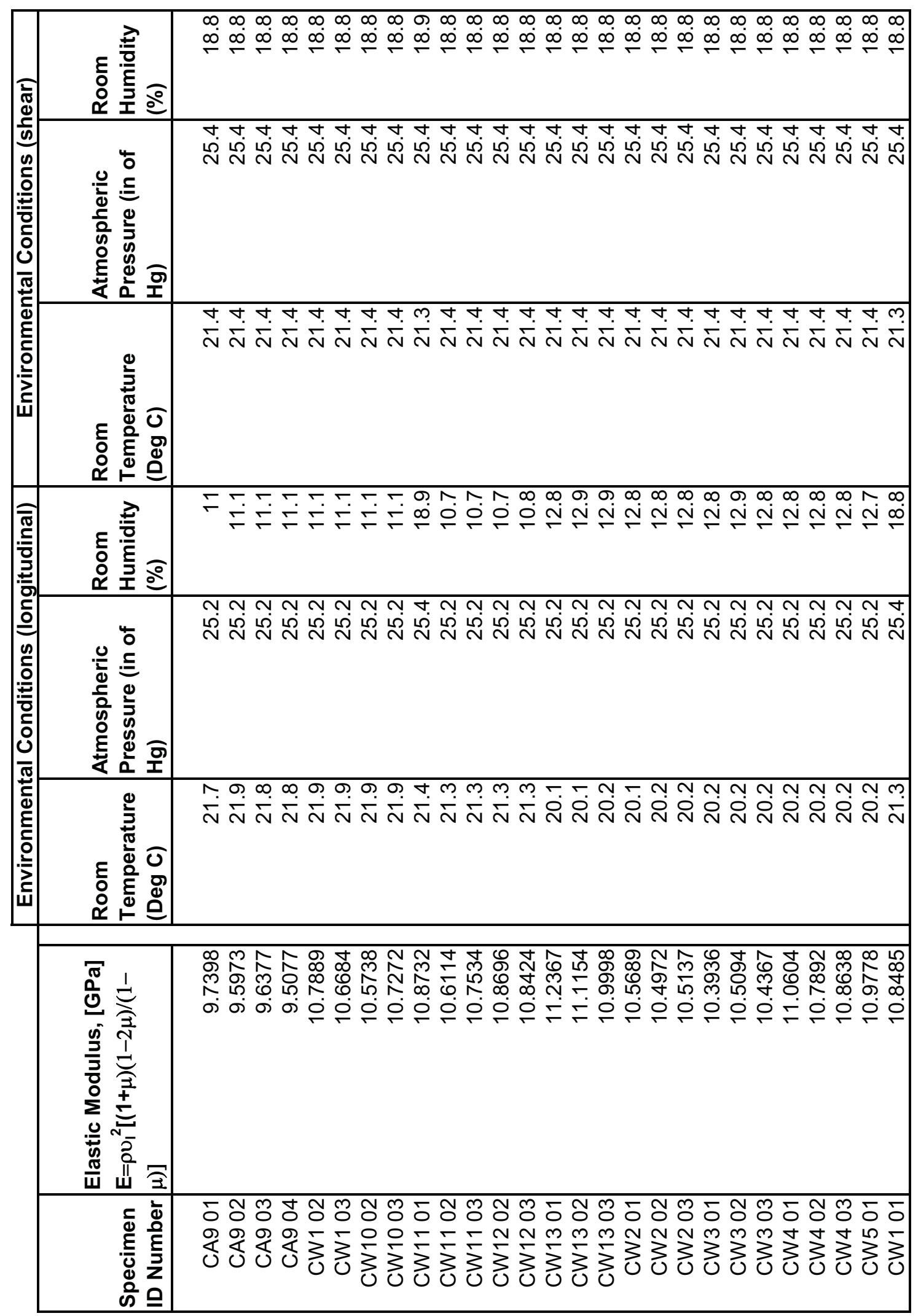




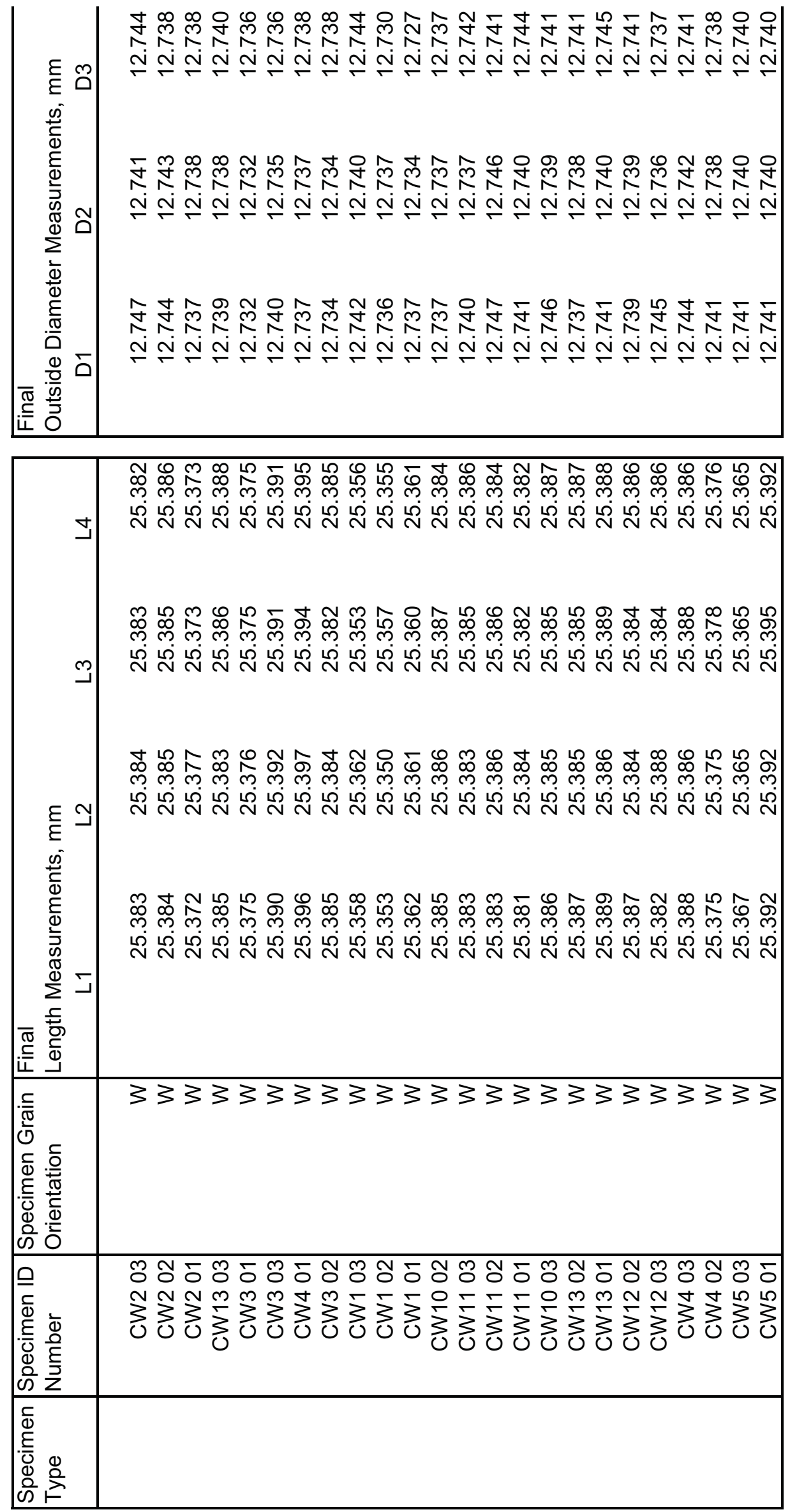




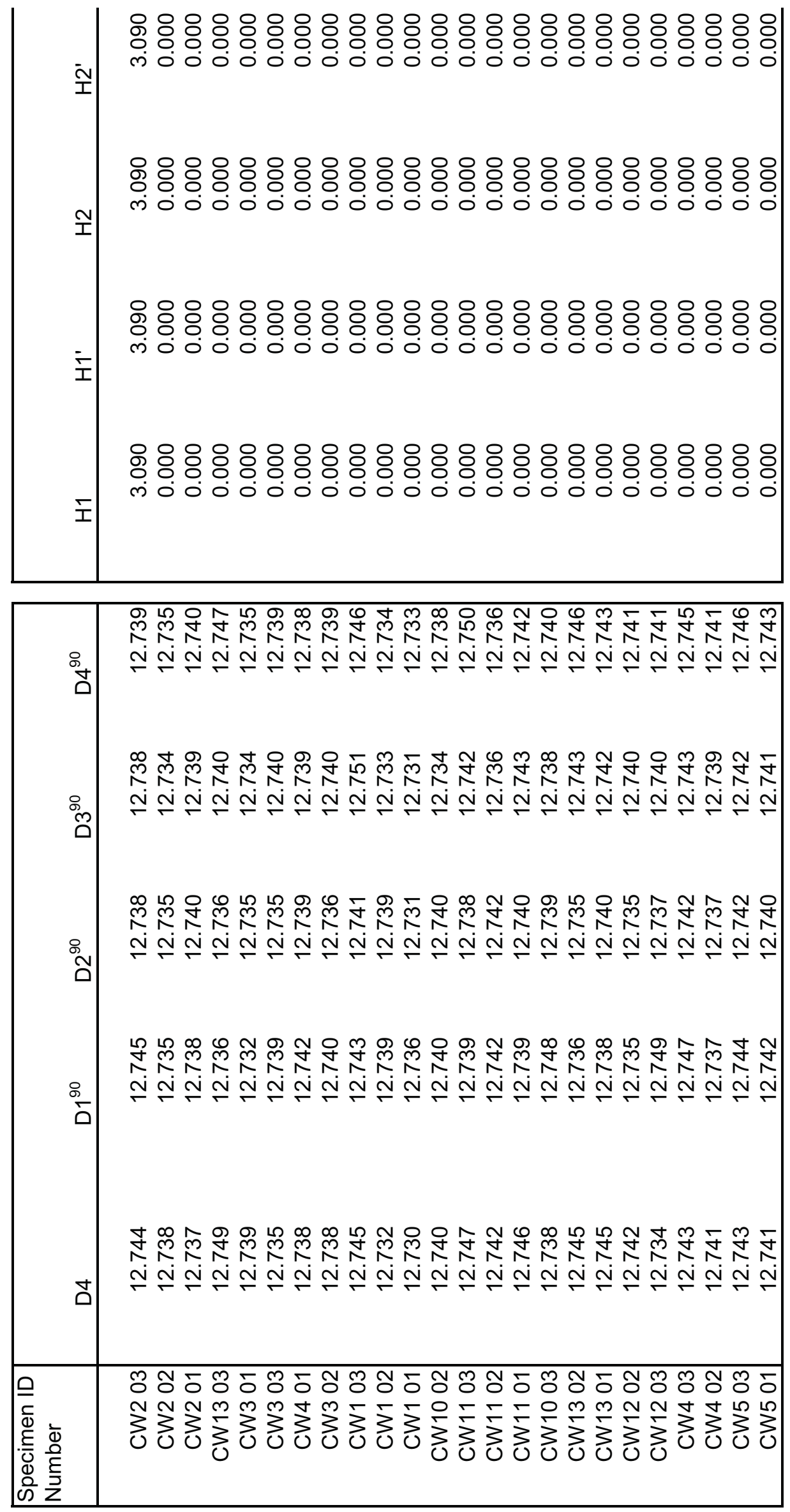




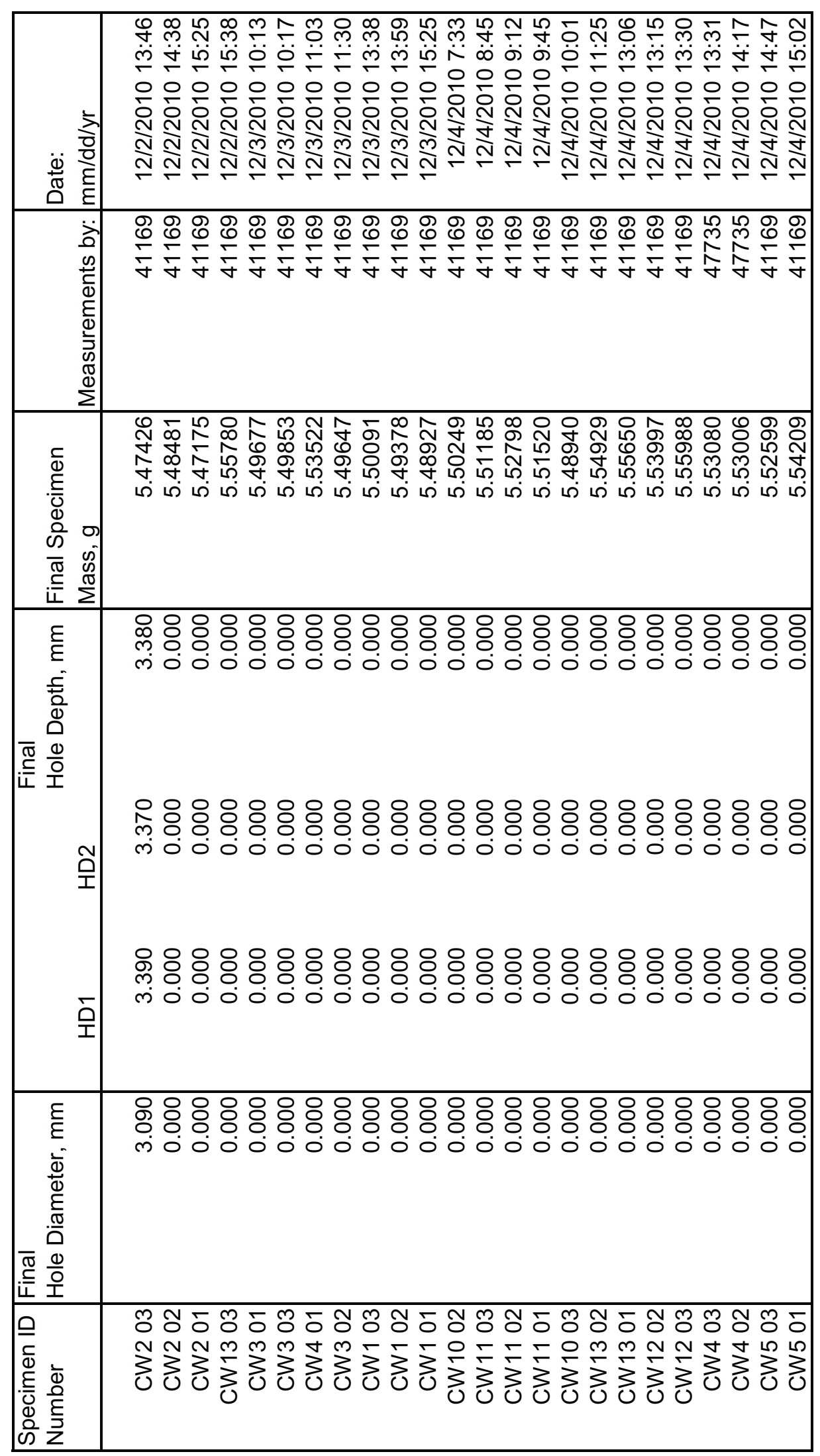




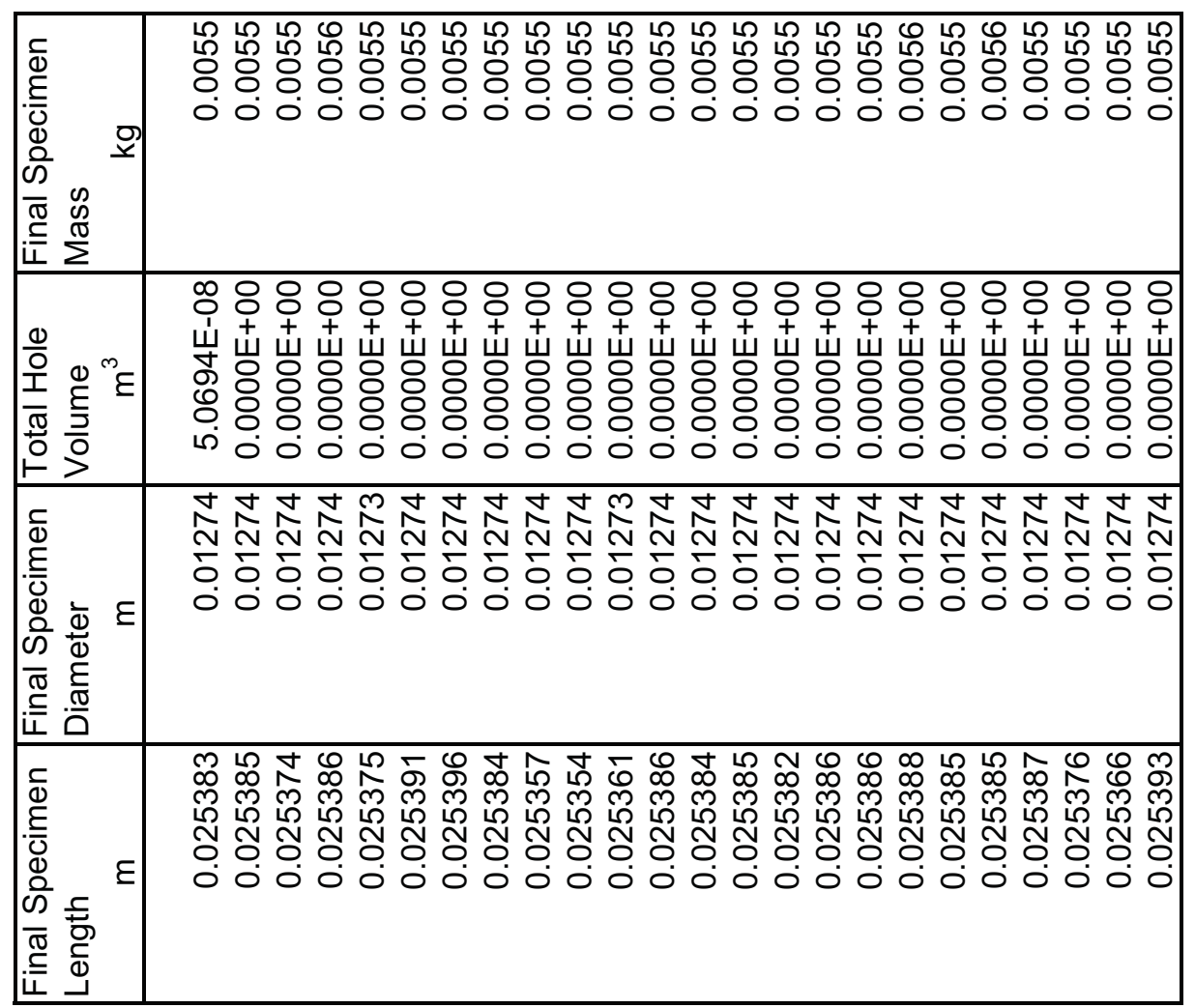

\begin{tabular}{|c|c|}
\hline 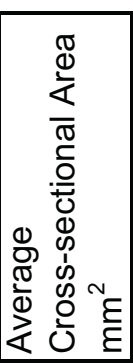 & 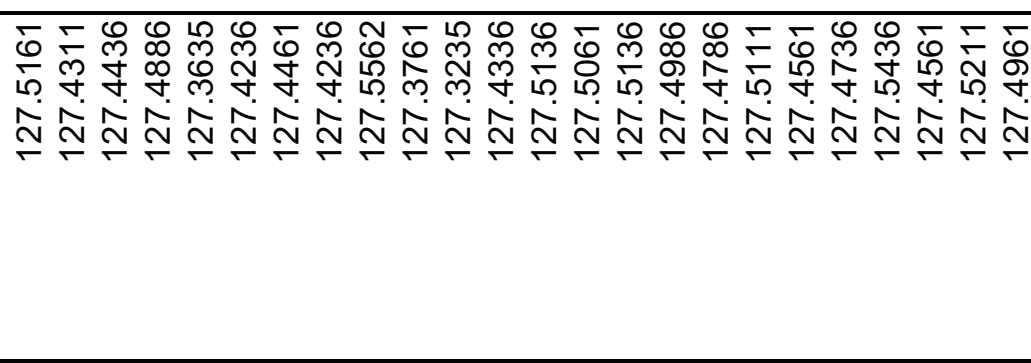 \\
\hline 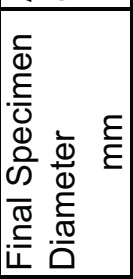 & 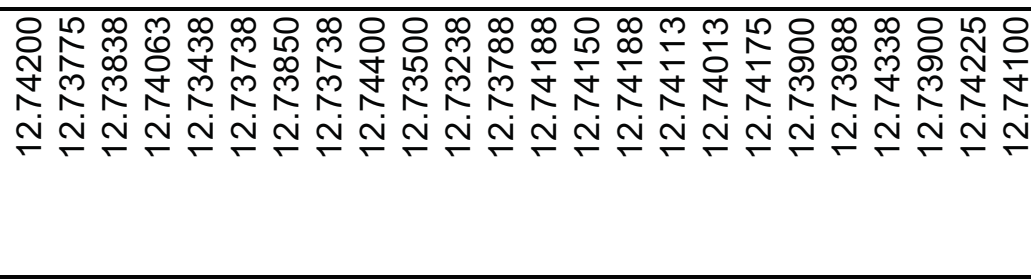 \\
\hline 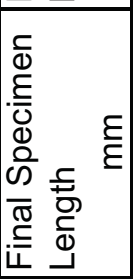 & 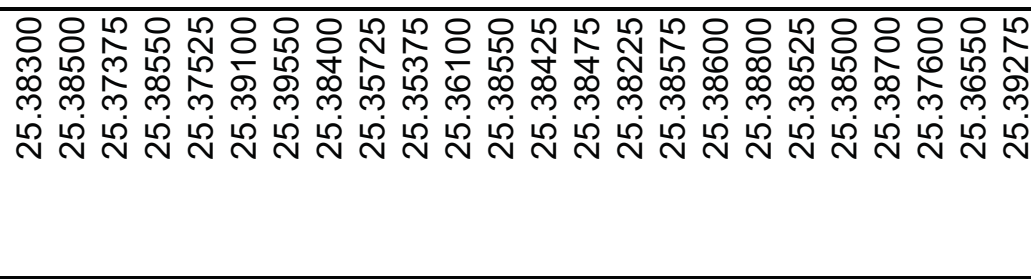 \\
\hline 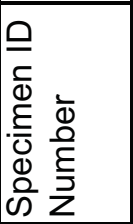 & 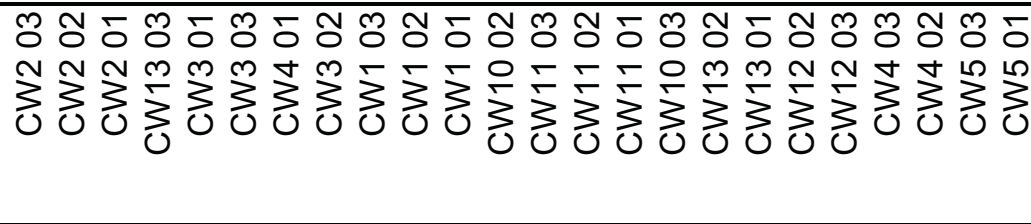 \\
\hline
\end{tabular}




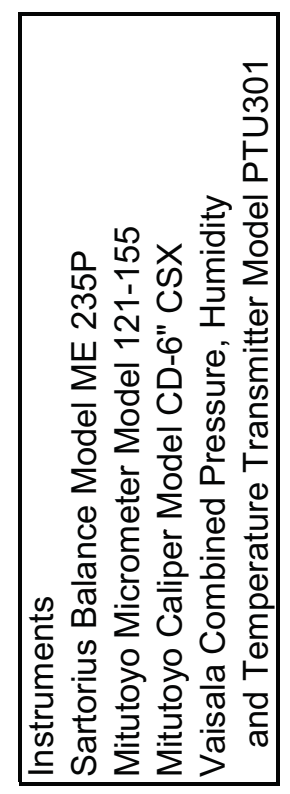

\begin{tabular}{|c|c|}
\hline 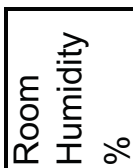 & 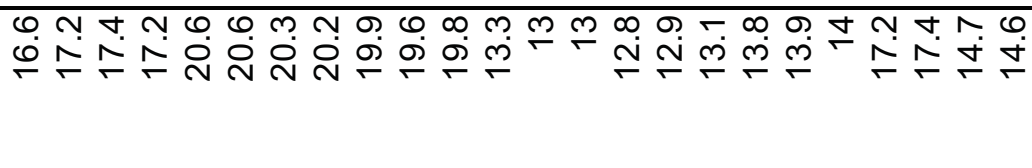 \\
\hline 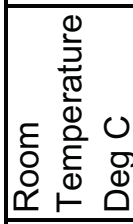 & 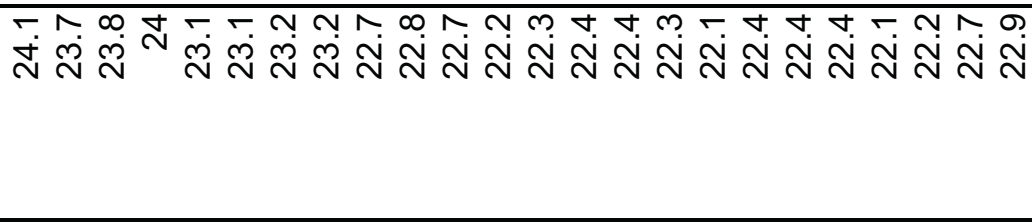 \\
\hline 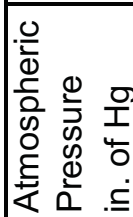 & 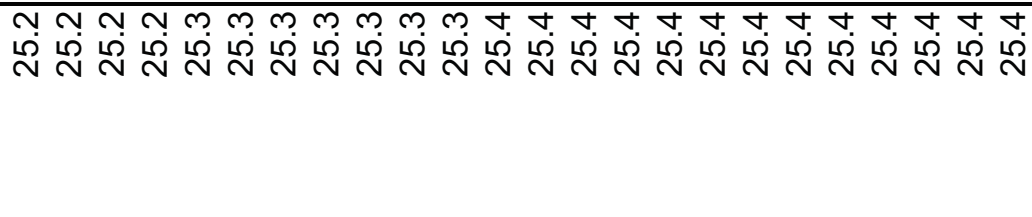 \\
\hline
\end{tabular}

\begin{tabular}{|c|c|}
\hline 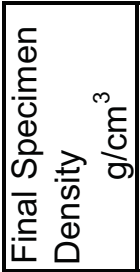 & 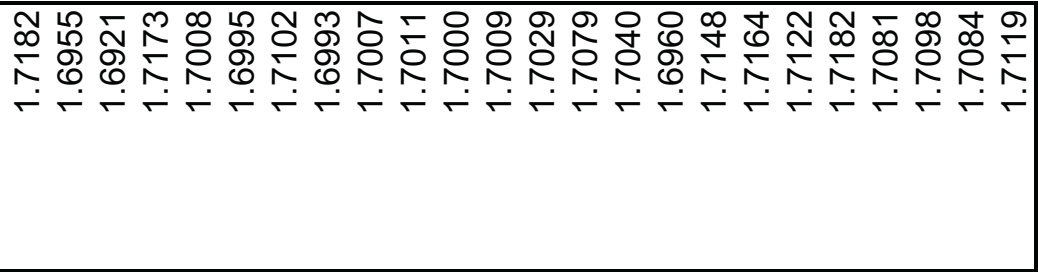 \\
\hline 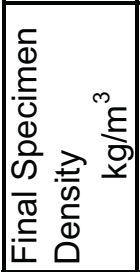 & 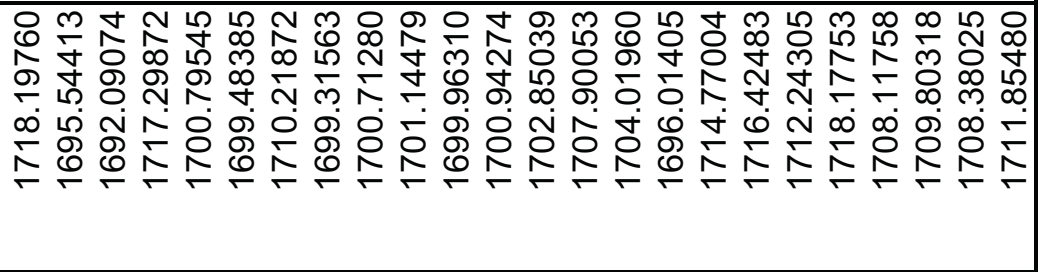 \\
\hline 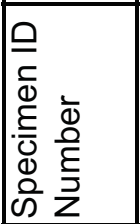 & 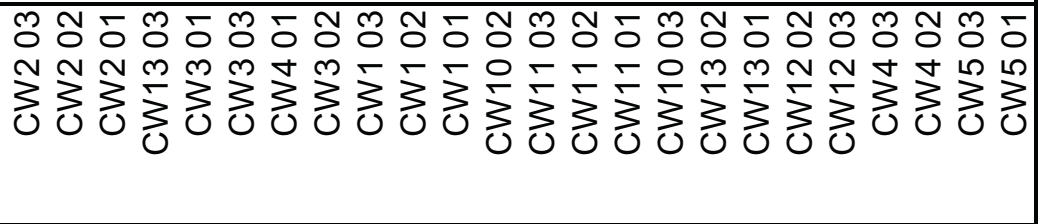 \\
\hline
\end{tabular}




$\begin{array}{ll}\text { Graphite Grade: } & \text { IG-110 } \\ \text { Graphite Manufacturer: } & \text { Toyo Tanso } \\ \text { Forming Process: } & \text { Isostatic-molded } \\ \text { Coke Particle Size: } & \text { Fine grain } \\ \text { Coke Type: } & \text { Petroleum coke filler, pitch binder } \\ \text { ASTM Class: } & \text { INHP } \\ \text { Specimen Geometry: } & \text { Cylinder }\end{array}$

Specimen ID \#'s:

EA1 01

EA1 02

EA2 01

EA2 02

EA3 01

EA3 02

EA4 01

EA4 02

EA5 01

EA5 02

EA6 01

EA6 02

EA7 01

EA7 02

EA8 01

EA8 02

EA9 01

EA9 02

EA10 01

EA10 02

EA11 01

EA11 02

EA12 01

EA12 02

EA13 01

EA13 02

EA14 01

EA14 02

EA15 01

EA15 02

EA16 01

EA16 02

EA17 01

EA17 02

EW01 01

EW01 02

EW01 03

EW01 04

EW02 01

EW02 02

EW02 03

EW02 04 


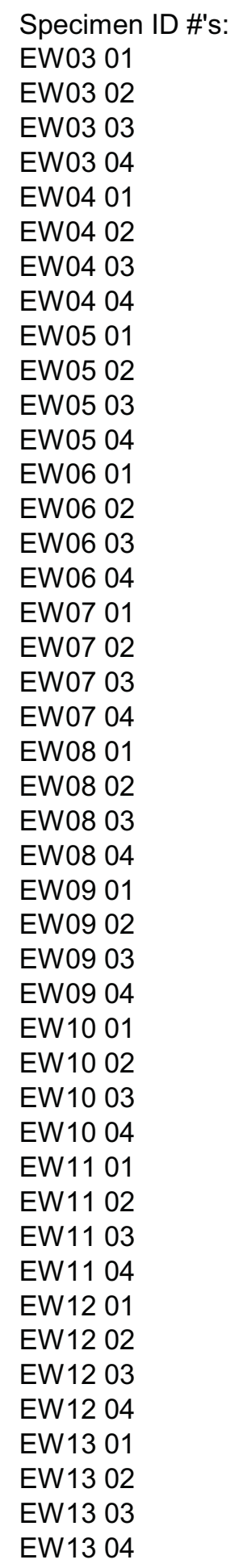




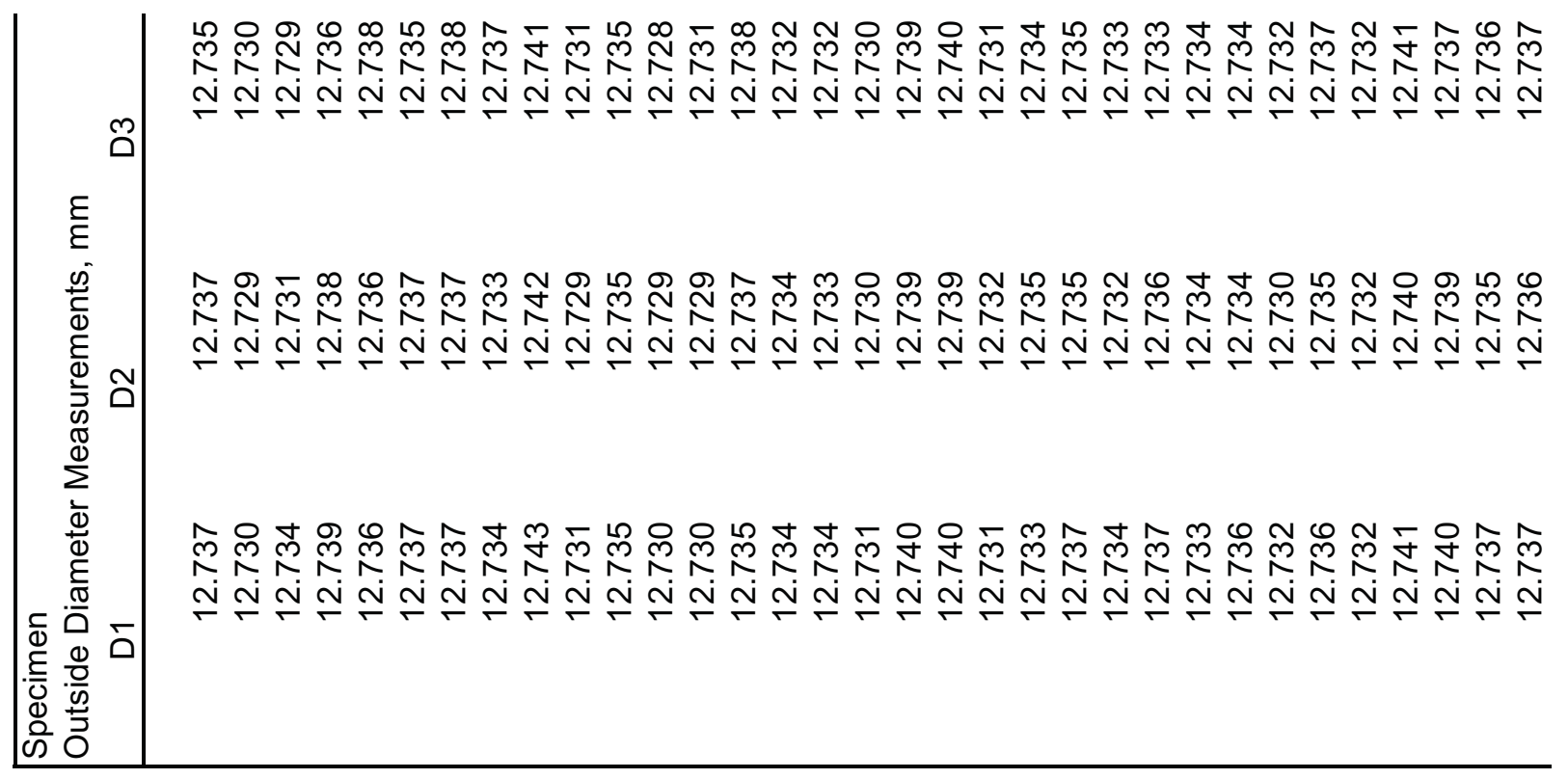

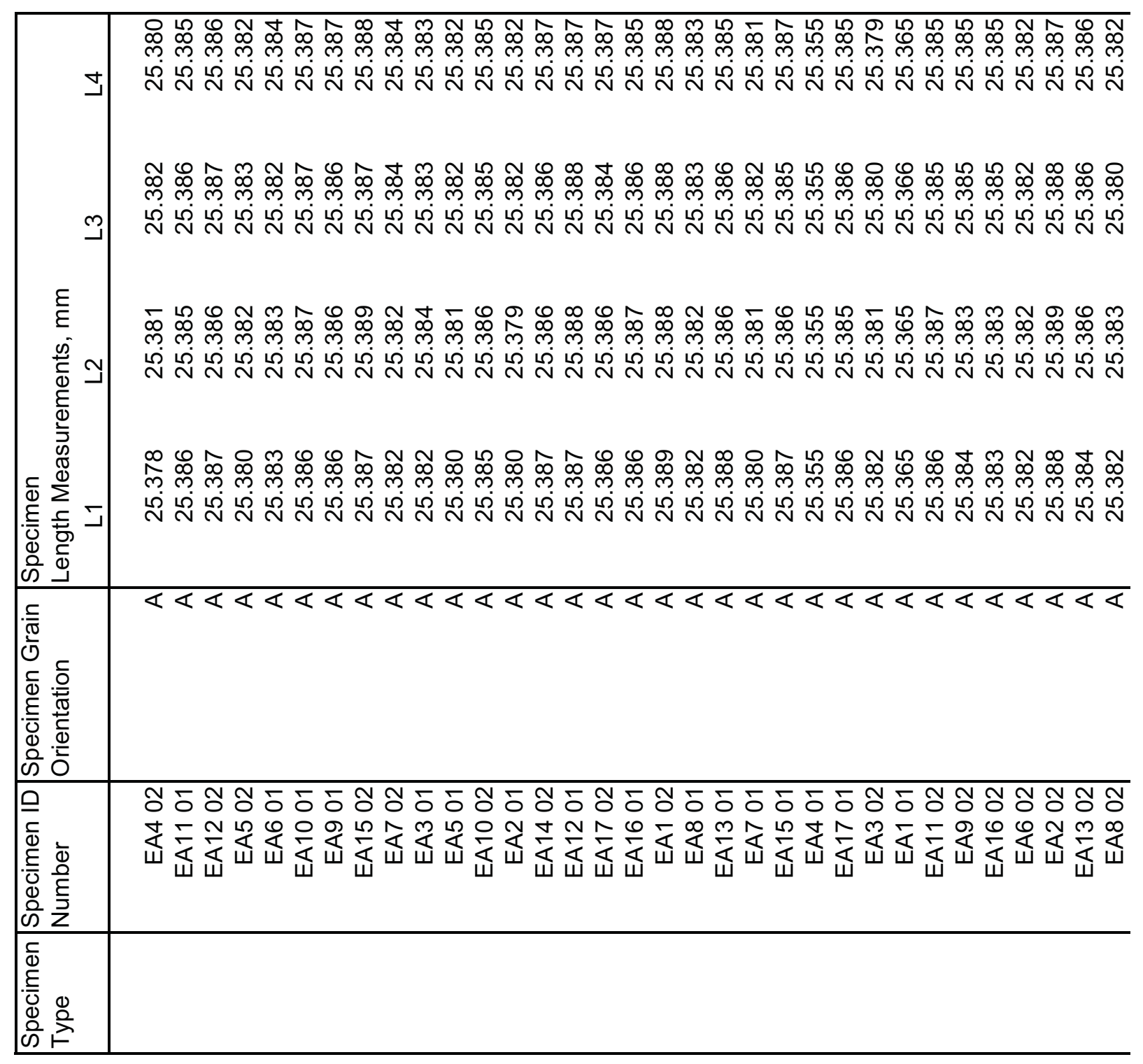




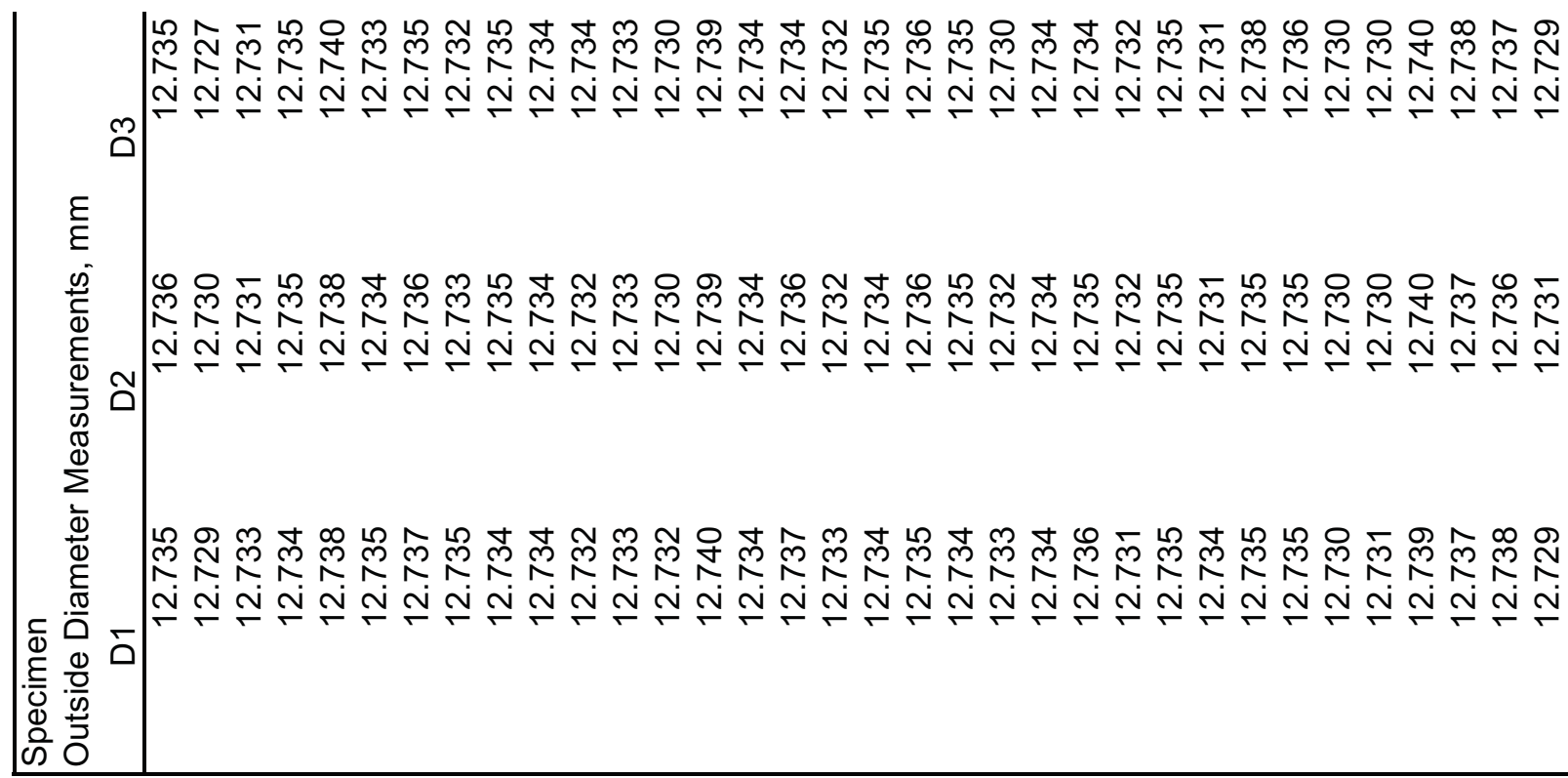

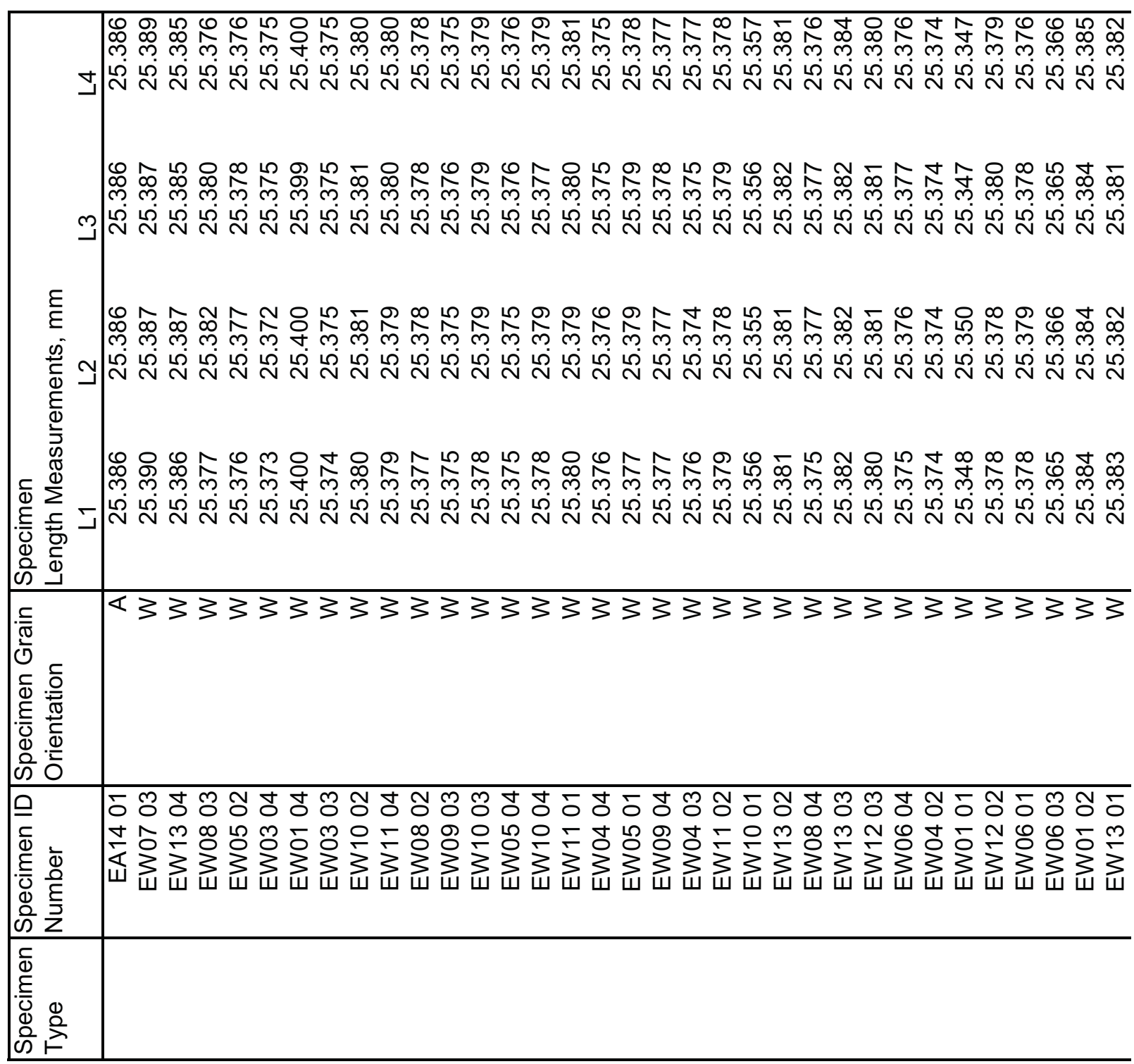




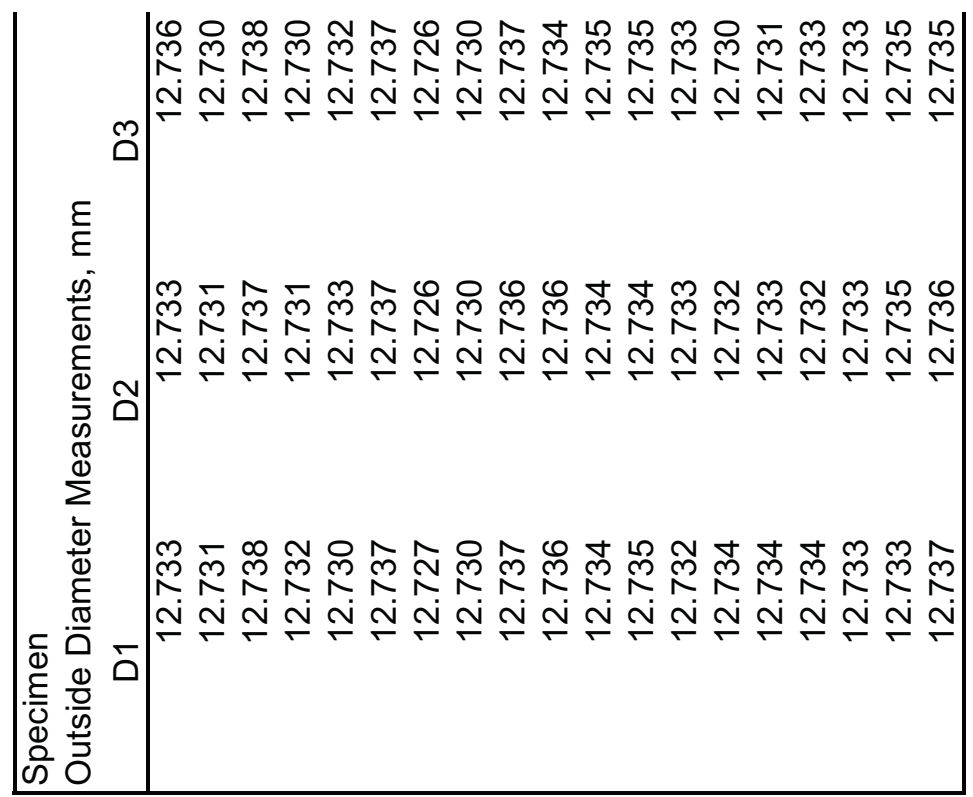

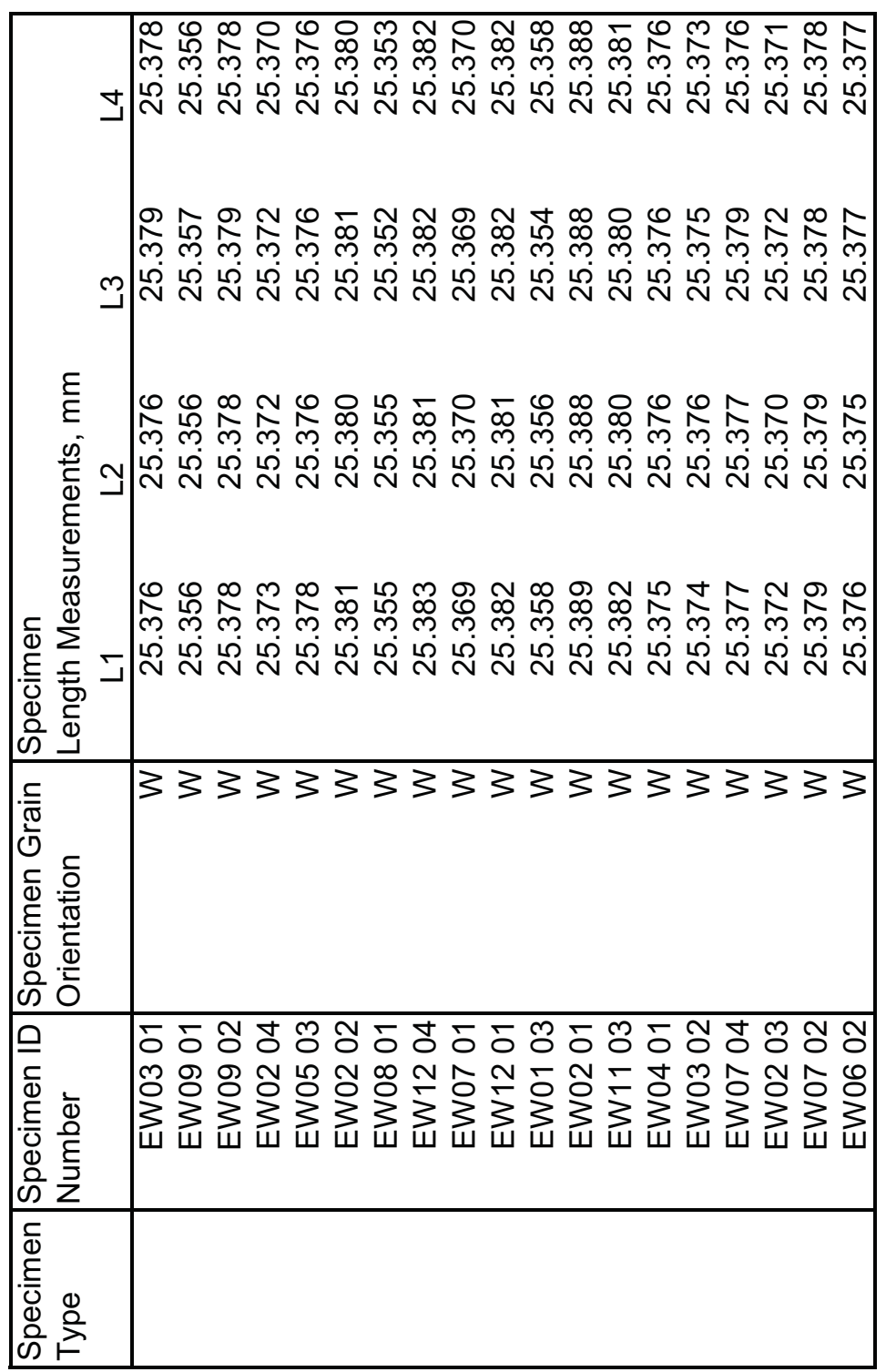




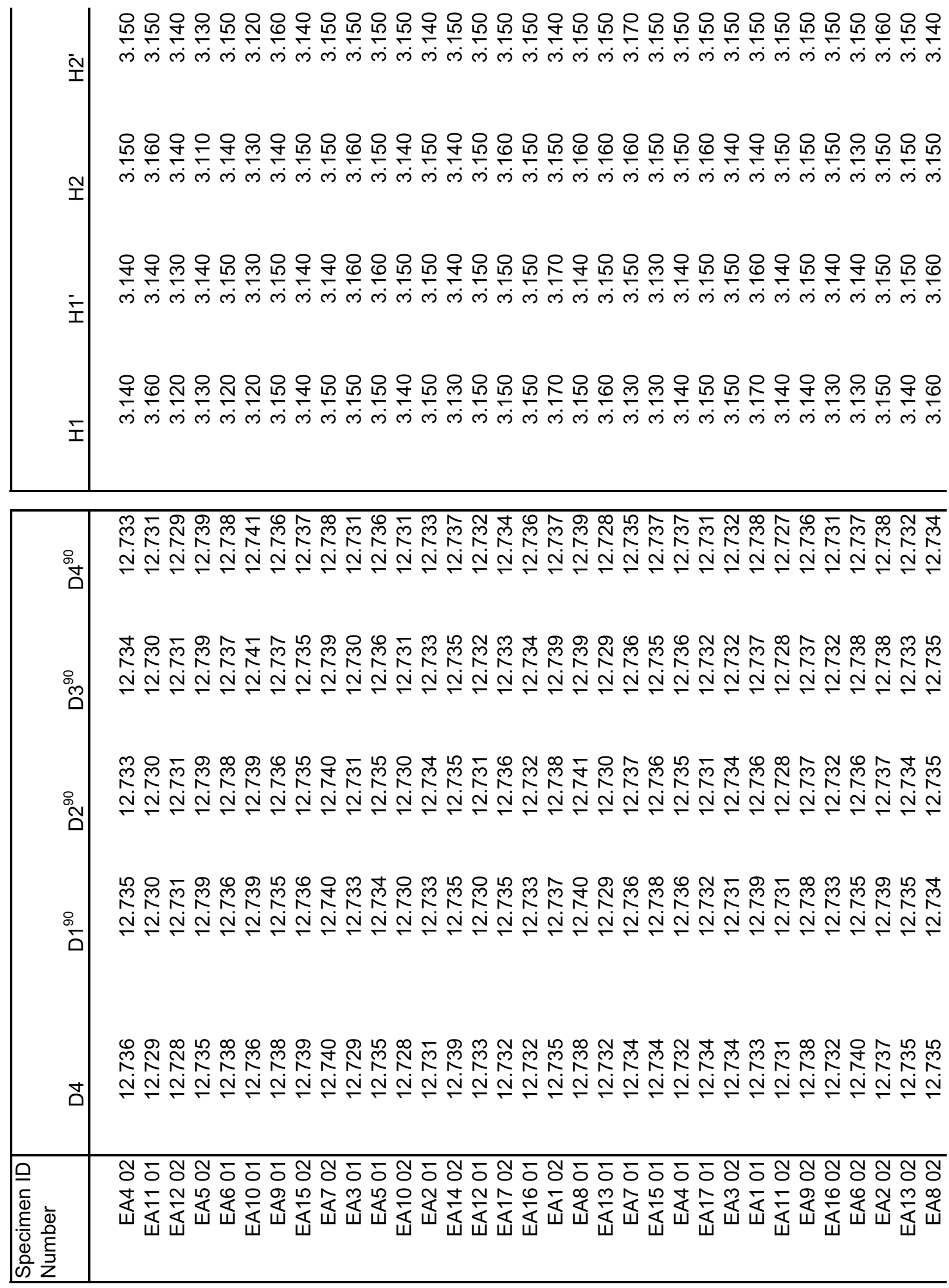




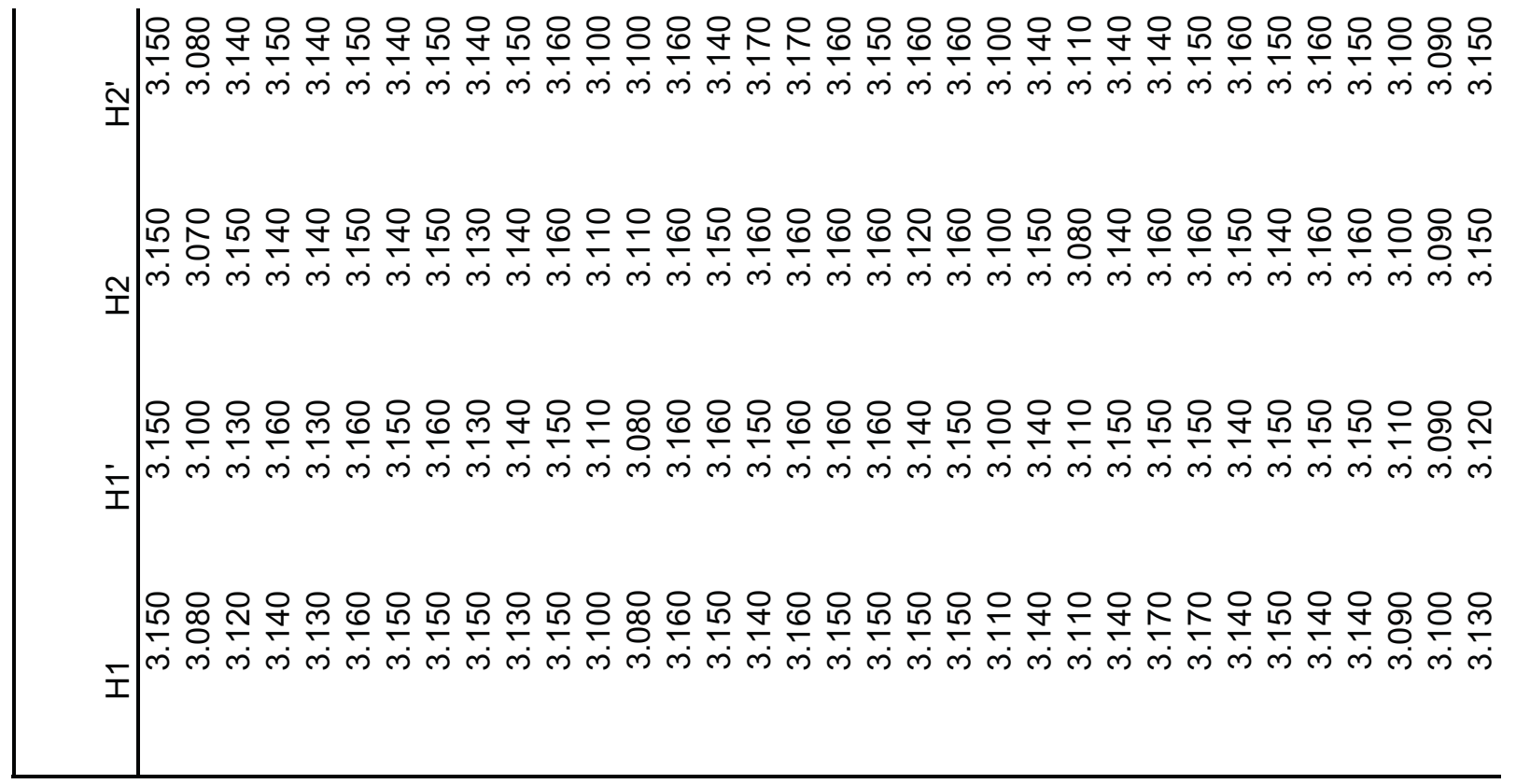

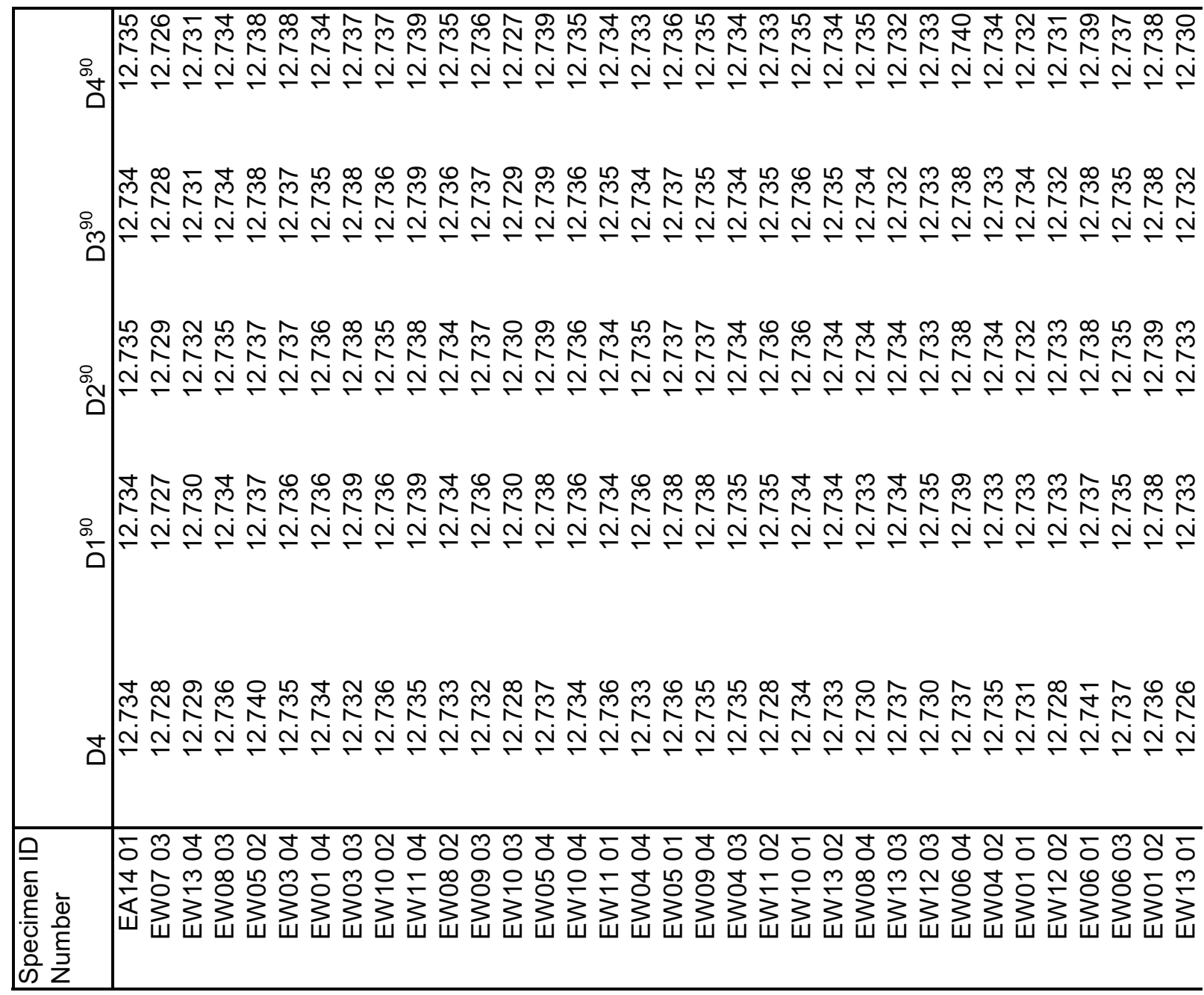




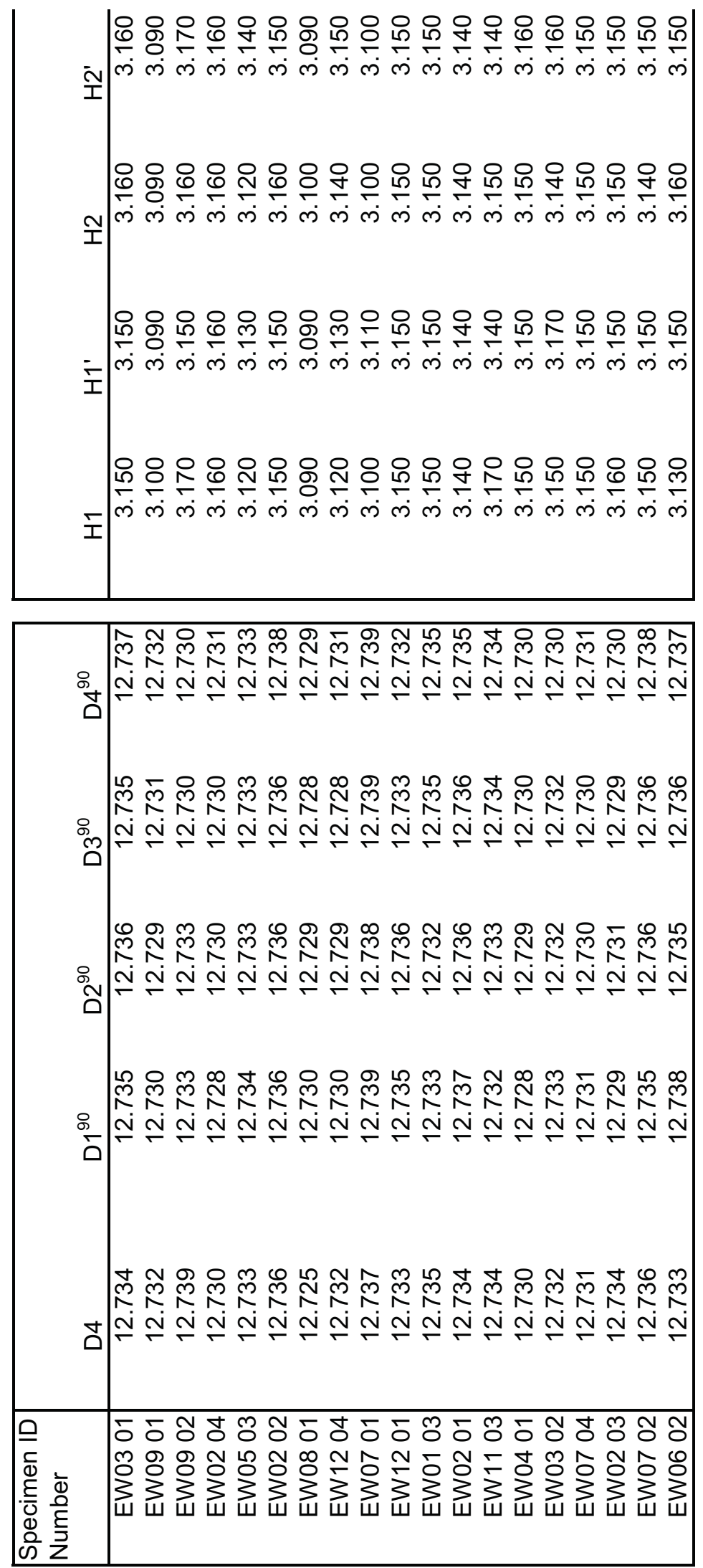




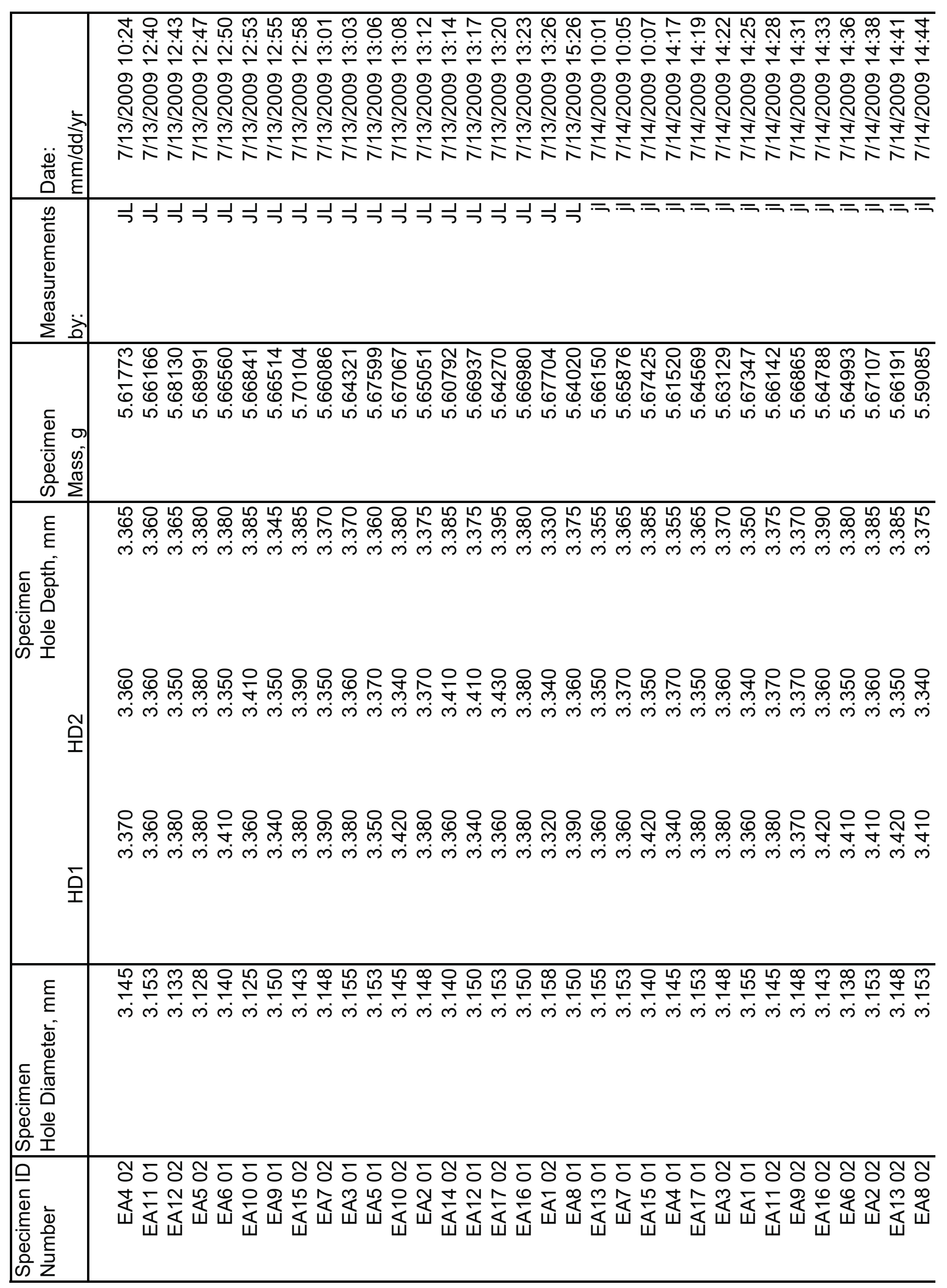




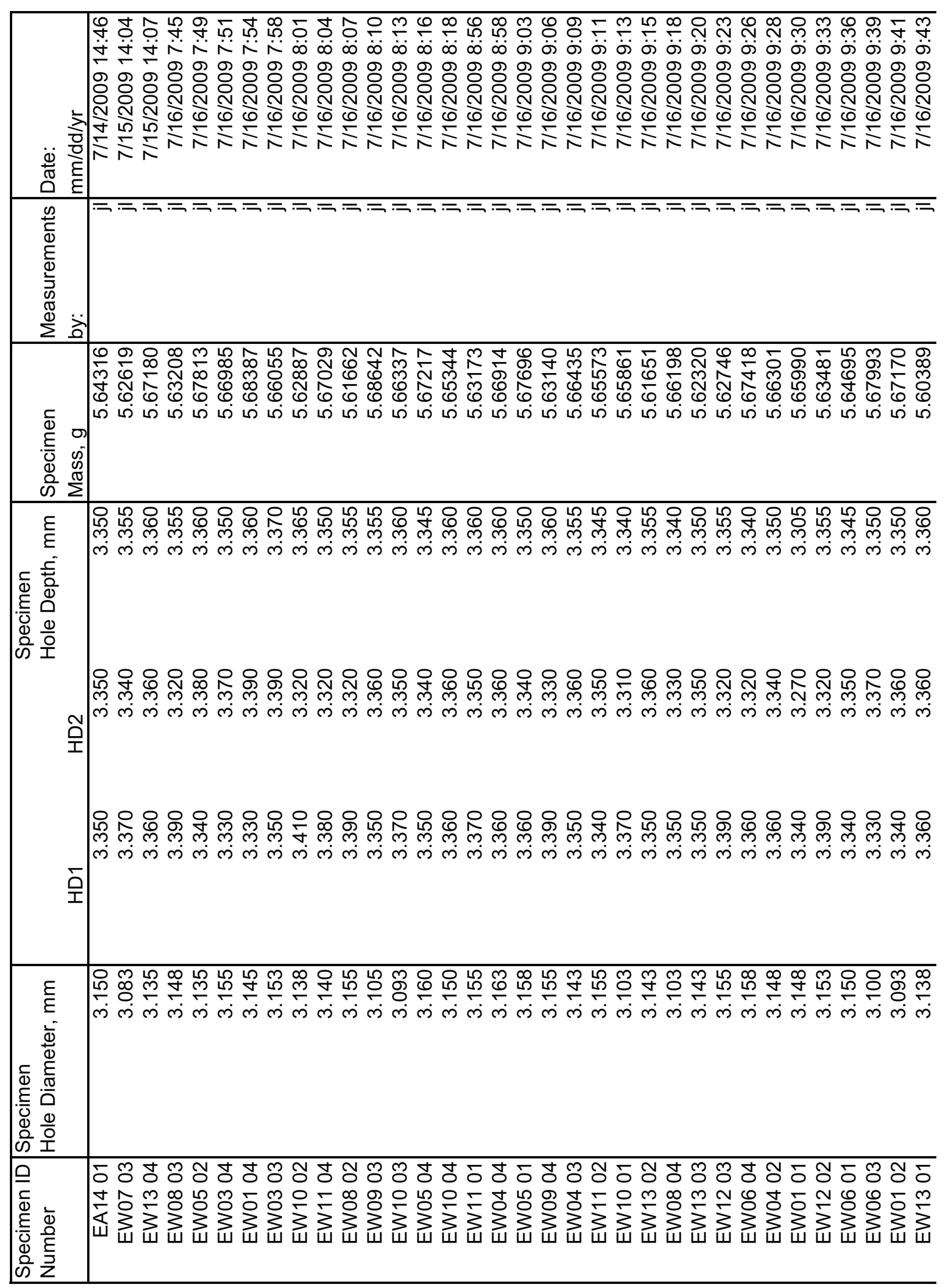




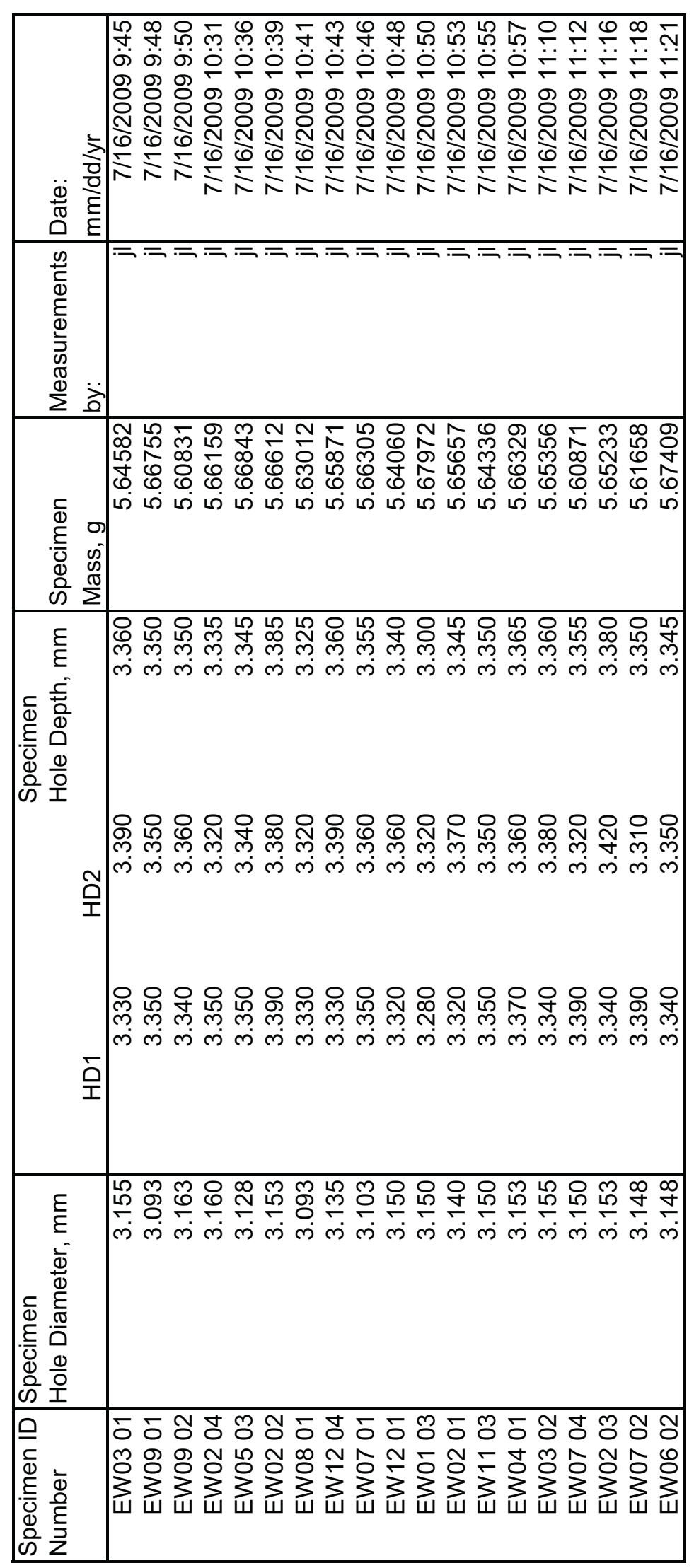




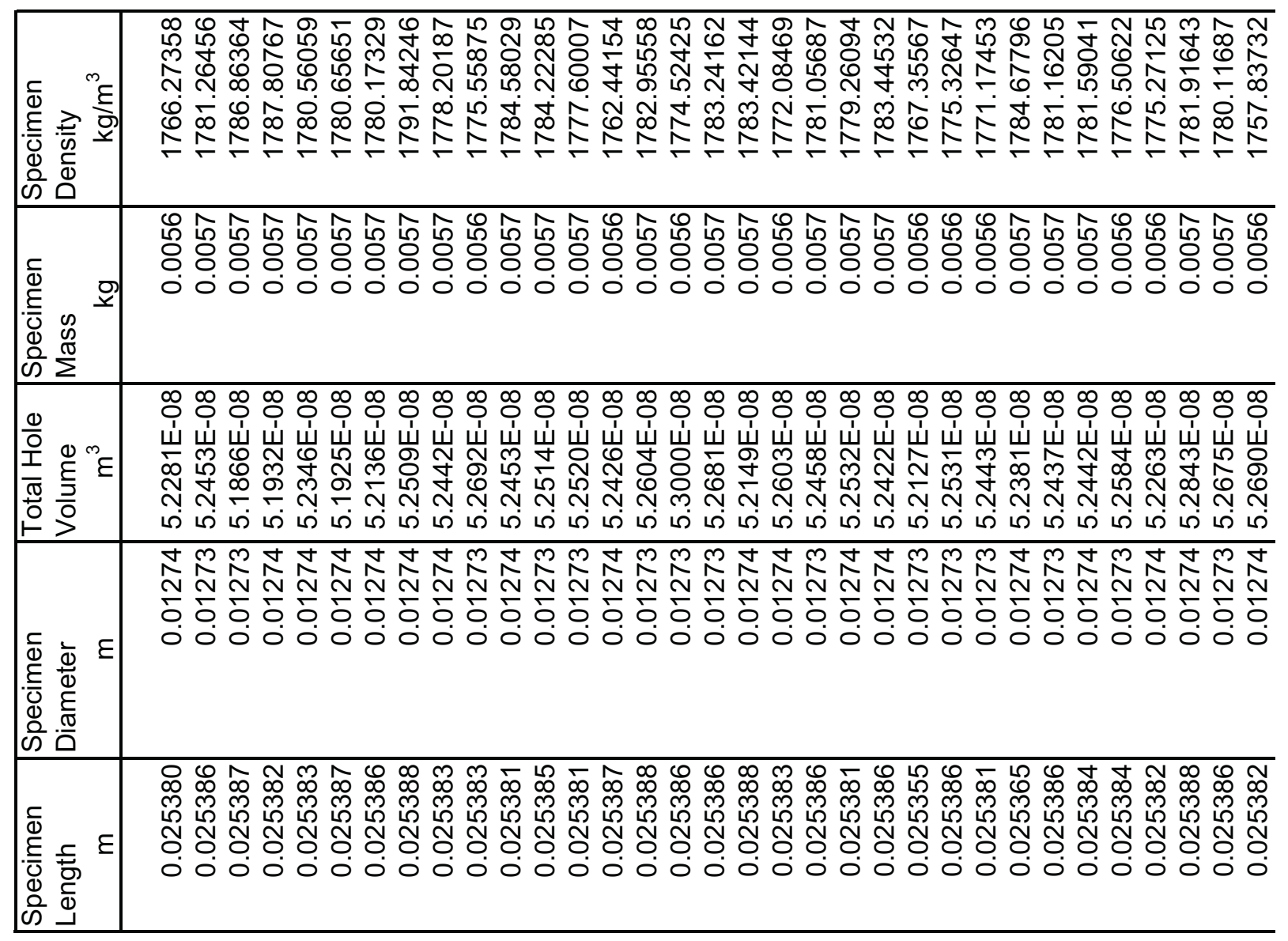

\begin{tabular}{|c|c|}
\hline 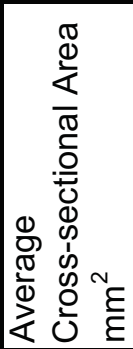 & 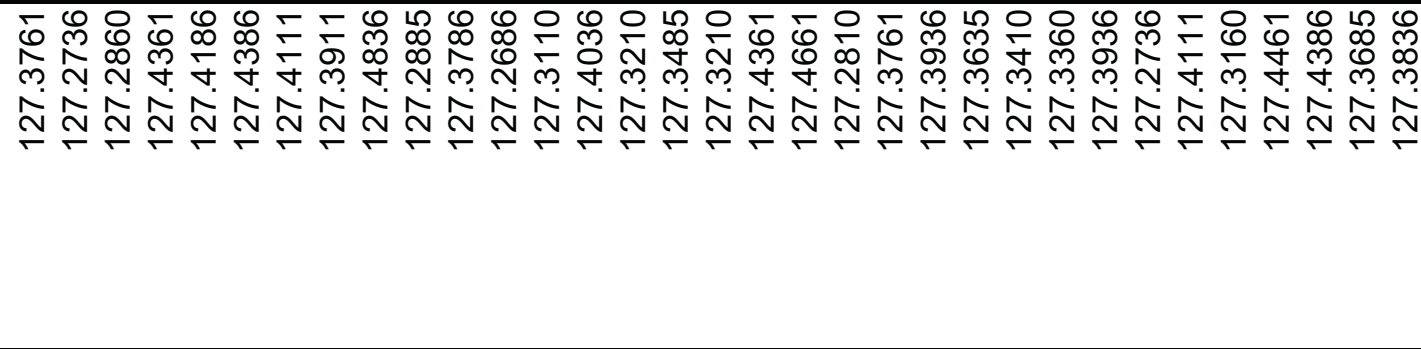 \\
\hline 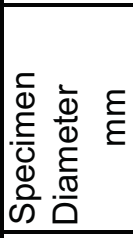 & 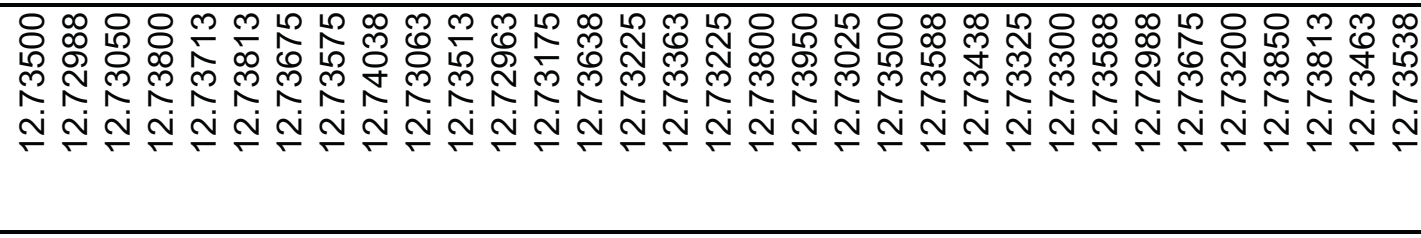 \\
\hline 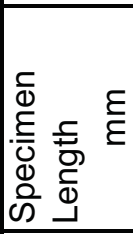 & 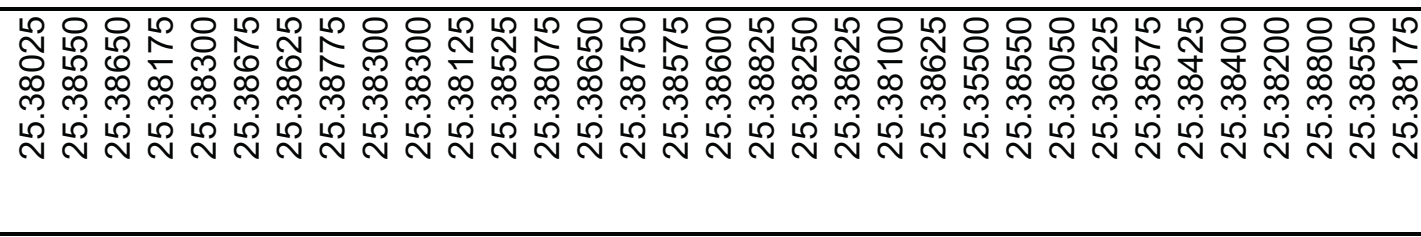 \\
\hline 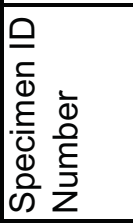 & 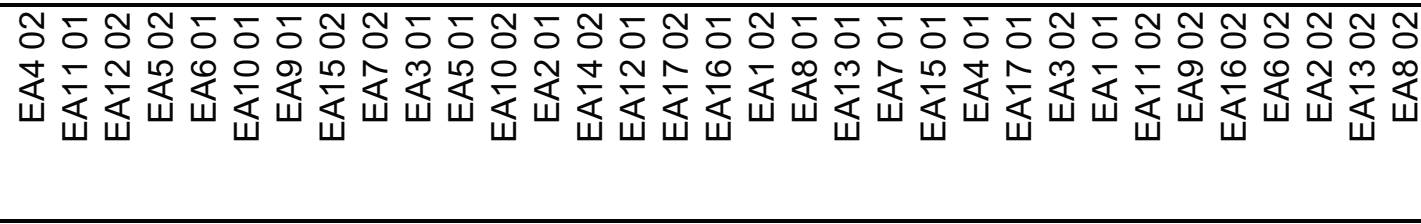 \\
\hline
\end{tabular}




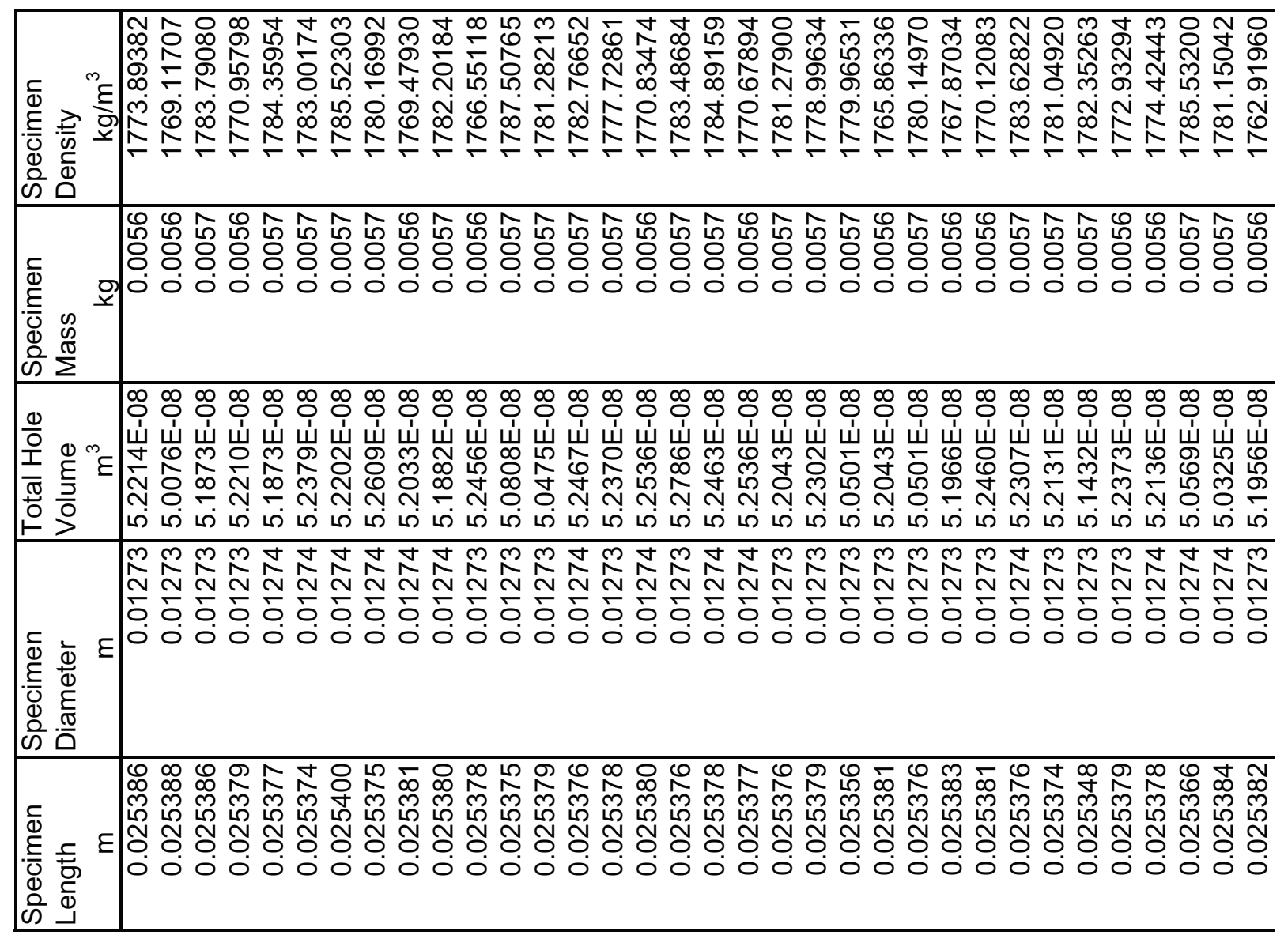

\begin{tabular}{|c|c|}
\hline 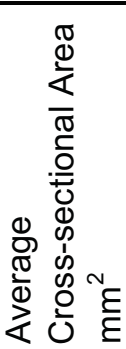 & 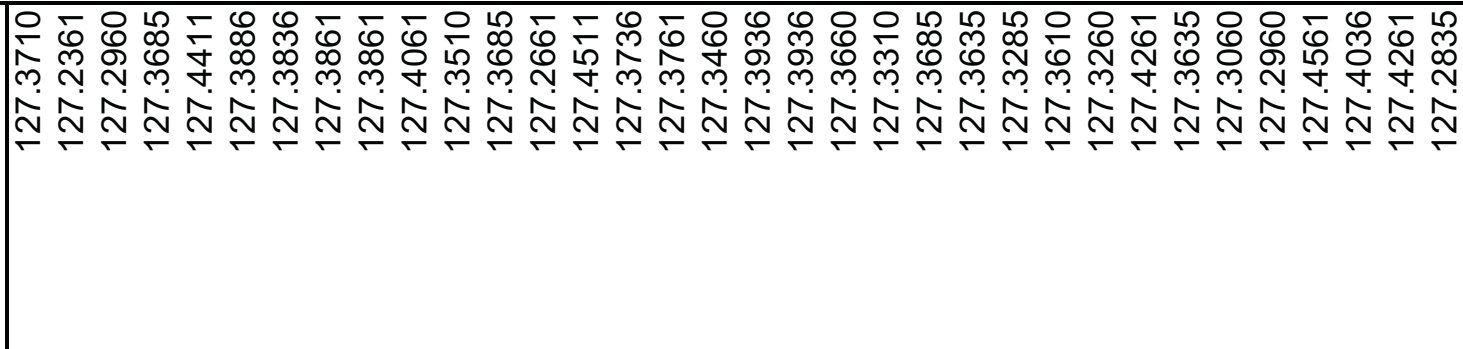 \\
\hline 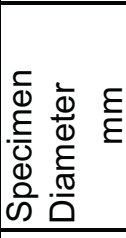 & 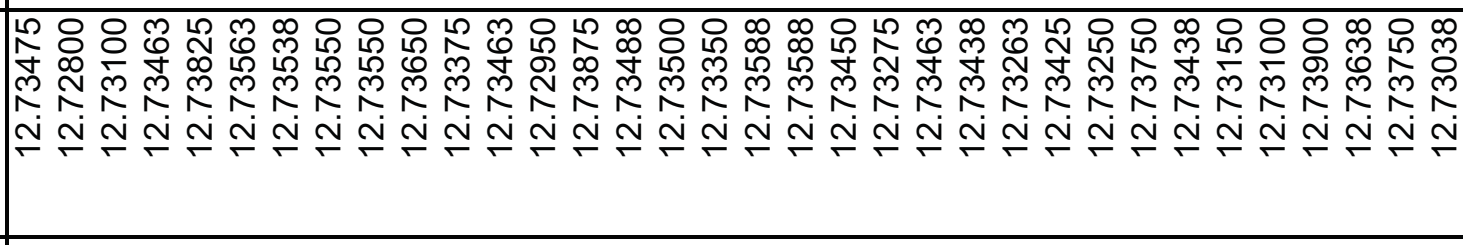 \\
\hline 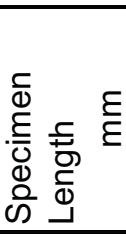 & 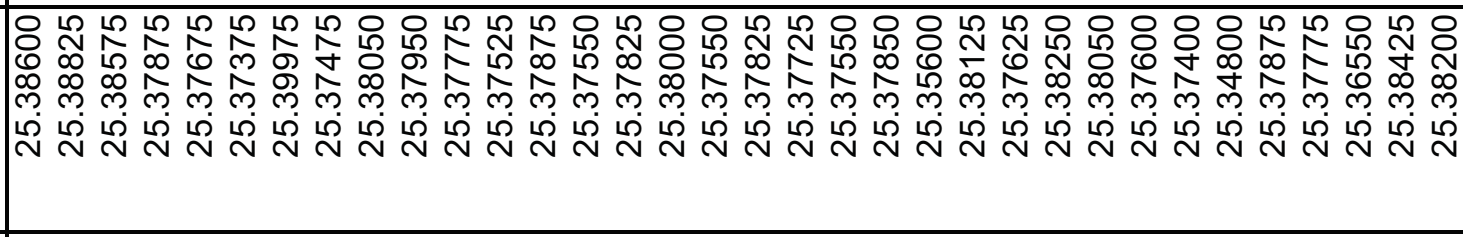 \\
\hline 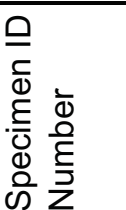 & 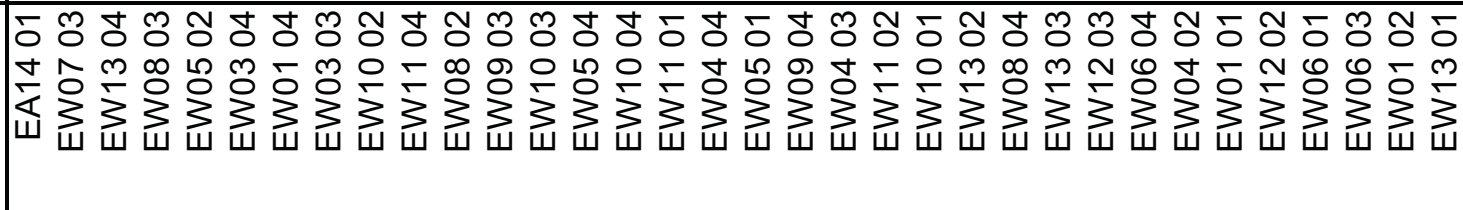 \\
\hline
\end{tabular}




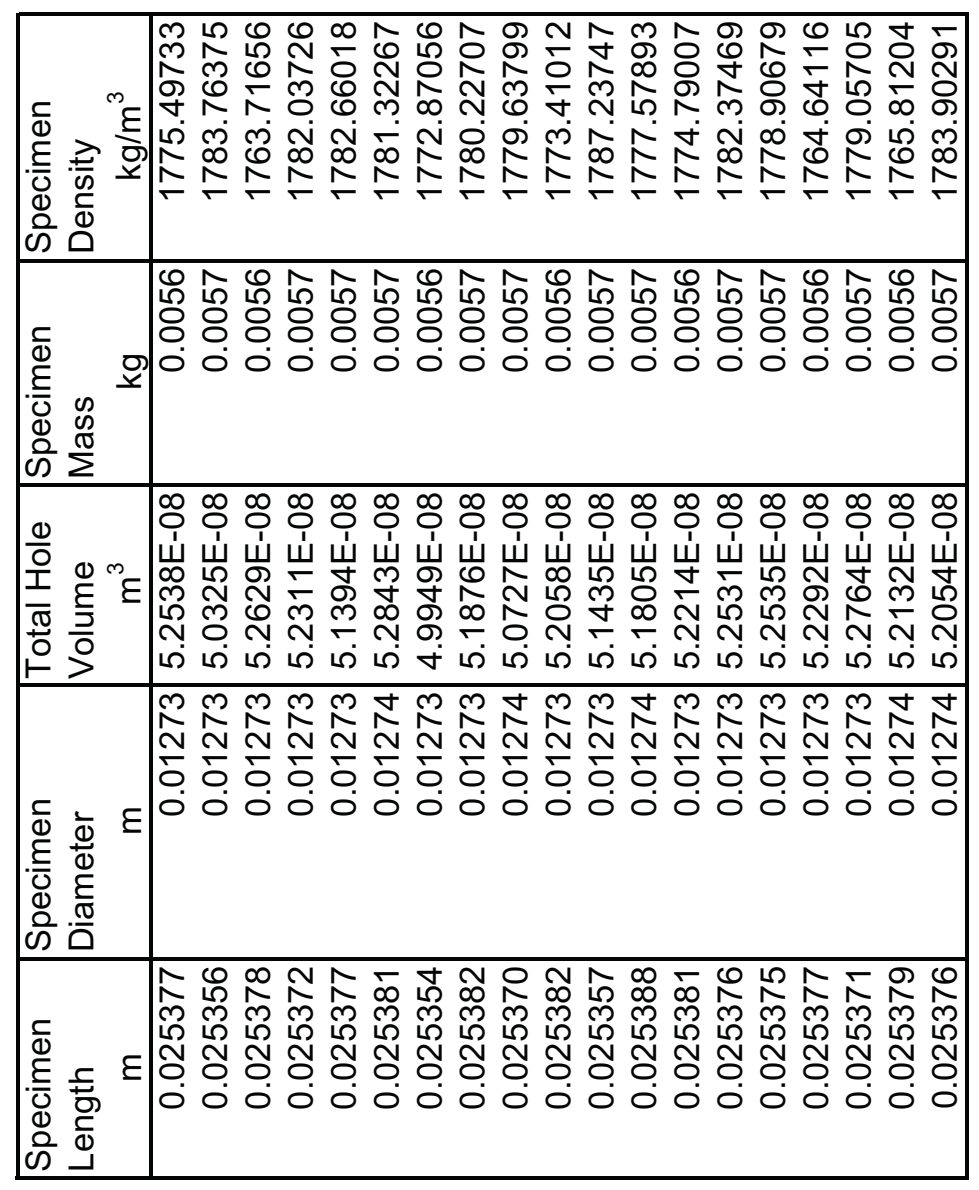

\begin{tabular}{|c|c|}
\hline 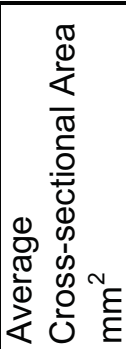 & 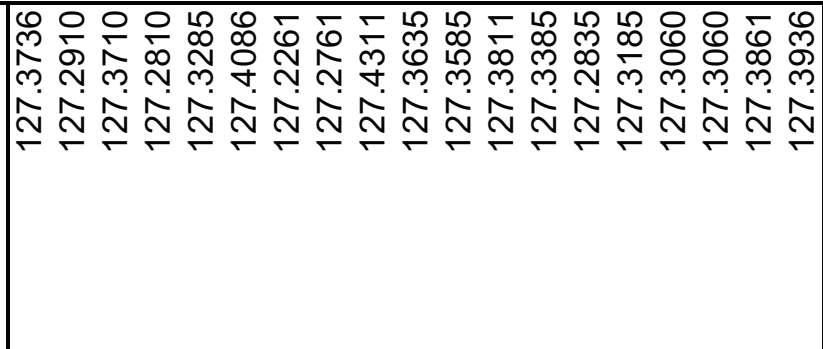 \\
\hline 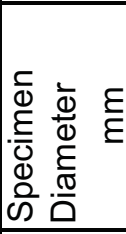 & 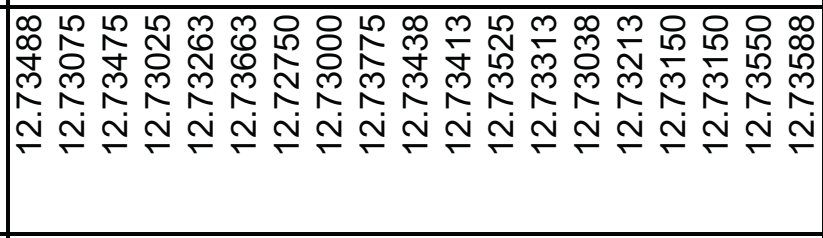 \\
\hline 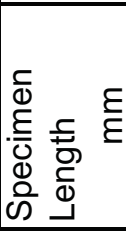 & 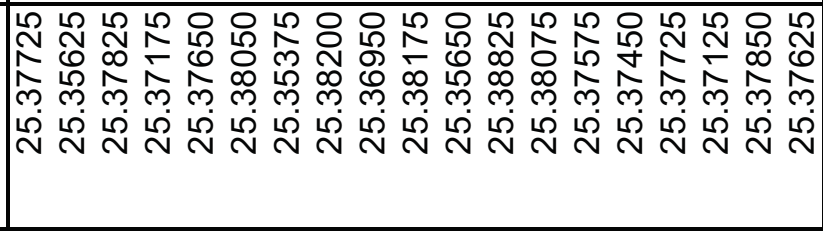 \\
\hline 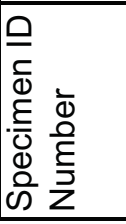 & 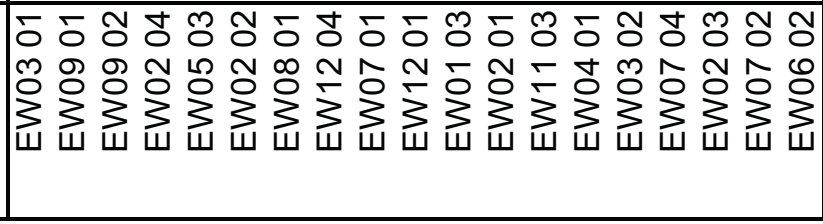 \\
\hline
\end{tabular}




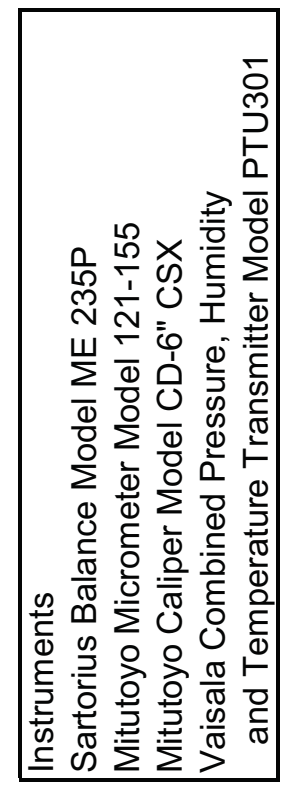

\begin{tabular}{|c|c|}
\hline 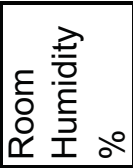 & 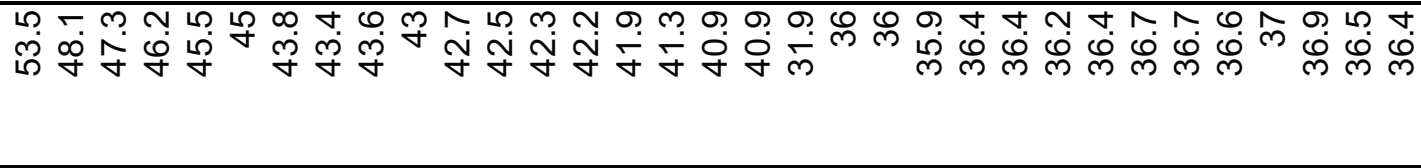 \\
\hline 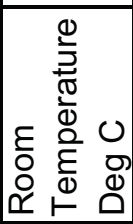 & 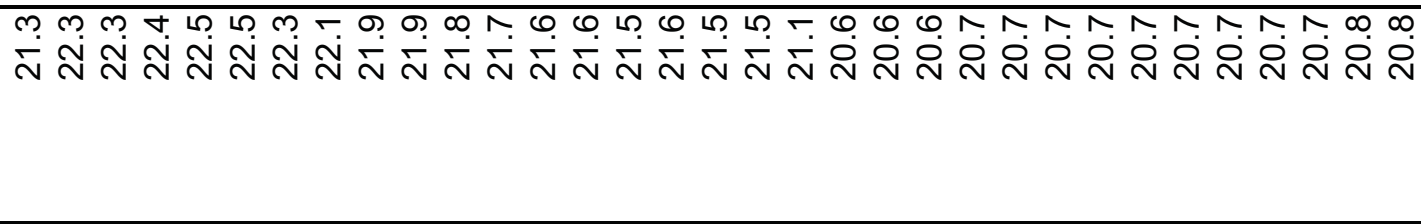 \\
\hline 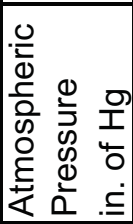 & 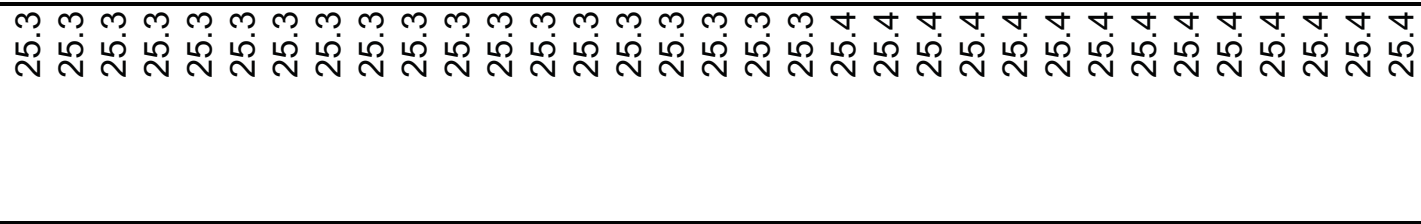 \\
\hline
\end{tabular}

\begin{tabular}{|c|c|}
\hline 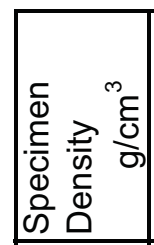 & 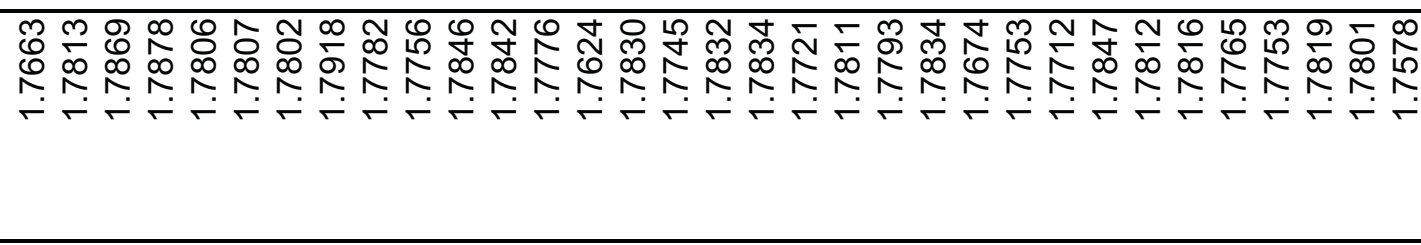 \\
\hline 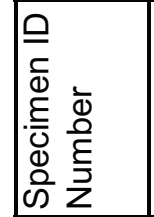 & 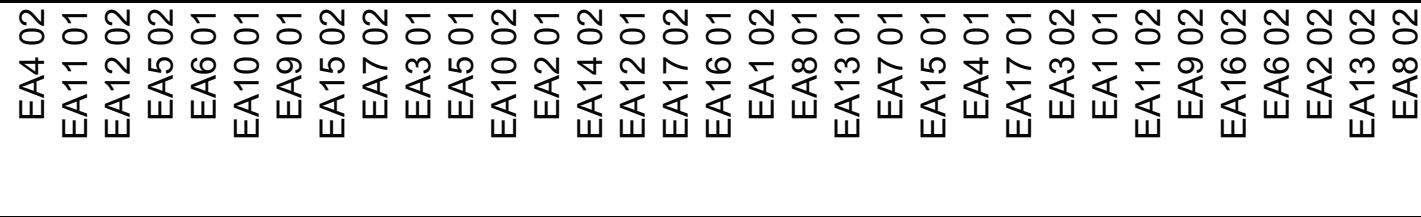 \\
\hline
\end{tabular}




\begin{tabular}{|c|c|}
\hline \multicolumn{2}{|r|}{ mm } \\
\hline 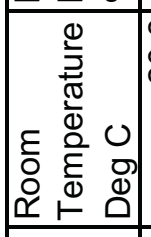 & 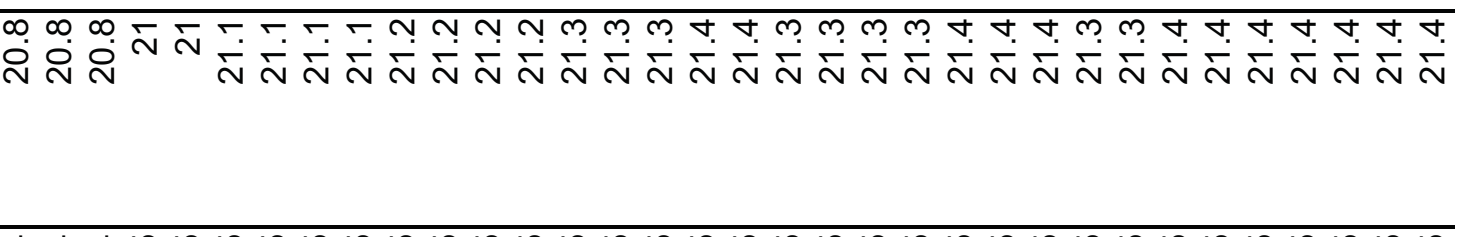 \\
\hline 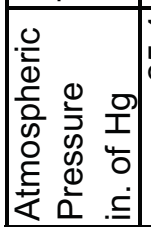 & 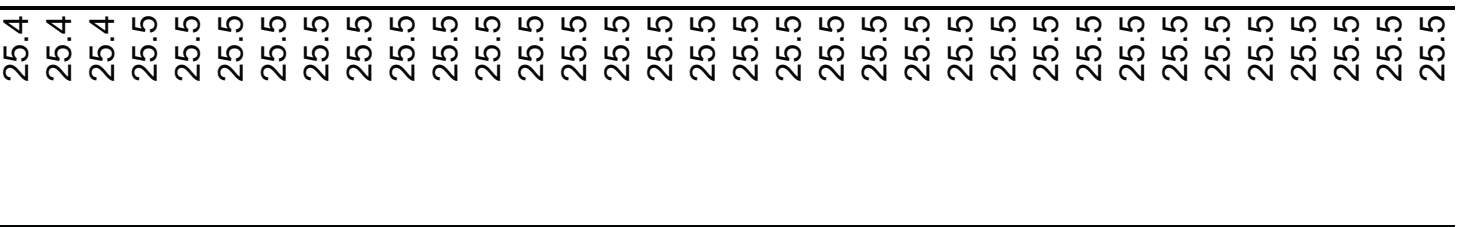 \\
\hline
\end{tabular}

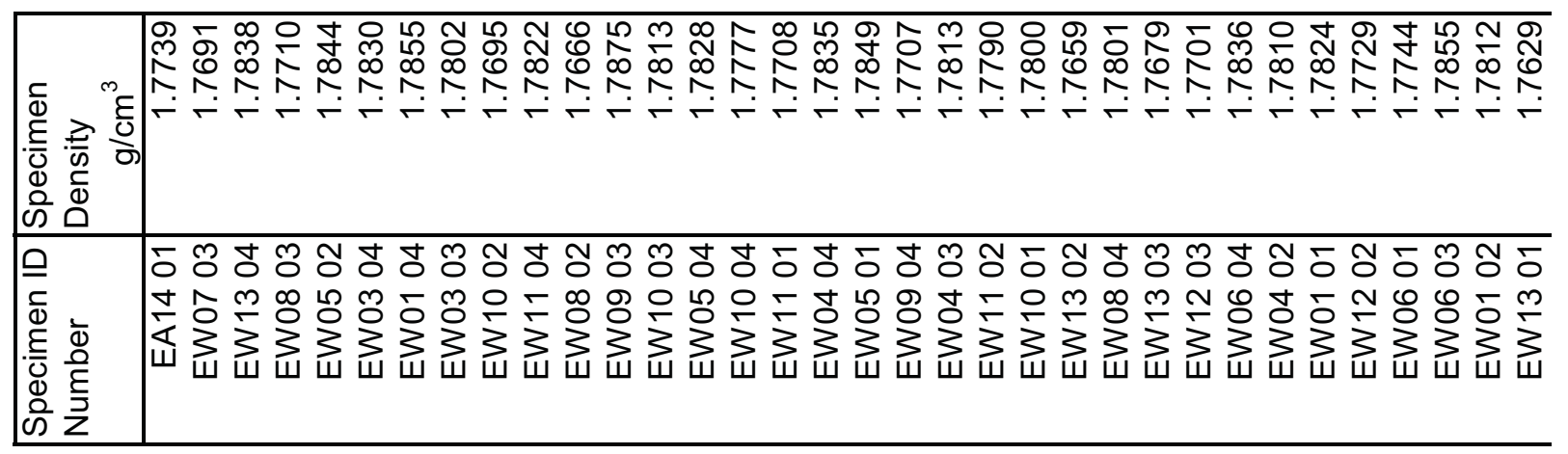



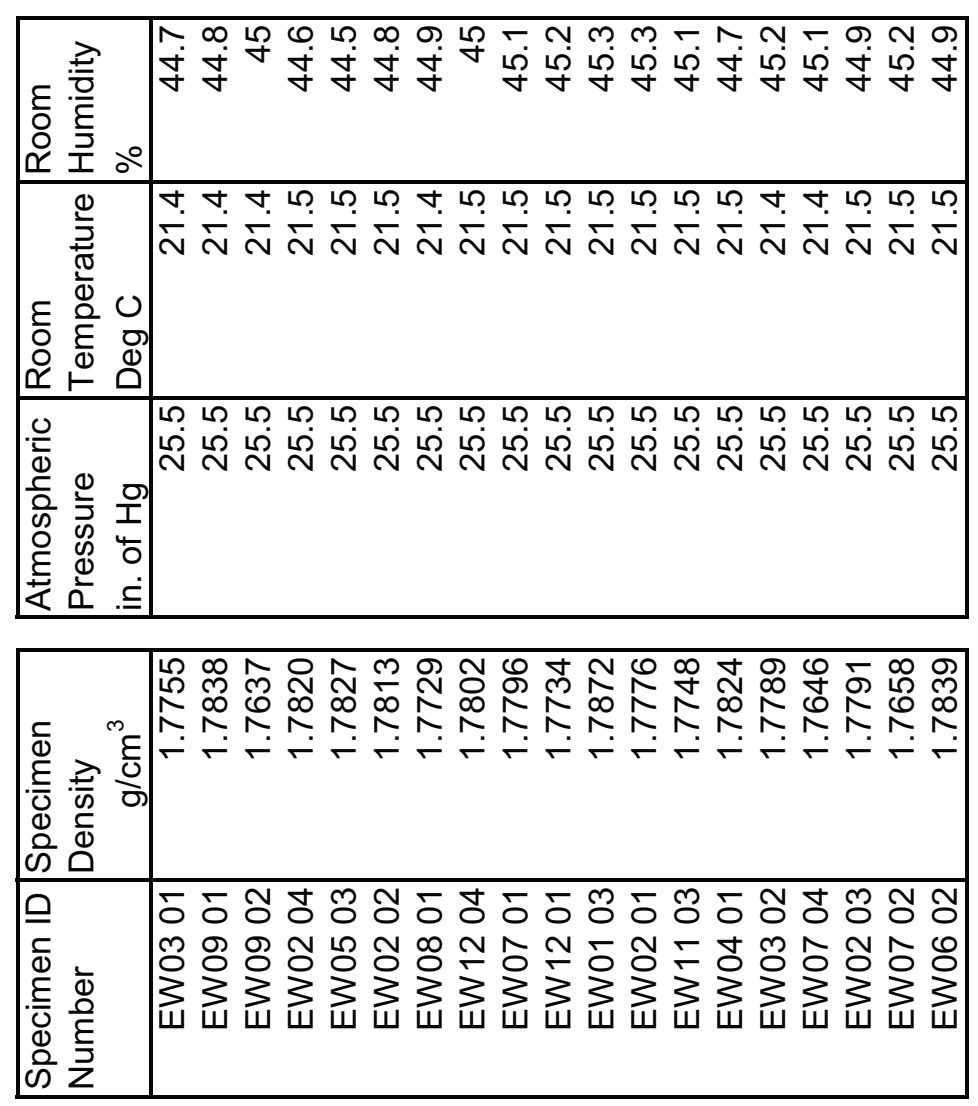


\begin{tabular}{|c|c|}
\hline 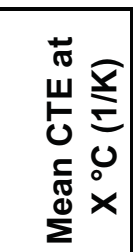 & 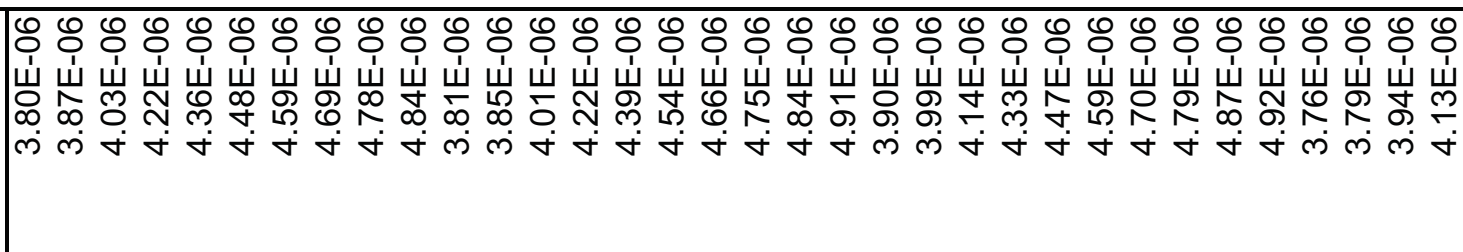 \\
\hline 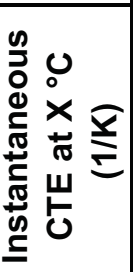 & 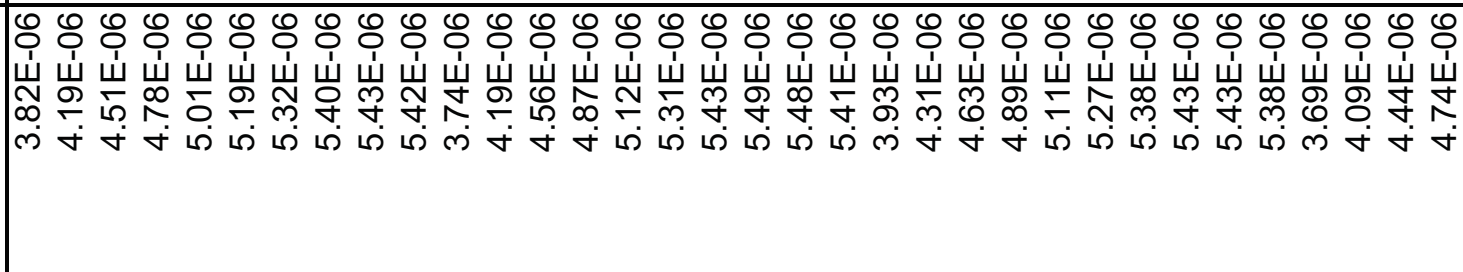 \\
\hline 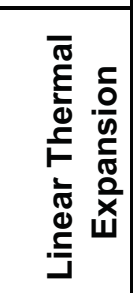 & 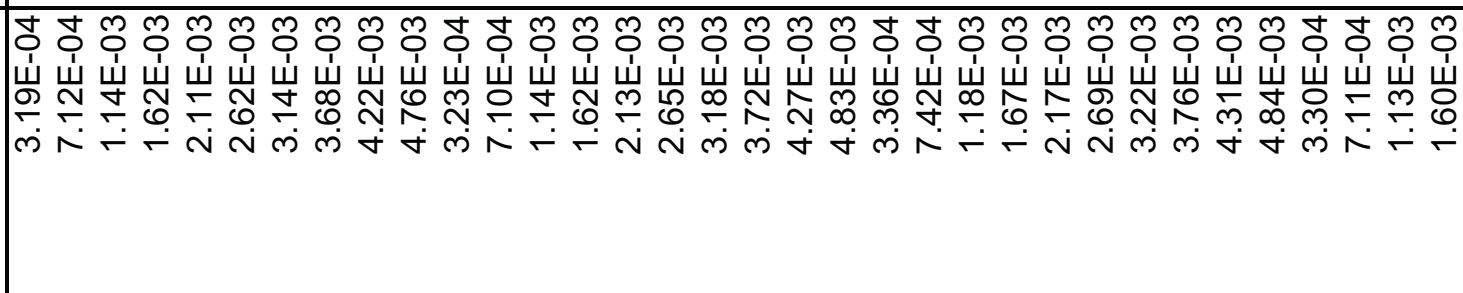 \\
\hline 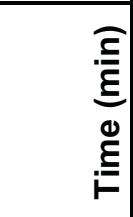 & 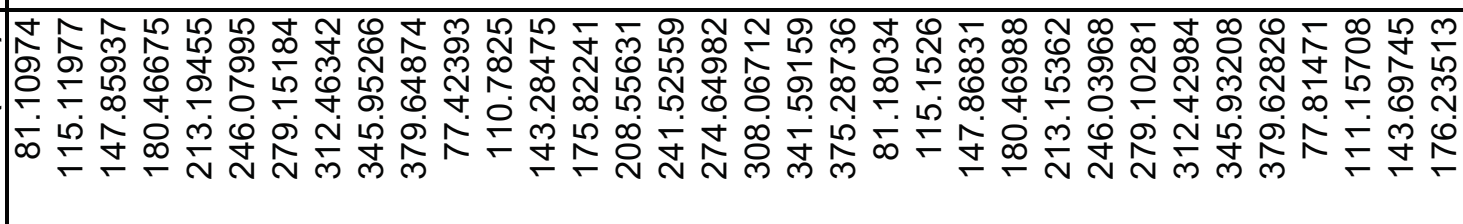 \\
\hline 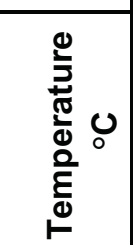 & 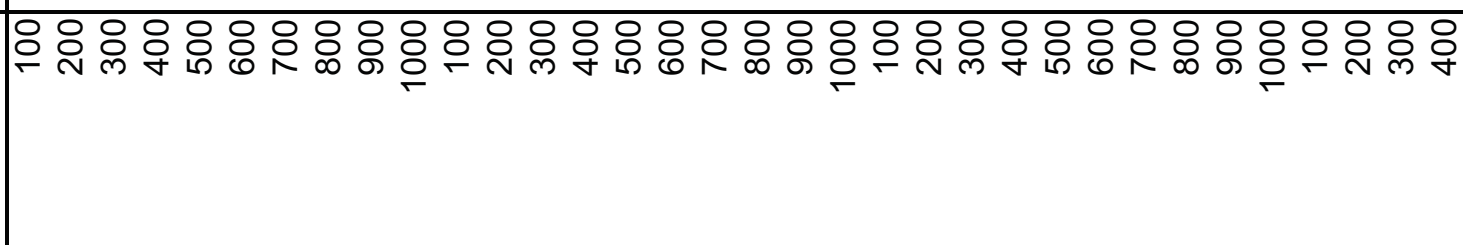 \\
\hline 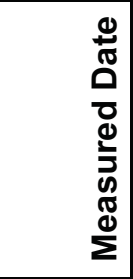 & 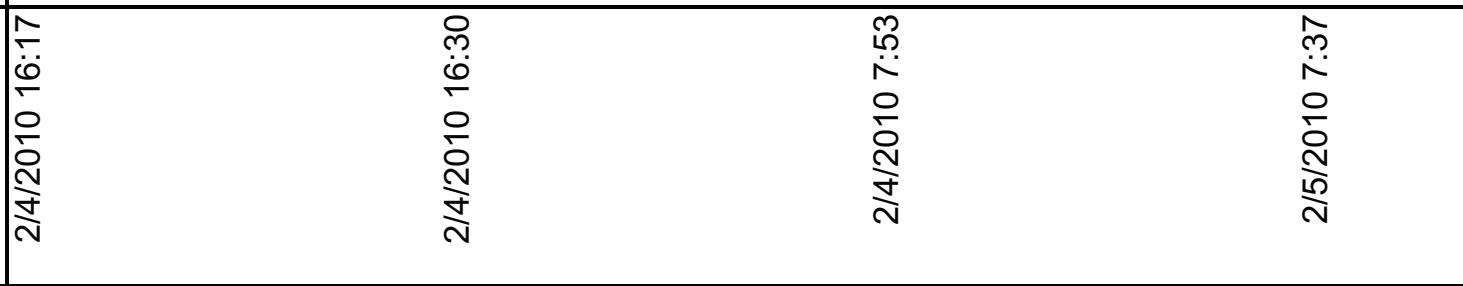 \\
\hline 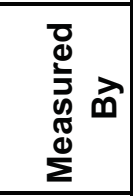 & $\begin{array}{ll}\stackrel{L}{\rho} & \stackrel{L}{\rho} \\
\stackrel{f}{f} & \stackrel{f}{f}\end{array}$ \\
\hline 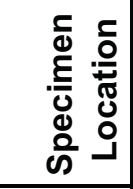 & \\
\hline 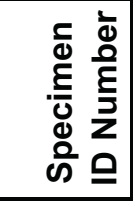 & $\mid \begin{array}{l}5 \\
0 \\
0 \\
\mathbb{4} \\
\end{array}$ \\
\hline
\end{tabular}




\begin{tabular}{|c|c|}
\hline 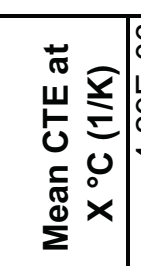 & 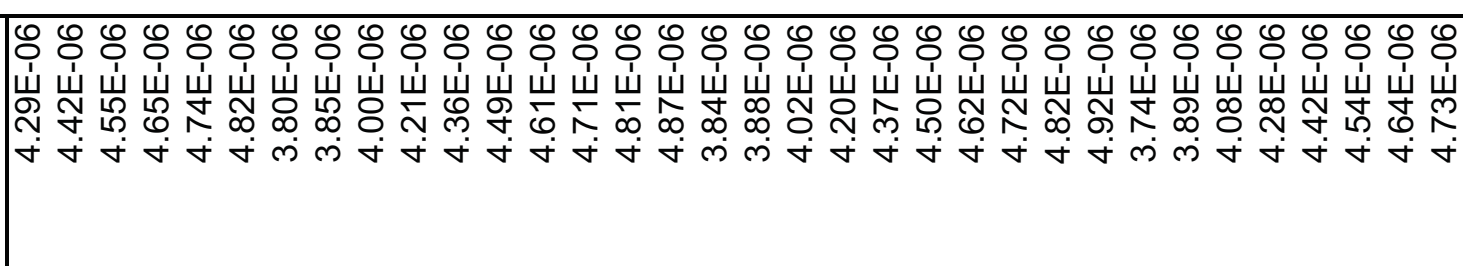 \\
\hline 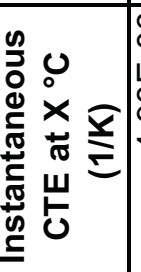 & 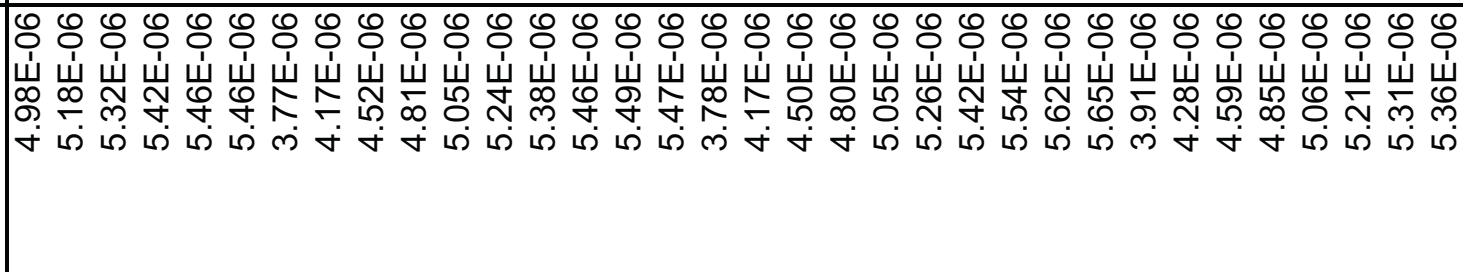 \\
\hline 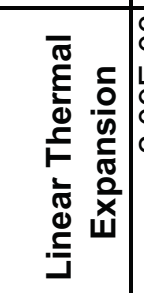 & 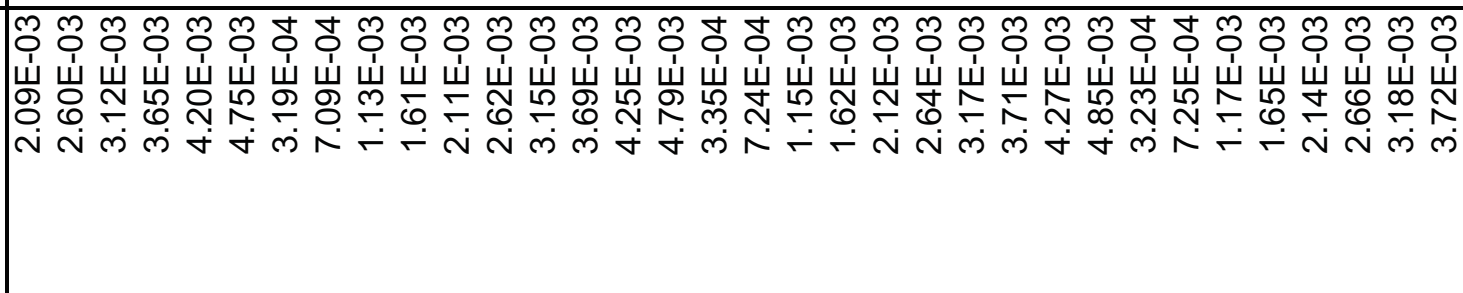 \\
\hline 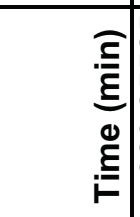 & 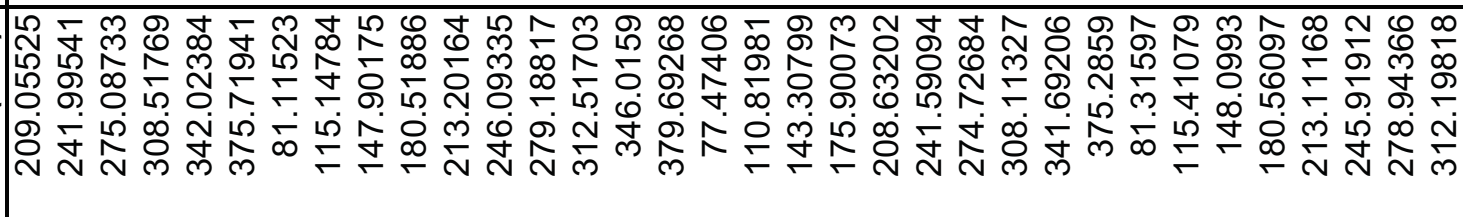 \\
\hline 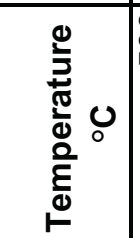 & 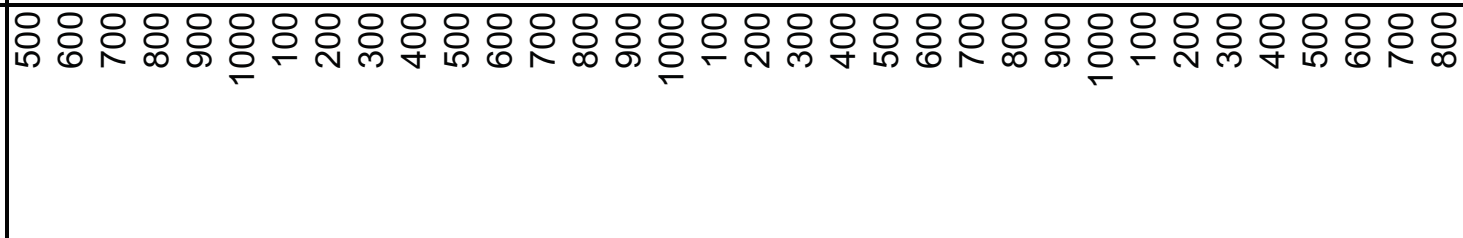 \\
\hline 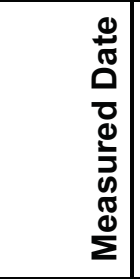 & 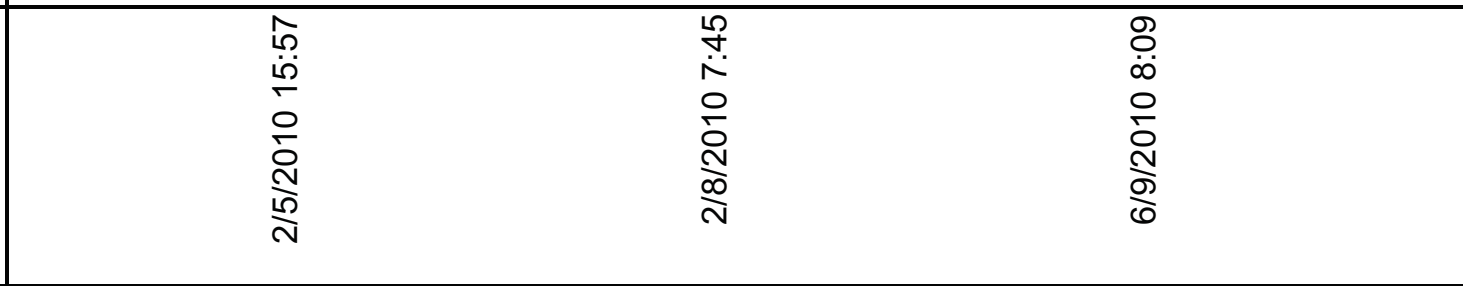 \\
\hline 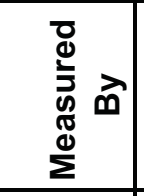 & 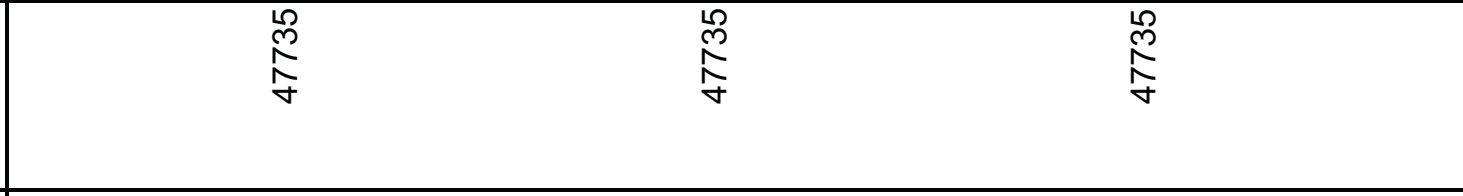 \\
\hline 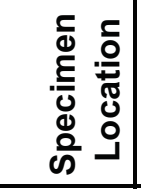 & \\
\hline 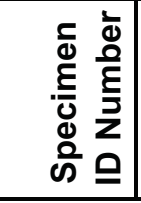 & $\sum_{\substack{\infty\\
}}^{0}$ \\
\hline
\end{tabular}




\begin{tabular}{|c|c|c|c|c|}
\hline 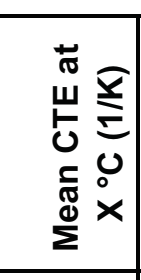 & \multicolumn{4}{|c|}{ 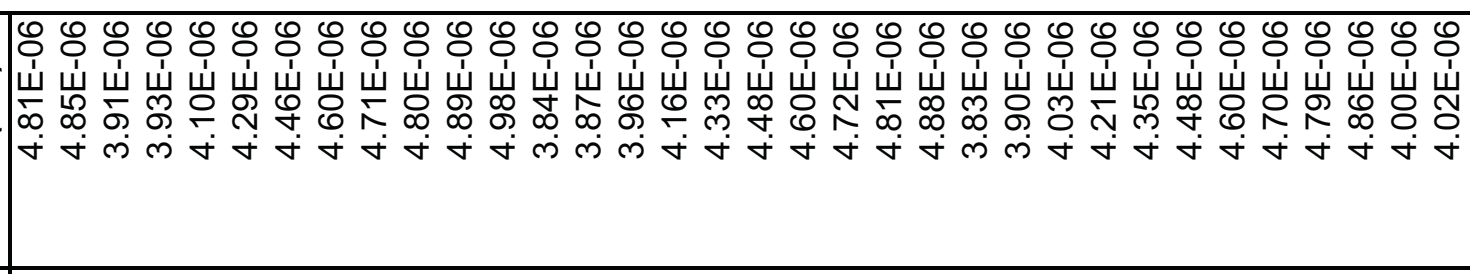 } \\
\hline 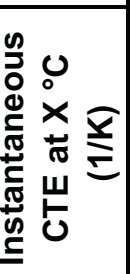 & 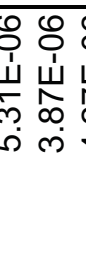 & 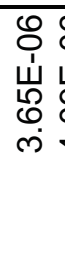 & 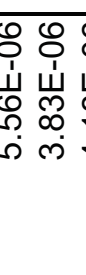 & 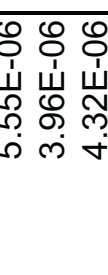 \\
\hline 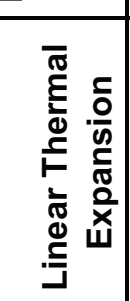 & & & & 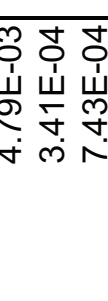 \\
\hline 产 & & 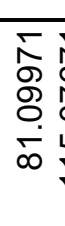 & 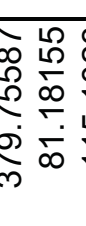 & 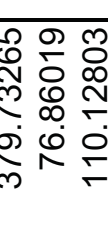 \\
\hline 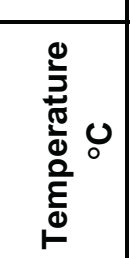 & : & 8 & 8 & 8:용 \\
\hline 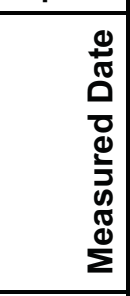 & 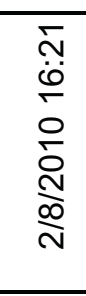 & 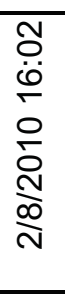 & 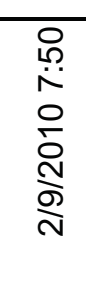 & 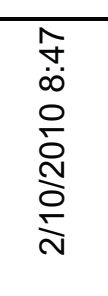 \\
\hline 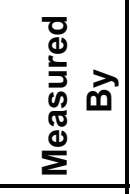 & 曽 & 兽 & 兽 & 惫 \\
\hline 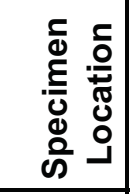 & & & & \\
\hline 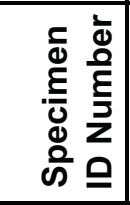 & $\begin{array}{l}\bar{o} \\
\text { Ñ } \\
\text { Du }\end{array}$ & $\begin{array}{l}\text { Oे } \\
\tilde{N} \\
\text { בै }\end{array}$ & 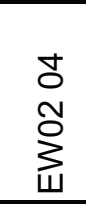 & 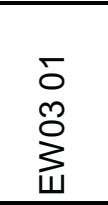 \\
\hline
\end{tabular}




\begin{tabular}{|c|c|}
\hline 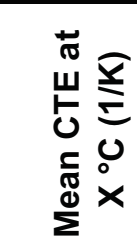 & 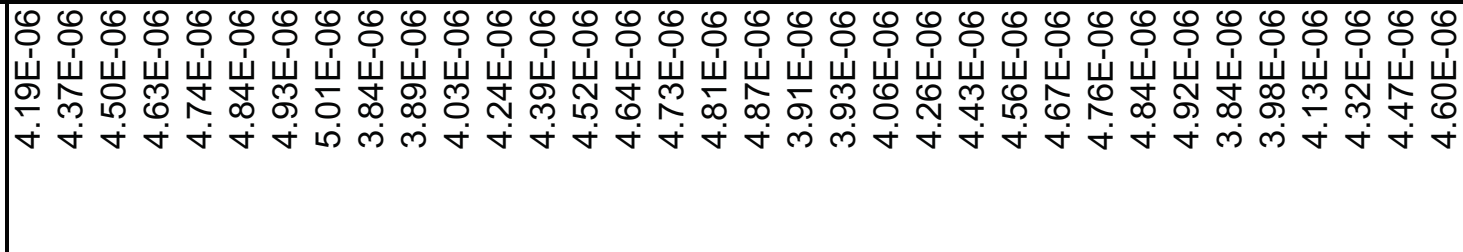 \\
\hline 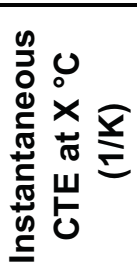 & 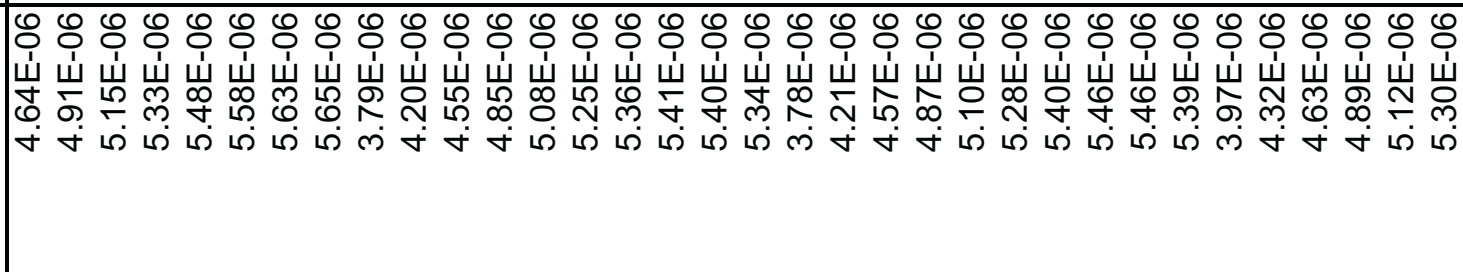 \\
\hline 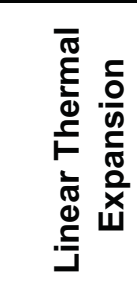 & 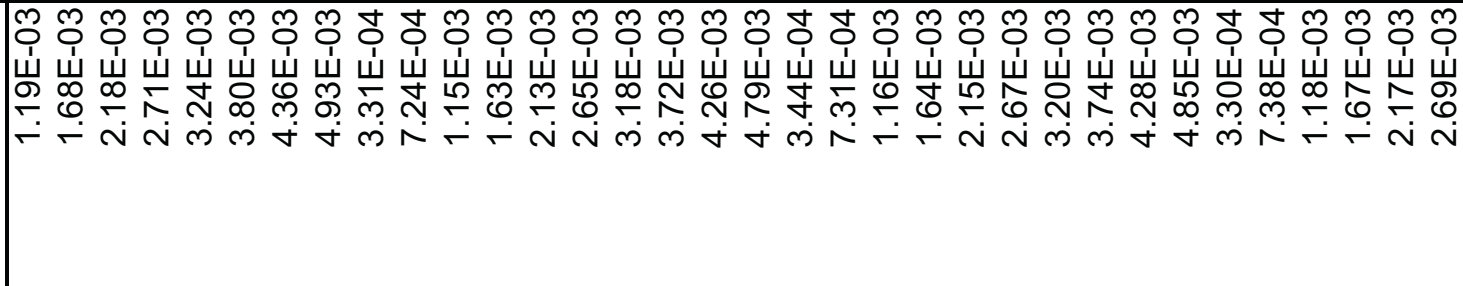 \\
\hline 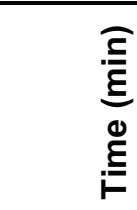 & 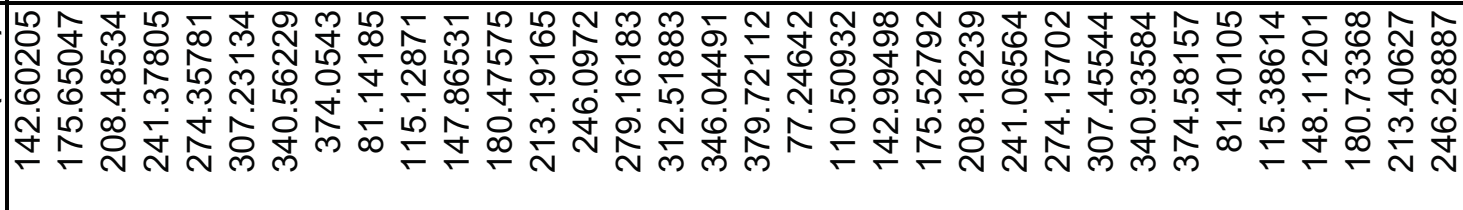 \\
\hline 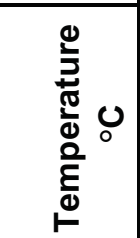 & 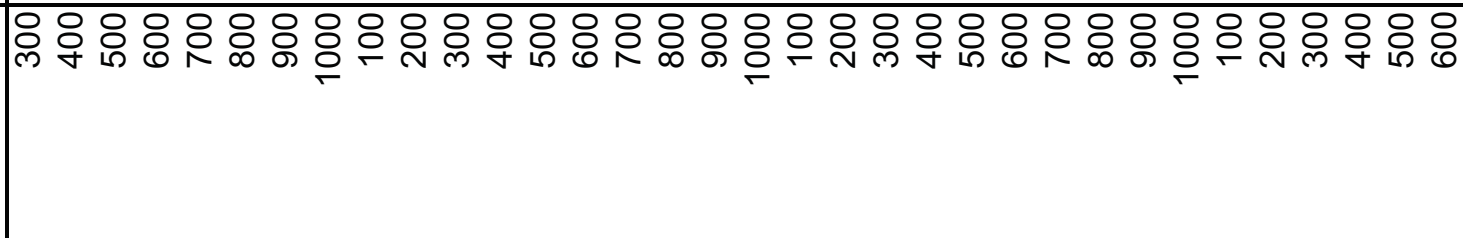 \\
\hline 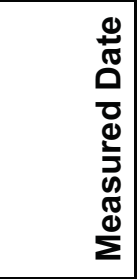 & 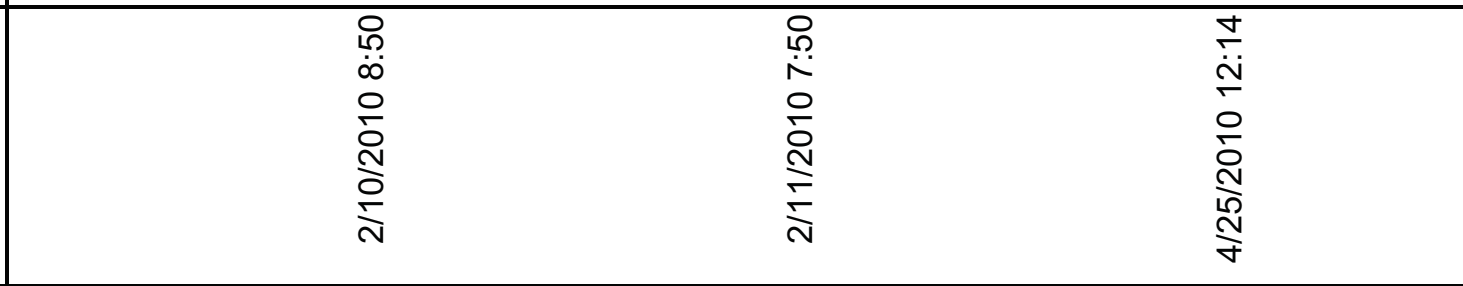 \\
\hline 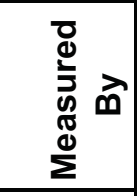 & $\stackrel{\stackrel{L}{N}}{\stackrel{f}{\sim}}$ \\
\hline 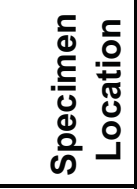 & \\
\hline 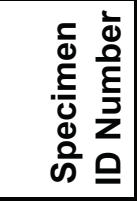 & $\begin{array}{l}\text { O } \\
\text { m } \\
\text { ○े }\end{array}$ \\
\hline
\end{tabular}




\begin{tabular}{|c|c|}
\hline 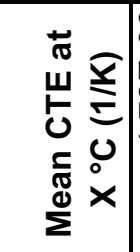 & 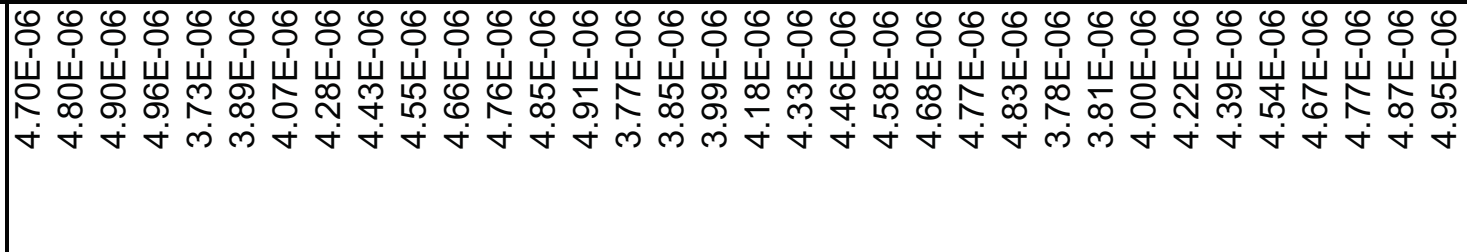 \\
\hline 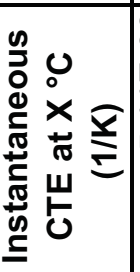 & 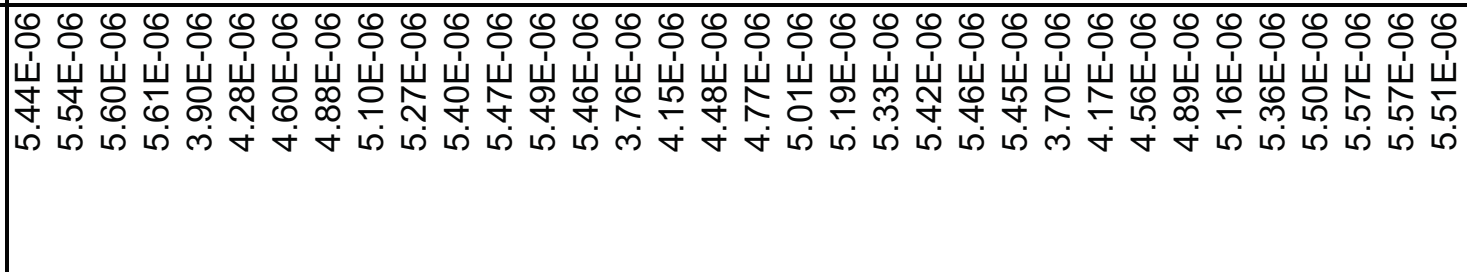 \\
\hline 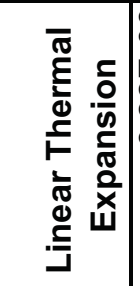 & 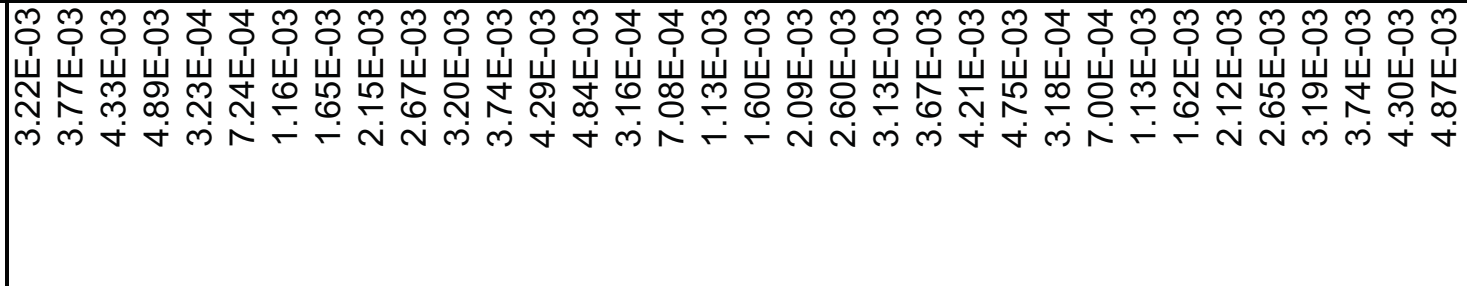 \\
\hline 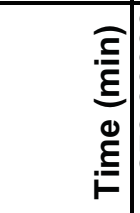 & 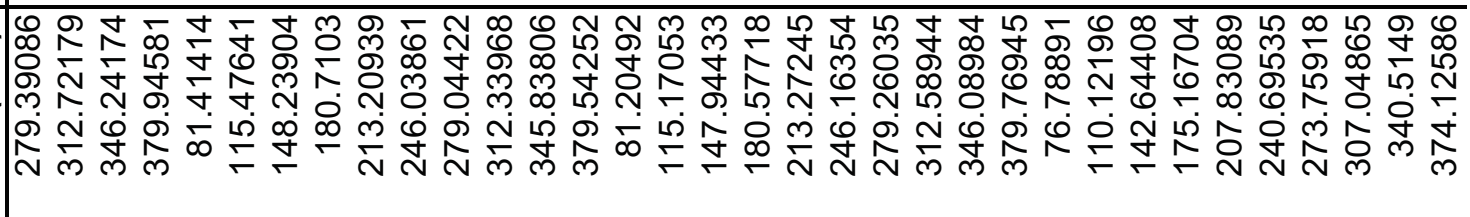 \\
\hline 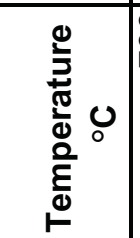 & 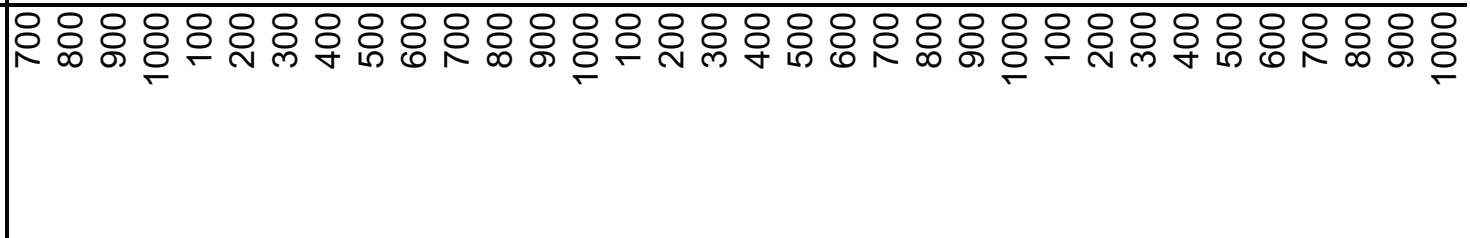 \\
\hline 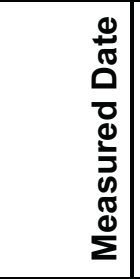 & 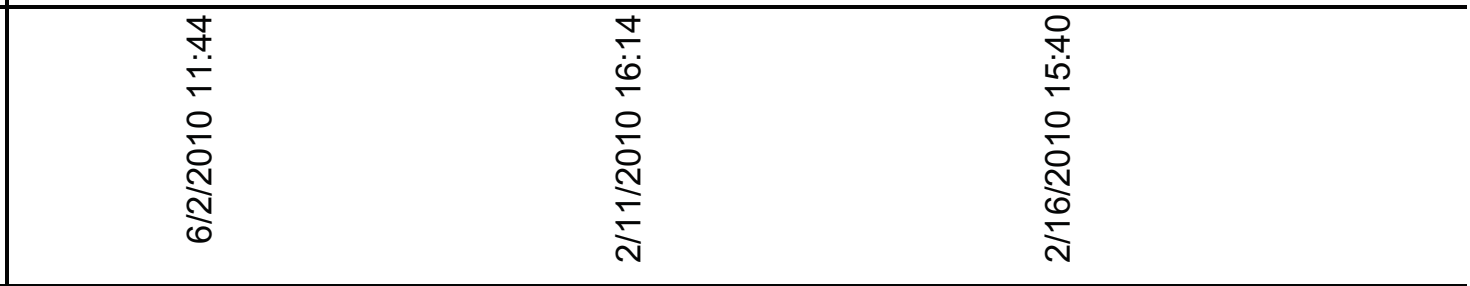 \\
\hline 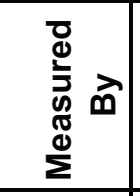 & $\begin{array}{l}\stackrel{\mathscr{L}}{\mathcal{N}} \\
\stackrel{\sim}{\sim}\end{array}$ \\
\hline 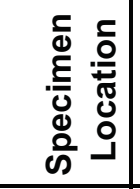 & \\
\hline 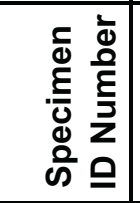 & 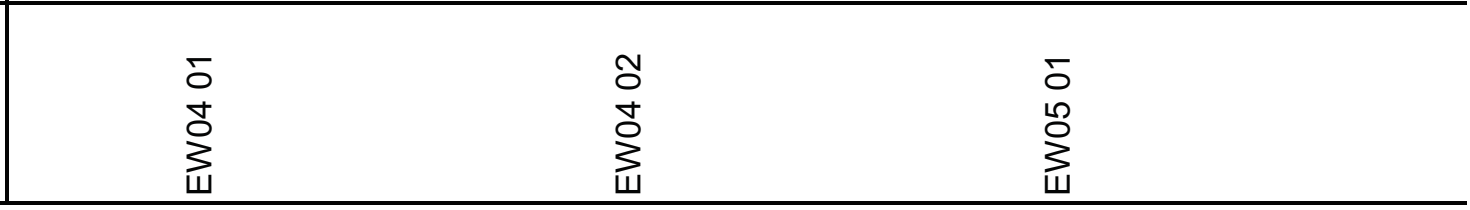 \\
\hline
\end{tabular}




\begin{tabular}{|c|c|c|c|}
\hline 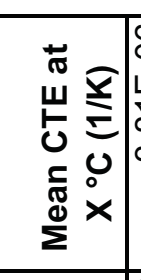 & 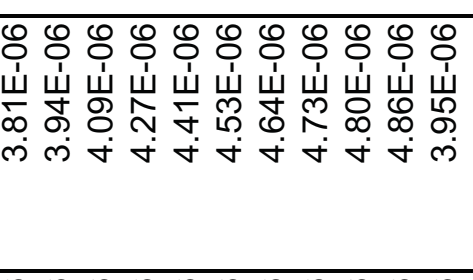 & 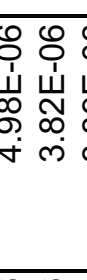 & 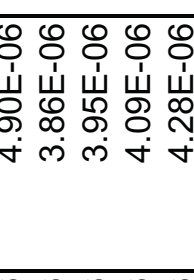 \\
\hline 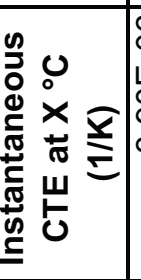 & 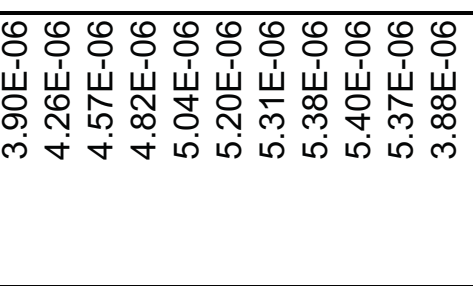 & 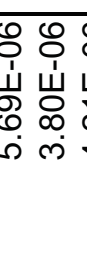 & $\begin{array}{ll}0 \\
0\end{array}$ \\
\hline 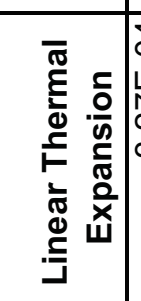 & 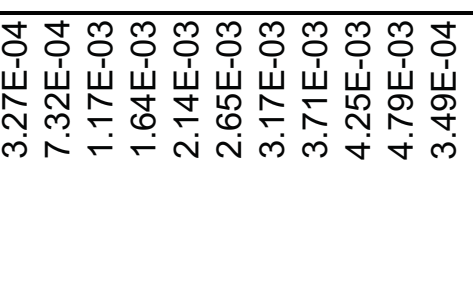 & 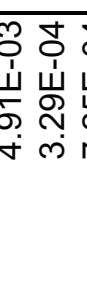 & 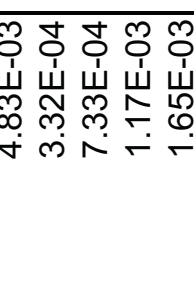 \\
\hline 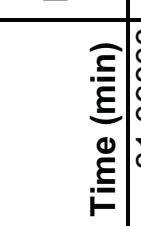 & 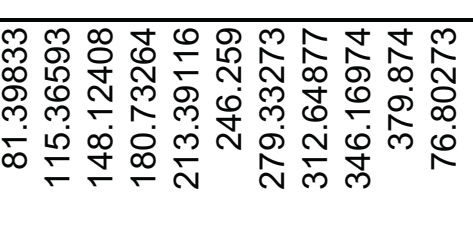 & 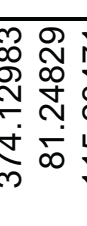 & 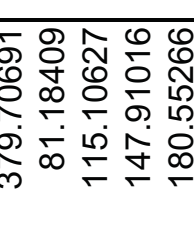 \\
\hline 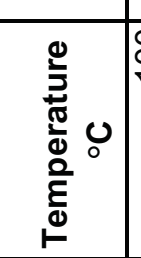 & 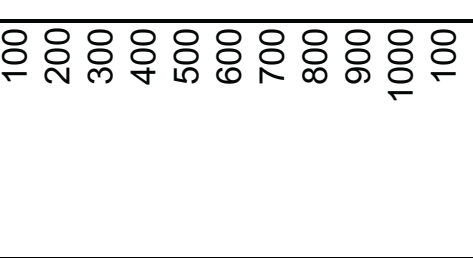 & 8 & 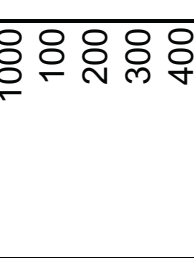 \\
\hline 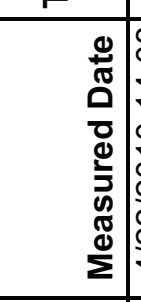 & 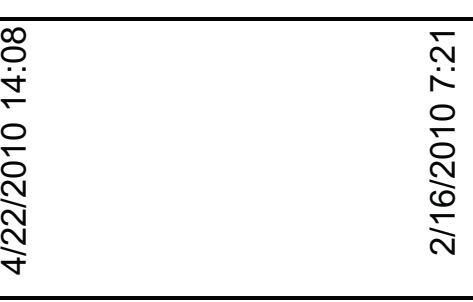 & 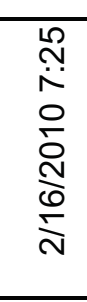 & 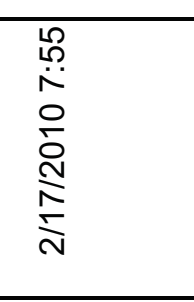 \\
\hline 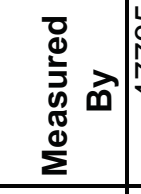 & 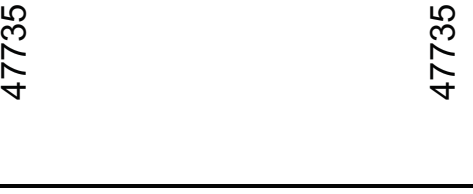 & 总 & 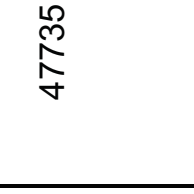 \\
\hline 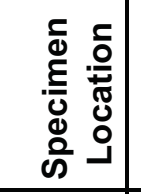 & & & \\
\hline 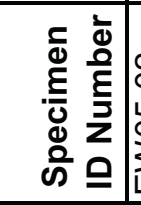 & 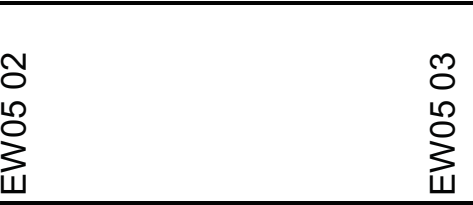 & $\begin{array}{l}\text { d } \\
\text { on } \\
\text { 离 }\end{array}$ & 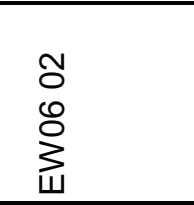 \\
\hline
\end{tabular}




\begin{tabular}{|c|c|}
\hline 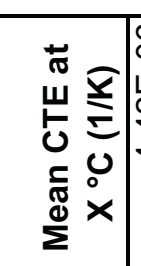 & 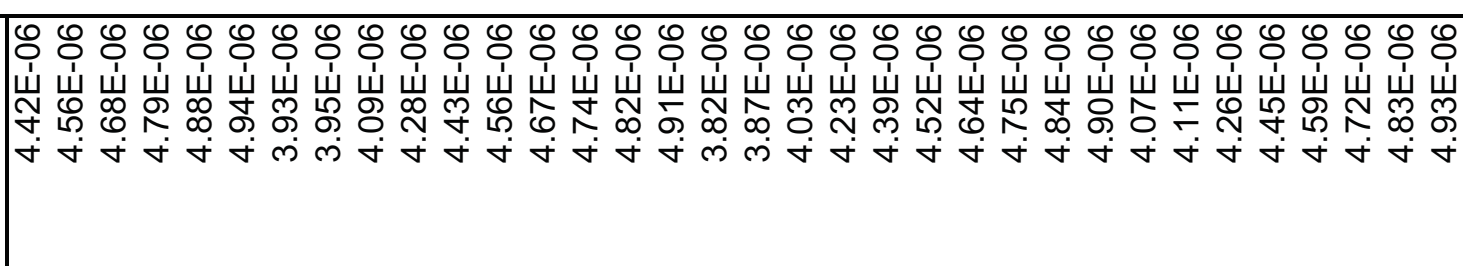 \\
\hline 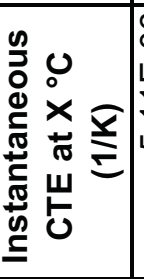 & 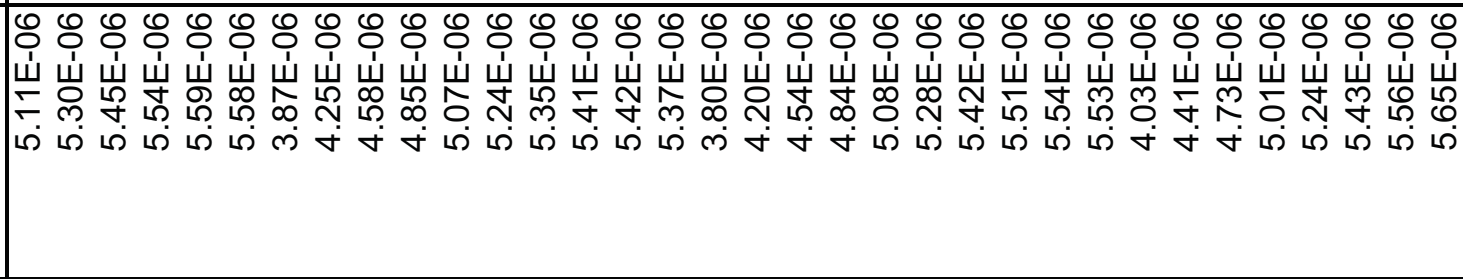 \\
\hline 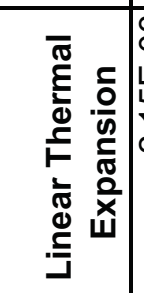 & 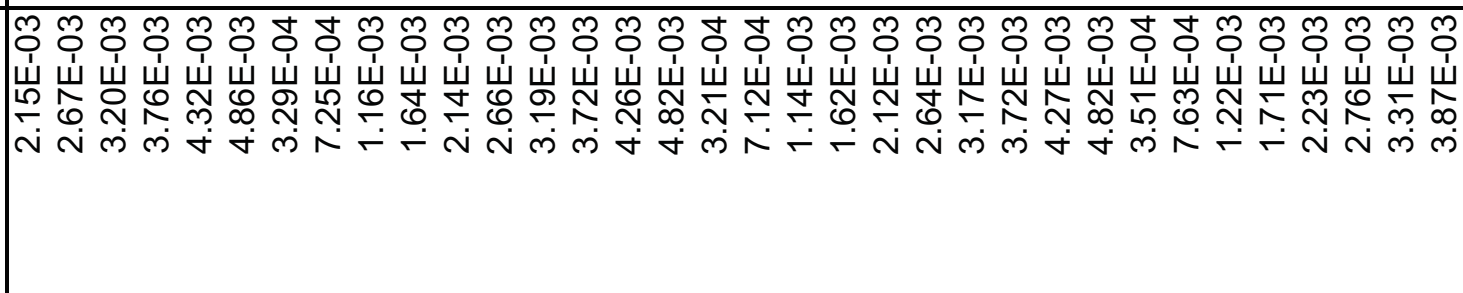 \\
\hline 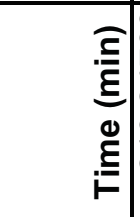 & 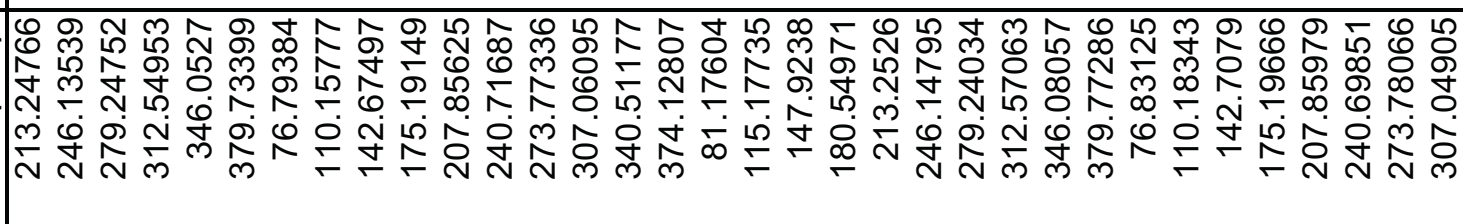 \\
\hline 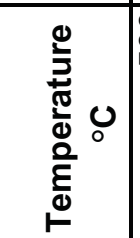 & 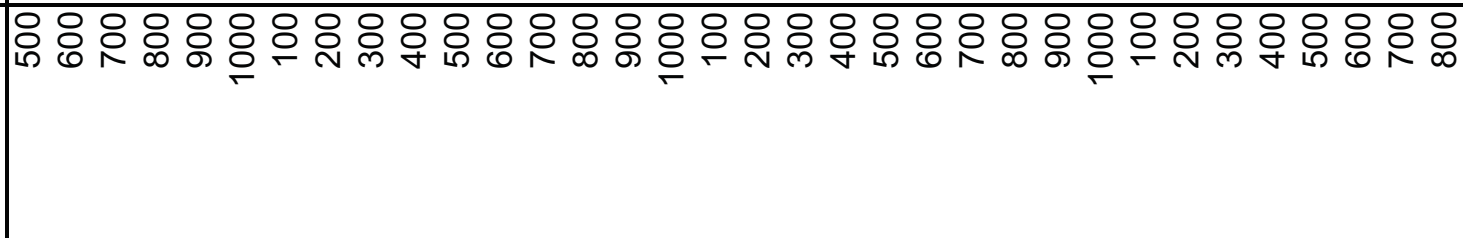 \\
\hline 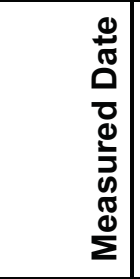 & 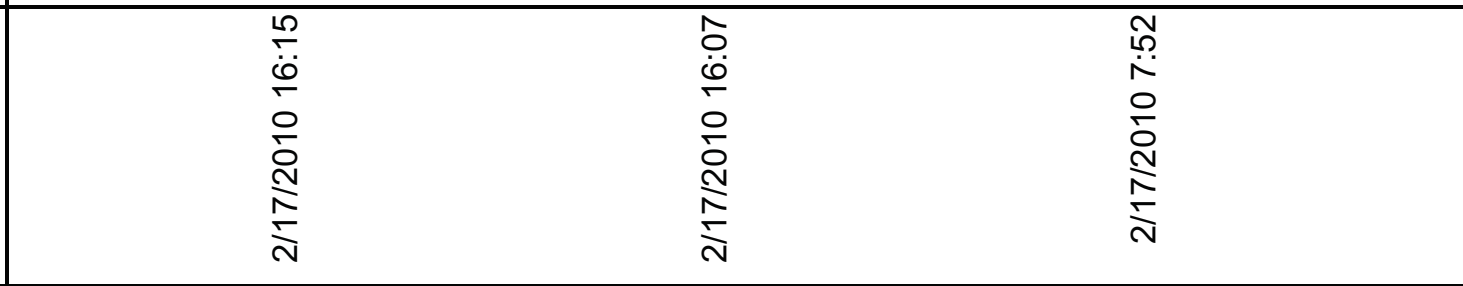 \\
\hline 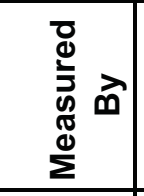 & 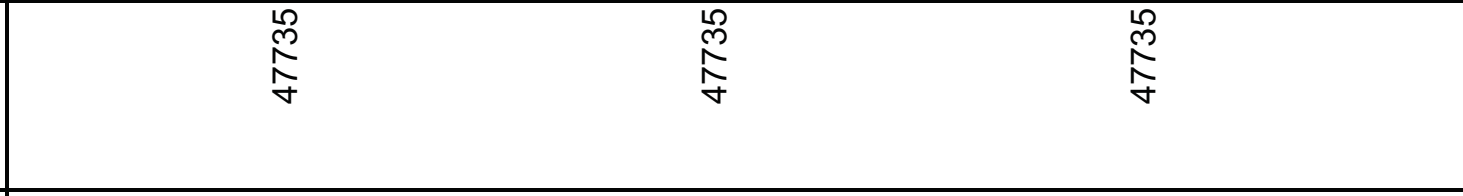 \\
\hline 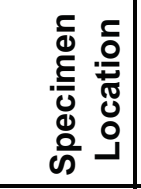 & \\
\hline 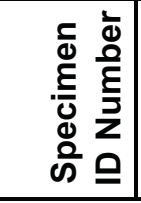 & 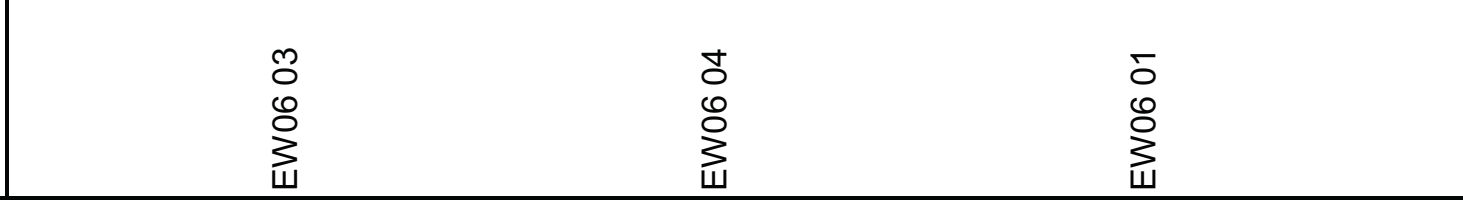 \\
\hline
\end{tabular}




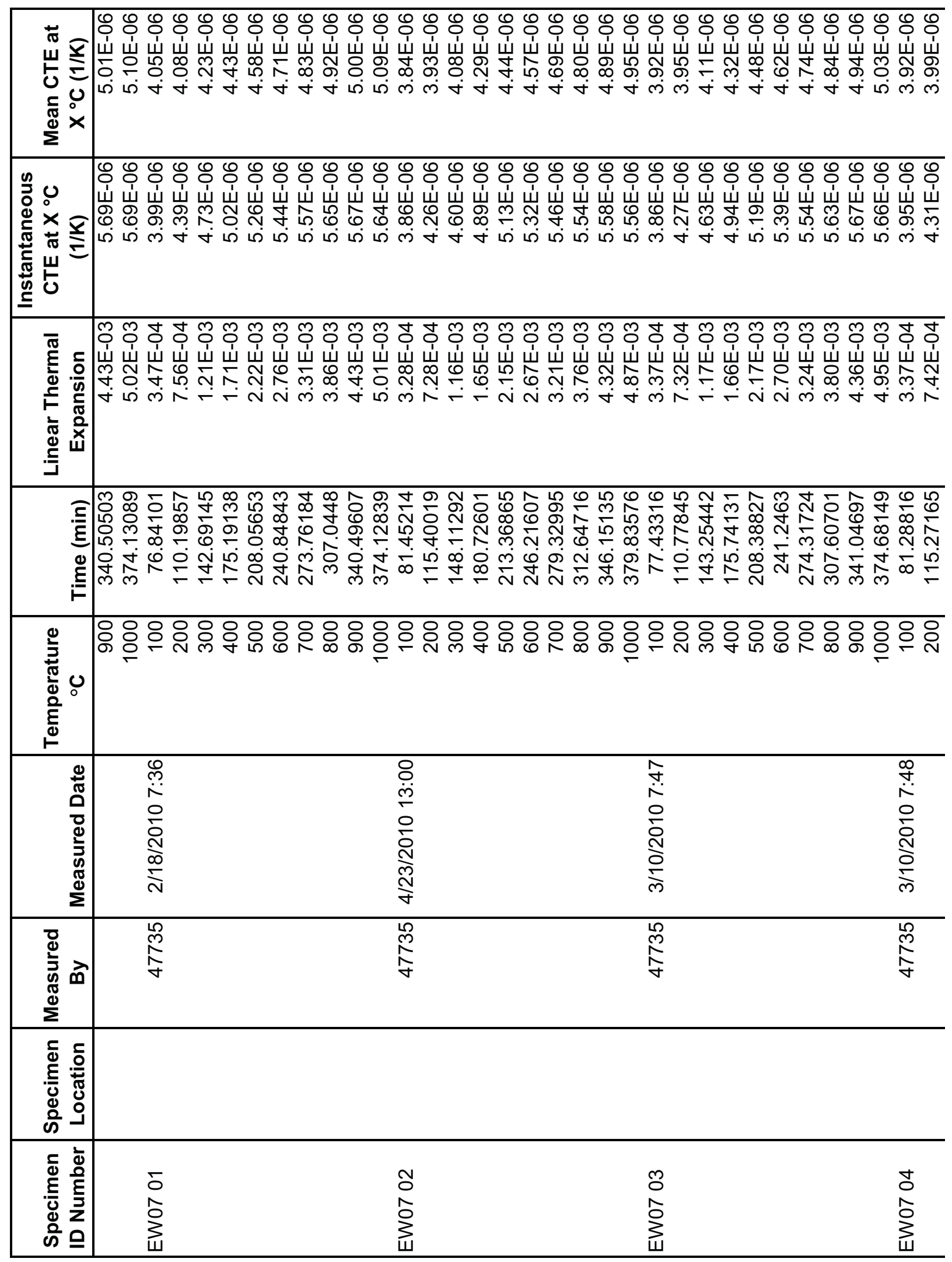




\begin{tabular}{|c|c|}
\hline 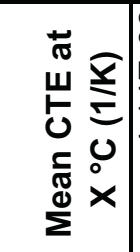 & 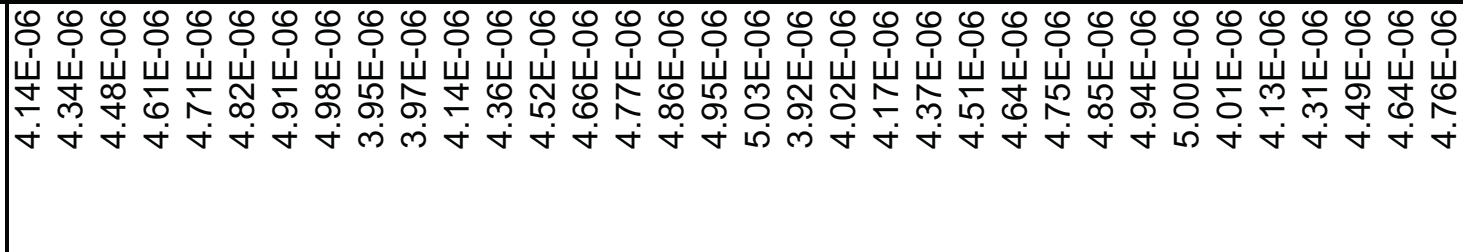 \\
\hline 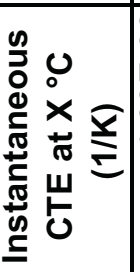 & 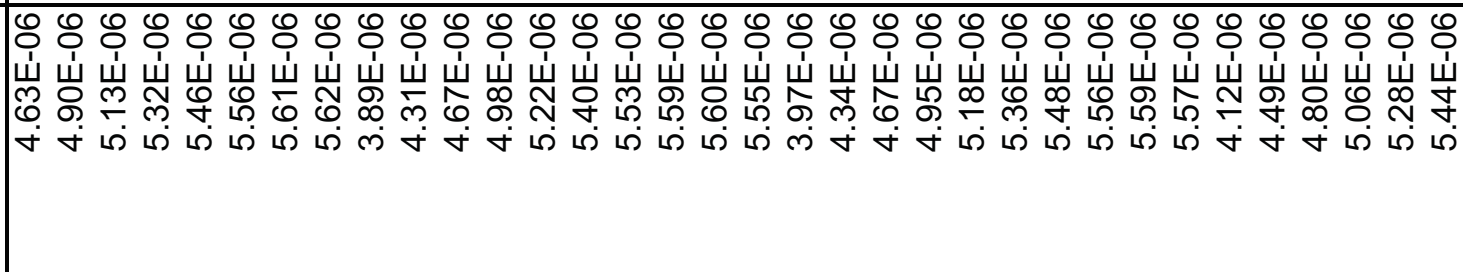 \\
\hline 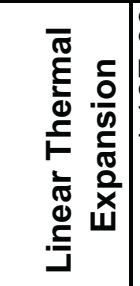 & 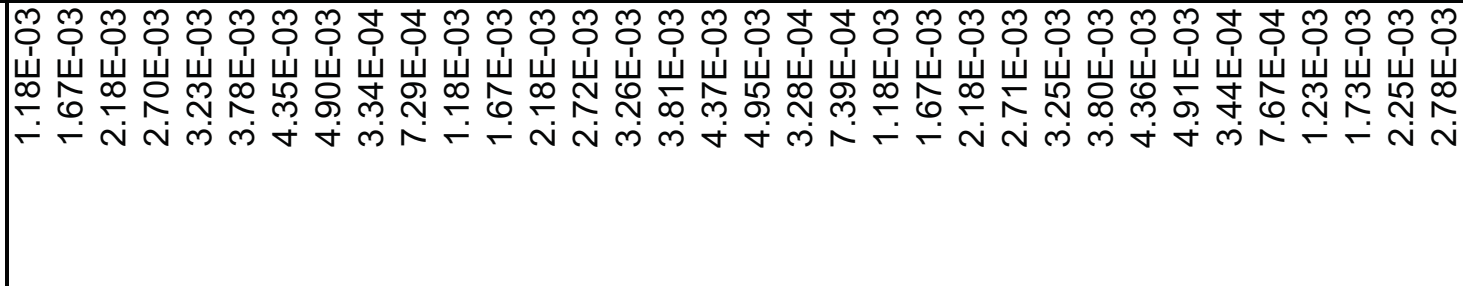 \\
\hline $\mid$ & 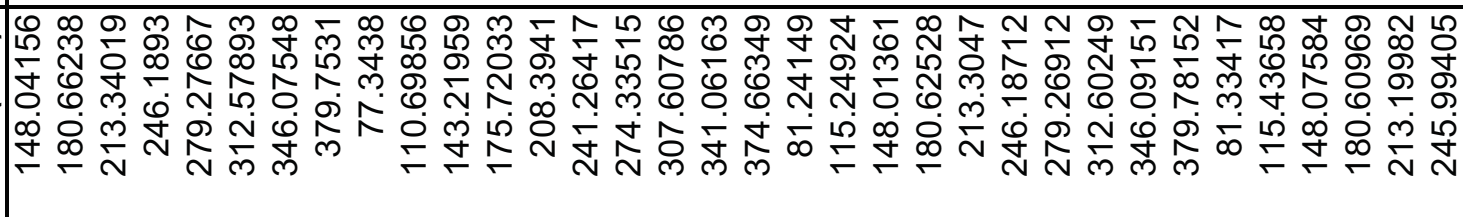 \\
\hline 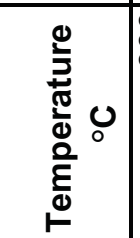 & 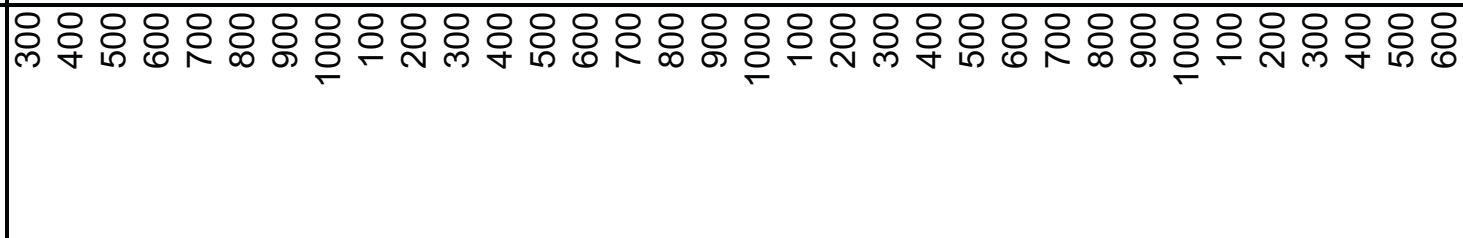 \\
\hline 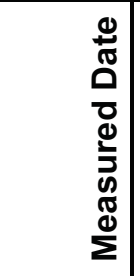 & 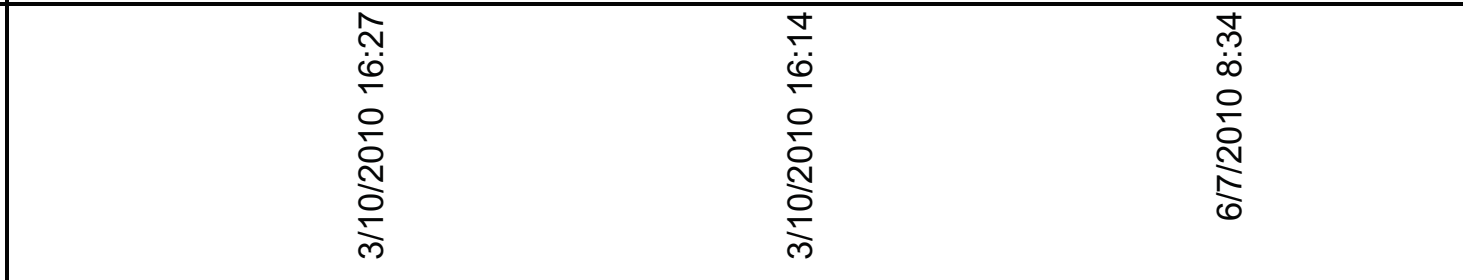 \\
\hline 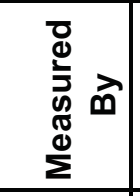 & 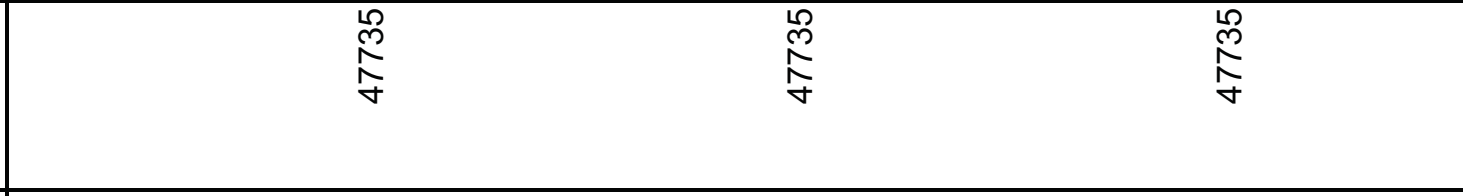 \\
\hline 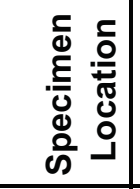 & \\
\hline 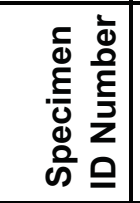 & 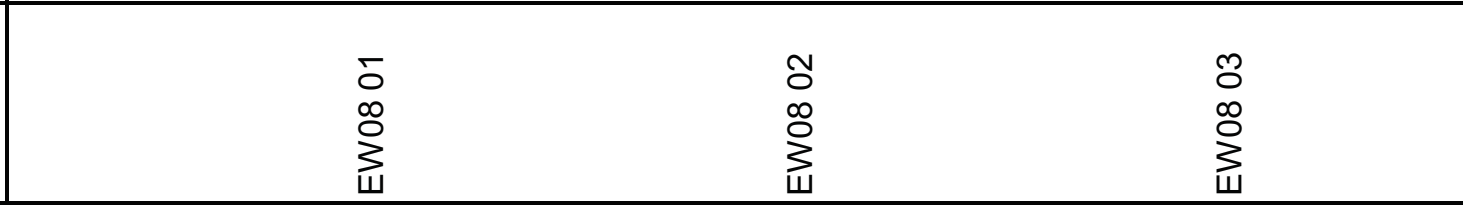 \\
\hline
\end{tabular}




\begin{tabular}{|c|c|}
\hline 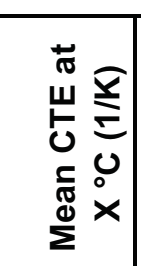 & 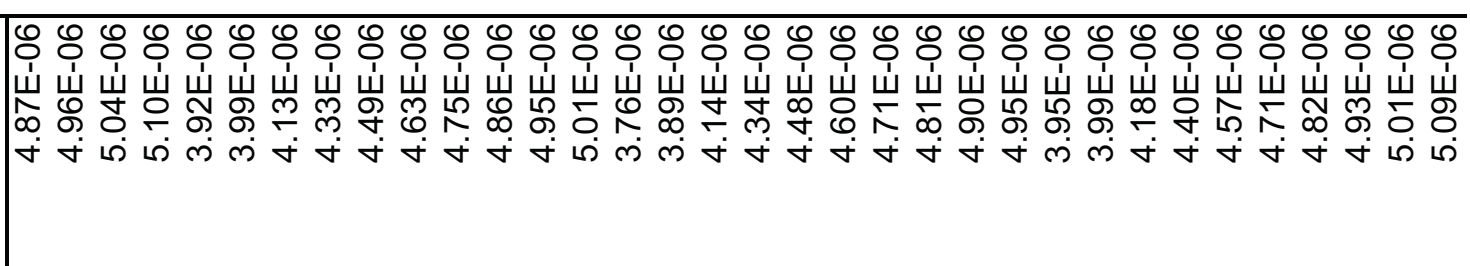 \\
\hline 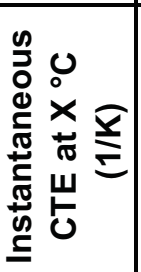 & 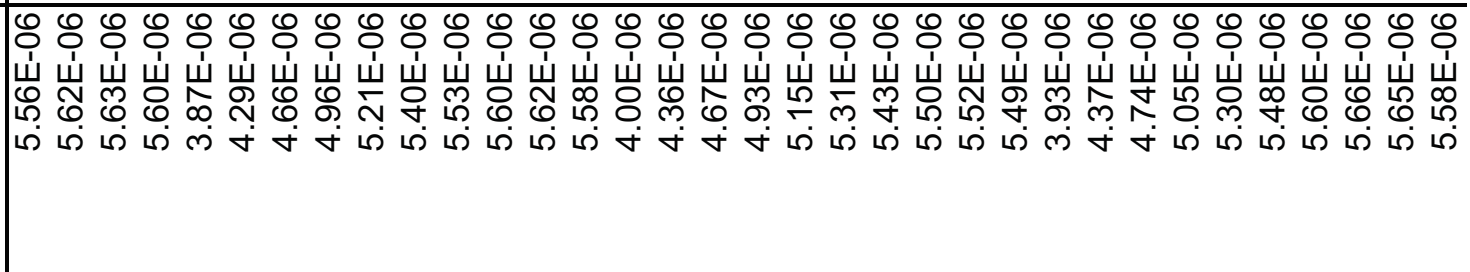 \\
\hline 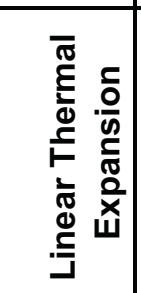 & 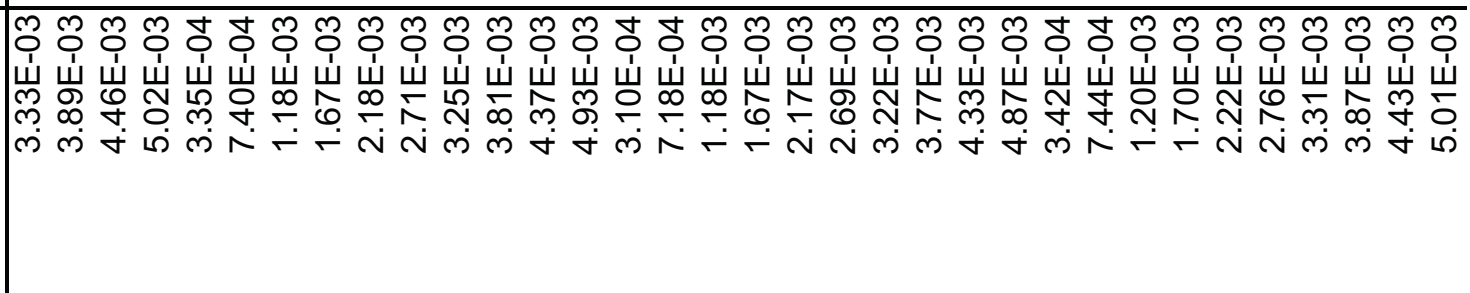 \\
\hline 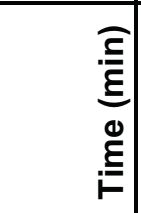 & 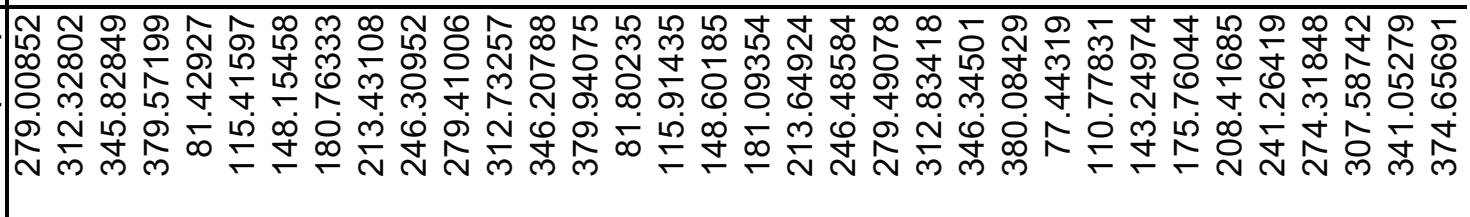 \\
\hline 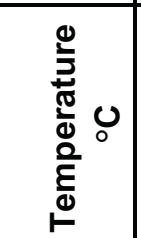 & 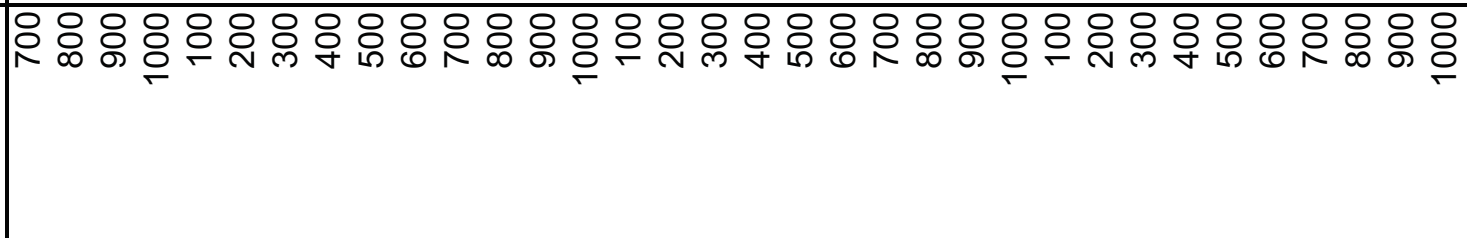 \\
\hline 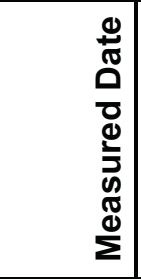 & 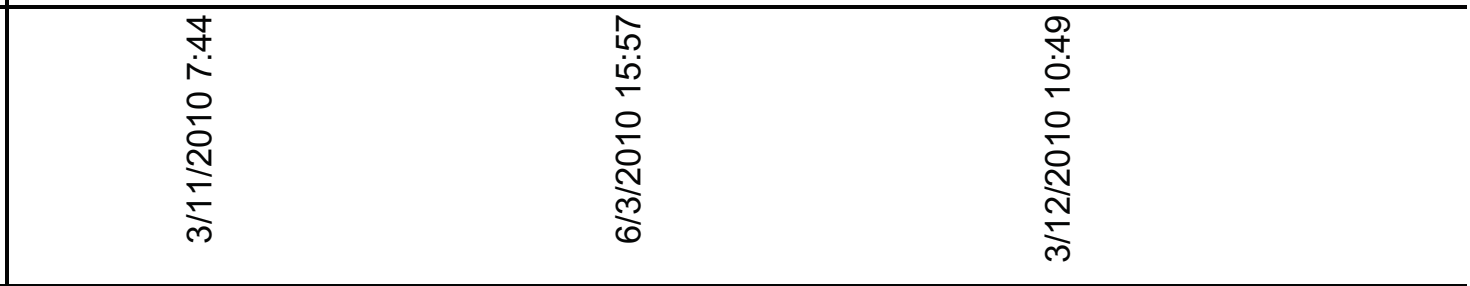 \\
\hline 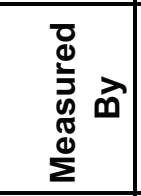 & 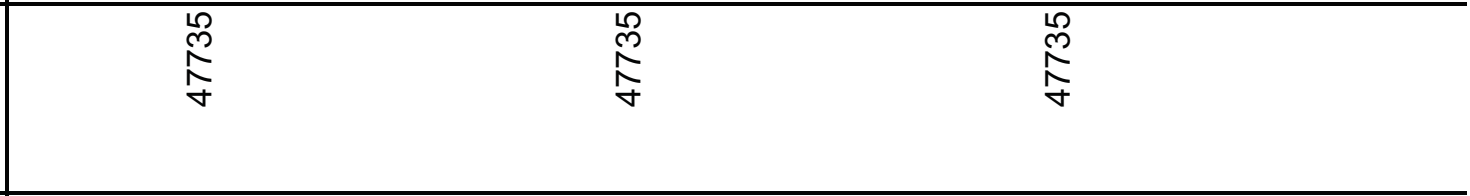 \\
\hline 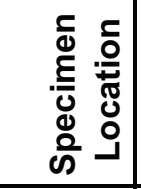 & \\
\hline 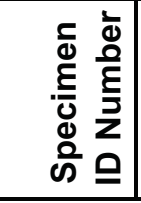 & 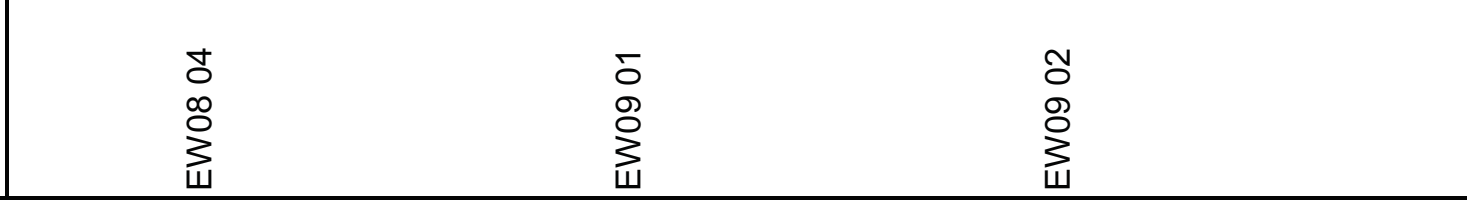 \\
\hline
\end{tabular}




\begin{tabular}{|c|c|c|c|}
\hline 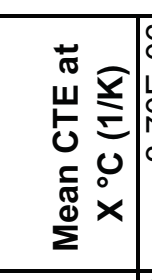 & 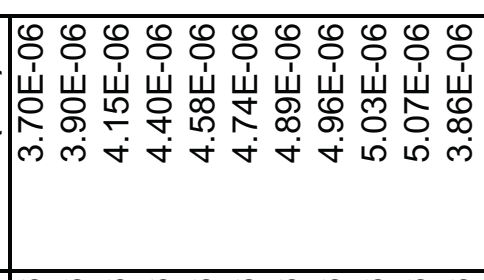 & 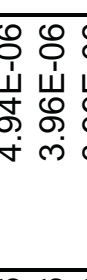 & $\begin{array}{ll}0 \\
0\end{array}$ \\
\hline 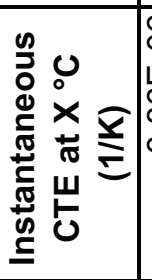 & 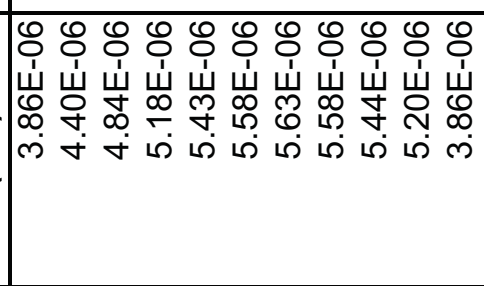 & 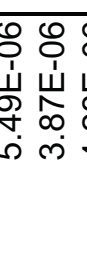 & 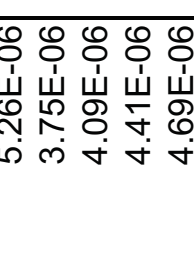 \\
\hline 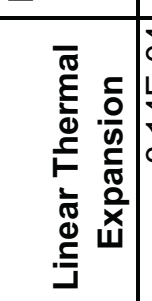 & 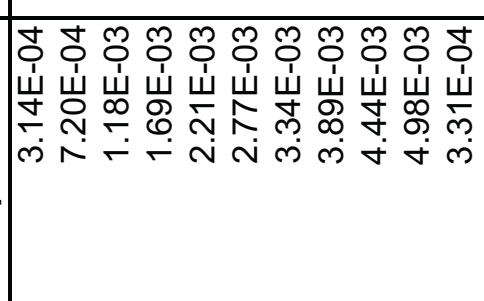 & 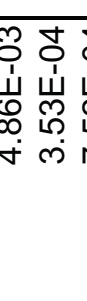 & 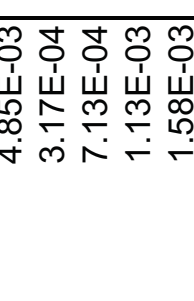 \\
\hline 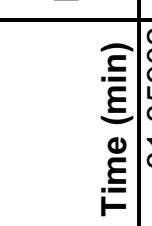 & 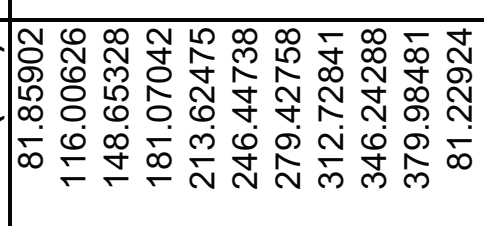 & 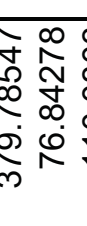 & 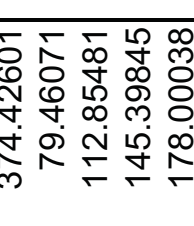 \\
\hline 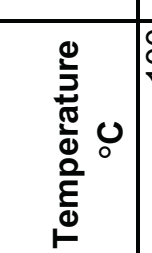 & 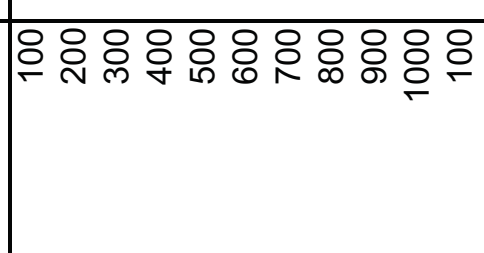 & 00 & 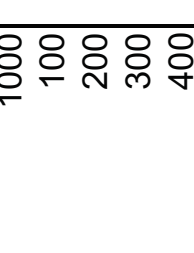 \\
\hline 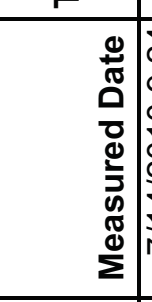 & 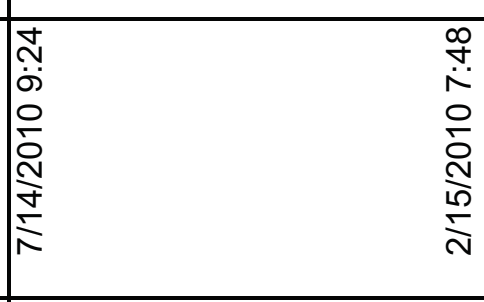 & 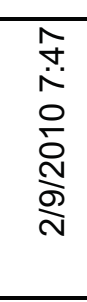 & 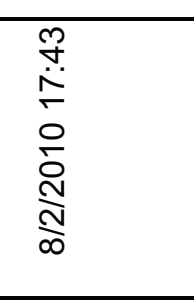 \\
\hline 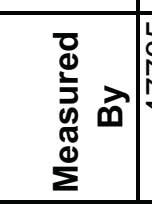 & 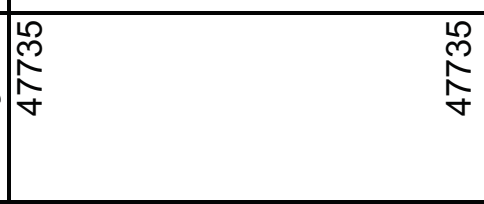 & 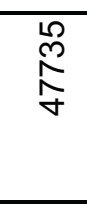 & 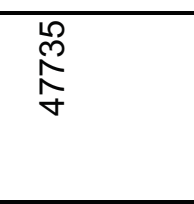 \\
\hline 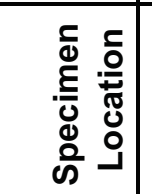 & & & \\
\hline 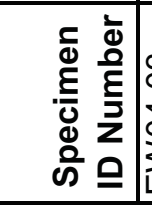 & 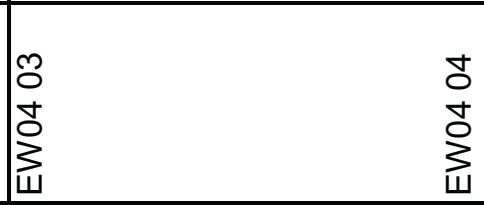 & 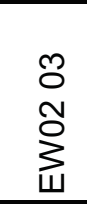 & $\begin{array}{l}\text { Oo } \\
\text { on } \\
\text { 离 }\end{array}$ \\
\hline
\end{tabular}




\begin{tabular}{|c|c|c|}
\hline 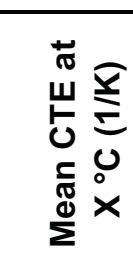 & 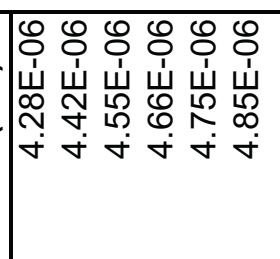 & 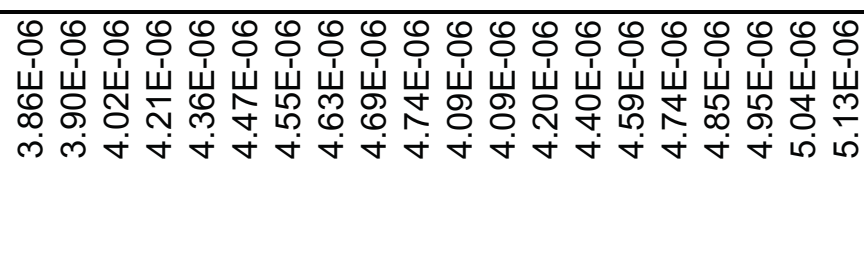 \\
\hline 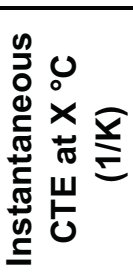 & 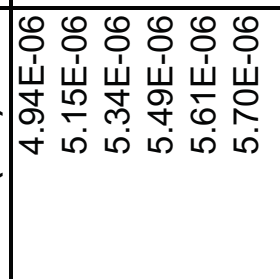 & 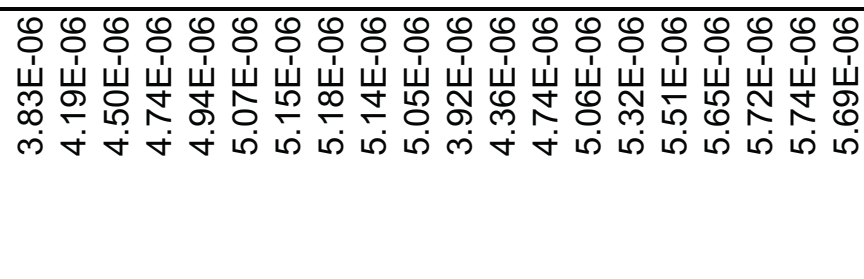 \\
\hline 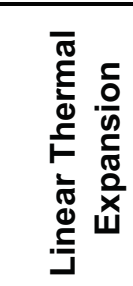 & 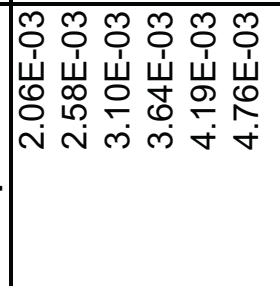 & 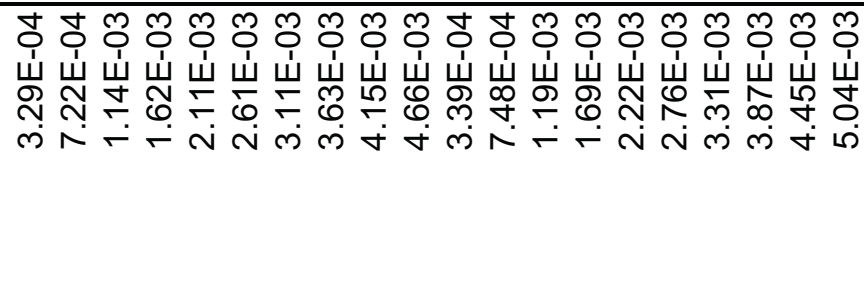 \\
\hline 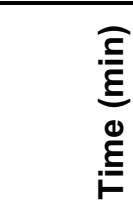 & 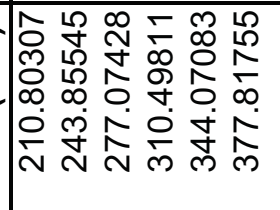 & 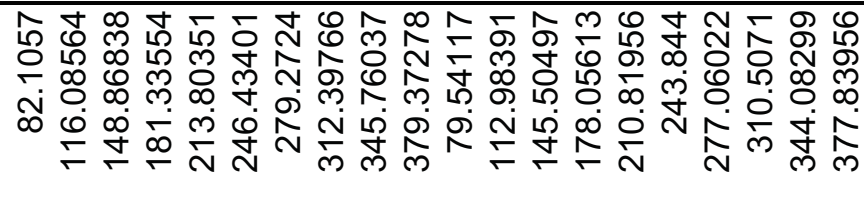 \\
\hline 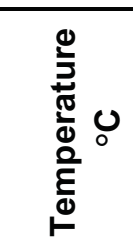 & 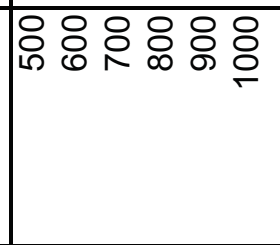 & 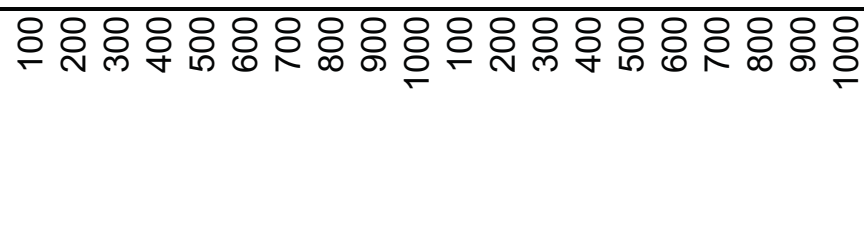 \\
\hline 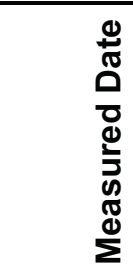 & & 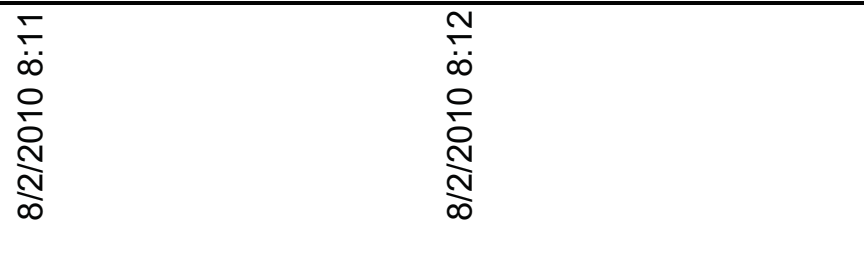 \\
\hline 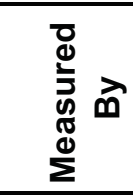 & & $\begin{array}{l}\stackrel{\mathscr{L}}{\stackrel{N}{f}} \\
\stackrel{f}{f}\end{array}$ \\
\hline 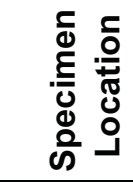 & & \\
\hline 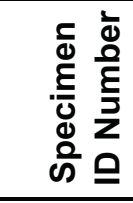 & & $\sum_{\substack{\circ \\
\vdots}}^{\circ}$ \\
\hline
\end{tabular}




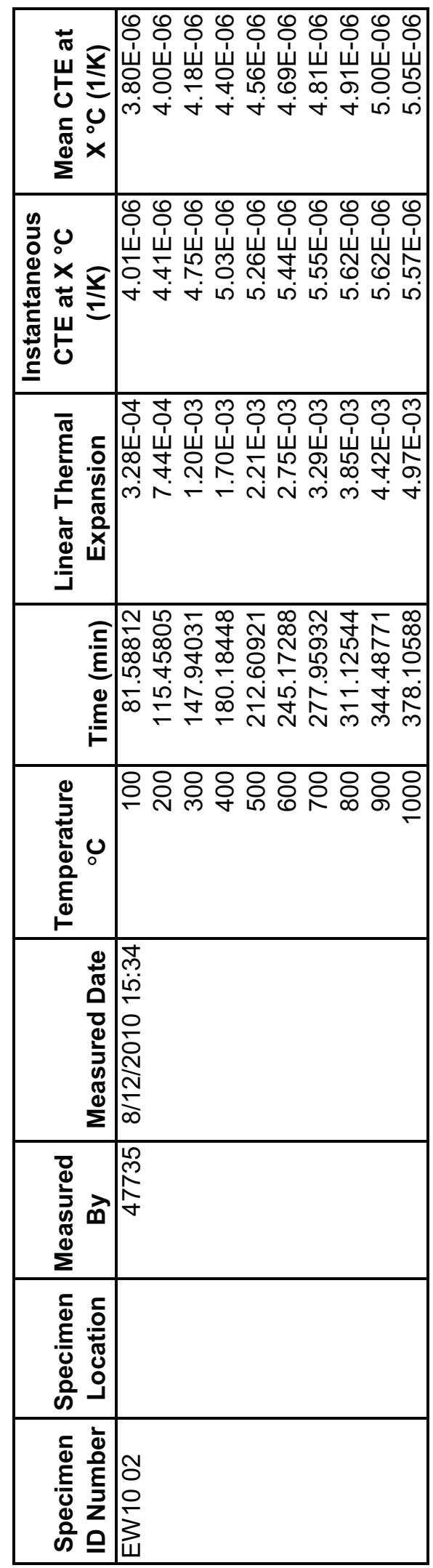




\begin{tabular}{|c|c|c|c|c|}
\hline 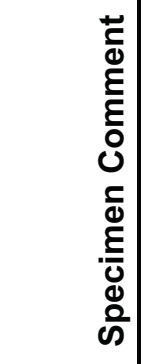 & & & & \\
\hline 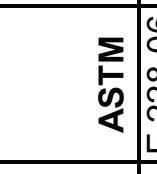 & \begin{tabular}{|l|}
0 \\
0 \\
1 \\
N \\
W \\
\end{tabular} & 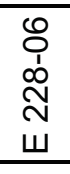 & 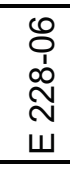 & $\begin{array}{l}\ddot{8} \\
0 \\
\stackrel{1}{N} \\
\text { w }\end{array}$ \\
\hline 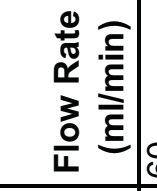 & 8 & 8 & 8 & 8 \\
\hline 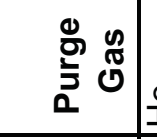 & $\underset{\Phi}{ \pm}$ & $\stackrel{\oplus}{I}$ & $\frac{0}{I}$ & $\stackrel{0}{I}$ \\
\hline 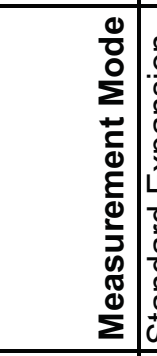 & 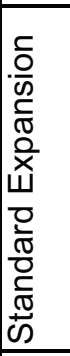 & 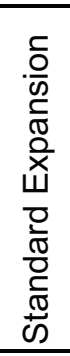 & $\begin{array}{l}\frac{c}{0} \\
\frac{0}{0} \\
\frac{0}{0} \\
\frac{0}{x} \\
w \\
\frac{0}{0} \\
\frac{0}{0} \\
\frac{0}{c} \\
\frac{w}{\omega 0} \\
\end{array}$ & 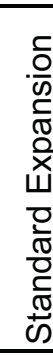 \\
\hline 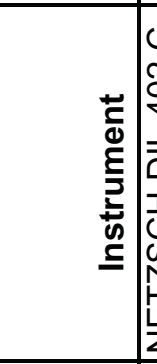 & 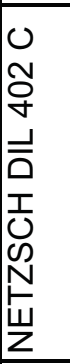 & 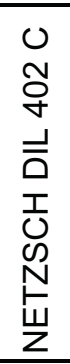 & 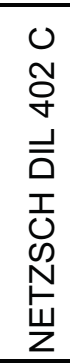 & 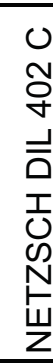 \\
\hline 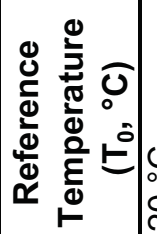 & $\begin{array}{l} \\
0 \\
0 \\
0 \\
\text { N }\end{array}$ & $\begin{array}{l}0 \\
0 \\
\text { N }\end{array}$ & $\begin{array}{l}0 \\
0 \\
\text { ON }\end{array}$ & $\begin{array}{l}0 \\
0 \\
\text { Oे }\end{array}$ \\
\hline 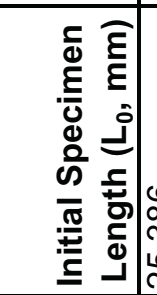 & 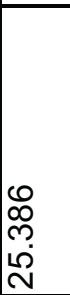 & $\begin{array}{l}\text { o } \\
\stackrel{\infty}{\infty} \\
\stackrel{N}{N}\end{array}$ & $\begin{array}{l}\infty \\
\stackrel{\infty}{\infty} \\
\stackrel{\rho}{N}\end{array}$ & 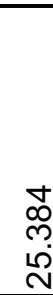 \\
\hline 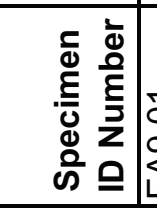 & \begin{tabular}{|l} 
\\
$\delta$ \\
0 \\
0 \\
W \\
\end{tabular} & 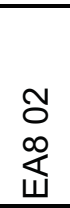 & $\begin{array}{l}5 \\
0 \\
\infty \\
\Psi \\
\end{array}$ & 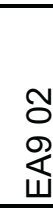 \\
\hline
\end{tabular}




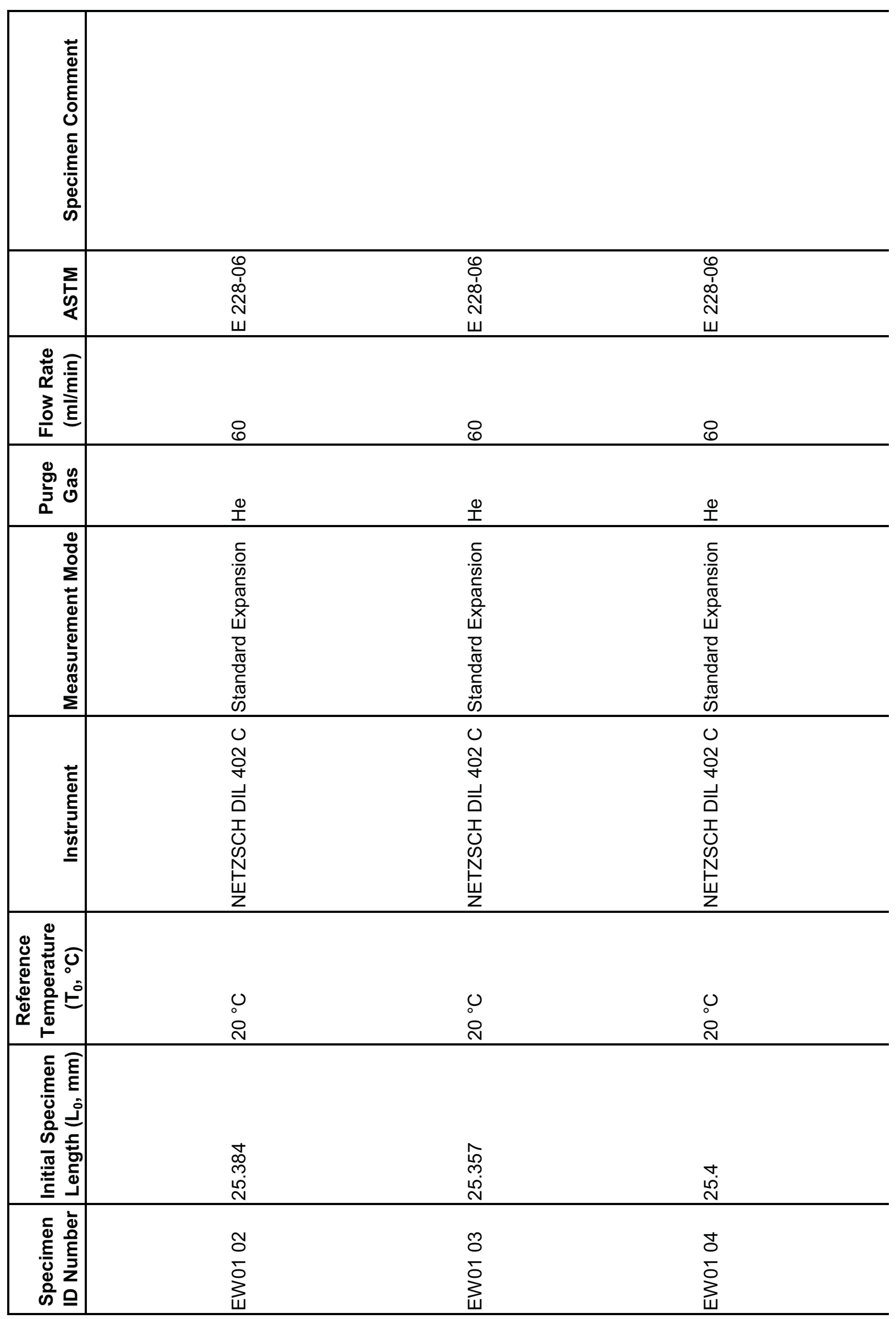

$\bar{n}$ 


\begin{tabular}{|c|c|c|c|c|}
\hline 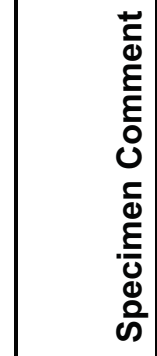 & & & & \\
\hline$\underset{\substack{\Sigma \\
\mathbf{S}}}{\Sigma}$ & $\begin{array}{l}\mathscr{Q} \\
0 \\
o \\
\text { N } \\
\Psi \\
\end{array}$ & $\begin{array}{l}\dot{Q} \\
0 \\
o \\
\text { N } \\
\Psi \\
\end{array}$ & 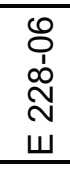 & 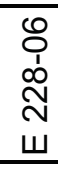 \\
\hline 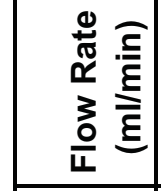 & 8 & 8 & 8 & 8 \\
\hline 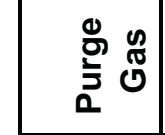 & $\frac{\Phi}{I}$ & 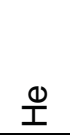 & 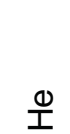 & 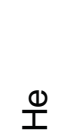 \\
\hline 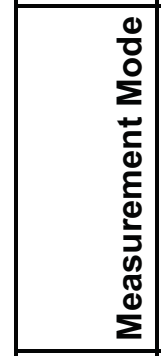 & 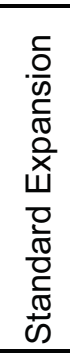 & 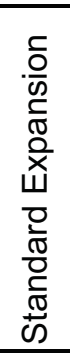 & 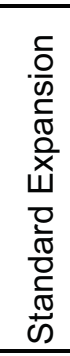 & 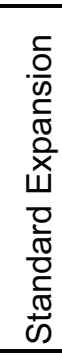 \\
\hline 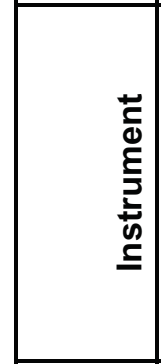 & 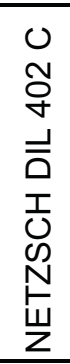 & 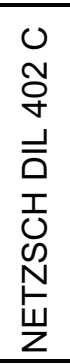 & 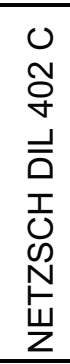 & 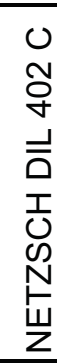 \\
\hline 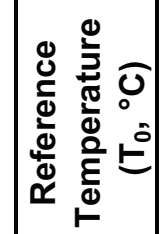 & $\begin{array}{l}0 \\
0 \\
\text { N }\end{array}$ & $\begin{array}{l}0 \\
0 \\
\text { N }\end{array}$ & $\begin{array}{l}0 \\
0 \\
\text { Oे }\end{array}$ & $\begin{array}{l}0 \\
0 \\
\text { N }\end{array}$ \\
\hline 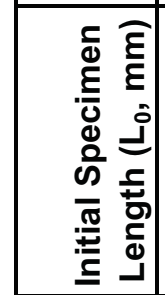 & 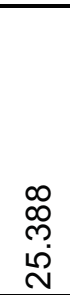 & $\begin{array}{l}\bar{\infty} \\
\stackrel{\infty}{\infty} \\
\stackrel{\rho}{.}\end{array}$ & 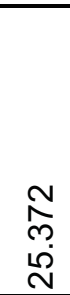 & $\begin{array}{l}\hat{N} \\
\stackrel{\rho}{\rho} \\
\text { Na }\end{array}$ \\
\hline 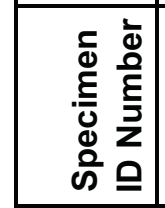 & $\begin{array}{l}\bar{o} \\
\tilde{N} \\
\sum_{u}\end{array}$ & $\begin{array}{l}\text { Oे } \\
\text { N } \\
\sum_{W} \\
\text { W }\end{array}$ & 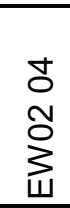 & $\begin{array}{l}\overline{0} \\
\text { Oू } \\
\sum_{w}\end{array}$ \\
\hline
\end{tabular}




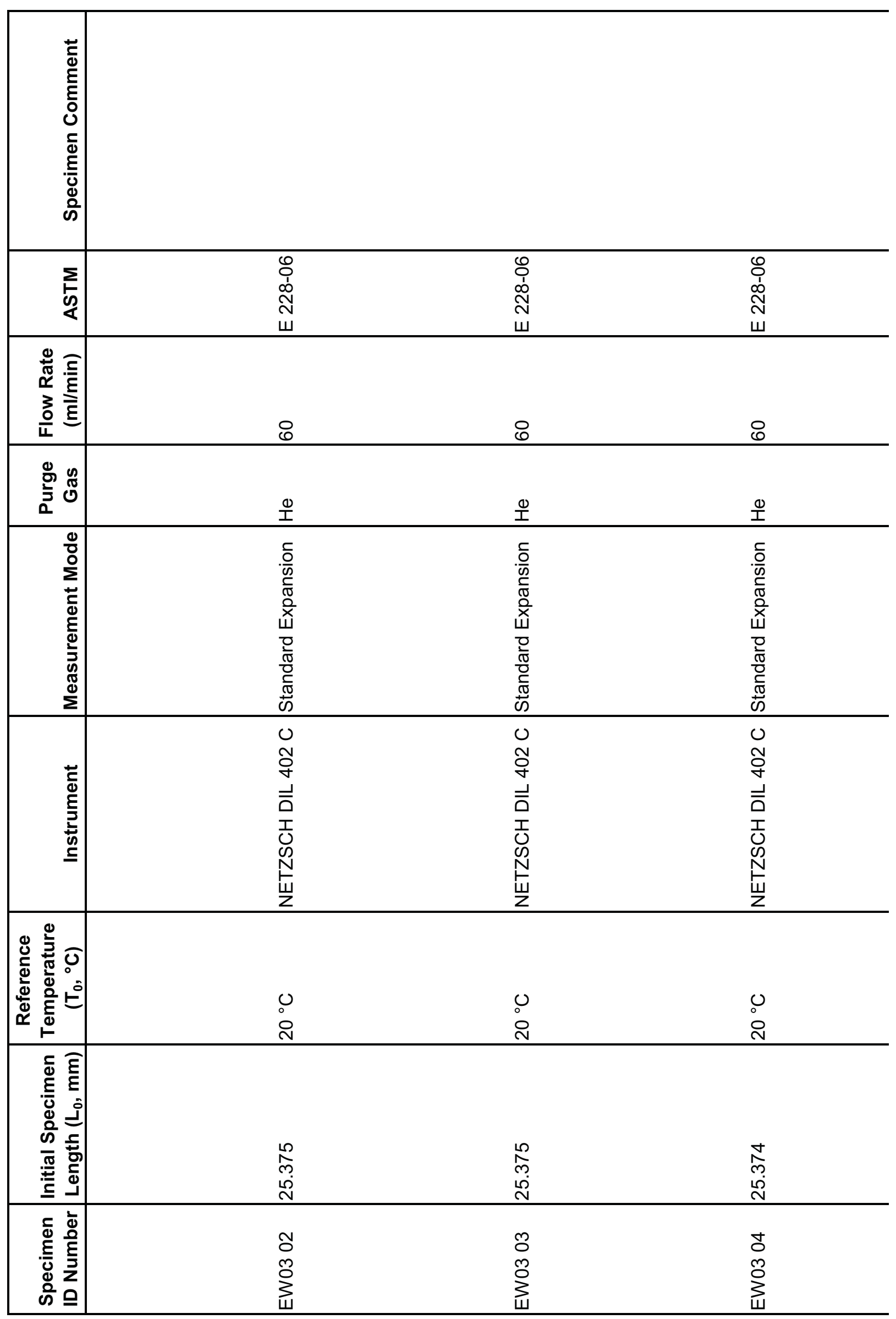




\begin{tabular}{|c|c|c|c|}
\hline 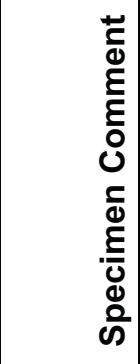 & & & \\
\hline 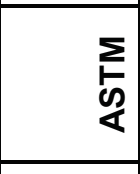 & $\begin{array}{l}\mathscr{Q} \\
0 \\
0 \\
\text { N } \\
w \\
\end{array}$ & $\begin{array}{l}\dot{Q} \\
0 \\
o \\
\text { N } \\
\Psi \\
\end{array}$ & $\begin{array}{l} \\
\\
0 \\
\text { Nิ } \\
\text { } \\
\end{array}$ \\
\hline 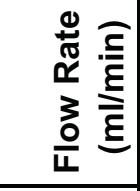 & 8 & 8 & 8 \\
\hline 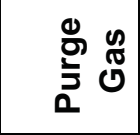 & $\stackrel{\oplus}{I}$ & $\frac{\circledast}{工}$ & $\stackrel{\oplus}{工}$ \\
\hline 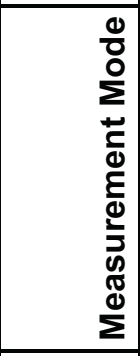 & 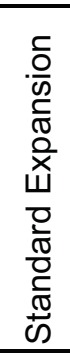 & 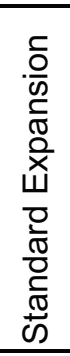 & 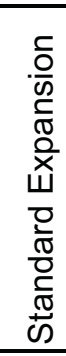 \\
\hline 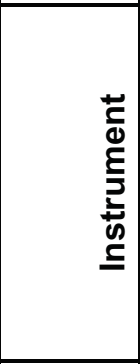 & 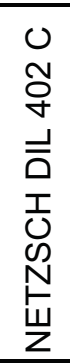 & 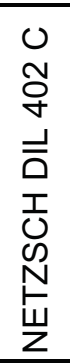 & 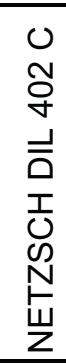 \\
\hline 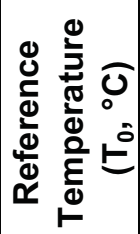 & $\begin{array}{l}0 \\
0 \\
\text { N }\end{array}$ & $\begin{array}{l}0 \\
0 \\
\text { N }\end{array}$ & $\begin{array}{l}0 \\
0 \\
\text { ON }\end{array}$ \\
\hline 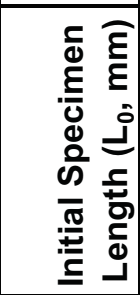 & $\begin{array}{l}\stackrel{0}{0} \\
\stackrel{m}{\stackrel{N}{N}}\end{array}$ & 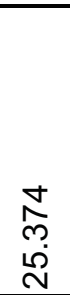 & $\begin{array}{l}\stackrel{\infty}{\infty} \\
\stackrel{\rho}{\sim} \\
\stackrel{\sim}{~}\end{array}$ \\
\hline 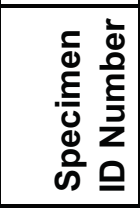 & 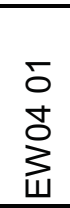 & $\begin{array}{l}\text { O } \\
\text { ¿े } \\
\text { 定 }\end{array}$ & 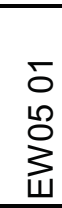 \\
\hline
\end{tabular}




\begin{tabular}{|c|c|c|c|c|}
\hline 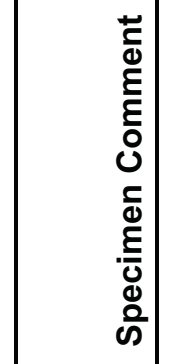 & & & & \\
\hline 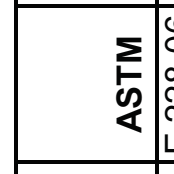 & \begin{tabular}{|l|}
0 \\
0 \\
1 \\
N \\
W \\
\end{tabular} & 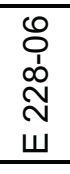 & 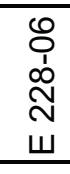 & 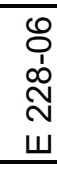 \\
\hline 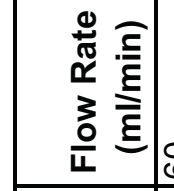 & 8 & 8 & 8 & 8 \\
\hline 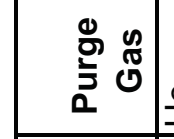 & $\underset{\Phi}{ \pm}$ & $\stackrel{\oplus}{I}$ & $\frac{0}{I}$ & $\stackrel{\oplus}{工}$ \\
\hline 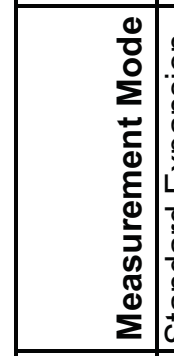 & 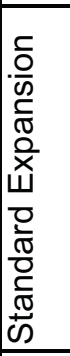 & 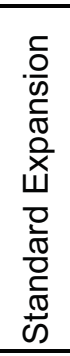 & $\begin{array}{l}\frac{c}{0} \\
\frac{0}{0} \\
\frac{0}{0} \\
\frac{0}{x} \\
w \\
\frac{0}{0} \\
\frac{0}{0} \\
\frac{0}{c} \\
\frac{w}{\omega 0} \\
\end{array}$ & 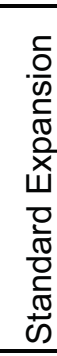 \\
\hline 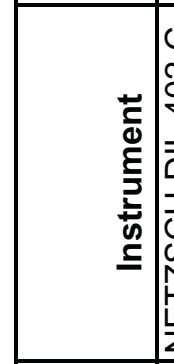 & 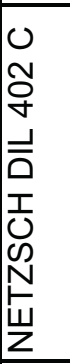 & 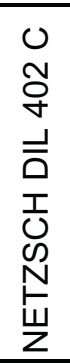 & 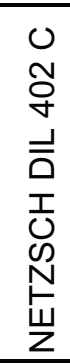 & 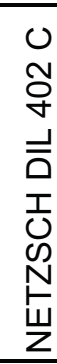 \\
\hline 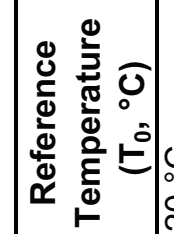 & $\begin{array}{l} \\
0 \\
0 \\
0 \\
\text { N }\end{array}$ & \begin{tabular}{l}
0 \\
0 \\
\multirow{N}{N}{}
\end{tabular} & $\begin{array}{l}0 \\
0 \\
\text { ON }\end{array}$ & $\begin{array}{l}0 \\
0 \\
0\end{array}$ \\
\hline 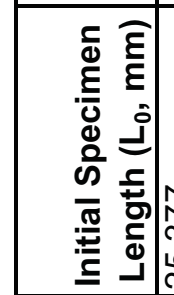 & 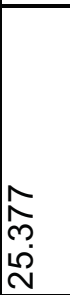 & $\begin{array}{l}\hat{N} \\
\stackrel{\omega}{\omega} \\
\stackrel{N}{N}\end{array}$ & 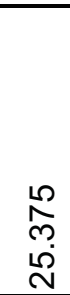 & 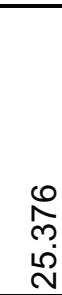 \\
\hline 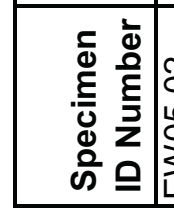 & 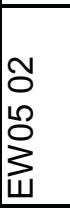 & 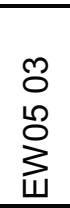 & 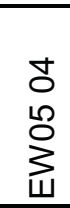 & 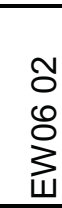 \\
\hline
\end{tabular}




\begin{tabular}{|c|c|c|c|}
\hline 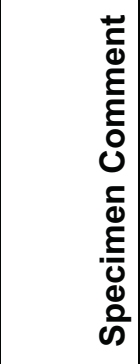 & & & \\
\hline 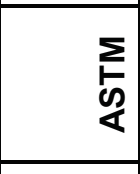 & 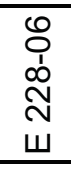 & 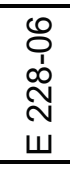 & 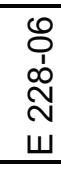 \\
\hline 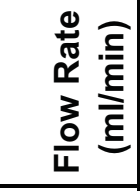 & 8 & 8 & 8 \\
\hline 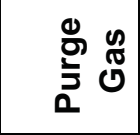 & $\frac{\Theta}{I}$ & $\stackrel{\oplus}{I}$ & $\frac{\Theta}{1}$ \\
\hline 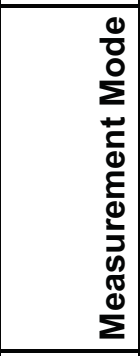 & 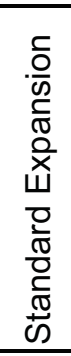 & 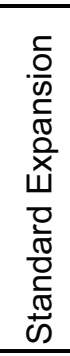 & 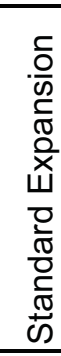 \\
\hline 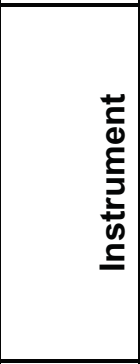 & 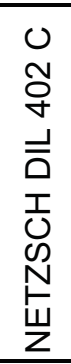 & 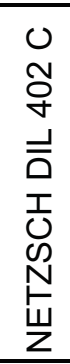 & 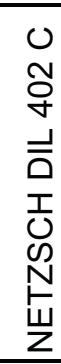 \\
\hline 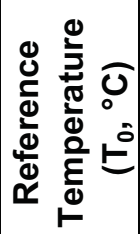 & \begin{tabular}{l}
0 \\
0 \\
\multirow{N}{*}{}
\end{tabular} & $\begin{array}{l}0 \\
0 \\
\text { Oे }\end{array}$ & $\begin{array}{l}0 \\
0 \\
\text { N }\end{array}$ \\
\hline 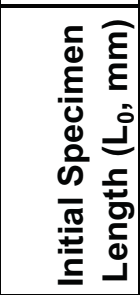 & 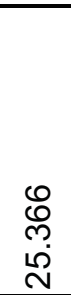 & $\begin{array}{l}0 \\
\stackrel{0}{0} \\
\stackrel{\rho}{N}\end{array}$ & 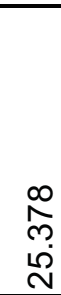 \\
\hline 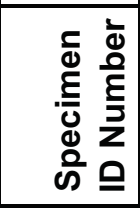 & 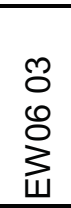 & 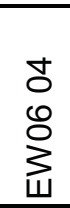 & $\begin{array}{l}\overline{0} \\
\stackrel{0}{0} \\
\sum_{\text {W }}\end{array}$ \\
\hline
\end{tabular}




\begin{tabular}{|c|c|c|c|c|}
\hline 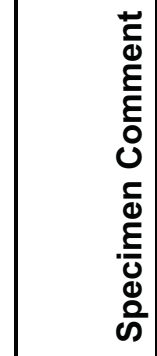 & & & & \\
\hline 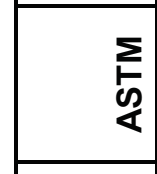 & $\begin{array}{l}\mathscr{Q} \\
0 \\
o \\
\text { N } \\
\Psi \\
\end{array}$ & $\begin{array}{l}\dot{Q} \\
0 \\
o \\
\text { N } \\
\Psi \\
\end{array}$ & 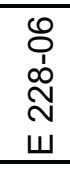 & 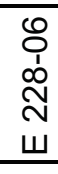 \\
\hline 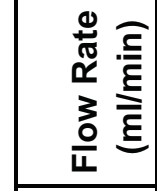 & 8 & 8 & 8 & 8 \\
\hline 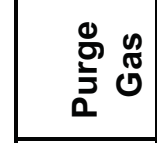 & $\stackrel{\oplus}{I}$ & $\stackrel{\oplus}{I}$ & $\underset{1}{\oplus}$ & $\frac{\oplus}{I}$ \\
\hline 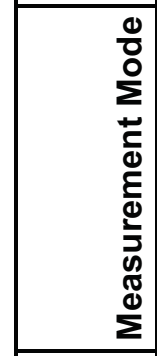 & 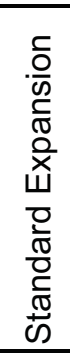 & 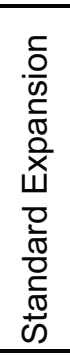 & 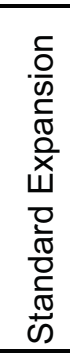 & 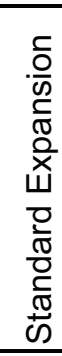 \\
\hline 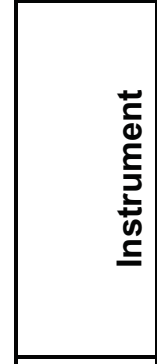 & 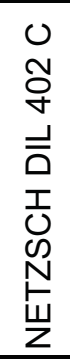 & 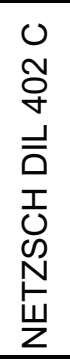 & 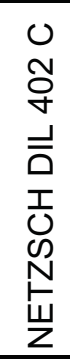 & 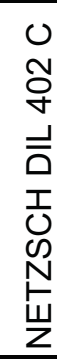 \\
\hline 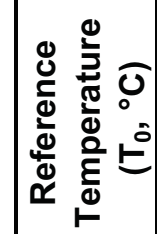 & $\begin{array}{l}0 \\
0 \\
\text { N }\end{array}$ & $\begin{array}{l}0 \\
0 \\
\text { N }\end{array}$ & $\begin{array}{l}0 \\
0 \\
\text { Oे }\end{array}$ & $\begin{array}{l}0 \\
0 \\
\text { N }\end{array}$ \\
\hline 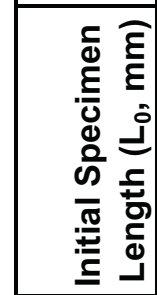 & 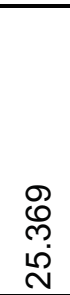 & $\begin{array}{l}\stackrel{\infty}{\infty} \\
\stackrel{\infty}{\infty} \\
\stackrel{n}{0}\end{array}$ & $\begin{array}{l}\infty \\
\infty \\
\infty \\
\stackrel{\rho}{N}\end{array}$ & $\begin{array}{l}\hat{N} \\
\stackrel{\rho}{\rho} \\
\text { Na }\end{array}$ \\
\hline 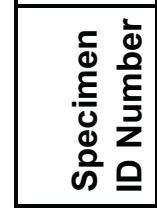 & $\begin{array}{l}\bar{\delta} \\
\hat{0} \\
\sum_{u}\end{array}$ & 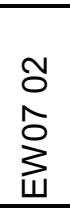 & $\begin{array}{l}0 \\
0 \\
\hat{0} \\
\text { 定 }\end{array}$ & $\begin{array}{l} \pm \\
\hat{0} \\
\sum_{W}^{3}\end{array}$ \\
\hline
\end{tabular}




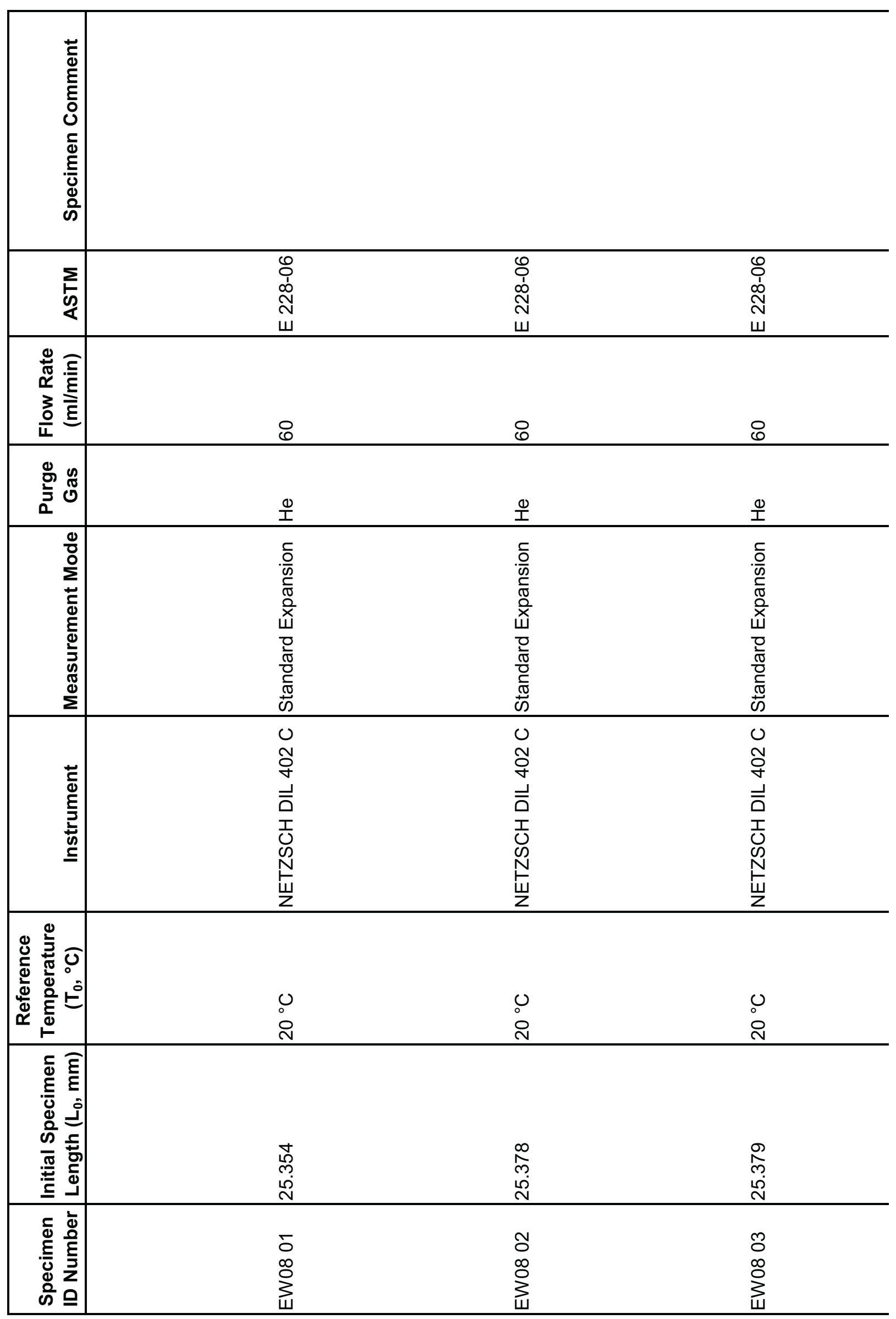




\begin{tabular}{|c|c|c|c|}
\hline 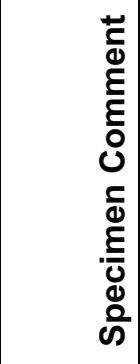 & & & \\
\hline 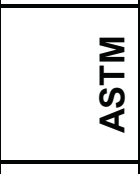 & $\begin{array}{l}\mathscr{Q} \\
0 \\
0 \\
\text { N } \\
w \\
\end{array}$ & 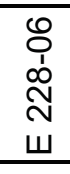 & $\begin{array}{l} \\
\\
0 \\
\text { Nิ } \\
\text { } \\
\end{array}$ \\
\hline 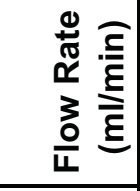 & 8 & 8 & 8 \\
\hline 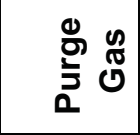 & $\stackrel{\oplus}{I}$ & $\frac{\Phi}{I}$ & $\stackrel{\oplus}{工}$ \\
\hline 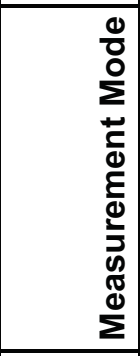 & 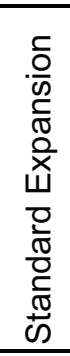 & 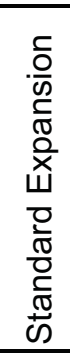 & 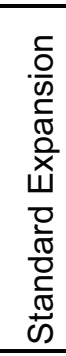 \\
\hline 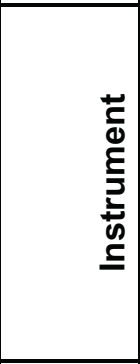 & 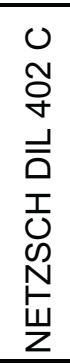 & 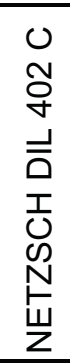 & 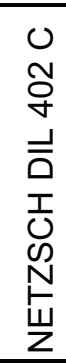 \\
\hline 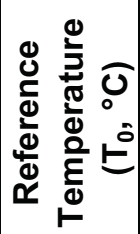 & $\begin{array}{l}0 \\
0 \\
\text { N }\end{array}$ & $\begin{array}{l}0 \\
0 \\
\text { Oे }\end{array}$ & $\begin{array}{l}0 \\
0 \\
\text { ON }\end{array}$ \\
\hline 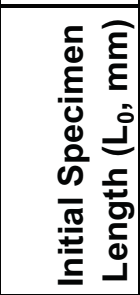 & $\begin{array}{l}0 \\
\stackrel{0}{0} \\
\stackrel{d}{d}\end{array}$ & $\begin{array}{l}\stackrel{0}{\rho} \\
\stackrel{\rho}{\rho} \\
\stackrel{N}{N}\end{array}$ & $\begin{array}{l}\stackrel{\infty}{\infty} \\
\stackrel{\rho}{\sim} \\
\stackrel{\sim}{~}\end{array}$ \\
\hline 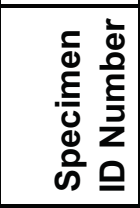 & 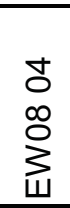 & 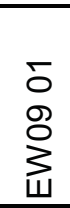 & $\begin{array}{l}\text { O } \\
\text { ஓे } \\
\sum_{\text {¿े }}\end{array}$ \\
\hline
\end{tabular}




\begin{tabular}{|c|c|c|c|c|}
\hline 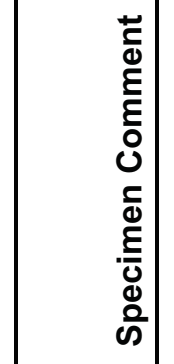 & & & & \\
\hline 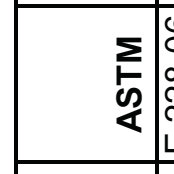 & \begin{tabular}{|l|}
0 \\
0 \\
1 \\
N \\
W \\
\end{tabular} & 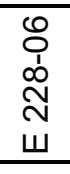 & 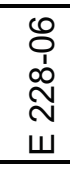 & 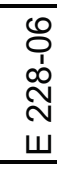 \\
\hline 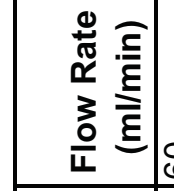 & 8 & 8 & 8 & 8 \\
\hline 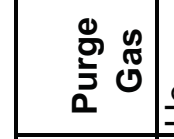 & $\underset{\Phi}{ \pm}$ & $\stackrel{\oplus}{I}$ & $\frac{0}{I}$ & $\stackrel{\oplus}{工}$ \\
\hline 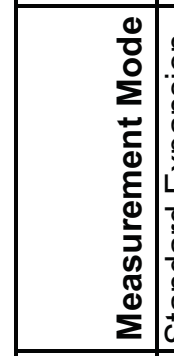 & 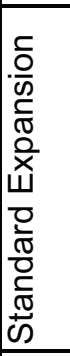 & 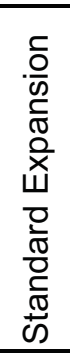 & $\begin{array}{l}\frac{c}{0} \\
\frac{0}{0} \\
\frac{0}{0} \\
\frac{0}{x} \\
w \\
\frac{0}{0} \\
\frac{0}{0} \\
\frac{0}{c} \\
\frac{w}{\omega 0} \\
\end{array}$ & 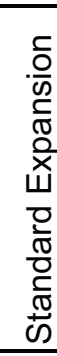 \\
\hline 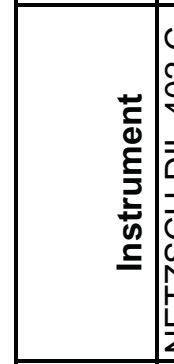 & 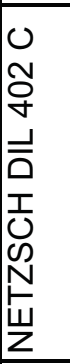 & 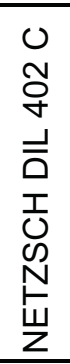 & 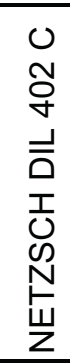 & 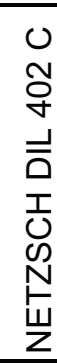 \\
\hline 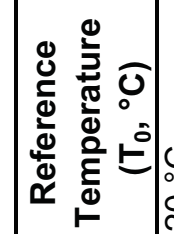 & $\begin{array}{l} \\
0 \\
0 \\
0 \\
\text { N }\end{array}$ & \begin{tabular}{l}
0 \\
0 \\
\multirow{N}{N}{}
\end{tabular} & $\begin{array}{l}0 \\
0 \\
\text { ON }\end{array}$ & $\begin{array}{l}0 \\
0 \\
0\end{array}$ \\
\hline 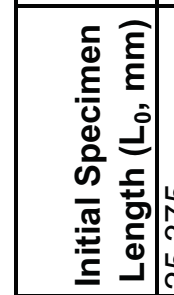 & 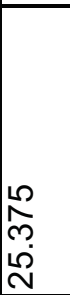 & $\begin{array}{l}\stackrel{R}{2} \\
\stackrel{\omega}{\rho} \\
\stackrel{d}{N}\end{array}$ & $\begin{array}{l}\bar{N} \\
\text { D. } \\
\stackrel{N}{N}\end{array}$ & 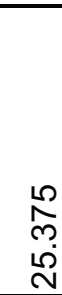 \\
\hline 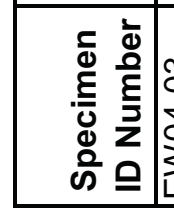 & \begin{tabular}{|l} 
\\
0 \\
0 \\
0 \\
\\
\\
\end{tabular} & 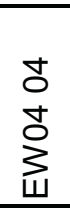 & 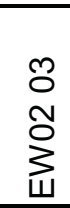 & 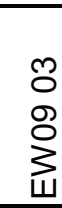 \\
\hline
\end{tabular}




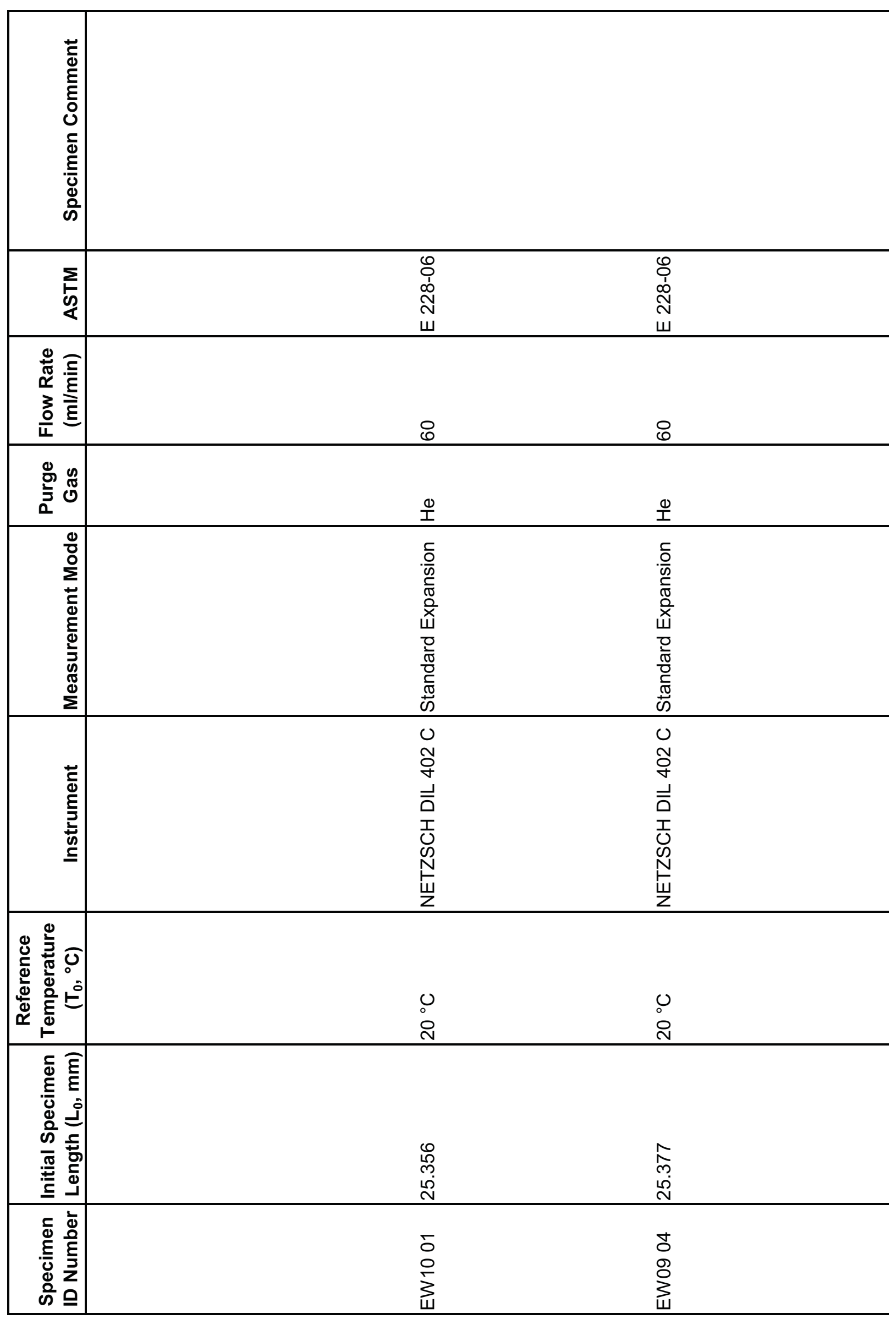




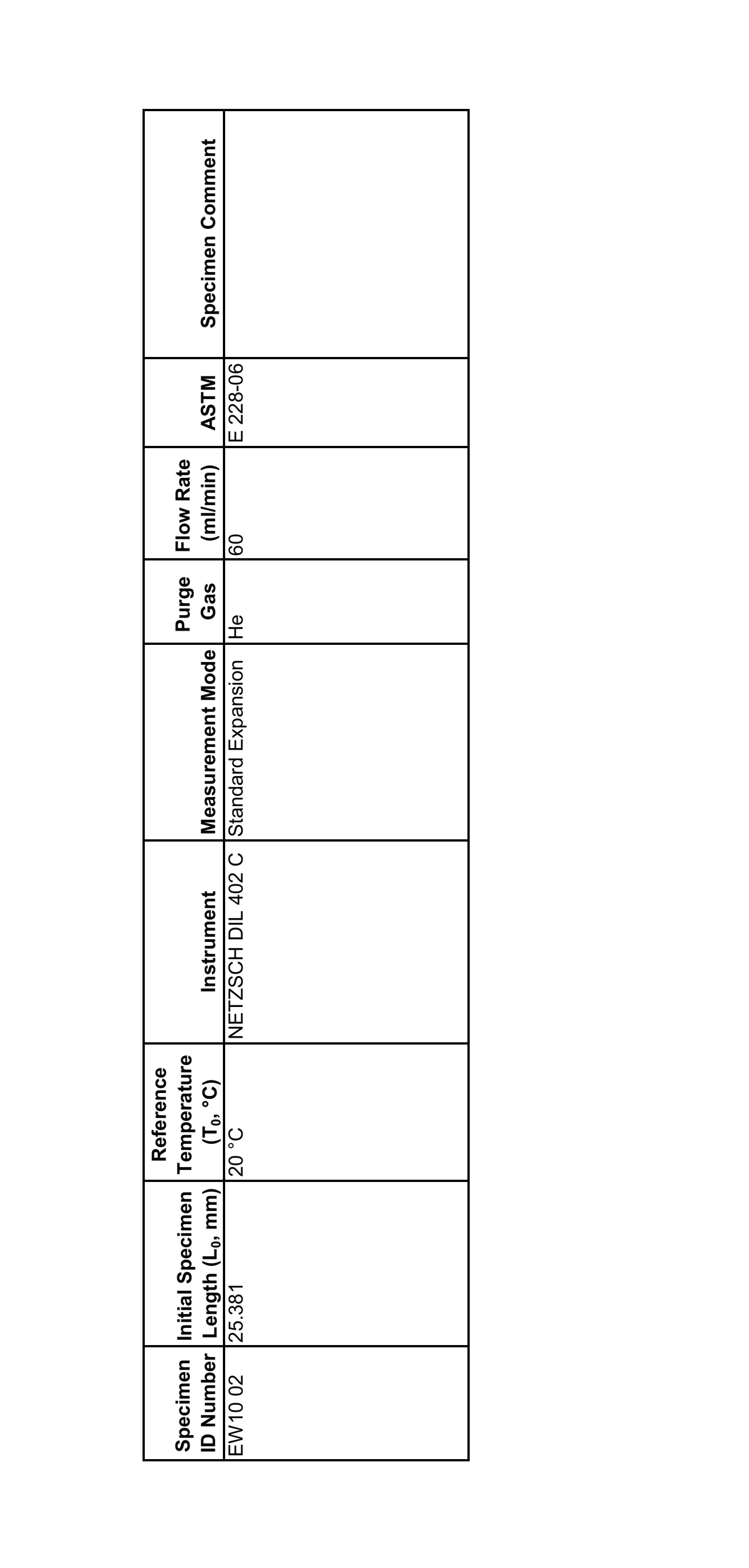




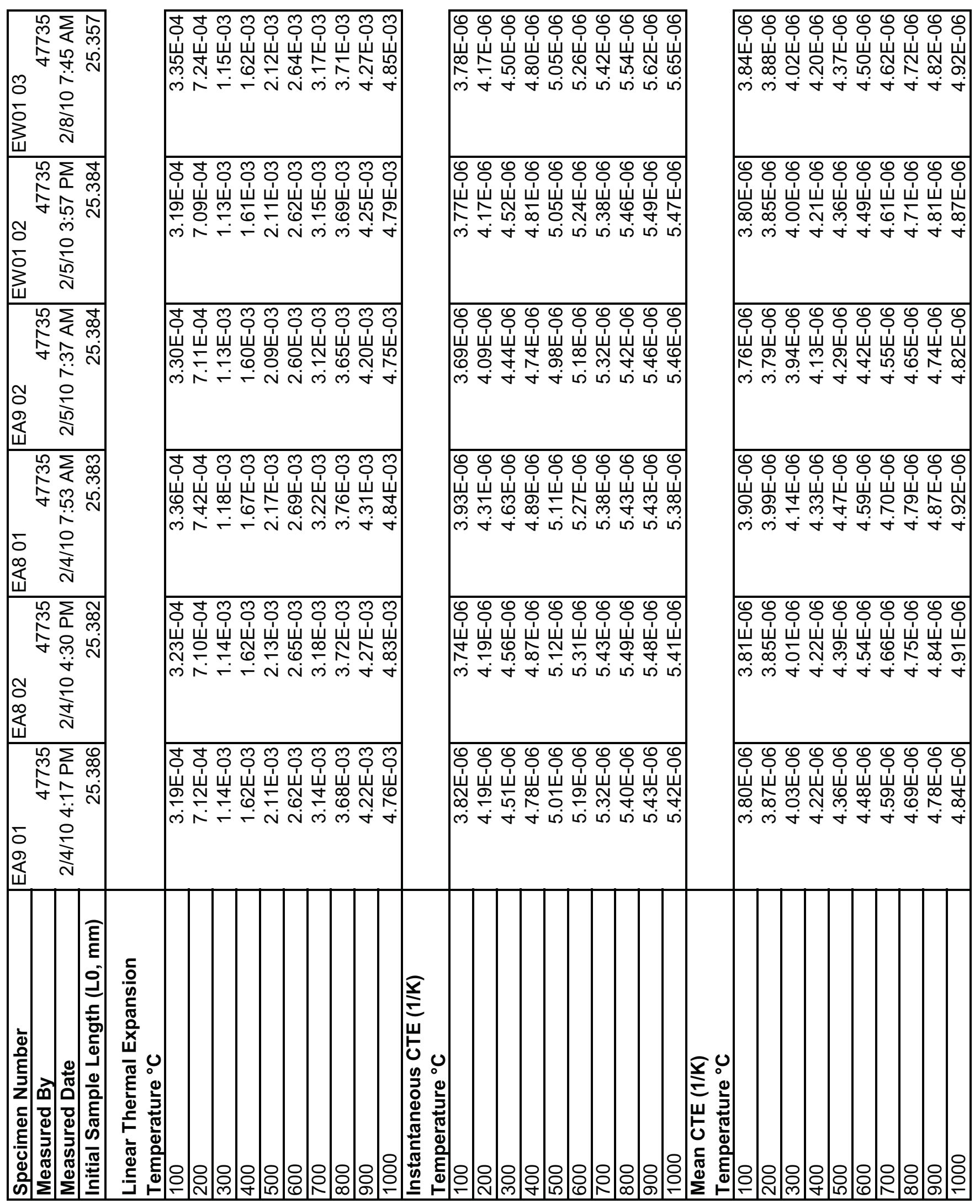




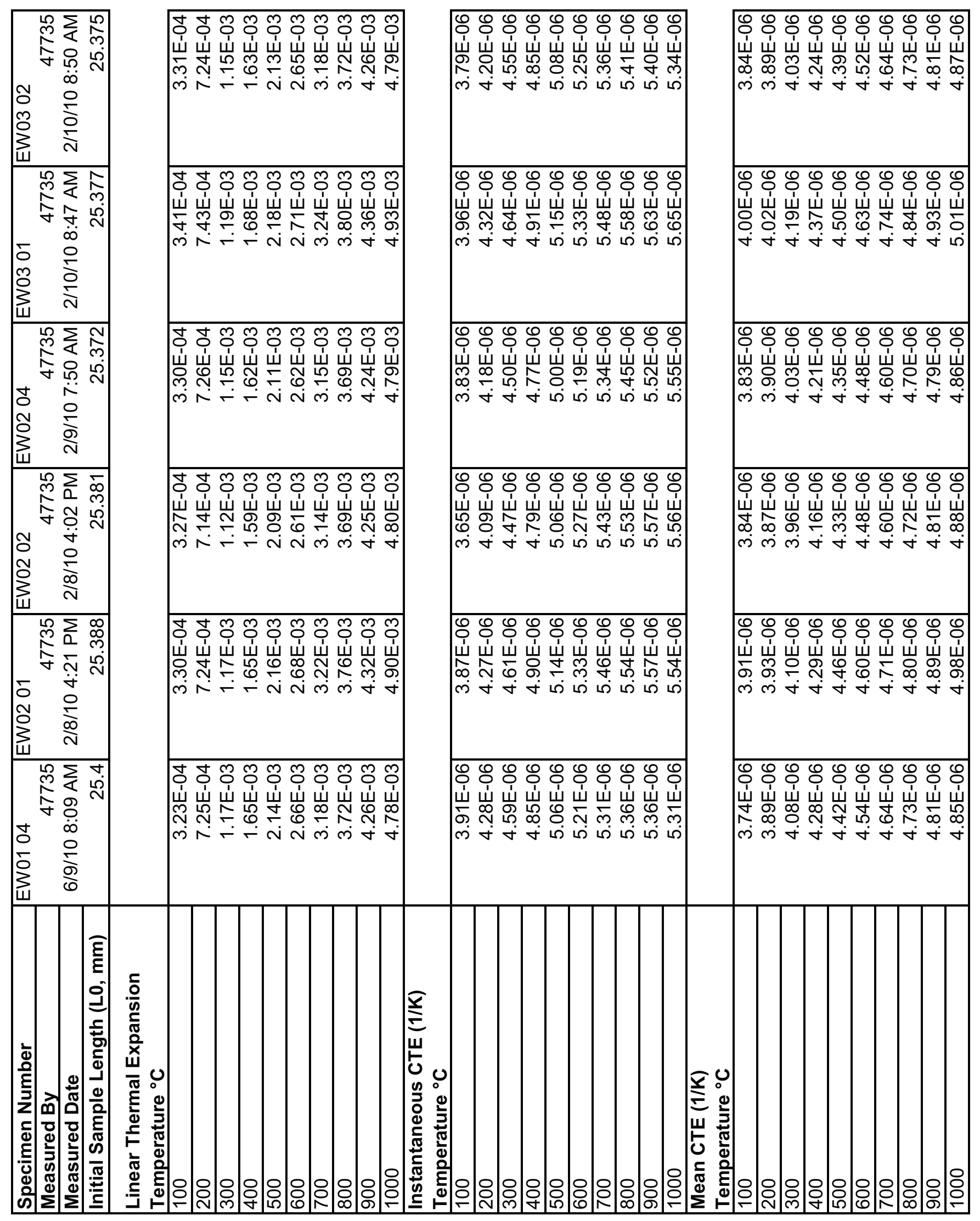




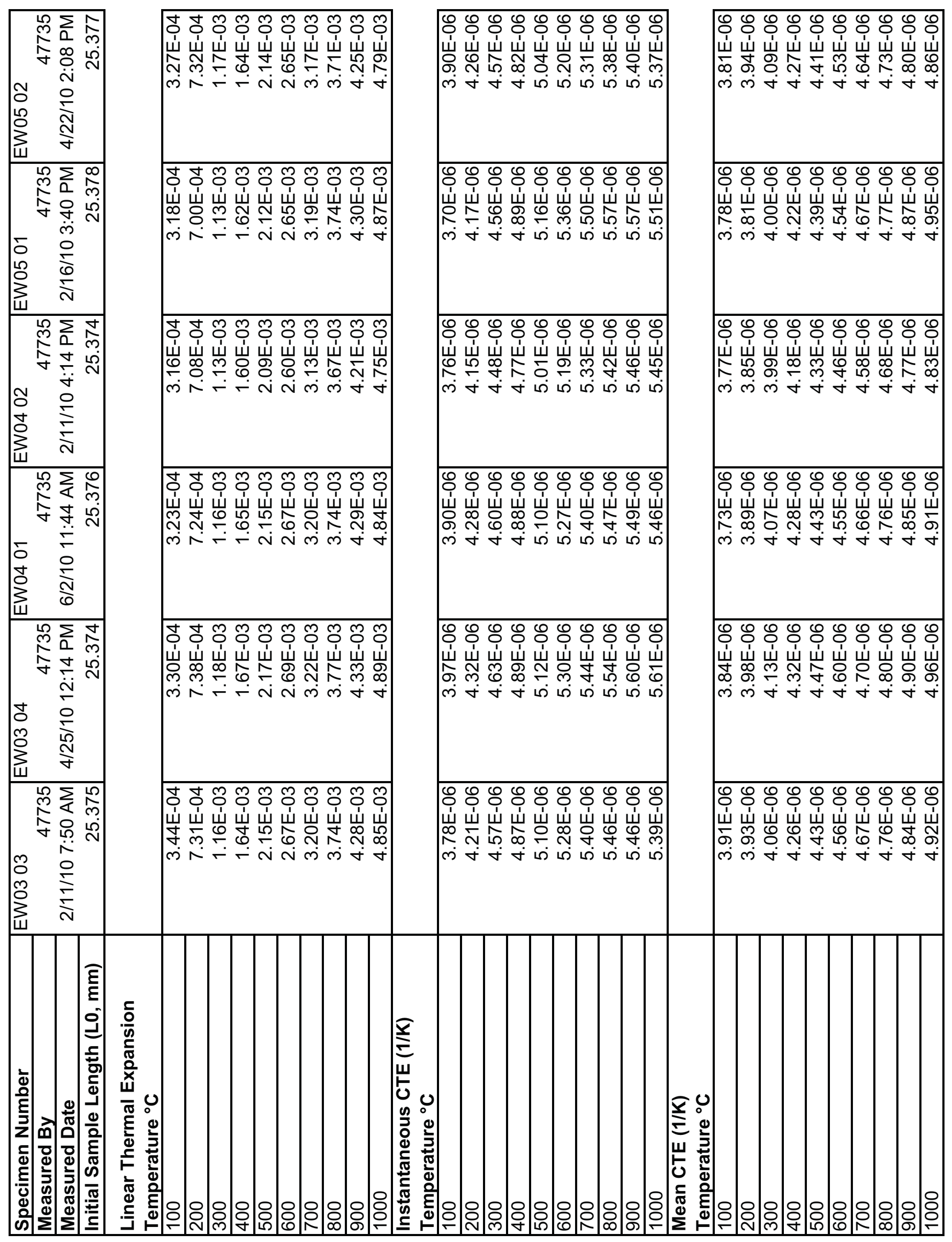




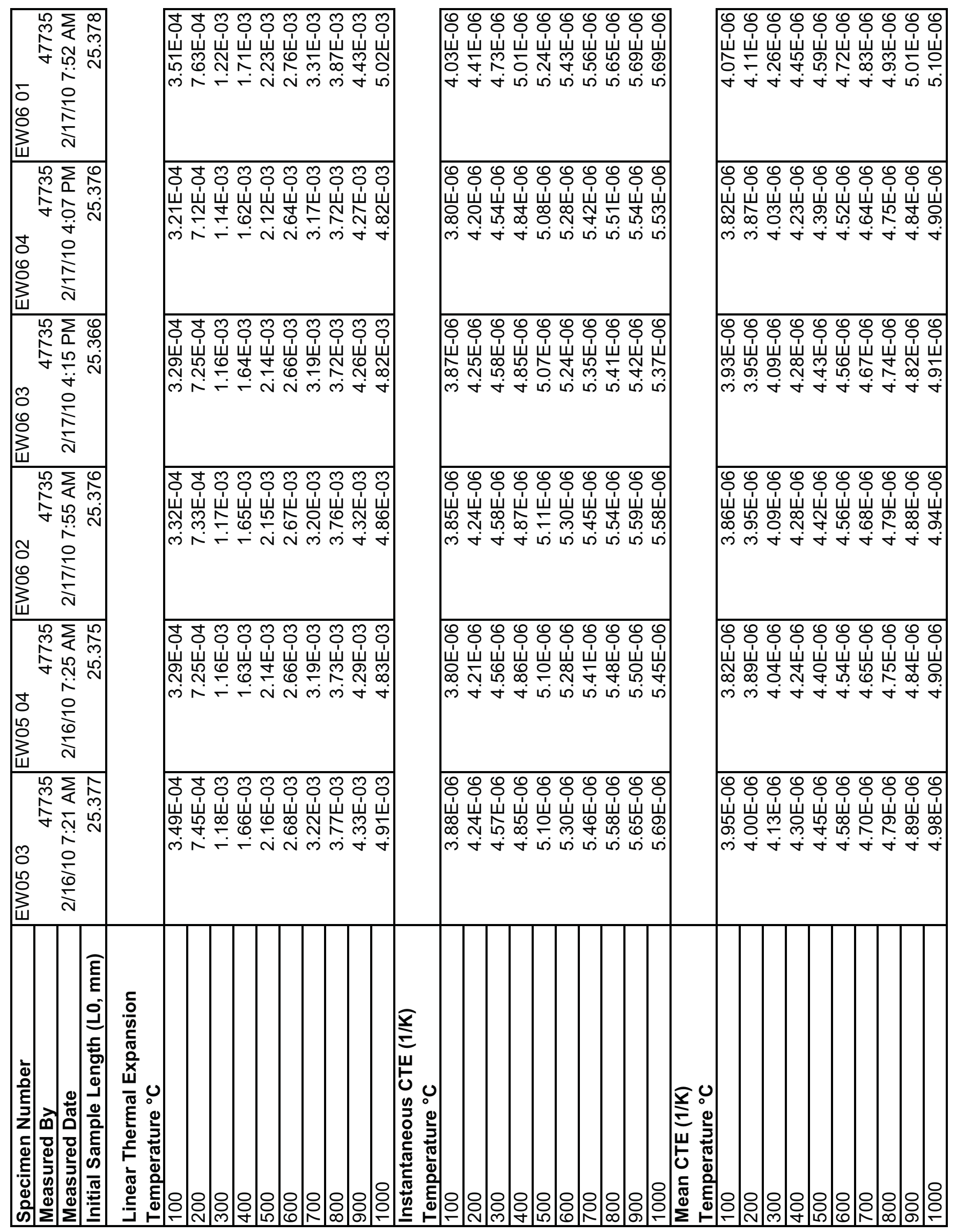




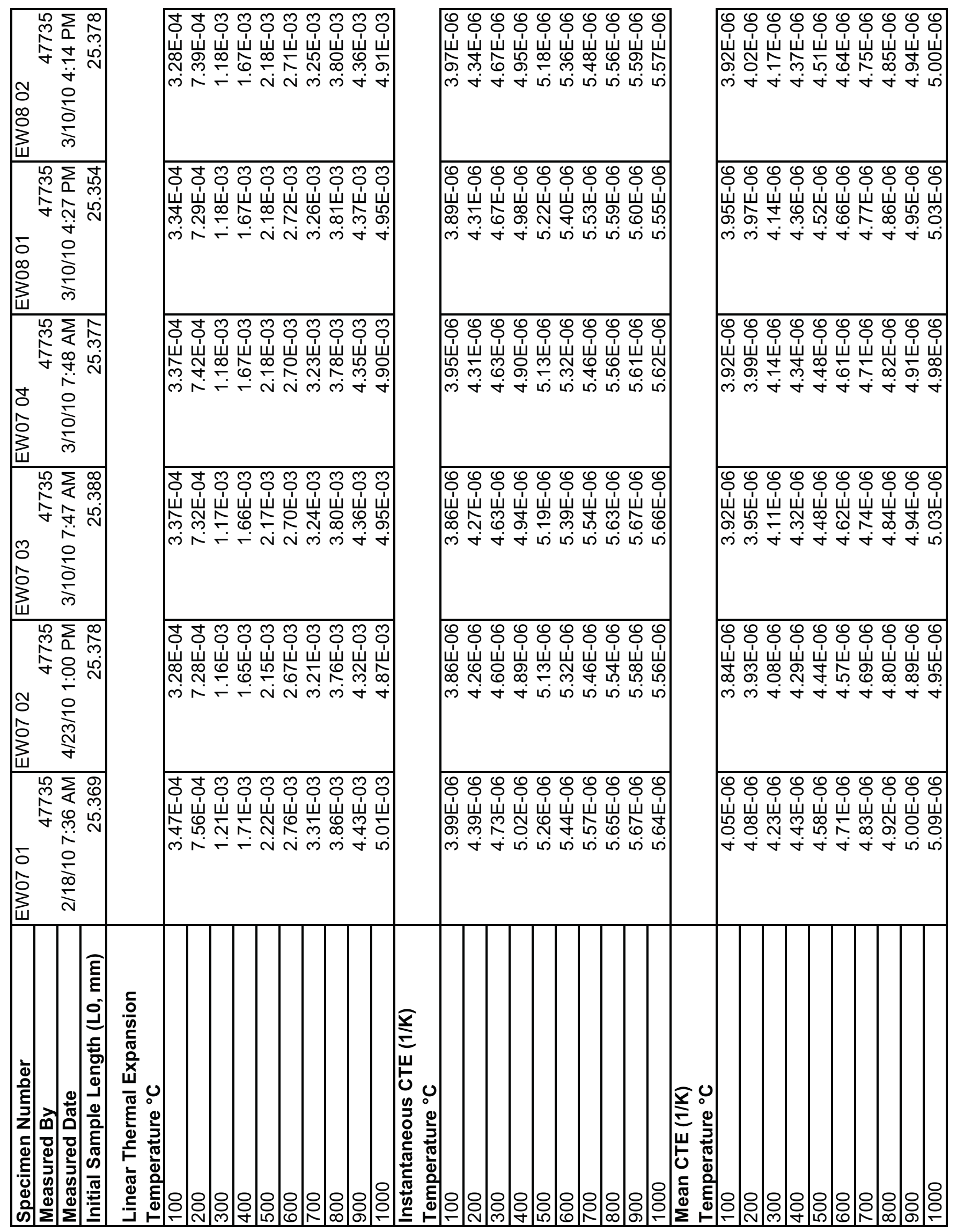




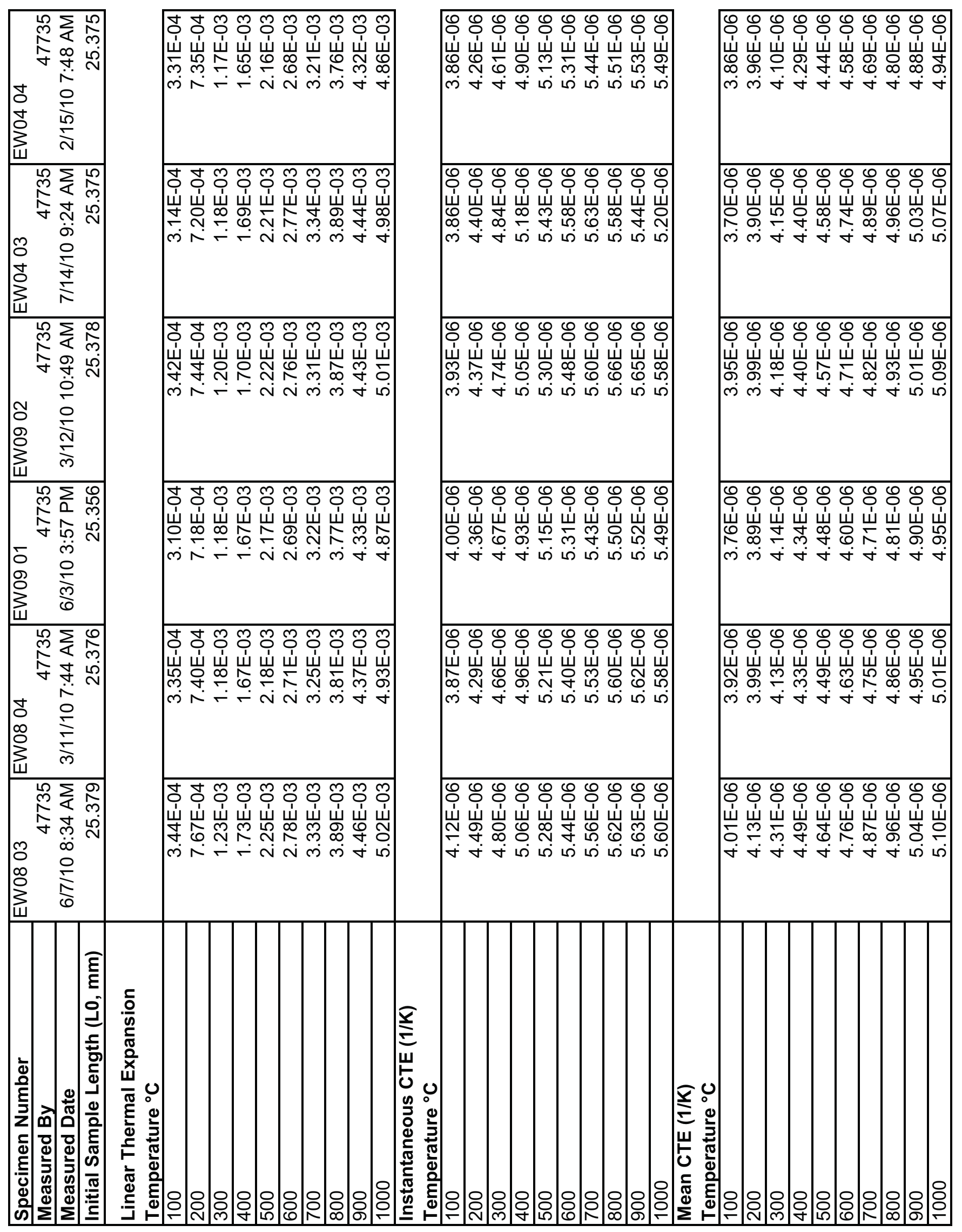




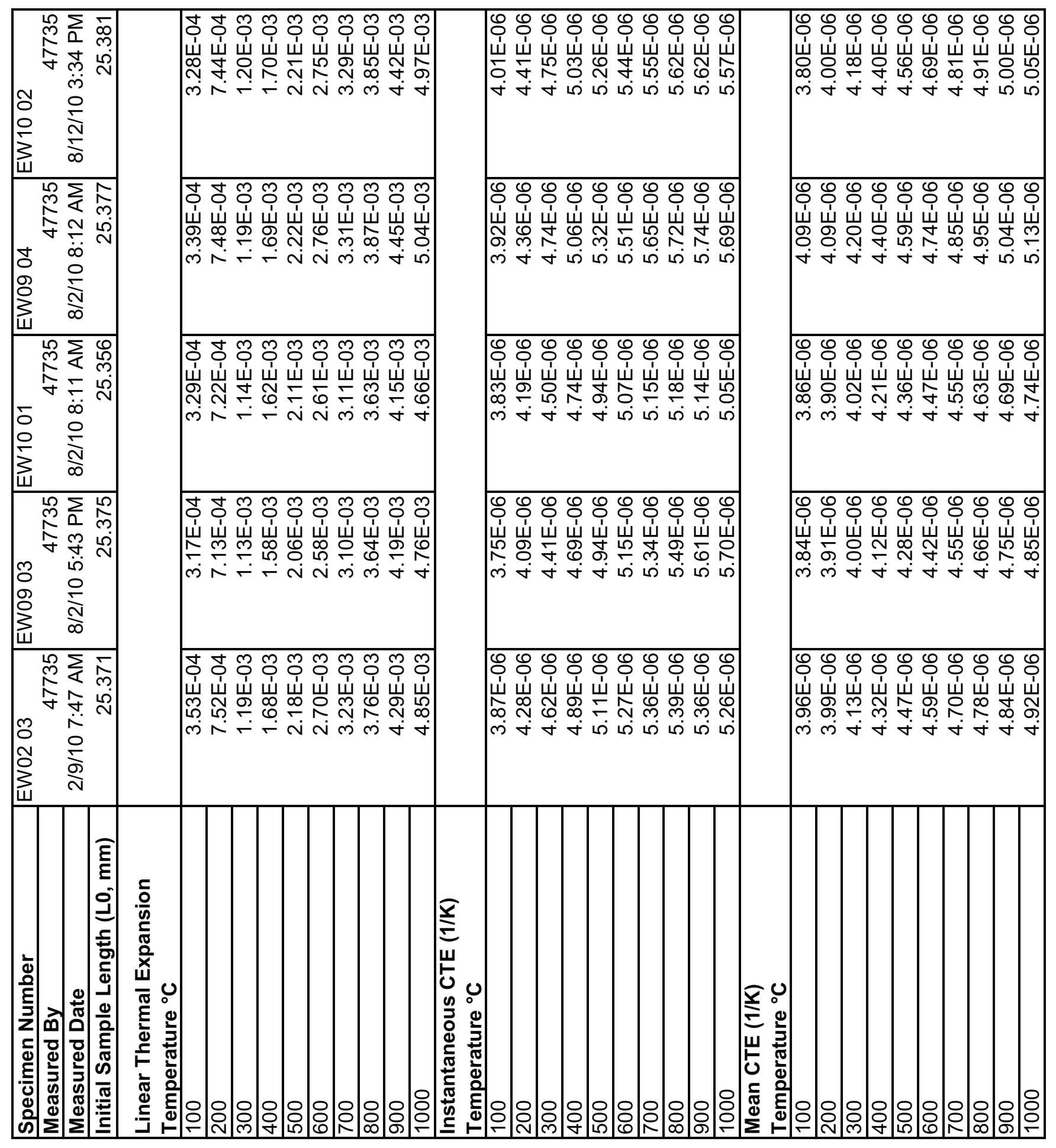




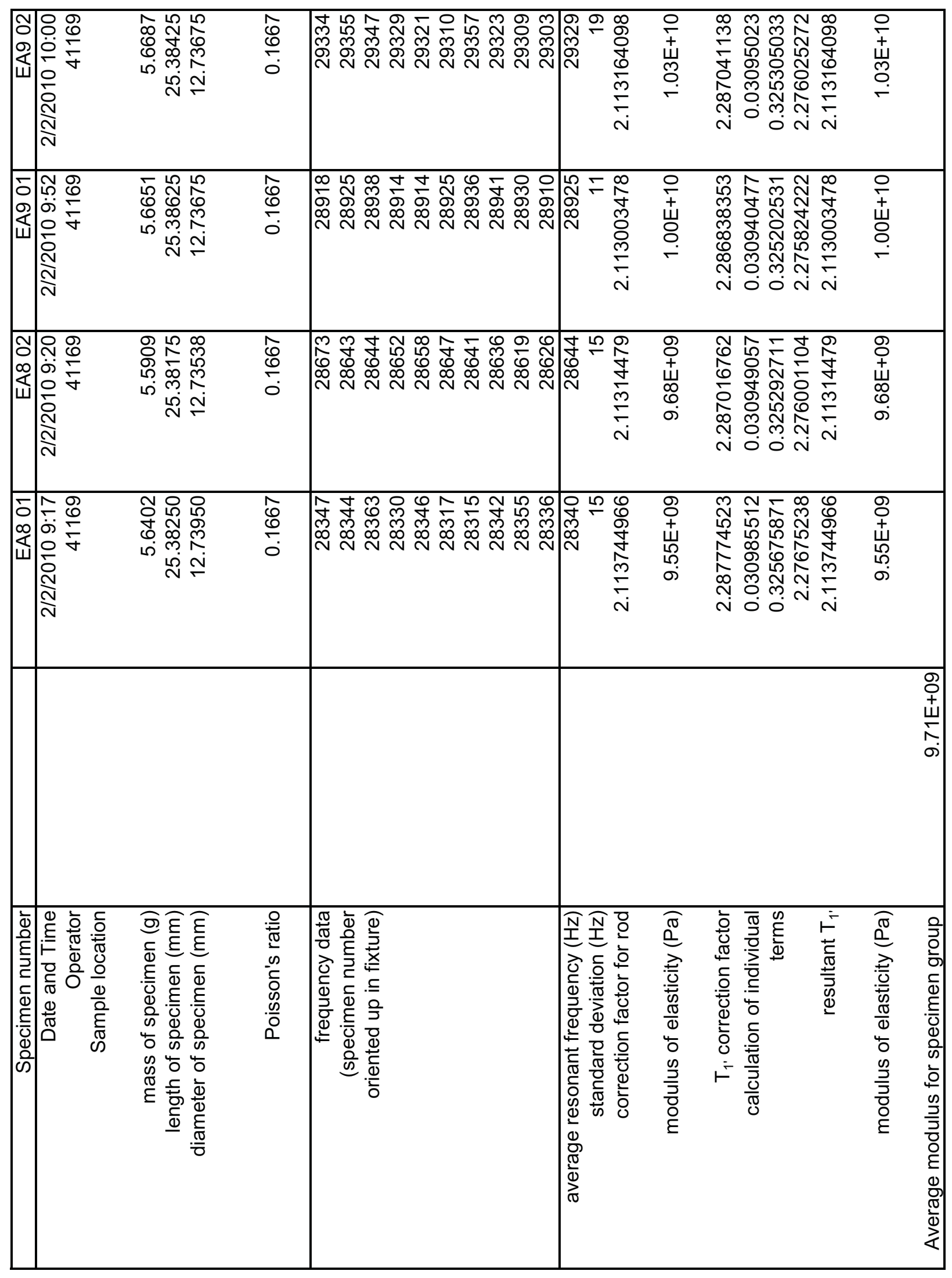




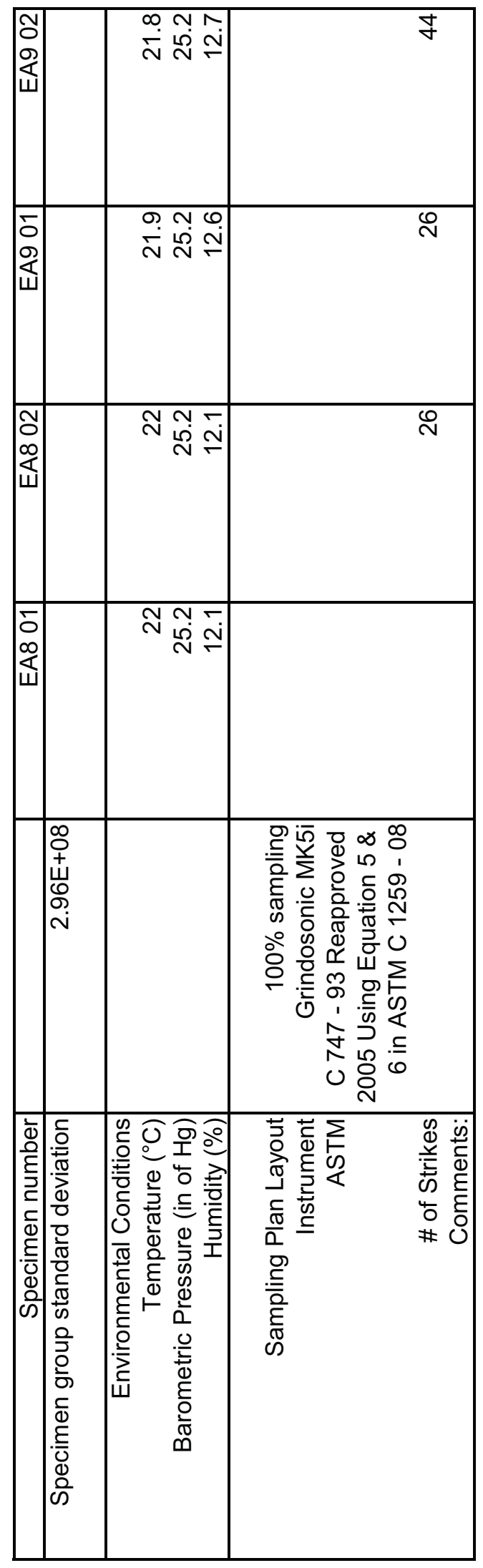




\begin{tabular}{|c|c|c|c|c|c|c|c|c|c|}
\hline 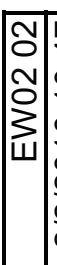 & 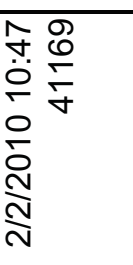 & 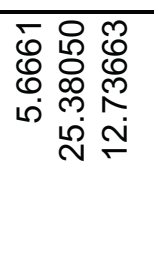 & $\begin{array}{l}\hat{\theta} \\
\frac{0}{0}\end{array}$ & 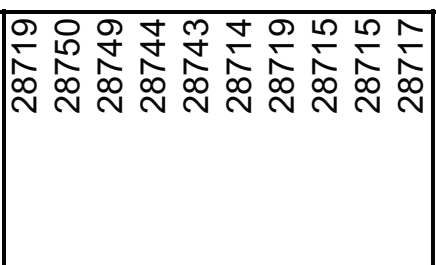 & 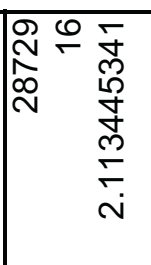 & $\begin{array}{l}\stackrel{9}{0} \\
+ \\
W \\
\infty \\
\infty \\
\infty\end{array}$ & 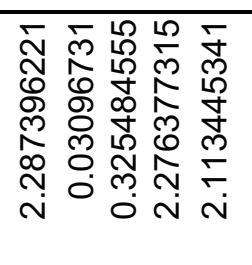 & 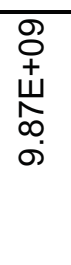 & \\
\hline 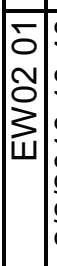 & 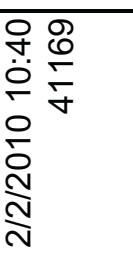 & 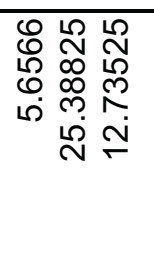 & $\begin{array}{l}\hat{0} \\
\stackrel{0}{0} \\
0\end{array}$ & 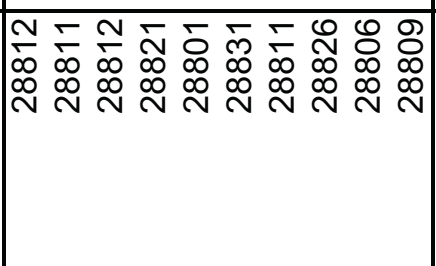 & 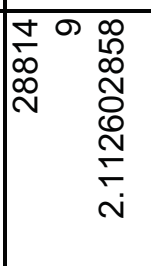 & $\begin{array}{l}\text { 8 } \\
+ \\
\dot{+} \\
\text { N } \\
\text { ஸ் }\end{array}$ & 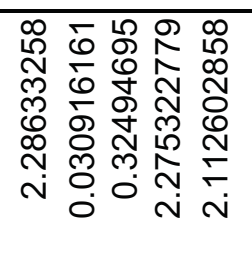 & $\begin{array}{l}\text { 8 } \\
+ \\
\dot{+} \\
\text { N } \\
\text { ஸ் }\end{array}$ & \\
\hline 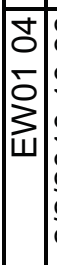 & 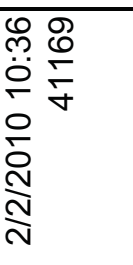 & 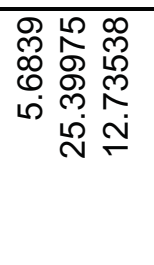 & $\begin{array}{l}\hat{\mathscr{\theta}} \\
\underline{0} \\
\dot{0}\end{array}$ & 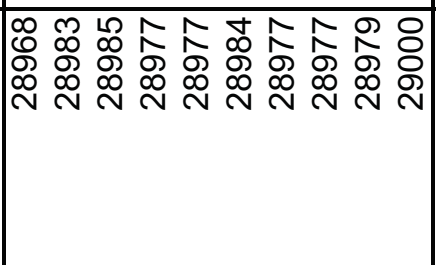 & 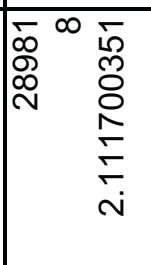 & $\begin{array}{l}\text { 음 } \\
+ \\
\stackrel{+}{\circ} \\
\stackrel{+}{-}\end{array}$ & 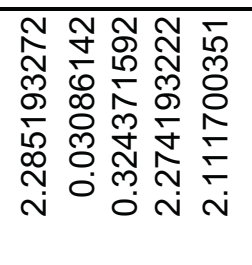 & $\begin{array}{l}\stackrel{\circ}{+} \\
\stackrel{+}{\sigma} \\
\stackrel{0}{\circ}\end{array}$ & \\
\hline 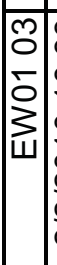 & 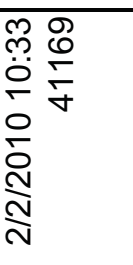 & 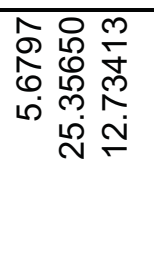 & $\begin{array}{l}\hat{\theta} \\
\stackrel{0}{0} \\
\vdots\end{array}$ & 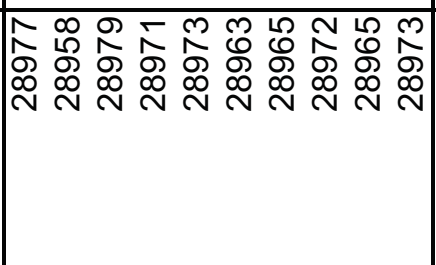 & 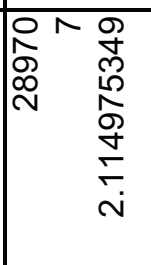 & 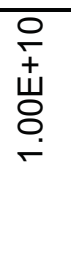 & 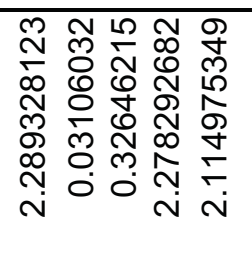 & $\begin{array}{l}\stackrel{\circ}{+} \\
+ \\
\dot{+} \\
\stackrel{\circ}{\circ}\end{array}$ & \\
\hline 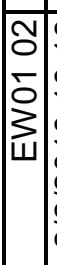 & 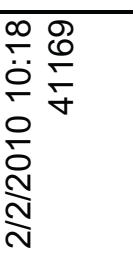 & 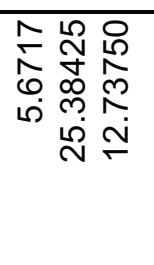 & $\begin{array}{l}\hat{\mathscr{\theta}} \\
\stackrel{0}{0}\end{array}$ & 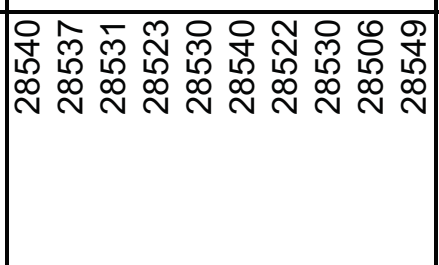 & 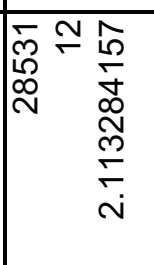 & \begin{tabular}{l}
8 \\
0 \\
+ \\
\multirow{+}{*}{} \\
0
\end{tabular} & 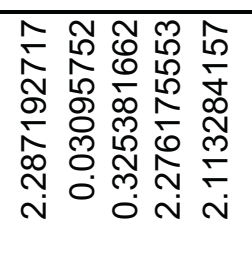 & \begin{tabular}{l} 
O \\
+ \\
$\Psi$ \\
\multirow{+}{+}{} \\
क
\end{tabular} & \\
\hline 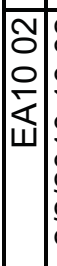 & 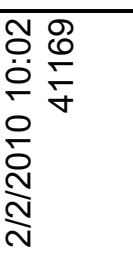 & 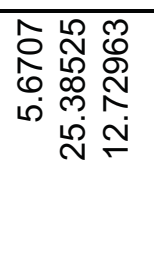 & $\begin{array}{l}\hat{0} \\
\stackrel{0}{0} \\
0\end{array}$ & 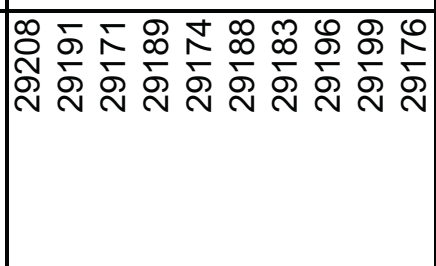 & 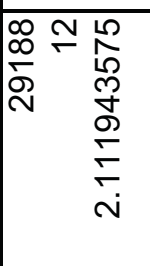 & $\begin{array}{l}\text { 음 } \\
\stackrel{+}{W} \\
\stackrel{ }{\circ}\end{array}$ & 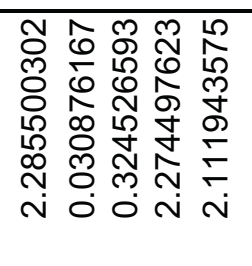 & $\begin{array}{l}\text { 음 } \\
+ \\
\stackrel{+}{N} \\
\stackrel{0}{-}\end{array}$ & \\
\hline 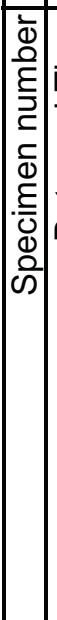 & 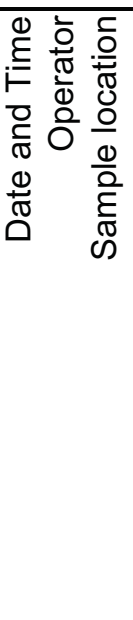 & 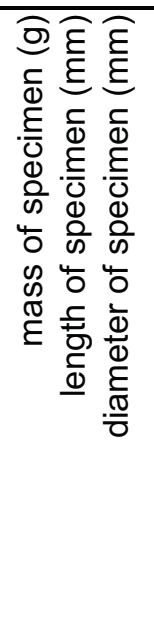 & $\begin{array}{l}.0 \\
\frac{0}{0} \\
\frac{0}{0} \\
-\frac{1}{0} \\
0 \\
0 \\
\frac{\infty}{0} \\
0 \\
0\end{array}$ & 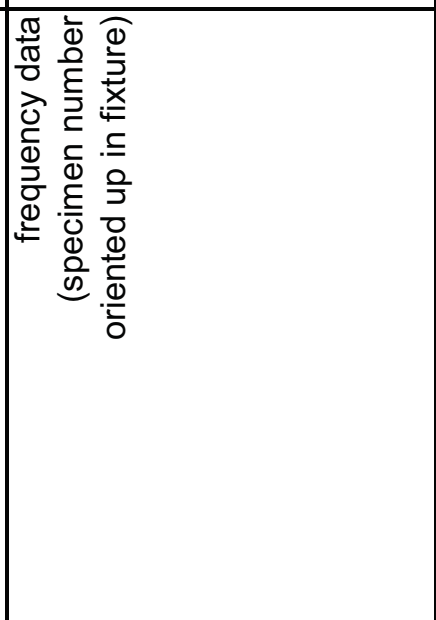 & 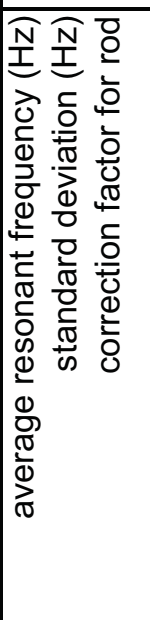 & 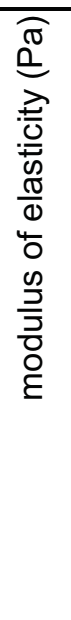 & 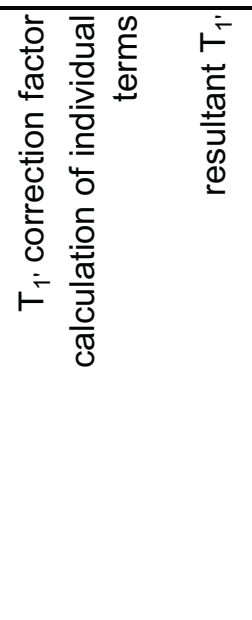 & 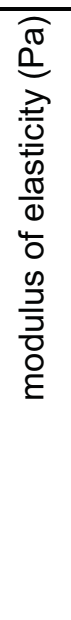 & 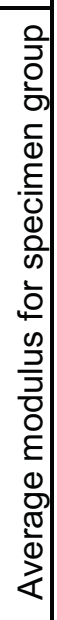 \\
\hline
\end{tabular}




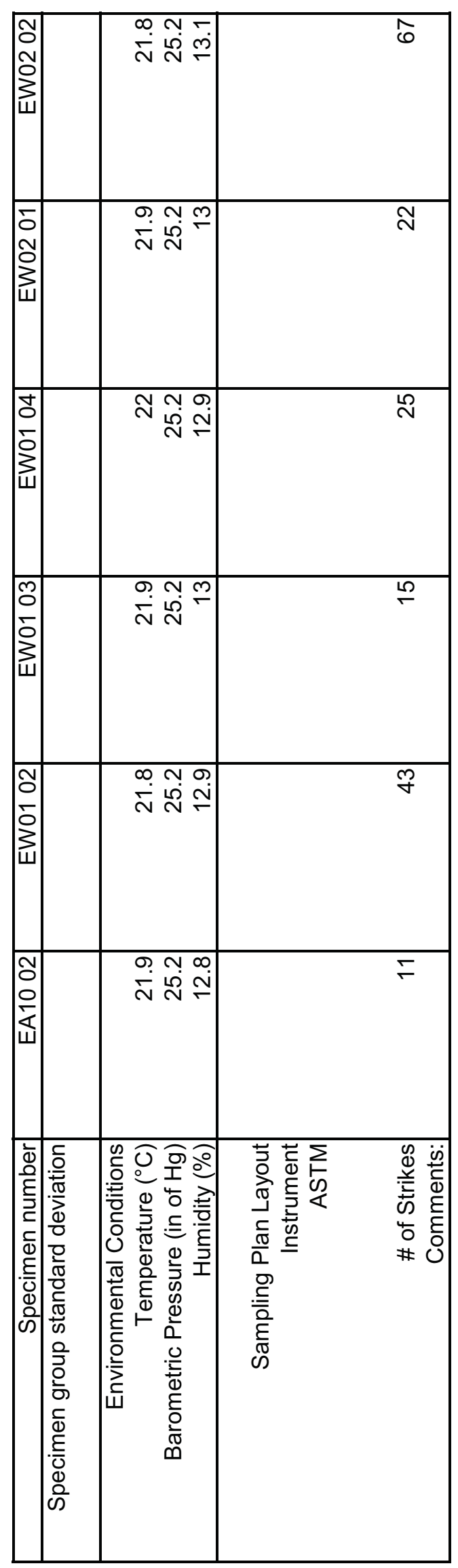




\begin{tabular}{|c|c|c|c|c|c|c|c|c|c|}
\hline \begin{tabular}{|l} 
\\
0 \\
$m$ \\
0 \\
3 \\
\end{tabular} & 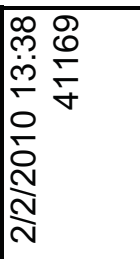 & 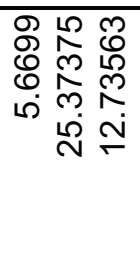 & $\begin{array}{l}\hat{\theta} \\
\ddot{0} \\
\dot{0}\end{array}$ & 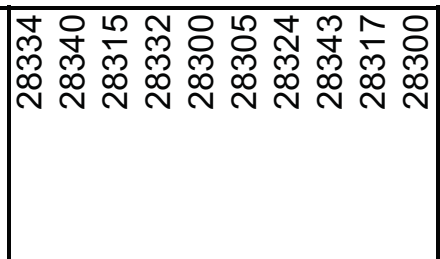 & 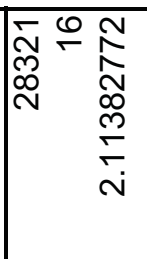 & 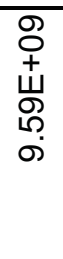 & 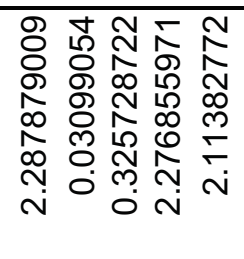 & 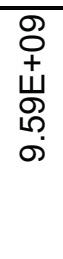 & \\
\hline 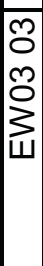 & 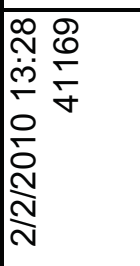 & 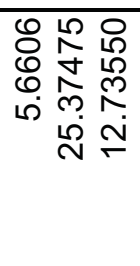 & $\begin{array}{l}\hat{\theta} \\
\stackrel{0}{0} \\
\vdots\end{array}$ & 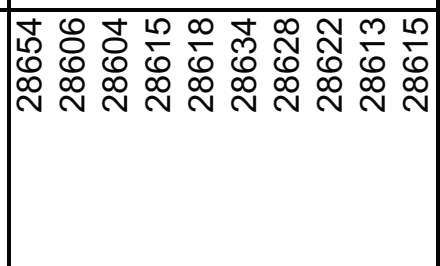 & 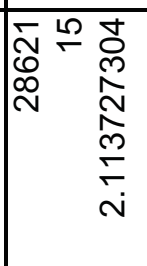 & $\begin{array}{l}8 \\
0 \\
+ \\
\infty \\
0 \\
0\end{array}$ & 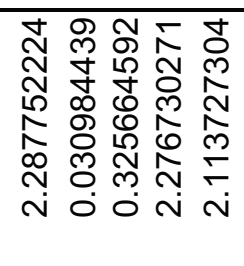 & $\begin{array}{l}8 \\
0 \\
+ \\
\infty \\
0 \\
0\end{array}$ & \\
\hline $\begin{array}{l}\text { No } \\
\text { O } \\
\text { O } \\
3 \\
\text { W } \\
\end{array}$ & 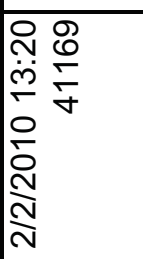 & 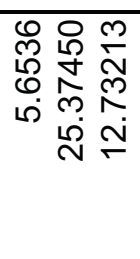 & $\begin{array}{l}\hat{\theta} \\
\ddot{0} \\
\dot{0}\end{array}$ & 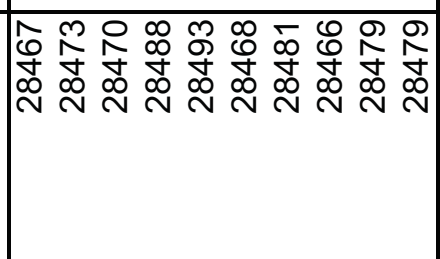 & 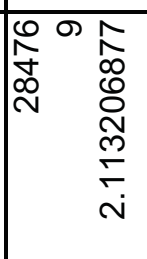 & $\begin{array}{l}8 \\
0 \\
+ \\
\pm \\
0 \\
0 \\
0\end{array}$ & 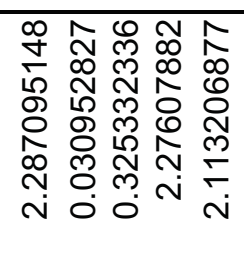 & $\begin{array}{l}8 \\
0 \\
+ \\
\dot{+} \\
0 \\
0 \\
0\end{array}$ & \\
\hline 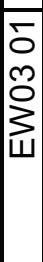 & 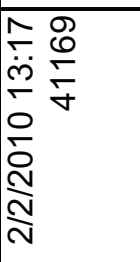 & 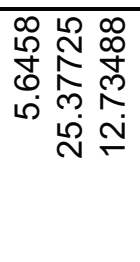 & $\begin{array}{l}\hat{\theta} \\
\grave{0} \\
\dot{0}\end{array}$ & 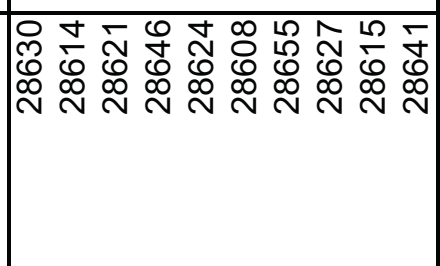 & 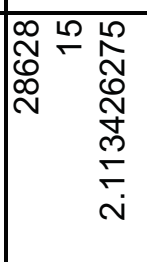 & $\begin{array}{l}8 \\
0 \\
+ \\
\bigsqcup \\
0 \\
0\end{array}$ & 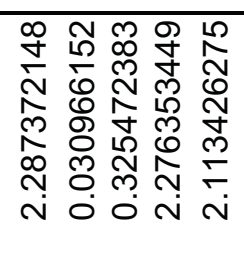 & $\begin{array}{l}8 \\
0 \\
+ \\
山 \\
0 \\
0\end{array}$ & \\
\hline 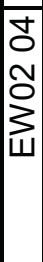 & 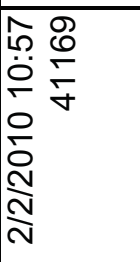 & 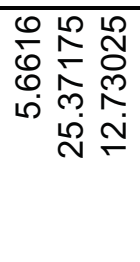 & $\begin{array}{l}\hat{\theta} \\
\stackrel{0}{0} \\
0\end{array}$ & 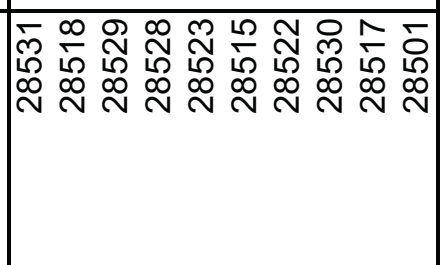 & 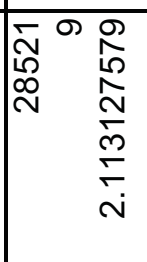 & $\begin{array}{l}\text { 8 } \\
+ \\
\stackrel{+}{N} \\
\text { a }\end{array}$ & 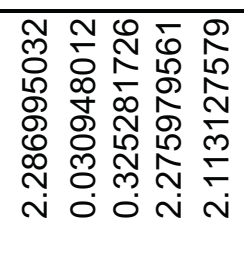 & $\begin{array}{l}\text { 8 } \\
+ \\
\stackrel{+}{N} \\
\text { o }\end{array}$ & \\
\hline 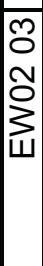 & 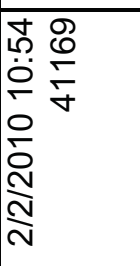 & 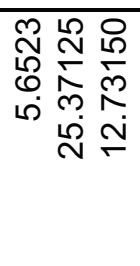 & $\begin{array}{l}\hat{\theta} \\
\stackrel{0}{0} \\
0\end{array}$ & 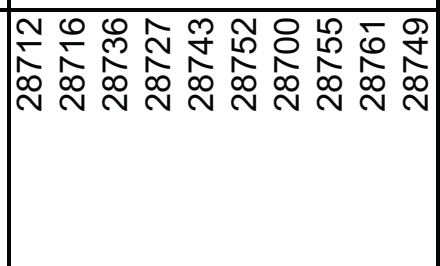 & 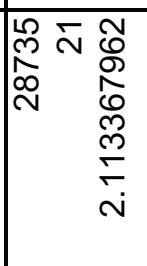 & $\begin{array}{l}8 \\
8 \\
+ \\
4 \\
\infty \\
\infty \\
0\end{array}$ & 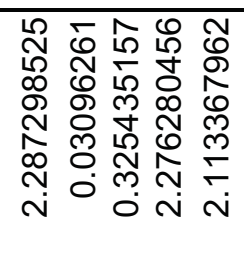 & $\begin{array}{l}8 \\
8 \\
+ \\
山 \\
\infty \\
\infty \\
0\end{array}$ & \\
\hline 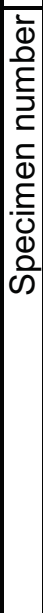 & 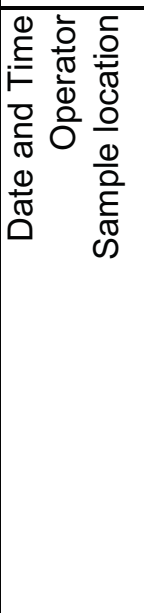 & 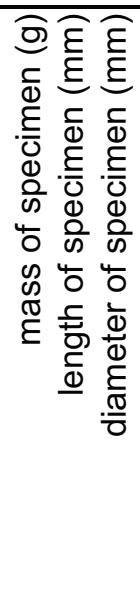 & 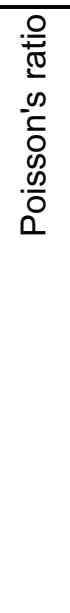 & 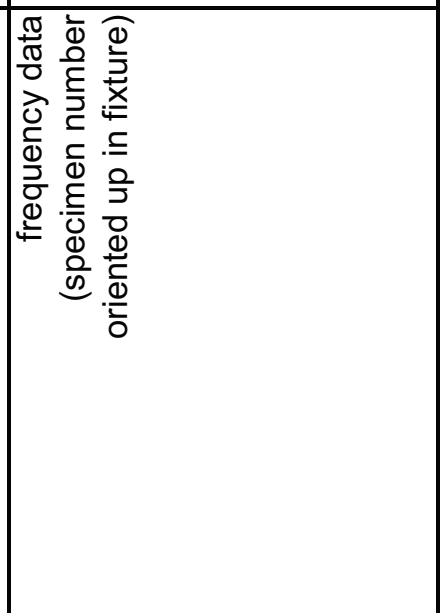 & 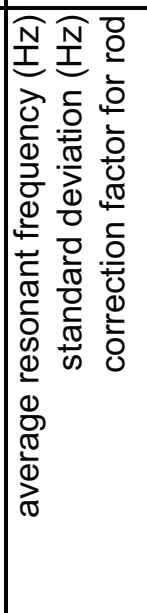 & 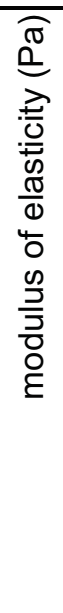 & 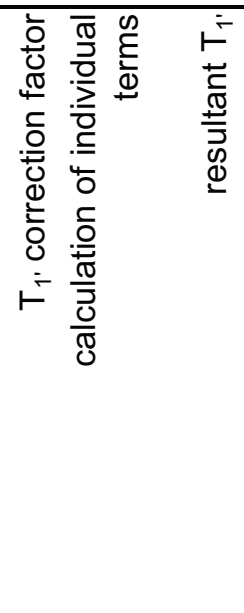 & 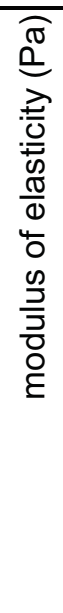 & 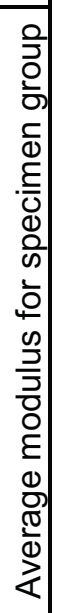 \\
\hline
\end{tabular}




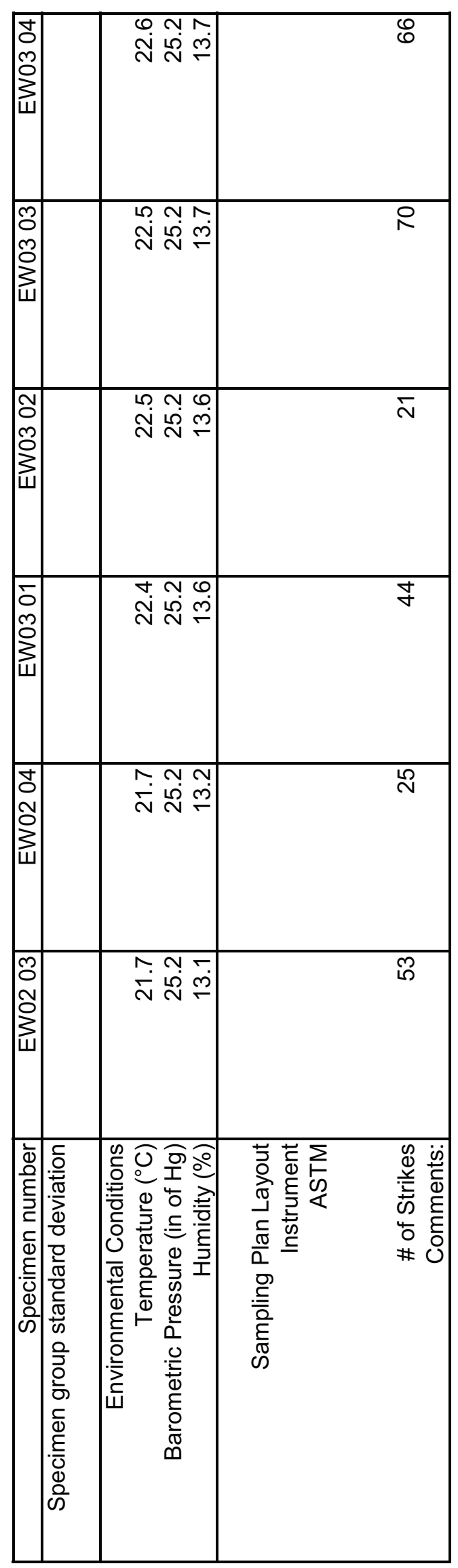




\begin{tabular}{|c|c|c|c|c|c|c|c|c|c|}
\hline 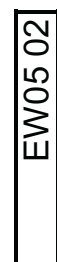 & 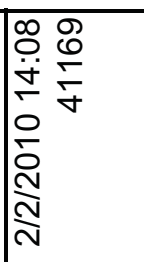 & 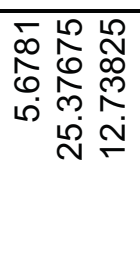 & $\begin{array}{l}\hat{\theta} \\
\grave{0}\end{array}$ & 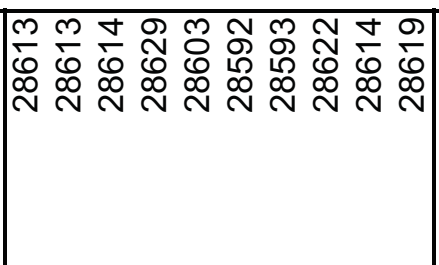 & 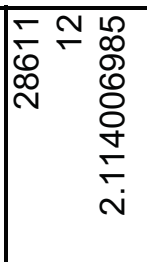 & $\begin{array}{l}8 \\
\\
+ \\
\dot{+} \\
\infty \\
0\end{array}$ & 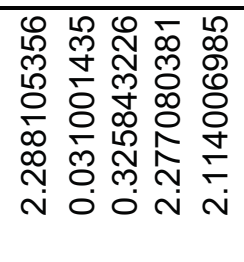 & $\begin{array}{l}8 \\
0 \\
+ \\
\dot{+} \\
\infty \\
0\end{array}$ & \\
\hline \begin{tabular}{|l|} 
\\
0 \\
0 \\
0 \\
3 \\
$w$ \\
\end{tabular} & 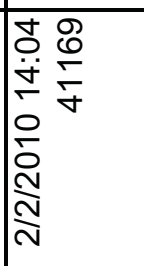 & 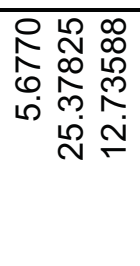 & $\begin{array}{l}\hat{\theta} \\
\stackrel{0}{0} \\
\vdots\end{array}$ & 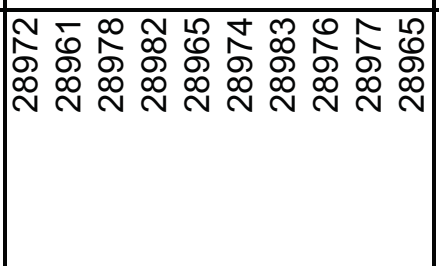 & 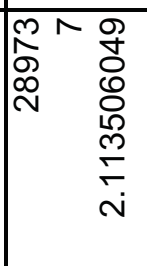 & 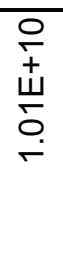 & 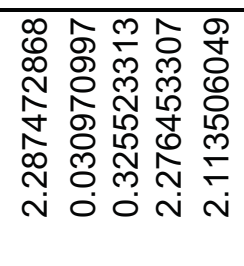 & 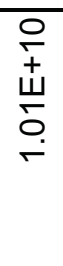 & \\
\hline 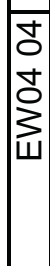 & 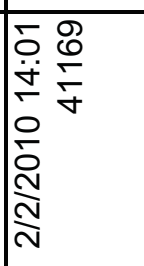 & 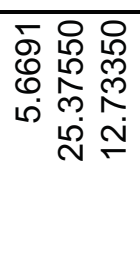 & $\begin{array}{l}\hat{\theta} \\
\ddot{0} \\
\dot{0}\end{array}$ & 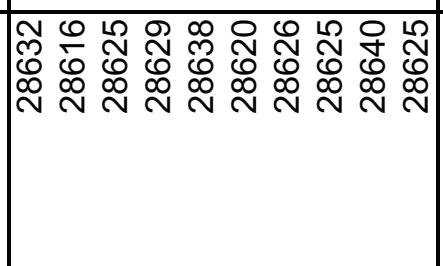 & 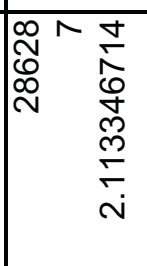 & $\begin{array}{l}8 \\
0 \\
+ \\
\dot{+} \\
\infty \\
0\end{array}$ & 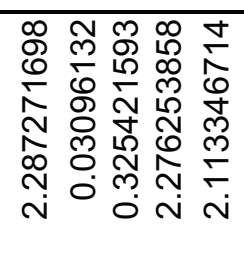 & $\begin{array}{l}\text { 8 } \\
+ \\
\dot{+} \\
\infty \\
\infty\end{array}$ & \\
\hline 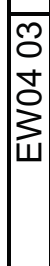 & 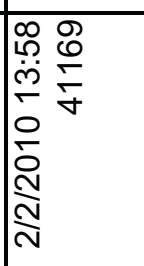 & 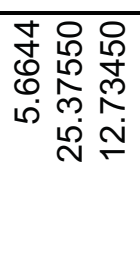 & $\frac{\hat{\theta}}{\mathscr{0}}$ & 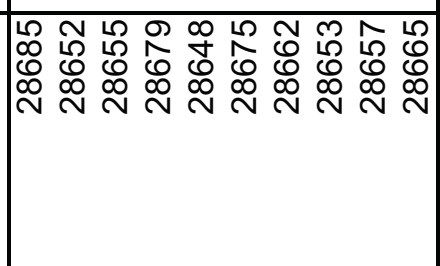 & 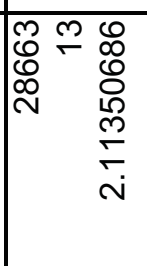 & $\begin{array}{l}\text { 8 } \\
+ \\
\stackrel{+}{N} \\
\infty \\
0\end{array}$ & 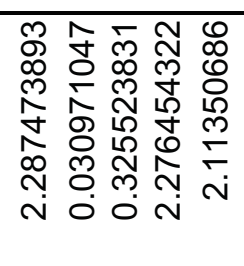 & $\begin{array}{l}8 \\
0 \\
+ \\
\stackrel{+}{N} \\
\infty \\
0\end{array}$ & \\
\hline 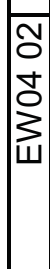 & 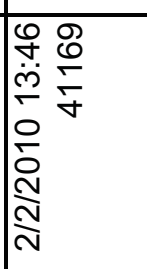 & 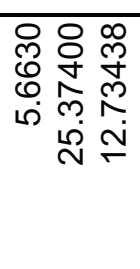 & $\begin{array}{l}\hat{\theta} \\
\frac{0}{0}\end{array}$ & 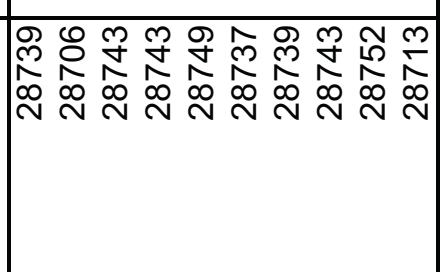 & 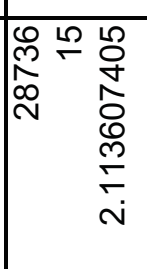 & $\begin{array}{l}\text { o } \\
+ \\
+ \\
\infty \\
\infty \\
0\end{array}$ & 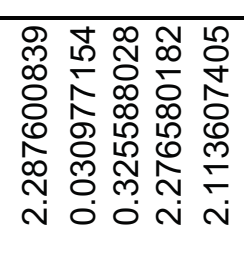 & $\begin{array}{l} \\
\\
+ \\
+ \\
\infty \\
\infty \\
0\end{array}$ & \\
\hline 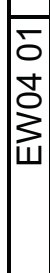 & 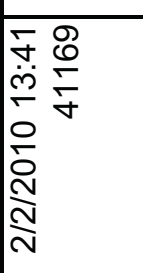 & 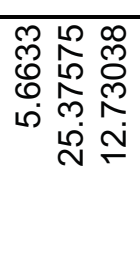 & $\begin{array}{l}\widehat{0} \\
\dddot{0} \\
\dot{0}\end{array}$ & 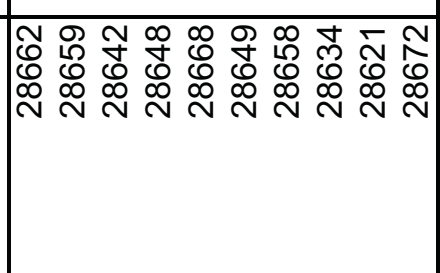 & 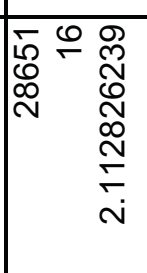 & $\begin{array}{l}8 \\
0 \\
+ \\
\stackrel{+}{\sim} \\
\infty \\
0\end{array}$ & 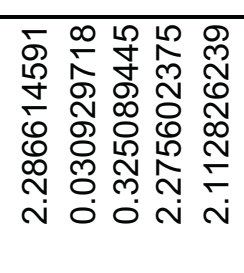 & $\begin{array}{l}\text { o } \\
+ \\
\dot{+} \\
\infty \\
\infty \\
0\end{array}$ & \\
\hline 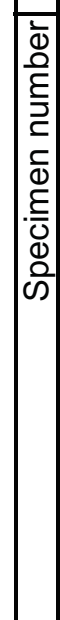 & 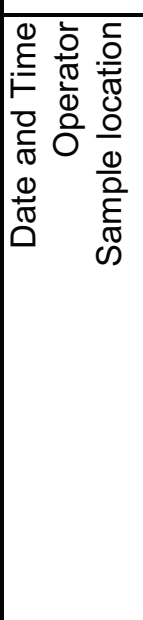 & 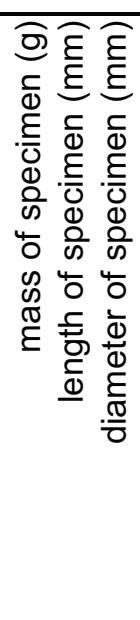 & 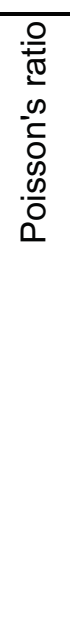 & 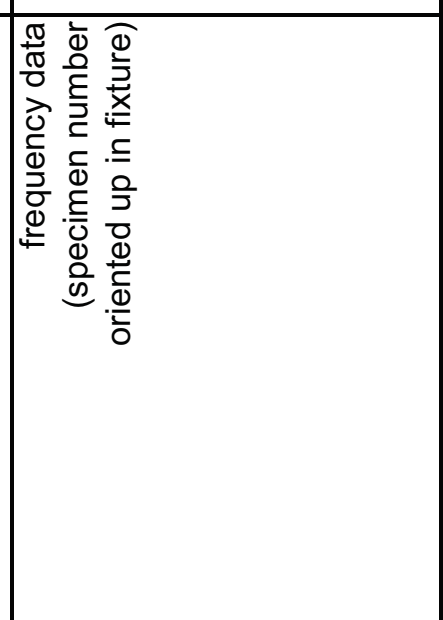 & 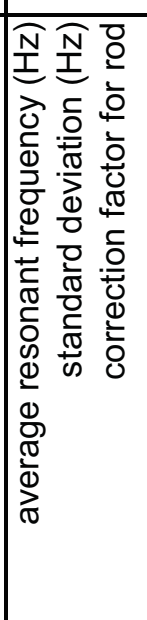 & 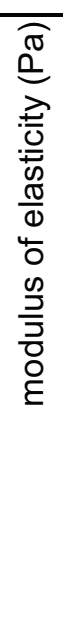 & 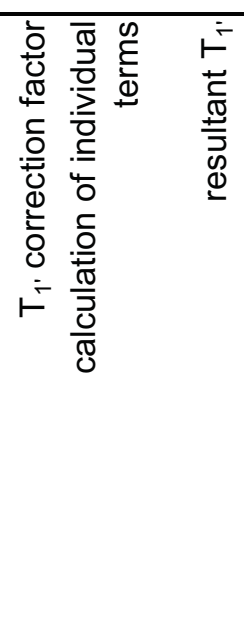 & 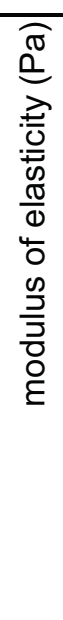 & 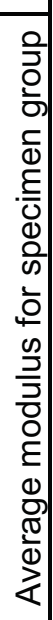 \\
\hline
\end{tabular}




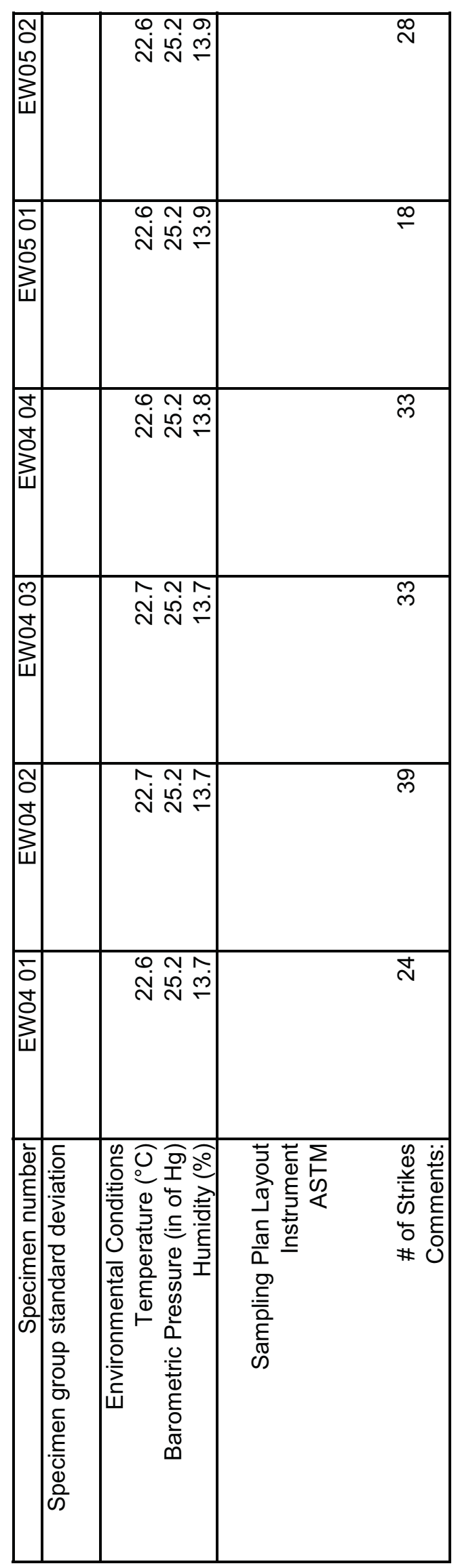

$m$ 


\begin{tabular}{|c|c|c|c|c|c|c|c|c|c|}
\hline \begin{tabular}{|l|} 
\\
0 \\
0 \\
8 \\
3 \\
\end{tabular} & 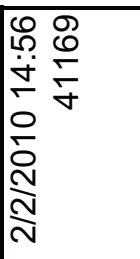 & 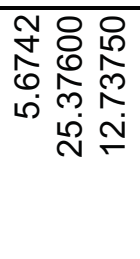 & $\begin{array}{l}\hat{\theta} \\
\ddot{0} \\
\dot{0}\end{array}$ & 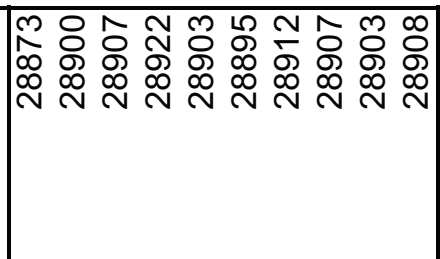 & 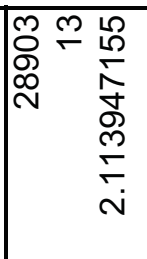 & 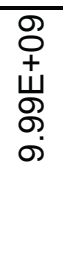 & 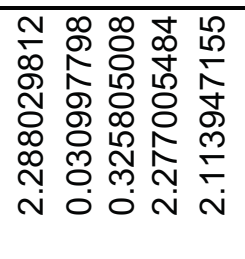 & 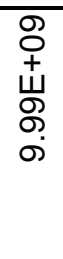 & \\
\hline $\begin{array}{l}m \\
0 \\
0 \\
8 \\
3 \\
w\end{array}$ & 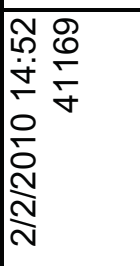 & 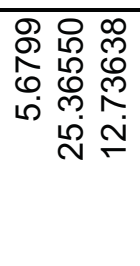 & $\begin{array}{l}\hat{\theta} \\
\stackrel{0}{0} \\
\vdots\end{array}$ & 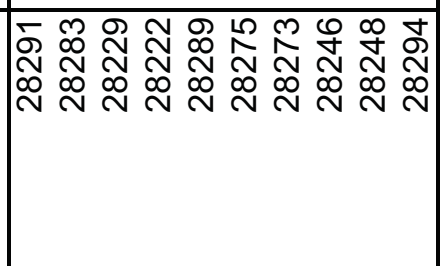 & 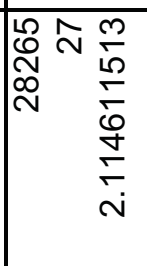 & $\begin{array}{l}8 \\
0 \\
+ \\
4 \\
0 \\
0 \\
0\end{array}$ & 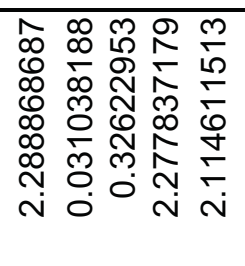 & 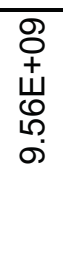 & \\
\hline \begin{tabular}{|l}
\multirow{2}{O}{} \\
0 \\
0 \\
3 \\
W \\
\end{tabular} & 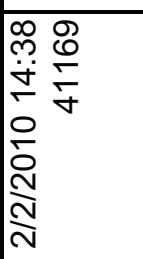 & 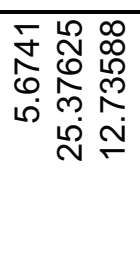 & $\begin{array}{l}\hat{\theta} \\
\ddot{0} \\
\dot{0}\end{array}$ & 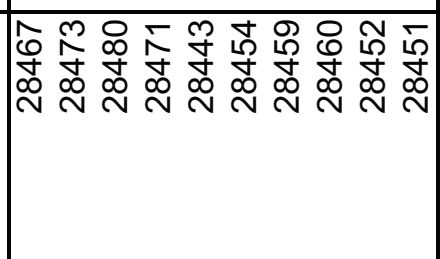 & 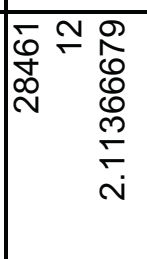 & 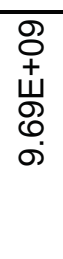 & 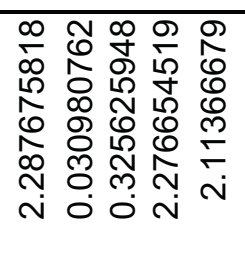 & $\begin{array}{l}8 \\
8 \\
+ \\
\dot{+} \\
8 \\
0\end{array}$ & \\
\hline $\begin{array}{l}5 \\
0 \\
0 \\
0 \\
3 \\
w\end{array}$ & 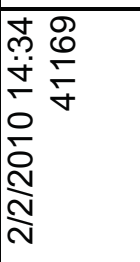 & 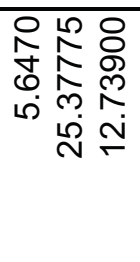 & $\begin{array}{l}\hat{\theta} \\
\grave{0} \\
\dot{0}\end{array}$ & 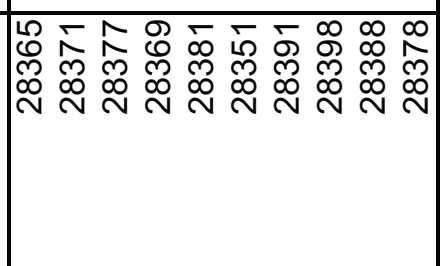 & 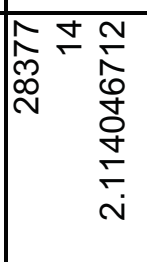 & 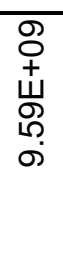 & 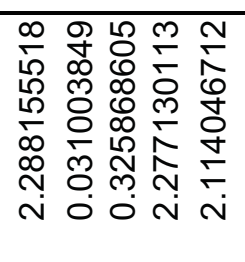 & 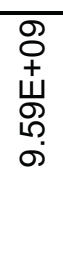 & \\
\hline $\begin{array}{l} \\
0 \\
0 \\
0 \\
3 \\
\\
\end{array}$ & 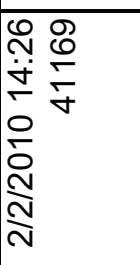 & 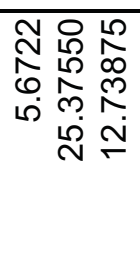 & $\begin{array}{l}\hat{\Theta} \\
\stackrel{\oplus}{0} \\
0\end{array}$ & 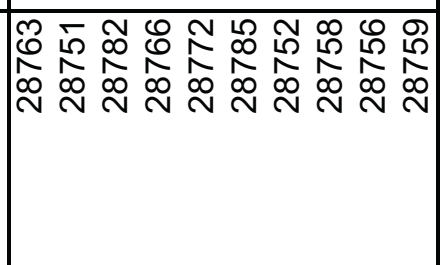 & 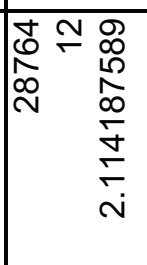 & $\begin{array}{l}\text { 8 } \\
+ \\
4 \\
\$ \\
\infty \\
0\end{array}$ & 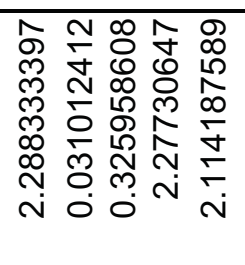 & $\begin{array}{l}\text { 8 } \\
+ \\
\stackrel{+}{\circ} \\
\infty \\
0\end{array}$ & \\
\hline 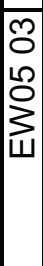 & 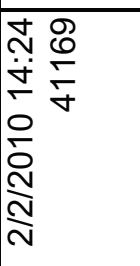 & 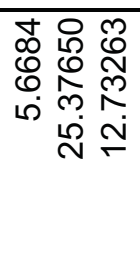 & $\begin{array}{l}\hat{\theta} \\
\stackrel{0}{0} \\
0\end{array}$ & 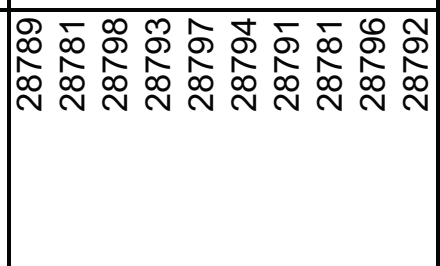 & 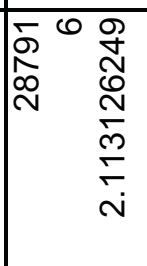 & $\begin{array}{l}\text { क् } \\
+ \\
\stackrel{+}{~} \\
\text { హ. } \\
\text { के }\end{array}$ & 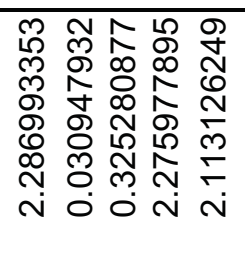 & 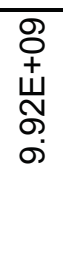 & \\
\hline 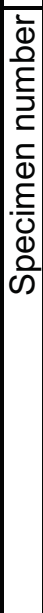 & 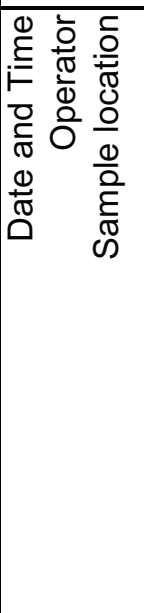 & 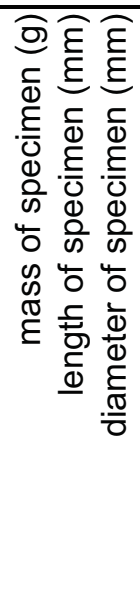 & 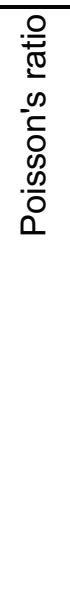 & 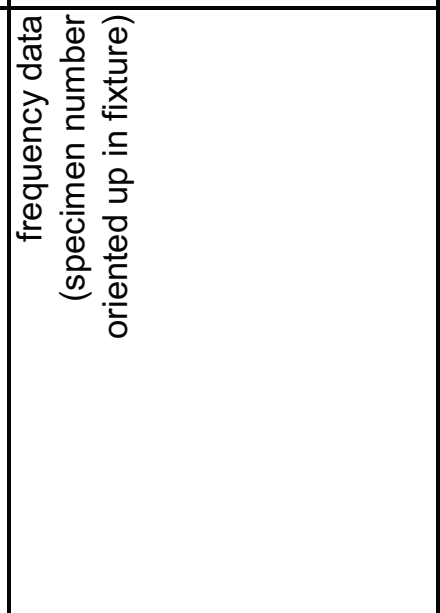 & 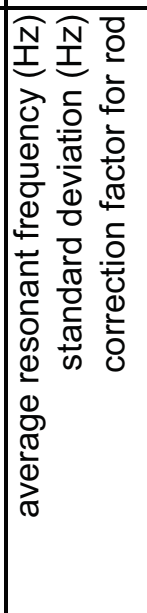 & 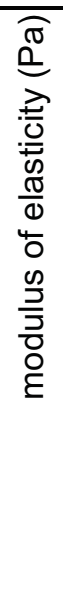 & 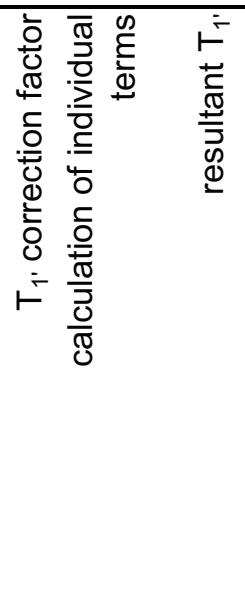 & 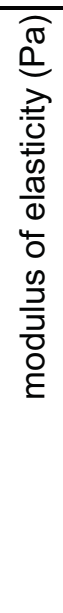 & 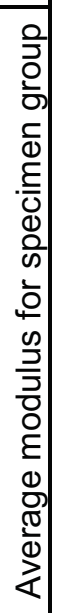 \\
\hline
\end{tabular}




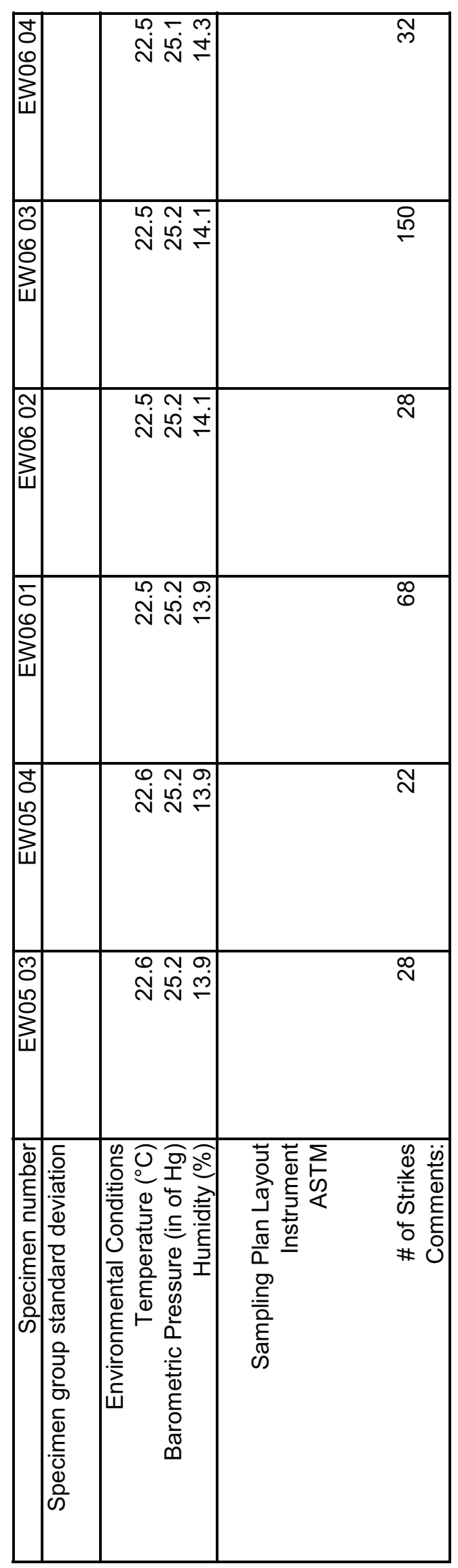




\begin{tabular}{|c|c|c|c|c|c|c|c|c|c|}
\hline 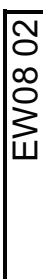 & 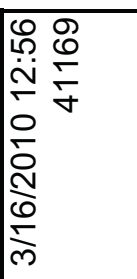 & 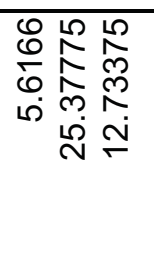 & $\begin{array}{l}\hat{\theta} \\
\vdots \\
0\end{array}$ & 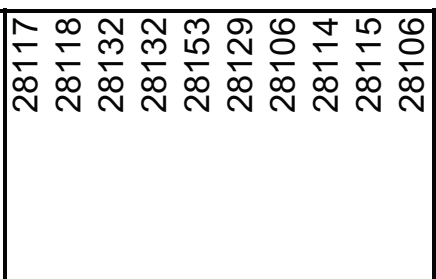 & 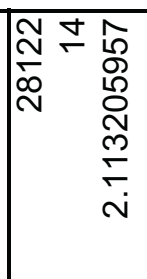 & $\begin{array}{l}8 \\
0 \\
+ \\
\stackrel{4}{0} \\
m \\
\infty\end{array}$ & 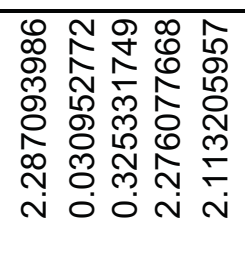 & 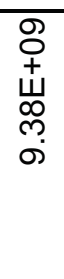 & \\
\hline $\begin{array}{l}\overline{0} \\
0 \\
0 \\
0 \\
\end{array}$ & 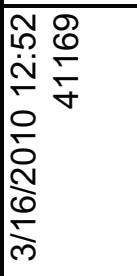 & 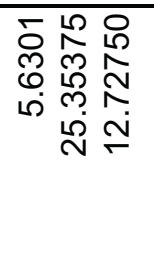 & $\begin{array}{l}\hat{0} \\
\vdots \\
0\end{array}$ & 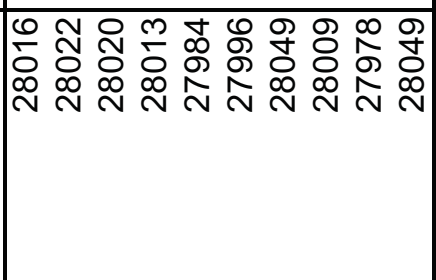 & 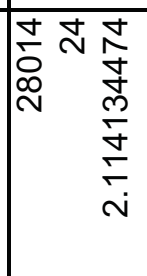 & $\begin{array}{l}\text { g } \\
+ \\
\stackrel{+}{N} \\
\text { లె } \\
\text { o }\end{array}$ & 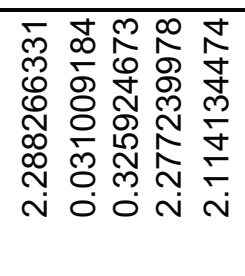 & $\begin{array}{l}\text { g } \\
+ \\
\stackrel{+}{\sim} \\
\text { ले } \\
\text { o. }\end{array}$ & \\
\hline 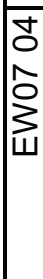 & 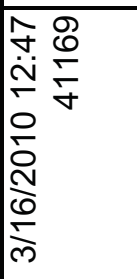 & 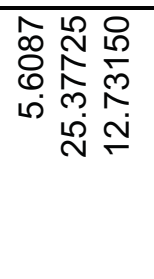 & $\begin{array}{l}\hat{0} \\
\stackrel{0}{0} \\
\vdots\end{array}$ & 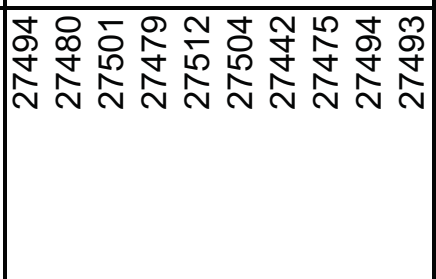 & 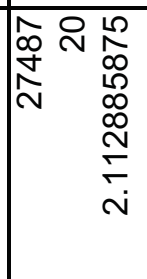 & 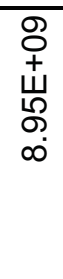 & 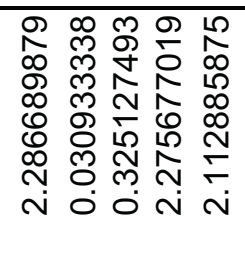 & 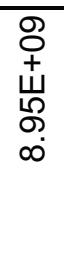 & \\
\hline 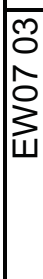 & 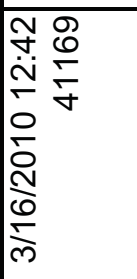 & 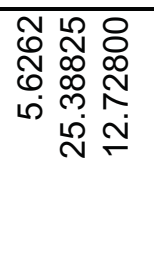 & $\begin{array}{l}\hat{\theta} \\
\vdots \\
\vdots \\
0\end{array}$ & 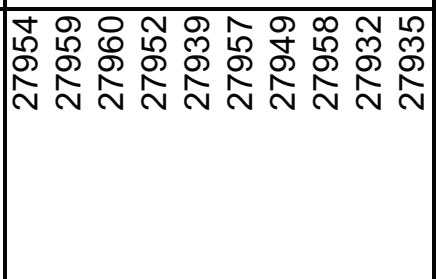 & 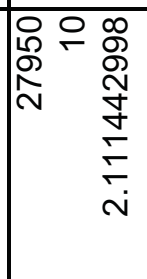 & 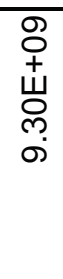 & 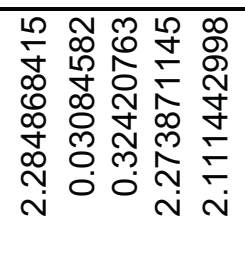 & $\begin{array}{l}\text { o } \\
+ \\
\text { 山े } \\
\text { m } \\
\text { o. }\end{array}$ & \\
\hline 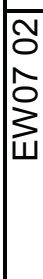 & 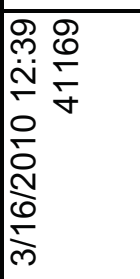 & 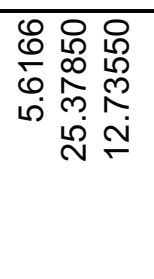 & $\begin{array}{l}\hat{\emptyset} \\
\stackrel{0}{0} \\
0\end{array}$ & 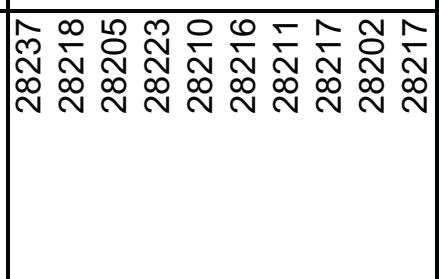 & 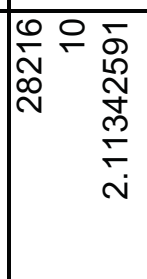 & $\begin{array}{l}8 \\
0 \\
+ \\
\stackrel{+}{0} \\
\stackrel{+}{+}\end{array}$ & 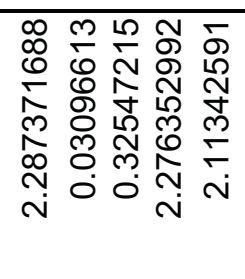 & 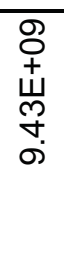 & \\
\hline 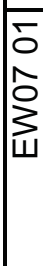 & 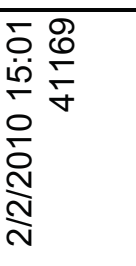 & 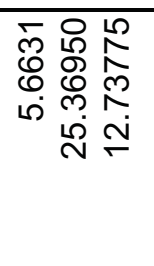 & $\begin{array}{l}\hat{0} \\
0 \\
\vdots \\
0\end{array}$ & 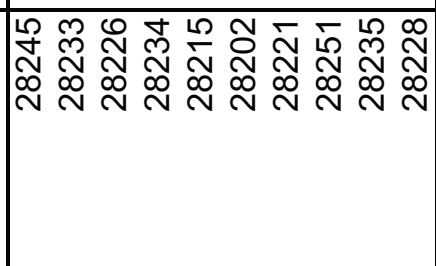 & 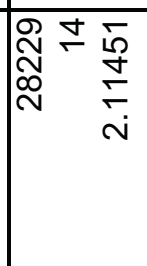 & $\begin{array}{l}\text { g } \\
+ \\
\stackrel{+}{\omega} \\
\dot{5} \\
0\end{array}$ & 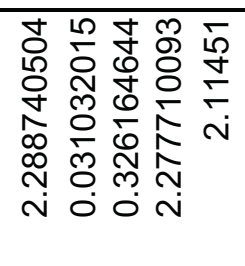 & 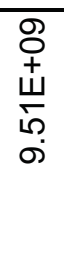 & \\
\hline 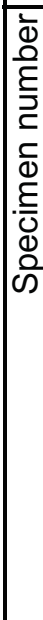 & 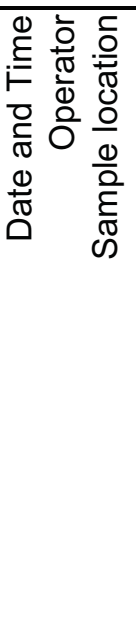 & 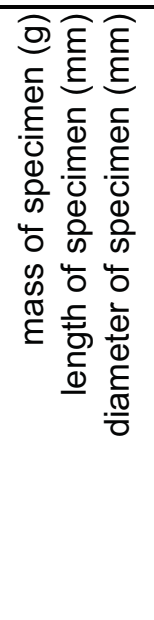 & 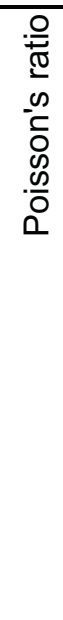 & 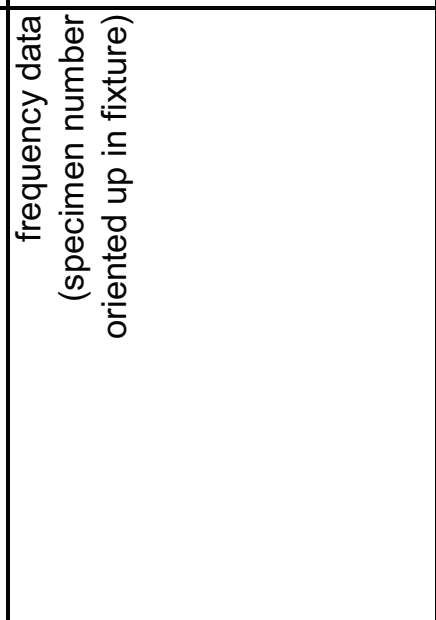 & 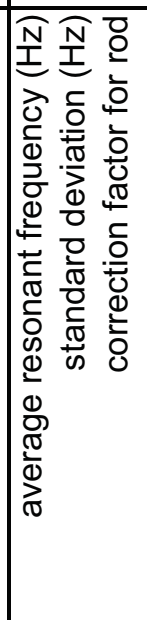 & 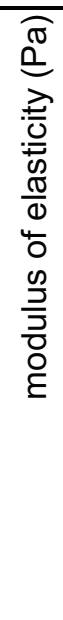 & 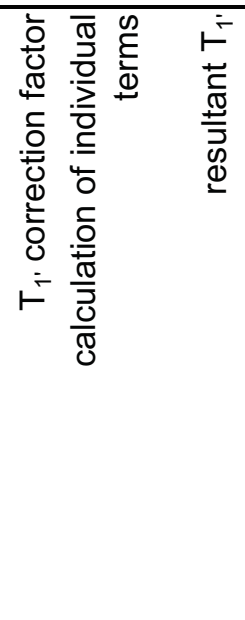 & 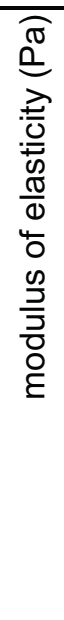 & 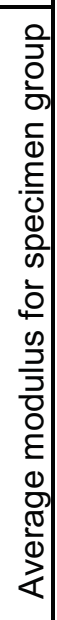 \\
\hline
\end{tabular}




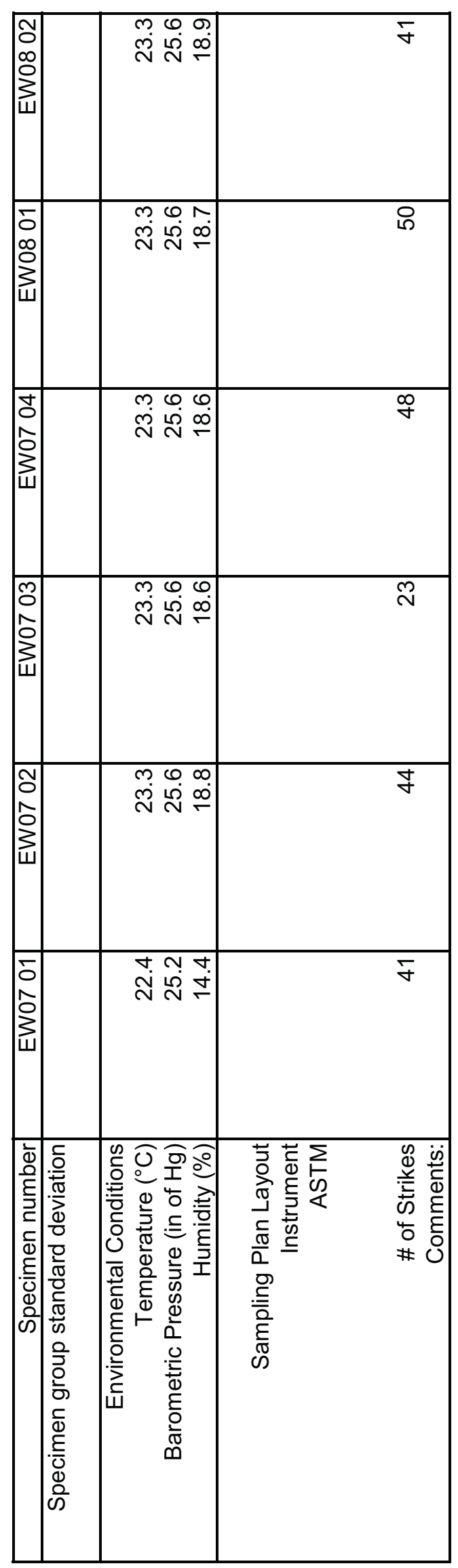




\begin{tabular}{|c|c|c|c|c|c|c|c|c|c|}
\hline \begin{tabular}{|l|}
5 \\
1 \\
1 \\
4 \\
\end{tabular} & 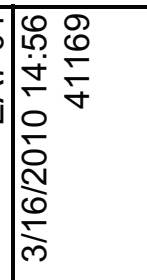 & 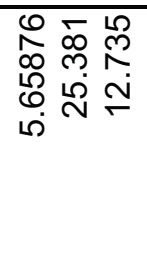 & $\begin{array}{l}\hat{\theta} \\
\stackrel{0}{0} \\
\vdots\end{array}$ & 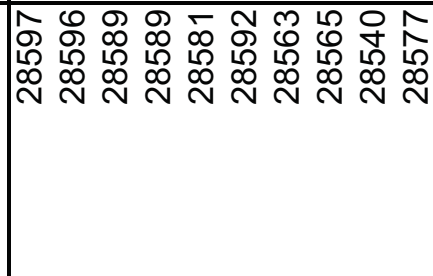 & 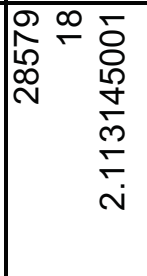 & 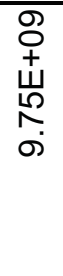 & 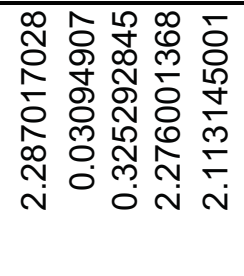 & 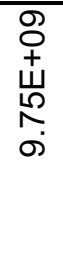 & \\
\hline 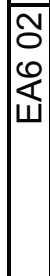 & 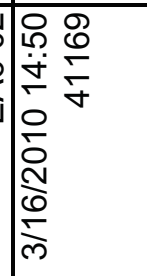 & 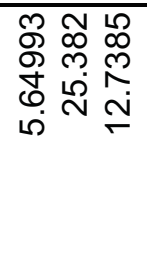 & $\begin{array}{l}\hat{\oplus} \\
\stackrel{0}{0} \\
\vdots\end{array}$ & 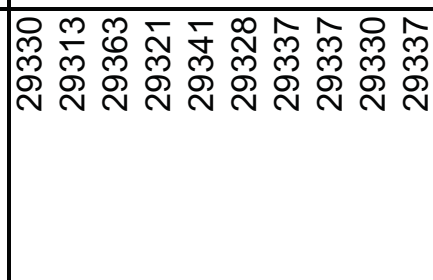 & 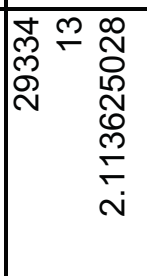 & 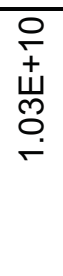 & 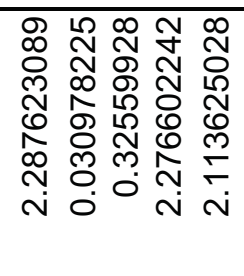 & 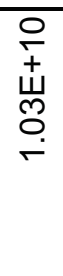 & \\
\hline 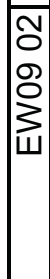 & 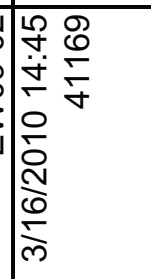 & 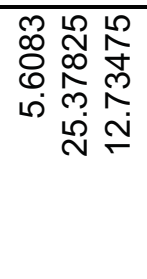 & $\begin{array}{l}\hat{\emptyset} \\
\dddot{0} \\
\vdots\end{array}$ & 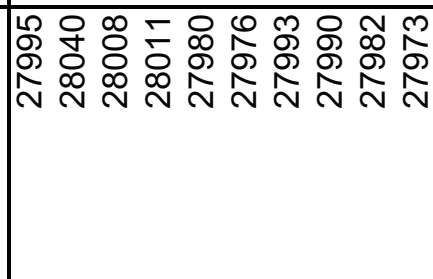 & 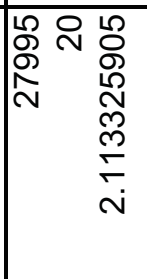 & \begin{tabular}{l}
8 \\
0 \\
+ \\
\multirow{N}{*}{} \\
o.
\end{tabular} & 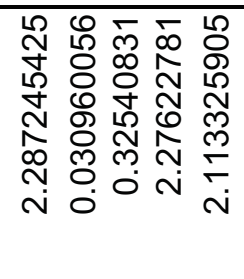 & 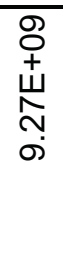 & \\
\hline 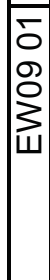 & 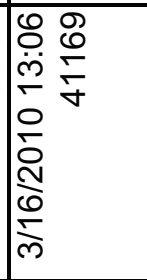 & 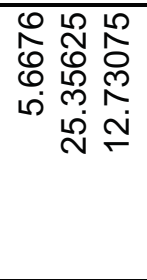 & $\begin{array}{l}\hat{\theta} \\
\vdots \\
0\end{array}$ & 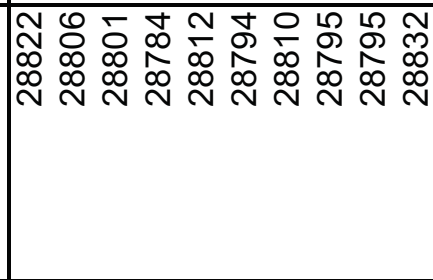 & 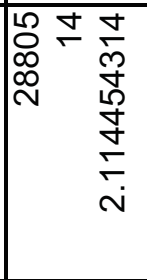 & 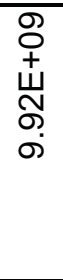 & 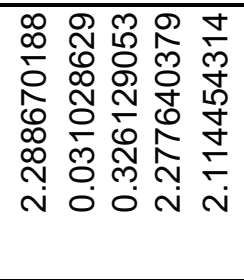 & 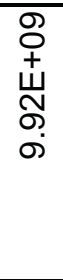 & \\
\hline 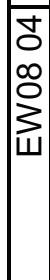 & 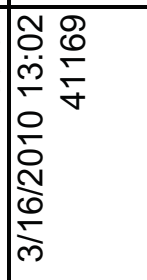 & 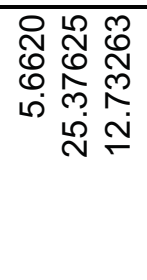 & $\begin{array}{l}\hat{\theta} \\
\vdots \\
0\end{array}$ & 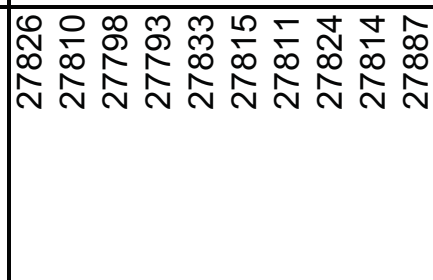 & 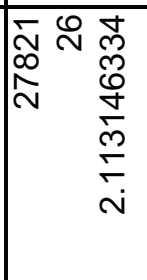 & 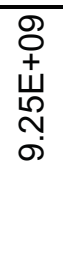 & 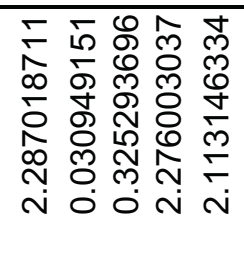 & 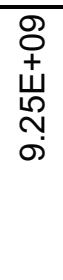 & \\
\hline $\begin{array}{l}\text { m } \\
0 \\
\infty \\
0 \\
\vdots \\
\text { w }\end{array}$ & 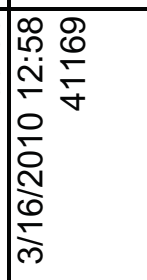 & 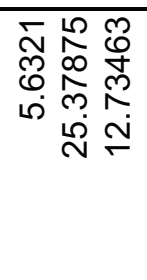 & $\begin{array}{l}\hat{0} \\
\vdots \\
\vdots\end{array}$ & 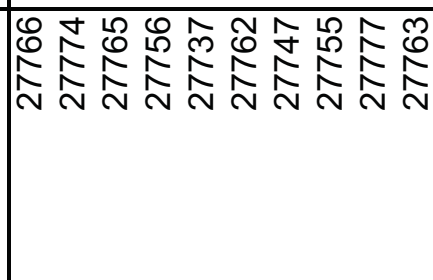 & 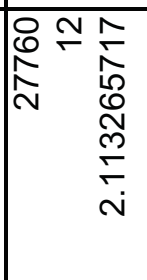 & $\begin{array}{l}8 \\
+ \\
4 \\
\stackrel{4}{0}\end{array}$ & 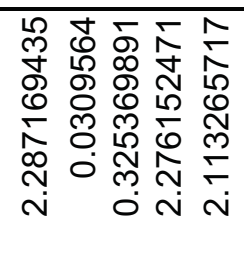 & $\begin{array}{l}8 \\
+ \\
+ \\
0 \\
0\end{array}$ & \\
\hline 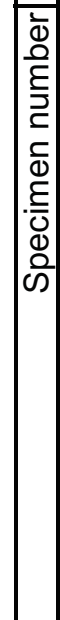 & 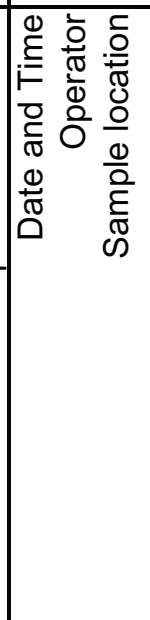 & 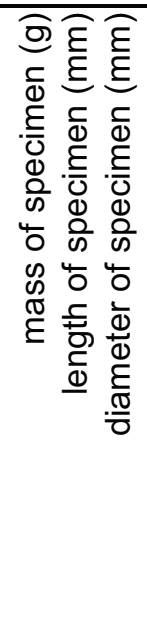 & 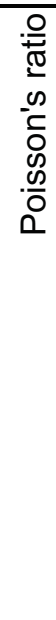 & 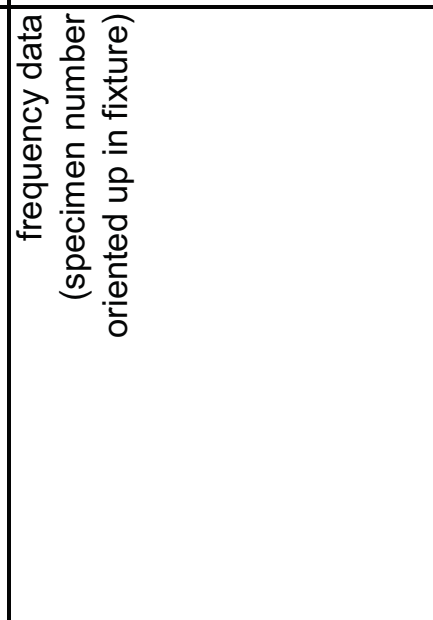 & 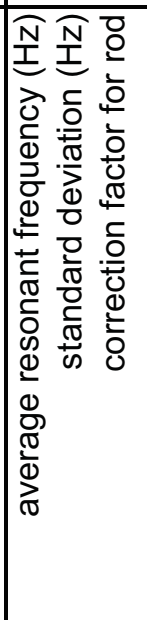 & 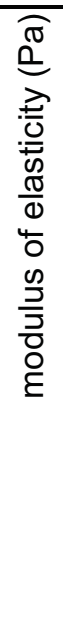 & 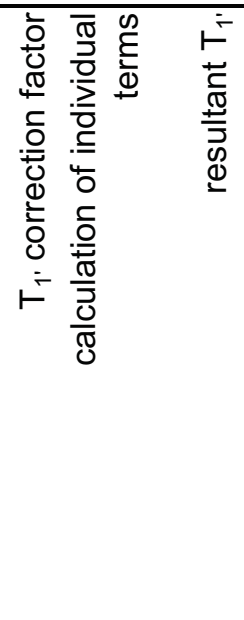 & 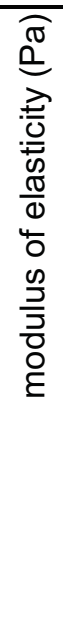 & 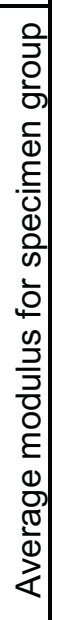 \\
\hline
\end{tabular}




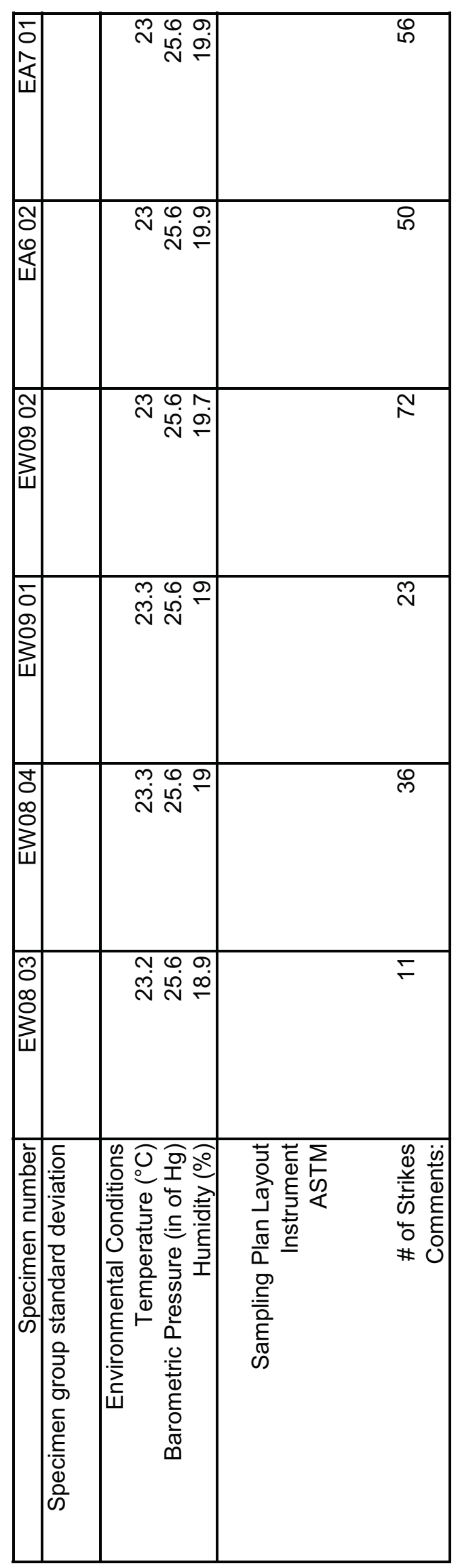




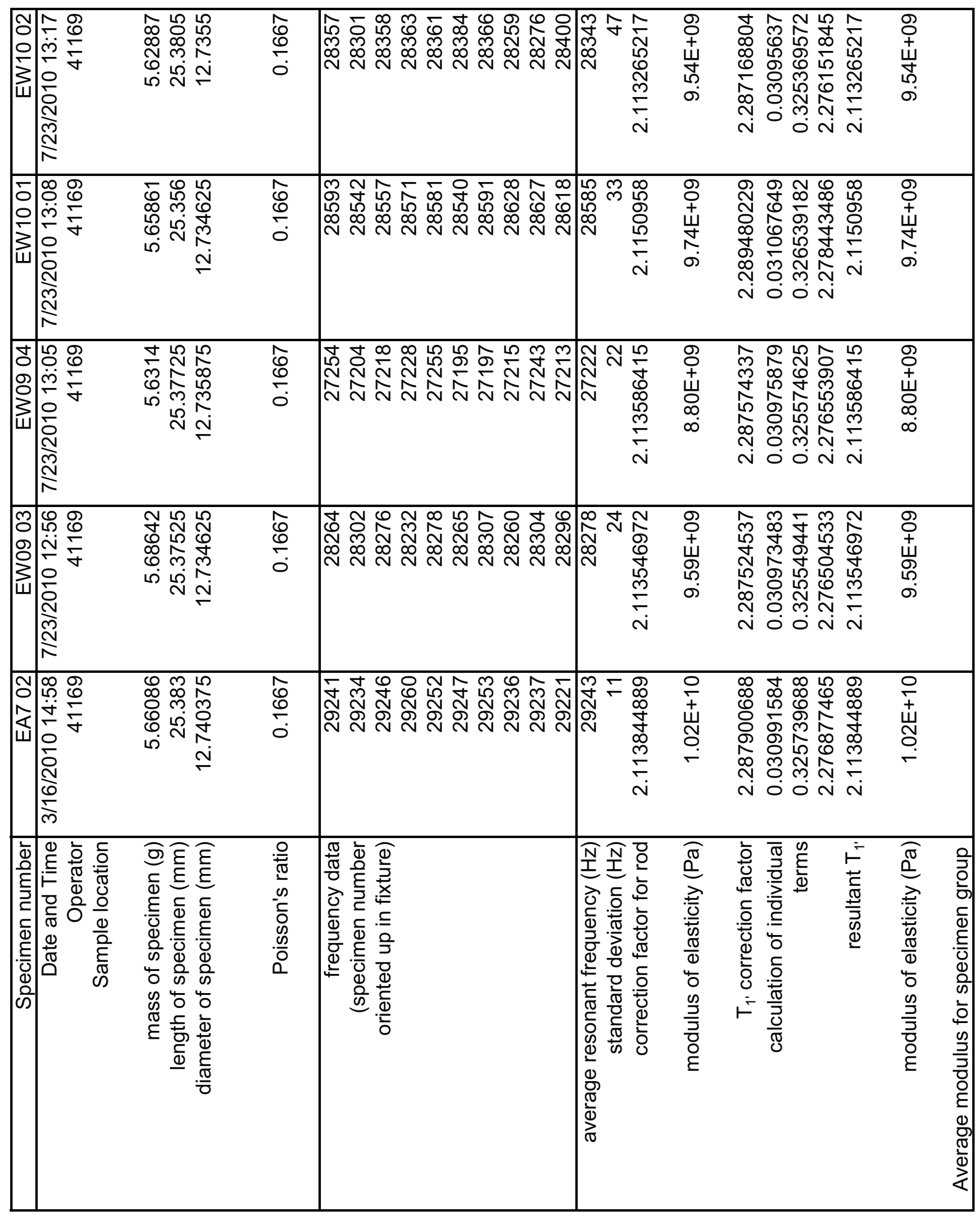




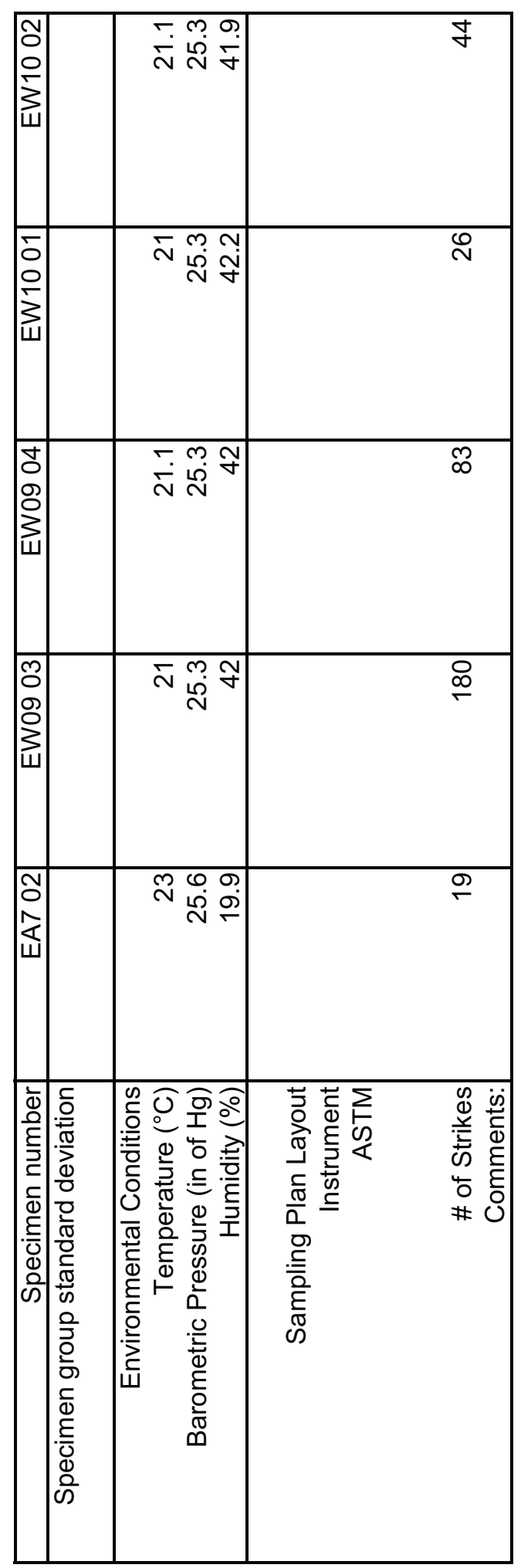




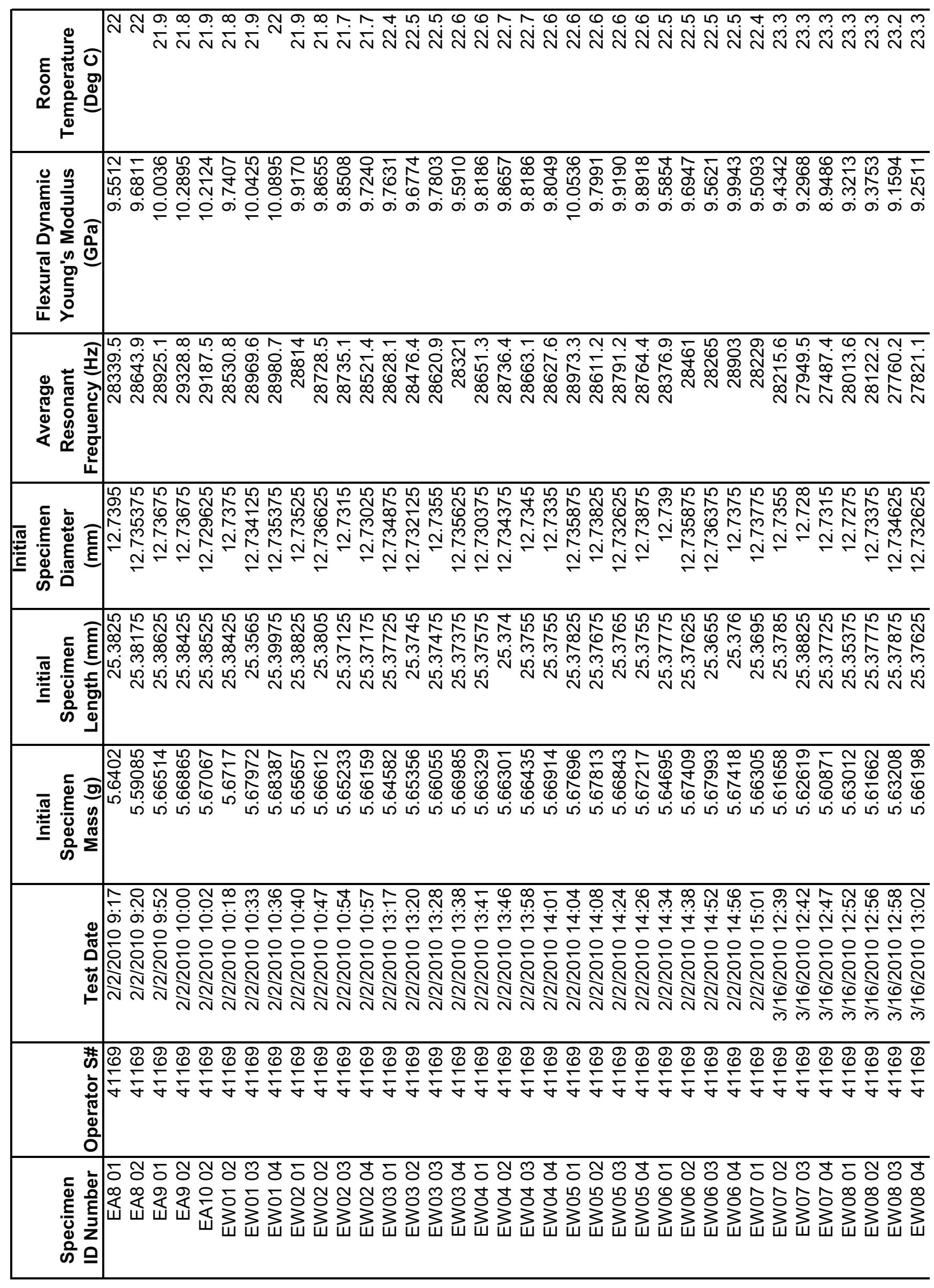




\begin{tabular}{|c|c|}
\hline 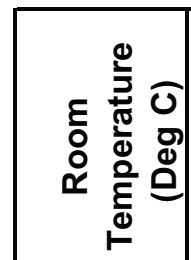 & 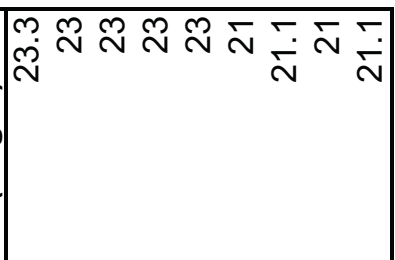 \\
\hline 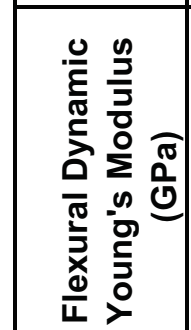 & 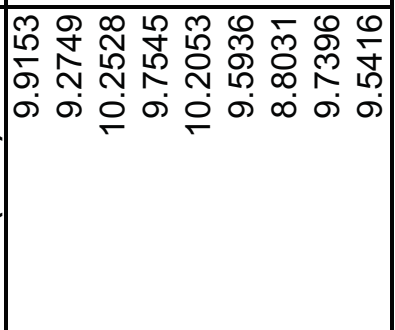 \\
\hline 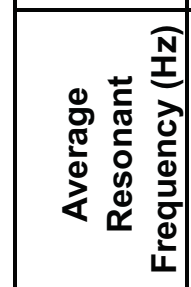 & 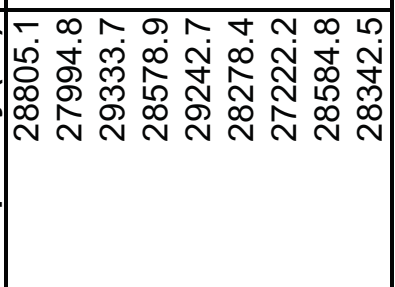 \\
\hline 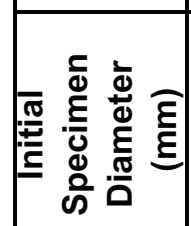 & 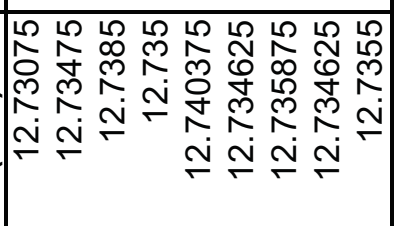 \\
\hline 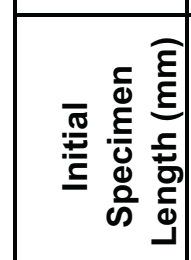 & 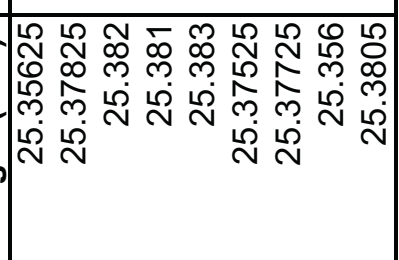 \\
\hline 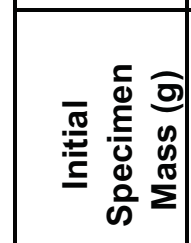 & 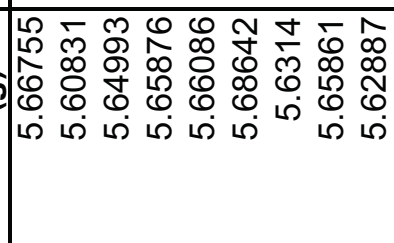 \\
\hline 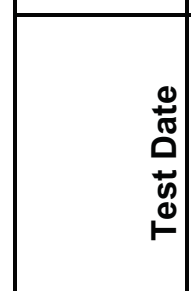 & 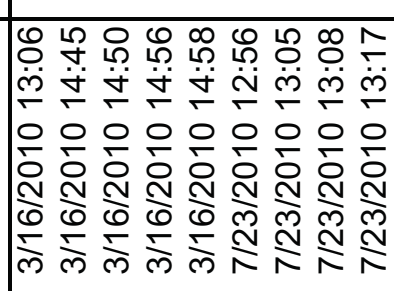 \\
\hline 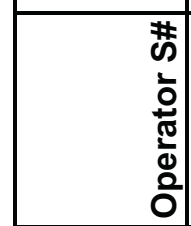 & 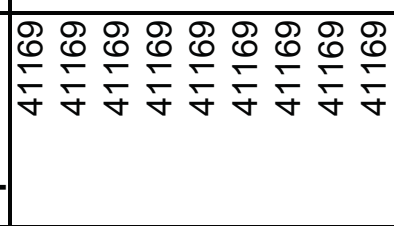 \\
\hline 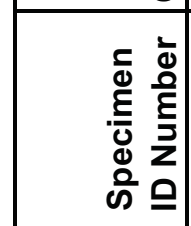 & 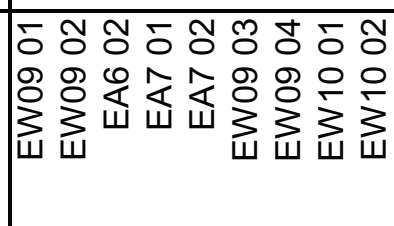 \\
\hline
\end{tabular}




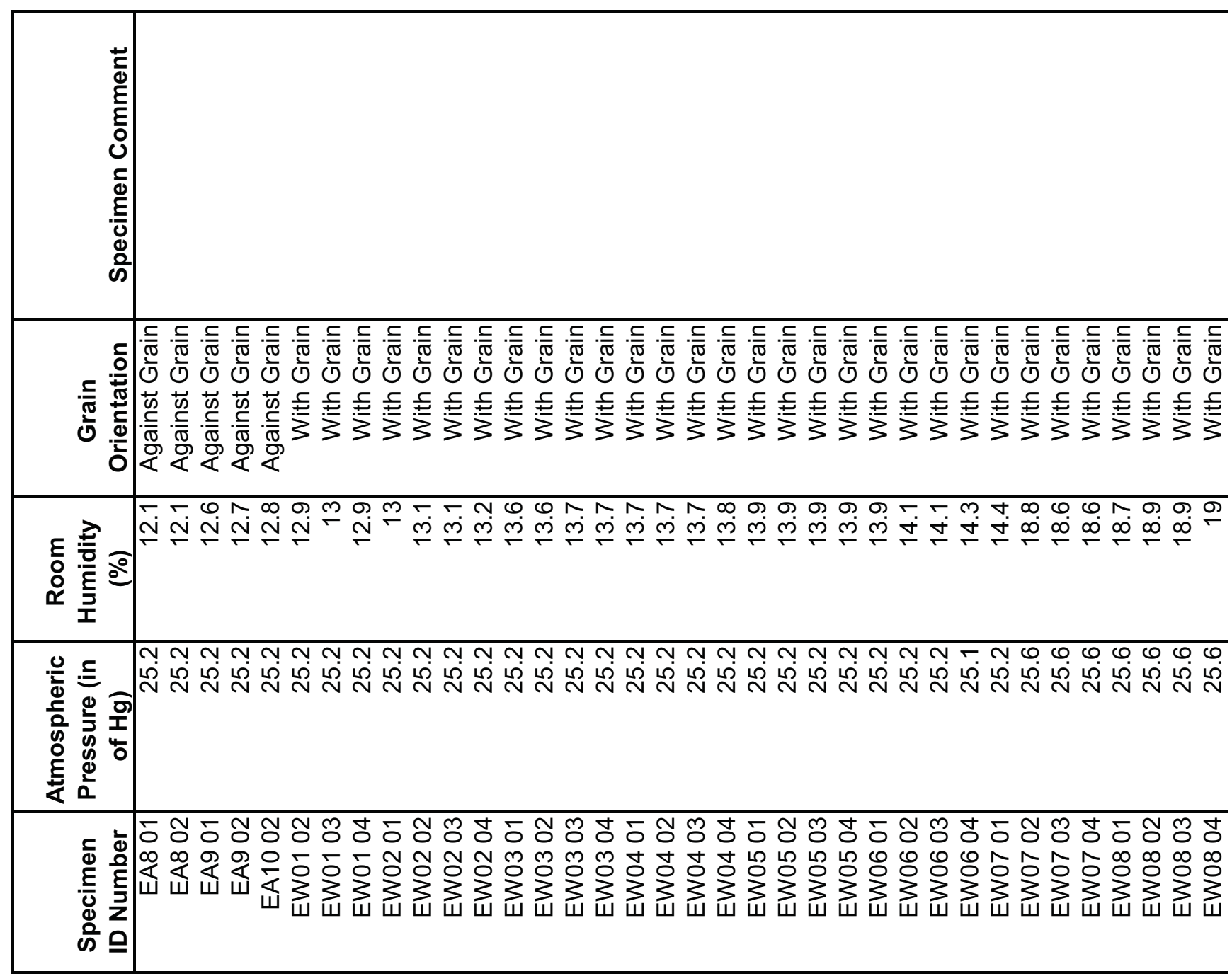




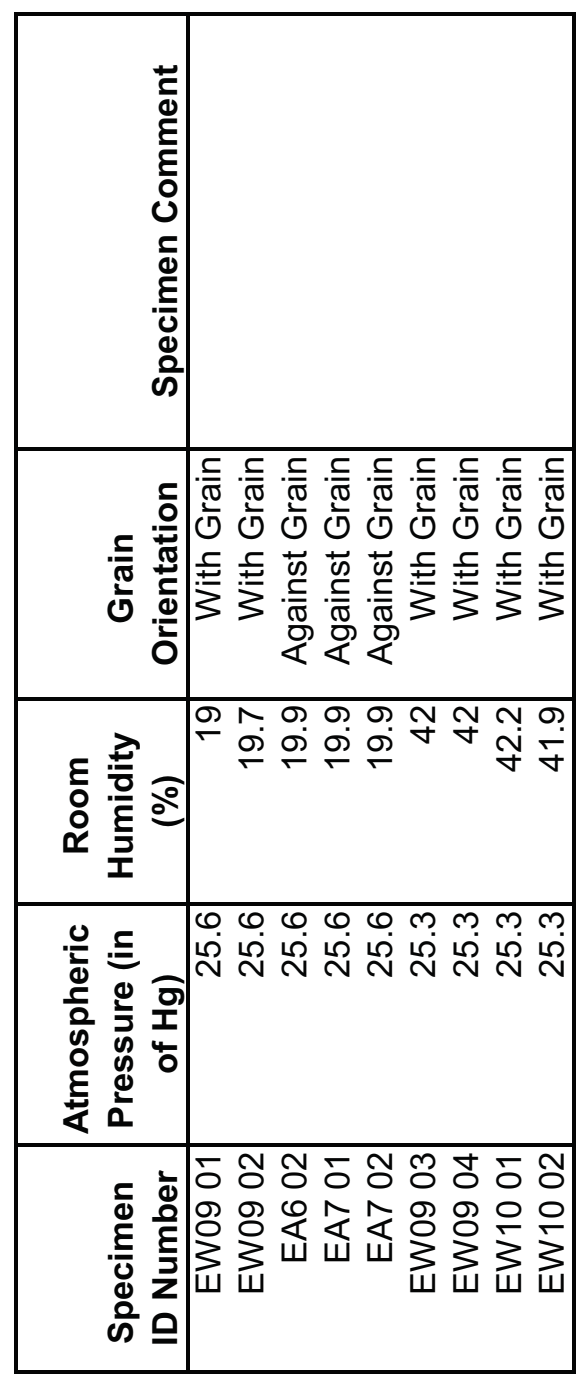




\begin{tabular}{|c|c|c|c|c|c|c|c|c|c|c|}
\hline \begin{tabular}{ll} 
& \\
$\approx$ \\
0 \\
$\infty$ \\
\hdashline \\
$\mathbb{C}$
\end{tabular} & 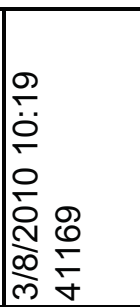 & 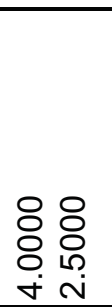 & & 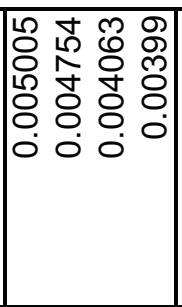 & 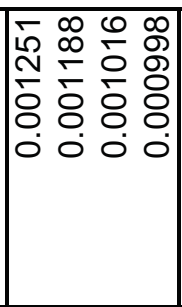 & & 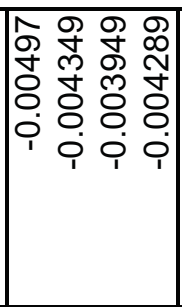 & 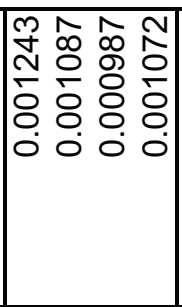 & & 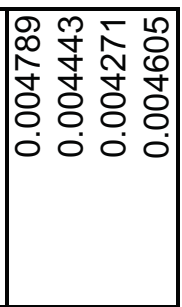 \\
\hline 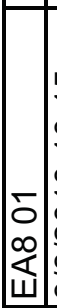 & 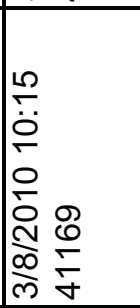 & 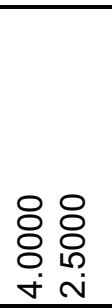 & & 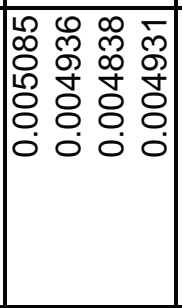 & 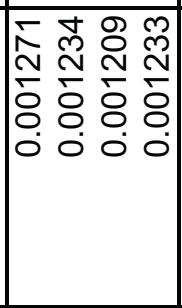 & & 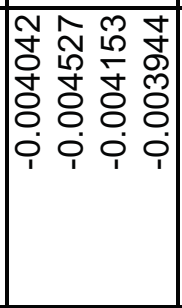 & 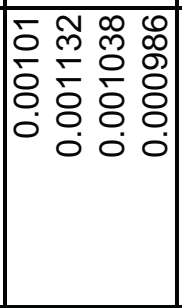 & & 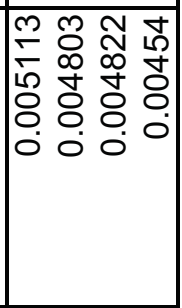 \\
\hline 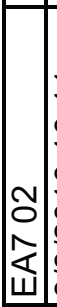 & 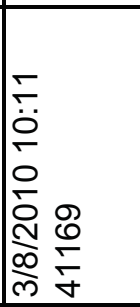 & 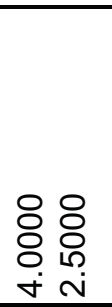 & & 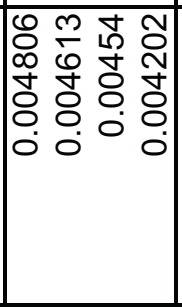 & 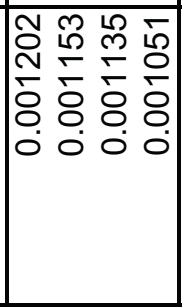 & & 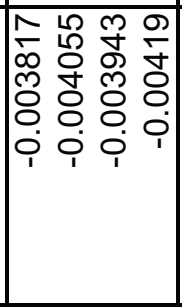 & 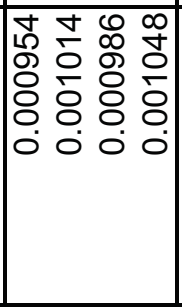 & & 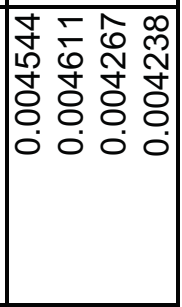 \\
\hline \begin{tabular}{|c} 
\\
\\
5 \\
0 \\
1 \\
\\
\end{tabular} & 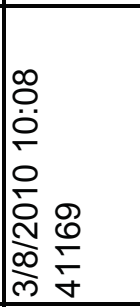 & 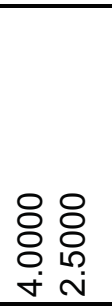 & & 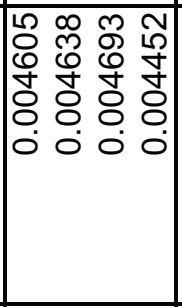 & 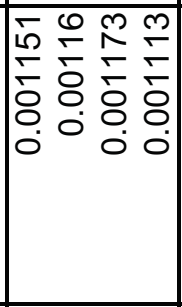 & & 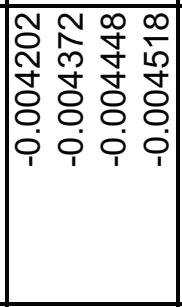 & 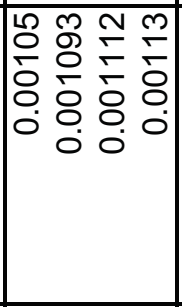 & & 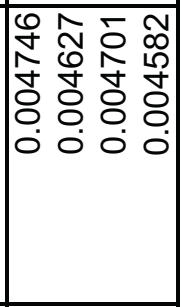 \\
\hline \begin{tabular}{|l|} 
\\
$\approx$ \\
0 \\
0 \\
$\mathbb{w}$ \\
$\varpi$
\end{tabular} & 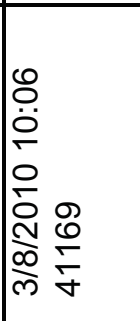 & 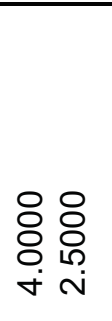 & & 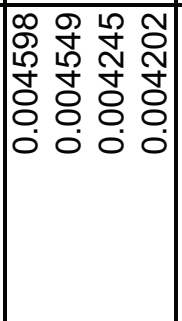 & 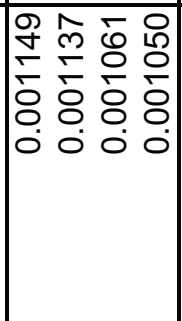 & & 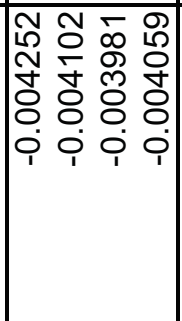 & 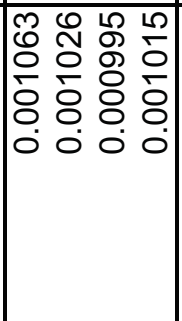 & & 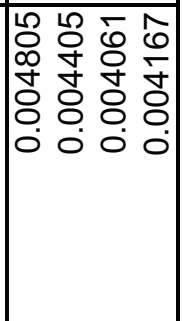 \\
\hline 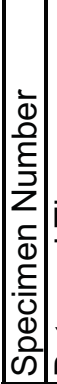 & 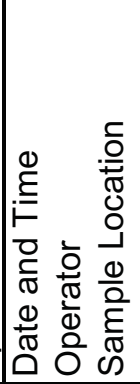 & 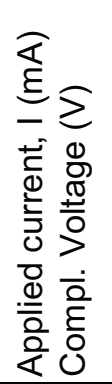 & 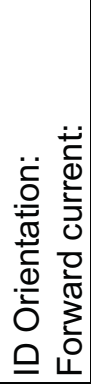 & 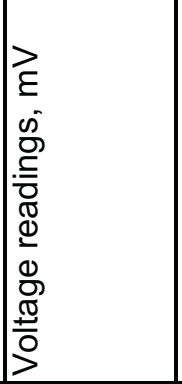 & 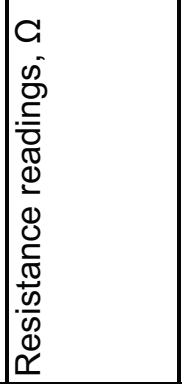 & 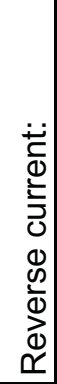 & 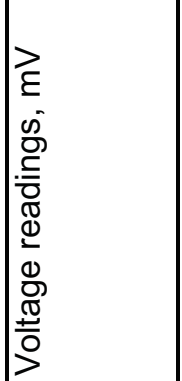 & 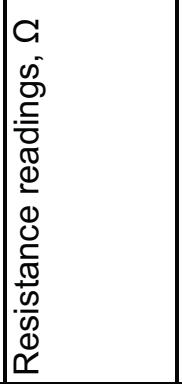 & 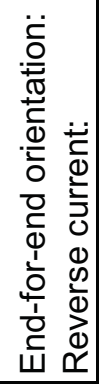 & 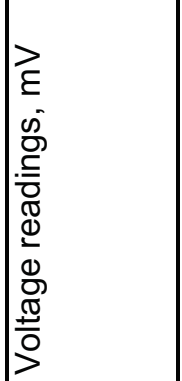 \\
\hline
\end{tabular}




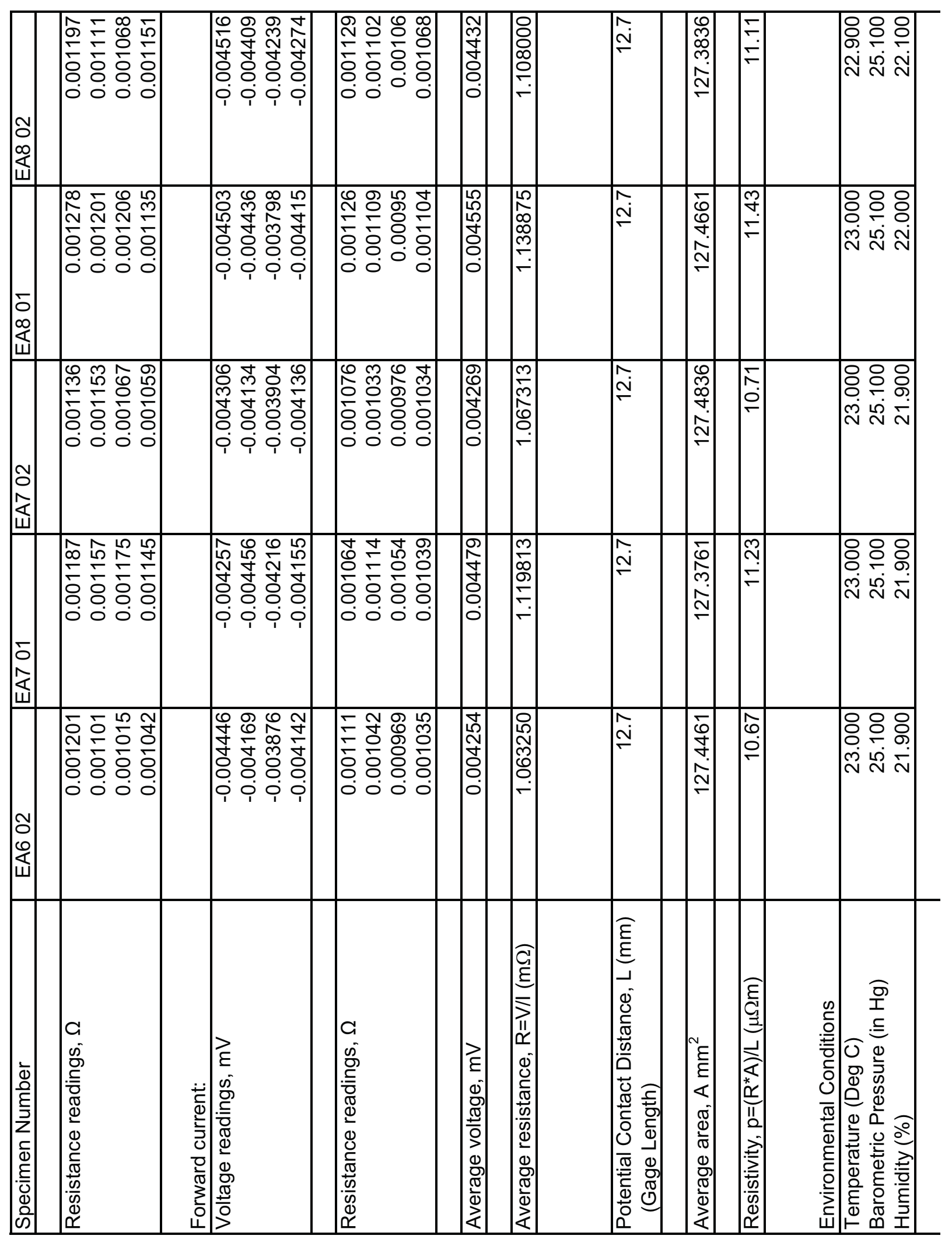




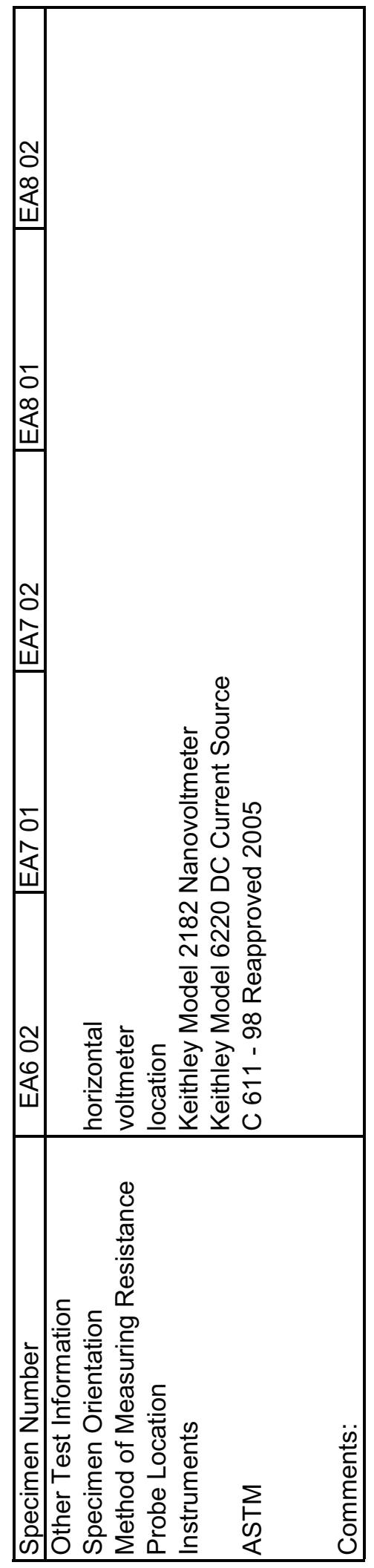




\begin{tabular}{|c|c|c|c|c|c|c|c|c|c|c|}
\hline 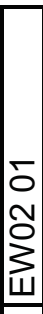 & 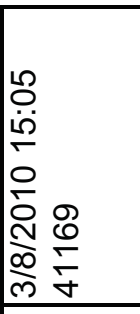 & 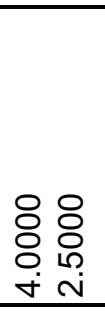 & & 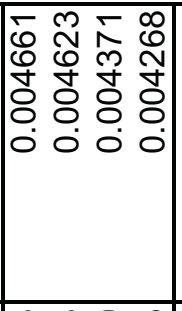 & 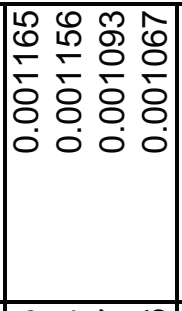 & & 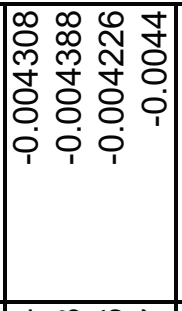 & 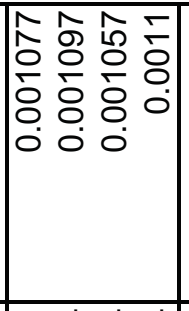 & & 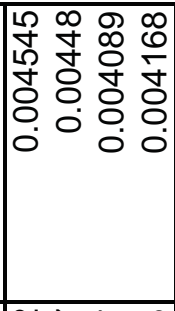 \\
\hline \begin{tabular}{|l} 
\\
0 \\
0 \\
\\
\\
\end{tabular} & 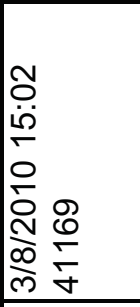 & 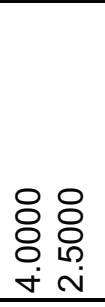 & & 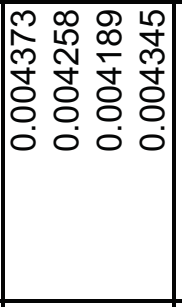 & 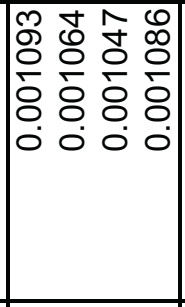 & & 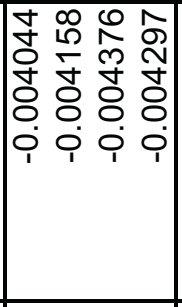 & 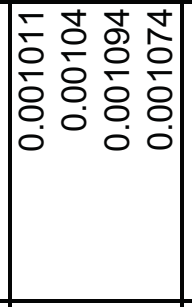 & & 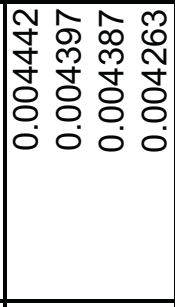 \\
\hline m & 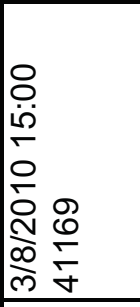 & 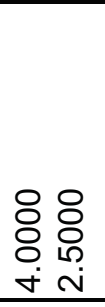 & & 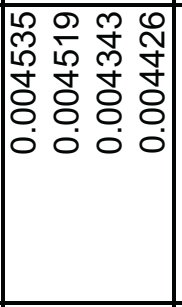 & 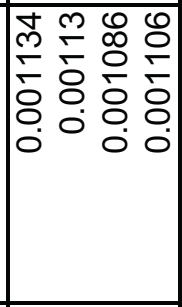 & & 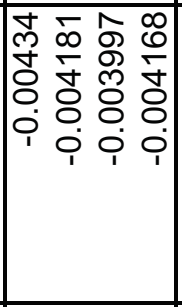 & 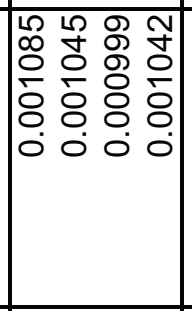 & & 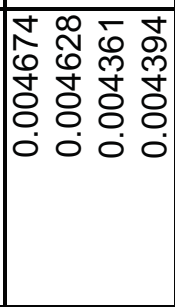 \\
\hline 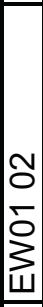 & 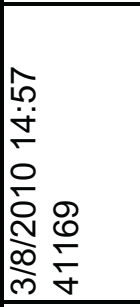 & 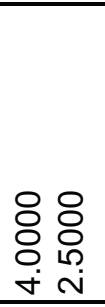 & & 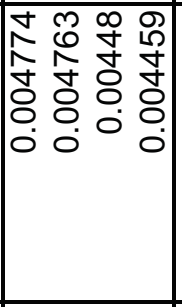 & 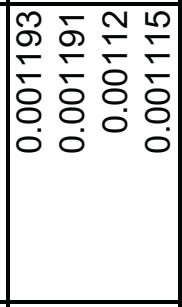 & & 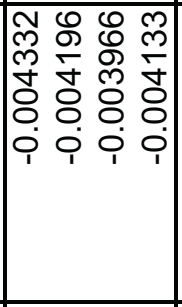 & 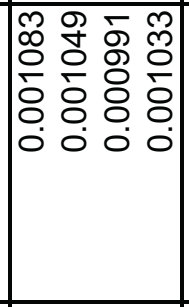 & & 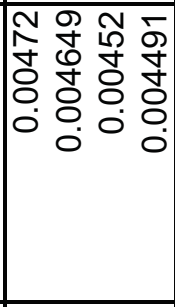 \\
\hline 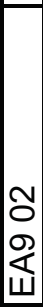 & 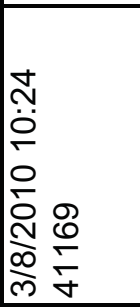 & 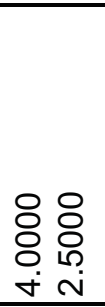 & & 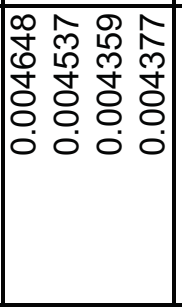 & 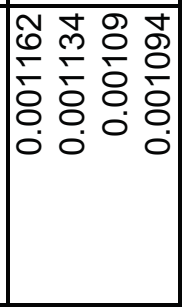 & & 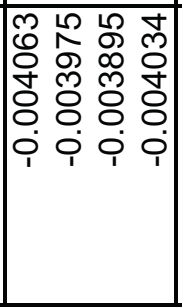 & 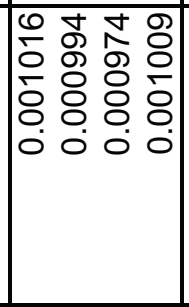 & & 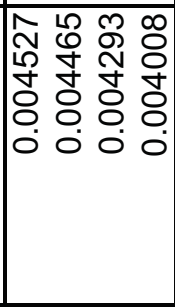 \\
\hline \begin{tabular}{|l} 
\\
0 \\
0 \\
\\
\end{tabular} & 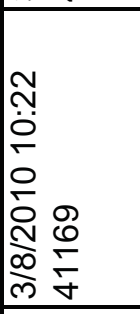 & $\begin{array}{l}8 \\
8 \\
8\end{array}$ & & 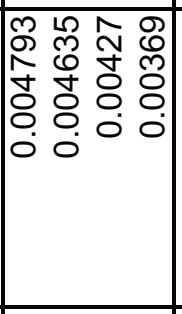 & 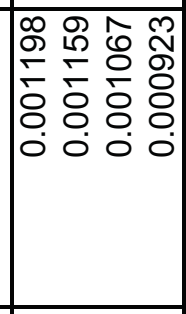 & & 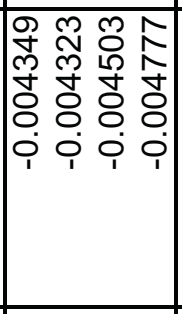 & 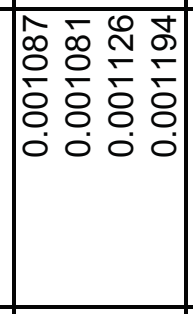 & & 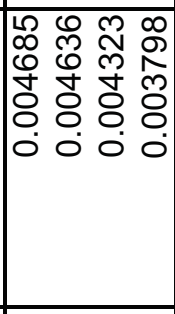 \\
\hline 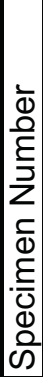 & 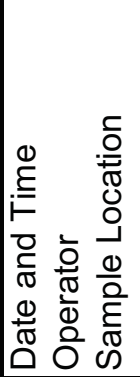 & 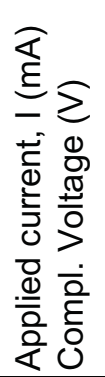 & 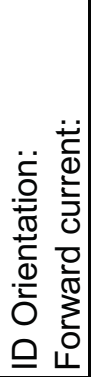 & 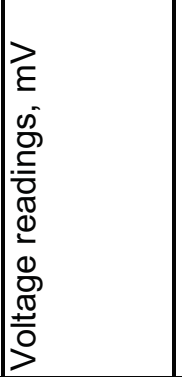 & 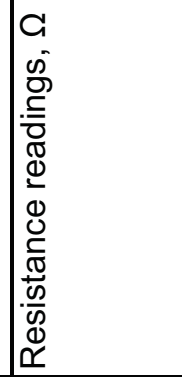 & 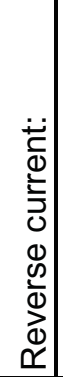 & 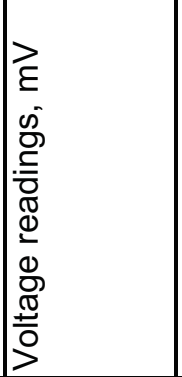 & 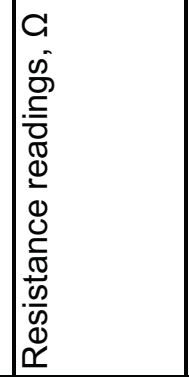 & 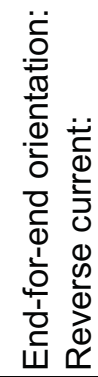 & 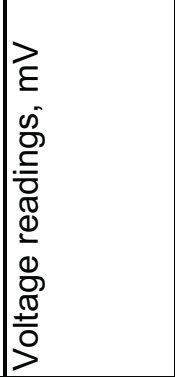 \\
\hline
\end{tabular}




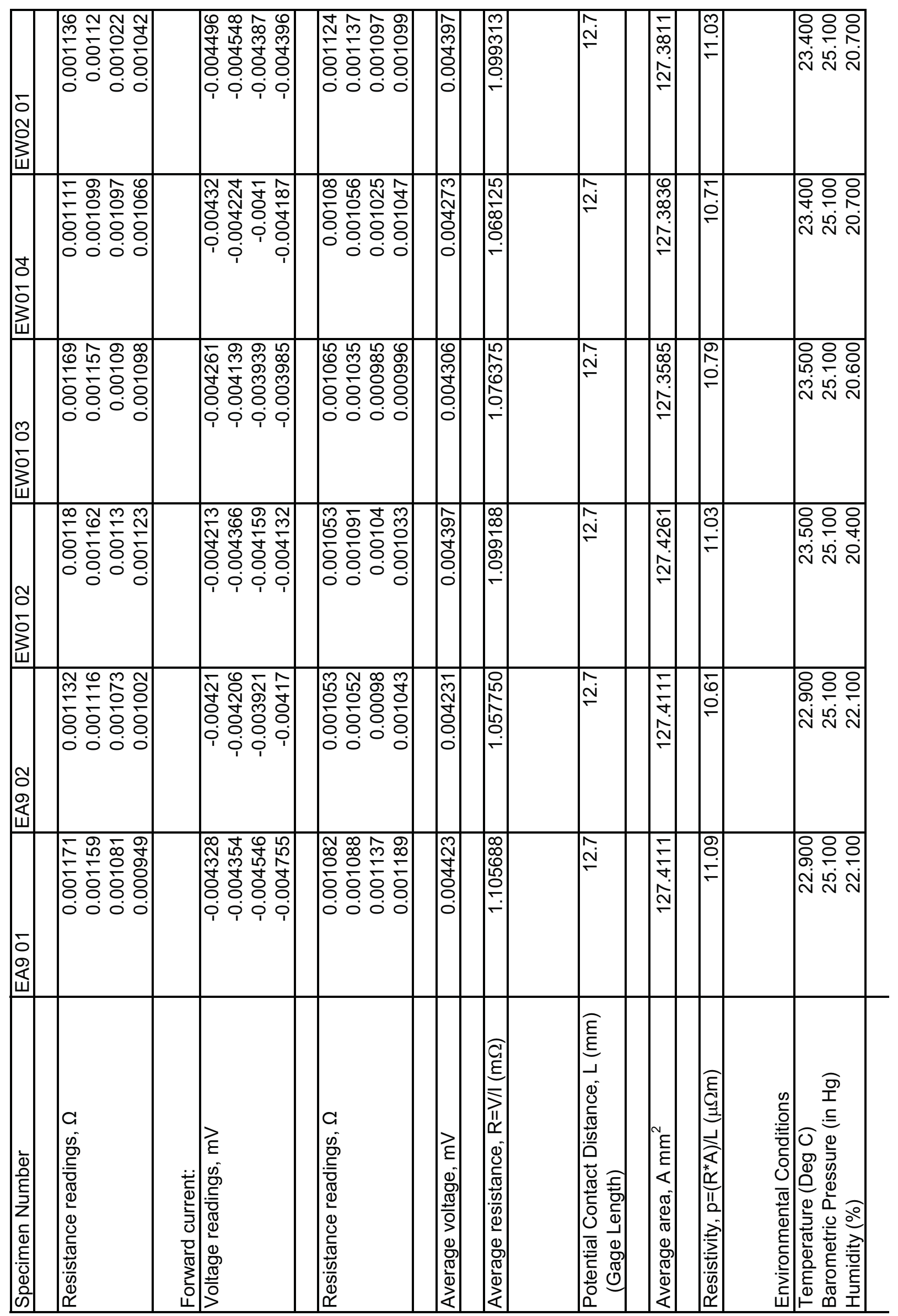




\begin{tabular}{|c|c|c|c|c|c|c|c|c|}
\hline 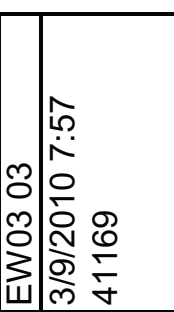 & 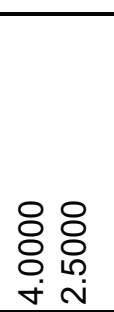 & & 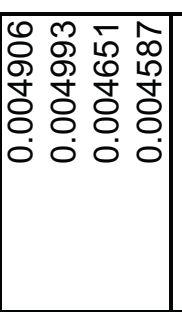 & 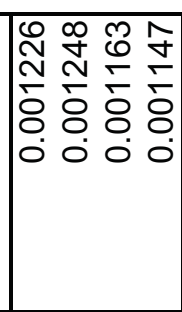 & 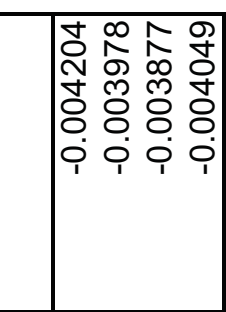 & 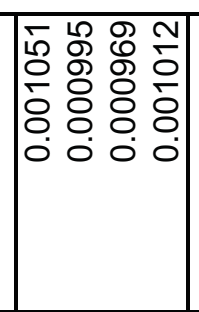 & & 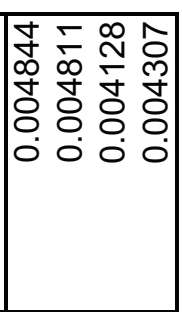 \\
\hline 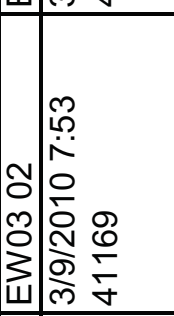 & 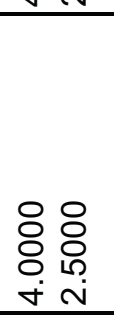 & & 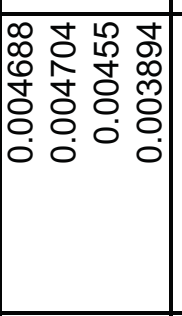 & 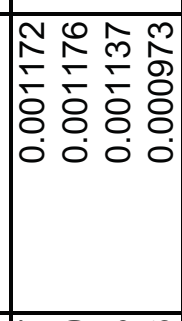 & 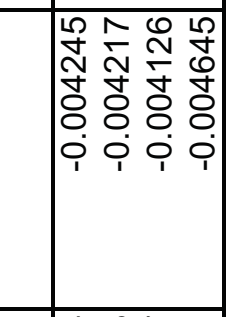 & 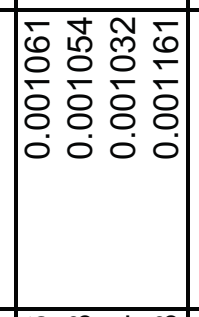 & & 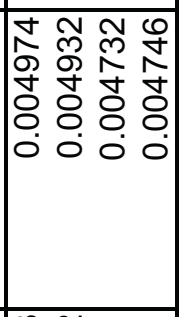 \\
\hline 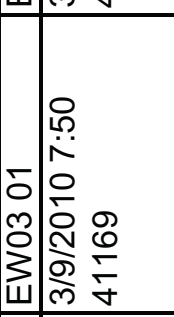 & 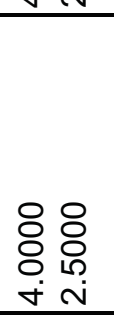 & & 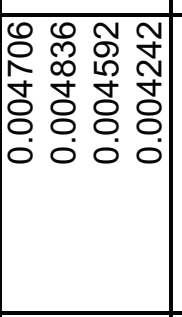 & 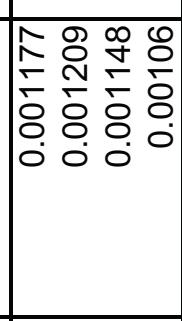 & 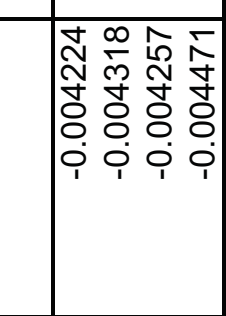 & 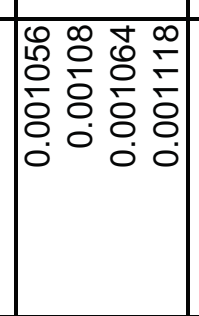 & & 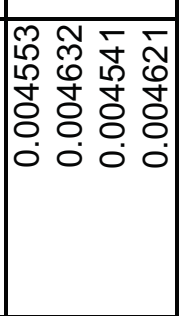 \\
\hline 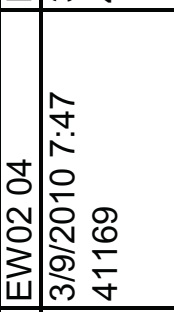 & 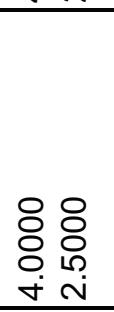 & & 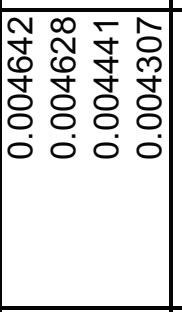 & 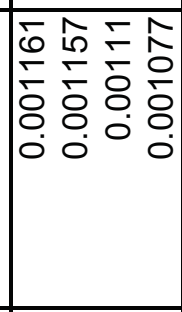 & 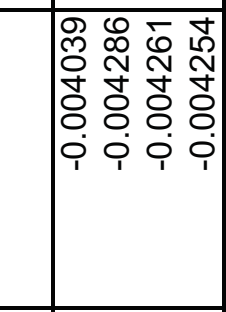 & $\left|\begin{array}{llll}\overline{0} & \overline{1} & \multicolumn{1}{|c}{} \\
\hdashline & 0 & 0 \\
8 & 0 & 0 & 0 \\
0 & 0 & 0 & 0 \\
0 & 0 & 0 & 0 \\
0 & 0 & 0 & 0\end{array}\right|$ & & 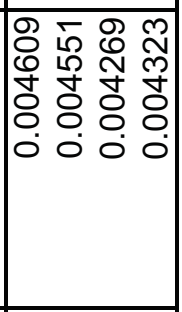 \\
\hline 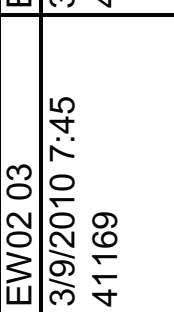 & 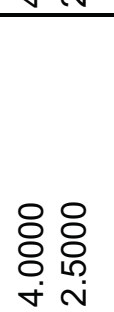 & & 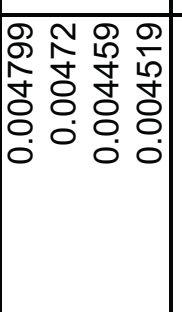 & 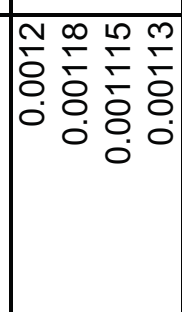 & 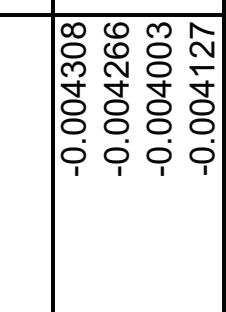 & 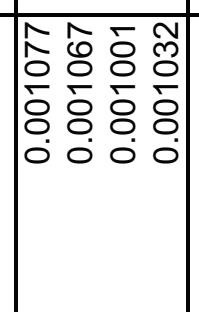 & & 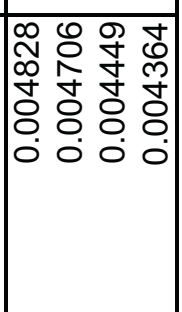 \\
\hline 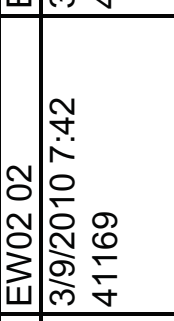 & 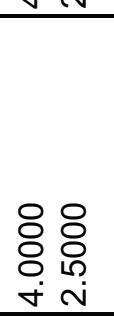 & & 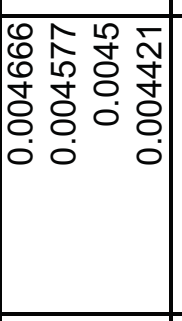 & 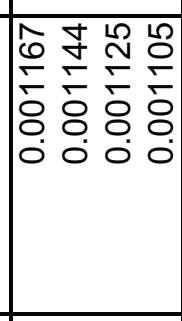 & 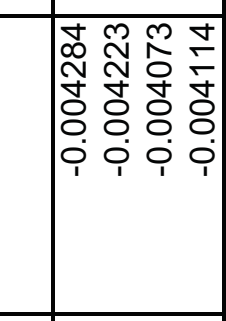 & 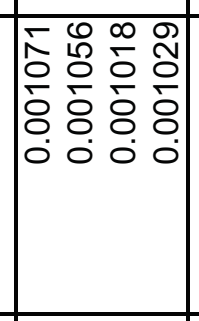 & & 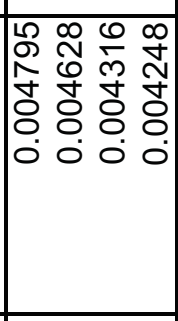 \\
\hline 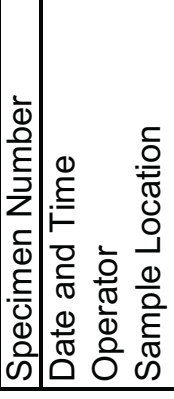 & 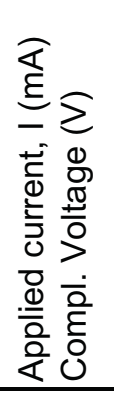 & 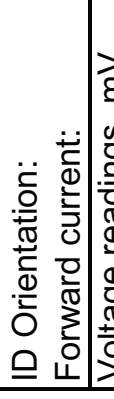 & 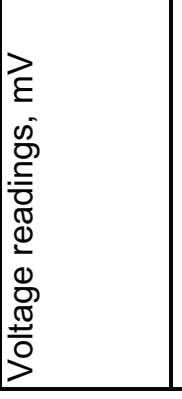 & 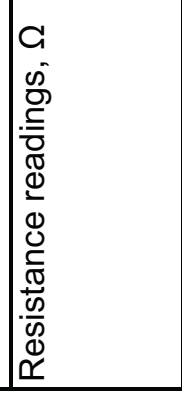 & 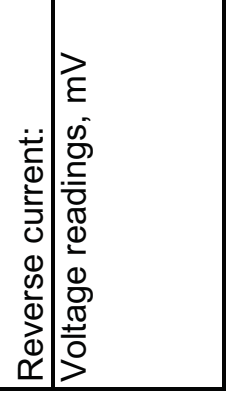 & 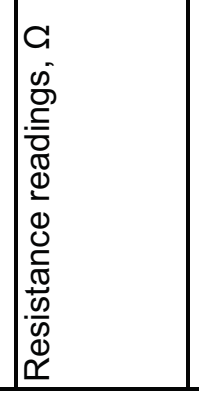 & 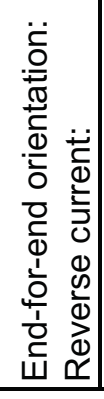 & 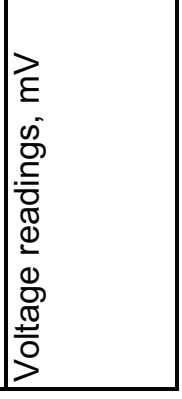 \\
\hline
\end{tabular}




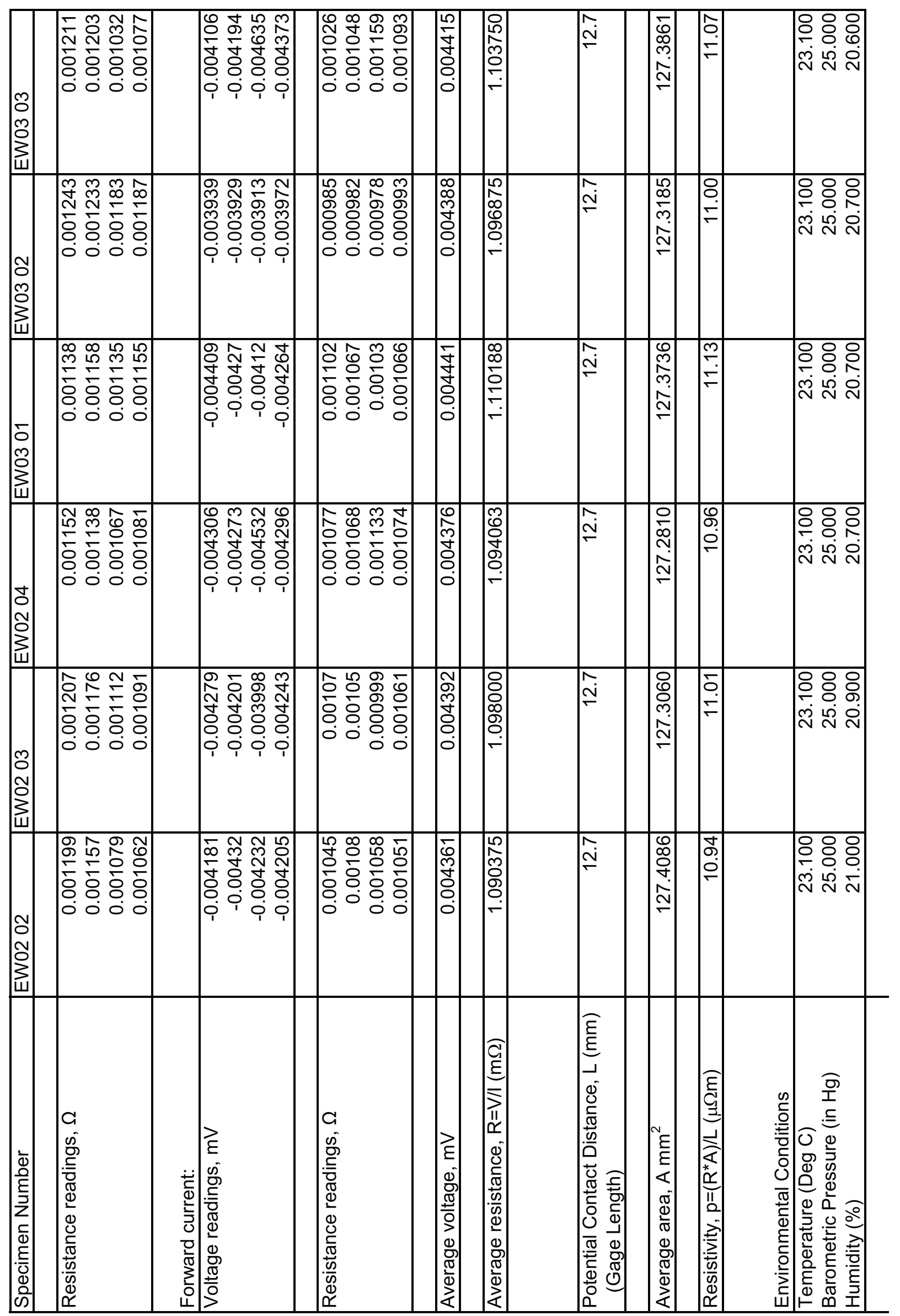




\begin{tabular}{|c|c|c|c|c|c|c|c|c|}
\hline 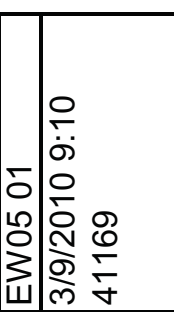 & 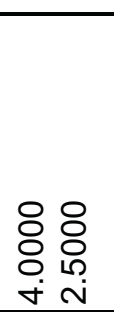 & & 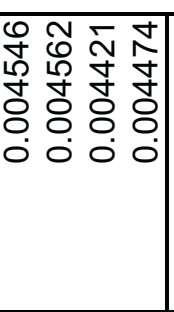 & 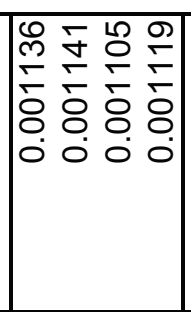 & 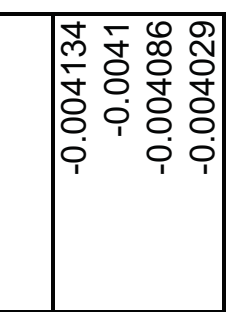 & 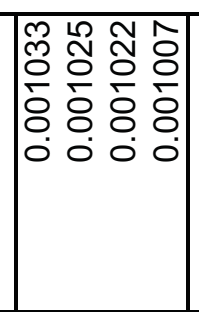 & & 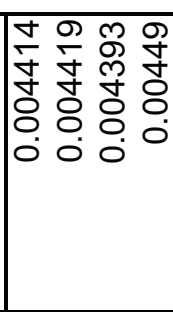 \\
\hline 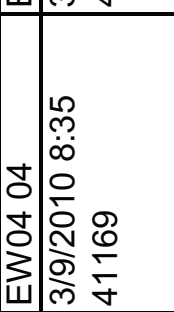 & 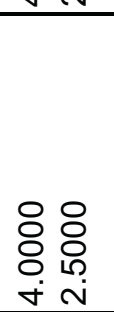 & & 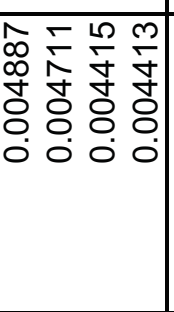 & 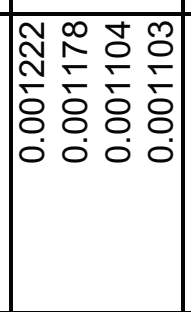 & 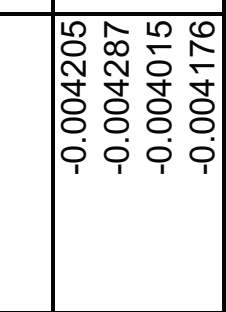 & 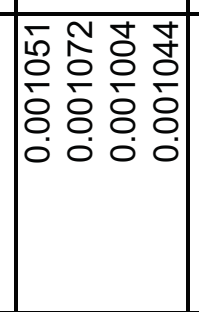 & & 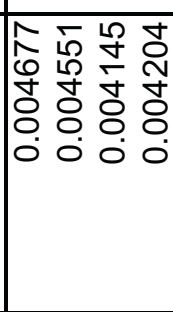 \\
\hline 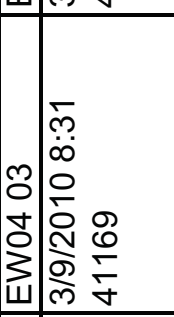 & 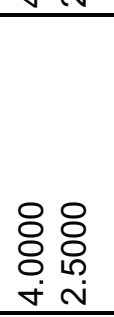 & & 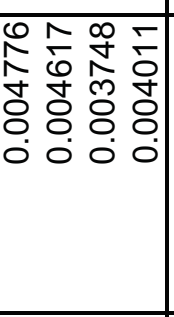 & 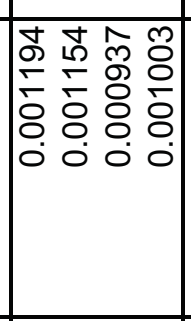 & 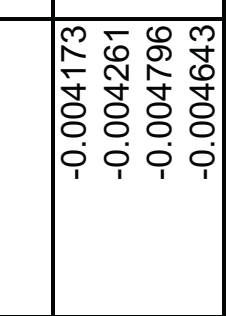 & 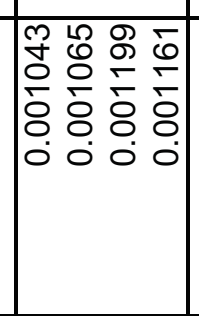 & & 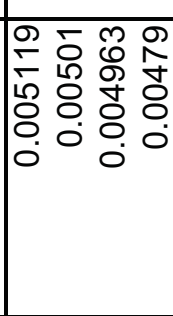 \\
\hline 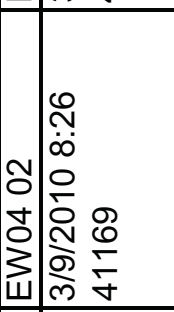 & 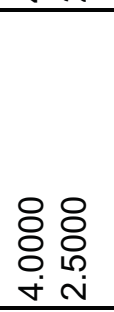 & & 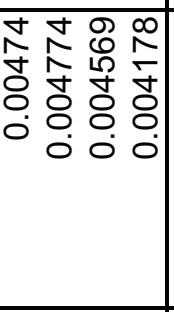 & 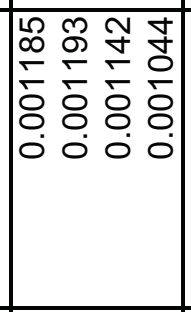 & 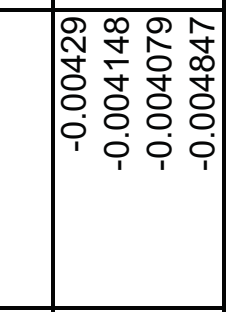 & 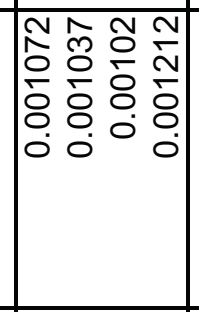 & & 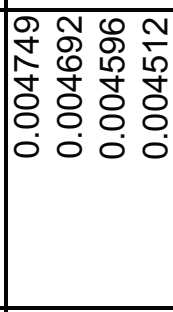 \\
\hline 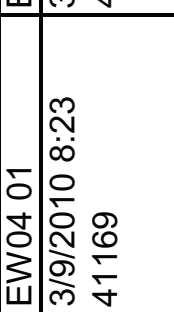 & 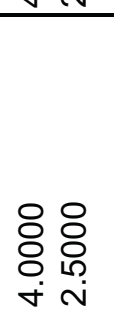 & & 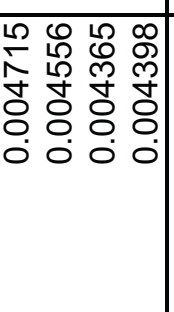 & 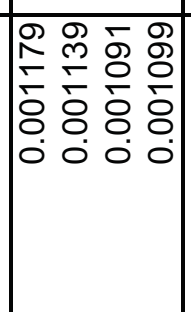 & 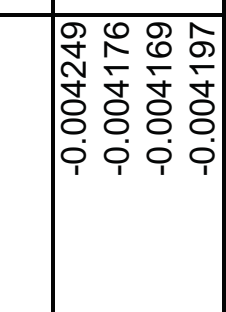 & 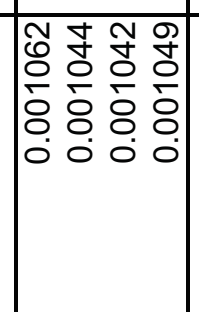 & & 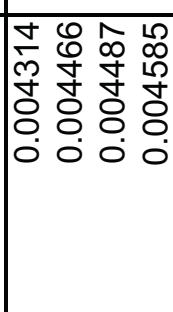 \\
\hline 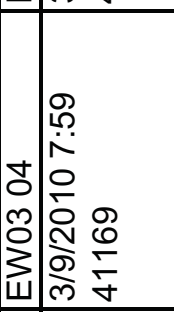 & 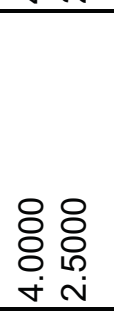 & & 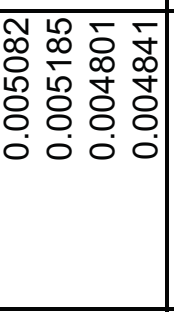 & 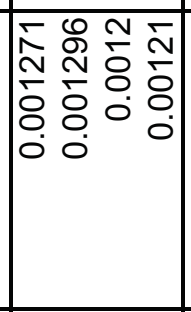 & 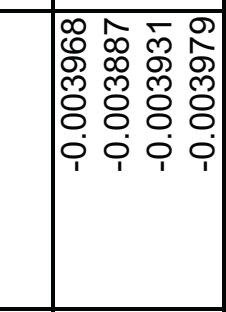 & 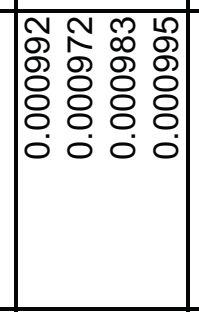 & & 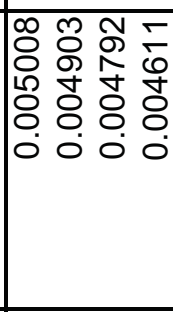 \\
\hline 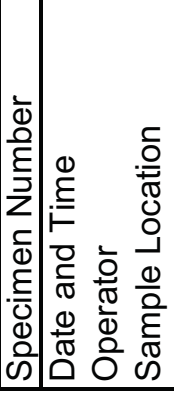 & 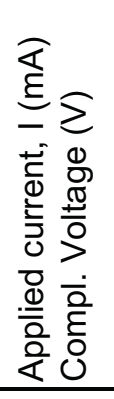 & 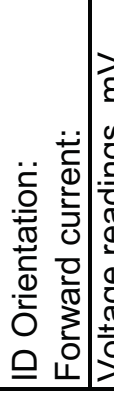 & 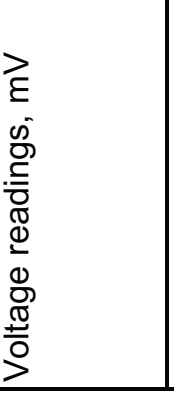 & 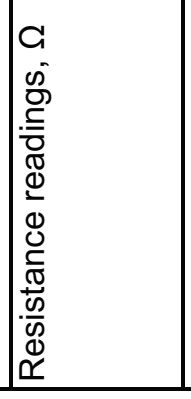 & 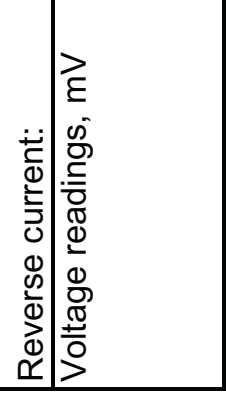 & 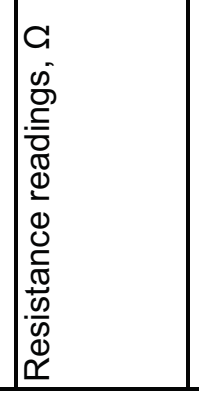 & 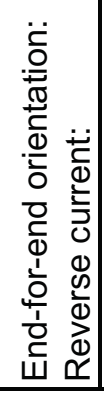 & 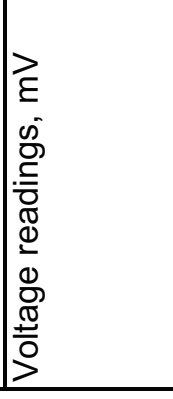 \\
\hline
\end{tabular}




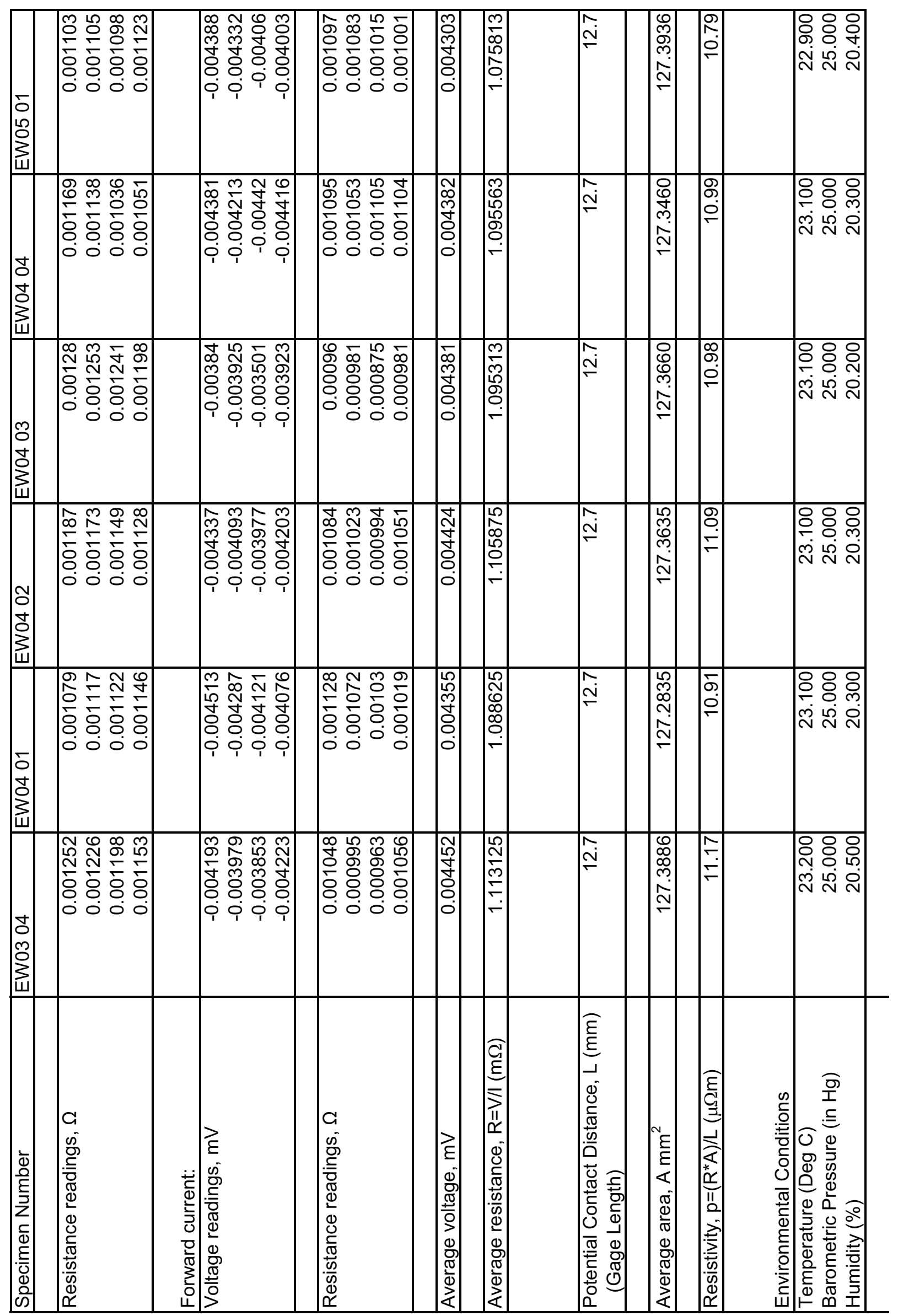




\begin{tabular}{|c|c|c|c|c|c|c|c|c|}
\hline 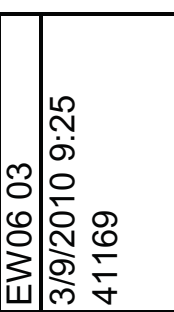 & 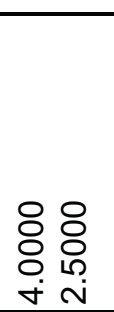 & & 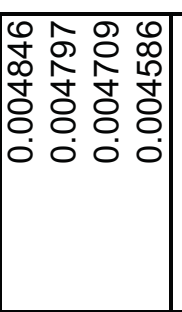 & 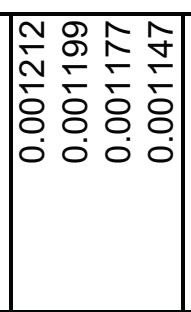 & 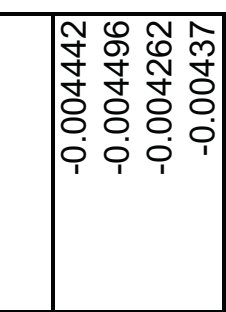 & 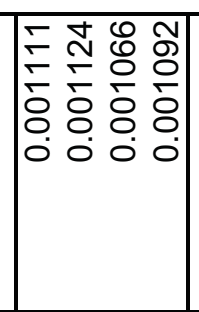 & & 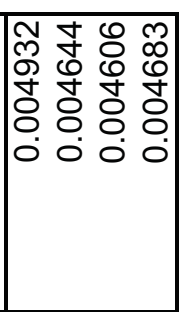 \\
\hline 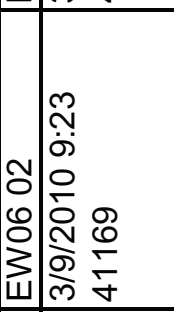 & 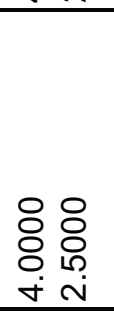 & & 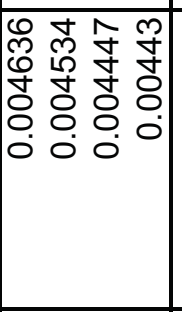 & 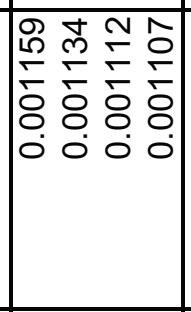 & 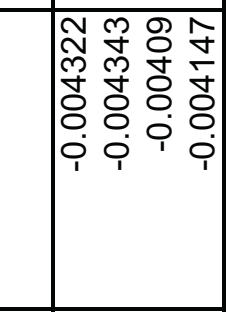 & 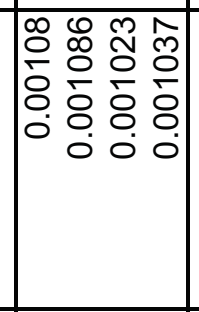 & & 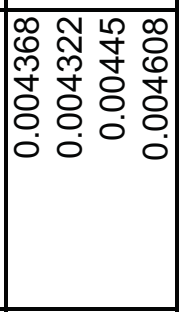 \\
\hline 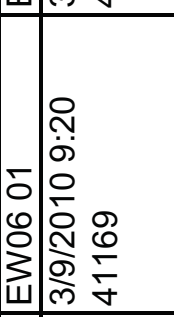 & 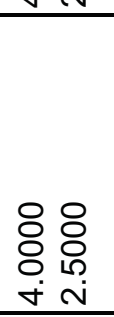 & & 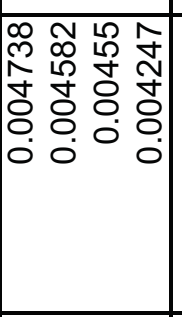 & 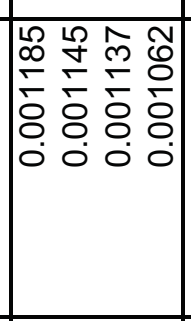 & 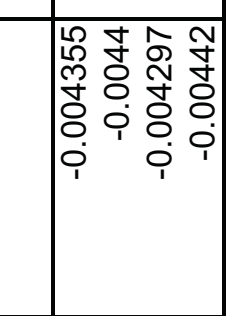 & 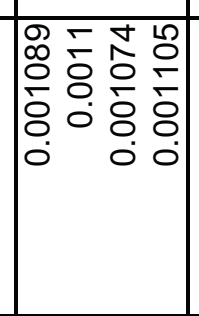 & & 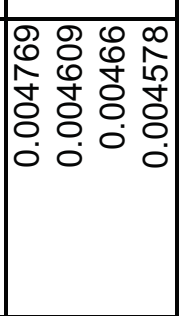 \\
\hline 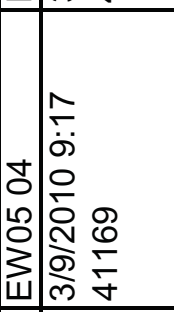 & 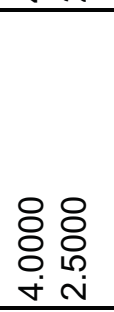 & & 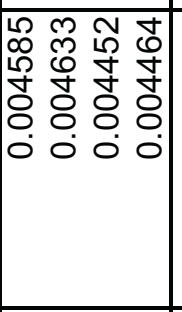 & 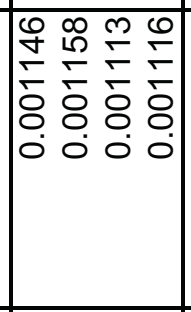 & 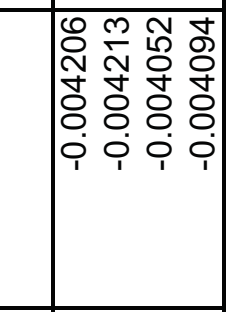 & 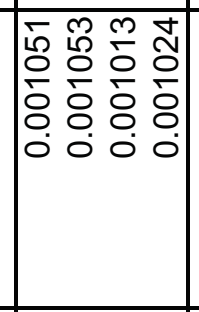 & & 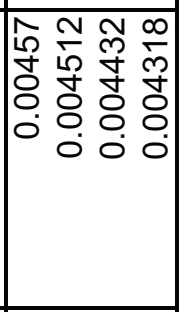 \\
\hline 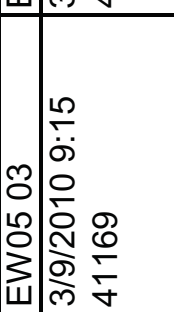 & 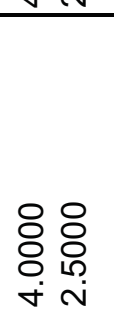 & & 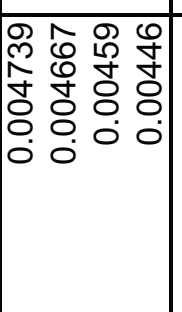 & 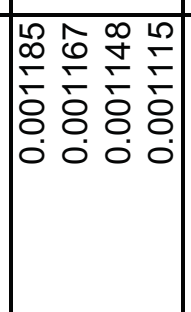 & 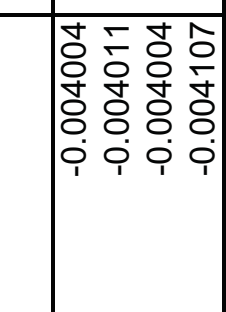 & 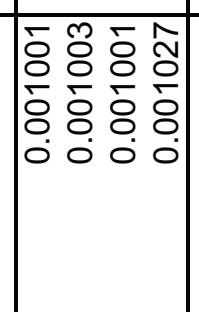 & & 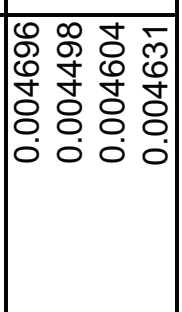 \\
\hline 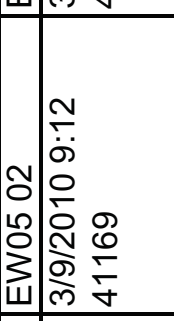 & 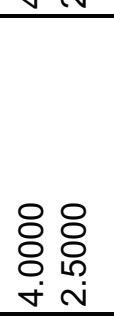 & & 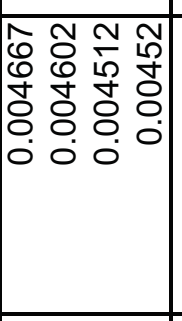 & 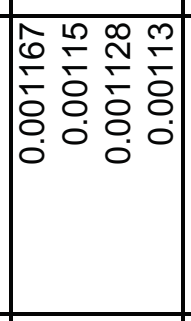 & 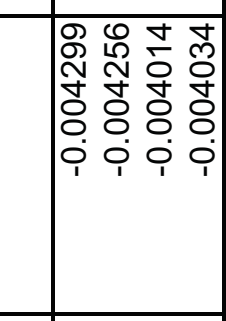 & 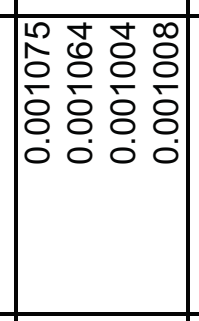 & & 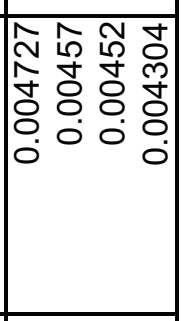 \\
\hline 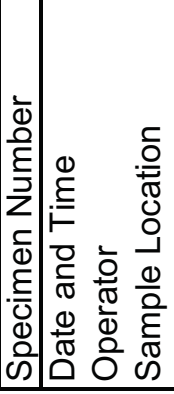 & 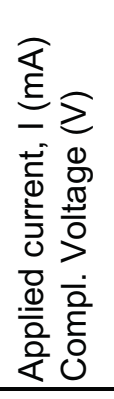 & 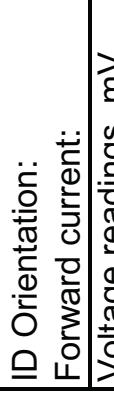 & 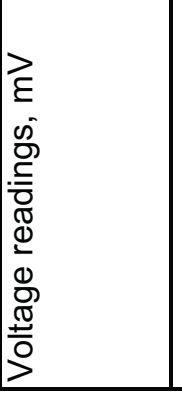 & 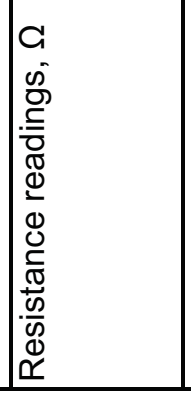 & 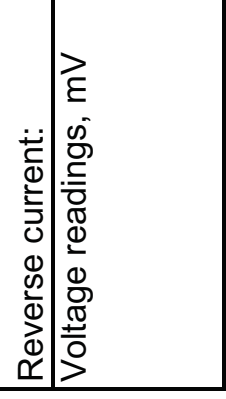 & 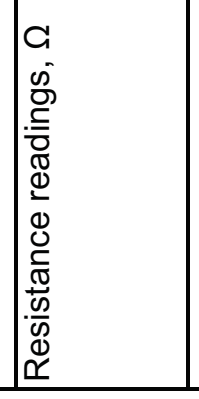 & 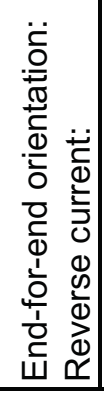 & 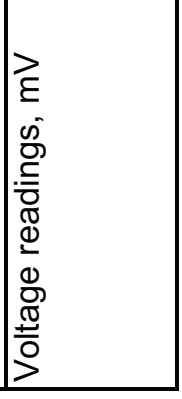 \\
\hline
\end{tabular}




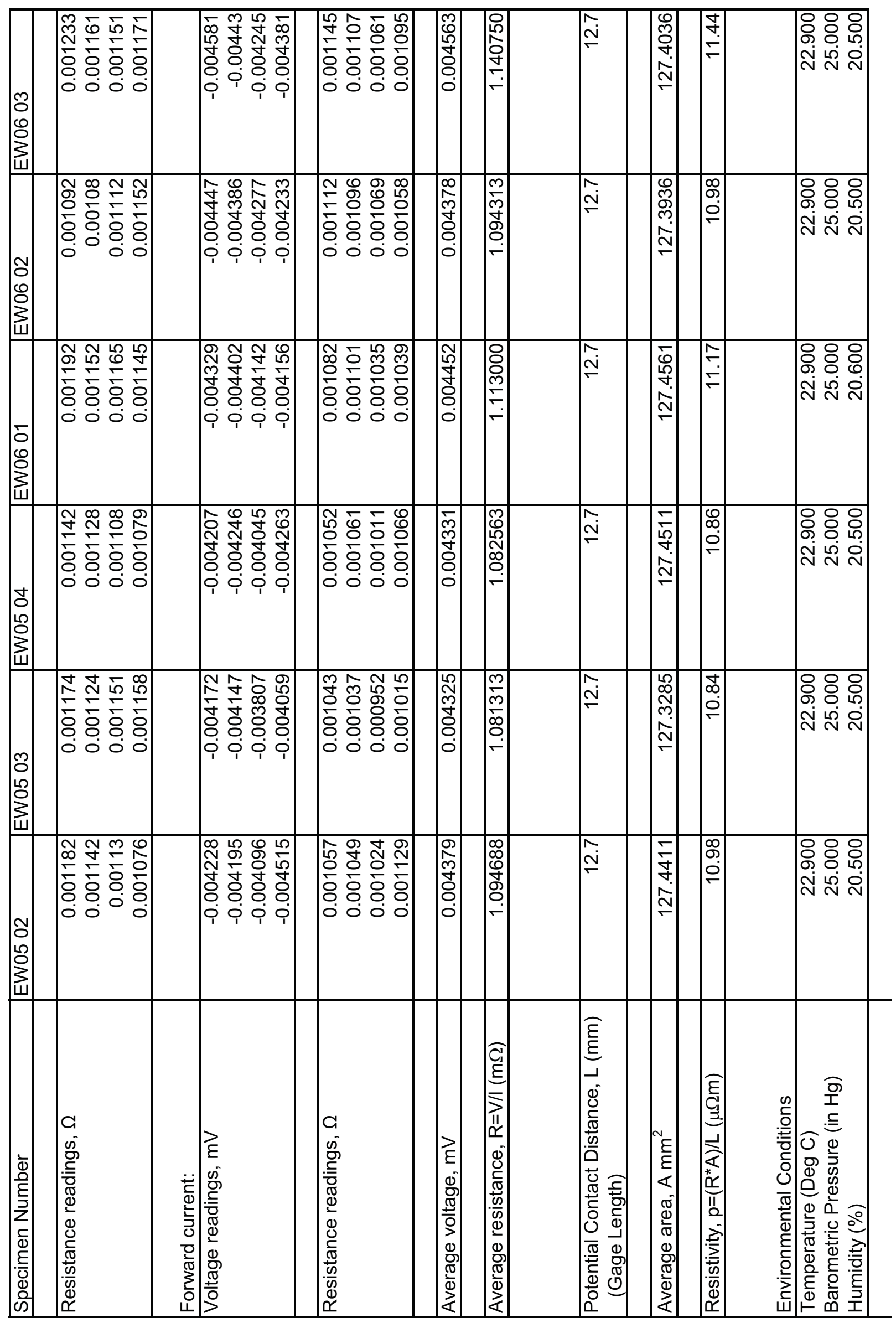




\begin{tabular}{|c|c|c|c|c|c|c|c|c|c|c|}
\hline $\mid \begin{array}{l}5 \\
0 \\
0 \\
0 \\
3 \\
\\
\end{array}$ & 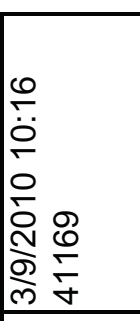 & 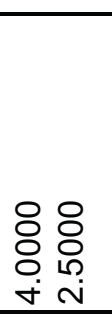 & & 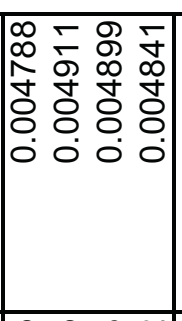 & 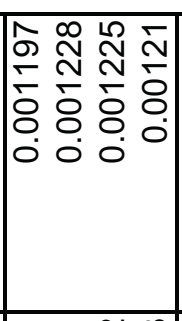 & & 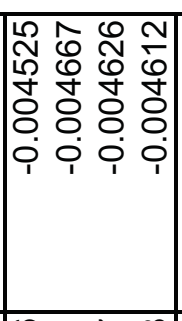 & 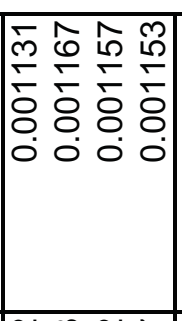 & & 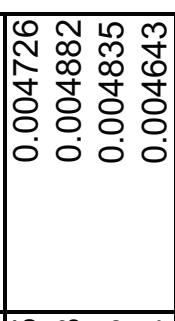 \\
\hline \begin{tabular}{|l} 
\\
0 \\
0 \\
0 \\
3 \\
\end{tabular} & 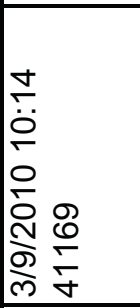 & 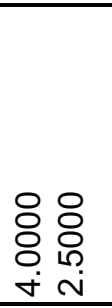 & & 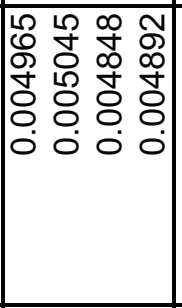 & 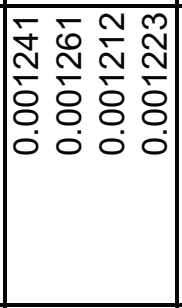 & & 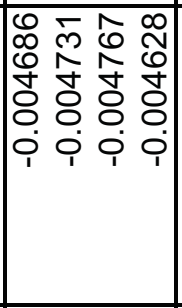 & 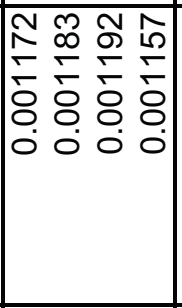 & & 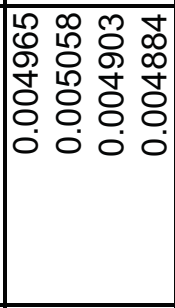 \\
\hline $\begin{array}{l}m \\
0 \\
1 \\
0 \\
3 \\
\\
\end{array}$ & 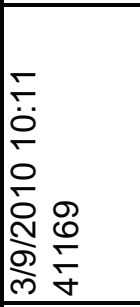 & 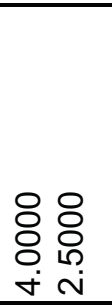 & & 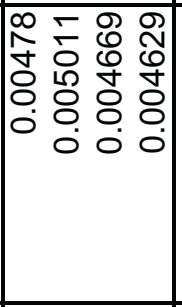 & 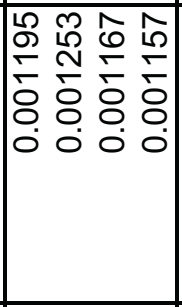 & & 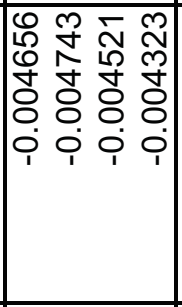 & 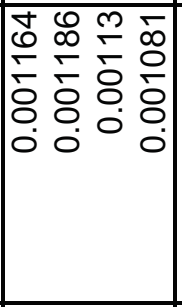 & & 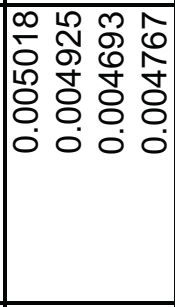 \\
\hline \begin{tabular}{|l} 
\\
\multirow{2}{*}{} \\
0 \\
0 \\
0 \\
3 \\
\\
\end{tabular} & 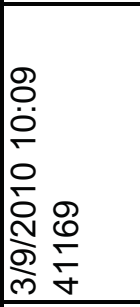 & 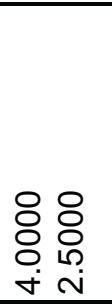 & & 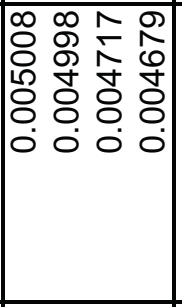 & 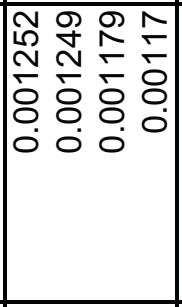 & & 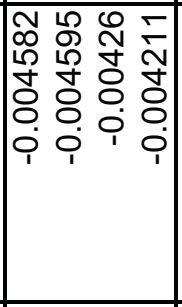 & 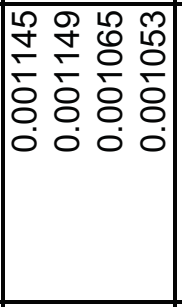 & & 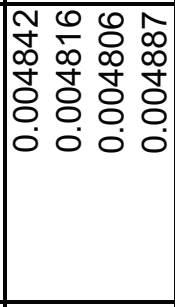 \\
\hline \begin{tabular}{|l} 
\\
$\delta$ \\
1 \\
0 \\
0 \\
3 \\
\end{tabular} & 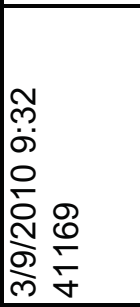 & $\begin{array}{l}8 \\
8 \\
8 \\
0 \\
\\
+\end{array}$ & & 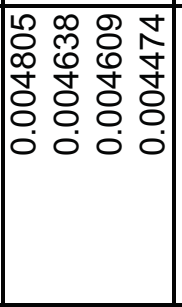 & 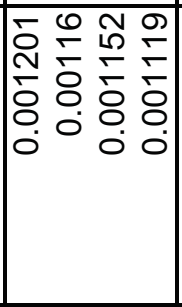 & & 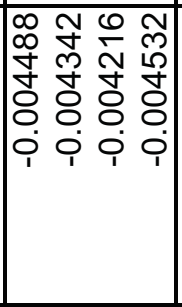 & 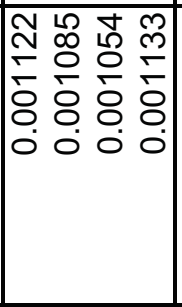 & & 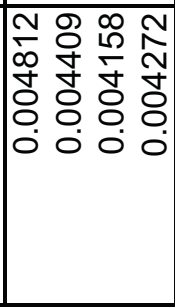 \\
\hline \begin{tabular}{|l} 
\\
0 \\
0 \\
9 \\
3 \\
\\
\end{tabular} & 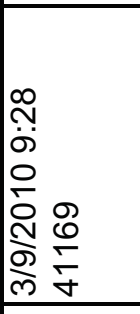 & 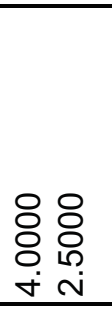 & & 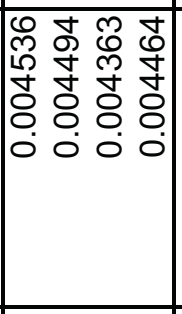 & 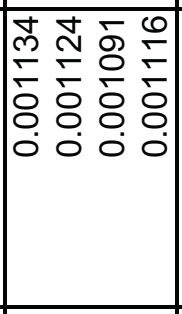 & & 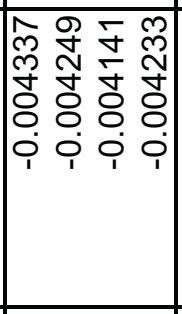 & 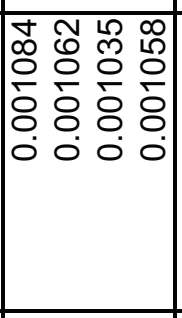 & & 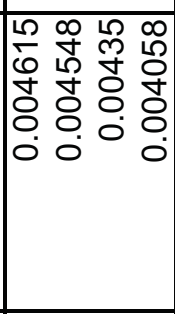 \\
\hline 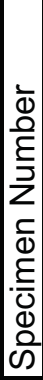 & 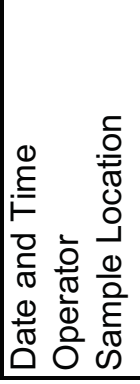 & 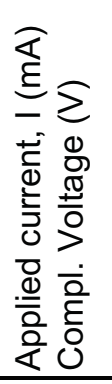 & 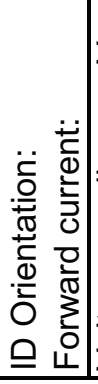 & 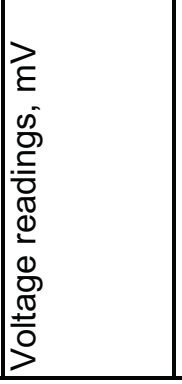 & 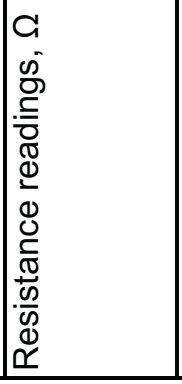 & 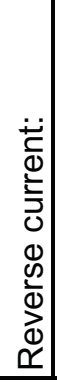 & 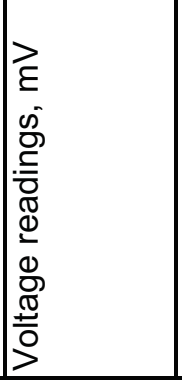 & 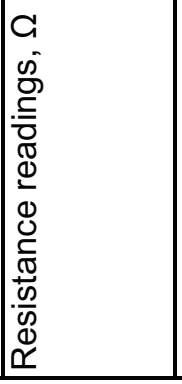 & 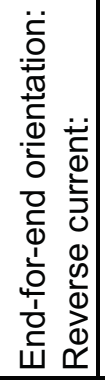 & 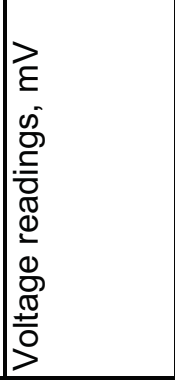 \\
\hline
\end{tabular}




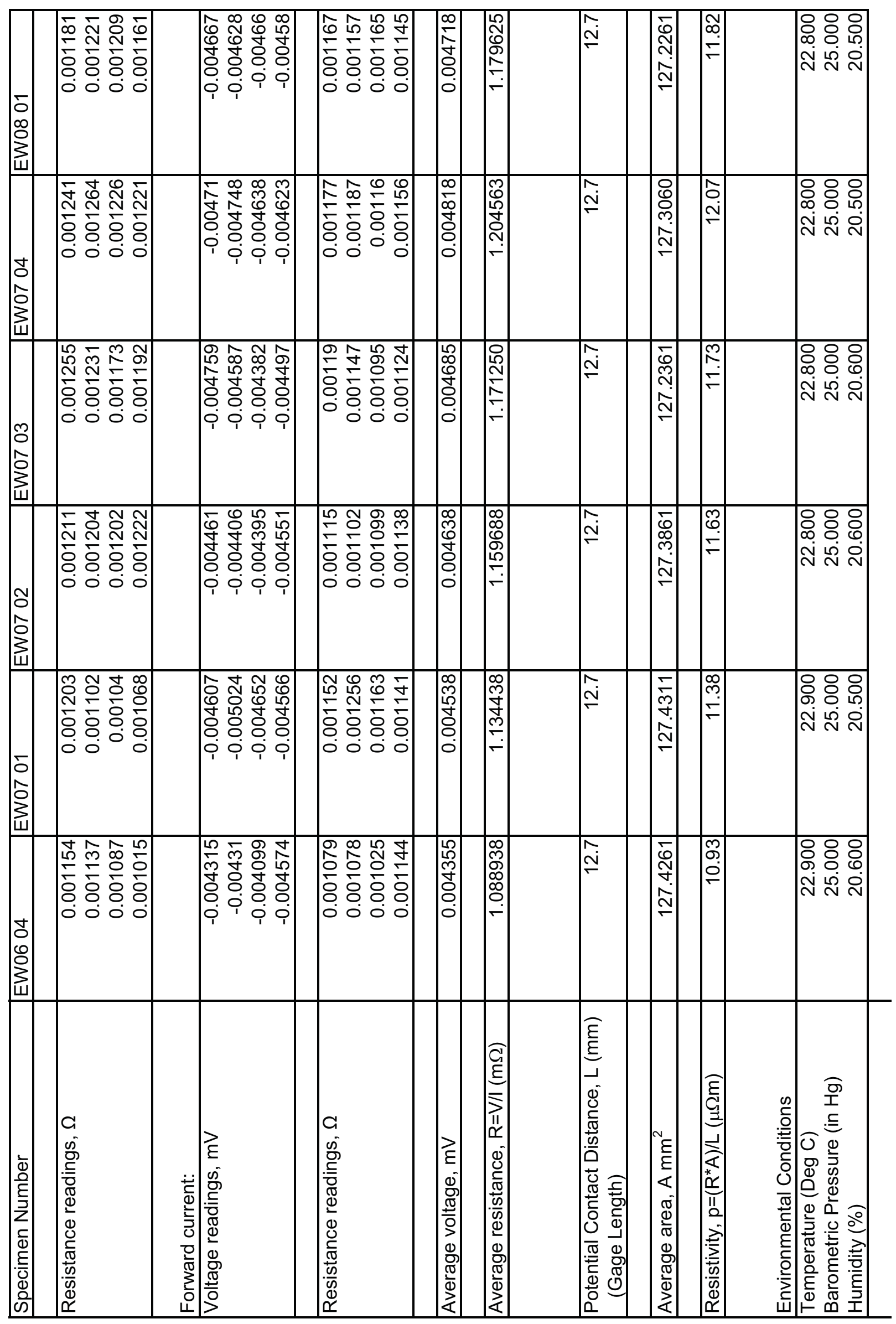




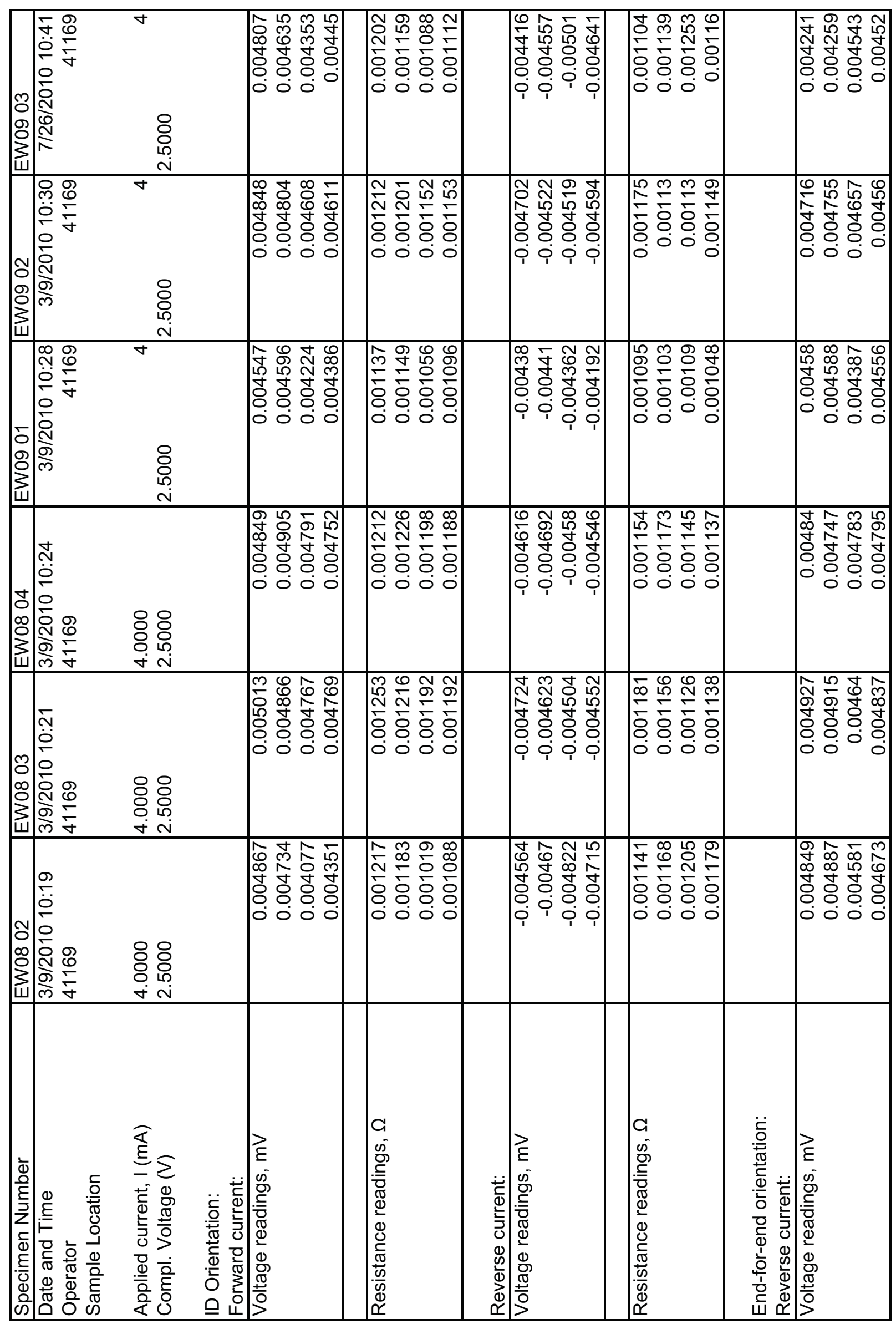




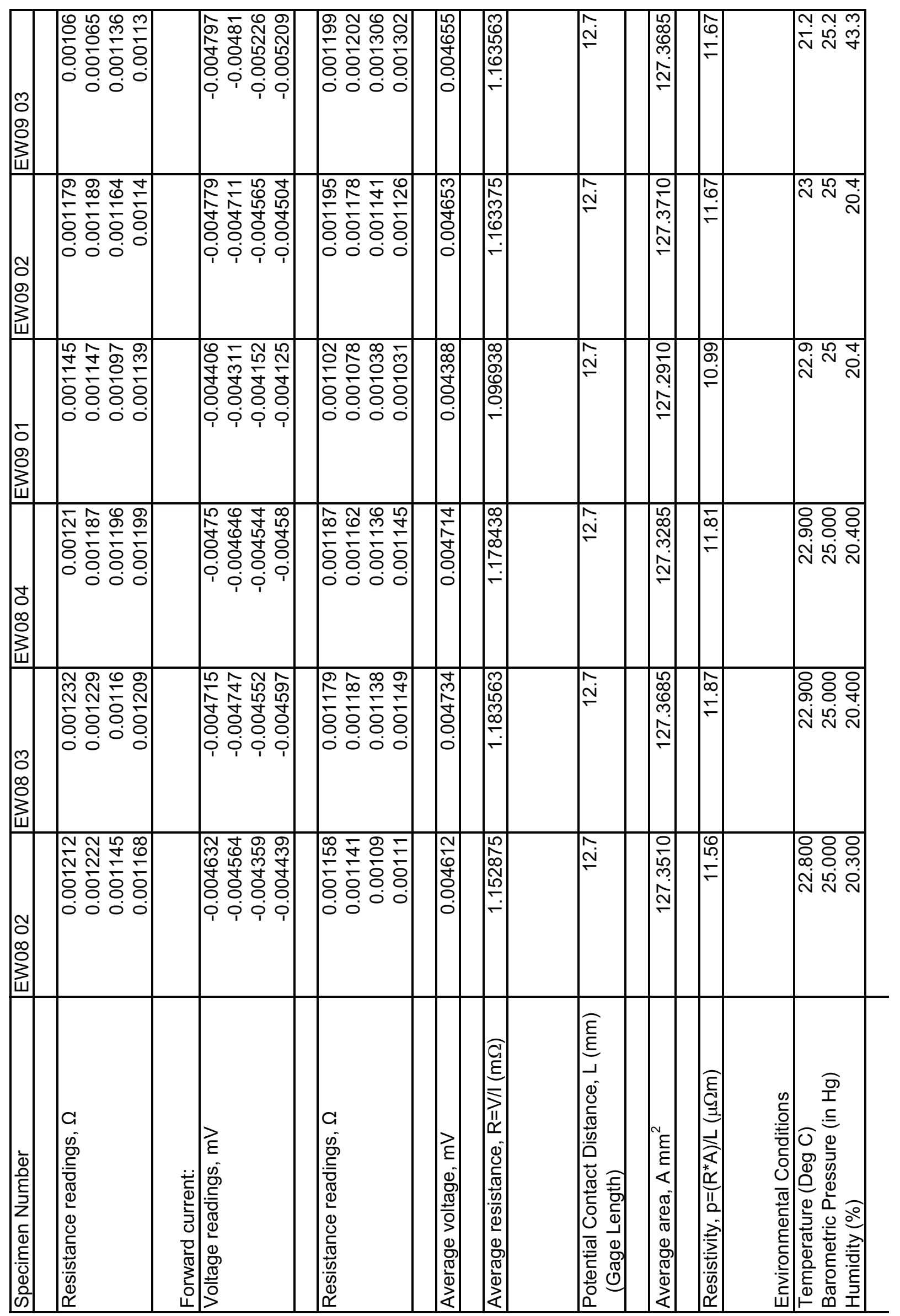




\begin{tabular}{|c|c|c|c|c|c|c|c|c|c|}
\hline 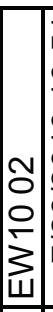 & 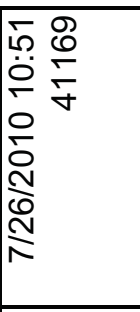 & $\begin{array}{l}8 \\
\stackrel{8}{0} \\
\stackrel{1}{0}\end{array}$ & & 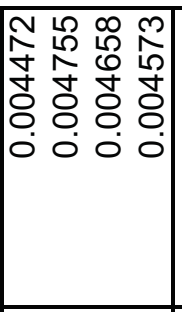 & 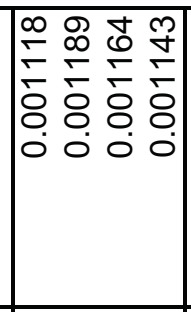 & 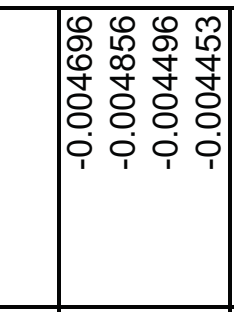 & 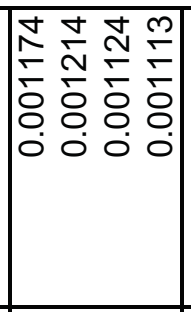 & & 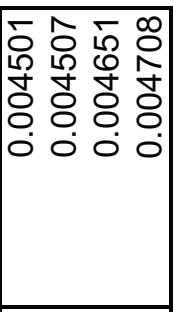 \\
\hline \begin{tabular}{|l|l} 
& \\
5 \\
0 \\
0 \\
0 \\
3 \\
4 \\
\end{tabular} & 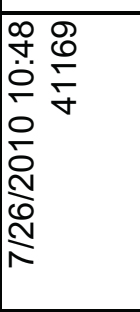 & $\begin{array}{l}8 \\
\text { চم } \\
\text { in }\end{array}$ & & 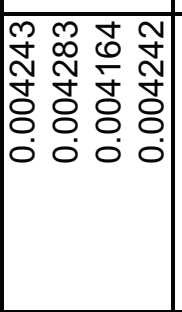 & 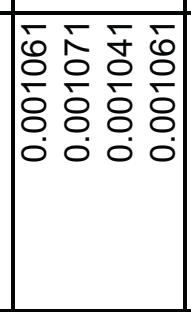 & 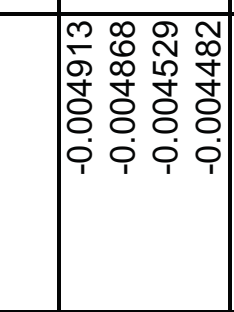 & 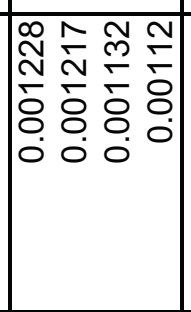 & & 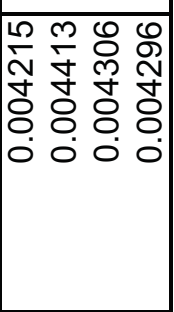 \\
\hline \begin{tabular}{|l|} 
\\
\\
8 \\
0 \\
3 \\
\end{tabular} & 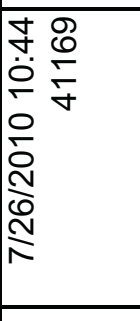 & $\begin{array}{l}8 \\
\stackrel{8}{0} \\
\stackrel{1}{0} \\
\text { ì }\end{array}$ & & 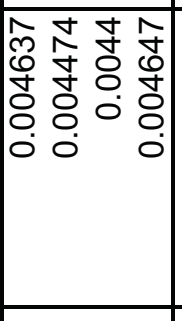 & 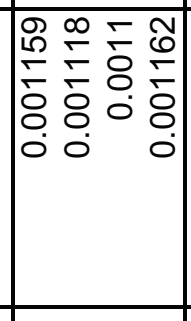 & 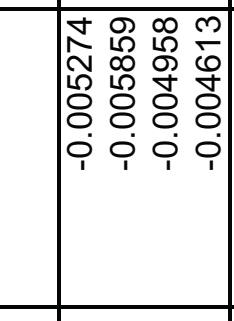 & 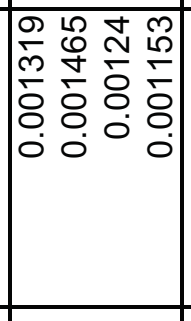 & & $\begin{array}{llll}0 & 0 & 0 \\
0 & 0 & 0 \\
1 & 0 & 0 \\
0 & 0 & 0 & \\
0 & 0 & 0 & 0 \\
0 & 0 & 0 & 0 \\
0 & 0 & 0 & 0\end{array}$ \\
\hline & 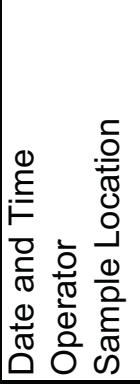 & 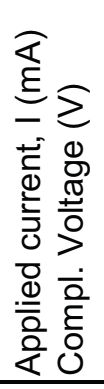 & 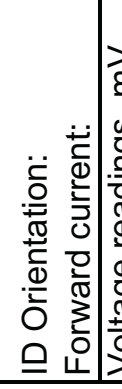 & 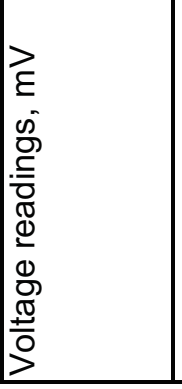 & 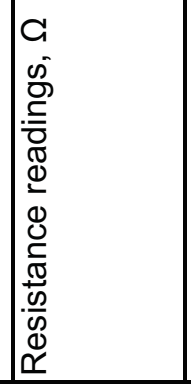 & 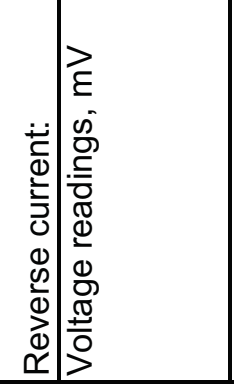 & 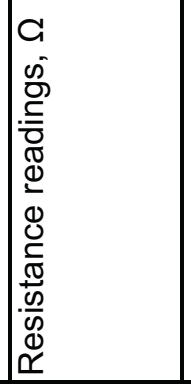 & 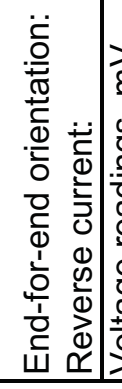 & 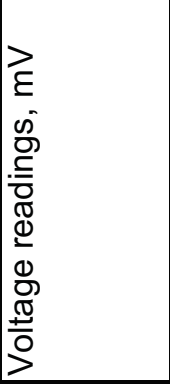 \\
\hline
\end{tabular}




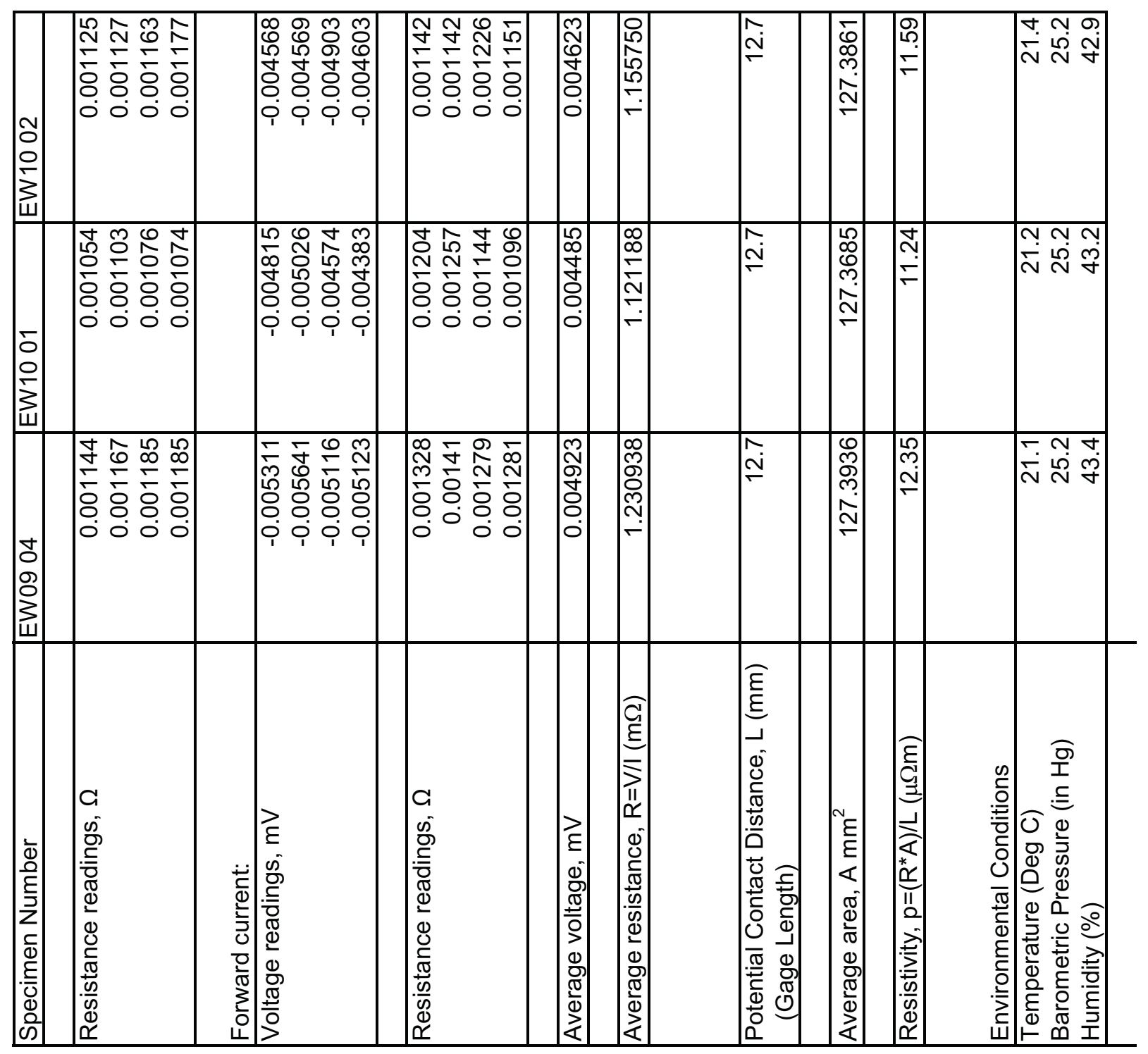




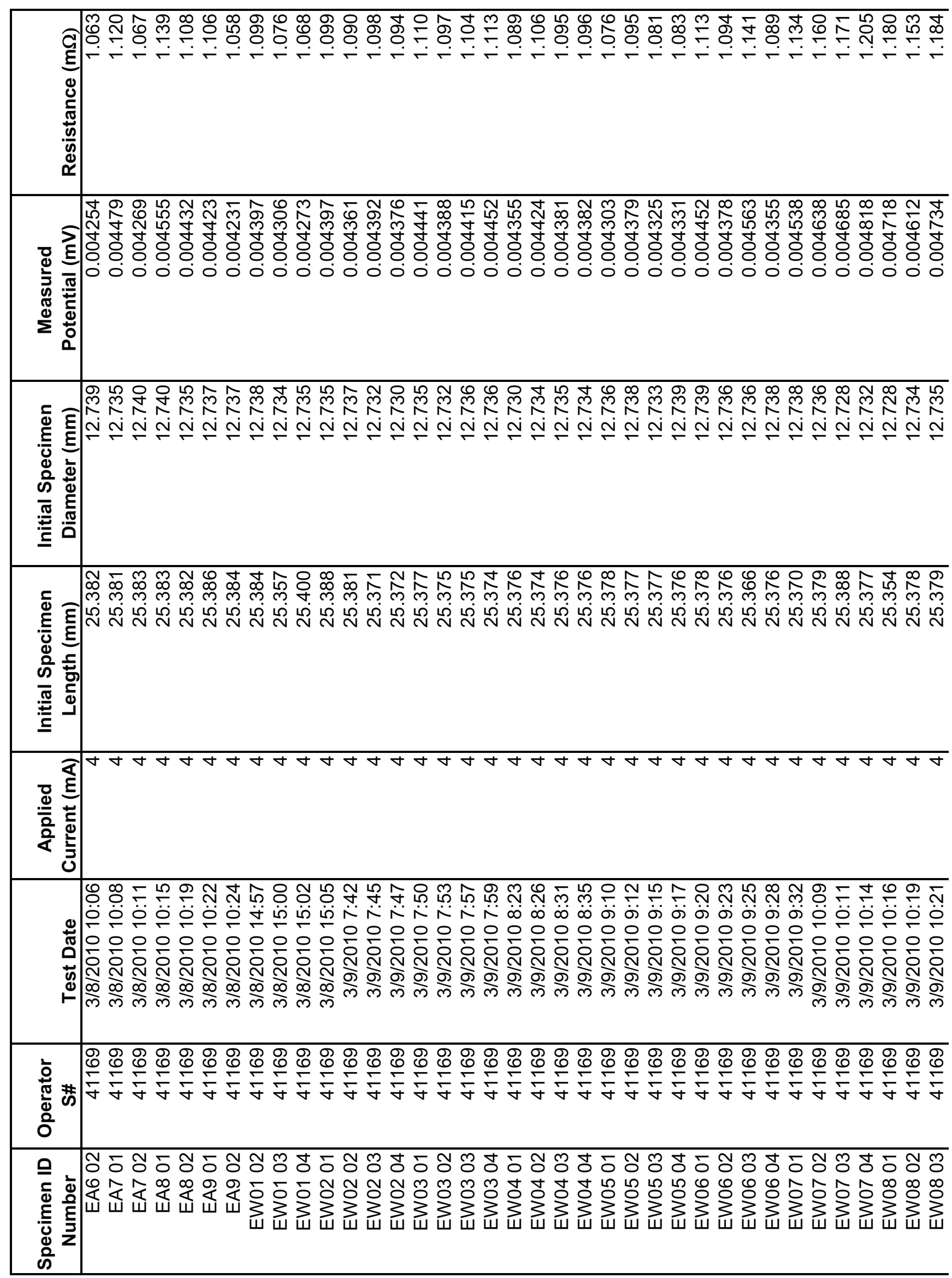




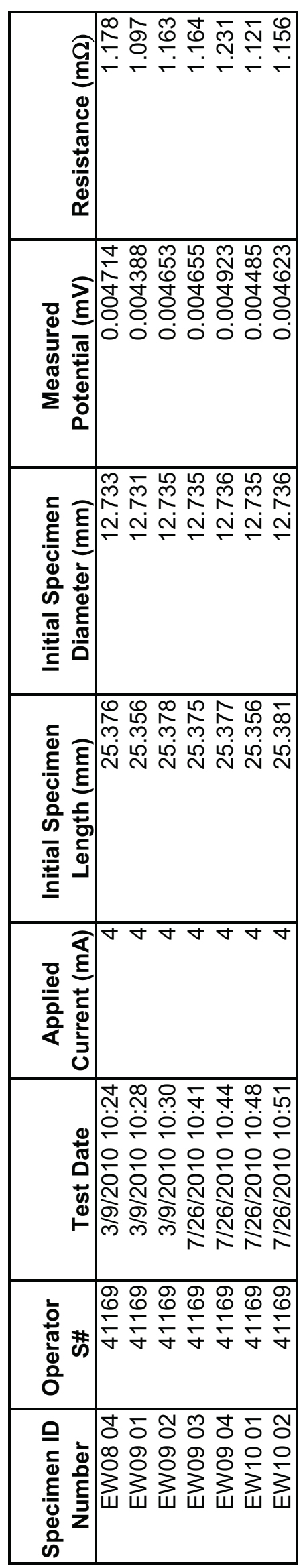




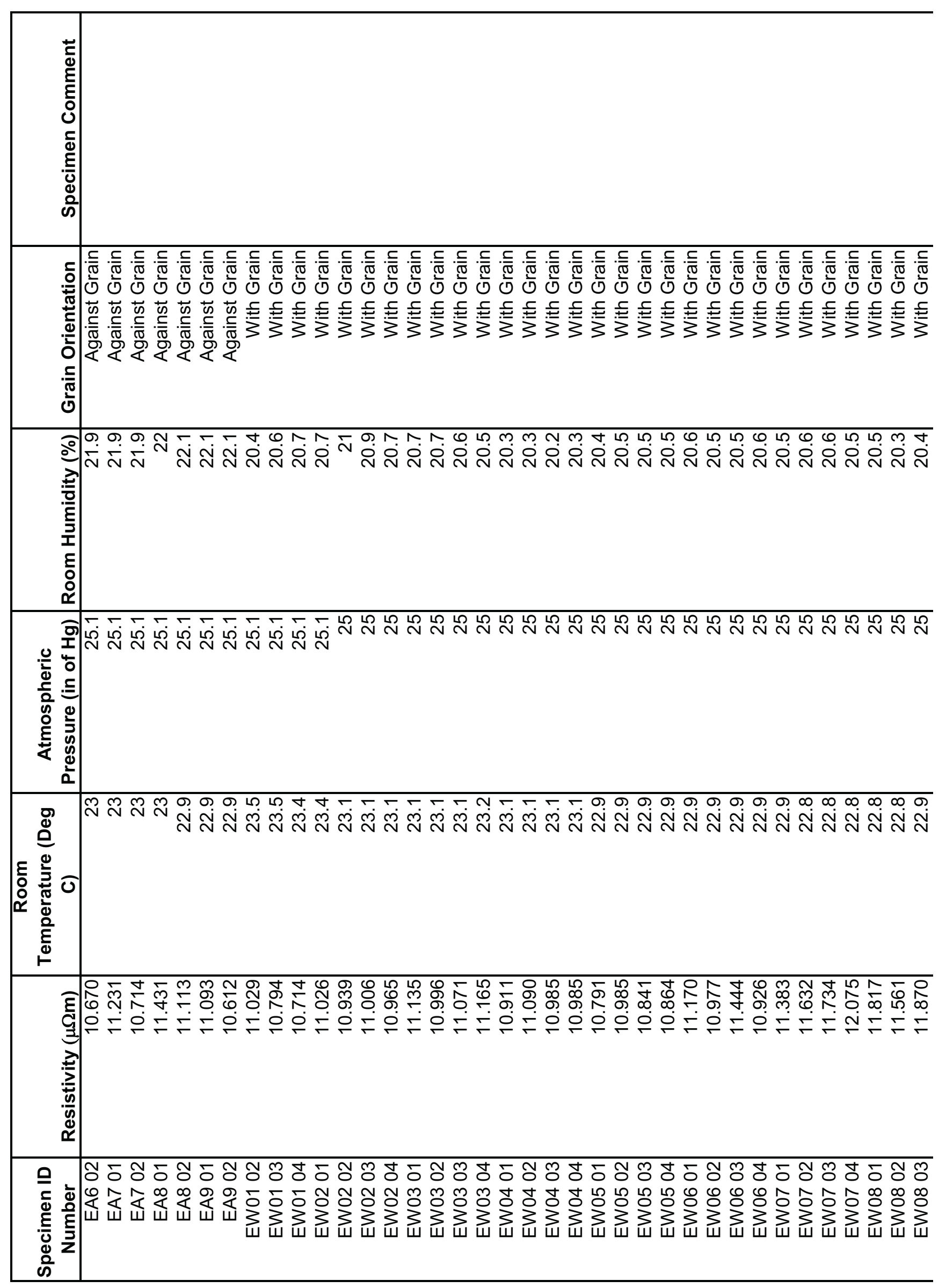




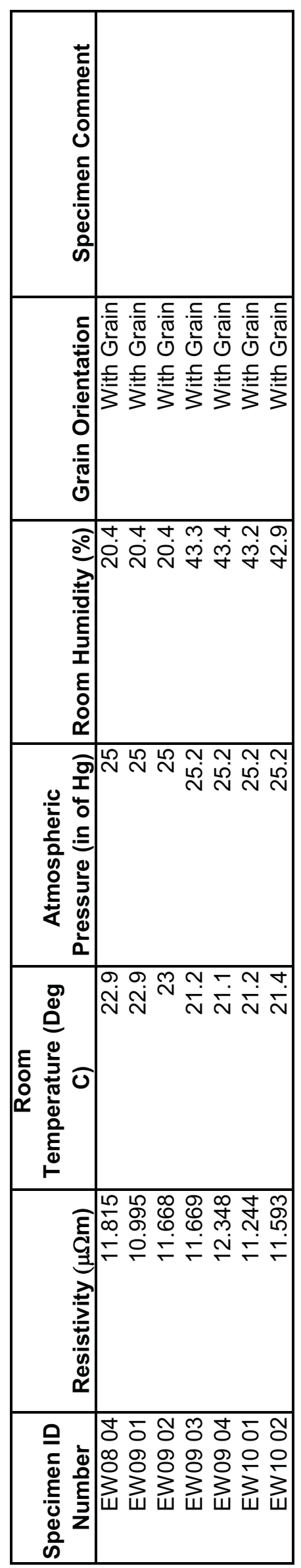




\begin{tabular}{|c|c|}
\hline 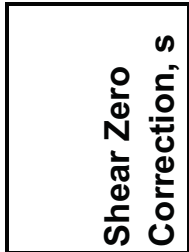 & 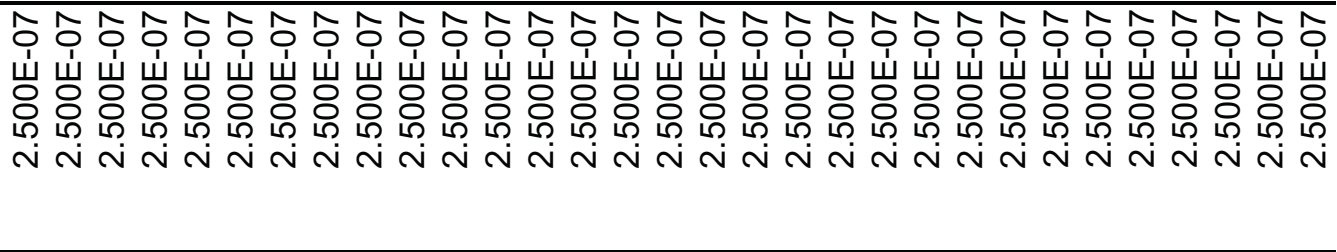 \\
\hline 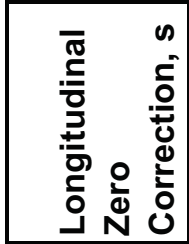 & 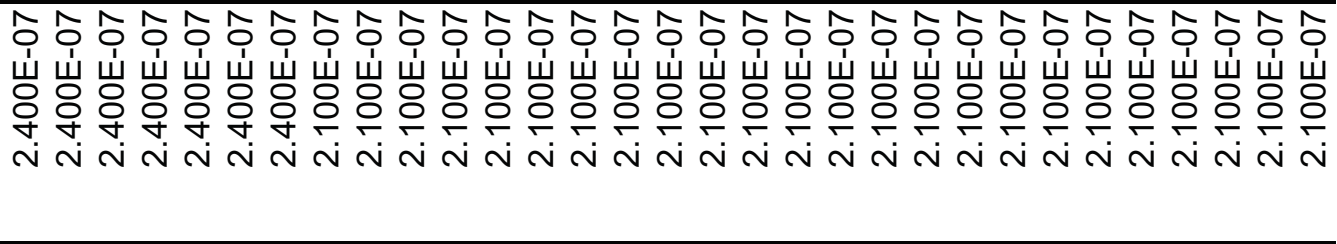 \\
\hline 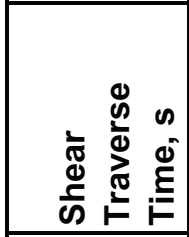 & 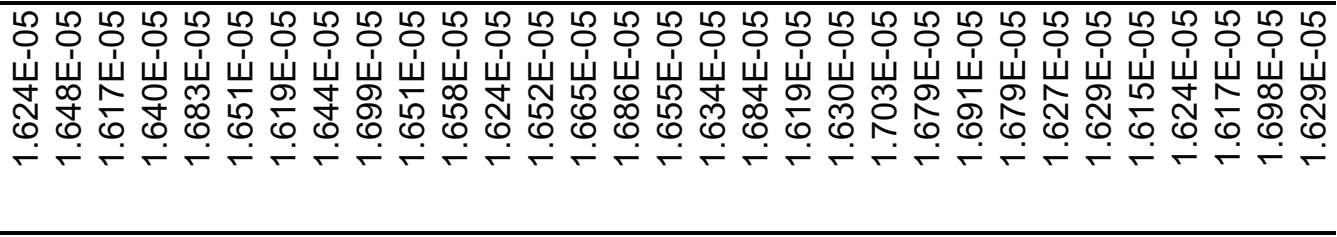 \\
\hline 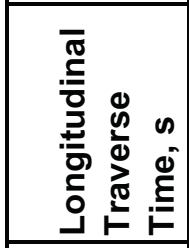 & 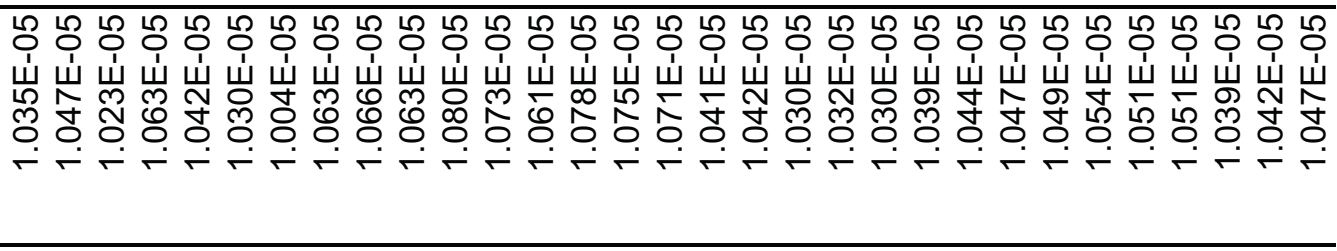 \\
\hline 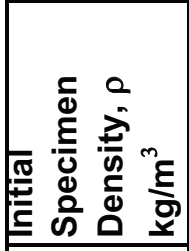 & 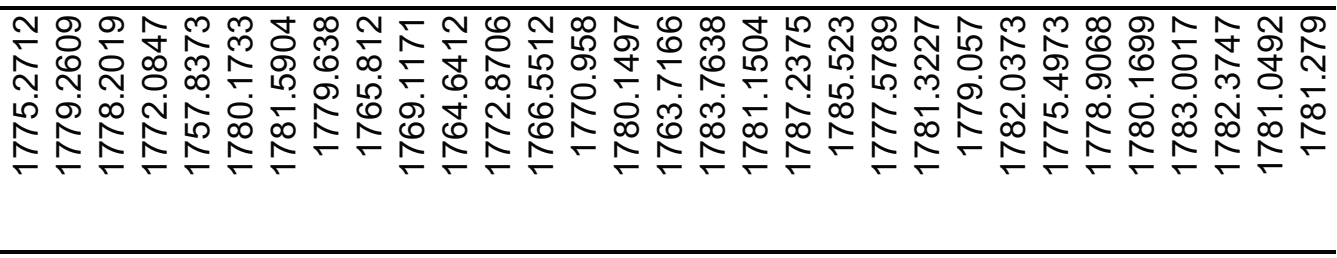 \\
\hline 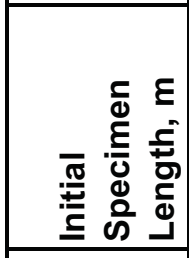 & 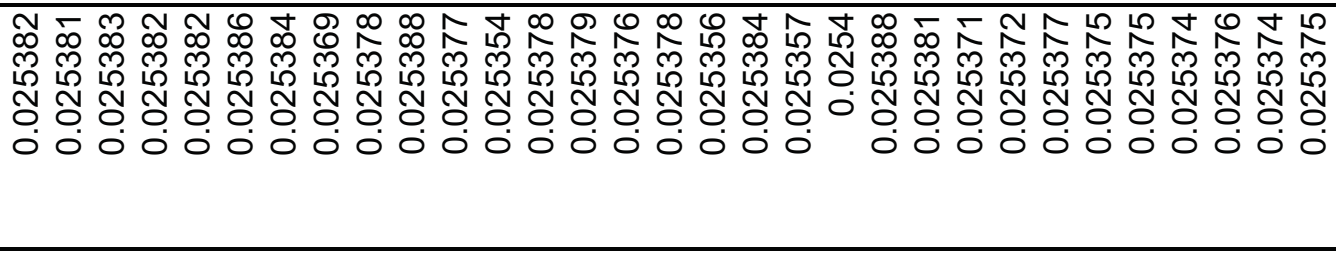 \\
\hline 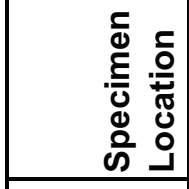 & \\
\hline $\begin{array}{l}\overline{0} \\
\frac{\pi}{2} \\
\frac{0}{0} \\
\text { 음 }\end{array}$ & 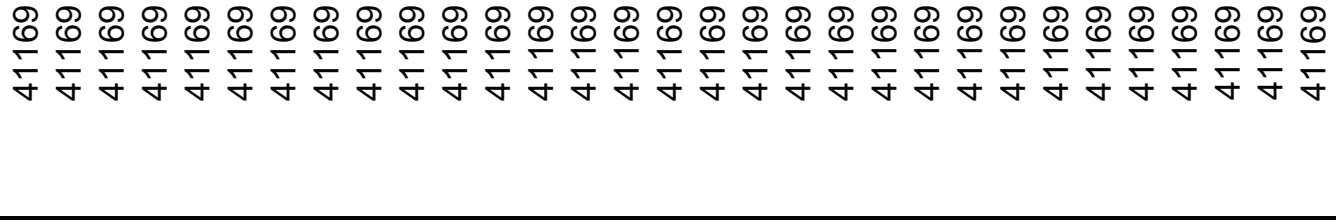 \\
\hline 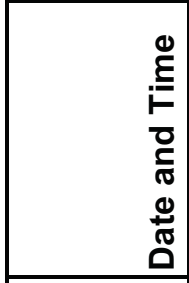 & 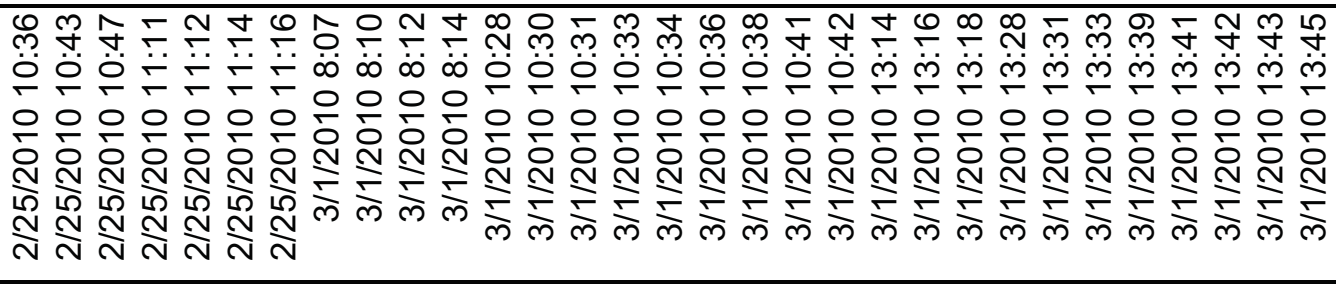 \\
\hline 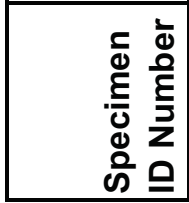 & 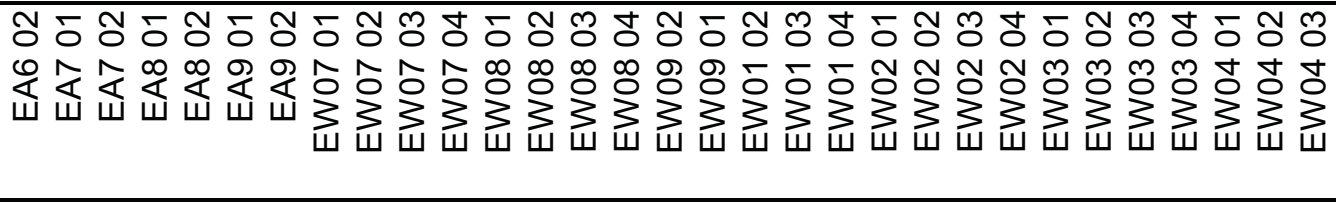 \\
\hline
\end{tabular}




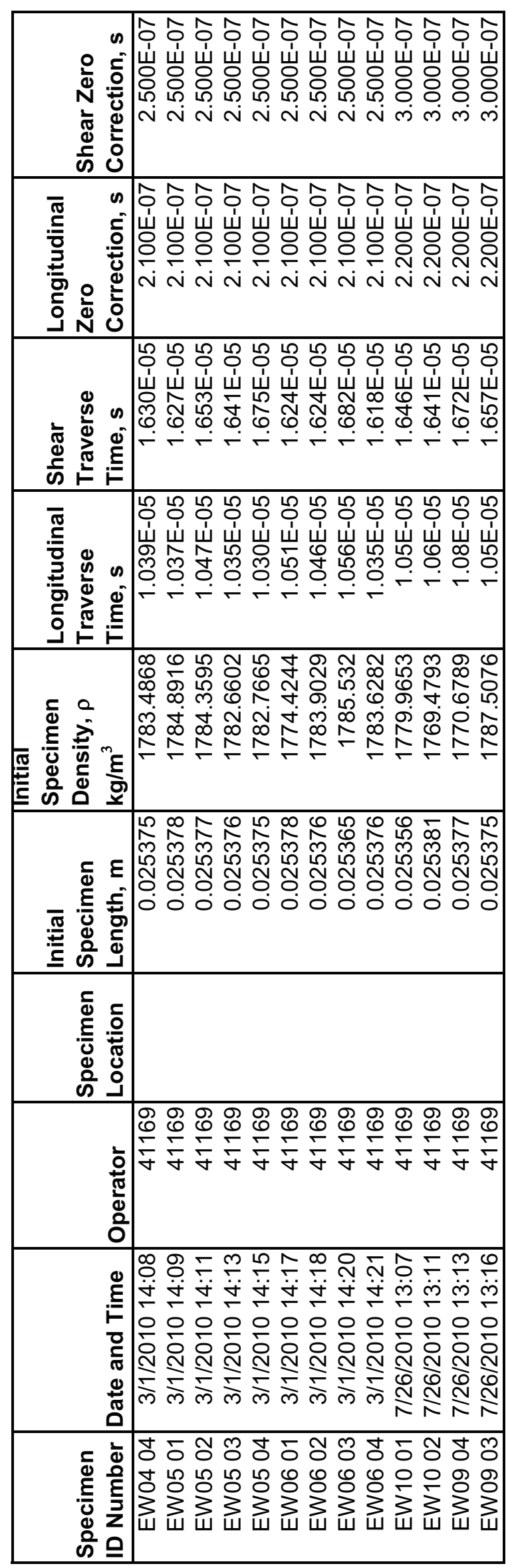




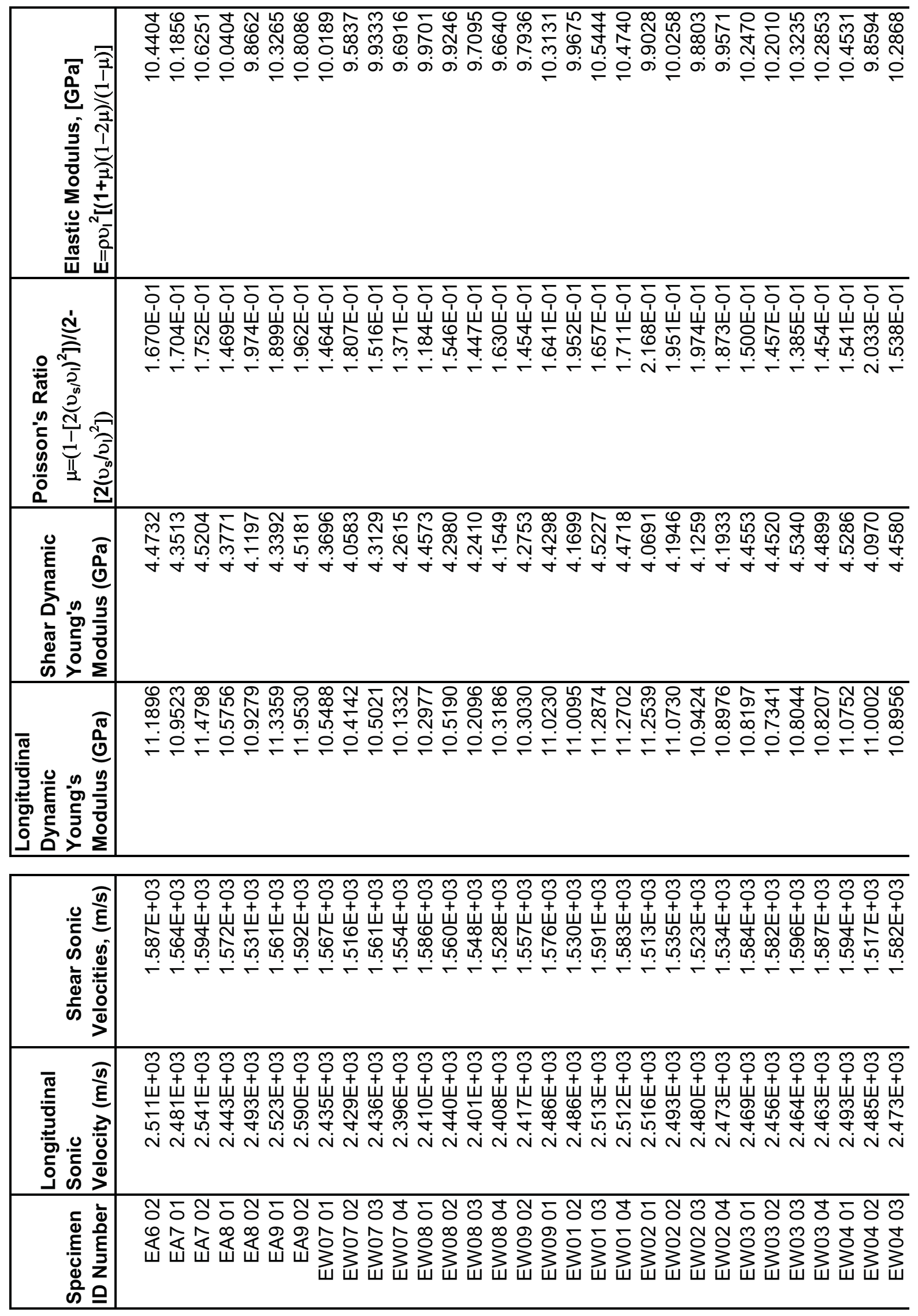



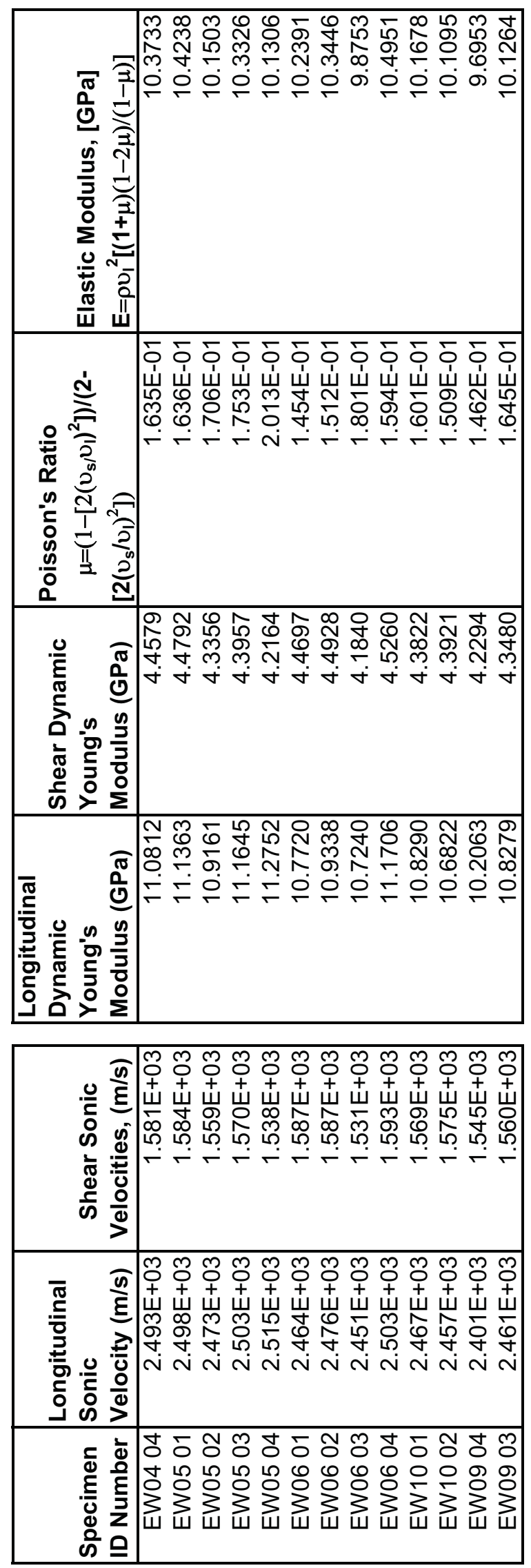


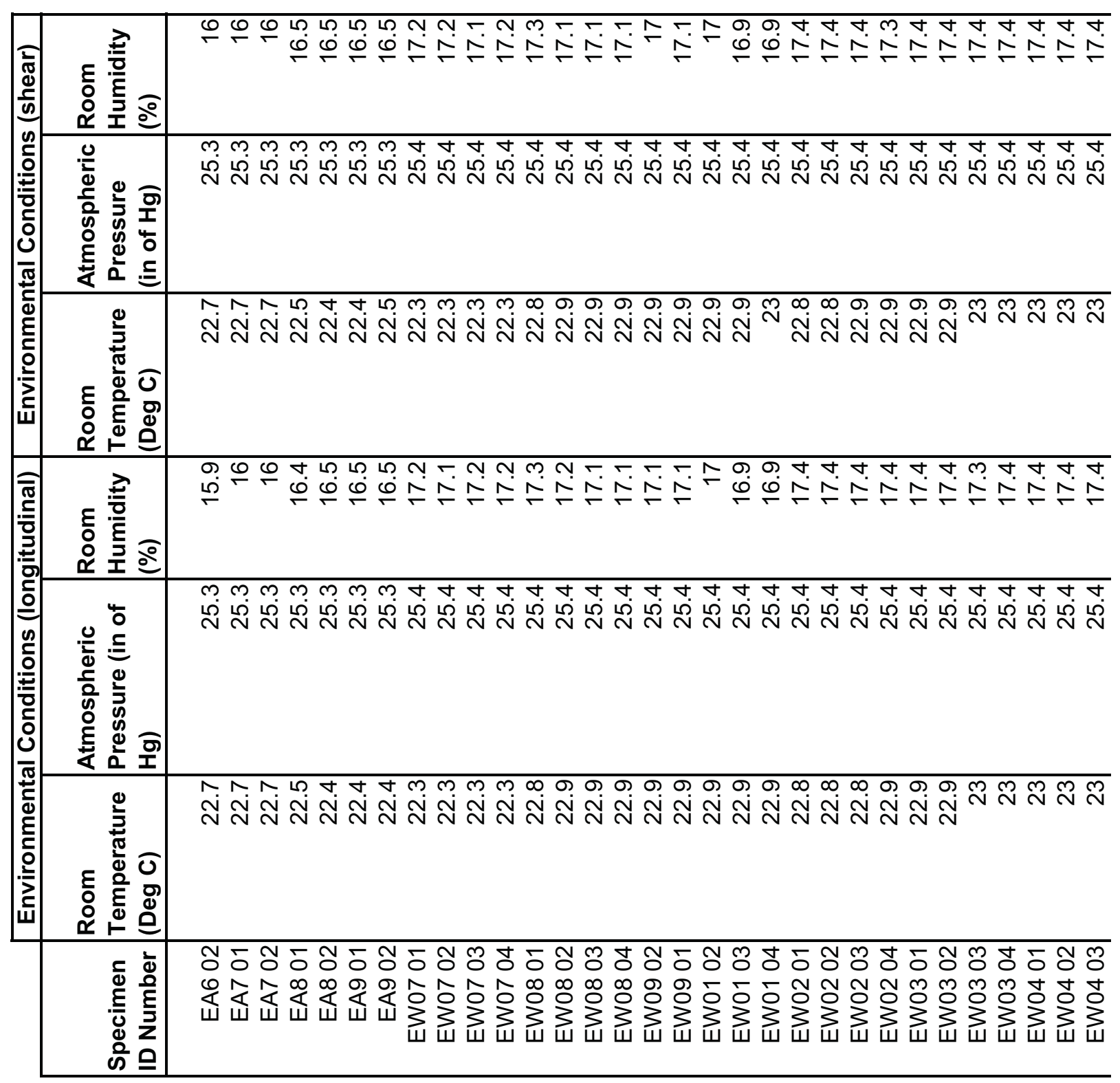




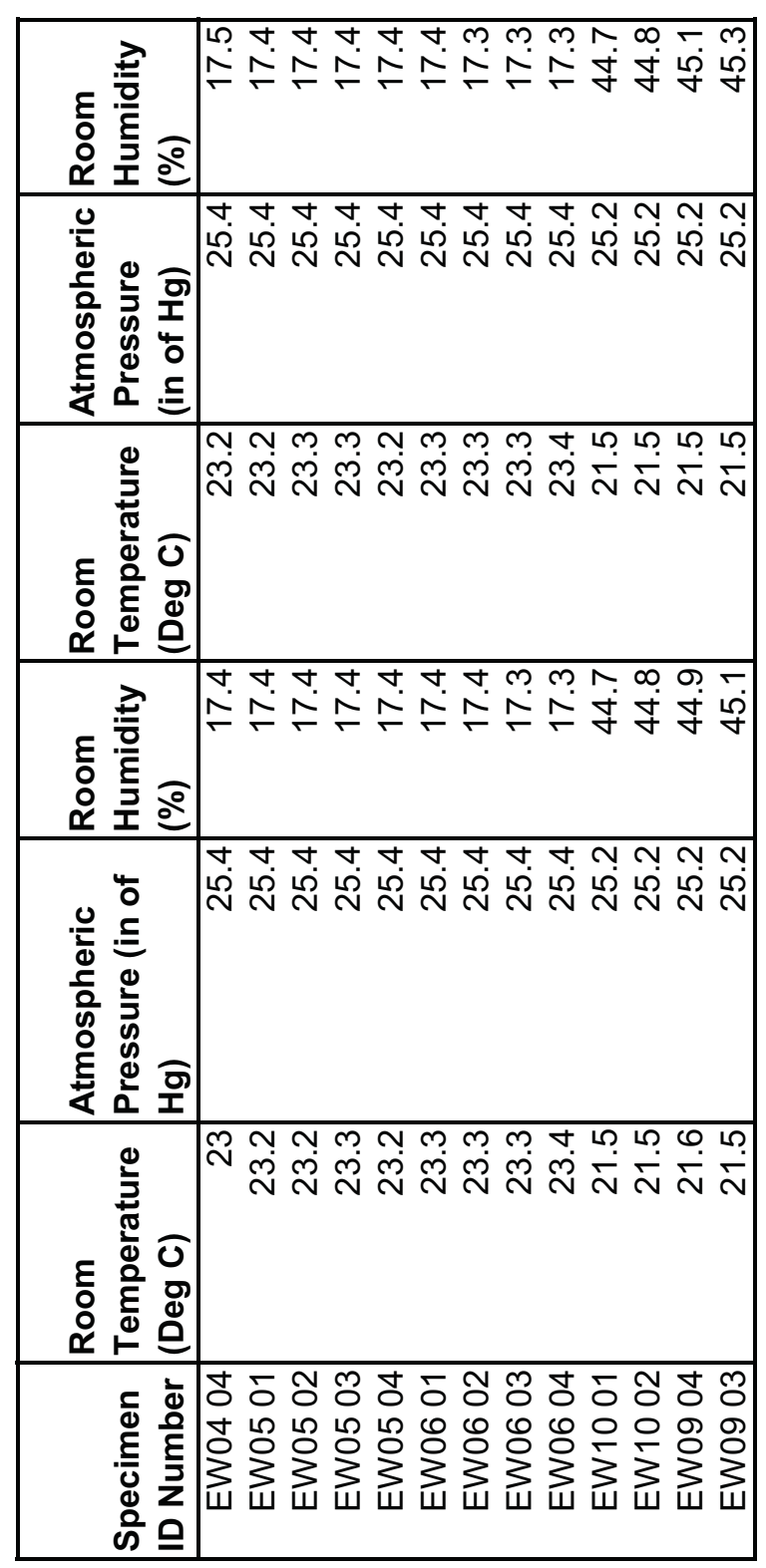




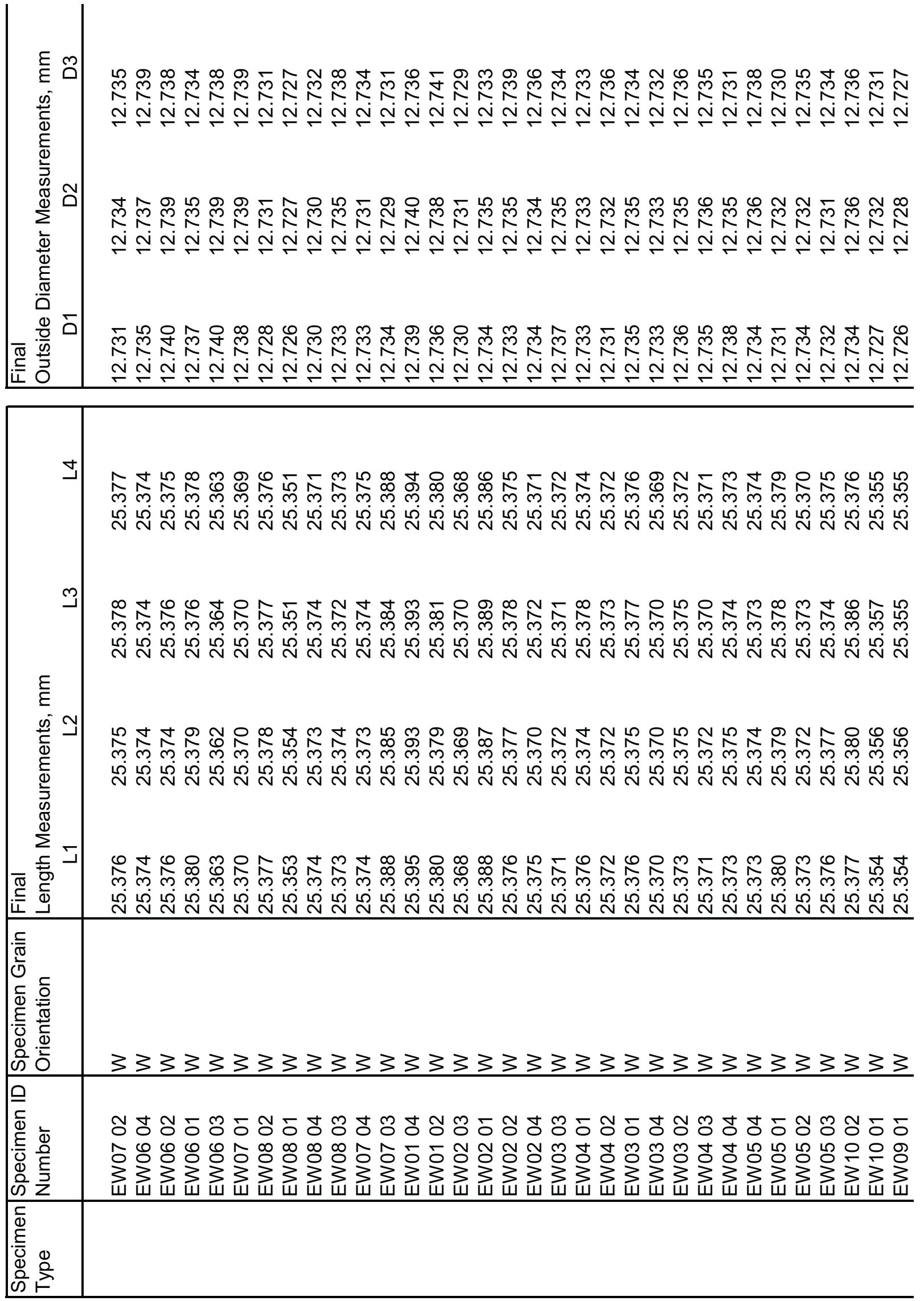



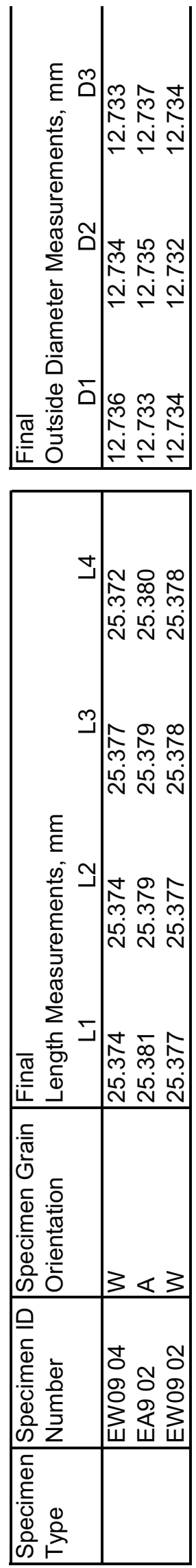


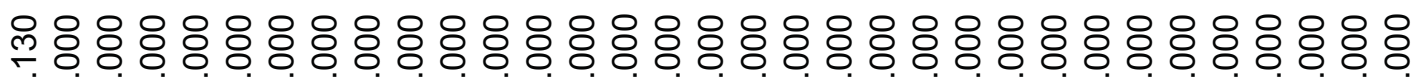

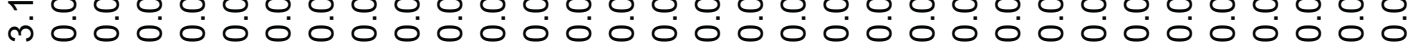

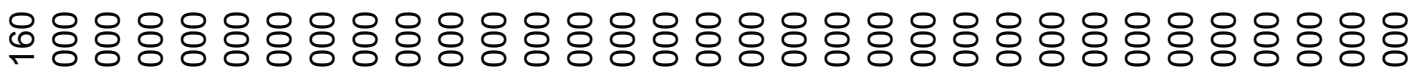

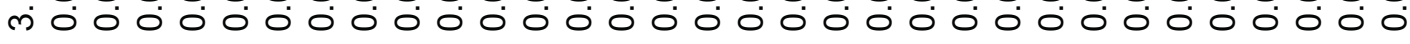

ㅇㅇㅇㅇㅇㅇㅇㅇㅇㅇㅇㅇㅇㅇㅇㅇㅇㅇㅇㅇㅇㅇㅇㅇㅇㅇㅇㅇㅇㅇㅇ mo 0.000000000000000000000000000000

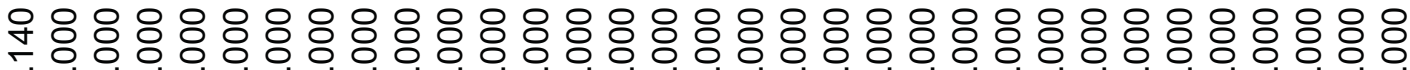
ำ.

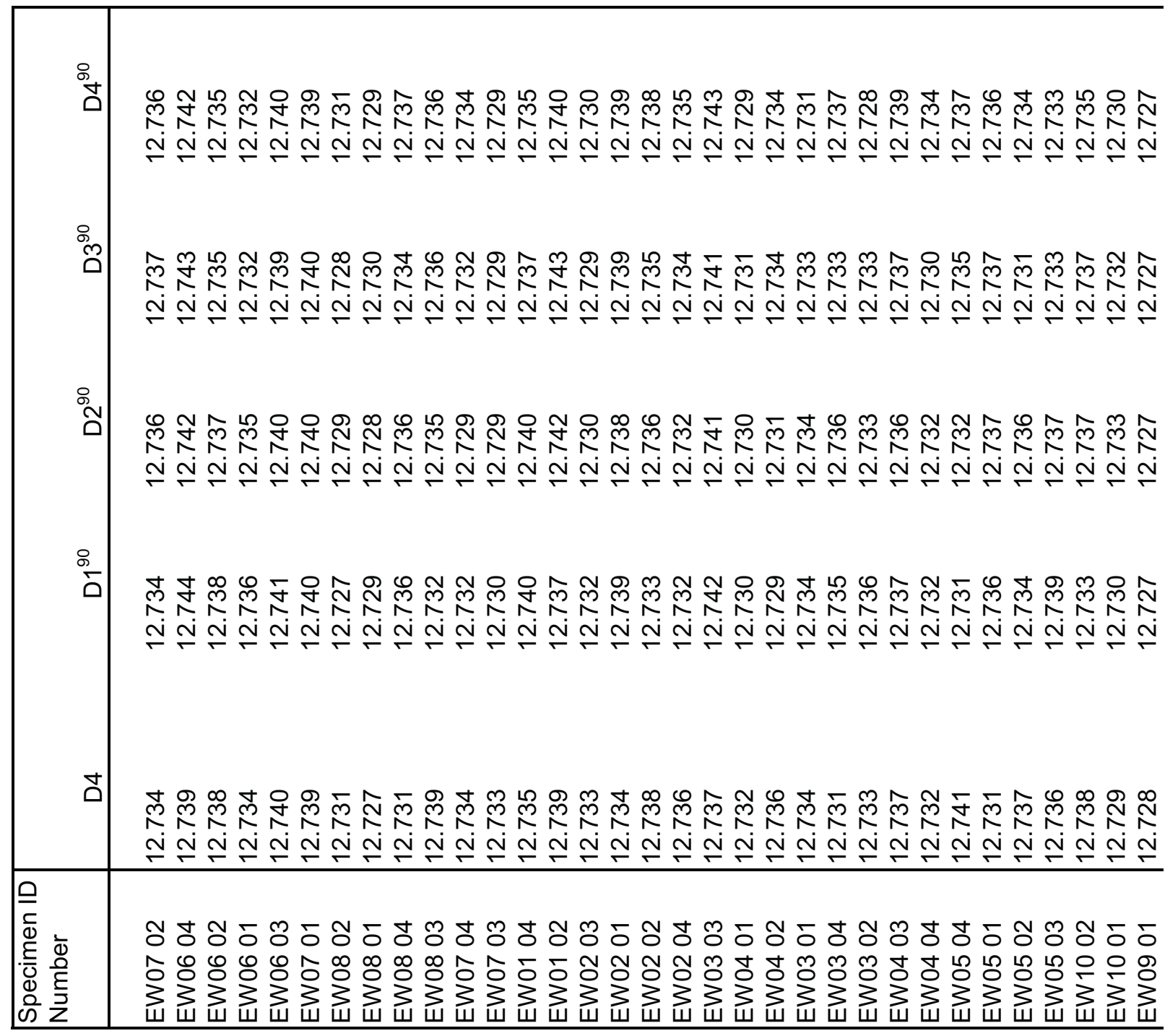




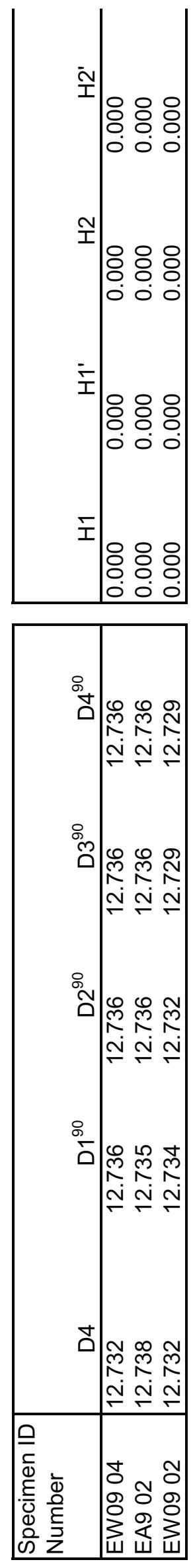

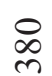




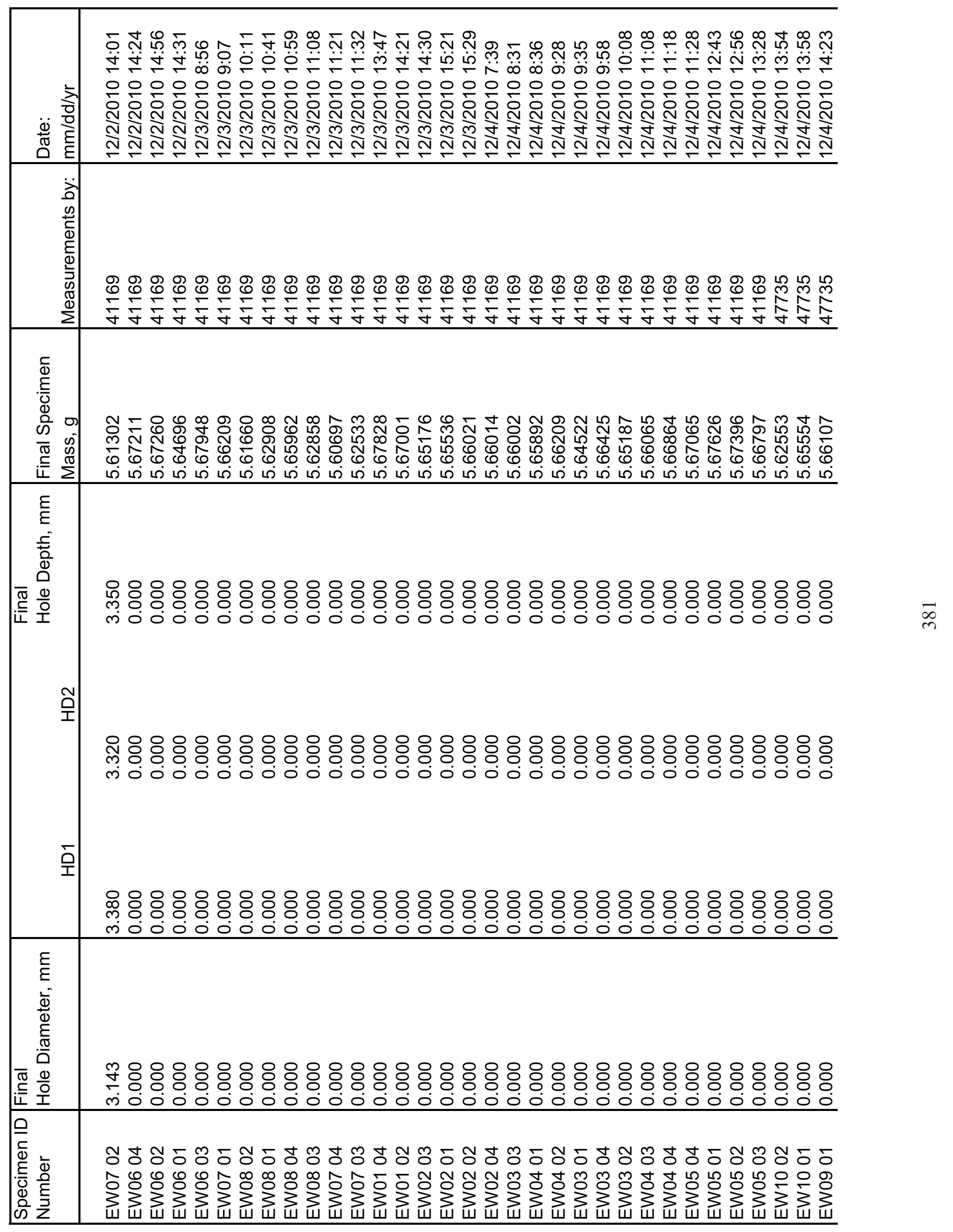




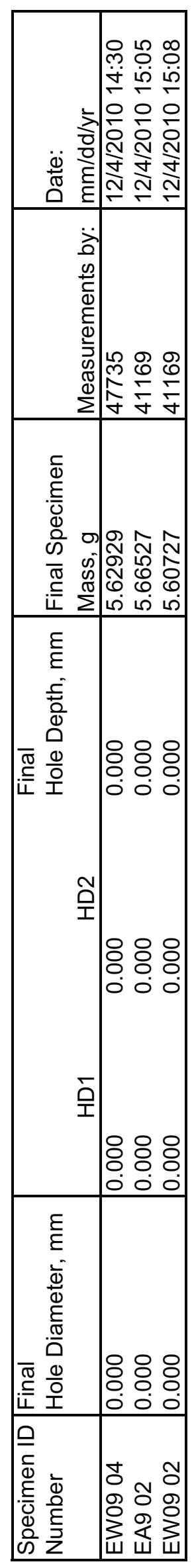




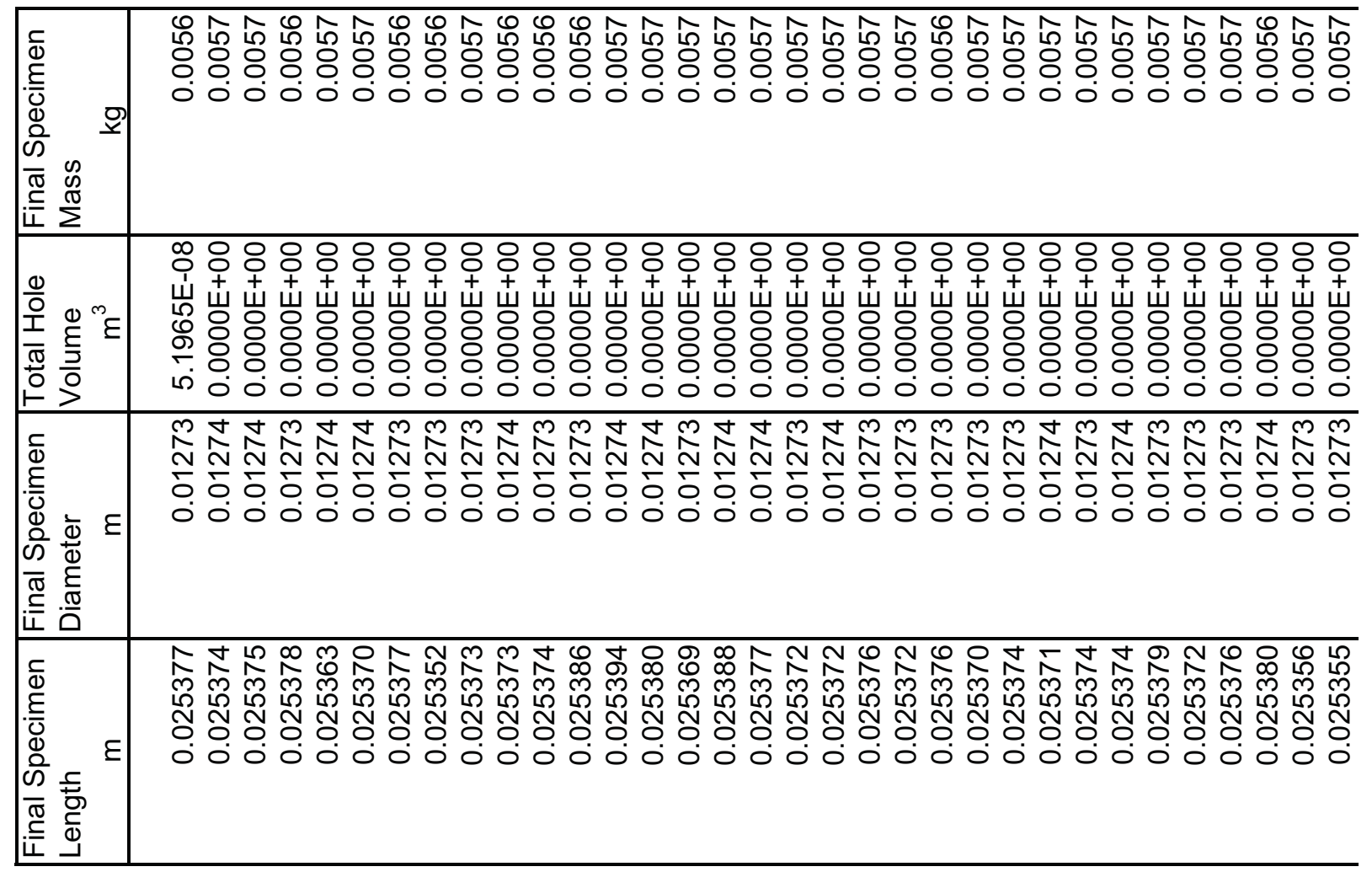

\begin{tabular}{|c|c|}
\hline 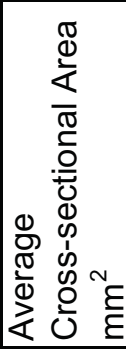 & 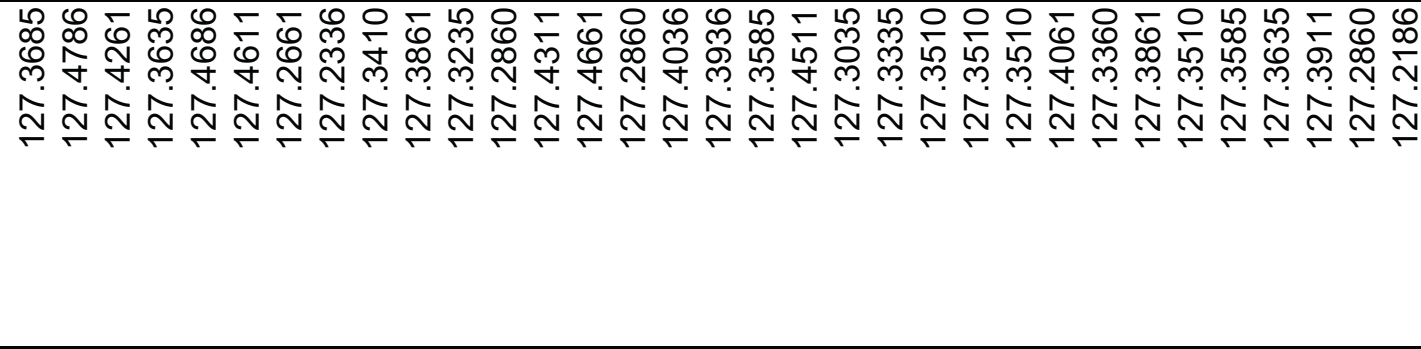 \\
\hline 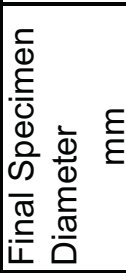 & 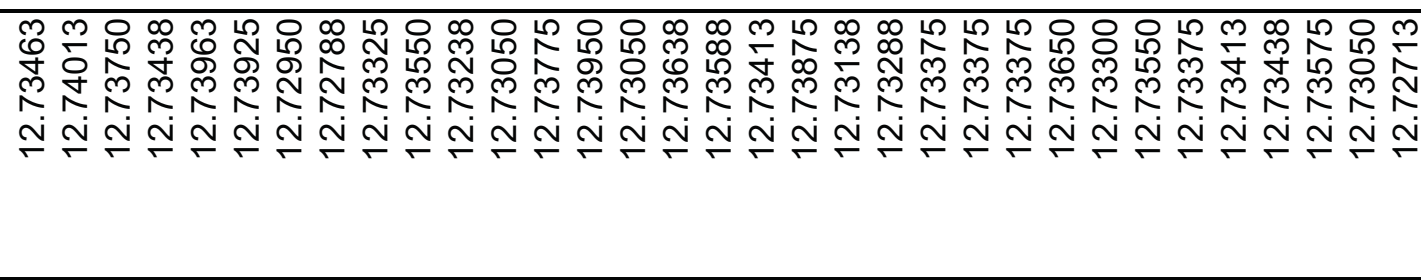 \\
\hline 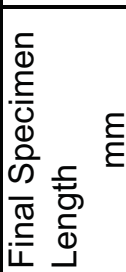 & 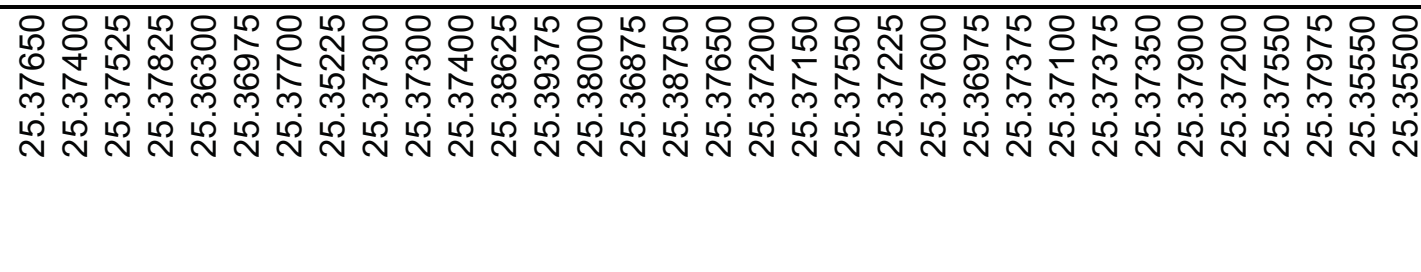 \\
\hline
\end{tabular}

\begin{tabular}{|c|c|}
\hline 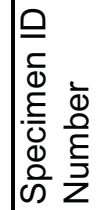 & 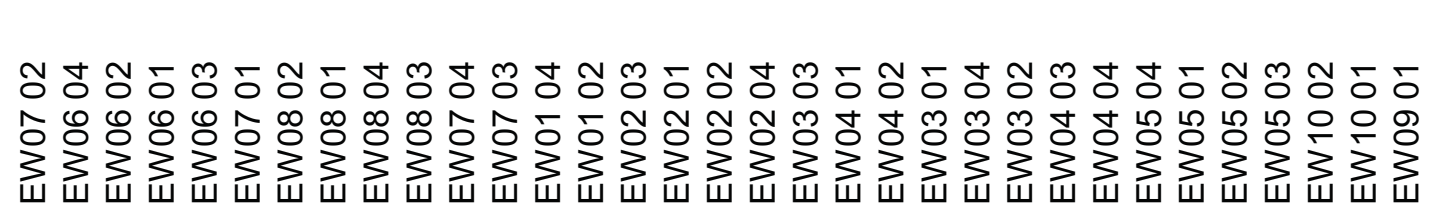 \\
\hline
\end{tabular}



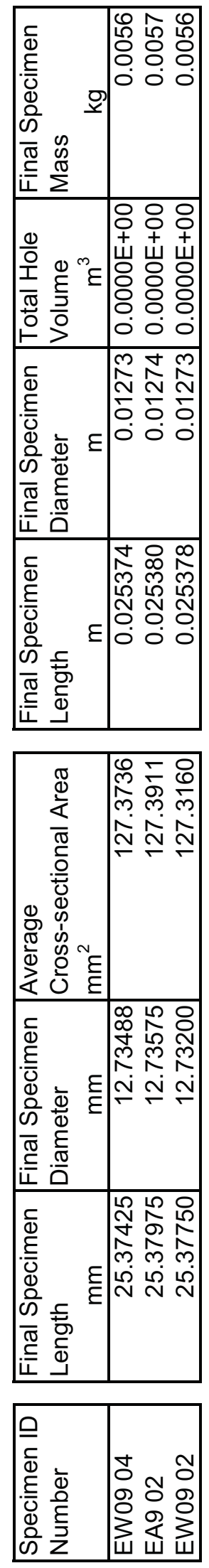


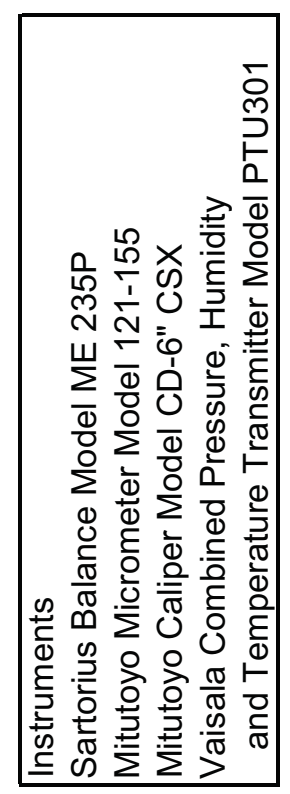

\begin{tabular}{|c|c|}
\hline & 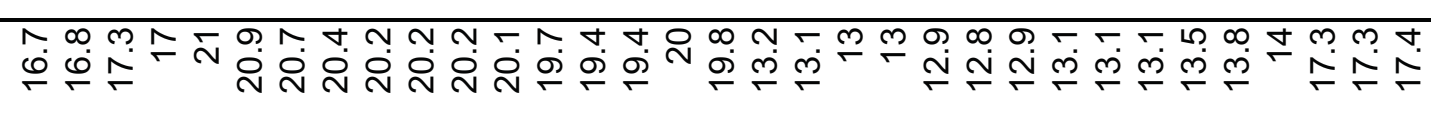 \\
\hline & 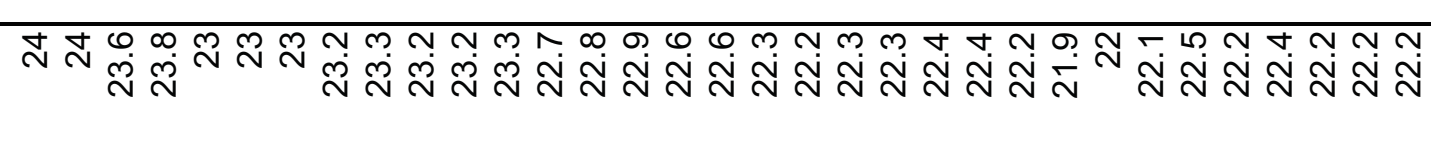 \\
\hline & 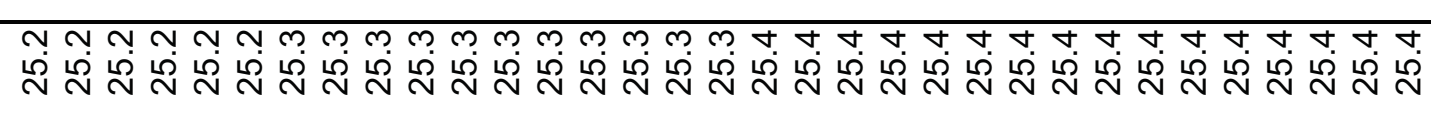 \\
\hline
\end{tabular}

\begin{tabular}{|c|c|}
\hline & 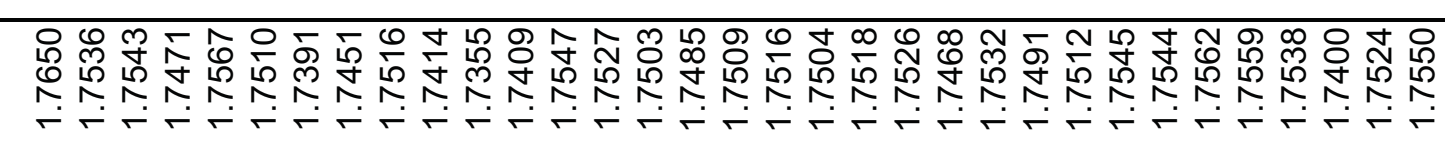 \\
\hline$\frac{\varepsilon}{2}$ & 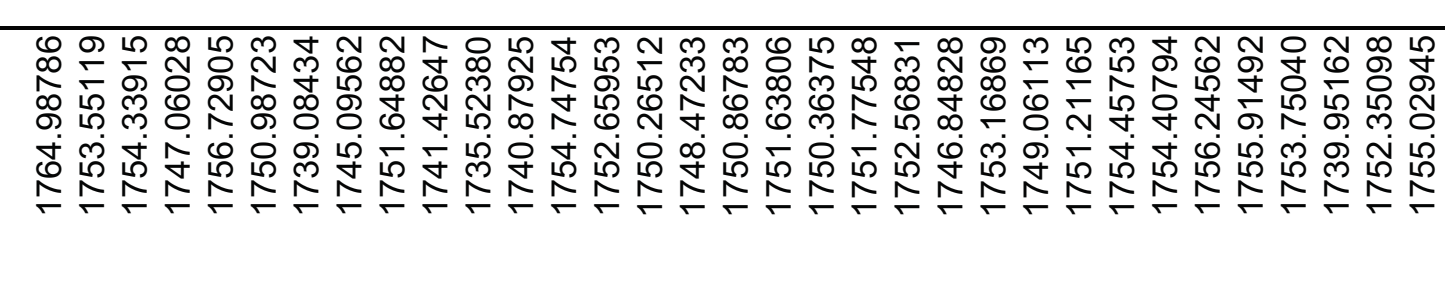 \\
\hline & 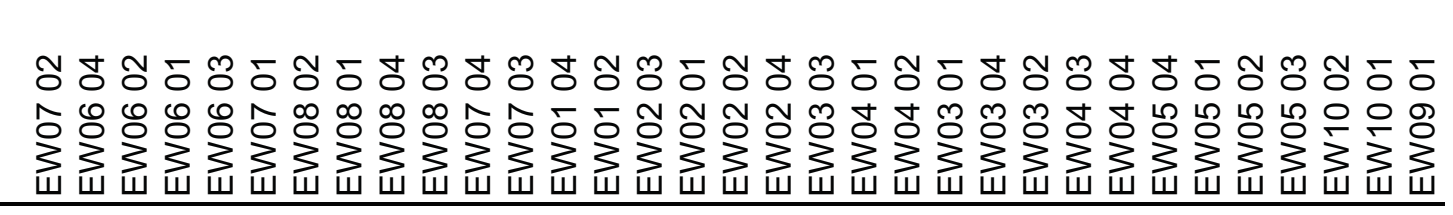 \\
\hline
\end{tabular}



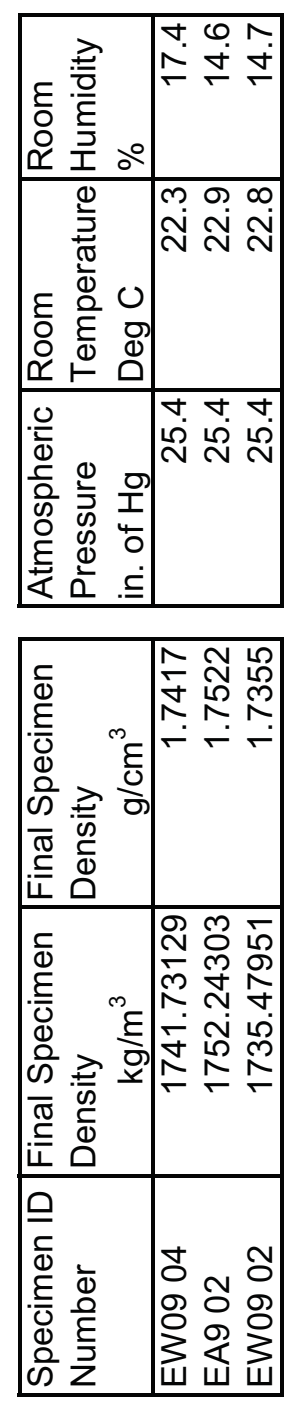


$\begin{array}{ll}\text { Graphite Grade: } & \text { IG-430 } \\ \text { Graphite Manufacturer: } & \text { Toyo Tanso } \\ \text { Forming Process: } & \text { Isostatic-molded } \\ \text { Coke Particle Size: } & \text { Fine grain } \\ \text { Coke Type: } & \text { Pitch coke } \\ \text { ASTM Class: } & \text { INHP } \\ \text { Specimen Geometry: } & \text { Cylinder }\end{array}$

Specimen ID \#'s:

FA1 01

FA1 02

FA2 01

FA2 02

FA3 01

FA3 02

FA4 01

FA4 02

FA5 01

FA5 02

FA6 01

FA6 02

FA7 01

FA7 02

FA8 01

FA8 02

FW01 01

FW01 02

FW01 03

FW01 04

FW02 01

FW02 02

FW02 03

FW02 04

FW03 01

FW03 02

FW03 03

FW03 04

FW04 01

FW04 02

FW04 03

FW04 04

FW05 01

FW05 02

FW05 03

FW05 04

FW06 01

FW06 02

FW06 03

FW06 04

FW07 01

FW07 02 


Specimen ID \#'s:
FW07 03
FW07 04
FW08 01
FW08 02
FW08 03
FW08 04
FW09 01
FW09 02
FW09 03
FW09 04
FW10 01
FW10 02
FW10 03
FW10 04
FW11 01
FW11 02
FW11 03
FW11 04
FW12 01
FW12 02
FW12 03
FW12 04
FW13 01
FW13 02
FW13 03
FW13 04
FW14 01
FW14 02
FW14 03
FW14 04
FW03 04A
FW03 02A
FA8 $01 A$
FA8 02A
FA7 02A
FW04 03A
FW01 01A
FW07 03A
FW02 04A
FW06 04B
FW08 01A
FA6 02A
FW02 04B
FW06 04C
FW08 01B




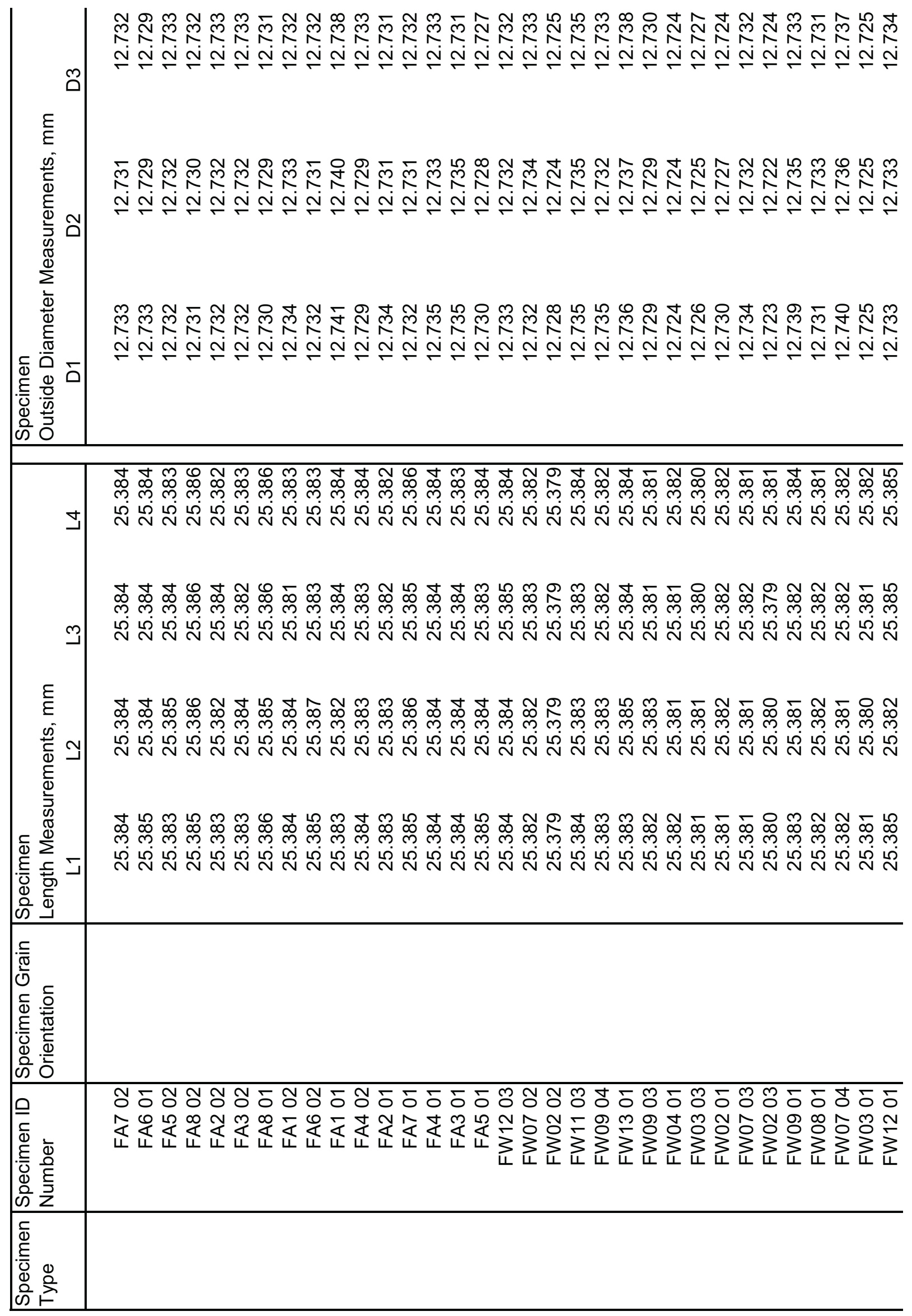




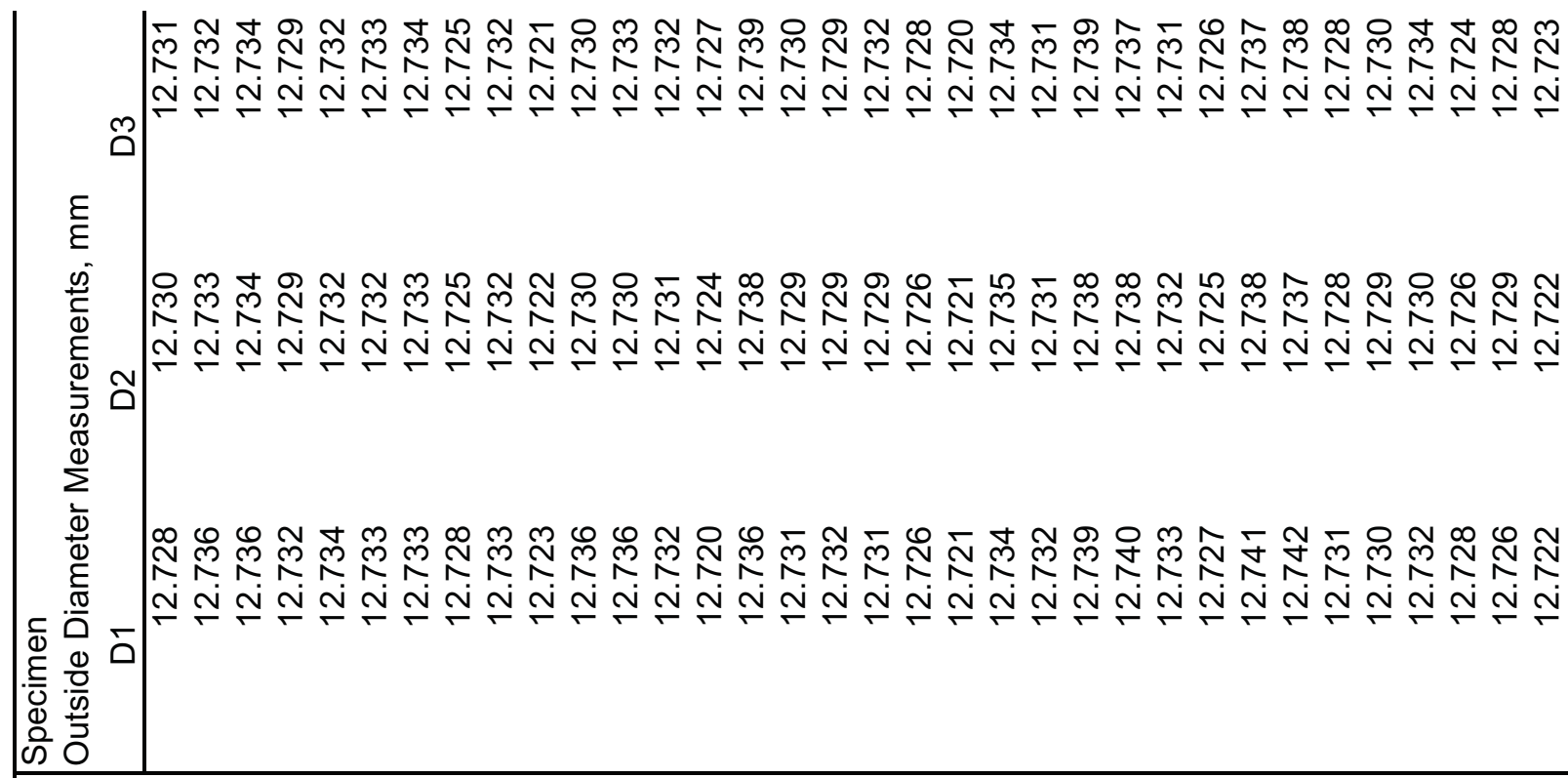

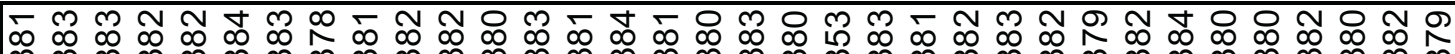

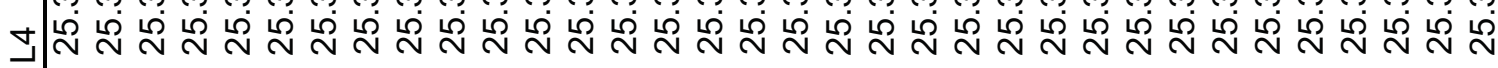

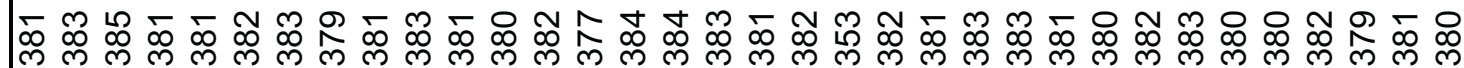
๑

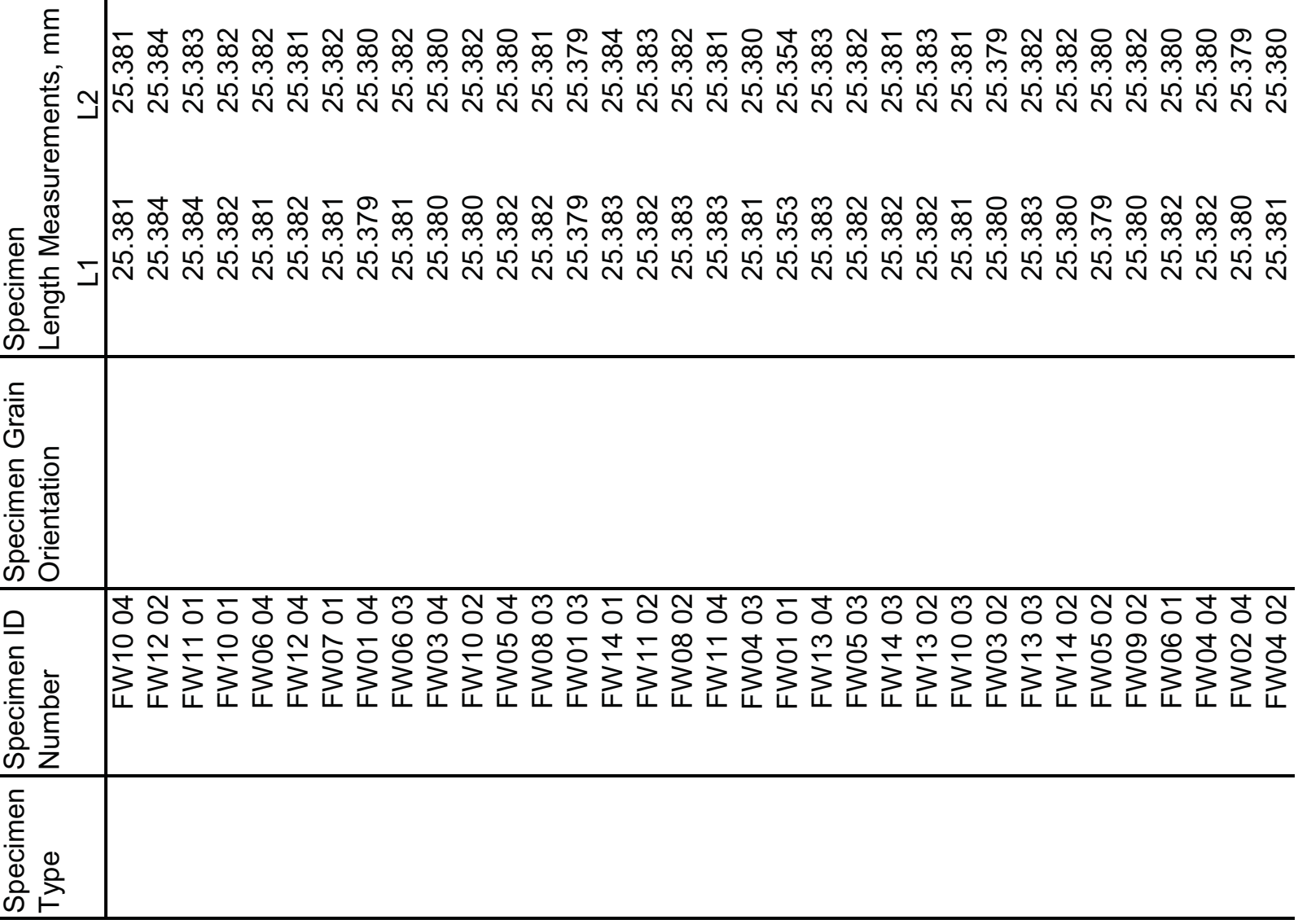




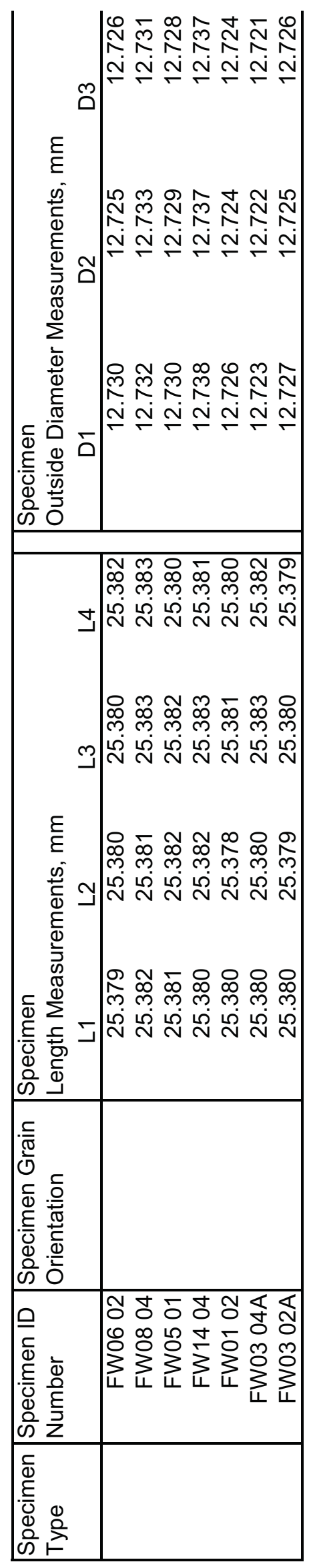




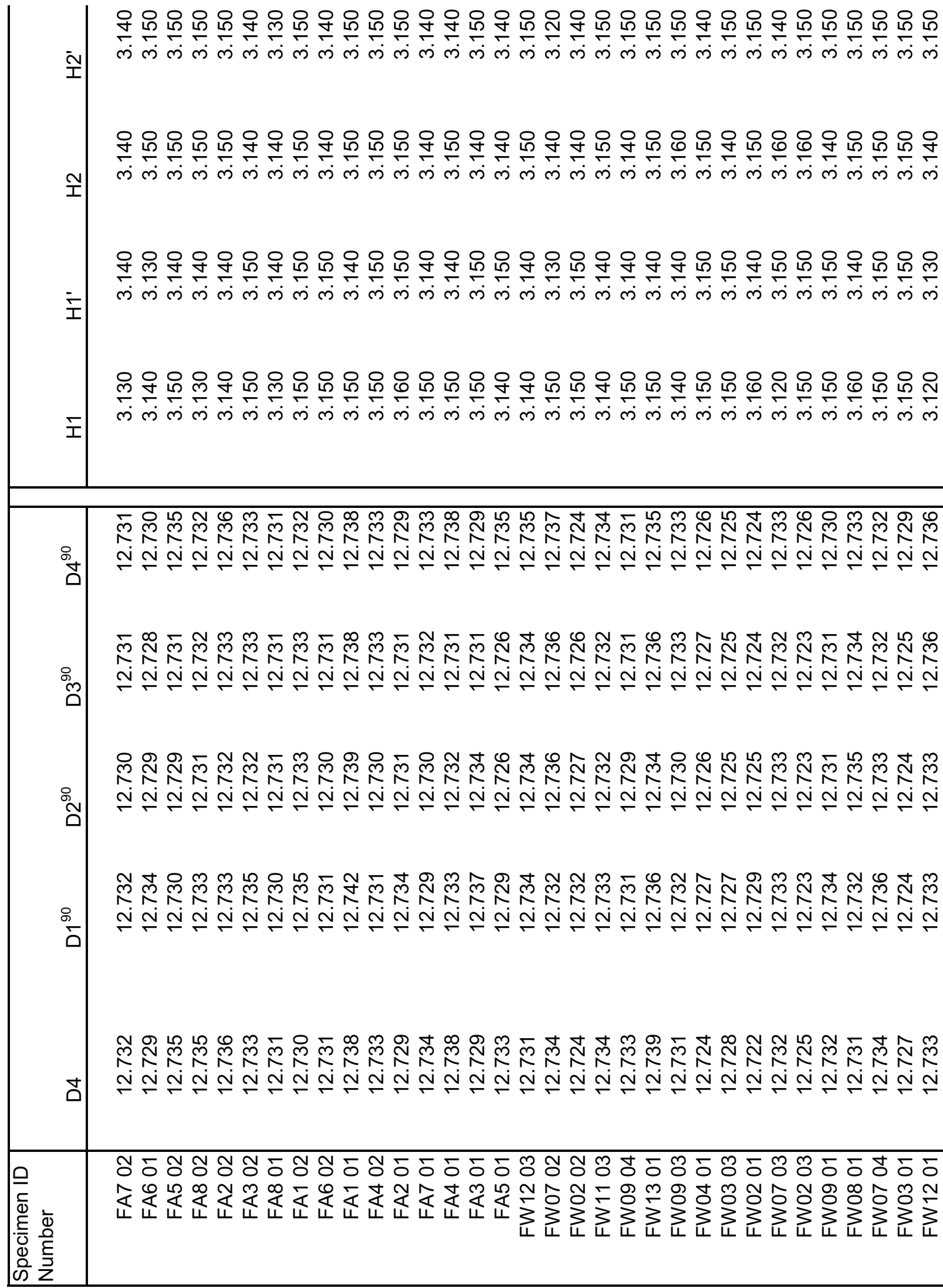




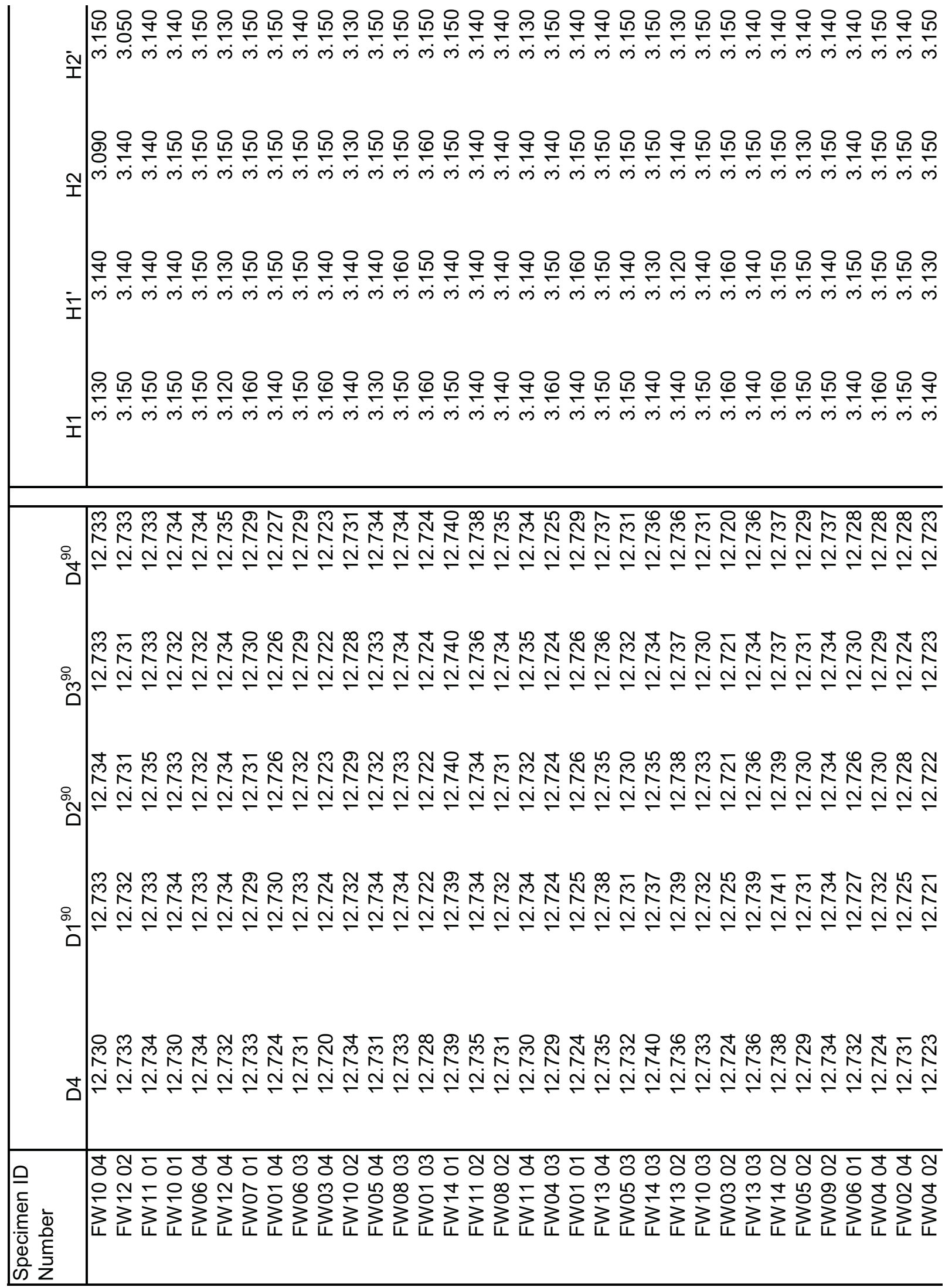




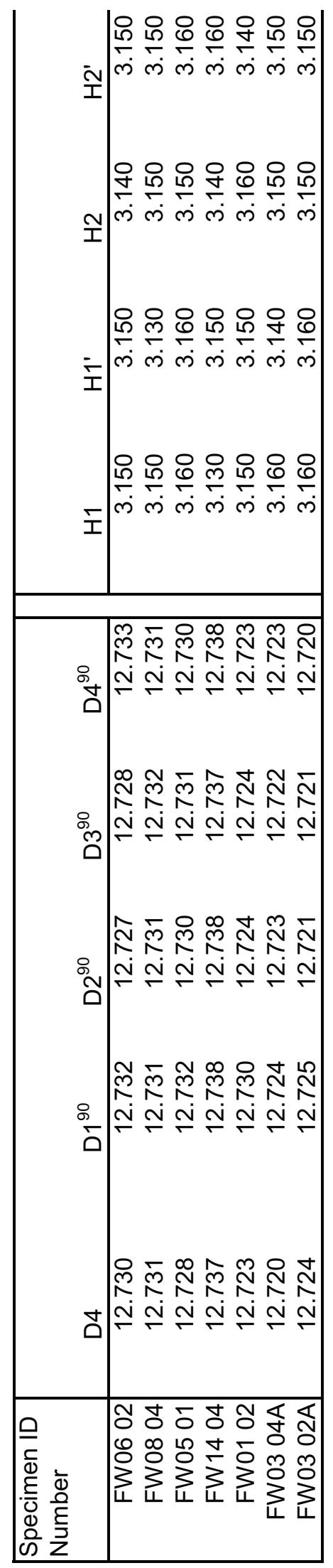




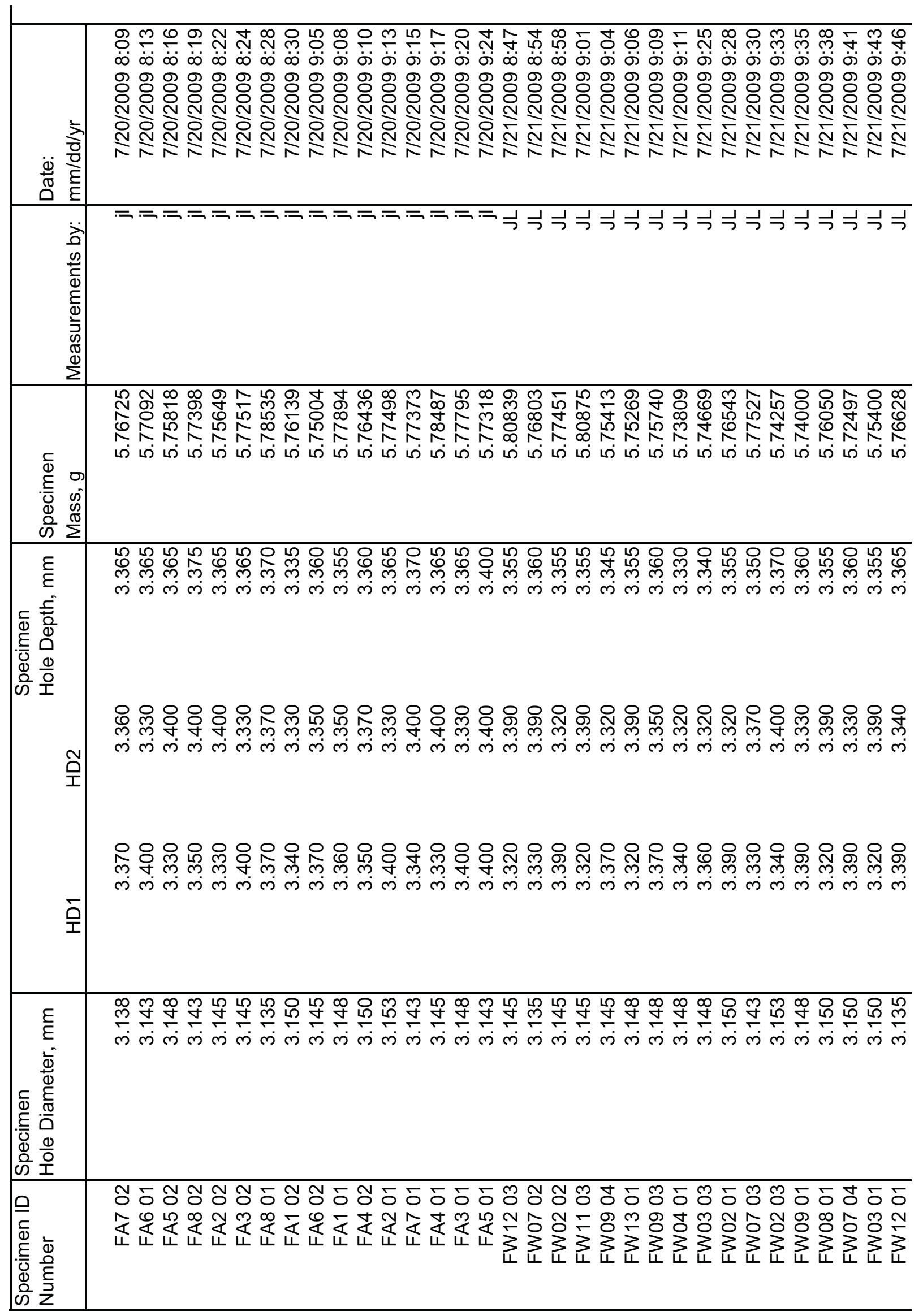




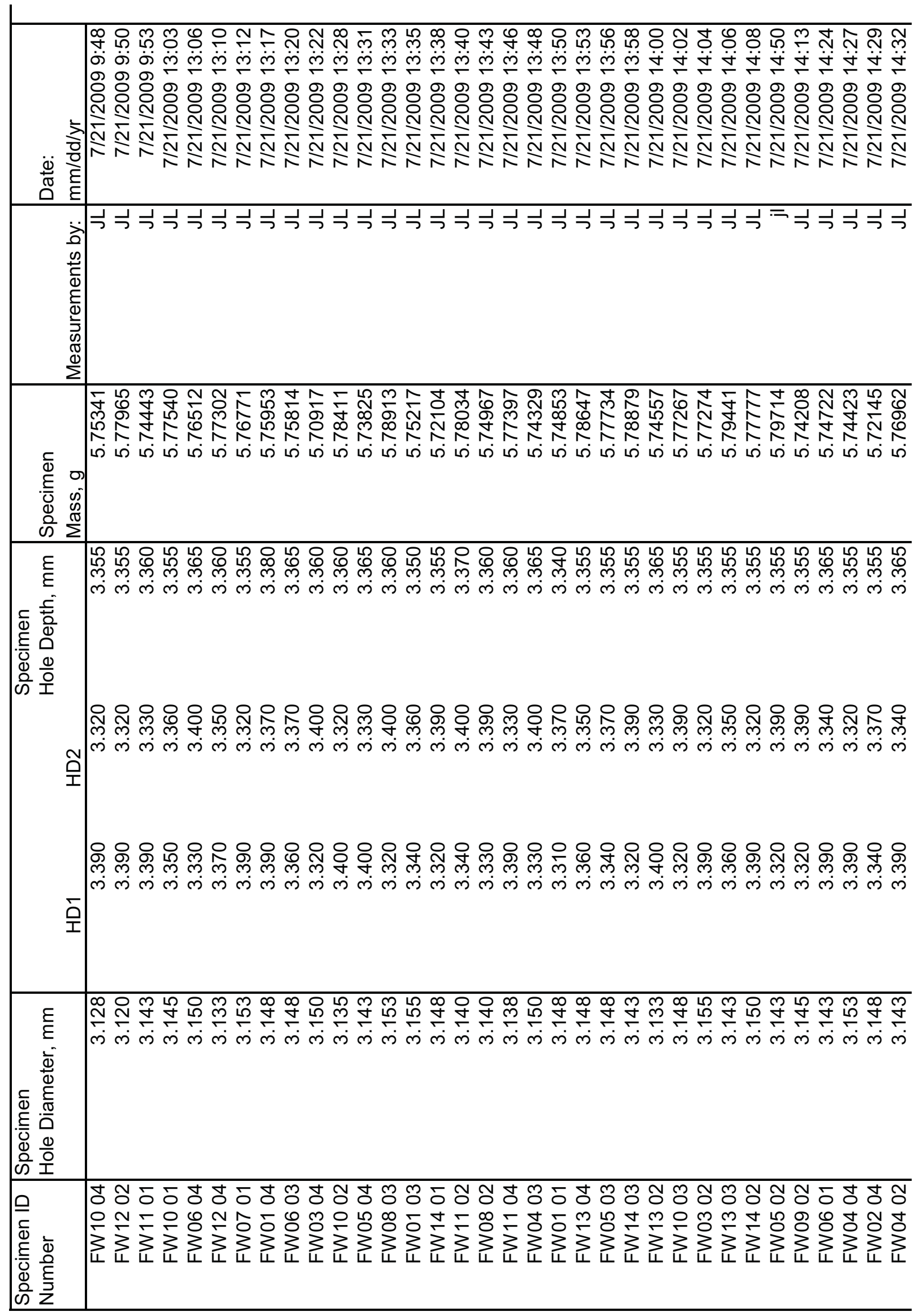




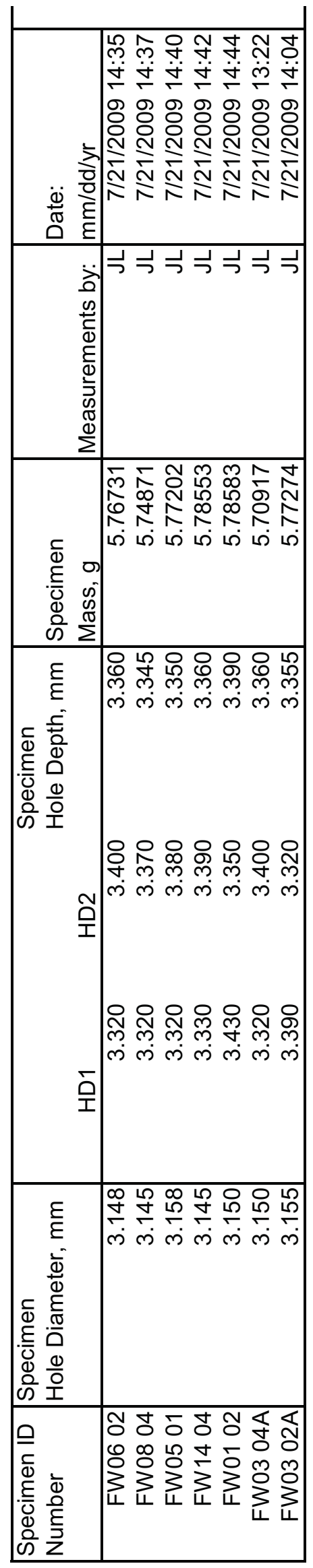




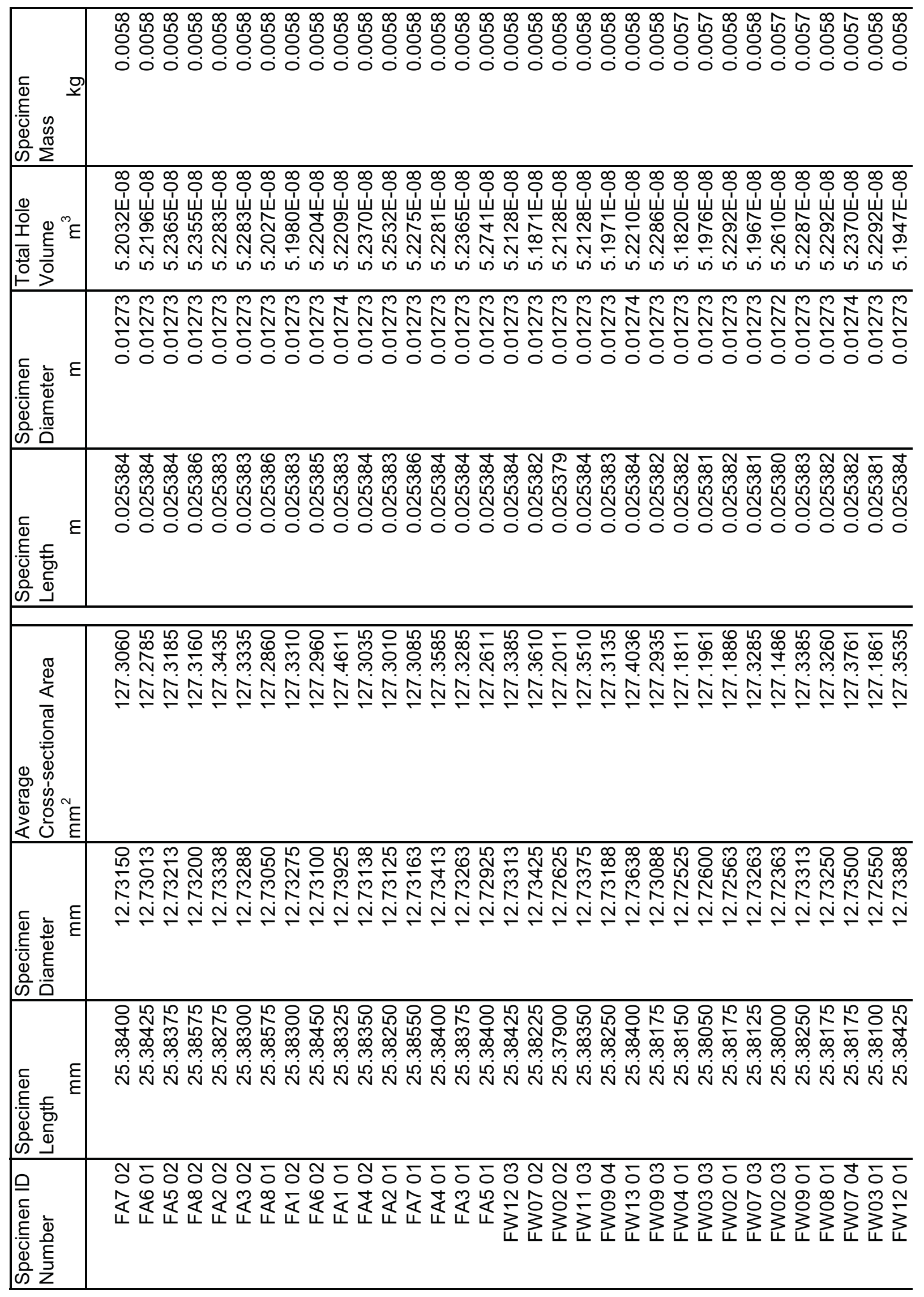




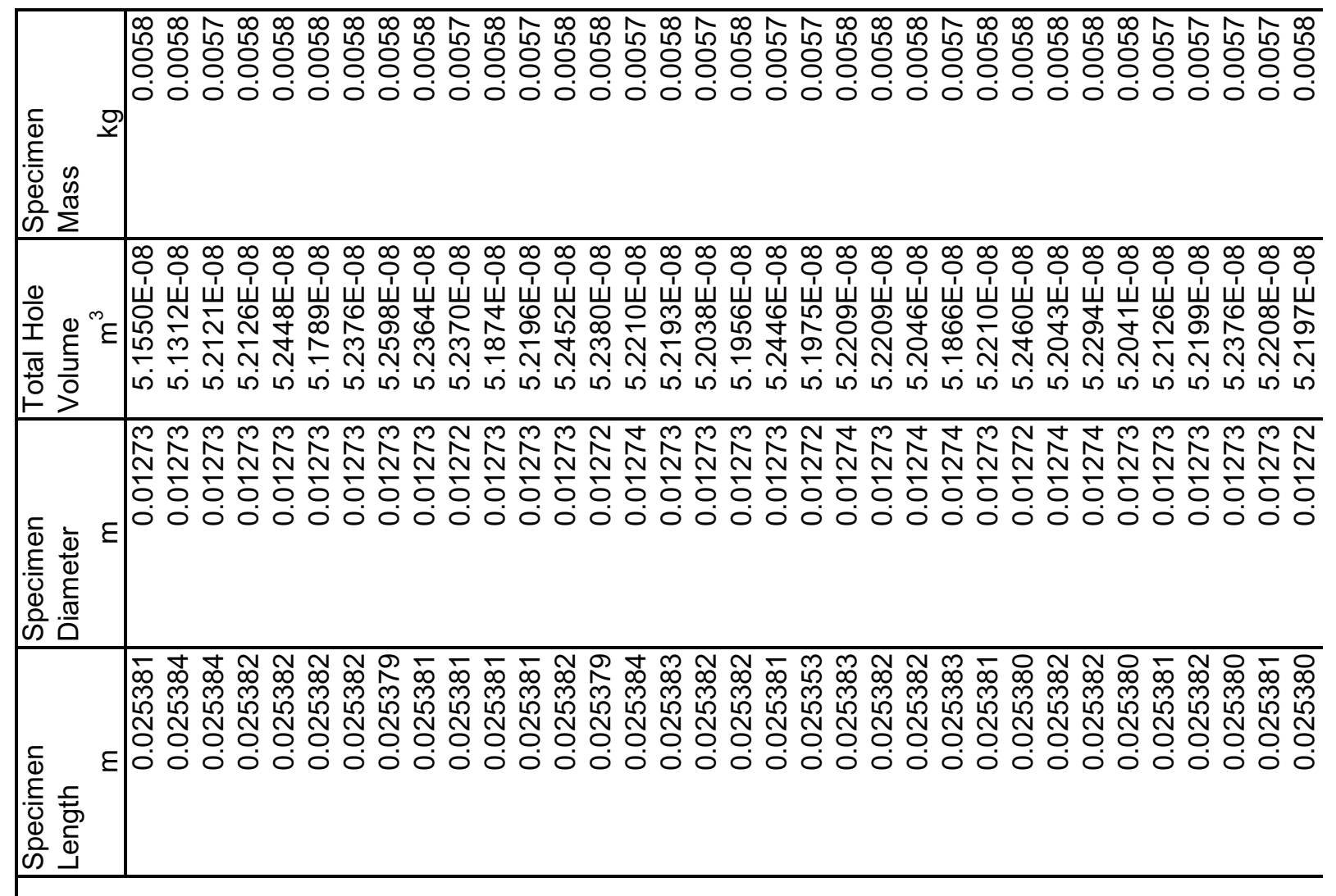

\begin{tabular}{|c|c|}
\hline 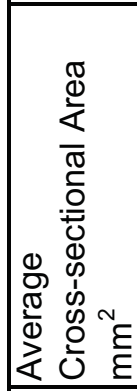 & 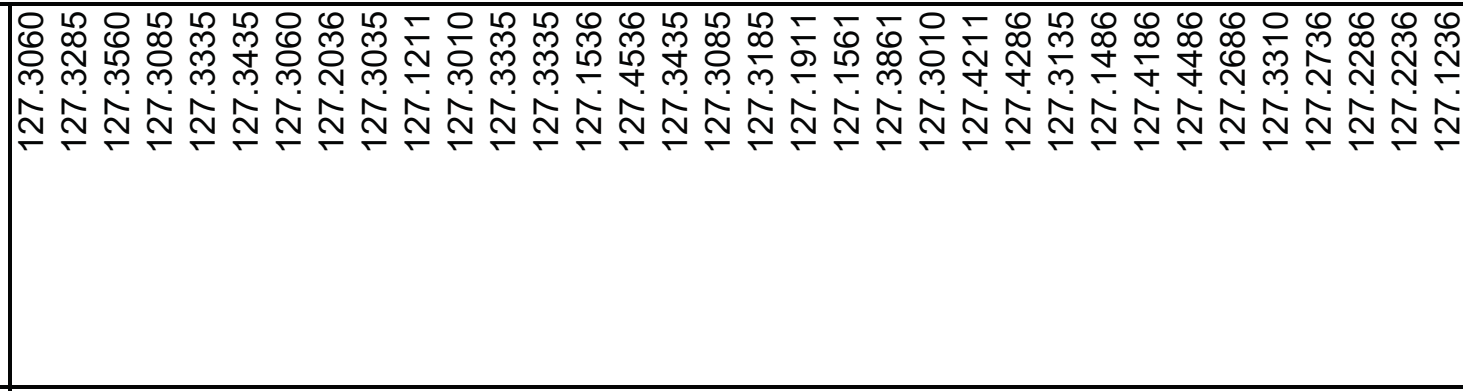 \\
\hline 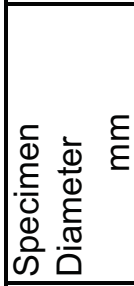 & 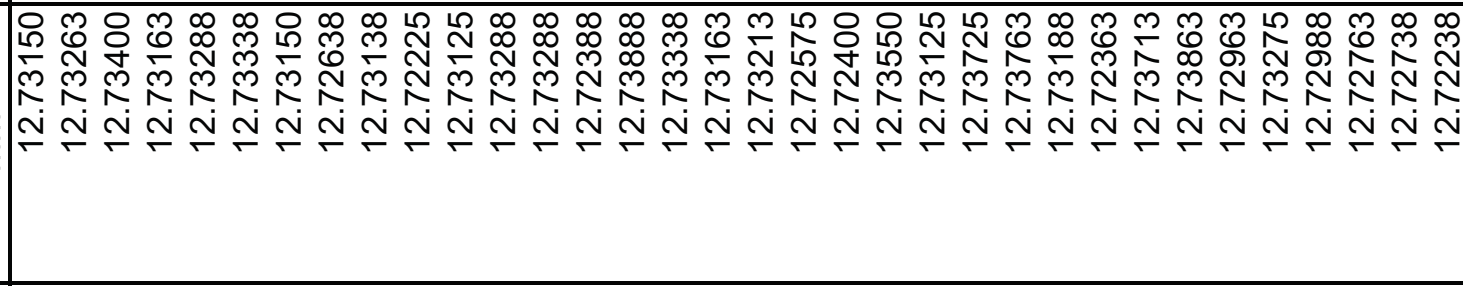 \\
\hline 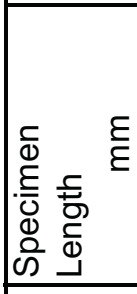 & 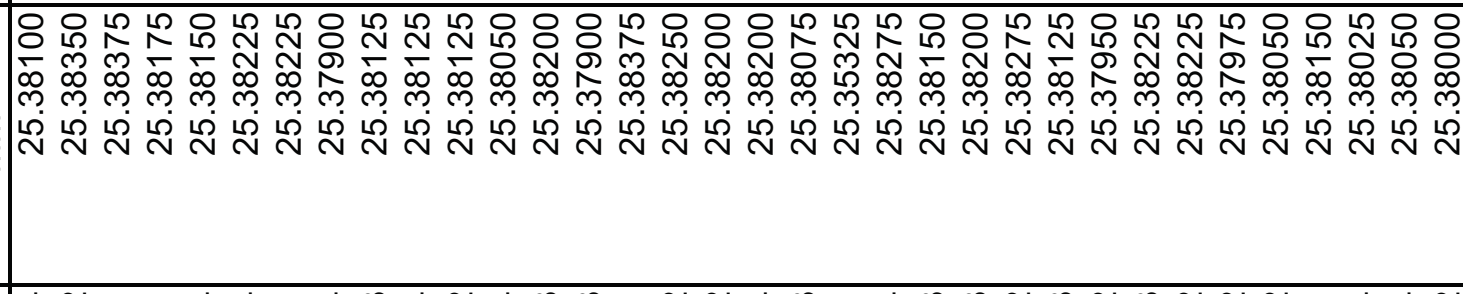 \\
\hline 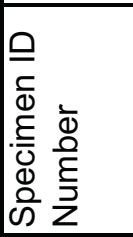 & 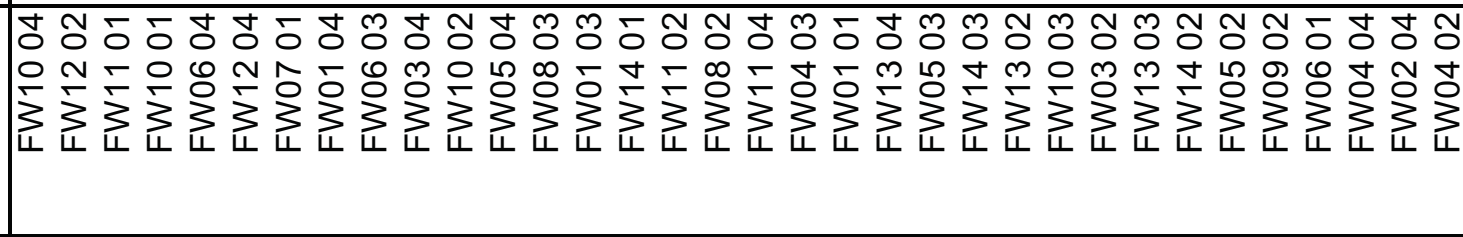 \\
\hline
\end{tabular}




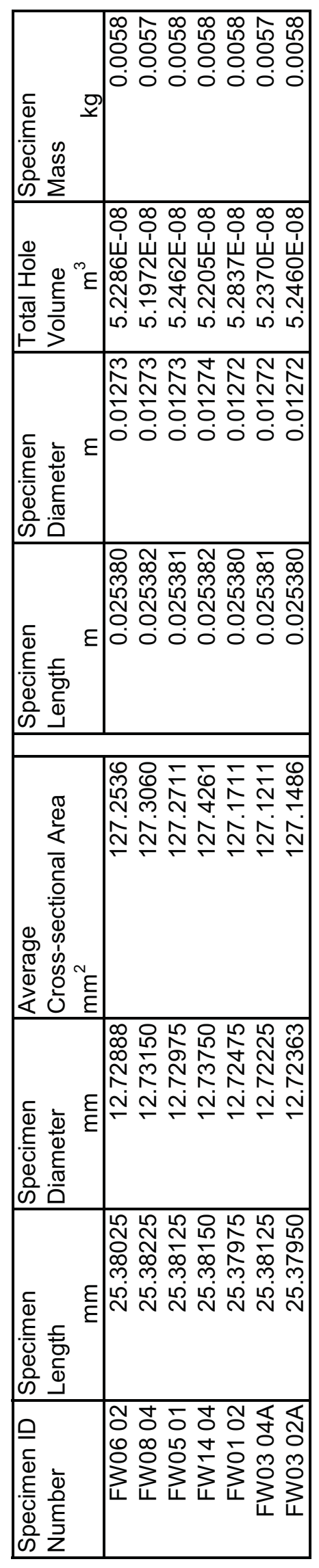




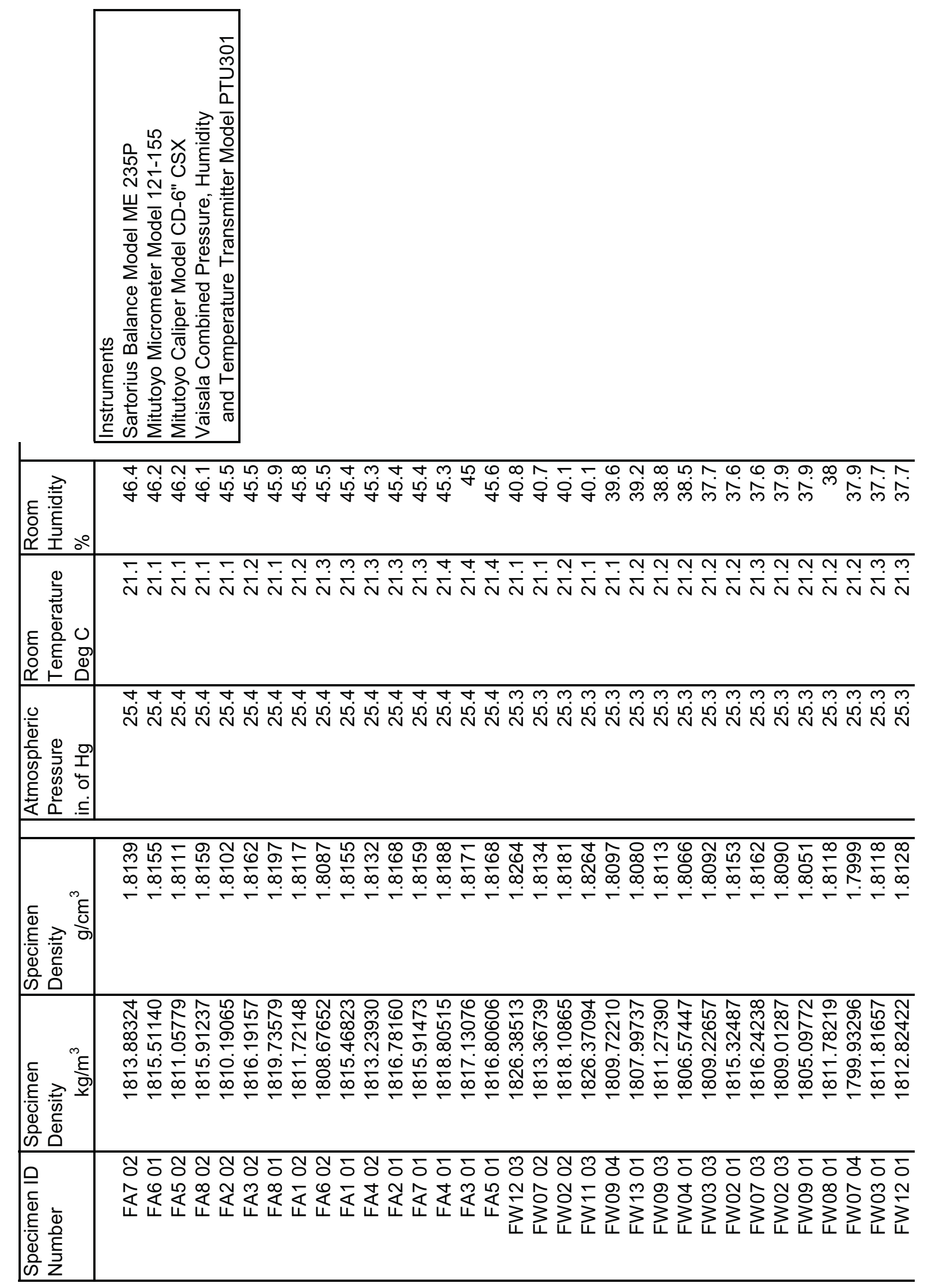




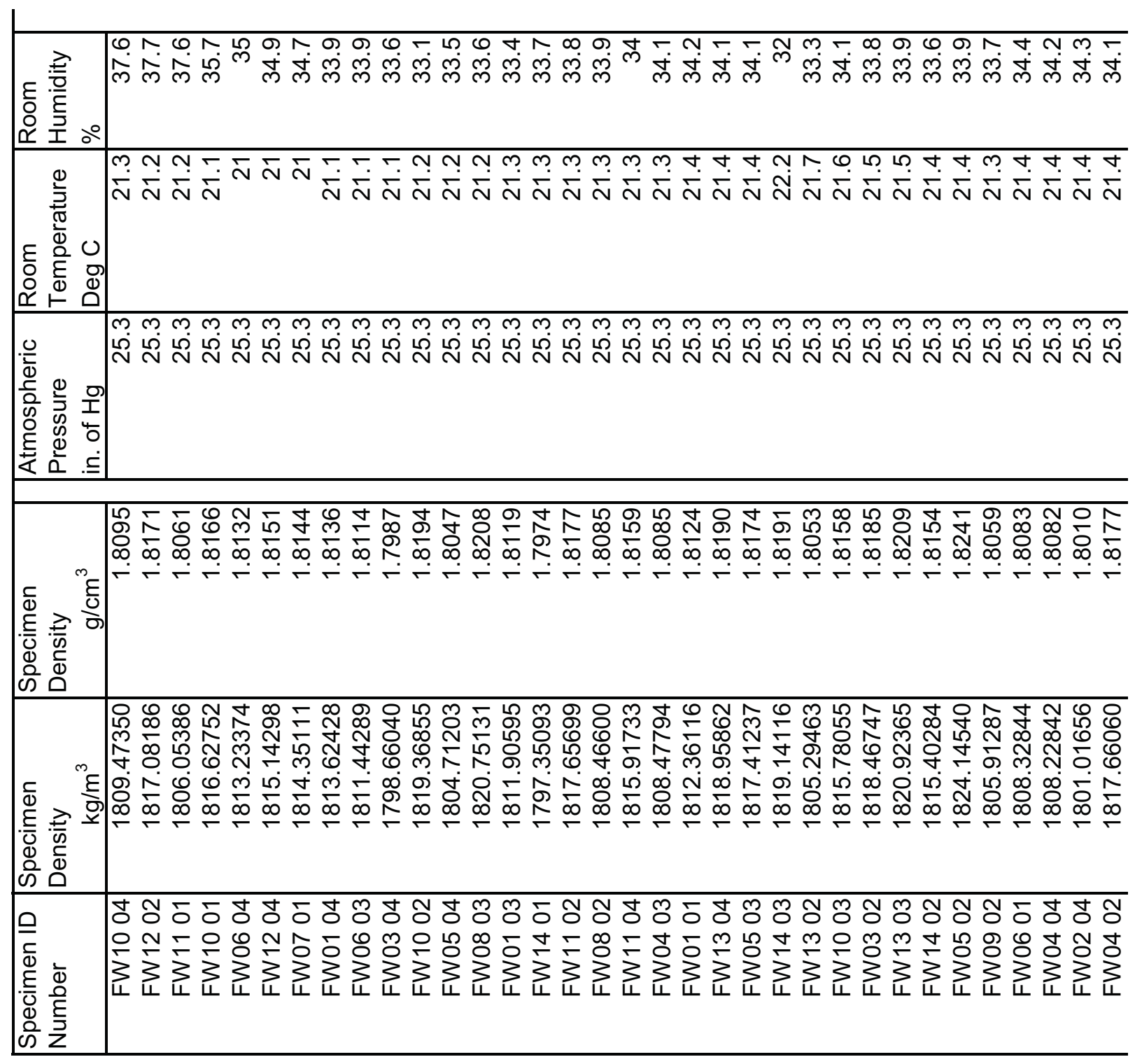




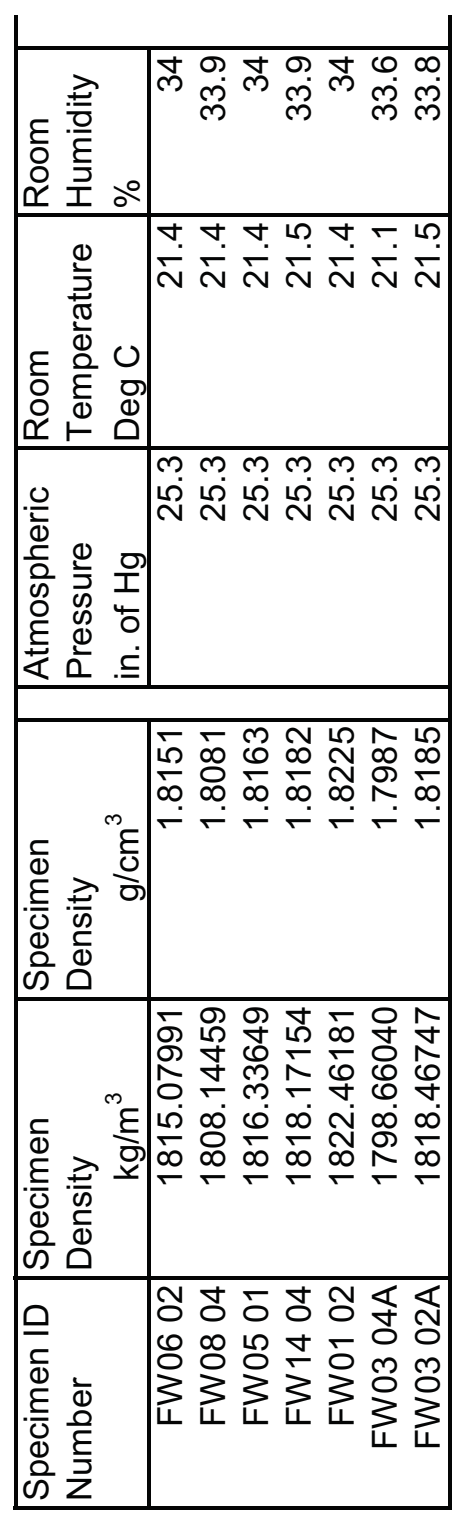




\begin{tabular}{|c|c|c|c|}
\hline 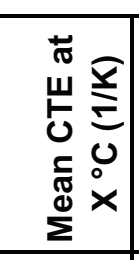 & \multicolumn{3}{|c|}{ 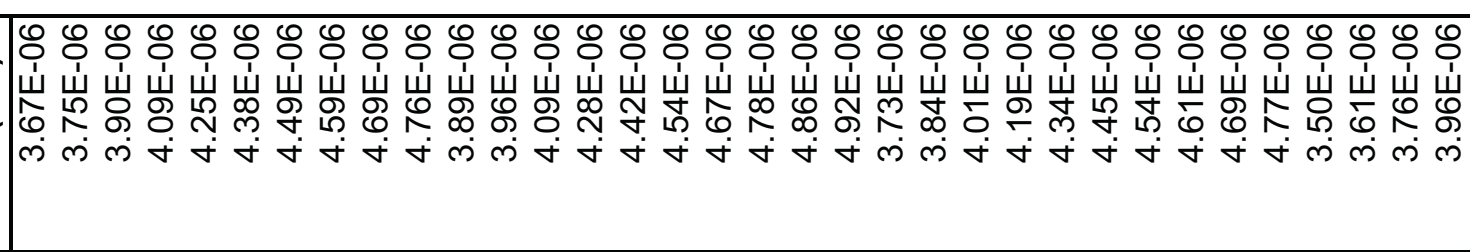 } \\
\hline 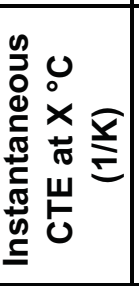 & \multicolumn{3}{|c|}{ 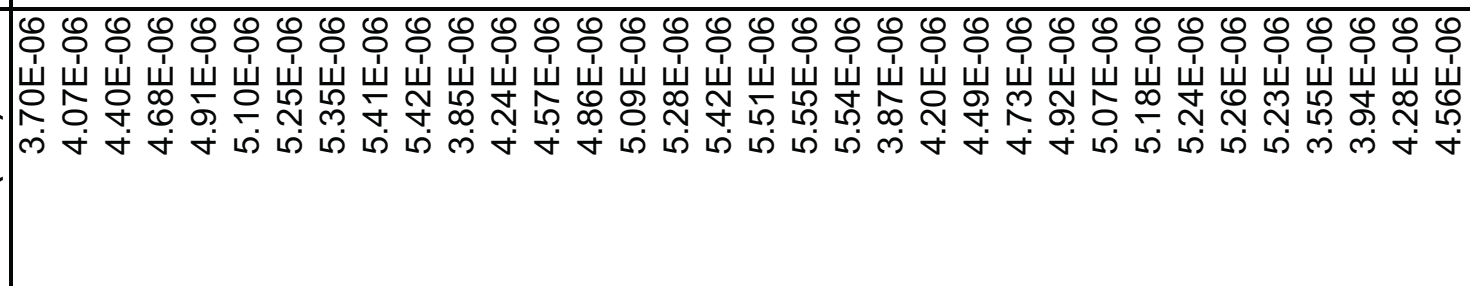 } \\
\hline 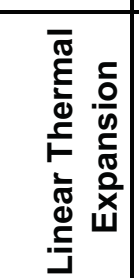 & \multicolumn{3}{|c|}{ 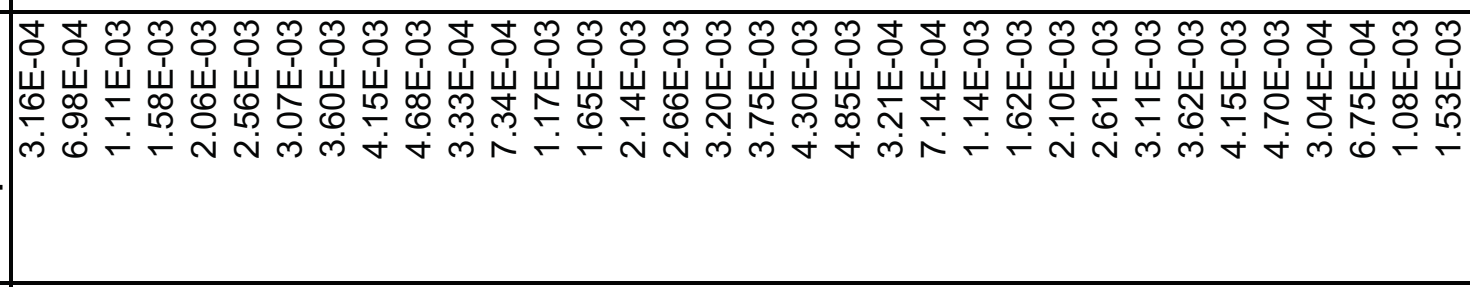 } \\
\hline 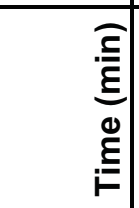 & \multicolumn{3}{|c|}{ 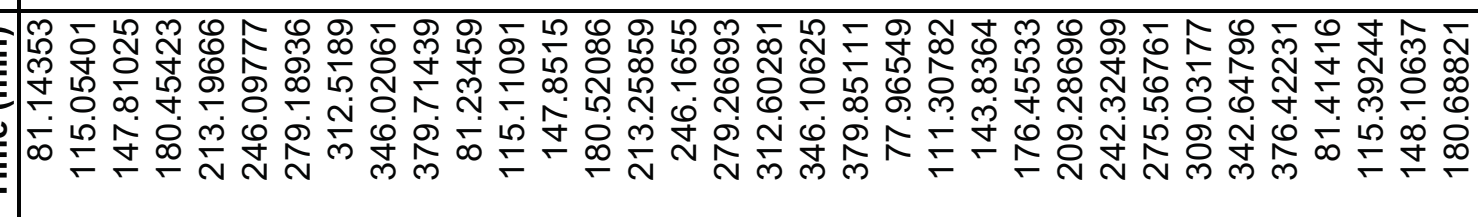 } \\
\hline 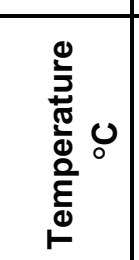 & \multicolumn{3}{|c|}{ 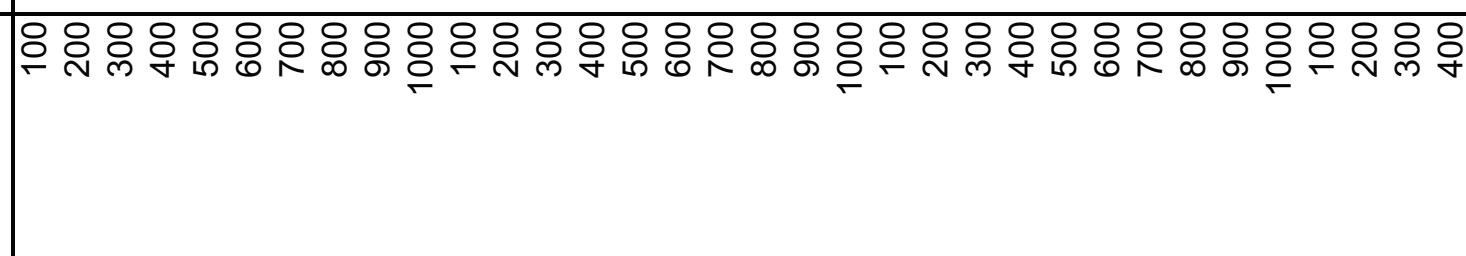 } \\
\hline 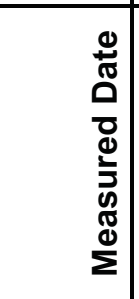 & 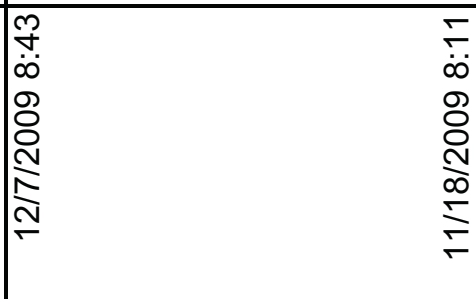 & 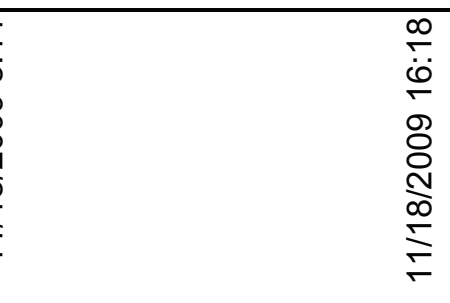 & 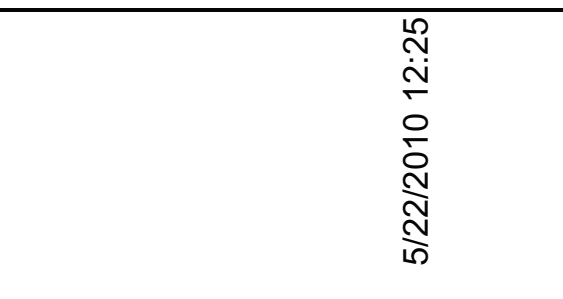 \\
\hline 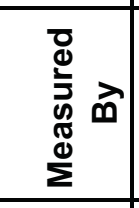 & 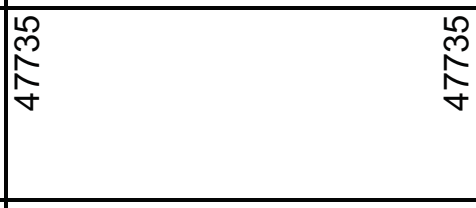 & $\begin{array}{l}8 \\
\frac{8}{6} \\
\frac{\pi}{4}\end{array}$ & \begin{tabular}{l}
$\mathscr{L}$ \\
$\stackrel{f}{f}$ \\
\multirow{f}{*}{}
\end{tabular} \\
\hline 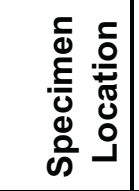 & & & \\
\hline 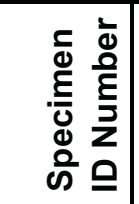 & 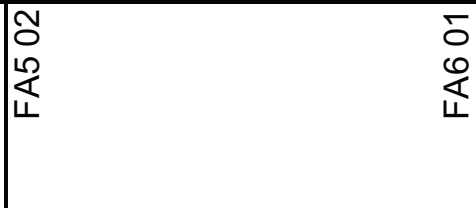 & 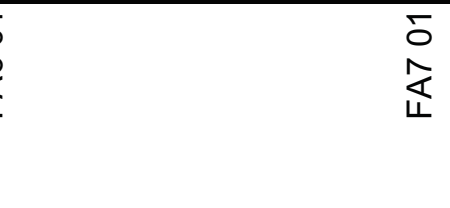 & 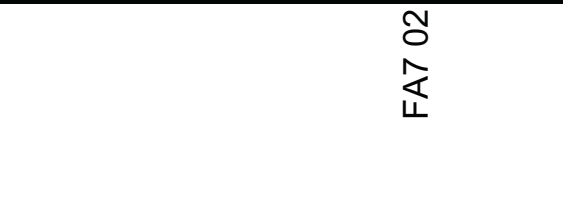 \\
\hline
\end{tabular}




\begin{tabular}{|c|c|}
\hline 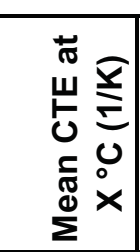 & 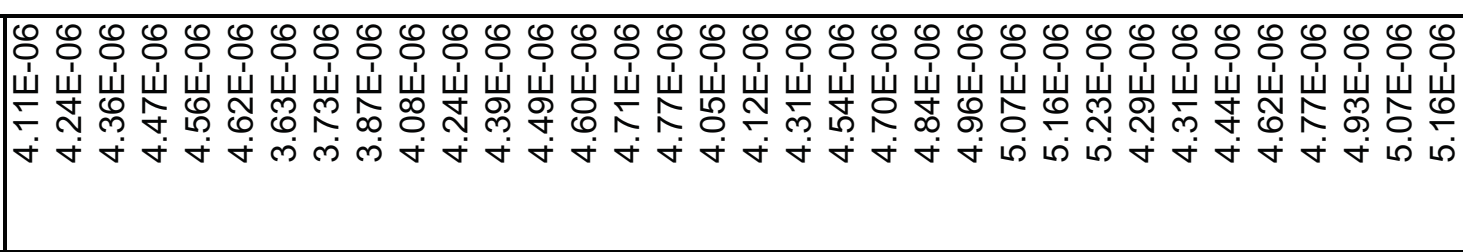 \\
\hline 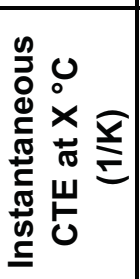 & 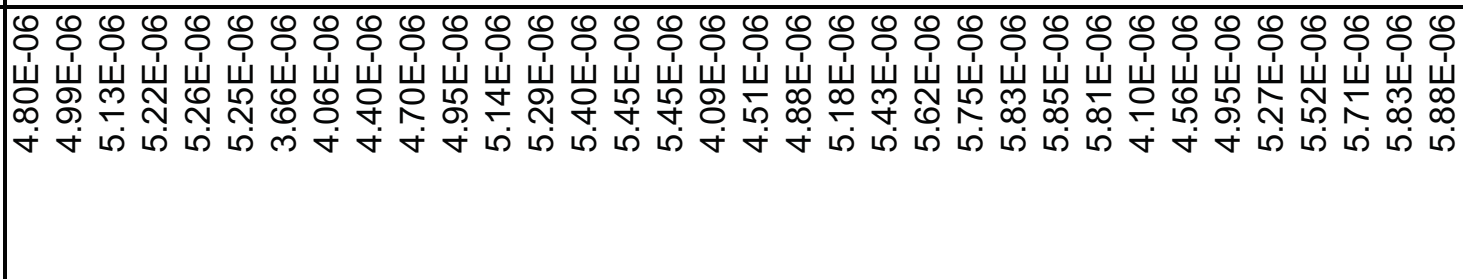 \\
\hline 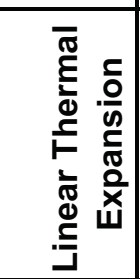 & 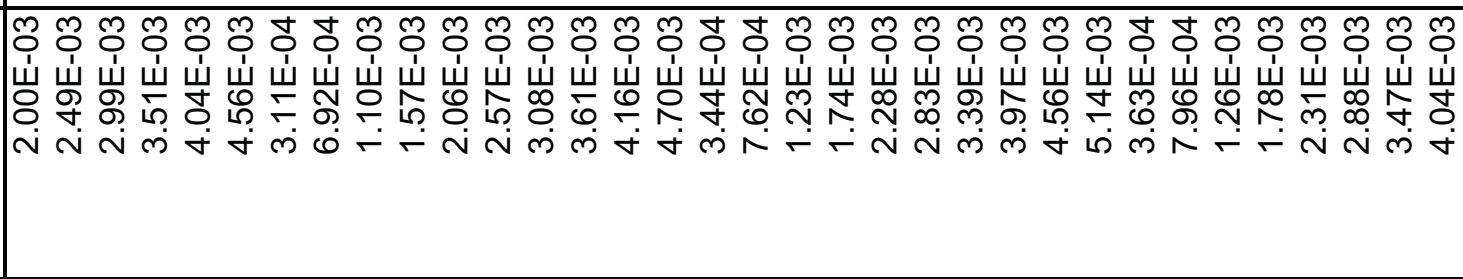 \\
\hline 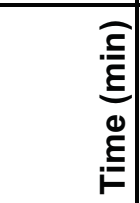 & 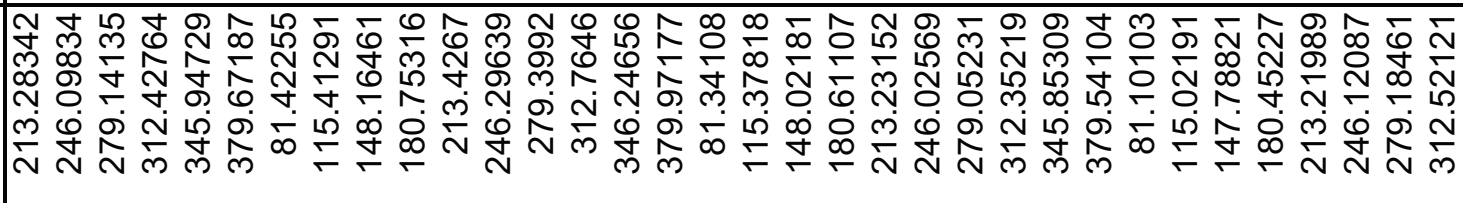 \\
\hline 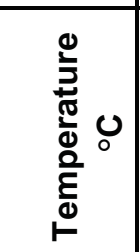 & 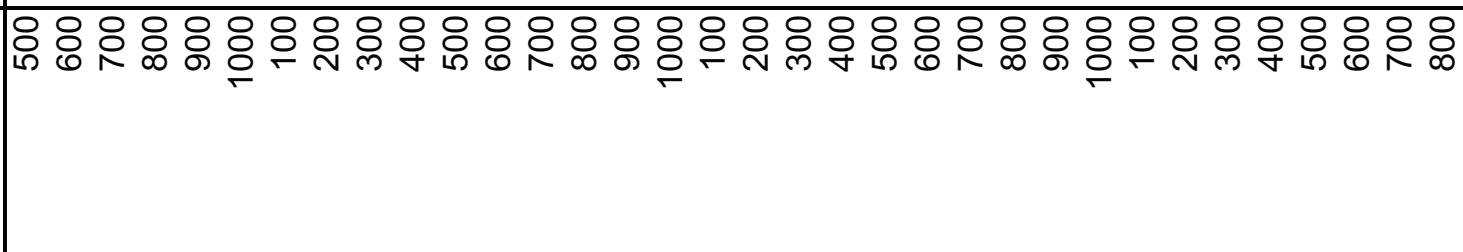 \\
\hline 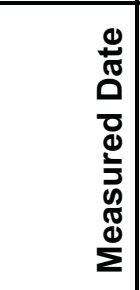 & 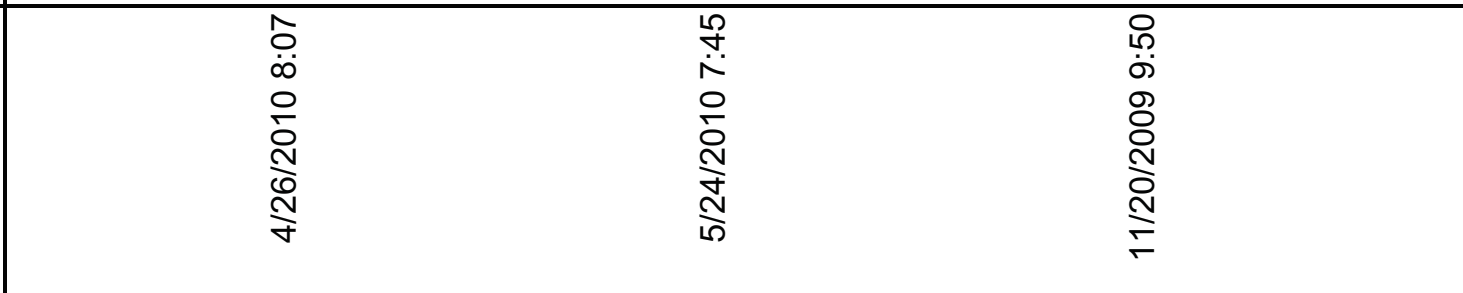 \\
\hline 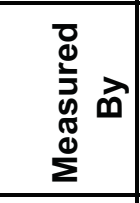 & 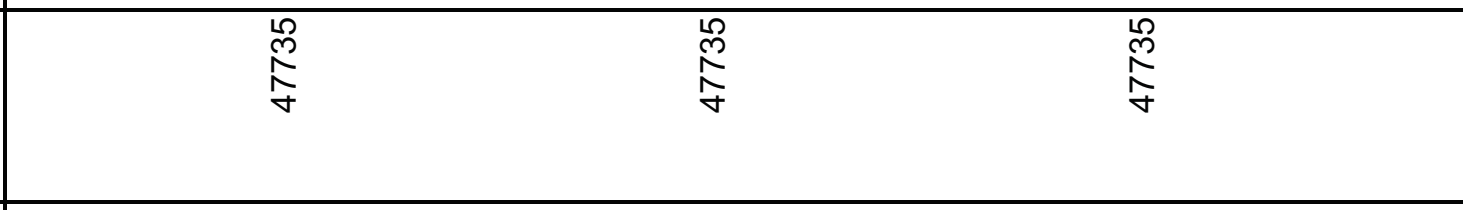 \\
\hline 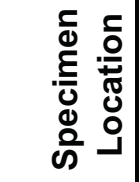 & \\
\hline 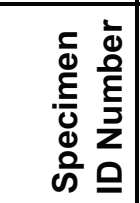 & $\sum_{\text {\} }^{5}} \\
$\hline
\end{tabular}




\begin{tabular}{|c|c|c|c|}
\hline 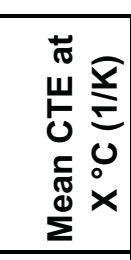 & 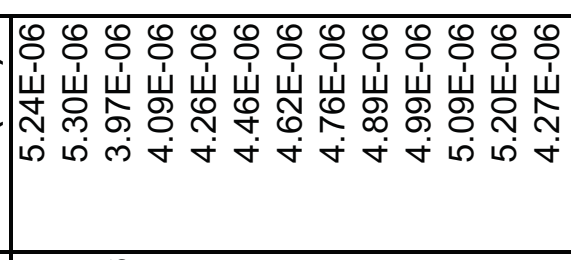 & 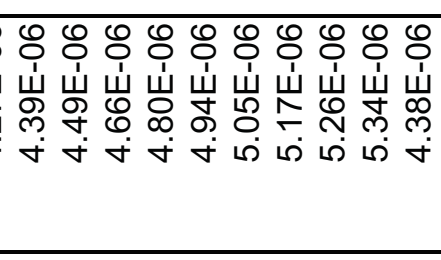 & 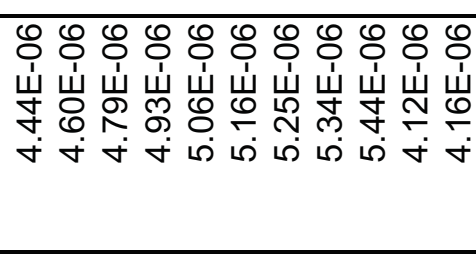 \\
\hline 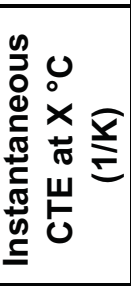 & 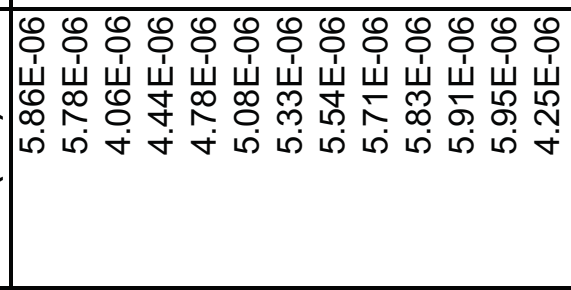 & 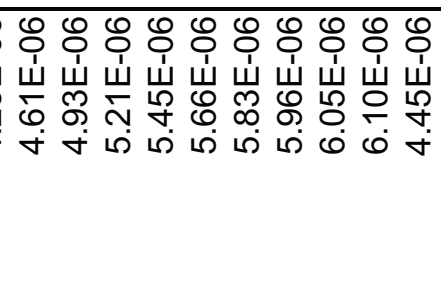 & 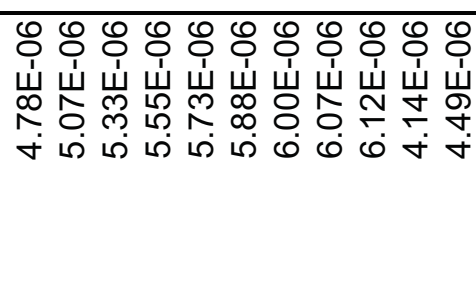 \\
\hline 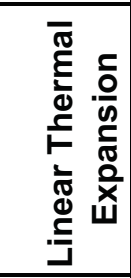 & 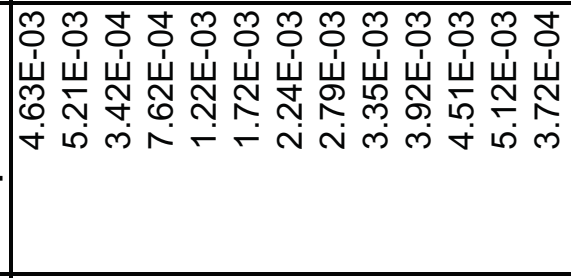 & 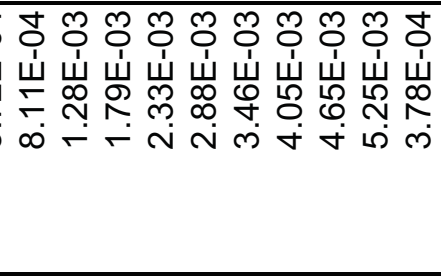 & 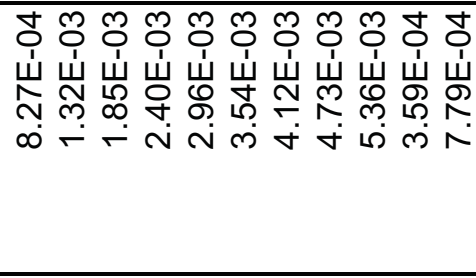 \\
\hline 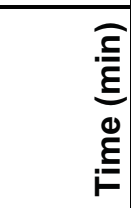 & 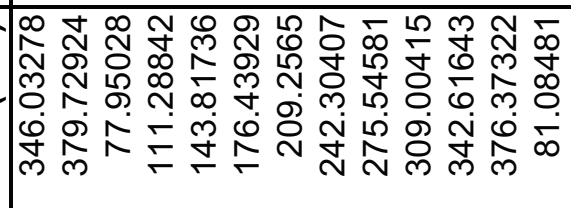 & 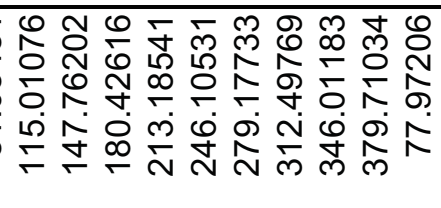 & 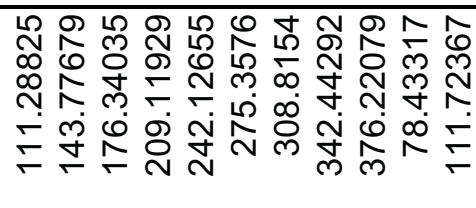 \\
\hline 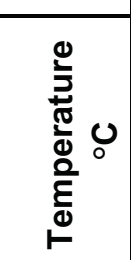 & 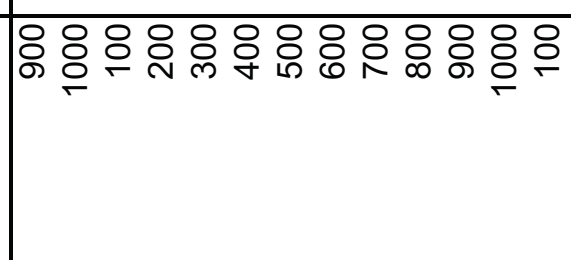 & 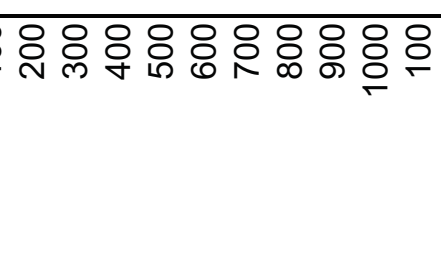 & 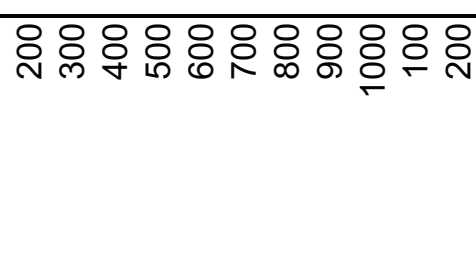 \\
\hline 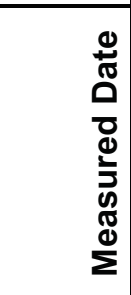 & 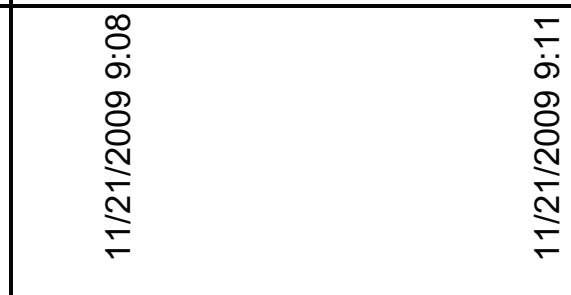 & 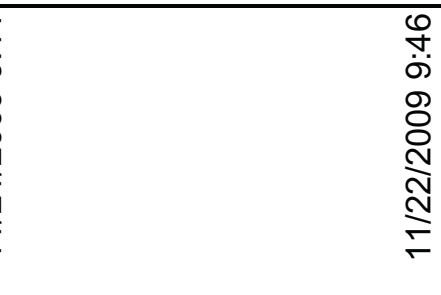 & 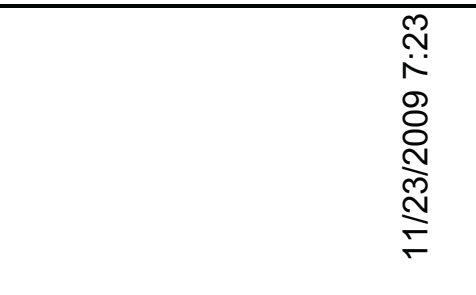 \\
\hline 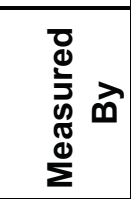 & $\begin{array}{l}\mathscr{8} \\
\frac{8}{7}\end{array}$ & $\begin{array}{l}\mathscr{8} \\
\frac{6}{q}\end{array}$ & $\begin{array}{l}\stackrel{8}{6} \\
\frac{\sigma}{q}\end{array}$ \\
\hline 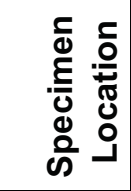 & & & \\
\hline 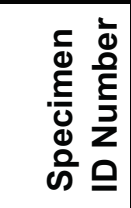 & $\sum_{i}^{0}$ & 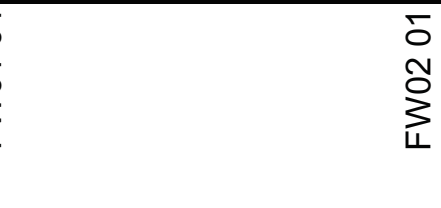 & 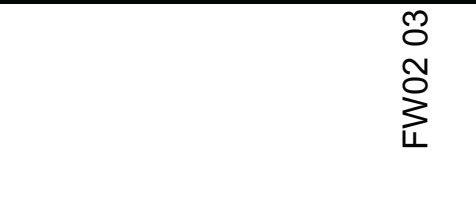 \\
\hline
\end{tabular}




\begin{tabular}{|c|c|}
\hline 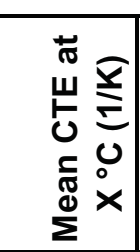 & 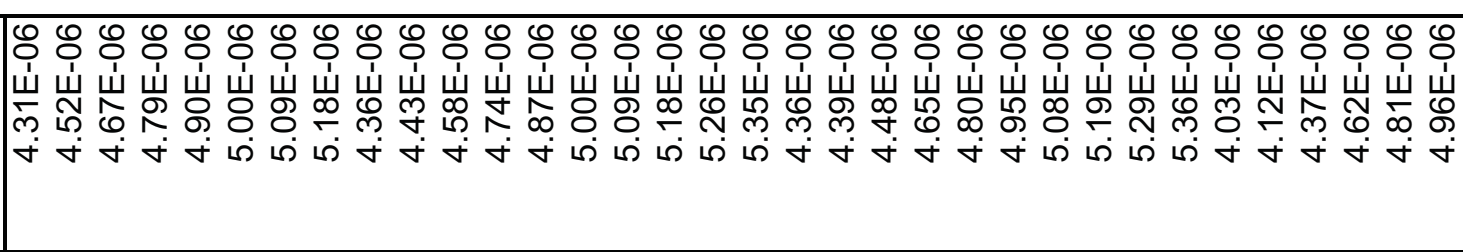 \\
\hline 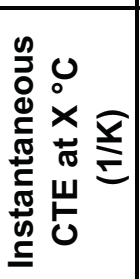 & 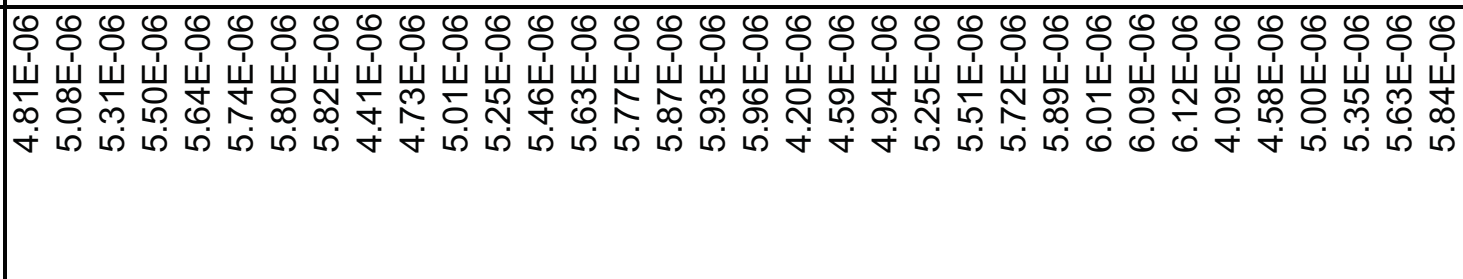 \\
\hline 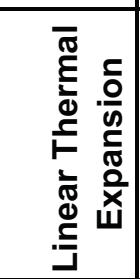 & 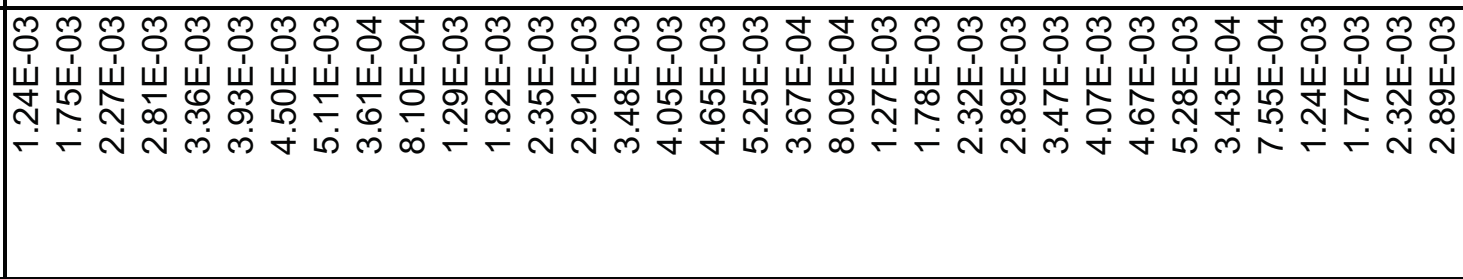 \\
\hline 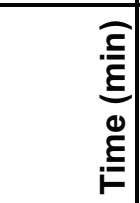 & 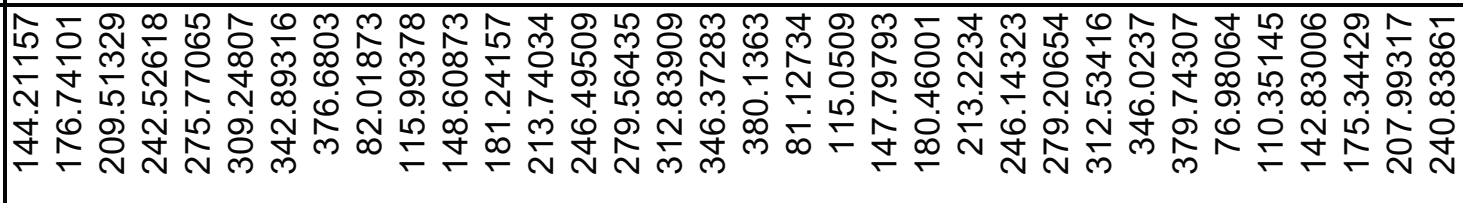 \\
\hline 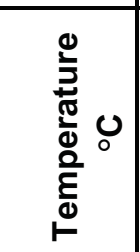 & 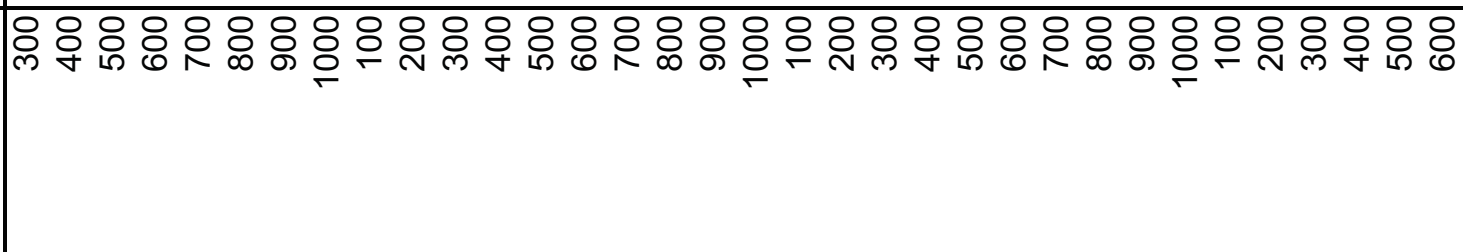 \\
\hline 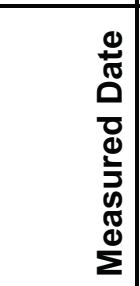 & 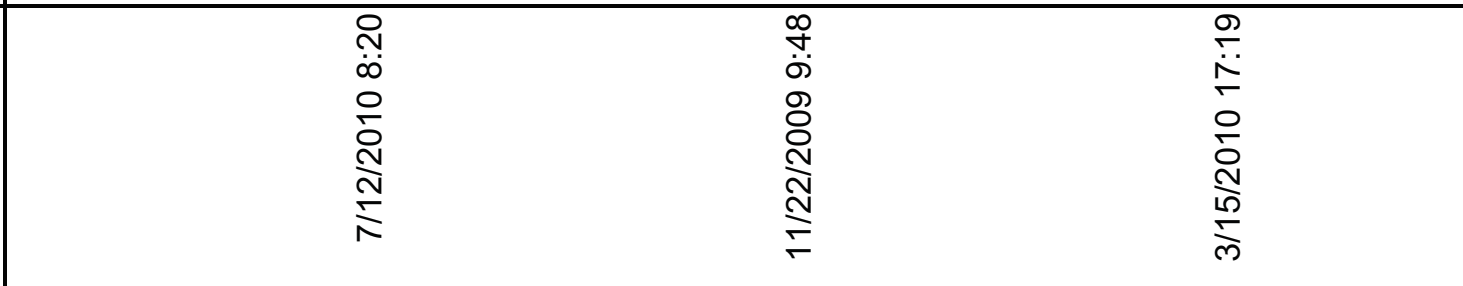 \\
\hline 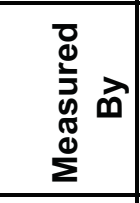 & 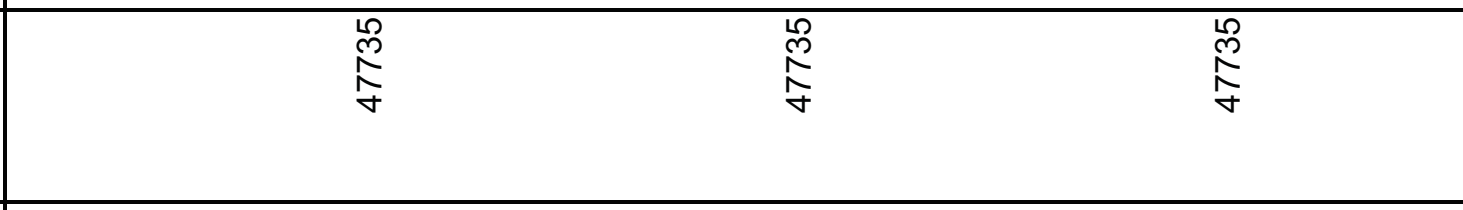 \\
\hline 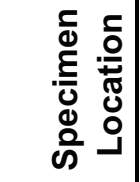 & \\
\hline 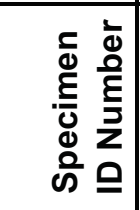 & 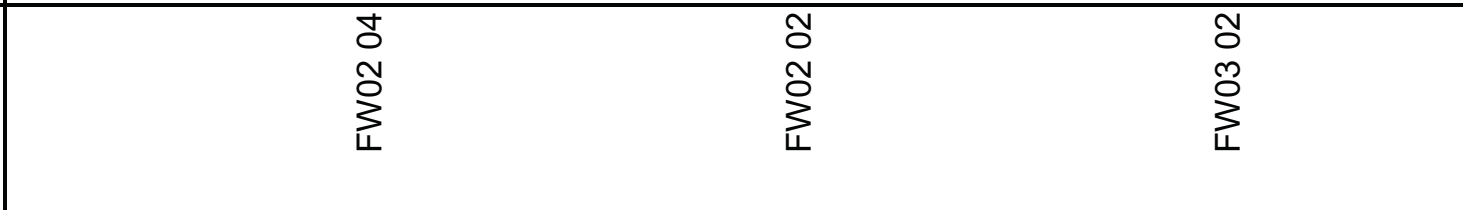 \\
\hline
\end{tabular}




\begin{tabular}{|c|c|c|}
\hline 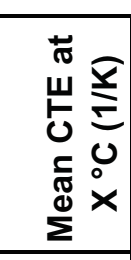 & 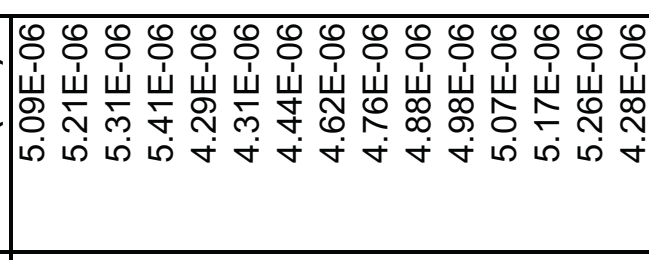 & 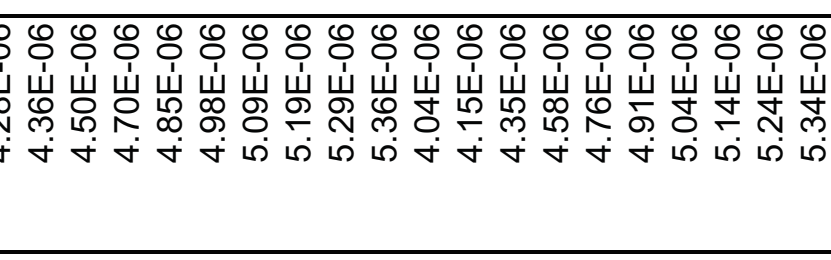 \\
\hline 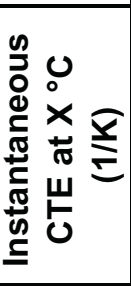 & 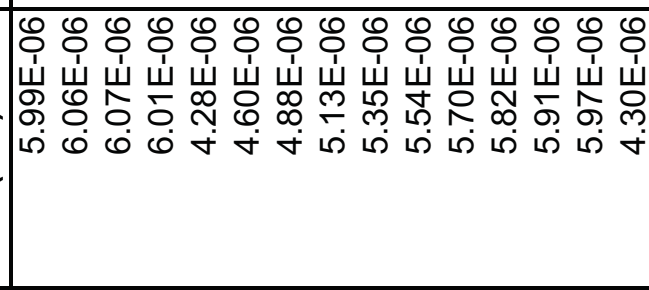 & 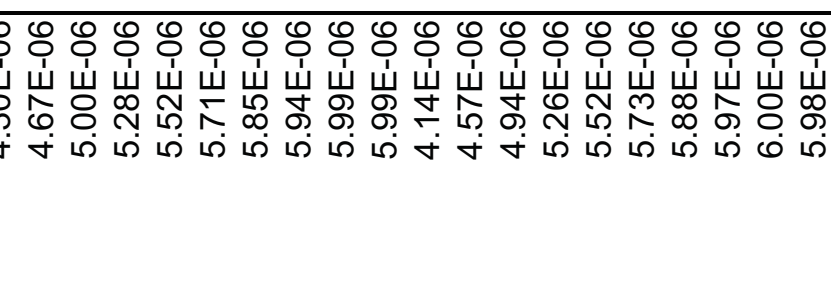 \\
\hline 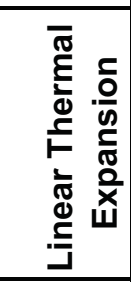 & 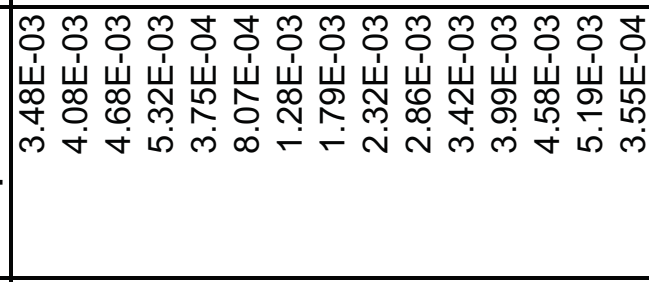 & 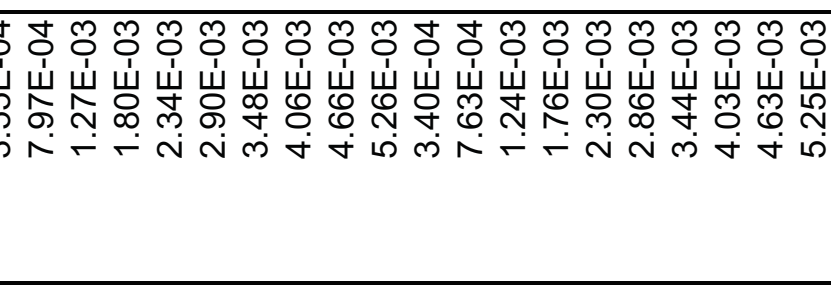 \\
\hline 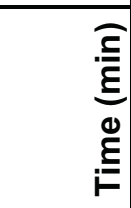 & 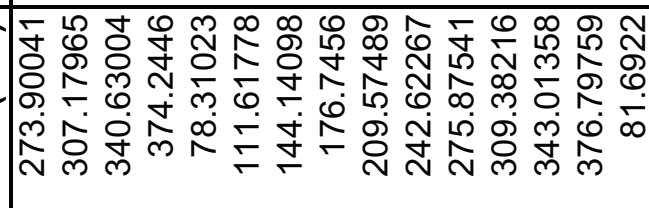 & 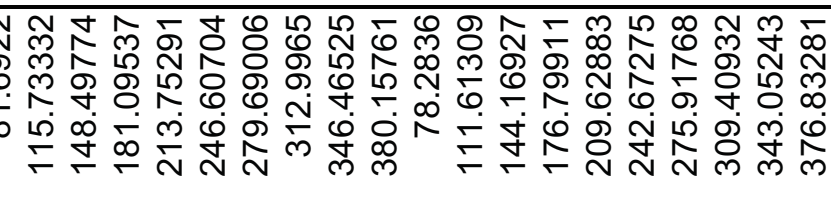 \\
\hline 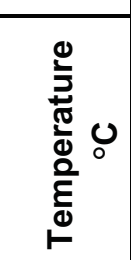 & 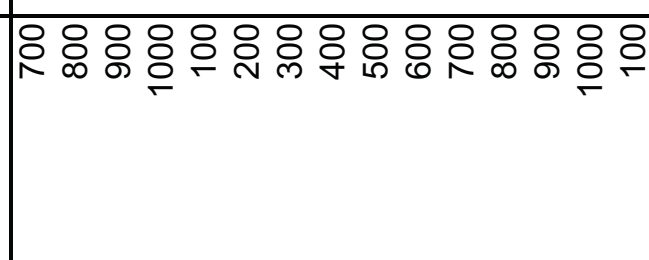 & 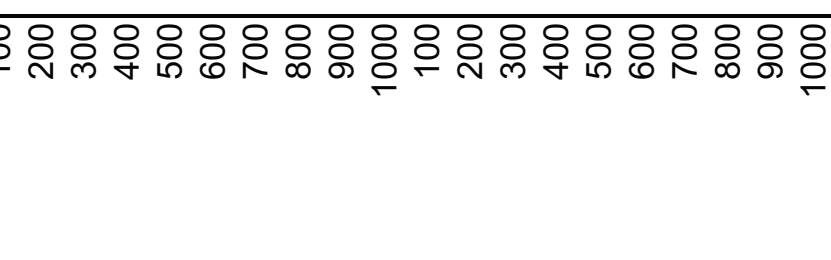 \\
\hline 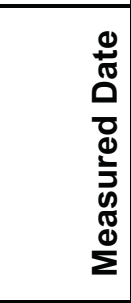 & 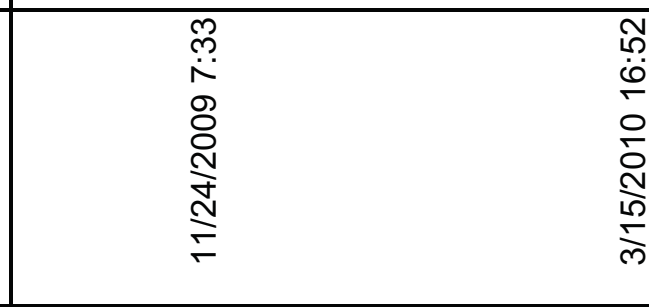 & 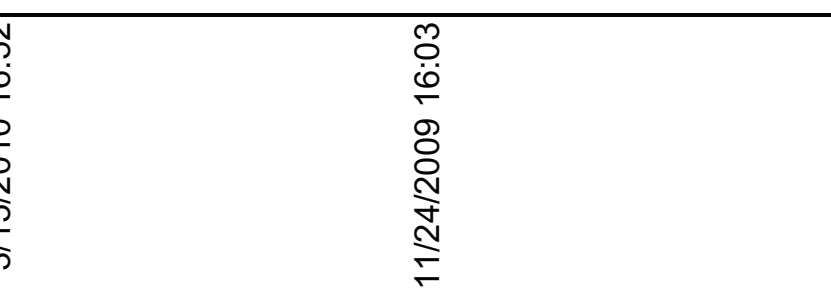 \\
\hline 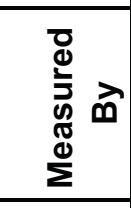 & 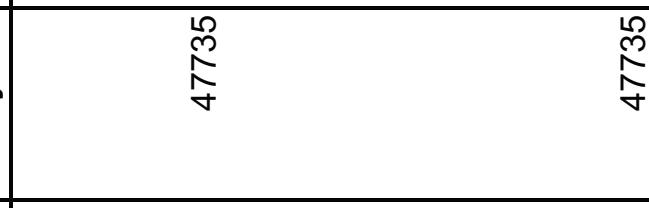 & $\begin{array}{l}\mathscr{L} \\
\stackrel{f}{f} \\
\end{array}$ \\
\hline 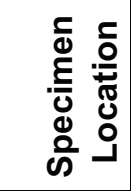 & & \\
\hline 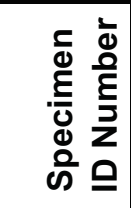 & 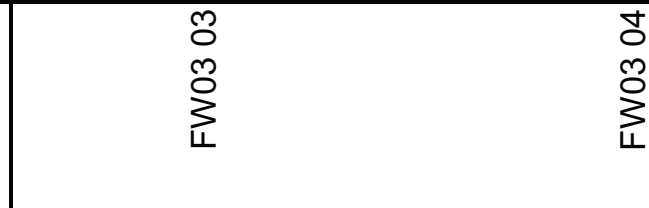 & $\begin{array}{l}5 \\
\delta \\
\$ \\
\sum_{1}\end{array}$ \\
\hline
\end{tabular}




\begin{tabular}{|c|c|}
\hline 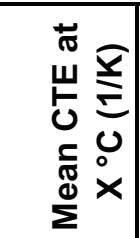 & 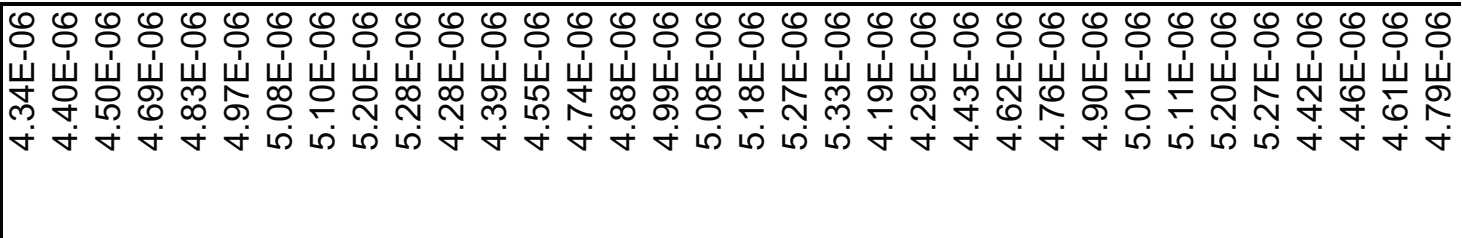 \\
\hline 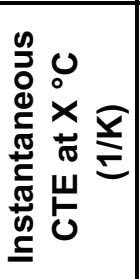 & 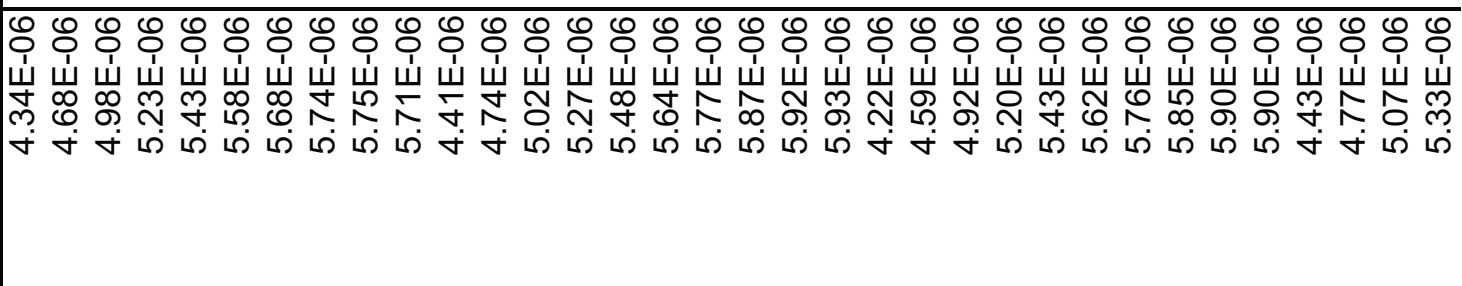 \\
\hline 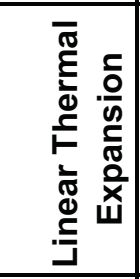 & 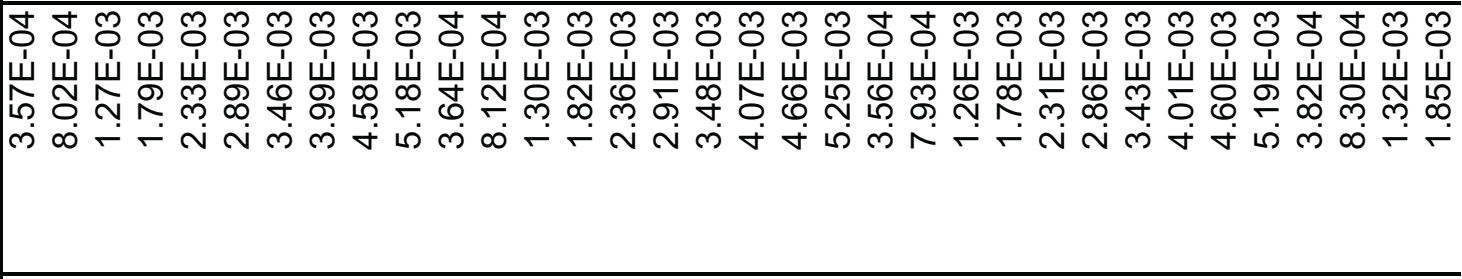 \\
\hline 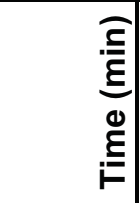 & 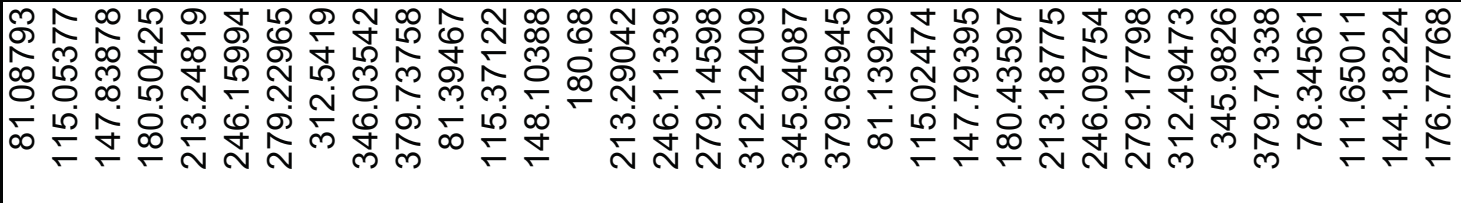 \\
\hline 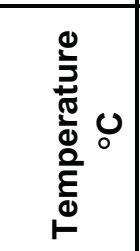 & 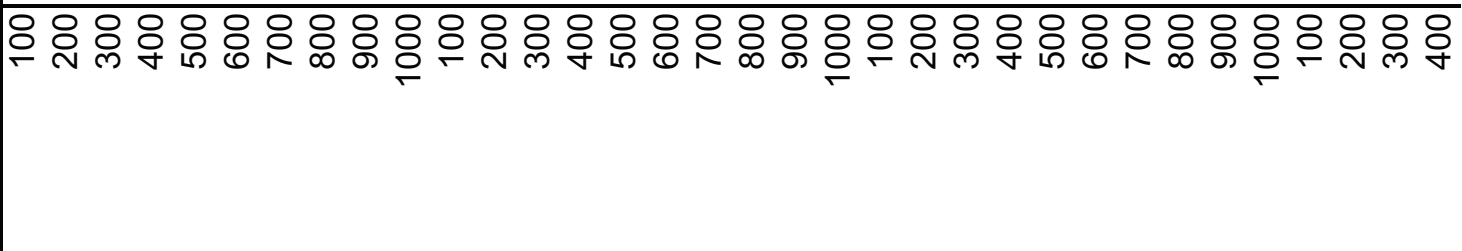 \\
\hline 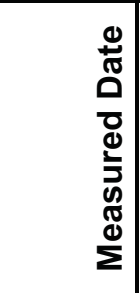 & 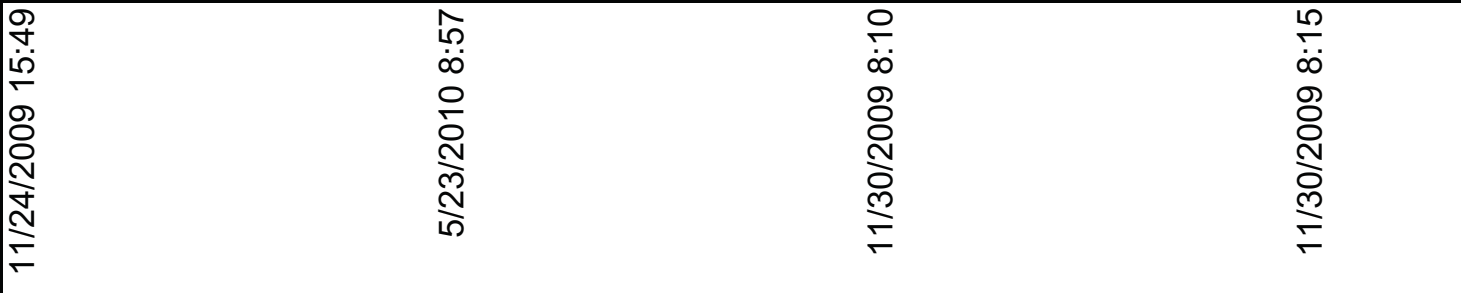 \\
\hline 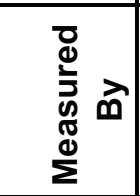 & 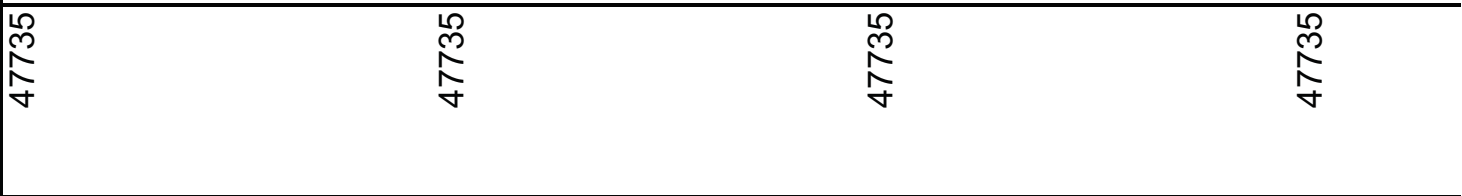 \\
\hline 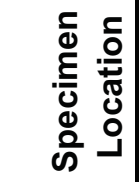 & \\
\hline 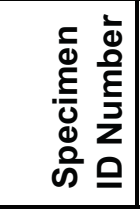 & 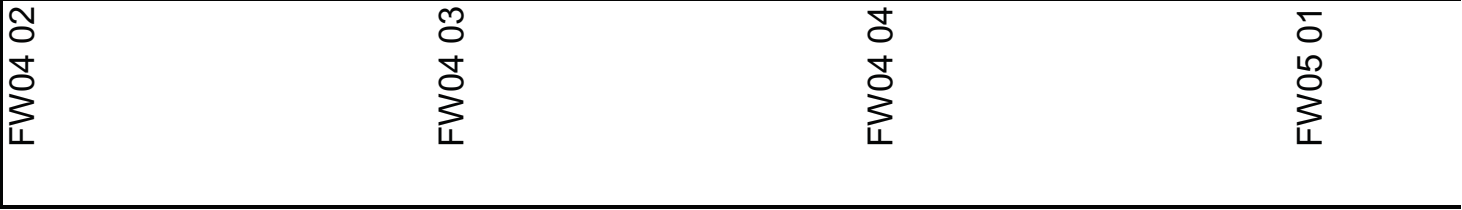 \\
\hline
\end{tabular}




\begin{tabular}{|c|c|}
\hline 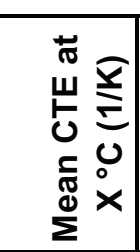 & 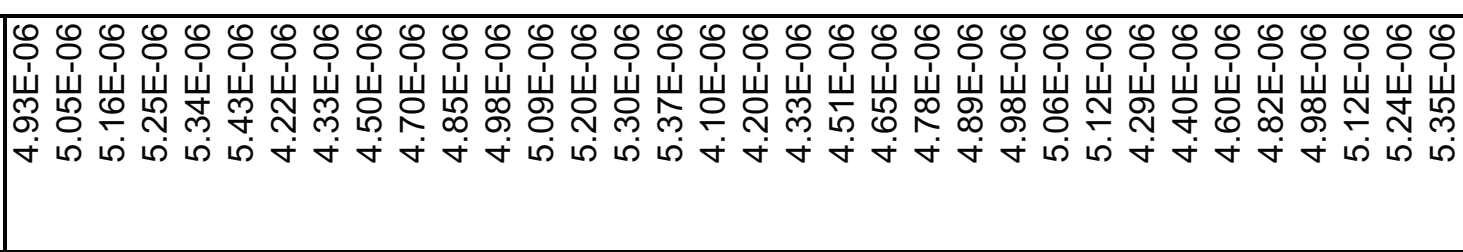 \\
\hline 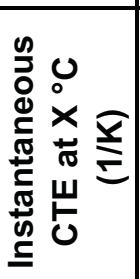 & 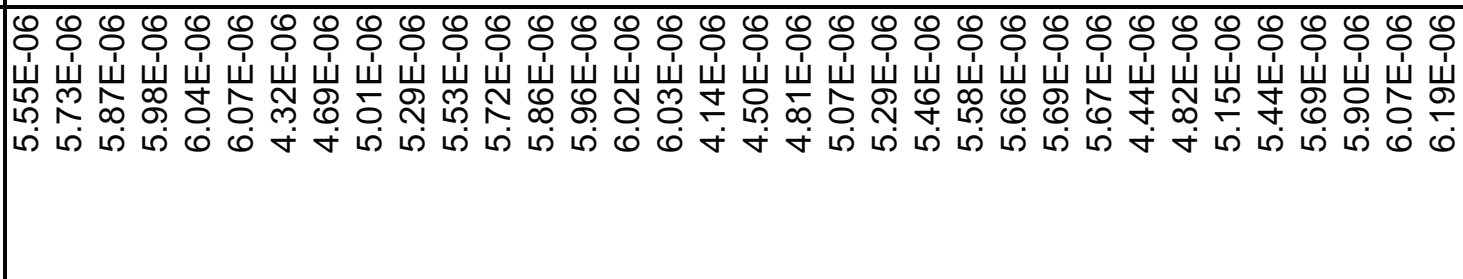 \\
\hline 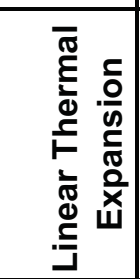 & 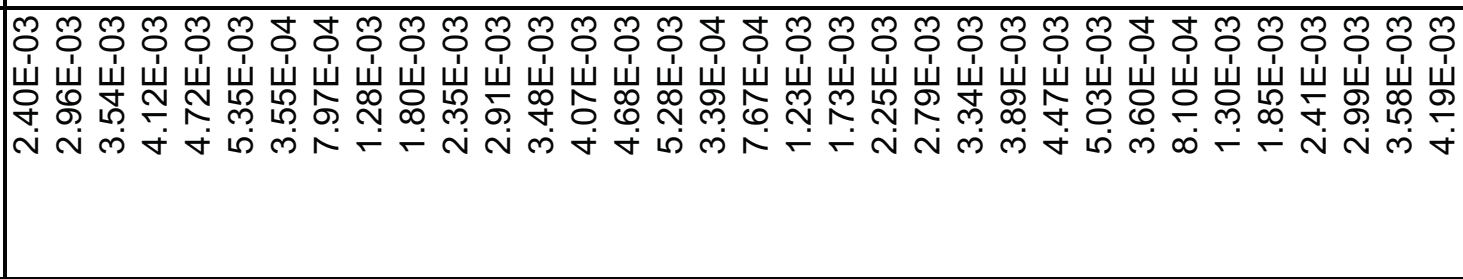 \\
\hline 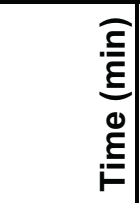 & 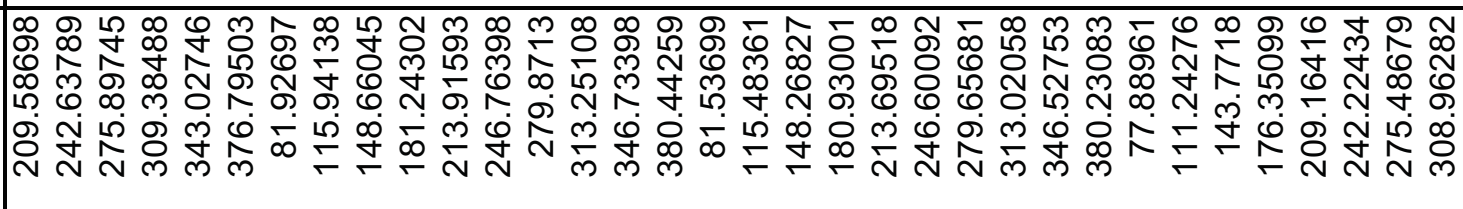 \\
\hline 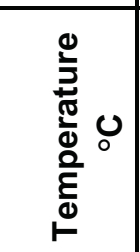 & 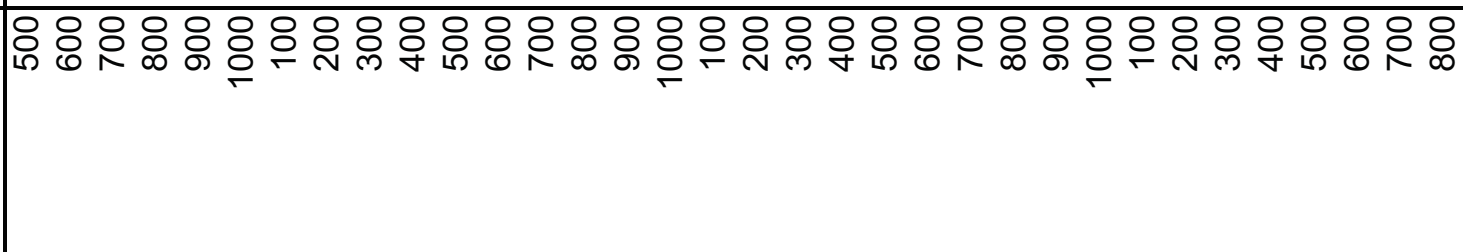 \\
\hline 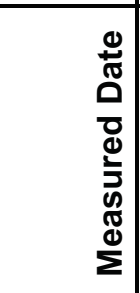 & 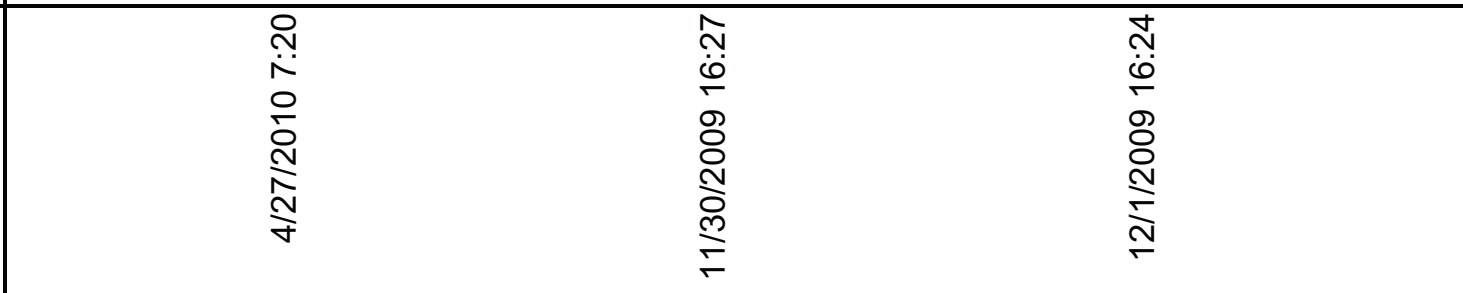 \\
\hline 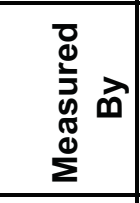 & 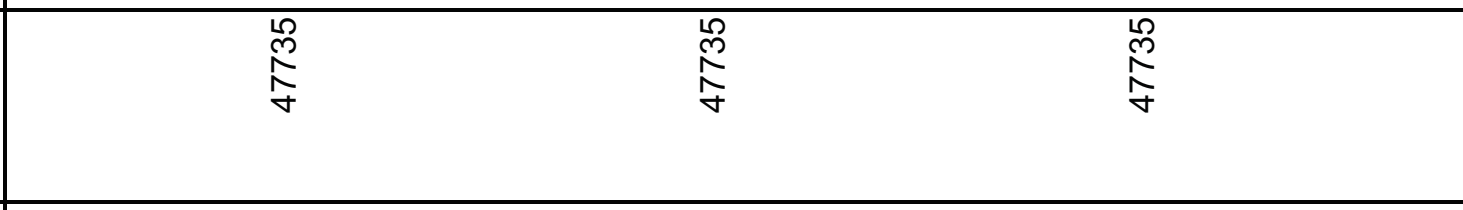 \\
\hline 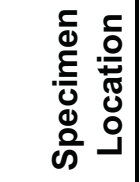 & \\
\hline 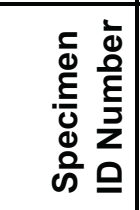 & 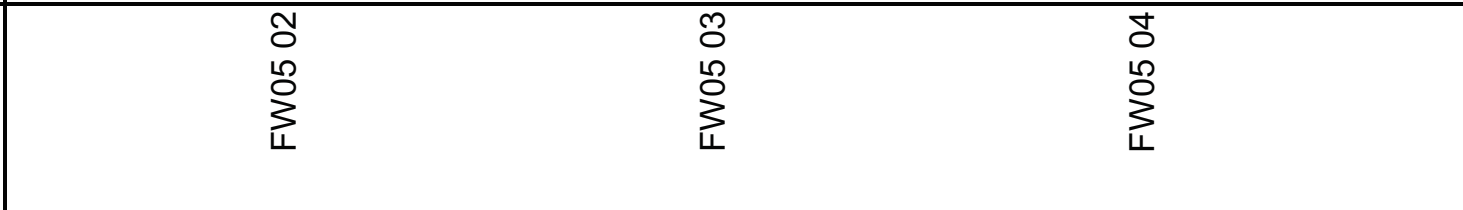 \\
\hline
\end{tabular}




\begin{tabular}{|c|c|c|c|}
\hline 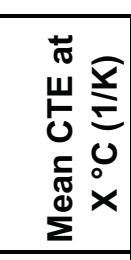 & \multicolumn{3}{|c|}{ 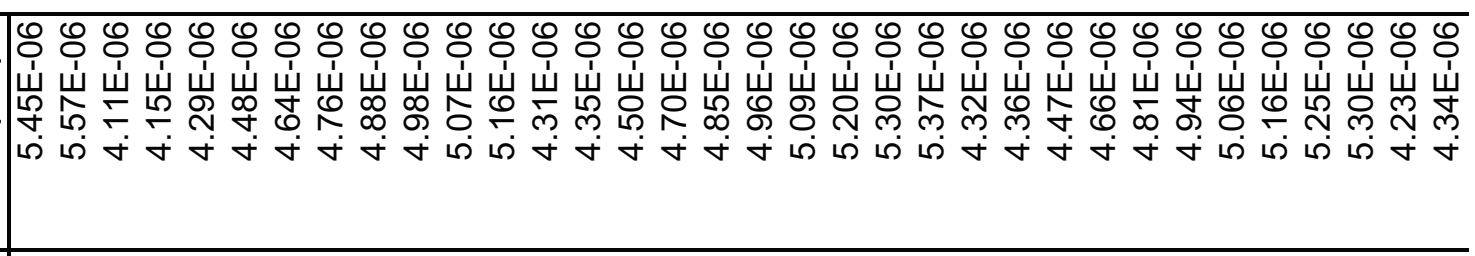 } \\
\hline 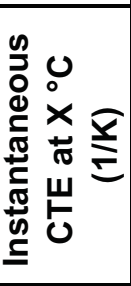 & \multicolumn{3}{|c|}{ 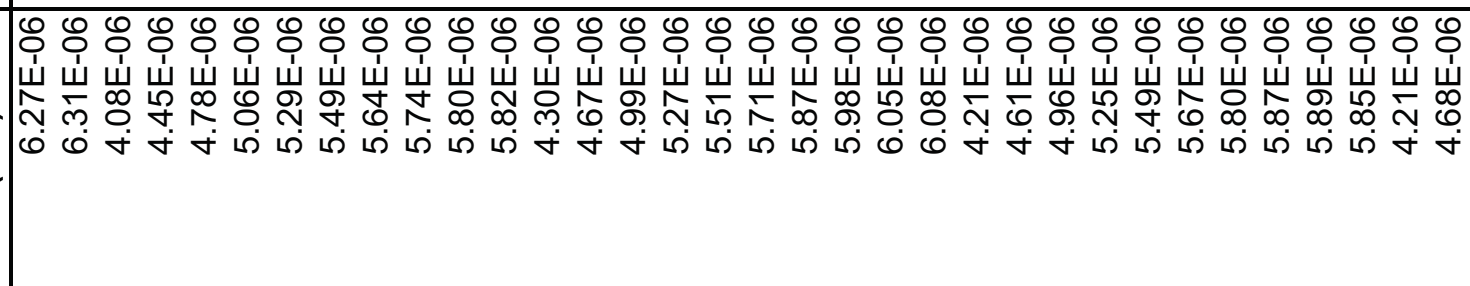 } \\
\hline 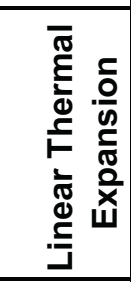 & \multicolumn{3}{|c|}{ 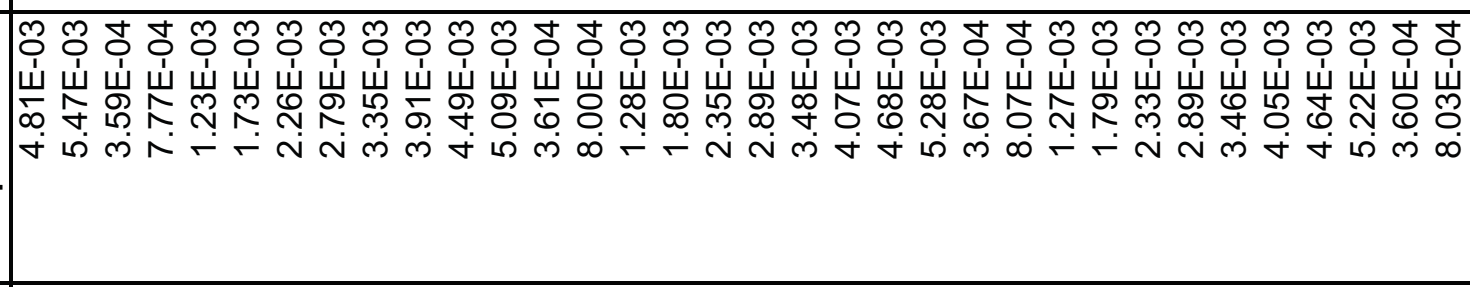 } \\
\hline$\stackrel{\widehat{\underline{\varepsilon}}}{\underline{\underline{\varepsilon}}}$ & \multicolumn{3}{|c|}{ 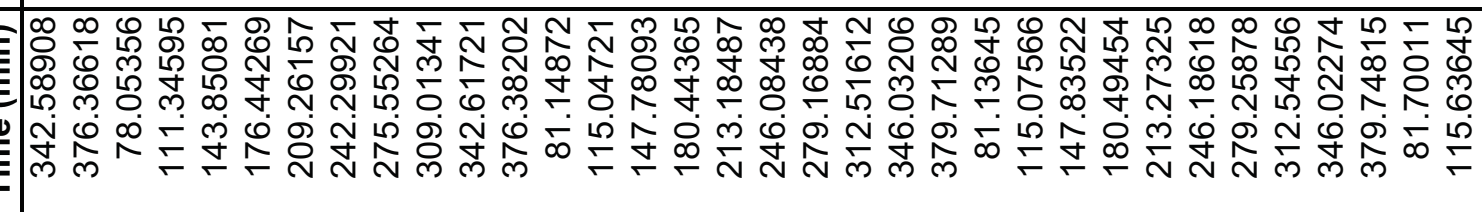 } \\
\hline 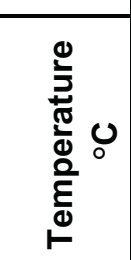 & \multicolumn{3}{|c|}{ 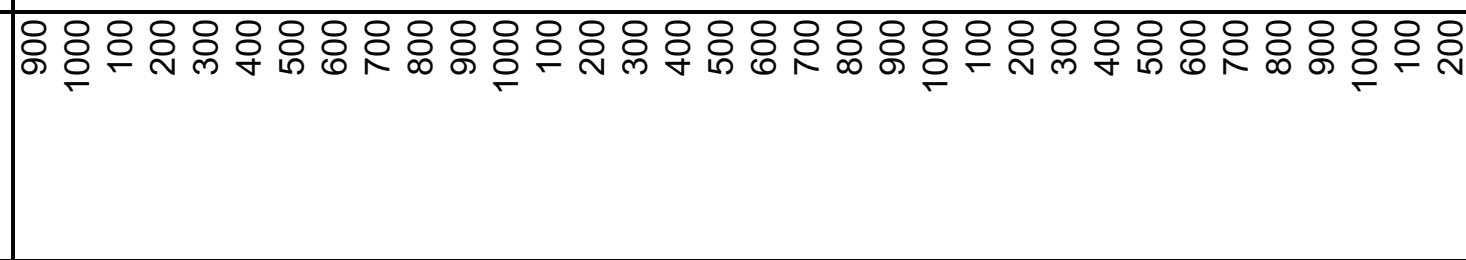 } \\
\hline 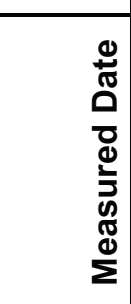 & 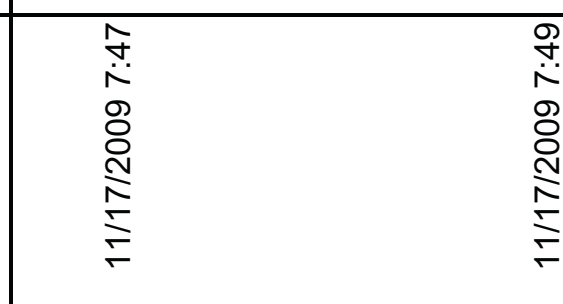 & 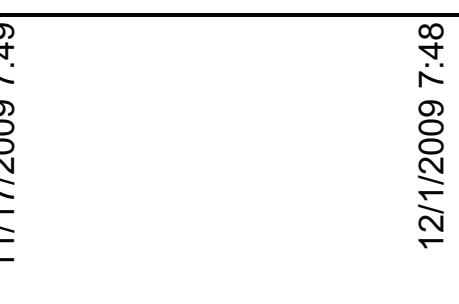 & 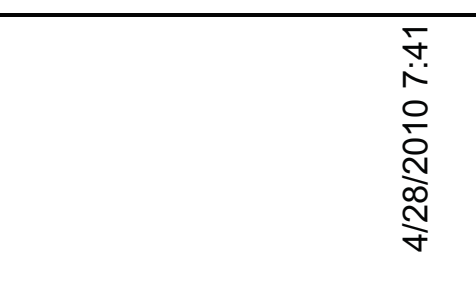 \\
\hline 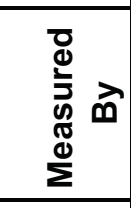 & $\begin{array}{l}8 \\
\frac{8}{4}\end{array}$ & 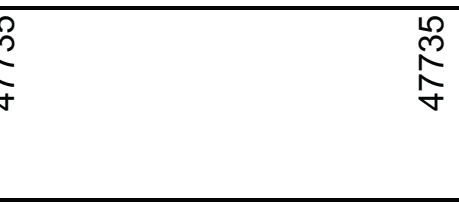 & $\begin{array}{l}\mathscr{L} \\
\stackrel{\rho}{f} \\
\end{array}$ \\
\hline 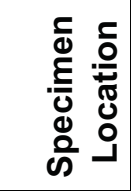 & & & \\
\hline 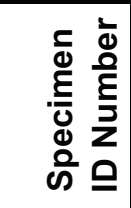 & 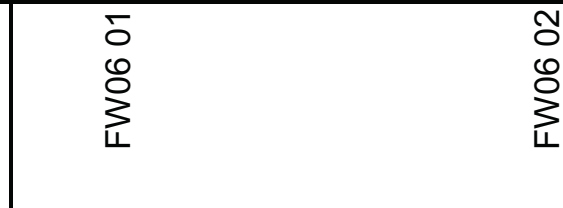 & $\begin{array}{l}0 \\
0 \\
0 \\
\sum_{L}\end{array}$ & $\begin{array}{l}0 \\
0 \\
0 \\
\vdots \\
\vdots \\
4\end{array}$ \\
\hline
\end{tabular}




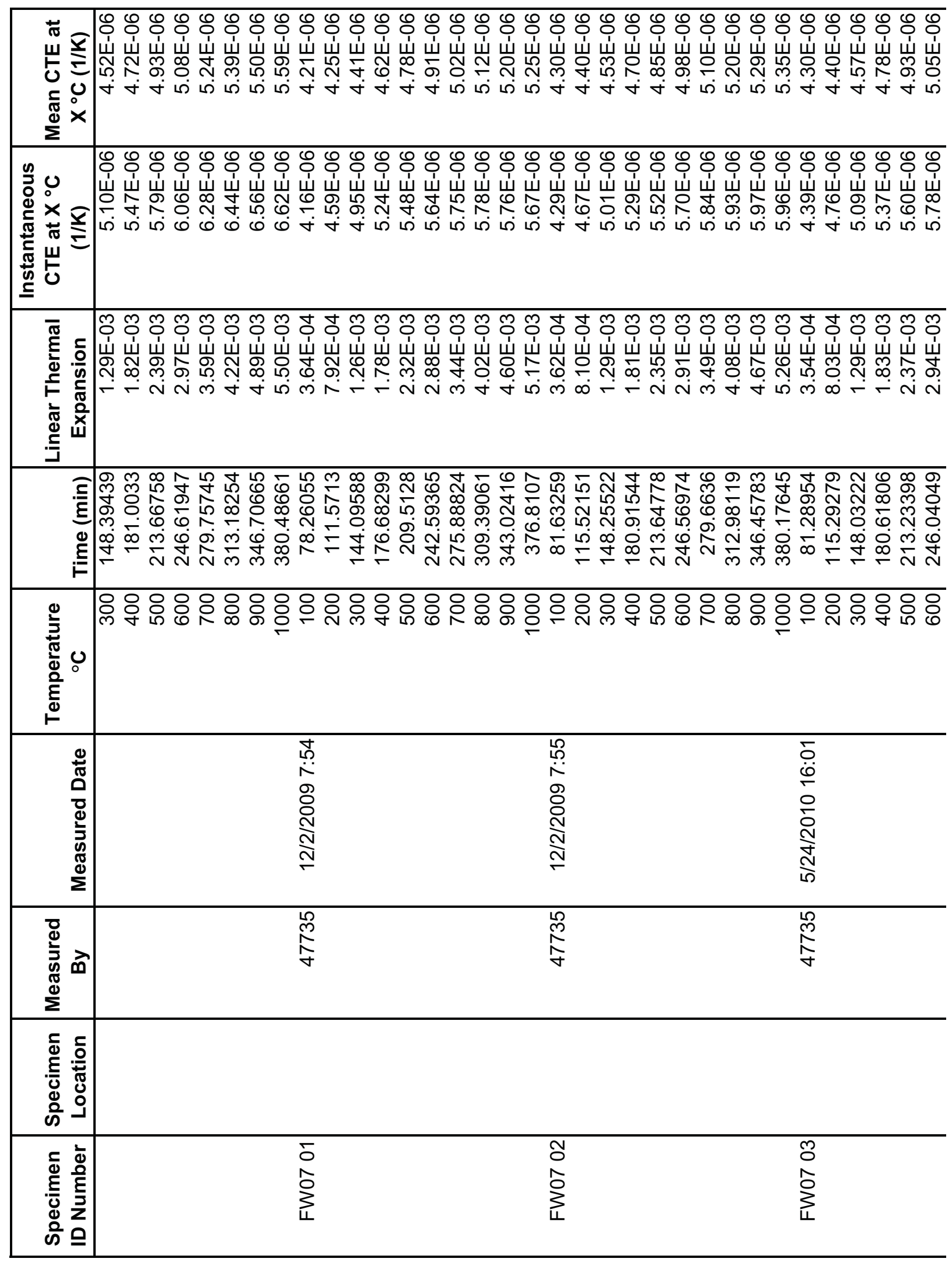




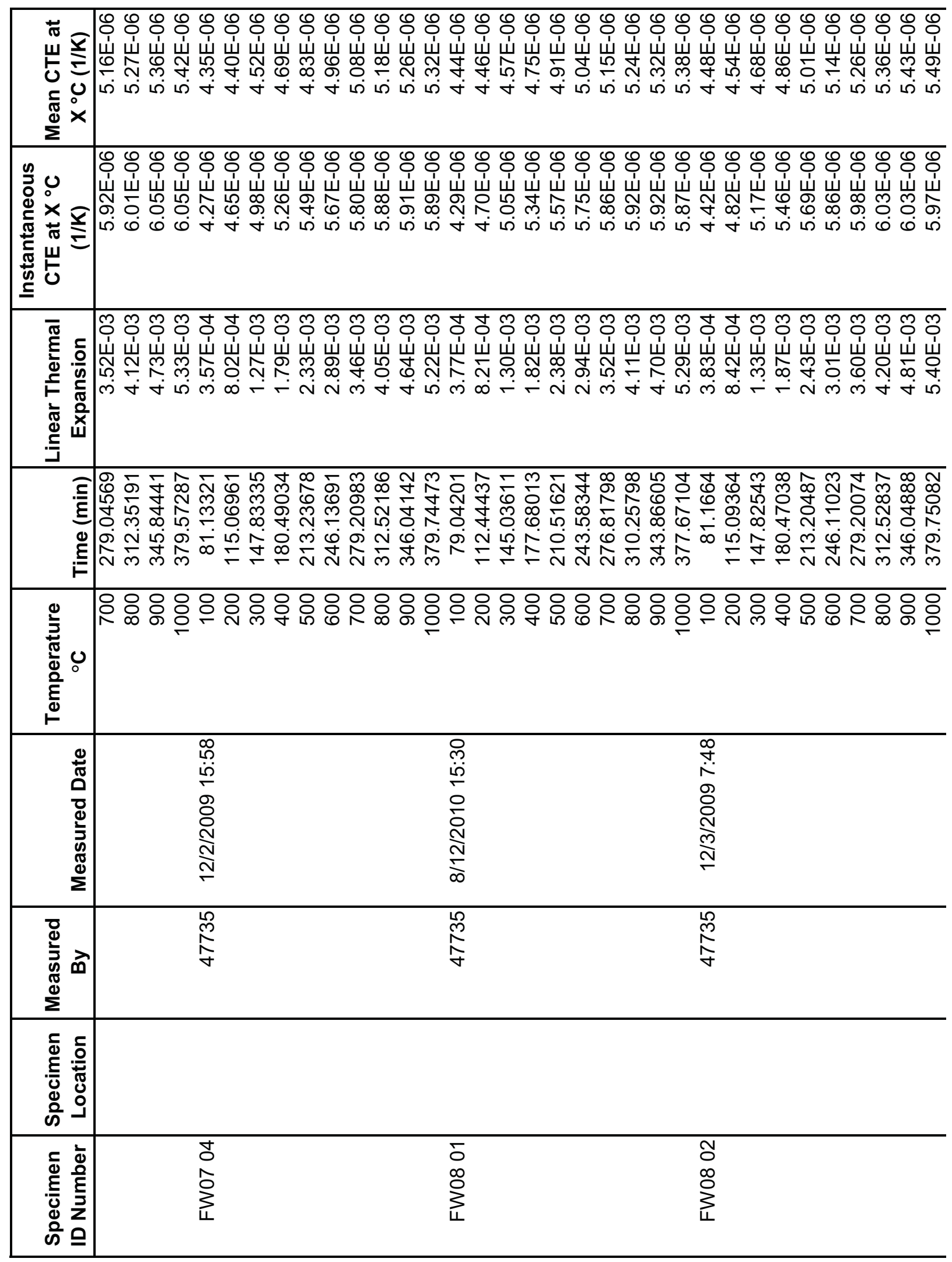




\begin{tabular}{|c|c|c|c|}
\hline 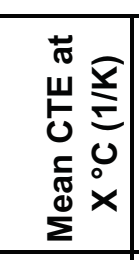 & \multicolumn{3}{|c|}{ 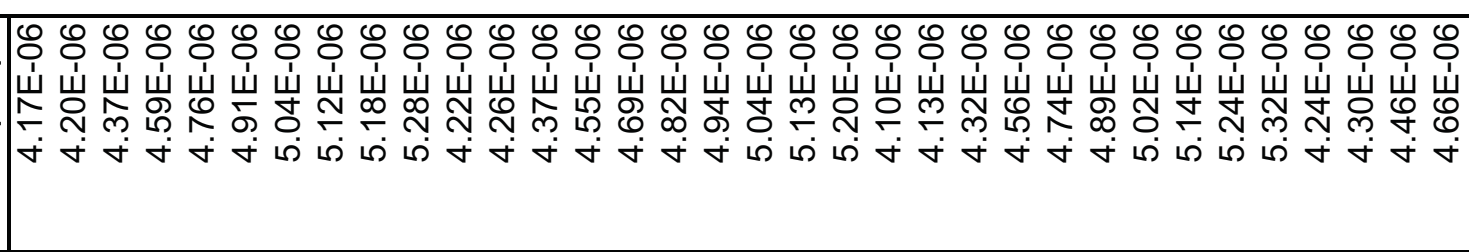 } \\
\hline 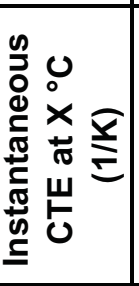 & \multicolumn{3}{|c|}{ 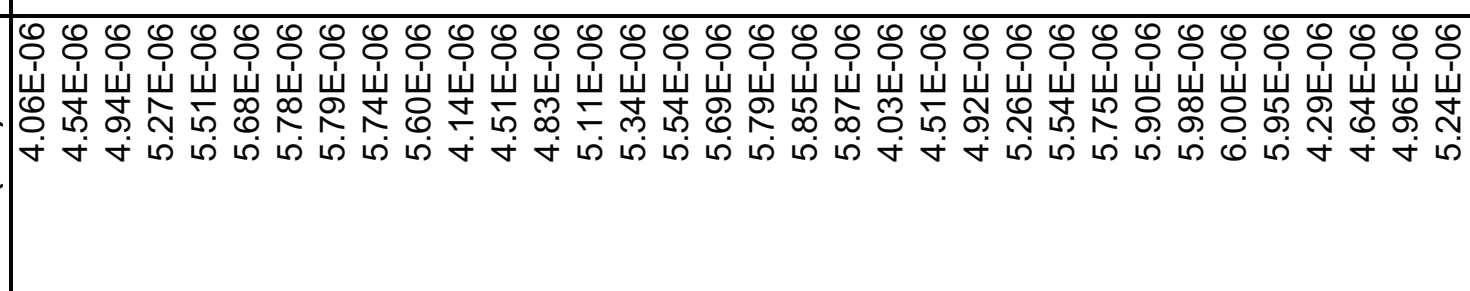 } \\
\hline 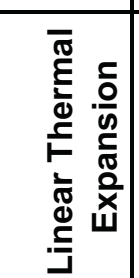 & \multicolumn{3}{|c|}{ 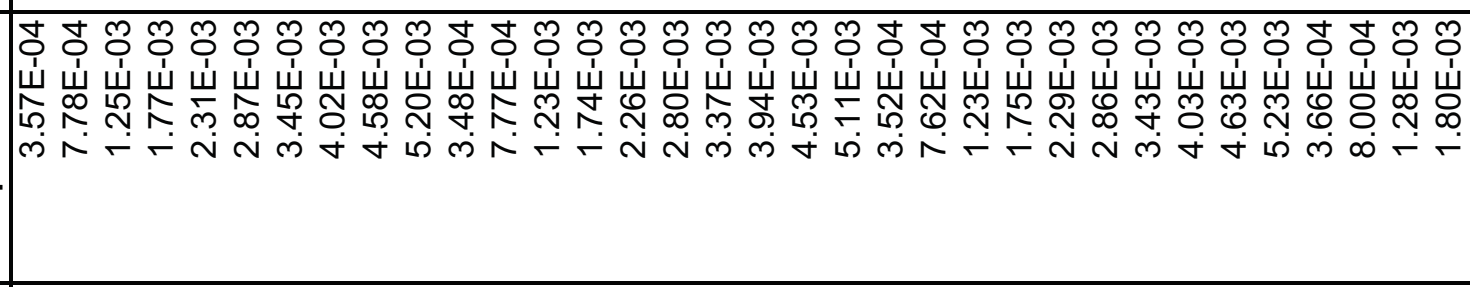 } \\
\hline 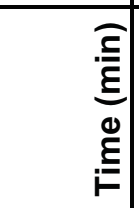 & \multicolumn{3}{|c|}{ 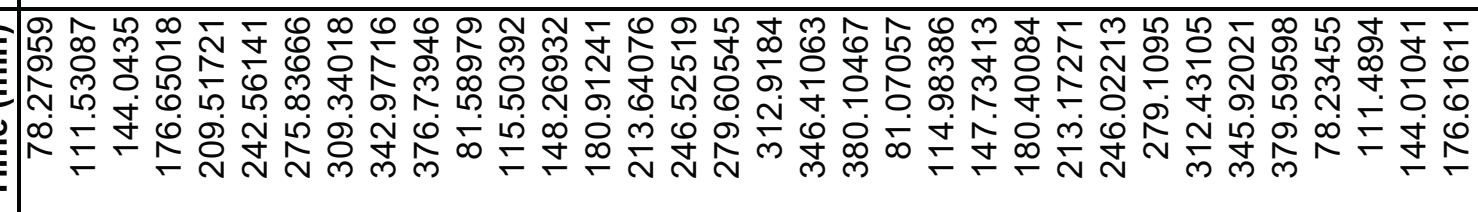 } \\
\hline 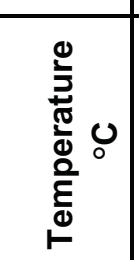 & \multicolumn{3}{|c|}{ 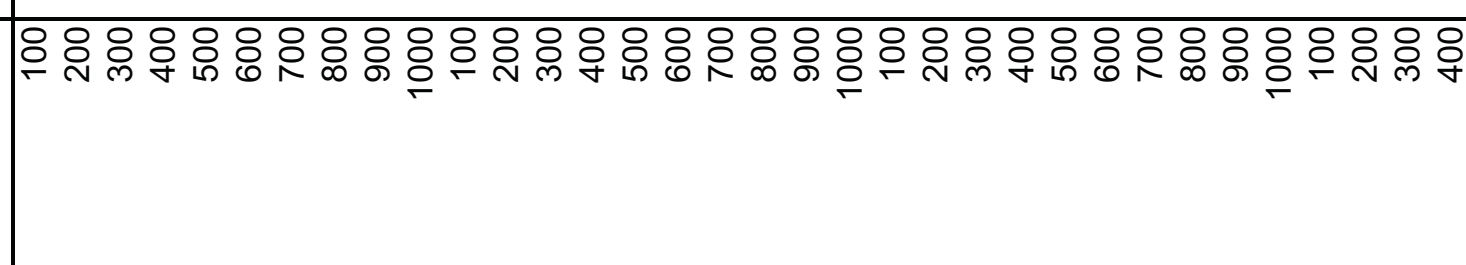 } \\
\hline 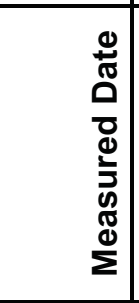 & 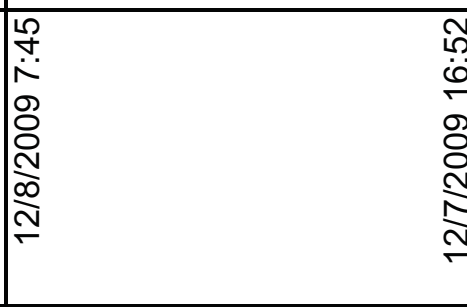 & 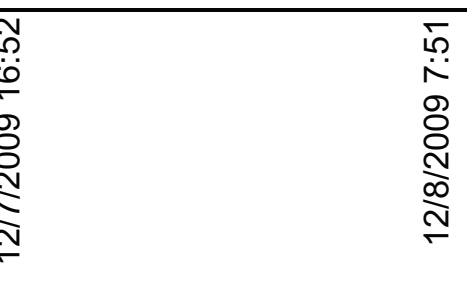 & 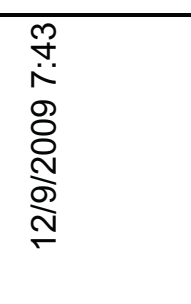 \\
\hline 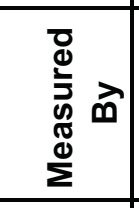 & 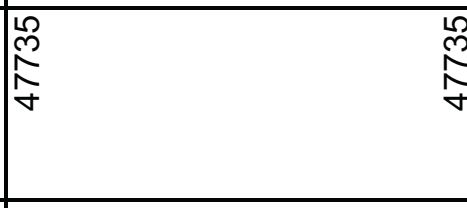 & 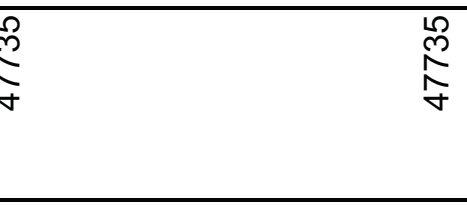 & 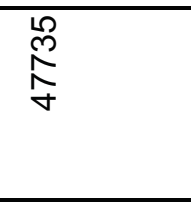 \\
\hline 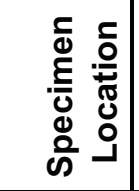 & & & \\
\hline 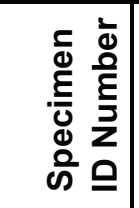 & $\begin{array}{l}\infty \\
0 \\
\infty \\
0 \\
3 \\
1 \\
1\end{array}$ & $\begin{array}{l}\bar{\delta} \\
\text { o } \\
\sum_{1} \\
\end{array}$ & 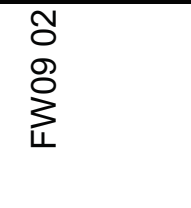 \\
\hline
\end{tabular}




\begin{tabular}{|c|c|}
\hline 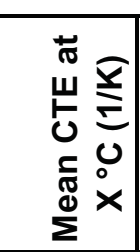 & 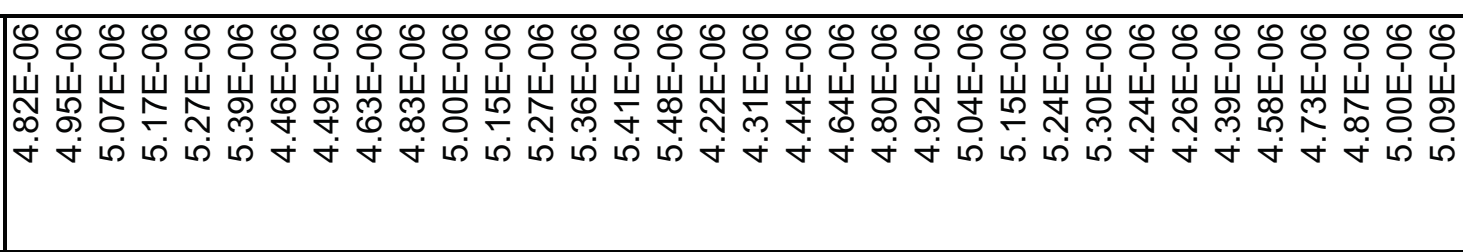 \\
\hline 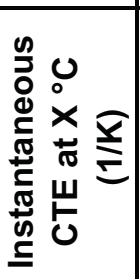 & 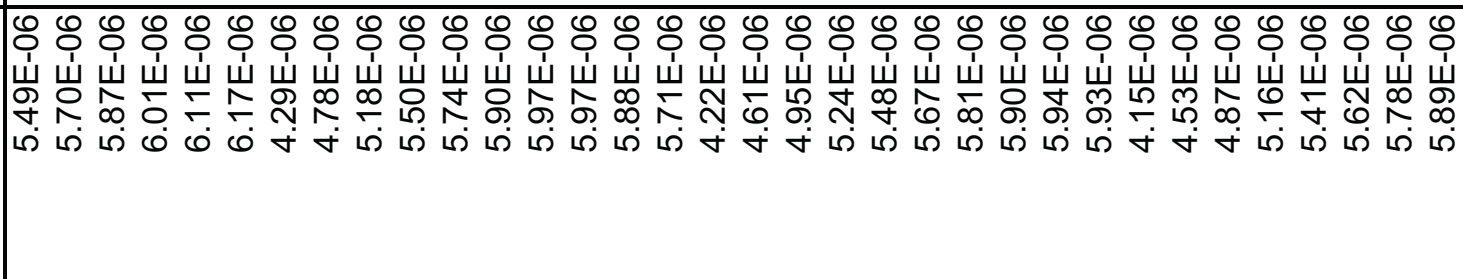 \\
\hline 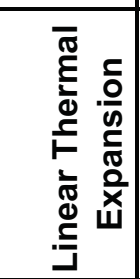 & 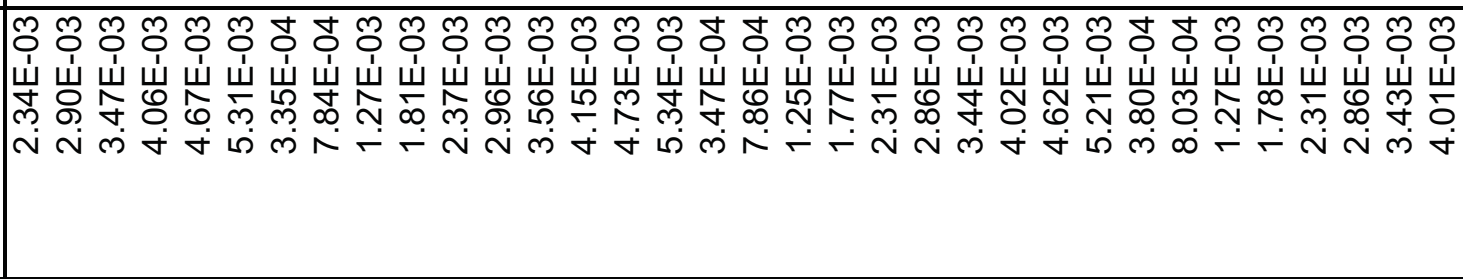 \\
\hline 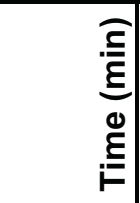 & 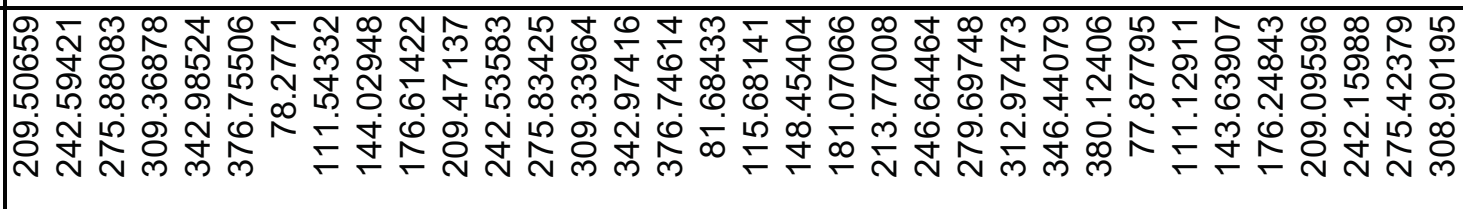 \\
\hline 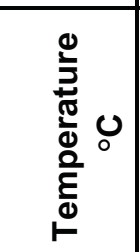 & 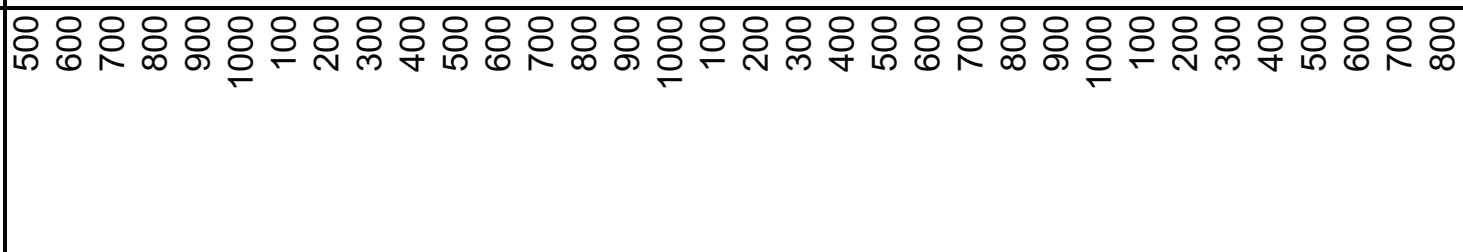 \\
\hline 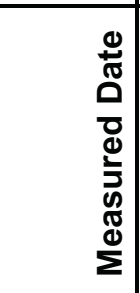 & 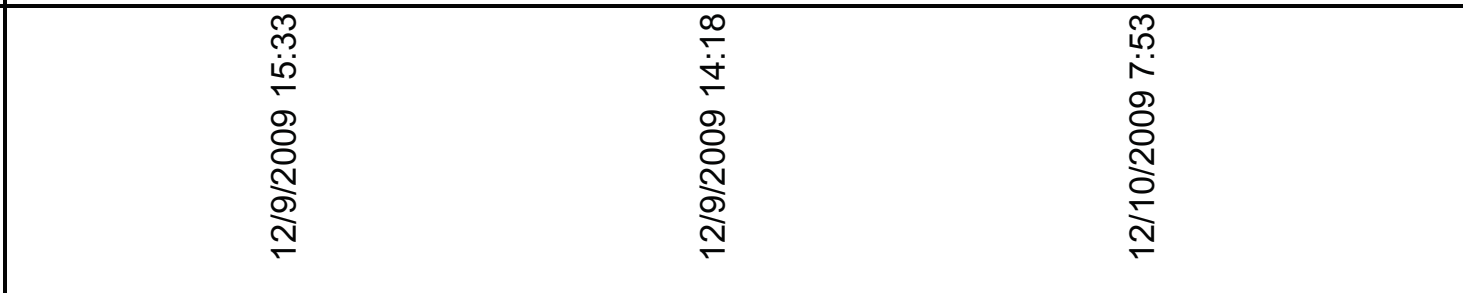 \\
\hline 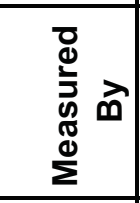 & 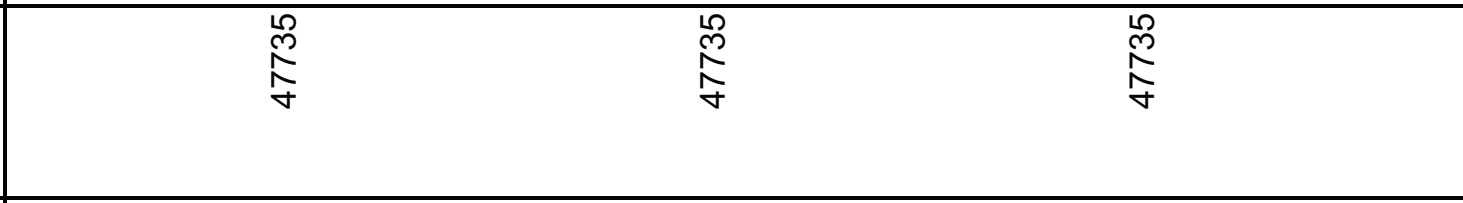 \\
\hline 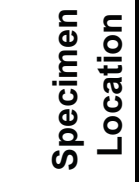 & \\
\hline 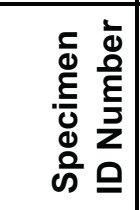 & $\begin{array}{l}\text { m } \\
0 \\
0 \\
0 \\
\sum_{4}\end{array}$ \\
\hline
\end{tabular}




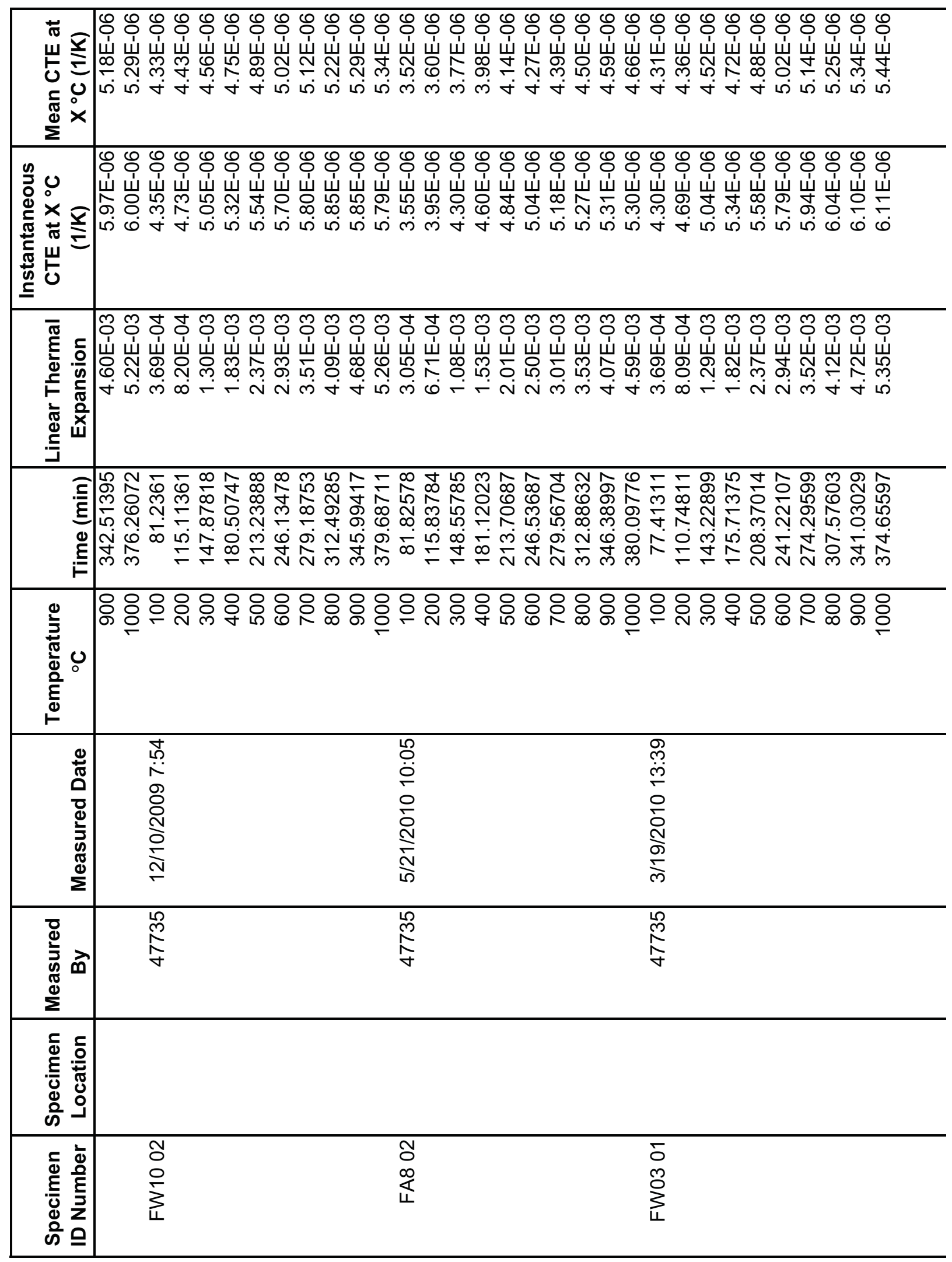




\begin{tabular}{|c|c|}
\hline 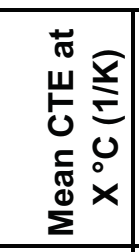 & 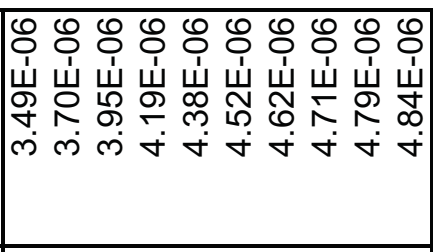 \\
\hline 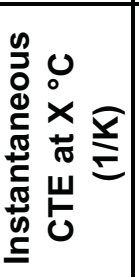 & 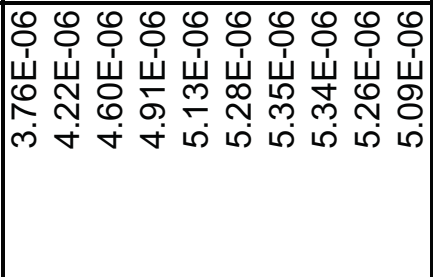 \\
\hline 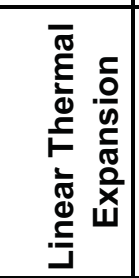 & 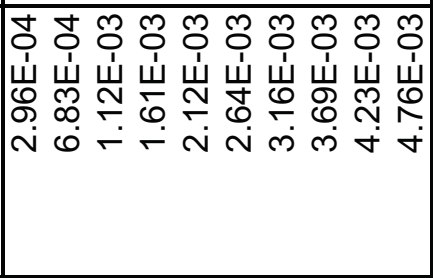 \\
\hline 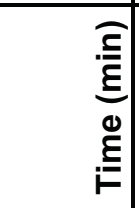 & 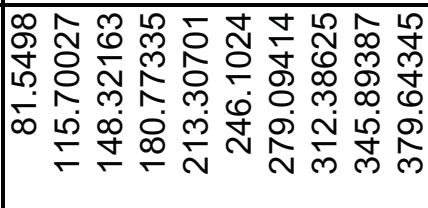 \\
\hline 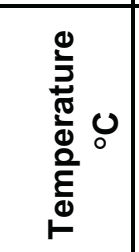 & 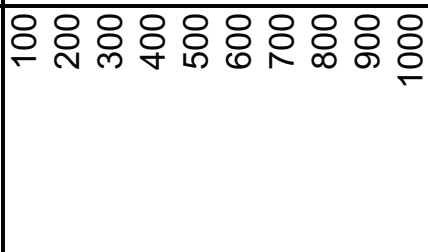 \\
\hline 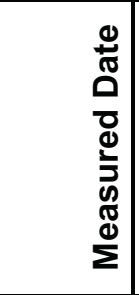 & 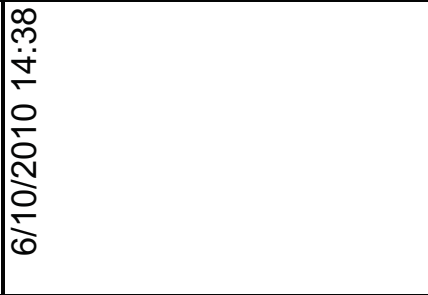 \\
\hline 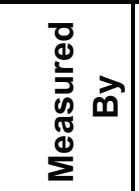 & 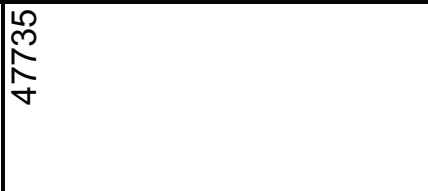 \\
\hline 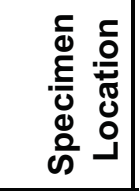 & \\
\hline 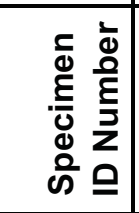 & 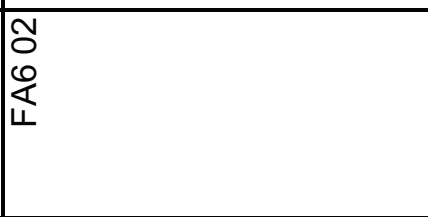 \\
\hline
\end{tabular}




\begin{tabular}{|c|c|c|c|c|}
\hline 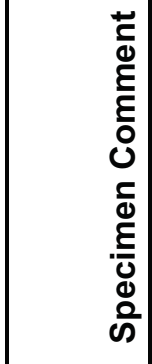 & & & & \\
\hline$\underset{\substack{\infty \\
\&}}{\sum}$ & 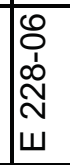 & \begin{tabular}{l}
0 \\
\\
$\infty$ \\
\multirow{N}{N}{} \\
$w$
\end{tabular} & 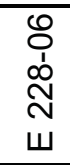 & 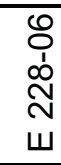 \\
\hline 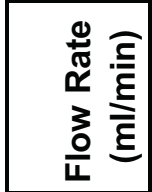 & 8 & 8 & 8 & 8 \\
\hline 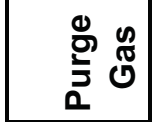 & $\frac{\oplus}{I}$ & $\frac{\Phi}{I}$ & $\frac{\infty}{I}$ & 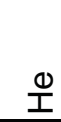 \\
\hline 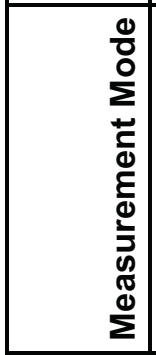 & 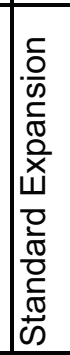 & 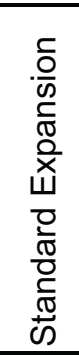 & 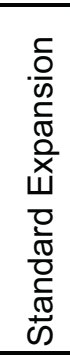 & 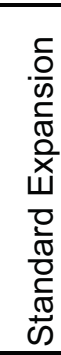 \\
\hline 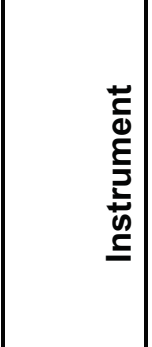 & 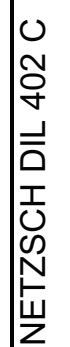 & 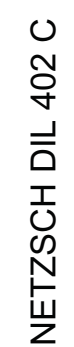 & 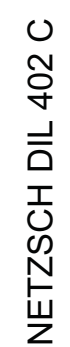 & 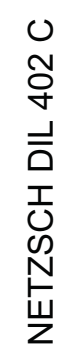 \\
\hline 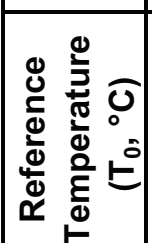 & . & $\begin{array}{l}0 \\
0 \\
0\end{array}$ & $\begin{array}{l}0 \\
0 \\
0\end{array}$ & $\begin{array}{l}0 \\
0 \\
0\end{array}$ \\
\hline 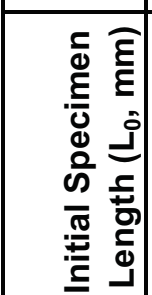 & 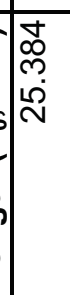 & 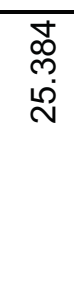 & $\begin{array}{l}\mathscr{\infty} \\
\stackrel{\infty}{ } \\
\stackrel{m}{N}\end{array}$ & 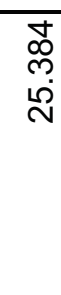 \\
\hline 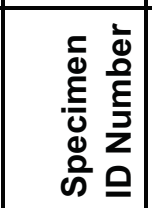 & $\begin{array}{l}\text { No } \\
0 \\
10 \\
\mathbb{4} \\
4\end{array}$ & 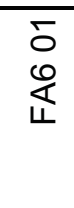 & $\begin{array}{l}\overline{0} \\
\text { 文 } \\
4\end{array}$ & 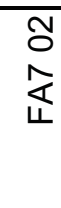 \\
\hline
\end{tabular}




\begin{tabular}{|c|c|c|c|}
\hline 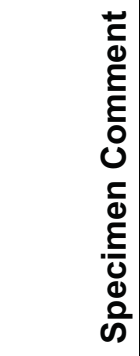 & & & \\
\hline$\underset{⿱ 乛 龰}{\stackrel{5}{8}}$ & 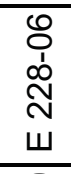 & 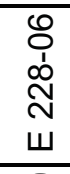 & 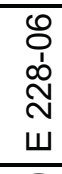 \\
\hline 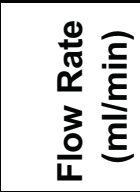 & 8 & 8 & 8 \\
\hline 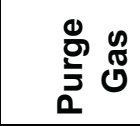 & $\frac{\Phi}{I}$ & $\stackrel{\oplus}{I}$ & $\stackrel{\oplus}{I}$ \\
\hline 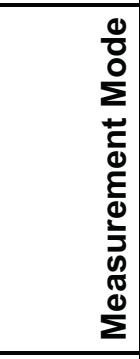 & 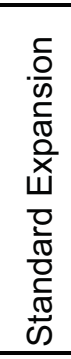 & 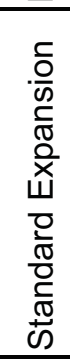 & 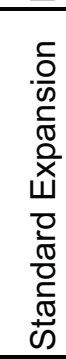 \\
\hline 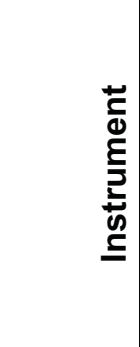 & 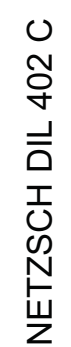 & 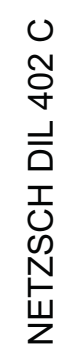 & 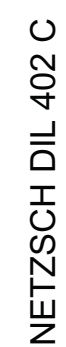 \\
\hline 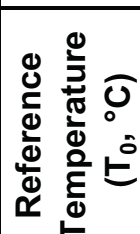 & $\begin{array}{l}0 \\
0 \\
\text { N }\end{array}$ & $\begin{array}{l}0 \\
0 \\
i\end{array}$ & $\begin{array}{l}0 \\
0 \\
0\end{array}$ \\
\hline 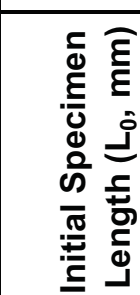 & 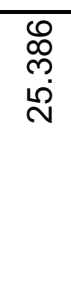 & 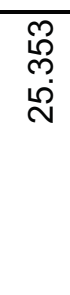 & $\begin{array}{l}\infty \\
\stackrel{\infty}{\infty} \\
\stackrel{\sim}{N}\end{array}$ \\
\hline 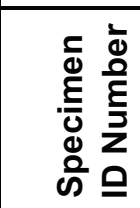 & $\begin{array}{l}\overline{0} \\
\text { o } \\
\text { 文 }\end{array}$ & $\begin{array}{l}5 \\
\vdots \\
\vdots \\
\end{array}$ & $\begin{array}{l}\text { No } \\
\sum_{\amalg}^{5}\end{array}$ \\
\hline
\end{tabular}




\begin{tabular}{|c|c|c|c|c|}
\hline 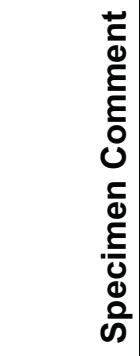 & & & & \\
\hline$\underset{⿱ 乛 龰}{\stackrel{5}{8}}$ & 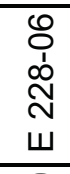 & $\begin{array}{l}\dot{8} \\
0 \\
o \\
\text { N } \\
w \\
\end{array}$ & 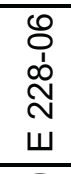 & 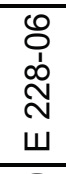 \\
\hline 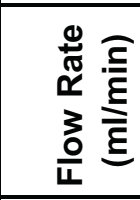 & 8 & 8 & 8 & 8 \\
\hline 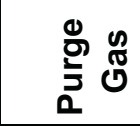 & $\frac{\infty}{I}$ & $\stackrel{\oplus}{I}$ & $\stackrel{\infty}{I}$ & 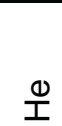 \\
\hline 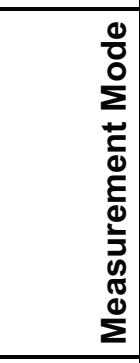 & 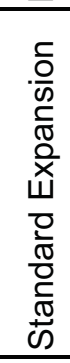 & 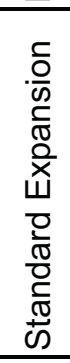 & 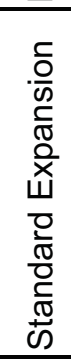 & 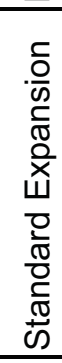 \\
\hline 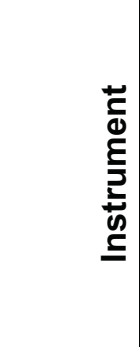 & 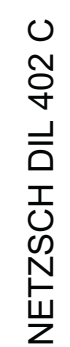 & 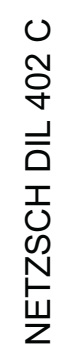 & 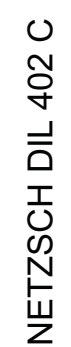 & 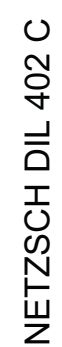 \\
\hline 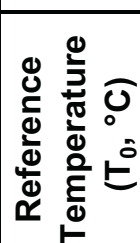 & $\begin{array}{l}0 \\
0 \\
i\end{array}$ & $\begin{array}{l}0 \\
0 \\
0\end{array}$ & $\begin{array}{l}0 \\
0 \\
0\end{array}$ & $\begin{array}{l}0 \\
0 \\
0\end{array}$ \\
\hline 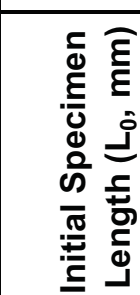 & $\begin{array}{l}\text { R } \\
\text { N } \\
\text { م. }\end{array}$ & 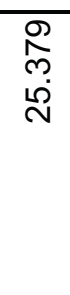 & $\begin{array}{l}\text { R } \\
\text { م. } \\
\stackrel{2}{N}\end{array}$ & 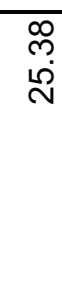 \\
\hline 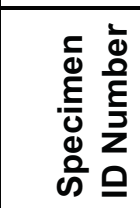 & $\begin{array}{l}0 \\
0 \\
\vdots \\
\vdots \\
1\end{array}$ & $\begin{array}{l}\text { d } \\
\sum_{1} \\
\vdots\end{array}$ & 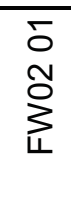 & $\begin{array}{l}\text { ô } \\
\tilde{N} \\
\sum_{\text {N }}\end{array}$ \\
\hline
\end{tabular}




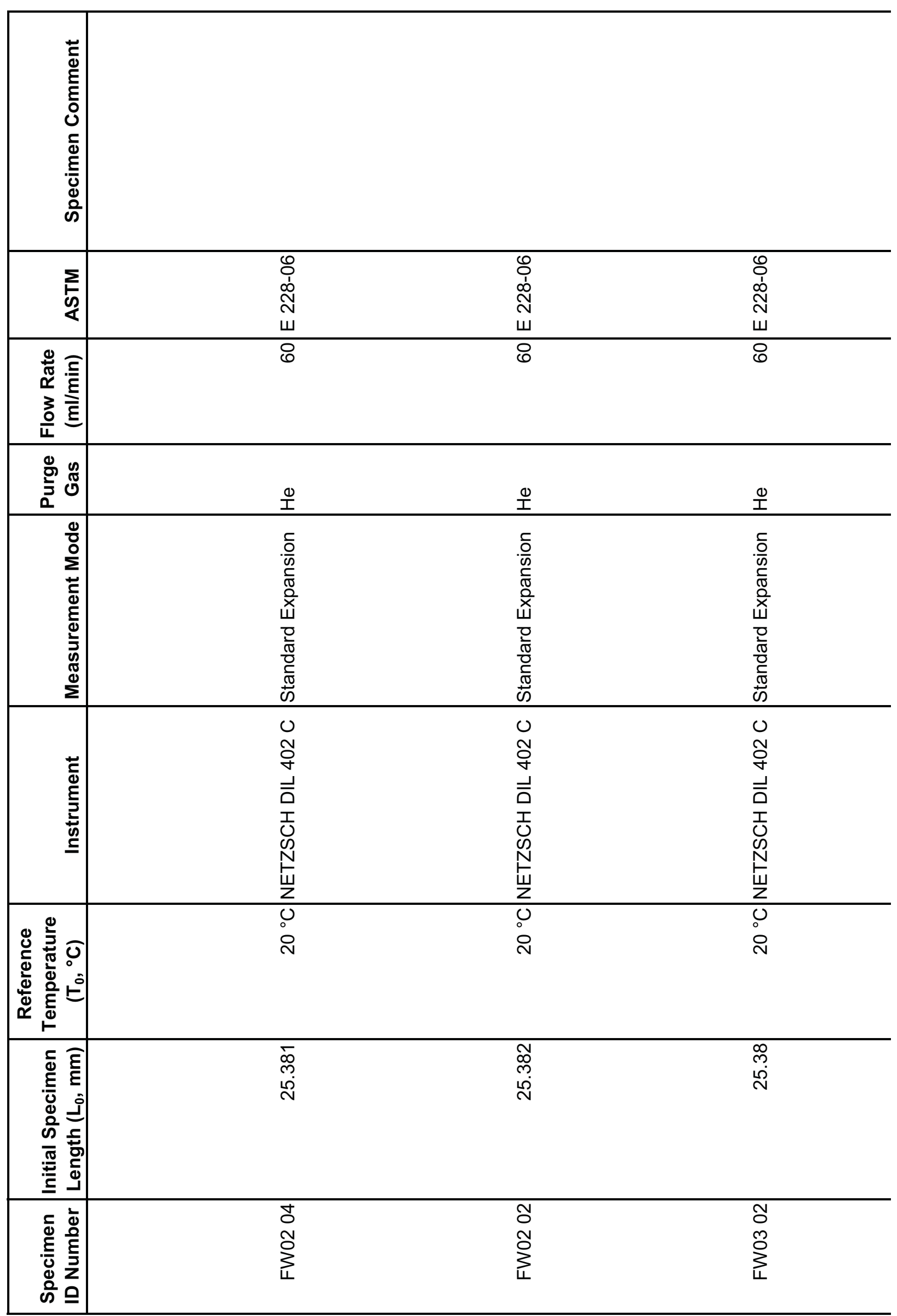




\begin{tabular}{|c|c|c|c|}
\hline 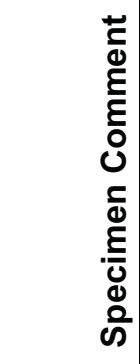 & & & \\
\hline$\underset{⿱ 乛 龰}{\stackrel{5}{8}}$ & 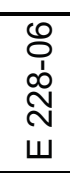 & 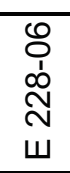 & 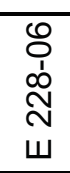 \\
\hline 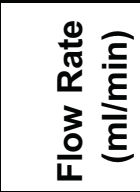 & 8 & 8 & 8 \\
\hline 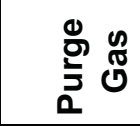 & $\stackrel{0}{I}$ & $\stackrel{\oplus}{I}$ & $\stackrel{\oplus}{工}$ \\
\hline 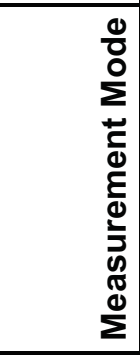 & 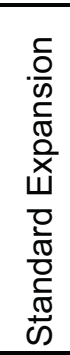 & 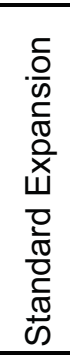 & 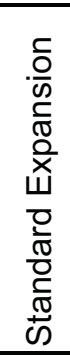 \\
\hline 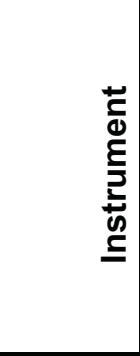 & 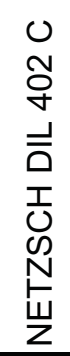 & 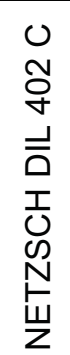 & 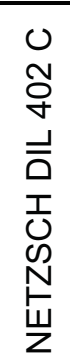 \\
\hline 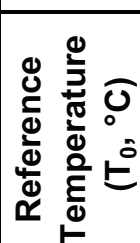 & 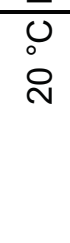 & $\begin{array}{l}0 \\
0 \\
\text { Oे }\end{array}$ & 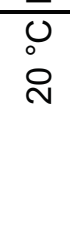 \\
\hline 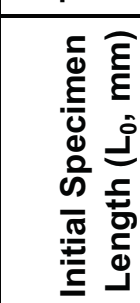 & $\begin{array}{l}\bar{\infty} \\
\stackrel{\infty}{\infty} \\
\stackrel{N}{N}\end{array}$ & $\begin{array}{l}\bar{\infty} \\
\stackrel{\infty}{\infty} \\
\stackrel{\sim}{N}\end{array}$ & $\begin{array}{l}\bar{\infty} \\
\stackrel{\infty}{\infty} \\
\stackrel{N}{N}\end{array}$ \\
\hline 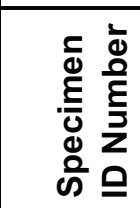 & $\begin{array}{l}m \\
0 \\
0 \\
0 \\
1\end{array}$ & $\begin{array}{l}\text { J } \\
\text { O } \\
\sum_{4}\end{array}$ & 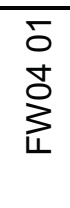 \\
\hline
\end{tabular}




\begin{tabular}{|c|c|c|c|c|}
\hline 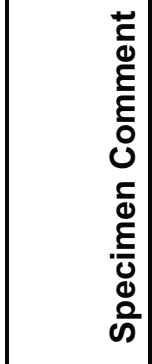 & & & & \\
\hline$\underset{\substack{\infty \\
\&}}{\sum}$ & 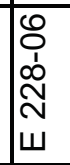 & \begin{tabular}{l}
0 \\
\\
$\infty$ \\
\multirow{N}{N}{} \\
$w$
\end{tabular} & 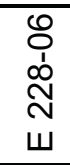 & 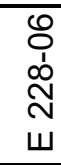 \\
\hline 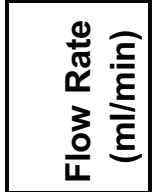 & 8 & 8 & 8 & 8 \\
\hline 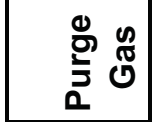 & $\frac{\oplus}{I}$ & $\frac{\Phi}{I}$ & $\frac{\infty}{I}$ & 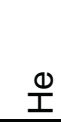 \\
\hline 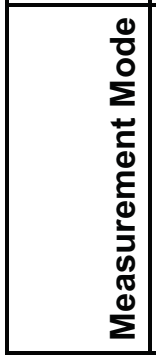 & 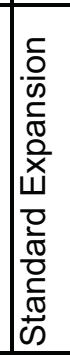 & 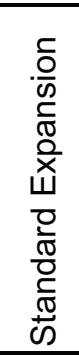 & 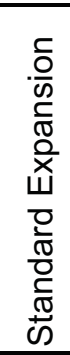 & 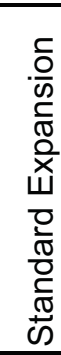 \\
\hline 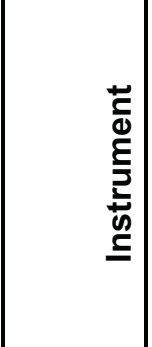 & 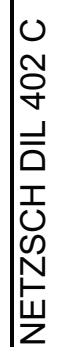 & 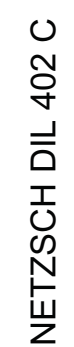 & 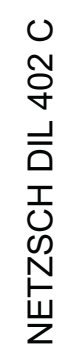 & 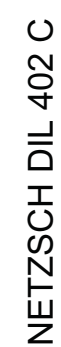 \\
\hline 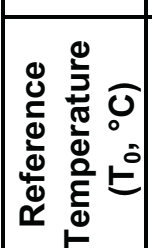 & $\begin{array}{l}0 \\
0 \\
0 \\
0 \\
0\end{array}$ & $\begin{array}{l}2 \\
0 \\
0 \\
O\end{array}$ & $\begin{array}{l}0 \\
0 \\
0\end{array}$ & $\begin{array}{l}0 \\
0 \\
0\end{array}$ \\
\hline 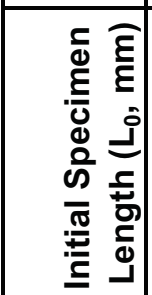 & $\mid \begin{array}{l}\infty \\
m \\
\infty\end{array}$ & $\begin{array}{l}\bar{\infty} \\
\stackrel{m}{ } \\
\stackrel{N}{N}\end{array}$ & $\begin{array}{l}\bar{\infty} \\
\stackrel{m}{\infty} \\
\stackrel{\rho}{N}\end{array}$ & $\begin{array}{l}\bar{\infty} \\
\stackrel{\infty}{\rho} \\
\stackrel{\rho}{N}\end{array}$ \\
\hline 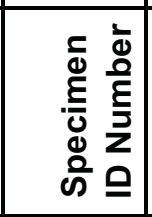 & $\begin{array}{l}\tilde{O} \\
0 \\
0 \\
3 \\
3 \\
4\end{array}$ & $\begin{array}{l}0 \\
0 \\
0 \\
0 \\
3\end{array}$ & $\begin{array}{l}\text { J } \\
0 \\
0 \\
\vdots \\
\end{array}$ & $\begin{array}{l}\bar{c} \\
\overline{0} \\
\sum_{1} \\
\text { L }\end{array}$ \\
\hline
\end{tabular}




\begin{tabular}{|c|c|c|c|}
\hline 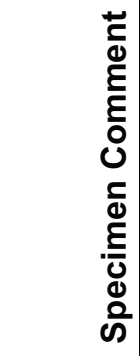 & & & \\
\hline$\underset{⿱ 乛 龰}{\stackrel{5}{8}}$ & 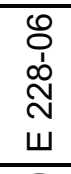 & 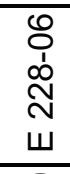 & 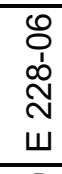 \\
\hline 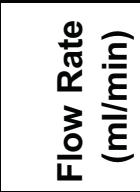 & 8 & 8 & 8 \\
\hline 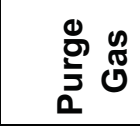 & $\frac{\Phi}{I}$ & $\stackrel{\oplus}{I}$ & $\stackrel{\oplus}{I}$ \\
\hline 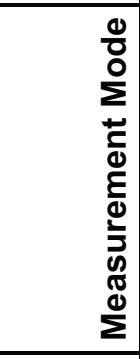 & 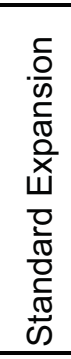 & 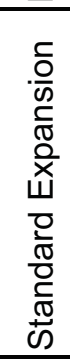 & 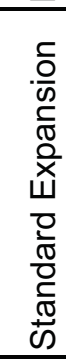 \\
\hline 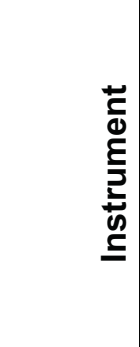 & 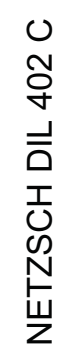 & 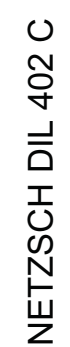 & 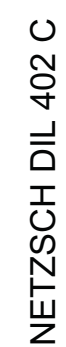 \\
\hline 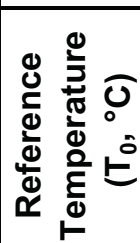 & $\begin{array}{l}0 \\
0 \\
\text { N }\end{array}$ & $\begin{array}{l}0 \\
0 \\
i\end{array}$ & $\begin{array}{l}0 \\
0 \\
0\end{array}$ \\
\hline 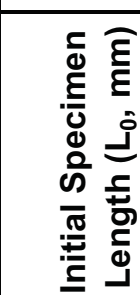 & $\begin{array}{l}\infty \\
\stackrel{\infty}{N} \\
\stackrel{N}{N}\end{array}$ & $\begin{array}{l}\bar{\infty} \\
\stackrel{\infty}{\infty} \\
\stackrel{N}{N}\end{array}$ & $\begin{array}{l}\bar{\infty} \\
m \\
\stackrel{\rho}{N}\end{array}$ \\
\hline 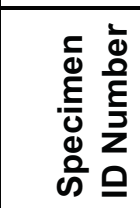 & $\begin{array}{l}\text { No } \\
0 \\
2 \\
3 \\
1\end{array}$ & $\begin{array}{l}0 \\
0 \\
0 \\
0 \\
3\end{array}$ & 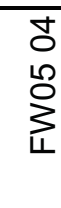 \\
\hline
\end{tabular}




\begin{tabular}{|c|c|c|c|c|}
\hline 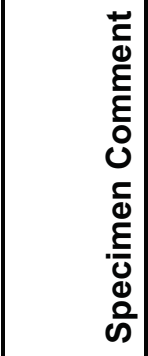 & & & & \\
\hline$\underset{\frac{5}{5}}{\frac{5}{2}}$ & 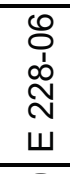 & $\begin{array}{l}\dot{8} \\
0 \\
o \\
\text { N } \\
w \\
\end{array}$ & 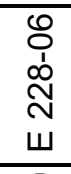 & 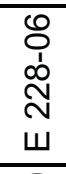 \\
\hline 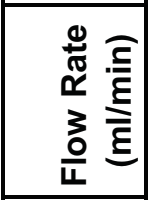 & 8 & 8 & 8 & 8 \\
\hline 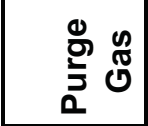 & $\frac{\infty}{I}$ & $\stackrel{\oplus}{I}$ & $\stackrel{\infty}{I}$ & 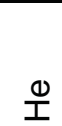 \\
\hline 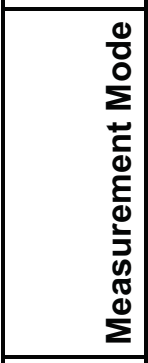 & 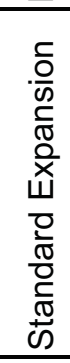 & 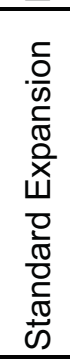 & 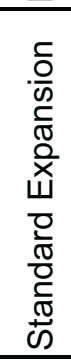 & 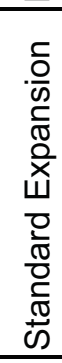 \\
\hline 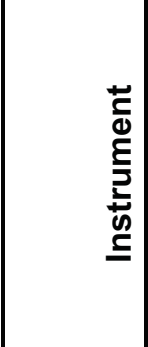 & 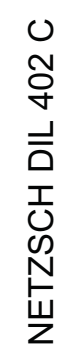 & 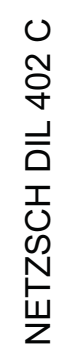 & 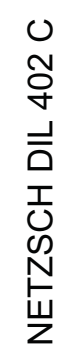 & 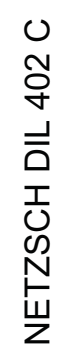 \\
\hline 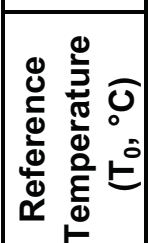 & $\begin{array}{l}0 \\
0 \\
0\end{array}$ & $\begin{array}{l}0 \\
0 \\
0\end{array}$ & $\begin{array}{l}0 \\
0 \\
0\end{array}$ & $\begin{array}{l}0 \\
0 \\
0 \\
0\end{array}$ \\
\hline 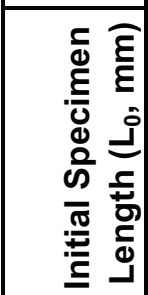 & $\begin{array}{l}\bar{\infty} \\
\text { m. } \\
\stackrel{N}{N}\end{array}$ & $\begin{array}{l}\infty \\
\stackrel{\infty}{\infty} \\
\stackrel{\sim}{N}\end{array}$ & 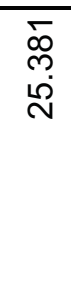 & $\begin{array}{l}\bar{\infty} \\
m \\
\stackrel{\rho}{\infty}\end{array}$ \\
\hline 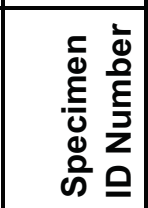 & $\begin{array}{l}5 \\
0 \\
0 \\
\vdots\end{array}$ & 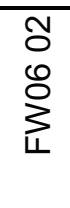 & $\begin{array}{l}0 \\
0 \\
0 \\
0 \\
1\end{array}$ & $\begin{array}{l}\text { J } \\
8 \\
8 \\
3\end{array}$ \\
\hline
\end{tabular}




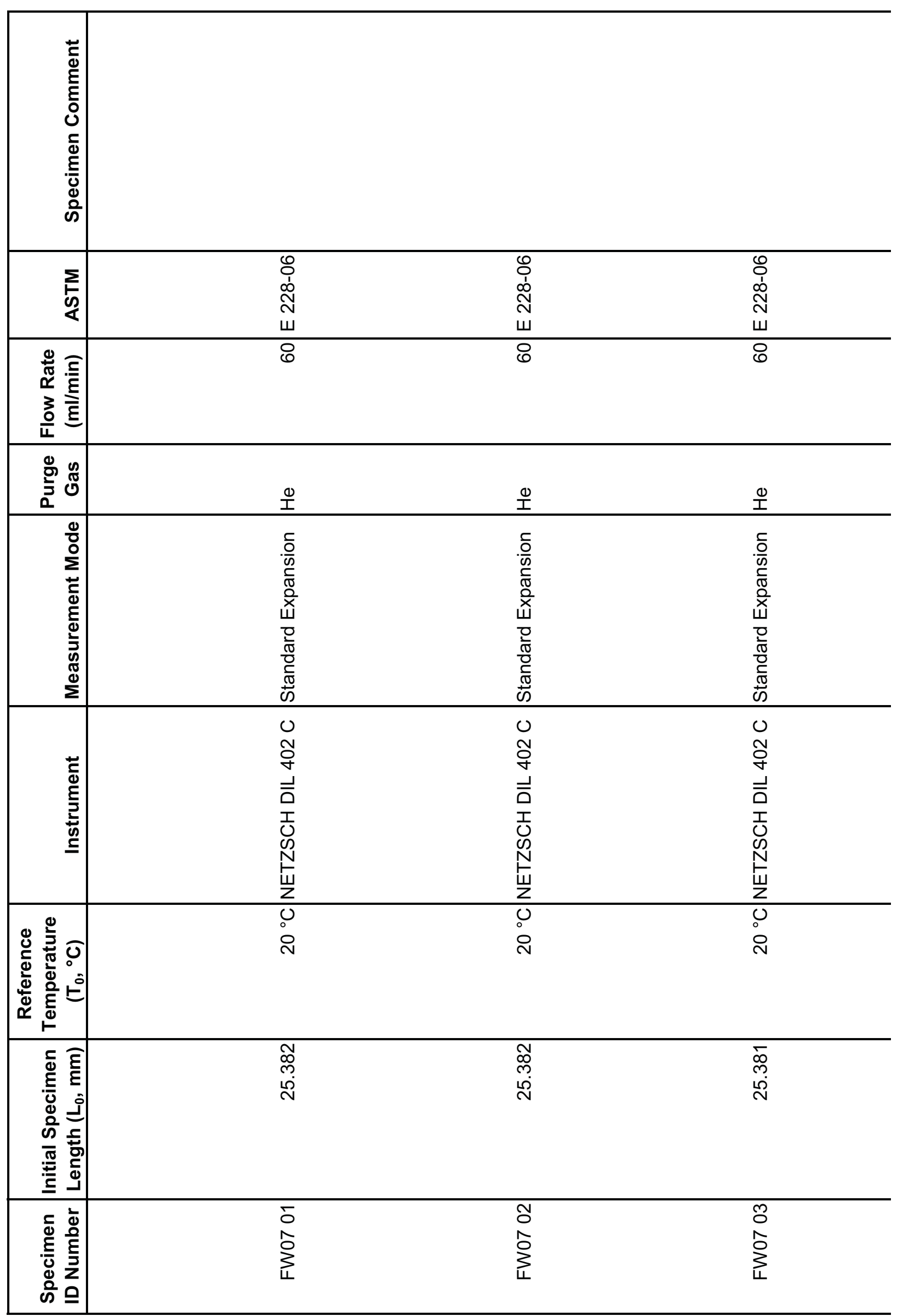




\begin{tabular}{|c|c|c|c|}
\hline 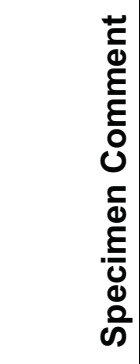 & & & \\
\hline$\underset{⿱ 乛 龰}{\stackrel{5}{8}}$ & 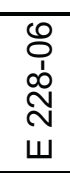 & 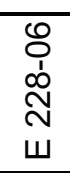 & 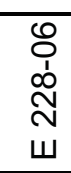 \\
\hline 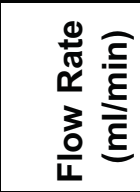 & 8 & 8 & 8 \\
\hline 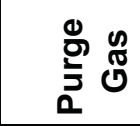 & $\stackrel{0}{I}$ & $\stackrel{\oplus}{I}$ & 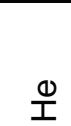 \\
\hline 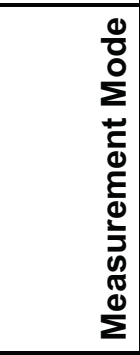 & 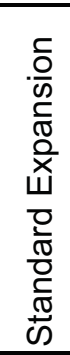 & 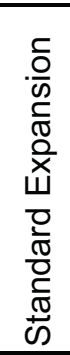 & 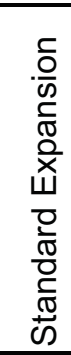 \\
\hline 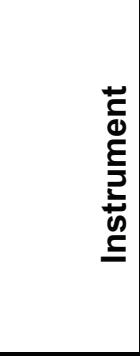 & 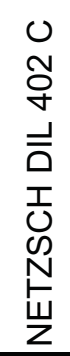 & 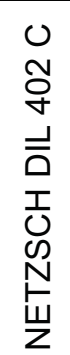 & 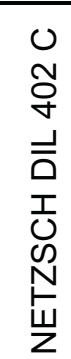 \\
\hline 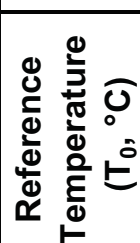 & 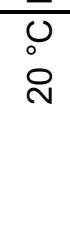 & $\begin{array}{l}0 \\
0 \\
\text { Oे }\end{array}$ & $\begin{array}{l}0 \\
0 \\
0\end{array}$ \\
\hline 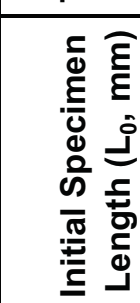 & $\begin{array}{l}\text { N } \\
\text { m. } \\
\stackrel{\sim}{N}\end{array}$ & $\begin{array}{l}\text { N } \\
\stackrel{\infty}{\infty} \\
\stackrel{\sim}{N}\end{array}$ & $\begin{array}{l}\text { N } \\
\text { ల్ } \\
\stackrel{\sim}{N}\end{array}$ \\
\hline 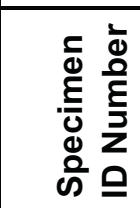 & $\begin{array}{l}\text { J } \\
0 \\
0 \\
\vdots \\
4\end{array}$ & $\begin{array}{l}\bar{\delta} \\
\infty \\
\sum_{1} \\
4\end{array}$ & $\begin{array}{l}\text { No } \\
\text { o } \\
0 \\
\sum_{4}\end{array}$ \\
\hline
\end{tabular}




\begin{tabular}{|c|c|c|c|c|}
\hline 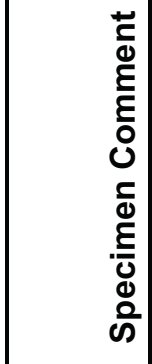 & & & & \\
\hline$\underset{\substack{\infty \\
\&}}{\sum}$ & 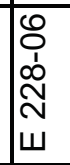 & \begin{tabular}{l}
0 \\
\\
$\infty$ \\
\multirow{N}{N}{} \\
$w$
\end{tabular} & 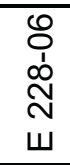 & 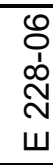 \\
\hline 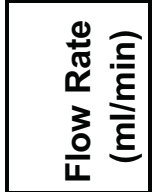 & 8 & 8 & 8 & 8 \\
\hline 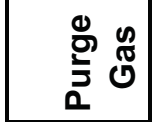 & $\frac{\oplus}{I}$ & $\frac{\Phi}{I}$ & $\frac{\infty}{I}$ & $\stackrel{\oplus}{I}$ \\
\hline 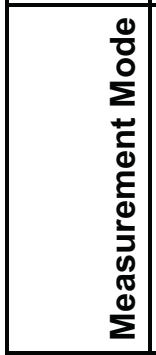 & 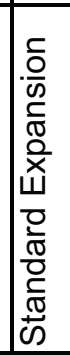 & 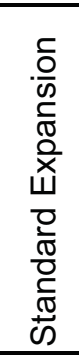 & 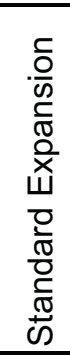 & 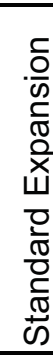 \\
\hline 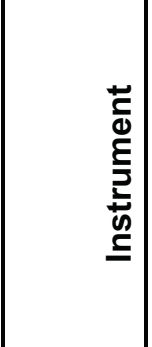 & 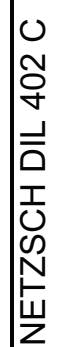 & 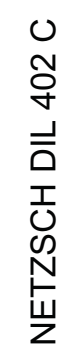 & 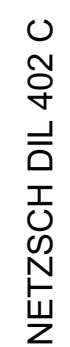 & 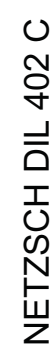 \\
\hline 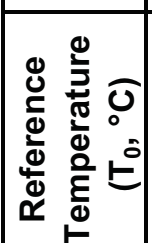 & . & $\begin{array}{l}0 \\
0 \\
0\end{array}$ & $\begin{array}{l}0 \\
0 \\
0\end{array}$ & \begin{tabular}{l}
0 \\
0 \\
0 \\
\multirow{N}{*}{}
\end{tabular} \\
\hline 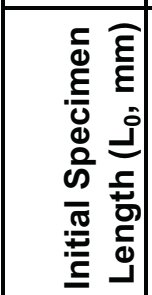 & $\mid \begin{array}{l}\infty \\
\infty \\
\infty \\
\infty \\
\end{array}$ & $\begin{array}{l}\text { N } \\
\stackrel{m}{ } \\
\stackrel{N}{N}\end{array}$ & $\begin{array}{l}\text { m } \\
\stackrel{m}{m} \\
\stackrel{N}{N}\end{array}$ & $\begin{array}{l}\bar{\infty} \\
\text { p. } \\
\stackrel{\rho}{N}\end{array}$ \\
\hline 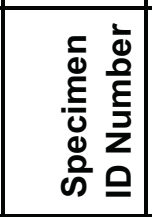 & $\begin{array}{l}\infty \\
0 \\
\infty \\
0 \\
3 \\
14\end{array}$ & $\begin{array}{l} \\
0 \\
0 \\
\sum_{4} \\
4\end{array}$ & $\begin{array}{l}5 \\
\delta \\
8 \\
\vdots \\
\end{array}$ & $\begin{array}{l}\text { N } \\
\text { O } \\
\text { ᄋ } \\
\sum_{4}\end{array}$ \\
\hline
\end{tabular}




\begin{tabular}{|c|c|c|c|}
\hline 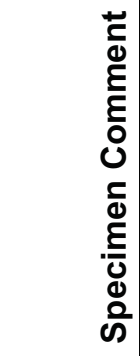 & & & \\
\hline$\underset{⿱ 乛 龰}{\stackrel{5}{8}}$ & 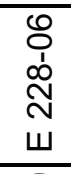 & 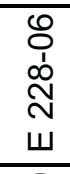 & 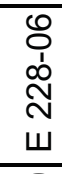 \\
\hline 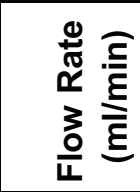 & 8 & 8 & 8 \\
\hline 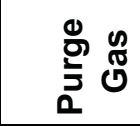 & $\frac{\Phi}{I}$ & $\stackrel{\oplus}{I}$ & $\stackrel{\oplus}{I}$ \\
\hline 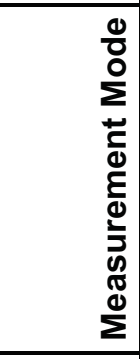 & 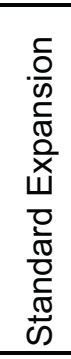 & 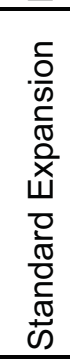 & 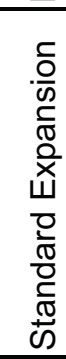 \\
\hline 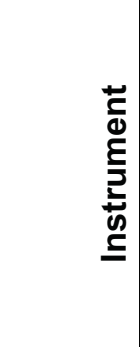 & 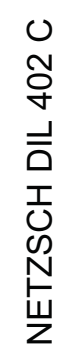 & 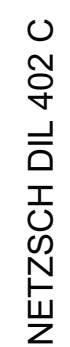 & 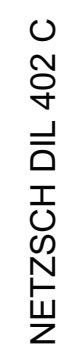 \\
\hline 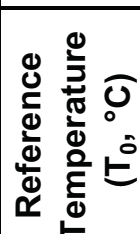 & $\begin{array}{l}0 \\
0 \\
\text { N }\end{array}$ & $\begin{array}{l}0 \\
0 \\
i\end{array}$ & $\begin{array}{l}0 \\
0 \\
0\end{array}$ \\
\hline 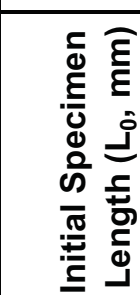 & $\begin{array}{l}\text { N } \\
\text { ల } \\
\stackrel{N}{N} \\
\stackrel{N}{N}\end{array}$ & $\begin{array}{l}\text { m } \\
\stackrel{\infty}{m} \\
\stackrel{\sim}{N}\end{array}$ & 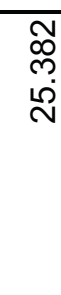 \\
\hline 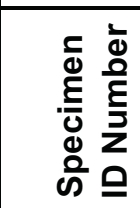 & $\begin{array}{l}0 \\
0 \\
8 \\
0 \\
3\end{array}$ & $\begin{array}{l} \\
0 \\
8 \\
0 \\
\vdots \\
\end{array}$ & $\begin{array}{l}\bar{\delta} \\
\sum_{u}^{\circ}\end{array}$ \\
\hline
\end{tabular}




\begin{tabular}{|c|c|c|c|}
\hline 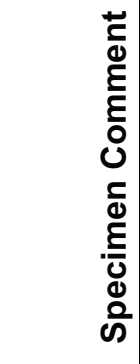 & & & \\
\hline$\underset{⿱ 乛 龰}{\stackrel{5}{8}}$ & 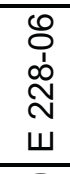 & $\begin{array}{l}\dot{8} \\
0 \\
o \\
\text { N } \\
w \\
\end{array}$ & 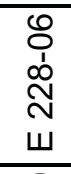 \\
\hline 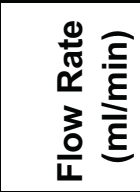 & 8 & 8 & 8 \\
\hline 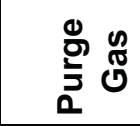 & $\frac{\infty}{I}$ & $\stackrel{\oplus}{I}$ & $\stackrel{\infty}{I}$ \\
\hline 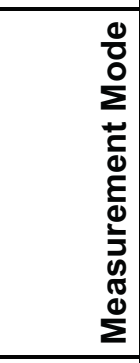 & 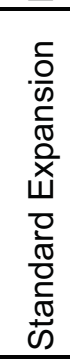 & 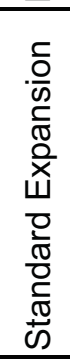 & 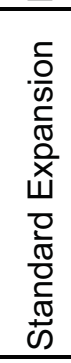 \\
\hline 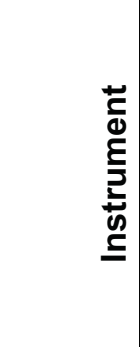 & 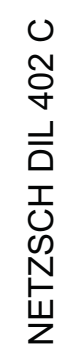 & 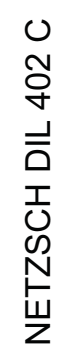 & 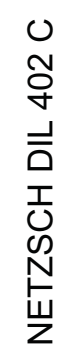 \\
\hline 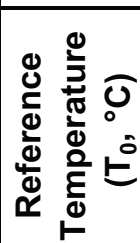 & $\begin{array}{l}0 \\
0 \\
i\end{array}$ & 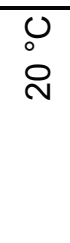 & $\begin{array}{l}0 \\
0 \\
\text { N }\end{array}$ \\
\hline 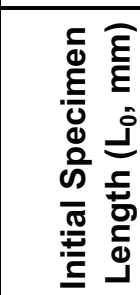 & $\begin{array}{l}\bar{\infty} \\
\text { m. } \\
\stackrel{N}{N}\end{array}$ & $\begin{array}{l}0 \\
\infty \\
\stackrel{0}{ } \\
\stackrel{\rho}{N}\end{array}$ & 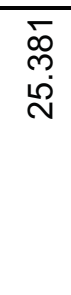 \\
\hline 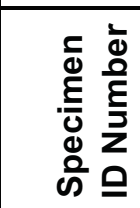 & 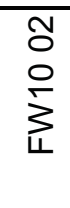 & 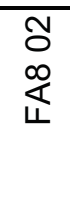 & $\begin{array}{l}5 \\
0 \\
0 \\
\sum_{1}\end{array}$ \\
\hline
\end{tabular}




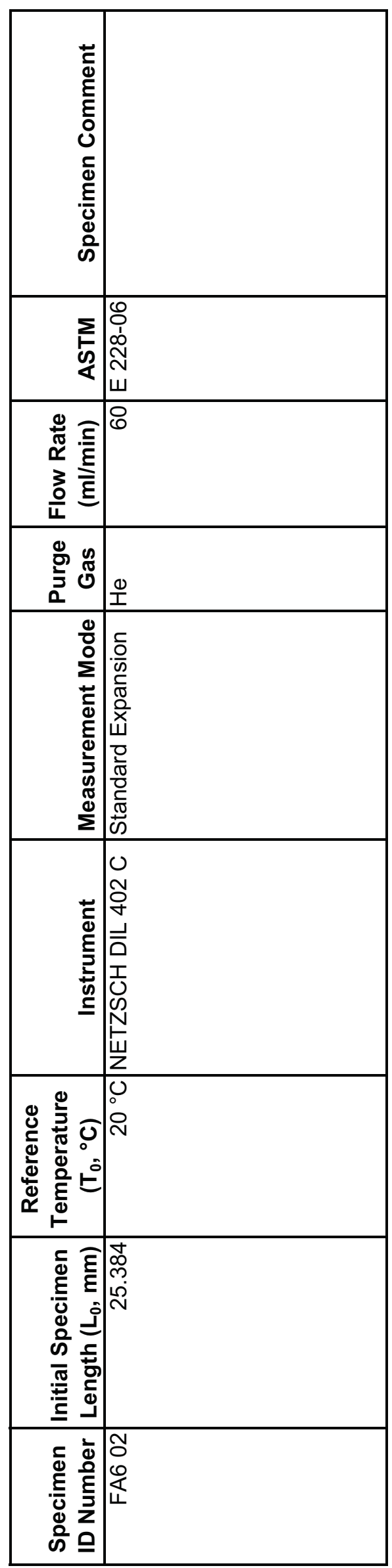




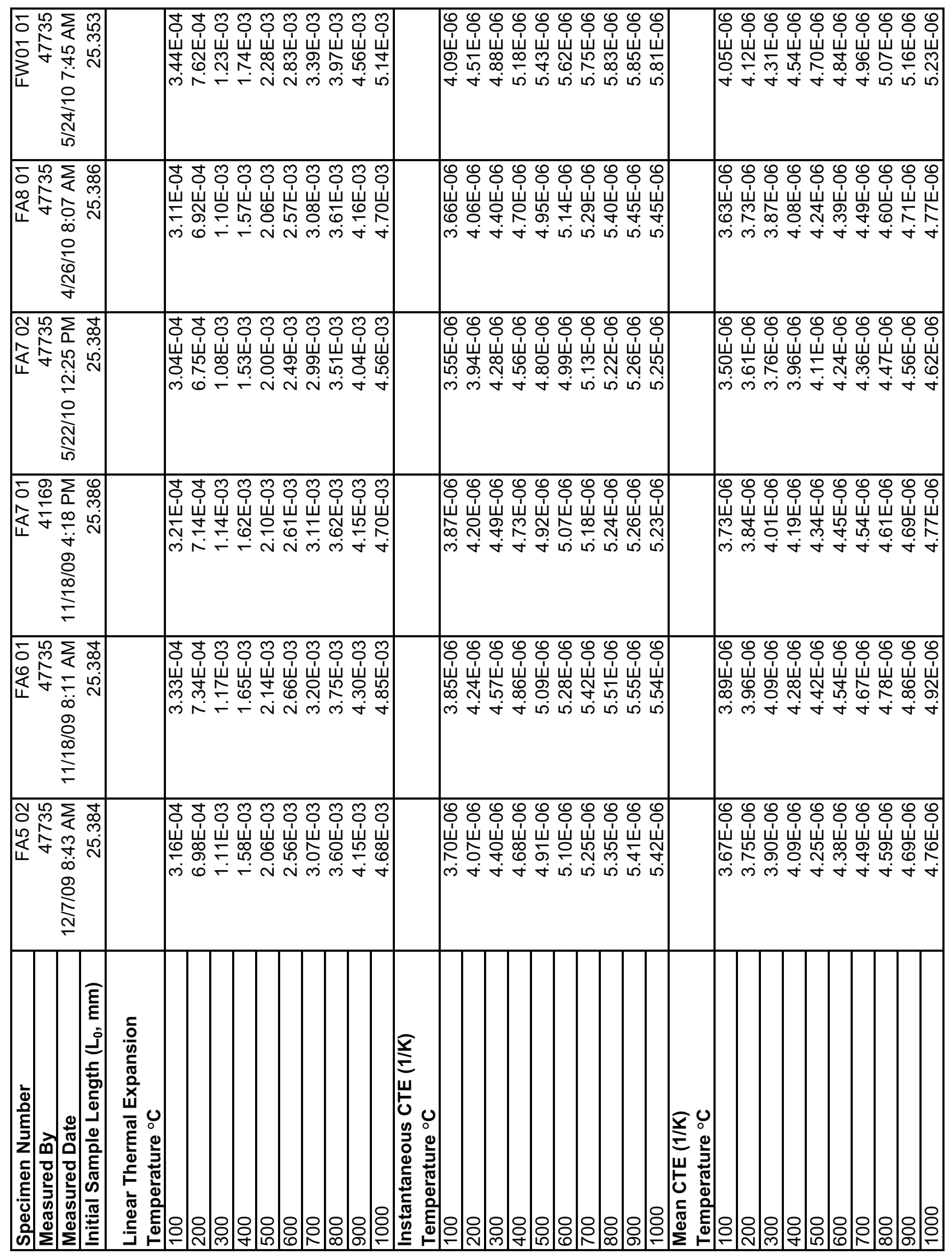




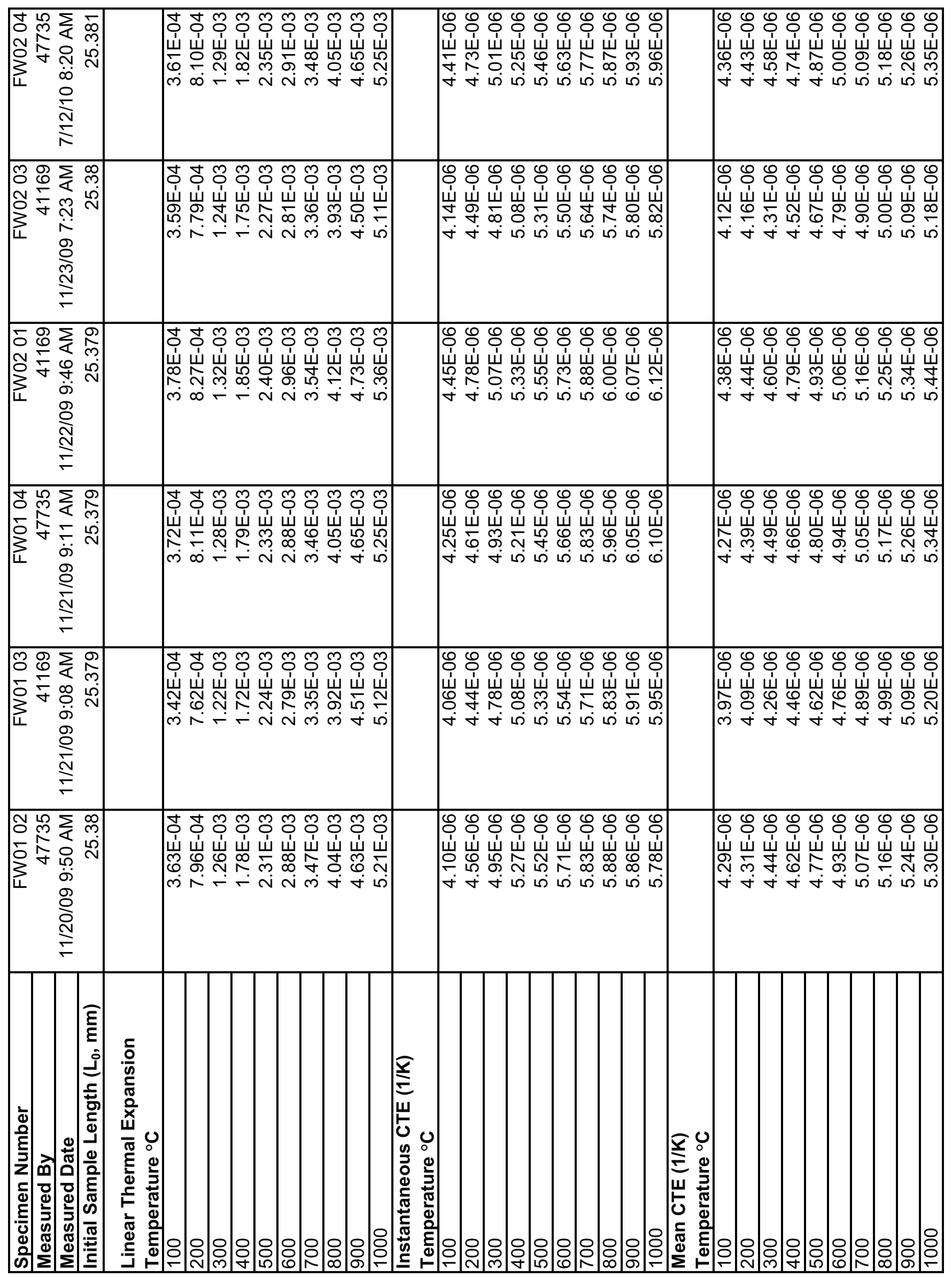




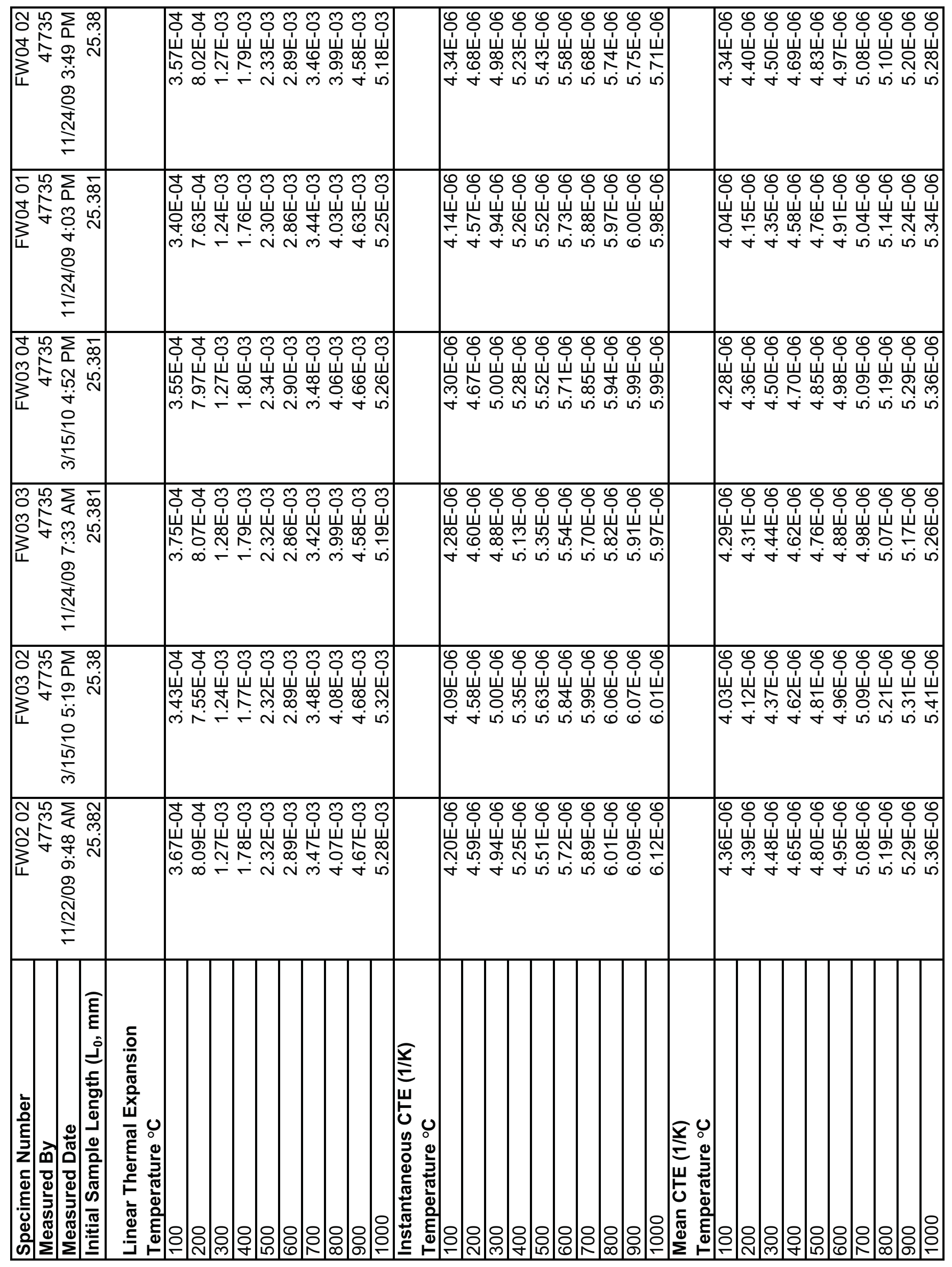




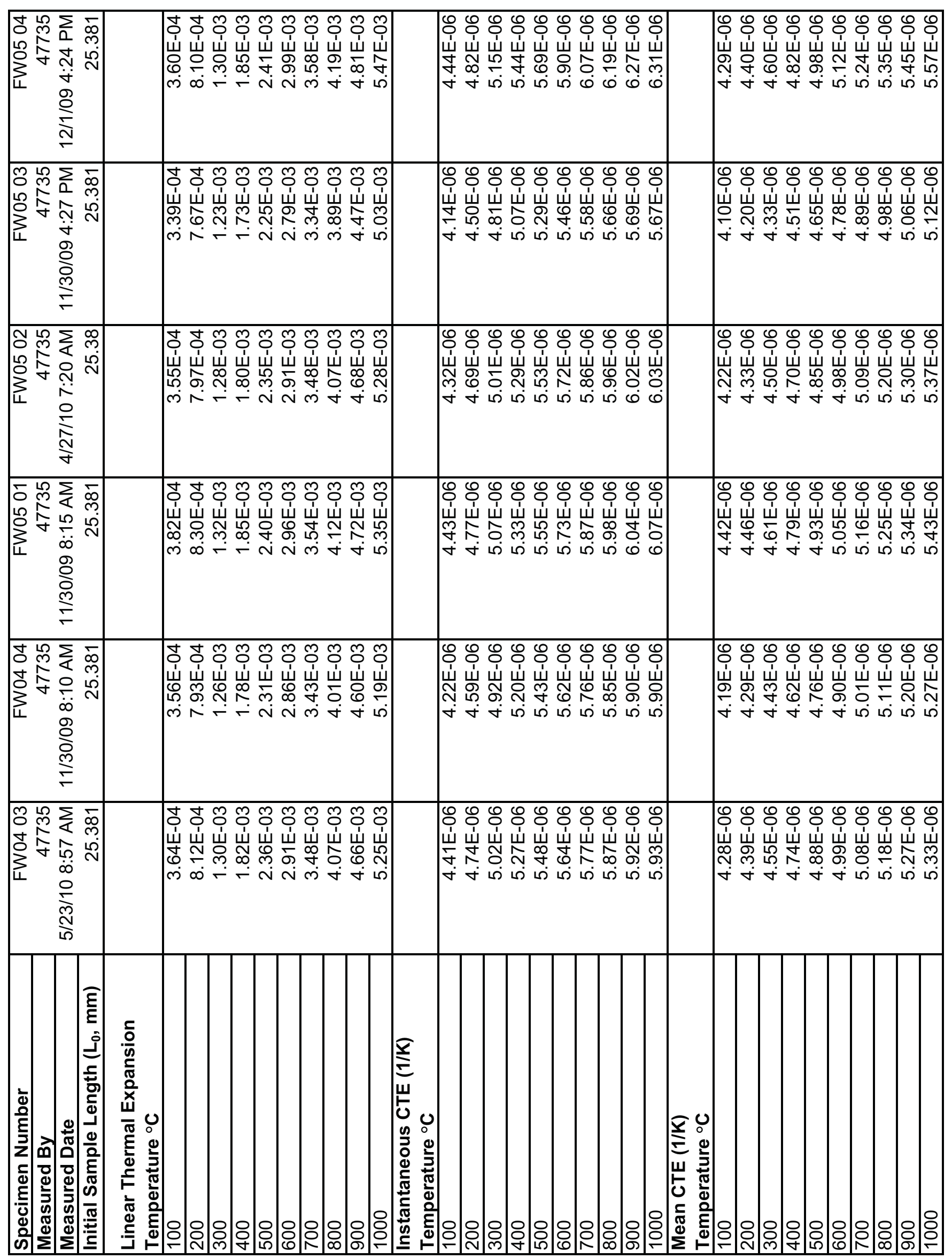




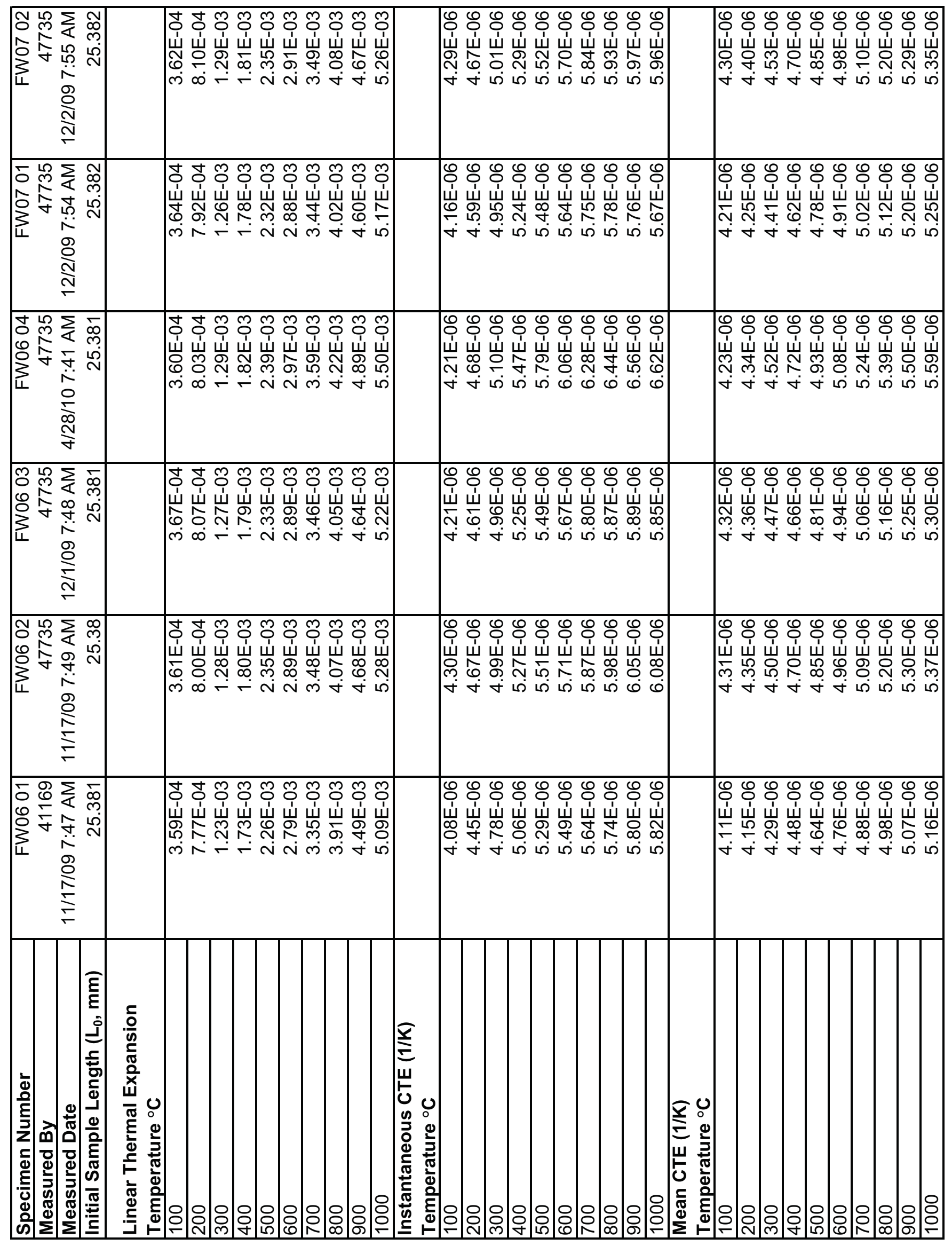




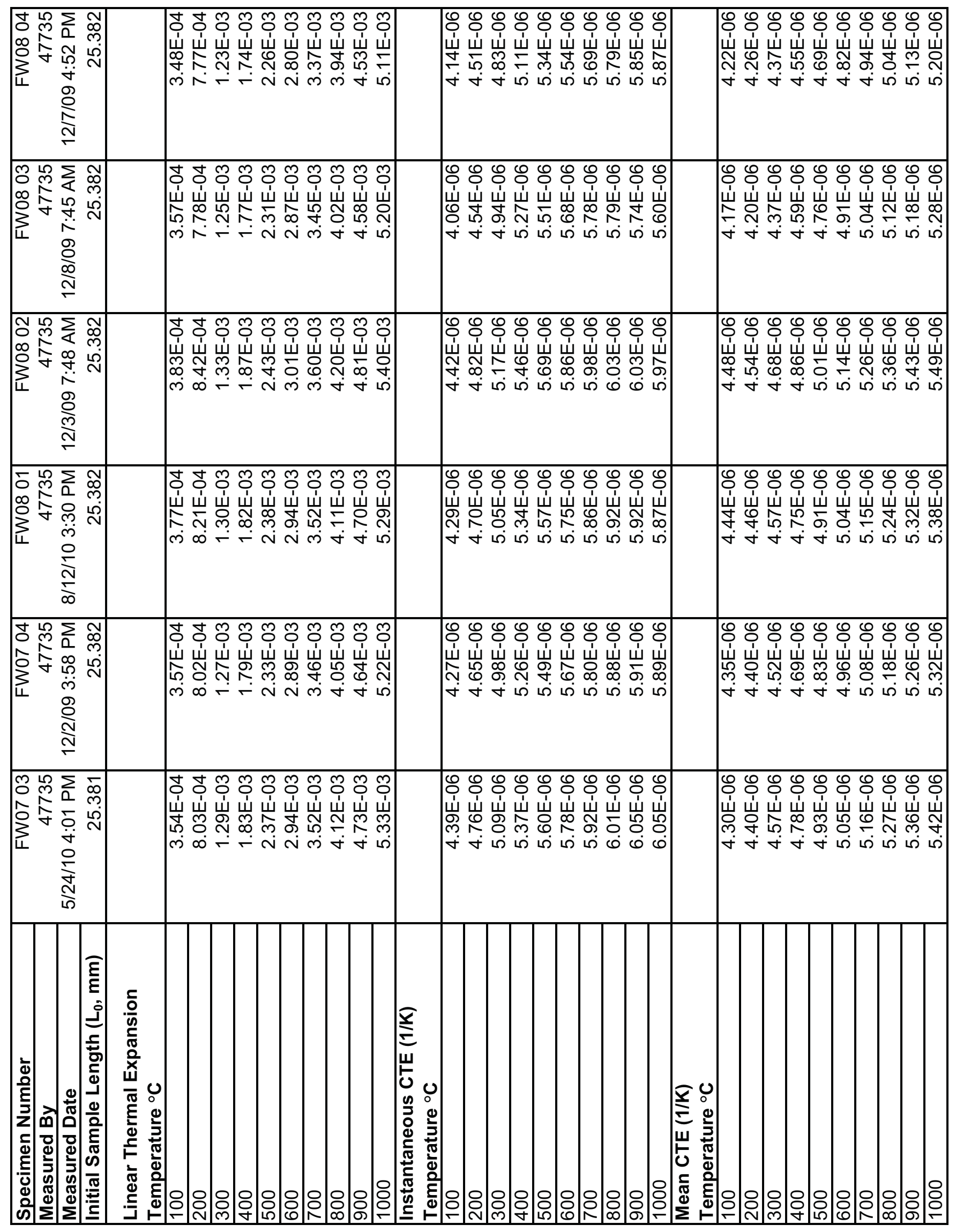




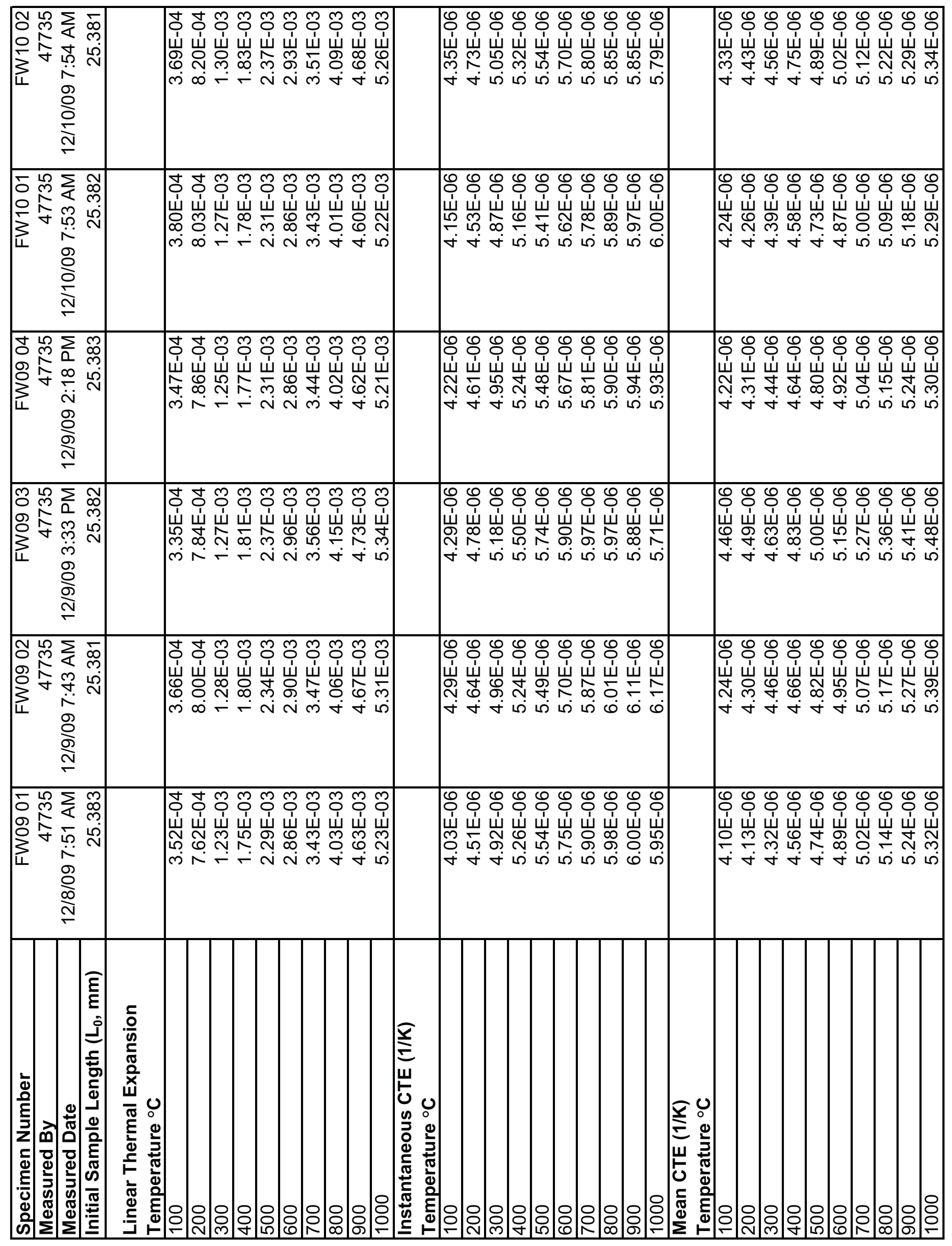




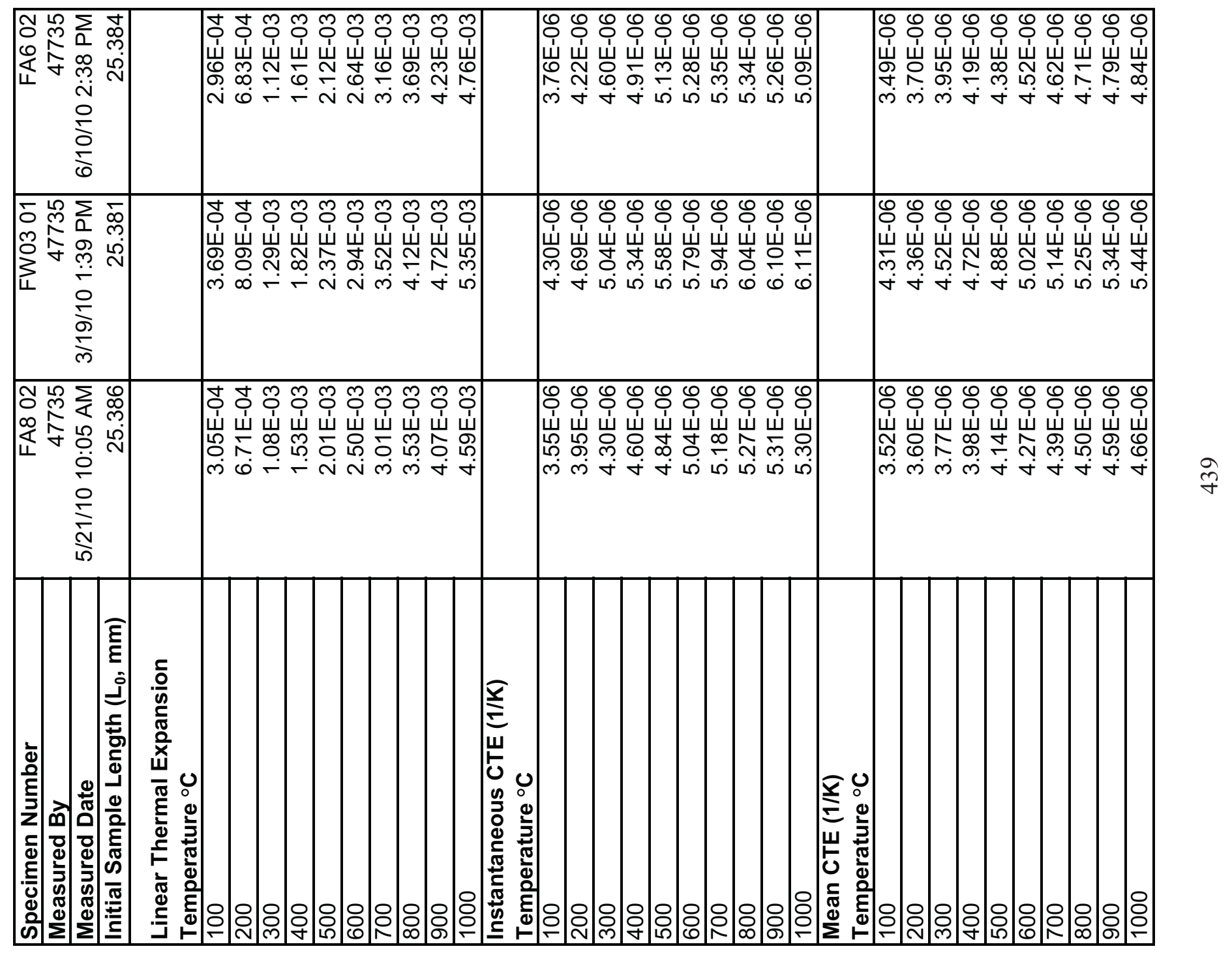




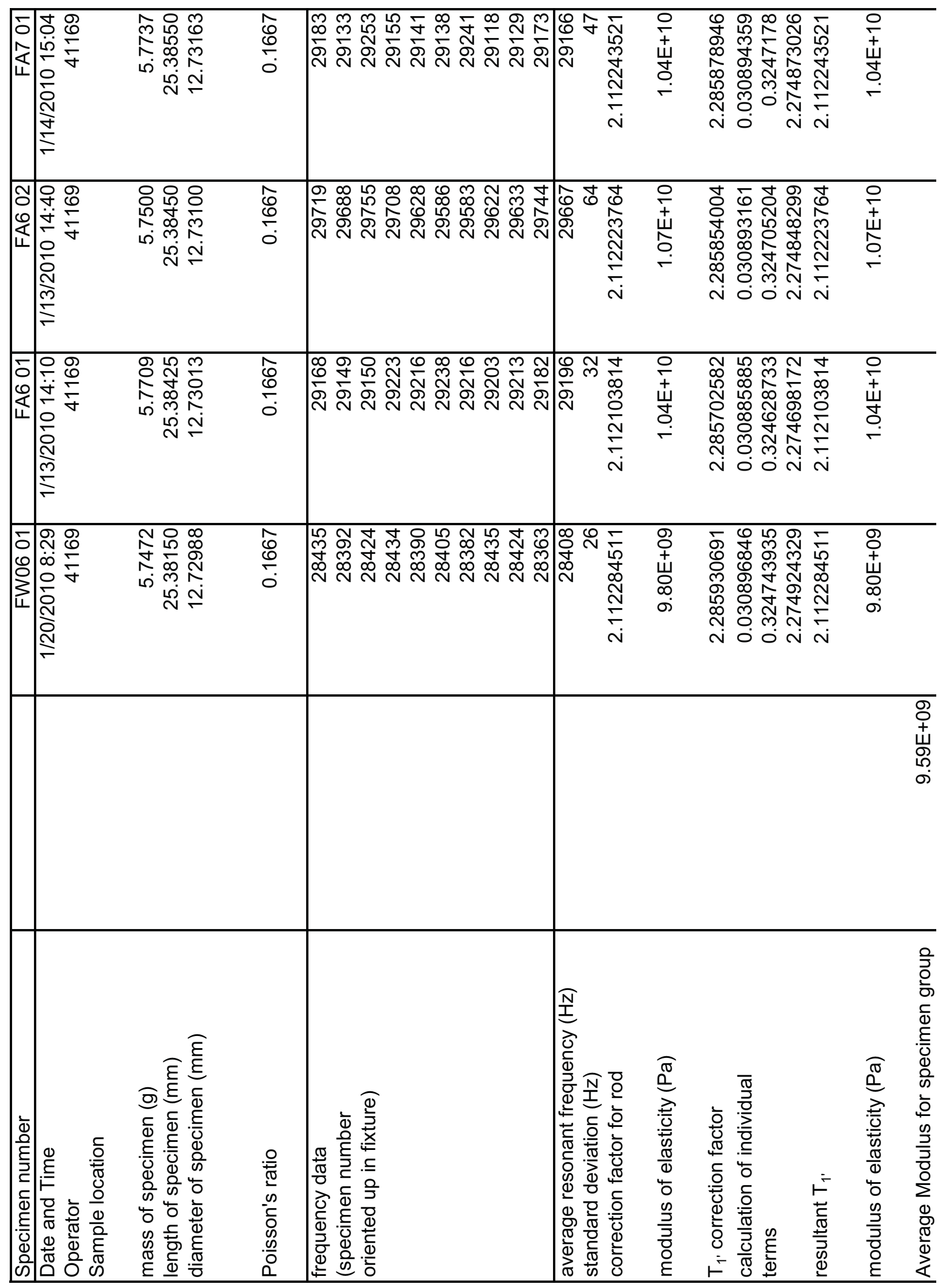




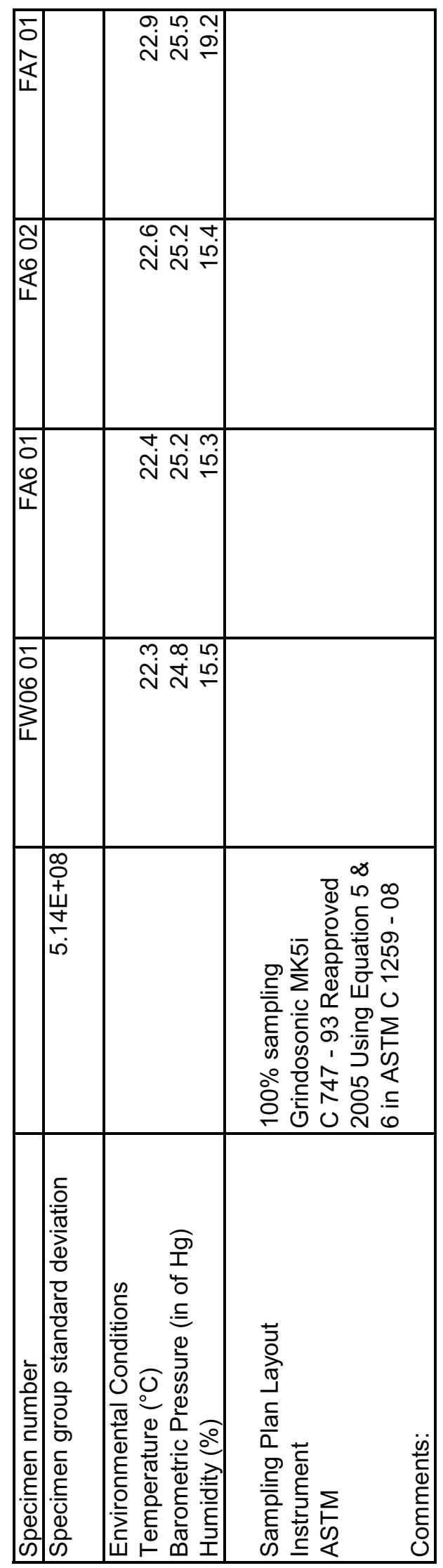




\begin{tabular}{|c|c|c|c|c|c|c|c|}
\hline 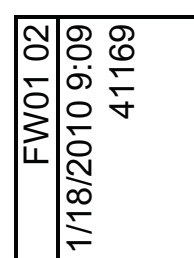 & 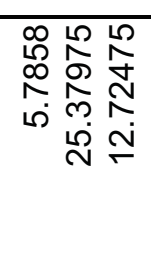 & 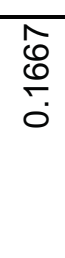 & 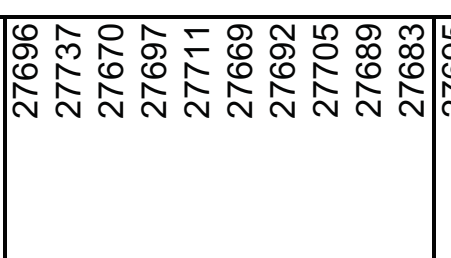 & 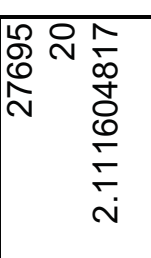 & 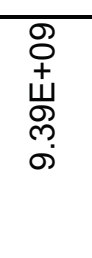 & 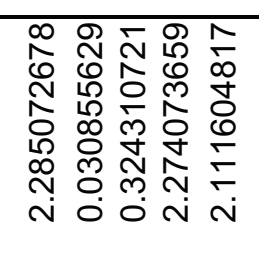 & 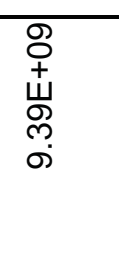 \\
\hline 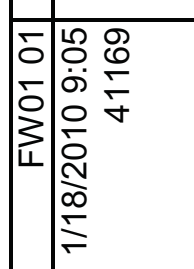 & 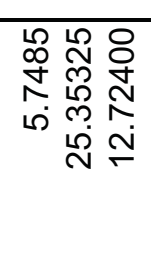 & $\begin{array}{l}\hat{0} \\
\stackrel{0}{0}\end{array}$ & 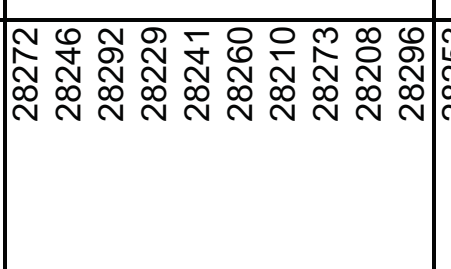 & 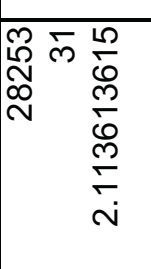 & 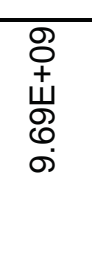 & 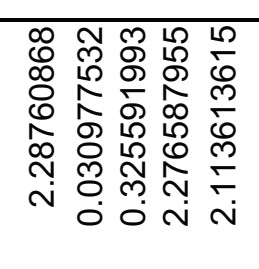 & 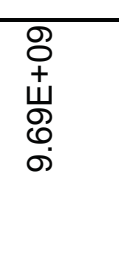 \\
\hline 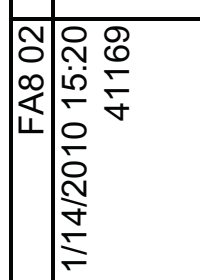 & 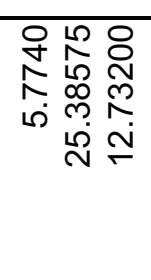 & 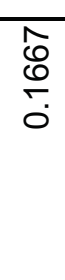 & 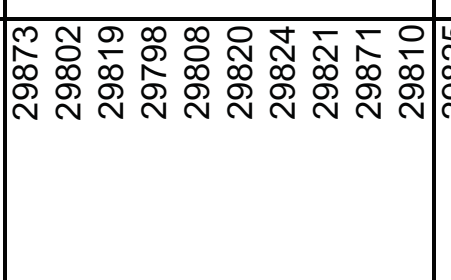 & 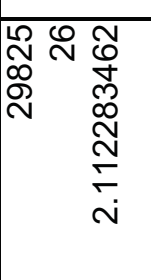 & 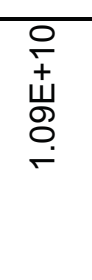 & 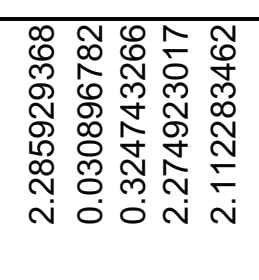 & $\begin{array}{l}\stackrel{0}{+} \\
\stackrel{+}{山} \\
\stackrel{2}{\circ}\end{array}$ \\
\hline 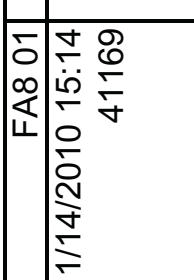 & 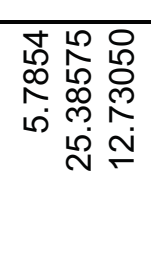 & 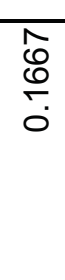 & 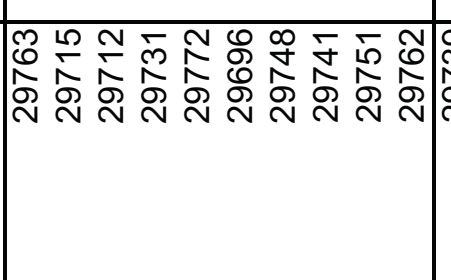 & 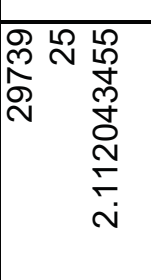 & 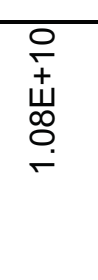 & 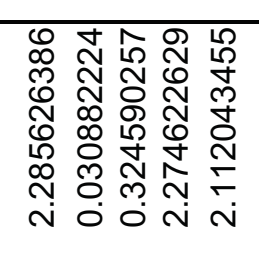 & 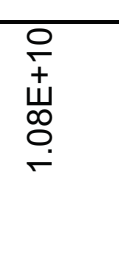 \\
\hline 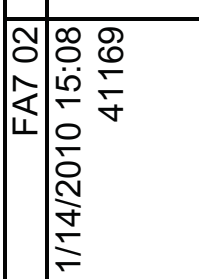 & 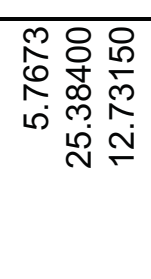 & $\begin{array}{l}T \\
\hat{0} \\
\stackrel{0}{0}\end{array}$ & 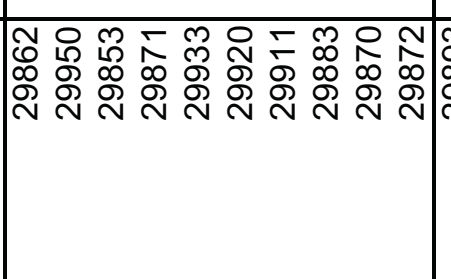 & 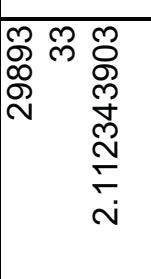 & 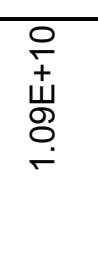 & 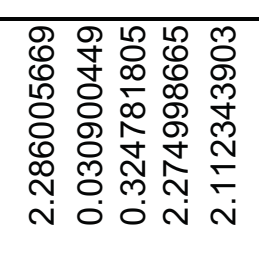 & 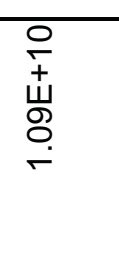 \\
\hline 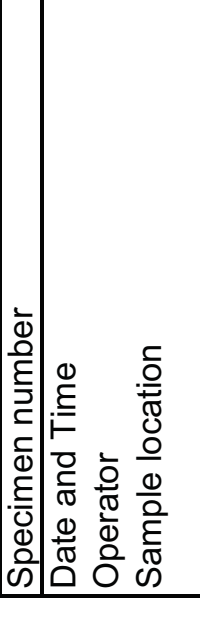 & 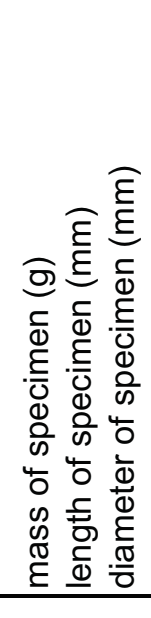 & 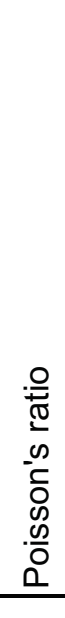 & 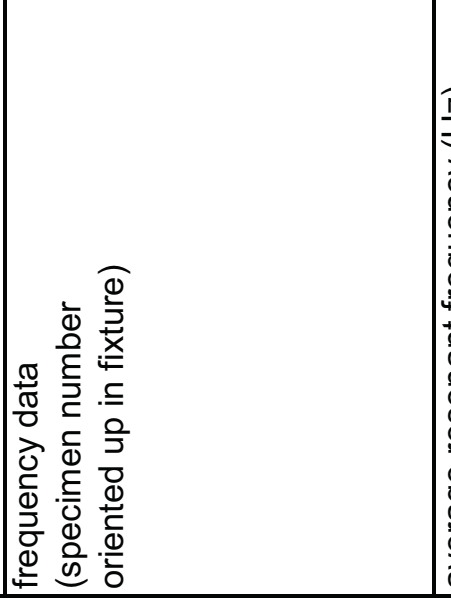 & 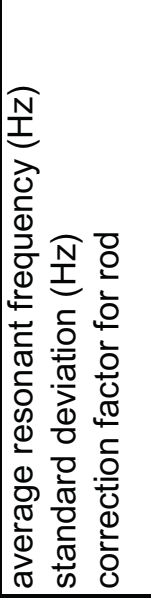 & 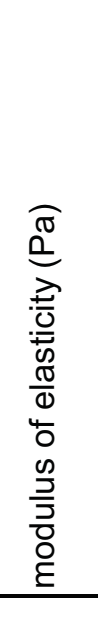 & 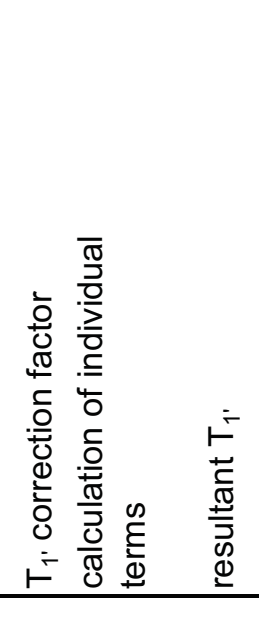 & 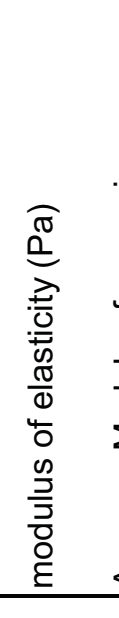 \\
\hline
\end{tabular}




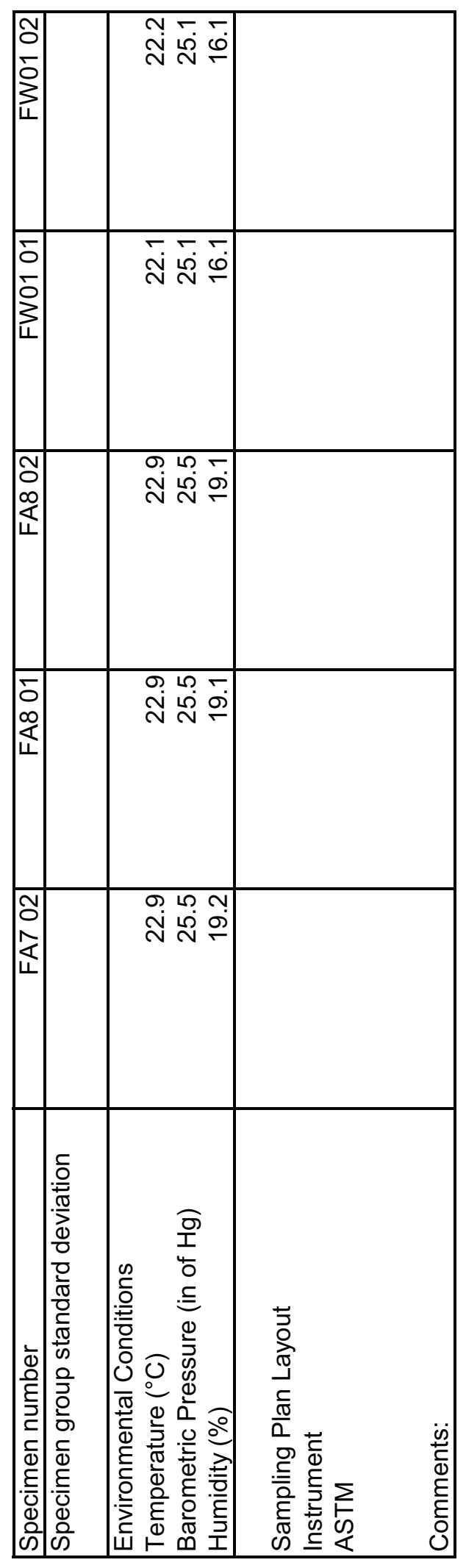




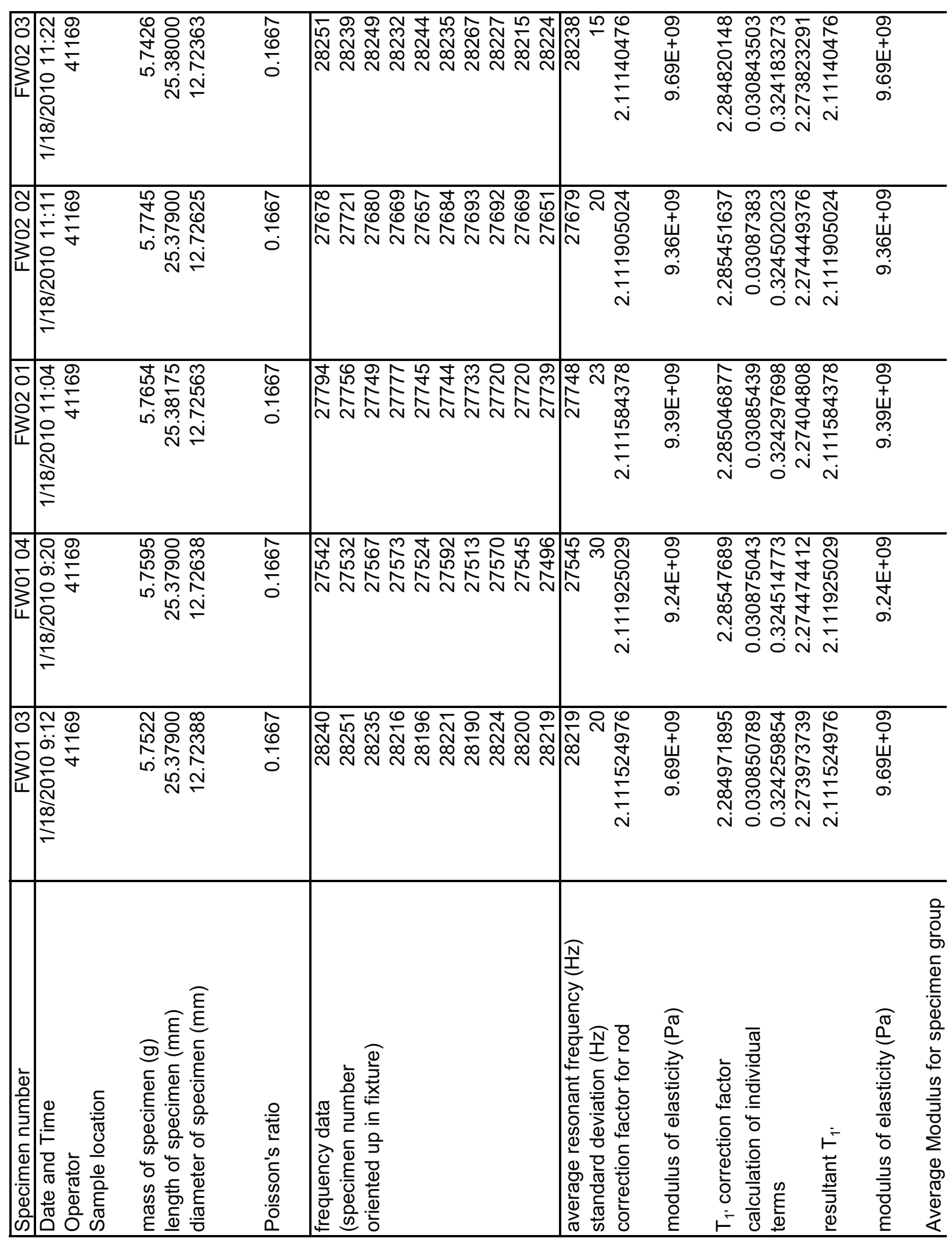




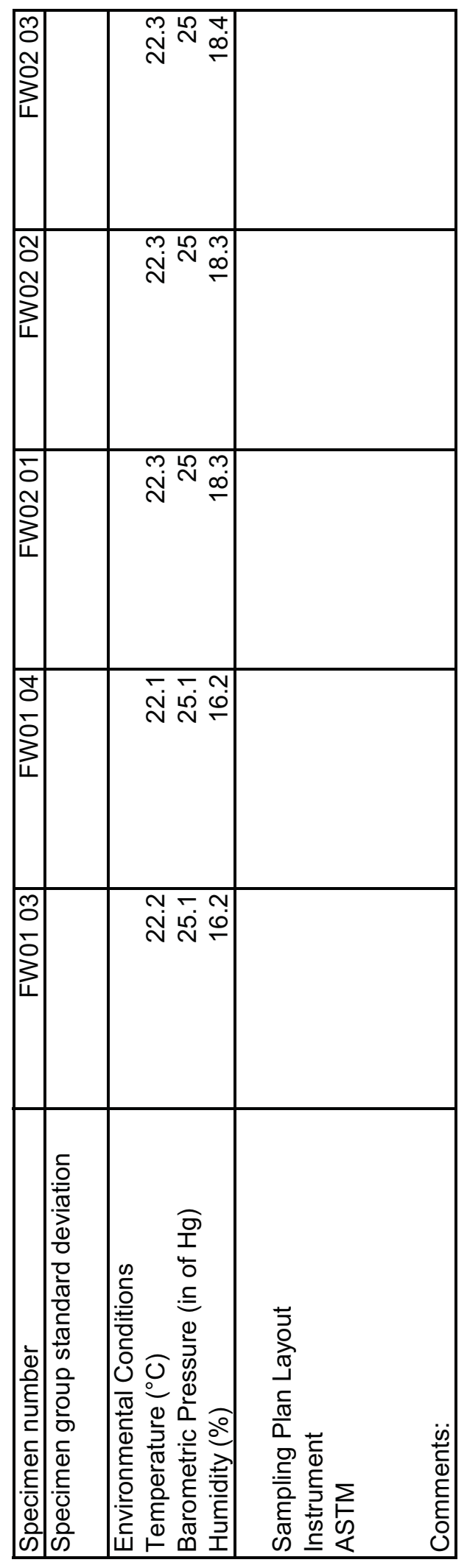




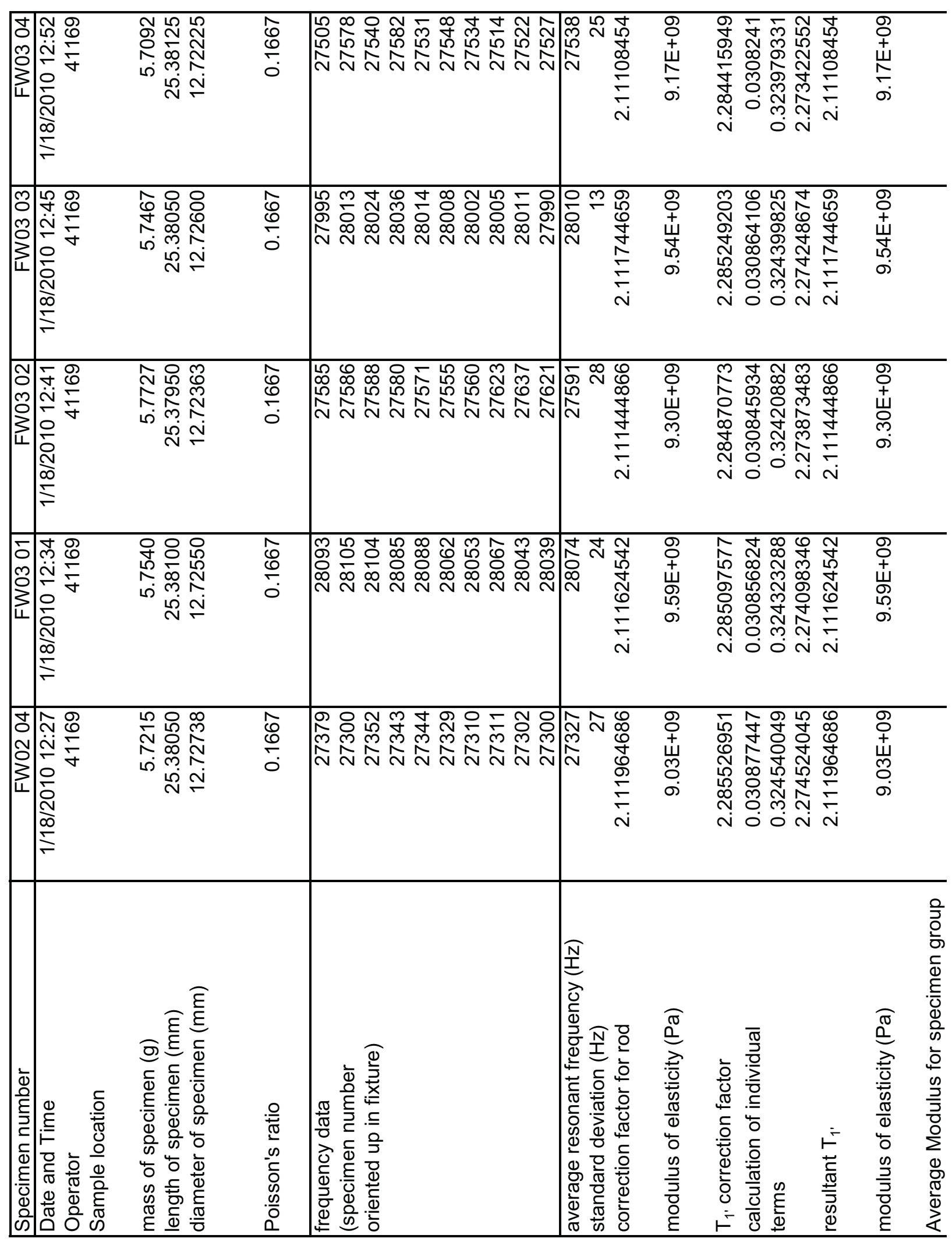




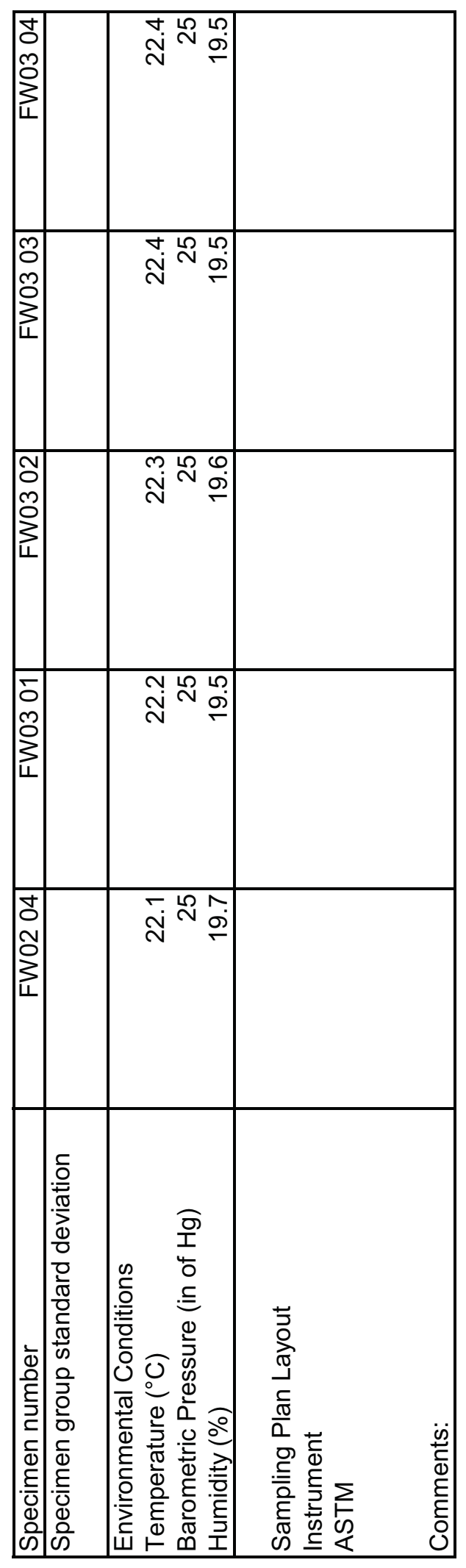




\begin{tabular}{|c|c|c|c|c|c|c|c|}
\hline 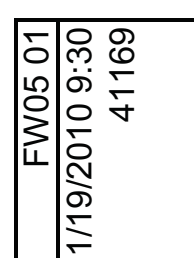 & 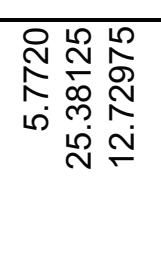 & $\begin{array}{l}\hat{0} \\
\stackrel{0}{0}\end{array}$ & 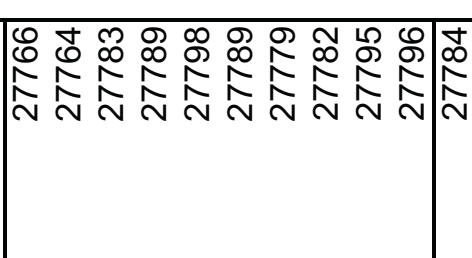 & 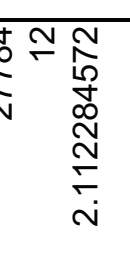 & 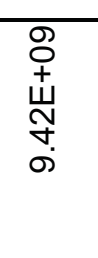 & 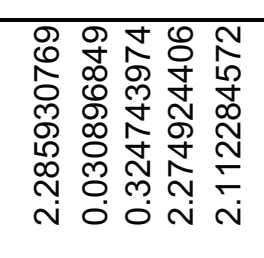 & 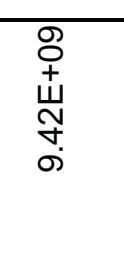 \\
\hline 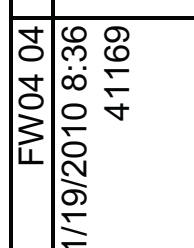 & 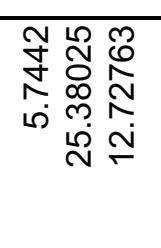 & 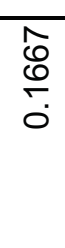 & 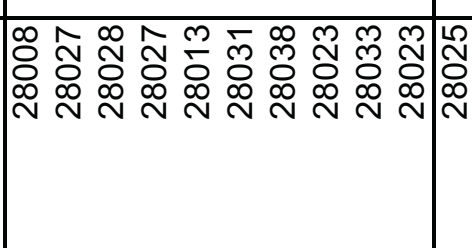 & 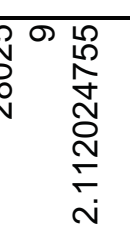 & 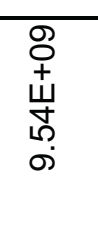 & 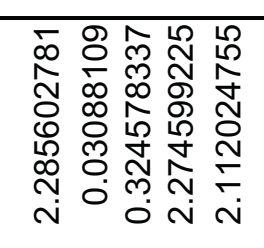 & 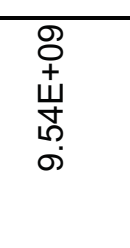 \\
\hline 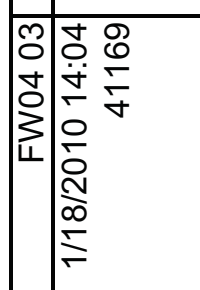 & 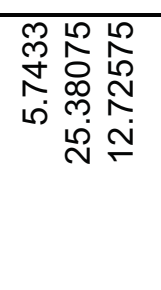 & $\begin{array}{l}\frac{\pi}{0} \\
\stackrel{0}{0}\end{array}$ & 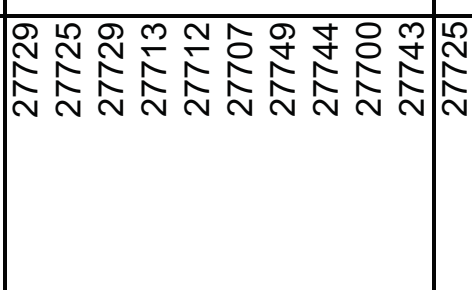 & 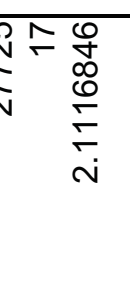 & 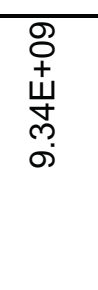 & 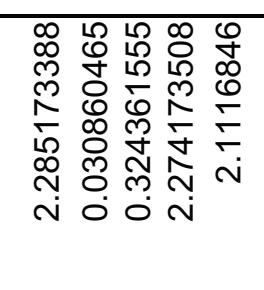 & 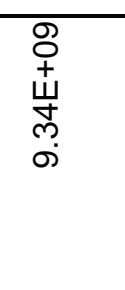 \\
\hline 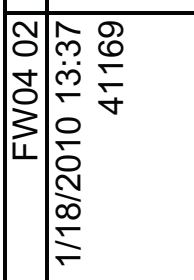 & 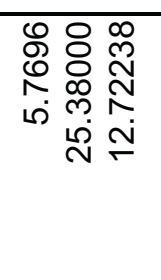 & $\begin{array}{l}\frac{\pi}{0} \\
\stackrel{\circ}{0}\end{array}$ & 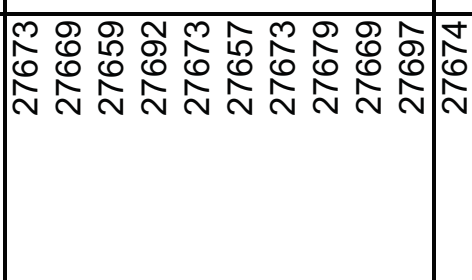 & 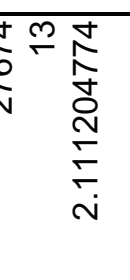 & 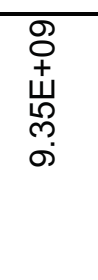 & 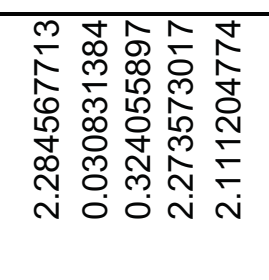 & 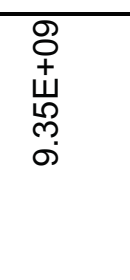 \\
\hline 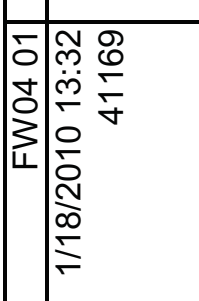 & 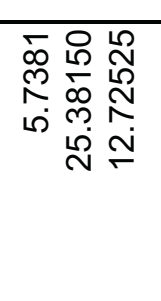 & $\begin{array}{l}\hat{0} \\
\stackrel{0}{0}\end{array}$ & 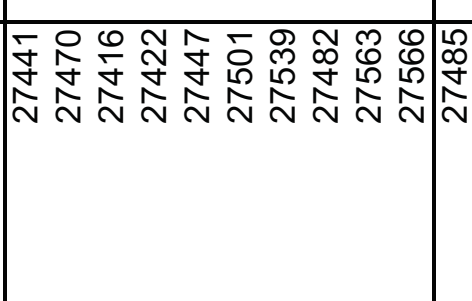 & 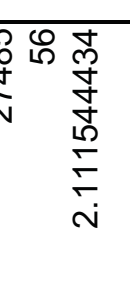 & 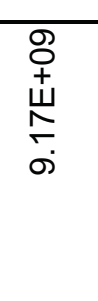 & 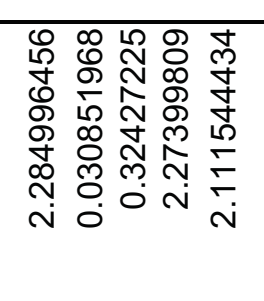 & $\begin{array}{l}\stackrel{8}{+} \\
\stackrel{+}{+} \\
\stackrel{5}{\circ} \\
\dot{0}\end{array}$ \\
\hline 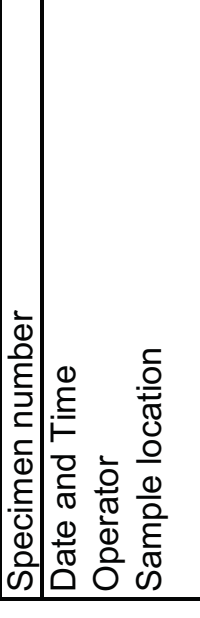 & 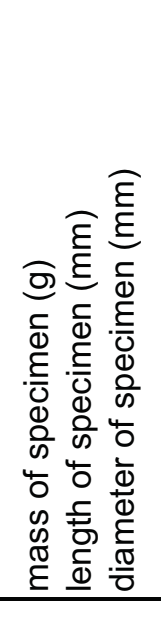 & 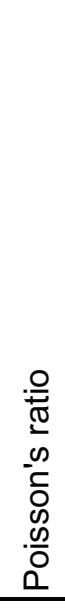 & 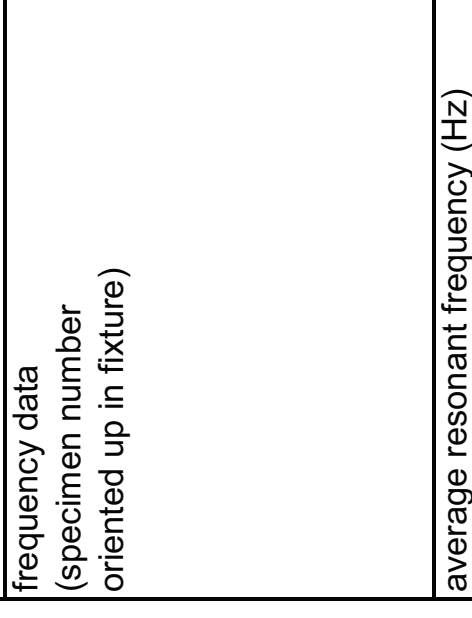 & 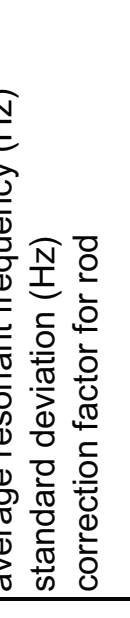 & 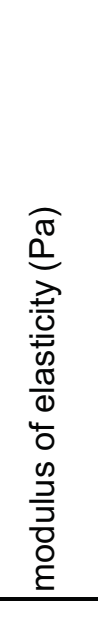 & 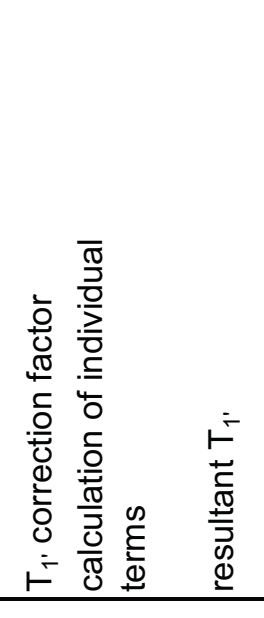 & 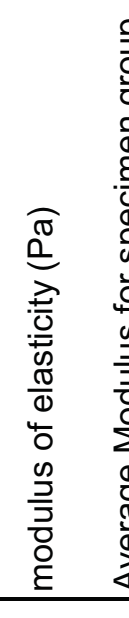 \\
\hline
\end{tabular}




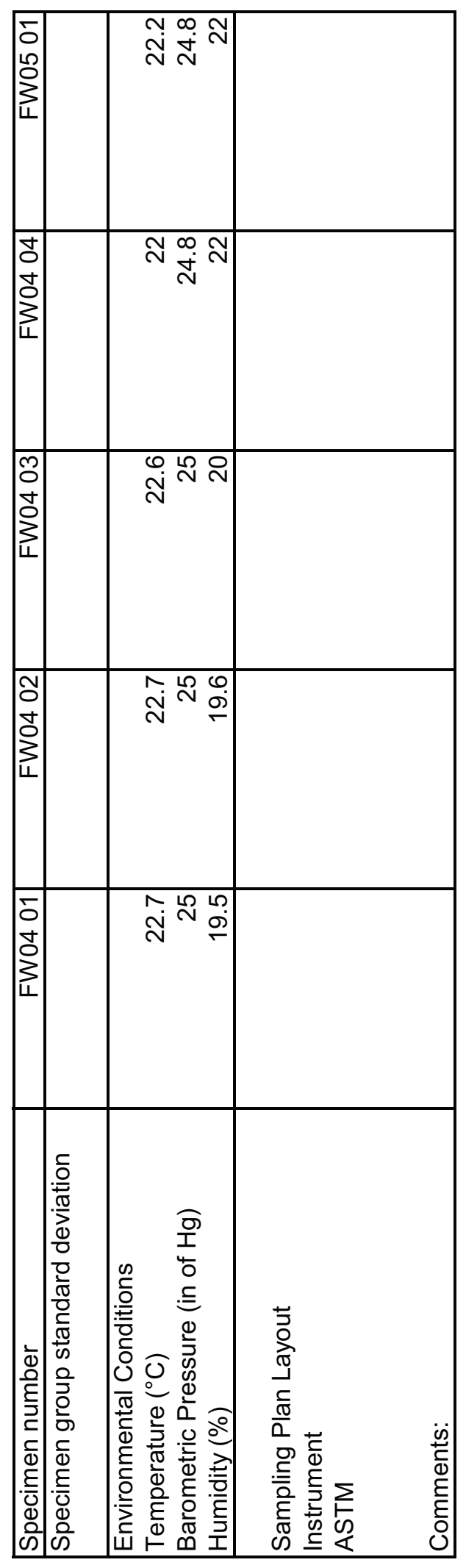




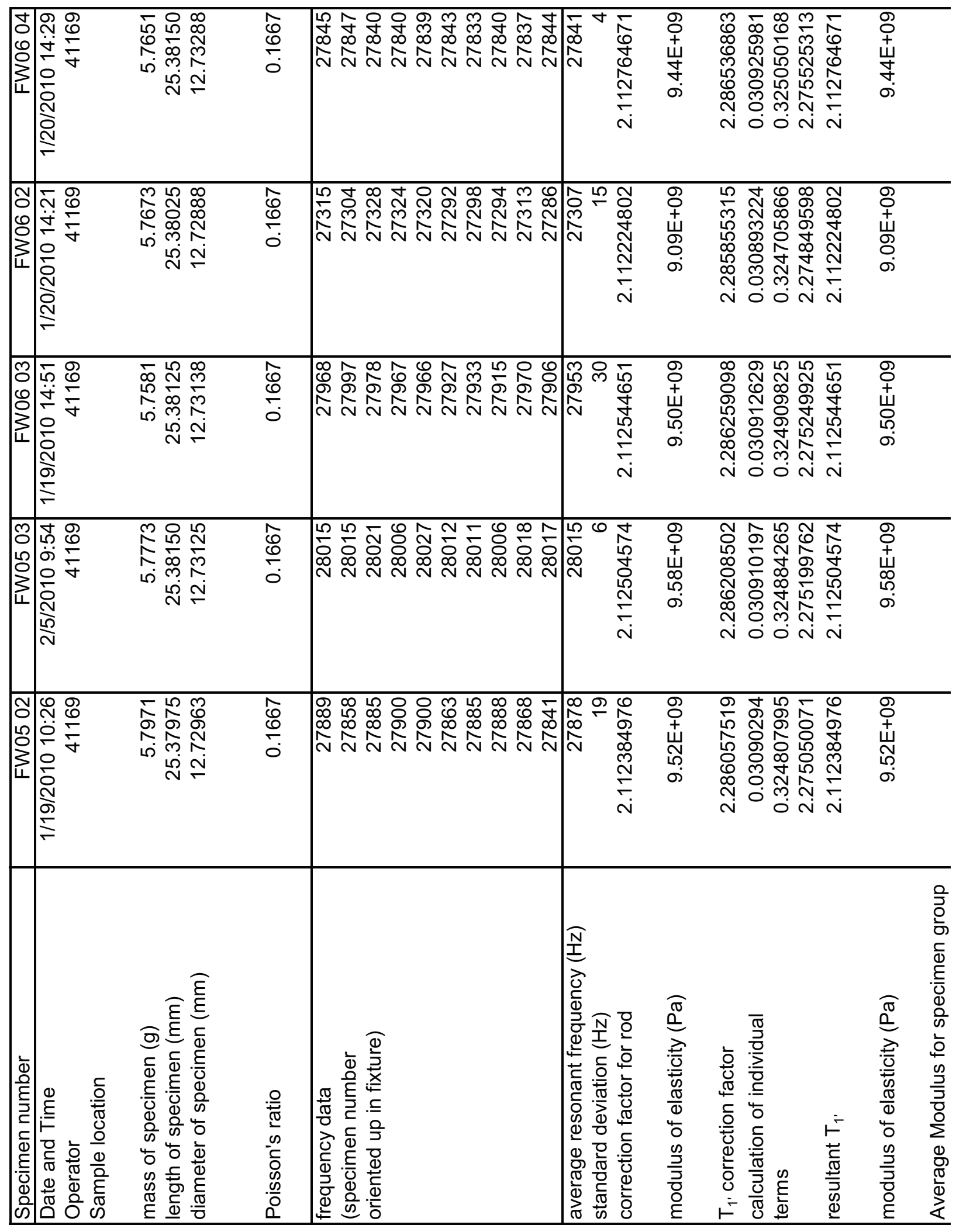




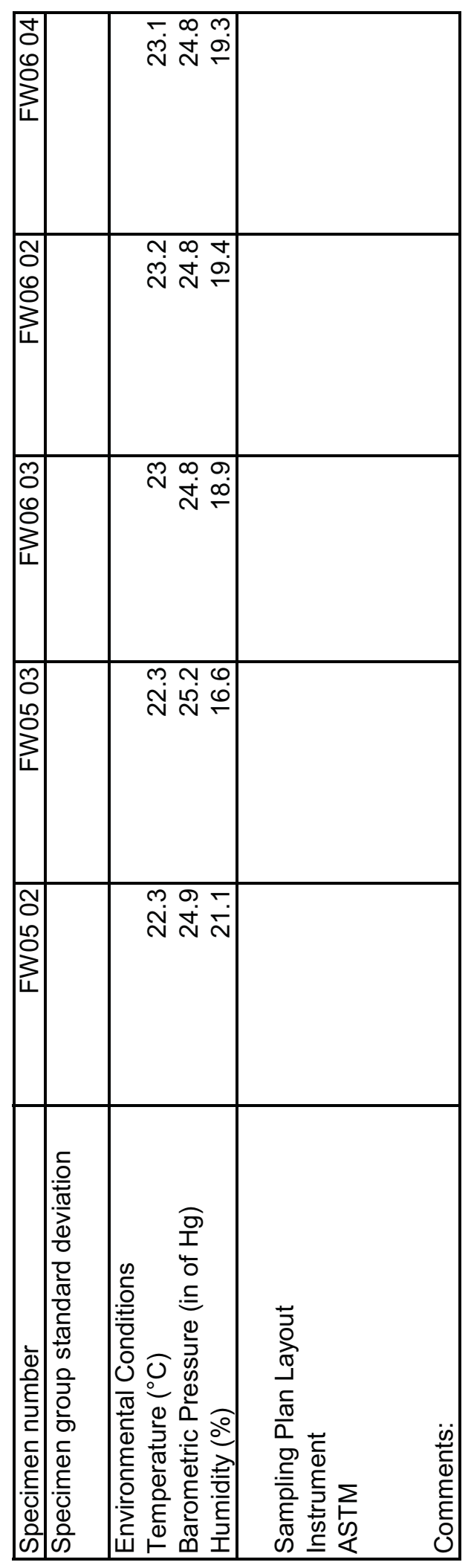

$\overline{7}$ 


\begin{tabular}{|c|c|c|c|c|c|c|c|}
\hline 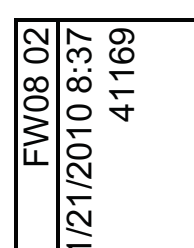 & 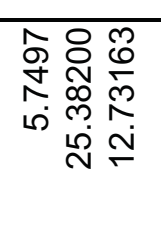 & $\begin{array}{l}\hat{0} \\
\stackrel{0}{0}\end{array}$ & 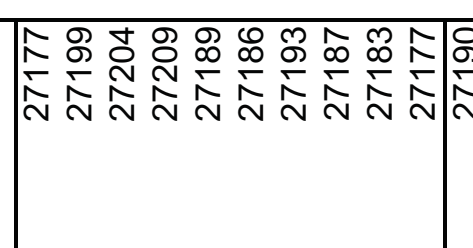 & Ț & 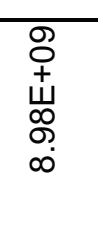 & 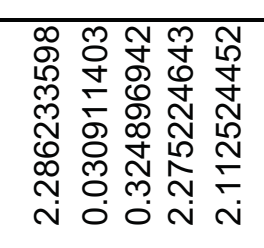 & 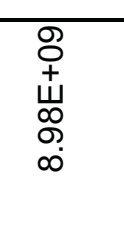 \\
\hline 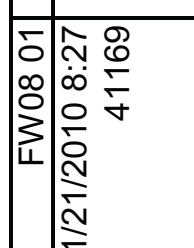 & 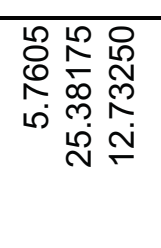 & 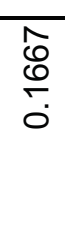 & 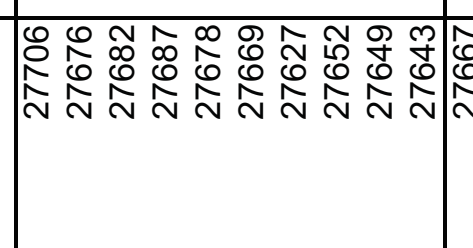 & 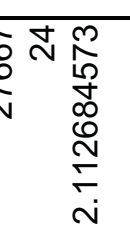 & 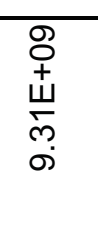 & 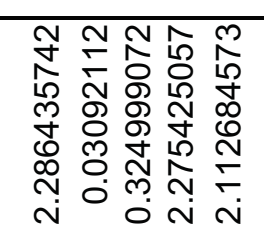 & 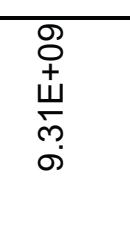 \\
\hline 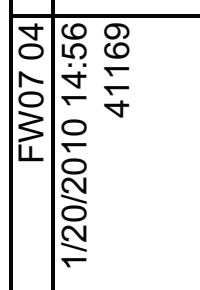 & 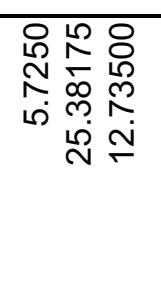 & $\begin{array}{l}\frac{\pi}{0} \\
\stackrel{0}{0}\end{array}$ & 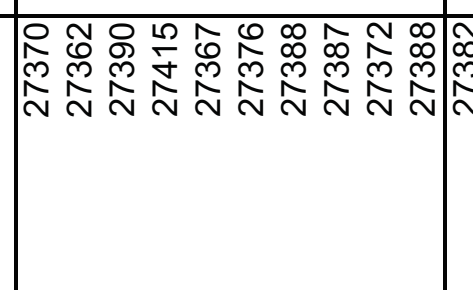 & 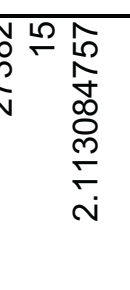 & 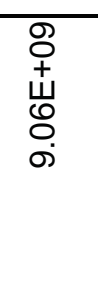 & 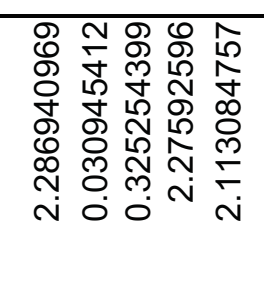 & 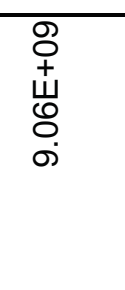 \\
\hline 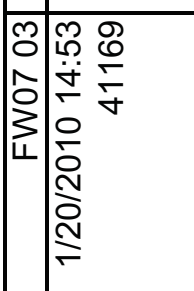 & 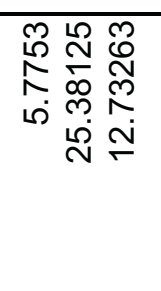 & $\begin{array}{l}\frac{\pi}{0} \\
\stackrel{\circ}{0}\end{array}$ & 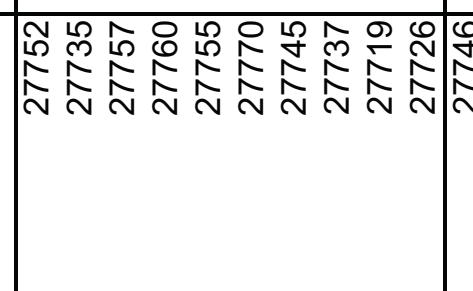 & 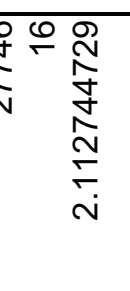 & 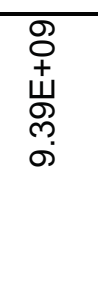 & 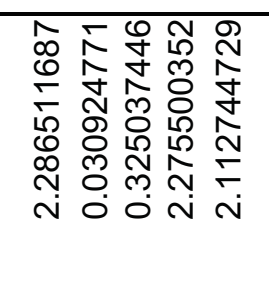 & 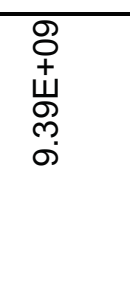 \\
\hline 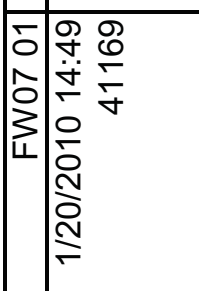 & 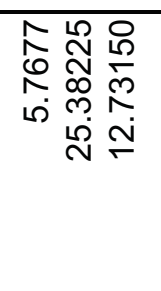 & $\begin{array}{l}\frac{1}{0} \\
\vdots \\
0\end{array}$ & 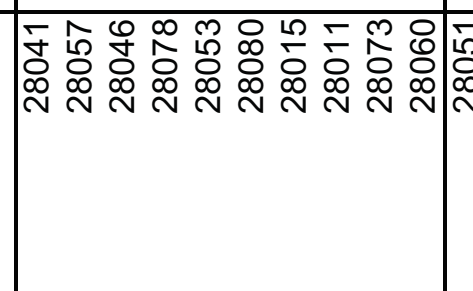 & 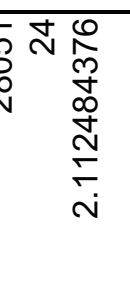 & 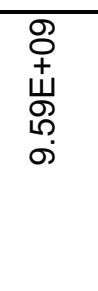 & 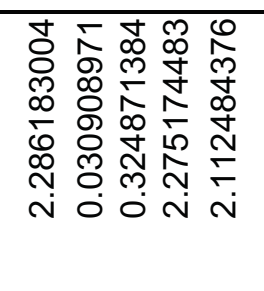 & 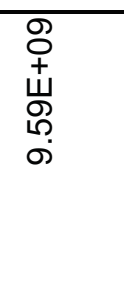 \\
\hline 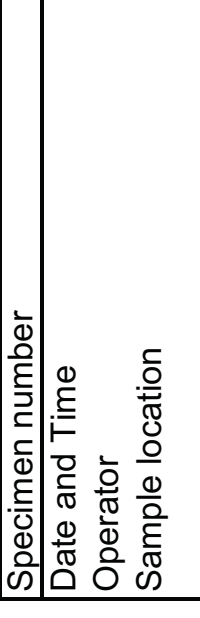 & 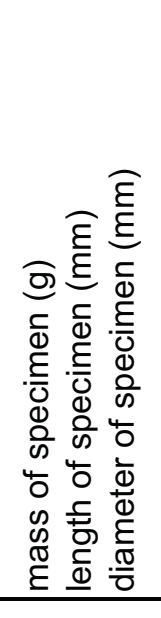 & 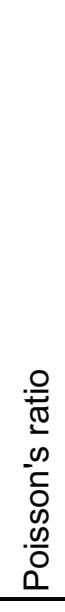 & 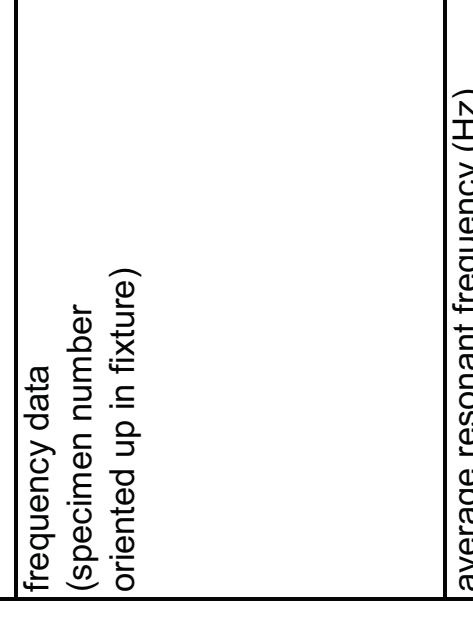 & 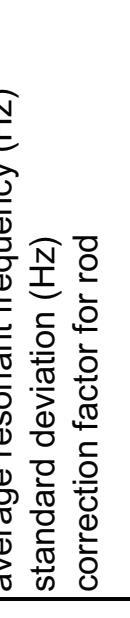 & 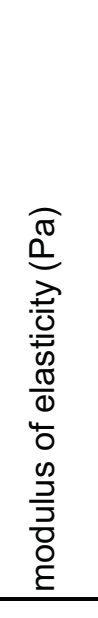 & 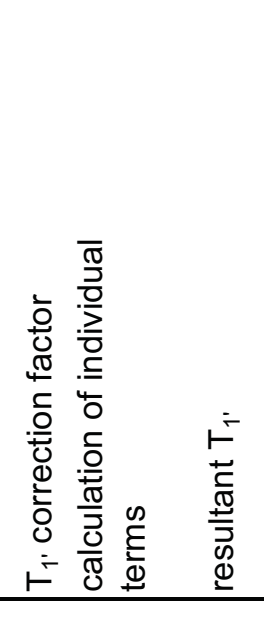 & 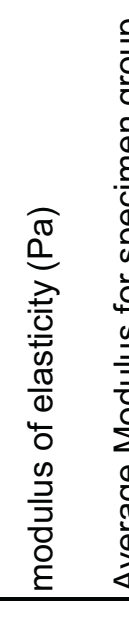 \\
\hline
\end{tabular}




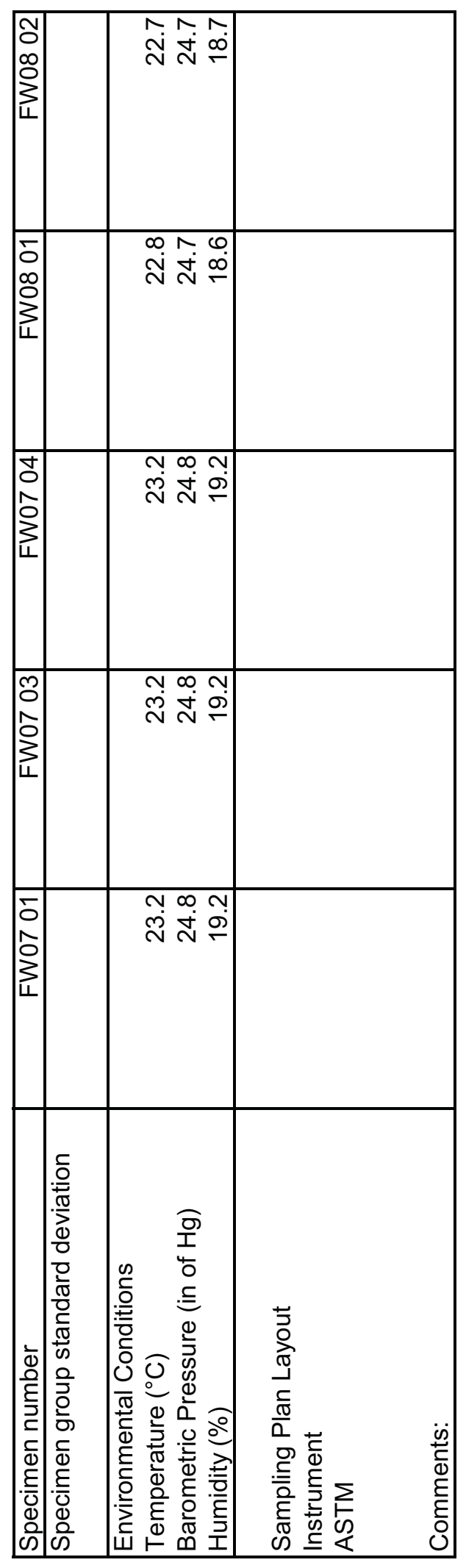




\begin{tabular}{|c|c|c|c|c|c|c|c|}
\hline 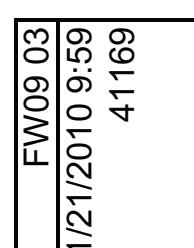 & 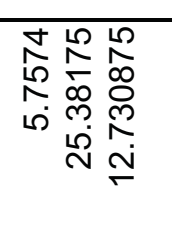 & $\begin{array}{l}T \\
\hat{0} \\
\stackrel{0}{0}\end{array}$ & |lll: & 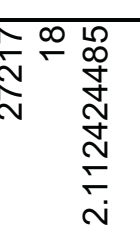 & 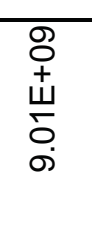 & 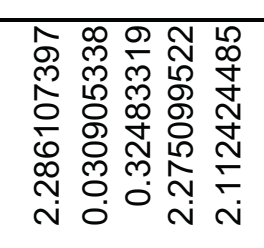 & $\begin{array}{l}\stackrel{O}{+} \\
\stackrel{+}{4} \\
\dot{0} \\
\dot{\sigma}\end{array}$ \\
\hline 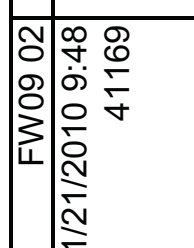 & 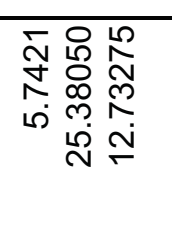 & $\begin{array}{l}T \\
\hat{0} \\
\stackrel{0}{0}\end{array}$ & 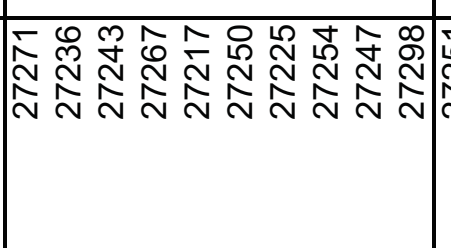 & Nָָ & 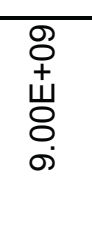 & 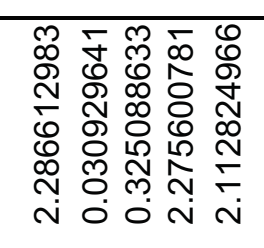 & 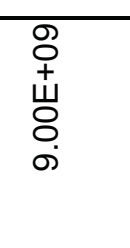 \\
\hline 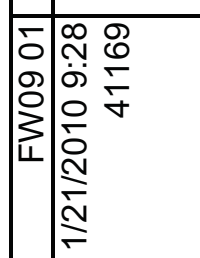 & 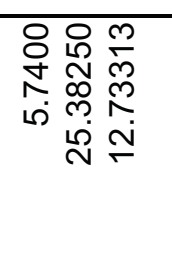 & $\begin{array}{l} \\
\hat{0} \\
\stackrel{0}{0}\end{array}$ & 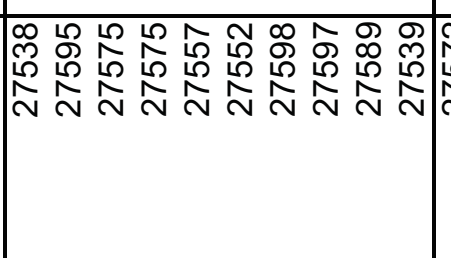 & 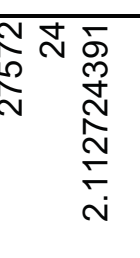 & 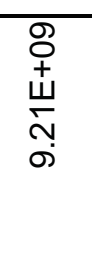 & 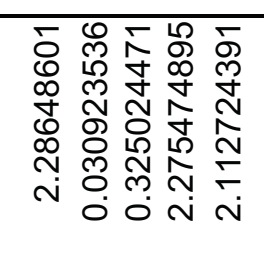 & 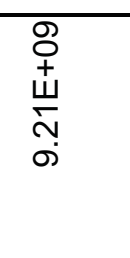 \\
\hline 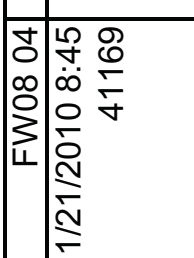 & 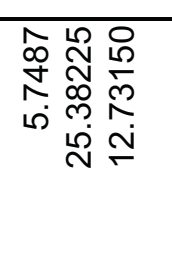 & $\begin{array}{l}\overline{0} \\
\hat{0} \\
\vdots \\
0\end{array}$ & 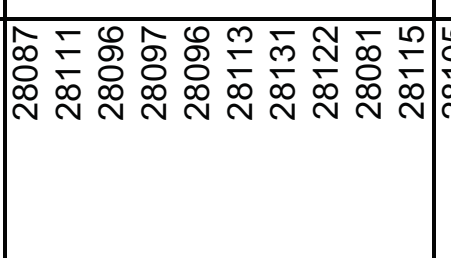 & 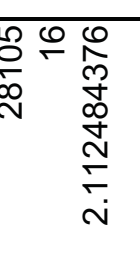 & 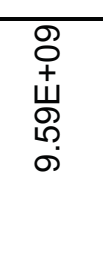 & 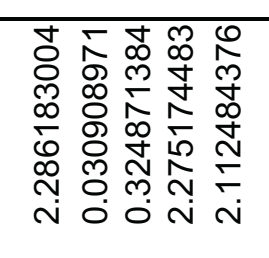 & 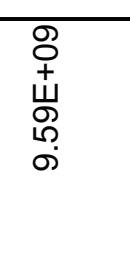 \\
\hline 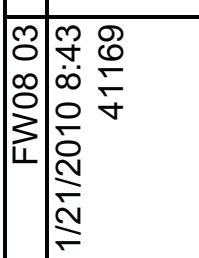 & 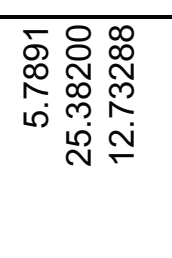 & $\begin{array}{l}\overline{0} \\
\stackrel{\leftrightarrow}{0} \\
\stackrel{0}{0}\end{array}$ & 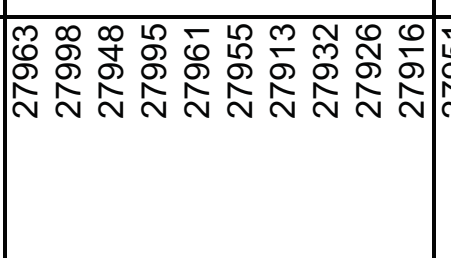 & 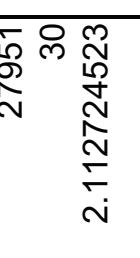 & 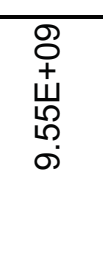 & 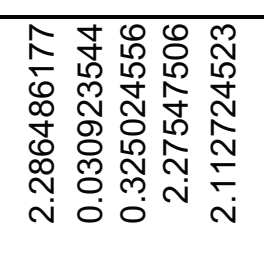 & 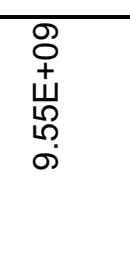 \\
\hline 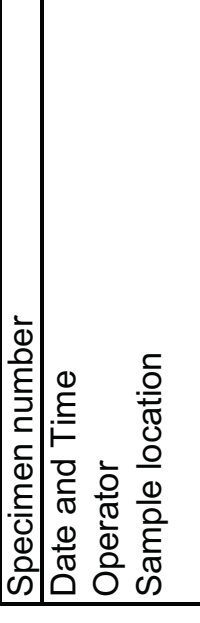 & 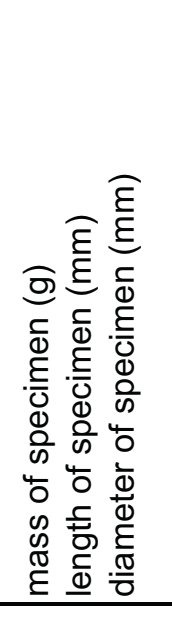 & 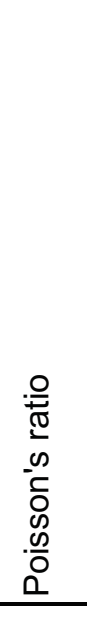 & 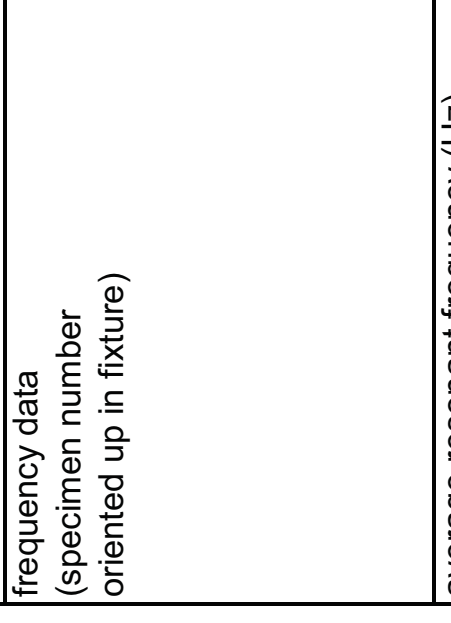 & 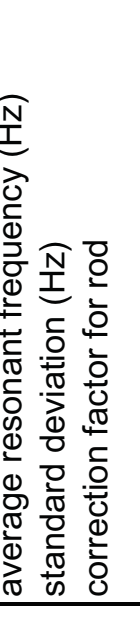 & 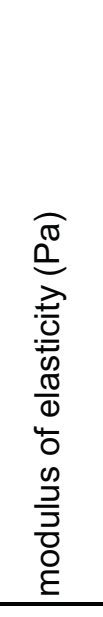 & 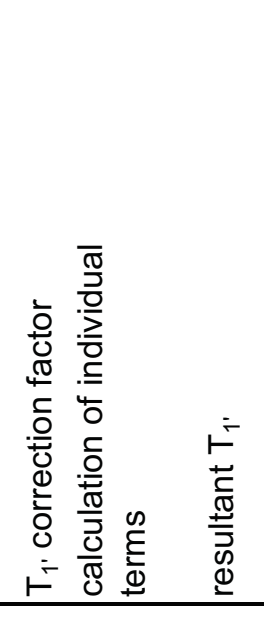 & 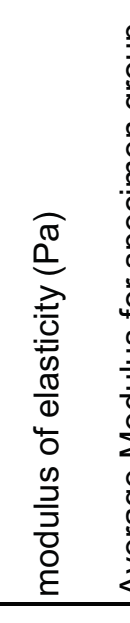 \\
\hline
\end{tabular}




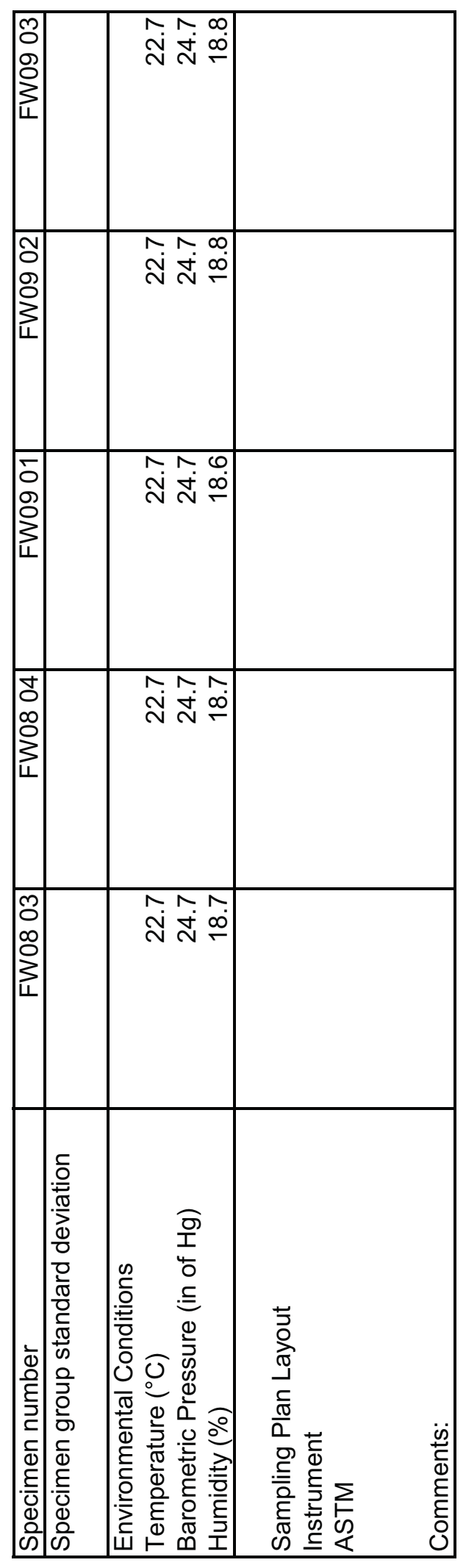




\begin{tabular}{|c|c|c|c|c|c|c|c|}
\hline 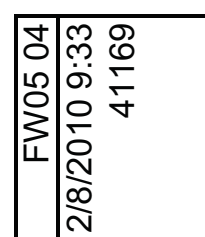 & 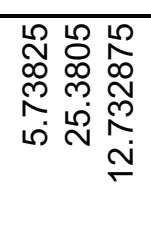 & 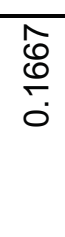 & 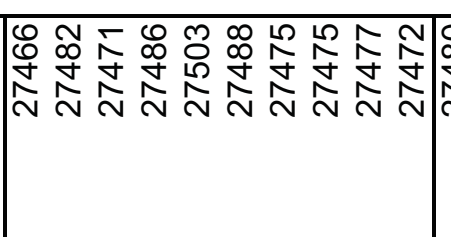 & 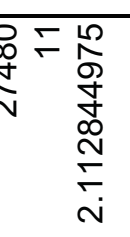 & $\begin{array}{l}\stackrel{8}{+} \\
\stackrel{4}{4} \\
\stackrel{4}{\circ} \\
\infty\end{array}$ & 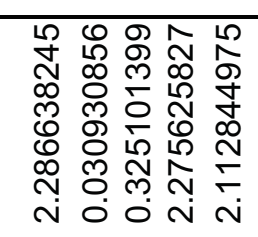 & 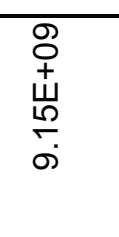 \\
\hline 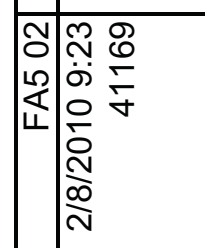 & 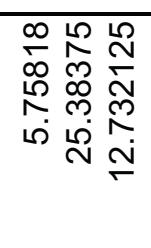 & $\begin{array}{l} \\
\hat{0} \\
\stackrel{0}{0}\end{array}$ & 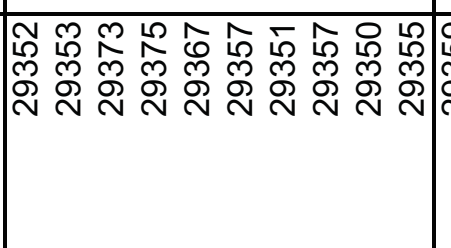 & 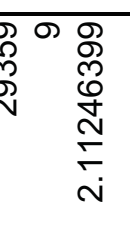 & 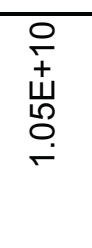 & 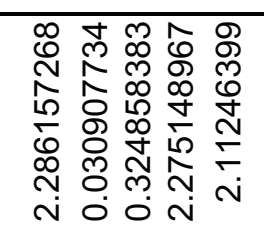 & 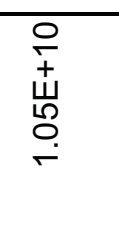 \\
\hline 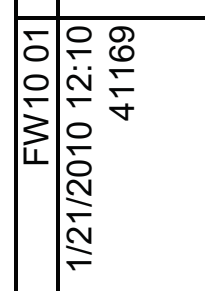 & 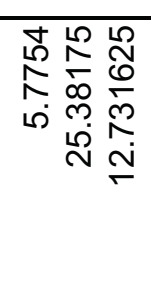 & $\begin{array}{l}T \\
\hat{0} \\
\stackrel{0}{0}\end{array}$ & 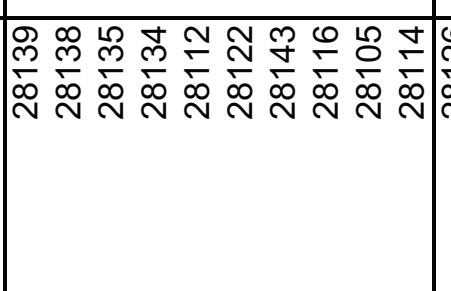 & 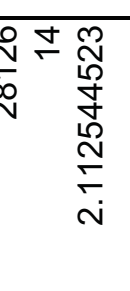 & 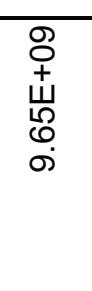 & 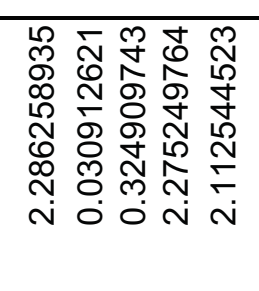 & 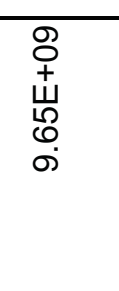 \\
\hline 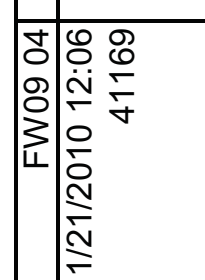 & 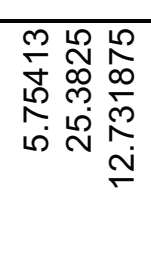 & 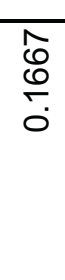 & 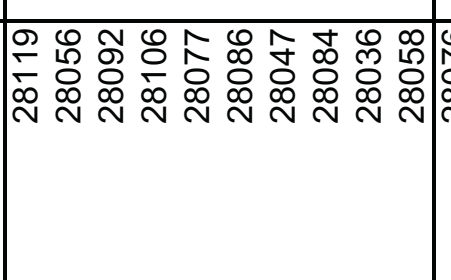 & 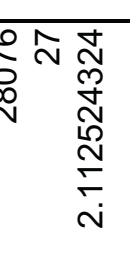 & 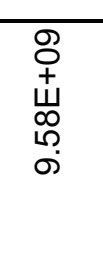 & 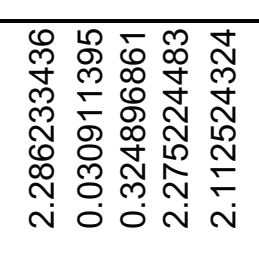 & 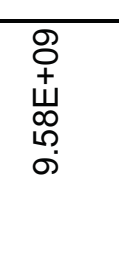 \\
\hline 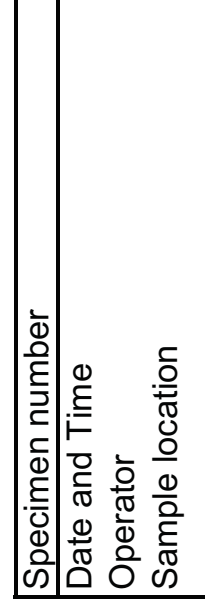 & 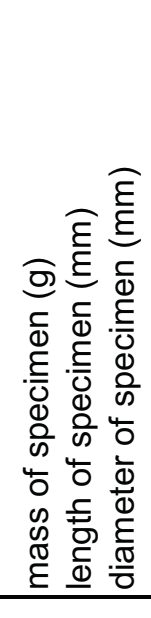 & 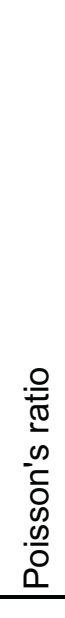 & 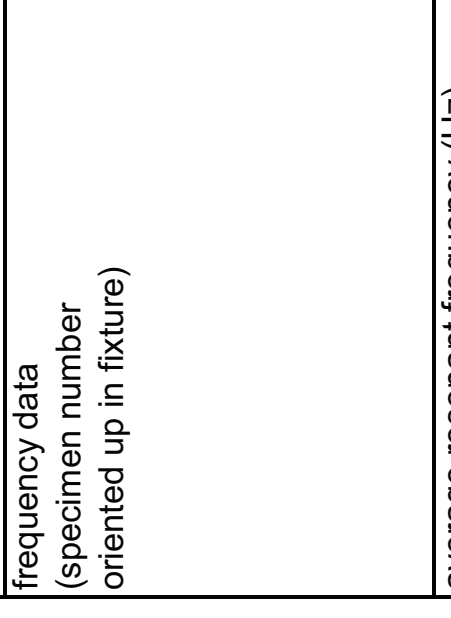 & 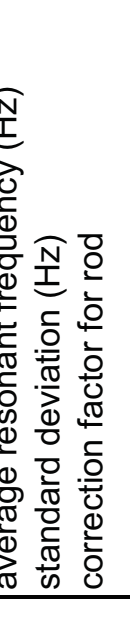 & 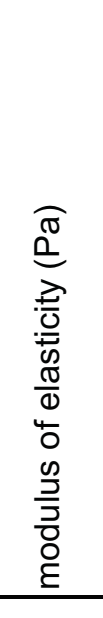 & 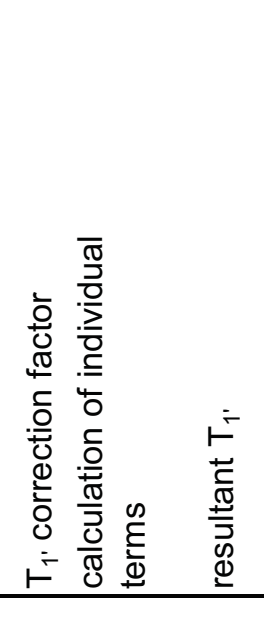 & 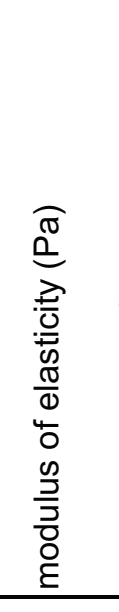 \\
\hline
\end{tabular}




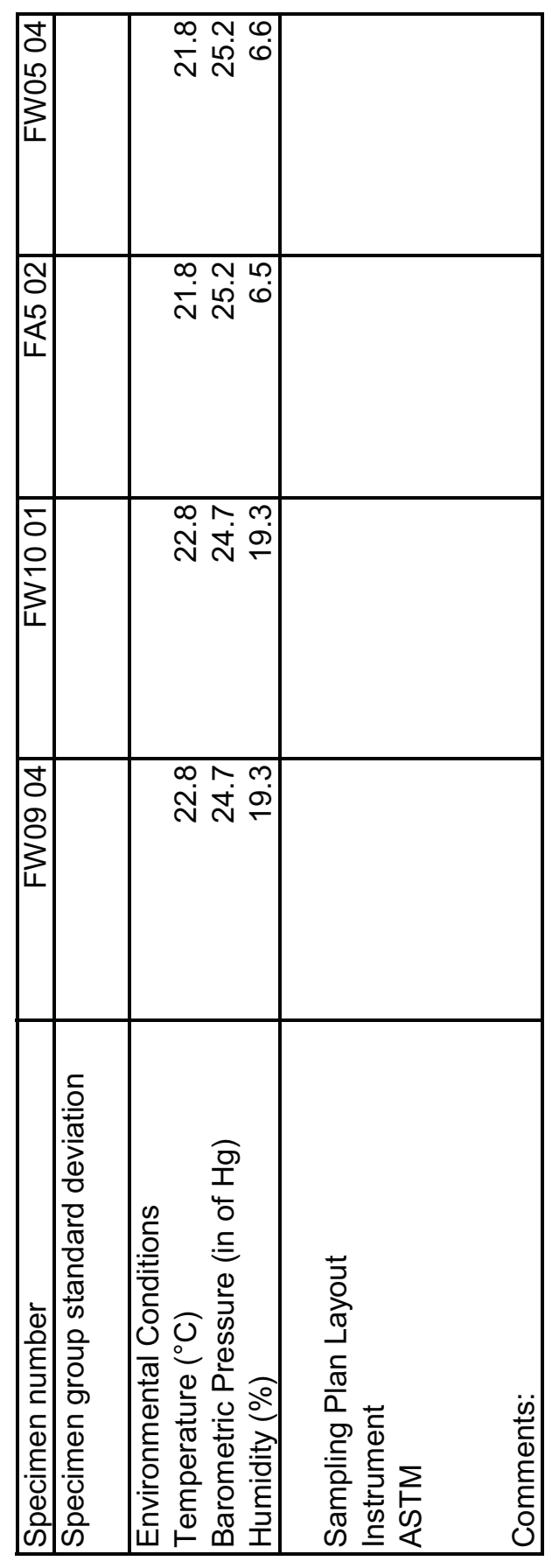

in 


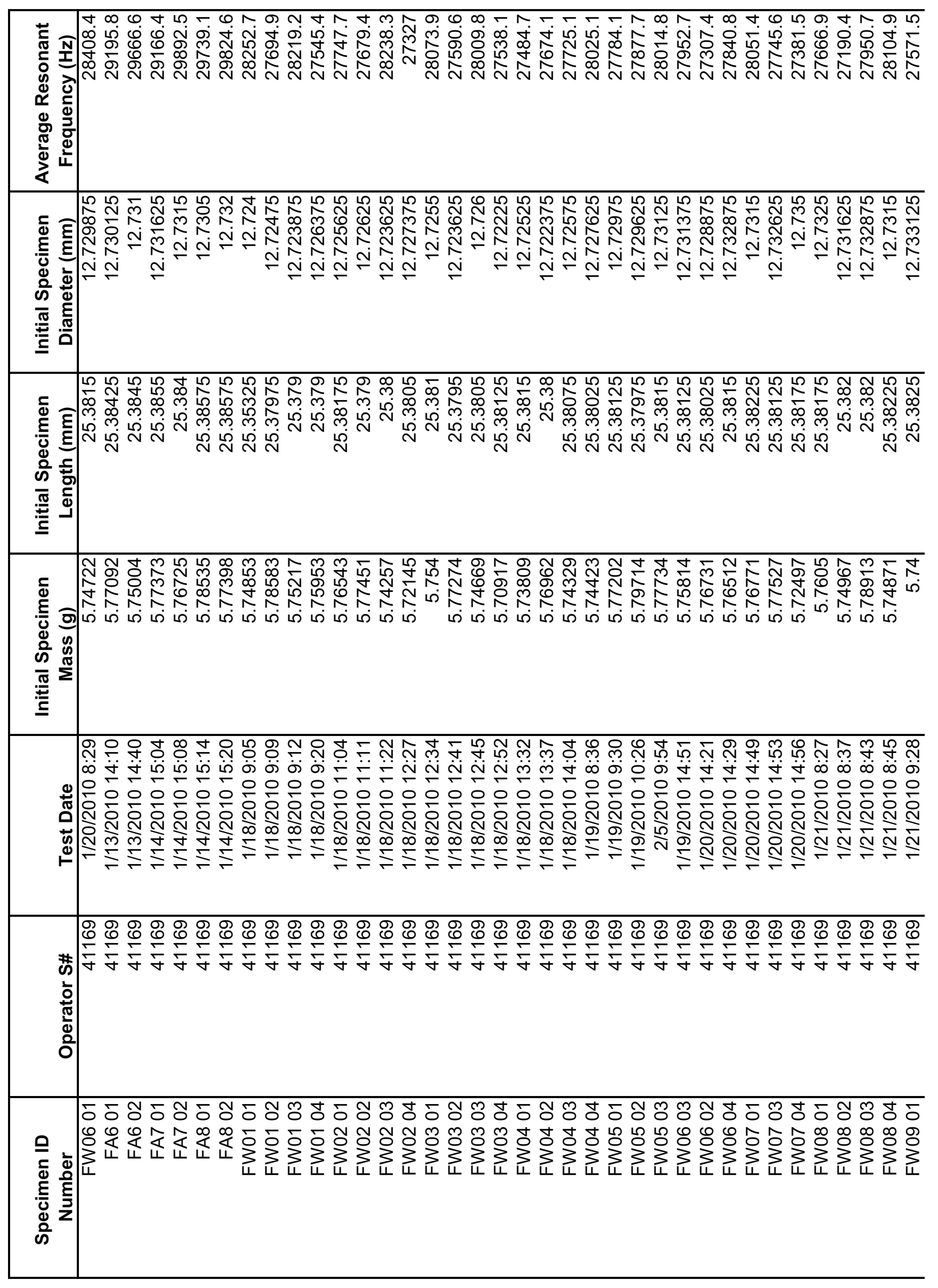




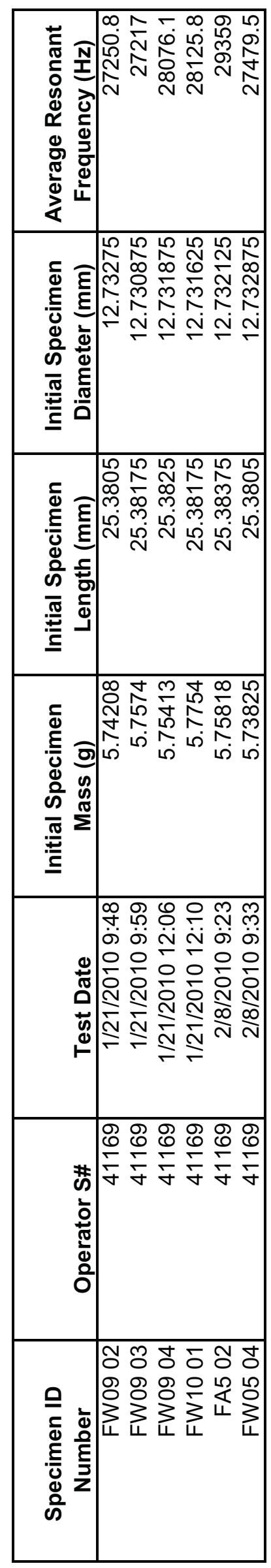




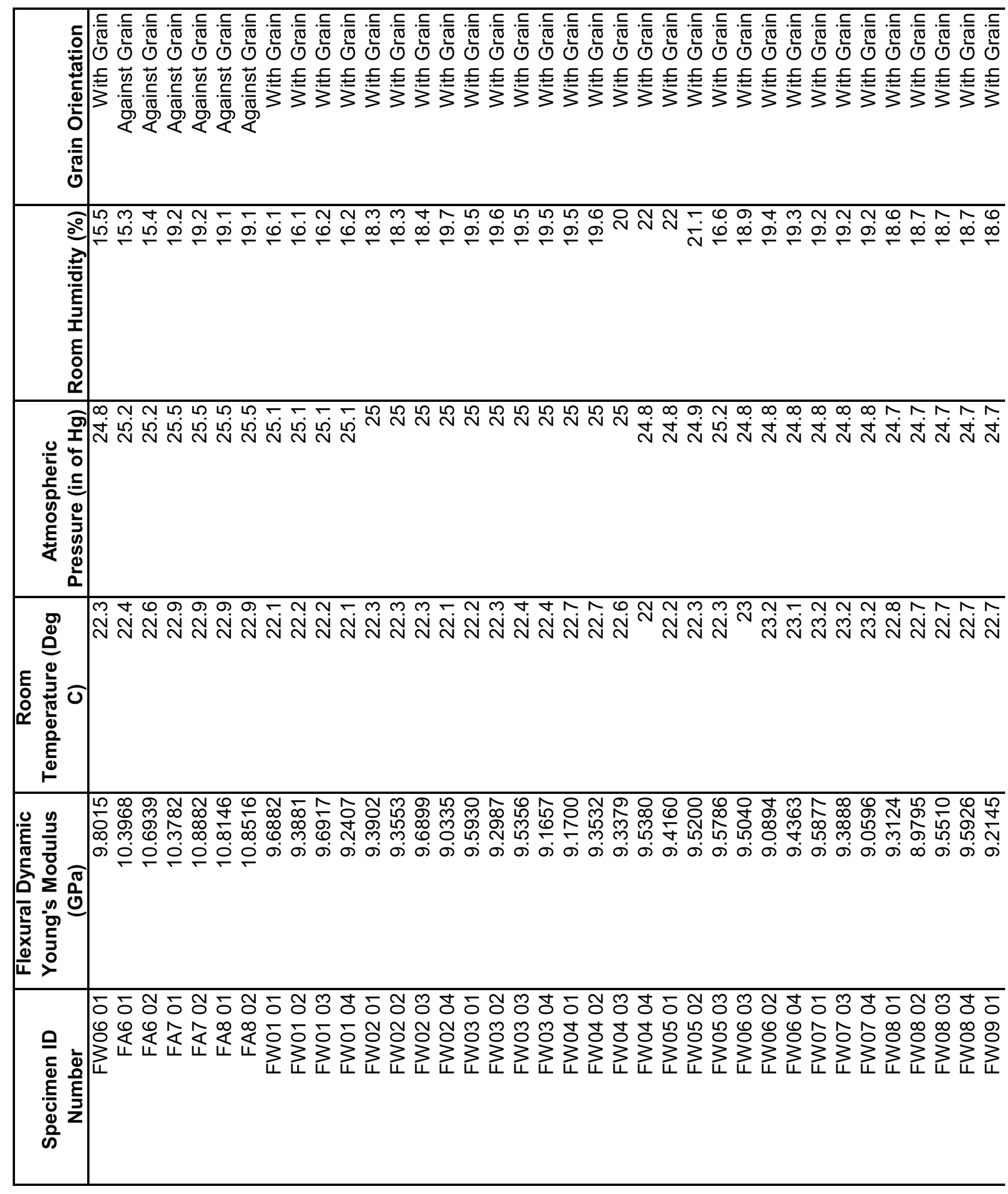




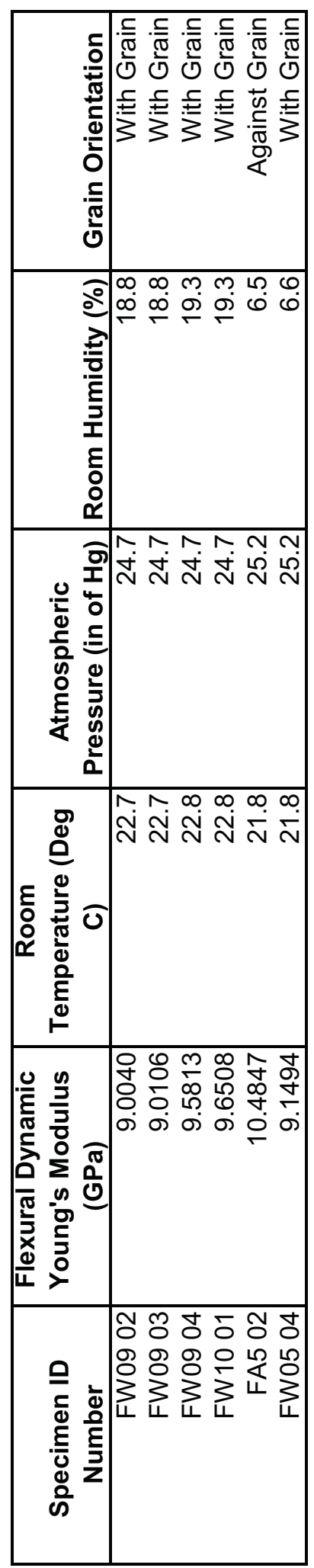




\begin{tabular}{|c|c|c|c|c|c|c|c|c|c|c|}
\hline \begin{tabular}{|l}
- \\
0 \\
$\infty$ \\
0 \\
$\mathbb{4}$ \\
\end{tabular} & 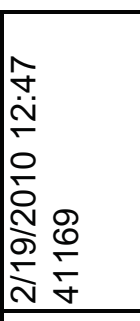 & 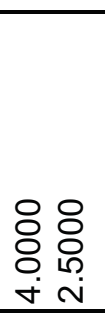 & & 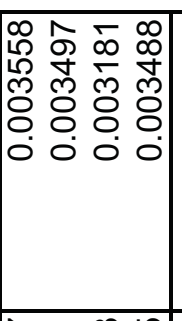 & 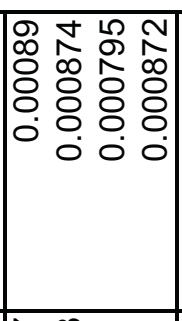 & & 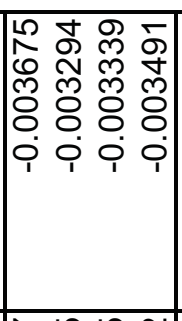 & 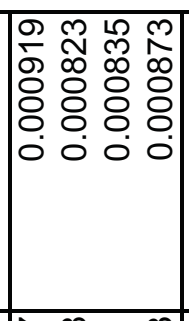 & & 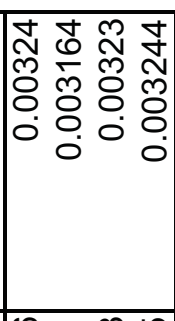 \\
\hline 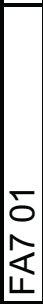 & 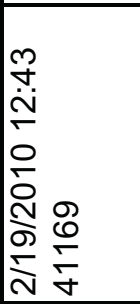 & 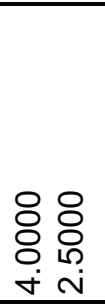 & & 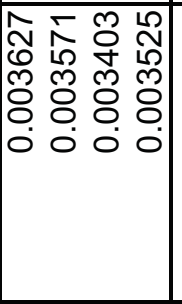 & 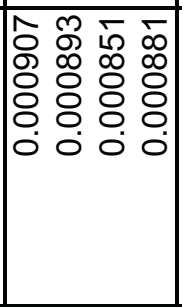 & & 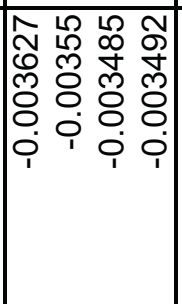 & 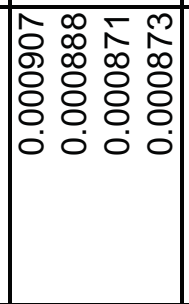 & & 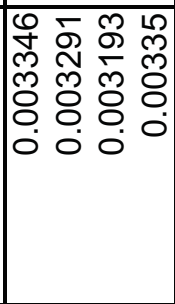 \\
\hline 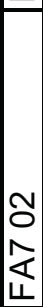 & 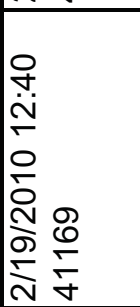 & $\begin{array}{l}8 \\
8\end{array}$ & & 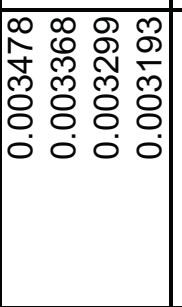 & 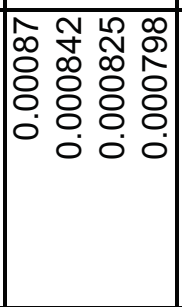 & & 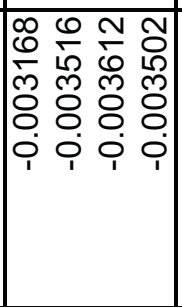 & 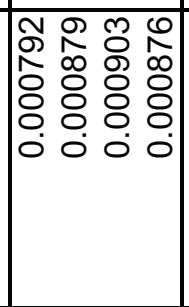 & & 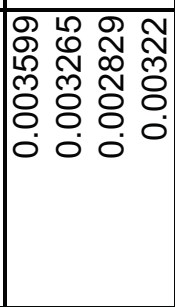 \\
\hline \begin{tabular}{|l} 
\\
5 \\
0 \\
0 \\
0 \\
\\
\end{tabular} & 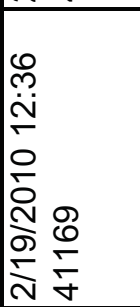 & 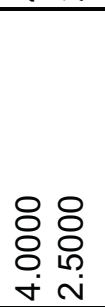 & & 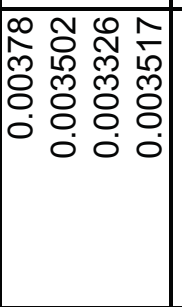 & 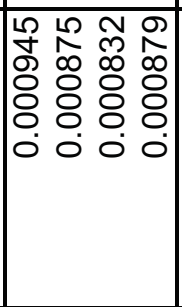 & & 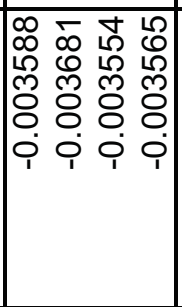 & 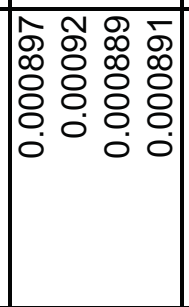 & & 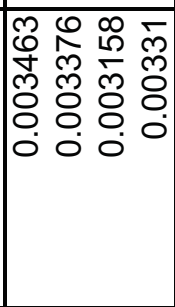 \\
\hline \begin{tabular}{|l} 
\\
\multirow{2}{*}{} \\
0 \\
10 \\
4 \\
\end{tabular} & 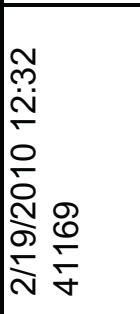 & 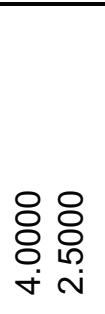 & & 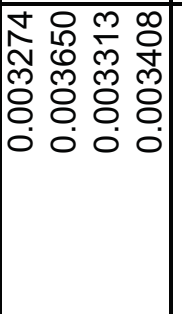 & 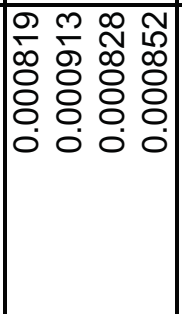 & & 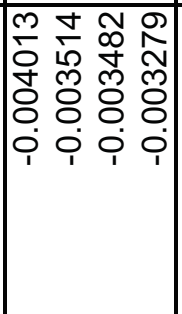 & 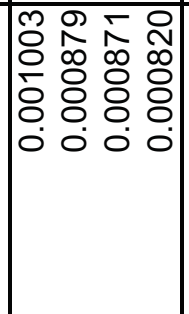 & & 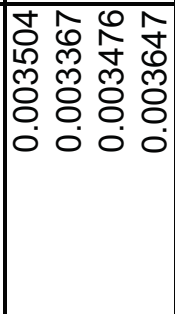 \\
\hline 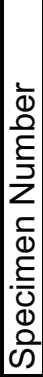 & 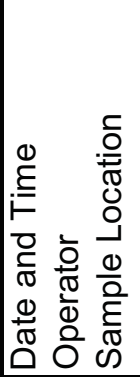 & 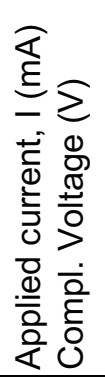 & 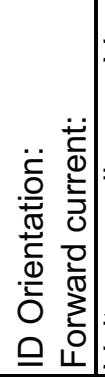 & 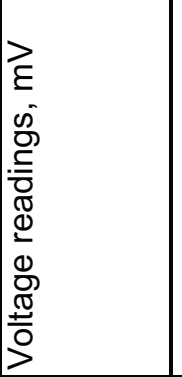 & 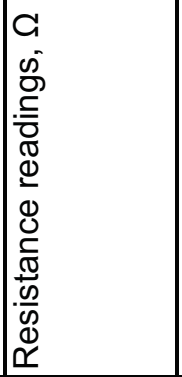 & 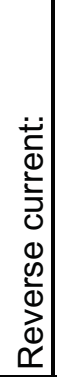 & 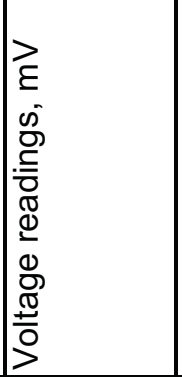 & 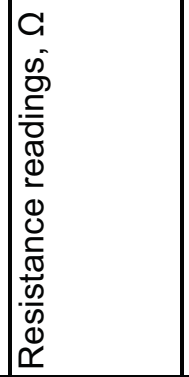 & 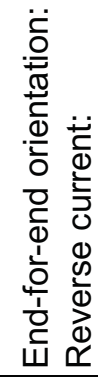 & 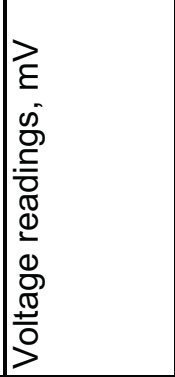 \\
\hline
\end{tabular}




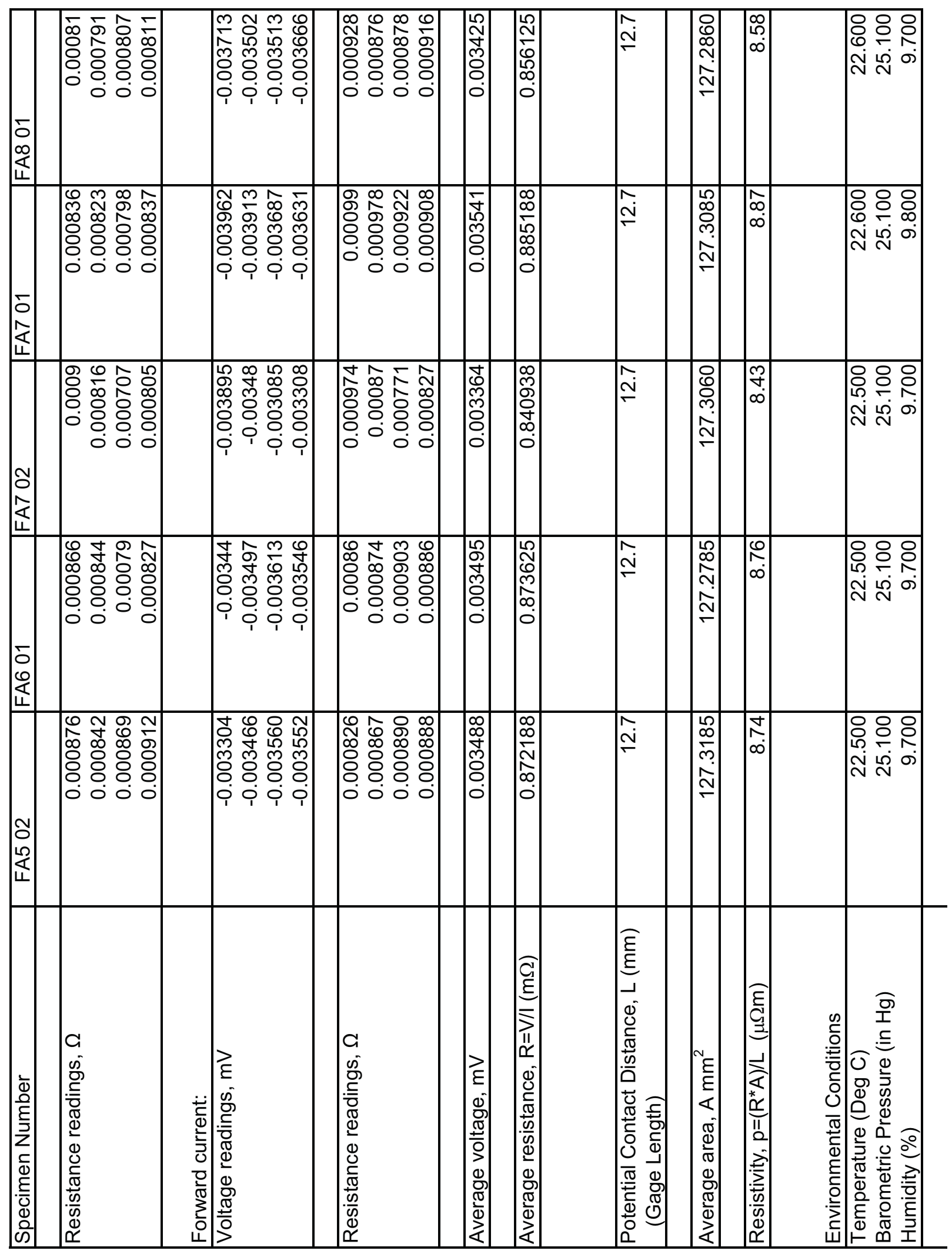




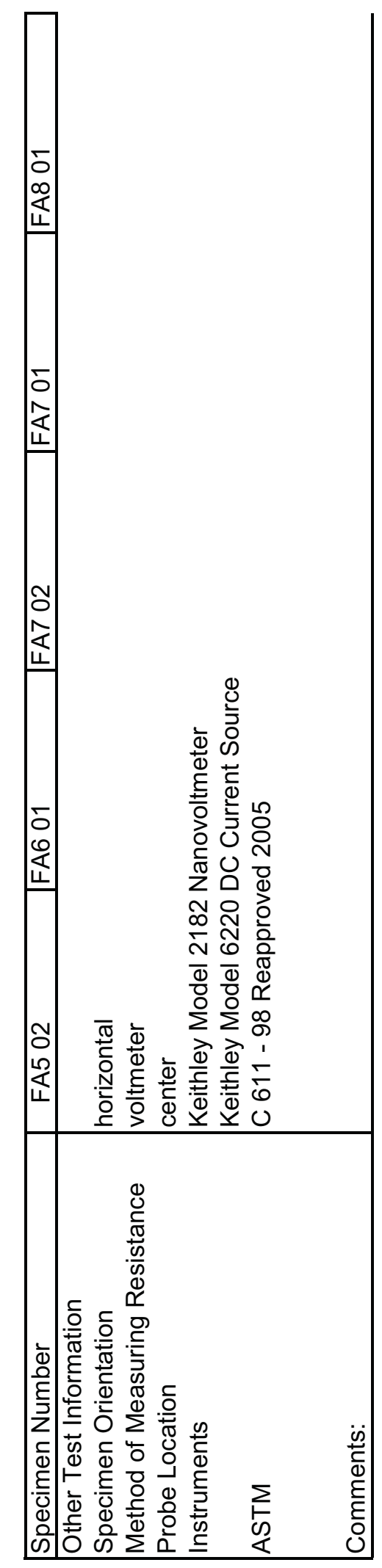




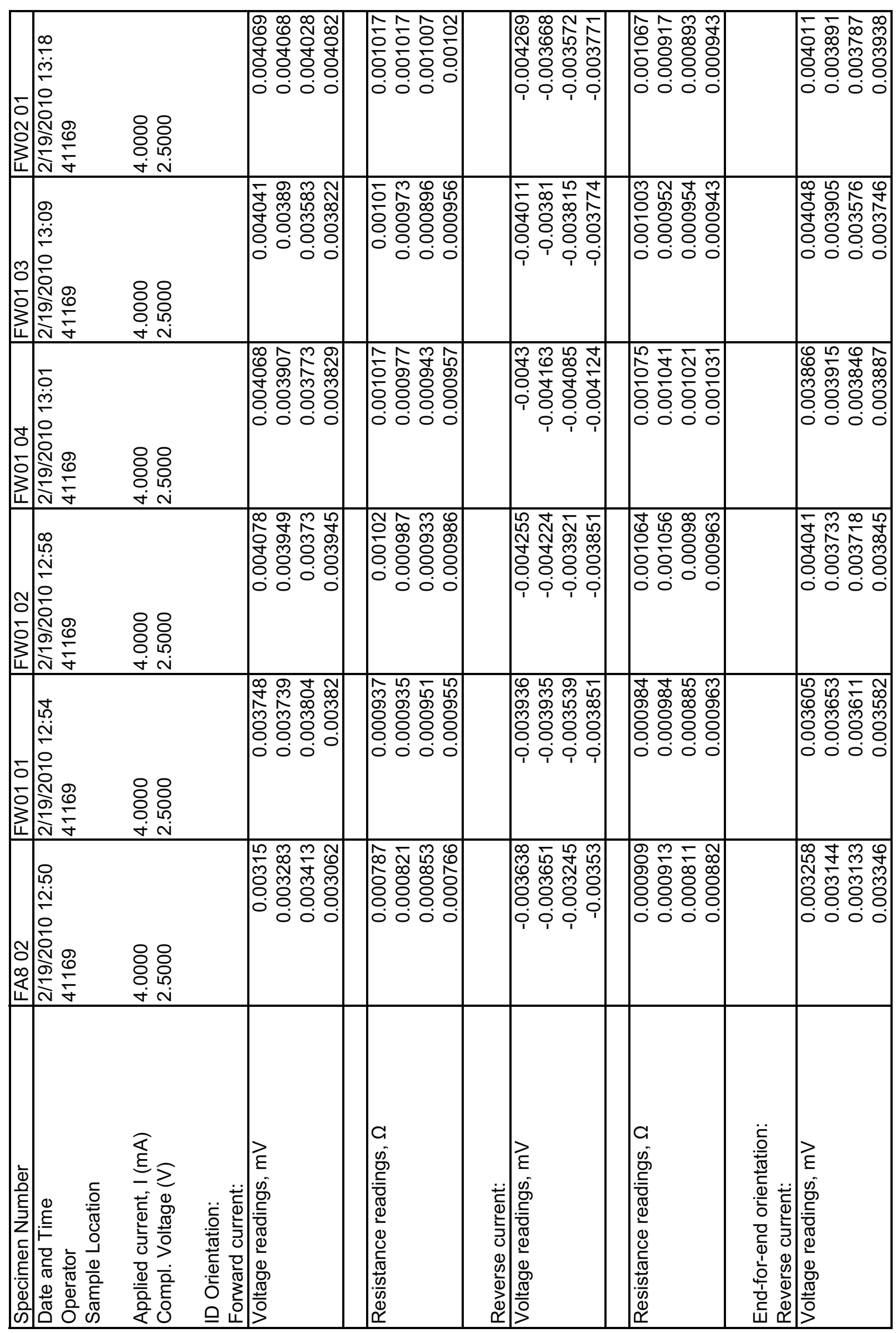




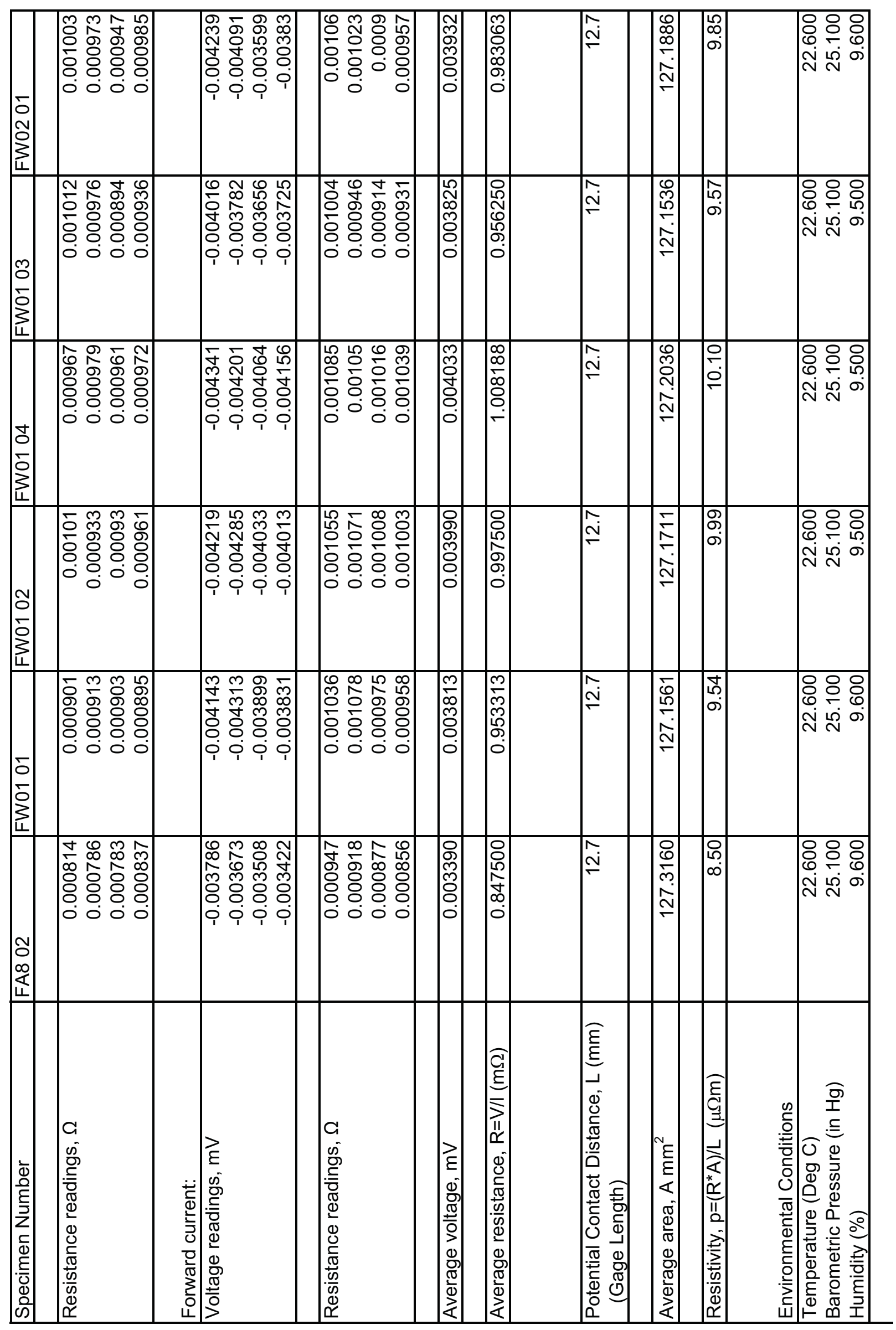




\begin{tabular}{|c|c|c|c|c|c|c|c|c|c|c|}
\hline \multicolumn{4}{|c|}{ 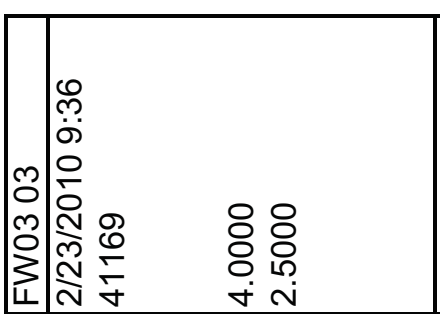 } & 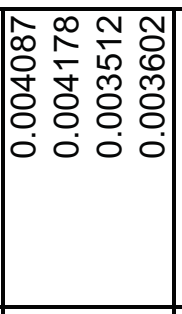 & 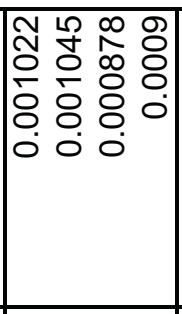 & & 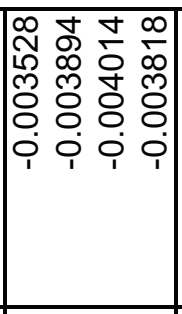 & 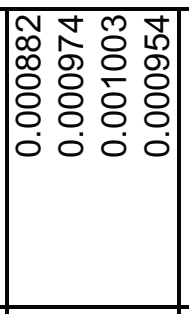 & & 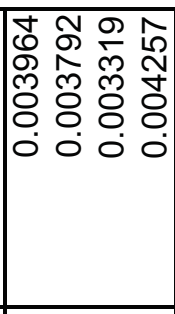 \\
\hline $\begin{array}{l} \\
0 \\
0 \\
m \\
0 \\
3 \\
\\
\end{array}$ & 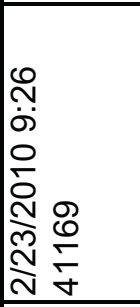 & $\begin{array}{l}8 \\
8 \\
8 \\
0 \\
\dot{\forall} \stackrel{\circ}{1} \\
\end{array}$ & & 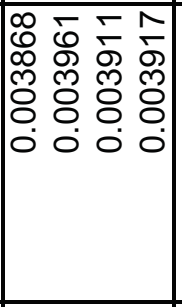 & 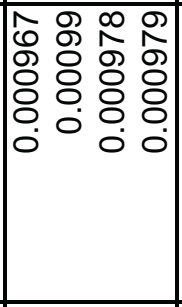 & & 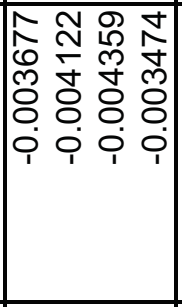 & 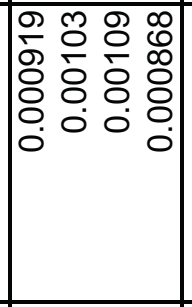 & & 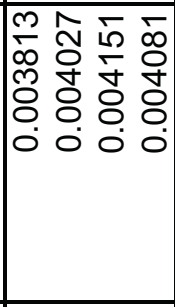 \\
\hline $\begin{array}{l}1 \\
\delta \\
0 \\
0 \\
0 \\
\\
\end{array}$ & 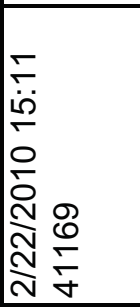 & 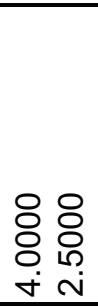 & & 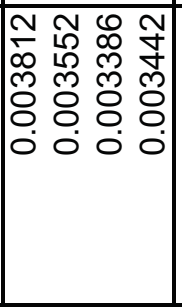 & 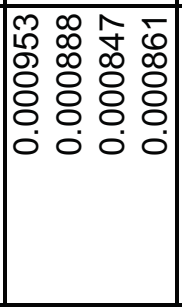 & & 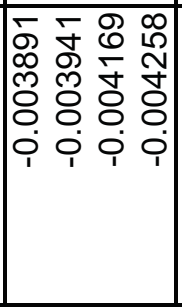 & 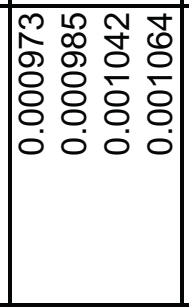 & & 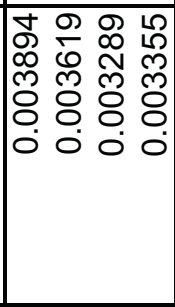 \\
\hline 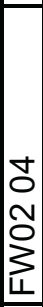 & 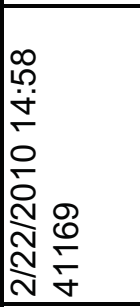 & 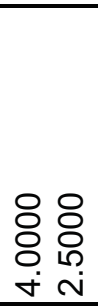 & & 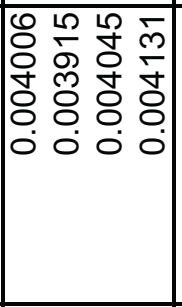 & 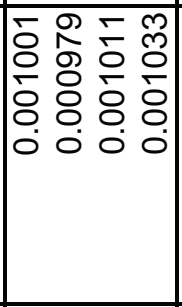 & & 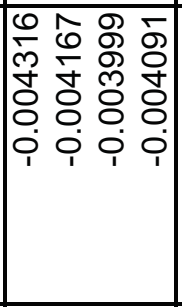 & 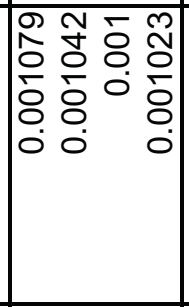 & & 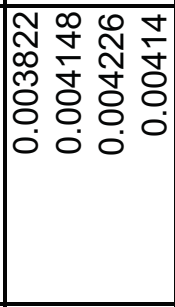 \\
\hline 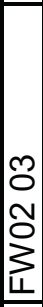 & 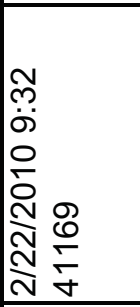 & 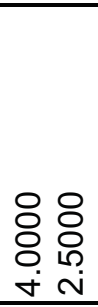 & & 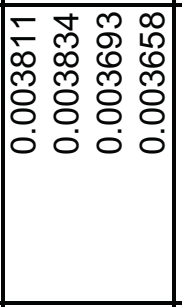 & 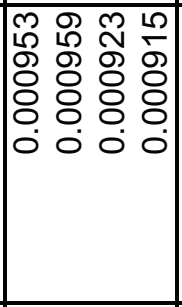 & & 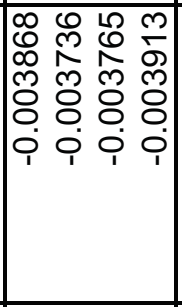 & 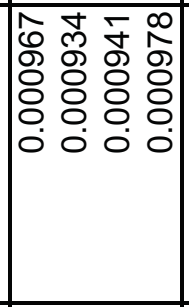 & & 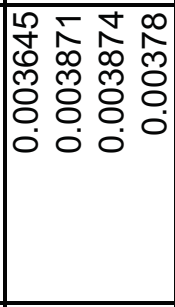 \\
\hline 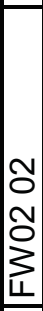 & 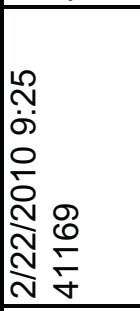 & \begin{tabular}{l}
8 \\
80 \\
80 \\
\hdashline \\
$\dot{\forall}$
\end{tabular} & & 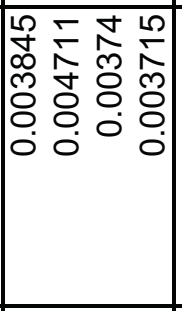 & 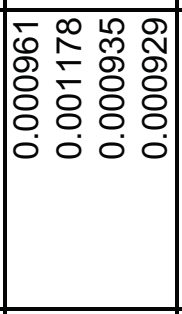 & & 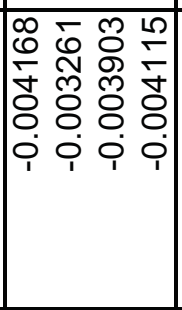 & 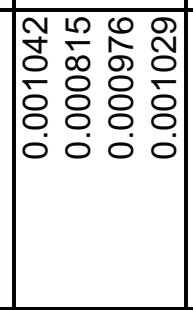 & & 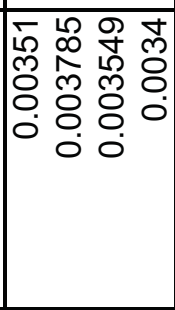 \\
\hline 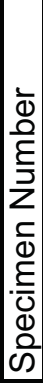 & 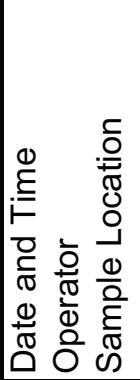 & 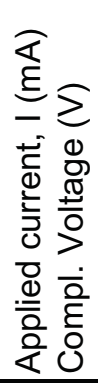 & 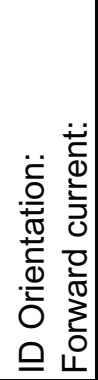 & 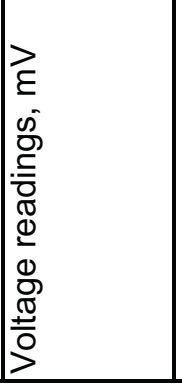 & 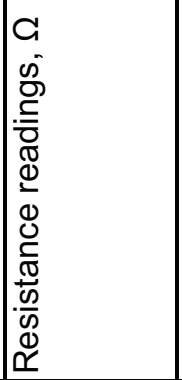 & 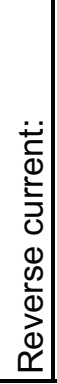 & 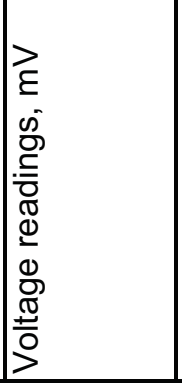 & 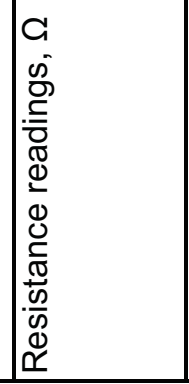 & 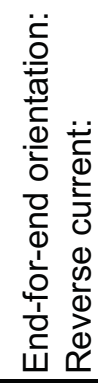 & 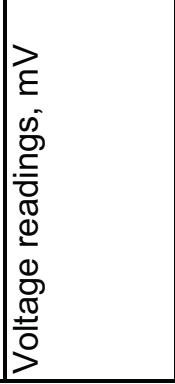 \\
\hline
\end{tabular}




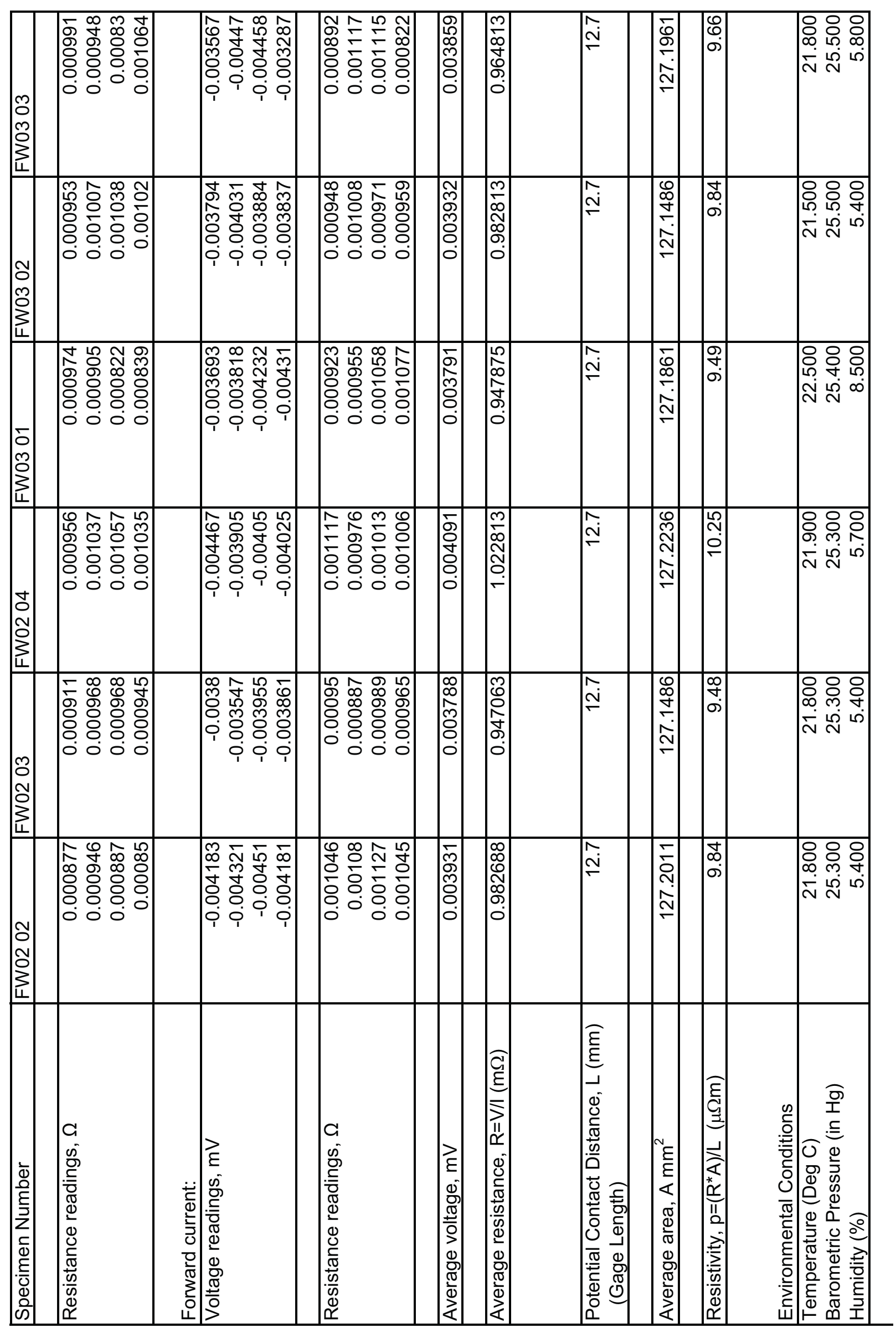




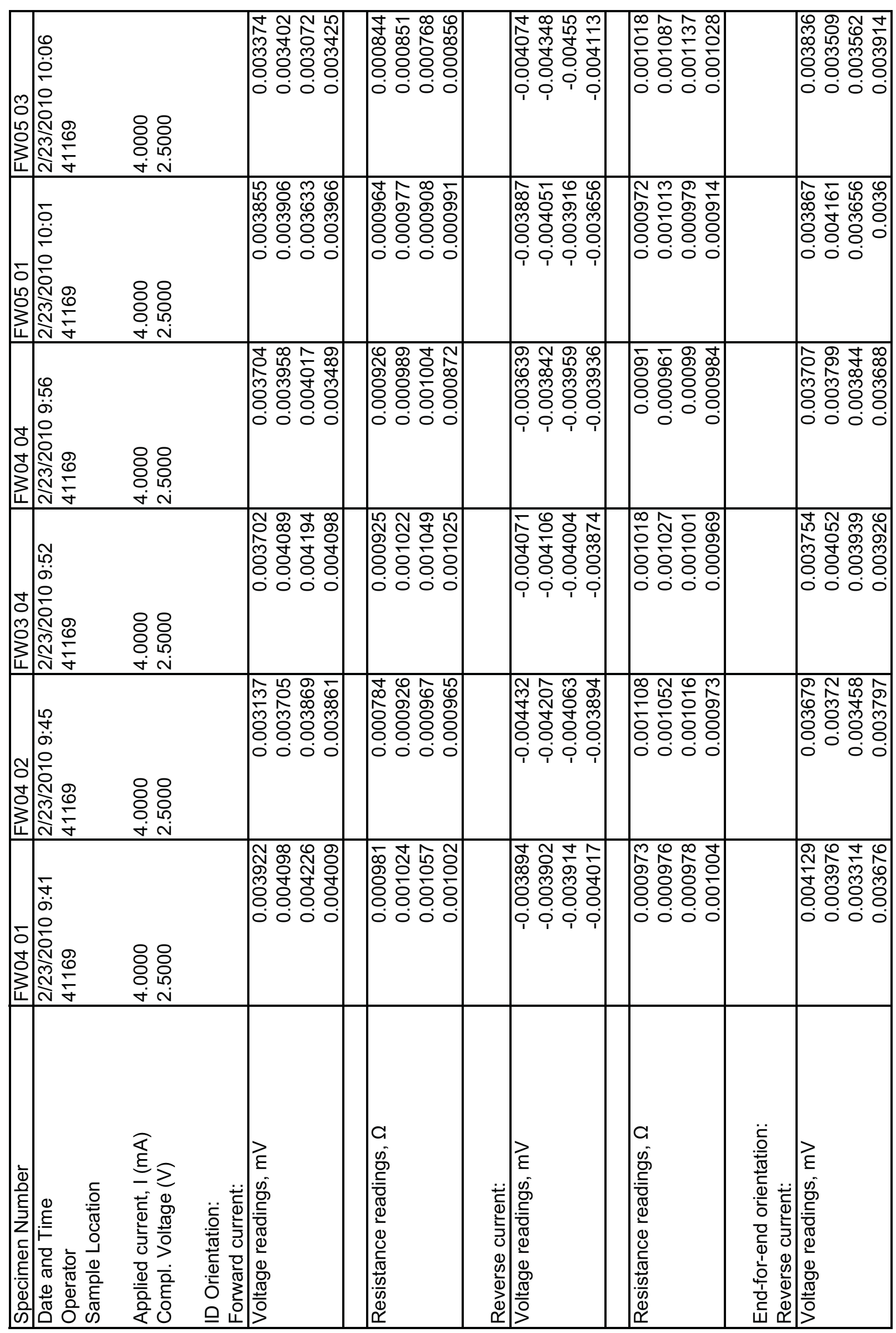




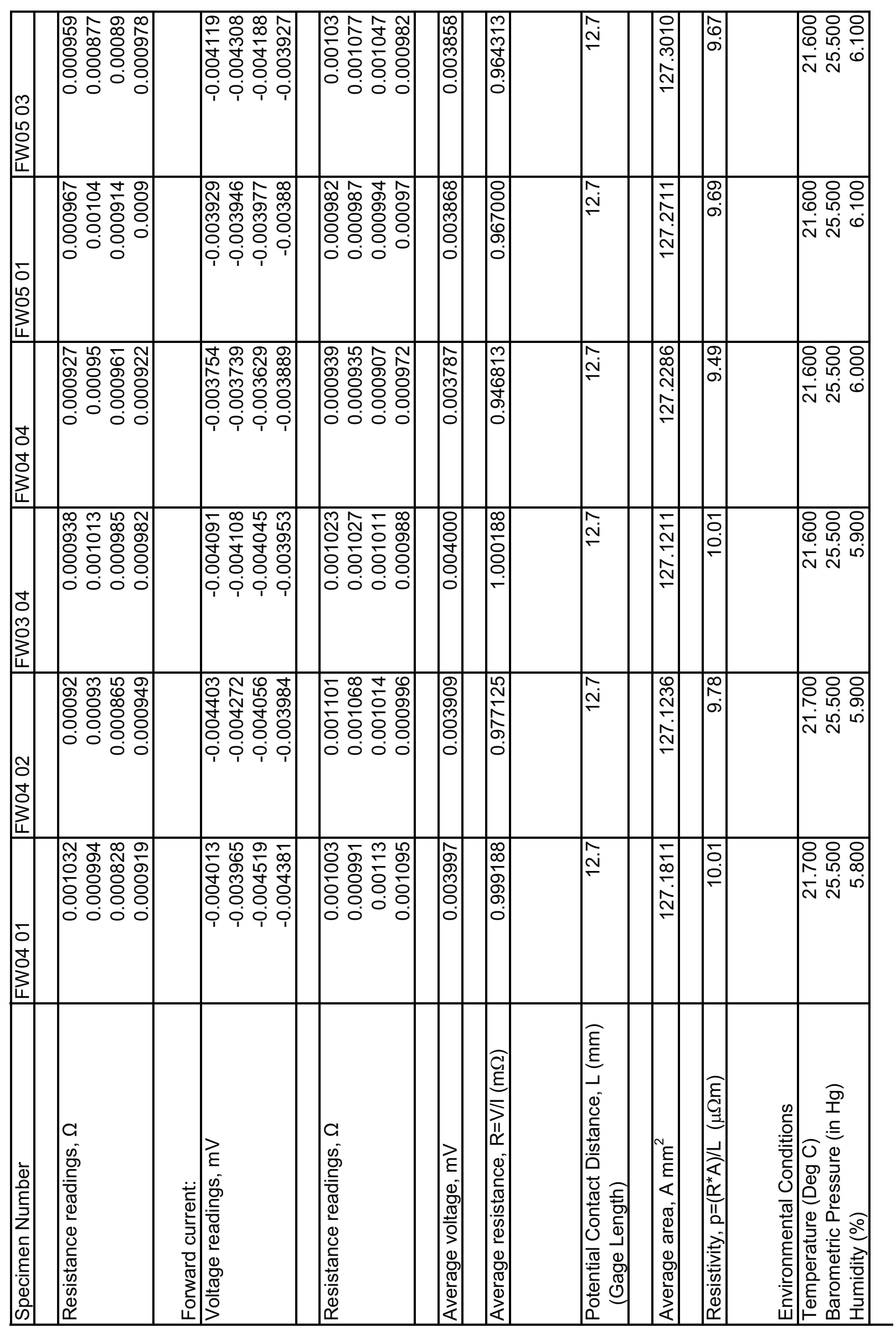




\begin{tabular}{|c|c|c|c|c|c|c|c|c|c|c|}
\hline \multicolumn{4}{|c|}{ 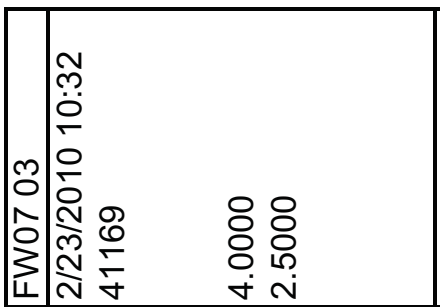 } & 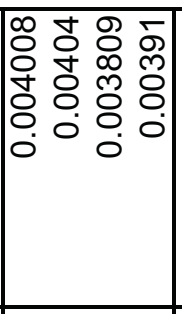 & 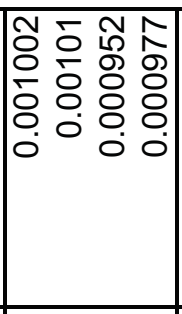 & & 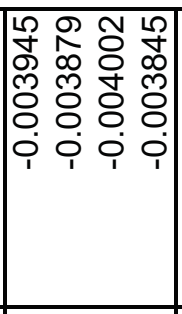 & 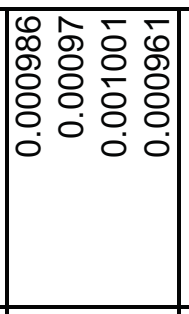 & & 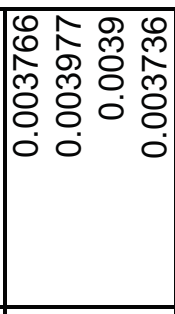 \\
\hline \begin{tabular}{|l} 
\\
5 \\
0 \\
0 \\
0 \\
3 \\
\end{tabular} & 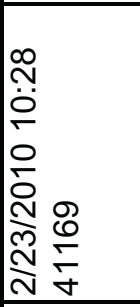 & $\begin{array}{l}8 \\
8 \\
8 \\
0 \\
\dot{\forall} \stackrel{\circ}{1} \\
\end{array}$ & & 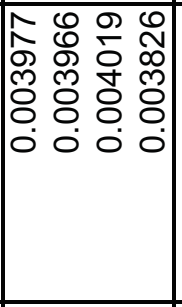 & 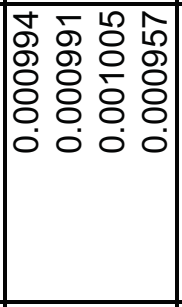 & & 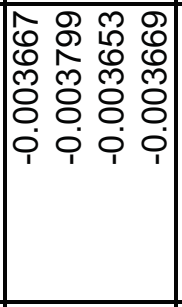 & 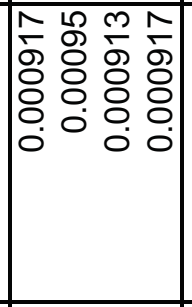 & & 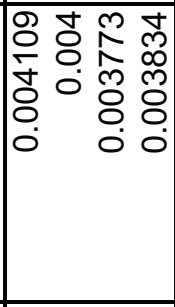 \\
\hline $\begin{array}{l} \\
\\
0 \\
0 \\
9 \\
3 \\
\end{array}$ & 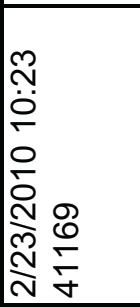 & 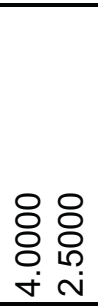 & & 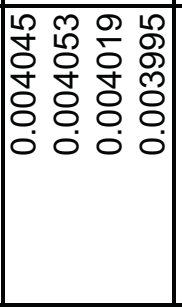 & 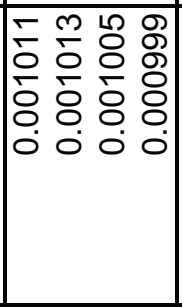 & & 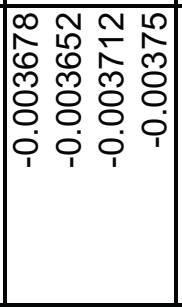 & 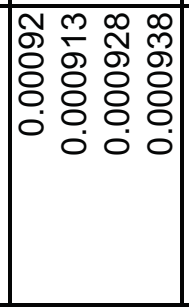 & & 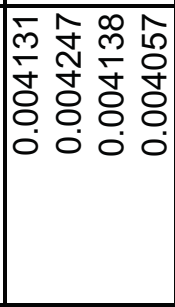 \\
\hline $\begin{array}{l}1 \\
0 \\
0 \\
0 \\
0 \\
3 \\
\\
\end{array}$ & 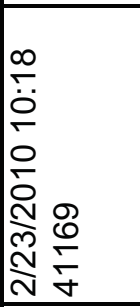 & 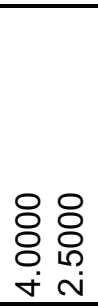 & & 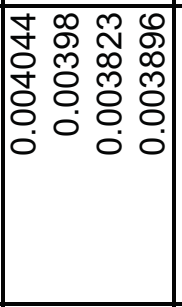 & 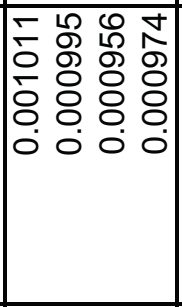 & & 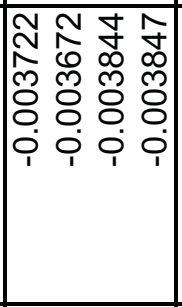 & 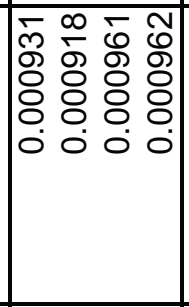 & & 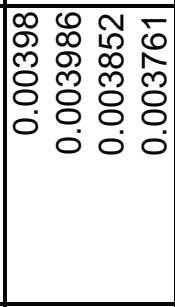 \\
\hline 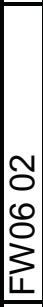 & 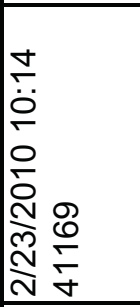 & 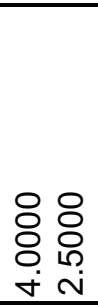 & & 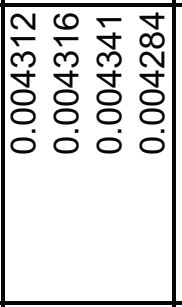 & 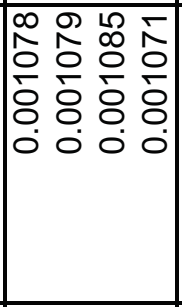 & & 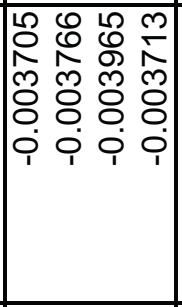 & 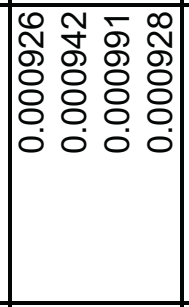 & & 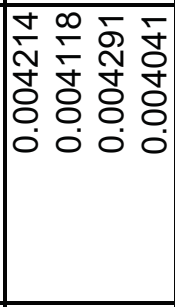 \\
\hline \begin{tabular}{|l} 
\\
\\
0 \\
0 \\
8 \\
8 \\
3 \\
\\
\end{tabular} & 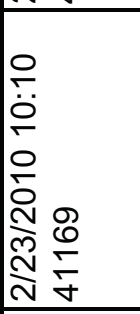 & \begin{tabular}{l}
8 \\
80 \\
80 \\
\hdashline \\
$\dot{\forall}$
\end{tabular} & & 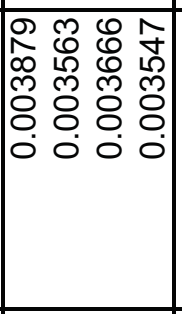 & 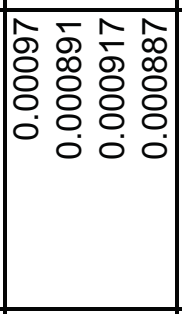 & & 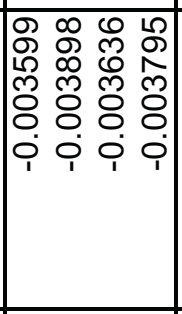 & 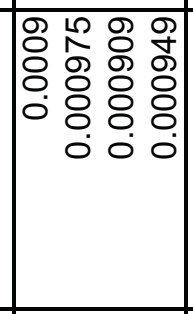 & & 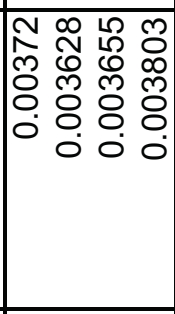 \\
\hline 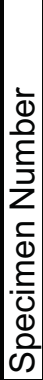 & 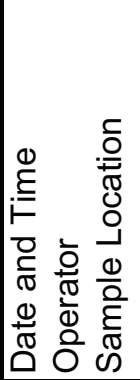 & 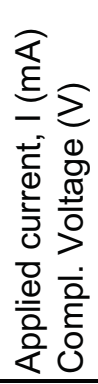 & 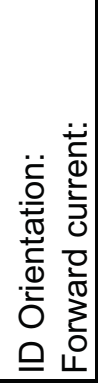 & 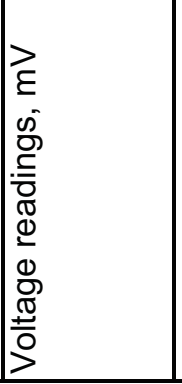 & 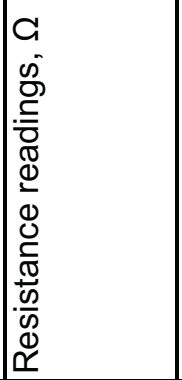 & 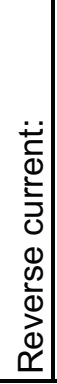 & 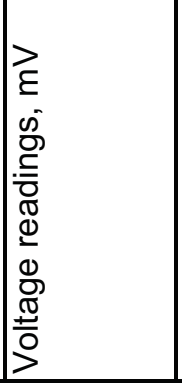 & 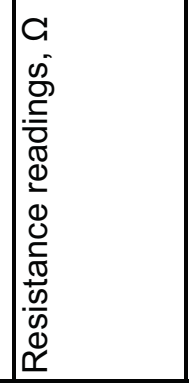 & 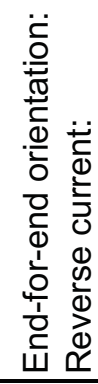 & 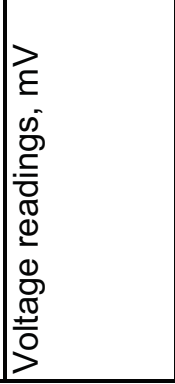 \\
\hline
\end{tabular}




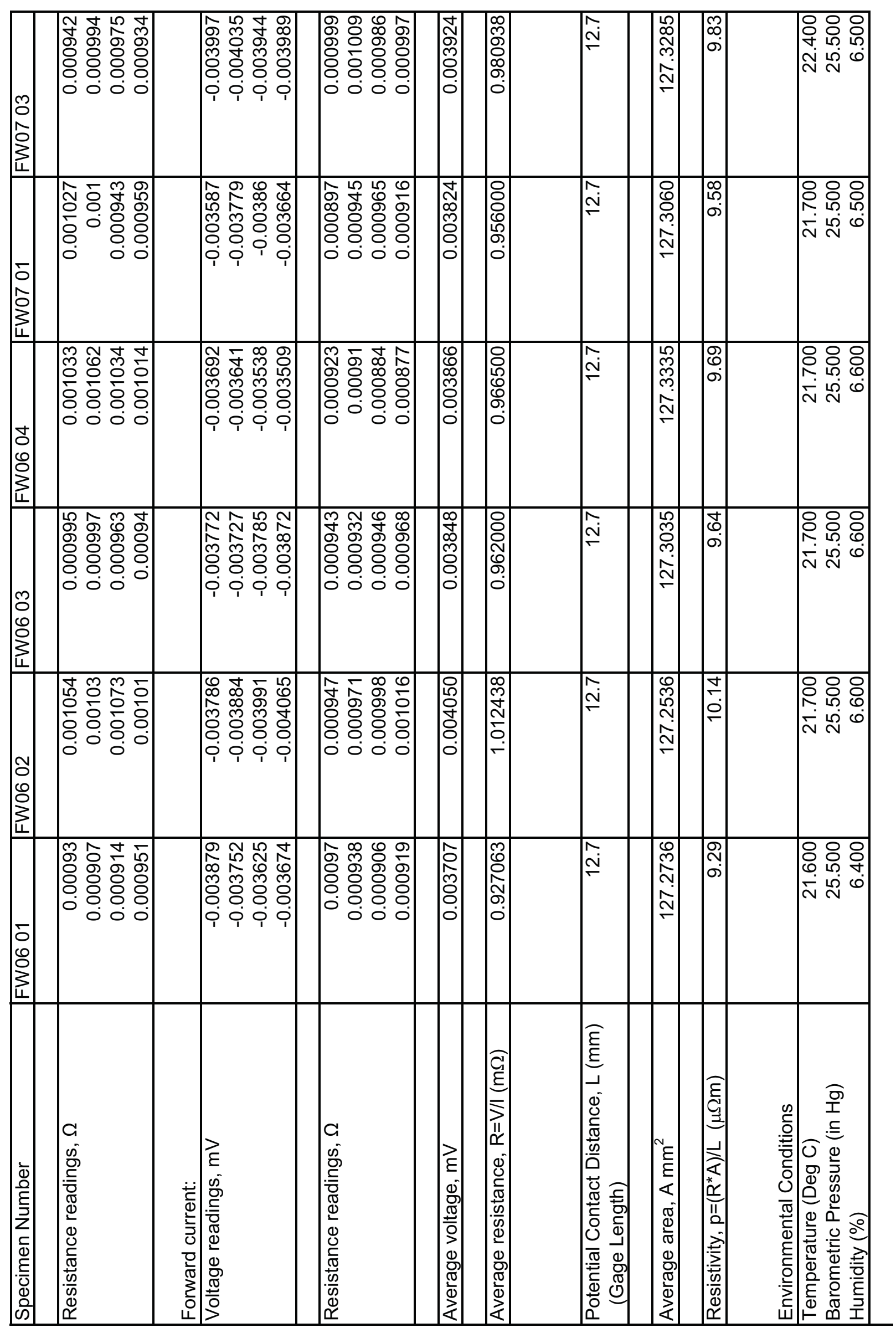




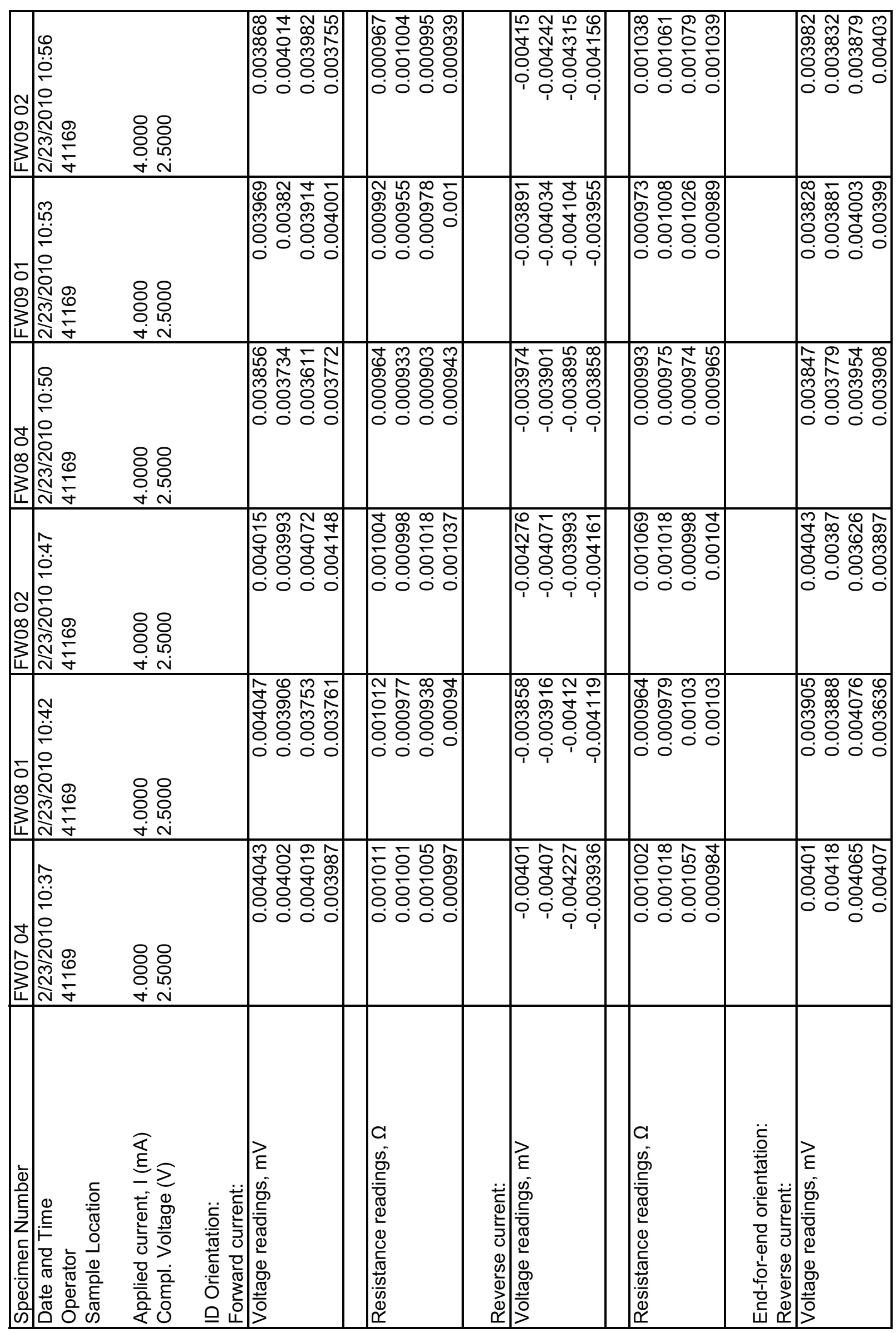




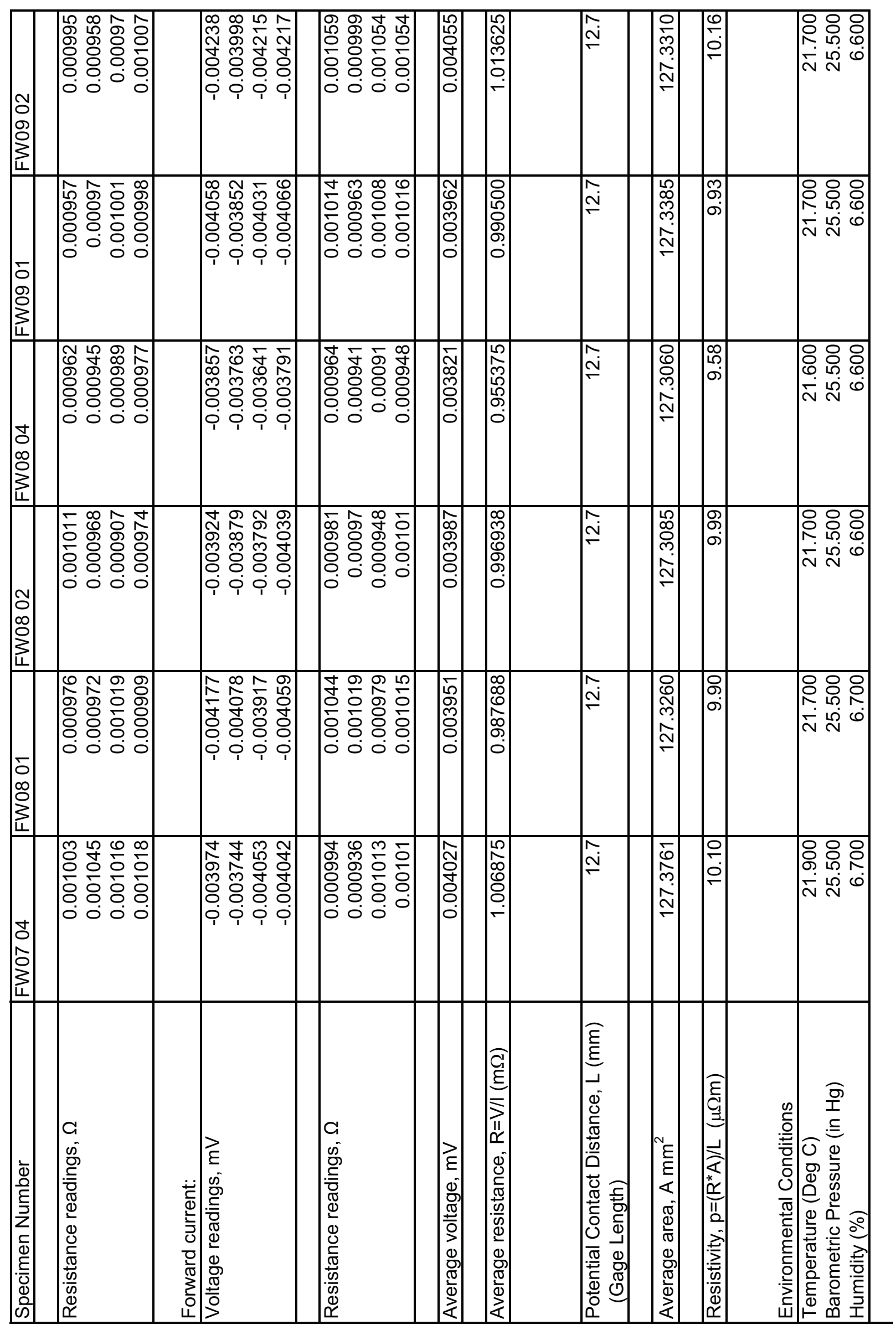




\begin{tabular}{|c|c|c|c|c|c|c|c|c|c|}
\hline $\begin{array}{l}\infty \\
0 \\
\infty \\
0 \\
\vdots \\
\\
\end{array}$ & 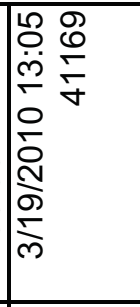 & 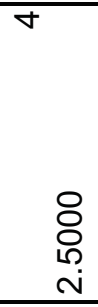 & & 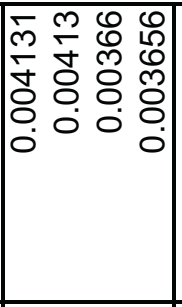 & 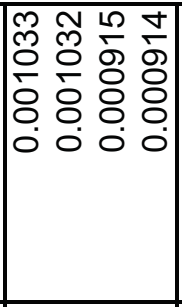 & 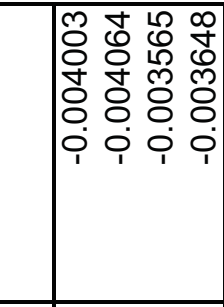 & 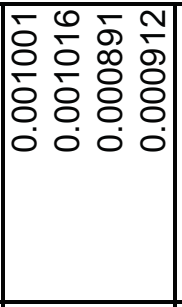 & & 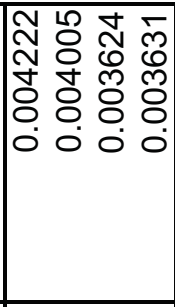 \\
\hline $\begin{array}{l} \\
0 \\
0 \\
0 \\
3 \\
\end{array}$ & 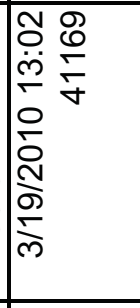 & $\begin{array}{l}8 \\
\circ \\
10 \\
\text { ํ. }\end{array}$ & & 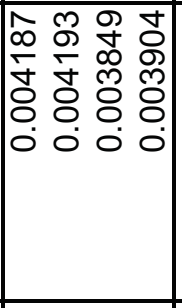 & 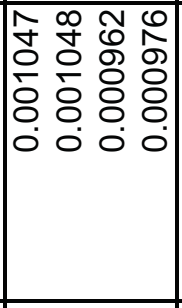 & 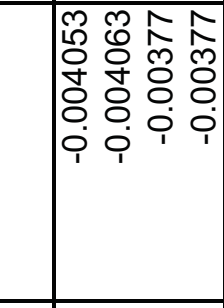 & 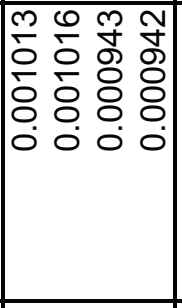 & & 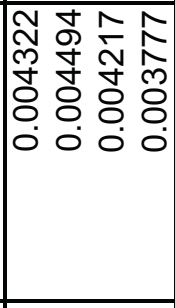 \\
\hline 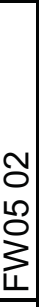 & 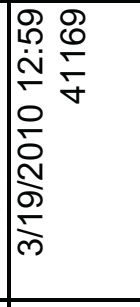 & $\begin{array}{l}8 \\
8 \\
i \\
i \\
\end{array}$ & & 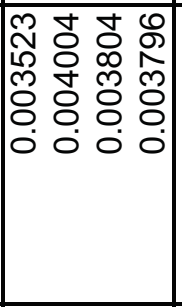 & 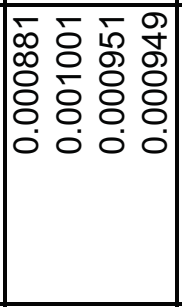 & 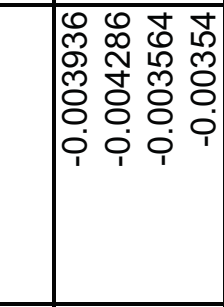 & 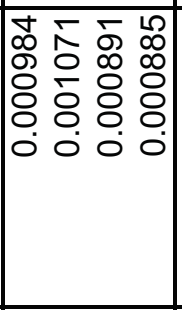 & & 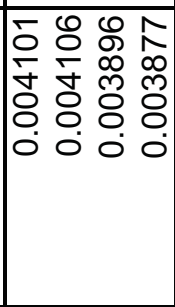 \\
\hline $\begin{array}{l}5 \\
0 \\
\frac{0}{2} \\
\\
\end{array}$ & 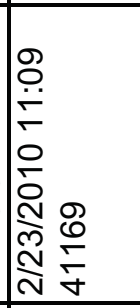 & $\begin{array}{l}8 \\
80 \\
80 \\
\circ \\
\dot{\forall}\end{array}$ & & 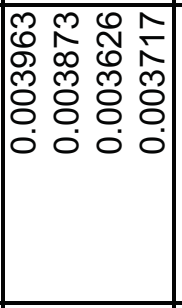 & 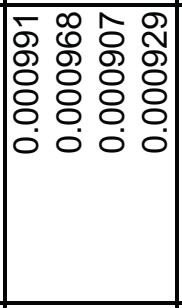 & 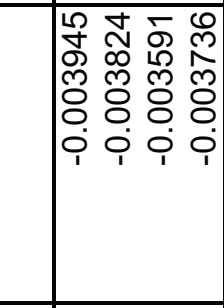 & 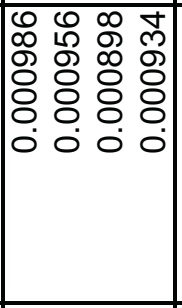 & & 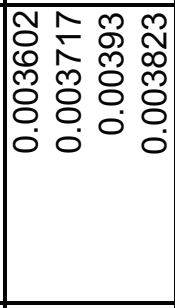 \\
\hline $\begin{array}{l} \\
\text { \& } \\
0 \\
0 \\
3 \\
\end{array}$ & 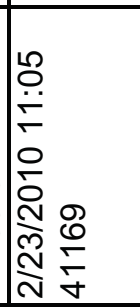 & $\begin{array}{l}8 \\
80 \\
8 \\
\\
\dot{1}\end{array}$ & & 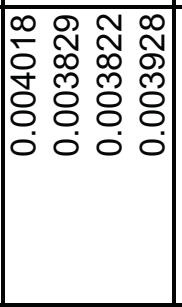 & 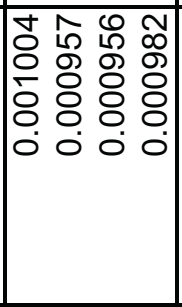 & 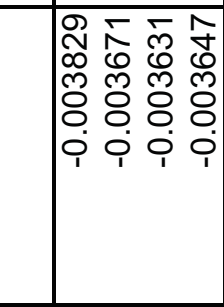 & 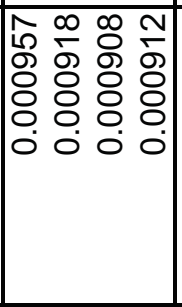 & & 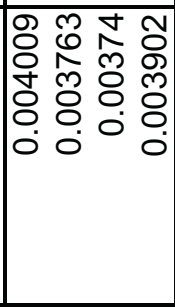 \\
\hline $\begin{array}{l}0 \\
0 \\
9 \\
0 \\
3 \\
\end{array}$ & 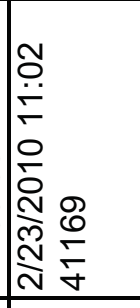 & 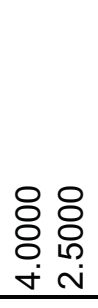 & & 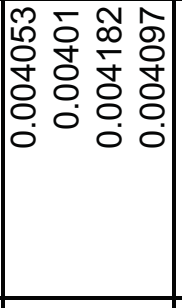 & 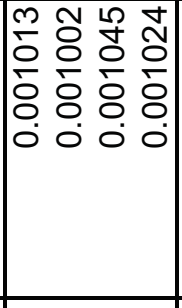 & 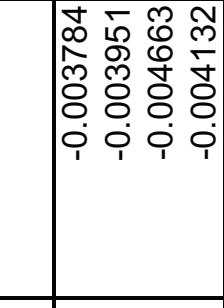 & 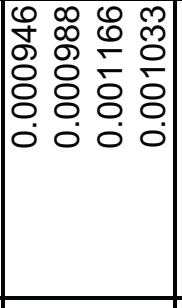 & & 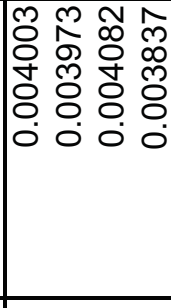 \\
\hline 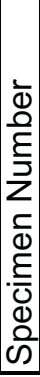 & 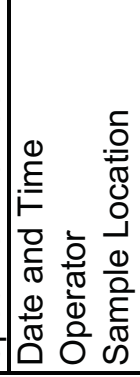 & 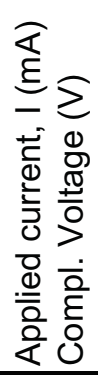 & 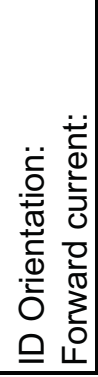 & 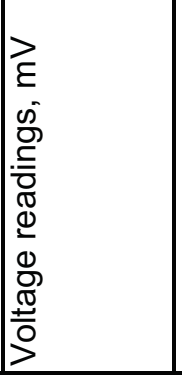 & 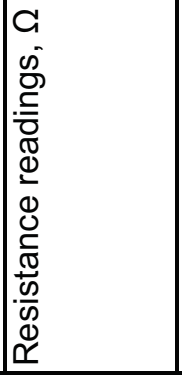 & 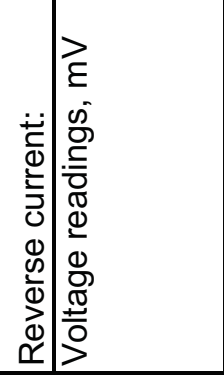 & 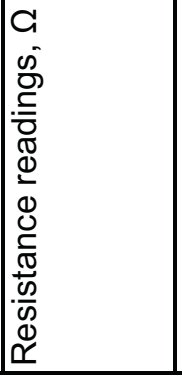 & 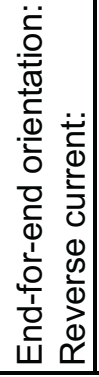 & 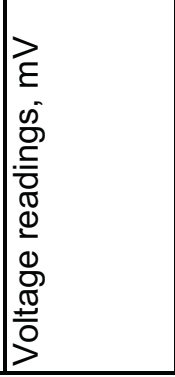 \\
\hline
\end{tabular}




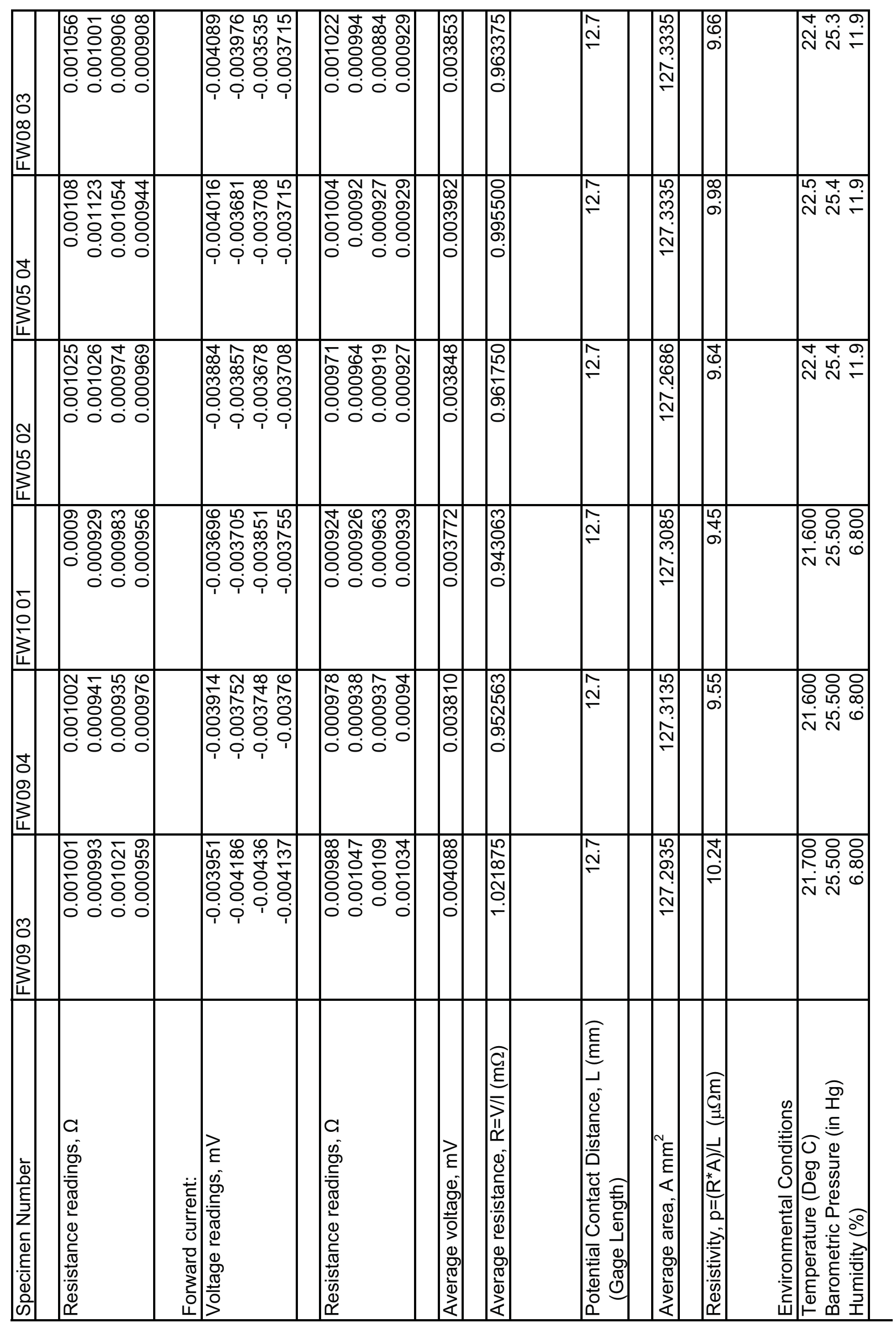




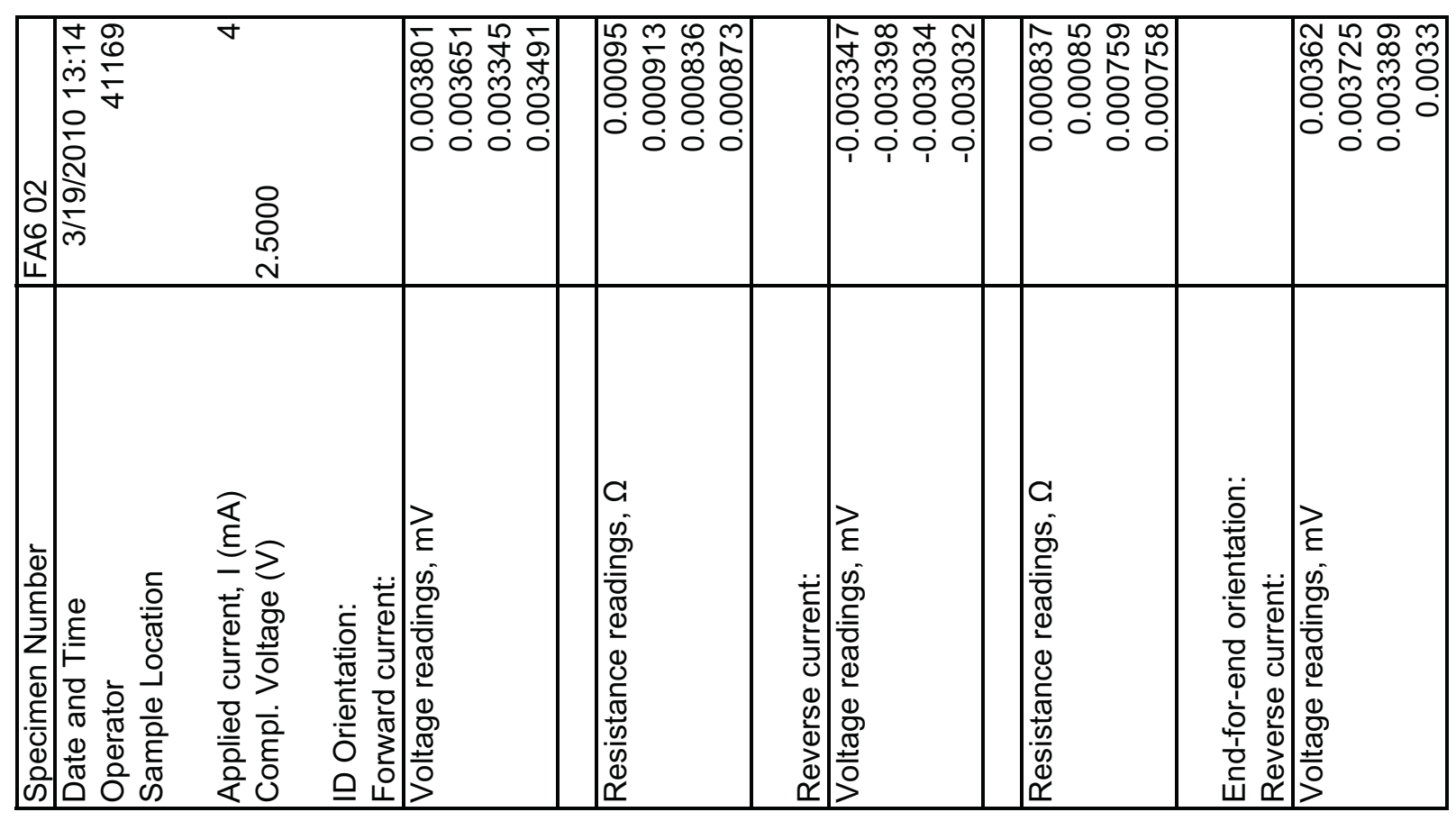




\begin{tabular}{|c|c|c|c|c|c|c|c|c|c|}
\hline$\left|\begin{array}{l}\mathfrak{v} \\
0 \\
0 \\
\mathbb{1}\end{array}\right|$ & 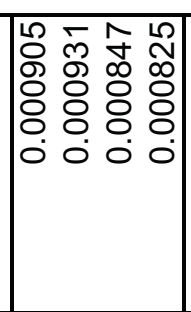 & 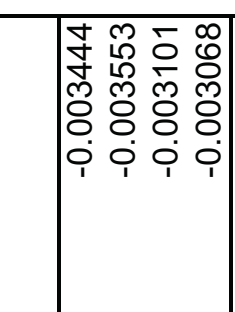 & 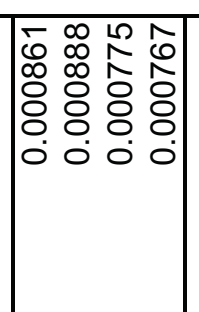 & 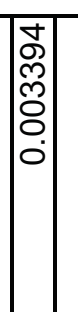 & $\left|\begin{array}{c}\infty \\
m \\
\infty \\
\infty \\
\infty \\
\infty \\
0\end{array}\right|$ & $\stackrel{\widehat{N}}{\mathfrak{N}}$ & 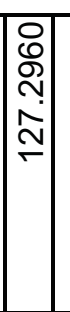 & $\left|\begin{array}{l}0 \\
\infty \\
\infty \\
\infty\end{array}\right|$ & N̦ \\
\hline 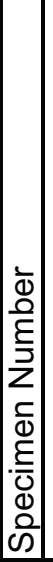 & 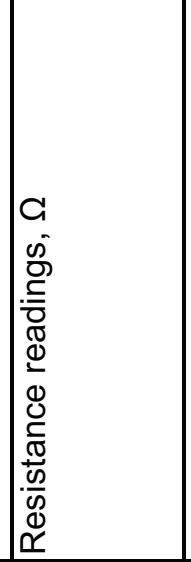 & 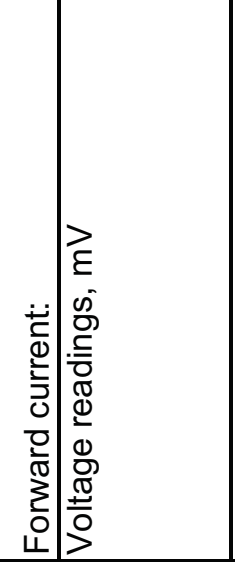 & 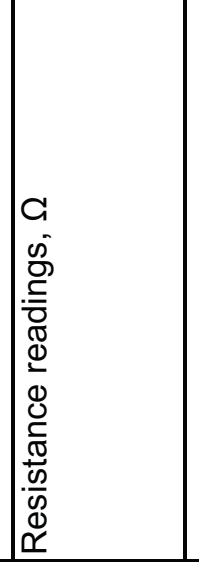 & 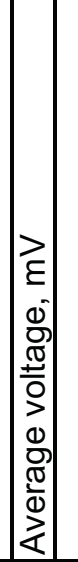 & 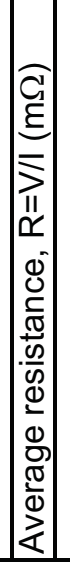 & 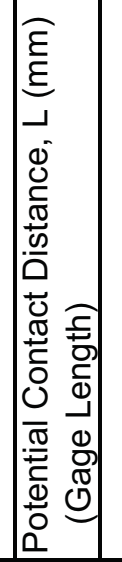 & 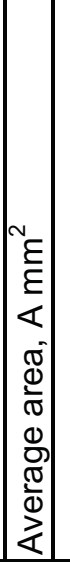 & 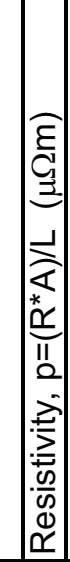 & 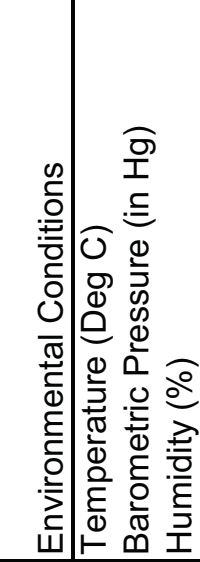 \\
\hline
\end{tabular}




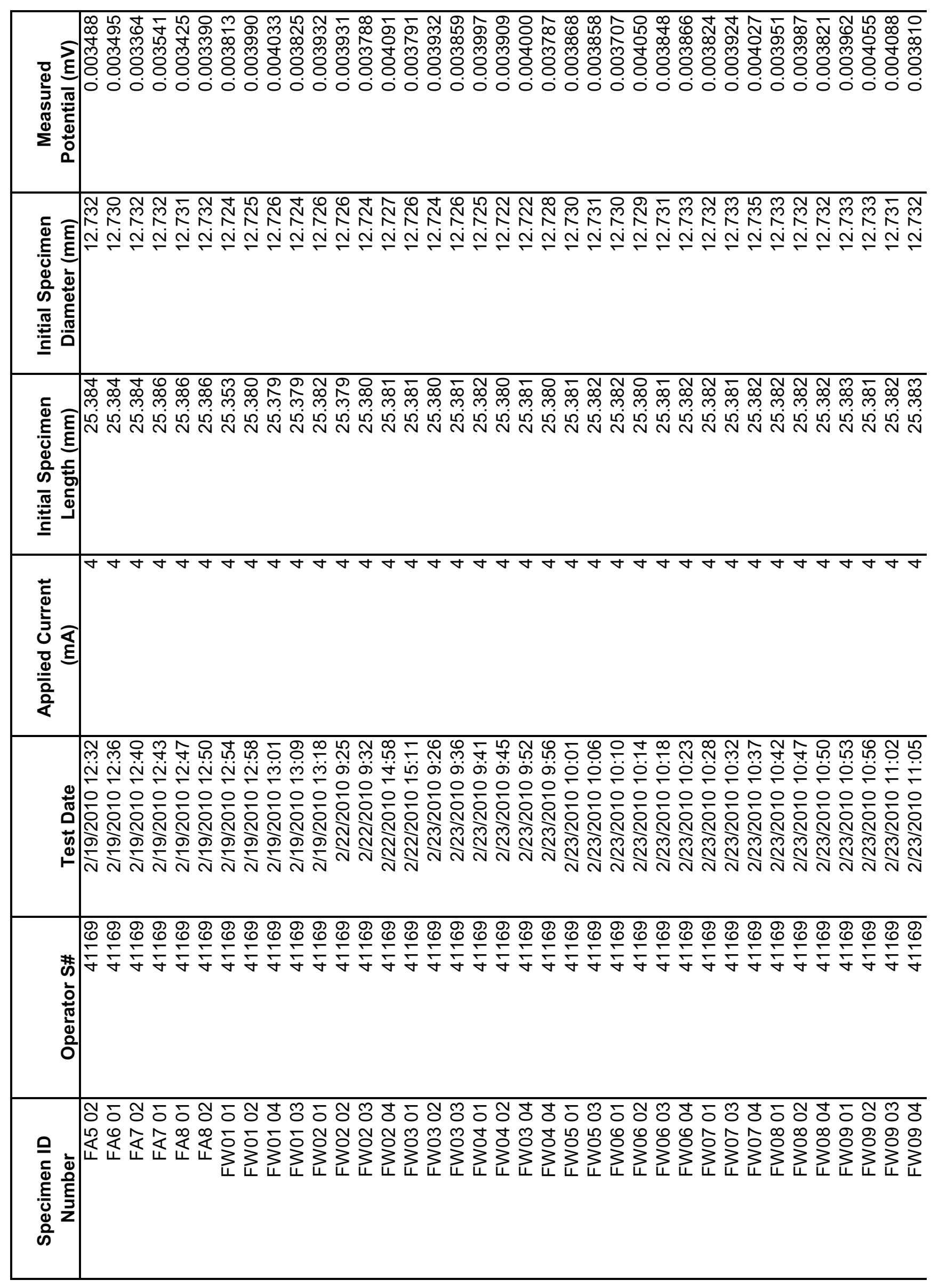




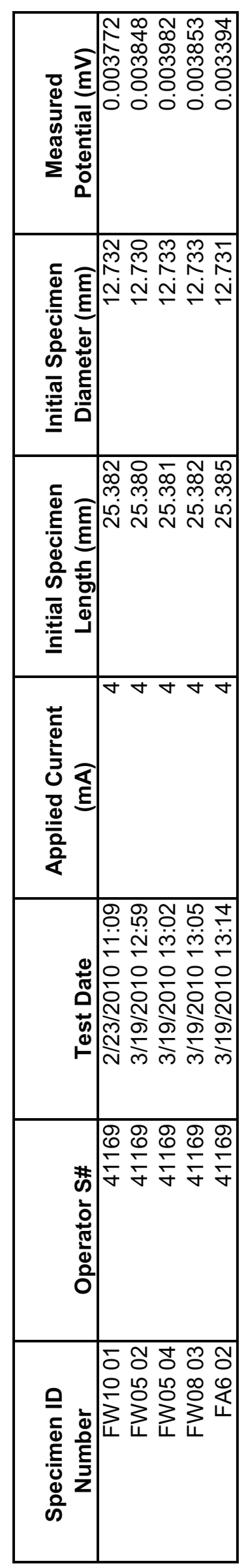




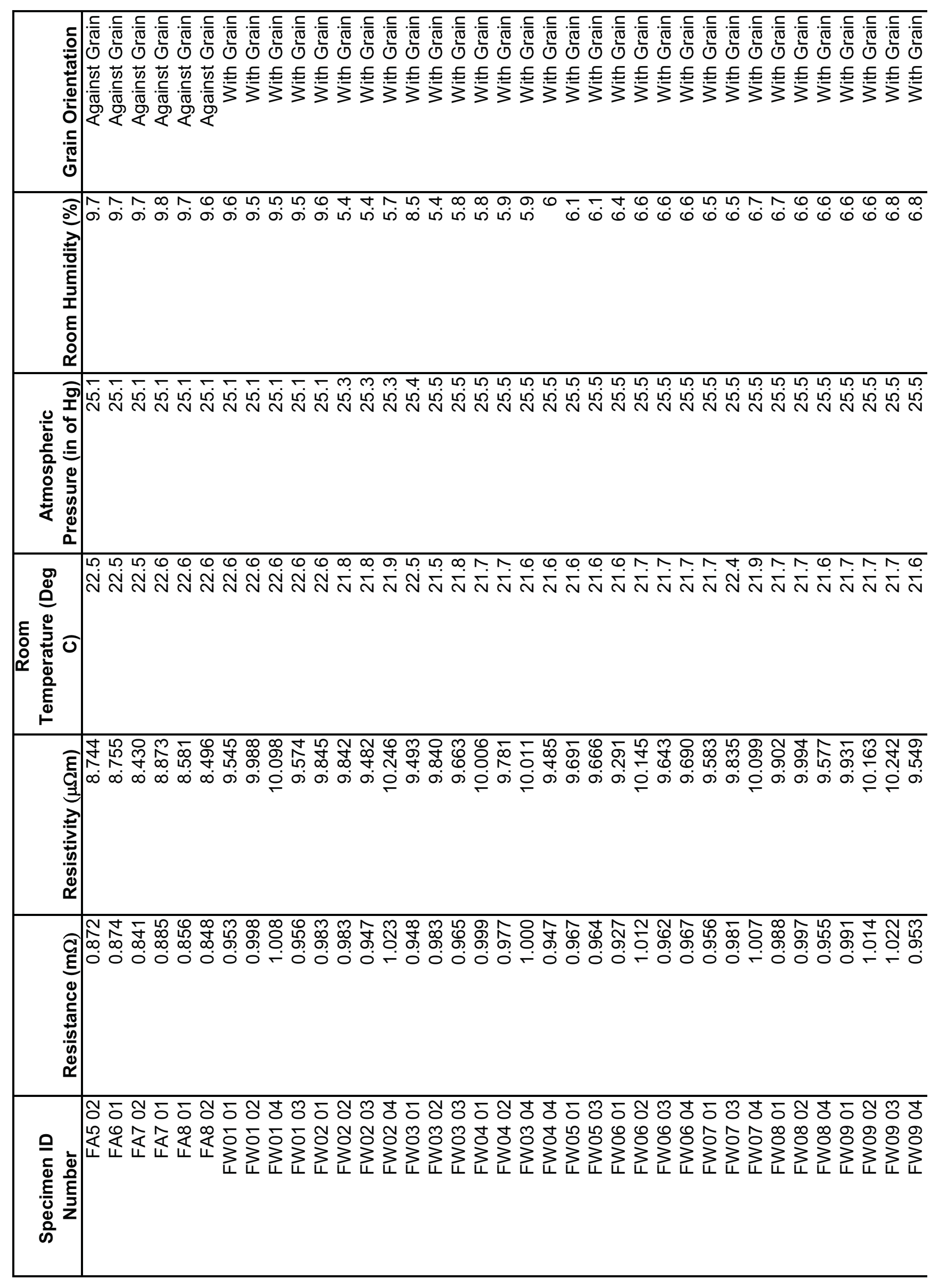




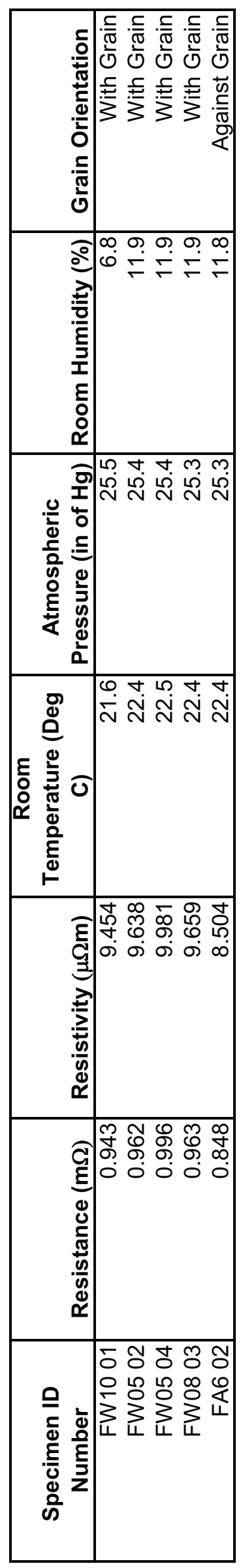




\begin{tabular}{|c|c|}
\hline 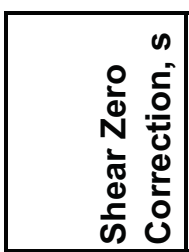 & 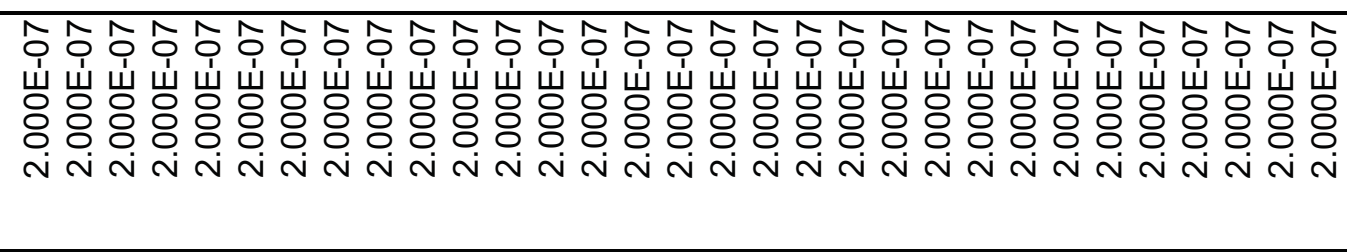 \\
\hline 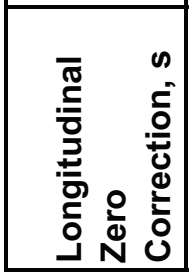 & 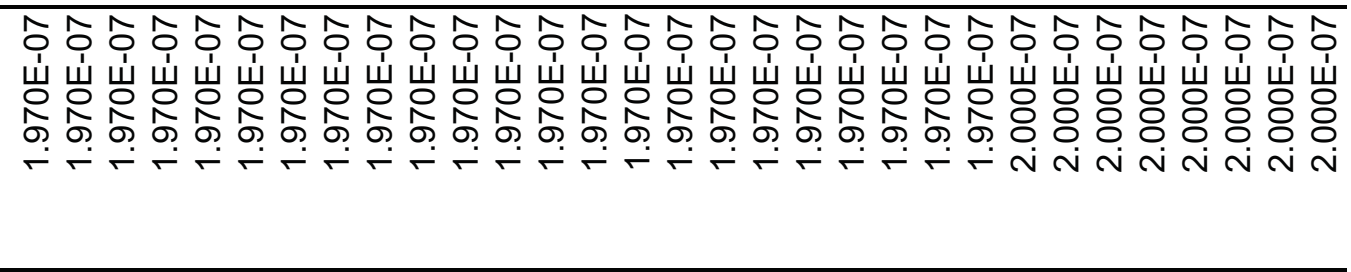 \\
\hline 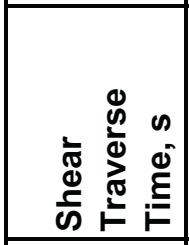 & 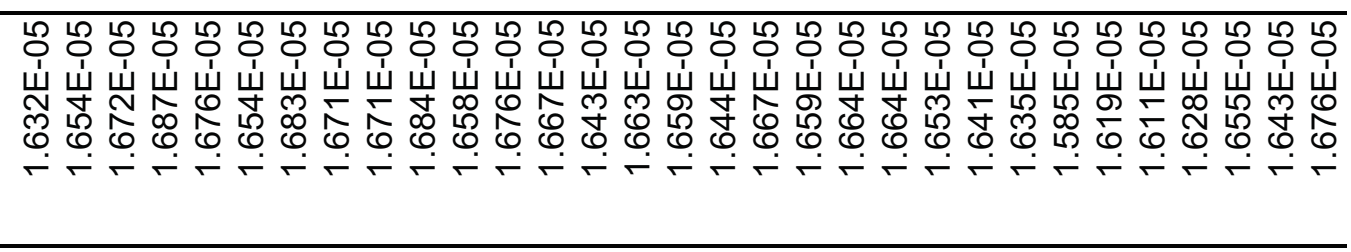 \\
\hline 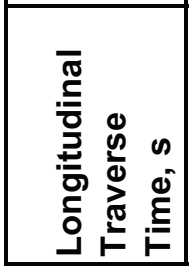 & 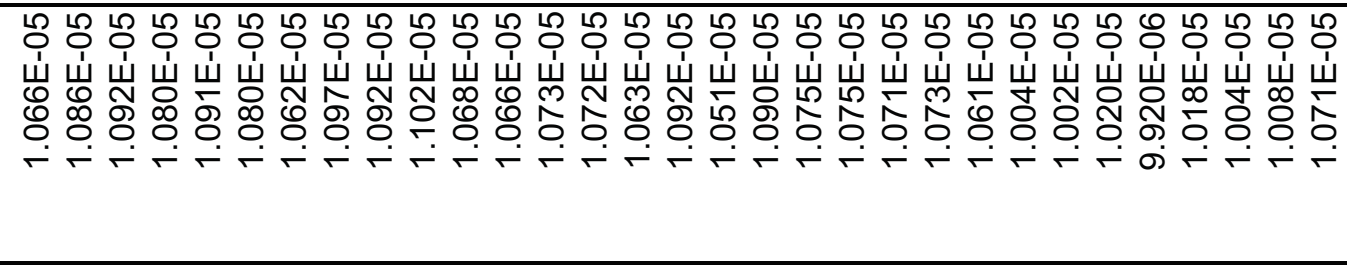 \\
\hline 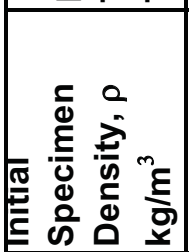 & 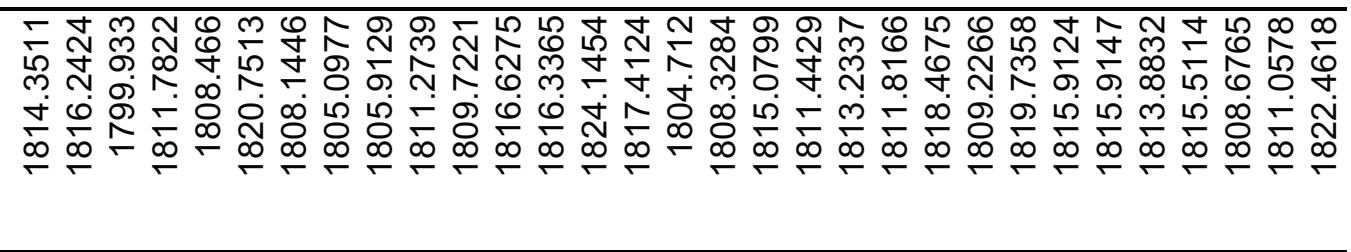 \\
\hline 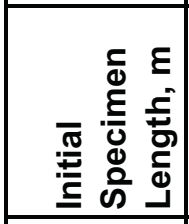 & 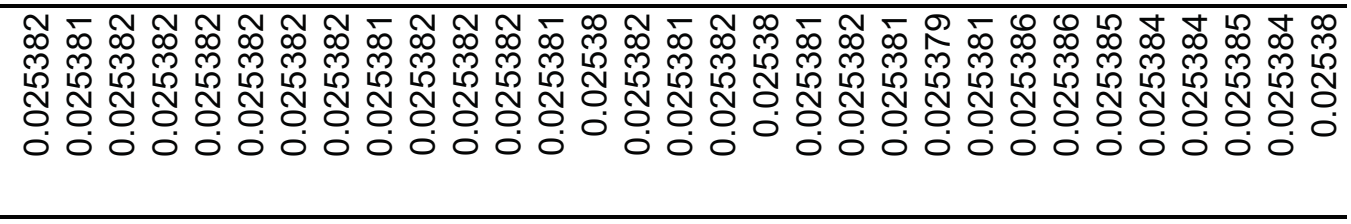 \\
\hline 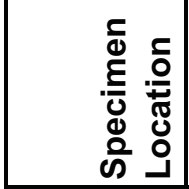 & \\
\hline $\begin{array}{l}\frac{0}{0} \\
\frac{\pi}{0} \\
\frac{2}{0} \\
\frac{0}{0} \\
\end{array}$ & 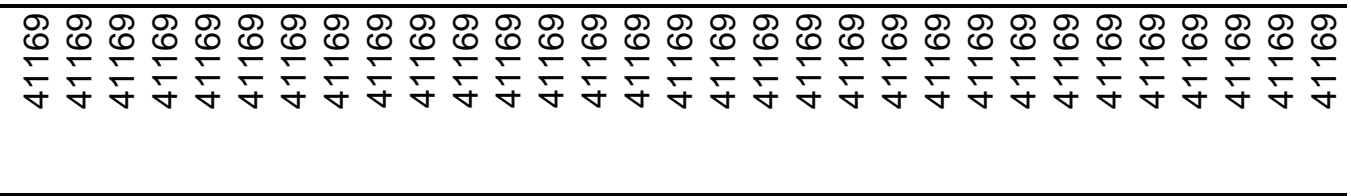 \\
\hline 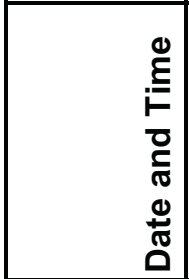 & 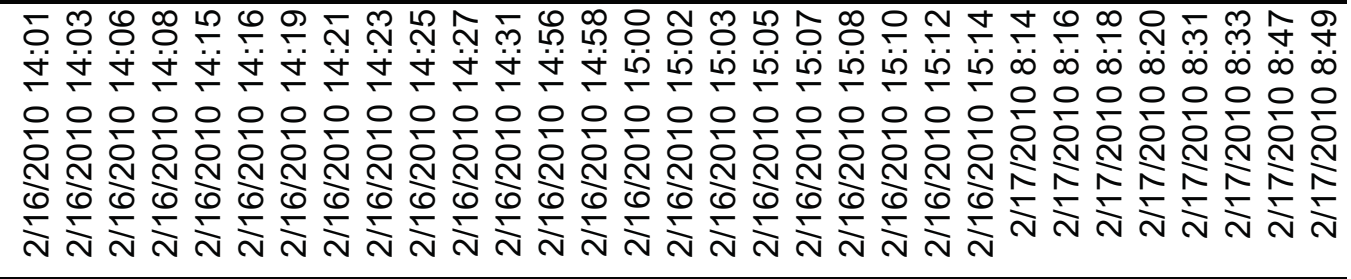 \\
\hline 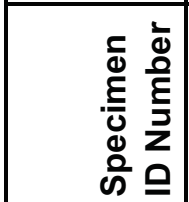 & 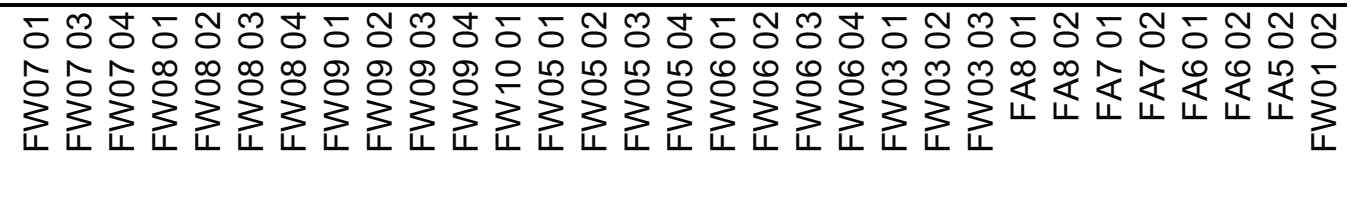 \\
\hline
\end{tabular}




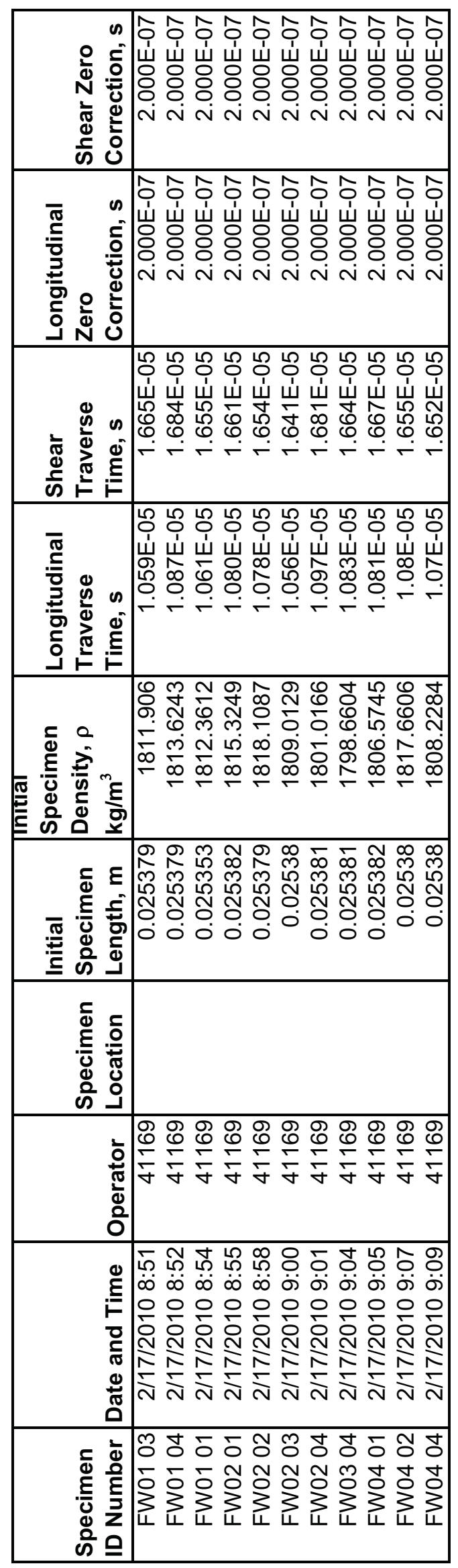




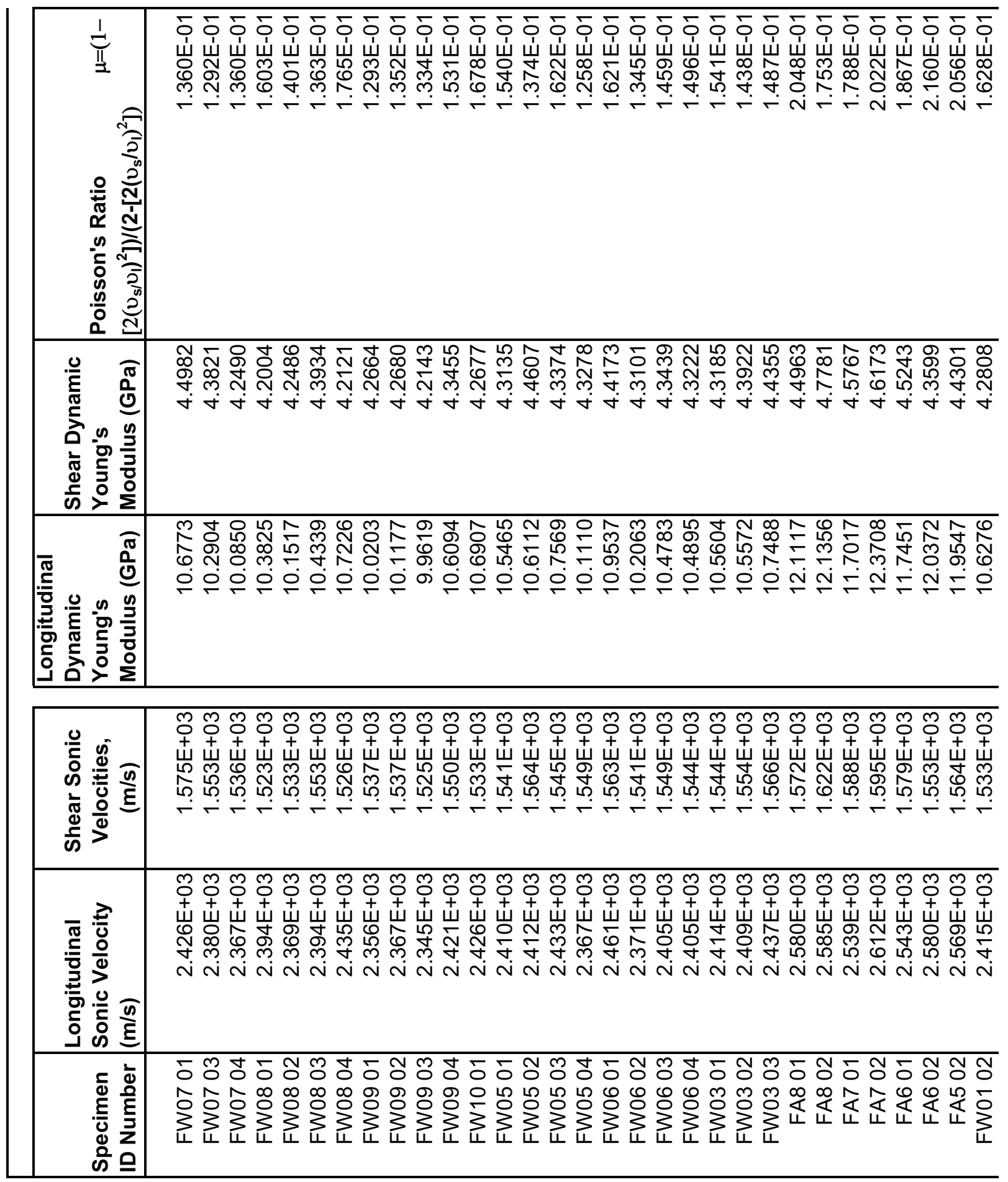




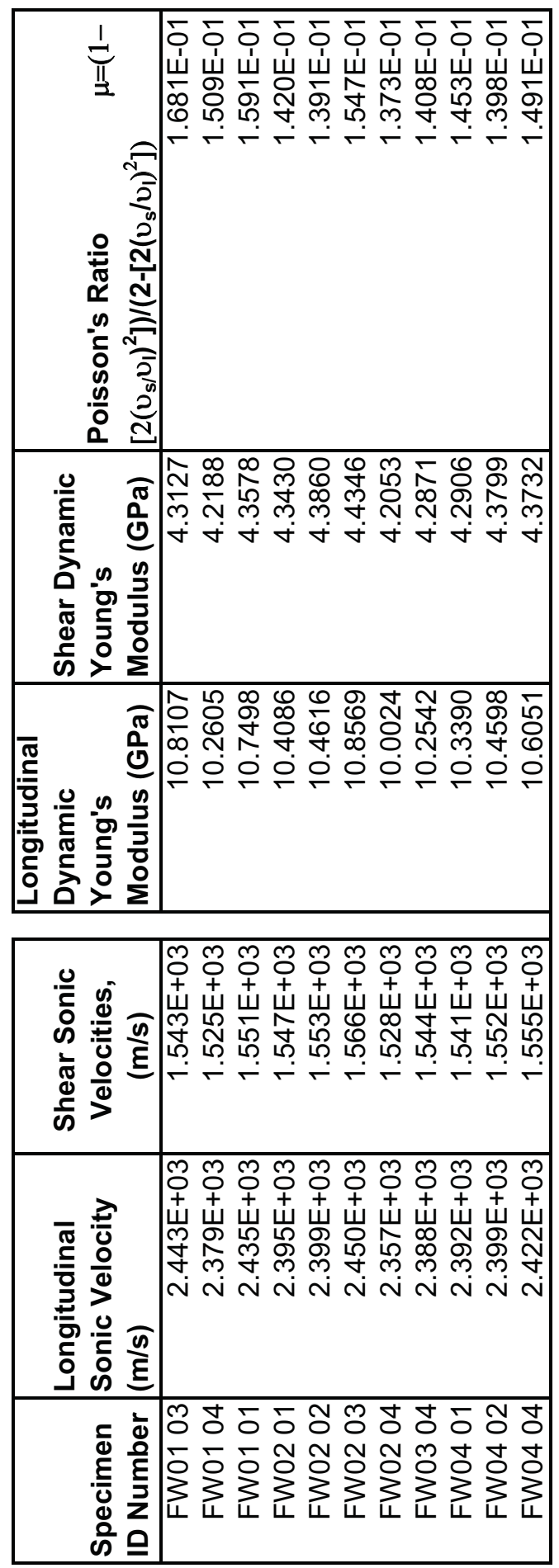




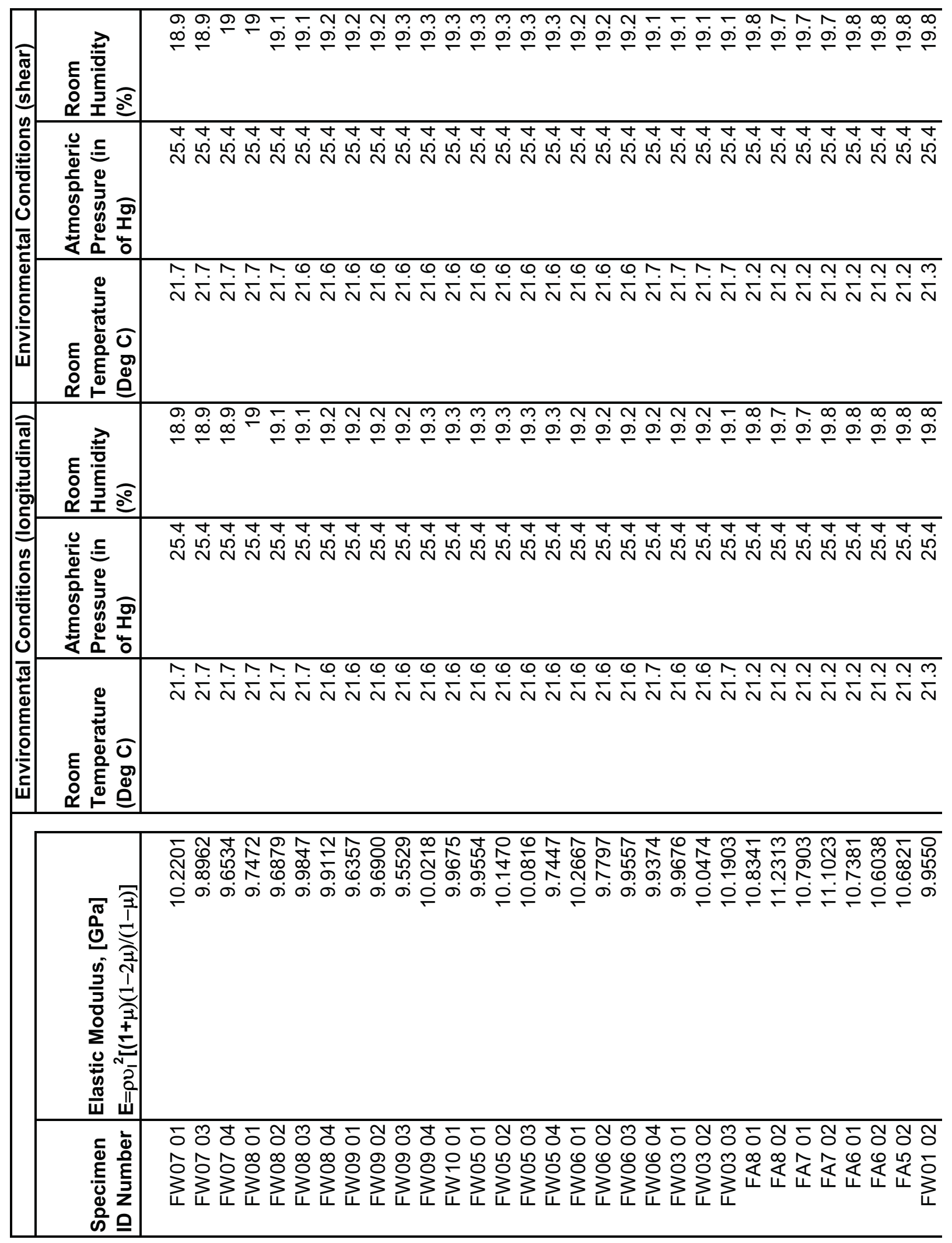




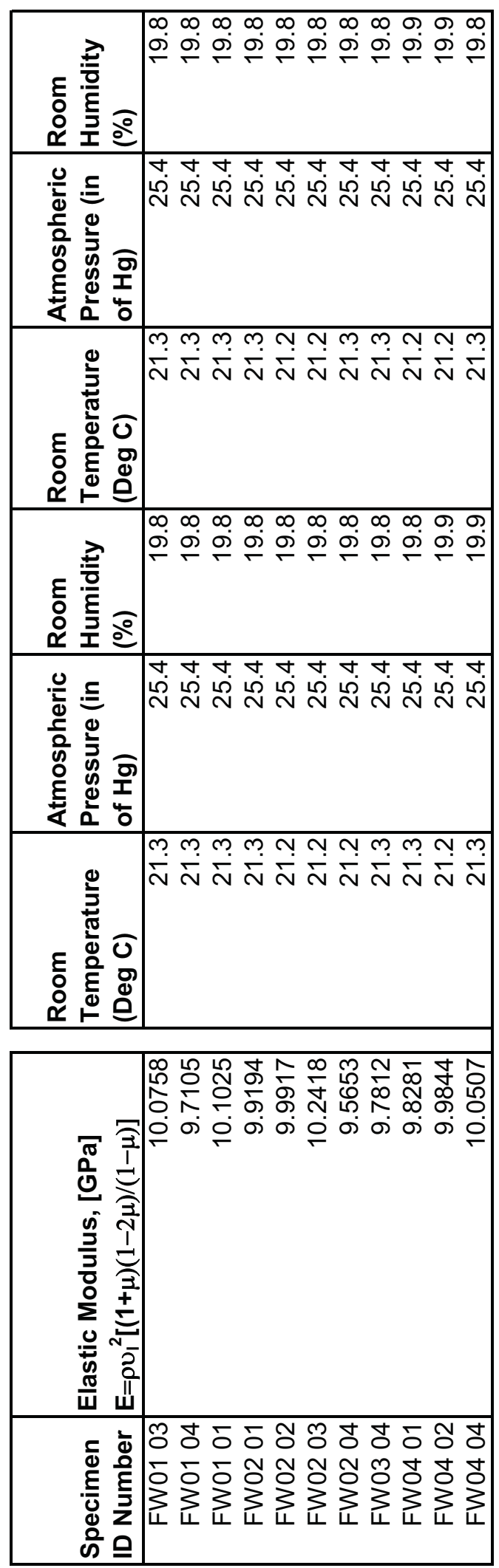




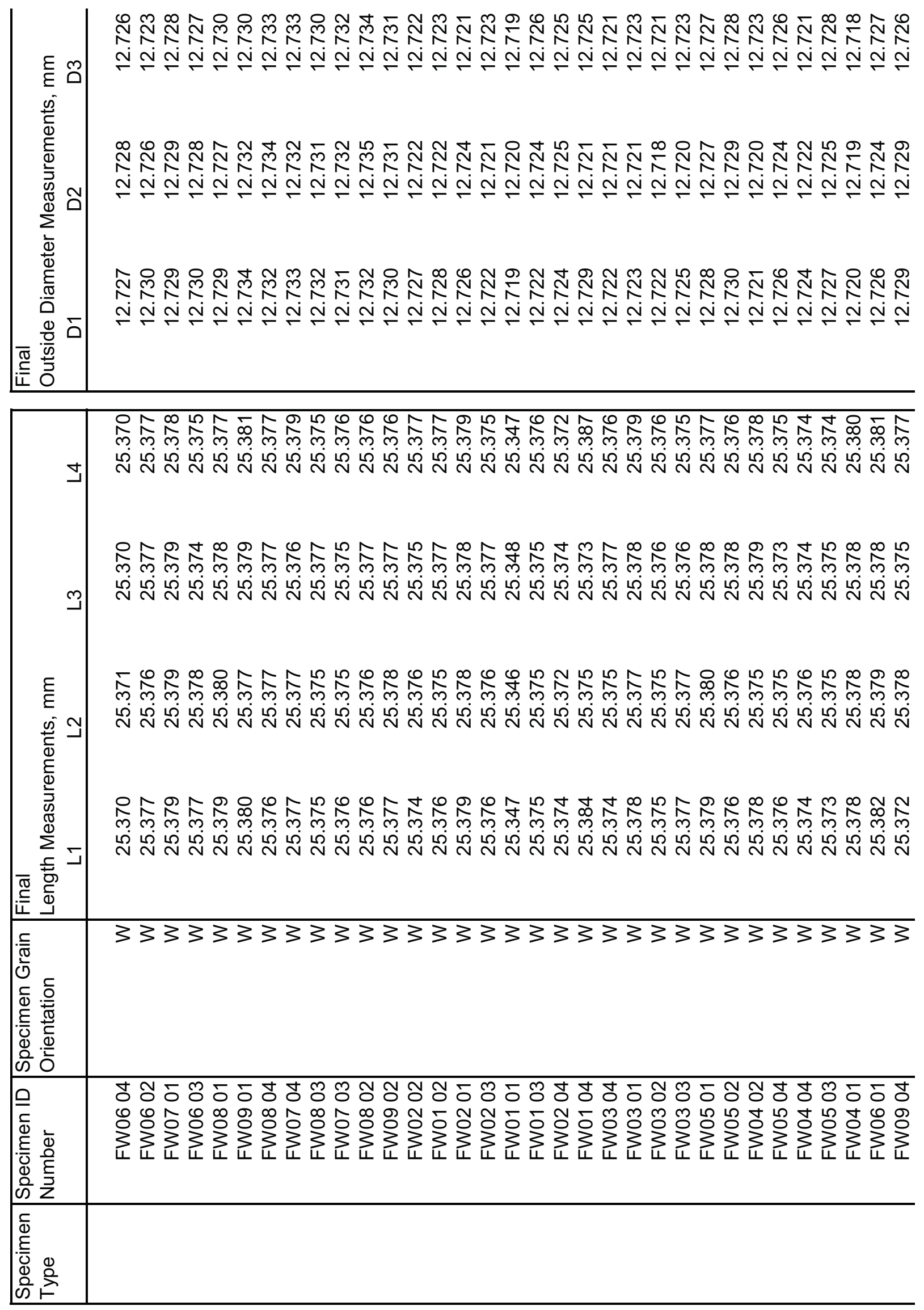



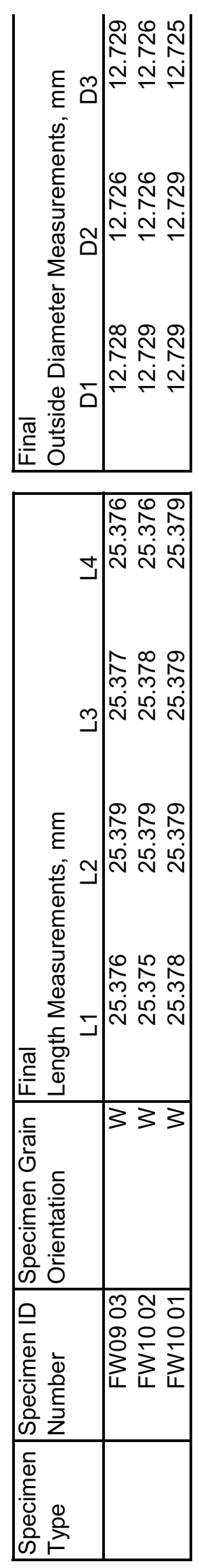

ஓ 


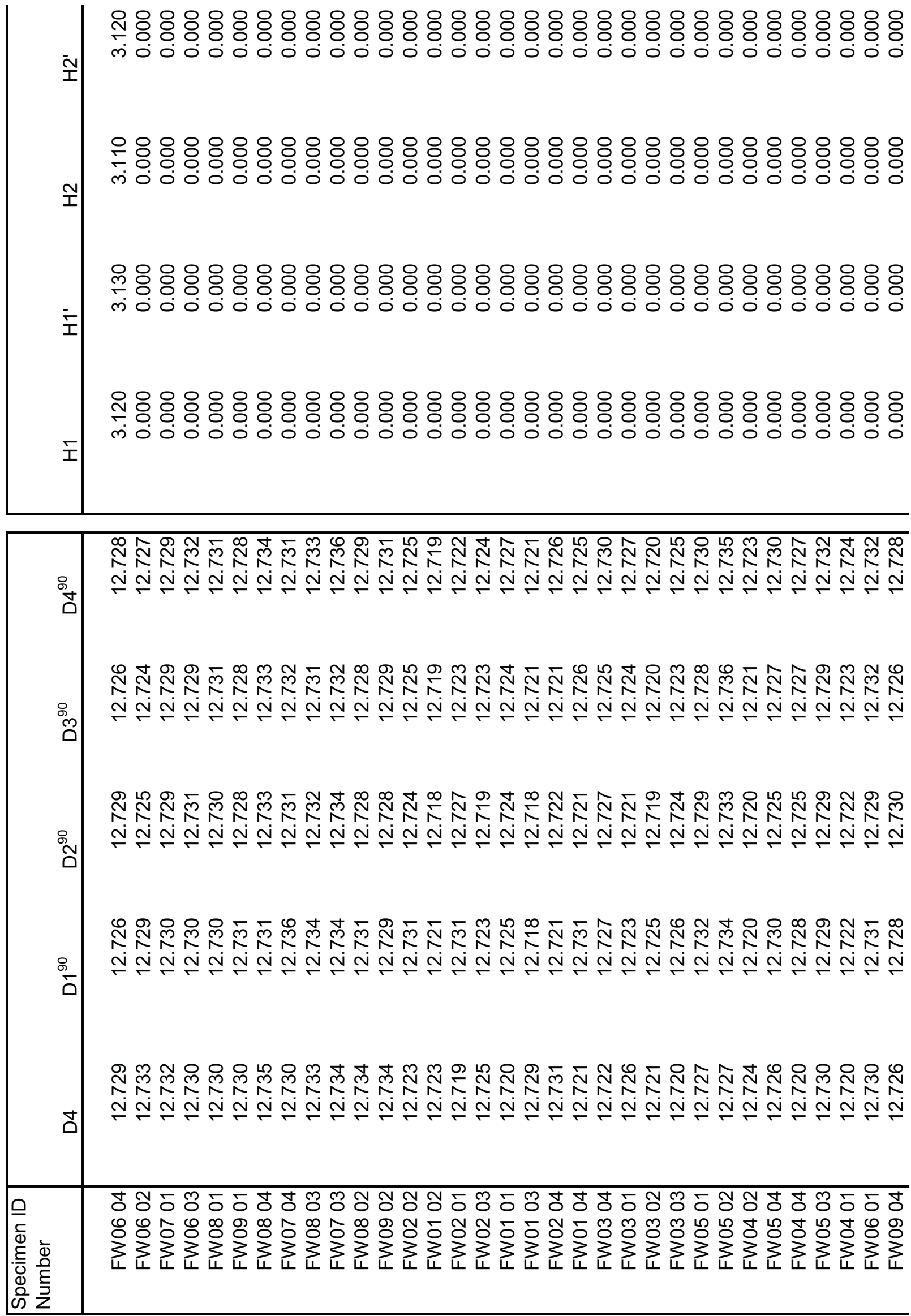




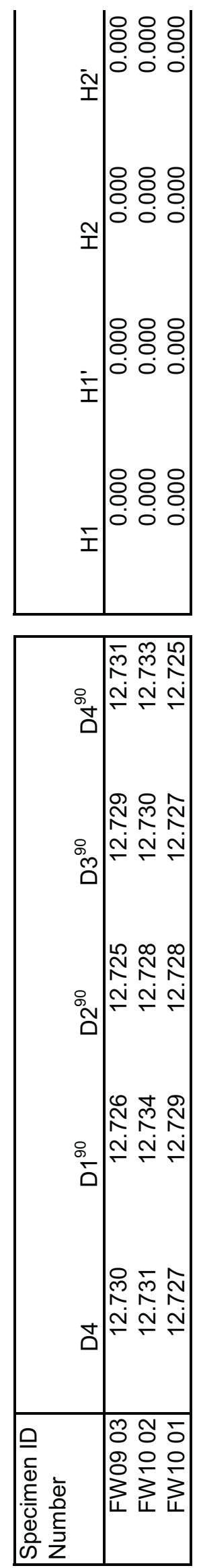




\begin{tabular}{|c|c|}
\hline 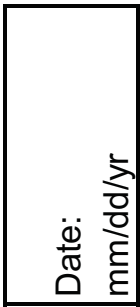 & 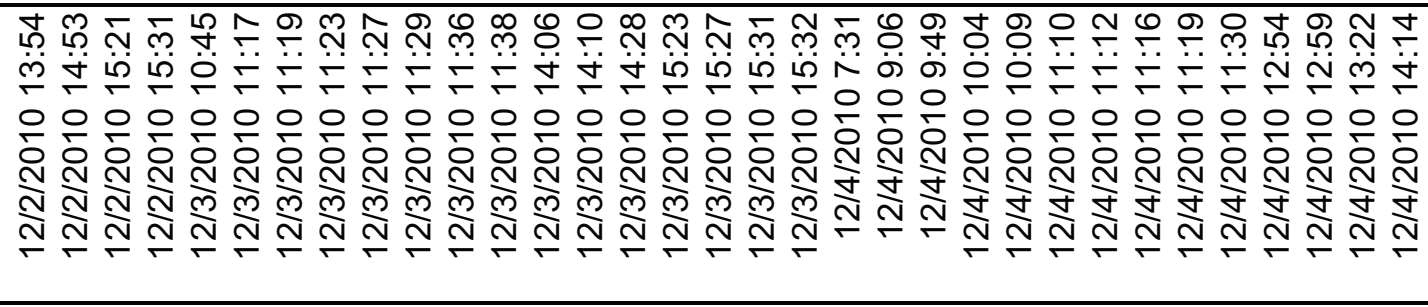 \\
\hline 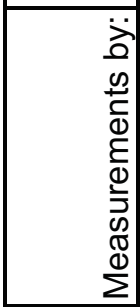 & 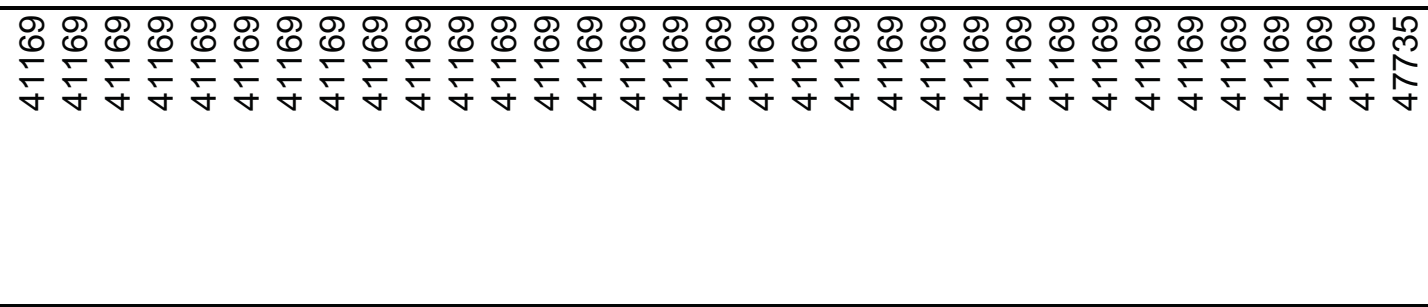 \\
\hline 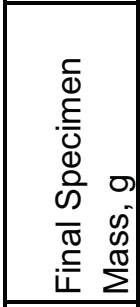 & 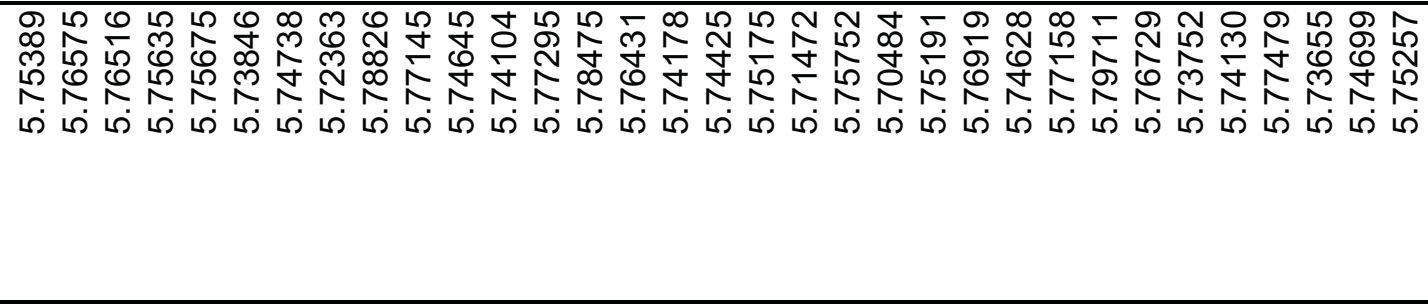 \\
\hline 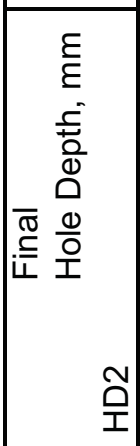 & 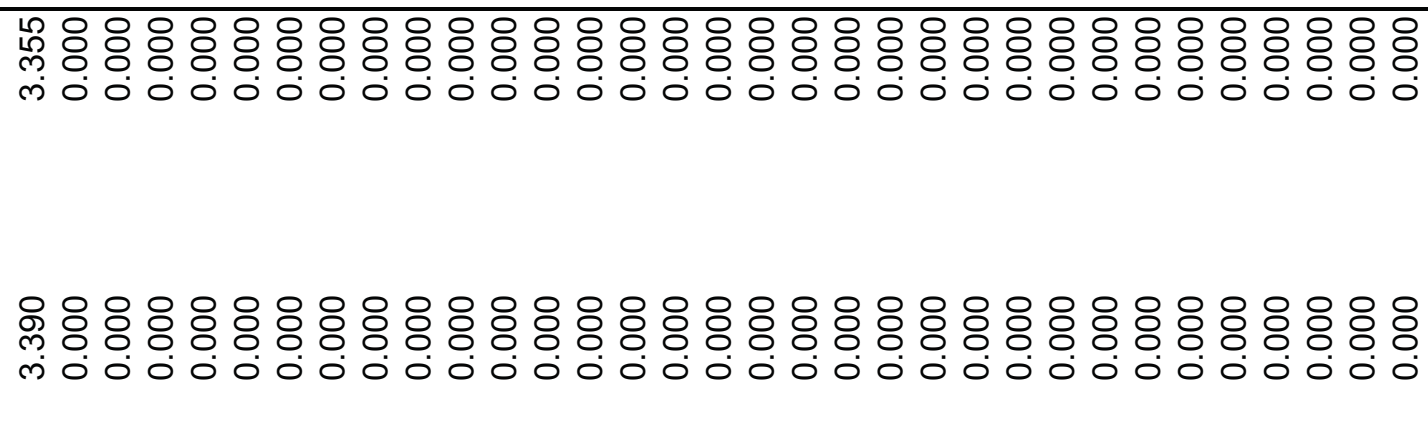 \\
\hline 呈 & 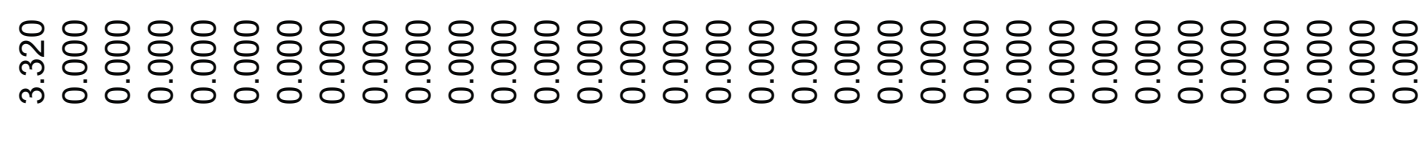 \\
\hline 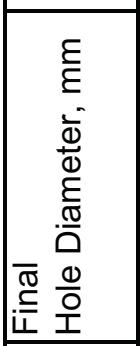 & 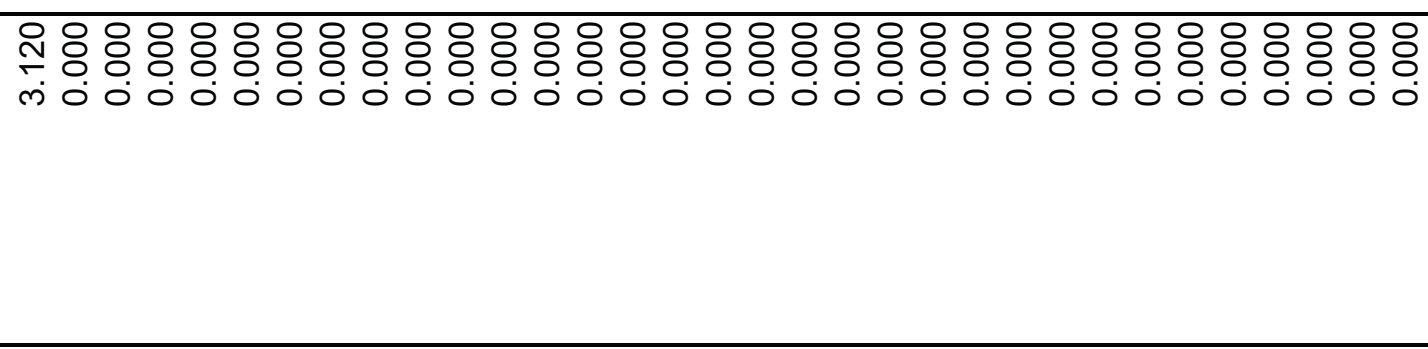 \\
\hline 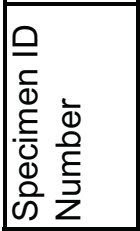 & 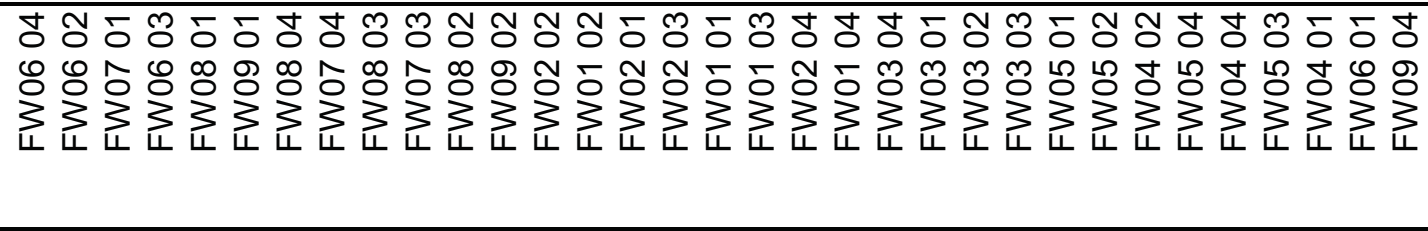 \\
\hline
\end{tabular}




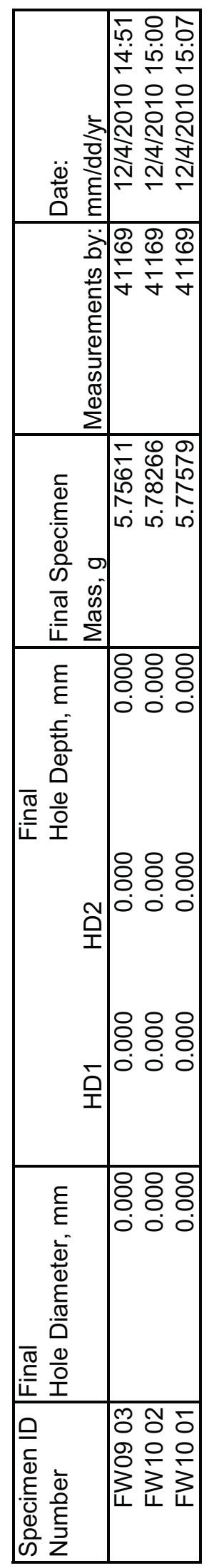




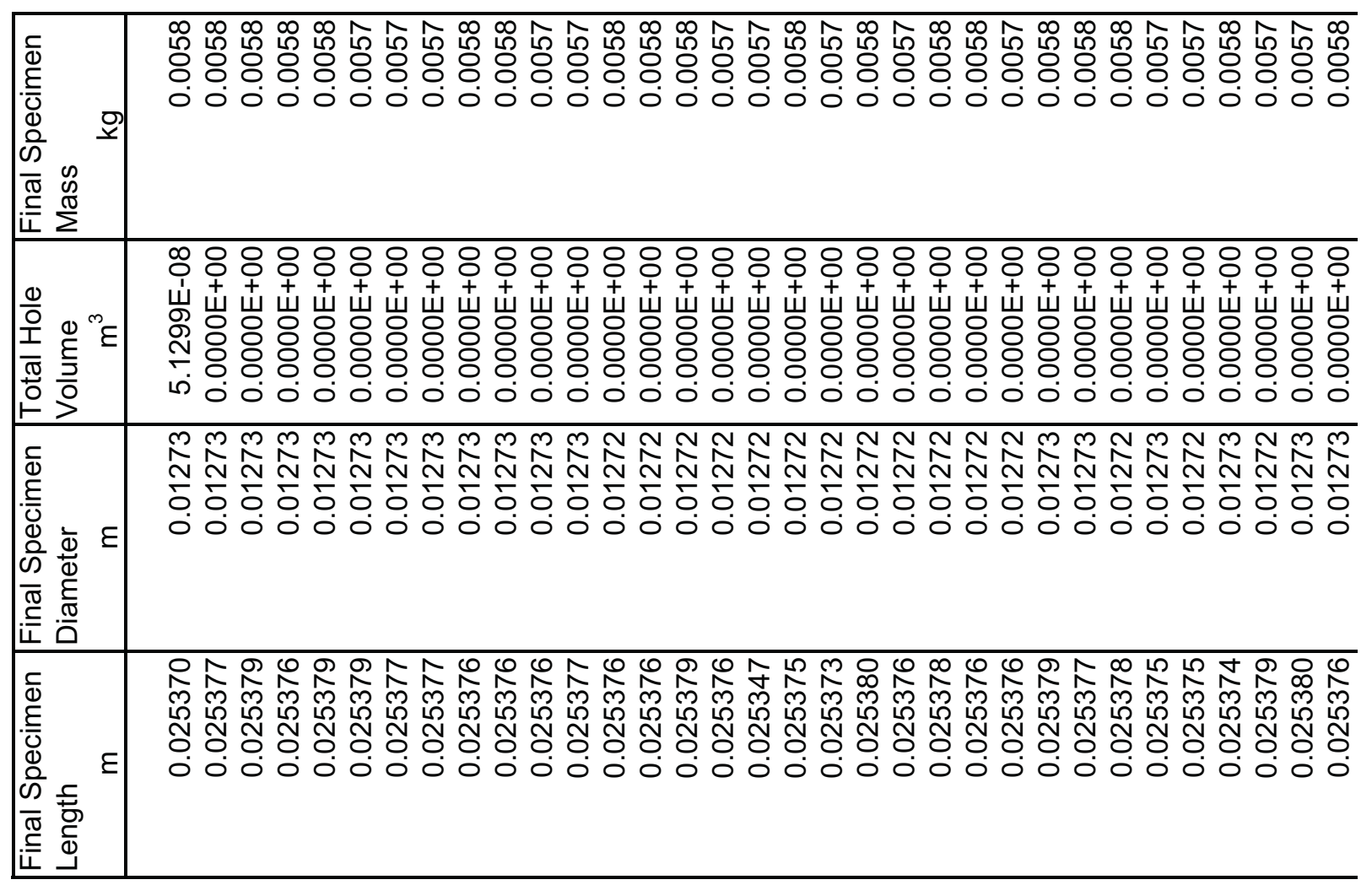

\begin{tabular}{|c|c|}
\hline 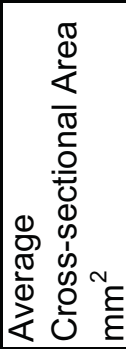 & 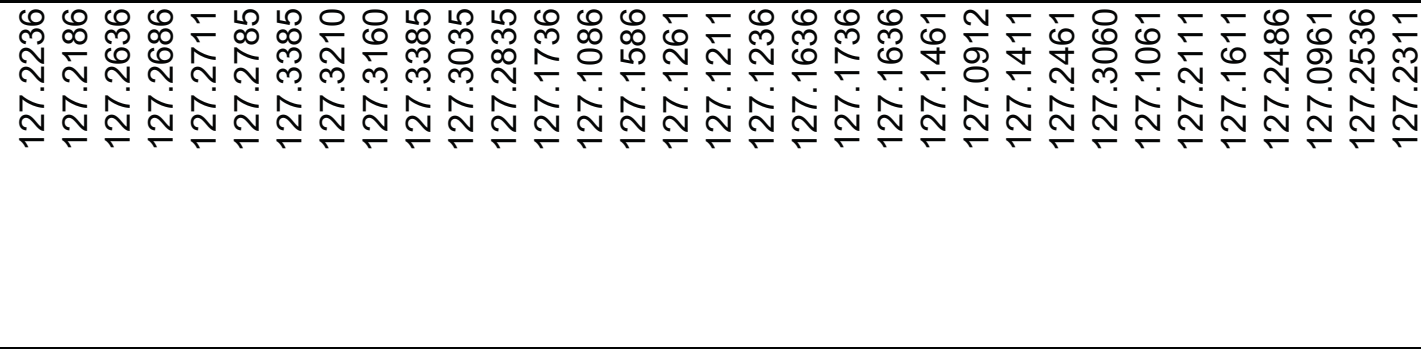 \\
\hline 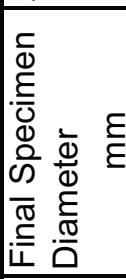 & 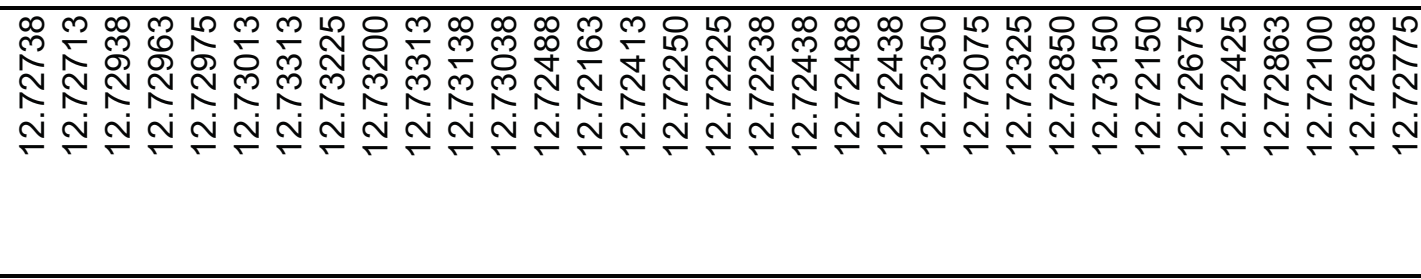 \\
\hline 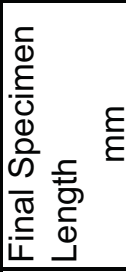 & 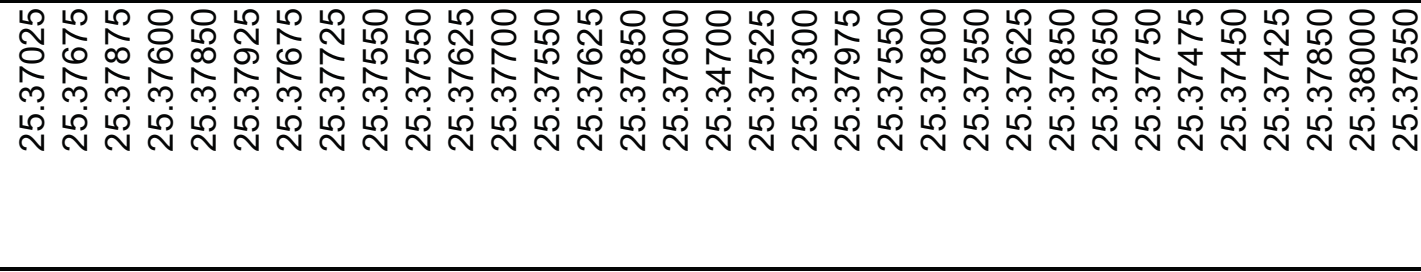 \\
\hline 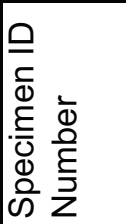 & 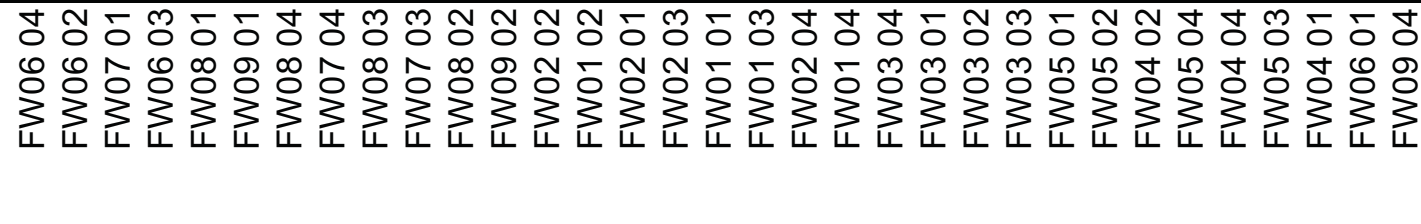 \\
\hline
\end{tabular}



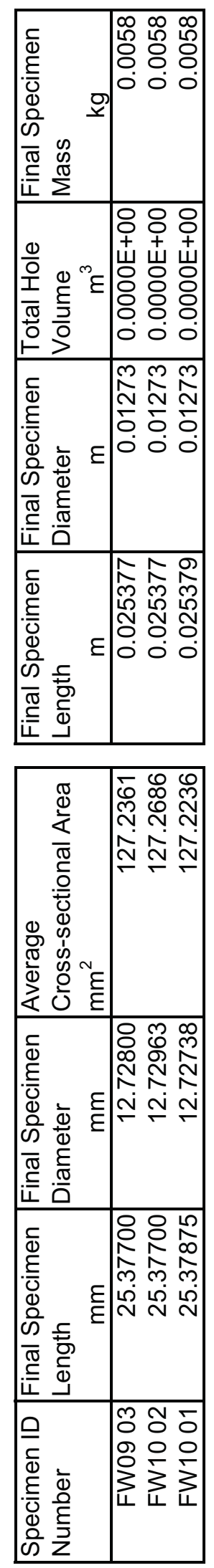


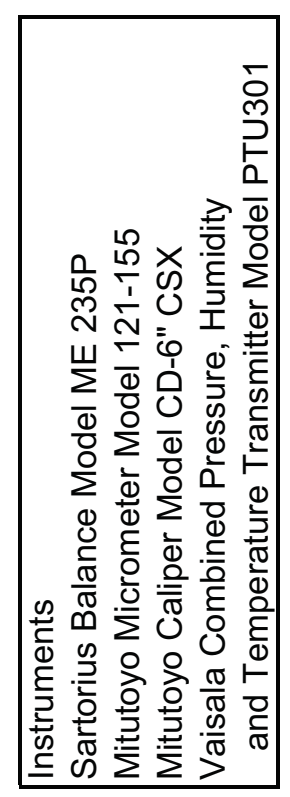

\begin{tabular}{|c|c|}
\hline 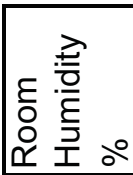 & 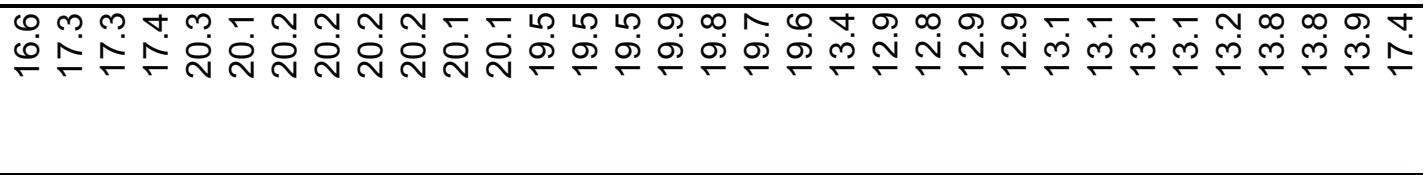 \\
\hline 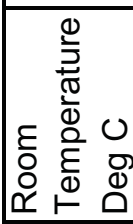 & 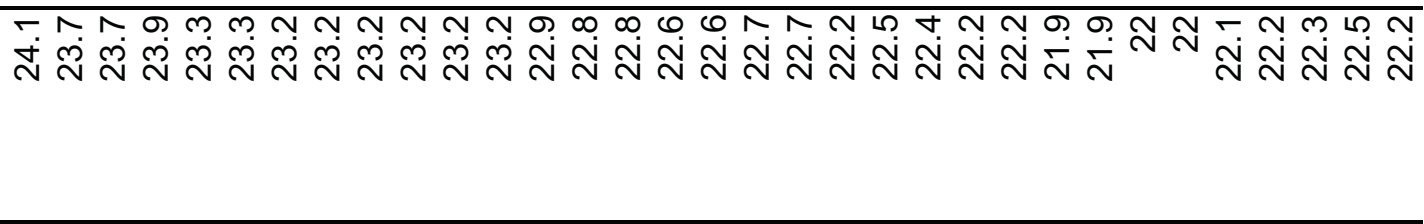 \\
\hline 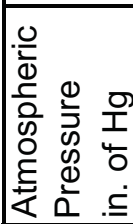 & 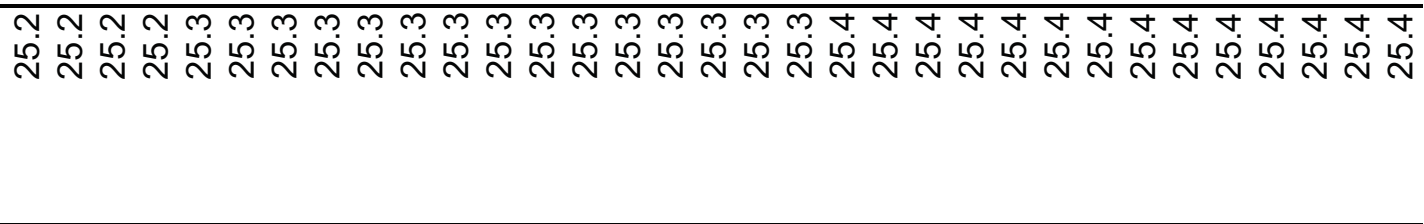 \\
\hline
\end{tabular}

\begin{tabular}{|c|c|}
\hline 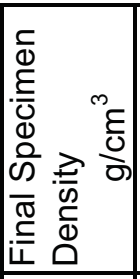 & 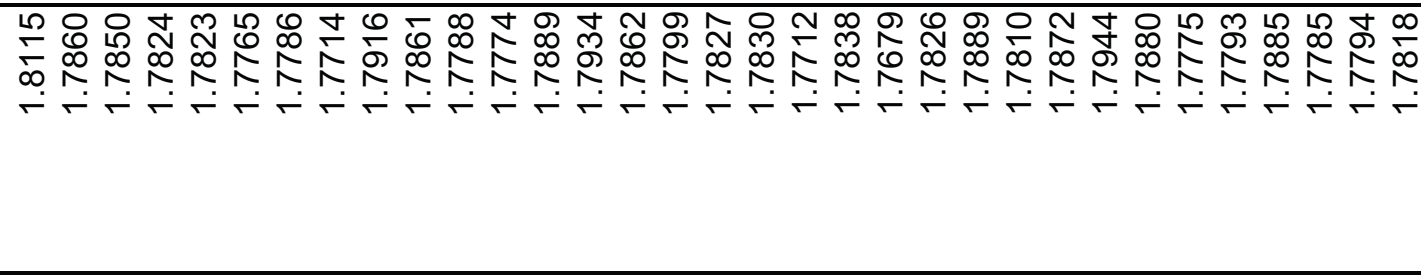 \\
\hline 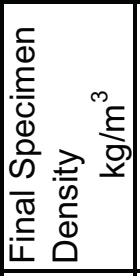 & 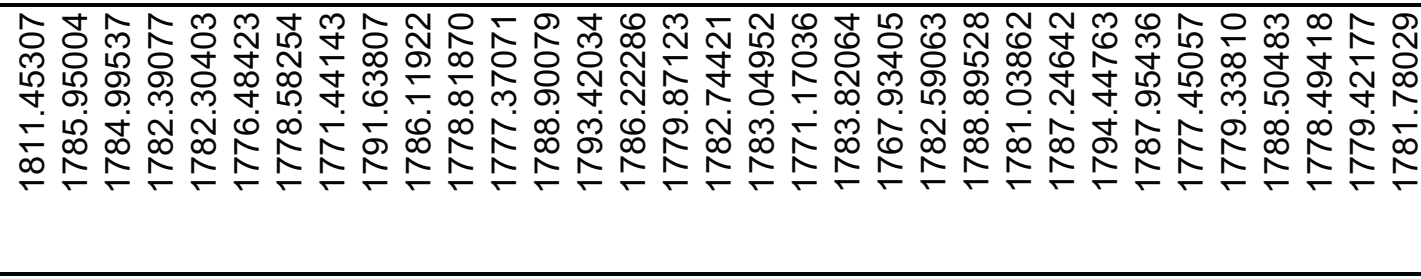 \\
\hline 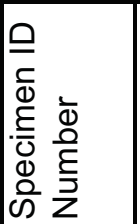 & 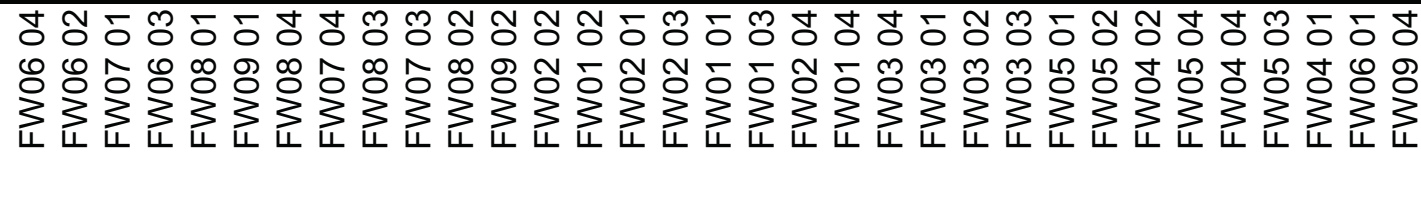 \\
\hline
\end{tabular}



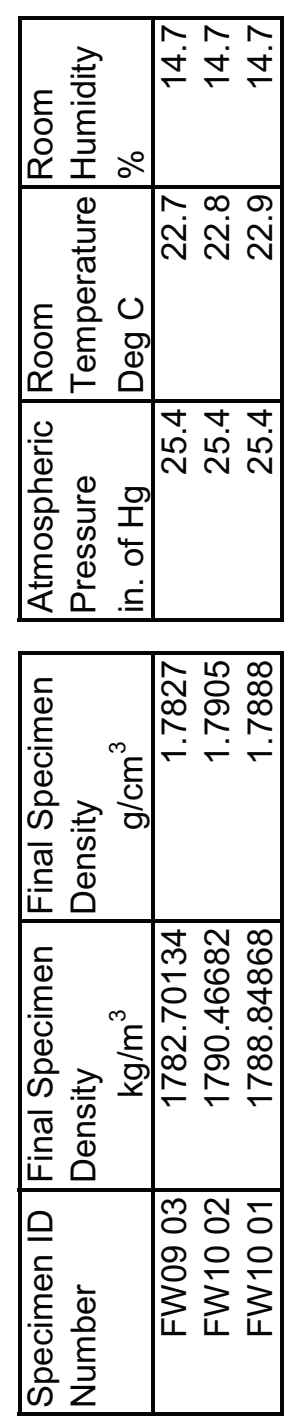


$\begin{array}{ll}\text { Graphite Grade: } & \text { NBG-17 } \\ \text { Graphite Manufacturer: } & \text { SGL Carbon Company } \\ \text { Forming Process: } & \text { Vibration molded } \\ \text { Coke Particle Size: } & \text { Medium grain } \\ \text { Coke Type: } & \text { Pitch coke filler, pitch binder } \\ \text { ASTM Class: } & \text { MNHP } \\ \text { Specimen Geometry: } & \text { Cylinder }\end{array}$

Specimen ID \#'s:

AL1 01

AL1 02

AL1 03

AL2 01

AL2 02

AL2 03

AL3 01

AL3 02

AL3 03

AL4 01

AL4 02

AL4 03

AL5 01

AL5 02

AL5 03

AL6 01

AL6 02

AL6 03

AL7 01

AL7 02

AL7 03

AL8 01

AL8 02

AL8 03

AL9 01

AL9 02

AL9 03

AL10 01

AL10 02

AL10 03

AL11 01

AL11 02

AL11 03

AL12 01

AL12 02

AL12 03

AL13 01

AL13 02

AL13 03

AP1 01

AP1 02

AP1 03 


\section{Specimen ID \#'s:}

AP2 01

AP2 02

AP2 03

AP3 01

AP3 02

AP3 03

AP4 01

AP4 02

AP4 03

AP5 01

AP5 02

AP5 03

AP6 01

AP6 02

AP6 03

AW1 01

AW1 02

AW1 03

AW10 01

AW10 02

AW10 03

AW11 01

AW11 02

AW11 03

AW12 01

AW12 02

AW12 03

AW13 01

AW13 02

AW13 03

AW14 01

AW14 02

AW14 03

AW15 01

AW15 02

AW15 03

AW16 01

AW16 02

AW16 03

AW2 01

AW2 02

AW2 03

AW3 01

AW3 02

AW3 03

AW4 01

AW4 02

AW4 03

AW5 01

AW5 02

AW5 03 
Specimen ID \#'s:

AW6 01

AW6 02

AW6 03

AW7 01

AW7 02

AW7 03

AW8 01

AW8 02

AW8 03

AW9 01

AW9 02

AW9 03 


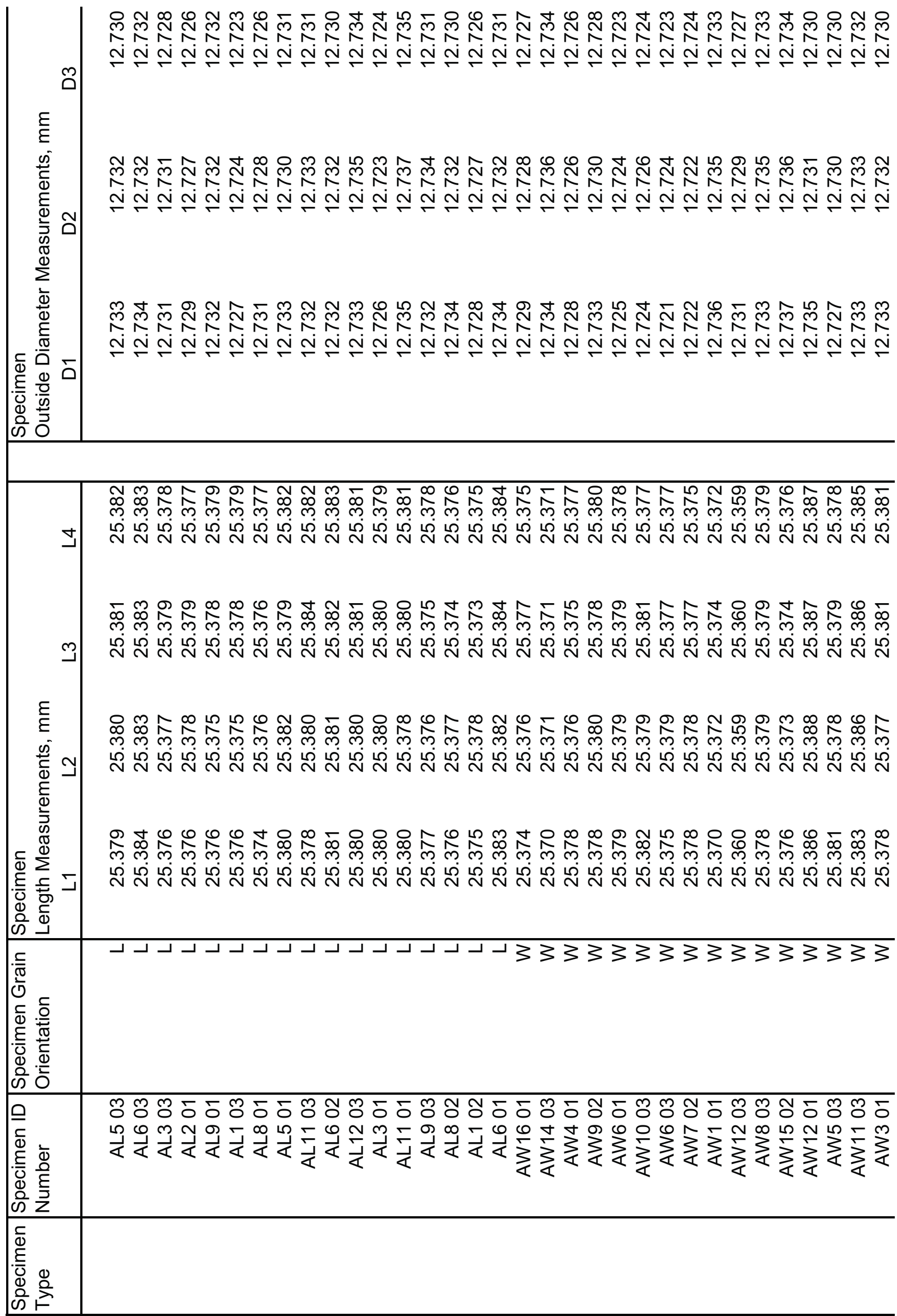




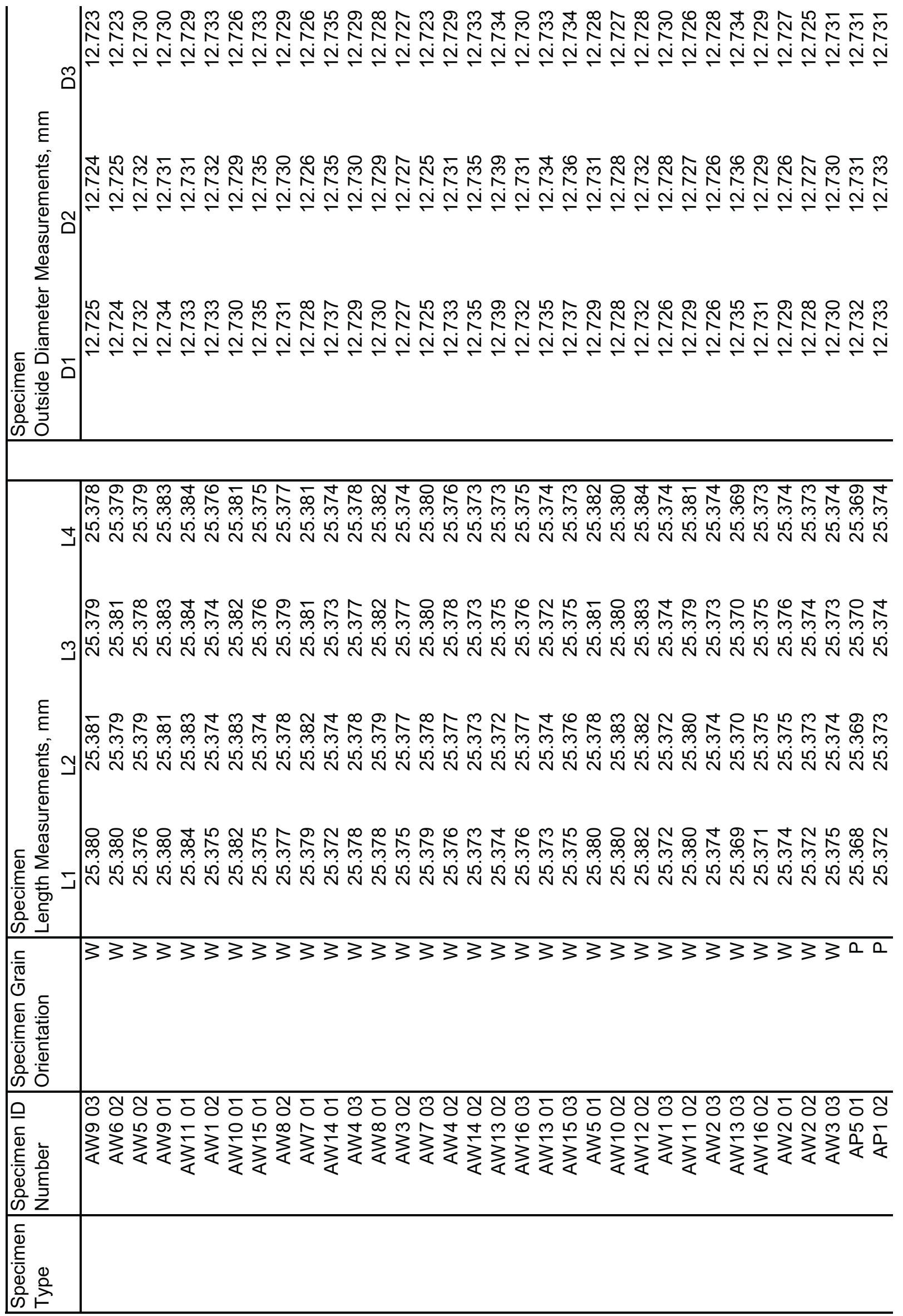




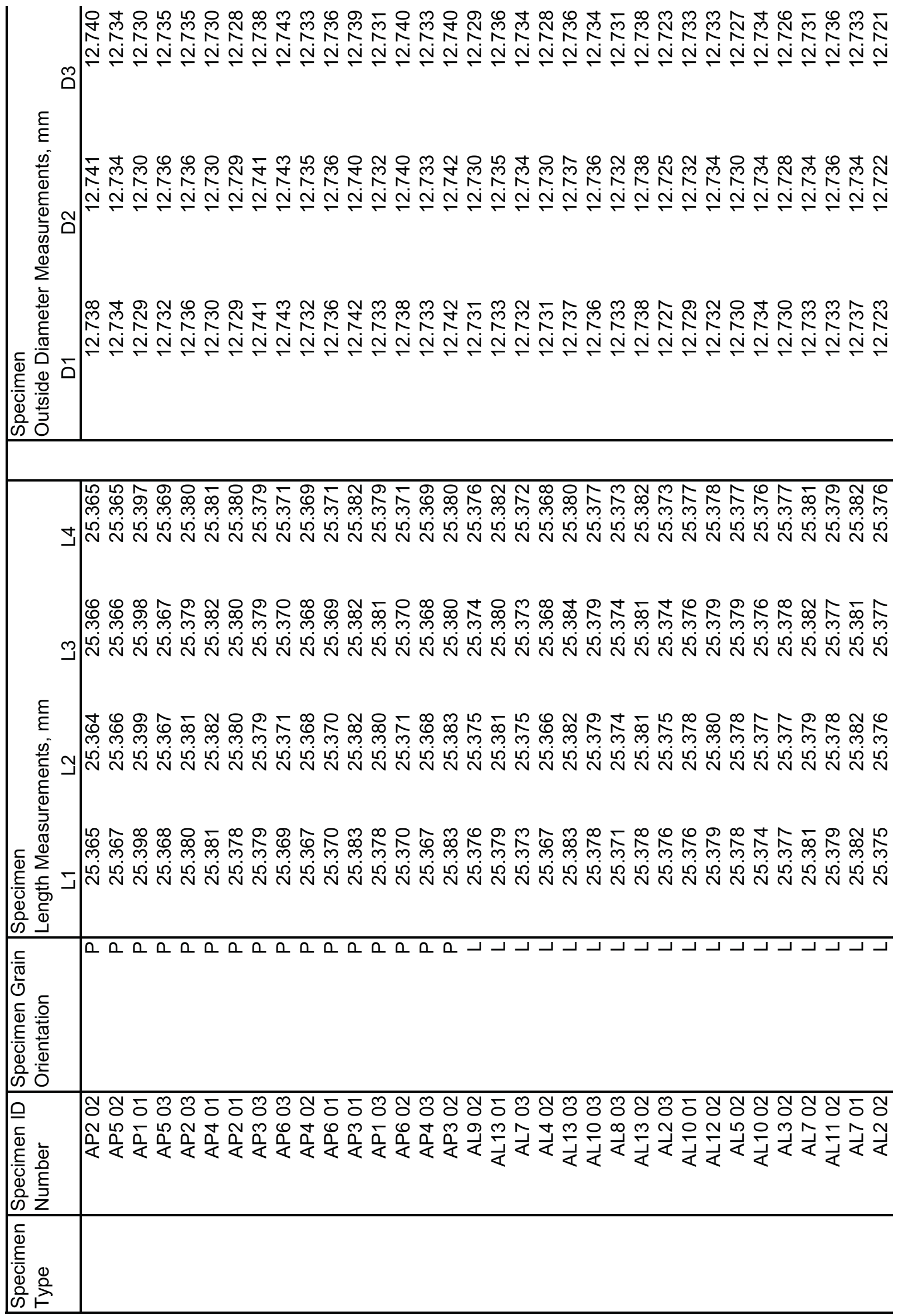




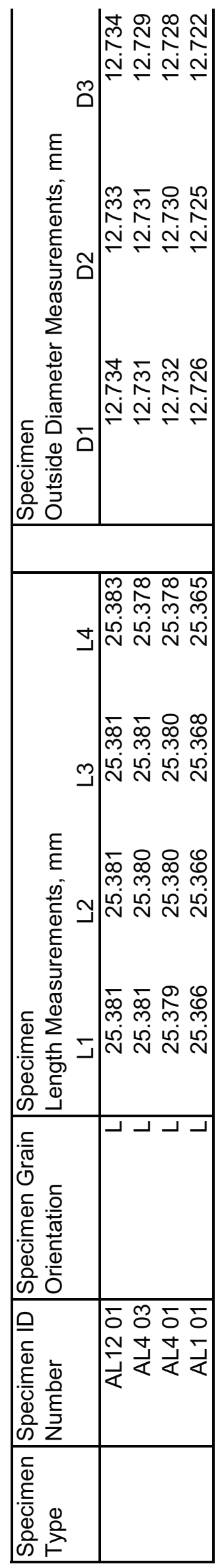




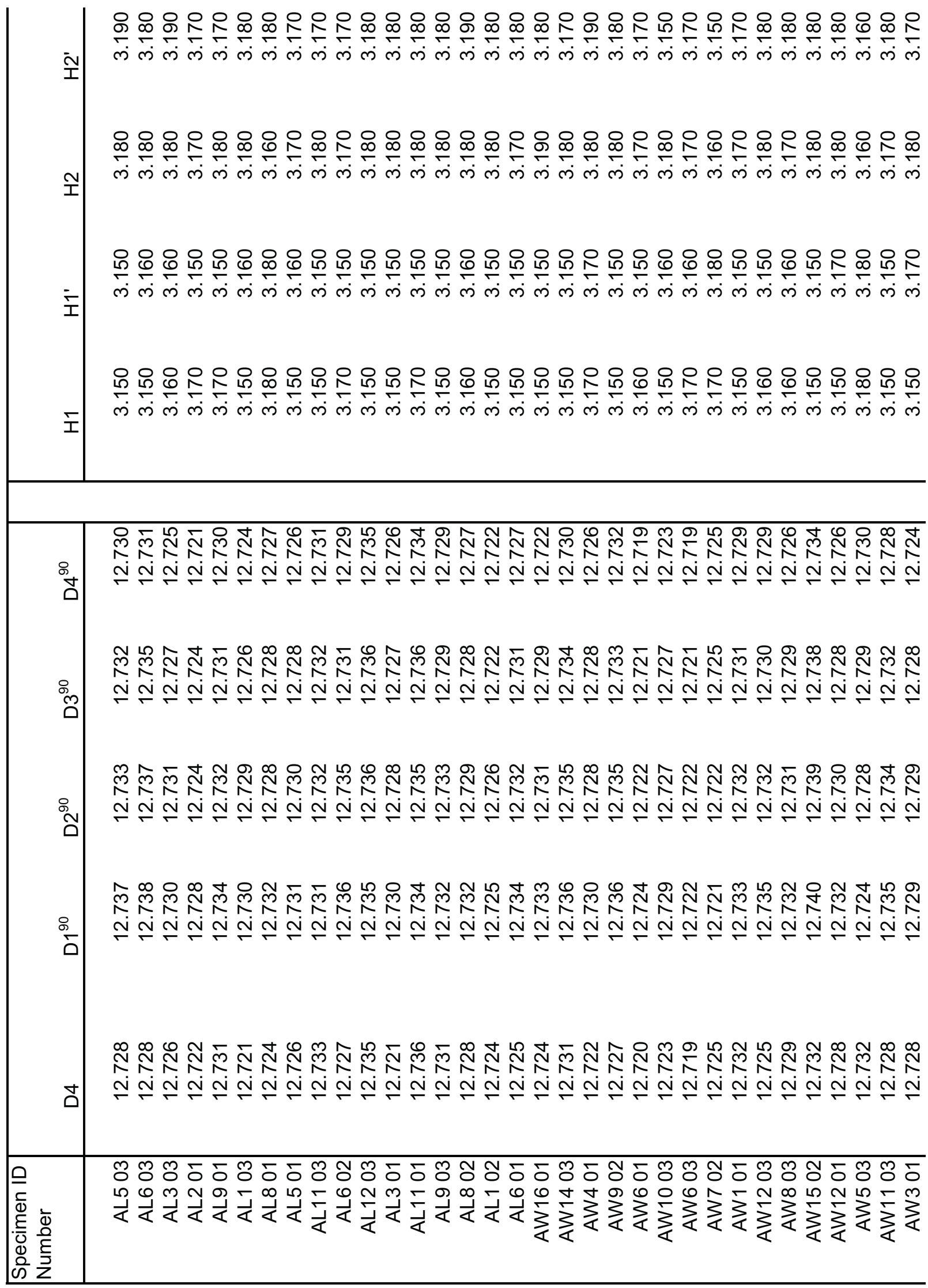




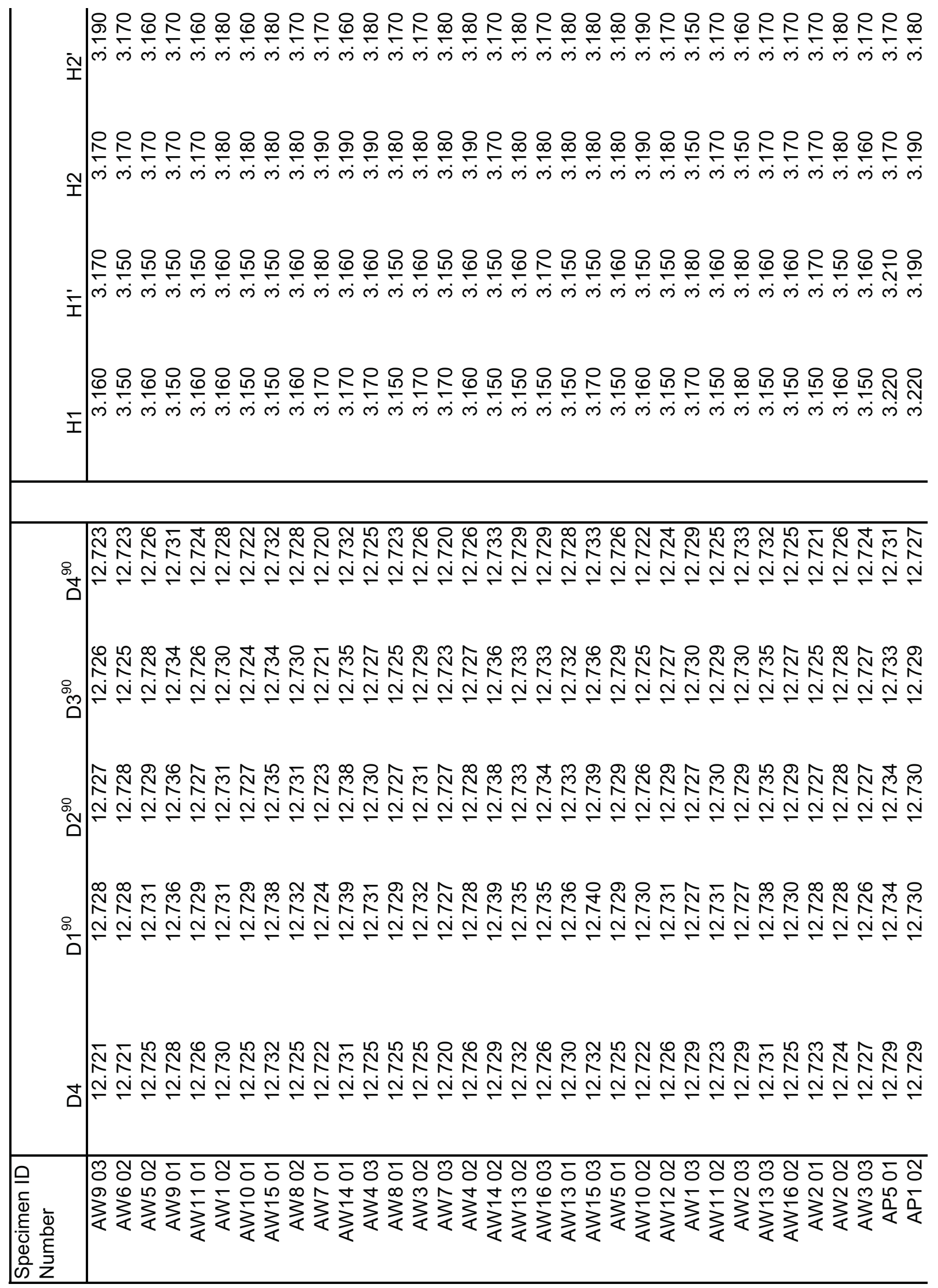




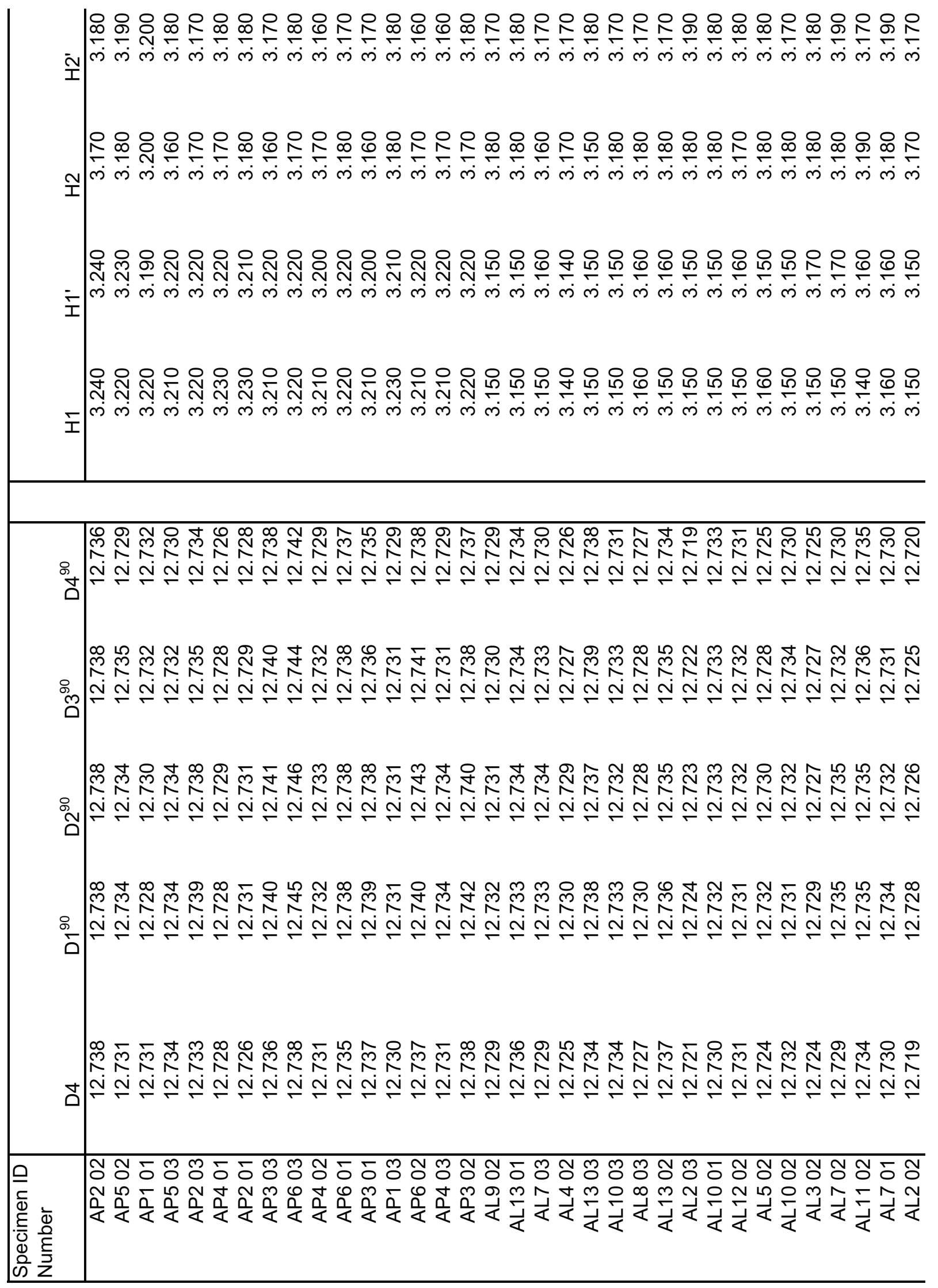




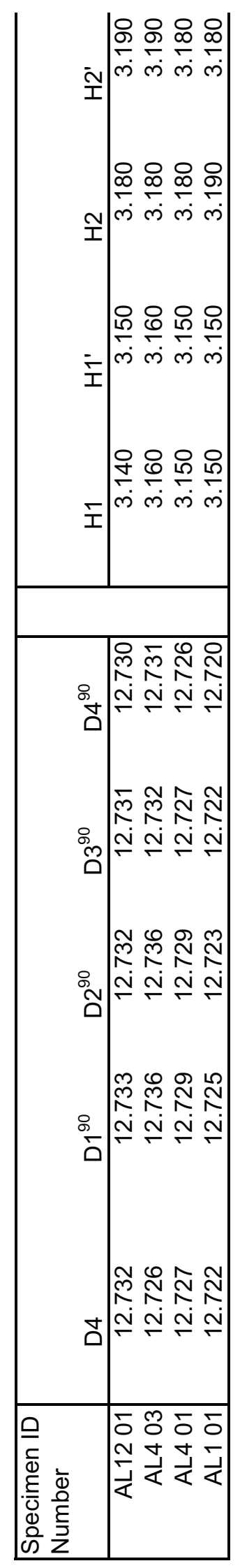

옹 


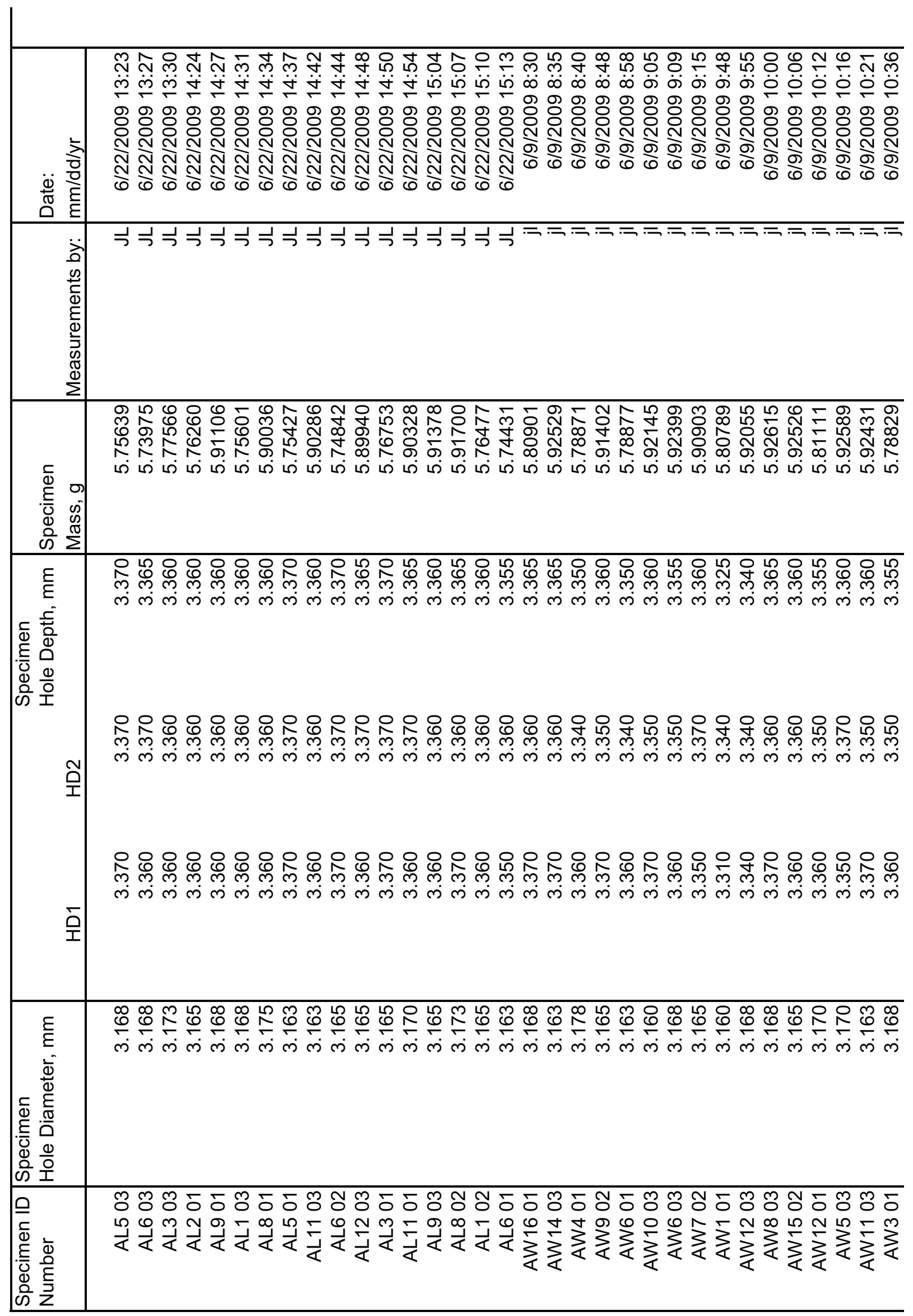




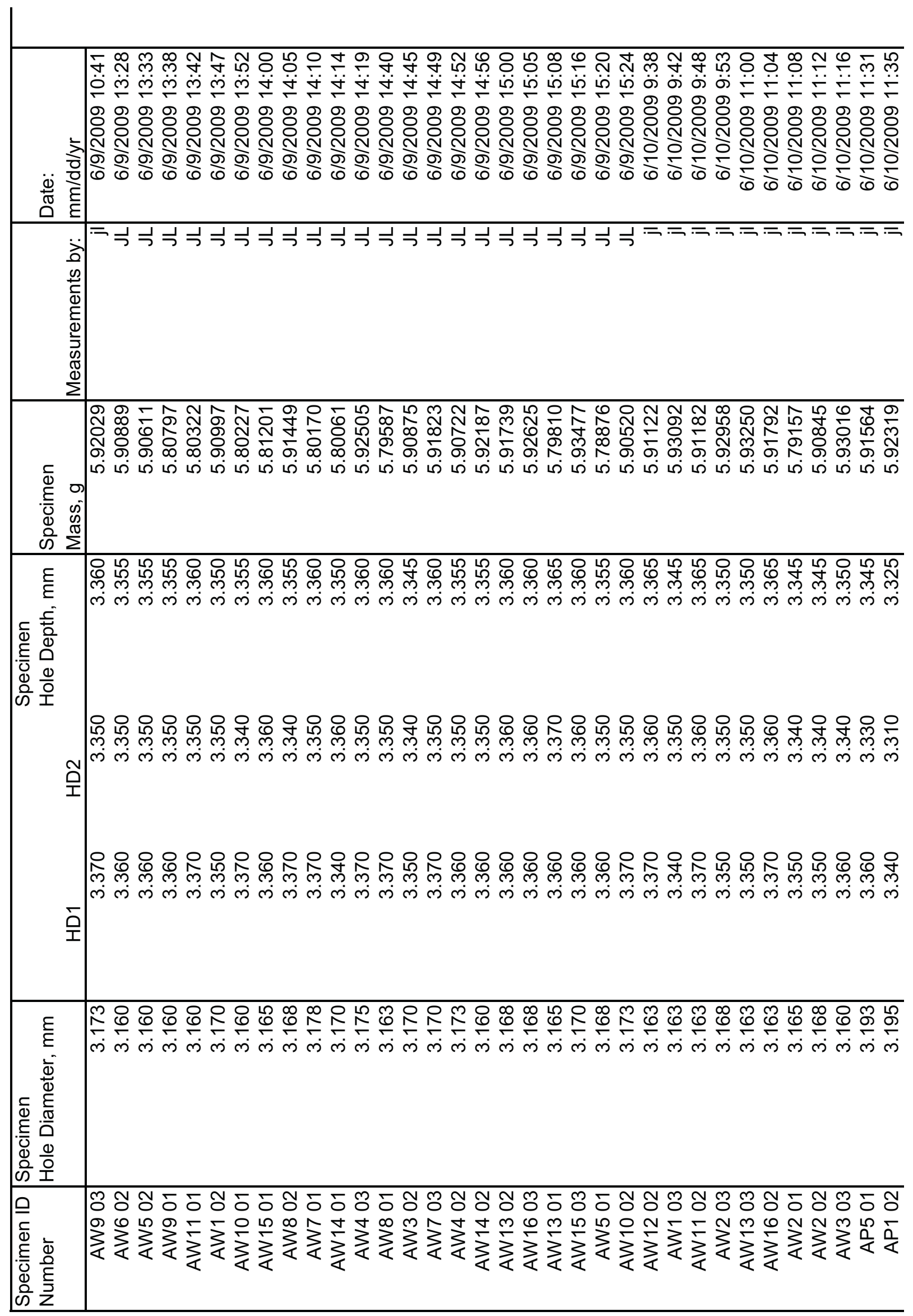




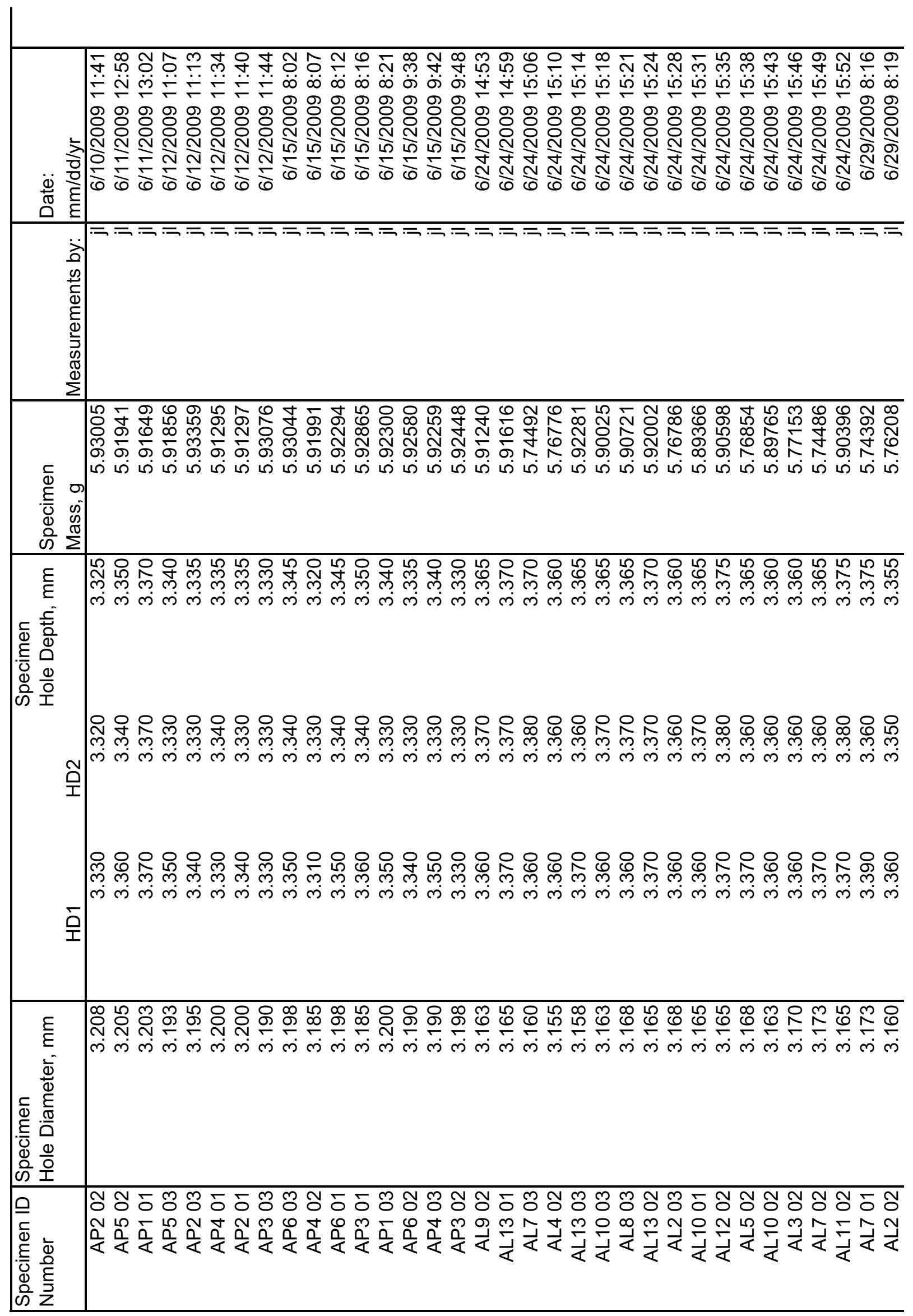




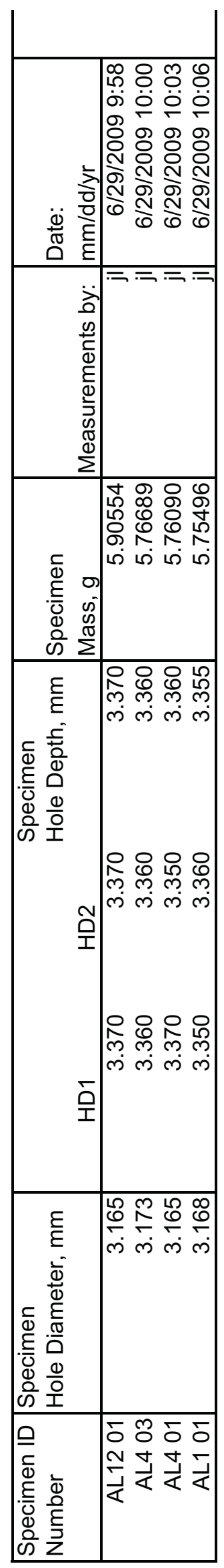

$\frac{n}{n}$ 


\begin{tabular}{|c|c|}
\hline 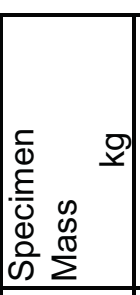 & 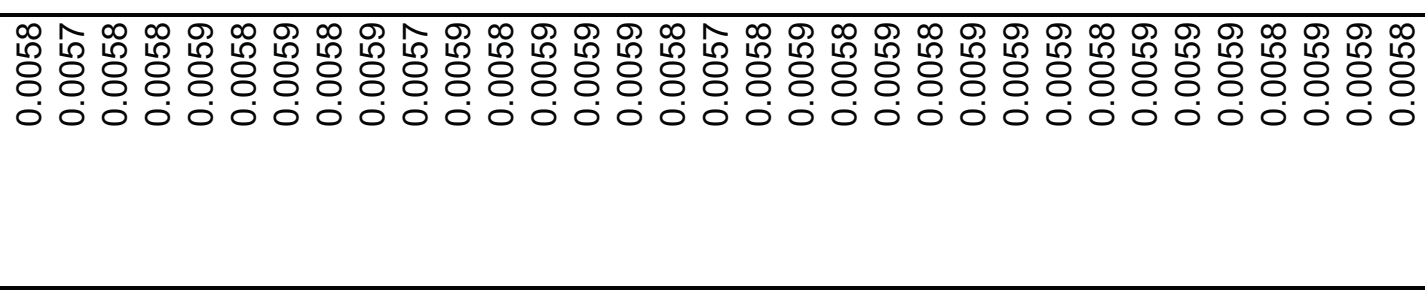 \\
\hline 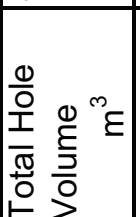 & 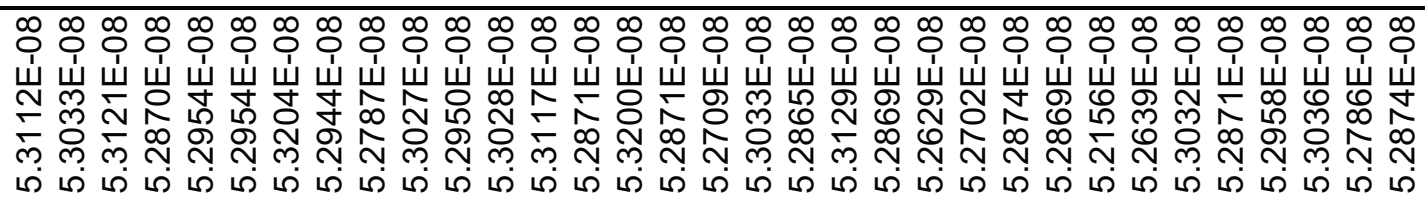 \\
\hline 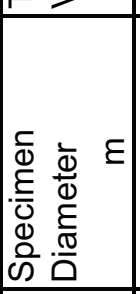 & 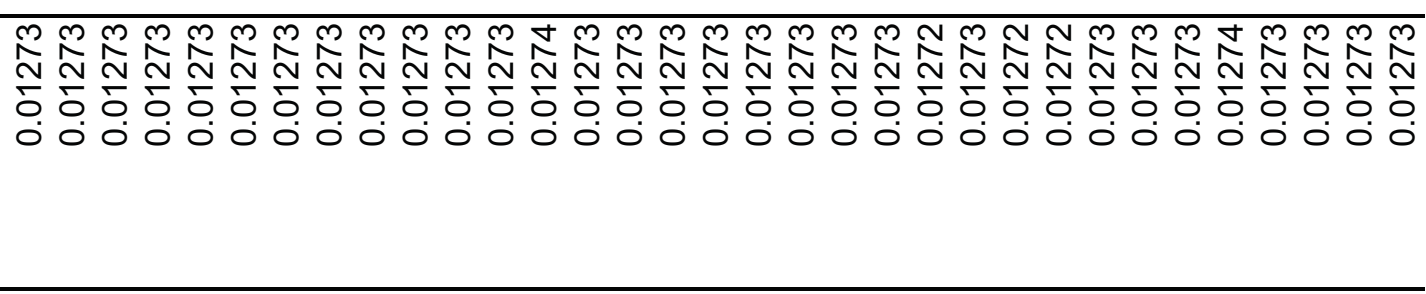 \\
\hline 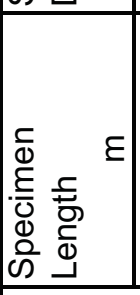 & 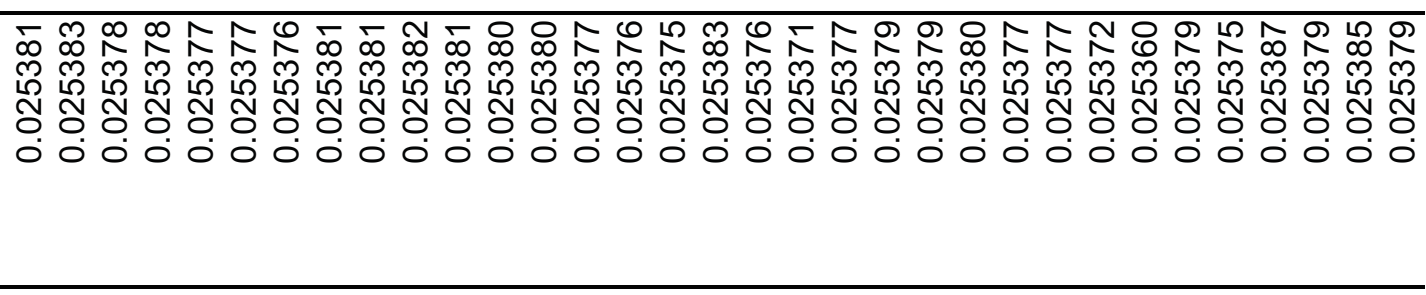 \\
\hline 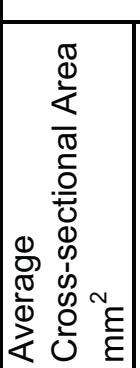 & 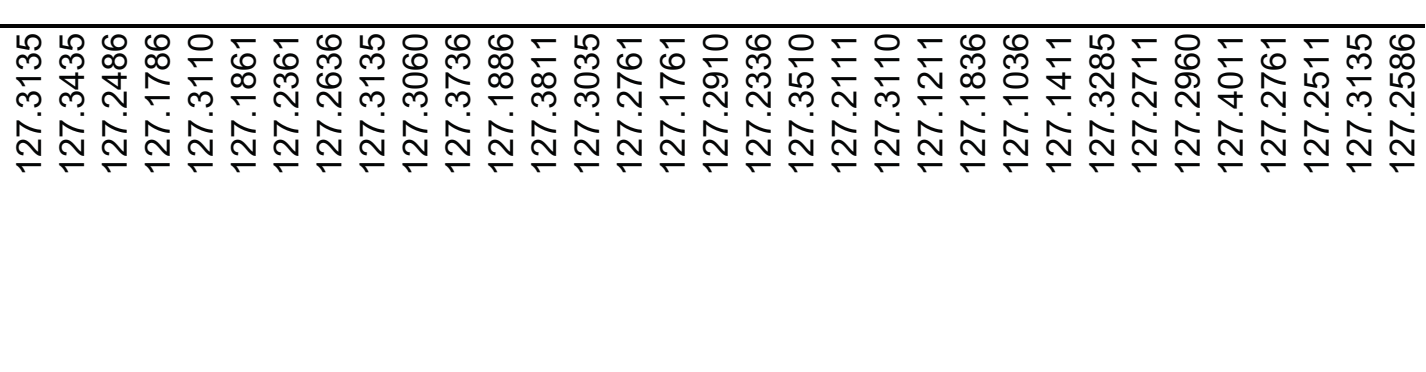 \\
\hline 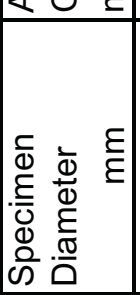 & 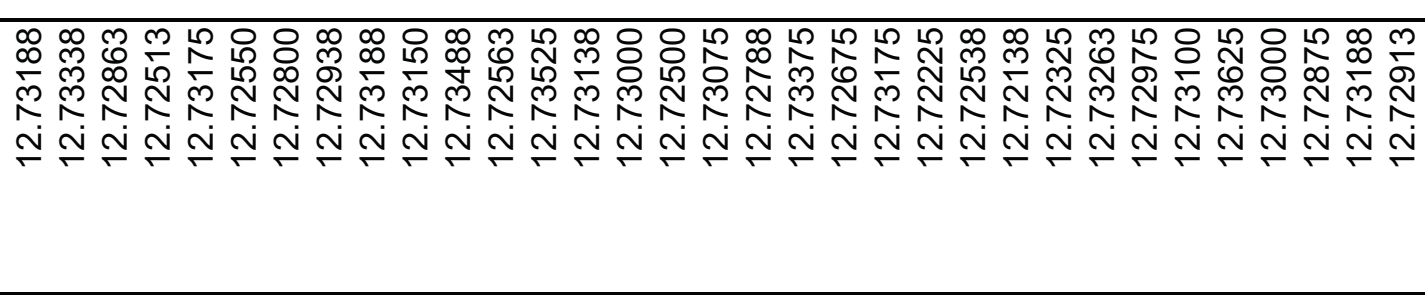 \\
\hline 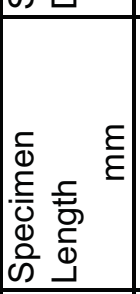 & 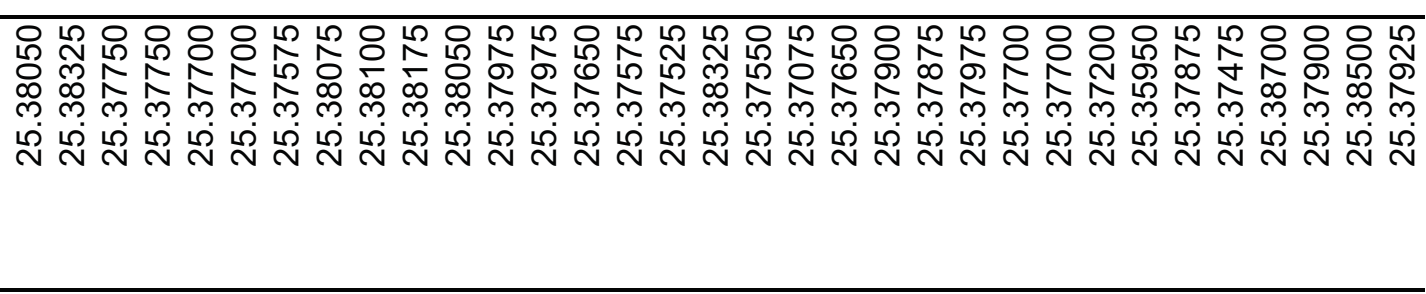 \\
\hline 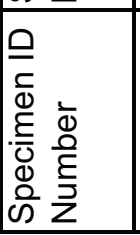 & 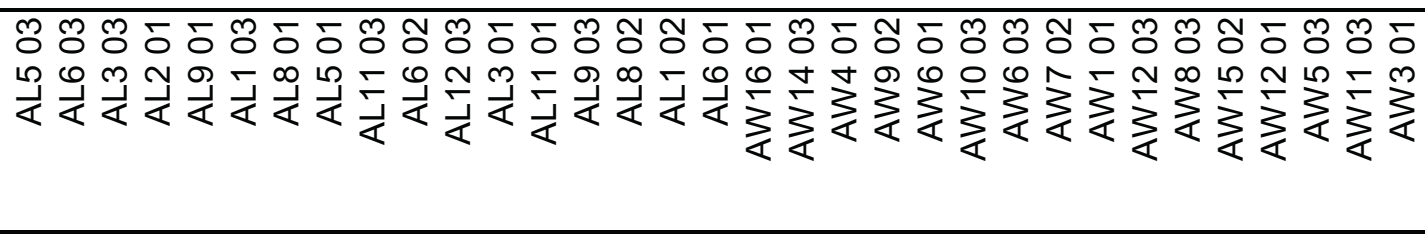 \\
\hline
\end{tabular}




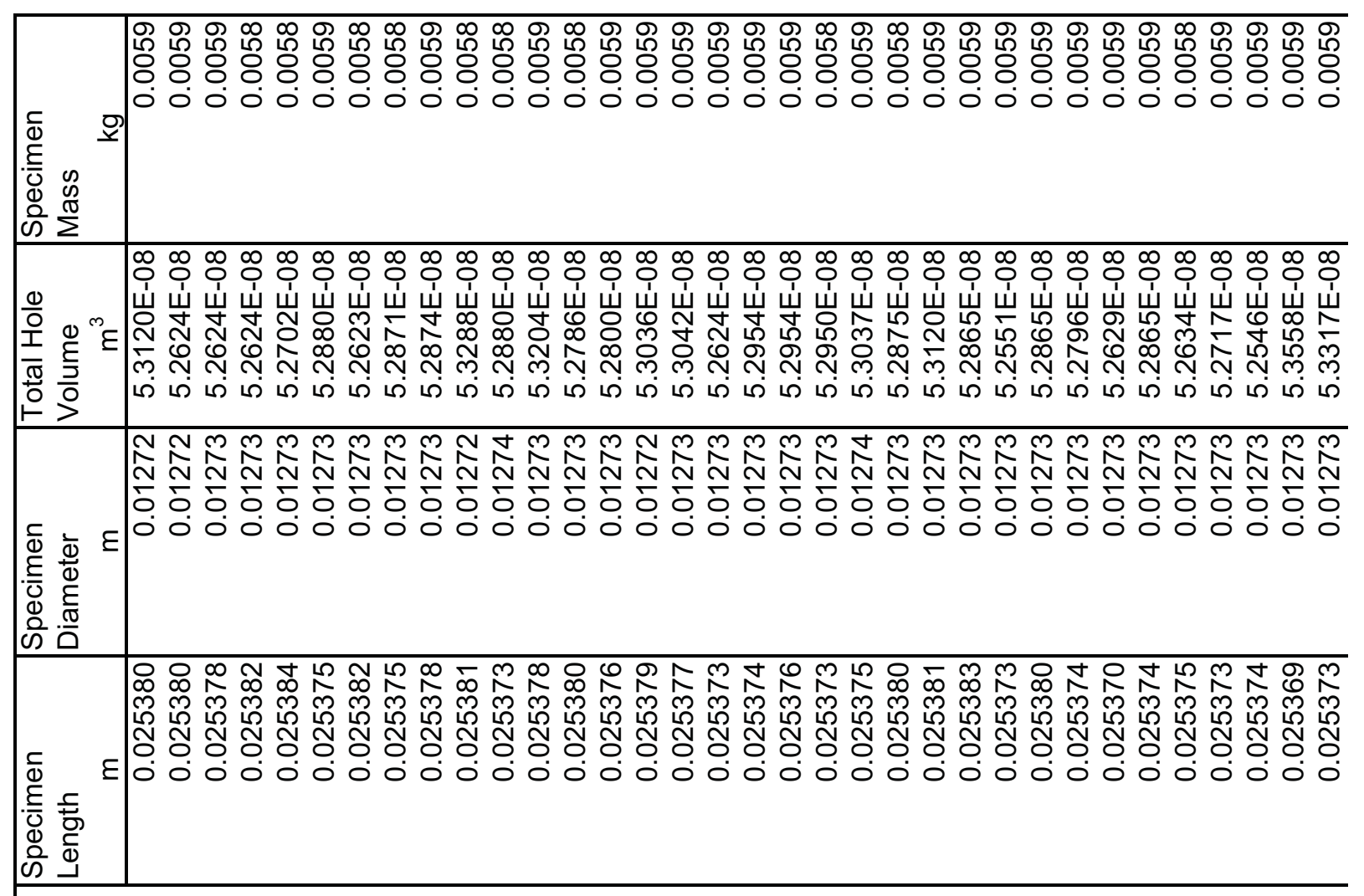

\begin{tabular}{|c|c|}
\hline 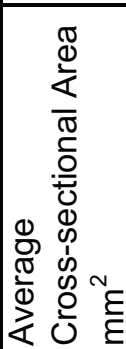 & 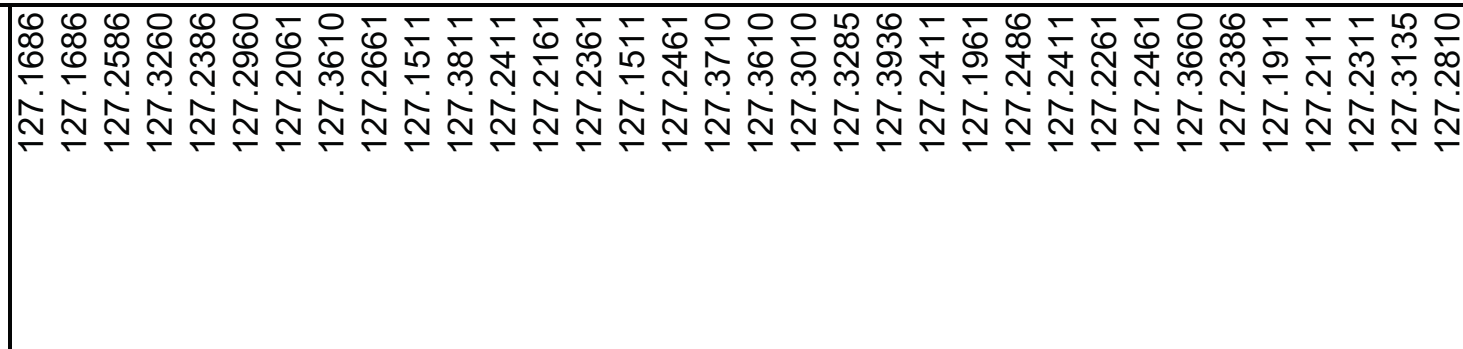 \\
\hline 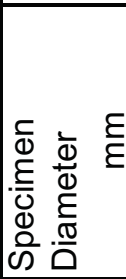 & 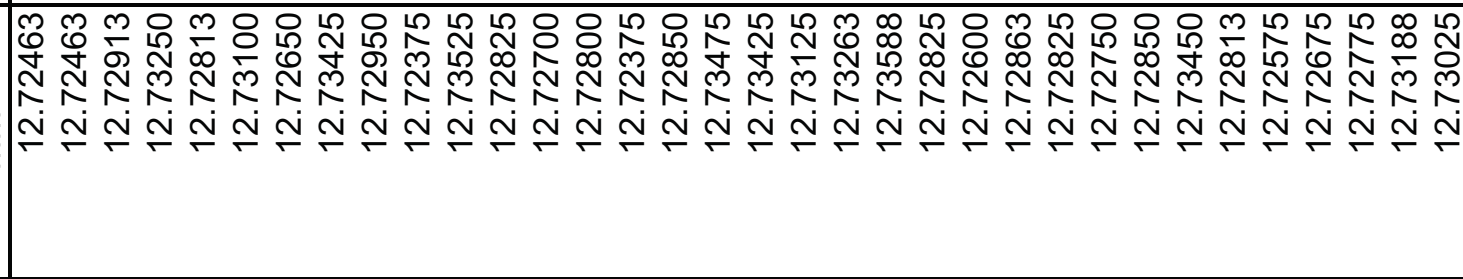 \\
\hline 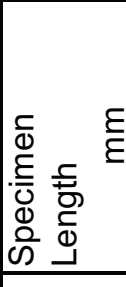 & 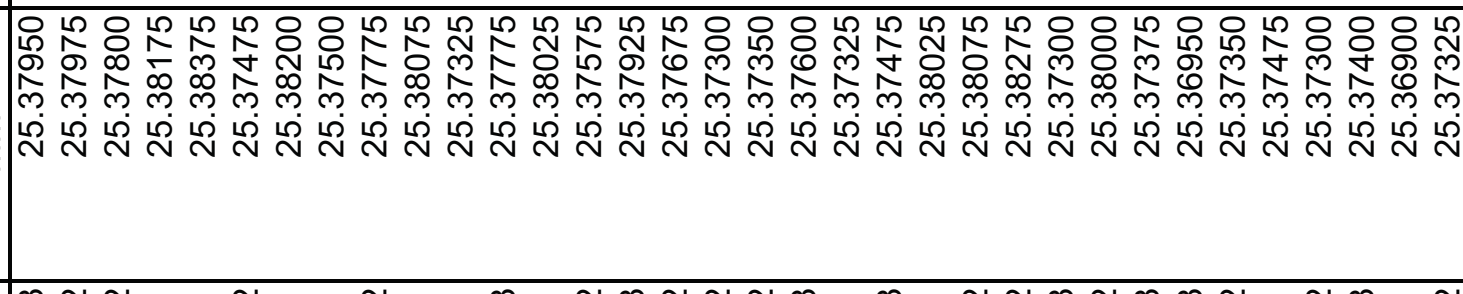 \\
\hline 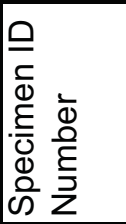 & 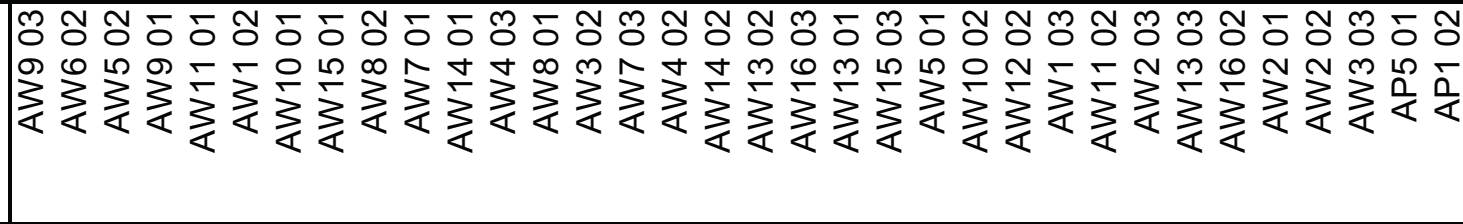 \\
\hline
\end{tabular}




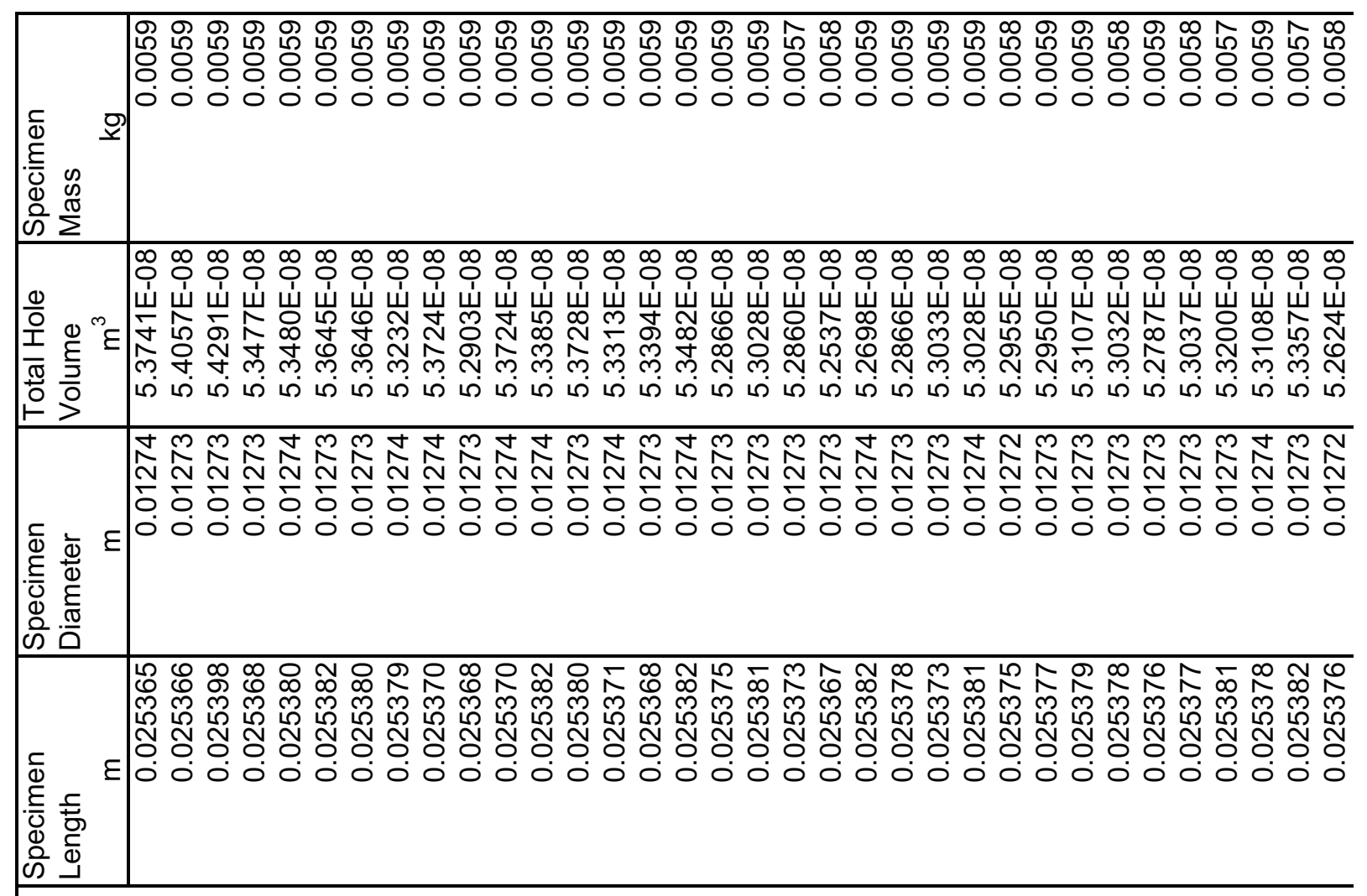

\begin{tabular}{|c|c|}
\hline 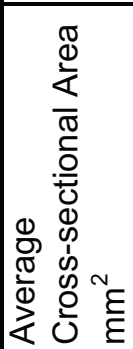 & 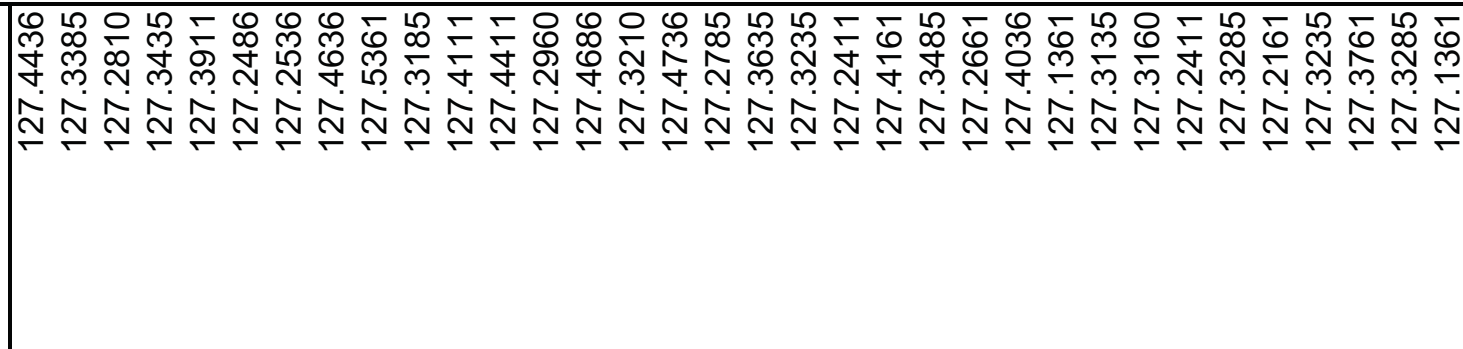 \\
\hline 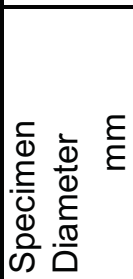 & 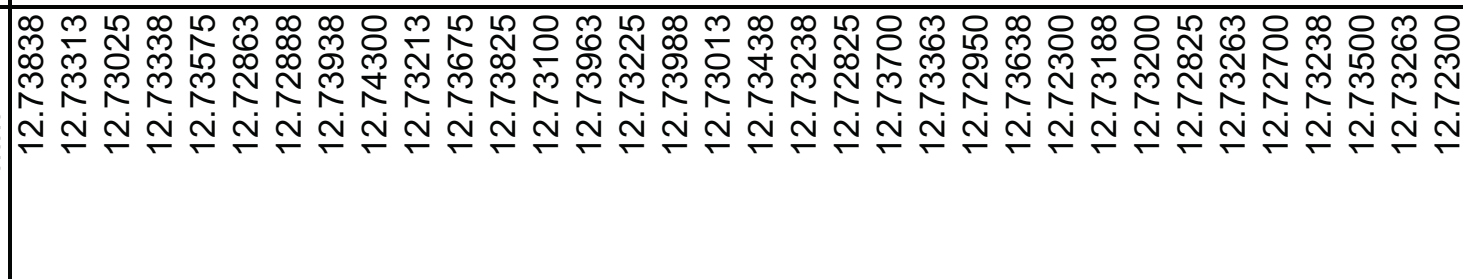 \\
\hline 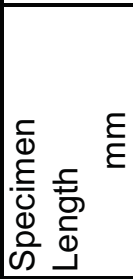 & 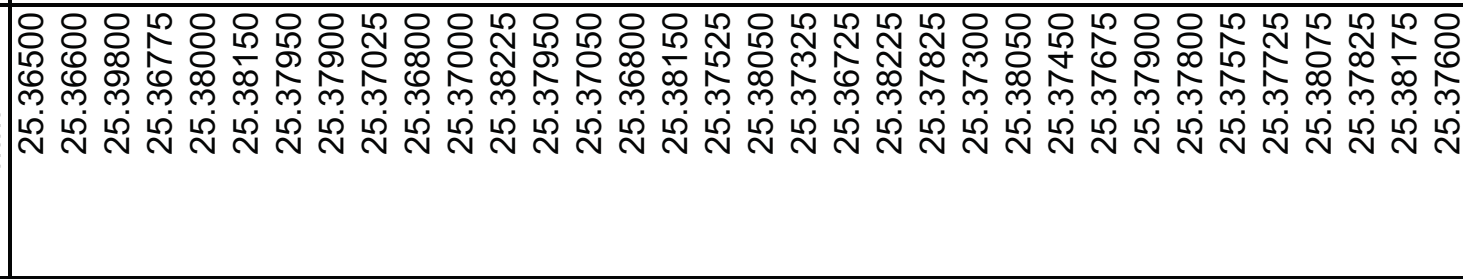 \\
\hline 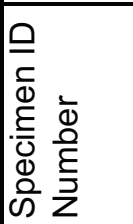 & 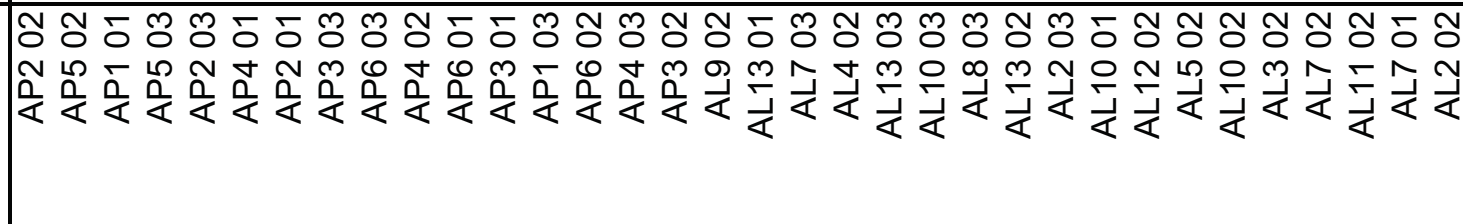 \\
\hline
\end{tabular}




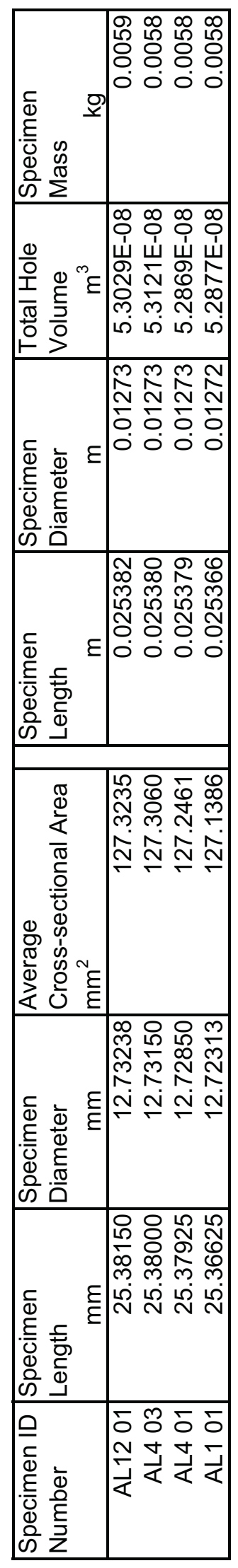

$\frac{1}{n}$ 


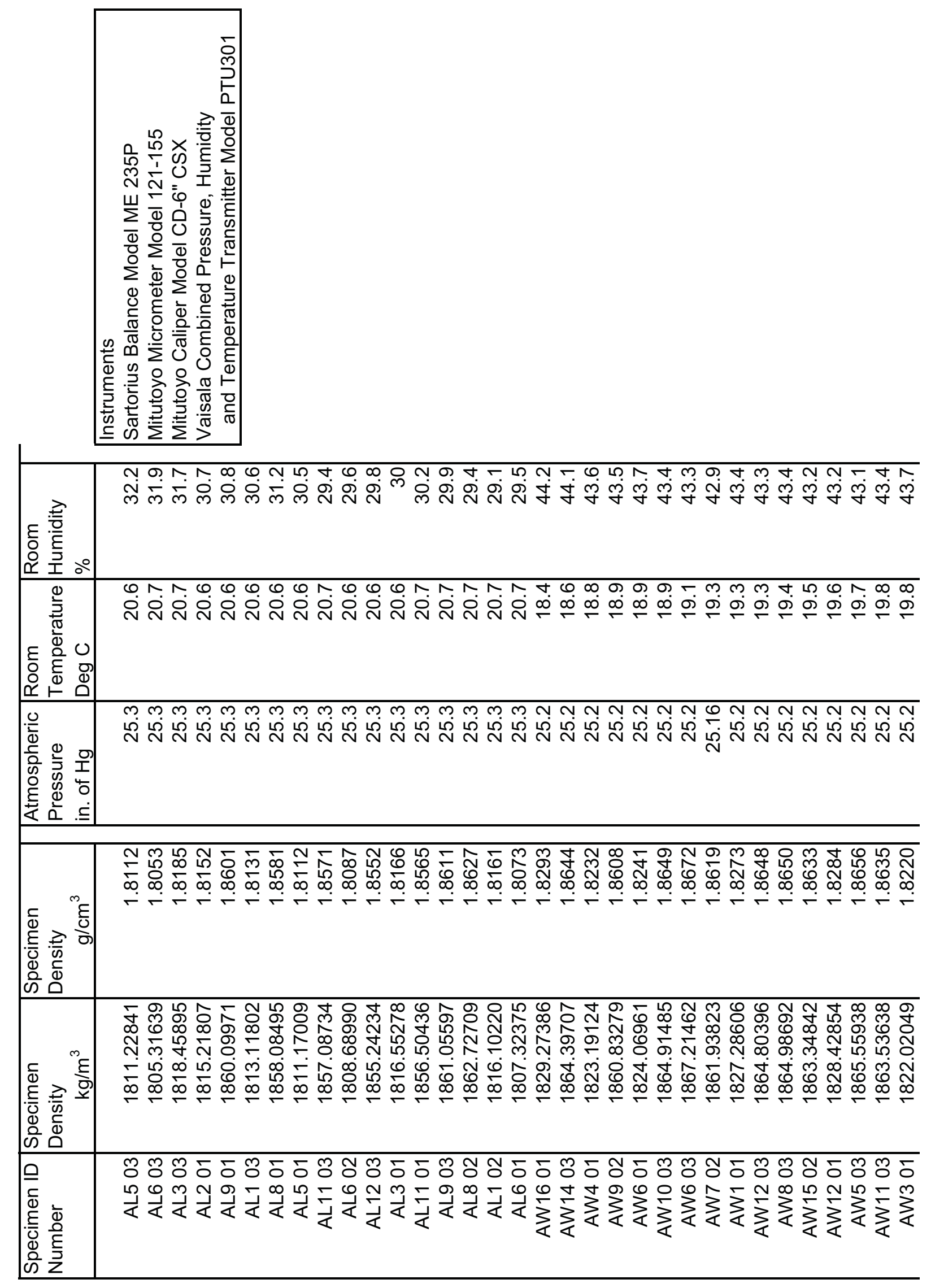




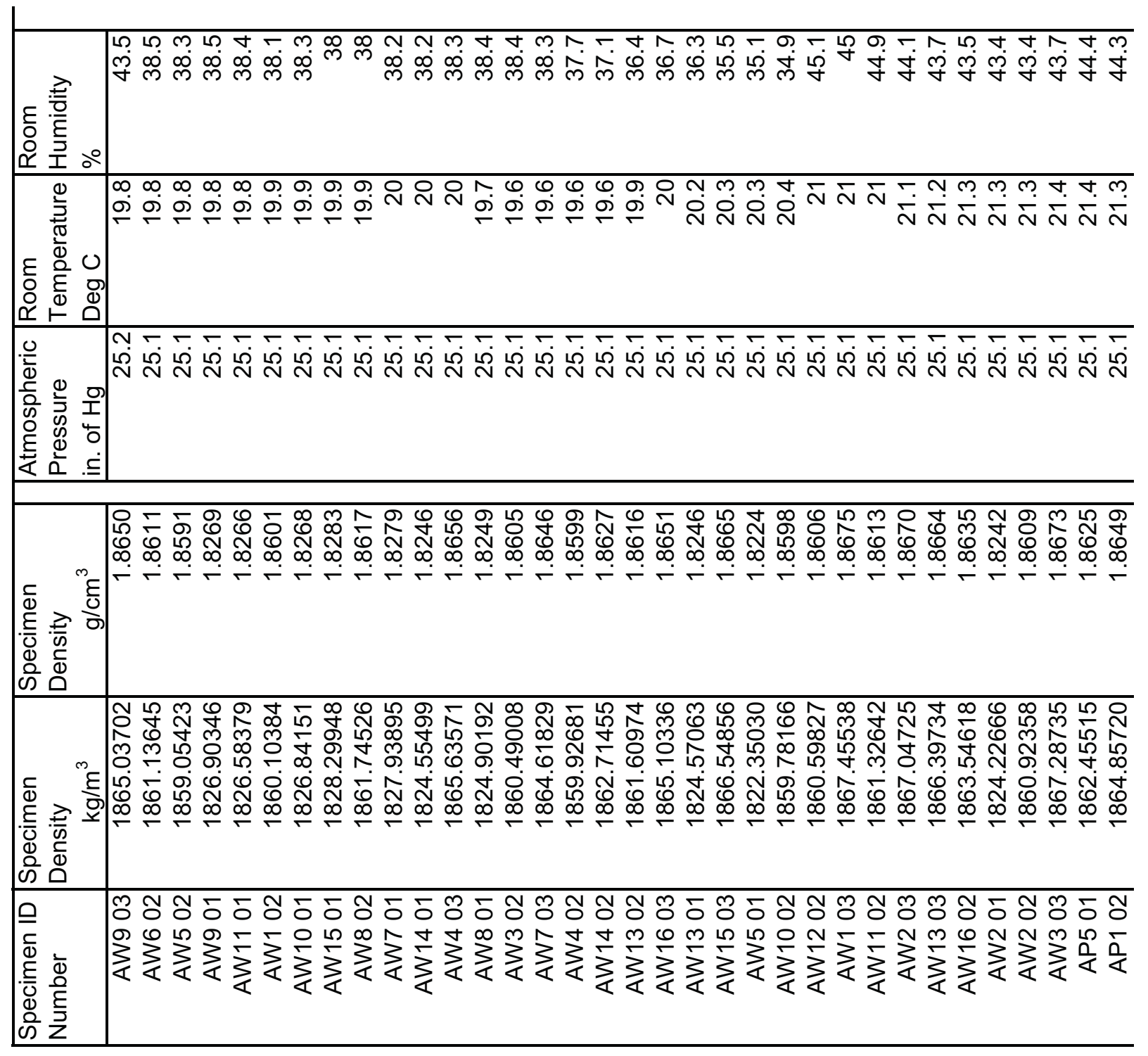




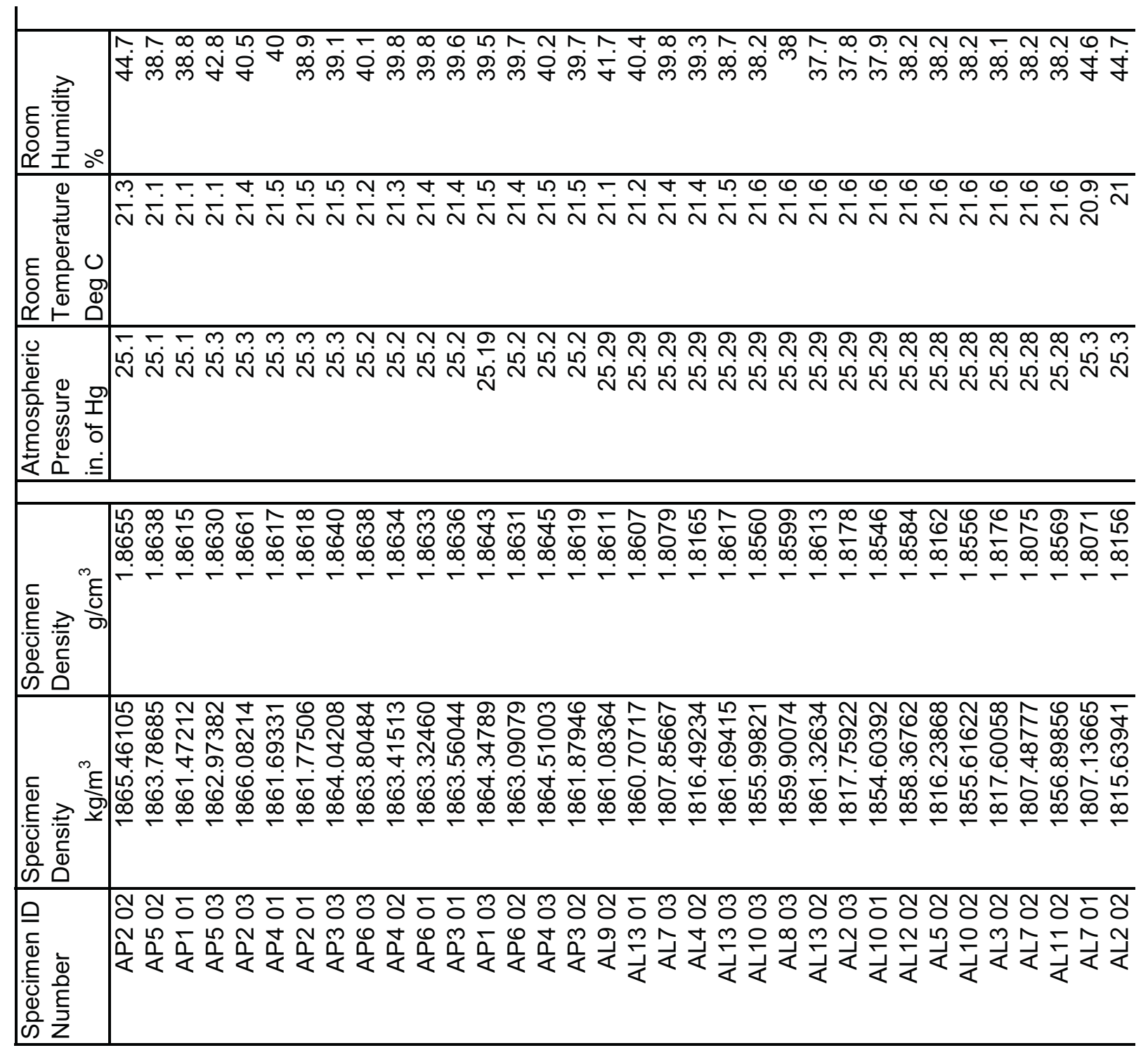




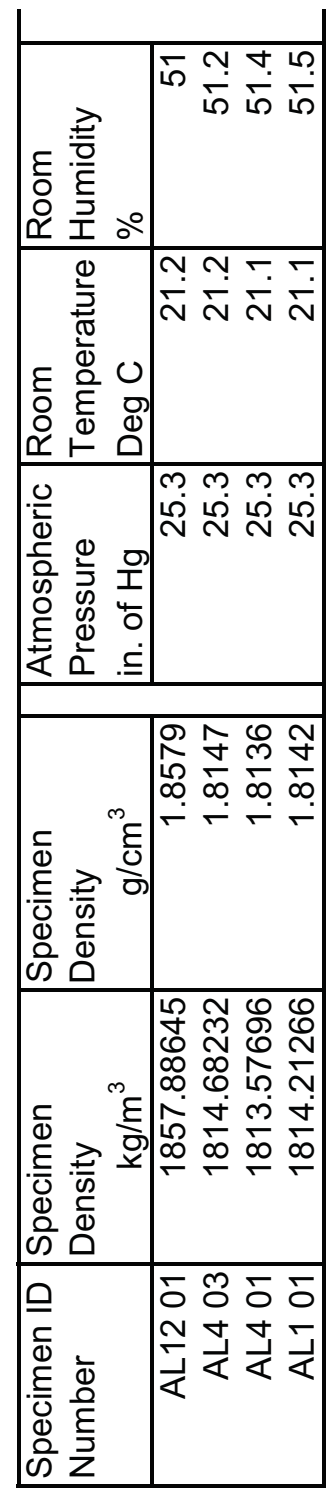

ำ 


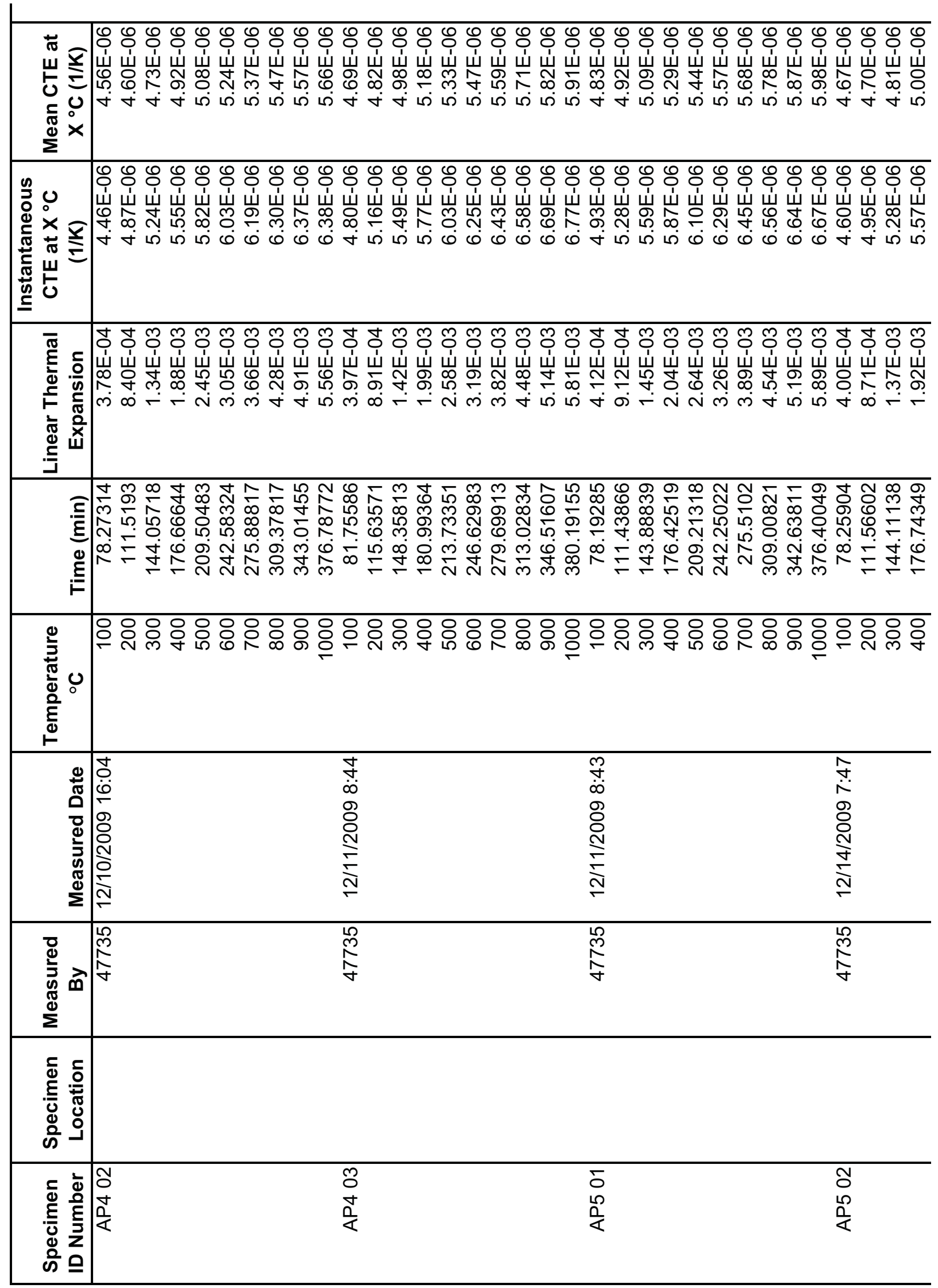




\begin{tabular}{|c|c|}
\hline 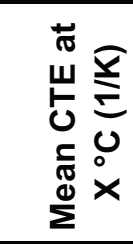 & 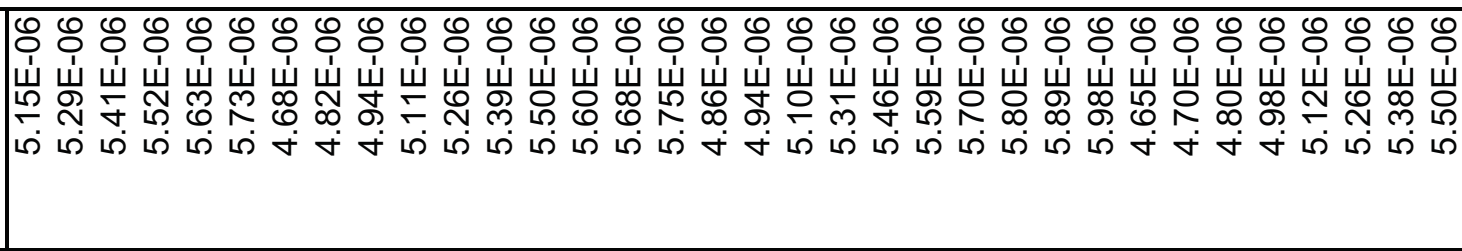 \\
\hline 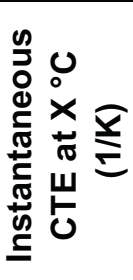 & 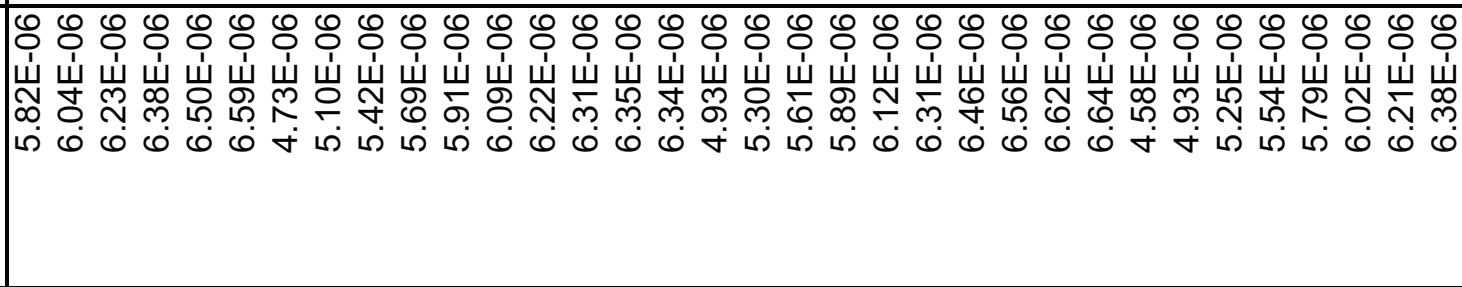 \\
\hline 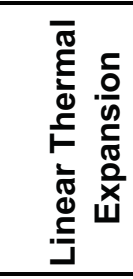 & 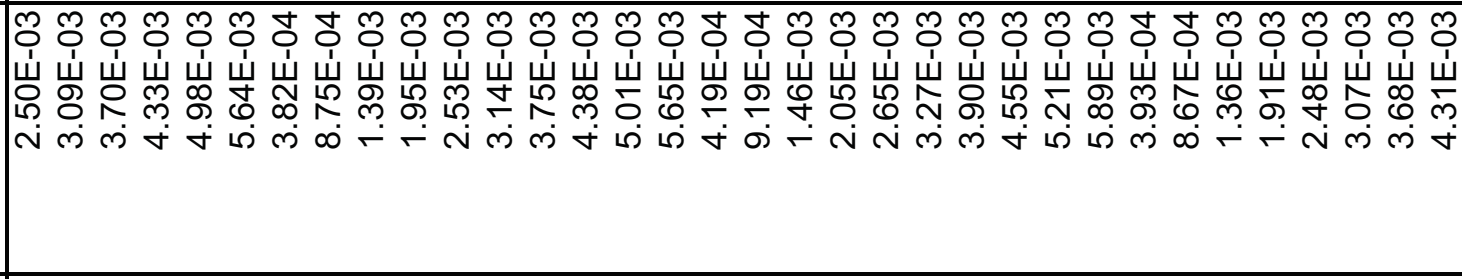 \\
\hline 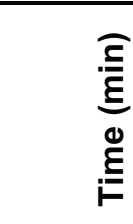 & 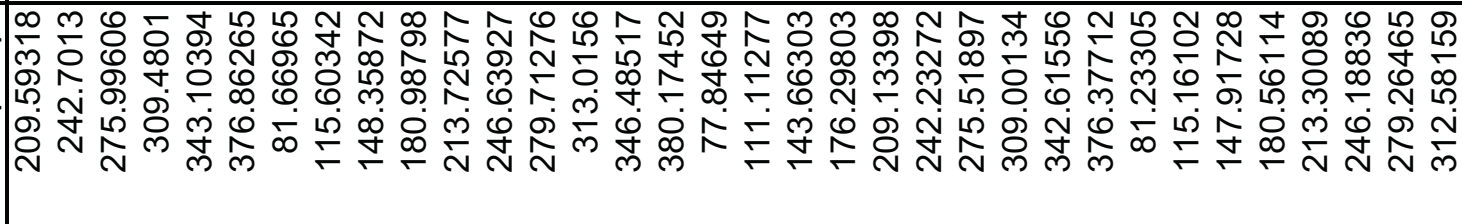 \\
\hline 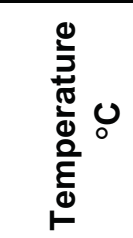 & 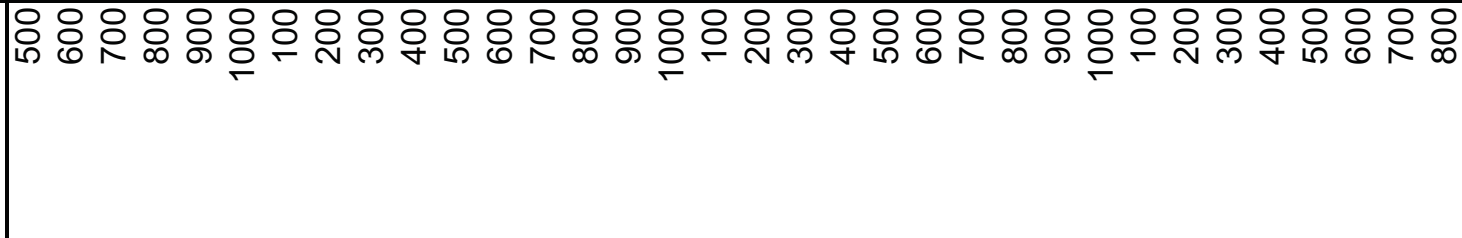 \\
\hline 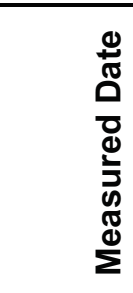 & 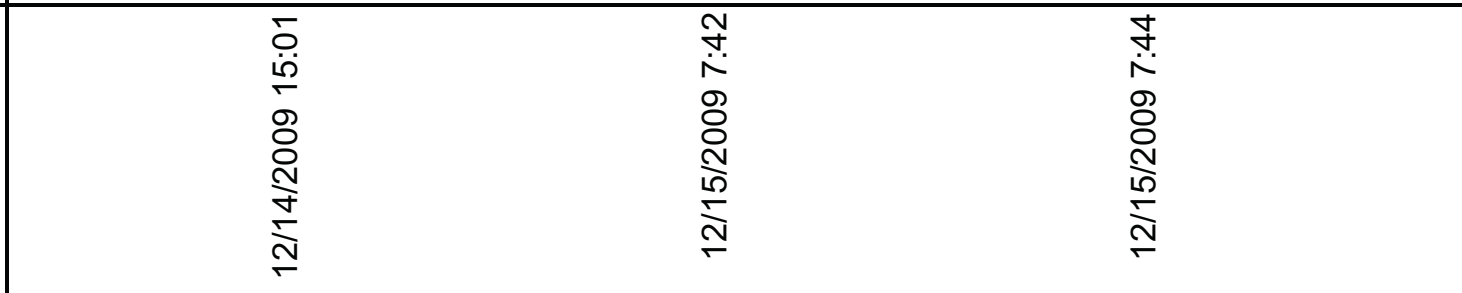 \\
\hline 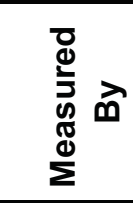 & 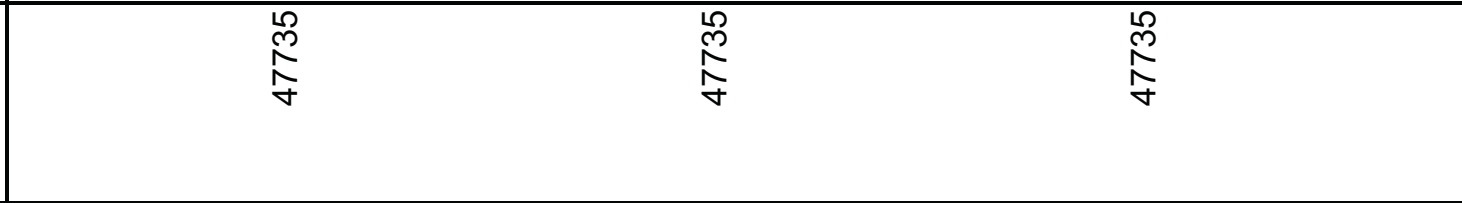 \\
\hline 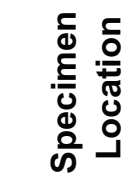 & \\
\hline 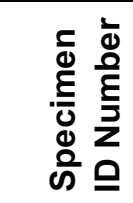 & $\begin{array}{l}\text { O } \\
0 \\
0 \\
\frac{1}{\alpha}\end{array}$ \\
\hline
\end{tabular}




\begin{tabular}{|c|c|}
\hline 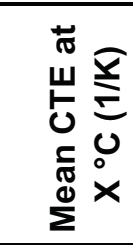 & 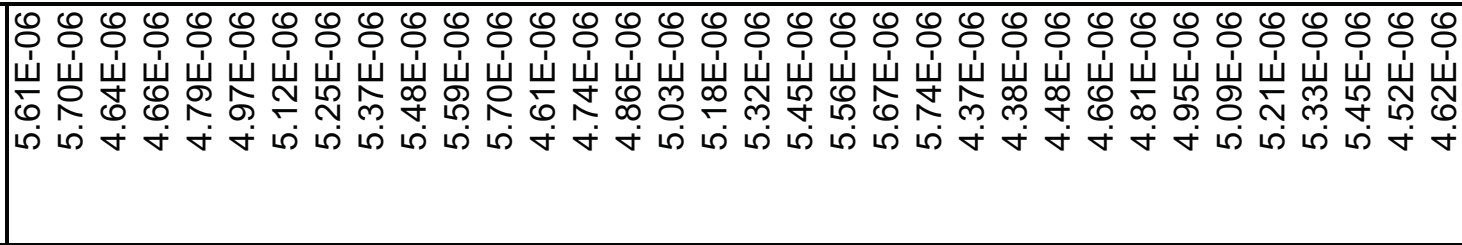 \\
\hline 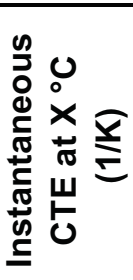 & 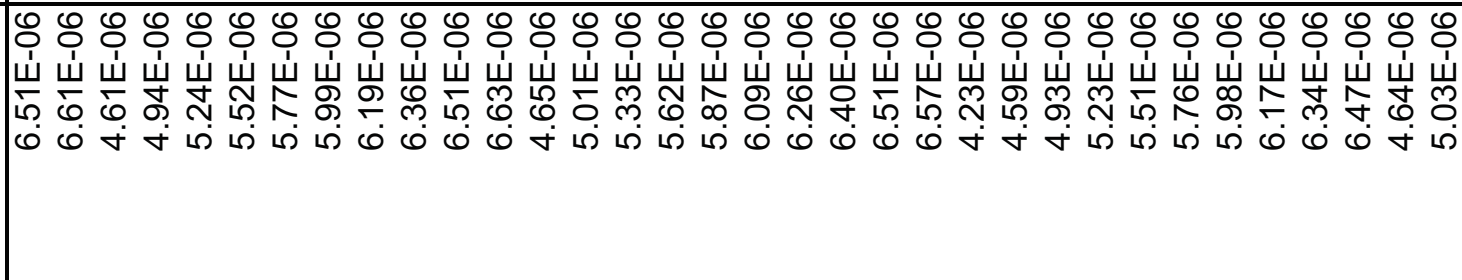 \\
\hline 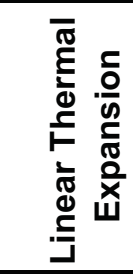 & 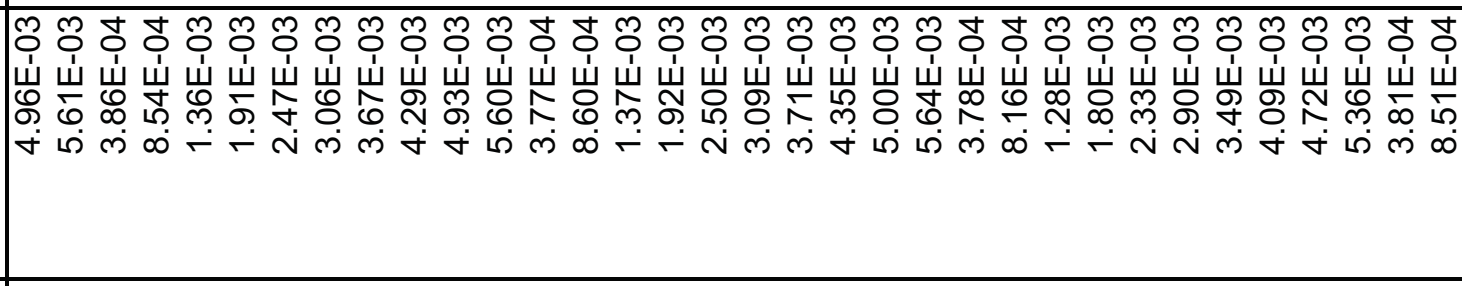 \\
\hline 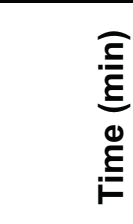 & 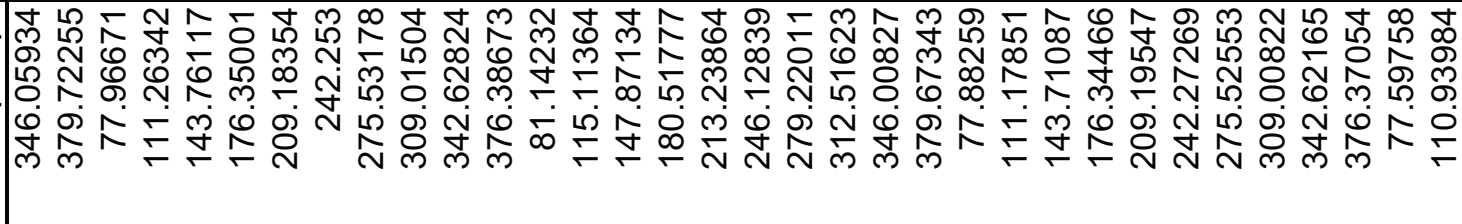 \\
\hline 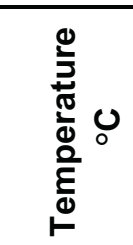 & 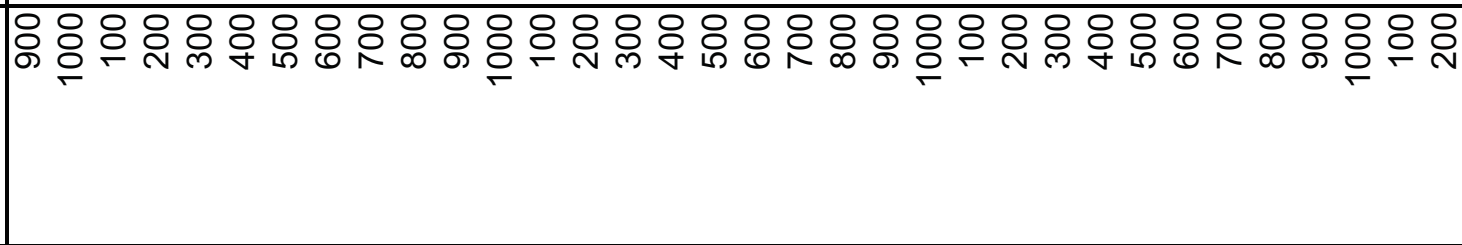 \\
\hline 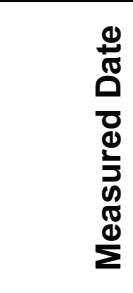 & 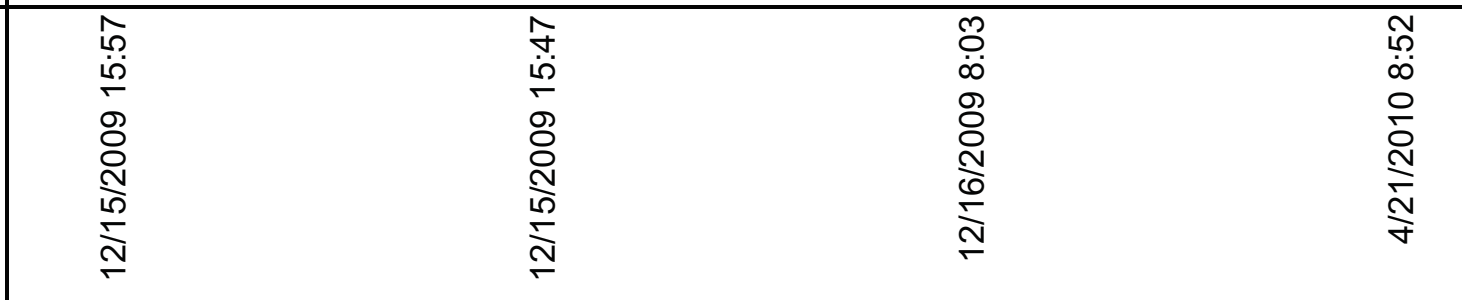 \\
\hline 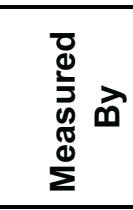 & $\begin{array}{ll}\stackrel{L}{\rho} & \stackrel{L}{\infty} \\
\stackrel{f}{f} & \stackrel{f}{f}\end{array}$ \\
\hline 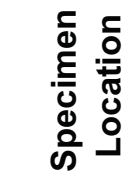 & \\
\hline 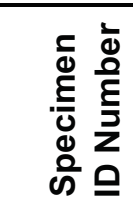 & 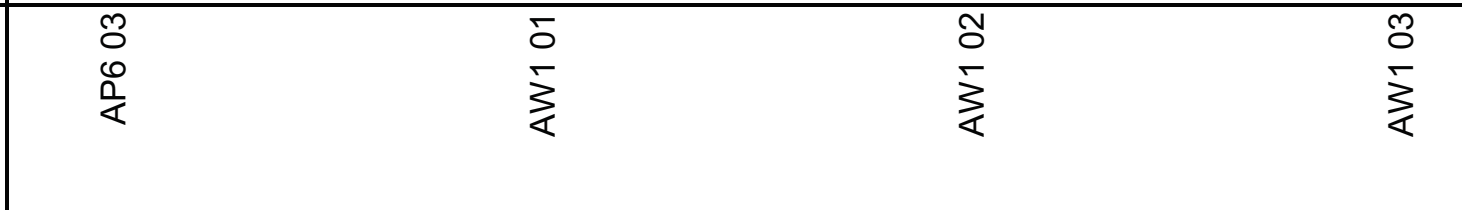 \\
\hline
\end{tabular}




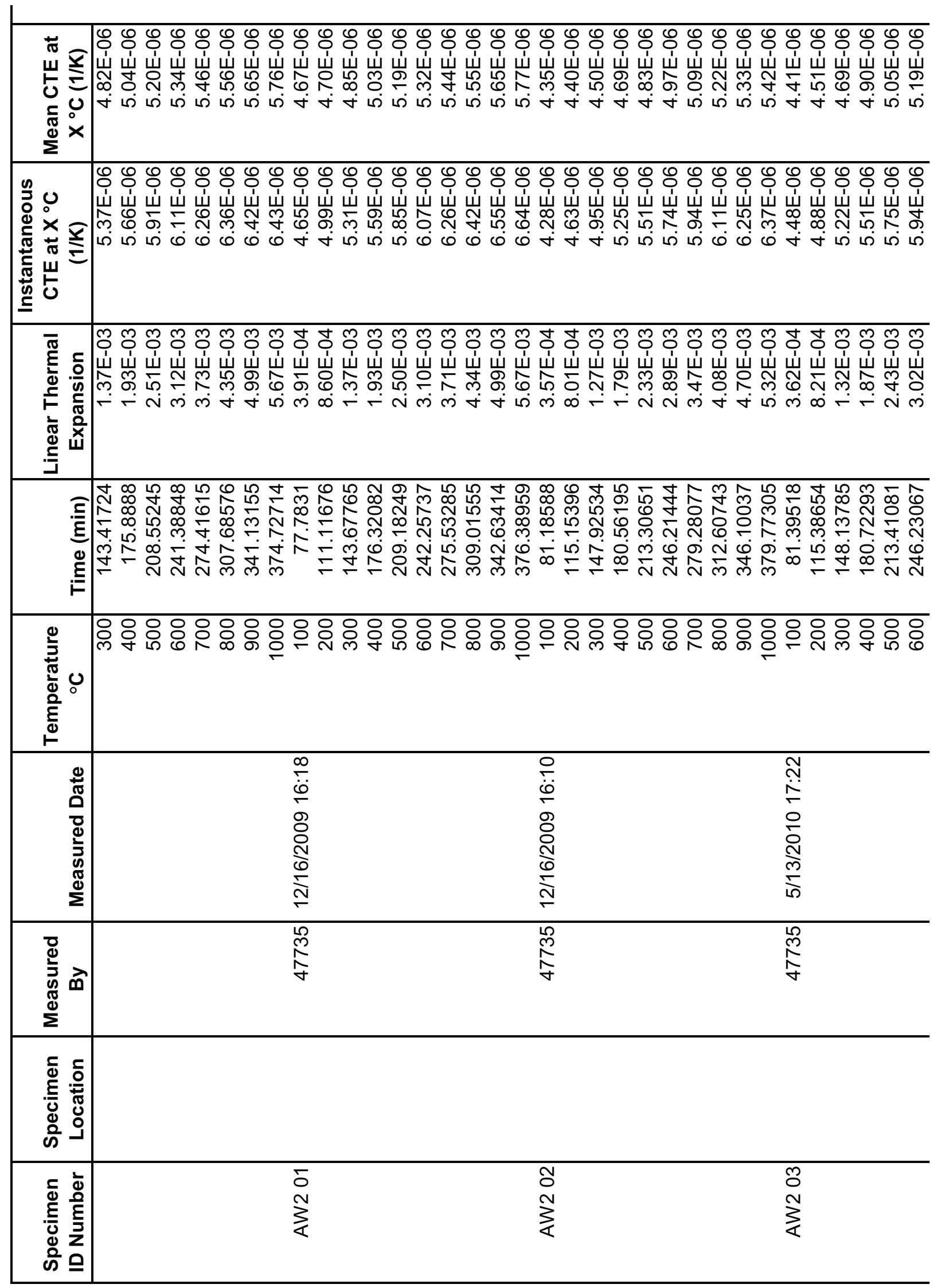




\begin{tabular}{|c|c|}
\hline 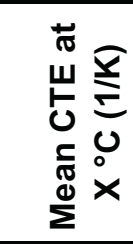 & 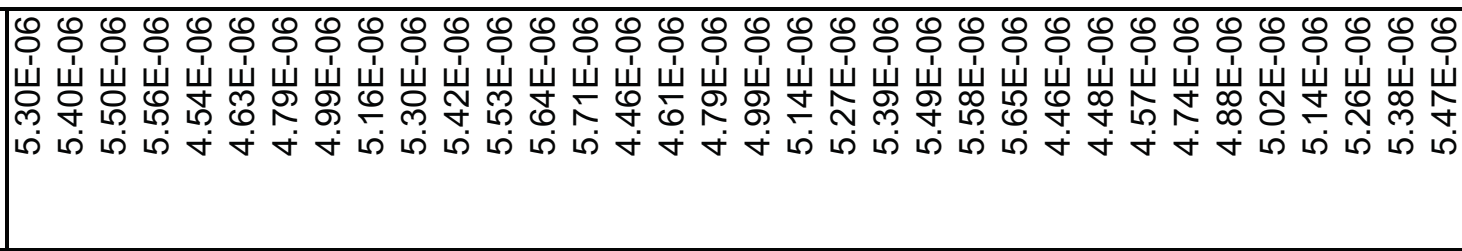 \\
\hline 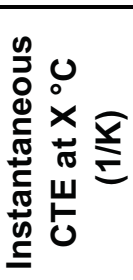 & 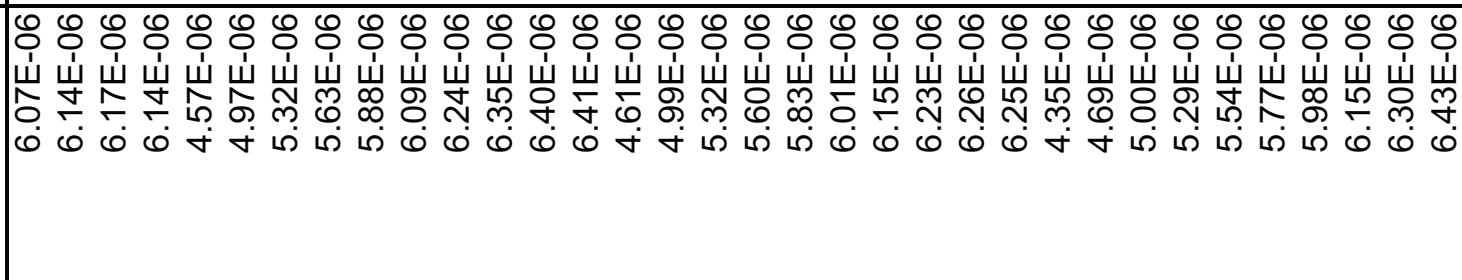 \\
\hline 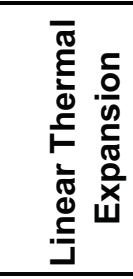 & 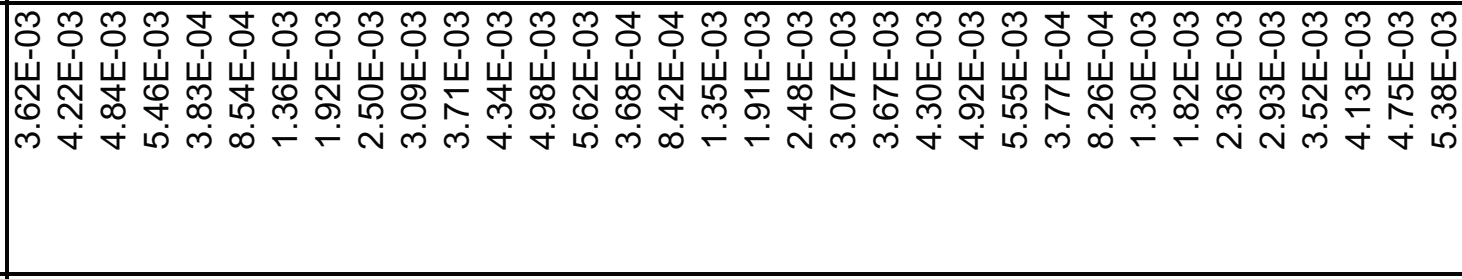 \\
\hline 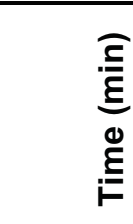 & 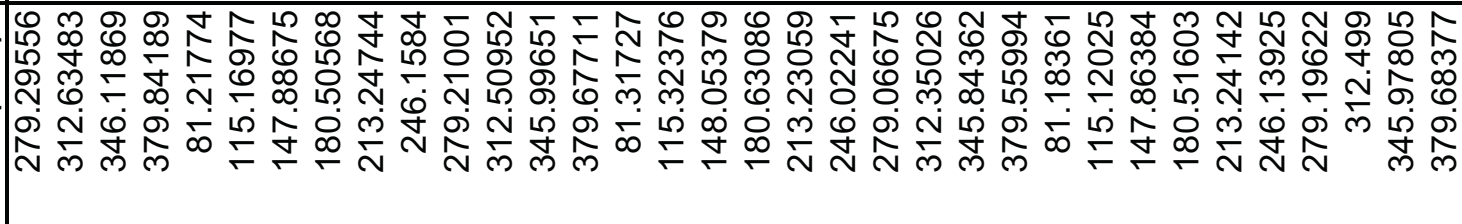 \\
\hline 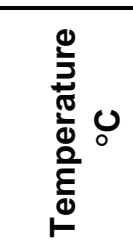 & 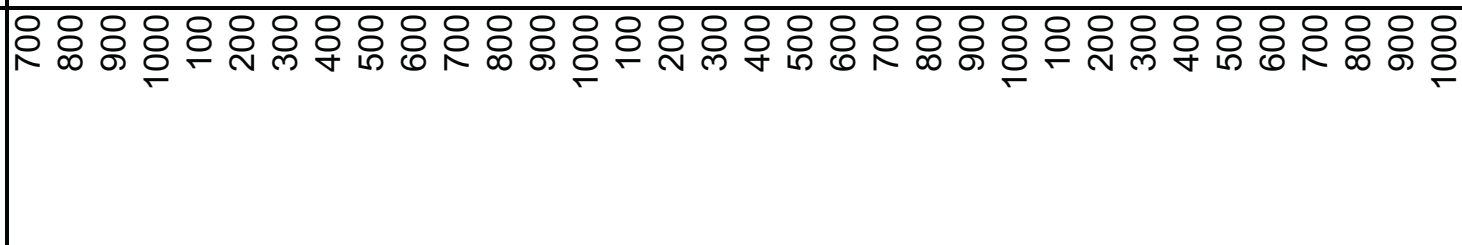 \\
\hline 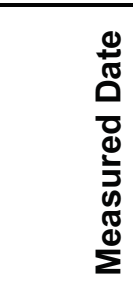 & 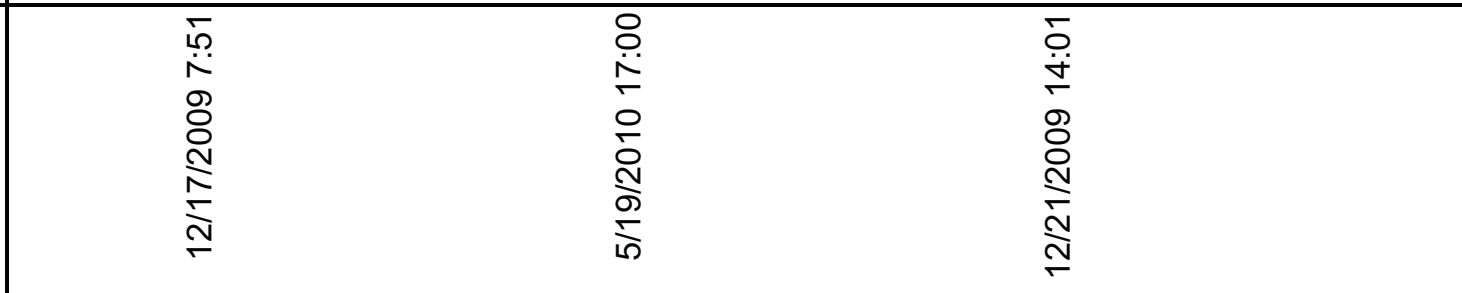 \\
\hline 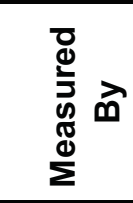 & 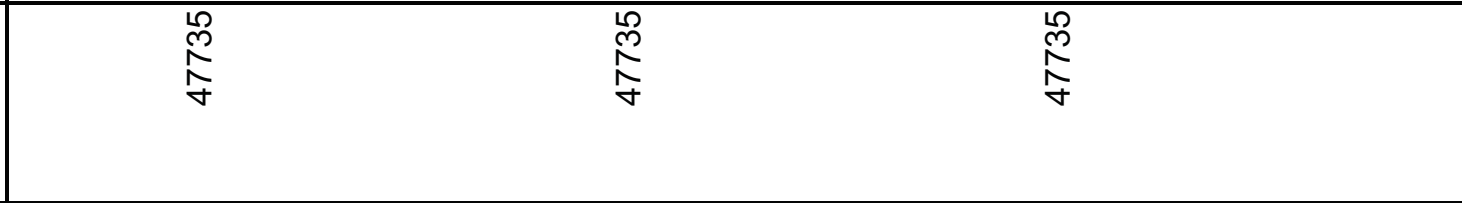 \\
\hline 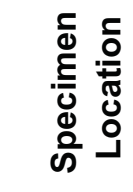 & \\
\hline 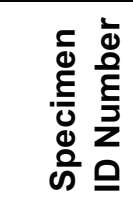 & 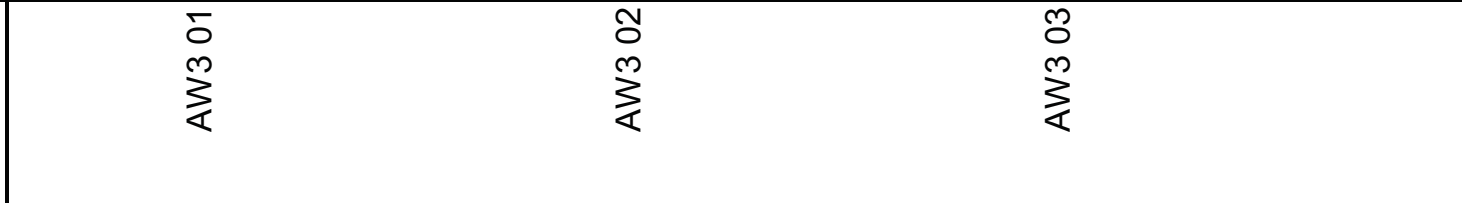 \\
\hline
\end{tabular}




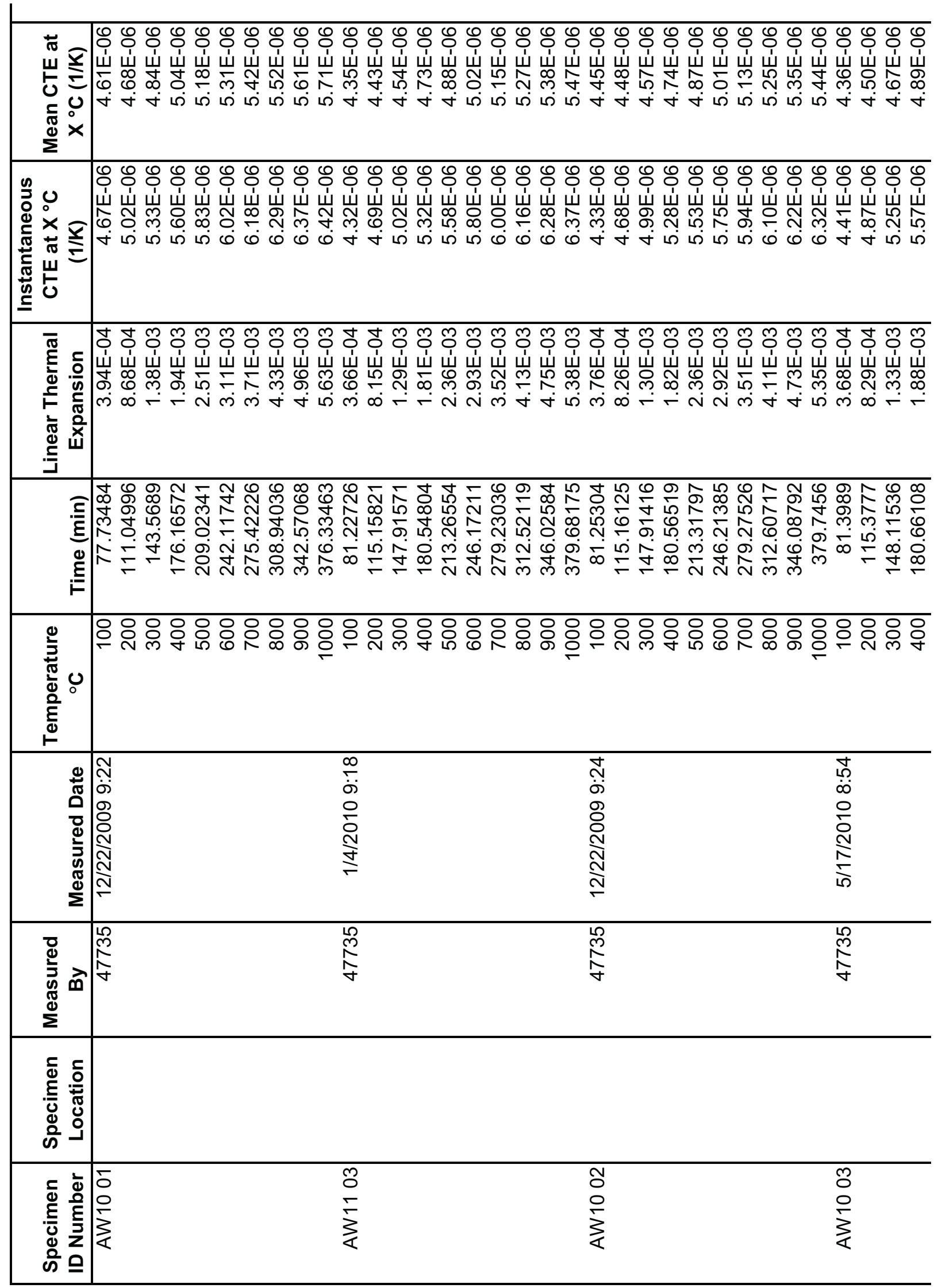




\begin{tabular}{|c|c|}
\hline 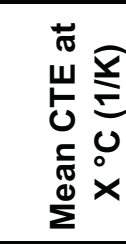 & 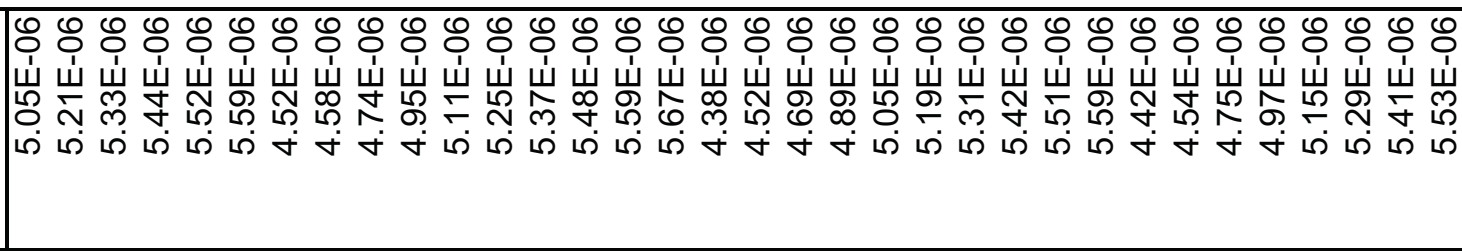 \\
\hline 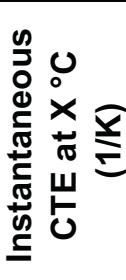 & 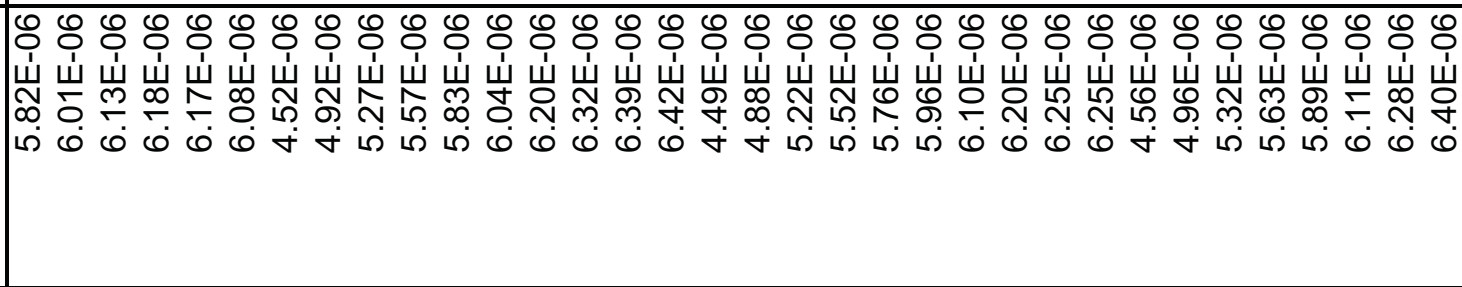 \\
\hline 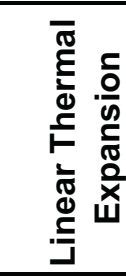 & 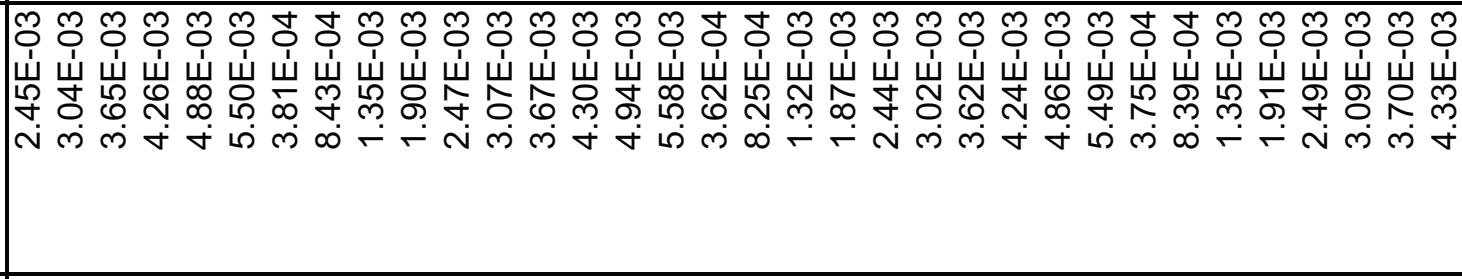 \\
\hline 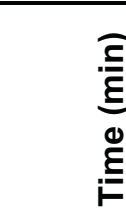 & 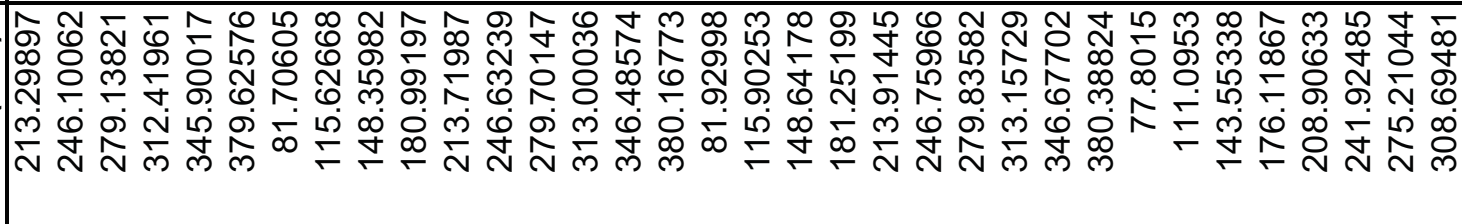 \\
\hline 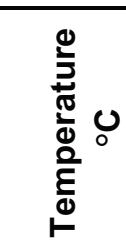 & 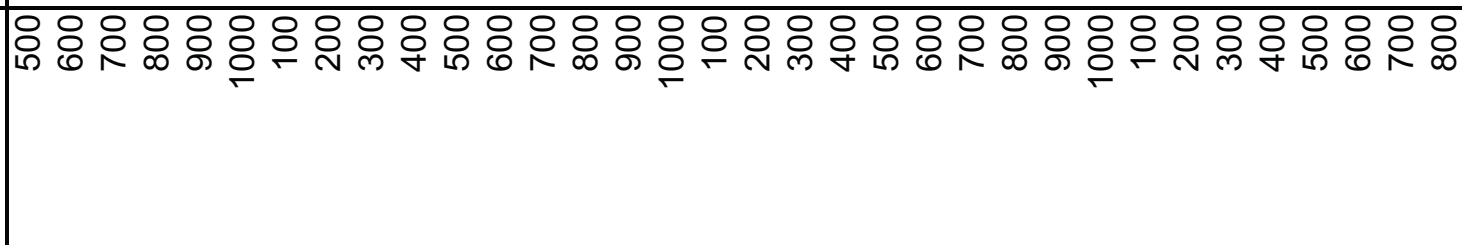 \\
\hline 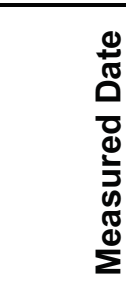 & 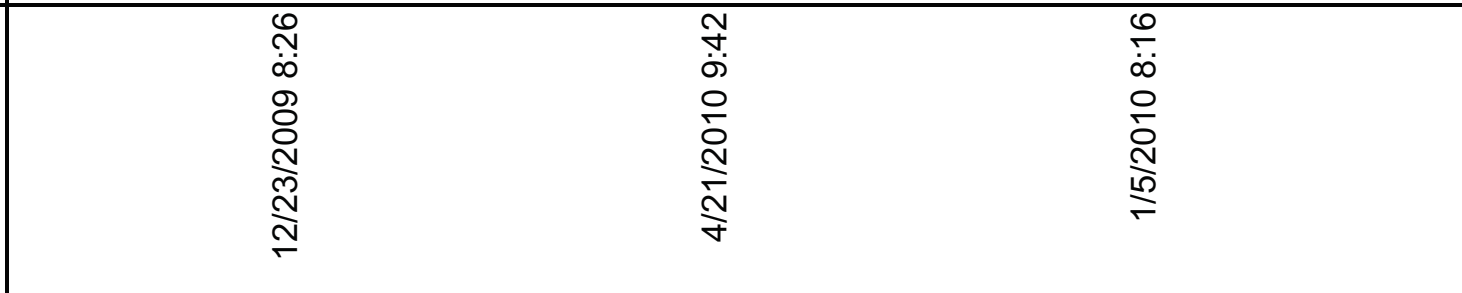 \\
\hline 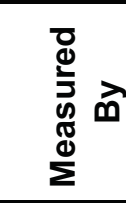 & 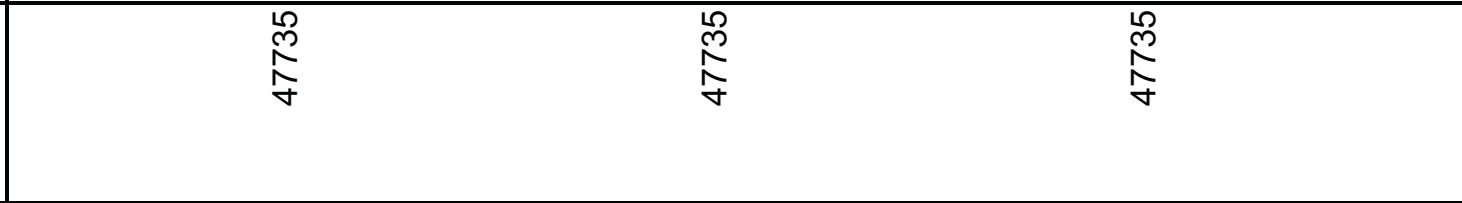 \\
\hline 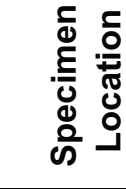 & \\
\hline 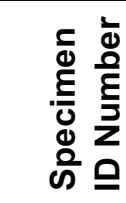 & $\stackrel{\substack{O \\
⿱}}{\vdots}$ \\
\hline
\end{tabular}




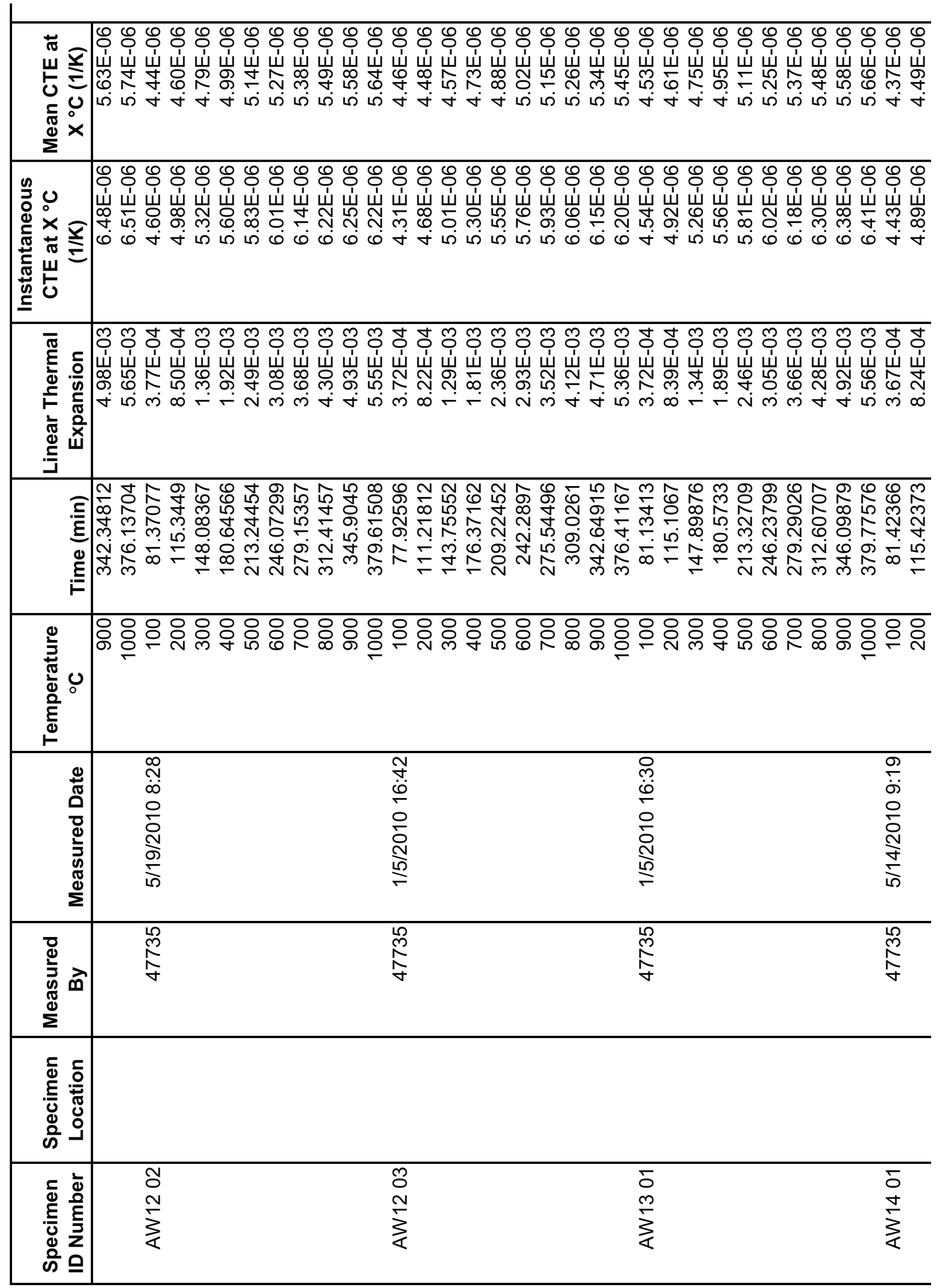




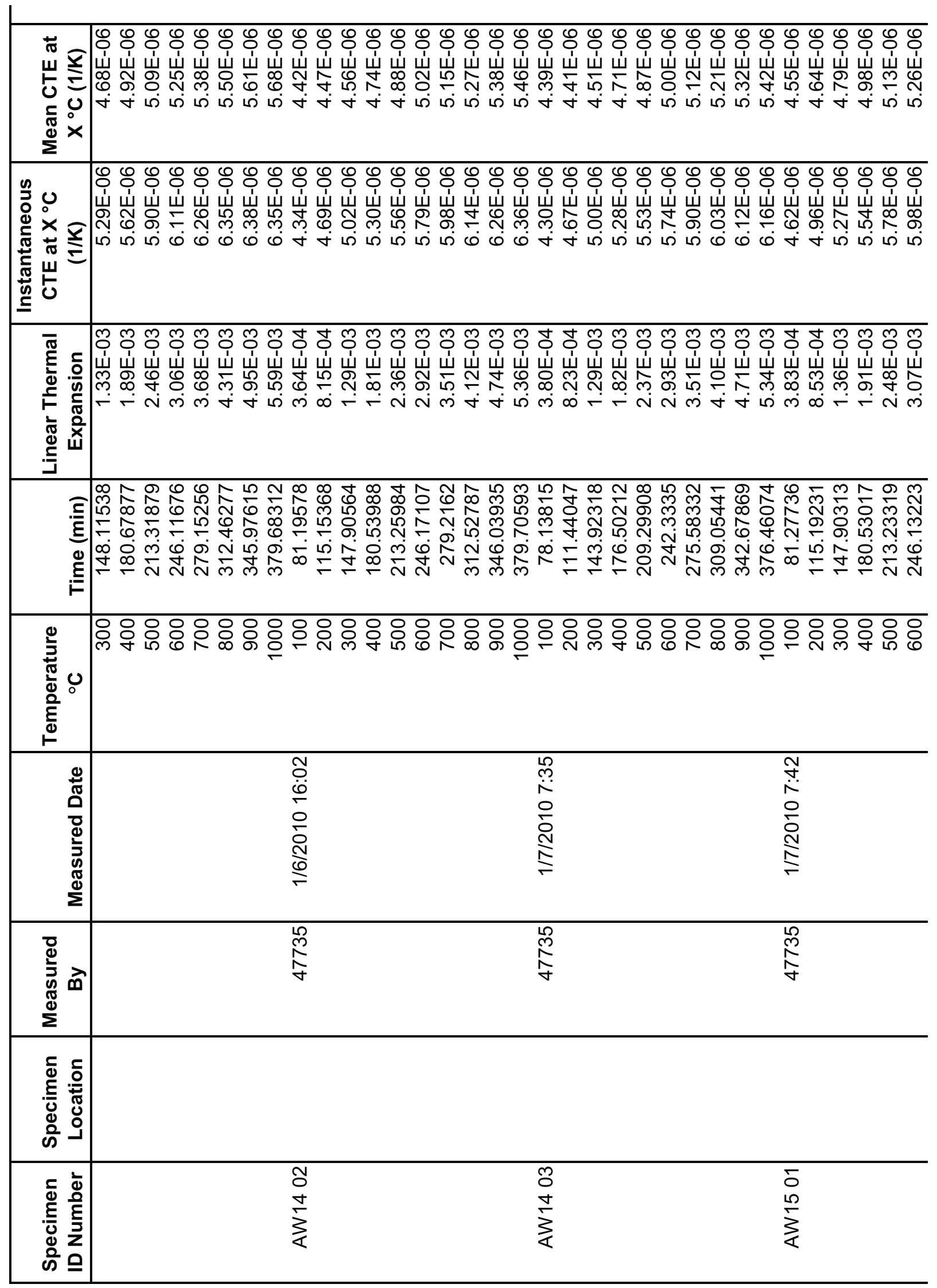




\begin{tabular}{|c|c|}
\hline 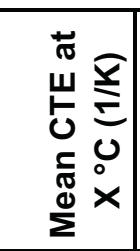 & 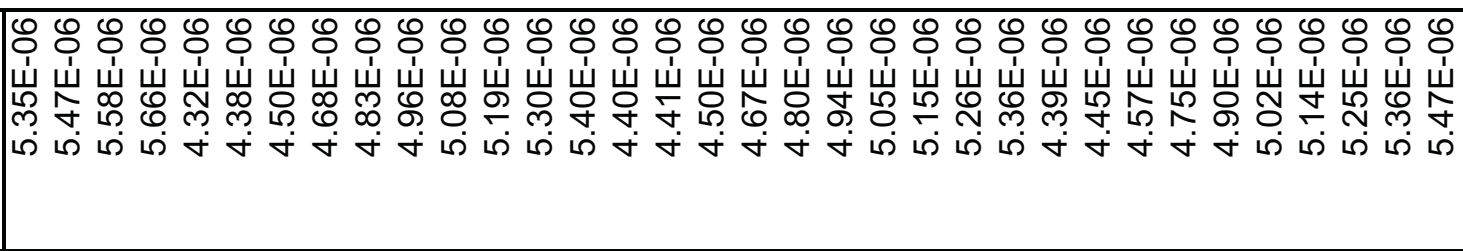 \\
\hline 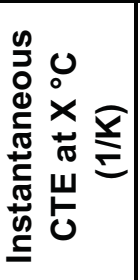 & 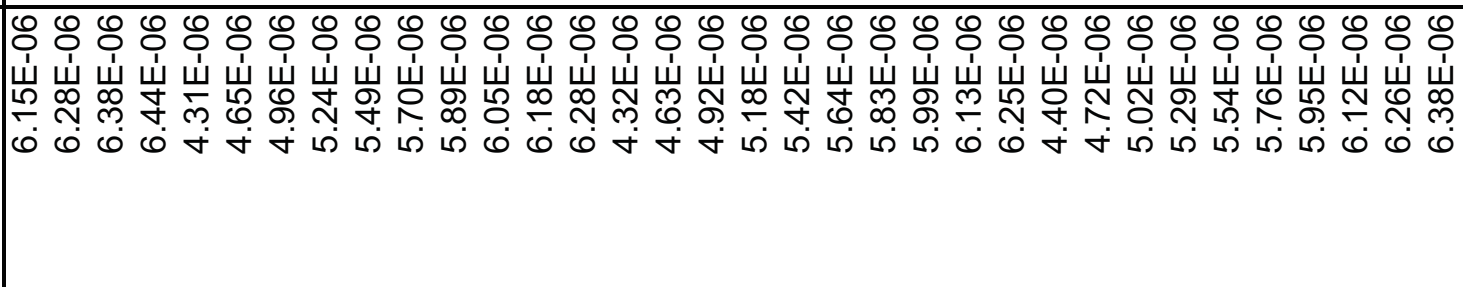 \\
\hline 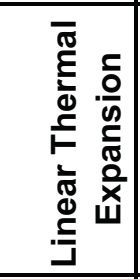 & 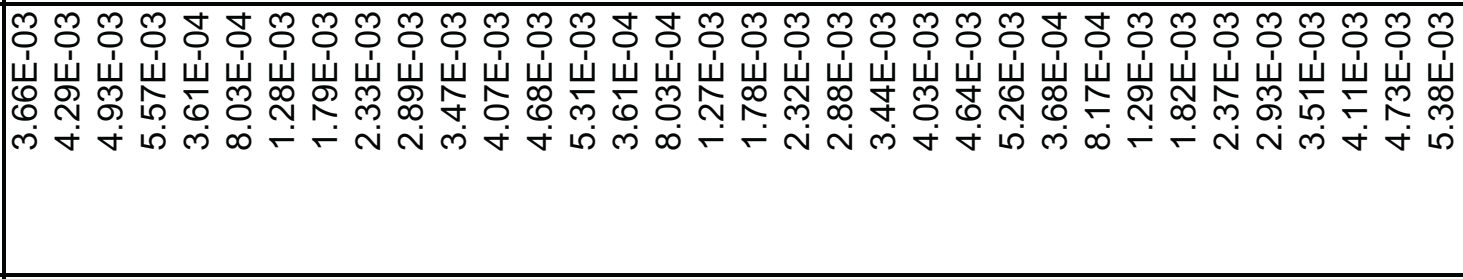 \\
\hline 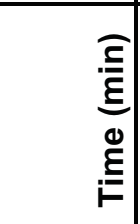 & 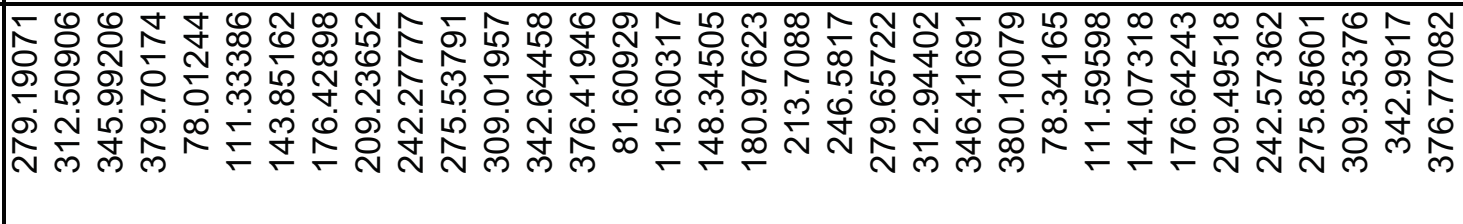 \\
\hline 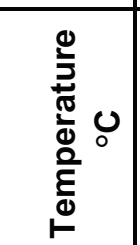 & 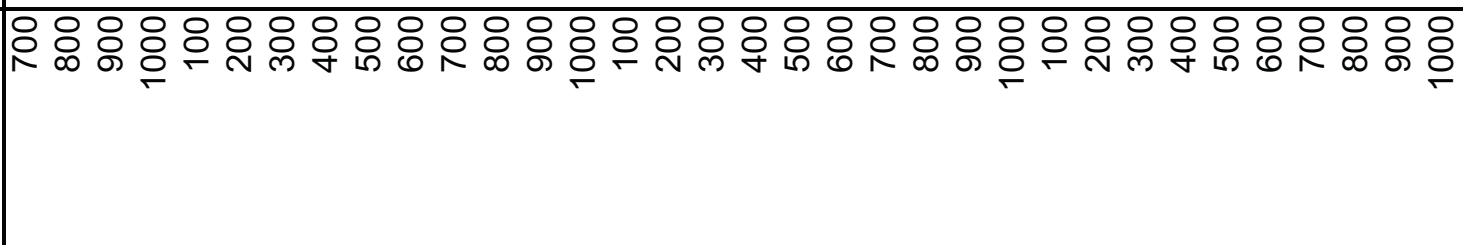 \\
\hline 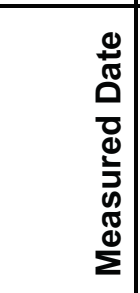 & 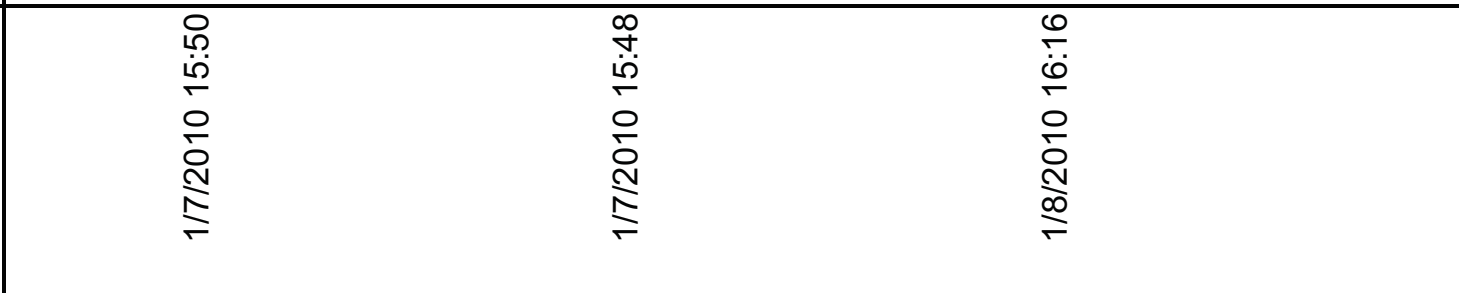 \\
\hline 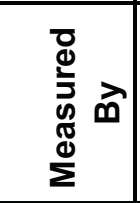 & 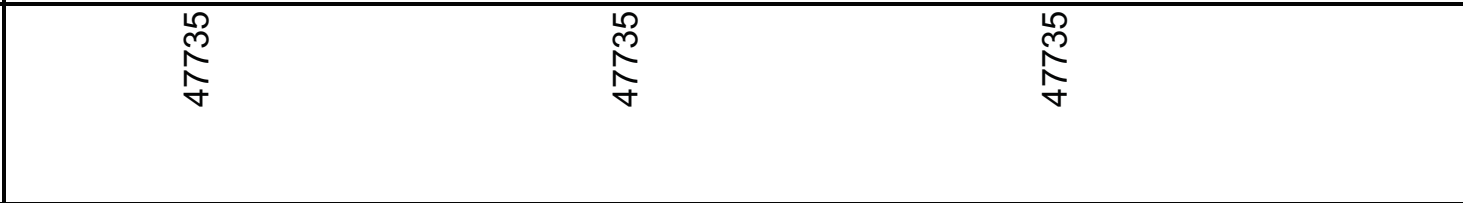 \\
\hline 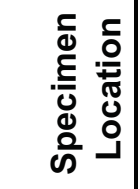 & \\
\hline 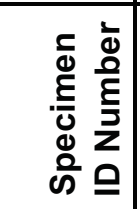 & 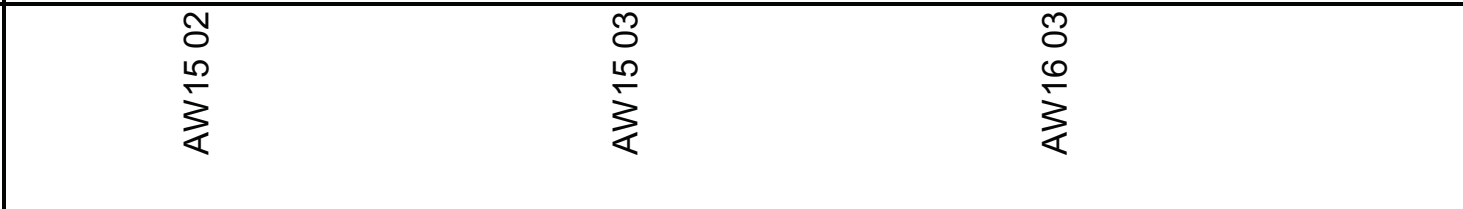 \\
\hline
\end{tabular}




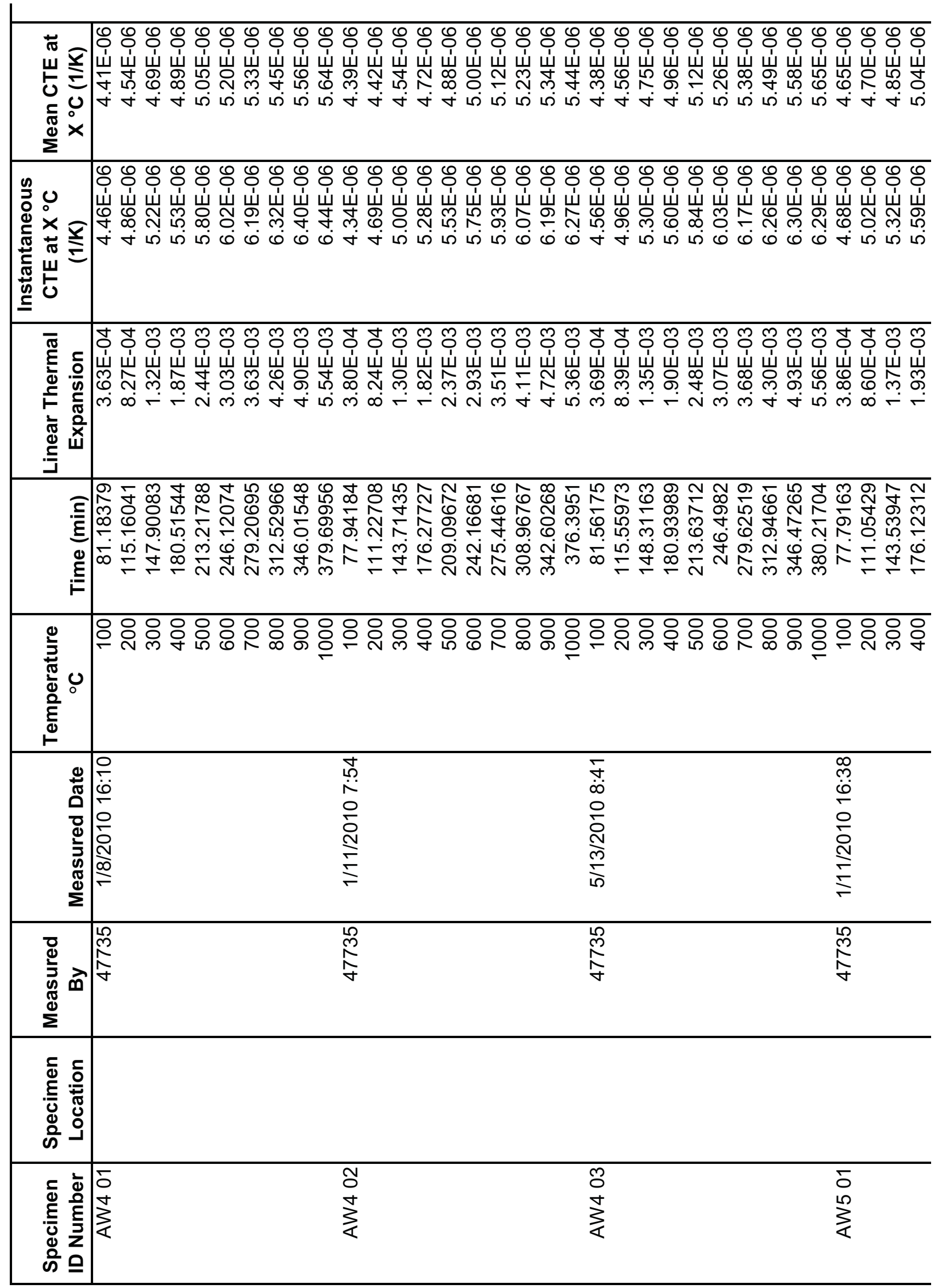




\begin{tabular}{|c|c|}
\hline 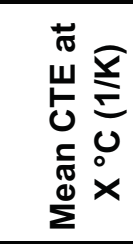 & 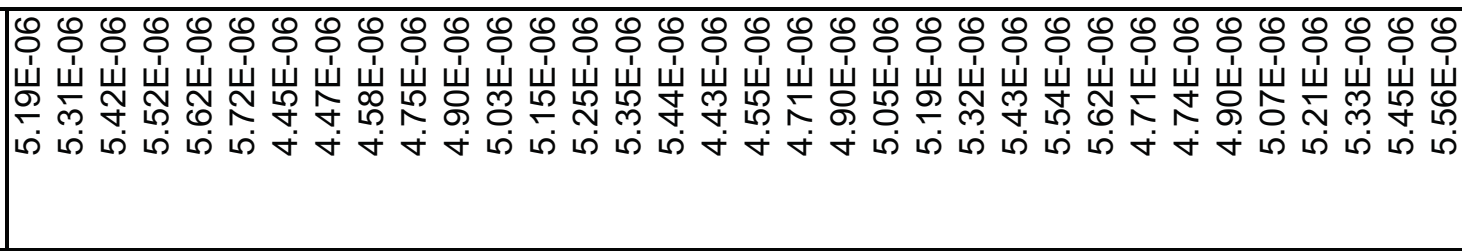 \\
\hline 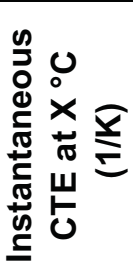 & 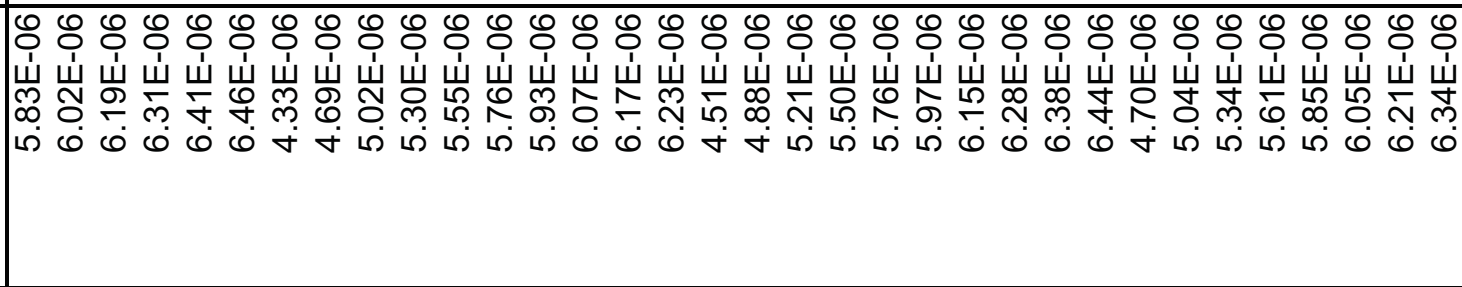 \\
\hline 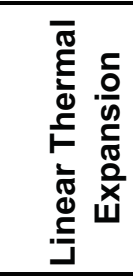 & 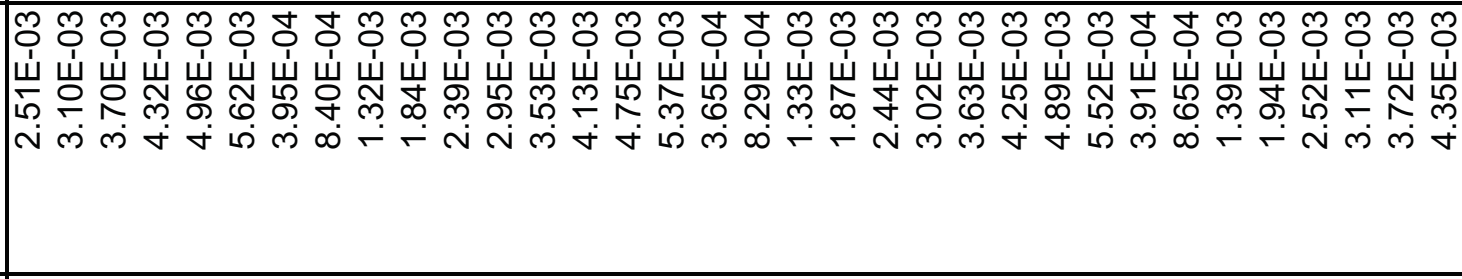 \\
\hline 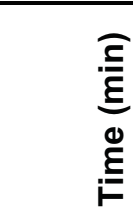 & 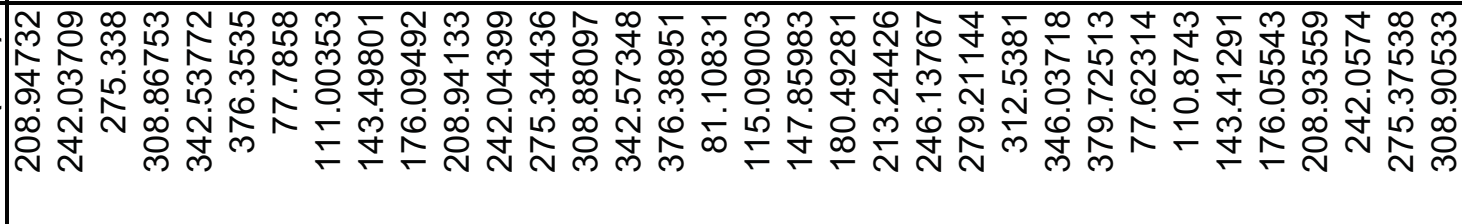 \\
\hline 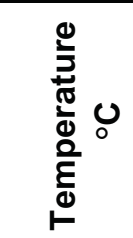 & 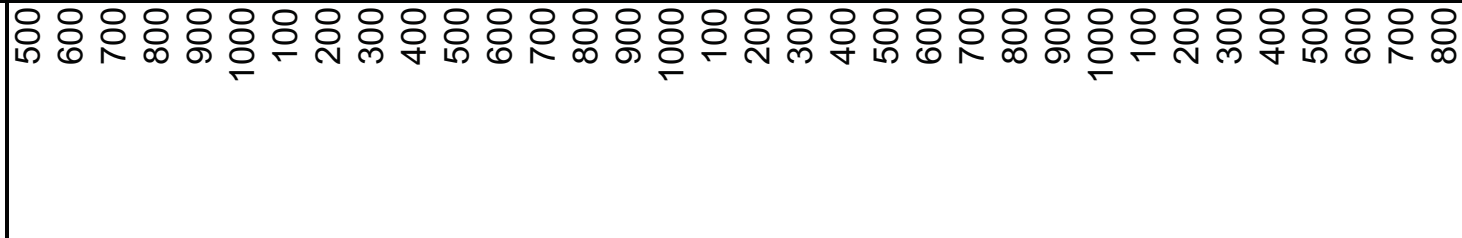 \\
\hline 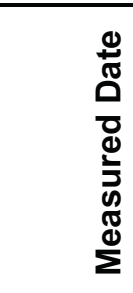 & 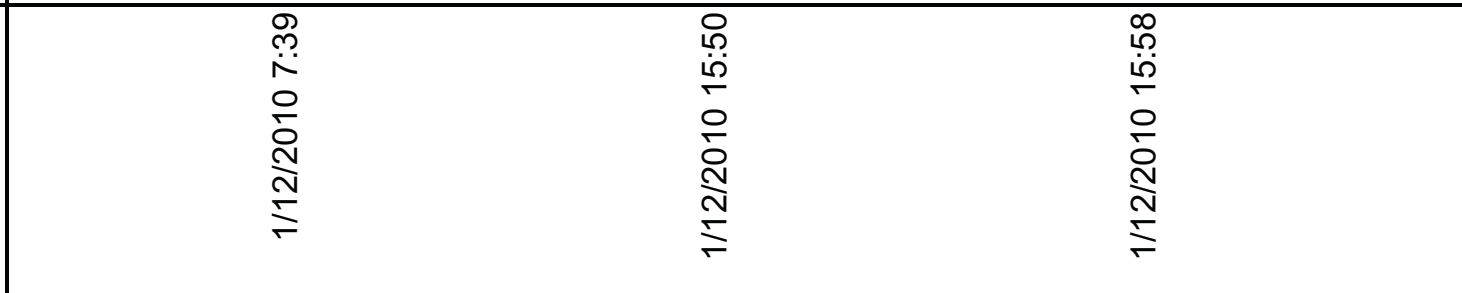 \\
\hline 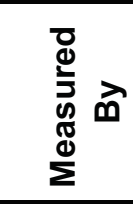 & 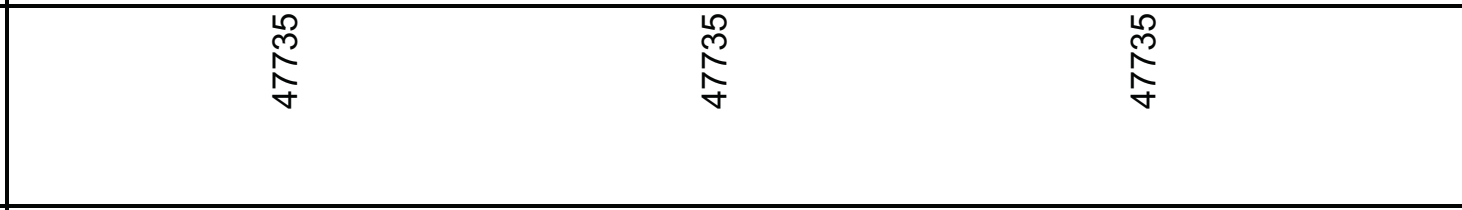 \\
\hline 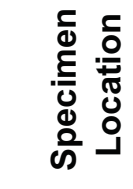 & \\
\hline 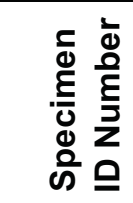 & $\begin{array}{l}\sigma \\
0 \\
\vdots\end{array}$ \\
\hline
\end{tabular}




\begin{tabular}{|c|c|}
\hline 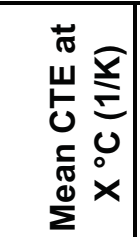 & 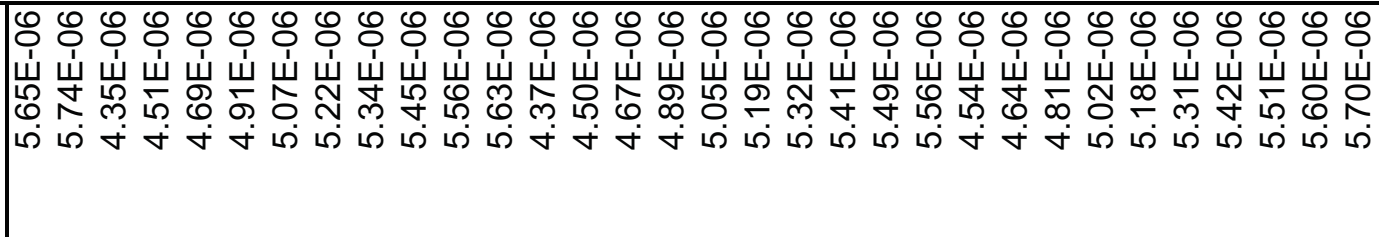 \\
\hline 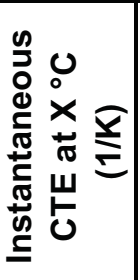 & 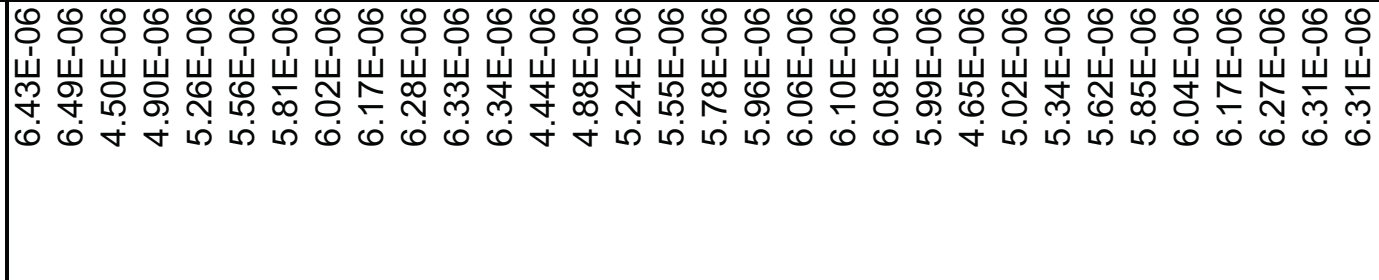 \\
\hline 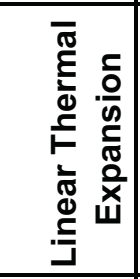 & 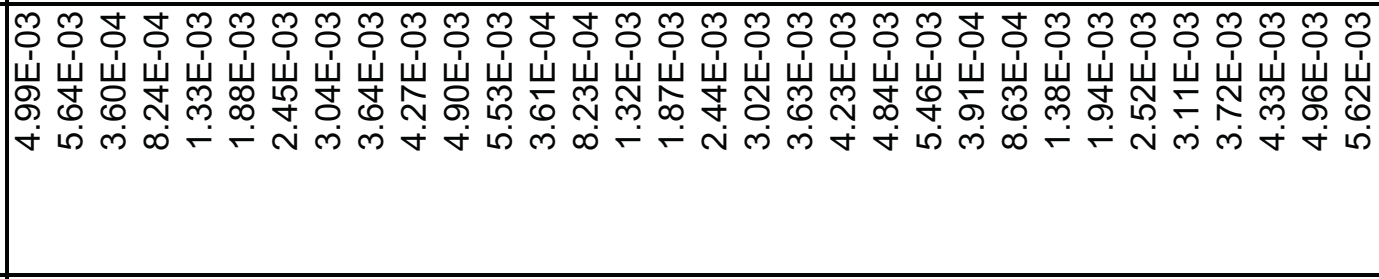 \\
\hline 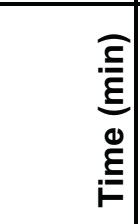 & 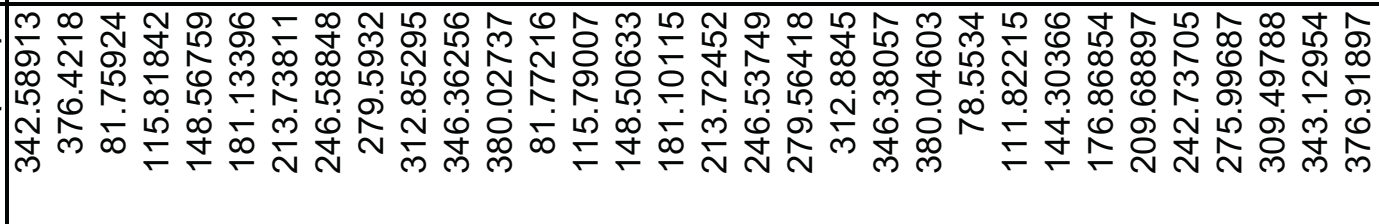 \\
\hline 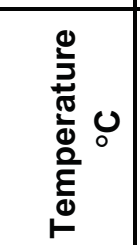 & 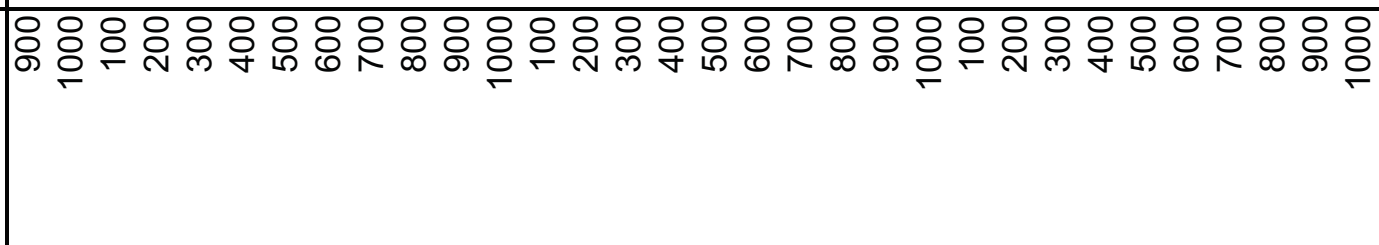 \\
\hline 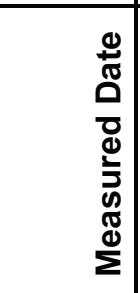 & 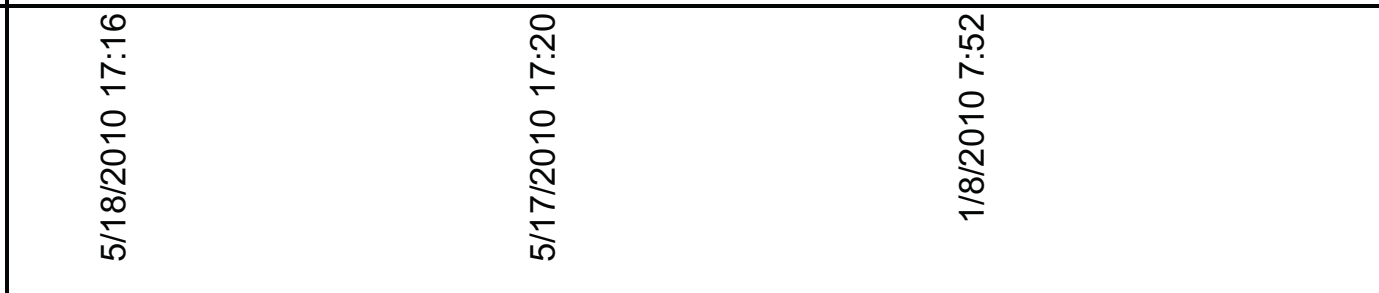 \\
\hline 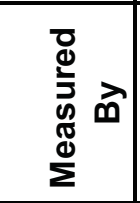 & 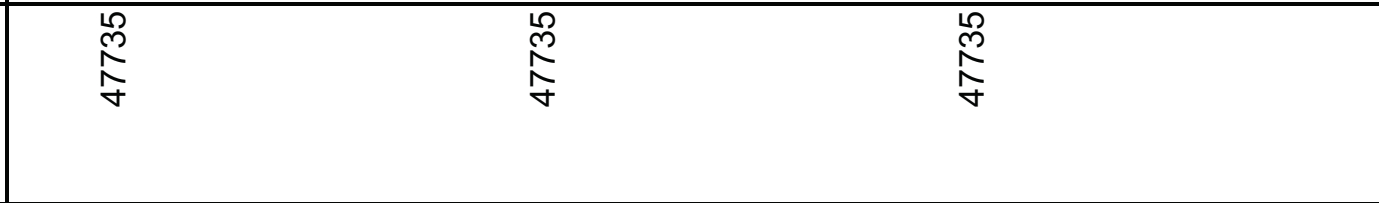 \\
\hline 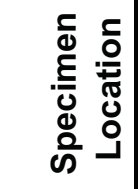 & \\
\hline 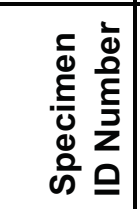 & 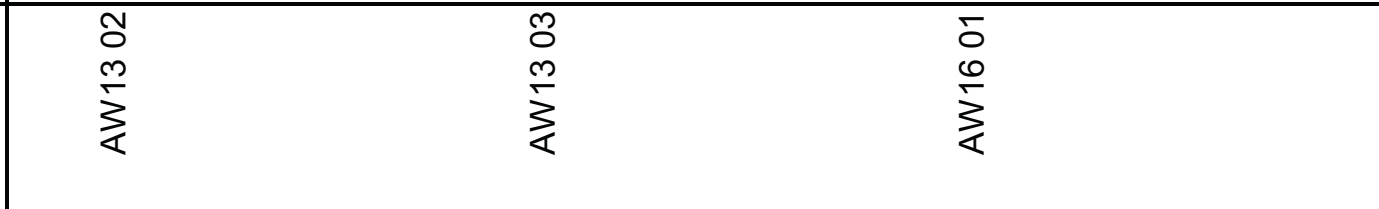 \\
\hline
\end{tabular}




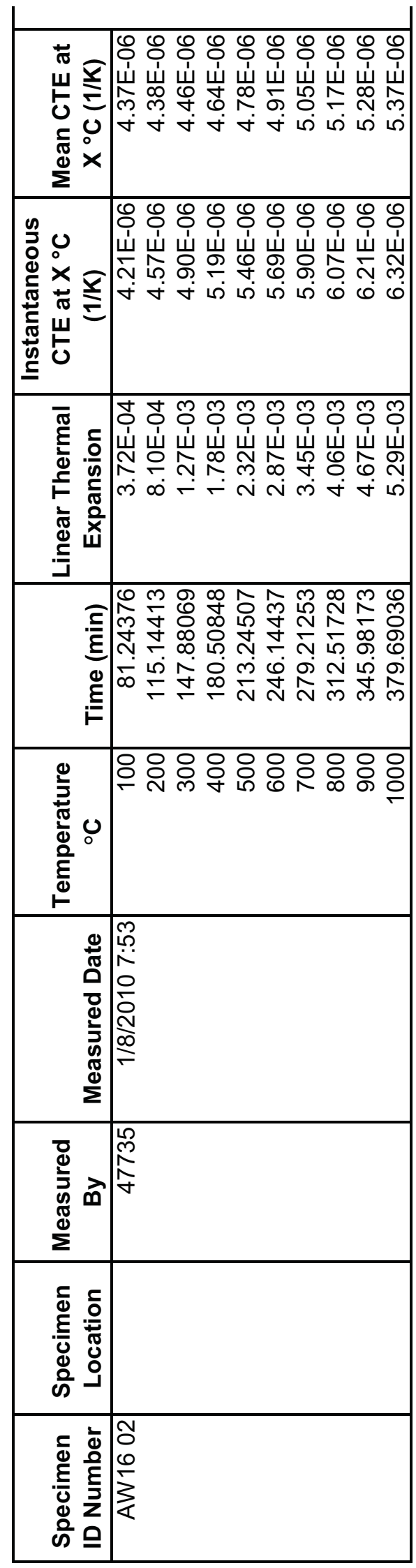




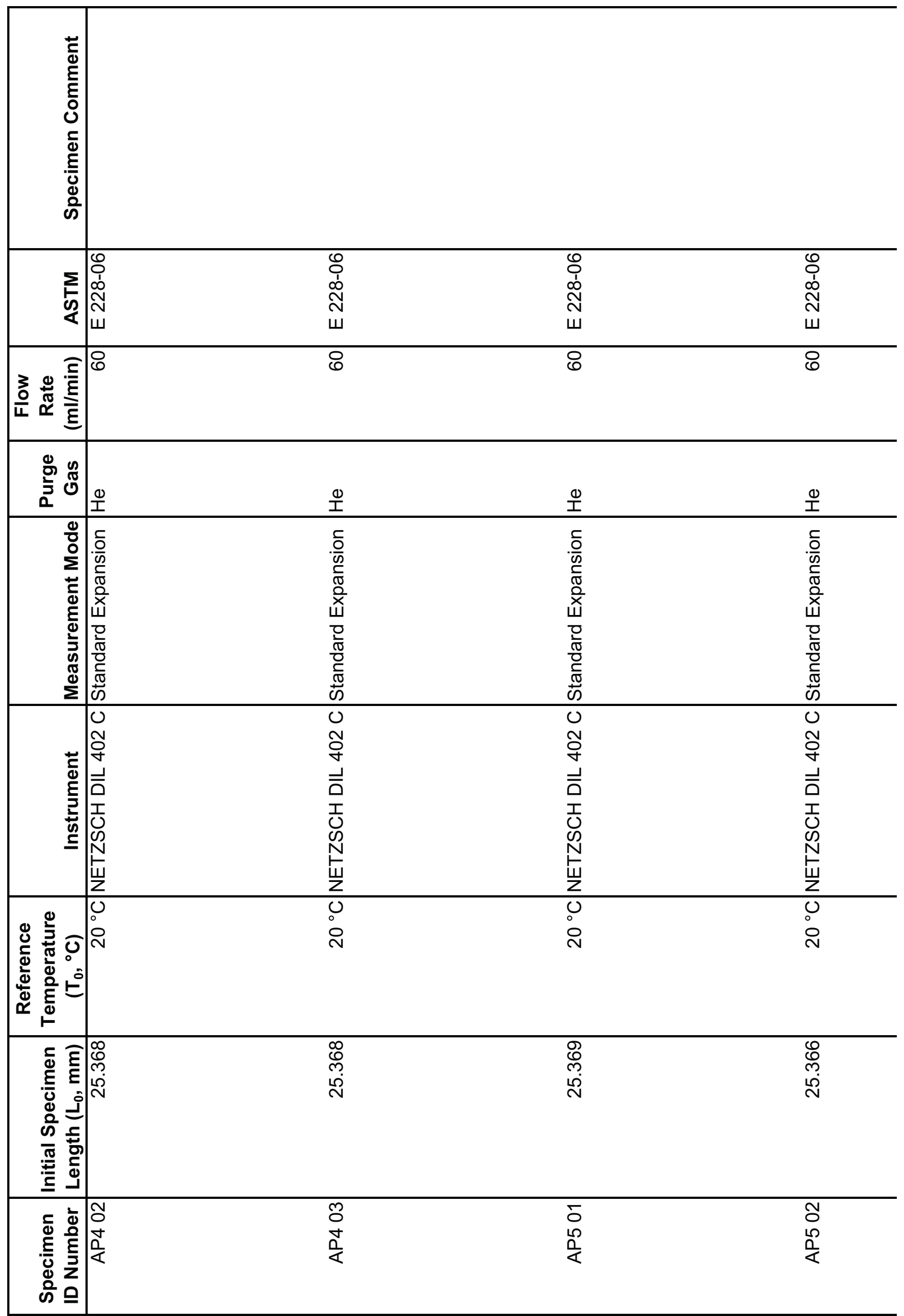




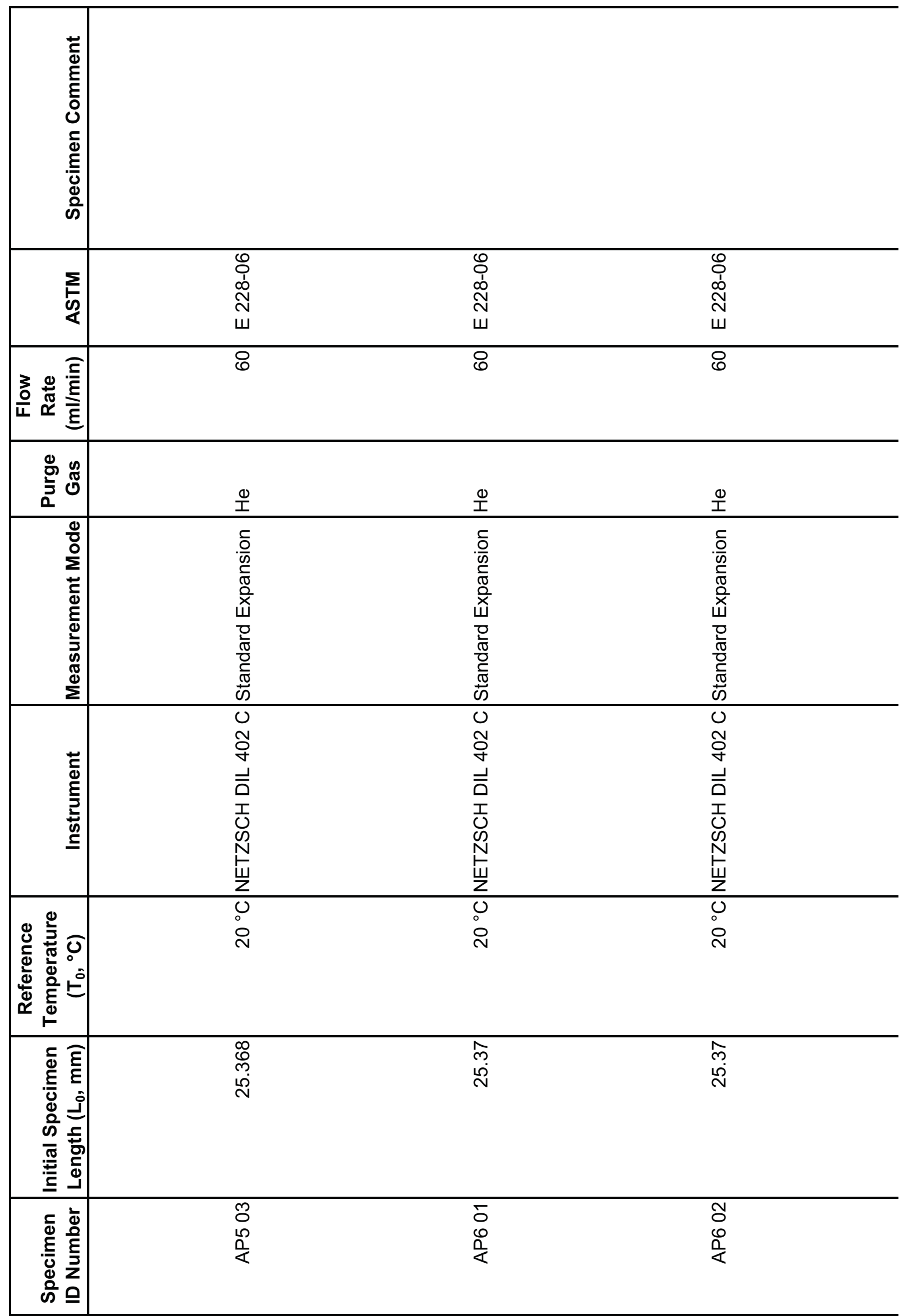




\begin{tabular}{|c|c|c|c|c|}
\hline 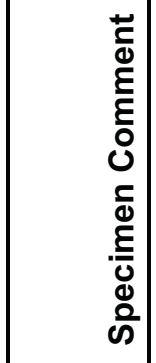 & & & & \\
\hline$\underset{\substack{\infty \\
\&}}{\sum}$ & $\begin{array}{l}\mathscr{Q} \\
0 \\
\stackrel{1}{0} \\
\text { W } \\
w\end{array}$ & $\begin{array}{l}0 \\
\\
1 \\
\stackrel{1}{ } \\
\text { N } \\
\text { W }\end{array}$ & 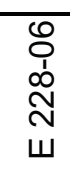 & 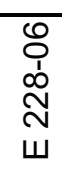 \\
\hline 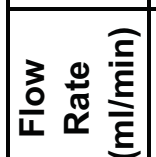 & 8 & 8 & 8 & 8 \\
\hline 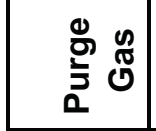 & $\stackrel{\oplus}{I}$ & $\stackrel{\oplus}{I}$ & $\stackrel{\oplus}{1}$ & $\stackrel{\oplus}{I}$ \\
\hline 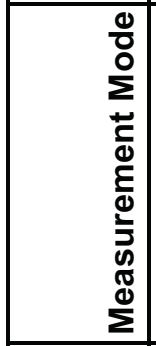 & 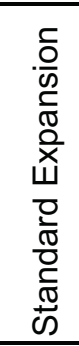 & 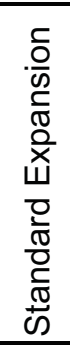 & $\begin{array}{l}\frac{c}{0} \\
\frac{0}{\omega} \\
\frac{0}{0} \\
\frac{0}{x} \\
w \\
\frac{0}{0} \\
\frac{0}{0} \\
\frac{0}{0} \\
\frac{w}{\omega}\end{array}$ & 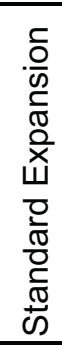 \\
\hline 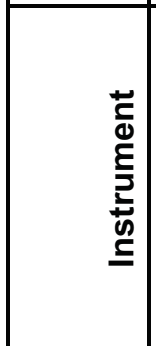 & 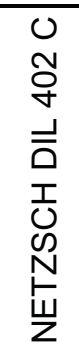 & 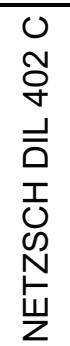 & 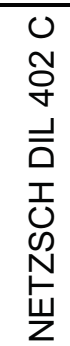 & 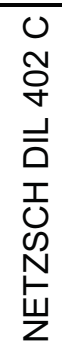 \\
\hline 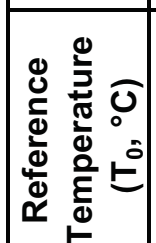 & $\begin{array}{l}0 \\
0 \\
0\end{array}$ & $\begin{array}{l}0 \\
0 \\
\text { N }\end{array}$ & 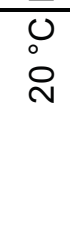 & $\begin{array}{l}\text { U } \\
\text { Oे }\end{array}$ \\
\hline 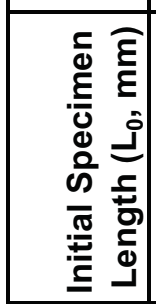 & $\begin{array}{l}\hat{N} \\
\stackrel{N}{N}\end{array}$ & $\begin{array}{l}\text { N } \\
\text { N } \\
\stackrel{N}{N}\end{array}$ & 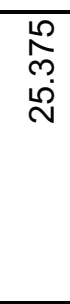 & $\begin{array}{l}\stackrel{m}{2} \\
\text { n. } \\
\stackrel{N}{N}\end{array}$ \\
\hline 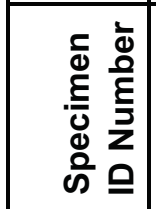 & $\begin{array}{l}30 \\
0 \\
0 \\
\frac{1}{4}\end{array}$ & $\begin{array}{l}\bar{\alpha} \\
\sum_{\alpha}\end{array}$ & $\frac{N}{\delta}$ & $\sum_{\frac{1}{2}}^{m}$ \\
\hline
\end{tabular}




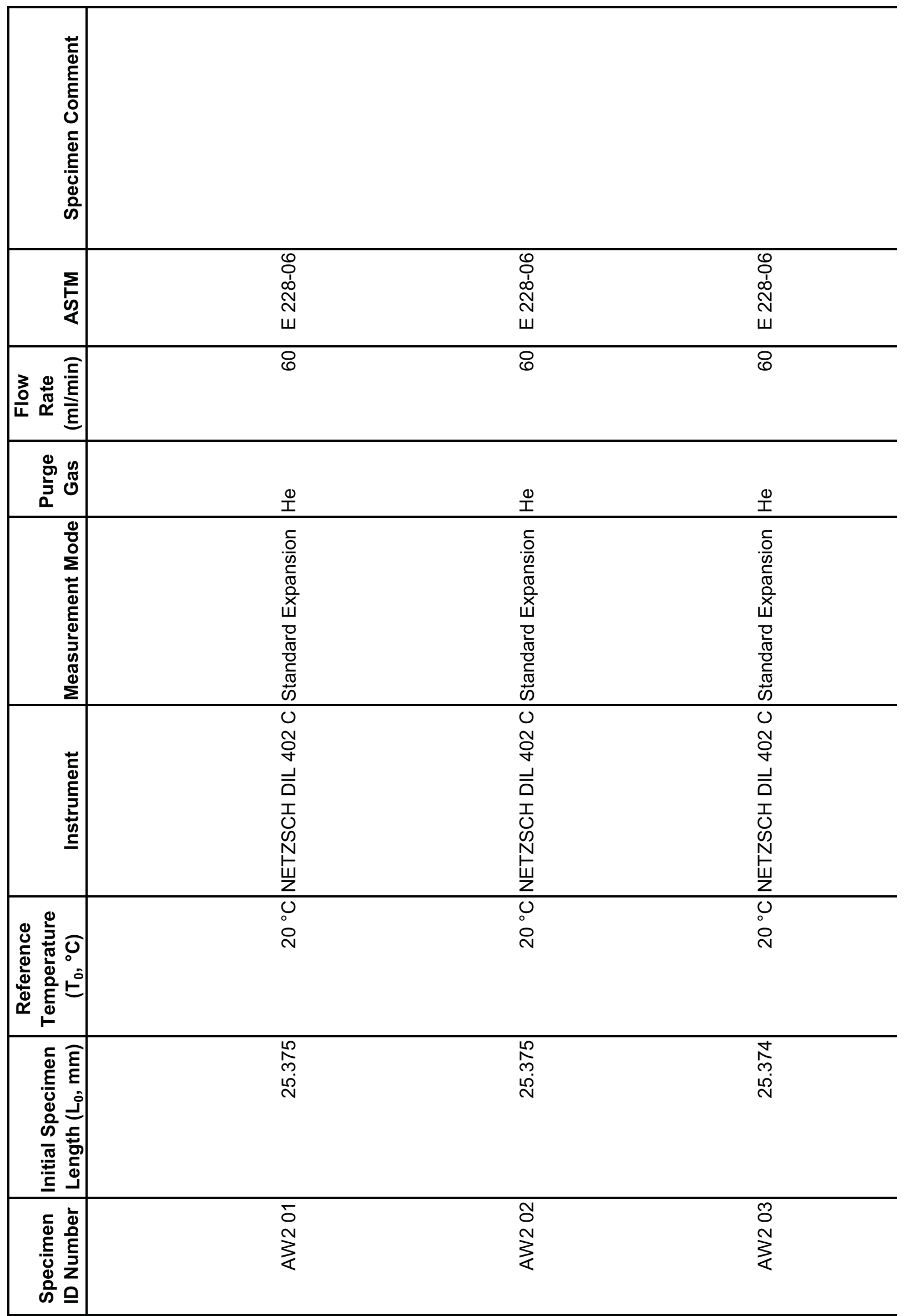




\begin{tabular}{|c|c|c|c|}
\hline 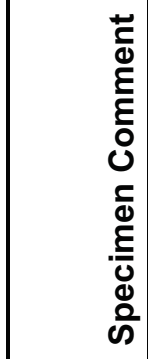 & & & \\
\hline$\underset{\frac{\Sigma}{2}}{\frac{2}{\alpha}}$ & $\begin{array}{l}\text { O } \\
0 \\
\text { Dे } \\
\text { W } \\
w\end{array}$ & \begin{tabular}{l}
$\stackrel{8}{0}$ \\
$\infty$ \\
\multirow{N}{0}{} \\
$w$
\end{tabular} & 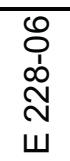 \\
\hline 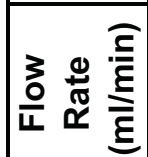 & 8 & 8 & 8 \\
\hline 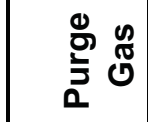 & $\stackrel{\oplus}{I}$ & 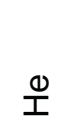 & $\stackrel{\oplus}{I}$ \\
\hline 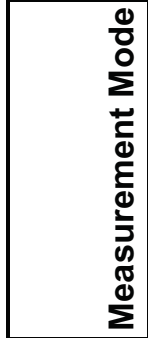 & 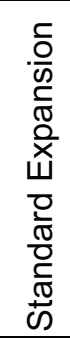 & 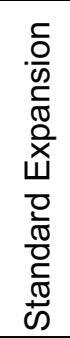 & 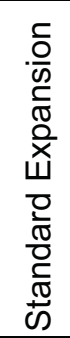 \\
\hline 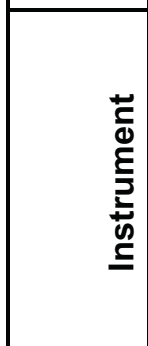 & 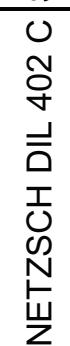 & 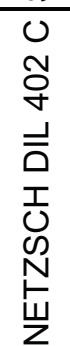 & 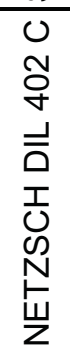 \\
\hline 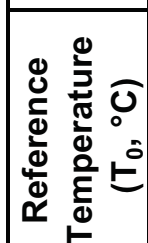 & $\begin{array}{l}0 \\
0 \\
\text { N }\end{array}$ & $\begin{array}{l}0 \\
0 \\
\text { Oे }\end{array}$ & 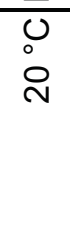 \\
\hline 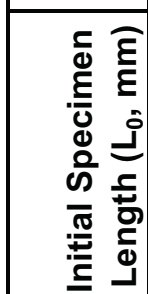 & 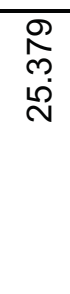 & 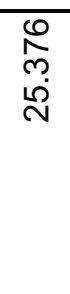 & $\begin{array}{l}\text { N } \\
\text { N } \\
\stackrel{\omega}{N}\end{array}$ \\
\hline 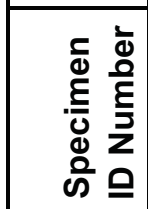 & $\begin{array}{l}5 \\
0 \\
3 \\
3\end{array}$ & $\begin{array}{l}\text { No } \\
\text { m } \\
\sum_{\alpha}\end{array}$ & $\begin{array}{l}0 \\
0 \\
m \\
3\end{array}$ \\
\hline
\end{tabular}




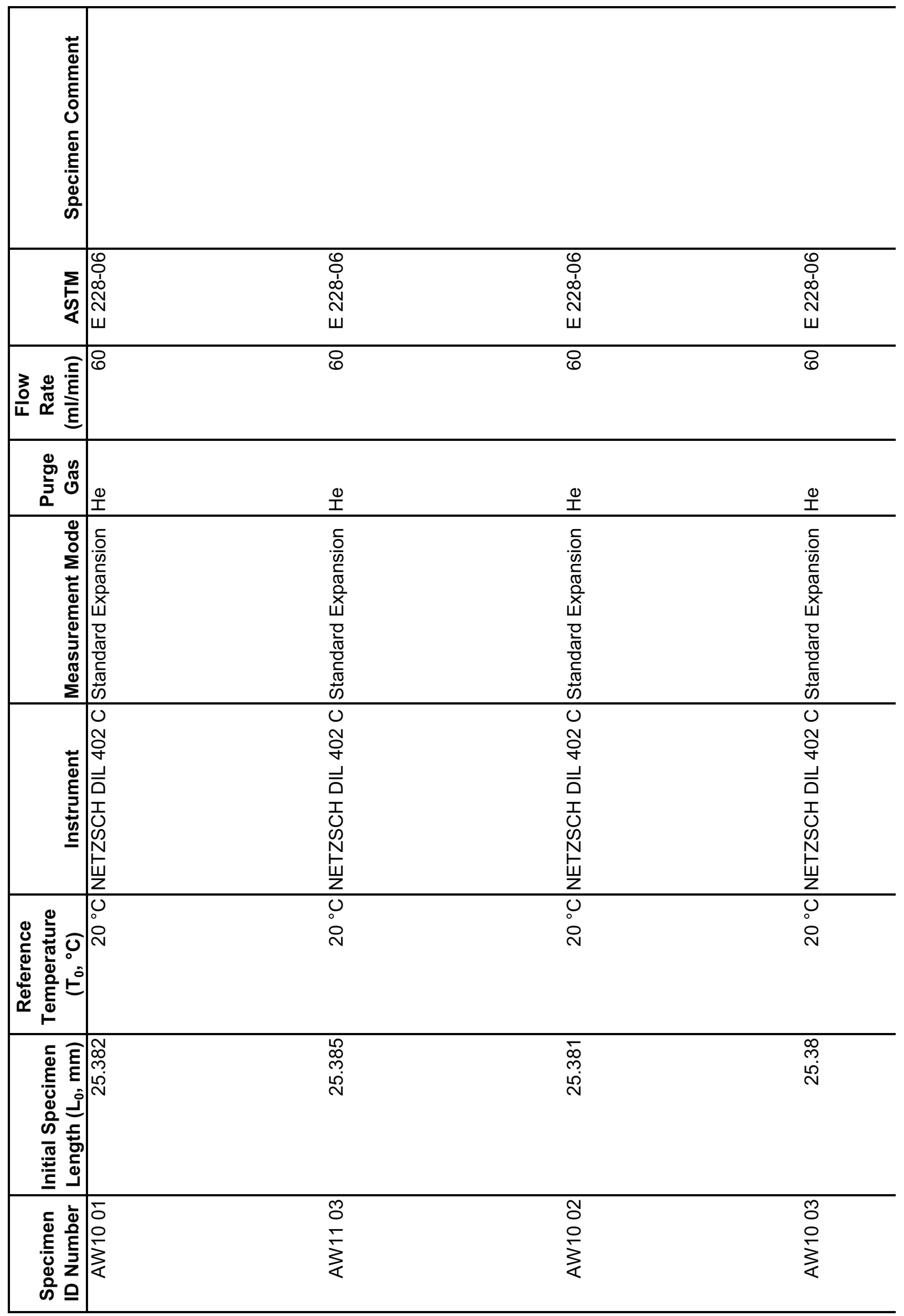




\begin{tabular}{|c|c|c|c|}
\hline 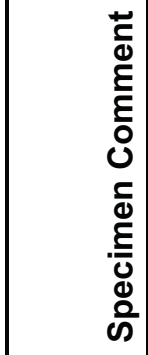 & & & \\
\hline$\underset{\frac{\Sigma}{5}}{\frac{5}{4}}$ & $\begin{array}{l}0 \\
0 \\
\dot{1} \\
\mathbb{N} \\
\text { w }\end{array}$ & \begin{tabular}{l} 
\\
\\
$\dot{1}$ \\
\multirow{N}{N}{} \\
$w$
\end{tabular} & 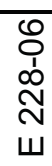 \\
\hline 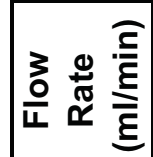 & 8 & 8 & 8 \\
\hline 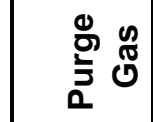 & $\stackrel{\oplus}{I}$ & $\stackrel{\oplus}{I}$ & $\stackrel{\oplus}{I}$ \\
\hline 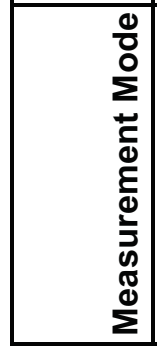 & 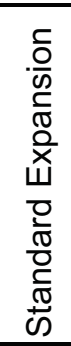 & 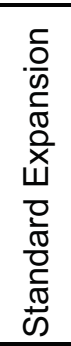 & 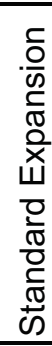 \\
\hline 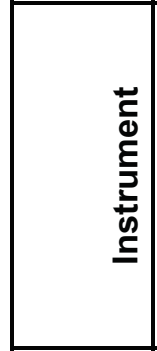 & 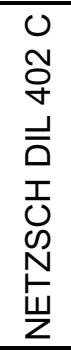 & 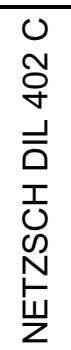 & 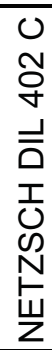 \\
\hline 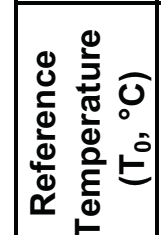 & $\begin{array}{l}\text { U } \\
0 \\
\text { Oे }\end{array}$ & $\begin{array}{l}\text { U } \\
\text { N }\end{array}$ & $\begin{array}{l}\overline{0} \\
\vdots \\
\stackrel{N}{ }\end{array}$ \\
\hline 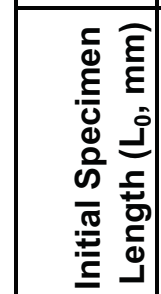 & 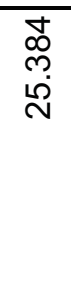 & $\begin{array}{l}\stackrel{m}{N} \\
m \\
\stackrel{D}{N}\end{array}$ & $\begin{array}{l}\hat{\infty} \\
\text { m. } \\
\stackrel{2}{N}\end{array}$ \\
\hline 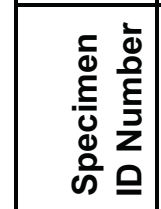 & $\begin{array}{l}\bar{\delta} \\
\sum_{\frac{1}{\alpha}} \\
\end{array}$ & $\sum_{\substack{\alpha\\
}}^{5}$ & \begin{tabular}{l}
$\overline{1}$ \\
\multirow{2}{N}{} \\
$\sum_{<}$
\end{tabular} \\
\hline
\end{tabular}




\begin{tabular}{|c|c|c|c|c|}
\hline 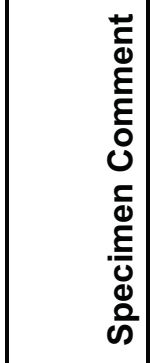 & & & & \\
\hline$\underset{\substack{\infty \\
\&}}{\sum}$ & $\begin{array}{l}\mathscr{Q} \\
0 \\
\stackrel{1}{0} \\
\text { W } \\
w\end{array}$ & $\begin{array}{l}0 \\
\\
1 \\
\stackrel{1}{ } \\
\text { N } \\
\Psi\end{array}$ & 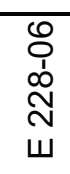 & 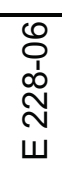 \\
\hline 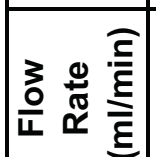 & 8 & 8 & 8 & 8 \\
\hline 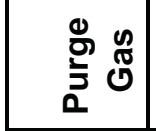 & $\stackrel{0}{I}$ & $\stackrel{\oplus}{I}$ & $\stackrel{0}{I}$ & $\stackrel{0}{I}$ \\
\hline 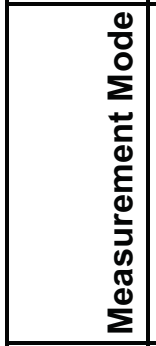 & 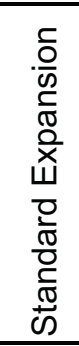 & 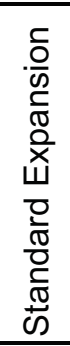 & 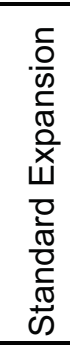 & 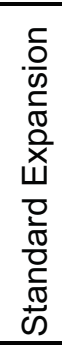 \\
\hline 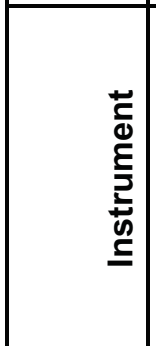 & 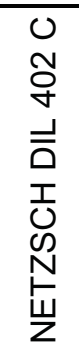 & 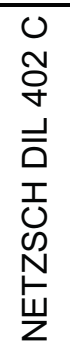 & 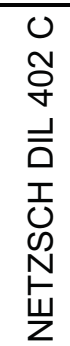 & 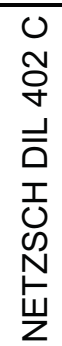 \\
\hline 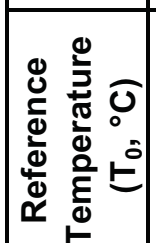 & $\begin{array}{l}0 \\
0 \\
0\end{array}$ & $\begin{array}{l}0 \\
0 \\
\text { N }\end{array}$ & 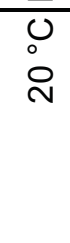 & $\begin{array}{l}\text { U } \\
\text { N }\end{array}$ \\
\hline 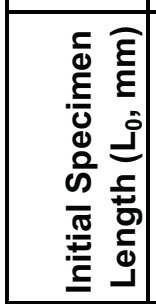 & $\begin{array}{l}\infty \\
\infty \\
\infty \\
\stackrel{\infty}{N} \\
\stackrel{N}{N}\end{array}$ & 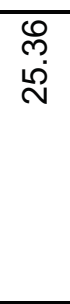 & 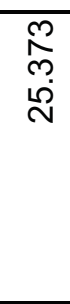 & 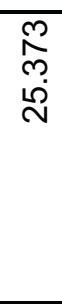 \\
\hline 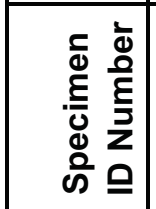 & 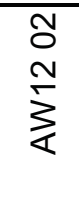 & 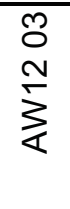 & $\begin{array}{l}\bar{\delta} \\
\sum_{\alpha}^{m} \\
\frac{m}{\alpha}\end{array}$ & $\sum_{\substack{\alpha\\
}}^{5}$ \\
\hline
\end{tabular}




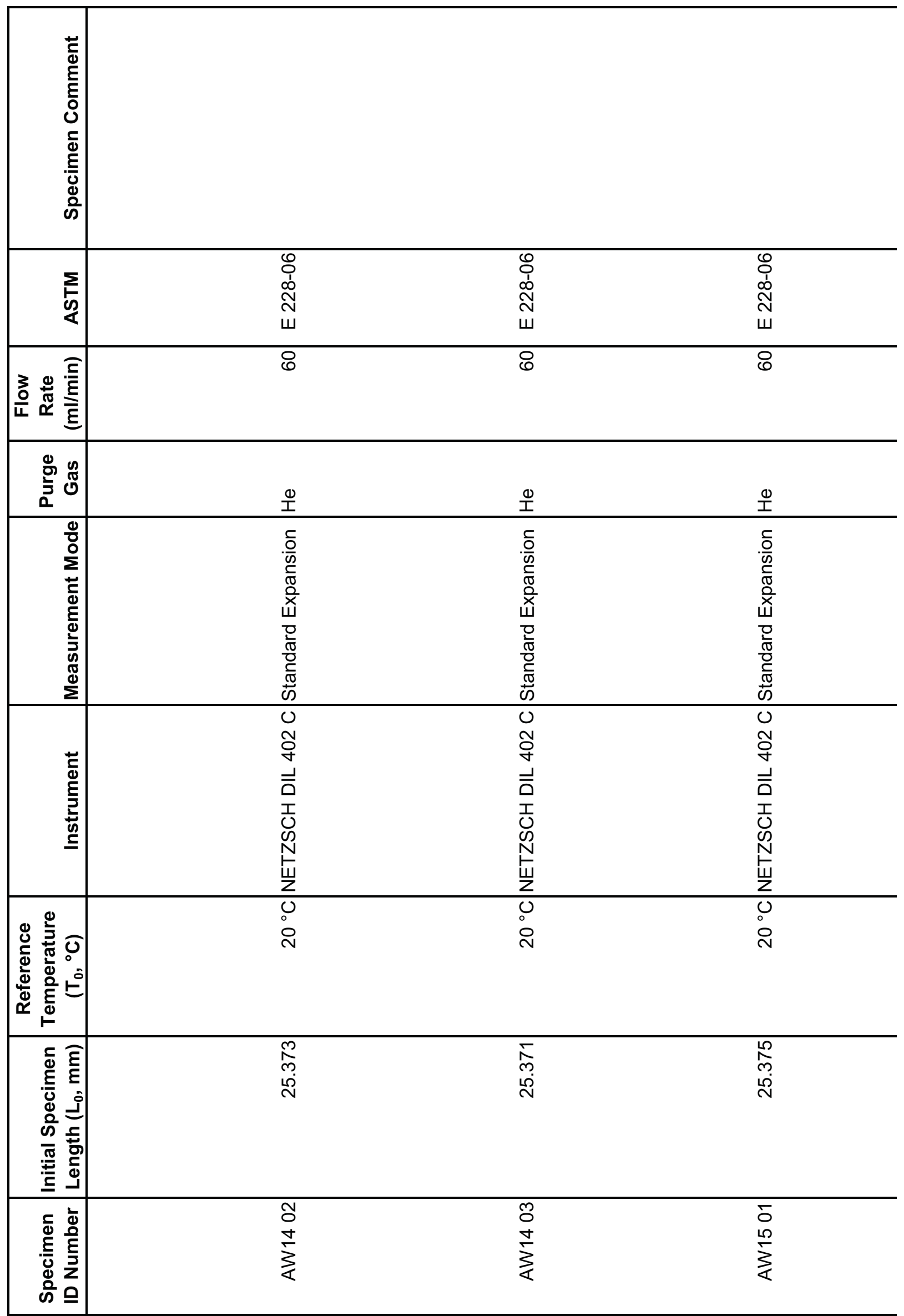




\begin{tabular}{|c|c|c|c|}
\hline 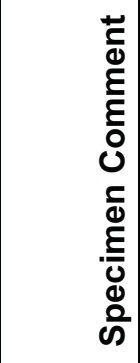 & & & \\
\hline 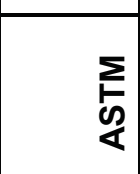 & 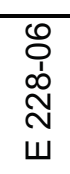 & 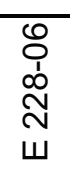 & 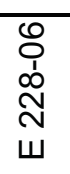 \\
\hline 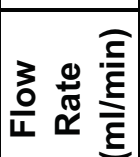 & 8 & 8 & 8 \\
\hline 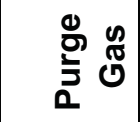 & 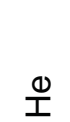 & 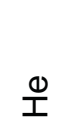 & $\stackrel{\oplus}{工}$ \\
\hline 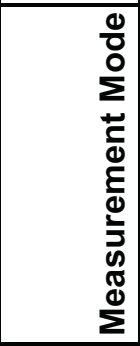 & 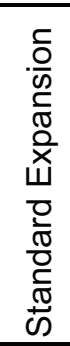 & 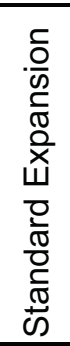 & 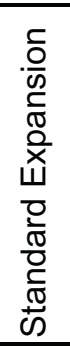 \\
\hline 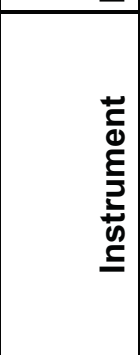 & 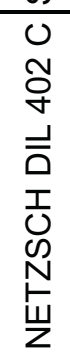 & 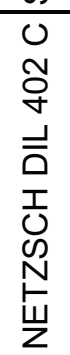 & 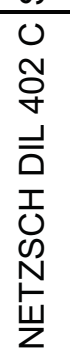 \\
\hline 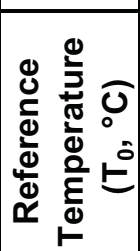 & $\begin{array}{l}\text { U } \\
\text { Oे }\end{array}$ & $\begin{array}{l}\text { Oे } \\
\text { N }\end{array}$ & $\begin{array}{l}0 \\
0 \\
\text { N }\end{array}$ \\
\hline 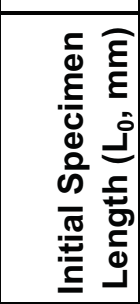 & 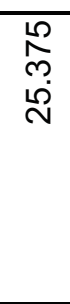 & 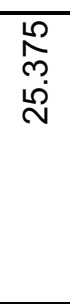 & 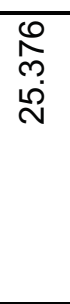 \\
\hline 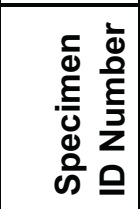 & $\begin{array}{l}0 \\
0 \\
\sum_{3}^{10} \\
\frac{10}{4}\end{array}$ & 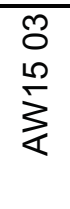 & $\begin{array}{l}0 \\
0 \\
0 \\
\sum_{<}^{0}\end{array}$ \\
\hline
\end{tabular}




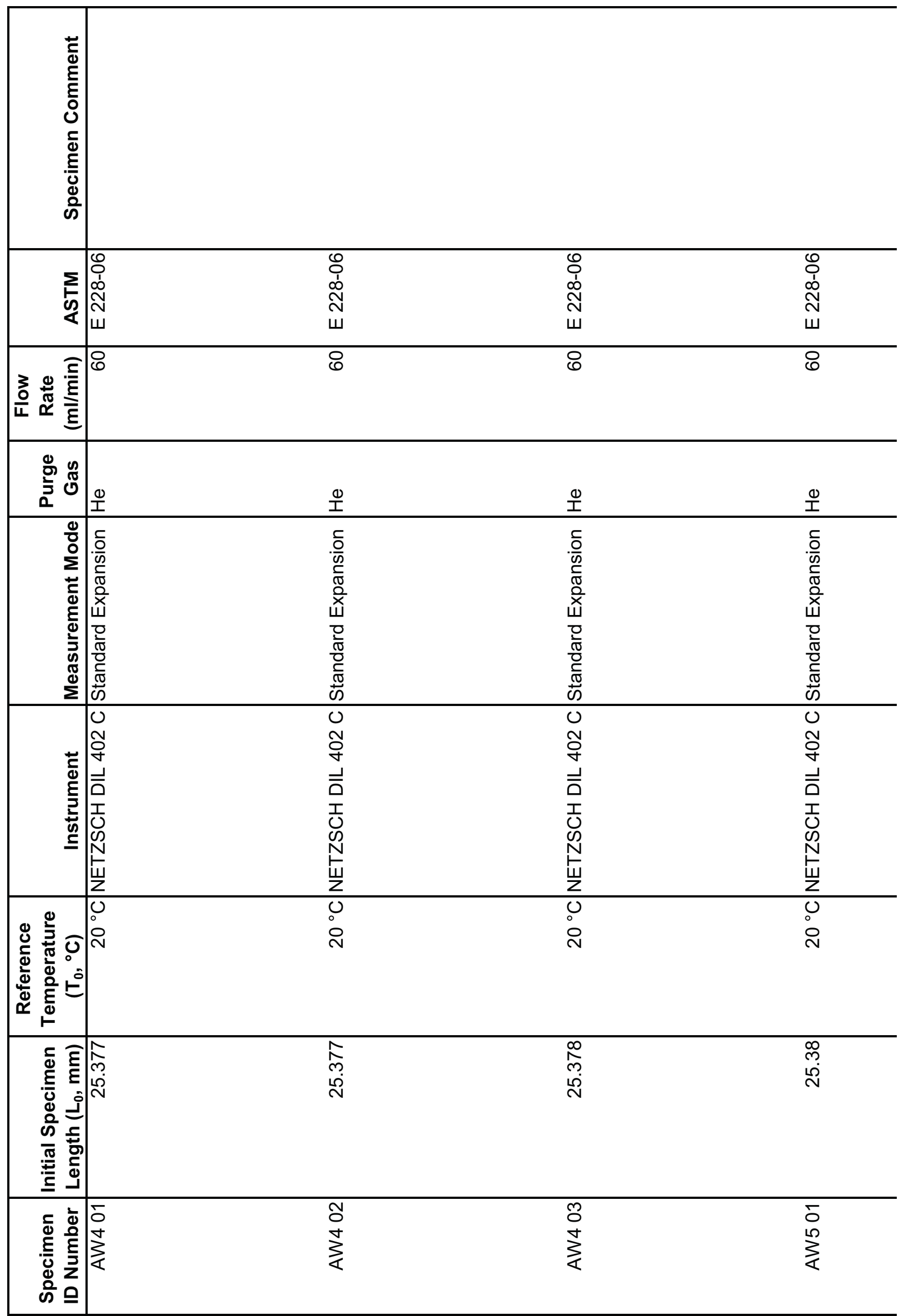




\begin{tabular}{|c|c|c|c|}
\hline 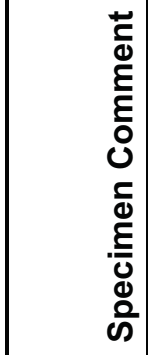 & & & \\
\hline$\underset{\frac{\Sigma}{5}}{\frac{5}{4}}$ & $\begin{array}{l}\mathbf{O} \\
0 \\
\stackrel{D}{N} \\
\text { w }\end{array}$ & $\begin{array}{l}\text { O } \\
\dot{1} \\
\text { N } \\
w\end{array}$ & 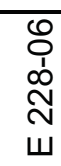 \\
\hline 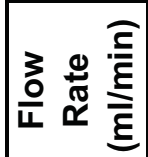 & 8 & 8 & 8 \\
\hline 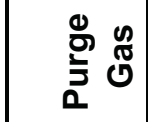 & $\stackrel{\oplus}{I}$ & $\stackrel{\infty}{I}$ & 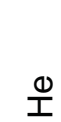 \\
\hline 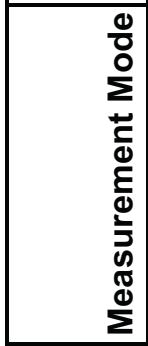 & 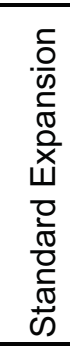 & 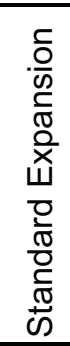 & 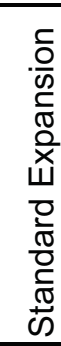 \\
\hline 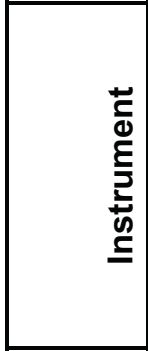 & 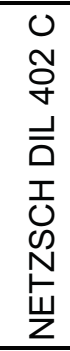 & 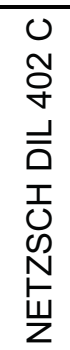 & 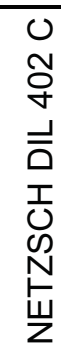 \\
\hline 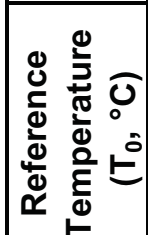 & $\begin{array}{l}0 \\
0 \\
\text { N }\end{array}$ & $\begin{array}{l}0 \\
0 \\
\text { Oे }\end{array}$ & $\begin{array}{l}0 \\
0 \\
\text { N }\end{array}$ \\
\hline 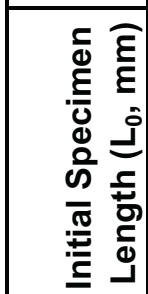 & $\begin{array}{l}\infty \\
\stackrel{\infty}{m} \\
\stackrel{\rho}{N}\end{array}$ & $\begin{array}{l}\text { o } \\
\stackrel{9}{0} \\
\stackrel{n}{N}\end{array}$ & 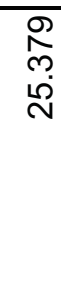 \\
\hline 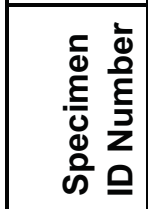 & $\begin{array}{l}N \\
\delta \\
3 \\
3 \\
3\end{array}$ & $\begin{array}{l}\text { m } \\
0 \\
3 \\
3\end{array}$ & $\begin{array}{l}\overline{0} \\
0 \\
\sum_{<}\end{array}$ \\
\hline
\end{tabular}




\begin{tabular}{|c|c|c|c|}
\hline 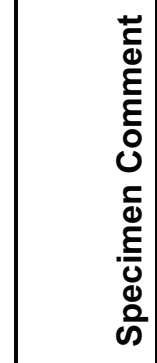 & & & \\
\hline 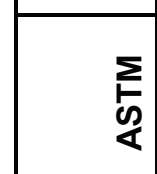 & 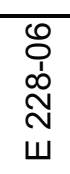 & 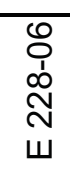 & 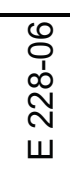 \\
\hline 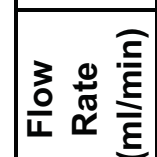 & 8 & 8 & 8 \\
\hline 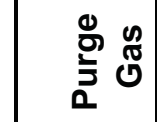 & $\stackrel{\oplus}{I}$ & 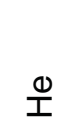 & 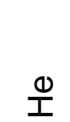 \\
\hline 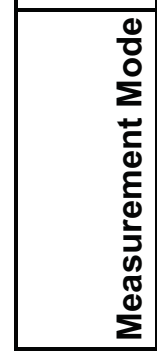 & 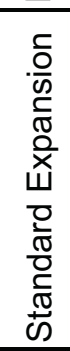 & 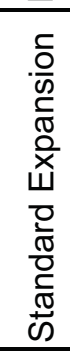 & 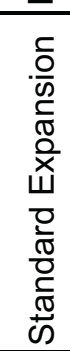 \\
\hline 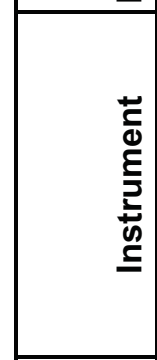 & 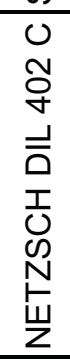 & 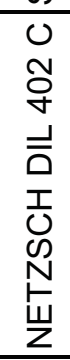 & 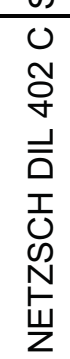 \\
\hline 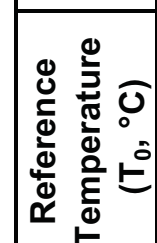 & $\begin{array}{l}\text { U } \\
\text { ¿े }\end{array}$ & $\begin{array}{l}\text { U } \\
\text { Oे }\end{array}$ & $\begin{array}{l}0 \\
0 \\
\text { N }\end{array}$ \\
\hline 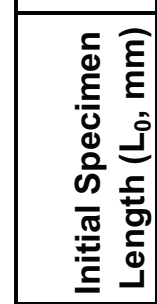 & 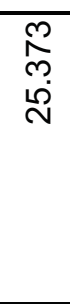 & 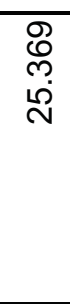 & 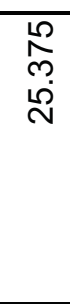 \\
\hline 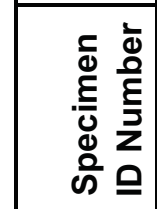 & $\begin{array}{l}\text { No } \\
\text { m } \\
\sum_{\alpha}\end{array}$ & $\begin{array}{l}m \\
\text { m } \\
m \\
\sum_{i}^{m}\end{array}$ & $\begin{array}{l}5 \\
0 \\
0 \\
3 \\
3\end{array}$ \\
\hline
\end{tabular}




$$
F
$$




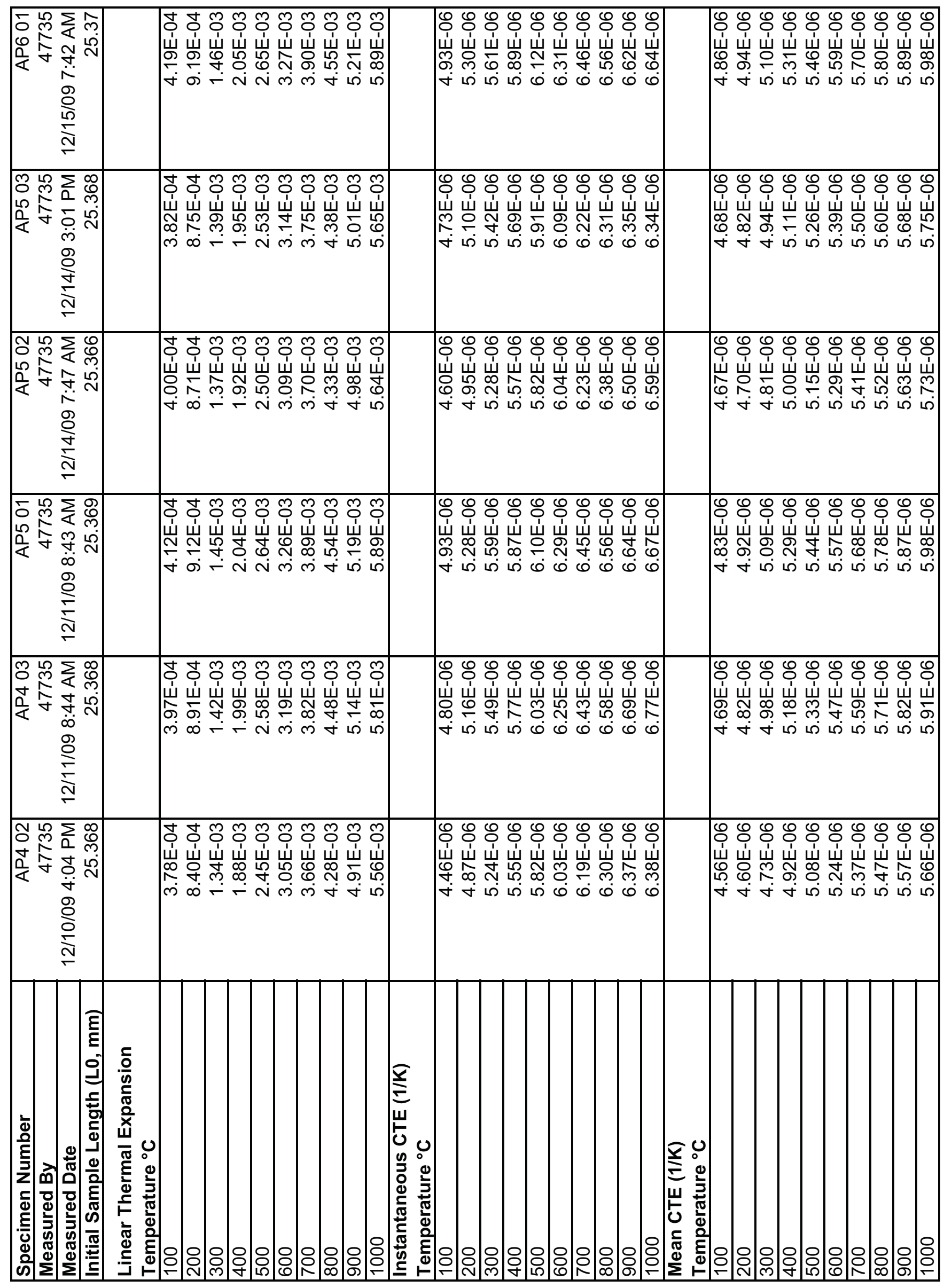




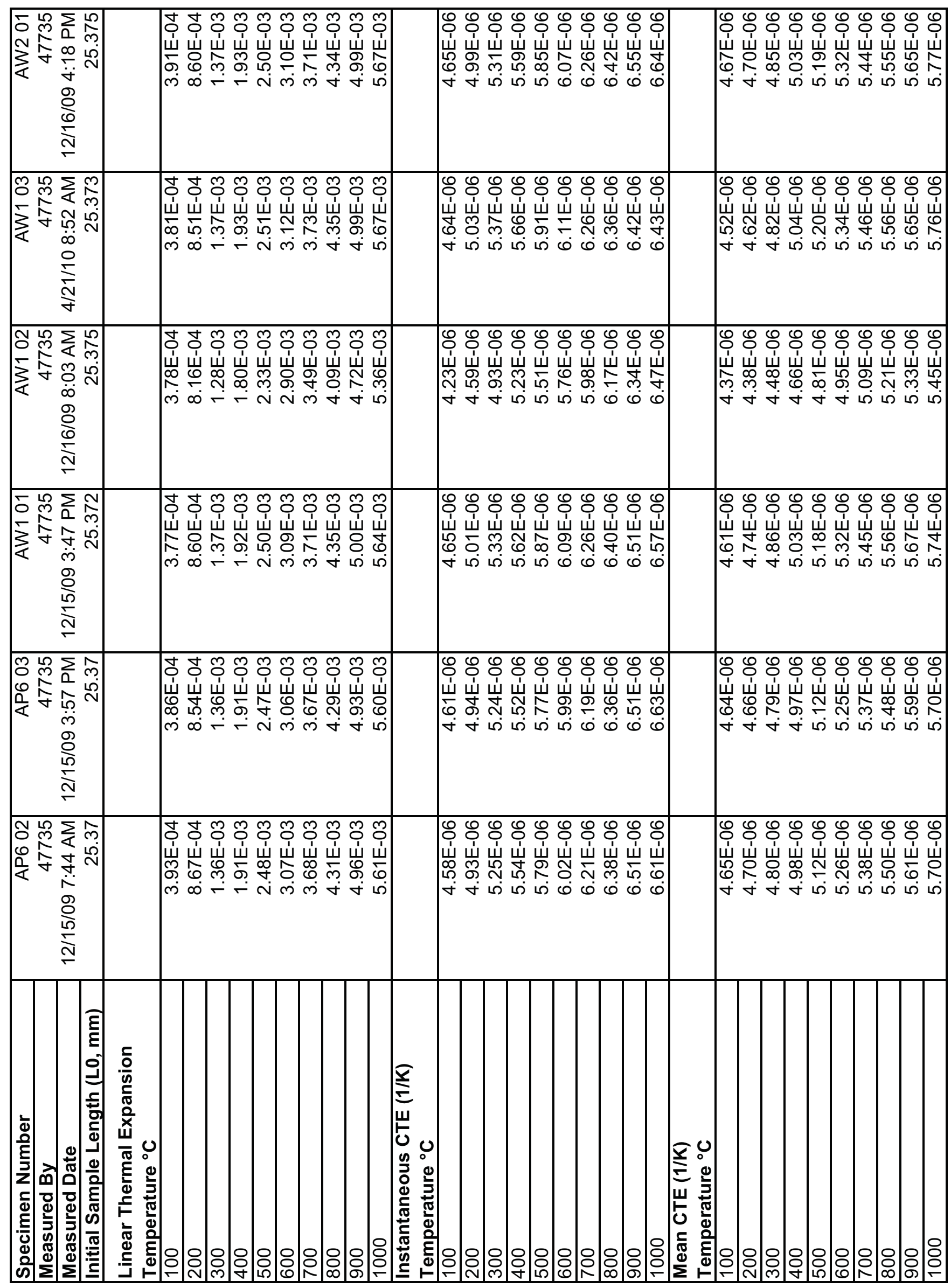




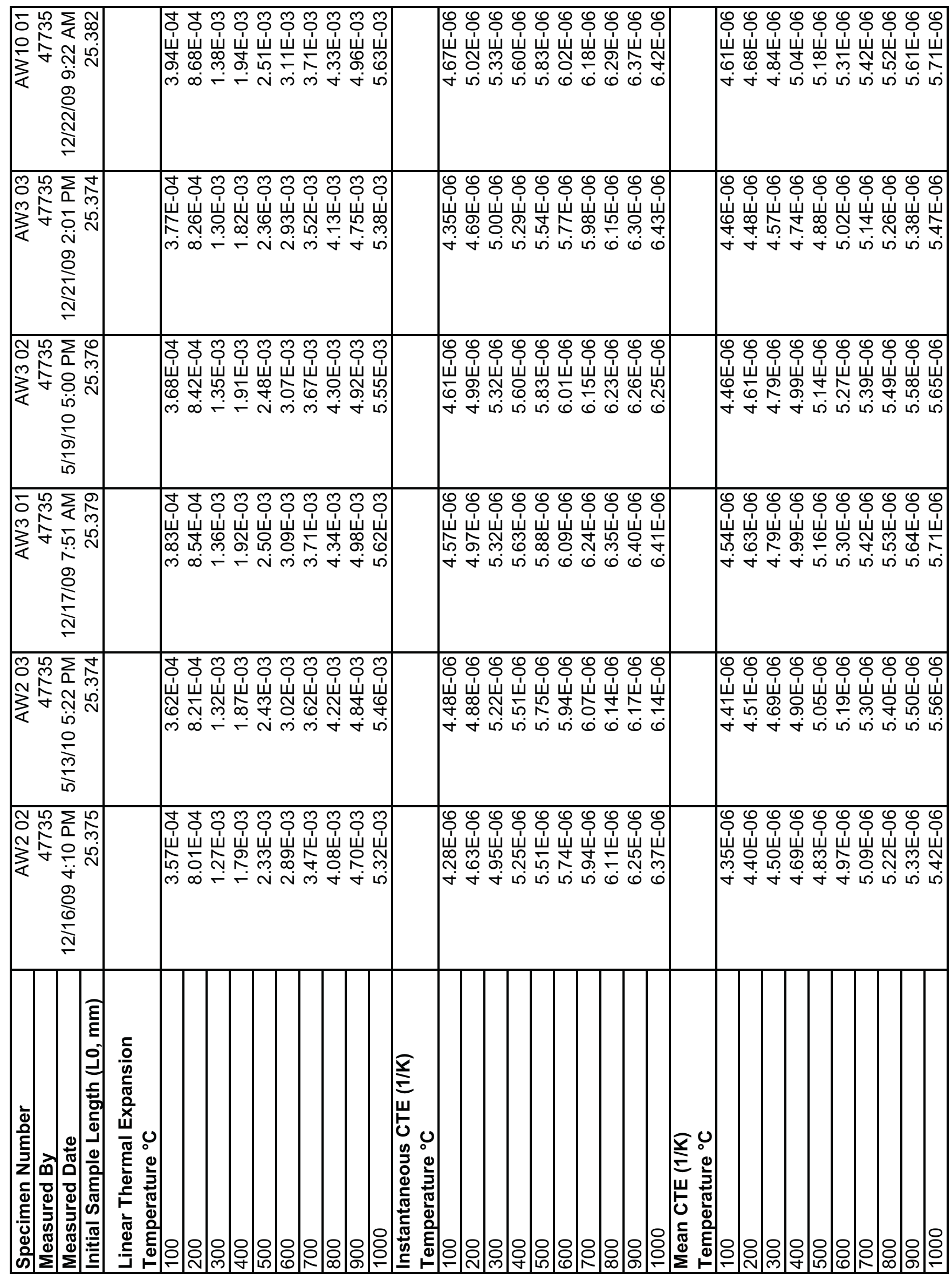




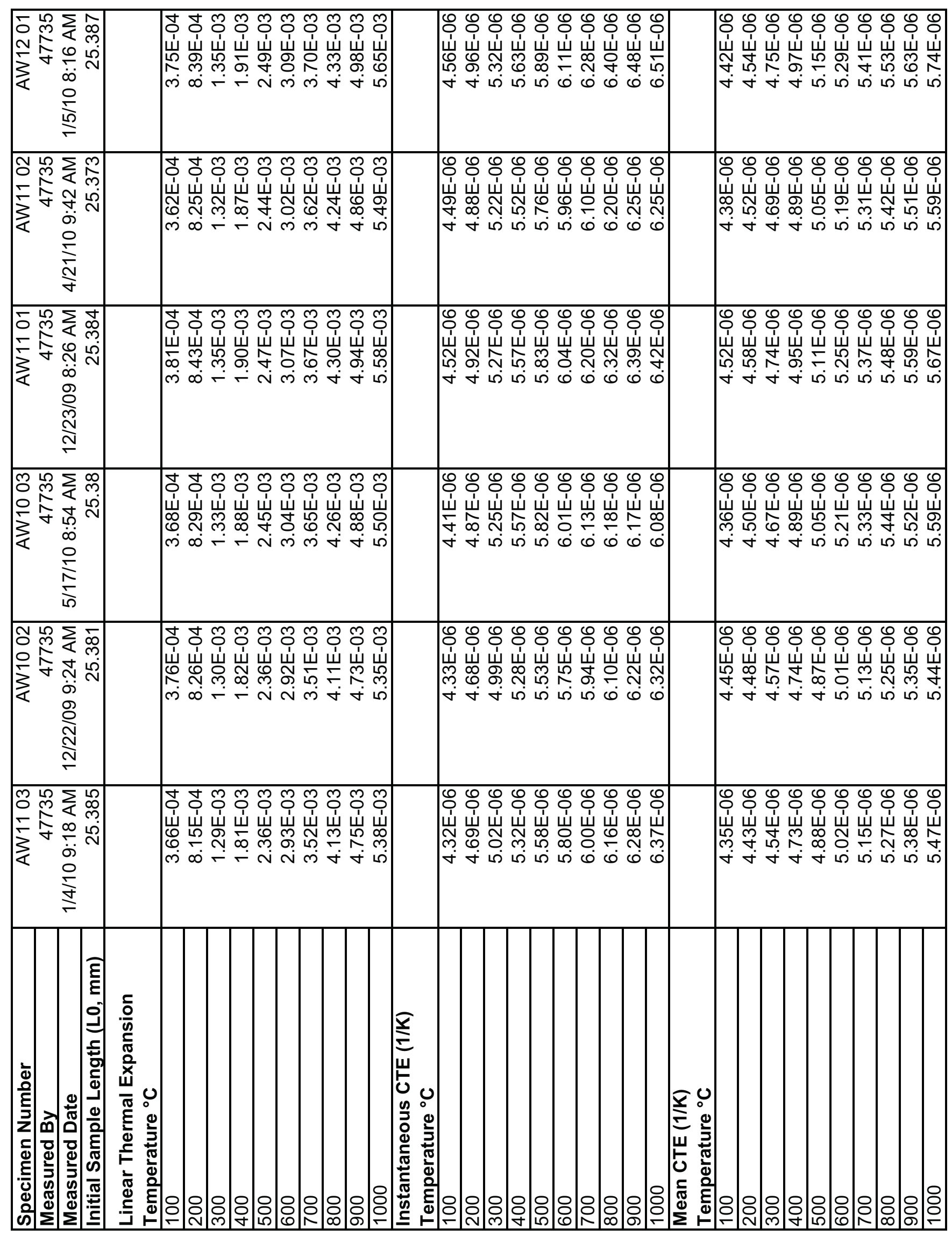




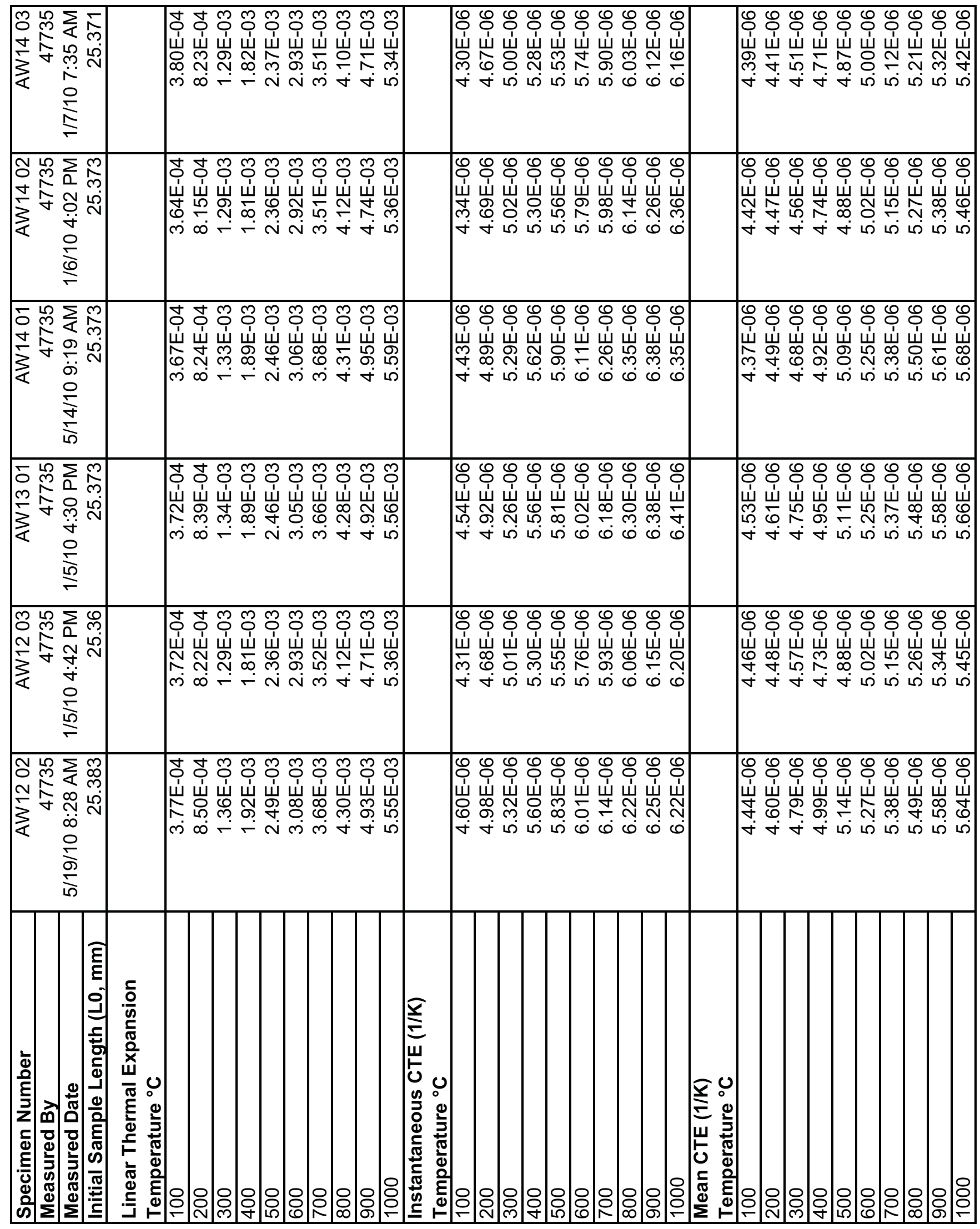




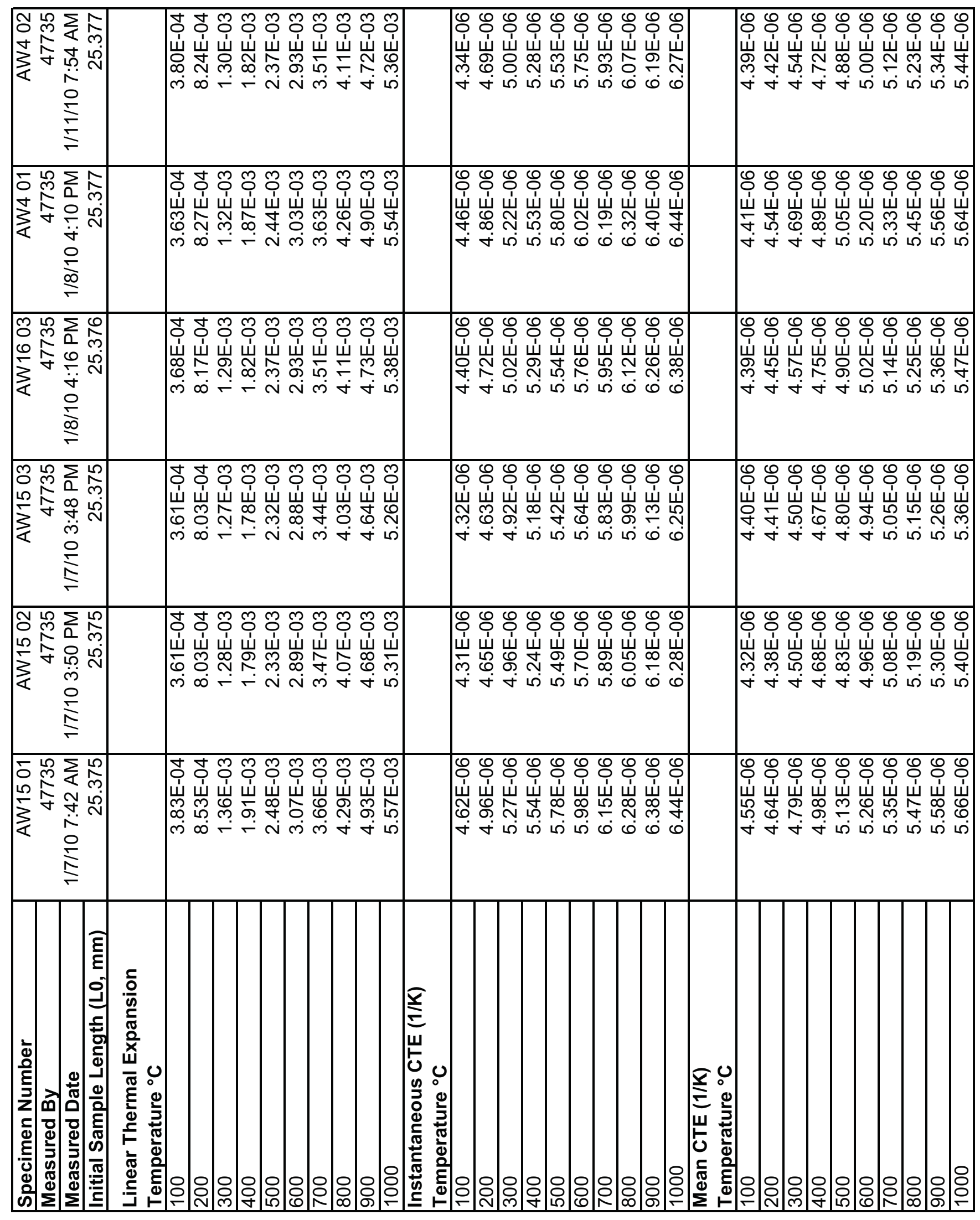




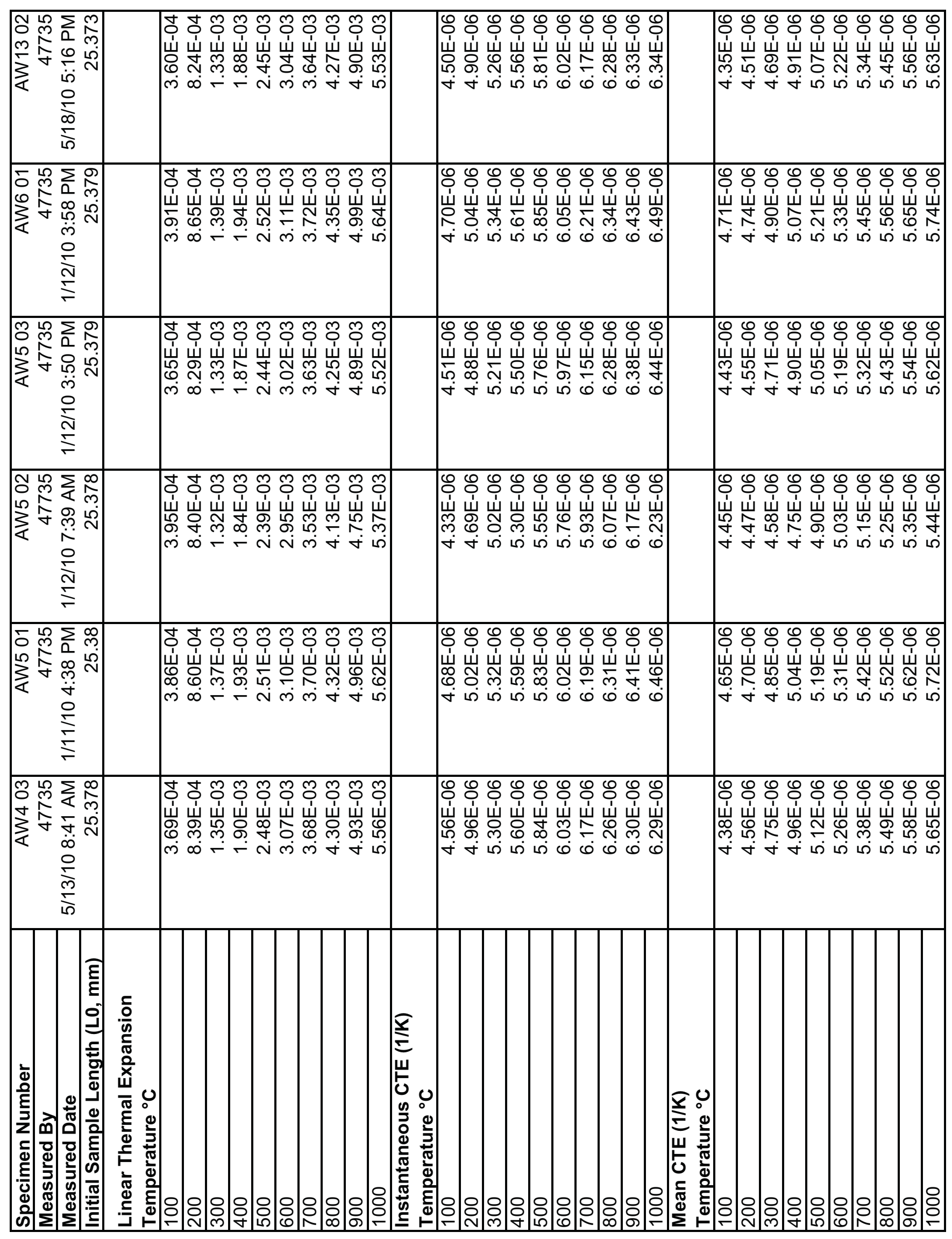




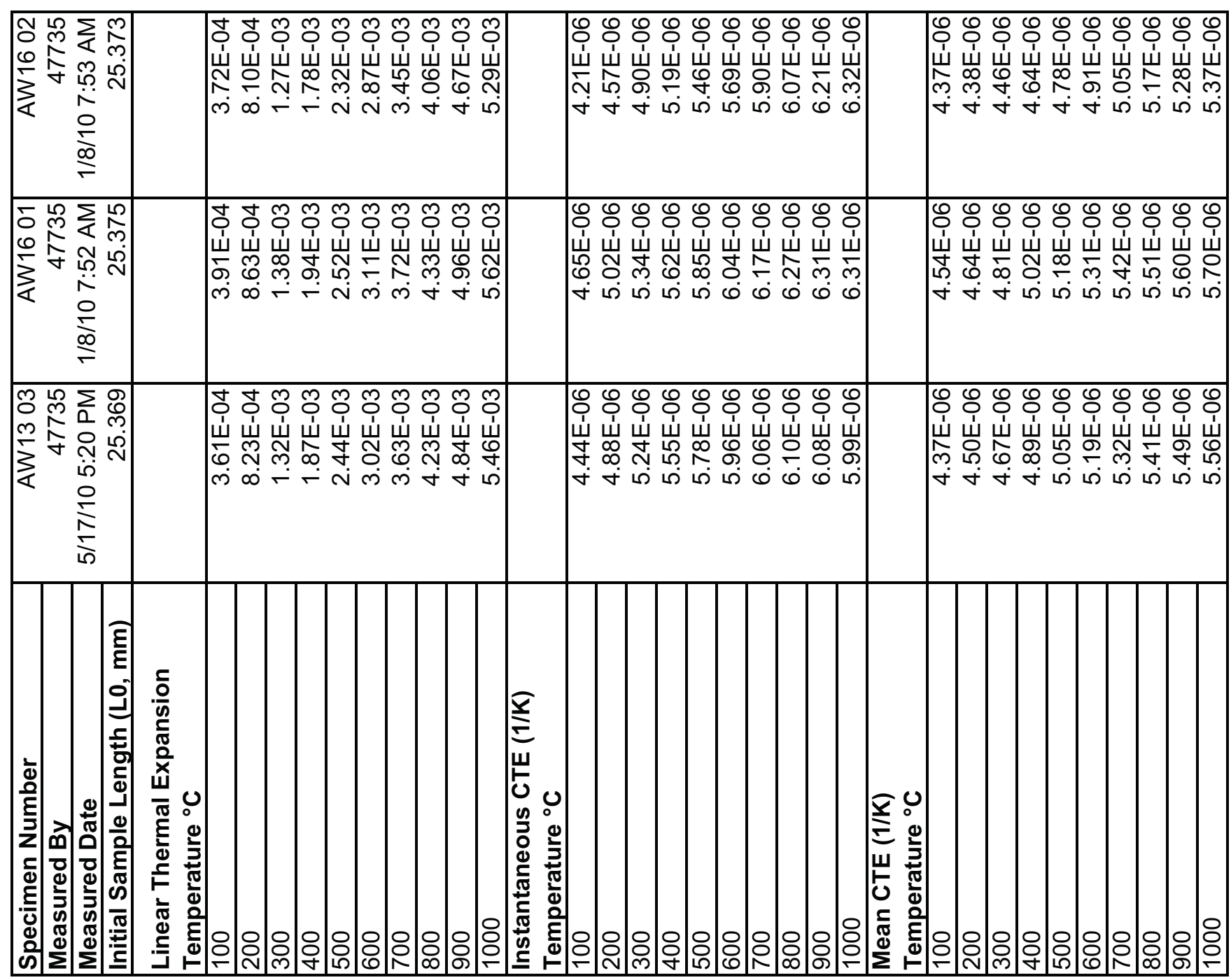




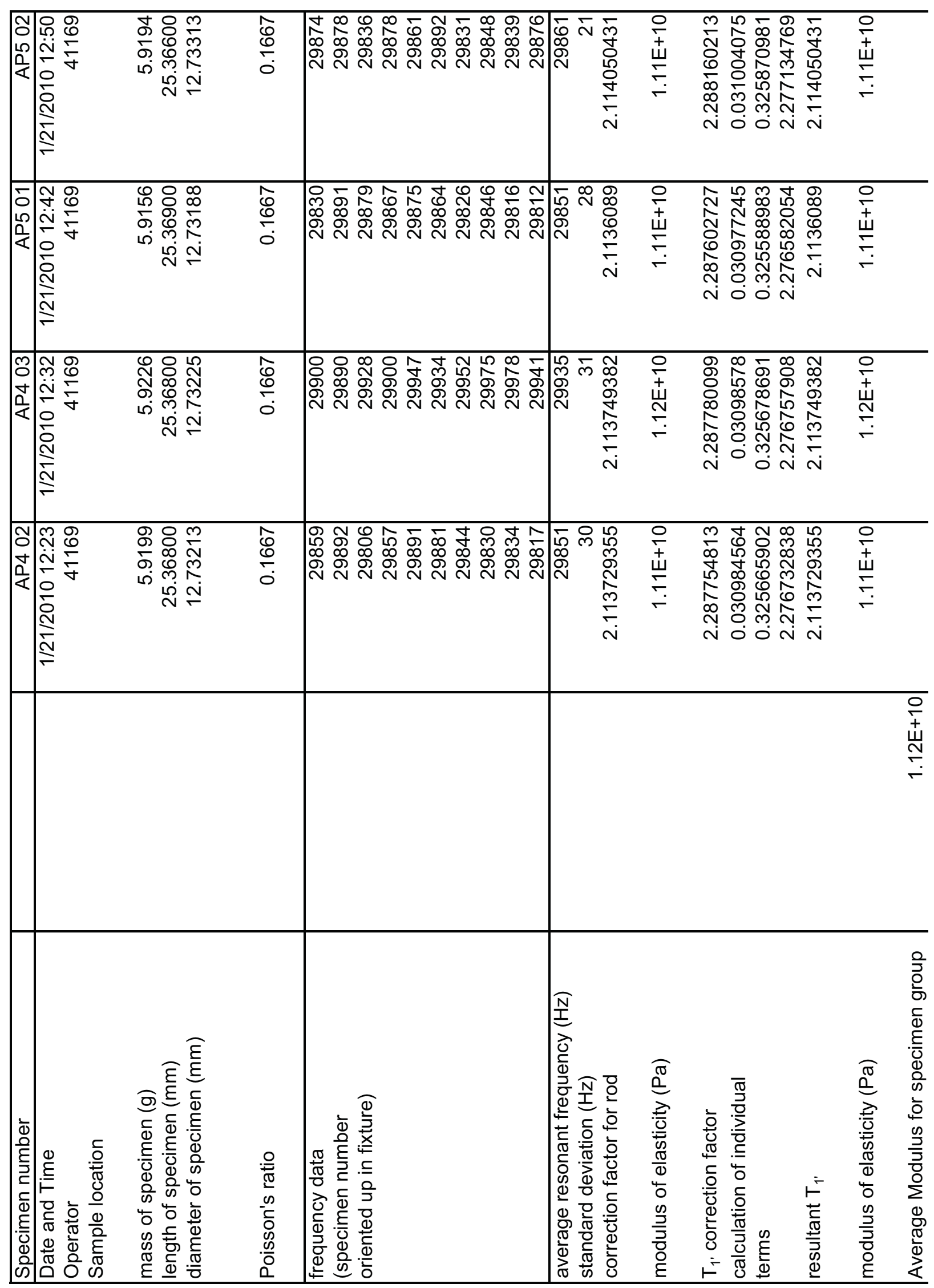




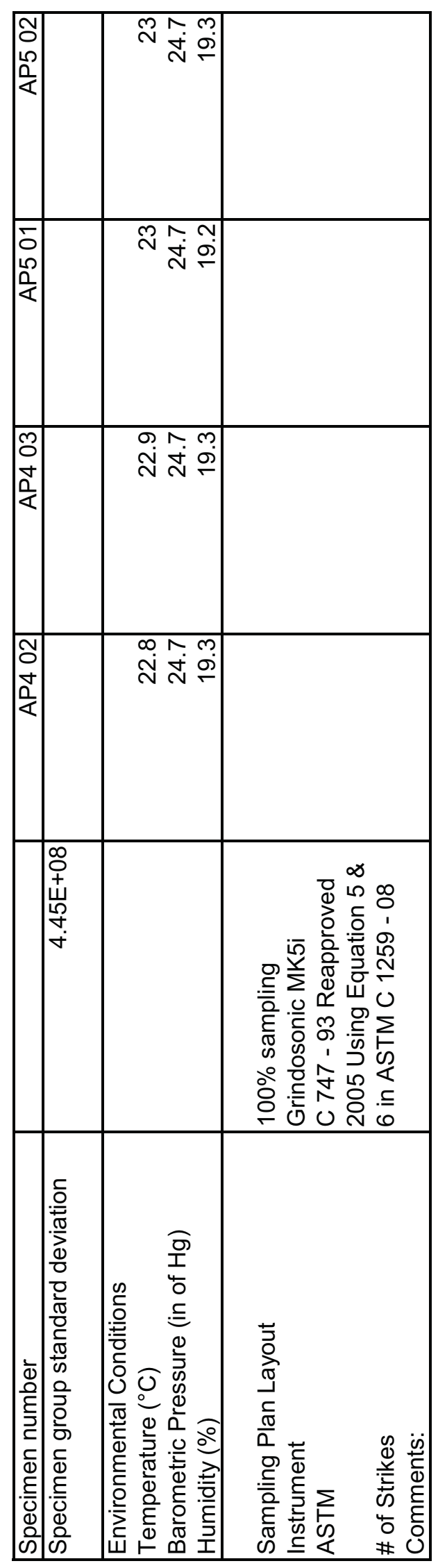

n 


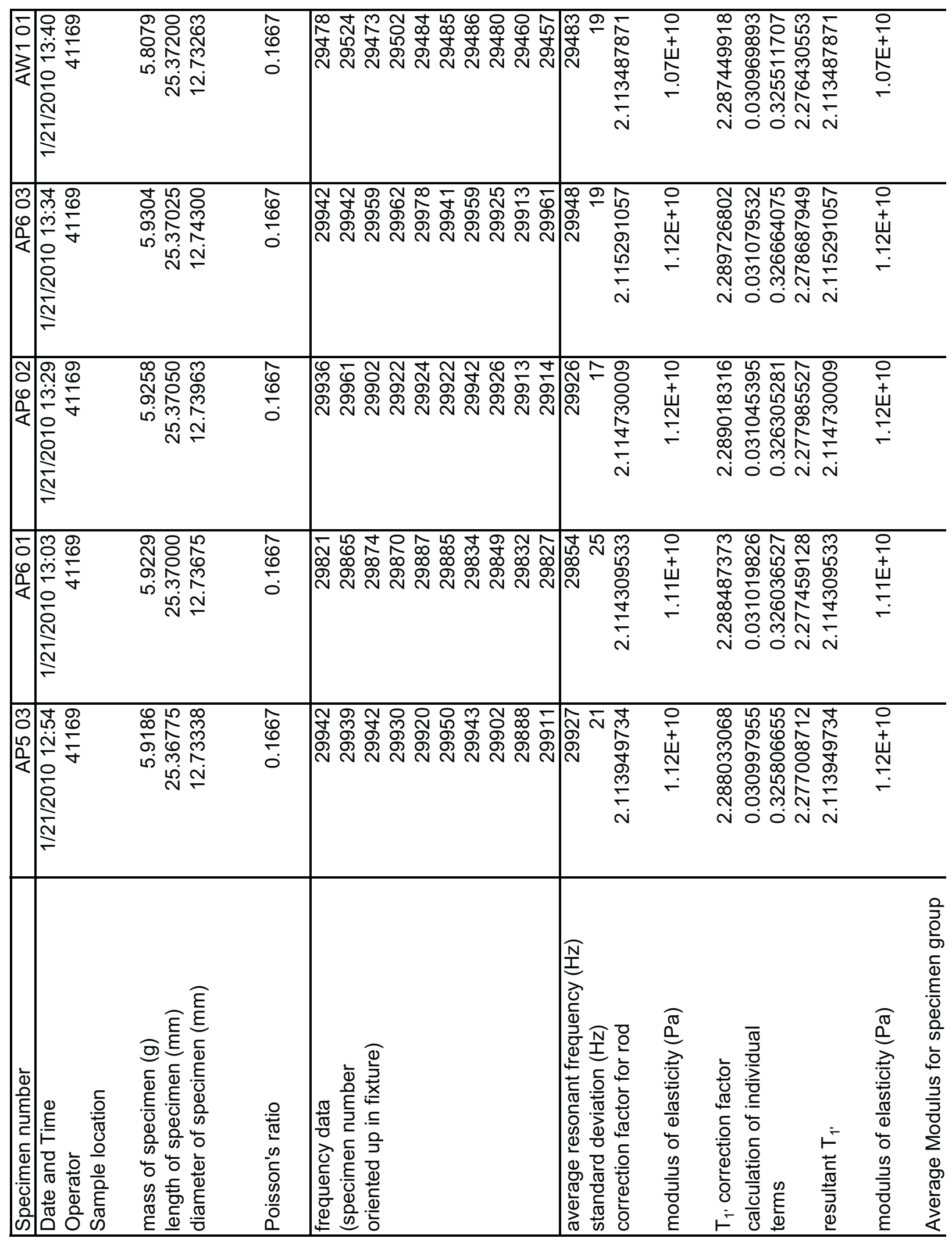




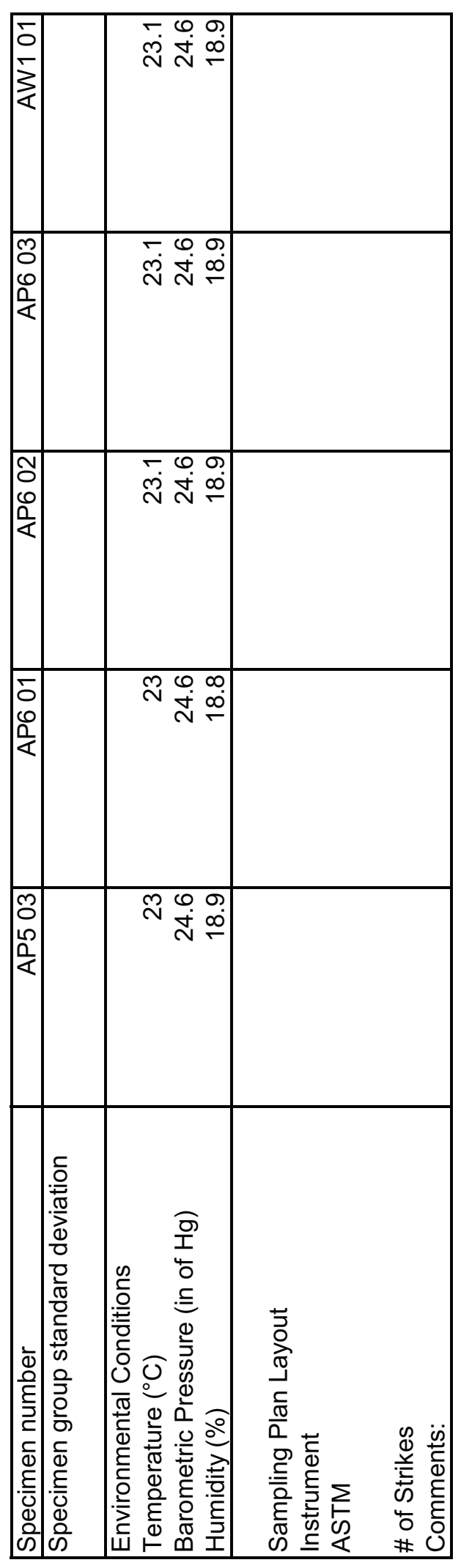

$\overline{6}$ 


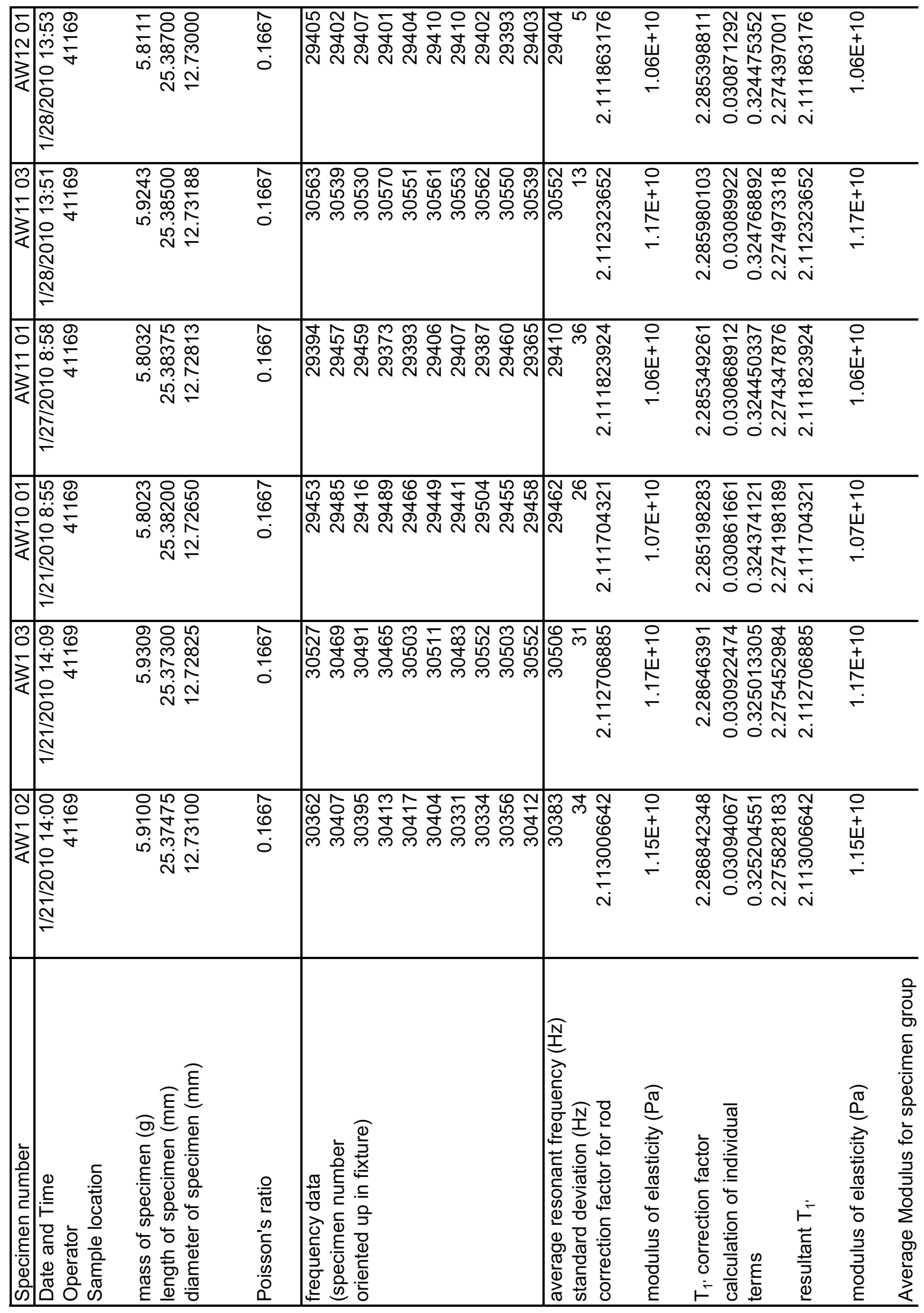




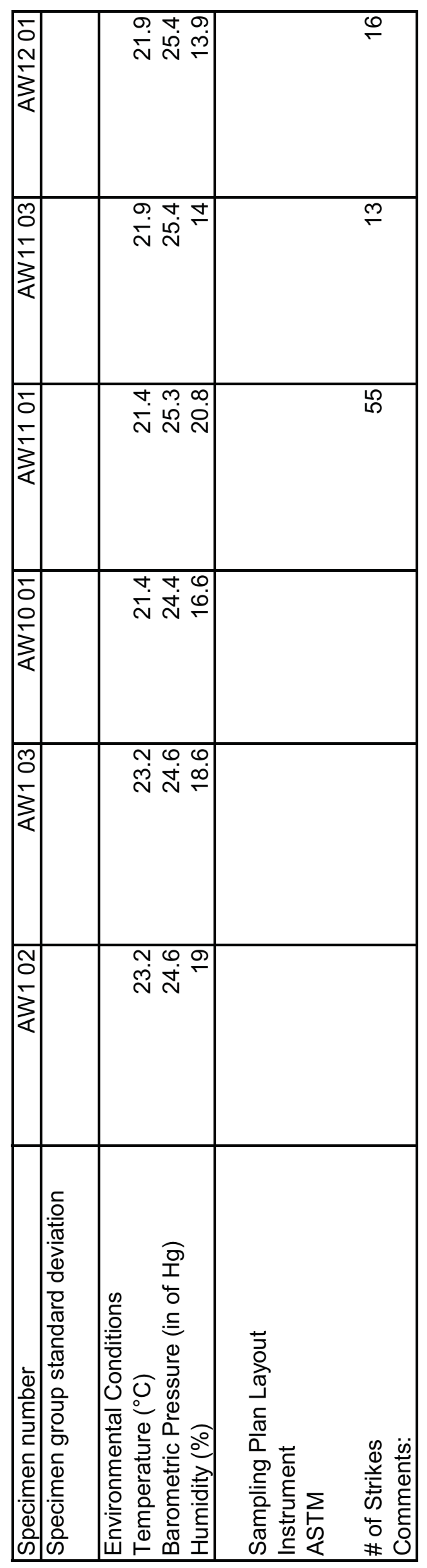

ถ 


\begin{tabular}{|c|c|c|c|c|c|c|c|c|c|}
\hline \begin{tabular}{|l} 
\\
0 \\
\\
$\vdots$ \\
3 \\
\\
4
\end{tabular} & 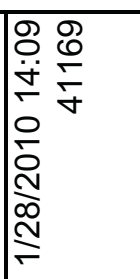 & 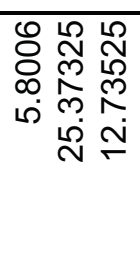 & $\begin{array}{l}\hat{\theta} \\
\frac{0}{0}\end{array}$ & 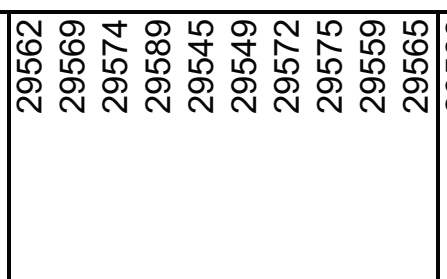 & 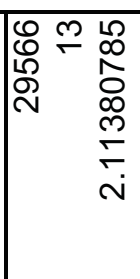 & 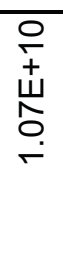 & 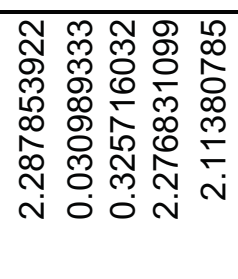 & 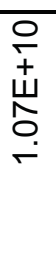 & \\
\hline 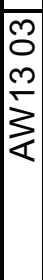 & 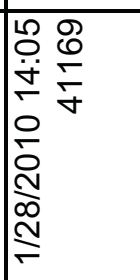 & 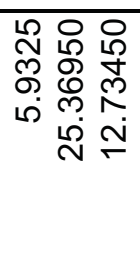 & $\begin{array}{l}\hat{\theta} \\
\grave{0} \\
\dot{0}\end{array}$ & 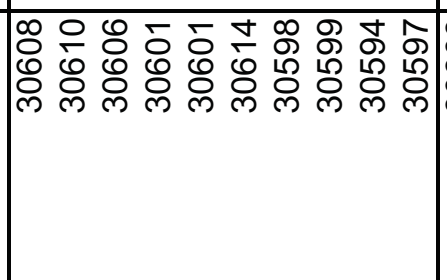 & 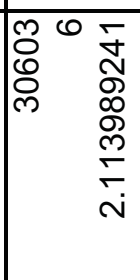 & 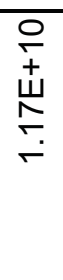 & 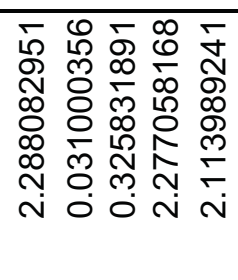 & 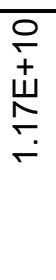 & \\
\hline 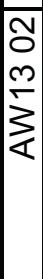 & 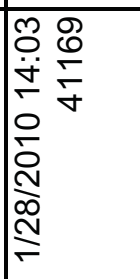 & 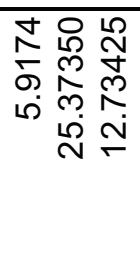 & $\begin{array}{l}\hat{\theta} \\
\stackrel{0}{0} \\
\vdots\end{array}$ & 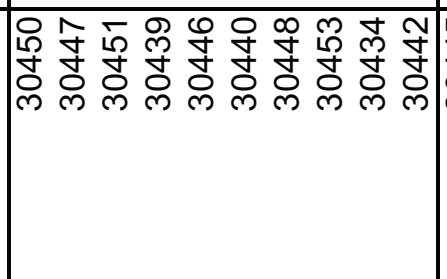 & 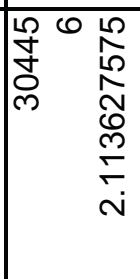 & 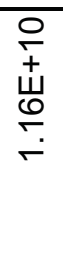 & 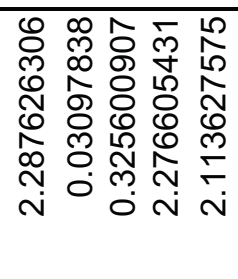 & 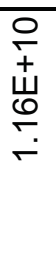 & \\
\hline \begin{tabular}{|l} 
\\
\\
0 \\
2 \\
\\
\\
\end{tabular} & 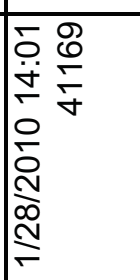 & 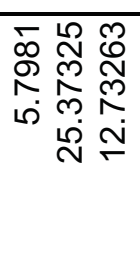 & $\begin{array}{l}\hat{\theta} \\
\qquad \\
0\end{array}$ & 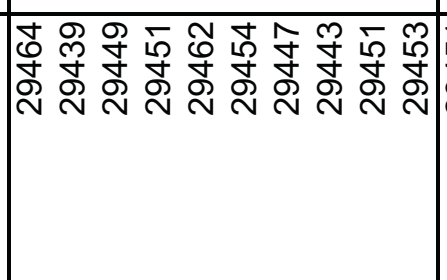 & 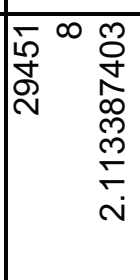 & 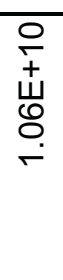 & 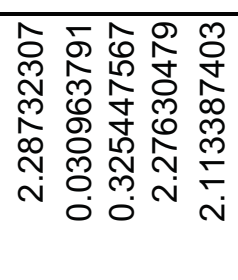 & 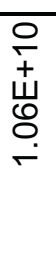 & \\
\hline 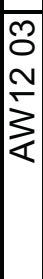 & 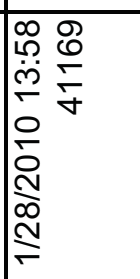 & 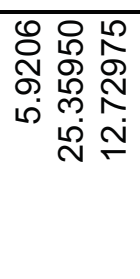 & $\begin{array}{l}\hat{\Theta} \\
\stackrel{\theta}{0}\end{array}$ & 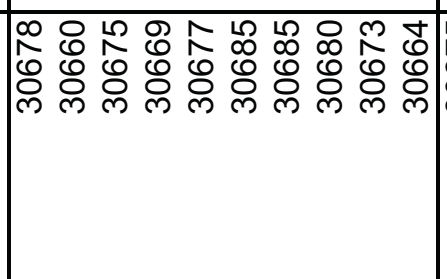 & 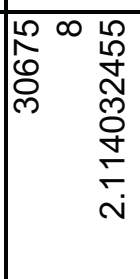 & 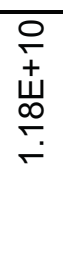 & 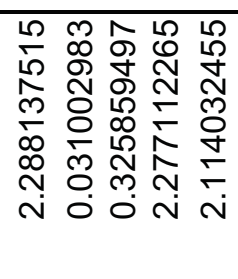 & 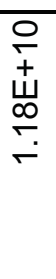 & \\
\hline 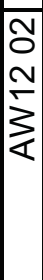 & 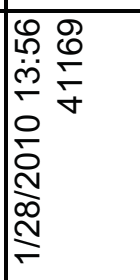 & 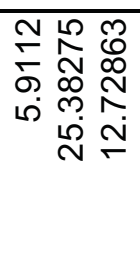 & $\begin{array}{l}\hat{\mathscr{\theta}} \\
\grave{0}\end{array}$ & 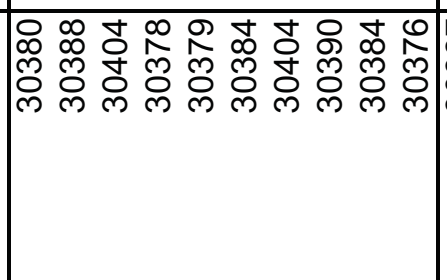 & 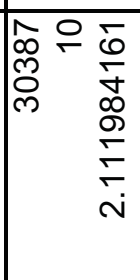 & 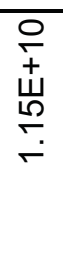 & 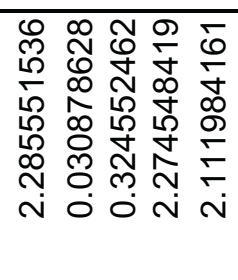 & 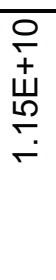 & \\
\hline 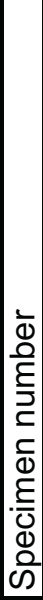 & 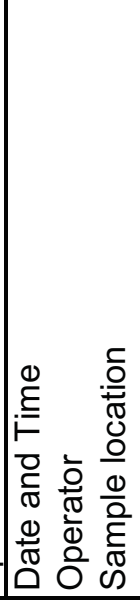 & 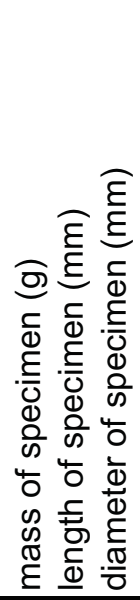 & $\begin{array}{l}.0 \\
\stackrel{0}{0} \\
-\infty \\
-\frac{1}{0} \\
0 \\
.00 \\
0 \\
0\end{array}$ & 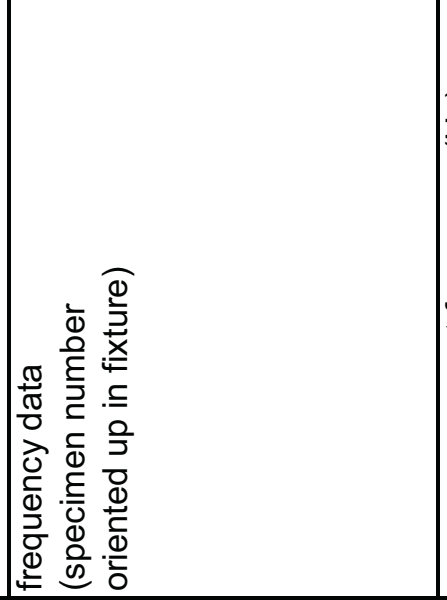 & 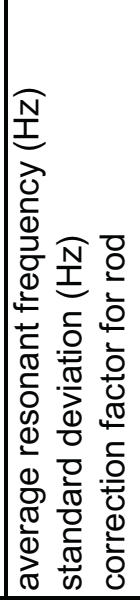 & 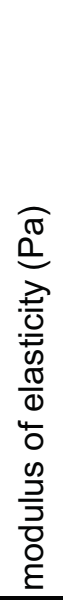 & 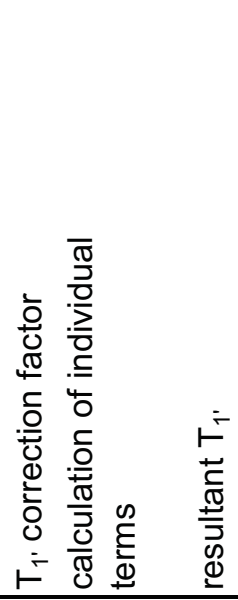 & 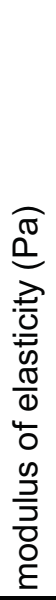 & 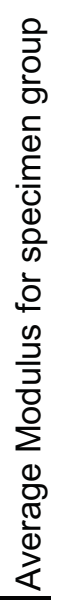 \\
\hline
\end{tabular}




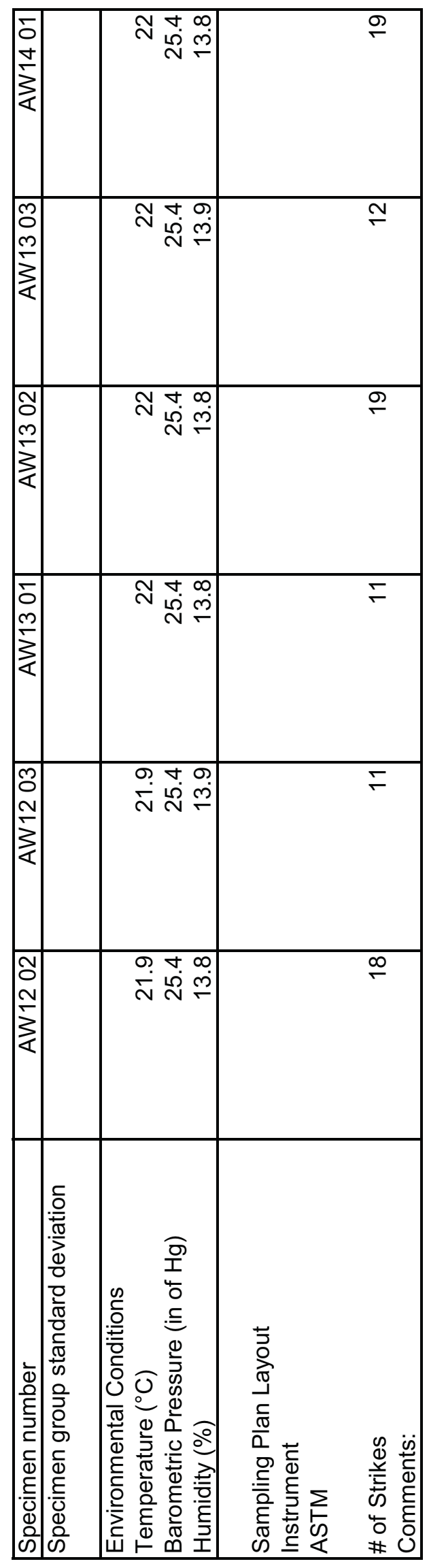




\begin{tabular}{|c|c|c|c|c|c|c|c|c|c|}
\hline \begin{tabular}{|l|} 
\\
0 \\
0 \\
\\
$\vdots$ \\
\\
\end{tabular} & 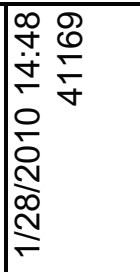 & 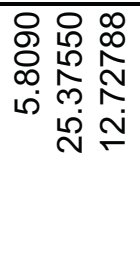 & $\begin{array}{l}\hat{0} \\
0 \\
0\end{array}$ & 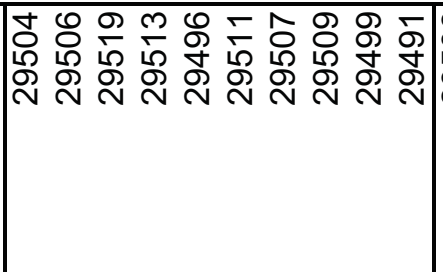 & 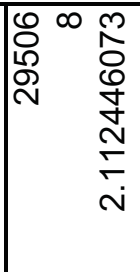 & 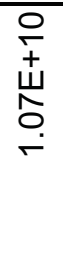 & 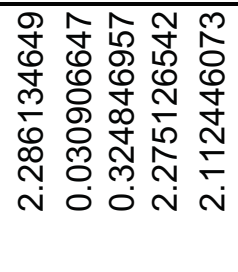 & 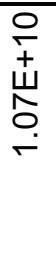 & \\
\hline $\begin{array}{l}m \\
0 \\
10 \\
2 \\
3 \\
4\end{array}$ & 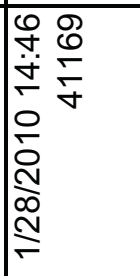 & 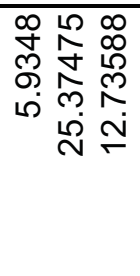 & $\begin{array}{l}\hat{0} \\
\stackrel{0}{0} \\
\vdots\end{array}$ & 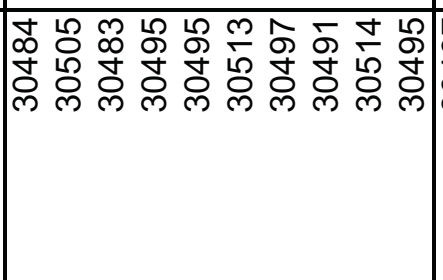 & 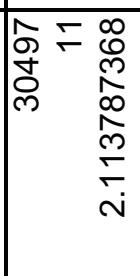 & 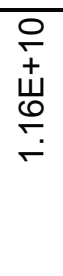 & 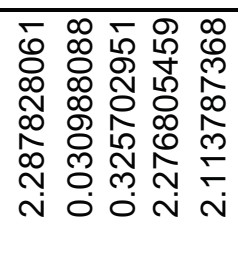 & 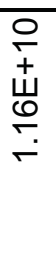 & \\
\hline 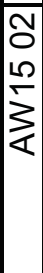 & 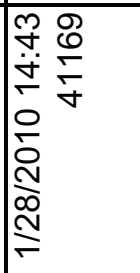 & 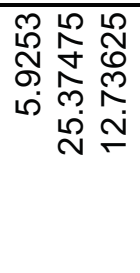 & $\begin{array}{l}\hat{0} \\
\stackrel{0}{0} \\
\vdots\end{array}$ & 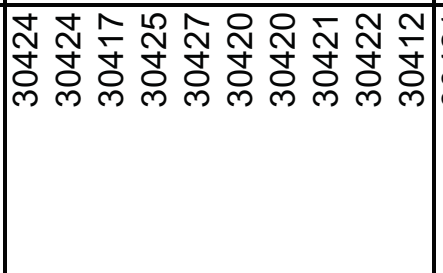 & 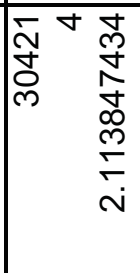 & 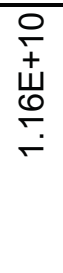 & 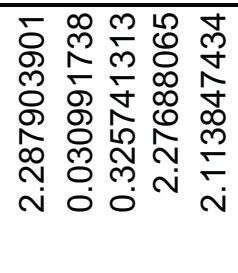 & 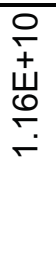 & \\
\hline $\begin{array}{l}5 \\
0 \\
10 \\
\frac{1}{3} \\
\frac{1}{4} \\
\end{array}$ & 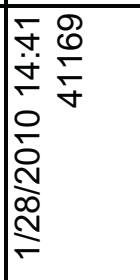 & 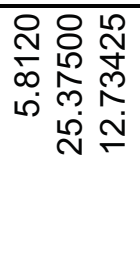 & $\begin{array}{l}\hat{0} \\
\stackrel{0}{0} \\
\vdots \\
0\end{array}$ & 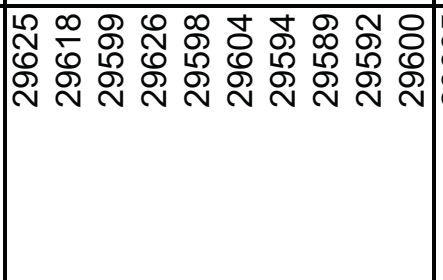 & 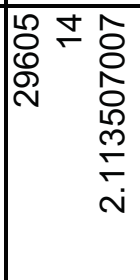 & 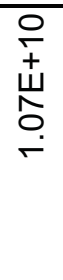 & 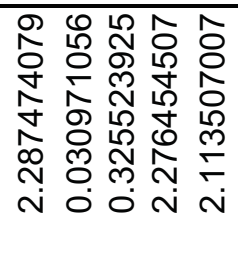 & 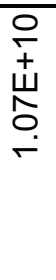 & \\
\hline 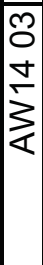 & 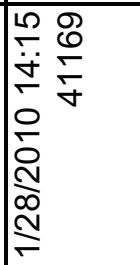 & 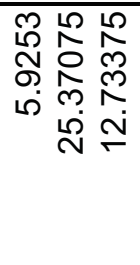 & $\begin{array}{l}\hat{0} \\
\stackrel{0}{0} \\
\vdots\end{array}$ & 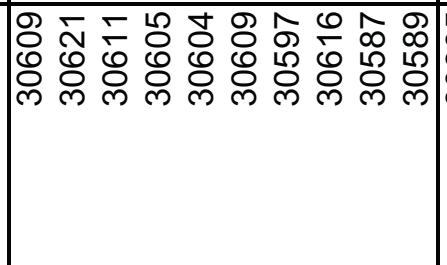 & 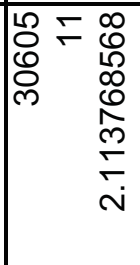 & 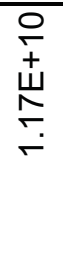 & 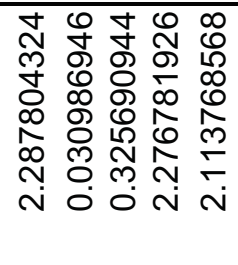 & 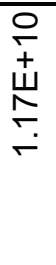 & \\
\hline 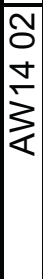 & 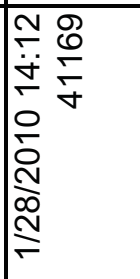 & 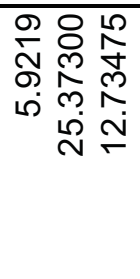 & $\begin{array}{l}\hat{0} \\
\stackrel{0}{0} \\
\vdots\end{array}$ & 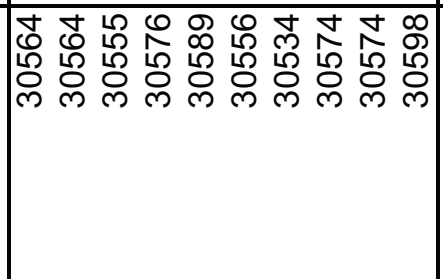 & 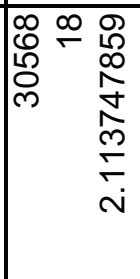 & 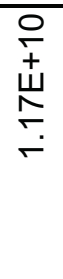 & 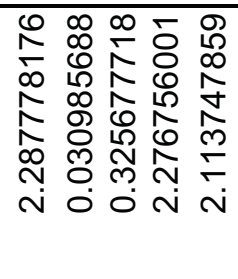 & 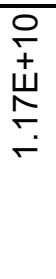 & \\
\hline 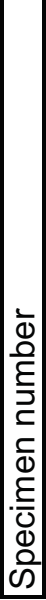 & 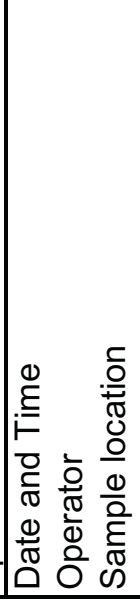 & 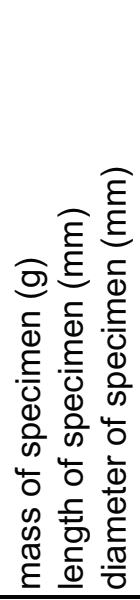 & 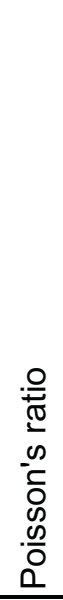 & 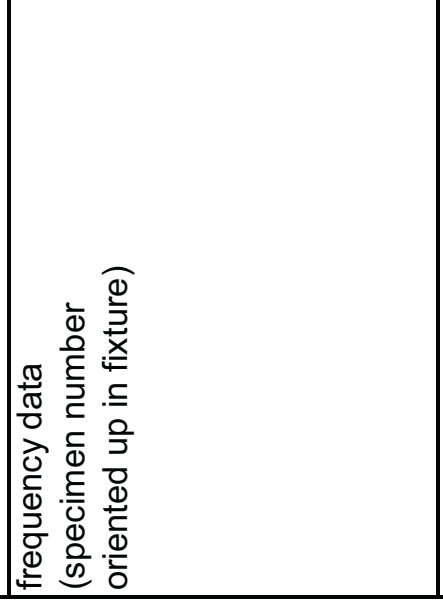 & 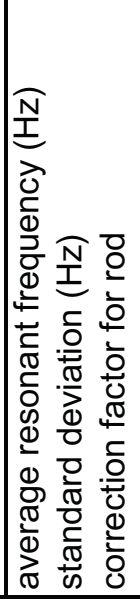 & 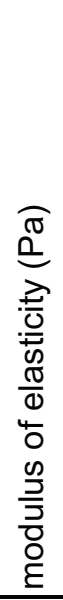 & 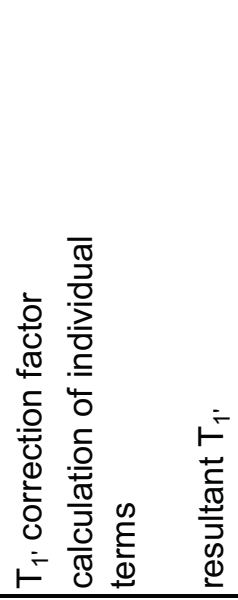 & 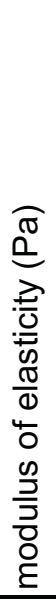 & 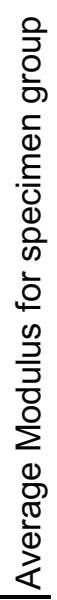 \\
\hline
\end{tabular}




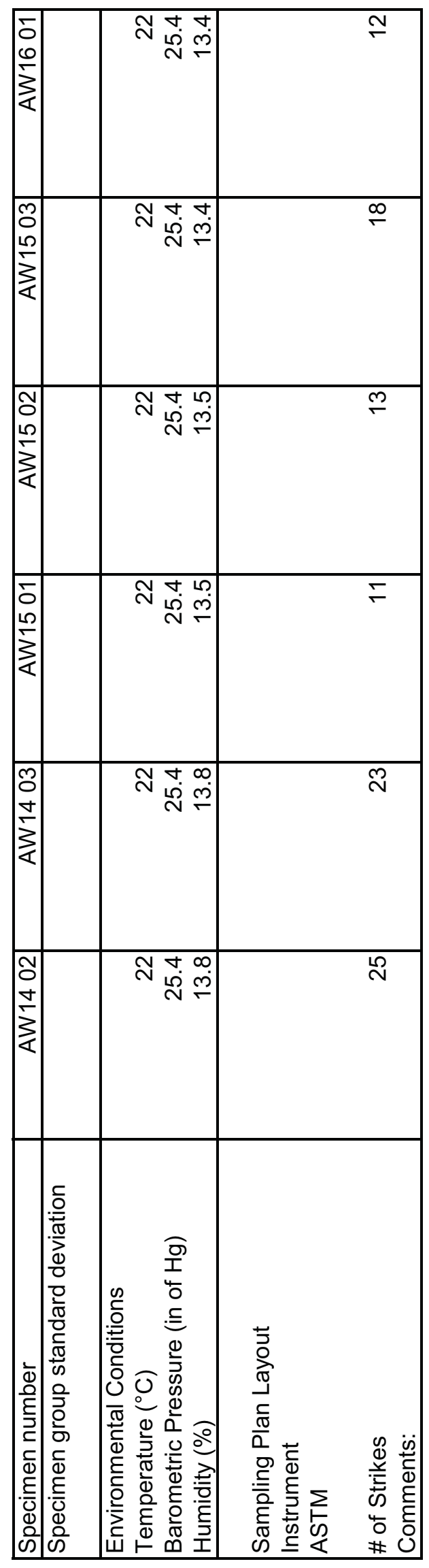

6 


\begin{tabular}{|c|c|c|c|c|c|c|c|c|c|}
\hline $\begin{array}{l}5 \\
0 \\
m \\
3 \\
\end{array}$ & 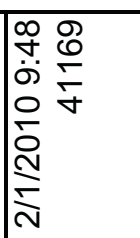 & 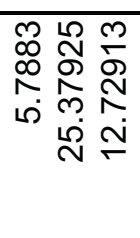 & $\begin{array}{c}\hat{\theta} \\
\underline{0} \\
\dot{0}\end{array}$ & 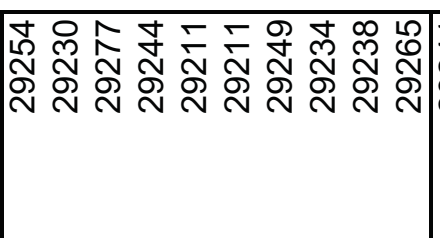 & 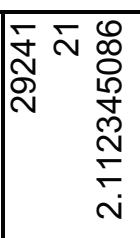 & 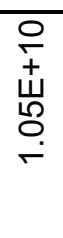 & 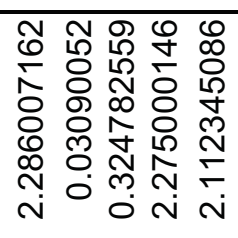 & 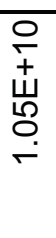 & \\
\hline 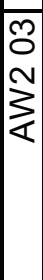 & 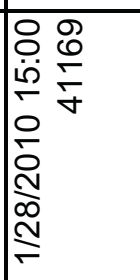 & 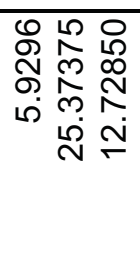 & $\begin{array}{l}\hat{\theta} \\
\grave{0} \\
\dot{0}\end{array}$ & 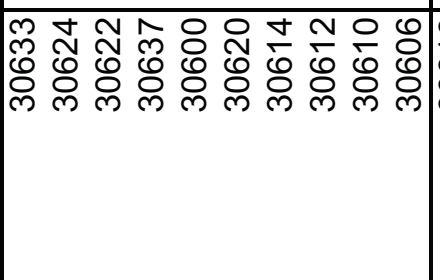 & 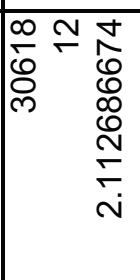 & $\begin{array}{l}\stackrel{\circ}{+} \\
\stackrel{+}{\rightleftarrows} \\
\stackrel{+}{\leftarrow}\end{array}$ & 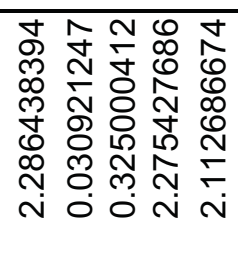 & 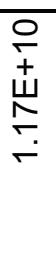 & \\
\hline 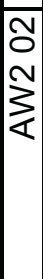 & 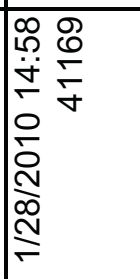 & 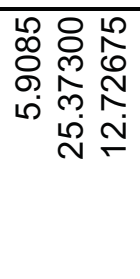 & $\begin{array}{l}\hat{\theta} \\
\grave{0} \\
\vdots\end{array}$ & 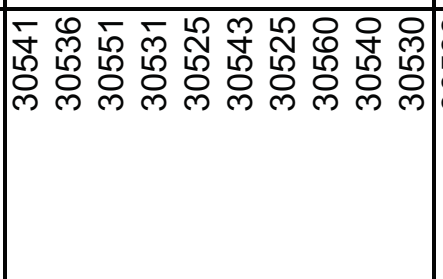 & 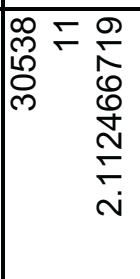 & 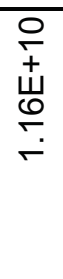 & 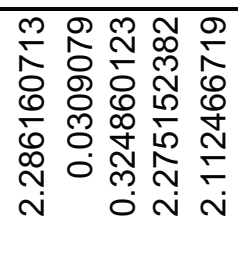 & 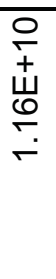 & \\
\hline $\begin{array}{l}- \\
0 \\
1 \\
3 \\
\vdots \\
\end{array}$ & 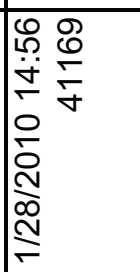 & 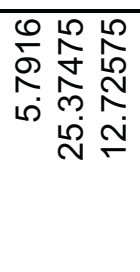 & $\begin{array}{l}\hat{\oplus} \\
\grave{0} \\
\vdots\end{array}$ & 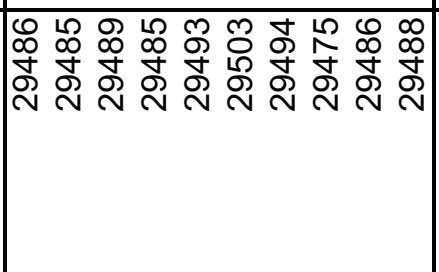 & 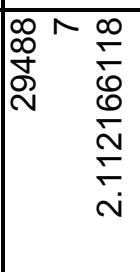 & 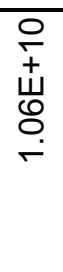 & 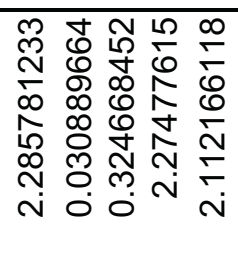 & 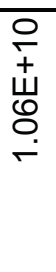 & \\
\hline $\begin{array}{l}m \\
0 \\
0 \\
0 \\
3 \\
3 \\
4\end{array}$ & 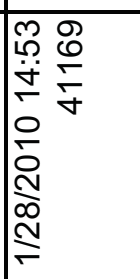 & 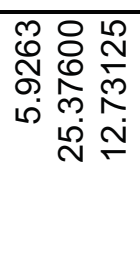 & $\begin{array}{l}\hat{\Theta} \\
\stackrel{\theta}{0}\end{array}$ & 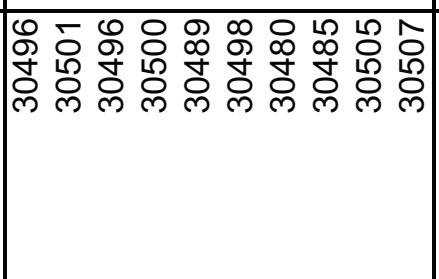 & 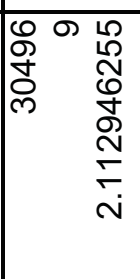 & 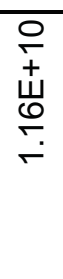 & 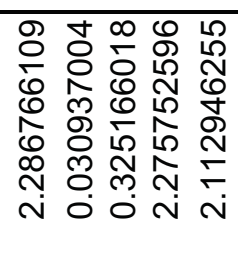 & 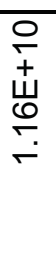 & \\
\hline $\begin{array}{l}0 \\
0 \\
0 \\
0 \\
\vdots \\
3 \\
4 \\
\end{array}$ & 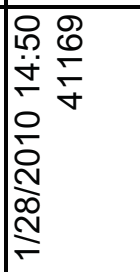 & 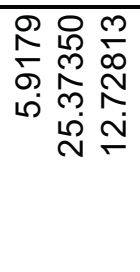 & $\begin{array}{l}\hat{\theta} \\
\stackrel{0}{0} \\
\vdots\end{array}$ & 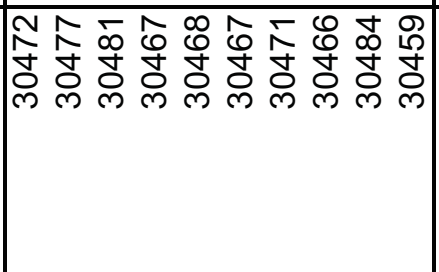 & 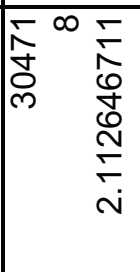 & 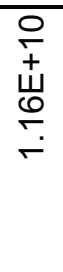 & 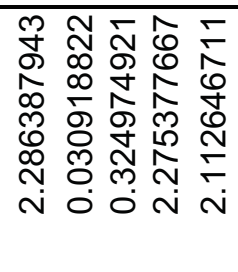 & 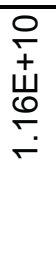 & \\
\hline 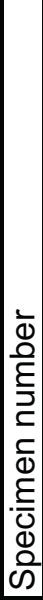 & 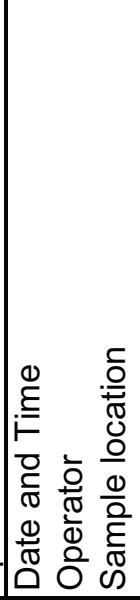 & 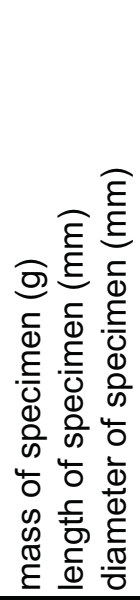 & $\begin{array}{l}.0 \\
\stackrel{0}{0} \\
-\infty \\
-\frac{1}{0} \\
0 \\
.00 \\
0 \\
0\end{array}$ & 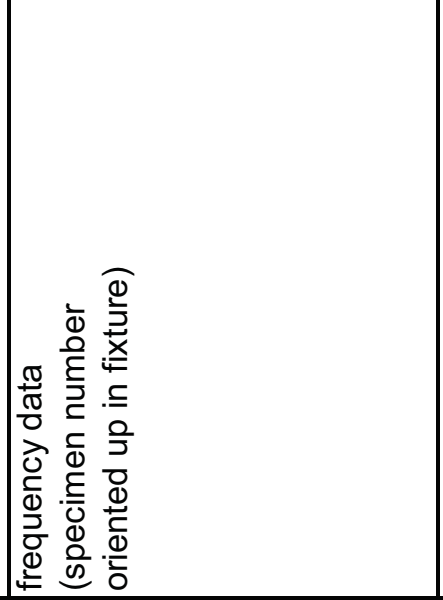 & 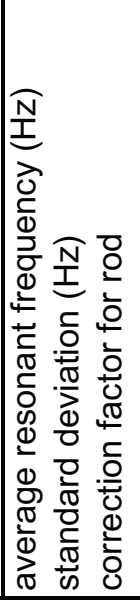 & 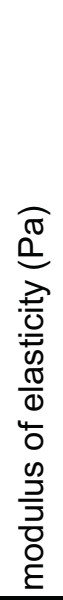 & 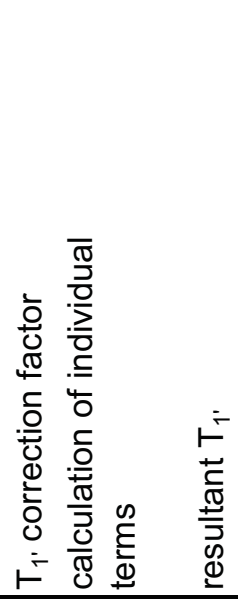 & 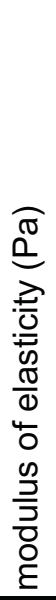 & 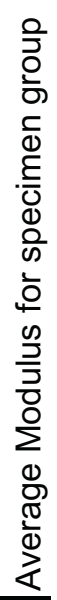 \\
\hline
\end{tabular}




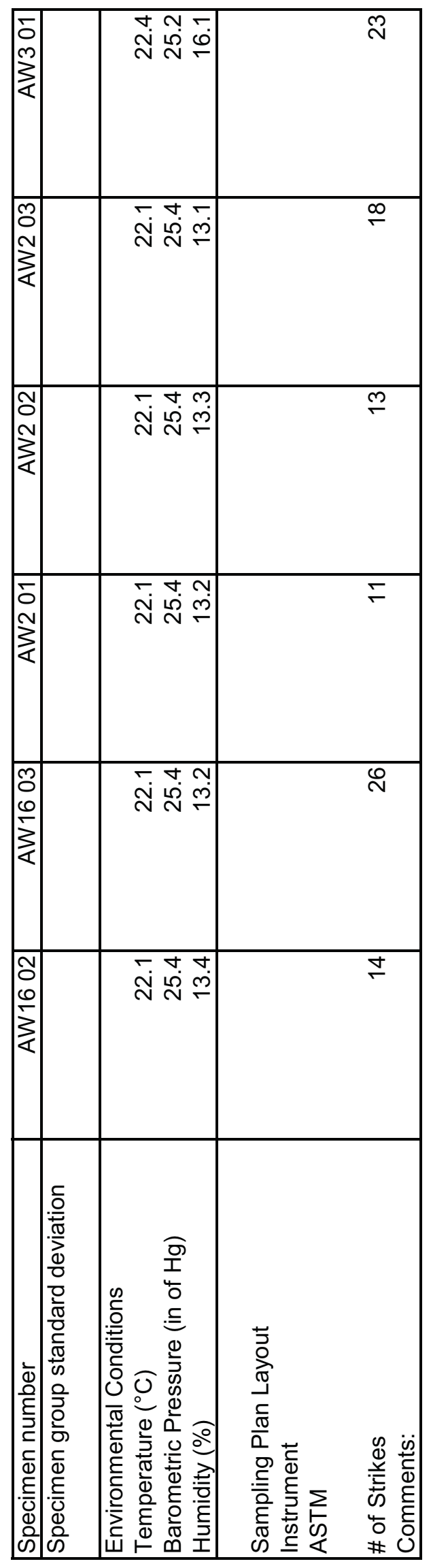

iे 


\begin{tabular}{|c|c|c|c|c|c|c|c|c|c|}
\hline \begin{tabular}{|c|} 
\\
0 \\
10 \\
10 \\
3 \\
\\
\end{tabular} & 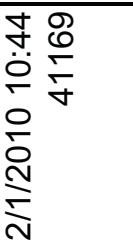 & 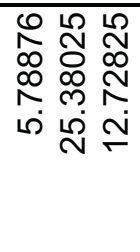 & $\begin{array}{l}\hat{\emptyset} \\
\stackrel{0}{0} \\
\vdots\end{array}$ & 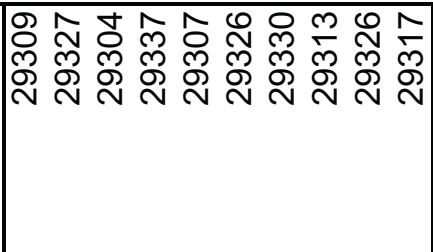 & 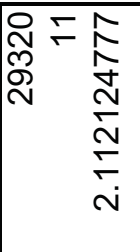 & 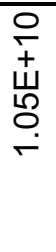 & 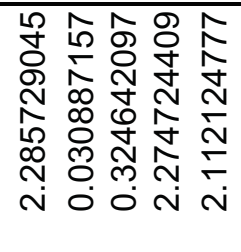 & 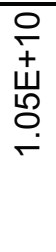 & \\
\hline 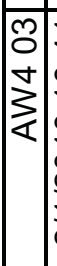 & 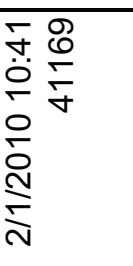 & 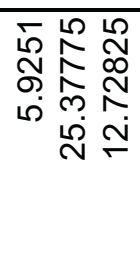 & $\begin{array}{l}\overline{0} \\
\stackrel{0}{0} \\
\dot{0}\end{array}$ & 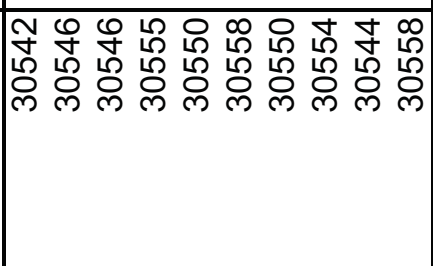 & 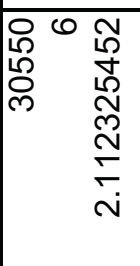 & 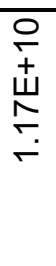 & 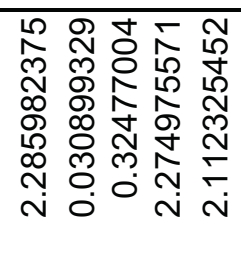 & 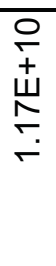 & \\
\hline 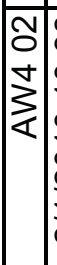 & 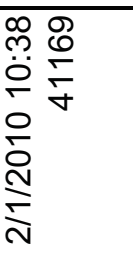 & 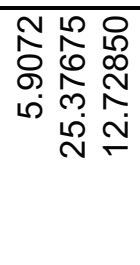 & $\begin{array}{l}\hat{0} \\
\stackrel{0}{0}\end{array}$ & 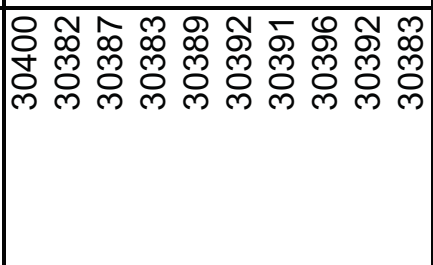 & 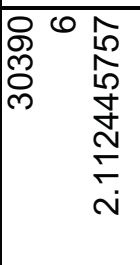 & 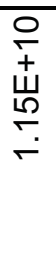 & 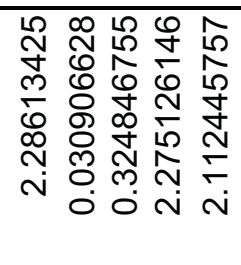 & 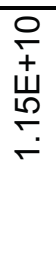 & \\
\hline 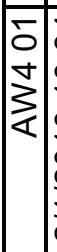 & 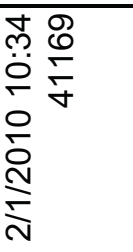 & 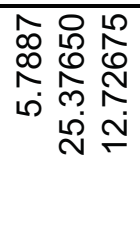 & $\begin{array}{l}\hat{\mathscr{\theta}} \\
\stackrel{\bullet}{0}\end{array}$ & 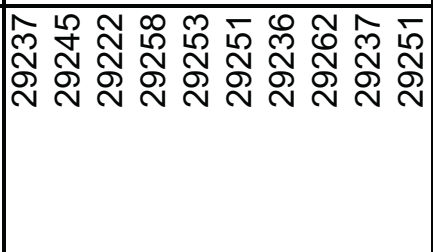 & 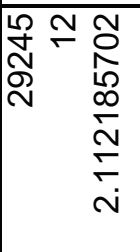 & 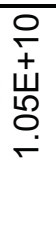 & 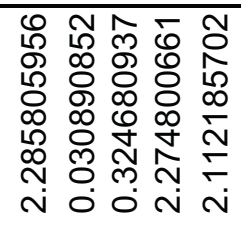 & 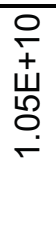 & \\
\hline 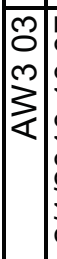 & 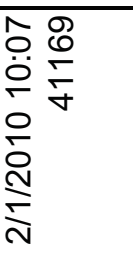 & 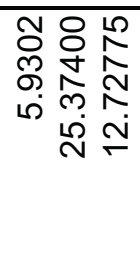 & $\begin{array}{l}\hat{\theta} \\
\stackrel{\emptyset}{0}\end{array}$ & 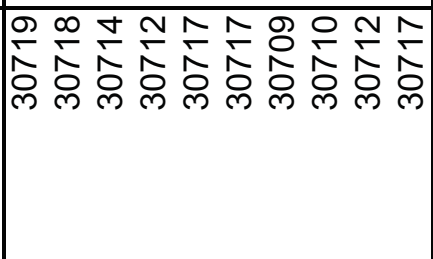 & 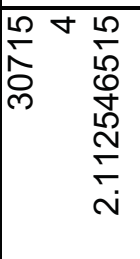 & $\begin{array}{l}\stackrel{0}{+} \\
\stackrel{+}{\varpi} \\
\stackrel{\infty}{\leftarrow}\end{array}$ & 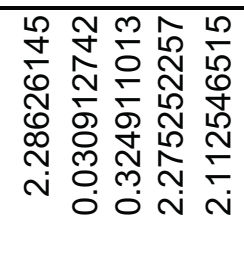 & $\begin{array}{l}\stackrel{0}{+} \\
\stackrel{+}{\varpi} \\
\stackrel{\infty}{\leftarrow}\end{array}$ & \\
\hline 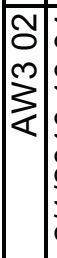 & 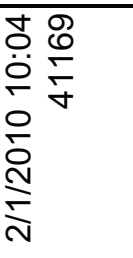 & 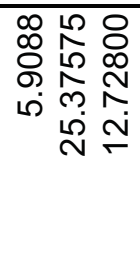 & $\begin{array}{l}\hat{\emptyset} \\
\emptyset \\
0\end{array}$ & 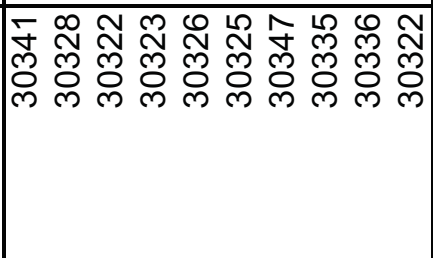 & 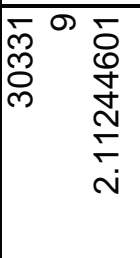 & 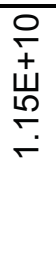 & 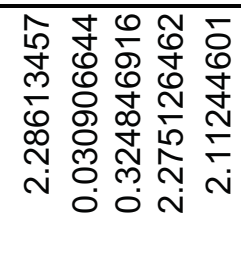 & $\begin{array}{l}\stackrel{0}{+} \\
\stackrel{+}{W} \\
\stackrel{5}{\leftarrow}\end{array}$ & \\
\hline 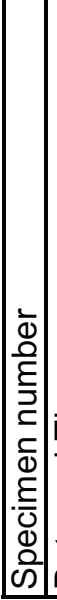 & 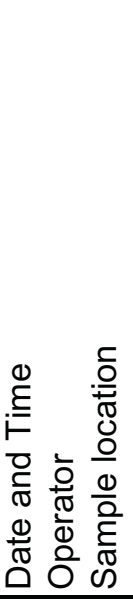 & 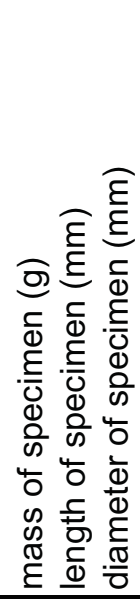 & 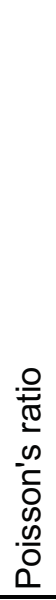 & 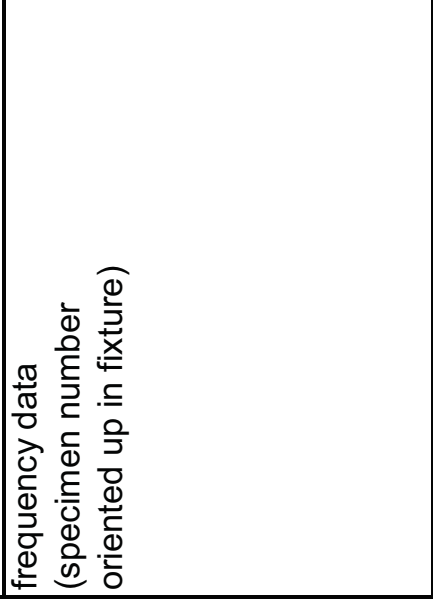 & 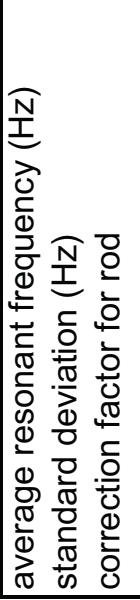 & 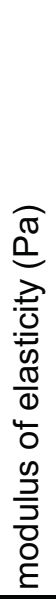 & 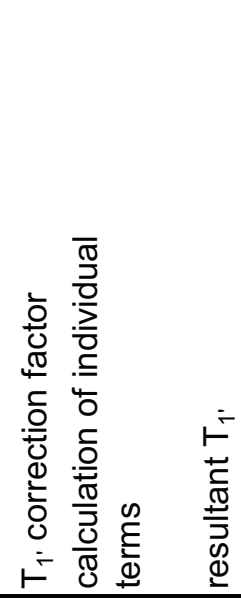 & 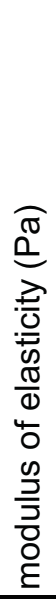 & 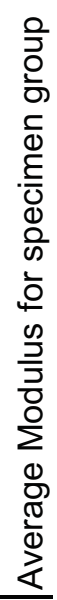 \\
\hline
\end{tabular}




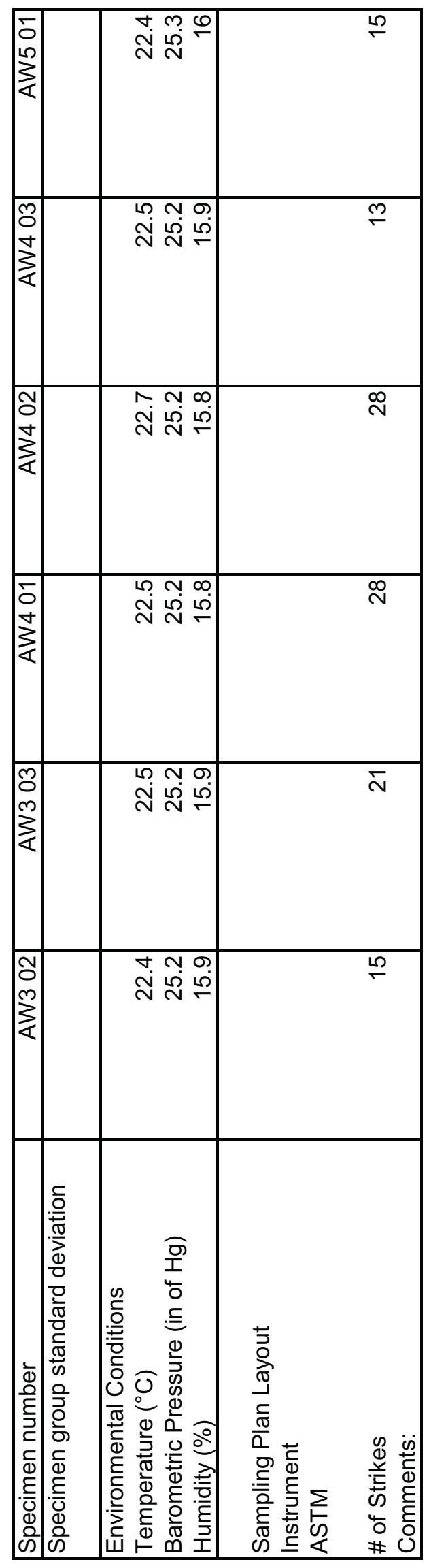




\begin{tabular}{|c|c|c|c|c|c|c|c|c|c|}
\hline 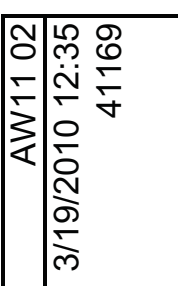 & 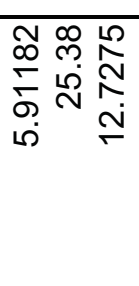 & $\begin{array}{l}\hat{\ddot{\theta}} \\
\stackrel{0}{0}\end{array}$ & 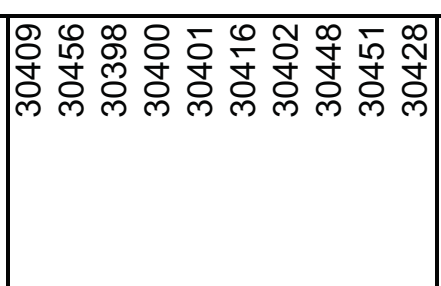 & 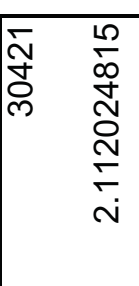 & 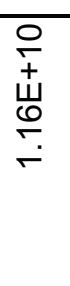 & \multicolumn{2}{|c|}{ 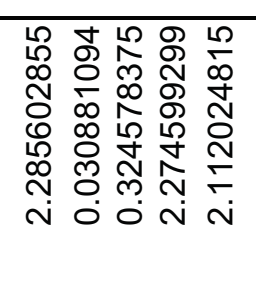 } & 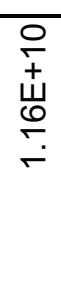 & \\
\hline 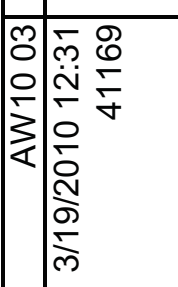 & 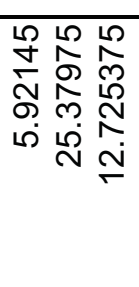 & $\begin{array}{l}\hat{\ddot{\theta}} \\
\stackrel{0}{0}\end{array}$ & 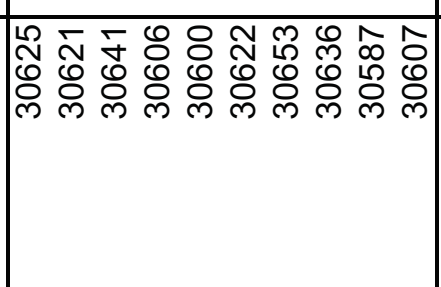 & 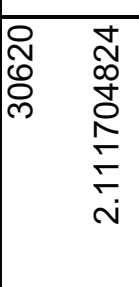 & 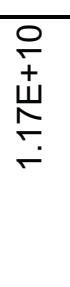 & 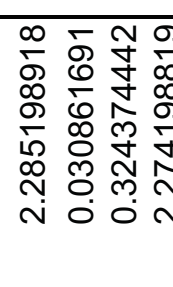 & & 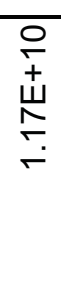 & \\
\hline 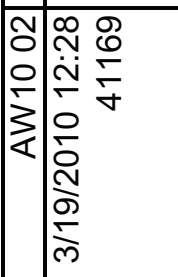 & 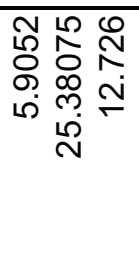 & $\begin{array}{l}\overline{0} \\
\stackrel{0}{0} \\
\vdots\end{array}$ & 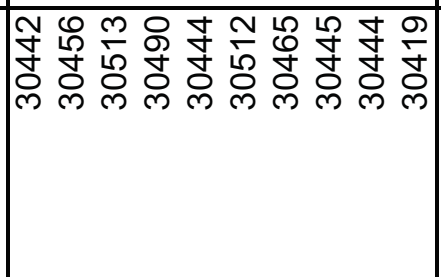 & 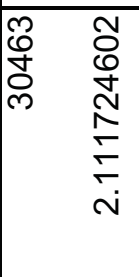 & 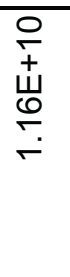 & 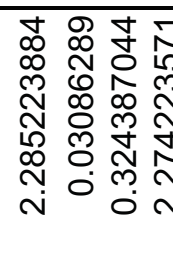 & & 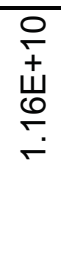 & \\
\hline 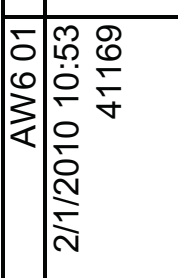 & 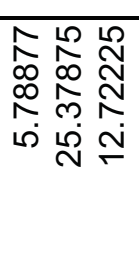 & $\begin{array}{l}\hat{\emptyset} \\
\stackrel{\Xi}{0} \\
0\end{array}$ & 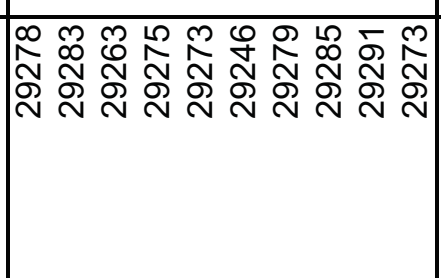 & 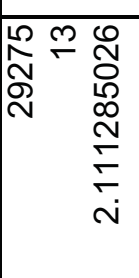 & 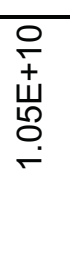 & 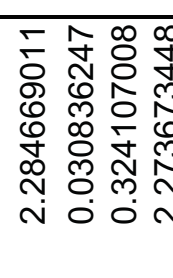 & & 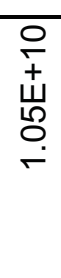 & \\
\hline 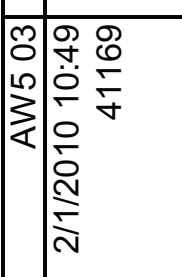 & 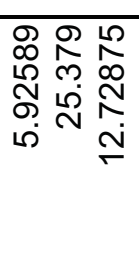 & $\begin{array}{l}\hat{\ddot{\theta}} \\
\stackrel{0}{0}\end{array}$ & 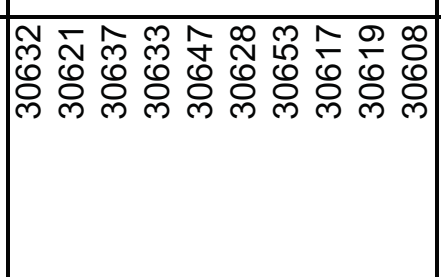 & 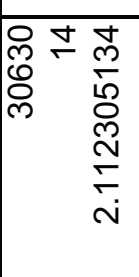 & 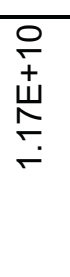 & 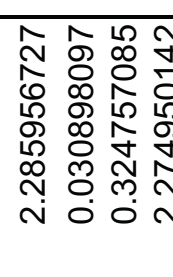 & 离 & $\begin{array}{l}\stackrel{\circ}{+} \\
\stackrel{+}{+} \\
\stackrel{5}{\leftarrow}\end{array}$ & \\
\hline 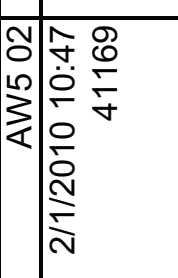 & 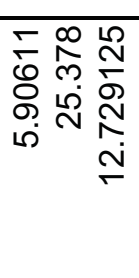 & $\begin{array}{l}\hat{0} \\
\stackrel{0}{0}\end{array}$ & 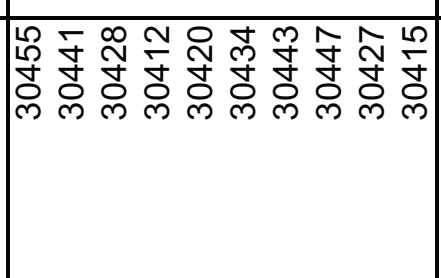 & 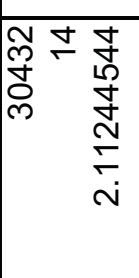 & 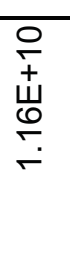 & 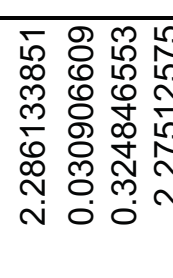 & 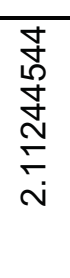 & 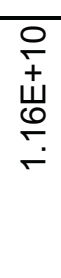 & \\
\hline 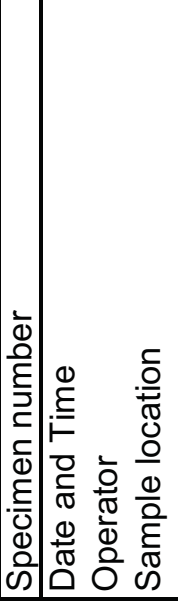 & 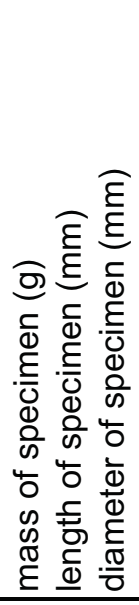 & 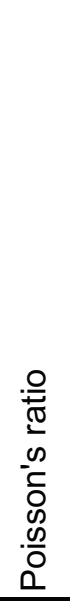 & 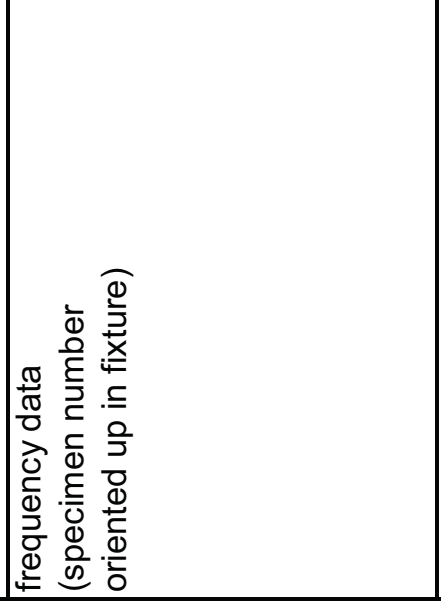 & 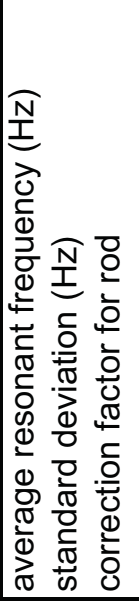 & 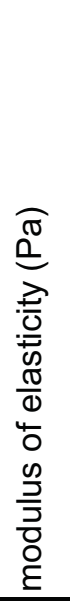 & 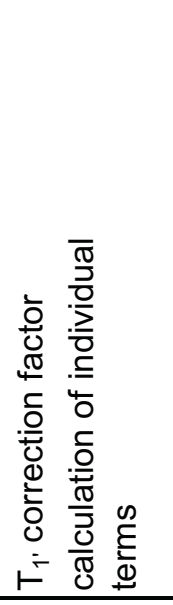 & 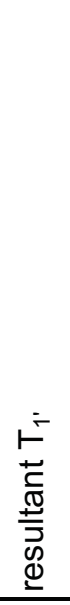 & 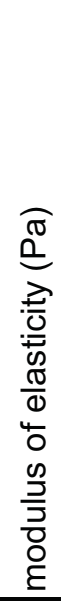 & 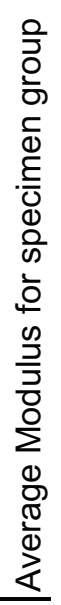 \\
\hline
\end{tabular}




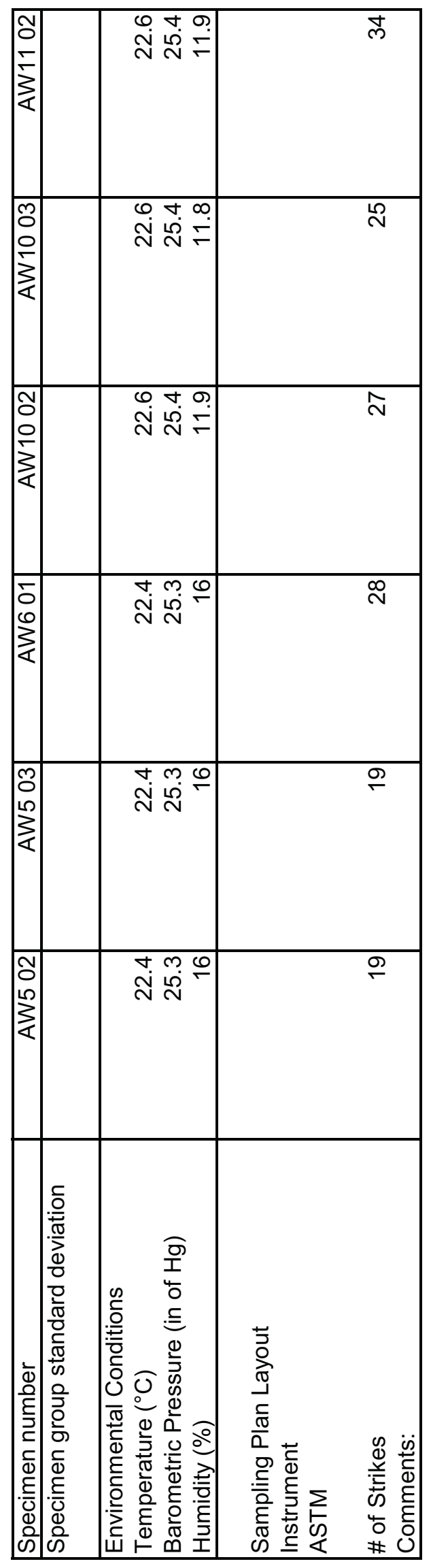

in 


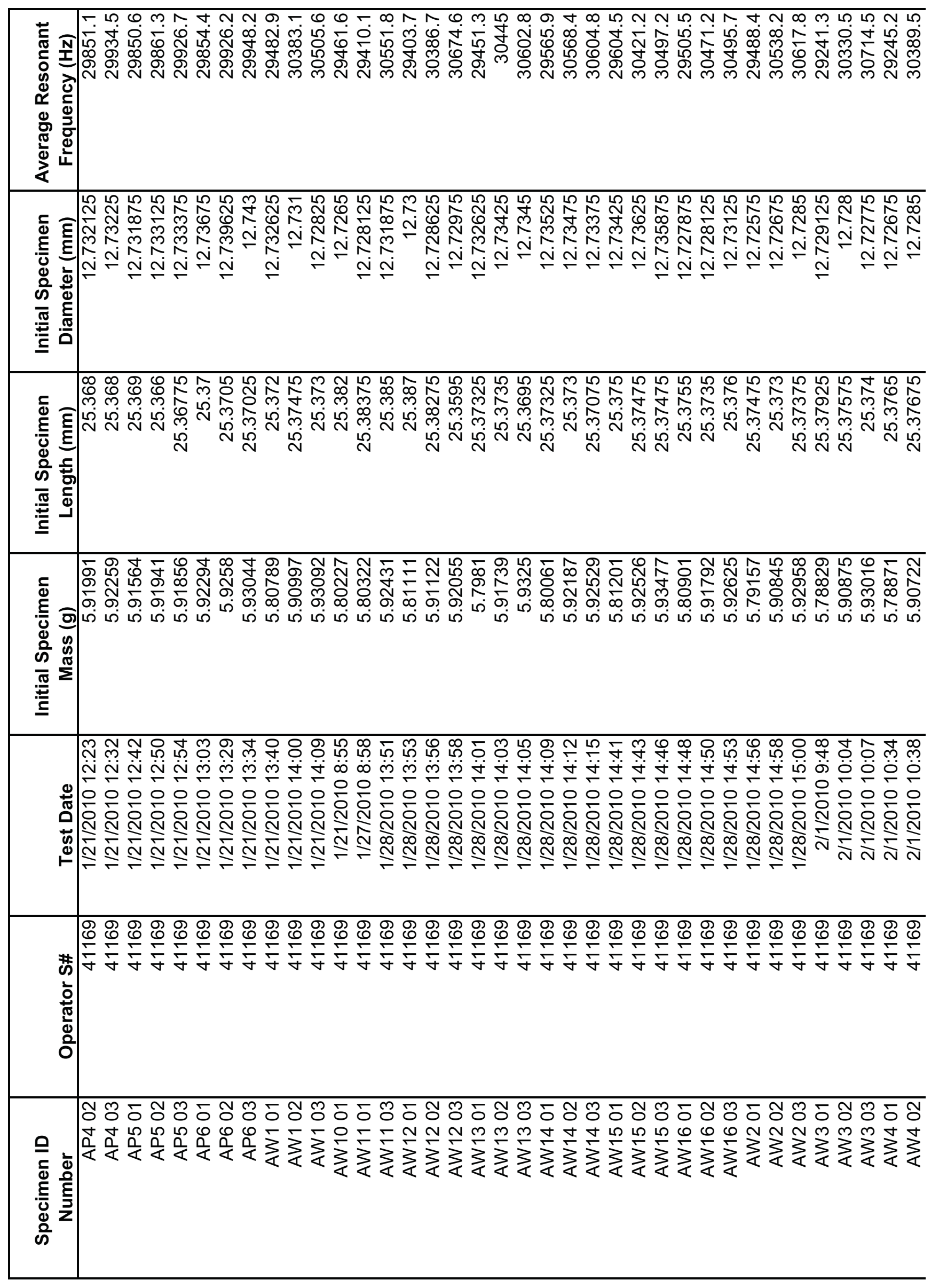




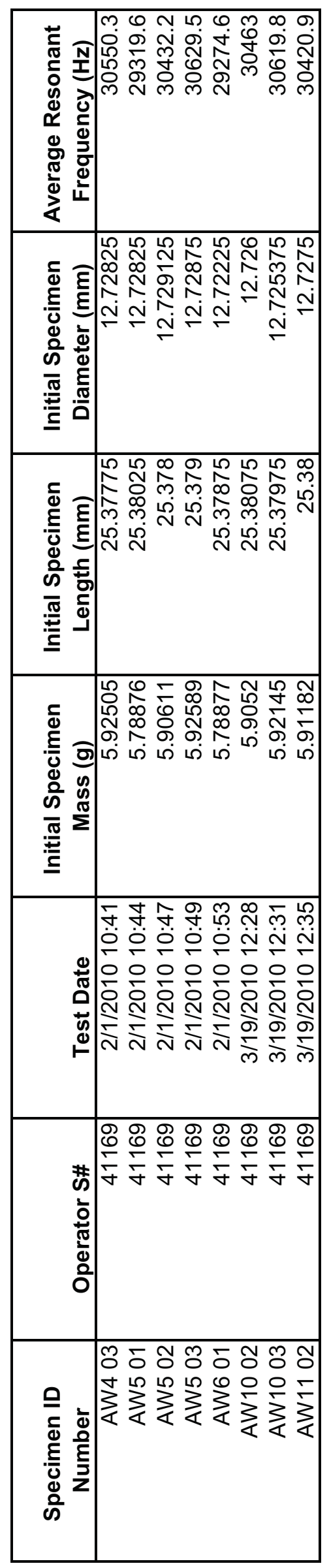




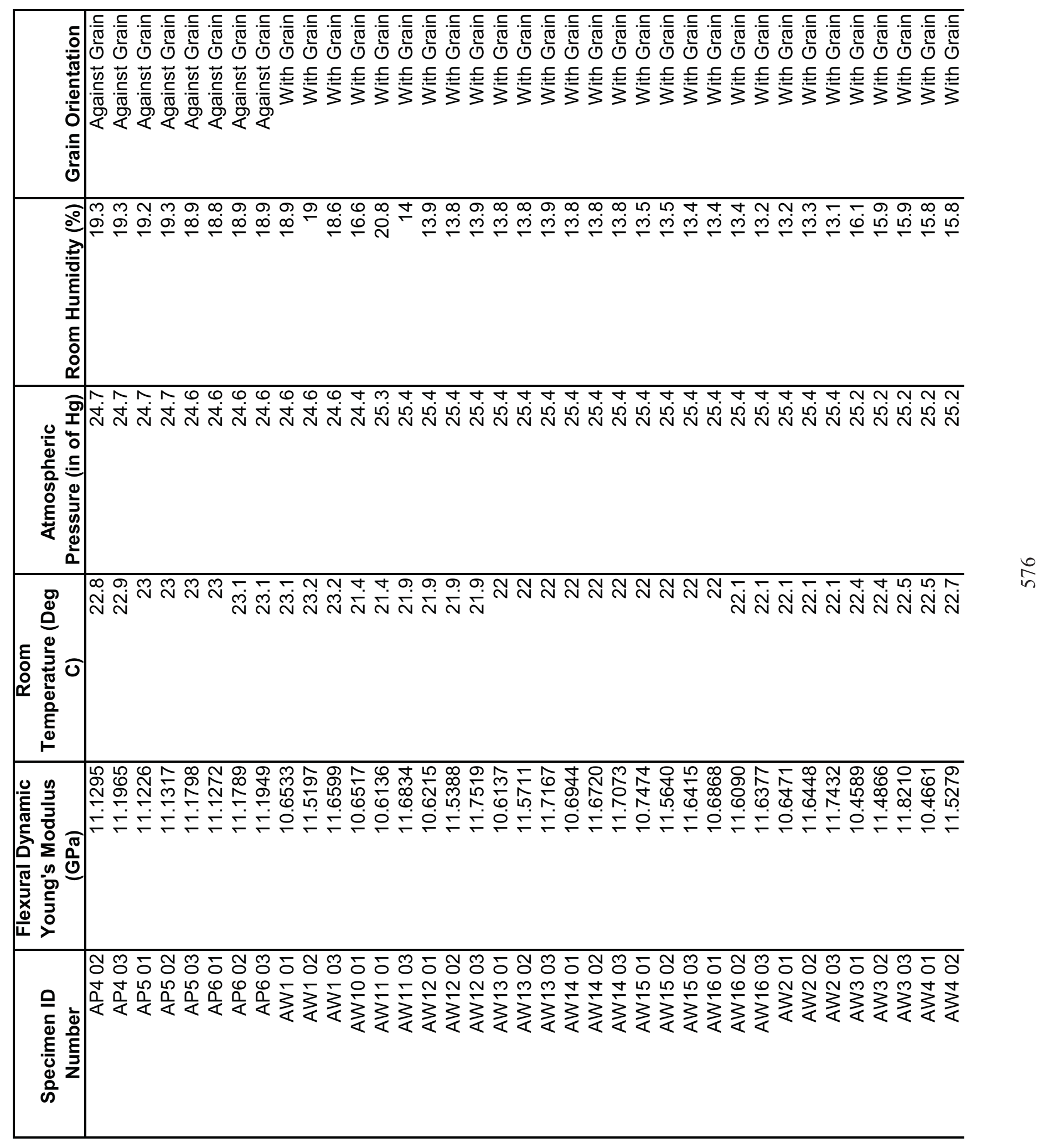




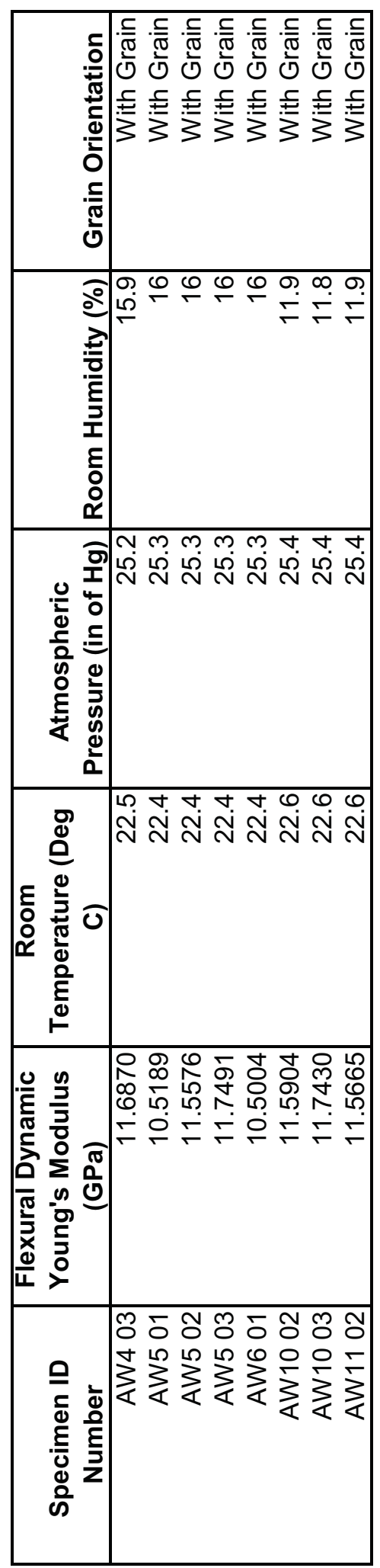

命 


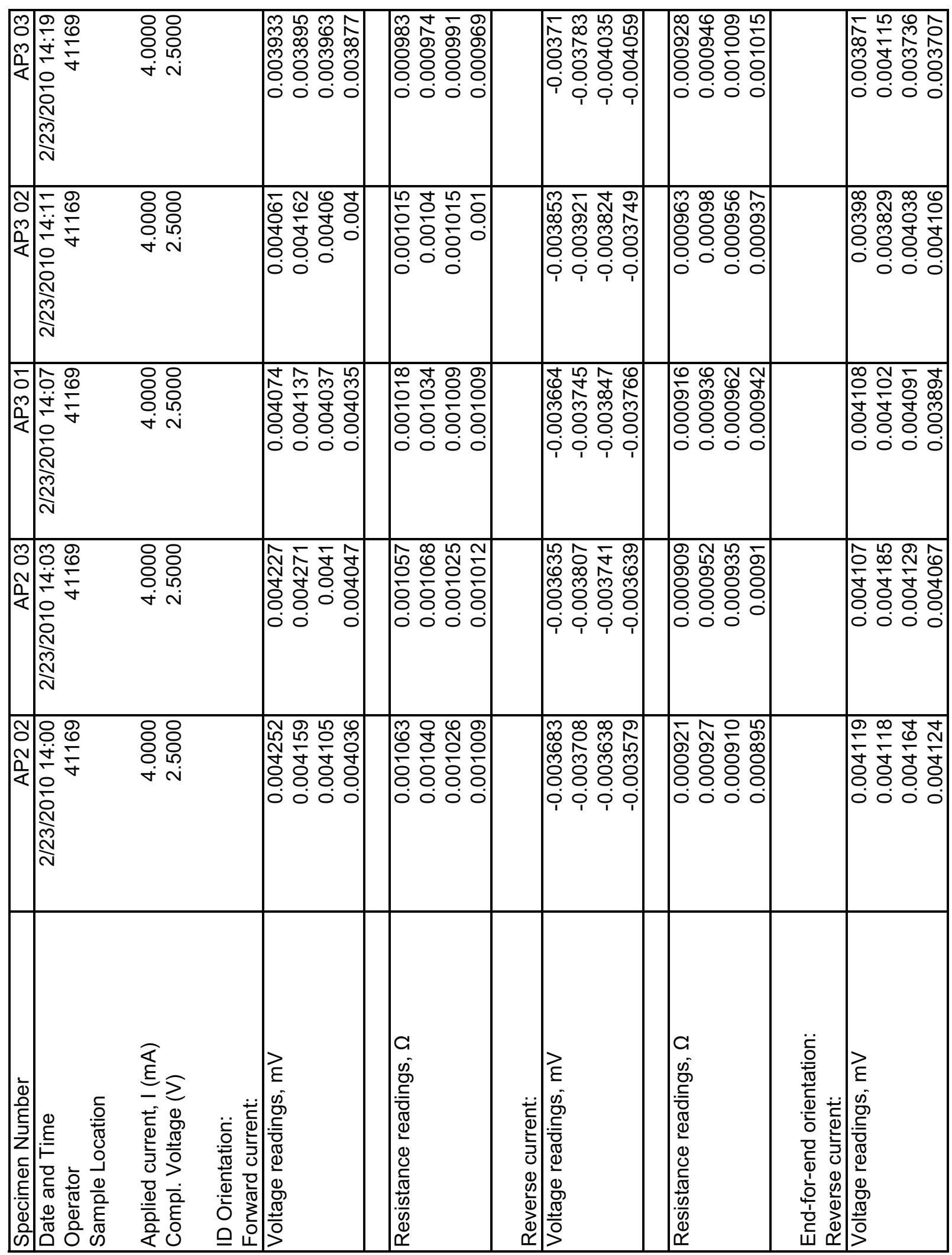




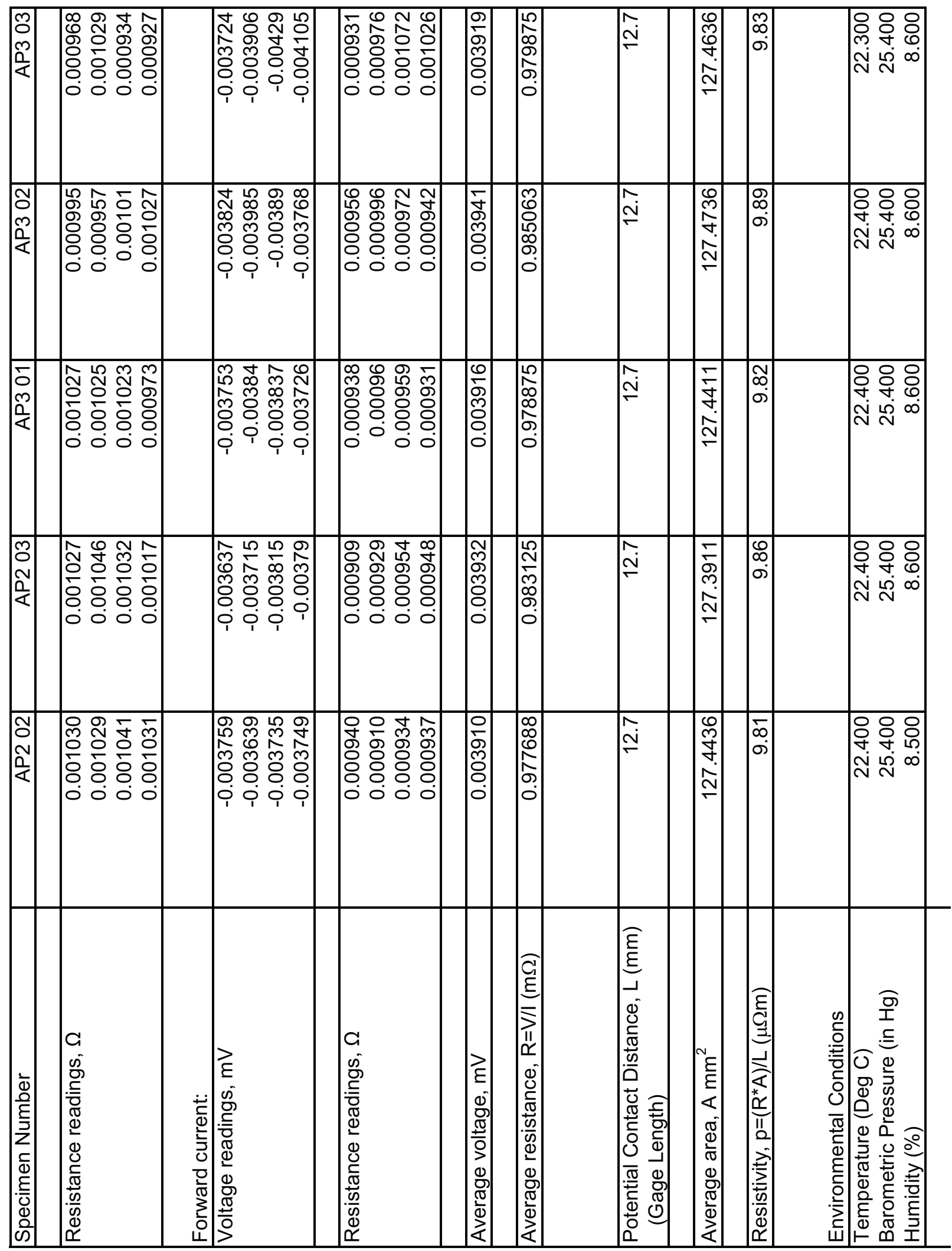




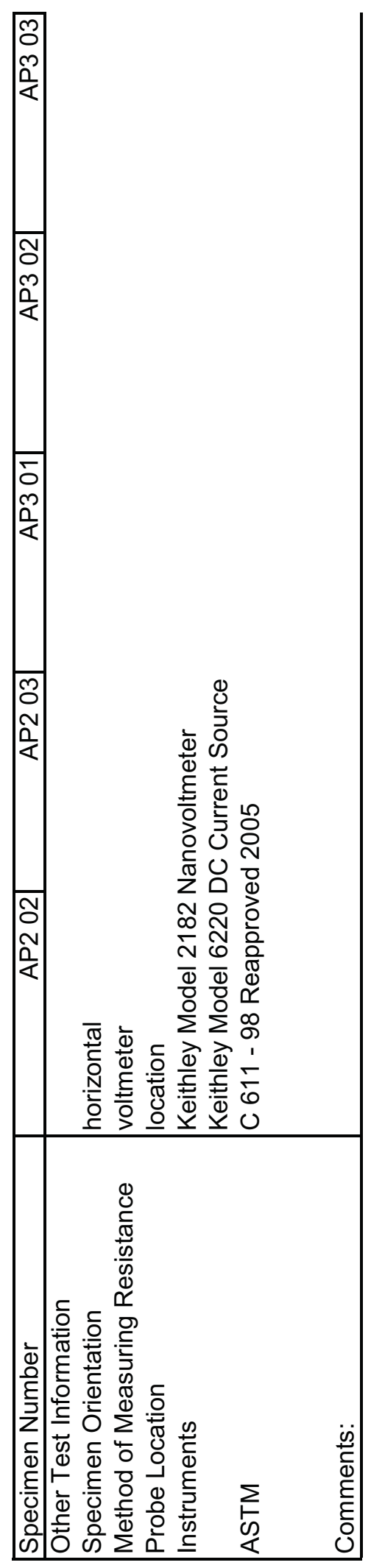

$\stackrel{\infty}{\infty}$ 


\begin{tabular}{|c|c|c|c|c|c|c|c|c|c|c|}
\hline \begin{tabular}{|l|}
5 \\
0 \\
0 \\
14 \\
\end{tabular} & 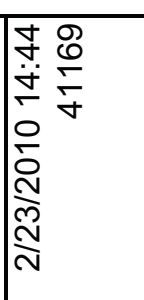 & 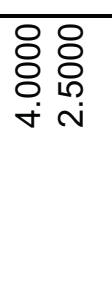 & & 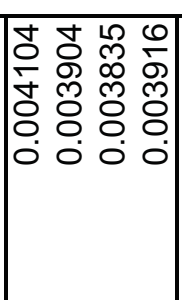 & 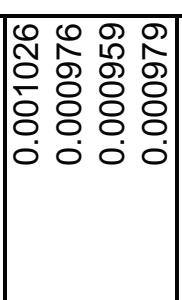 & & 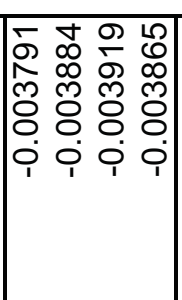 & 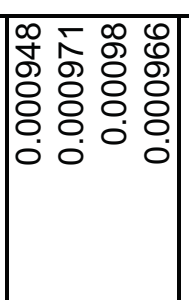 & & 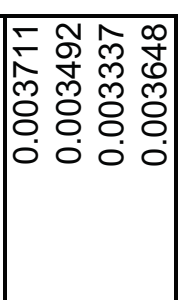 \\
\hline $\begin{array}{l}m \\
0 \\
0 \\
0 \\
\alpha\end{array}$ & 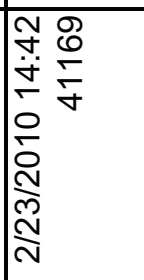 & 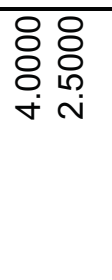 & & 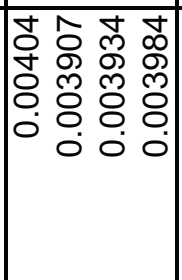 & 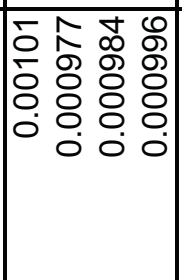 & & 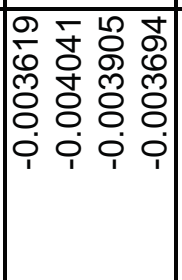 & 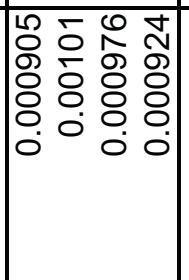 & & 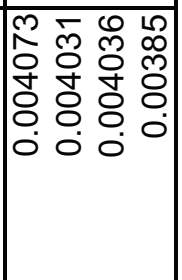 \\
\hline 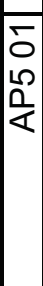 & 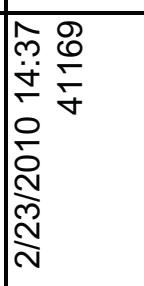 & 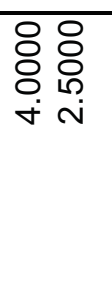 & & 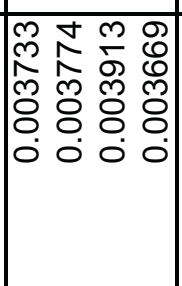 & 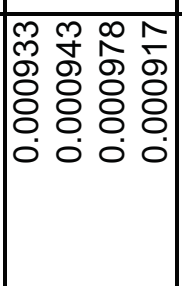 & & 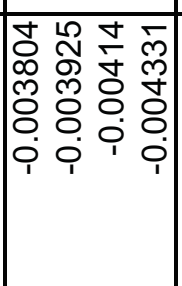 & 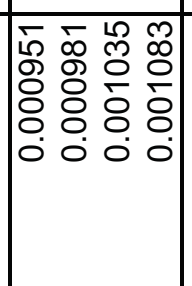 & & 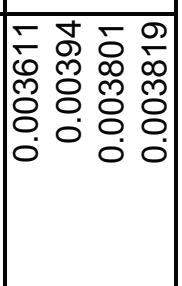 \\
\hline 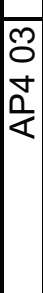 & 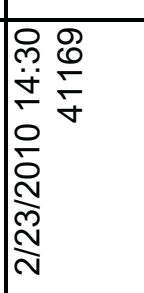 & 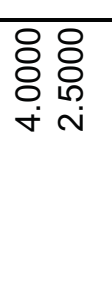 & & 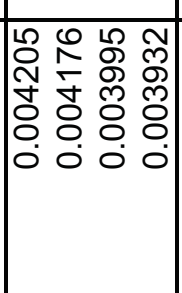 & 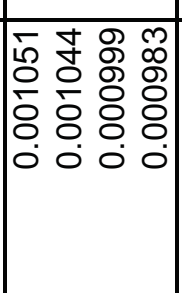 & & 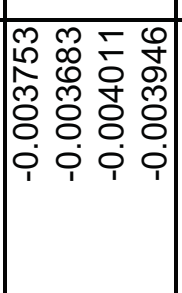 & 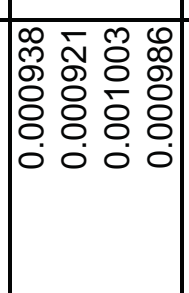 & & 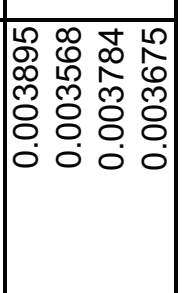 \\
\hline 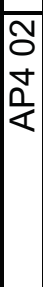 & 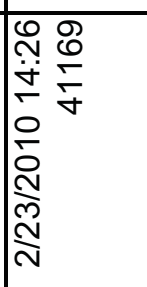 & $\begin{array}{l}88 \\
880 \\
\circ \text { 우 } \\
\dot{\forall} \text { i }\end{array}$ & & 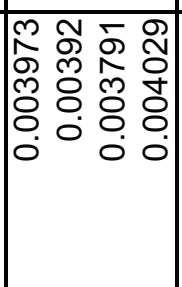 & 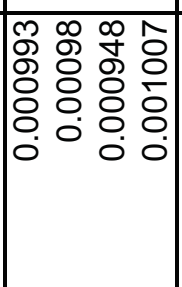 & & 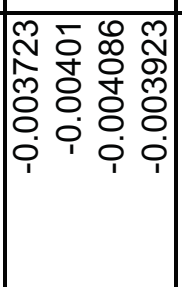 & 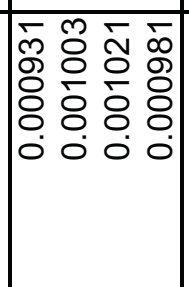 & & 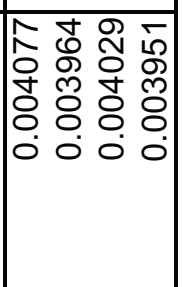 \\
\hline $\begin{array}{l}\overline{0} \\
\overline{1} \\
2 \\
\end{array}$ & 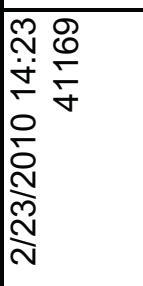 & 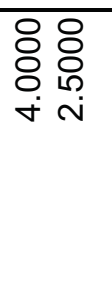 & & 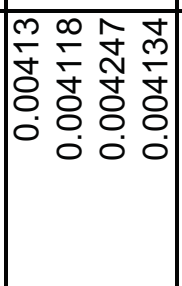 & 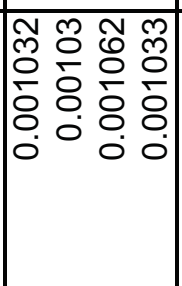 & & 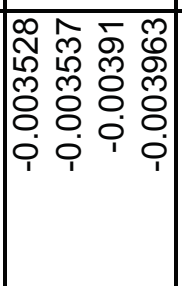 & 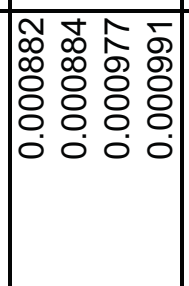 & & 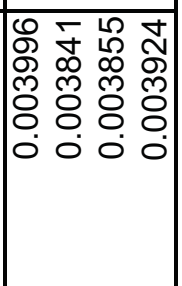 \\
\hline 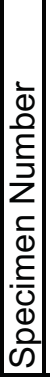 & 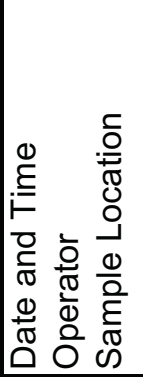 & 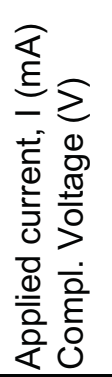 & 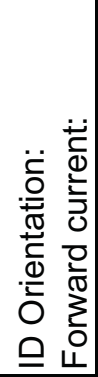 & 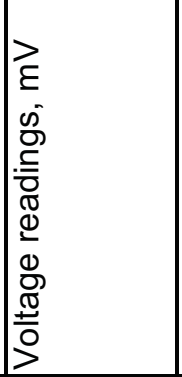 & 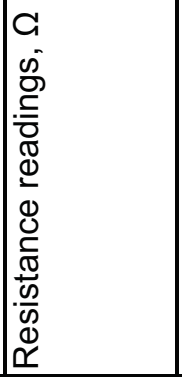 & 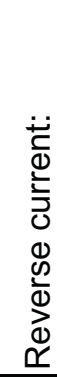 & 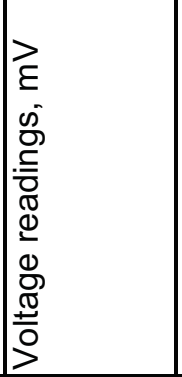 & 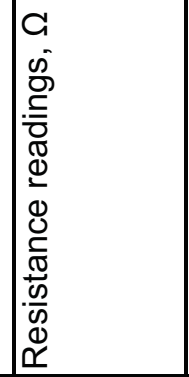 & 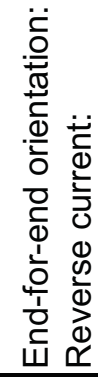 & 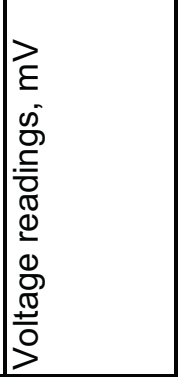 \\
\hline
\end{tabular}




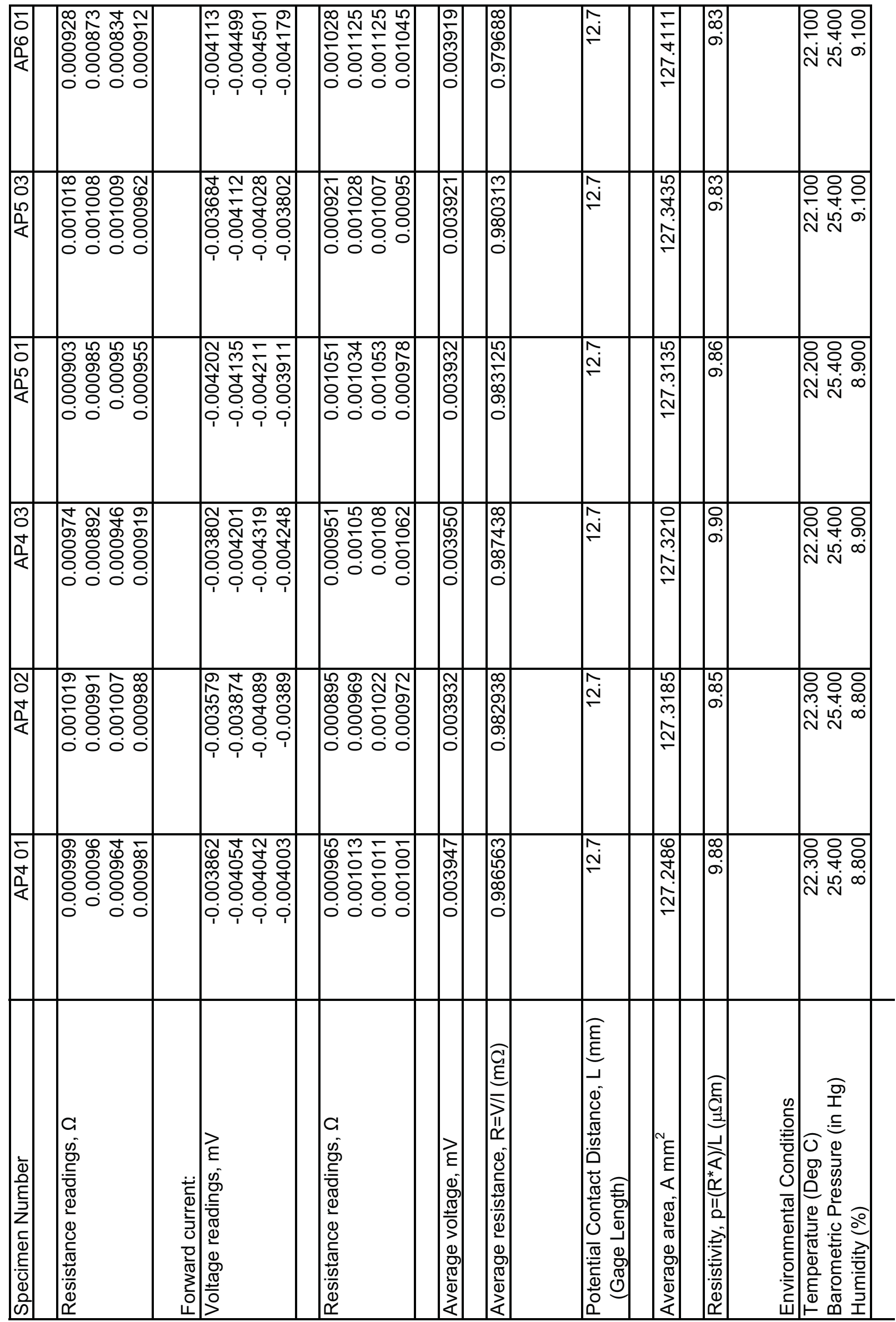




\begin{tabular}{|c|c|c|c|c|c|c|c|c|c|c|}
\hline \begin{tabular}{|l|} 
\\
0 \\
$N$ \\
3 \\
\\
\end{tabular} & 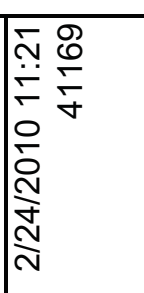 & 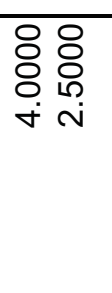 & & 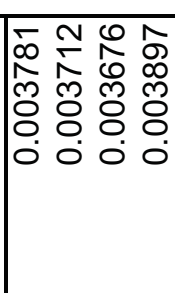 & 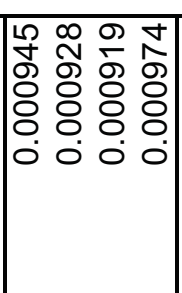 & & 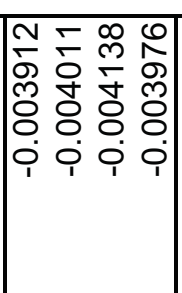 & 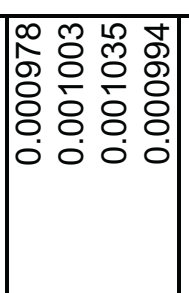 & & 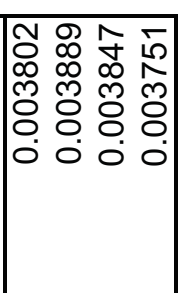 \\
\hline $\begin{array}{l}m \\
0 \\
\vdots \\
3 \\
1\end{array}$ & 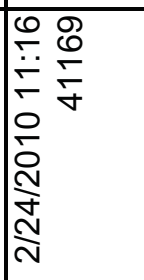 & 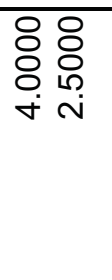 & & 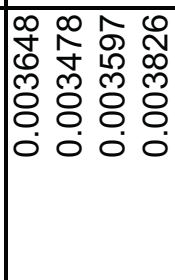 & 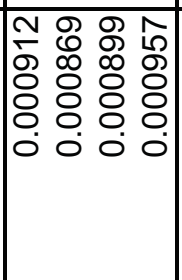 & & 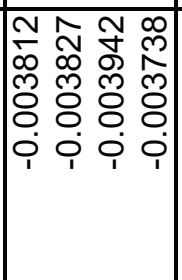 & 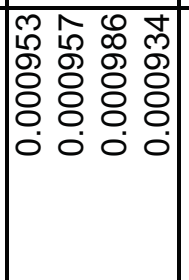 & & 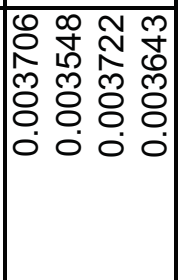 \\
\hline 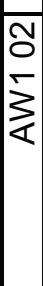 & 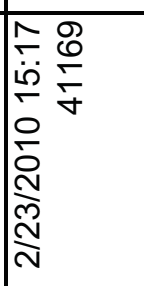 & 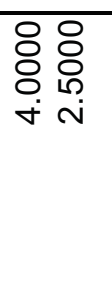 & & 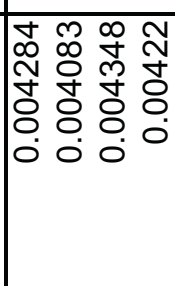 & 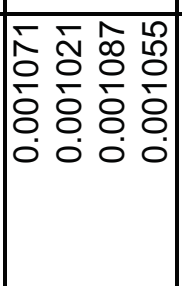 & & 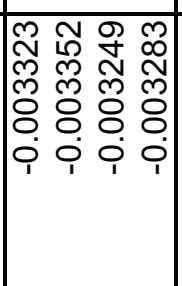 & 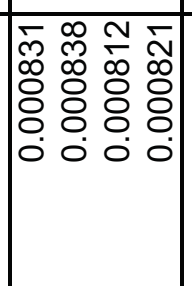 & & 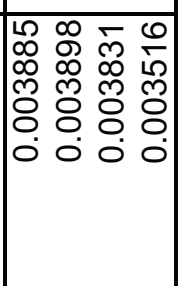 \\
\hline \begin{tabular}{|l}
5 \\
\\
$\vdots$ \\
$\vdots$ \\
\end{tabular} & 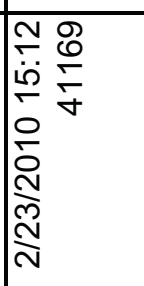 & 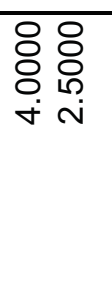 & & 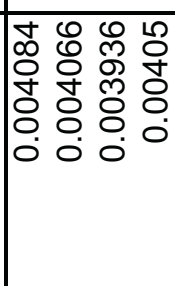 & 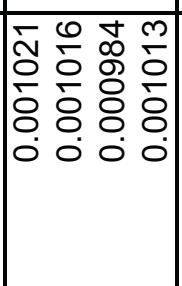 & & 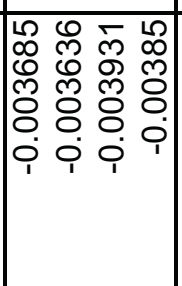 & 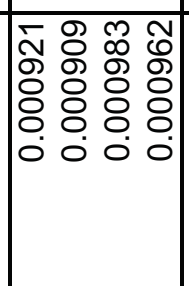 & & 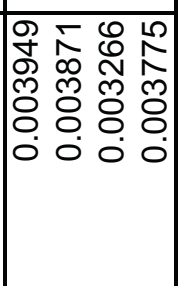 \\
\hline $\begin{array}{l}0 \\
0 \\
0 \\
0 \\
\alpha \\
<\end{array}$ & 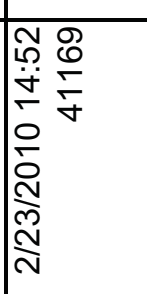 & 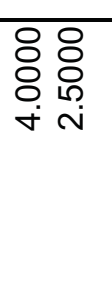 & & 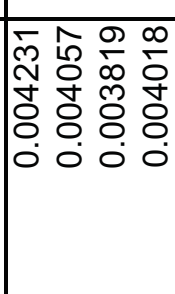 & 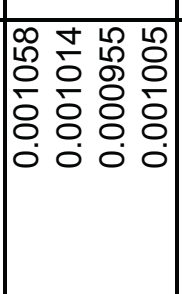 & & 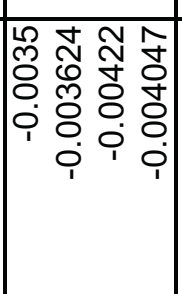 & 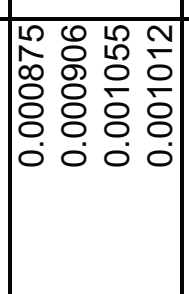 & & 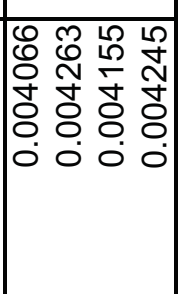 \\
\hline 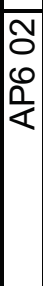 & 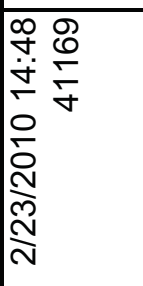 & 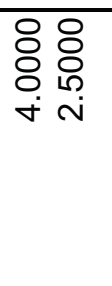 & & 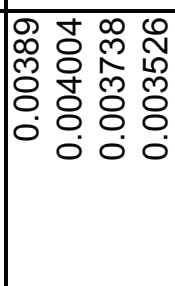 & 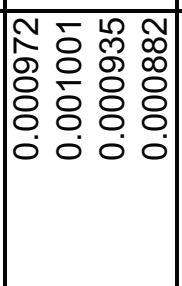 & & 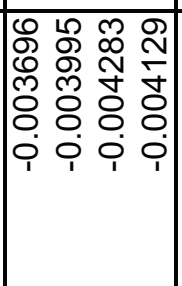 & 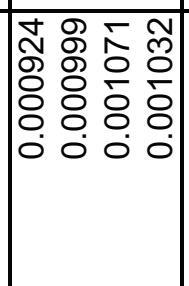 & & 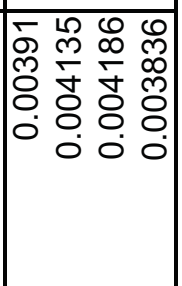 \\
\hline 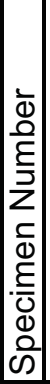 & 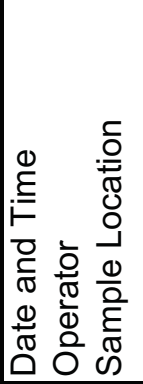 & 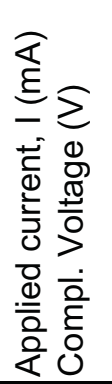 & 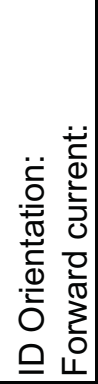 & 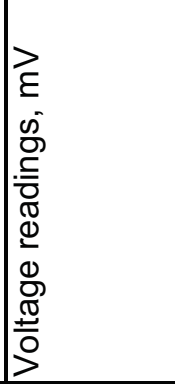 & 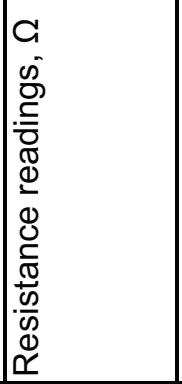 & 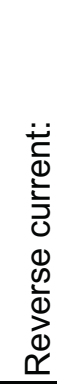 & 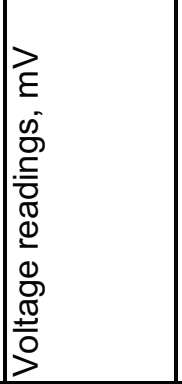 & 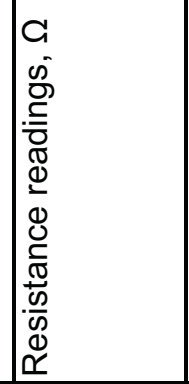 & 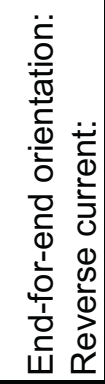 & 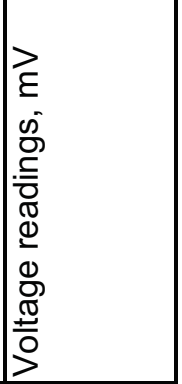 \\
\hline
\end{tabular}




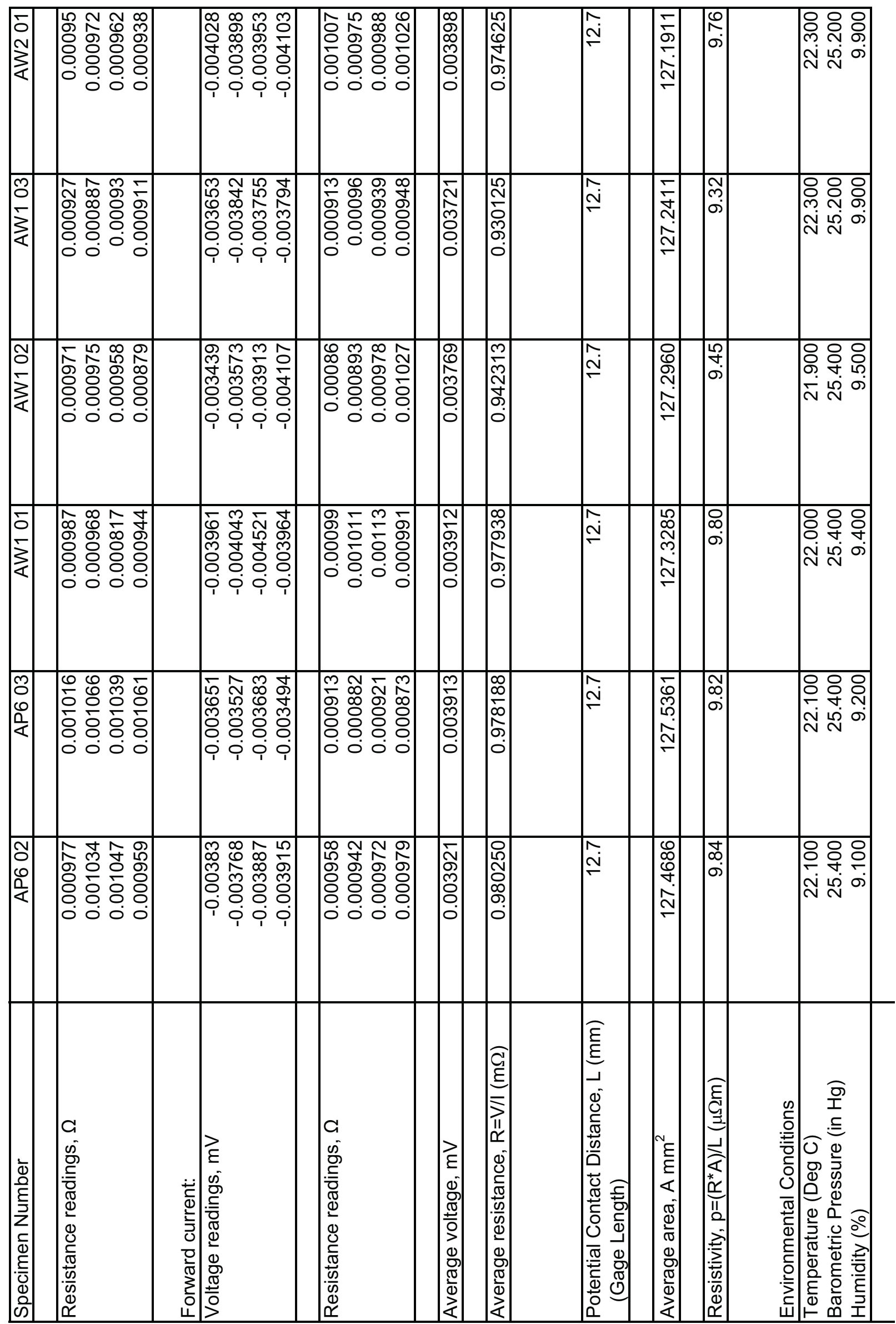




\begin{tabular}{|c|c|c|c|c|c|c|c|c|c|c|}
\hline & 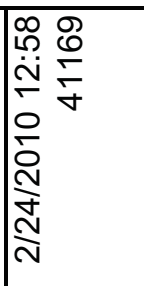 & \begin{tabular}{l} 
용 \\
80.0 \\
\hdashline ஸ
\end{tabular} & & 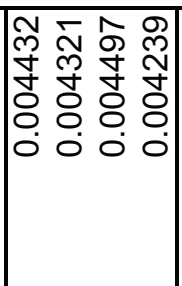 & 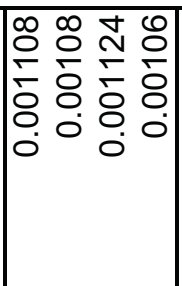 & & 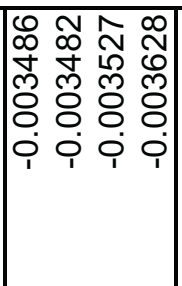 & 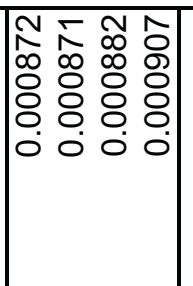 & & 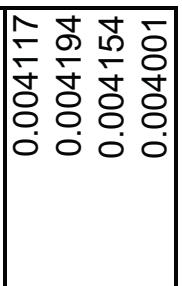 \\
\hline $\begin{array}{l}m \\
0 \\
m \\
3 \\
\vdots\end{array}$ & 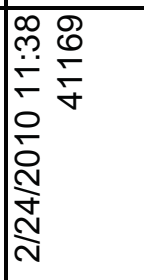 & 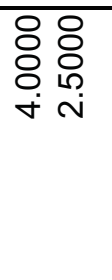 & & 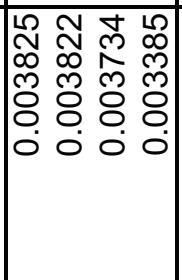 & 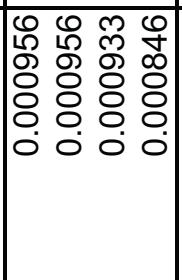 & & 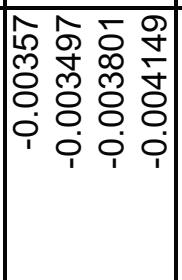 & 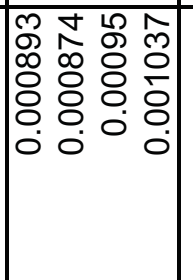 & & 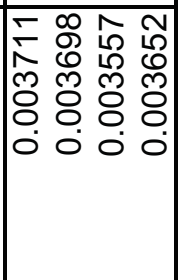 \\
\hline 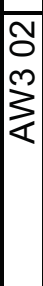 & 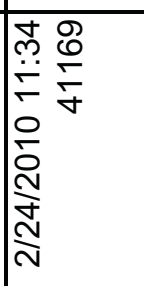 & $\begin{array}{l}88 \\
880 \\
\circ \text { 유 } \\
\dot{\forall} \text { ஸi }\end{array}$ & & 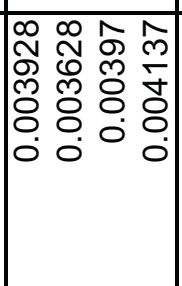 & 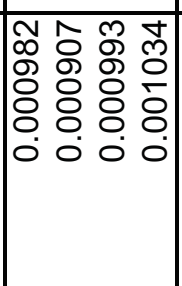 & & 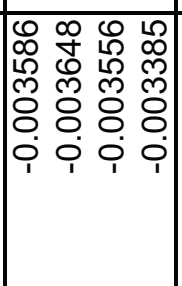 & 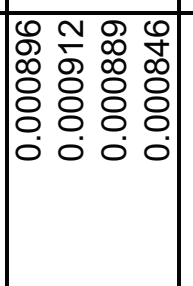 & & 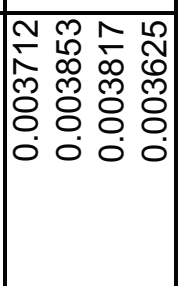 \\
\hline 西 & 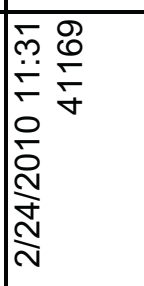 & $\begin{array}{l}\circ 8 \\
880 \\
8 \\
\circ \text { ஸ } \\
\dot{\forall}\end{array}$ & & 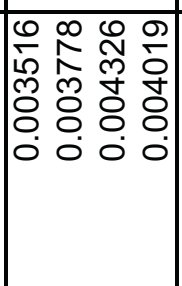 & 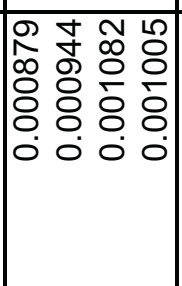 & & 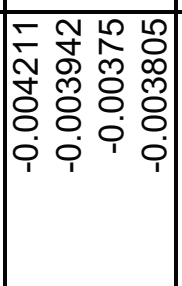 & 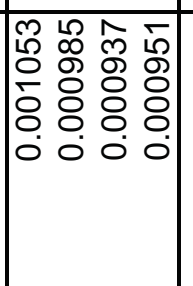 & & 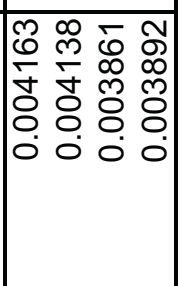 \\
\hline On & 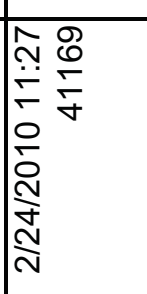 & 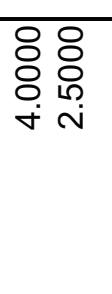 & & 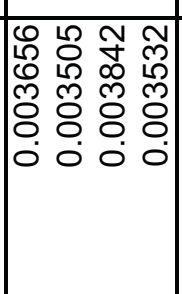 & 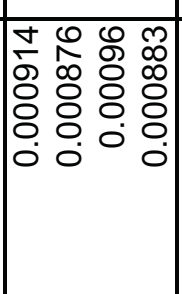 & & 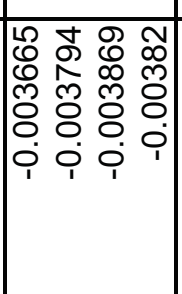 & 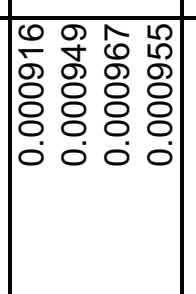 & & 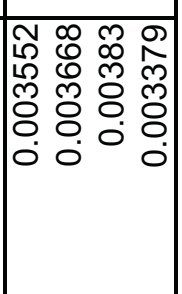 \\
\hline 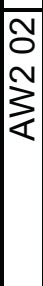 & 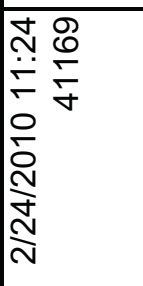 & 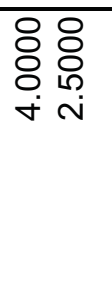 & & 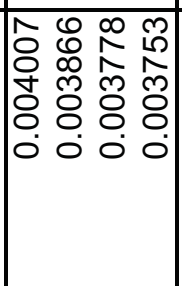 & 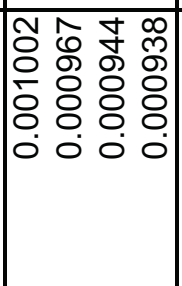 & & 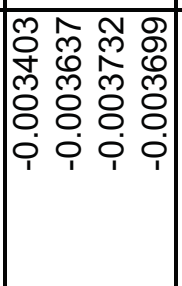 & 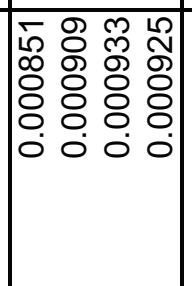 & & 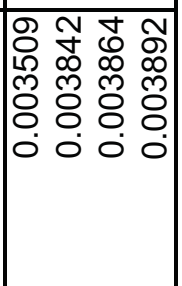 \\
\hline 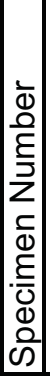 & 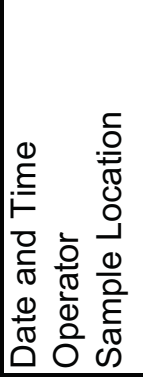 & 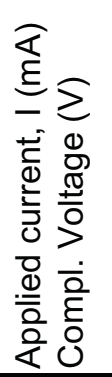 & 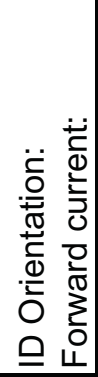 & 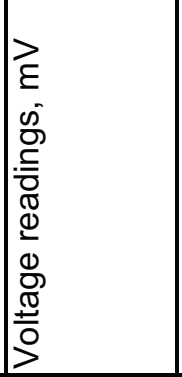 & 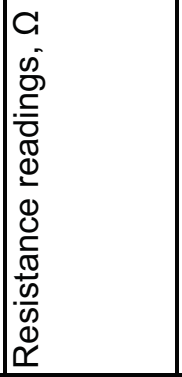 & 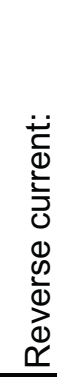 & 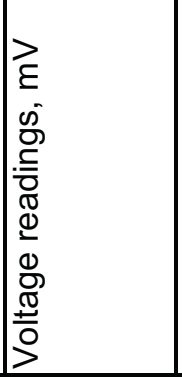 & 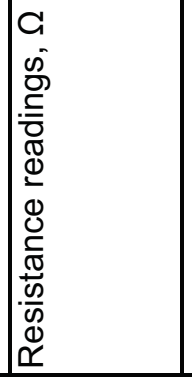 & 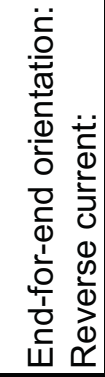 & 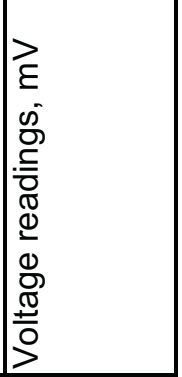 \\
\hline
\end{tabular}




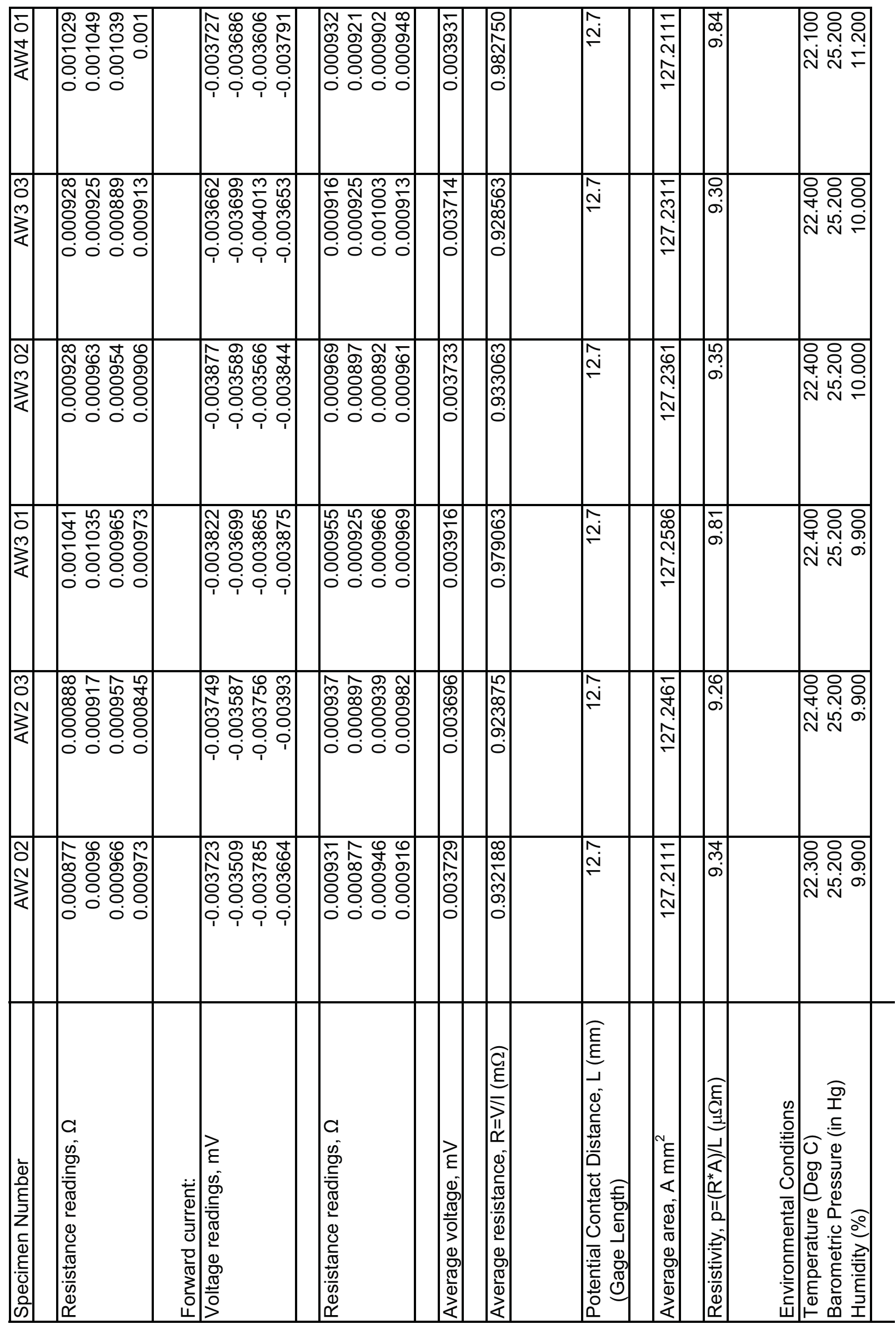




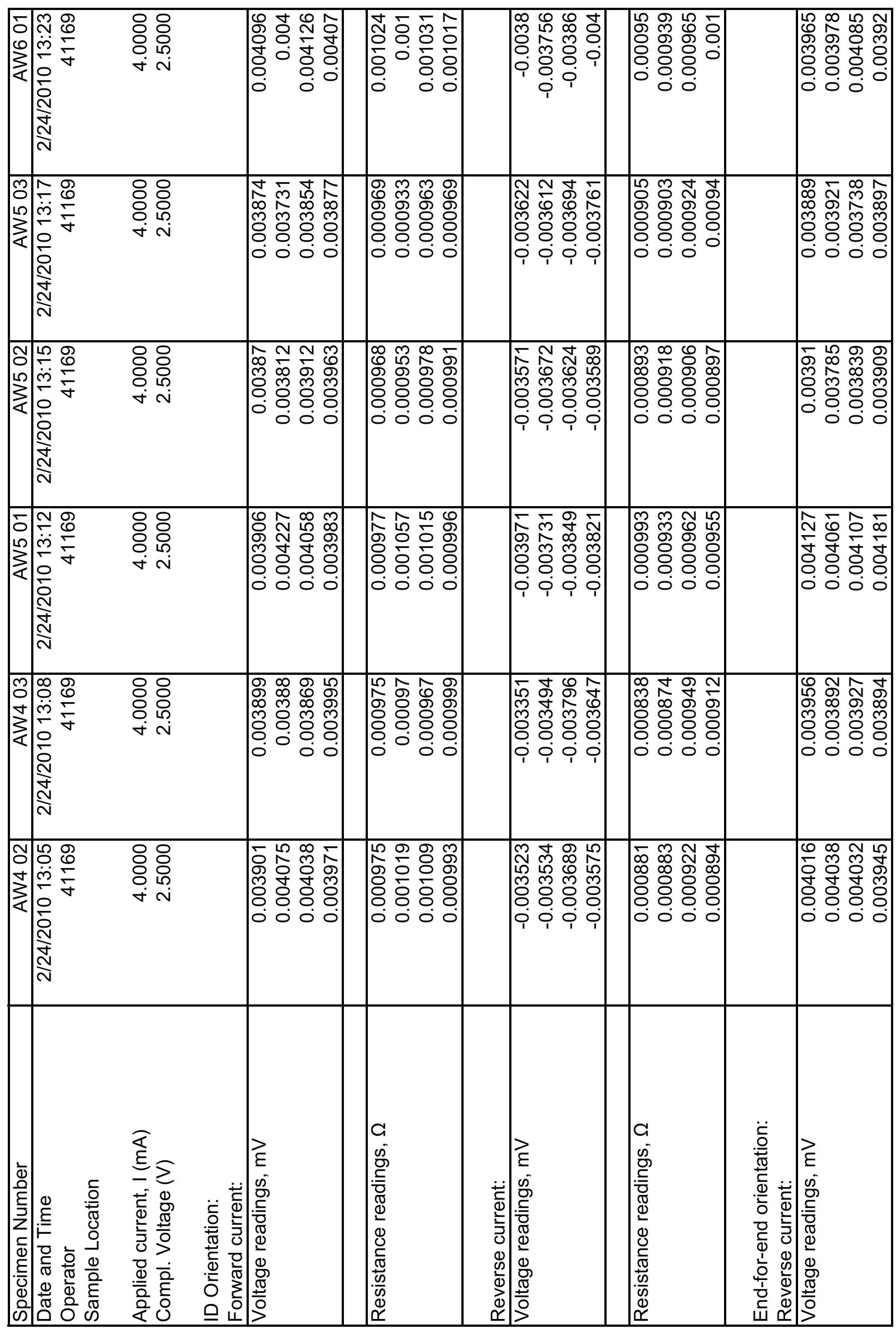




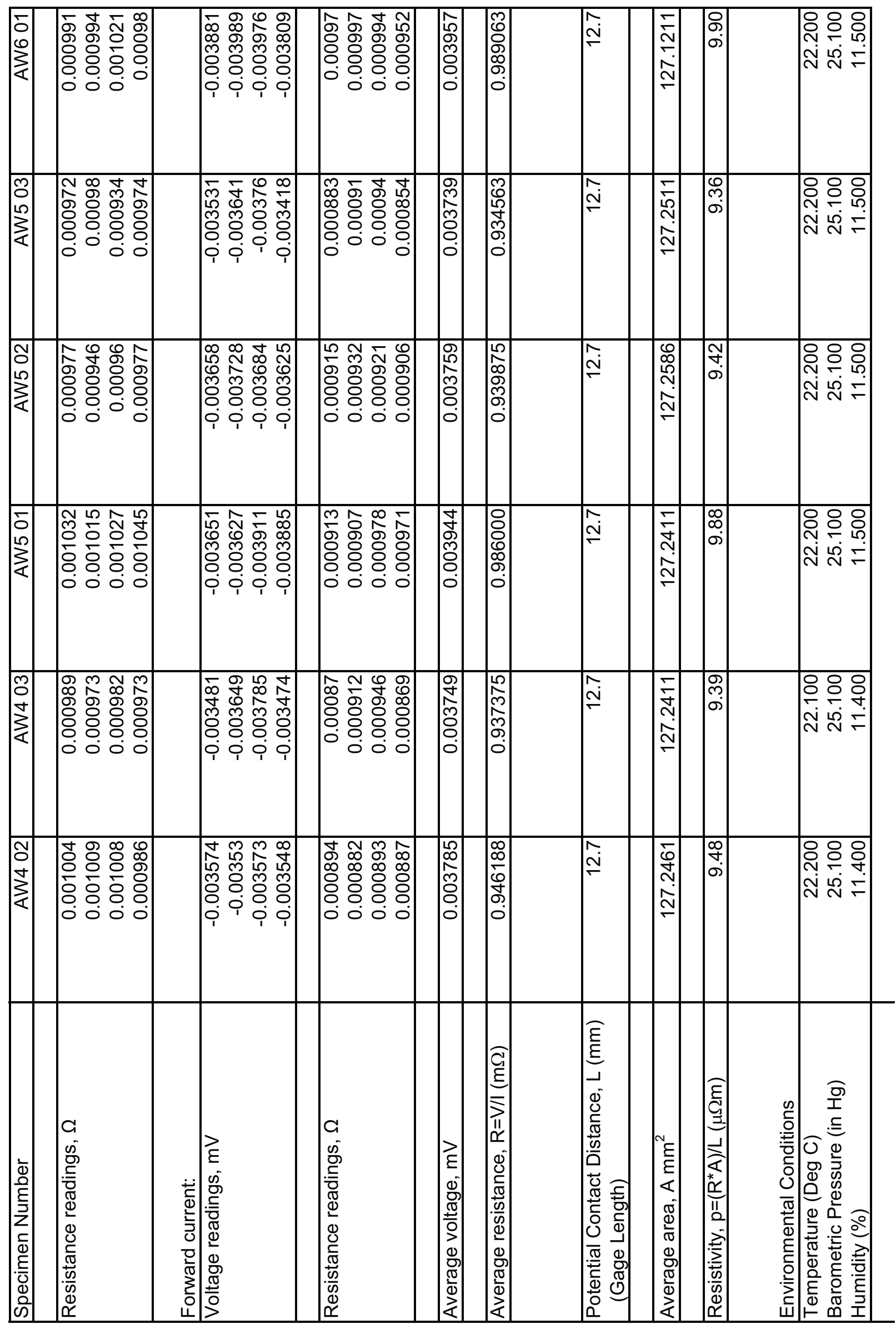




\begin{tabular}{|c|c|c|c|c|c|c|c|c|c|c|}
\hline & 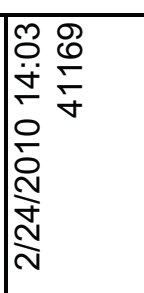 & 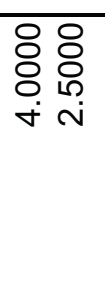 & & 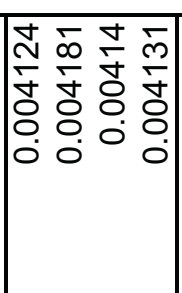 & 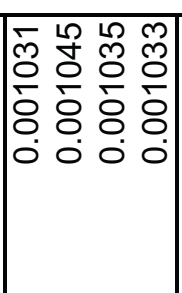 & & 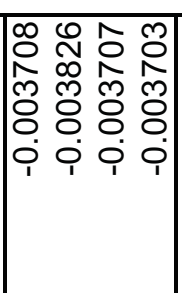 & 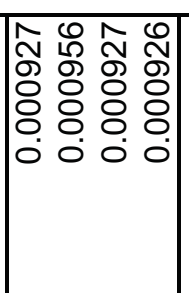 & & 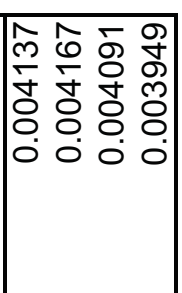 \\
\hline $\begin{array}{l}m \\
0 \\
1 \\
3 \\
3 \\
1\end{array}$ & 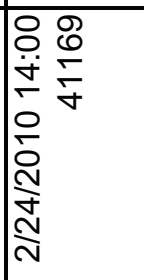 & 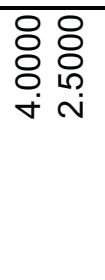 & & 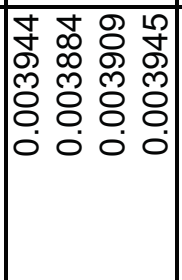 & 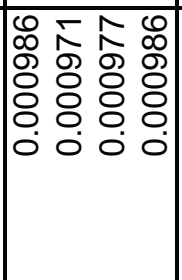 & & 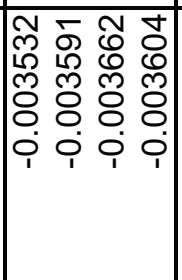 & 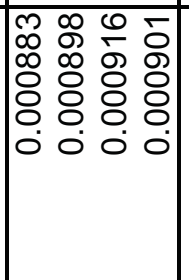 & & 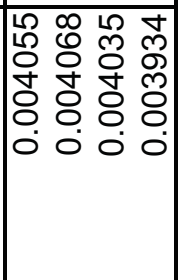 \\
\hline $\mid \begin{array}{c}N \\
0 \\
1 \\
3 \\
3 \\
\end{array}$ & 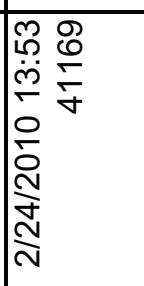 & 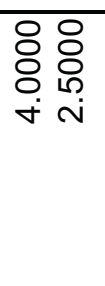 & & 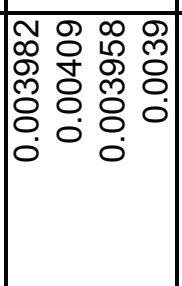 & 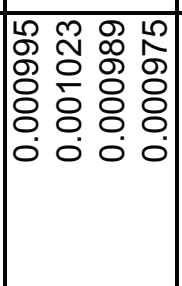 & & 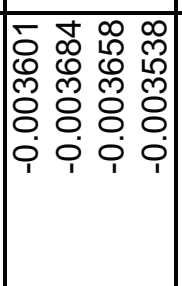 & 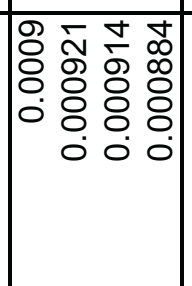 & & 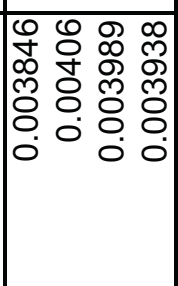 \\
\hline $\begin{array}{l}5 \\
0 \\
1 \\
3 \\
1 \\
4\end{array}$ & 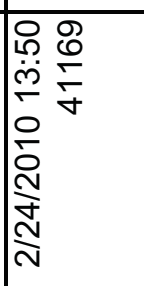 & 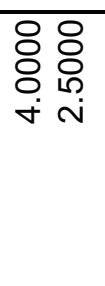 & & 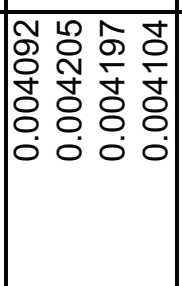 & 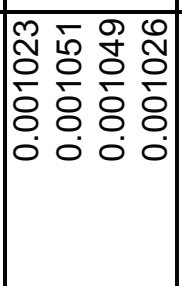 & & 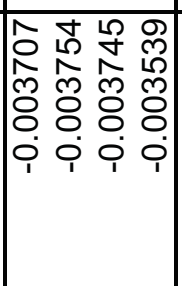 & 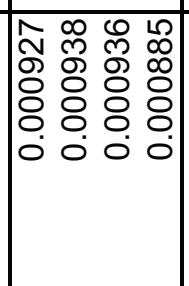 & & 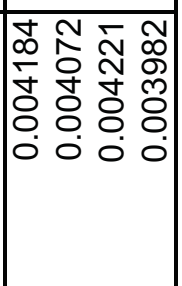 \\
\hline $\begin{array}{l}m \\
0 \\
0 \\
3 \\
3\end{array}$ & 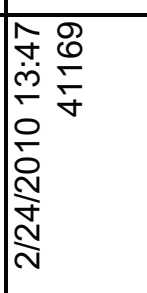 & 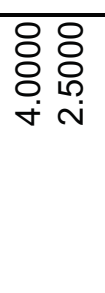 & & 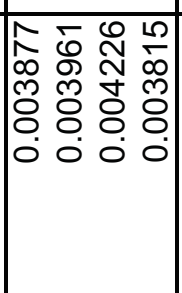 & 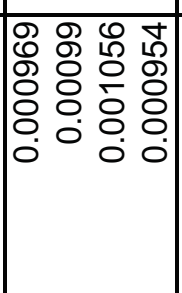 & & 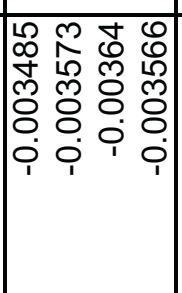 & 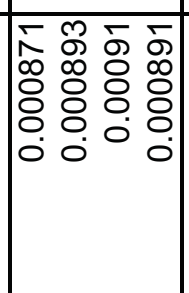 & & 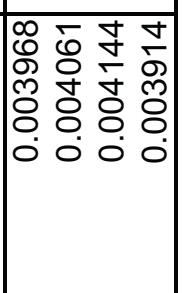 \\
\hline \begin{tabular}{|l}
\multirow{2}{*}{} \\
0 \\
0 \\
3 \\
\\
\end{tabular} & 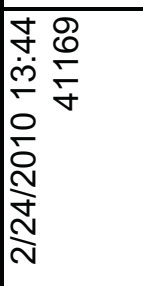 & 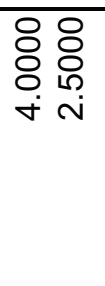 & & 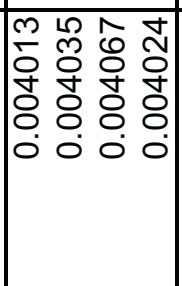 & 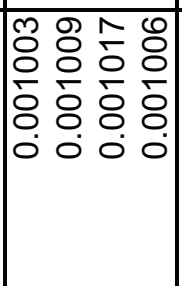 & & 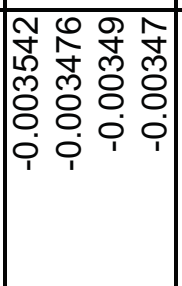 & 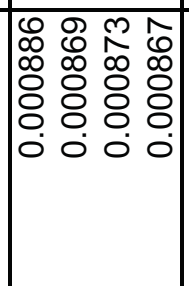 & & 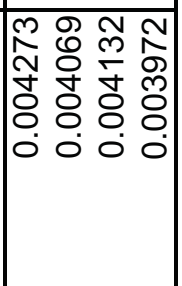 \\
\hline 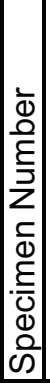 & 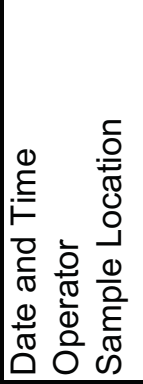 & 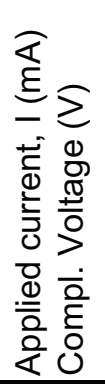 & 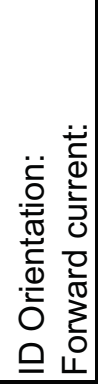 & 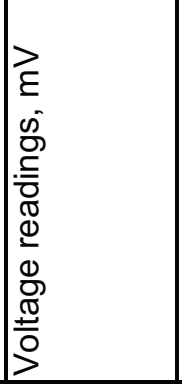 & 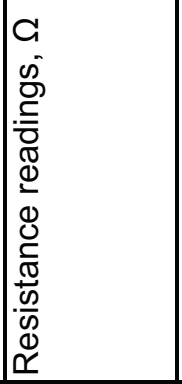 & 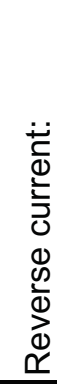 & 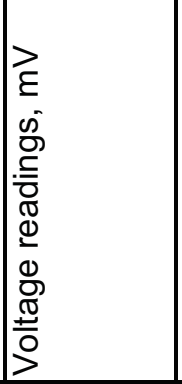 & 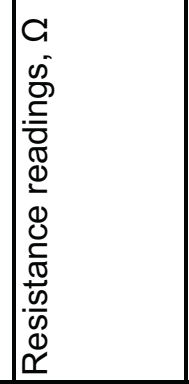 & 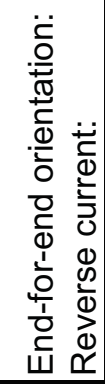 & 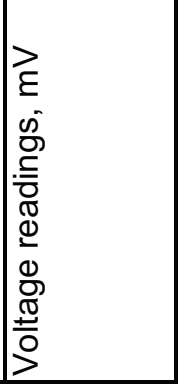 \\
\hline
\end{tabular}




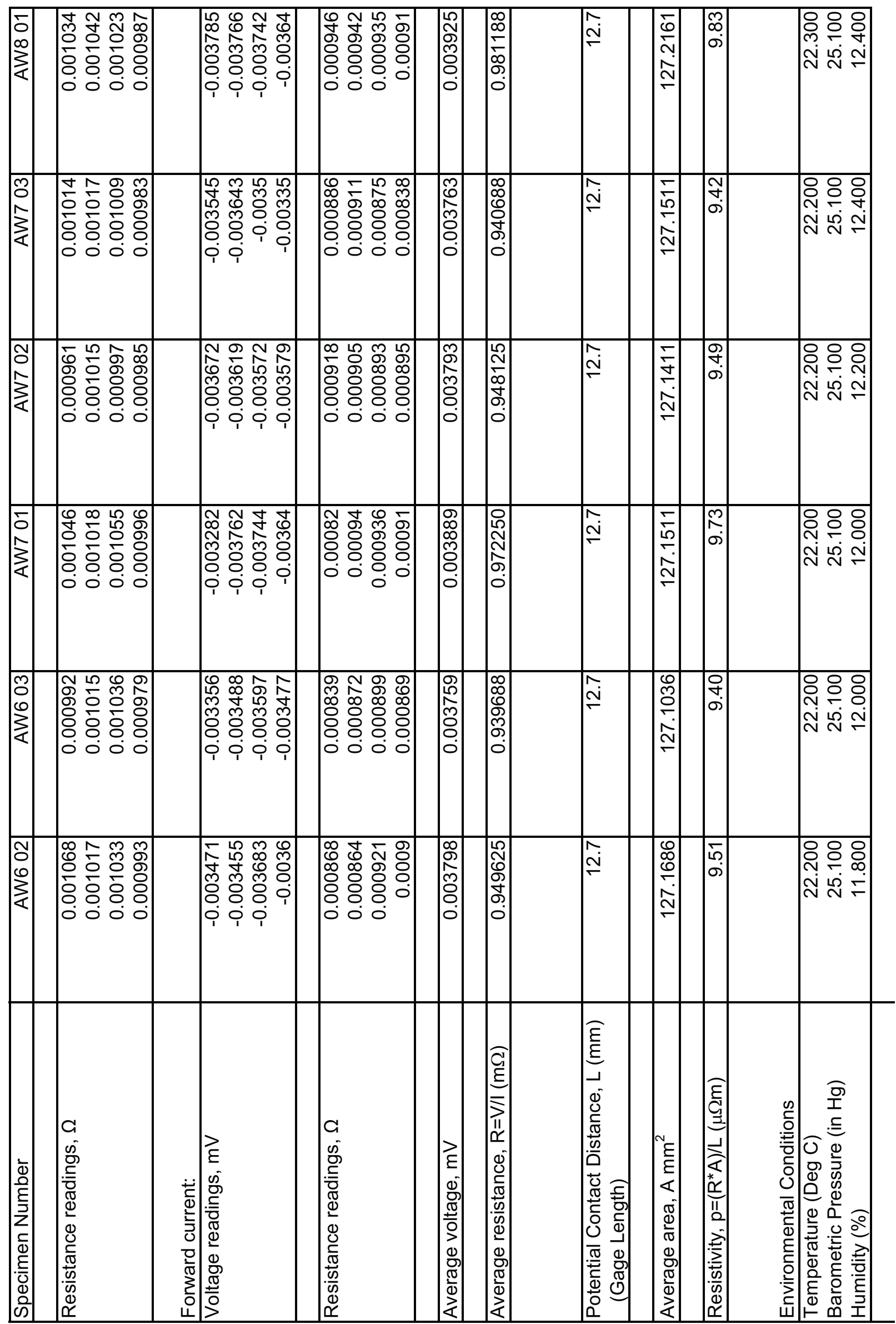




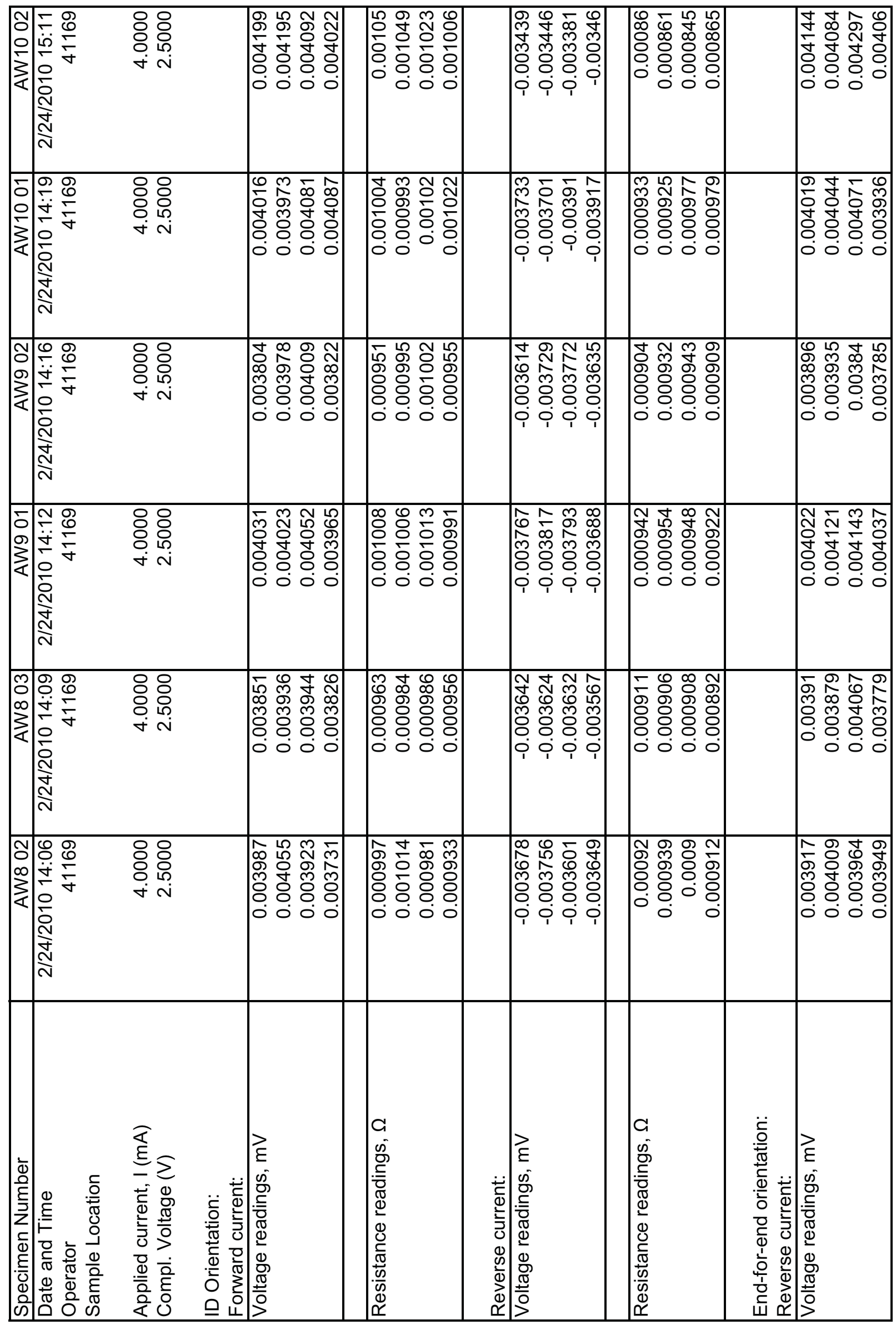




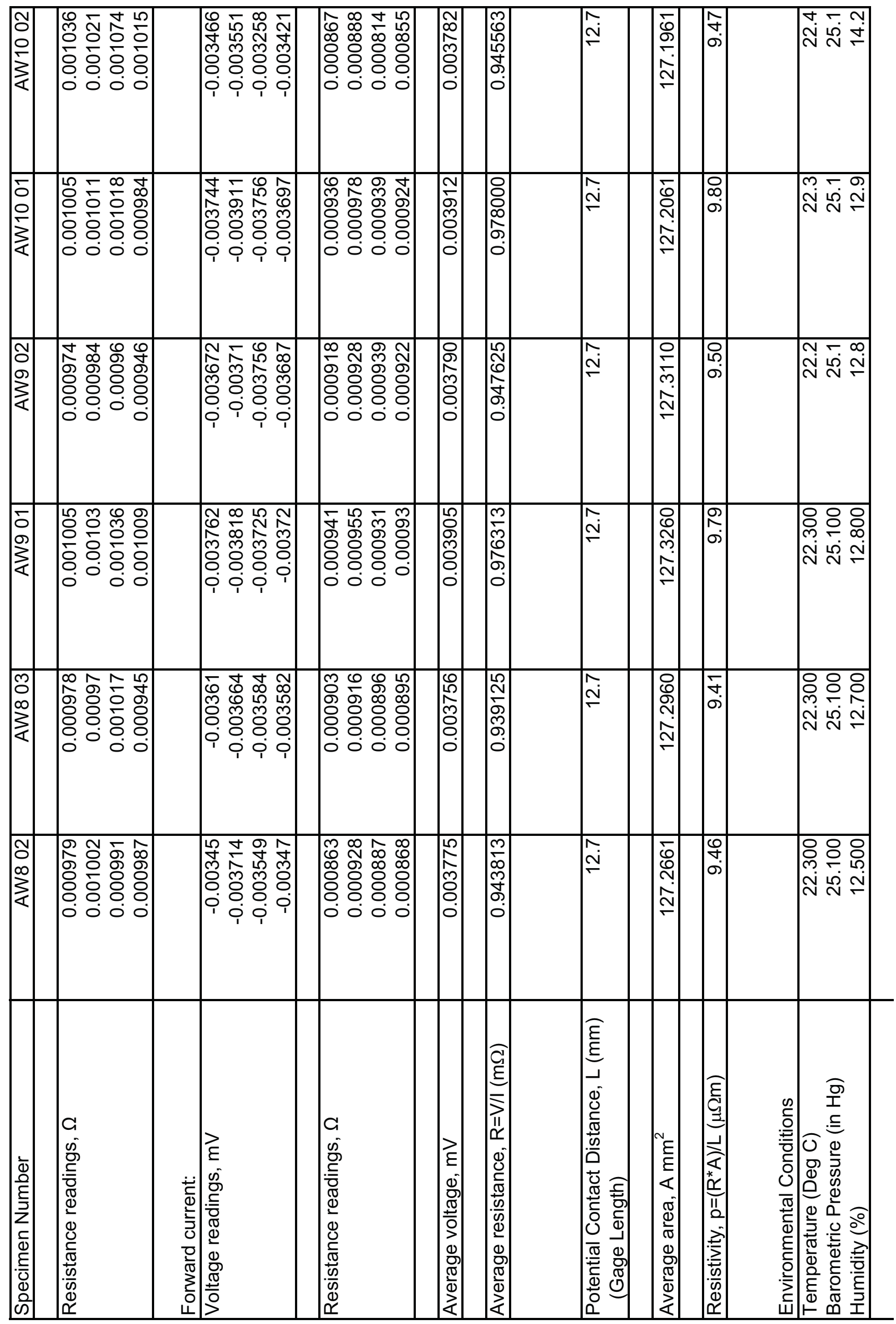




\begin{tabular}{|c|c|c|c|c|c|c|c|c|c|c|}
\hline 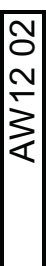 & 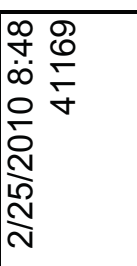 & $\begin{array}{l}\circ 8 \\
88 \\
8 \\
\circ\end{array}$ & & 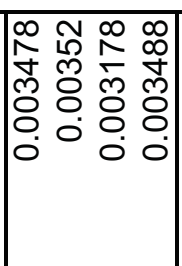 & 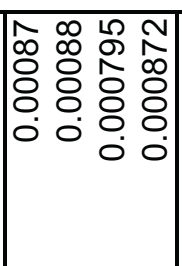 & & 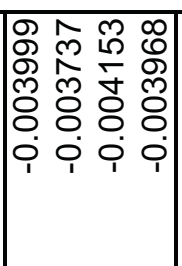 & 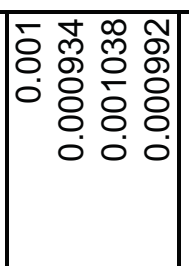 & & 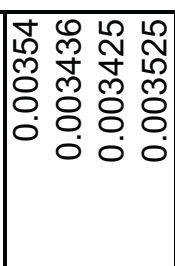 \\
\hline \begin{tabular}{|l|} 
\\
\\
2 \\
\hdashline \\
$\vdots$ \\
$\vdots$ \\
\\
\end{tabular} & 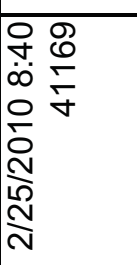 & $\begin{array}{l}88 \\
88 \\
8 \\
\circ \text { ஸ } \\
\dot{\forall}\end{array}$ & & 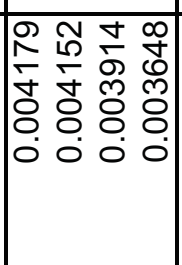 & 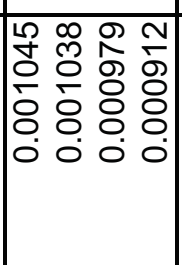 & & 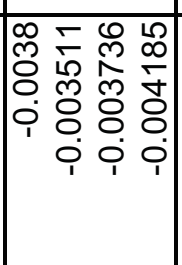 & 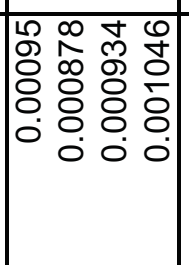 & & 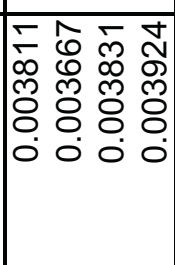 \\
\hline \begin{tabular}{|l|l}
0 \\
0 \\
$\vdots$ \\
$\vdots$ \\
\\
\end{tabular} & 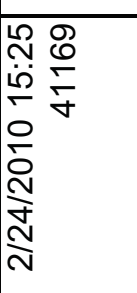 & 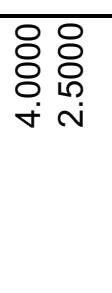 & & 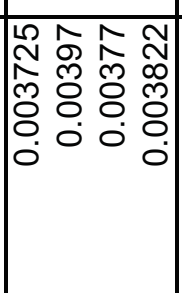 & 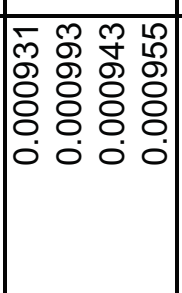 & & 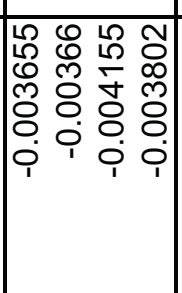 & 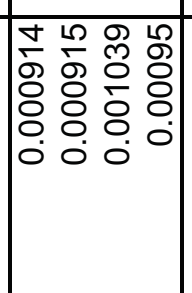 & & 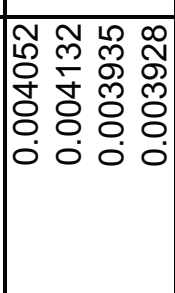 \\
\hline 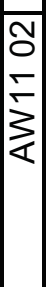 & 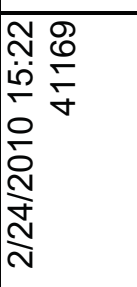 & $\begin{array}{l}\circ 8 \\
880 \\
\circ \text { 유 } \\
\dot{\forall} \text { ஸi }\end{array}$ & & 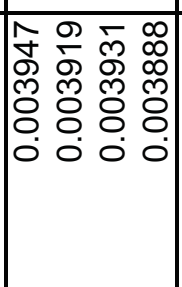 & 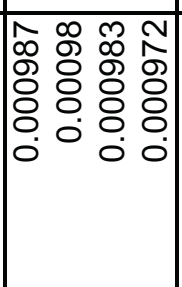 & & 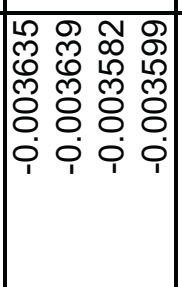 & 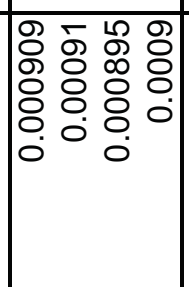 & & 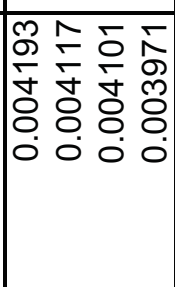 \\
\hline 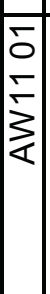 & 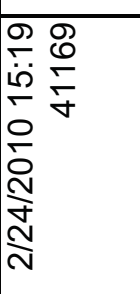 & $\begin{array}{l}88 \\
880 \\
\circ \text { 유 } \\
\dot{\forall} \text { i }\end{array}$ & & 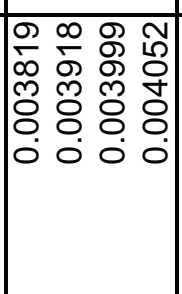 & 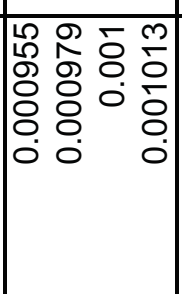 & & 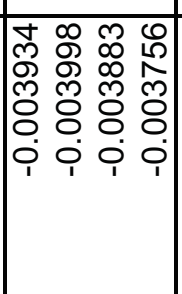 & 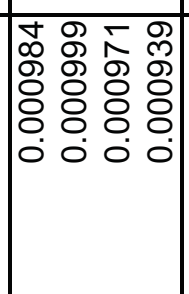 & & 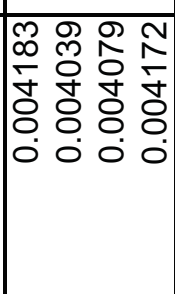 \\
\hline \begin{tabular}{|l|} 
\\
0 \\
0 \\
\\
\\
\\
\end{tabular} & 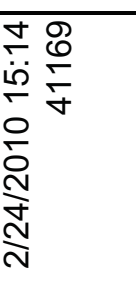 & $\begin{array}{l}88 \\
880 \\
\circ \\
\circ \text { 유 }\end{array}$ & & 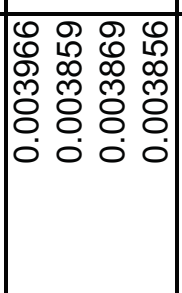 & 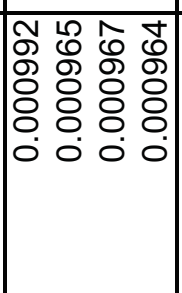 & & 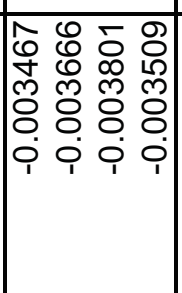 & 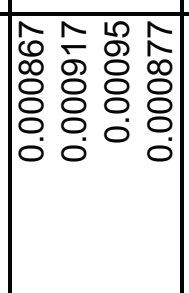 & & 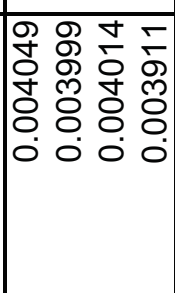 \\
\hline 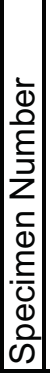 & 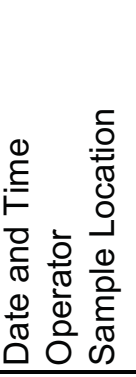 & 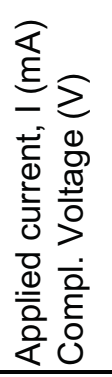 & 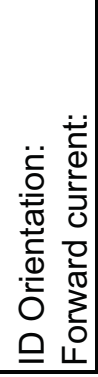 & 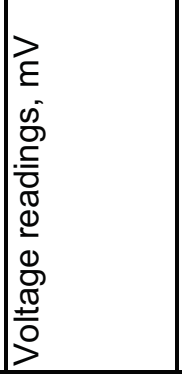 & 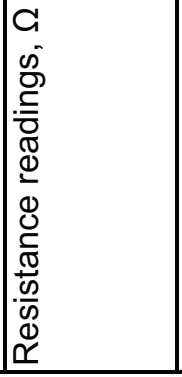 & 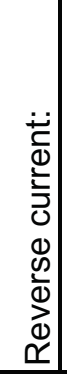 & 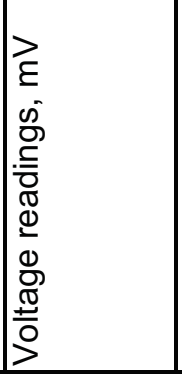 & 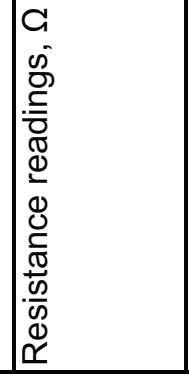 & 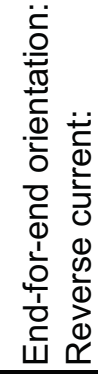 & 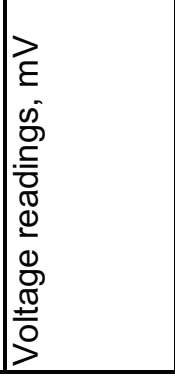 \\
\hline
\end{tabular}




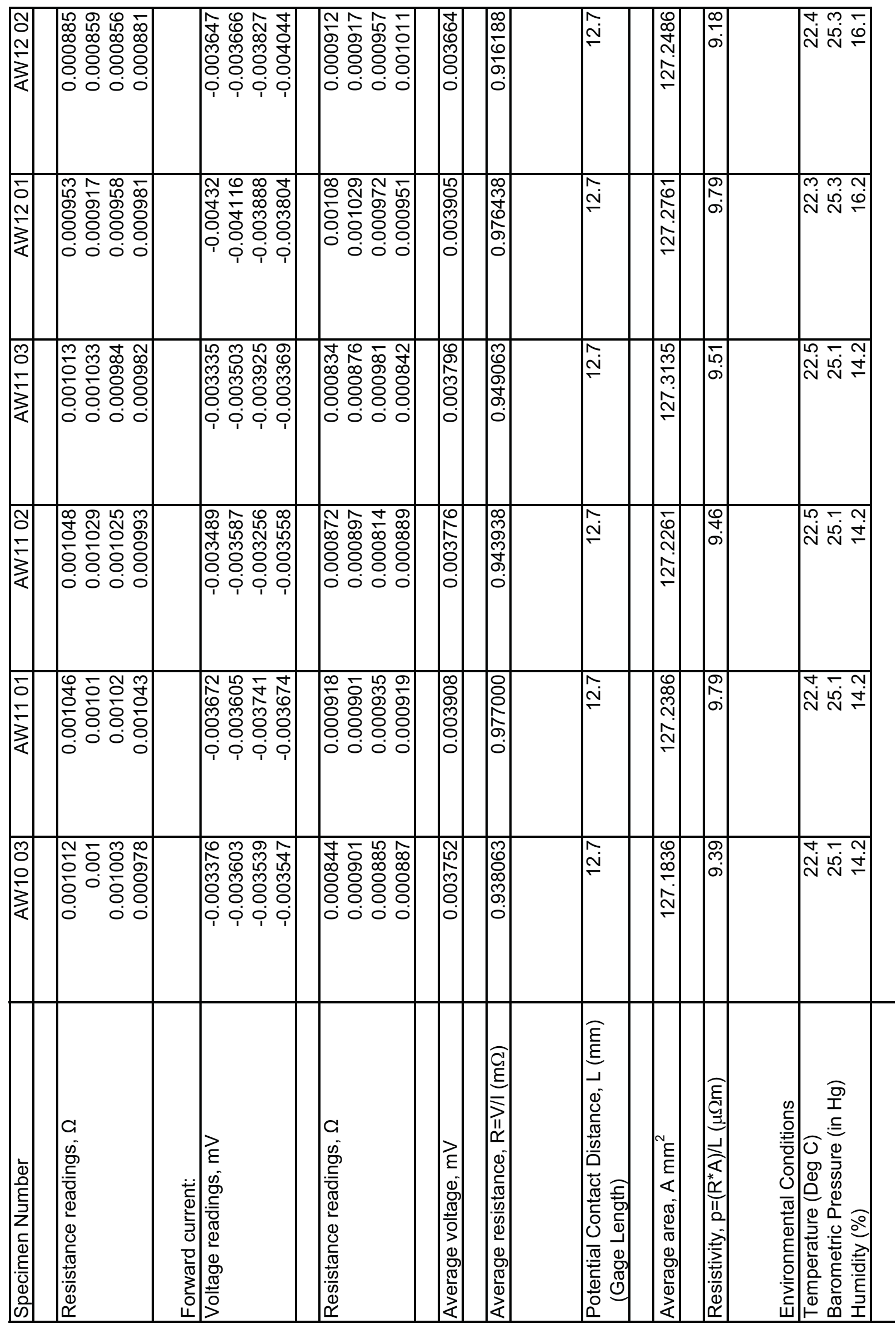




\begin{tabular}{|c|c|c|c|c|c|c|c|c|c|c|}
\hline 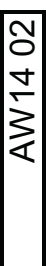 & 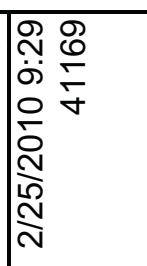 & $\begin{array}{l}\circ 8 \\
88 \\
8 \\
\circ\end{array}$ & & 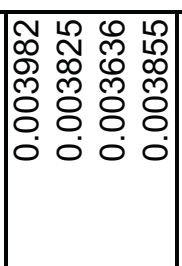 & 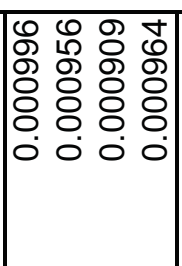 & & 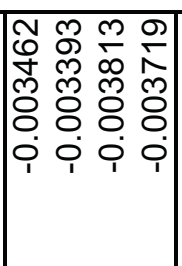 & 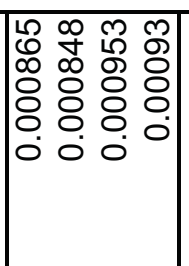 & & 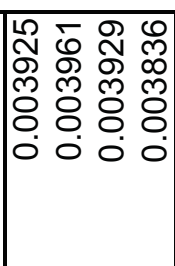 \\
\hline \begin{tabular}{|l|} 
\\
0 \\
0 \\
\\
3 \\
\\
\end{tabular} & 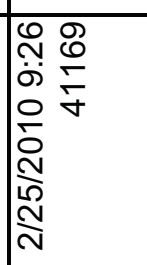 & $\begin{array}{l}88 \\
88 \\
8 \\
\circ \text { ஸ } \\
\dot{\forall}\end{array}$ & & 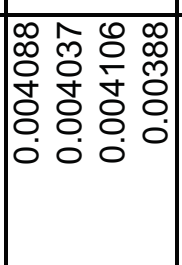 & 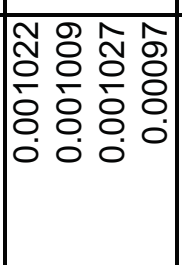 & & 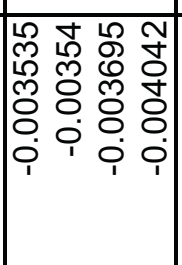 & 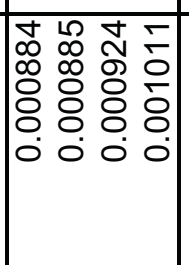 & & 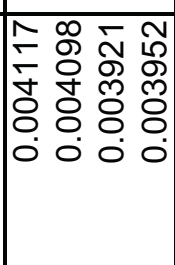 \\
\hline \begin{tabular}{|l|} 
\\
0 \\
$m$ \\
$\vdots$ \\
3 \\
$\frac{1}{\alpha}$ \\
\end{tabular} & 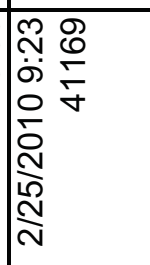 & 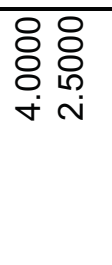 & & 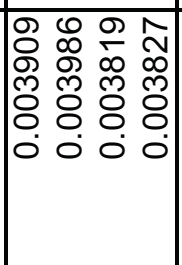 & 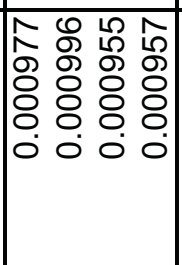 & & 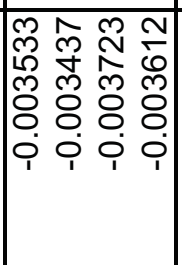 & 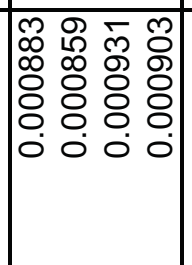 & & 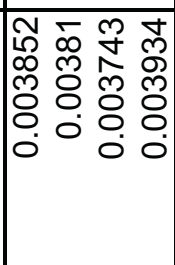 \\
\hline 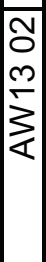 & 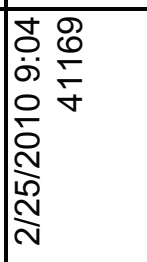 & 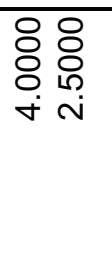 & & 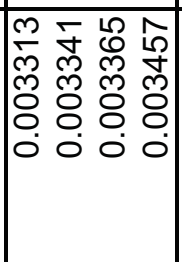 & 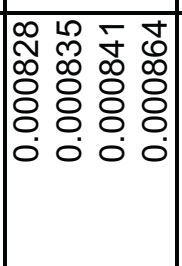 & & 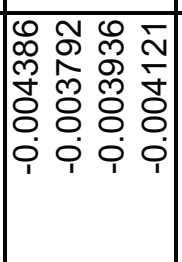 & 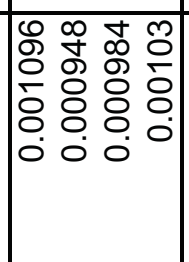 & & 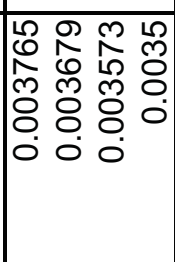 \\
\hline 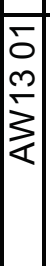 & 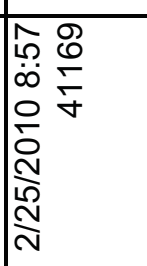 & $\begin{array}{l}88 \\
880 \\
\circ \text { 유 } \\
\dot{\forall} \text { i }\end{array}$ & & 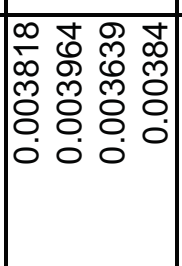 & 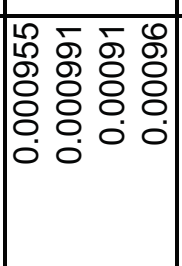 & & 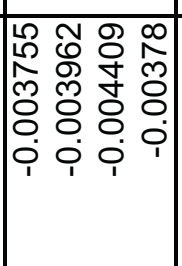 & 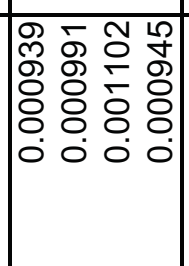 & & 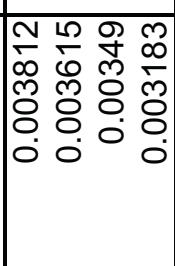 \\
\hline 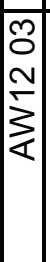 & 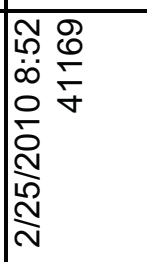 & 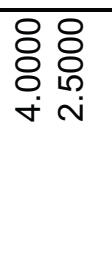 & & 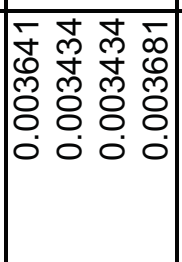 & 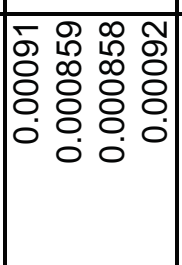 & & 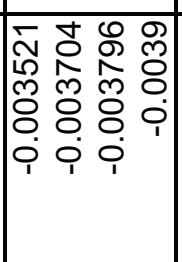 & 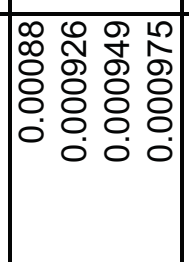 & & 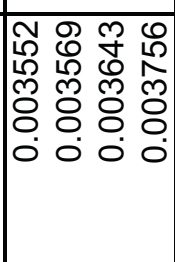 \\
\hline 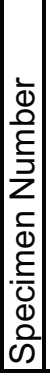 & 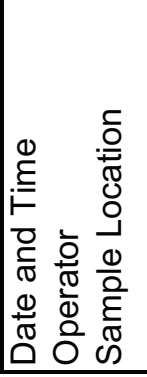 & 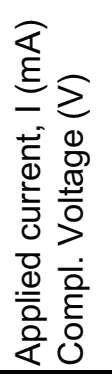 & 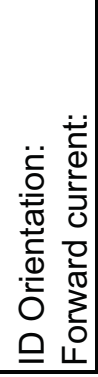 & 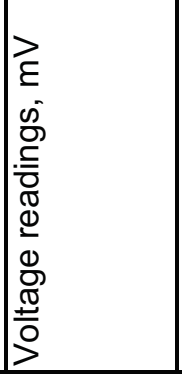 & 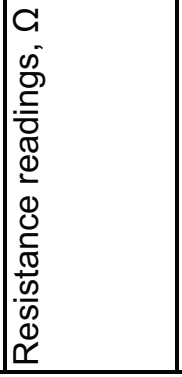 & 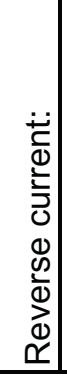 & 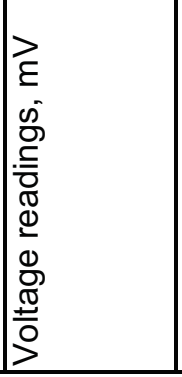 & 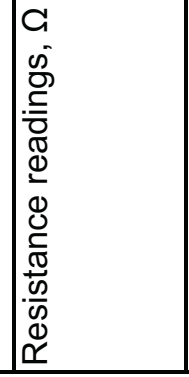 & 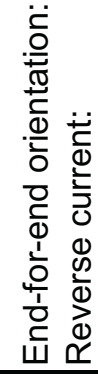 & 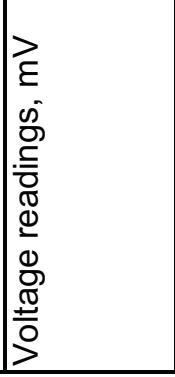 \\
\hline
\end{tabular}




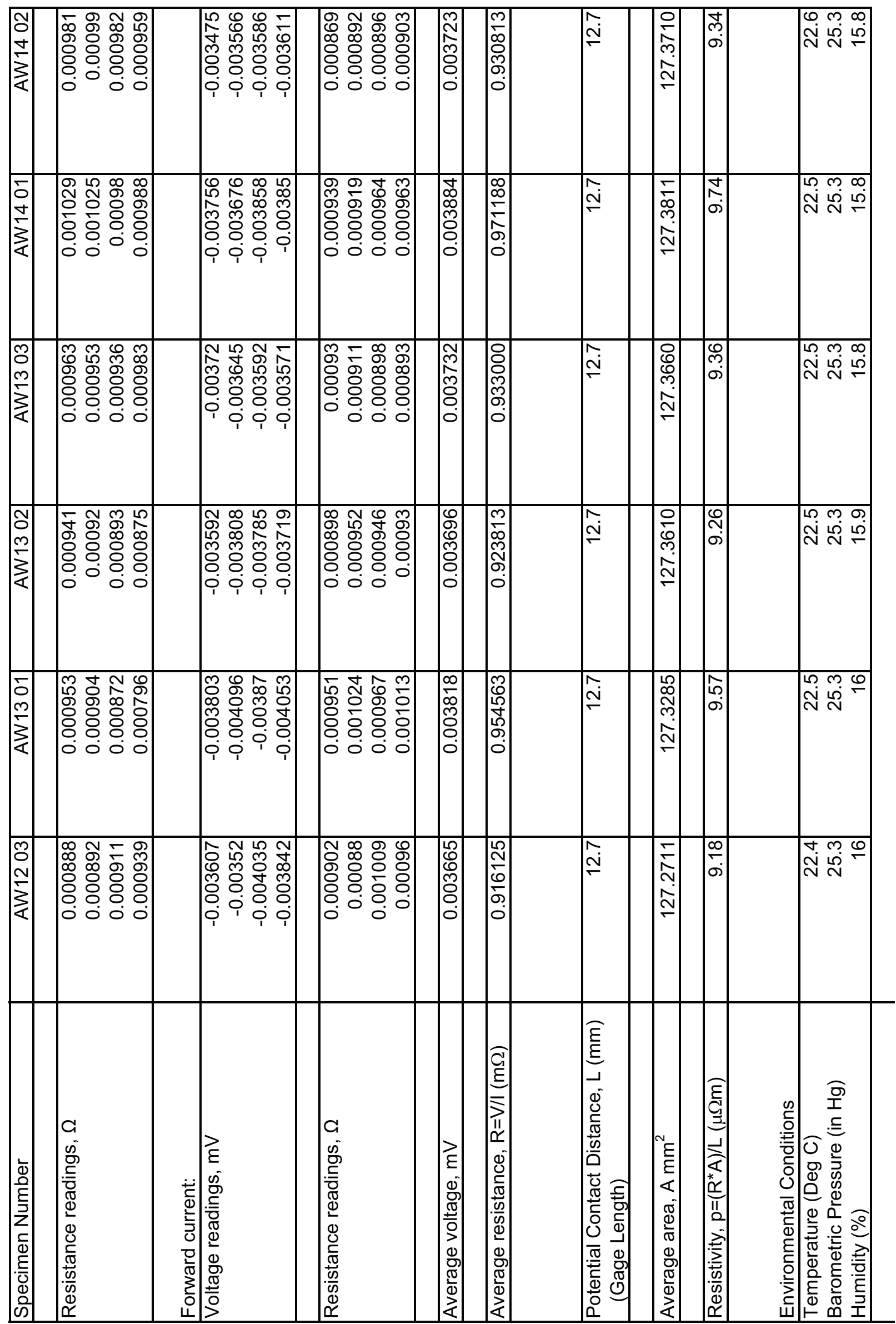




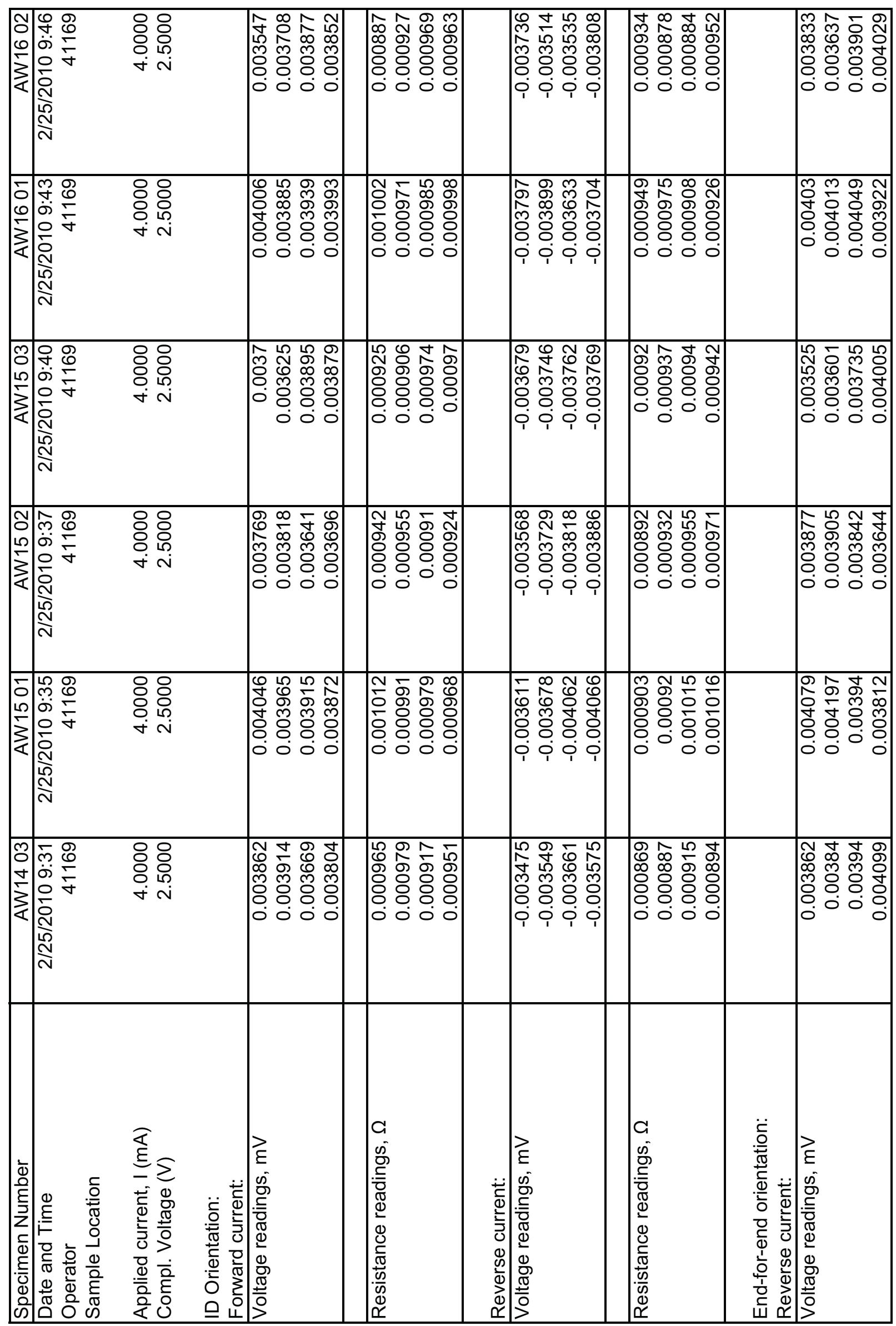




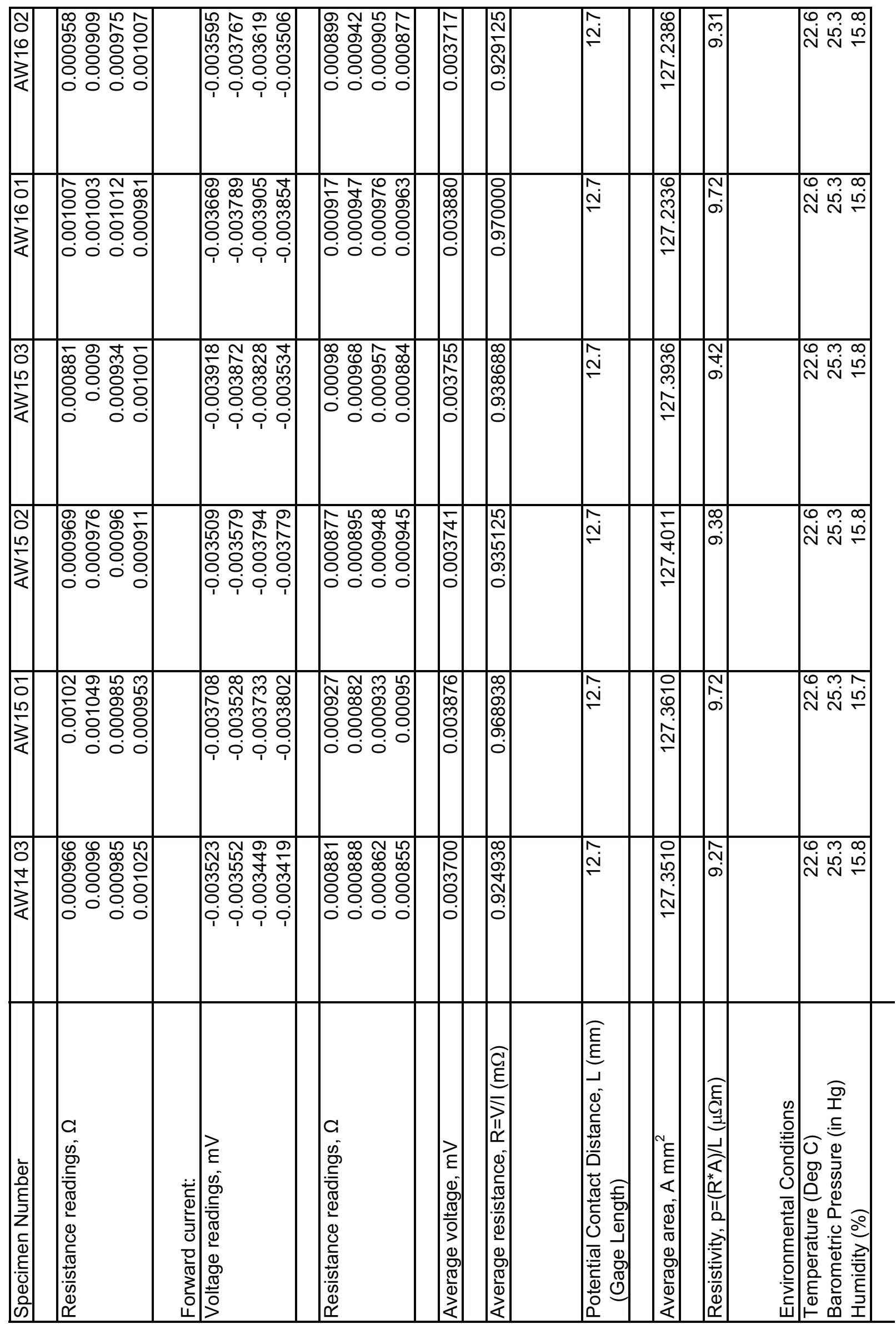




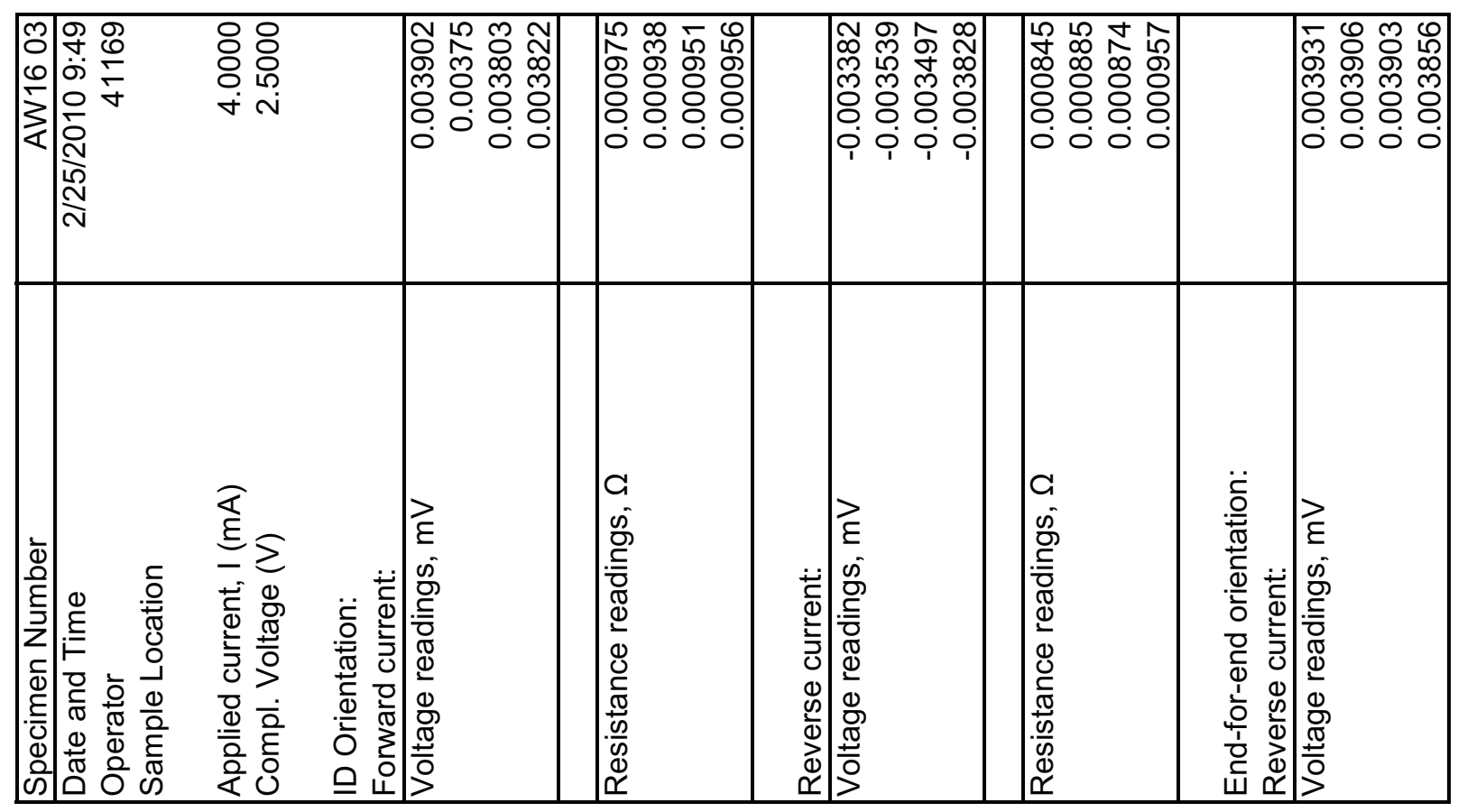




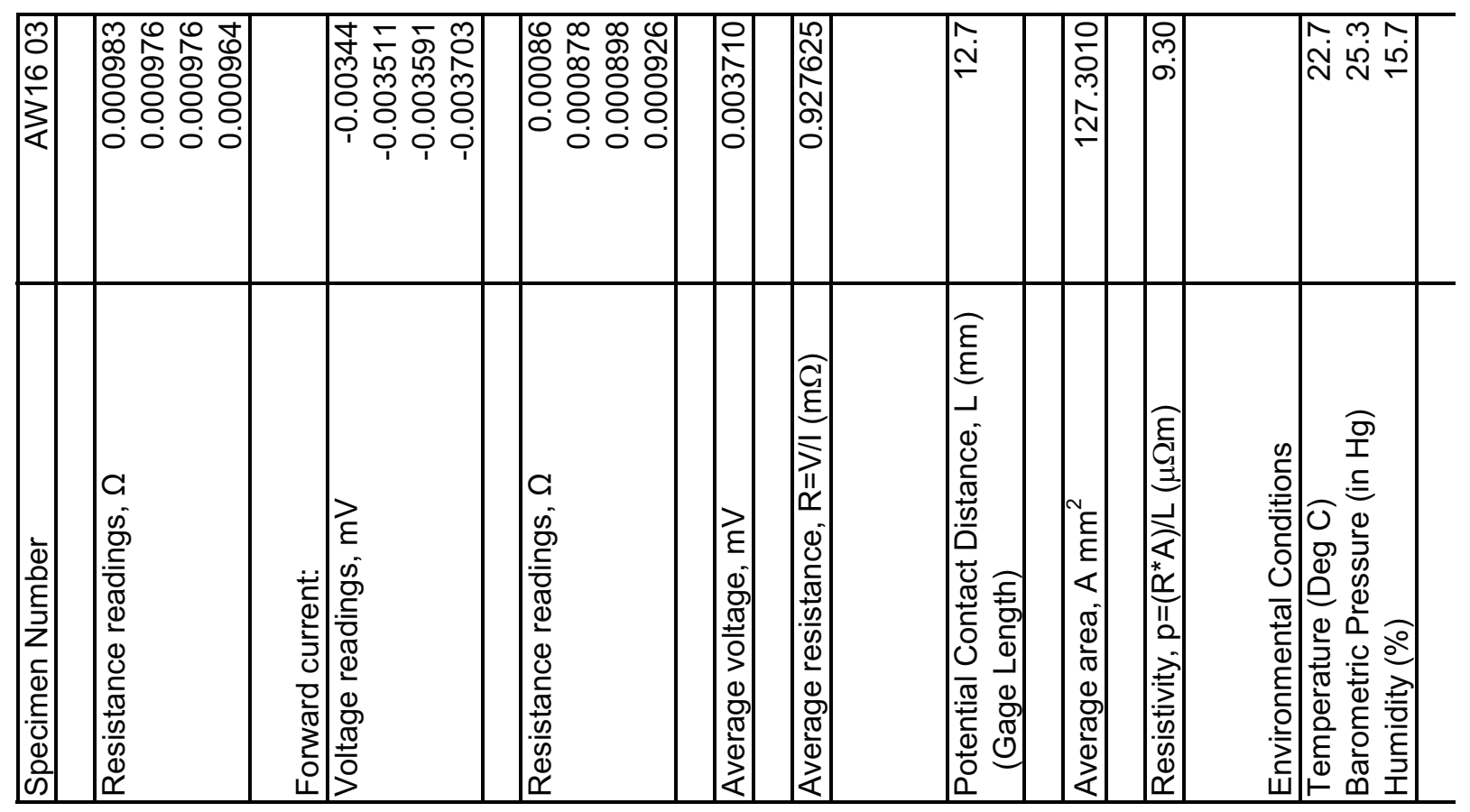




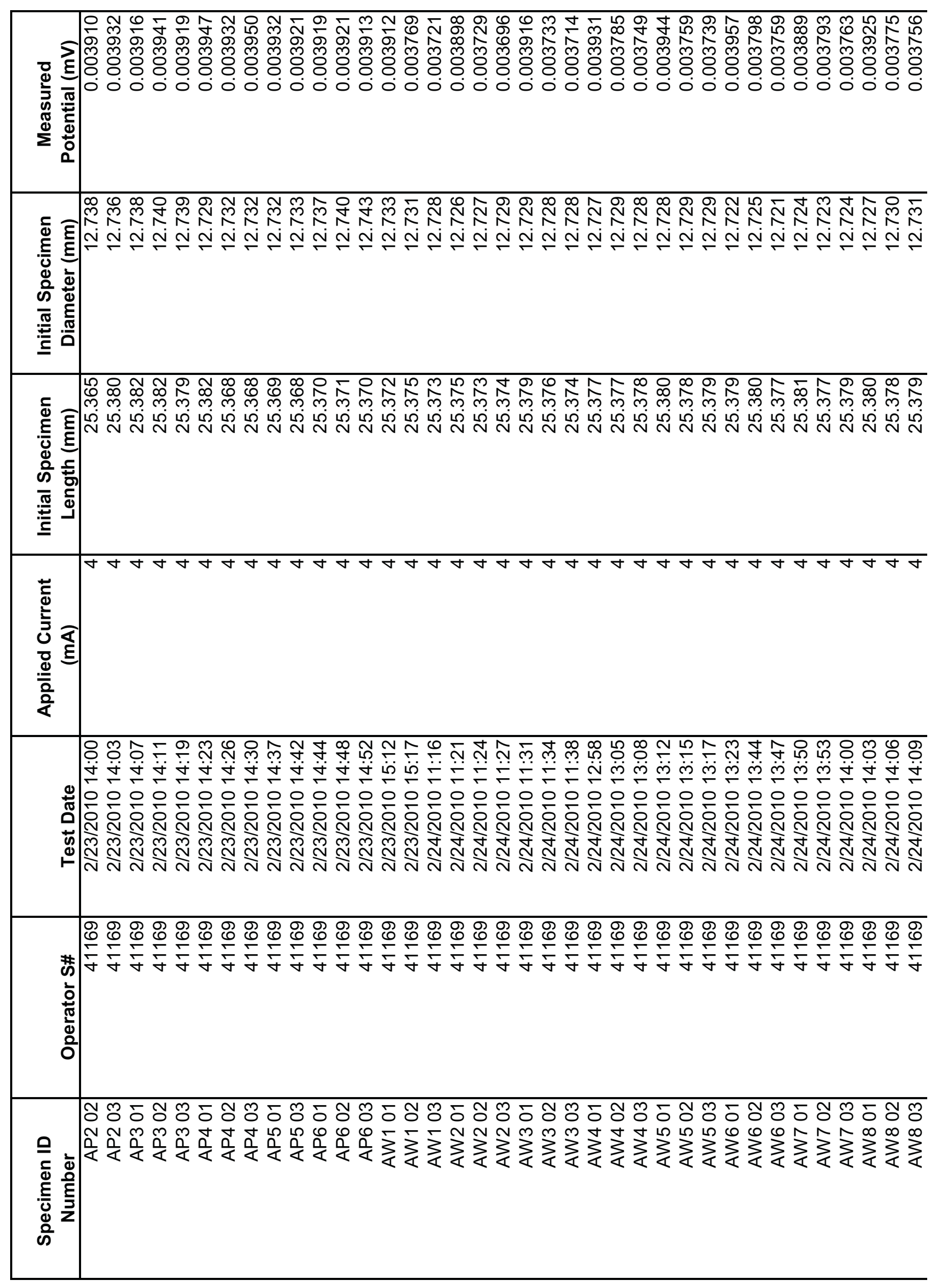




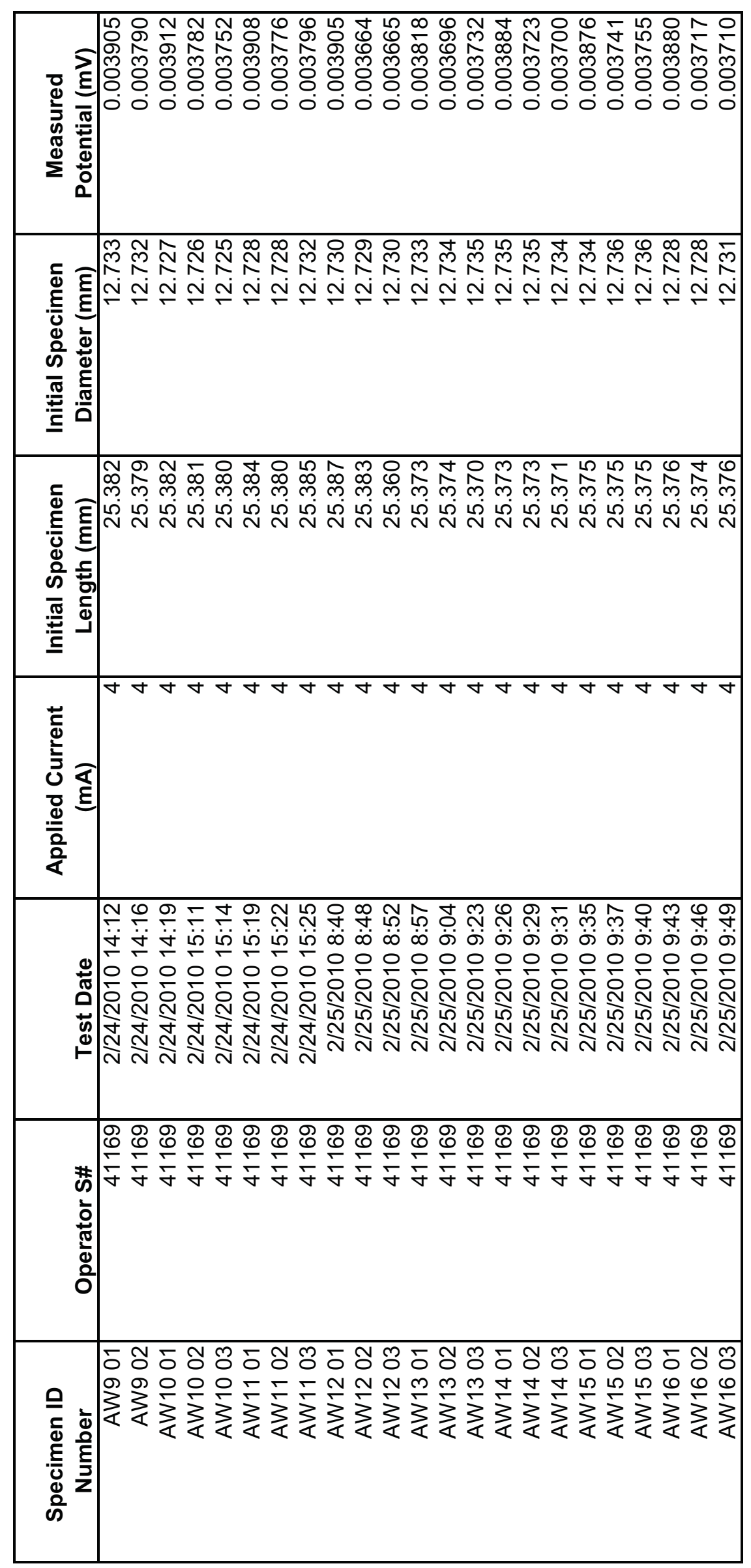




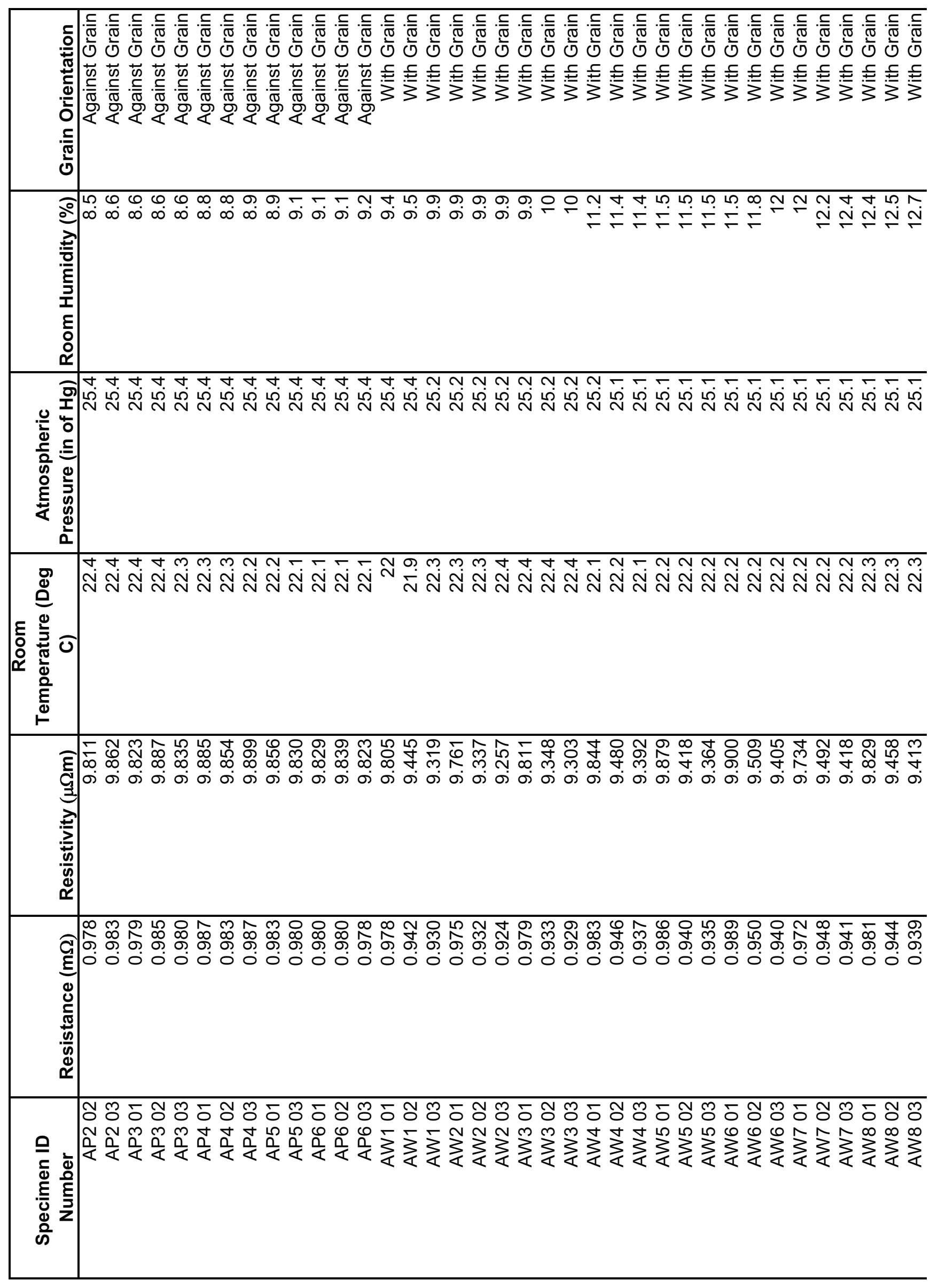




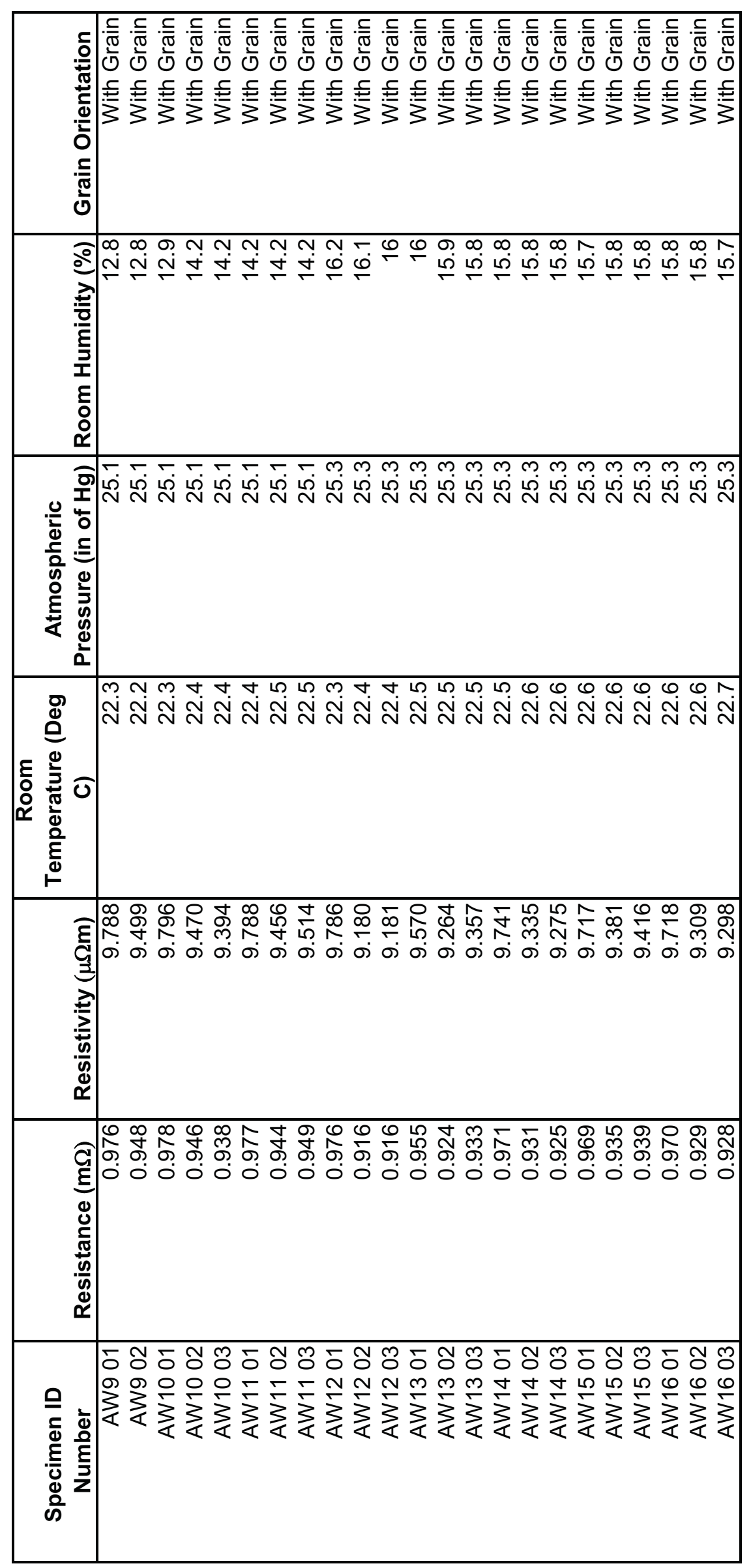




\begin{tabular}{|c|c|}
\hline 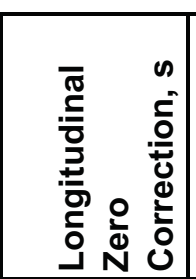 & 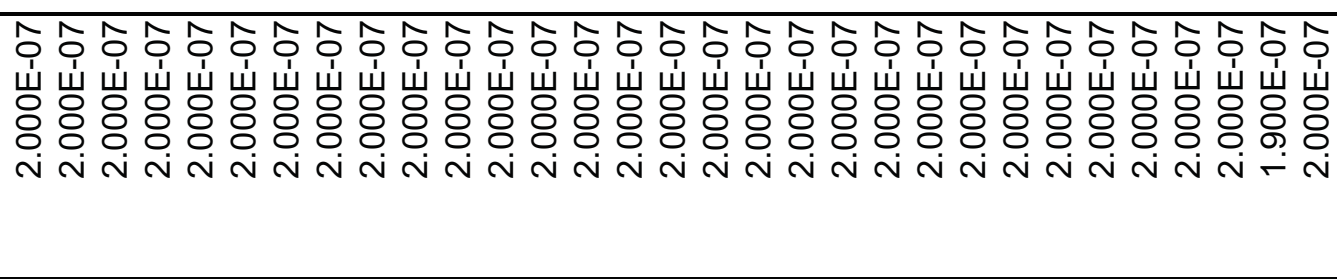 \\
\hline 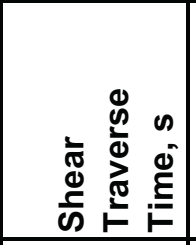 & 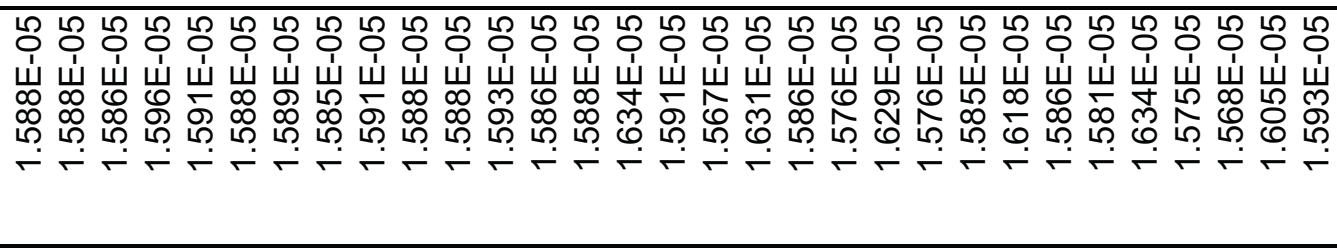 \\
\hline 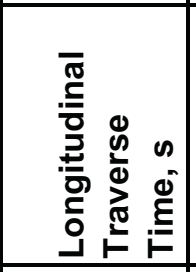 & 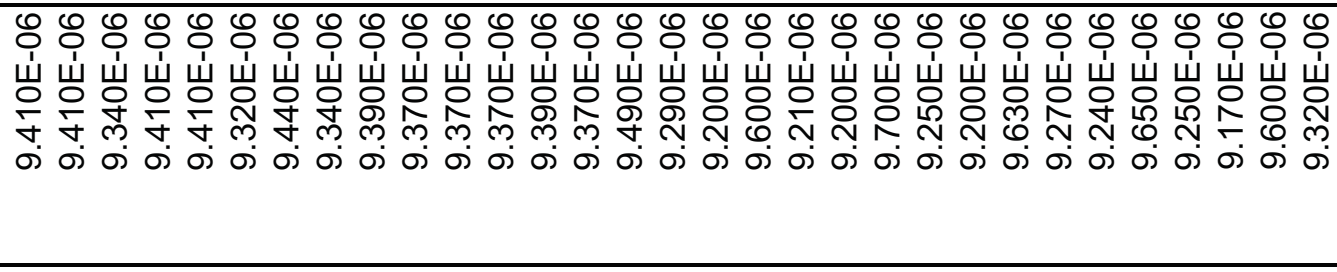 \\
\hline 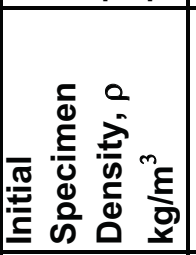 & 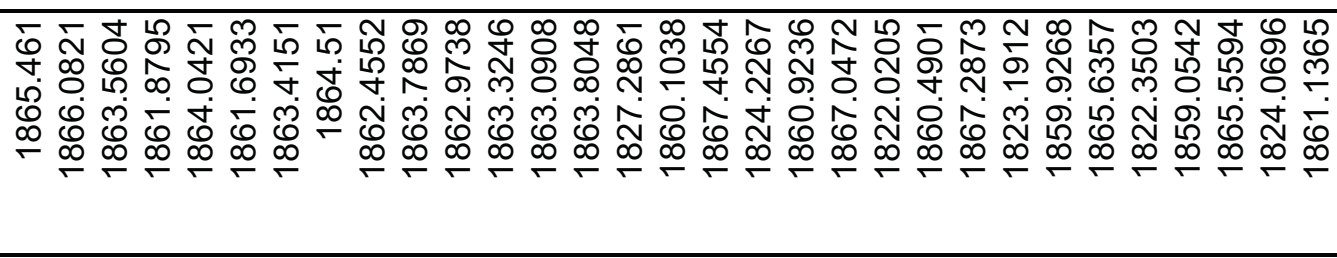 \\
\hline 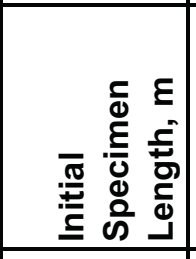 & 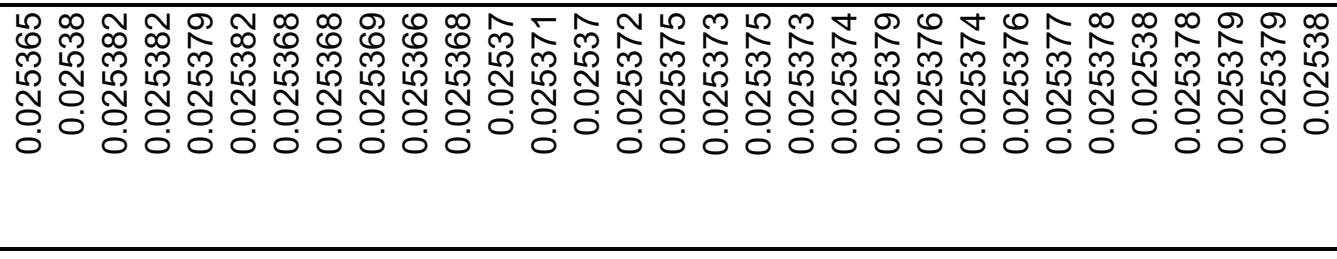 \\
\hline 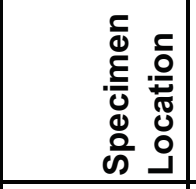 & \\
\hline $\begin{array}{l}\frac{0}{0} \\
\frac{\pi}{0} \\
\frac{0}{0} \\
\text { Oे }\end{array}$ & 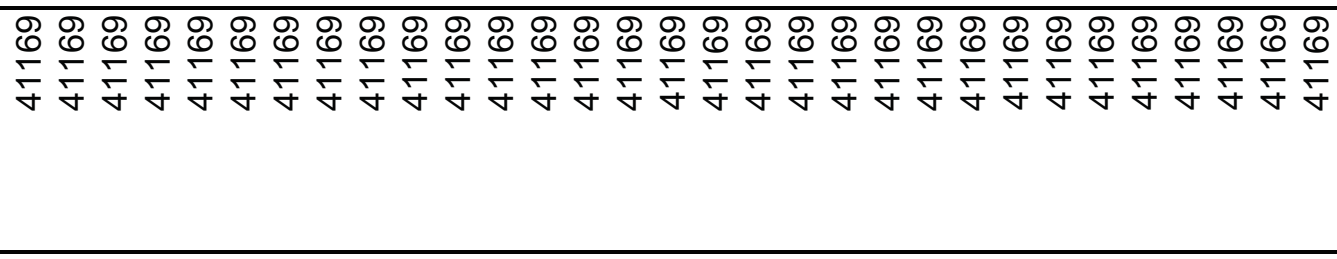 \\
\hline 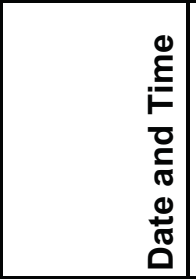 & 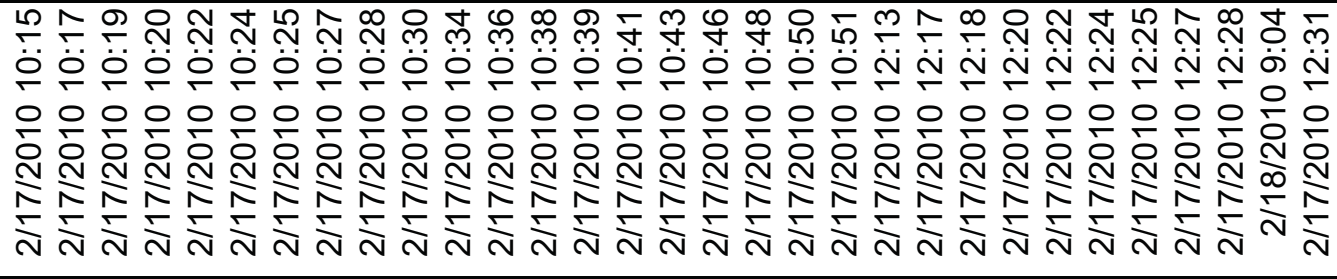 \\
\hline 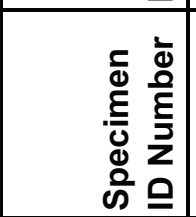 & 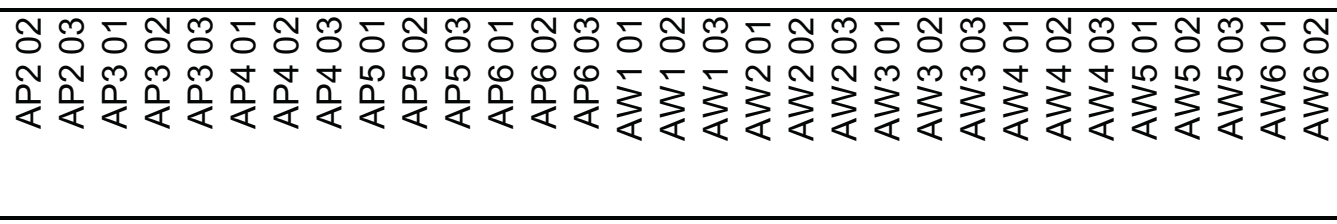 \\
\hline
\end{tabular}




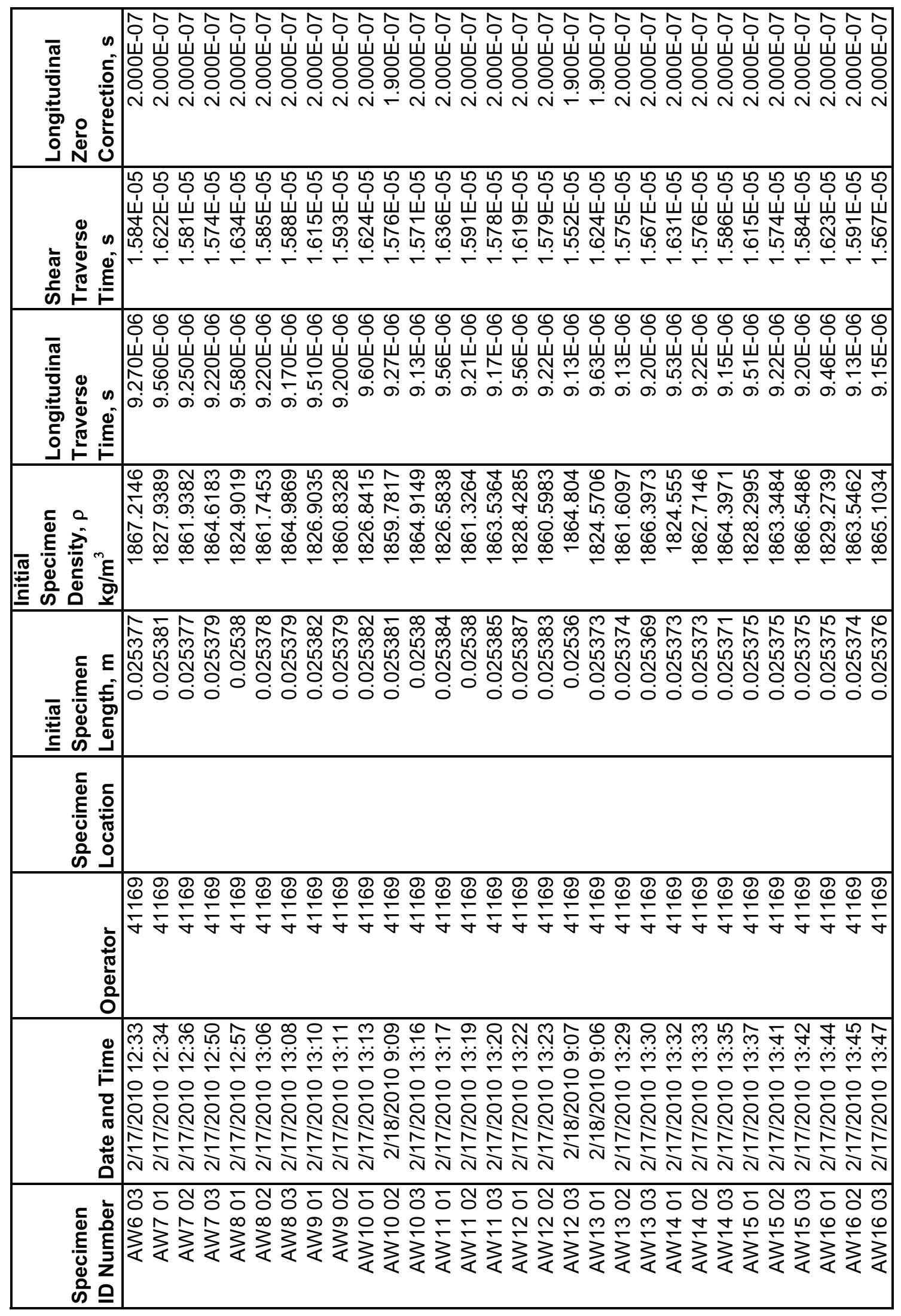




\begin{tabular}{|c|c|}
\hline 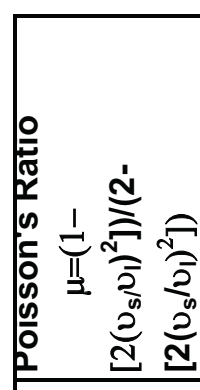 & 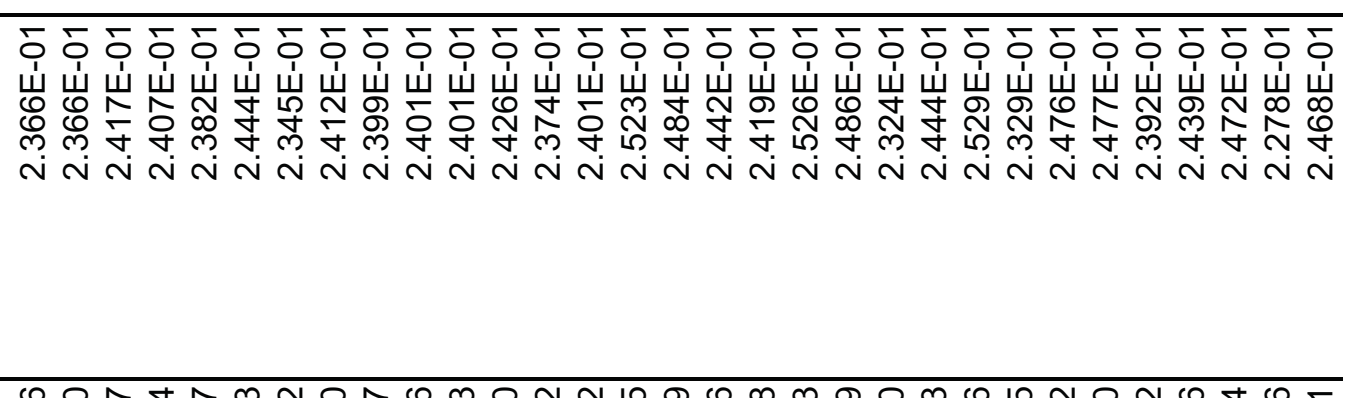 \\
\hline 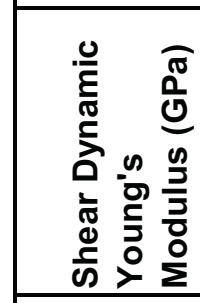 & 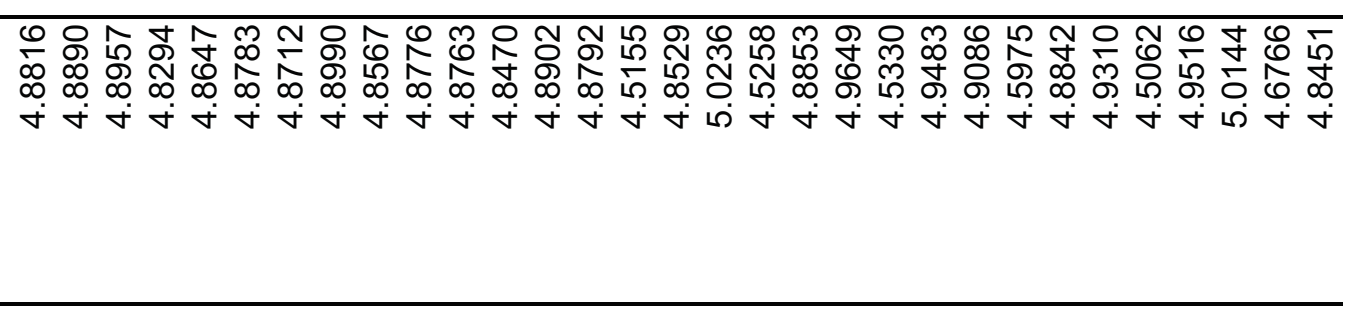 \\
\hline 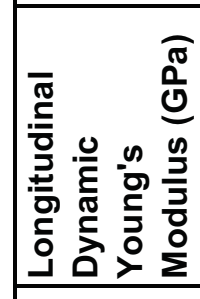 & 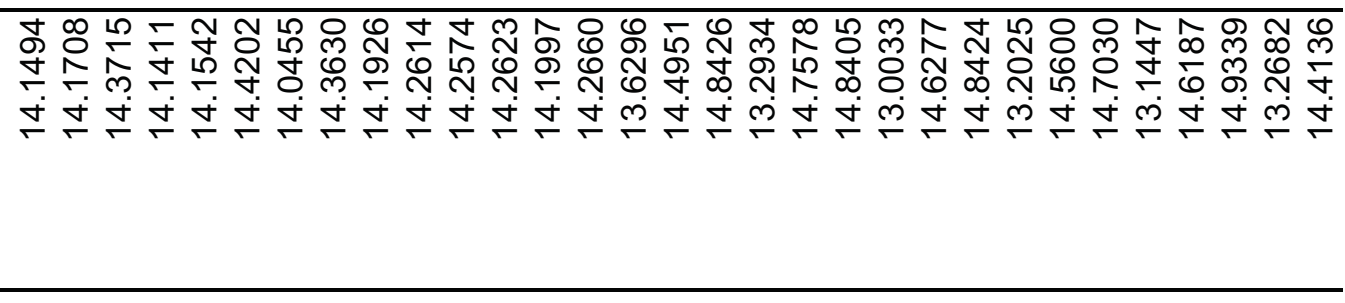 \\
\hline 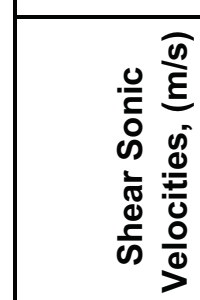 & 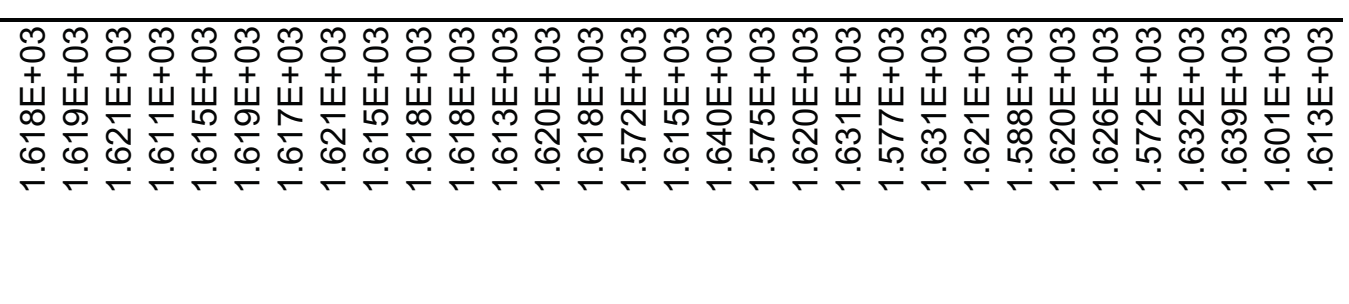 \\
\hline 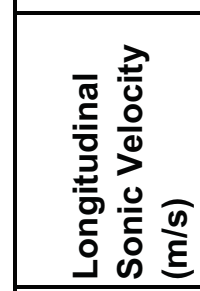 & 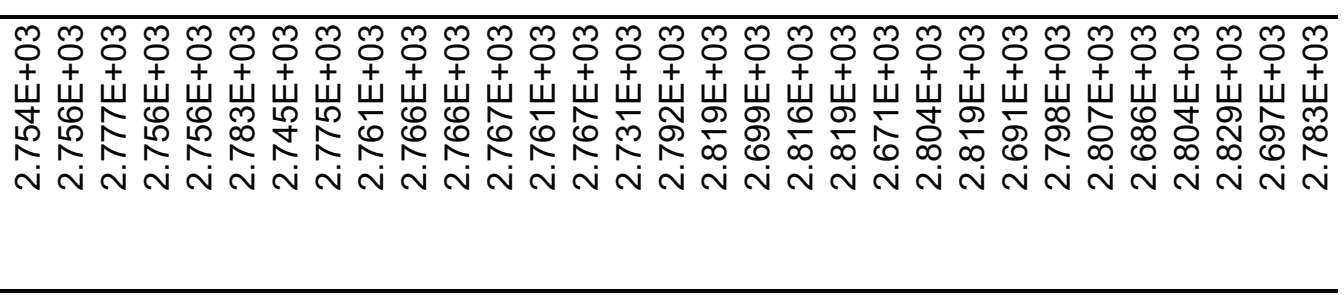 \\
\hline 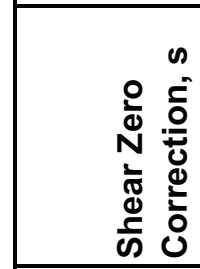 & 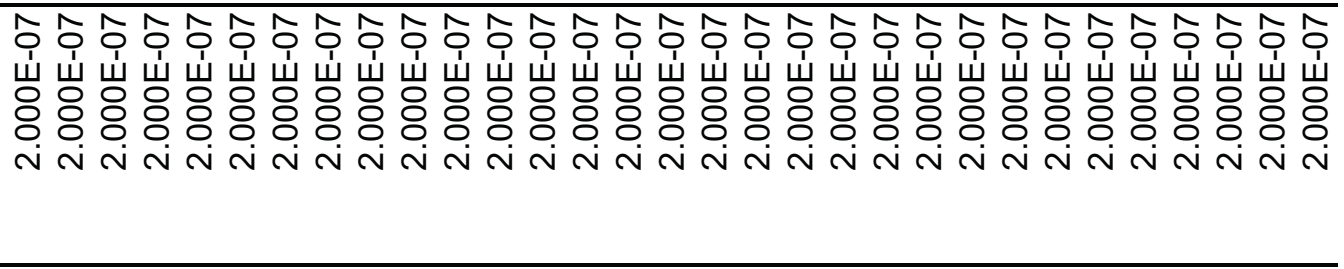 \\
\hline 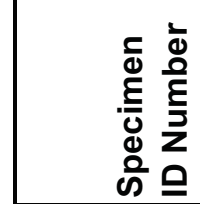 & 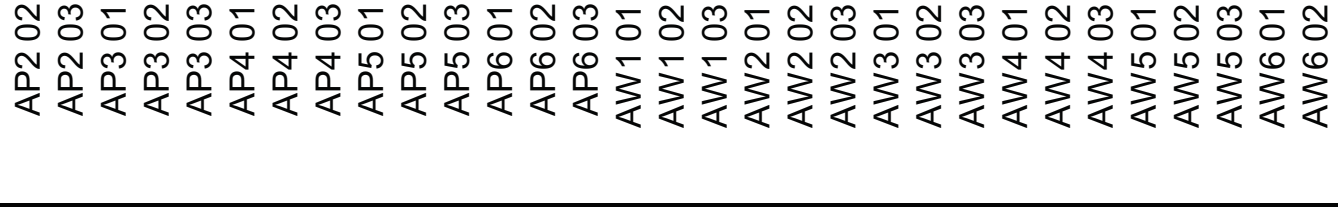 \\
\hline
\end{tabular}




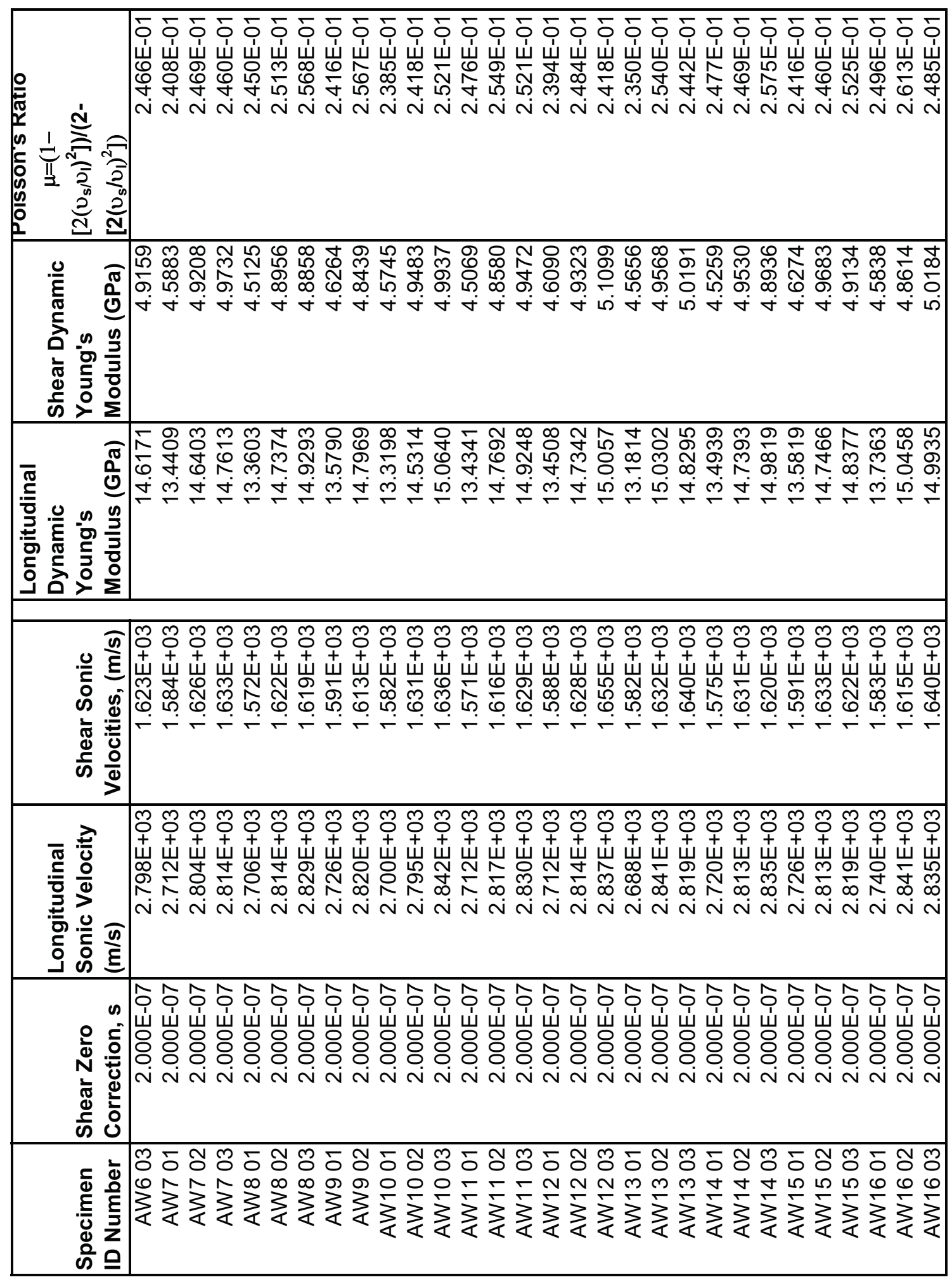




\begin{tabular}{|c|c|c|}
\hline 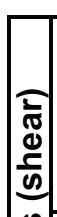 & 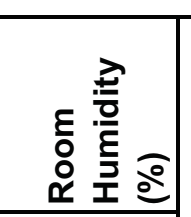 & 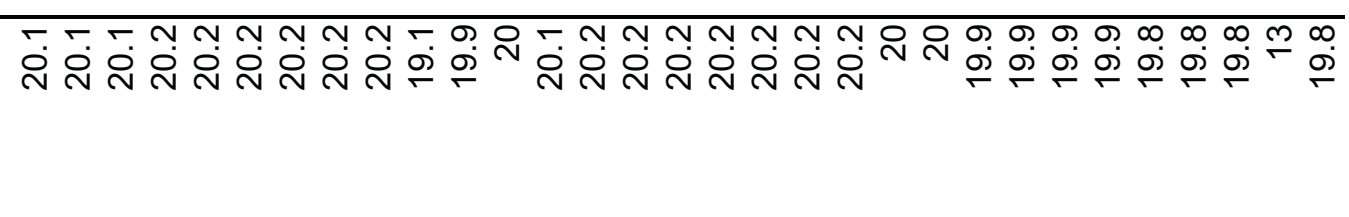 \\
\hline & 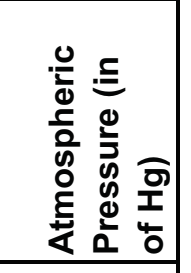 & 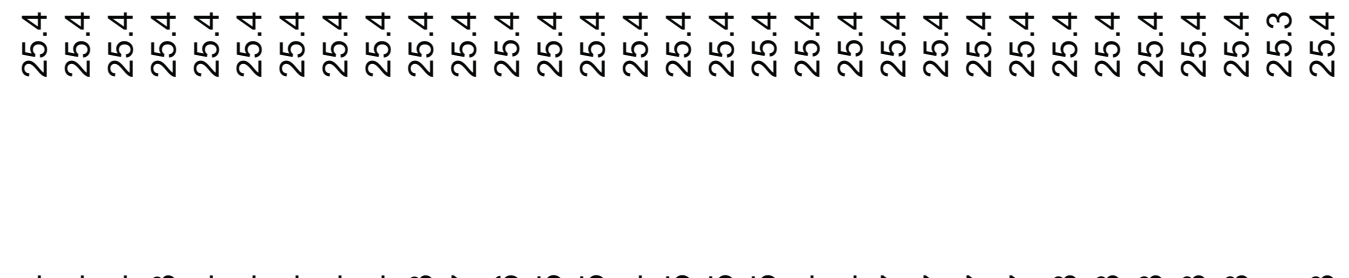 \\
\hline | & 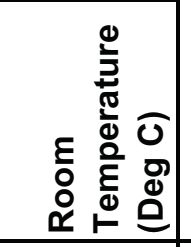 & 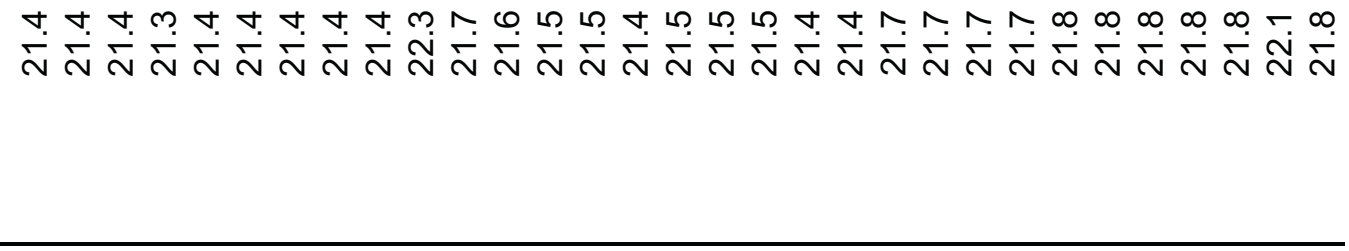 \\
\hline बิ & 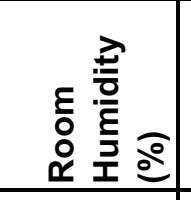 & 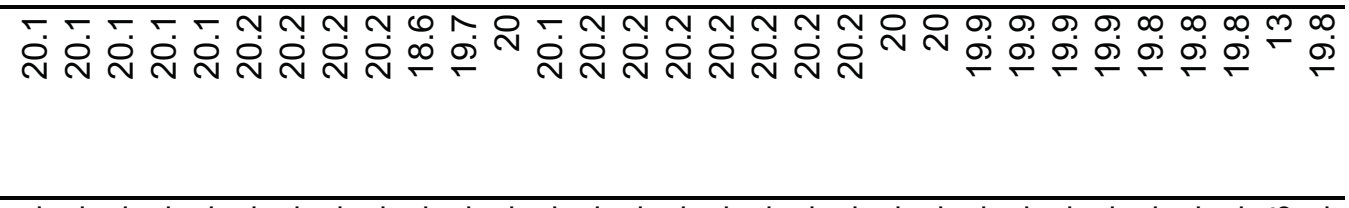 \\
\hline$\left|\begin{array}{c}0 \\
0 \\
c\end{array}\right|$ & 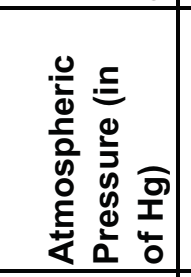 & 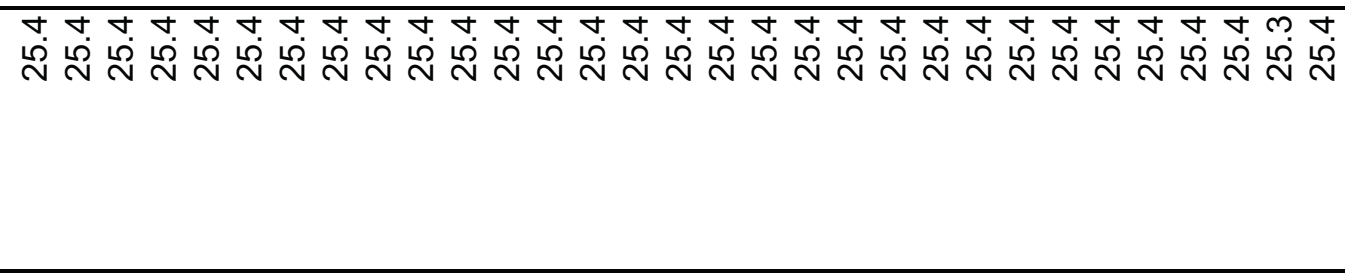 \\
\hline & 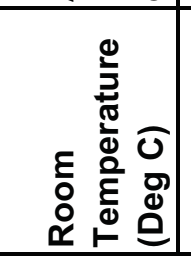 & 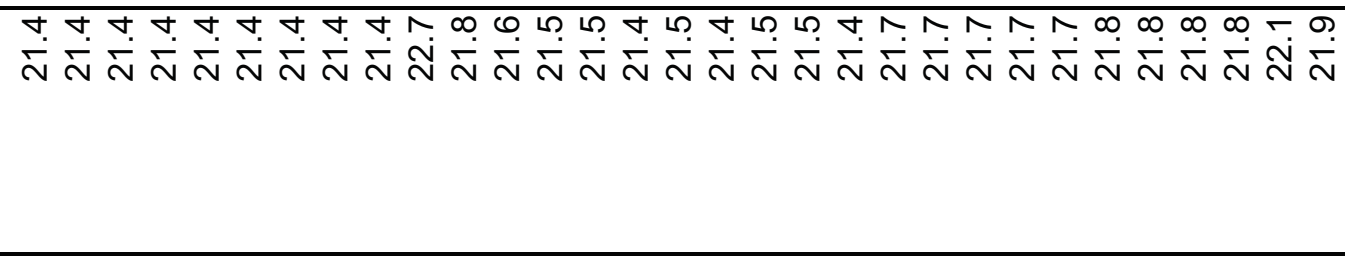 \\
\hline & 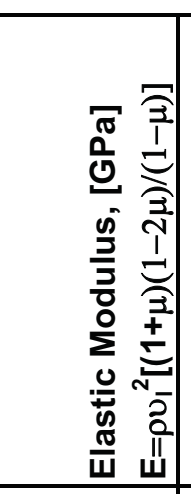 & 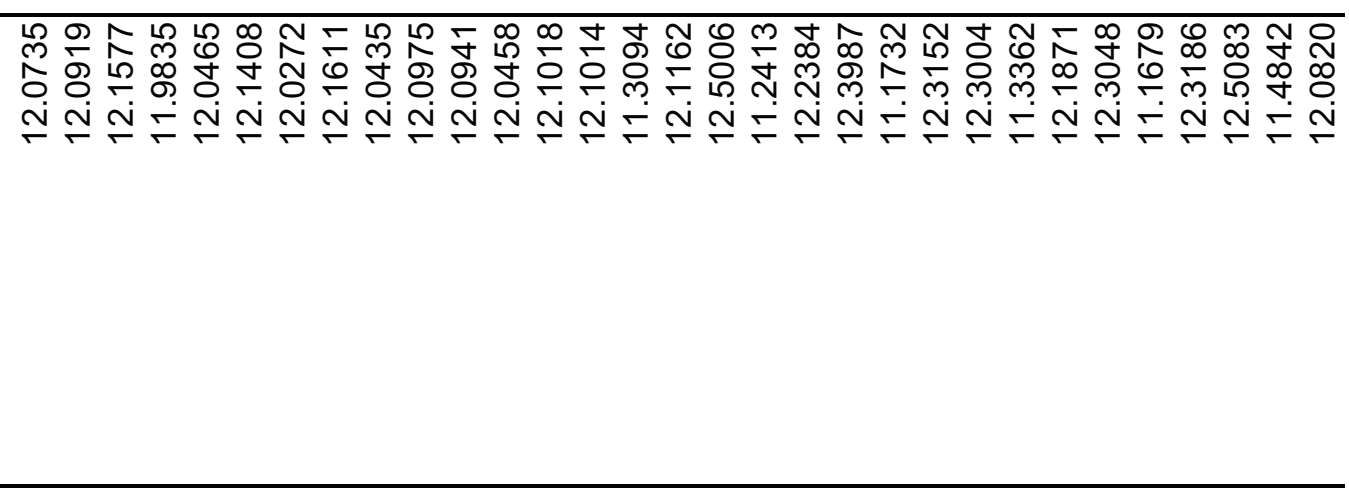 \\
\hline & 至 & 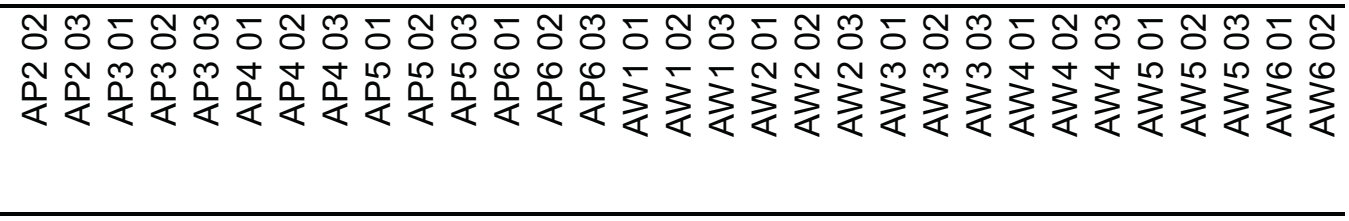 \\
\hline
\end{tabular}




\begin{tabular}{|c|c|}
\hline 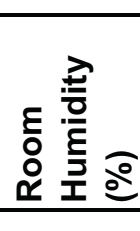 & 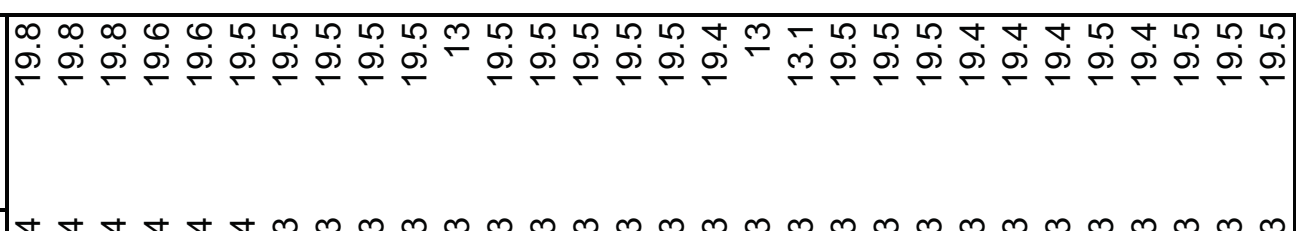 \\
\hline 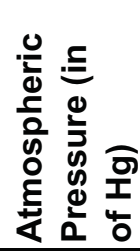 & के \\
\hline 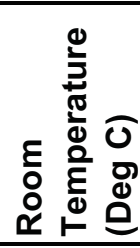 & 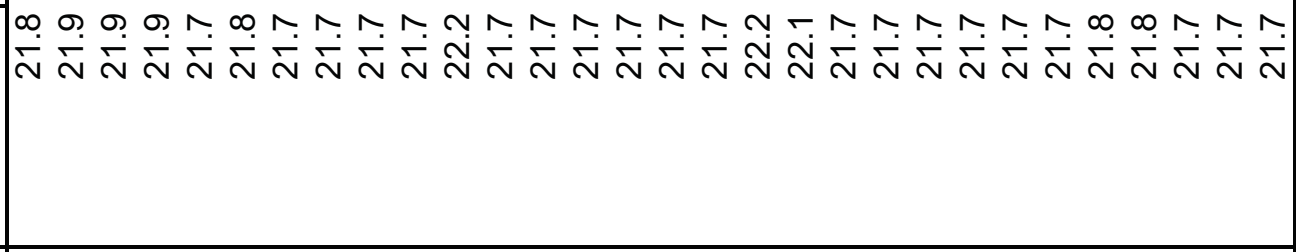 \\
\hline 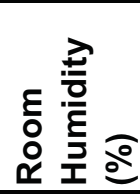 & 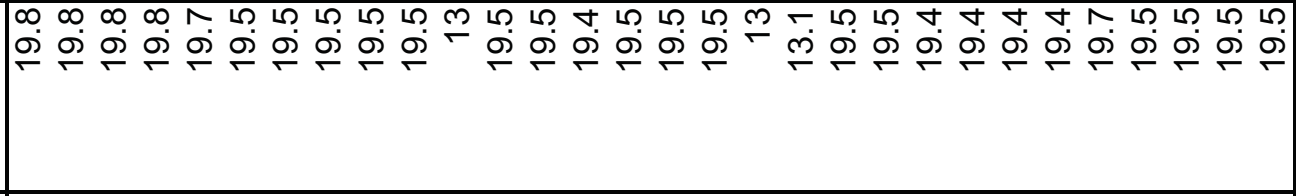 \\
\hline 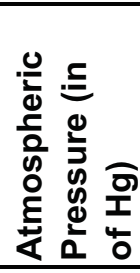 & 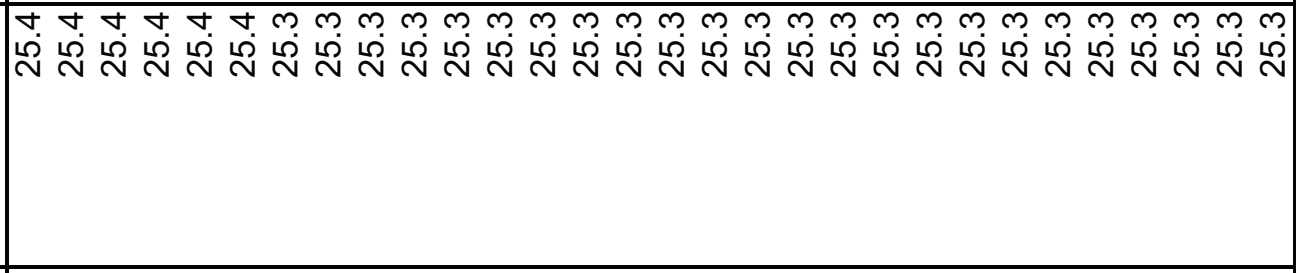 \\
\hline 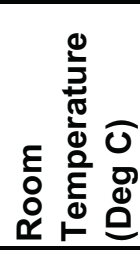 & 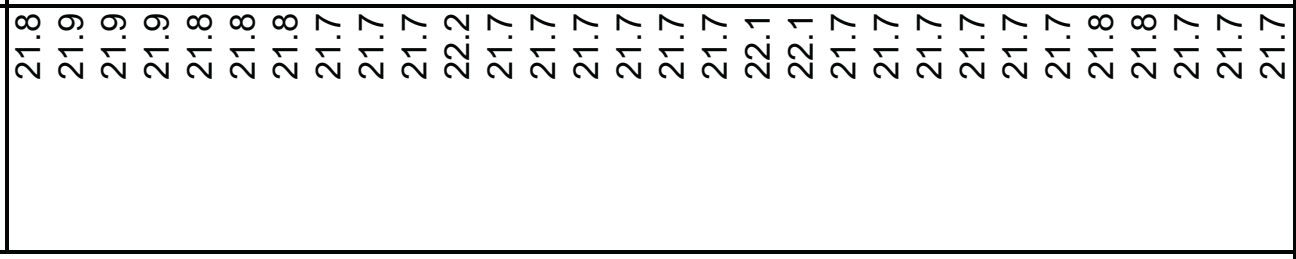 \\
\hline 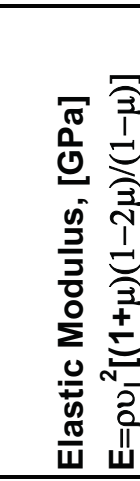 & 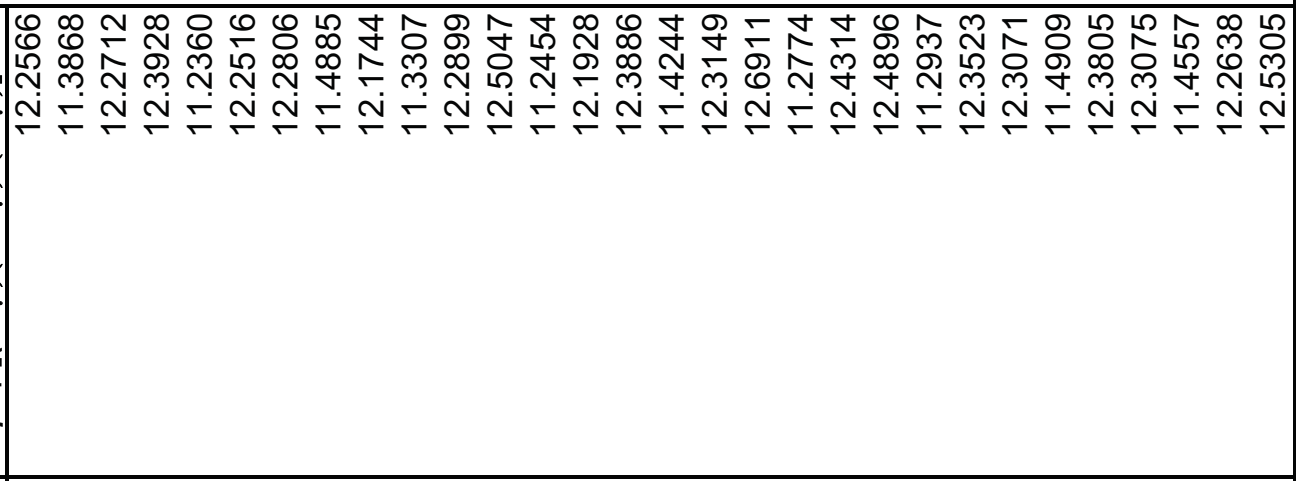 \\
\hline 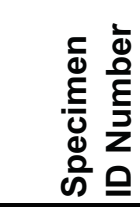 & 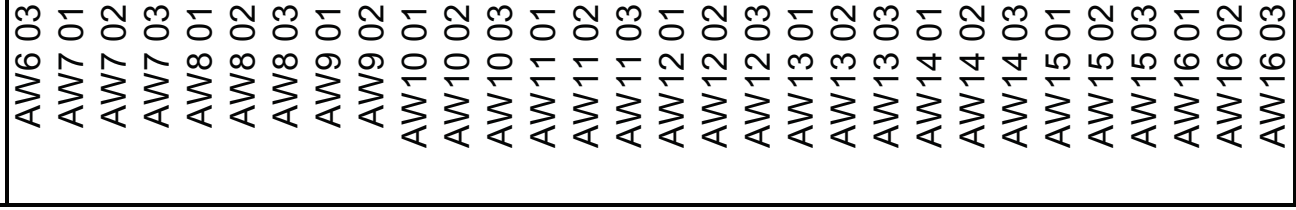 \\
\hline
\end{tabular}




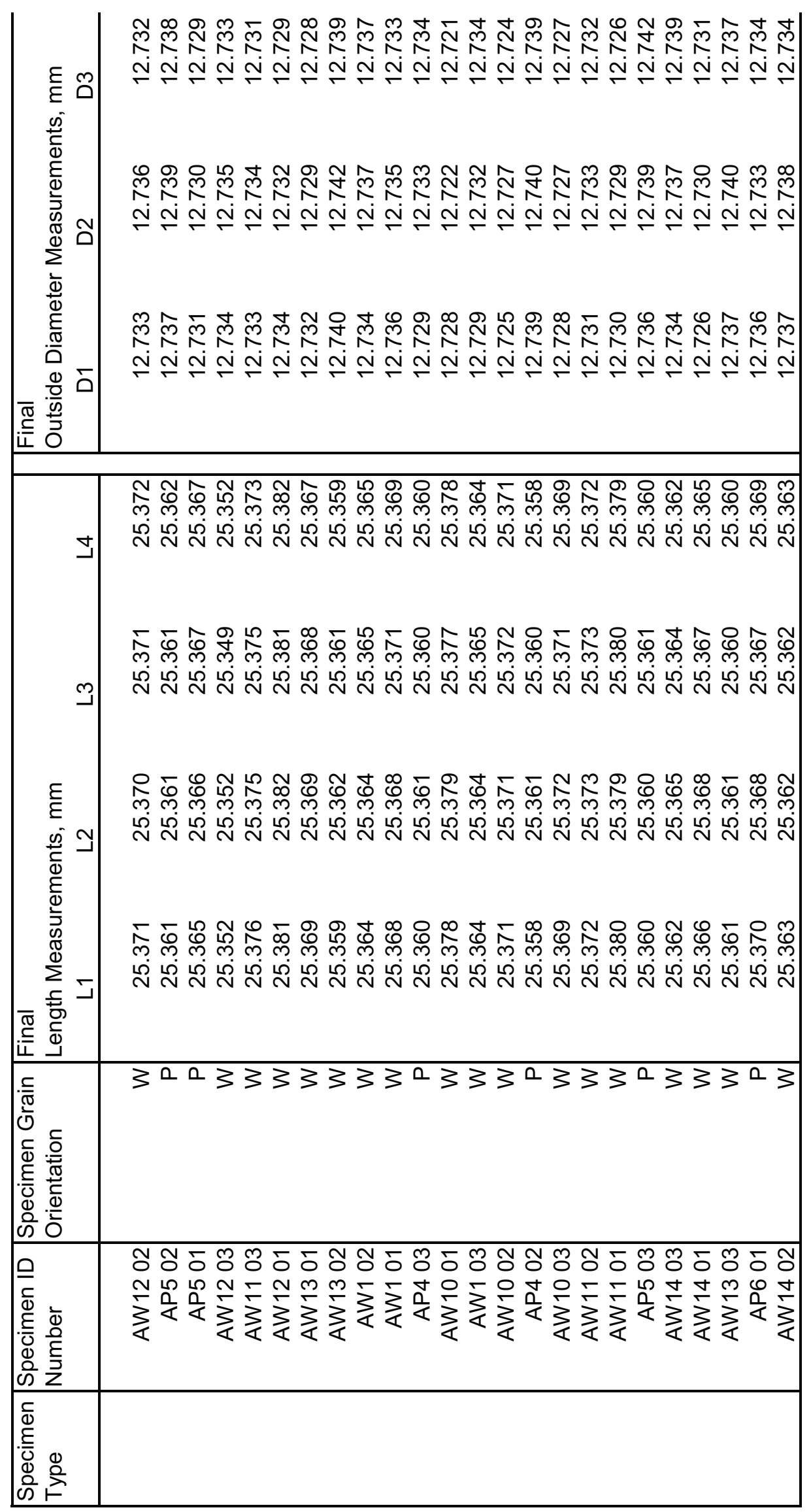




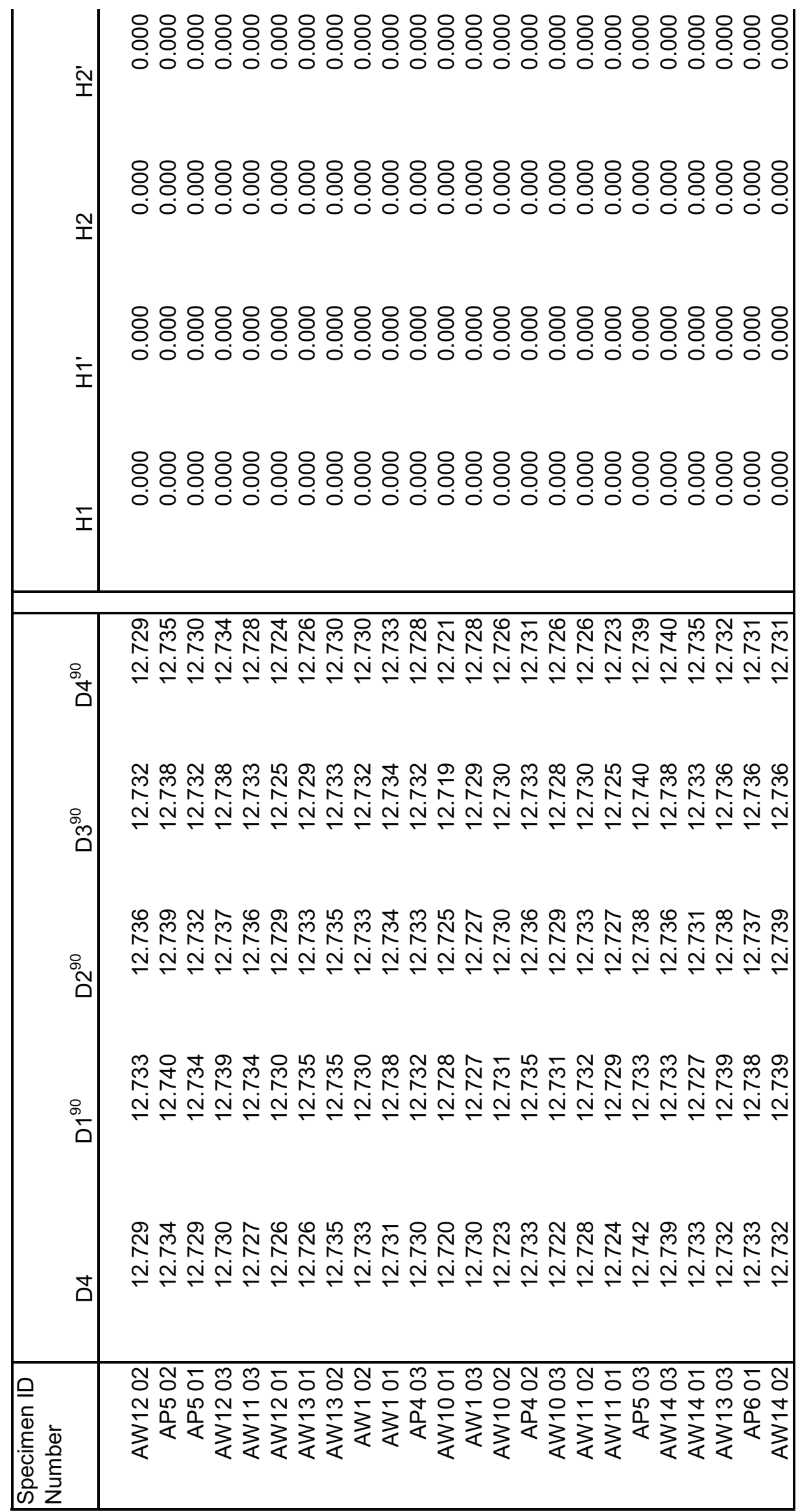




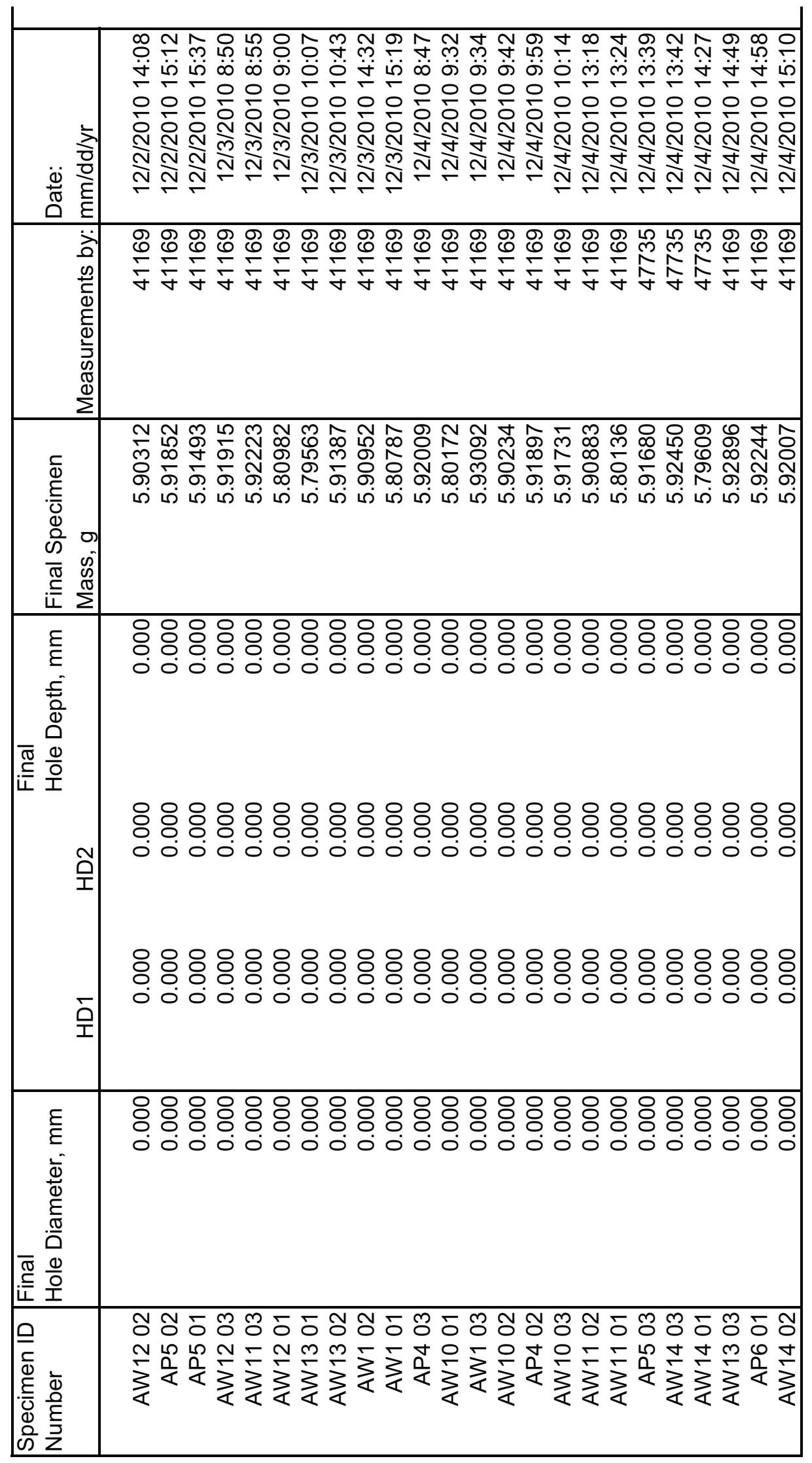




\begin{tabular}{|c|c|}
\hline 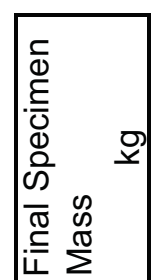 & 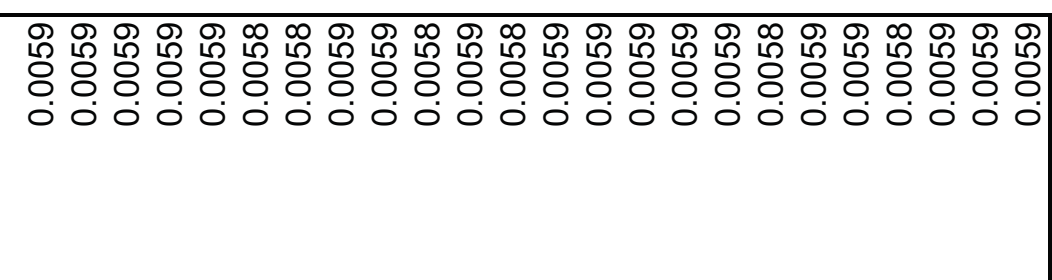 \\
\hline 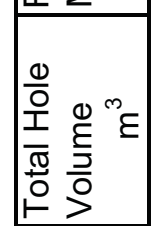 & 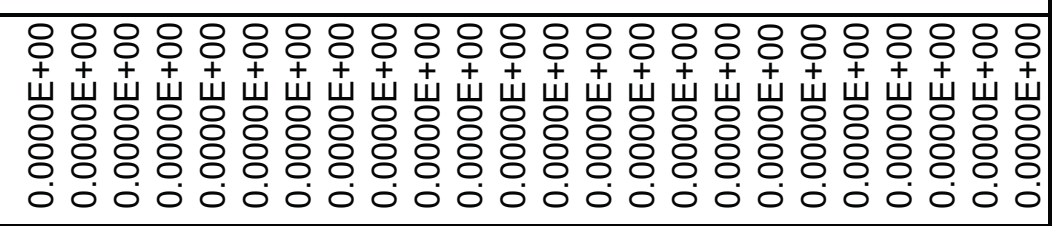 \\
\hline 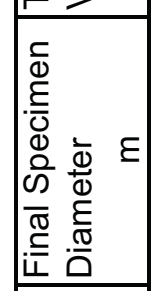 & 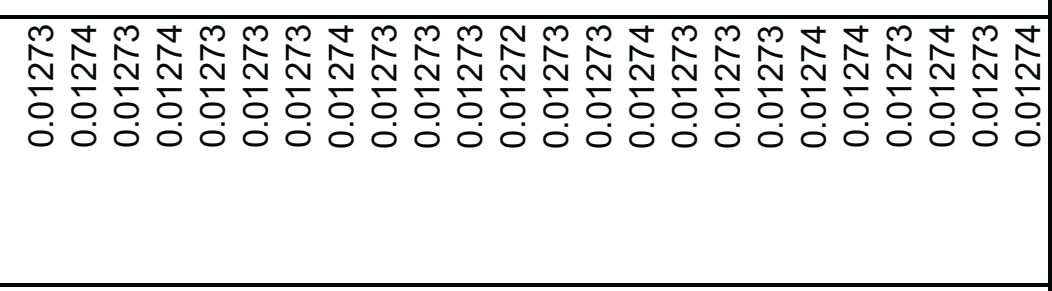 \\
\hline & 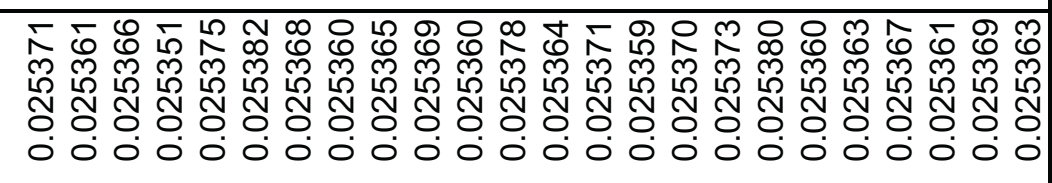 \\
\hline 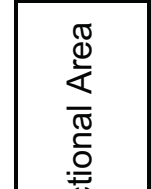 & 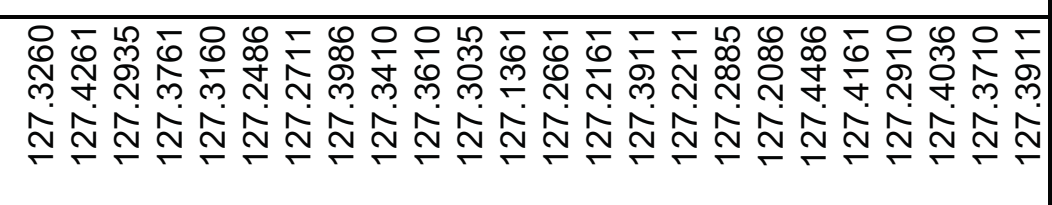 \\
\hline & 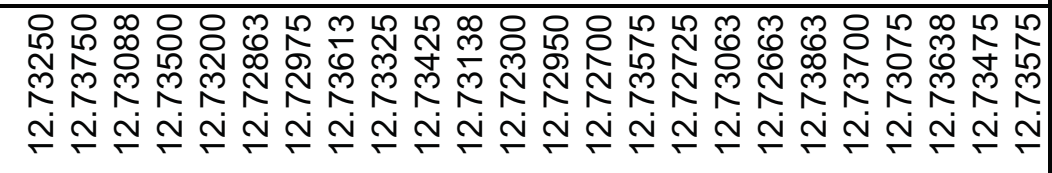 \\
\hline$\underline{\varepsilon}$ & 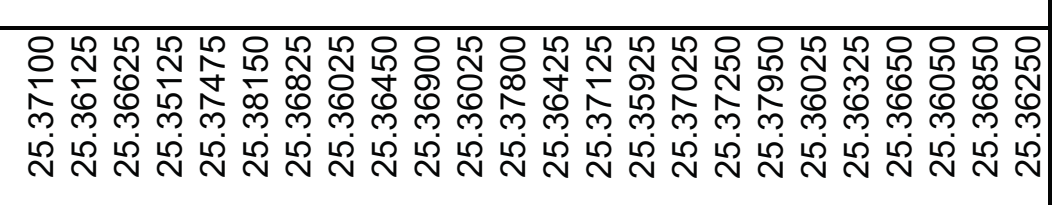 \\
\hline 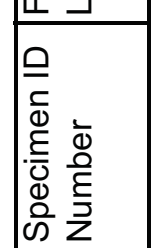 & 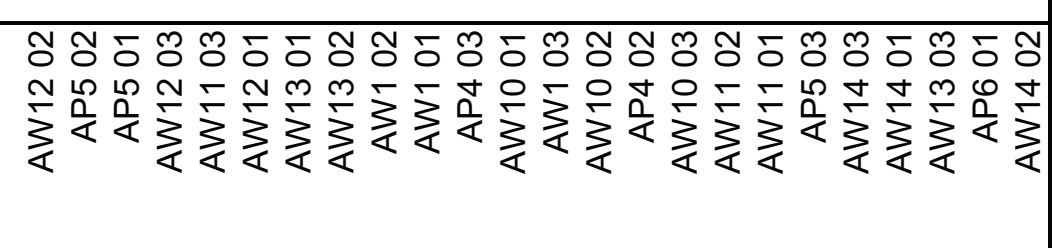 \\
\hline
\end{tabular}




\begin{tabular}{|c|c|}
\hline : & 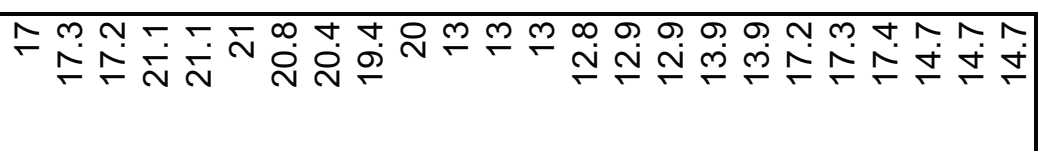 \\
\hline 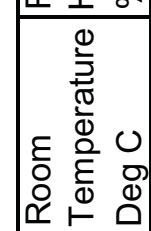 & 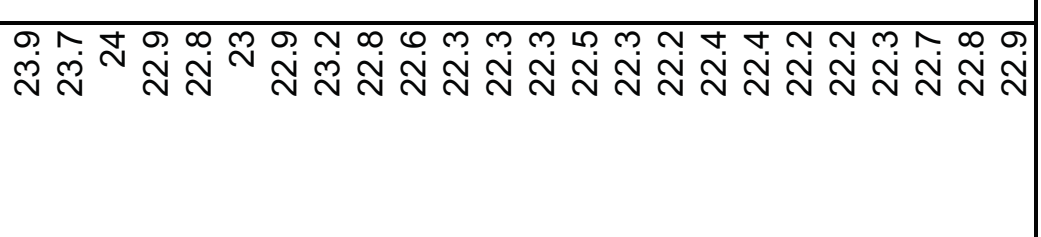 \\
\hline 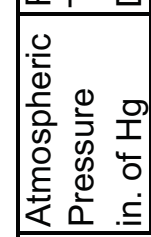 & 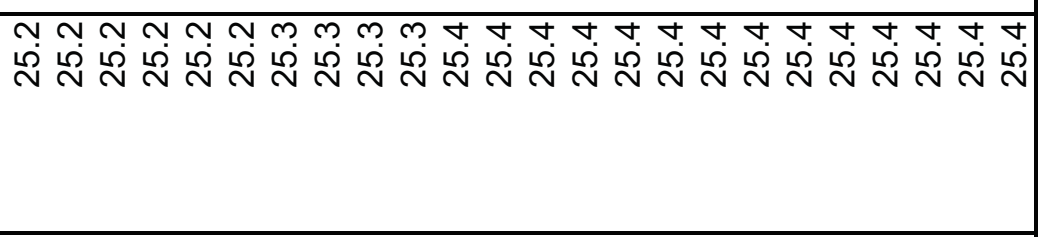 \\
\hline 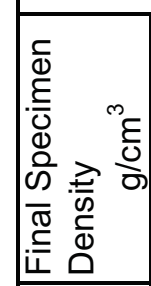 & 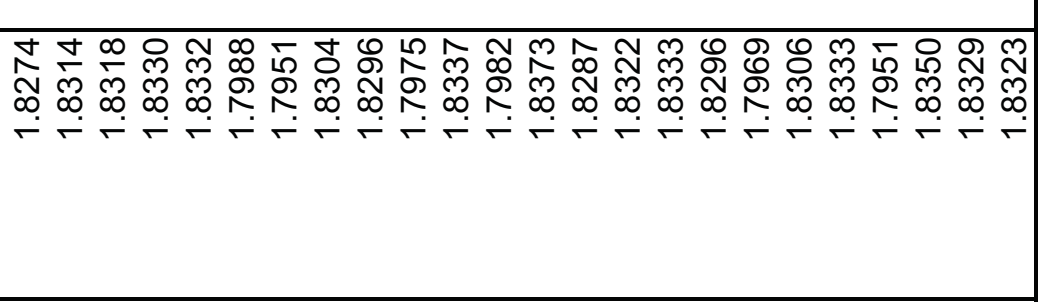 \\
\hline 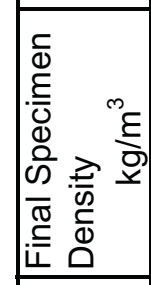 & 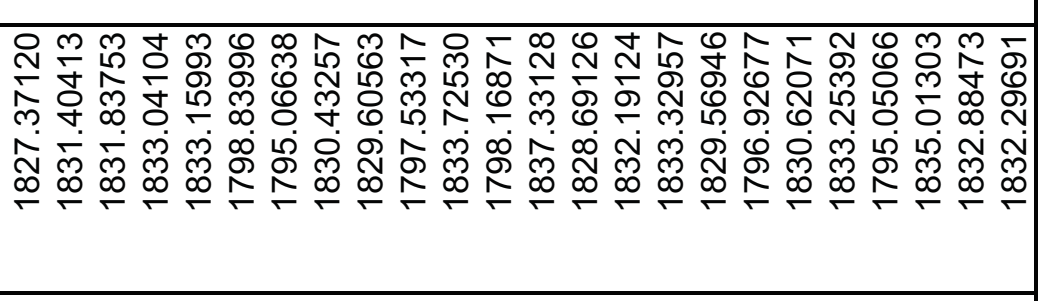 \\
\hline 离 & 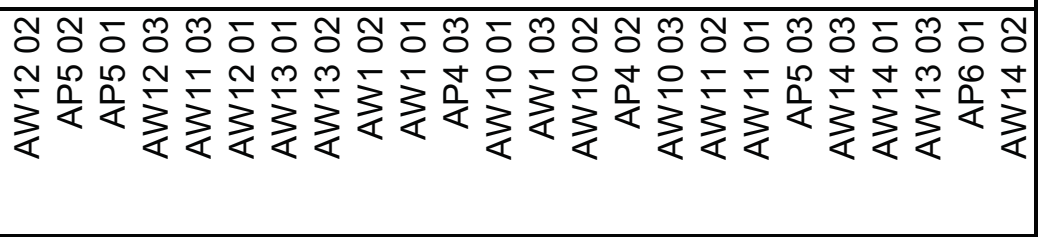 \\
\hline
\end{tabular}




$\begin{array}{ll}\text { Graphite Grade: } & \text { NBG-18 } \\ \text { Graphite Manufacturer: } & \text { SGL Carbon Company } \\ \text { Forming Process: } & \text { Vibration molded } \\ \text { Coke Particle Size: } & \text { Medium grain } \\ \text { Coke Type: } & \text { Pitch coke filler, pitch binder } \\ \text { ASTM Class: } & \text { MNHP } \\ \text { Specimen Geometry: } & \text { Cylinder }\end{array}$

Specimen ID \#'s:

BP1 01

BP1 02

BP1 03

BP2 01

BP2 02

BP2 03

BP3 01

BP3 02

BP3 03

BP4 01

BP4 02

BP4 03

BP5 01

BP5 02

BP5 03

BP6 01

BP6 02

BP6 03

BW1 01

BW1 02

BW1 03

BW2 01

BW2 02

BW2 03

BW3 01

BW3 02

BW3 03

BW4 01

BW4 02

BW4 03

BW5 01

BW5 02

BW5 03

BW6 01

BW6 02

BW6 03

BW7 01

BW7 02

BW7 03

BW8 01

BW8 02

BW8 03 


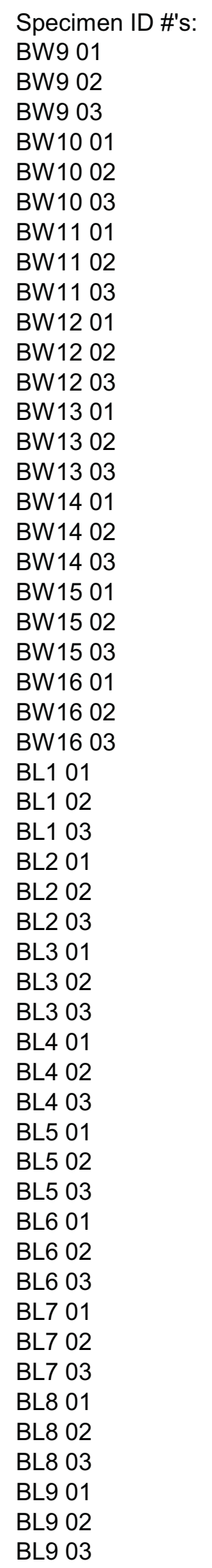


Specimen ID \#'s:

BL10 01

BL10 02

BL10 03

BL11 01

BL11 02

BL11 03

BL12 01

BL12 02

BL12 03

BL13 01

BL13 02

BL13 03 


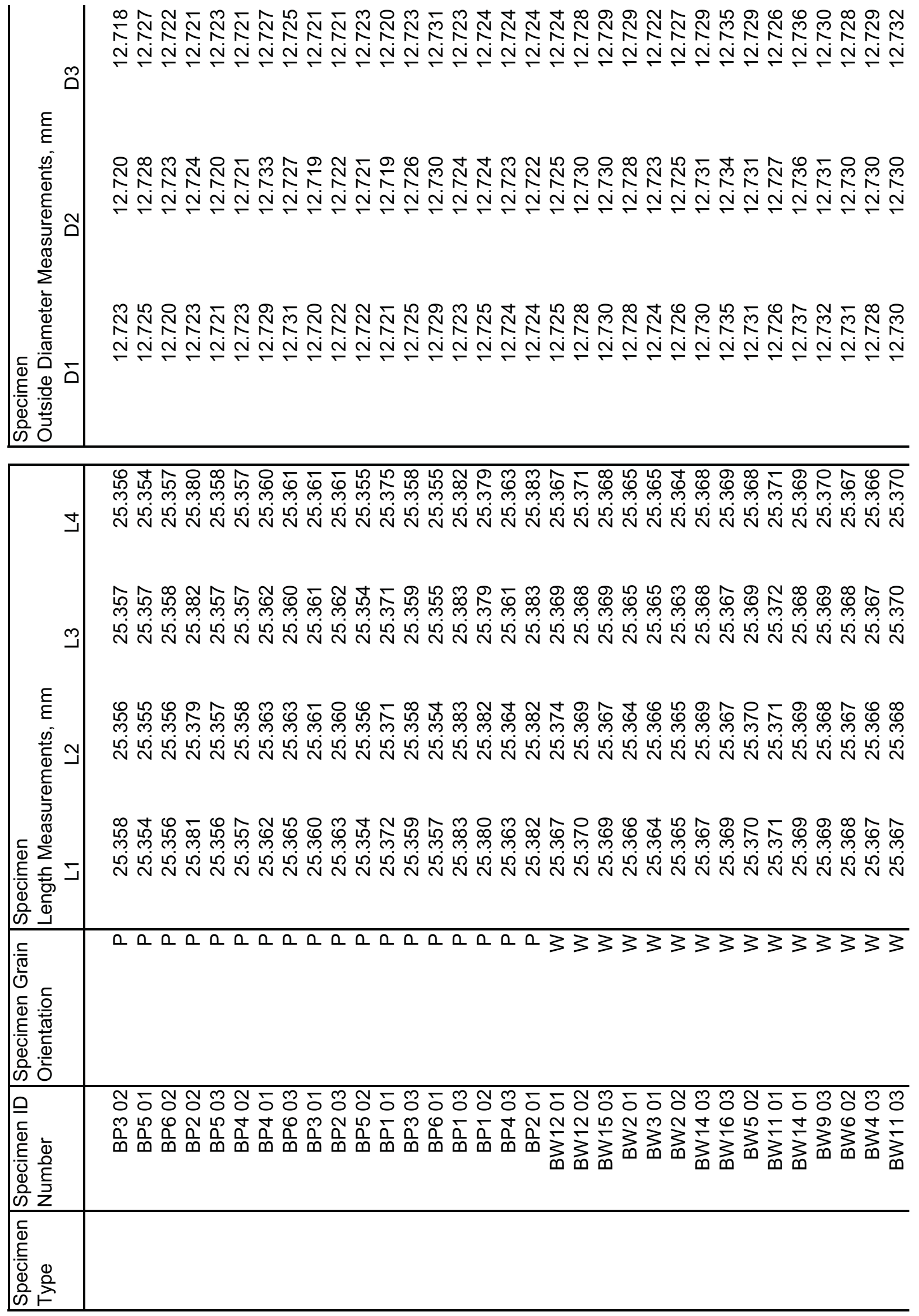




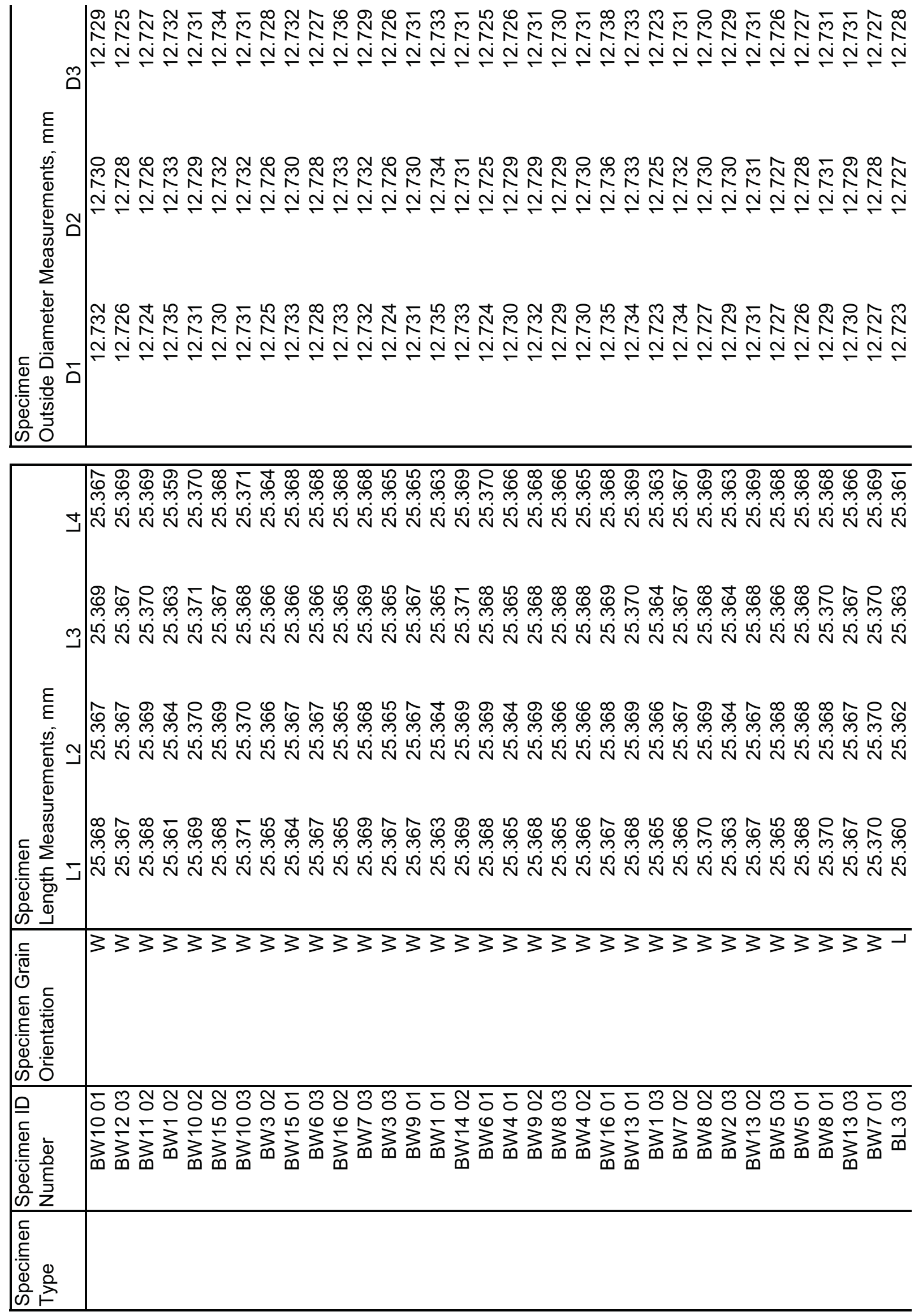




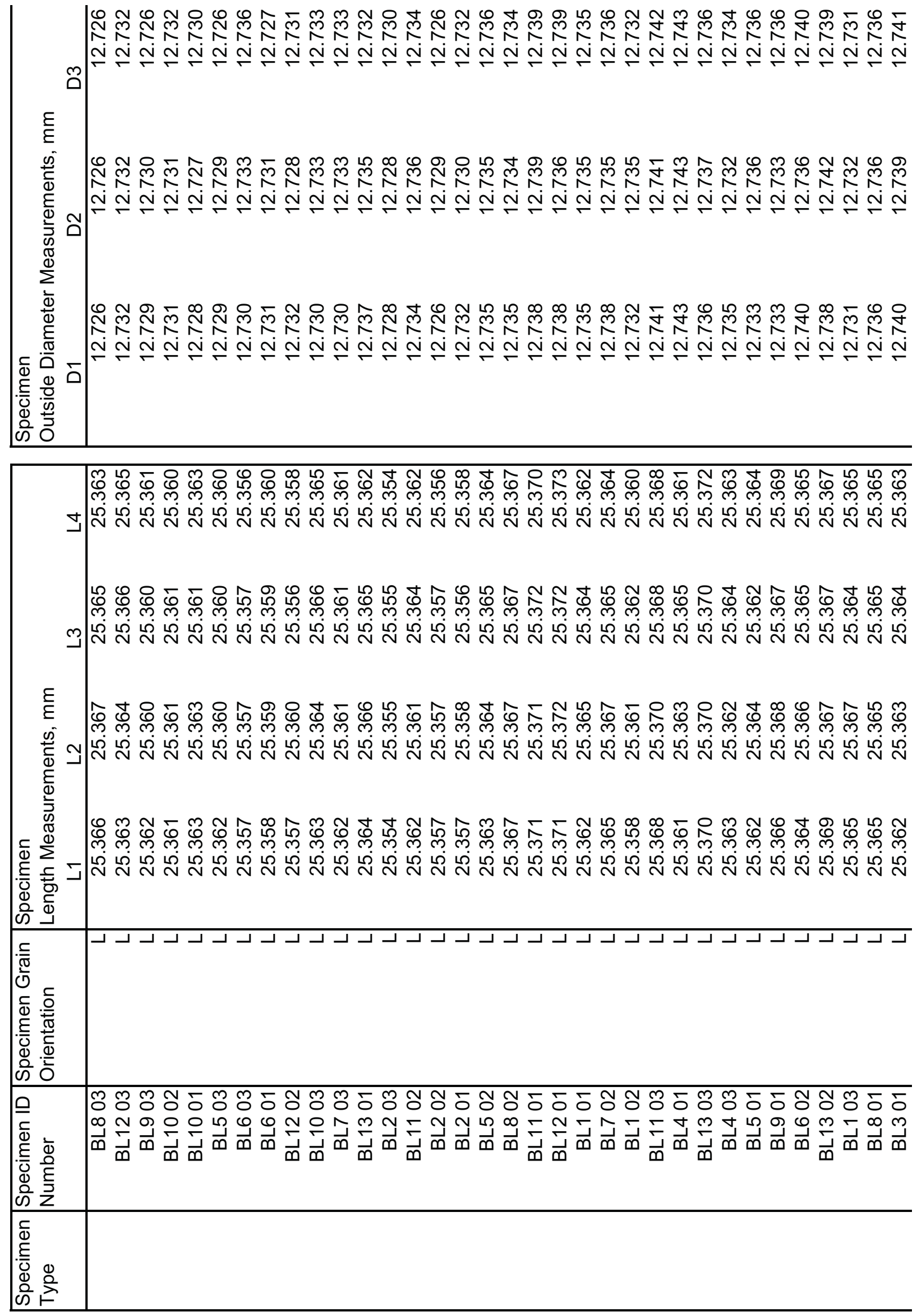



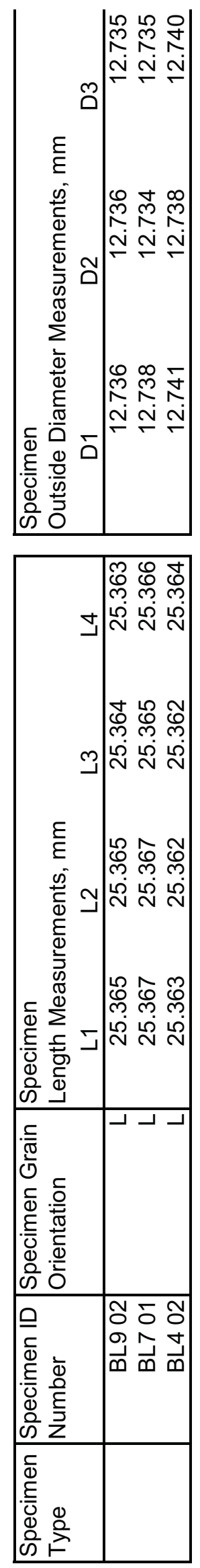


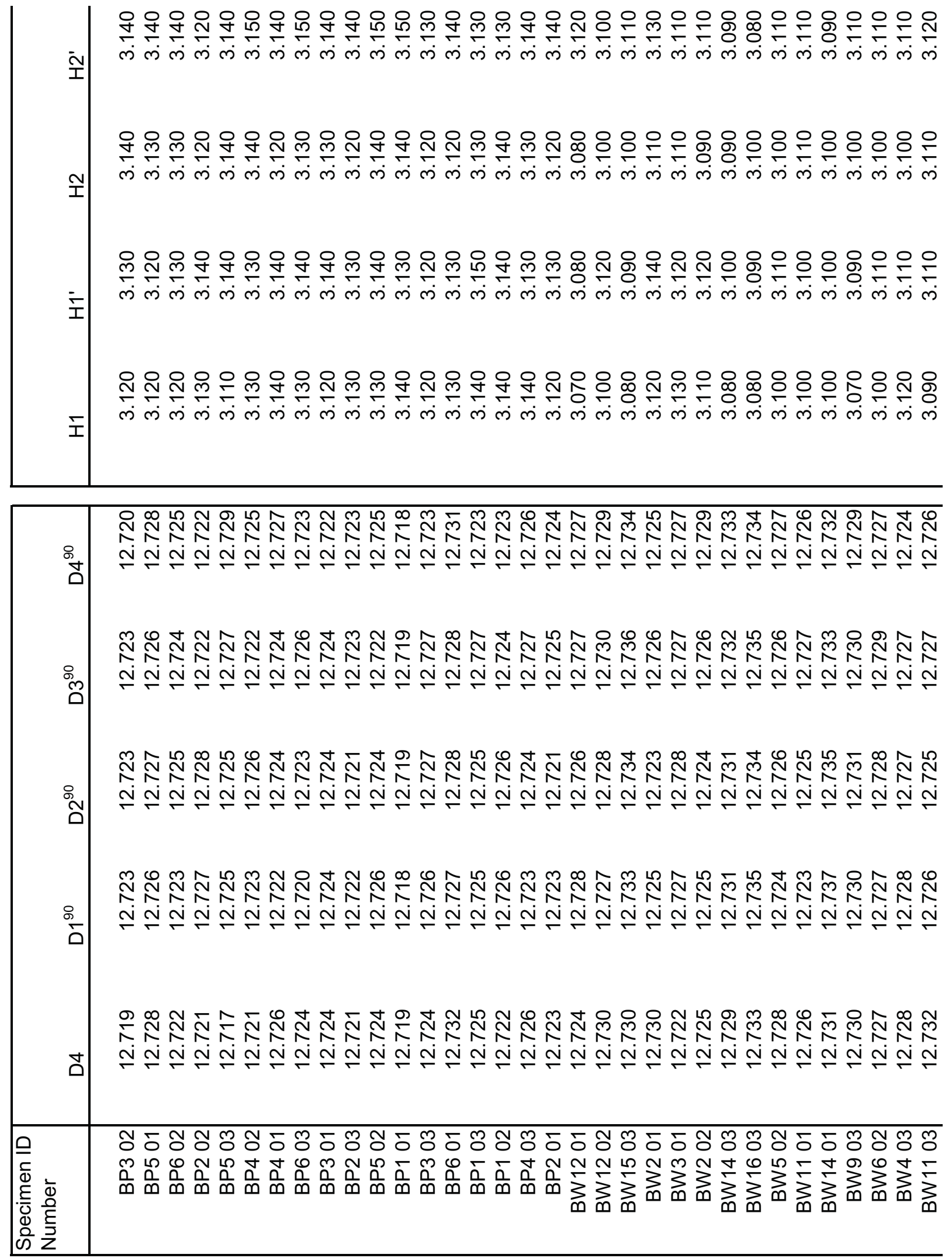




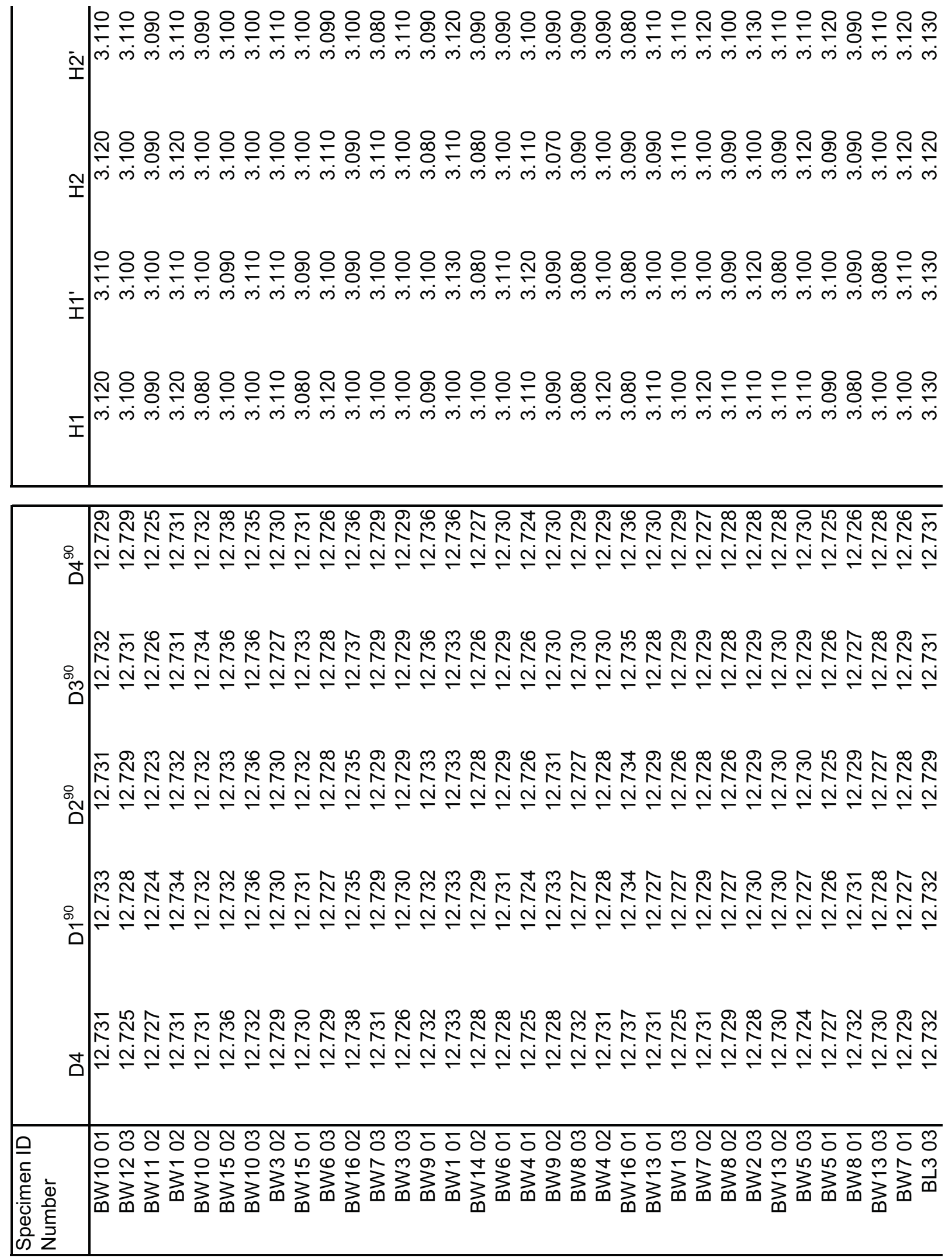




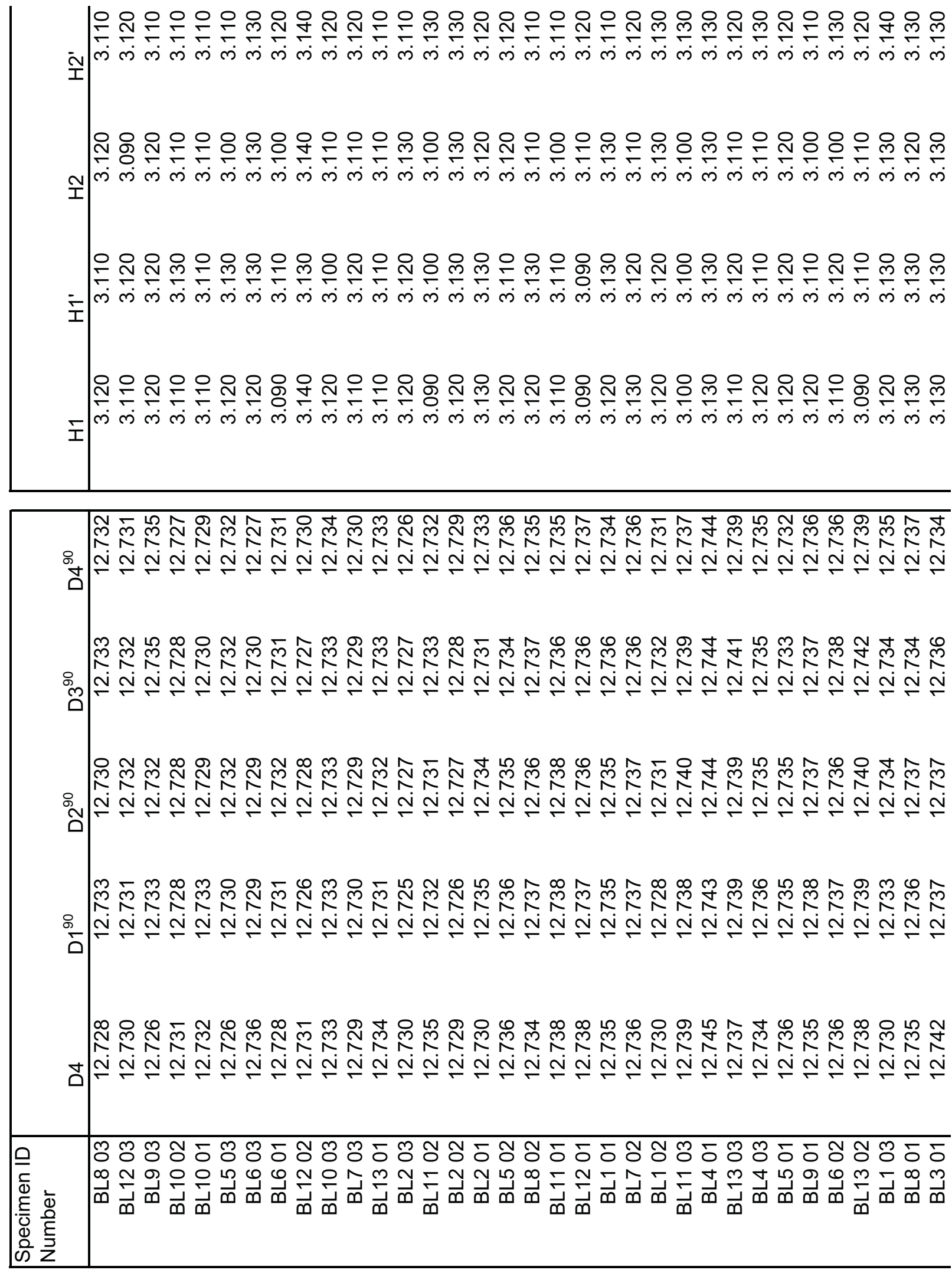



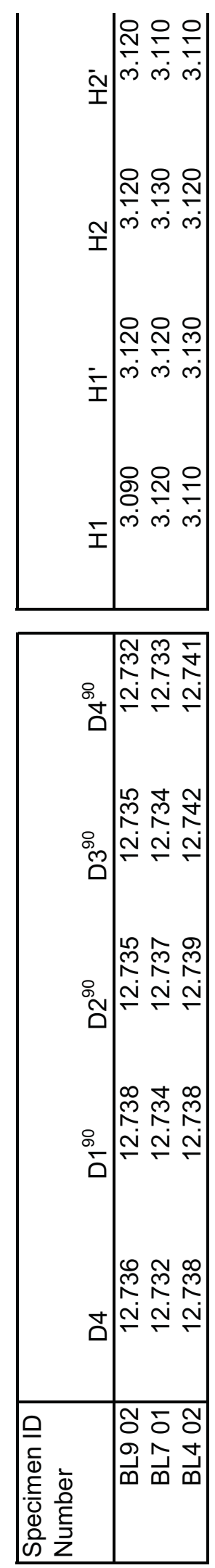


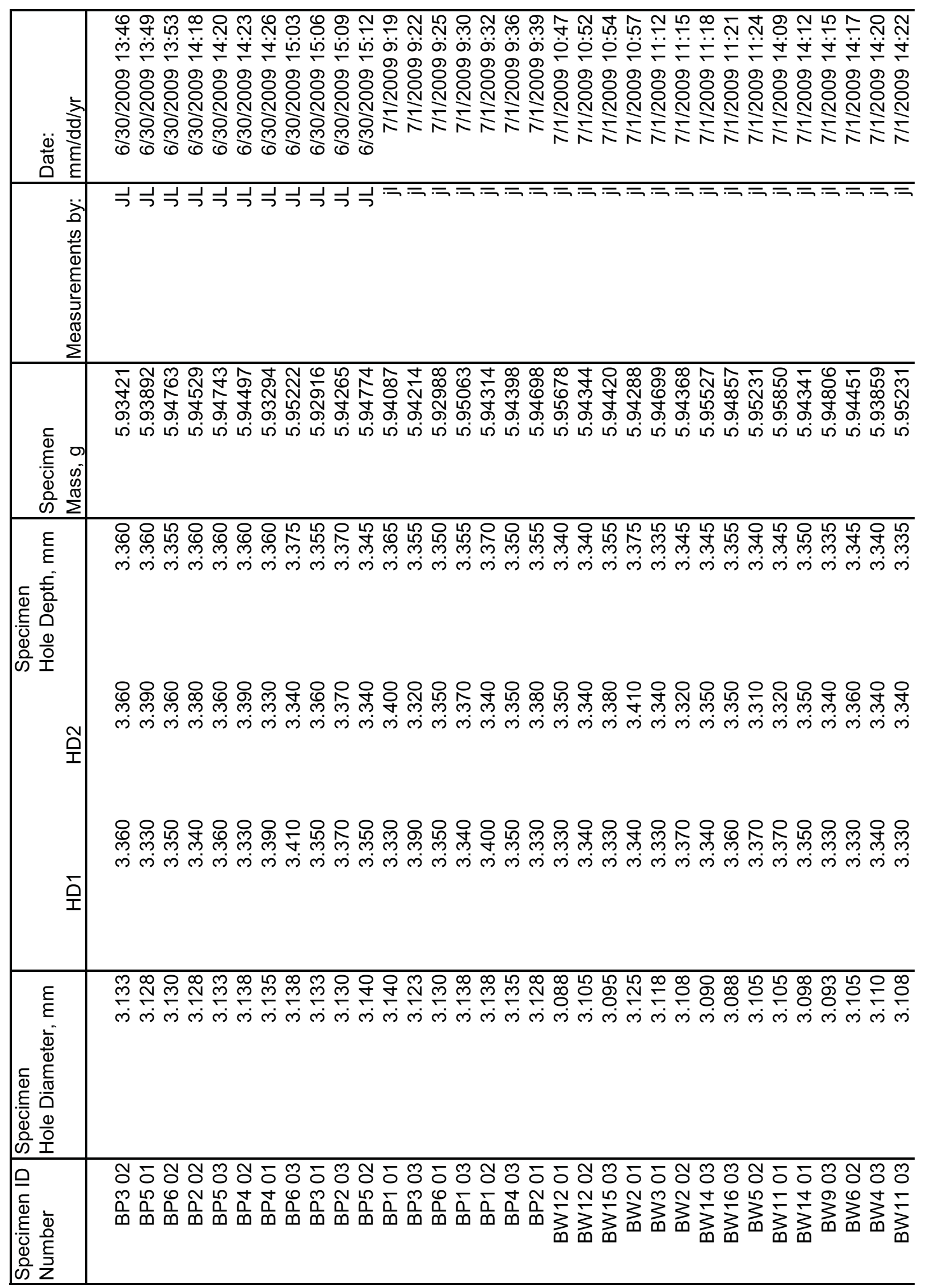




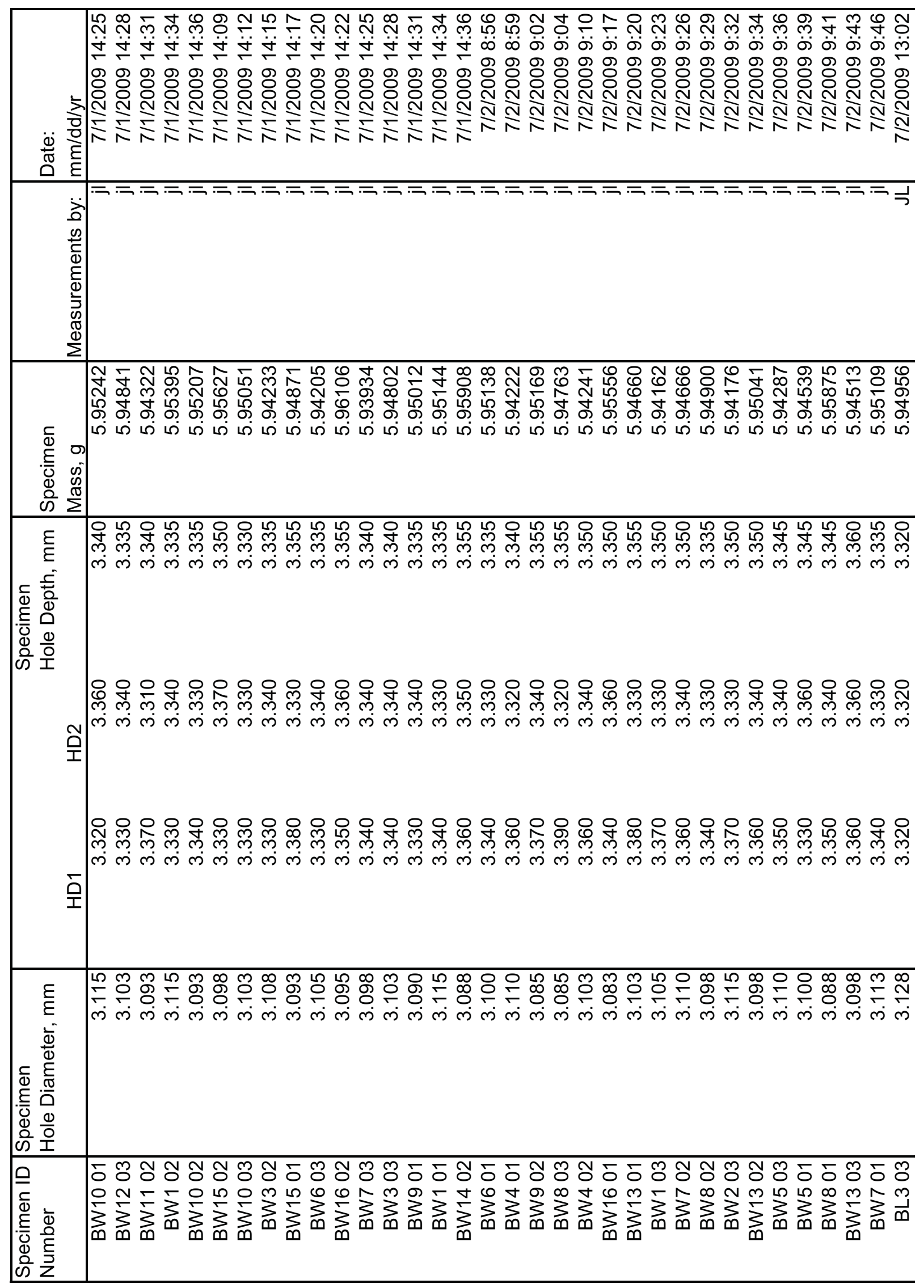




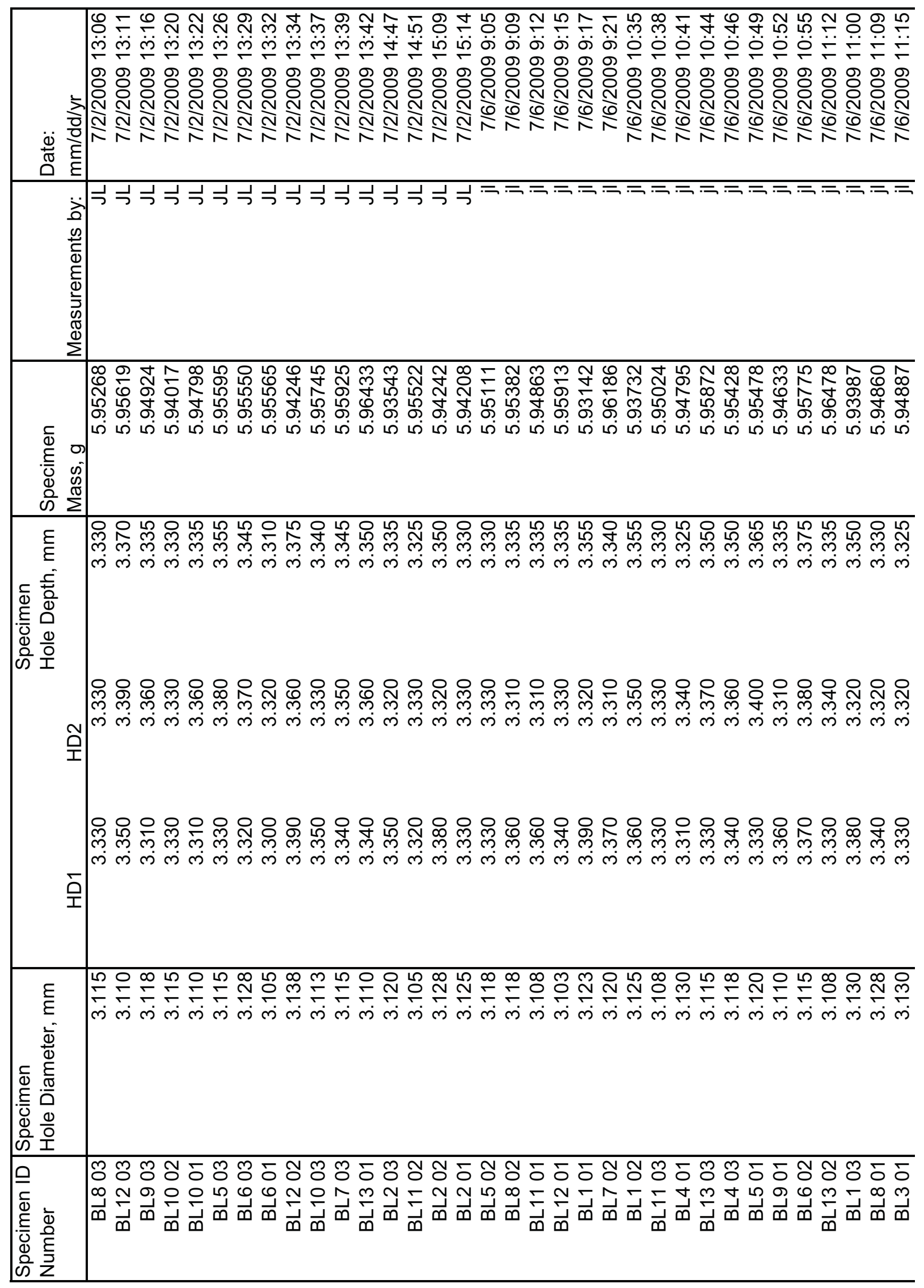




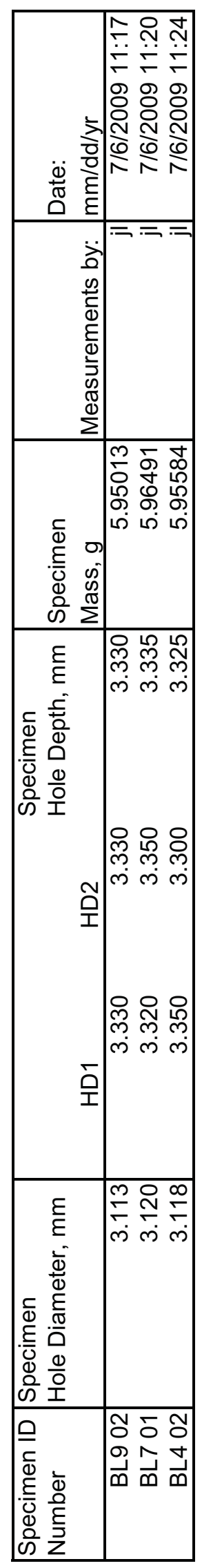

శ్రి 


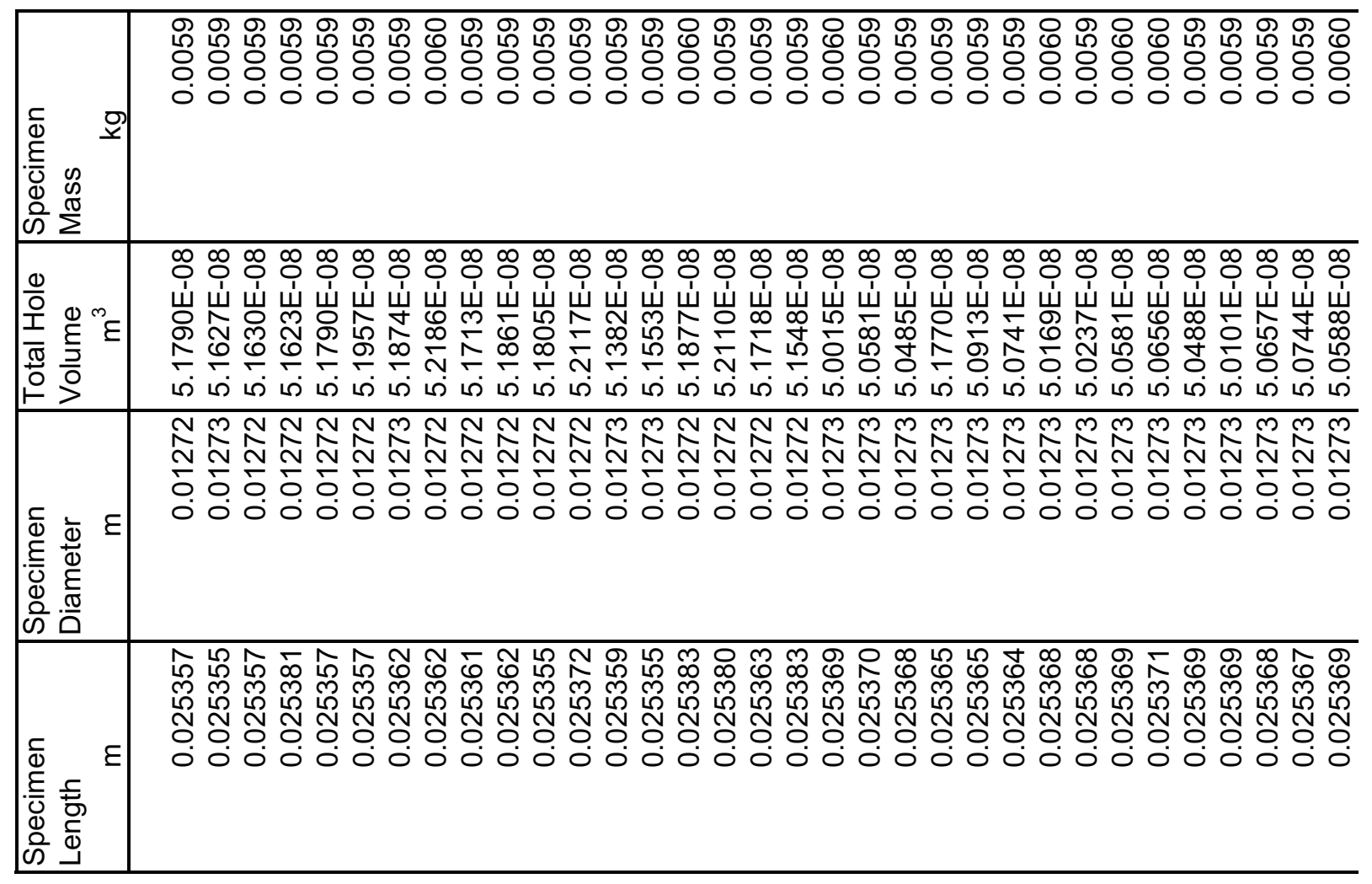

\begin{tabular}{|c|c|}
\hline 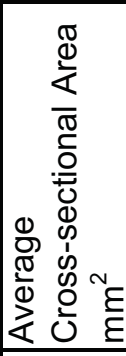 & 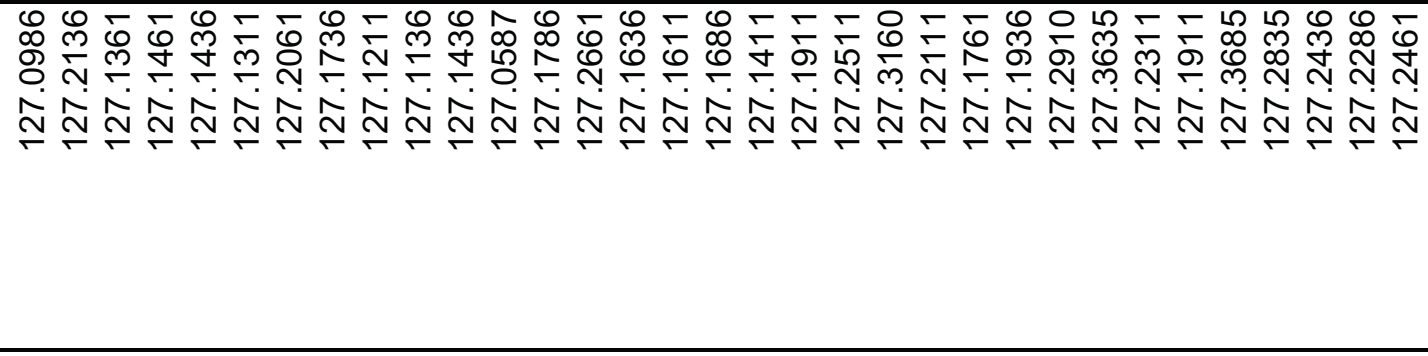 \\
\hline 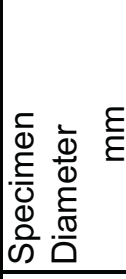 & 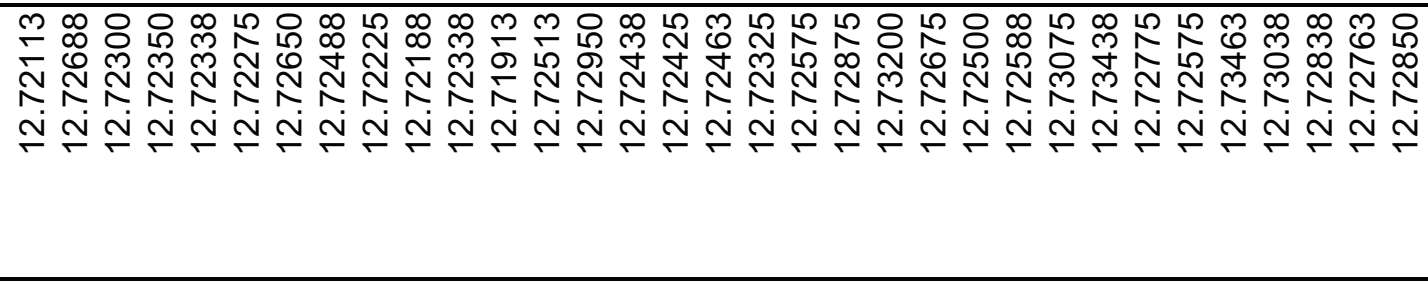 \\
\hline 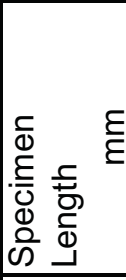 & 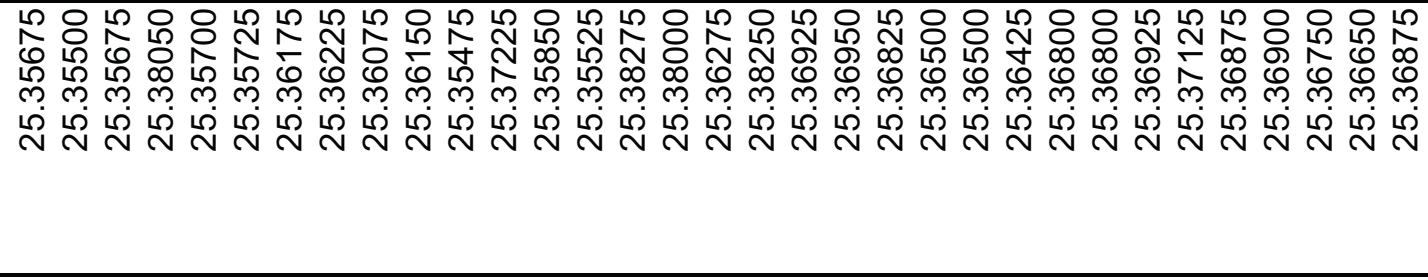 \\
\hline 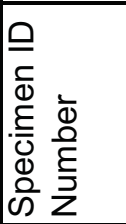 & 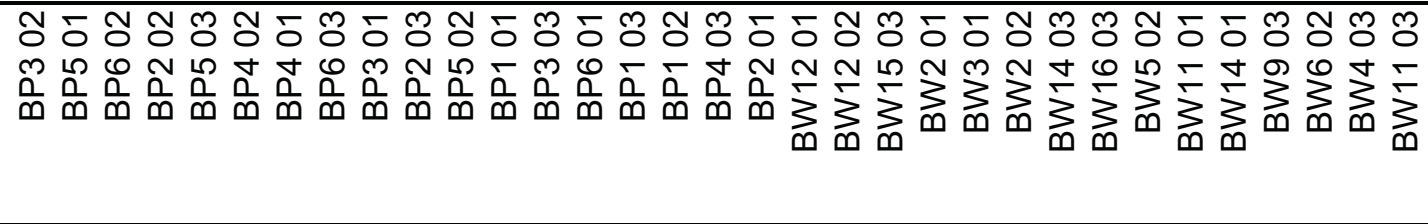 \\
\hline
\end{tabular}




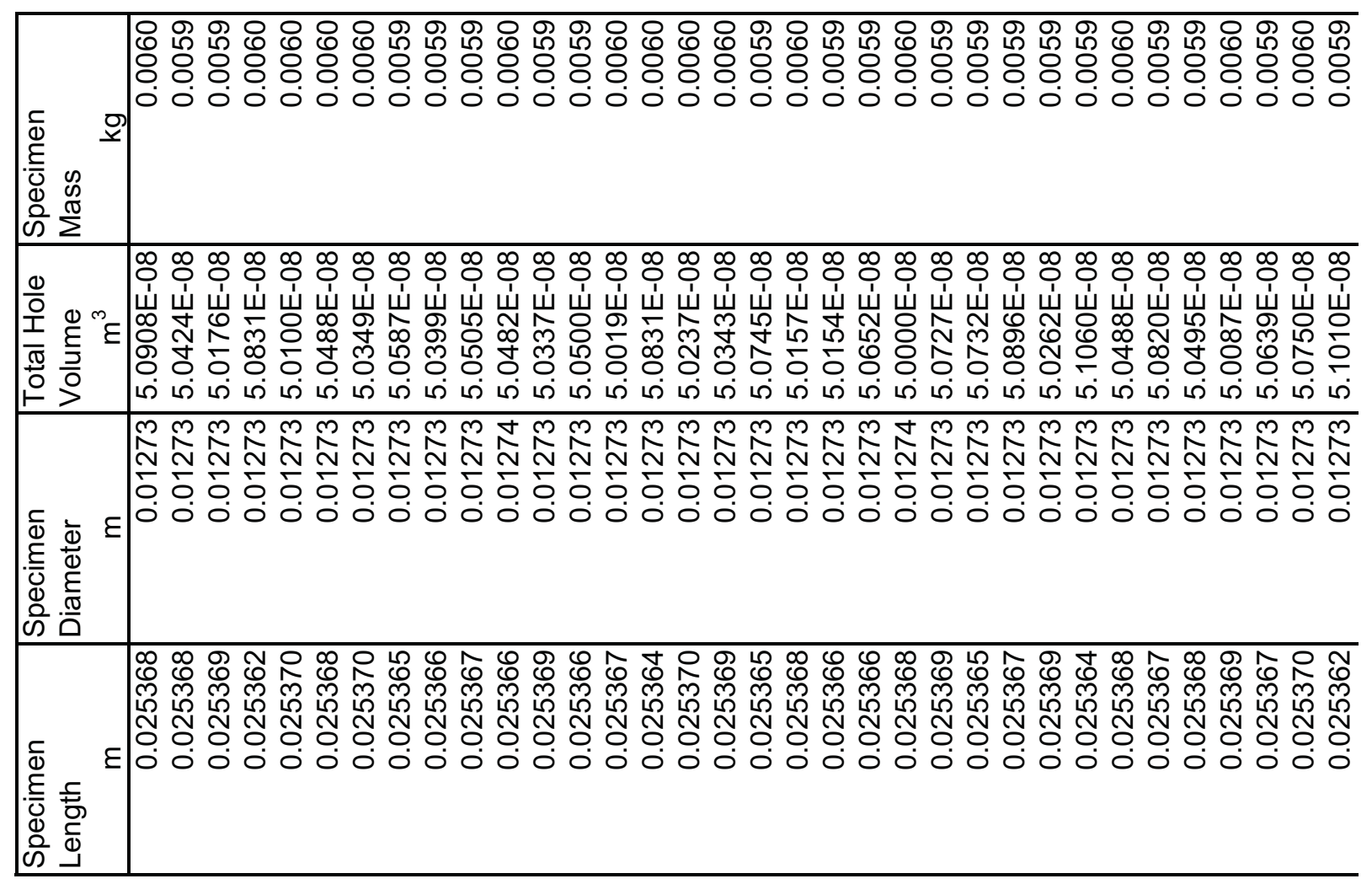

\begin{tabular}{|c|c|}
\hline 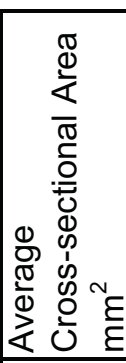 & 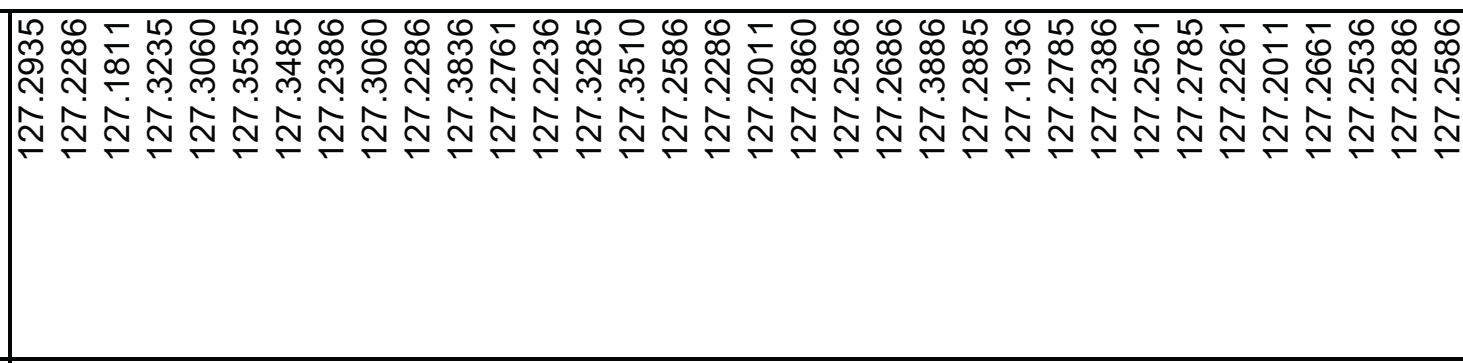 \\
\hline 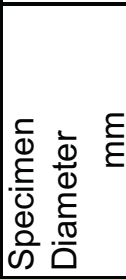 & 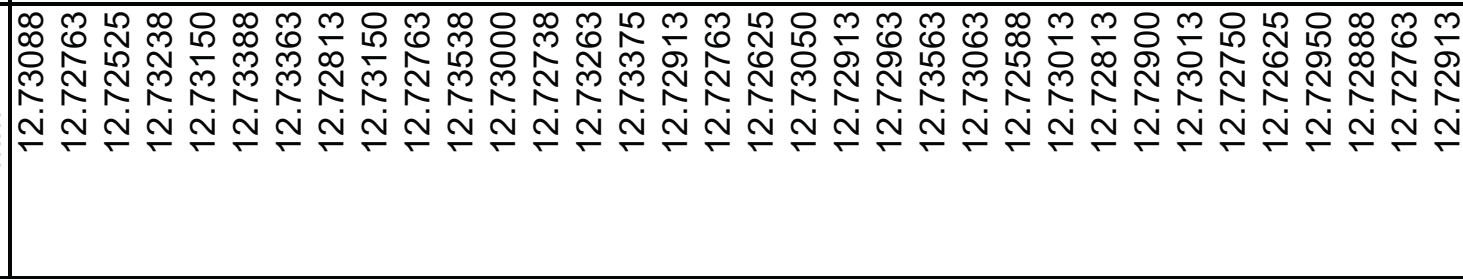 \\
\hline 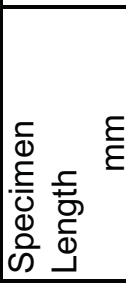 & 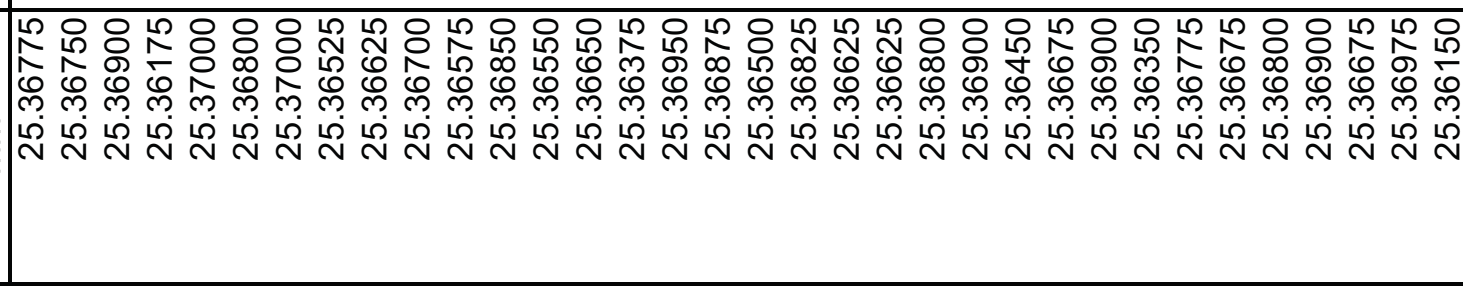 \\
\hline 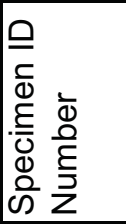 & 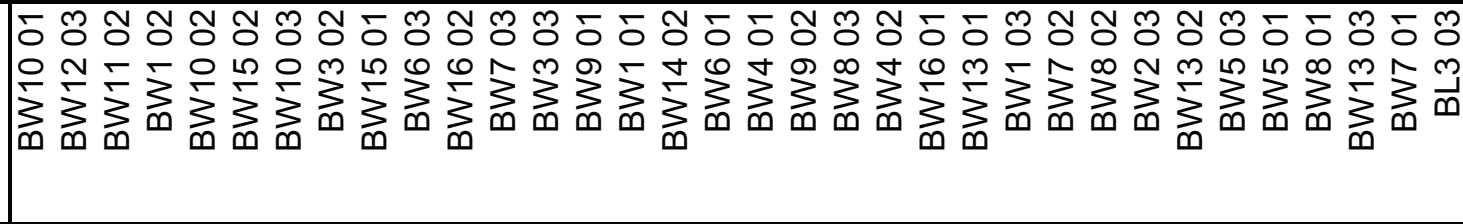 \\
\hline
\end{tabular}




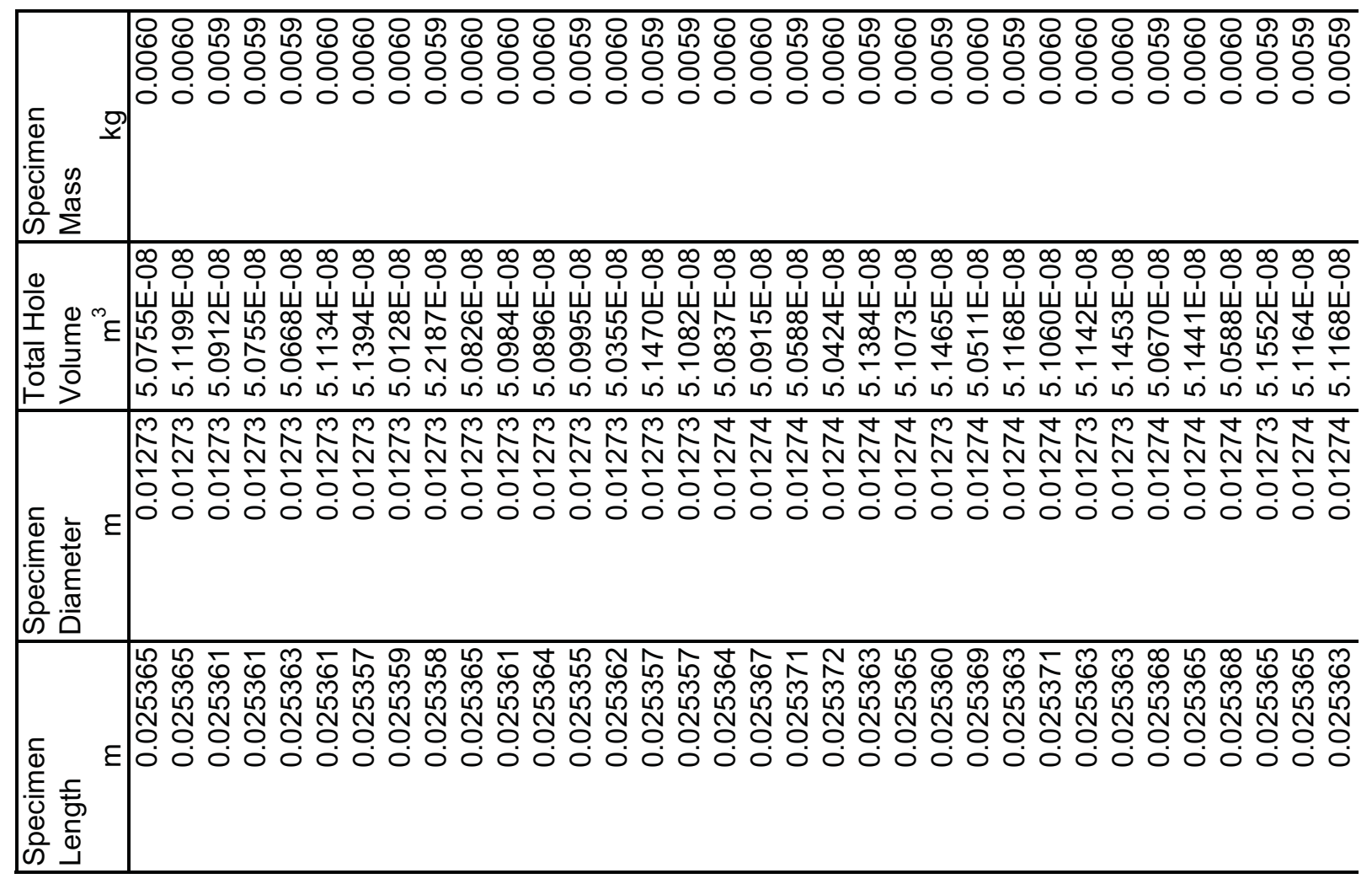

\begin{tabular}{|c|c|}
\hline 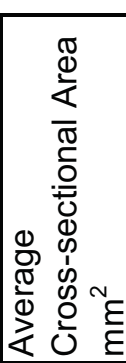 & 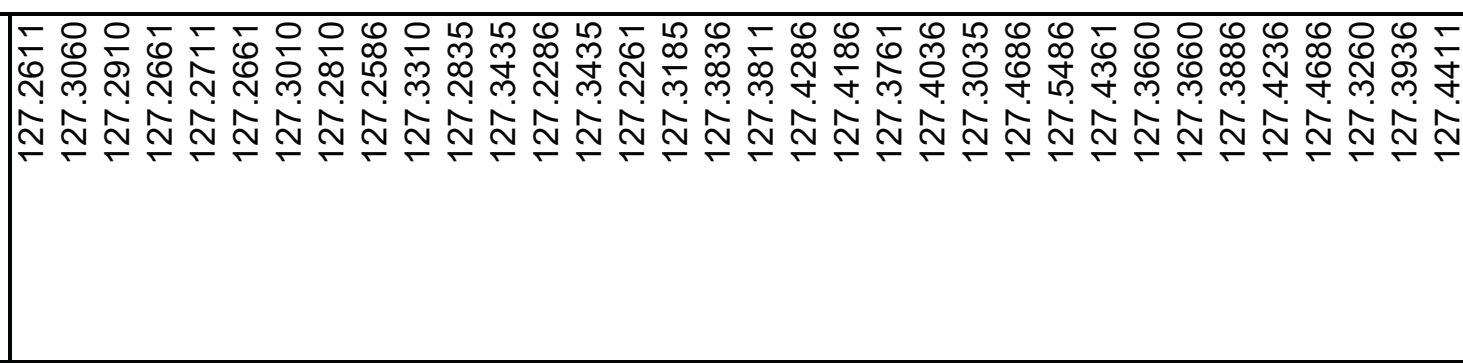 \\
\hline 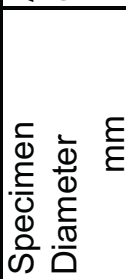 & 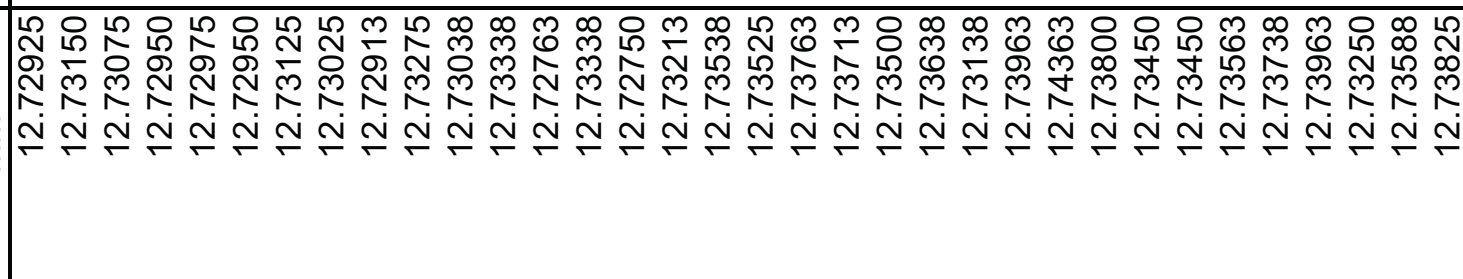 \\
\hline 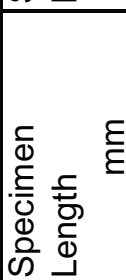 & 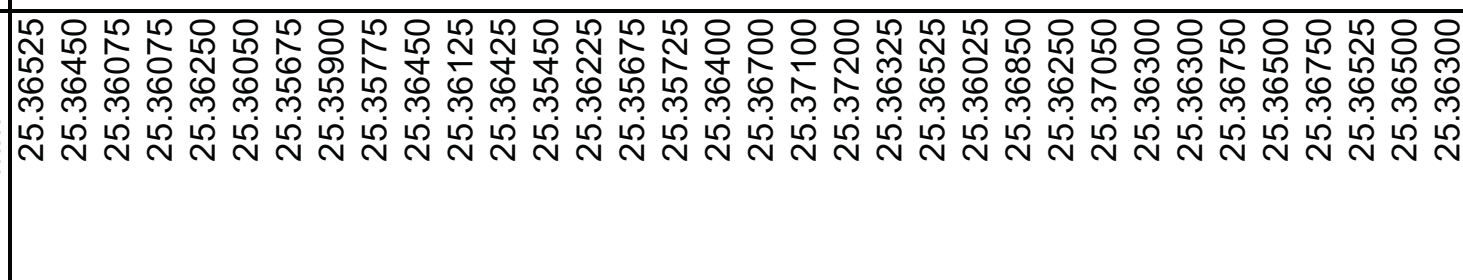 \\
\hline 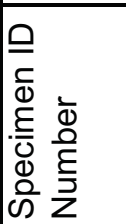 & 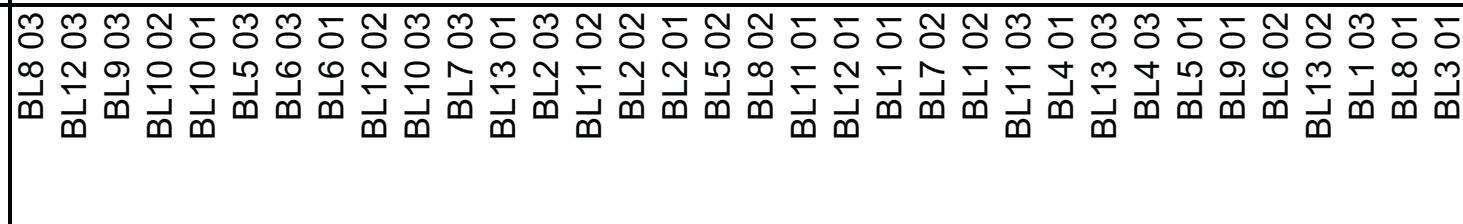 \\
\hline
\end{tabular}



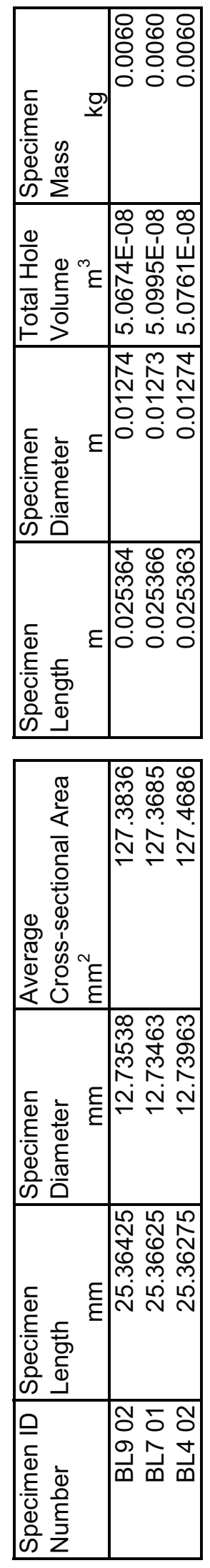


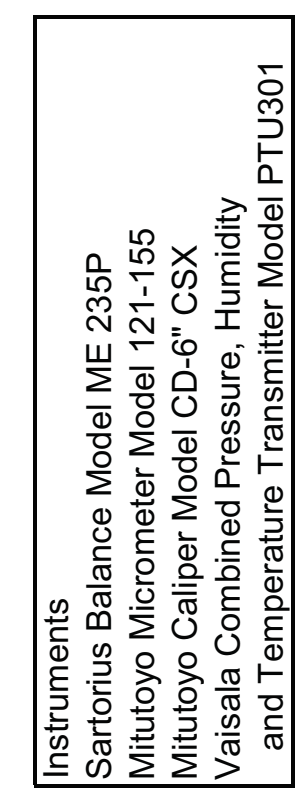

\begin{tabular}{|c|c|}
\hline 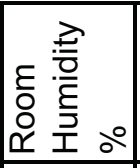 & 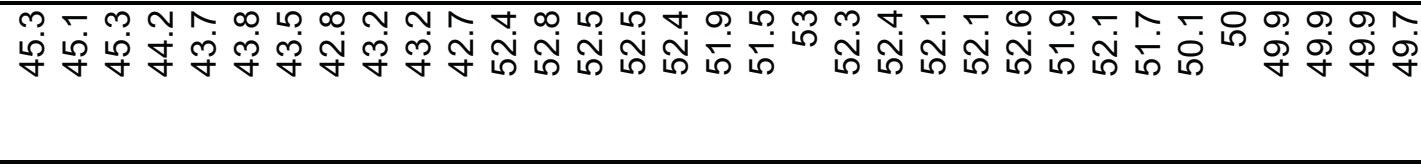 \\
\hline 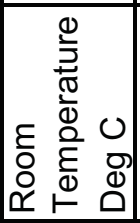 & 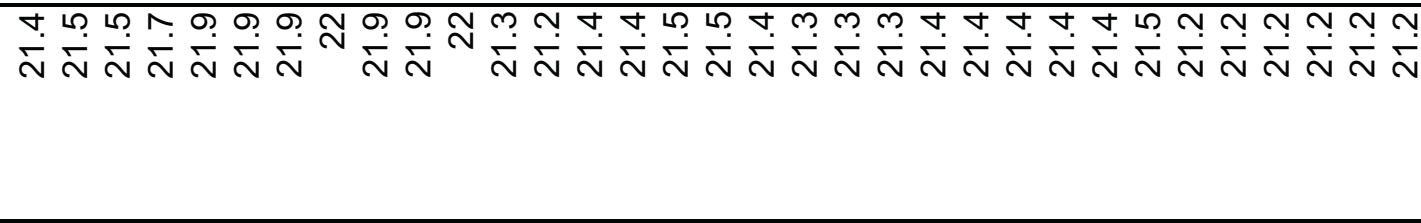 \\
\hline 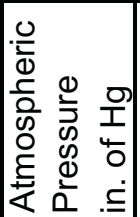 & 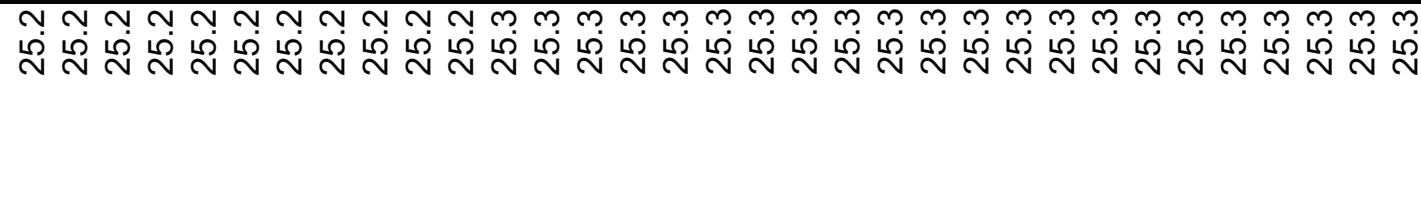 \\
\hline
\end{tabular}

\begin{tabular}{|c|c|}
\hline 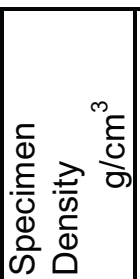 & 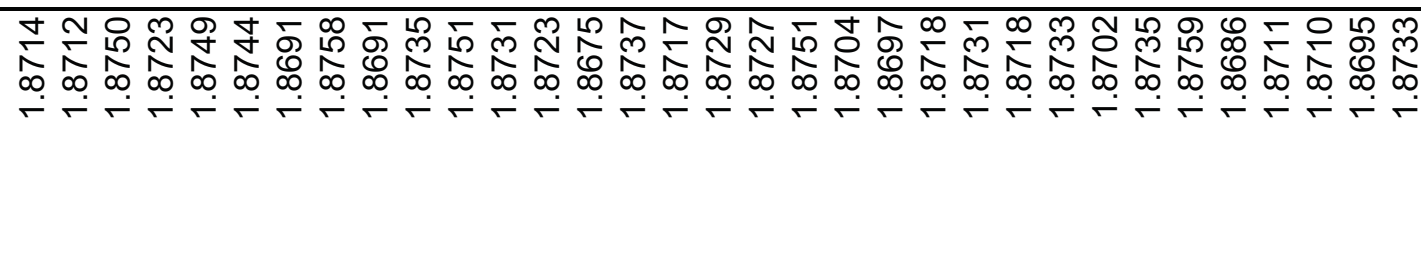 \\
\hline 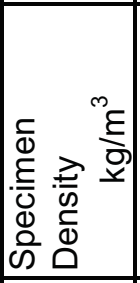 & 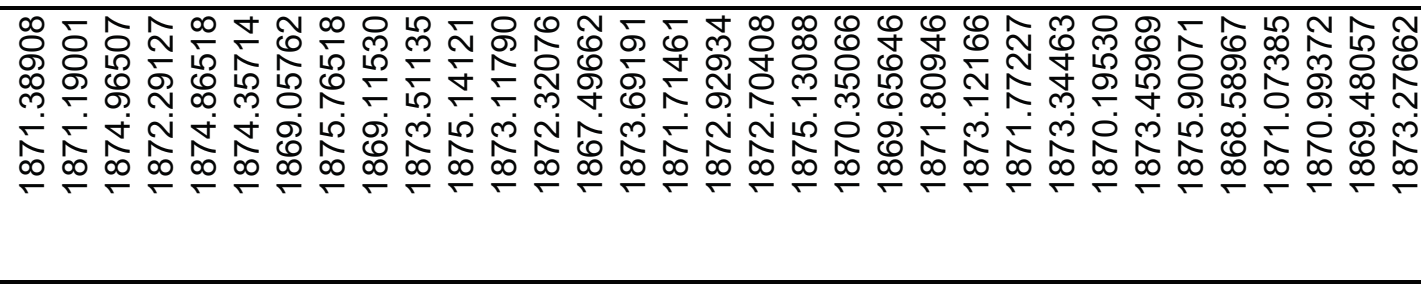 \\
\hline 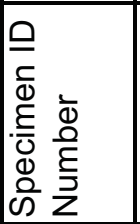 & 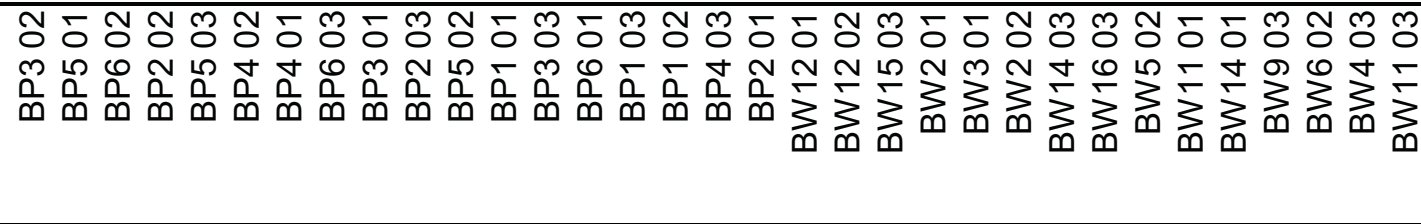 \\
\hline
\end{tabular}




\begin{tabular}{|c|c|}
\hline 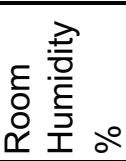 & 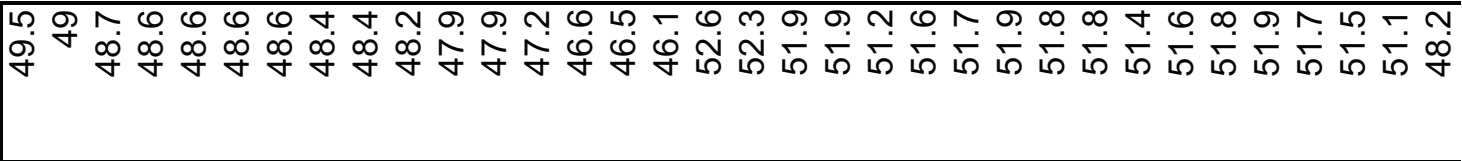 \\
\hline 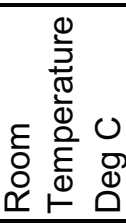 & 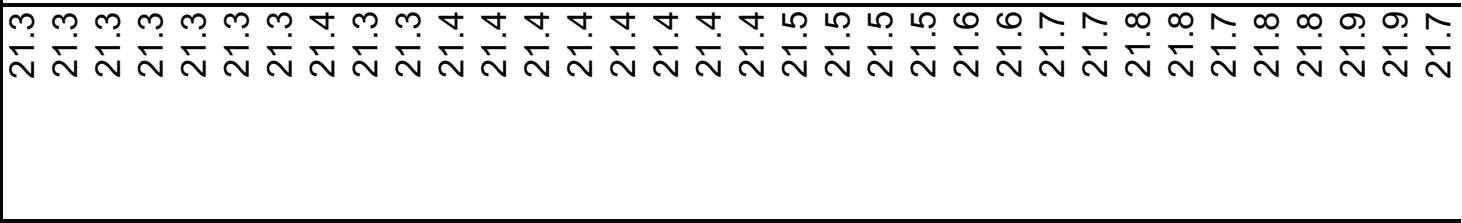 \\
\hline 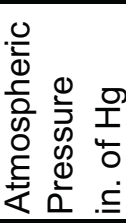 & 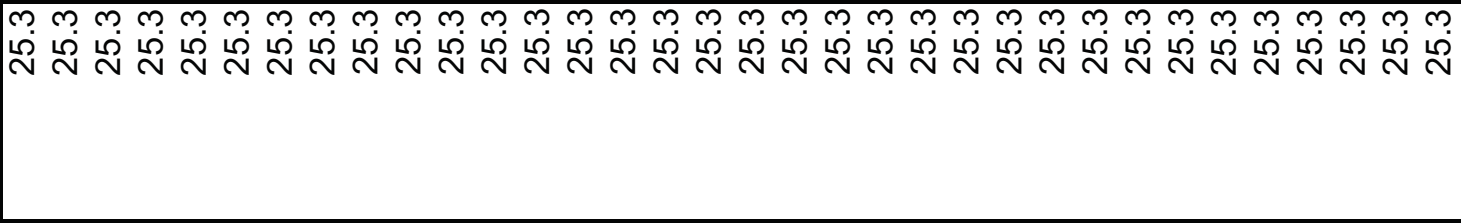 \\
\hline
\end{tabular}

\begin{tabular}{|c|c|}
\hline 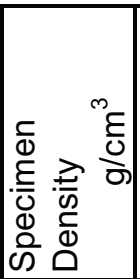 & 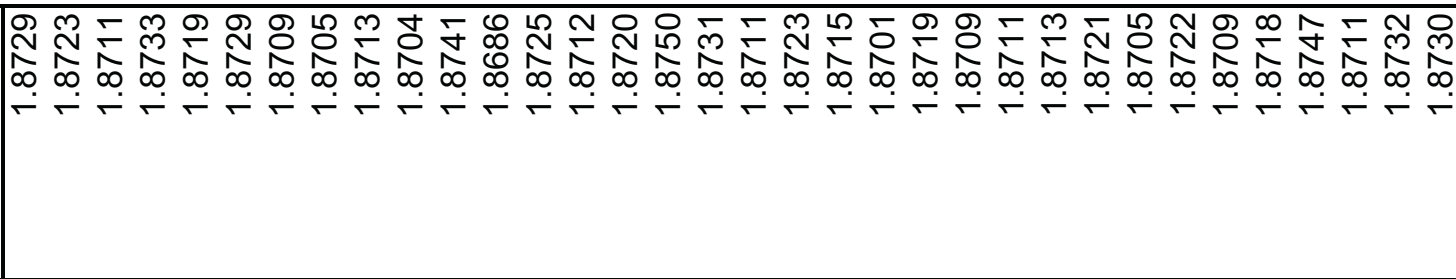 \\
\hline 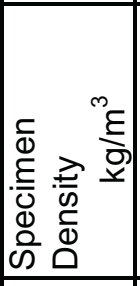 & 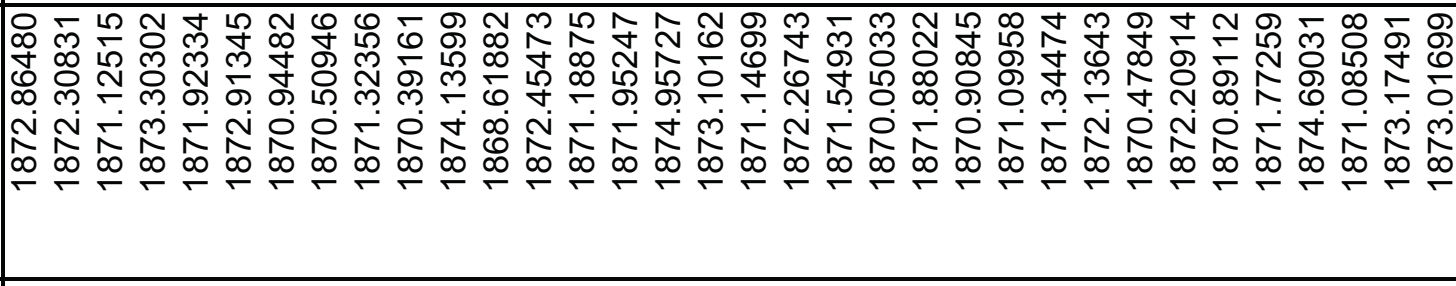 \\
\hline 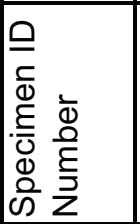 & 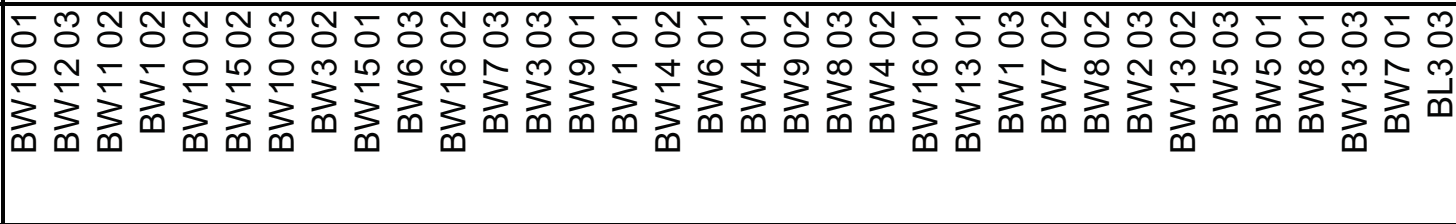 \\
\hline
\end{tabular}




\begin{tabular}{|c|c|}
\hline 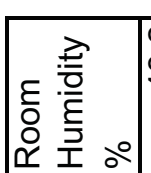 & 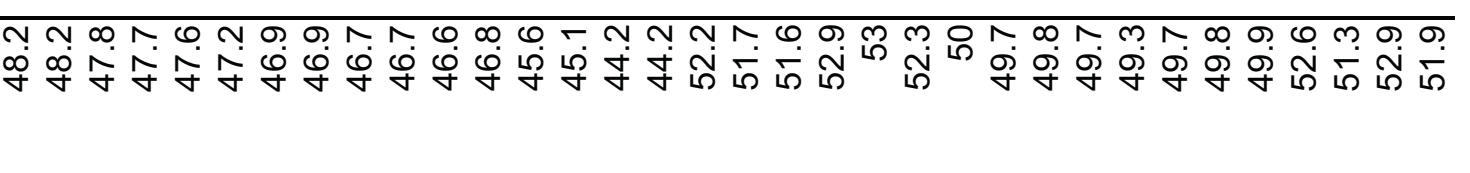 \\
\hline 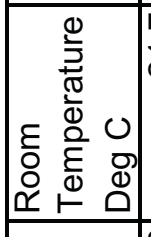 & 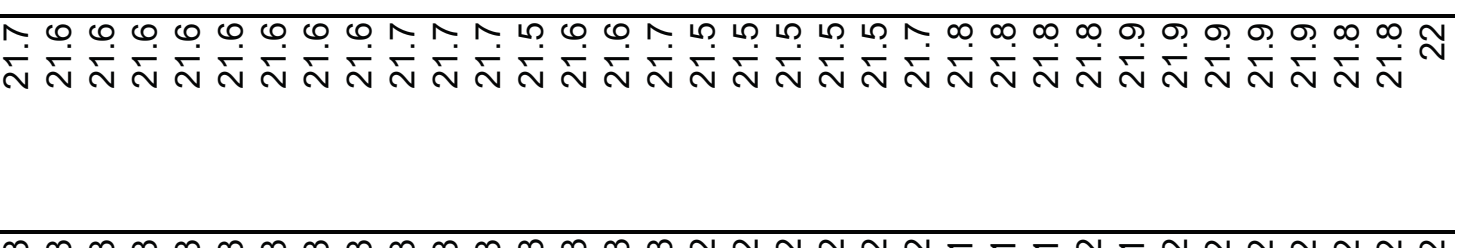 \\
\hline 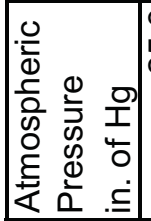 & 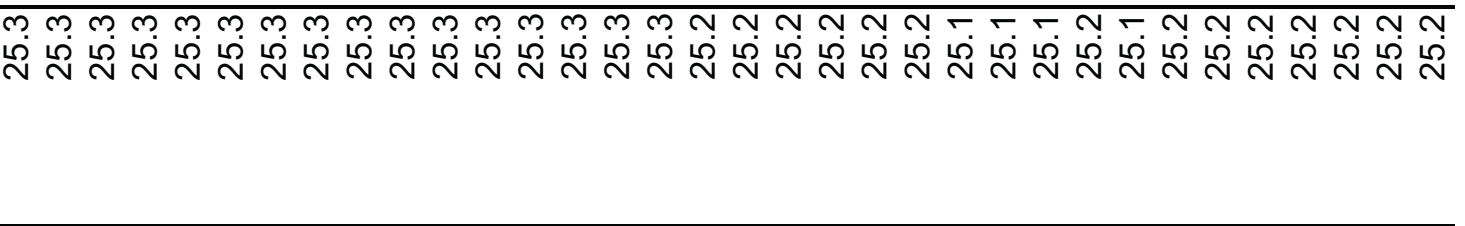 \\
\hline
\end{tabular}

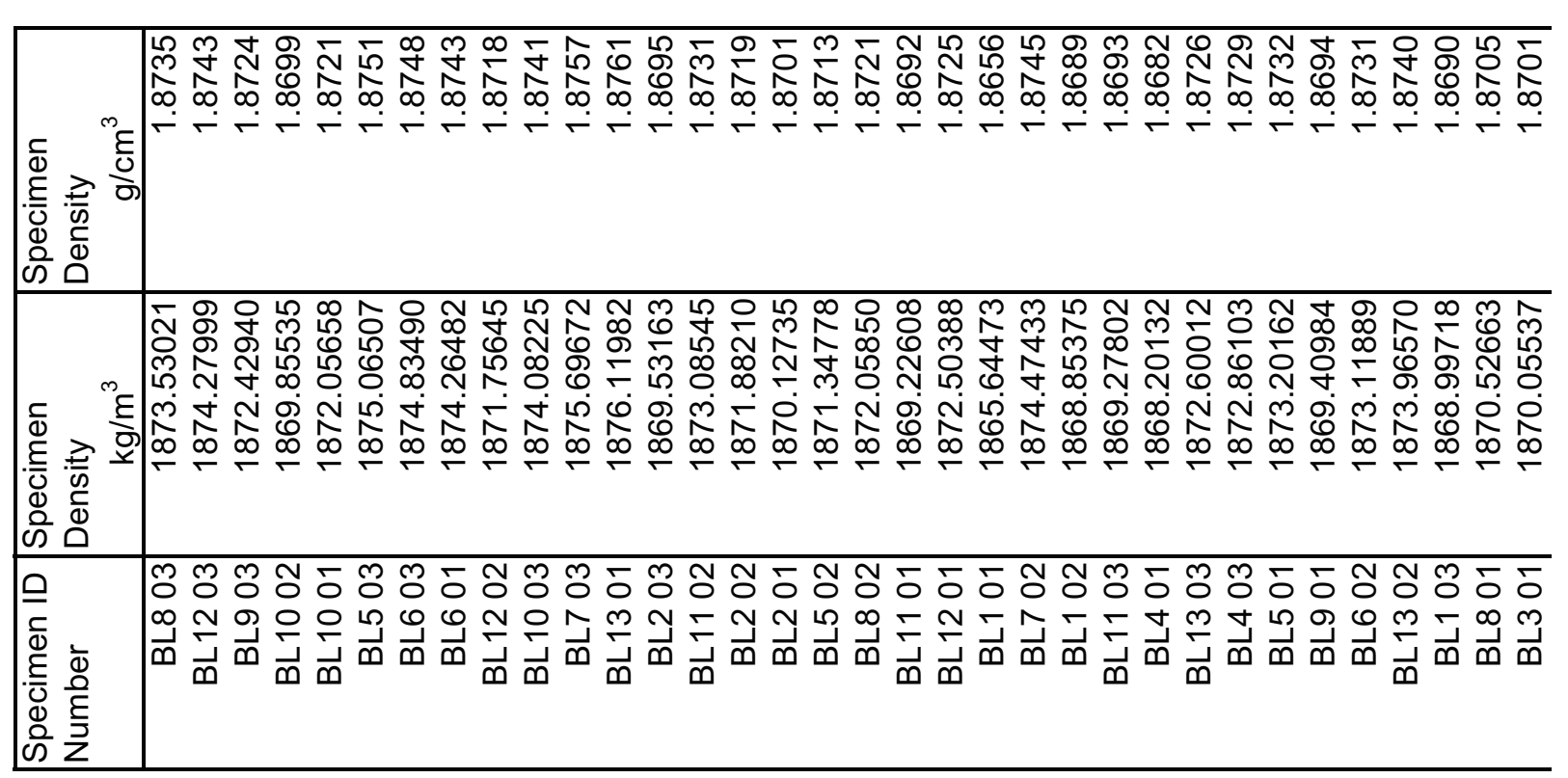



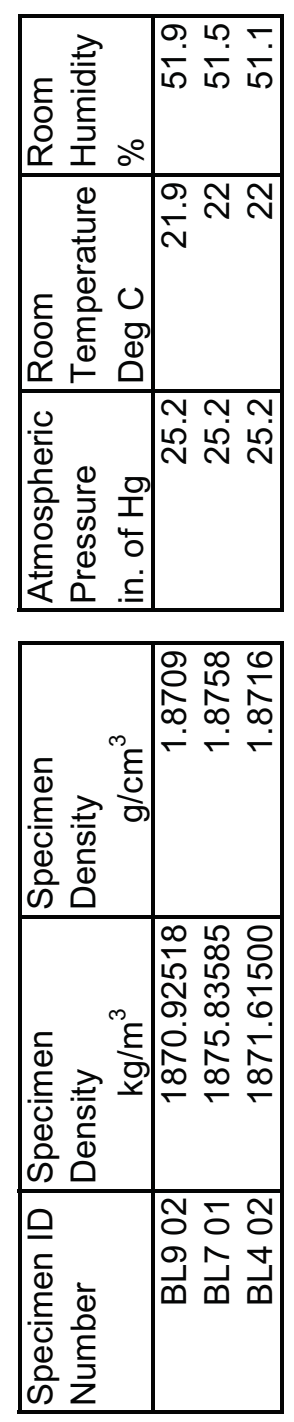


\begin{tabular}{|c|c|}
\hline 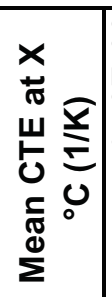 & 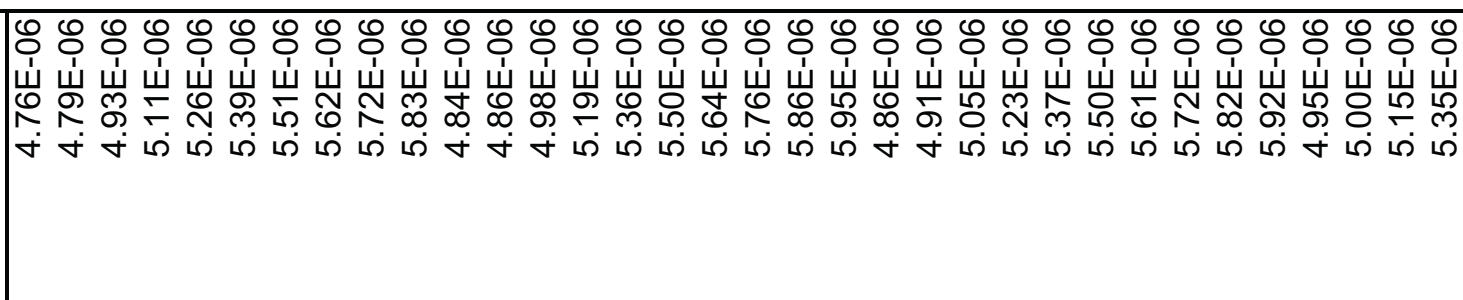 \\
\hline 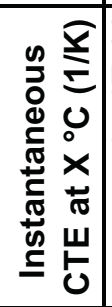 & 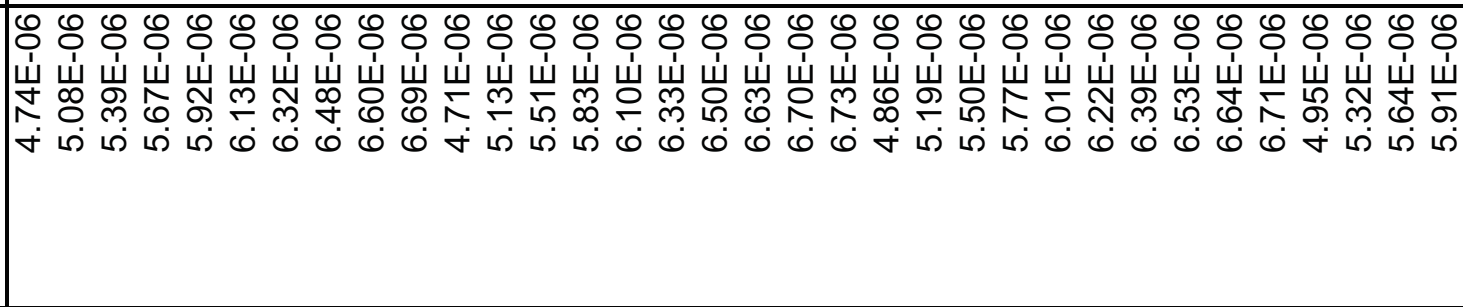 \\
\hline 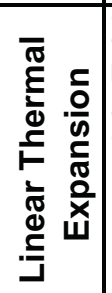 & 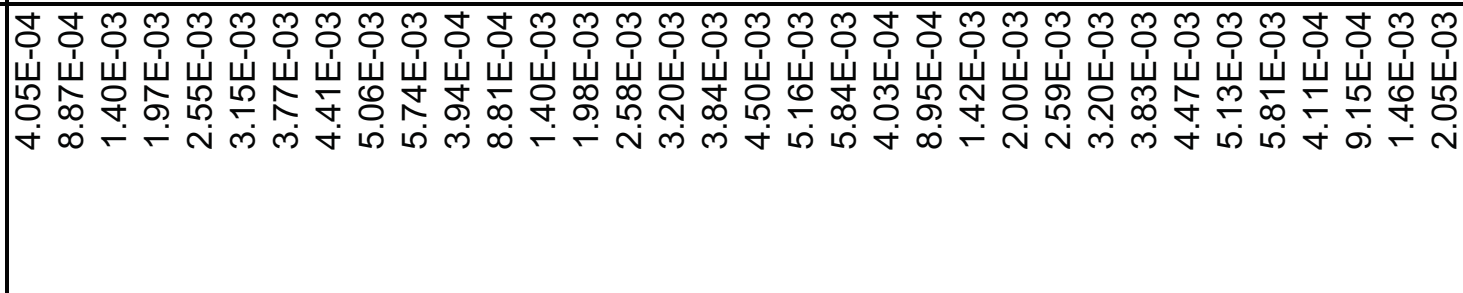 \\
\hline 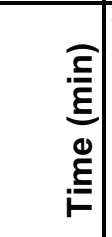 & 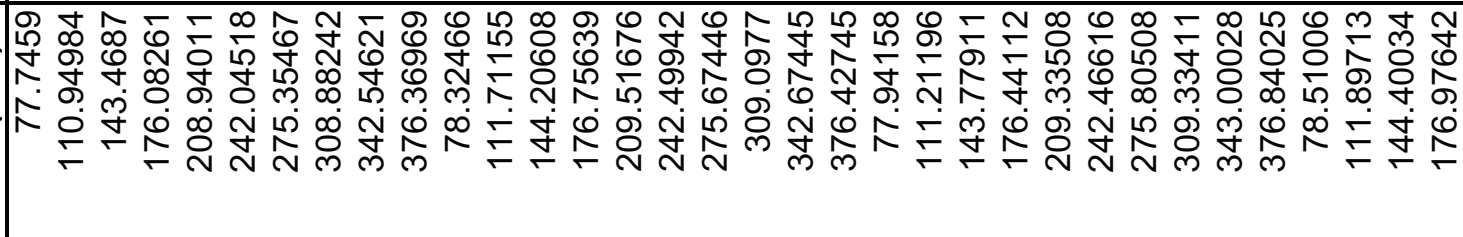 \\
\hline 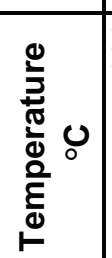 & 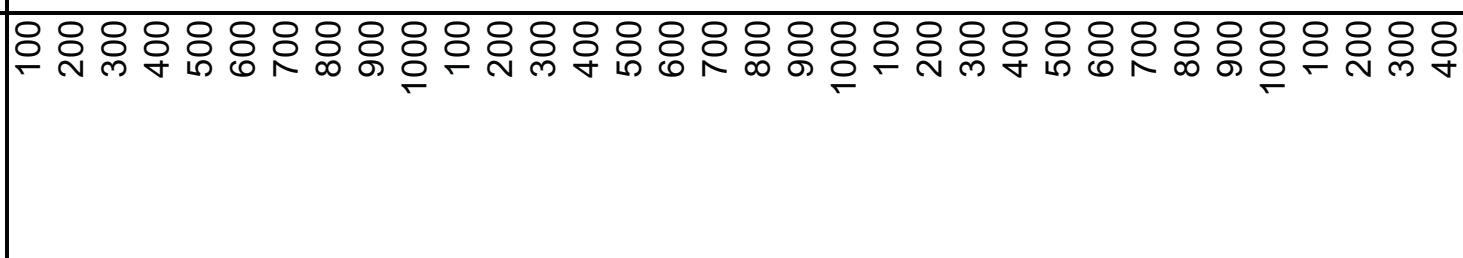 \\
\hline 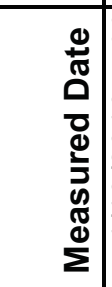 & 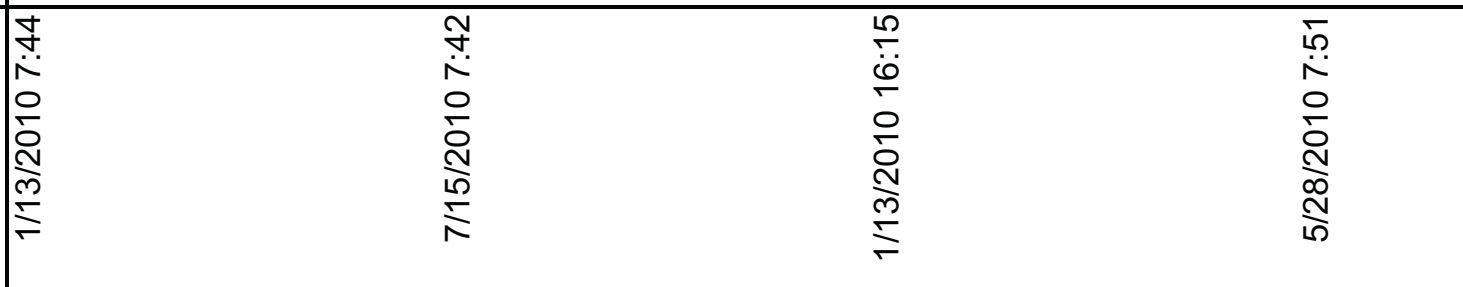 \\
\hline 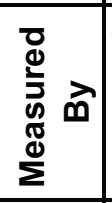 & $\sum_{3}^{\infty}$ \\
\hline 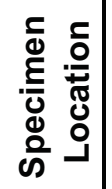 & \\
\hline 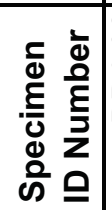 & 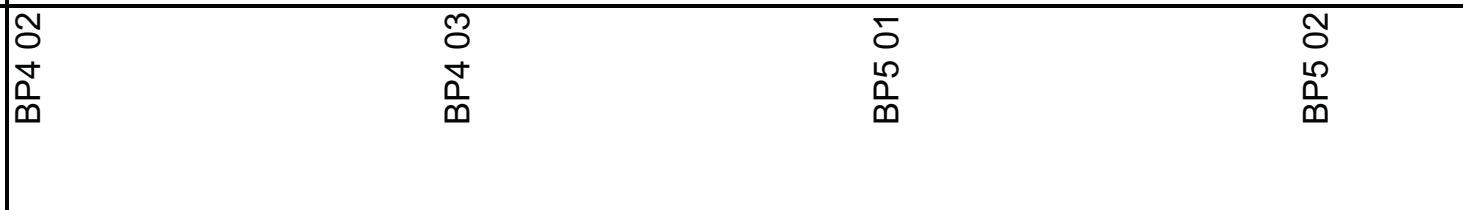 \\
\hline
\end{tabular}




\begin{tabular}{|c|c|}
\hline 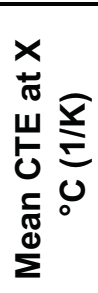 & 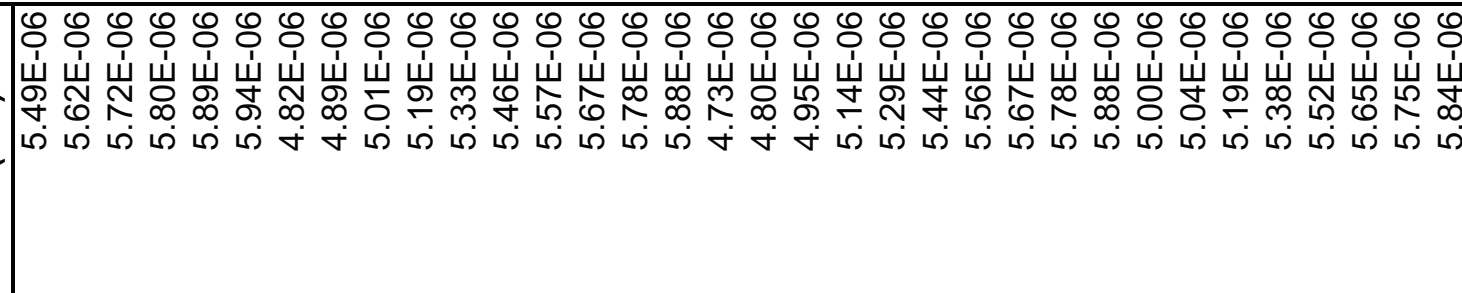 \\
\hline 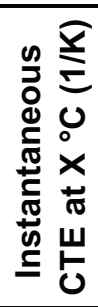 & 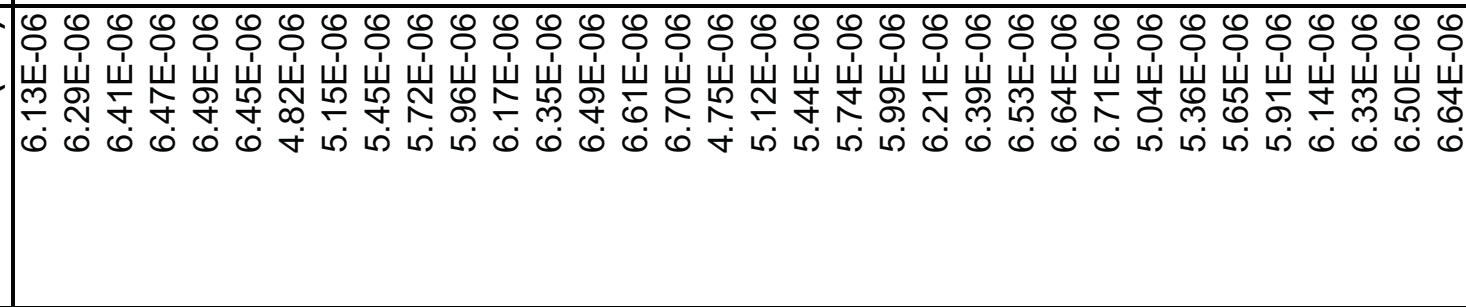 \\
\hline 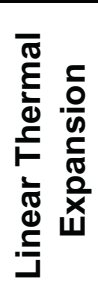 & 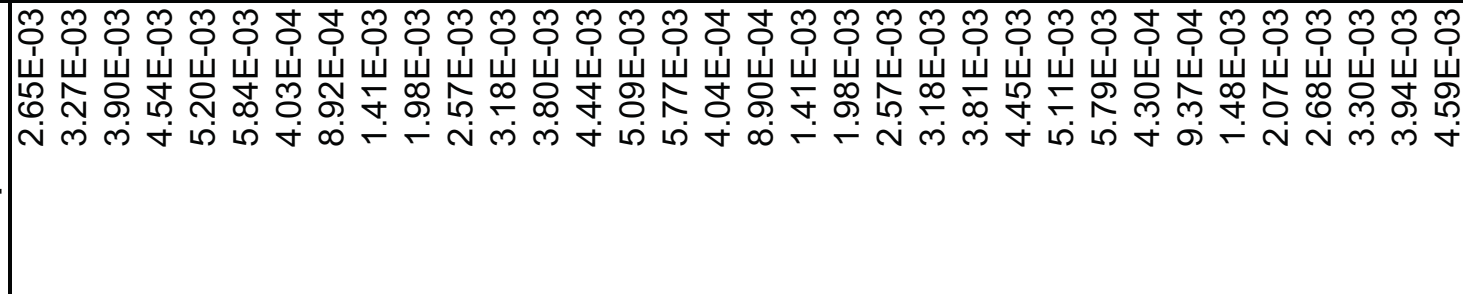 \\
\hline 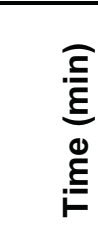 & 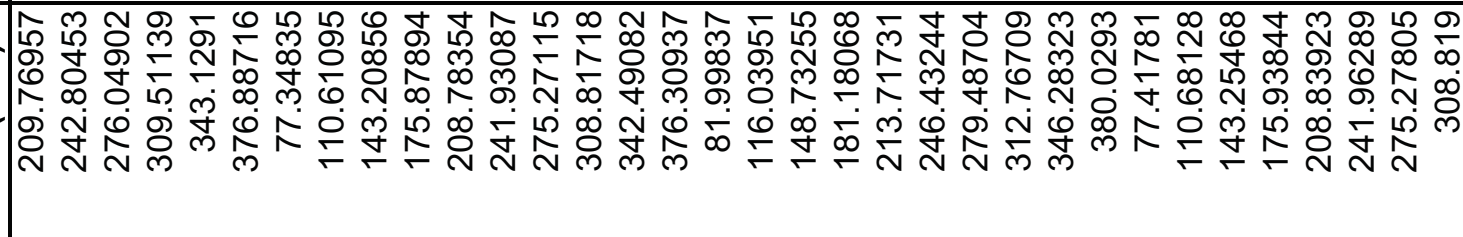 \\
\hline 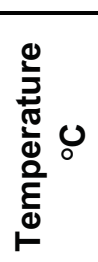 & 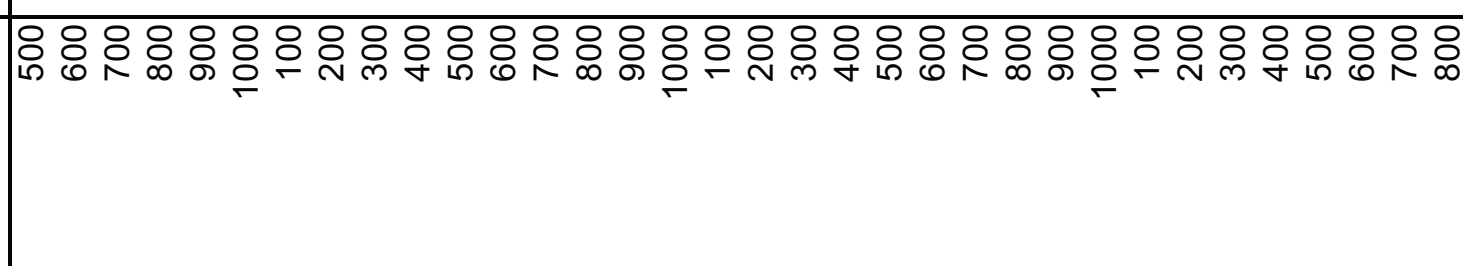 \\
\hline 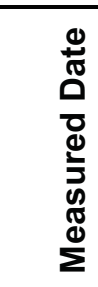 & 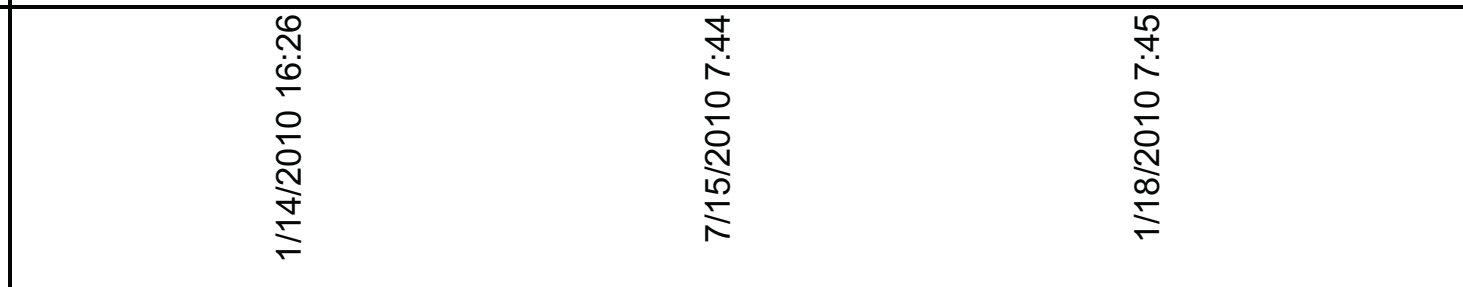 \\
\hline 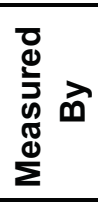 & $\frac{0}{3}$ \\
\hline 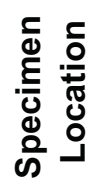 & \\
\hline 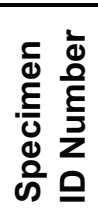 & $\begin{array}{l}5 \\
\sum_{0}\end{array}$ \\
\hline
\end{tabular}




\begin{tabular}{|c|c|}
\hline 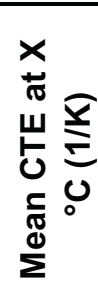 & 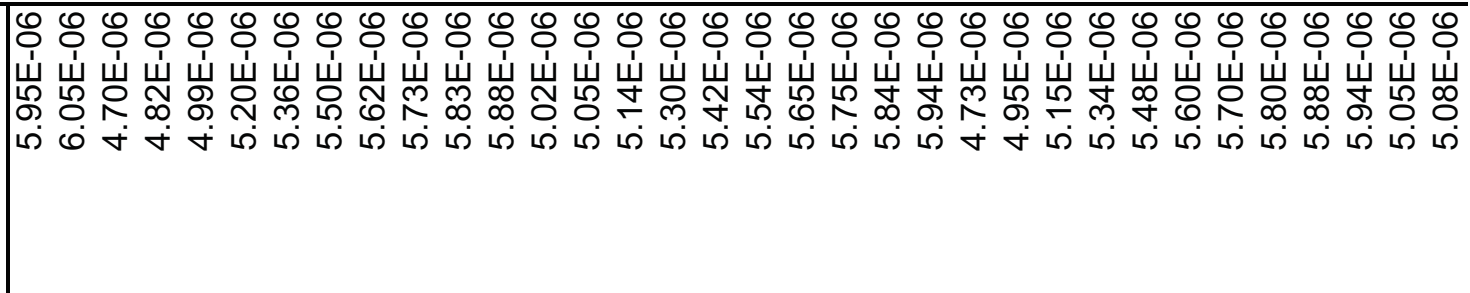 \\
\hline 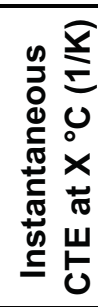 & 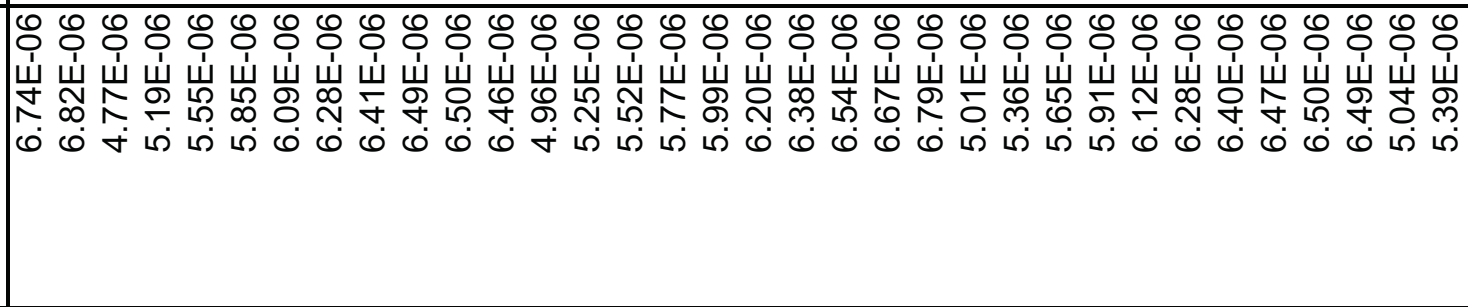 \\
\hline 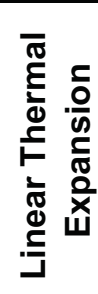 & 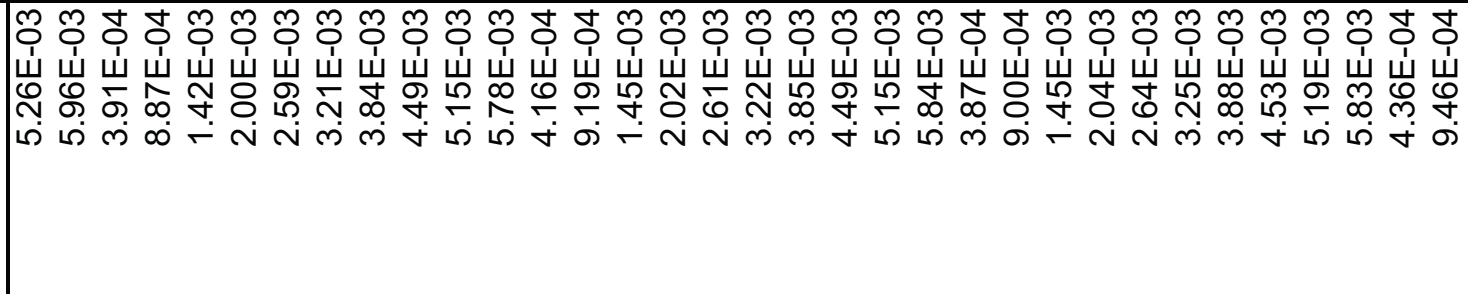 \\
\hline 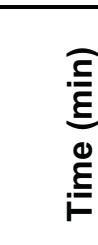 & 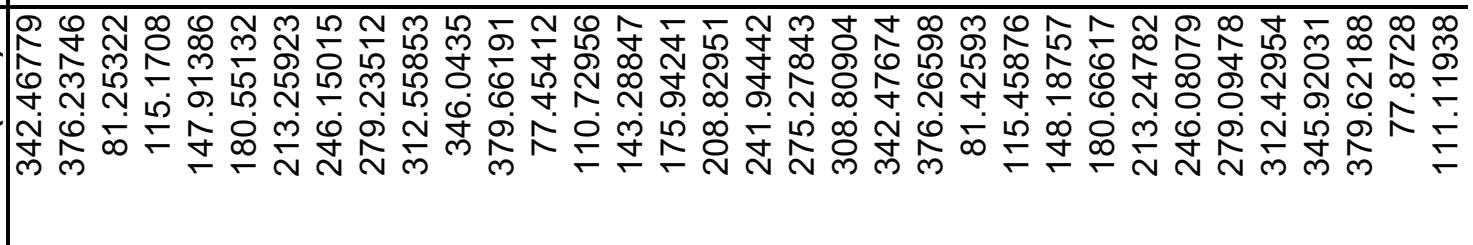 \\
\hline 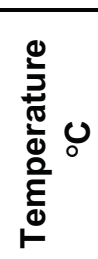 & 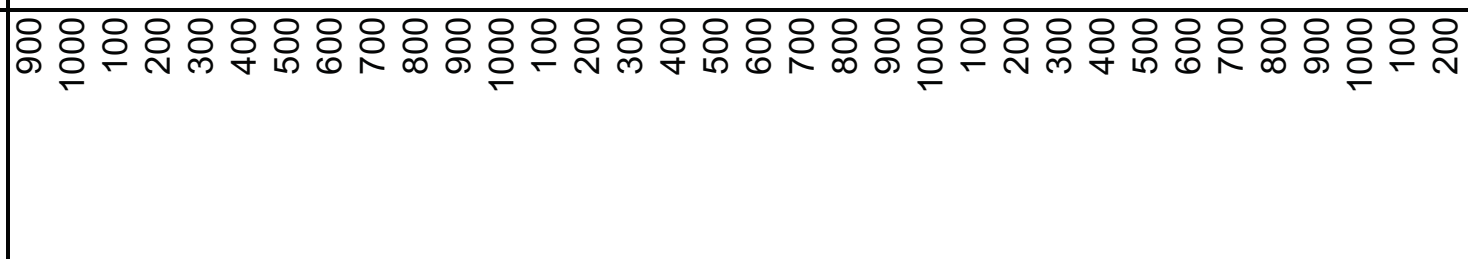 \\
\hline 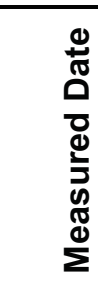 & 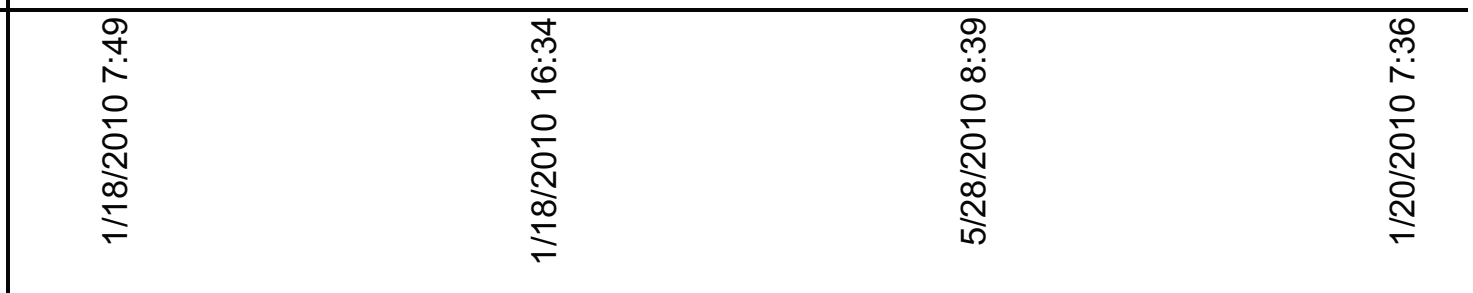 \\
\hline 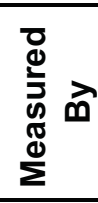 & $\frac{n}{3}$ \\
\hline 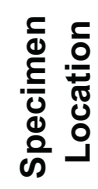 & \\
\hline 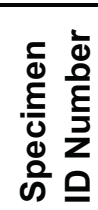 & $\begin{array}{l}\overline{0} \\
\frac{1}{\infty}\end{array}$ \\
\hline
\end{tabular}




\begin{tabular}{|c|c|}
\hline 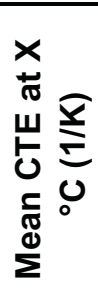 & 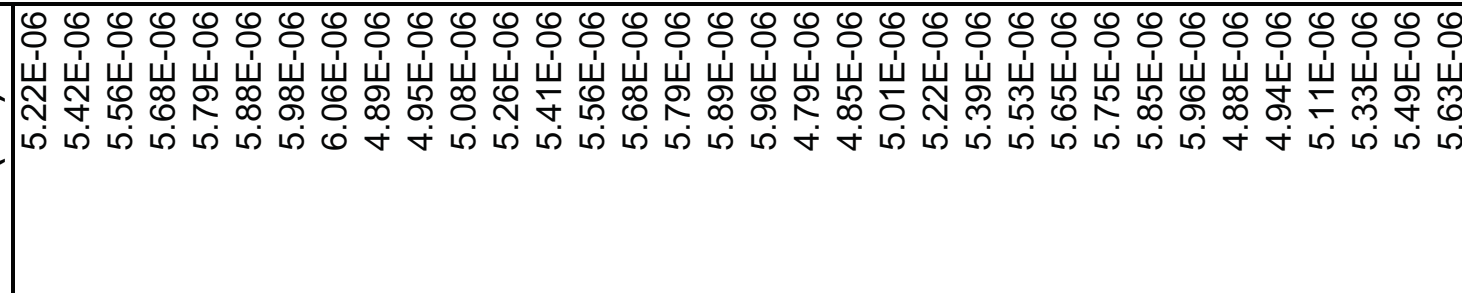 \\
\hline 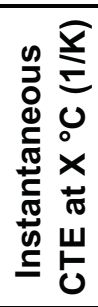 & 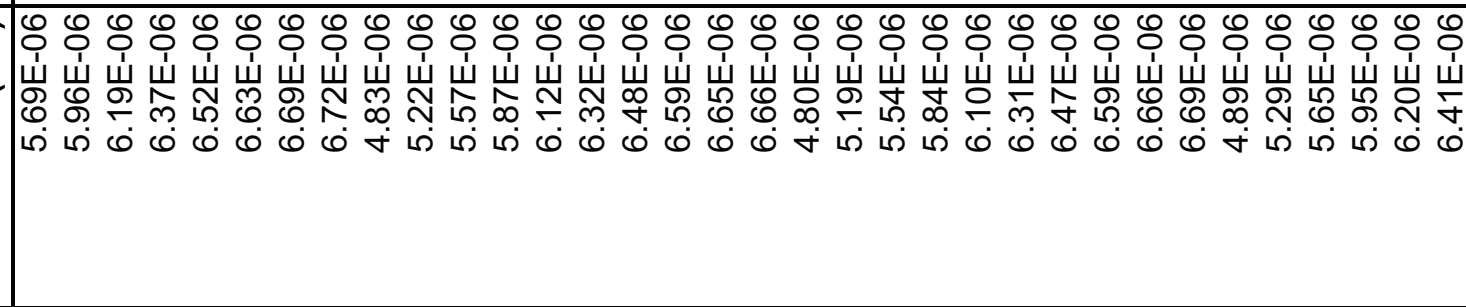 \\
\hline 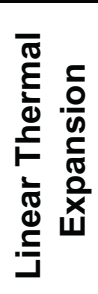 & 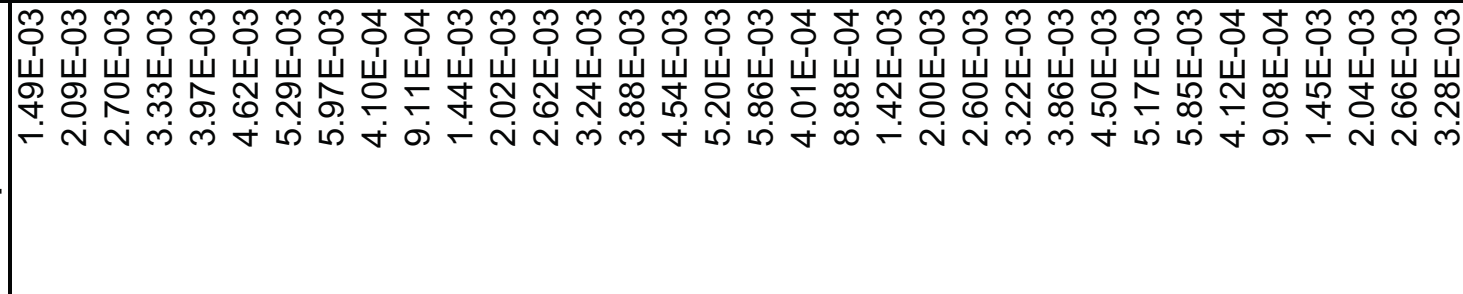 \\
\hline 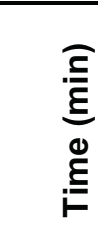 & 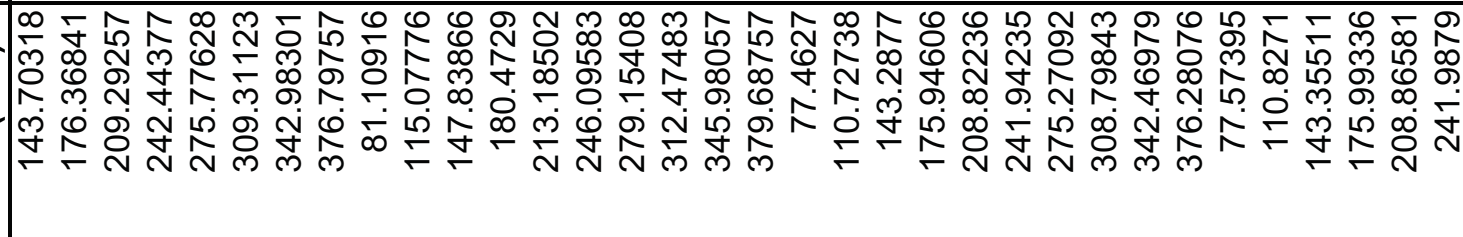 \\
\hline 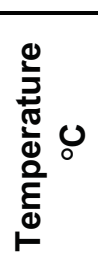 & 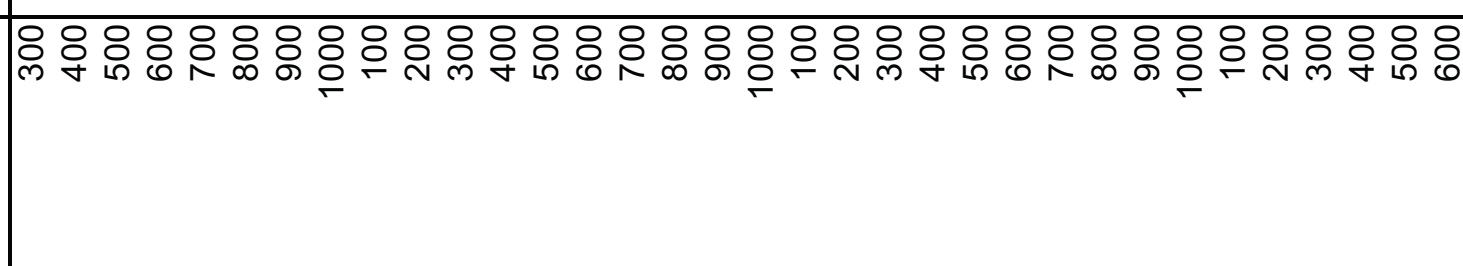 \\
\hline 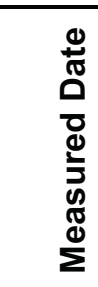 & 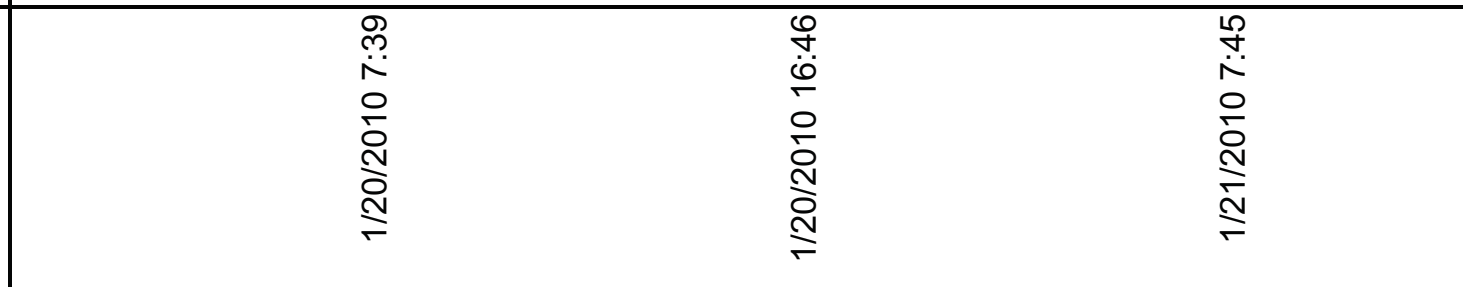 \\
\hline 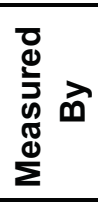 & $\sum_{3}^{\infty}$ \\
\hline 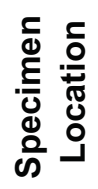 & \\
\hline 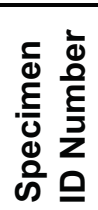 & 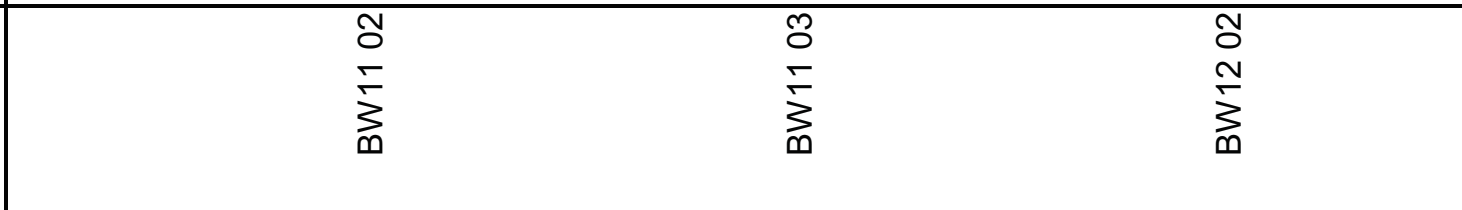 \\
\hline
\end{tabular}




\begin{tabular}{|c|c|}
\hline 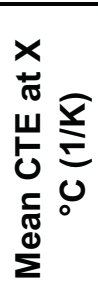 & 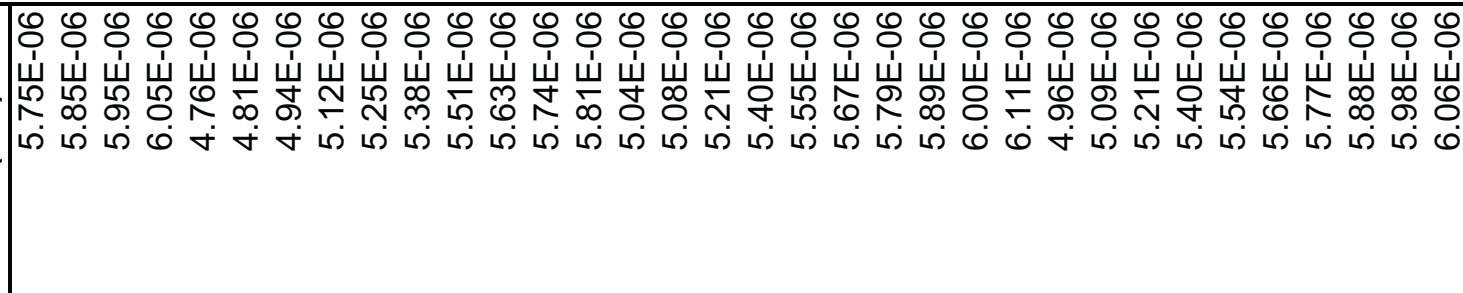 \\
\hline 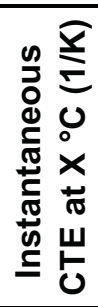 & 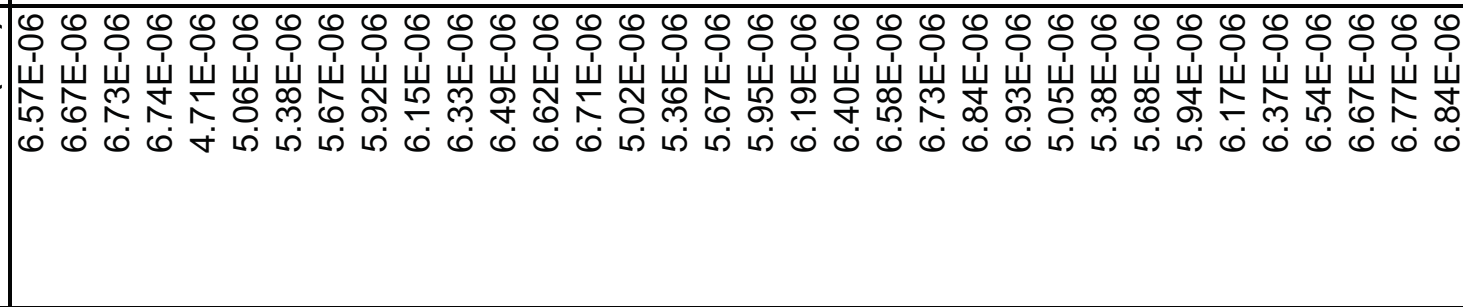 \\
\hline 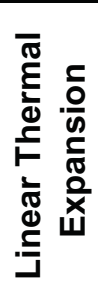 & 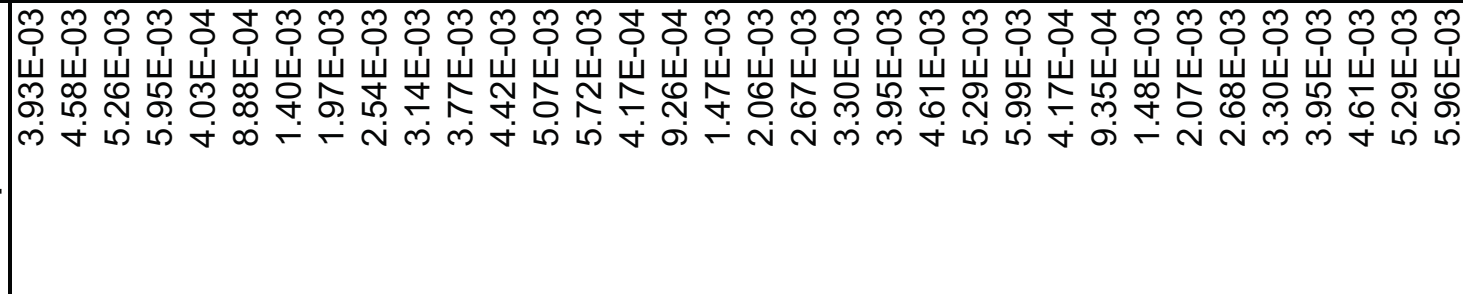 \\
\hline 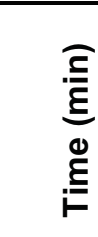 & 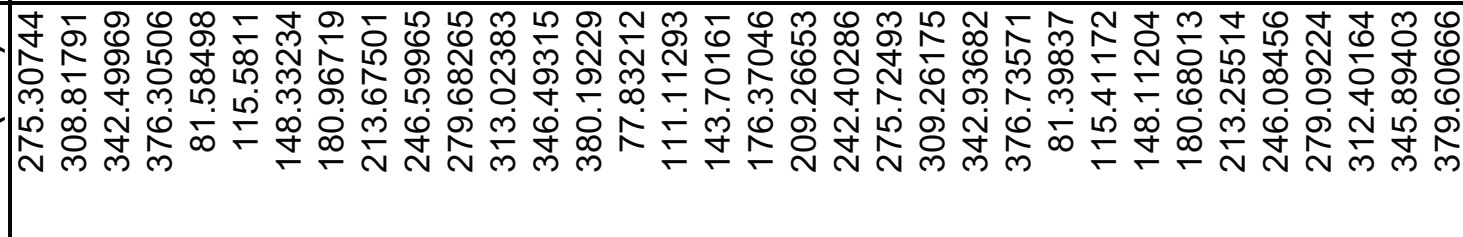 \\
\hline 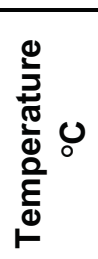 & 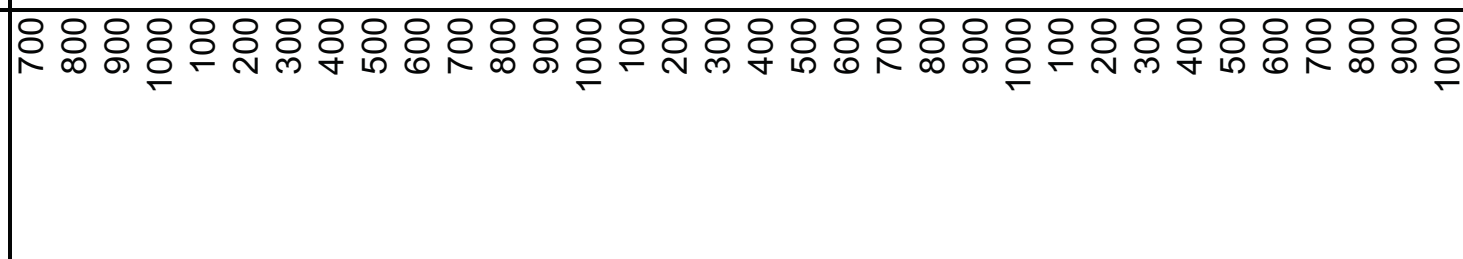 \\
\hline 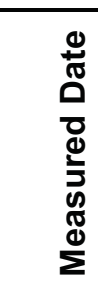 & 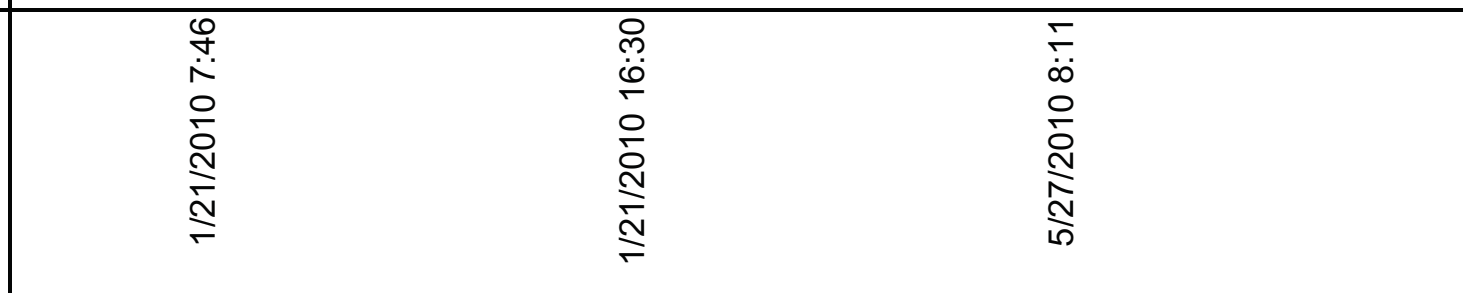 \\
\hline 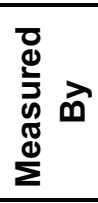 & $\sum_{3}^{\infty}$ \\
\hline 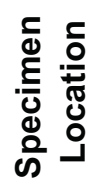 & \\
\hline 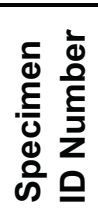 & $\stackrel{\substack{\infty \\
⿱ 亠 乂}}{\stackrel{\infty}{\infty}}$ \\
\hline
\end{tabular}




\begin{tabular}{|c|c|}
\hline 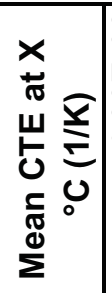 & 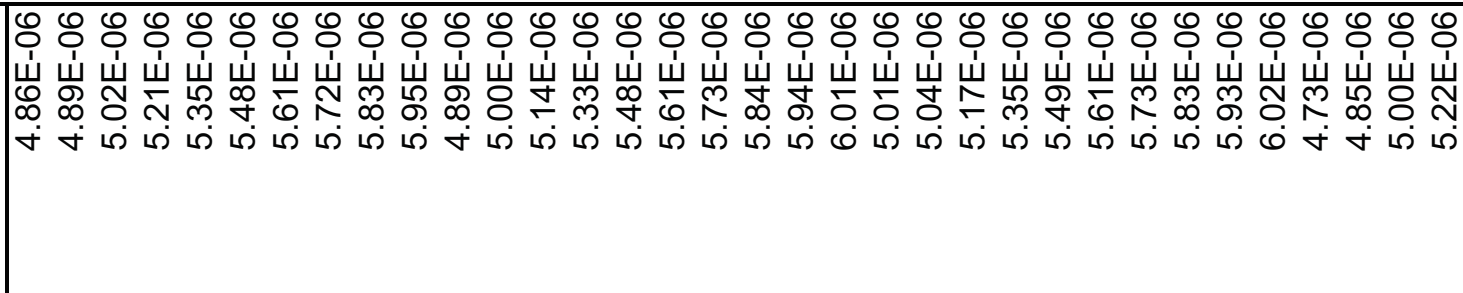 \\
\hline 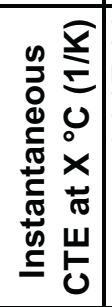 & 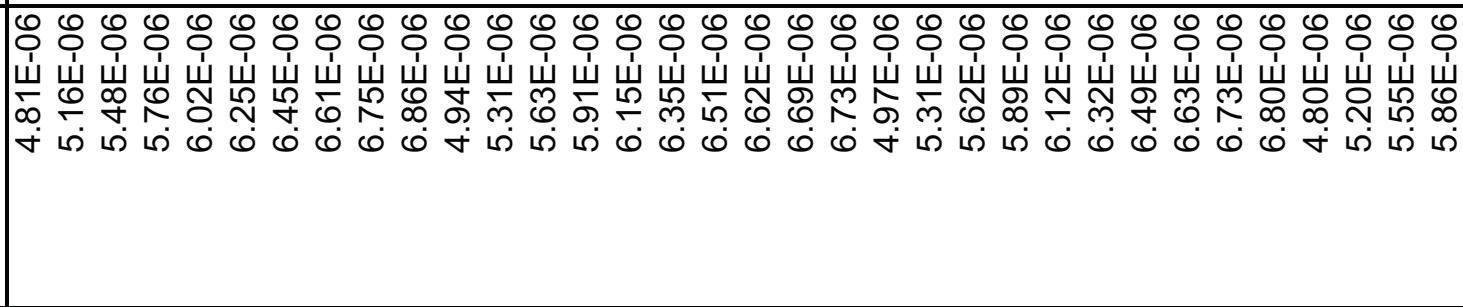 \\
\hline 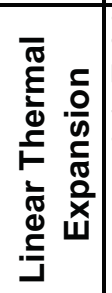 & 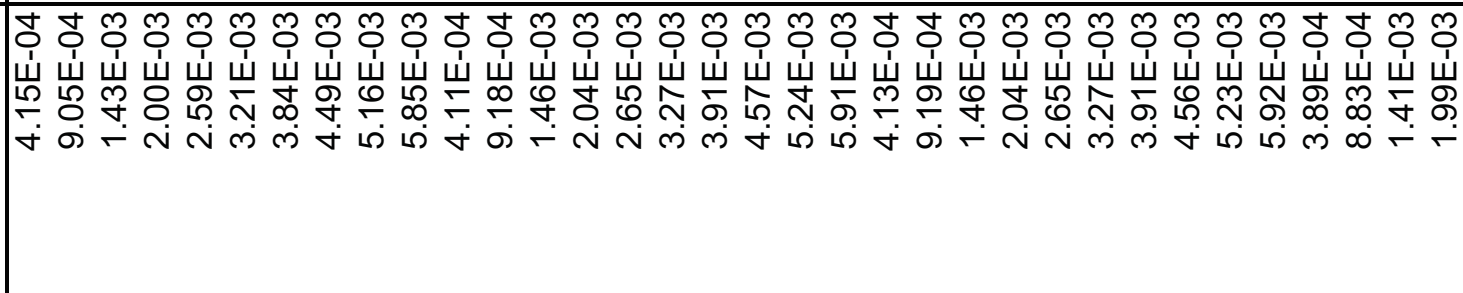 \\
\hline 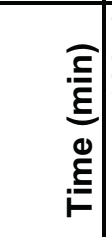 & 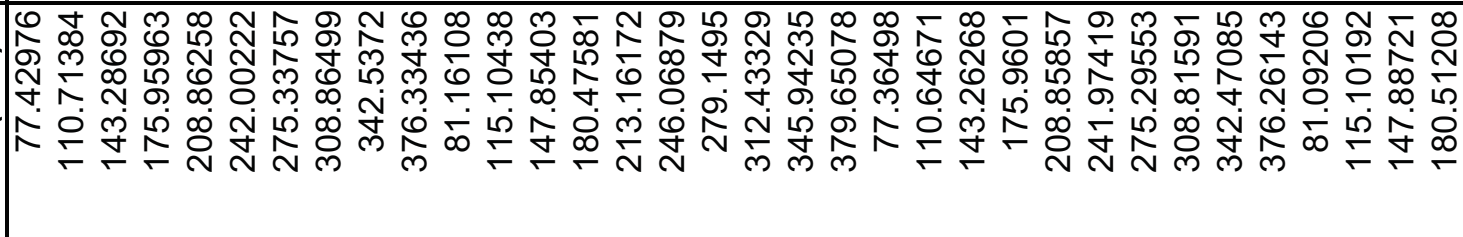 \\
\hline 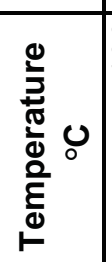 & 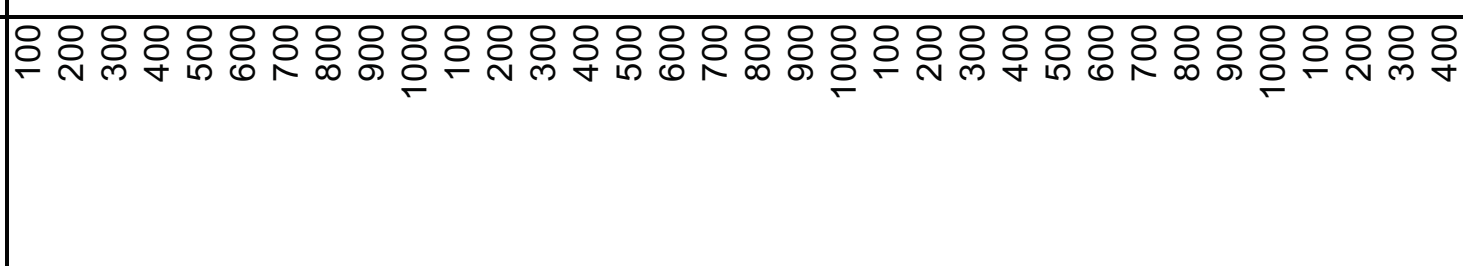 \\
\hline 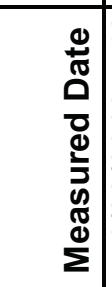 & 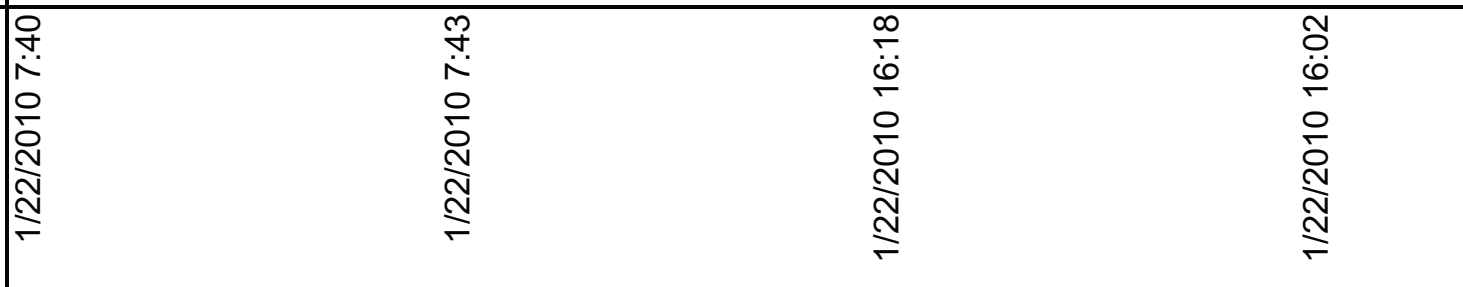 \\
\hline 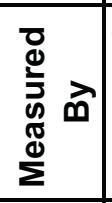 & $\sum_{3}^{\infty}$ \\
\hline 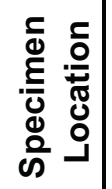 & \\
\hline 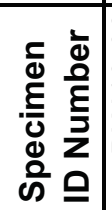 & $\sum_{\substack{\infty \\
m}}^{\infty}$ \\
\hline
\end{tabular}




\begin{tabular}{|c|c|}
\hline 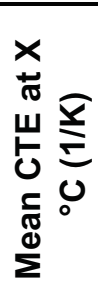 & 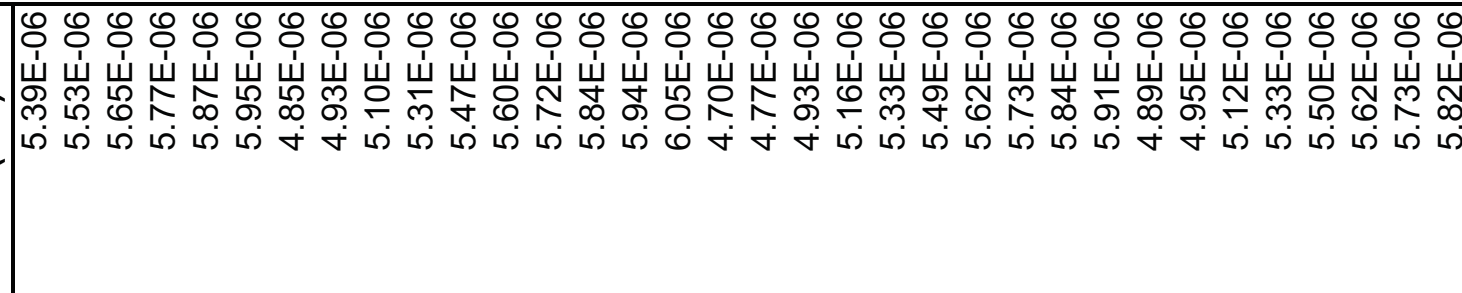 \\
\hline 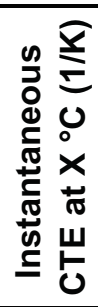 & 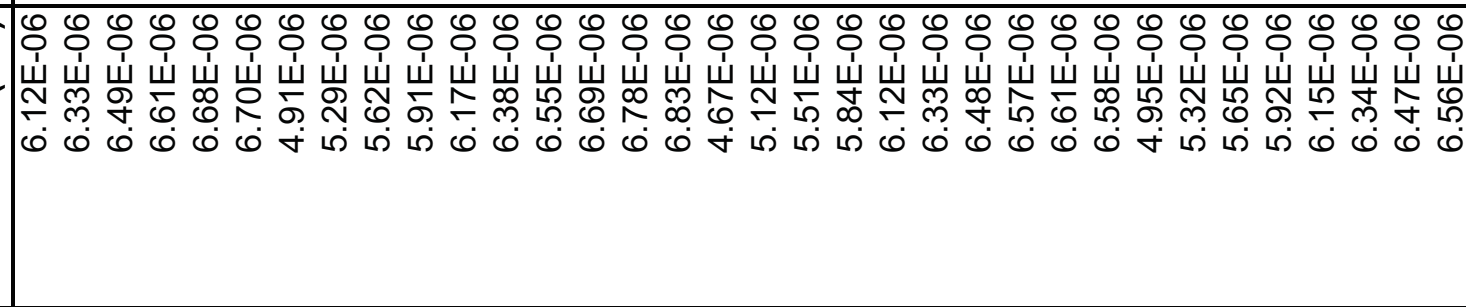 \\
\hline 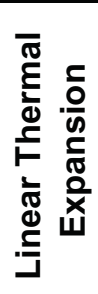 & 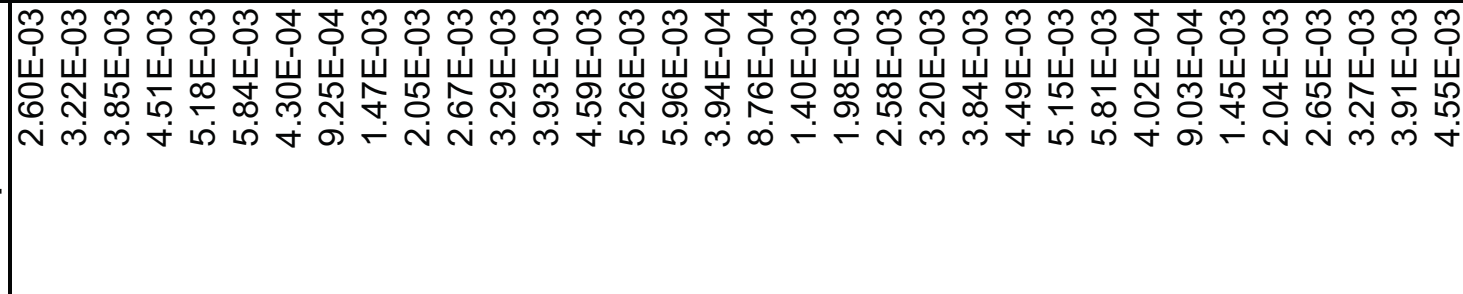 \\
\hline 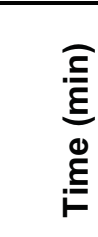 & 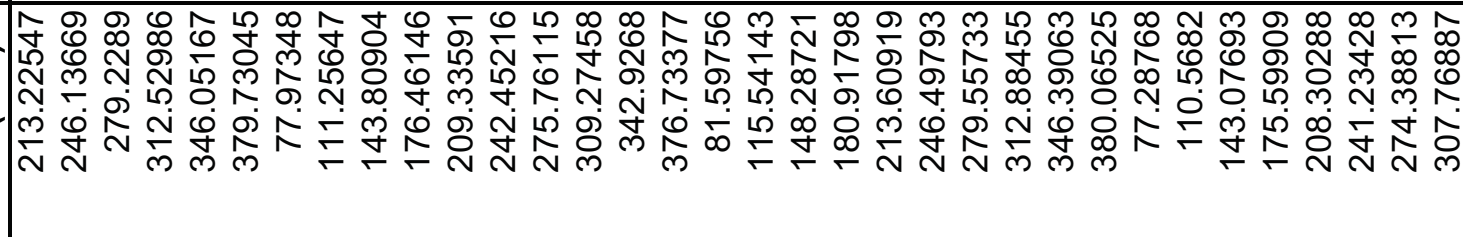 \\
\hline 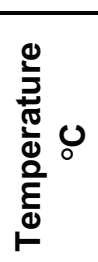 & 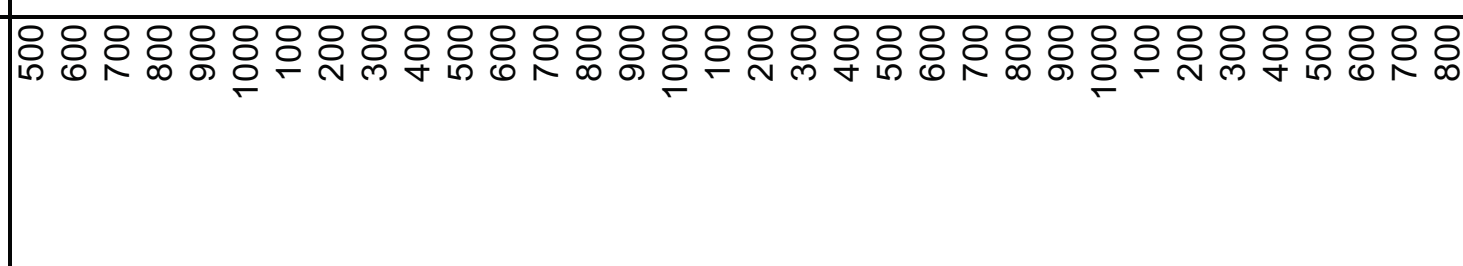 \\
\hline 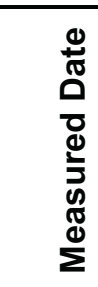 & 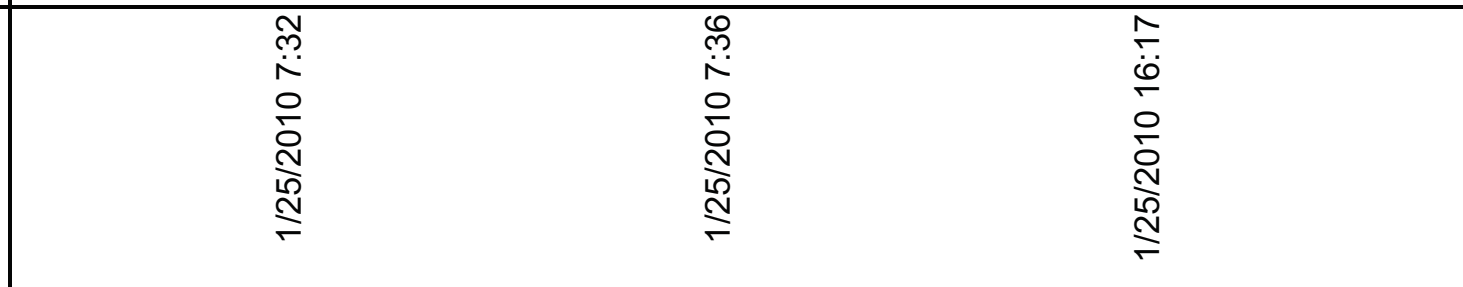 \\
\hline 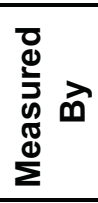 & $\frac{n}{3}$ \\
\hline 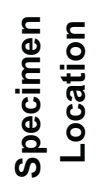 & \\
\hline 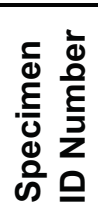 & $\begin{array}{l} \\
5 \\
10 \\
\infty\end{array}$ \\
\hline
\end{tabular}




\begin{tabular}{|c|c|}
\hline 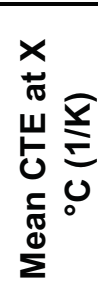 & 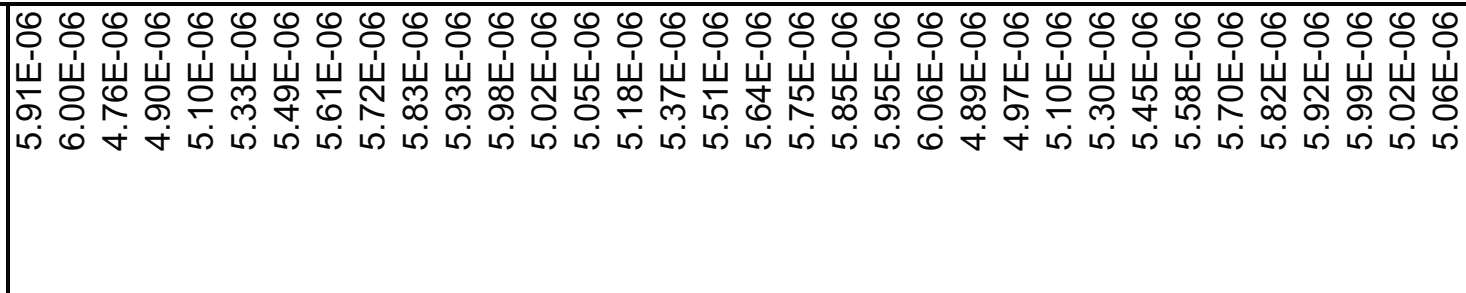 \\
\hline 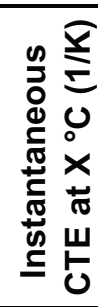 & 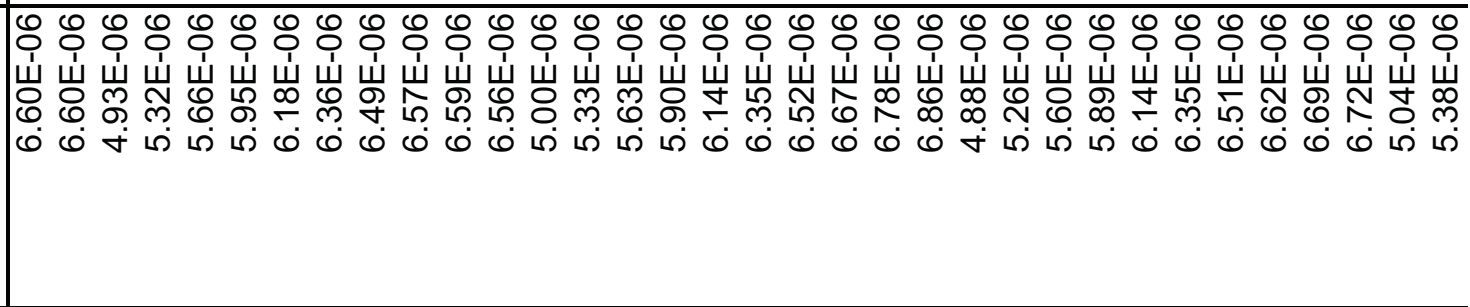 \\
\hline 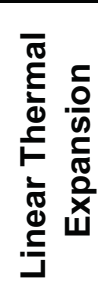 & 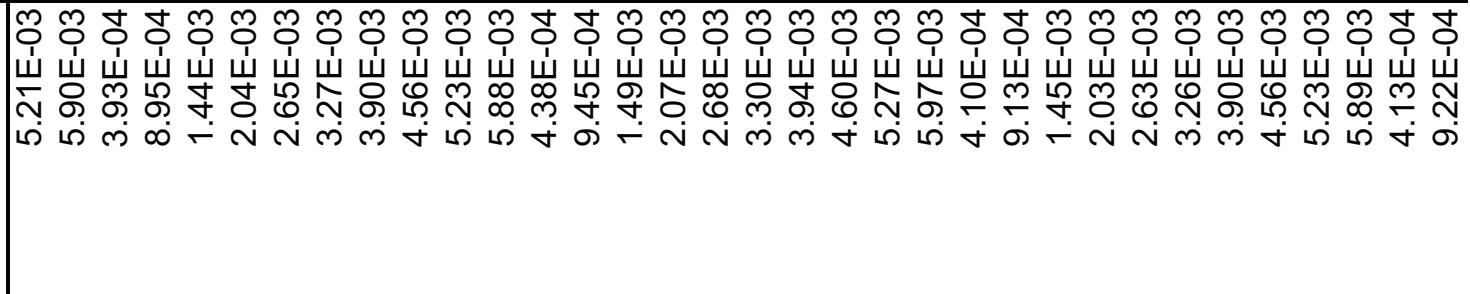 \\
\hline 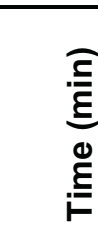 & 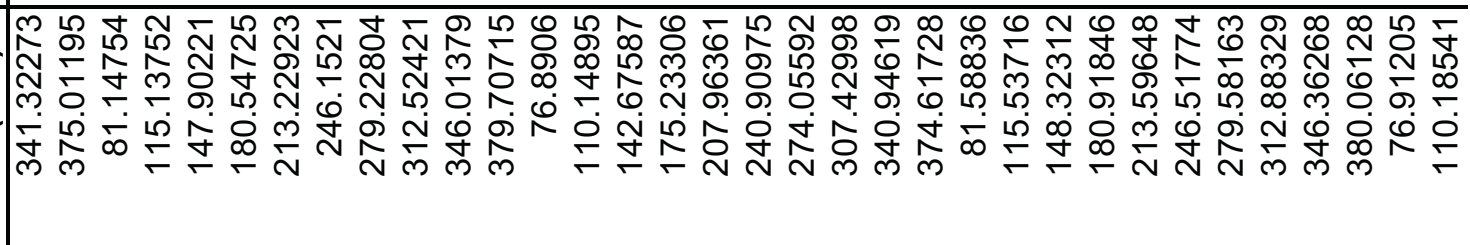 \\
\hline 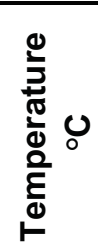 & 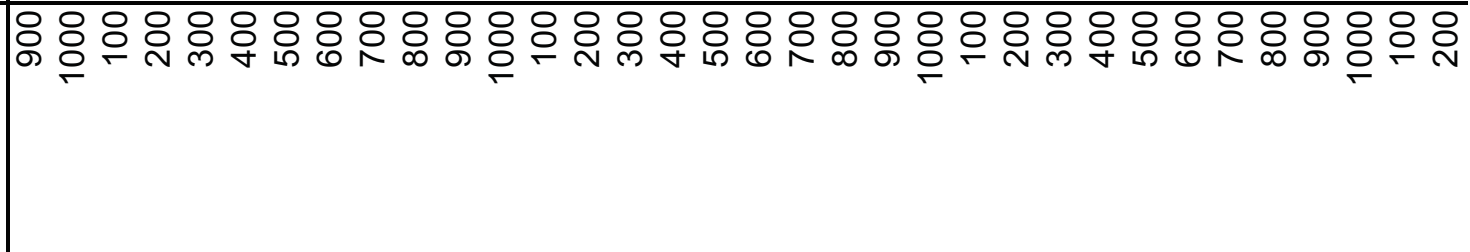 \\
\hline 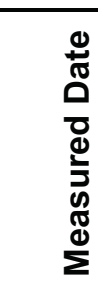 & 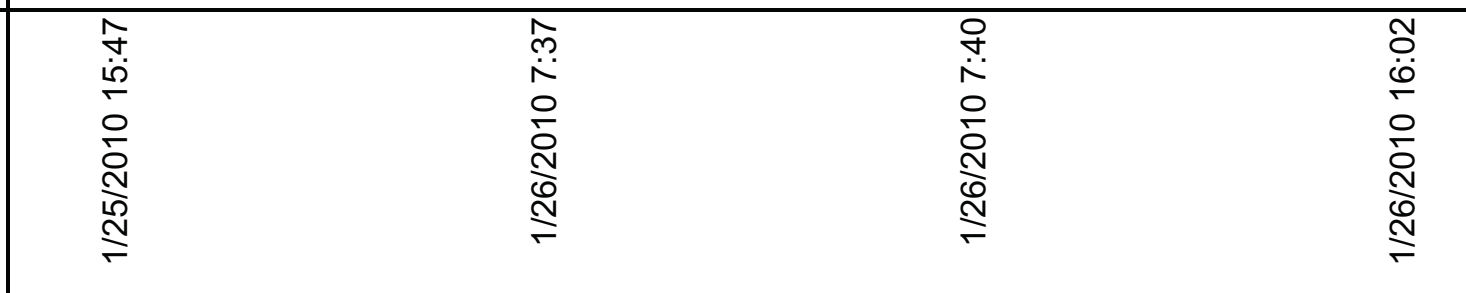 \\
\hline 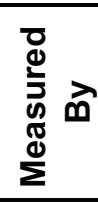 & $\frac{0}{0}$ \\
\hline 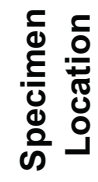 & \\
\hline 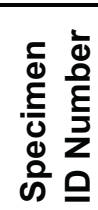 & $\begin{array}{l}5 \\
0 \\
\sum_{0}^{0}\end{array}$ \\
\hline
\end{tabular}




\begin{tabular}{|c|c|}
\hline 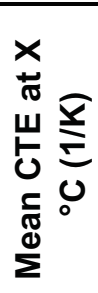 & 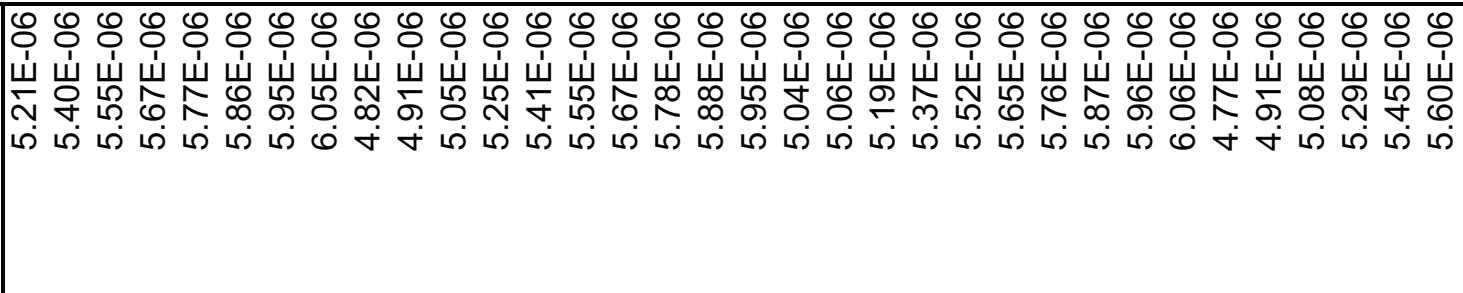 \\
\hline 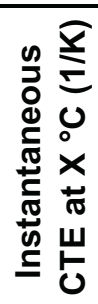 & 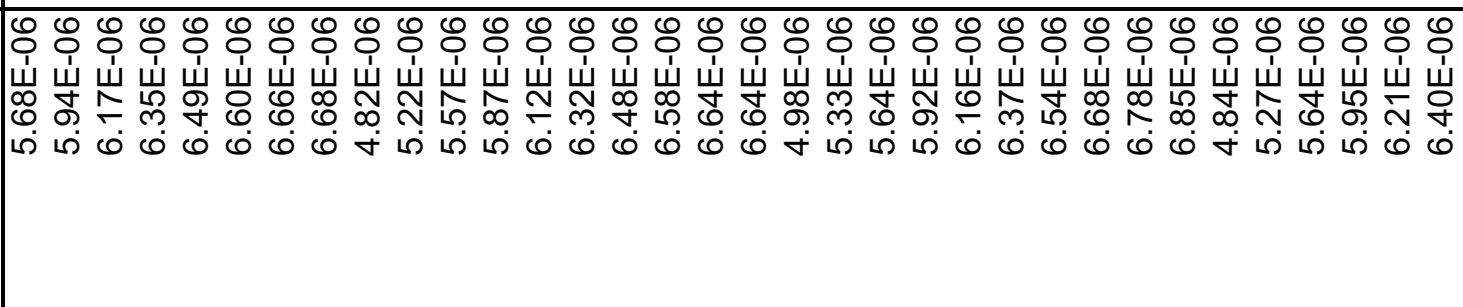 \\
\hline 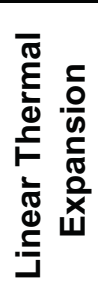 & 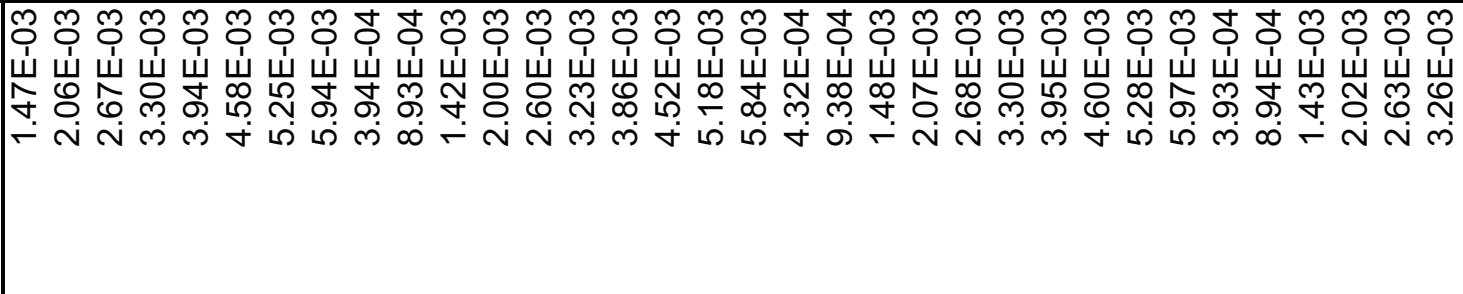 \\
\hline 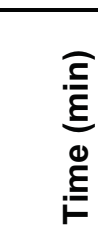 & 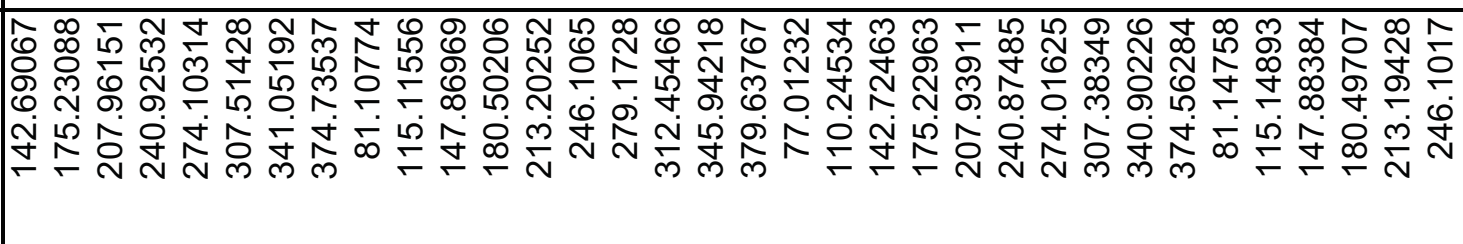 \\
\hline 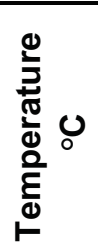 & 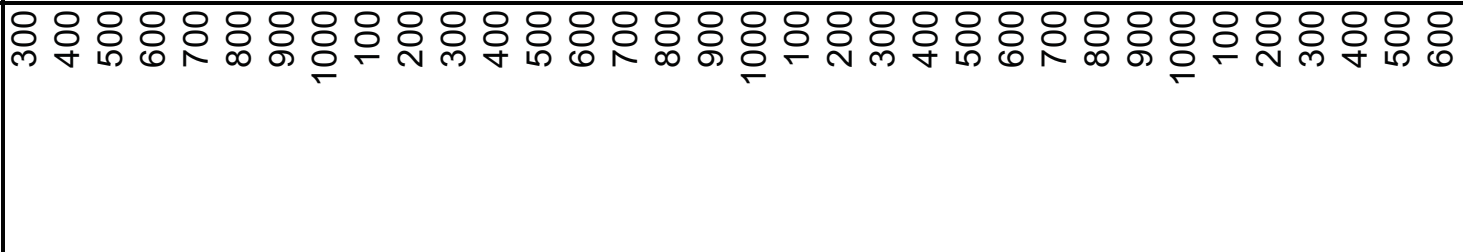 \\
\hline 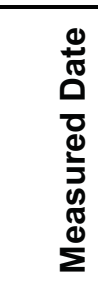 & 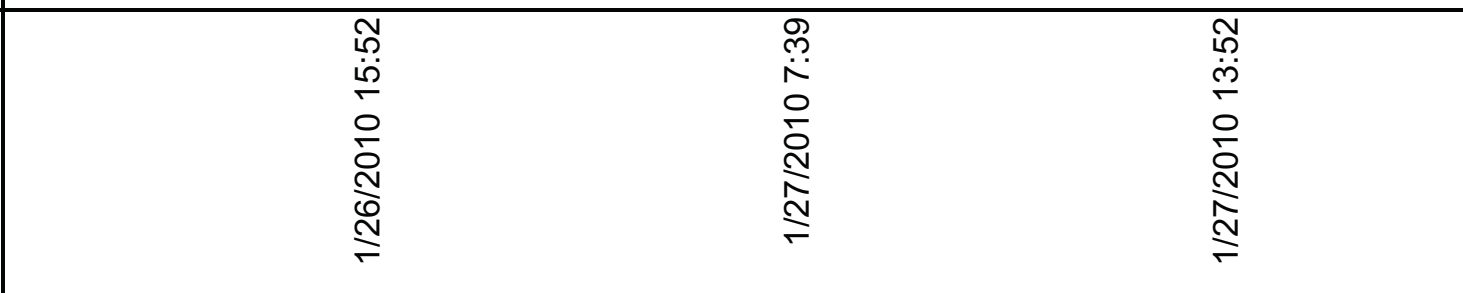 \\
\hline 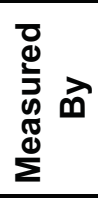 & $\sum_{3}^{\infty}$ \\
\hline 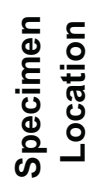 & \\
\hline 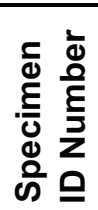 & 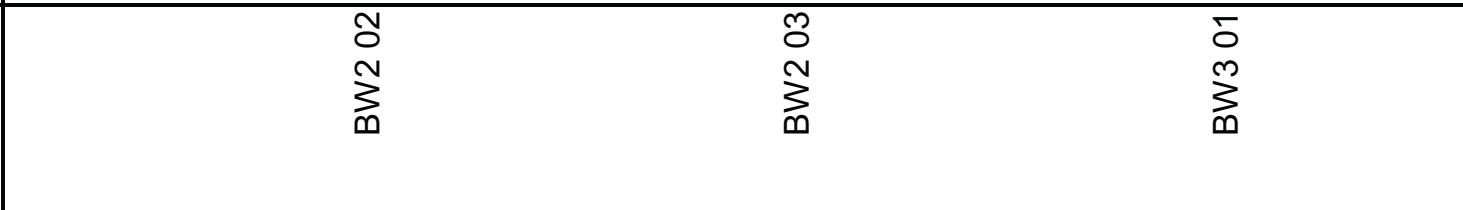 \\
\hline
\end{tabular}




\begin{tabular}{|c|c|}
\hline 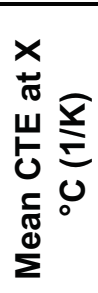 & 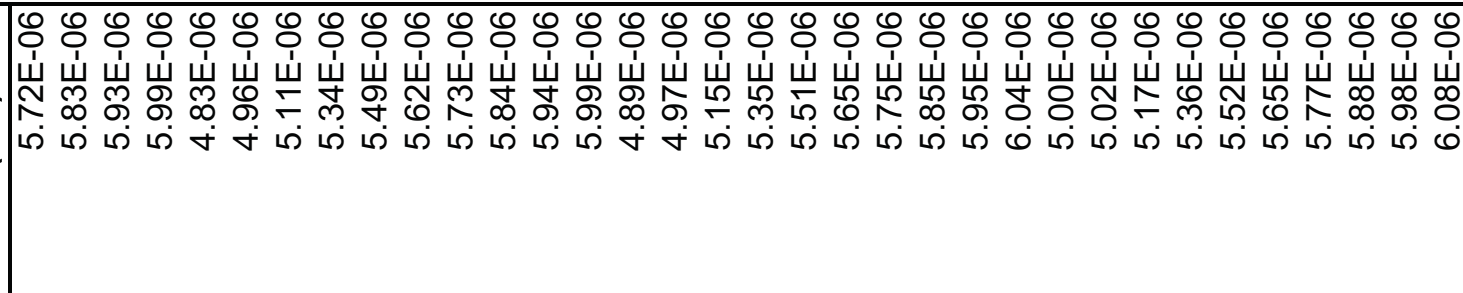 \\
\hline 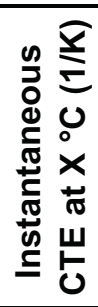 & 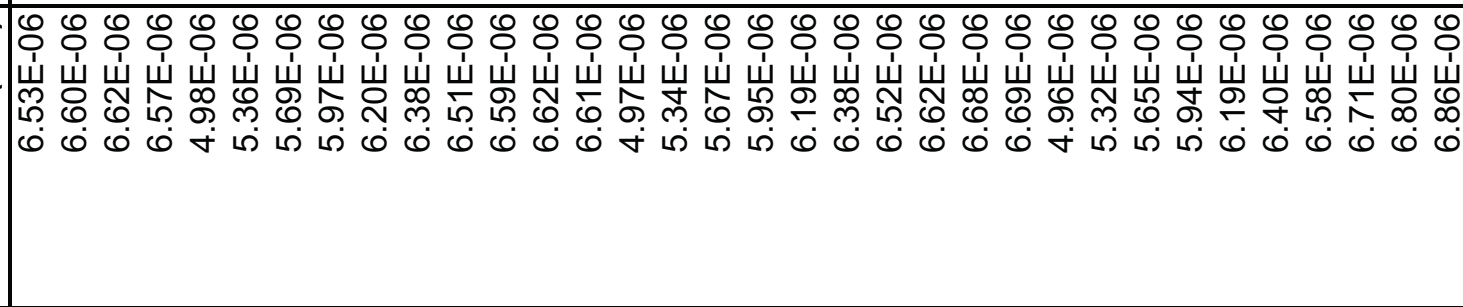 \\
\hline 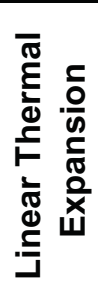 & 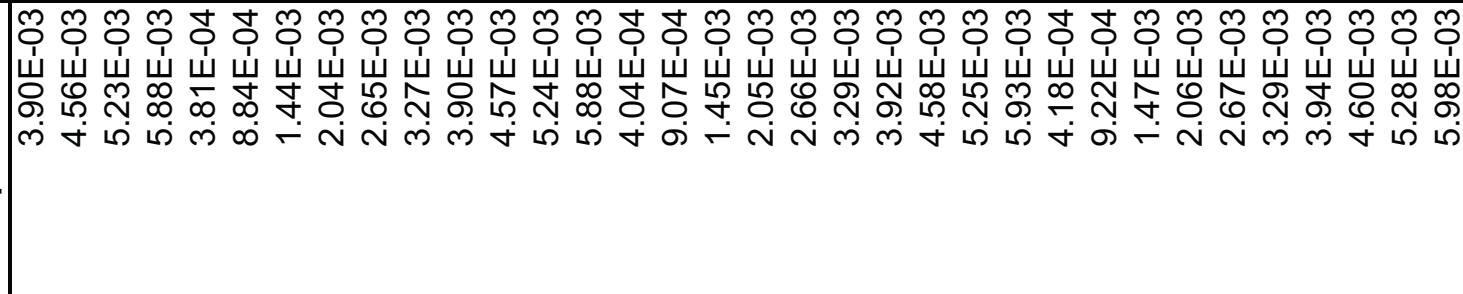 \\
\hline 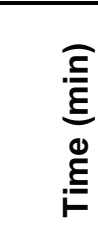 & 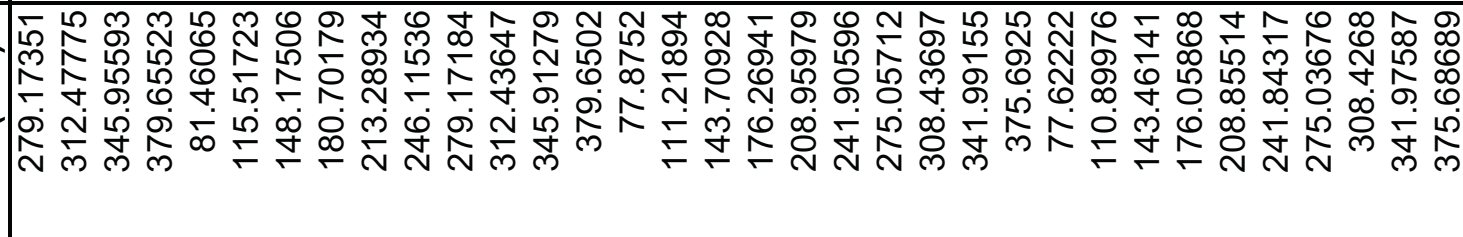 \\
\hline 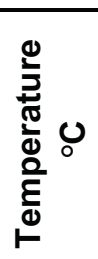 & 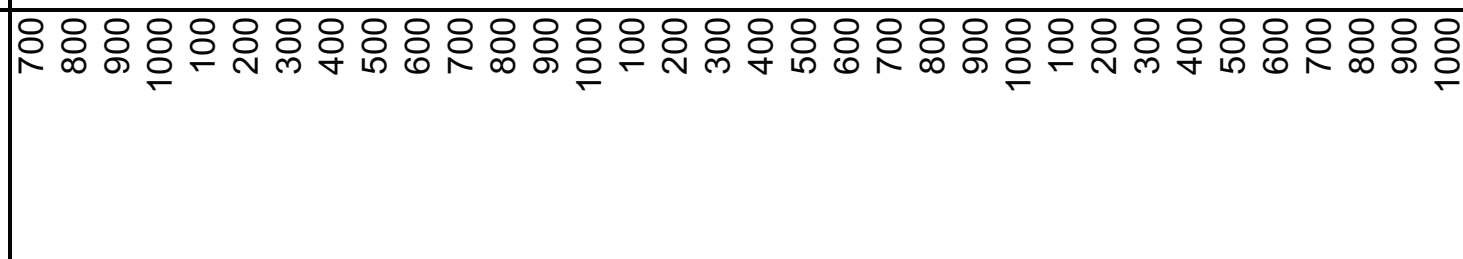 \\
\hline 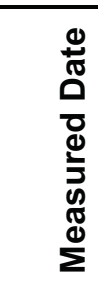 & 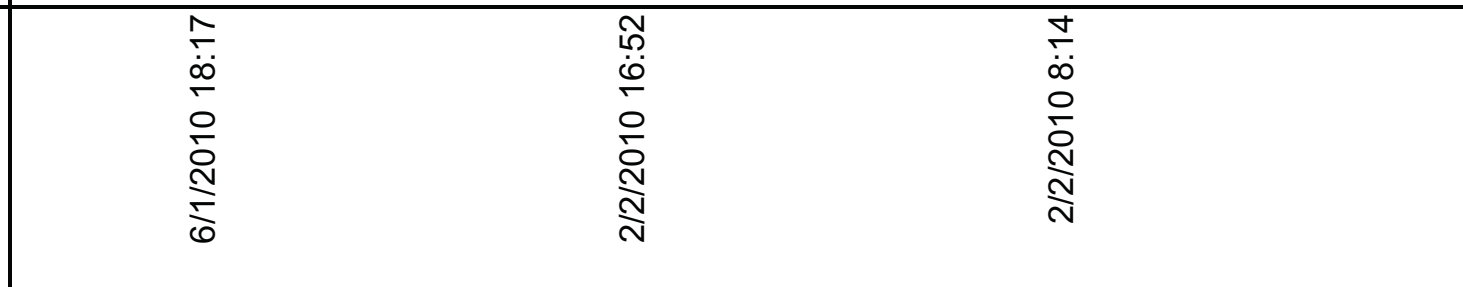 \\
\hline 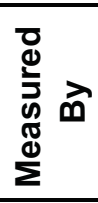 & $\sum_{3}^{\infty}$ \\
\hline 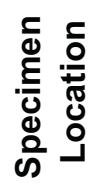 & \\
\hline 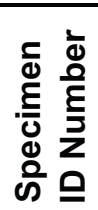 & $\begin{array}{l}m \\
m \\
m\end{array}$ \\
\hline
\end{tabular}




\begin{tabular}{|c|c|}
\hline 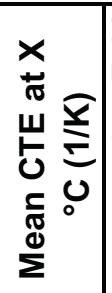 & 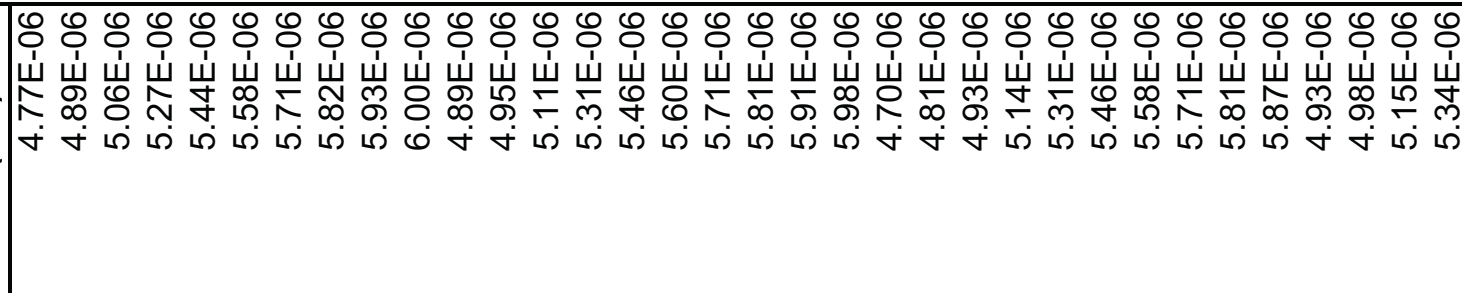 \\
\hline 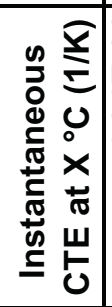 & 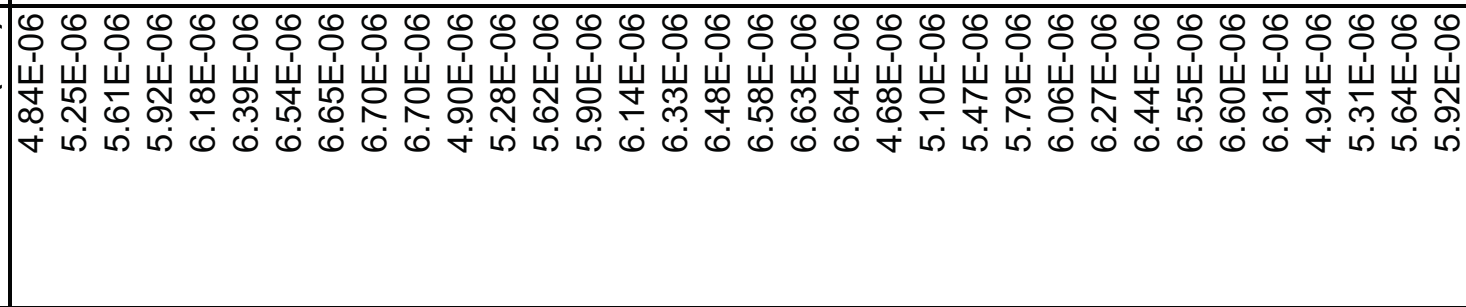 \\
\hline 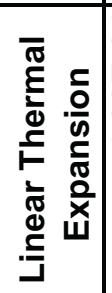 & 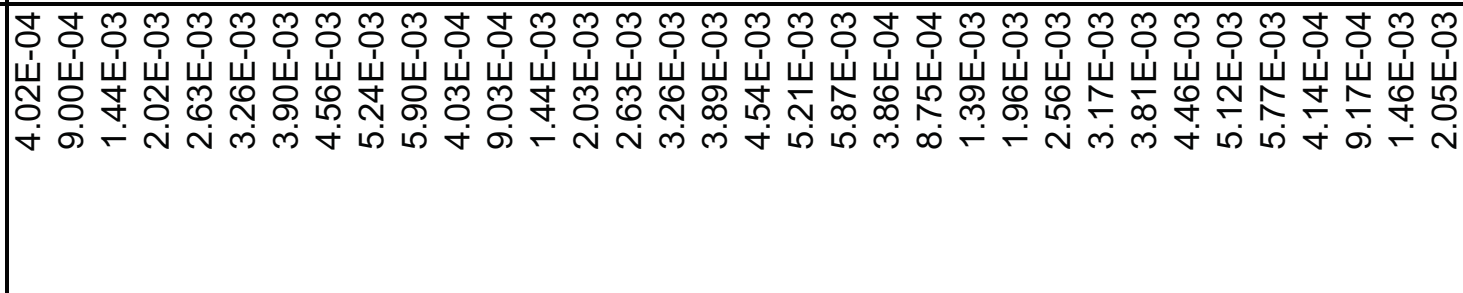 \\
\hline 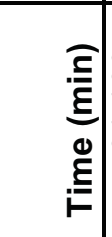 & 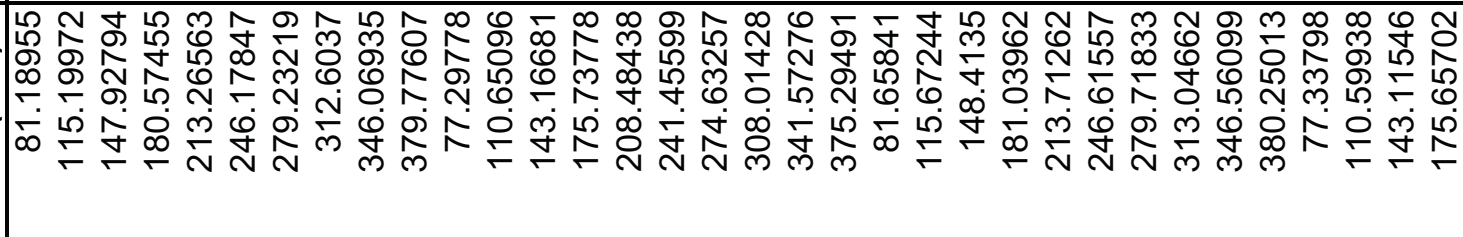 \\
\hline 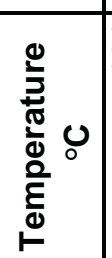 & 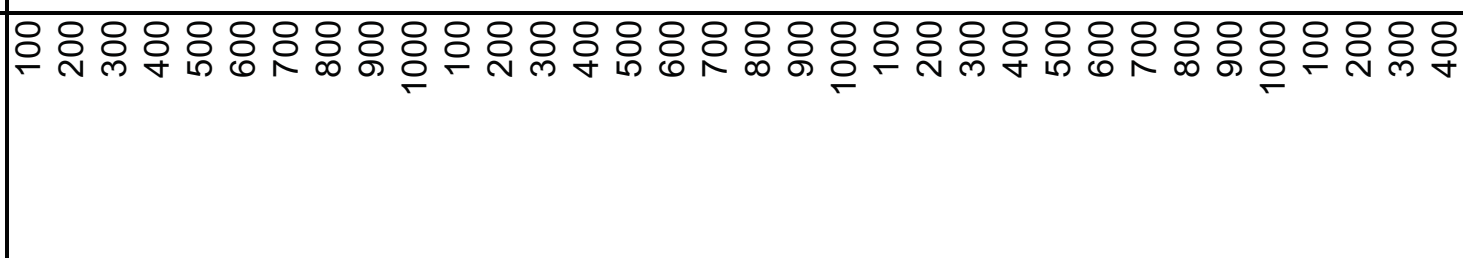 \\
\hline 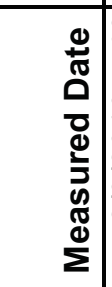 & 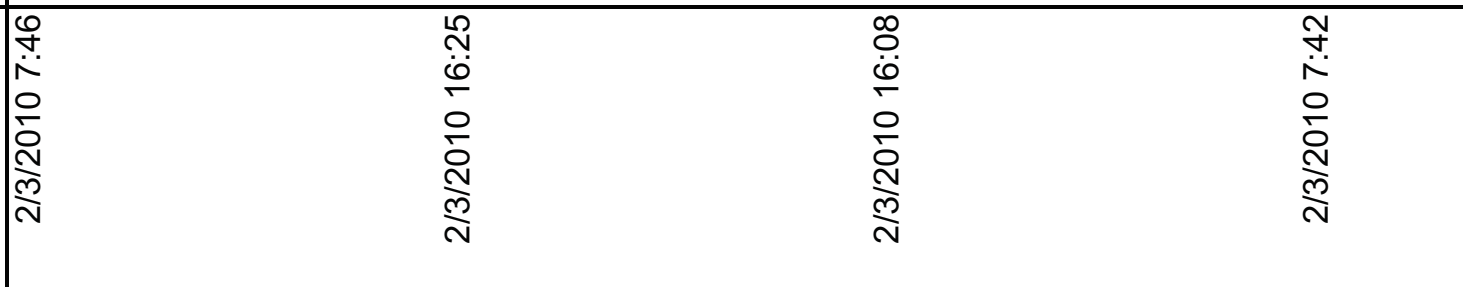 \\
\hline 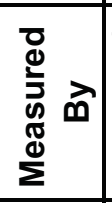 & $\frac{0}{3}$ \\
\hline 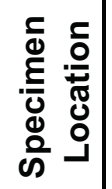 & \\
\hline 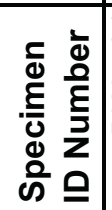 & $\sum_{\substack{\infty \\
0 \\
\infty}}^{\infty}$ \\
\hline
\end{tabular}




\begin{tabular}{|c|c|}
\hline 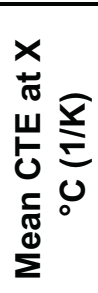 & 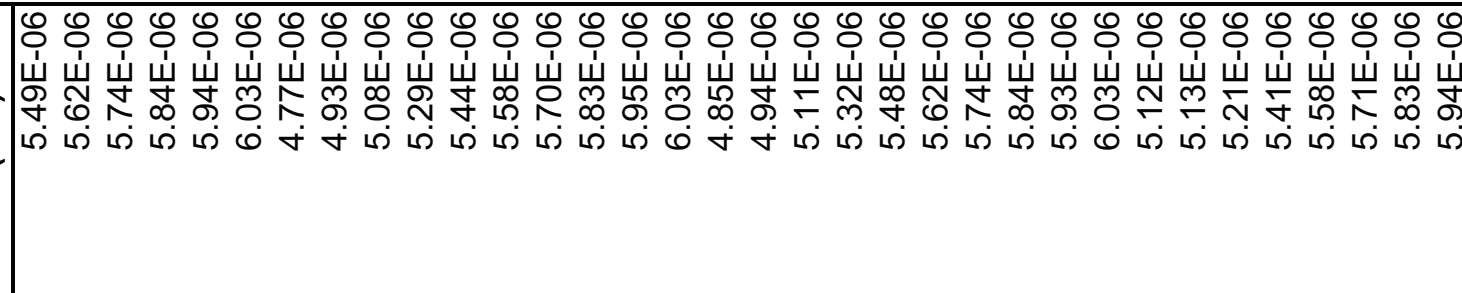 \\
\hline 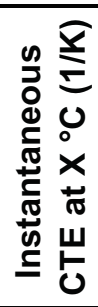 & 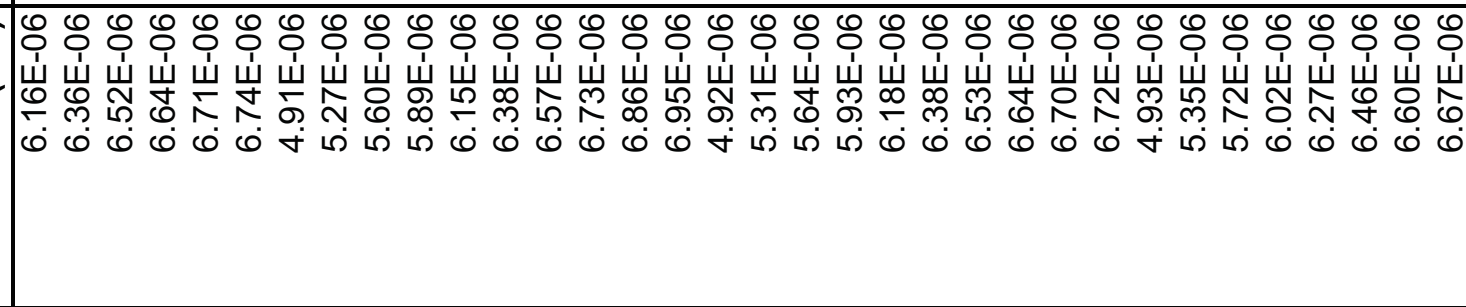 \\
\hline 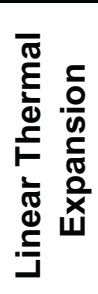 & 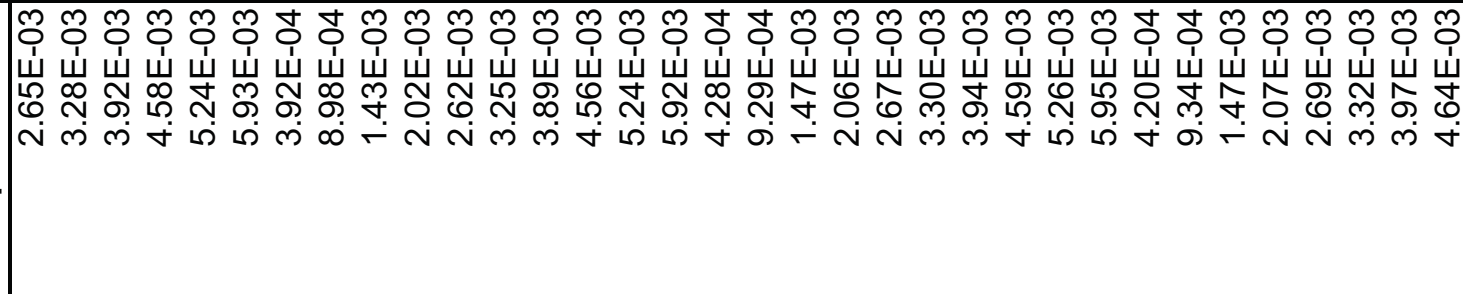 \\
\hline 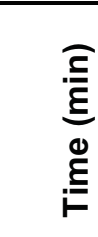 & 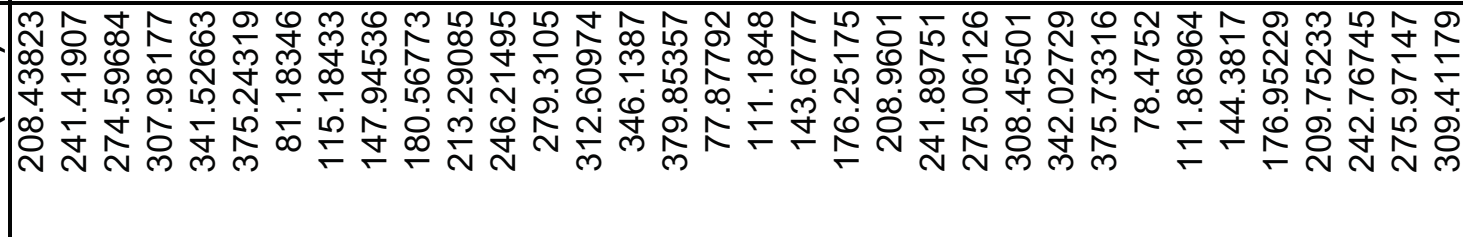 \\
\hline 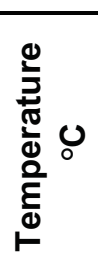 & 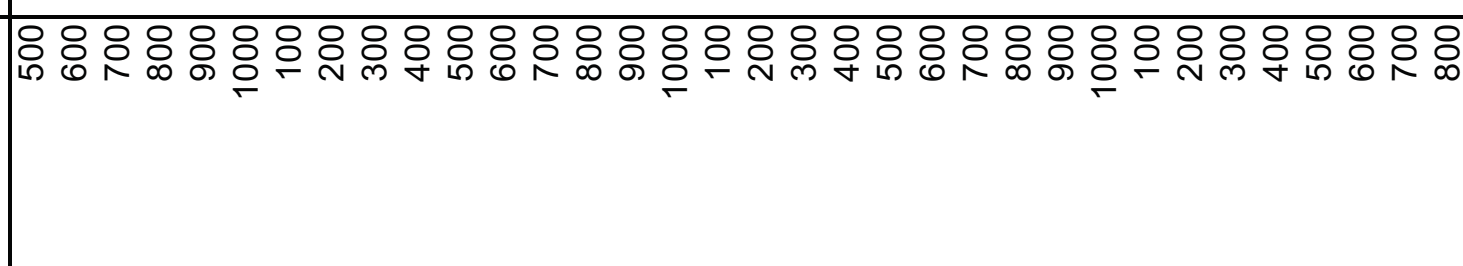 \\
\hline 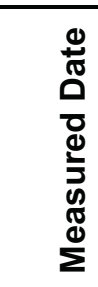 & 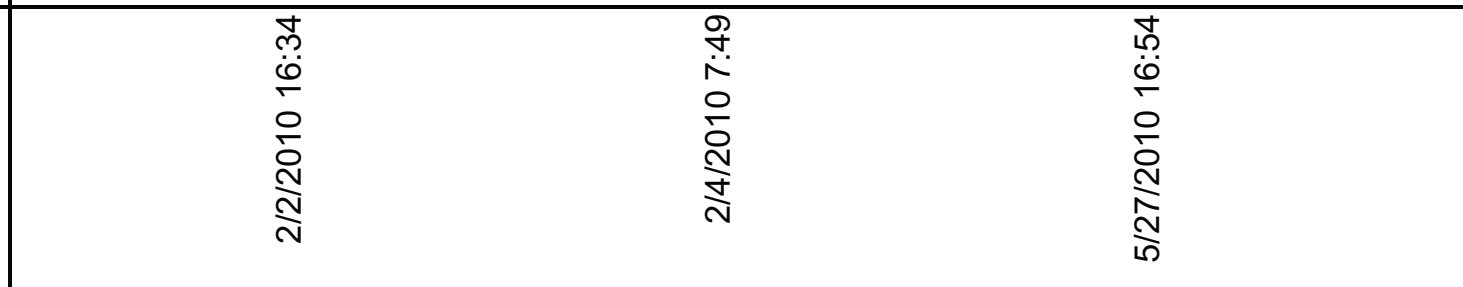 \\
\hline 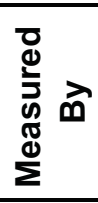 & $\frac{0}{0}$ \\
\hline 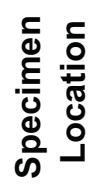 & \\
\hline 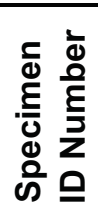 & $\begin{array}{l}\text { N } \\
\text { 음 } \\
\stackrel{\infty}{\infty}\end{array}$ \\
\hline
\end{tabular}




\begin{tabular}{|c|c|}
\hline 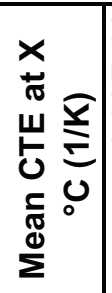 & 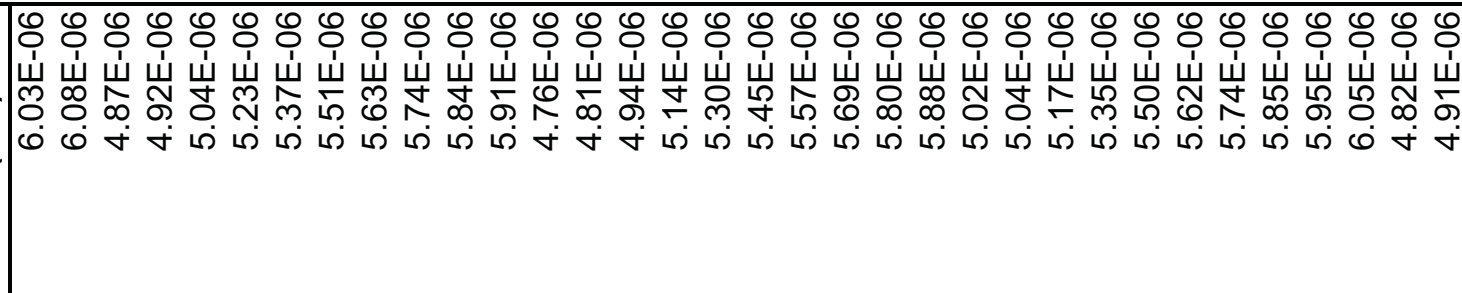 \\
\hline 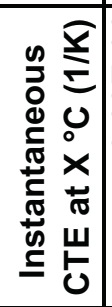 & 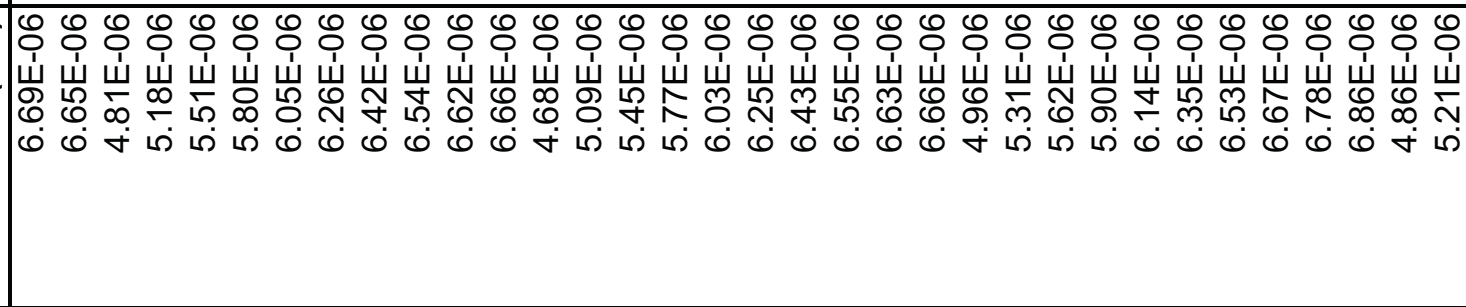 \\
\hline 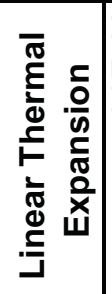 & 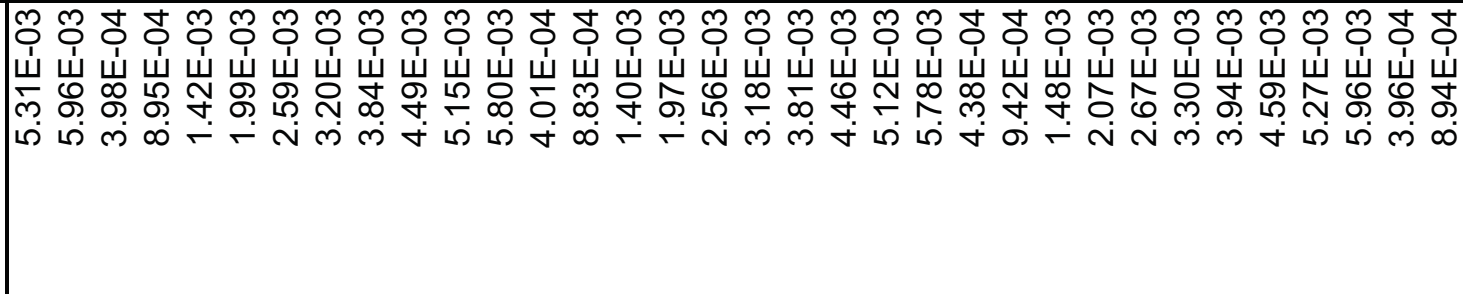 \\
\hline 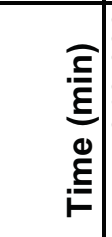 & 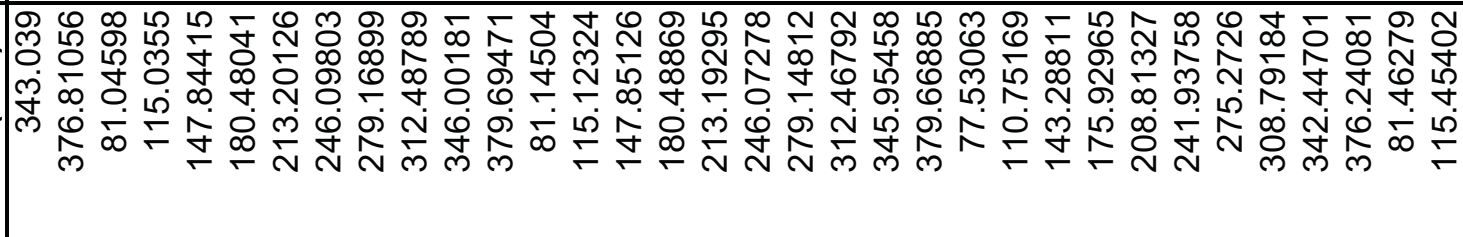 \\
\hline 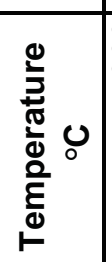 & 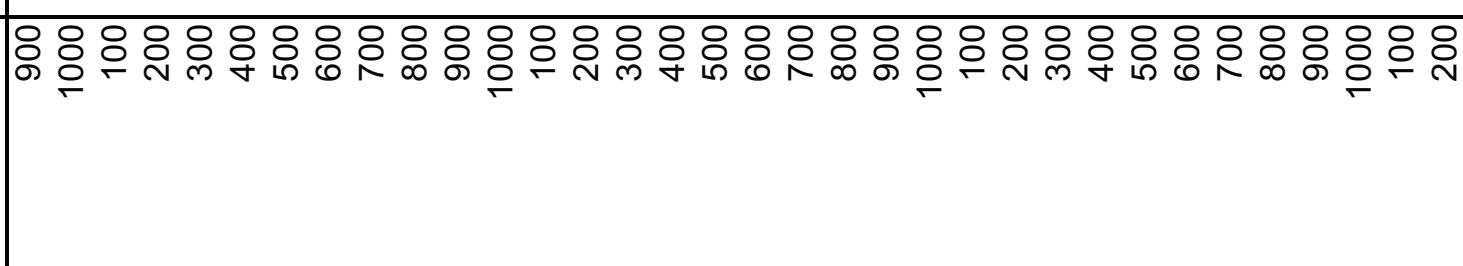 \\
\hline 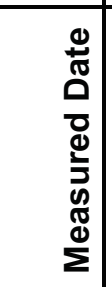 & 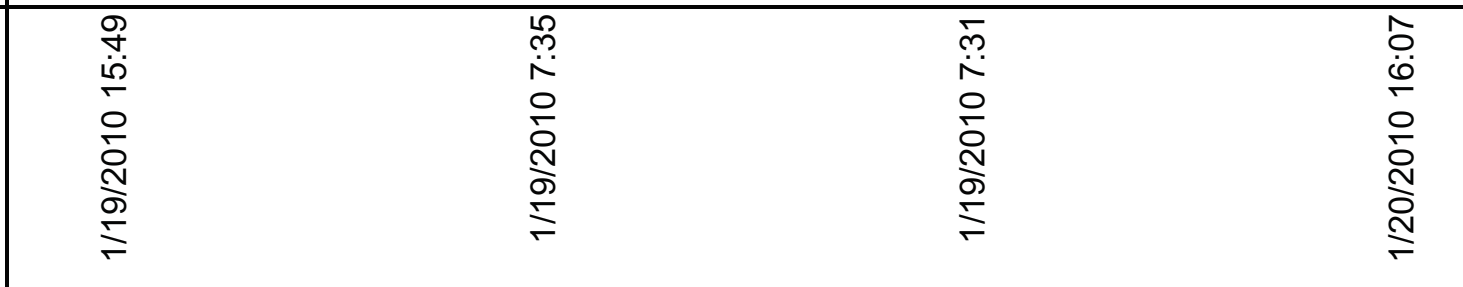 \\
\hline 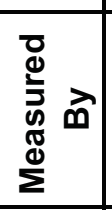 & $\frac{n}{3}$ \\
\hline 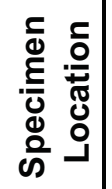 & \\
\hline 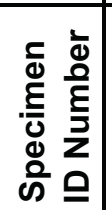 & $\begin{array}{l}5 \\
\vdots \\
\vdots \\
\end{array}$ \\
\hline
\end{tabular}




\begin{tabular}{|c|c|}
\hline 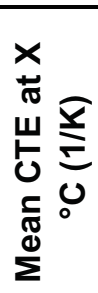 & 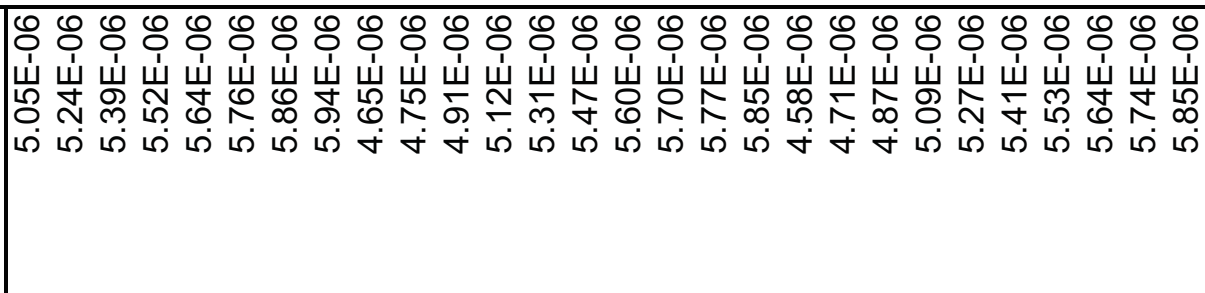 \\
\hline 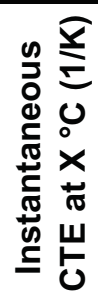 & 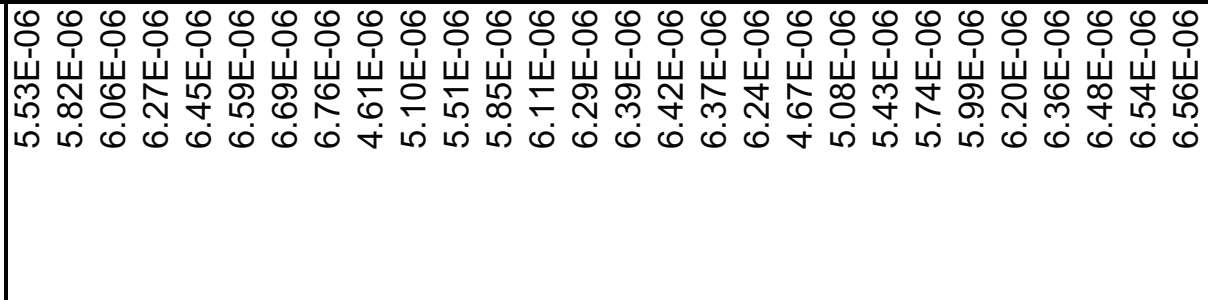 \\
\hline 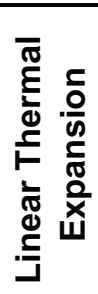 & 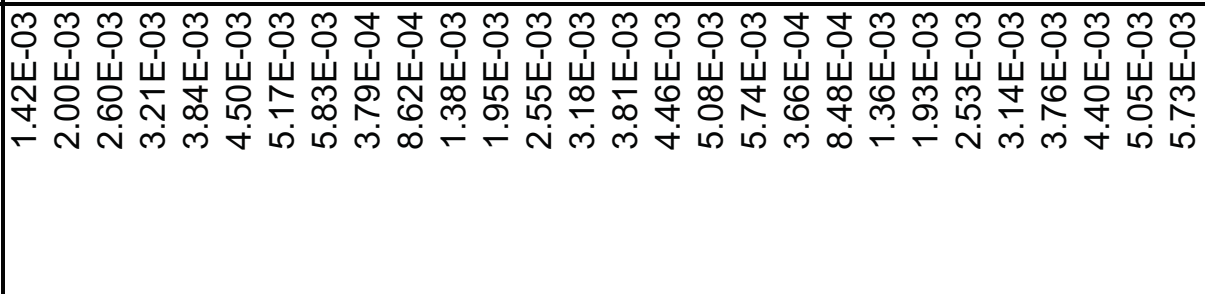 \\
\hline 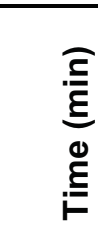 & 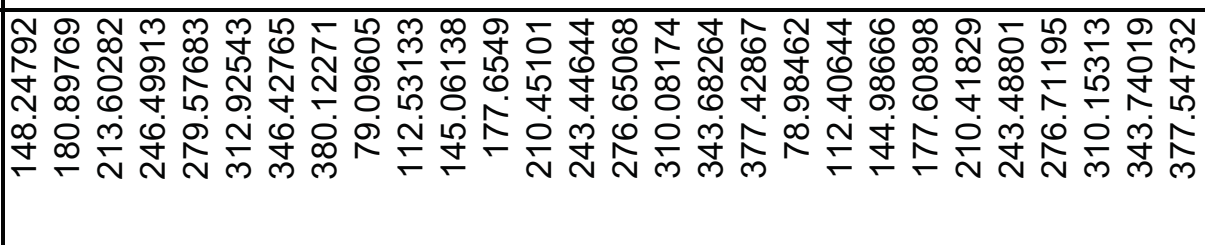 \\
\hline 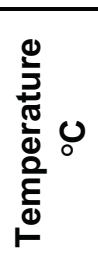 & 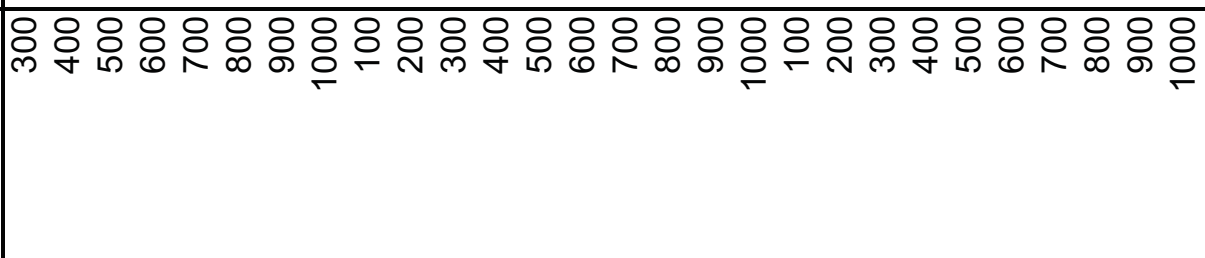 \\
\hline 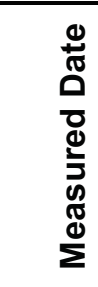 & 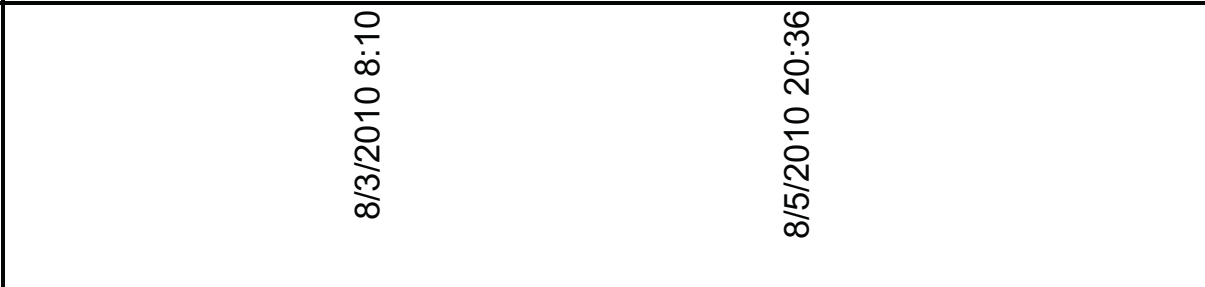 \\
\hline 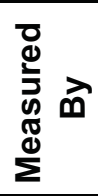 & $\frac{0}{0}$ \\
\hline 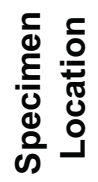 & \\
\hline 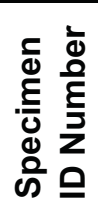 & \begin{tabular}{l}
$\overline{0}$ \\
\hdashline \\
0 \\
0 \\
$\infty$
\end{tabular} \\
\hline
\end{tabular}




\begin{tabular}{|c|c|}
\hline 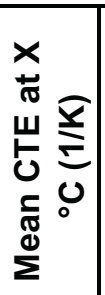 & 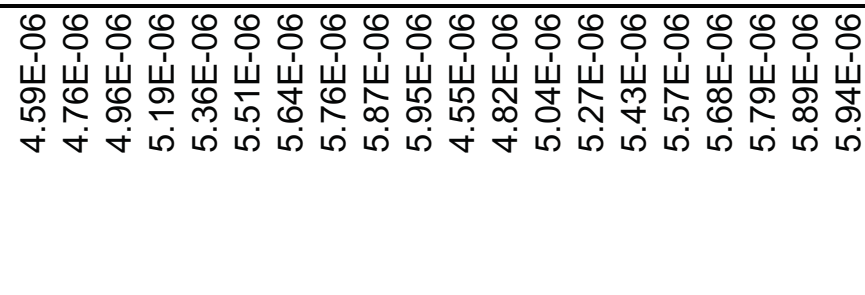 \\
\hline 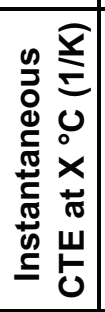 & 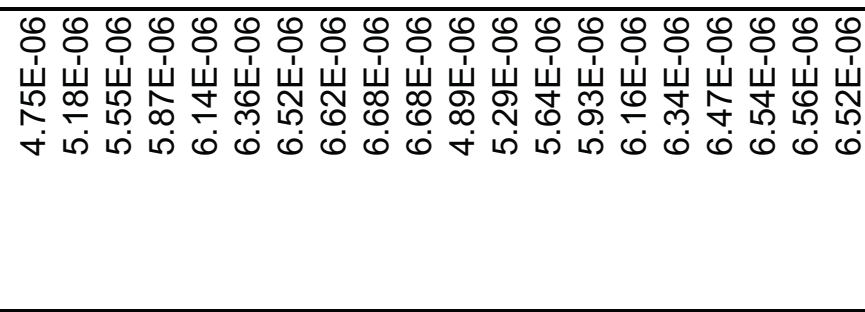 \\
\hline 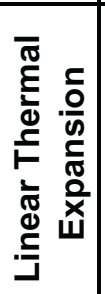 & 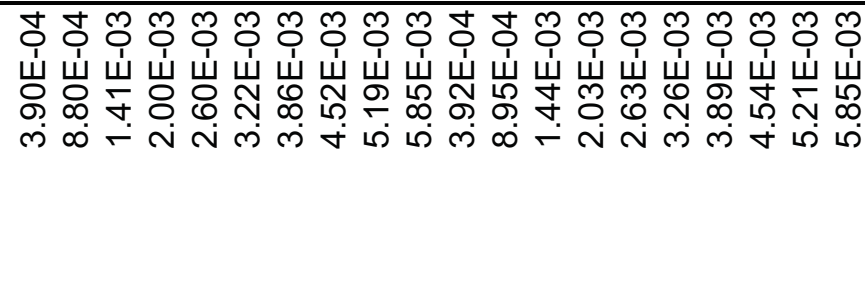 \\
\hline 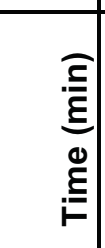 & 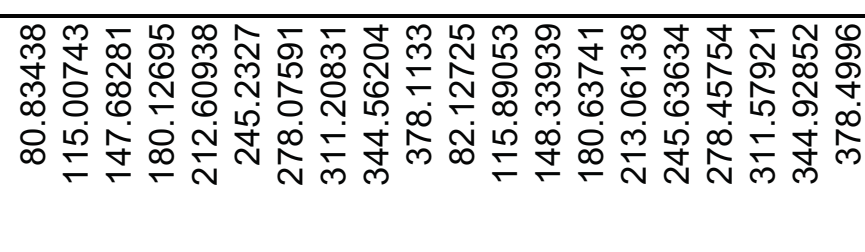 \\
\hline 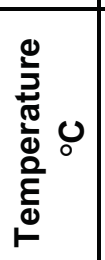 & 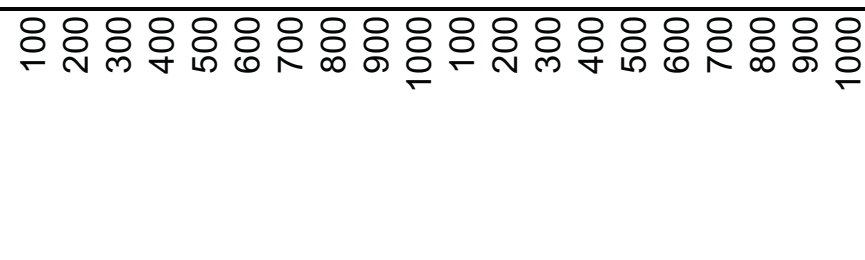 \\
\hline 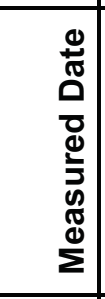 & 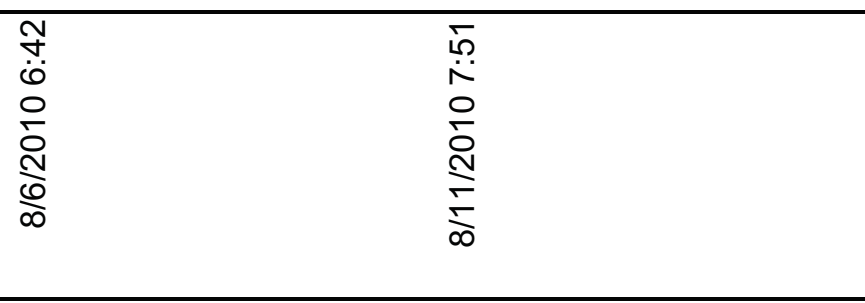 \\
\hline 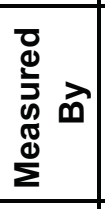 & $\frac{\infty}{3}$ \\
\hline 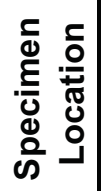 & \\
\hline 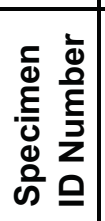 & $\begin{array}{l}\text { mo } \\
\infty \\
0 \\
0\end{array}$ \\
\hline
\end{tabular}




\begin{tabular}{|c|c|c|c|c|}
\hline 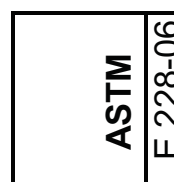 & 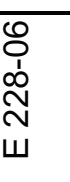 & 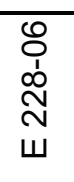 & $\begin{array}{l}\text { o } \\
\dot{0} \\
\stackrel{\infty}{N} \\
\text { w }\end{array}$ & $\begin{array}{l}\text { O } \\
0 \\
o \\
\text { N } \\
w\end{array}$ \\
\hline 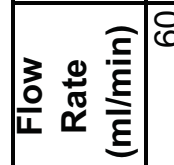 & 8 & 8 & 8 & 8 \\
\hline 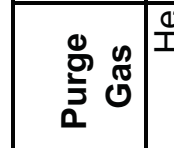 & 䨝 & $\frac{\Theta}{1}$ & $\frac{\Phi}{I}$ & 雳 \\
\hline 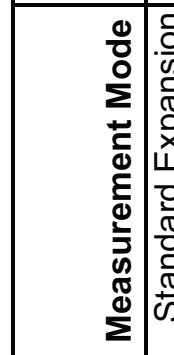 & 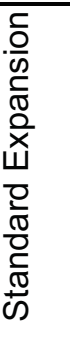 & 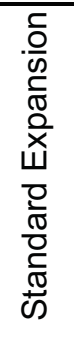 & 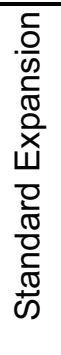 & 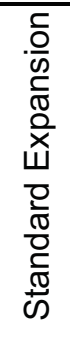 \\
\hline 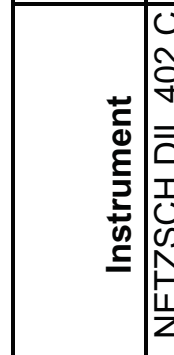 & 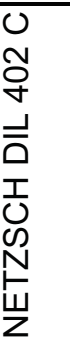 & 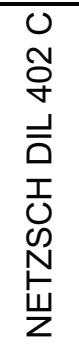 & 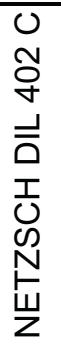 & 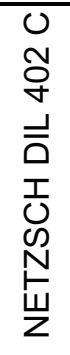 \\
\hline 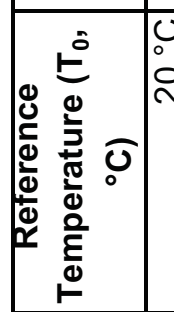 & $\begin{array}{l}\text { U } \\
\text { iv }\end{array}$ & $\begin{array}{l}0 \\
0 \\
\text { N }\end{array}$ & $\begin{array}{l}0 \\
\vdots \\
i\end{array}$ & $\begin{array}{l}0 \\
0 \\
i\end{array}$ \\
\hline 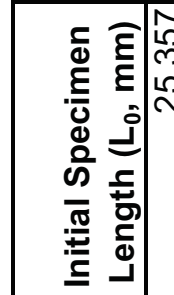 & & 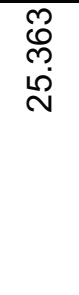 & 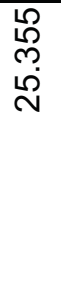 & $\begin{array}{l}\text { L } \\
\stackrel{n}{\infty} \\
\stackrel{\sim}{N}\end{array}$ \\
\hline 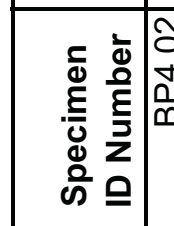 & & $\begin{array}{l}\text { mo } \\
0 \\
0 \\
0 \\
0\end{array}$ & $\begin{array}{l}\overline{0} \\
10 \\
0 \\
0\end{array}$ & $\begin{array}{l}\tilde{O} \\
\text { No } \\
0 \\
0\end{array}$ \\
\hline
\end{tabular}




\begin{tabular}{|c|c|c|c|}
\hline 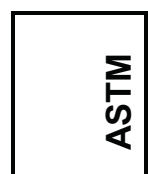 & 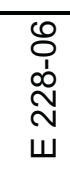 & 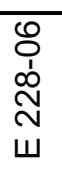 & 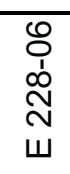 \\
\hline 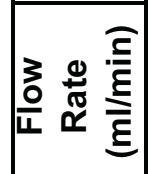 & 8 & 8 & 8 \\
\hline 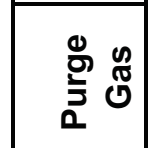 & $\frac{\Phi}{1}$ & $\frac{0}{1}$ & $\frac{\oplus}{1}$ \\
\hline 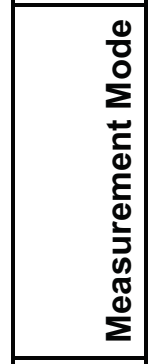 & 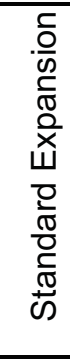 & 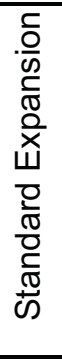 & 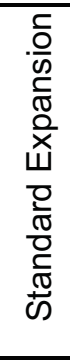 \\
\hline 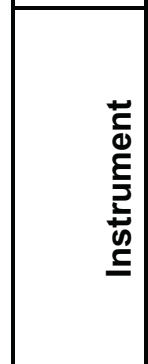 & 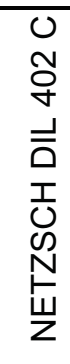 & 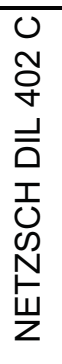 & 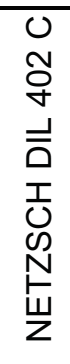 \\
\hline 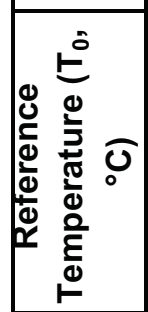 & $\begin{array}{l}0 \\
0 \\
\text { N }\end{array}$ & $\begin{array}{l}0 \\
\vdots \\
i\end{array}$ & $\begin{array}{l}0 \\
0 \\
\text { N }\end{array}$ \\
\hline 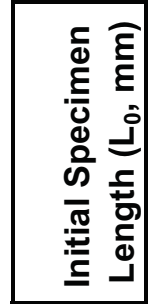 & 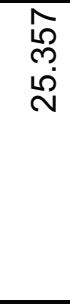 & $\begin{array}{l}\text { ָै } \\
\text { ले } \\
\text { مै }\end{array}$ & 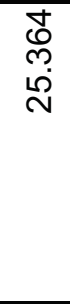 \\
\hline 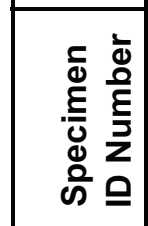 & $\begin{array}{l}\widetilde{O} \\
0 \\
\varnothing \\
0\end{array}$ & $\begin{array}{l}0 \\
0 \\
0 \\
0 \\
0\end{array}$ & $\sum_{\substack{\infty\\
}}^{\bar{c}}$ \\
\hline
\end{tabular}




\begin{tabular}{|c|c|c|c|c|}
\hline$\underset{⿱ 亠 䒑}{\mathbf{g}}$ & 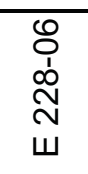 & 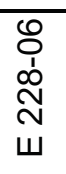 & 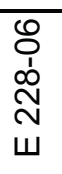 & $\begin{array}{l}\mathscr{Q} \\
\dot{1} \\
\text { N̦ } \\
w \\
w\end{array}$ \\
\hline 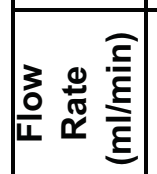 & 8 & 8 & 8 & 8 \\
\hline 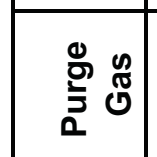 & $\frac{\Phi}{I}$ & $\stackrel{\oplus}{1}$ & $\stackrel{\oplus}{\mathbf{I}}$ & $\stackrel{\Phi}{I}$ \\
\hline 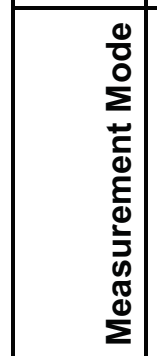 & 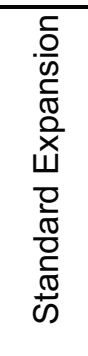 & 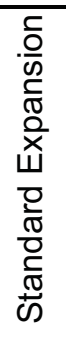 & 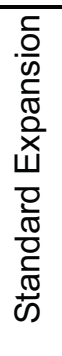 & 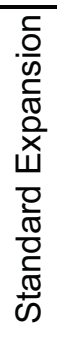 \\
\hline 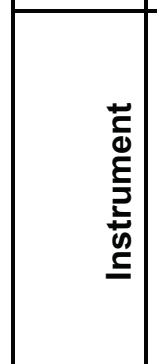 & 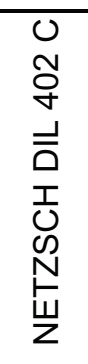 & 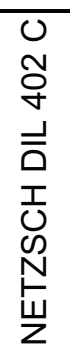 & 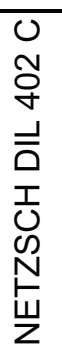 & 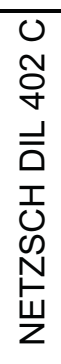 \\
\hline 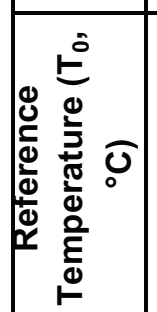 & $\begin{array}{l}0 \\
0 \\
O \\
N\end{array}$ & $\begin{array}{l}0 \\
0 \\
\text { Oे }\end{array}$ & $\begin{array}{l}\text { O } \\
\text { N }\end{array}$ & $\begin{array}{l}0 \\
0 \\
0 \\
\text { N }\end{array}$ \\
\hline 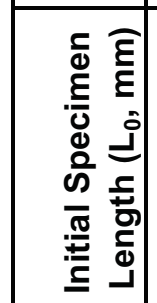 & 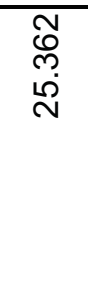 & $\begin{array}{l}\hat{\mathrm{p}} \\
\mathrm{m} \\
\stackrel{\omega}{\mathrm{N}}\end{array}$ & 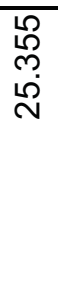 & 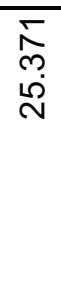 \\
\hline 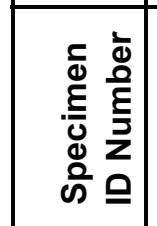 & $\begin{array}{l}\tilde{O} \\
\sum_{\tilde{m}}\end{array}$ & $\begin{array}{l}0 \\
0 \\
\llcorner \\
0 \\
0\end{array}$ & $\begin{array}{l}\overline{0} \\
0 \\
0 \\
0\end{array}$ & $\begin{array}{l}\bar{\delta} \\
\sum_{0}^{\frac{j}{j}}\end{array}$ \\
\hline
\end{tabular}




\begin{tabular}{|c|c|c|c|}
\hline$\underset{\substack{g \\
\&}}{\Sigma}$ & 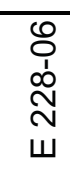 & $\begin{array}{l}\text { O্ } \\
0 \\
\text { Dे } \\
\text { W }\end{array}$ & 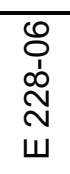 \\
\hline 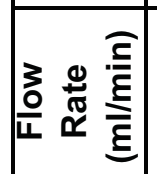 & 8 & 8 & 8 \\
\hline 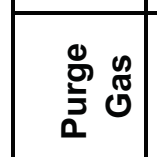 & $\stackrel{\oplus}{I}$ & $\stackrel{\oplus}{\Phi}$ & $\stackrel{\oplus}{I}$ \\
\hline 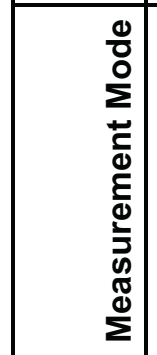 & 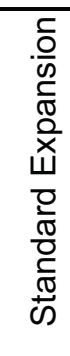 & 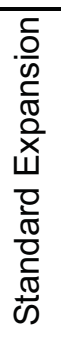 & 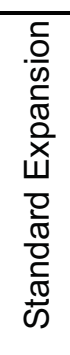 \\
\hline 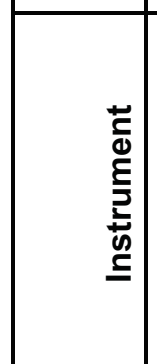 & 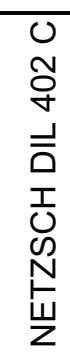 & 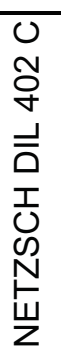 & 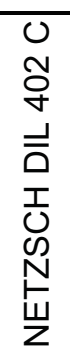 \\
\hline 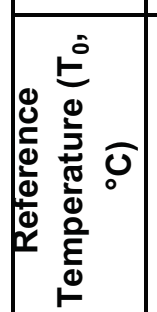 & $\begin{array}{l}\text { U } \\
\text { N }\end{array}$ & $\begin{array}{l}0 \\
\vdots \\
\text { N }\end{array}$ & $\begin{array}{l}\text { U } \\
\text { Oे }\end{array}$ \\
\hline 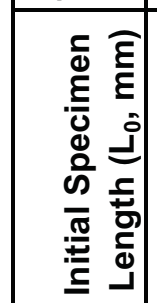 & $\begin{array}{l}\text { \&్ } \\
\text { क्. } \\
\text { ஸें }\end{array}$ & 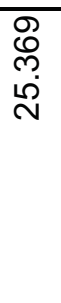 & 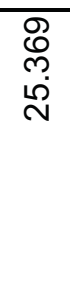 \\
\hline 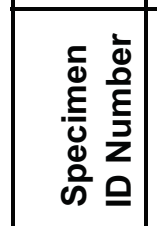 & 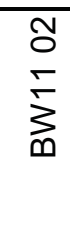 & 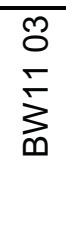 & $\sum_{\substack{\text { N } \\
\text { N }\\
}}$ \\
\hline
\end{tabular}




\begin{tabular}{|c|c|c|c|}
\hline$\underset{⿱ ㇒}{\mathbf{g}}$ & 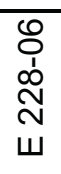 & 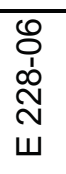 & $\begin{array}{l}0 \\
\\
\dot{1} \\
\text { N } \\
w\end{array}$ \\
\hline 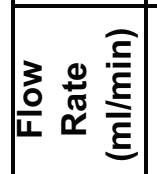 & 8 & 8 & 8 \\
\hline 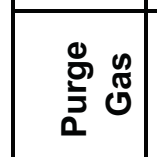 & $\frac{\Phi}{I}$ & $\stackrel{\oplus}{I}$ & $\frac{\Theta}{I}$ \\
\hline 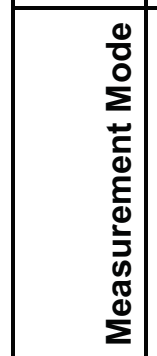 & 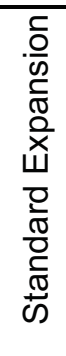 & 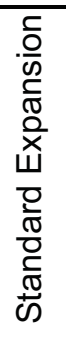 & 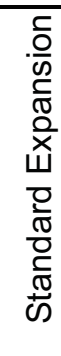 \\
\hline 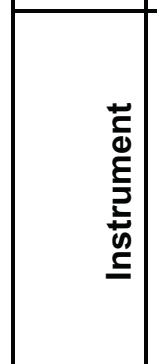 & 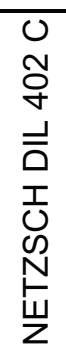 & 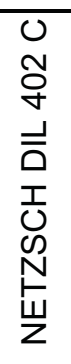 & 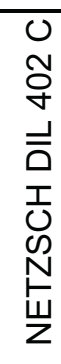 \\
\hline 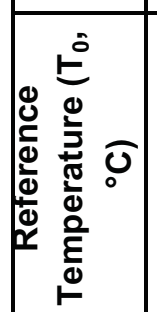 & $\begin{array}{l}0 \\
0 \\
\text { Q }\end{array}$ & $\begin{array}{l}0 \\
0 \\
\text { Q }\end{array}$ & $\begin{array}{l}0 \\
0 \\
i\end{array}$ \\
\hline 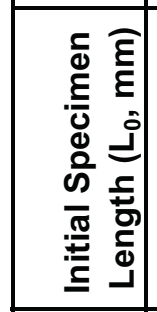 & $\begin{array}{l}\hat{0} \\
\text { N } \\
\stackrel{N}{N}\end{array}$ & 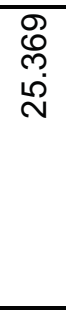 & 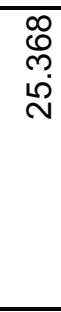 \\
\hline 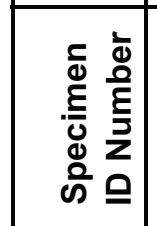 & $\begin{array}{l}\text { Oo } \\
\text { N } \\
\sum_{0}^{N}\end{array}$ & $\begin{array}{l}\bar{c} \\
\sum_{m}^{m}\end{array}$ & $\begin{array}{l}\text { No } \\
\sum_{\infty}^{m}\end{array}$ \\
\hline
\end{tabular}




\begin{tabular}{|c|c|c|c|c|}
\hline 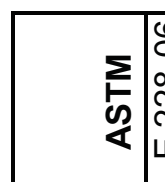 & 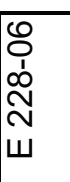 & 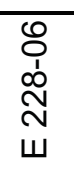 & 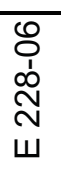 & 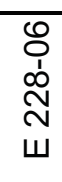 \\
\hline 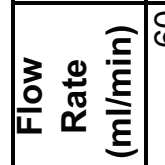 & 8 & 8 & 8 & 8 \\
\hline 竞 & $\stackrel{\oplus}{I}$ & $\frac{\Theta}{1}$ & $\frac{\Phi}{I}$ & $\frac{0}{1}$ \\
\hline 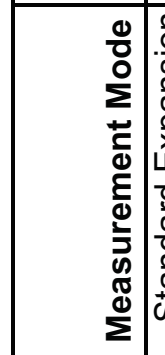 & 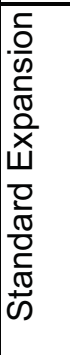 & 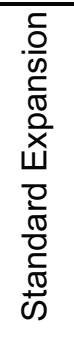 & 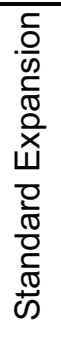 & 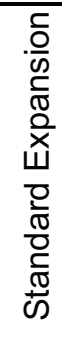 \\
\hline 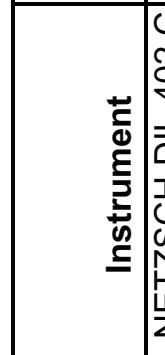 & 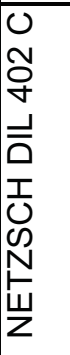 & 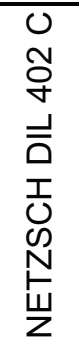 & 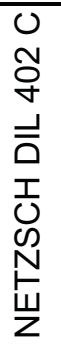 & 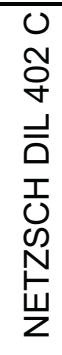 \\
\hline 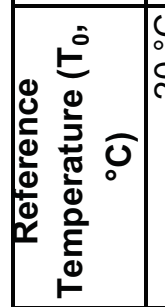 & $\begin{array}{l}0 \\
0 \\
\text { N }\end{array}$ & $\begin{array}{l}0 \\
0 \\
\text { N }\end{array}$ & $\begin{array}{l}0 \\
\vdots \\
i\end{array}$ & $\begin{array}{l}0 \\
0 \\
i\end{array}$ \\
\hline 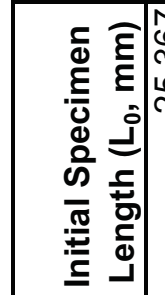 & 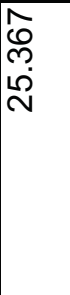 & 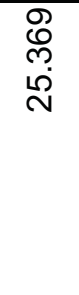 & 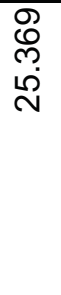 & $\begin{array}{l}\infty \\
0 \\
0 \\
\text { m. } \\
\stackrel{D}{N}\end{array}$ \\
\hline 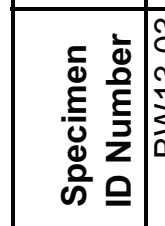 & 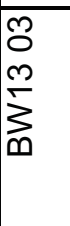 & $\begin{array}{l}\bar{\delta} \\
\dot{J} \\
\sum_{0}\end{array}$ & 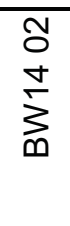 & $\begin{array}{l}0 \\
0 \\
\dot{\square} \\
\vdots \\
\vdots\end{array}$ \\
\hline
\end{tabular}




\begin{tabular}{|c|c|c|c|}
\hline$\underset{\substack{\underline{2} \\
\&}}{\mathbf{g}}$ & $\begin{array}{l}0 \\
\\
1 \\
\stackrel{1}{ } \\
\text { N } \\
\Psi\end{array}$ & 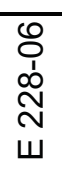 & 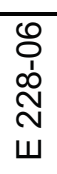 \\
\hline 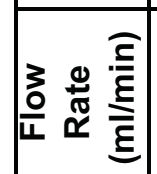 & 8 & 8 & 8 \\
\hline 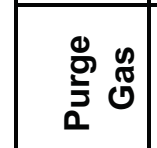 & $\frac{\Phi}{I}$ & $\frac{\mathbb{1}}{1}$ & $\frac{\mathbb{O}}{I}$ \\
\hline 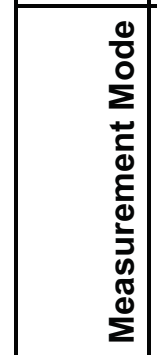 & 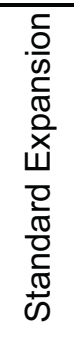 & 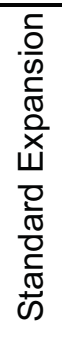 & 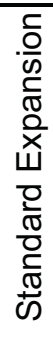 \\
\hline 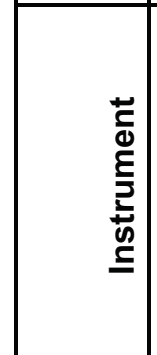 & 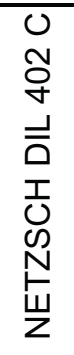 & 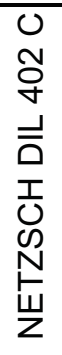 & 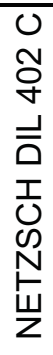 \\
\hline 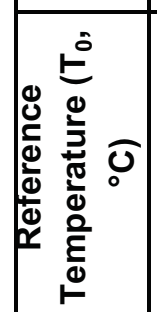 & $\begin{array}{l}0 \\
\vdots \\
\text { Nे }\end{array}$ & $\begin{array}{l}0 \\
0 \\
\text { N }\end{array}$ & $\begin{array}{l}0 \\
0 \\
0 \\
N\end{array}$ \\
\hline 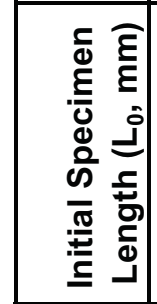 & 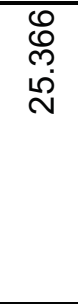 & $\begin{array}{l}\infty \\
0 \\
\text { m } \\
\stackrel{n}{N}\end{array}$ & $\begin{array}{l}\infty \\
\infty \\
\infty \\
\stackrel{\rho}{\rho} \\
\text { N }\end{array}$ \\
\hline 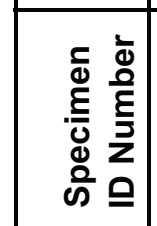 & $\begin{array}{l}\bar{\delta} \\
\sum_{0}^{\infty} \\
\end{array}$ & 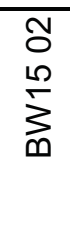 & $\begin{array}{l}\text { m } \\
0 \\
\sum_{0}^{10}\end{array}$ \\
\hline
\end{tabular}




\begin{tabular}{|c|c|c|c|c|}
\hline$\underset{⿱ 亠 䒑}{\mathbf{g}}$ & 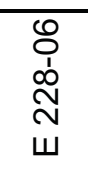 & 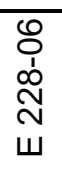 & 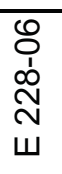 & $\begin{array}{l}\mathscr{Q} \\
0 \\
\text { Ǹ } \\
\text { w }\end{array}$ \\
\hline 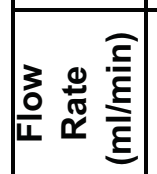 & 8 & 8 & 8 & 8 \\
\hline 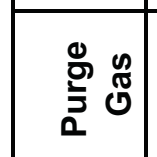 & $\frac{\Phi}{I}$ & $\stackrel{\oplus}{\perp}$ & $\stackrel{\oplus}{\mathbf{I}}$ & $\stackrel{\Phi}{I}$ \\
\hline 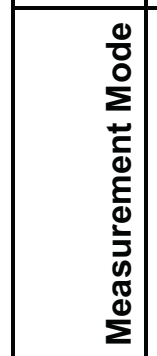 & 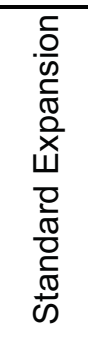 & 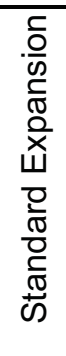 & 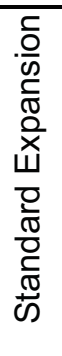 & 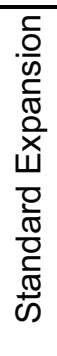 \\
\hline 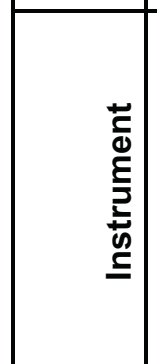 & 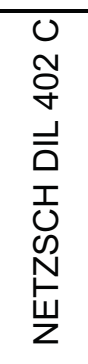 & 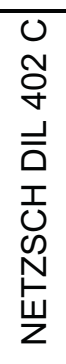 & 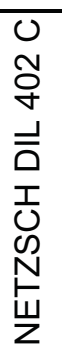 & 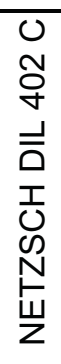 \\
\hline 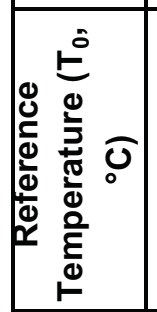 & $\begin{array}{l}0 \\
0 \\
O \\
N\end{array}$ & $\begin{array}{l}\text { U } \\
0 \\
\text { N }\end{array}$ & $\begin{array}{l}0 \\
0 \\
0\end{array}$ & $\begin{array}{l}0 \\
0 \\
0\end{array}$ \\
\hline 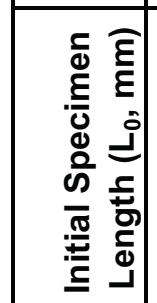 & $\begin{array}{l}\infty \\
0 \\
0 \\
\text { Dे } \\
\text { N }\end{array}$ & 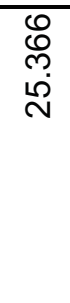 & 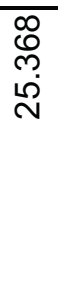 & 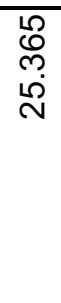 \\
\hline 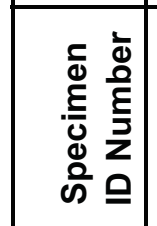 & $\begin{array}{l}\overline{0} \\
0 \\
\vdots \\
\vdots\end{array}$ & $\begin{array}{l}\text { No } \\
0 \\
0 \\
\sum_{0}\end{array}$ & $\begin{array}{l}\text { o } \\
0 \\
0 \\
\sum_{0}\end{array}$ & $\begin{array}{l}\bar{\delta} \\
\text { No } \\
\text { ñ }\end{array}$ \\
\hline
\end{tabular}




\begin{tabular}{|c|c|c|c|}
\hline$\underset{\substack{\sum \\
\&}}{\sum}$ & 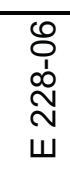 & 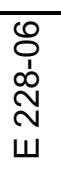 & 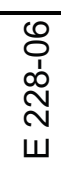 \\
\hline 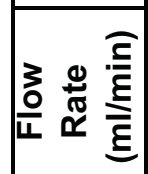 & 8 & 8 & 8 \\
\hline 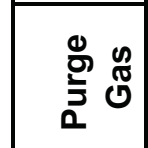 & $\frac{\Phi}{1}$ & $\frac{\Phi}{I}$ & $\frac{\Theta}{1}$ \\
\hline 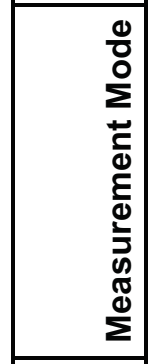 & 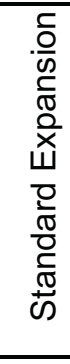 & 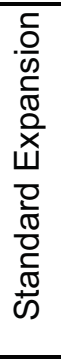 & 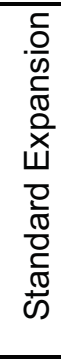 \\
\hline 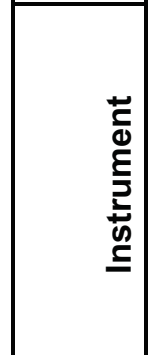 & 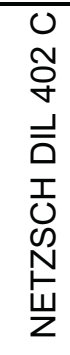 & 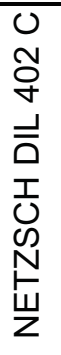 & 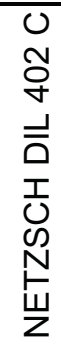 \\
\hline 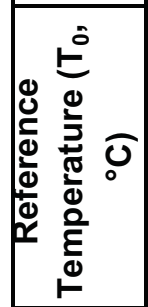 & 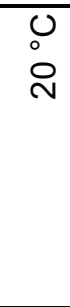 & $\begin{array}{l}0 \\
0 \\
\text { N }\end{array}$ & $\begin{array}{l}0 \\
\vdots \\
\text { N }\end{array}$ \\
\hline 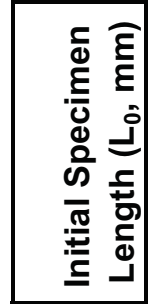 & 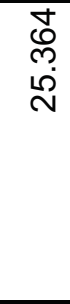 & 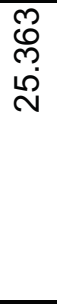 & $\begin{array}{l}\stackrel{0}{0} \\
0 \\
\stackrel{\infty}{0}\end{array}$ \\
\hline 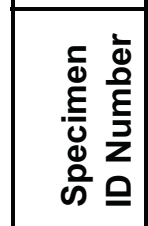 & $\begin{array}{l}\text { N } \\
\text { No } \\
\text { in }\end{array}$ & $\begin{array}{l}\text { Õ } \\
\text { N } \\
\text { jom }\end{array}$ & $\begin{array}{l}\bar{c} \\
m \\
\sum_{0}\end{array}$ \\
\hline
\end{tabular}




\begin{tabular}{|c|c|c|c|}
\hline$\underset{⿱ ㇒}{\mathbf{g}}$ & 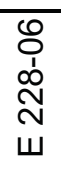 & 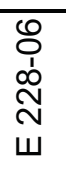 & 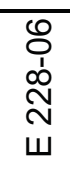 \\
\hline 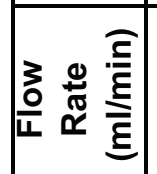 & 8 & 8 & 8 \\
\hline 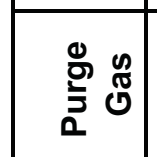 & $\stackrel{\Phi}{I}$ & $\stackrel{\oplus}{\Phi}$ & $\stackrel{\oplus}{1}$ \\
\hline 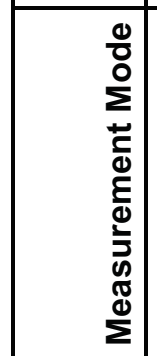 & 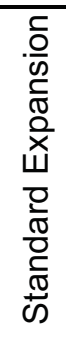 & 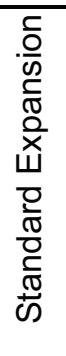 & 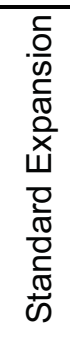 \\
\hline 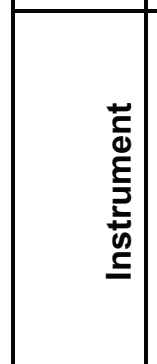 & 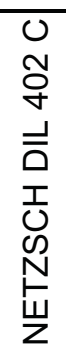 & 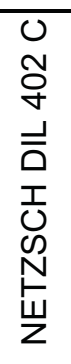 & 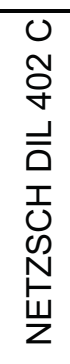 \\
\hline 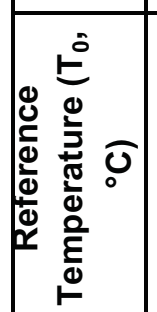 & $\begin{array}{l}0 \\
0 \\
\text { Oे }\end{array}$ & $\begin{array}{l}0 \\
0 \\
\text { Oे }\end{array}$ & $\begin{array}{l}0 \\
0 \\
\text { Oे }\end{array}$ \\
\hline 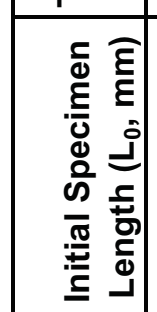 & $\begin{array}{l}\mathbb{Q} \\
\stackrel{0}{ } \\
\stackrel{\rho}{N}\end{array}$ & 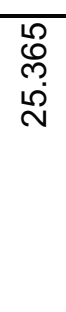 & 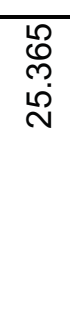 \\
\hline 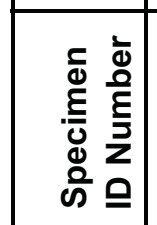 & $\begin{array}{l}\text { m } \\
\text { m } \\
\sum_{0}\end{array}$ & $\begin{array}{l}\overline{0} \\
\sum_{\infty}^{+}\end{array}$ & $\begin{array}{l}\text { õ } \\
\text { m } \\
\sum_{0}\end{array}$ \\
\hline
\end{tabular}




\begin{tabular}{|c|c|c|c|c|}
\hline 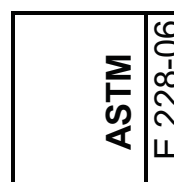 & 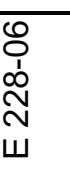 & 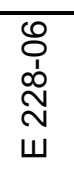 & $\begin{array}{l}\text { o } \\
\dot{0} \\
\stackrel{\infty}{N} \\
\text { w }\end{array}$ & $\begin{array}{l}\text { O } \\
0 \\
o \\
\text { N } \\
w\end{array}$ \\
\hline 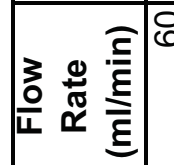 & 8 & 8 & 8 & 8 \\
\hline 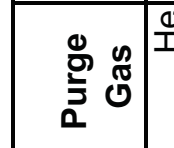 & 䨝 & $\frac{\Theta}{1}$ & 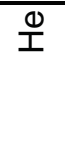 & 雳 \\
\hline 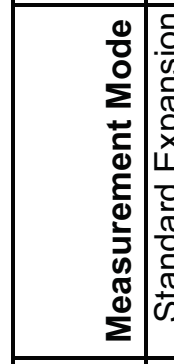 & 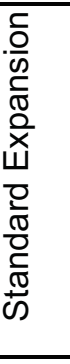 & 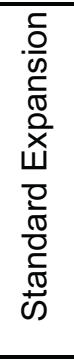 & 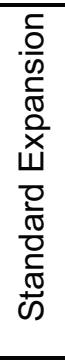 & 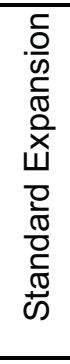 \\
\hline 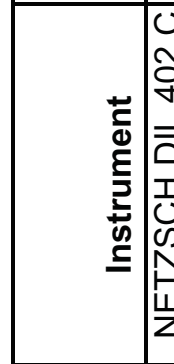 & 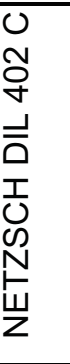 & 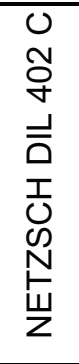 & 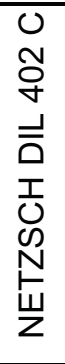 & 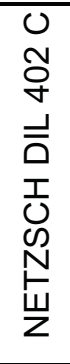 \\
\hline 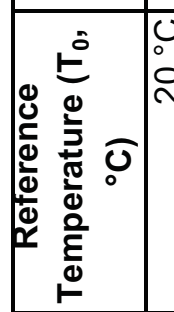 & $\begin{array}{l}\text { U } \\
\text { iv }\end{array}$ & $\begin{array}{l}0 \\
0 \\
\text { N }\end{array}$ & $\begin{array}{l}\text { U } \\
\text { N }\end{array}$ & $\begin{array}{l}0 \\
0 \\
i\end{array}$ \\
\hline 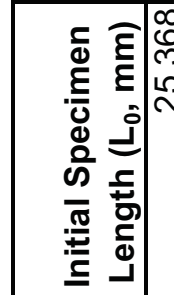 & & 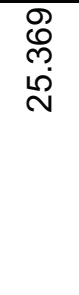 & $\begin{array}{l}\hat{0} \\
\text { p. } \\
\stackrel{2}{\infty}\end{array}$ & 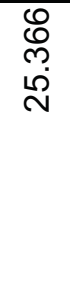 \\
\hline 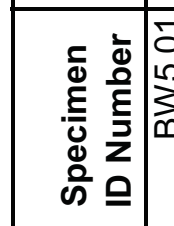 & & 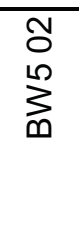 & $\begin{array}{l}0 \\
0 \\
\sum_{0}^{n} \\
\text { m }\end{array}$ & $\begin{array}{l}\text { Oे } \\
0 \\
\sum_{0}^{+}\end{array}$ \\
\hline
\end{tabular}




\begin{tabular}{|c|c|c|c|}
\hline$\underset{\substack{g \\
\&}}{\Sigma}$ & 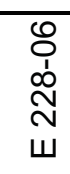 & 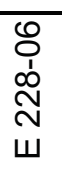 & 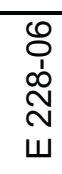 \\
\hline 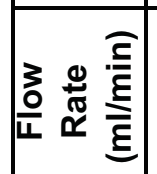 & 8 & 8 & 8 \\
\hline 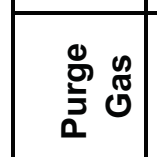 & $\stackrel{\Phi}{I}$ & $\stackrel{\oplus}{\perp}$ & $\stackrel{\oplus}{I}$ \\
\hline 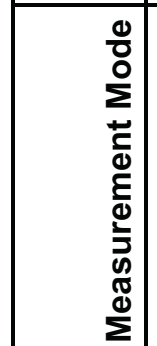 & 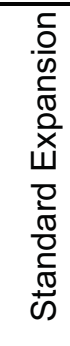 & 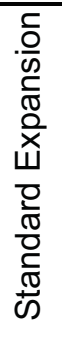 & 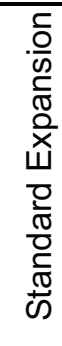 \\
\hline 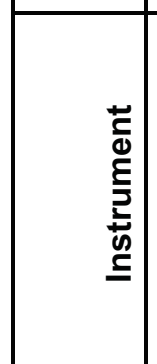 & 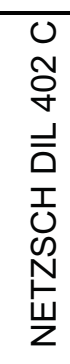 & 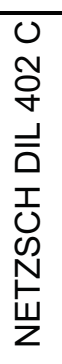 & 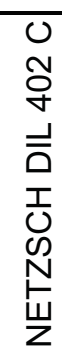 \\
\hline 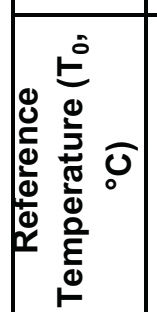 & $\begin{array}{l}\text { U } \\
\text { N }\end{array}$ & $\begin{array}{l}\text { U } \\
\text { N }\end{array}$ & $\begin{array}{l}\text { U } \\
\text { N }\end{array}$ \\
\hline 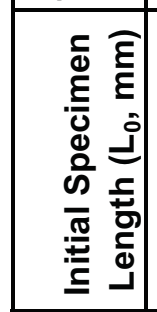 & $\begin{array}{l}\mathscr{O} \\
\stackrel{0}{ } \\
\stackrel{\infty}{N}\end{array}$ & 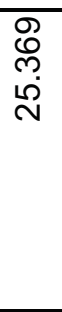 & $\begin{array}{l}\hat{m} \\
\stackrel{N}{N}\end{array}$ \\
\hline 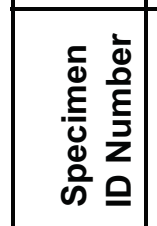 & $\begin{array}{l}\text { J } \\
\text { J } \\
\sum_{\infty}^{+}\end{array}$ & $\begin{array}{l}\delta \\
0 \\
0 \\
\sum_{0}\end{array}$ & $\begin{array}{l}\text { No } \\
\text { O } \\
\text { ऐo }\end{array}$ \\
\hline
\end{tabular}




\begin{tabular}{|c|c|c|c|c|}
\hline$\underset{⿱ ㇒}{\mathbf{g}}$ & 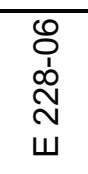 & 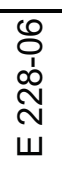 & 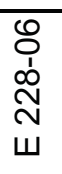 & $\begin{array}{l}\text { O } \\
0 \\
\text { N̦ } \\
w\end{array}$ \\
\hline 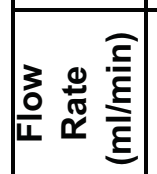 & 8 & 8 & 8 & 8 \\
\hline 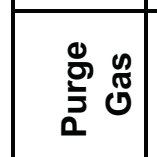 & $\frac{\Phi}{I}$ & $\stackrel{\oplus}{\perp}$ & $\stackrel{\oplus}{\mathbf{I}}$ & 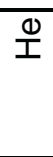 \\
\hline 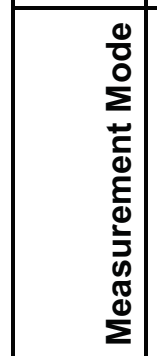 & 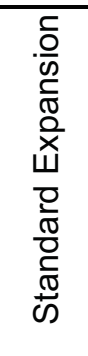 & 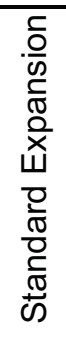 & 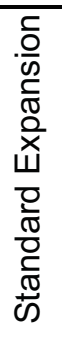 & 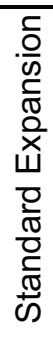 \\
\hline 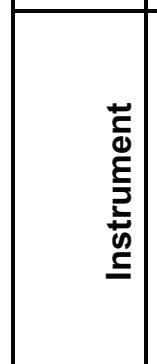 & 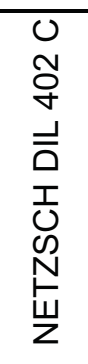 & 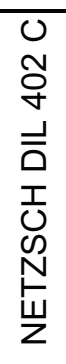 & 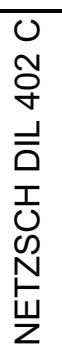 & 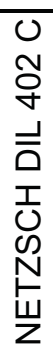 \\
\hline 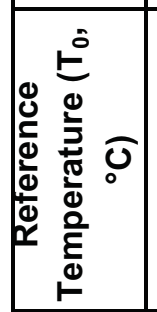 & $\begin{array}{l}0 \\
0 \\
O \\
N\end{array}$ & $\begin{array}{l}\text { U } \\
0 \\
\text { N }\end{array}$ & $\begin{array}{l}0 \\
0 \\
0\end{array}$ & $\begin{array}{l}0 \\
0 \\
0 \\
N\end{array}$ \\
\hline 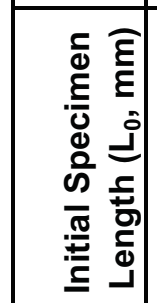 & $\begin{array}{l}\hat{m} \\
\stackrel{N}{\text { N }}\end{array}$ & $\begin{array}{l}0 \\
0 \\
\stackrel{0}{0} \\
\stackrel{\omega}{N}\end{array}$ & $\begin{array}{l}\text { 志 } \\
\text { ळ. } \\
\stackrel{\text { N }}{ }\end{array}$ & $\begin{array}{l}\text { \& } \\
\text { m. } \\
\text { مैं }\end{array}$ \\
\hline 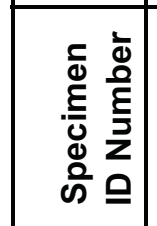 & $\begin{array}{l}\text { O } \\
0 \\
0 \\
\sum_{0}\end{array}$ & $\begin{array}{l}\bar{\delta} \\
\text { O } \\
\sum_{0}^{\prime}\end{array}$ & $\sum_{\substack{\infty \\
0}}^{\infty}$ & $\begin{array}{l}\bar{j} \\
\text { N } \\
\sum_{\infty}\end{array}$ \\
\hline
\end{tabular}




\begin{tabular}{|c|c|c|}
\hline 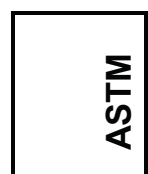 & 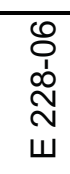 & 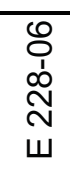 \\
\hline 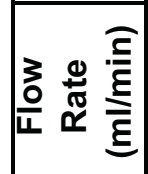 & 8 & 8 \\
\hline 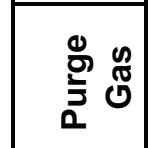 & 雪 & $\frac{\oplus}{I}$ \\
\hline 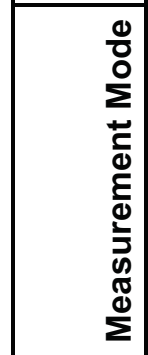 & 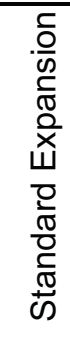 & 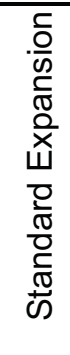 \\
\hline 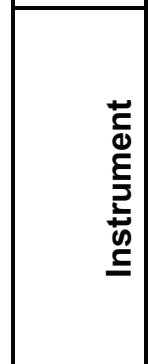 & 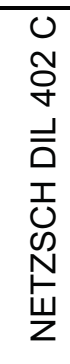 & 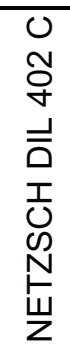 \\
\hline 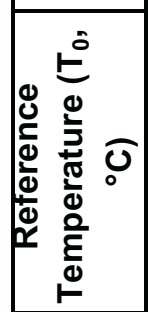 & $\begin{array}{l}0 \\
0 \\
2\end{array}$ & $\begin{array}{l}0 \\
0 \\
\text { N }\end{array}$ \\
\hline 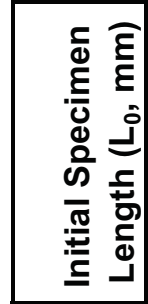 & 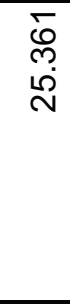 & 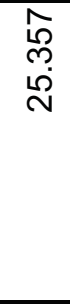 \\
\hline 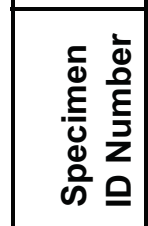 & $\begin{array}{l}\overline{0} \\
m \\
0 \\
0\end{array}$ & $\begin{array}{l}\tilde{O} \\
\tilde{m} \\
\tilde{0} \\
0\end{array}$ \\
\hline
\end{tabular}




\begin{tabular}{|c|c|c|}
\hline$\underset{\substack{\mathbf{g} \\
\mathbf{g}}}{\mathbf{\Sigma}}$ & $\begin{array}{l}0 \\
\\
o \\
\text { N } \\
\text { w }\end{array}$ & $\begin{array}{l}0 \\
0 \\
0 \\
\text { Nै } \\
\text { w }\end{array}$ \\
\hline 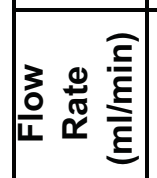 & 8 & 8 \\
\hline 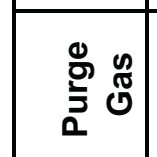 & $\frac{\Phi}{I}$ & $\frac{\Phi}{I}$ \\
\hline 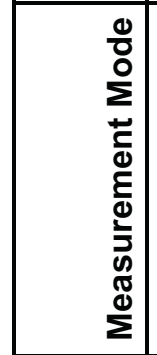 & 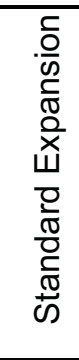 & 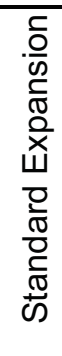 \\
\hline 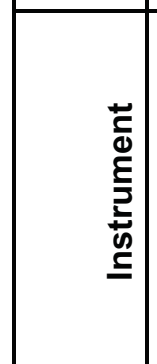 & 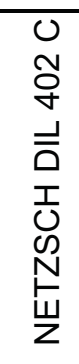 & 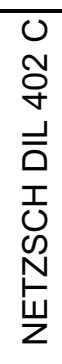 \\
\hline 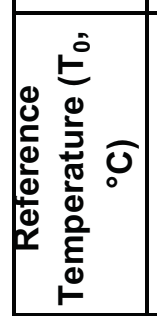 & $\begin{array}{l}\text { U } \\
\vdots \\
\text { N }\end{array}$ & $\begin{array}{l}0 \\
0 \\
\text { N }\end{array}$ \\
\hline 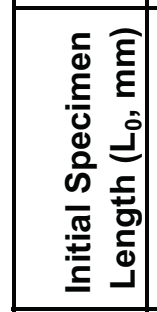 & 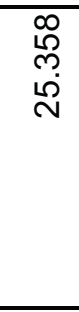 & 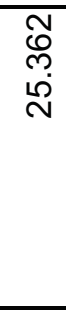 \\
\hline 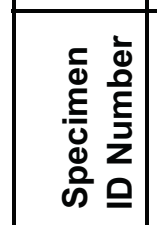 & $\begin{array}{l}0 \\
0 \\
0 \\
0 \\
0\end{array}$ & $\begin{array}{l}\bar{\delta} \\
\vdots \\
0 \\
0\end{array}$ \\
\hline
\end{tabular}




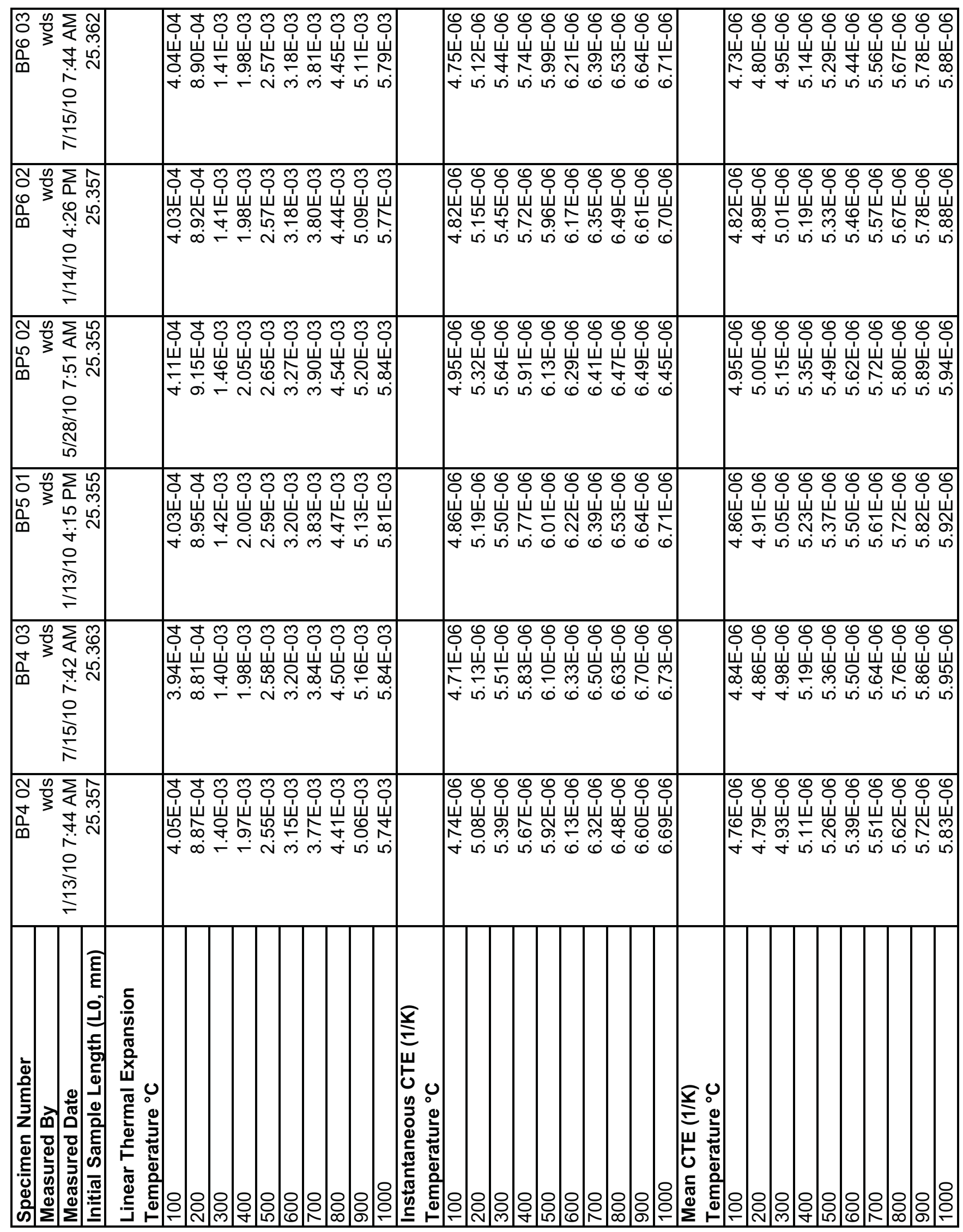




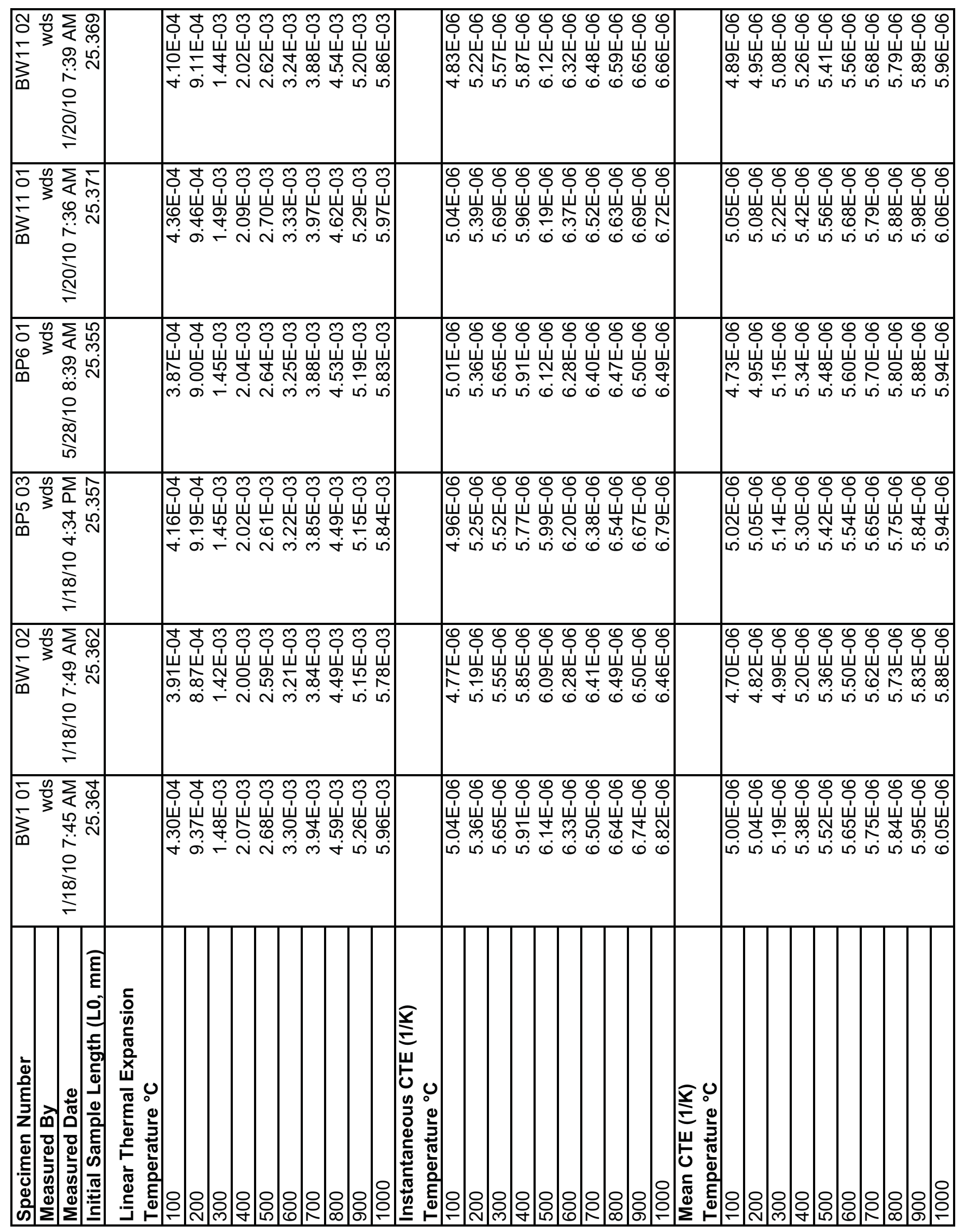




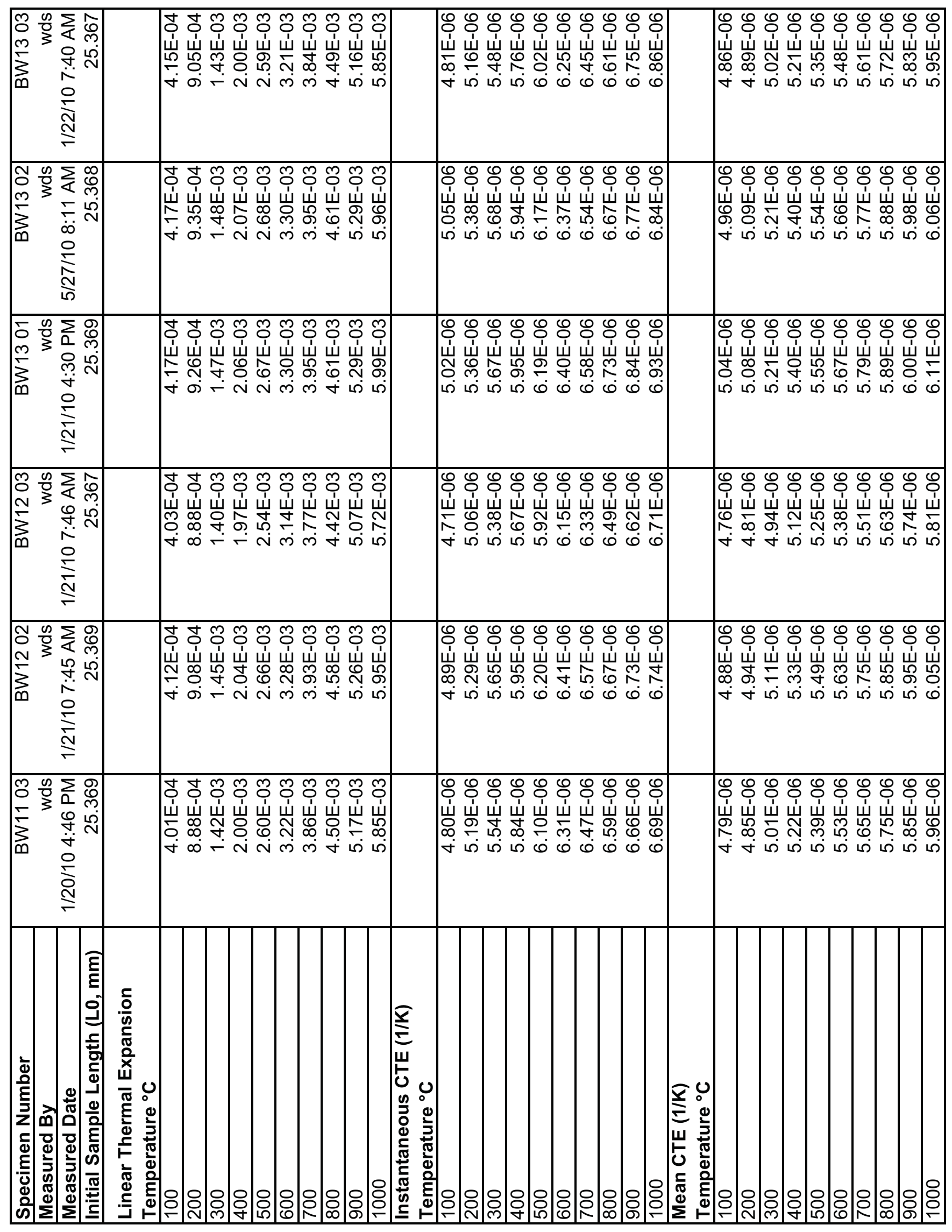




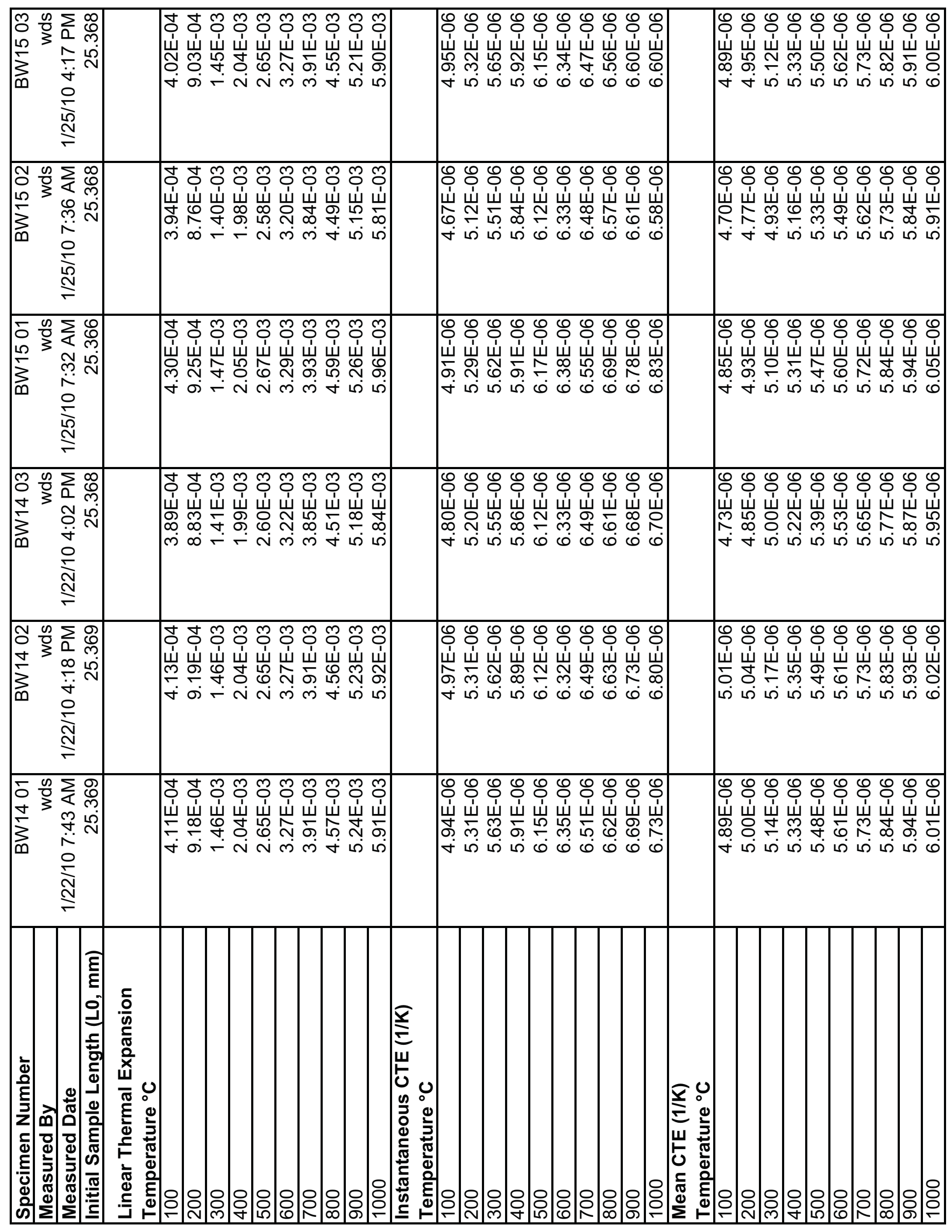




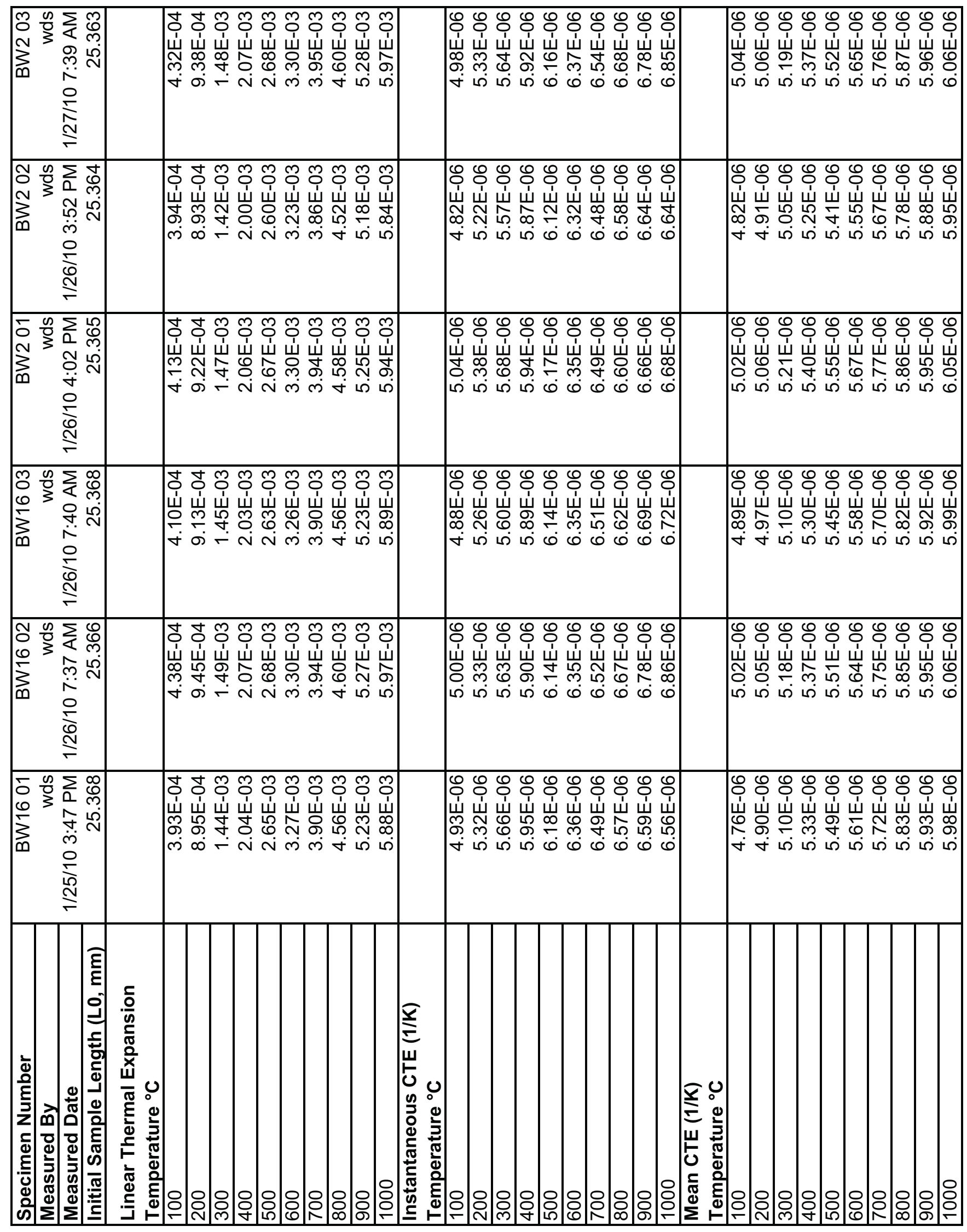




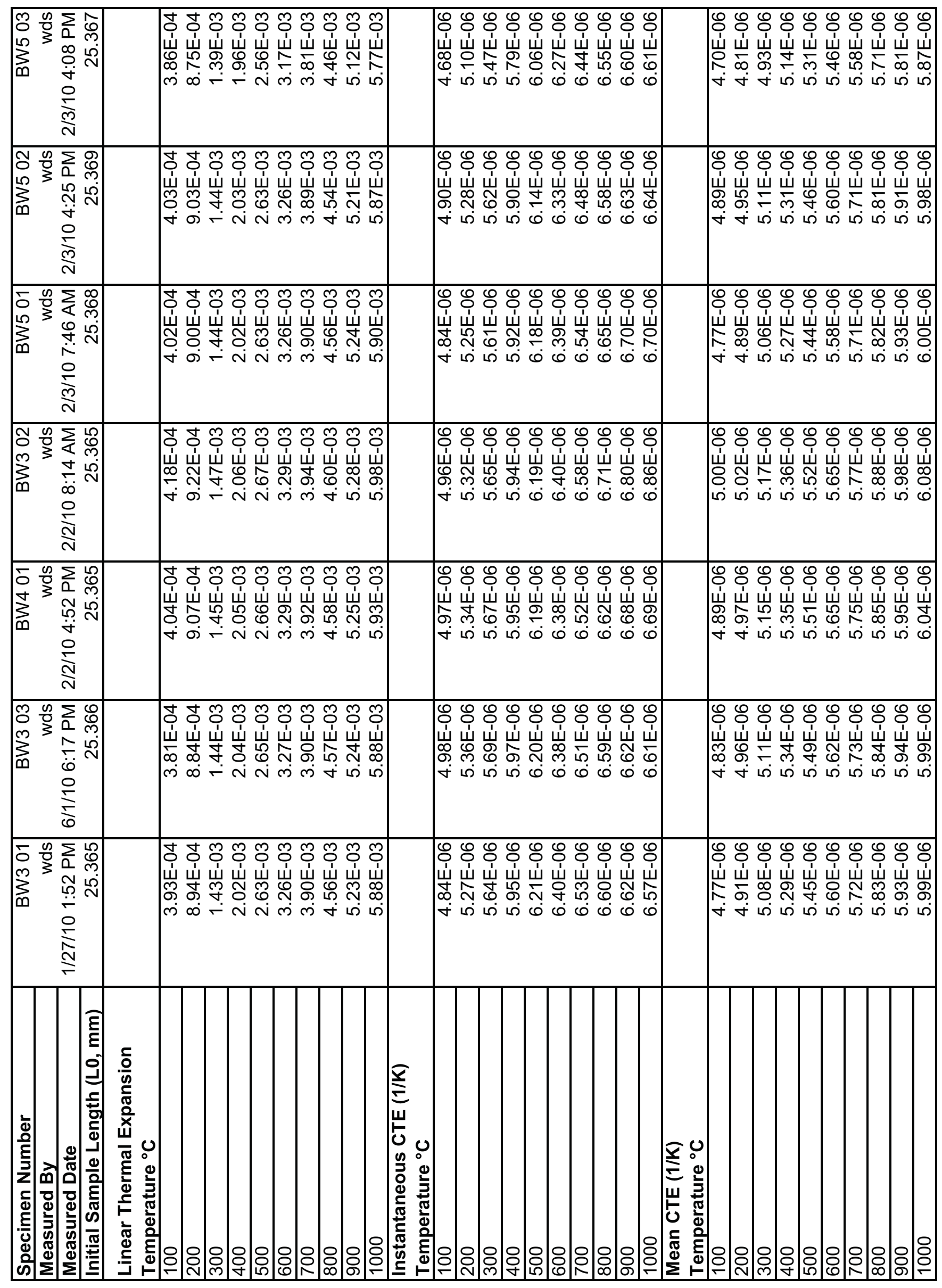




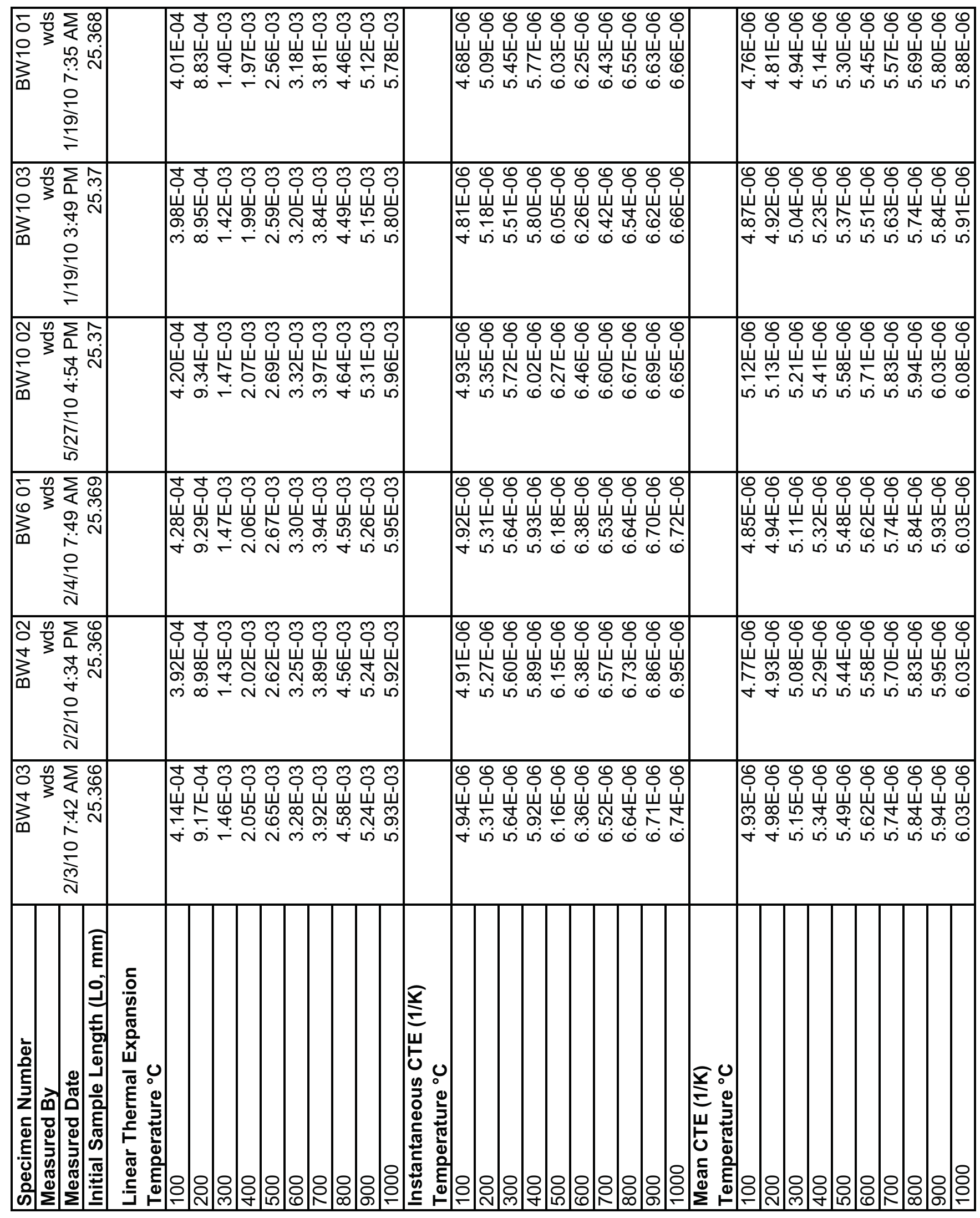




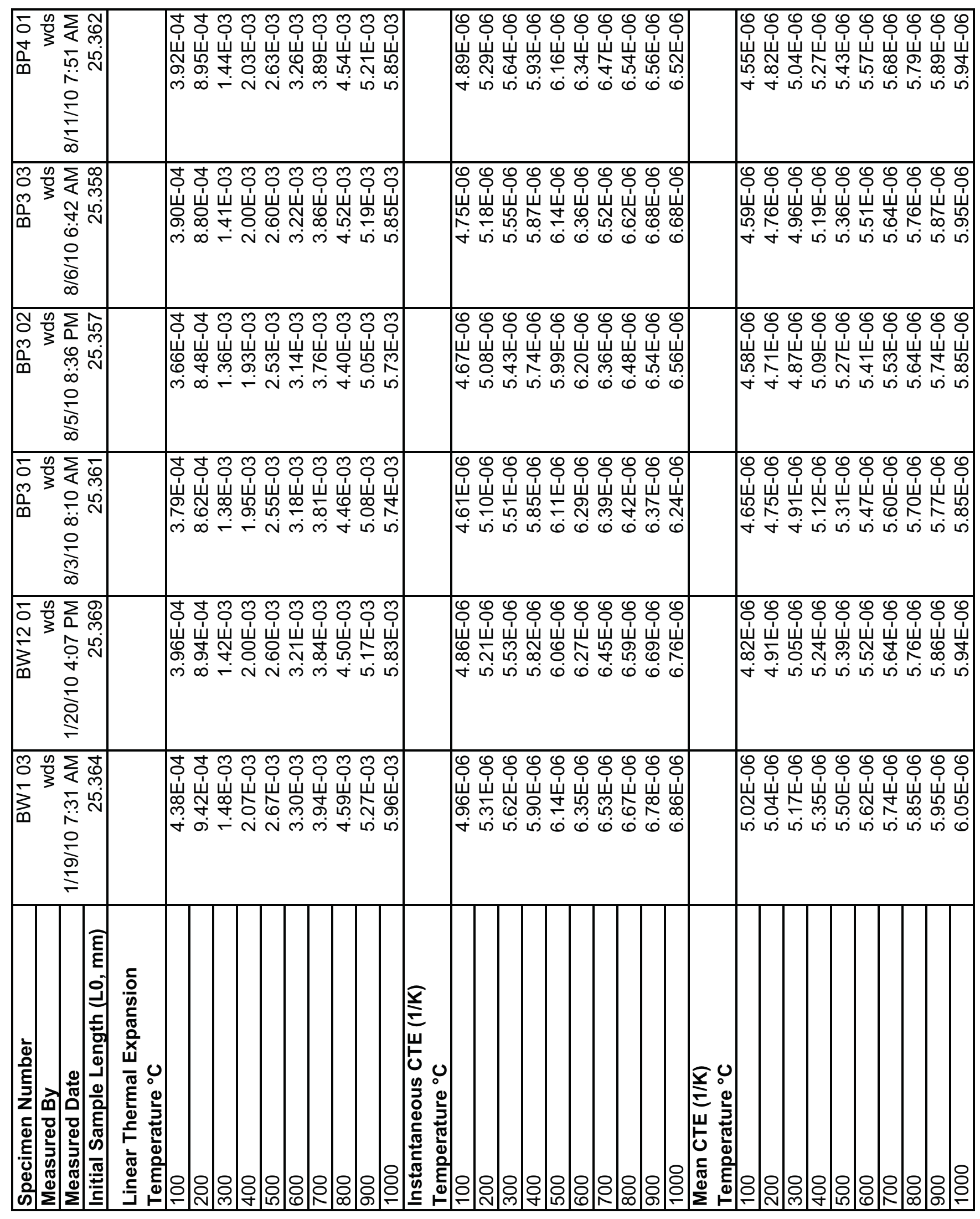




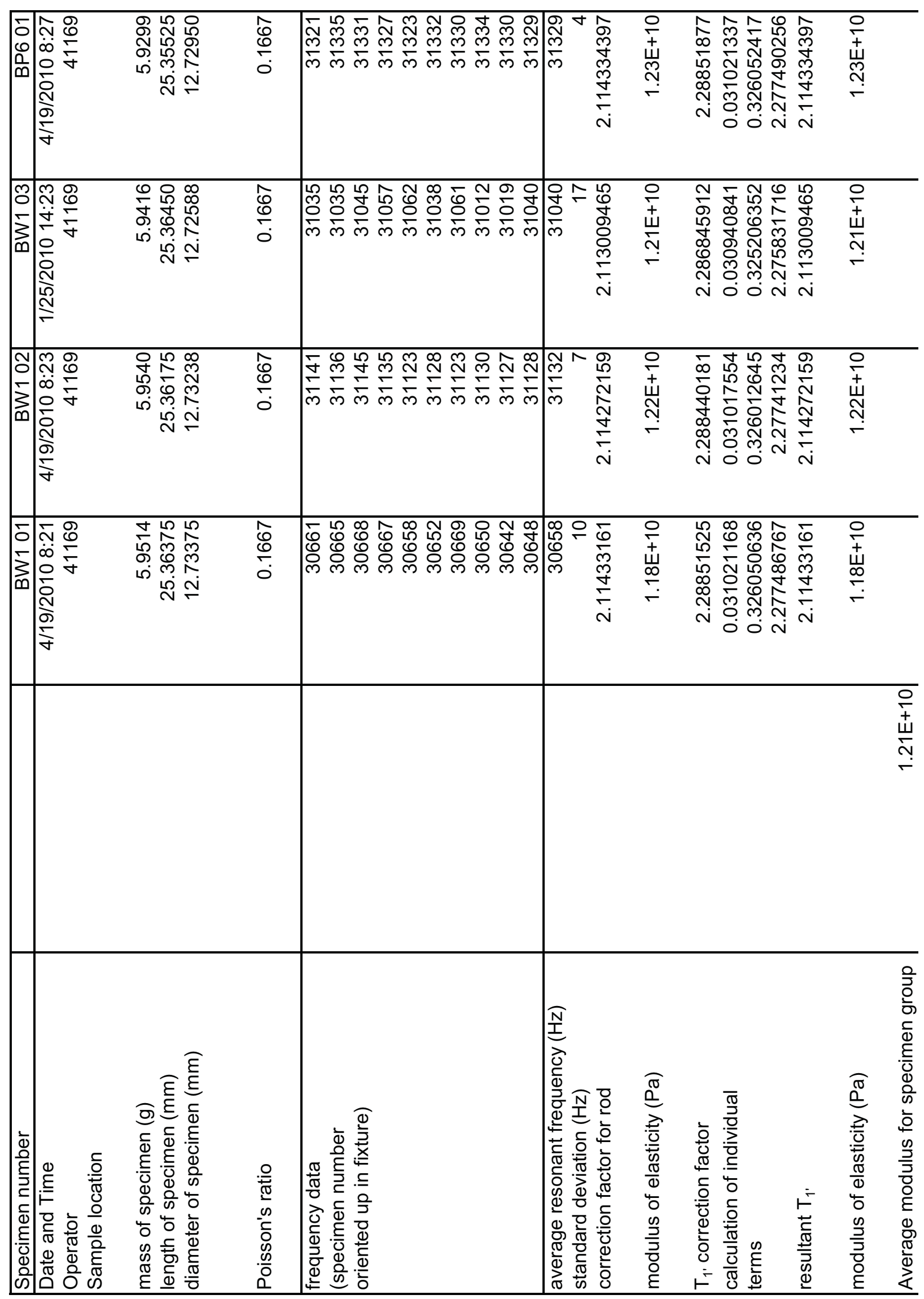




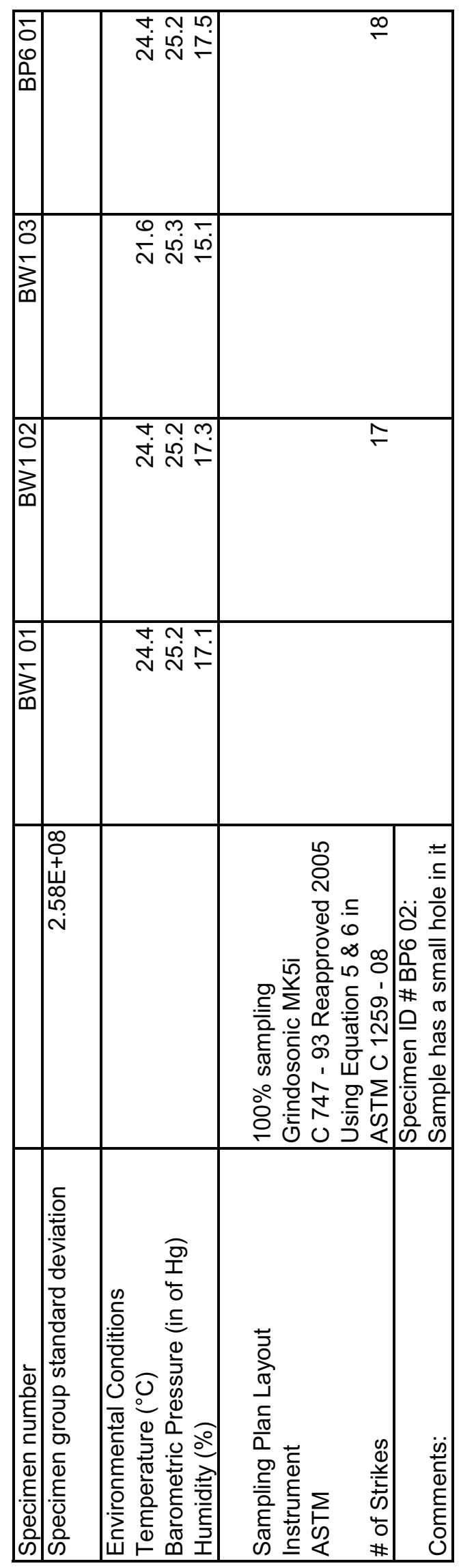




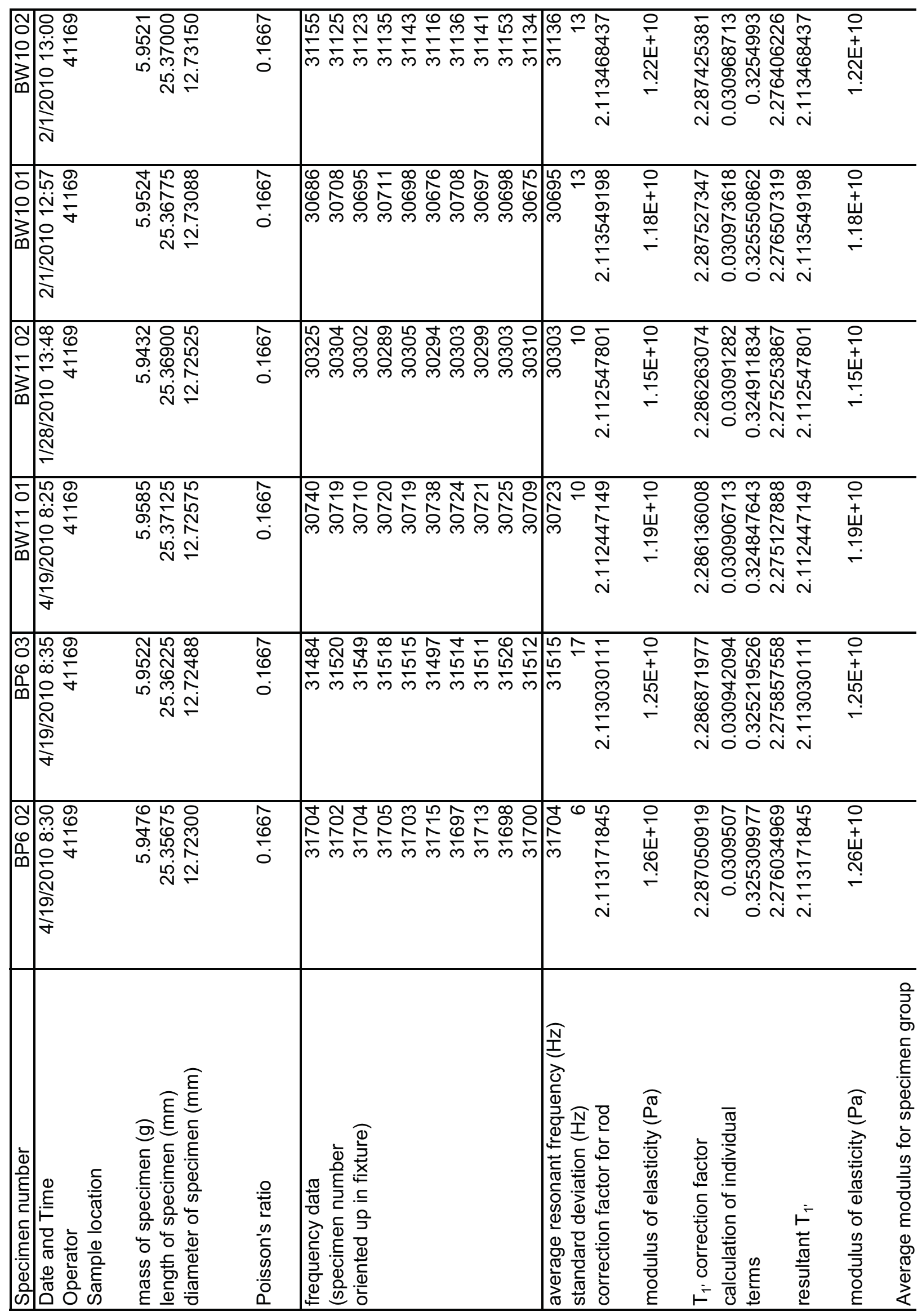




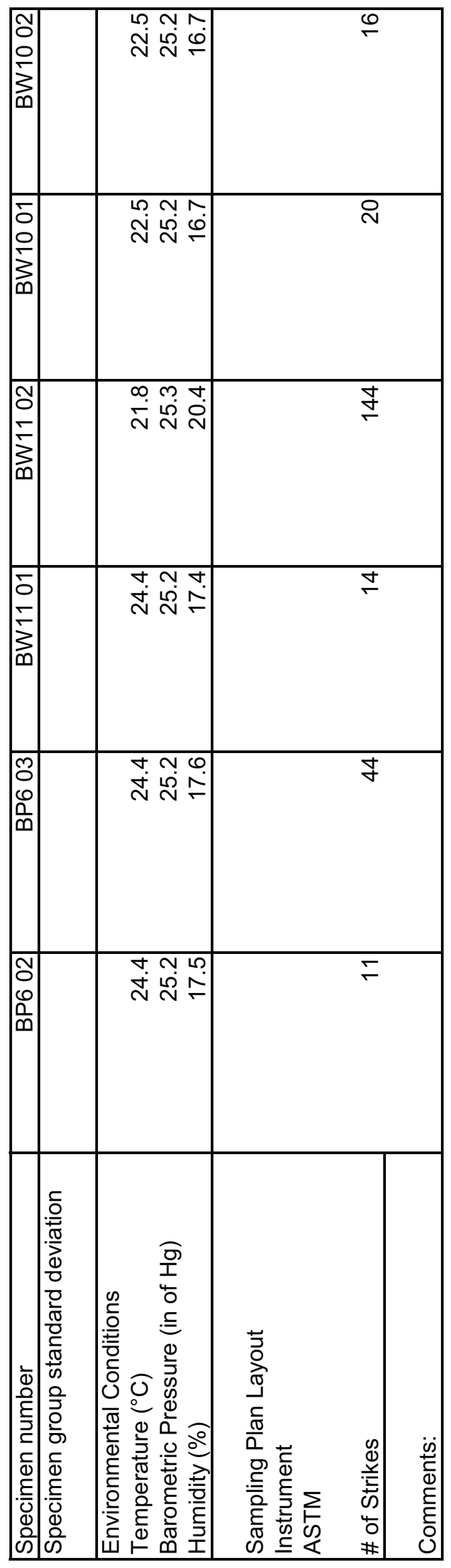




\begin{tabular}{|c|c|c|c|c|c|c|c|c|c|}
\hline $\begin{array}{l}m \\
0 \\
\infty \\
0 \\
\infty\end{array}$ & 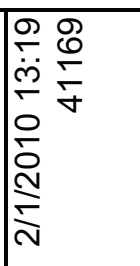 & 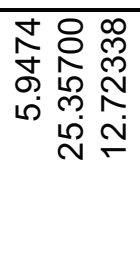 & $\begin{array}{l}\hat{\theta} \\
\grave{0} \\
\vdots\end{array}$ & 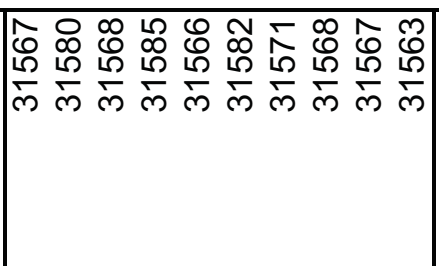 & 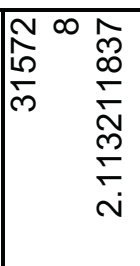 & 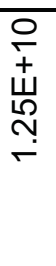 & 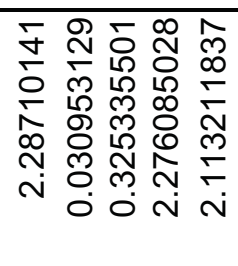 & 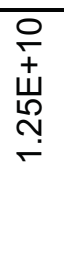 & \\
\hline 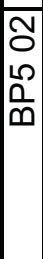 & 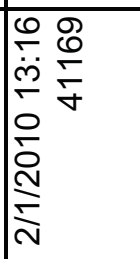 & 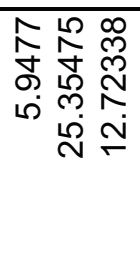 & $\begin{array}{l}\hat{\Theta} \\
\stackrel{0}{0}\end{array}$ & 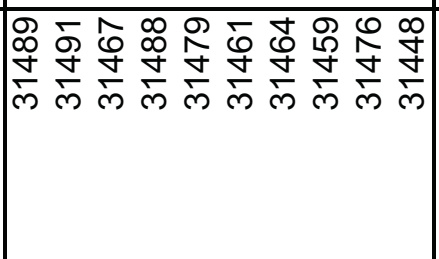 & 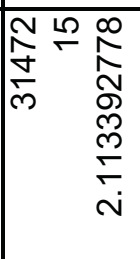 & 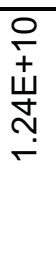 & 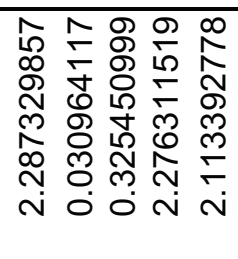 & 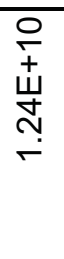 & \\
\hline $\begin{array}{l}m \\
0 \\
j \\
0 \\
0\end{array}$ & 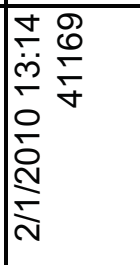 & 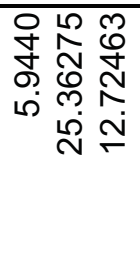 & $\begin{array}{l}\hat{\mathscr{\theta}} \\
\underline{0} \\
\vdots\end{array}$ & 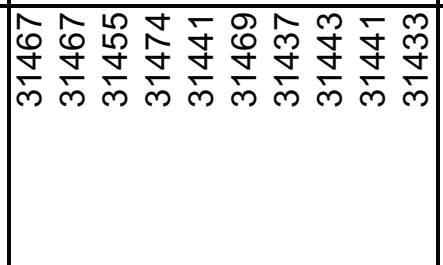 & 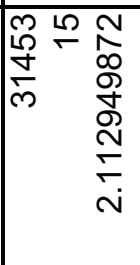 & $\begin{array}{l}\stackrel{ }{+} \\
\stackrel{+}{+} \\
\stackrel{\sim}{\leftarrow}\end{array}$ & 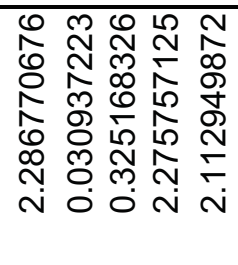 & $\begin{array}{l}\text { 움 } \\
\stackrel{+}{\ddagger} \\
\stackrel{+}{\sim}\end{array}$ & \\
\hline$\left[\begin{array}{l}5 \\
10 \\
0 \\
0\end{array}\right.$ & 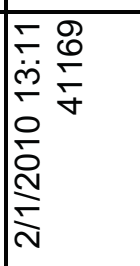 & 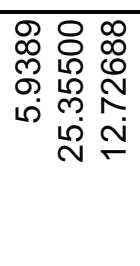 & $\frac{\widehat{o}}{\mathscr{E}}$ & 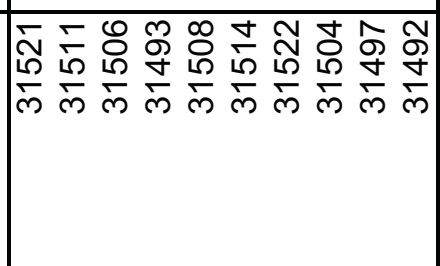 & 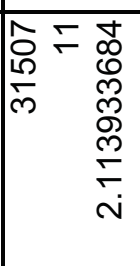 & $\begin{array}{l}\stackrel{ }{+} \\
\stackrel{+}{+} \\
\stackrel{+}{\leftarrow}\end{array}$ & 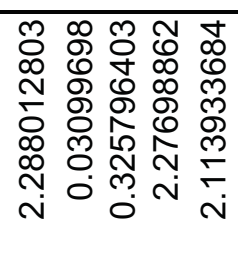 & 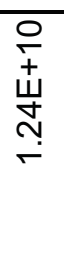 & \\
\hline 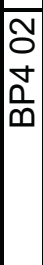 & 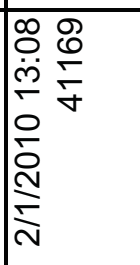 & 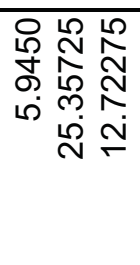 & $\begin{array}{l}\hat{\theta} \\
\stackrel{0}{0} \\
\vdots\end{array}$ & 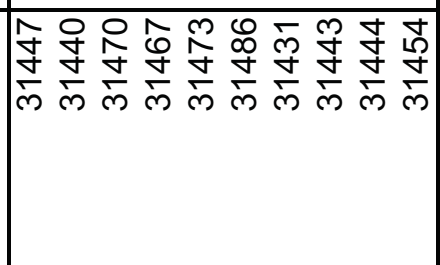 & 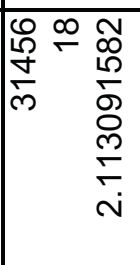 & 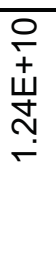 & 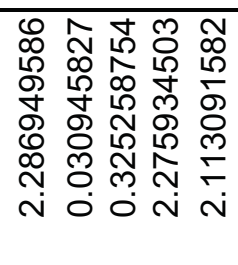 & 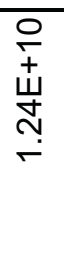 & \\
\hline ma & 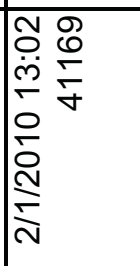 & 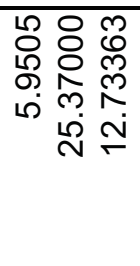 & $\begin{array}{l}\hat{\mathscr{\theta}} \\
\stackrel{0}{0} \\
\vdots\end{array}$ & 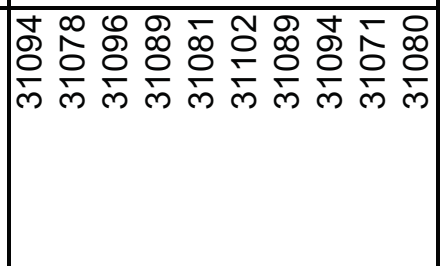 & 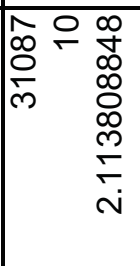 & 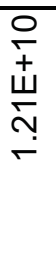 & 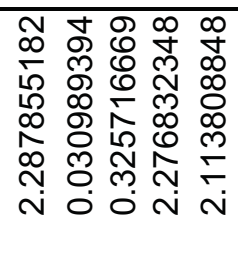 & 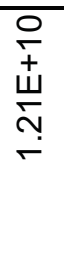 & \\
\hline 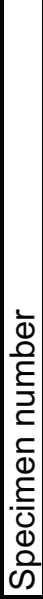 & 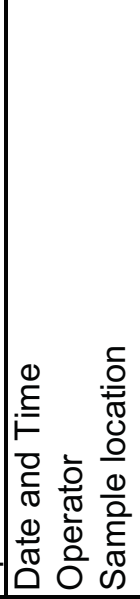 & 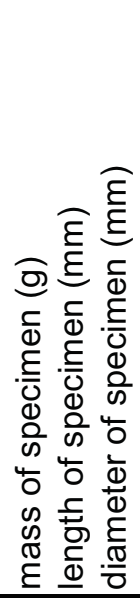 & 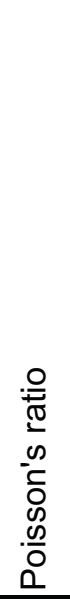 & 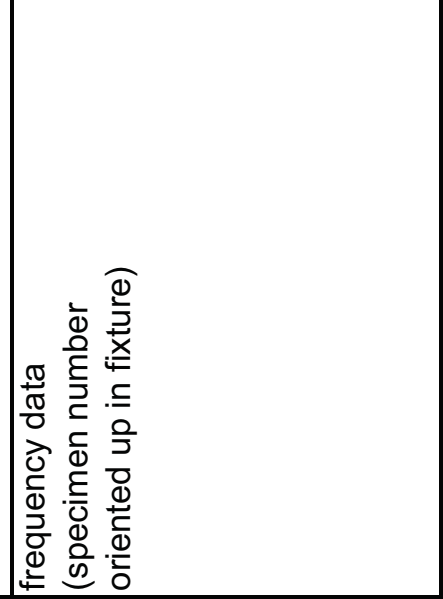 & 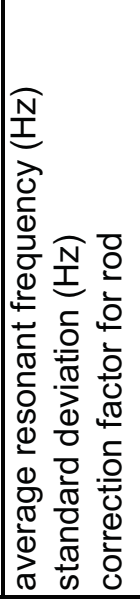 & 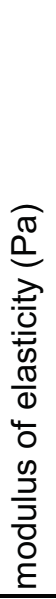 & 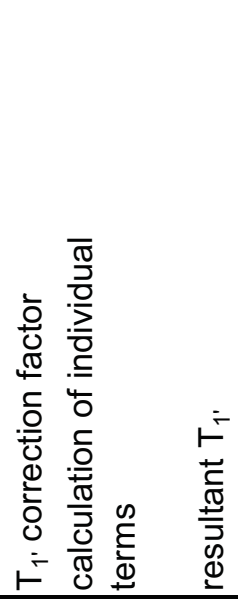 & 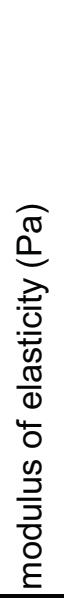 & 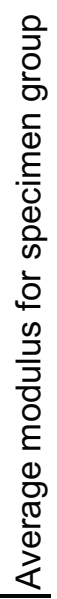 \\
\hline
\end{tabular}




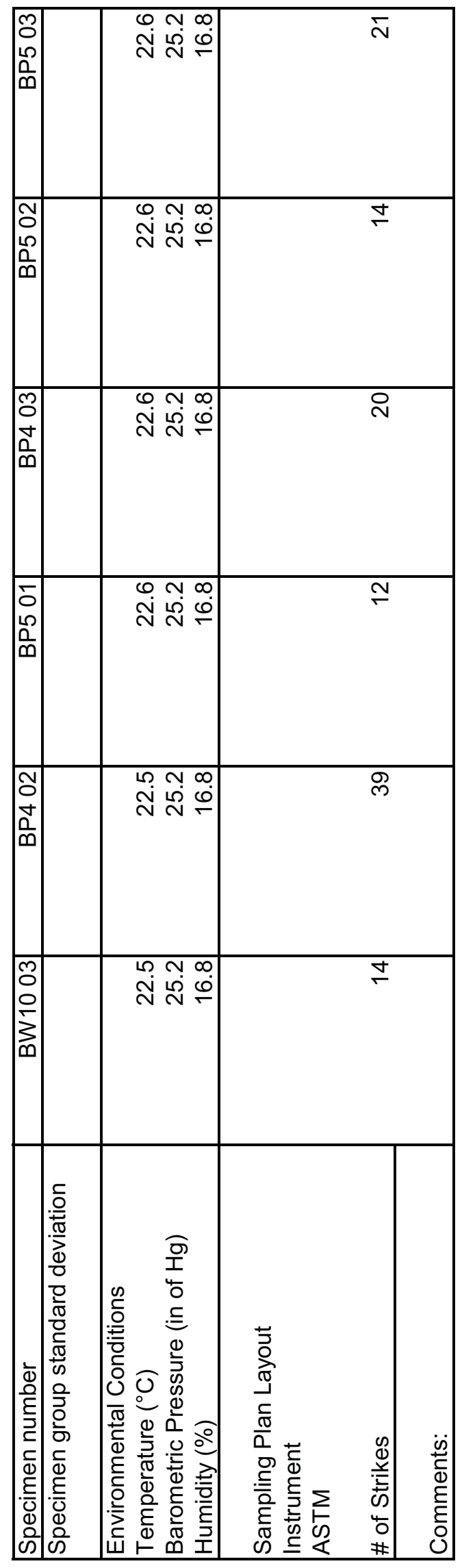




\begin{tabular}{|c|c|c|c|c|c|c|c|c|c|}
\hline $\begin{array}{l}m \\
0 \\
m \\
\sum_{0}\end{array}$ & 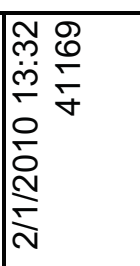 & 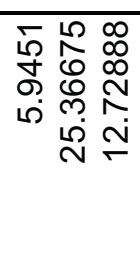 & $\begin{array}{l}\hat{\theta} \\
\stackrel{0}{0}\end{array}$ & 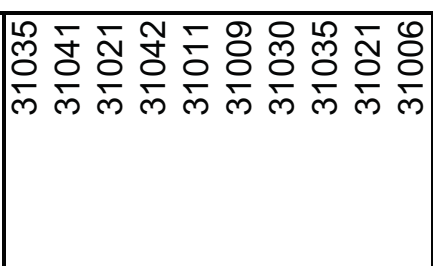 & 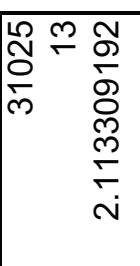 & $\begin{array}{l}\text { 음 } \\
\stackrel{+}{\varpi} \\
\stackrel{N}{+}\end{array}$ & 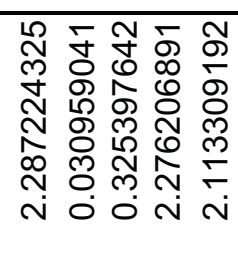 & 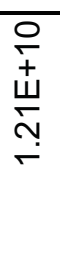 & \\
\hline$\sum_{\infty}^{\infty}$ & 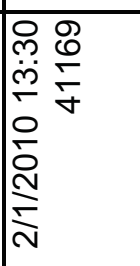 & 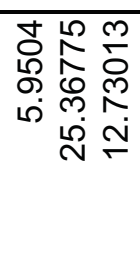 & $\begin{array}{l}\overline{0} \\
\stackrel{\emptyset}{0} \\
0\end{array}$ & 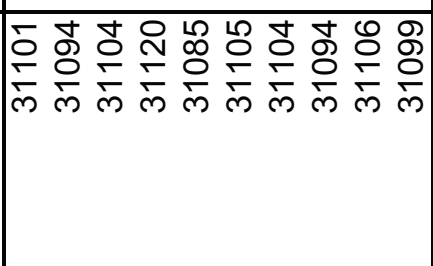 & 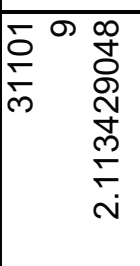 & $\begin{array}{l}\text { 음 } \\
+ \\
\stackrel{+}{\grave{~}}\end{array}$ & 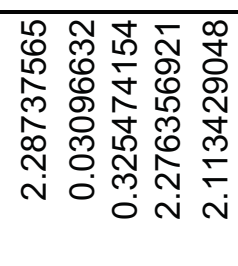 & $\begin{array}{l}\text { 옴 } \\
\stackrel{+}{W} \\
\stackrel{N}{\leftarrow}\end{array}$ & \\
\hline $\begin{array}{l}5 \\
\vdots \\
m \\
\vdots \\
\infty\end{array}$ & 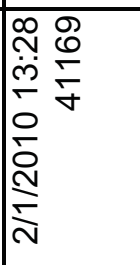 & 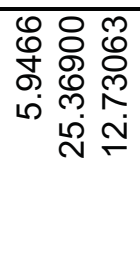 & $\begin{array}{l}\hat{\theta} \\
\stackrel{0}{0}\end{array}$ & 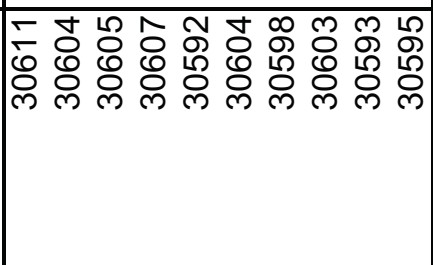 & 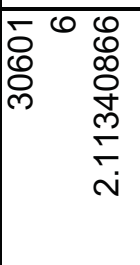 & 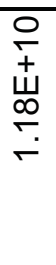 & 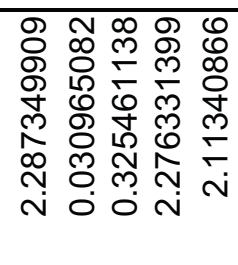 & 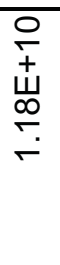 & \\
\hline 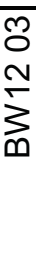 & 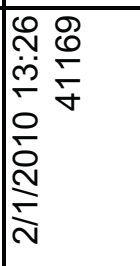 & 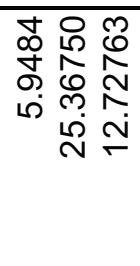 & $\begin{array}{l}\hat{\theta} \\
\stackrel{\emptyset}{0}\end{array}$ & 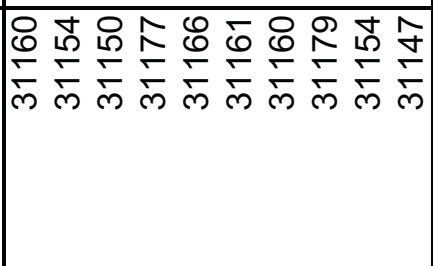 & 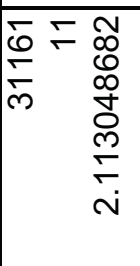 & 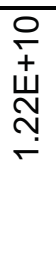 & 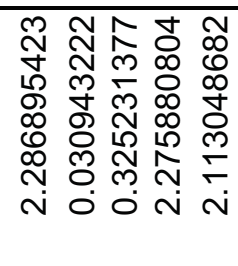 & $\begin{array}{l}\text { 음 } \\
\stackrel{+}{\underset{N}{N}} \\
\stackrel{-}{-}\end{array}$ & \\
\hline 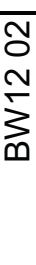 & 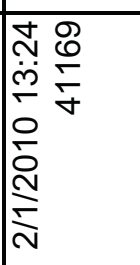 & 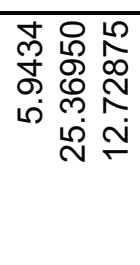 & $\begin{array}{l}\overline{0} \\
\stackrel{0}{0} \\
0\end{array}$ & 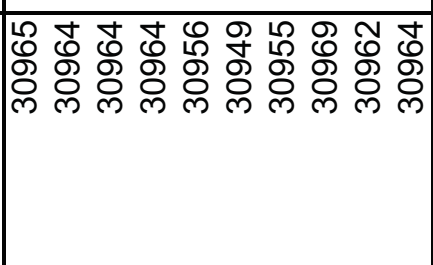 & 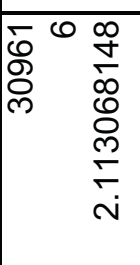 & $\begin{array}{l}\text { 음 } \\
+ \\
\text { Ш } \\
\stackrel{\text { }}{\leftarrow}\end{array}$ & 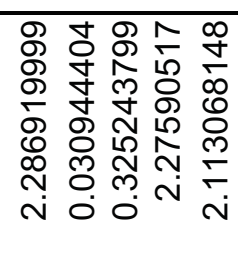 & 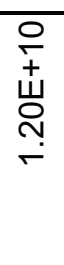 & \\
\hline 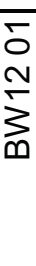 & 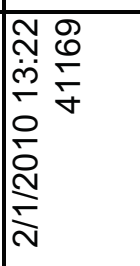 & 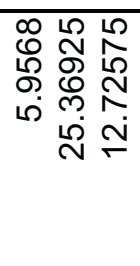 & $\begin{array}{l}\hat{\theta} \\
\stackrel{0}{0}\end{array}$ & 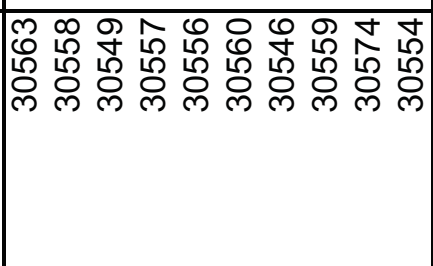 & 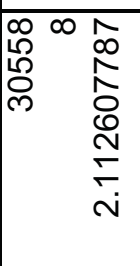 & 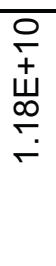 & 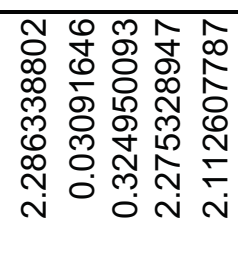 & 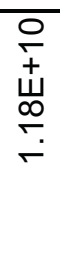 & \\
\hline 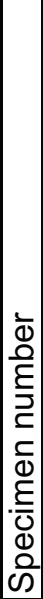 & 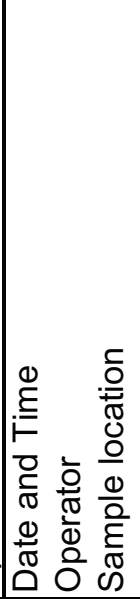 & 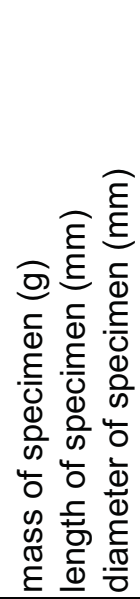 & 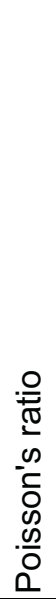 & 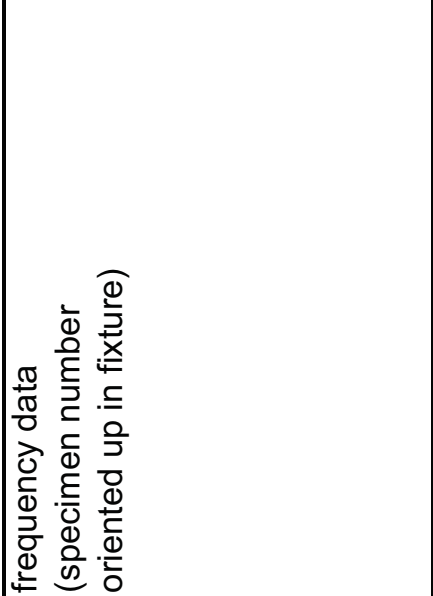 & 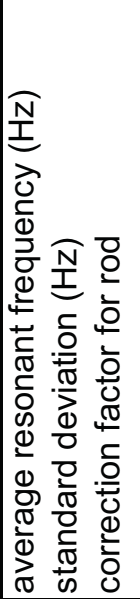 & $\begin{array}{l}\frac{\pi}{\sigma} \\
0 \\
\frac{1}{0} \\
\frac{0}{0} \\
\frac{0}{0} \\
\frac{\pi}{0} \\
\frac{0}{0} \\
\frac{0}{0} \\
\frac{0}{5} \\
\frac{5}{0} \\
\frac{0}{\varepsilon}\end{array}$ & 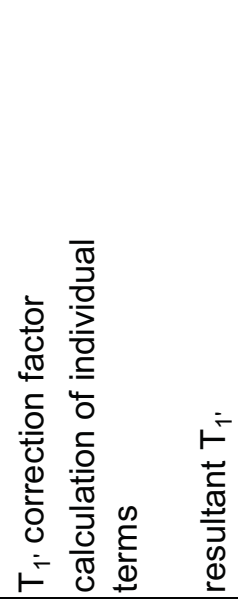 & 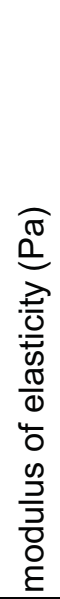 & 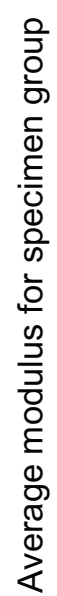 \\
\hline
\end{tabular}




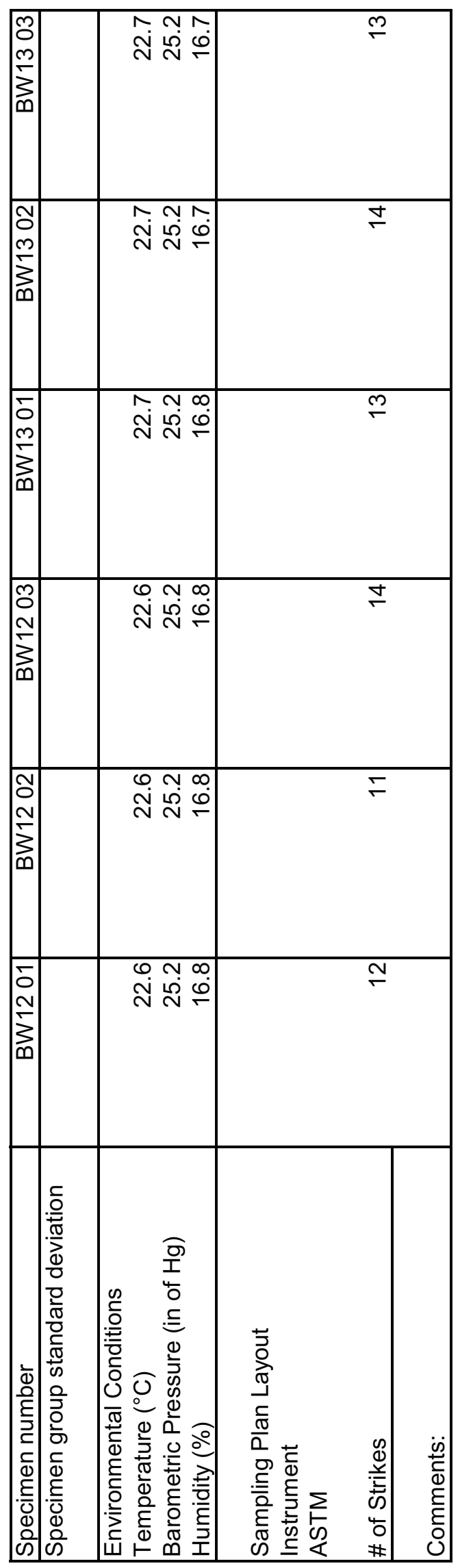




\begin{tabular}{|c|c|c|c|c|c|c|c|c|c|}
\hline $\begin{array}{l}m \\
0 \\
0 \\
\vdots \\
0 \\
0\end{array}$ & 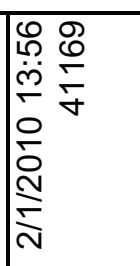 & 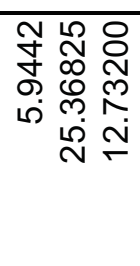 & $\begin{array}{l}\hat{\theta} \\
\stackrel{0}{0}\end{array}$ & 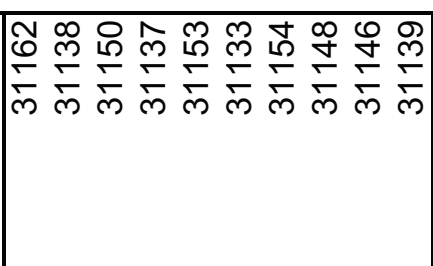 & 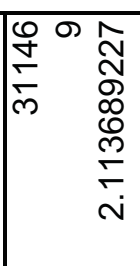 & 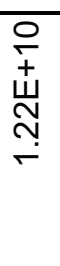 & 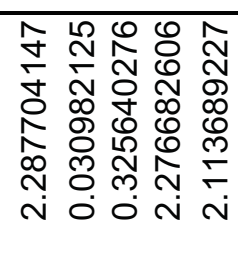 & $\begin{array}{l}\text { 옴 } \\
\stackrel{+}{\sim} \\
\stackrel{N}{\leftarrow}\end{array}$ & \\
\hline$\sum_{\infty}^{\infty}$ & 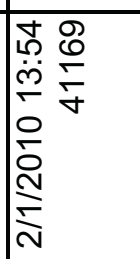 & 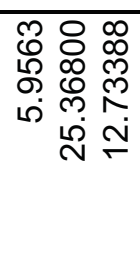 & $\begin{array}{l}\overline{0} \\
\stackrel{\emptyset}{0} \\
0\end{array}$ & 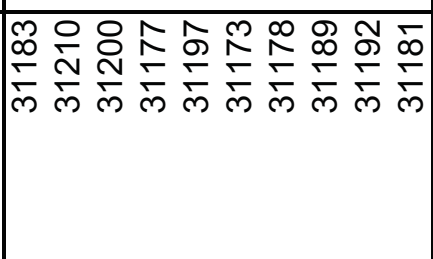 & 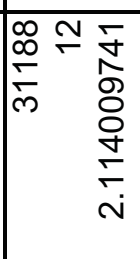 & $\begin{array}{l}\text { 온 } \\
\stackrel{+}{N} \\
\stackrel{N}{r}\end{array}$ & 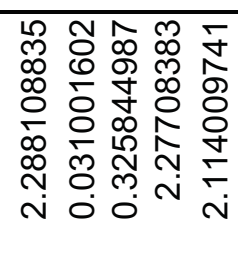 & 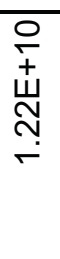 & \\
\hline $\begin{array}{l}5 \\
\\
1 \\
\vdots \\
\vdots \\
0\end{array}$ & 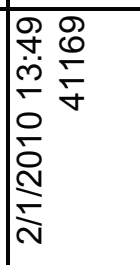 & 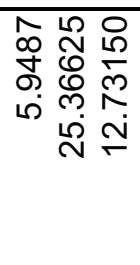 & $\begin{array}{l}\hat{\theta} \\
\stackrel{0}{0}\end{array}$ & 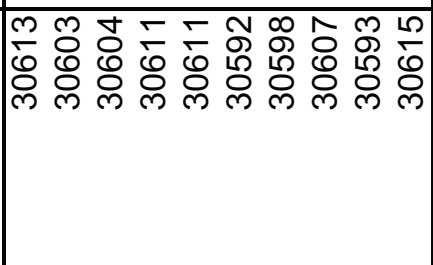 & 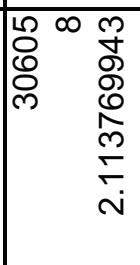 & 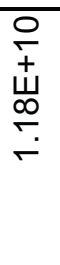 & 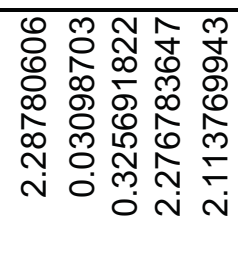 & 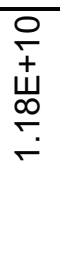 & \\
\hline 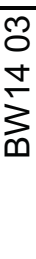 & 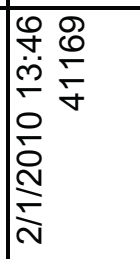 & 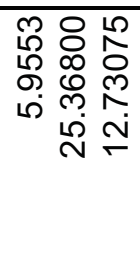 & $\begin{array}{l}\hat{\theta} \\
\stackrel{\emptyset}{0}\end{array}$ & 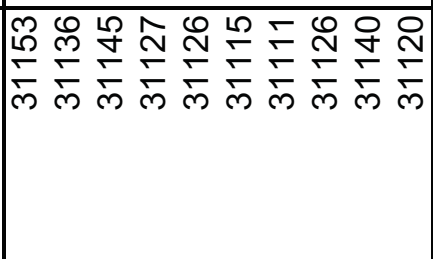 & 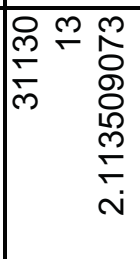 & $\begin{array}{l}\stackrel{ }{+} \\
\stackrel{+}{N} \\
\stackrel{N}{r}\end{array}$ & 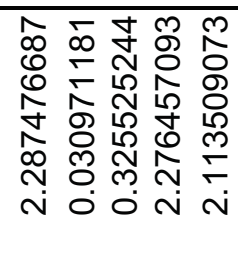 & $\begin{array}{l}\text { 음 } \\
\stackrel{+}{\underset{N}{N}} \\
\stackrel{-}{-}\end{array}$ & \\
\hline 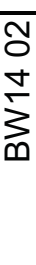 & 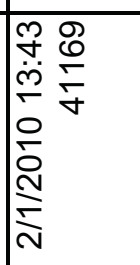 & 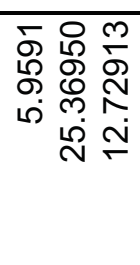 & $\begin{array}{l}\overline{0} \\
\stackrel{0}{0}\end{array}$ & 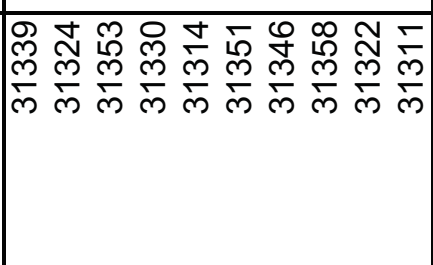 & 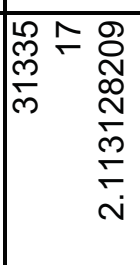 & 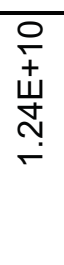 & 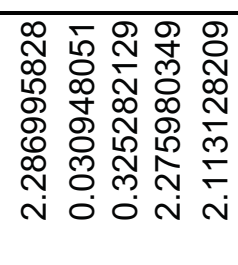 & $\begin{array}{l}\stackrel{O}{+} \\
\stackrel{+}{+} \\
\stackrel{+}{+}\end{array}$ & \\
\hline$\frac{5}{5}$ & 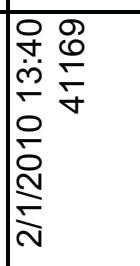 & 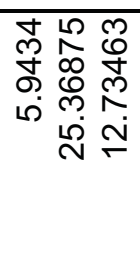 & $\begin{array}{l}\hat{\theta} \\
\stackrel{0}{0}\end{array}$ & 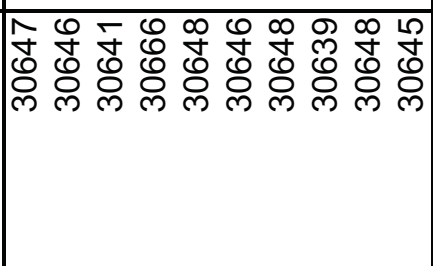 & 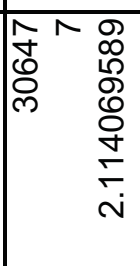 & 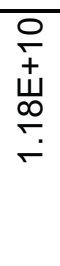 & 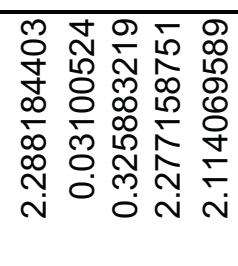 & 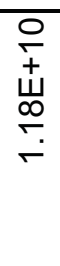 & \\
\hline 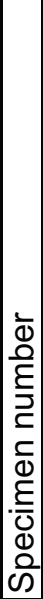 & 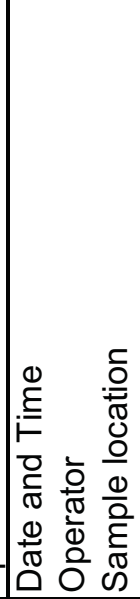 & 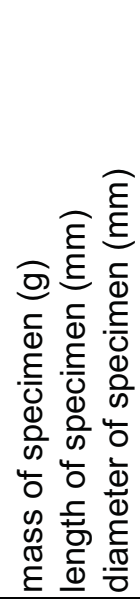 & 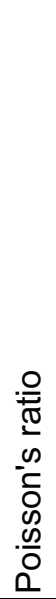 & 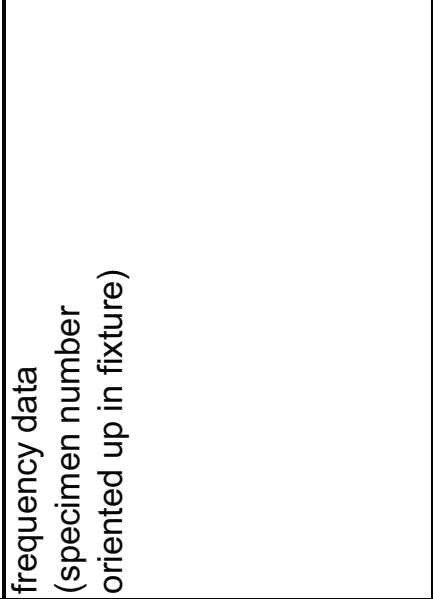 & 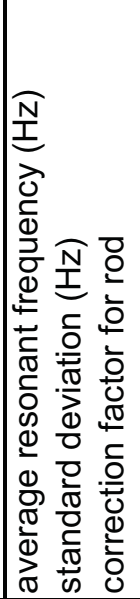 & 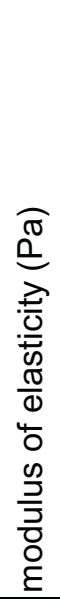 & 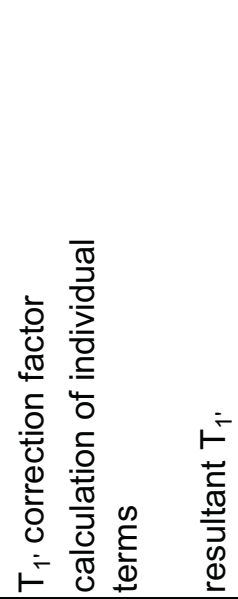 & 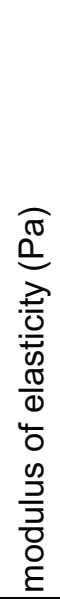 & 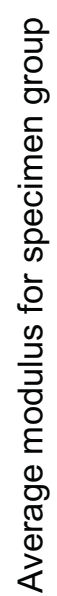 \\
\hline
\end{tabular}




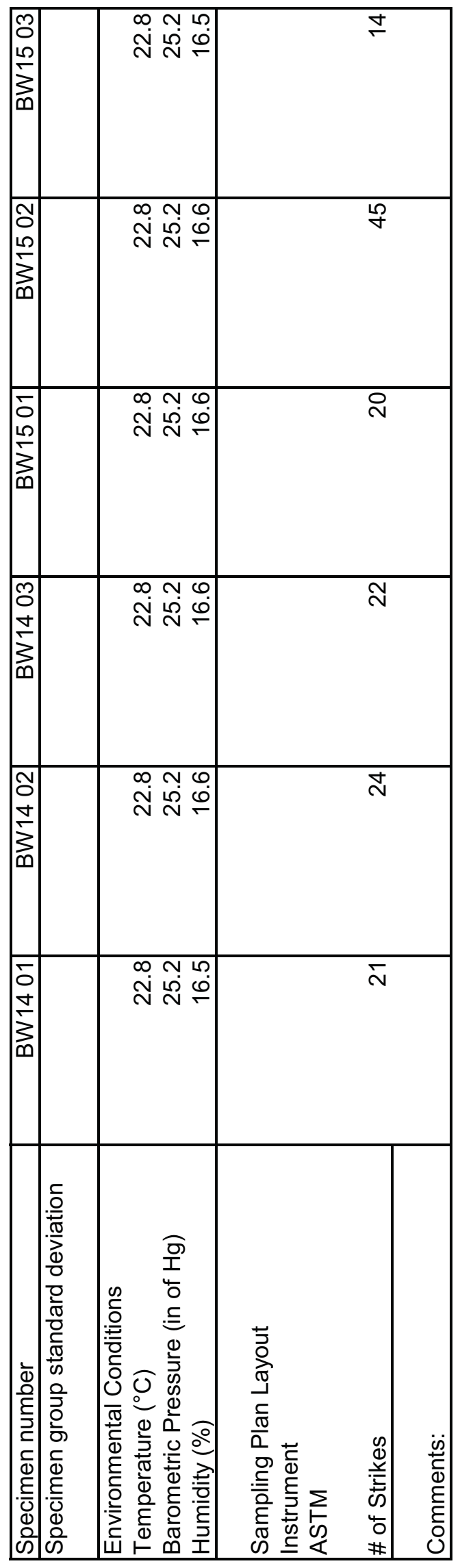




\begin{tabular}{|c|c|c|c|c|c|c|c|c|c|}
\hline $\bar{\phi}$ & 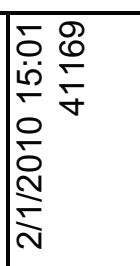 & 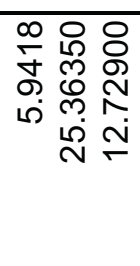 & $\begin{array}{l}\hat{\theta} \\
\stackrel{0}{0}\end{array}$ & 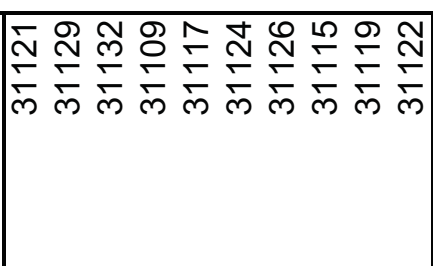 & 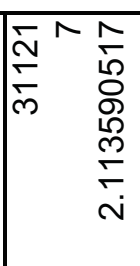 & $\begin{array}{l}\text { 옴 } \\
\stackrel{+}{W} \\
\stackrel{N}{r}\end{array}$ & 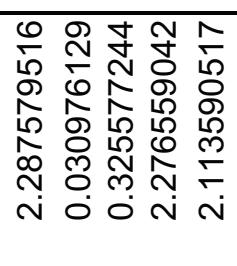 & 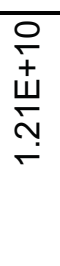 & \\
\hline$\widetilde{N}$ & 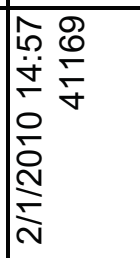 & 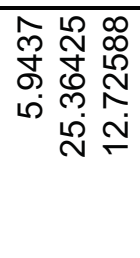 & $\begin{array}{l}\overline{0} \\
\stackrel{\emptyset}{0} \\
0\end{array}$ & 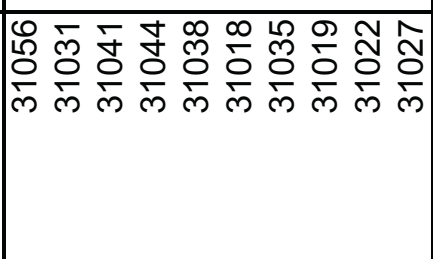 & 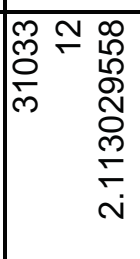 & $\begin{array}{l}\text { 옴 } \\
\stackrel{+}{W} \\
\stackrel{N}{r}\end{array}$ & 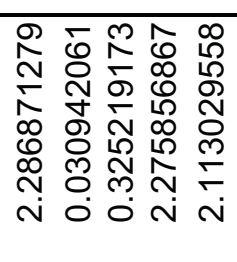 & $\begin{array}{l}\text { 옴 } \\
\stackrel{+}{\varpi} \\
\stackrel{N}{r}\end{array}$ & \\
\hline 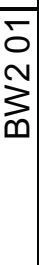 & 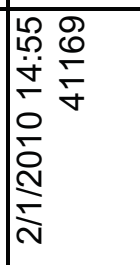 & 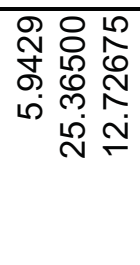 & $\begin{array}{l}\hat{\theta} \\
\stackrel{\Xi}{0} \\
0\end{array}$ & 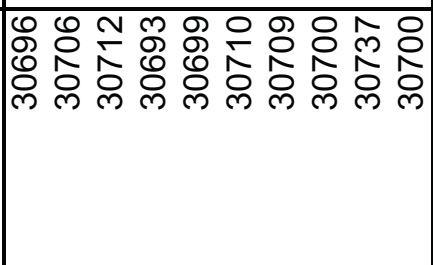 & 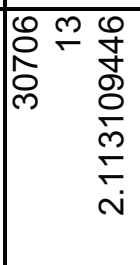 & 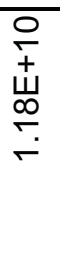 & 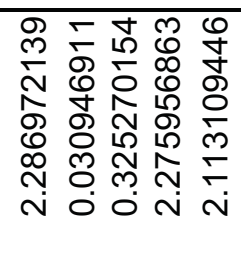 & 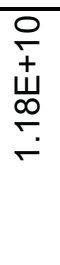 & \\
\hline $\begin{array}{l}m \\
0 \\
0 \\
\vdots \\
\vdots \\
0\end{array}$ & 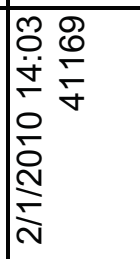 & 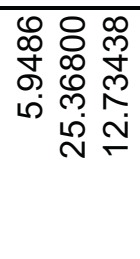 & $\begin{array}{l}\hat{\theta} \\
\stackrel{\emptyset}{0}\end{array}$ & 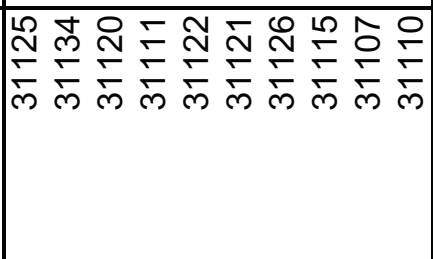 & 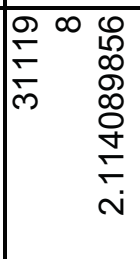 & $\begin{array}{l}\stackrel{ }{+} \\
\stackrel{+}{\sim} \\
\stackrel{N}{r}\end{array}$ & 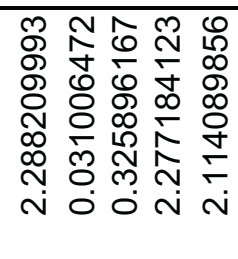 & $\begin{array}{l}\stackrel{\circ}{+} \\
\stackrel{+}{N} \\
\stackrel{\sim}{r}\end{array}$ & \\
\hline \begin{tabular}{l}
\multirow{2}{0}{} \\
0 \\
$\frac{0}{3}$ \\
$\vdots$
\end{tabular} & 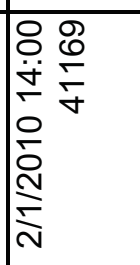 & 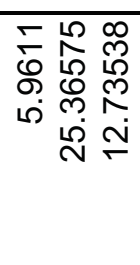 & $\begin{array}{l}\overline{0} \\
\stackrel{0}{0}\end{array}$ & 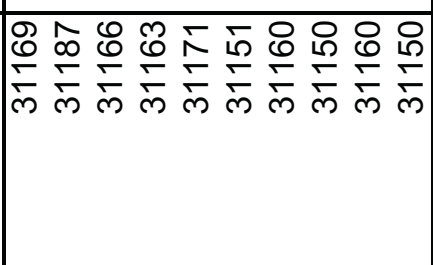 & 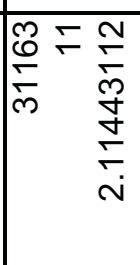 & 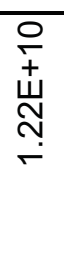 & 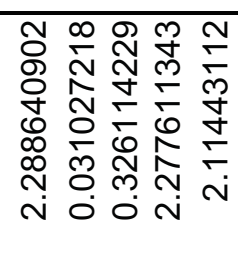 & 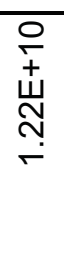 & \\
\hline $\begin{array}{l}5 \\
\vdots \\
\vdots \\
\infty\end{array}$ & 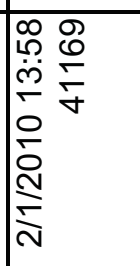 & 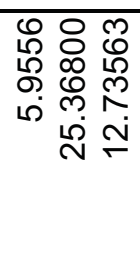 & $\begin{array}{l}\hat{\theta} \\
\stackrel{0}{0}\end{array}$ & 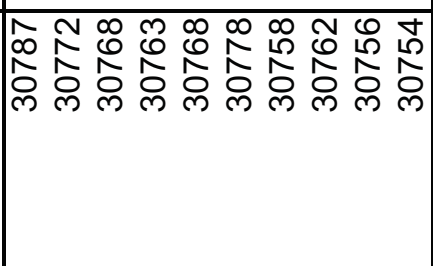 & 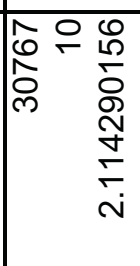 & 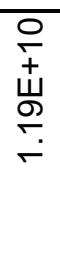 & 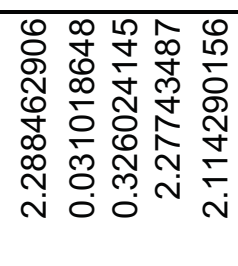 & 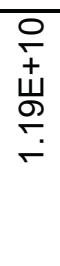 & \\
\hline 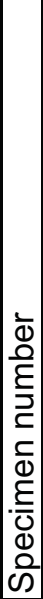 & 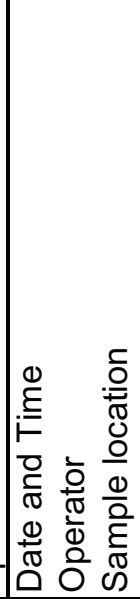 & 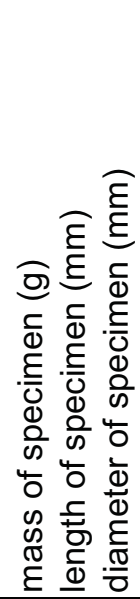 & 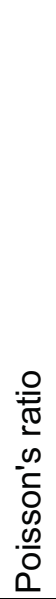 & 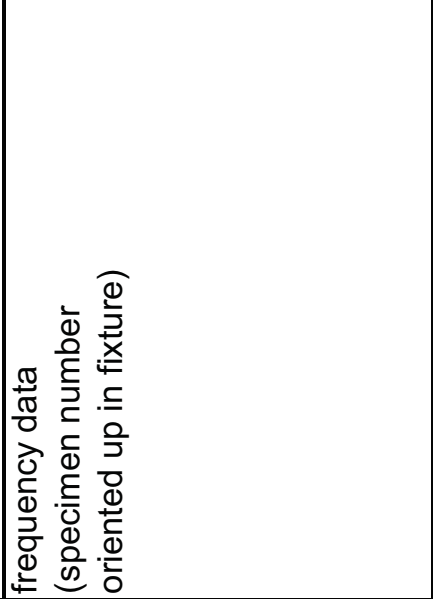 & 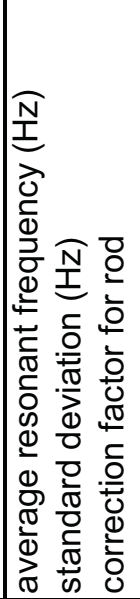 & 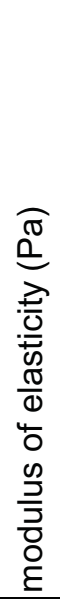 & 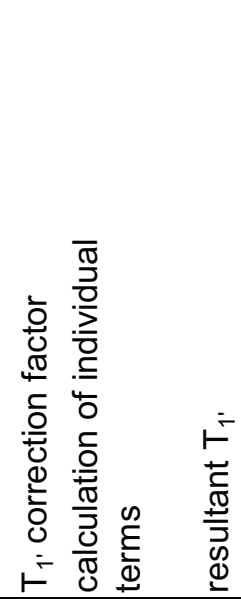 & 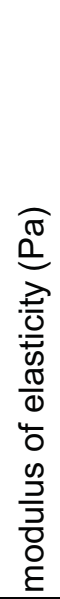 & 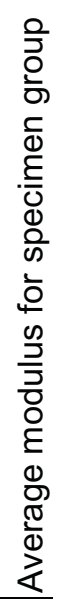 \\
\hline
\end{tabular}




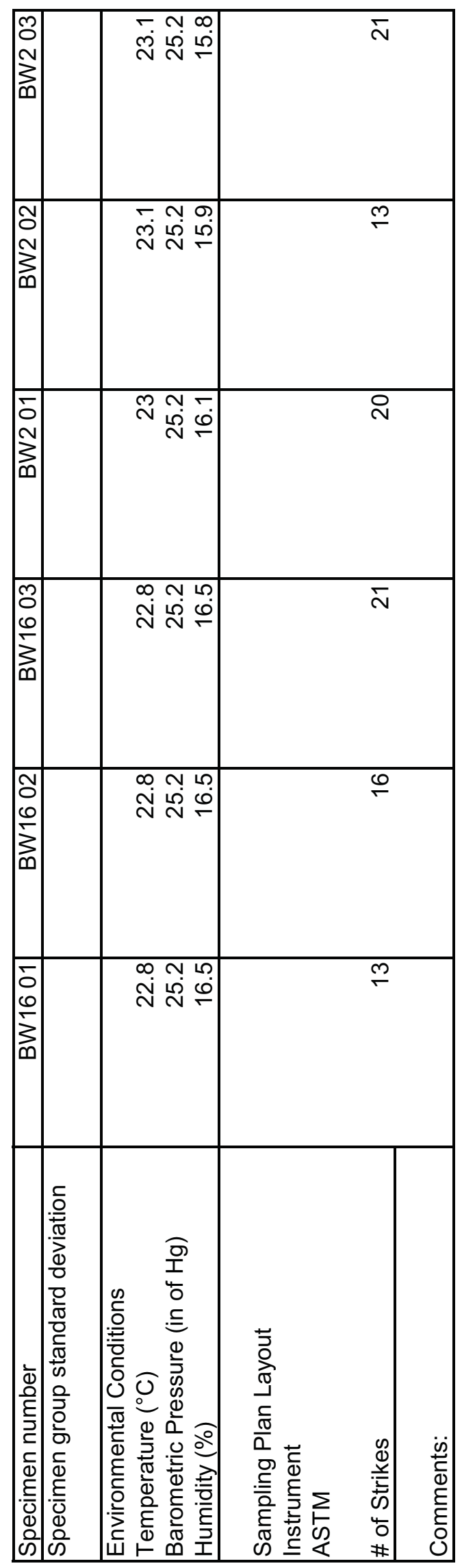




\begin{tabular}{|c|c|c|c|c|c|c|c|c|c|}
\hline ஜ & 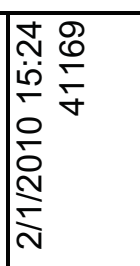 & 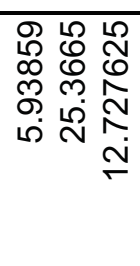 & $\begin{array}{l}\hat{\theta} \\
\stackrel{0}{0}\end{array}$ & 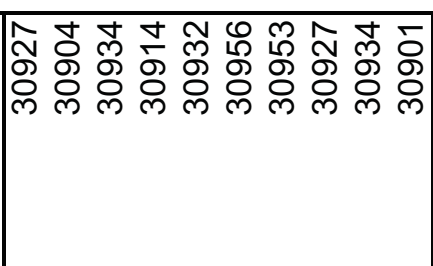 & 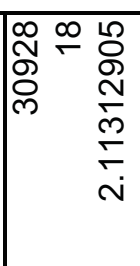 & $\begin{array}{l}\text { 음 } \\
+ \\
\text { 山े } \\
\stackrel{+}{+}\end{array}$ & 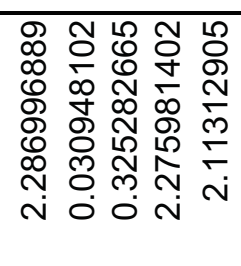 & $\begin{array}{l}\text { 옴 } \\
\stackrel{+}{\circ} \\
\stackrel{N}{r}\end{array}$ & \\
\hline$\widetilde{N}$ & 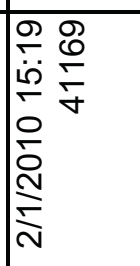 & 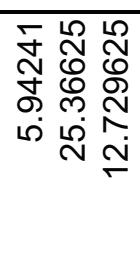 & $\begin{array}{l}\hat{\theta} \\
\stackrel{\emptyset}{0} \\
\vdots\end{array}$ & 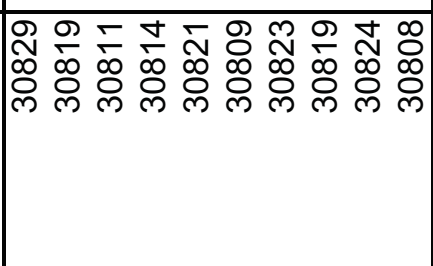 & 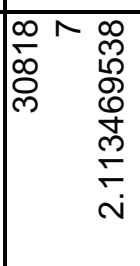 & $\begin{array}{l}\text { 음 } \\
+ \\
\text { 山े } \\
\stackrel{\circ}{\leftarrow}\end{array}$ & 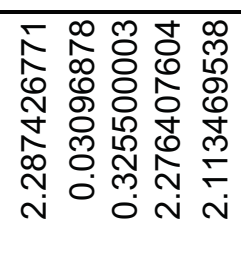 & 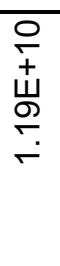 & \\
\hline $\begin{array}{l}\bar{\delta} \\
+\end{array}$ & 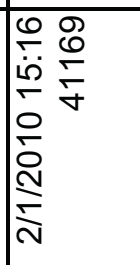 & 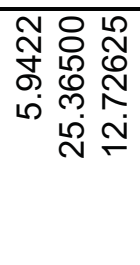 & $\begin{array}{l}\hat{\theta} \\
\stackrel{0}{0}\end{array}$ & 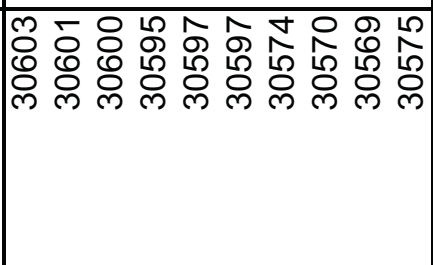 & 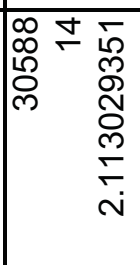 & $\begin{array}{l}\text { 음 } \\
+ \\
\stackrel{W}{ } \\
\stackrel{5}{r}\end{array}$ & 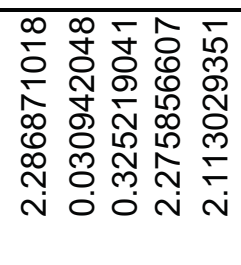 & 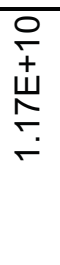 & \\
\hline g & 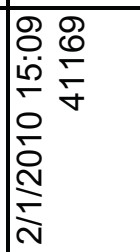 & 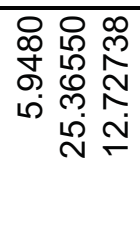 & $\begin{array}{l}\hat{\theta} \\
\stackrel{\emptyset}{0}\end{array}$ & 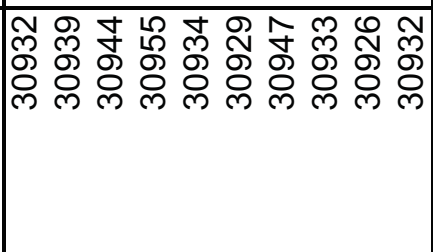 & 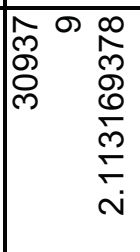 & $\begin{array}{l}\text { 음 } \\
\text { 屯் } \\
\text { ஸे }\end{array}$ & 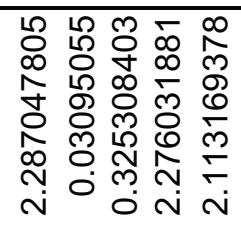 & 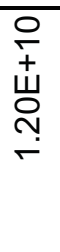 & \\
\hline$\sum_{\substack{N \\
\text { M } \\
m}}^{2}$ & 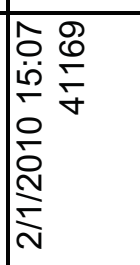 & 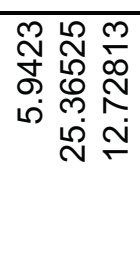 & $\begin{array}{l}\overline{0} \\
\stackrel{0}{0}\end{array}$ & 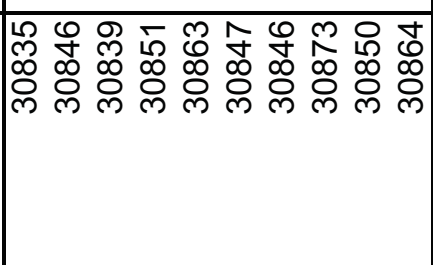 & 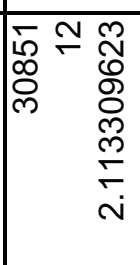 & 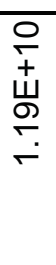 & 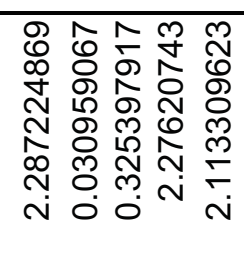 & 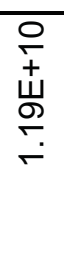 & \\
\hline$\underset{\infty}{\infty}$ & 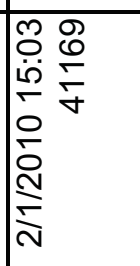 & 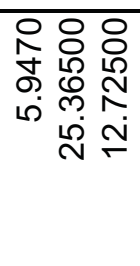 & $\begin{array}{l}\hat{\emptyset} \\
\stackrel{\emptyset}{0} \\
0\end{array}$ & 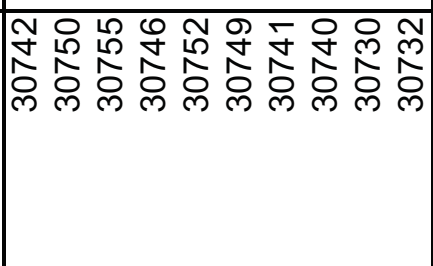 & 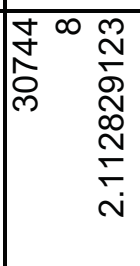 & $\begin{array}{l}\text { 음 } \\
+ \\
\text { 山े } \\
\stackrel{\circ}{\leftarrow}\end{array}$ & 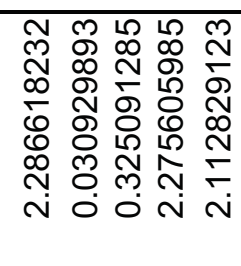 & 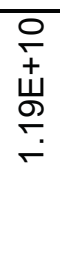 & \\
\hline 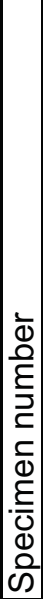 & 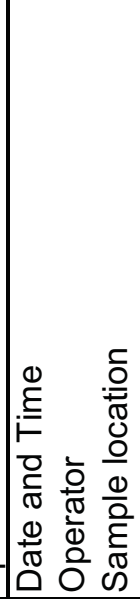 & 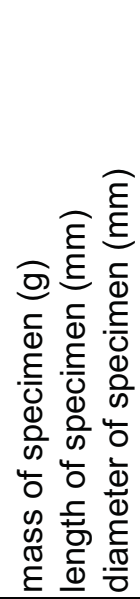 & 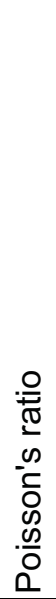 & 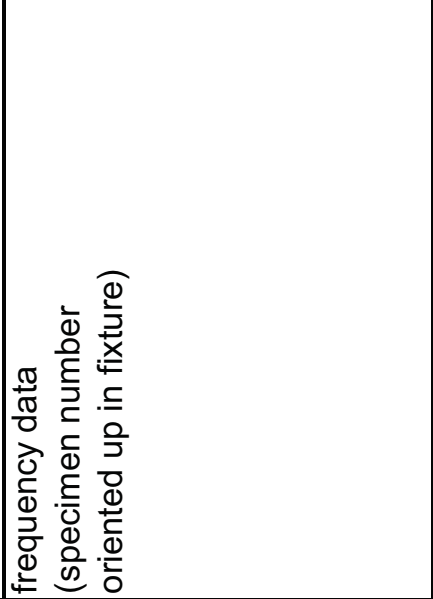 & 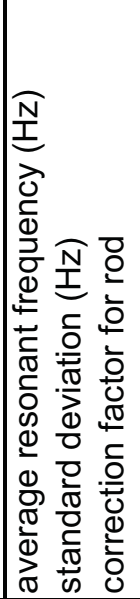 & $\begin{array}{l}\frac{\pi}{\sigma} \\
0 \\
\frac{1}{0} \\
\frac{0}{0} \\
\frac{0}{0} \\
\frac{\pi}{0} \\
\frac{0}{0} \\
\frac{0}{0} \\
\frac{0}{5} \\
\frac{5}{0} \\
\frac{0}{\varepsilon}\end{array}$ & 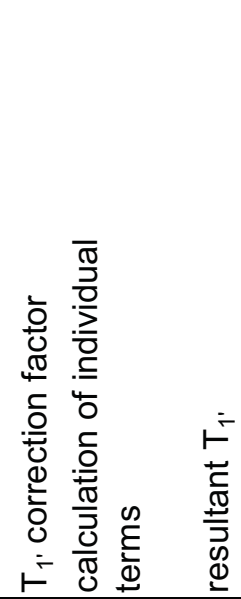 & 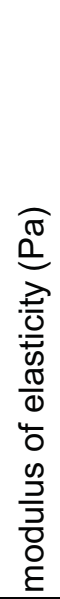 & 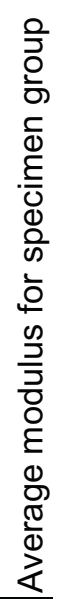 \\
\hline
\end{tabular}




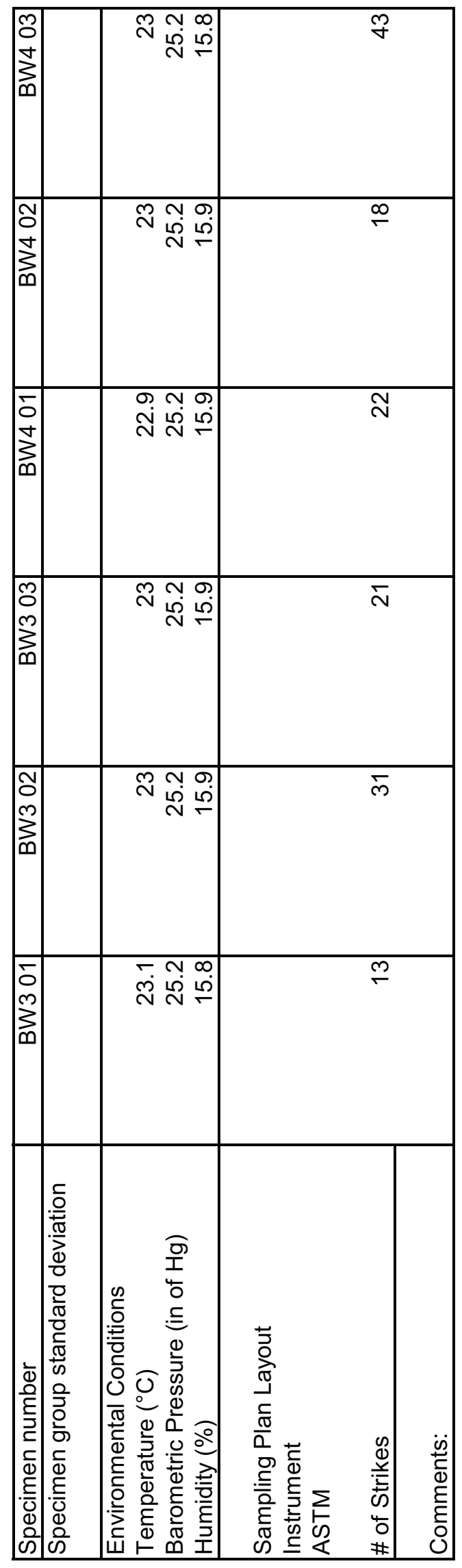




\begin{tabular}{|c|c|c|c|c|c|c|c|c|c|}
\hline $\begin{array}{l}\tilde{0} \\
\infty \\
0 \\
\infty\end{array}$ & 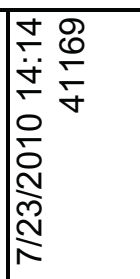 & 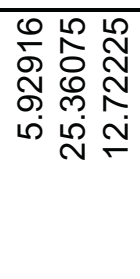 & $\begin{array}{l}\hat{0} \\
\stackrel{0}{0} \\
0\end{array}$ & 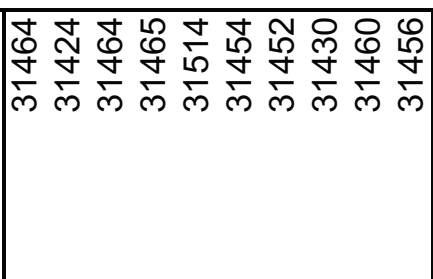 & 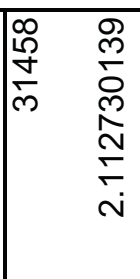 & $\begin{array}{l}\stackrel{ }{+} \\
\stackrel{+}{+} \\
\stackrel{+}{+}\end{array}$ & 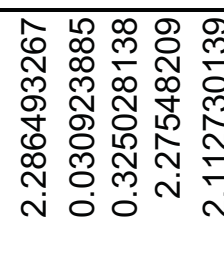 & $\begin{array}{l}\stackrel{O}{+} \\
\stackrel{+}{+} \\
\stackrel{+}{\leftarrow}\end{array}$ & \\
\hline$\frac{m}{\infty}$ & 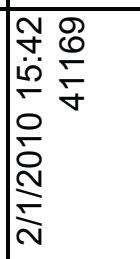 & 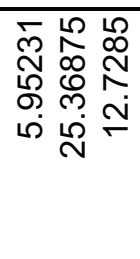 & $\begin{array}{l}\overline{0} \\
\stackrel{0}{0}\end{array}$ & 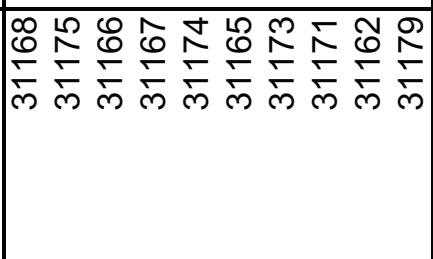 & 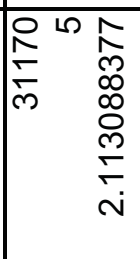 & 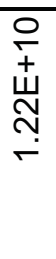 & 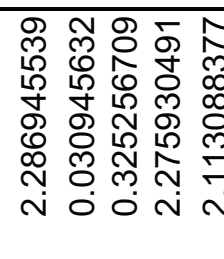 & $\begin{array}{l}\text { 음 } \\
\stackrel{+}{\sim} \\
\stackrel{N}{\leftarrow}\end{array}$ & \\
\hline $\begin{array}{l}\tilde{0} \\
0 \\
0 \\
0 \\
0\end{array}$ & 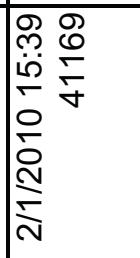 & 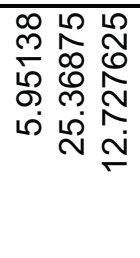 & $\begin{array}{l}\hat{0} \\
\stackrel{0}{0} \\
0\end{array}$ & 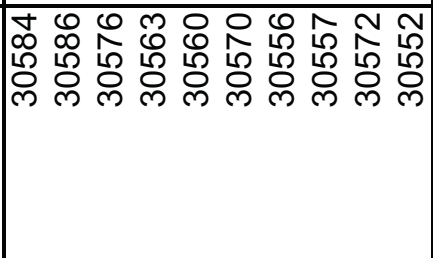 & 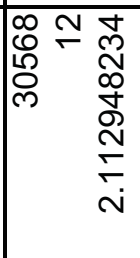 & $\begin{array}{l}\stackrel{0}{+} \\
\stackrel{+}{\rightleftarrows} \\
\stackrel{+}{\leftarrow}\end{array}$ & 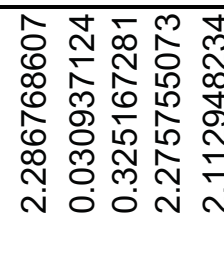 & $\begin{array}{l}\stackrel{\circ}{+} \\
\stackrel{+}{\rightleftarrows} \\
\stackrel{5}{\leftarrow}\end{array}$ & \\
\hline $\begin{array}{l}m \\
0 \\
1 \\
3 \\
0 \\
0\end{array}$ & 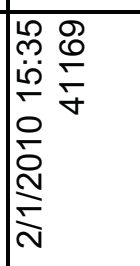 & 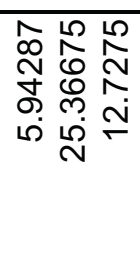 & $\begin{array}{l}\hat{\emptyset} \\
\stackrel{\emptyset}{0}\end{array}$ & 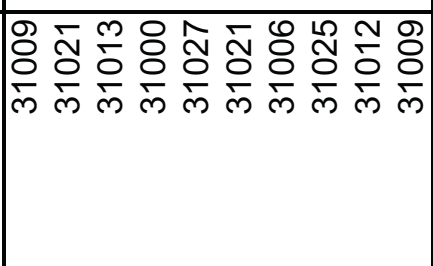 & 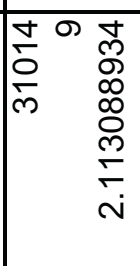 & 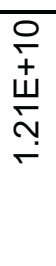 & 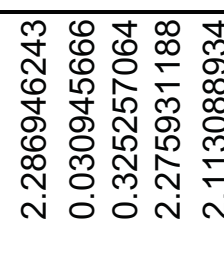 & $\begin{array}{l}\stackrel{\circ}{+} \\
\stackrel{+}{\varpi} \\
\stackrel{N}{\leftarrow}\end{array}$ & \\
\hline $\begin{array}{l}\text { No } \\
1 \\
1 \\
\infty \\
\infty\end{array}$ & 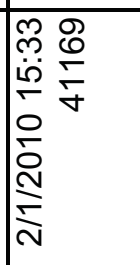 & 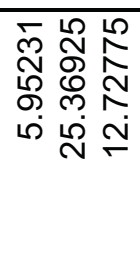 & $\begin{array}{l}\hat{0} \\
\stackrel{0}{0} \\
\vdots\end{array}$ & 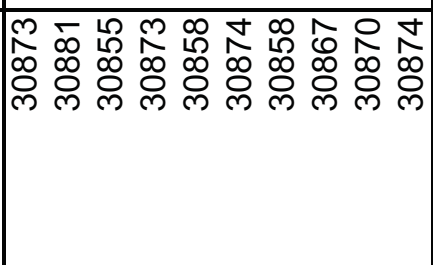 & 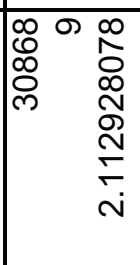 & $\begin{array}{l}\text { 음 } \\
\text { யे } \\
\text { ஸ̣ }\end{array}$ & 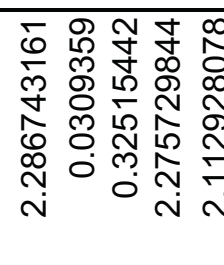 & 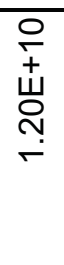 & \\
\hline $\begin{array}{l}5 \\
0 \\
10 \\
0 \\
0\end{array}$ & 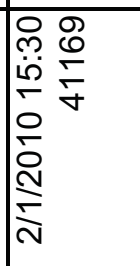 & 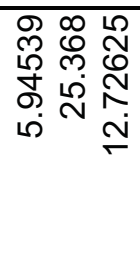 & $\begin{array}{l}\hat{\theta} \\
\stackrel{\emptyset}{0}\end{array}$ & 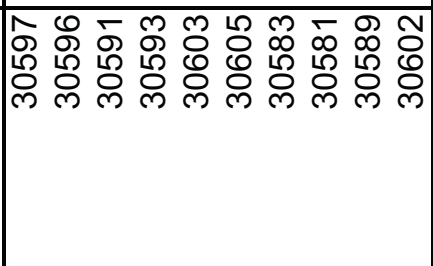 & 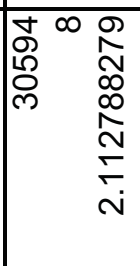 & 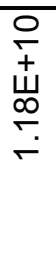 & 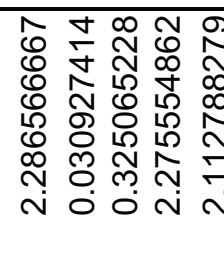 & 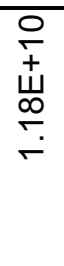 & \\
\hline 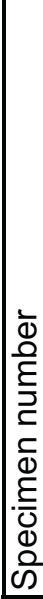 & 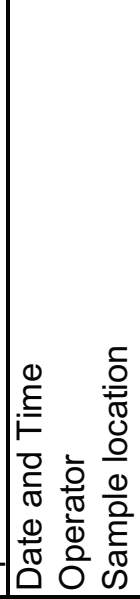 & 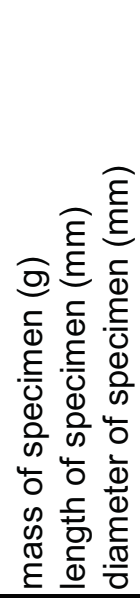 & 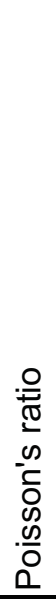 & 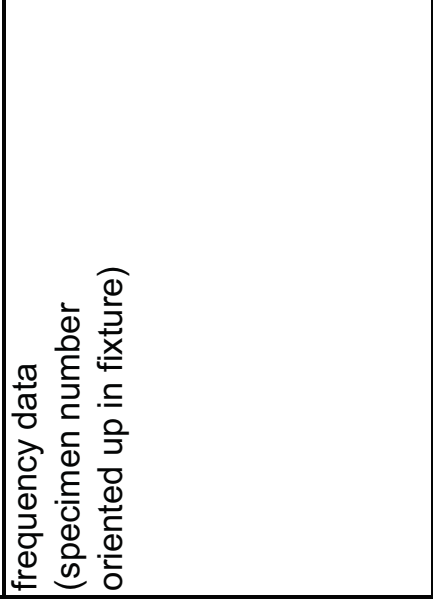 & 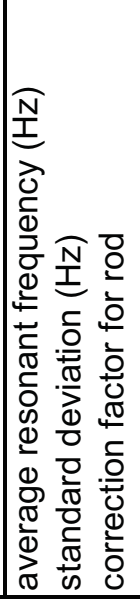 & 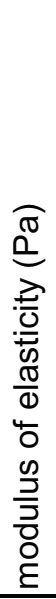 & 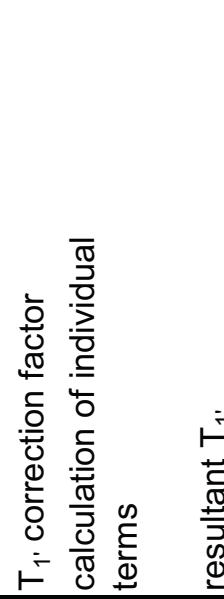 & 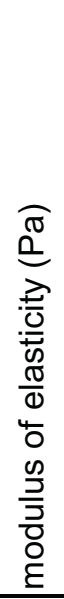 & 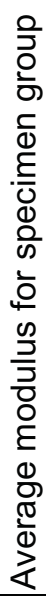 \\
\hline
\end{tabular}




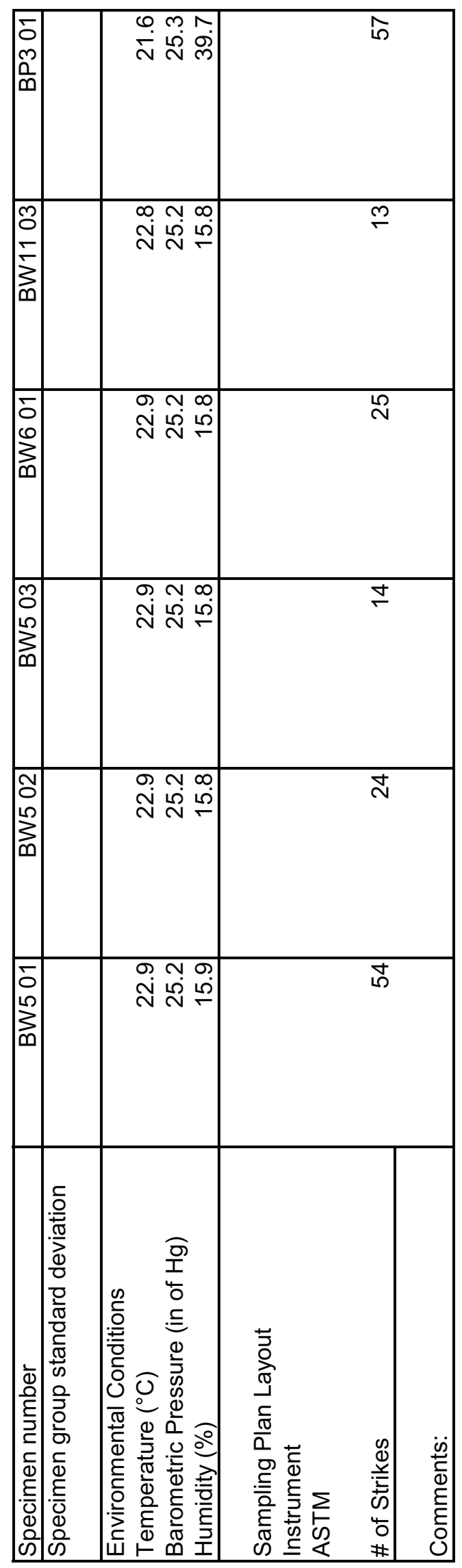




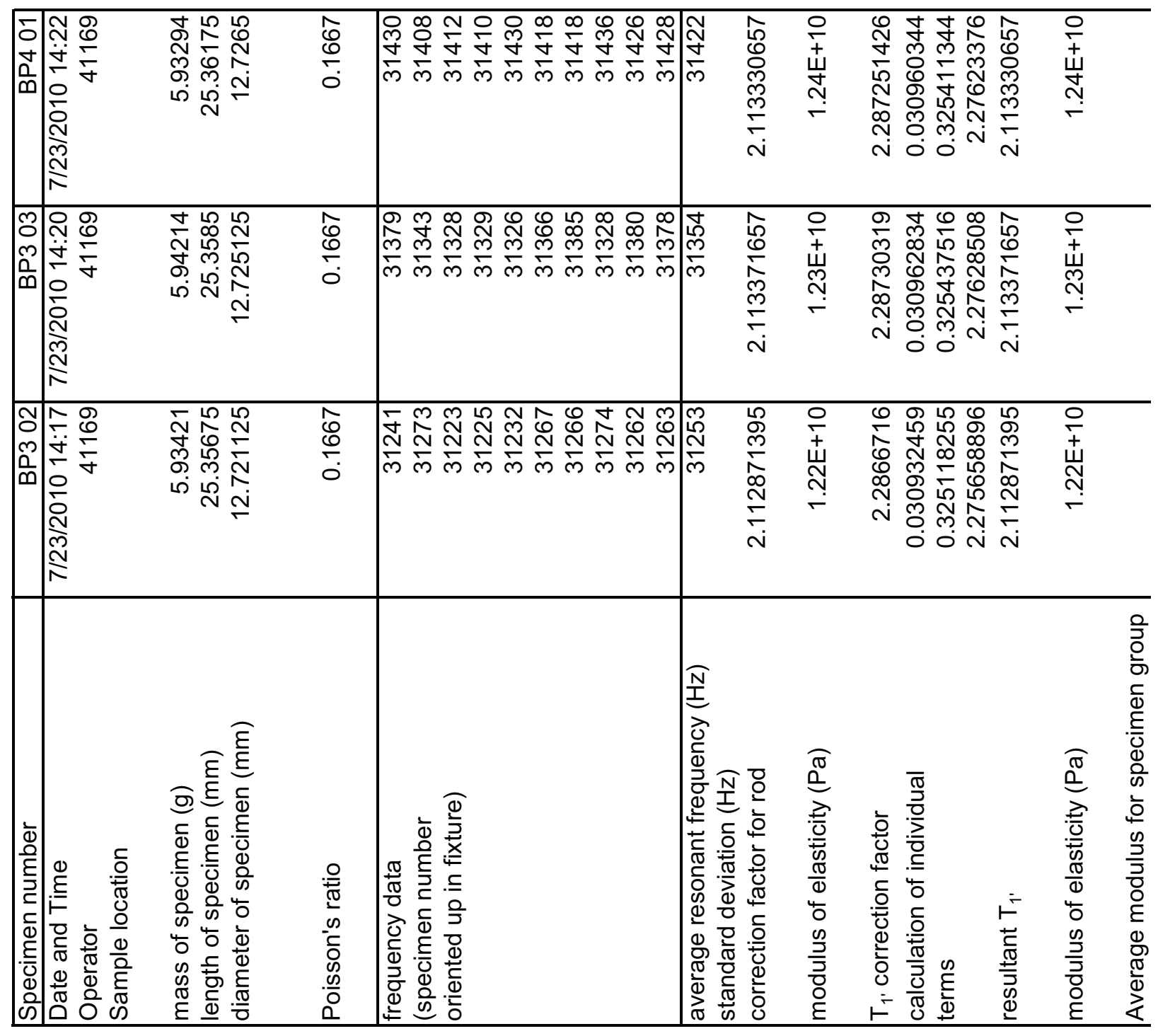




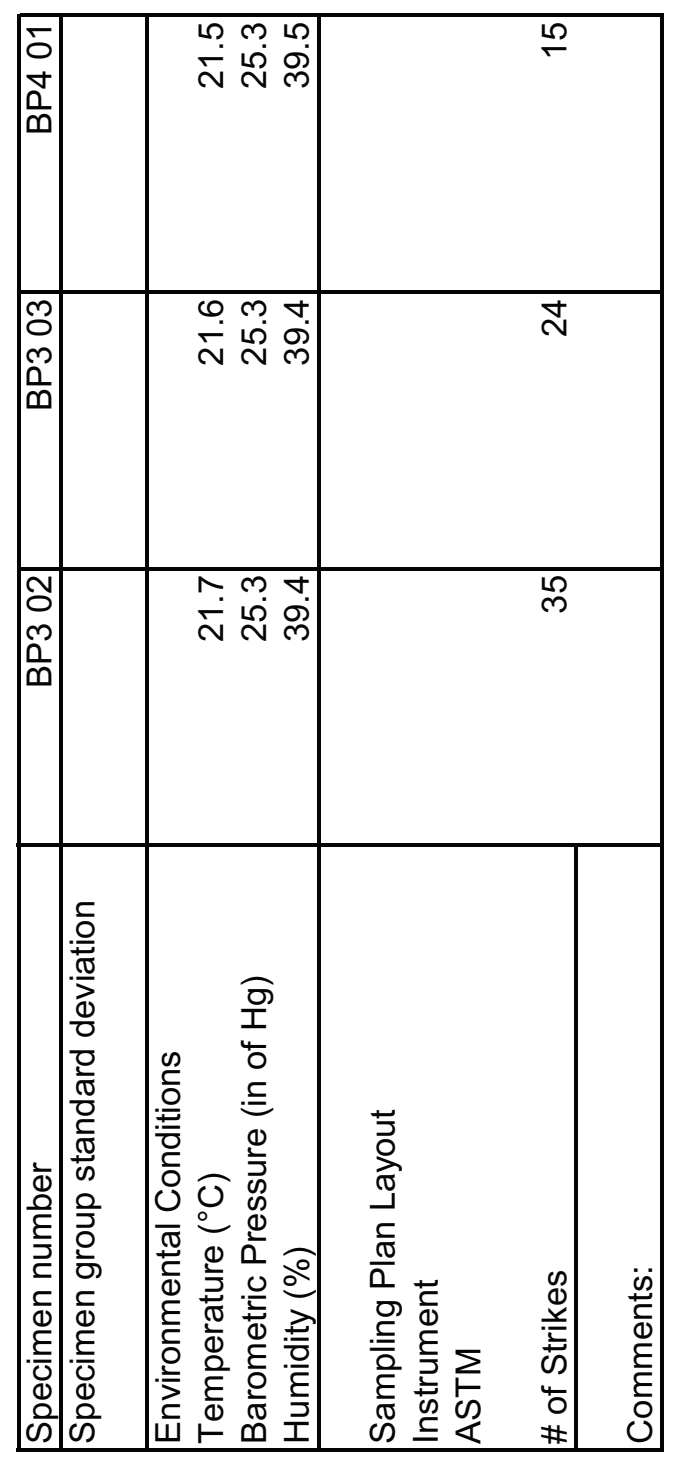




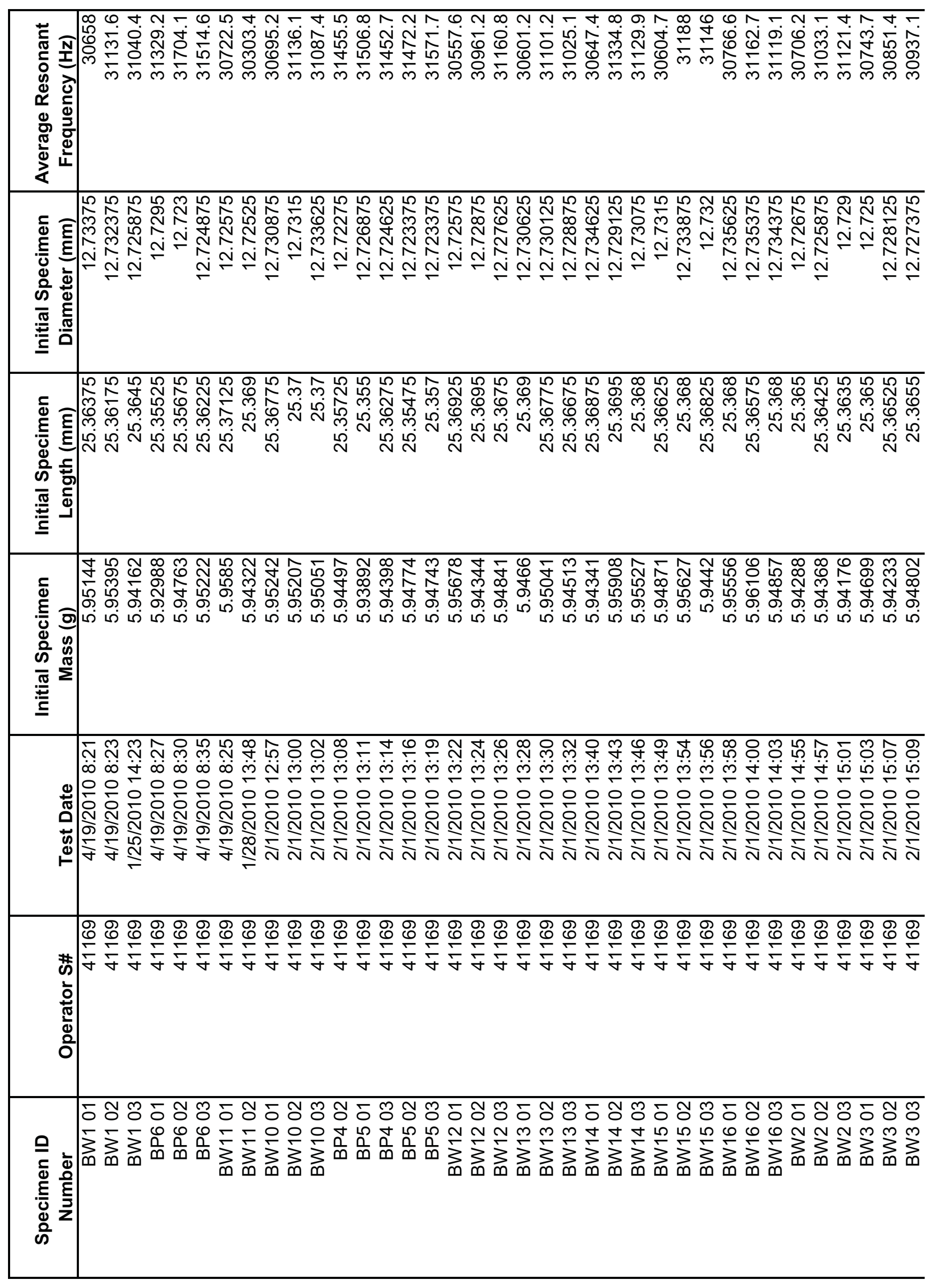




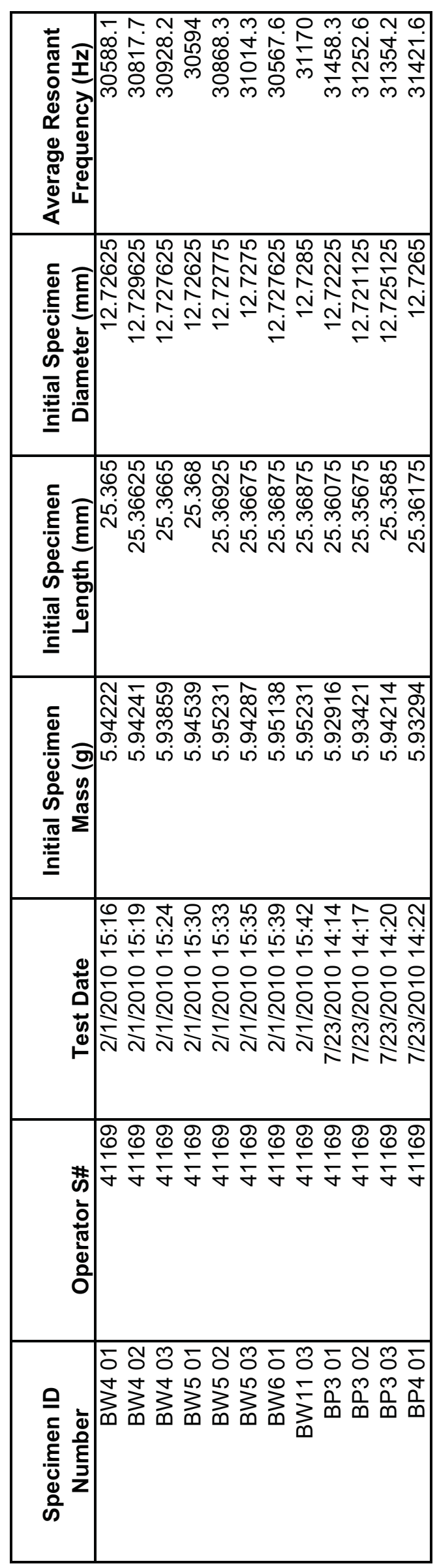




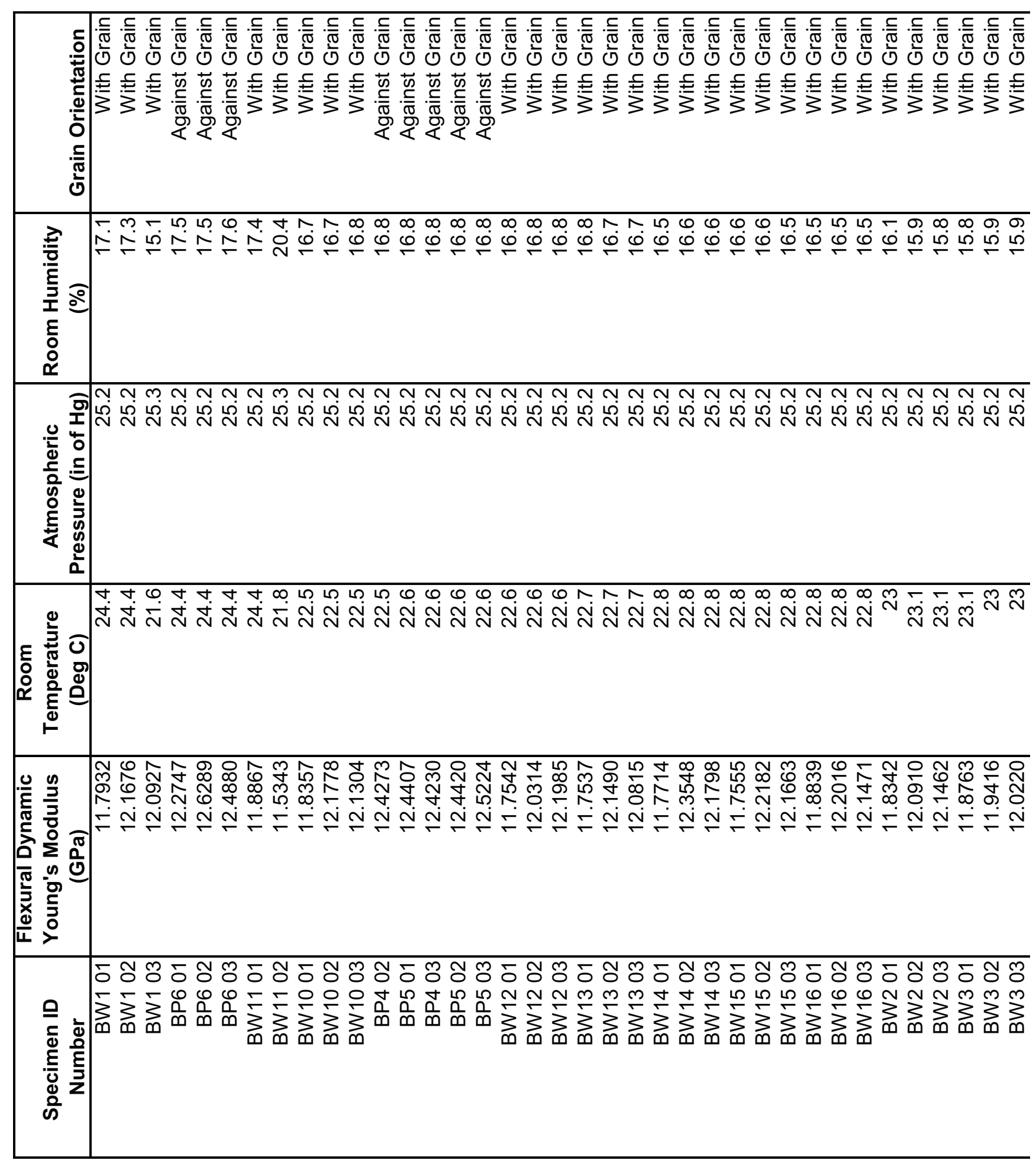




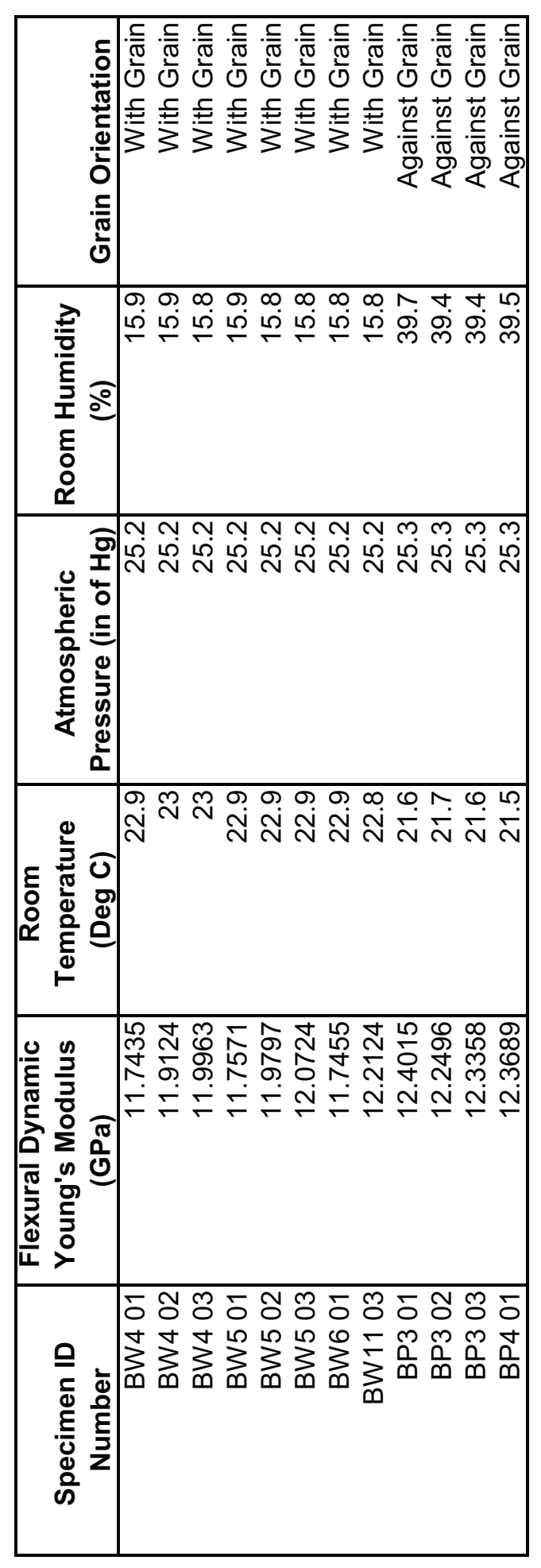




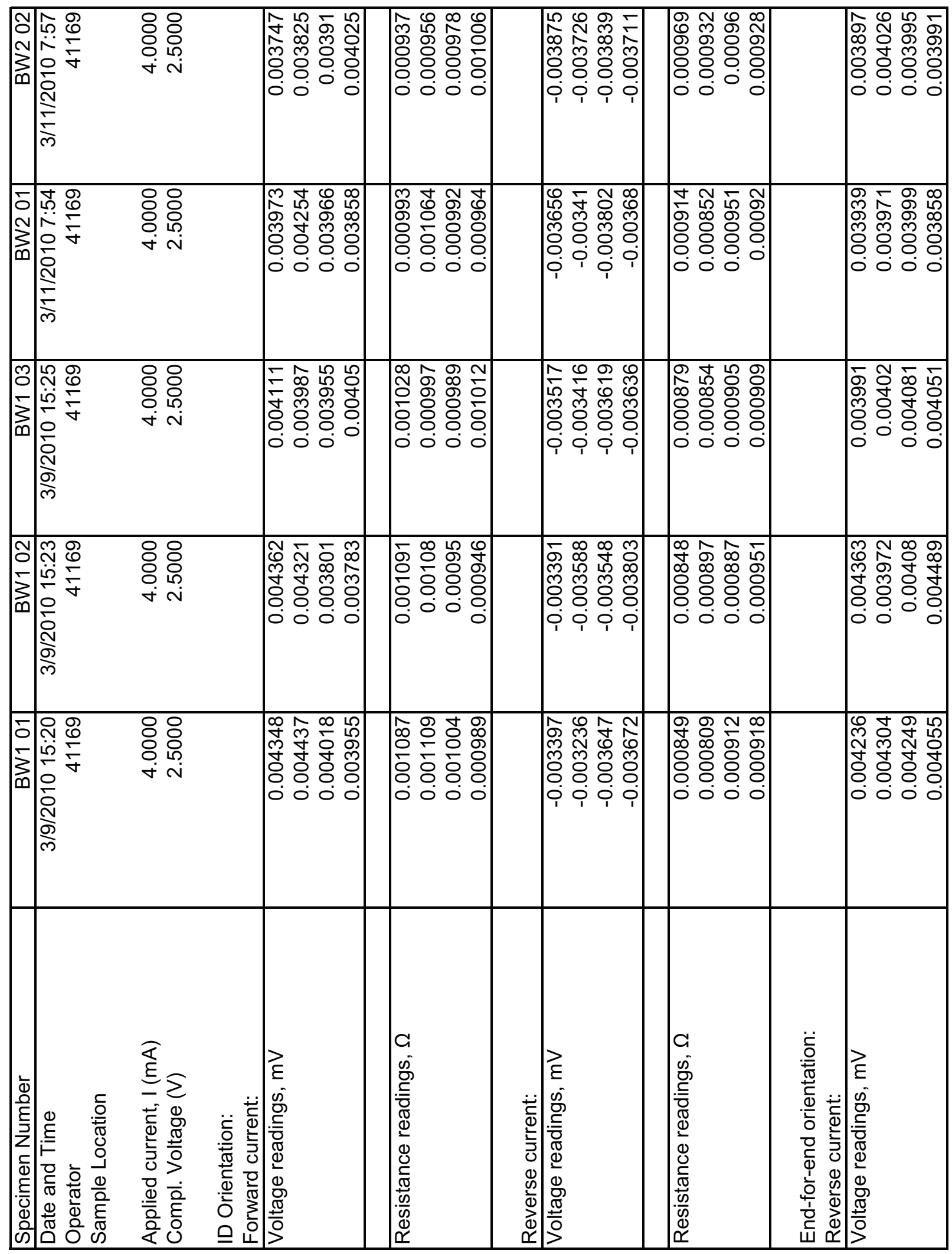




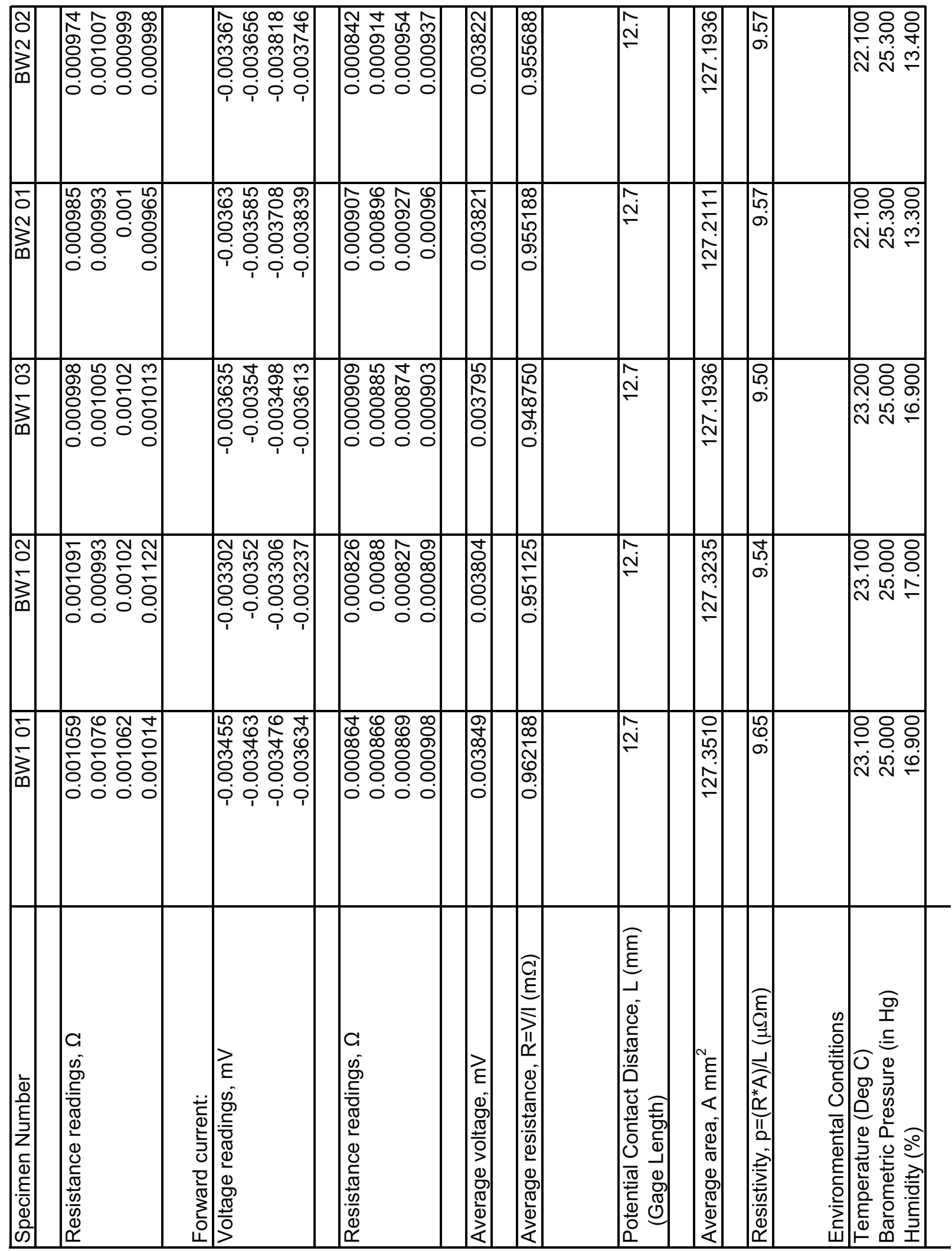




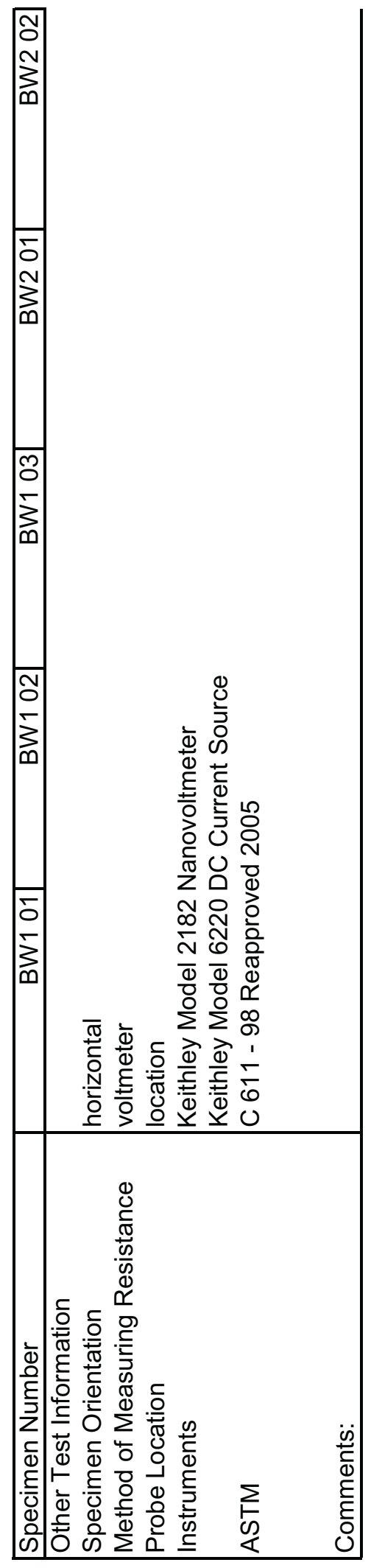

웅 


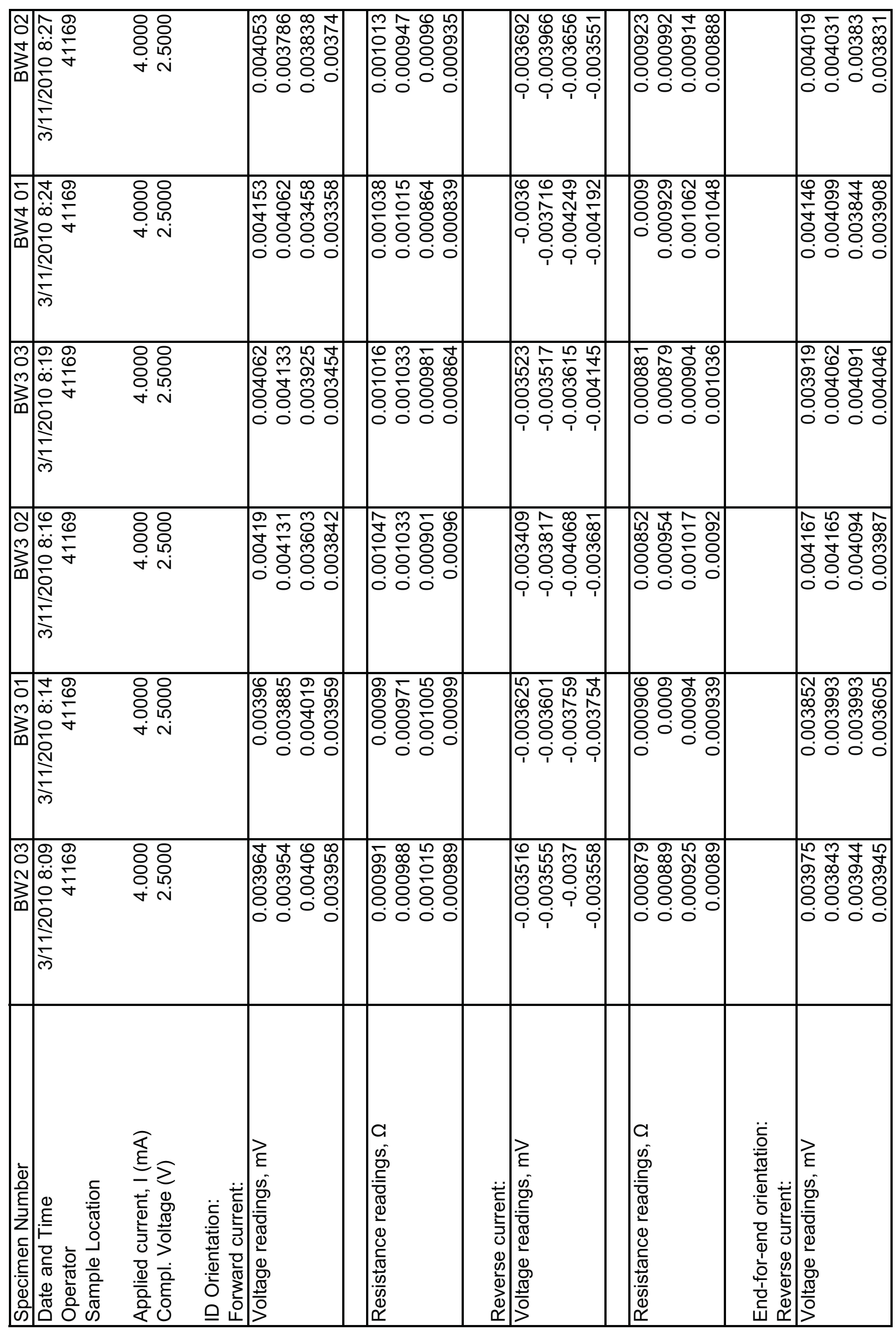




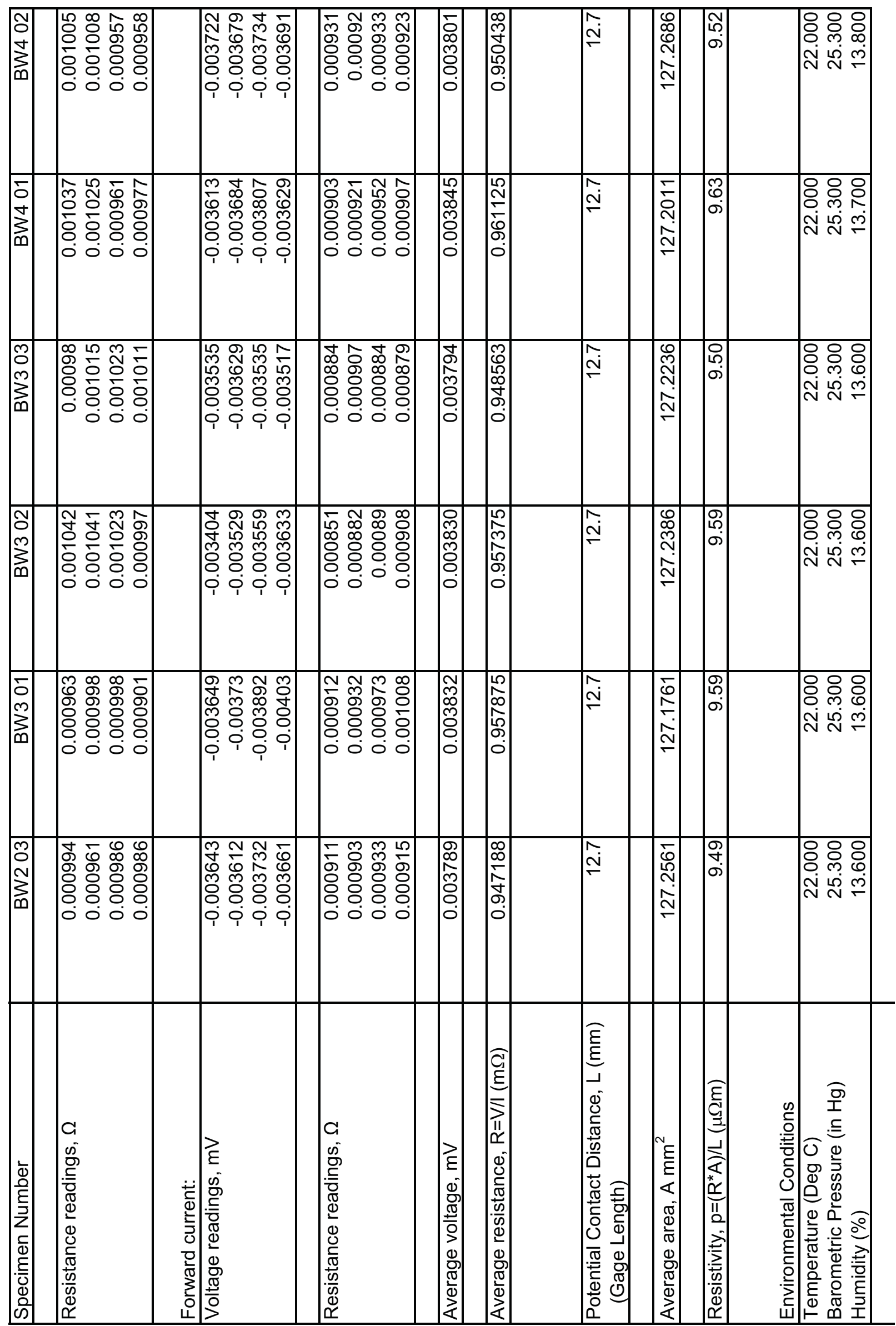




\begin{tabular}{|c|c|c|c|c|c|c|c|c|c|}
\hline \begin{tabular}{|l|} 
\\
0 \\
0 \\
3 \\
0 \\
\end{tabular} & 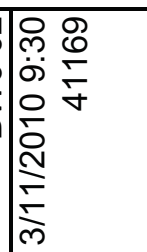 & 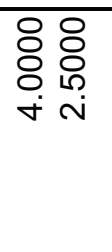 & & 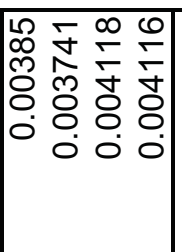 & 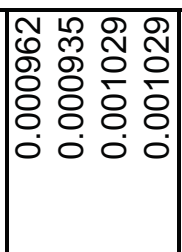 & 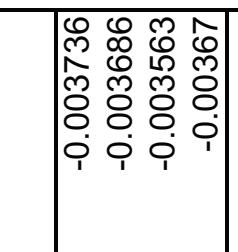 & 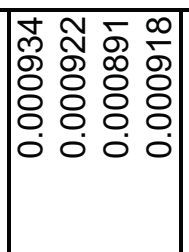 & & 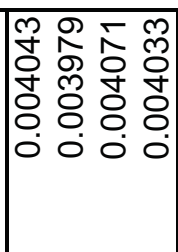 \\
\hline \begin{tabular}{|l|} 
\\
0 \\
0 \\
0 \\
0 \\
\end{tabular} & 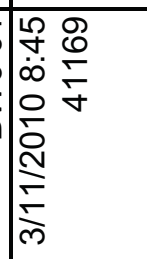 & 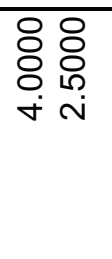 & & 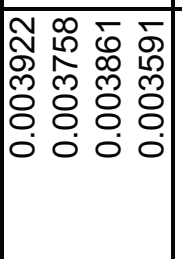 & 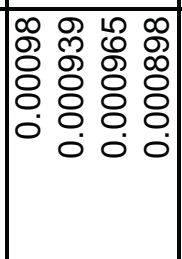 & 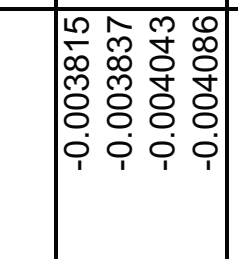 & 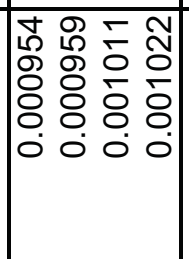 & & 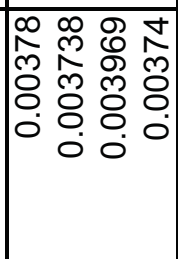 \\
\hline \begin{tabular}{|l|} 
\\
0 \\
0 \\
10 \\
0 \\
0
\end{tabular} & 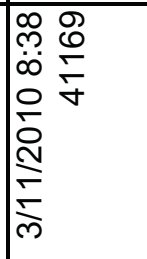 & 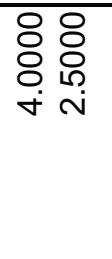 & & 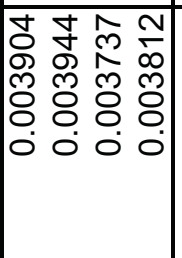 & 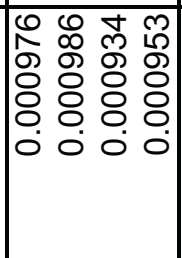 & 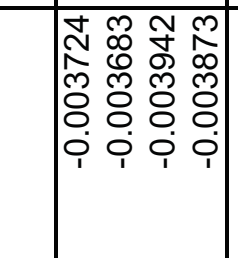 & 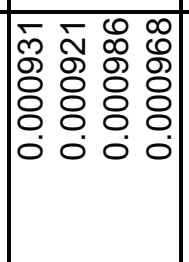 & & 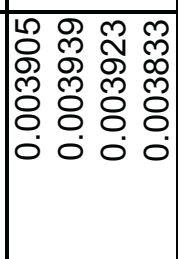 \\
\hline 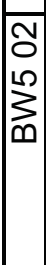 & 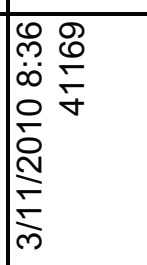 & 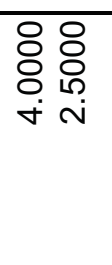 & & 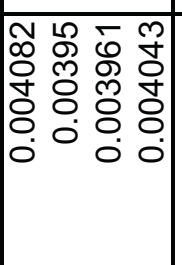 & 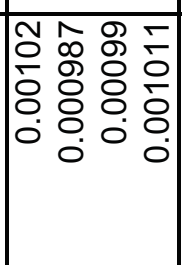 & 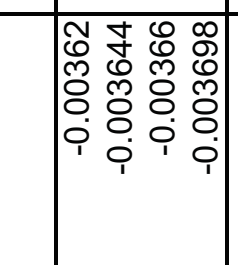 & 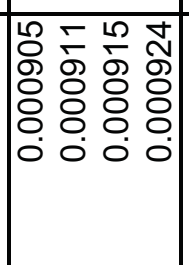 & & 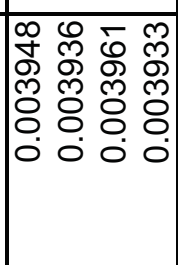 \\
\hline \begin{tabular}{|l|} 
\\
0 \\
10 \\
3 \\
0 \\
\end{tabular} & 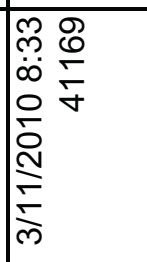 & 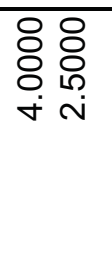 & & 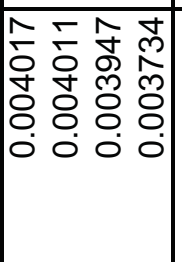 & 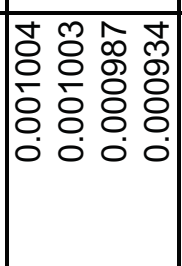 & 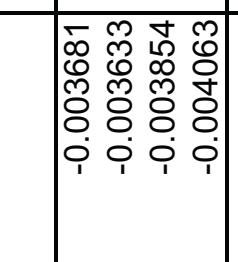 & 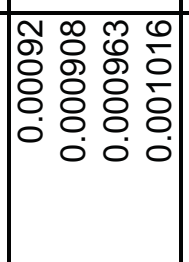 & & 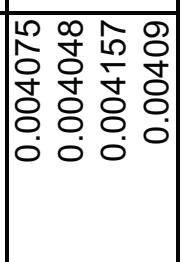 \\
\hline $\begin{array}{l}m \\
0 \\
\vdots \\
\vdots \\
\vdots\end{array}$ & 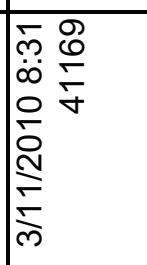 & 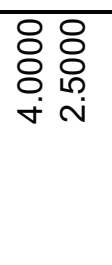 & & 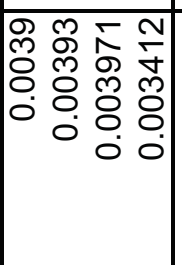 & 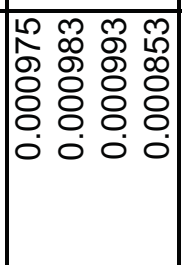 & 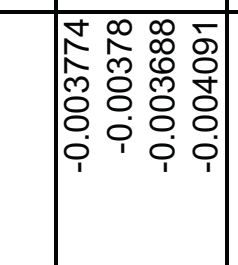 & 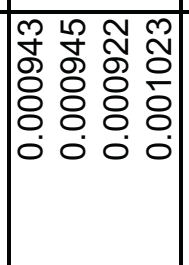 & & 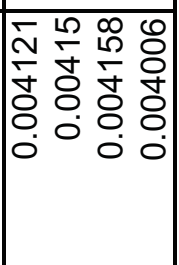 \\
\hline 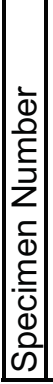 & 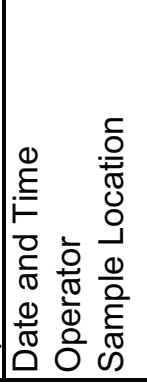 & 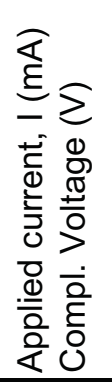 & 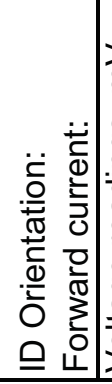 & 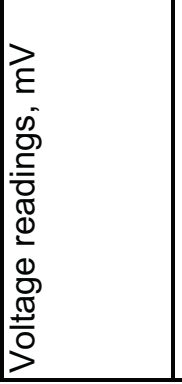 & 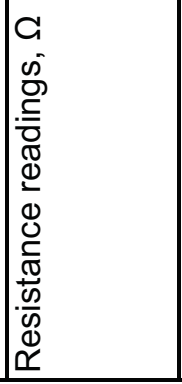 & 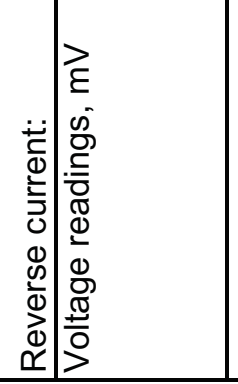 & 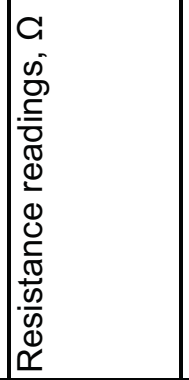 & 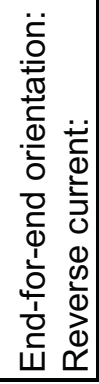 & 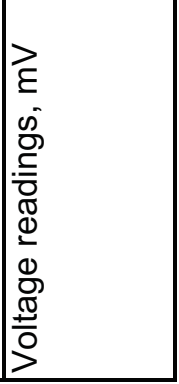 \\
\hline
\end{tabular}




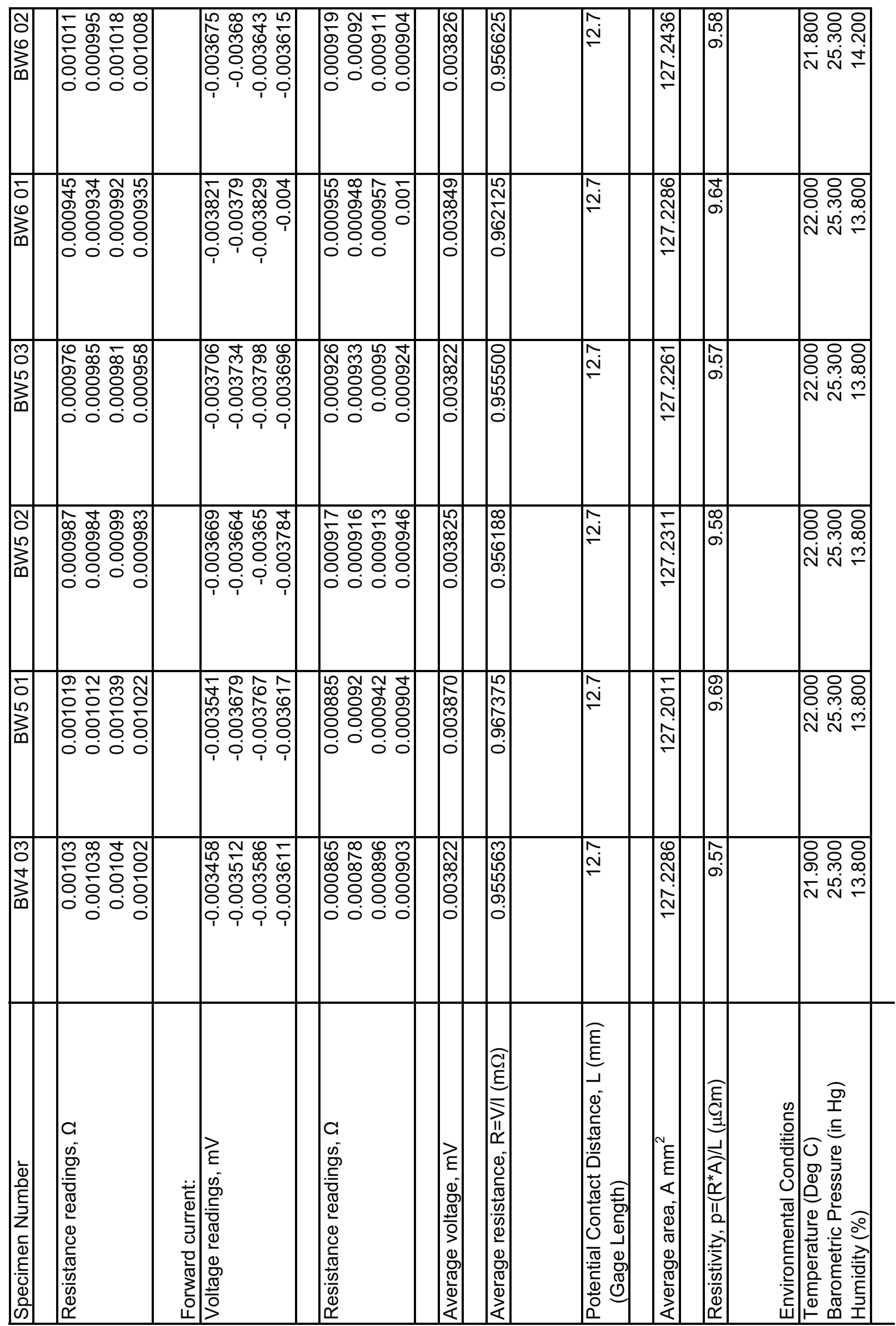




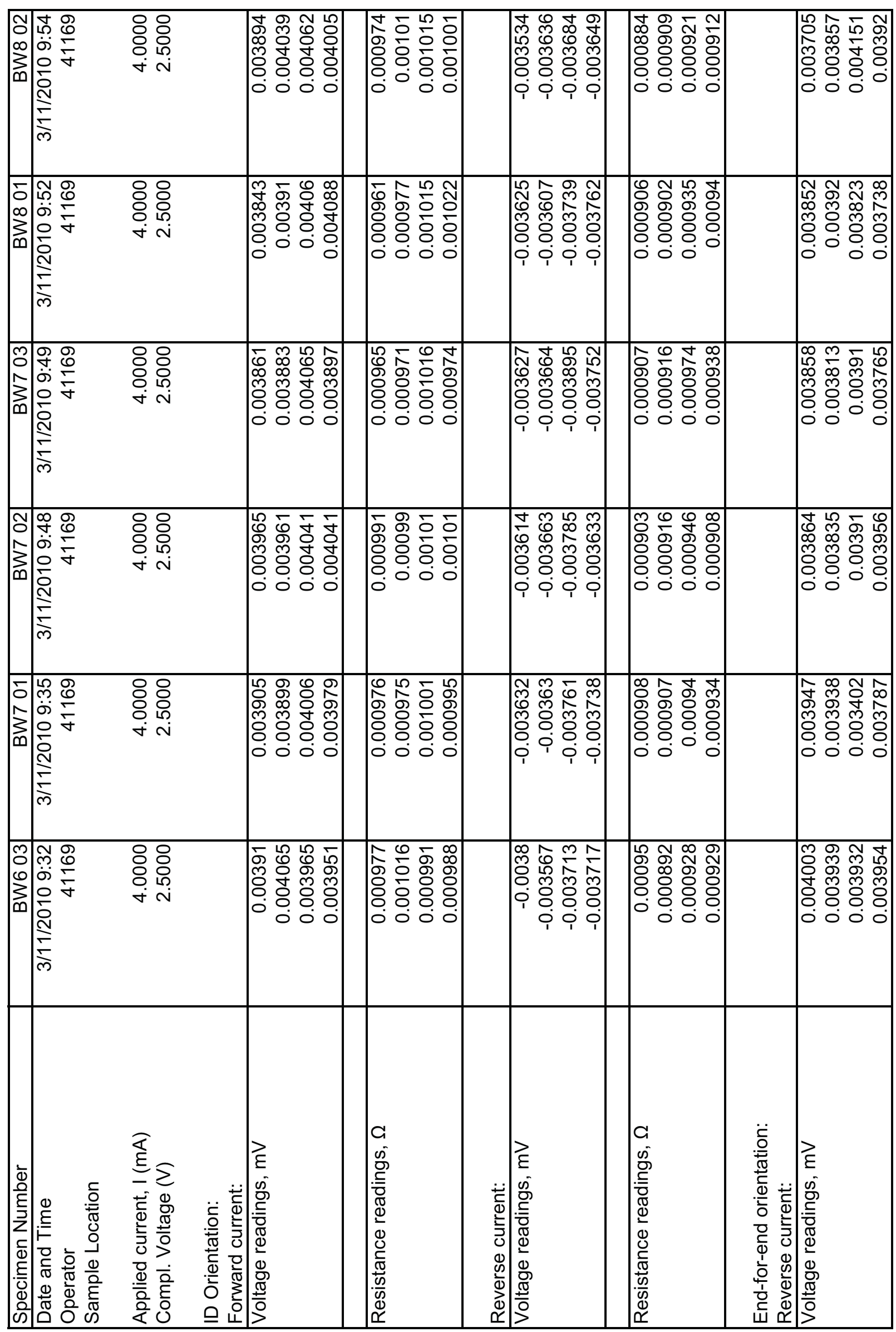




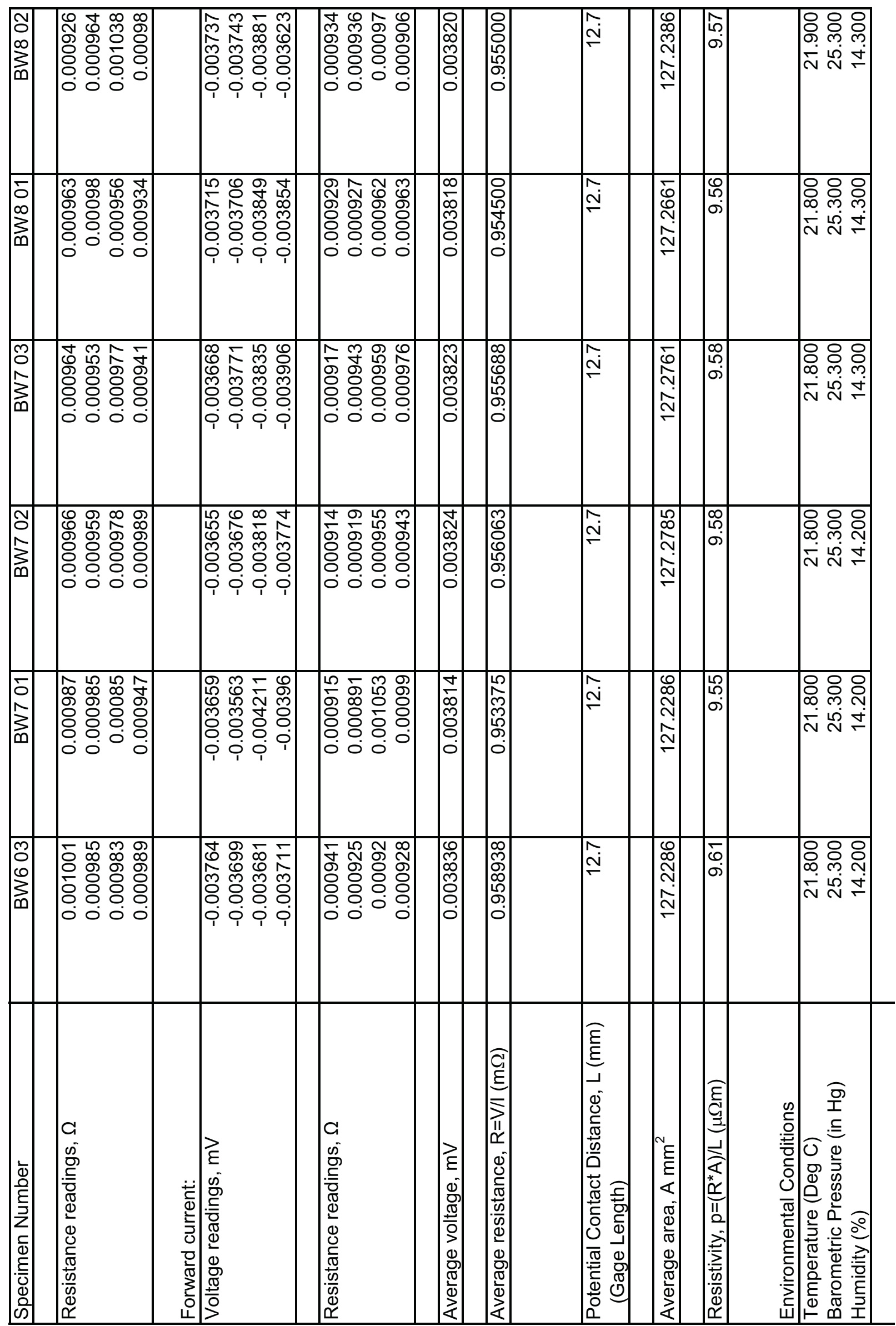




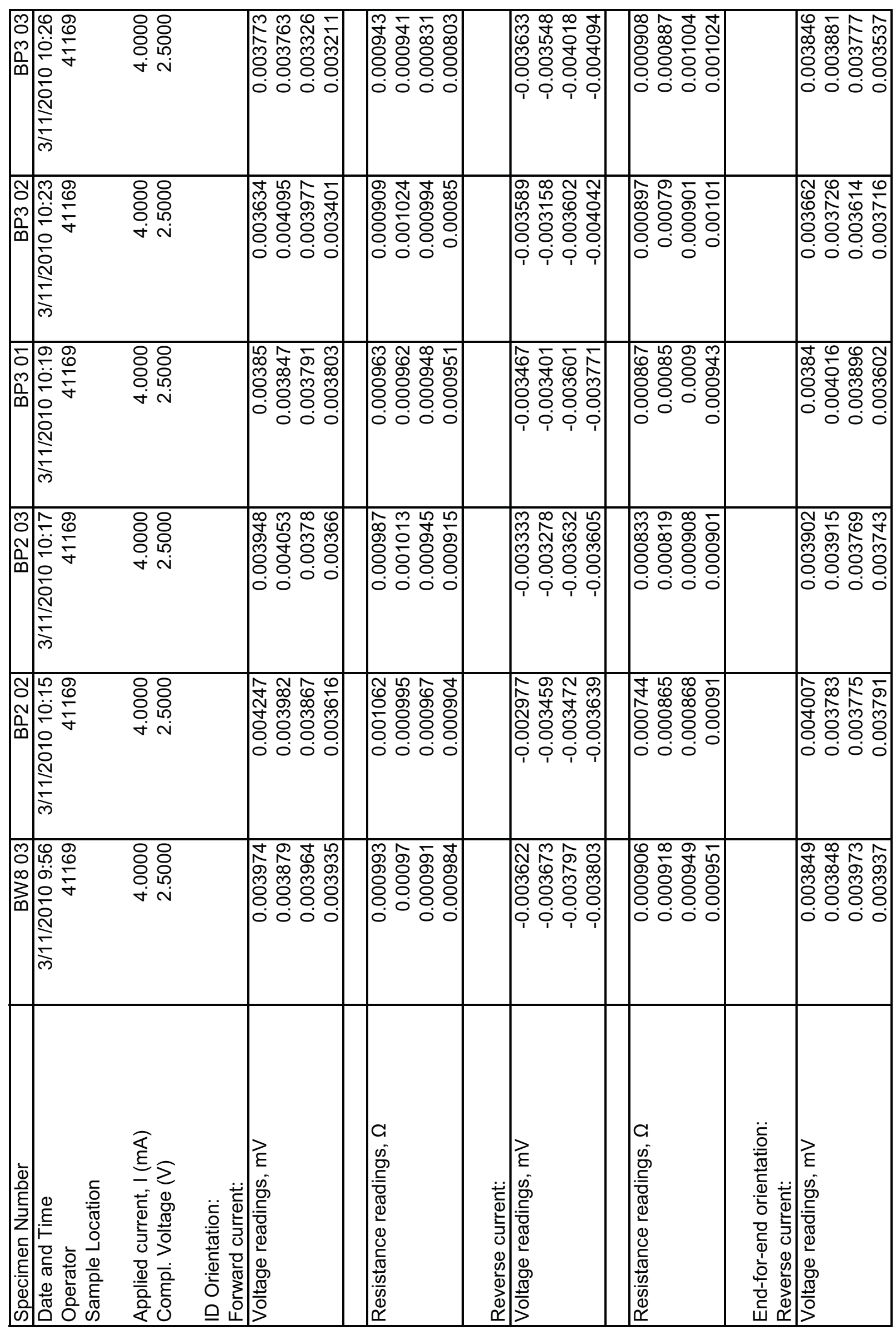




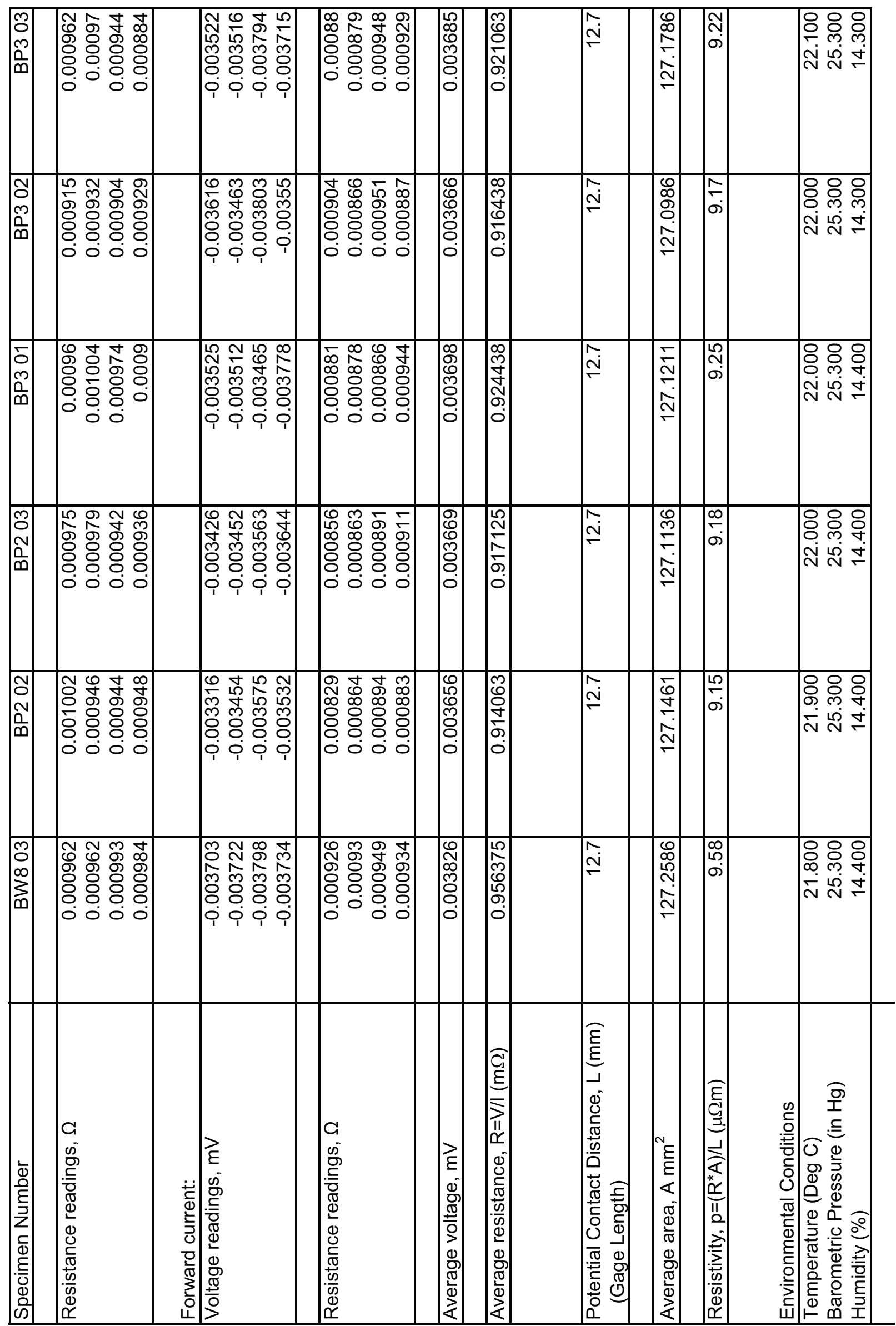




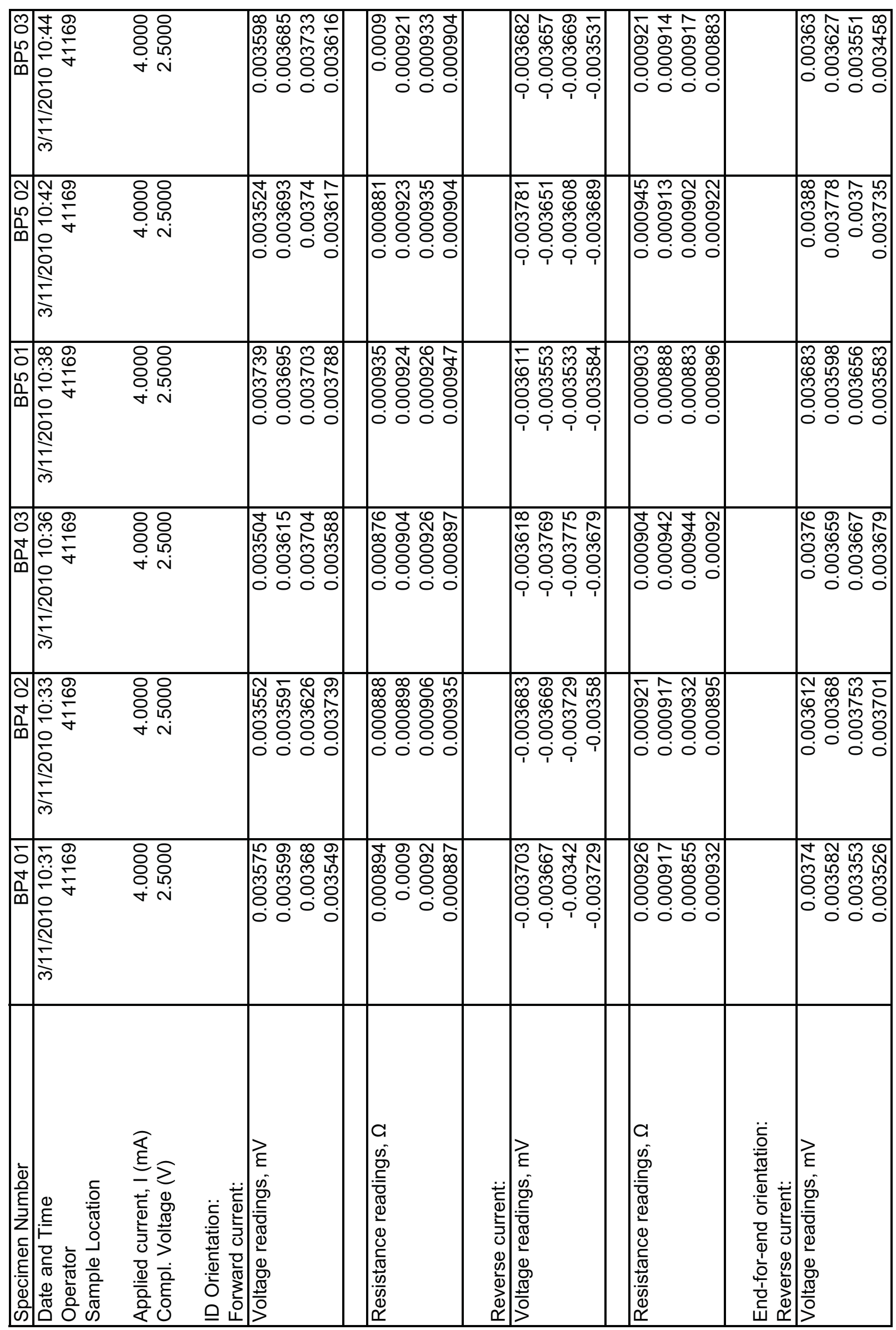




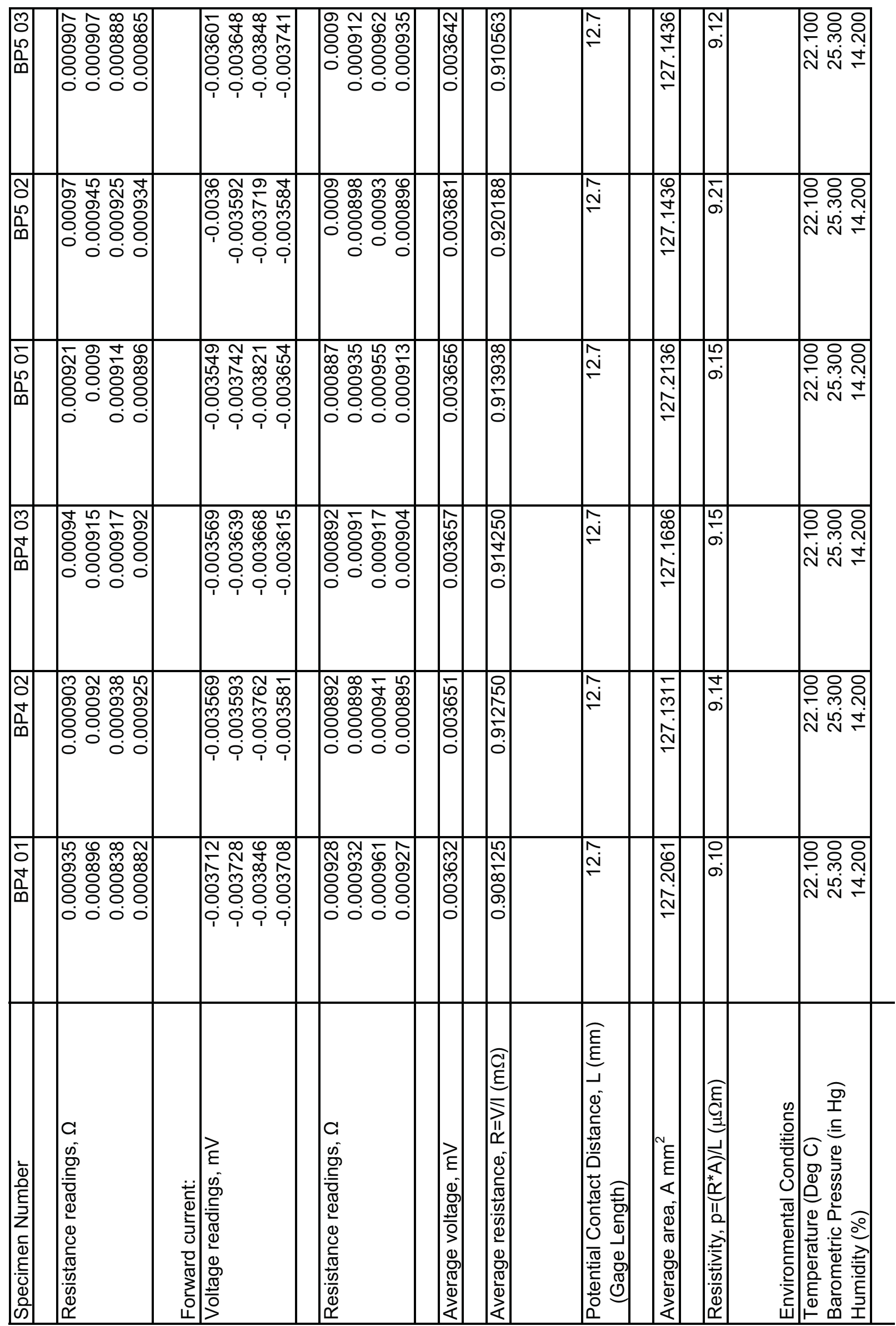




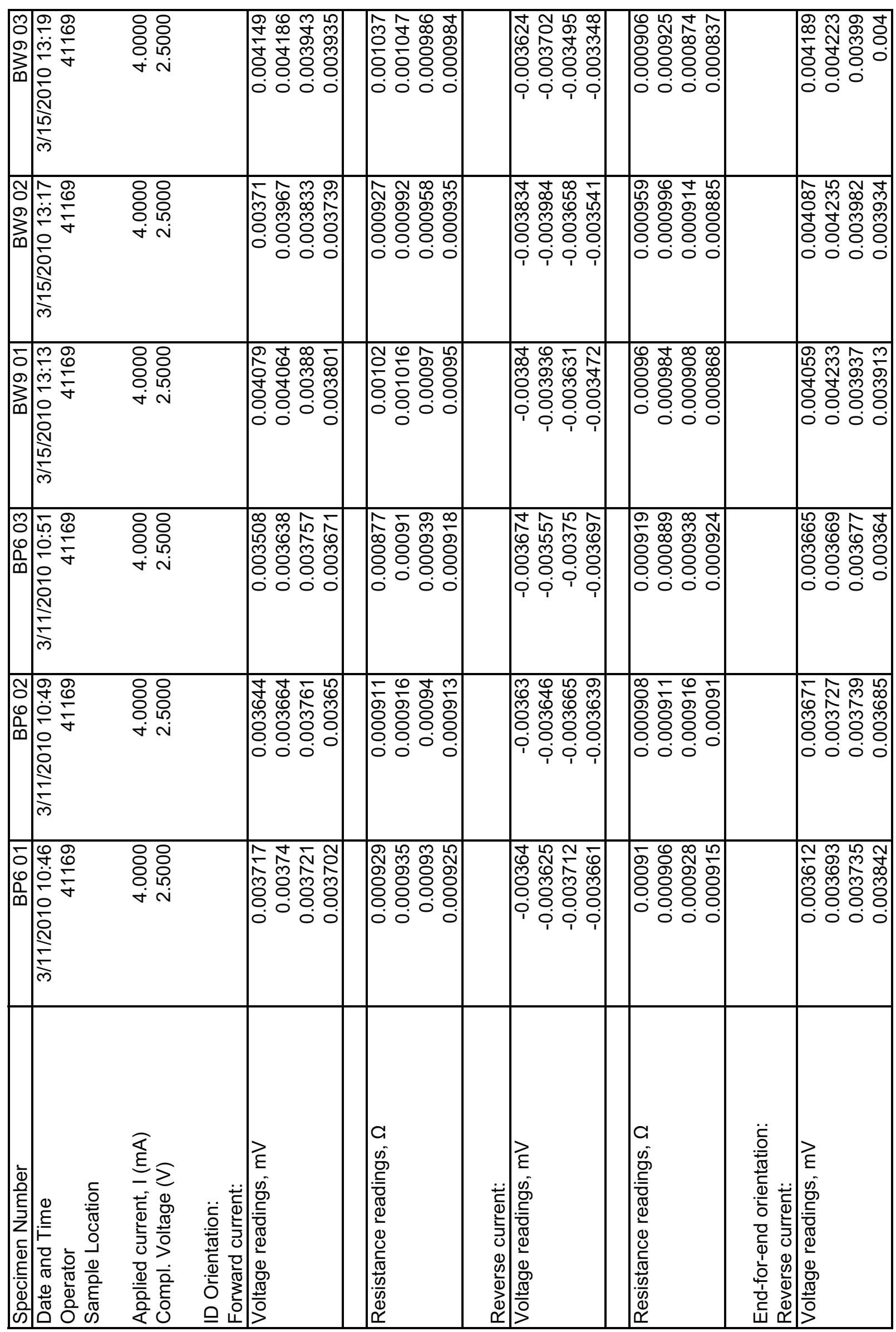




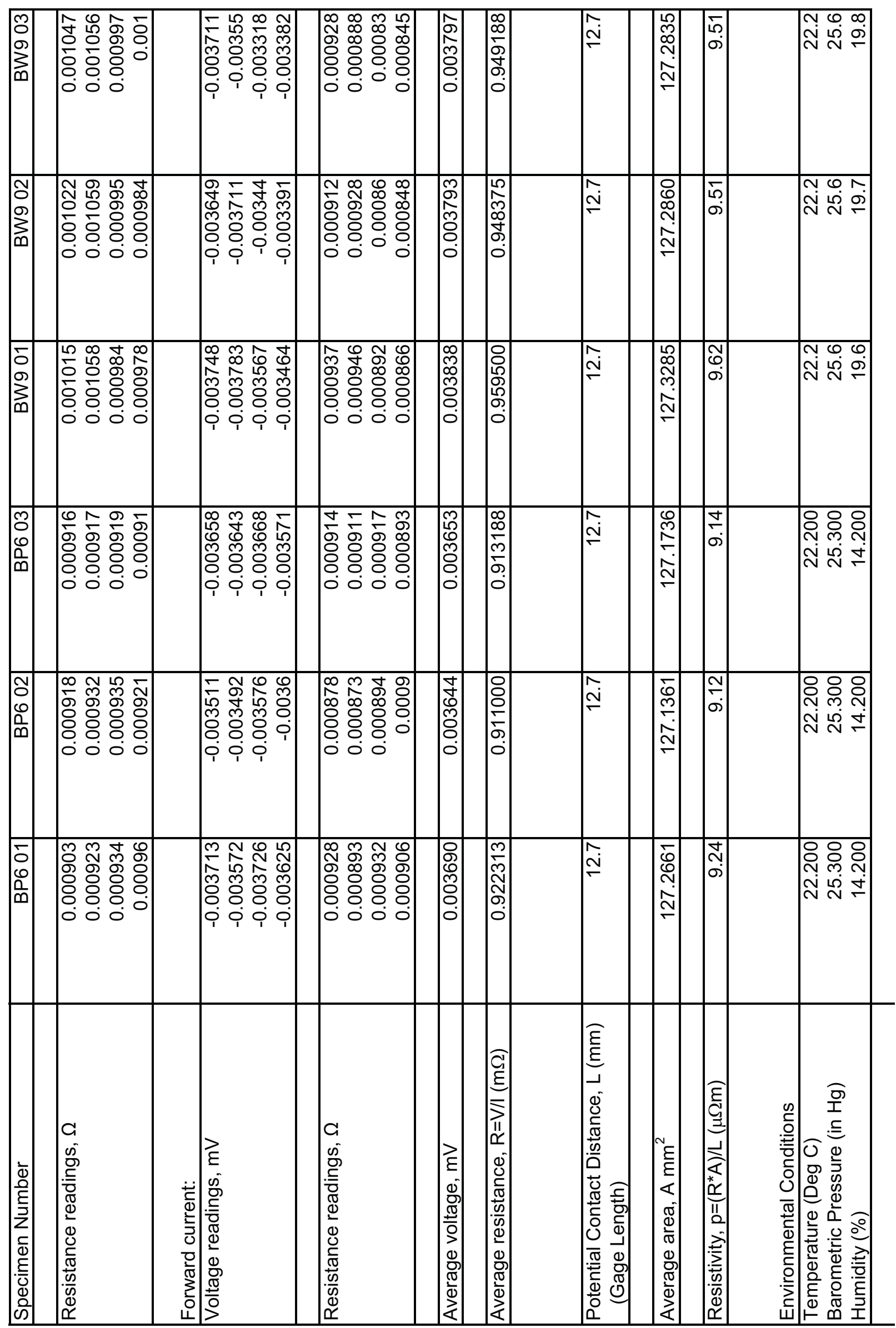




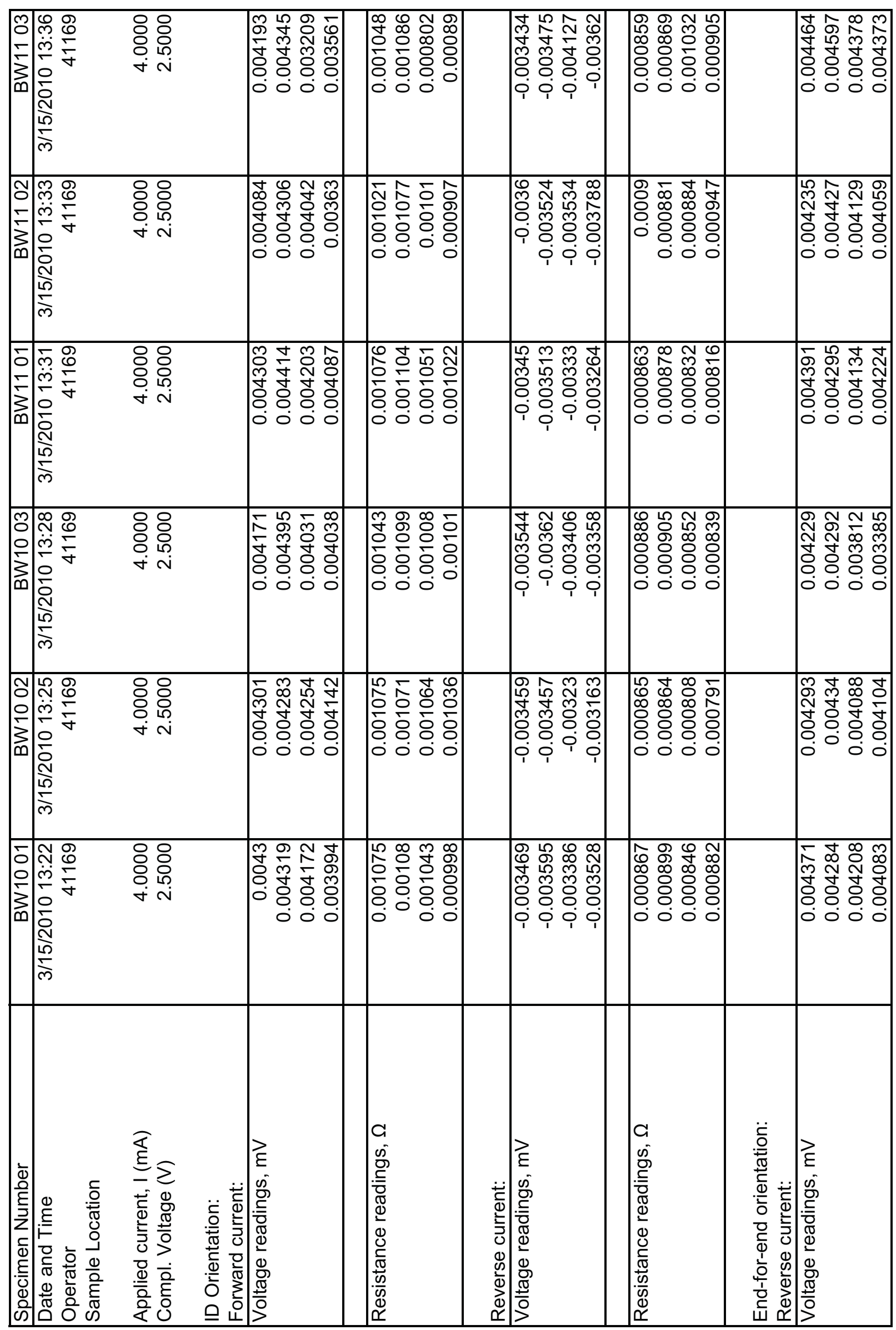




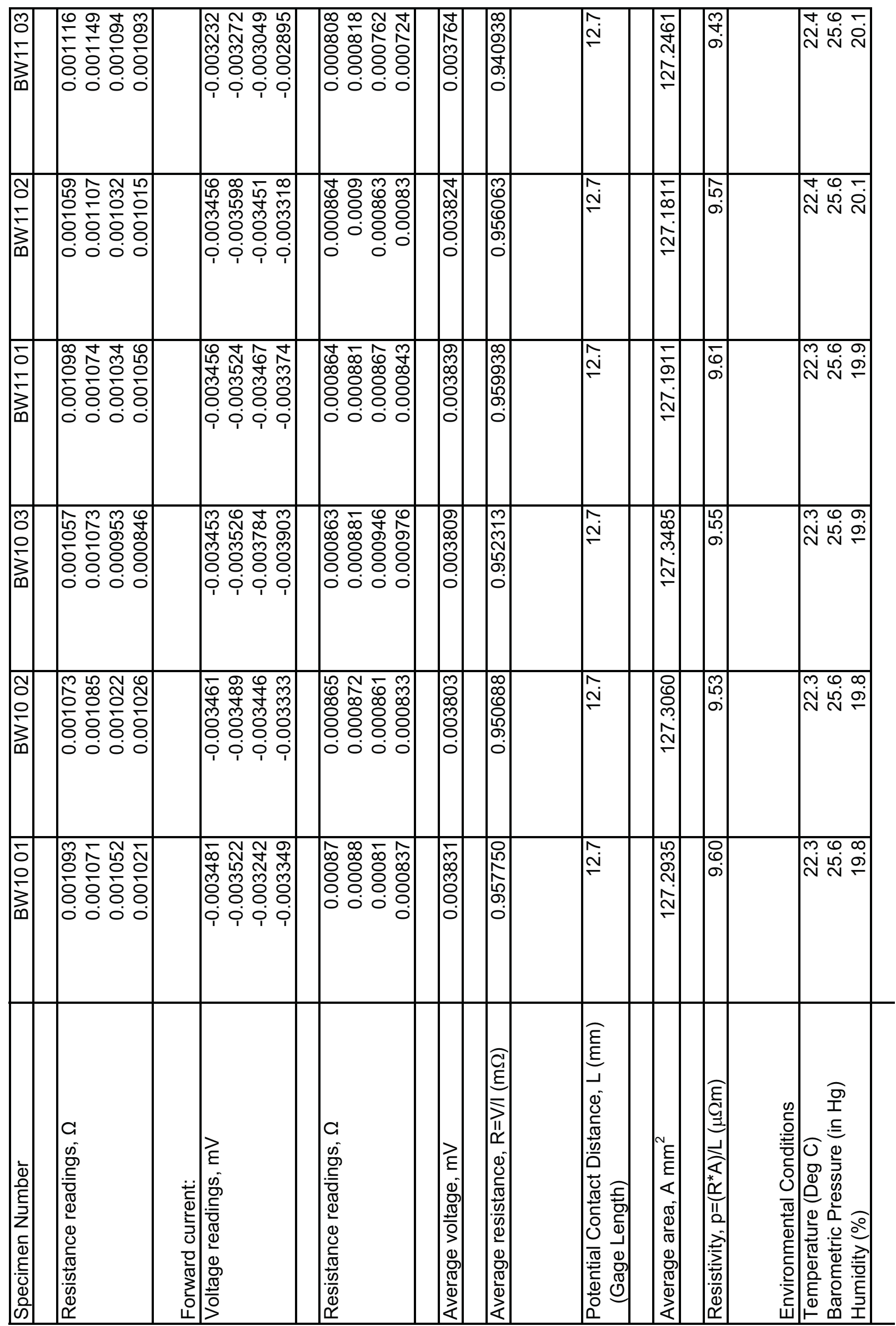




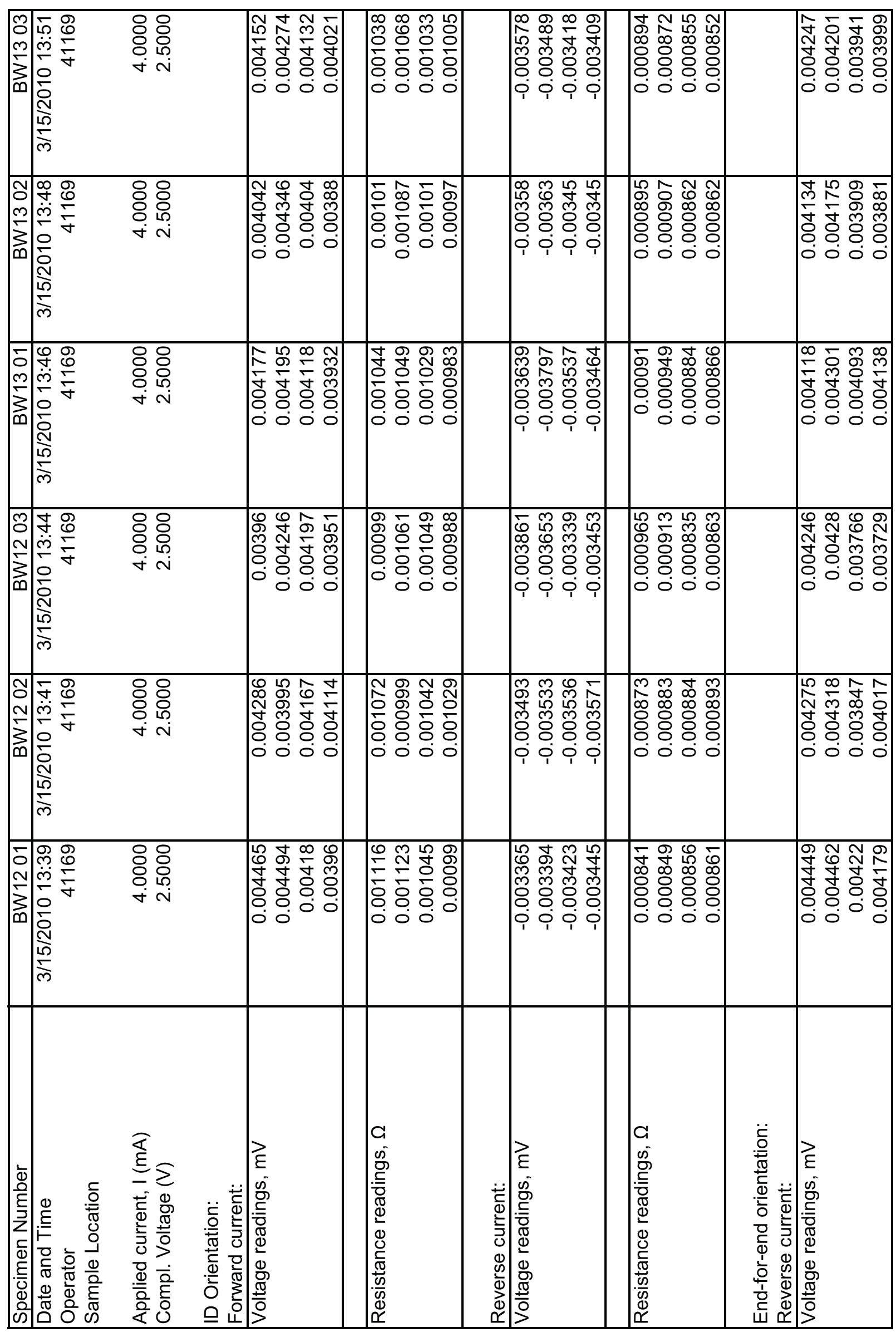




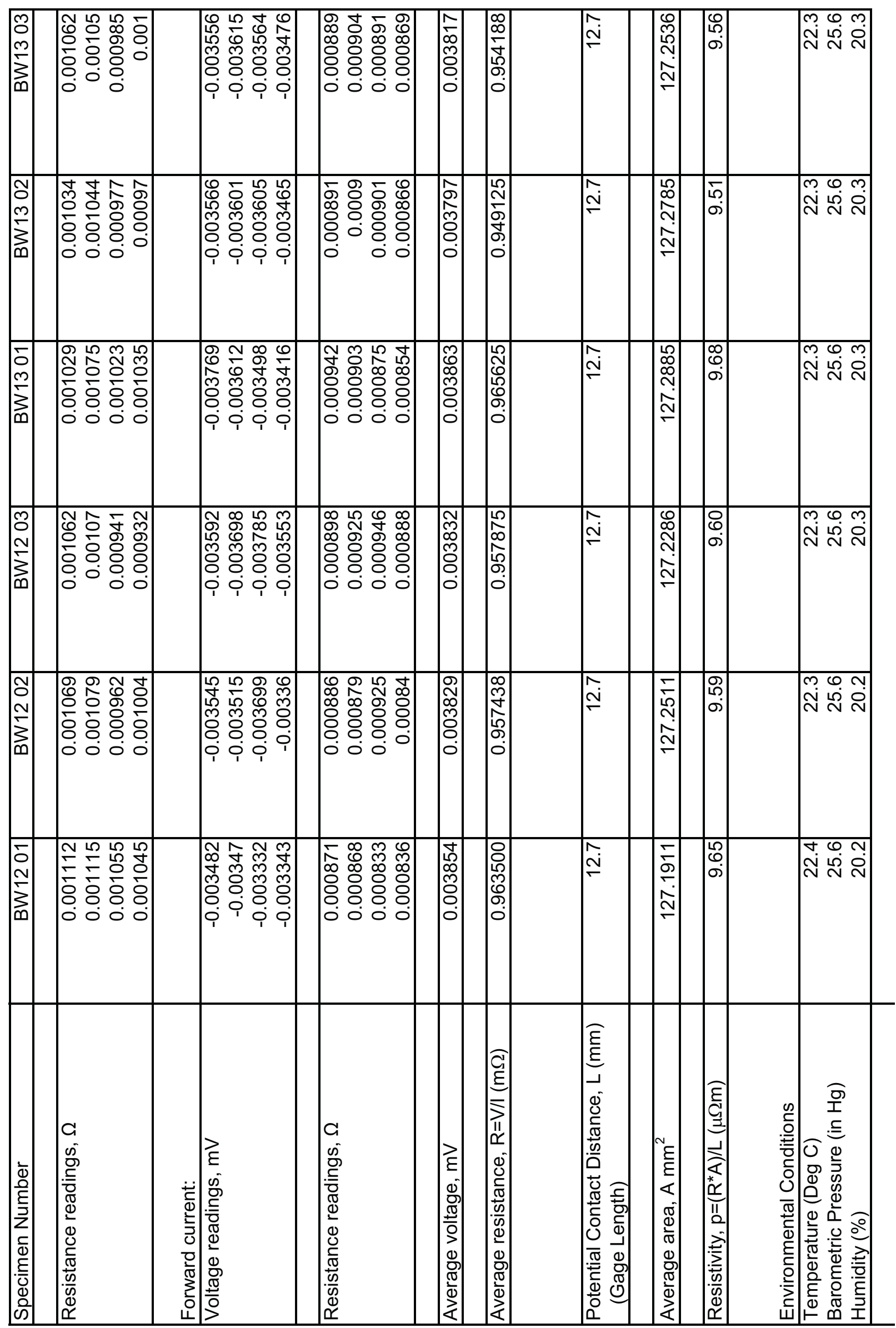




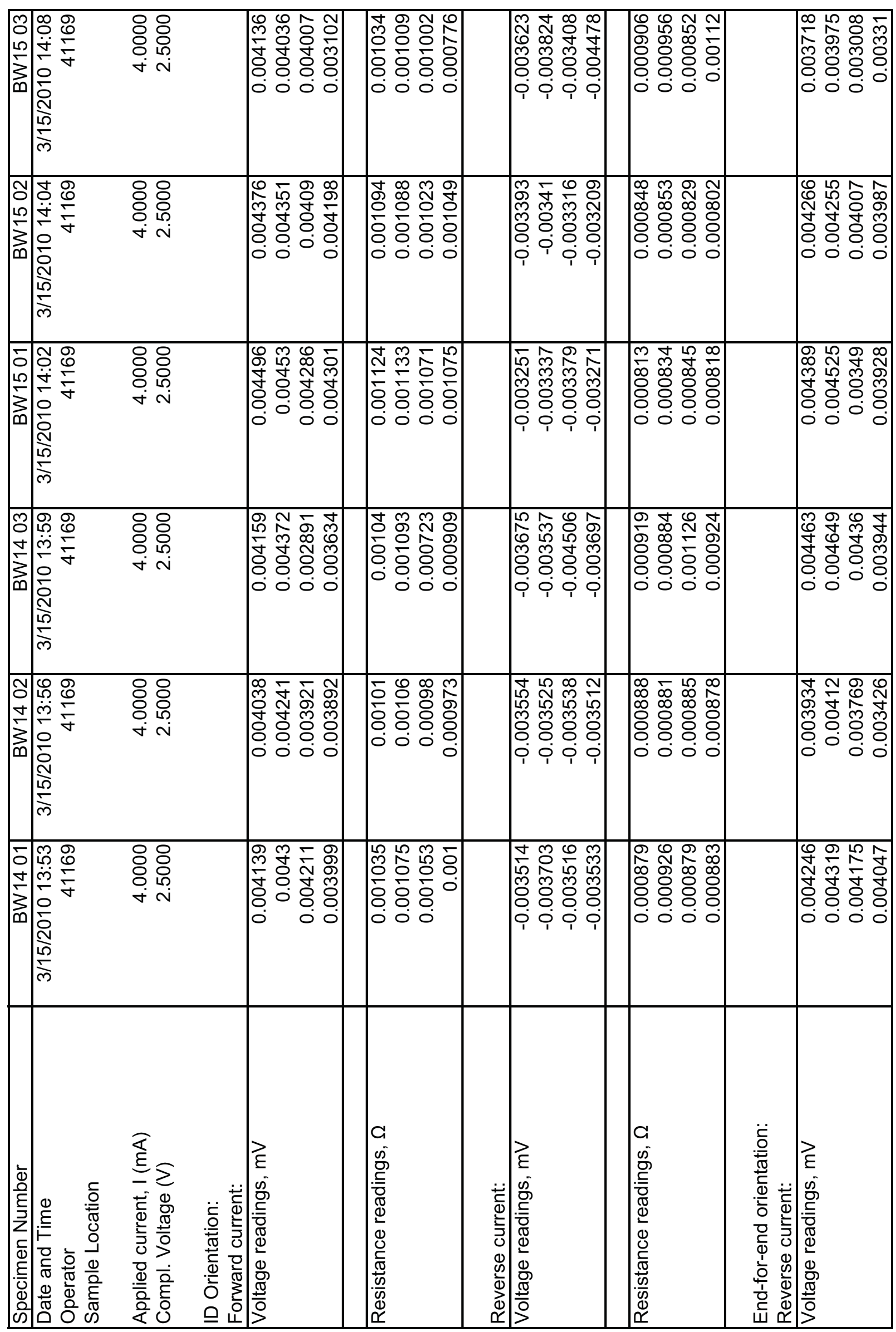




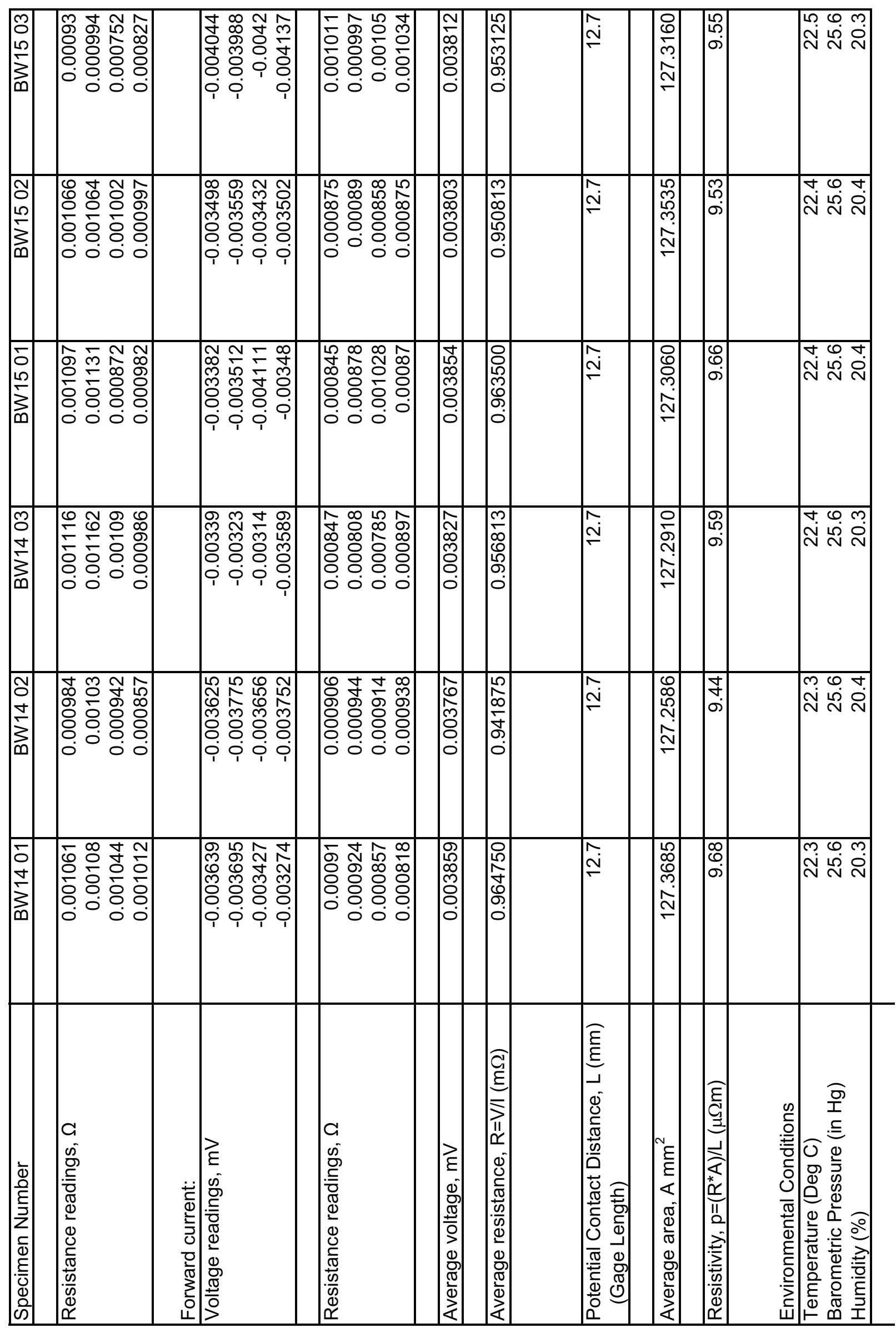




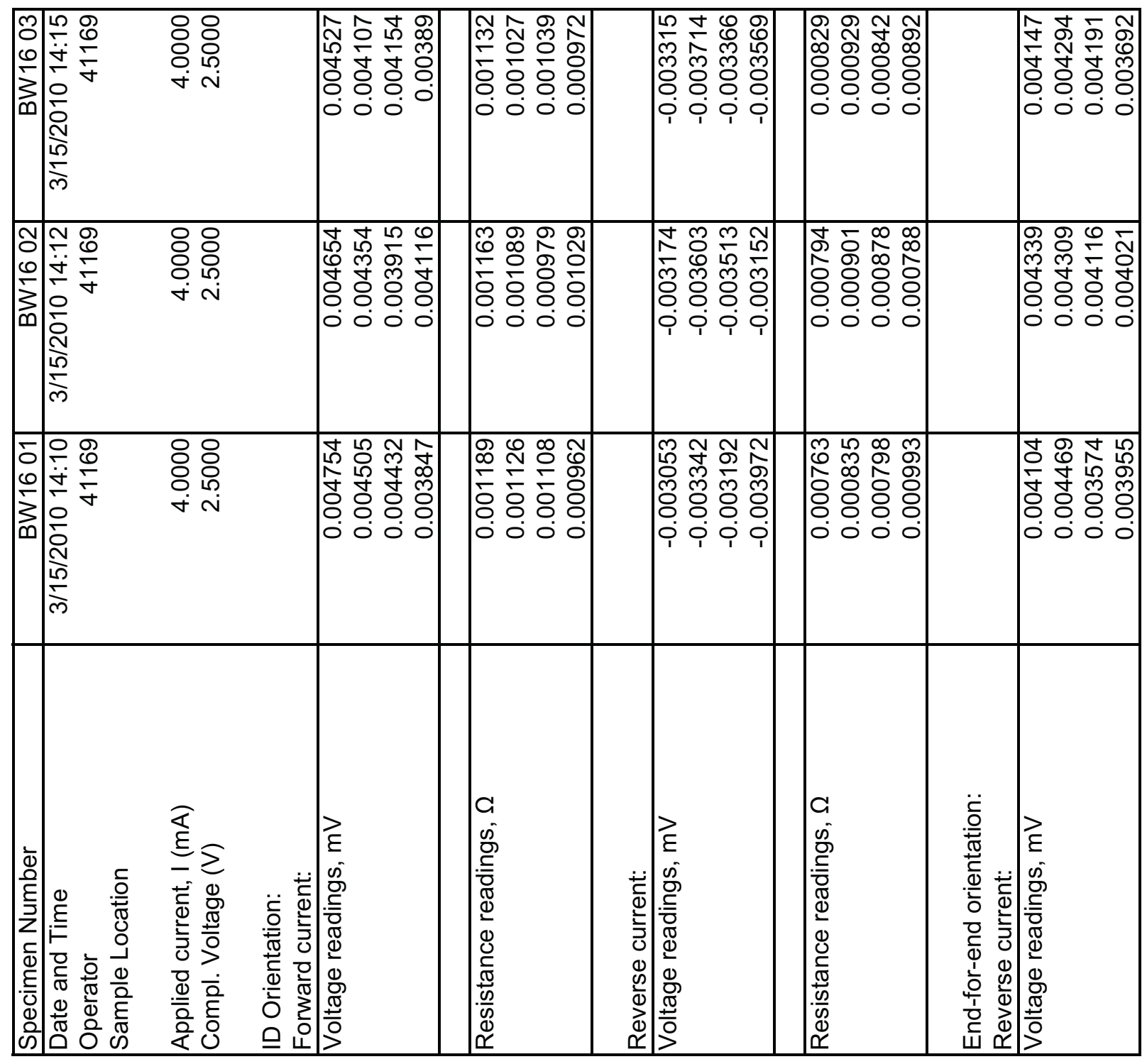




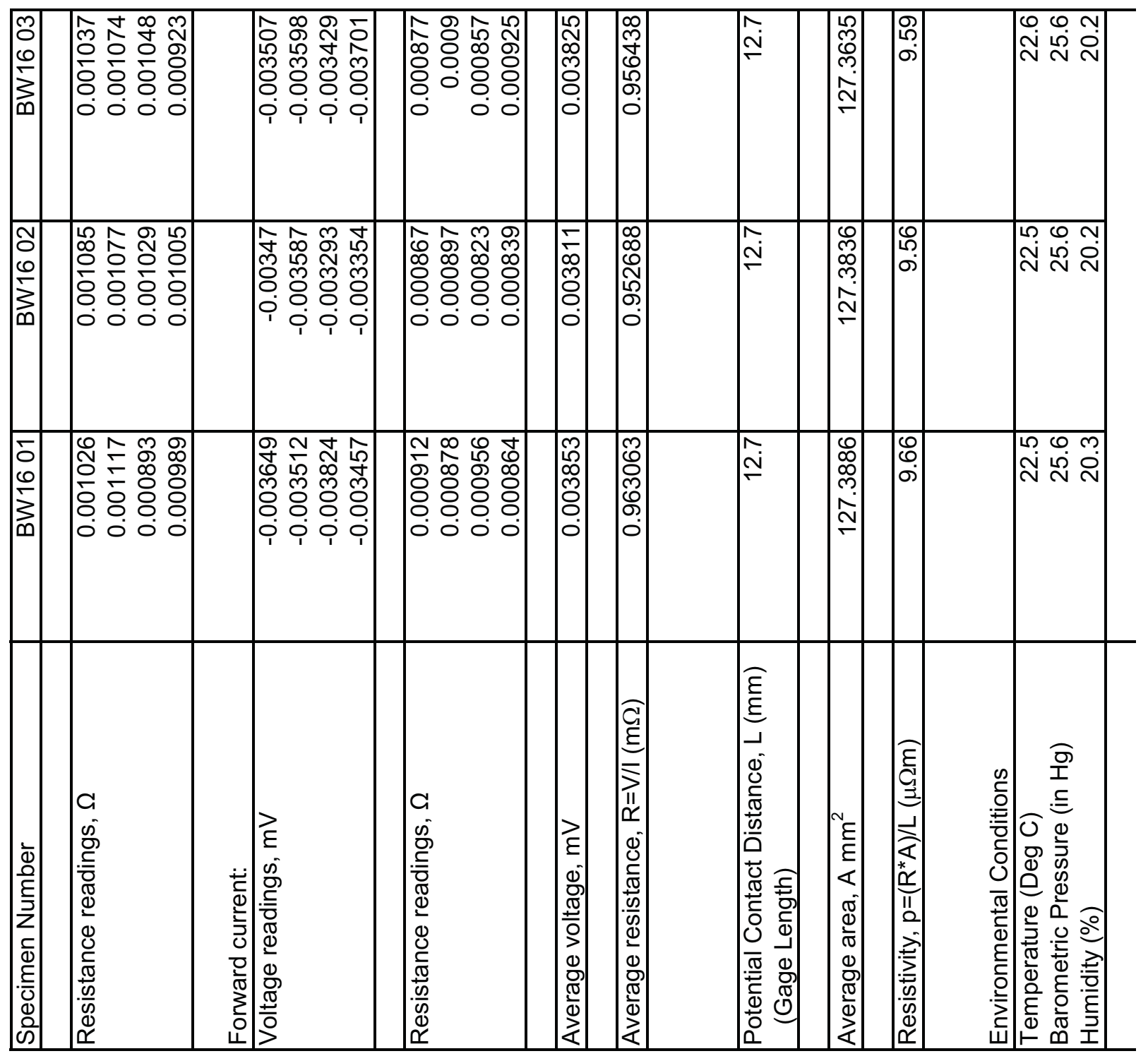




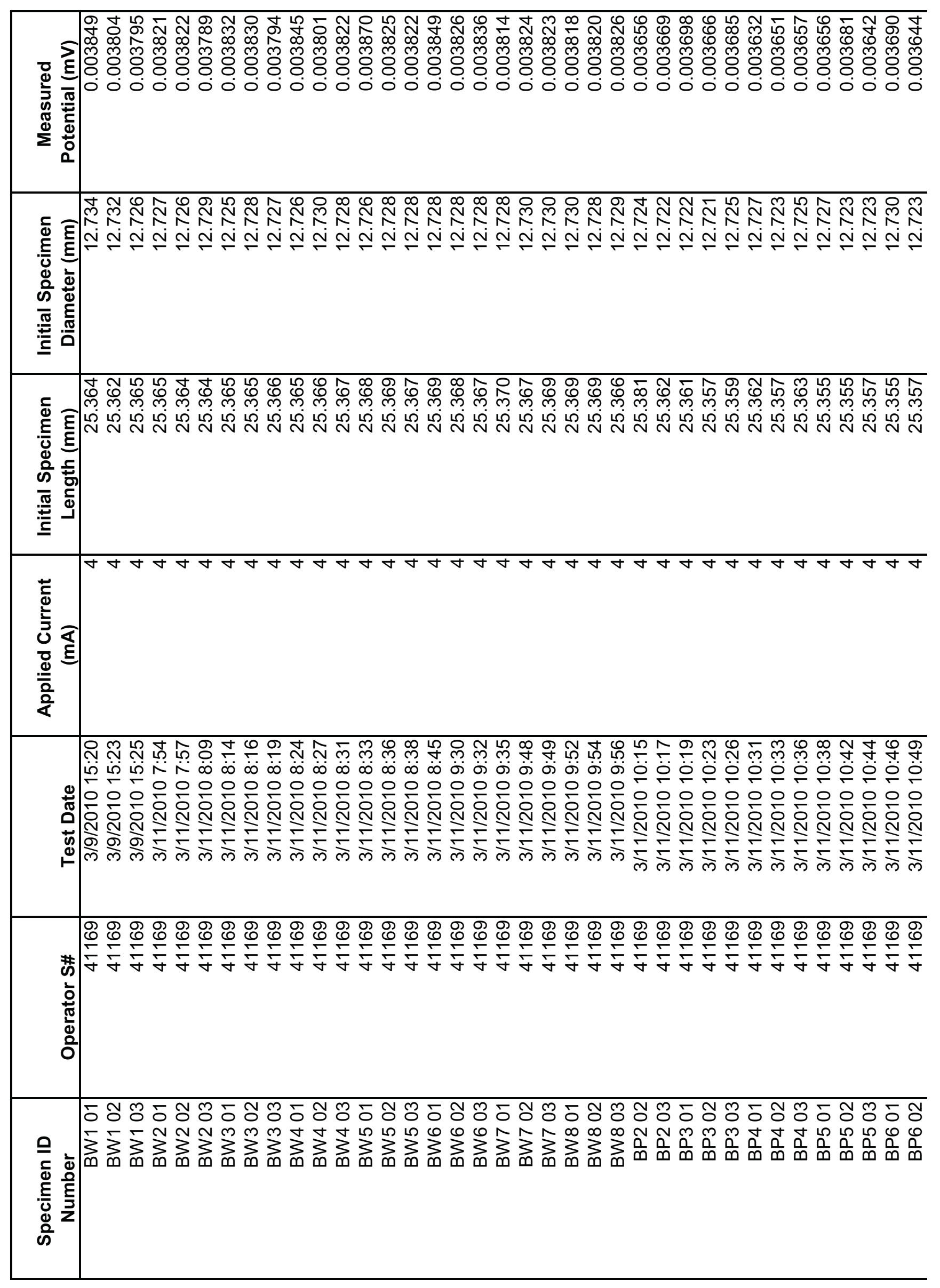




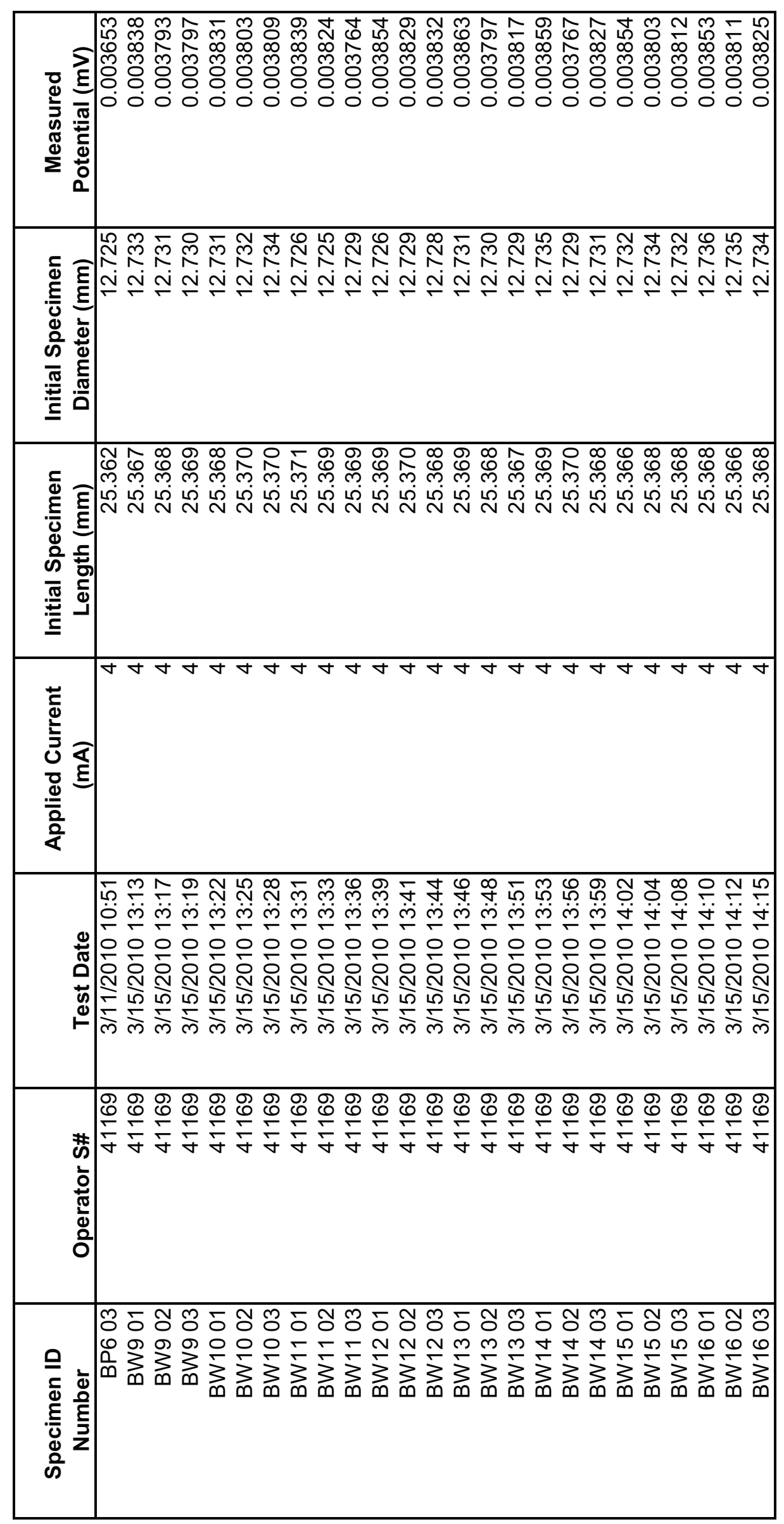




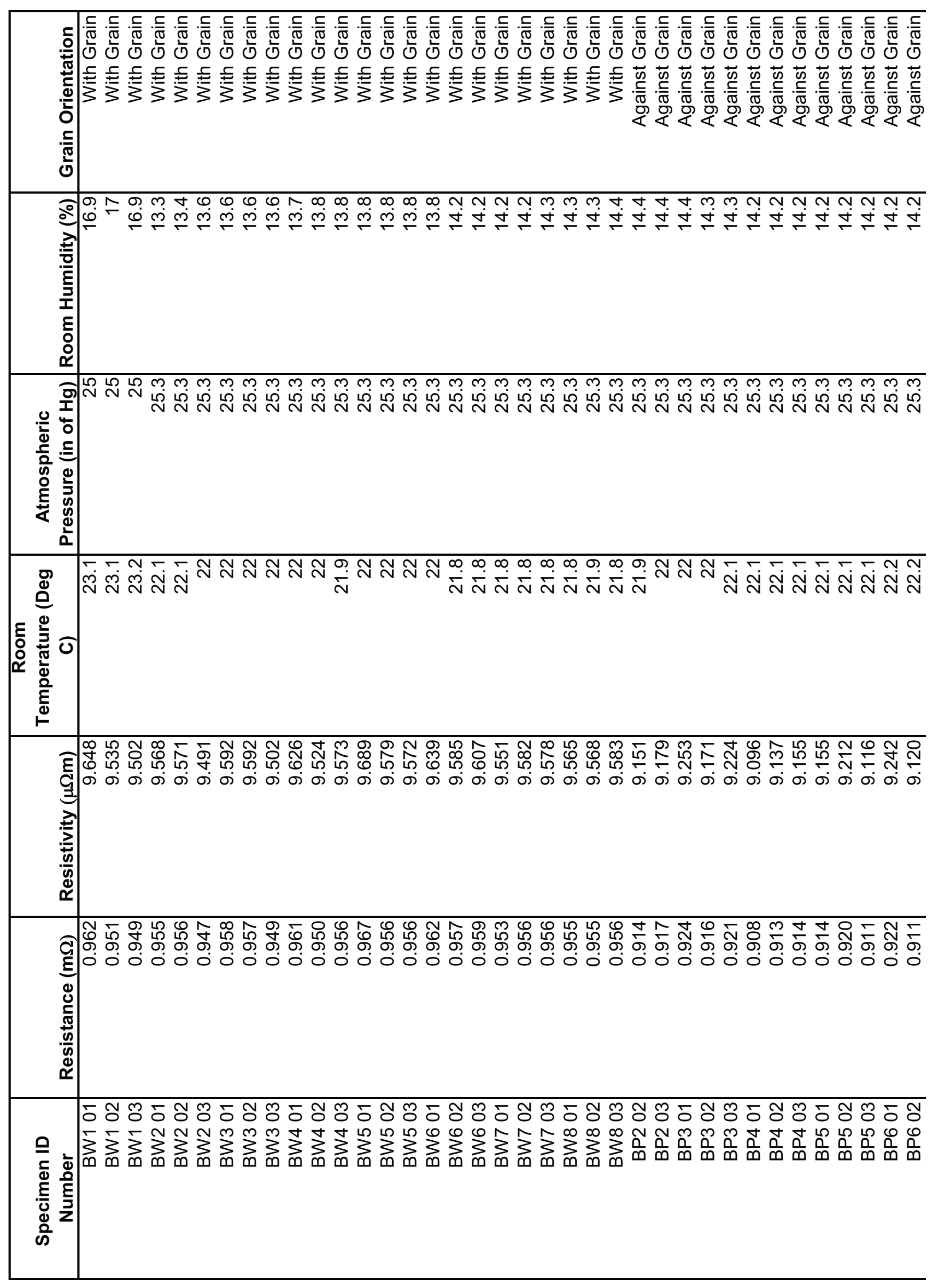




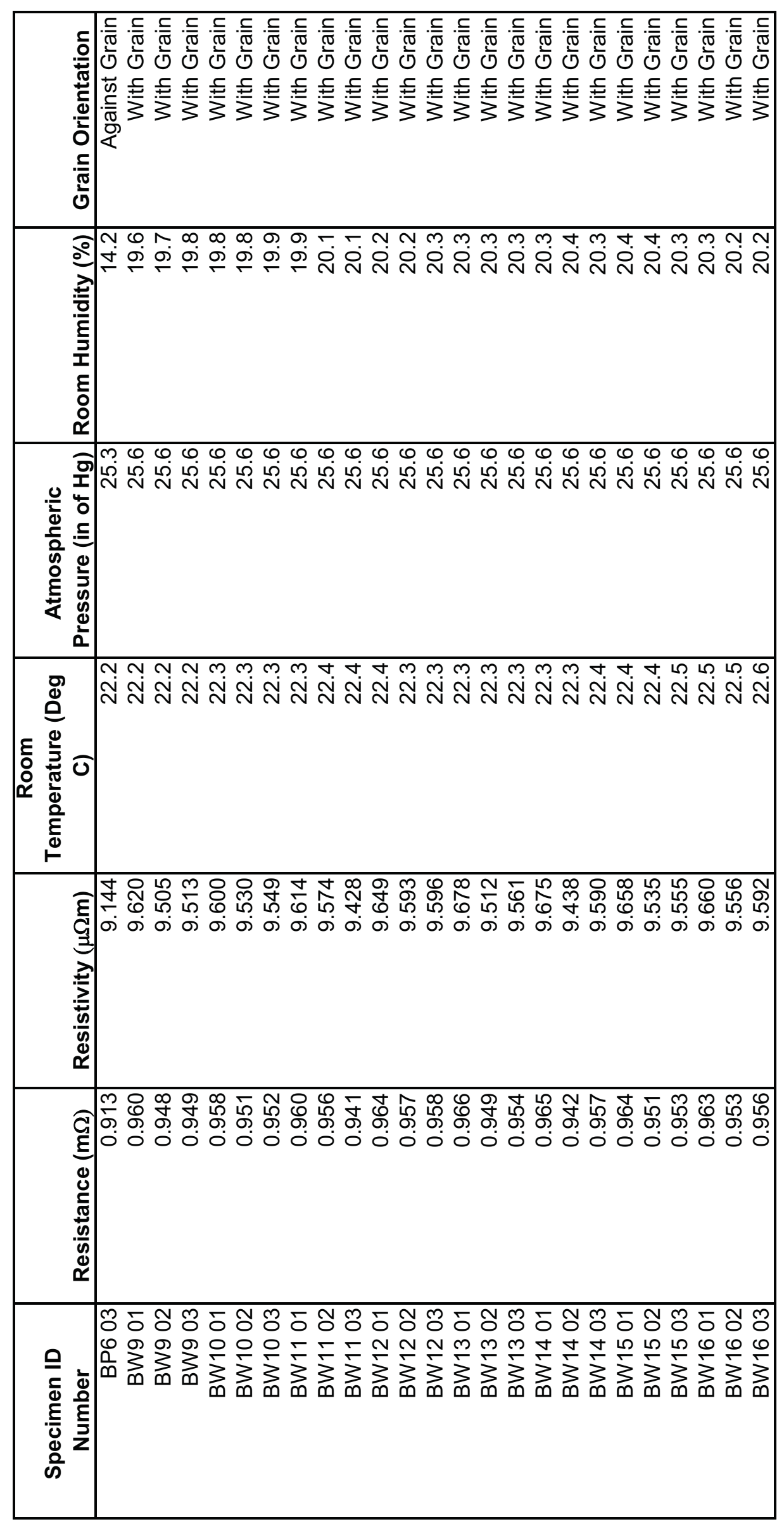




\begin{tabular}{|c|c|}
\hline 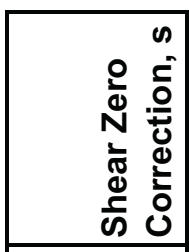 & 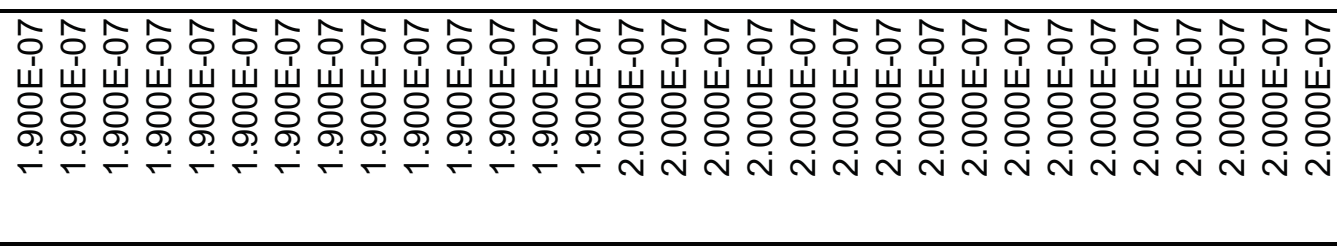 \\
\hline 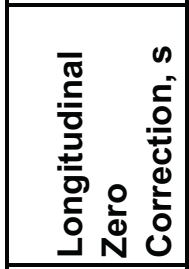 & 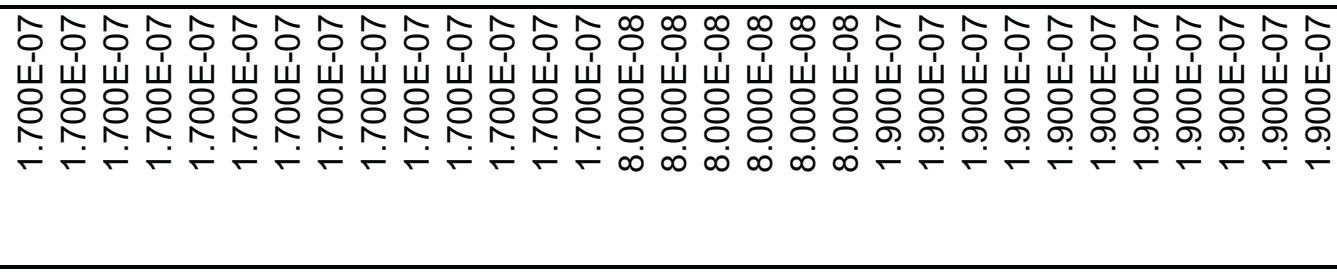 \\
\hline 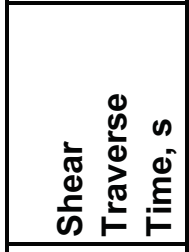 & 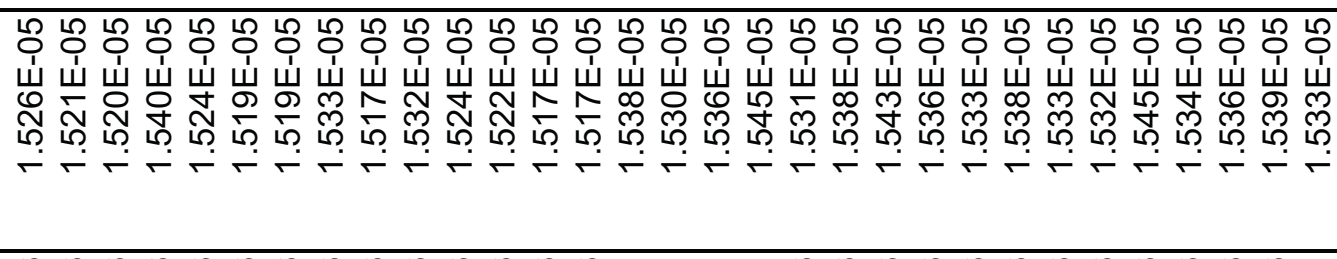 \\
\hline 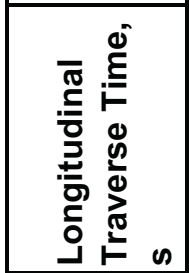 & 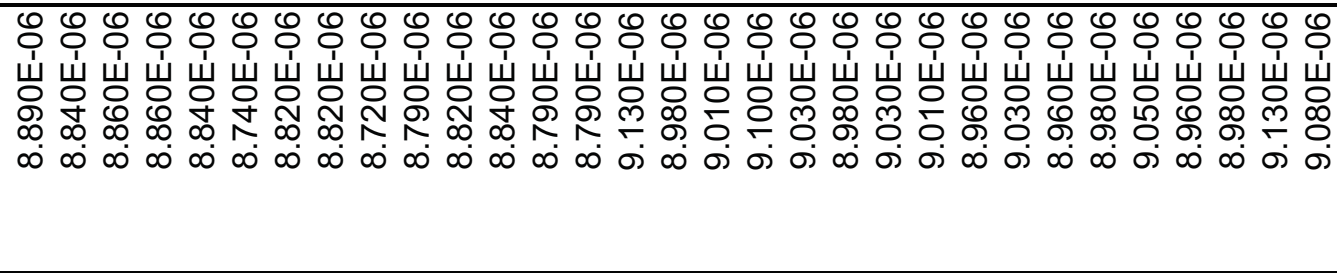 \\
\hline 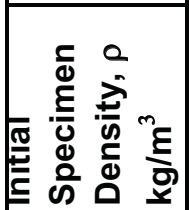 & 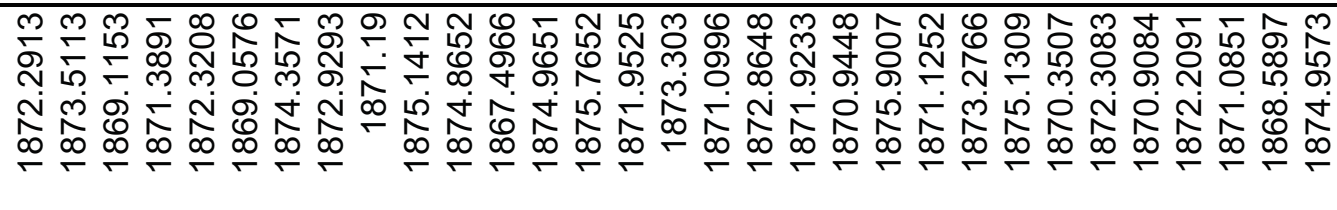 \\
\hline 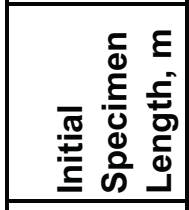 & 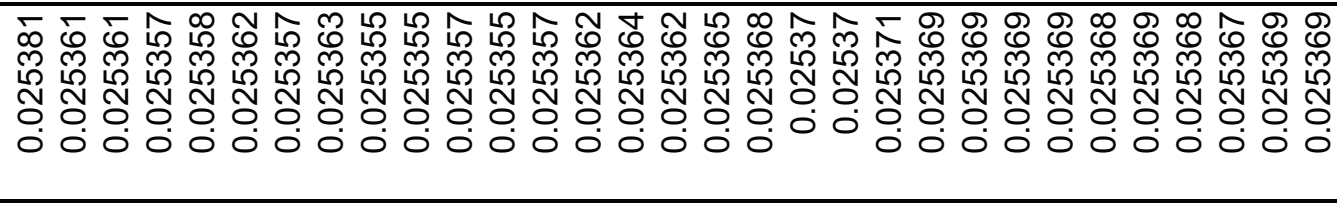 \\
\hline 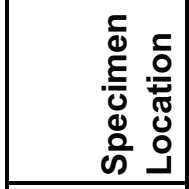 & \\
\hline $\begin{array}{l}\stackrel{2}{0} \\
\stackrel{0}{\frac{T}{0}} \\
\frac{0}{0} \\
\stackrel{0}{0} \\
\end{array}$ & 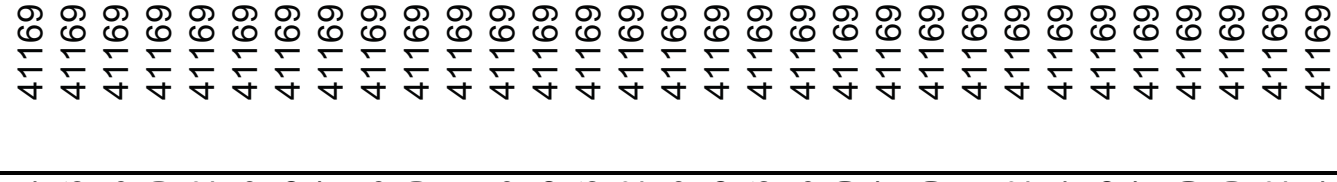 \\
\hline 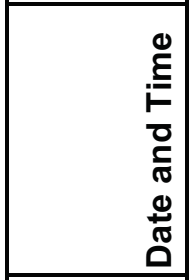 & 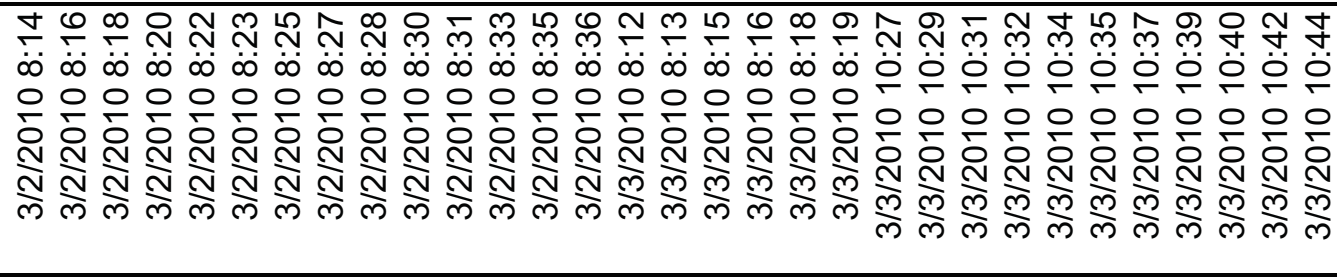 \\
\hline 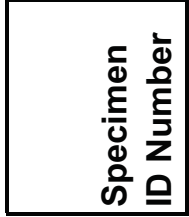 & 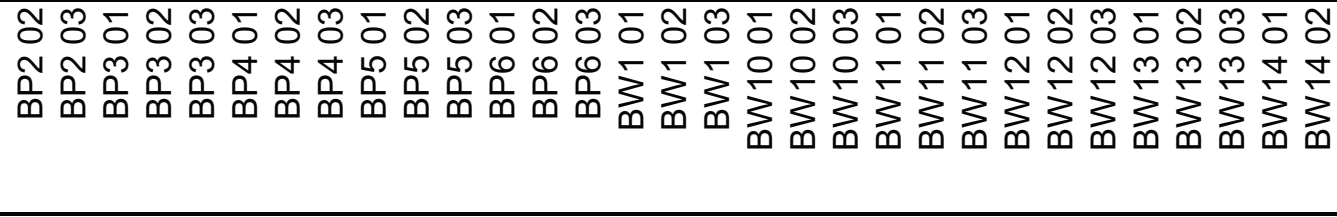 \\
\hline
\end{tabular}




\begin{tabular}{|c|c|}
\hline 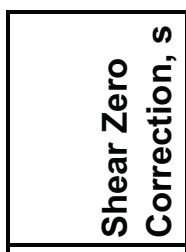 & 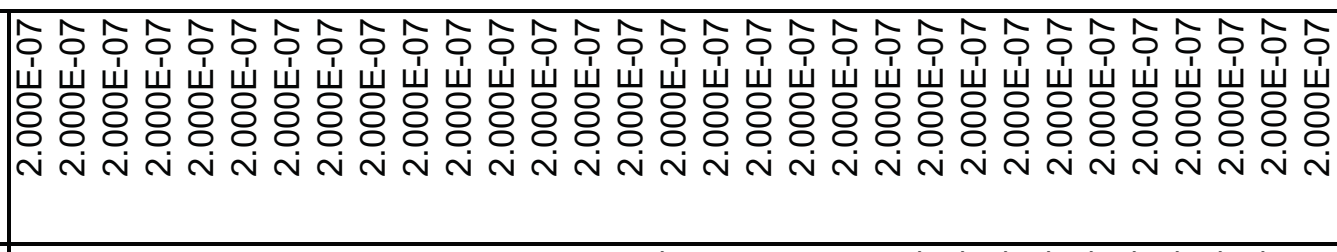 \\
\hline 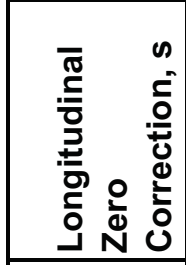 & 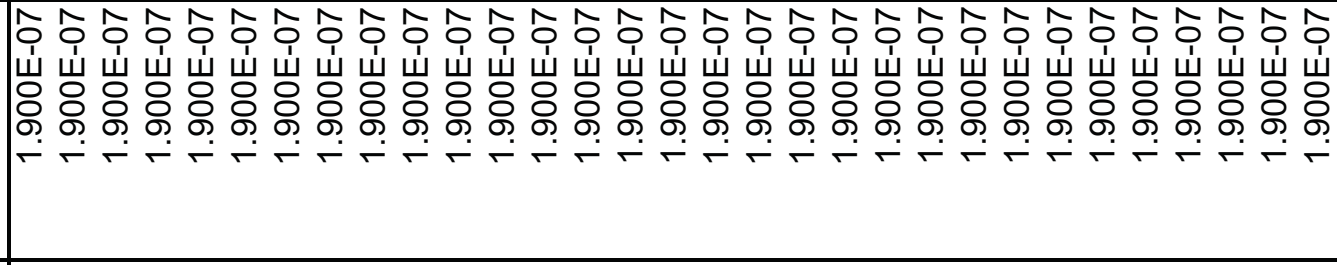 \\
\hline 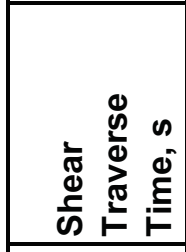 & 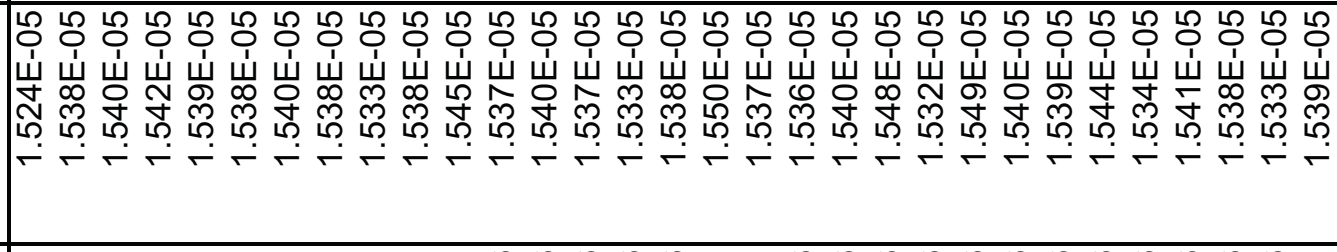 \\
\hline 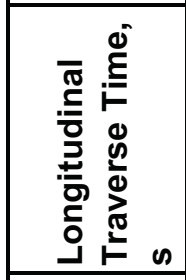 & 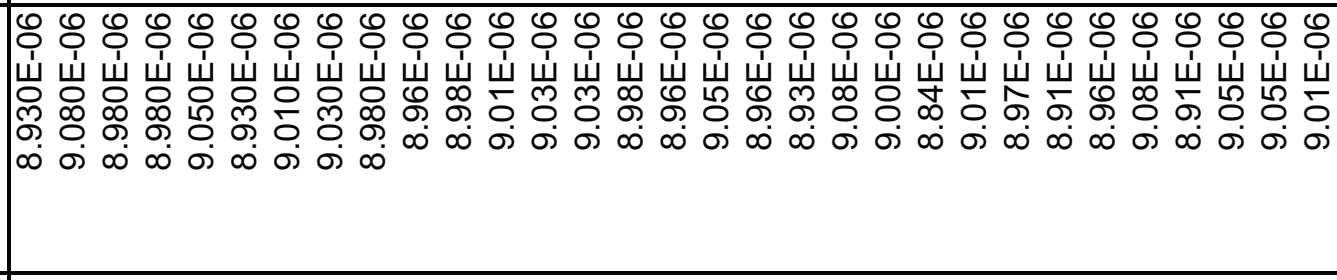 \\
\hline 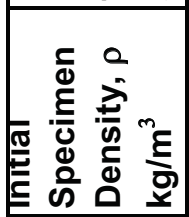 & 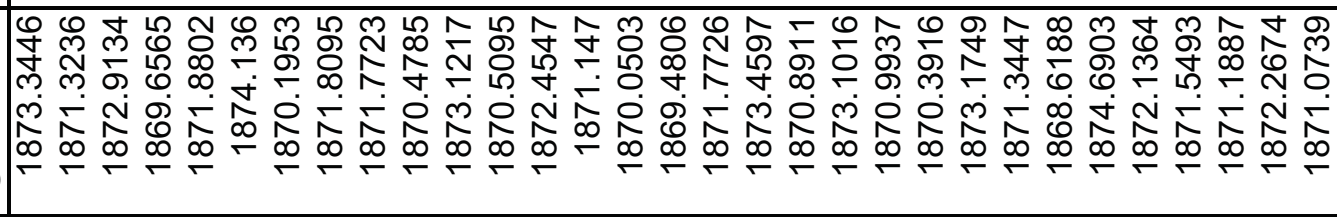 \\
\hline 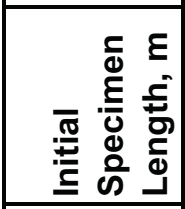 & 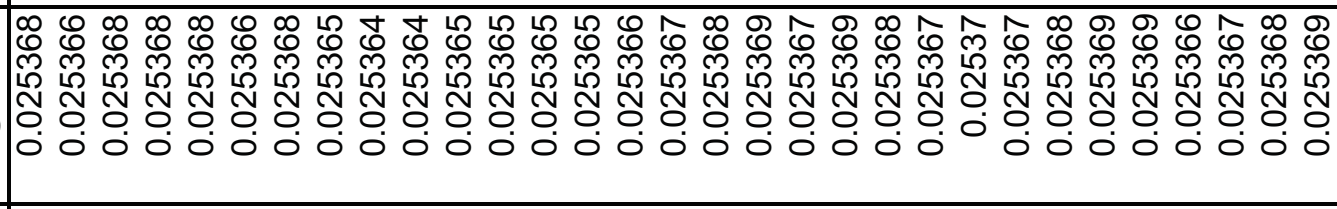 \\
\hline 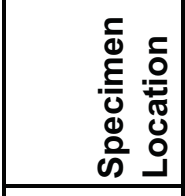 & \\
\hline 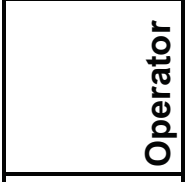 & 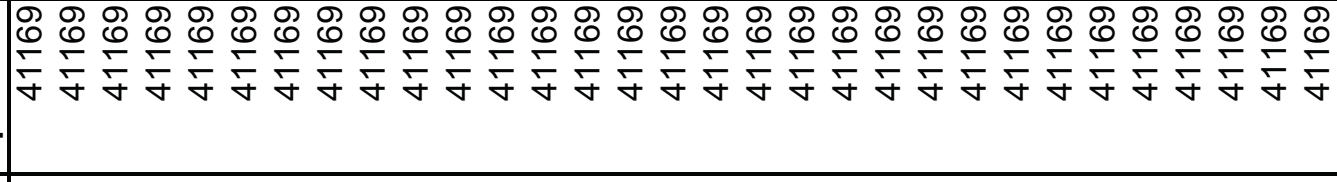 \\
\hline 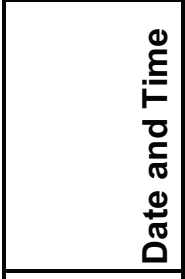 & 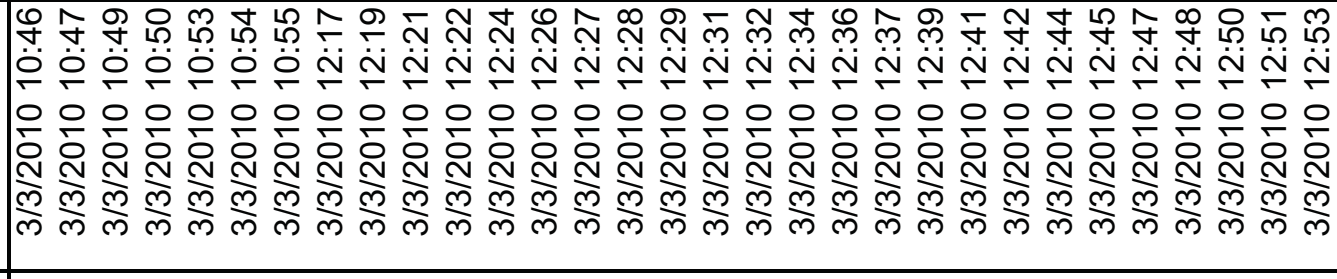 \\
\hline 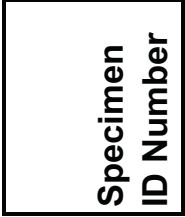 & 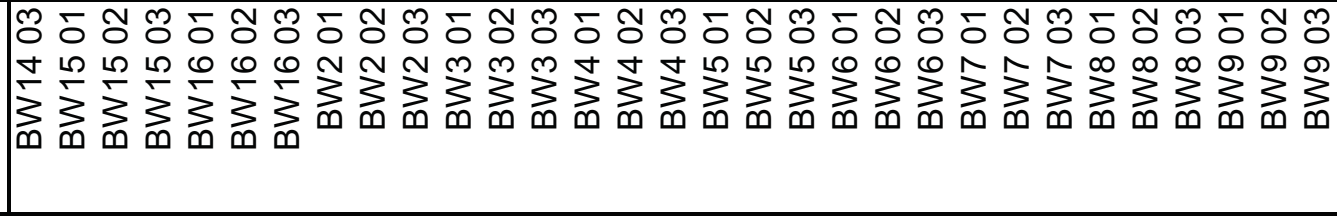 \\
\hline
\end{tabular}




\begin{tabular}{|c|c|}
\hline 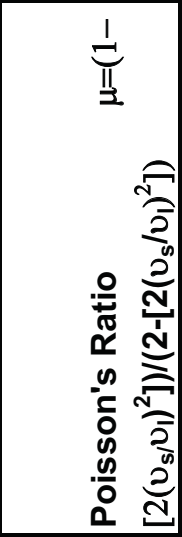 & 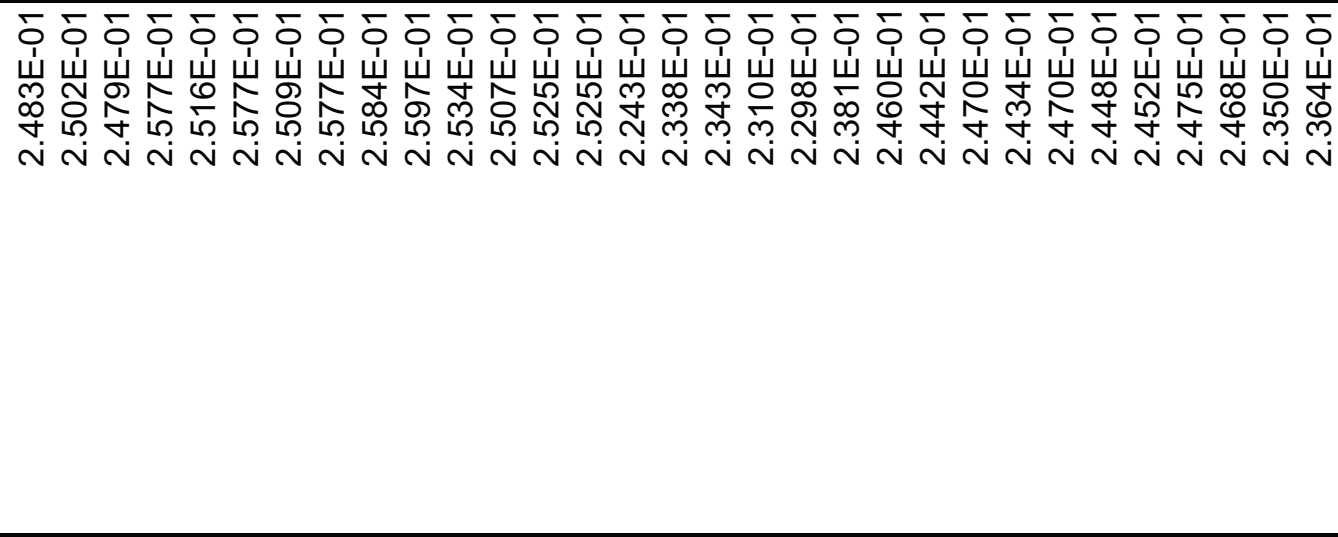 \\
\hline 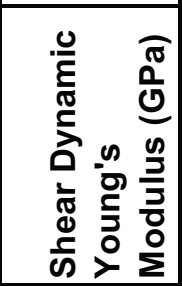 & 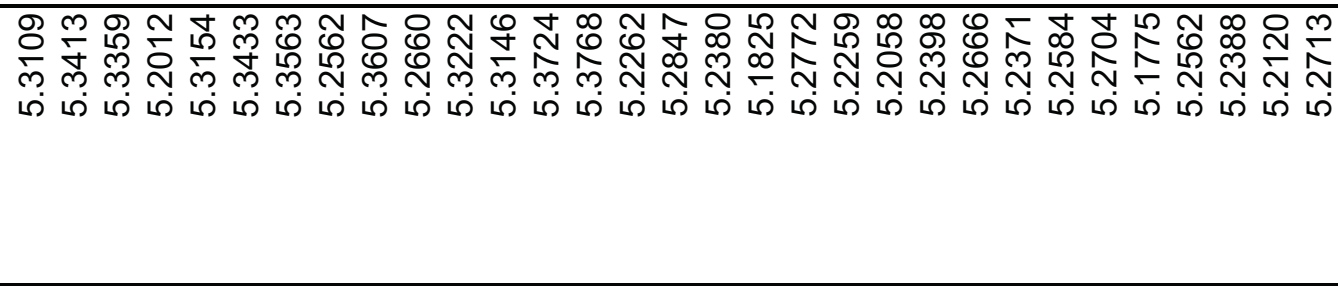 \\
\hline 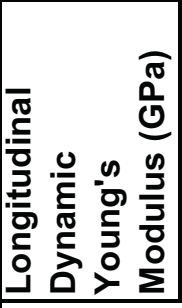 & 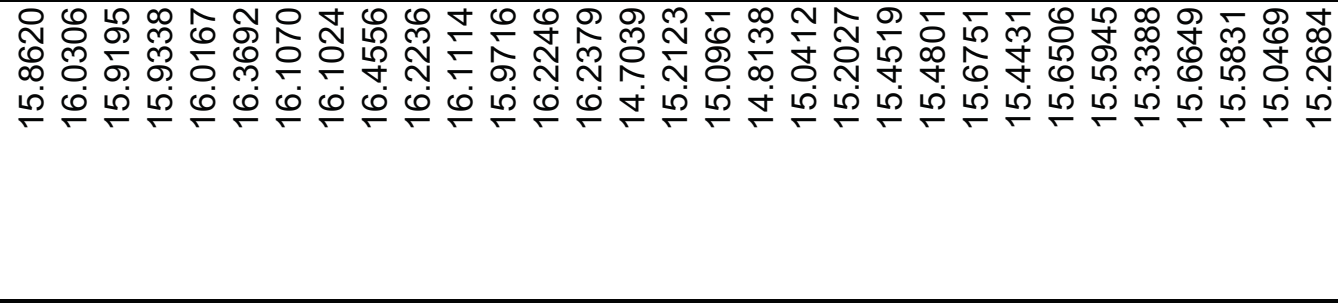 \\
\hline 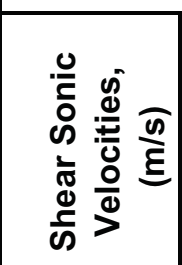 & 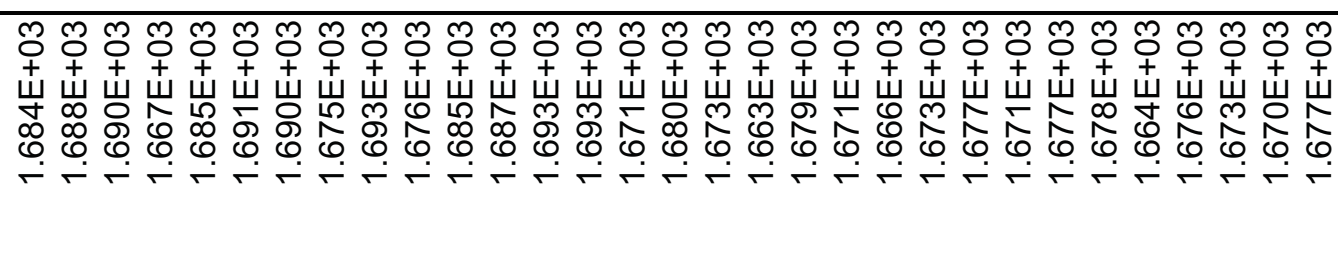 \\
\hline 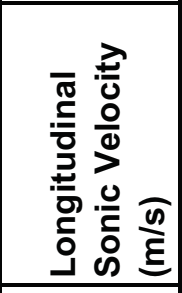 & 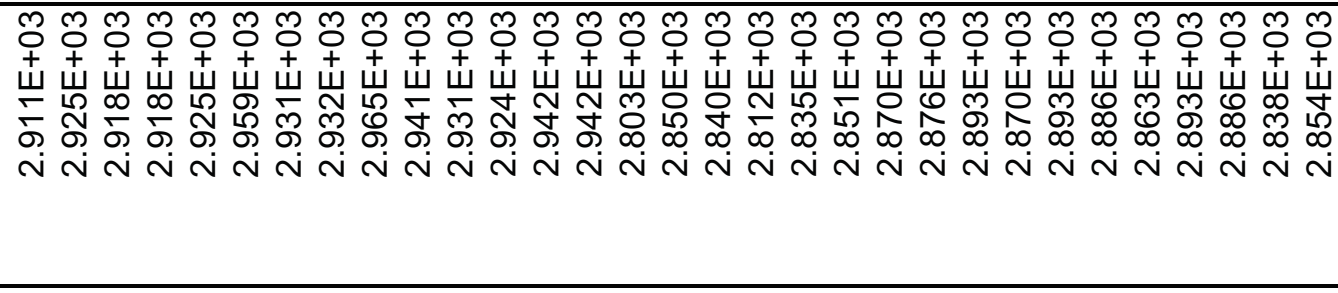 \\
\hline 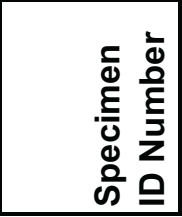 & 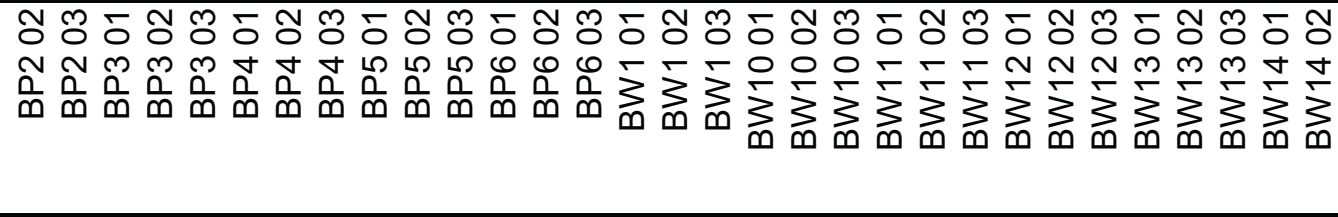 \\
\hline
\end{tabular}




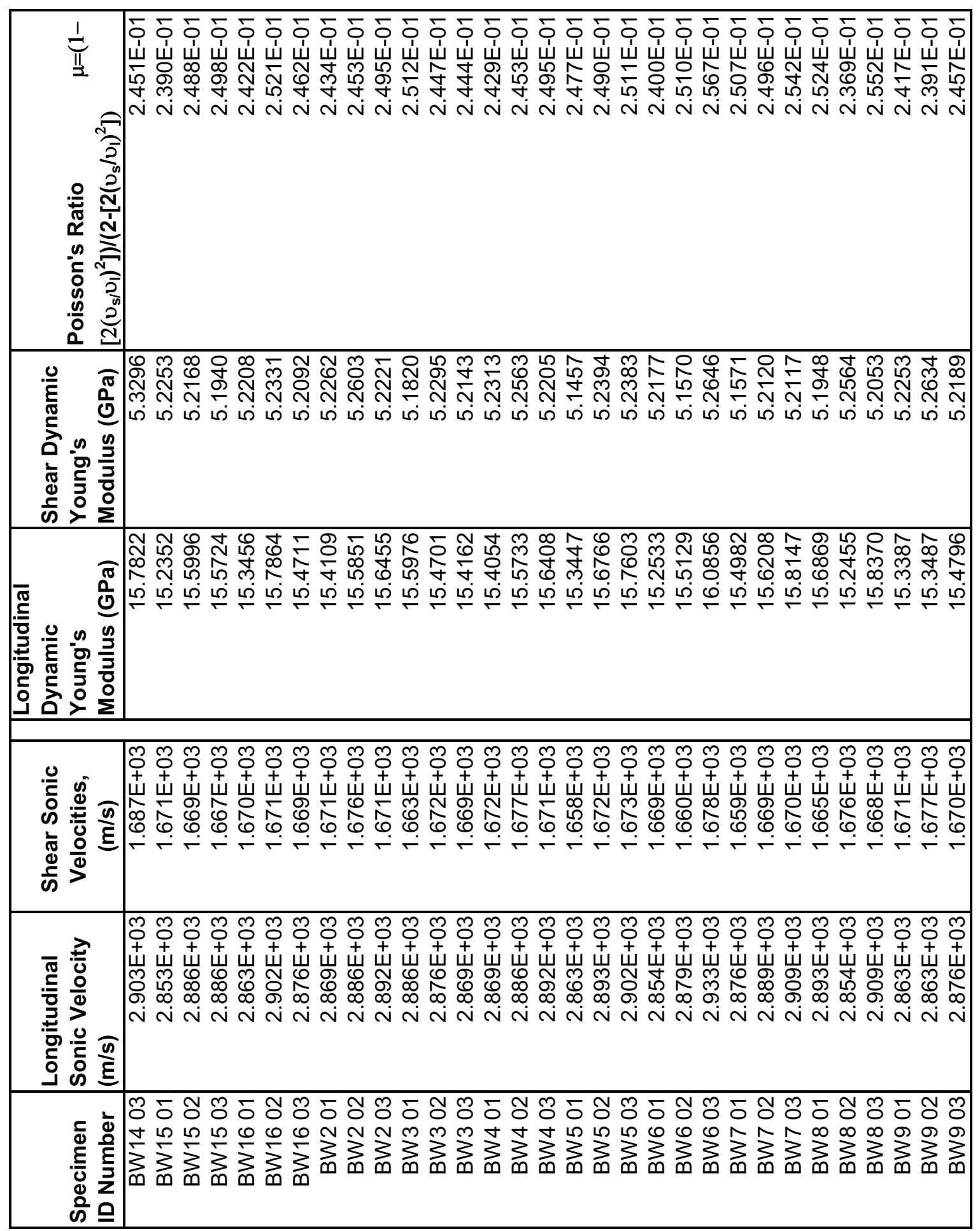




\begin{tabular}{|c|c|}
\hline 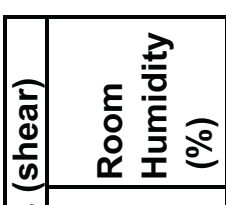 & 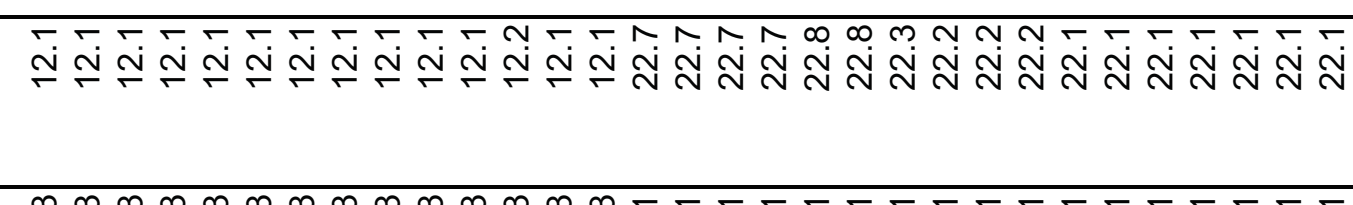 \\
\hline 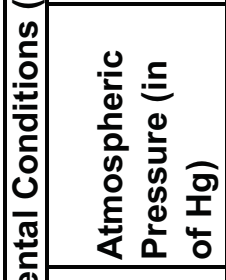 & 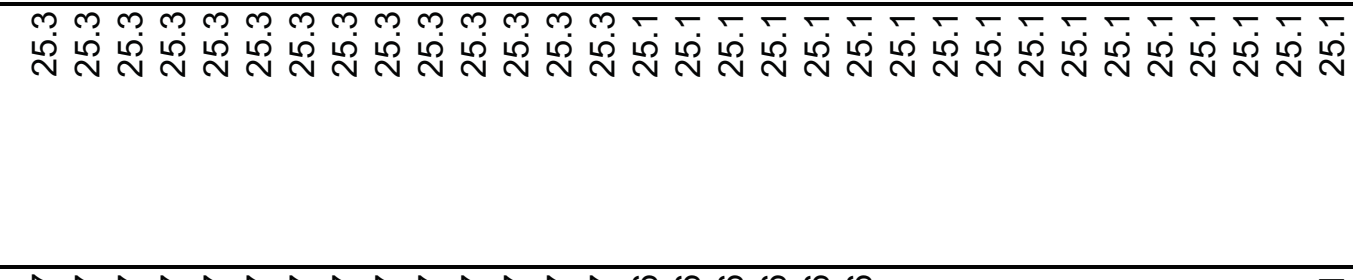 \\
\hline 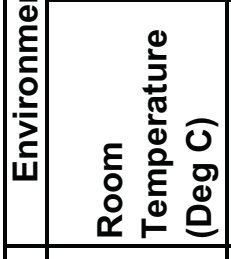 & มี \\
\hline 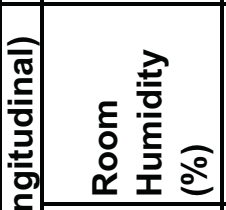 & 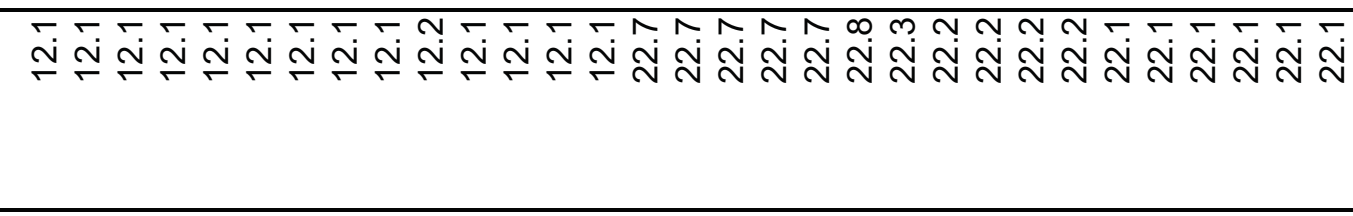 \\
\hline 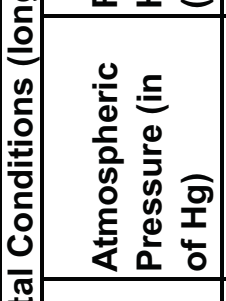 & 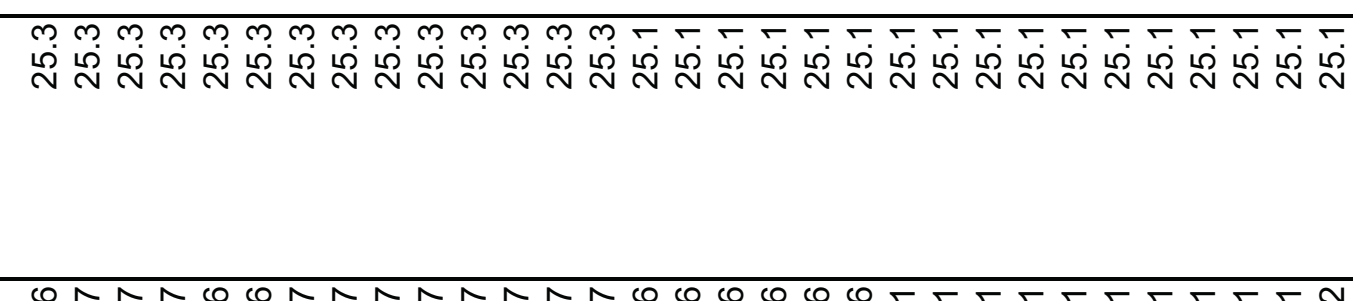 \\
\hline 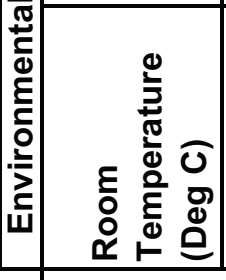 & 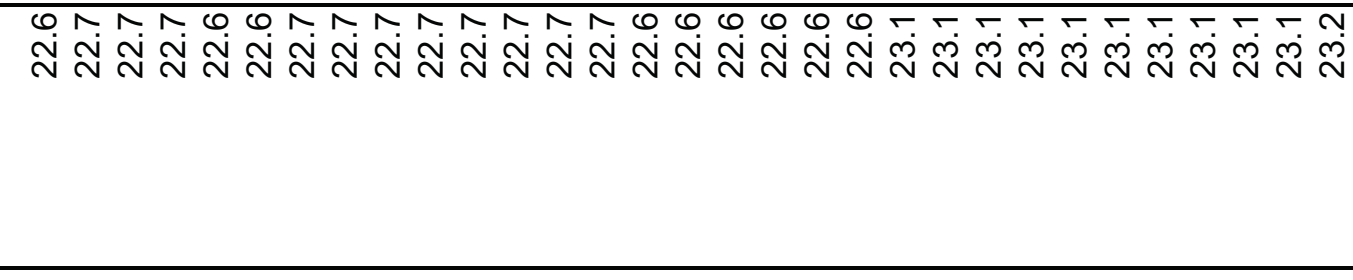 \\
\hline 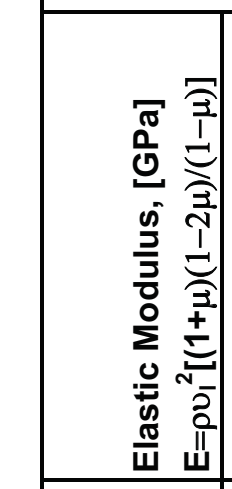 & 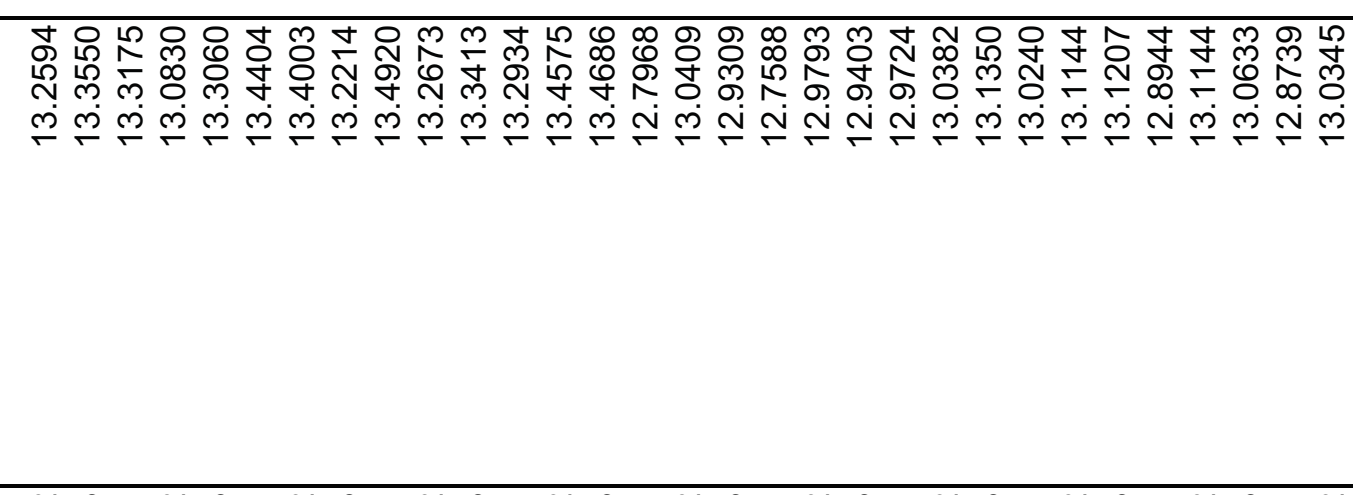 \\
\hline 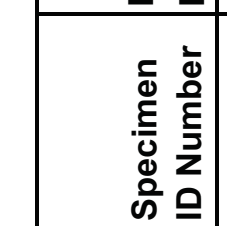 & 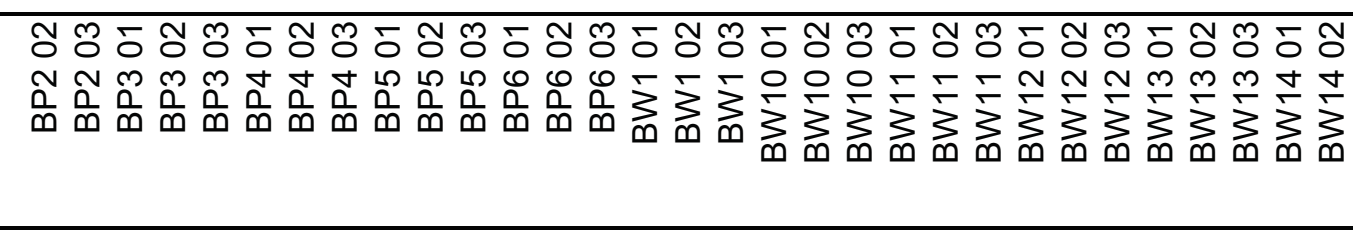 \\
\hline
\end{tabular}




\begin{tabular}{|c|c|}
\hline 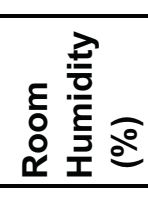 & 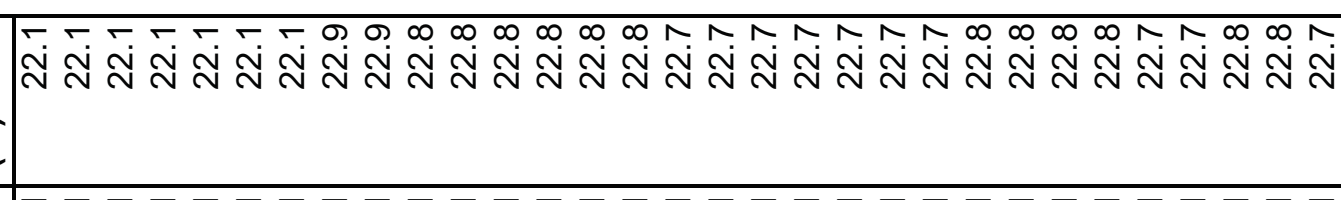 \\
\hline 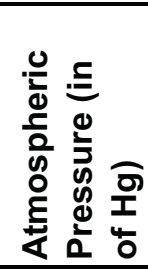 & क人⿱宀 \\
\hline 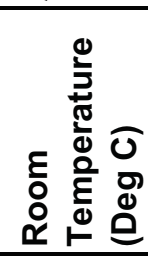 & 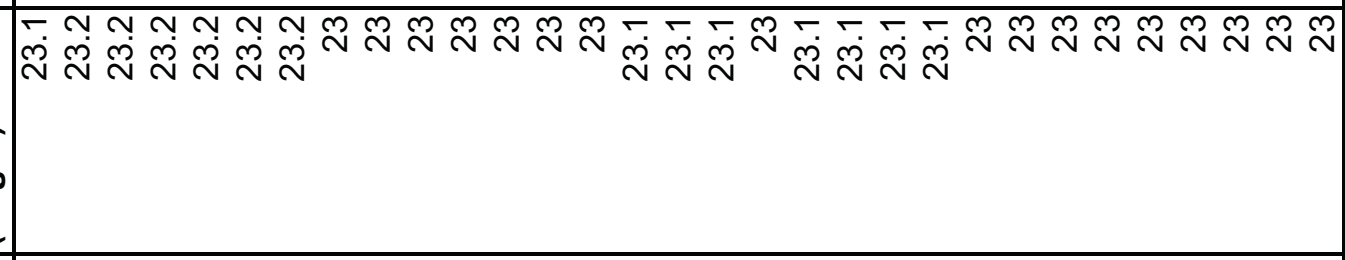 \\
\hline 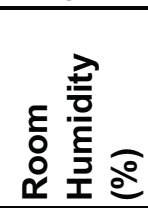 & 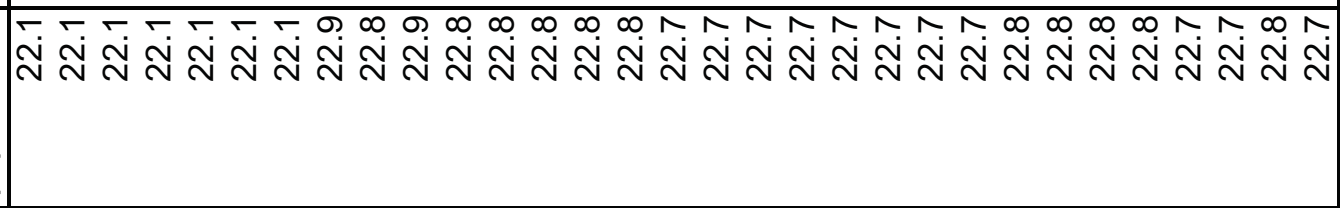 \\
\hline 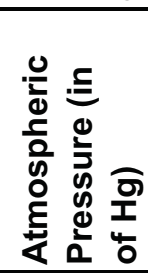 & مैं \\
\hline 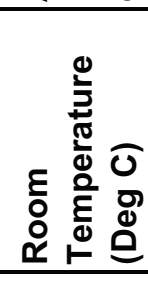 & 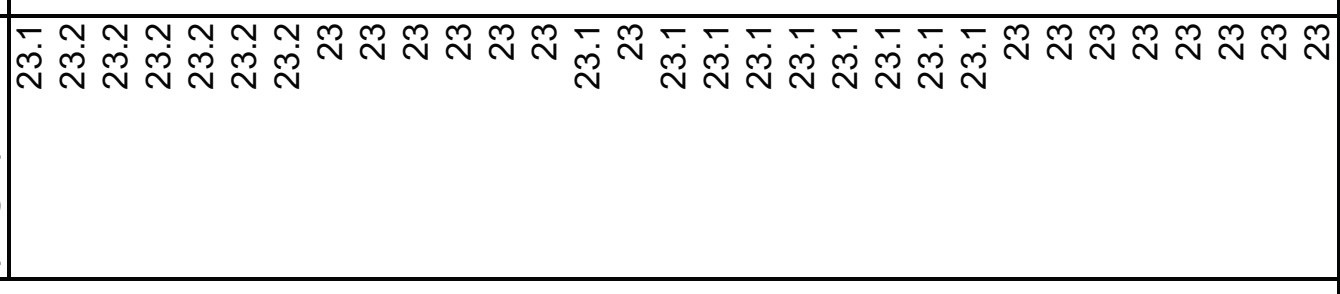 \\
\hline 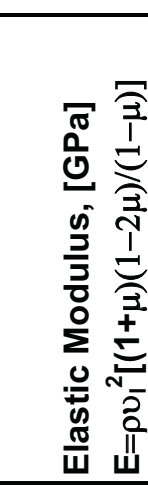 & 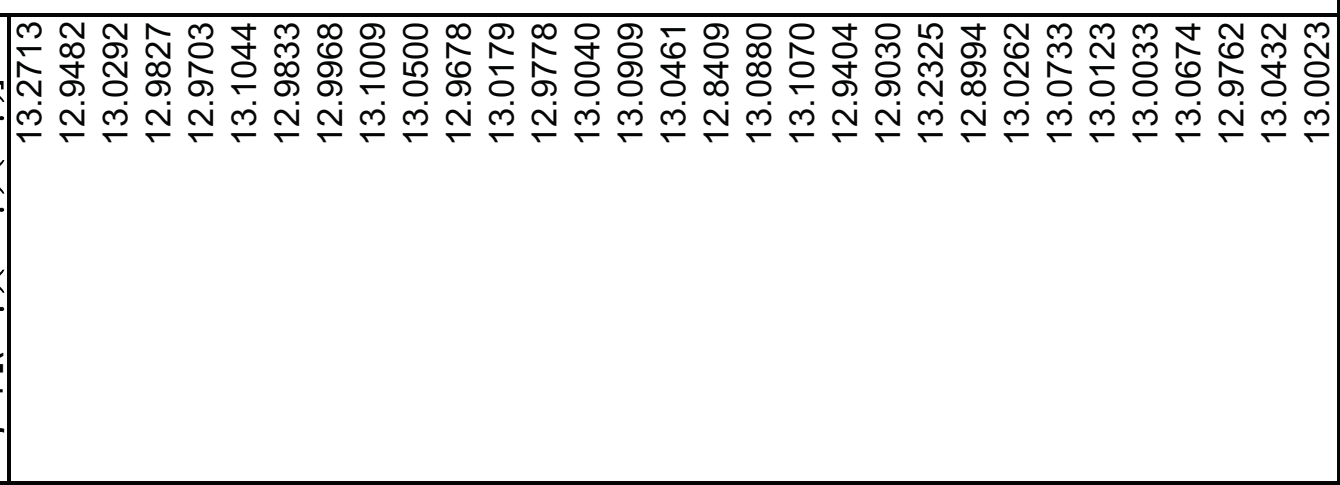 \\
\hline 这 & 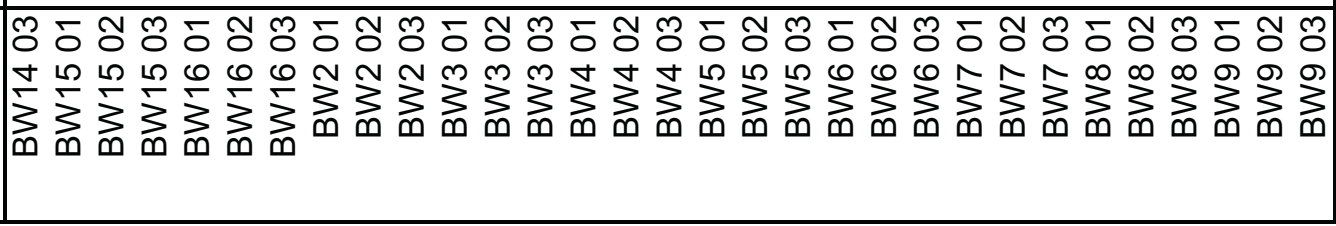 \\
\hline
\end{tabular}




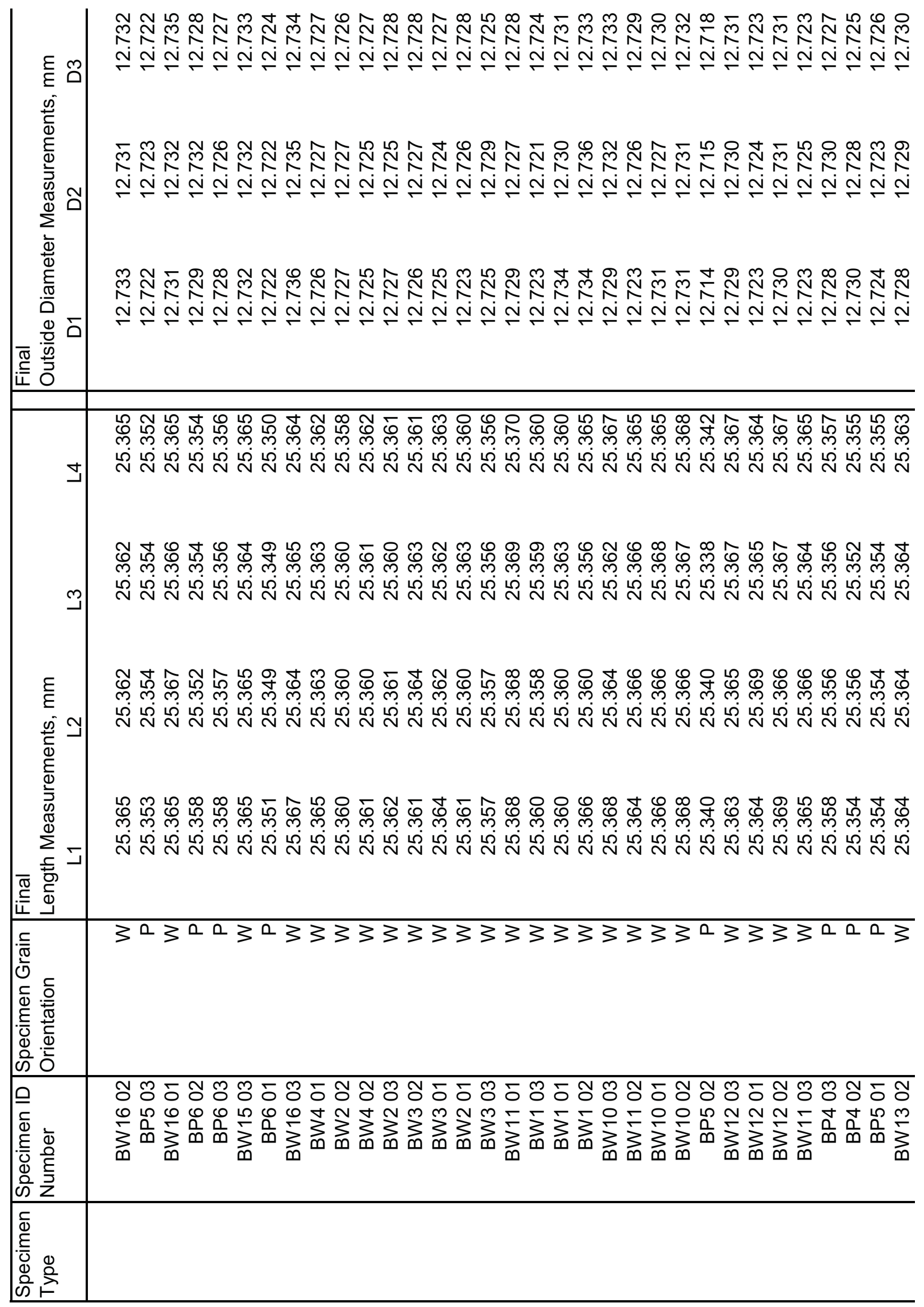




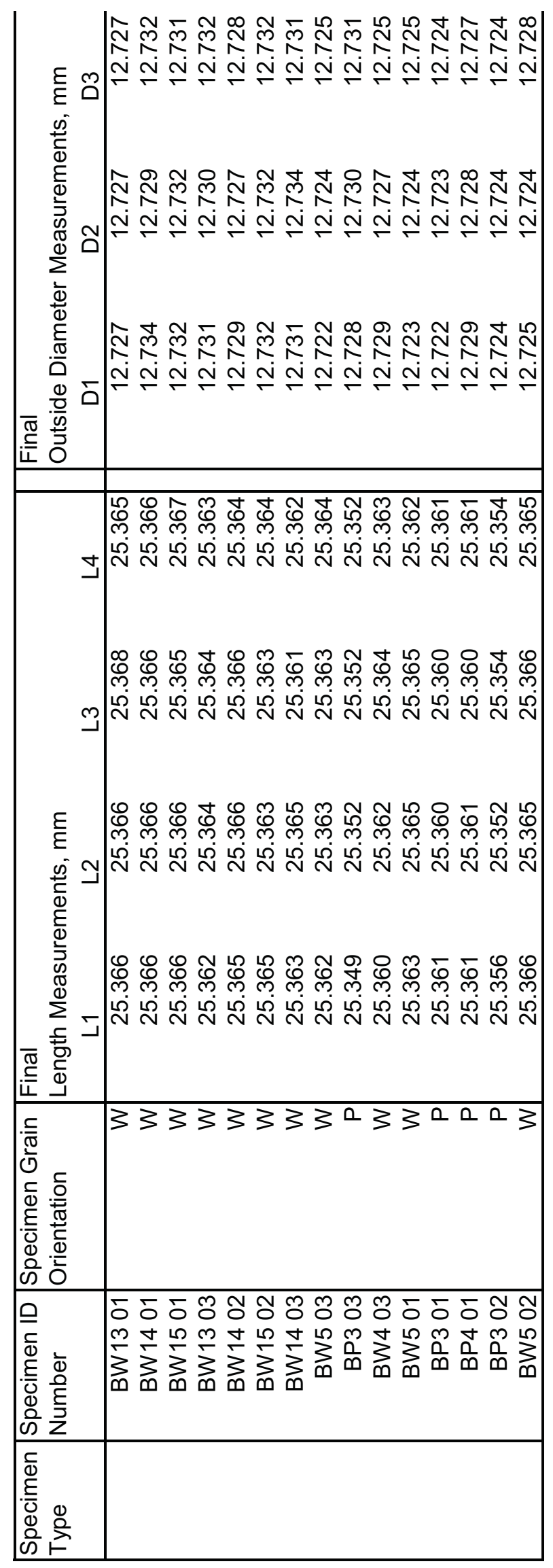




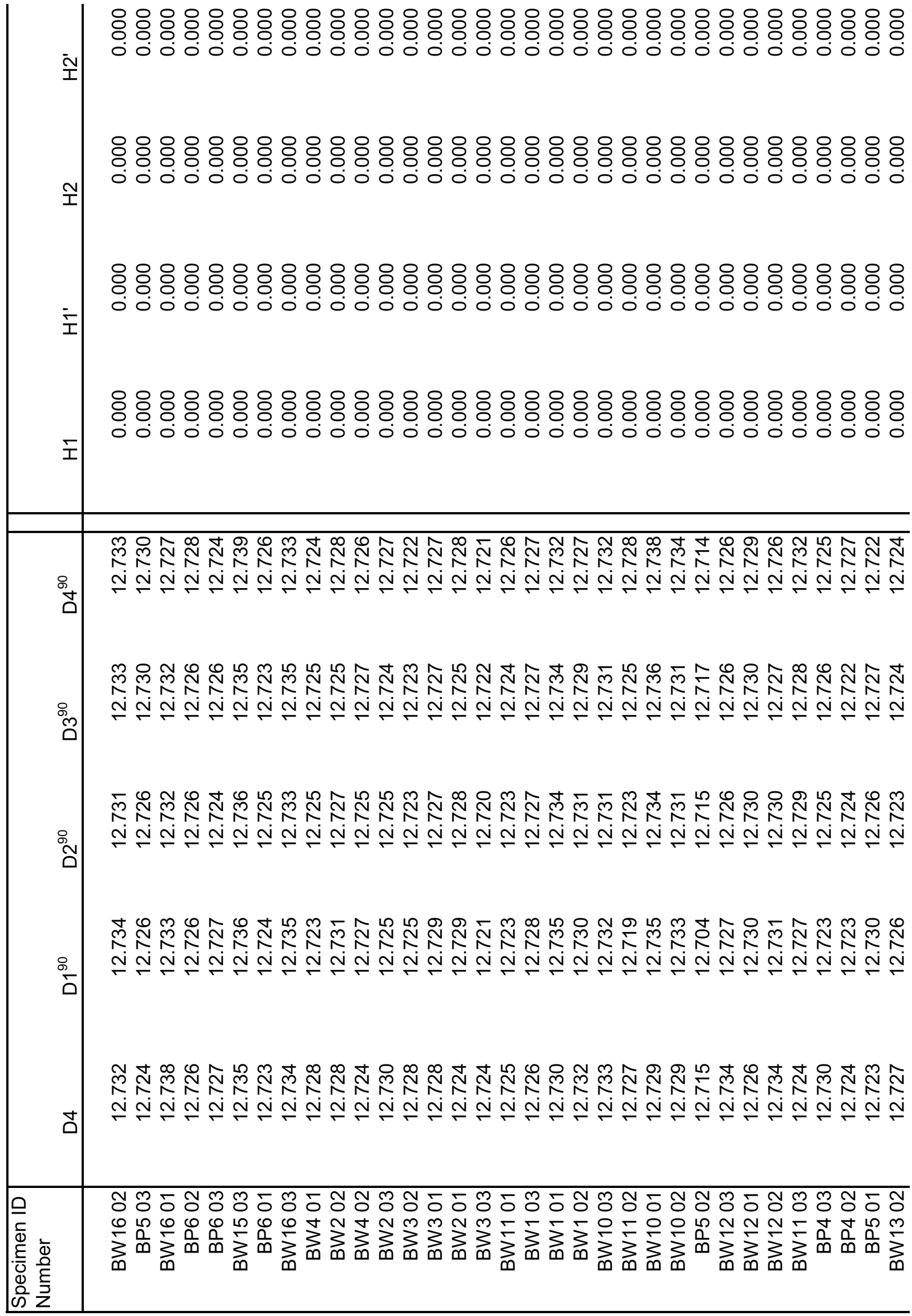




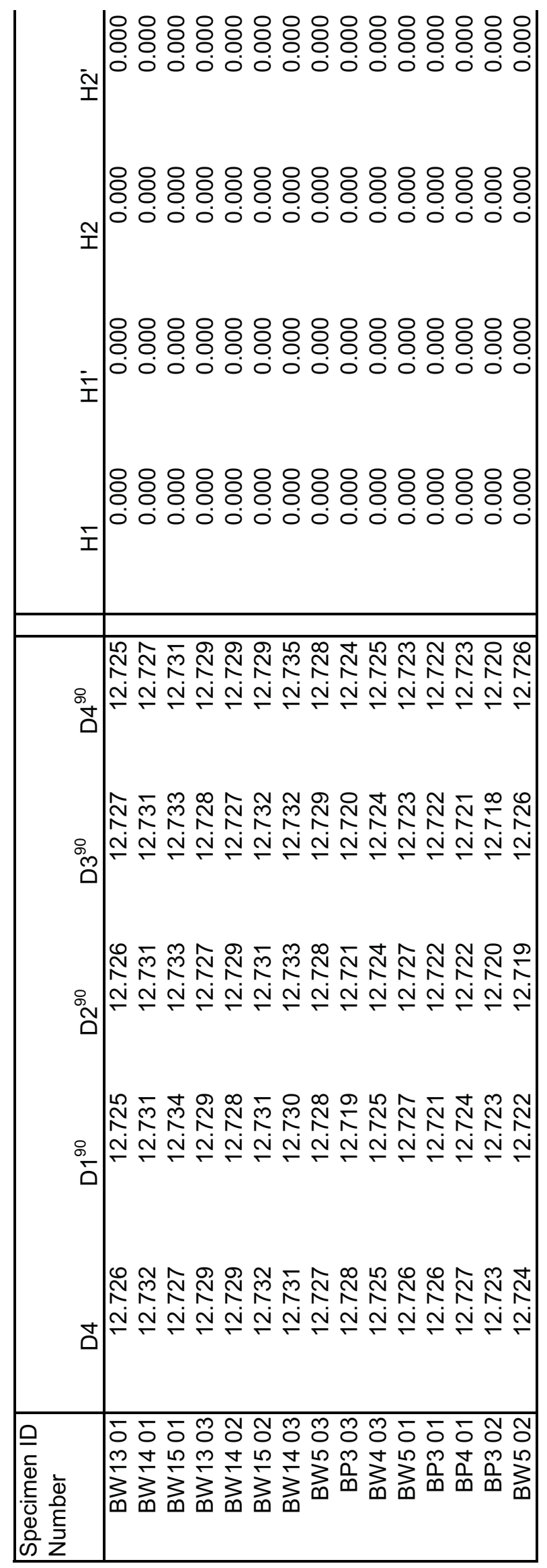




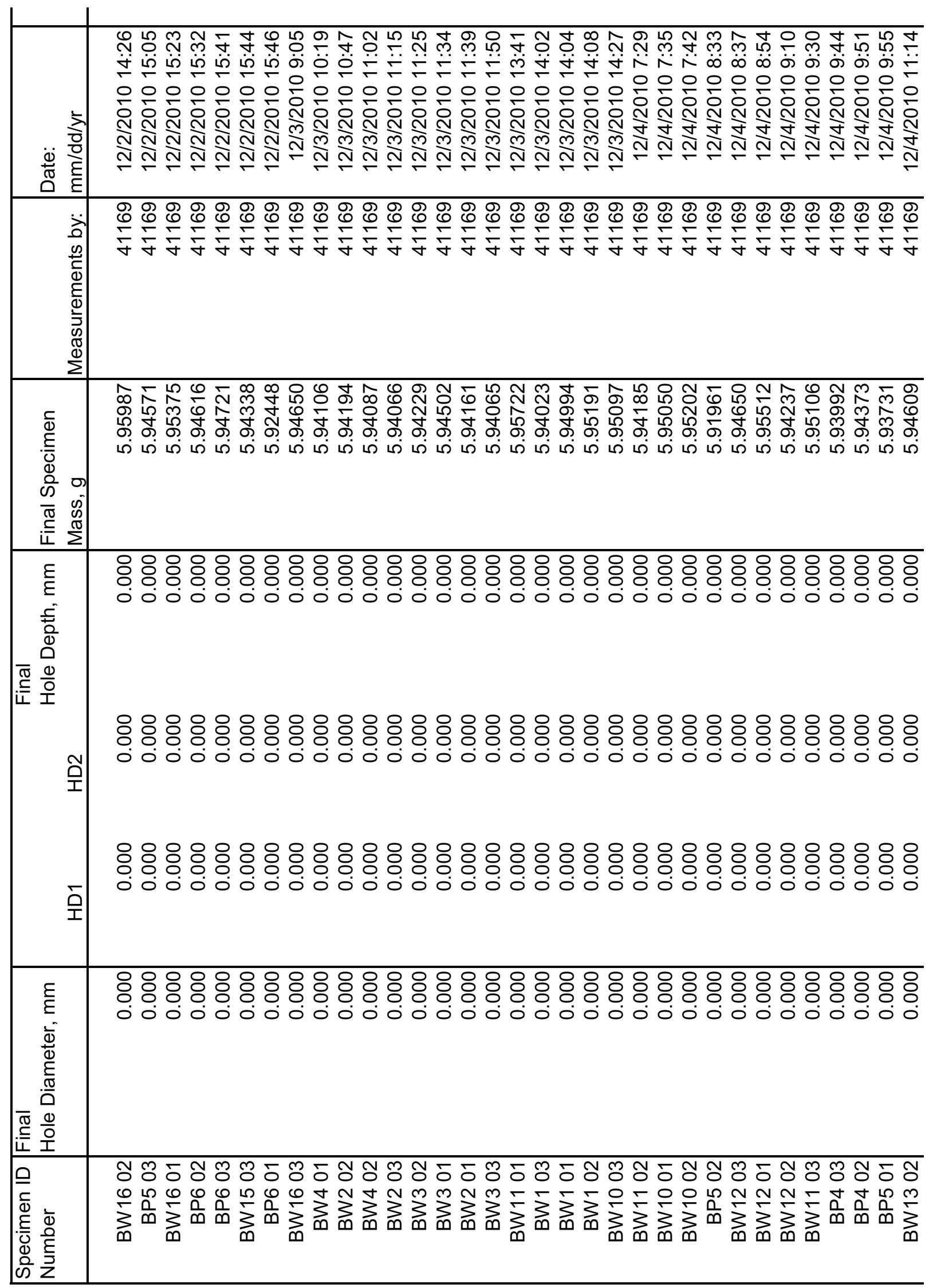




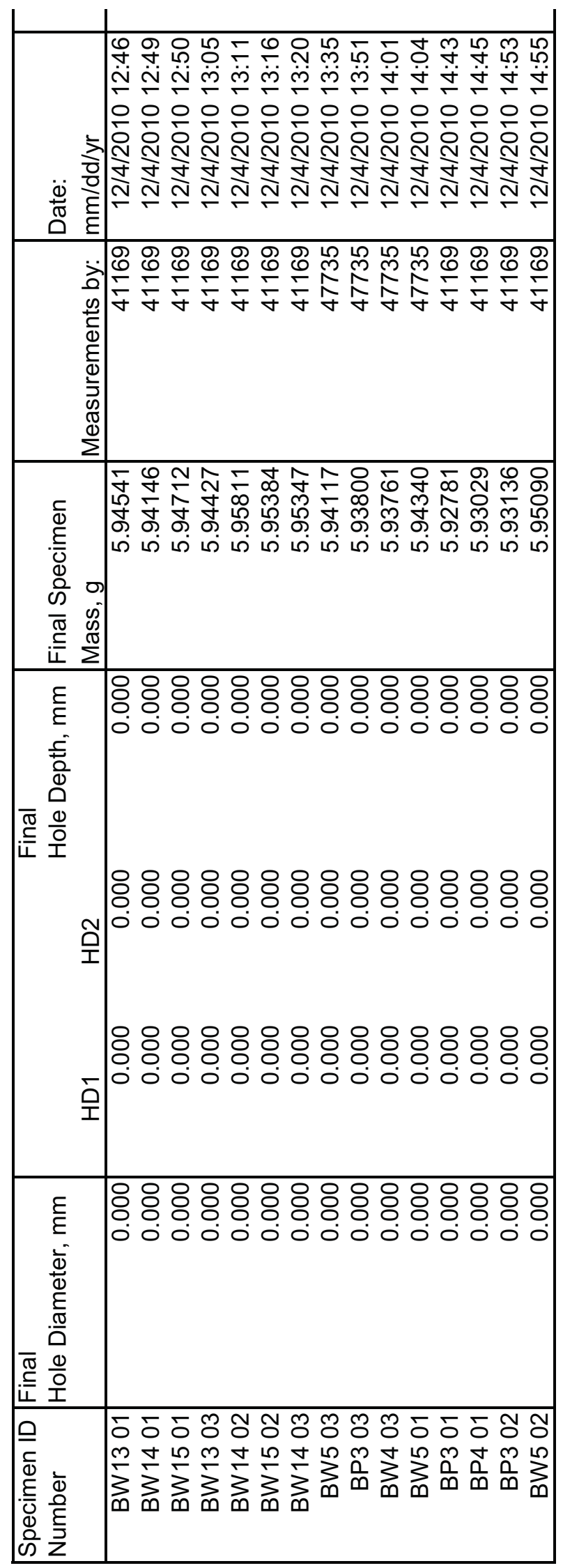




\begin{tabular}{|c|c|}
\hline$\stackrel{9}{9}$ & 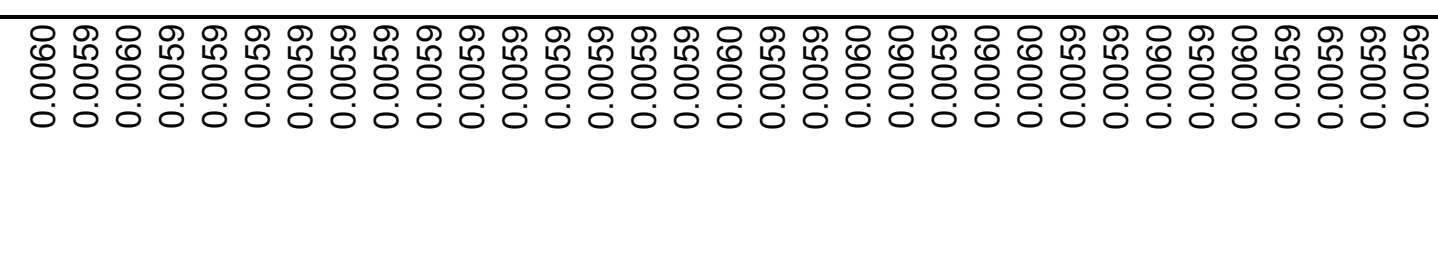 \\
\hline 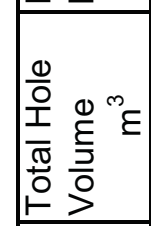 & 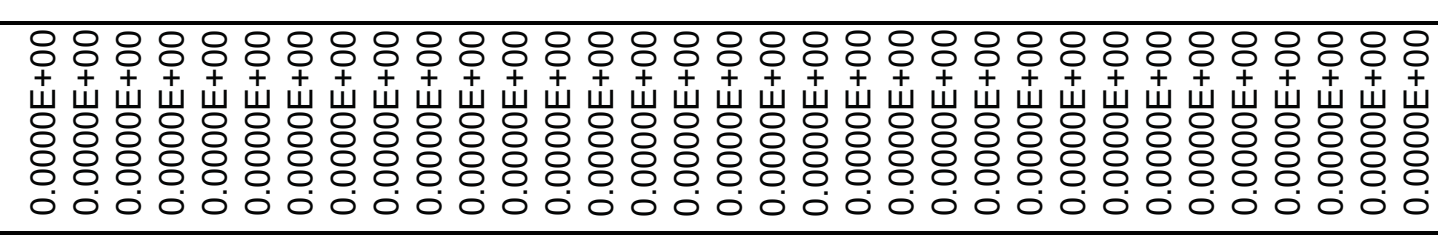 \\
\hline$\varepsilon$ & 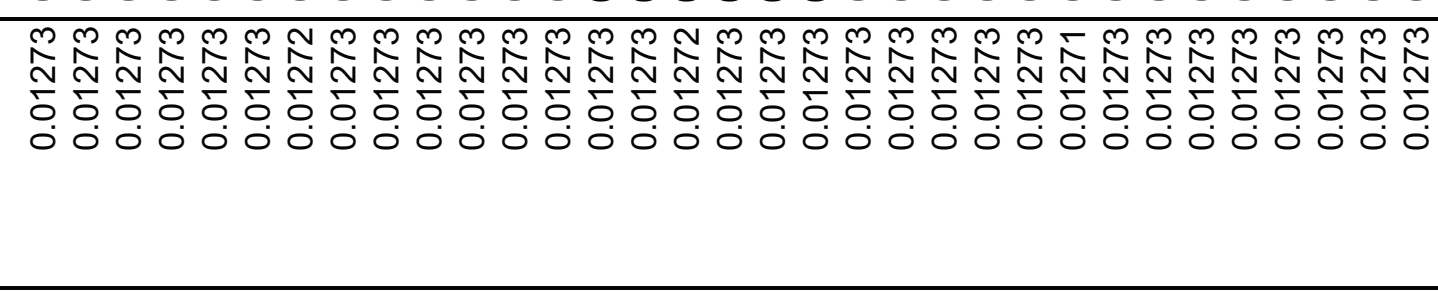 \\
\hline$\varepsilon$ & 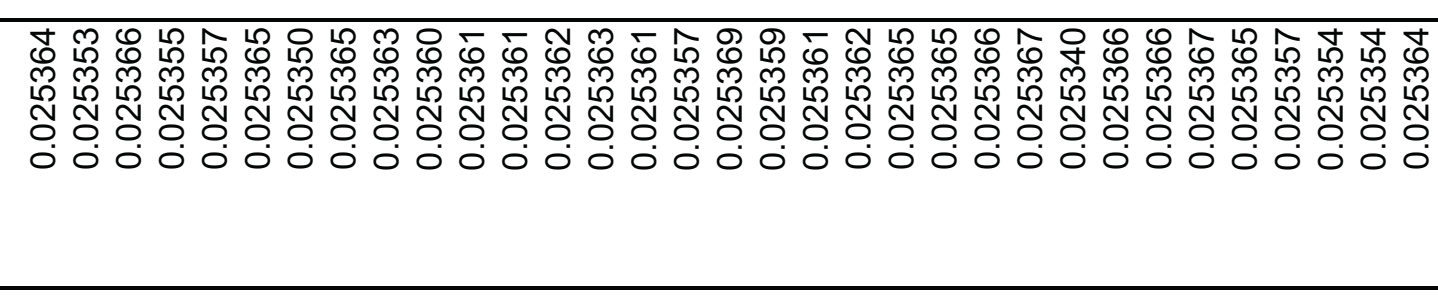 \\
\hline 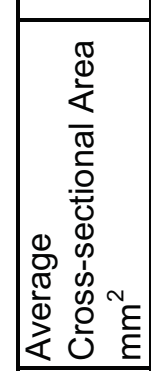 & 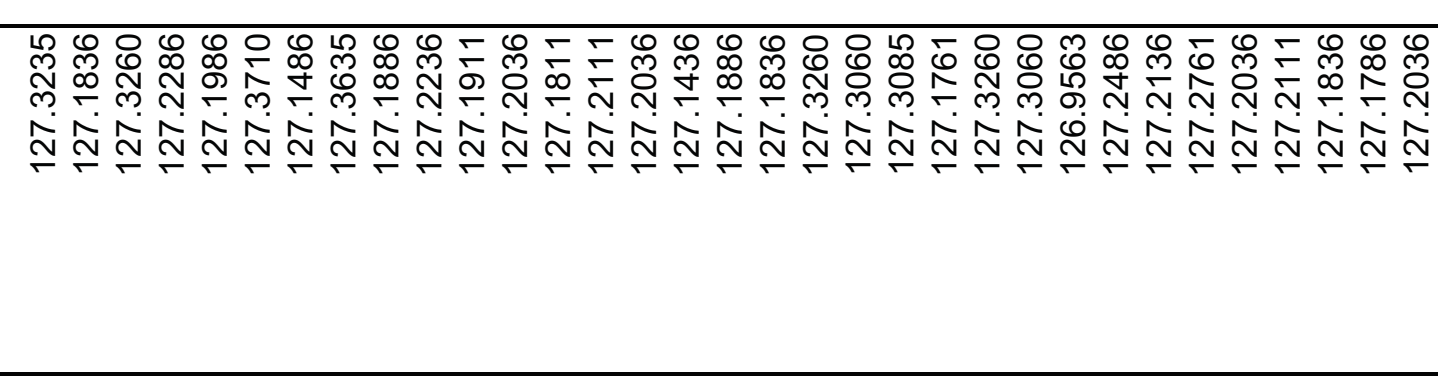 \\
\hline$\frac{\bar{s}}{\bar{w}}$ & 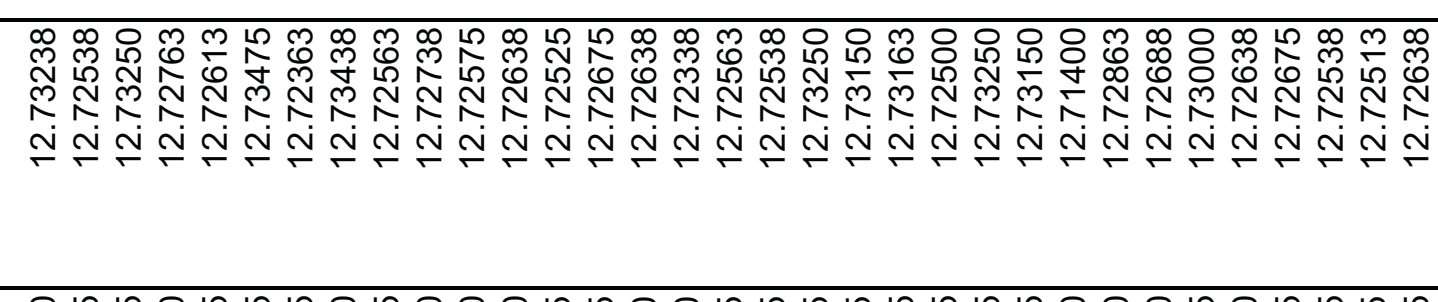 \\
\hline$\frac{\varepsilon}{b}$ & 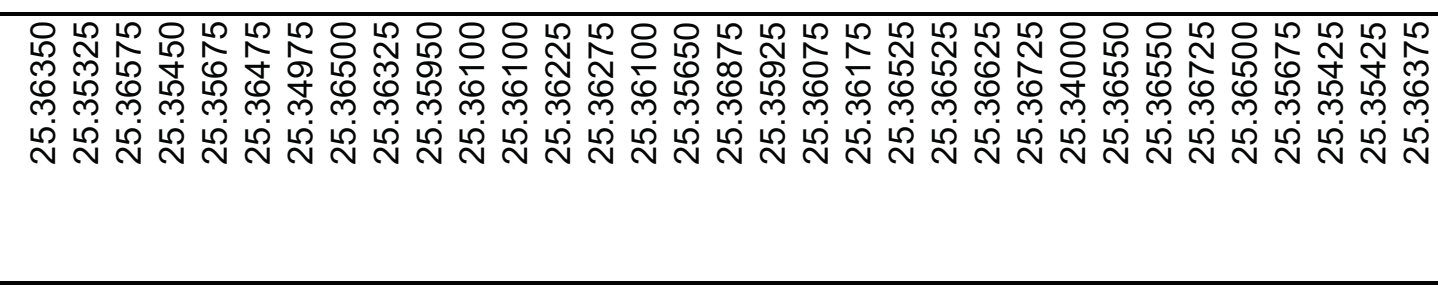 \\
\hline 离 & 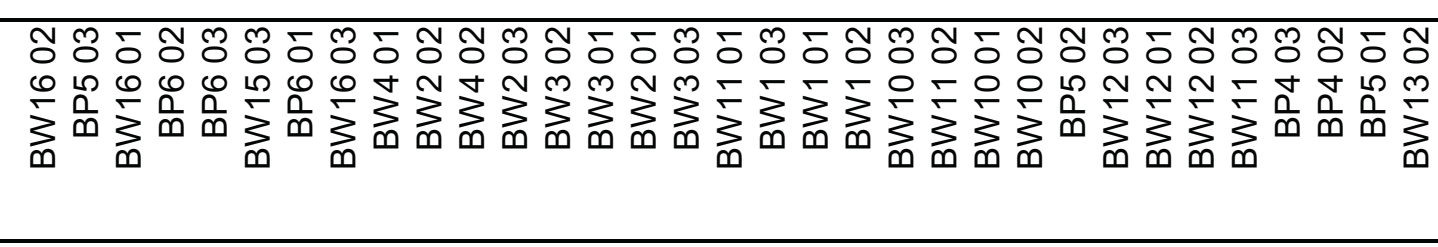 \\
\hline
\end{tabular}




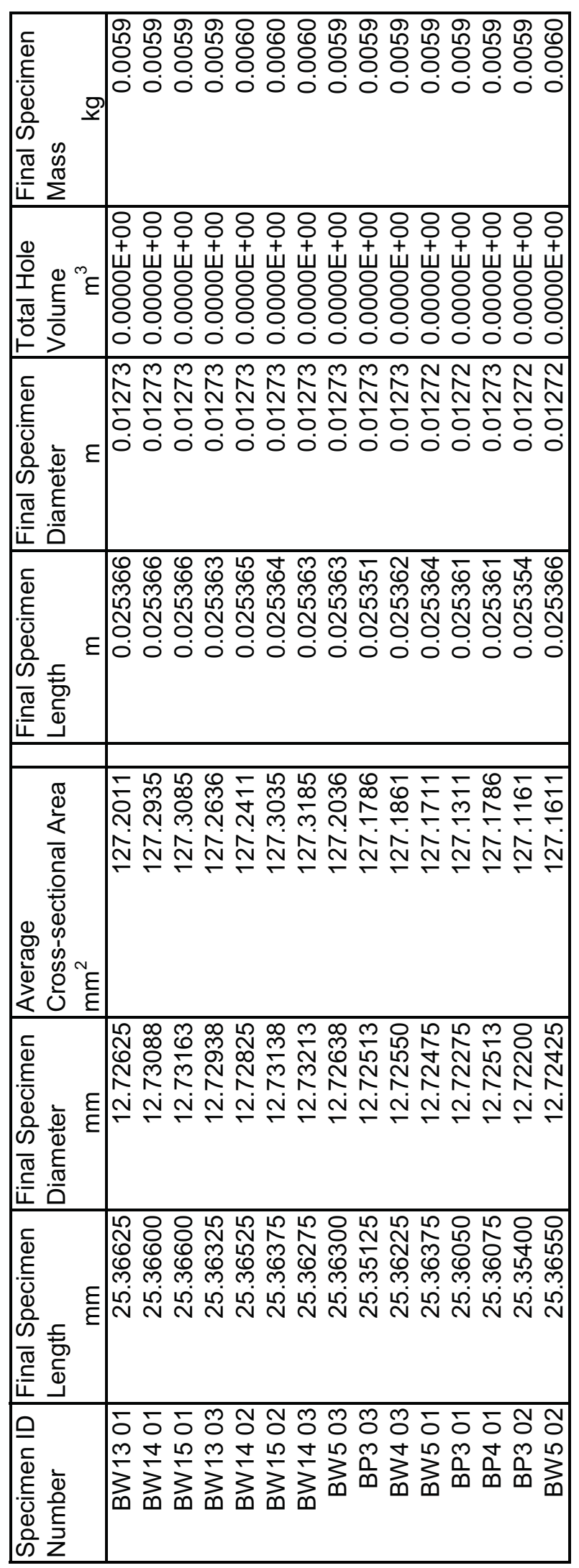




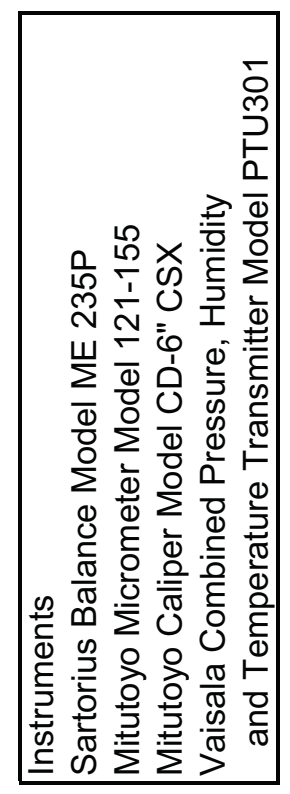

\begin{tabular}{|c|c|}
\hline 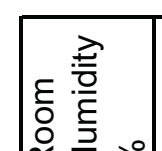 & 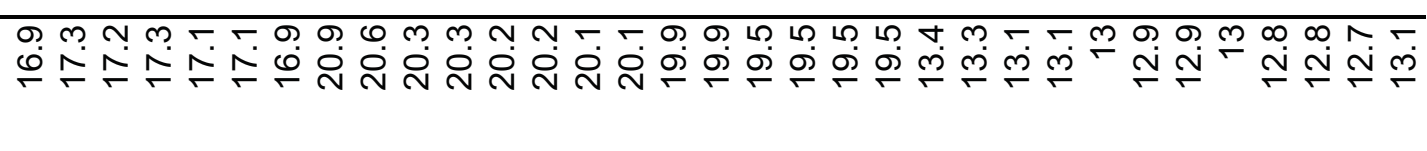 \\
\hline 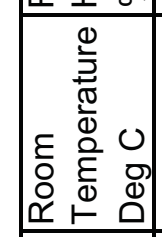 & 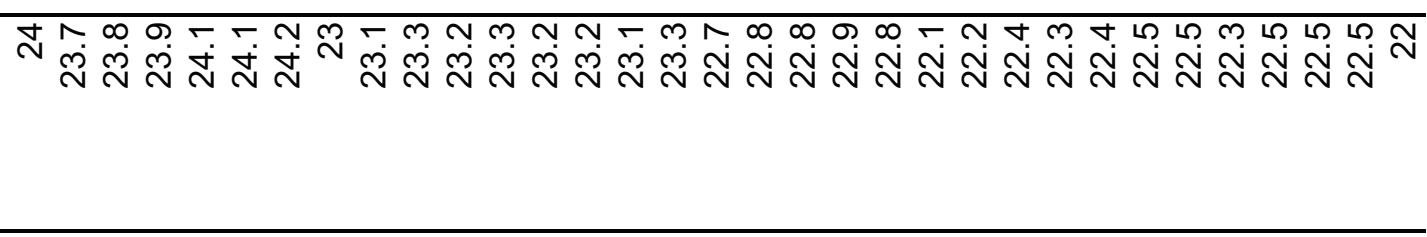 \\
\hline 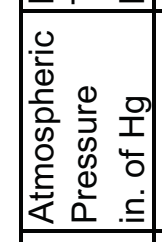 & 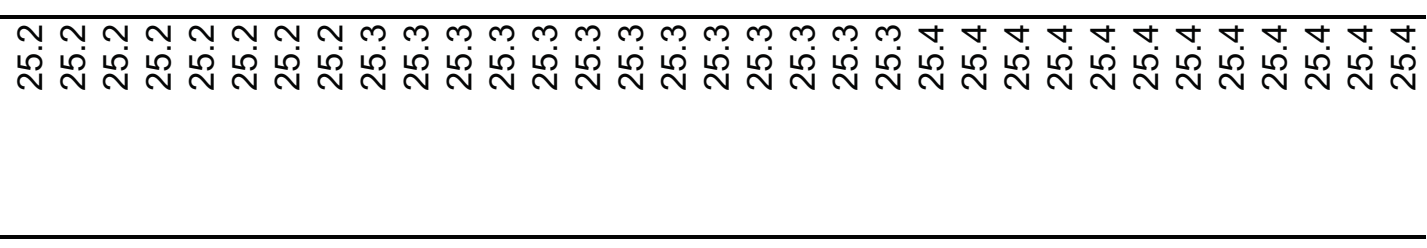 \\
\hline 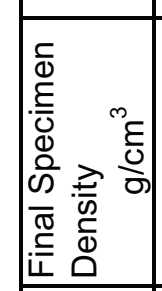 & 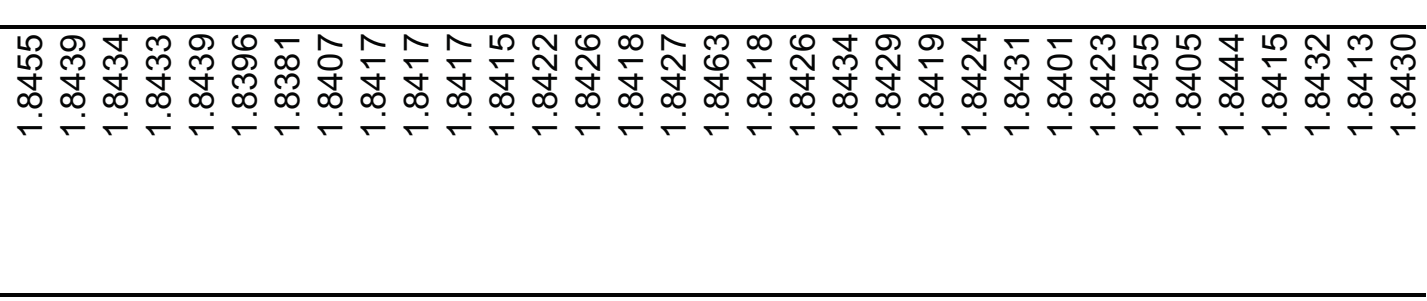 \\
\hline 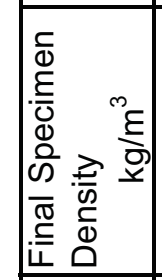 & 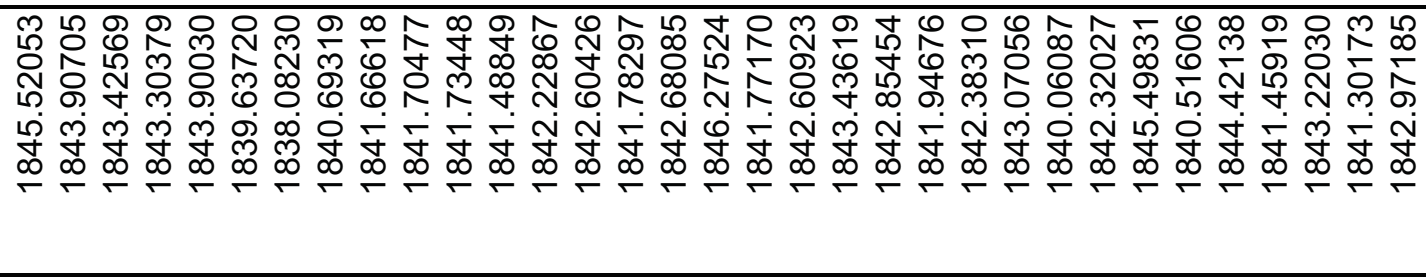 \\
\hline 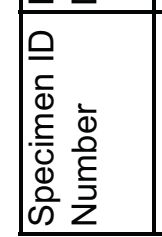 & 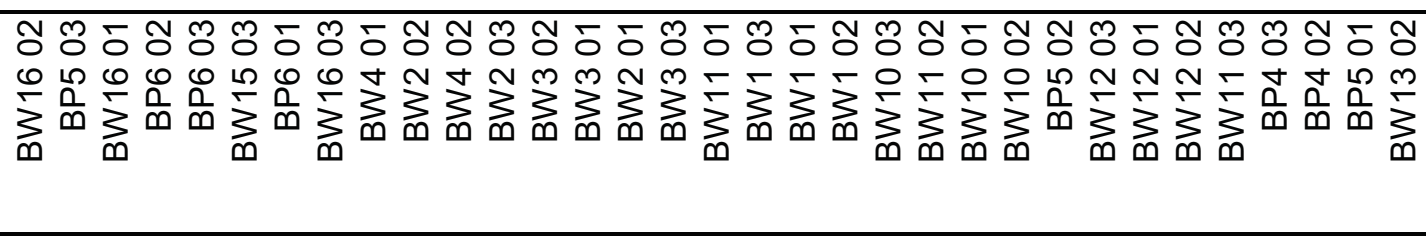 \\
\hline
\end{tabular}




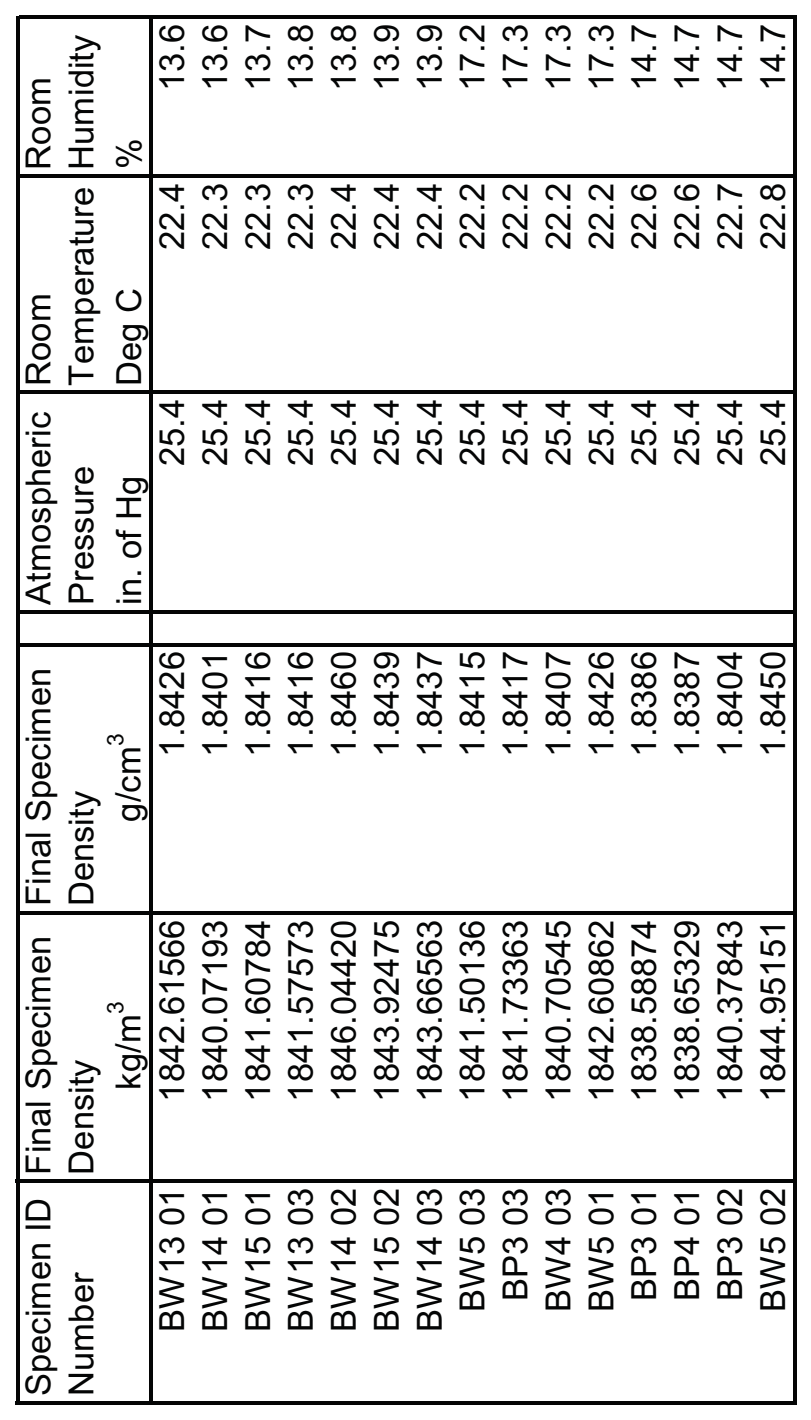


Graphite Grade:

PCEA

Graphite Manufacturer: Graftech International

Forming Process: Extruded

Coke Particle Size: Medium grain

Coke Type:

ASTM Class:

Pitch coke filler, pitch binder

Specimen Geometry: Cylinder

Specimen ID \#'s:

DA4 02

DA6 03

DA6 01

DA5 03

DA6 02

DA2 03

DA2 02

DA7 02

DA4 01

DA4 03

DA5 01

DA1 02

DA3 03

DA2 01

DA3 02

DA5 02

DA1 01

DA1 03

DA7 01

DA3 01

DA7 03

DW5 02

DW6 03

DW4 04

DW17 03

DW8 02

DW9 01

DW14 01

DW5 01

DW10 03

DW1 04

DW2 01

DW9 02

DW1 03

DW12 01

DW15 04

DW11 04

DW8 03

DW10 04

DW13 04

DW7 04

DW15 03 


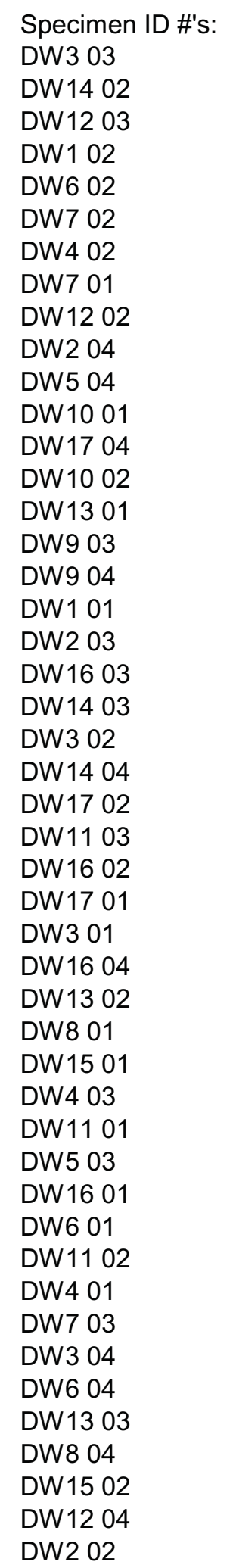




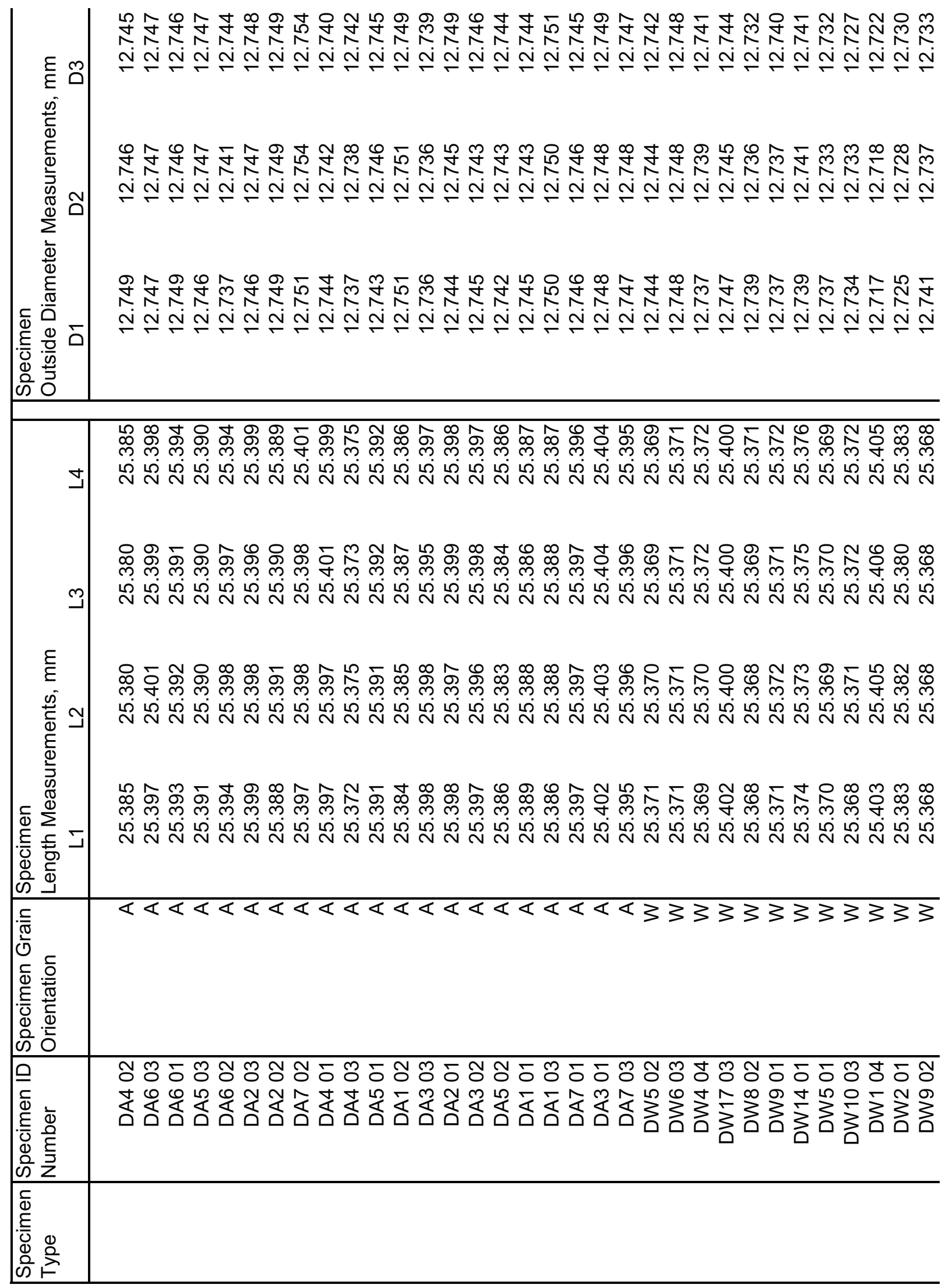




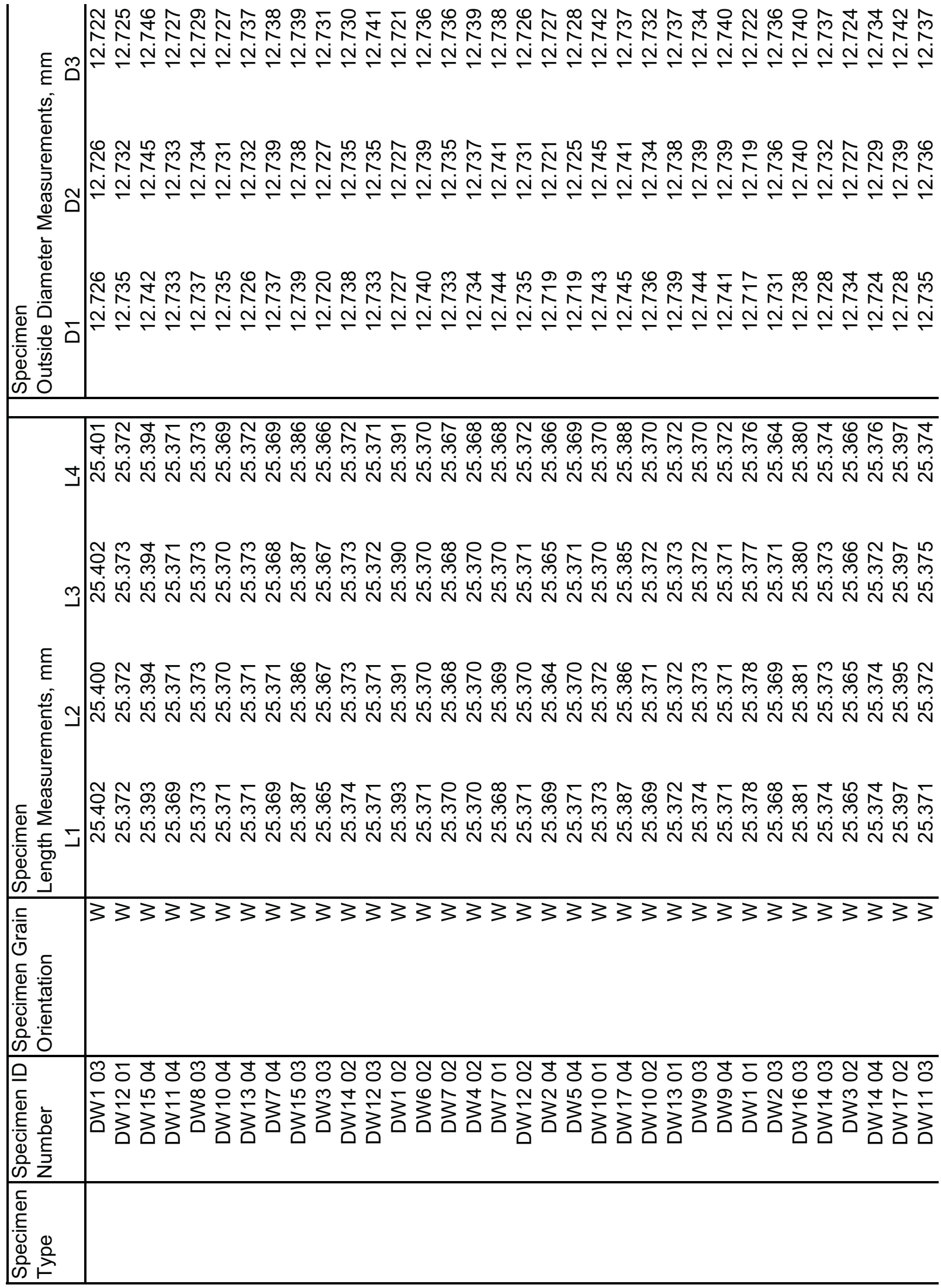




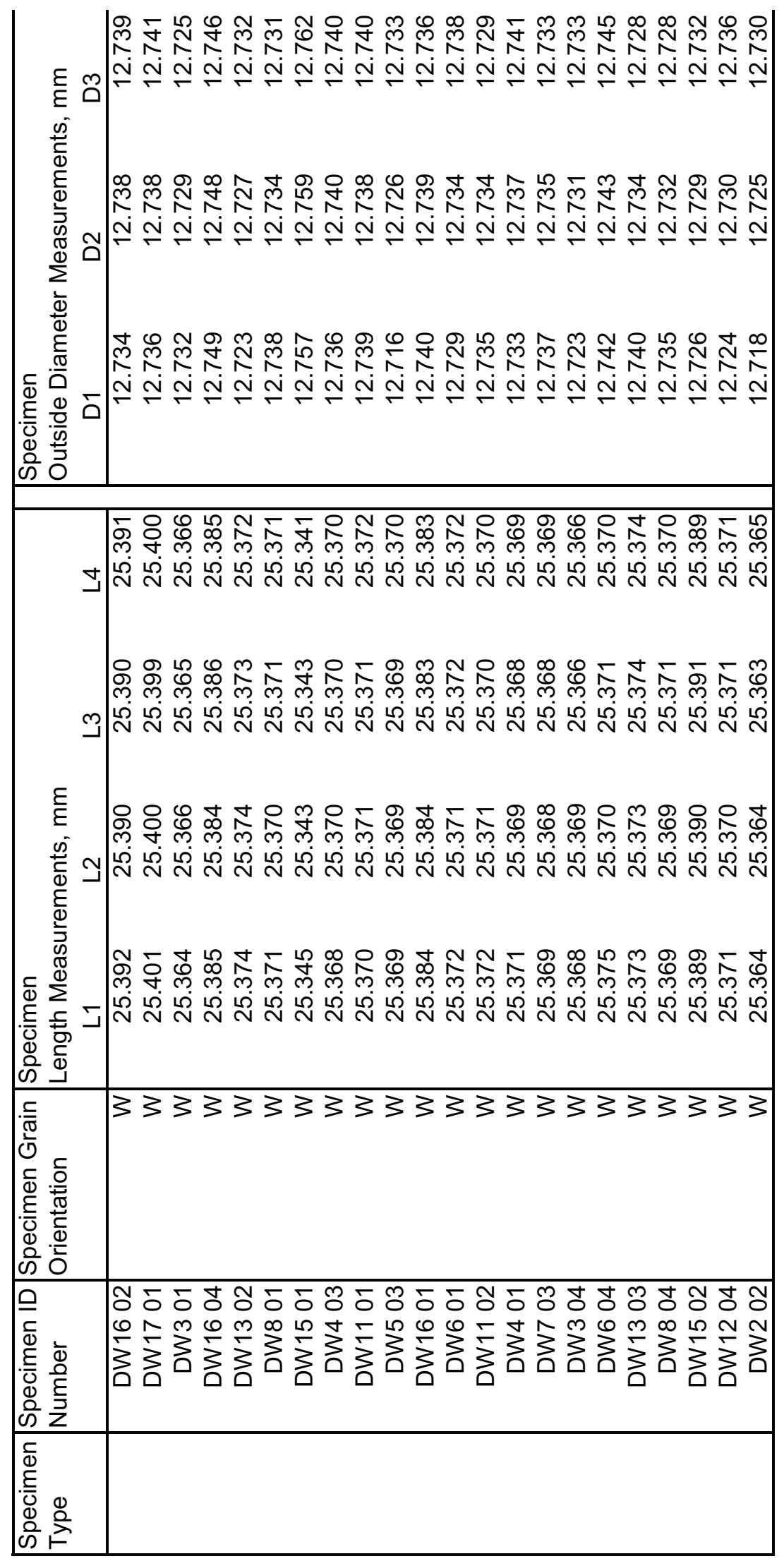




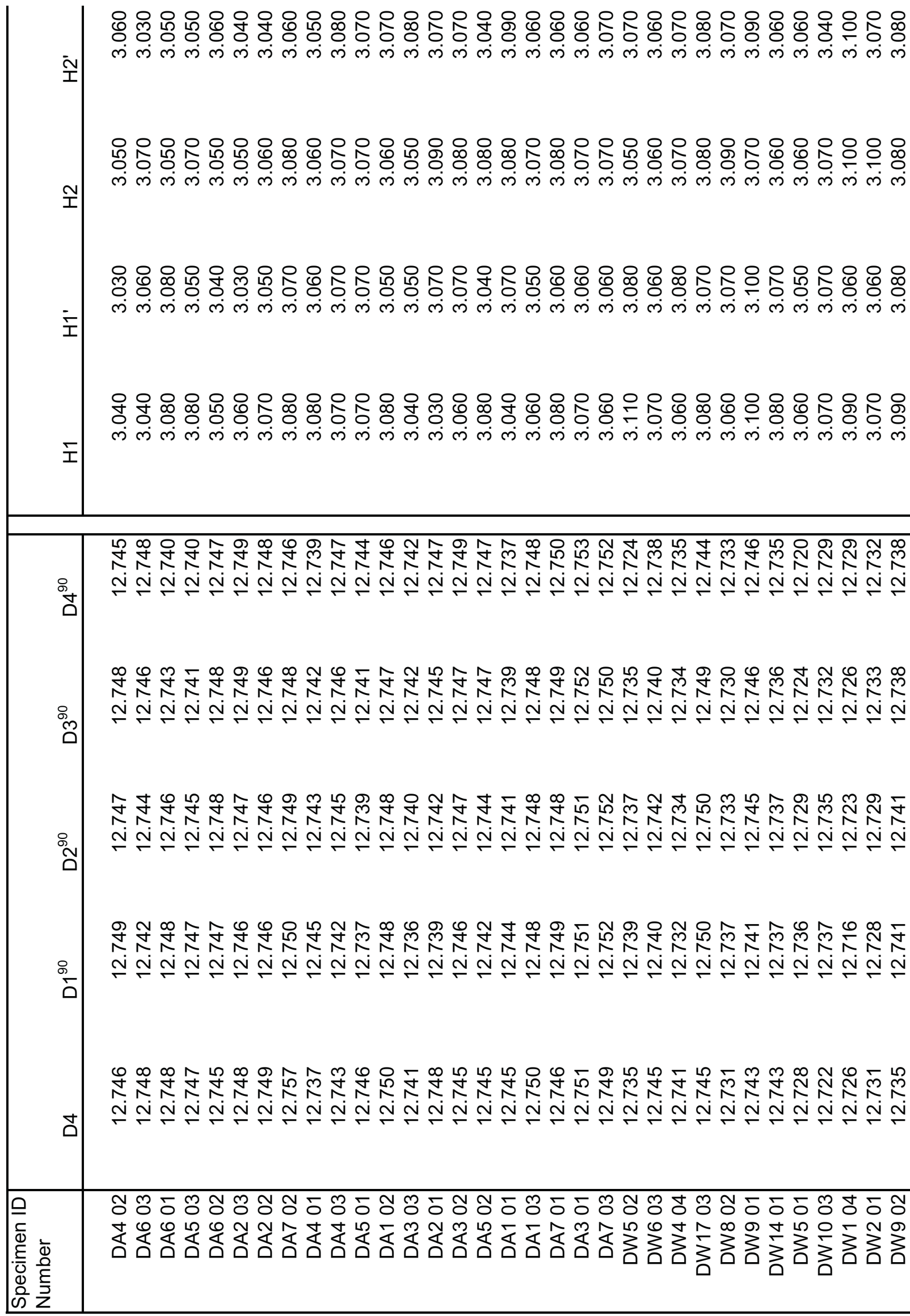




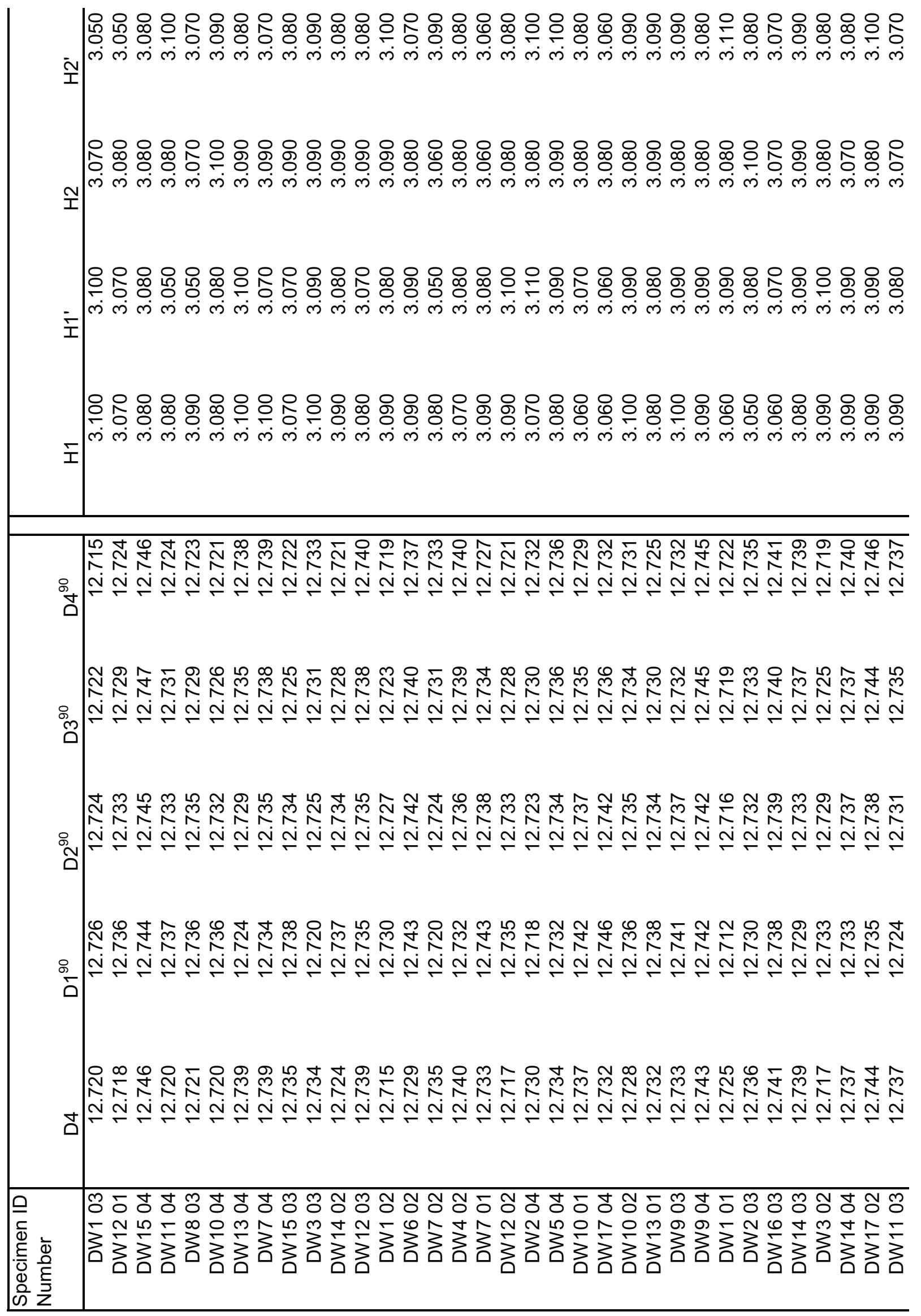




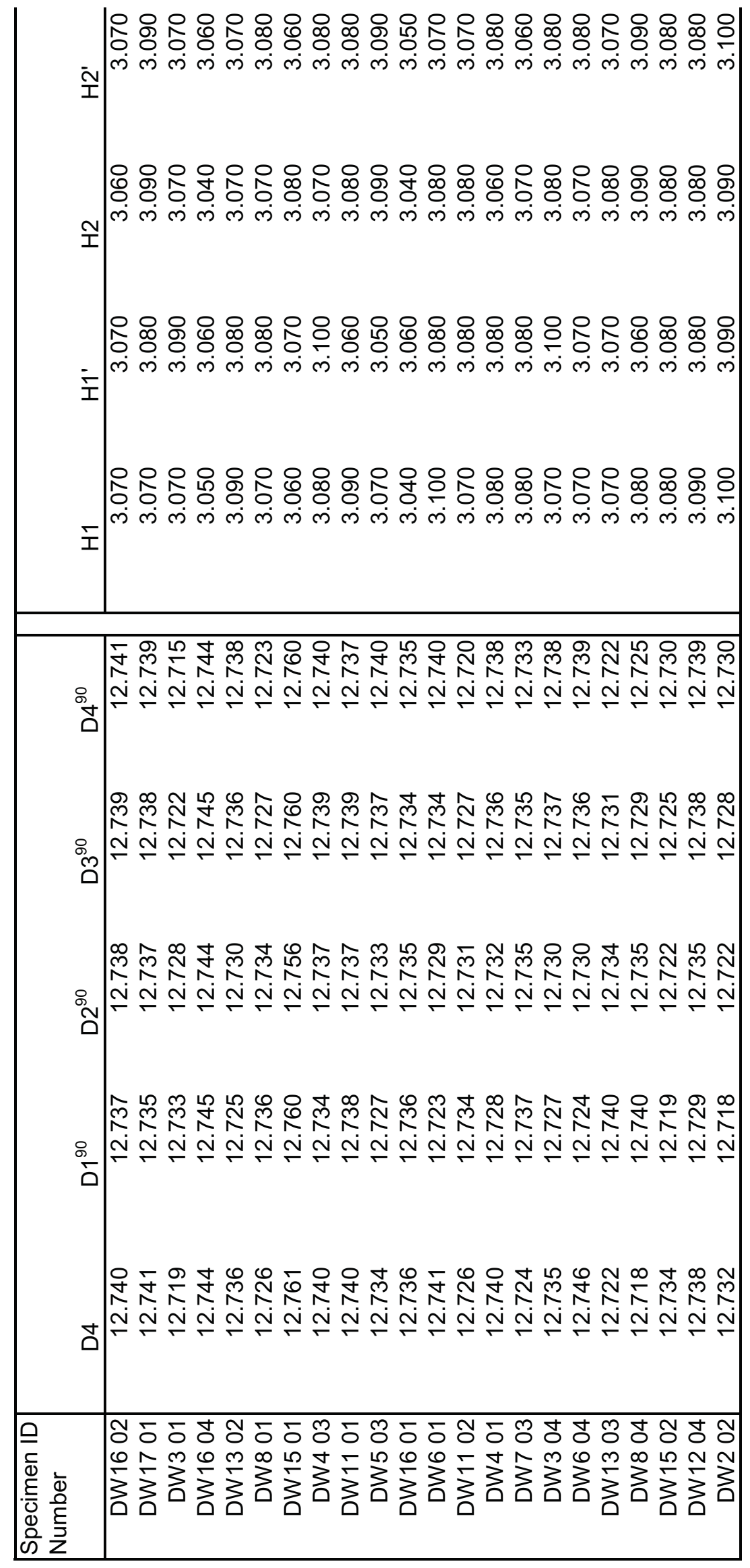




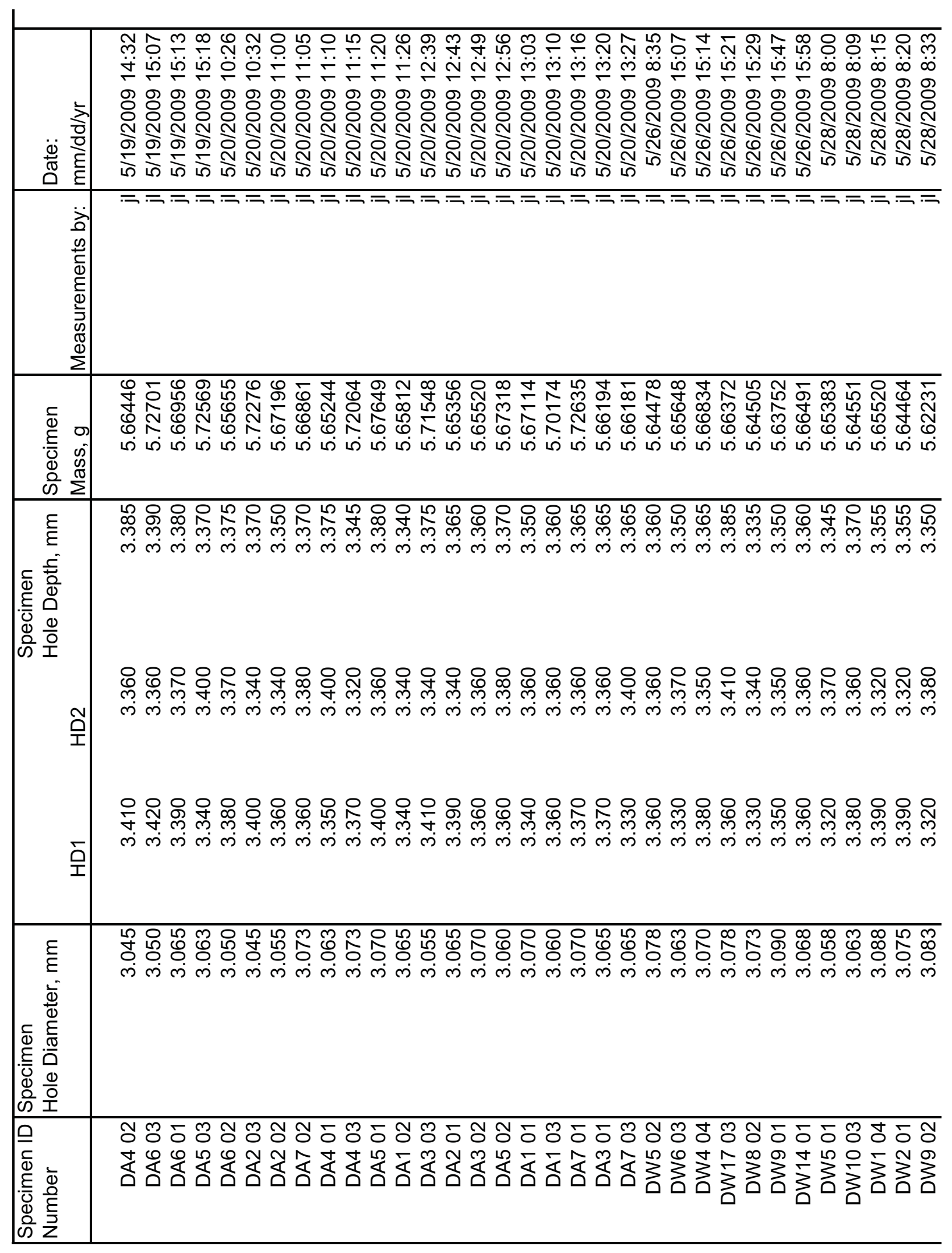




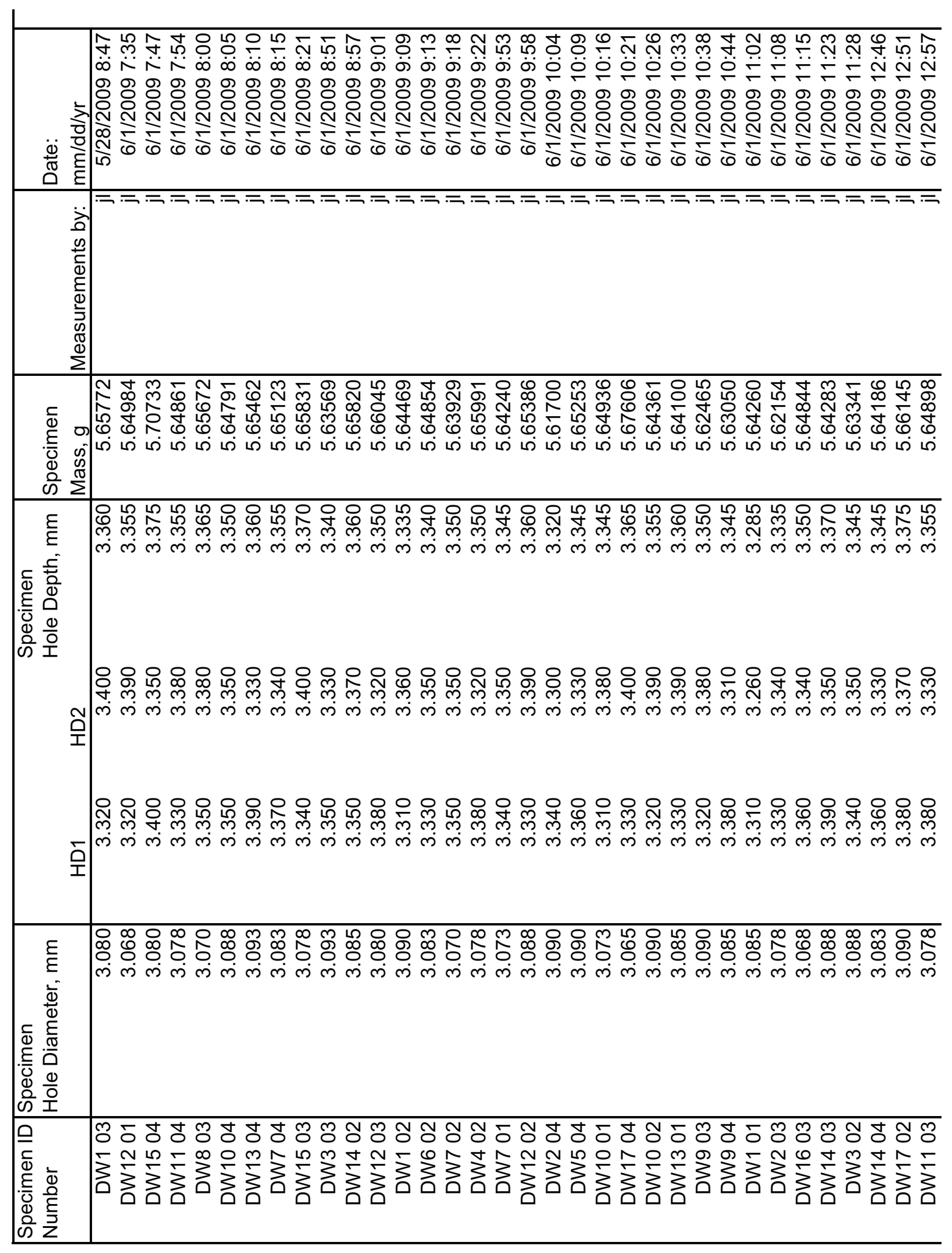




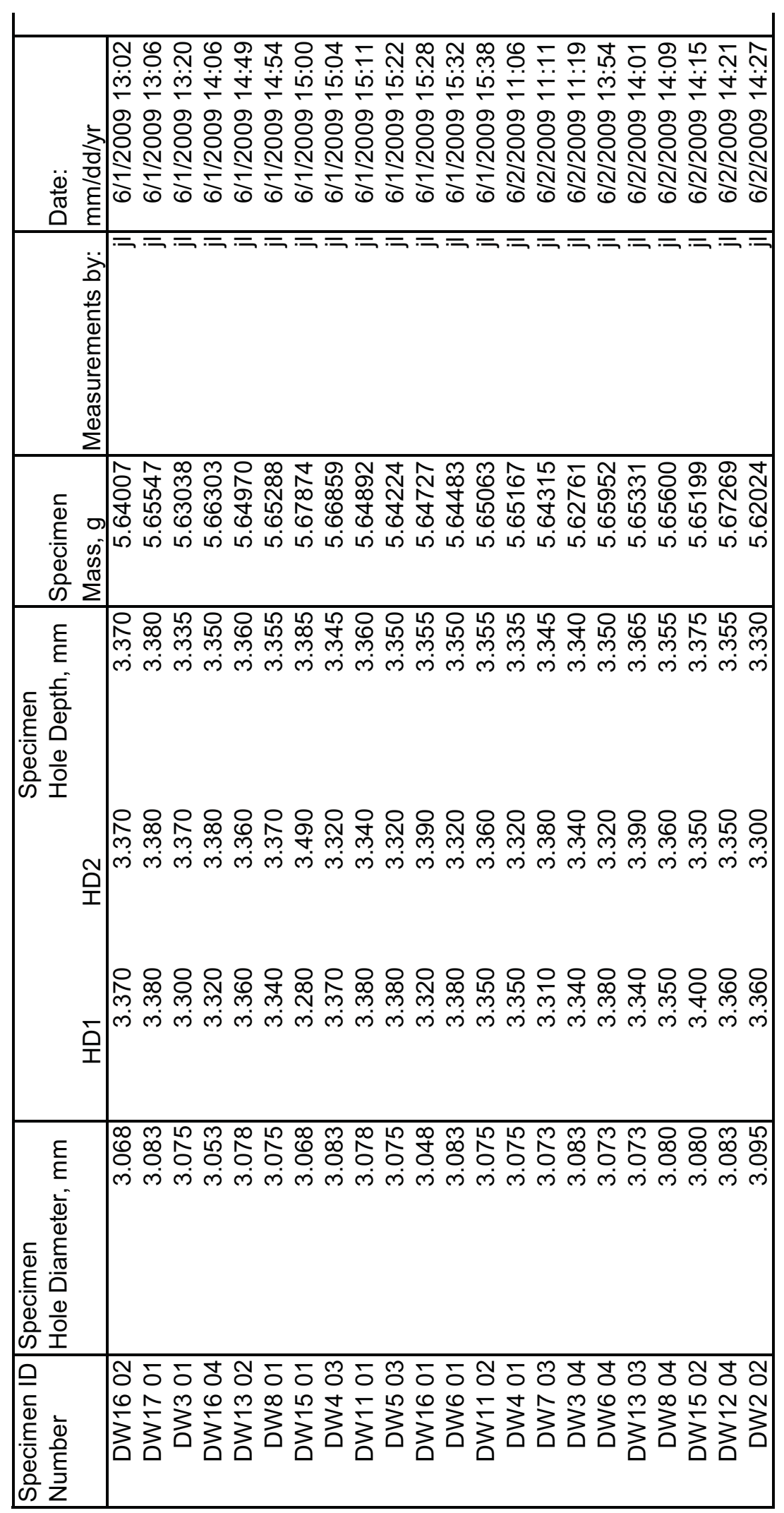




\begin{tabular}{|c|c|}
\hline 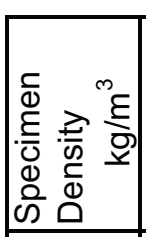 & 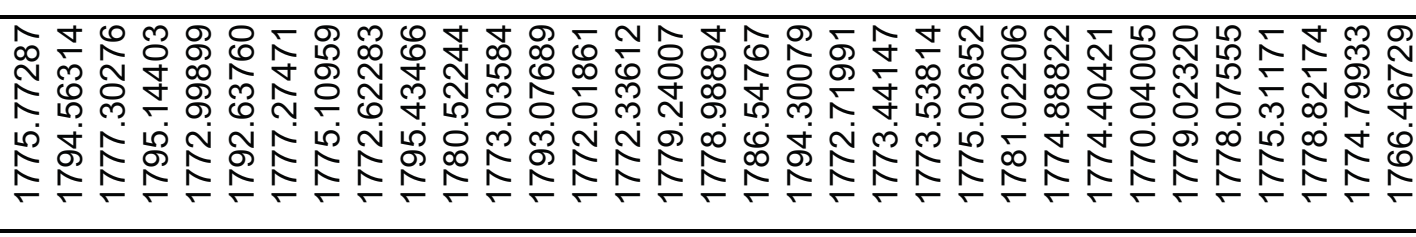 \\
\hline & 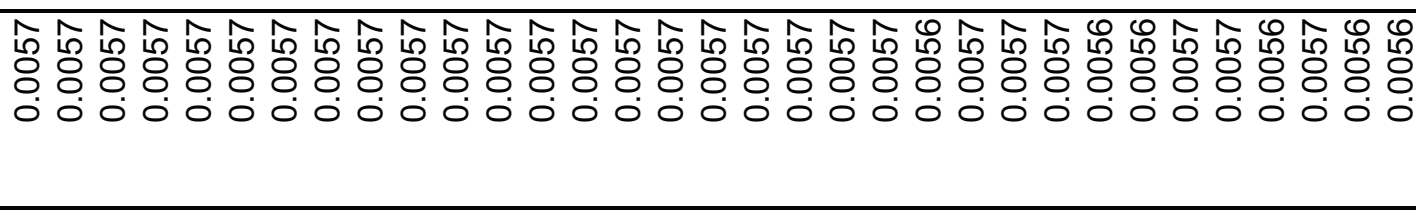 \\
\hline$E^{\prime \prime} E$ & 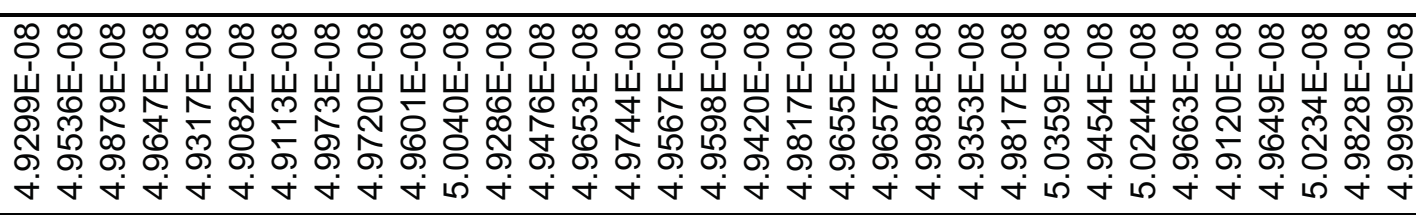 \\
\hline 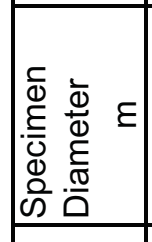 & 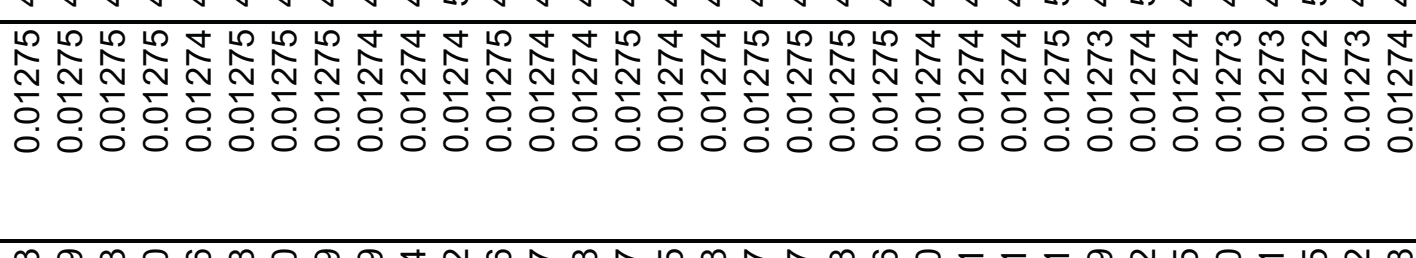 \\
\hline & 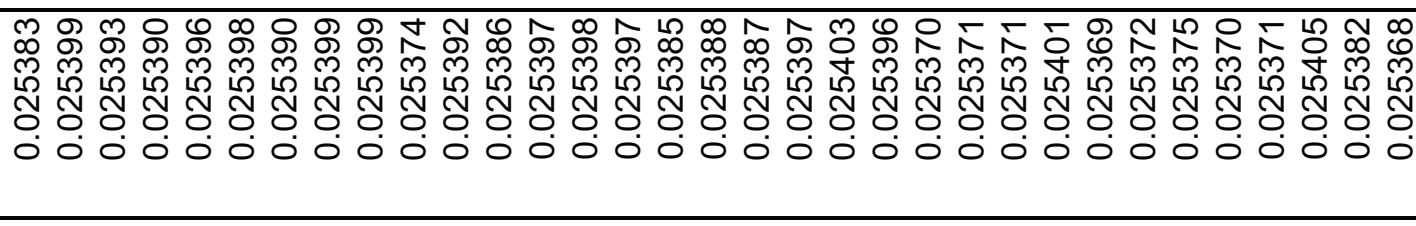 \\
\hline 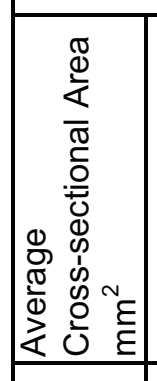 & 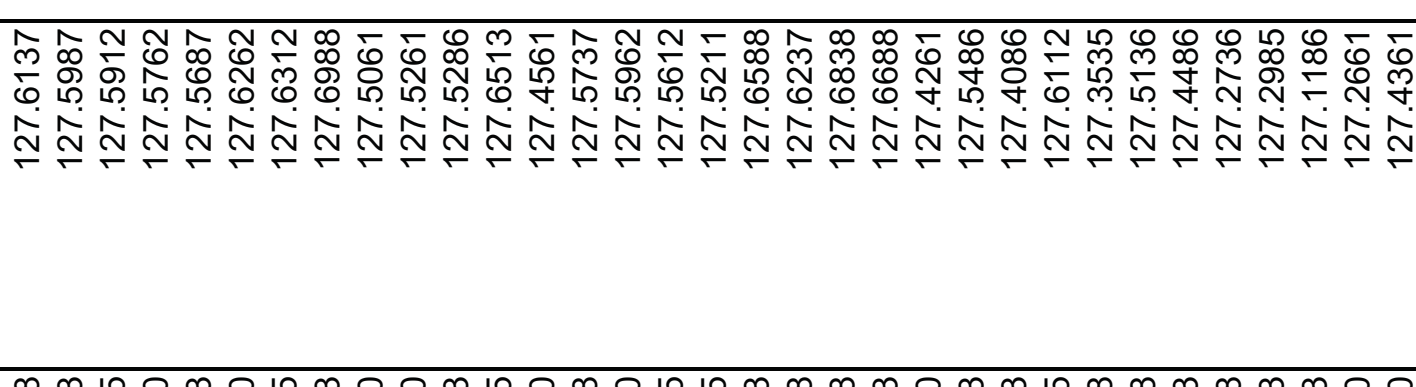 \\
\hline 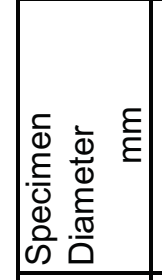 & 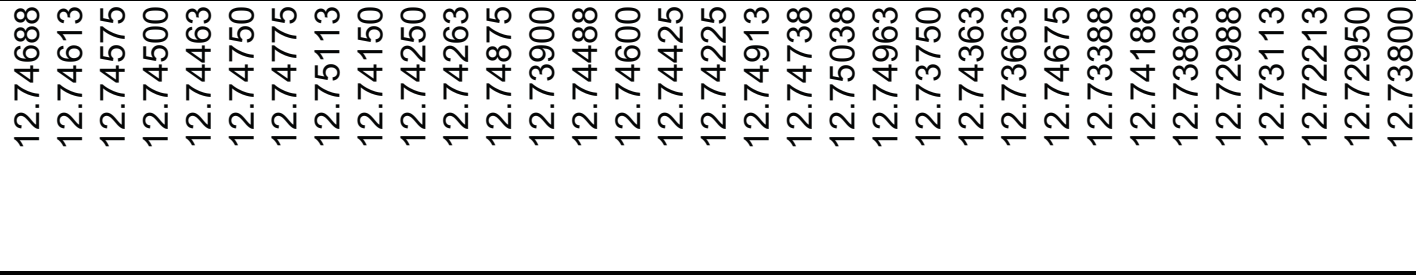 \\
\hline 흥 & 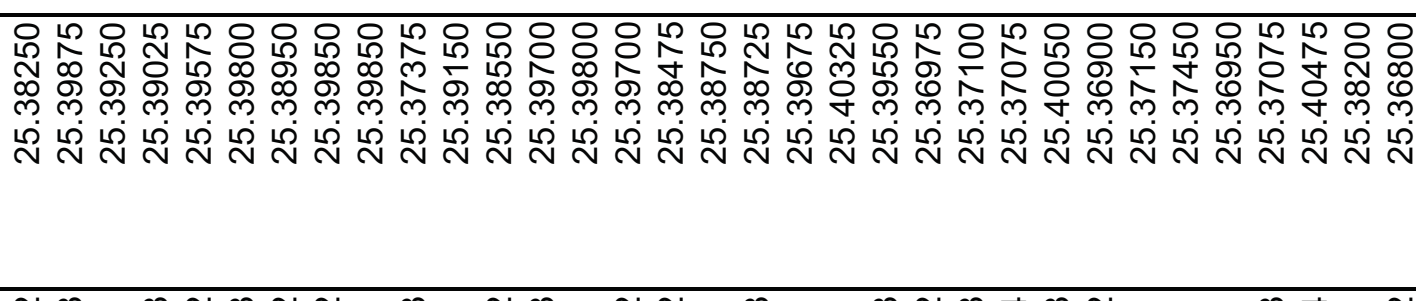 \\
\hline$=0$ & 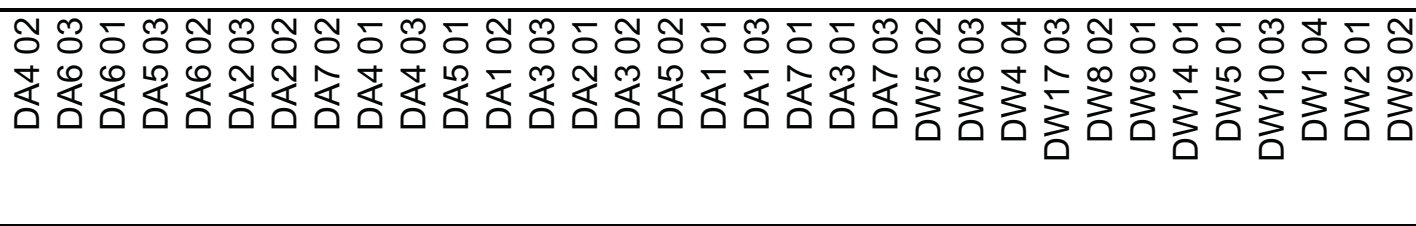 \\
\hline
\end{tabular}




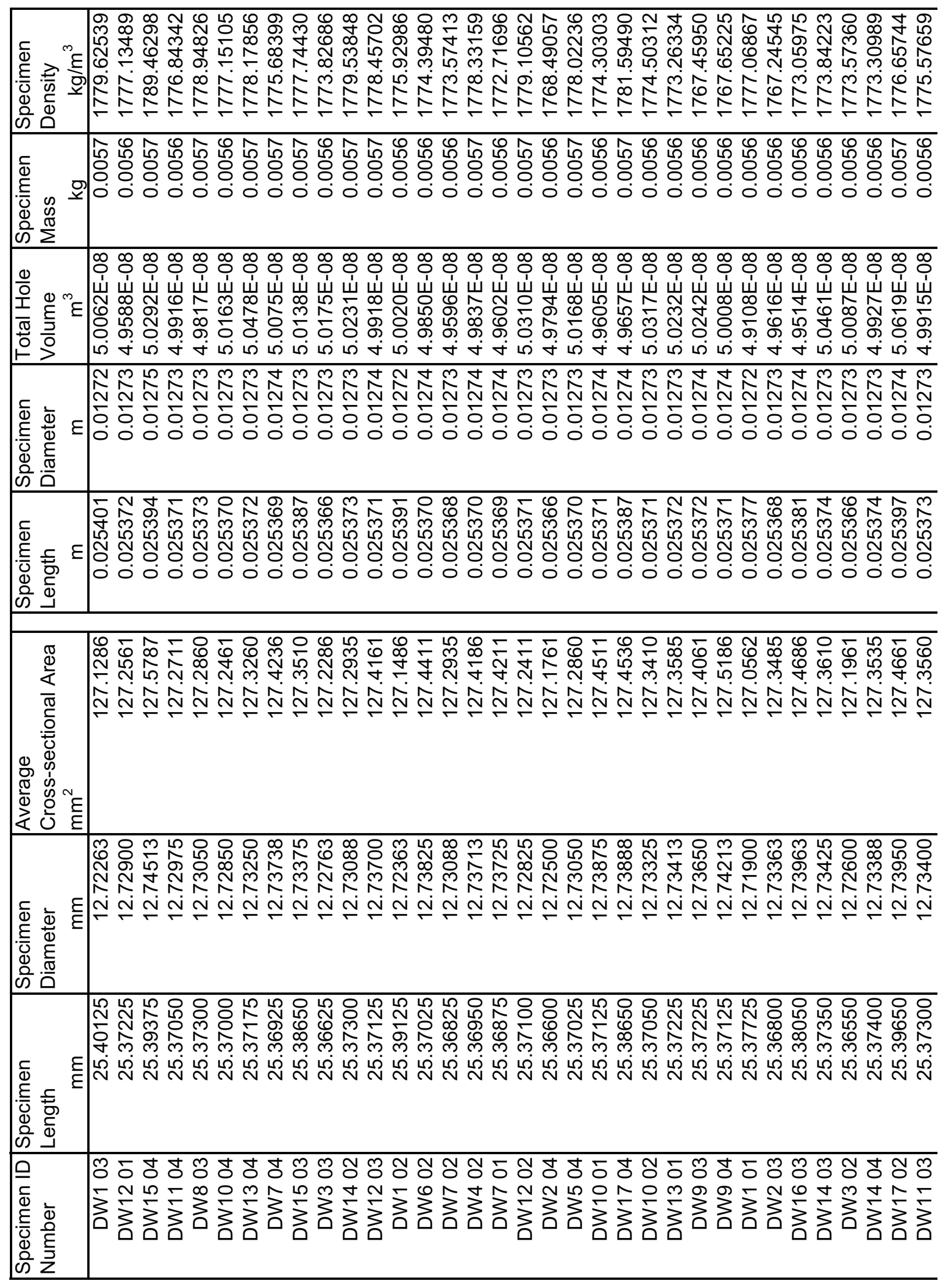




\begin{tabular}{|c|c|}
\hline 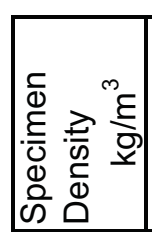 & 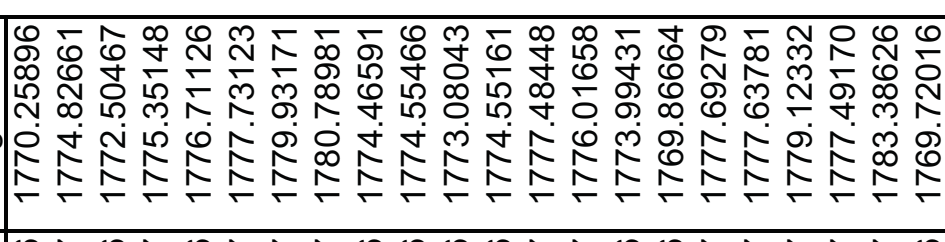 \\
\hline 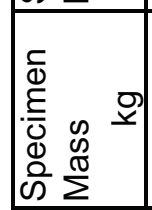 & 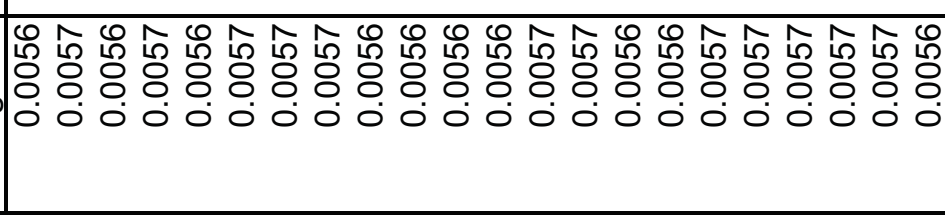 \\
\hline $\mathbb{\Phi}_{\xi}^{\prime \prime} E$ & 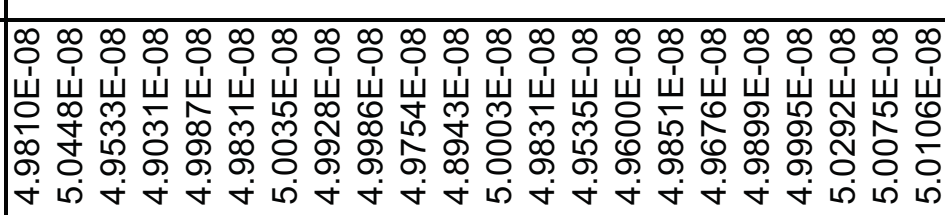 \\
\hline 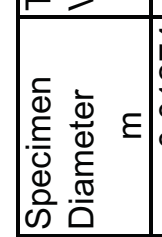 & 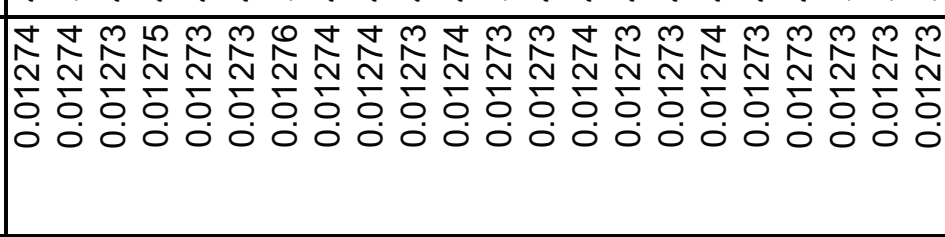 \\
\hline & 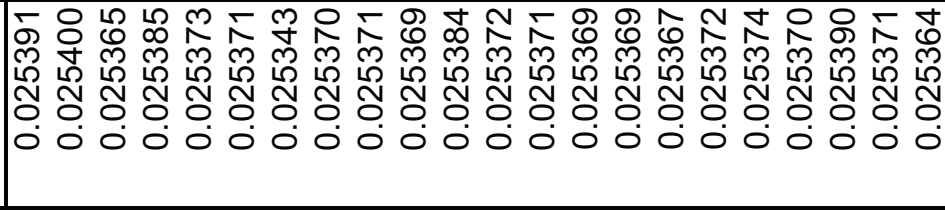 \\
\hline 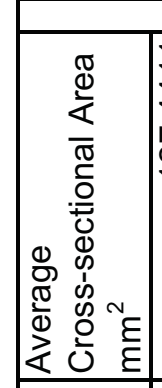 & 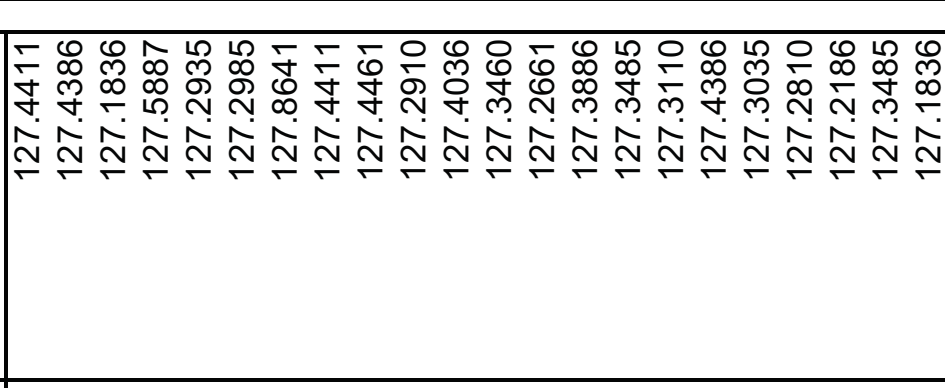 \\
\hline 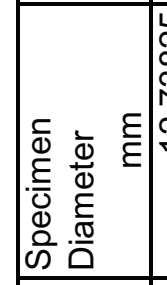 & 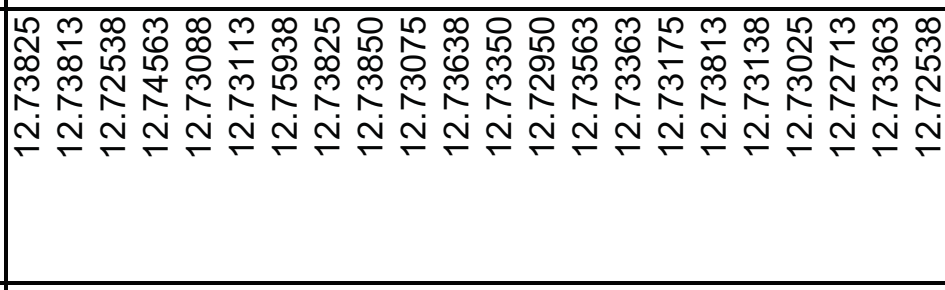 \\
\hline 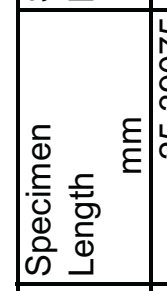 & 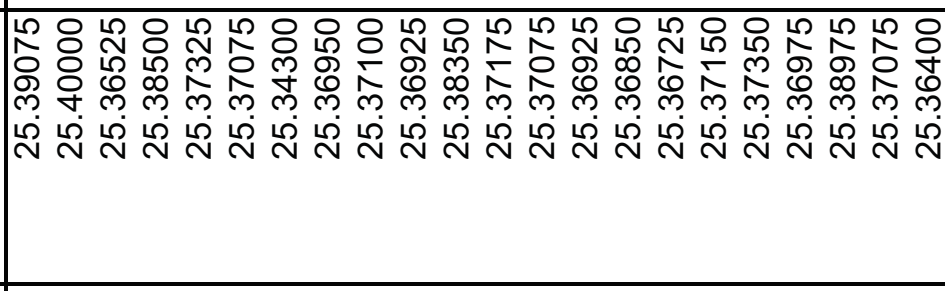 \\
\hline 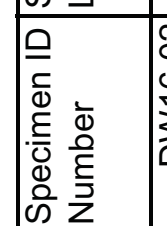 & 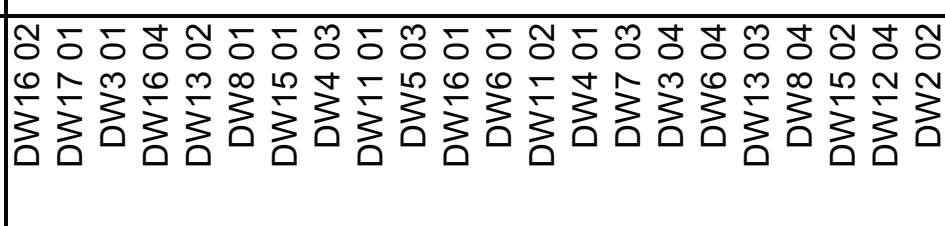 \\
\hline
\end{tabular}




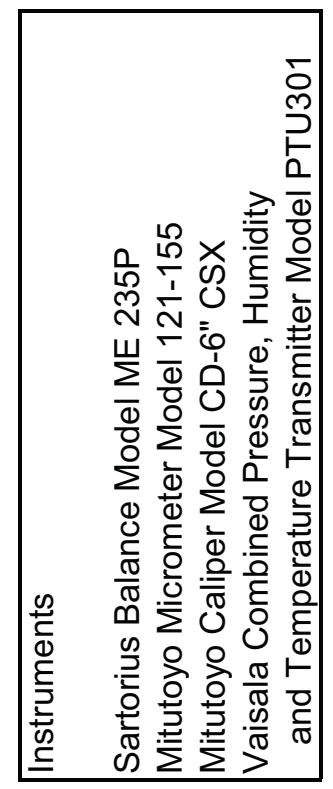

\begin{tabular}{|c|c|}
\hline 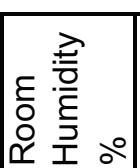 & 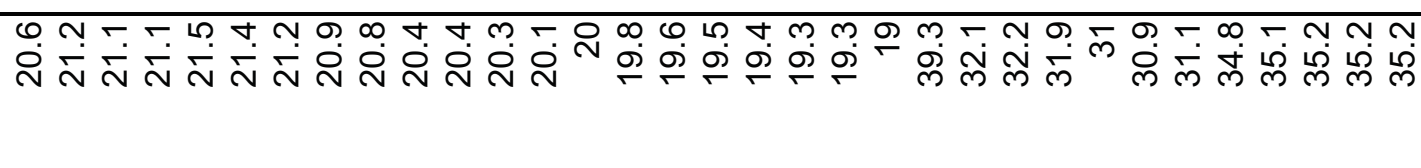 \\
\hline 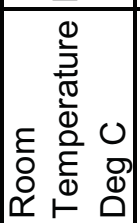 & 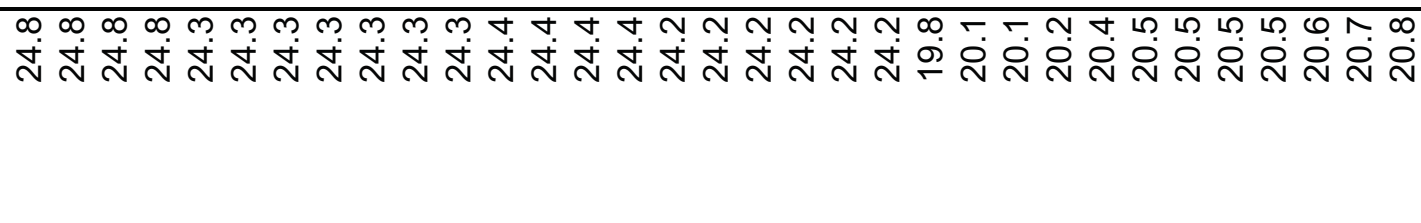 \\
\hline 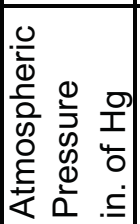 & 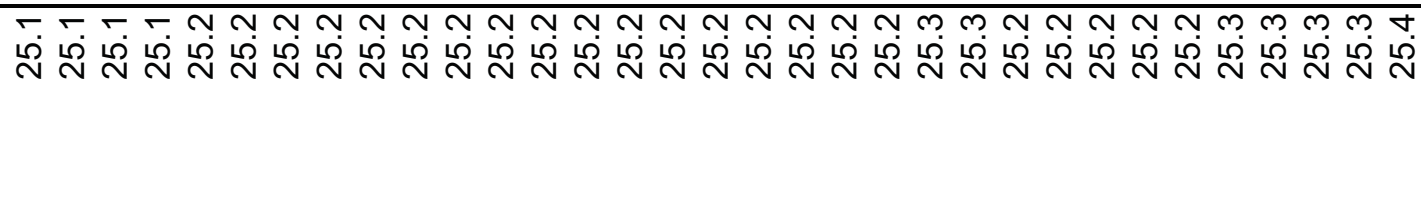 \\
\hline
\end{tabular}

\begin{tabular}{|c|c|}
\hline 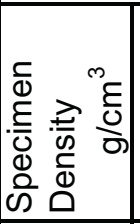 & 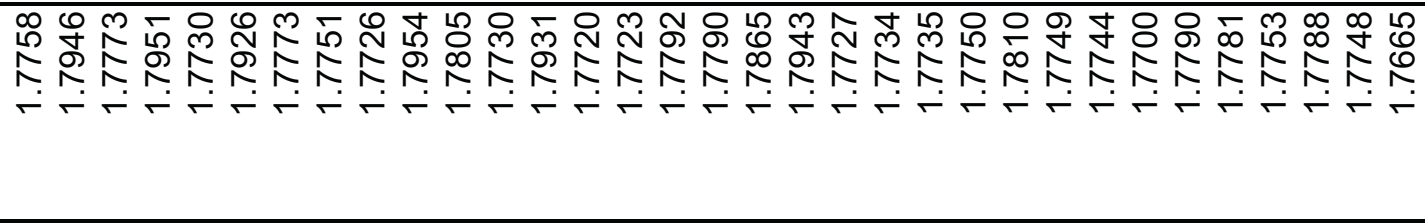 \\
\hline 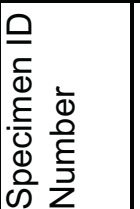 & 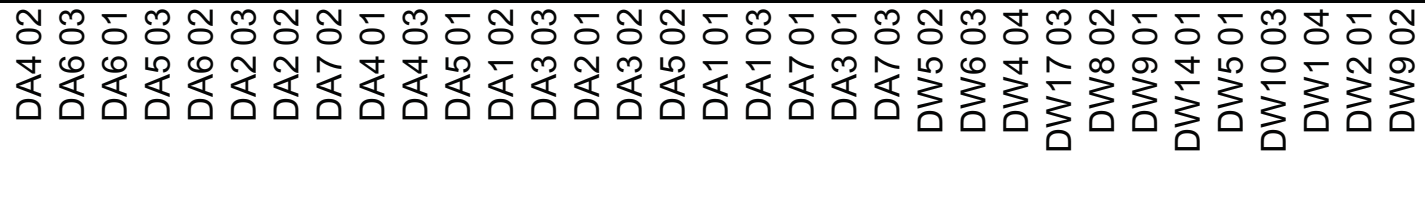 \\
\hline
\end{tabular}




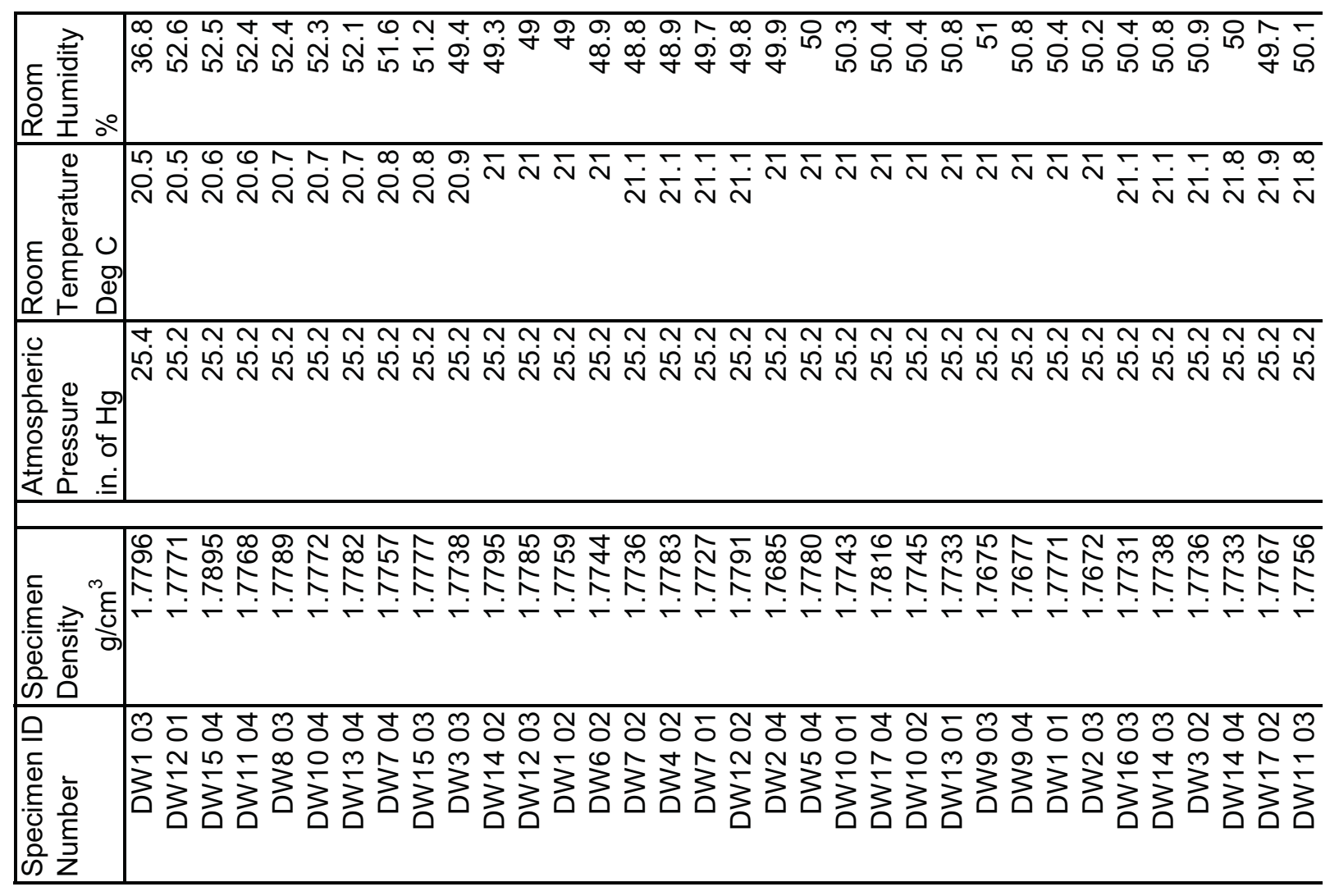




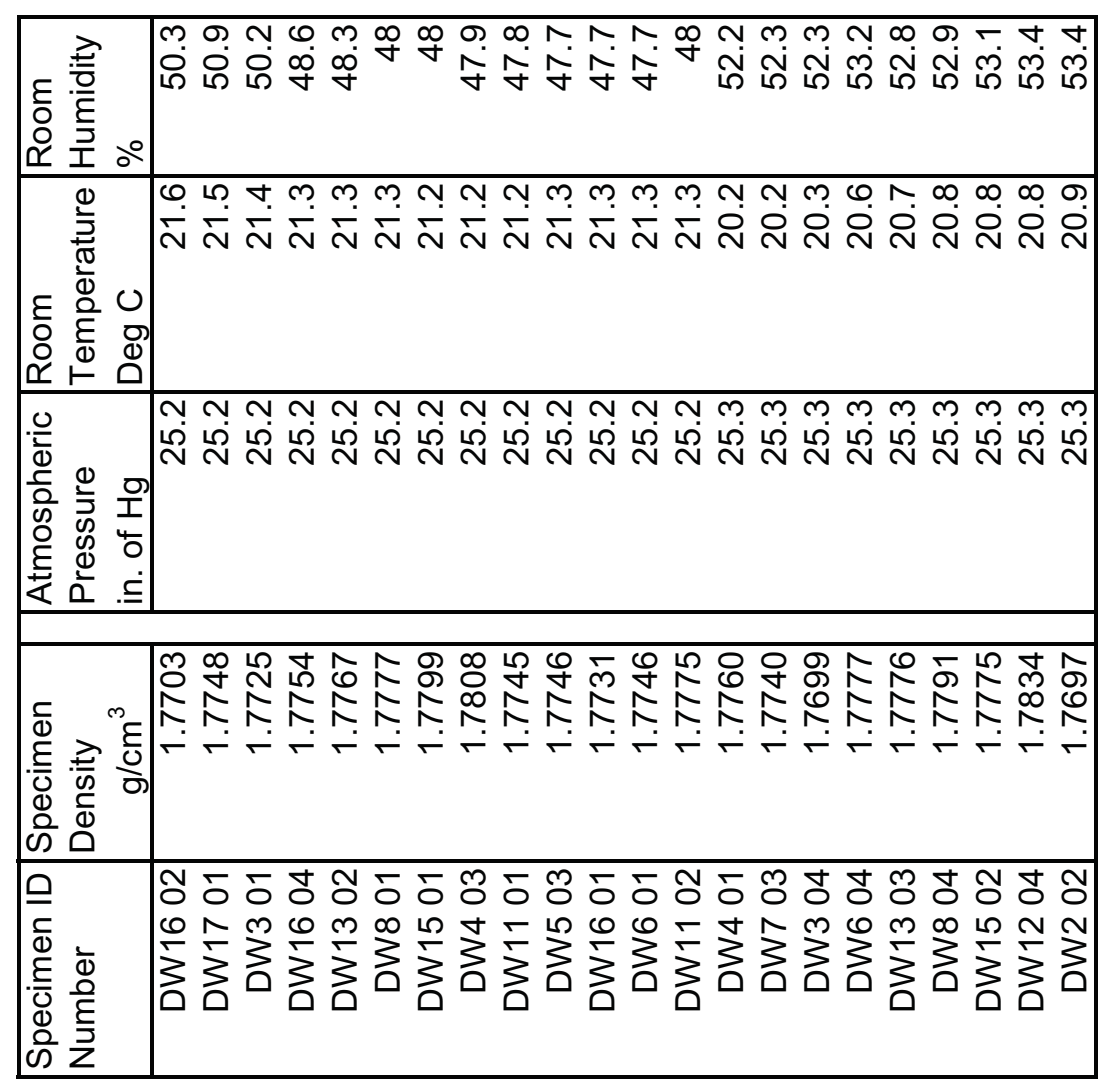




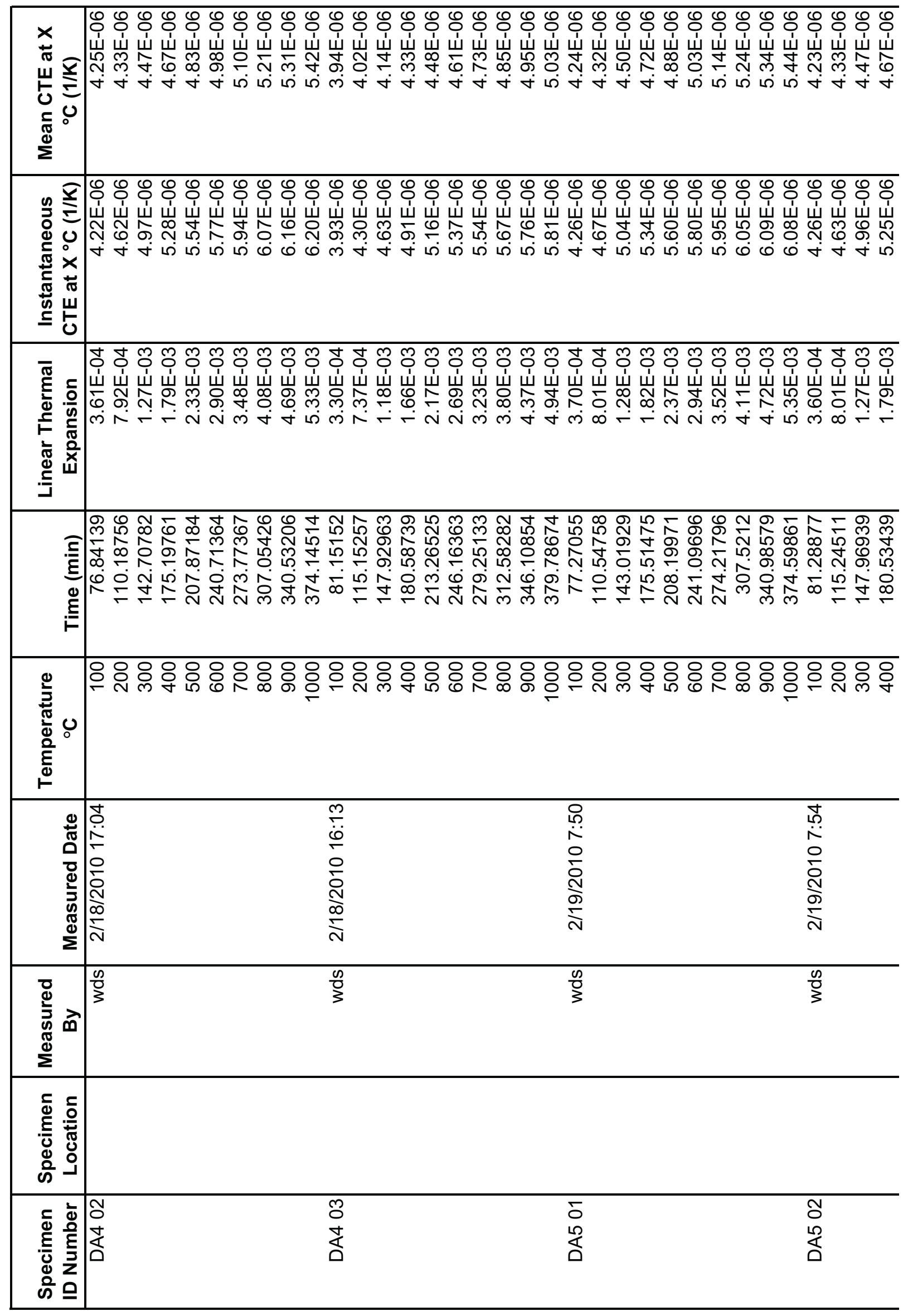




\begin{tabular}{|c|c|}
\hline 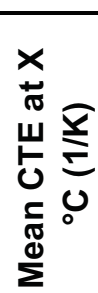 & 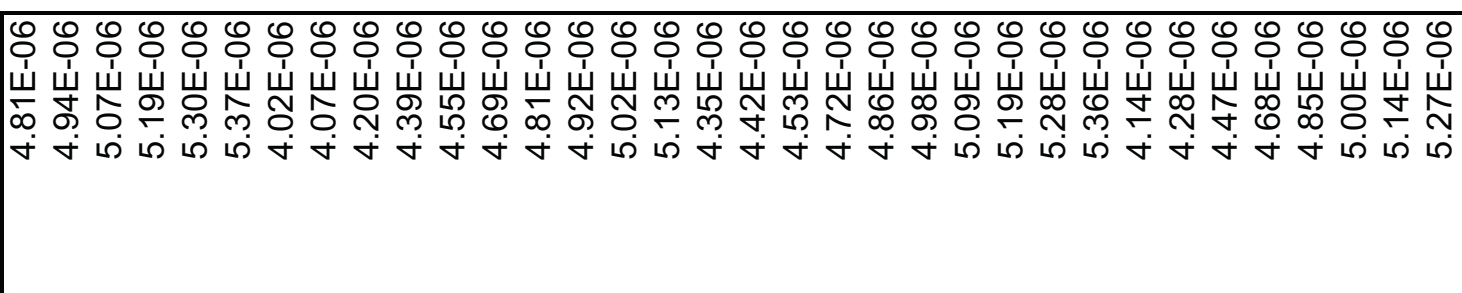 \\
\hline 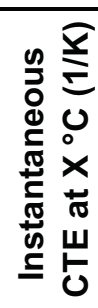 & 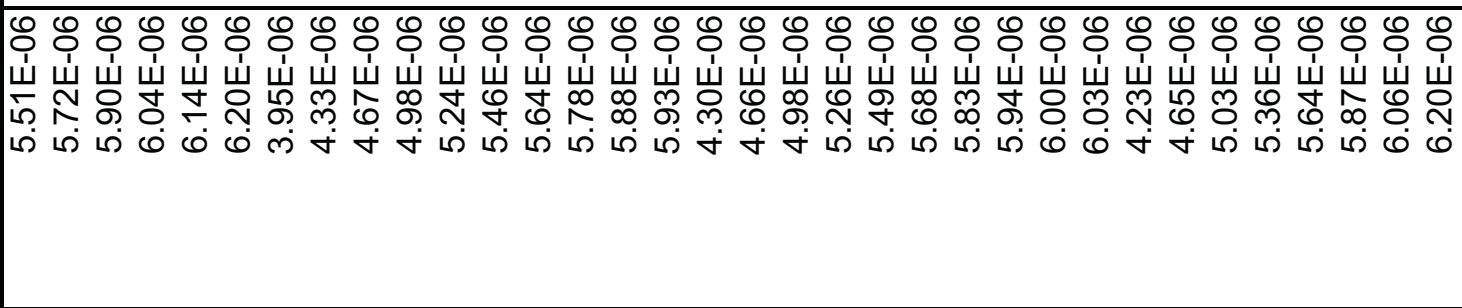 \\
\hline 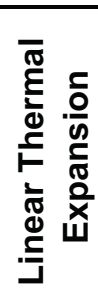 & 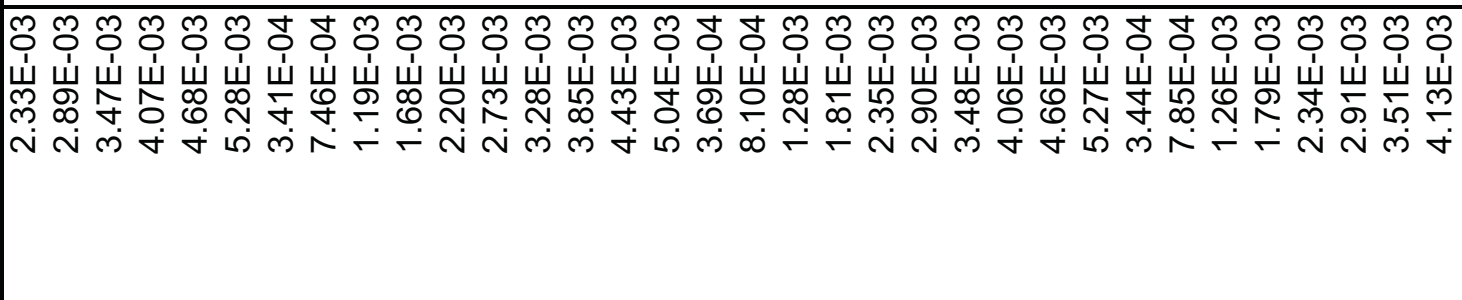 \\
\hline 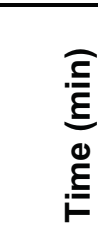 & 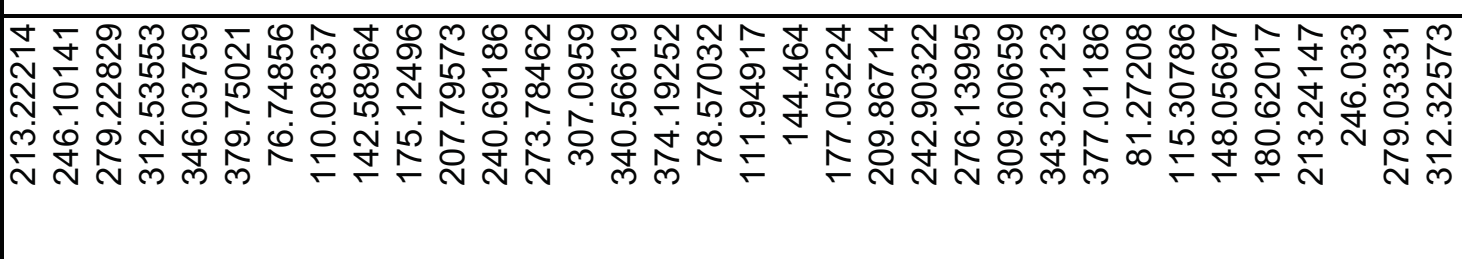 \\
\hline 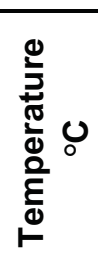 & 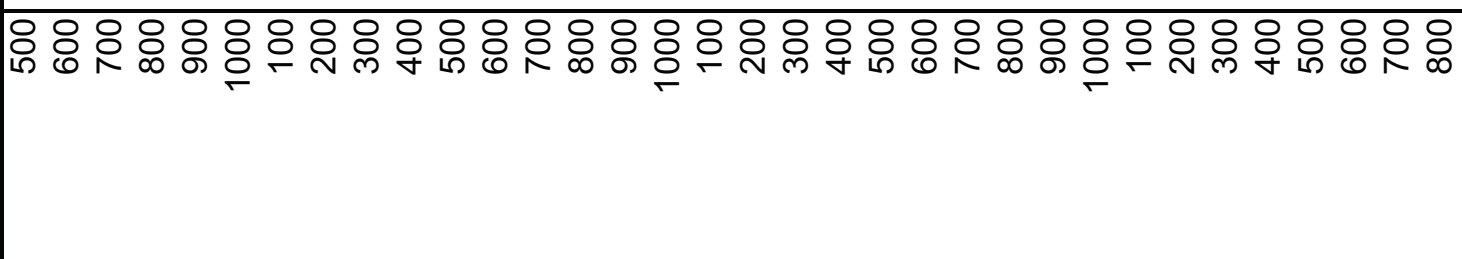 \\
\hline 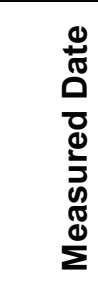 & 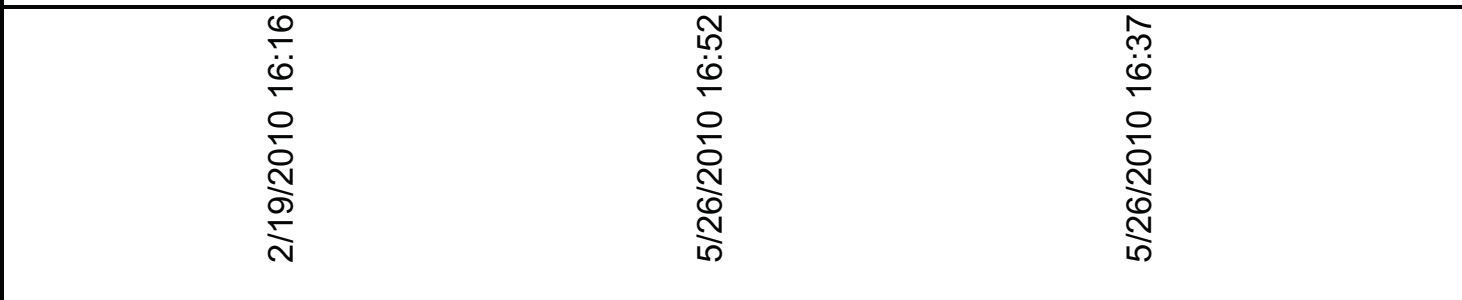 \\
\hline 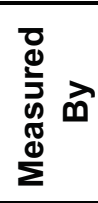 & $\frac{n}{0}$ \\
\hline 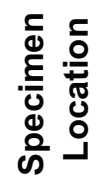 & \\
\hline 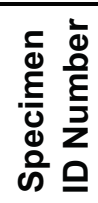 & $\begin{array}{l}5 \\
\\
0 \\
0\end{array}$ \\
\hline
\end{tabular}




\begin{tabular}{|c|c|}
\hline 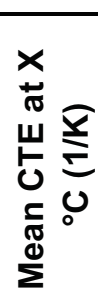 & 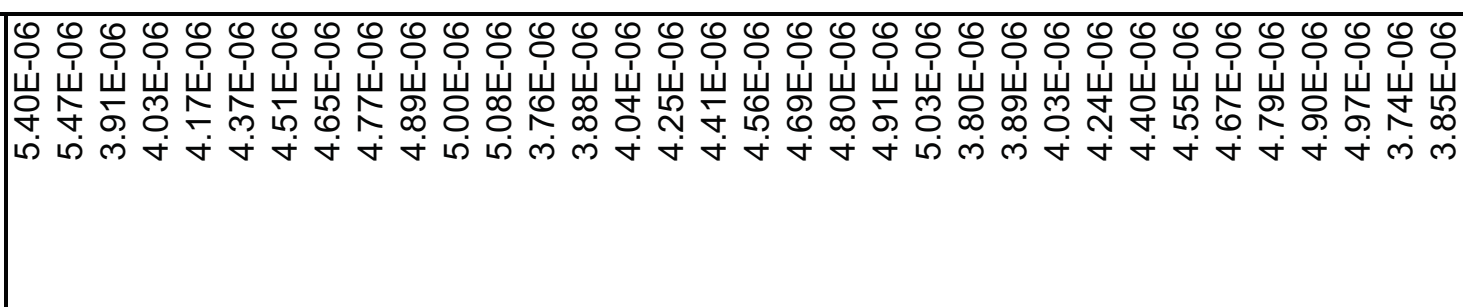 \\
\hline 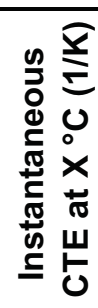 & 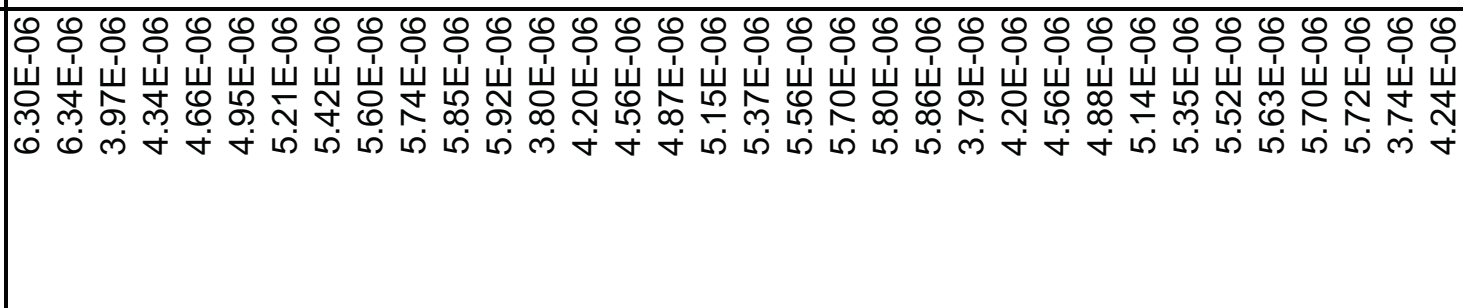 \\
\hline 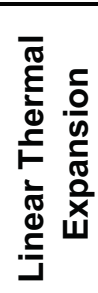 & 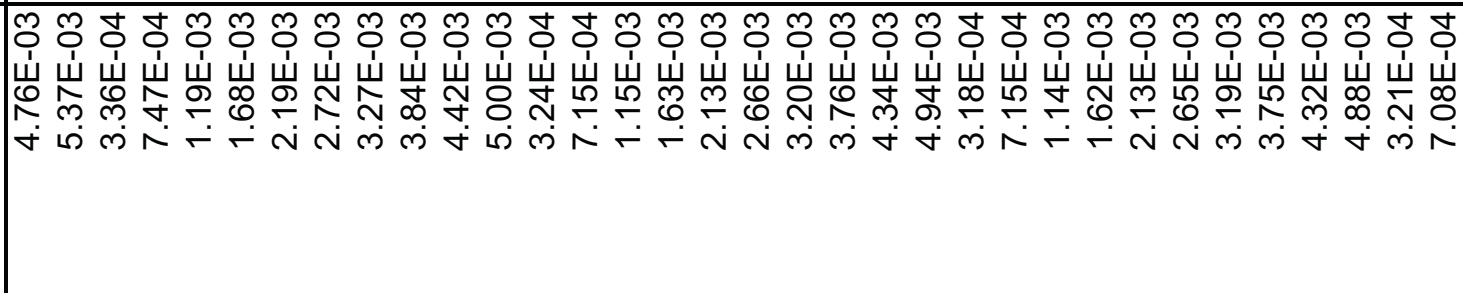 \\
\hline 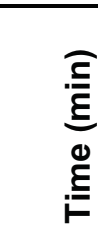 & 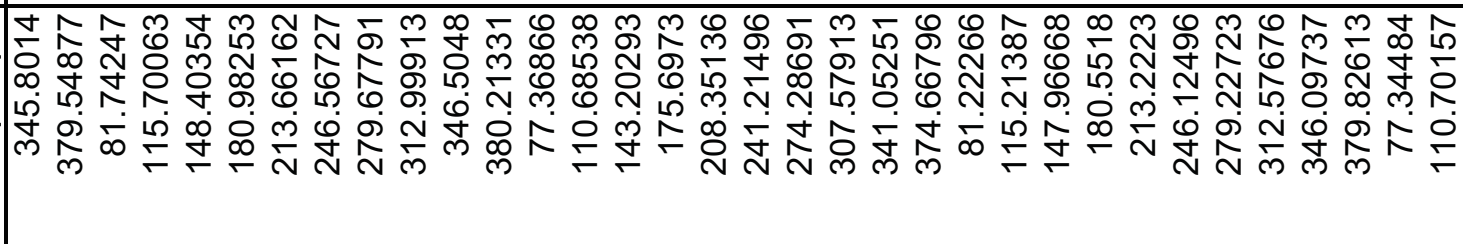 \\
\hline 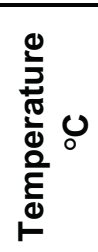 & 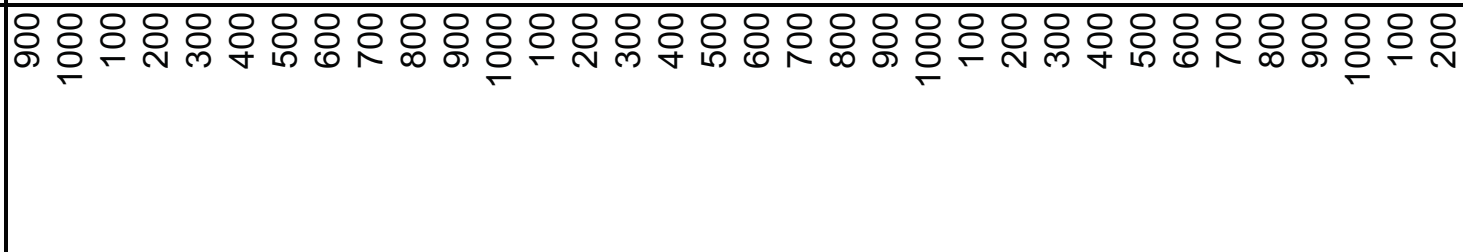 \\
\hline 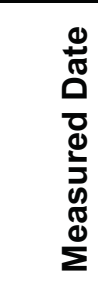 & 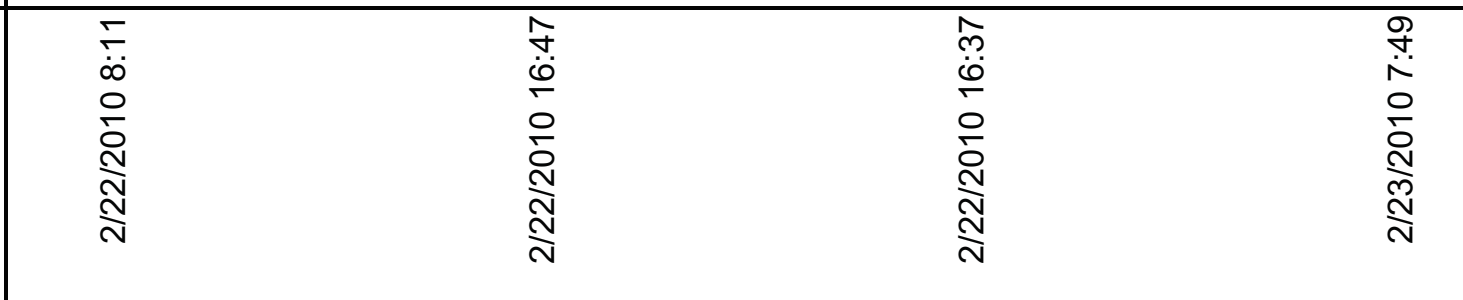 \\
\hline 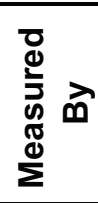 & $\frac{\infty}{0}$ \\
\hline 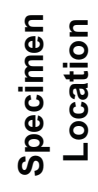 & \\
\hline 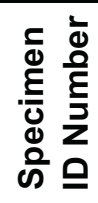 & $\begin{array}{l}5 \\
\stackrel{0}{\circ} \\
\vdots \\
\vdots\end{array}$ \\
\hline
\end{tabular}




\begin{tabular}{|c|c|}
\hline 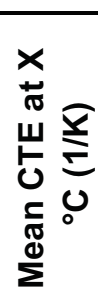 & 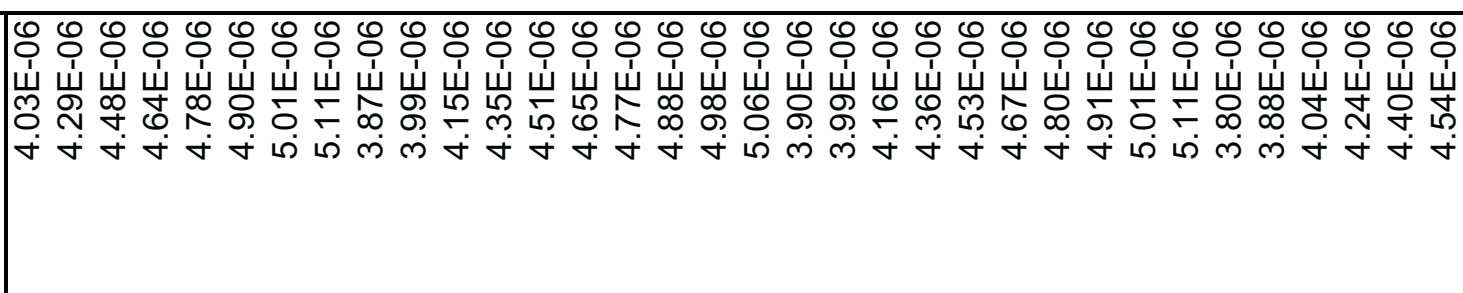 \\
\hline 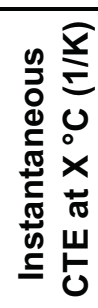 & 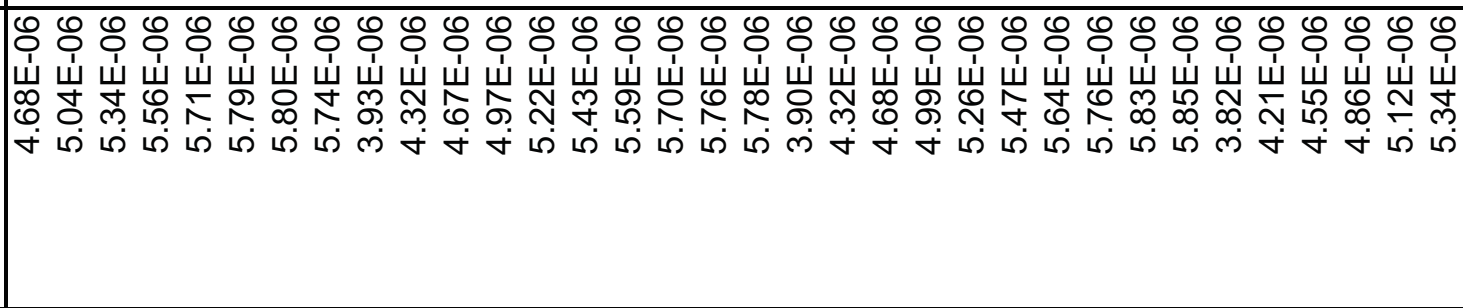 \\
\hline 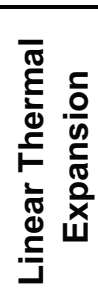 & 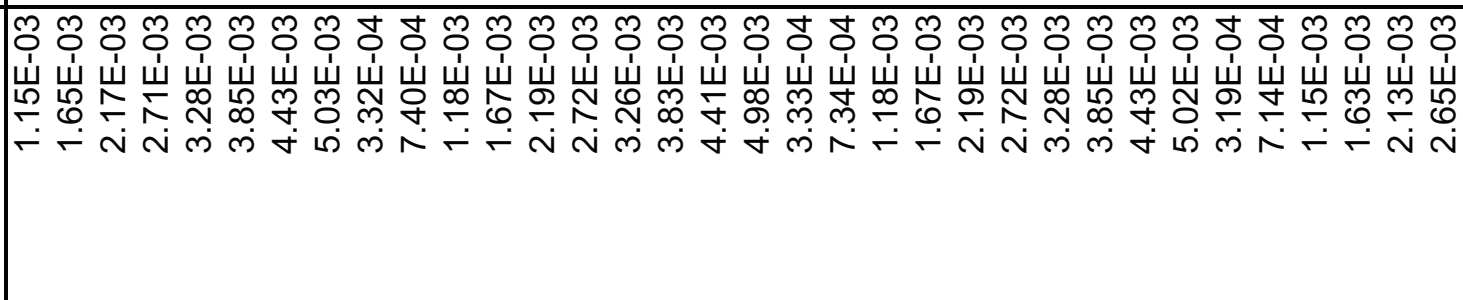 \\
\hline 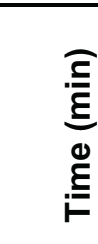 & 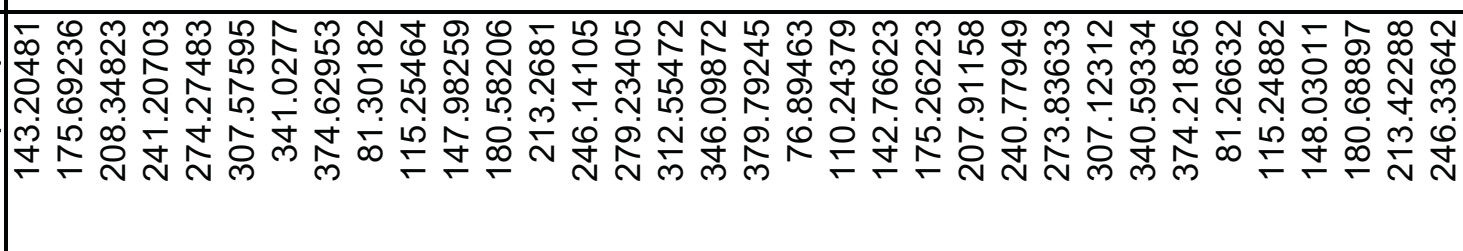 \\
\hline 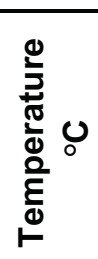 & 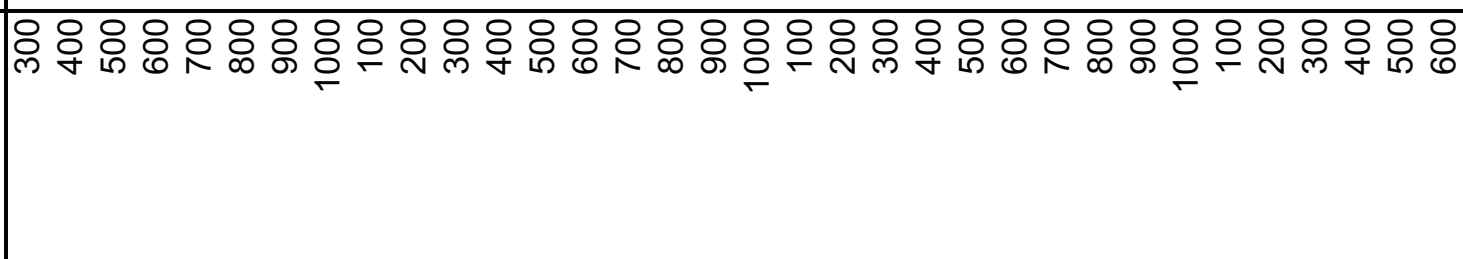 \\
\hline 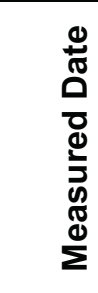 & 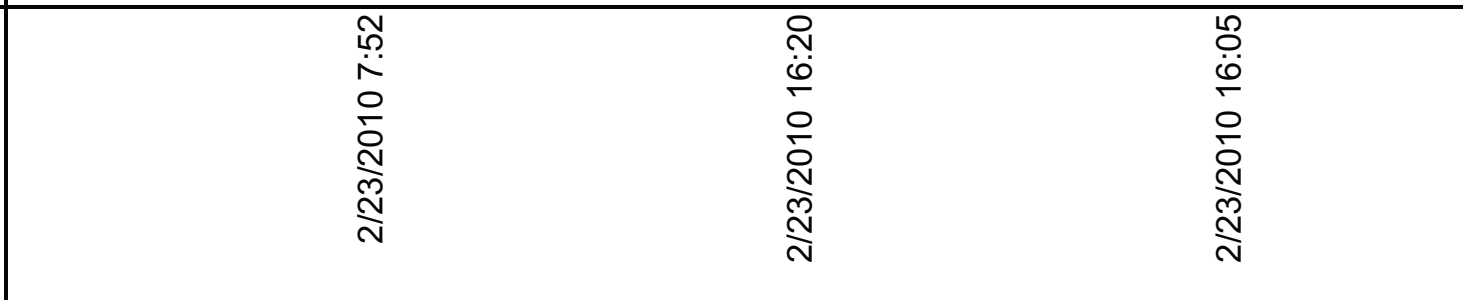 \\
\hline 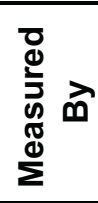 & $\frac{n}{0}$ \\
\hline 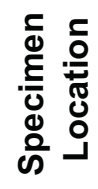 & \\
\hline 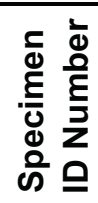 & 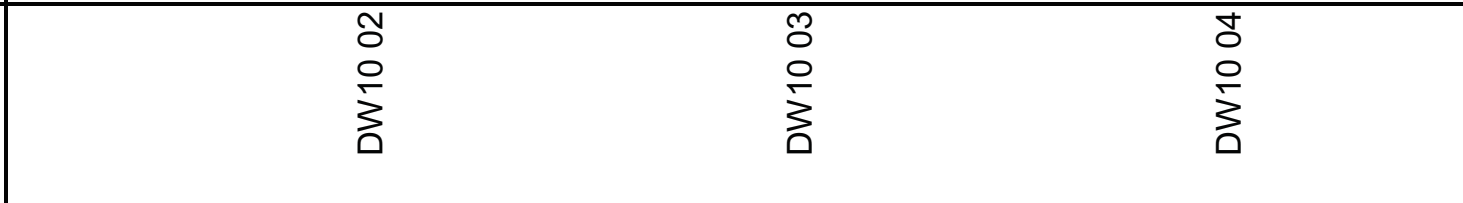 \\
\hline
\end{tabular}




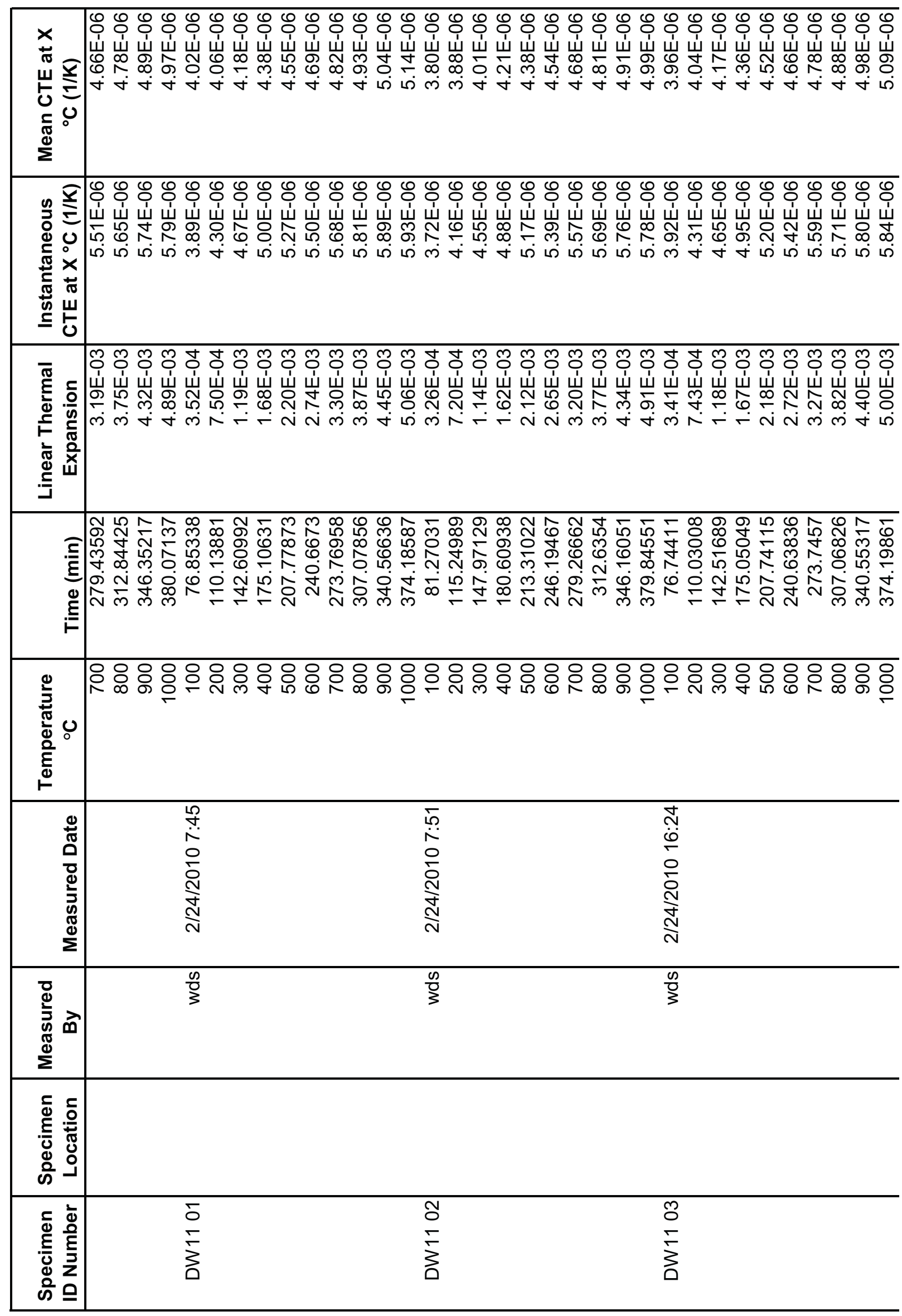




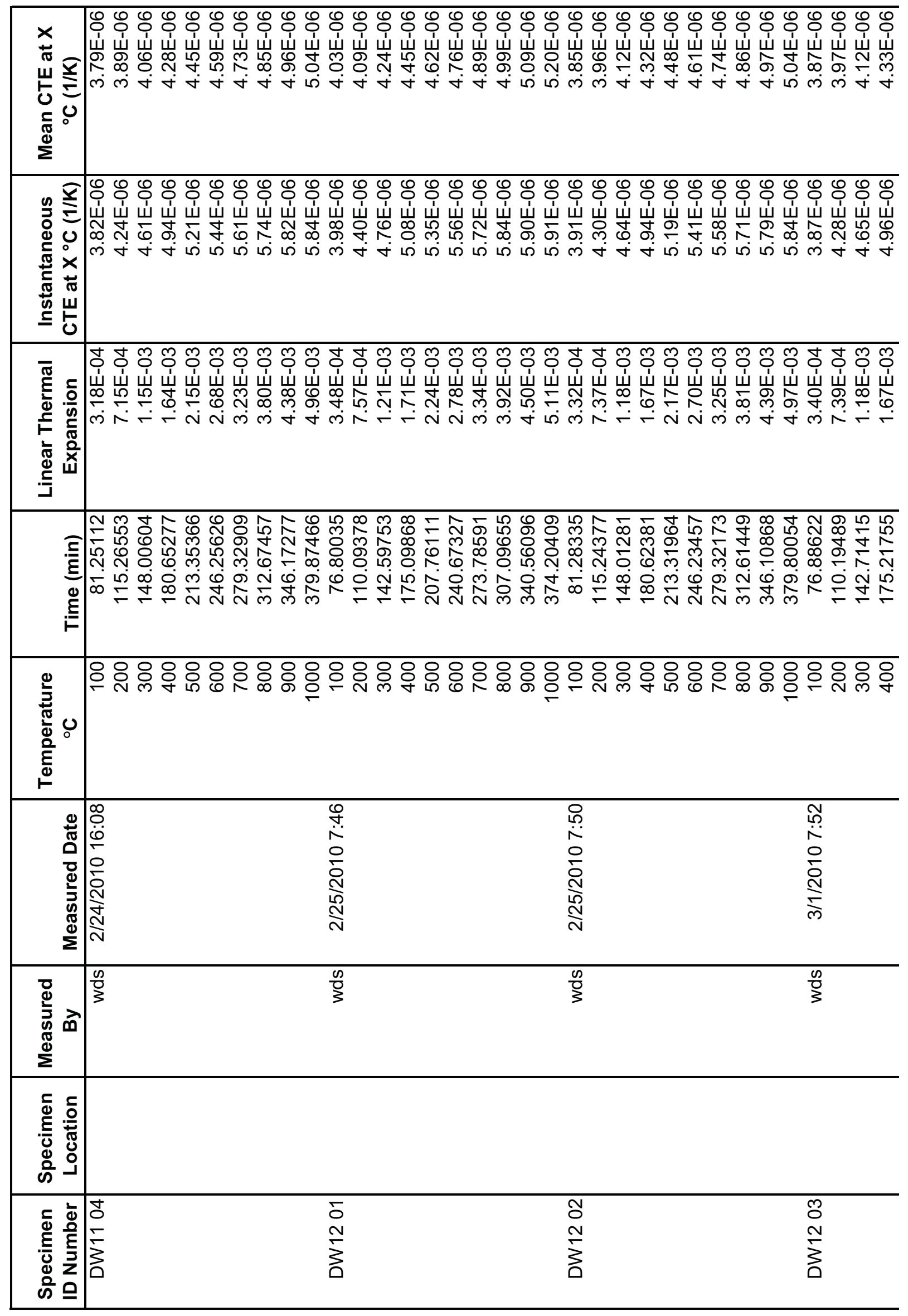




\begin{tabular}{|c|c|}
\hline 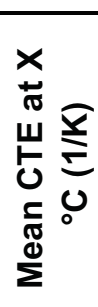 & 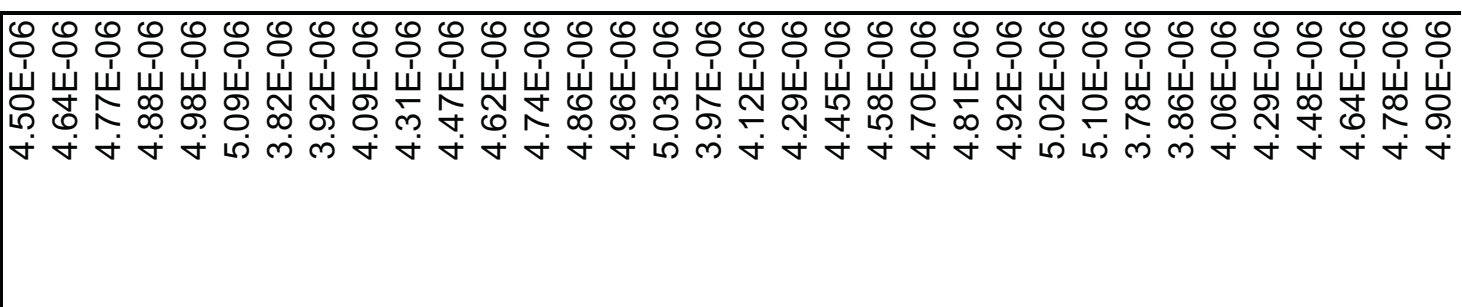 \\
\hline 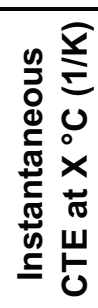 & 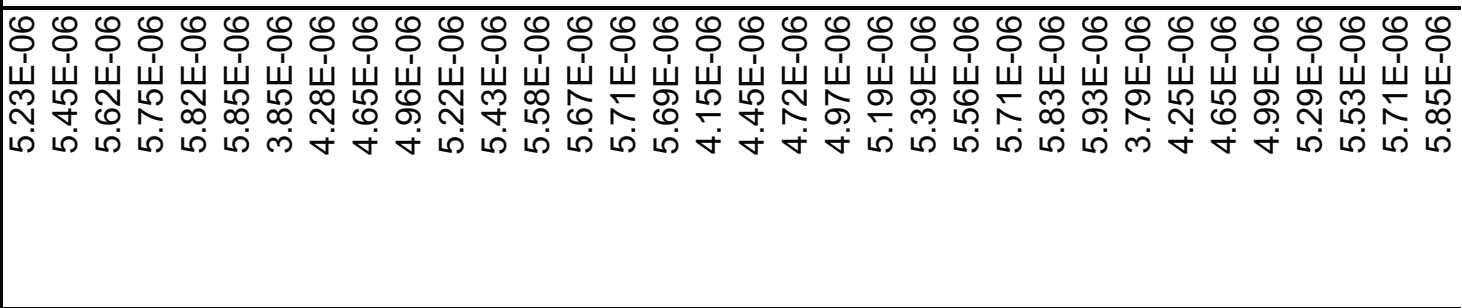 \\
\hline 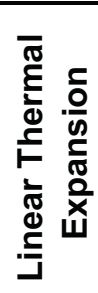 & 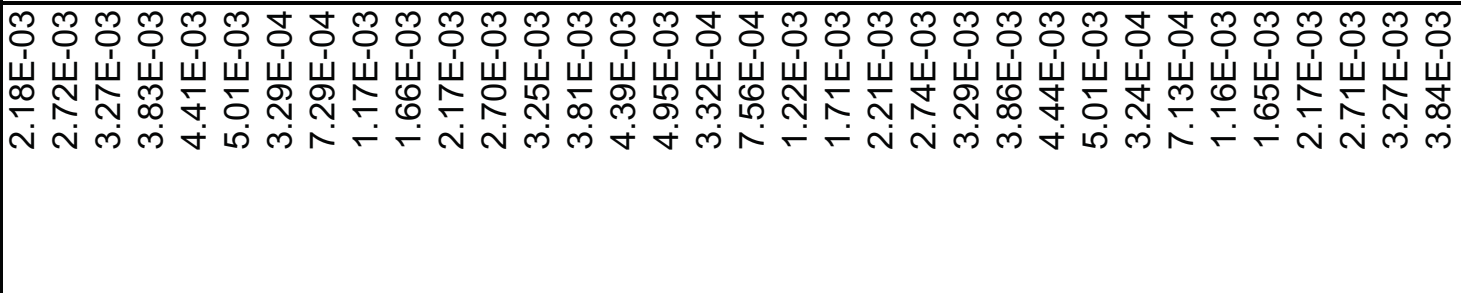 \\
\hline 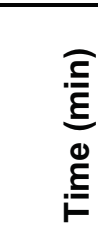 & 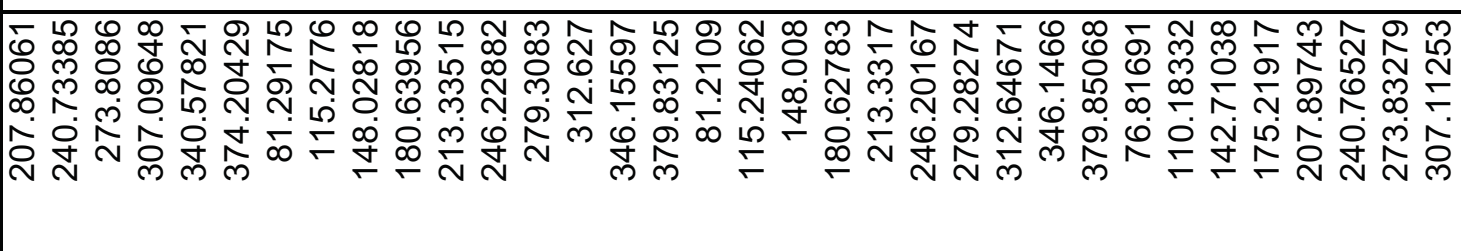 \\
\hline 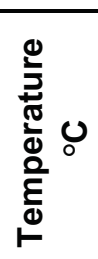 & 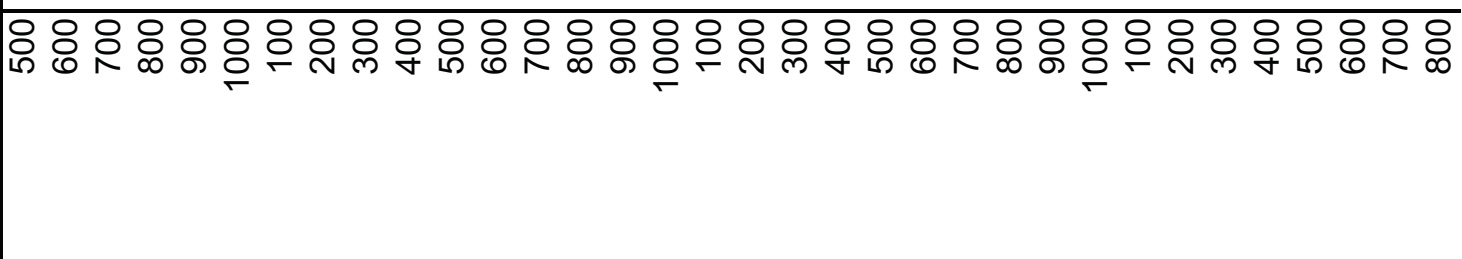 \\
\hline 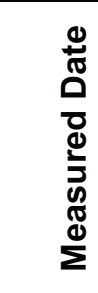 & 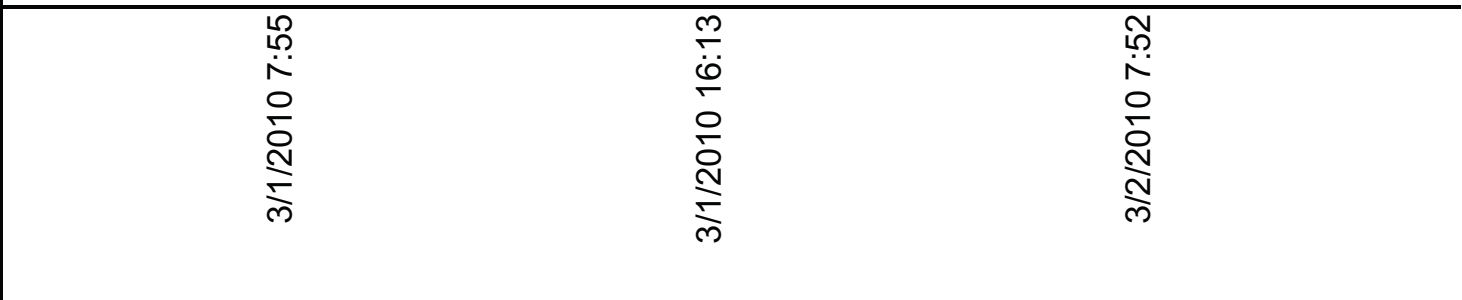 \\
\hline 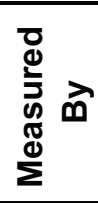 & $\frac{0}{0}$ \\
\hline 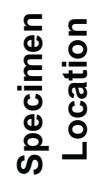 & \\
\hline 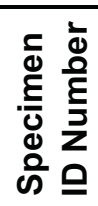 & 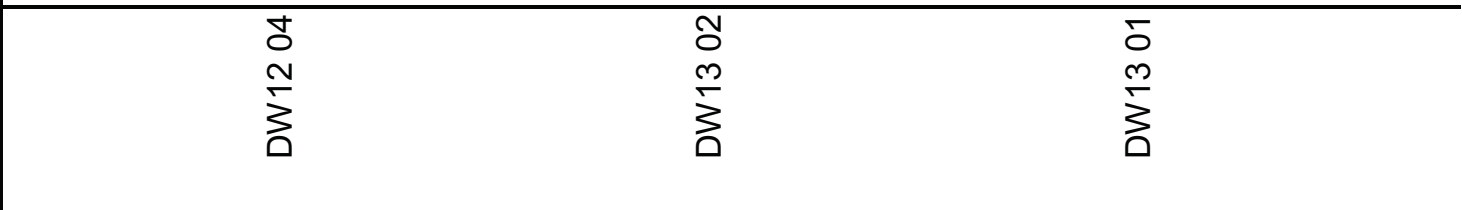 \\
\hline
\end{tabular}




\begin{tabular}{|c|c|}
\hline 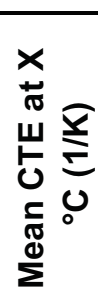 & 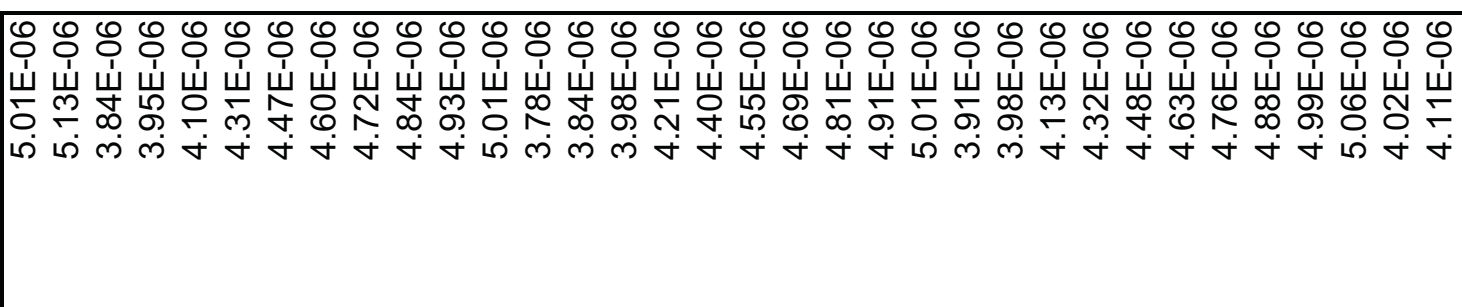 \\
\hline 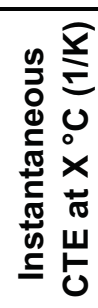 & 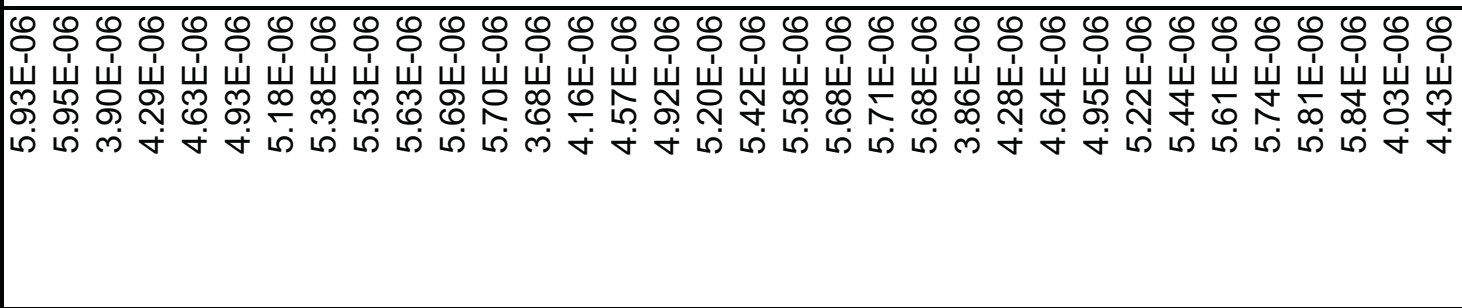 \\
\hline 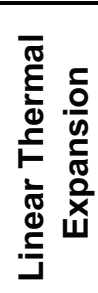 & 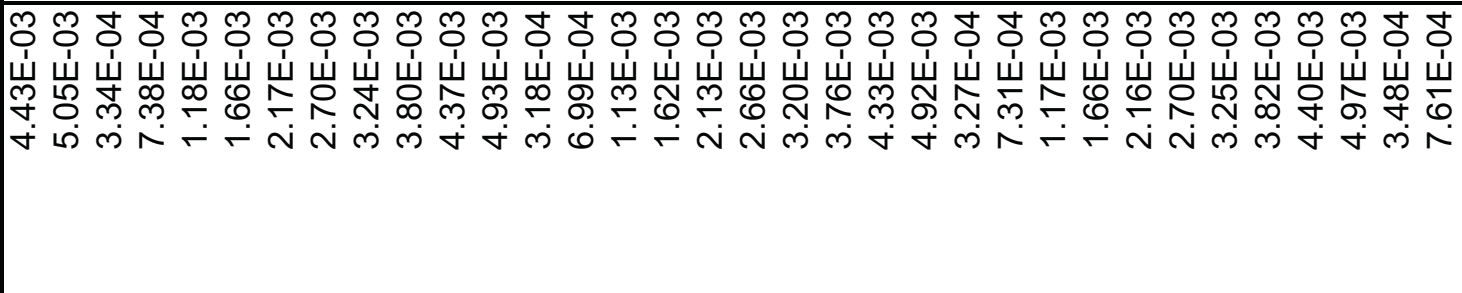 \\
\hline 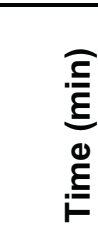 & 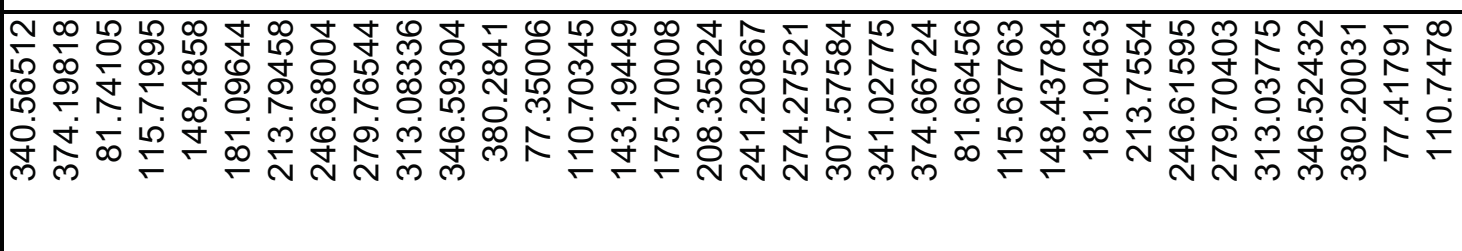 \\
\hline 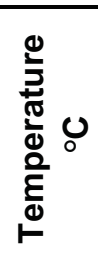 & 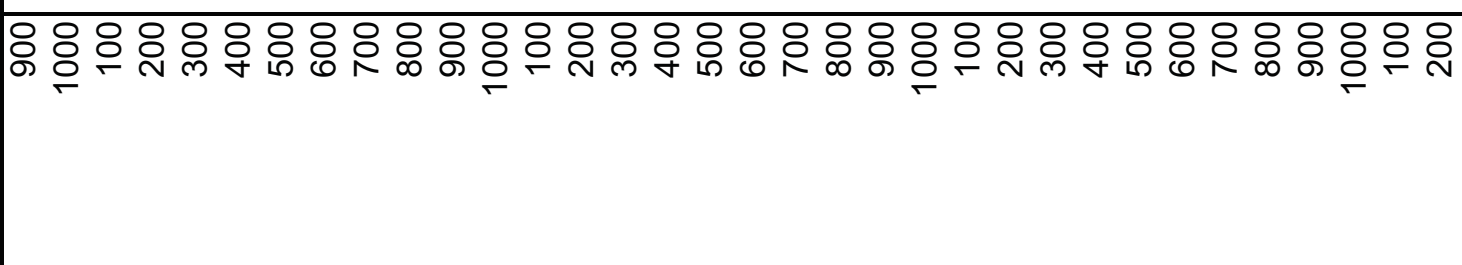 \\
\hline 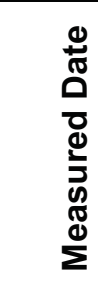 & 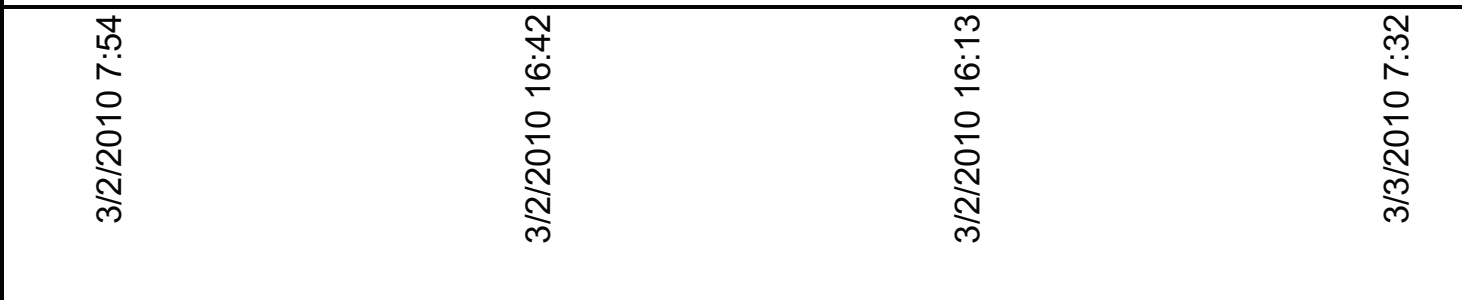 \\
\hline 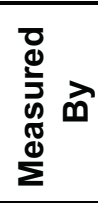 & $\frac{1}{3}$ \\
\hline 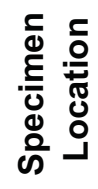 & \\
\hline 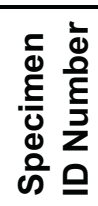 & 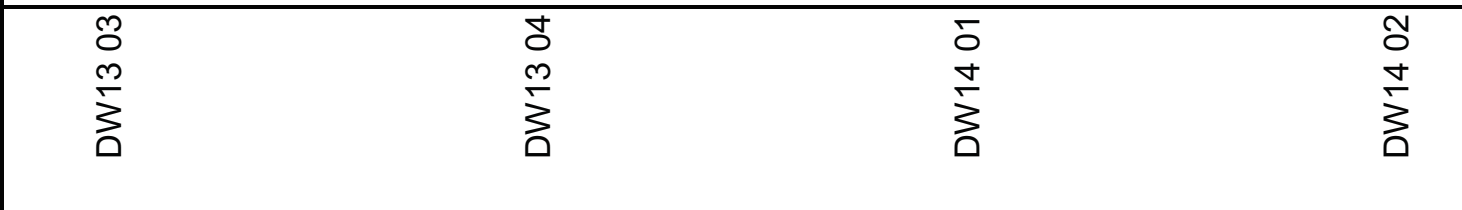 \\
\hline
\end{tabular}




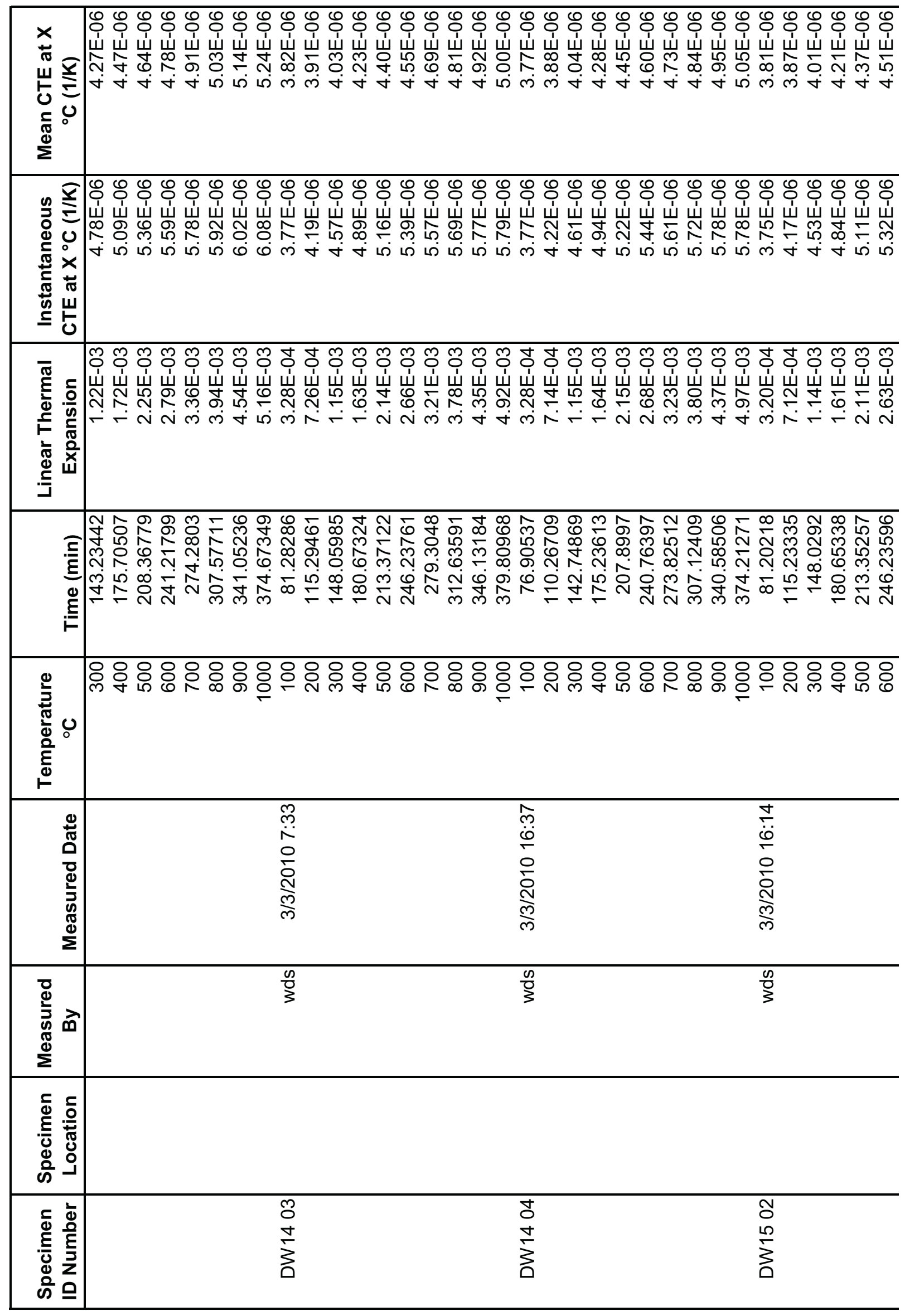




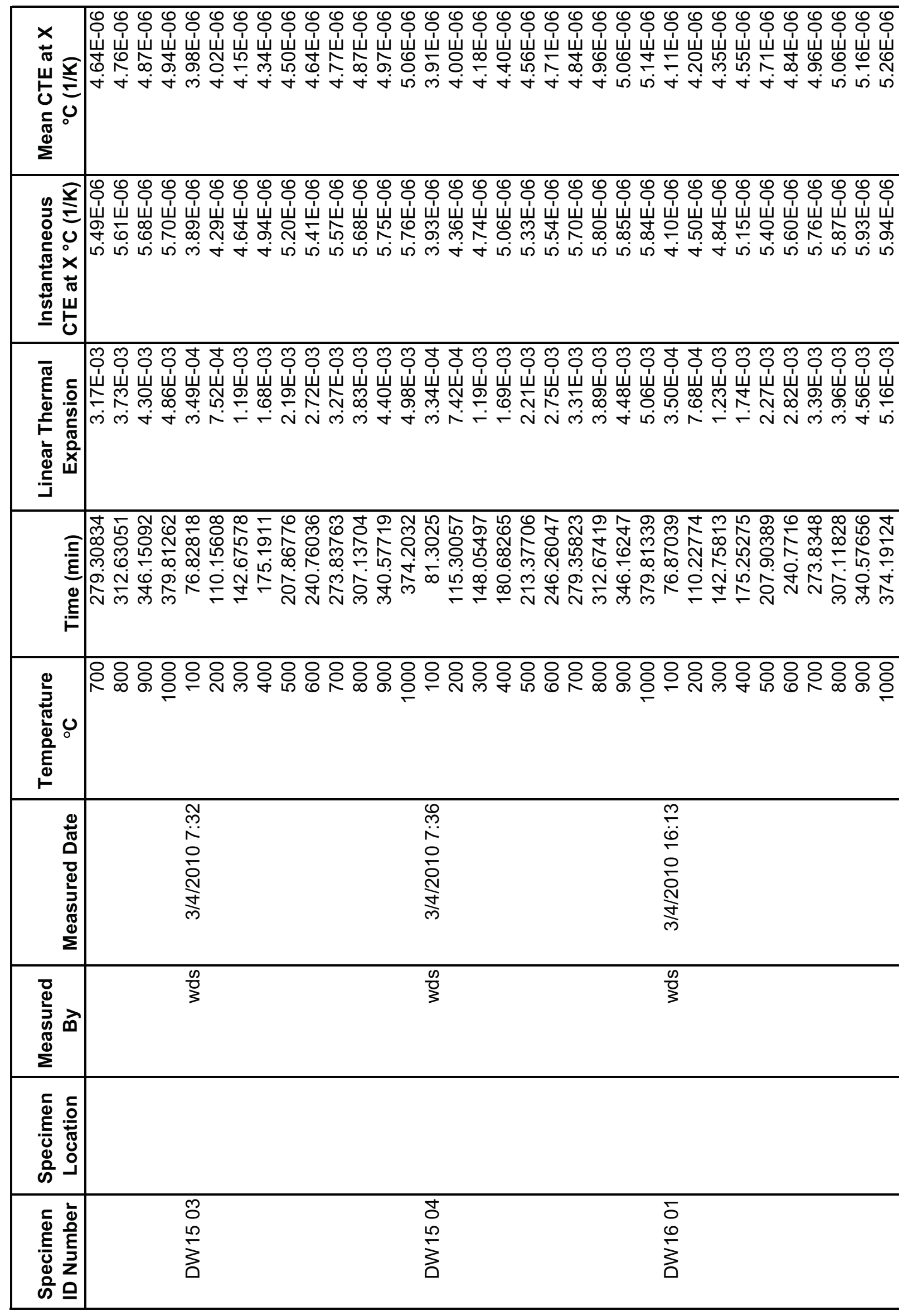




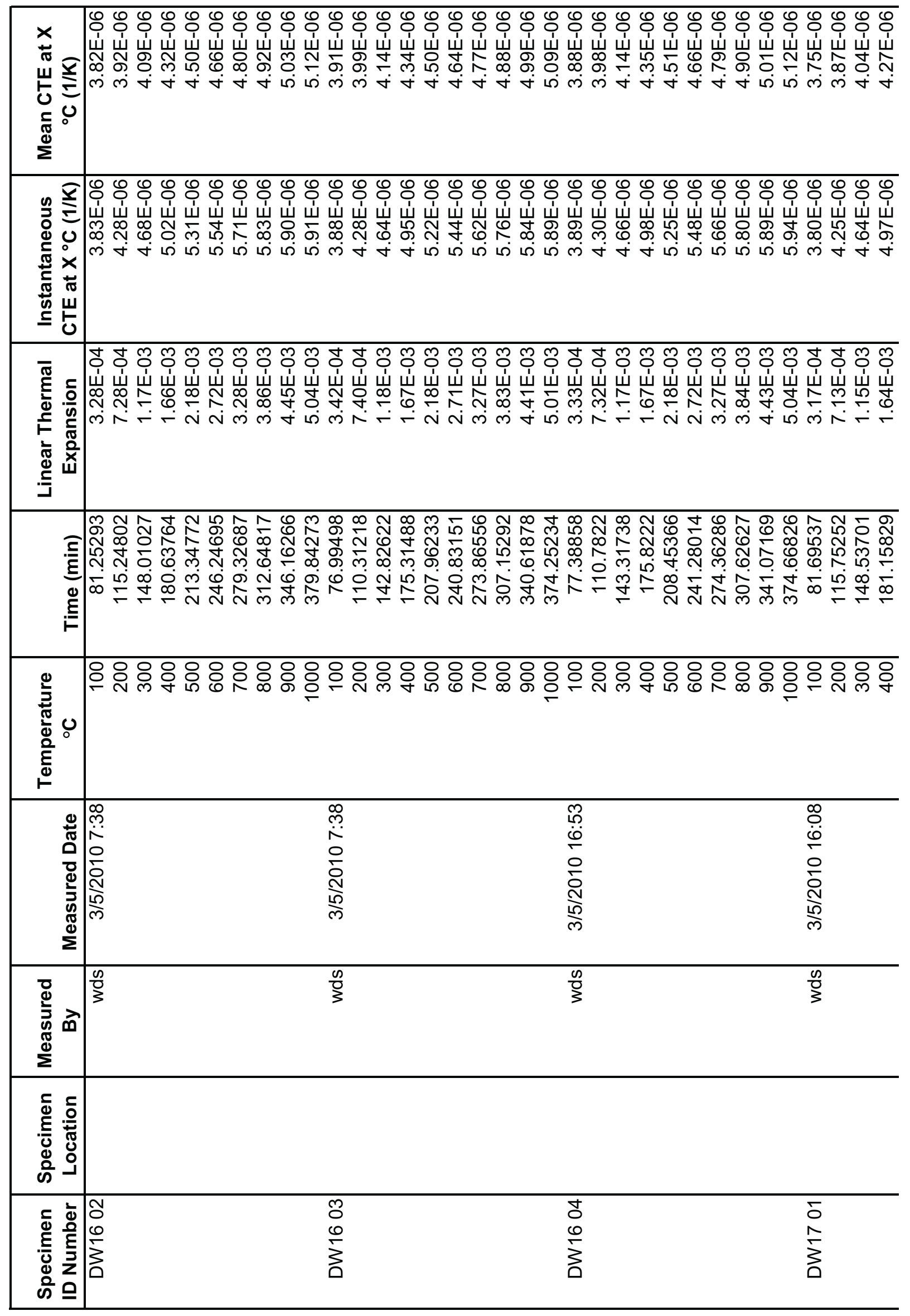




\begin{tabular}{|c|c|}
\hline 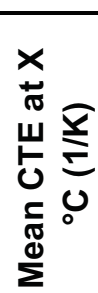 & 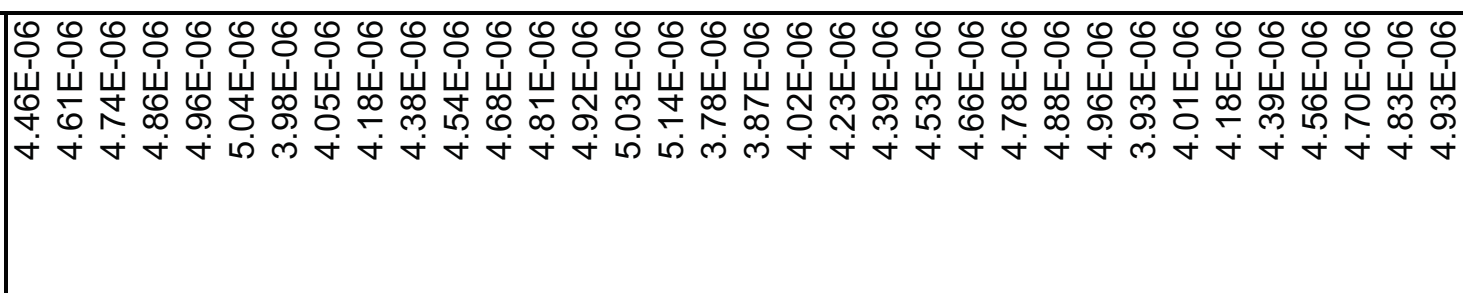 \\
\hline 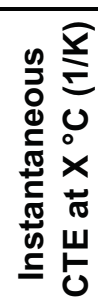 & 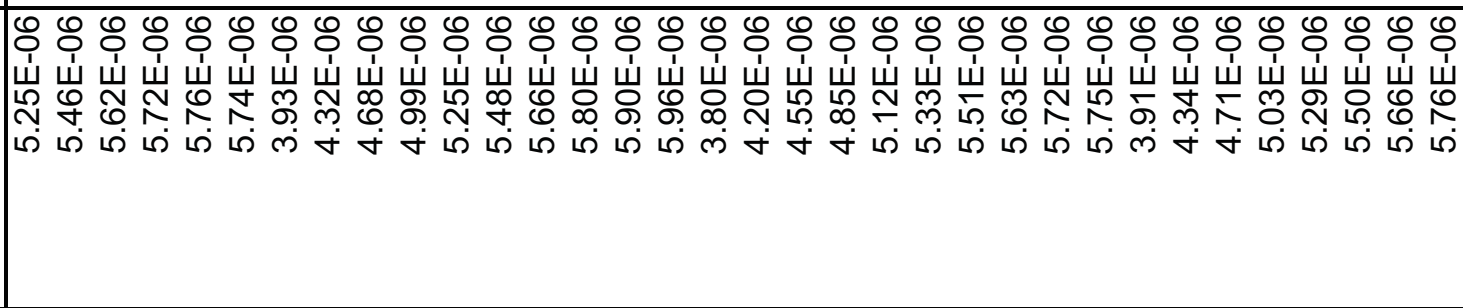 \\
\hline 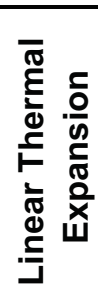 & 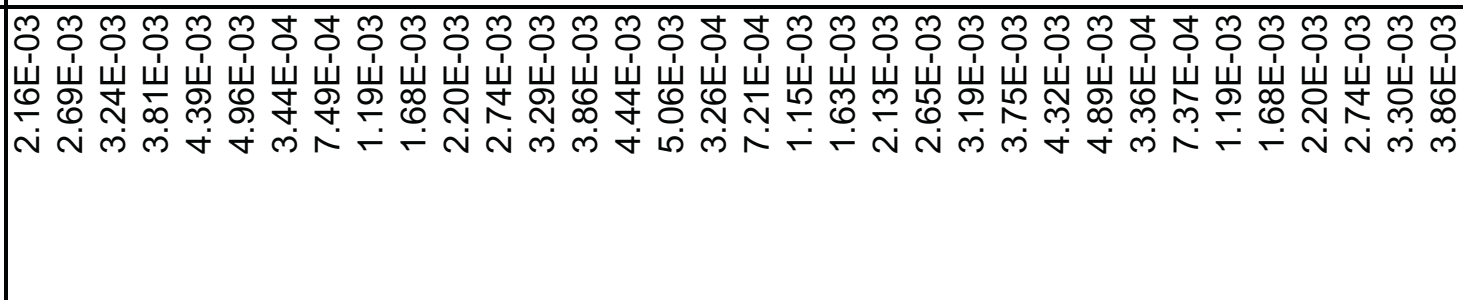 \\
\hline 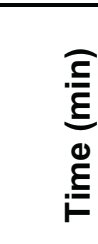 & 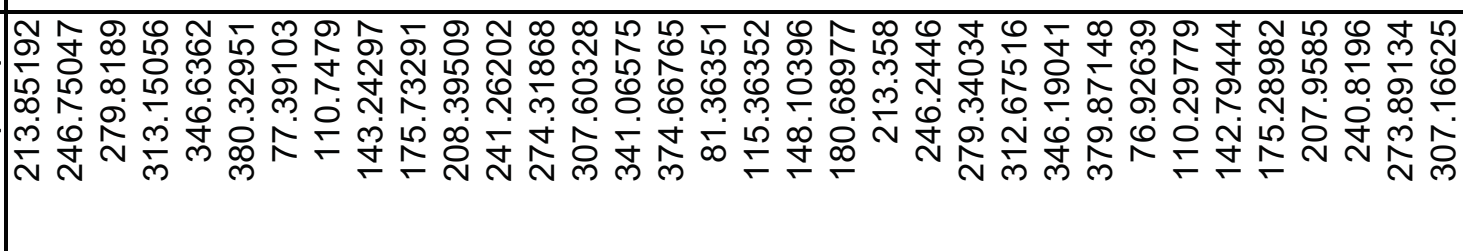 \\
\hline 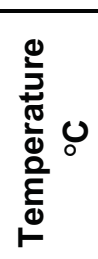 & 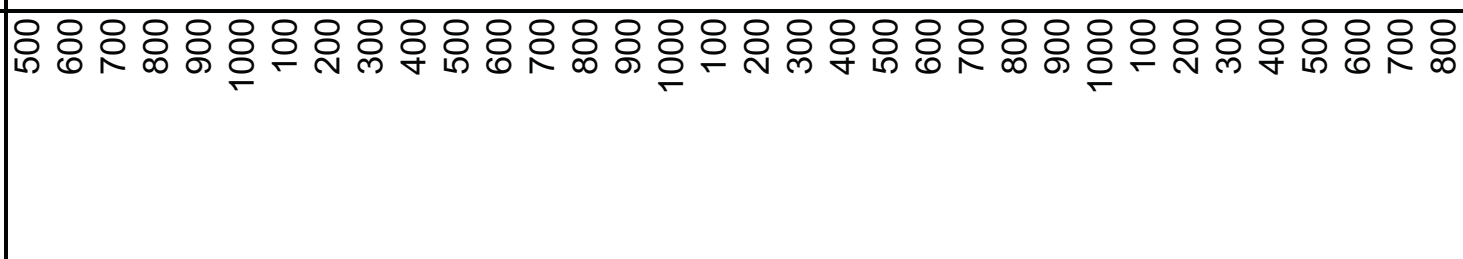 \\
\hline 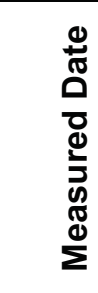 & 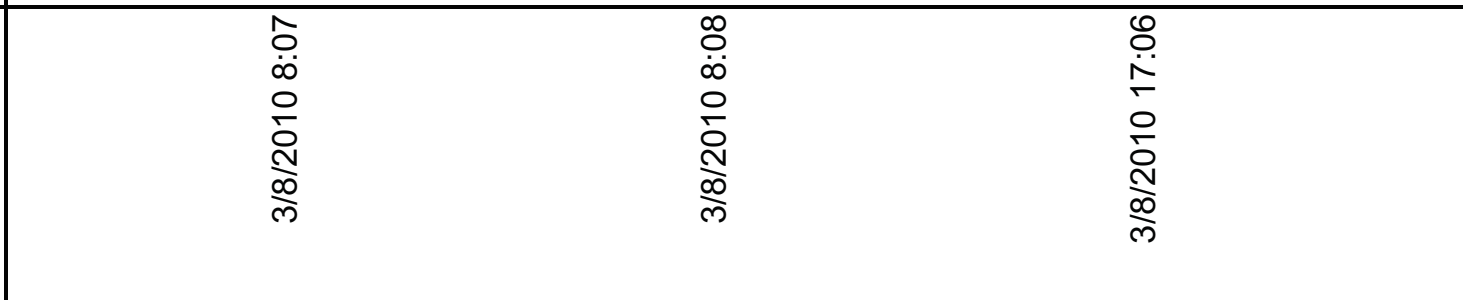 \\
\hline 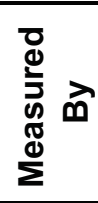 & $\frac{n}{0}$ \\
\hline 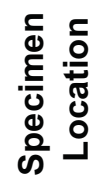 & \\
\hline 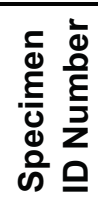 & $\stackrel{\text { J }}{\stackrel{1}{2}}$ \\
\hline
\end{tabular}




\begin{tabular}{|c|c|}
\hline 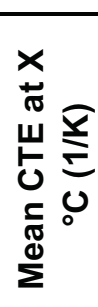 & 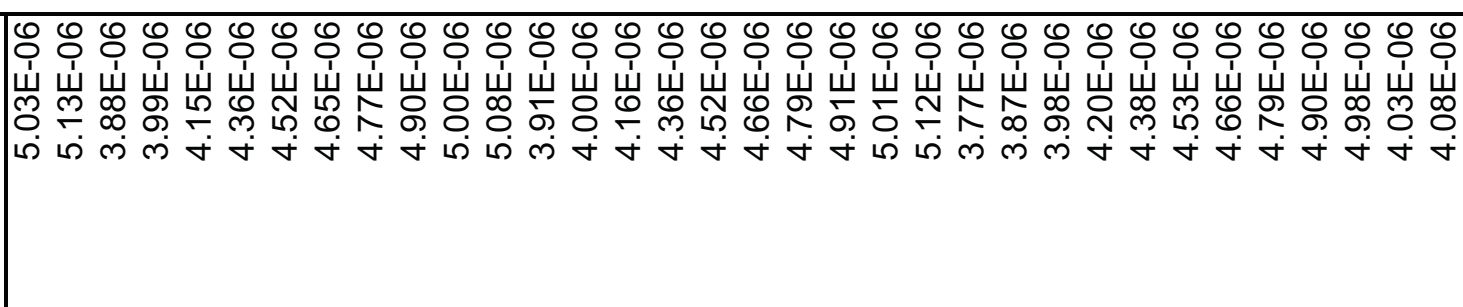 \\
\hline 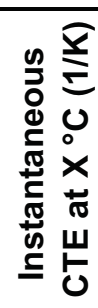 & 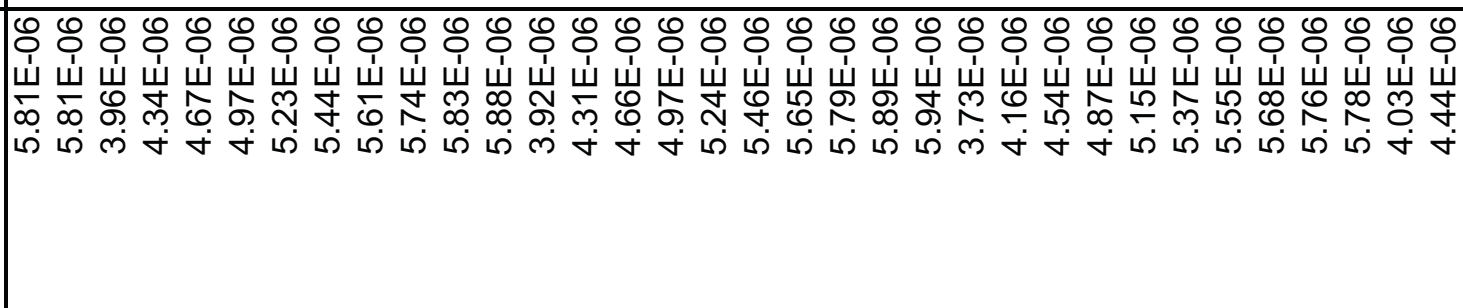 \\
\hline 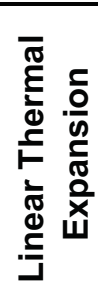 & 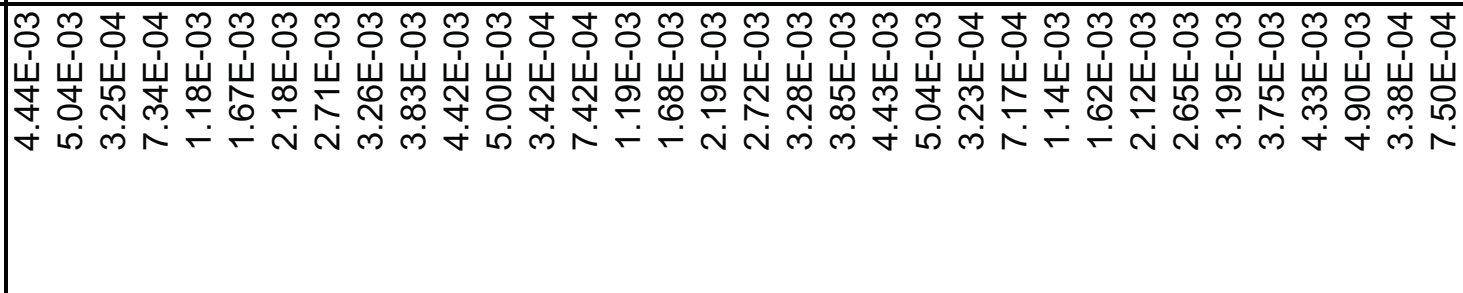 \\
\hline 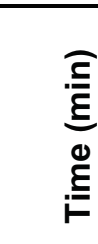 & 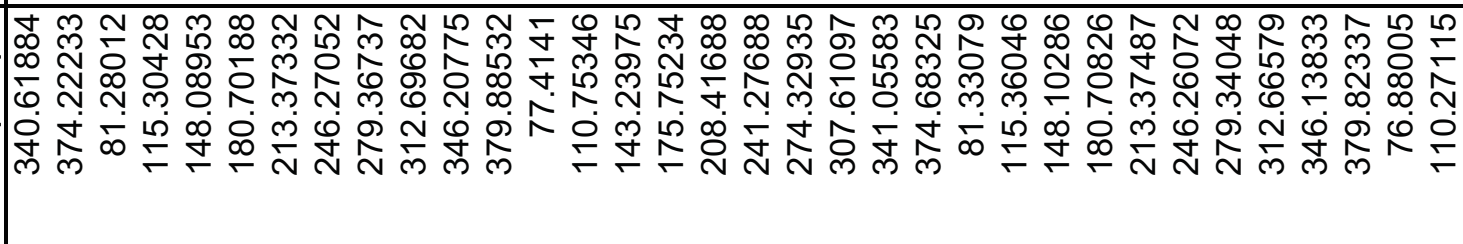 \\
\hline 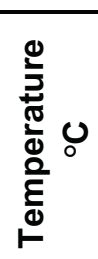 & 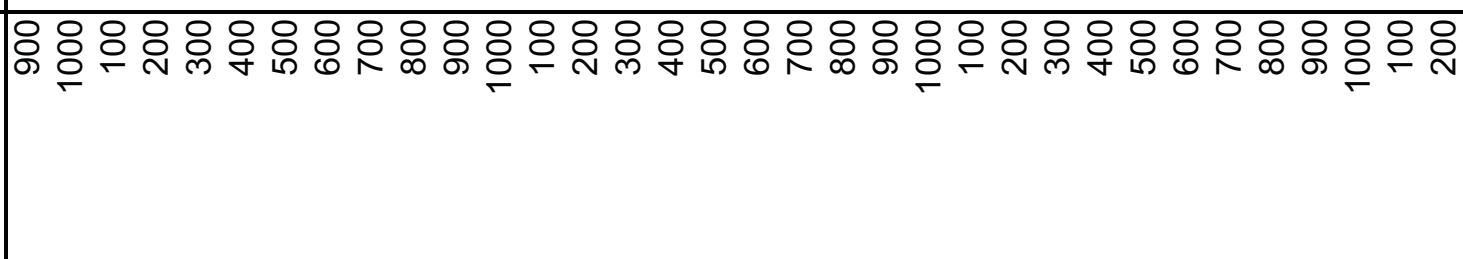 \\
\hline 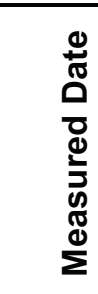 & 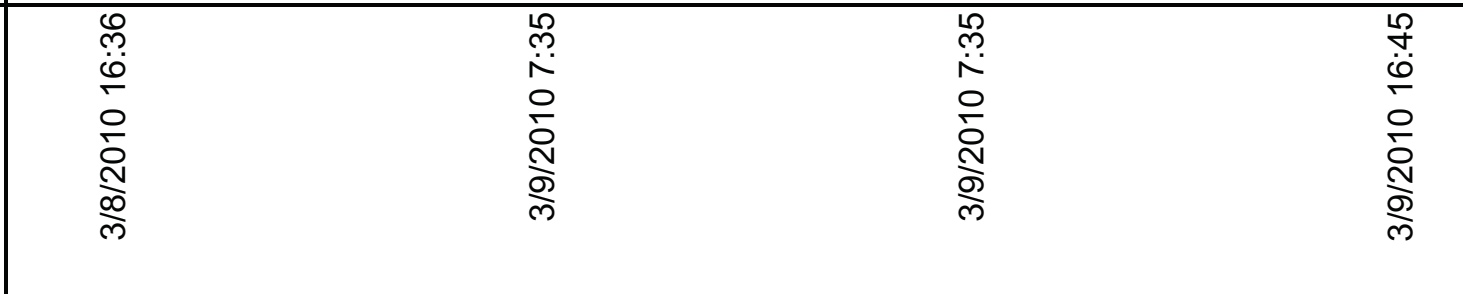 \\
\hline 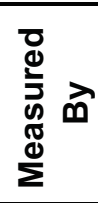 & $\frac{0}{3}$ \\
\hline 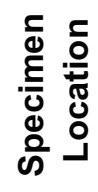 & \\
\hline 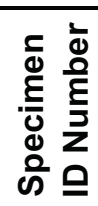 & 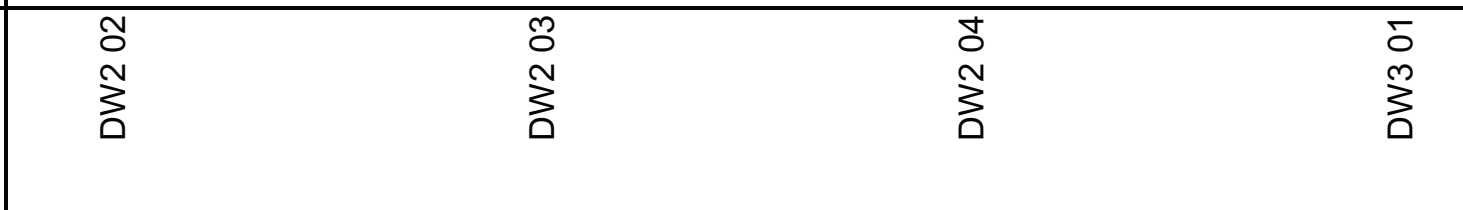 \\
\hline
\end{tabular}




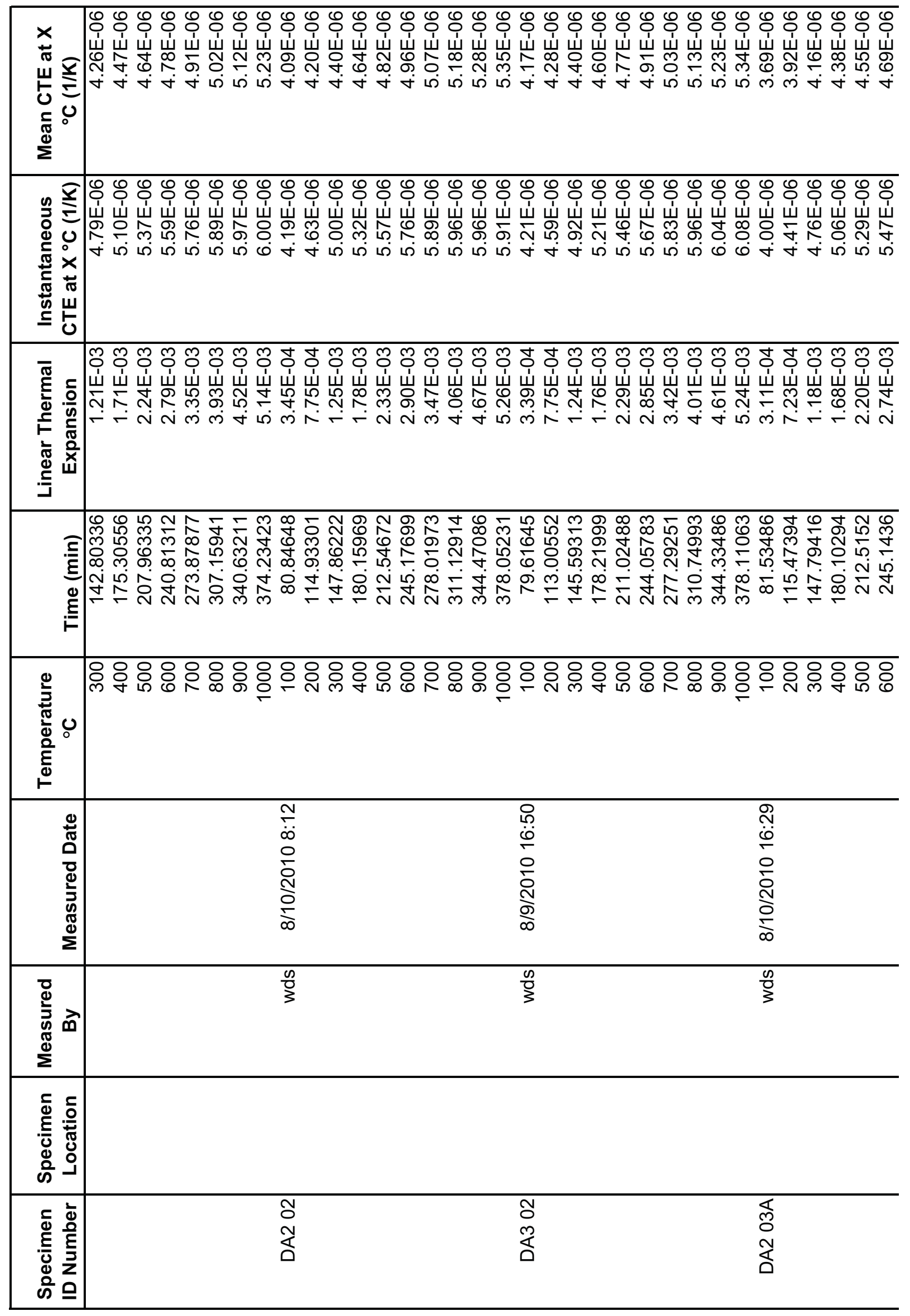




\begin{tabular}{|c|c|}
\hline 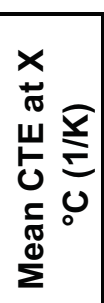 & 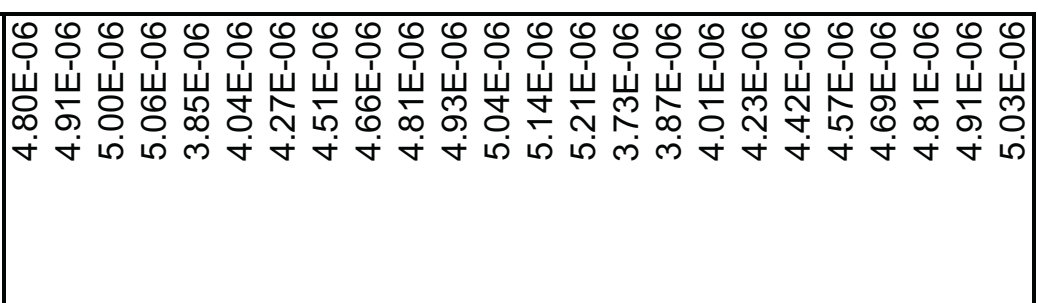 \\
\hline 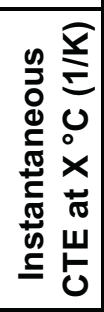 & 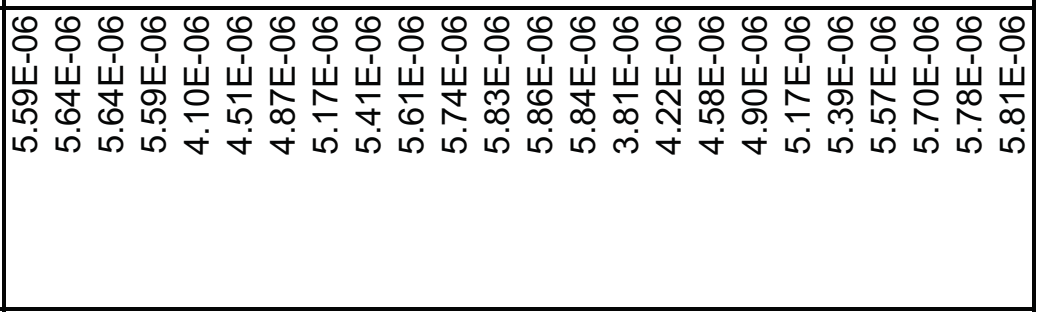 \\
\hline 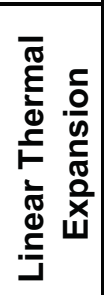 & 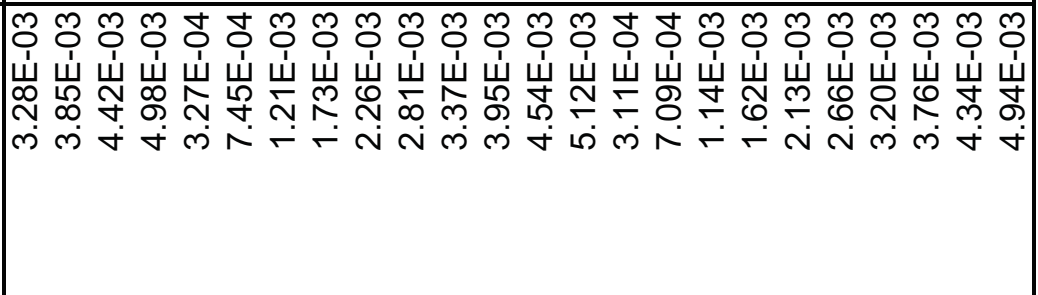 \\
\hline 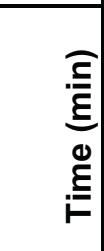 & 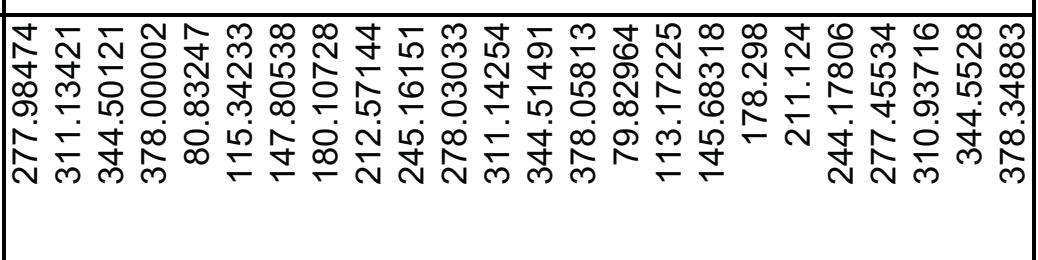 \\
\hline 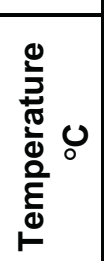 & 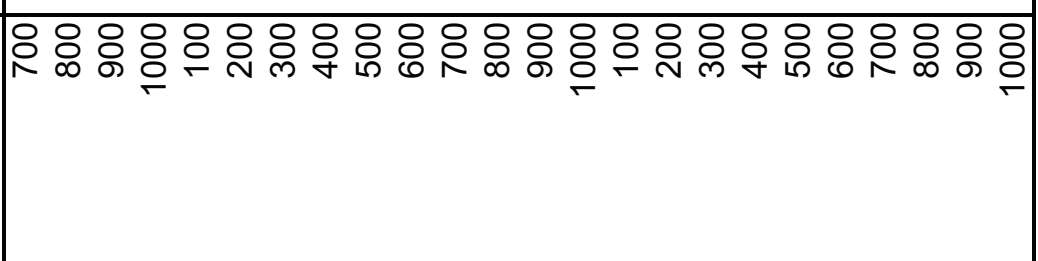 \\
\hline 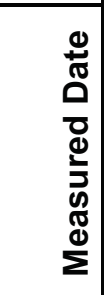 & 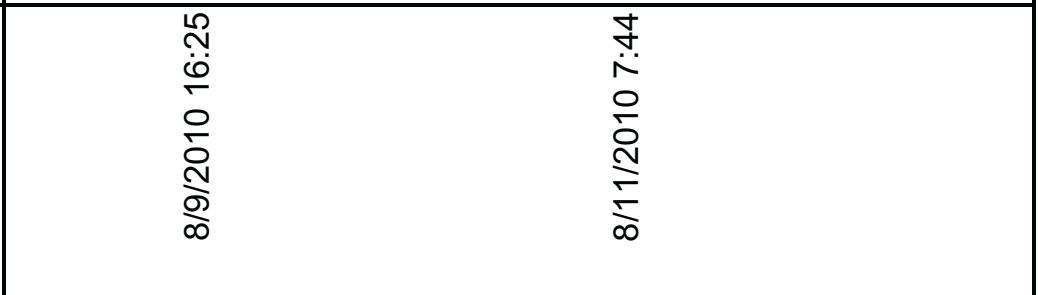 \\
\hline 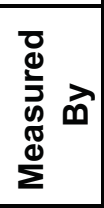 & $\sum_{3}^{\infty}$ \\
\hline 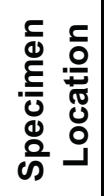 & \\
\hline 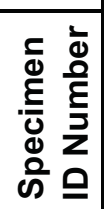 & 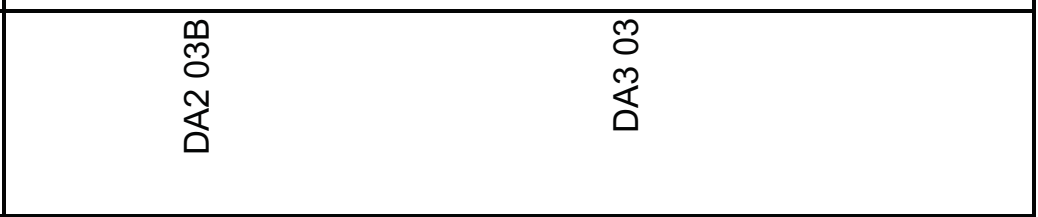 \\
\hline
\end{tabular}




\begin{tabular}{|c|c|c|c|c|}
\hline 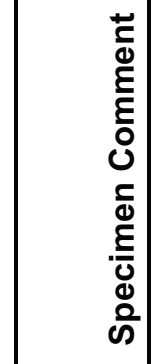 & & & & \\
\hline$\underset{⿱ 亠 䒑}{Q}$ & 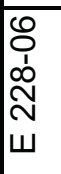 & $\begin{array}{l}0 \\
\\
1 \\
\infty \\
\text { N } \\
w\end{array}$ & 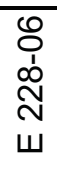 & 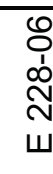 \\
\hline 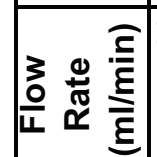 & 8 & 8 & 8 & 8 \\
\hline 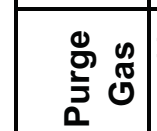 & $\Phi$ & $\frac{\oplus}{1}$ & $\stackrel{\Phi}{I}$ & $\stackrel{\oplus}{I}$ \\
\hline 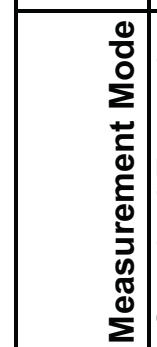 & 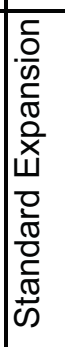 & 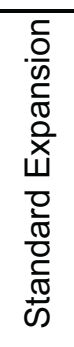 & 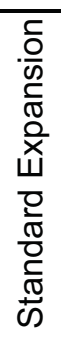 & 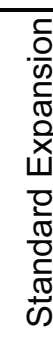 \\
\hline 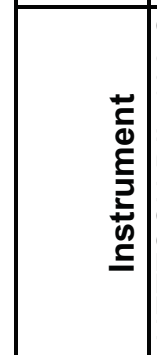 & 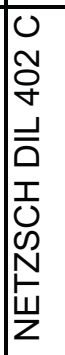 & 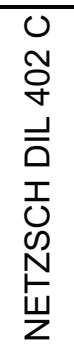 & 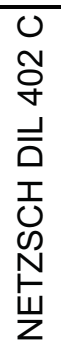 & 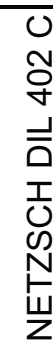 \\
\hline 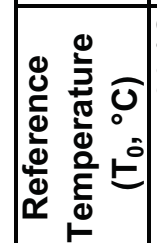 & o & $\begin{array}{l}0 \\
0 \\
2\end{array}$ & $\begin{array}{l}0 \\
0 \\
i\end{array}$ & $\begin{array}{l}0 \\
0 \\
0\end{array}$ \\
\hline 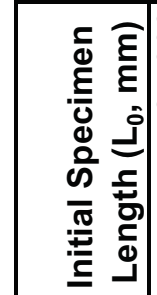 & & 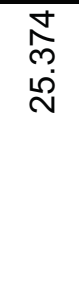 & 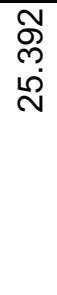 & 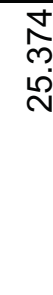 \\
\hline 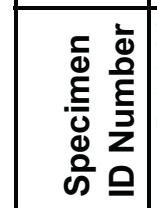 & 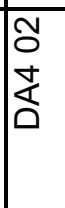 & $\begin{array}{l}\text { m } \\
0 \\
+ \\
\Delta\end{array}$ & $\begin{array}{l}\overline{0} \\
\text { L̊ } \\
0\end{array}$ & 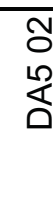 \\
\hline
\end{tabular}




\begin{tabular}{|c|c|c|c|}
\hline 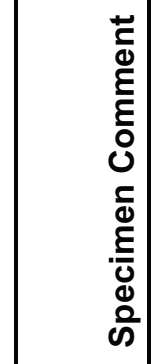 & & & \\
\hline$\underset{\substack{2 \\
\&}}{\sum}$ & $\begin{array}{l}\text { Oे } \\
\dot{1} \\
\text { N } \\
\text { w }\end{array}$ & 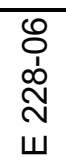 & 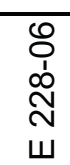 \\
\hline 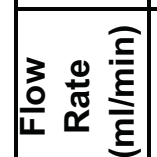 & 8 & 8 & 8 \\
\hline 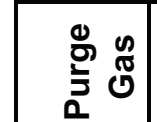 & $\frac{\Phi}{1}$ & $\frac{\Phi}{I}$ & $\frac{\Phi}{I}$ \\
\hline 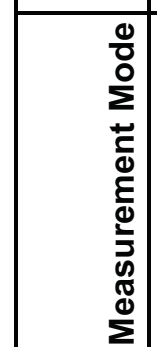 & 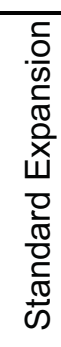 & 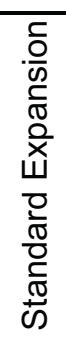 & 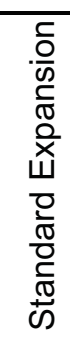 \\
\hline 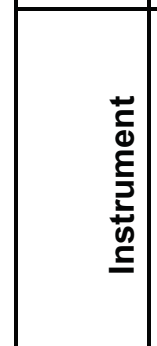 & 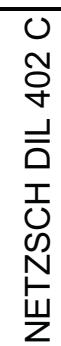 & 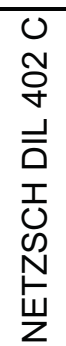 & 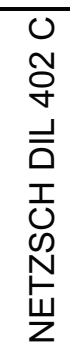 \\
\hline 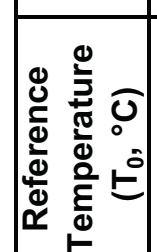 & $\begin{array}{l}0 \\
\vdots \\
\stackrel{N}{0}\end{array}$ & $\begin{array}{l}\text { U } \\
\text { Oे }\end{array}$ & $\begin{array}{l}\text { U } \\
0 \\
\text { N }\end{array}$ \\
\hline 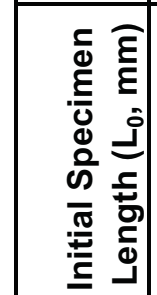 & $\begin{array}{l}\text { o } \\
\dot{\rho} \\
\stackrel{\rho}{N}\end{array}$ & 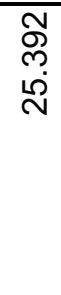 & 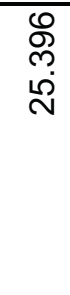 \\
\hline 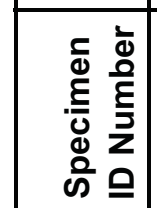 & 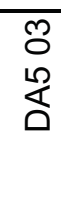 & $\begin{array}{l}\bar{\delta} \\
0 \\
0\end{array}$ & 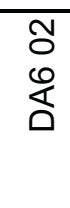 \\
\hline
\end{tabular}




\begin{tabular}{|c|c|c|c|c|}
\hline 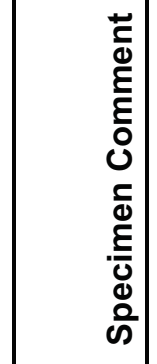 & & & & \\
\hline$\underset{⿱ 乛 龰}{\mathbf{c}}$ & 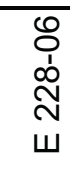 & $\begin{array}{l}\text { Oo } \\
0 \\
\text { N } \\
\text { w }\end{array}$ & 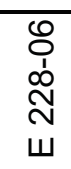 & 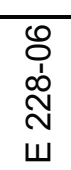 \\
\hline 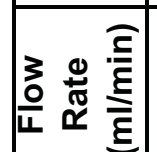 & 8 & 8 & 8 & 8 \\
\hline \begin{tabular}{ll}
0 \\
\multirow{3}{*}{} \\
0
\end{tabular} & 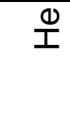 & 里 & $\frac{\Phi}{I}$ & $\frac{\mathbb{O}}{I}$ \\
\hline 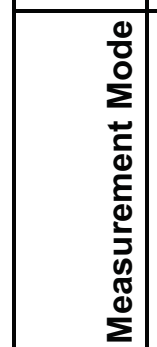 & 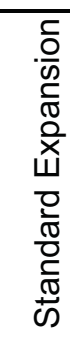 & 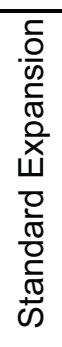 & 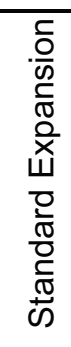 & 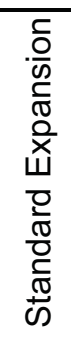 \\
\hline 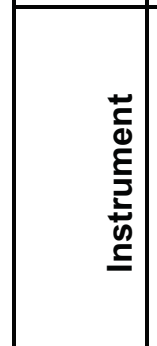 & 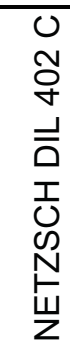 & 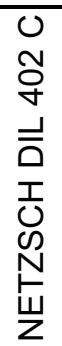 & 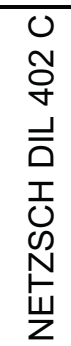 & 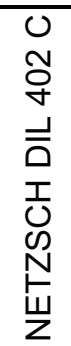 \\
\hline 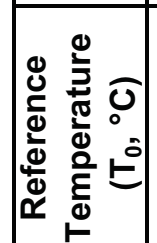 & $\begin{array}{l}0 \\
0 \\
0 \\
N\end{array}$ & $\begin{array}{l}\text { U } \\
\text { N }\end{array}$ & $\begin{array}{l}0 \\
0 \\
0\end{array}$ & $\begin{array}{l}0 \\
0 \\
\text { Oे }\end{array}$ \\
\hline 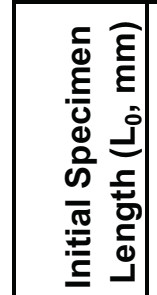 & $\begin{array}{l}\hat{\text { Dे }} \\
\text { dֶ } \\
\text { N. }\end{array}$ & $\begin{array}{l}\text { î } \\
\hat{N} \\
\stackrel{\omega}{\hat{~}}\end{array}$ & 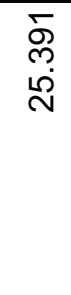 & $\begin{array}{l}\bar{N} \\
\hat{N} \\
\stackrel{d}{N}\end{array}$ \\
\hline 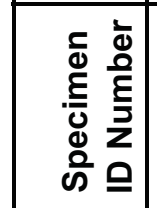 & \begin{tabular}{l}
5 \\
0 \\
\multirow{\alpha}{\Delta}{}
\end{tabular} & $\begin{array}{l}\overline{0} \\
\bar{z} \\
\overline{3}\end{array}$ & $\begin{array}{l}\text { No } \\
\vdots \\
\vdots \\
\vdots\end{array}$ & $\begin{array}{l}\overline{0} \\
\vdots \\
\vdots \\
\vdots\end{array}$ \\
\hline
\end{tabular}




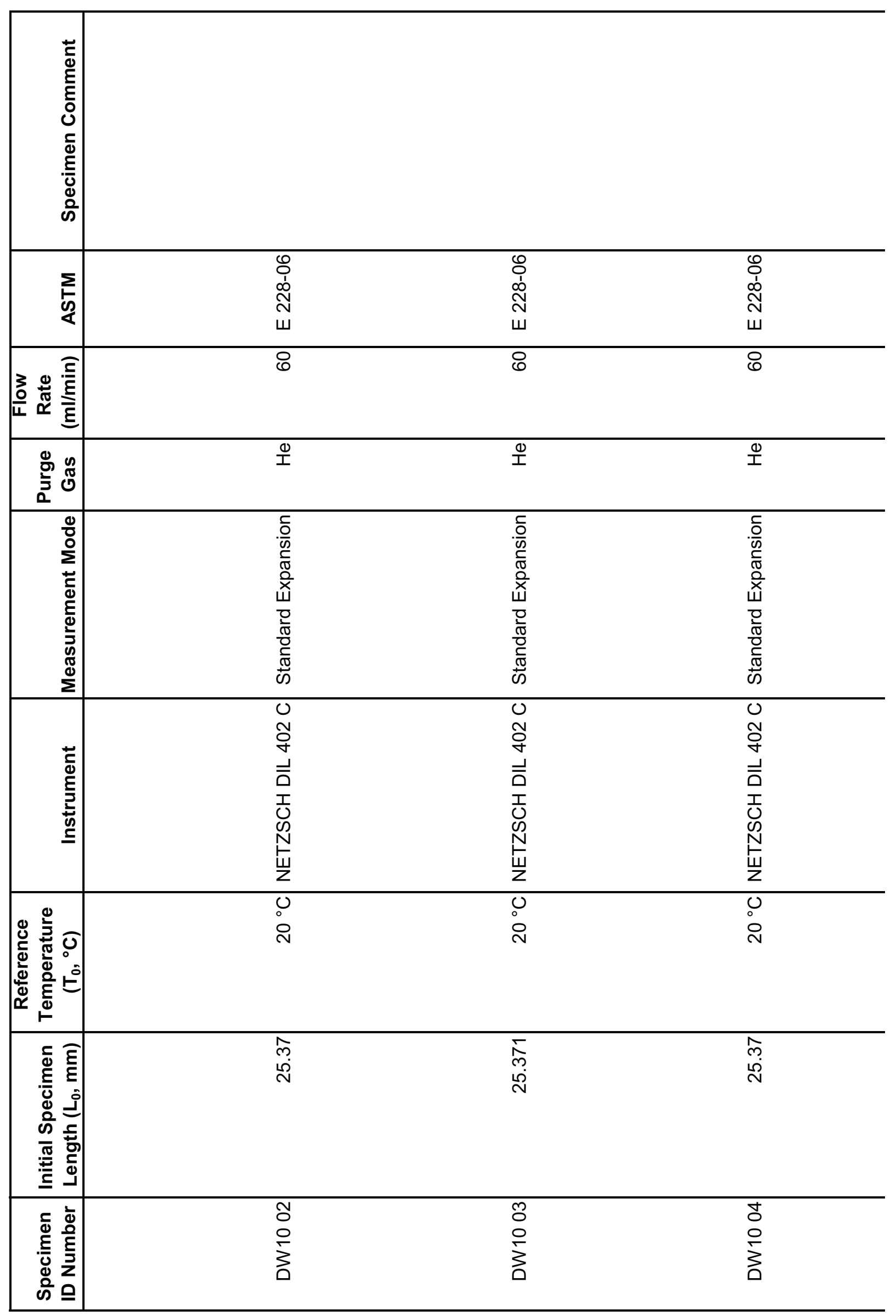

용 


\begin{tabular}{|c|c|c|c|}
\hline 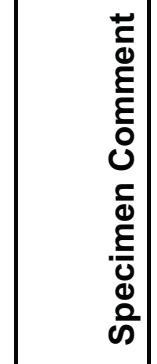 & & & \\
\hline 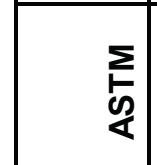 & 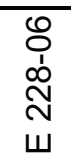 & 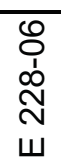 & 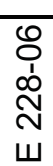 \\
\hline 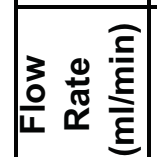 & 8 & 8 & 8 \\
\hline 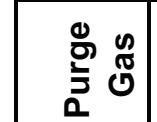 & $\frac{O}{I}$ & $\frac{\Phi}{I}$ & $\stackrel{\oplus}{I}$ \\
\hline 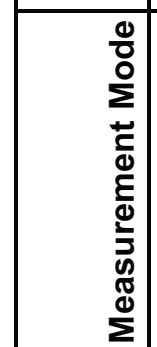 & 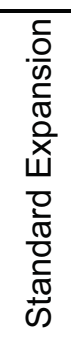 & 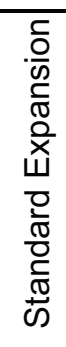 & 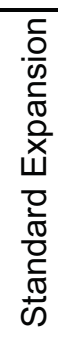 \\
\hline 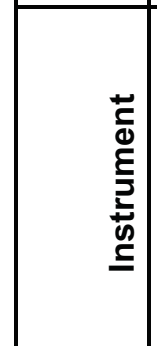 & 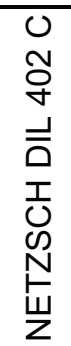 & 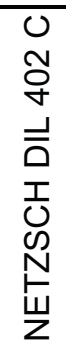 & 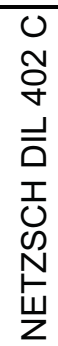 \\
\hline 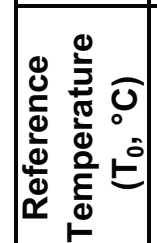 & $\begin{array}{l}0 \\
0 \\
\text { Oे }\end{array}$ & 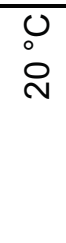 & $\begin{array}{l}0 \\
0 \\
0\end{array}$ \\
\hline 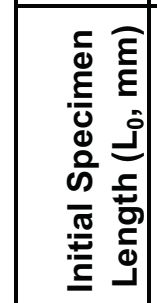 & $\begin{array}{l}\bar{\Gamma} \\
\hat{n} \\
\stackrel{\rho}{N}\end{array}$ & $\begin{array}{l}\bar{N} \\
m \\
\stackrel{n}{N}\end{array}$ & $\begin{array}{l}\stackrel{m}{N} \\
m \\
\stackrel{n}{N}\end{array}$ \\
\hline 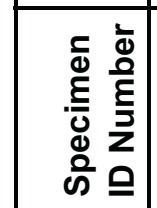 & $\begin{array}{l}\overline{0} \\
\frac{5}{5} \\
\vdots\end{array}$ & $\begin{array}{l}\mathcal{N} \\
0 \\
5 \\
\vdots \\
\vdots\end{array}$ & $\begin{array}{l}9 \\
0 \\
5 \\
\vdots\end{array}$ \\
\hline
\end{tabular}




\begin{tabular}{|c|c|c|c|c|}
\hline 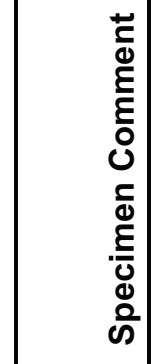 & & & & \\
\hline 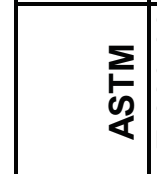 & 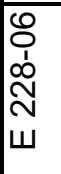 & $\begin{array}{l}0 \\
\\
1 \\
\infty \\
\text { N } \\
w\end{array}$ & 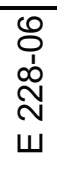 & 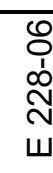 \\
\hline 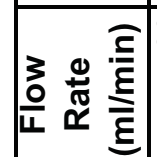 & 8 & 8 & 8 & 8 \\
\hline 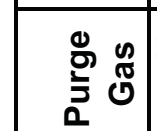 & $\Phi$ & $\frac{\oplus}{1}$ & $\stackrel{\Phi}{I}$ & $\stackrel{\oplus}{I}$ \\
\hline 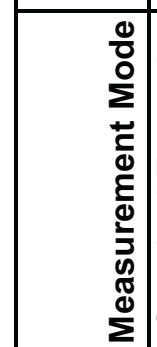 & 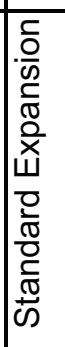 & 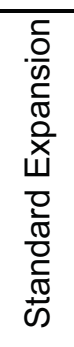 & 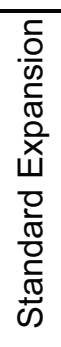 & 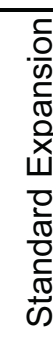 \\
\hline 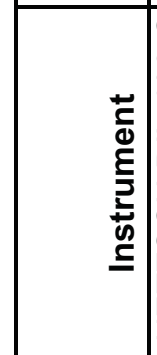 & 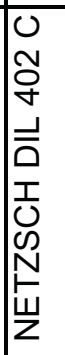 & 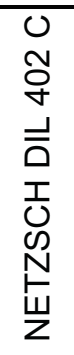 & 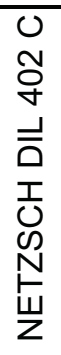 & 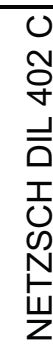 \\
\hline 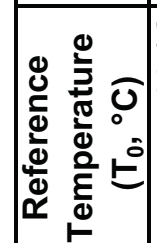 & o & $\begin{array}{l}0 \\
0 \\
2\end{array}$ & $\begin{array}{l}0 \\
0 \\
i\end{array}$ & $\begin{array}{l}0 \\
0 \\
0\end{array}$ \\
\hline 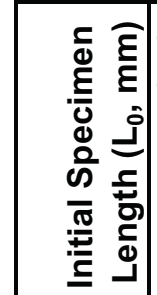 & مิ & $\begin{array}{l}\text { N } \\
\text { N } \\
\stackrel{d}{N}\end{array}$ & 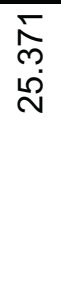 & 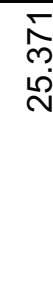 \\
\hline 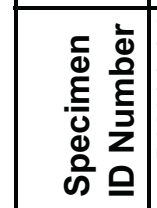 & 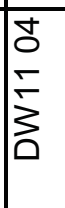 & $\begin{array}{l}\bar{\delta} \\
\stackrel{N}{3} \\
\text { a }\end{array}$ & 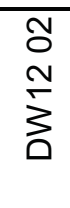 & $\begin{array}{l}\text { mo } \\
\stackrel{2}{N} \\
\vdots \\
\vdots\end{array}$ \\
\hline
\end{tabular}




\begin{tabular}{|c|c|c|c|}
\hline 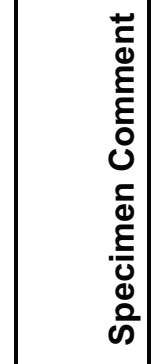 & & & \\
\hline 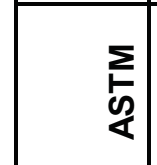 & $\begin{array}{l}\text { Oo } \\
0 \\
\text { D } \\
\text { w }\end{array}$ & 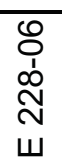 & 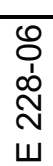 \\
\hline 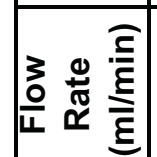 & 8 & 8 & 8 \\
\hline $\begin{array}{l}0 \\
0 \\
0\end{array}$ & $\frac{\Phi}{I}$ & 里 & $\frac{\Theta}{I}$ \\
\hline 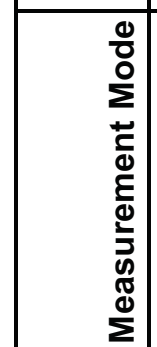 & 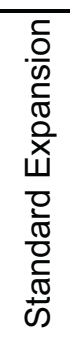 & 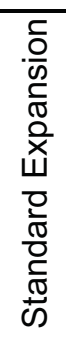 & 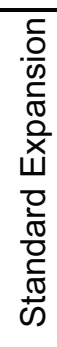 \\
\hline 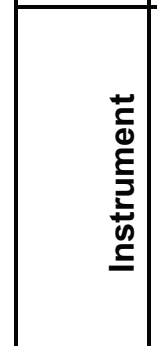 & 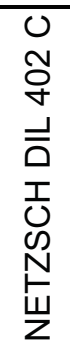 & 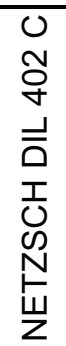 & 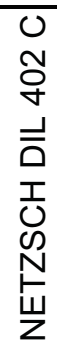 \\
\hline 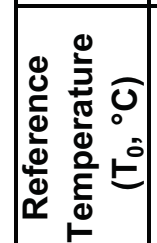 & $\begin{array}{l}0 \\
0 \\
\text { Oे }\end{array}$ & $\begin{array}{l}0 \\
0 \\
\text { Q }\end{array}$ & $\begin{array}{l}0 \\
\vdots \\
\text { N }\end{array}$ \\
\hline 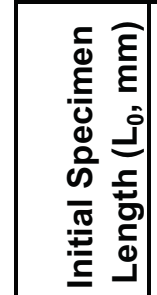 & 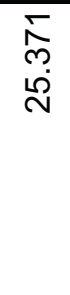 & $\begin{array}{l}\text { No } \\
\hat{N} \\
\stackrel{d}{N}\end{array}$ & $\begin{array}{l}\text { Na } \\
\text { 岁 } \\
\stackrel{\sim}{N}\end{array}$ \\
\hline 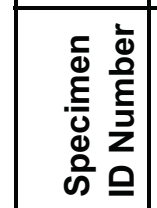 & $\begin{array}{l}\text { J } \\
\stackrel{N}{5} \\
\vdots\end{array}$ & $\begin{array}{l}\text { N } \\
\sum_{0}^{m}\end{array}$ & $\begin{array}{l}\bar{o} \\
\sum_{0}^{m}\end{array}$ \\
\hline
\end{tabular}




\begin{tabular}{|c|c|c|c|c|}
\hline 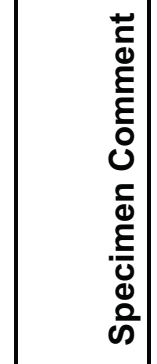 & & & & \\
\hline$\underset{\frac{\sum}{2}}{\frac{\sum}{\alpha}}$ & 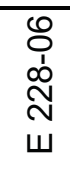 & 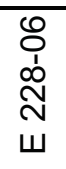 & 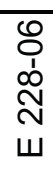 & 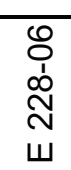 \\
\hline 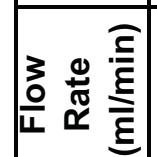 & 8 & 8 & 8 & 8 \\
\hline 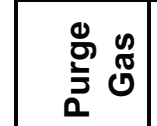 & $\frac{\oplus}{I}$ & $\frac{\Theta}{1}$ & 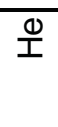 & $\stackrel{\oplus}{I}$ \\
\hline 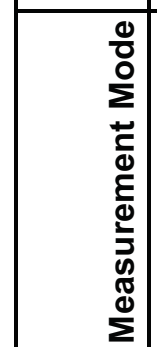 & 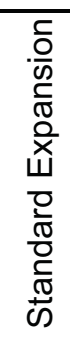 & 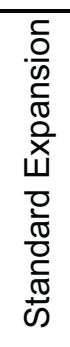 & 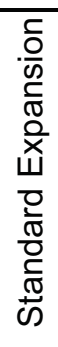 & 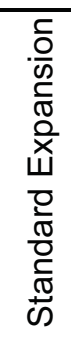 \\
\hline 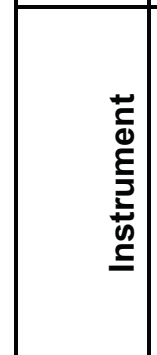 & 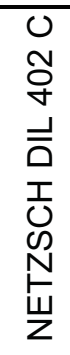 & 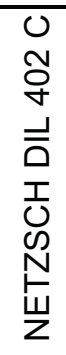 & 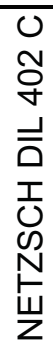 & 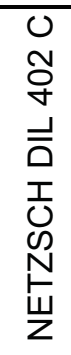 \\
\hline 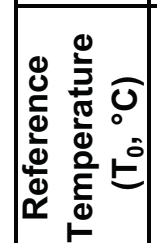 & $\begin{array}{l}0 \\
0 \\
0\end{array}$ & $\begin{array}{l}0 \\
0 \\
\text { Dे }\end{array}$ & $\begin{array}{l}0 \\
0 \\
0\end{array}$ & $\begin{array}{l}0 \\
0 \\
0\end{array}$ \\
\hline 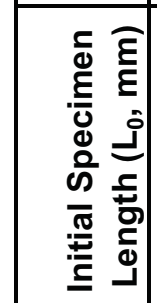 & $\begin{array}{l}\text { M } \\
\text { n. } \\
\stackrel{N}{N}\end{array}$ & $\begin{array}{l}\text { N } \\
\text { n. } \\
\stackrel{N}{N}\end{array}$ & 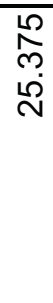 & 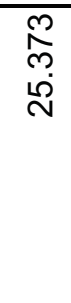 \\
\hline 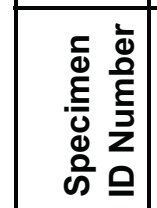 & $\begin{array}{l}m \\
\text { m } \\
m \\
3 \\
\vdots\end{array}$ & $\begin{array}{l}\text { J } \\
m \\
\text { m }\end{array}$ & $\begin{array}{l}\bar{\delta} \\
\vdots \\
\vdots \\
\vdots\end{array}$ & \begin{tabular}{l}
$\mathcal{N}$ \\
0 \\
\multirow{J}{3}{} \\
$\vdots$ \\
0
\end{tabular} \\
\hline
\end{tabular}




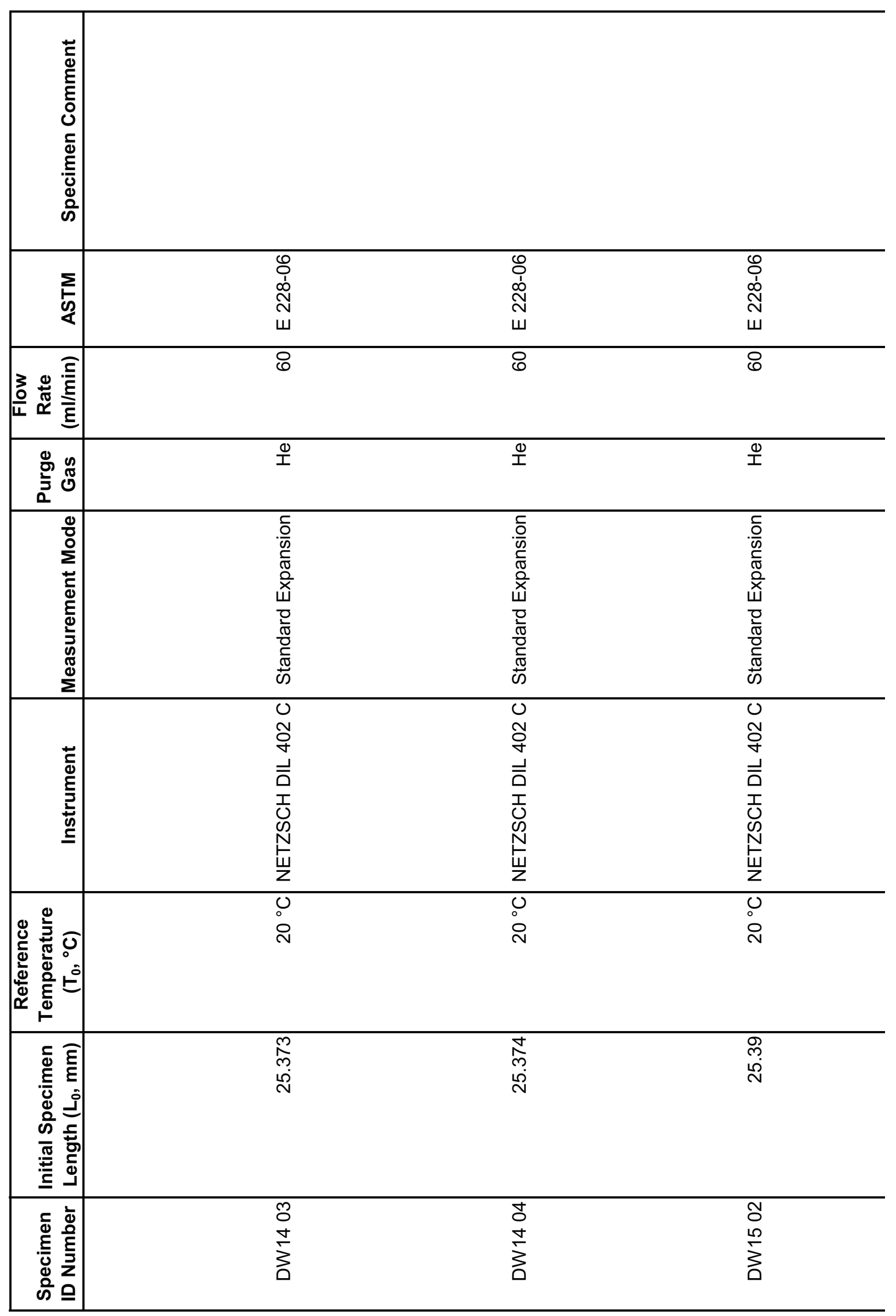




\begin{tabular}{|c|c|c|c|}
\hline 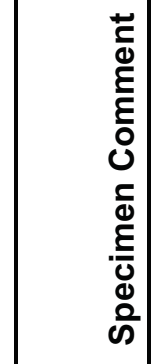 & & & \\
\hline 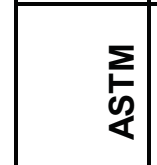 & 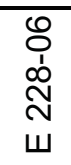 & 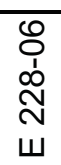 & 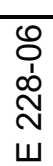 \\
\hline 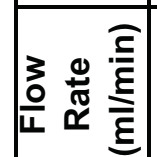 & 8 & 8 & 8 \\
\hline 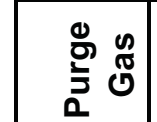 & $\frac{O}{I}$ & $\frac{\Phi}{I}$ & $\stackrel{\oplus}{I}$ \\
\hline 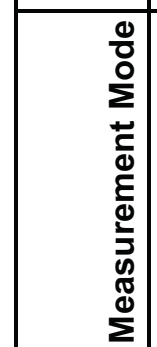 & 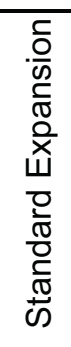 & 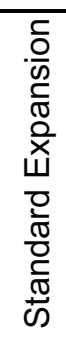 & 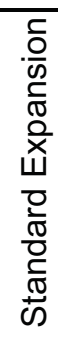 \\
\hline 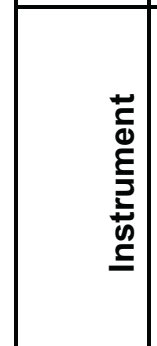 & 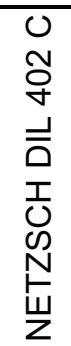 & 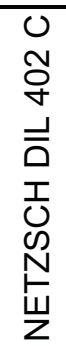 & 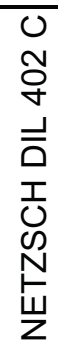 \\
\hline 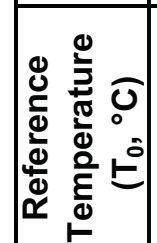 & $\begin{array}{l}0 \\
0 \\
\text { Oे }\end{array}$ & 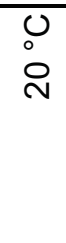 & $\begin{array}{l}0 \\
0 \\
0\end{array}$ \\
\hline 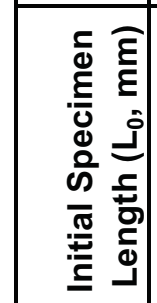 & $\begin{array}{l}\hat{\infty} \\
\stackrel{\infty}{0} \\
\stackrel{\rho}{\rho}\end{array}$ & 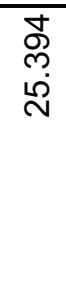 & $\begin{array}{l}\text { Dे } \\
\text { m. } \\
\stackrel{\text { Dे }}{\text { L }}\end{array}$ \\
\hline 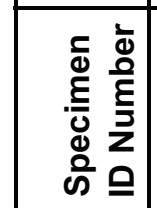 & $\begin{array}{l}0 \\
0 \\
0 \\
10 \\
3\end{array}$ & $\begin{array}{l}5 \\
0 \\
10 \\
\vdots \\
\vdots\end{array}$ & $\begin{array}{l}5 \\
0 \\
\vdots \\
\vdots \\
0\end{array}$ \\
\hline
\end{tabular}




\begin{tabular}{|c|c|c|c|c|}
\hline 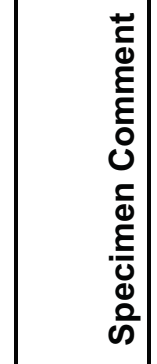 & & & & \\
\hline 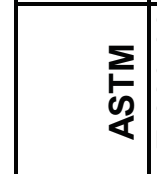 & 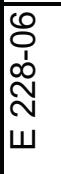 & $\begin{array}{l}0 \\
\\
1 \\
\infty \\
\text { N } \\
w\end{array}$ & 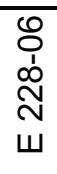 & 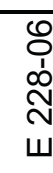 \\
\hline 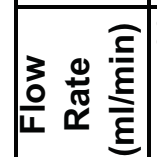 & 8 & 8 & 8 & 8 \\
\hline 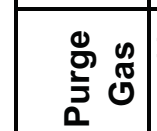 & $\Phi$ & $\frac{\oplus}{1}$ & $\stackrel{\Phi}{I}$ & $\stackrel{\oplus}{I}$ \\
\hline 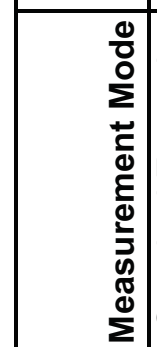 & 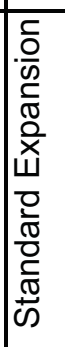 & 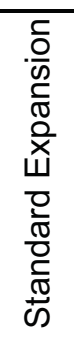 & 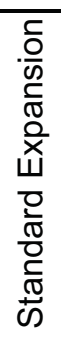 & 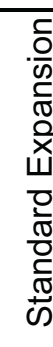 \\
\hline 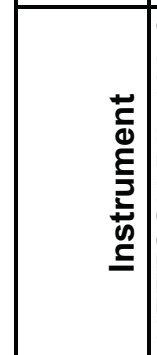 & 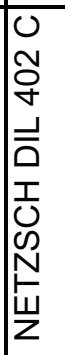 & 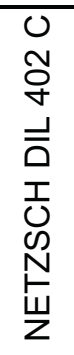 & 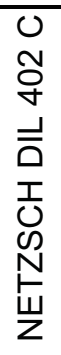 & 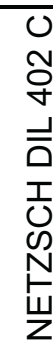 \\
\hline 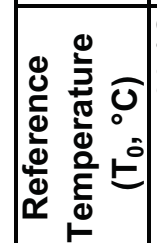 & o & $\begin{array}{l}0 \\
0 \\
2\end{array}$ & $\begin{array}{l}0 \\
0 \\
i\end{array}$ & $\begin{array}{l}0 \\
0 \\
0\end{array}$ \\
\hline 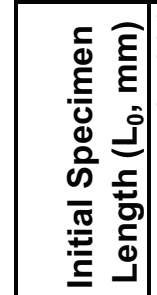 & & 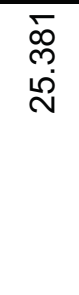 & 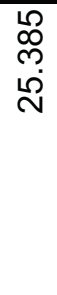 & مै \\
\hline 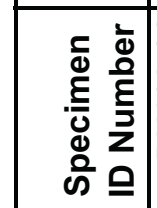 & $\begin{array}{l}\approx \\
0 \\
0 \\
3 \\
3 \\
0\end{array}$ & $\begin{array}{l}08 \\
0 \\
0 \\
3 \\
0\end{array}$ & $\begin{array}{l}0 \\
0 \\
0 \\
\vdots \\
\vdots\end{array}$ & \begin{tabular}{l}
$\bar{o}$ \\
\multirow{1}{c}{} \\
$\vdots$
\end{tabular} \\
\hline
\end{tabular}




\begin{tabular}{|c|c|c|c|}
\hline 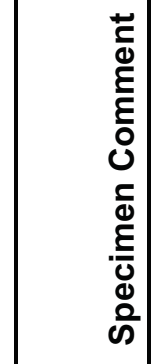 & & & \\
\hline 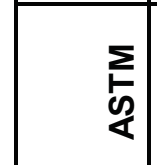 & $\begin{array}{l}\text { Oo } \\
0 \\
\text { D } \\
\text { w }\end{array}$ & 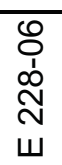 & 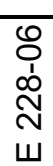 \\
\hline 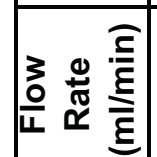 & 8 & 8 & 8 \\
\hline $\begin{array}{l}0 \\
0 \\
0\end{array}$ & $\frac{\Phi}{I}$ & 里 & $\frac{\Theta}{I}$ \\
\hline 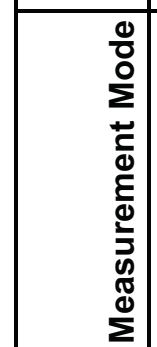 & 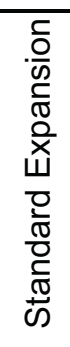 & 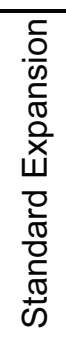 & 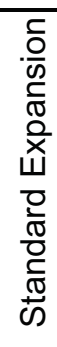 \\
\hline 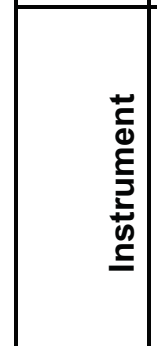 & 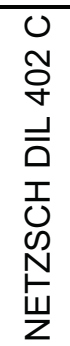 & 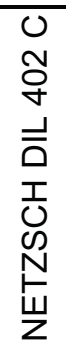 & 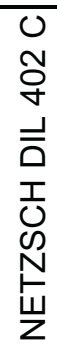 \\
\hline 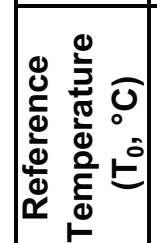 & $\begin{array}{l}0 \\
0 \\
\text { Oे }\end{array}$ & $\begin{array}{l}0 \\
0 \\
\text { Q }\end{array}$ & $\begin{array}{l}0 \\
\vdots \\
\text { N }\end{array}$ \\
\hline 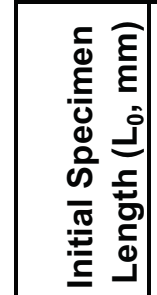 & $\begin{array}{l}\text { Oి } \\
\text { ల్ } \\
\stackrel{d}{d}\end{array}$ & $\begin{array}{l}\widehat{\infty} \\
\text { o } \\
\stackrel{\sim}{N}\end{array}$ & $\begin{array}{l}\text { o } \\
\stackrel{0}{\sim} \\
\stackrel{\sim}{\sim}\end{array}$ \\
\hline 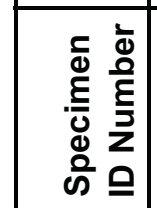 & \begin{tabular}{l} 
N \\
\multirow{2}{人}{} \\
$\vdots$ \\
$\vdots$
\end{tabular} & $\begin{array}{l}\text { J } \\
\hat{N} \\
\sum\end{array}$ & $\begin{array}{l}\bar{o} \\
\stackrel{1}{1} \\
\text { aे }\end{array}$ \\
\hline
\end{tabular}




\begin{tabular}{|c|c|c|c|c|}
\hline 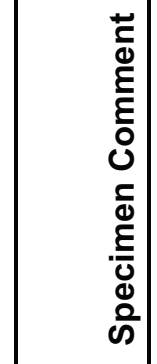 & & & & \\
\hline$\underset{\frac{\sum}{2}}{\frac{\sum}{\alpha}}$ & 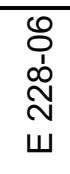 & 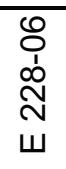 & $\begin{array}{l}\stackrel{0}{0} \\
\dot{1} \\
\stackrel{1}{N} \\
w\end{array}$ & 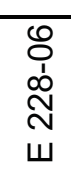 \\
\hline 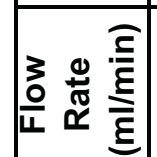 & 8 & 8 & 8 & 8 \\
\hline 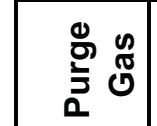 & $\frac{\oplus}{I}$ & $\frac{\Theta}{1}$ & $\stackrel{\oplus}{1}$ & $\stackrel{\oplus}{I}$ \\
\hline 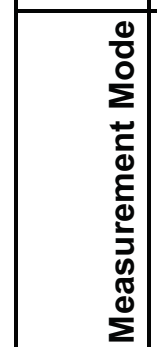 & 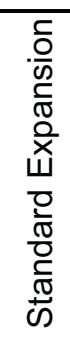 & 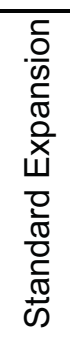 & 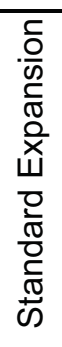 & 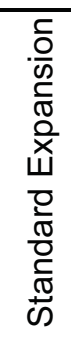 \\
\hline 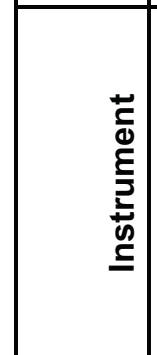 & 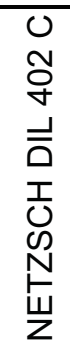 & 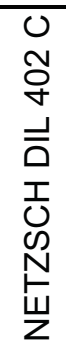 & 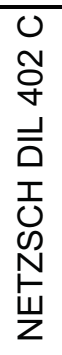 & 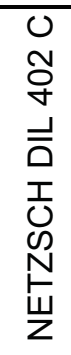 \\
\hline 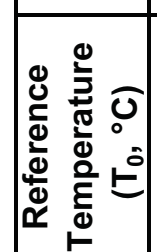 & $\begin{array}{l}\text { O } \\
\text { Oे }\end{array}$ & $\begin{array}{l}\text { U } \\
\text { Oे }\end{array}$ & $\begin{array}{l}\text { O } \\
\text { Oे }\end{array}$ & $\begin{array}{l}0 \\
0 \\
\text { Dे }\end{array}$ \\
\hline 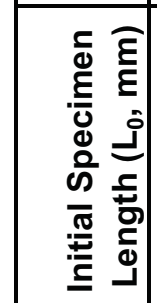 & 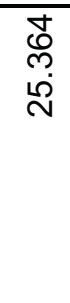 & $\begin{array}{l}\infty \\
0 \\
0 \\
\text { D. } \\
\stackrel{N}{N}\end{array}$ & $\begin{array}{l}\text { O } \\
\stackrel{0}{0} \\
\stackrel{\omega}{N}\end{array}$ & 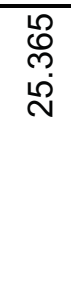 \\
\hline 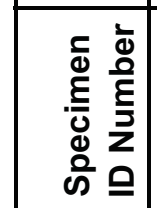 & 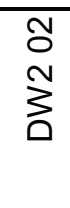 & 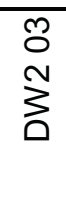 & $\begin{array}{l}\text { J } \\
\text { N } \\
\text { à }\end{array}$ & $\begin{array}{l}\overline{0} \\
\text { m } \\
\vdots\end{array}$ \\
\hline
\end{tabular}




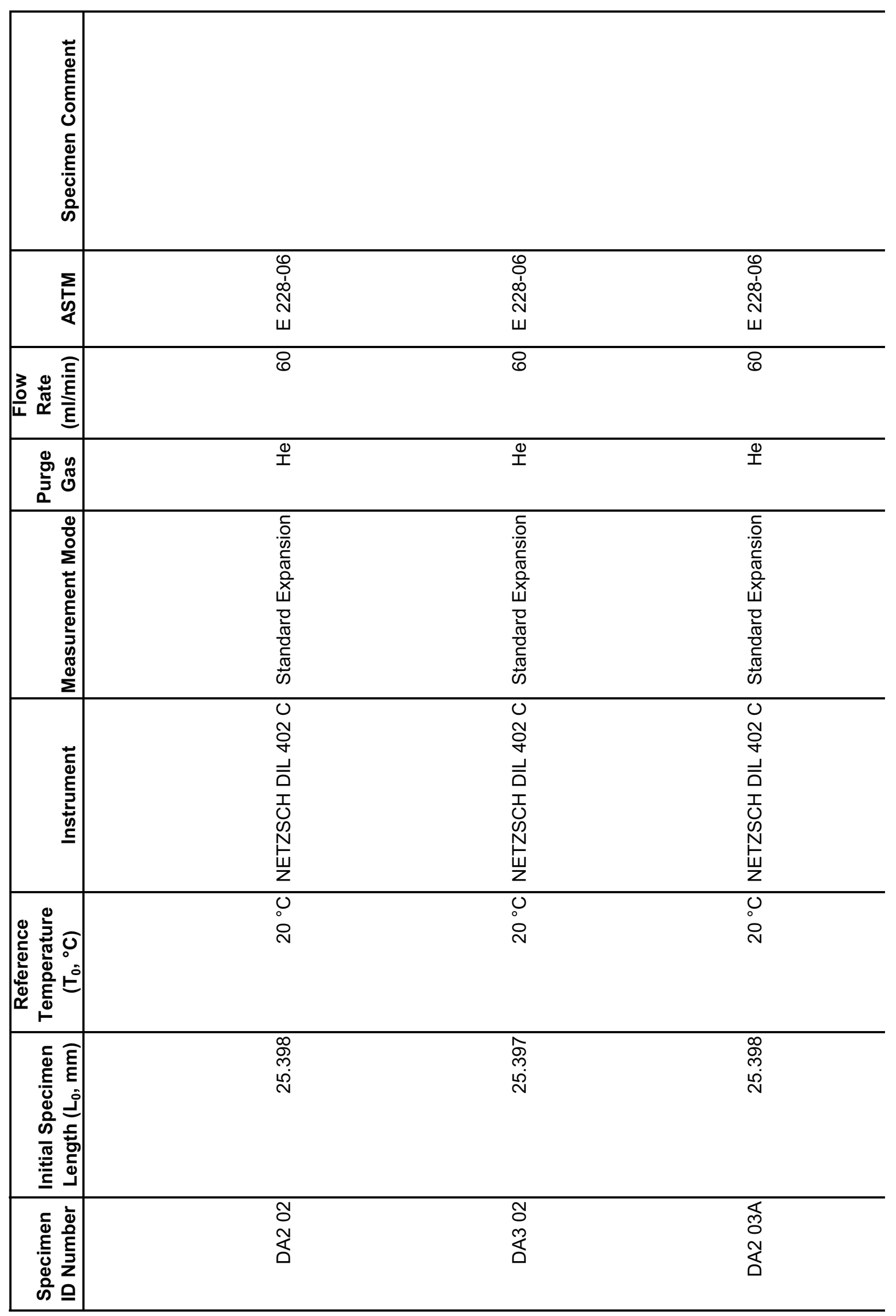




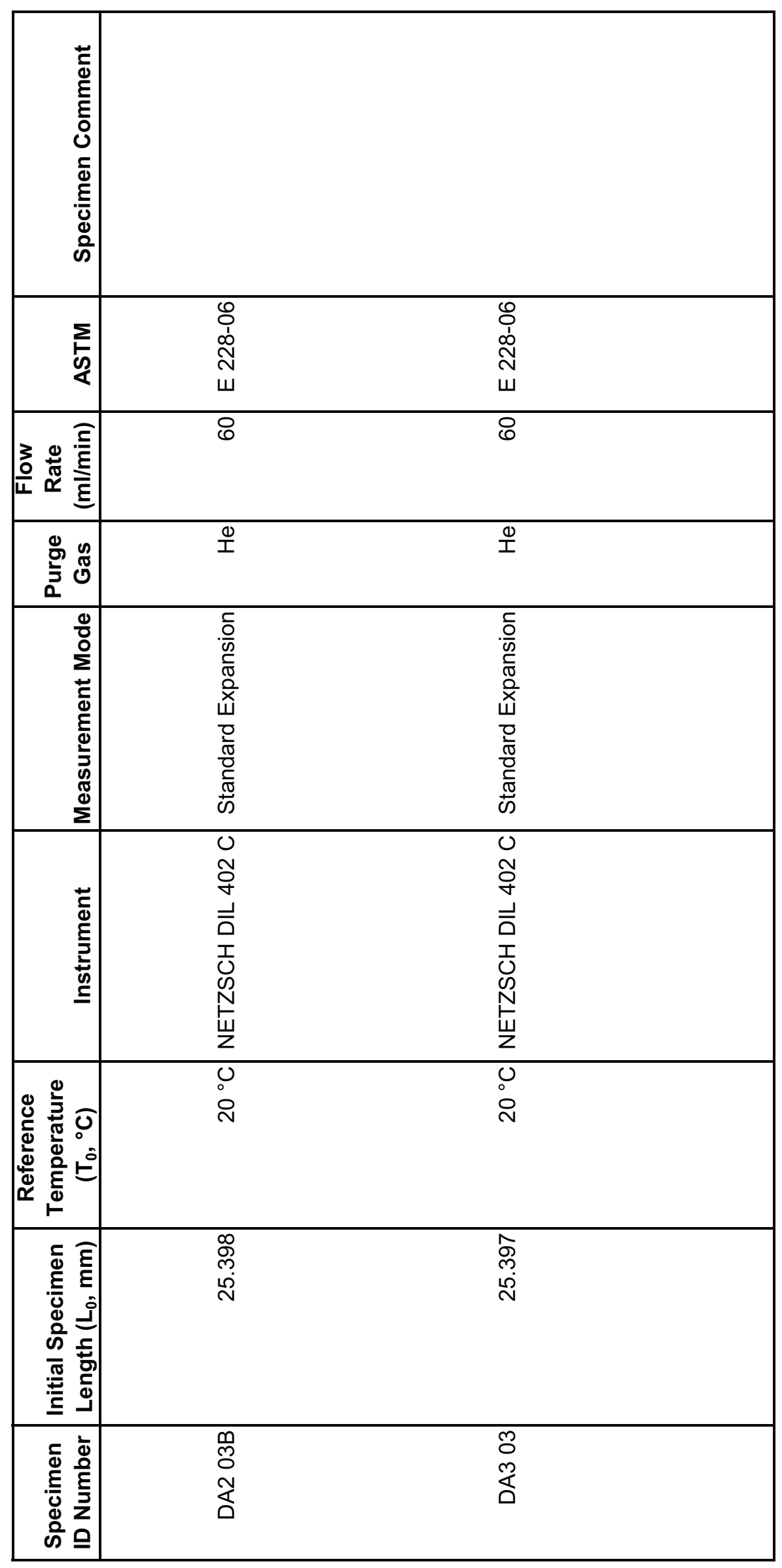




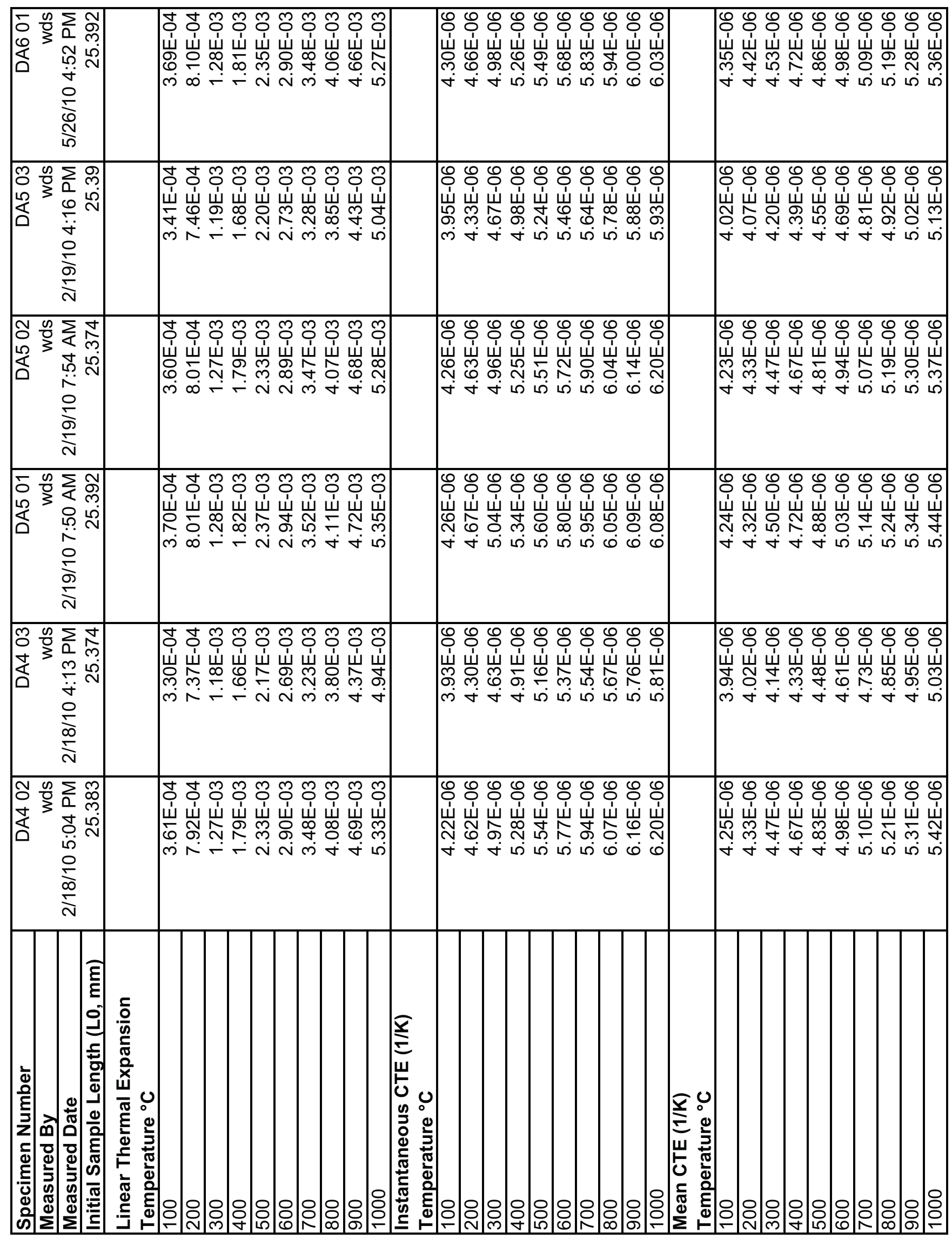




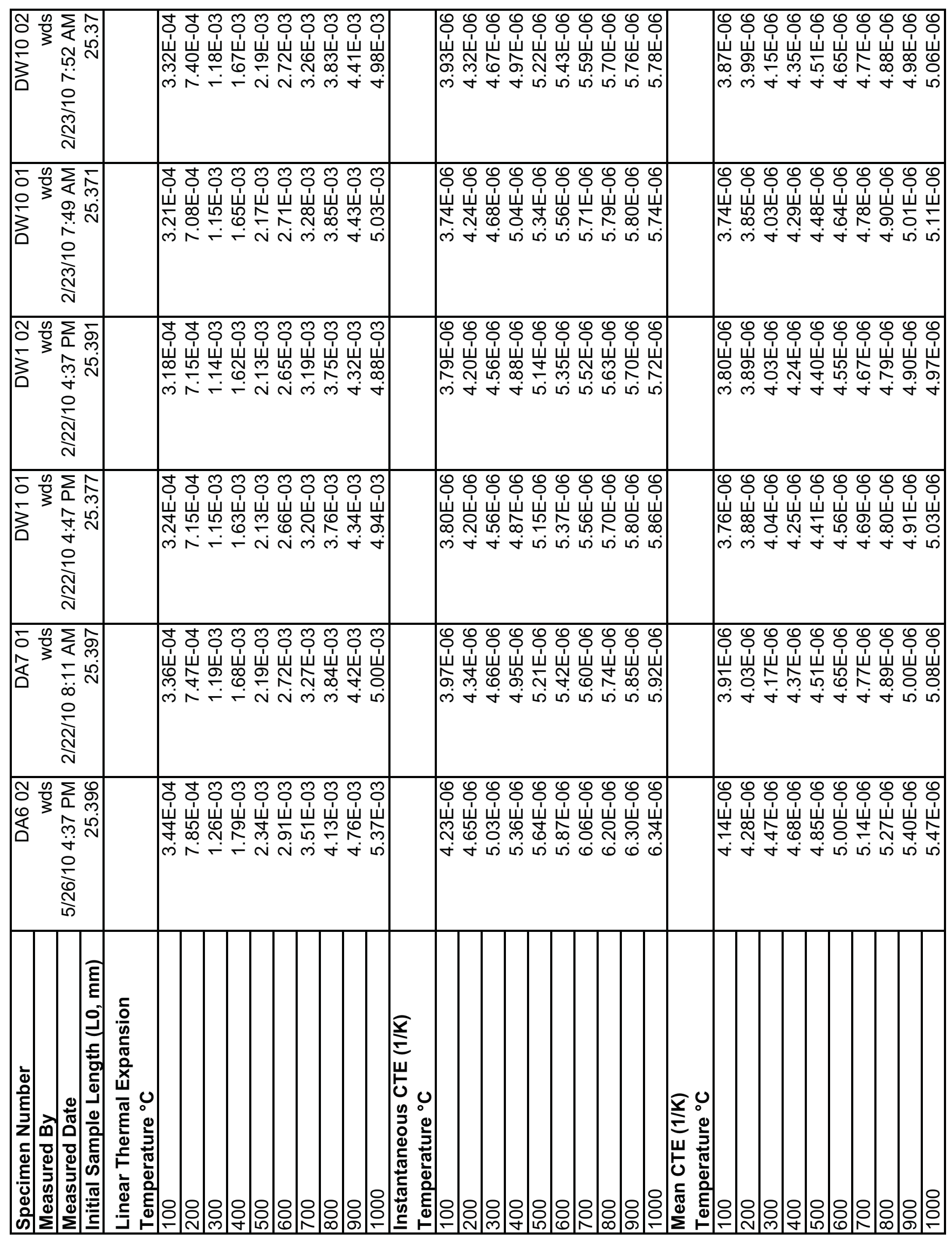




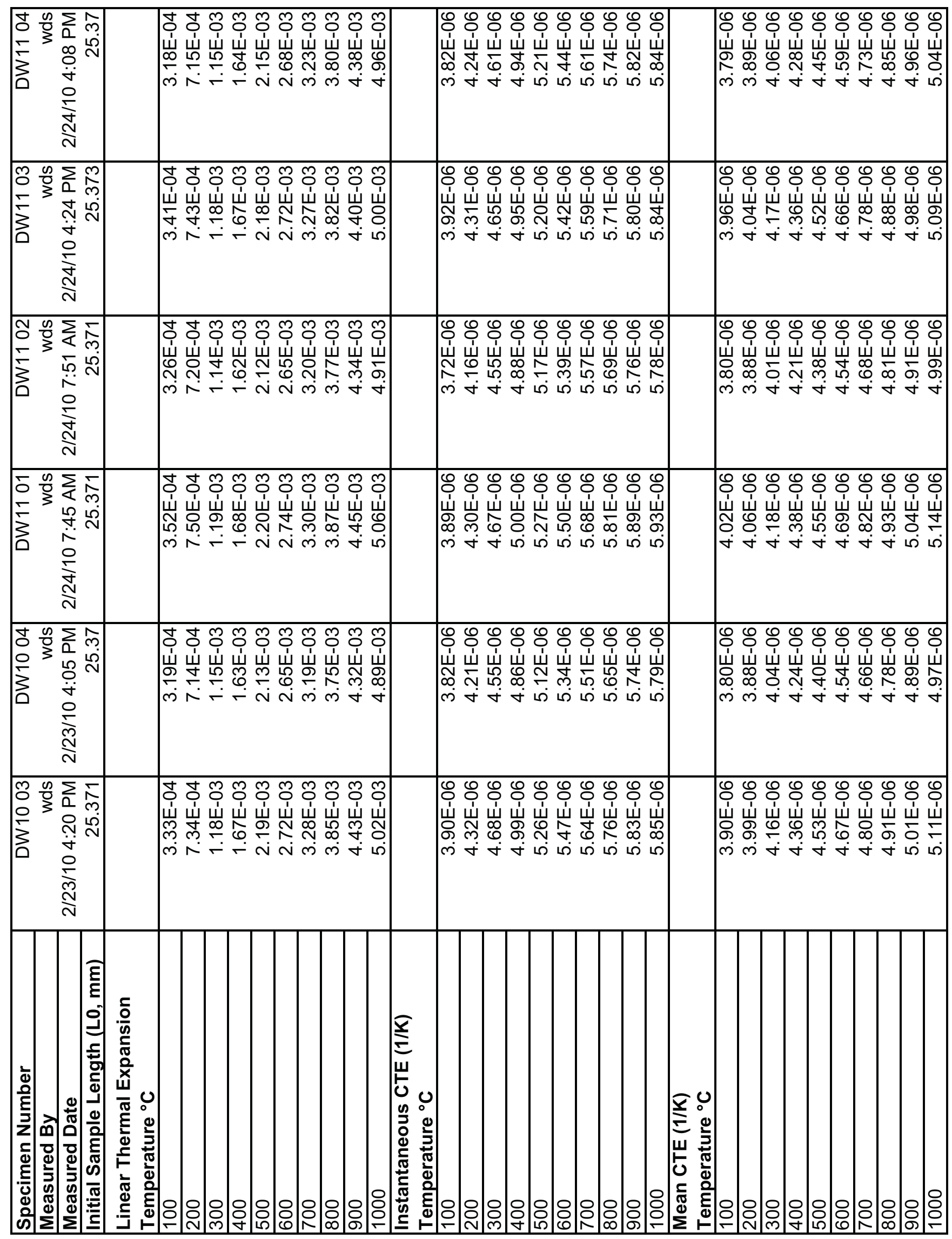




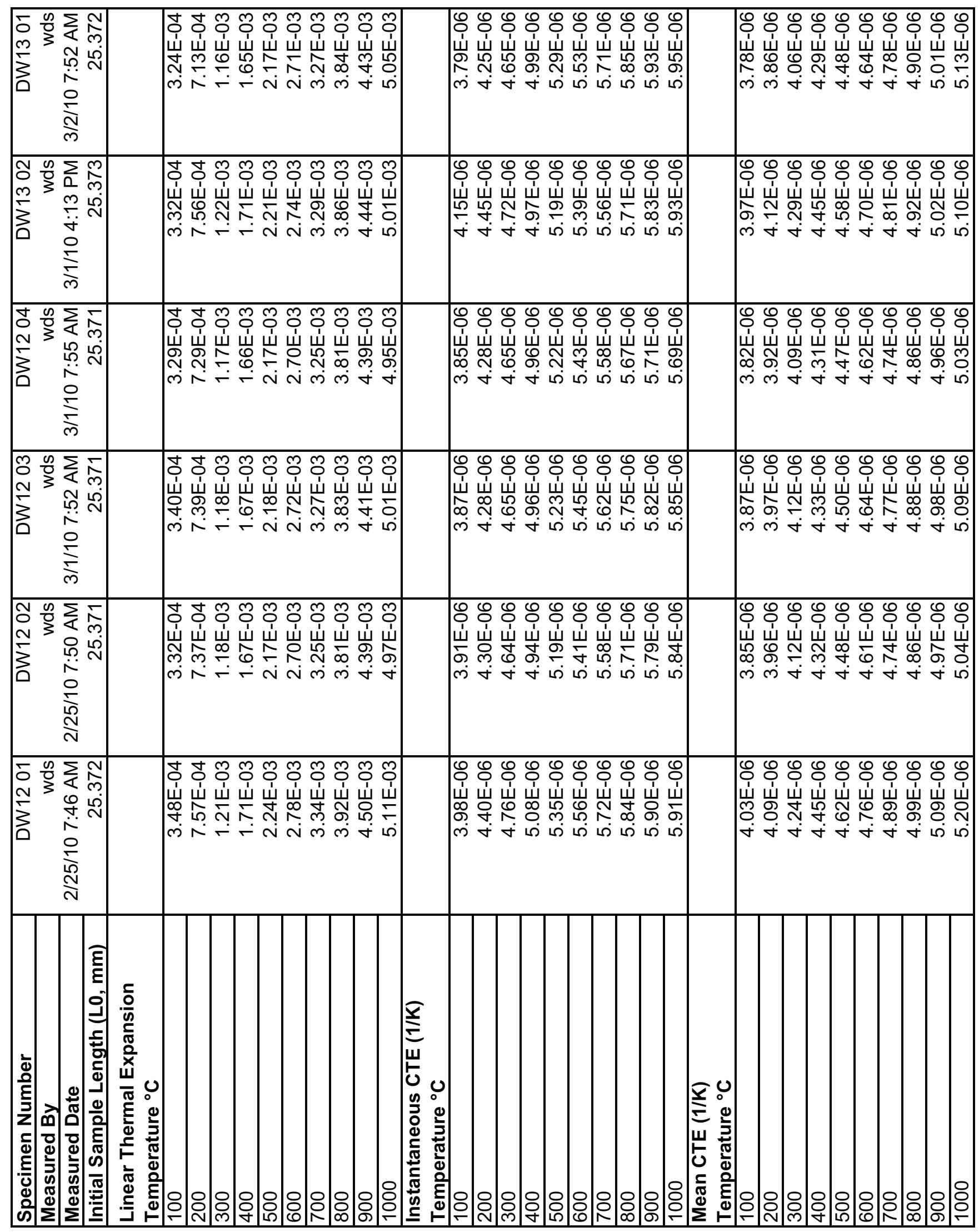




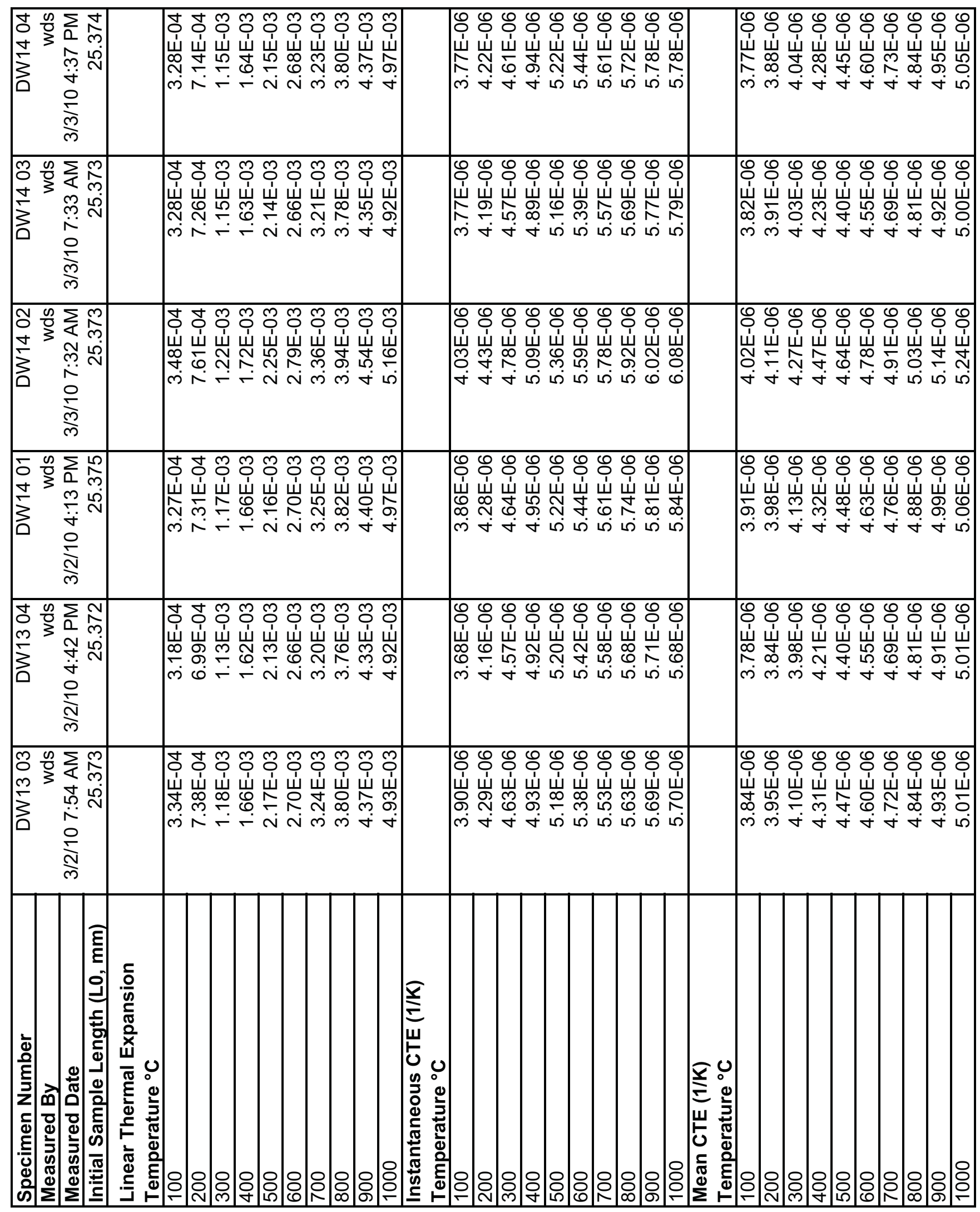




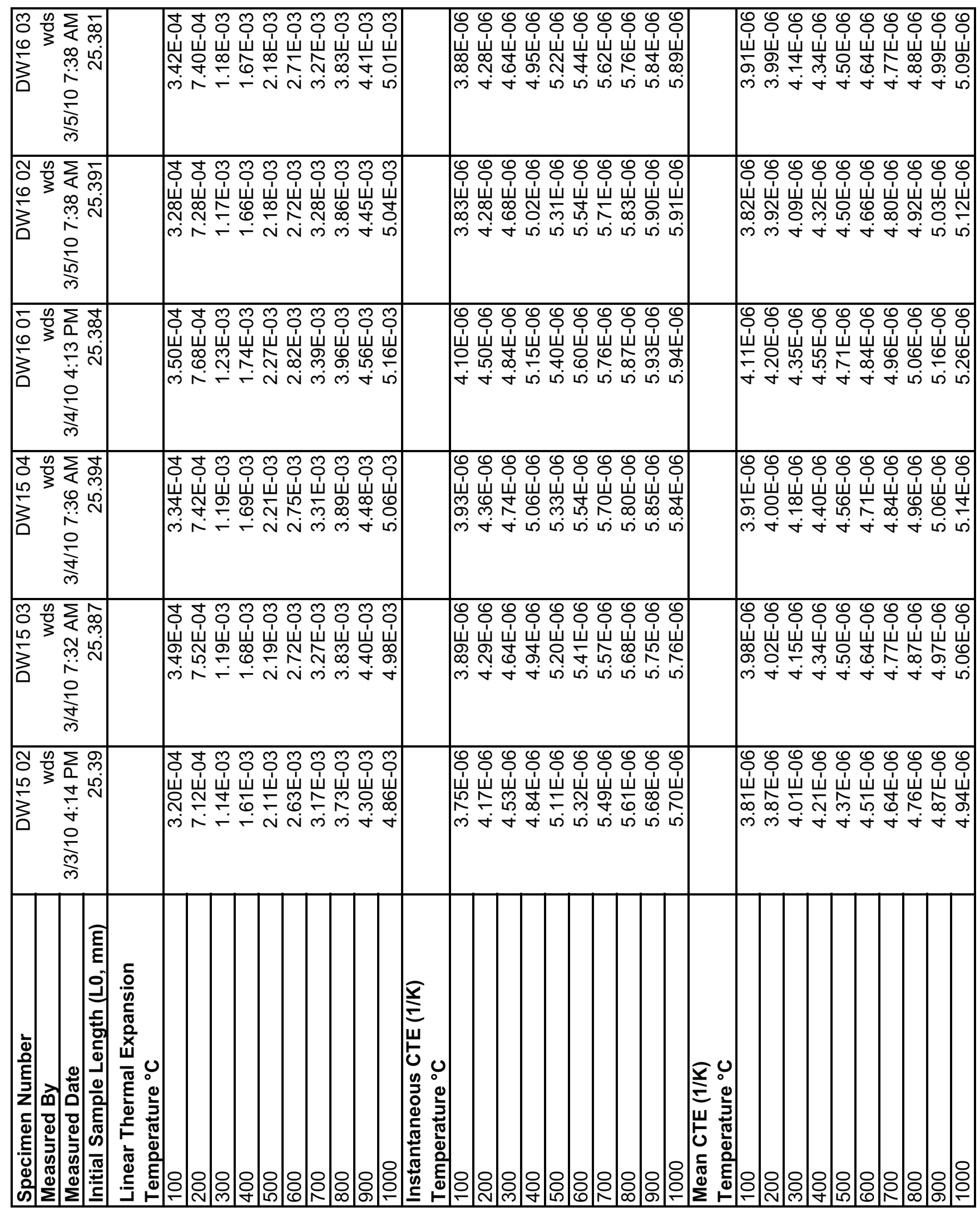




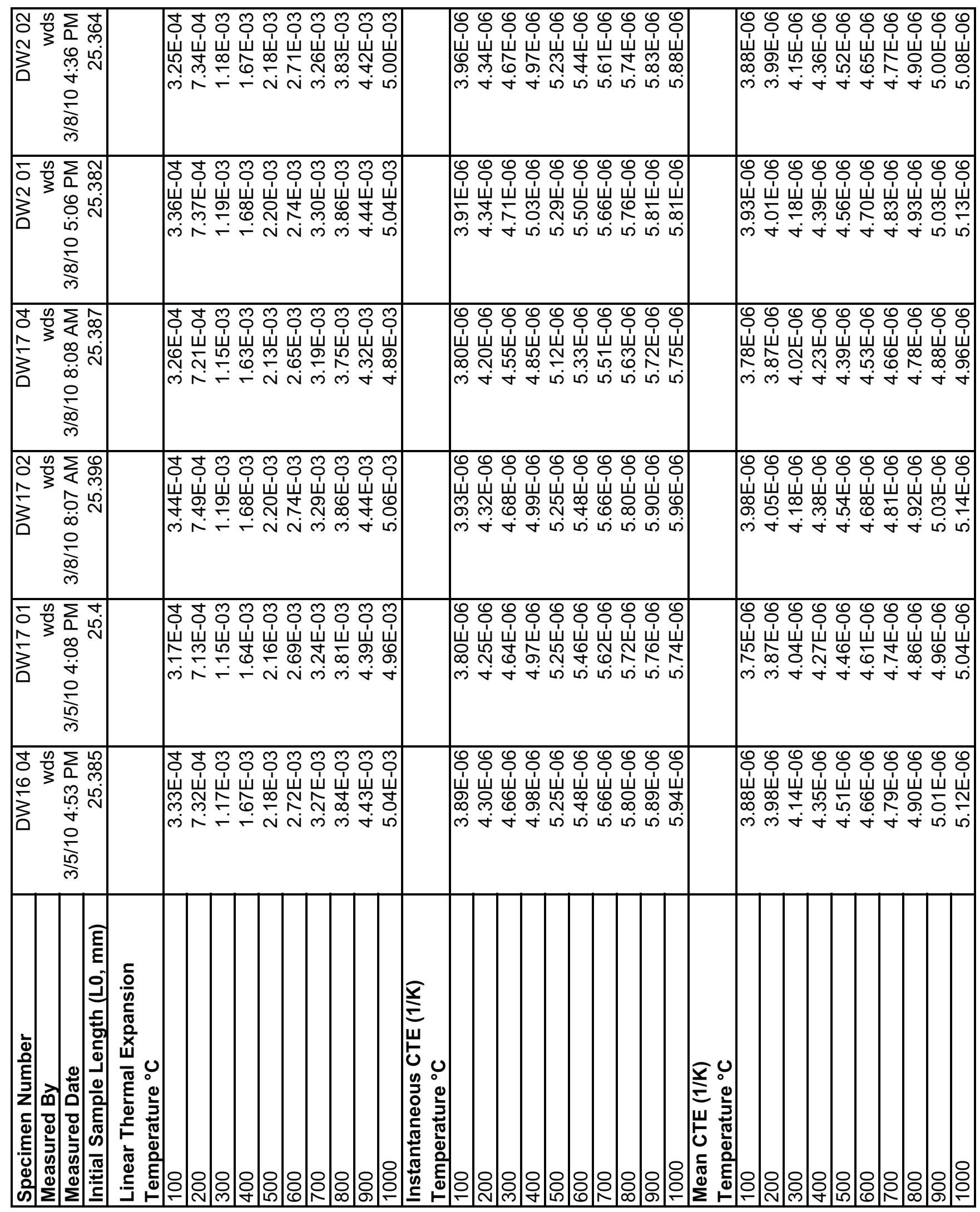




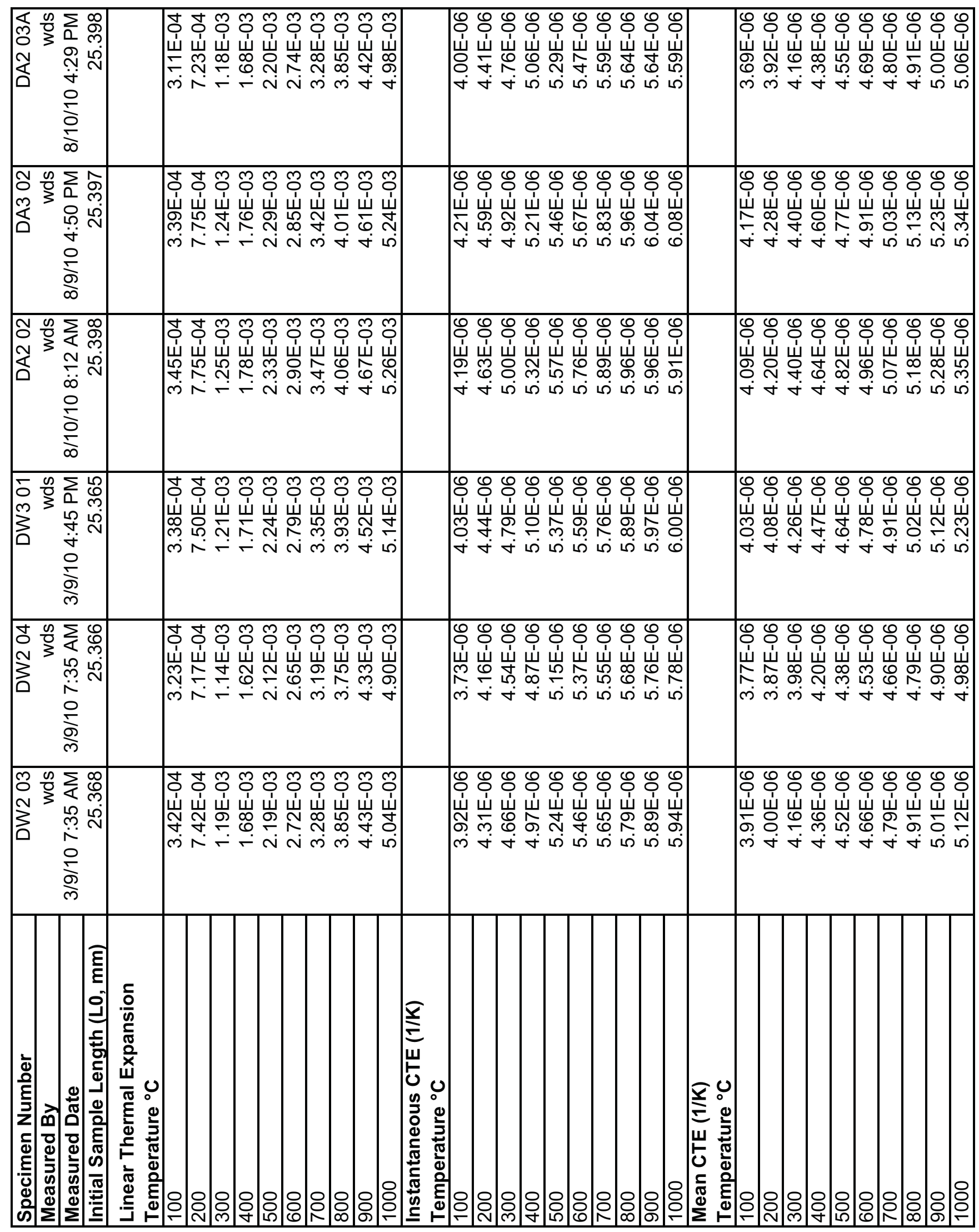




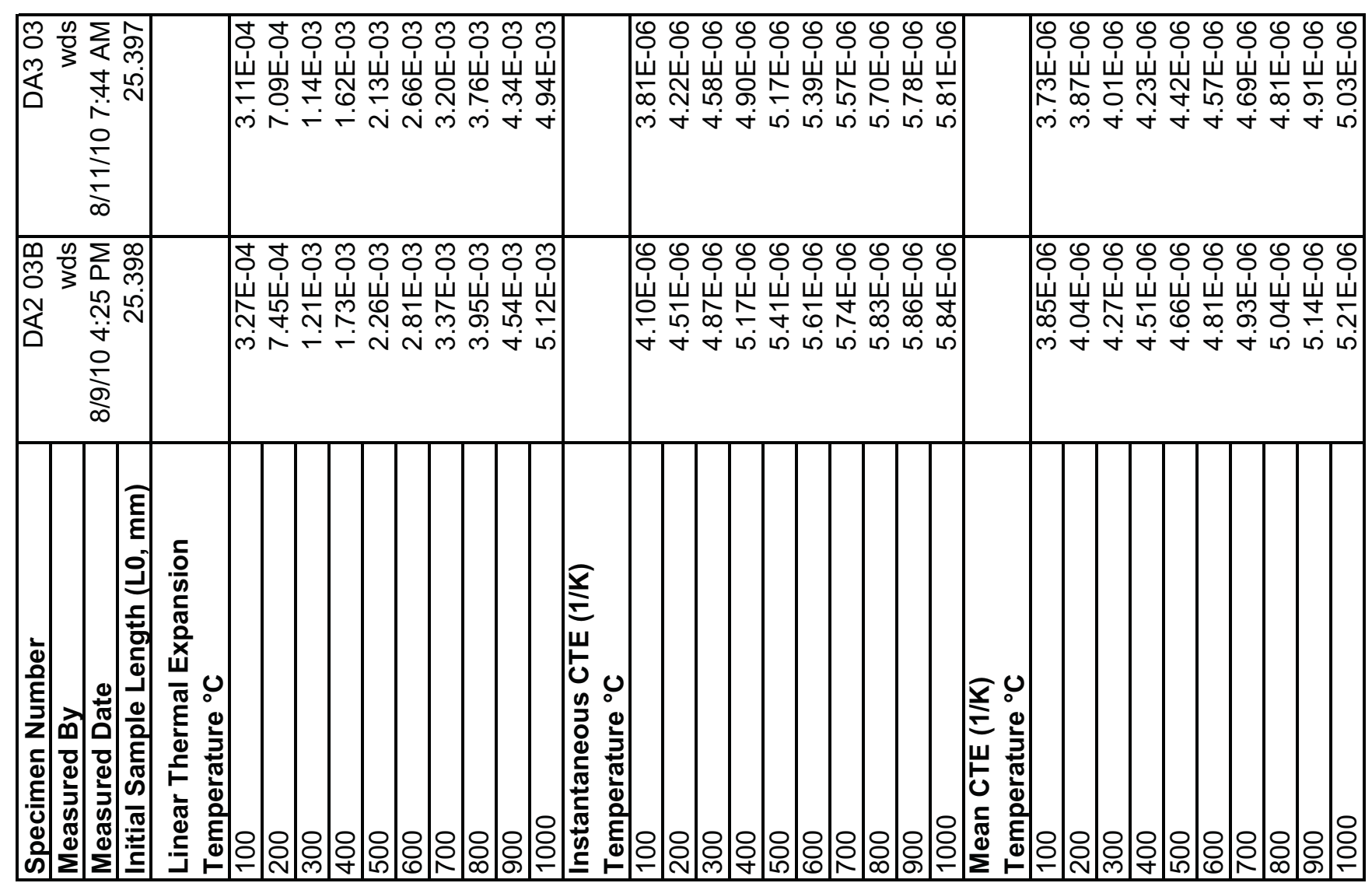




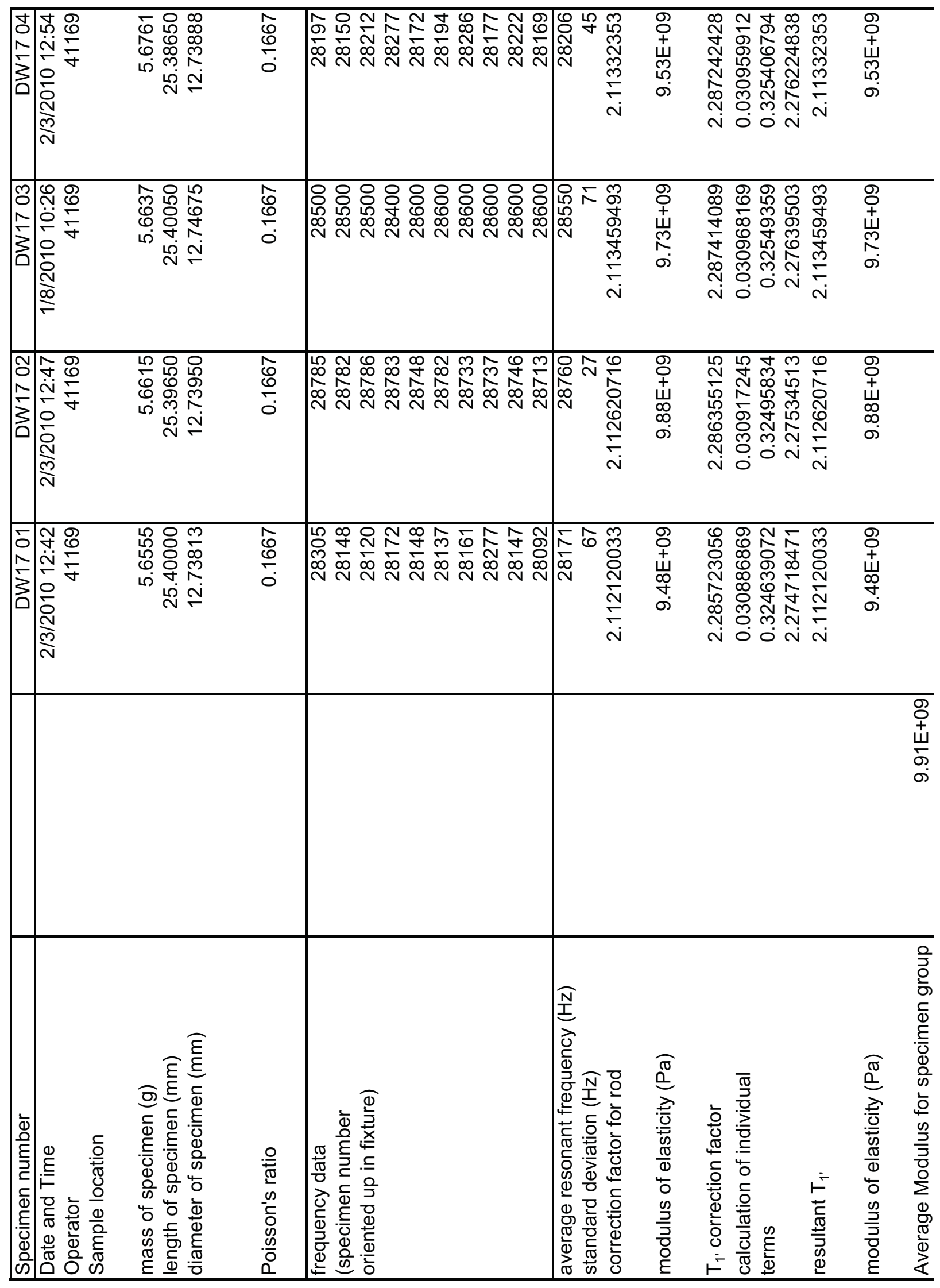




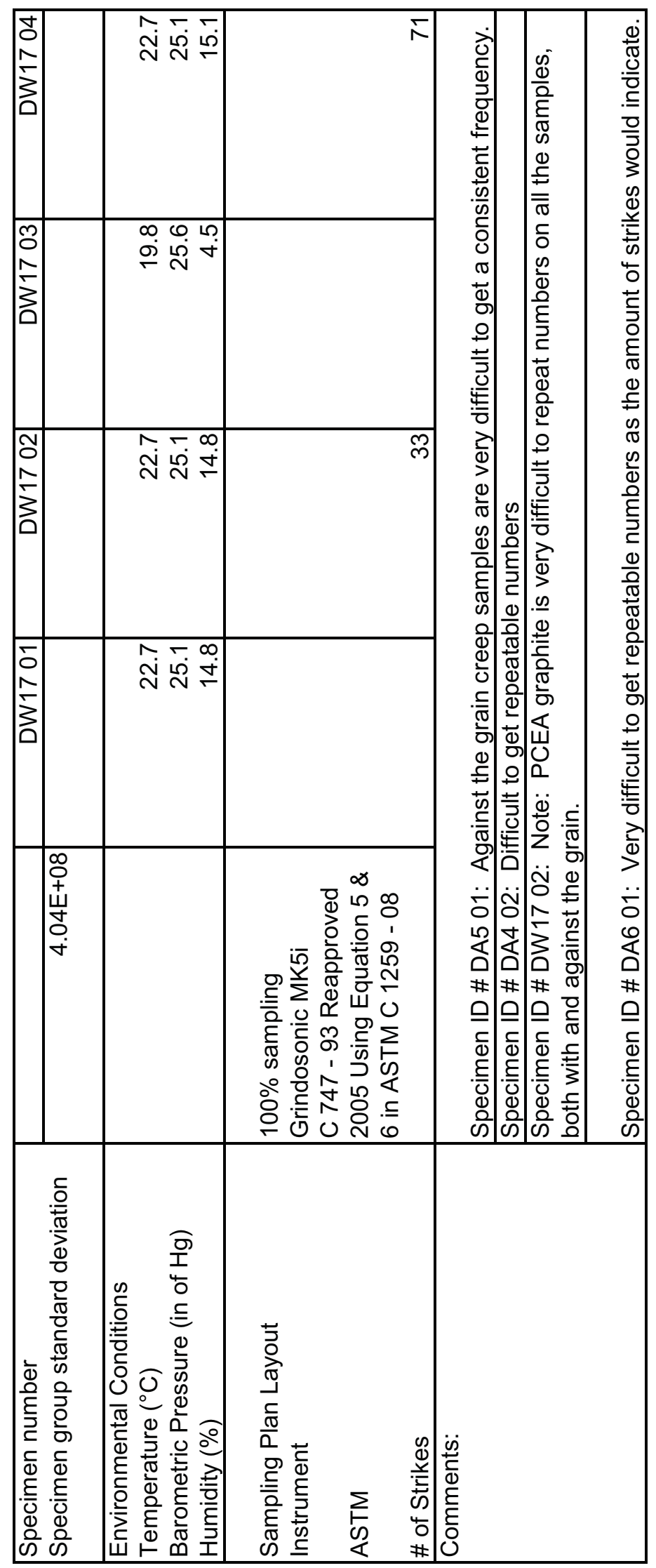




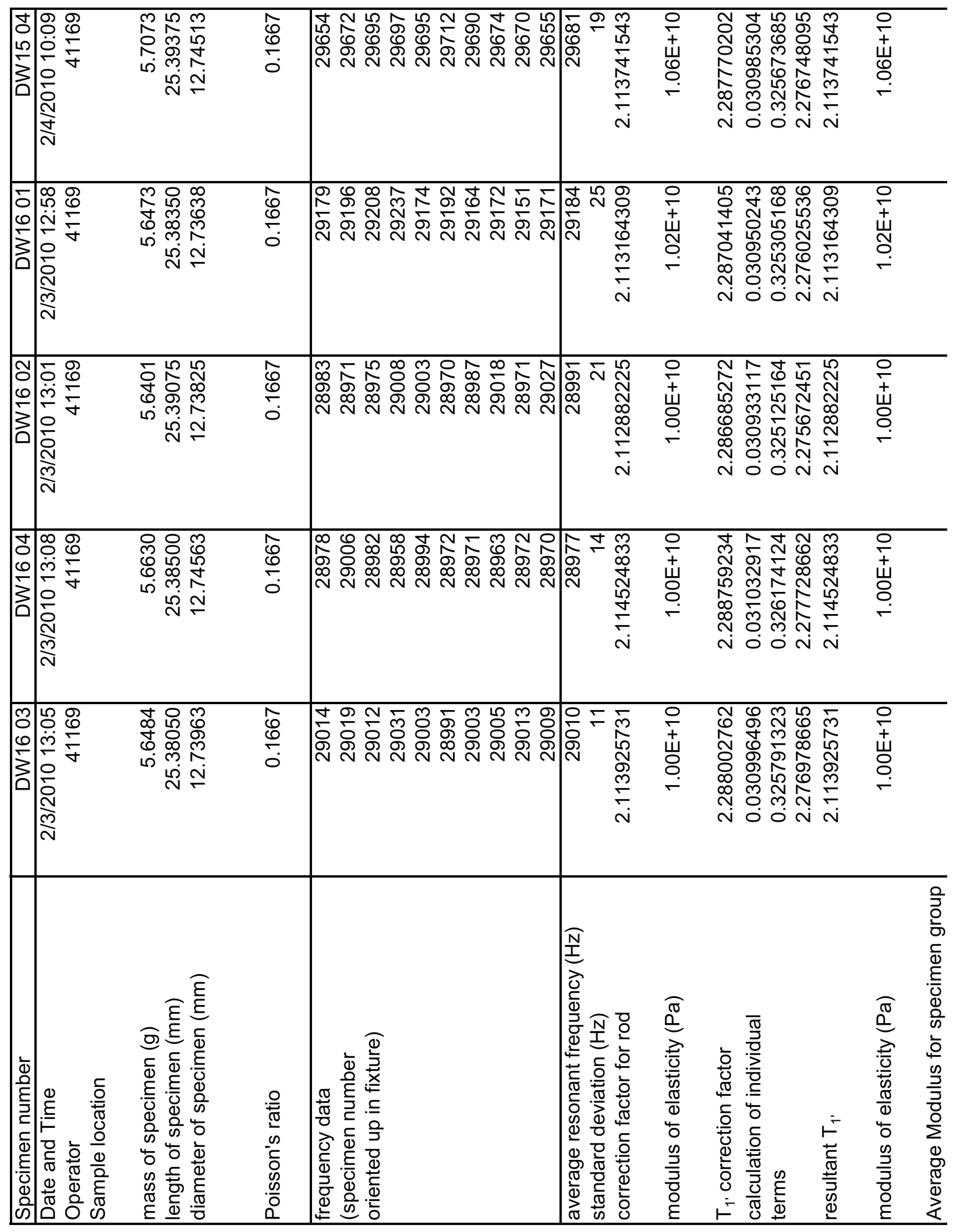




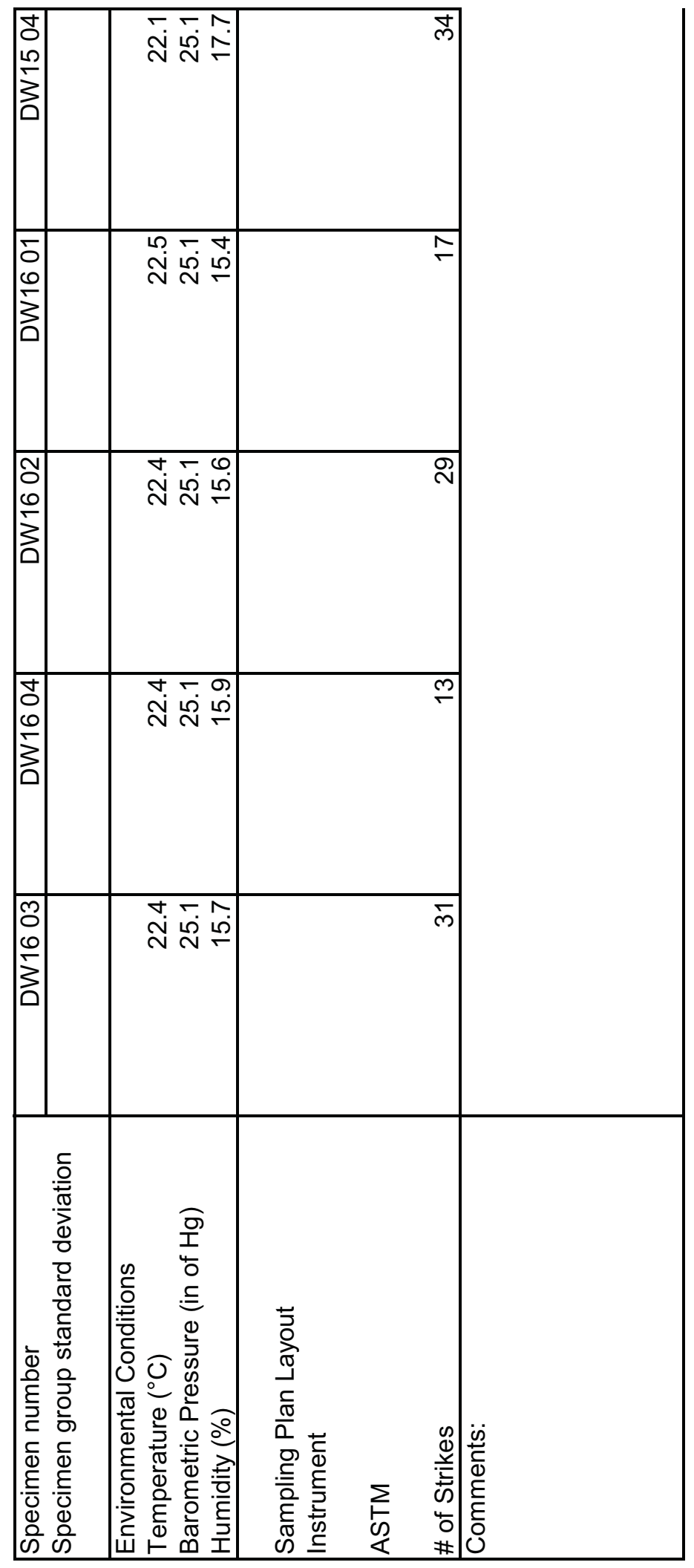




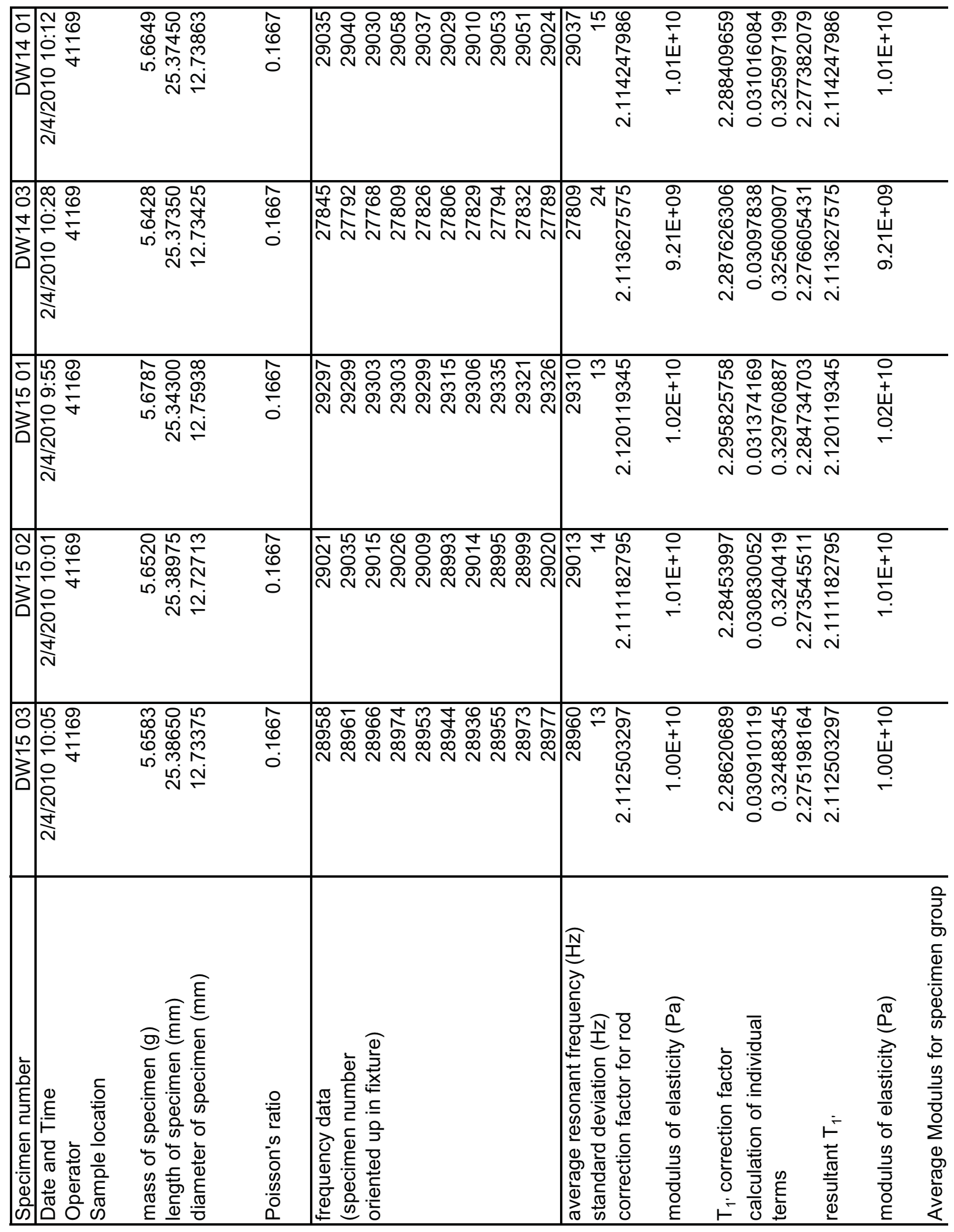




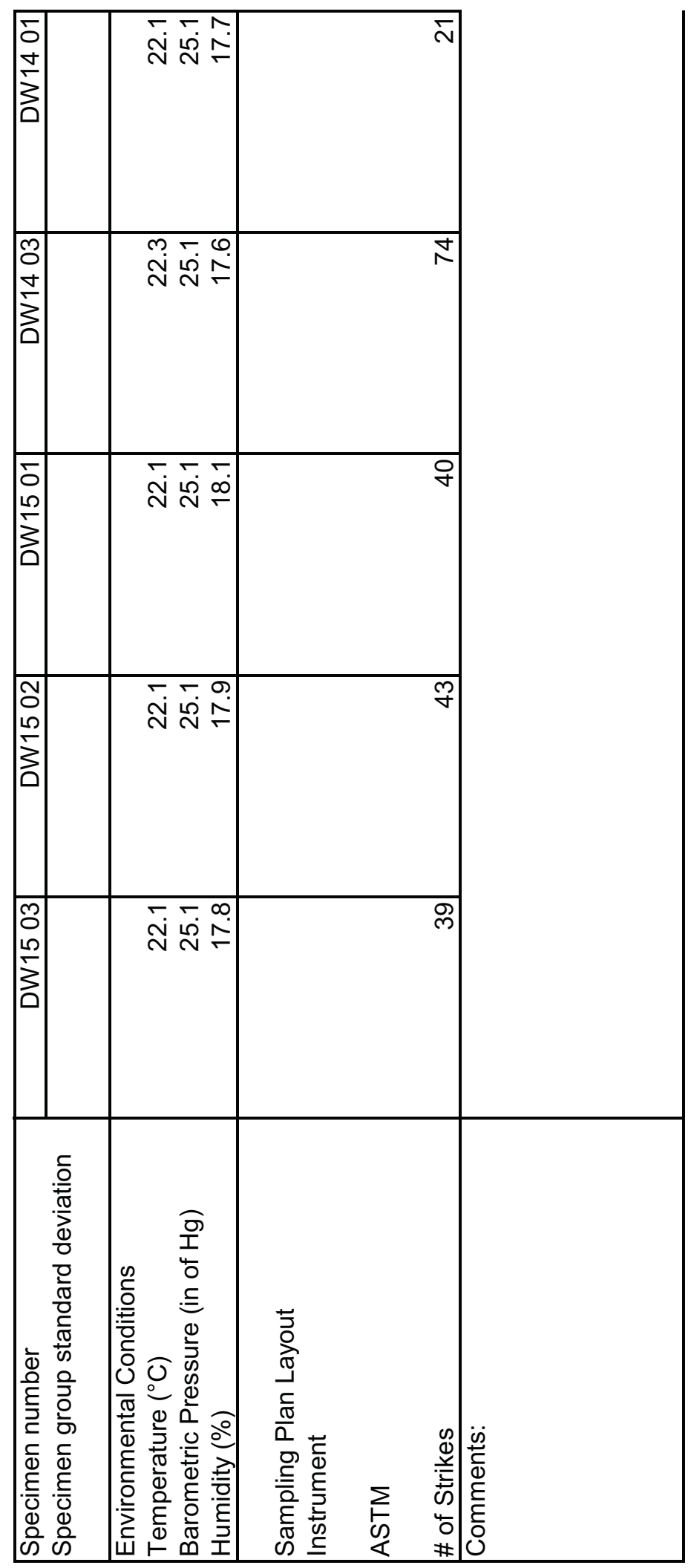




\begin{tabular}{|c|c|c|c|c|c|c|c|c|c|}
\hline \begin{tabular}{l|} 
\\
0 \\
$v$ \\
$\vdots$ \\
$\vdots$ \\
0
\end{tabular} & 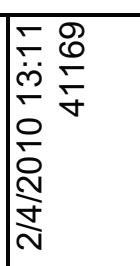 & 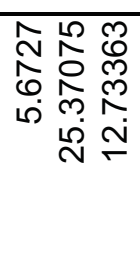 & $\begin{array}{l}\hat{0} \\
\stackrel{0}{0} \\
\dot{0}\end{array}$ & 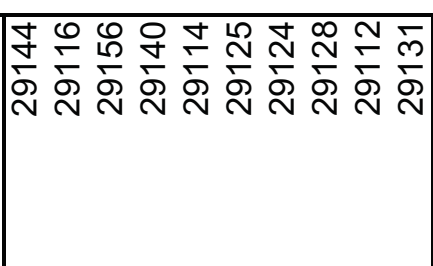 & 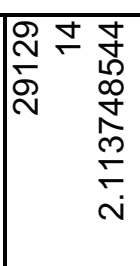 & $\begin{array}{l}\stackrel{0}{+} \\
\stackrel{+}{\sim} \\
\text { O } \\
\stackrel{-}{\leftarrow}\end{array}$ & 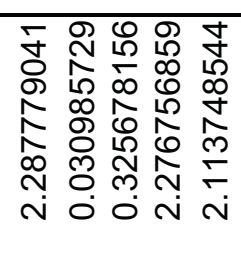 & $\begin{array}{l}\text { OO } \\
\stackrel{+}{W} \\
\stackrel{\text { O̦ }}{\circ}\end{array}$ & \\
\hline $\begin{array}{l}5 \\
0 \\
m \\
\vdots \\
\vdots \\
0\end{array}$ & 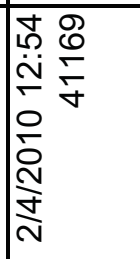 & 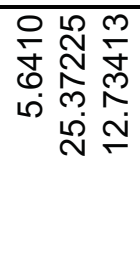 & $\begin{array}{l}\overline{0} \\
\stackrel{0}{0} \\
\vdots\end{array}$ & 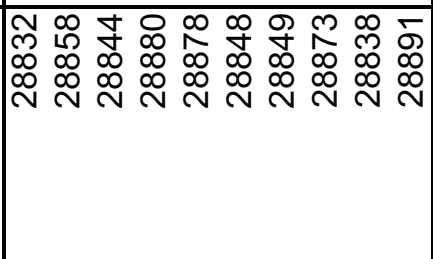 & 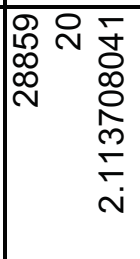 & 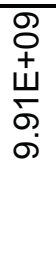 & 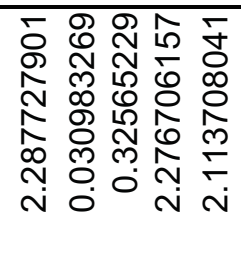 & 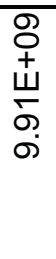 & \\
\hline $\begin{array}{l}\bar{N} \\
0 \\
m \\
\vdots \\
\vdots \\
0\end{array}$ & 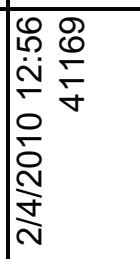 & 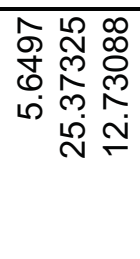 & $\begin{array}{l}\hat{\theta} \\
\stackrel{\oplus}{0} \\
\dot{0}\end{array}$ & 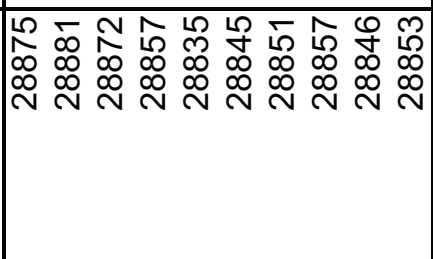 & 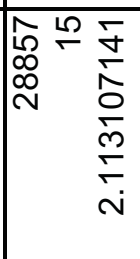 & 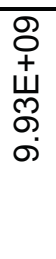 & 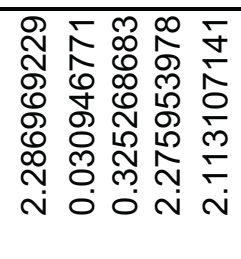 & $\begin{array}{l}\text { क् } \\
+ \\
\dot{+} \\
\text { ळ̆ } \\
\text { aे }\end{array}$ & \\
\hline $\begin{array}{l}m \\
0 \\
m \\
\vdots \\
\vdots \\
0\end{array}$ & 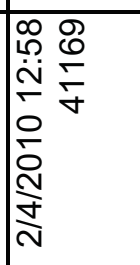 & 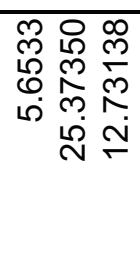 & $\begin{array}{l}\hat{\theta} \\
\stackrel{0}{0}\end{array}$ & 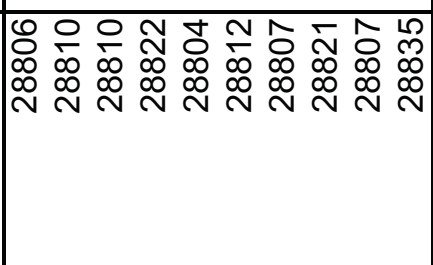 & 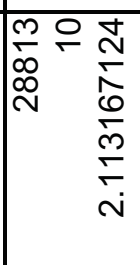 & 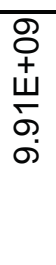 & 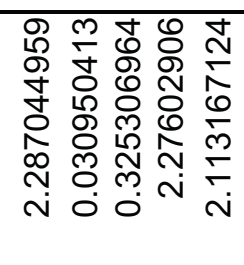 & 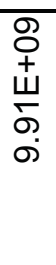 & \\
\hline $\begin{array}{l}\text { J } \\
\text { m } \\
\vdots \\
\vdots\end{array}$ & 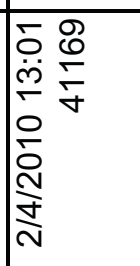 & 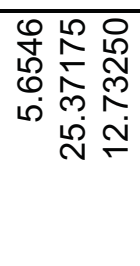 & $\begin{array}{l}\overline{0} \\
\stackrel{0}{0} \\
\vdots\end{array}$ & 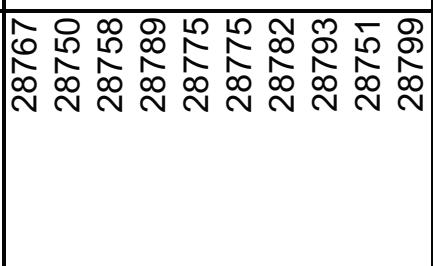 & 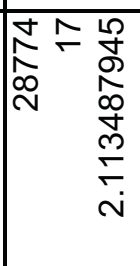 & $\begin{array}{l}\text { क } \\
+ \\
\dot{1} \\
\infty \\
\infty \\
\infty\end{array}$ & 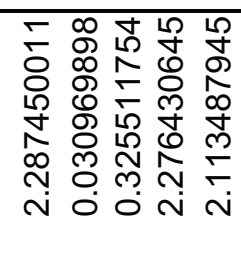 & $\begin{array}{l}\text { ச } \\
+ \\
+ \\
\infty \\
\infty \\
0\end{array}$ & \\
\hline 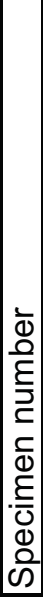 & 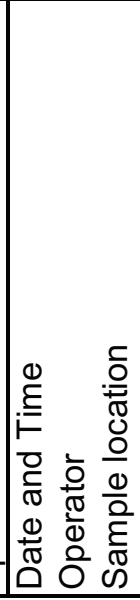 & 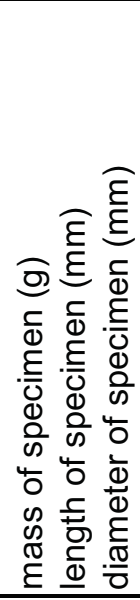 & 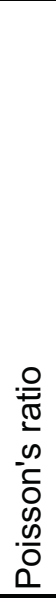 & 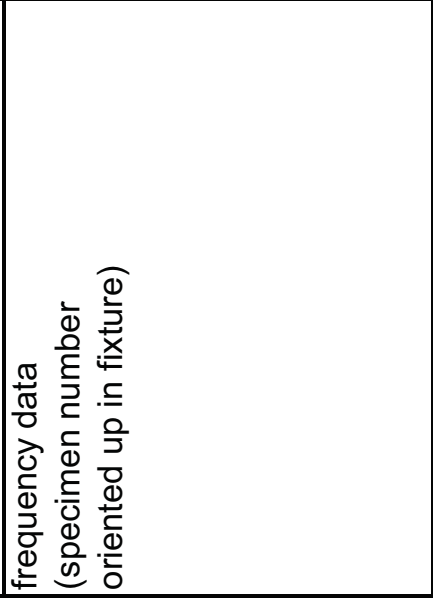 & 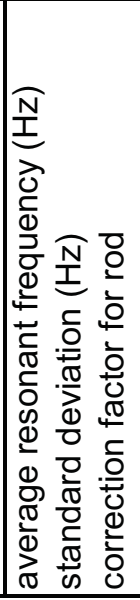 & $\begin{array}{l}\pi \\
\pi \\
0 \\
\frac{\pi}{0} \\
0 \\
\frac{0}{0} \\
\frac{\pi}{0} \\
0 \\
40 \\
0 \\
\frac{0}{5} \\
\frac{5}{0} \\
\frac{0}{\varepsilon}\end{array}$ & 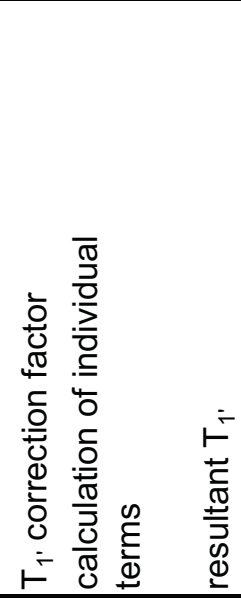 & 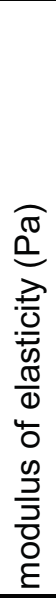 & 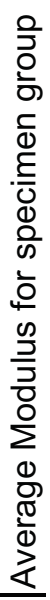 \\
\hline
\end{tabular}




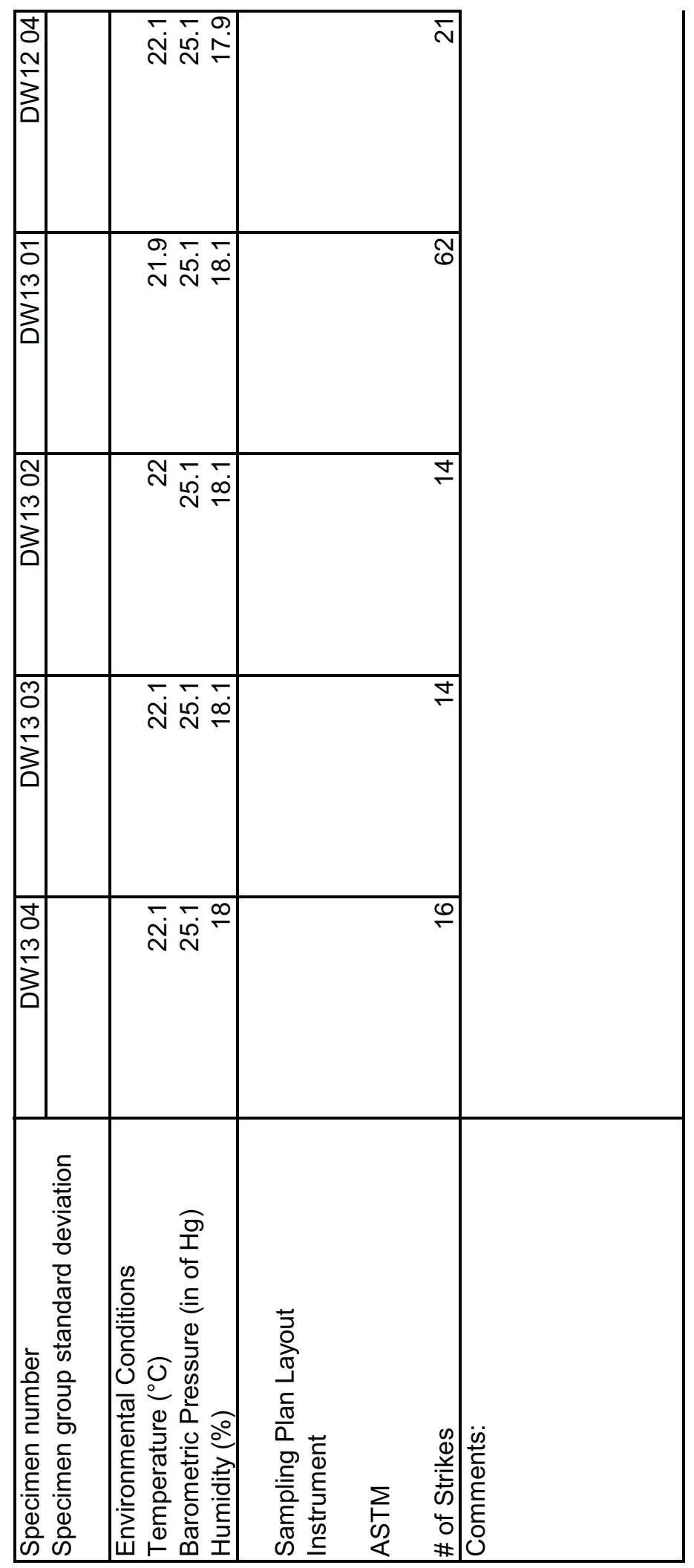




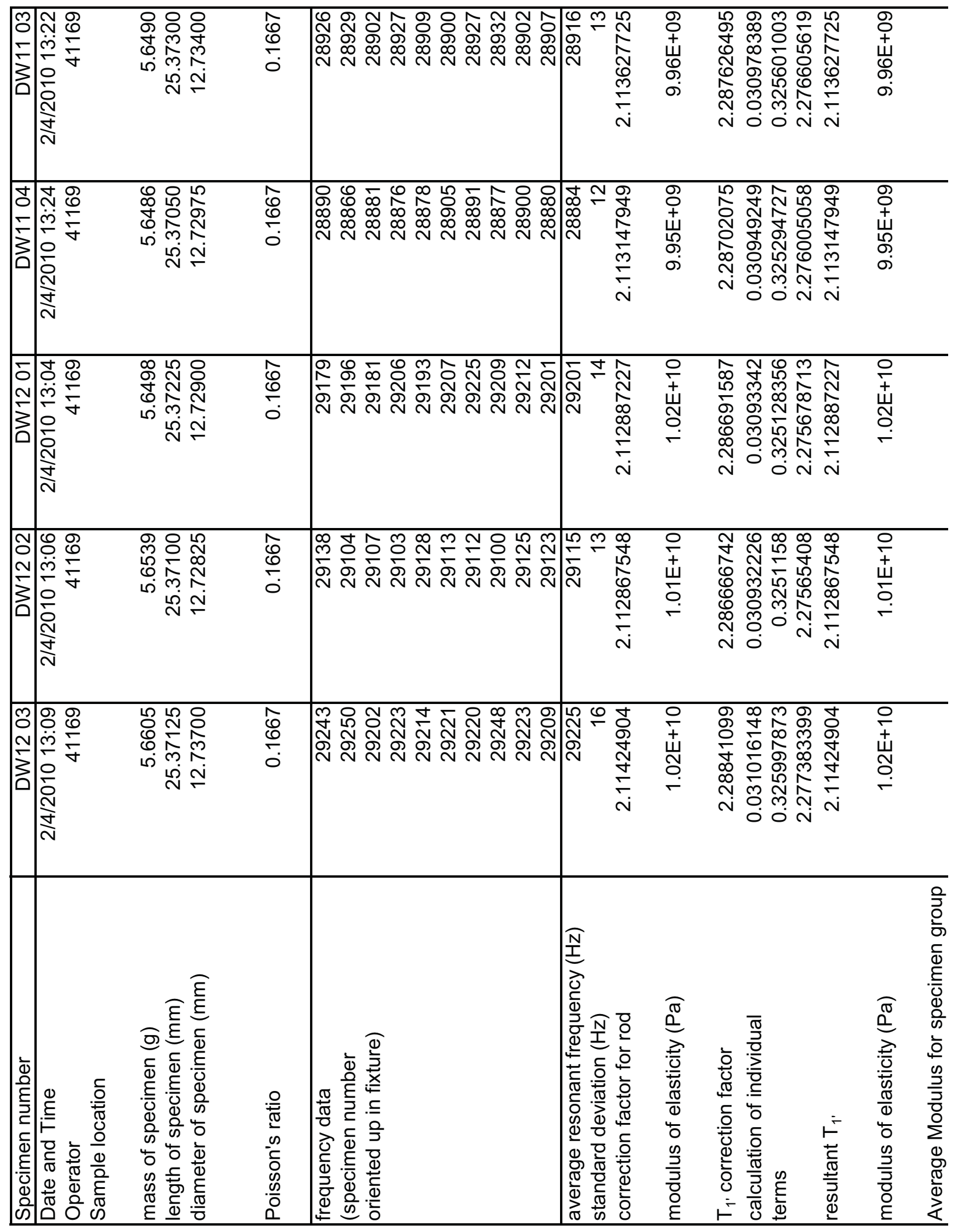




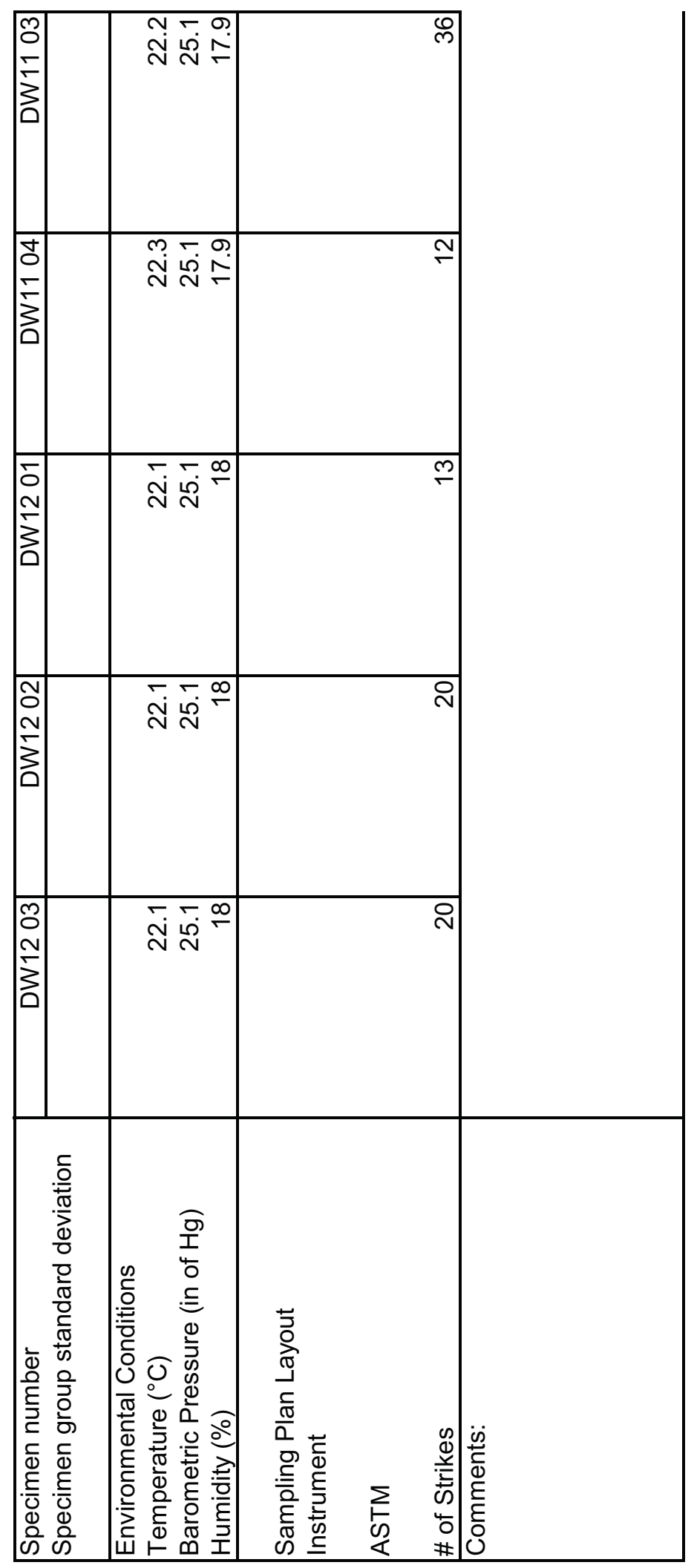




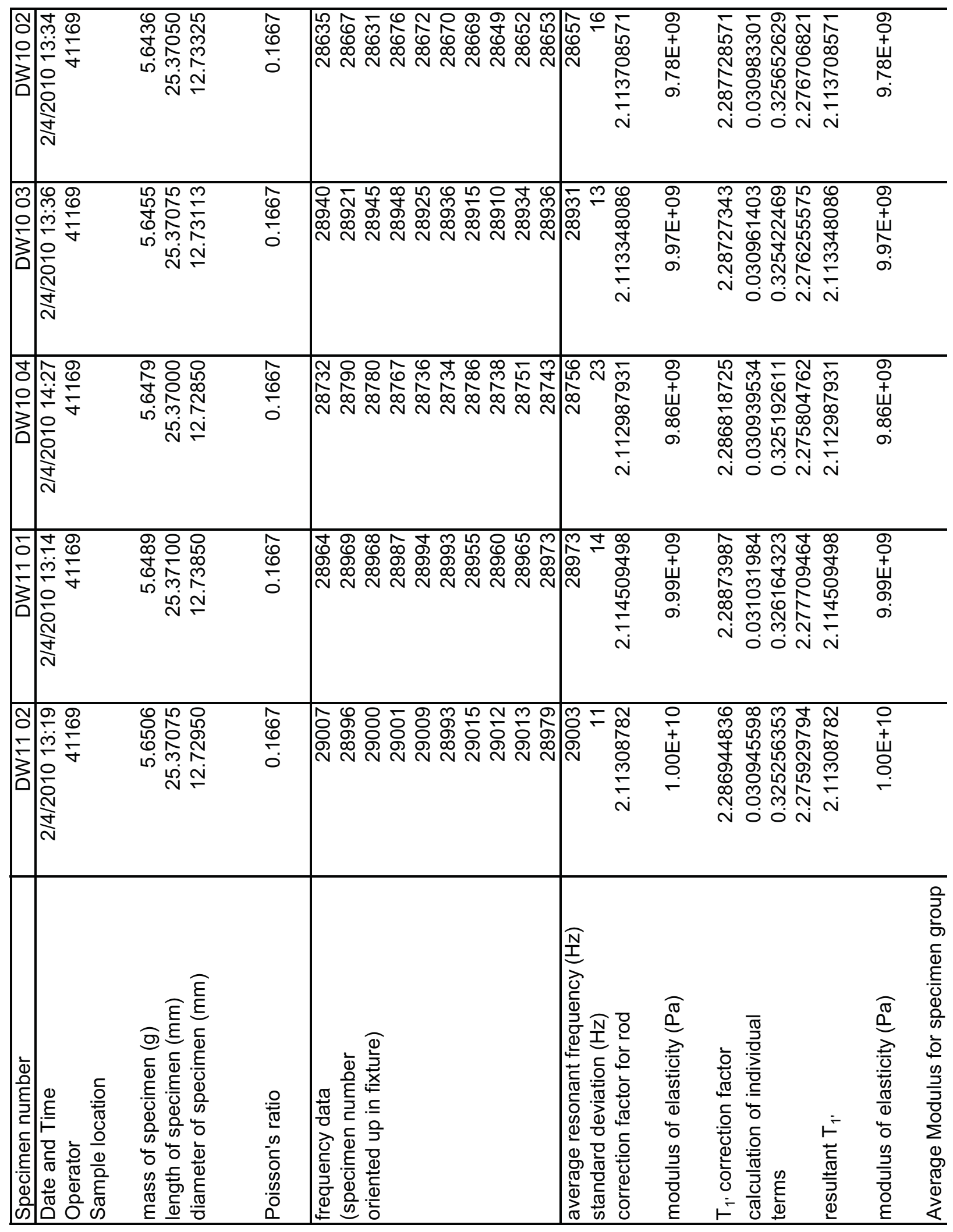




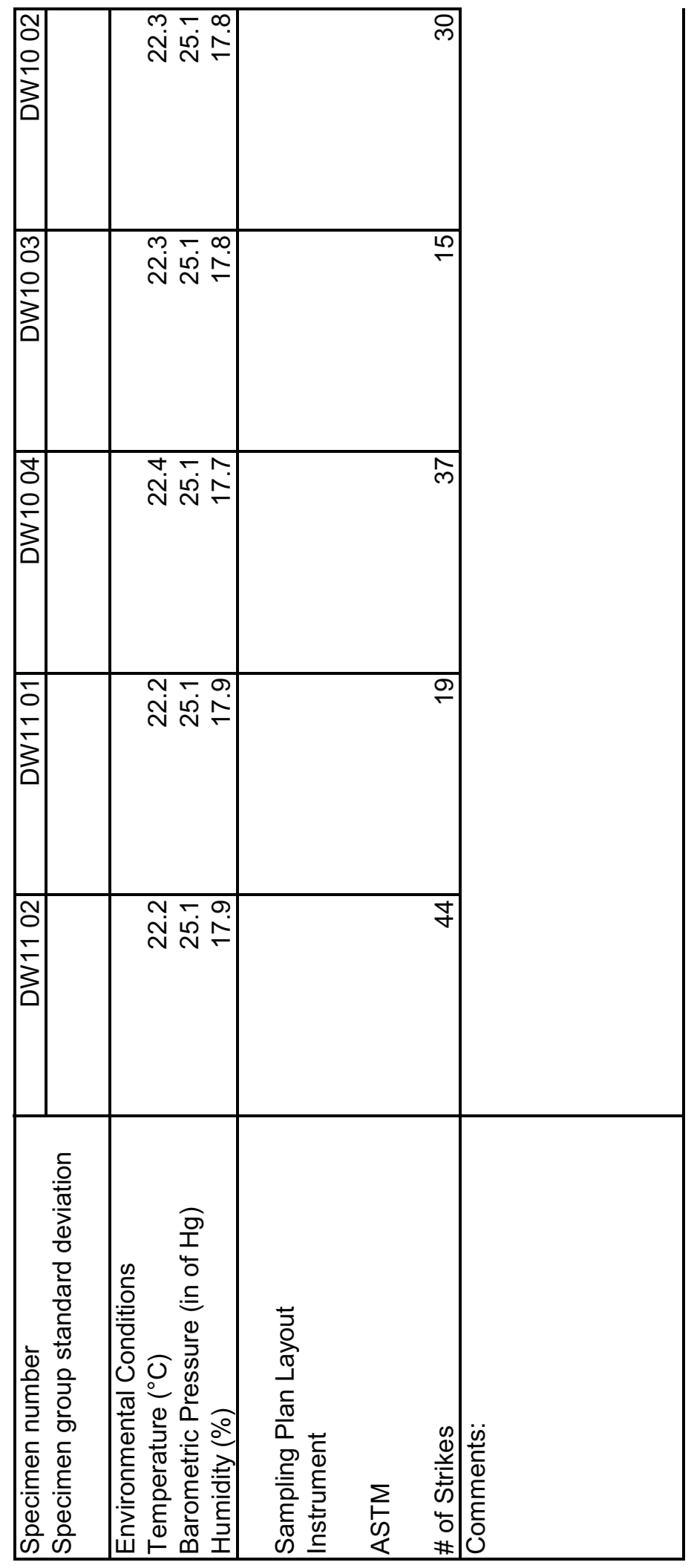




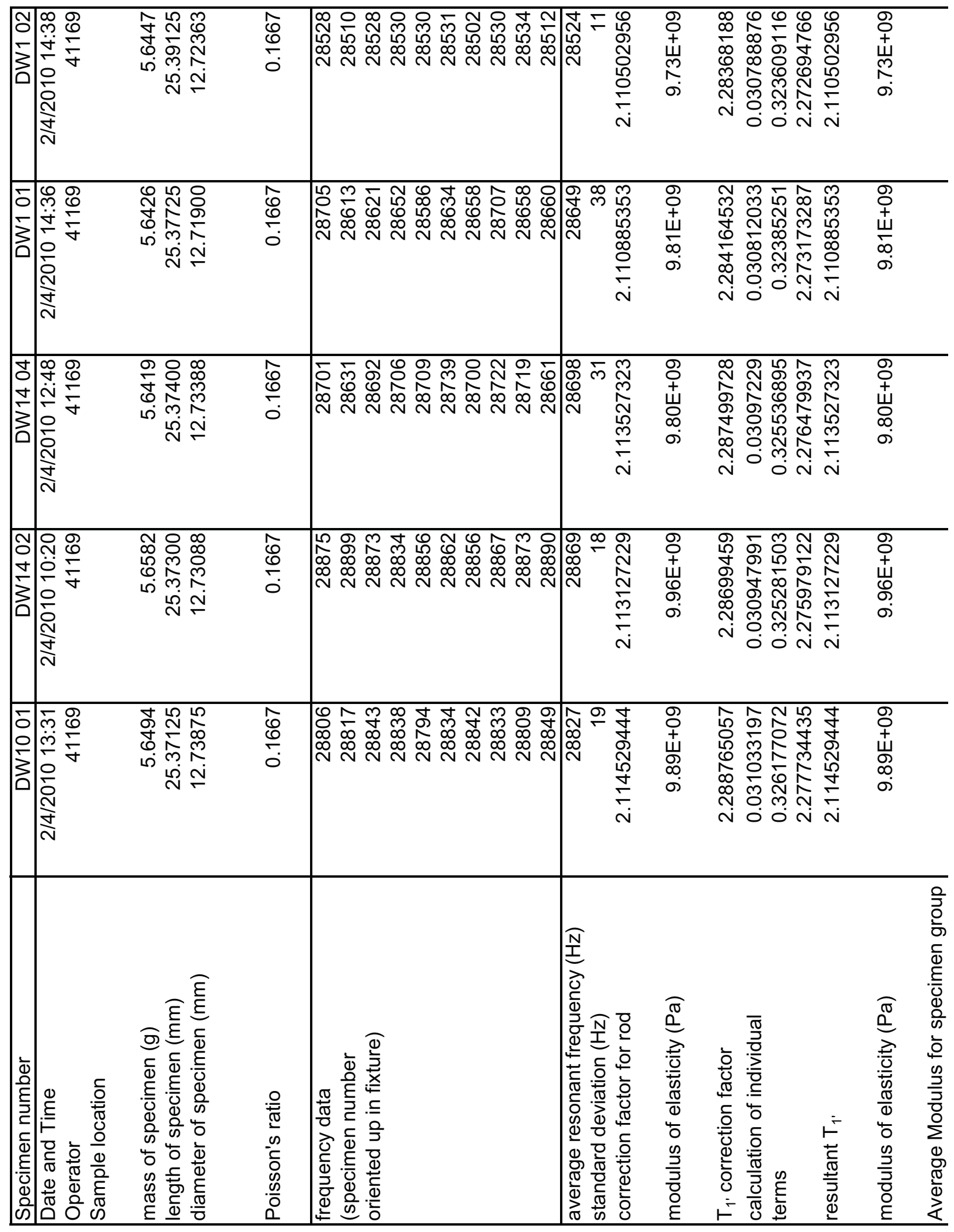




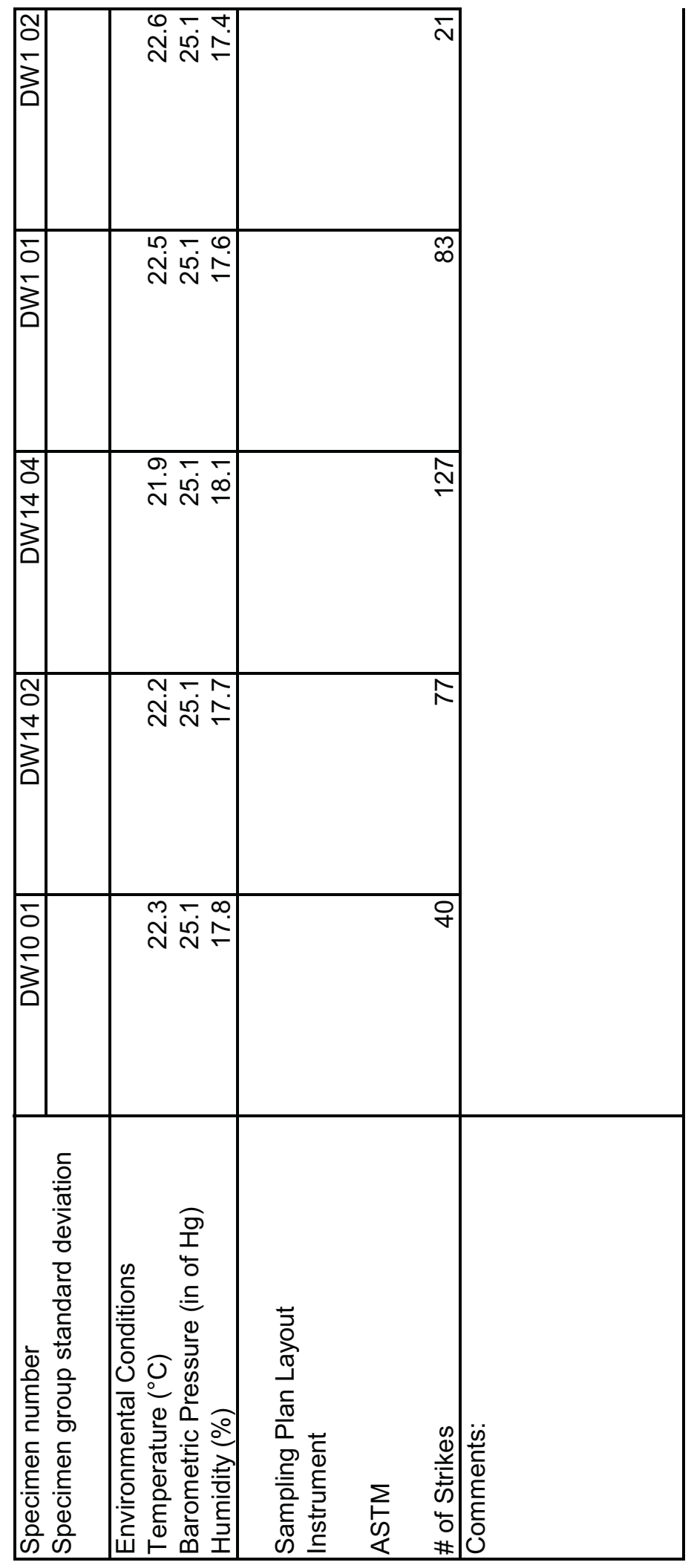

$=$ 


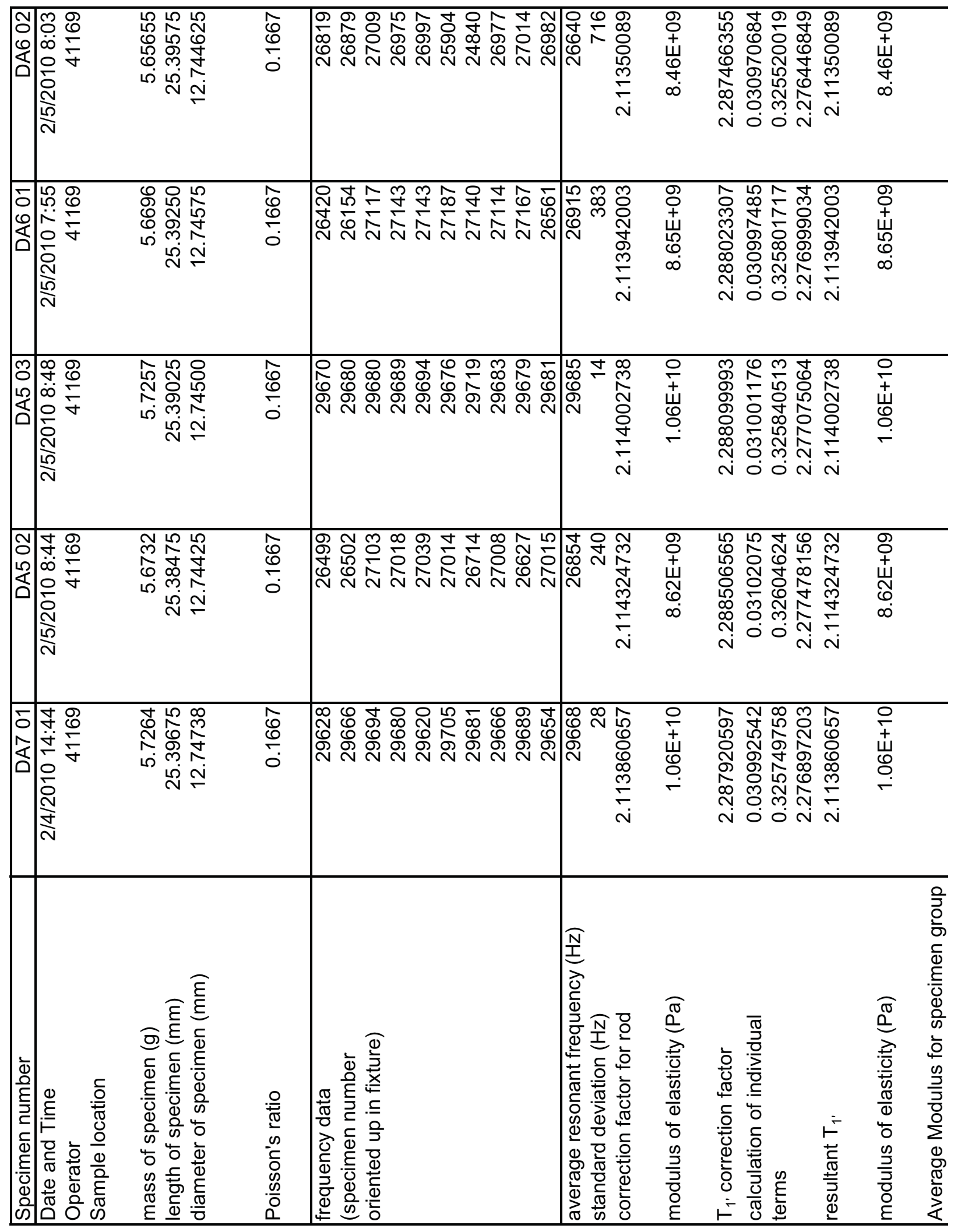




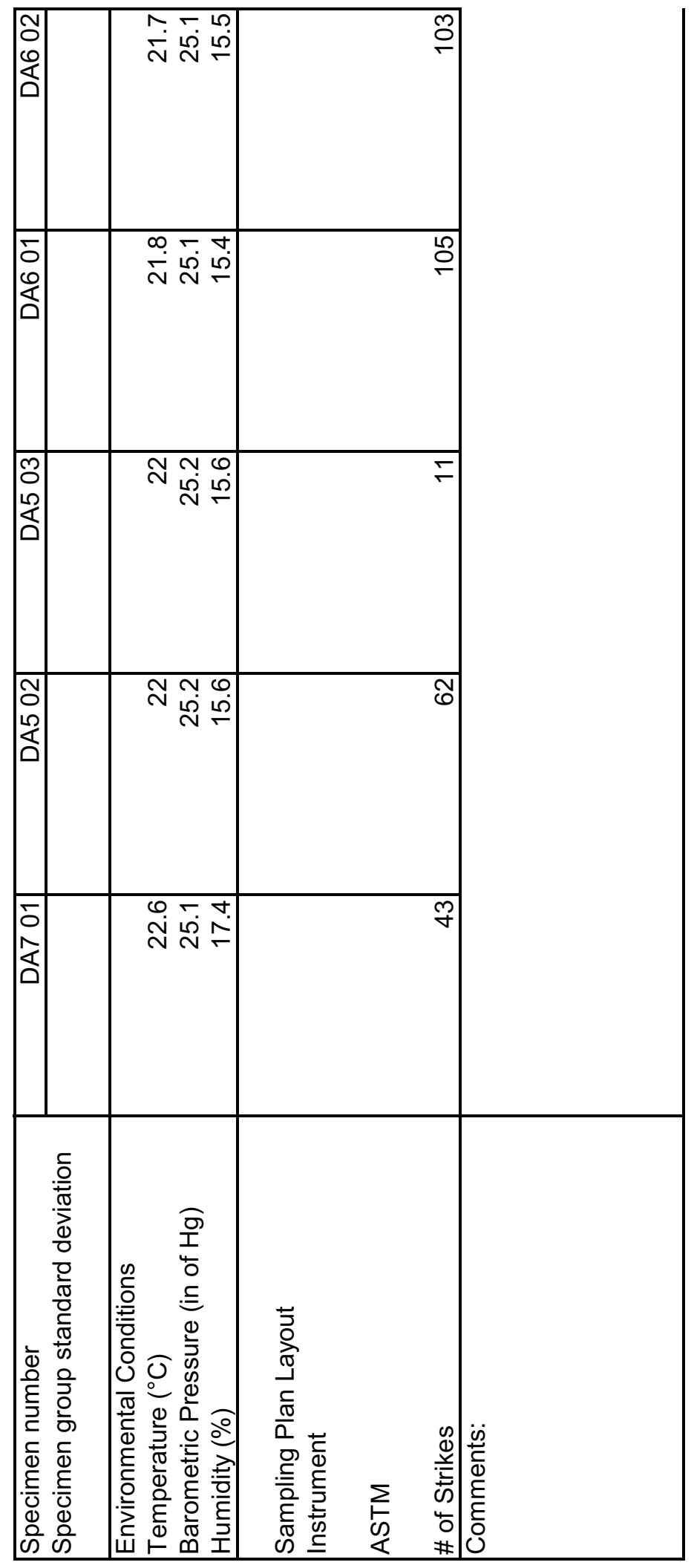

$\frac{m}{\infty}$ 


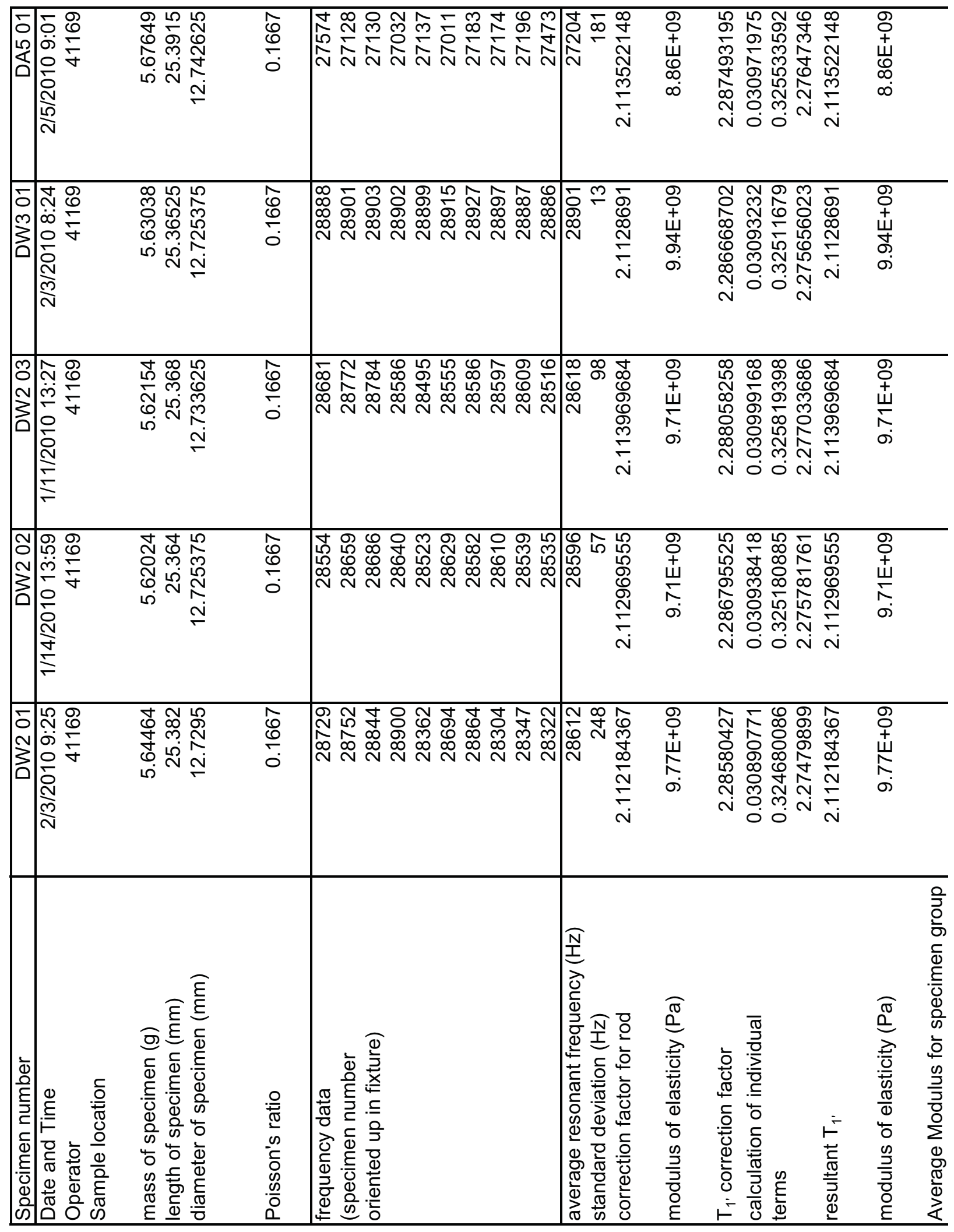




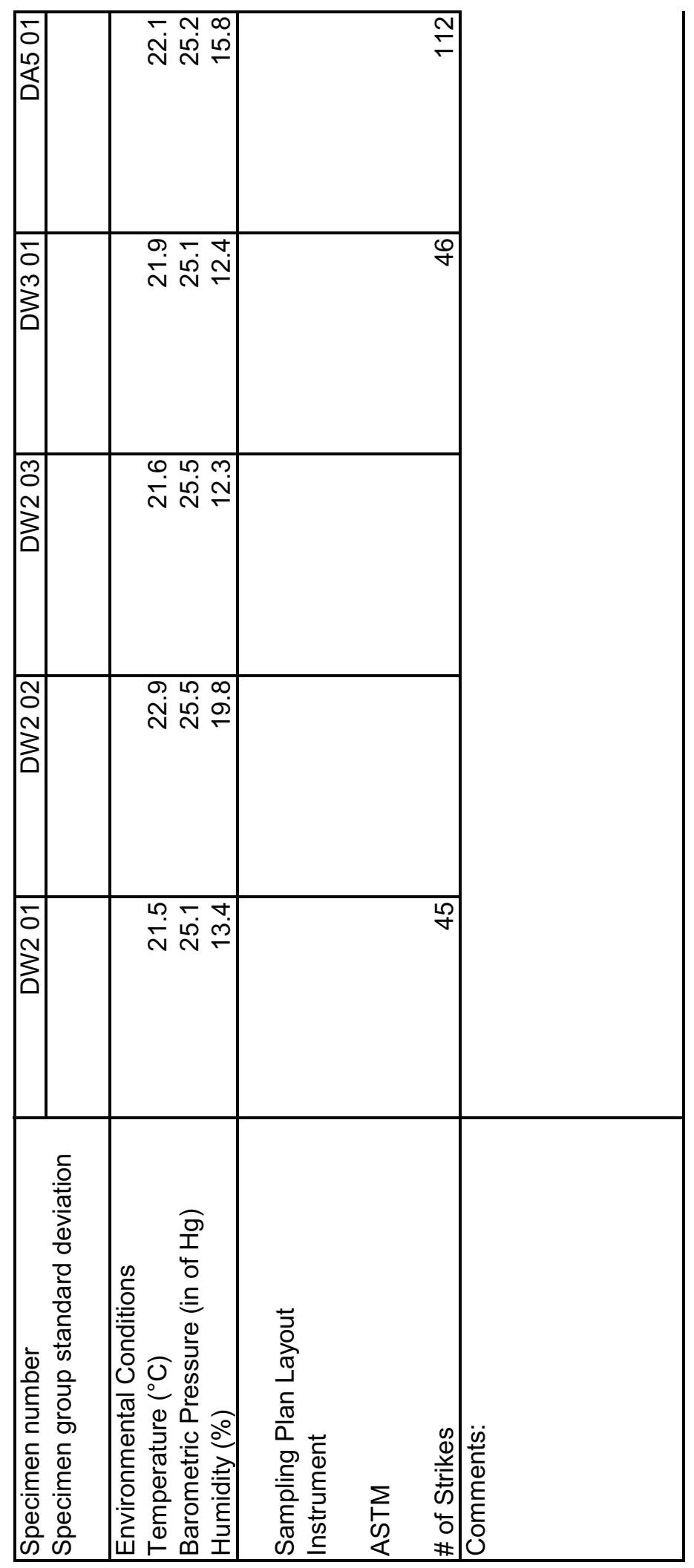




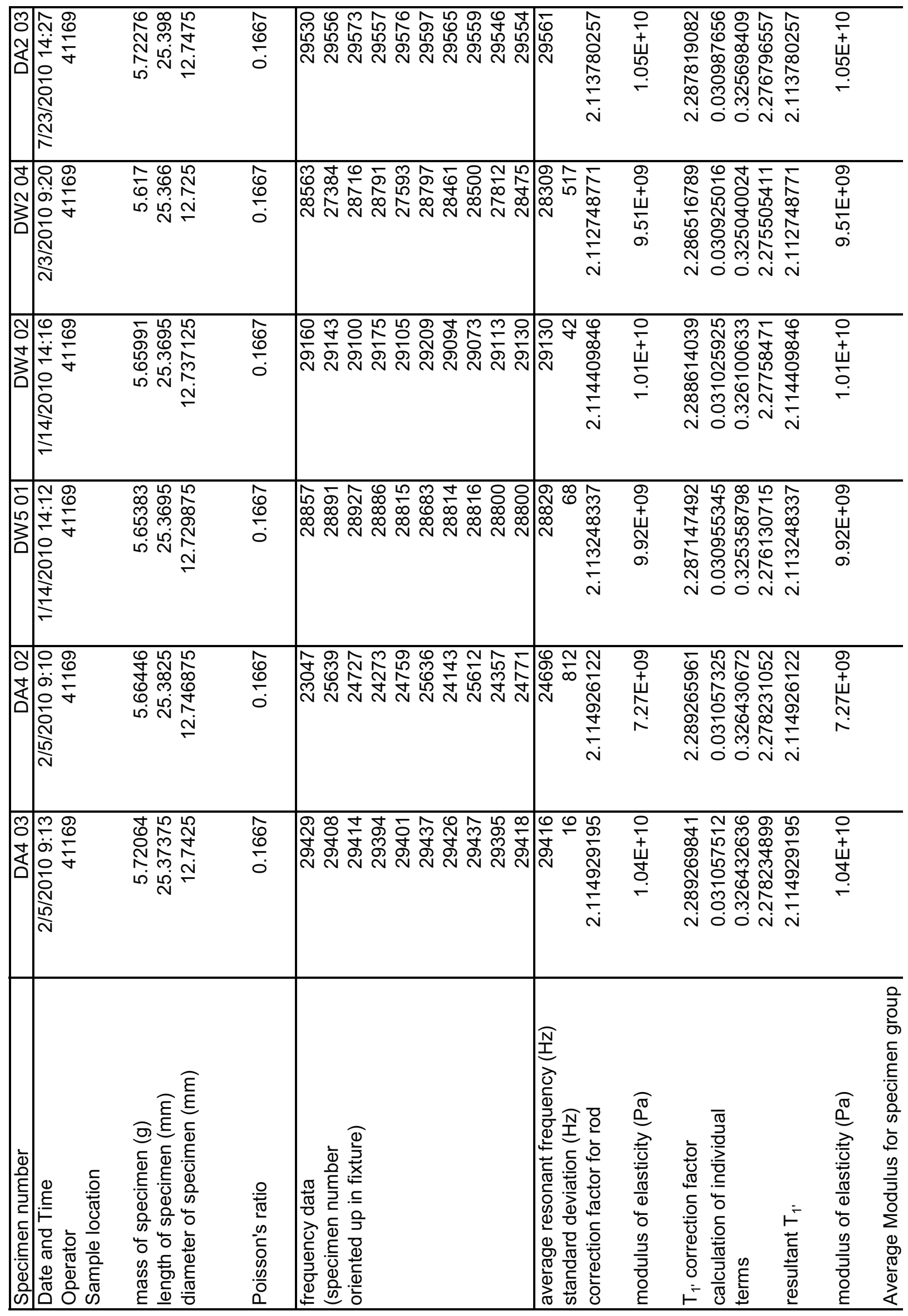




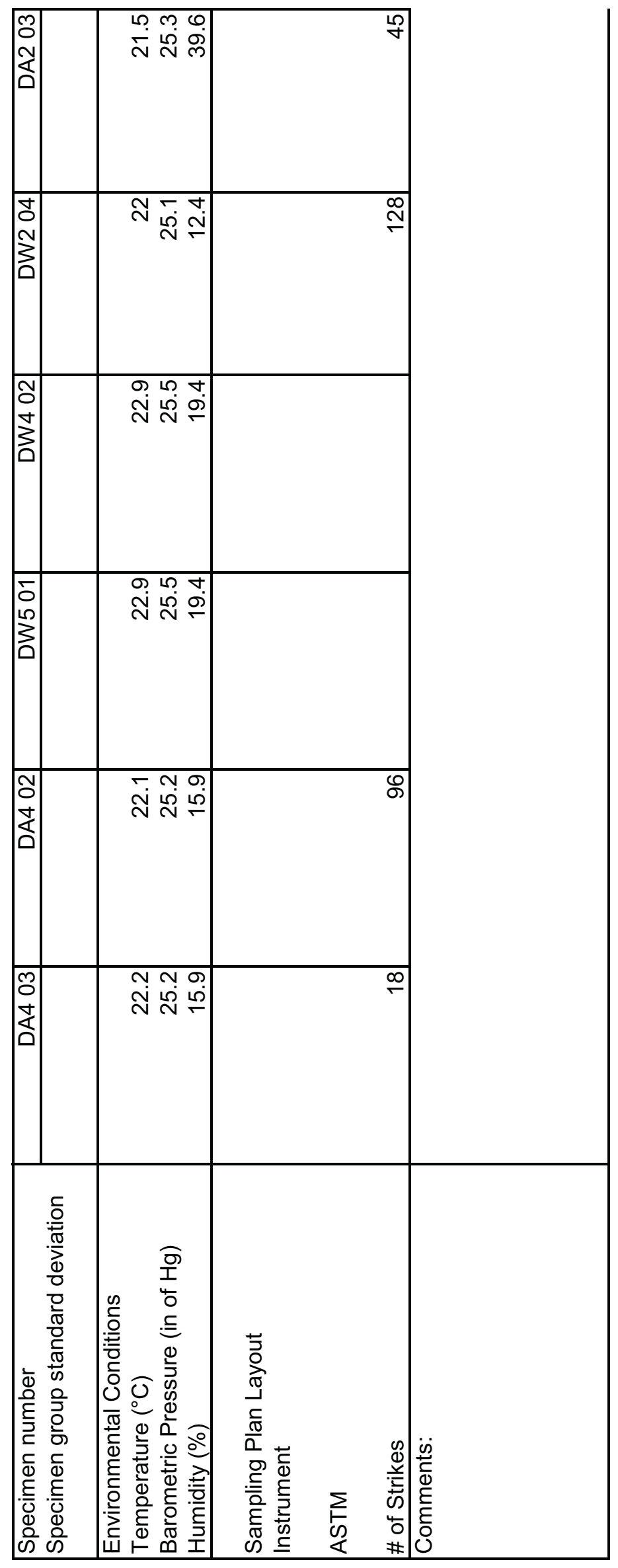

$\frac{1}{\infty}$ 


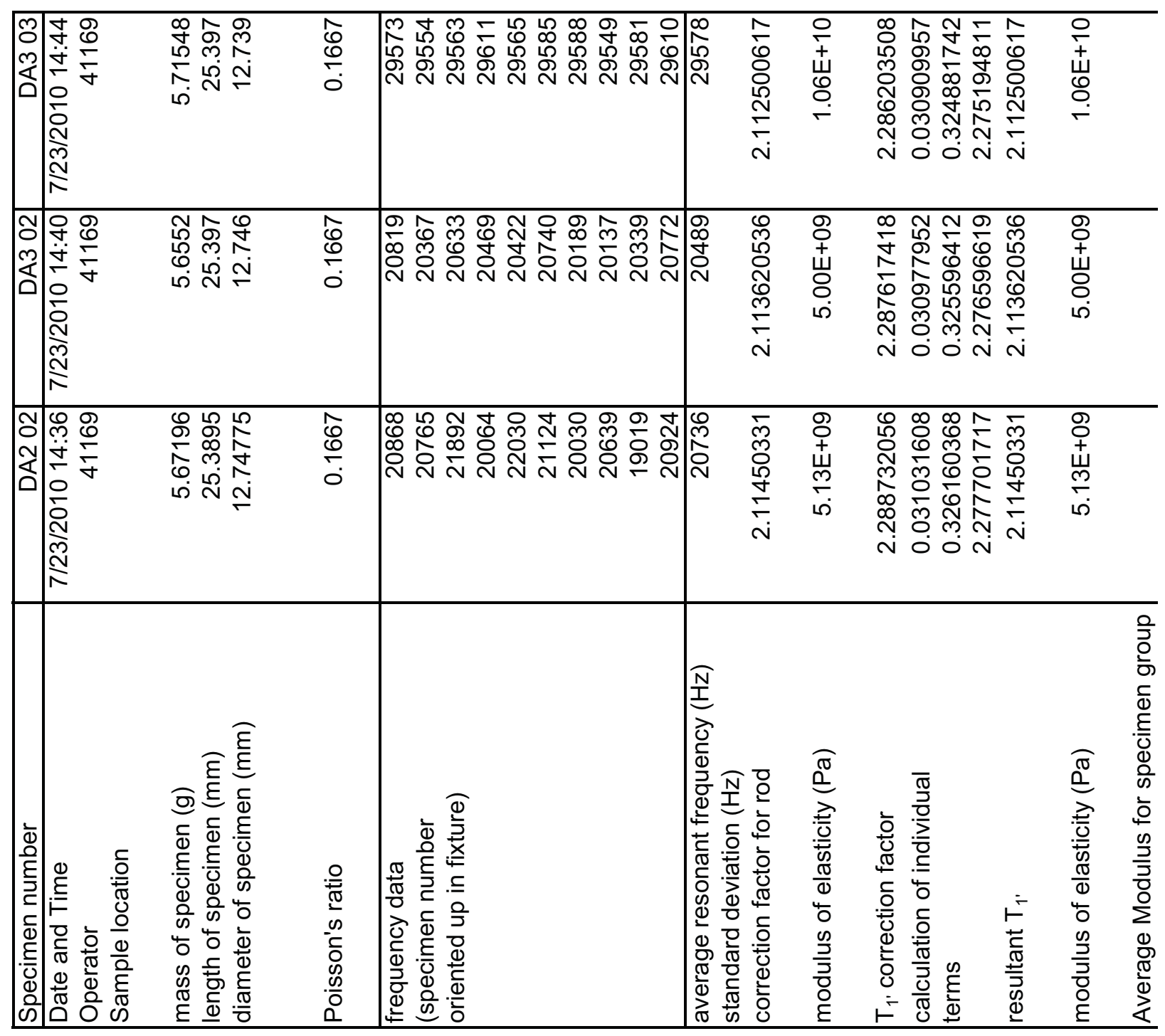




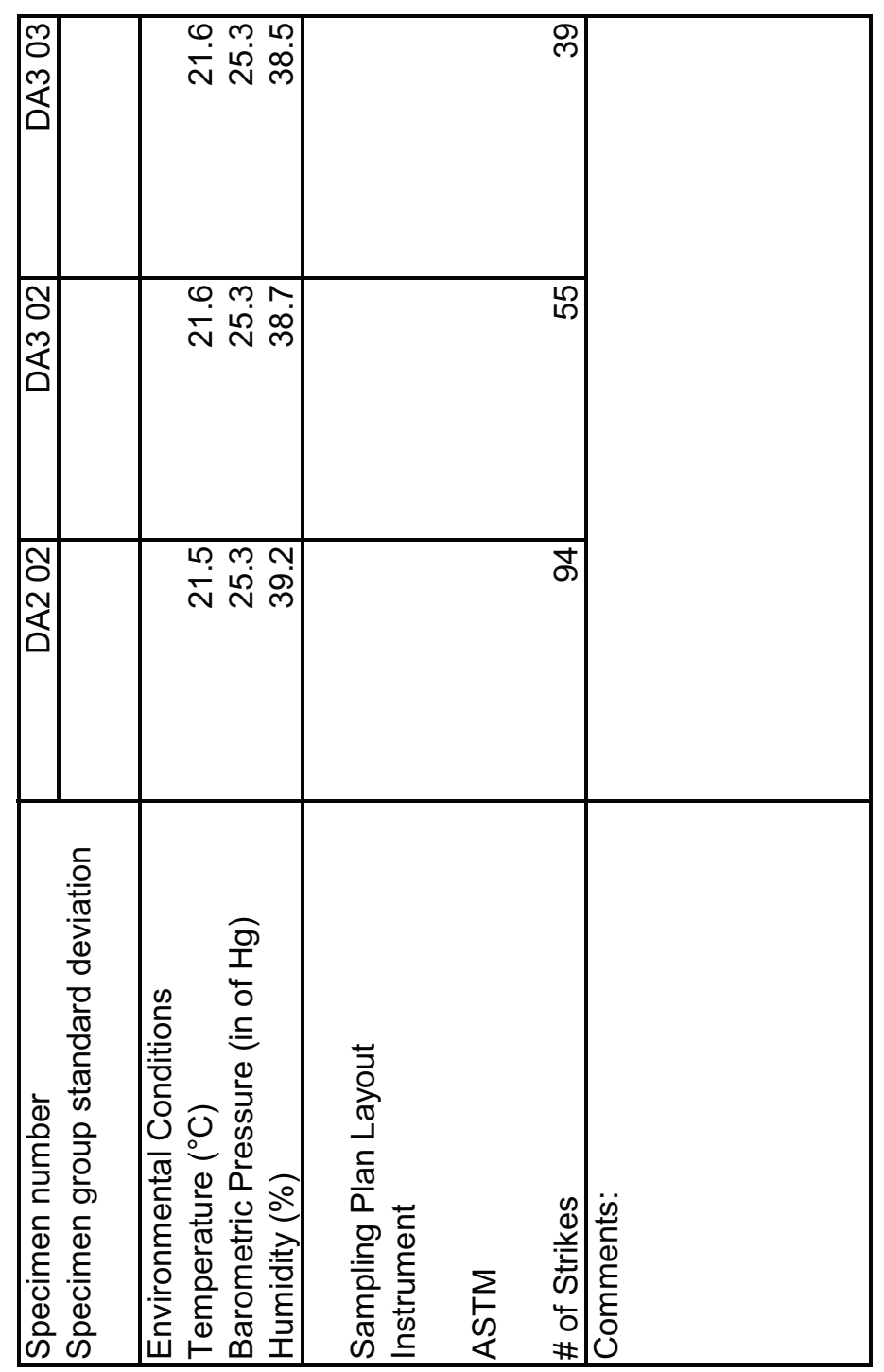




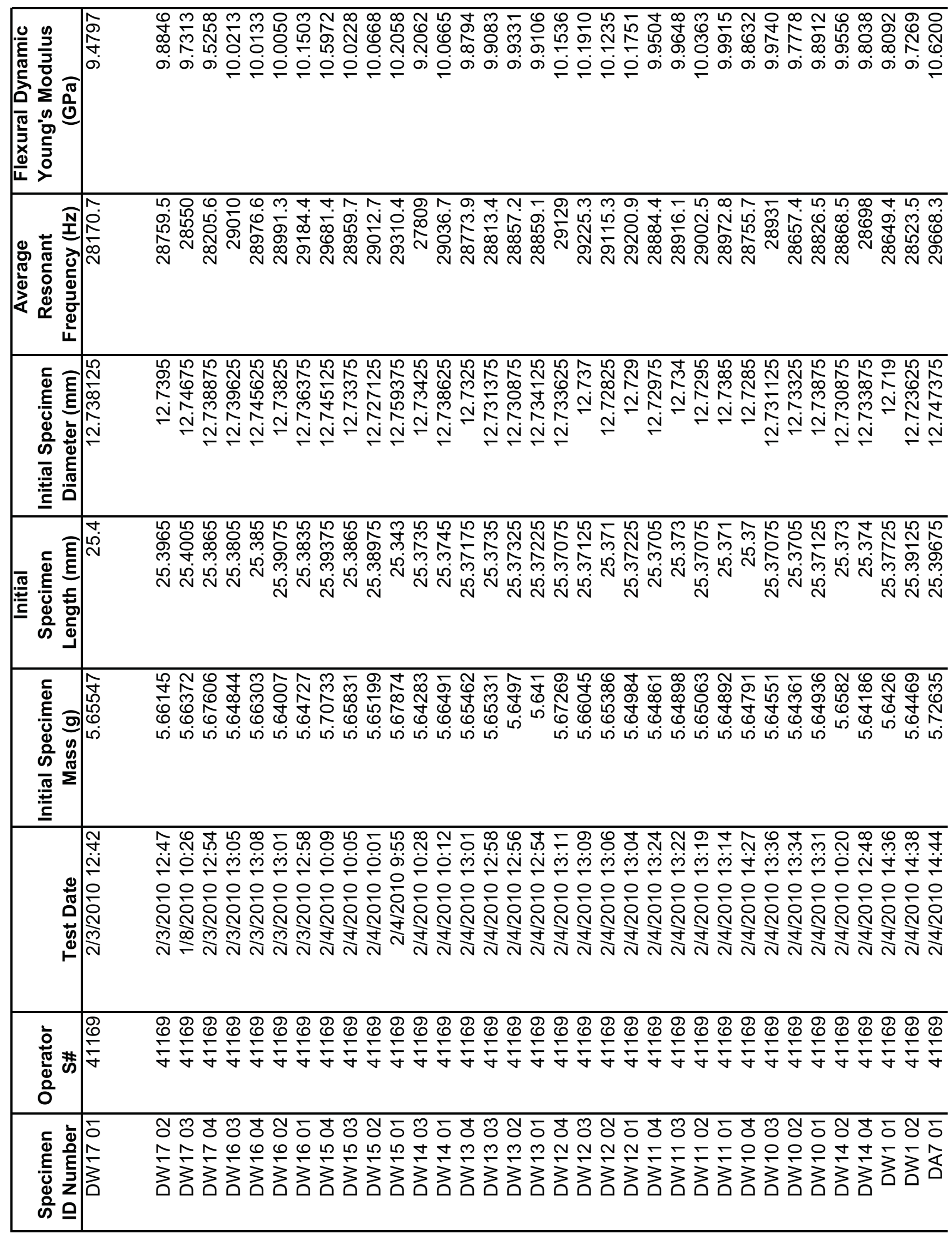




\begin{tabular}{|c|c|c|c|c|}
\hline 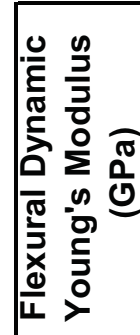 & 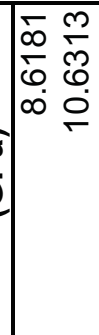 & $\begin{array}{l}\infty \\
\text { M }\end{array}$ & 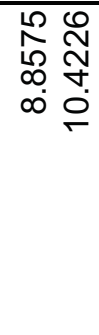 & 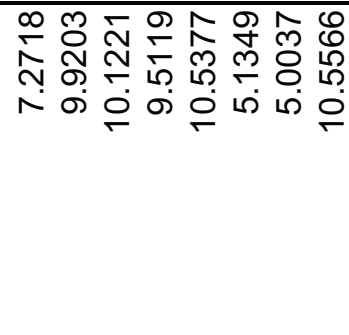 \\
\hline 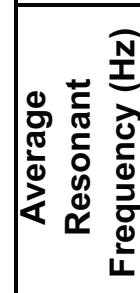 & 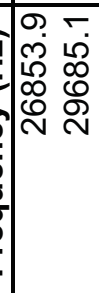 & $\begin{array}{l}0 \\
\end{array}$ & 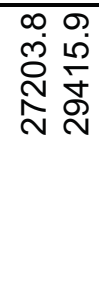 & 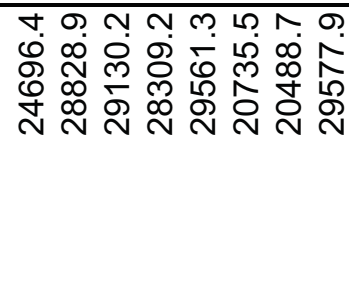 \\
\hline 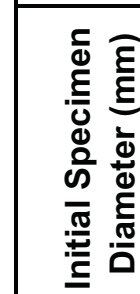 & 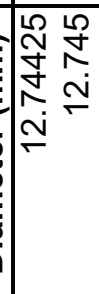 & 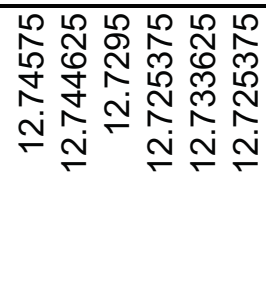 & 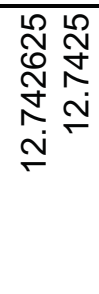 & 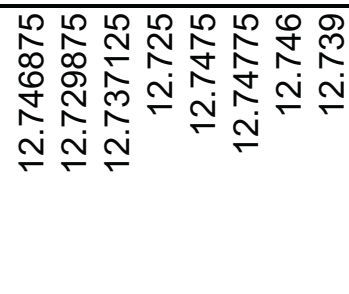 \\
\hline 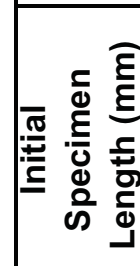 & 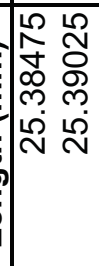 & 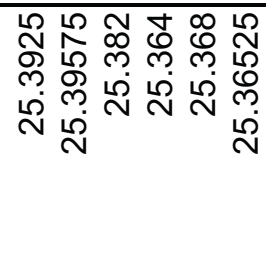 & 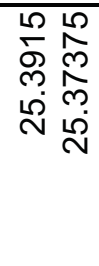 & 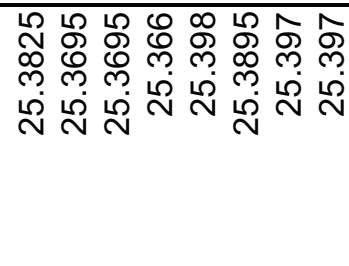 \\
\hline 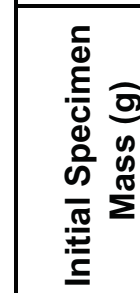 & 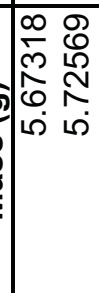 & 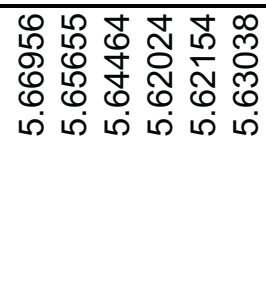 & 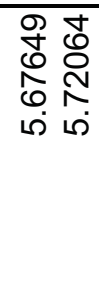 & 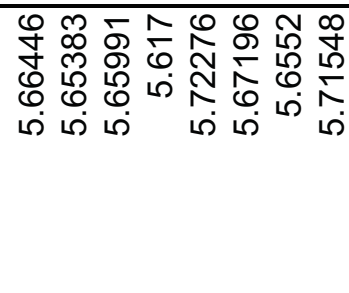 \\
\hline 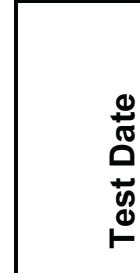 & 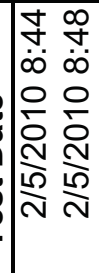 & 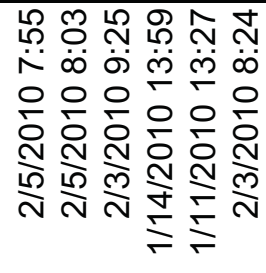 & 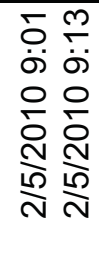 & 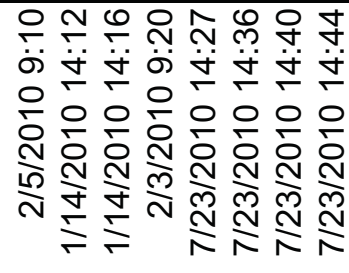 \\
\hline 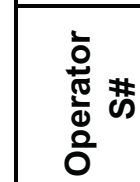 & 舟 & 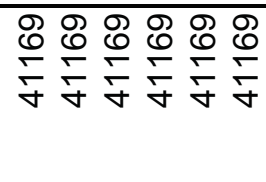 & 电 & 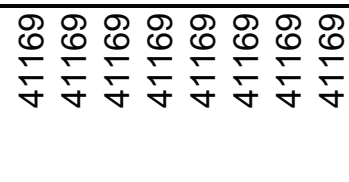 \\
\hline 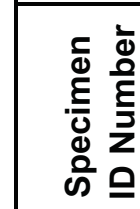 & $\begin{array}{ll}10 & 0 \\
0 & 0 \\
10 & 10 \\
0 & 0 \\
0 & 0\end{array}$ & 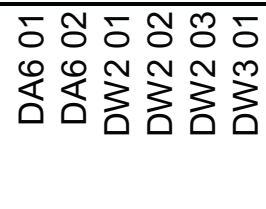 & 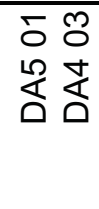 & 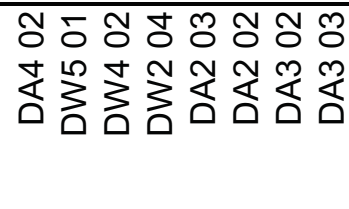 \\
\hline
\end{tabular}




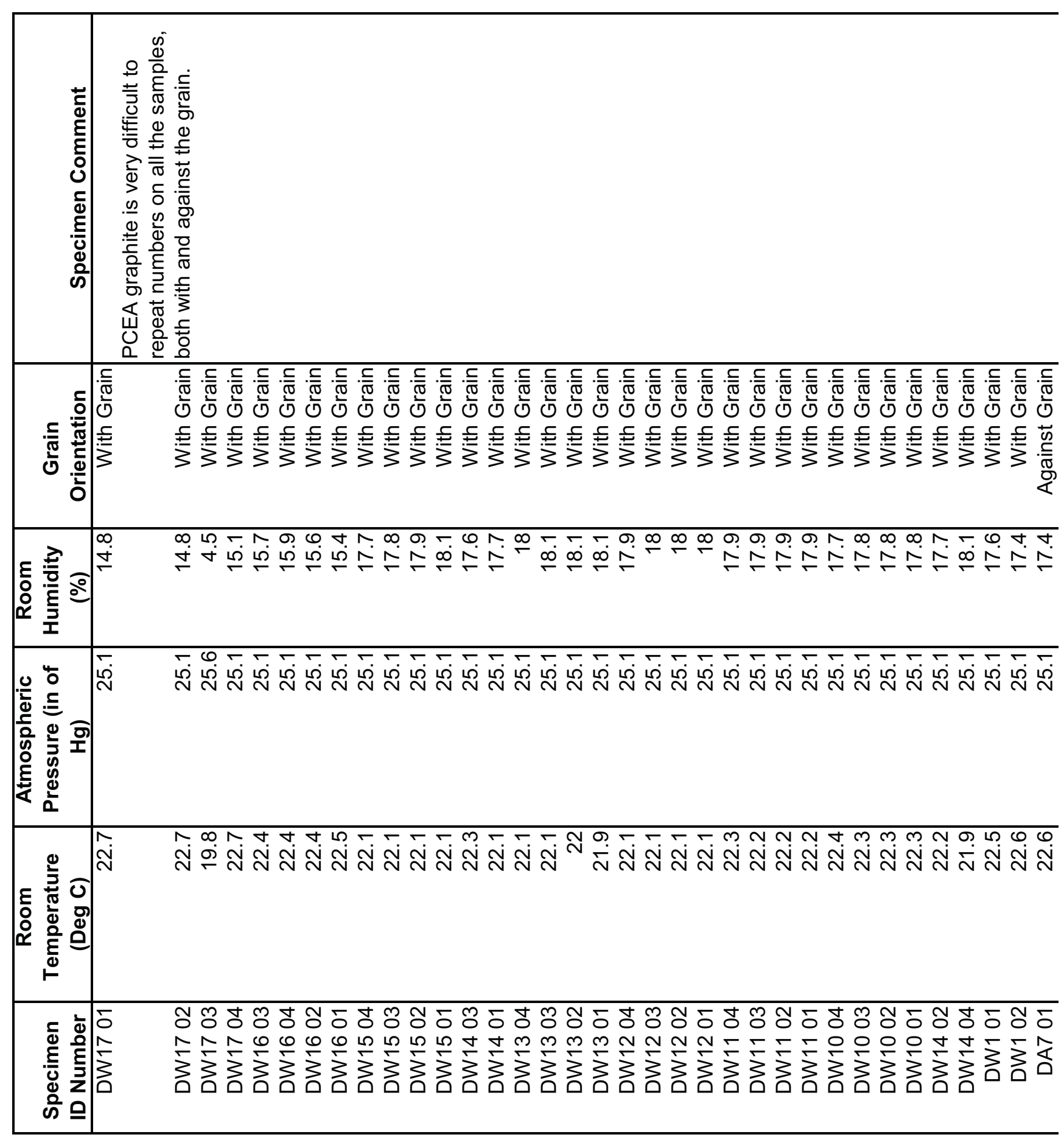




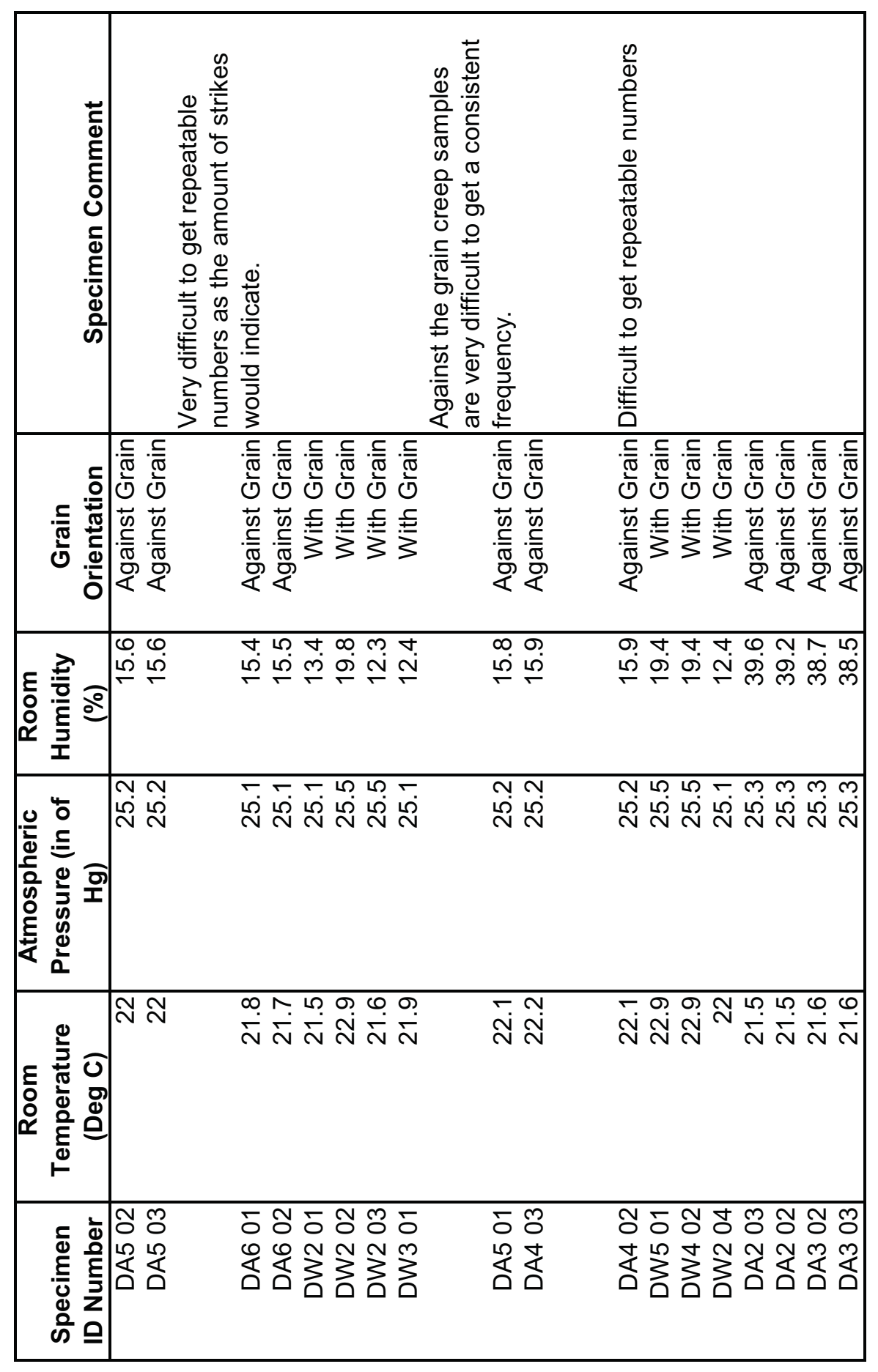




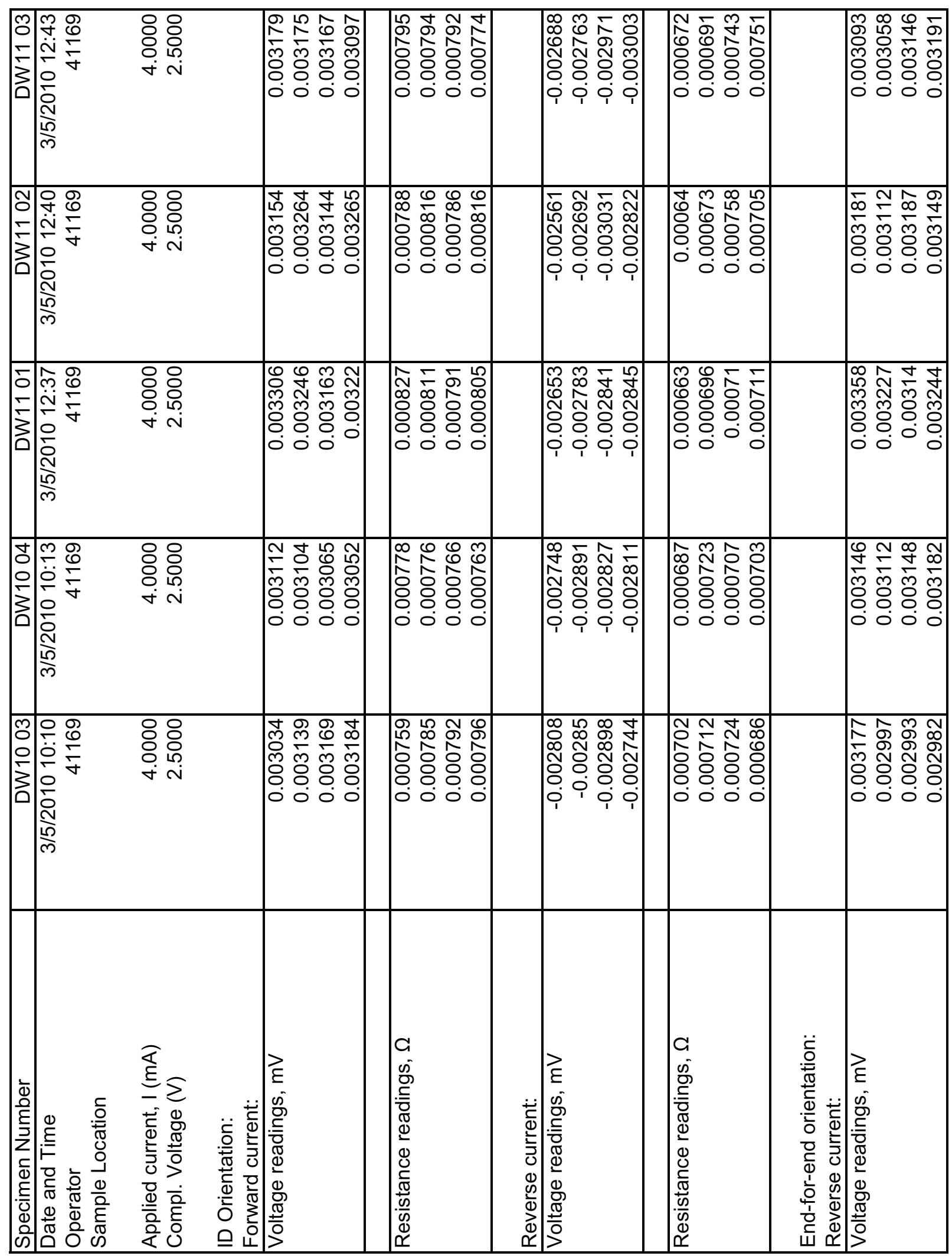




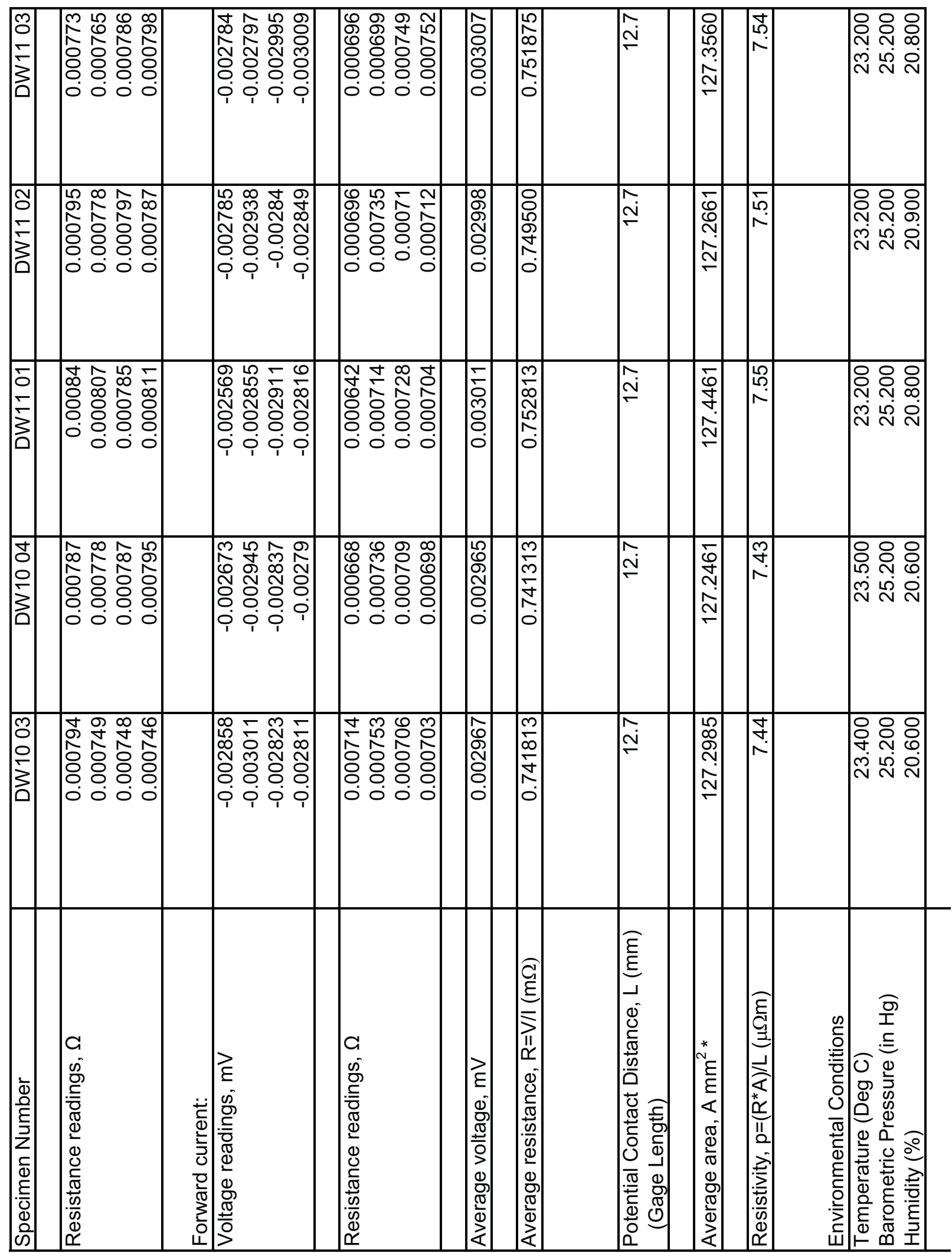




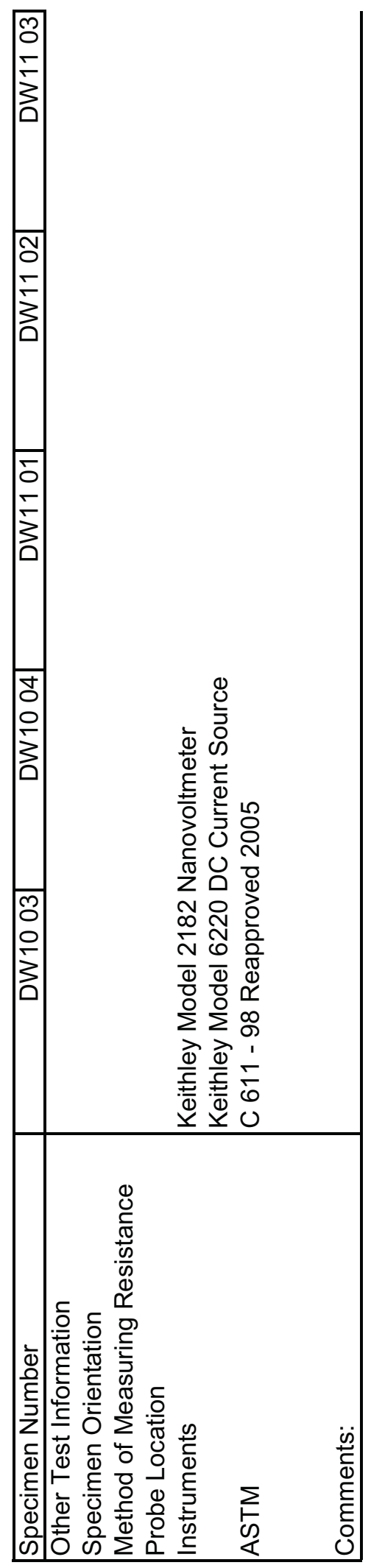




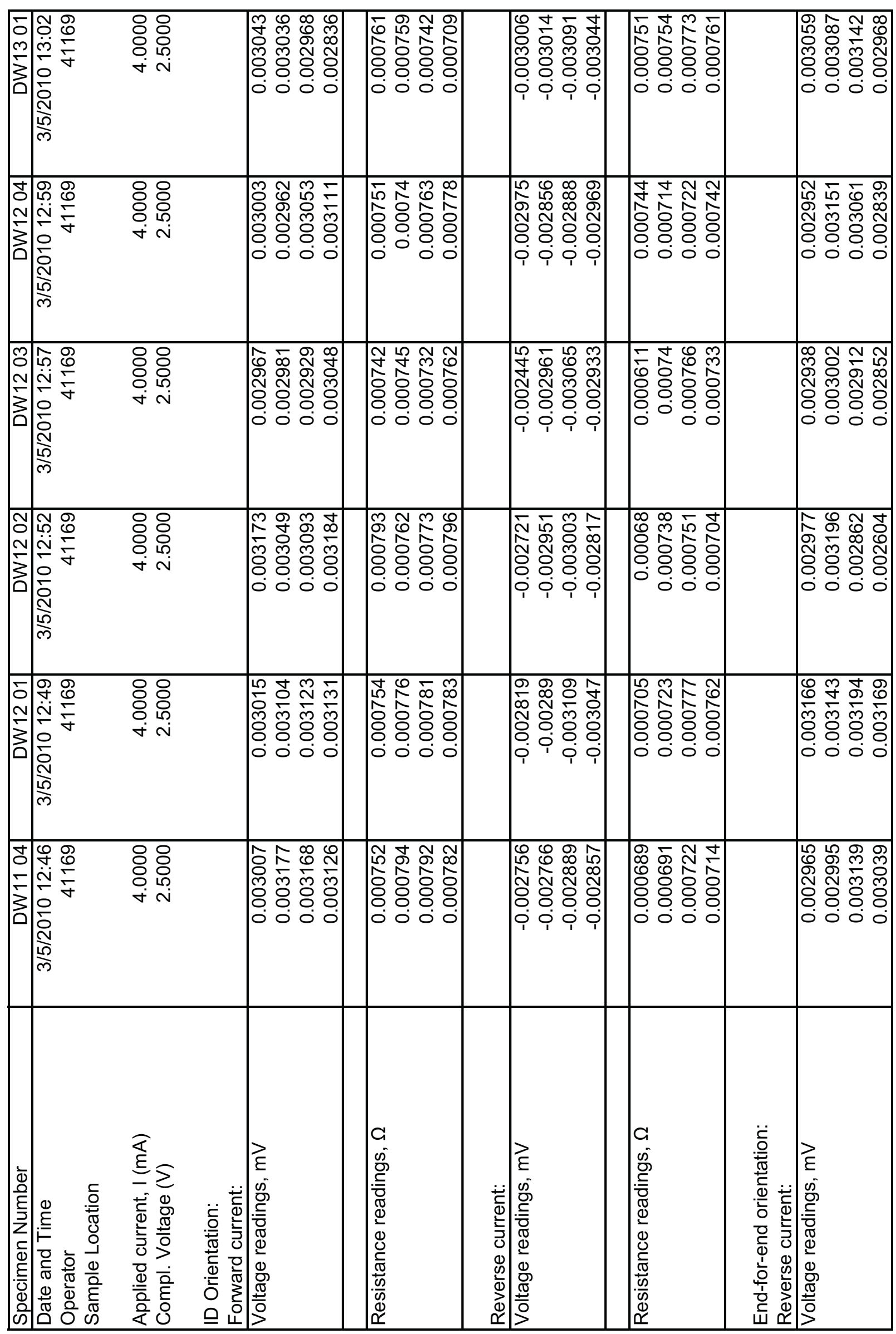




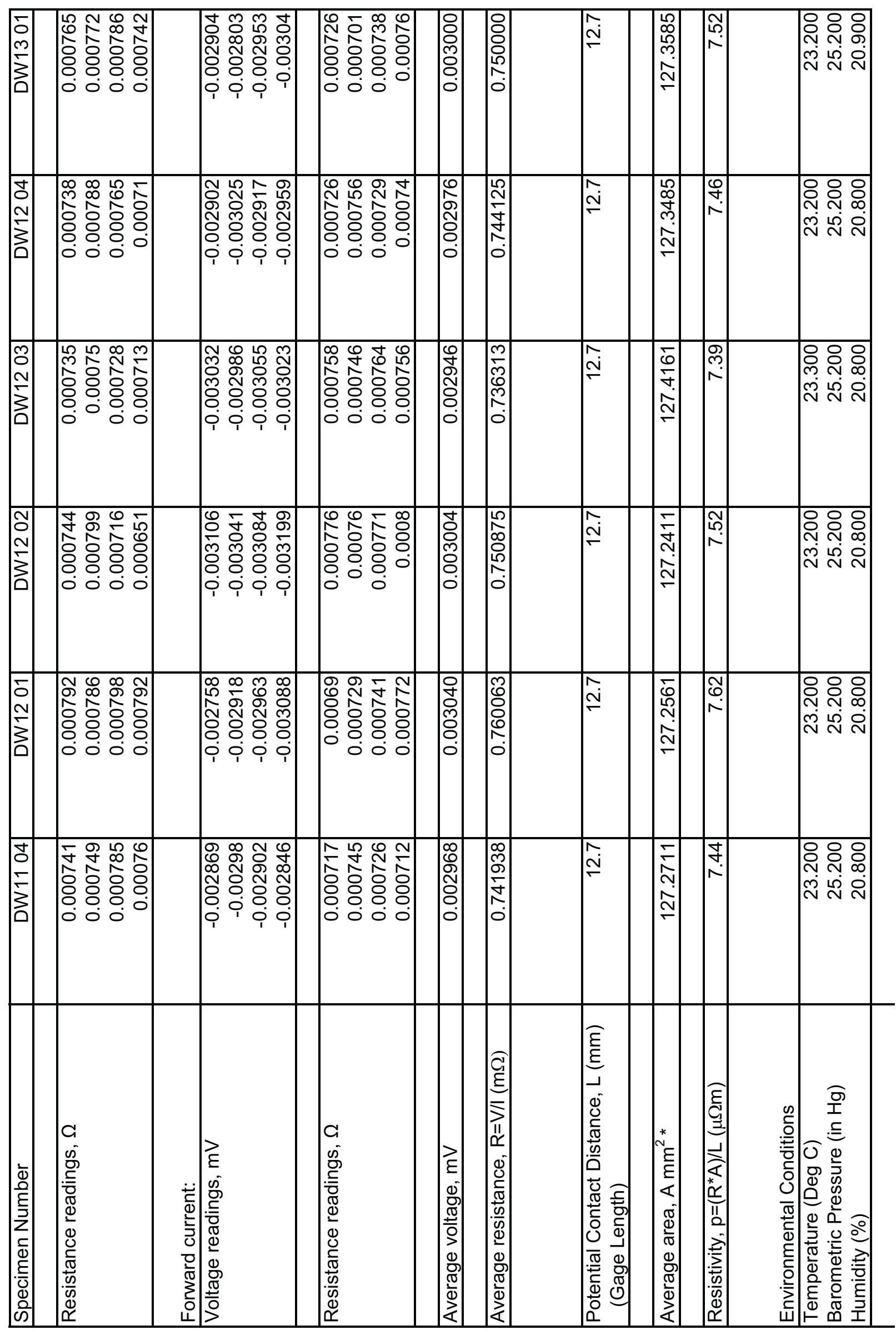




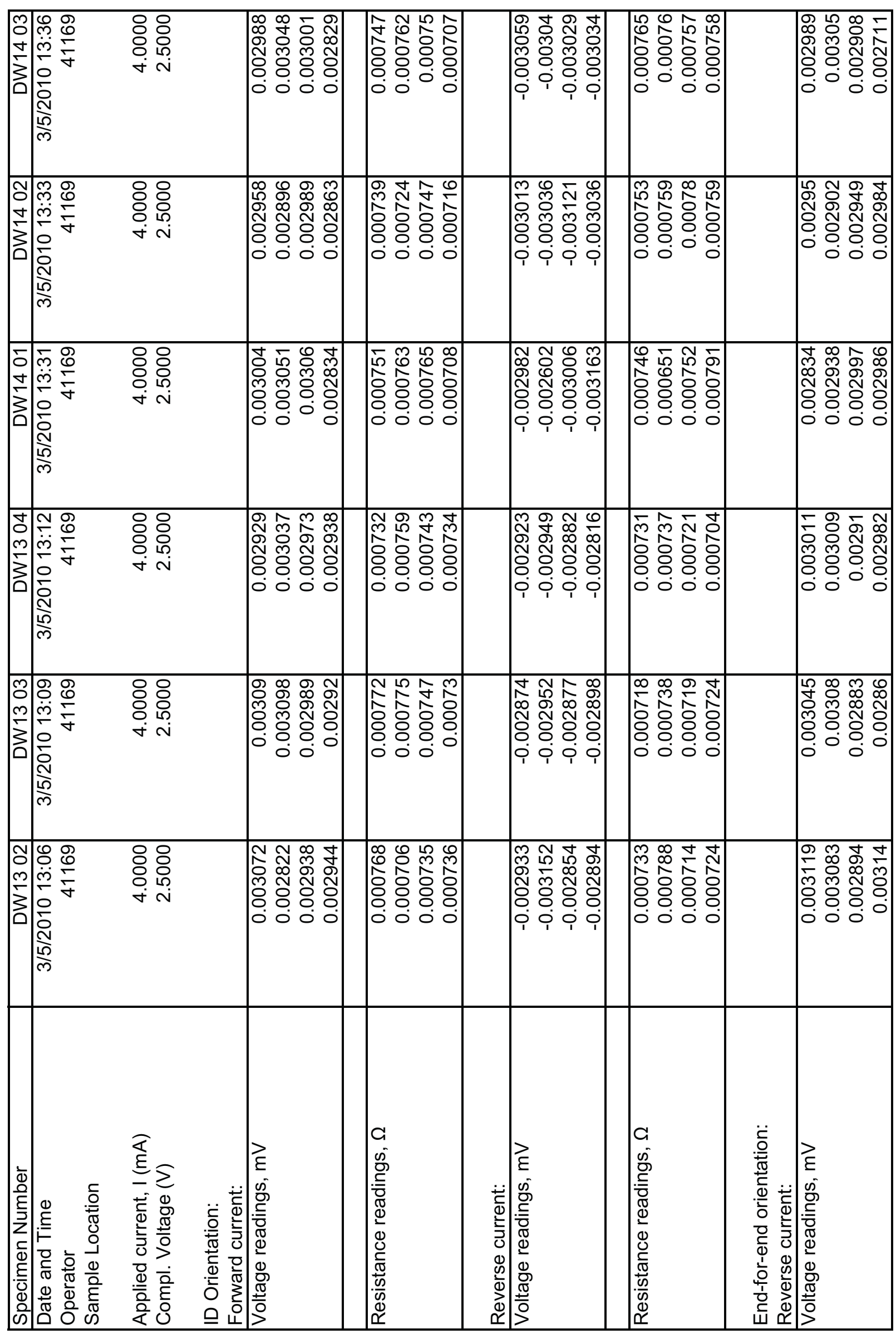




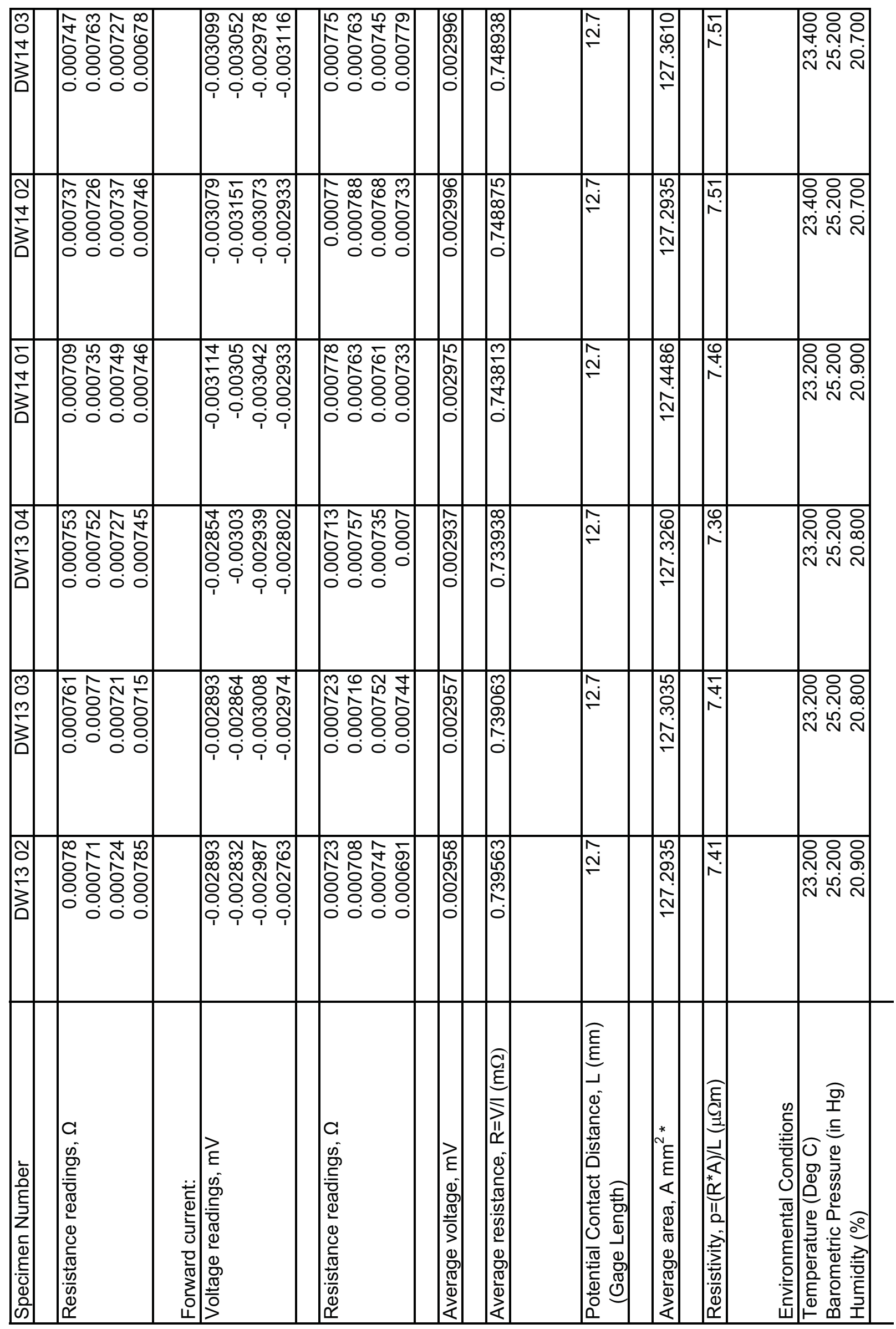




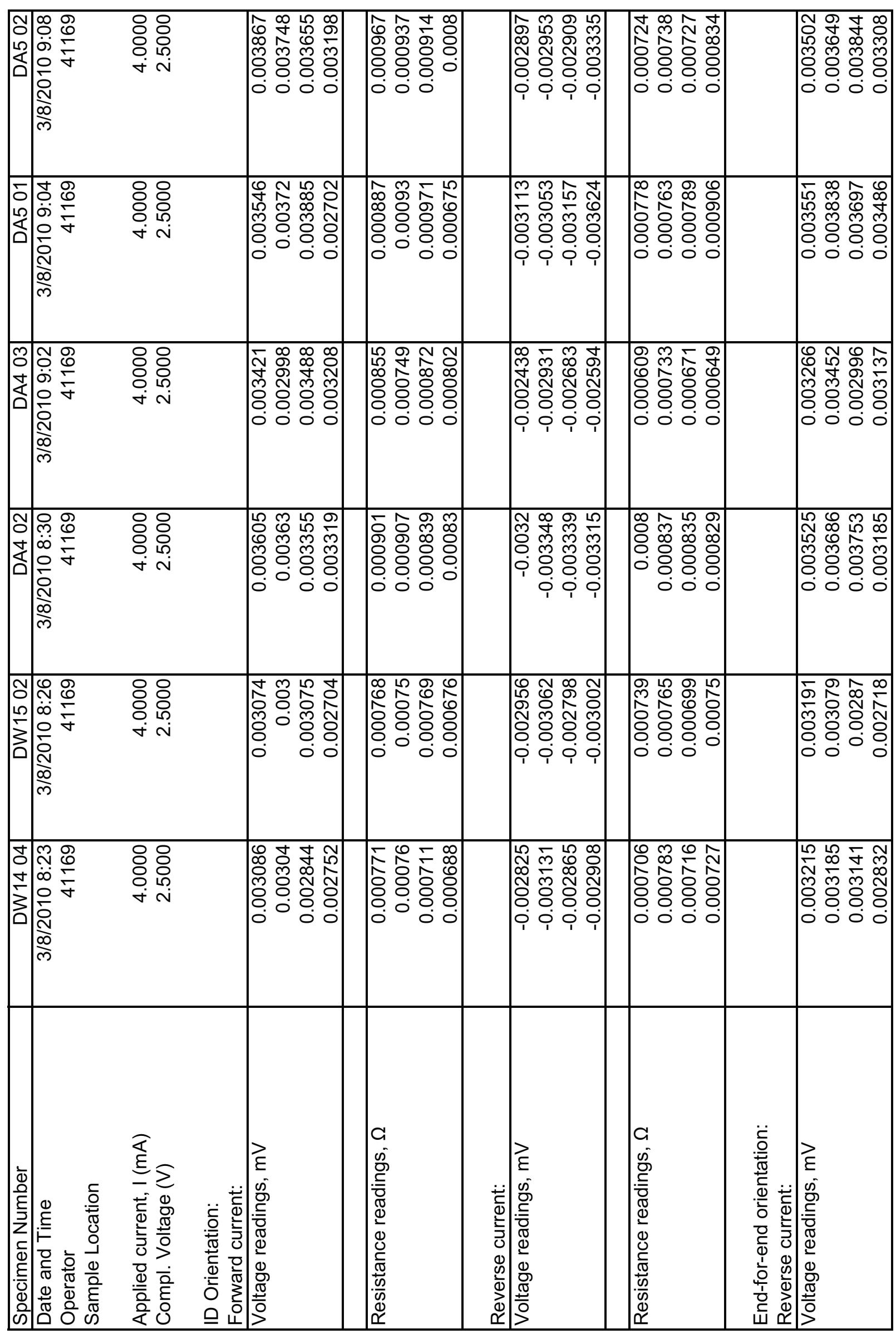




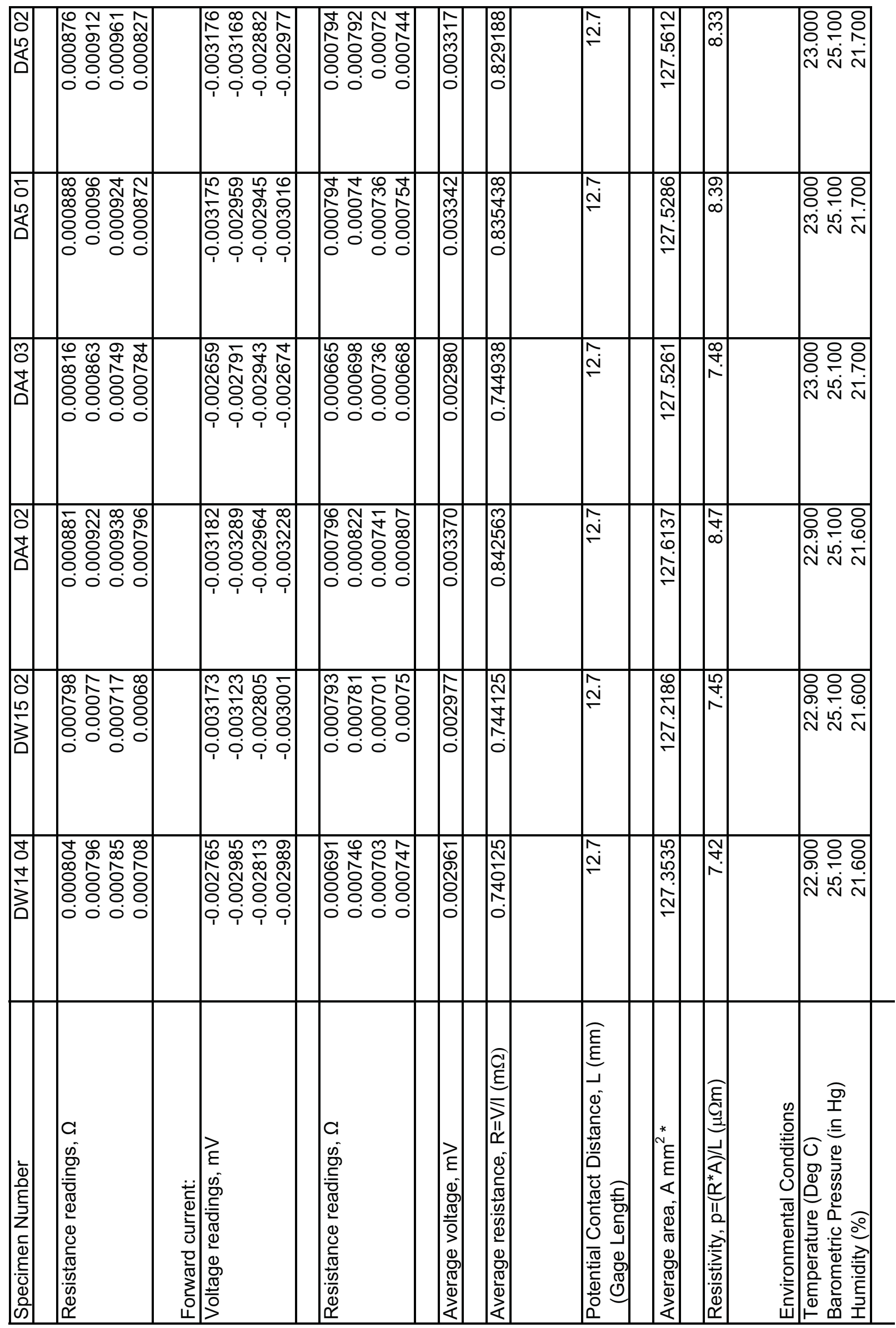




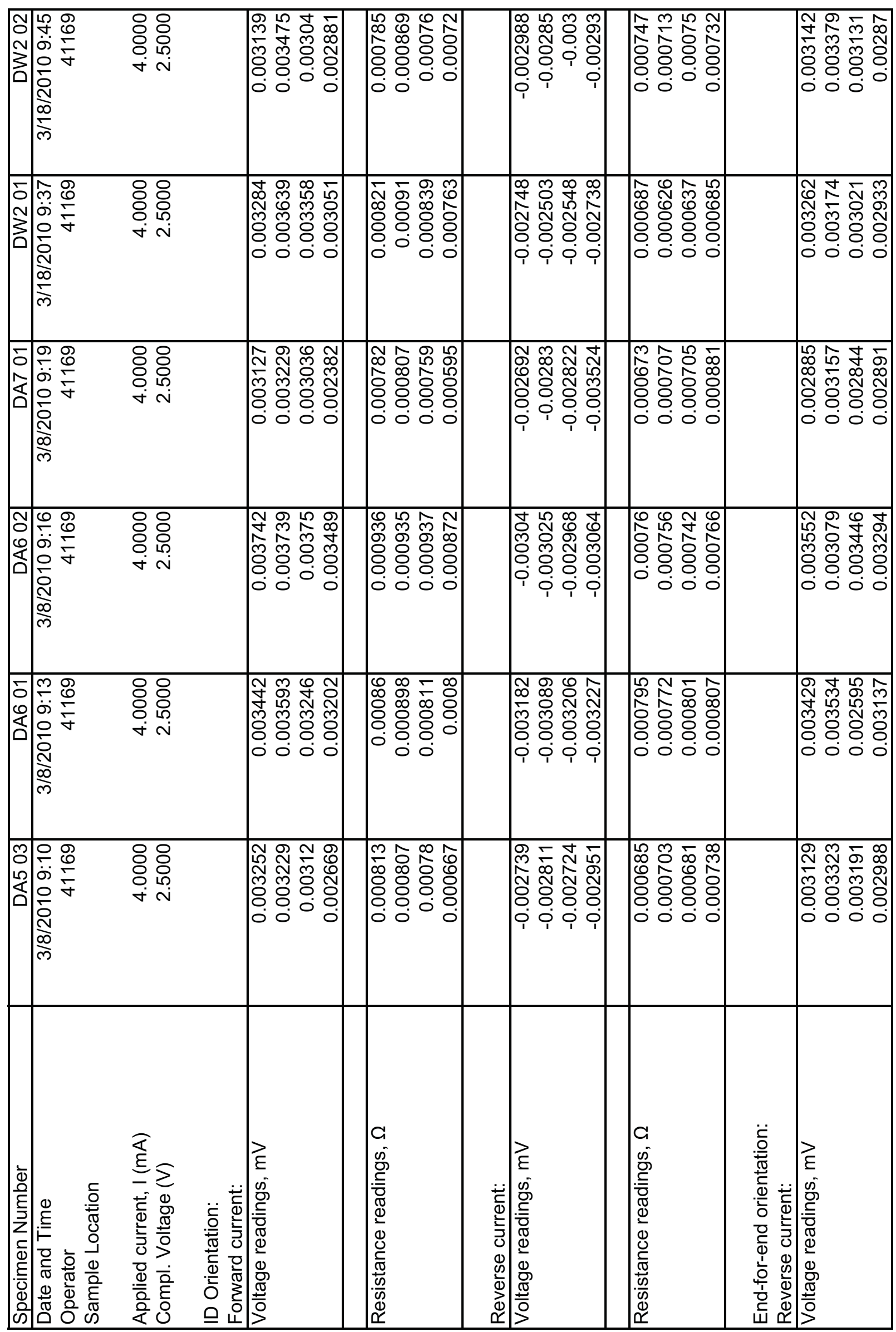




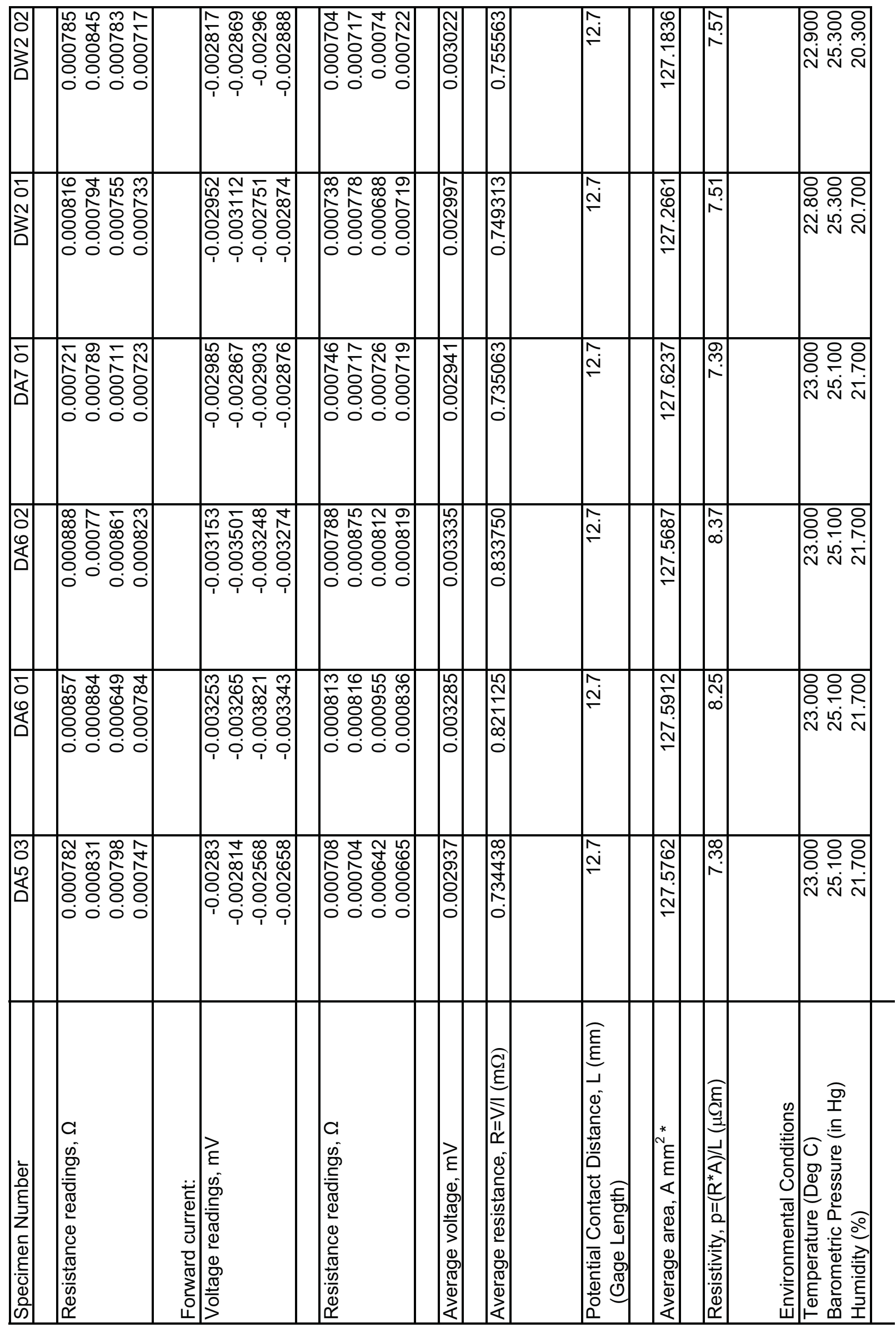




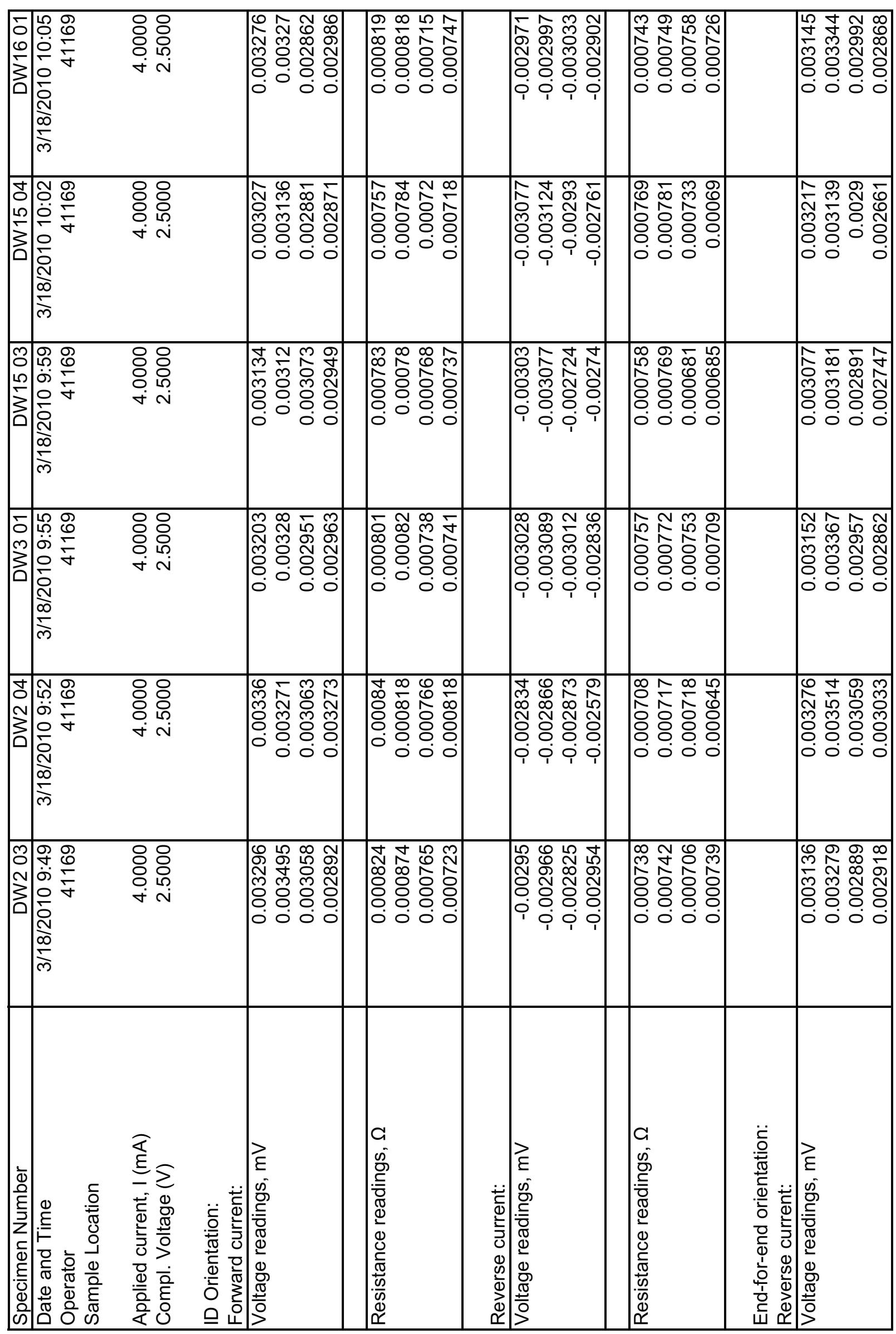




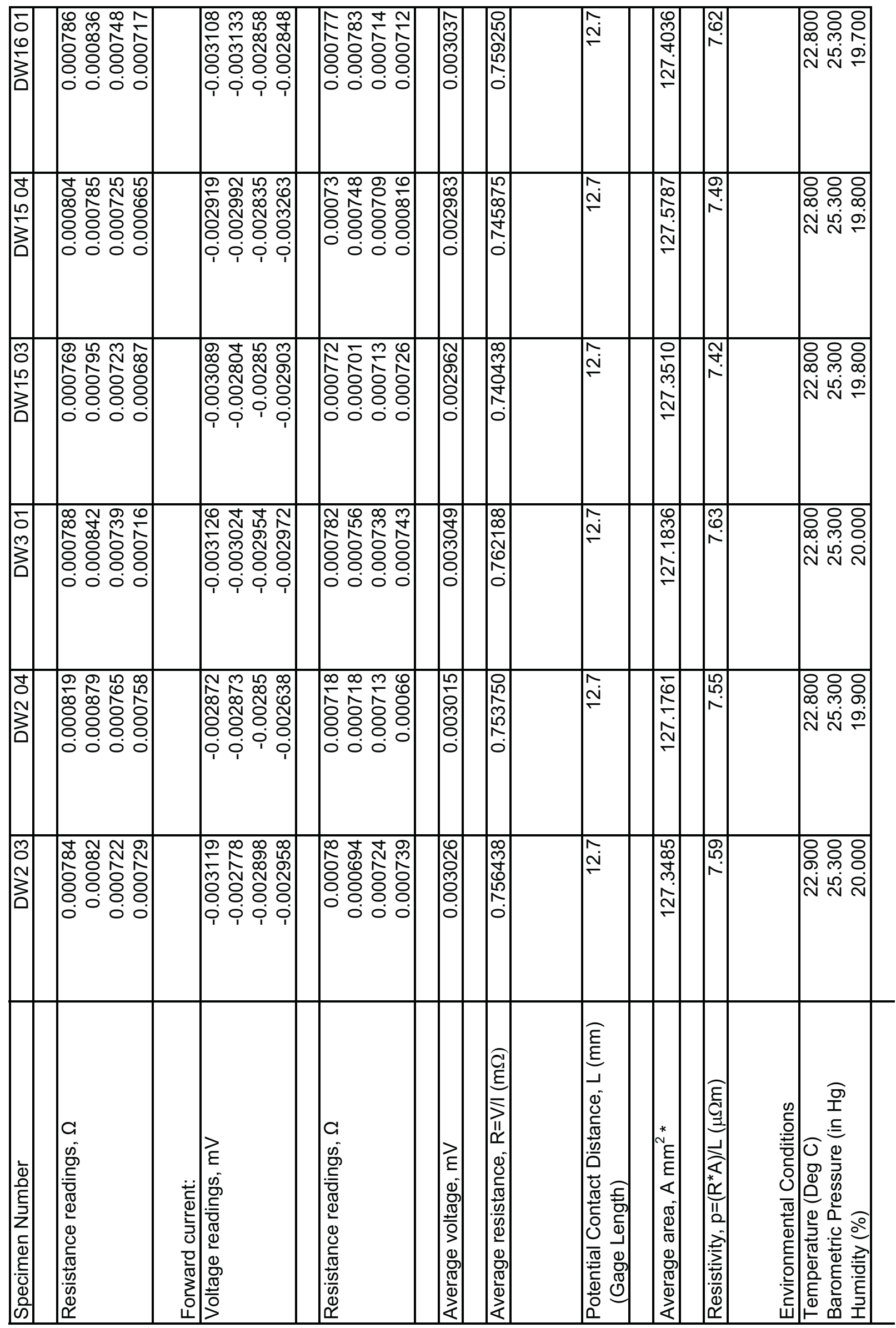




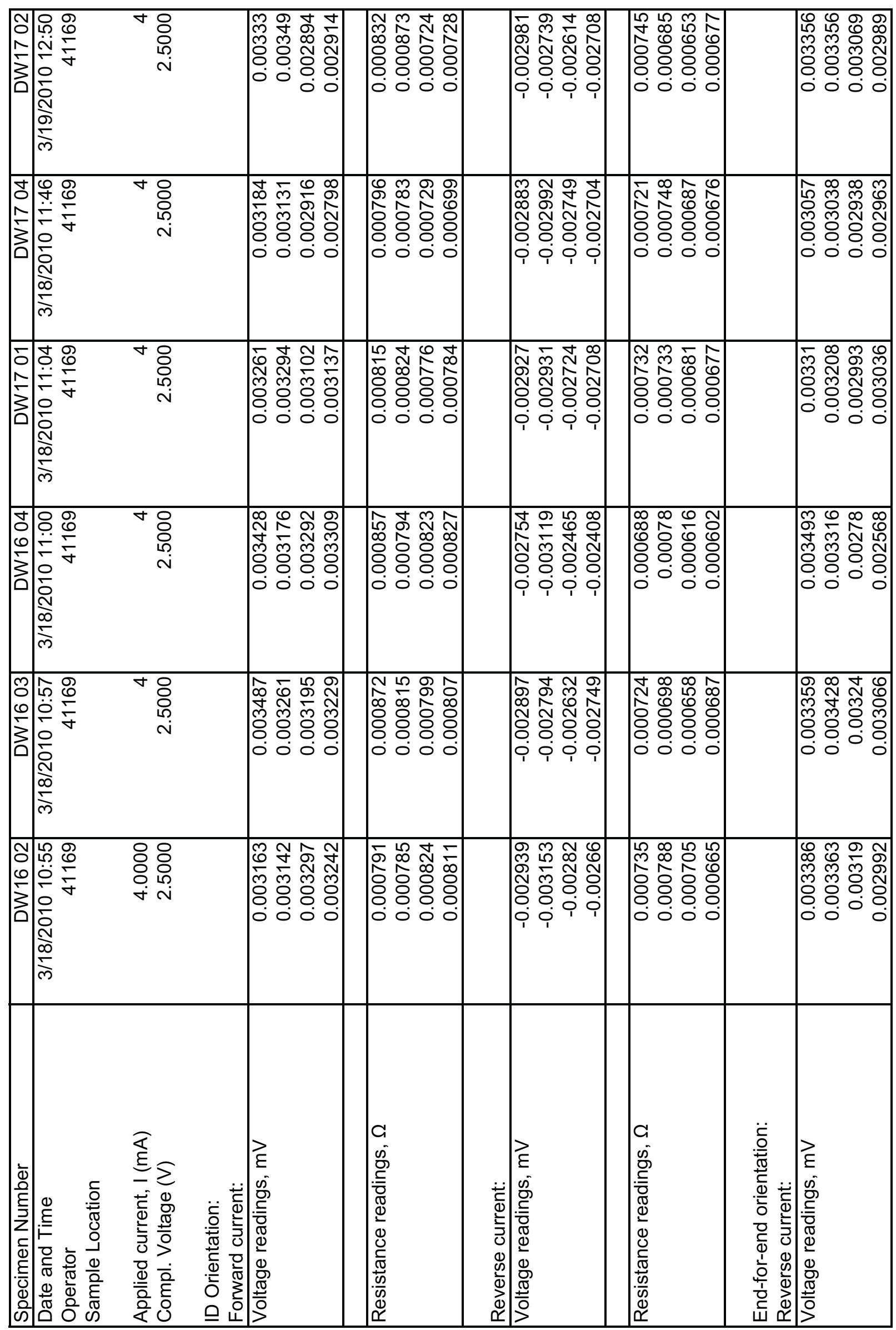




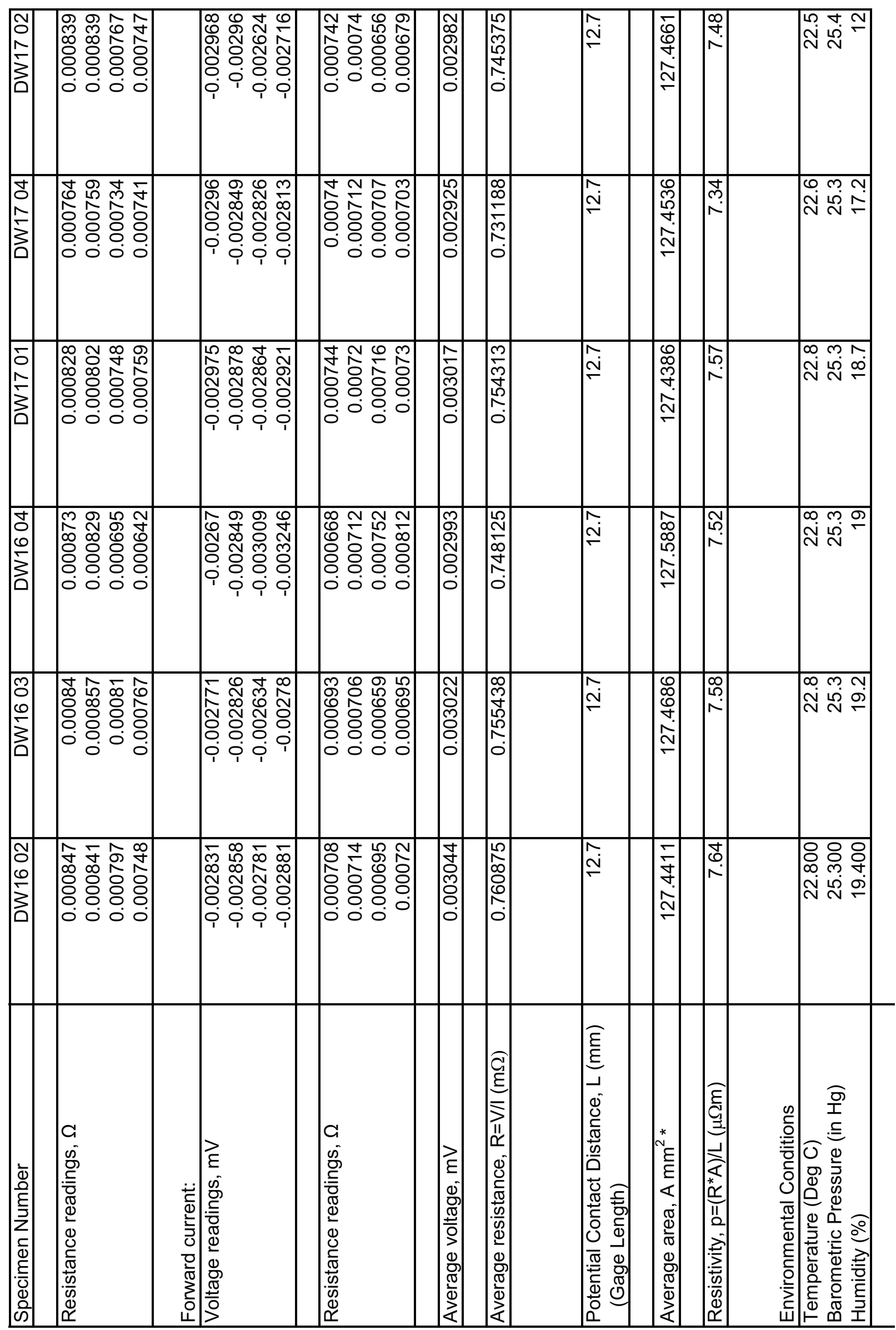




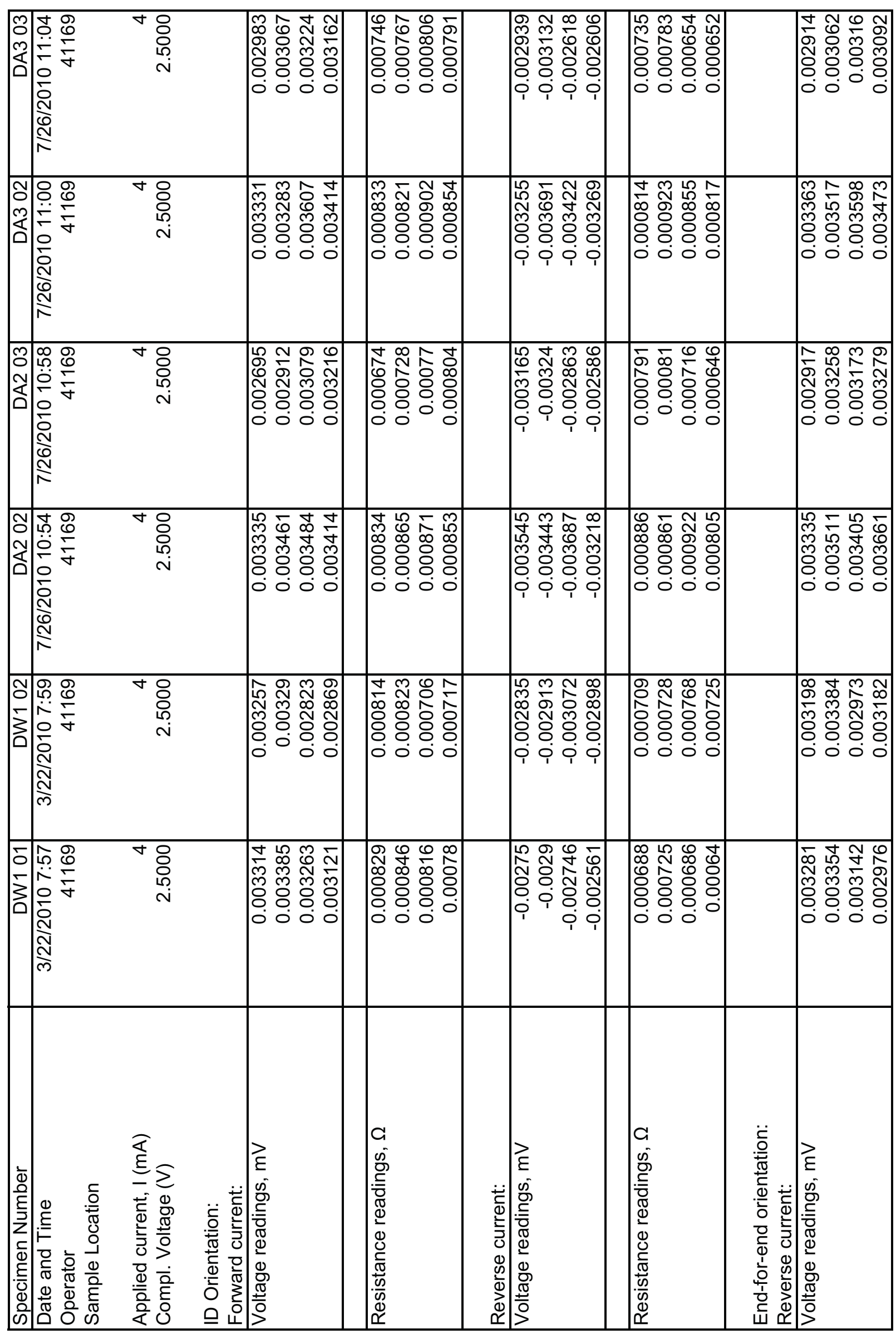




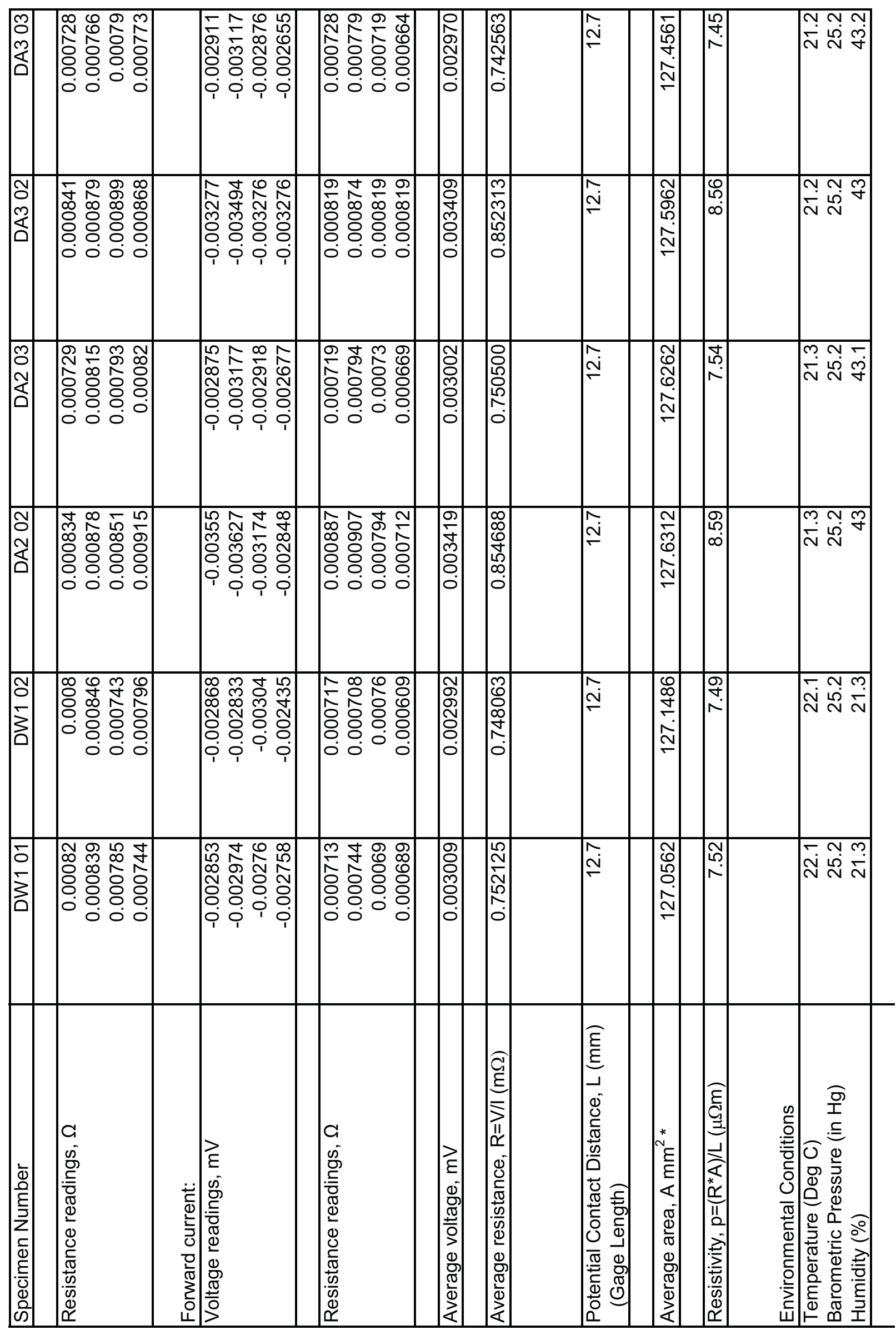




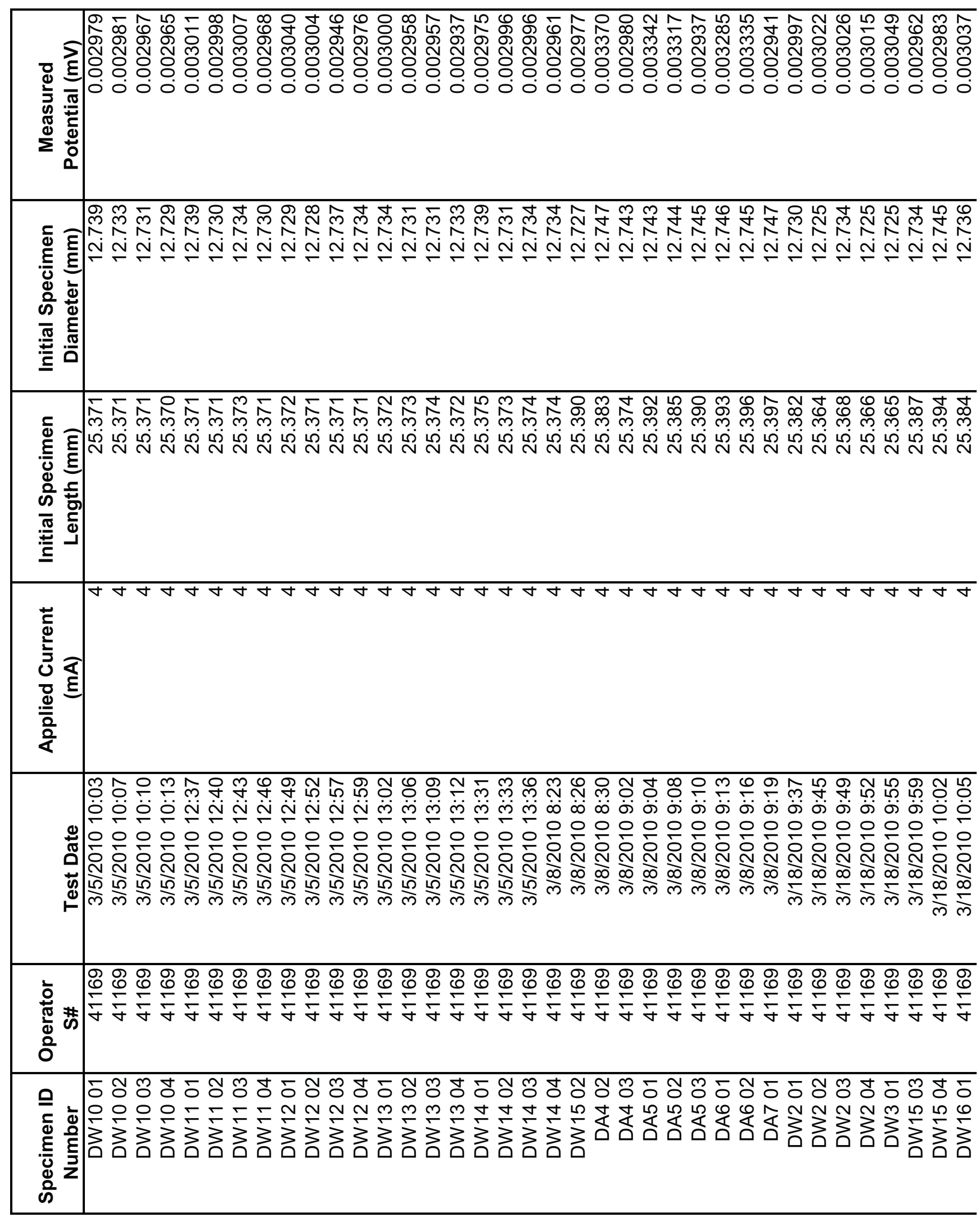




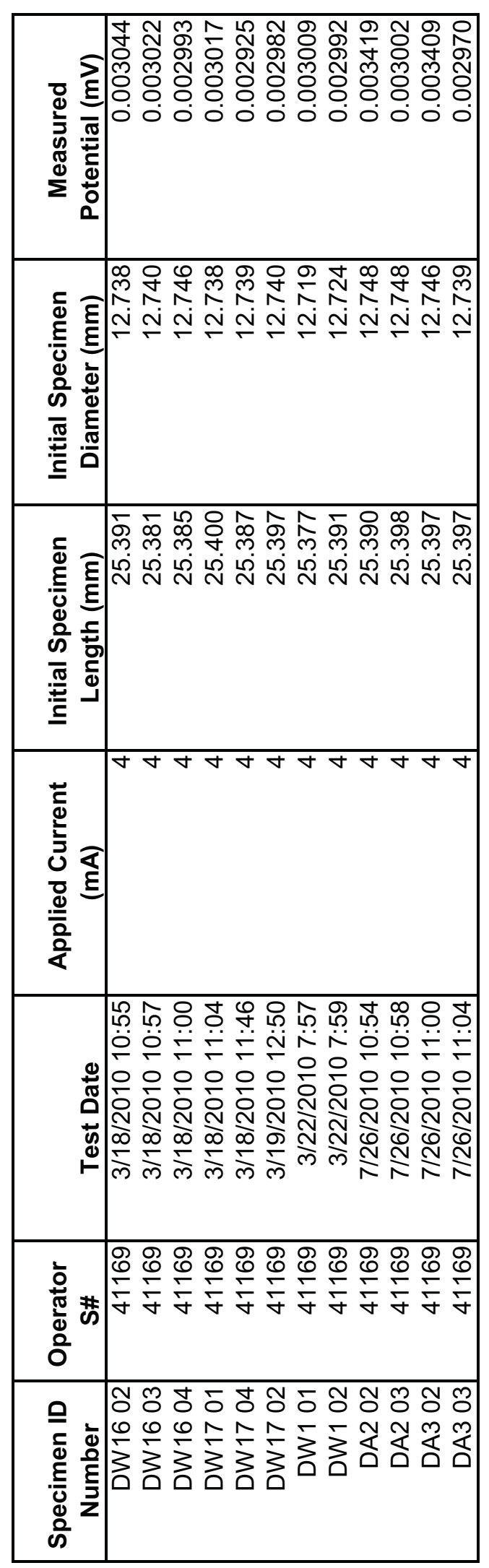




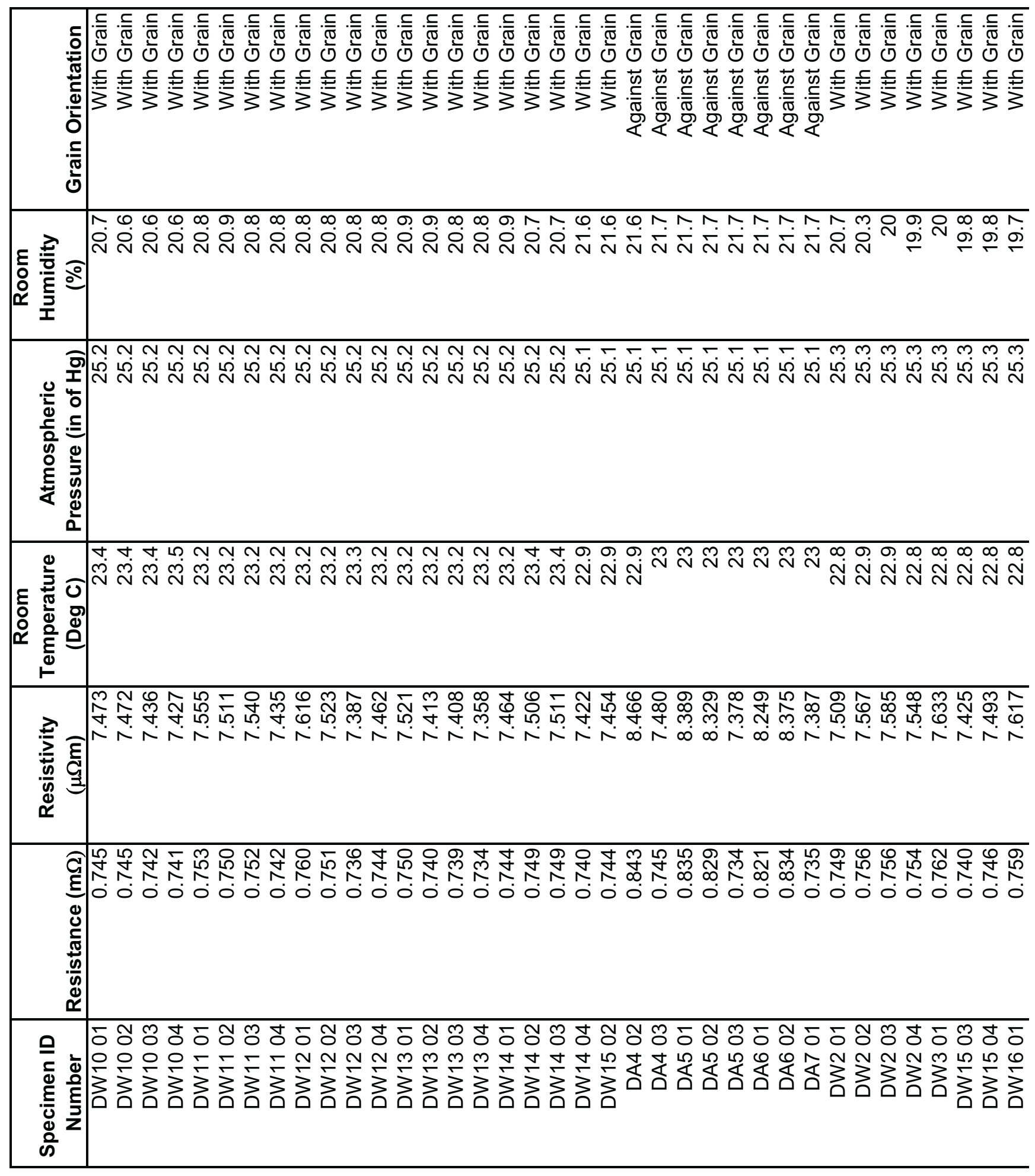




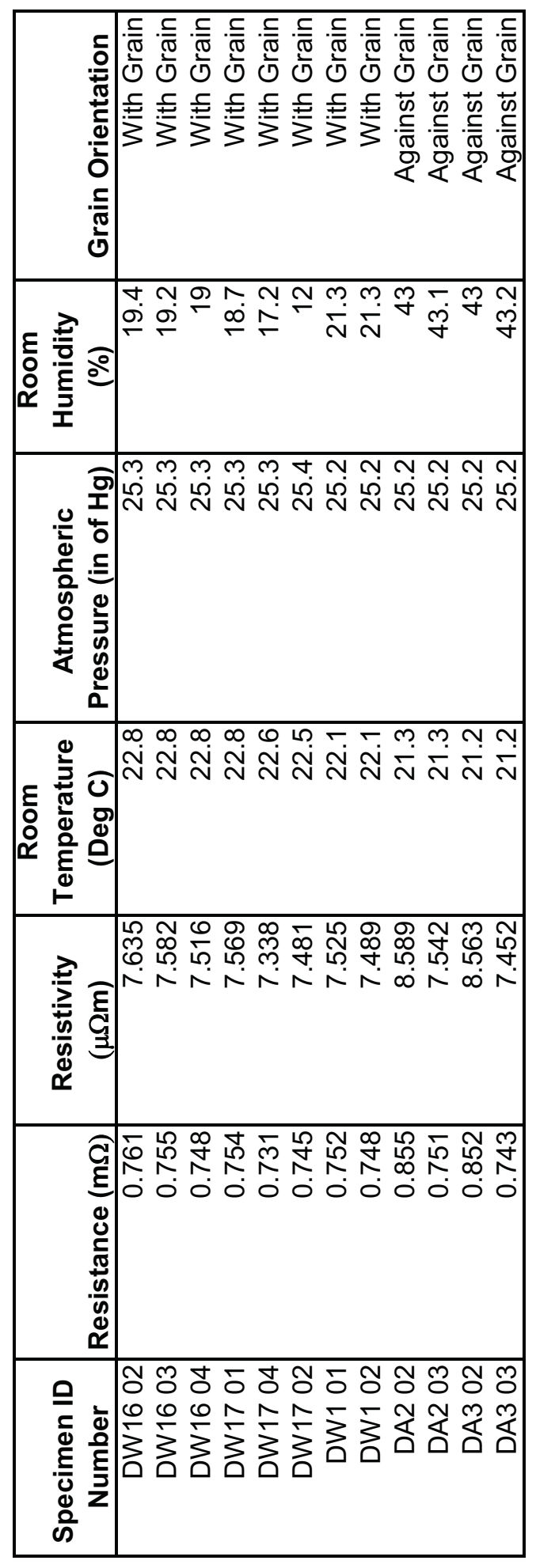




\begin{tabular}{|c|c|}
\hline 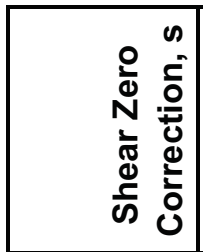 & 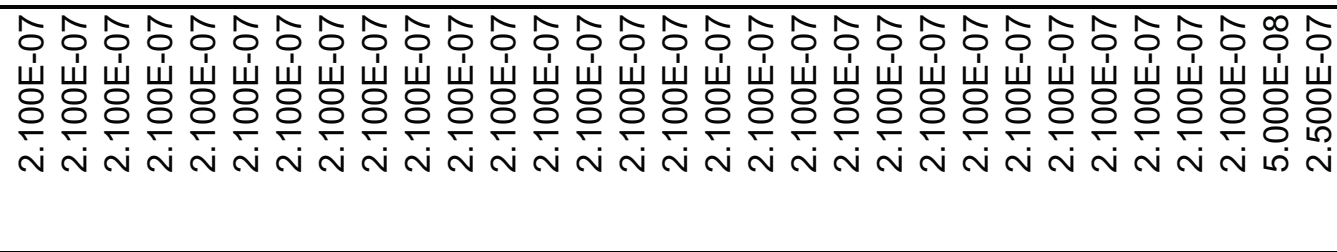 \\
\hline 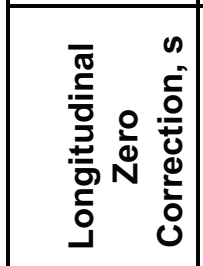 & 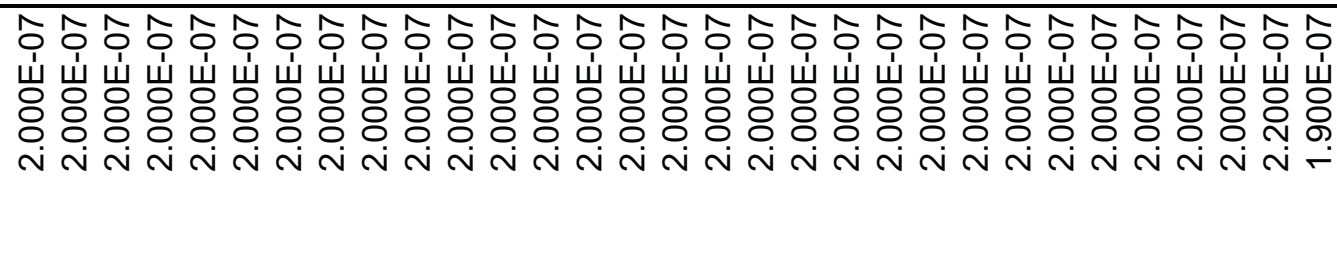 \\
\hline 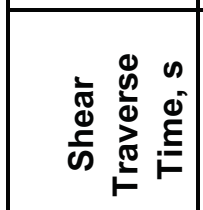 & 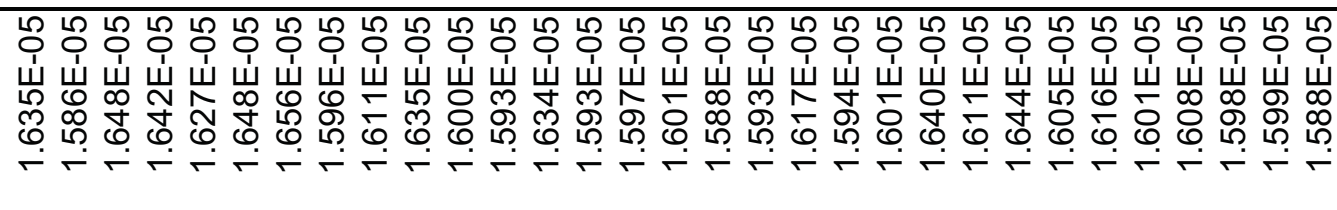 \\
\hline 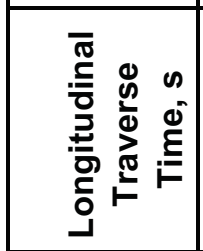 & 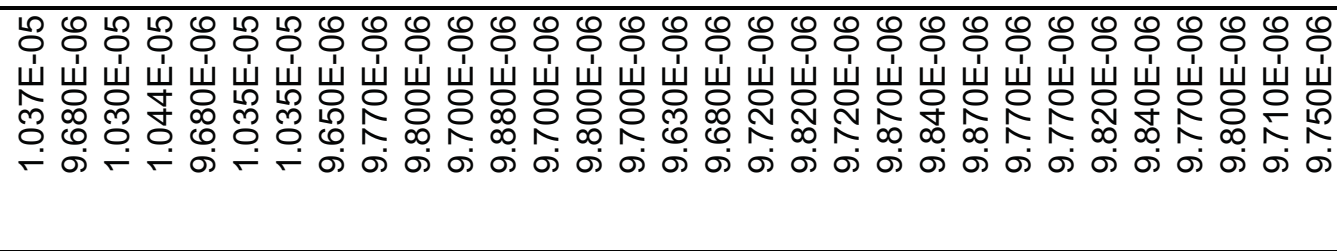 \\
\hline 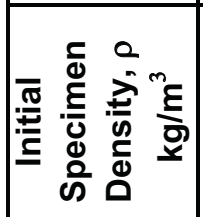 & 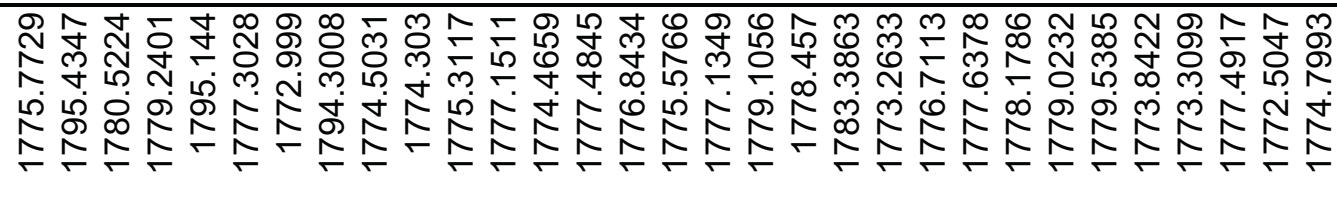 \\
\hline 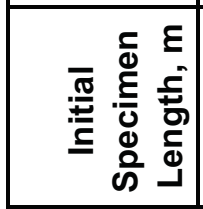 & 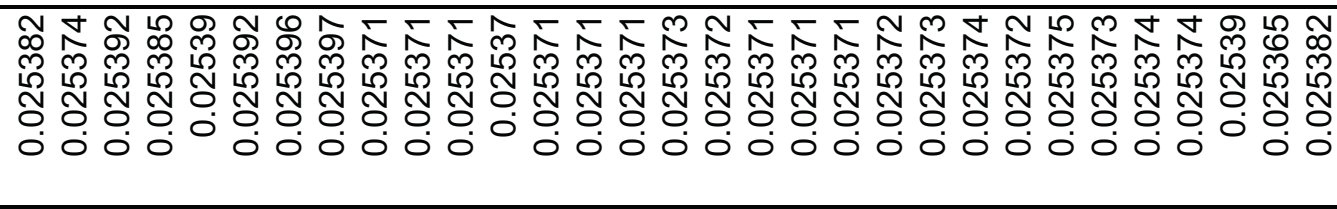 \\
\hline 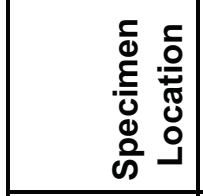 & \\
\hline $\begin{array}{l}\frac{1}{0} \\
\frac{0}{0} \\
\frac{\pi}{0} \\
\stackrel{0}{0}\end{array}$ & 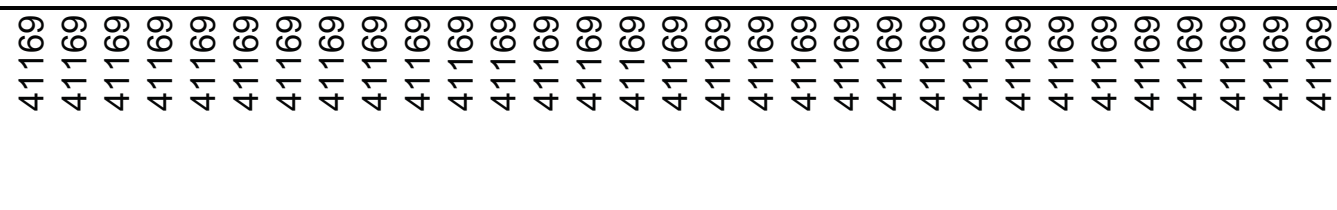 \\
\hline 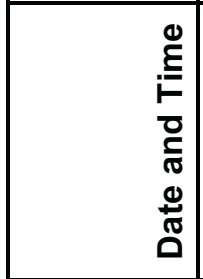 & 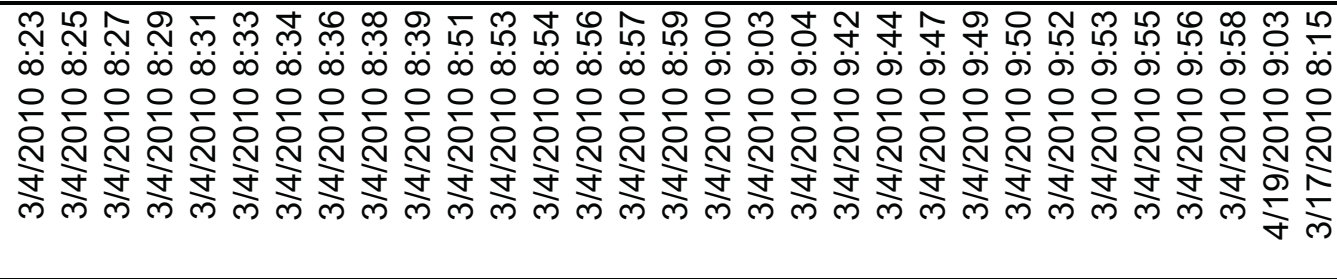 \\
\hline 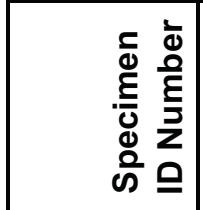 & 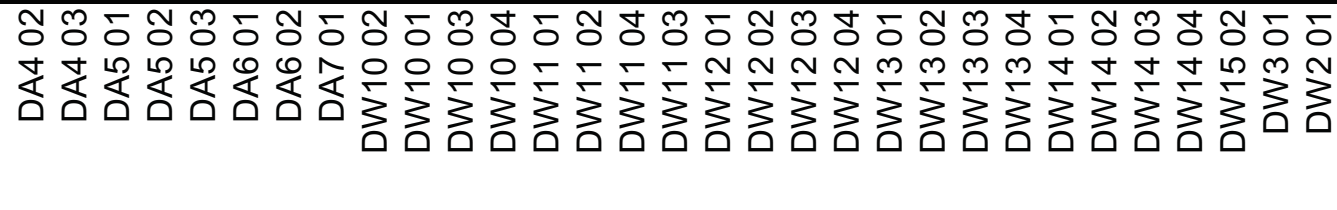 \\
\hline
\end{tabular}




\begin{tabular}{|c|c|}
\hline 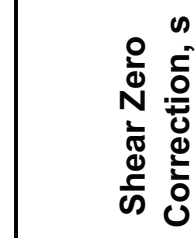 & 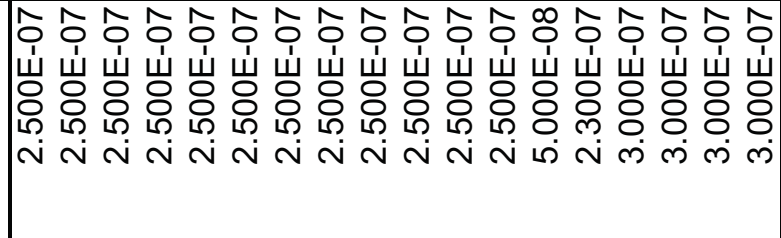 \\
\hline 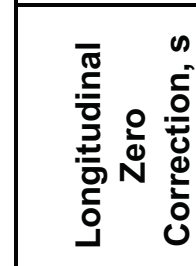 & 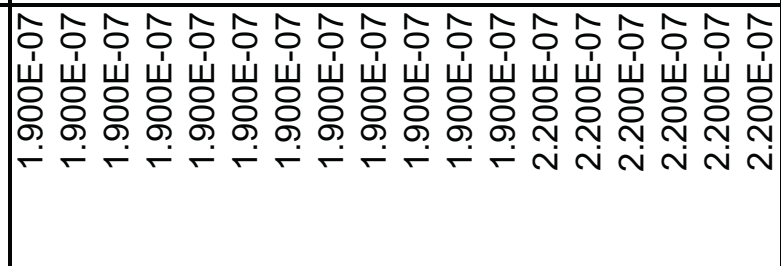 \\
\hline 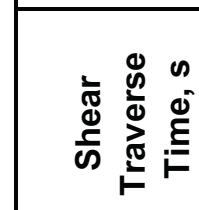 & 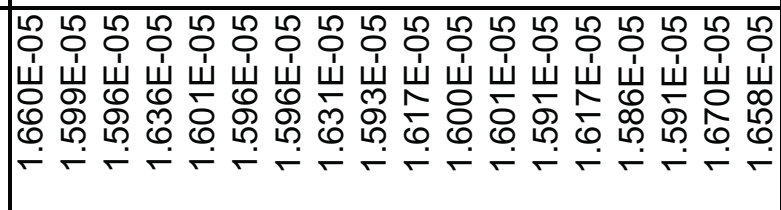 \\
\hline 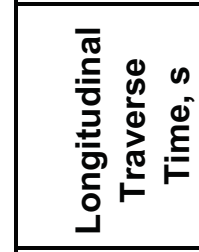 & 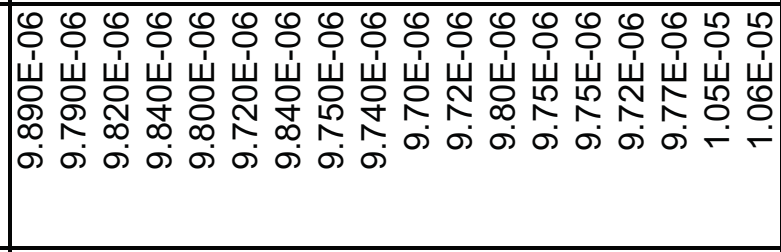 \\
\hline 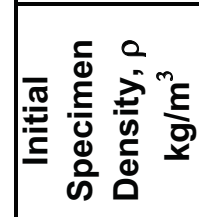 & 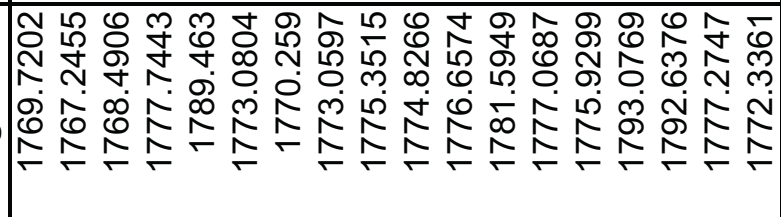 \\
\hline 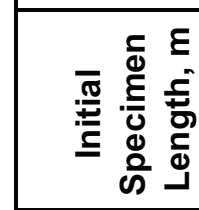 & 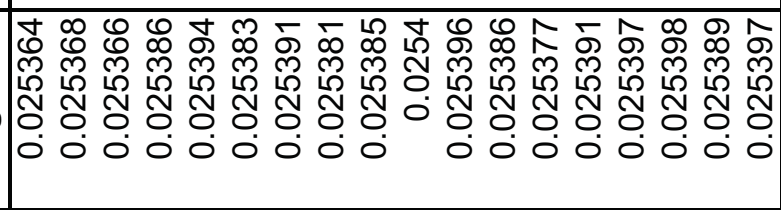 \\
\hline 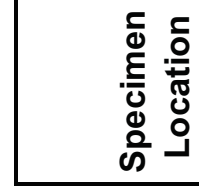 & \\
\hline $\begin{array}{l}\frac{1}{0} \\
\frac{\pi}{2} \\
\frac{0}{0} \\
\text { Oे }\end{array}$ & 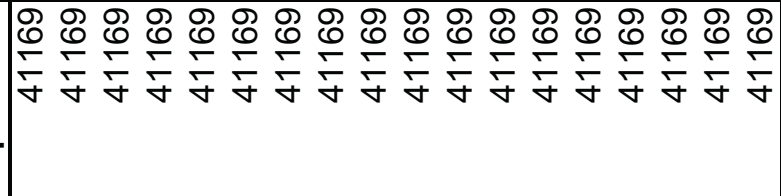 \\
\hline 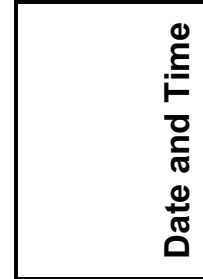 & 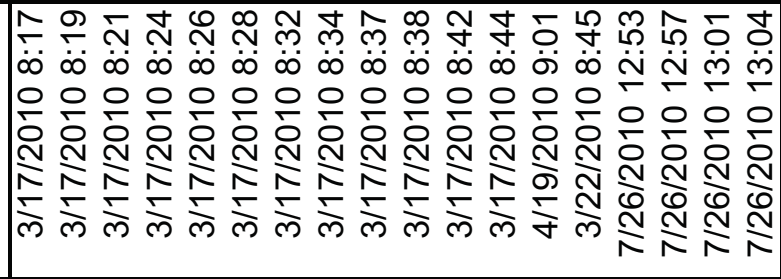 \\
\hline 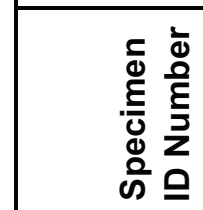 & 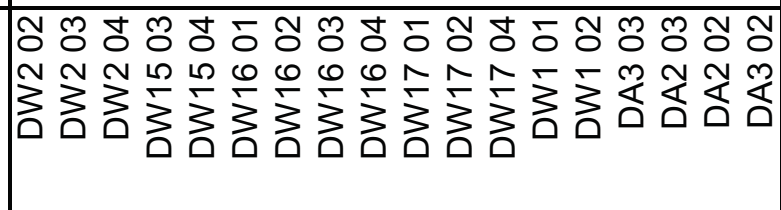 \\
\hline
\end{tabular}




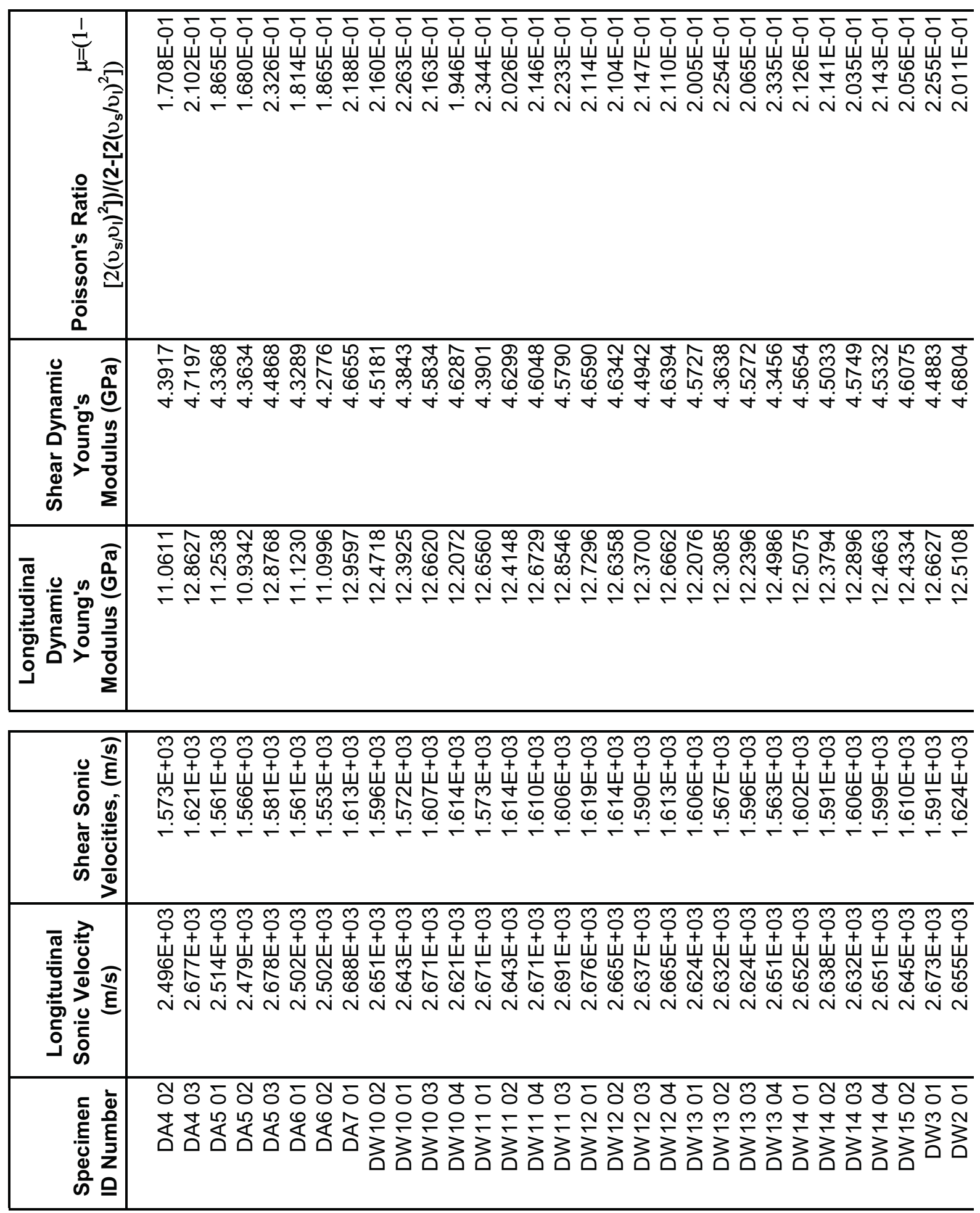



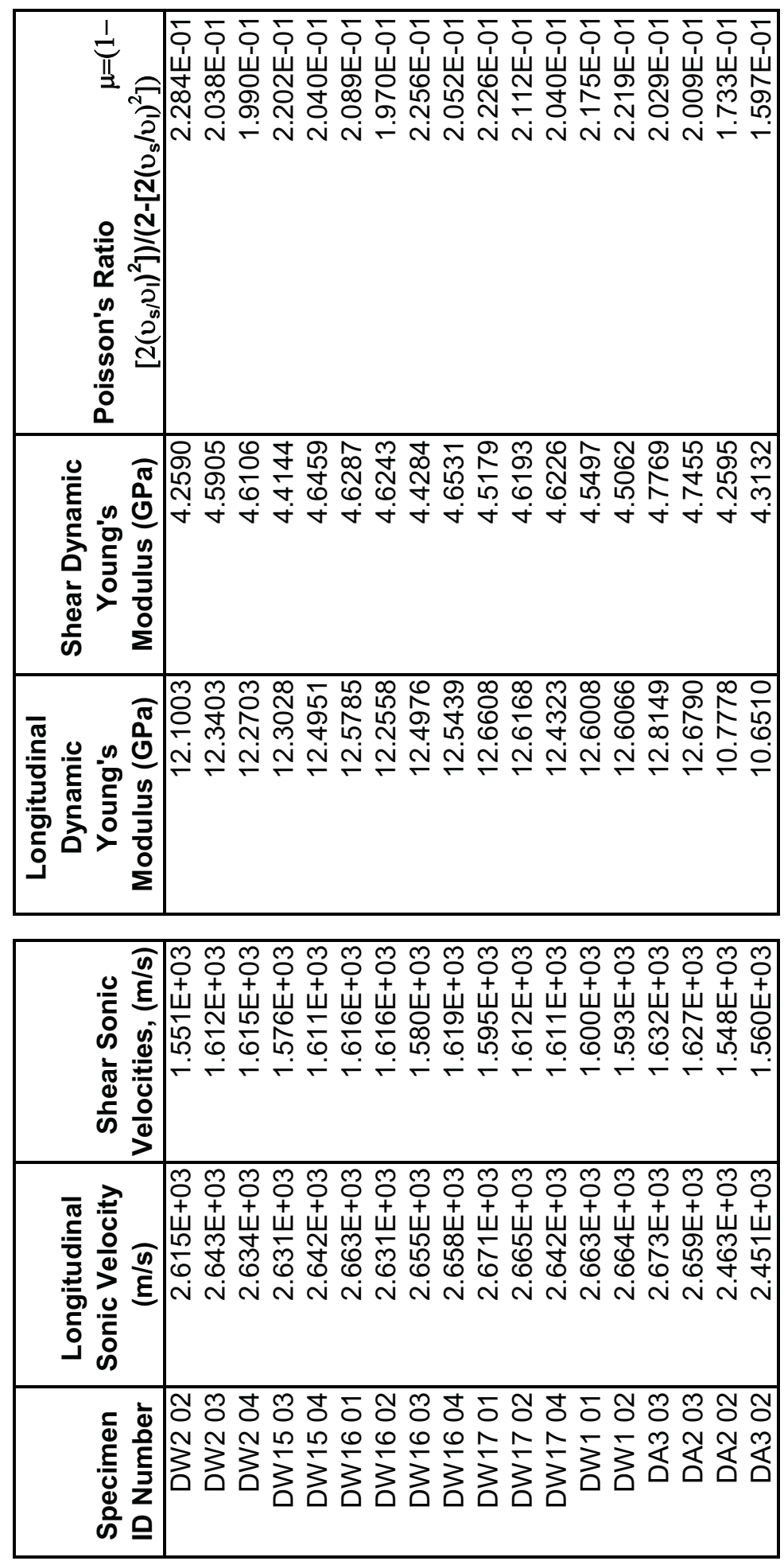


\begin{tabular}{|c|c|c|}
\hline & 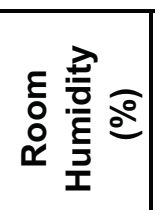 & 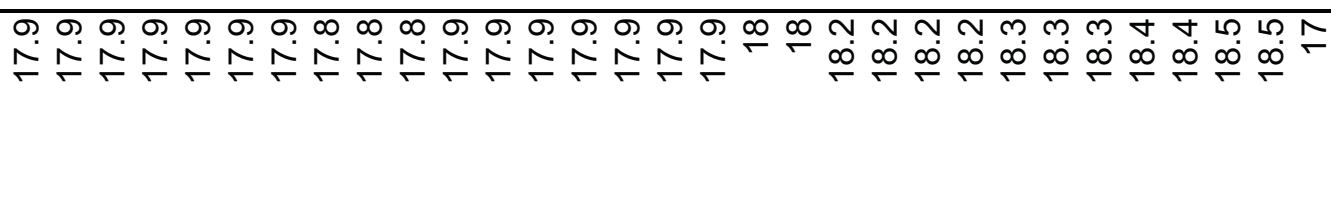 \\
\hline 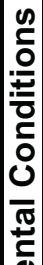 & 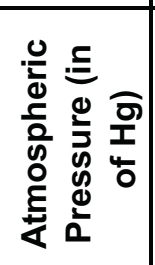 & 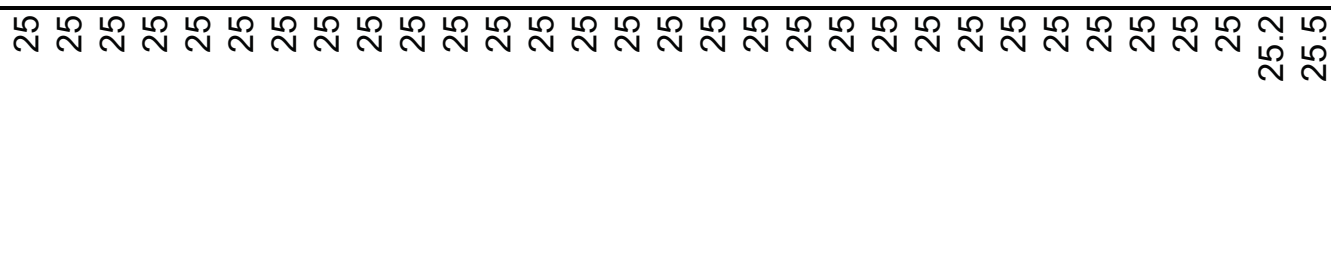 \\
\hline है & 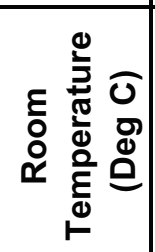 & 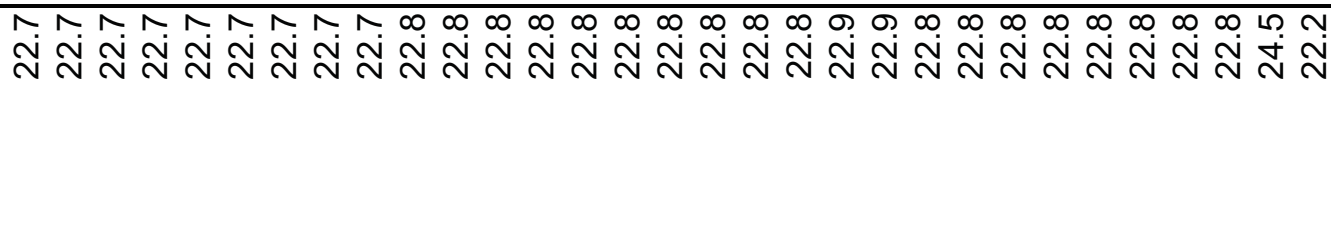 \\
\hline 产 & 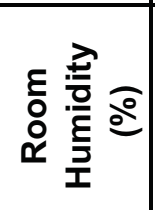 & 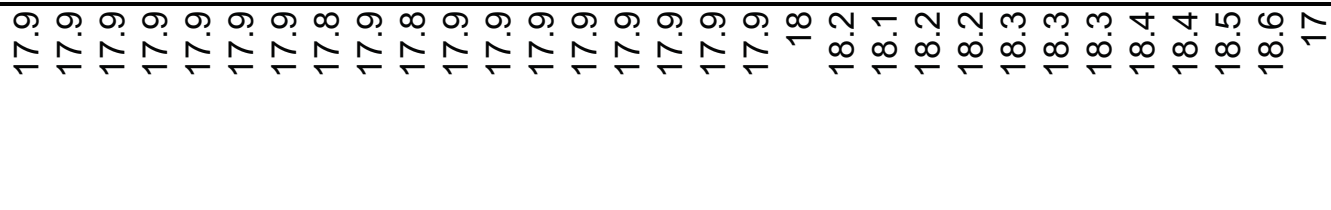 \\
\hline $\begin{array}{l}0 \\
0 \\
0 \\
0 \\
0 \\
\vdots \\
0 \\
0 \\
0 \\
0 \\
\frac{0}{\sigma}\end{array}$ & 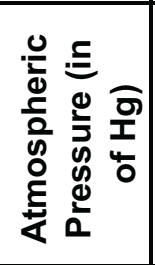 & 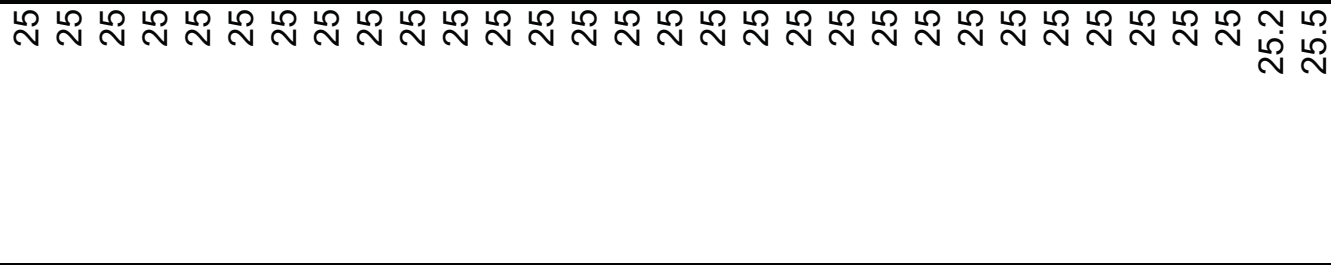 \\
\hline 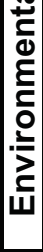 & 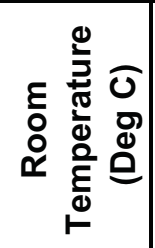 & 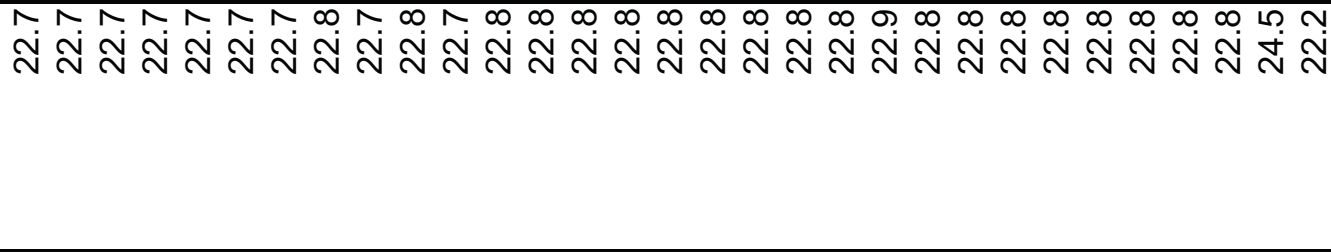 \\
\hline
\end{tabular}

\begin{tabular}{|c|c|}
\hline 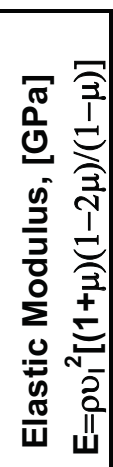 & 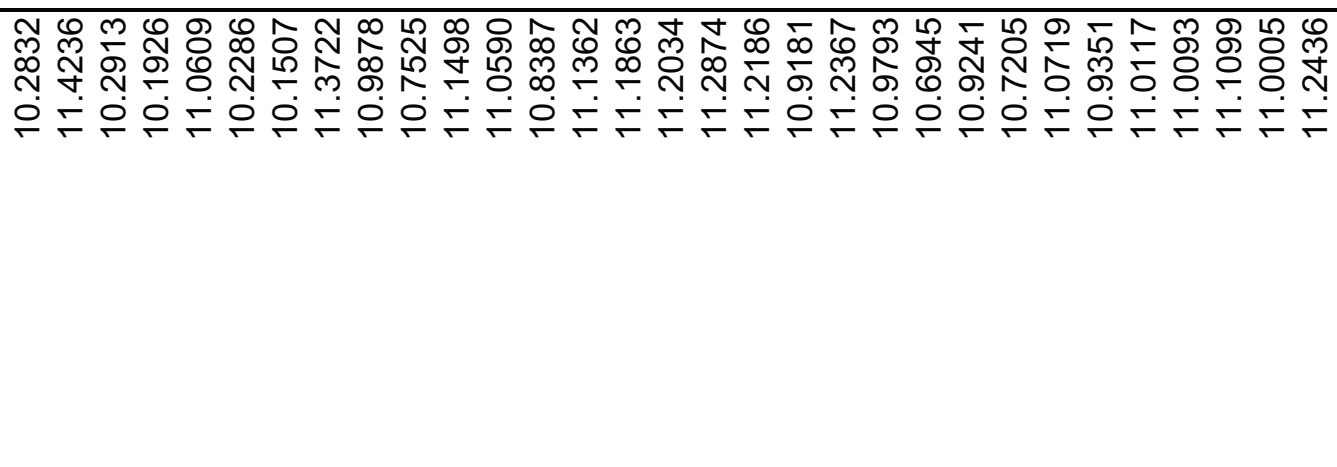 \\
\hline 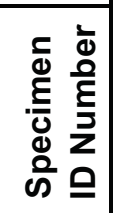 & 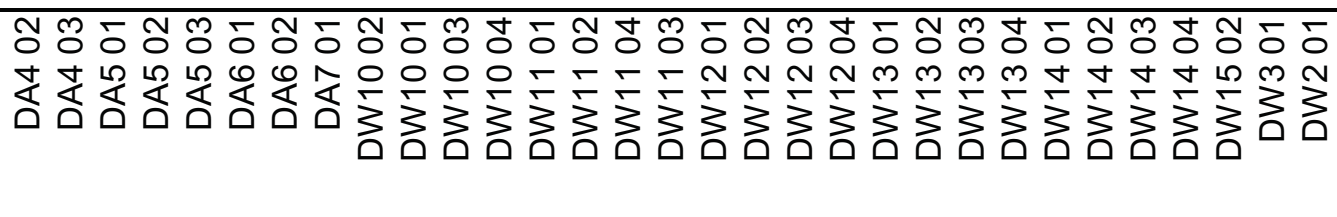 \\
\hline
\end{tabular}




\begin{tabular}{|c|c|}
\hline 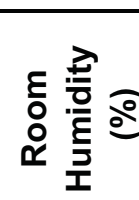 & 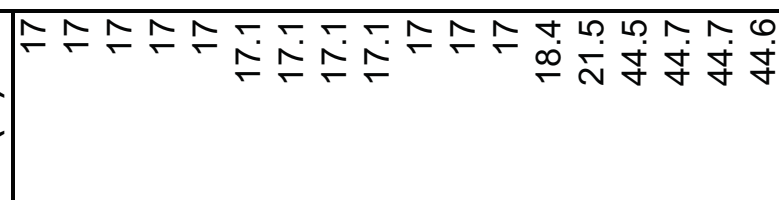 \\
\hline 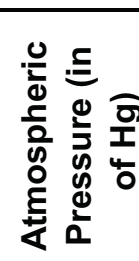 & 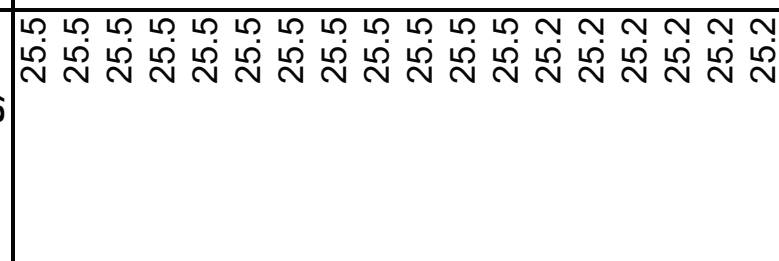 \\
\hline 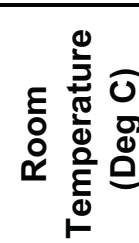 & 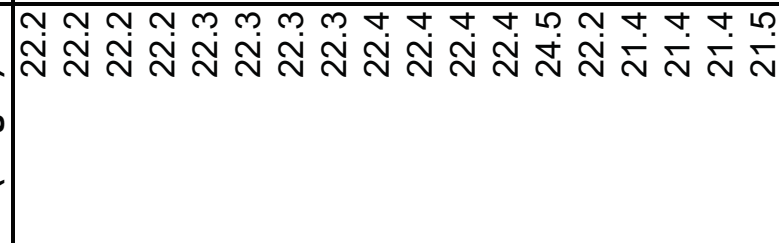 \\
\hline 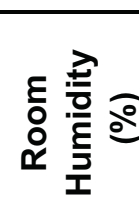 & Fร \\
\hline 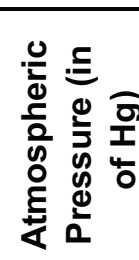 & 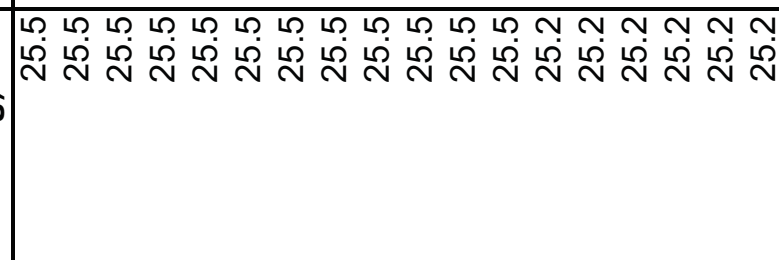 \\
\hline 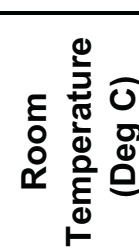 & 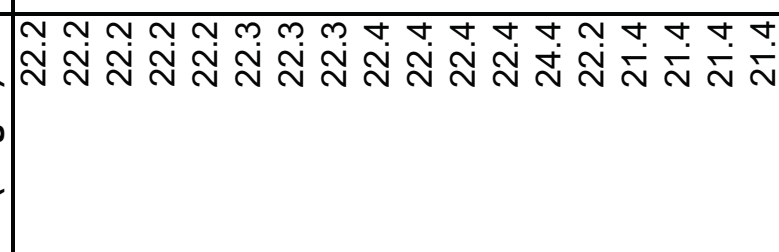 \\
\hline
\end{tabular}

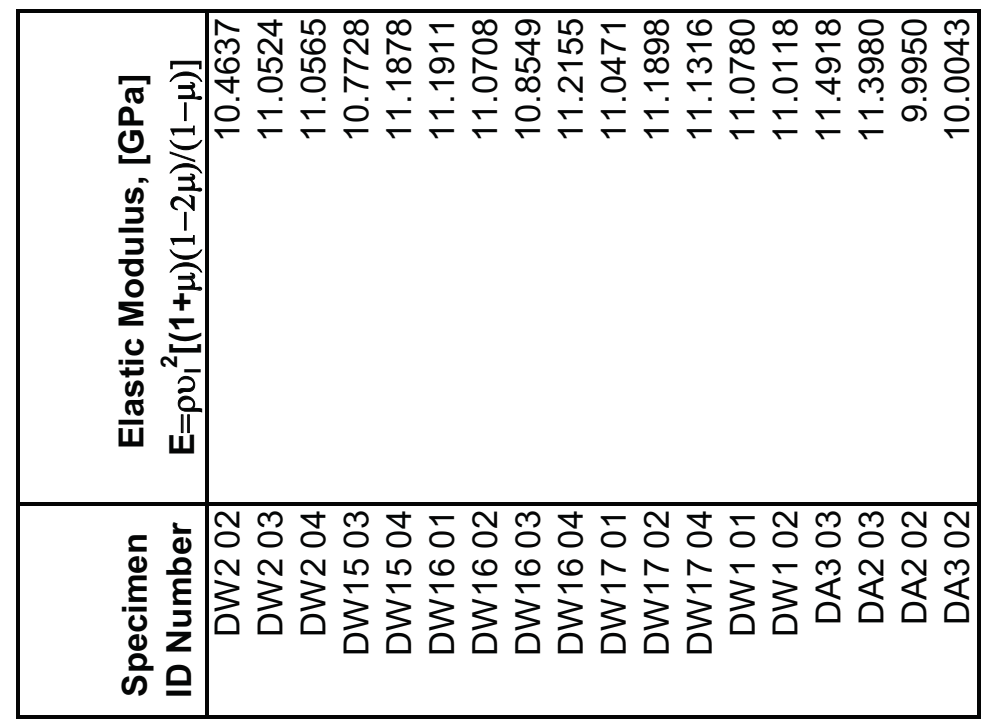




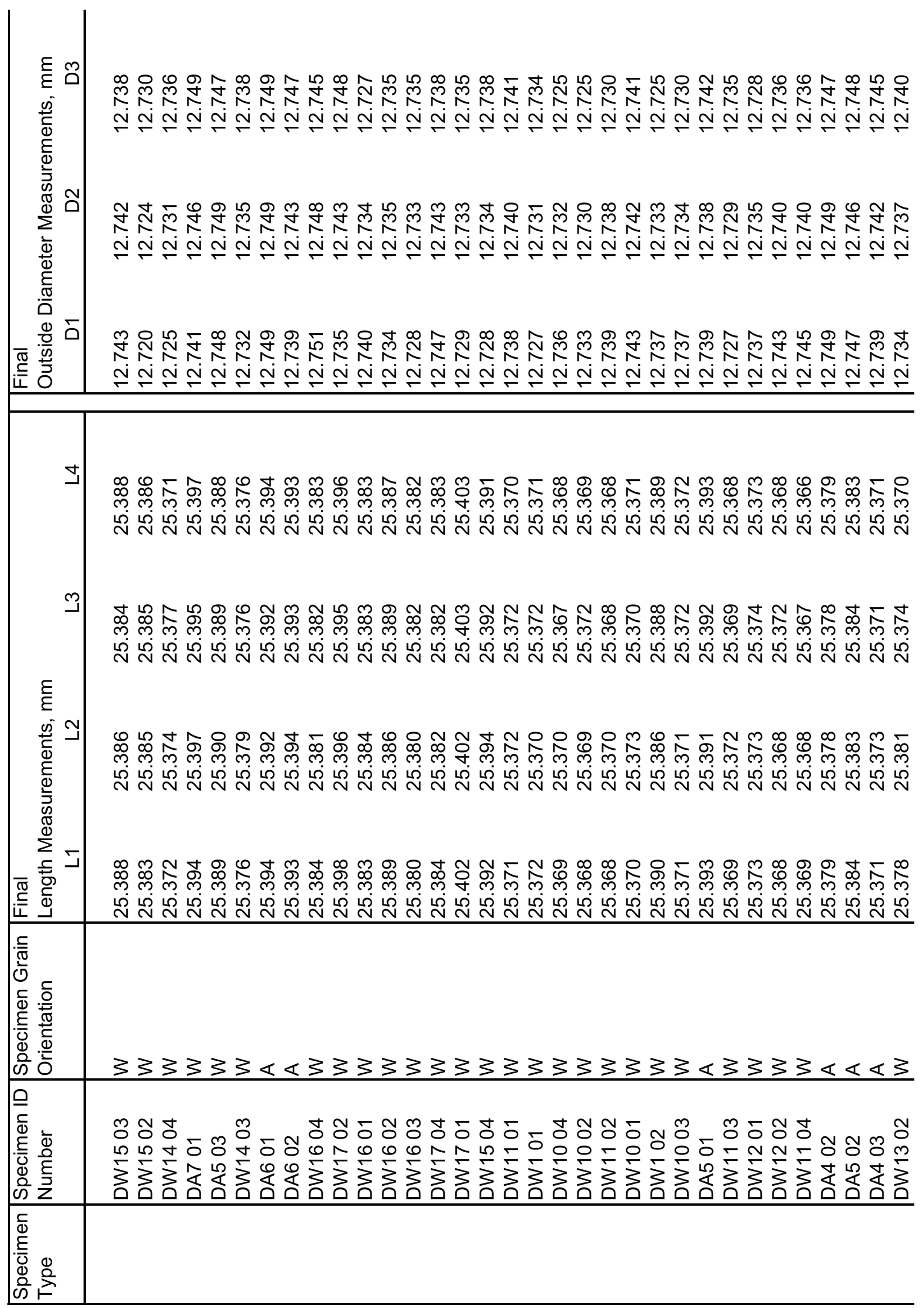




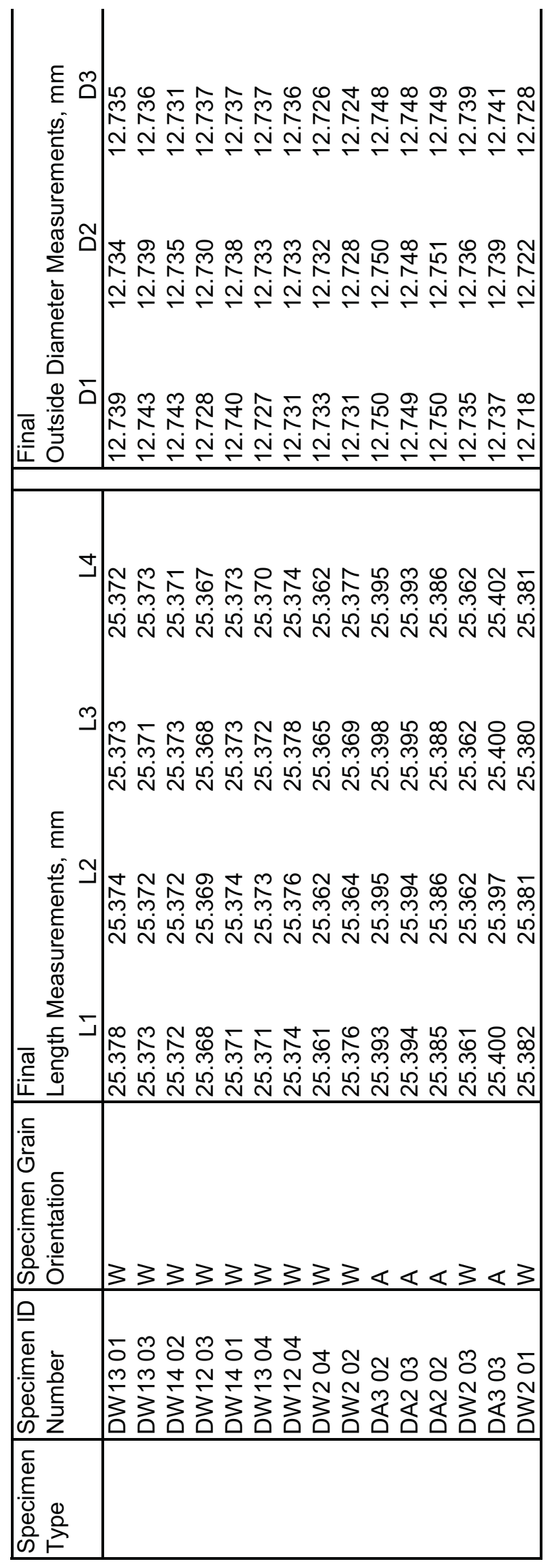




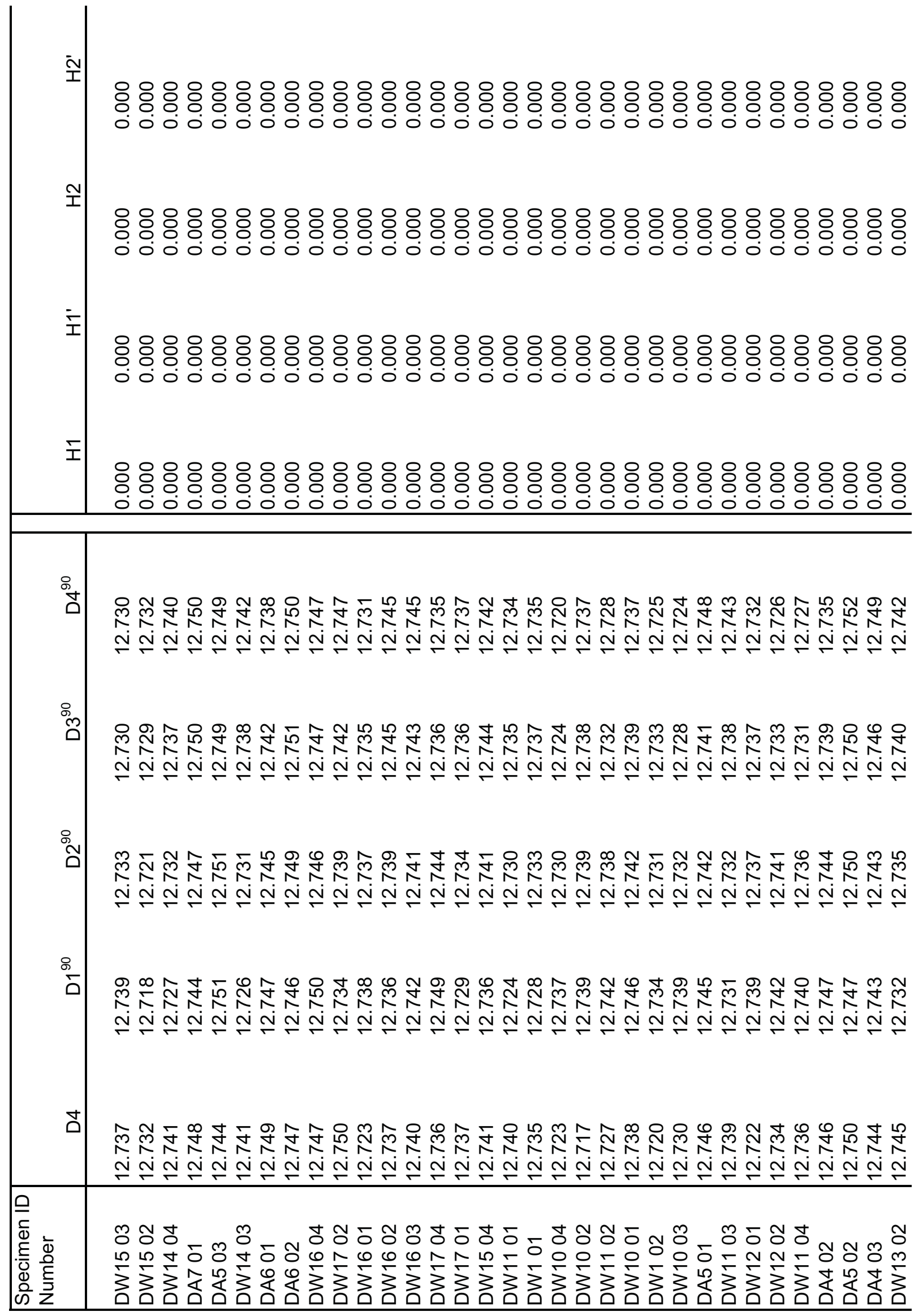




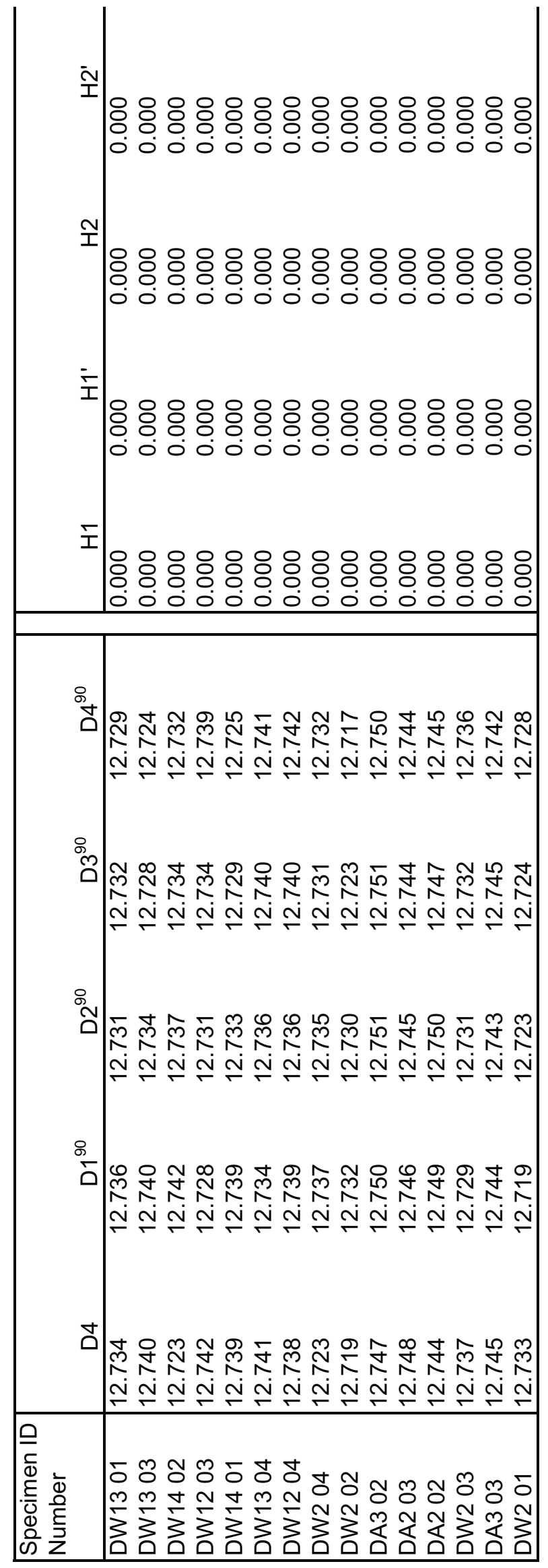




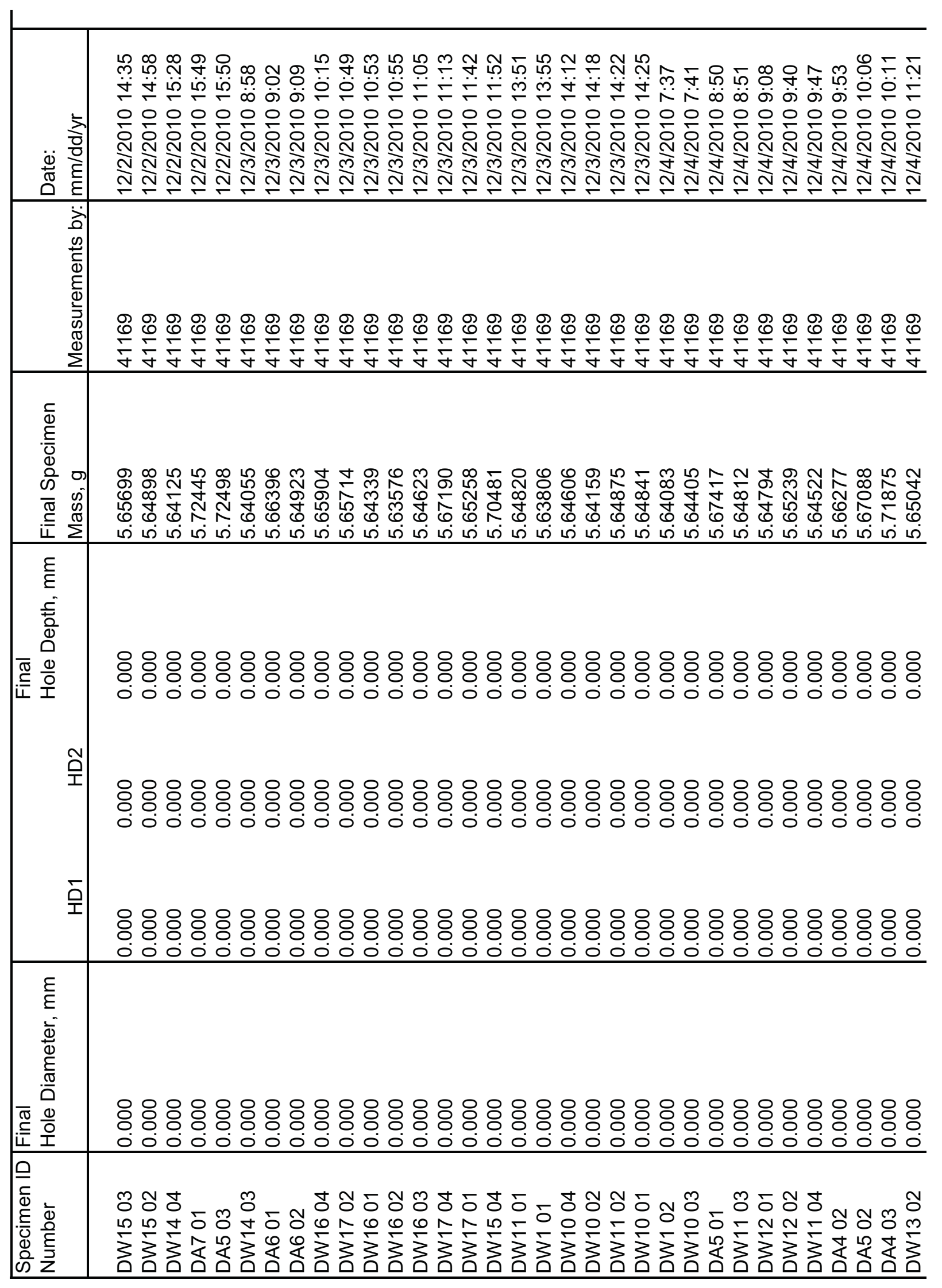




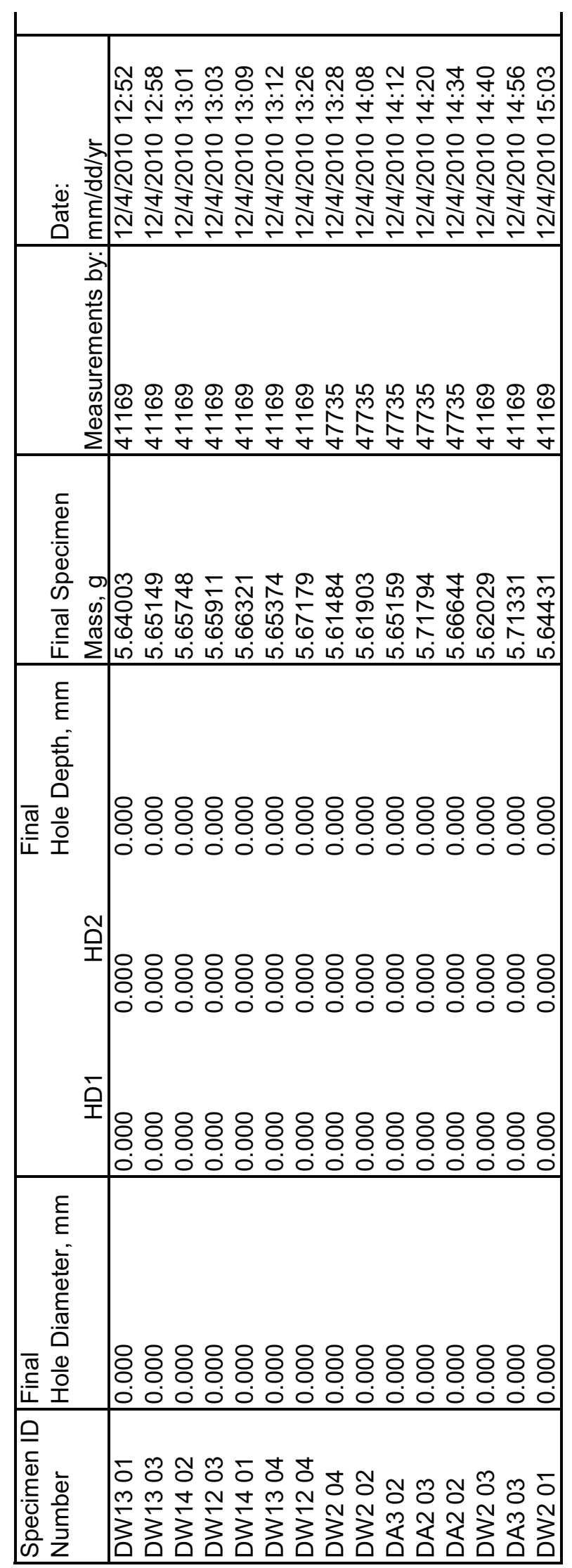




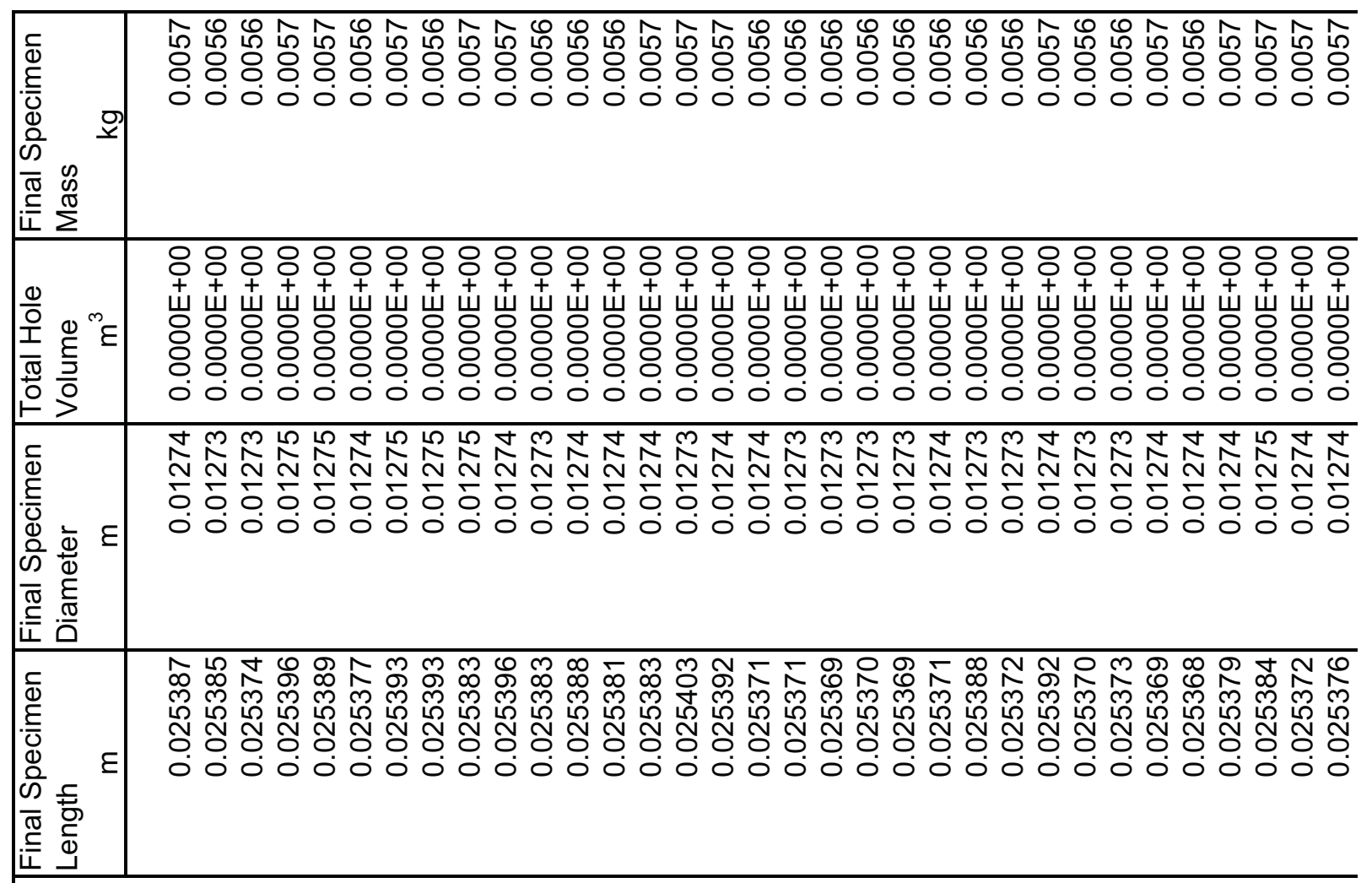

\begin{tabular}{|c|c|}
\hline 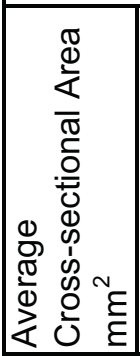 & 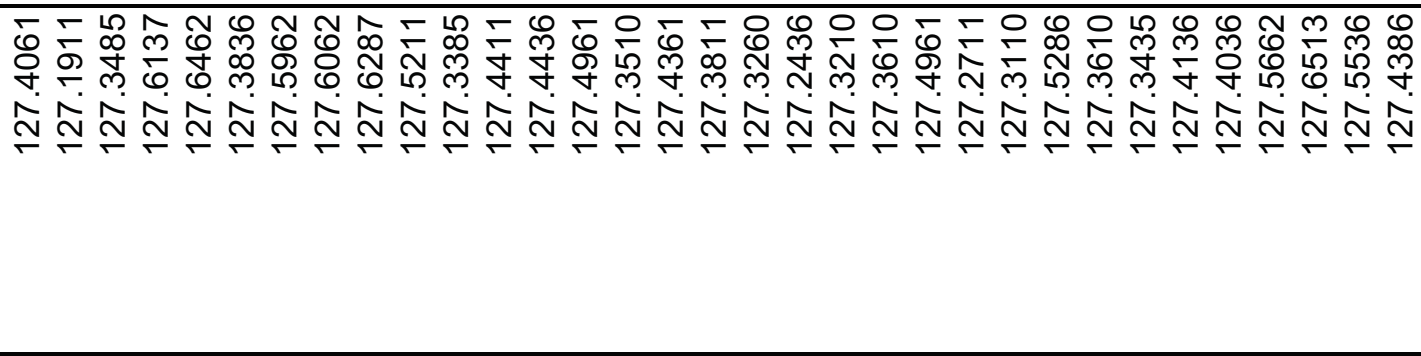 \\
\hline 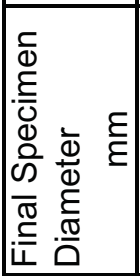 & 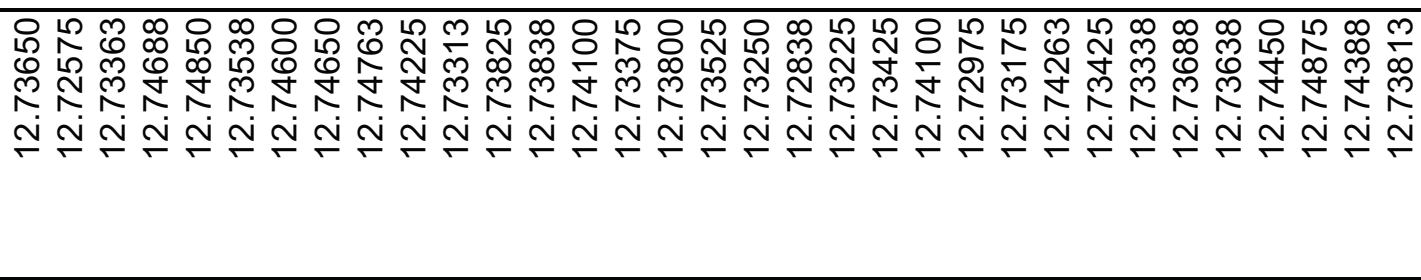 \\
\hline 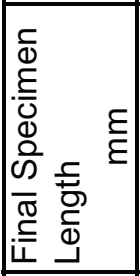 & 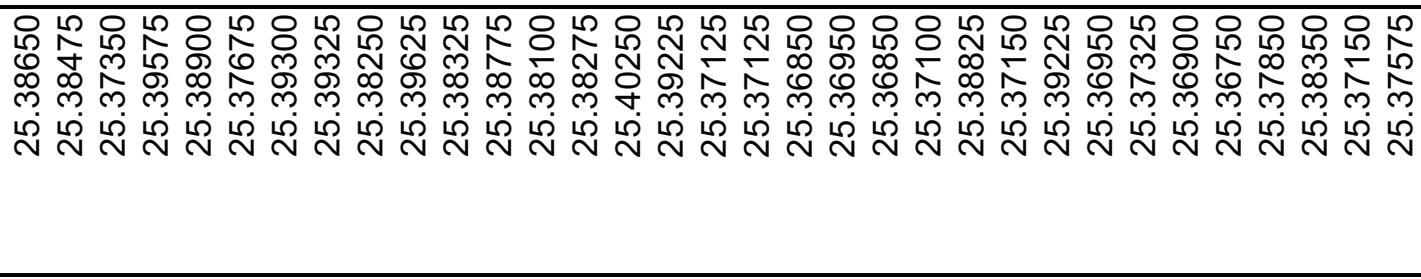 \\
\hline 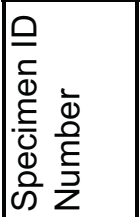 & 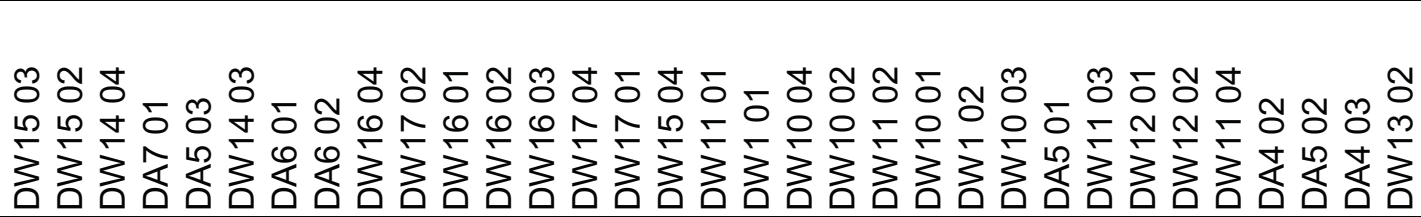 \\
\hline
\end{tabular}




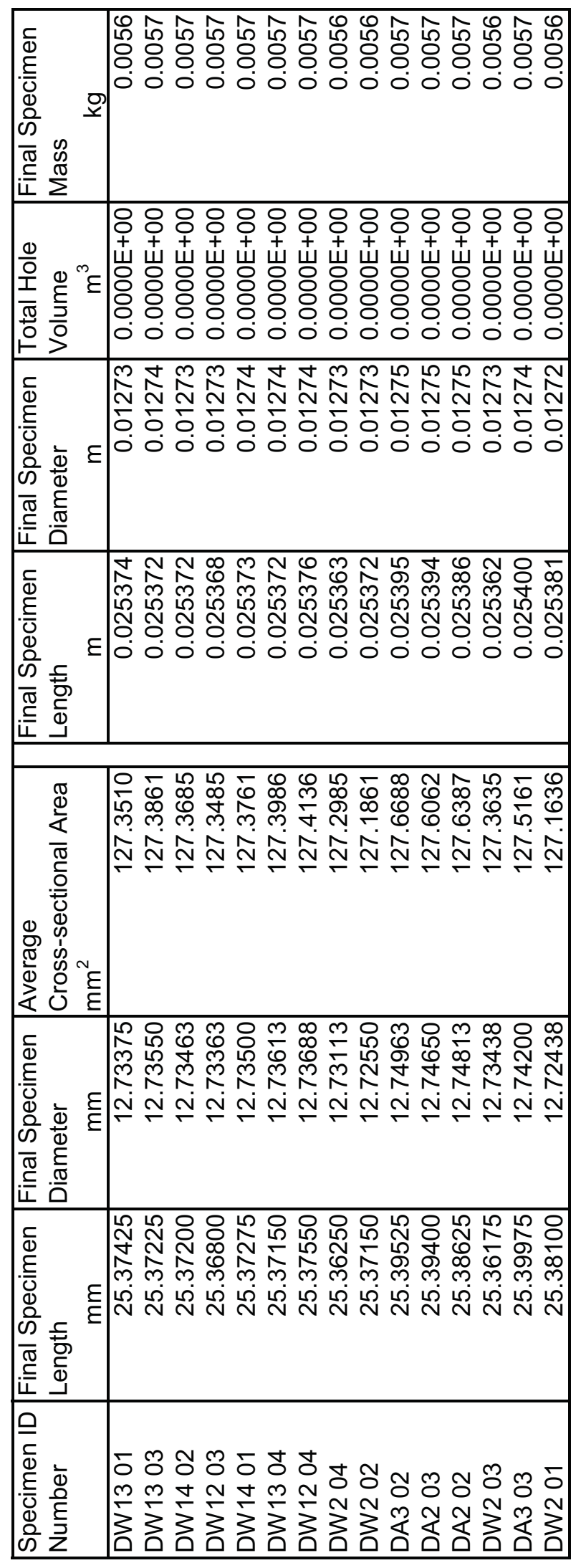




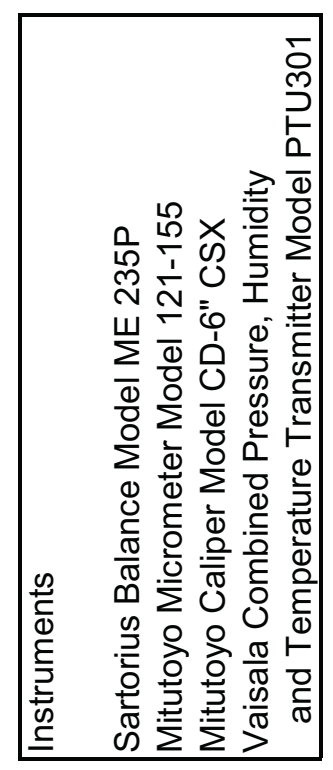

\begin{tabular}{|c|c|}
\hline 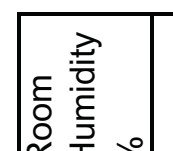 & ז. \\
\hline 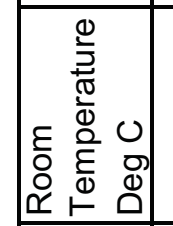 & 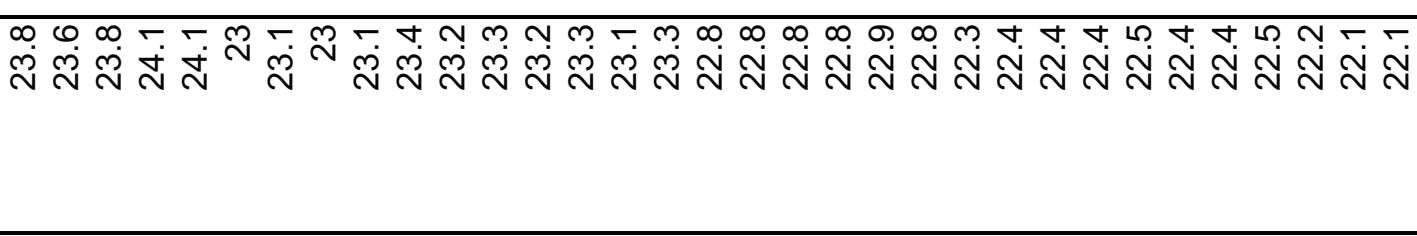 \\
\hline 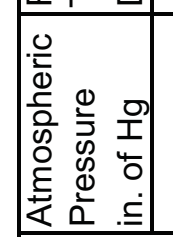 & 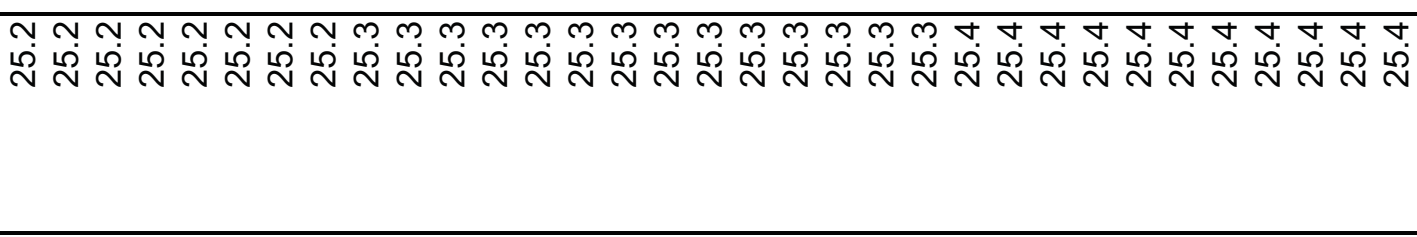 \\
\hline 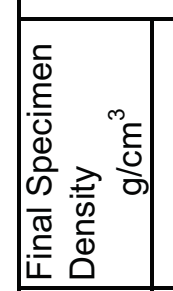 & 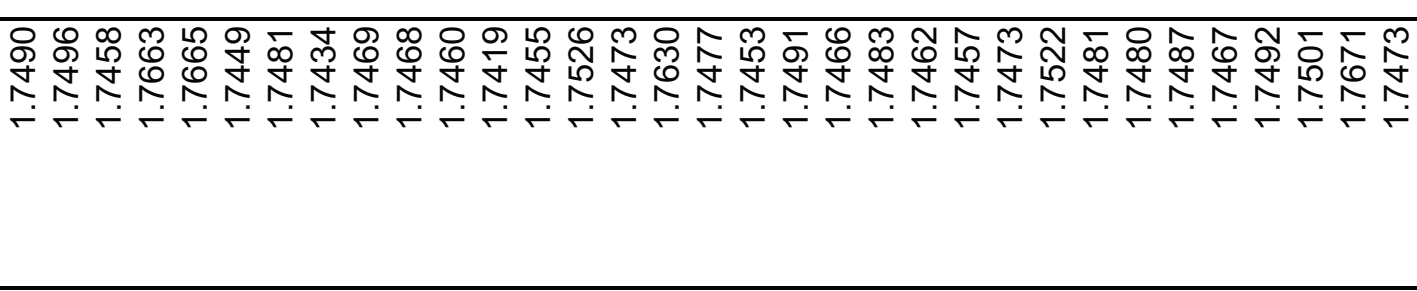 \\
\hline 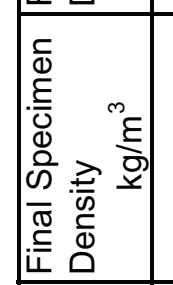 & 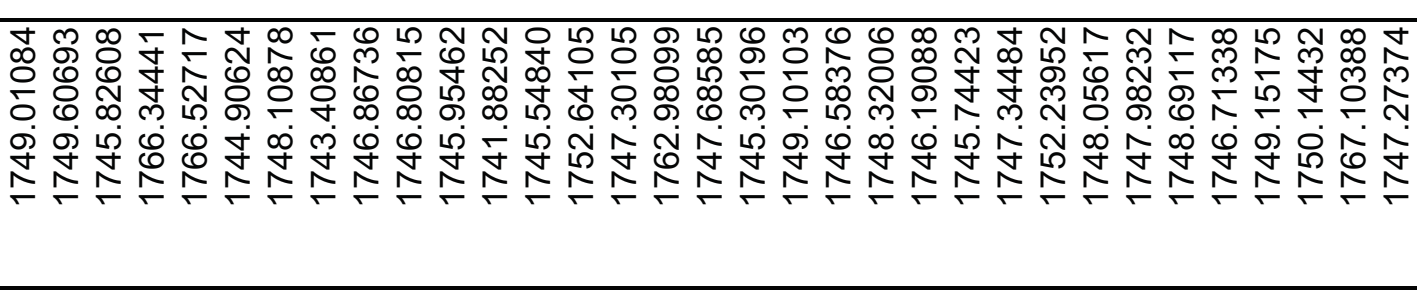 \\
\hline 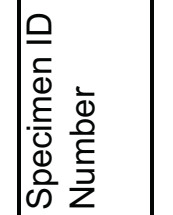 & 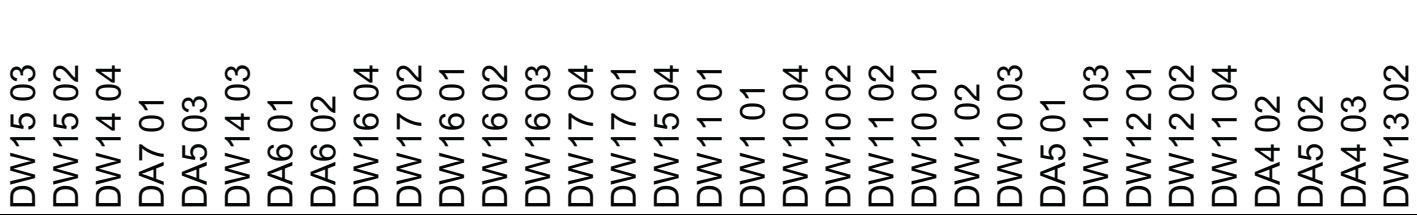 \\
\hline
\end{tabular}




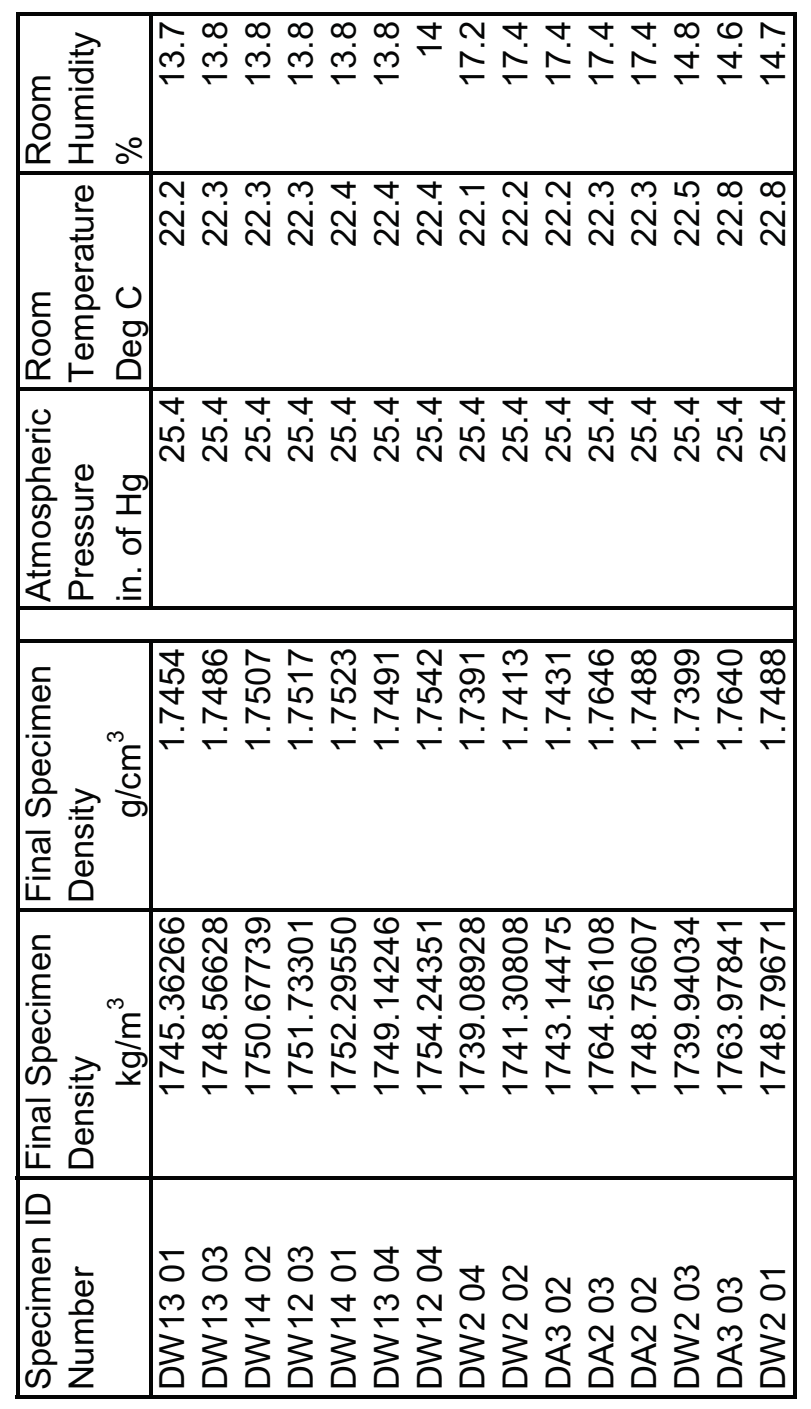




$\begin{array}{ll}\text { Graphite Grade: } & 2114 \\ \text { Graphite Manufacturer: } & \text { Mersen (Carbone Lorraine USA) } \\ \text { Forming Process: } & \text { Isostatic-molded } \\ \text { Coke Particle Size: } & \text { Fine grain } \\ \text { Coke Type: } & \text { Pitch coke filler, pitch binder } \\ \text { ASTM Class: } & \text { INHP } \\ \text { Specimen Geometry: } & \text { Cylinder }\end{array}$

Specimen ID \#'s:

TP01

TP02

TP03

TP04

TP05

TP06

TP07

TP08

TP09

TP10

TP11

TP12

TP13

TP14

TP15

TP16

TP17

TP18

TP19

TP20

TP21

TP22

TP23

TP24

TP25

TP26

TP27

TP28

TP29

TP30

TP31

TP32

TP33

TP34

TP35

TP36

TP37

TP38

TP39

TP40

TP41

TP42 
Specimen ID \#'s:

TP43

TP44

TP45

TP46

TP47

TP48 


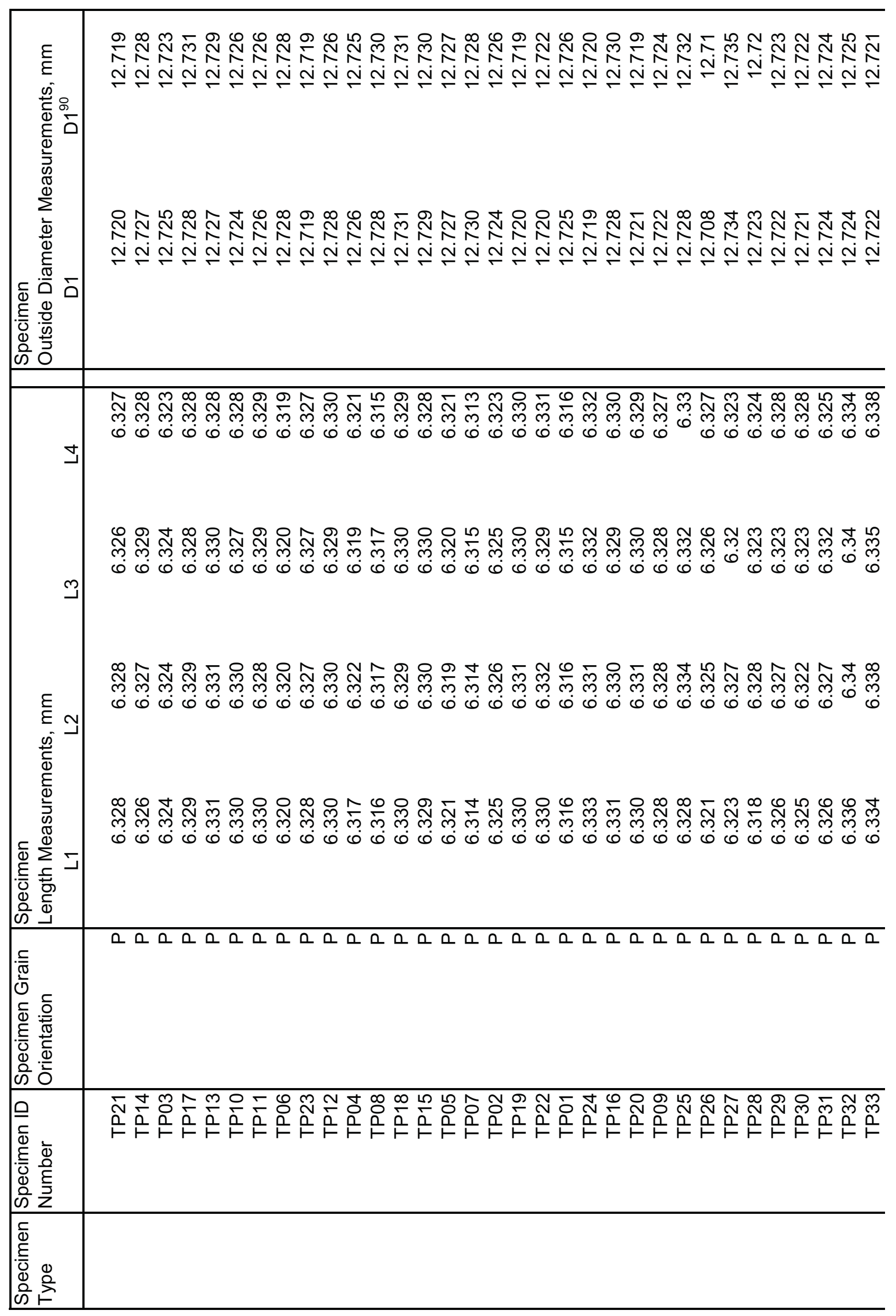




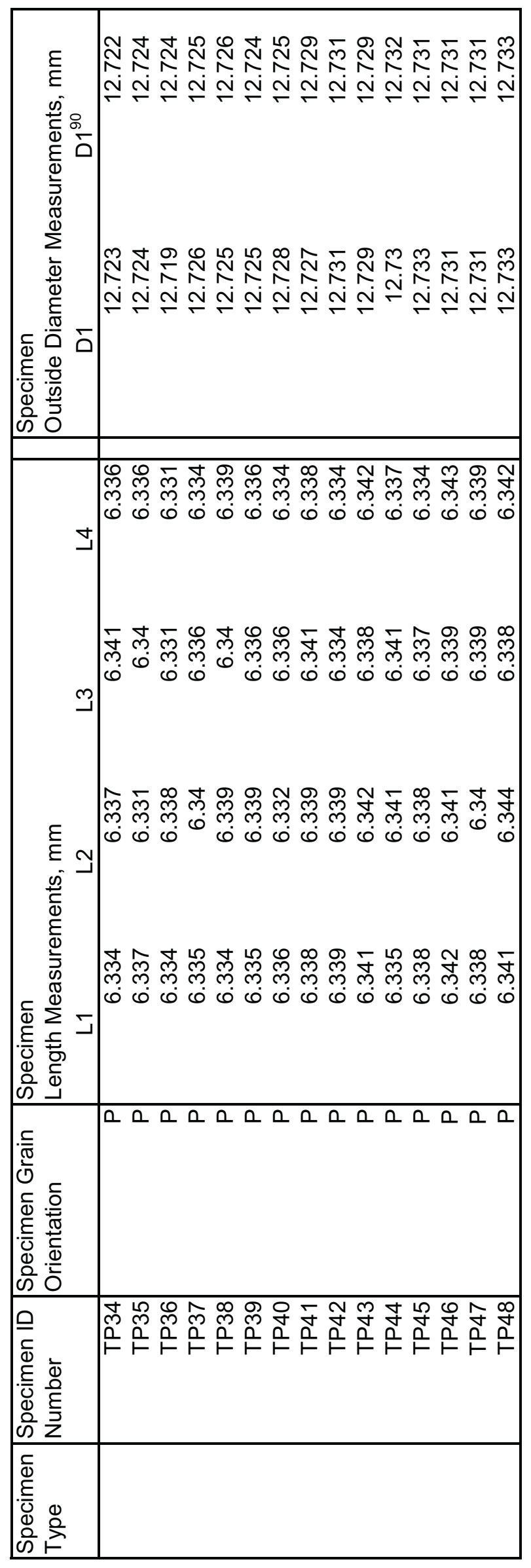




\begin{tabular}{|c|c|}
\hline 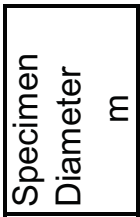 & 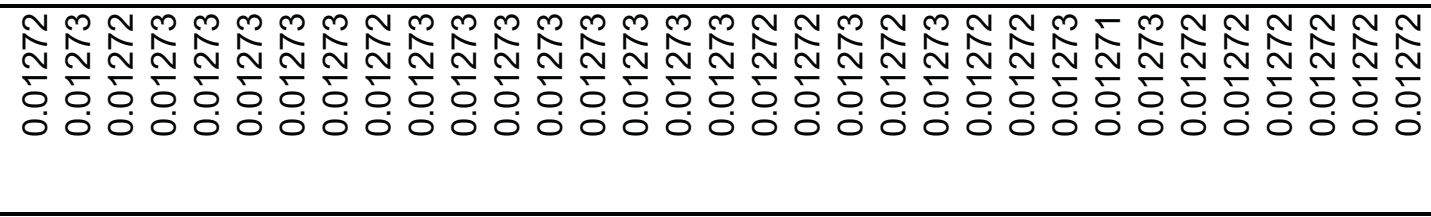 \\
\hline 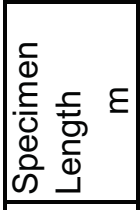 & 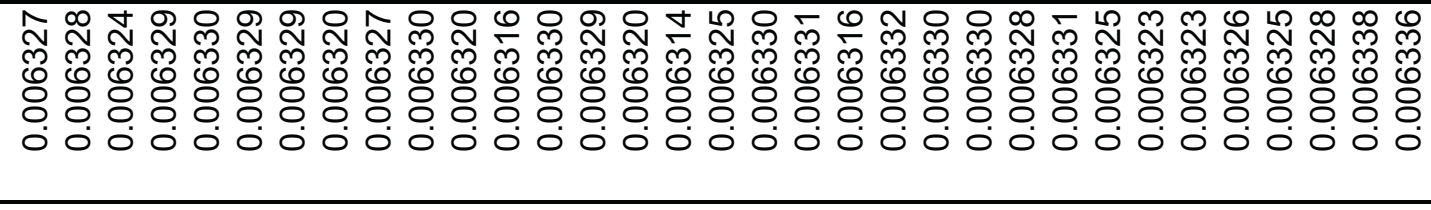 \\
\hline 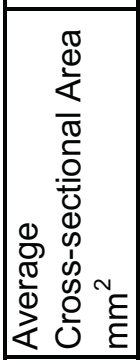 & 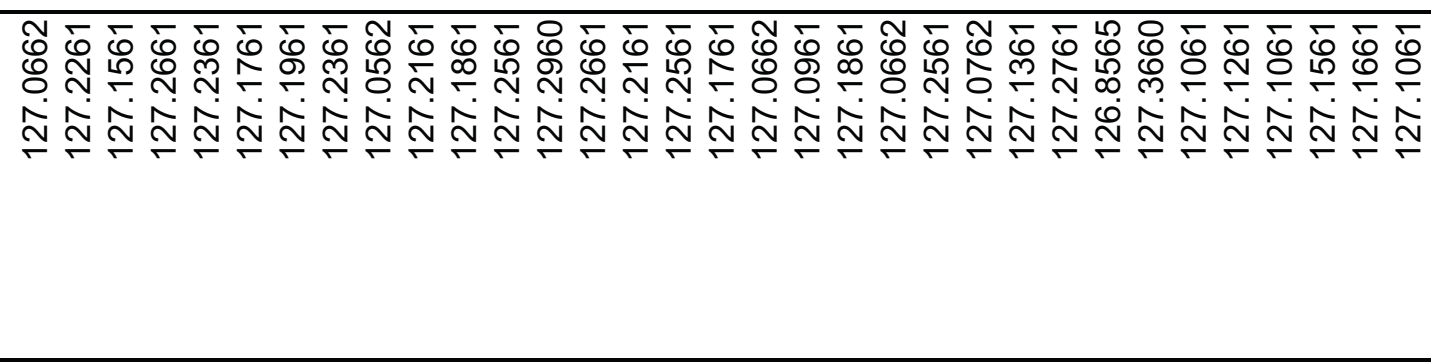 \\
\hline 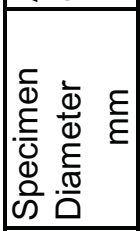 & 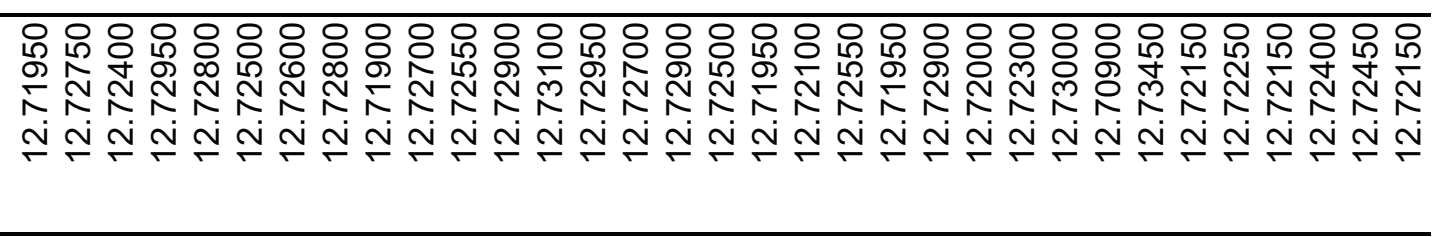 \\
\hline 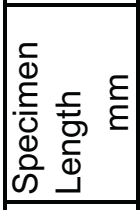 & 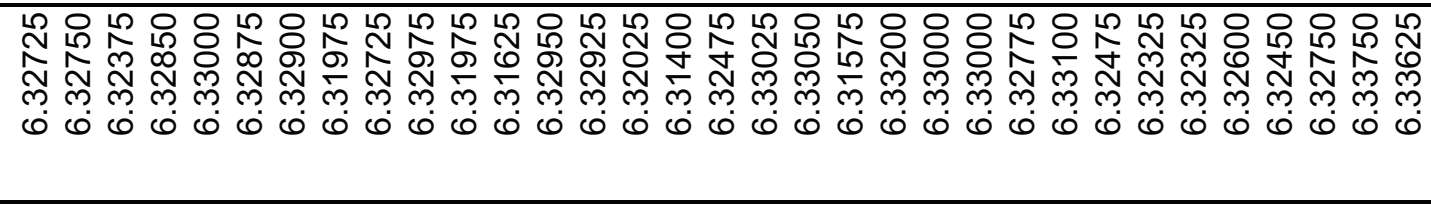 \\
\hline 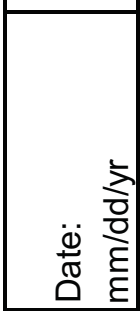 & 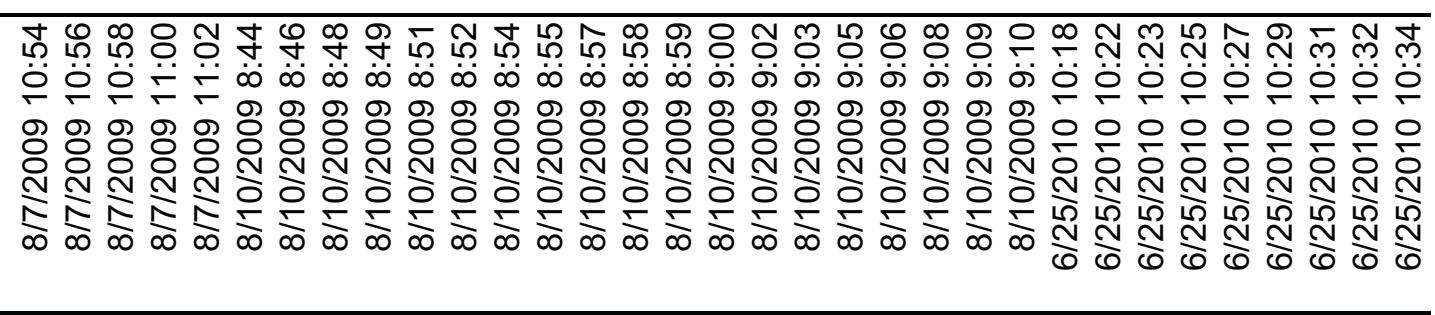 \\
\hline 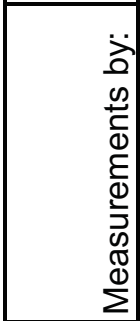 & 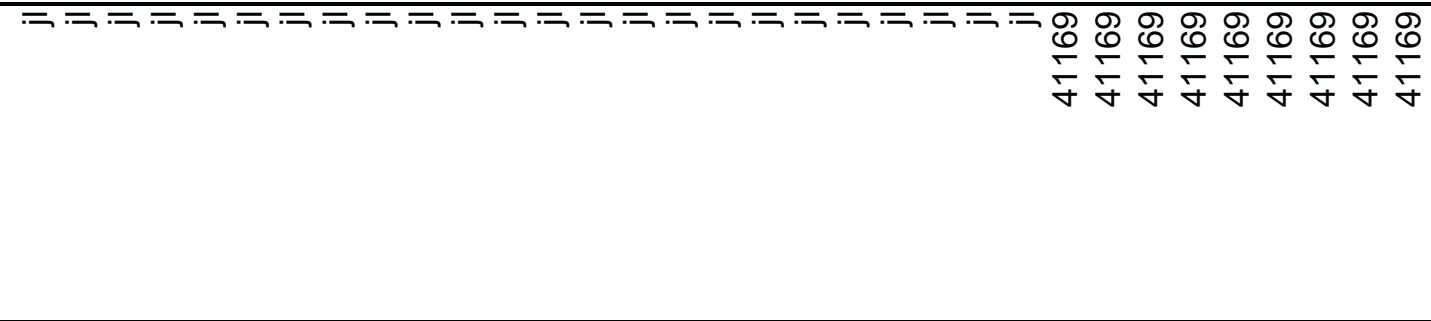 \\
\hline 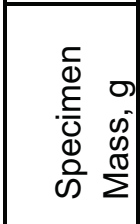 & 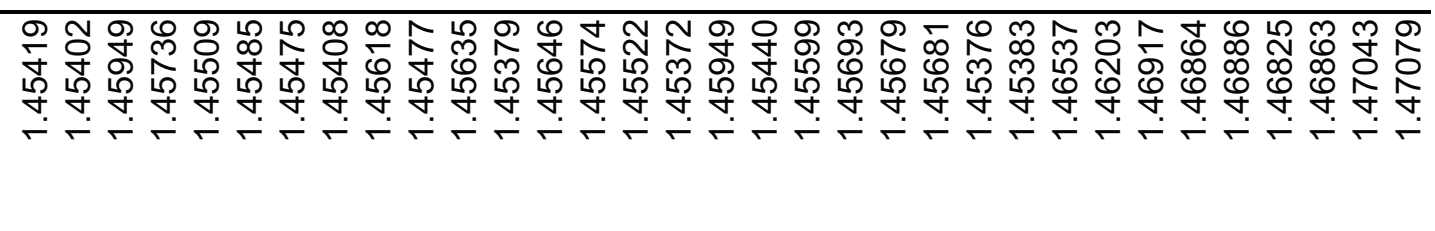 \\
\hline 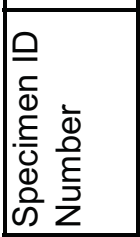 & 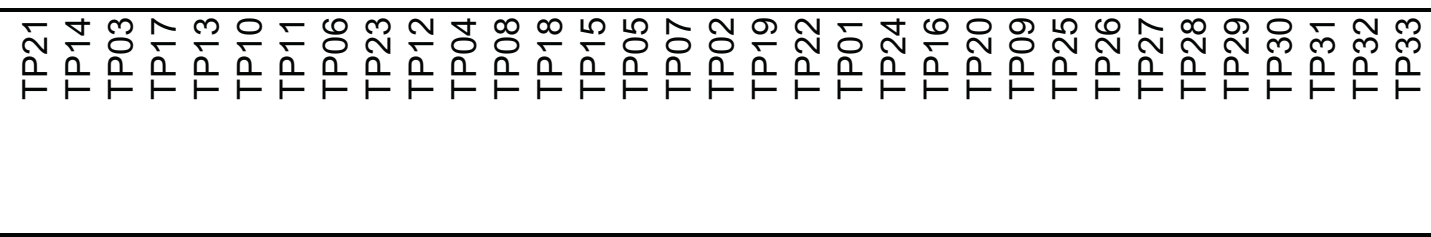 \\
\hline
\end{tabular}




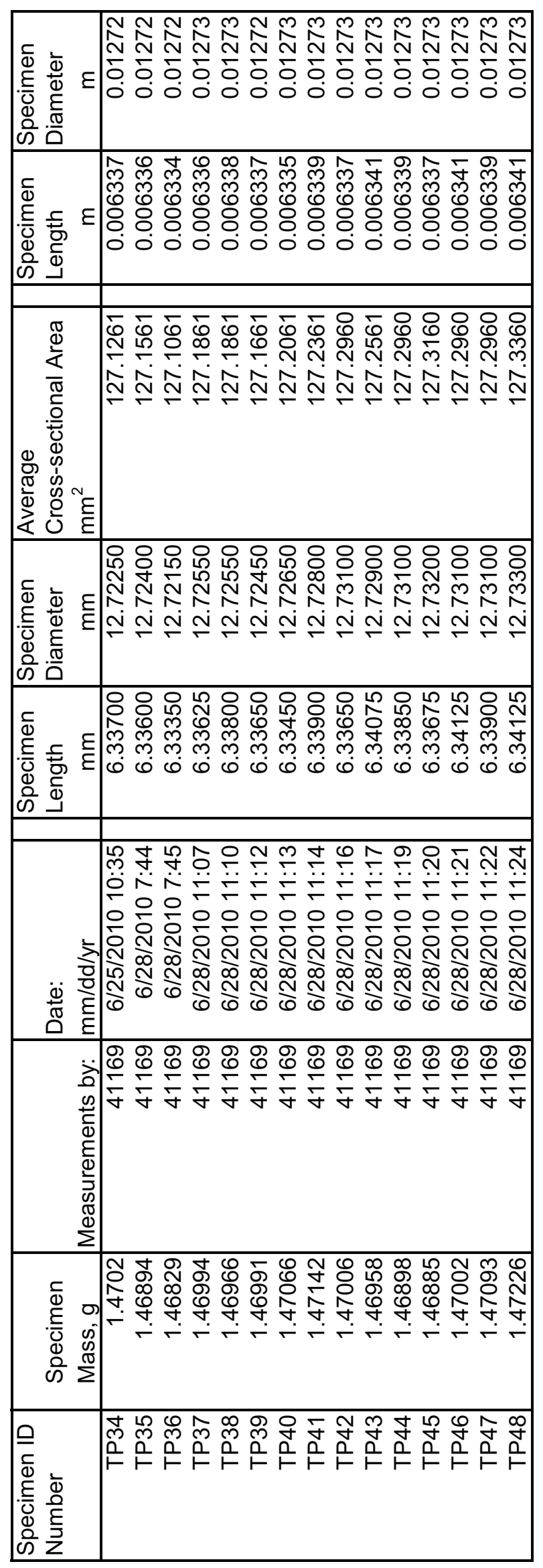




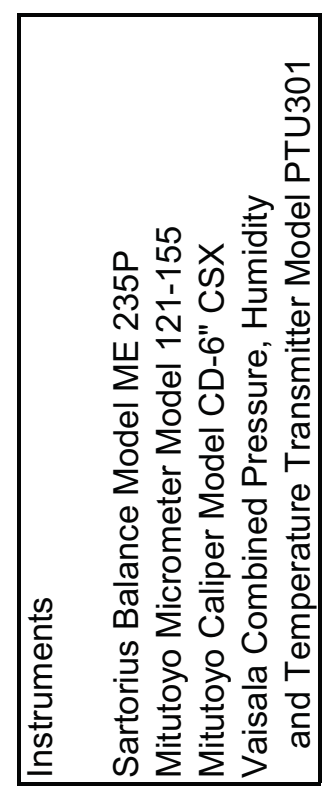

\begin{tabular}{|c|c|}
\hline \multicolumn{2}{|r|}{ 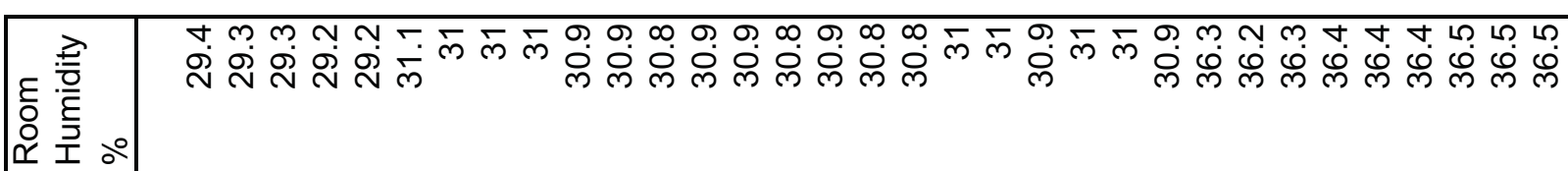 } \\
\hline & 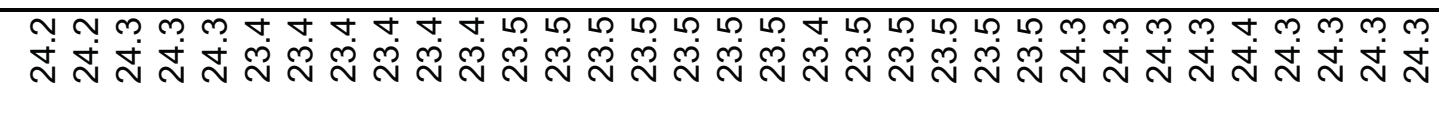 \\
\hline & 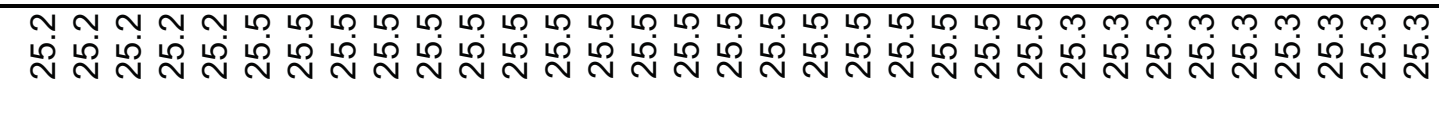 \\
\hline & 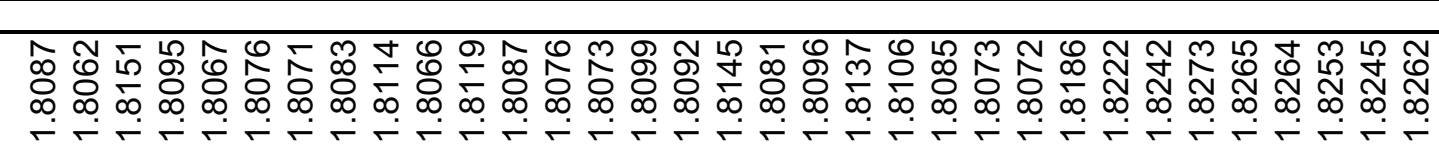 \\
\hline & 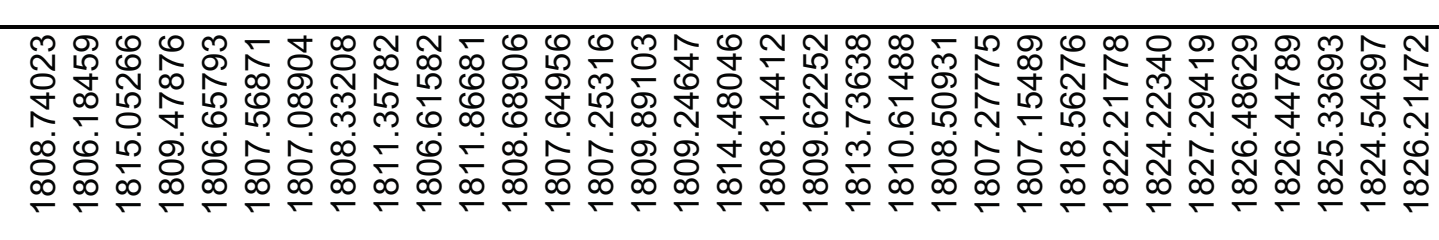 \\
\hline & 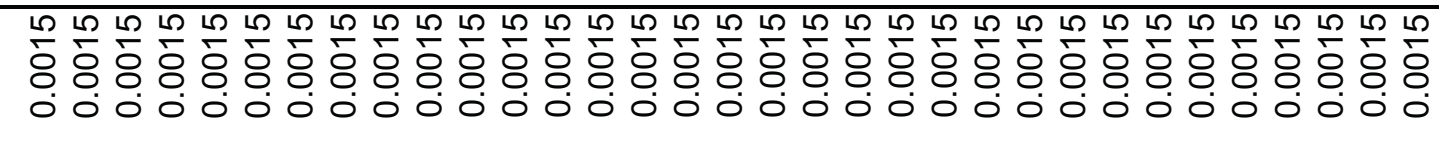 \\
\hline & 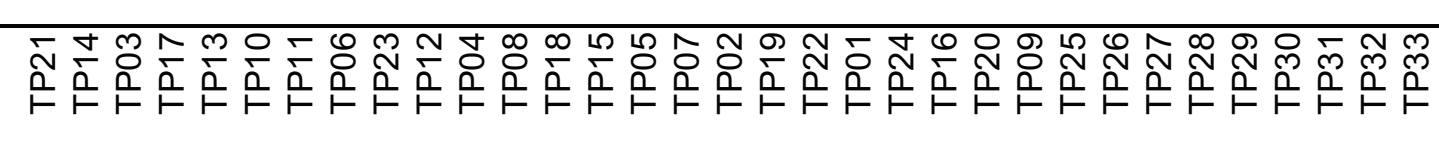 \\
\hline & \\
\hline
\end{tabular}




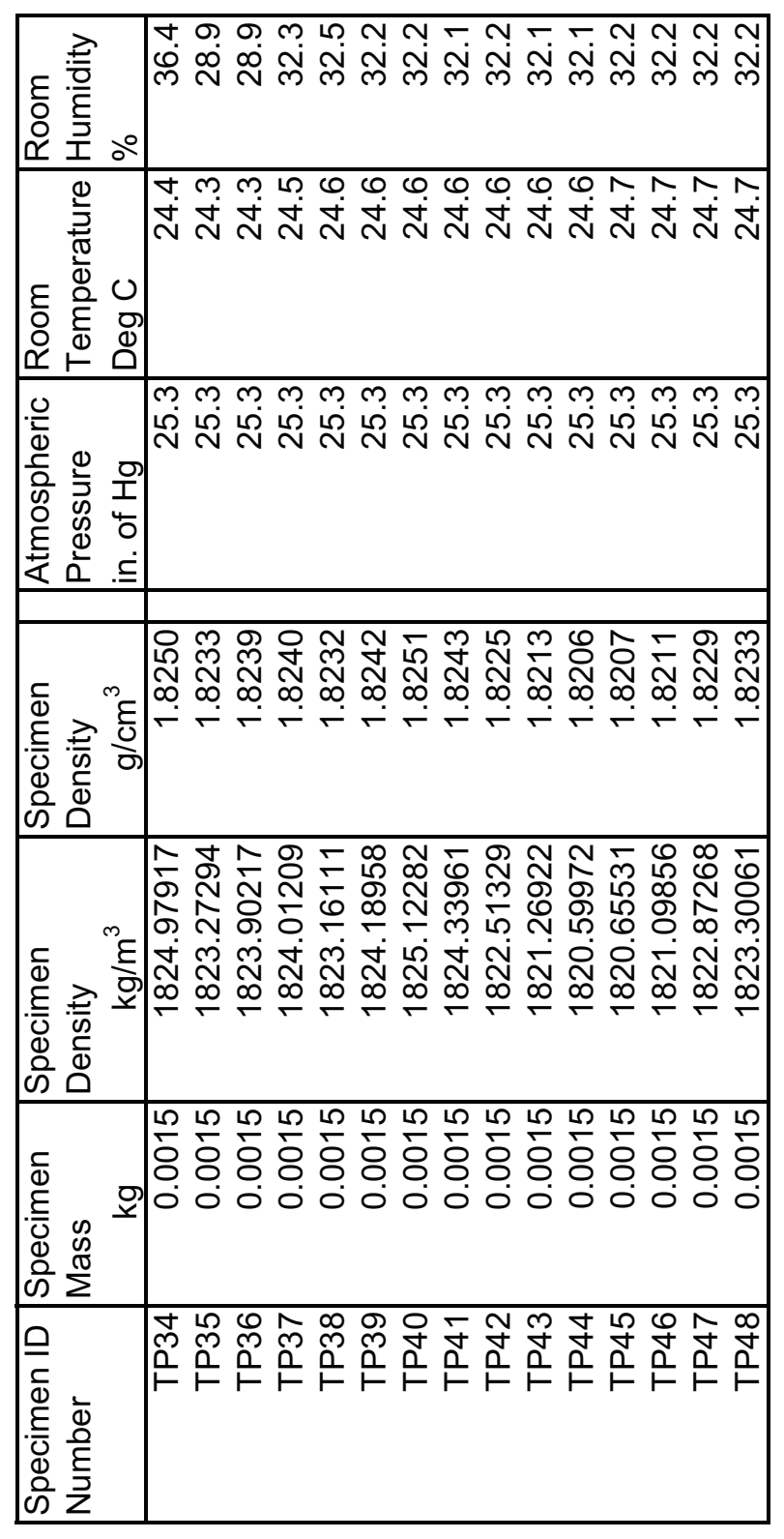




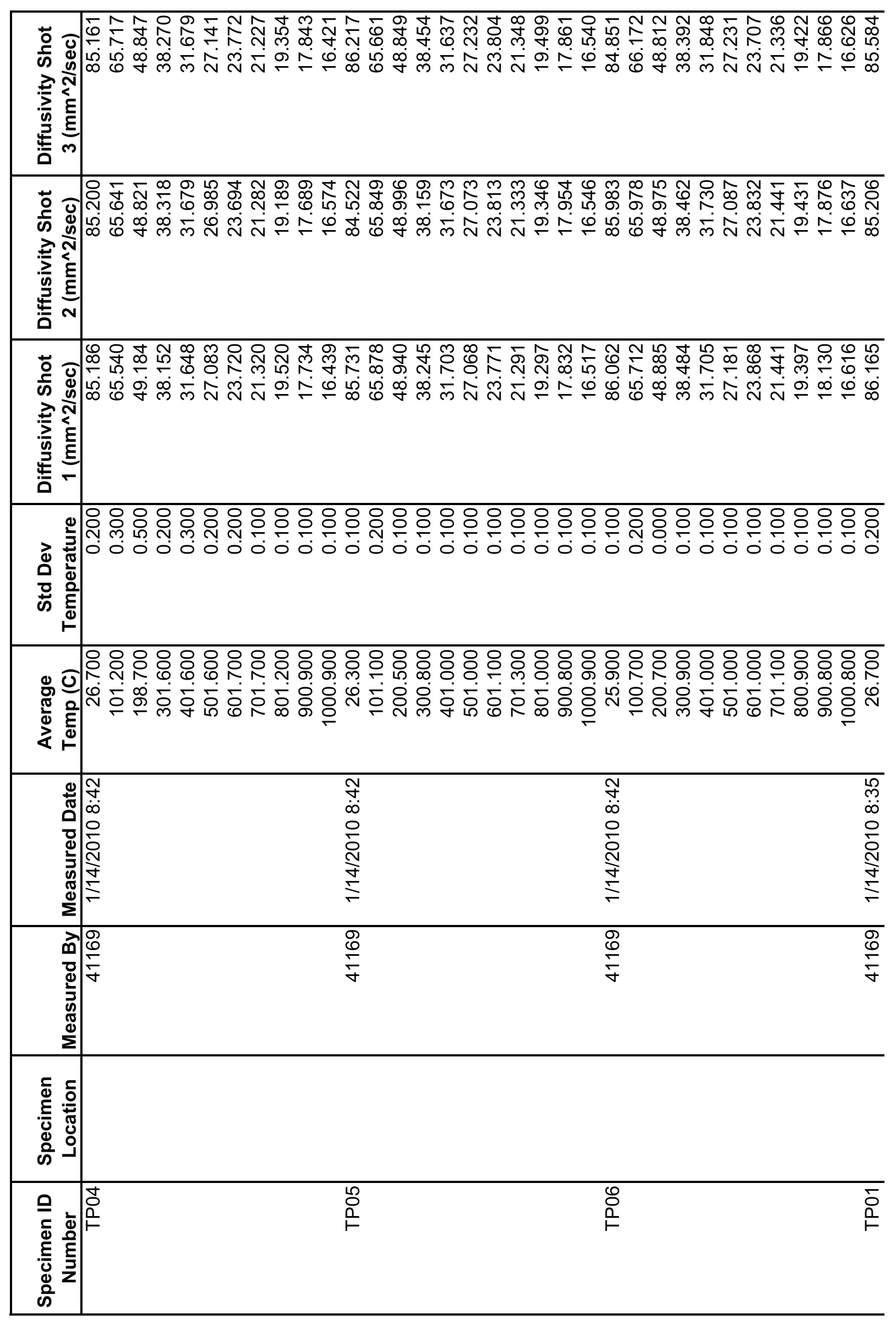




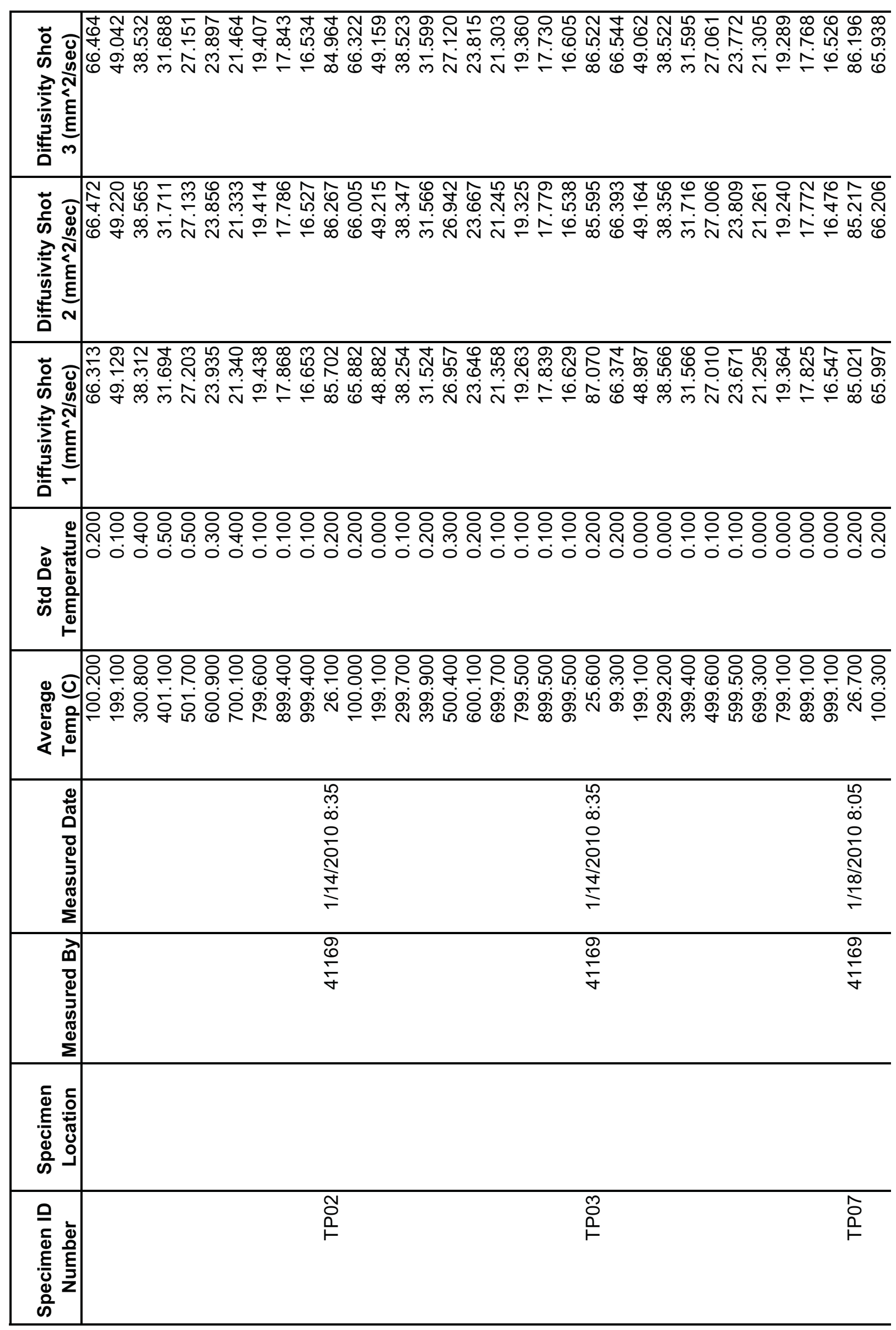




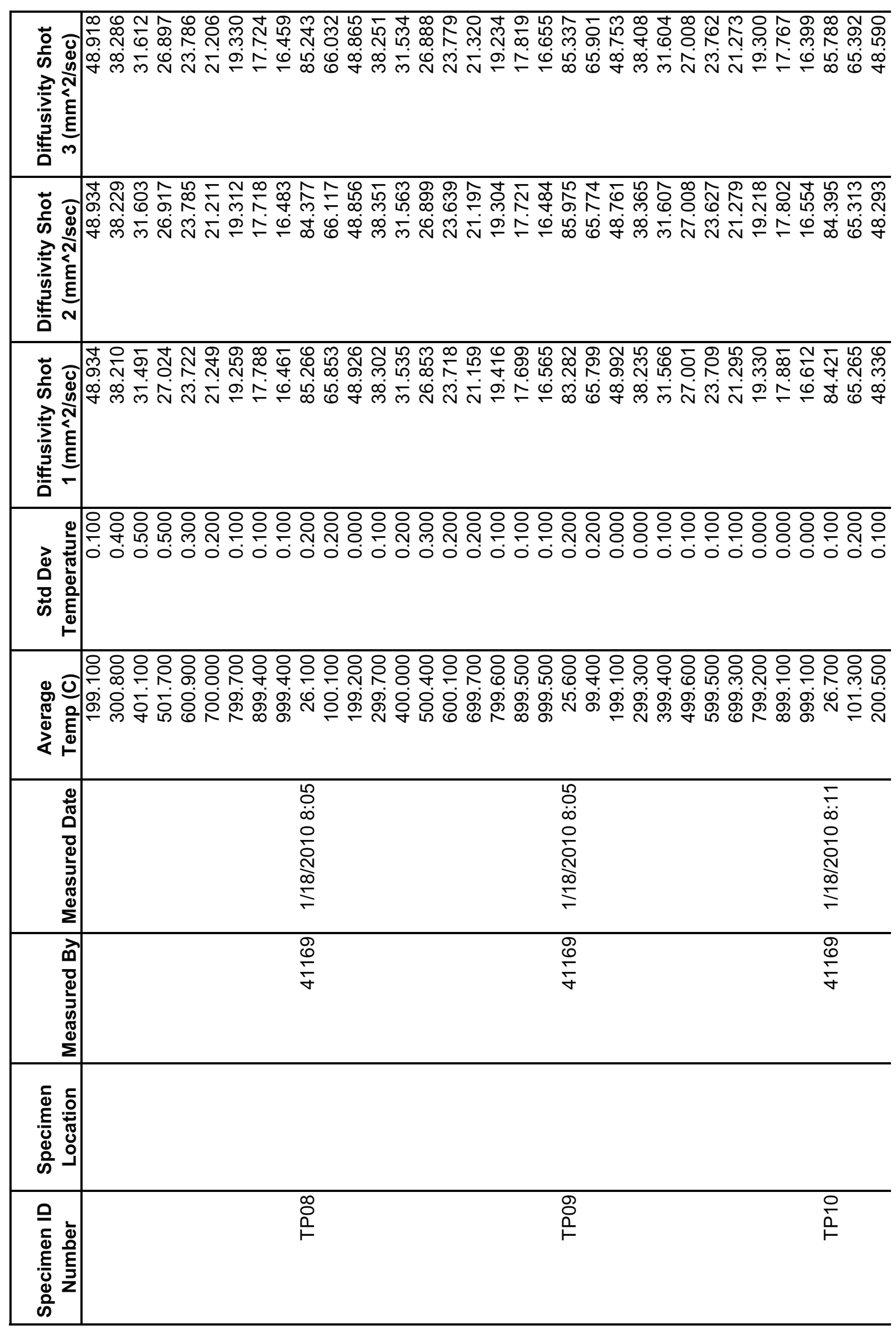




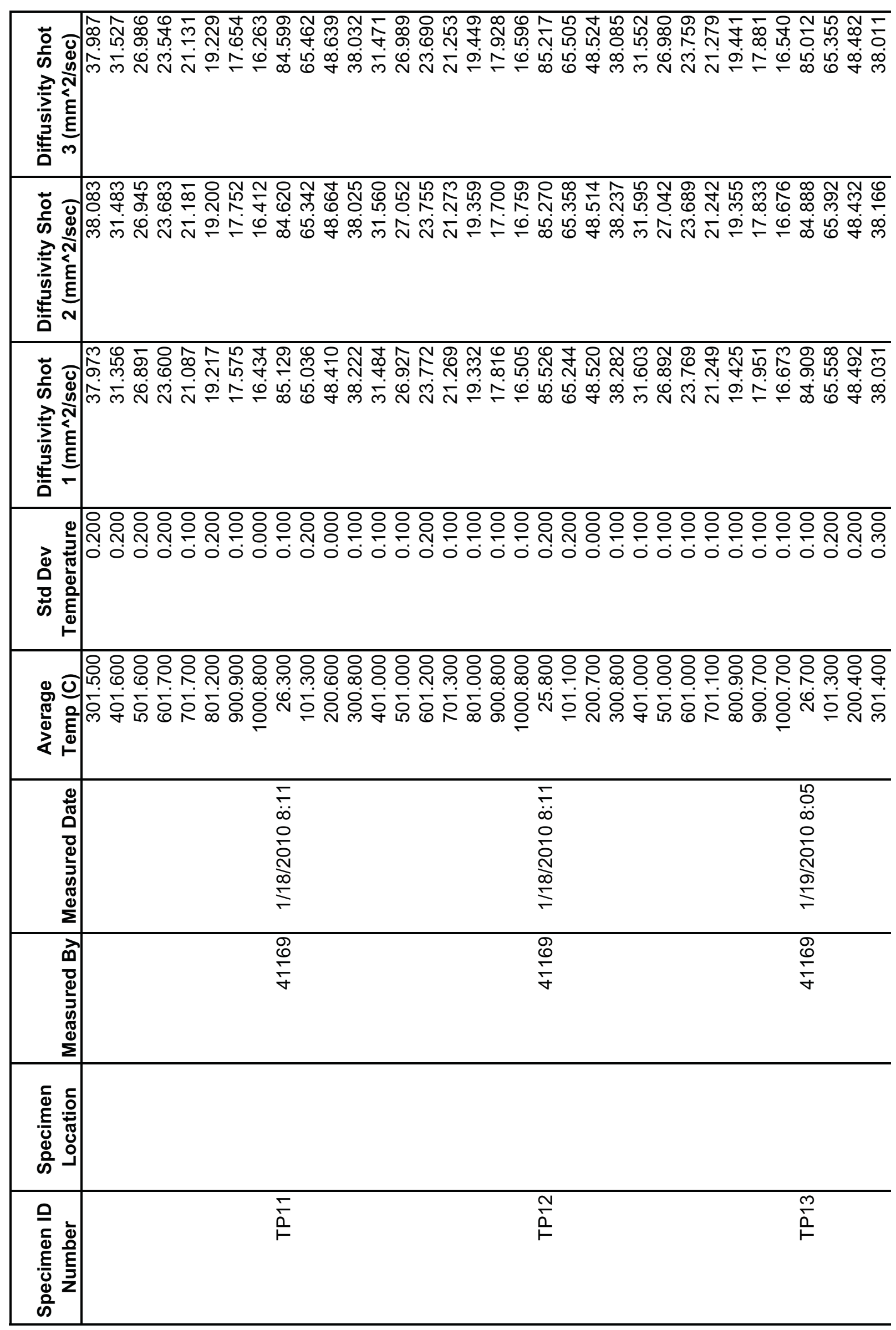




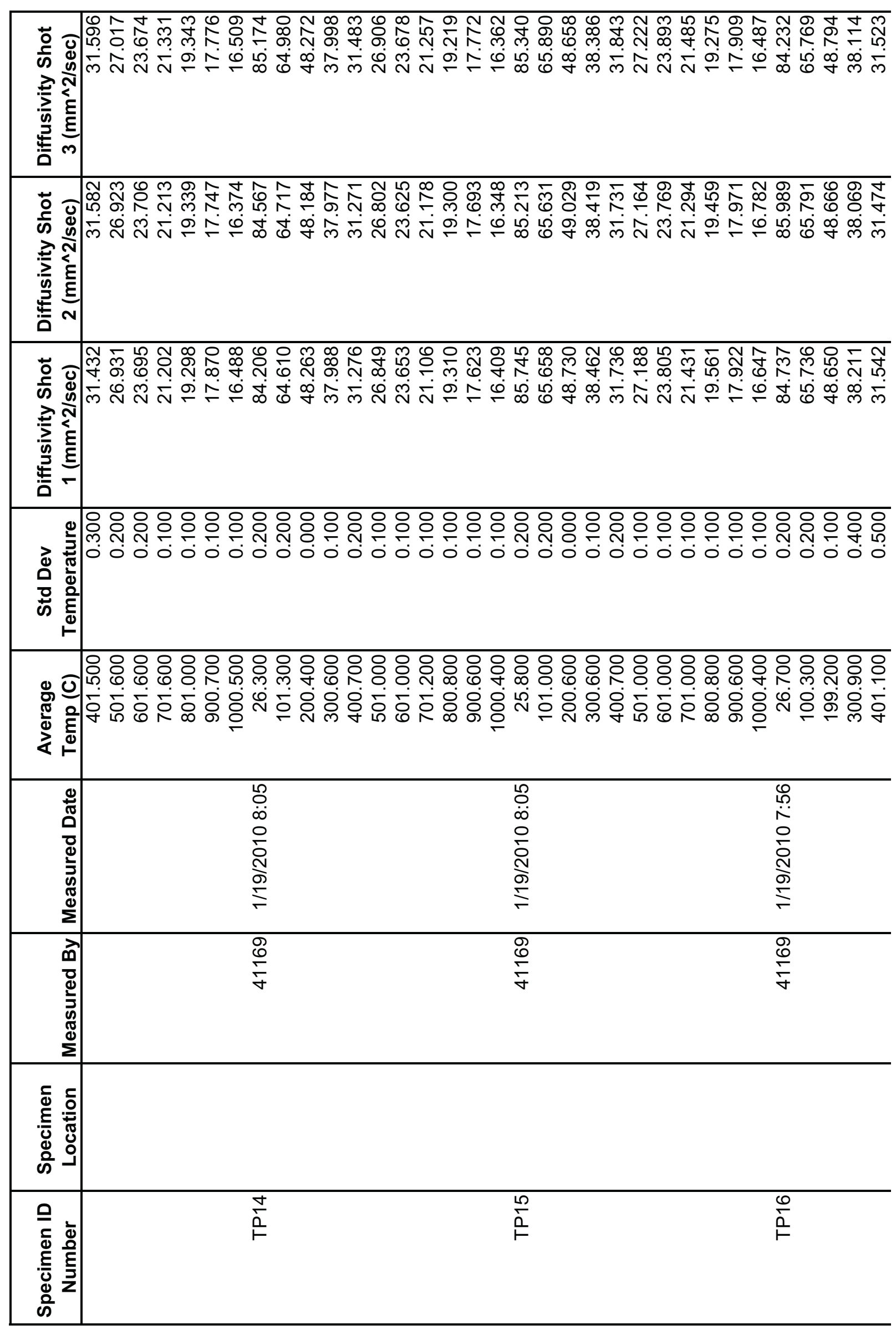




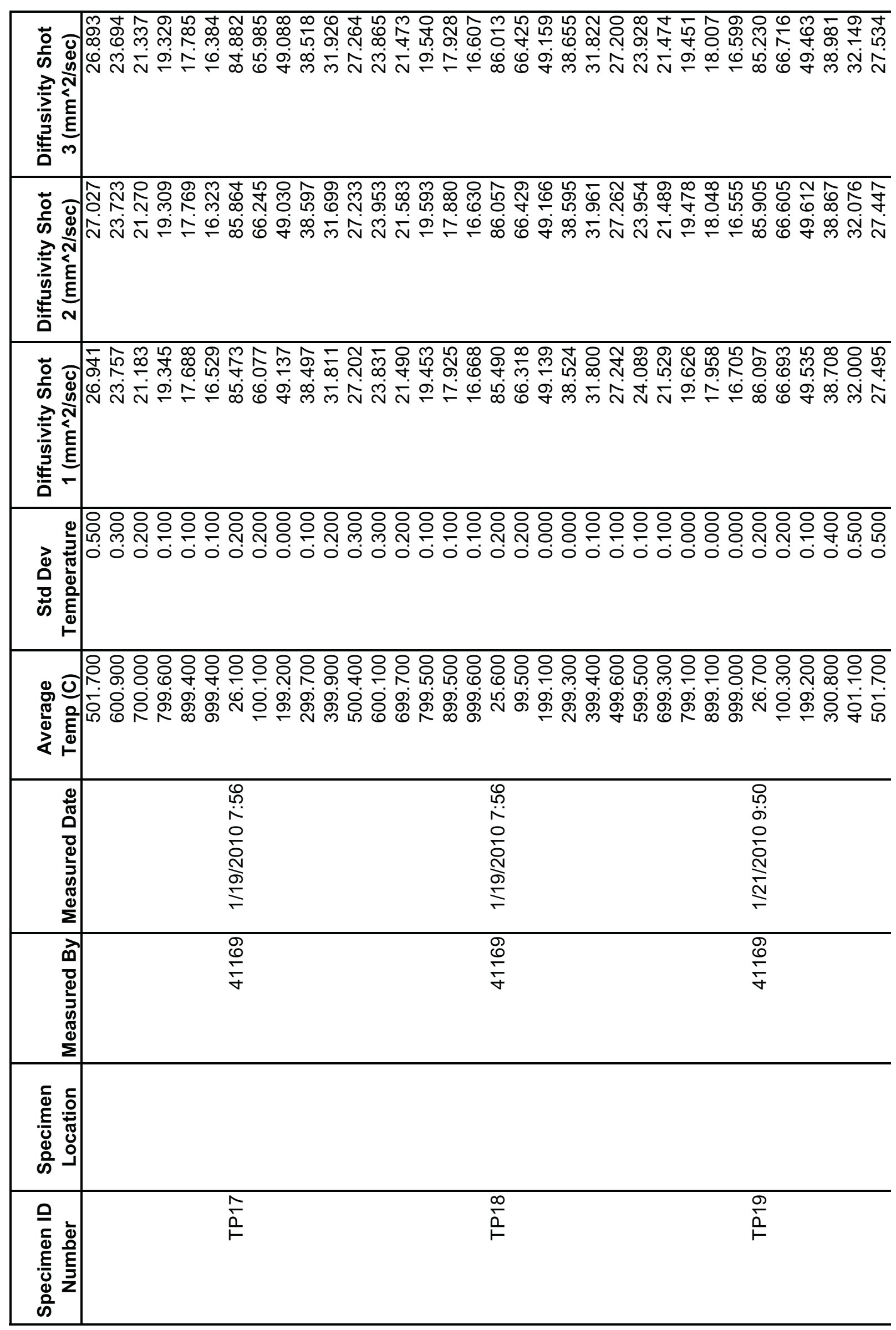




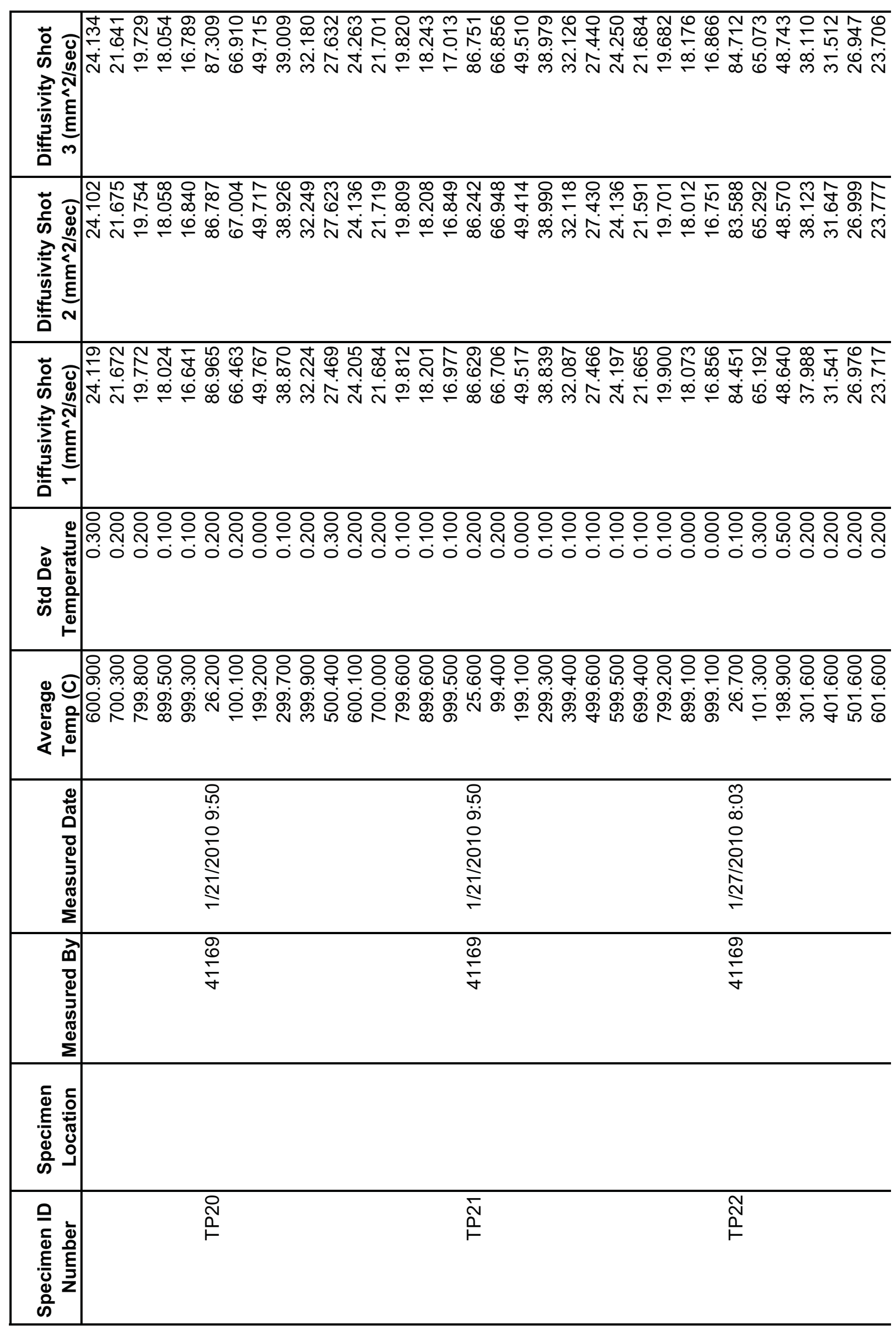




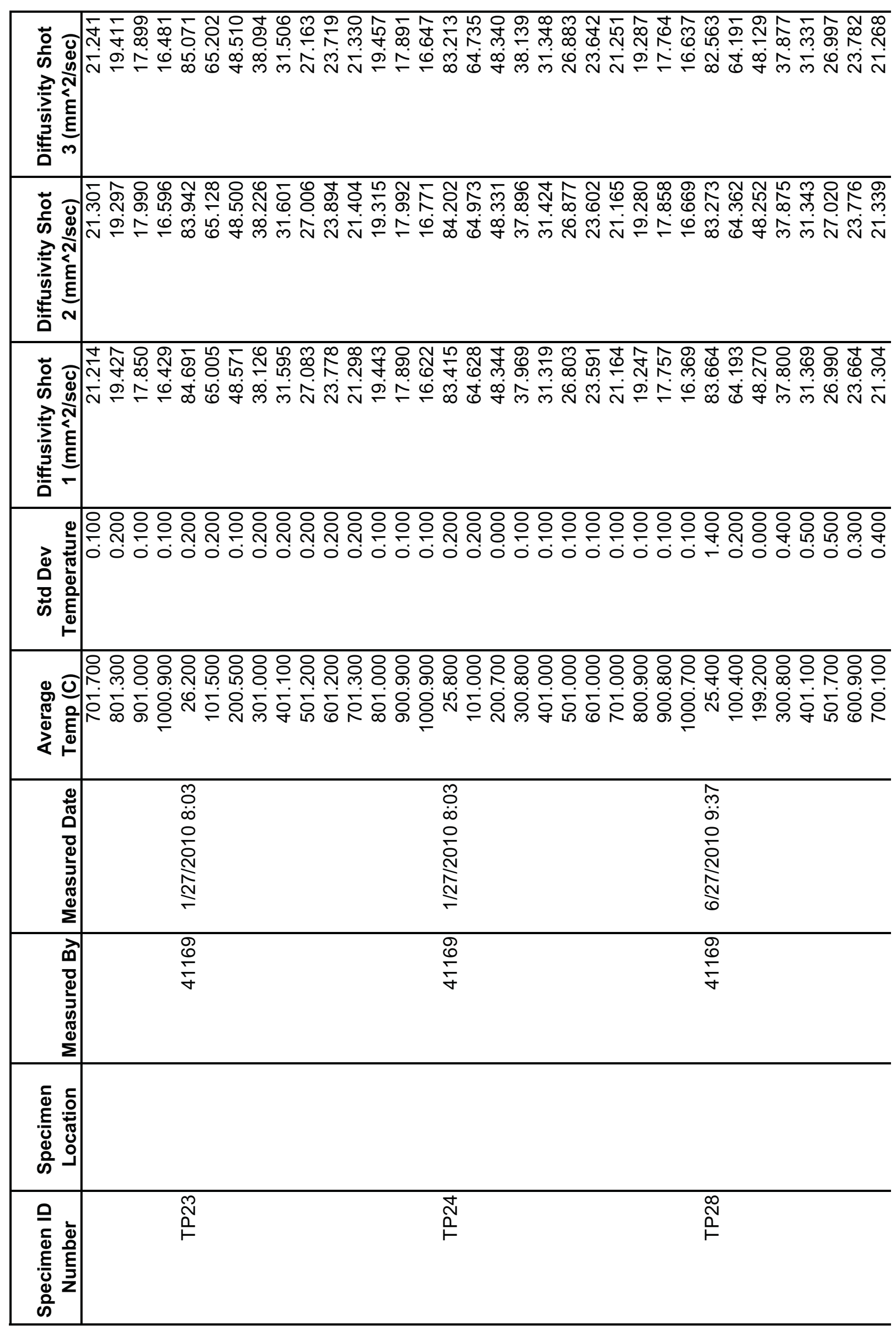




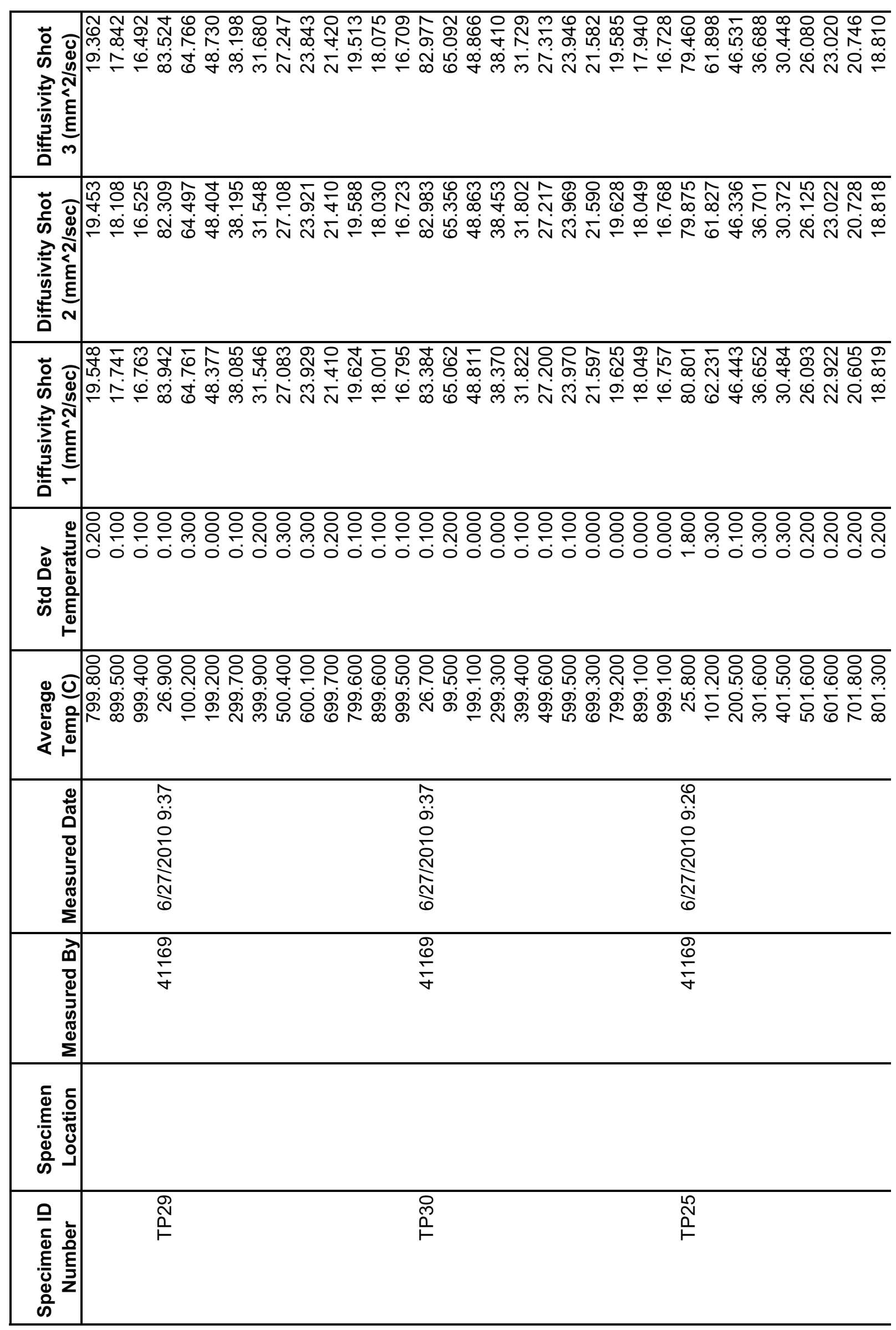




\begin{tabular}{|c|c|}
\hline 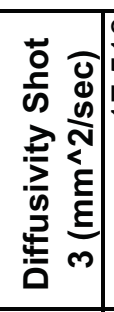 & 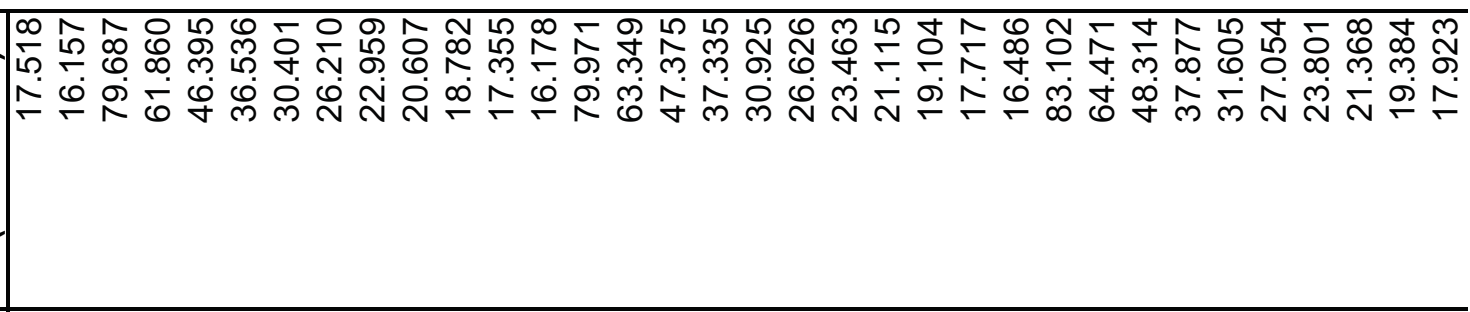 \\
\hline 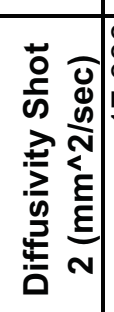 & 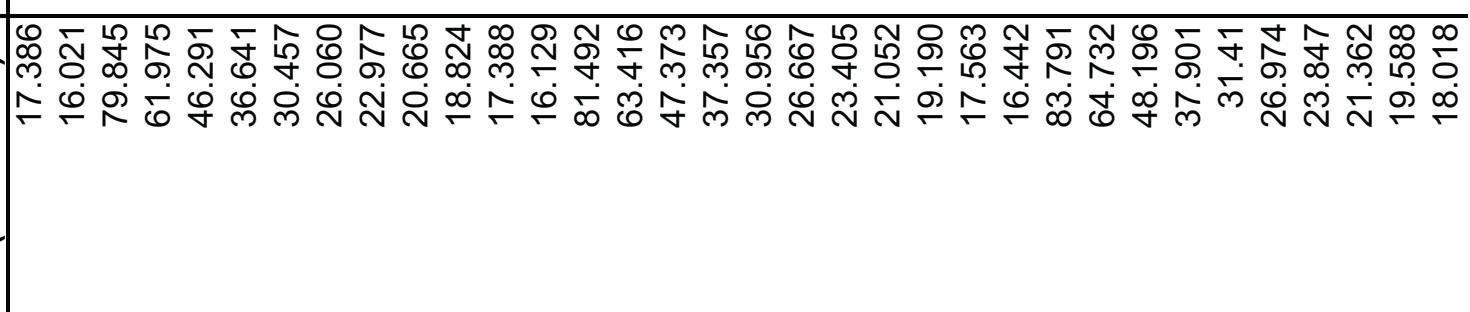 \\
\hline 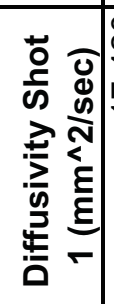 & 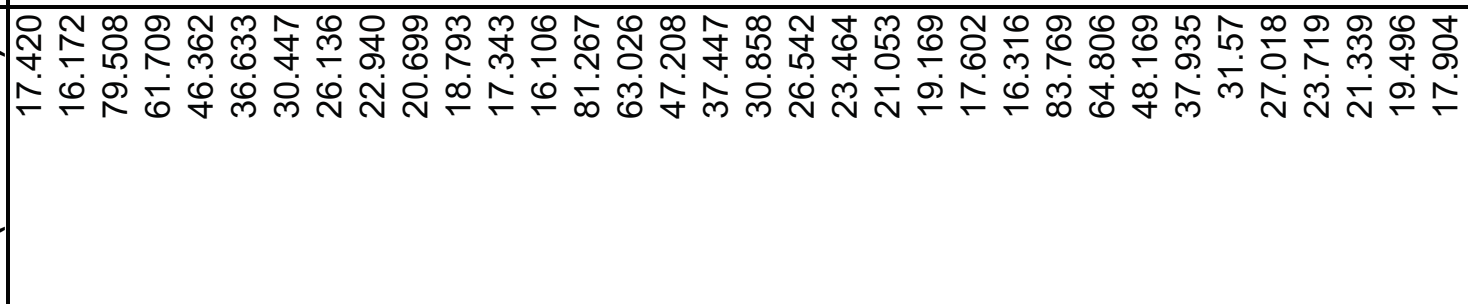 \\
\hline 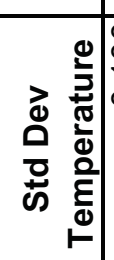 & 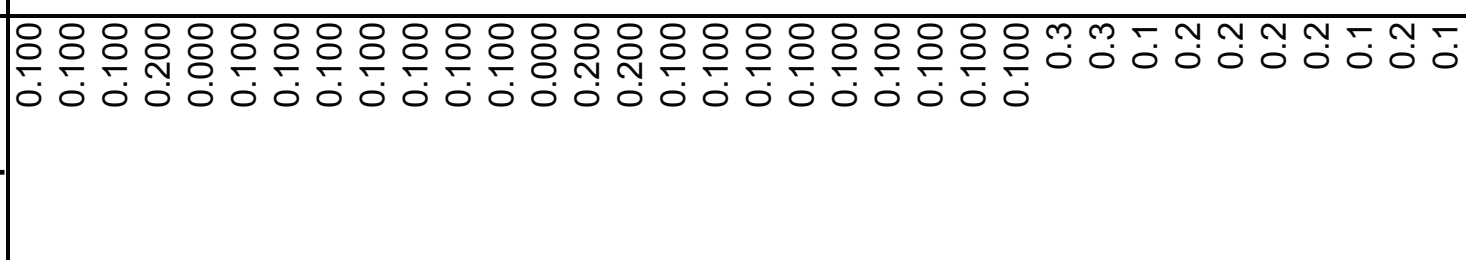 \\
\hline 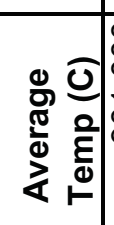 & 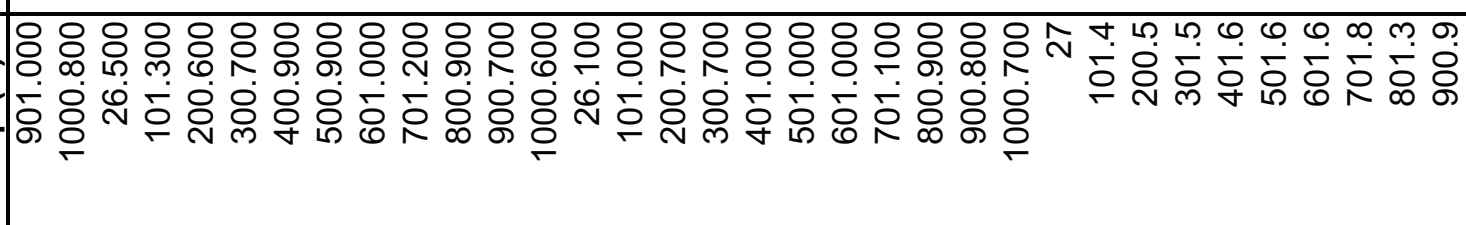 \\
\hline 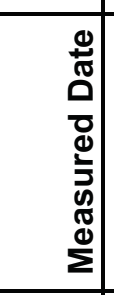 & 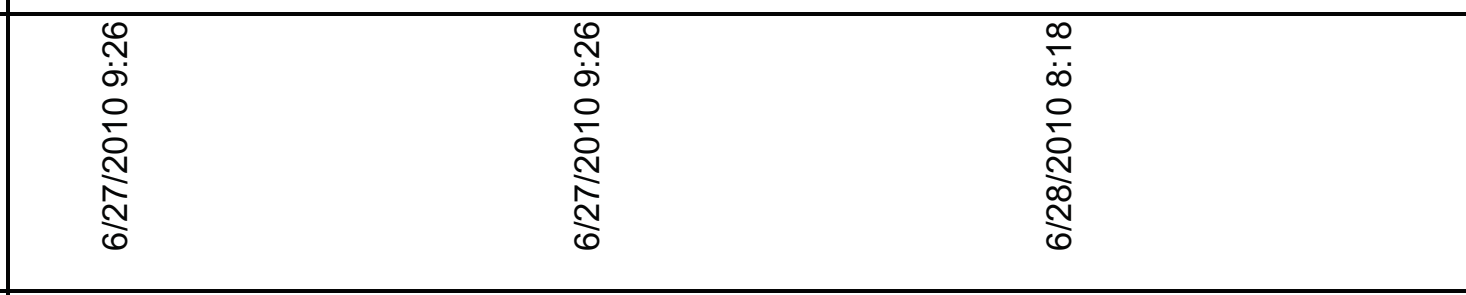 \\
\hline 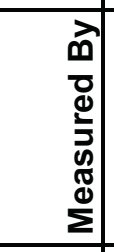 & 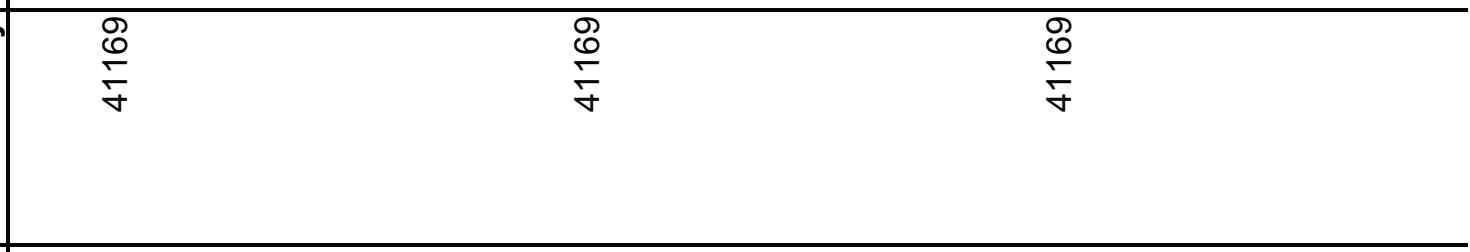 \\
\hline 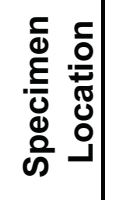 & \\
\hline 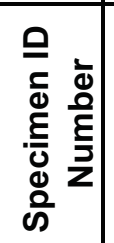 & 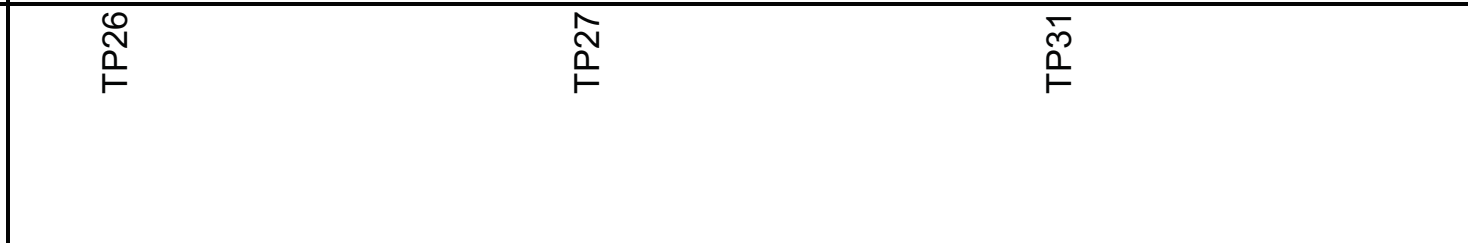 \\
\hline
\end{tabular}




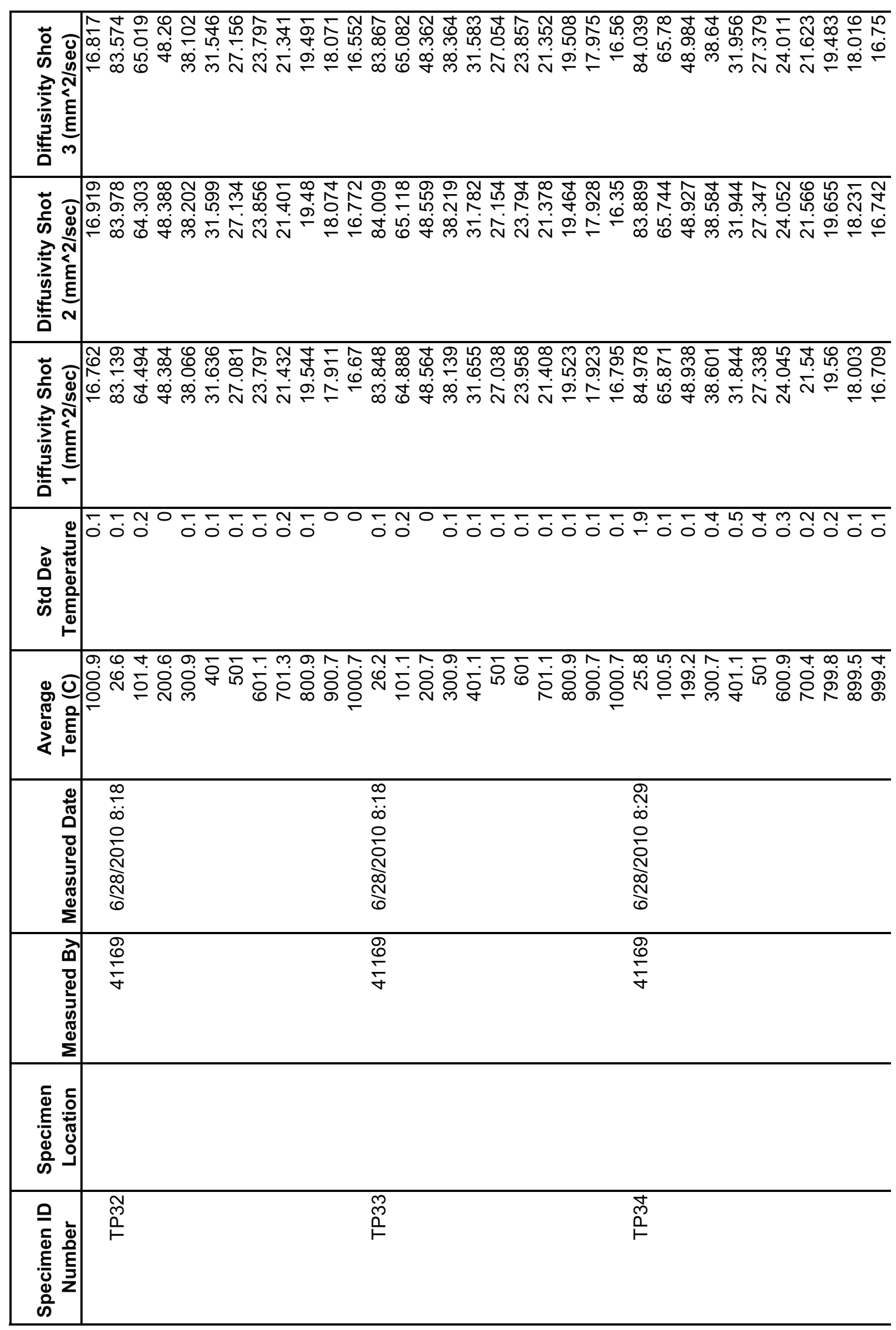




\begin{tabular}{|c|c|}
\hline 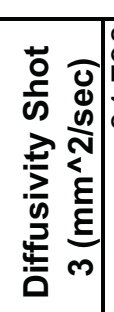 & 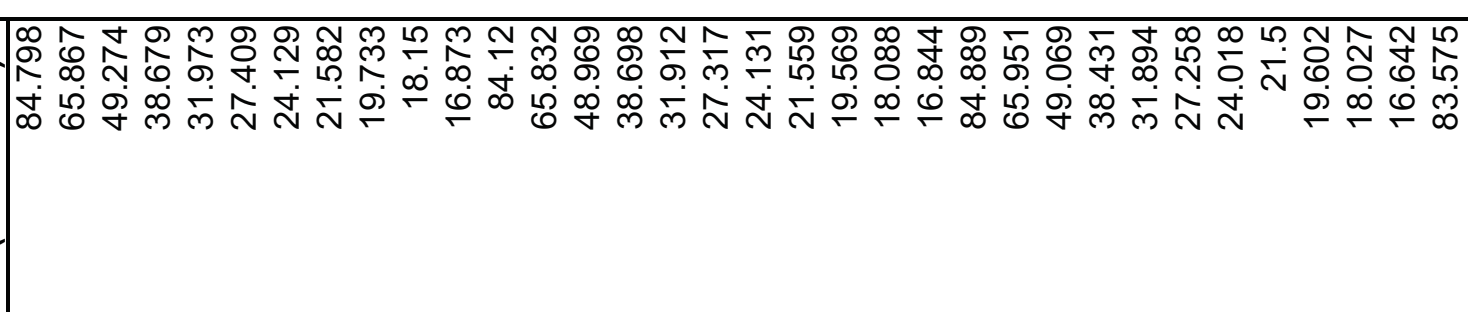 \\
\hline 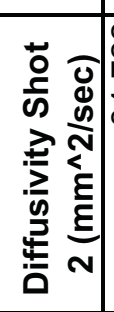 & 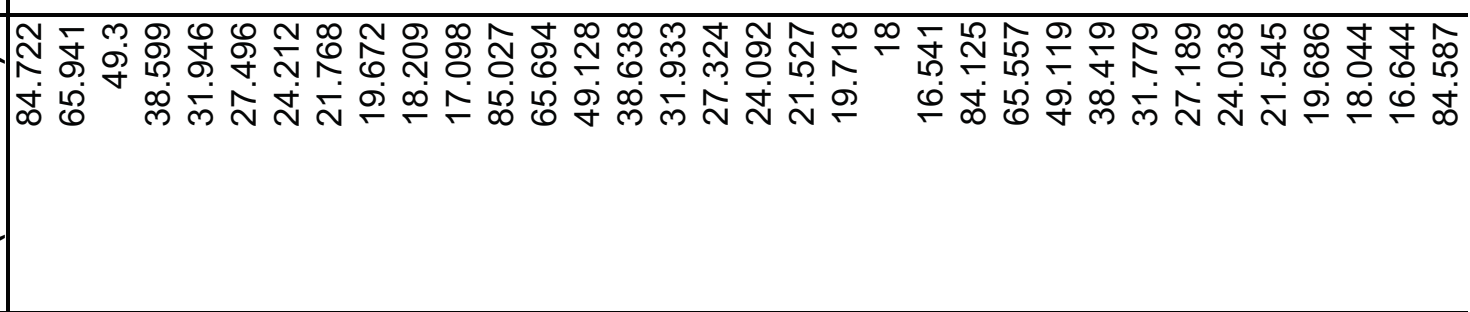 \\
\hline 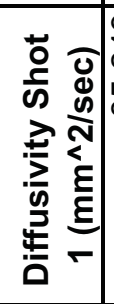 & 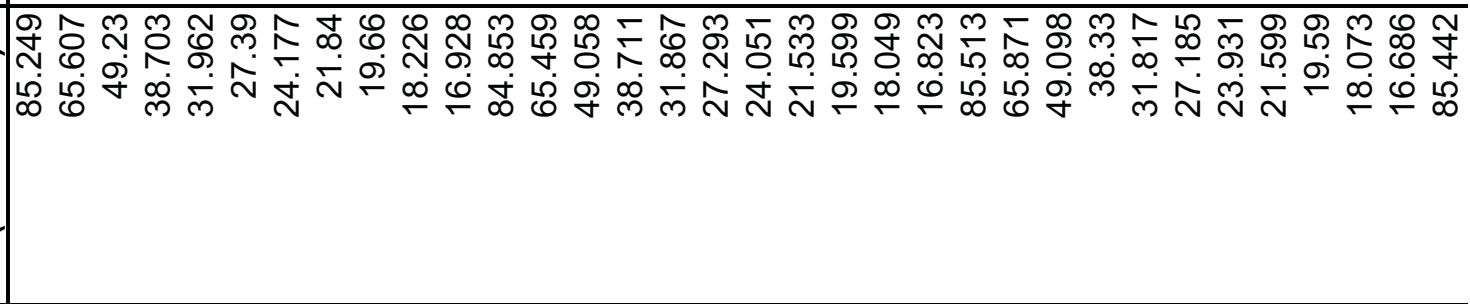 \\
\hline 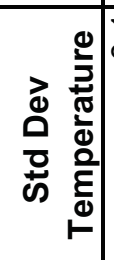 & 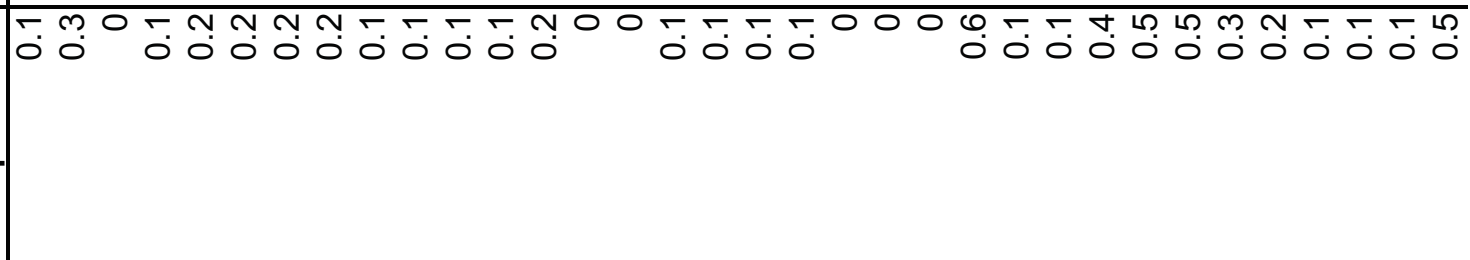 \\
\hline 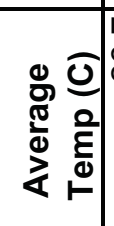 & 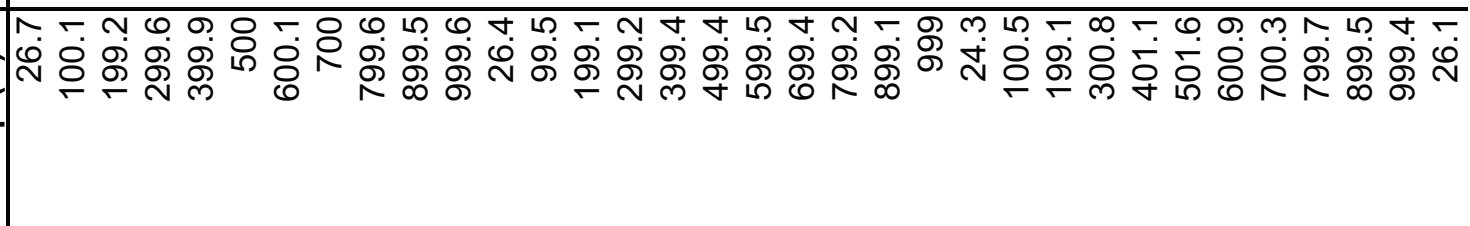 \\
\hline 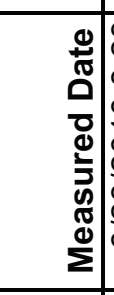 & 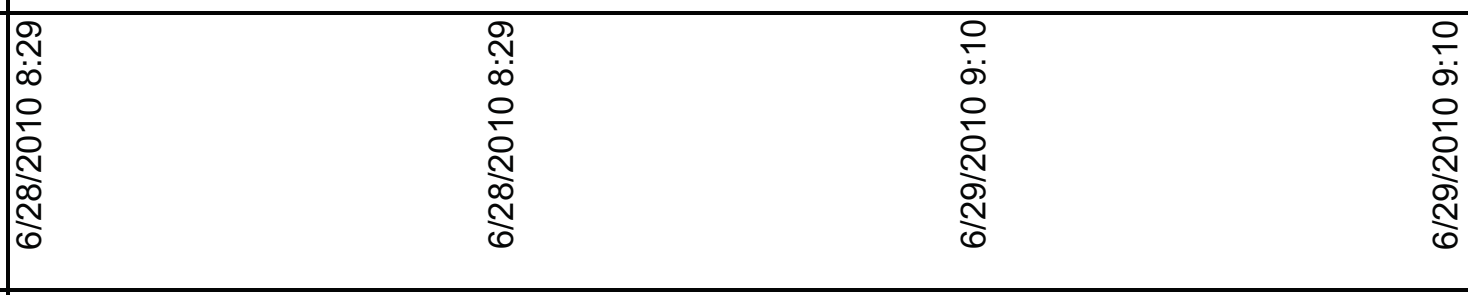 \\
\hline 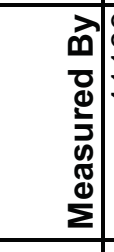 & 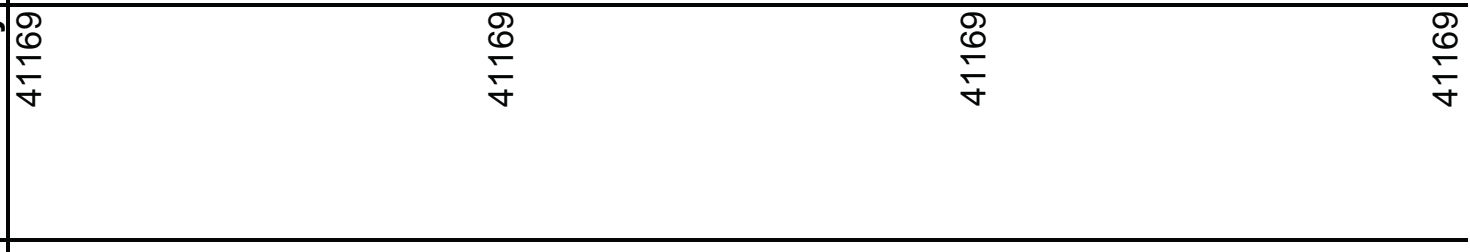 \\
\hline 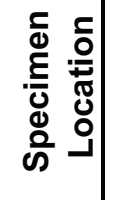 & \\
\hline 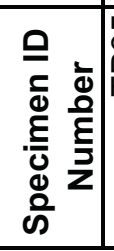 & 岕 \\
\hline
\end{tabular}




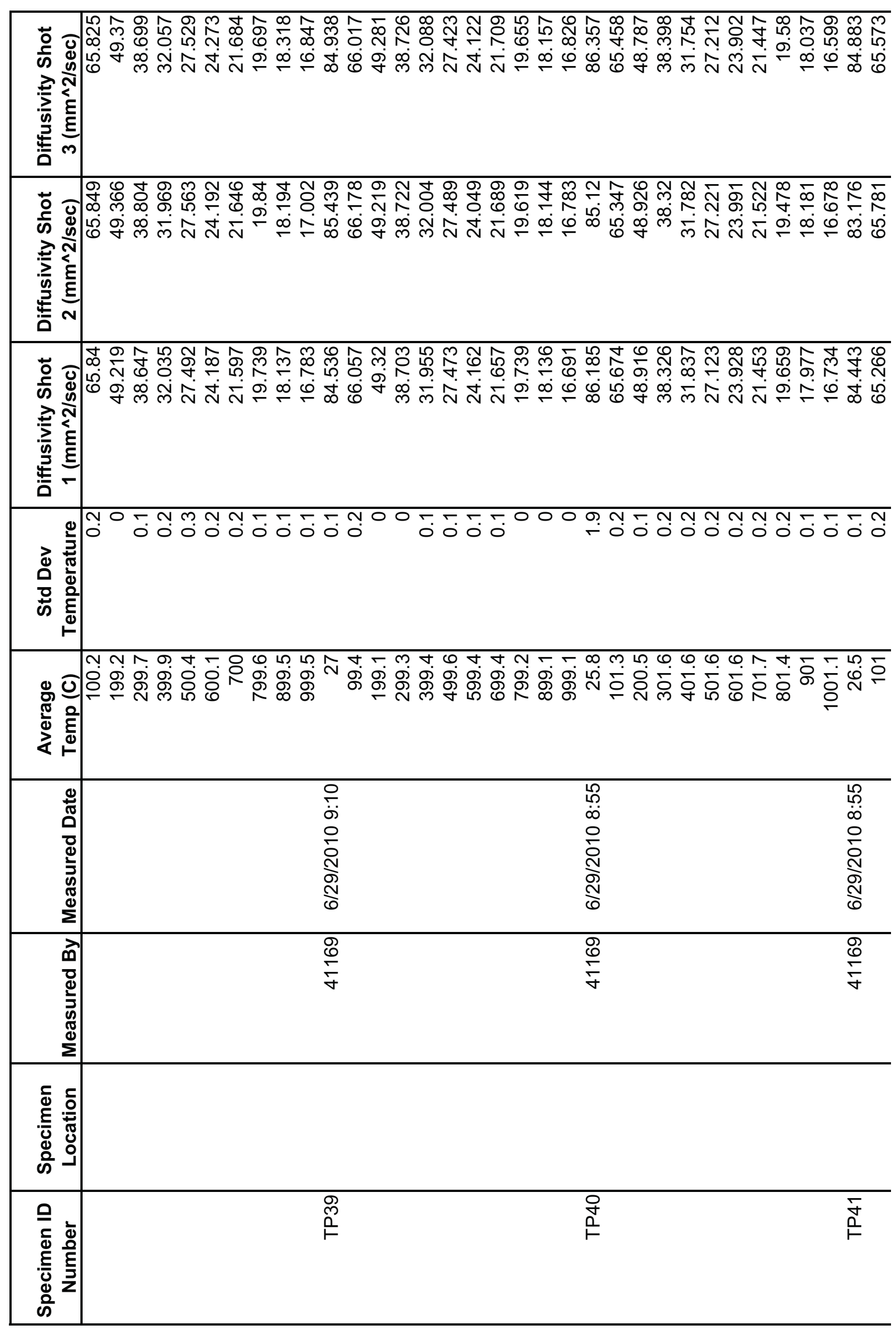




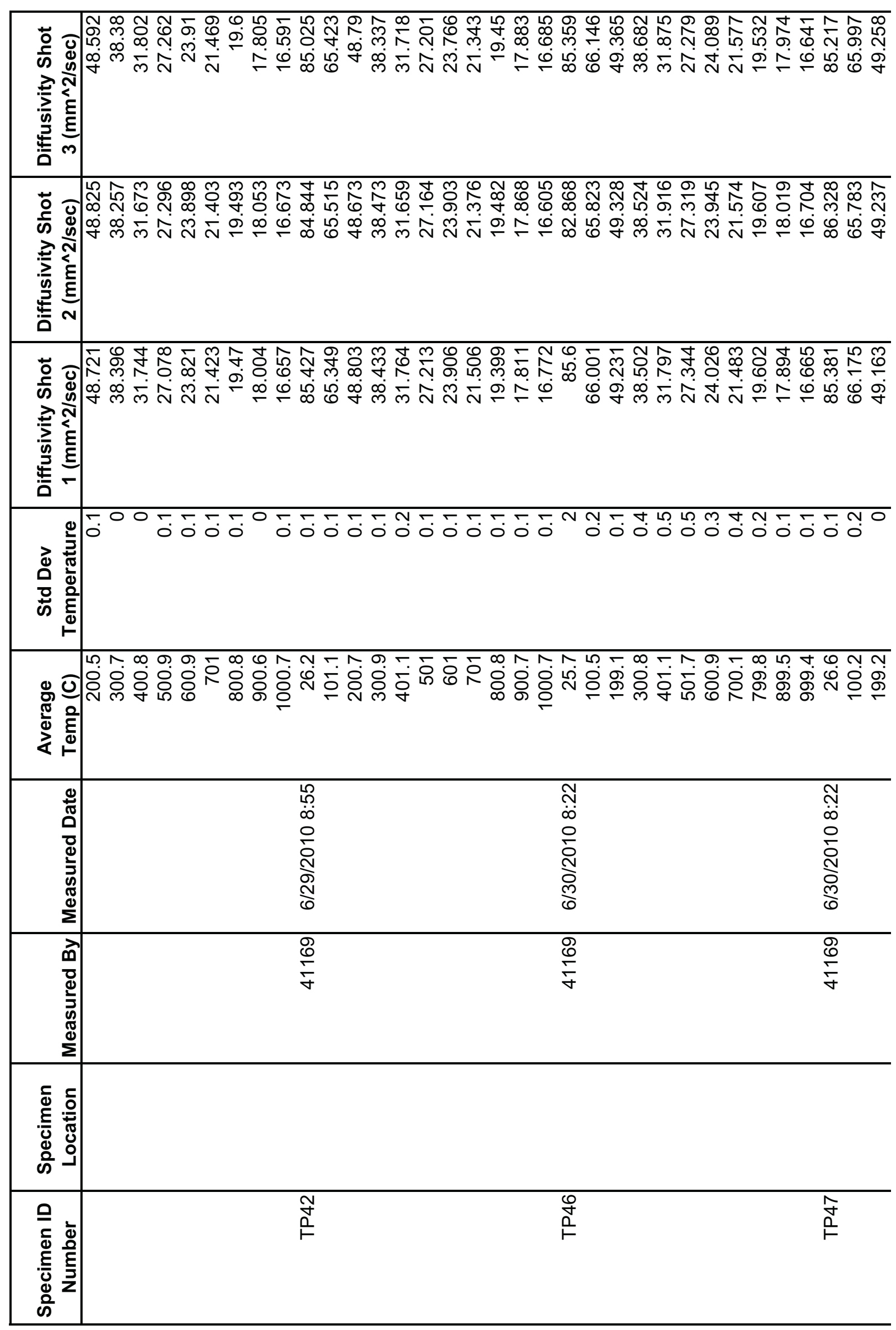




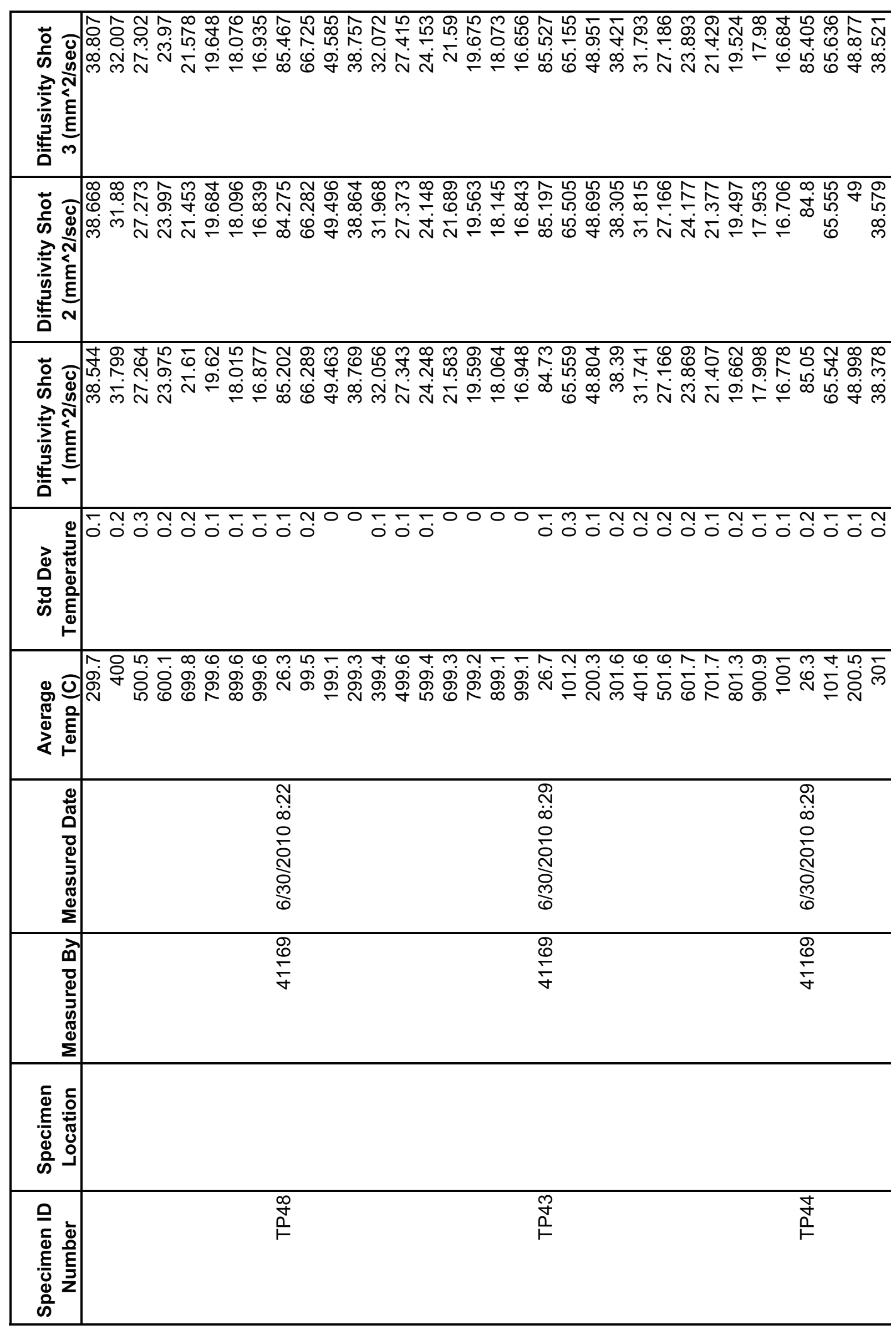




\begin{tabular}{|c|c|}
\hline 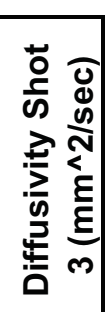 & 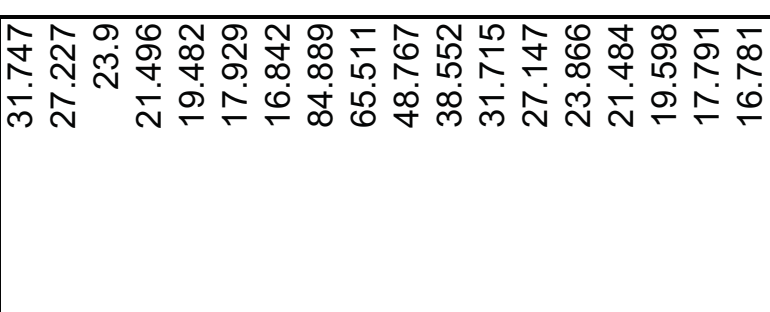 \\
\hline 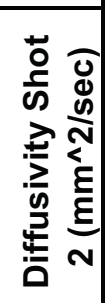 & 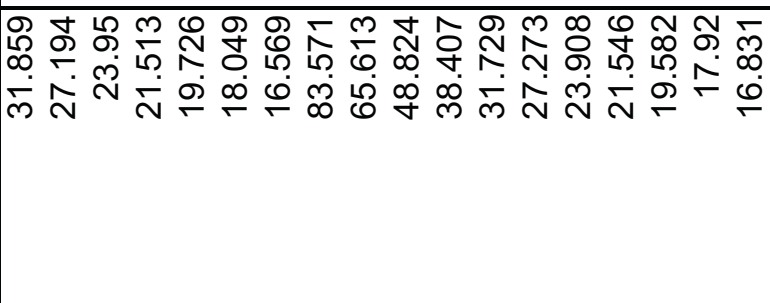 \\
\hline 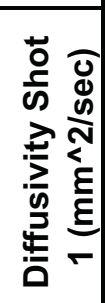 & 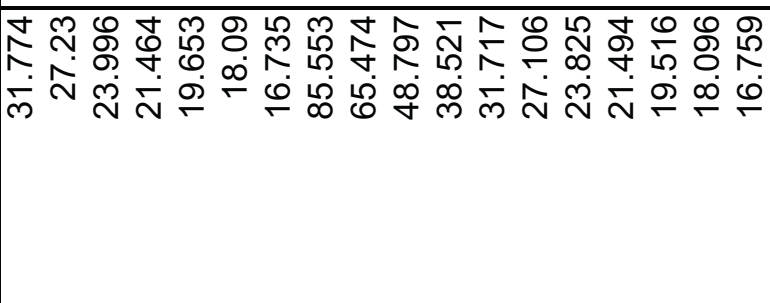 \\
\hline 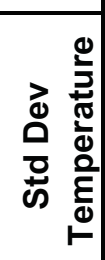 & 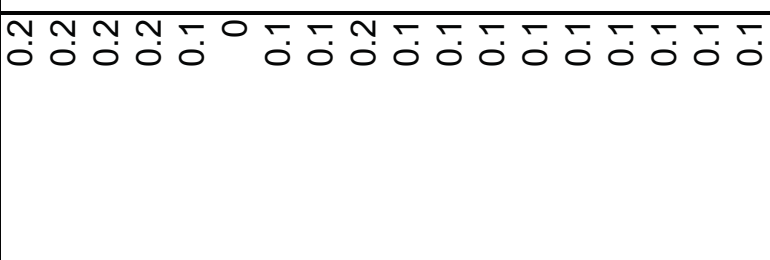 \\
\hline 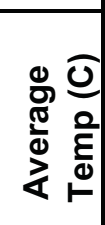 & 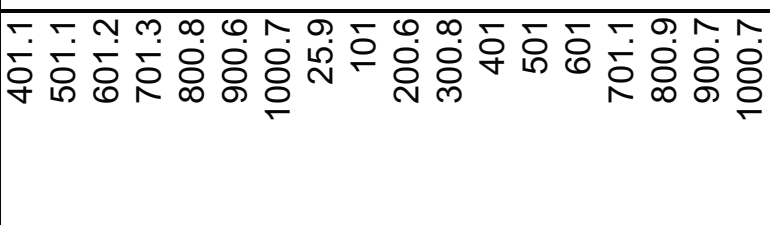 \\
\hline 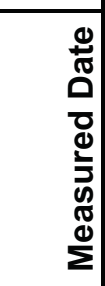 & 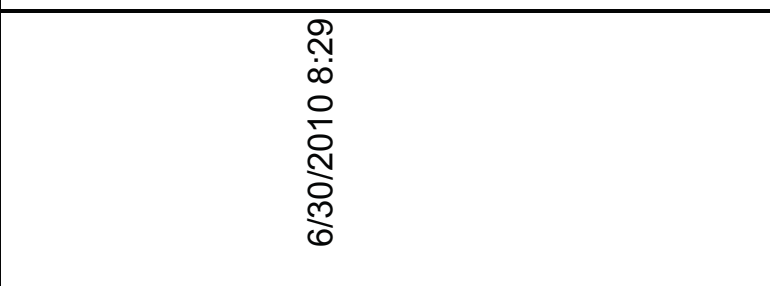 \\
\hline 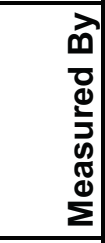 & $\begin{array}{l}\frac{8}{8} \\
\frac{6}{7}\end{array}$ \\
\hline 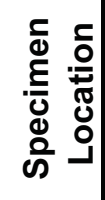 & \\
\hline 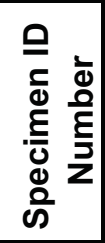 & $\begin{array}{l}\text { 导 } \\
1 \\
1\end{array}$ \\
\hline
\end{tabular}




\begin{tabular}{|c|c|c|c|c|}
\hline$\underset{5}{5}$ & 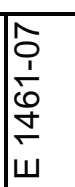 & 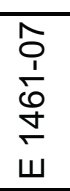 & $\begin{array}{l}\hat{o} \\
\frac{1}{1} \\
\dot{0} \\
\dot{+} \\
w\end{array}$ & 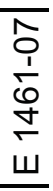 \\
\hline 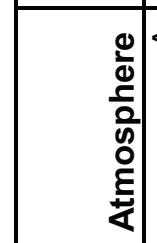 & & 交 & $\bar{z}$ & $\overline{\frac{1}{\alpha}}$ \\
\hline 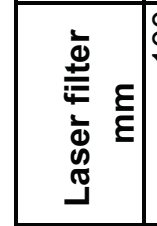 & & $\stackrel{\circ}{\circ}$ & 음 & 음 \\
\hline 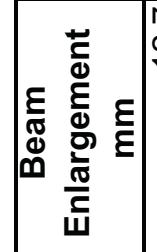 & & $\stackrel{\stackrel{N}{\sim}}{\sim}$ & 犬্ & $\stackrel{\stackrel{N}{\mathfrak{N}}}{ }$ \\
\hline 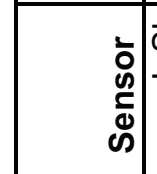 & & की & $\begin{array}{l}\text { की } \\
\text { की }\end{array}$ & $\begin{array}{l}\text { की } \\
\text { 足 }\end{array}$ \\
\hline 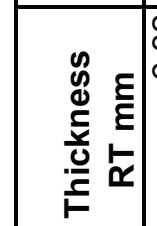 & & $\begin{array}{l}\widetilde{N} \\
\widetilde{0}\end{array}$ & ్ָల్ర్ల్ & స్ల్ర \\
\hline 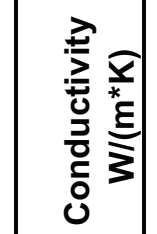 & & & & \\
\hline 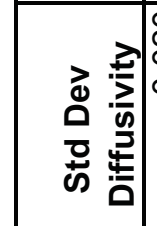 & : & $\begin{array}{c}\text { No } \\
\infty \\
0\end{array}$ & 0 & \\
\hline 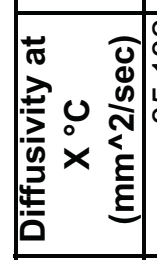 & 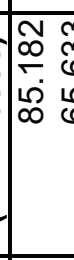 & 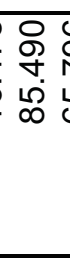 & $\begin{array}{l}\mathbb{C}_{0} \\
\infty \\
\infty\end{array}$ & $\begin{array}{l}\bar{N} \\
0 \\
0 \\
\infty \\
\infty\end{array}$ \\
\hline 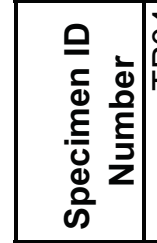 & & $\begin{array}{l}\text { 员 } \\
0\end{array}$ & م & @ \\
\hline
\end{tabular}




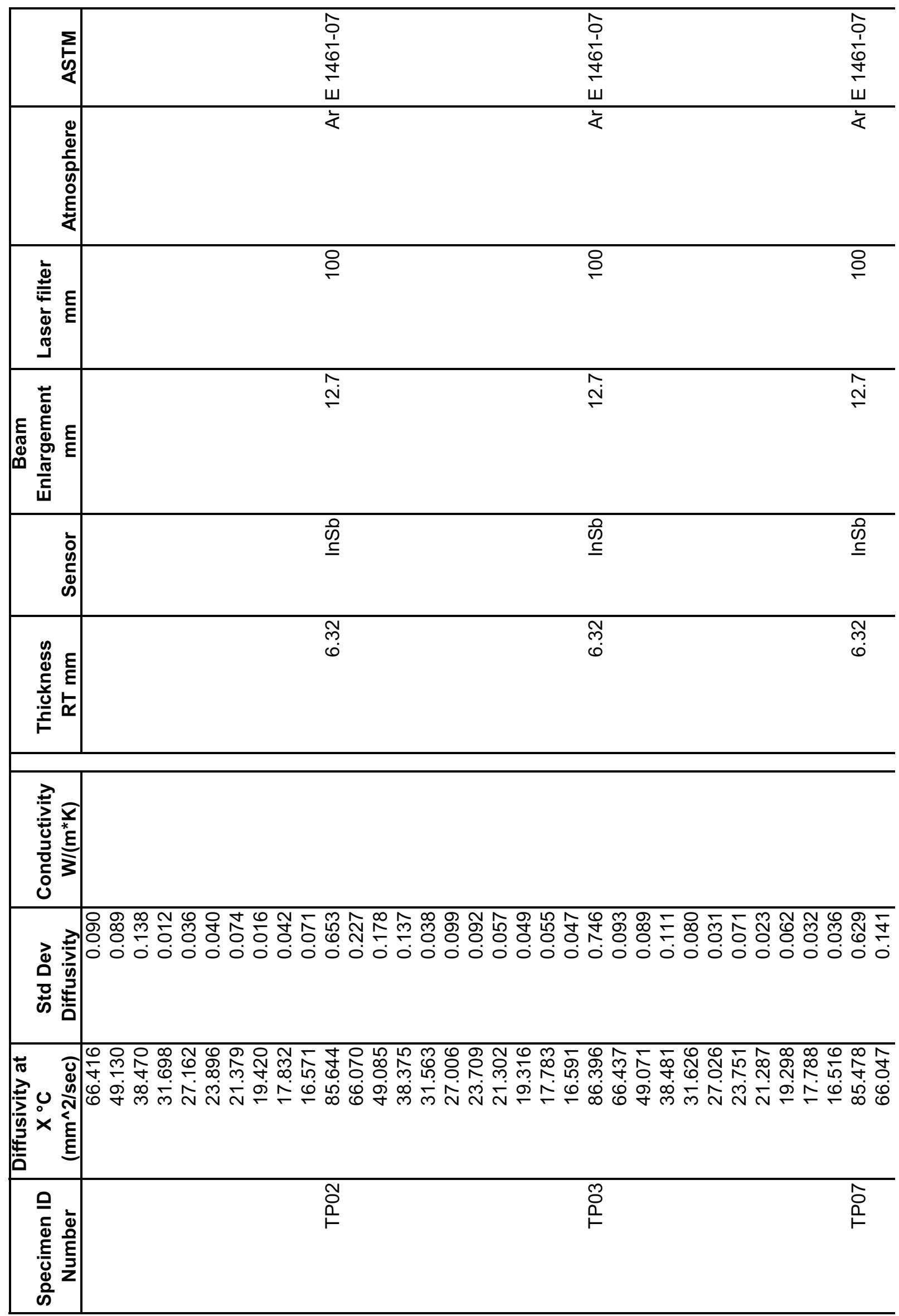




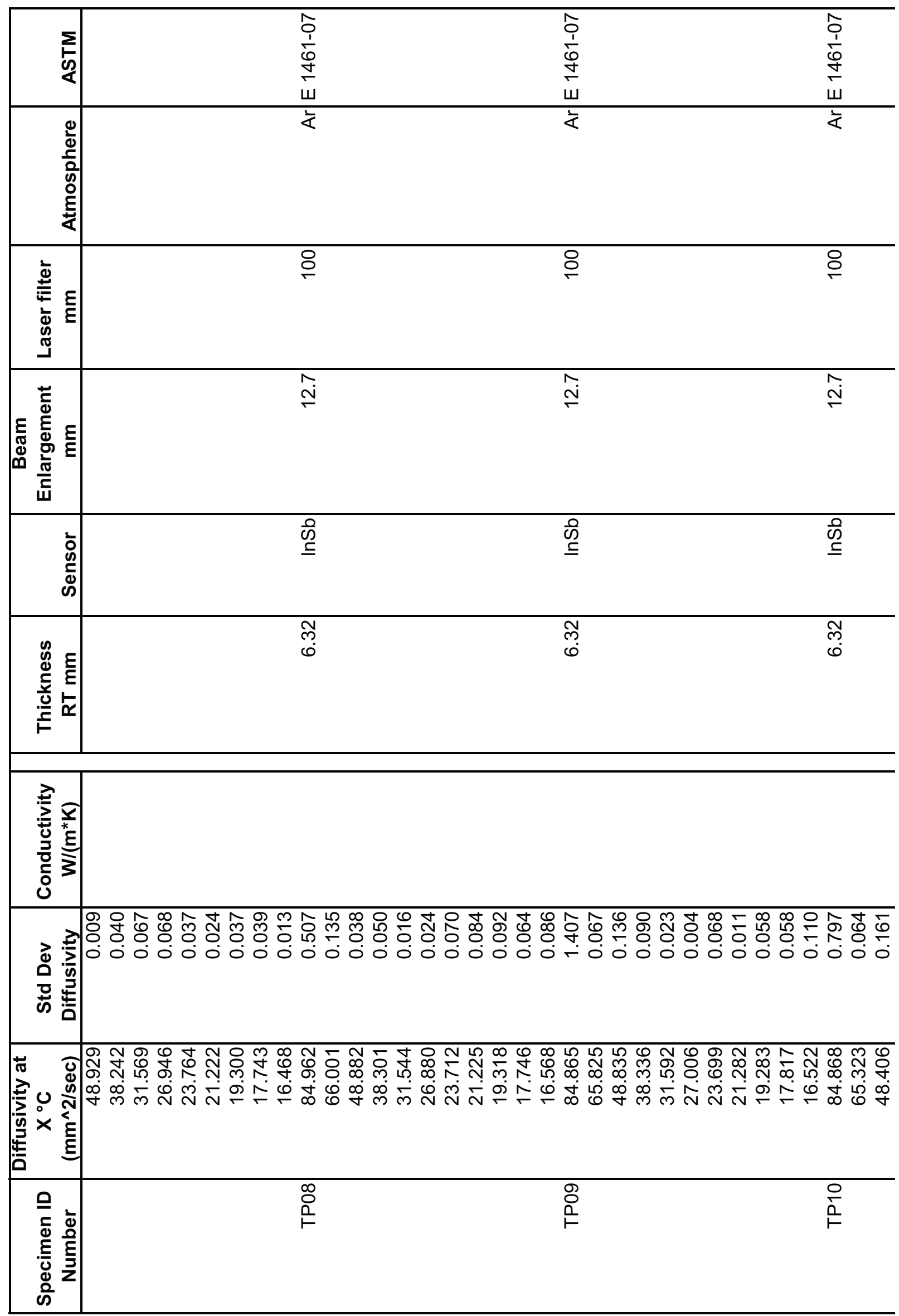




\begin{tabular}{|c|c|c|c|}
\hline 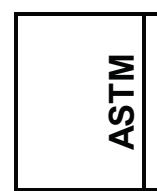 & $\begin{array}{l}\hat{o} \\
\dot{1} \\
\dot{0} \\
\dot{+} \\
\dot{w}\end{array}$ & $\begin{array}{l}\hat{o} \\
\frac{1}{1} \\
\frac{1}{5} \\
\dot{\omega}\end{array}$ & $\begin{array}{l}\hat{o} \\
\frac{1}{0} \\
\frac{0}{+} \\
\dot{\omega}\end{array}$ \\
\hline 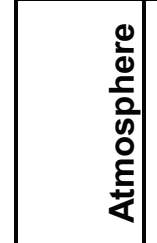 & 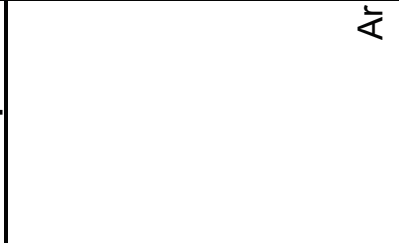 & ¿ & ¿ \\
\hline 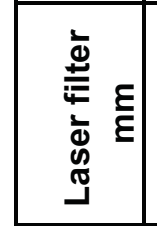 & $\stackrel{\circ}{\circ}$ & 음 & 음 \\
\hline 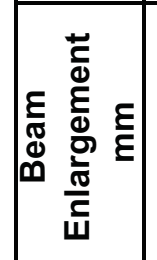 & $\stackrel{\stackrel{N}{\mathrm{~N}}}{ }$ & $\stackrel{\widetilde{N}}{\stackrel{N}{N}}$ & $\stackrel{\widehat{N}}{\stackrel{N}{N}}$ \\
\hline 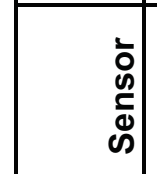 & $\begin{array}{l}\text { की } \\
\text { की }\end{array}$ & 吕 & $\begin{array}{l}\text { की } \\
\text { 足 }\end{array}$ \\
\hline 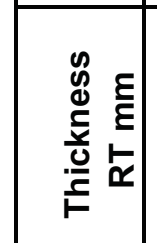 & $\begin{array}{l}\widetilde{N} \\
\widetilde{N}\end{array}$ & స్ల్ర & స్ల్ల \\
\hline 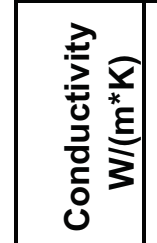 & & & \\
\hline 䠞 & 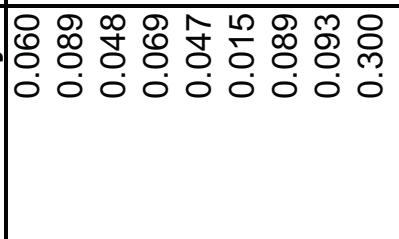 & 0 & 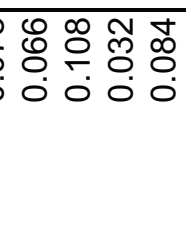 \\
\hline 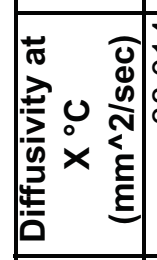 & 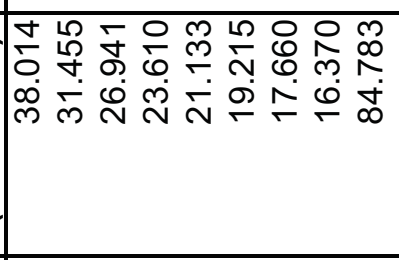 & @i & 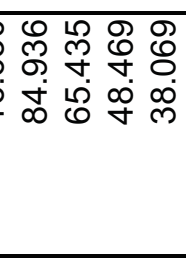 \\
\hline 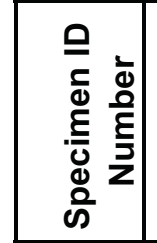 & $\bar{\Sigma}$ & $\frac{N}{n}$ & $\sum_{n}^{m}$ \\
\hline
\end{tabular}




\begin{tabular}{|c|c|c|c|}
\hline 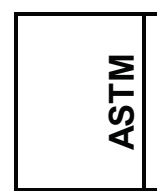 & $\begin{array}{l}\frac{\hat{s}}{1} \\
\frac{1}{o} \\
\frac{\sigma}{w}\end{array}$ & $\begin{array}{l}\hat{o} \\
\frac{1}{0} \\
\dot{6} \\
\dot{+} \\
w\end{array}$ & 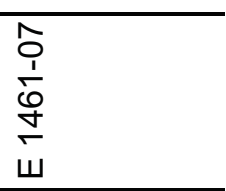 \\
\hline 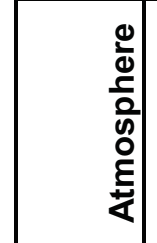 & 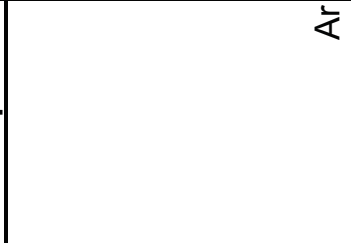 & ¿ & ¿ \\
\hline 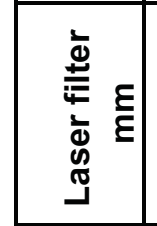 & 음 & 음 & 음 \\
\hline 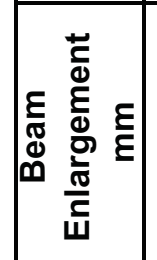 & 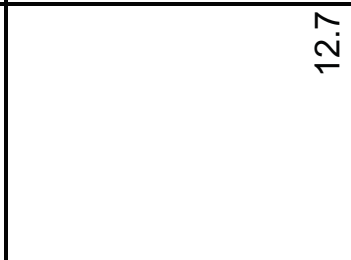 & $\stackrel{\widehat{N}}{\stackrel{N}{N}}$ & $\stackrel{\widehat{N}}{\stackrel{N}{N}}$ \\
\hline 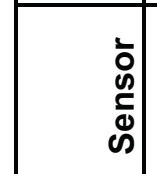 & $\begin{array}{l}\text { की } \\
\text { की }\end{array}$ & की & $\begin{array}{l}\text { की } \\
\text { की }\end{array}$ \\
\hline 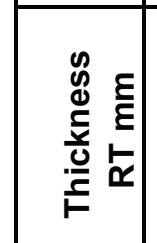 & స్ల్ల & స్ల్రా & స్ల \\
\hline 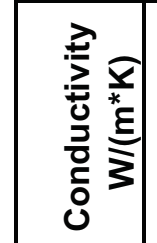 & & & \\
\hline 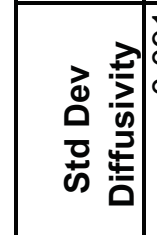 & 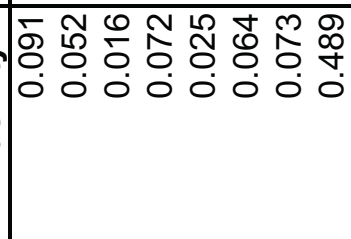 & 0 & 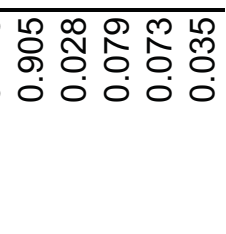 \\
\hline 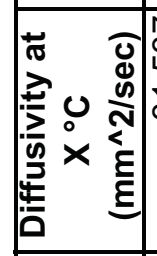 & 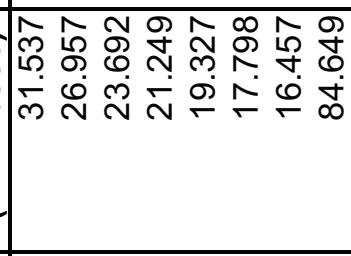 & $\stackrel{\circ}{\circ}$ & 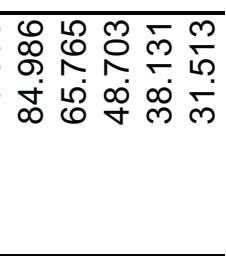 \\
\hline 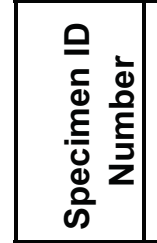 & 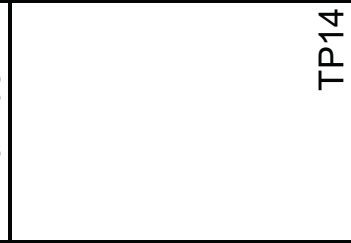 & $\frac{10}{2}$ & 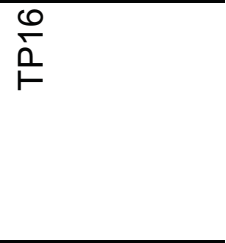 \\
\hline
\end{tabular}




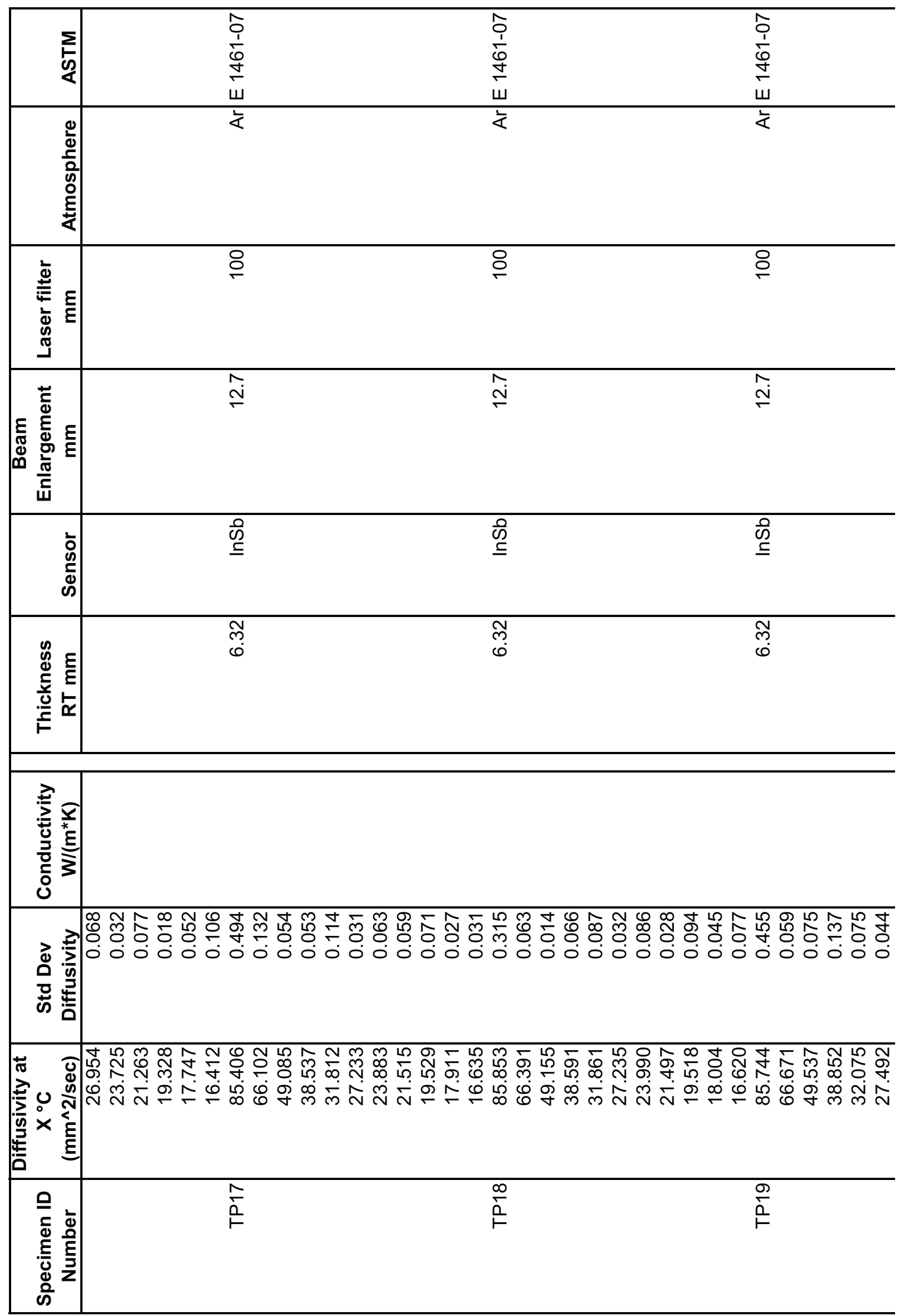




\begin{tabular}{|c|c|c|c|}
\hline 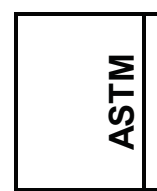 & $\begin{array}{l}\hat{o} \\
\frac{1}{o} \\
\frac{\sigma}{+} \\
\dot{w}\end{array}$ & 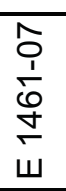 & $\begin{array}{l}\hat{o} \\
\frac{1}{o} \\
\frac{1}{+} \\
\dot{w}\end{array}$ \\
\hline 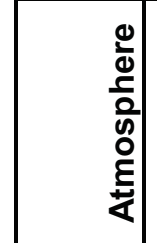 & . & ¿ & ¿ \\
\hline 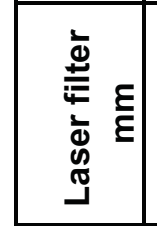 & $\stackrel{\circ}{\circ}$ & 음 & $\stackrel{\circ}{\circ}$ \\
\hline 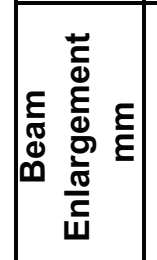 & $\widehat{\widehat{N}}$ & $\widehat{\stackrel{N}{\mathrm{~N}}}$ & $\stackrel{\widetilde{\mathfrak{N}}}{\widehat{N}}$ \\
\hline 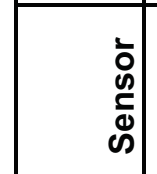 & $\begin{array}{l}\text { की } \\
\text { की }\end{array}$ & $\begin{array}{l}\text { की } \\
\text { की }\end{array}$ & 吕 \\
\hline 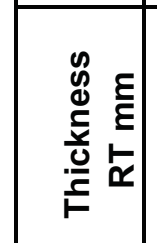 & ్ָల్ల & $\begin{array}{l}\widetilde{N} \\
\widetilde{0}\end{array}$ & ్ָల్ల \\
\hline 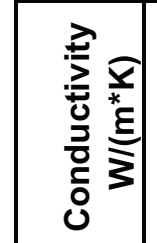 & & & \\
\hline 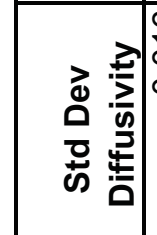 & 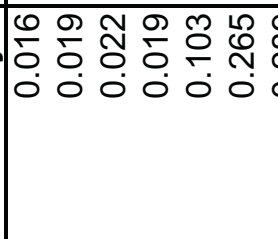 & 0 & 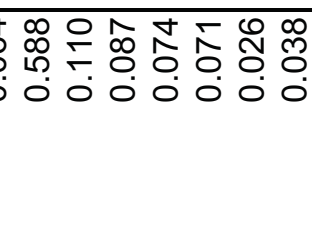 \\
\hline 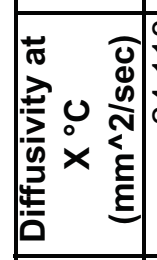 & 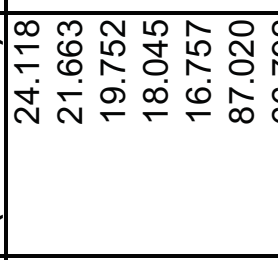 & $\begin{array}{l}0 \\
0 \\
0 \\
\infty\end{array}$ & 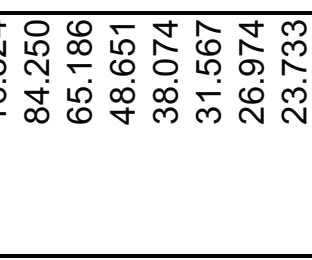 \\
\hline 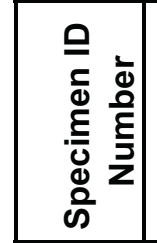 & 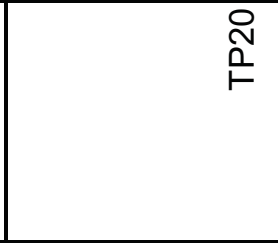 & 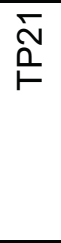 & $\begin{array}{l}\text { N } \\
\text { N }\end{array}$ \\
\hline
\end{tabular}




\begin{tabular}{|c|c|c|c|}
\hline 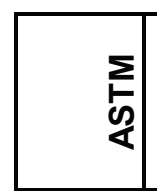 & $\begin{array}{l}\hat{o} \\
\frac{1}{0} \\
\dot{0} \\
\dot{+} \\
\end{array}$ & 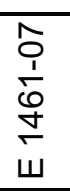 & $\begin{array}{l}\frac{\hat{1}}{1} \\
\frac{1}{6} \\
\frac{g}{\leftarrow} \\
\dot{w}\end{array}$ \\
\hline 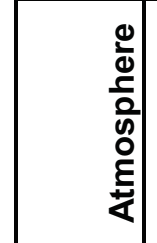 & 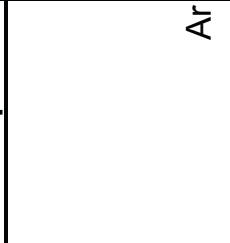 & ¿ & ¿ \\
\hline 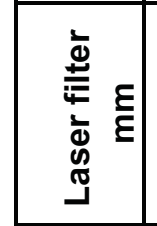 & $\stackrel{8}{\circ}$ & $\stackrel{\circ}{\circ}$ & 음 \\
\hline 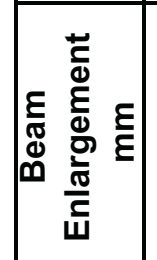 & $\stackrel{\widehat{N}}{\stackrel{N}{N}}$ & 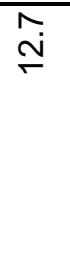 & 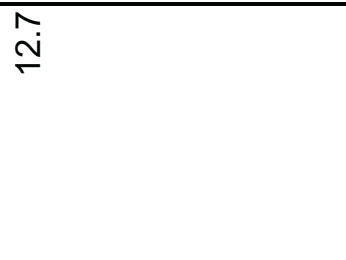 \\
\hline 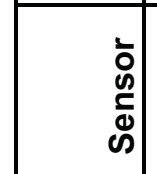 & $\begin{array}{l}\text { की } \\
\text { की }\end{array}$ & $\begin{array}{l}\text { के } \\
\text { की }\end{array}$ & की \\
\hline 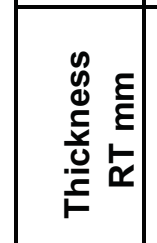 & ్ֶָ & $\begin{array}{l}\sqrt[N]{0} \\
\text { ט. }\end{array}$ & స్ల్ర \\
\hline 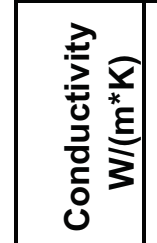 & & & \\
\hline 䠞 & $\begin{array}{ll}0 \\
0\end{array}$ & $\overbrace{0}^{2}$ & 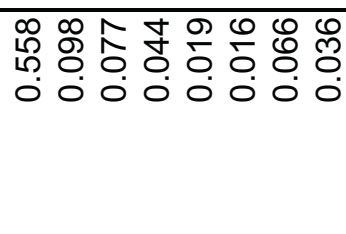 \\
\hline 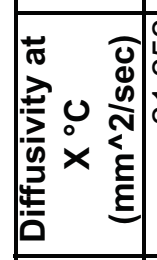 & 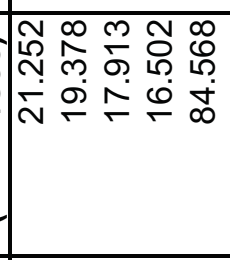 & $\begin{array}{ll}0 \\
0 & 1 \\
10 & 1 \\
\infty & 0\end{array}$ & 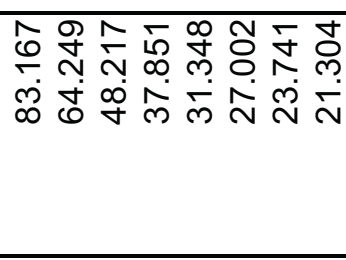 \\
\hline 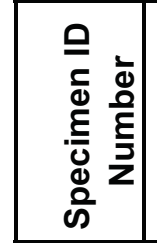 & $\begin{array}{l}\tilde{N} \\
\stackrel{n}{n}\end{array}$ & $\begin{array}{l}\stackrel{⿱}{N} \\
\stackrel{n}{F}\end{array}$ & 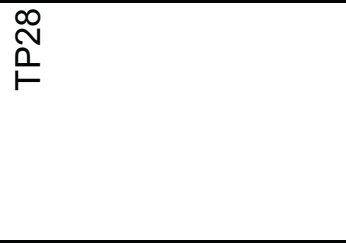 \\
\hline
\end{tabular}




\begin{tabular}{|c|c|c|c|}
\hline 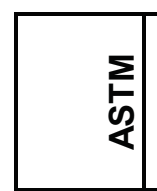 & $\begin{array}{l}\hat{o} \\
\frac{1}{\dot{\varphi}} \\
\stackrel{+}{\leftarrow} \\
\dot{\omega}\end{array}$ & 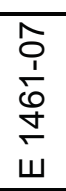 & $\begin{array}{l}\hat{o} \\
\frac{1}{o} \\
\frac{1}{+} \\
\dot{w}\end{array}$ \\
\hline 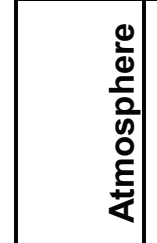 & 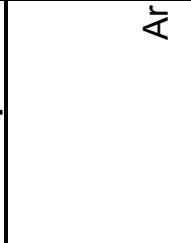 & ¿ & 交 \\
\hline 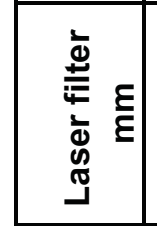 & $\stackrel{\square}{\circ}$ & 음 & $\stackrel{\circ}{\circ}$ \\
\hline 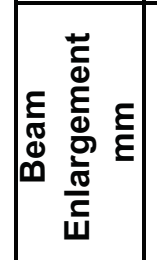 & 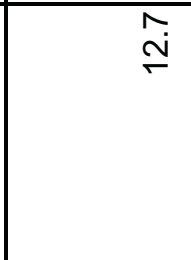 & $\stackrel{\widehat{N}}{\stackrel{N}{N}}$ & $\stackrel{\widetilde{N}}{\widehat{N}}$ \\
\hline $\begin{array}{l}\bar{\vdots} \\
0 \\
\stackrel{5}{\Phi} \\
\infty\end{array}$ & $\begin{array}{l}\text { की } \\
\text { की }\end{array}$ & $\begin{array}{l}\text { की } \\
\text { की }\end{array}$ & 吕 \\
\hline 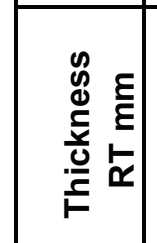 & 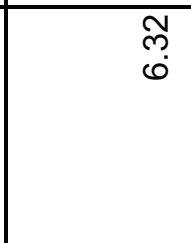 & $\begin{array}{l}\widetilde{N} \\
\text { ర్ }\end{array}$ & $\underset{0}{\widetilde{N}}$ \\
\hline 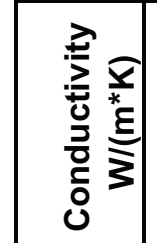 & & & \\
\hline 䠞 & 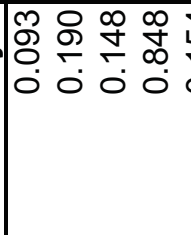 & 0 & 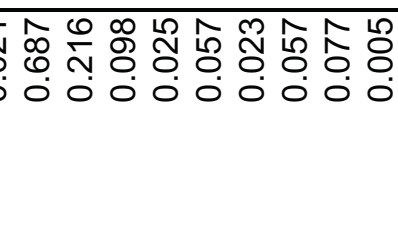 \\
\hline 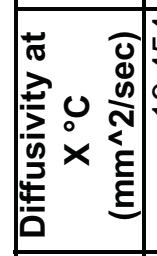 & 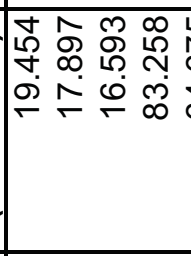 & $\ddot{\infty}$ & 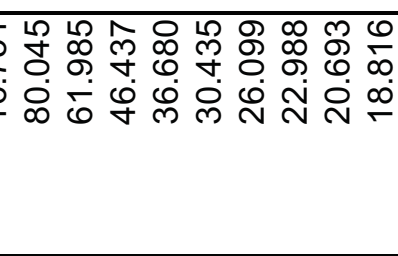 \\
\hline 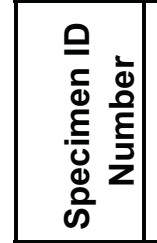 & 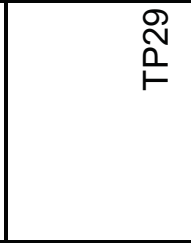 & $\begin{array}{l}\text { Oo } \\
\hat{n}\end{array}$ & $\begin{array}{l}\stackrel{\mathscr{N}}{N} \\
\stackrel{N}{F}\end{array}$ \\
\hline
\end{tabular}




\begin{tabular}{|c|c|c|c|}
\hline 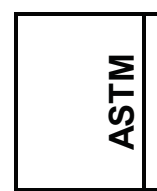 & $\frac{\hat{o}}{\frac{1}{o}}$ & $\begin{array}{l}\hat{o} \\
\frac{1}{1} \\
\dot{o} \\
\dot{w} \\
\end{array}$ & $\begin{array}{l}\frac{\hat{1}}{1} \\
\frac{1}{6} \\
\frac{G}{\omega} \\
\end{array}$ \\
\hline 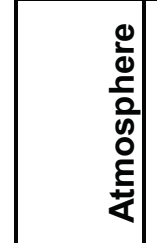 & 交 & ¿ & ¿ \\
\hline 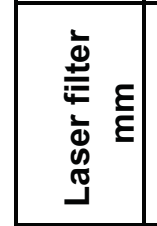 & $\stackrel{\circ}{\circ}$ & $\stackrel{8}{\circ}$ & 음 \\
\hline 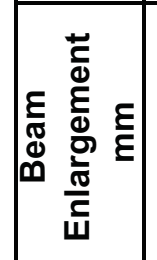 & $\stackrel{\widehat{N}}{\sim}$ & 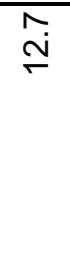 & $\stackrel{\stackrel{N}{\sim}}{\stackrel{N}{*}}$ \\
\hline $\begin{array}{l}\vdots \\
\vdots \\
\stackrel{\omega}{0} \\
\infty \\
\infty\end{array}$ & $\begin{array}{l}\text { के } \\
\text { की }\end{array}$ & $\begin{array}{l}\text { की } \\
\text { की }\end{array}$ & $\begin{array}{l}\text { की } \\
\text { की }\end{array}$ \\
\hline 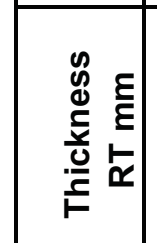 & $\begin{array}{l}\widetilde{N} \\
\stackrel{0}{0}\end{array}$ & $\begin{array}{l}\mathbb{N} \\
\widetilde{N} \\
0\end{array}$ & 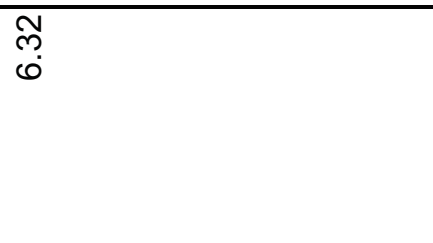 \\
\hline 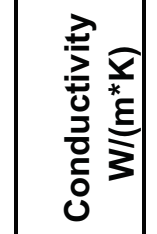 & & & \\
\hline 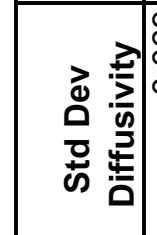 & : & 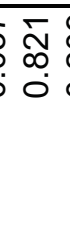 & 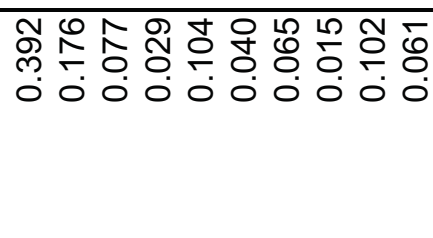 \\
\hline 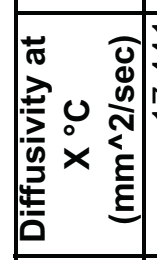 & 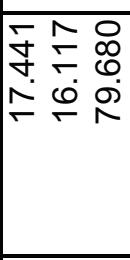 & $\begin{array}{l}3 \circ \\
\vdots \\
\vdots \\
\vdots \\
\vdots\end{array}$ & 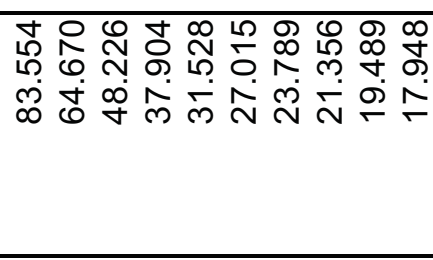 \\
\hline 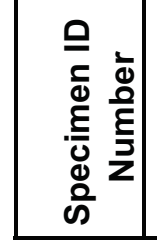 & $\begin{array}{l}\stackrel{Q}{\Omega} \\
\stackrel{2}{R}\end{array}$ & $\begin{array}{l}\hat{N} \\
\hat{N}\end{array}$ & $\begin{array}{c}\bar{m} \\
\stackrel{n}{p}\end{array}$ \\
\hline
\end{tabular}




\begin{tabular}{|c|c|c|c|}
\hline$\underset{\substack{\Sigma \\
\mathbf{c}}}{\mathbf{s}}$ & 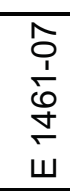 & 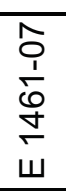 & 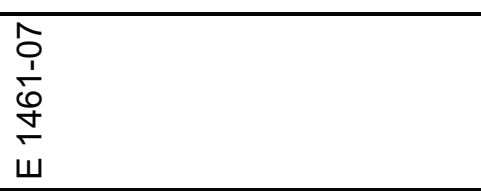 \\
\hline 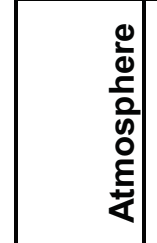 & 交 & ¿ & ¿ \\
\hline 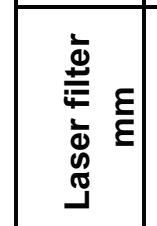 & $\stackrel{\circ}{\circ}$ & 우 & $\stackrel{8}{\circ}$ \\
\hline 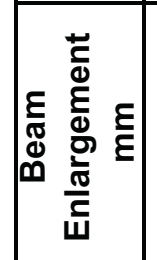 & $\stackrel{\hat{N}}{\stackrel{N}{N}}$ & $\stackrel{\widehat{N}}{\stackrel{N}{N}}$ & $\stackrel{\widehat{N}}{\stackrel{N}{N}}$ \\
\hline $\begin{array}{l}\bar{\vdots} \\
0 \\
\bar{\Phi} \\
\infty\end{array}$ & $\begin{array}{l}\text { की } \\
\text { की }\end{array}$ & $\begin{array}{l}\text { की } \\
\text { की }\end{array}$ & $\begin{array}{l}\text { की } \\
\text { की }\end{array}$ \\
\hline 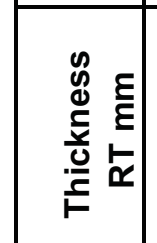 & స్ల్ల & $\begin{array}{l}\widetilde{N} \\
\text { ర్ }\end{array}$ & $\begin{array}{l}\widetilde{N} \\
\text { }\end{array}$ \\
\hline 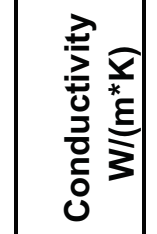 & & & \\
\hline 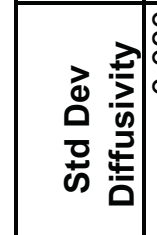 & : & 0 & 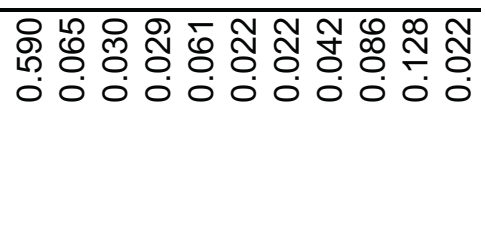 \\
\hline 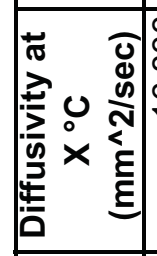 & 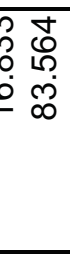 & & 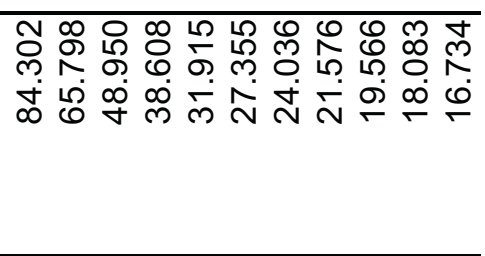 \\
\hline 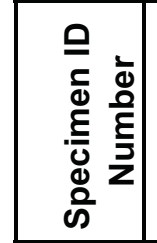 & $\begin{array}{l}\tilde{N} \\
\stackrel{N}{F}\end{array}$ & 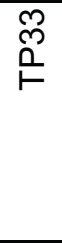 & 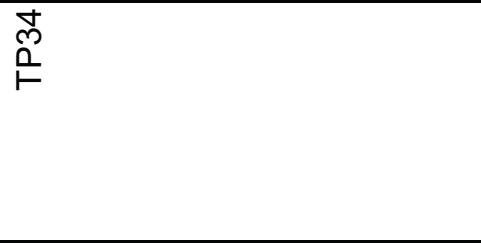 \\
\hline
\end{tabular}




\begin{tabular}{|c|c|c|c|c|}
\hline$\underset{5}{5}$ & 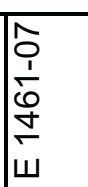 & 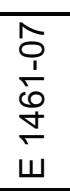 & $\begin{array}{l}\hat{o} \\
\frac{1}{1} \\
\dot{0} \\
\dot{+} \\
w\end{array}$ & 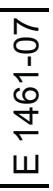 \\
\hline 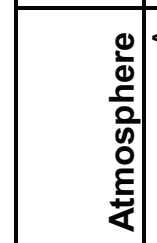 & & 交 & $\bar{z}$ & $\overline{\frac{i}{4}}$ \\
\hline 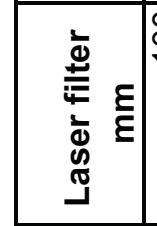 & & $\stackrel{\circ}{\circ}$ & 음 & 음 \\
\hline 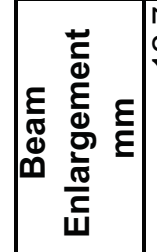 & $\hat{\stackrel{N}{\grave{j}}}$ & $\stackrel{\stackrel{N}{\sim}}{\sim}$ & 犬্ & $\stackrel{\stackrel{N}{ }}{ }$ \\
\hline 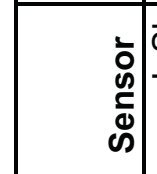 & 吕 & की & $\begin{array}{l}\text { की } \\
\text { की }\end{array}$ & $\begin{array}{l}\text { की } \\
\text { Dी }\end{array}$ \\
\hline 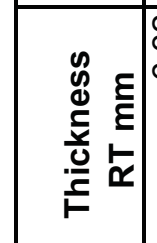 & & $\begin{array}{l}\widetilde{N} \\
\widetilde{0}\end{array}$ & ్ָల్ర్ల్ & ్ָల్ \\
\hline 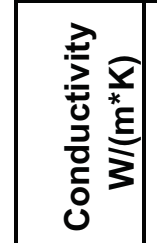 & & & & \\
\hline 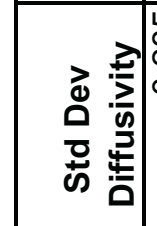 & 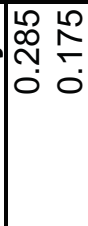 & 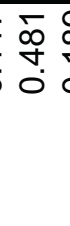 & 0 & \\
\hline 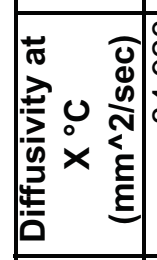 & 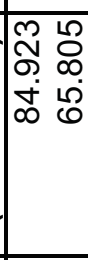 & $\begin{array}{l}\widehat{\otimes} \\
\dot{\phi} \\
\dot{\infty}\end{array}$ & $\begin{array}{l}\infty \\
\dot{+} \\
\dot{\infty}\end{array}$ & $\begin{array}{l}0 \\
\tilde{D} \\
0 \\
0 \\
\infty\end{array}$ \\
\hline 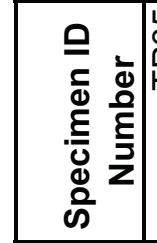 & 歺 & $\begin{array}{l}\stackrel{0}{\infty} \\
\hat{n}\end{array}$ & م) & 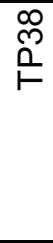 \\
\hline
\end{tabular}




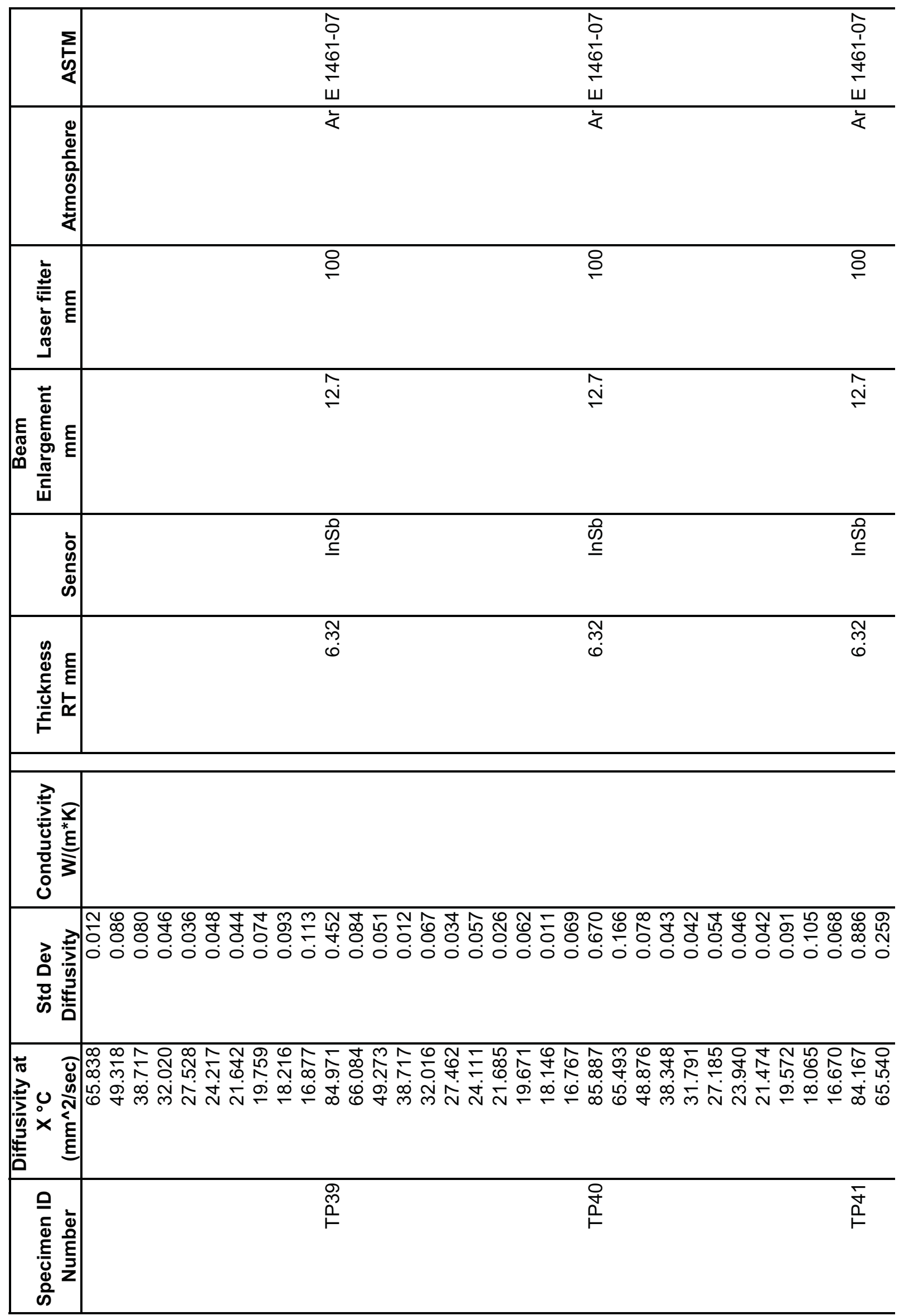




\begin{tabular}{|c|c|c|c|}
\hline 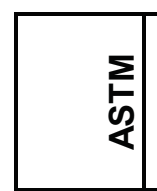 & $\begin{array}{l}\hat{T} \\
\frac{1}{o} \\
\frac{\delta}{+} \\
w\end{array}$ & 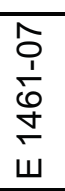 & $\begin{array}{l}\hat{o} \\
\frac{1}{o} \\
\frac{0}{+} \\
\dot{w}\end{array}$ \\
\hline 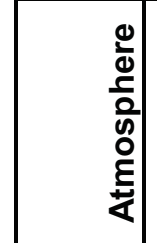 & ¿ & ¿ & ¿ \\
\hline 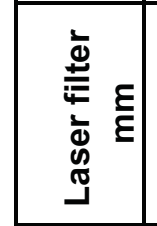 & $\stackrel{\circ}{\circ}$ & 음 & 음 \\
\hline 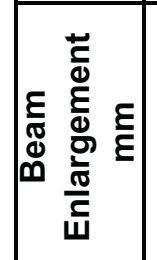 & 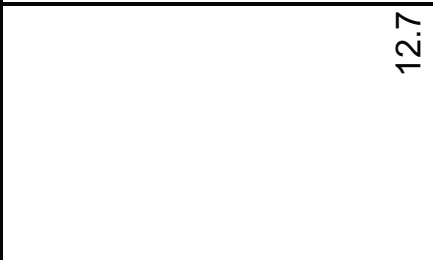 & $\widehat{\stackrel{\sim}{\mathrm{N}}}$ & $\stackrel{\widehat{N}}{\stackrel{N}{N}}$ \\
\hline 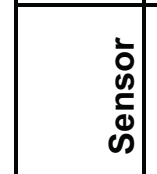 & $\begin{array}{l}\text { की } \\
\text { की }\end{array}$ & $\begin{array}{l}\text { की } \\
\text { की }\end{array}$ & $\begin{array}{l}\text { की } \\
\text { 足 }\end{array}$ \\
\hline 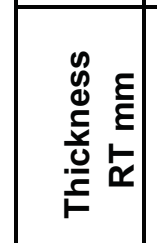 & $\begin{array}{l}\widetilde{N} \\
\text { ల్ }\end{array}$ & $\begin{array}{l}\widetilde{N} \\
\stackrel{N}{0}\end{array}$ & స్ల్ల \\
\hline 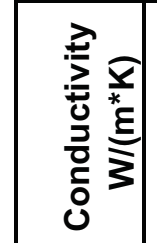 & & & \\
\hline 䠞 & 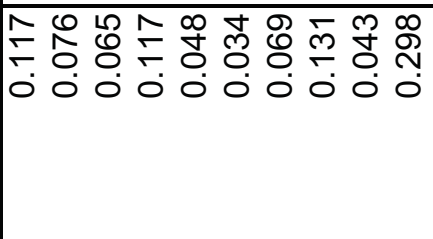 & $\stackrel{\text { Pִ }}{\rightarrow}$ & 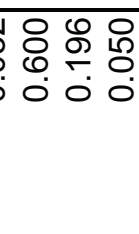 \\
\hline 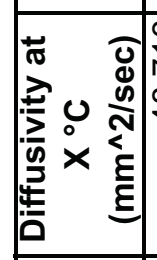 & 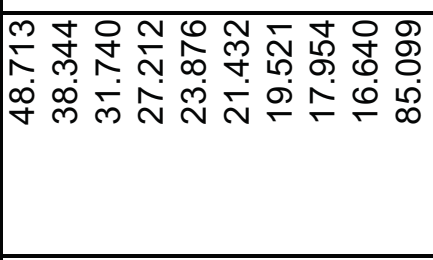 & \& & 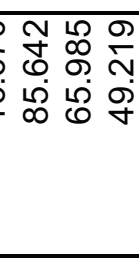 \\
\hline 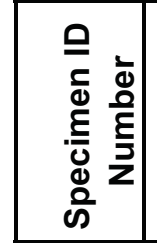 & 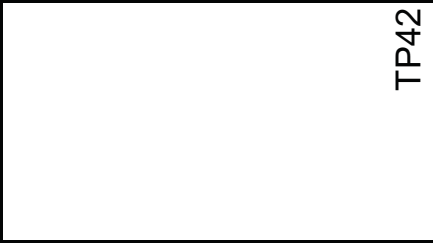 & 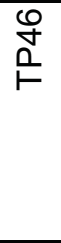 & $\begin{array}{l}\text { 咅 } \\
\hat{R}\end{array}$ \\
\hline
\end{tabular}




\begin{tabular}{|c|c|c|c|}
\hline 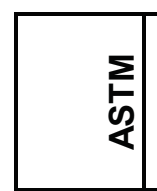 & $\begin{array}{l}\hat{o} \\
\dot{1} \\
\dot{0} \\
\dot{+} \\
\dot{w}\end{array}$ & $\begin{array}{l}\hat{o} \\
\frac{1}{1} \\
\frac{1}{5} \\
\dot{\omega}\end{array}$ & $\begin{array}{l}\hat{o} \\
\frac{1}{0} \\
\frac{0}{+} \\
\dot{\omega}\end{array}$ \\
\hline 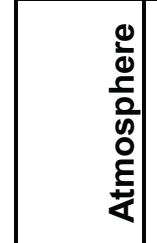 & 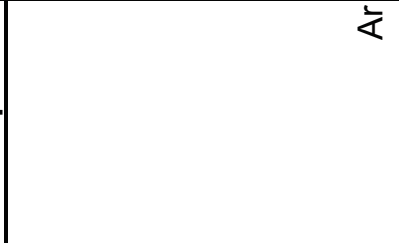 & ¿ & ¿ \\
\hline 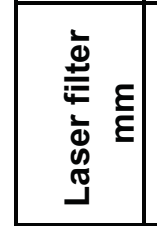 & $\stackrel{\circ}{\circ}$ & 음 & 음 \\
\hline 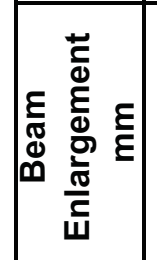 & $\stackrel{\stackrel{N}{\mathrm{~N}}}{ }$ & $\stackrel{\widetilde{N}}{\stackrel{N}{N}}$ & $\stackrel{\widehat{N}}{\stackrel{N}{N}}$ \\
\hline 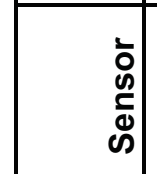 & $\begin{array}{l}\text { की } \\
\text { की }\end{array}$ & 吕 & $\begin{array}{l}\text { की } \\
\text { 足 }\end{array}$ \\
\hline 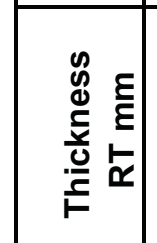 & $\begin{array}{l}\widetilde{N} \\
\widetilde{N}\end{array}$ & స్ల్ర & స్ల్ల \\
\hline 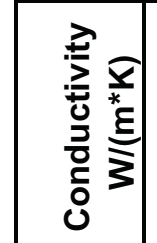 & & & \\
\hline 䠞 & 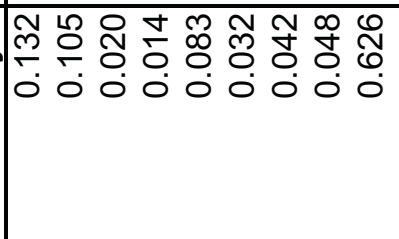 & 0 & 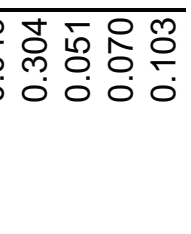 \\
\hline 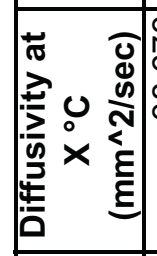 & 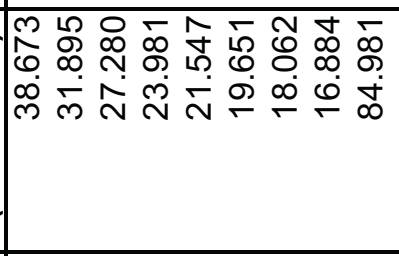 & & 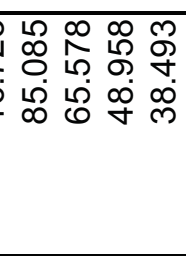 \\
\hline 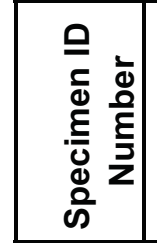 & 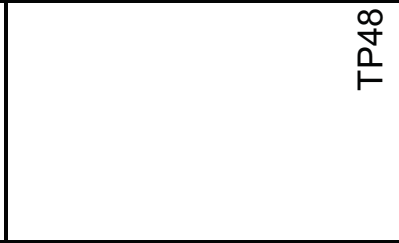 & $\begin{array}{l}\underbrace{m}_{0} \\
\stackrel{2}{r}\end{array}$ & $\begin{array}{l}\text { 吉 } \\
\stackrel{1}{R}\end{array}$ \\
\hline
\end{tabular}




\begin{tabular}{|c|c|}
\hline $\begin{array}{l}\frac{\Sigma}{5} \\
\frac{5}{\alpha}\end{array}$ & 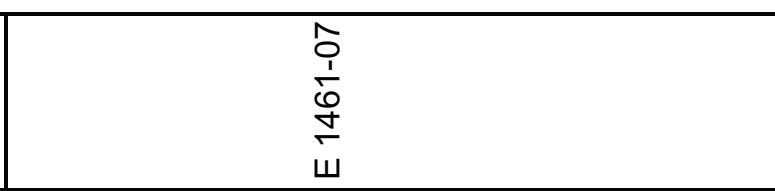 \\
\hline 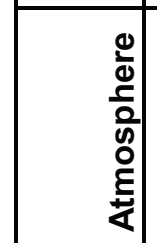 & 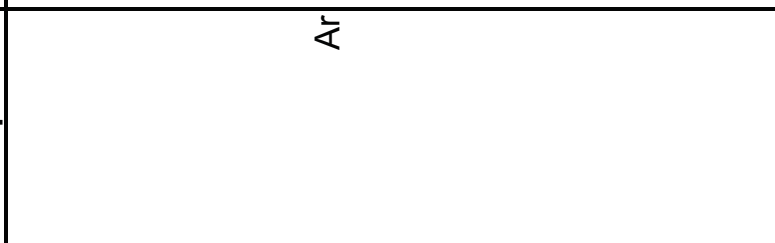 \\
\hline 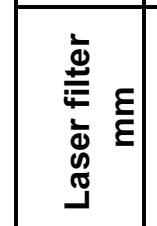 & 웅 \\
\hline 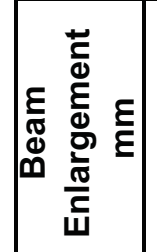 & $\stackrel{\widehat{N}}{\stackrel{N}{N}}$ \\
\hline $\begin{array}{l}\vdots \\
0 \\
0 \\
\infty \\
\infty\end{array}$ & $\begin{array}{l}\text { की } \\
\text { की }\end{array}$ \\
\hline 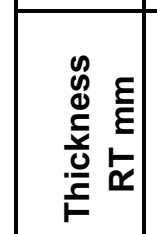 & $\begin{array}{l}\widetilde{N} \\
\text { W. }\end{array}$ \\
\hline 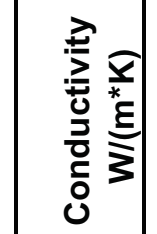 & \\
\hline 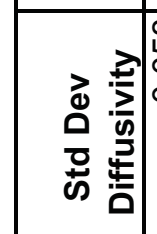 & 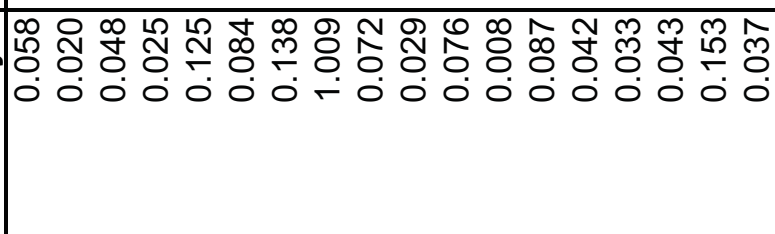 \\
\hline 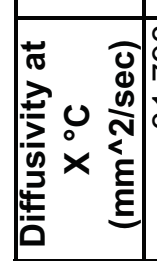 & 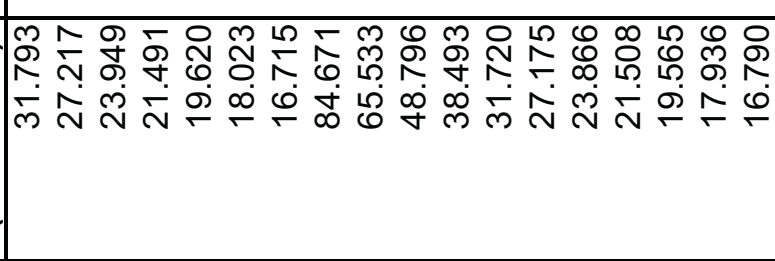 \\
\hline 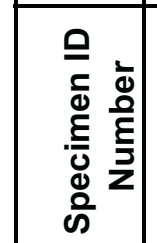 & $\begin{array}{l}e_{0}^{8} \\
0\end{array}$ \\
\hline
\end{tabular}




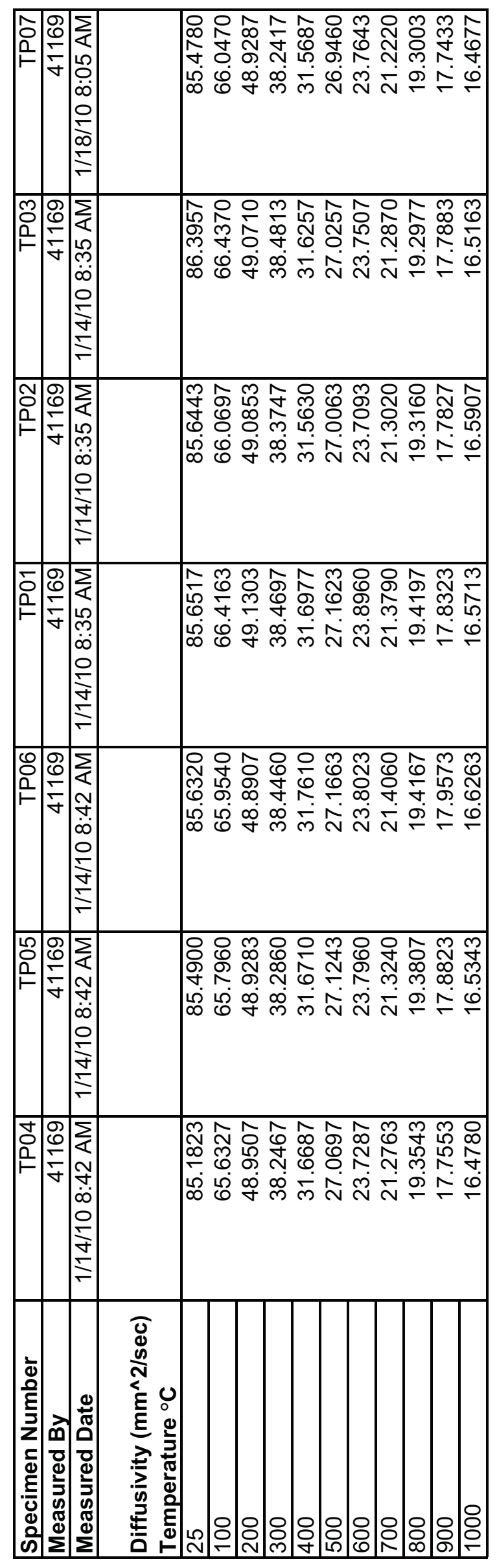




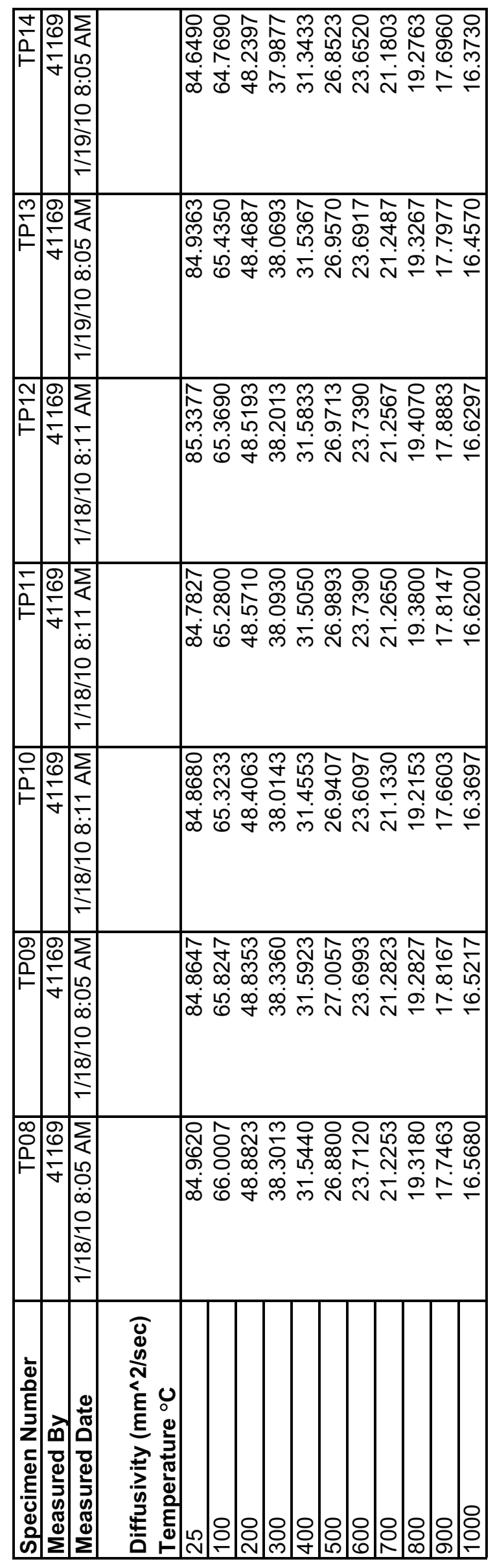




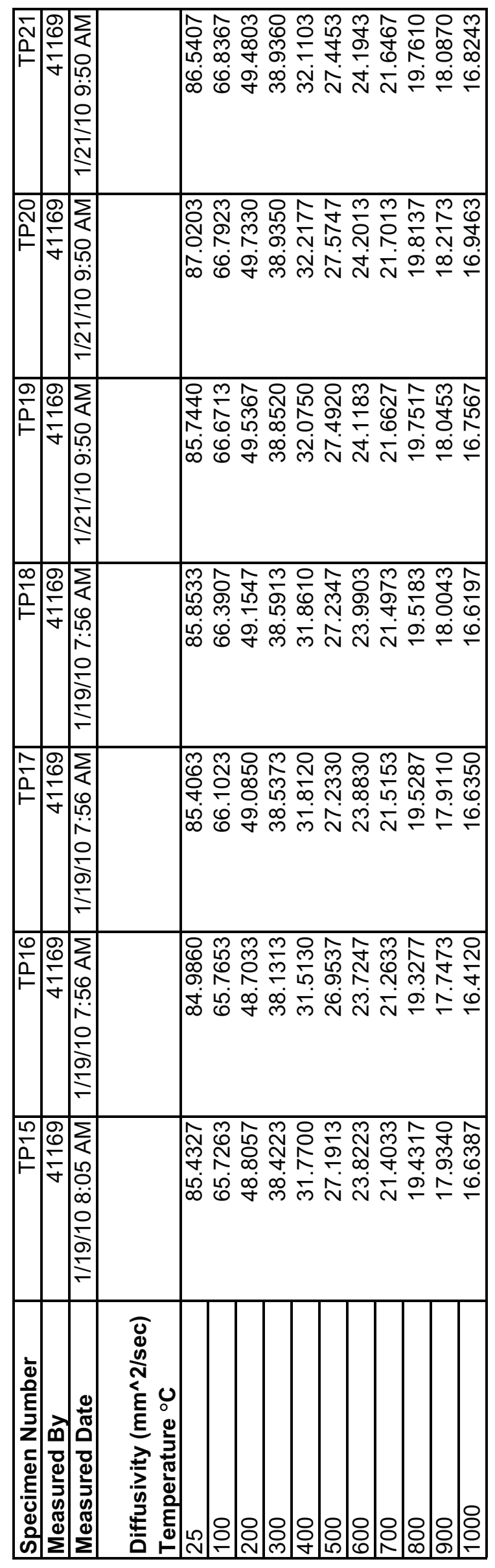




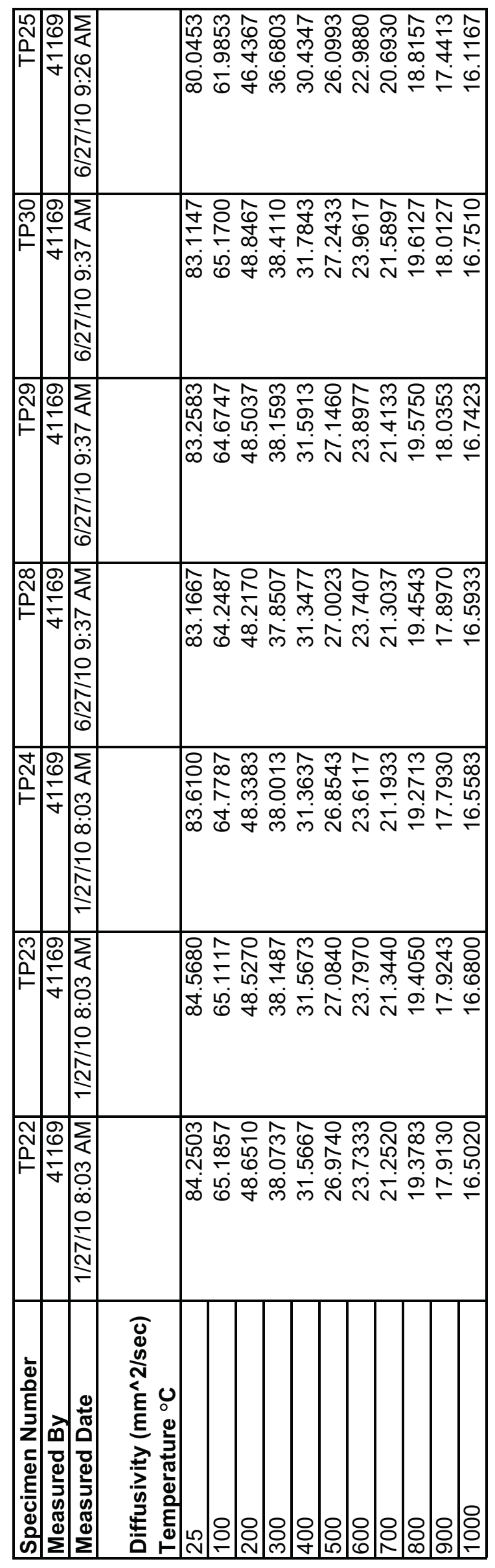




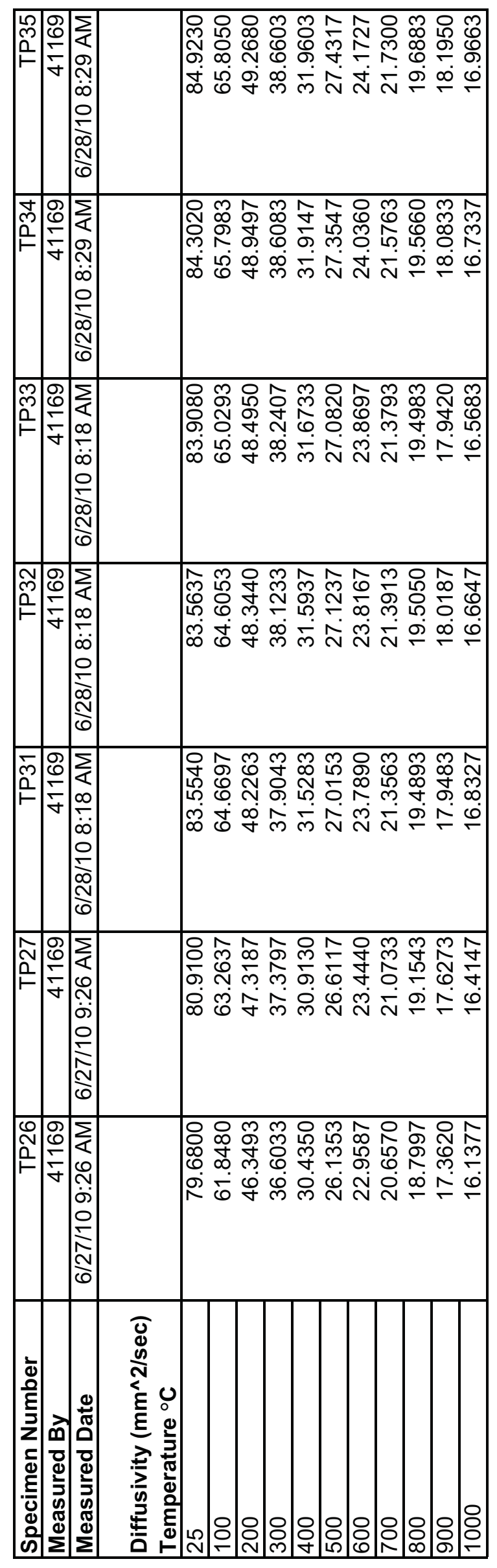




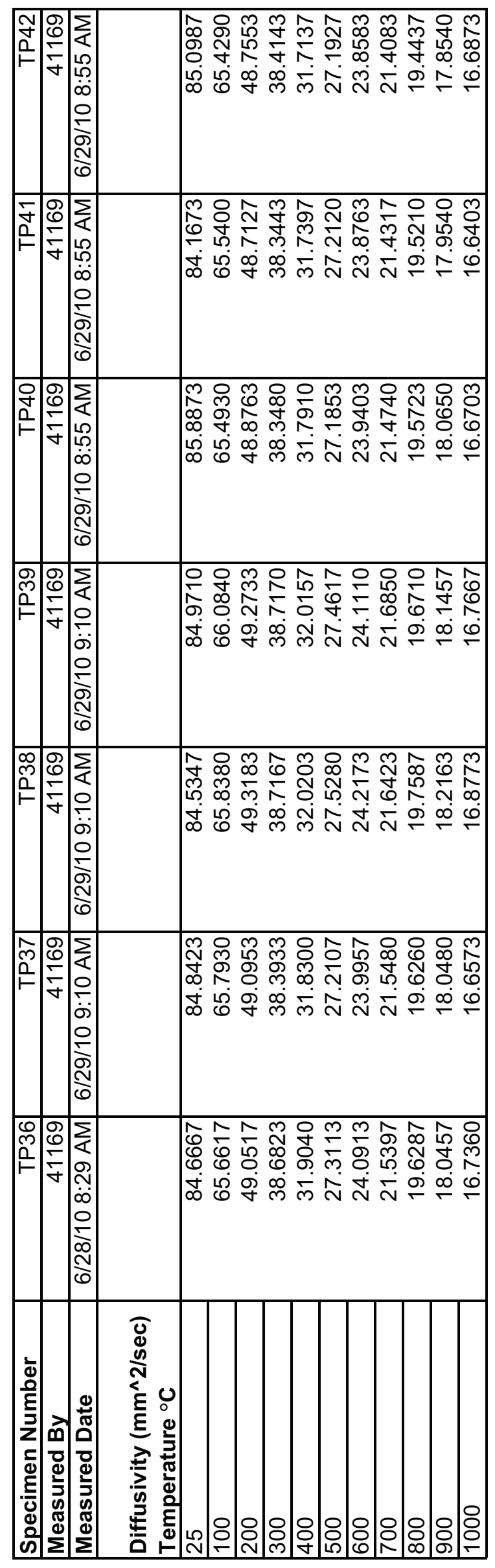




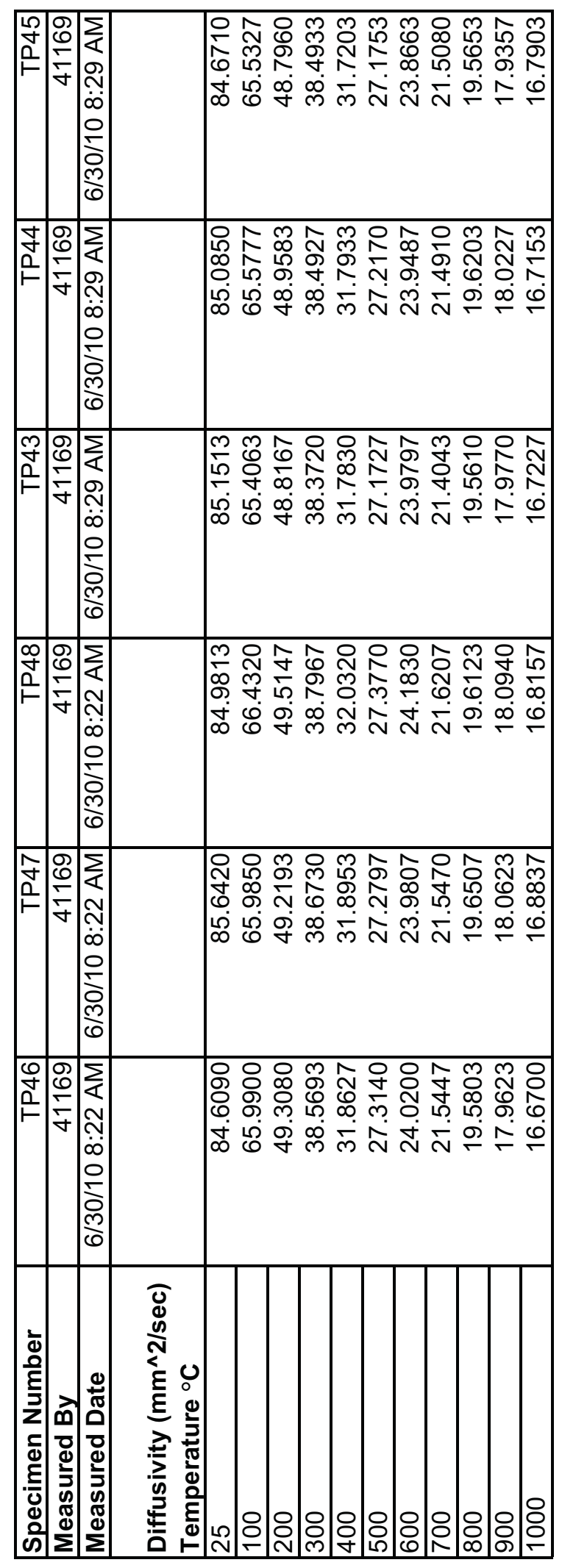




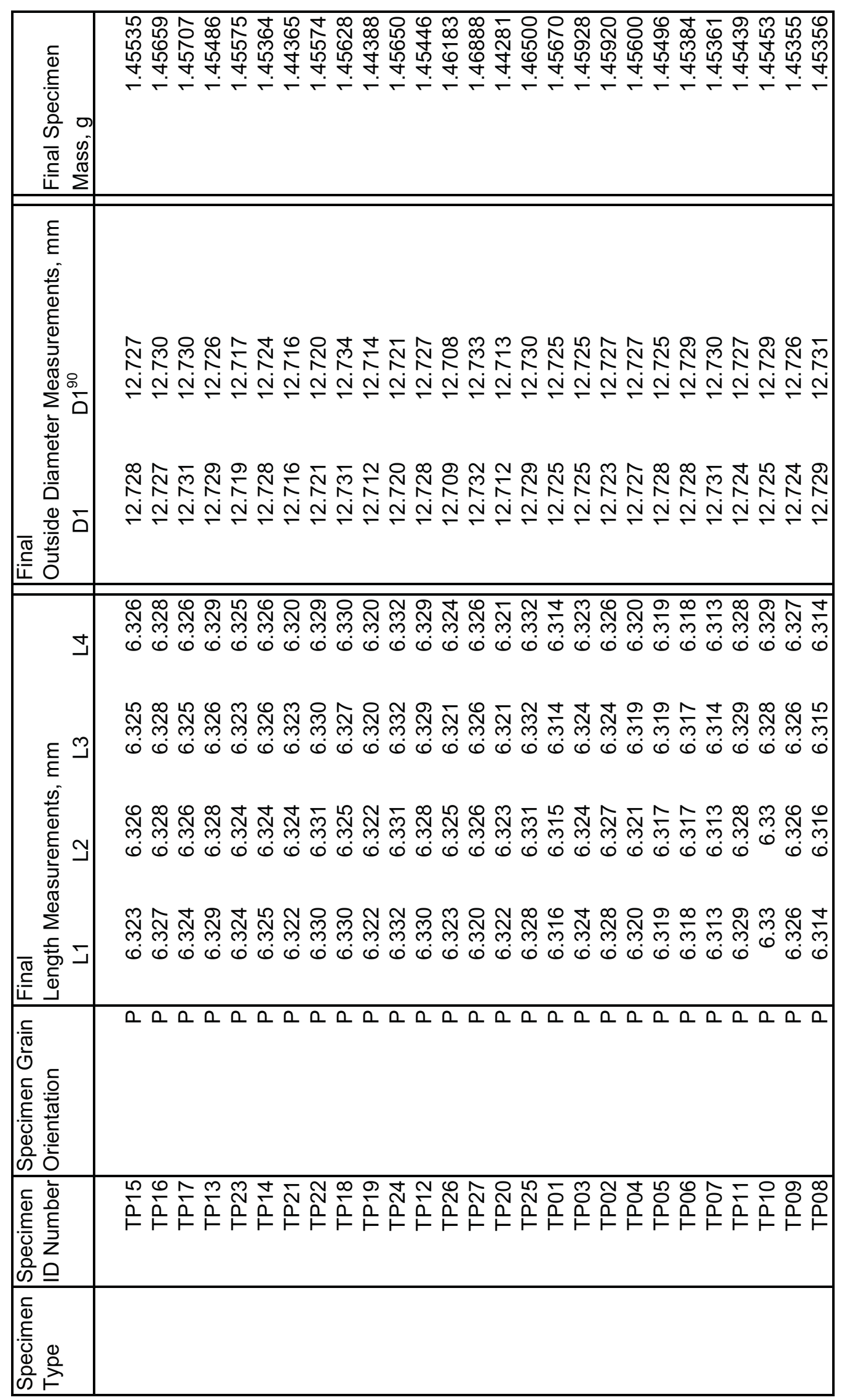




\begin{tabular}{|c|c|}
\hline 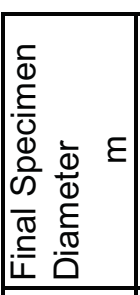 & 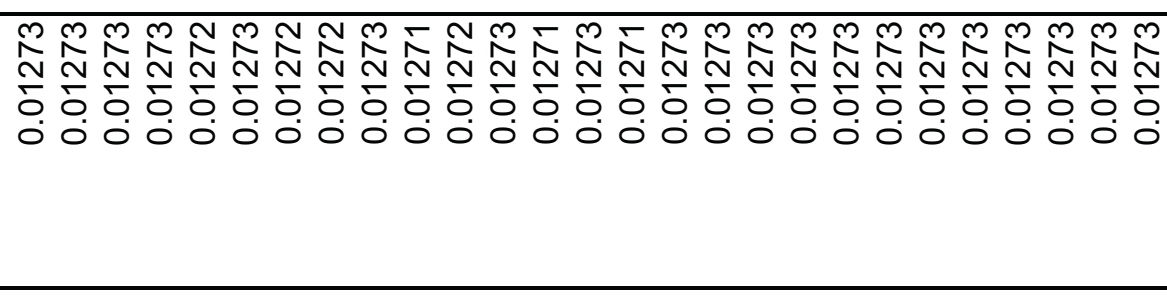 \\
\hline 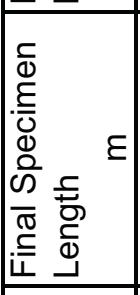 & 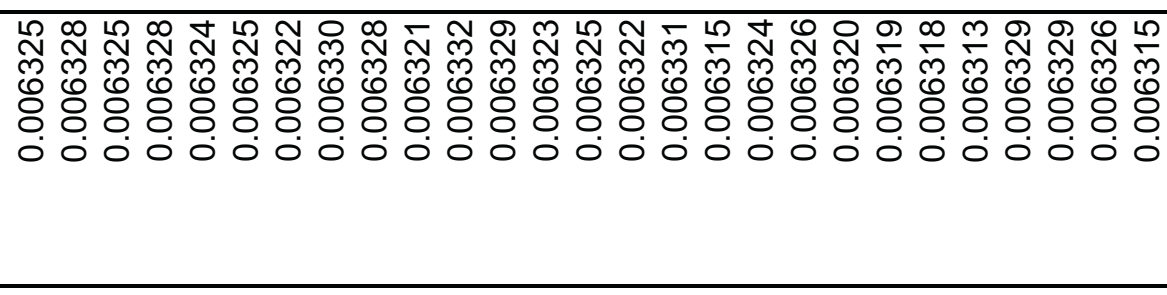 \\
\hline 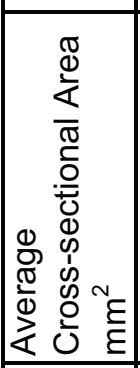 & 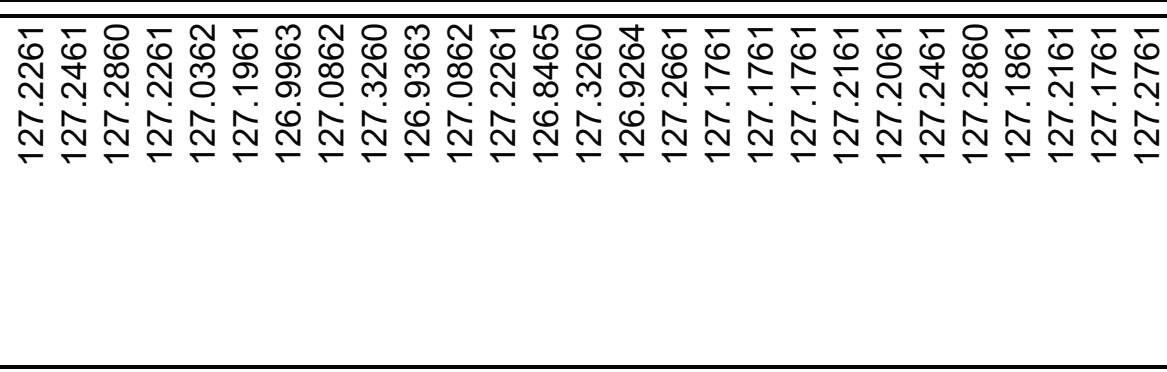 \\
\hline 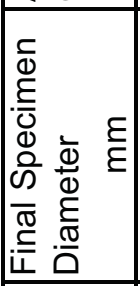 & 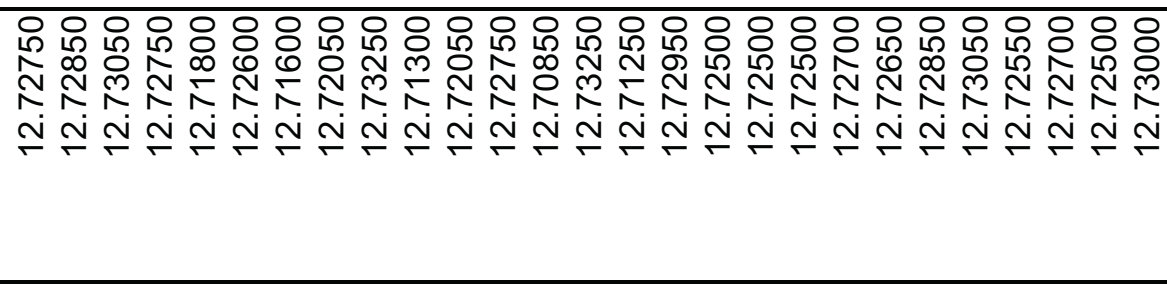 \\
\hline 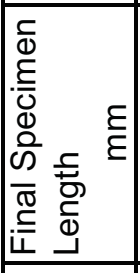 & 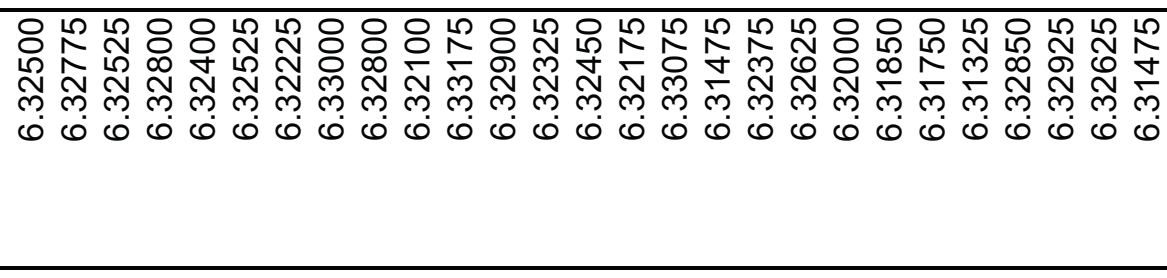 \\
\hline 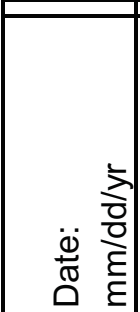 & 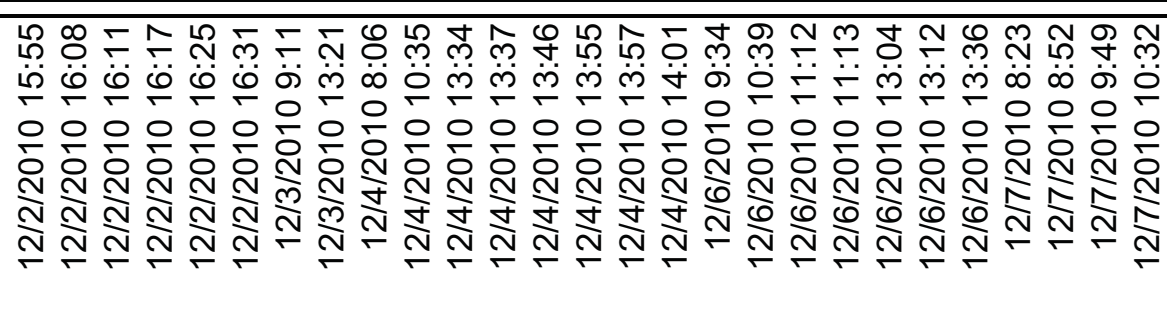 \\
\hline 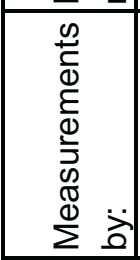 & 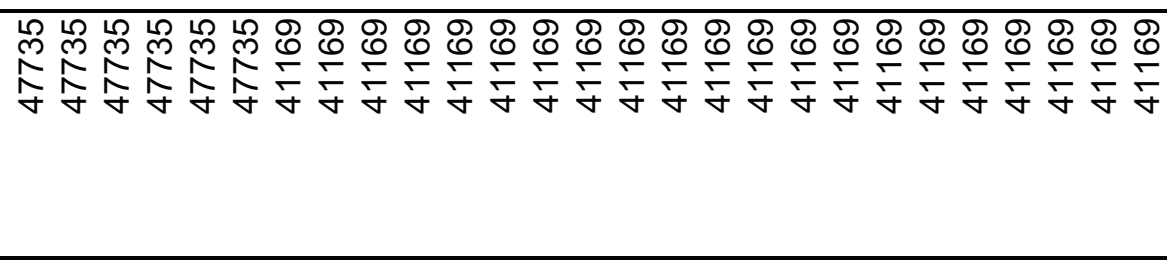 \\
\hline 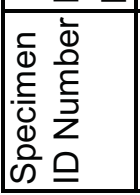 & 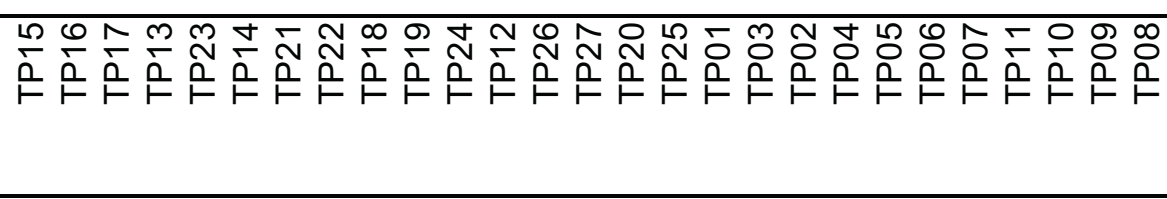 \\
\hline
\end{tabular}




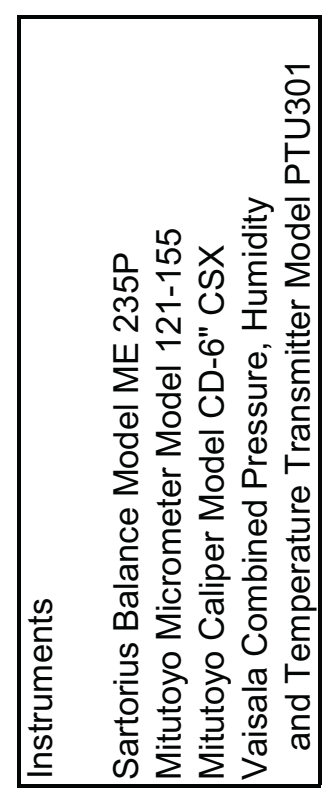

\begin{tabular}{|c|c|}
\hline 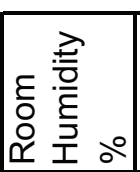 & 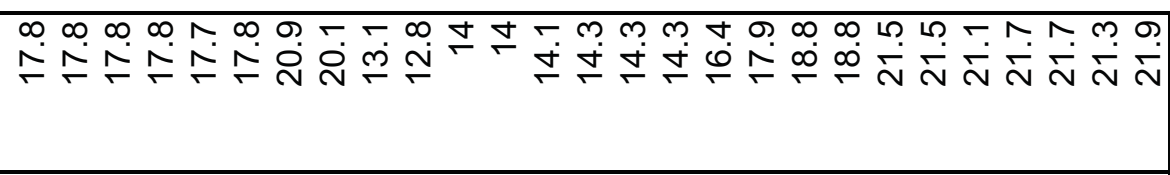 \\
\hline 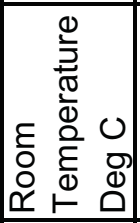 & 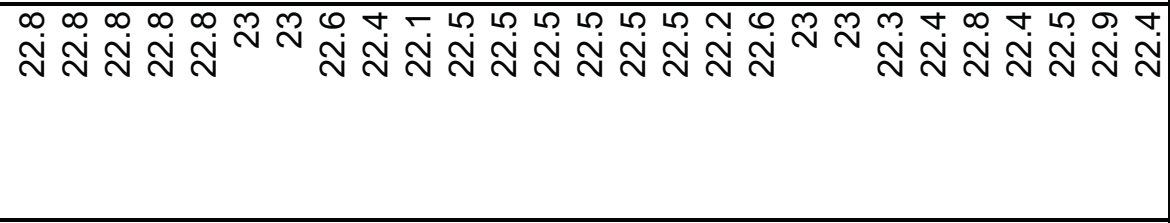 \\
\hline 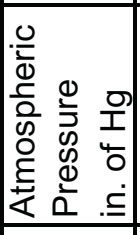 & 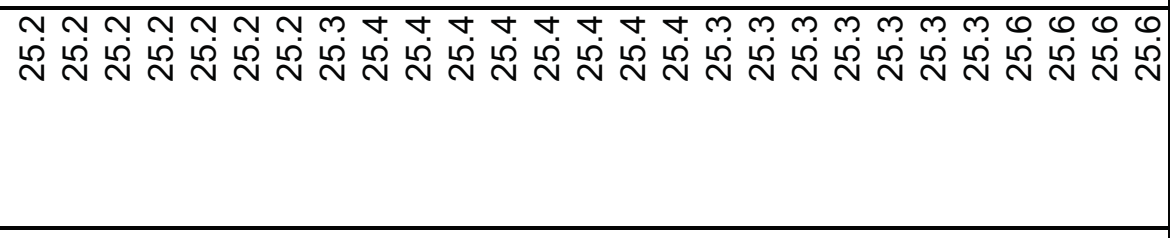 \\
\hline 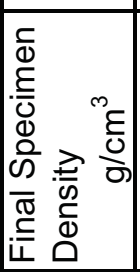 & 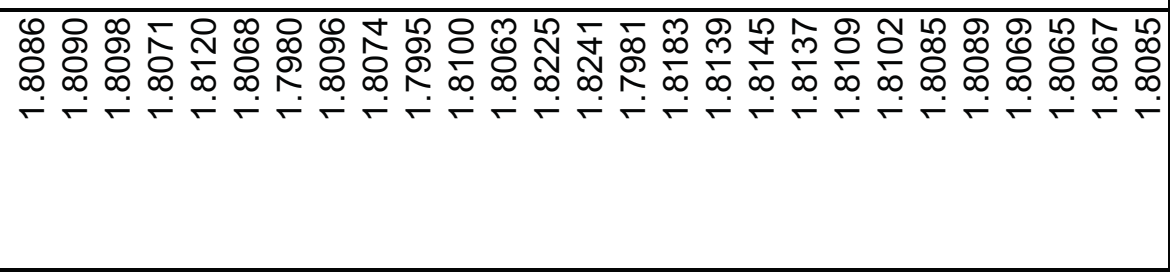 \\
\hline 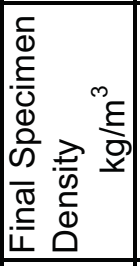 & 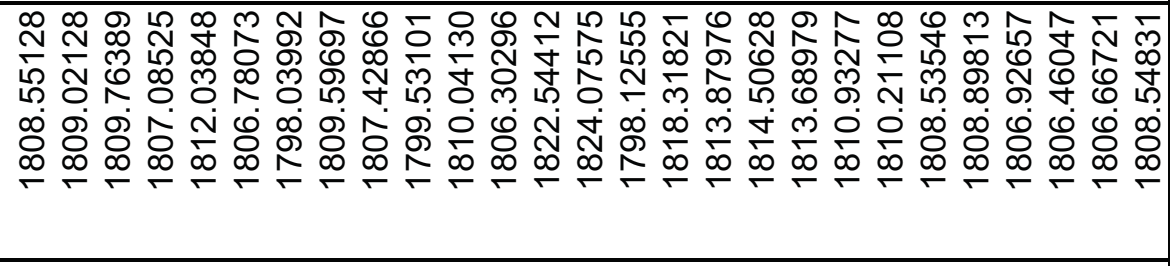 \\
\hline 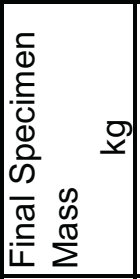 & 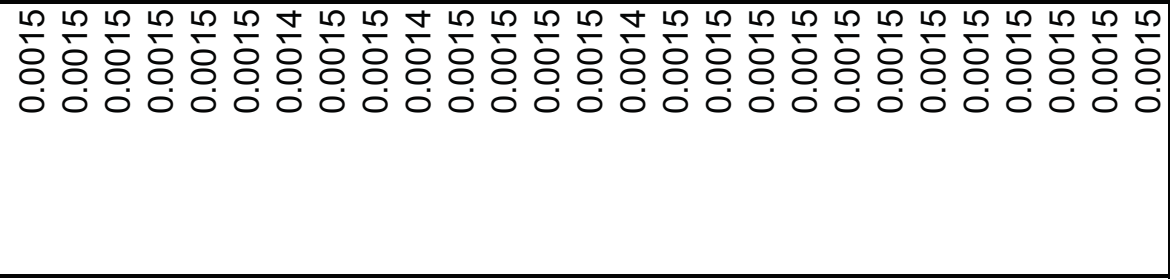 \\
\hline 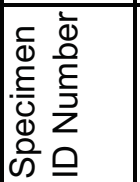 & 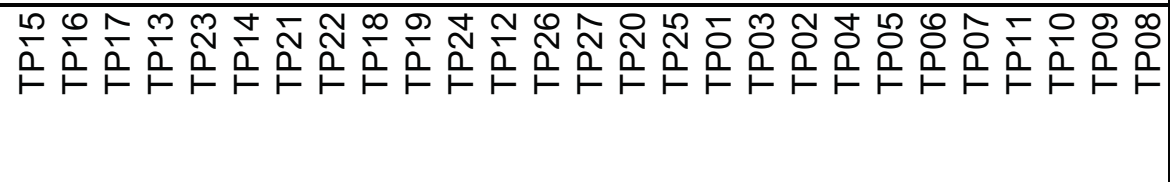 \\
\hline
\end{tabular}




$\begin{array}{ll}\text { Graphite Grade: } & \text { A3 MATRIX } \\ \text { Graphite Manufacturer: } & \text { ORNL } \\ \text { Forming Process: } & \text { Unknown } \\ \text { Coke Particle Size: } & \text { Unknown } \\ \text { Coke Type: } & \text { Unknown } \\ \text { ASTM Class: } & \text { Unknown } \\ \text { Specimen Geometry: } & \text { Cylinder }\end{array}$

Specimen ID \#'s:

H461

$\mathrm{H} 462$

$\mathrm{H} 471$

$\mathrm{H} 472$

$\mathrm{H} 481$

$\mathrm{H} 482$

$\mathrm{H} 491$

$\mathrm{H} 492$

$\mathrm{H} 501$

H502

H511

H512

H521

H522

H531

H532

H541

H542

H551

H552

H561

H562

H571

H572

H581

H582

H591

H592

$\mathrm{H} 601$

$\mathrm{H} 602$ 


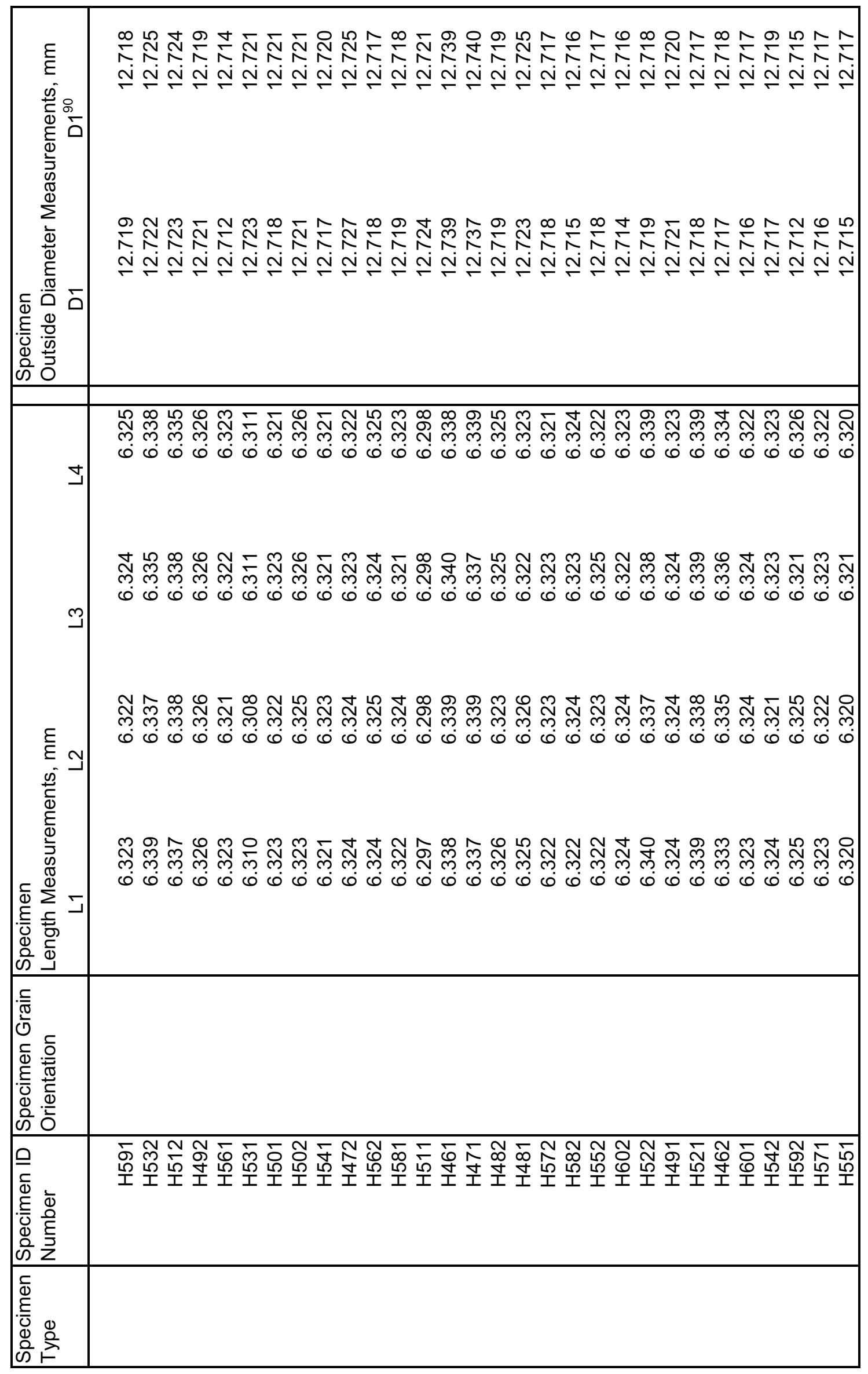




\begin{tabular}{|c|c|}
\hline 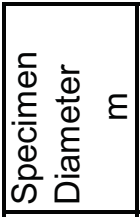 & 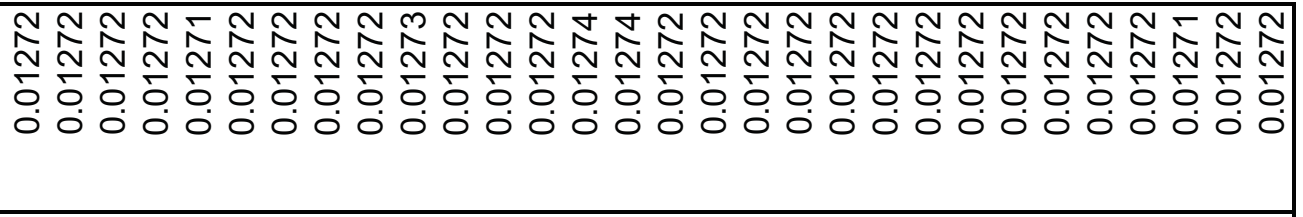 \\
\hline 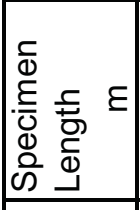 & 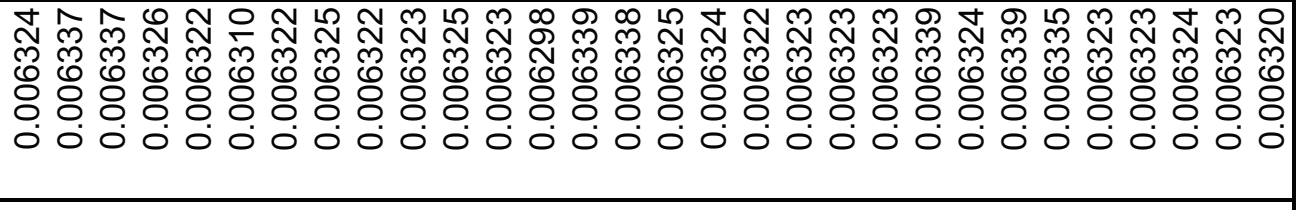 \\
\hline 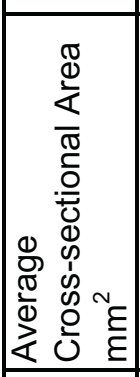 & 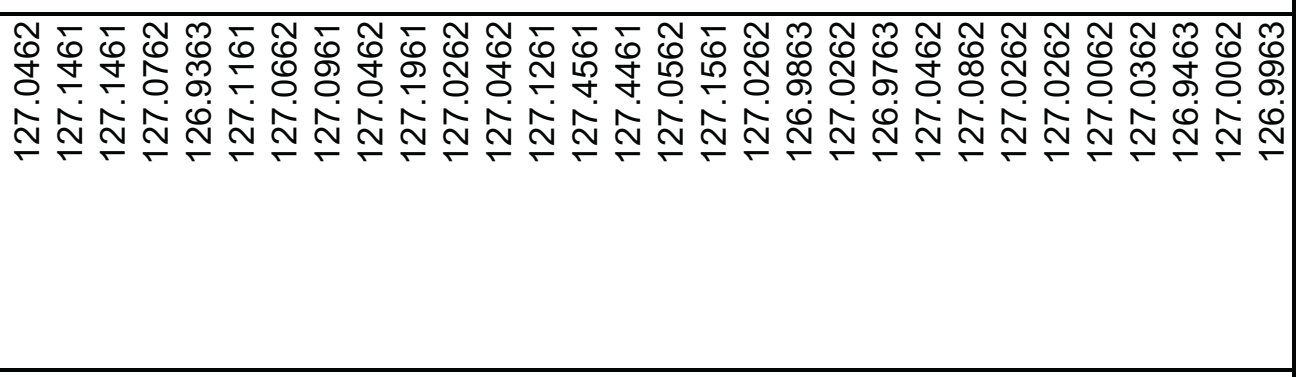 \\
\hline 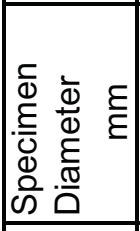 & 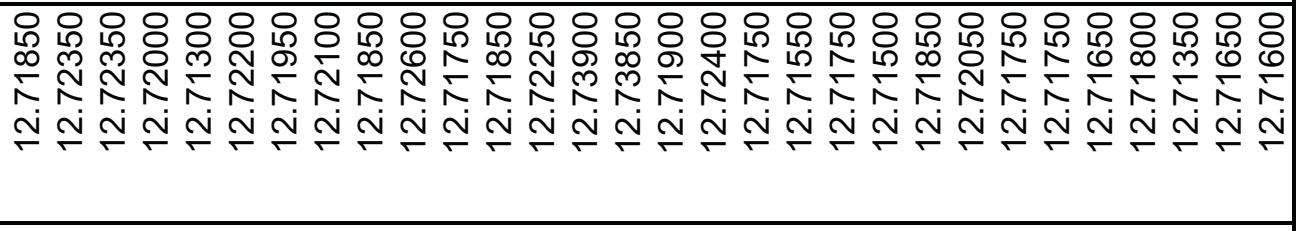 \\
\hline 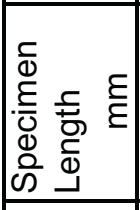 & 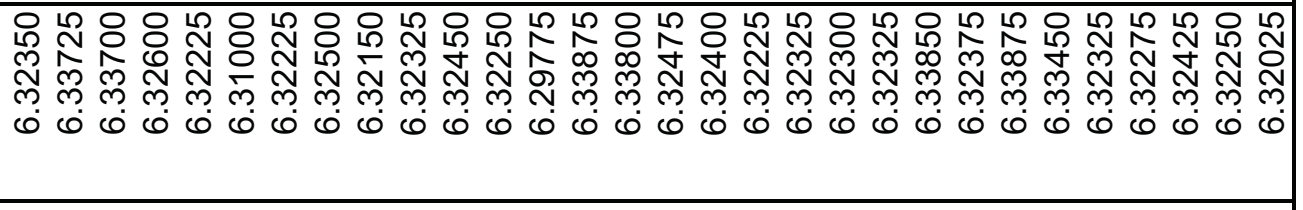 \\
\hline 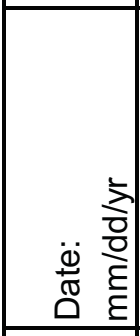 & 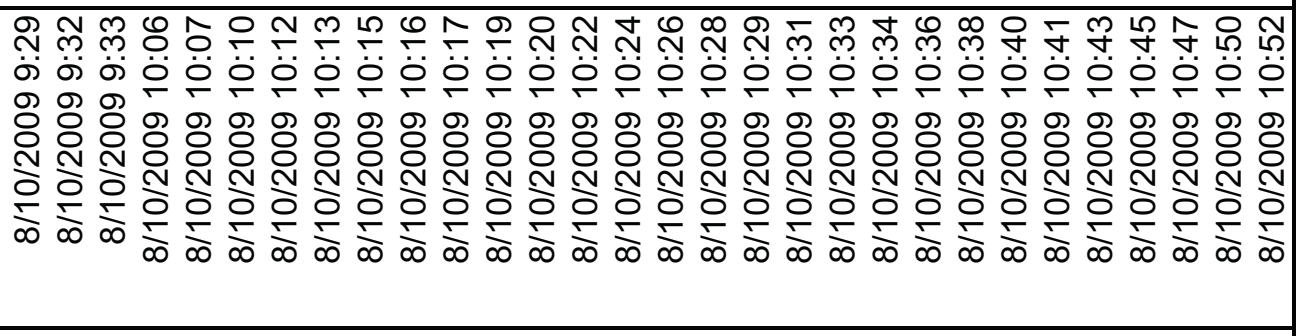 \\
\hline 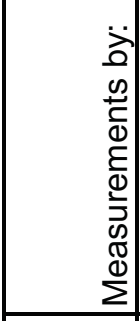 & $=\overline{=}=$ \\
\hline 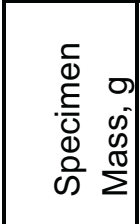 & 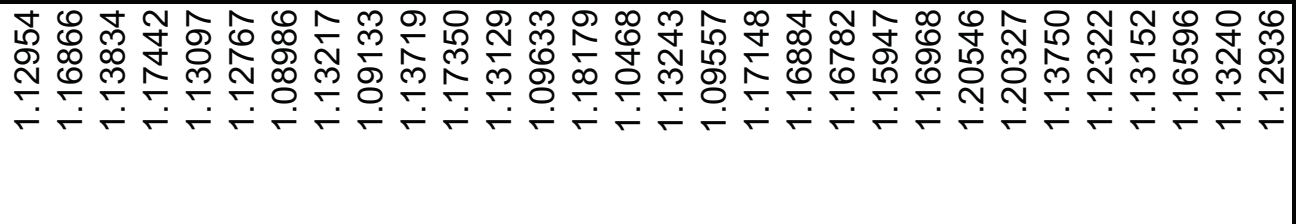 \\
\hline 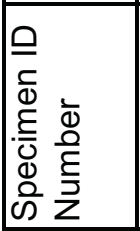 & 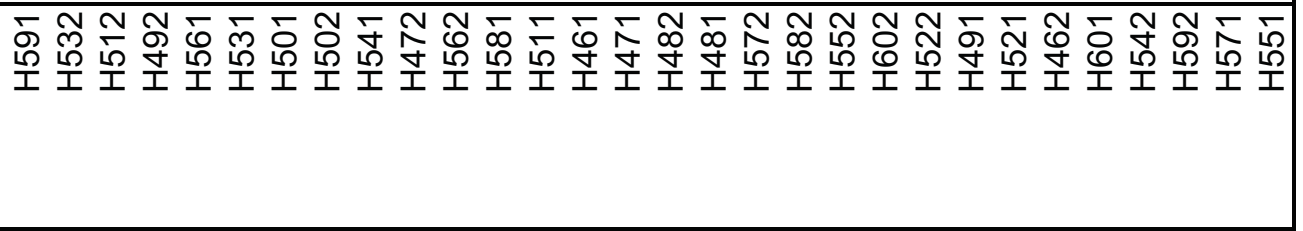 \\
\hline
\end{tabular}




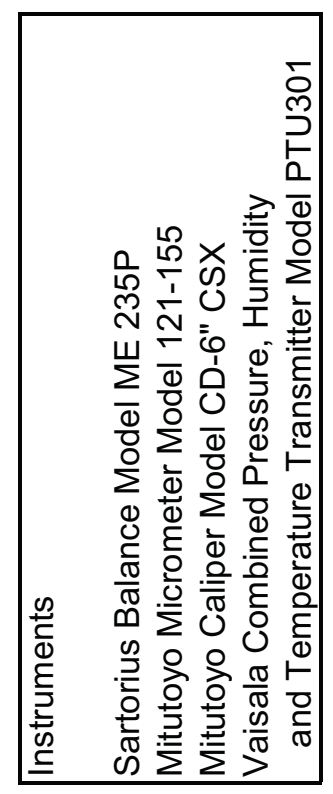

\begin{tabular}{|c|c|}
\hline 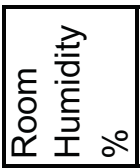 & 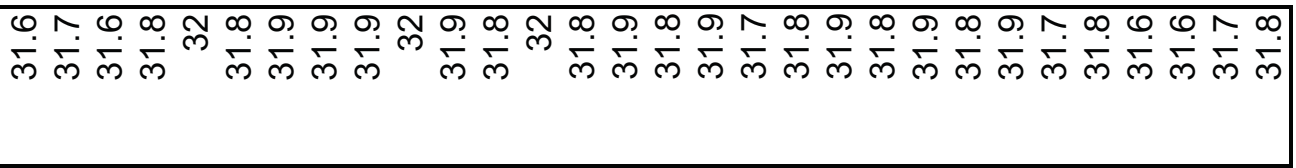 \\
\hline 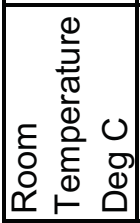 & 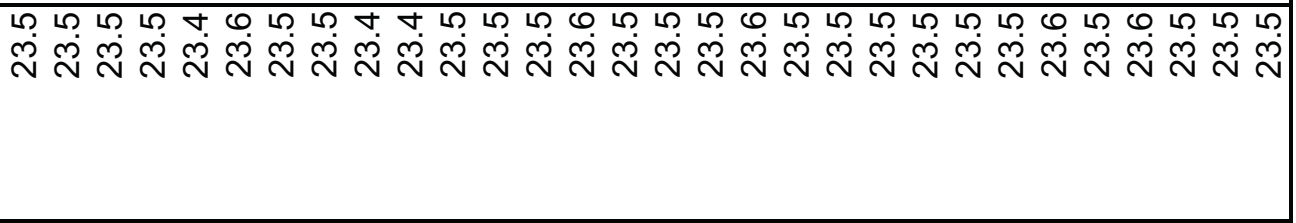 \\
\hline 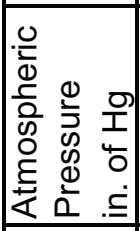 & 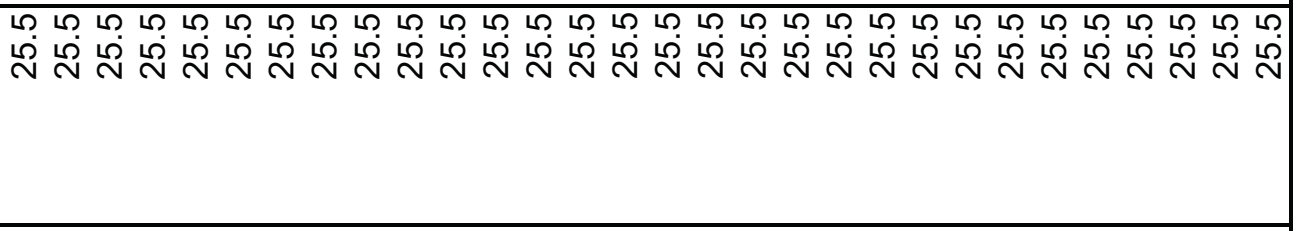 \\
\hline 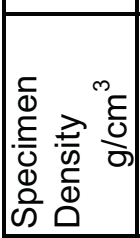 & 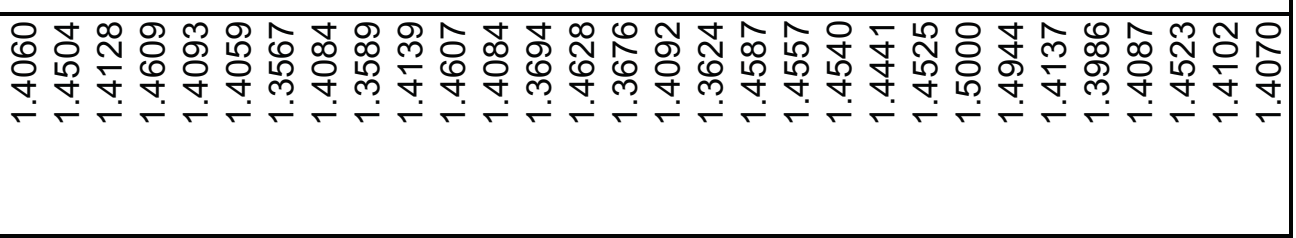 \\
\hline 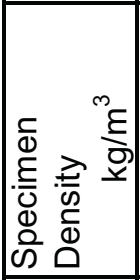 & 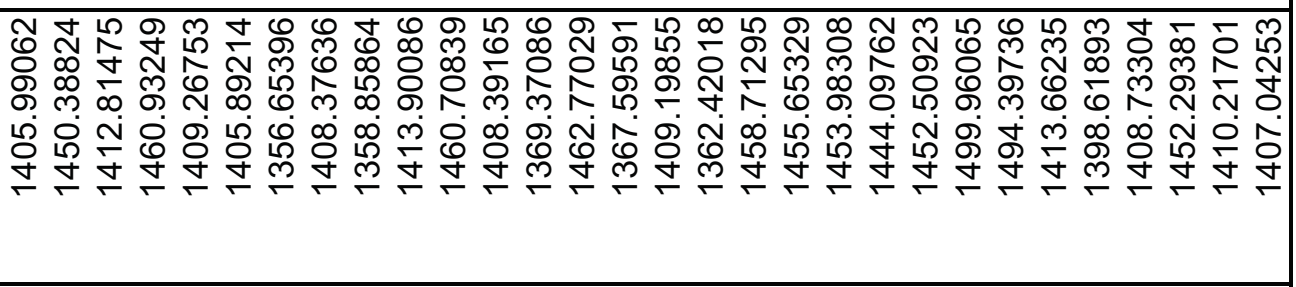 \\
\hline 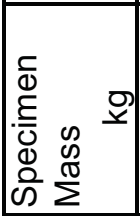 & 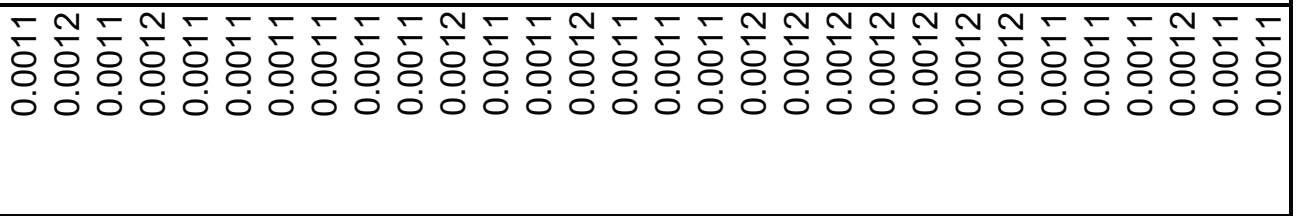 \\
\hline 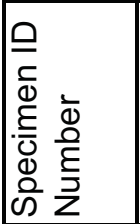 & 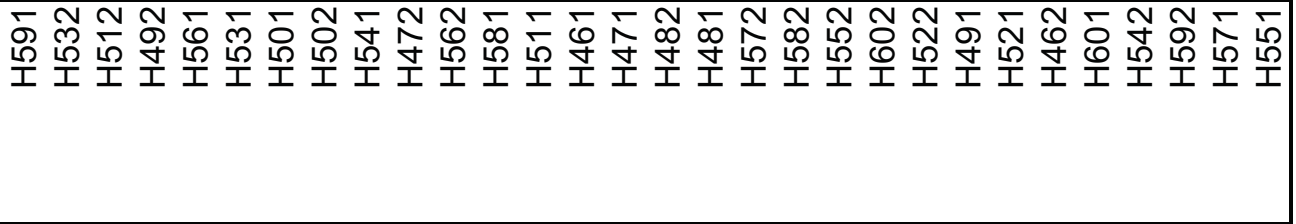 \\
\hline
\end{tabular}




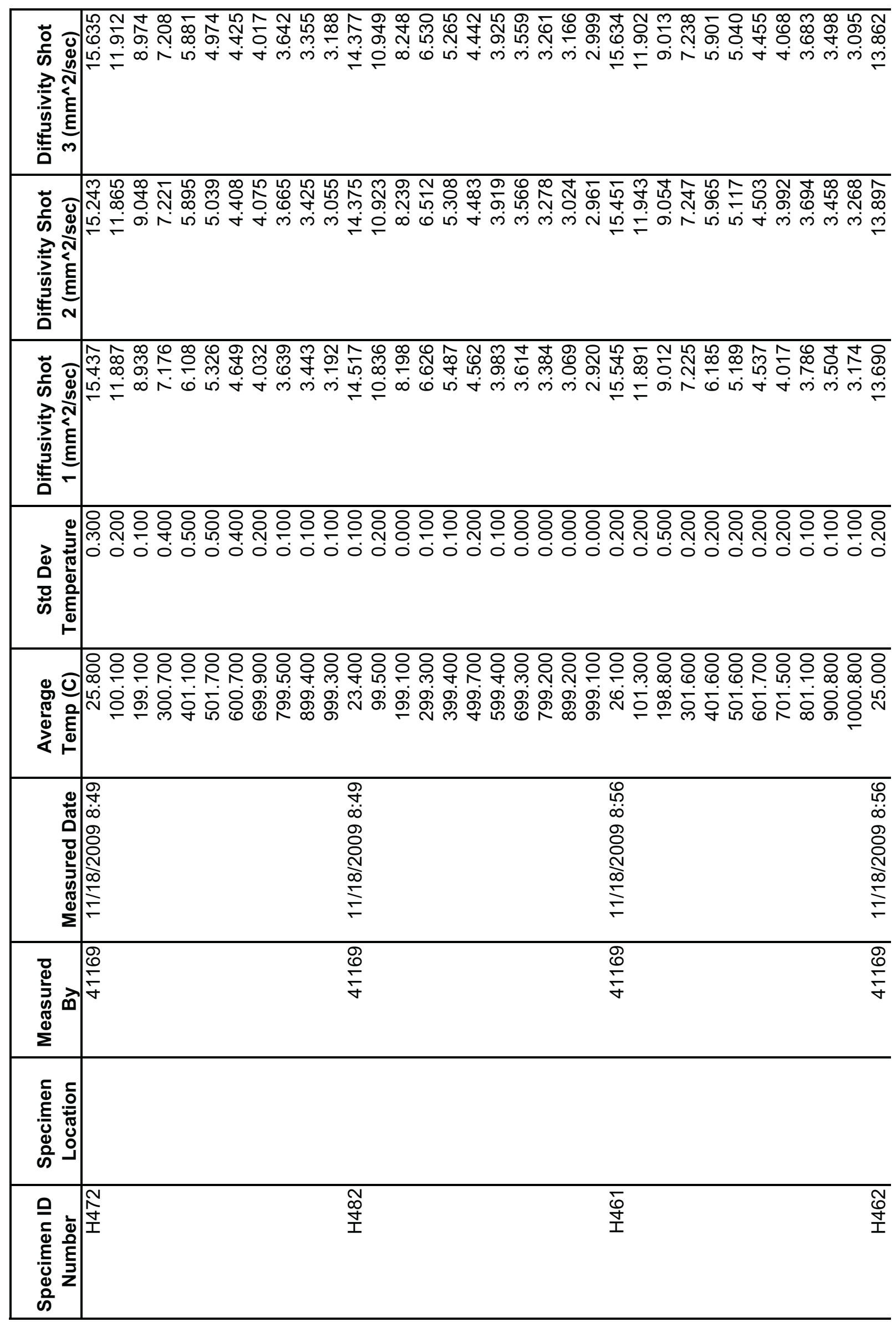




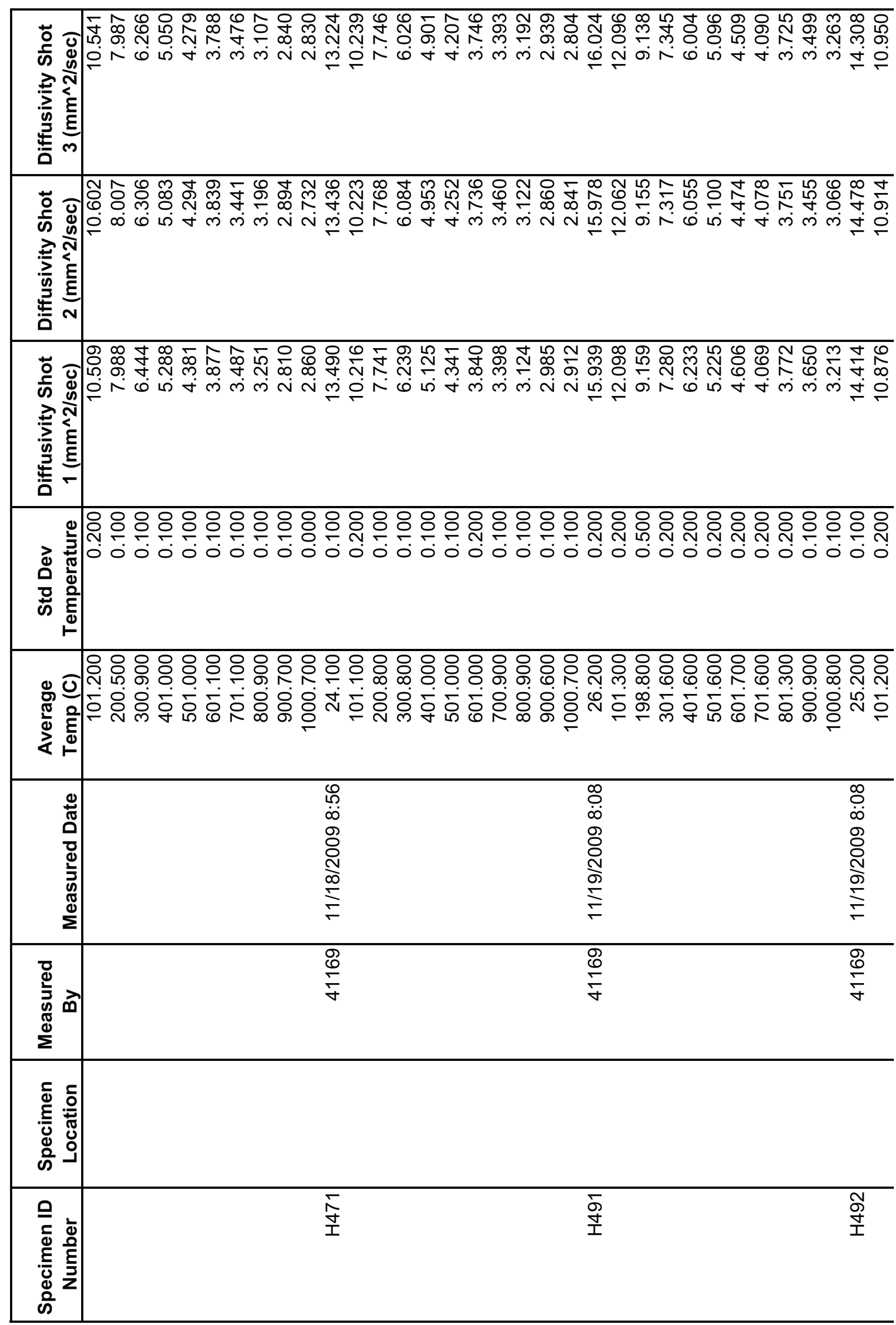




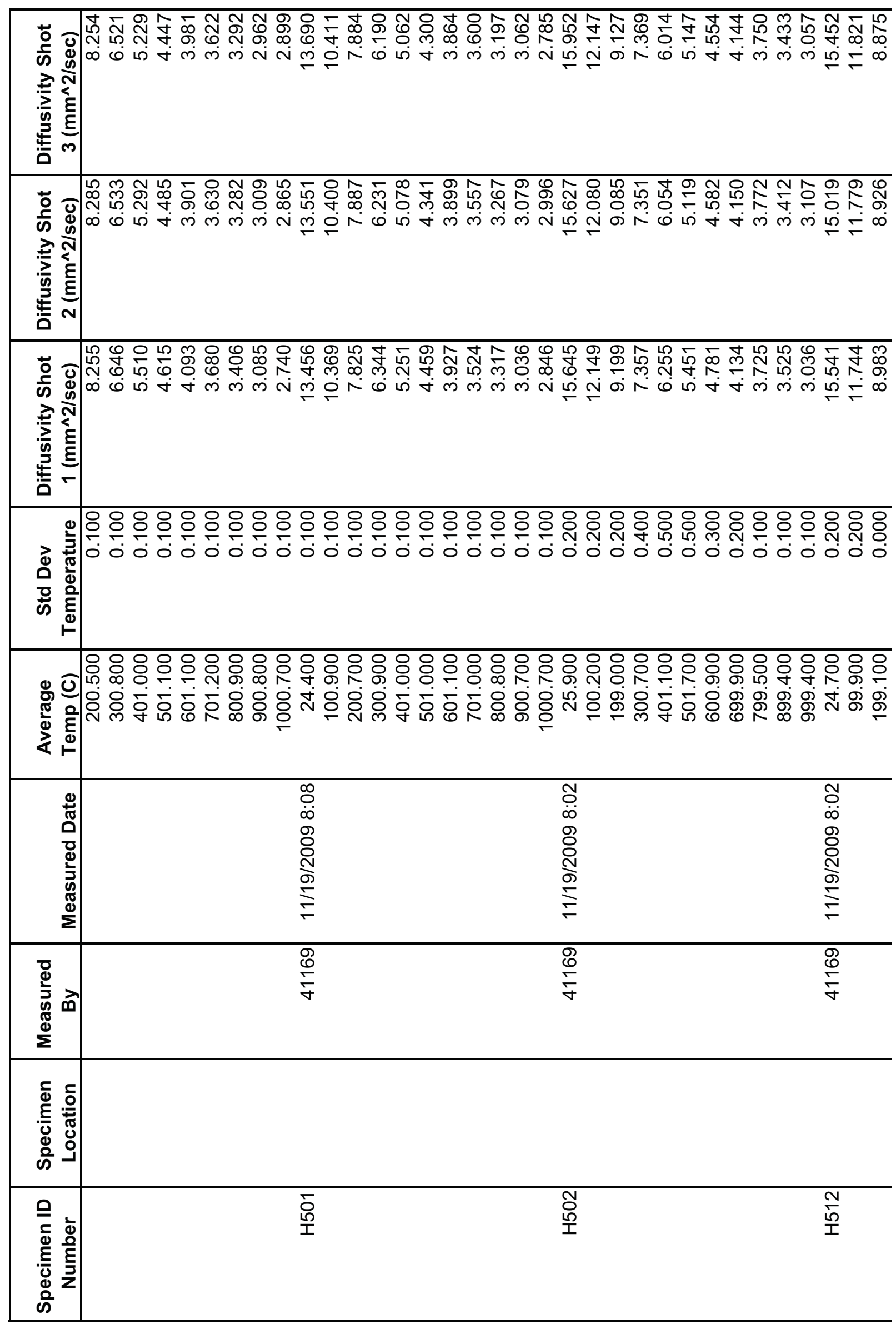




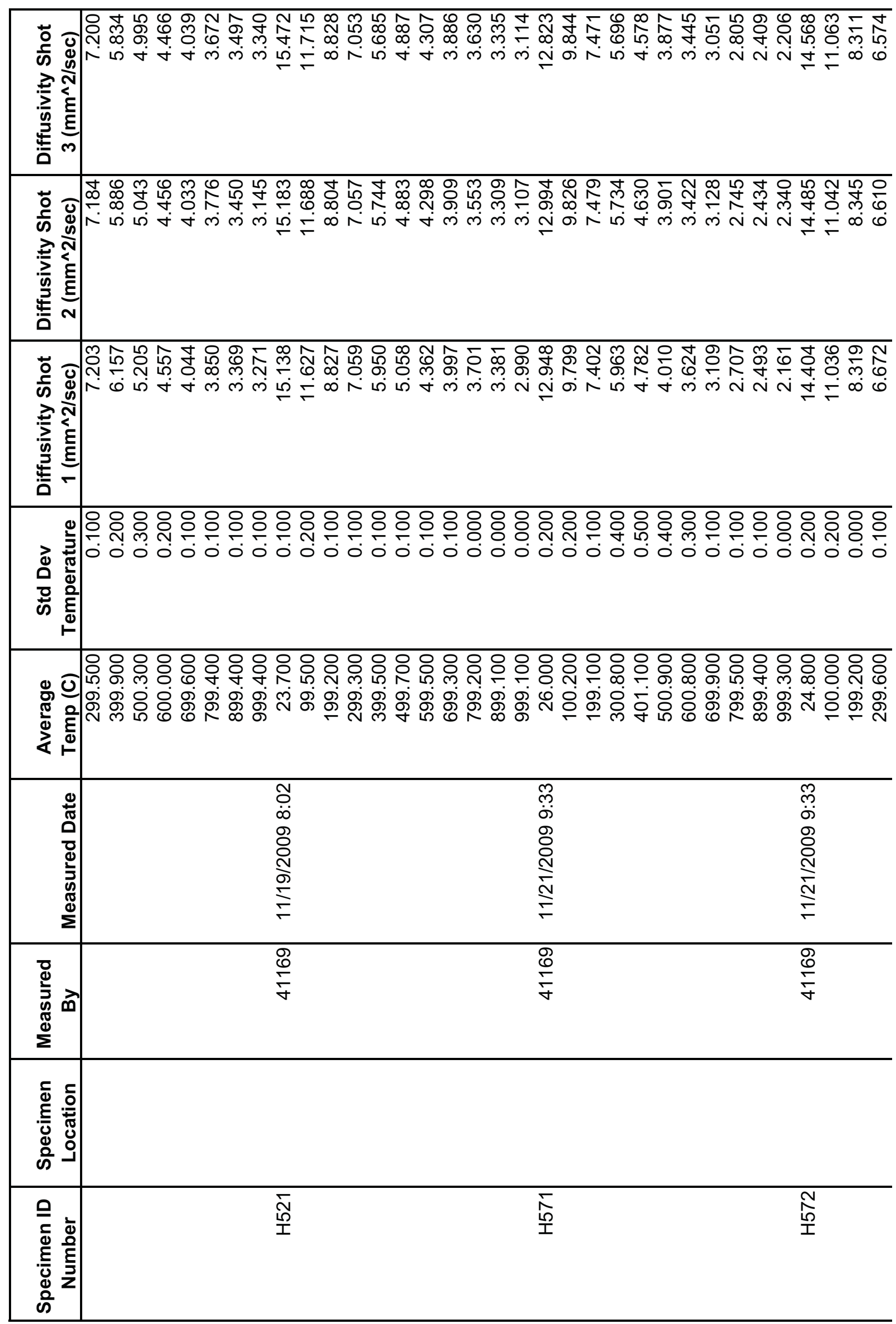




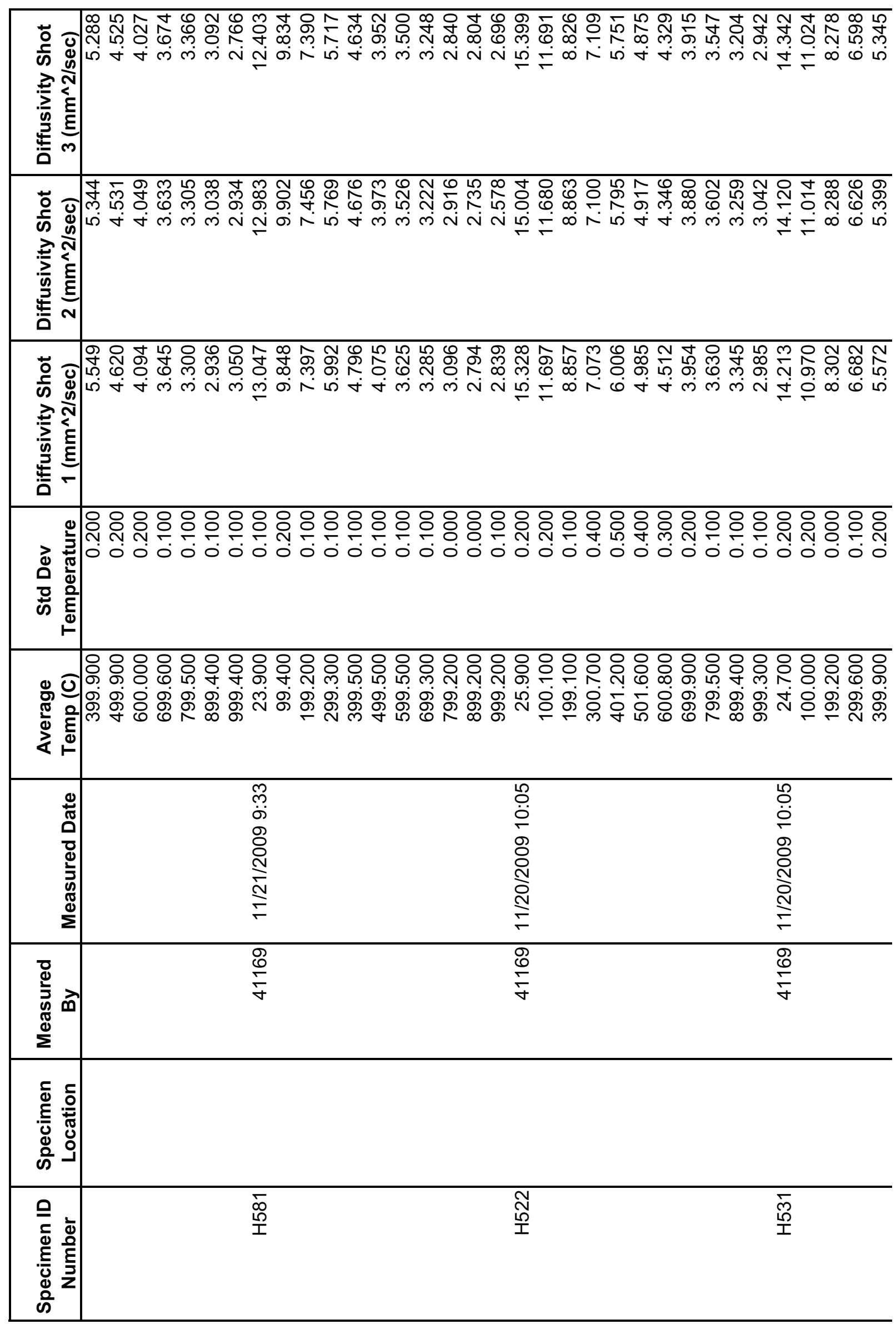




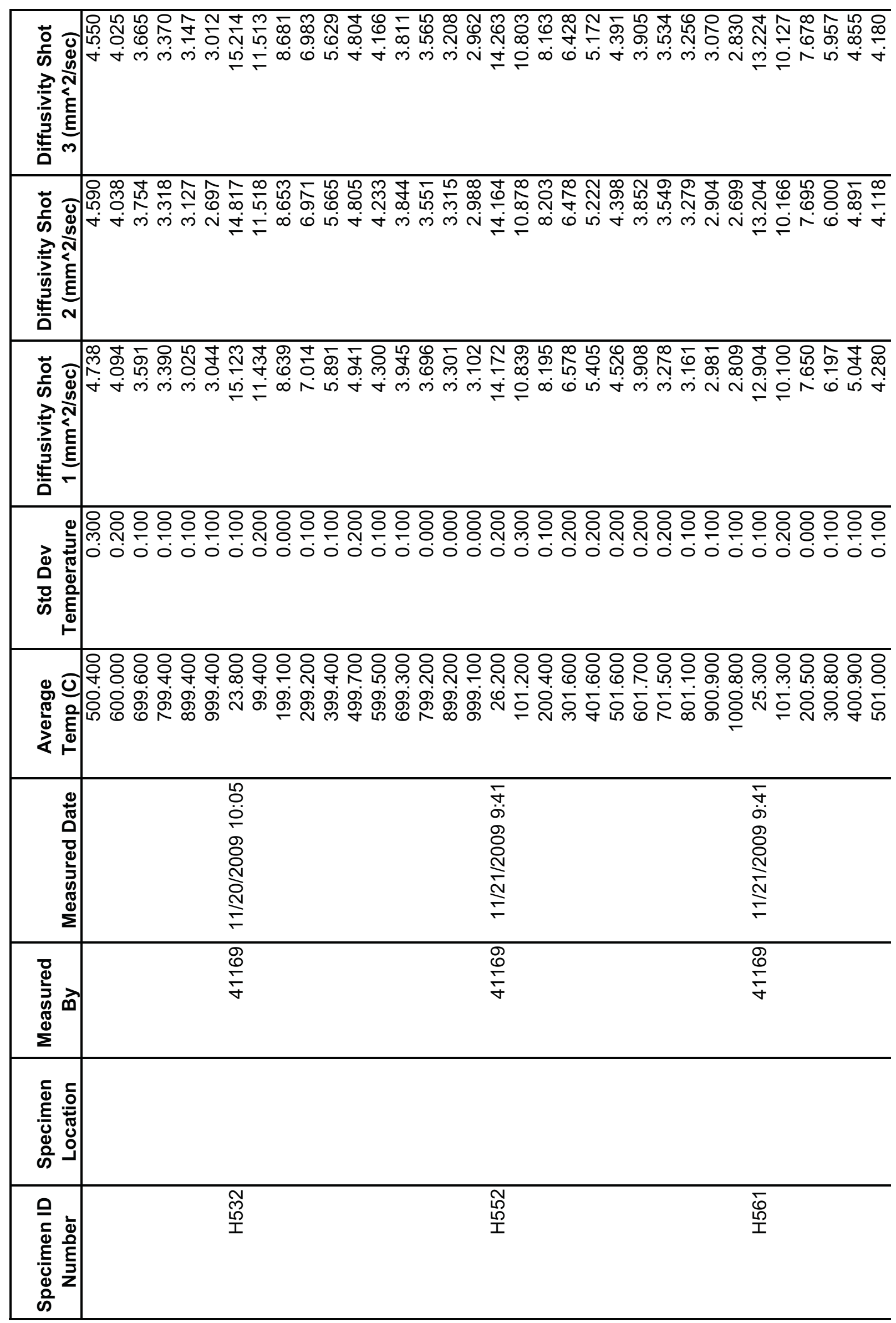




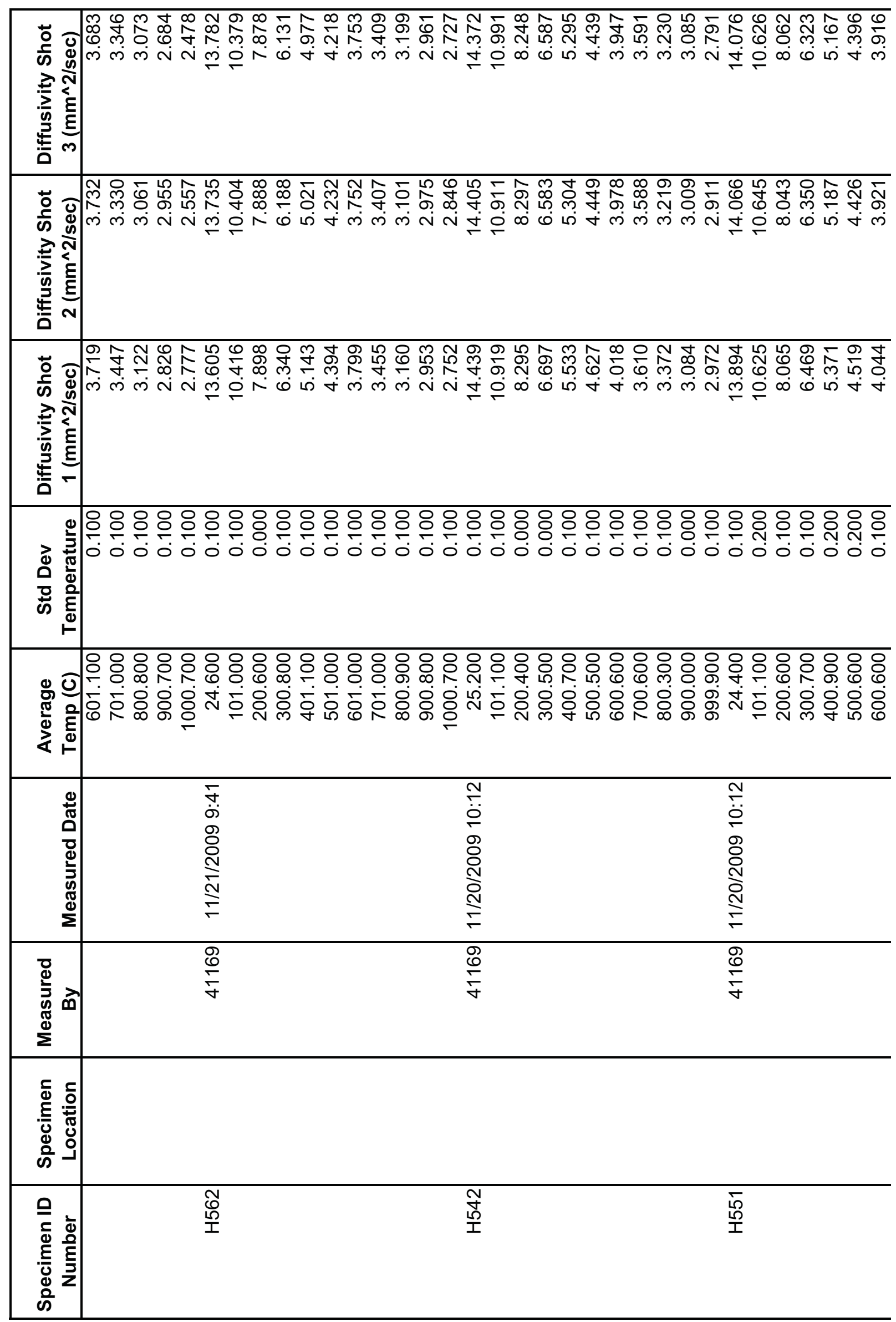




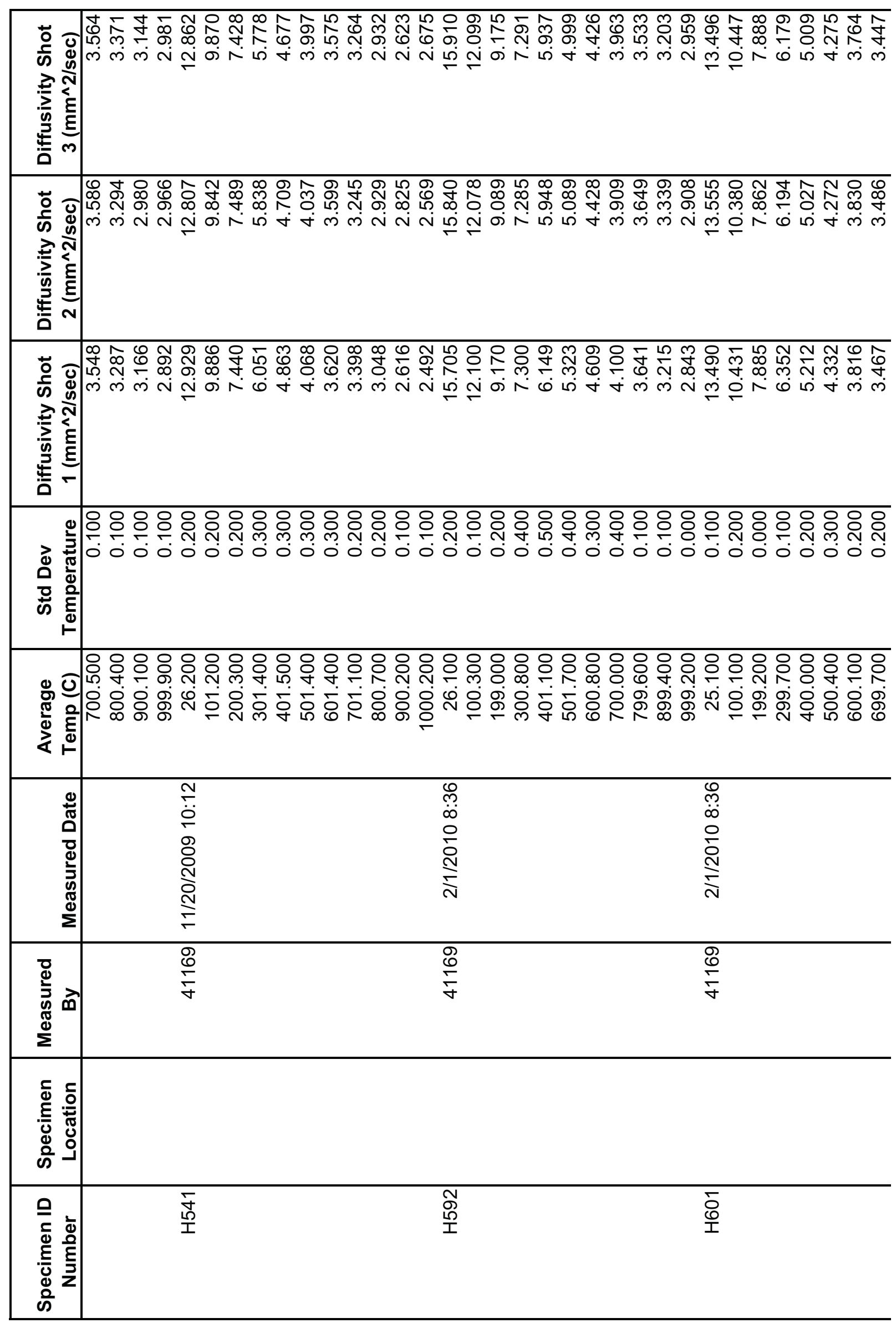




\begin{tabular}{|c|c|}
\hline 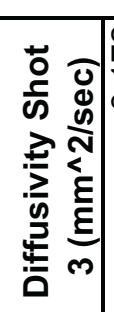 & 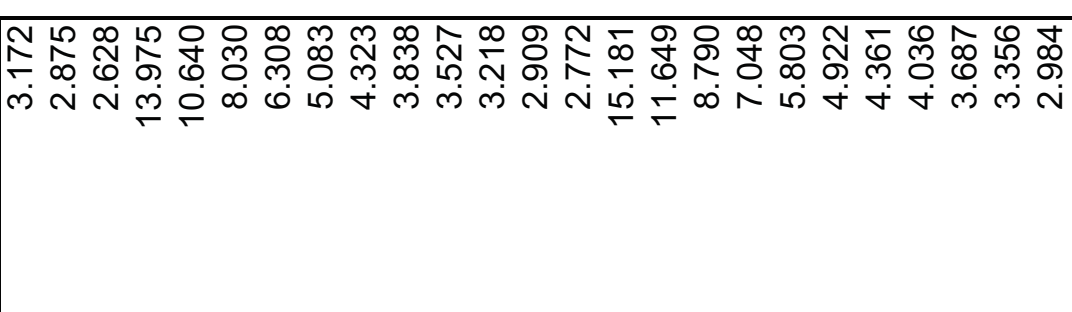 \\
\hline 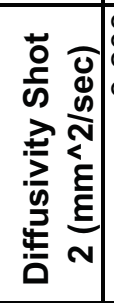 & 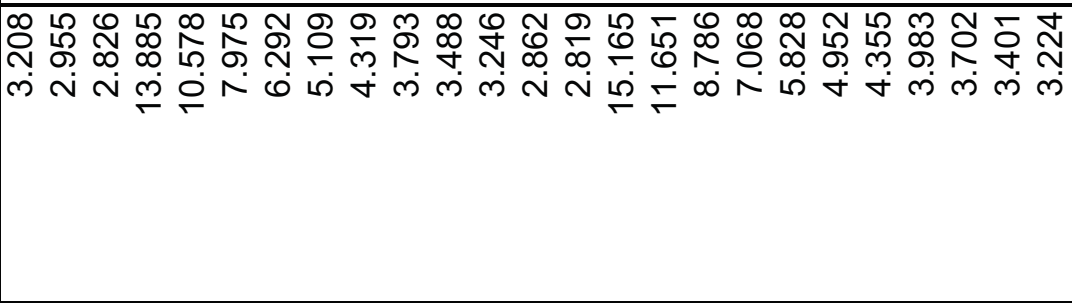 \\
\hline 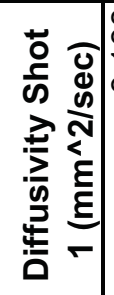 & 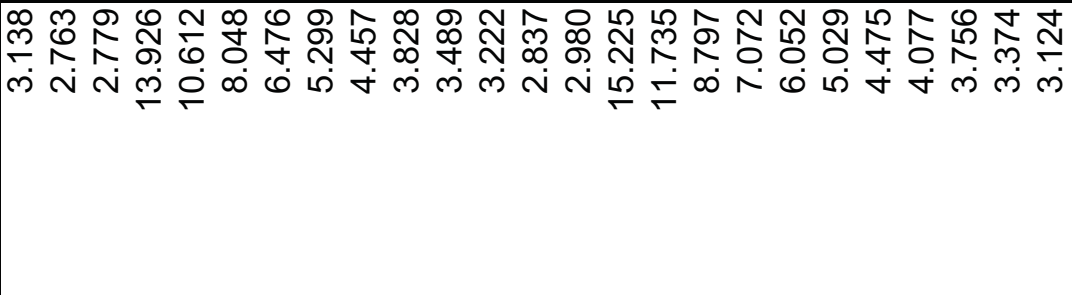 \\
\hline 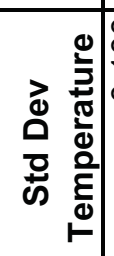 & 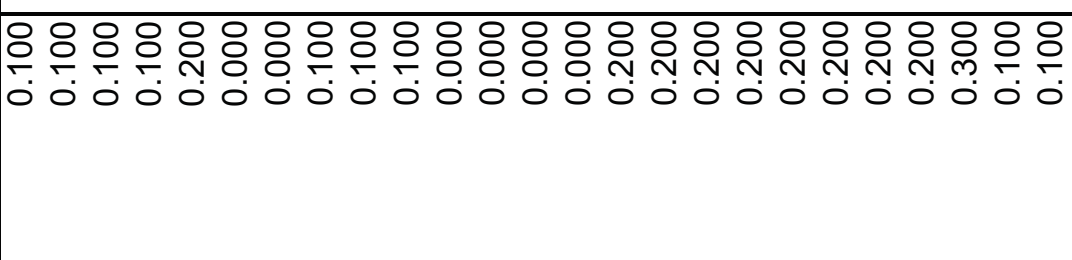 \\
\hline 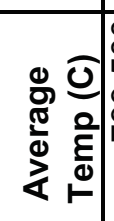 & 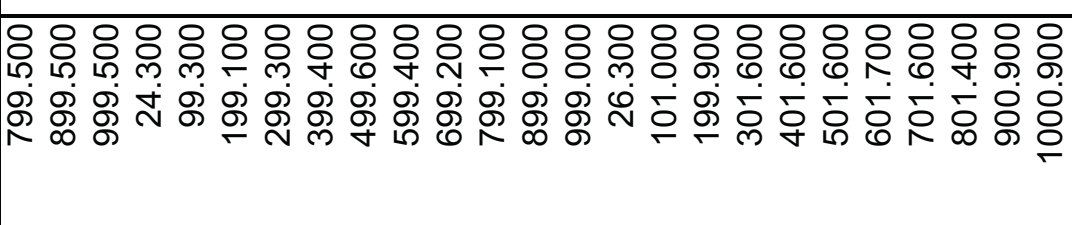 \\
\hline 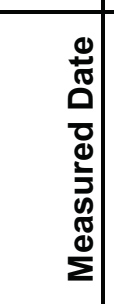 & 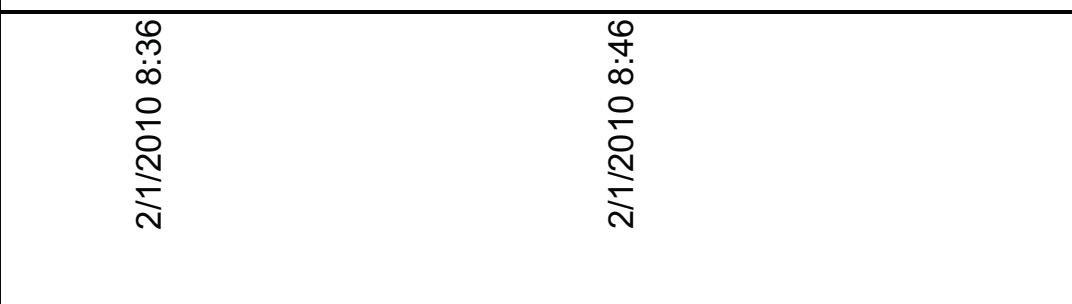 \\
\hline 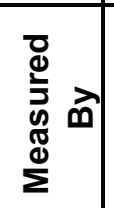 & 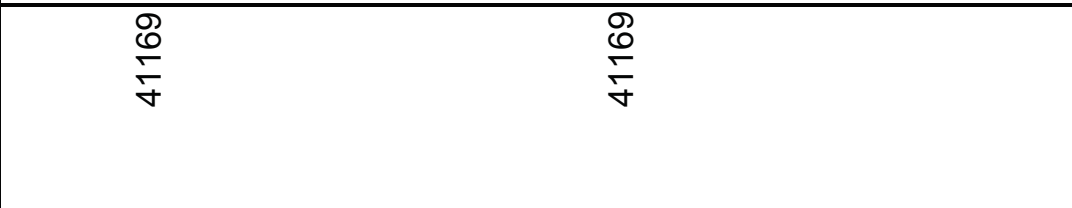 \\
\hline 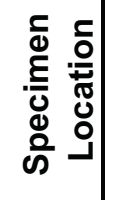 & \\
\hline 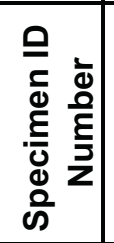 & 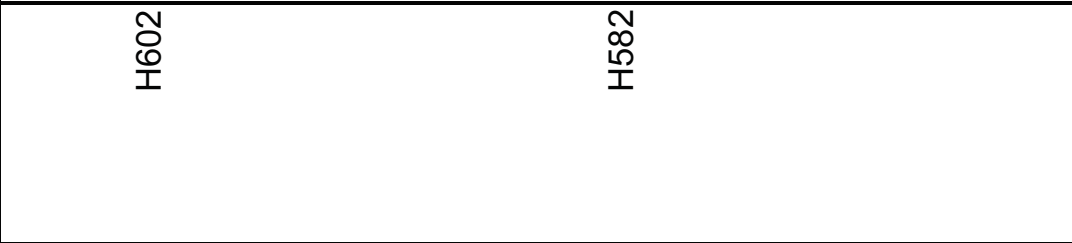 \\
\hline
\end{tabular}




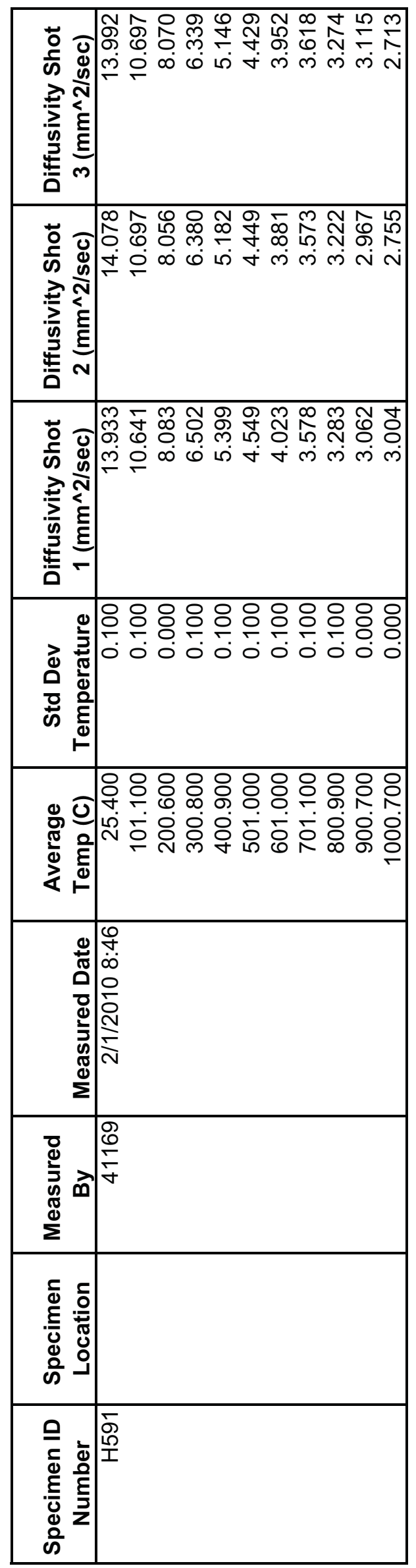




\begin{tabular}{|c|c|c|c|c|}
\hline 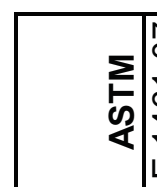 & 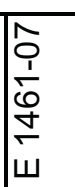 & 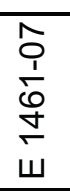 & $\begin{array}{l}\hat{o} \\
\frac{1}{o} \\
\stackrel{+}{+} \\
\dot{w}\end{array}$ & 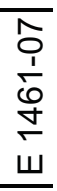 \\
\hline 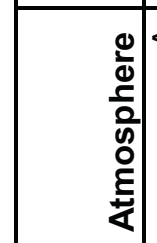 & & 交 & $\bar{z}$ & $\overline{\frac{i}{4}}$ \\
\hline 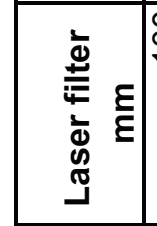 & & $\stackrel{\circ}{\circ}$ & 음 & 음 \\
\hline 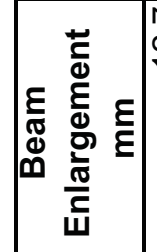 & & $\stackrel{\stackrel{N}{\sim}}{\sim}$ & $\stackrel{\stackrel{N}{\mathrm{~N}}}{ }$ & $\stackrel{\stackrel{N}{\mathfrak{N}}}{ }$ \\
\hline 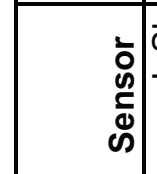 & 角 & की & 吕 & $\begin{array}{l}\text { की } \\
\underline{\underline{D}}\end{array}$ \\
\hline 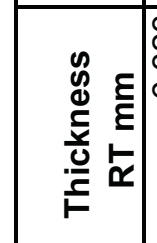 & & 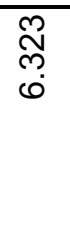 & 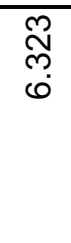 & 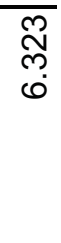 \\
\hline 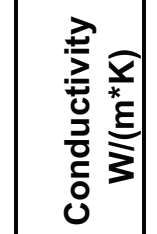 & & & & \\
\hline 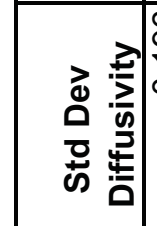 & 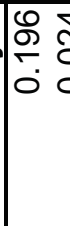 & 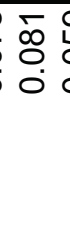 & $\begin{array}{l}\pi \\
\delta \\
\\
\end{array}$ & \\
\hline 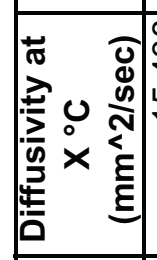 & 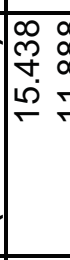 & $\begin{array}{l}\stackrel{\mathscr{N}}{\forall} \\
\stackrel{\sim}{\sim}\end{array}$ & 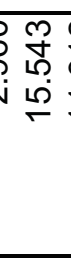 & 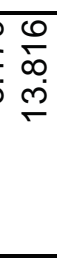 \\
\hline 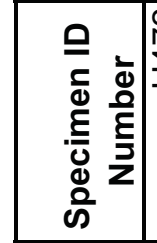 & & 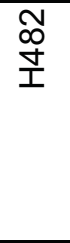 & $\begin{array}{l}\bar{o} \\
\stackrel{+}{I}\end{array}$ & $\begin{array}{l}\tilde{O} \\
\stackrel{0}{I}\end{array}$ \\
\hline
\end{tabular}




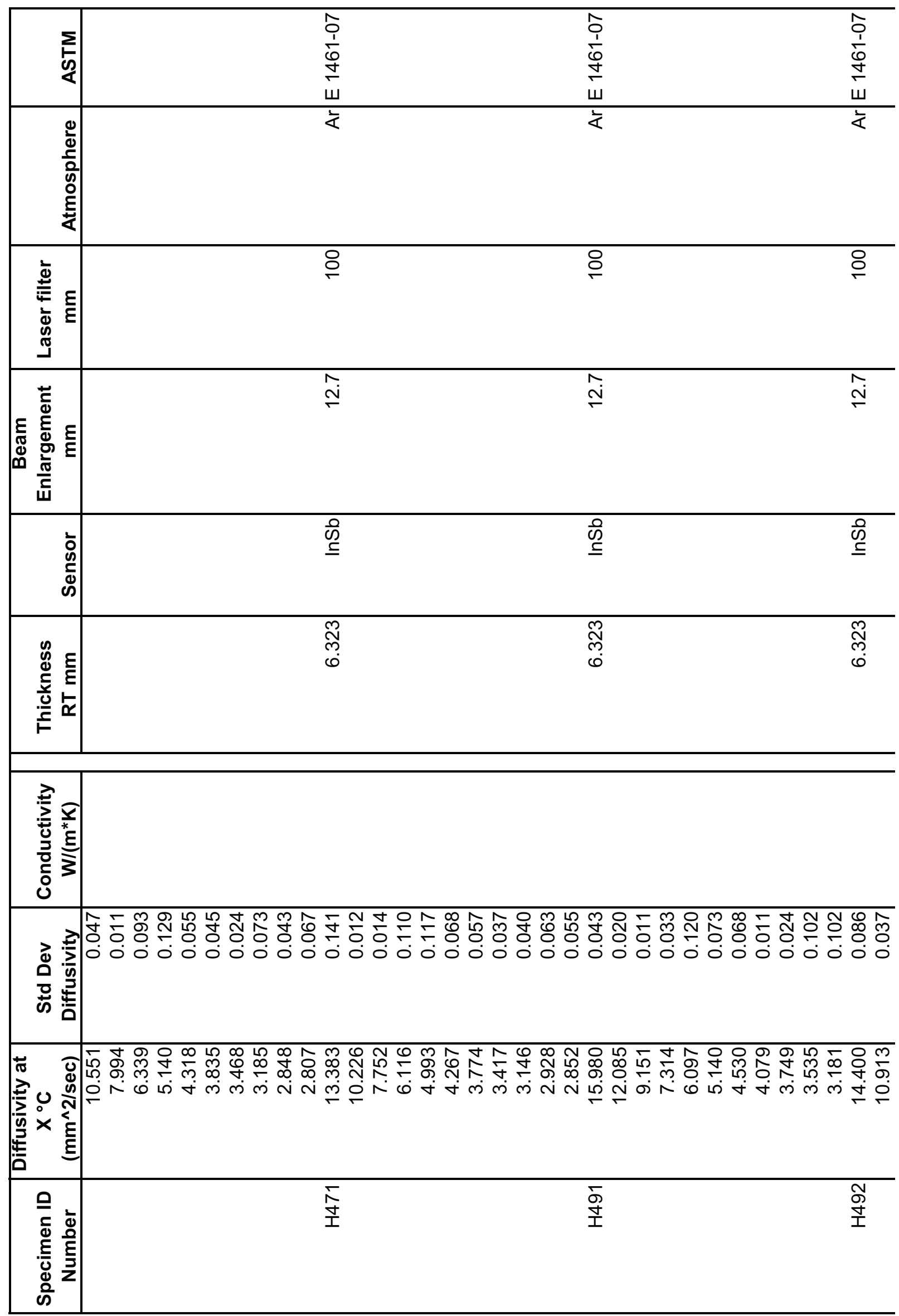




\begin{tabular}{|c|c|c|c|}
\hline 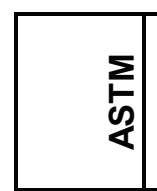 & $\begin{array}{l}\hat{o} \\
\frac{1}{0} \\
\frac{1}{+} \\
\dot{\omega}\end{array}$ & $\begin{array}{l}\hat{o} \\
\frac{1}{o} \\
\frac{\sigma}{\leftarrow} \\
\dot{W}\end{array}$ & $\begin{array}{l}\hat{o} \\
\frac{1}{o} \\
\frac{0}{+} \\
\dot{w}\end{array}$ \\
\hline 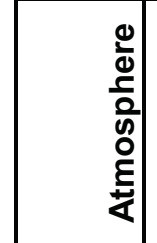 & 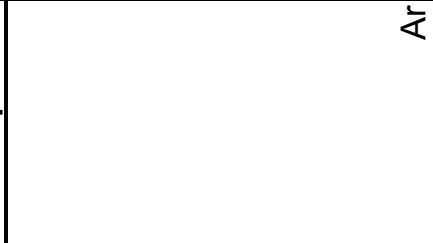 & 广 & ¿ \\
\hline 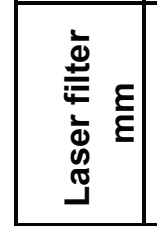 & 음 & 음 & 음 \\
\hline 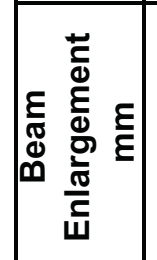 & $\stackrel{\widehat{N}}{\widehat{N}}$ & $\stackrel{\widetilde{N}}{\widetilde{N}}$ & $\stackrel{\widehat{N}}{\stackrel{N}{N}}$ \\
\hline 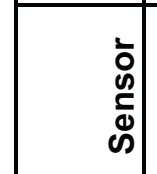 & $\begin{array}{l}\text { की } \\
\text { की }\end{array}$ & $\begin{array}{l}\text { 吕 } \\
\underline{\underline{n}}\end{array}$ & $\begin{array}{l}\text { की } \\
\text { 足 }\end{array}$ \\
\hline 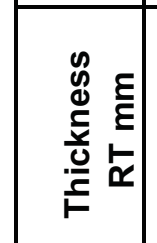 & 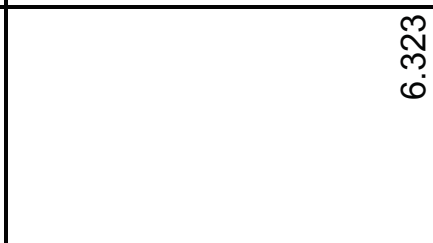 & 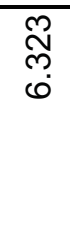 & $\begin{array}{c}\text { ల్లె } \\
0\end{array}$ \\
\hline 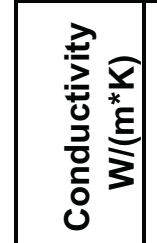 & & & \\
\hline 䠞 & 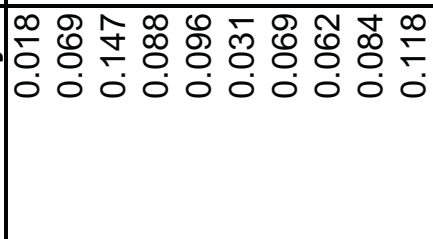 & $\begin{array}{l}\infty \\
\infty \\
\vdots \\
\end{array}$ & 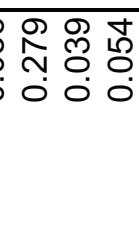 \\
\hline 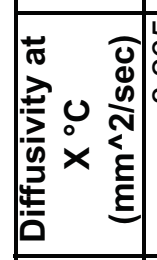 & 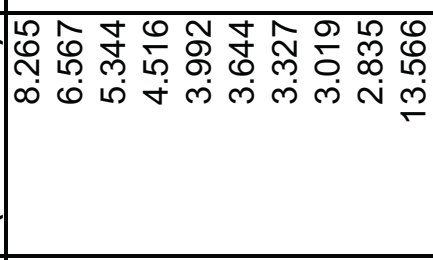 & 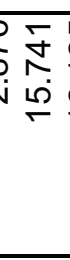 & 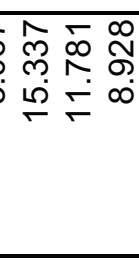 \\
\hline 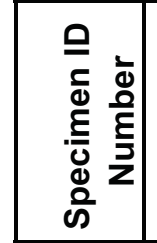 & $\overline{\bar{\delta}}$ & $\begin{array}{l}\text { 令 } \\
\text { 胥 }\end{array}$ & $\frac{N}{\frac{N}{1}}$ \\
\hline
\end{tabular}




\begin{tabular}{|c|c|c|c|}
\hline 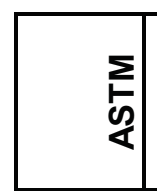 & 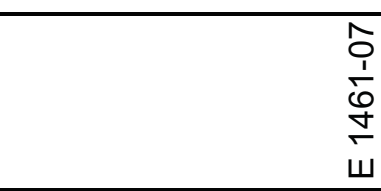 & 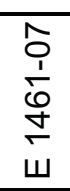 & $\begin{array}{l}\hat{o} \\
\frac{1}{0} \\
\frac{0}{+} \\
\dot{\omega}\end{array}$ \\
\hline 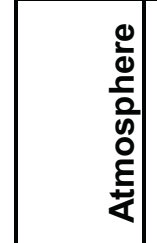 & 这 & 文 & ¿ \\
\hline 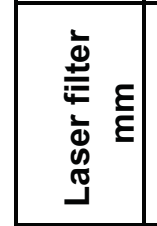 & 음 & 음 & 음 \\
\hline 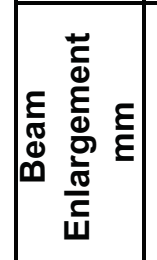 & $\stackrel{\widehat{N}}{\widehat{N}}$ & $\stackrel{\widetilde{N}}{\widehat{N}}$ & $\stackrel{\widehat{N}}{\stackrel{N}{N}}$ \\
\hline 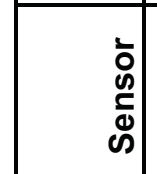 & $\begin{array}{l}\text { की } \\
\text { की }\end{array}$ & $\begin{array}{l}\text { 吊 } \\
\underline{\underline{n}}\end{array}$ & $\begin{array}{l}\text { की } \\
\text { 足 }\end{array}$ \\
\hline 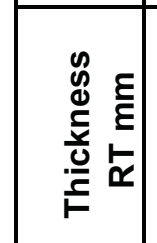 & $\underset{\widetilde{N}}{\stackrel{\widetilde{N}}{6}}$ & $\begin{array}{c}\widetilde{N} \\
0 \\
0\end{array}$ & $\begin{array}{l}\stackrel{\widetilde{N}}{0} \\
0\end{array}$ \\
\hline 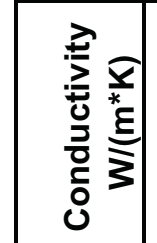 & & & \\
\hline 䠞 & 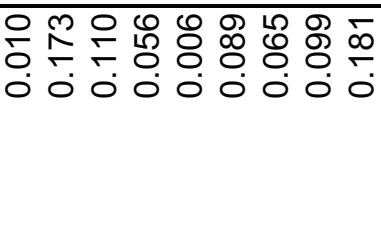 & $\begin{array}{l}x \\
\vdots \\
\vdots \\
0 \\
0\end{array}$ & 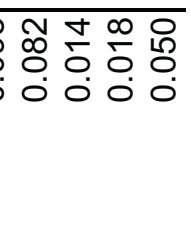 \\
\hline 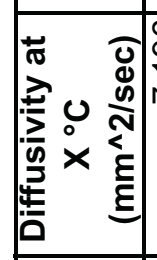 & 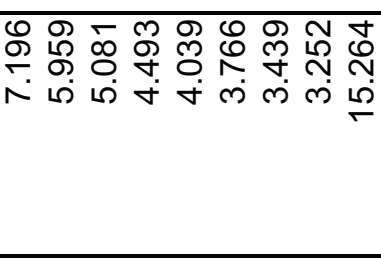 & 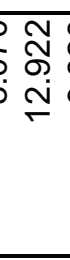 & 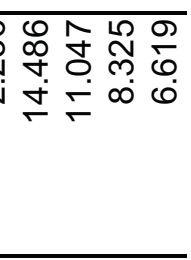 \\
\hline 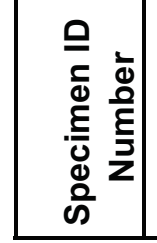 & $\begin{array}{l}\bar{N} \\
\text { W్ }\end{array}$ & $\underset{\hat{\delta}}{\bar{\phi}}$ & 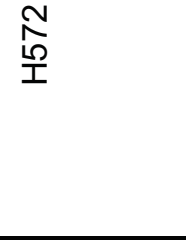 \\
\hline
\end{tabular}




\begin{tabular}{|c|c|c|c|}
\hline 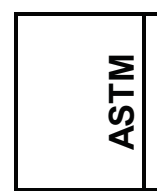 & $\begin{array}{l}\frac{0}{1} \\
\frac{1}{0} \\
\frac{1}{+} \\
\dot{w}\end{array}$ & 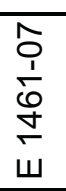 & 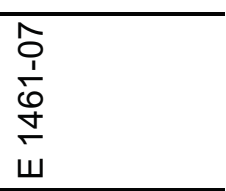 \\
\hline 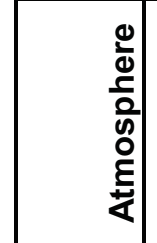 & 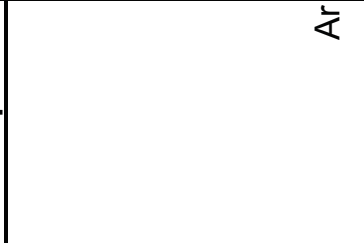 & ¿ & ¿ \\
\hline 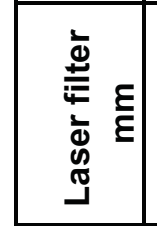 & $\stackrel{\square}{\circ}$ & 음 & 음 \\
\hline 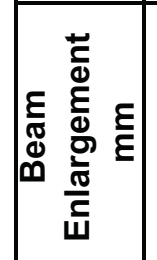 & 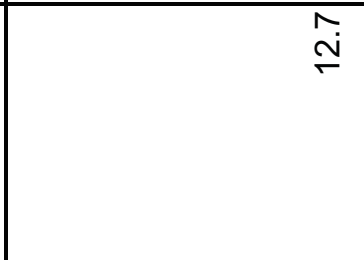 & $\stackrel{\widehat{N}}{\widehat{N}}$ & $\stackrel{\widehat{N}}{\stackrel{N}{N}}$ \\
\hline 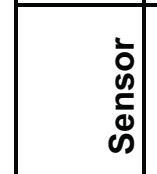 & $\begin{array}{l}\text { की } \\
\text { की }\end{array}$ & $\begin{array}{l}\text { की } \\
\text { की }\end{array}$ & $\begin{array}{l}\text { की } \\
\text { की }\end{array}$ \\
\hline 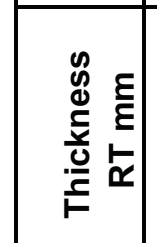 & 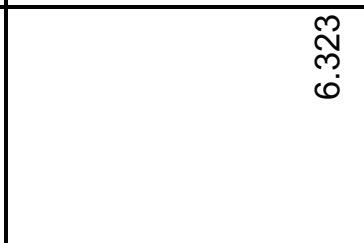 & $\begin{array}{l}\widetilde{N} \\
\text { లై } \\
0\end{array}$ & 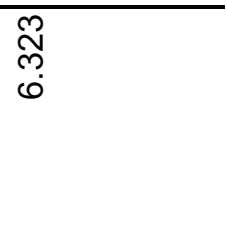 \\
\hline 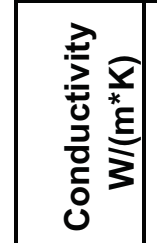 & & & \\
\hline 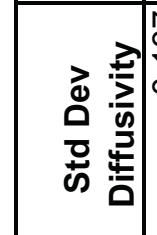 & 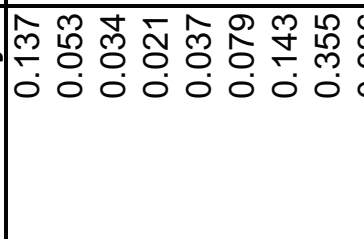 & 0 & 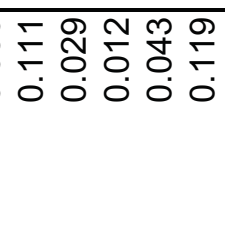 \\
\hline 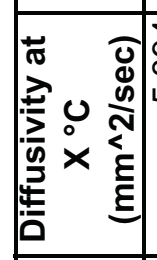 & 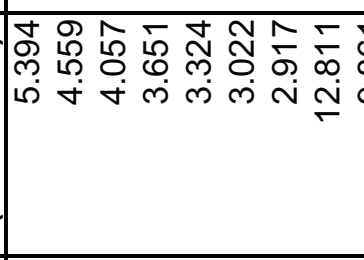 & 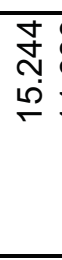 & 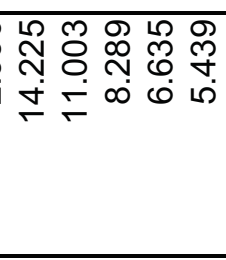 \\
\hline 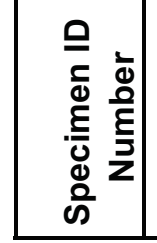 & $\begin{array}{l}\bar{\delta} \\
\stackrel{\rho}{I} \\
\stackrel{p}{I}\end{array}$ & 옴 & $\begin{array}{l}\overline{\tilde{M}} \\
\text { 足 }\end{array}$ \\
\hline
\end{tabular}




\begin{tabular}{|c|c|c|c|}
\hline 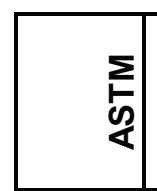 & $\begin{array}{l}\frac{\hat{o}}{1} \\
\frac{1}{0} \\
\frac{\sigma}{+} \\
\dot{\omega}\end{array}$ & $\begin{array}{l}\hat{o} \\
\frac{1}{o} \\
\frac{\sigma}{\leftarrow} \\
\dot{w}\end{array}$ & $\begin{array}{l}\hat{o} \\
\frac{1}{o} \\
\frac{0}{+} \\
\dot{w}\end{array}$ \\
\hline 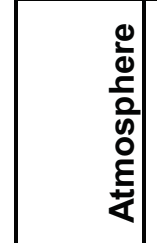 & ¿ & « & ¿ \\
\hline 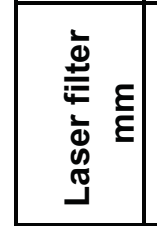 & 음 & 음 & 음 \\
\hline 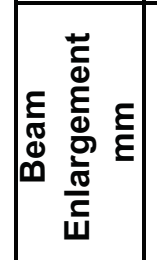 & $\stackrel{\widehat{N}}{\widehat{N}}$ & 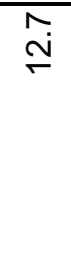 & $\stackrel{\widehat{N}}{\stackrel{N}{N}}$ \\
\hline 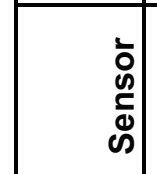 & $\begin{array}{l}\text { की } \\
\text { की }\end{array}$ & $\begin{array}{l}\text { की } \\
\text { Фी }\end{array}$ & $\begin{array}{l}\text { की } \\
\text { 足 }\end{array}$ \\
\hline 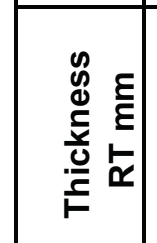 & $\underset{\widetilde{N}}{\stackrel{\widetilde{N}}{6}}$ & 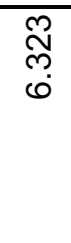 & $\begin{array}{l}\underset{N}{\tilde{N}} \\
0\end{array}$ \\
\hline 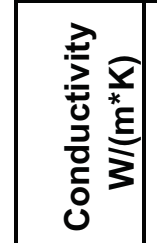 & & & \\
\hline 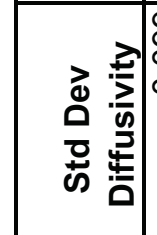 & 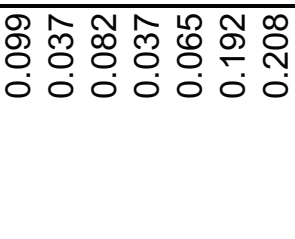 & 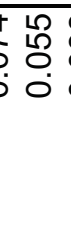 & 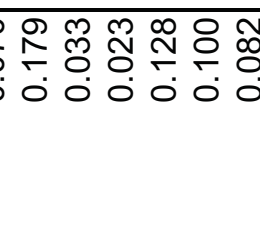 \\
\hline 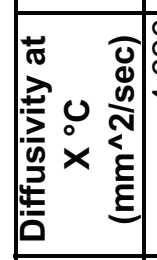 & 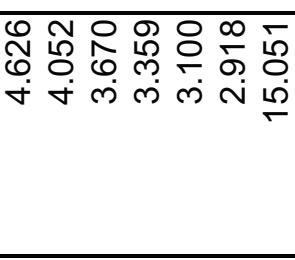 & : & 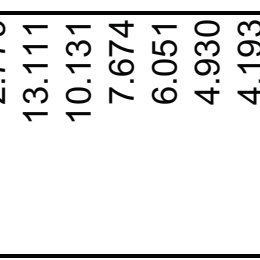 \\
\hline 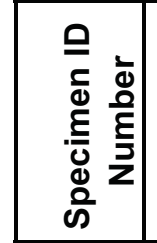 & 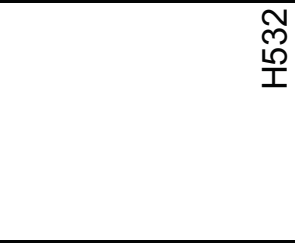 & $\begin{array}{l}\text { N } \\
\text { 兽 }\end{array}$ & $\begin{array}{l}\bar{\delta} \\
\text { 足 }\end{array}$ \\
\hline
\end{tabular}




\begin{tabular}{|c|c|c|c|}
\hline 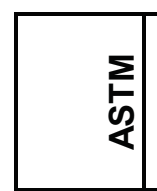 & $\begin{array}{l}\frac{0}{1} \\
\frac{1}{0} \\
\frac{1}{+} \\
\dot{w}\end{array}$ & 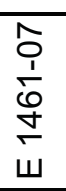 & $\begin{array}{l}\hat{o} \\
\frac{1}{o} \\
\frac{1}{+} \\
\dot{w}\end{array}$ \\
\hline 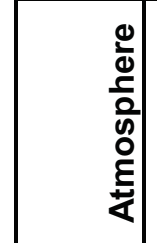 & 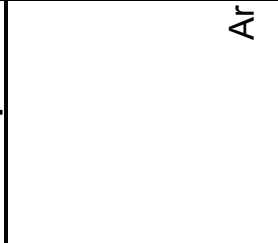 & ¿ & ¿ \\
\hline 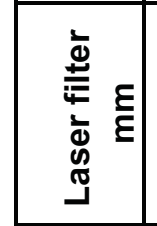 & $\stackrel{\circ}{\circ}$ & 음 & $\stackrel{\circ}{\circ}$ \\
\hline 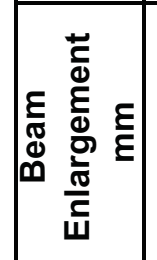 & $\widehat{\widehat{N}}$ & $\widehat{\stackrel{N}{\mathrm{~N}}}$ & $\stackrel{\widetilde{\mathfrak{N}}}{\widehat{N}}$ \\
\hline 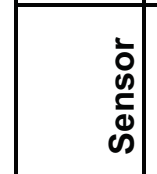 & $\begin{array}{l}\text { की } \\
\text { की }\end{array}$ & $\begin{array}{l}\text { की } \\
\text { की }\end{array}$ & 吕 \\
\hline 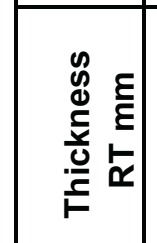 & $\begin{array}{l}\widetilde{N} \\
\widetilde{N} \\
0\end{array}$ & 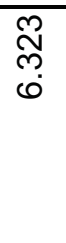 & $\begin{array}{l}\stackrel{N}{N} \\
0 \\
0\end{array}$ \\
\hline 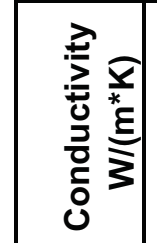 & & & \\
\hline 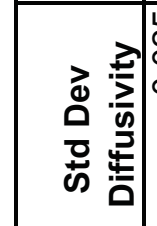 & 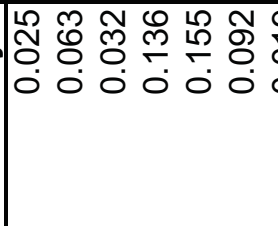 & 0 & 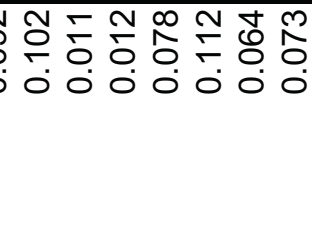 \\
\hline 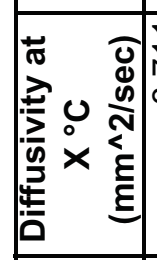 & 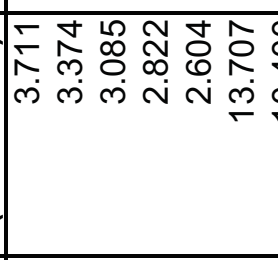 & f & 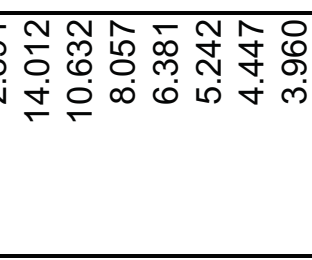 \\
\hline 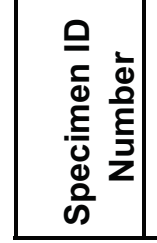 & $\begin{array}{l}\mathbb{N} \\
\text { 足 }\end{array}$ & 莡 & $\begin{array}{l}\overline{5} \\
\text { 足 }\end{array}$ \\
\hline
\end{tabular}




\begin{tabular}{|c|c|c|c|}
\hline 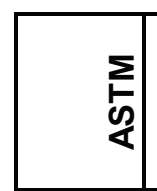 & $\begin{array}{l}\hat{o} \\
\frac{1}{0} \\
\dot{0} \\
\dot{+} \\
\end{array}$ & 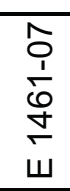 & $\begin{array}{l}\frac{\hat{1}}{1} \\
\frac{1}{6} \\
\frac{g}{\leftarrow} \\
\dot{w}\end{array}$ \\
\hline 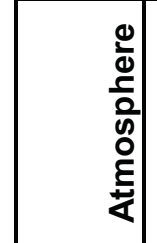 & 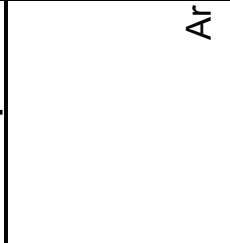 & ¿ & ¿ \\
\hline 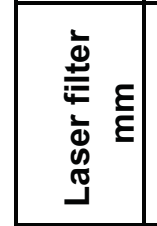 & $\stackrel{8}{\circ}$ & $\stackrel{2}{\circ}$ & 음 \\
\hline 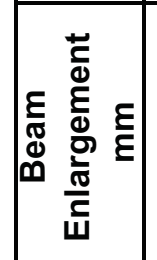 & 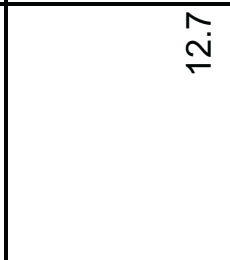 & 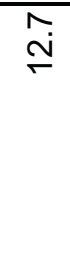 & $\stackrel{\widehat{N}}{\hat{N}}$ \\
\hline 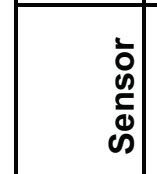 & $\begin{array}{l}\text { की } \\
\text { की }\end{array}$ & $\begin{array}{l}\text { 足 } \\
\text { क्ञ }\end{array}$ & की \\
\hline 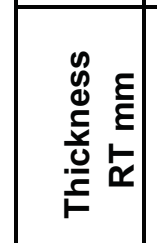 & 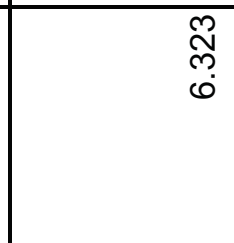 & 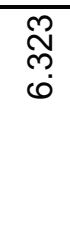 & 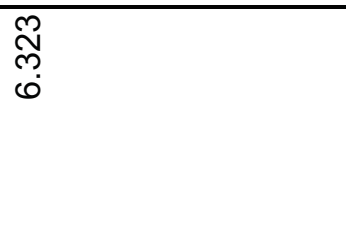 \\
\hline 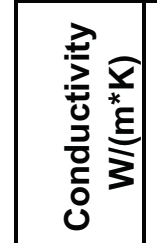 & & & \\
\hline 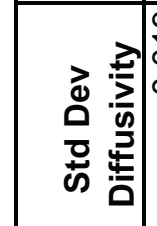 & 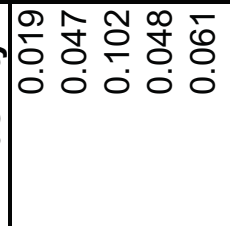 & $\begin{array}{l}\frac{1}{0} \\
\frac{0}{0}\end{array}$ & $\begin{array}{l}0 \\
0 \\
0 \\
0 \\
0\end{array}$ \\
\hline 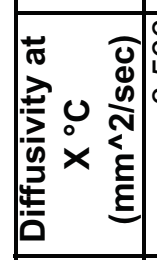 & 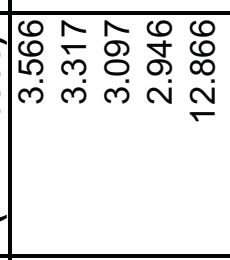 & 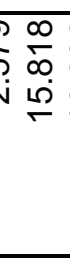 & 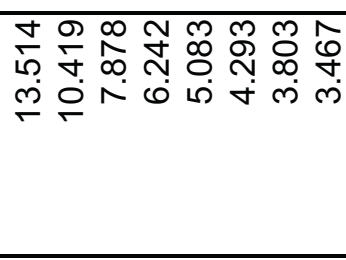 \\
\hline 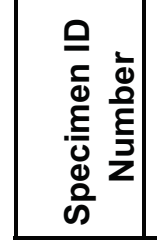 & $\begin{array}{l}\text { 它 } \\
\text { to }\end{array}$ & $\begin{array}{l}\text { 令 } \\
\text { 茥 }\end{array}$ & $\begin{array}{l}\bar{\phi} \\
\text { ष }\end{array}$ \\
\hline
\end{tabular}




\begin{tabular}{|c|c|c|}
\hline 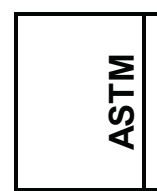 & 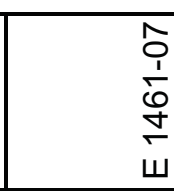 & 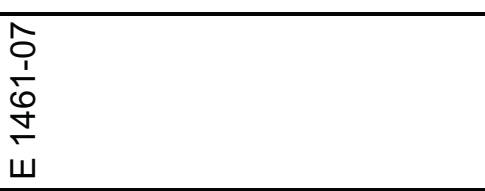 \\
\hline 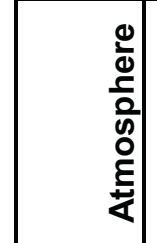 & 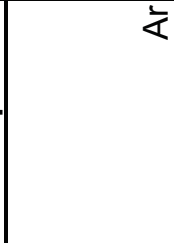 & $\frac{1}{4}$ \\
\hline 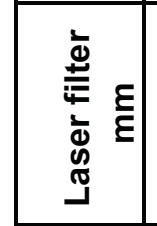 & 음 & $\stackrel{8}{\circ}$ \\
\hline 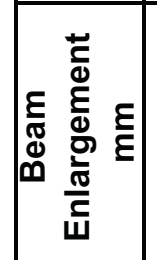 & $\stackrel{\widehat{N}}{\mathrm{~N}}$ & $\widehat{\stackrel{N}{N}}$ \\
\hline $\begin{array}{l}\text { bे } \\
\dot{0} \\
\bar{\Phi} \\
\text { ஸे }\end{array}$ & $\begin{array}{l}\text { की } \\
\text { की }\end{array}$ & $\begin{array}{l}\text { 吕 } \\
\text { की }\end{array}$ \\
\hline 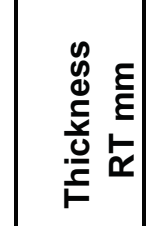 & 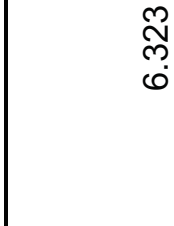 & $\begin{array}{l}\underset{N}{\tilde{N}} \\
0 \\
0\end{array}$ \\
\hline 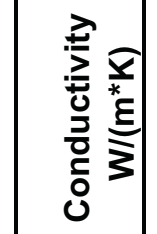 & & \\
\hline के & $\mid \begin{array}{llll}0 & 0 \\
0 & 0 & 0 & 0 \\
0 & 0 & 0 & 0 \\
0 & 0 & 0 & 0 \\
0 & 0 & 0 & 0 \\
0\end{array}$ & 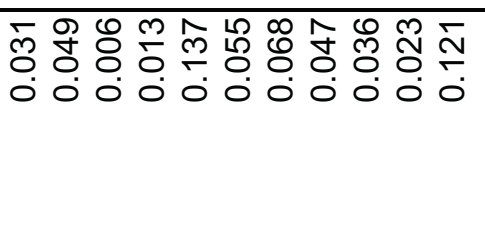 \\
\hline 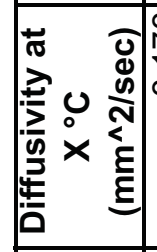 & 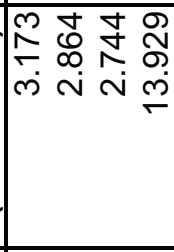 & 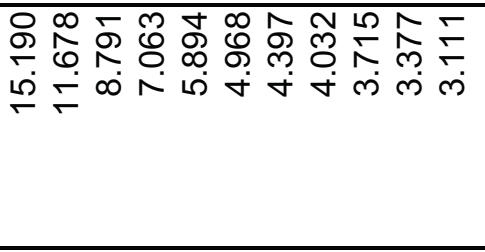 \\
\hline 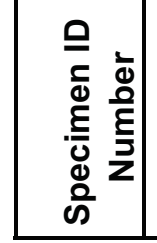 & $\begin{array}{l}\mathcal{N} \\
\text { ㅇ }\end{array}$ & 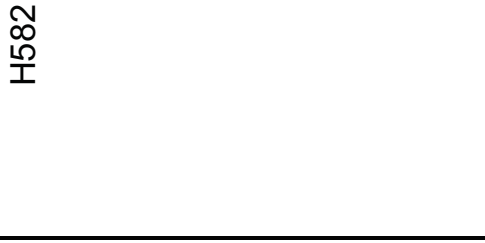 \\
\hline
\end{tabular}




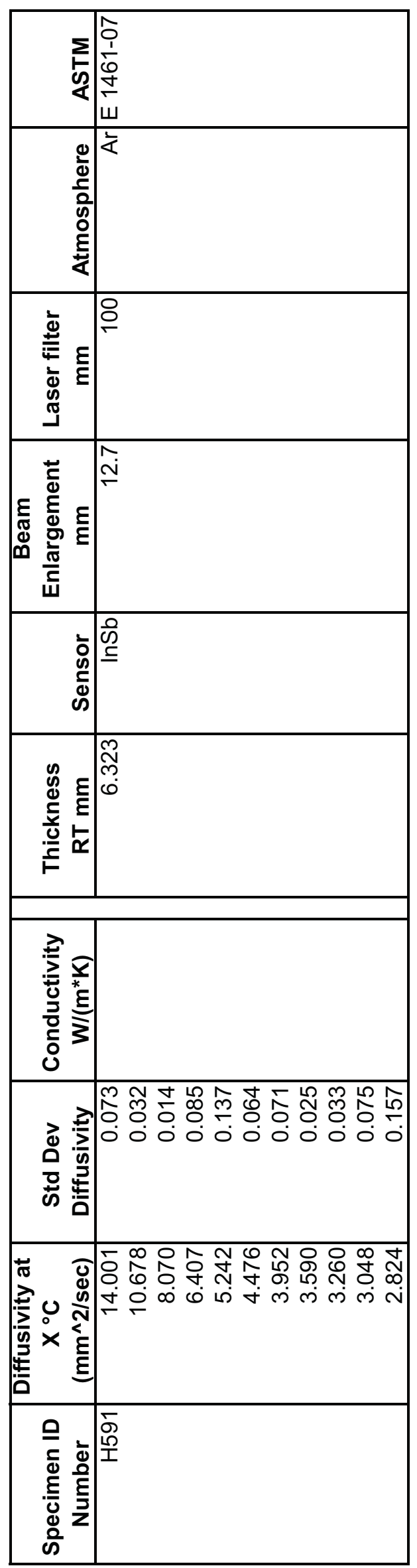




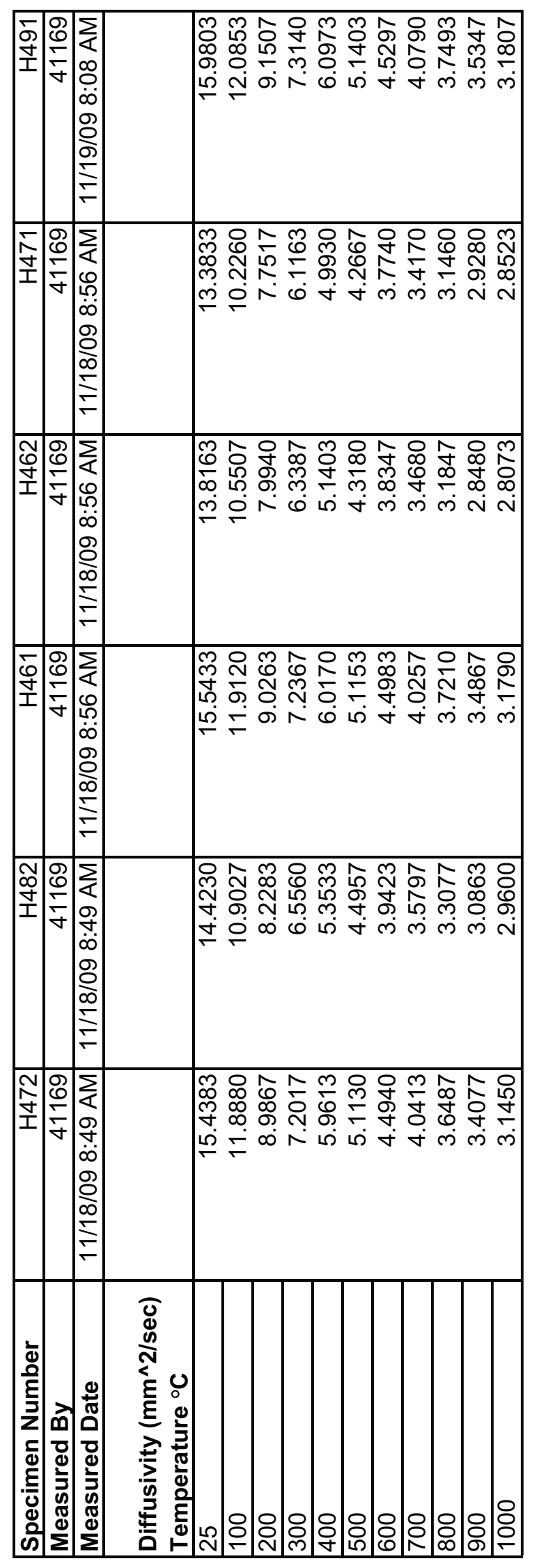




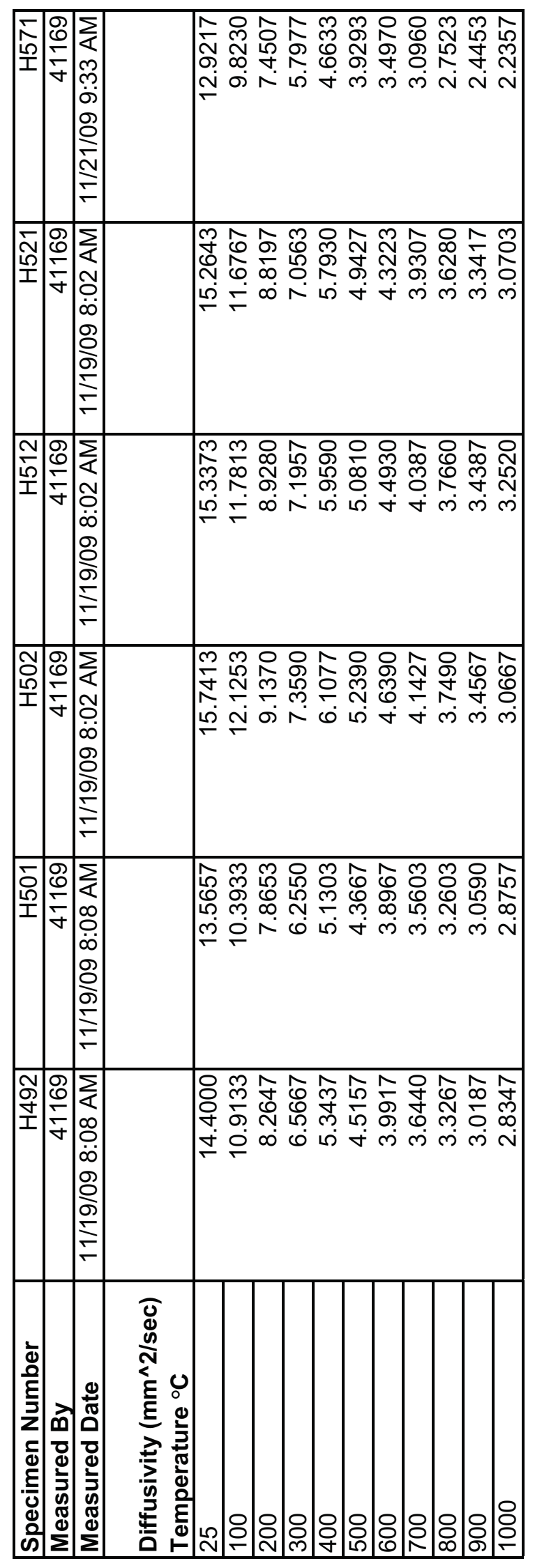




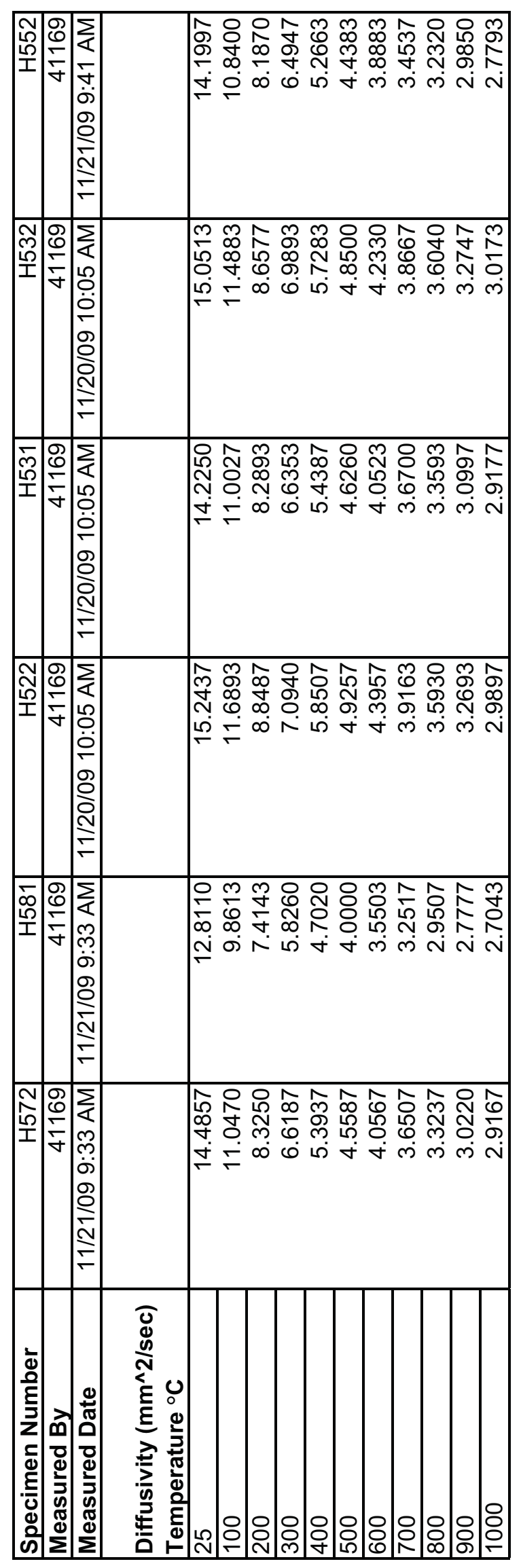




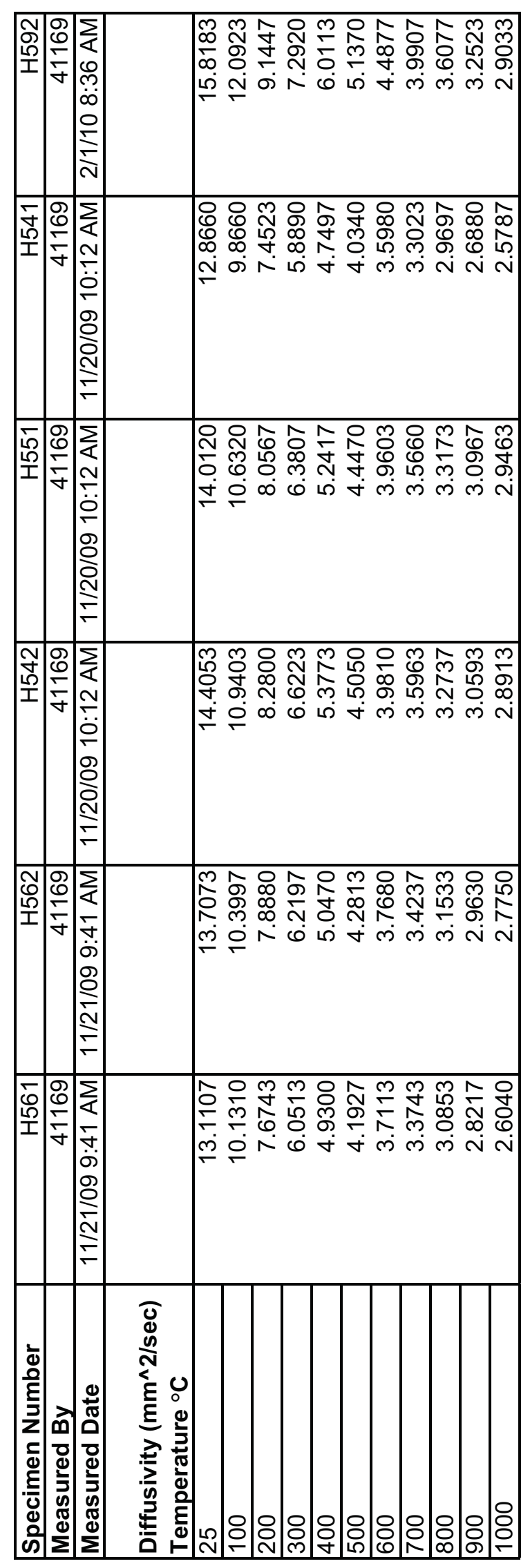




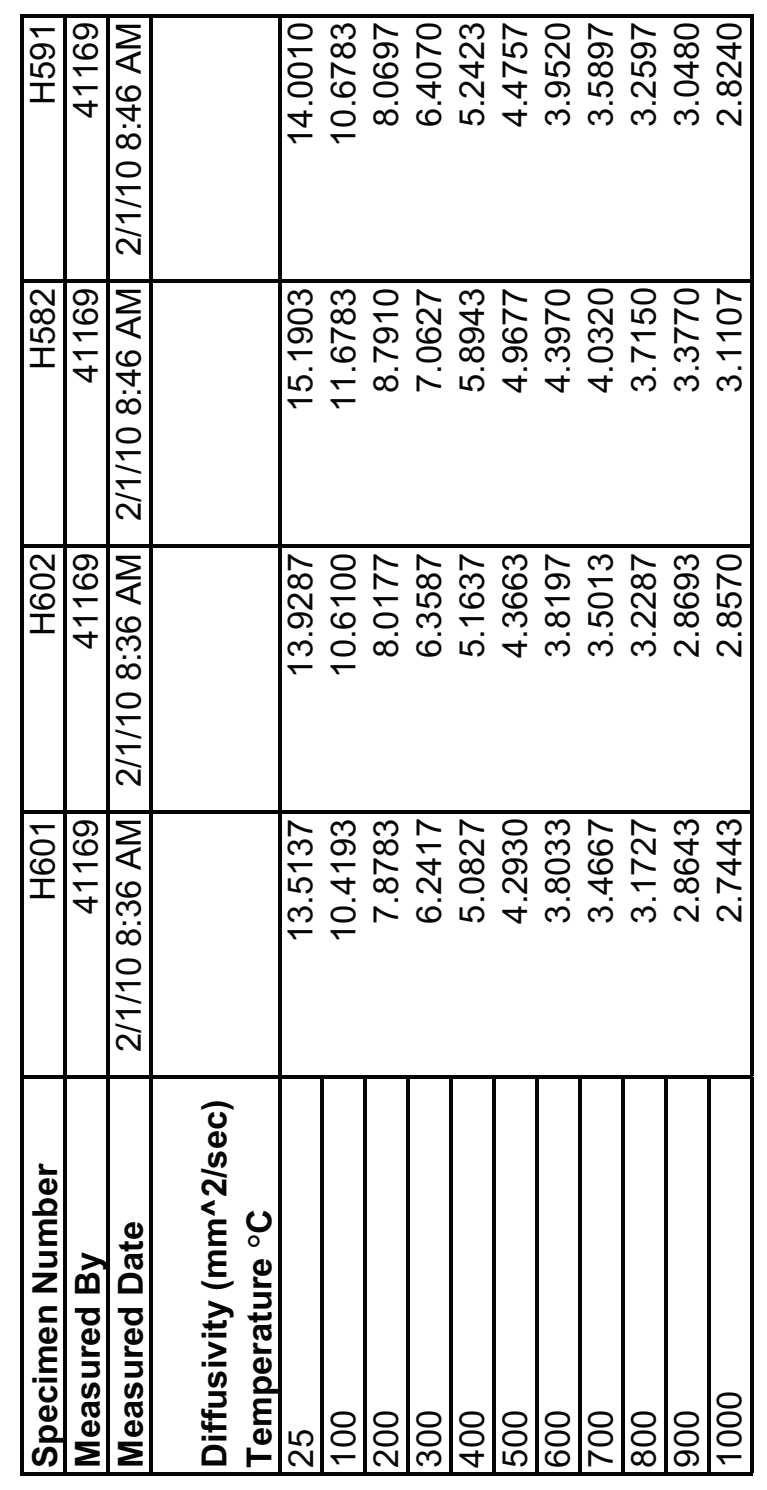




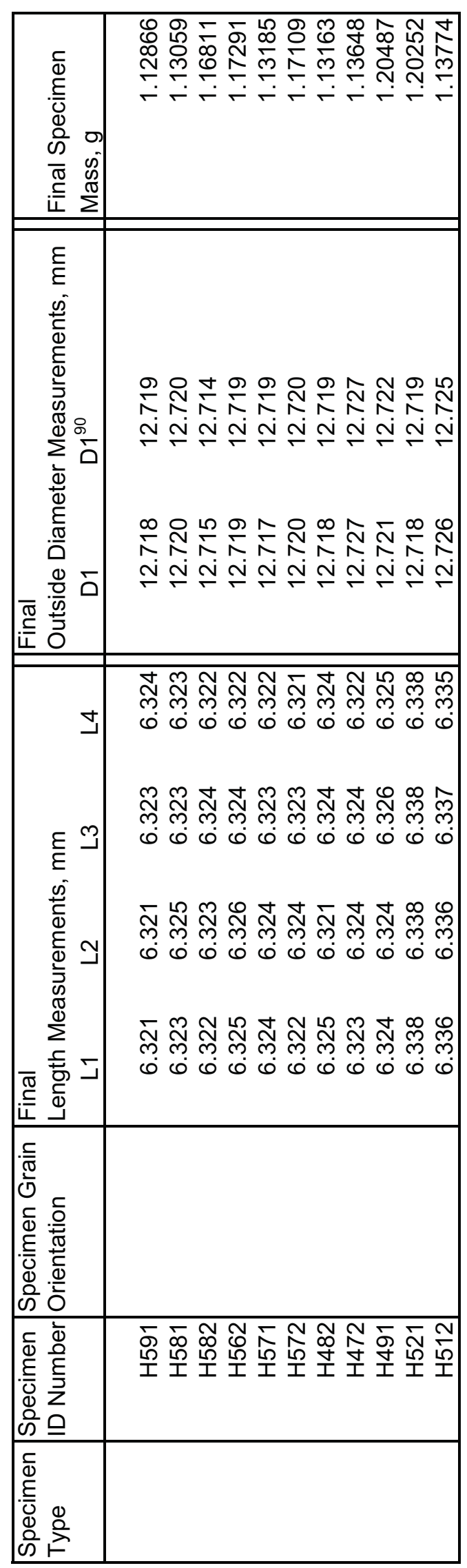




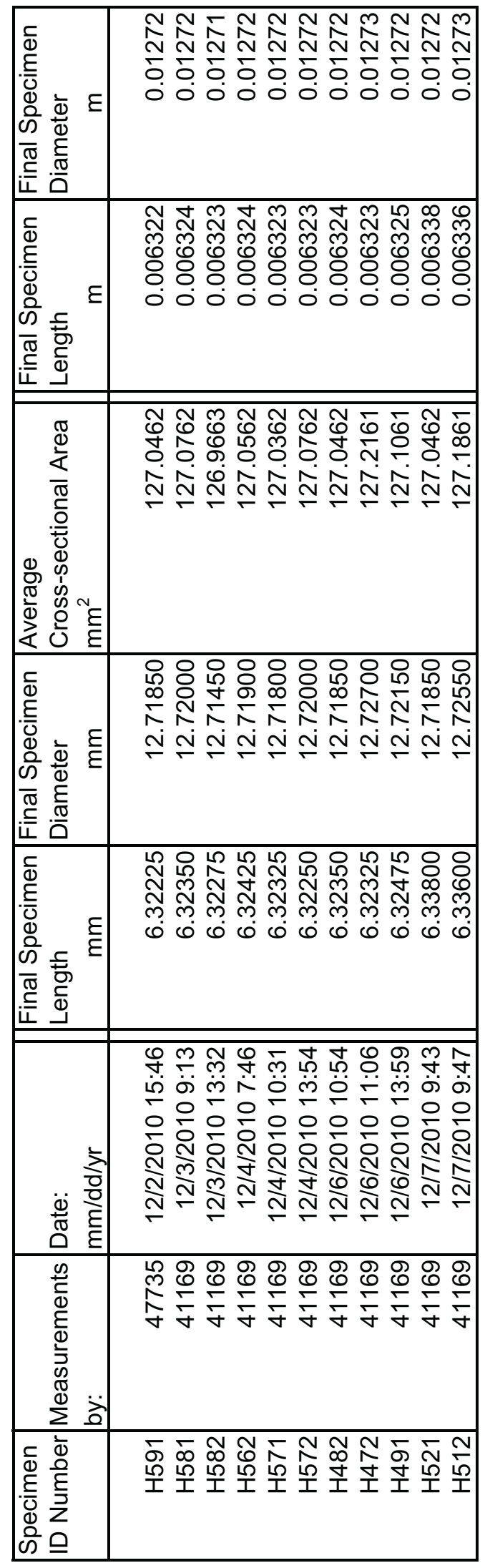




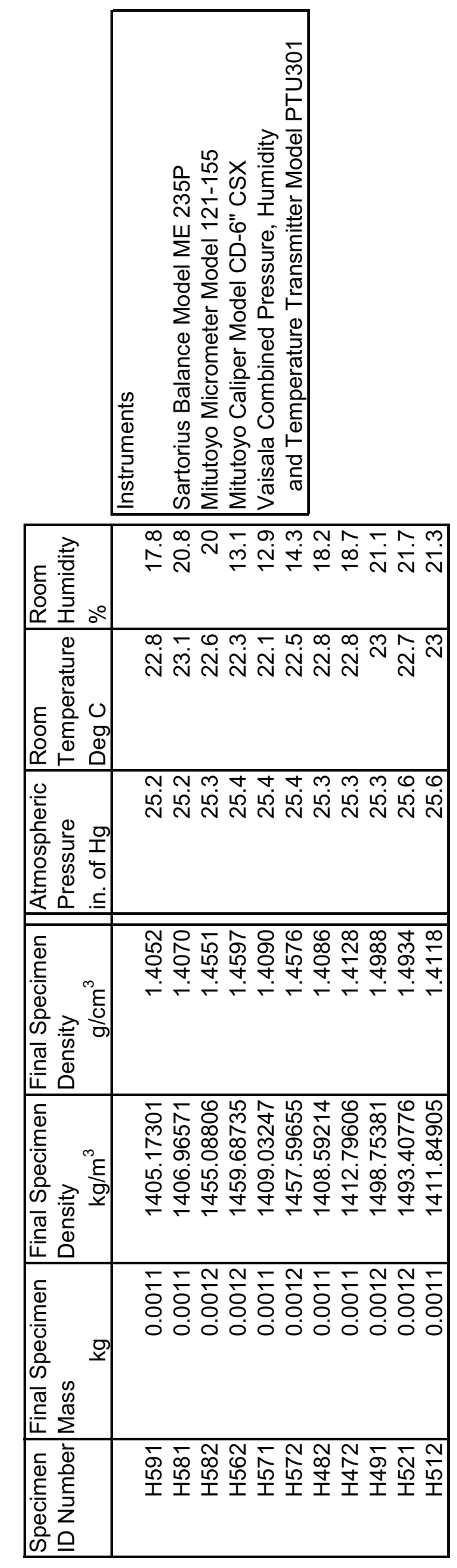




$\begin{array}{ll}\text { Graphite Grade: } & \text { BAN } \\ \text { Graphite Manufacturer: } & \text { Graftech International } \\ \text { Forming Process: } & \text { Extruded } \\ \text { Coke Particle Size: } & \text { Medium grain } \\ \text { Coke Type: } & \text { Petroleum (needle) coke } \\ \text { ASTM Class: } & \text { ENHP } \\ \text { Specimen Geometry: } & \text { Cylinder }\end{array}$

Specimen ID \#'s:

RW1 01

RW1 02

RW1 03

RW1 04

RW1 05

RW1 06

RW1 07

RW1 08

RW1 09

RW1 10

RW2 01

RW2 02

RW2 03

RW2 04

RW2 05

RW2 06

RW2 07

RW2 08

RW2 09

RW2 10

RW4 01

RW4 02

RW4 03

RW4 04

RW4 05

RW4 06

RW4 07

RW4 08

RW4 09

RW4 10

RW5 01

RW5 02

RW5 03

RW5 04

RW5 05

RW5 06

RW5 07

RW5 08

RW5 09

RW5 10

RW6 01

RW6 02 
Specimen ID \#'s:

RW6 03

RW6 04

RW6 05

RW6 06

RW6 07

RW6 08

RW6 09

RW6 10 


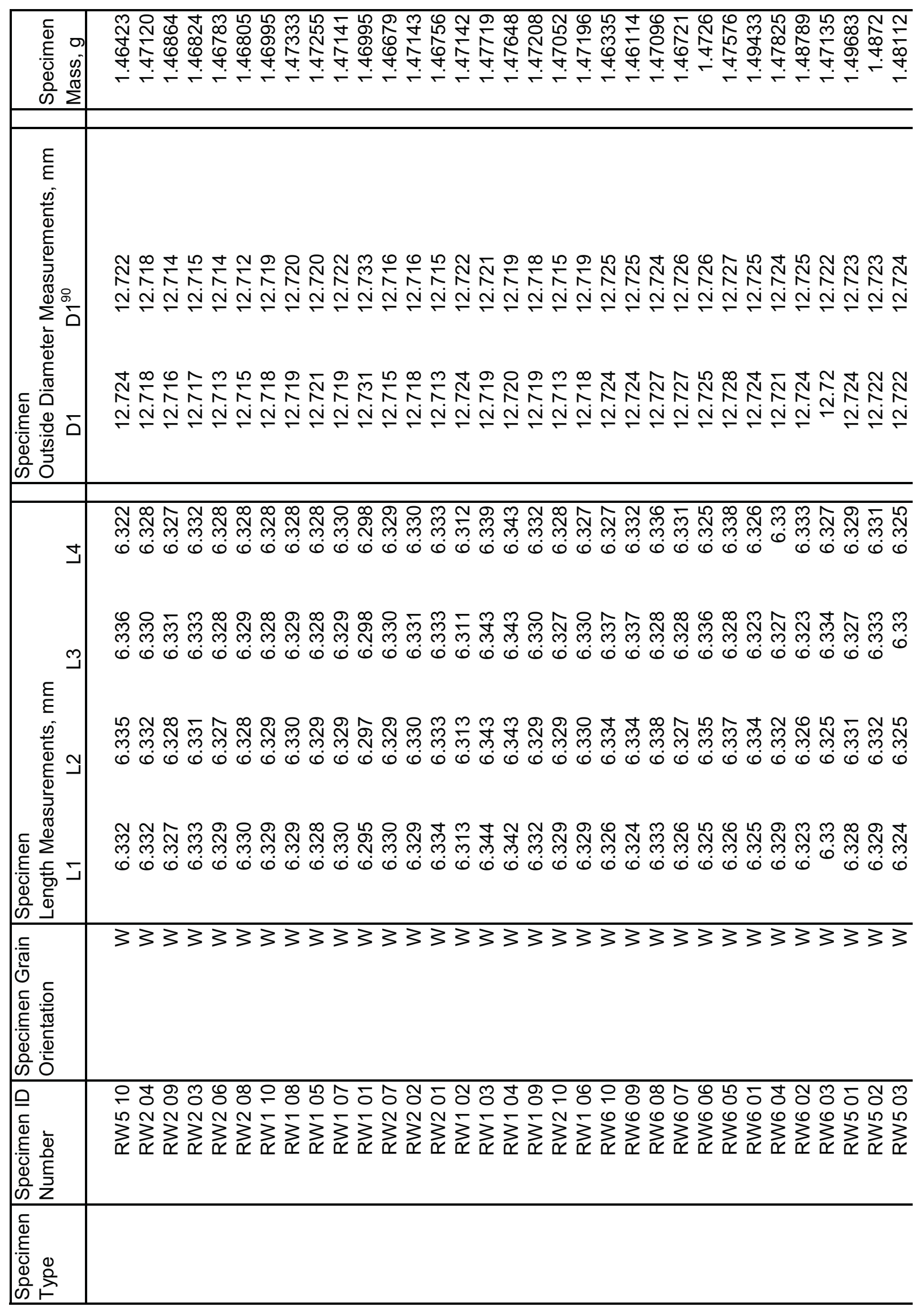




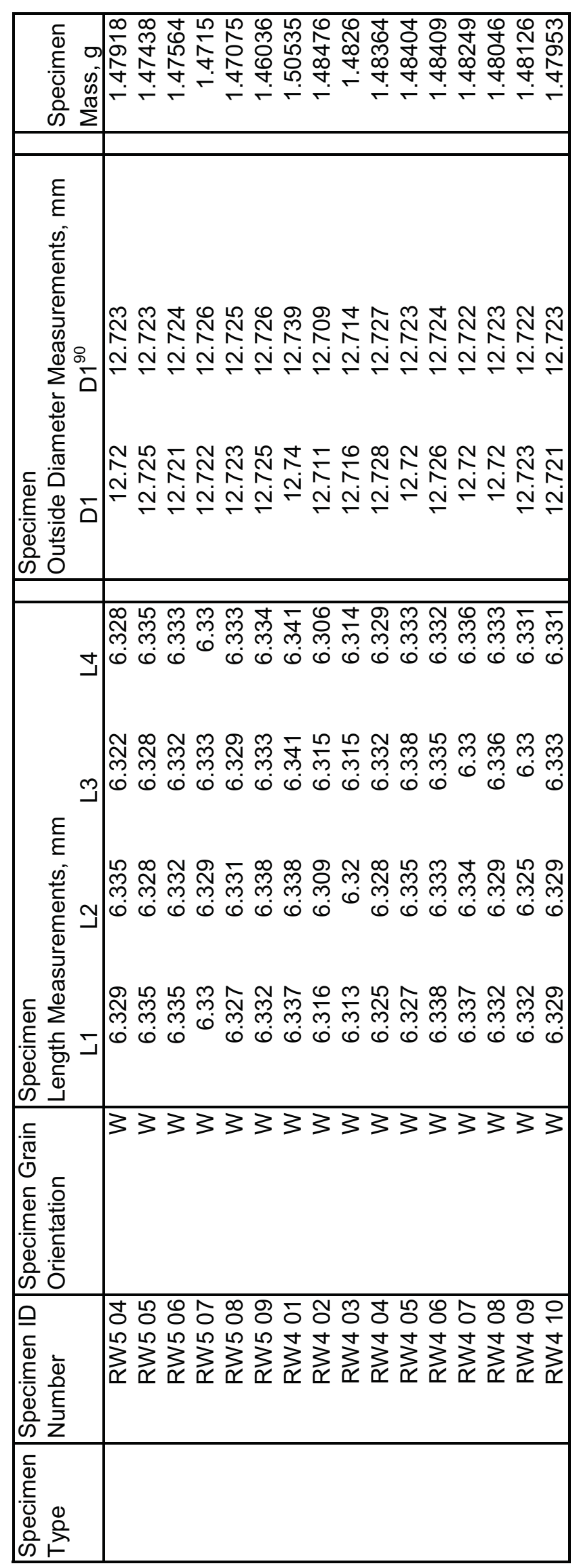




\begin{tabular}{|c|c|}
\hline 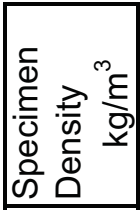 & 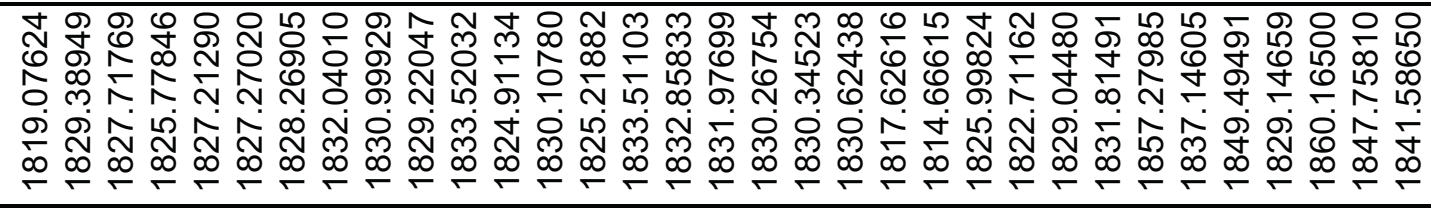 \\
\hline 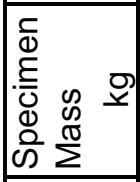 & 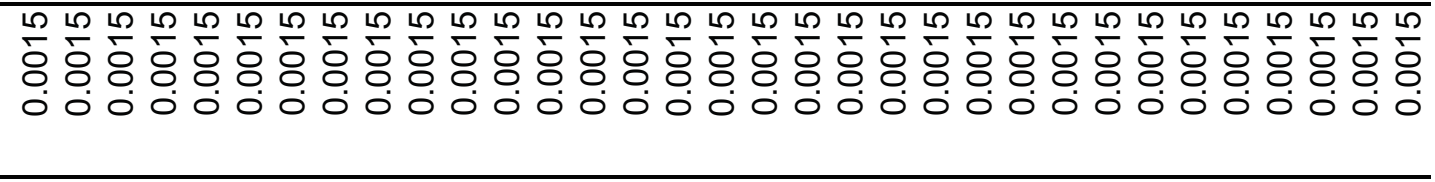 \\
\hline 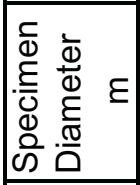 & 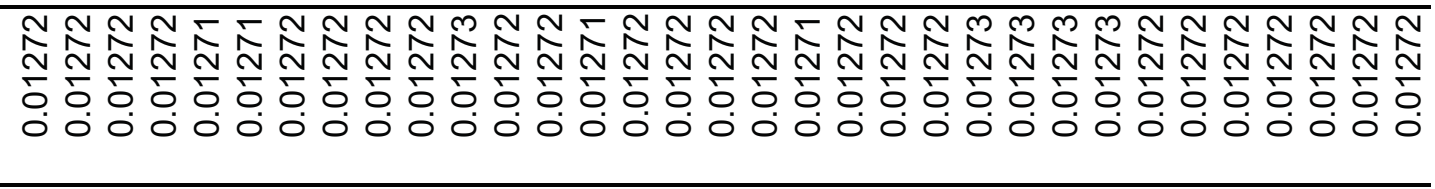 \\
\hline 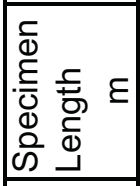 & 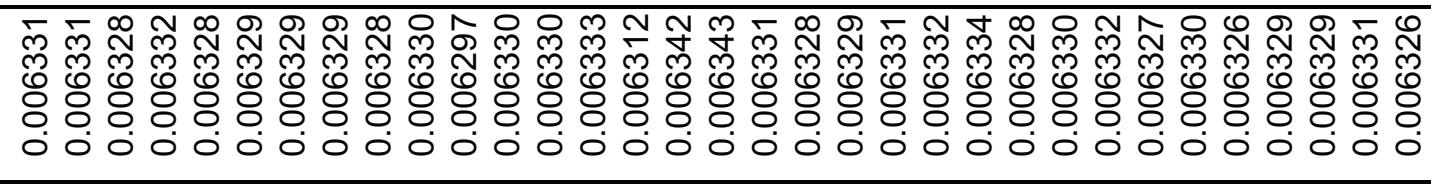 \\
\hline 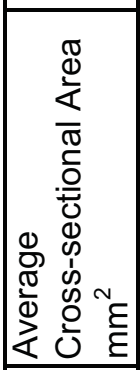 & 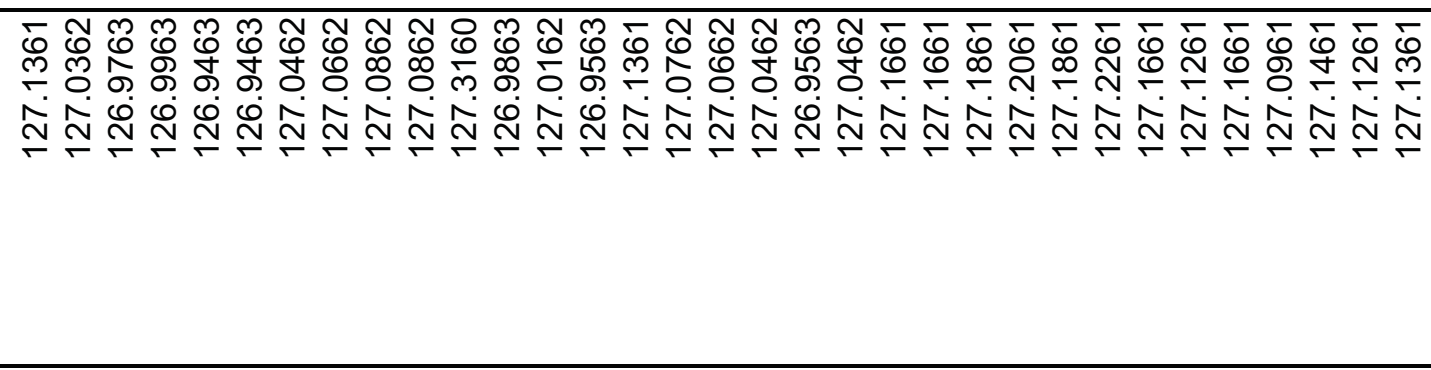 \\
\hline 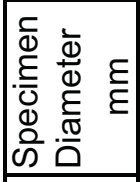 & 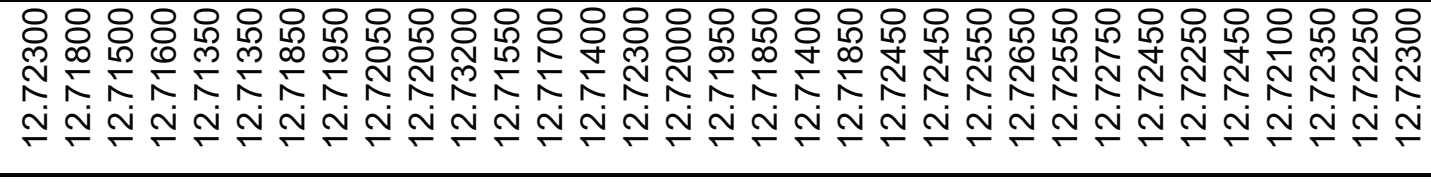 \\
\hline 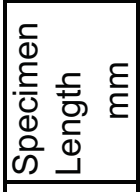 & 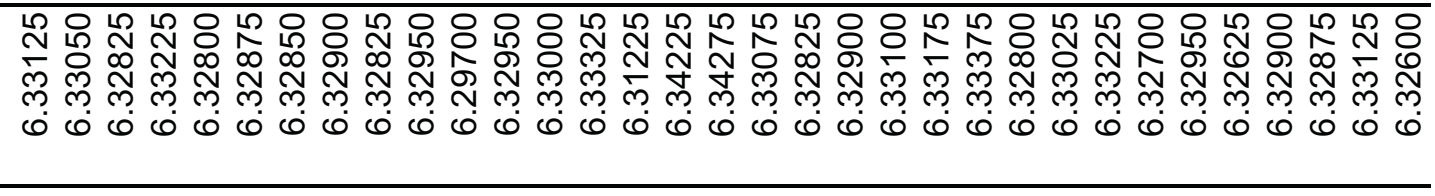 \\
\hline 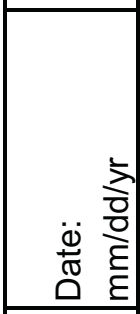 & 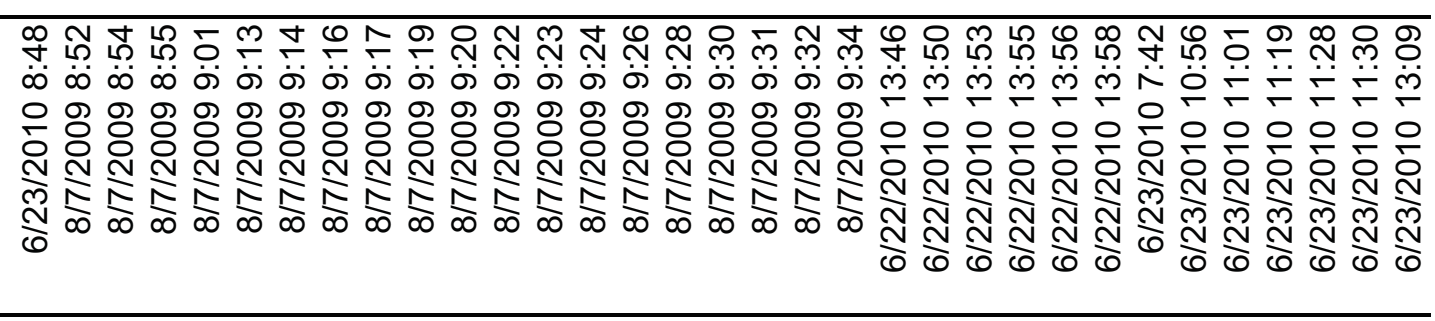 \\
\hline 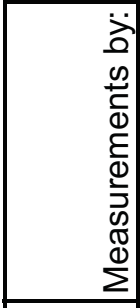 & 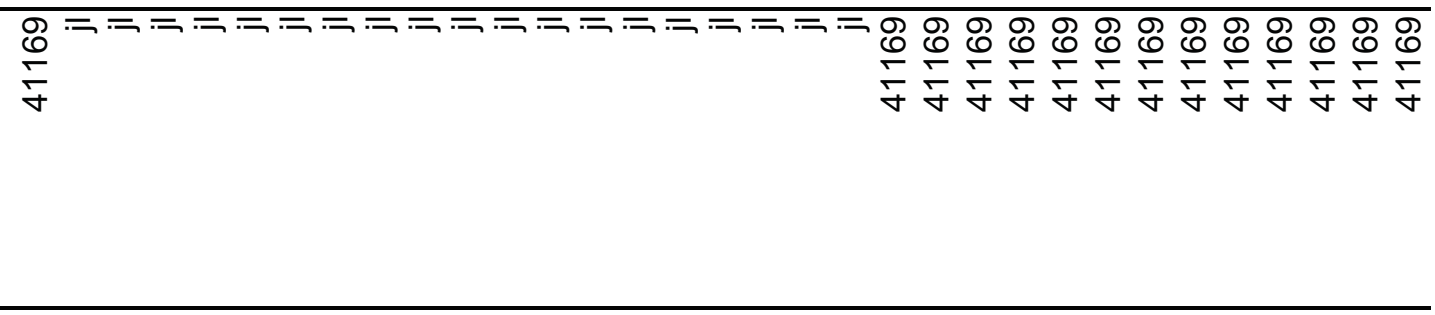 \\
\hline 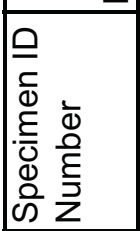 & 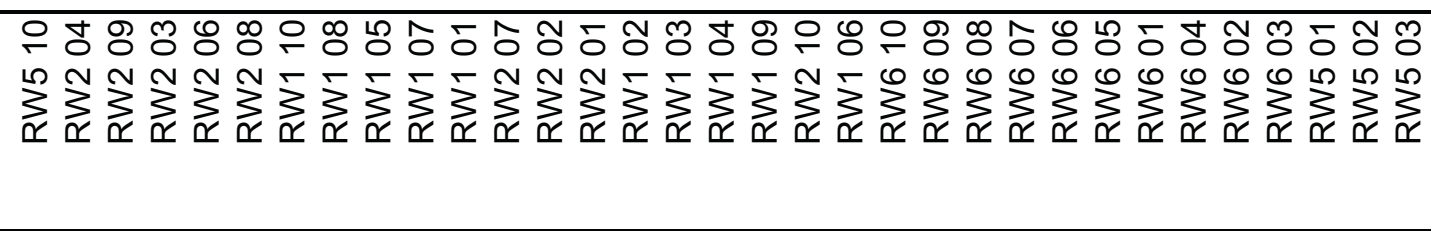 \\
\hline
\end{tabular}




\begin{tabular}{|c|c|}
\hline 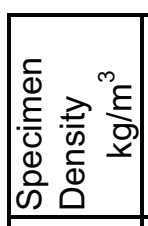 & 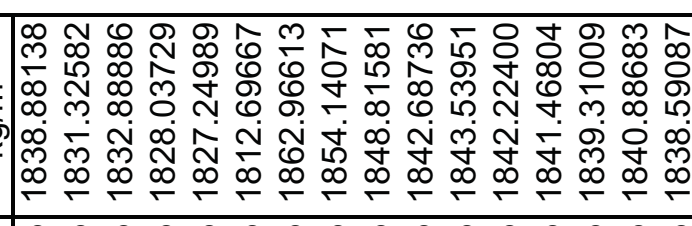 \\
\hline 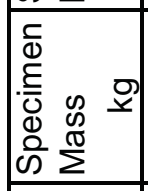 & 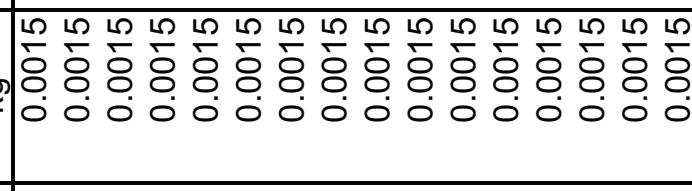 \\
\hline 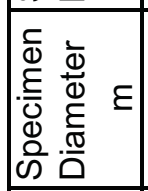 & 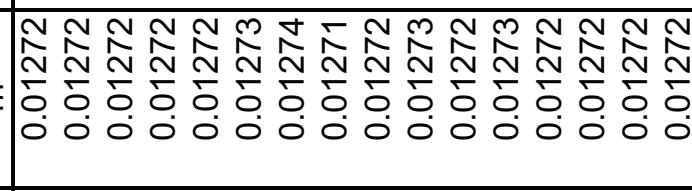 \\
\hline 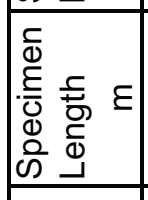 & 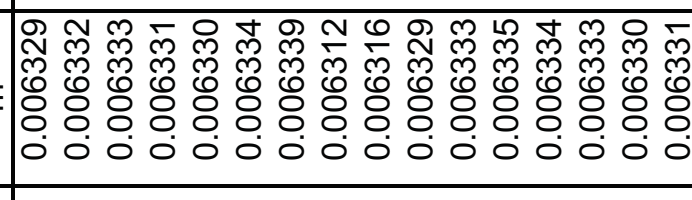 \\
\hline 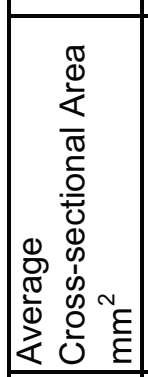 & 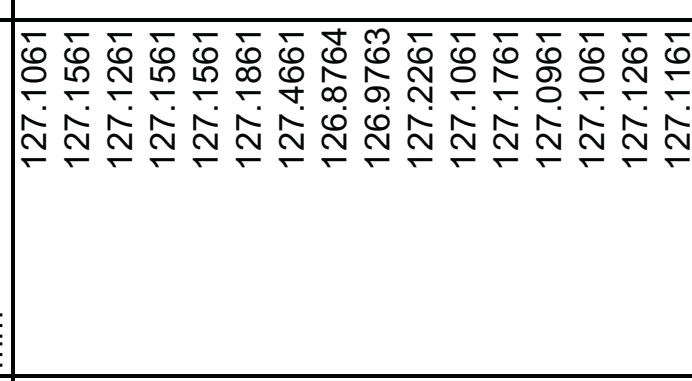 \\
\hline 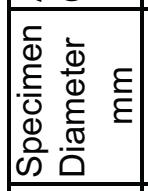 & 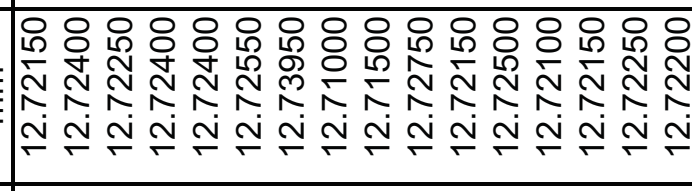 \\
\hline 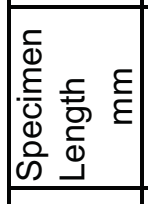 & 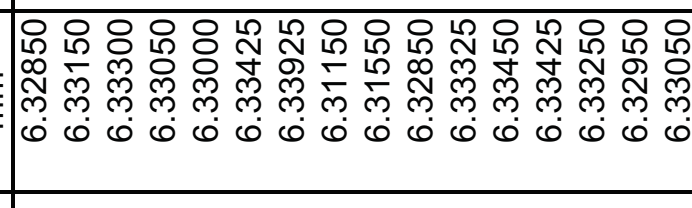 \\
\hline 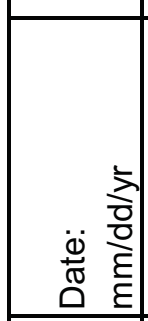 & 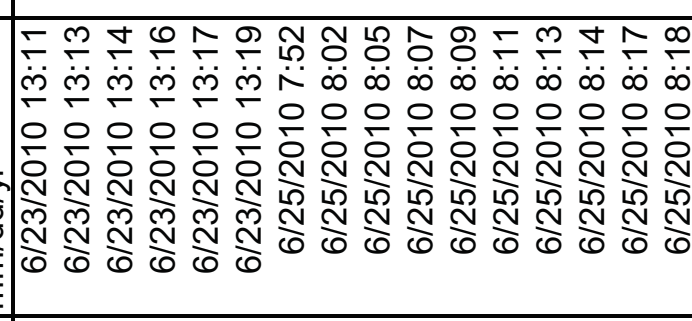 \\
\hline 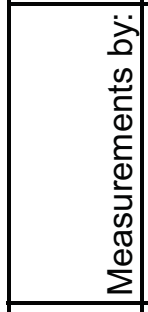 & : \\
\hline 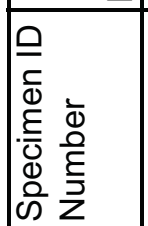 & 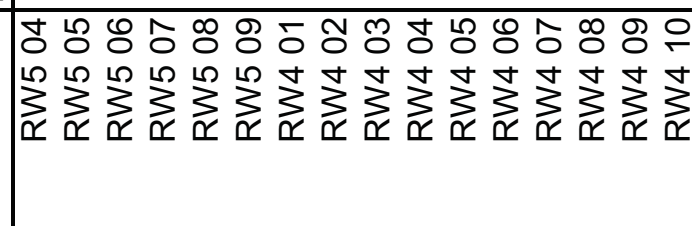 \\
\hline
\end{tabular}




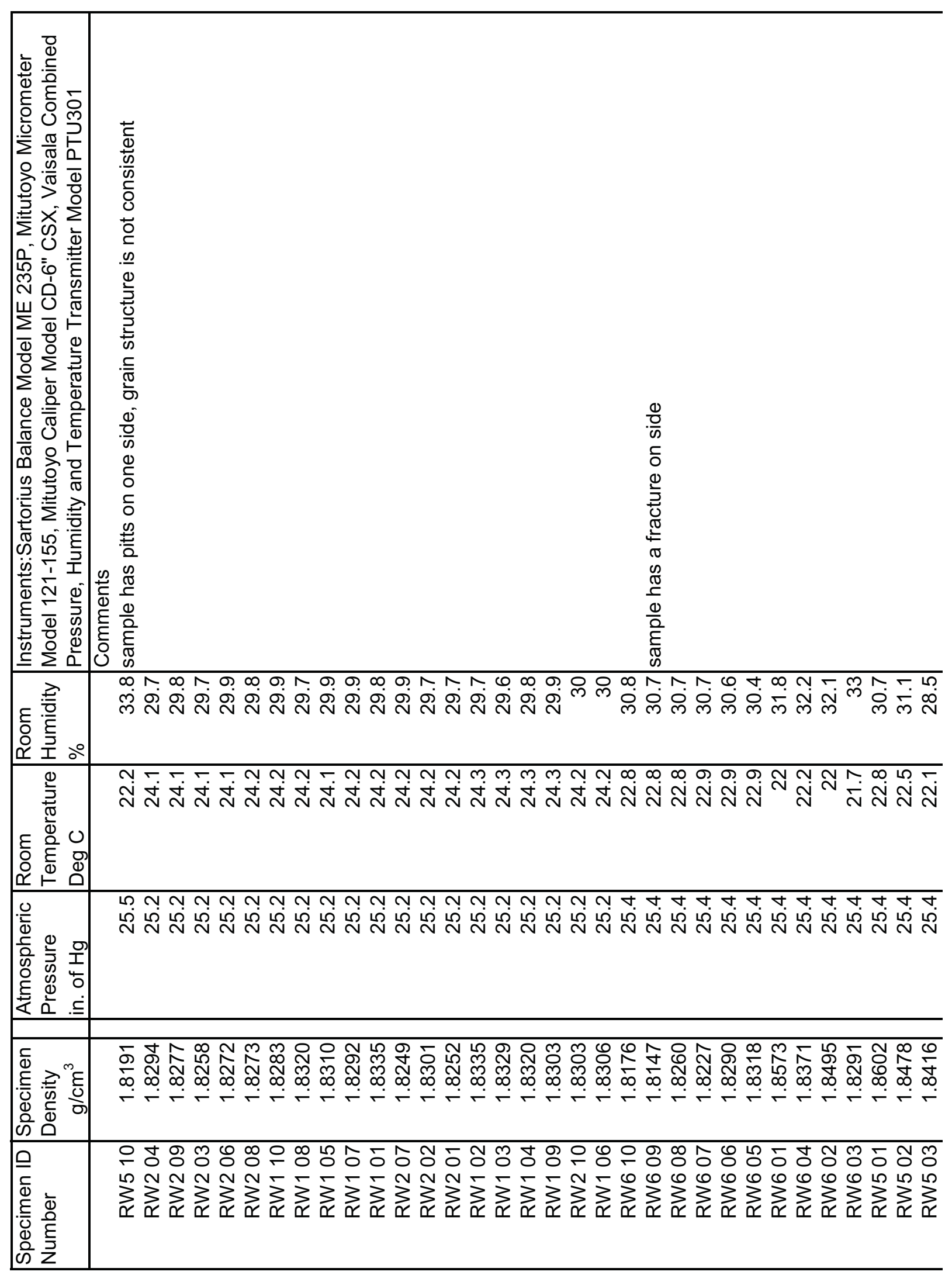




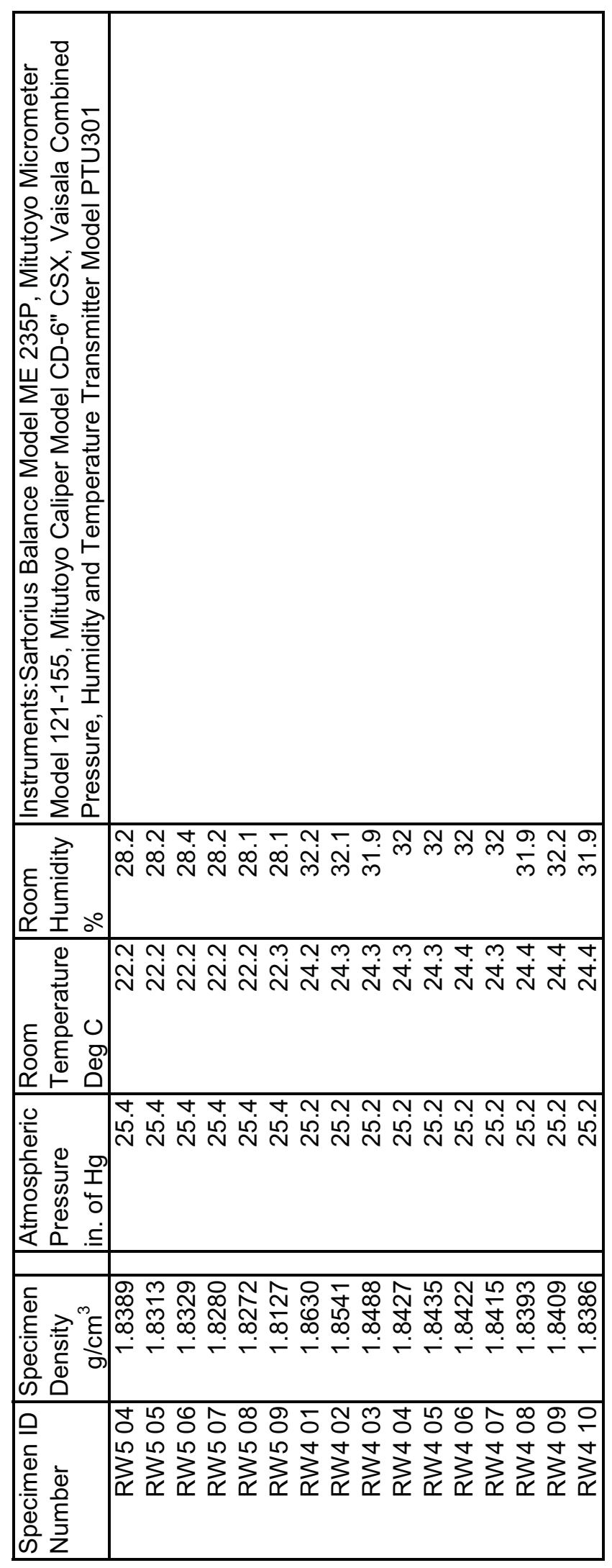




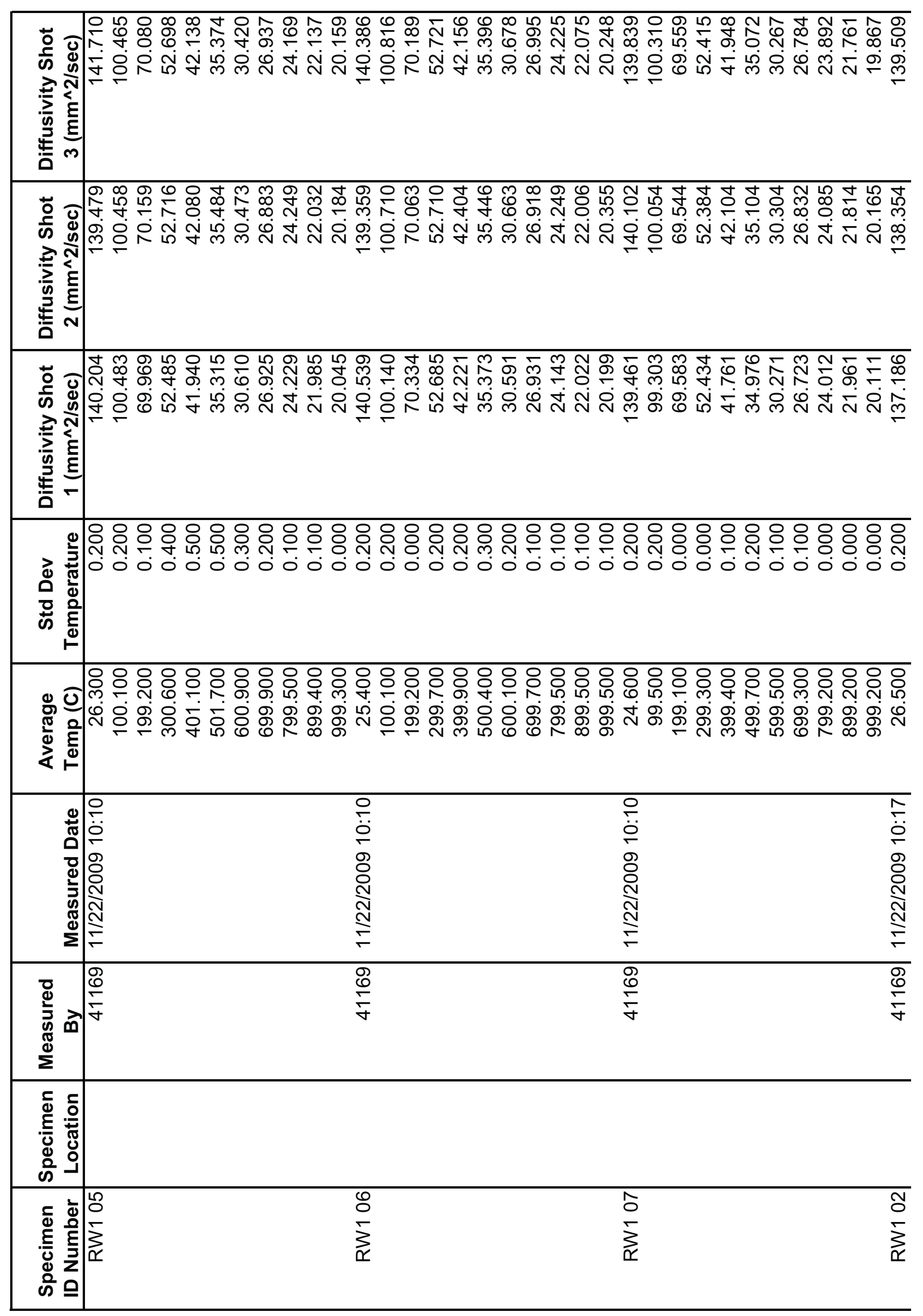




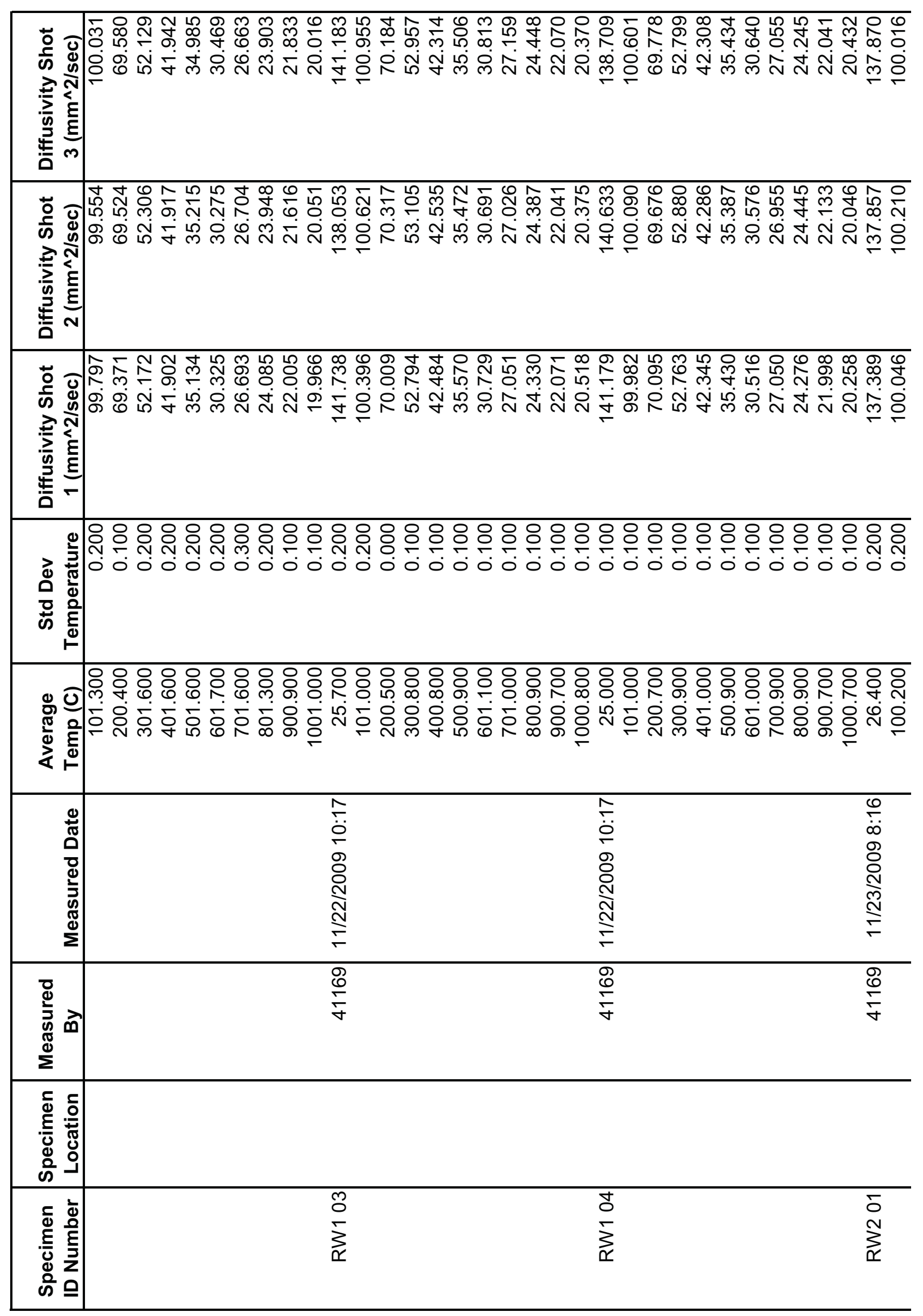




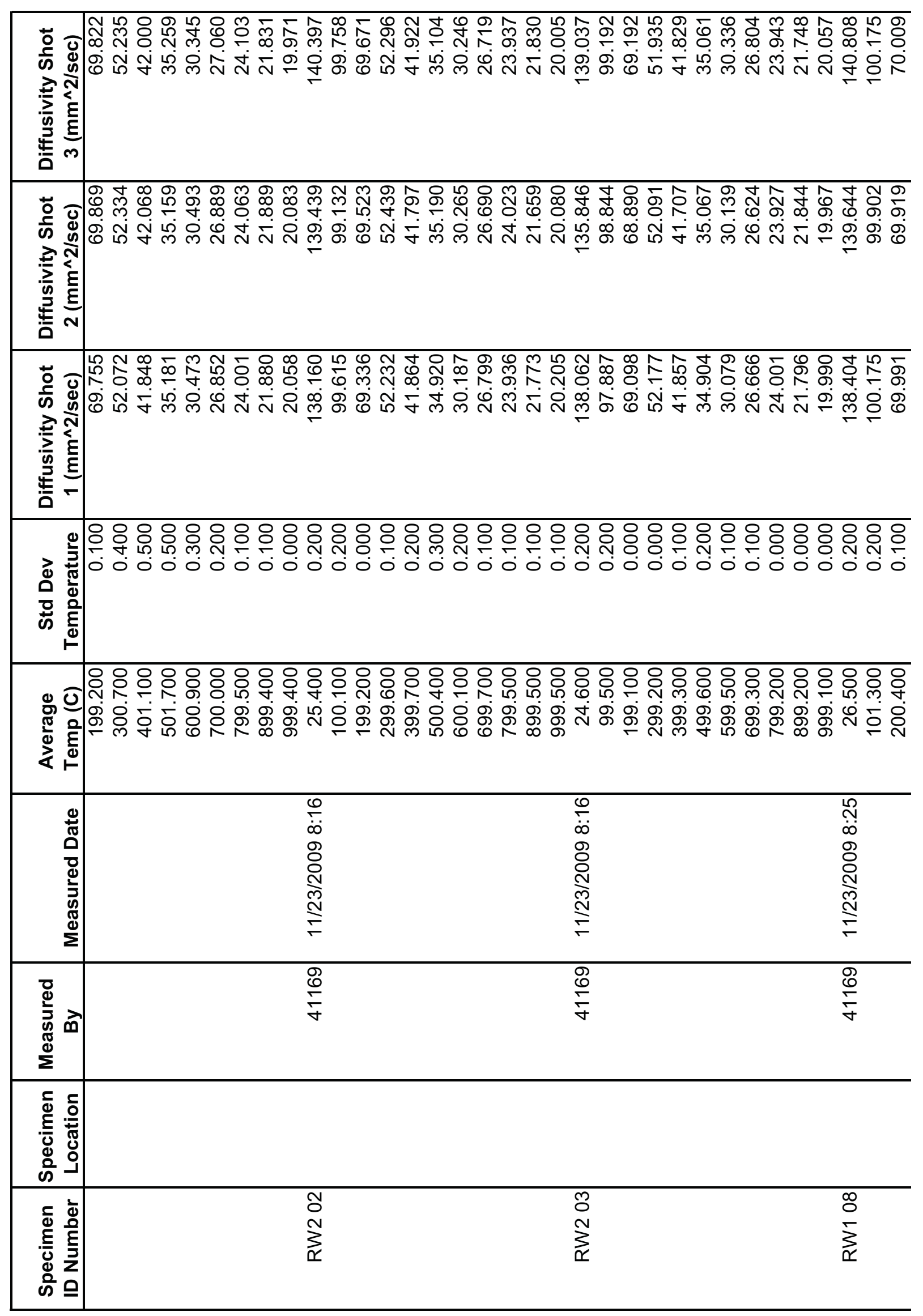




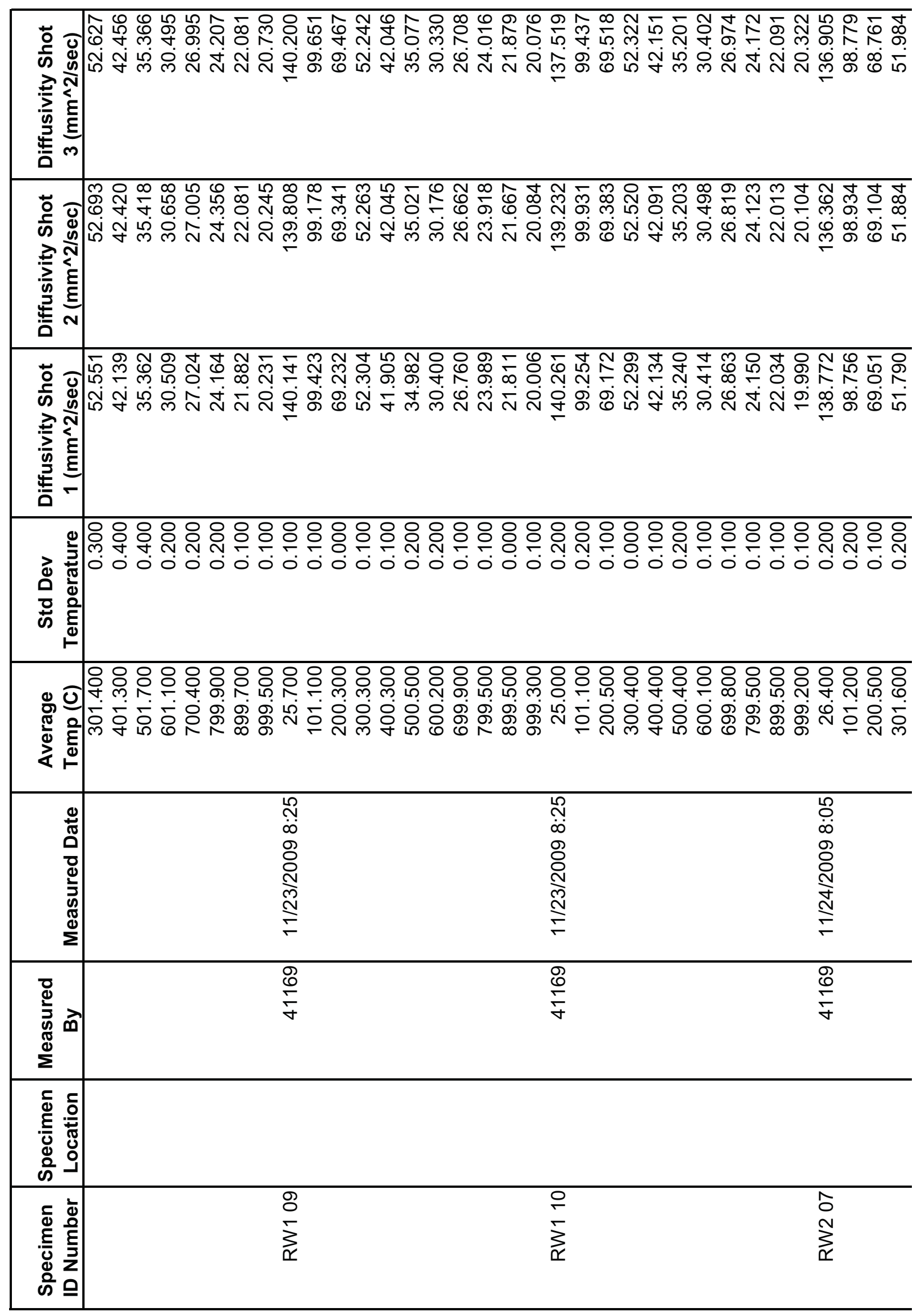




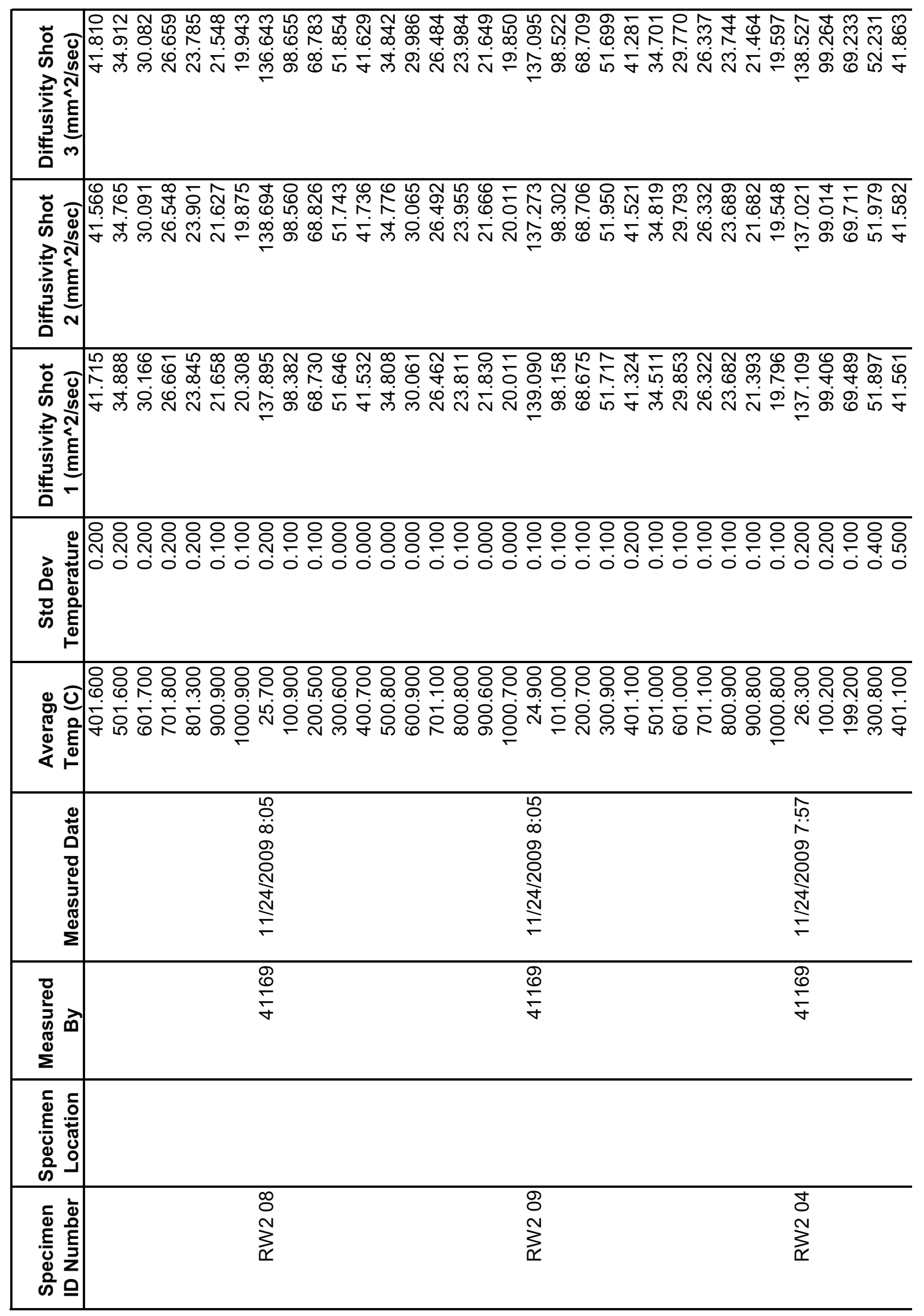




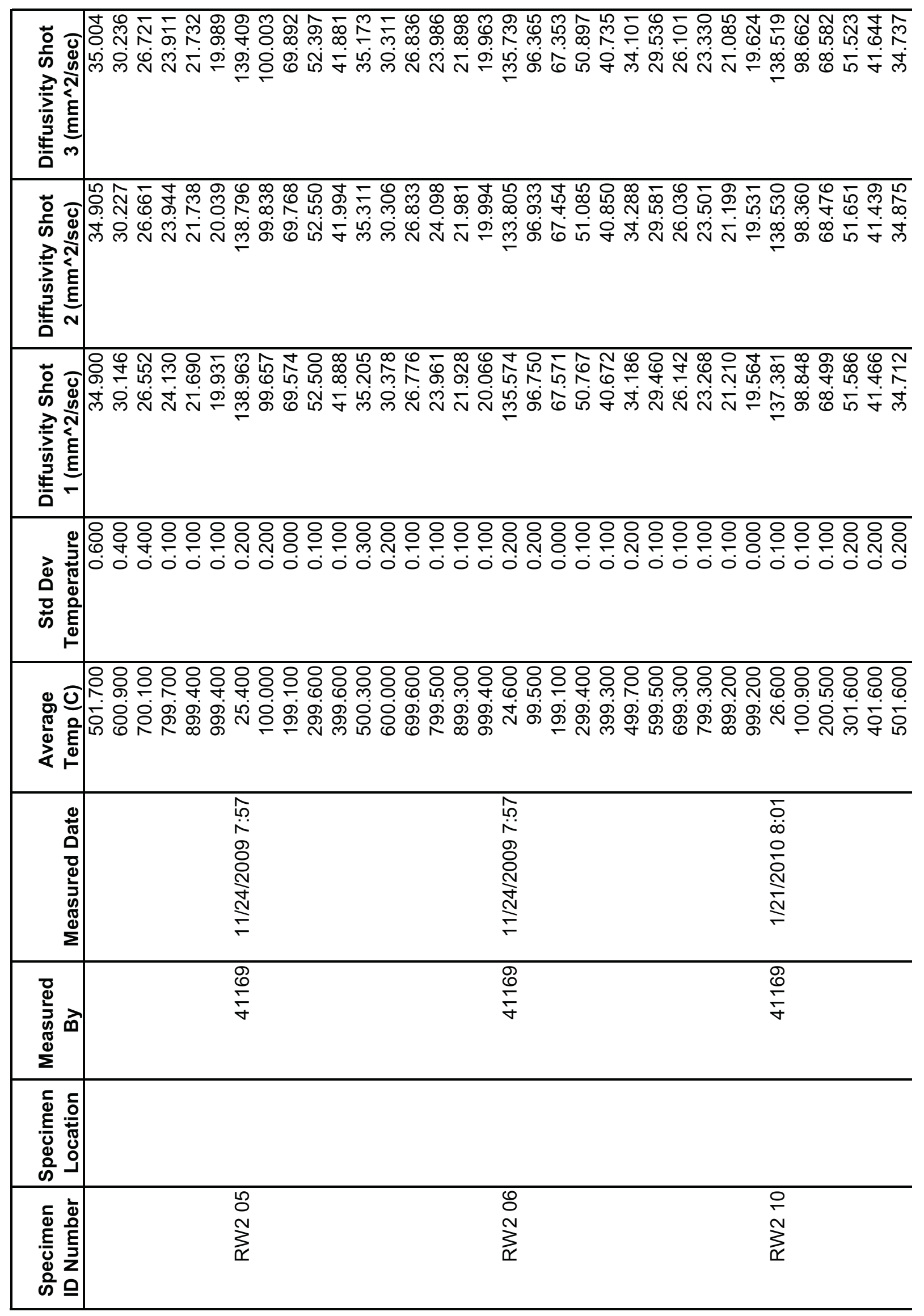




\begin{tabular}{|c|c|c|}
\hline 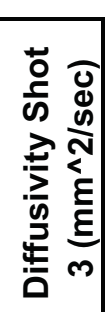 & 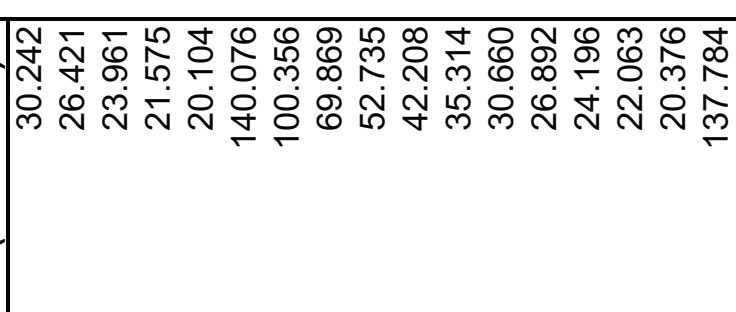 & 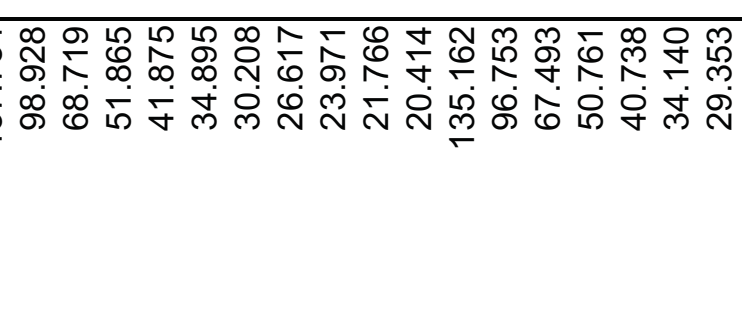 \\
\hline 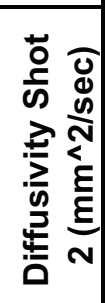 & 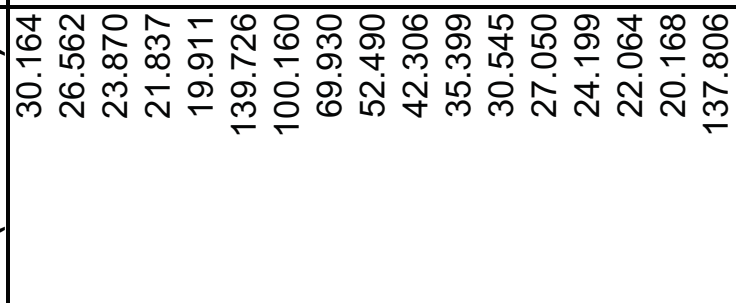 & 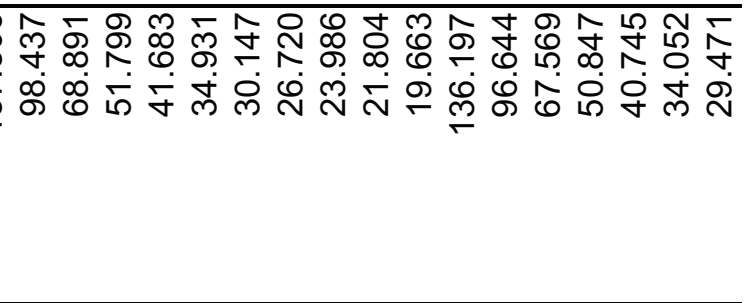 \\
\hline 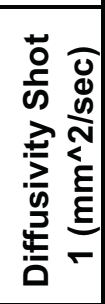 & 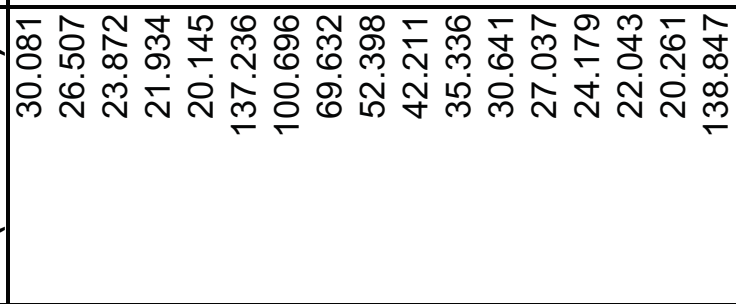 & 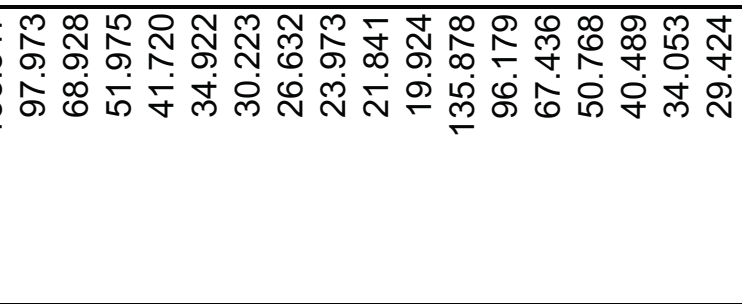 \\
\hline 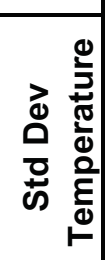 & 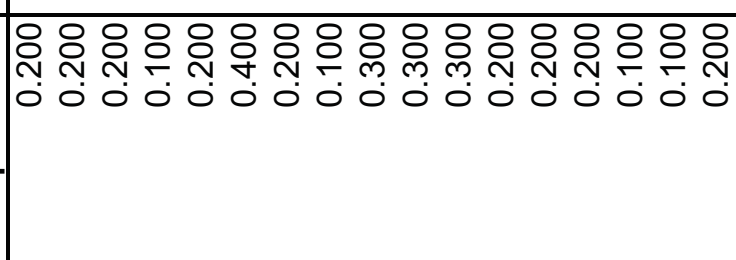 & 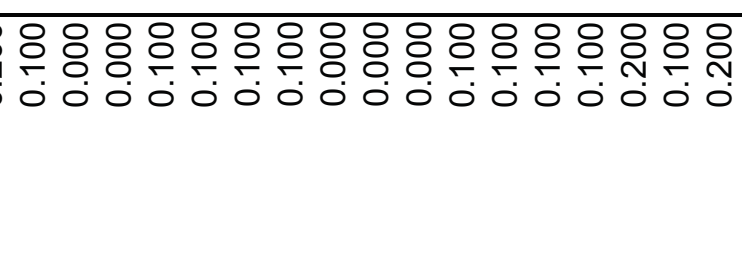 \\
\hline 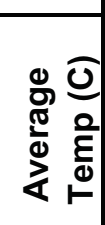 & 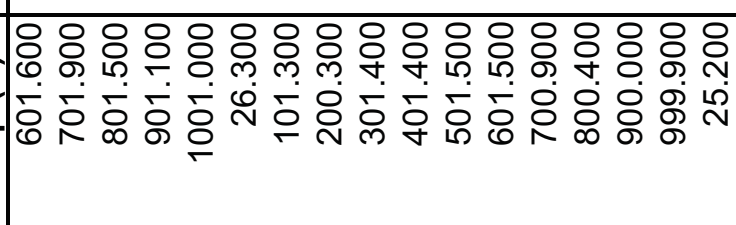 & 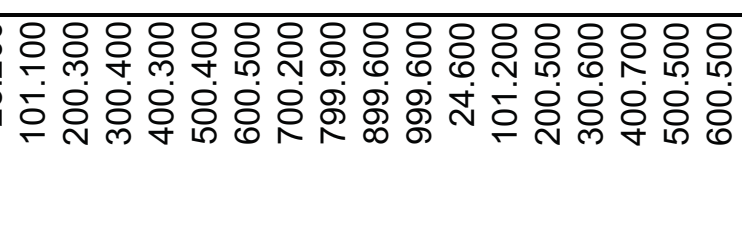 \\
\hline 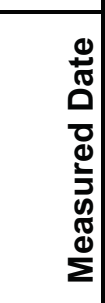 & 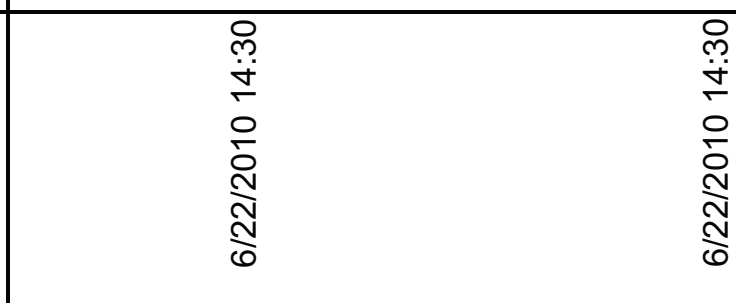 & 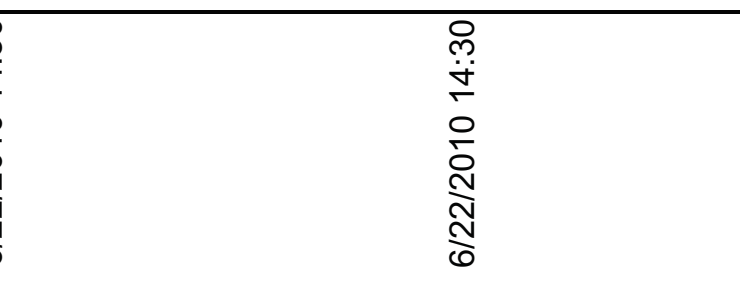 \\
\hline 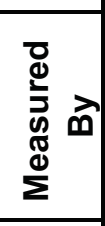 & $\begin{array}{l}\dot{b} \\
\frac{8}{6}\end{array}$ & 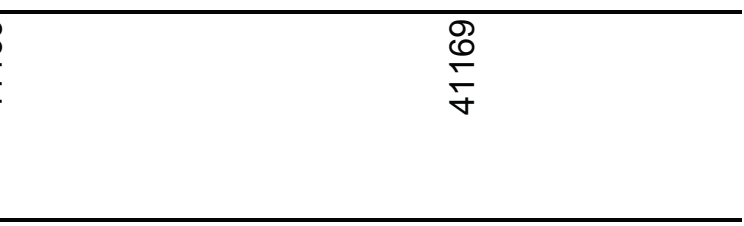 \\
\hline 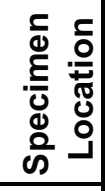 & & \\
\hline 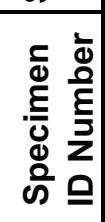 & 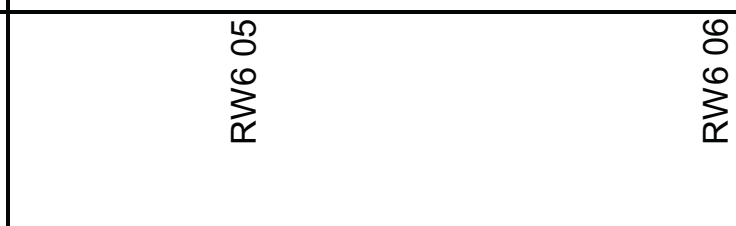 & \begin{tabular}{l}
1 \\
\\
0 \\
0 \\
\hdashline
\end{tabular} \\
\hline
\end{tabular}




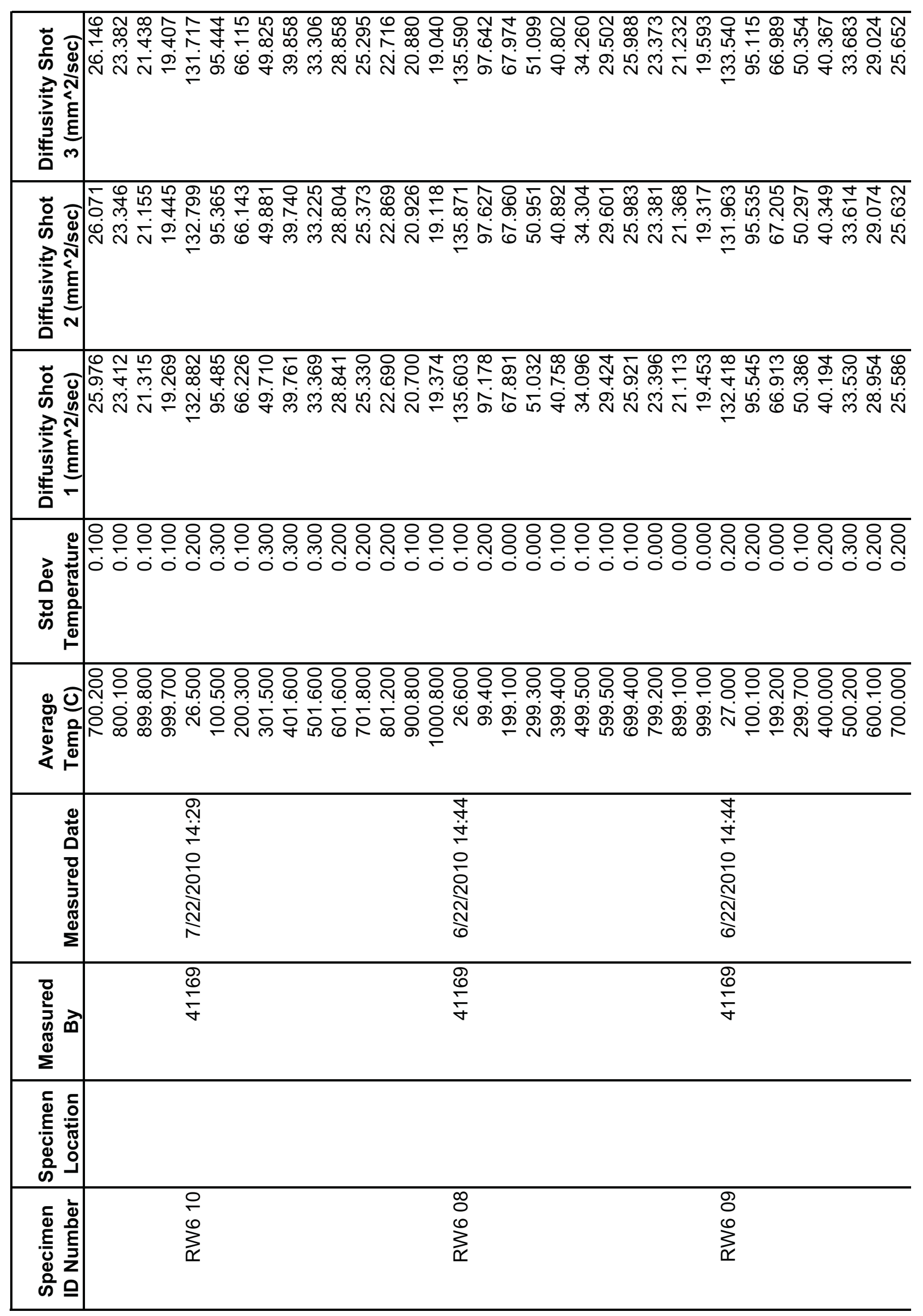




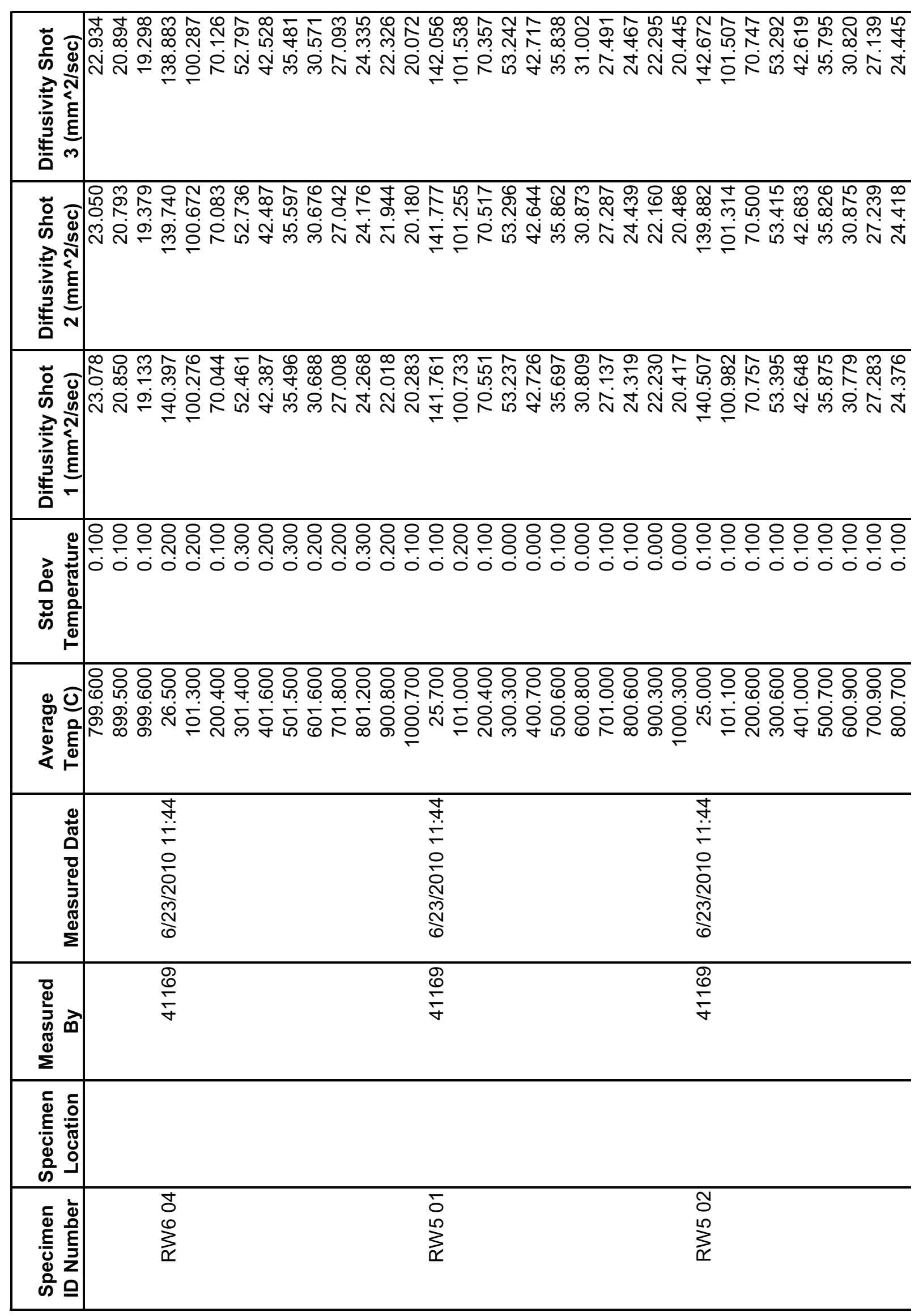




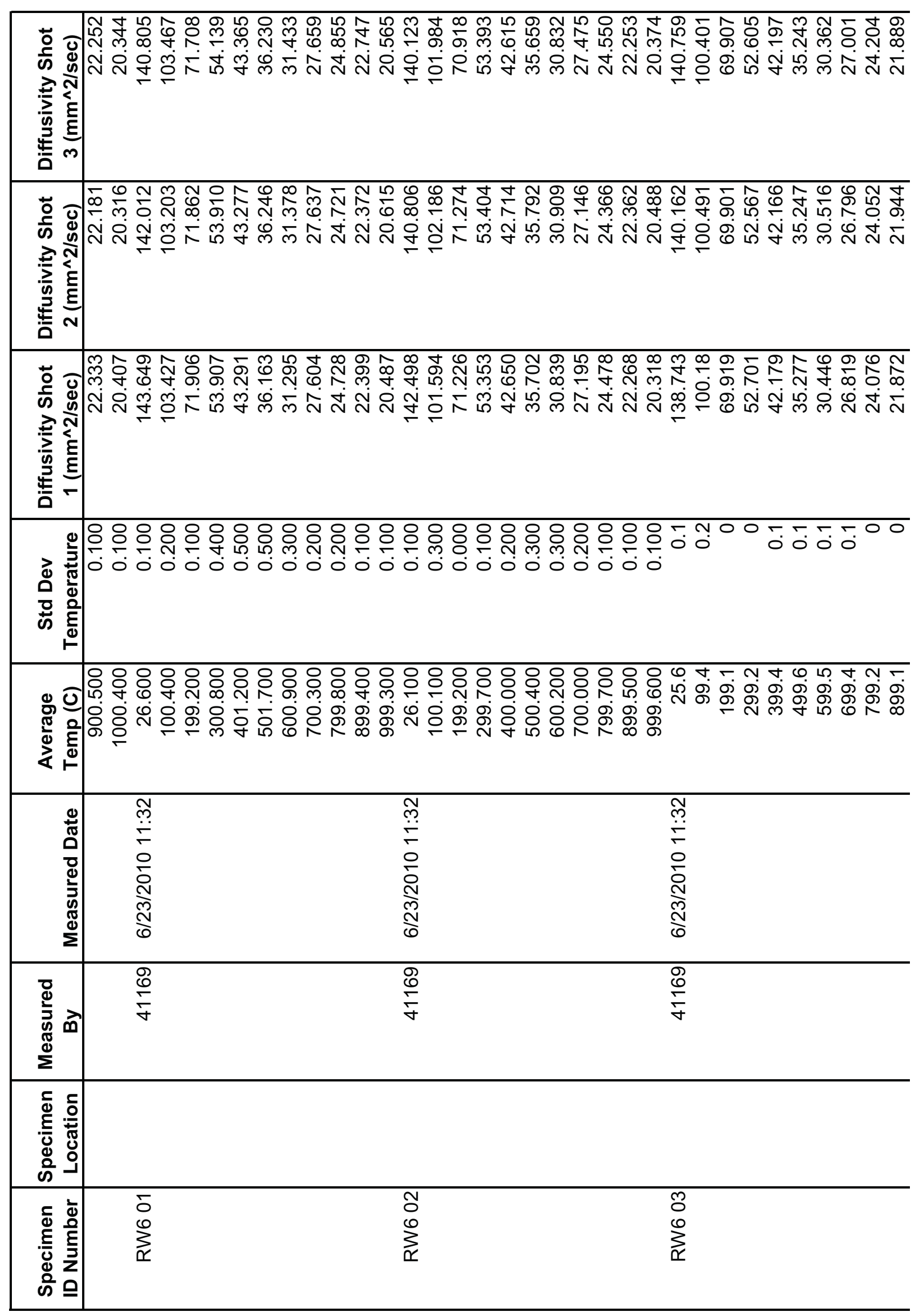




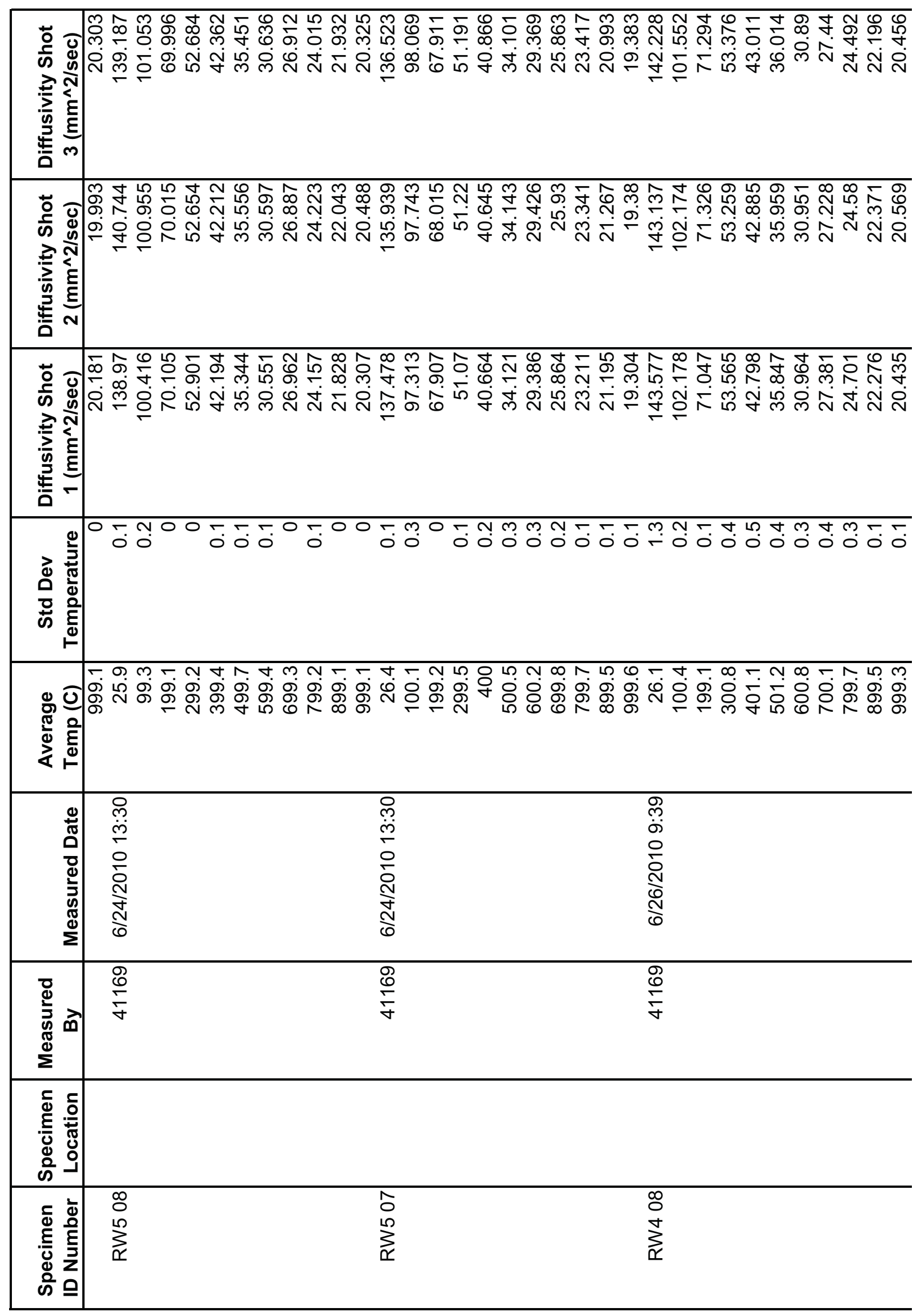




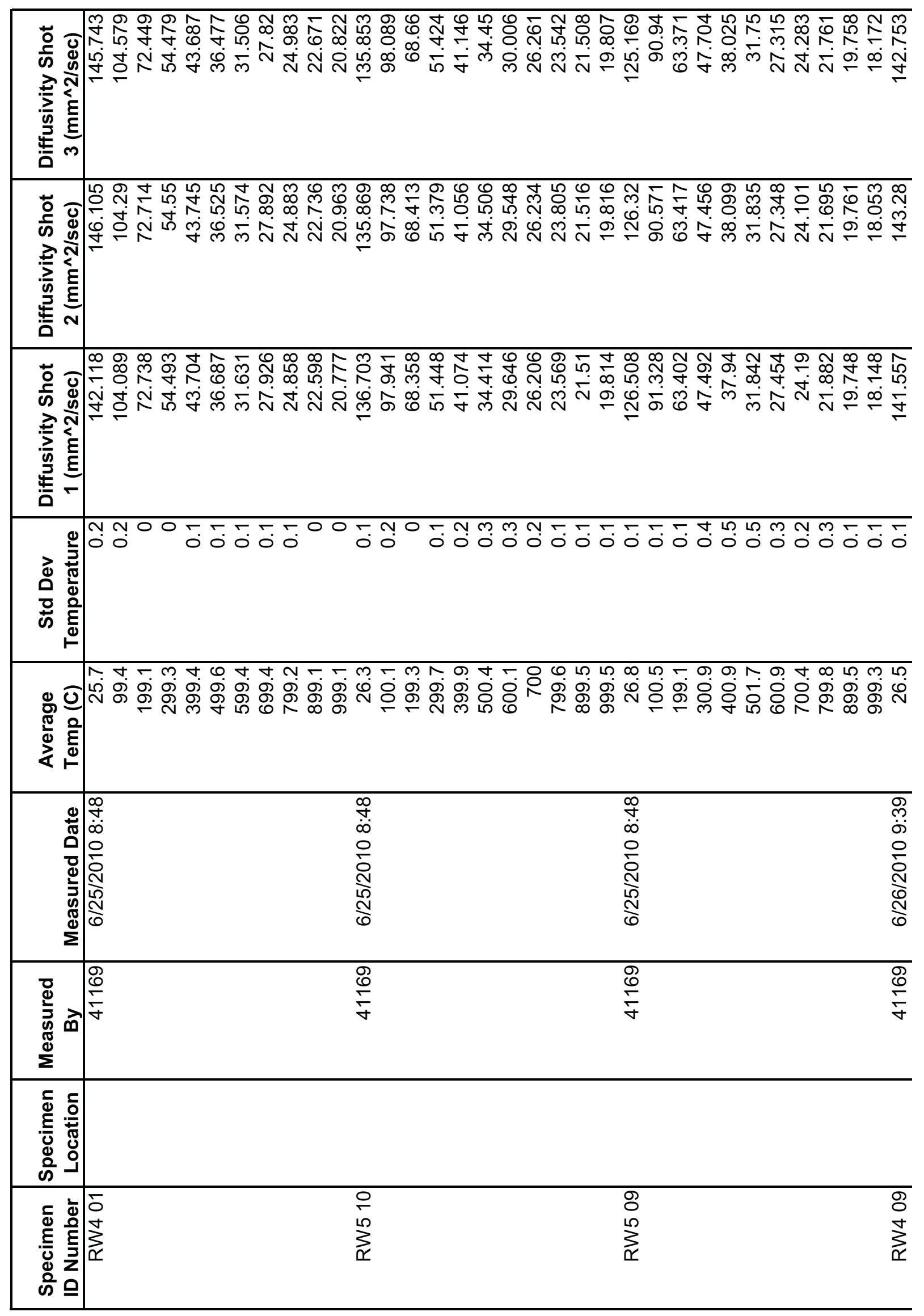




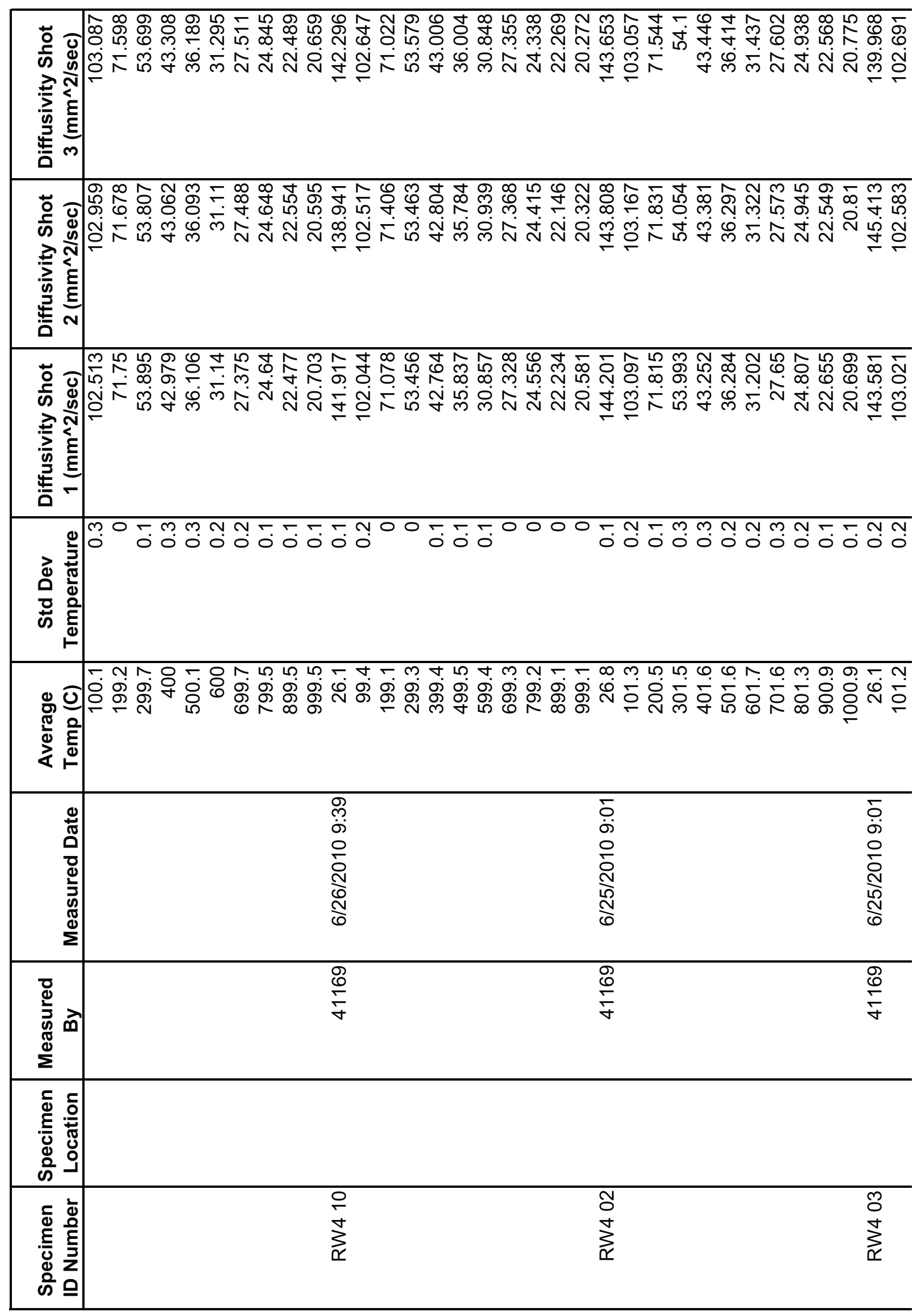




\begin{tabular}{|c|c|}
\hline 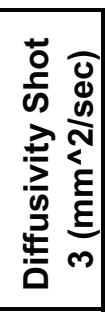 & 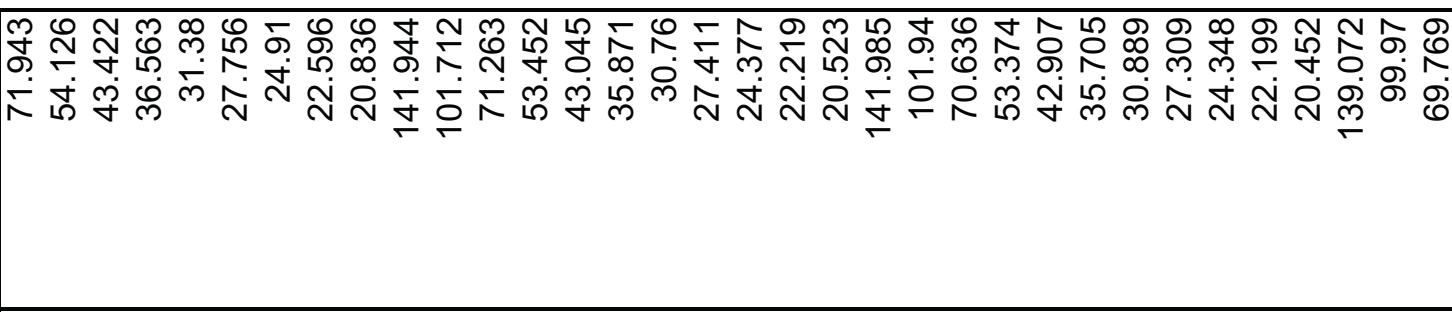 \\
\hline 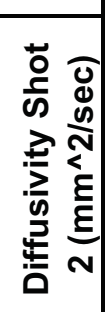 & 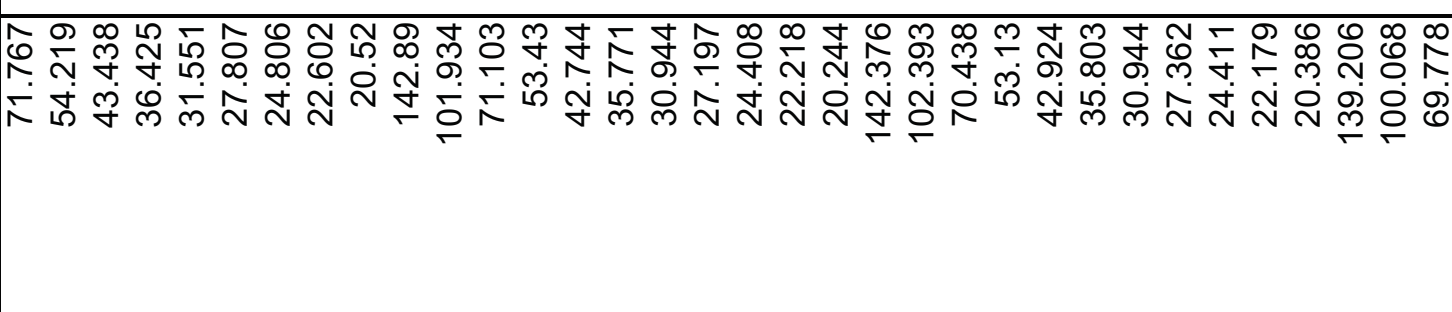 \\
\hline 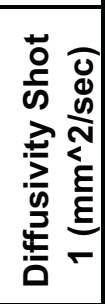 & 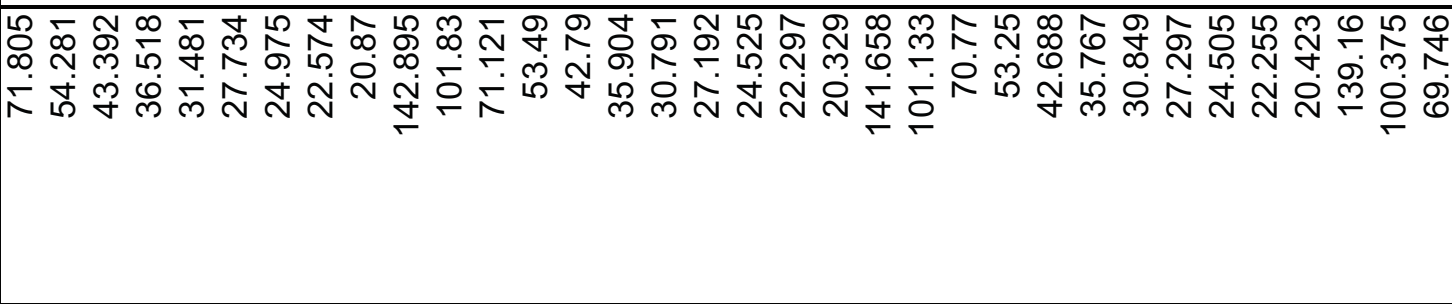 \\
\hline 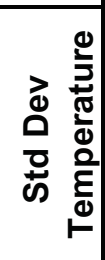 & 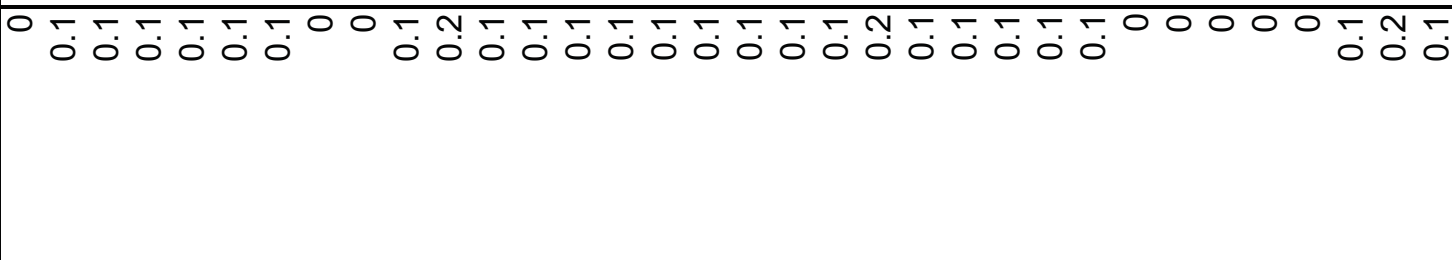 \\
\hline 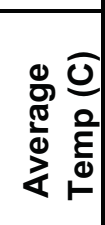 & 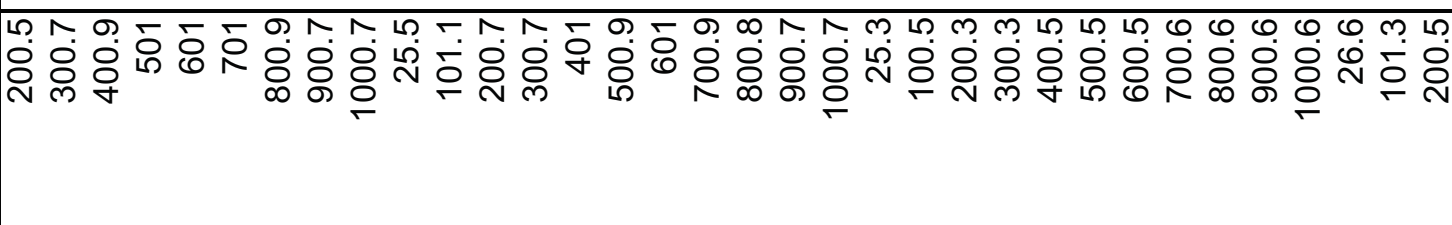 \\
\hline 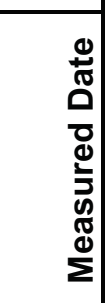 & 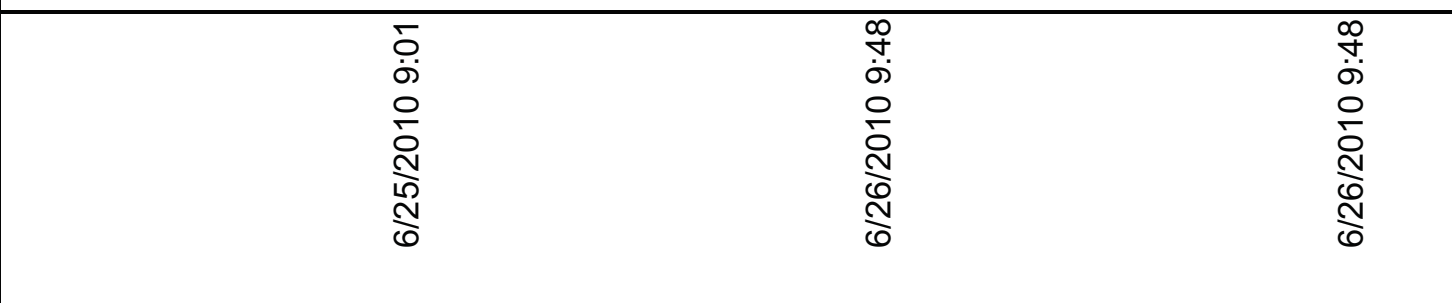 \\
\hline 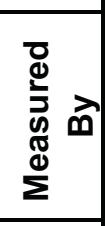 & 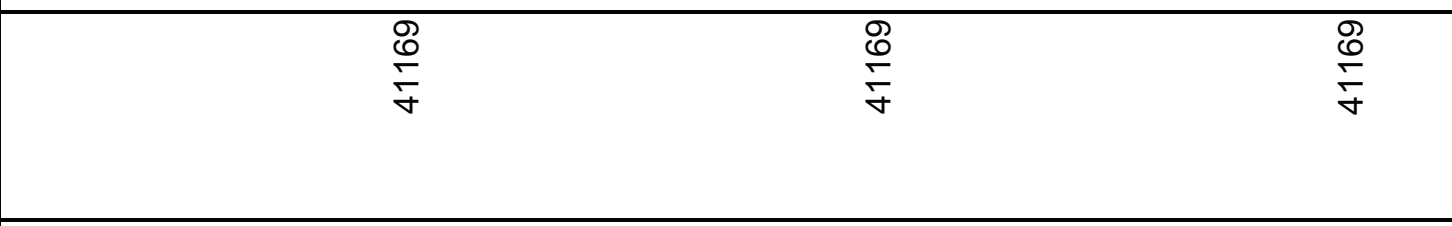 \\
\hline 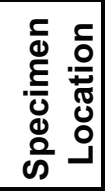 & \\
\hline 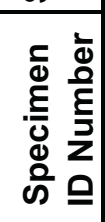 & $\begin{array}{l}l_{0}^{2} \\
\sum_{x}^{+}\end{array}$ \\
\hline
\end{tabular}




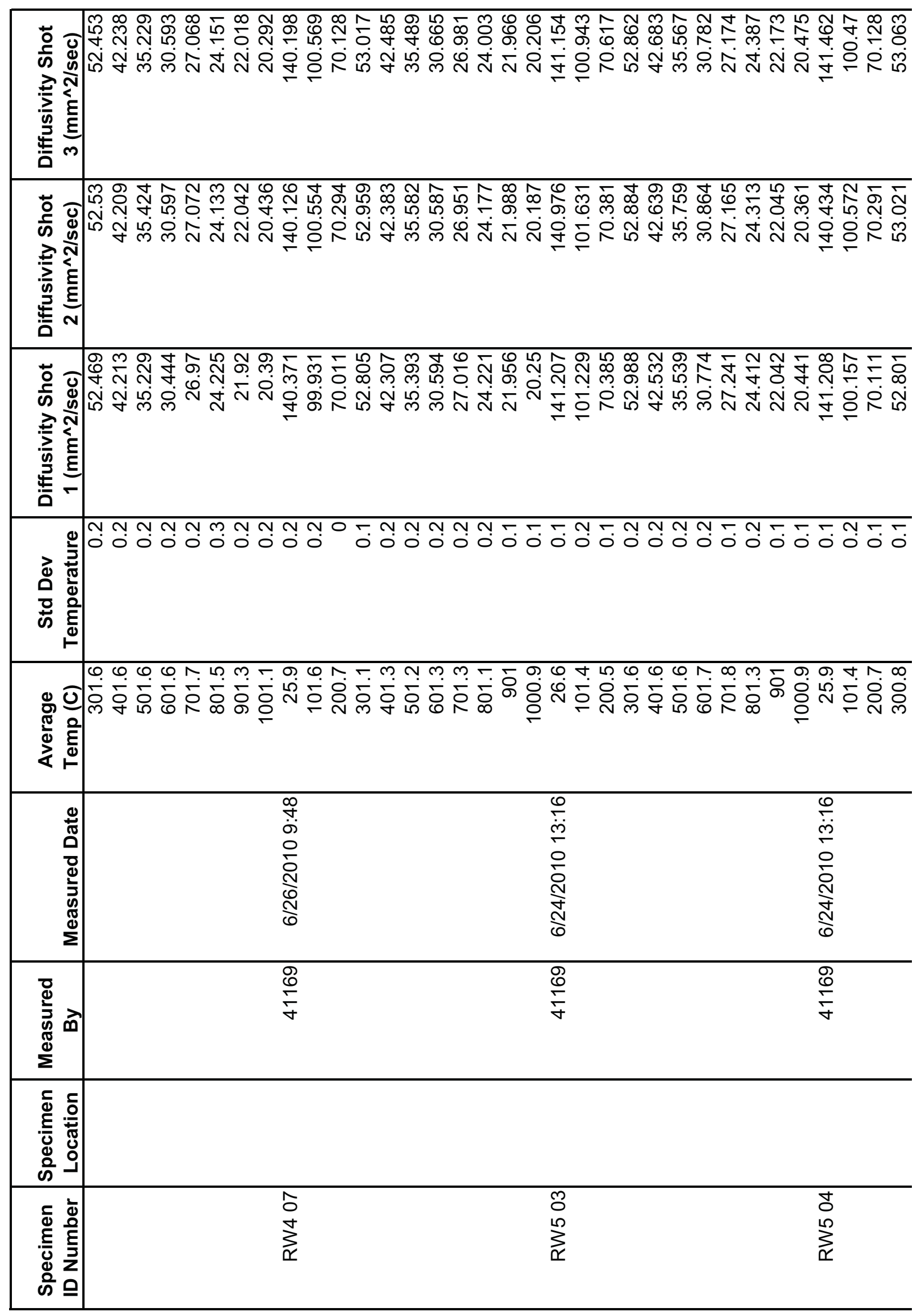




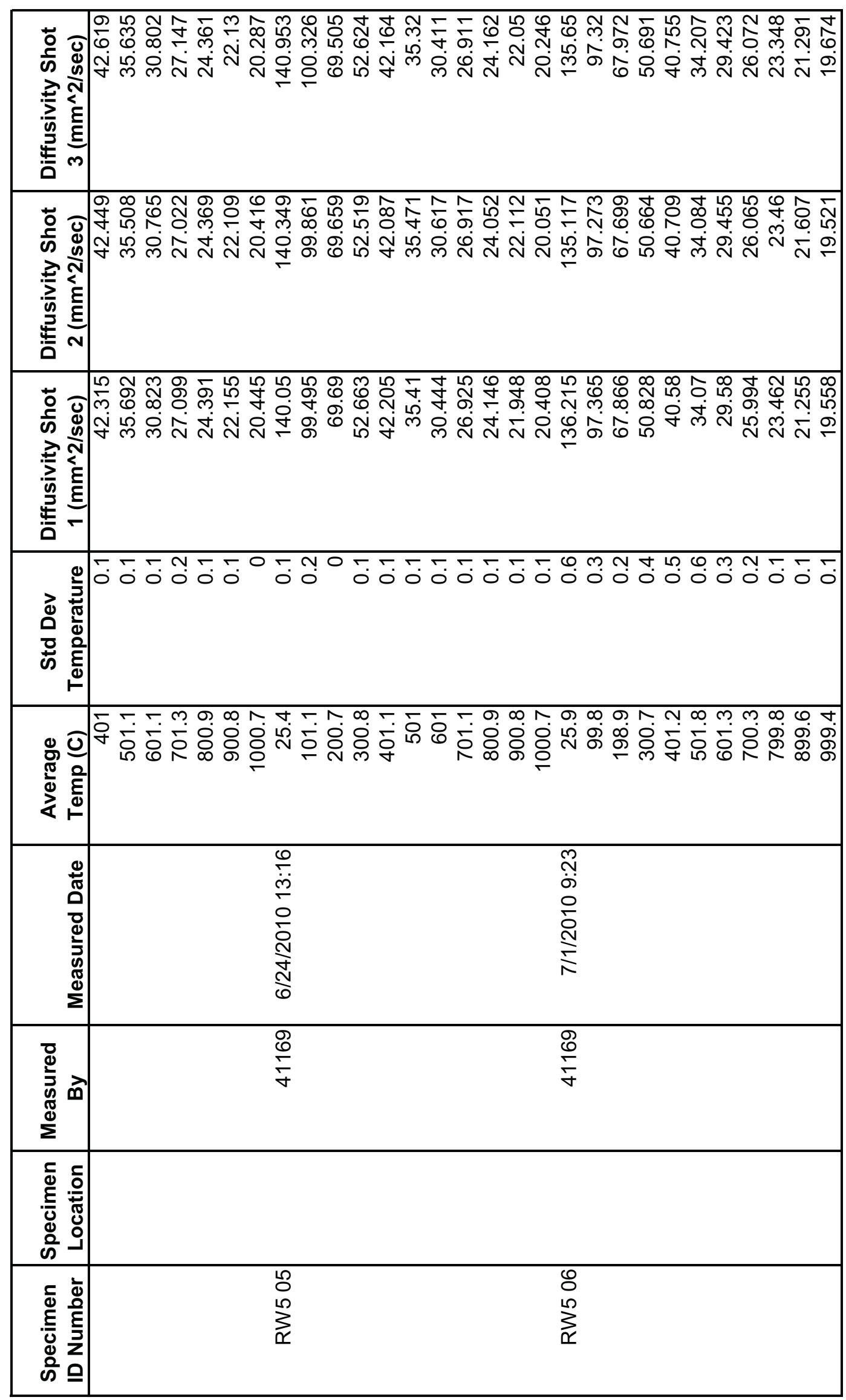




\begin{tabular}{|c|c|c|c|c|}
\hline 妾 & 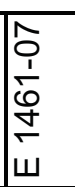 & $\begin{array}{l}\hat{O} \\
\frac{1}{0} \\
\stackrel{0}{+} \\
\dot{w}\end{array}$ & $\begin{array}{l}\hat{o} \\
\frac{1}{0} \\
\stackrel{+}{+} \\
\dot{w}\end{array}$ & 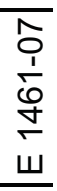 \\
\hline 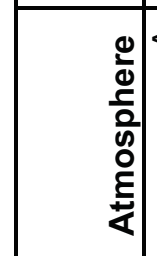 & & $\overline{\dot{z}}$ & 交 & $\bar{k}$ \\
\hline 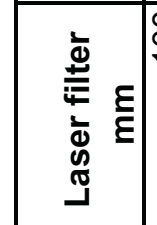 & & 음 & 음 & 음 \\
\hline 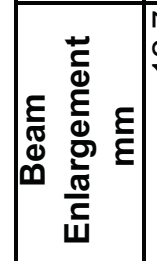 & & $\stackrel{\stackrel{N}{\sim}}{\sim}$ & $\stackrel{\stackrel{N}{ }}{ }$ & 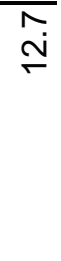 \\
\hline 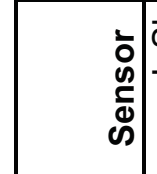 & & $\begin{array}{l}\text { की } \\
\text { की }\end{array}$ & $\begin{array}{l}\text { की } \\
\text { D }\end{array}$ & की \\
\hline 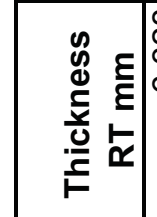 & & 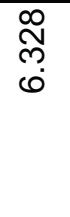 & $\begin{array}{l}\infty \\
\stackrel{\infty}{0} \\
0 \\
0\end{array}$ & 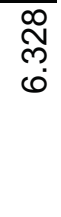 \\
\hline 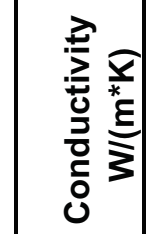 & & & & \\
\hline 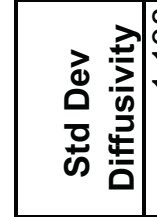 & & $\mathcal{F}$ & 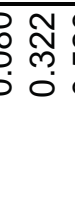 & \\
\hline 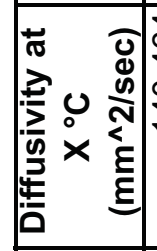 & 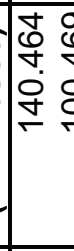 & \begin{tabular}{l}
$\mathscr{2}$ \\
0 \\
0 \\
\hdashline
\end{tabular} & 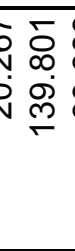 & 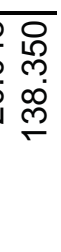 \\
\hline 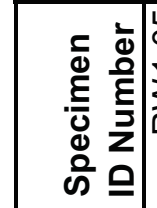 & & $\begin{array}{l}8 \\
\sum_{\mathbb{1}}\end{array}$ & $\begin{array}{l}\hat{O} \\
\sum_{\alpha \times)}^{5}\end{array}$ & $\begin{array}{l}\bar{\delta} \\
\sum_{\mathbb{\alpha}}^{\mathcal{N}}\end{array}$ \\
\hline
\end{tabular}




\begin{tabular}{|c|c|c|c|}
\hline 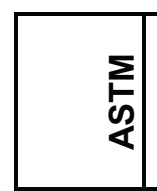 & $\begin{array}{l}\hat{o} \\
\frac{1}{0} \\
\dot{0} \\
\dot{5} \\
\end{array}$ & $\begin{array}{l}\hat{S} \\
\frac{1}{1} \\
\frac{0}{5} \\
\dot{w}\end{array}$ & 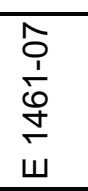 \\
\hline 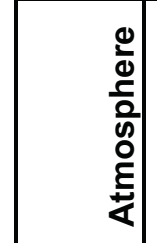 & 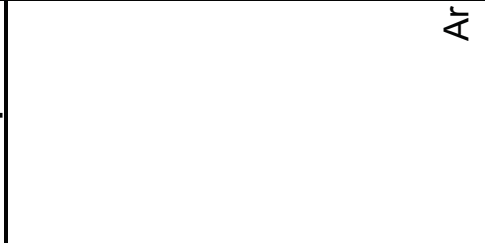 & $\frac{1}{\alpha}$ & 广 \\
\hline 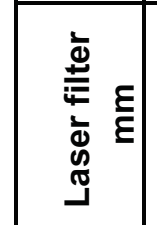 & 음 & 음 & 음 \\
\hline 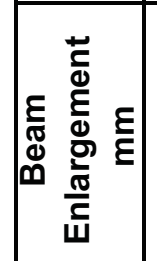 & $\stackrel{\widehat{N}}{\stackrel{N}{N}}$ & 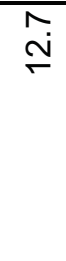 & 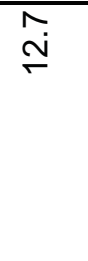 \\
\hline $\begin{array}{l}\vdots \\
\vdots \\
0 \\
\bar{d} \\
\infty\end{array}$ & $\begin{array}{l}\text { की } \\
\text { की }\end{array}$ & 吕 & $\begin{array}{l}\text { की } \\
\text { की }\end{array}$ \\
\hline 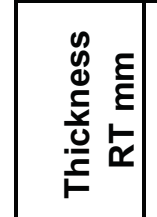 & 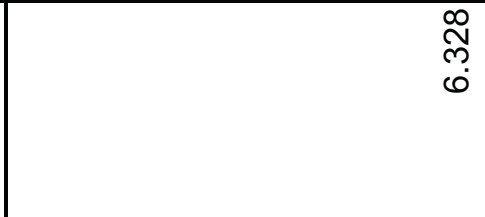 & $\begin{array}{l}\stackrel{\infty}{N} \\
\tilde{\omega} \\
0\end{array}$ & 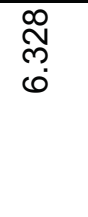 \\
\hline 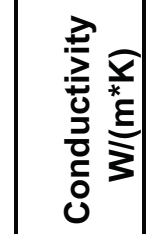 & & & \\
\hline 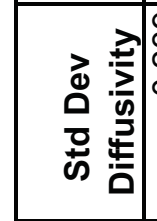 & 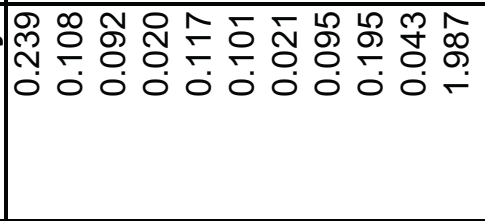 & $\stackrel{\sim}{\sim}$ & 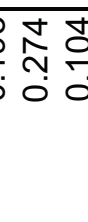 \\
\hline 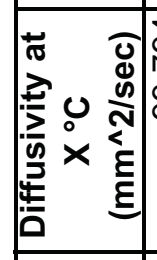 & 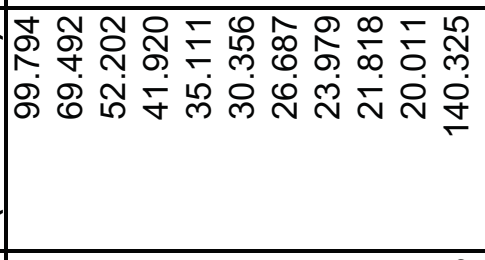 & 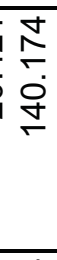 & 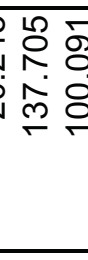 \\
\hline 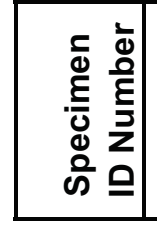 & 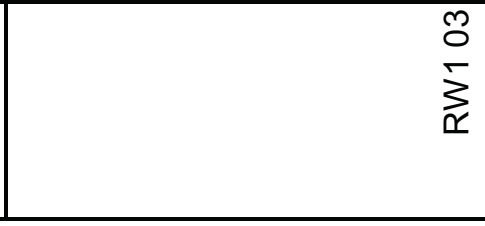 & $\sum_{\substack{x\\
}}^{+}$ & 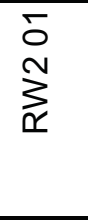 \\
\hline
\end{tabular}




\begin{tabular}{|c|c|c|c|}
\hline 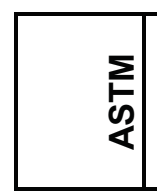 & 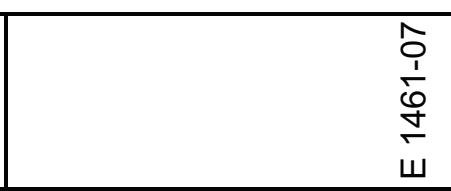 & $\begin{array}{l}\hat{o} \\
\frac{1}{0} \\
\stackrel{0}{+} \\
\dot{\omega}\end{array}$ & $\begin{array}{l}\frac{\hat{1}}{1} \\
\frac{1}{0} \\
\frac{1}{4} \\
\dot{w}\end{array}$ \\
\hline 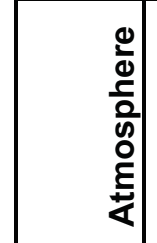 & 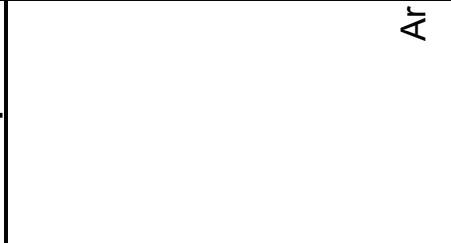 & 衣 & 交 \\
\hline 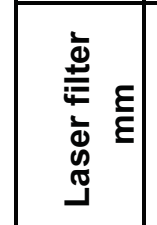 & 음 & 음 & 음 \\
\hline 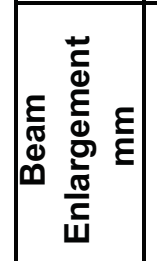 & 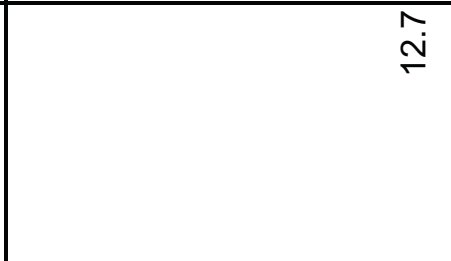 & $\stackrel{\stackrel{N}{ }}{ }$ & $\stackrel{\widetilde{N}}{\stackrel{N}{N}}$ \\
\hline $\begin{array}{l}\bar{b} \\
\vdots \\
心 \\
\Phi \\
\infty\end{array}$ & $\begin{array}{l}\text { की } \\
\text { की }\end{array}$ & $\begin{array}{l}\text { की } \\
\text { I }\end{array}$ & $\begin{array}{l}\text { की } \\
\text { की }\end{array}$ \\
\hline 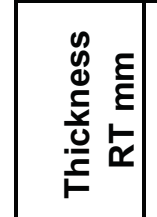 & $\begin{array}{l}\stackrel{\infty}{\infty} \\
\tilde{N} \\
\oplus\end{array}$ & $\begin{array}{l}\stackrel{\infty}{N} \\
\underset{0}{0}\end{array}$ & 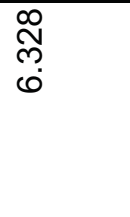 \\
\hline 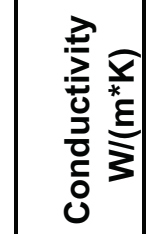 & & & \\
\hline 焉 & 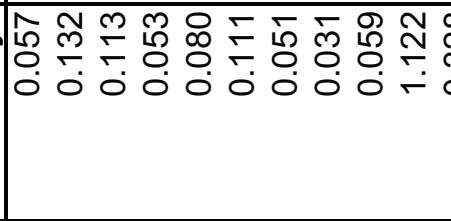 & 它 & 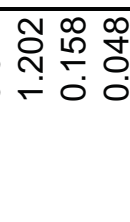 \\
\hline 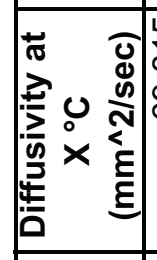 & 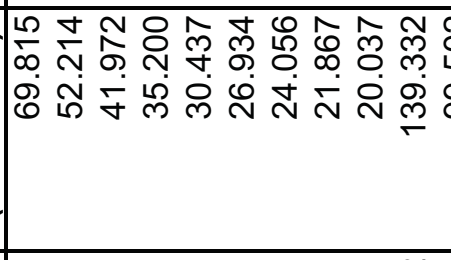 & 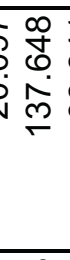 & 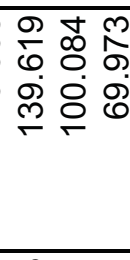 \\
\hline 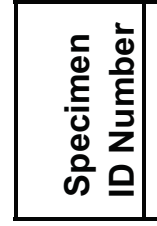 & $\sum_{\substack{x \\
N}}^{N}$ & 艿 & $\sum_{\substack{x \\
\infty}}^{\infty}$ \\
\hline
\end{tabular}




\begin{tabular}{|c|c|c|c|}
\hline 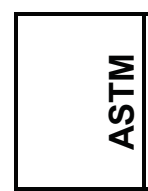 & $\begin{array}{l}\hat{o} \\
\dot{1} \\
\dot{0} \\
\dot{+} \\
\dot{w}\end{array}$ & $\begin{array}{l}\hat{o} \\
\frac{1}{0} \\
\stackrel{0}{+} \\
\dot{\omega}\end{array}$ & $\begin{array}{l}\hat{o} \\
\frac{1}{0} \\
\stackrel{0}{+} \\
\dot{w}\end{array}$ \\
\hline 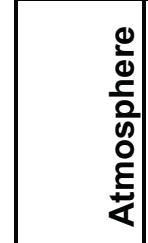 & 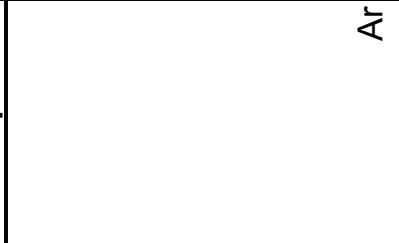 & 文 & $\frac{1}{4}$ \\
\hline 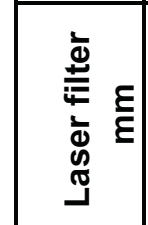 & 음 & $\stackrel{\circ}{\circ}$ & 음 \\
\hline 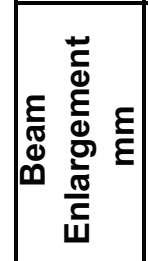 & $\stackrel{\widehat{N}}{\stackrel{N}{N}}$ & $\stackrel{\stackrel{N}{\sim}}{\mathrm{N}}$ & $\stackrel{\stackrel{\sim}{\sim}}{ }$ \\
\hline 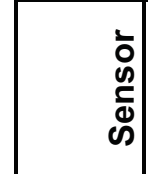 & $\begin{array}{l}\text { की } \\
\text { की }\end{array}$ & $\begin{array}{l}\text { की } \\
\text { I }\end{array}$ & $\begin{array}{l}\text { की } \\
\text { 足 }\end{array}$ \\
\hline 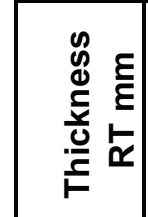 & 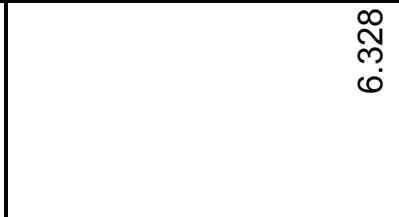 & $\begin{array}{l}\stackrel{\infty}{N} \\
\underset{0}{0}\end{array}$ & $\begin{array}{l}\stackrel{\infty}{\tilde{N}} \\
\dot{\omega}\end{array}$ \\
\hline 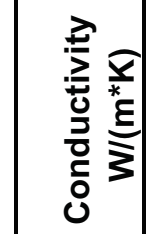 & & & \\
\hline 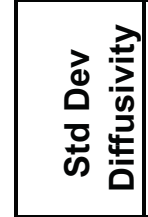 & 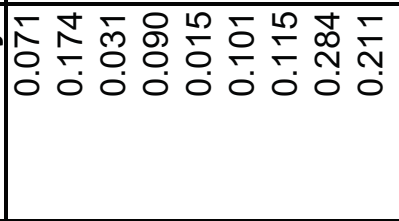 & 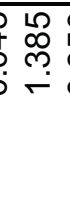 & 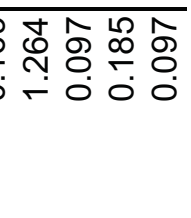 \\
\hline 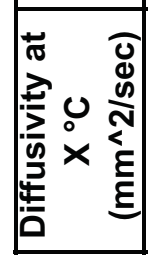 & 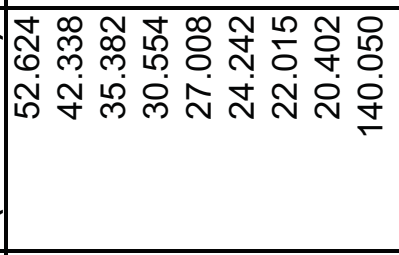 & 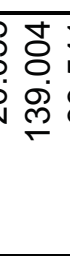 & 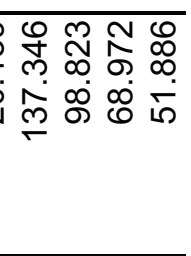 \\
\hline 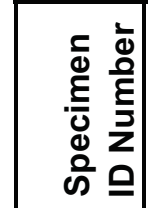 & $\begin{array}{l}8 \\
\\
\sum_{\mathbb{1}}\end{array}$ & $\frac{\sum_{x}^{\circ}}{\sum_{x}}$ & 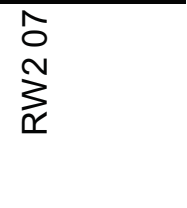 \\
\hline
\end{tabular}




\begin{tabular}{|c|c|c|c|}
\hline 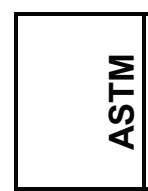 & $\begin{array}{l}\hat{O} \\
\frac{1}{0} \\
\stackrel{0}{+} \\
\dot{w}\end{array}$ & 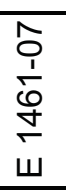 & $\begin{array}{l}\hat{O} \\
\frac{1}{0} \\
\frac{0}{+} \\
\dot{w}\end{array}$ \\
\hline $\begin{array}{c}0 \\
\frac{0}{0} \\
\frac{5}{0} \\
0 \\
0 \\
\frac{1}{4}\end{array}$ & 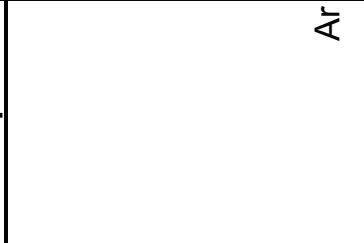 & $\frac{1}{\alpha}$ & 交 \\
\hline 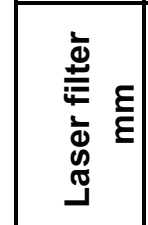 & $\stackrel{\circ}{\circ}$ & 음 & 음 \\
\hline 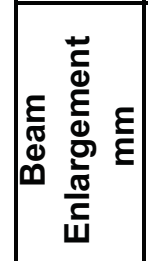 & $\stackrel{\widehat{N}}{\stackrel{N}{S}}$ & 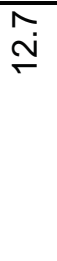 & $\stackrel{\stackrel{N}{N}}{ }$ \\
\hline 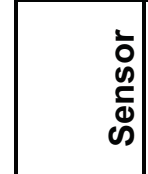 & $\begin{array}{l}\text { की } \\
\text { की }\end{array}$ & $\begin{array}{l}\text { की } \\
\text { क् }\end{array}$ & $\begin{array}{l}\text { की } \\
\text { Ф }\end{array}$ \\
\hline 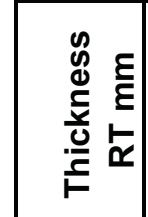 & $\begin{array}{l}\stackrel{\infty}{\infty} \\
\tilde{N} \\
i\end{array}$ & 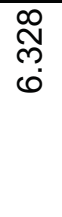 & 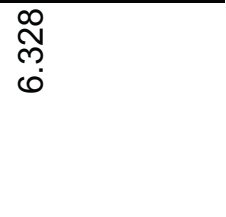 \\
\hline 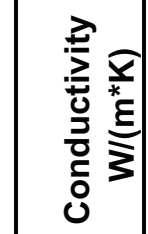 & & & \\
\hline 焉竞 & 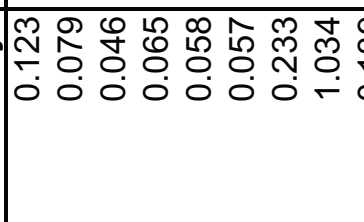 & - & 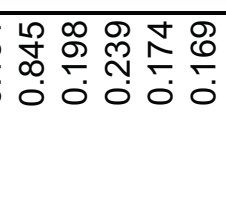 \\
\hline 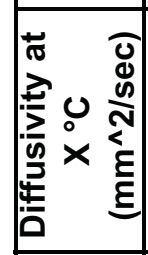 & 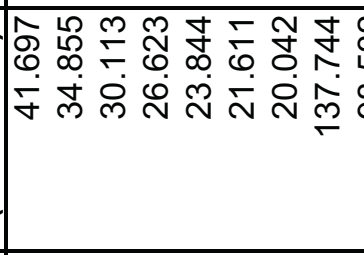 & $\begin{array}{l}\bar{\alpha} \\
\dot{m} \\
\underline{m}\end{array}$ & 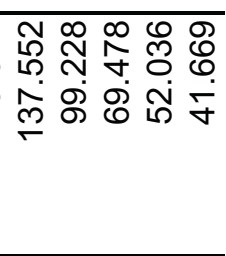 \\
\hline 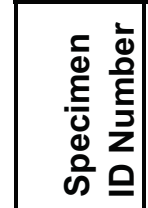 & $\begin{array}{l}\sum_{\infty}^{\infty} \\
\sum_{\alpha}^{N} \\
\end{array}$ & 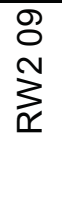 & 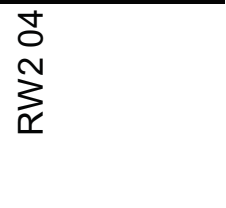 \\
\hline
\end{tabular}




\begin{tabular}{|c|c|c|c|}
\hline 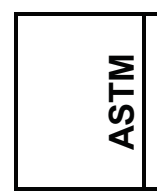 & $\begin{array}{l}\frac{0}{1} \\
\frac{1}{0} \\
\frac{1}{+} \\
\dot{w}\end{array}$ & $\begin{array}{l}\hat{o} \\
\frac{1}{0} \\
\stackrel{0}{+} \\
\dot{\omega}\end{array}$ & $\begin{array}{l}\hat{0} \\
\frac{1}{0} \\
\stackrel{0}{+} \\
\dot{\omega}\end{array}$ \\
\hline 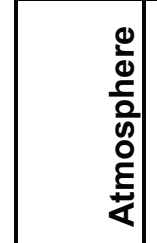 & 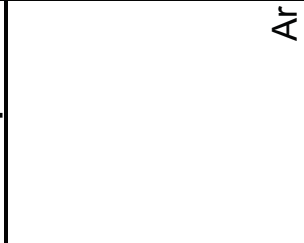 & 㐫 & $\frac{1}{4}$ \\
\hline 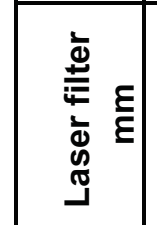 & 음 & 음 & 음 \\
\hline 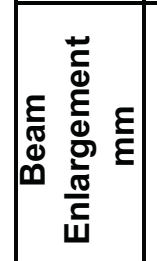 & $\stackrel{\widetilde{N}}{\stackrel{N}{N}}$ & 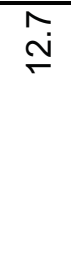 & $\stackrel{\stackrel{\sim}{\sim}}{ }$ \\
\hline 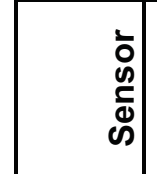 & $\begin{array}{l}\text { की } \\
\text { की }\end{array}$ & 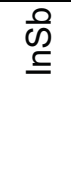 & $\begin{array}{l}\text { की } \\
\text { 足 }\end{array}$ \\
\hline 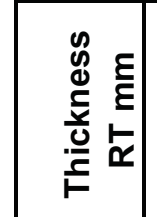 & 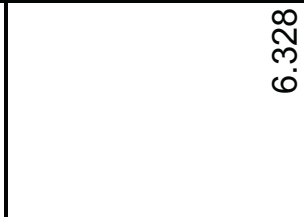 & $\begin{array}{l}\stackrel{\infty}{N} \\
\underset{0}{0}\end{array}$ & $\begin{array}{l}\stackrel{\infty}{0} \\
\underset{N}{0} \\
0\end{array}$ \\
\hline 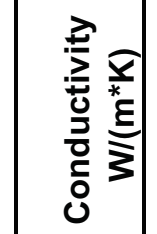 & & & \\
\hline 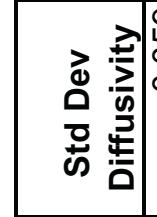 & 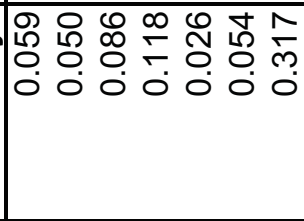 & 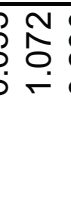 & 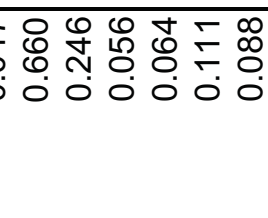 \\
\hline 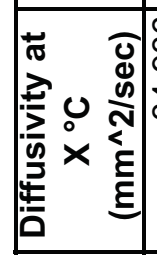 & 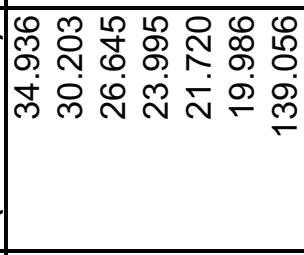 & 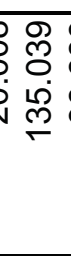 & 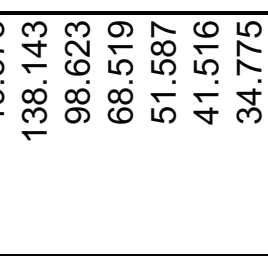 \\
\hline 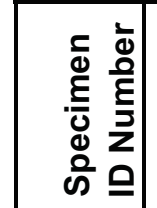 & 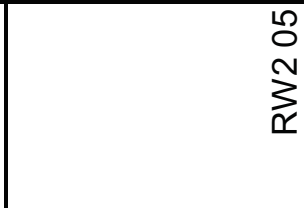 & $\begin{array}{l}8 \\
\\
\sum_{\alpha \times}^{N}\end{array}$ & 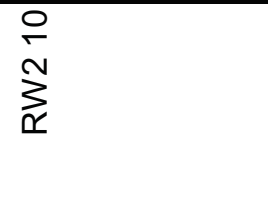 \\
\hline
\end{tabular}




\begin{tabular}{|c|c|c|c|}
\hline 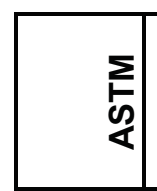 & $\begin{array}{l}\hat{o} \\
\frac{1}{0} \\
\dot{o} \\
\dot{w} \\
\end{array}$ & $\begin{array}{l}\hat{o} \\
\frac{1}{1} \\
\dot{o} \\
\dot{+} \\
w\end{array}$ & 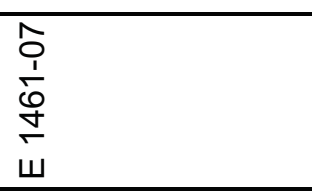 \\
\hline 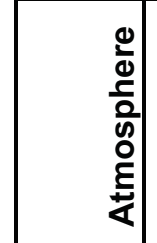 & 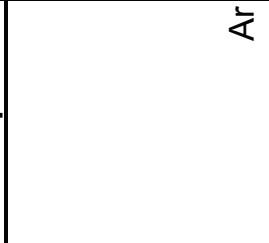 & 㐫 & $\dot{z}$ \\
\hline 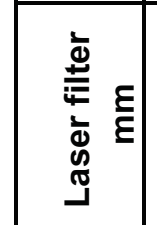 & 음 & 음 & 음 \\
\hline 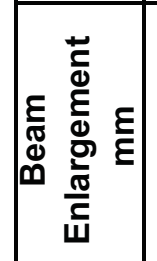 & $\stackrel{\widehat{N}}{\stackrel{N}{N}}$ & 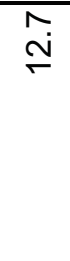 & 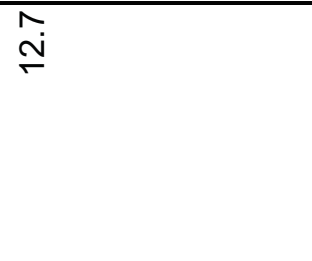 \\
\hline $\begin{array}{l}\bar{b} \\
0 \\
\stackrel{5}{\Phi} \\
\infty\end{array}$ & $\begin{array}{l}\text { की } \\
\text { की }\end{array}$ & 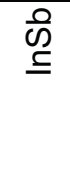 & $\begin{array}{l}\text { की } \\
\text { 足 }\end{array}$ \\
\hline 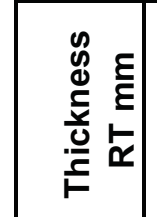 & $\begin{array}{c}\text { ্ָ } \\
\underset{0}{0}\end{array}$ & $\begin{array}{l}\infty \\
\underset{N}{\infty} \\
0\end{array}$ & 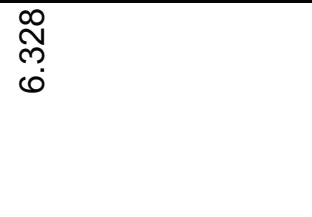 \\
\hline 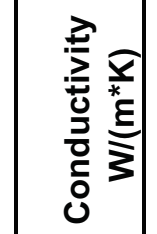 & & & \\
\hline 焉 & 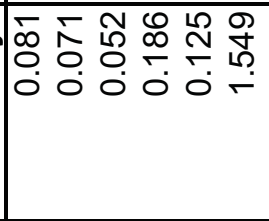 & $\begin{array}{l}0 \\
0 \\
0 \\
0 \\
0\end{array}$ & $\begin{array}{l}0 \\
0\end{array}$ \\
\hline 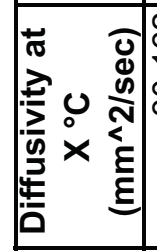 & 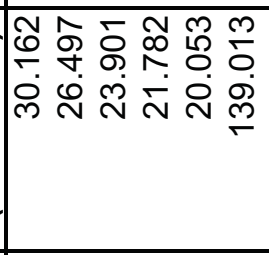 & 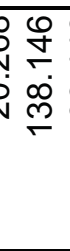 & 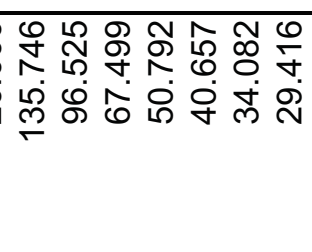 \\
\hline 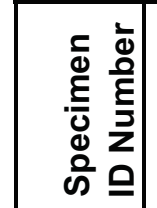 & 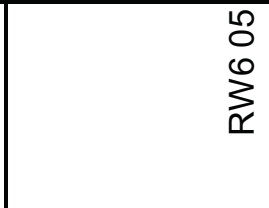 & $\begin{array}{l}8 \\
8 \\
0 \\
0 \\
0\end{array}$ & 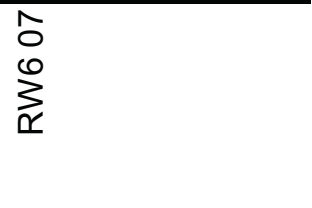 \\
\hline
\end{tabular}




\begin{tabular}{|c|c|c|c|}
\hline$\underset{\Sigma}{\Sigma}$ & $\begin{array}{l}\hat{o} \\
\dot{1} \\
\dot{6} \\
\dot{+} \\
\dot{\omega}\end{array}$ & $\begin{array}{l}\hat{O} \\
\frac{1}{1} \\
\frac{\sigma}{5} \\
\dot{\omega}\end{array}$ & $\begin{array}{l}\hat{o} \\
\frac{1}{0} \\
\dot{0} \\
\dot{W} \\
\end{array}$ \\
\hline 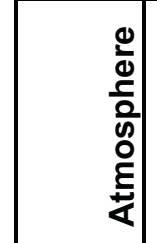 & 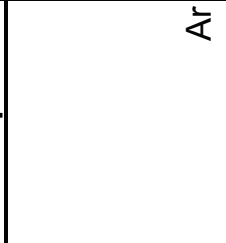 & ¿ & $\dot{k}$ \\
\hline 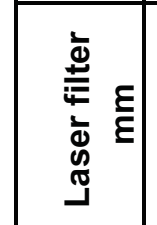 & 응 & 음 & 음 \\
\hline 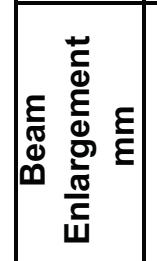 & $\stackrel{\widehat{N}}{\stackrel{N}{N}}$ & 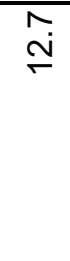 & $\stackrel{\stackrel{N}{\sim}}{ }$ \\
\hline $\begin{array}{l}\bar{b} \\
0 \\
\stackrel{0}{\Phi} \\
心\end{array}$ & $\begin{array}{l}\text { की } \\
\text { की }\end{array}$ & $\begin{array}{l}\text { की } \\
\text { की }\end{array}$ & 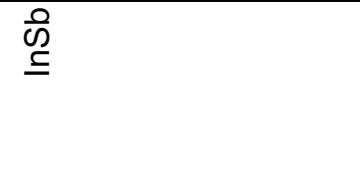 \\
\hline 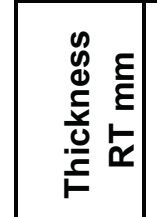 & $\begin{array}{c}\text { ্ָ } \\
\underset{0}{0}\end{array}$ & 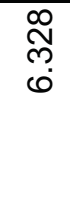 & $\begin{array}{l}\infty \\
\widetilde{N} \\
0\end{array}$ \\
\hline 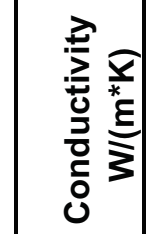 & & & \\
\hline 焉 & 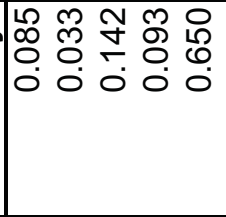 & $\begin{array}{l}\frac{8}{R} \\
\stackrel{0}{\circ} \\
\end{array}$ & 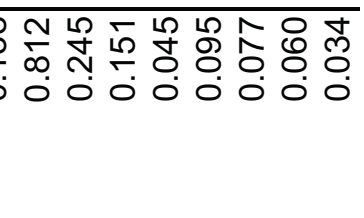 \\
\hline 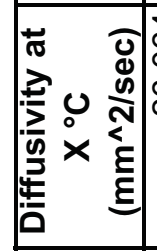 & 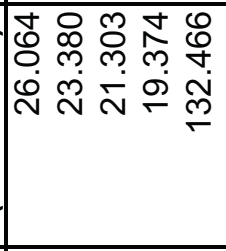 & 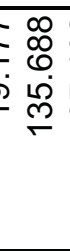 & 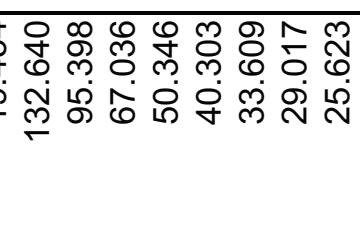 \\
\hline 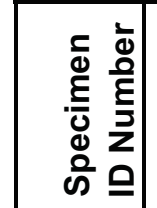 & 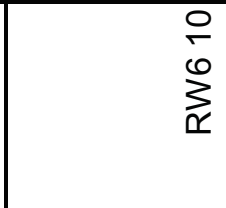 & \begin{tabular}{l}
$0^{\infty}$ \\
0 \\
0 \\
0 \\
\hdashline
\end{tabular} & $\begin{array}{l}8 \\
8 \\
0 \\
0 \\
01\end{array}$ \\
\hline
\end{tabular}




\begin{tabular}{|c|c|c|c|}
\hline$\underset{\Sigma}{\Sigma}$ & $\begin{array}{l}\hat{o} \\
\dot{1} \\
\dot{6} \\
\dot{+} \\
\dot{\omega}\end{array}$ & $\begin{array}{l}\hat{o} \\
\dot{1} \\
\frac{0}{6} \\
\dot{+} \\
\dot{w} \\
\end{array}$ & 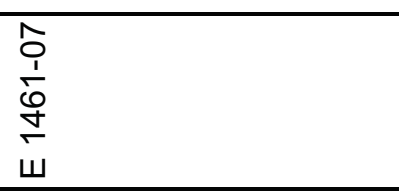 \\
\hline 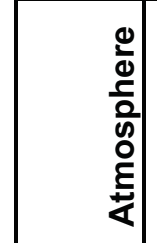 & 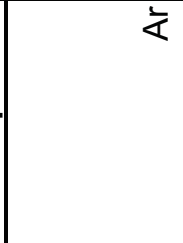 & ¿ & 交 \\
\hline 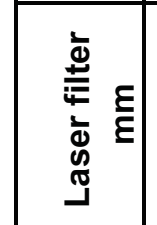 & 응 & 음 & 응 \\
\hline 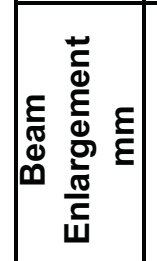 & $\stackrel{\widehat{N}}{\stackrel{N}{N}}$ & $\stackrel{\stackrel{N}{\sim}}{ }$ & $\stackrel{\stackrel{N}{N}}{ }$ \\
\hline $\begin{array}{l}\bar{b} \\
0 \\
\stackrel{0}{\Phi} \\
心\end{array}$ & $\begin{array}{l}\text { की } \\
\text { की }\end{array}$ & $\begin{array}{l}\text { की } \\
\text { 足 }\end{array}$ & $\begin{array}{l}\text { की } \\
\text { I }\end{array}$ \\
\hline 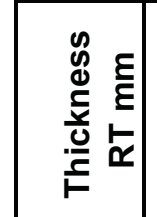 & $\begin{array}{c}\text { ্ָ } \\
\underset{0}{0}\end{array}$ & 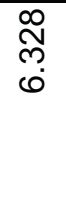 & 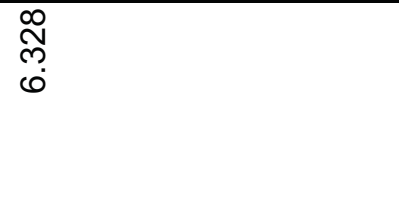 \\
\hline 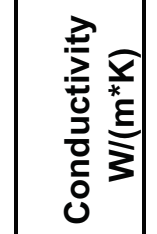 & & & \\
\hline 焉 & 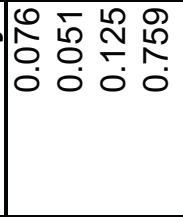 & $\dot{0}$ & 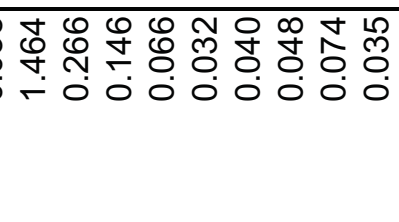 \\
\hline 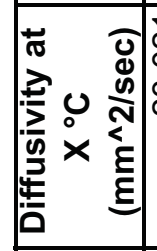 & 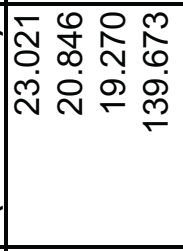 & 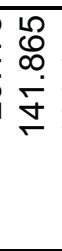 & 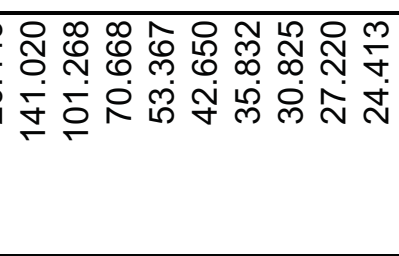 \\
\hline 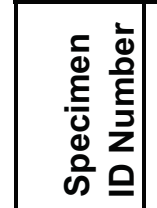 & 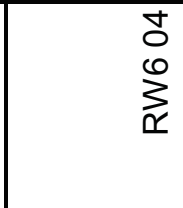 & 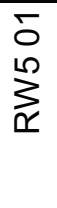 & $\begin{array}{l}\text { No } \\
\text { 年 } \\
\sum_{x=1}\end{array}$ \\
\hline
\end{tabular}




\begin{tabular}{|c|c|c|c|}
\hline$\underset{\Sigma}{\Sigma}$ & $\begin{array}{l}\hat{S} \\
\frac{1}{\delta} \\
\stackrel{\sigma}{\leftarrow} \\
\text { W }\end{array}$ & 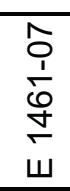 & 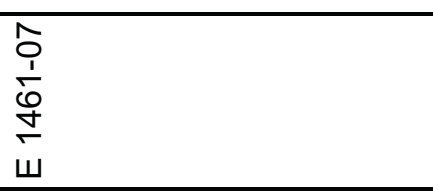 \\
\hline 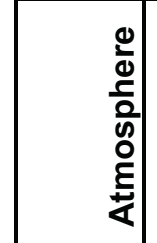 & 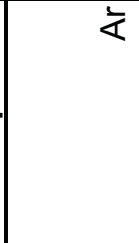 & ¿ & 文 \\
\hline 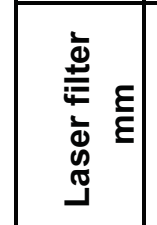 & 음 & 음 & 음 \\
\hline 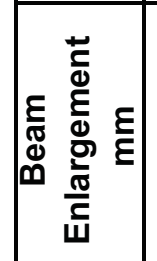 & $\stackrel{\widetilde{N}}{\stackrel{N}{N}}$ & $\stackrel{\grave{N}}{\stackrel{N}{N}}$ & $\stackrel{\widetilde{N}}{\stackrel{N}{N}}$ \\
\hline 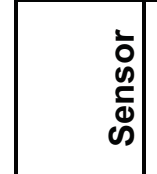 & की & $\begin{array}{l}\text { की } \\
\text { की }\end{array}$ & $\begin{array}{l}\text { की } \\
\text { की }\end{array}$ \\
\hline 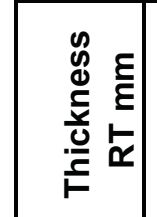 & 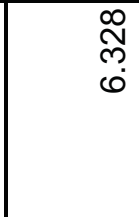 & 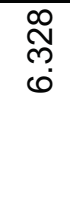 & $\begin{array}{l}\stackrel{\infty}{\infty} \\
\tilde{N} \\
i\end{array}$ \\
\hline 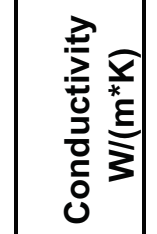 & & & \\
\hline 焉 & 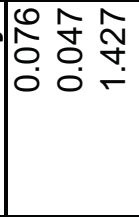 & ָָ ָָ & 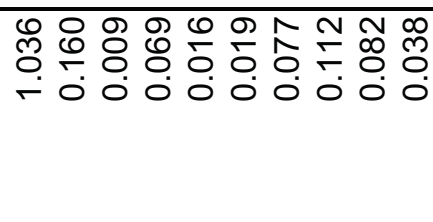 \\
\hline 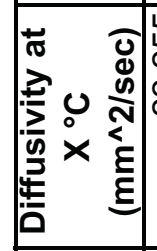 & 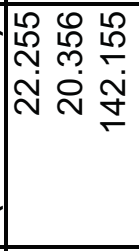 & b & 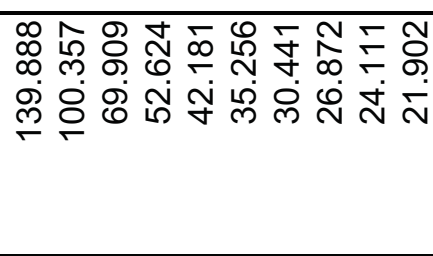 \\
\hline 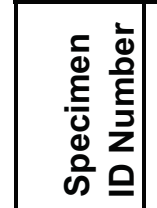 & $\begin{array}{l}\bar{\delta} \\
\sum_{x<}^{0}\end{array}$ & 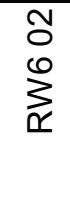 & $\begin{array}{l}0 \\
0 \\
0 \\
0 \\
0\end{array}$ \\
\hline
\end{tabular}




\begin{tabular}{|c|c|c|c|}
\hline$\underset{\substack{5 \\
\mathbf{c}}}{\Sigma}$ & 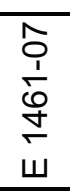 & $\begin{array}{l}\hat{o} \\
\dot{1} \\
\frac{1}{6} \\
\dot{+} \\
\dot{w} \\
\end{array}$ & 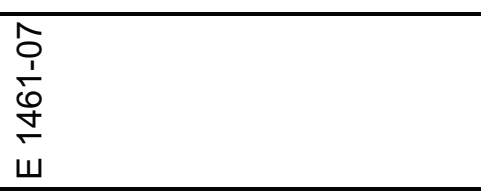 \\
\hline 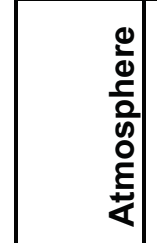 & $\frac{1}{4}$ & ¿ & 文 \\
\hline 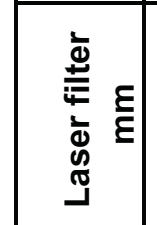 & 음 & 음 & 음 \\
\hline 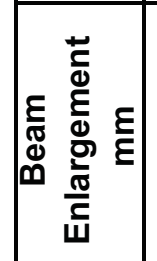 & $\stackrel{\stackrel{\leftrightarrow}{\sim}}{ }$ & $\stackrel{\stackrel{N}{\sim}}{\text { N }}$ & $\stackrel{\grave{N}}{\stackrel{N}{N}}$ \\
\hline 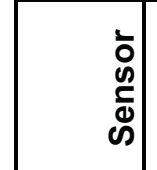 & $\begin{array}{l}\text { की } \\
\text { 足 }\end{array}$ & $\begin{array}{l}\text { की } \\
\text { 足 }\end{array}$ & $\begin{array}{l}\text { की } \\
\text { की }\end{array}$ \\
\hline 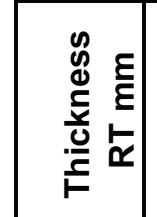 & 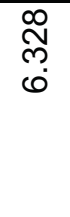 & 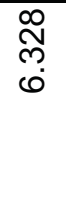 & $\begin{array}{l}\stackrel{\infty}{\widetilde{N}} \\
\text { }\end{array}$ \\
\hline 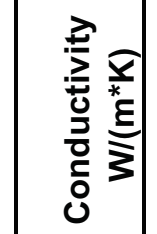 & & & \\
\hline 焉 & $\begin{array}{l}\infty \\
0 \\
0 \\
0 \\
0\end{array}$ & $\hat{0}$ & 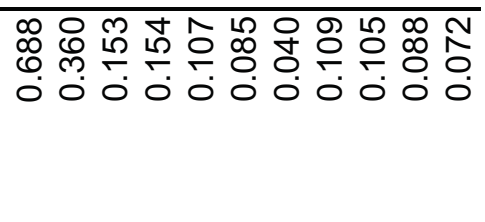 \\
\hline 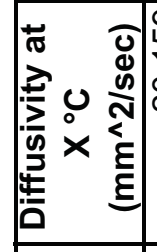 & 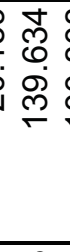 & $\begin{array}{l}\text { f } \\
0 \\
0 \\
\stackrel{0}{\circ}\end{array}$ & 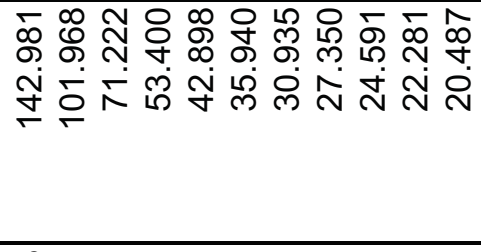 \\
\hline 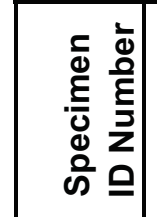 & $\begin{array}{l}{ }^{\infty} \\
0 \\
\sum_{x=1}^{n}\end{array}$ & 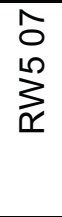 & 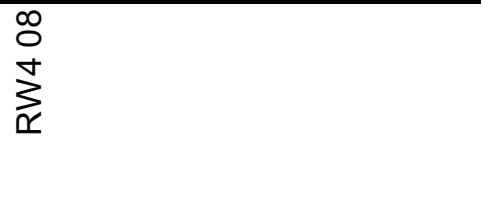 \\
\hline
\end{tabular}




\begin{tabular}{|c|c|c|c|c|}
\hline $\begin{array}{l}\Sigma \\
\vdots \\
\\
\end{array}$ & & $\begin{array}{l}\hat{o} \\
\frac{1}{1} \\
\dot{\sigma} \\
\dot{L} \\
\end{array}$ & 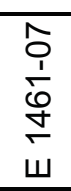 & 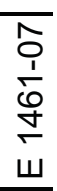 \\
\hline 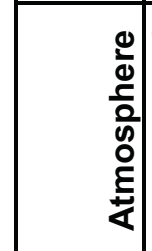 & & $\bar{k}$ & $\dot{z}$ & $\bar{k}$ \\
\hline 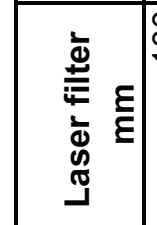 & 8 & 음 & 음 & 음 \\
\hline 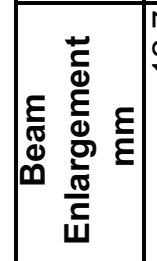 & $\stackrel{\hat{N}}{\underline{N}}$ & $\stackrel{\widehat{N}}{\stackrel{N}{N}}$ & $\stackrel{\stackrel{N}{\sim}}{ }$ & 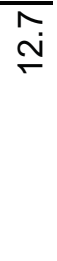 \\
\hline \begin{tabular}{l|} 
\\
$\vdots$ \\
$\grave{d}$ \\
$\grave{d}$
\end{tabular} & & 吕 & 吕 & $\begin{array}{l}\text { की } \\
\text { क् }\end{array}$ \\
\hline 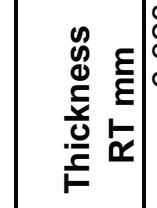 & & $\begin{array}{l}\stackrel{\infty}{N} \\
\underset{\omega}{0}\end{array}$ & $\begin{array}{l}\stackrel{\infty}{N} \\
\underset{0}{0}\end{array}$ & 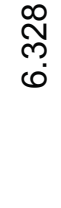 \\
\hline 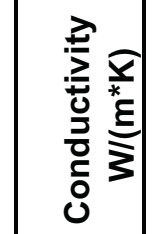 & & & & \\
\hline 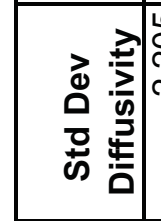 & & 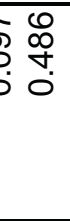 & 蛋 & $\left\{\begin{array}{l}0 \\
\infty \\
\infty \\
0 \\
0\end{array}\right.$ \\
\hline 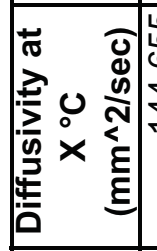 & 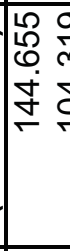 & 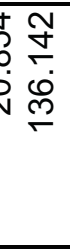 & 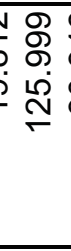 & 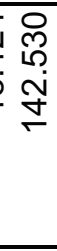 \\
\hline 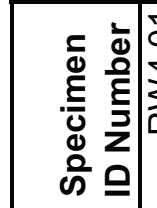 & & $\sum_{i \infty}^{\circ}$ & $\begin{array}{l}8 \\
0 \\
\sum_{x=1}^{n}\end{array}$ & $\begin{array}{l}8 \\
8 \\
\sum_{\mathbb{1}}^{+}\end{array}$ \\
\hline
\end{tabular}




\begin{tabular}{|c|c|c|c|}
\hline$\underset{\Sigma}{\Sigma}$ & $\begin{array}{l}\hat{o} \\
\frac{1}{1} \\
\dot{0} \\
\frac{1}{\omega} \\
\end{array}$ & $\begin{array}{l}\hat{S} \\
\frac{1}{1} \\
\frac{0}{5} \\
\dot{w}\end{array}$ & 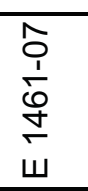 \\
\hline 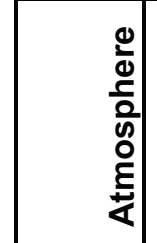 & 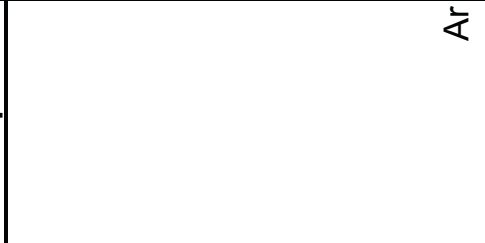 & $\frac{1}{\alpha}$ & 广 \\
\hline 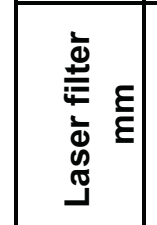 & 음 & $\stackrel{8}{\circ}$ & 음 \\
\hline 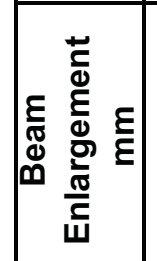 & $\stackrel{\widehat{N}}{\stackrel{N}{N}}$ & 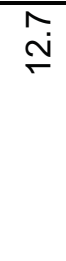 & 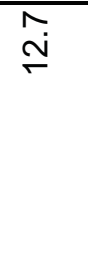 \\
\hline $\begin{array}{l}\bar{b} \\
\vdots \\
心 \\
\Phi \\
\infty\end{array}$ & $\begin{array}{l}\text { की } \\
\text { की }\end{array}$ & 吊 & $\begin{array}{l}\text { की } \\
\text { 足 }\end{array}$ \\
\hline 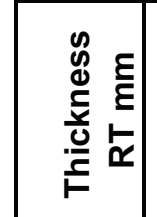 & 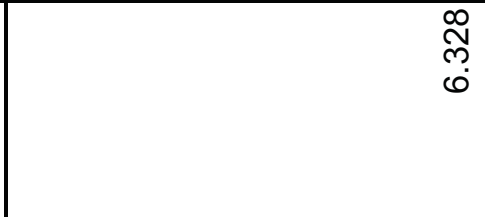 & $\begin{array}{l}\stackrel{\infty}{N} \\
\tilde{\omega} \\
0\end{array}$ & 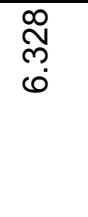 \\
\hline 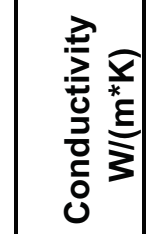 & & & \\
\hline 焉 & 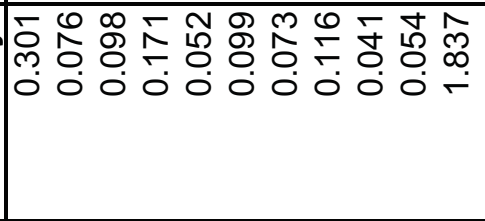 & ; & : \\
\hline 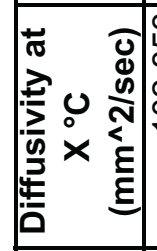 & 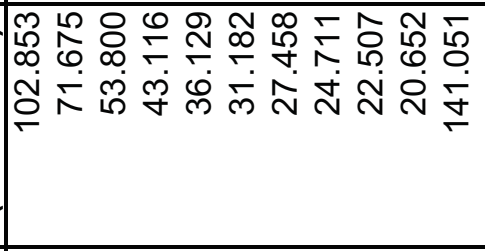 & $\begin{array}{l}\infty \\
\infty \\
\stackrel{\infty}{\sigma} \\
\stackrel{9}{+}\end{array}$ & 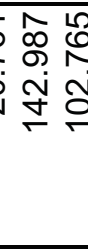 \\
\hline 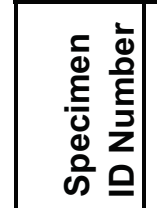 & 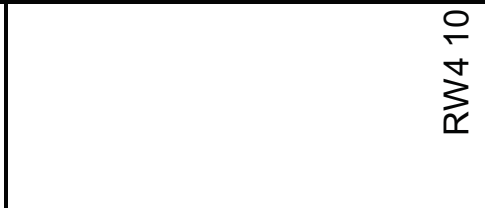 & $\begin{array}{l}\text { O } \\
\text { Oे } \\
\sum_{\alpha=}^{+}\end{array}$ & $\begin{array}{l}\text { O } \\
\text { O } \\
\sum_{\text {X }}^{+}\end{array}$ \\
\hline
\end{tabular}




\begin{tabular}{|c|c|c|c|}
\hline 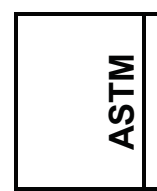 & 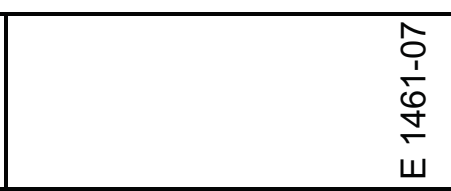 & $\begin{array}{l}\hat{o} \\
\frac{1}{0} \\
\stackrel{0}{+} \\
\dot{\omega}\end{array}$ & $\begin{array}{l}\hat{o} \\
\frac{1}{0} \\
\stackrel{0}{+} \\
\dot{\omega}\end{array}$ \\
\hline 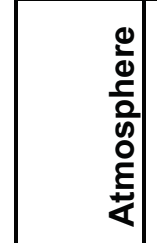 & 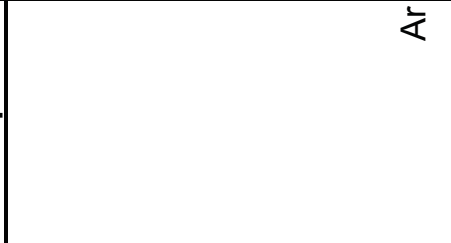 & 衣 & 交 \\
\hline 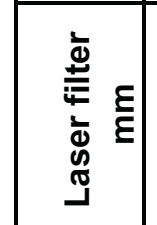 & 음 & 음 & 음 \\
\hline 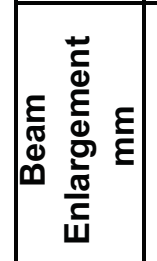 & 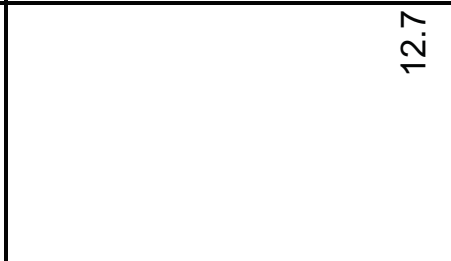 & $\stackrel{\stackrel{N}{ }}{ }$ & $\stackrel{\stackrel{\sim}{\sim}}{ }$ \\
\hline $\begin{array}{l}\vdots \\
\vdots \\
0 \\
\bar{d} \\
\infty\end{array}$ & $\begin{array}{l}\text { की } \\
\text { की }\end{array}$ & 吕 & $\begin{array}{l}\text { की } \\
\text { की }\end{array}$ \\
\hline 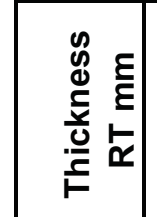 & $\begin{array}{l}\stackrel{\infty}{\infty} \\
\tilde{N} \\
\oplus\end{array}$ & $\begin{array}{l}\stackrel{\infty}{N} \\
\underset{0}{0}\end{array}$ & 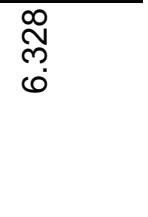 \\
\hline 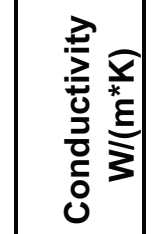 & & & \\
\hline 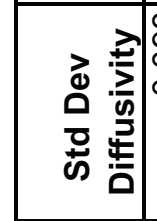 & 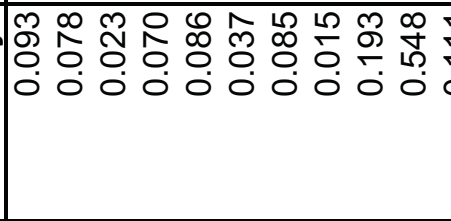 & $\begin{array}{l}0 \\
0 \\
0 \\
0 \\
0 \\
0\end{array}$ & 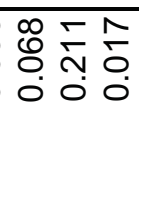 \\
\hline 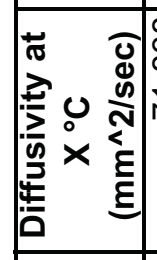 & 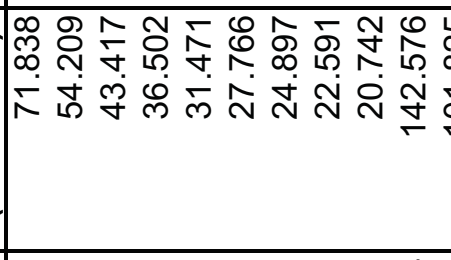 & 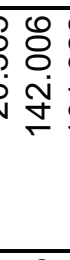 & 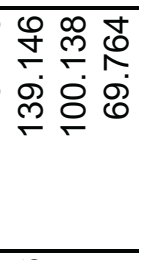 \\
\hline 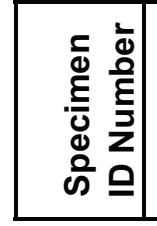 & $\begin{array}{l}0 \\
0 \\
\sum_{x}^{+}\end{array}$ & 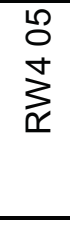 & $\begin{array}{l}8 \\
\sum_{\not<}^{+} \\
+\end{array}$ \\
\hline
\end{tabular}




\begin{tabular}{|c|c|c|c|}
\hline 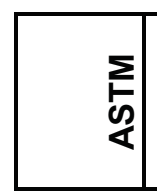 & $\begin{array}{l}\hat{o} \\
\dot{1} \\
\dot{0} \\
\dot{+} \\
\dot{w}\end{array}$ & $\begin{array}{l}\hat{o} \\
\frac{1}{0} \\
\stackrel{0}{+} \\
\dot{\omega}\end{array}$ & $\begin{array}{l}\hat{o} \\
\frac{1}{0} \\
\stackrel{0}{+} \\
\dot{w}\end{array}$ \\
\hline 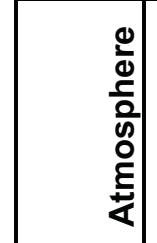 & 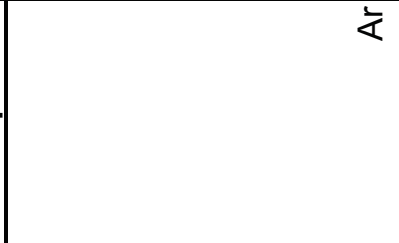 & 文 & 交 \\
\hline 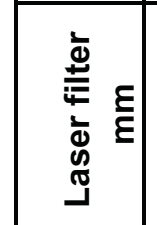 & 음 & $\stackrel{\circ}{\circ}$ & 음 \\
\hline 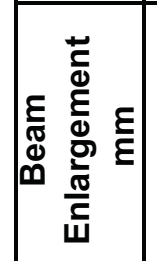 & $\stackrel{\widehat{N}}{\stackrel{N}{N}}$ & $\stackrel{\stackrel{N}{\sim}}{\mathrm{N}}$ & $\stackrel{\stackrel{\sim}{\sim}}{ }$ \\
\hline $\begin{array}{l}\bar{b} \\
0 \\
\stackrel{5}{\Phi} \\
\infty\end{array}$ & $\begin{array}{l}\text { की } \\
\text { की }\end{array}$ & $\begin{array}{l}\text { की } \\
\text { I }\end{array}$ & $\begin{array}{l}\text { की } \\
\text { 足 }\end{array}$ \\
\hline 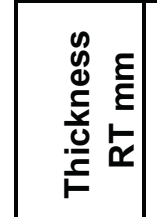 & 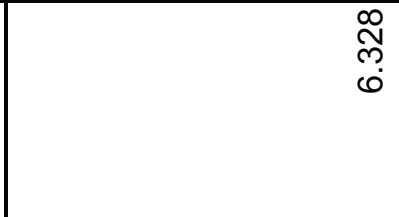 & $\begin{array}{l}\stackrel{\infty}{N} \\
\underset{0}{0}\end{array}$ & $\begin{array}{l}\stackrel{\infty}{\tilde{N}} \\
\dot{\omega}\end{array}$ \\
\hline 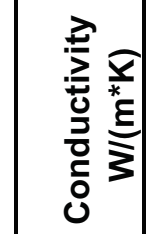 & & & \\
\hline 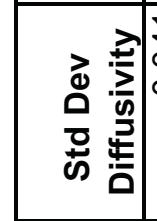 & 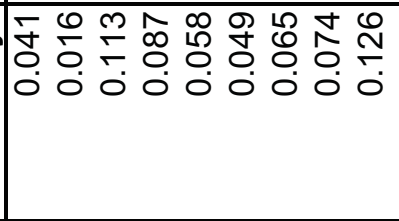 & 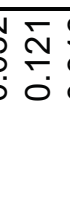 & 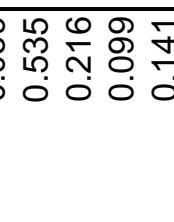 \\
\hline 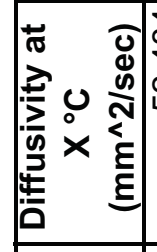 & 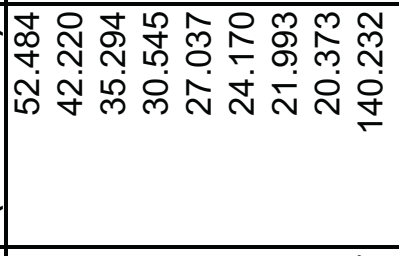 & ț & 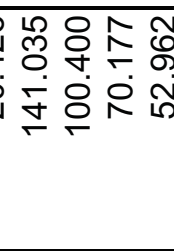 \\
\hline 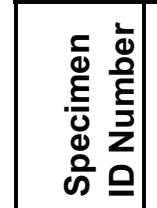 & $\begin{array}{l}\text { Tे } \\
\text { O } \\
\sum_{\text {I }}^{+}\end{array}$ & $\begin{array}{l}\text { m } \\
\text { m } \\
\sum_{\alpha=1}^{n}\end{array}$ & 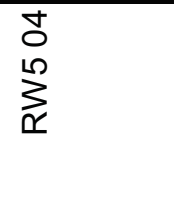 \\
\hline
\end{tabular}




\begin{tabular}{|c|c|c|}
\hline 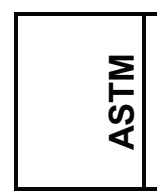 & $\begin{array}{l}\frac{\hat{1}}{1} \\
\frac{1}{6} \\
\frac{1}{\leftarrow} \\
\dot{\omega}\end{array}$ & 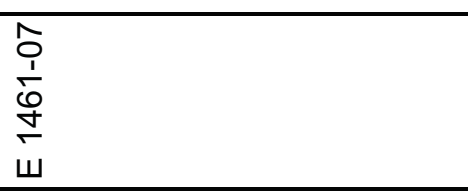 \\
\hline 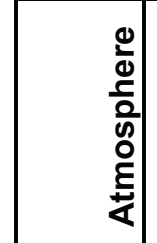 & 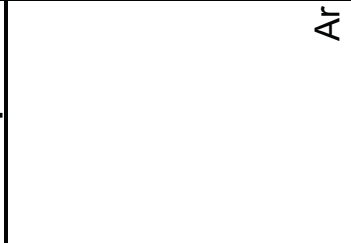 & 㐫 \\
\hline 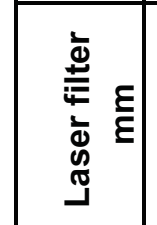 & $\stackrel{0}{\circ}$ & 음 \\
\hline 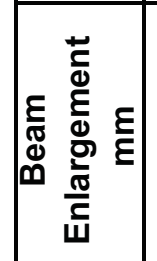 & $\stackrel{\bar{N}}{\stackrel{N}{N}}$ & 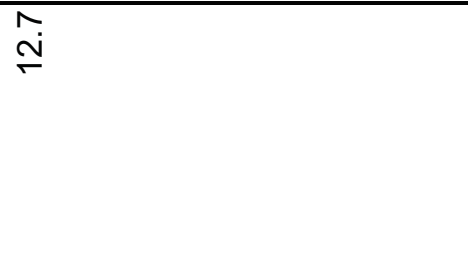 \\
\hline 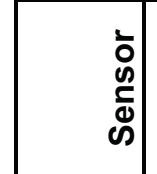 & $\begin{array}{l}\text { की } \\
\text { ? }\end{array}$ & $\begin{array}{l}\text { की } \\
\text { Ф }\end{array}$ \\
\hline 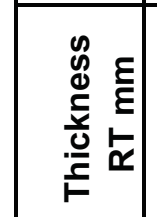 & 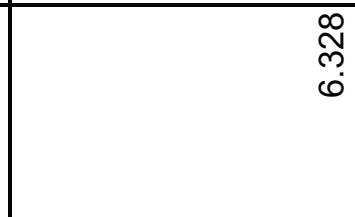 & 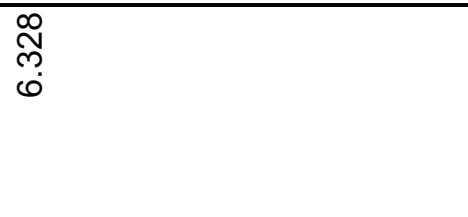 \\
\hline 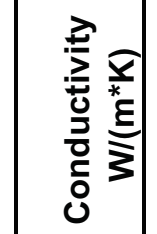 & & \\
\hline 焉 & 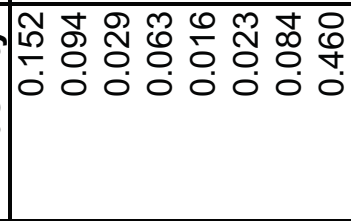 & 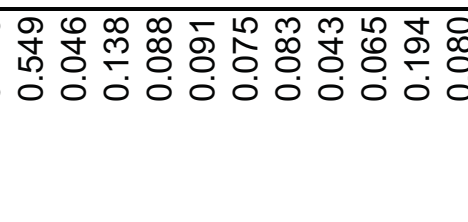 \\
\hline 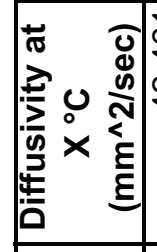 & 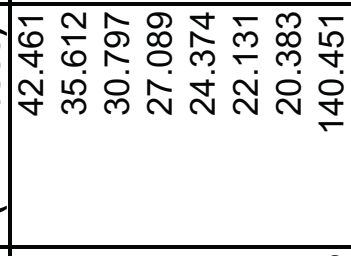 & 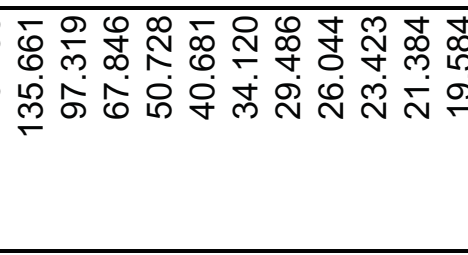 \\
\hline 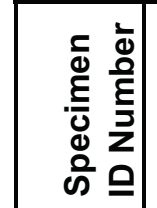 & 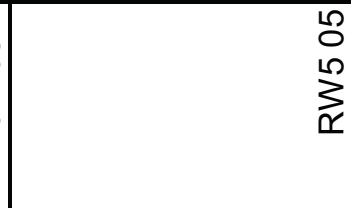 & $\begin{array}{l}8 \\
8 \\
\sum_{x=1}^{0}\end{array}$ \\
\hline
\end{tabular}




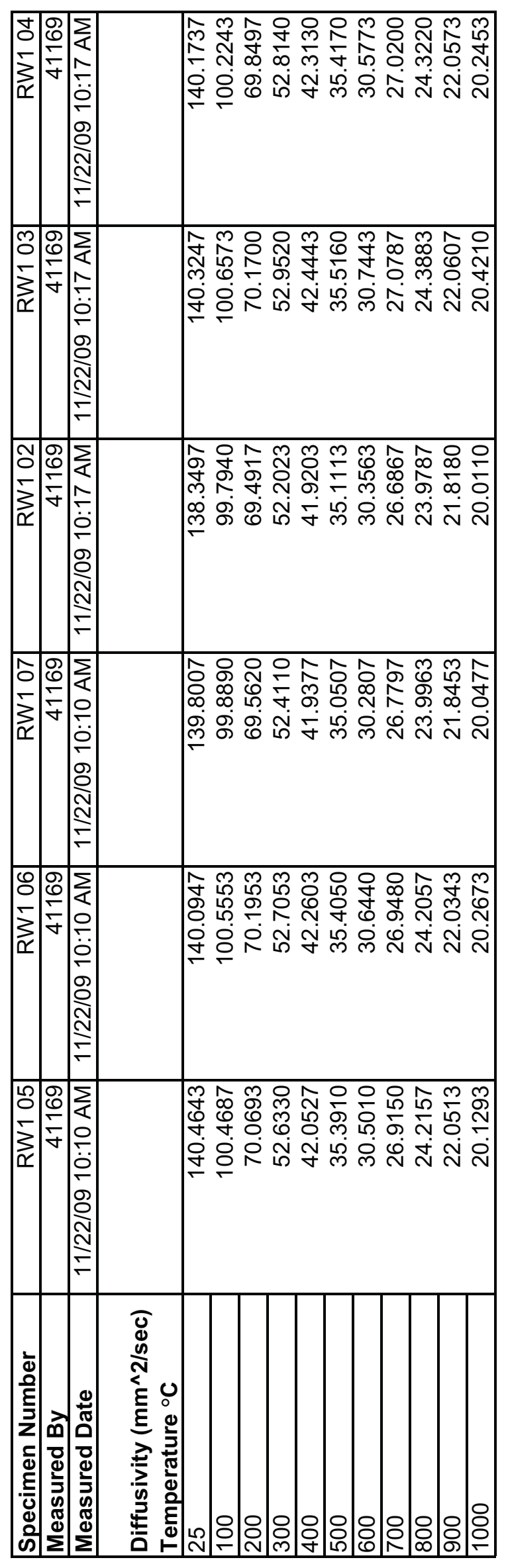




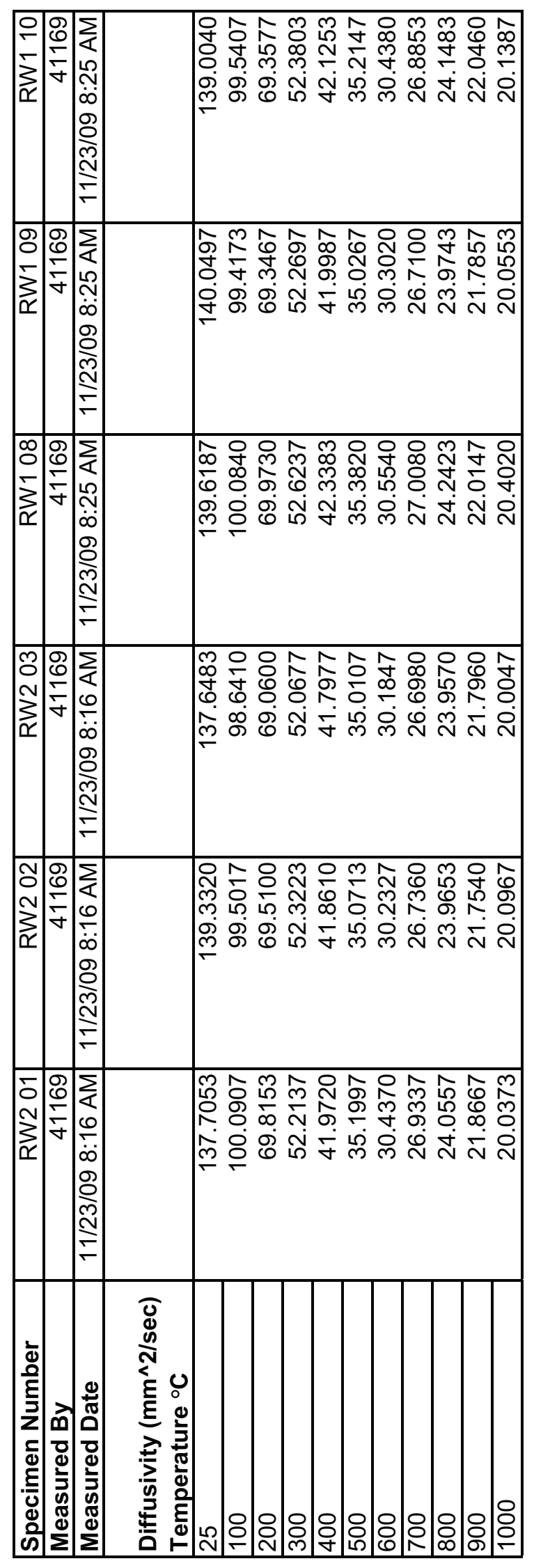




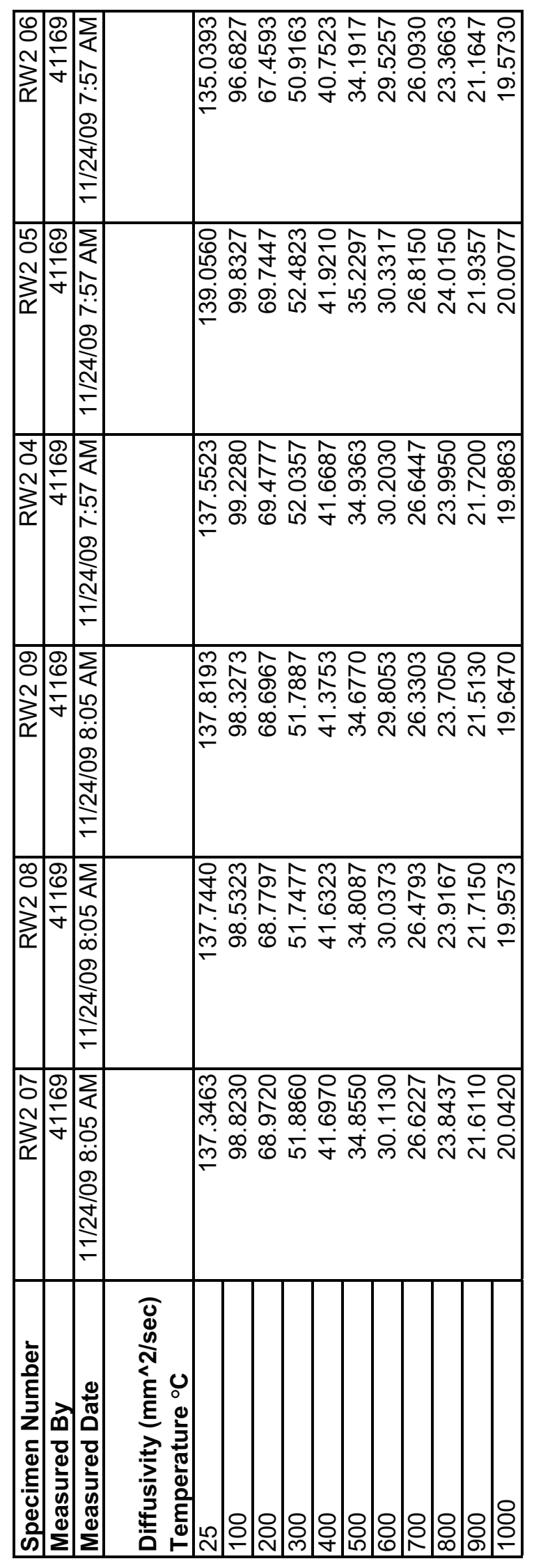




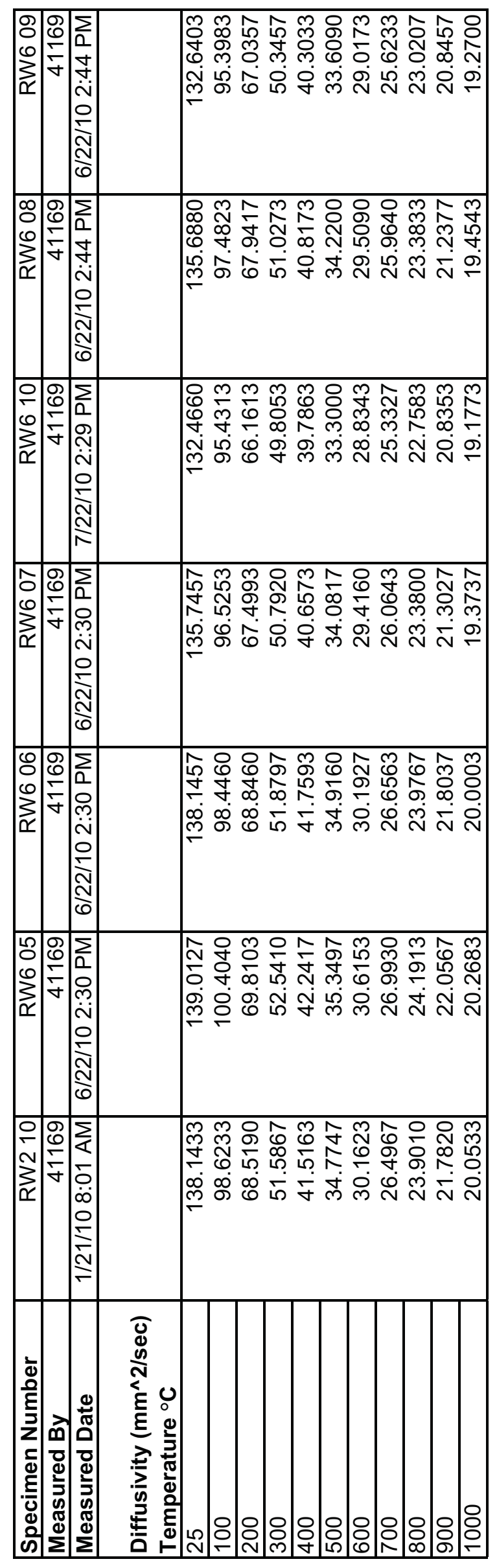




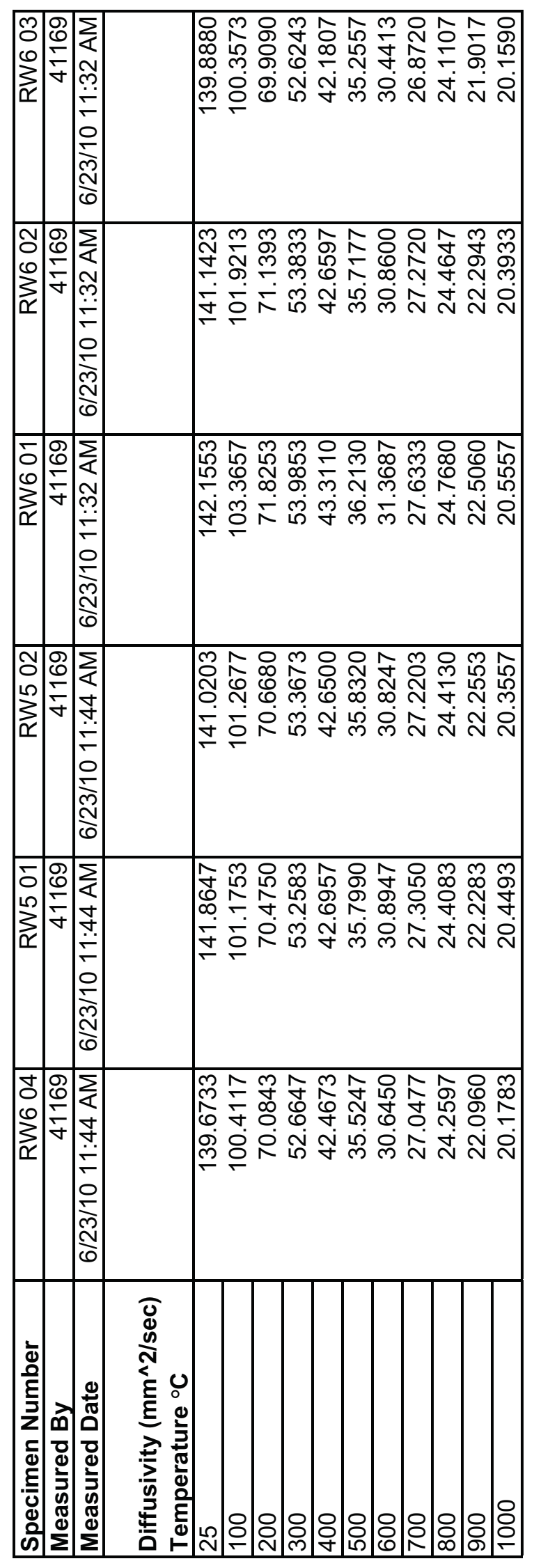




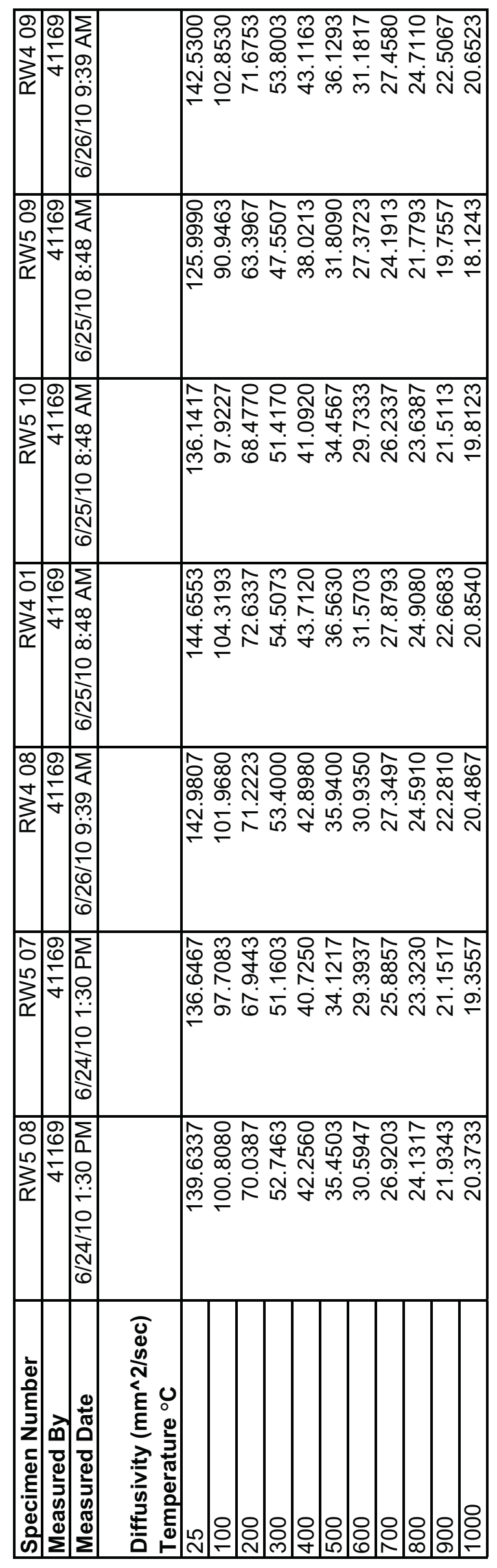




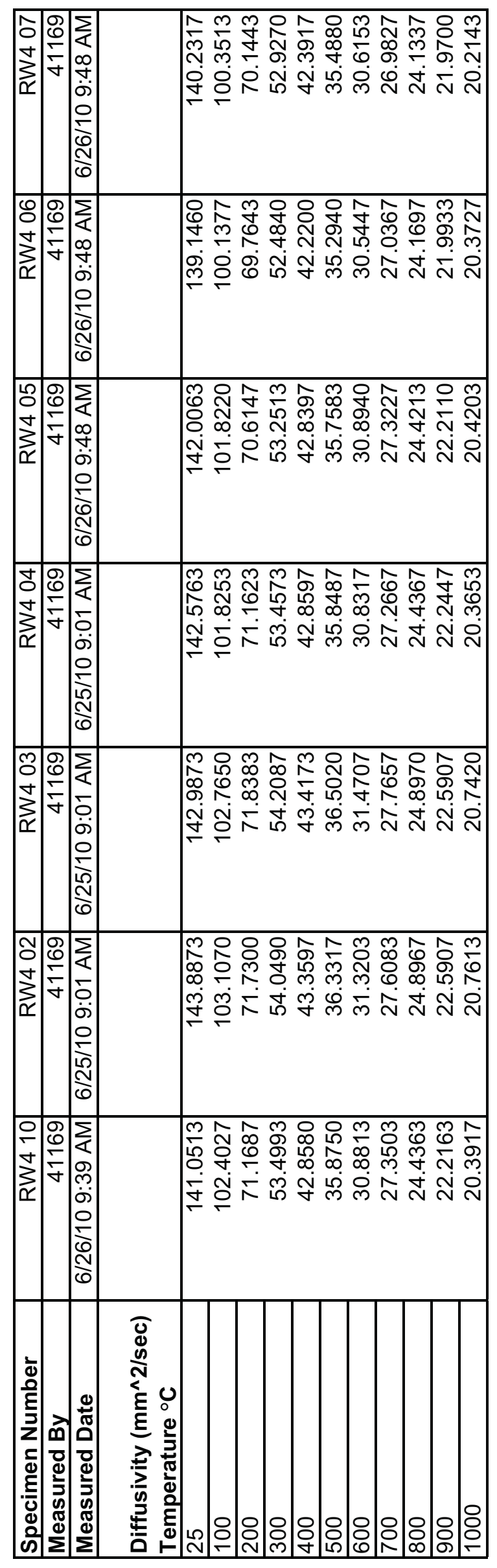




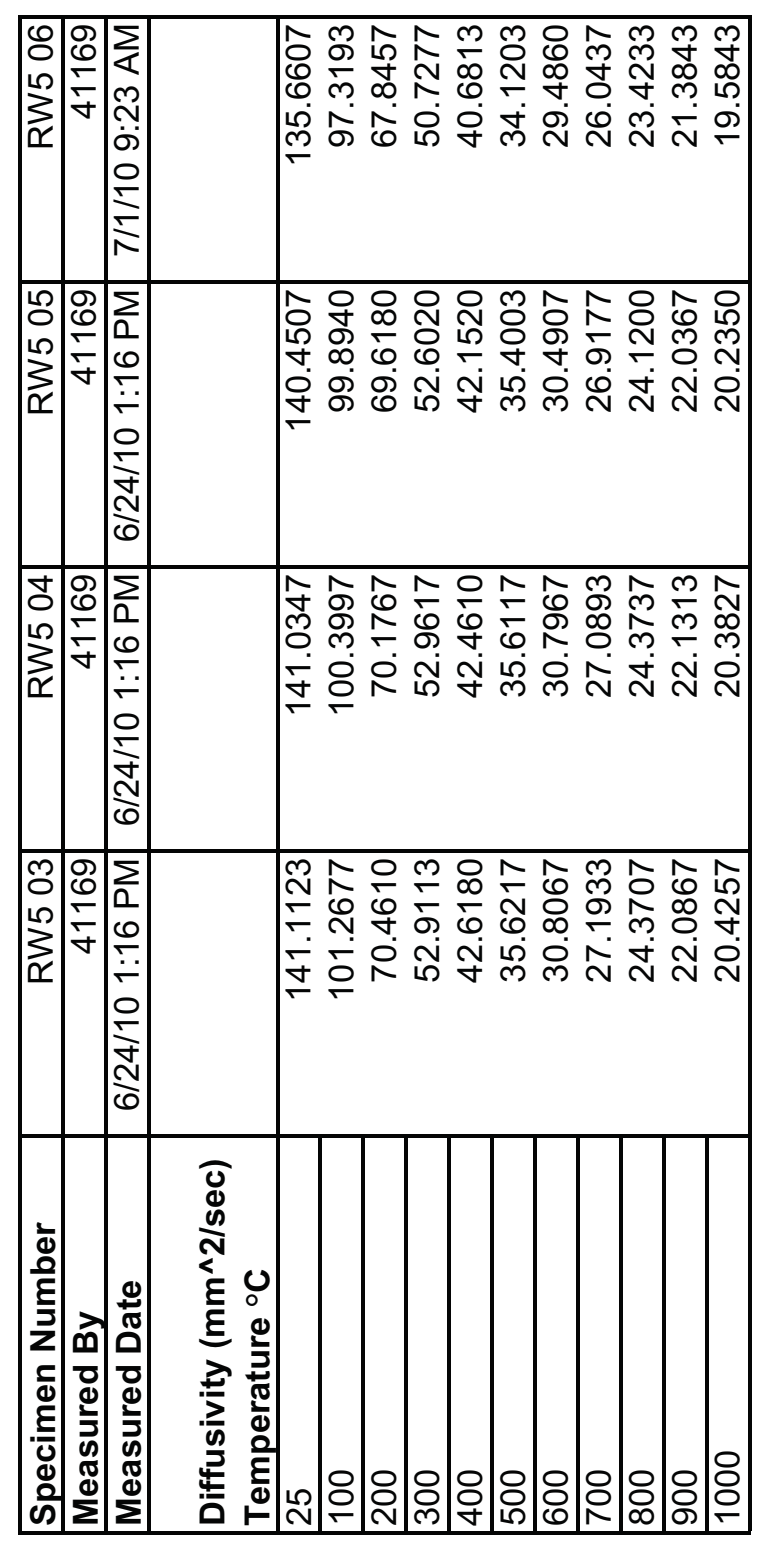




\begin{tabular}{|c|c|}
\hline 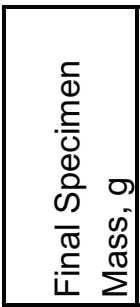 & 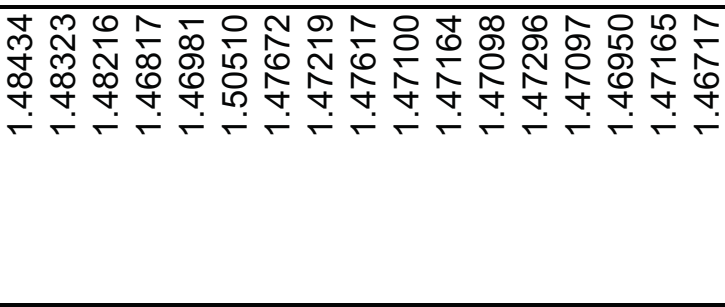 \\
\hline 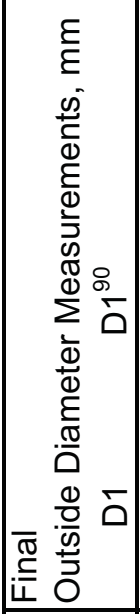 & 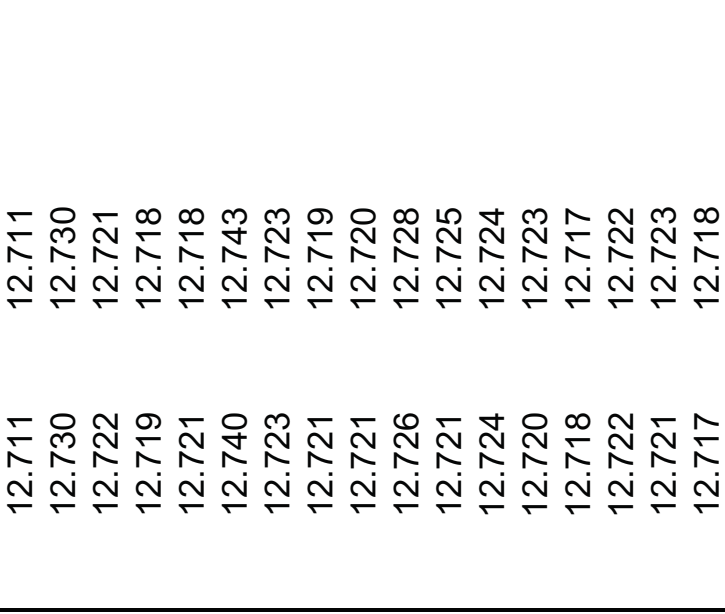 \\
\hline 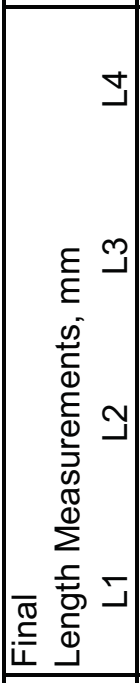 & 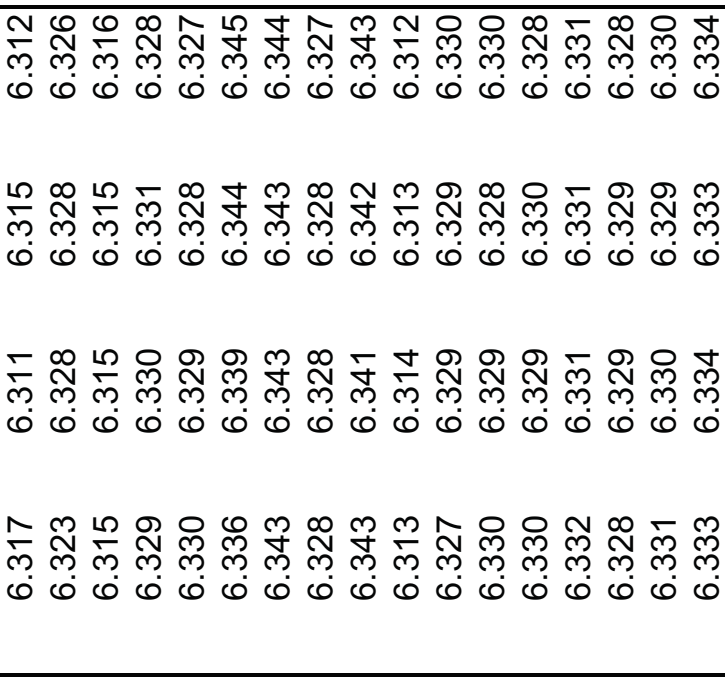 \\
\hline 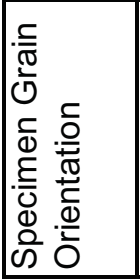 & 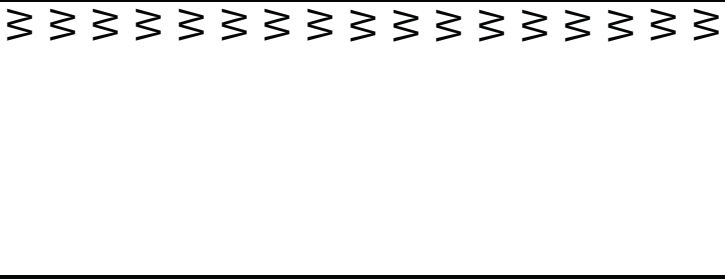 \\
\hline 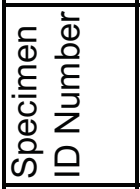 & 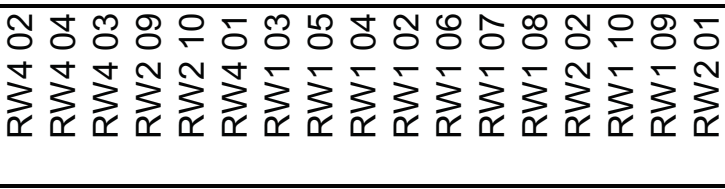 \\
\hline 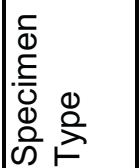 & \\
\hline
\end{tabular}




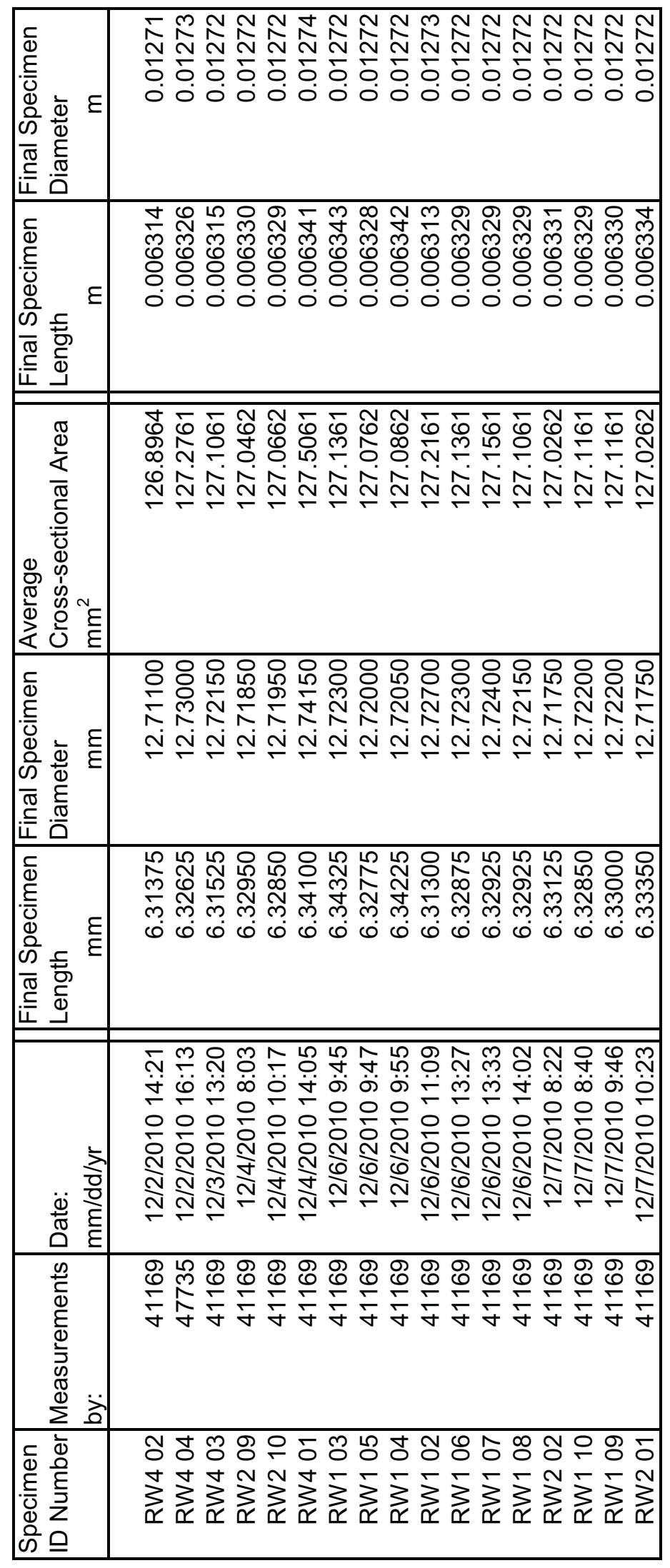




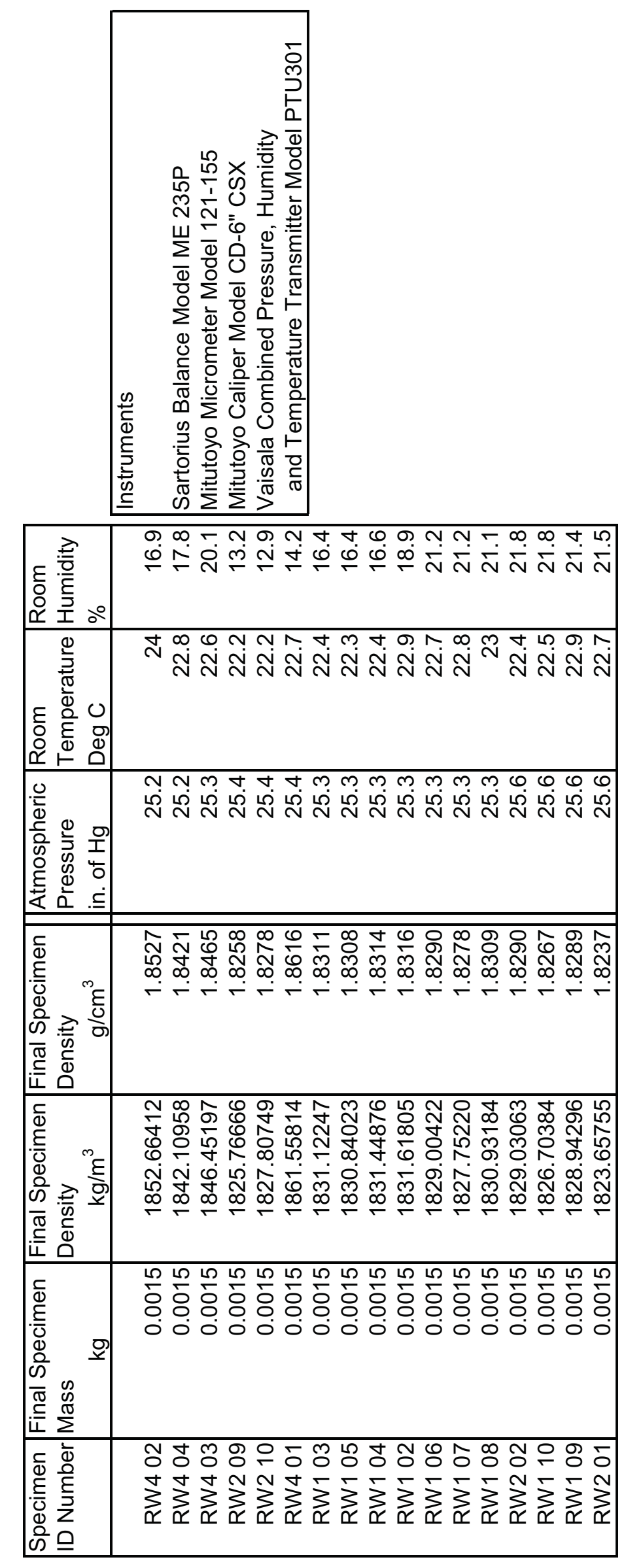




$\begin{array}{ll}\text { Graphite Grade: } & \mathrm{H}-451 \\ \text { Graphite Manufacturer: } & \text { SGL Carbon Company } \\ \text { Forming Process: } & \text { Extruded } \\ \text { Coke Particle Size: } & \text { Medium grain } \\ \text { Coke Type: } & \text { Petroleum coke } \\ \text { ASTM Class: } & \text { ENHP } \\ \text { Specimen Geometry: } & \text { Cylinder }\end{array}$

Specimen ID \#'s:

CA11 01

CA11 02

CA11 03

CA11 04

CA11 05

CA11 06

CA11 07

CA11 08

CA11 09

CA11 10

CA11 11

CA11 12

CW14 01

CW14 02

CW14 03

CW14 04

CW14 05

CW14 06

CW14 07

CW14 08

CW14 09

CW14 10 


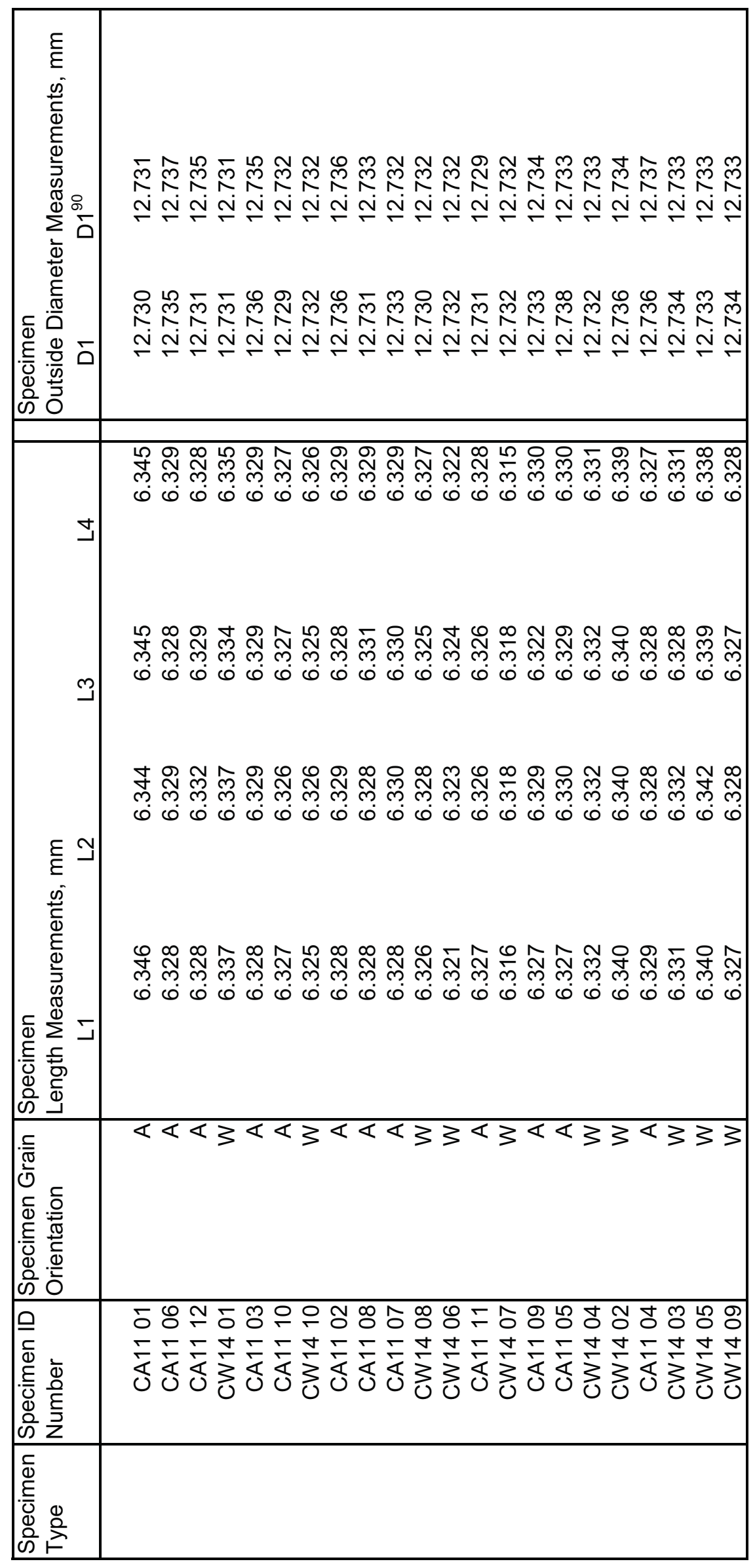




\begin{tabular}{|c|c|}
\hline 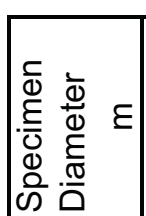 & 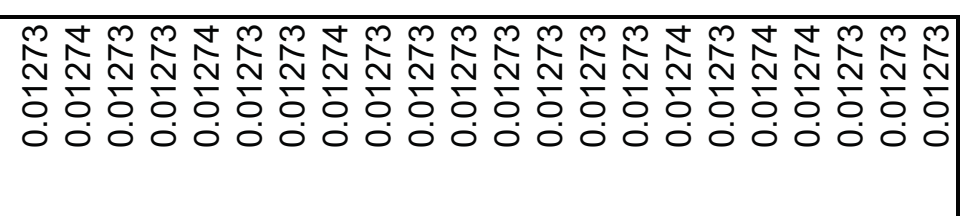 \\
\hline 喜 & 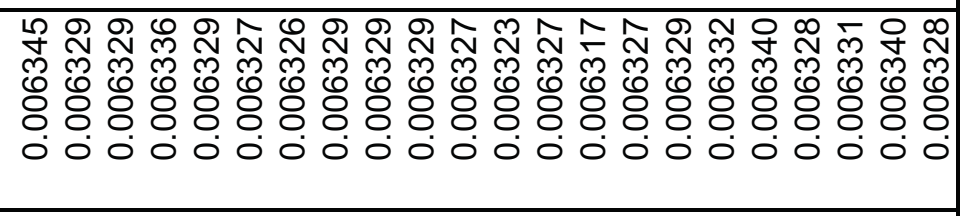 \\
\hline 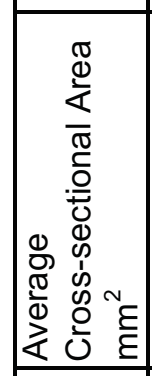 & 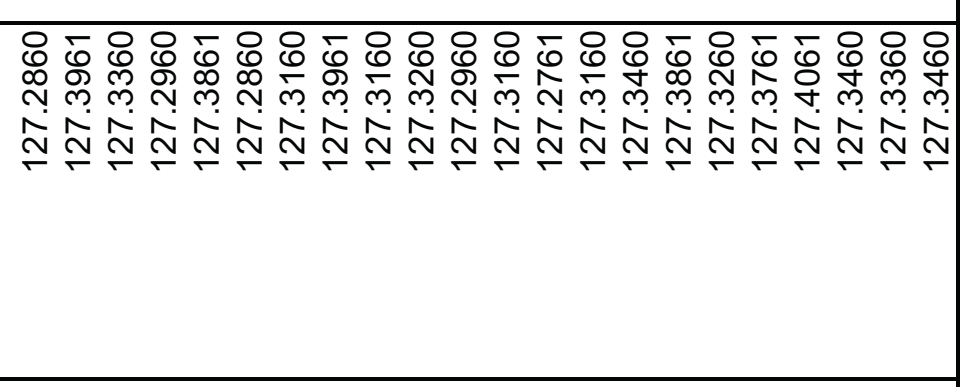 \\
\hline 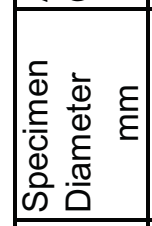 & 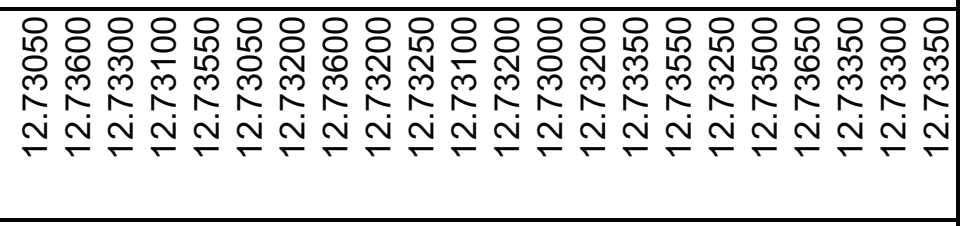 \\
\hline 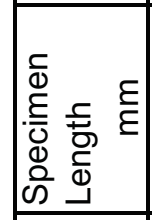 & 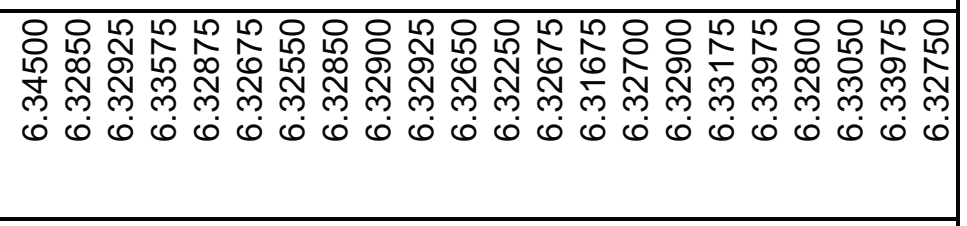 \\
\hline 这 & 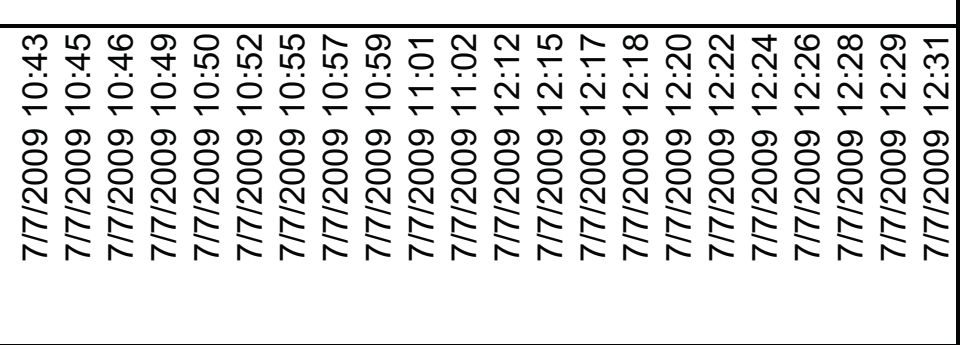 \\
\hline & 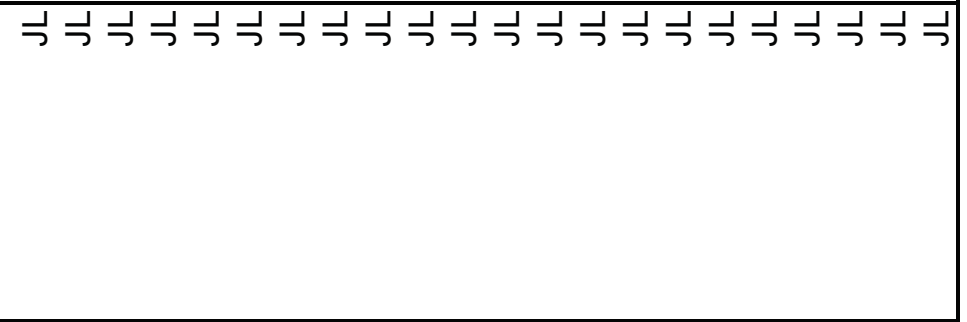 \\
\hline 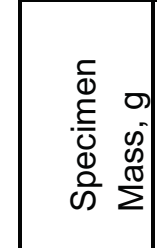 & 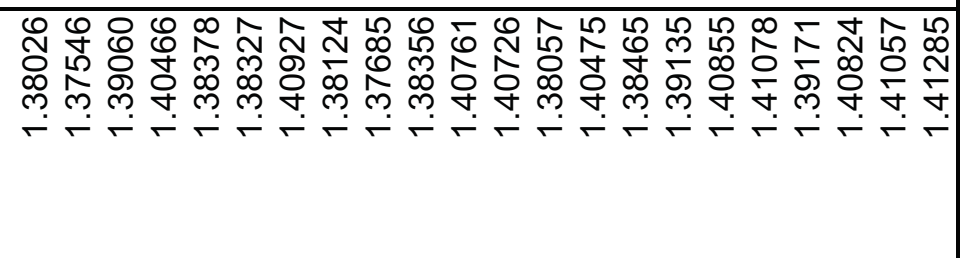 \\
\hline 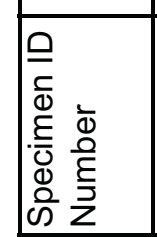 & 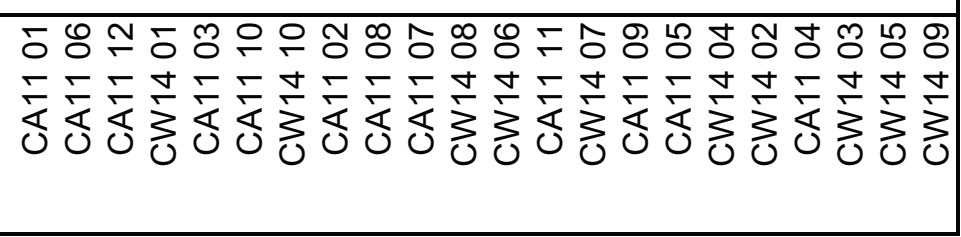 \\
\hline
\end{tabular}




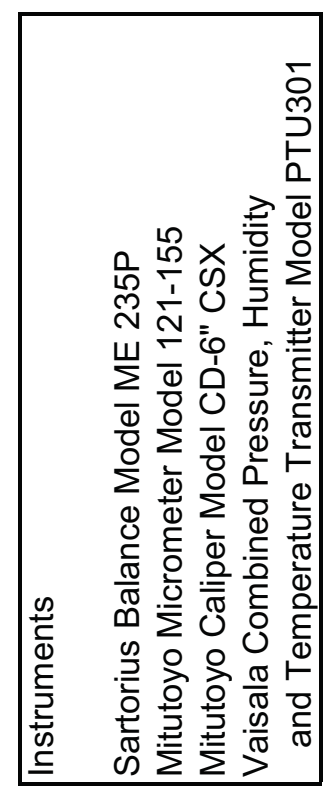

\begin{tabular}{|c|c|}
\hline 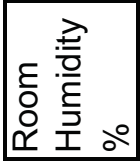 & 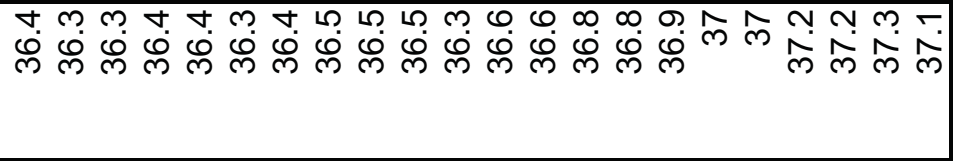 \\
\hline 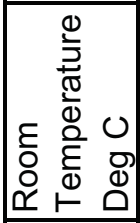 & 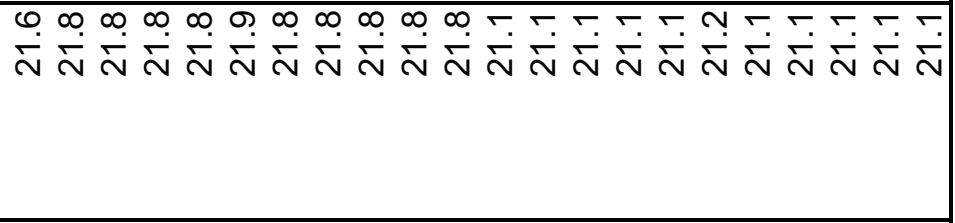 \\
\hline 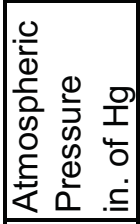 & 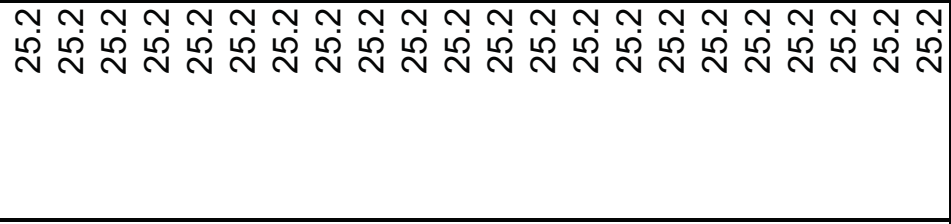 \\
\hline 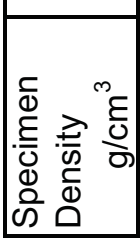 & 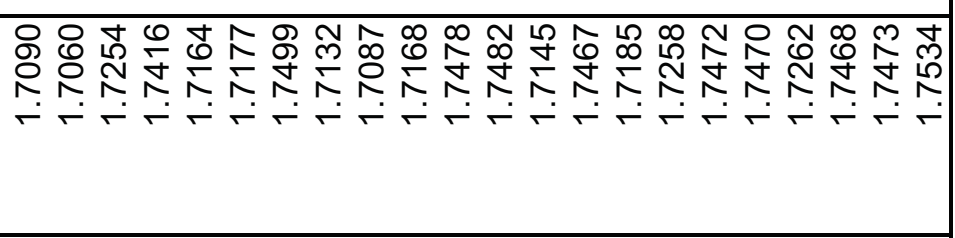 \\
\hline 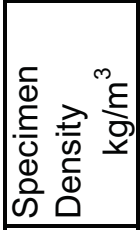 & 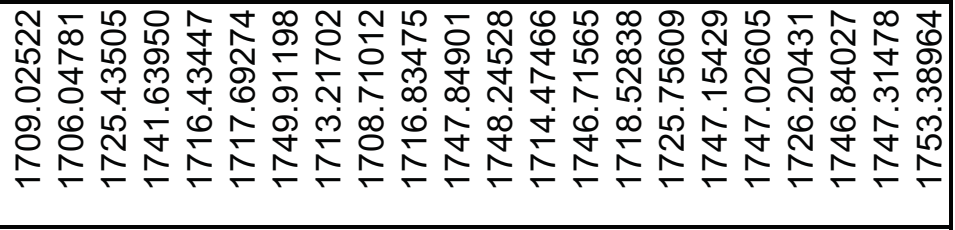 \\
\hline 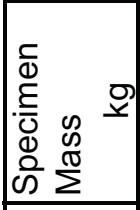 & 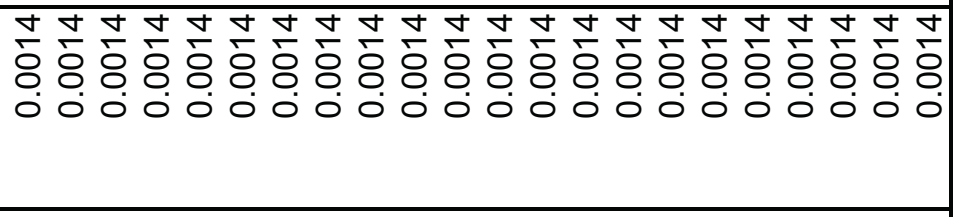 \\
\hline 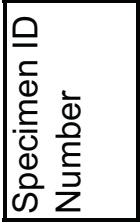 & 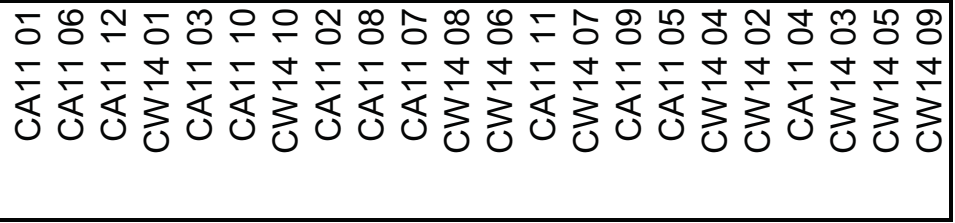 \\
\hline
\end{tabular}




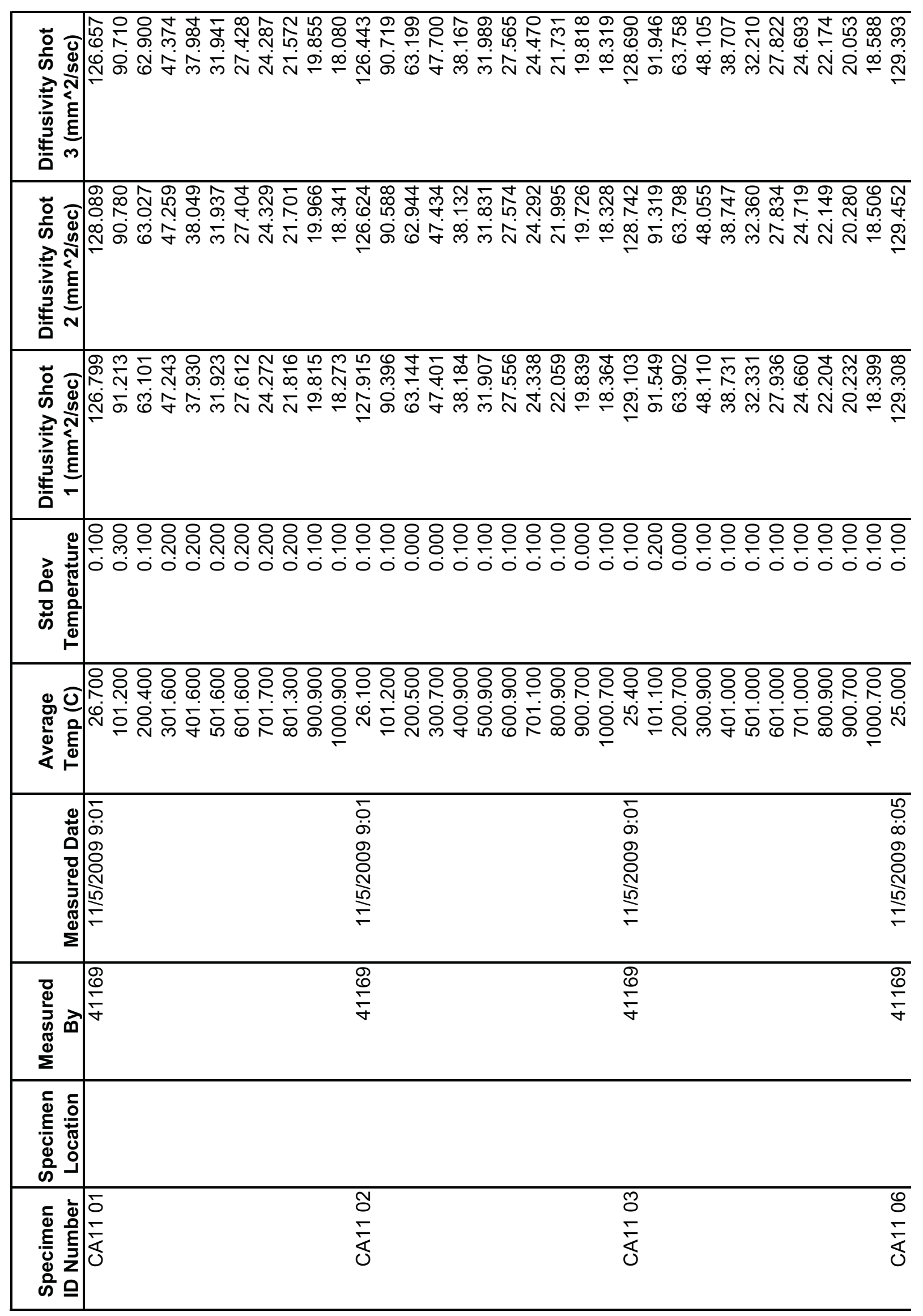




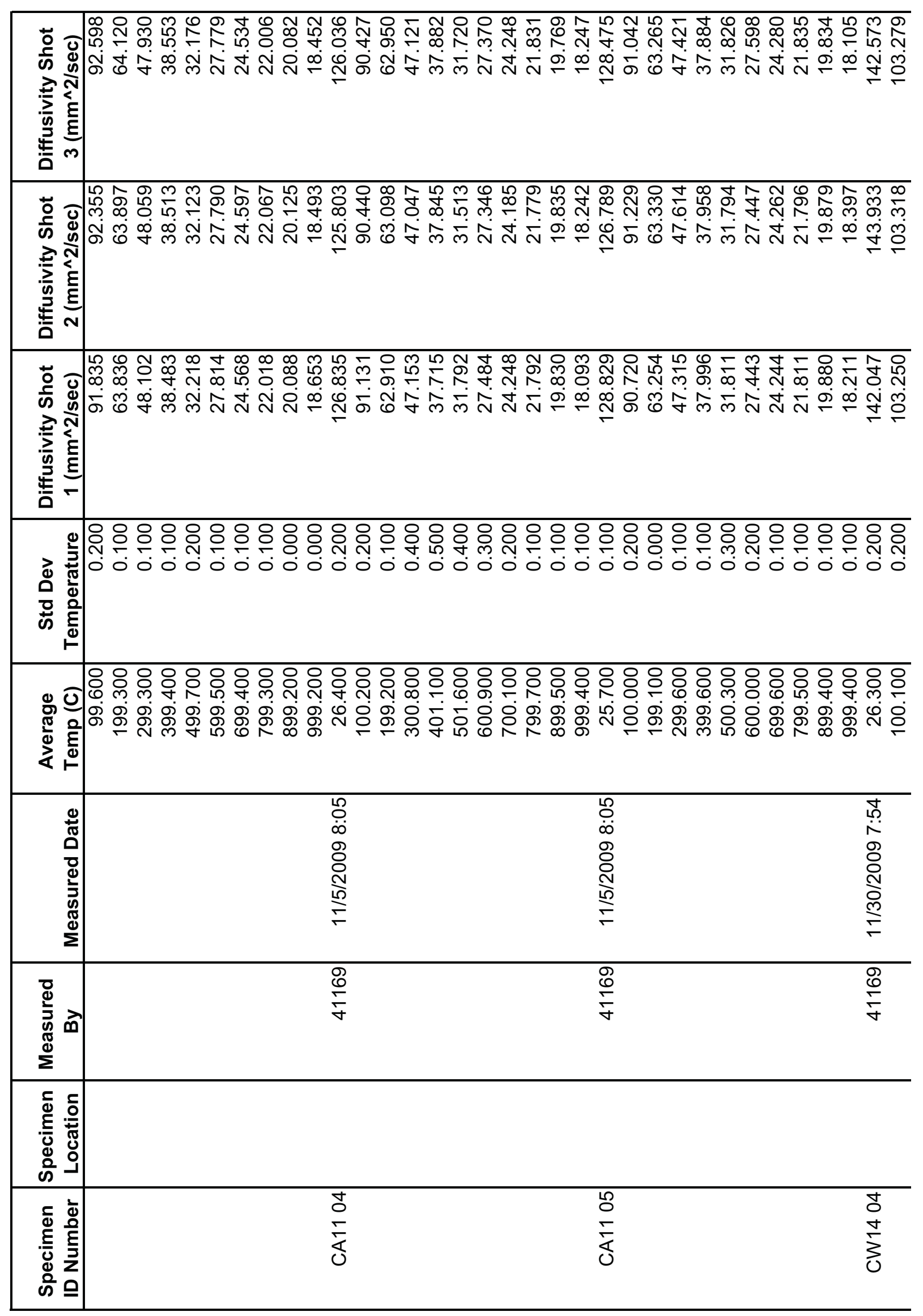




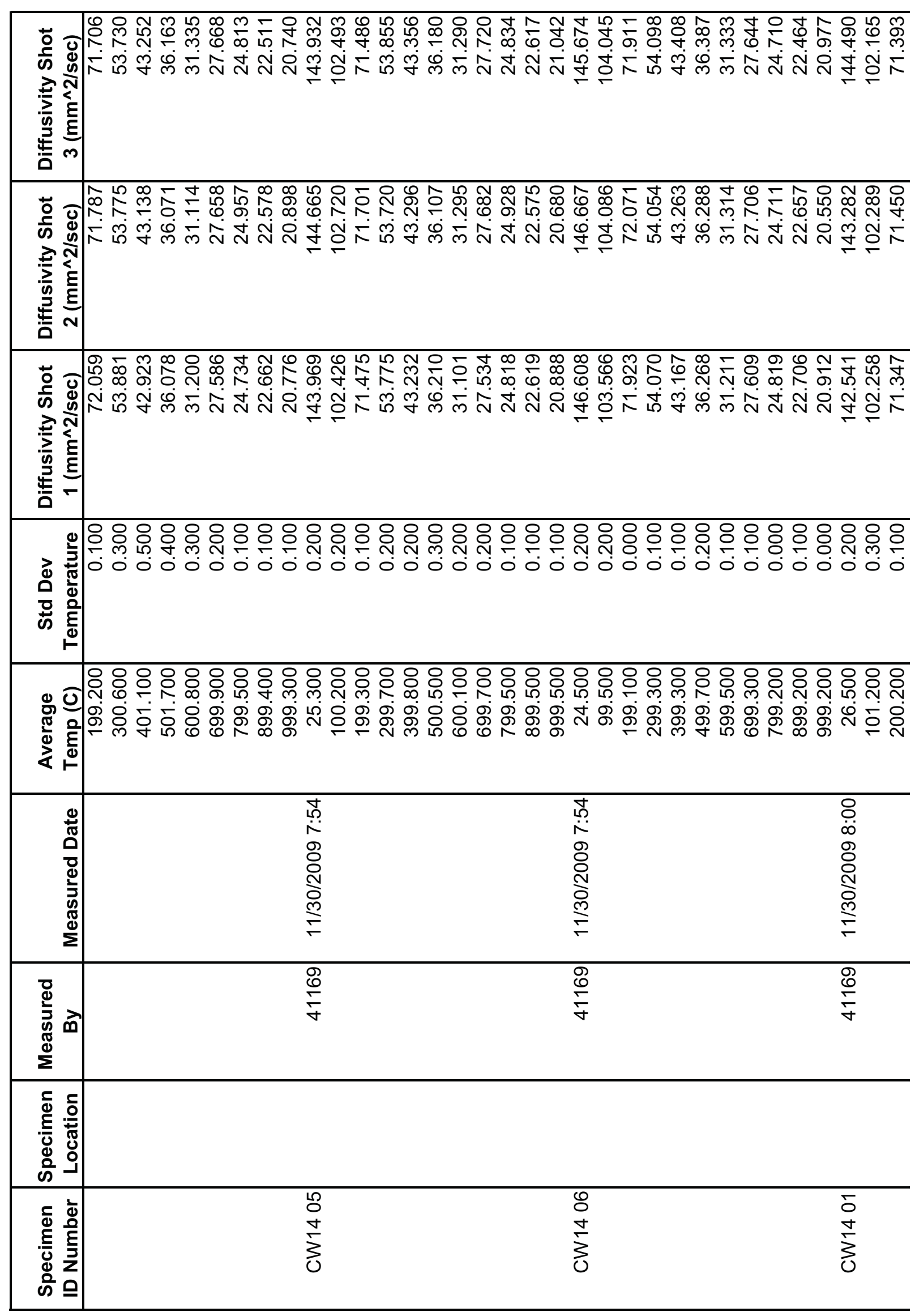




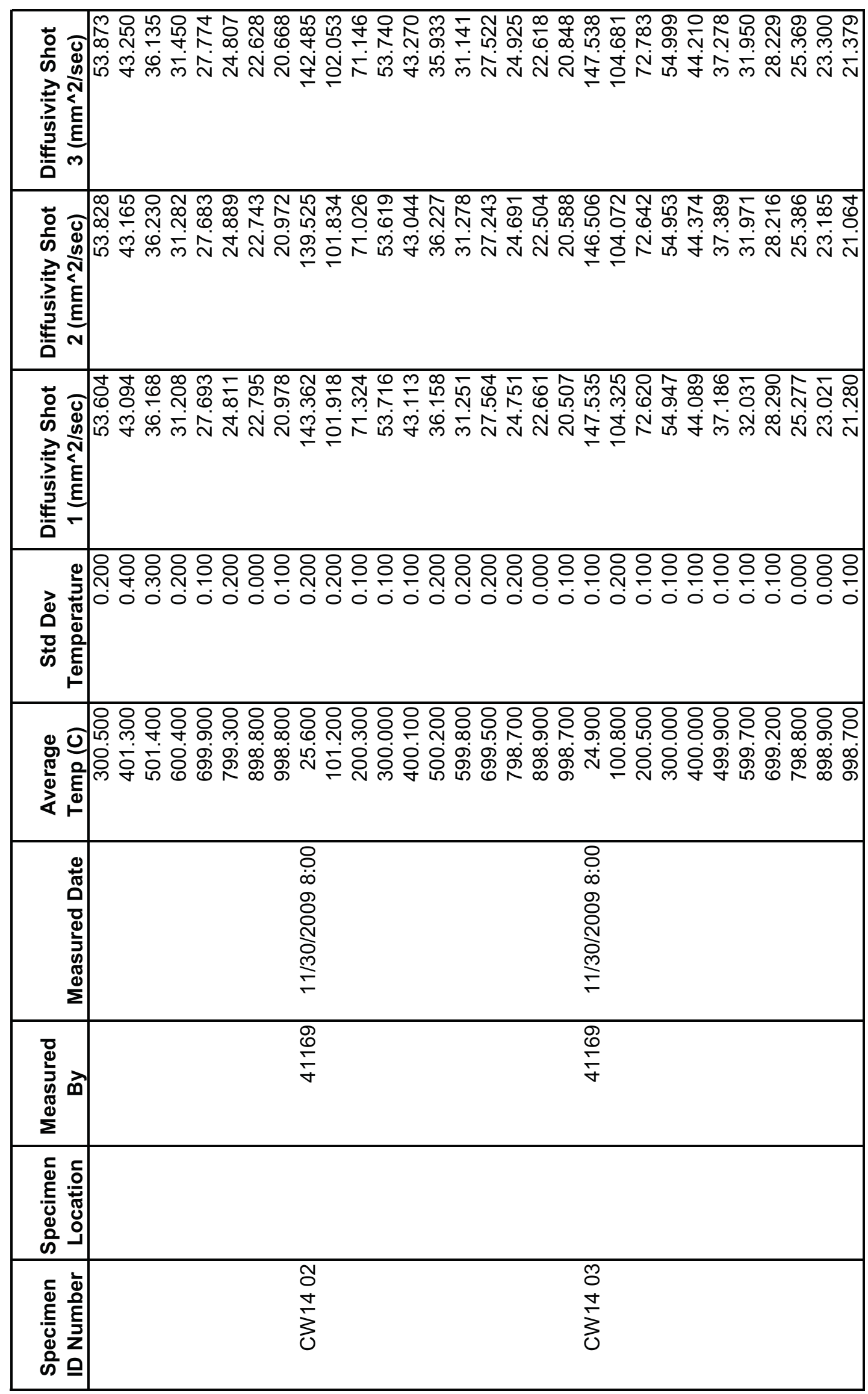




\begin{tabular}{|c|c|c|c|c|}
\hline 妾 & 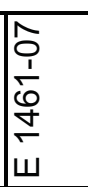 & 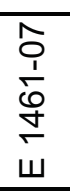 & 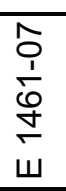 & 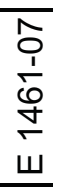 \\
\hline 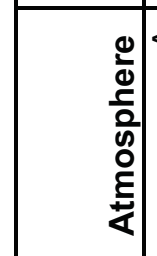 & & $\overline{\dot{z}}$ & $\bar{k}$ & $\bar{i}$ \\
\hline 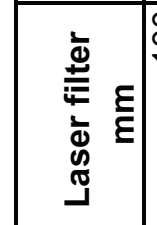 & 음 & 음 & 음 & 8 \\
\hline 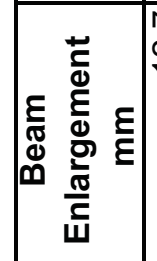 & 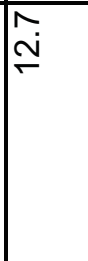 & $\stackrel{\stackrel{N}{\sim}}{\sim}$ & $\stackrel{\widehat{N}}{\stackrel{N}{S}}$ & 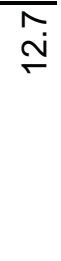 \\
\hline 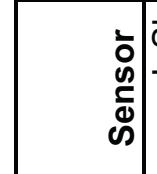 & 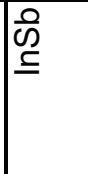 & $\begin{array}{l}\text { की } \\
\text { की }\end{array}$ & $\begin{array}{l}\text { की } \\
\text { क्ञ }\end{array}$ & की \\
\hline 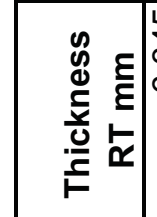 & ف & 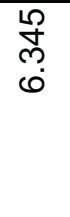 & ़ְ & 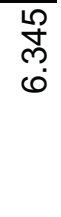 \\
\hline 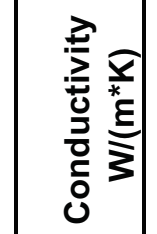 & & & & \\
\hline 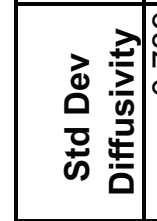 & 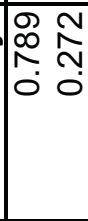 & $\begin{array}{c}0 \\
0 \\
0 \\
0\end{array}$ & Nָ & SO \\
\hline 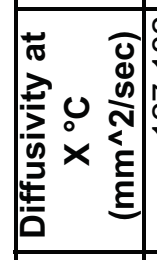 & 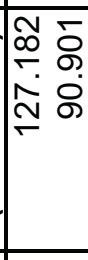 & $\begin{array}{l}\stackrel{\circ}{\circ} \\
\stackrel{\sim}{\sim} \\
\sim\end{array}$ & 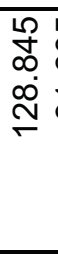 & 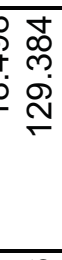 \\
\hline 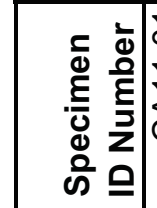 & 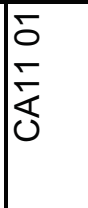 & $\begin{array}{l}\frac{N}{O} \\
\frac{\Gamma}{\delta}\end{array}$ & 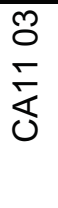 & $\begin{array}{l}8 \\
\frac{8}{\pi} \\
\delta\end{array}$ \\
\hline
\end{tabular}




\begin{tabular}{|c|c|c|c|}
\hline 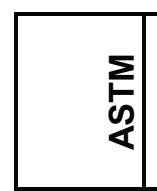 & $\begin{array}{l}\hat{o} \\
\frac{1}{0} \\
\dot{0} \\
\dot{5} \\
\end{array}$ & $\begin{array}{l}\hat{S} \\
\frac{1}{1} \\
\frac{0}{5} \\
\dot{w}\end{array}$ & 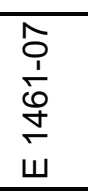 \\
\hline $\begin{array}{c}0 \\
\frac{0}{0} \\
\frac{5}{0} \\
0 \\
0 \\
\frac{1}{4}\end{array}$ & 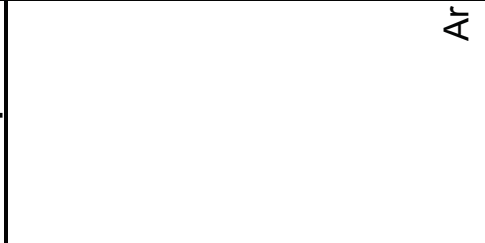 & $\frac{1}{\alpha}$ & 广 \\
\hline 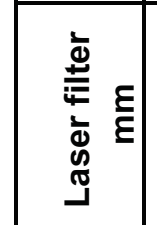 & 음 & $\stackrel{8}{\circ}$ & 음 \\
\hline 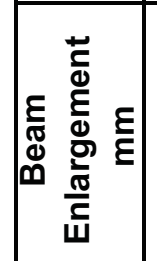 & $\stackrel{\widehat{N}}{\stackrel{N}{N}}$ & 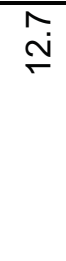 & 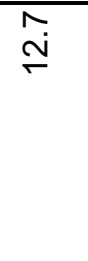 \\
\hline $\begin{array}{l}\dot{b} \\
\vdots \\
\Phi \\
\Phi \\
\infty\end{array}$ & $\begin{array}{l}\text { की } \\
\text { की }\end{array}$ & 吕 & $\begin{array}{l}\text { की } \\
\text { की }\end{array}$ \\
\hline 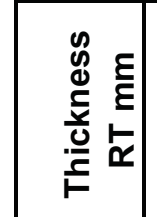 & 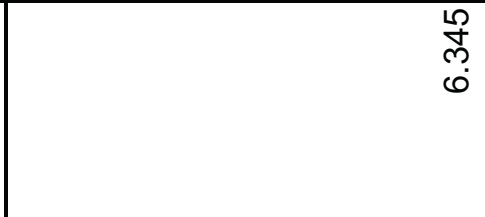 & 㐌 & 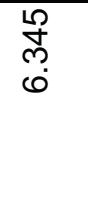 \\
\hline 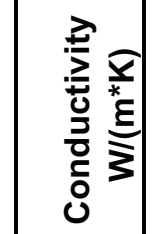 & & & \\
\hline 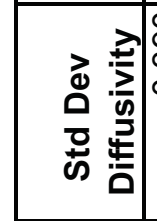 & 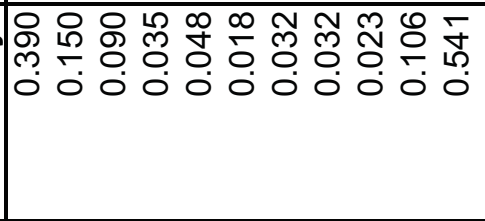 & $\stackrel{\circ}{-}$ & 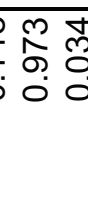 \\
\hline 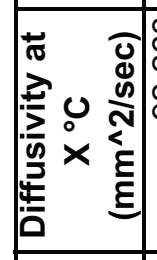 & 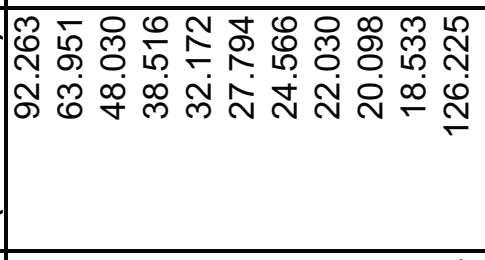 & $\begin{array}{l}\overline{\tilde{m}} \\
0 \\
\stackrel{\infty}{N} \\
\text { N }\end{array}$ & 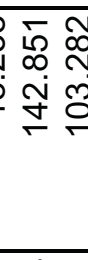 \\
\hline 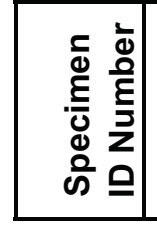 & 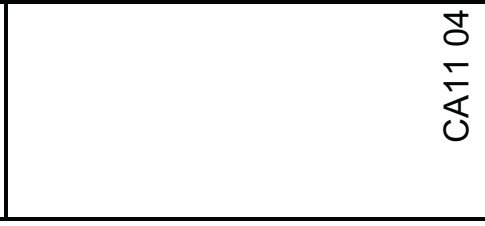 & $\frac{\stackrel{2}{O}}{\frac{\pi}{\delta}}$ & $\begin{array}{l}0 \\
0 \\
j \\
j \\
0\end{array}$ \\
\hline
\end{tabular}




\begin{tabular}{|c|c|c|c|}
\hline 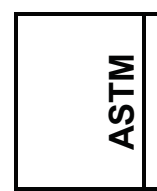 & 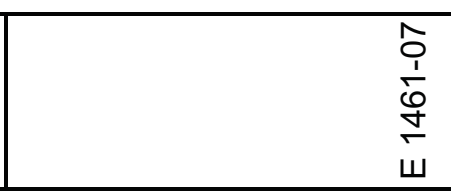 & $\begin{array}{l}\hat{o} \\
\frac{1}{0} \\
\stackrel{0}{+} \\
\dot{\omega}\end{array}$ & $\begin{array}{l}\frac{\hat{1}}{1} \\
\frac{1}{0} \\
\frac{1}{4} \\
\dot{w}\end{array}$ \\
\hline 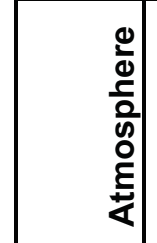 & 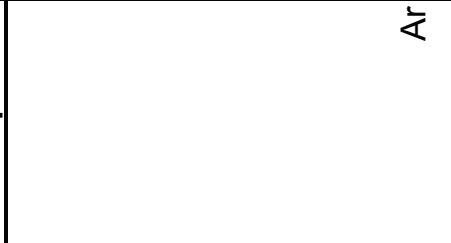 & 衣 & $\bar{z}$ \\
\hline 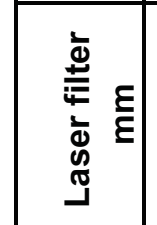 & 음 & 음 & 음 \\
\hline 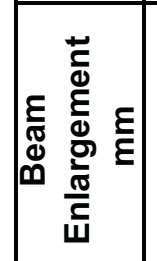 & 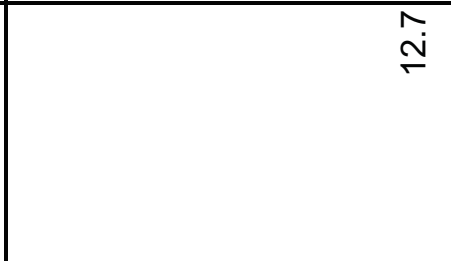 & $\stackrel{\stackrel{N}{ }}{ }$ & $\stackrel{\widetilde{N}}{\stackrel{N}{N}}$ \\
\hline $\begin{array}{l}\vdots \\
\vdots \\
0 \\
\bar{d} \\
\infty\end{array}$ & $\begin{array}{l}\text { की } \\
\text { की }\end{array}$ & 吕 & $\begin{array}{l}\text { की } \\
\text { की }\end{array}$ \\
\hline 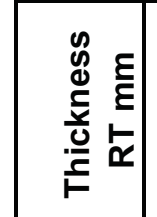 & 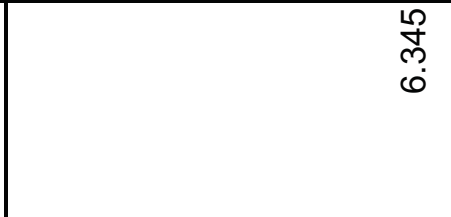 & 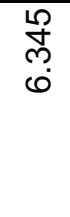 & 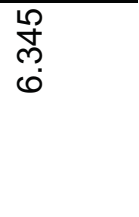 \\
\hline 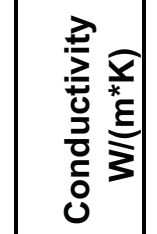 & & & \\
\hline 焉 & 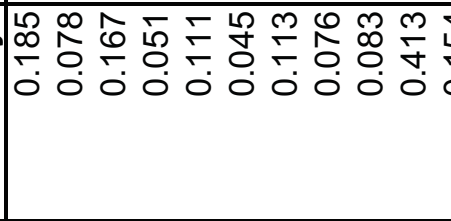 & 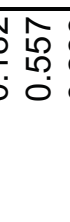 & $\begin{array}{lll} & 10 & 1 \\
0 & 0 & 0 \\
0 & 0 & 0 \\
0 & 0 & 0 \\
0 & 0\end{array}$ \\
\hline 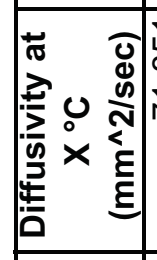 & 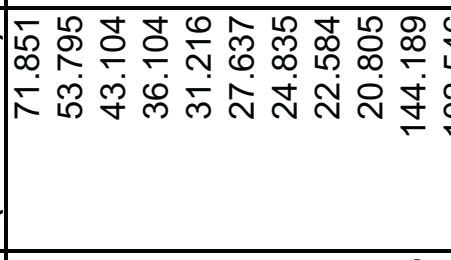 & 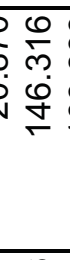 & 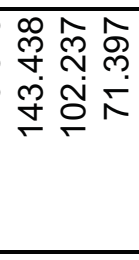 \\
\hline 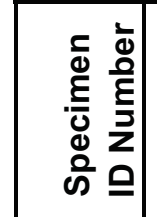 & 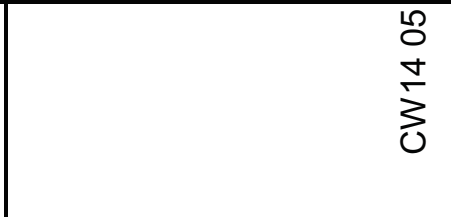 & $\begin{array}{l}8 \\
0 \\
j \\
j \\
0\end{array}$ & $\begin{array}{l}\overline{0} \\
\dot{j} \\
\dot{j}\end{array}$ \\
\hline
\end{tabular}




\begin{tabular}{|c|c|c|}
\hline $\begin{array}{c}\Sigma \\
5 \\
\&\end{array}$ & $\begin{array}{l}\hat{o} \\
\frac{1}{1} \\
\dot{\sigma} \\
\dot{\omega} \\
\omega\end{array}$ & $\begin{array}{l}\hat{0} \\
\frac{1}{0} \\
\dot{0} \\
\dot{+} \\
\end{array}$ \\
\hline 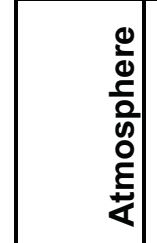 & 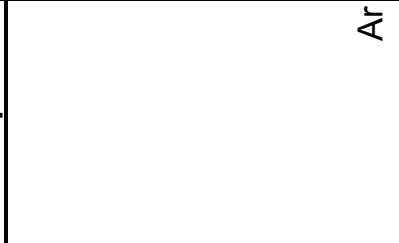 & 衣 \\
\hline 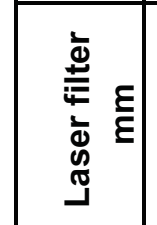 & 음 & $\stackrel{8}{\circ}$ \\
\hline 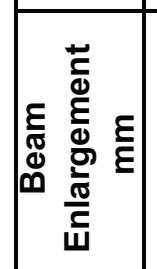 & $\stackrel{\stackrel{N}{\mathfrak{N}}}{ }$ & $\stackrel{\stackrel{\mathfrak{N}}{\mathrm{N}}}{ }$ \\
\hline $\begin{array}{l}\dot{b} \\
\vdots \\
\Phi \\
\Phi \\
\infty\end{array}$ & 吕 & $\begin{array}{l}\text { 吕 } \\
\underline{\underline{n}}\end{array}$ \\
\hline 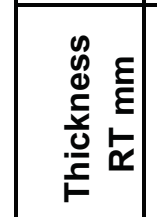 & 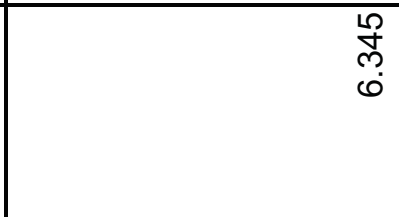 & 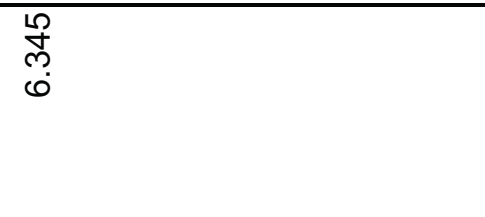 \\
\hline 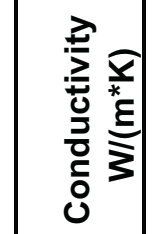 & & \\
\hline 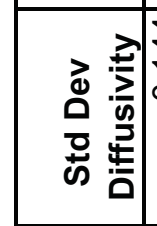 & 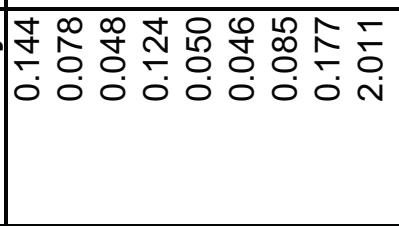 & 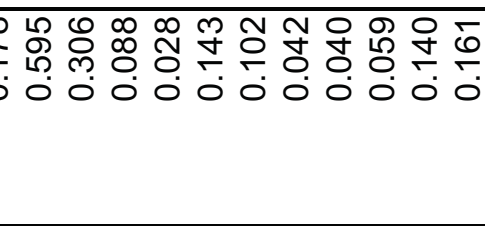 \\
\hline 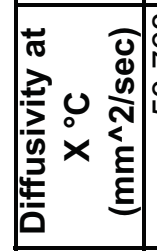 & $\begin{array}{l}0 \\
\infty\end{array}$ & 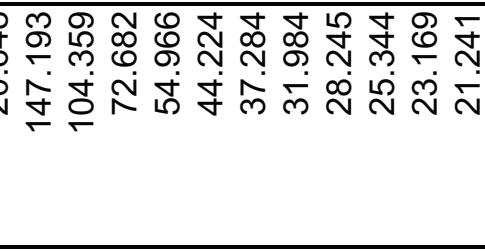 \\
\hline 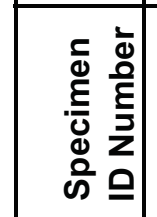 & $\begin{array}{l}\text { N } \\
\text { O } \\
\sum_{0}^{+}\end{array}$ & 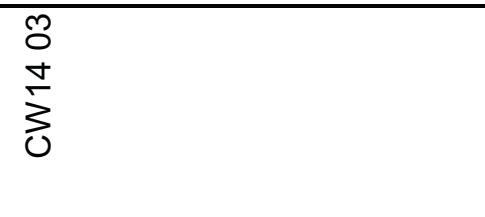 \\
\hline
\end{tabular}




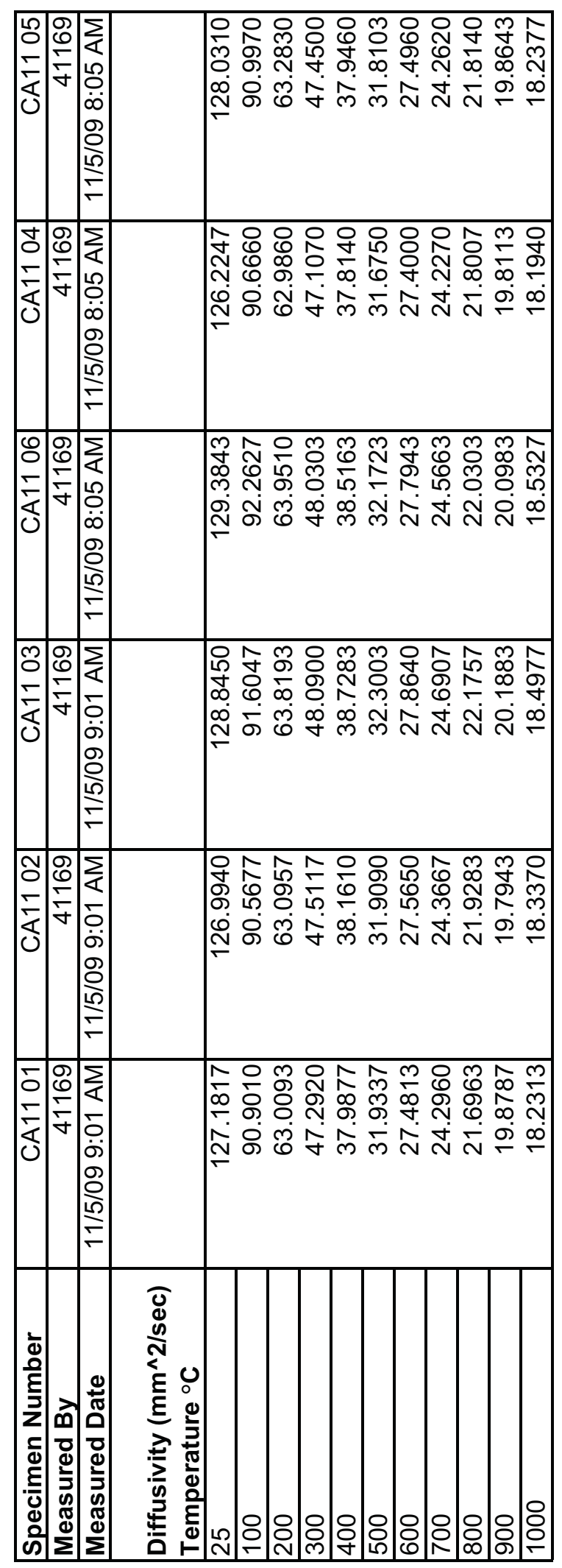




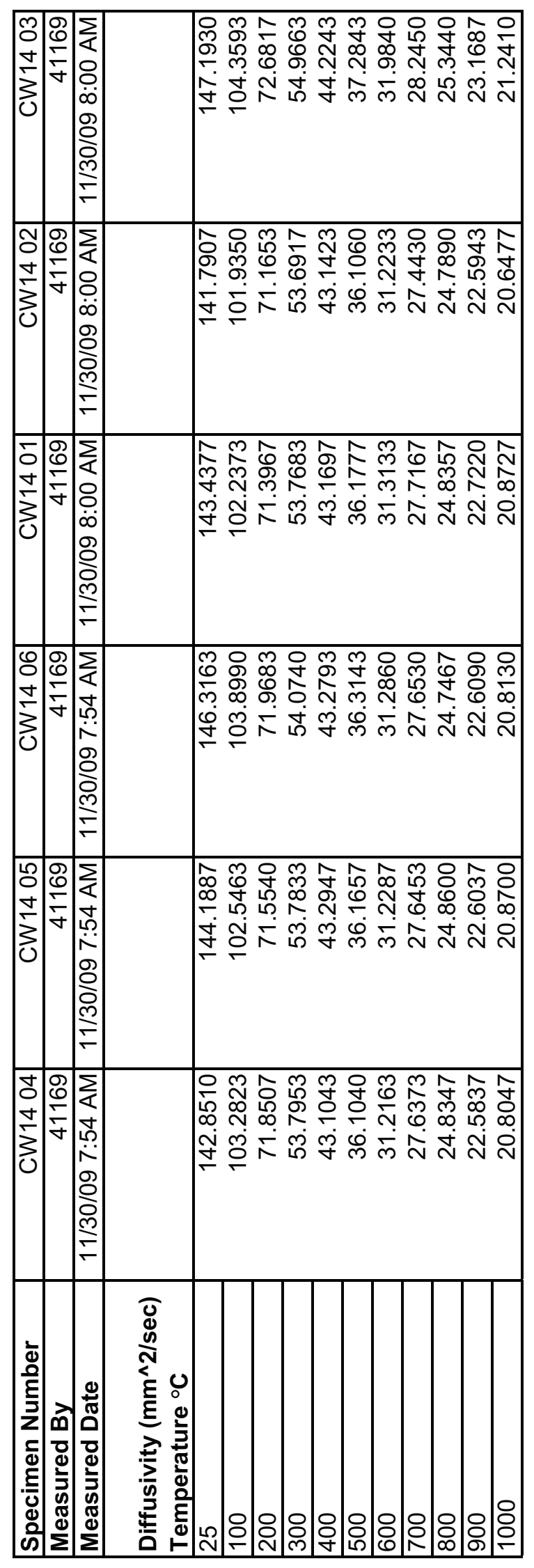




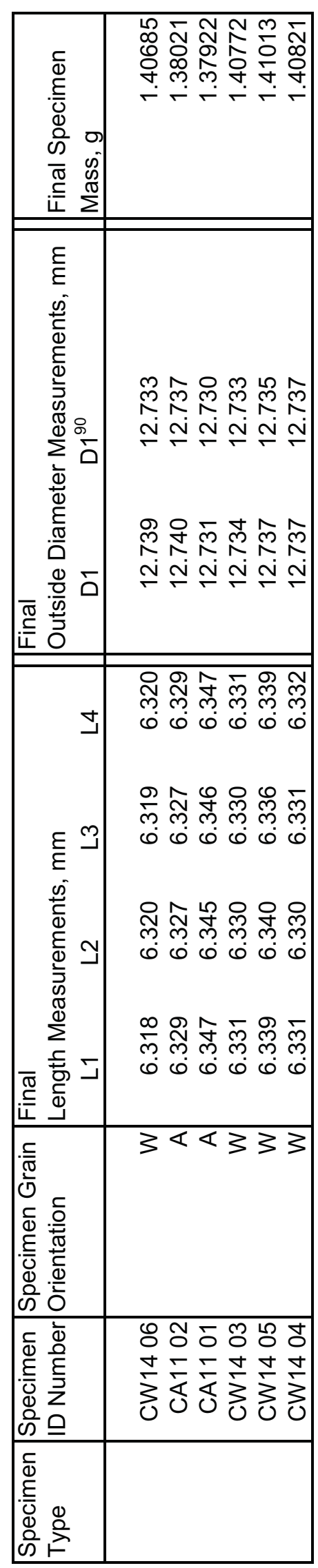




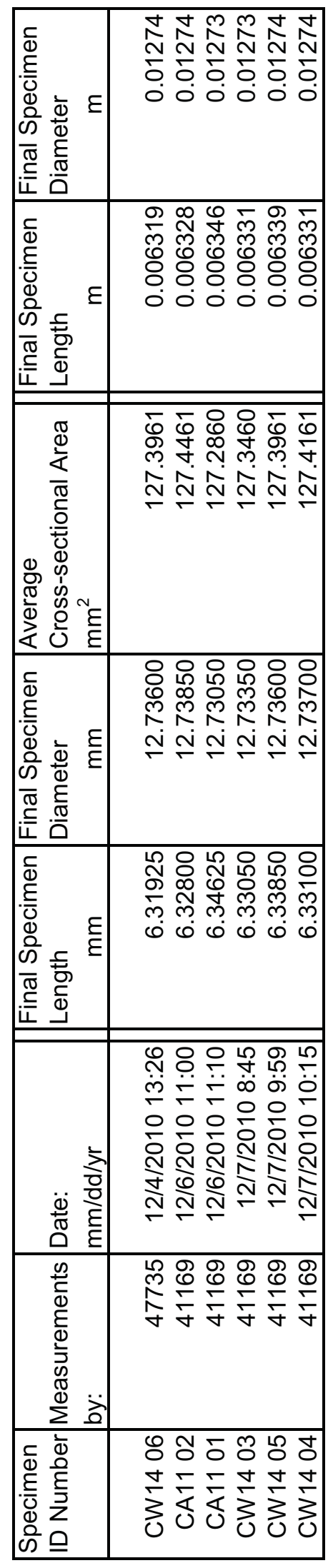




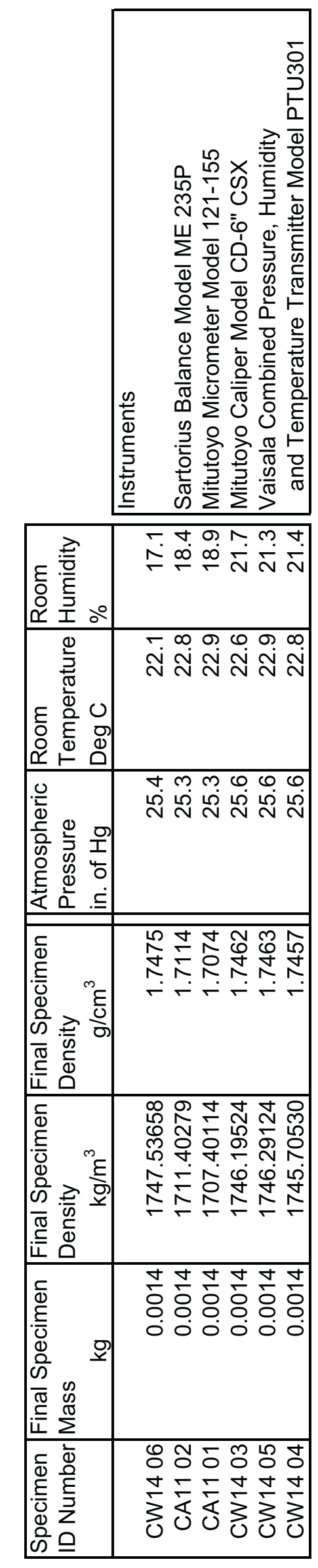

응 


$\begin{array}{ll}\text { Graphite Grade: } & \text { HLM } \\ \text { Graphite Manufacturer: } & \text { SGL Carbon Company } \\ \text { Forming Process: } & \text { Extruded } \\ \text { Coke Particle Size: } & \text { Medium grain } \\ \text { Coke Type: } & \text { Petroleum coke } \\ \text { ASTM Class: } & \text { ENHP } \\ \text { Specimen Geometry: } & \text { Cylinder }\end{array}$

Specimen ID \#'s:

J1 01

$\mathrm{J} 102$

J1 03

$\mathrm{J} 104$

J1 05

J1 06

$\mathrm{J} 107$

J1 08

J1 09

J1 10

J1 11

J1 12

J2 01

$\mathrm{J} 202$

$\mathrm{J} 203$

$\mathrm{J} 204$

J2 05

J2 06

J2 07

J2 08

J2 09

J2 10

J2 11

J2 12

J1 SPARE

J2 SPARE 


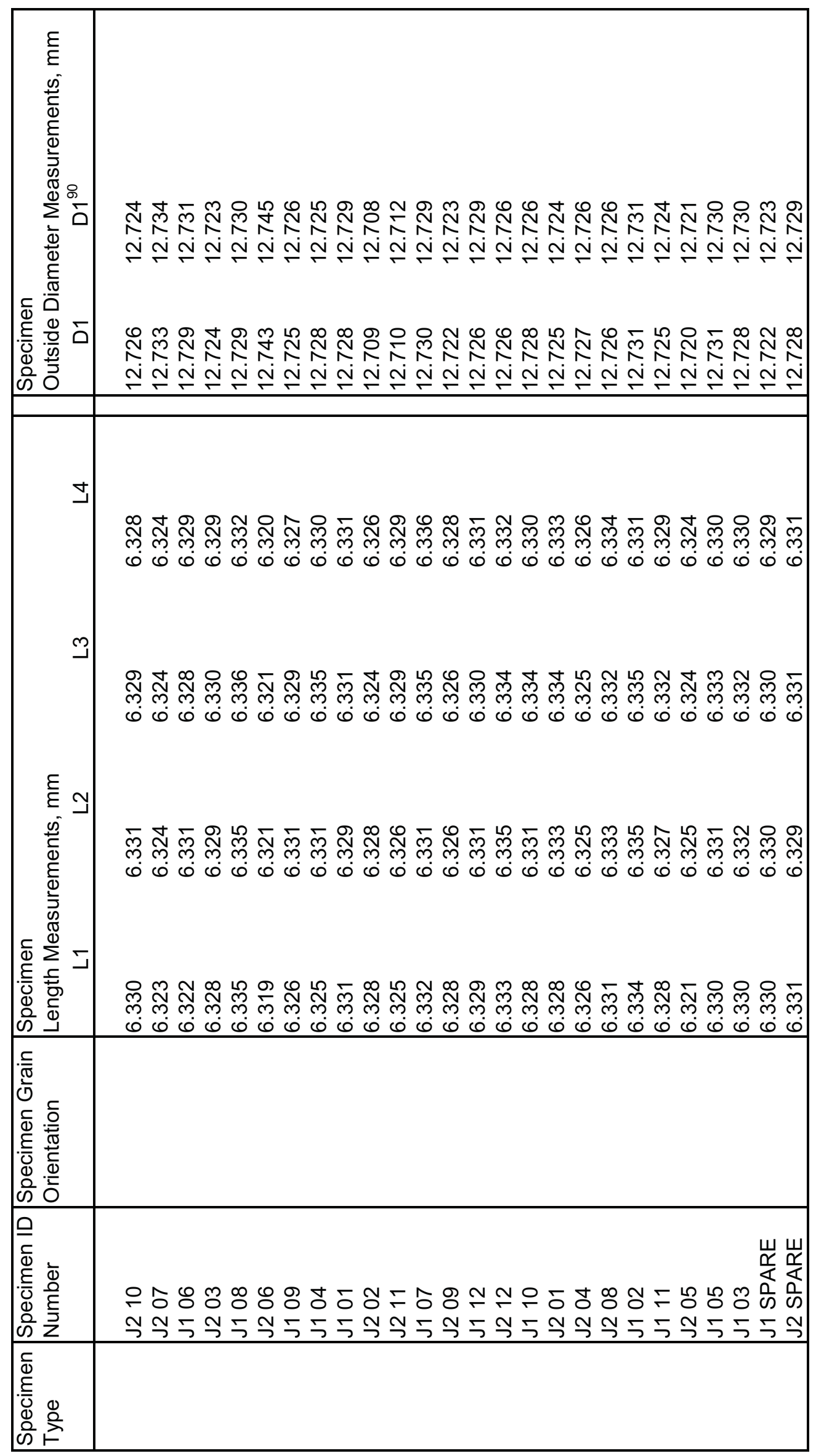




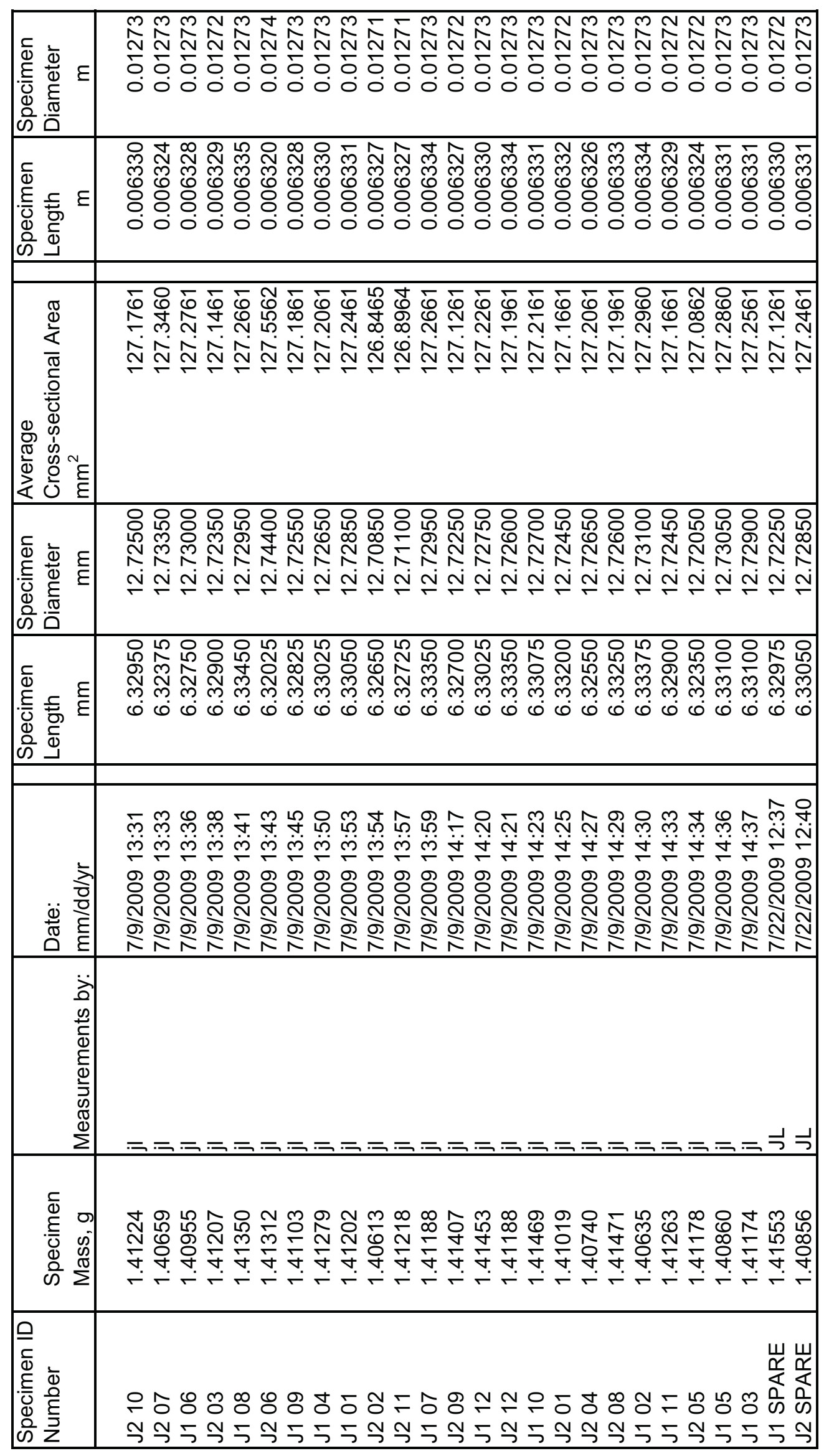




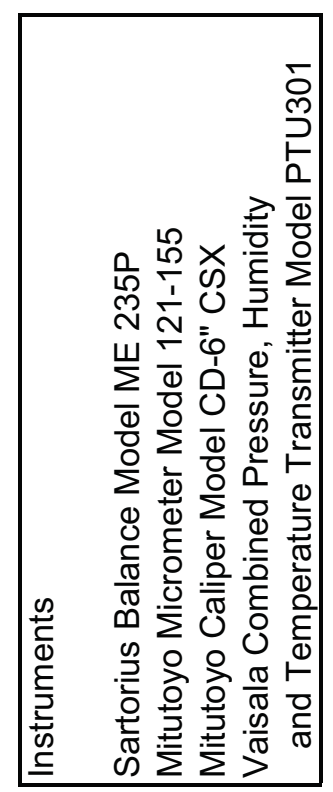

\begin{tabular}{|c|c|}
\hline 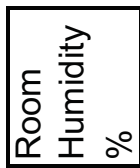 & 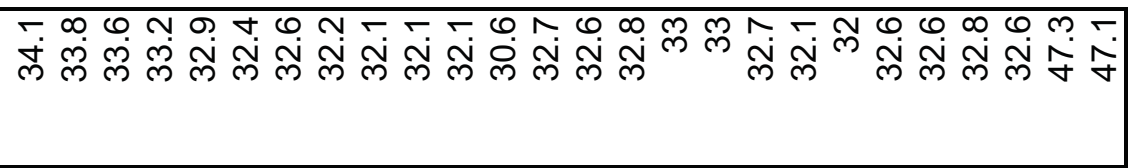 \\
\hline 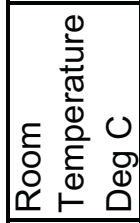 & 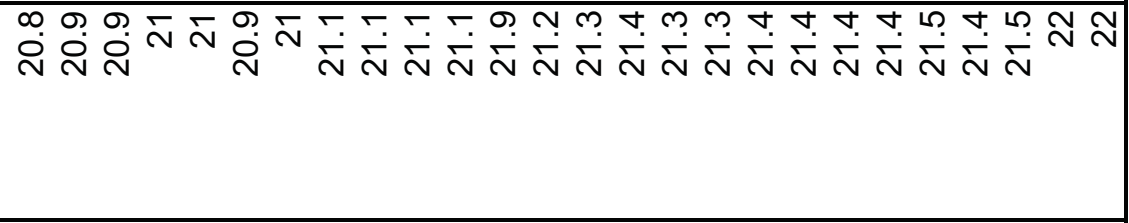 \\
\hline 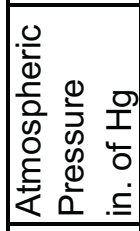 & 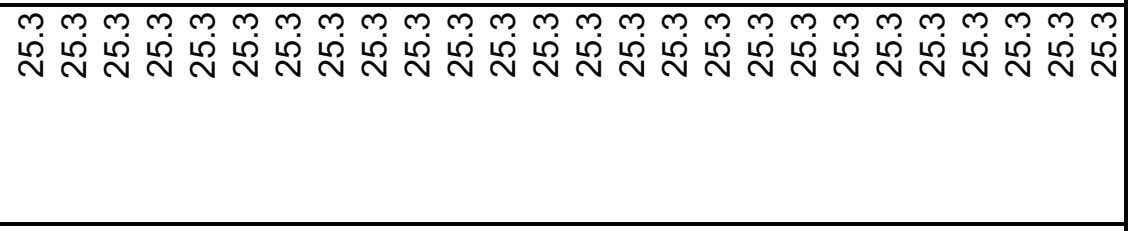 \\
\hline 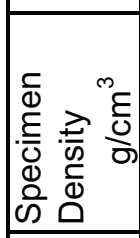 & 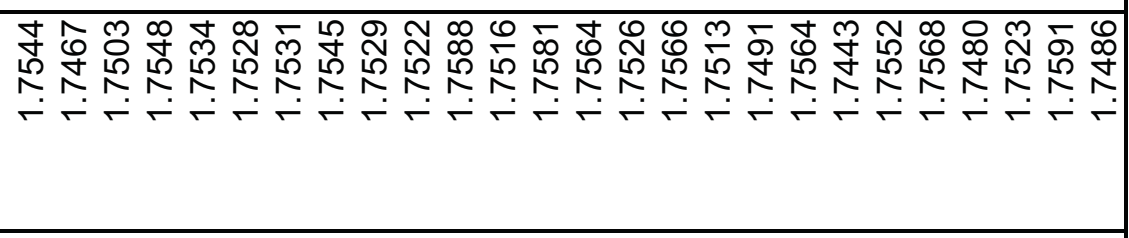 \\
\hline 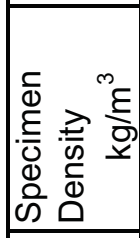 & 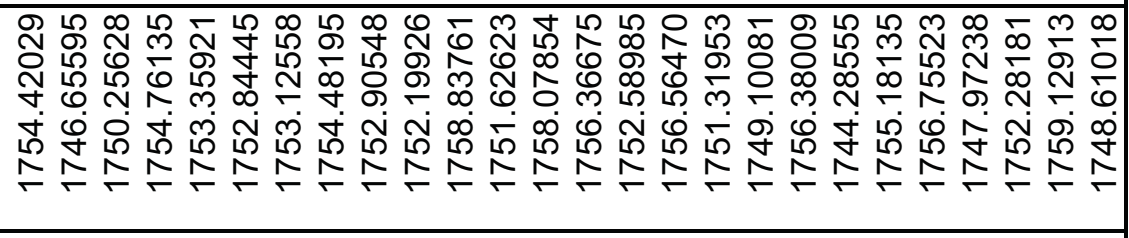 \\
\hline 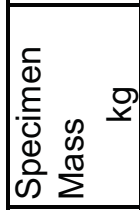 & 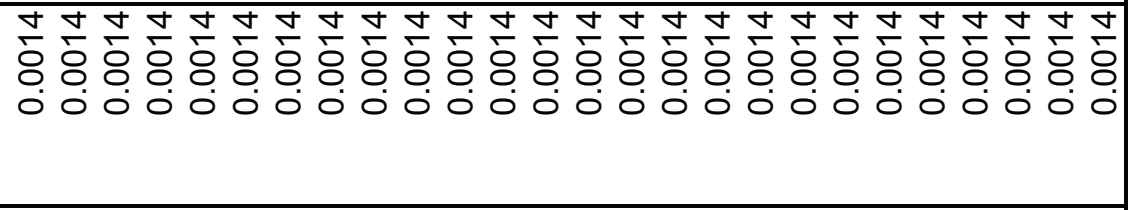 \\
\hline 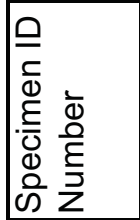 & 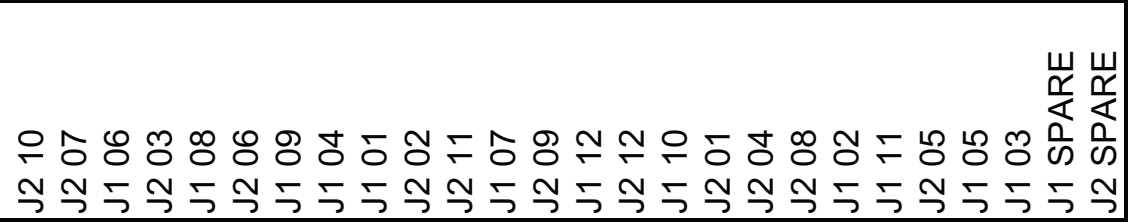 \\
\hline
\end{tabular}




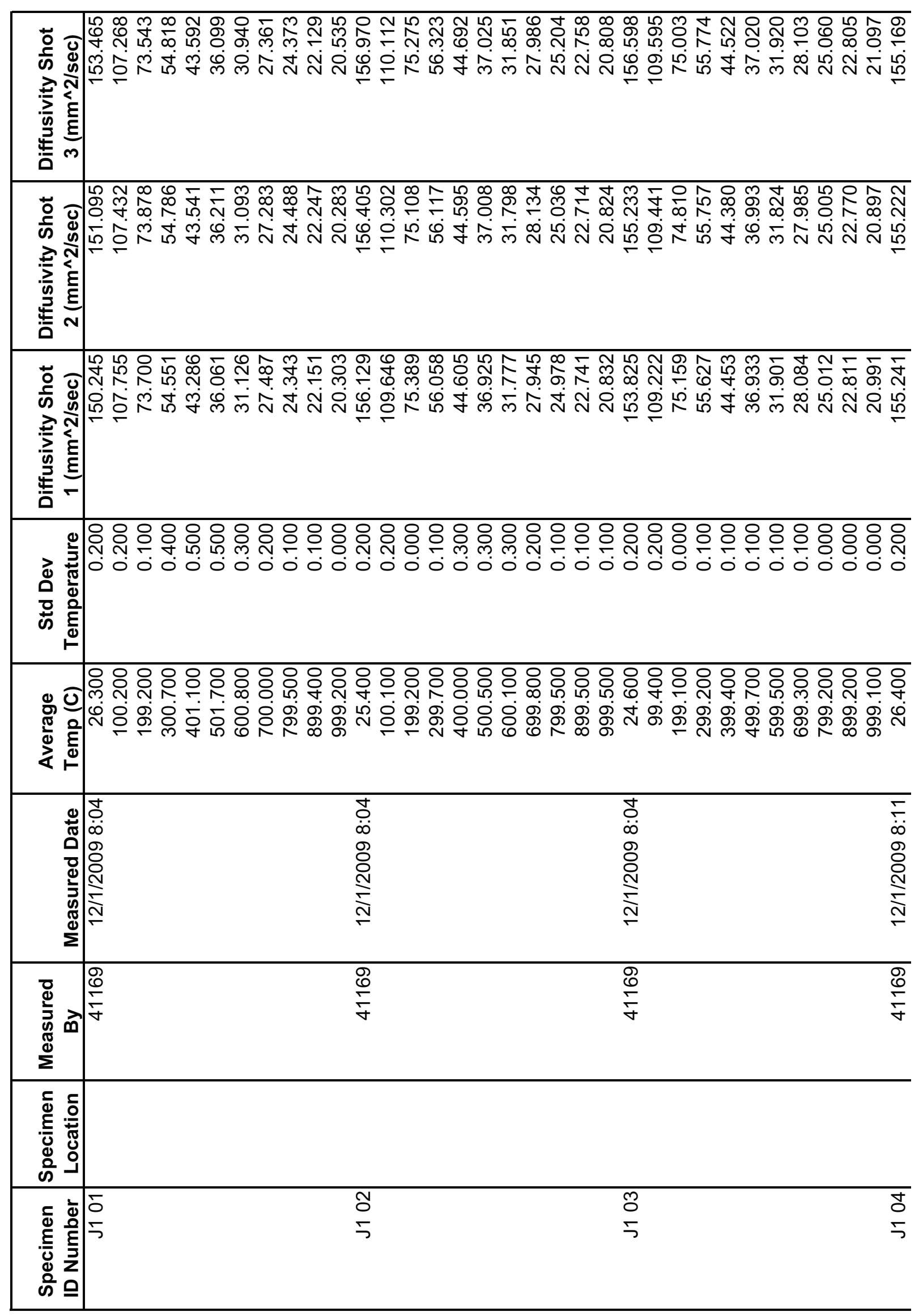




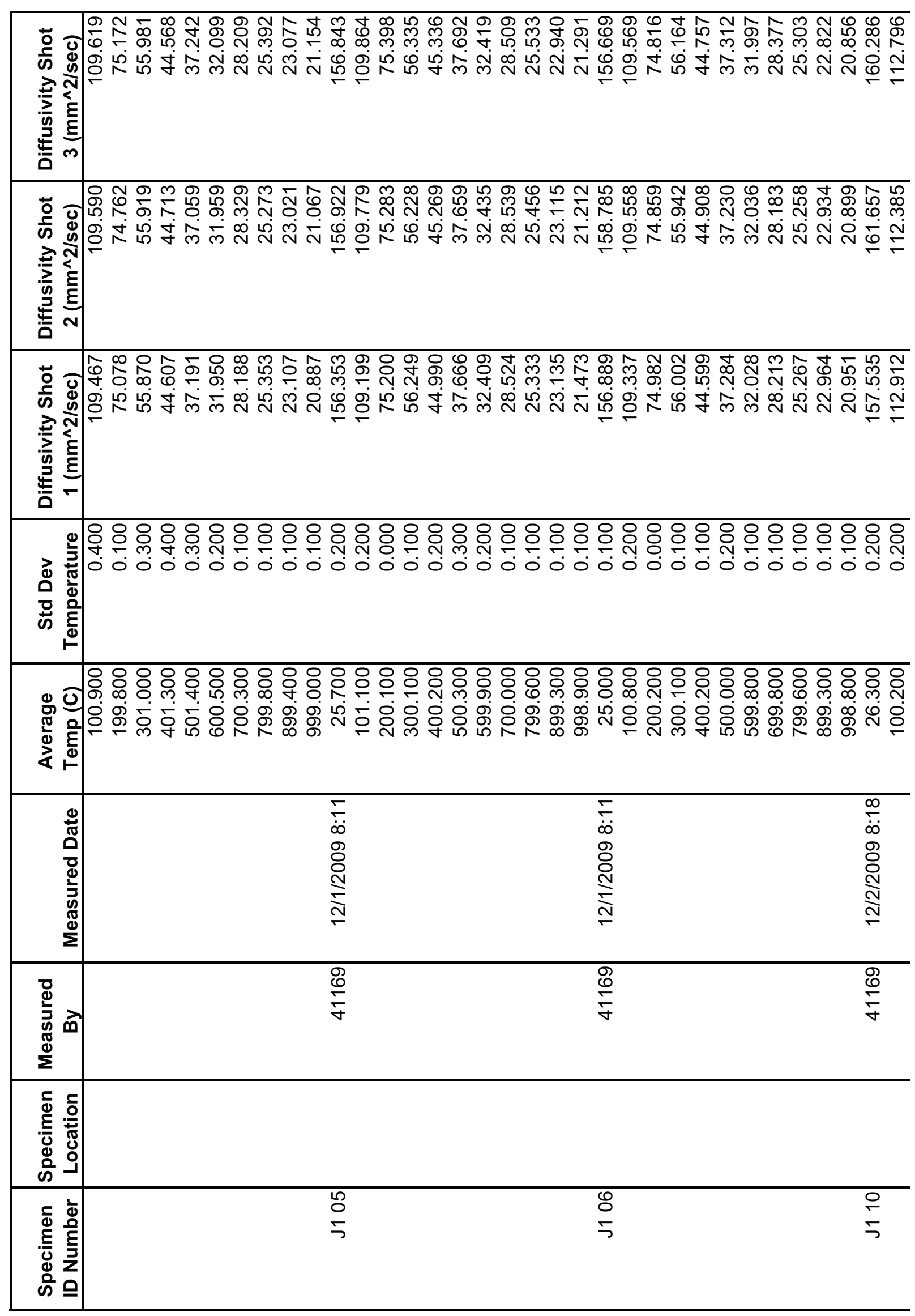




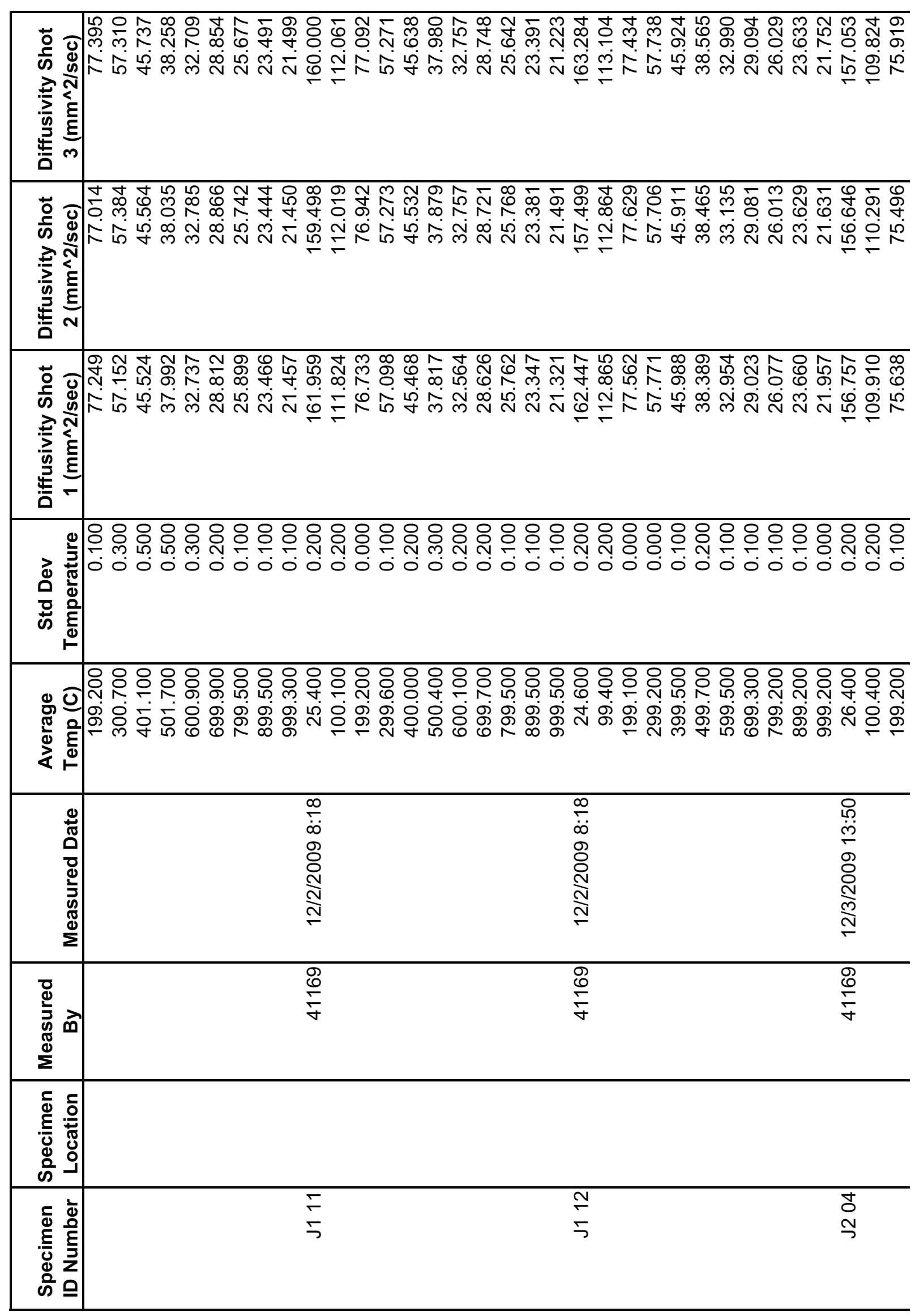




\begin{tabular}{|c|c|c|}
\hline 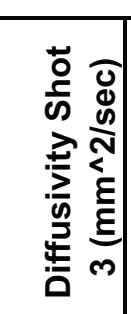 & \multicolumn{2}{|c|}{ 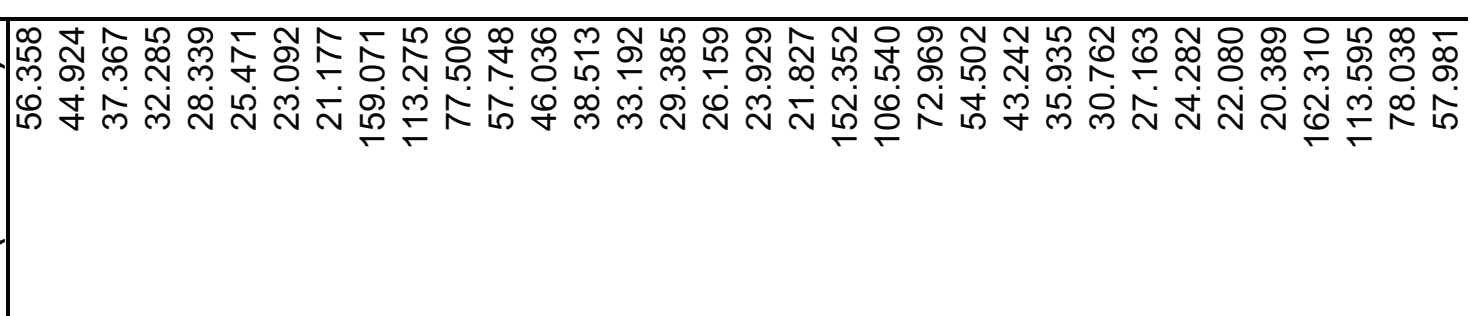 } \\
\hline 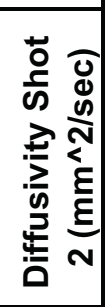 & 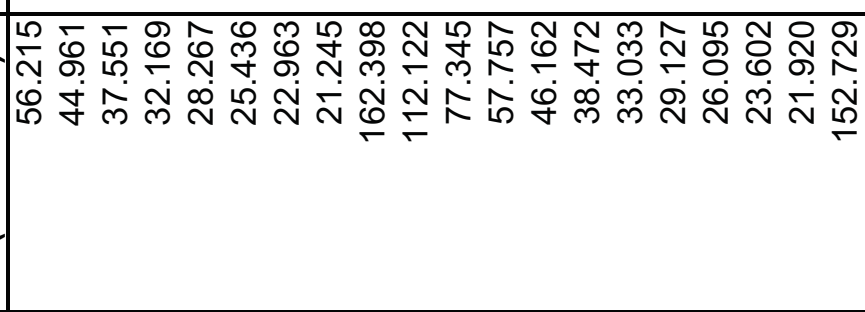 & 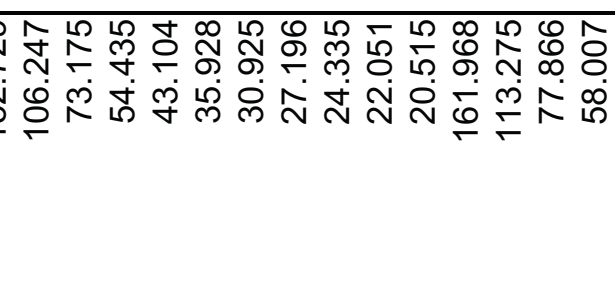 \\
\hline 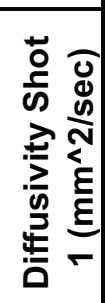 & 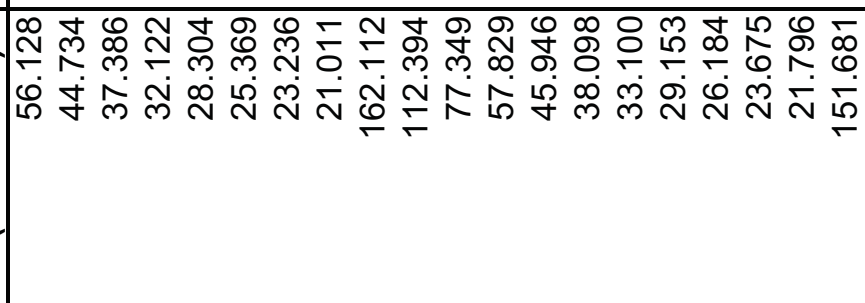 & 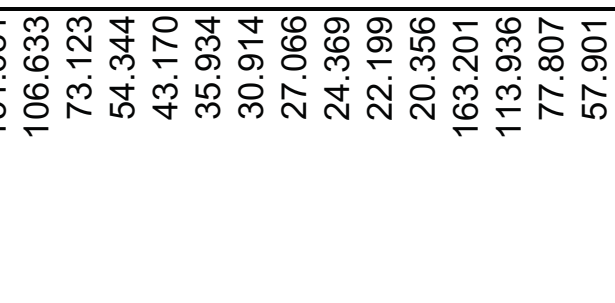 \\
\hline 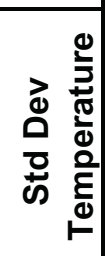 & 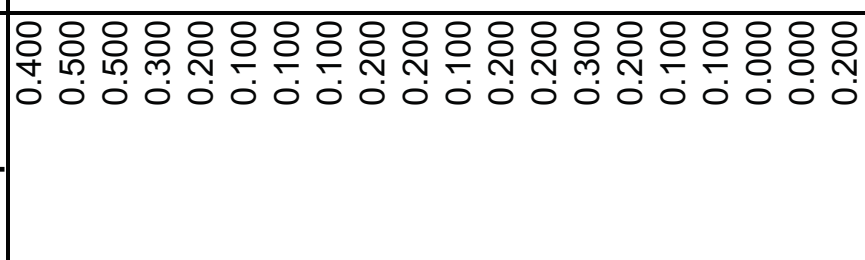 & 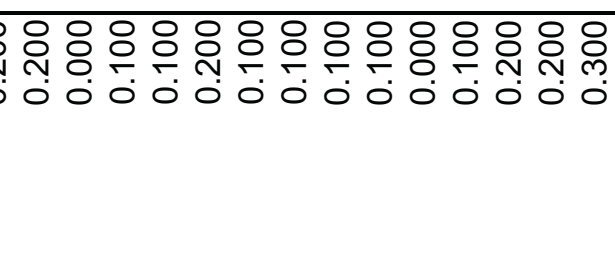 \\
\hline 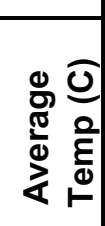 & \begin{tabular}{|l} 
\\
\end{tabular} & 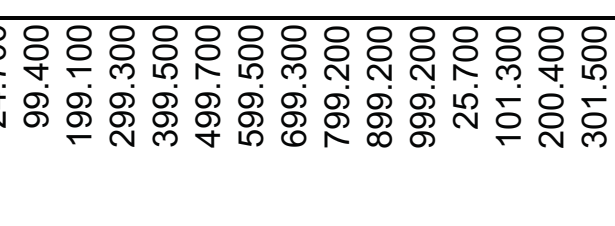 \\
\hline 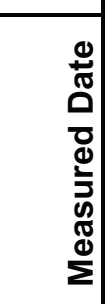 & 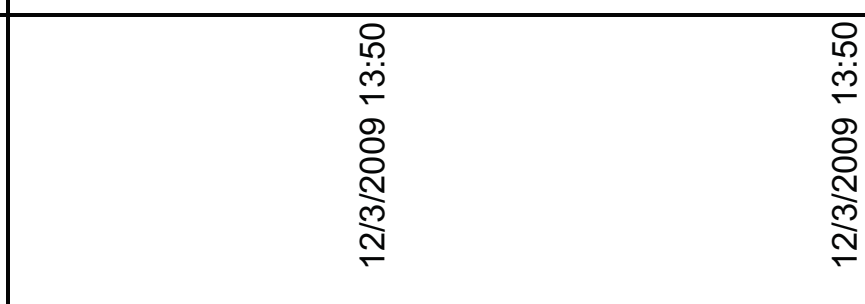 & 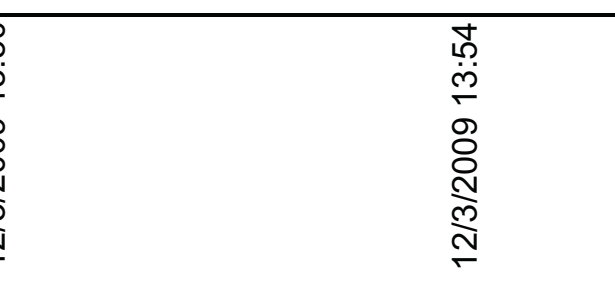 \\
\hline 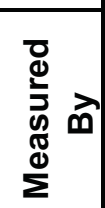 & $\begin{array}{l}8 \\
\frac{8}{\sigma} \\
\dot{\sigma}\end{array}$ & $\begin{array}{l}\stackrel{8}{\circ} \\
\frac{\sigma}{\sigma}\end{array}$ \\
\hline 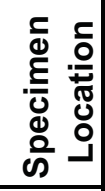 & & \\
\hline 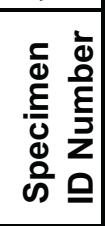 & $\begin{array}{l}\text { L } \\
\text { N }\end{array}$ & $\begin{array}{l}\bar{\delta} \\
\text { N }\end{array}$ \\
\hline
\end{tabular}




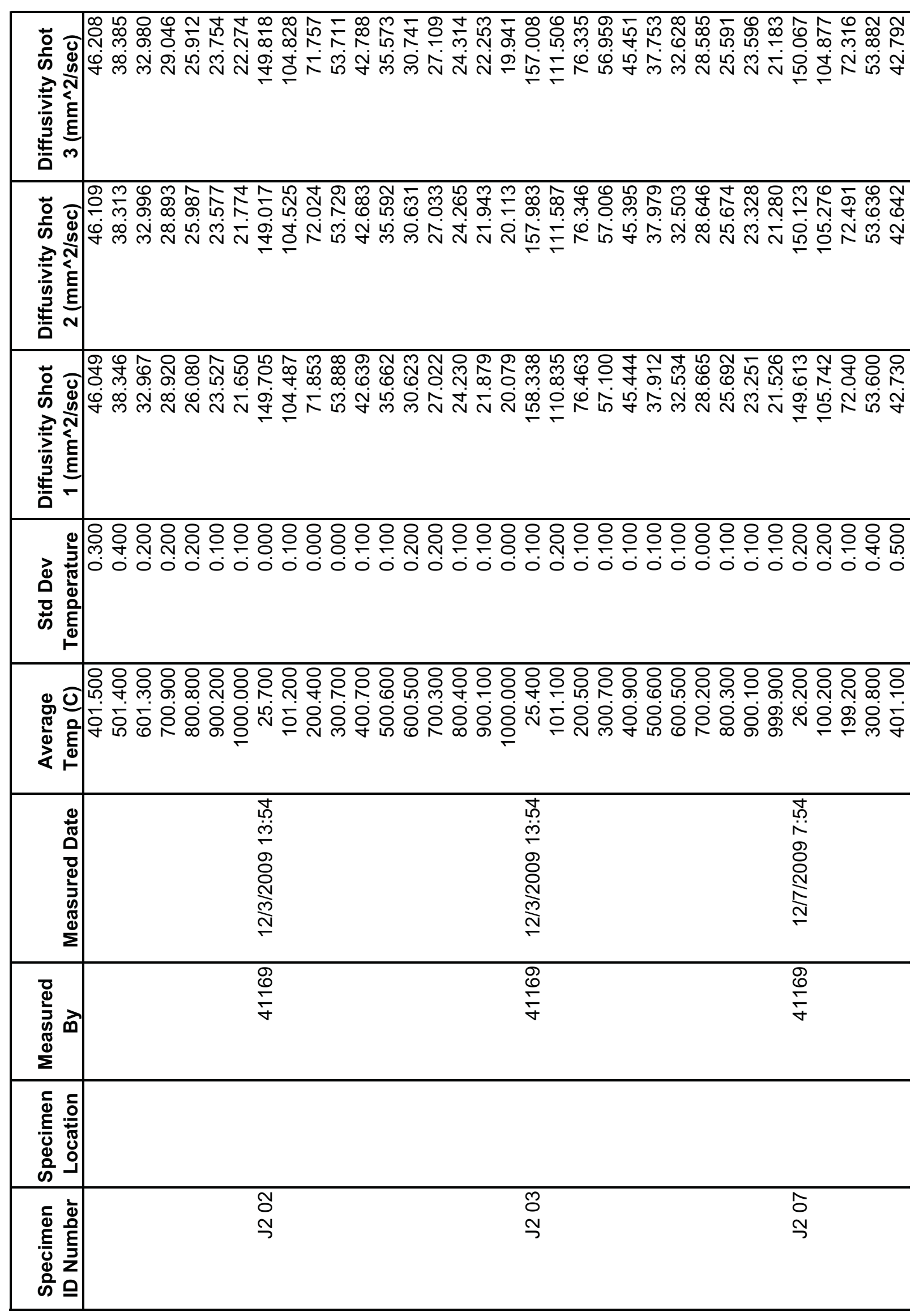




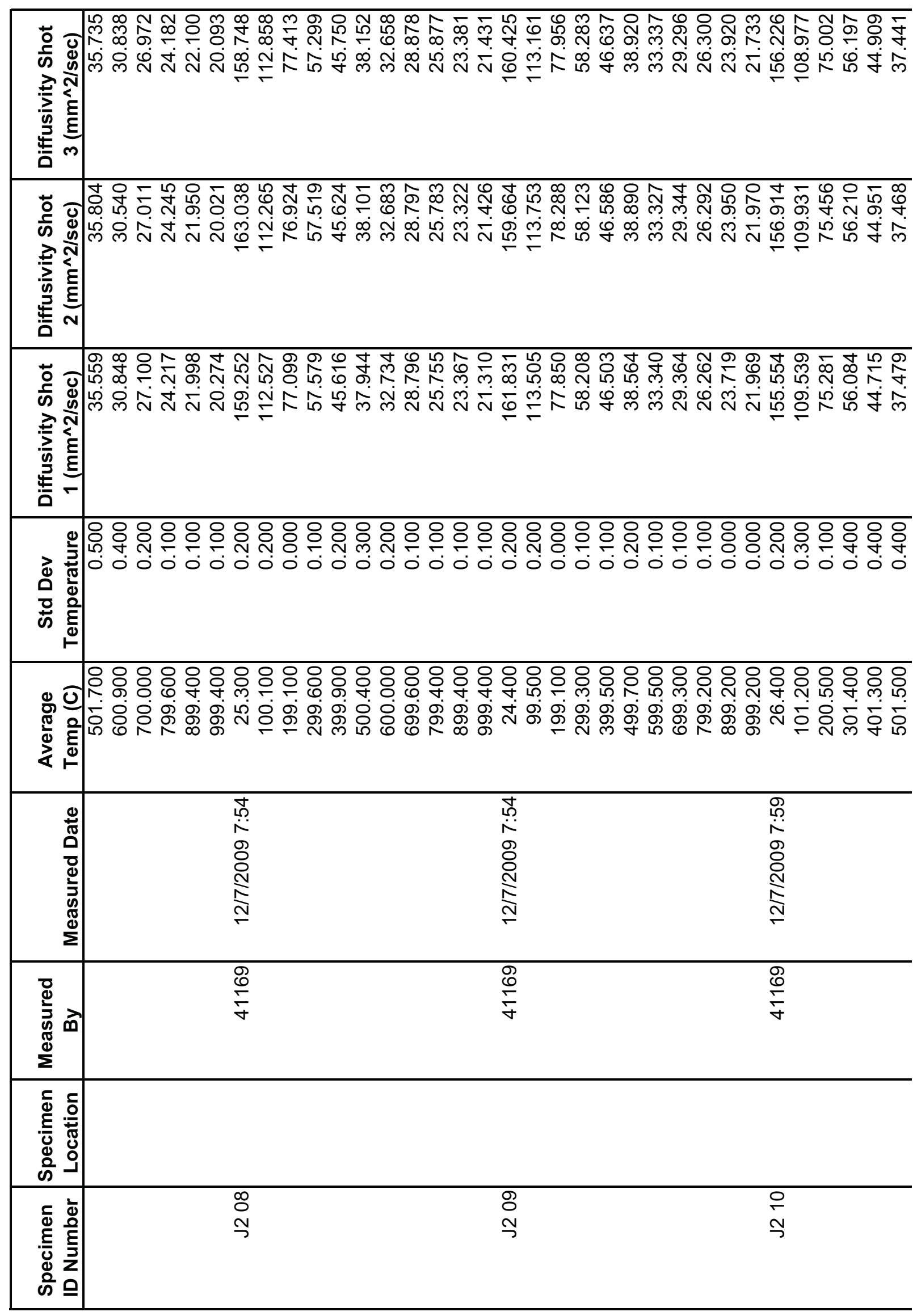




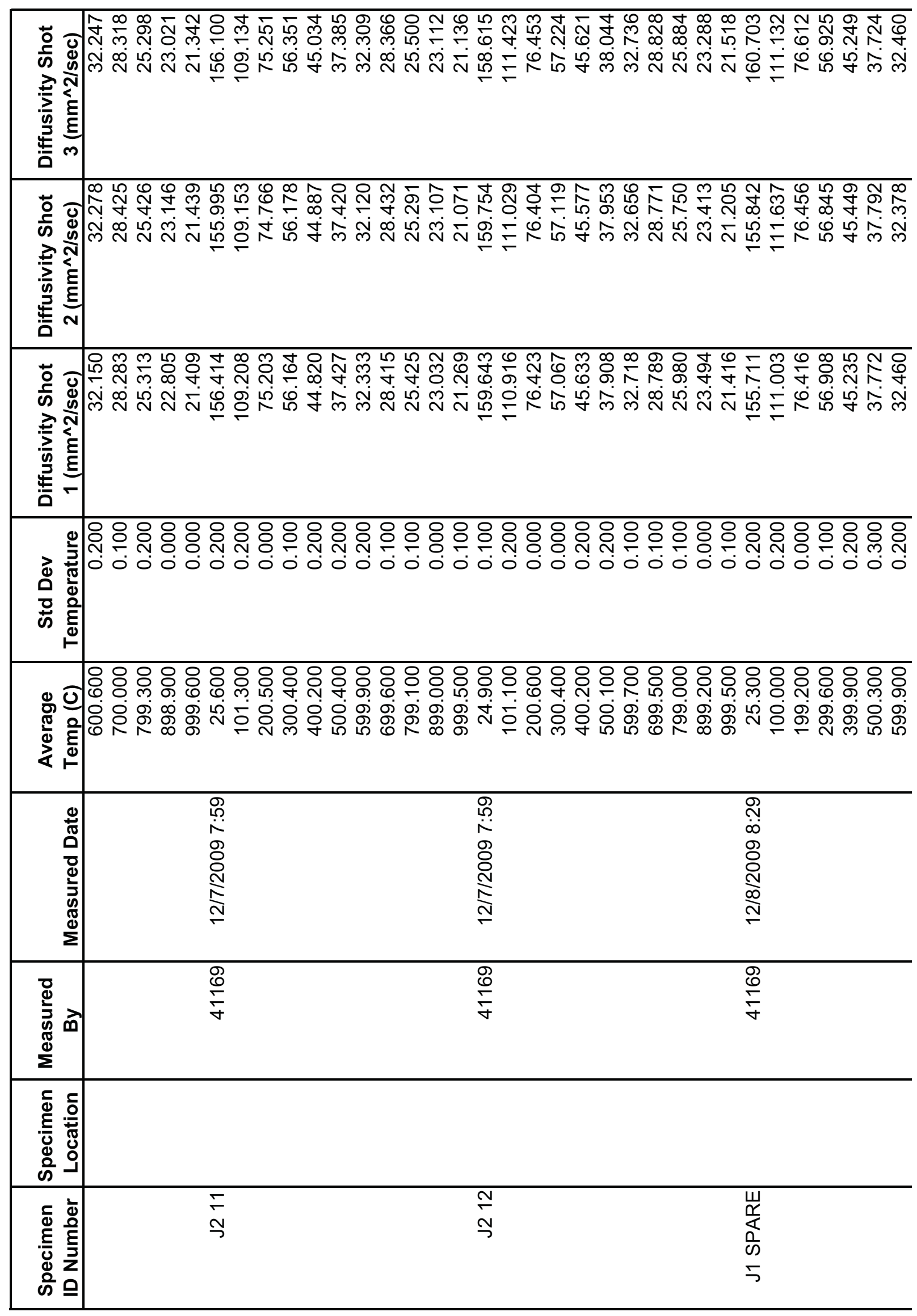




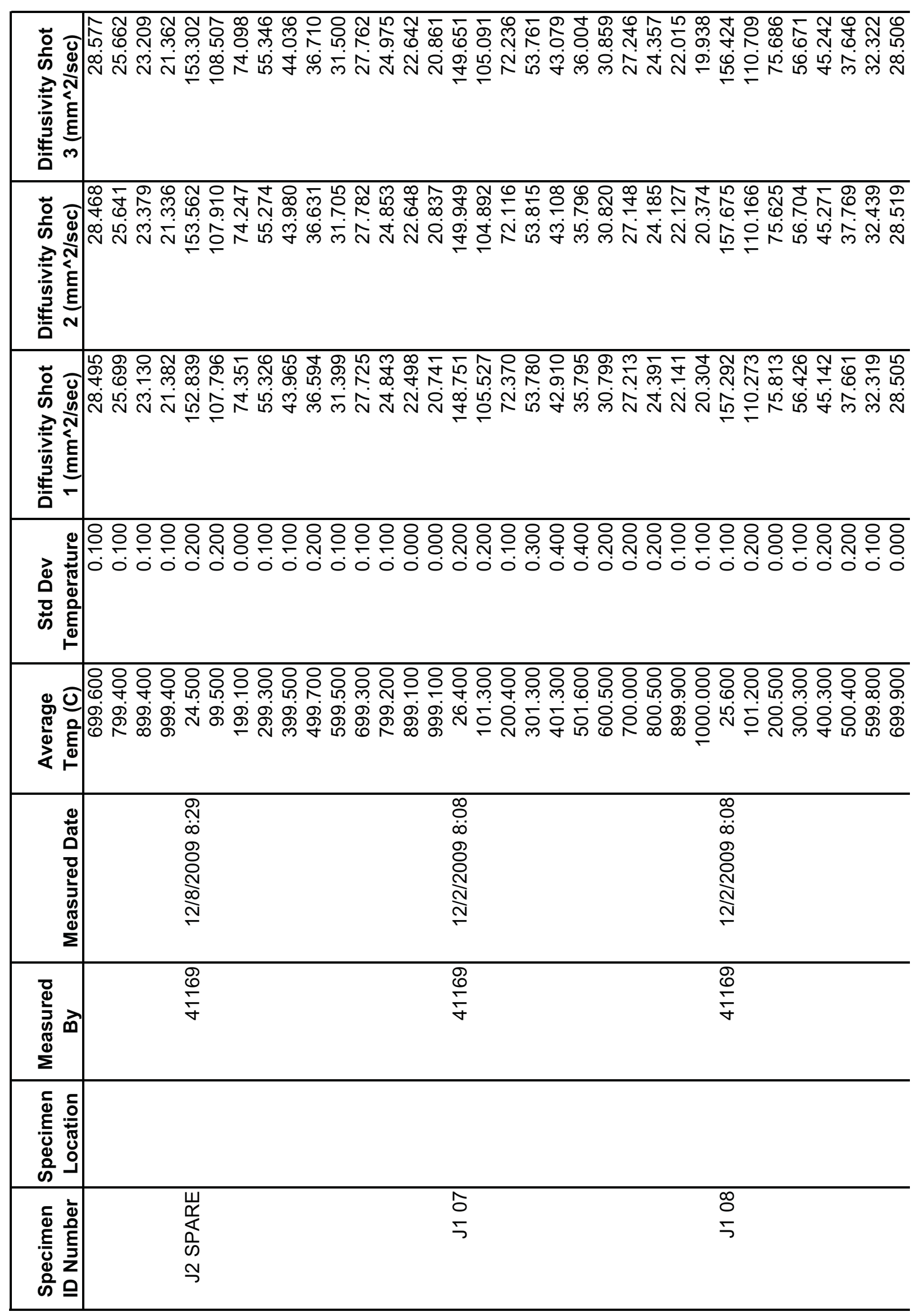




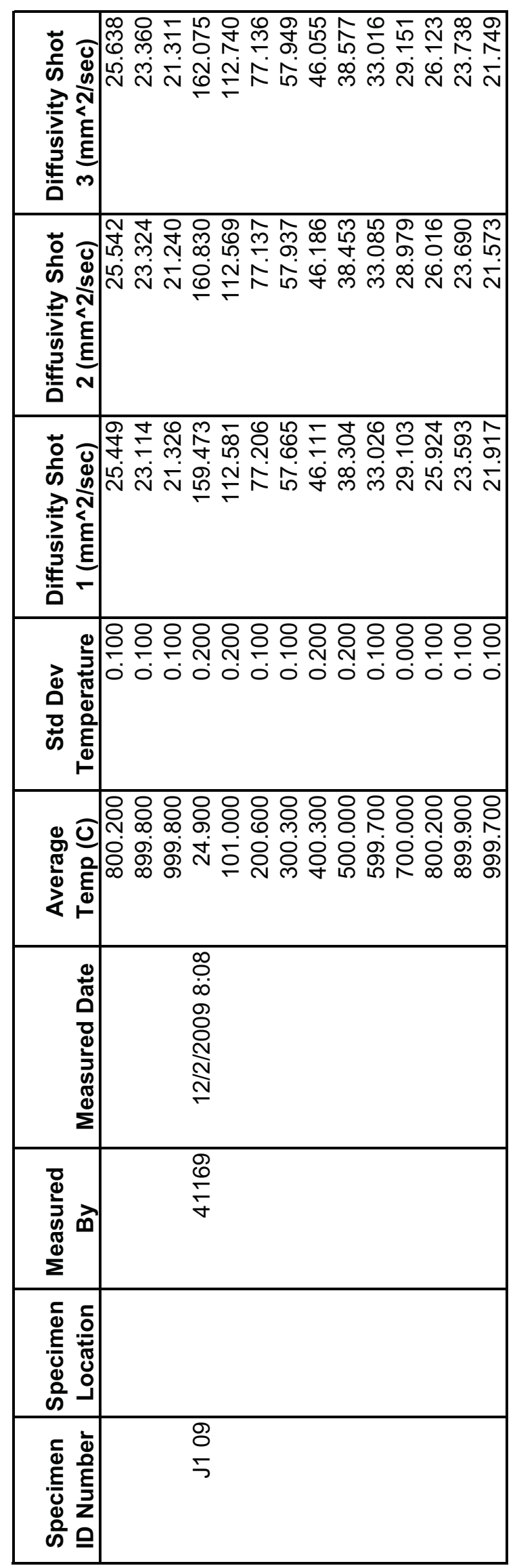




\begin{tabular}{|c|c|c|c|c|}
\hline$\underset{5}{5}$ & & $\begin{array}{l}\hat{O} \\
\frac{1}{0} \\
\stackrel{0}{+} \\
\dot{w}\end{array}$ & 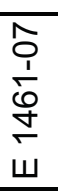 & 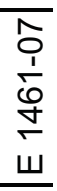 \\
\hline \begin{tabular}{|c|} 
\\
$\frac{0}{0}$ \\
$\frac{5}{0}$ \\
0 \\
0 \\
$\frac{\xi}{<}$
\end{tabular} & & 交 & $\bar{k}$ & $\bar{k}$ \\
\hline 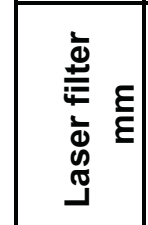 & & $\stackrel{8}{\circ}$ & 음 & 음 \\
\hline 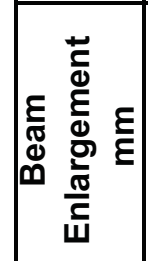 & 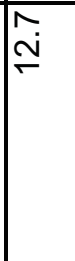 & $\stackrel{\stackrel{N}{\sim}}{\sim}$ & $\stackrel{\widehat{N}}{\stackrel{N}{Y}}$ & 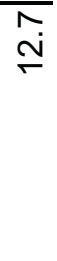 \\
\hline 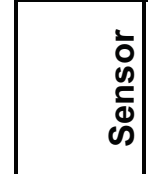 & & $\begin{array}{l}\text { की } \\
\text { की }\end{array}$ & $\begin{array}{l}\text { की } \\
\text { क्ञ }\end{array}$ & की \\
\hline 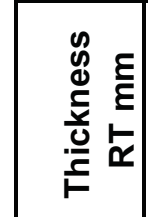 & & 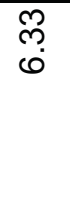 & 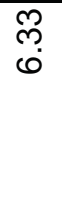 & 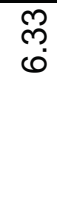 \\
\hline 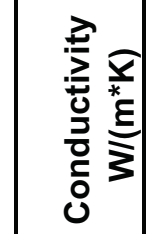 & & & & \\
\hline 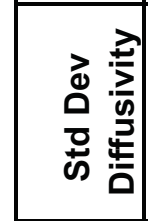 & 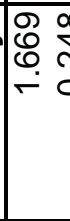 & Oे & $\stackrel{m}{\leftarrow}$ & 㐱 \\
\hline 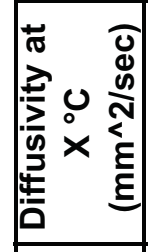 & $\begin{array}{ll}2 \\
0 \\
0 \\
0\end{array}$ & $\begin{array}{l}\bar{\delta} \\
0 \\
0 \\
\sim \\
\sim\end{array}$ & 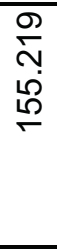 & 今. \\
\hline 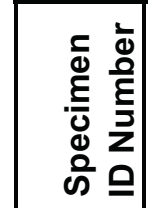 & & $\begin{array}{l}0 \\
\sigma \\
5\end{array}$ & $\begin{array}{l}\text { \% } \\
5\end{array}$ & $\begin{array}{l}5 \\
5 \\
5\end{array}$ \\
\hline
\end{tabular}




\begin{tabular}{|c|c|c|c|}
\hline 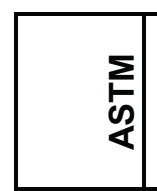 & 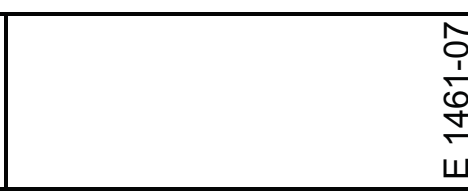 & 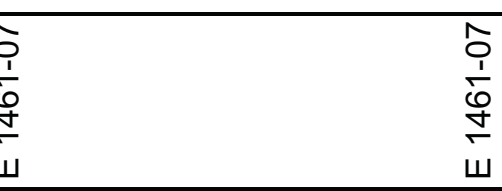 & 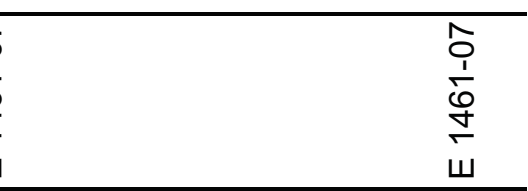 \\
\hline 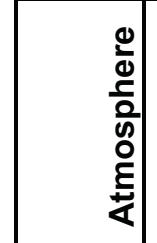 & 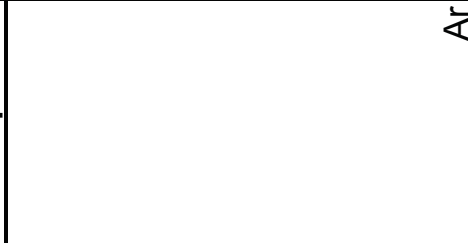 & 这 & 这 \\
\hline 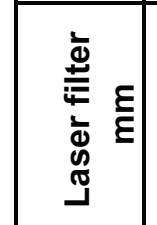 & $\stackrel{8}{\square}$ & 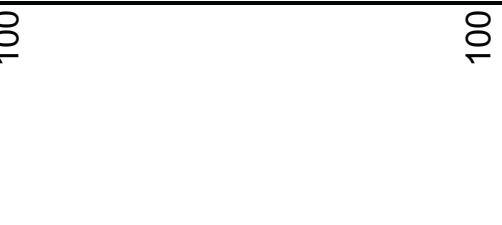 & 음 \\
\hline 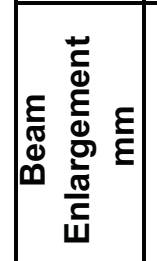 & $\stackrel{\mathbb{N}}{\stackrel{N}{\sim}}$ & $\stackrel{\bar{N}}{\stackrel{N}{N}}$ & $\stackrel{\Upsilon}{\stackrel{N}{N}}$ \\
\hline 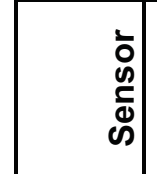 & & $\begin{array}{l}\text { की } \\
\text { की }\end{array}$ & $\begin{array}{l}\text { की } \\
\text { की }\end{array}$ \\
\hline 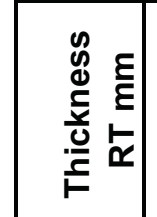 & & लु & 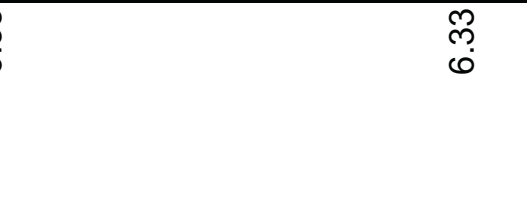 \\
\hline 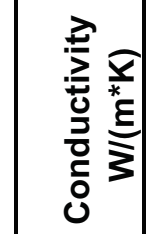 & & & \\
\hline 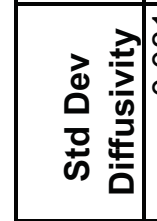 & 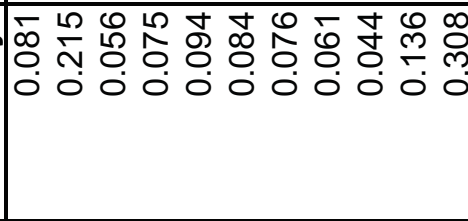 & 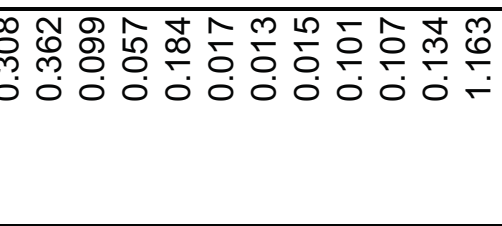 & 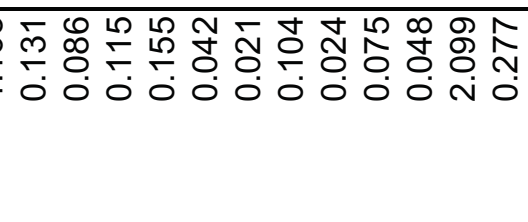 \\
\hline 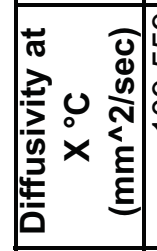 & 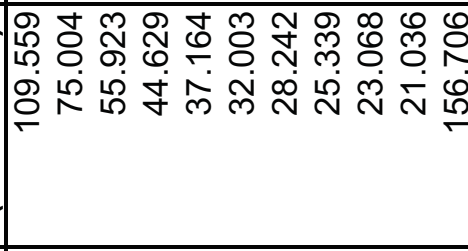 & 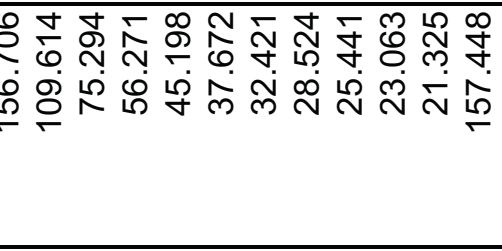 & 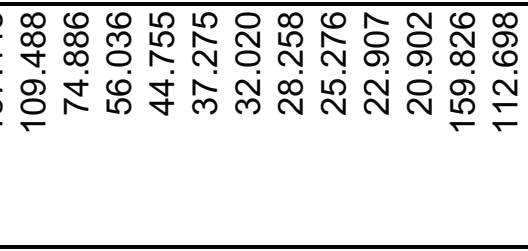 \\
\hline 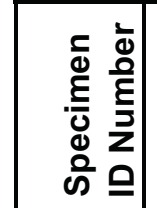 & & $\begin{array}{l}8 \\
5 \\
5\end{array}$ & $\begin{array}{l}\text { 음 } \\
5\end{array}$ \\
\hline
\end{tabular}




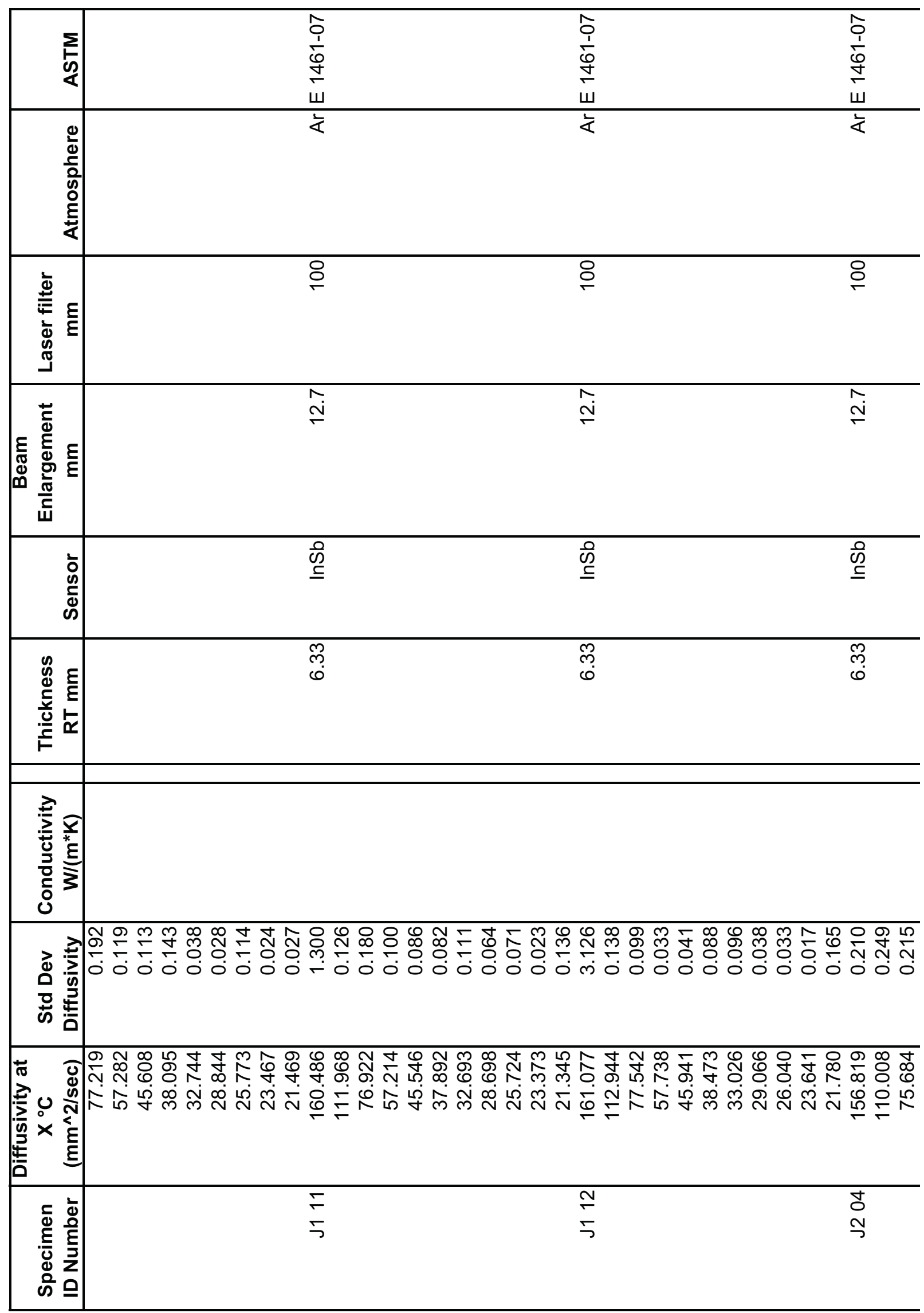




\begin{tabular}{|c|c|c|c|}
\hline 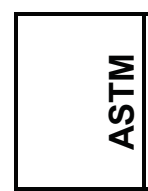 & $\begin{array}{l}\hat{o} \\
\dot{1} \\
\dot{0} \\
\dot{+} \\
\dot{w}\end{array}$ & $\begin{array}{l}\hat{D} \\
\frac{1}{1} \\
\dot{0} \\
\frac{1}{\omega} \\
\end{array}$ & $\begin{array}{l}\hat{O} \\
\frac{1}{0} \\
\dot{0} \\
\dot{+} \\
\end{array}$ \\
\hline 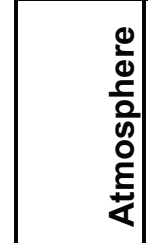 & 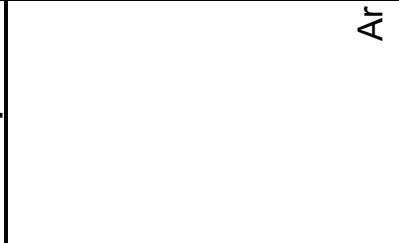 & $\frac{1}{\alpha}$ & ¿ \\
\hline 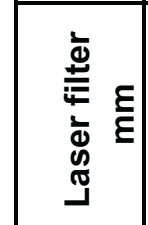 & 음 & 음 & 음 \\
\hline 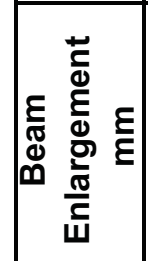 & $\stackrel{\widehat{N}}{\stackrel{N}{N}}$ & 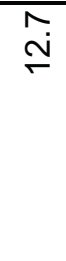 & $\widehat{\stackrel{N}{\underline{N}}}$ \\
\hline 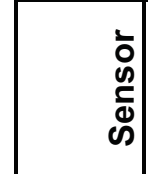 & $\begin{array}{l}\text { की } \\
\text { की }\end{array}$ & 吊 & की \\
\hline 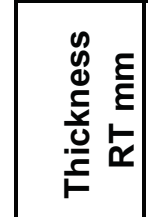 & 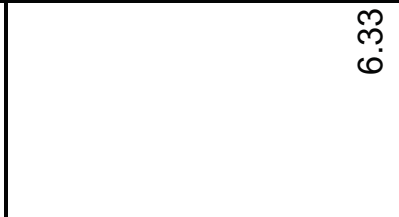 & m & 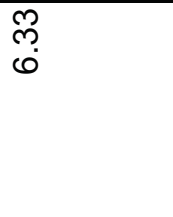 \\
\hline 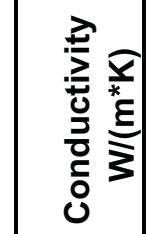 & & & \\
\hline 永辛 & 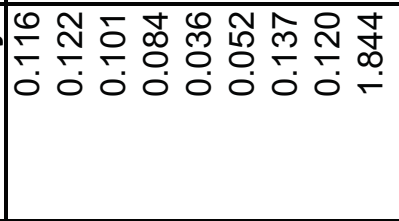 & هُ & 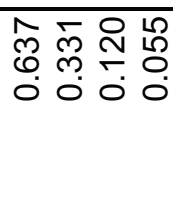 \\
\hline 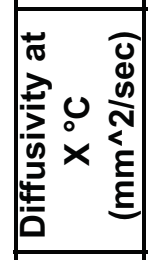 & 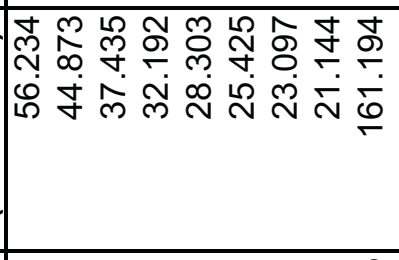 & 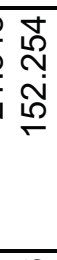 & 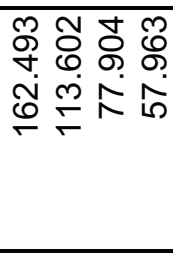 \\
\hline 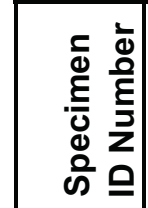 & $\begin{array}{l}\text { ஜ } \\
\text { N̦ }\end{array}$ & $\begin{array}{l}8 \\
\stackrel{8}{7}\end{array}$ & $\begin{array}{l}\bar{\delta} \\
\text { N }\end{array}$ \\
\hline
\end{tabular}




\begin{tabular}{|c|c|c|c|}
\hline$\underset{\Sigma}{\Sigma}$ & $\begin{array}{l}\hat{O} \\
\frac{1}{0} \\
\stackrel{0}{+} \\
\dot{w}\end{array}$ & $\begin{array}{l}\hat{o} \\
\frac{1}{0} \\
\stackrel{0}{+} \\
\dot{\omega}\end{array}$ & 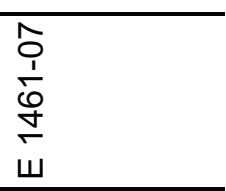 \\
\hline 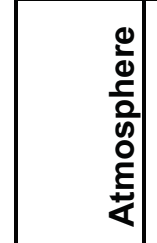 & 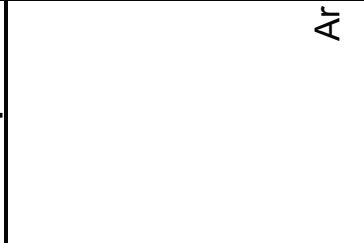 & 衣 & 交 \\
\hline 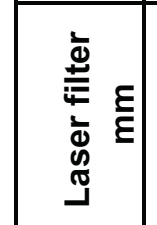 & $\stackrel{\circ}{\circ}$ & 8 & 음 \\
\hline 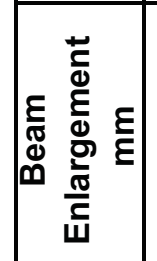 & $\stackrel{\widehat{N}}{\stackrel{N}{S}}$ & $\stackrel{\stackrel{N}{\sim}}{ }$ & $\stackrel{\stackrel{N}{\sim}}{\underline{N}}$ \\
\hline $\begin{array}{l}\vdots \\
\vdots \\
0 \\
⿱ 亠 凶 \\
\infty\end{array}$ & $\begin{array}{l}\text { की } \\
\text { की }\end{array}$ & $\begin{array}{l}\text { की } \\
\text { I }\end{array}$ & $\begin{array}{l}\text { की } \\
\text { Ф }\end{array}$ \\
\hline 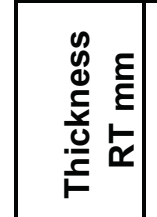 & 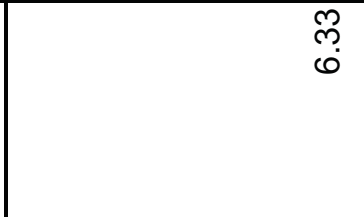 & लm & 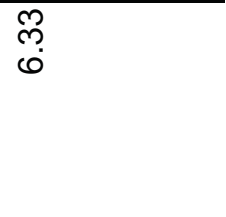 \\
\hline 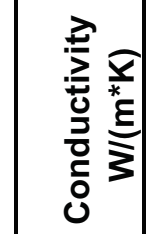 & & & \\
\hline 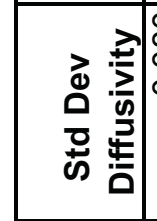 & 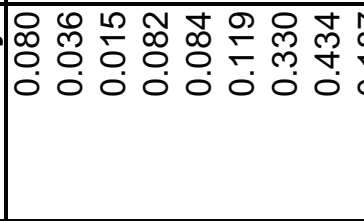 & 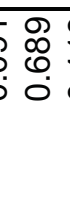 & 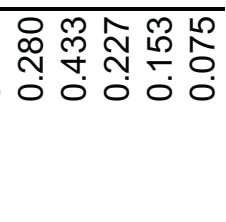 \\
\hline 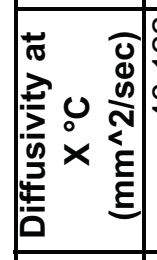 & 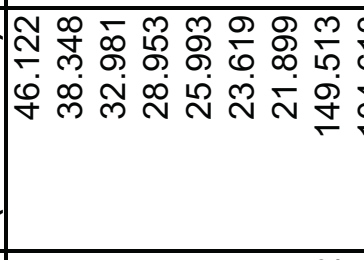 & 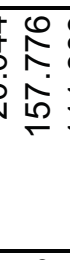 & 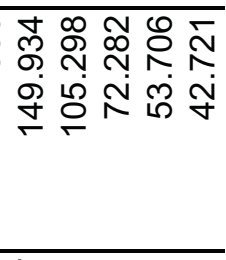 \\
\hline 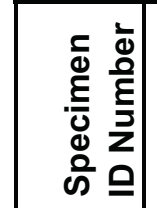 & $\begin{array}{l}\tilde{N} \\
\text { N }\end{array}$ & $\begin{array}{l}\text { 乃 } \\
\text { N }\end{array}$ & $\begin{array}{l}\mathbf{D} \\
\text { N }\end{array}$ \\
\hline
\end{tabular}




\begin{tabular}{|c|c|c|c|}
\hline$\underset{\Sigma}{\Sigma}$ & $\begin{array}{l}\hat{o} \\
\frac{1}{0} \\
\dot{o} \\
\dot{w} \\
\end{array}$ & $\begin{array}{l}\hat{o} \\
\frac{1}{0} \\
\stackrel{0}{+} \\
\dot{\omega}\end{array}$ & 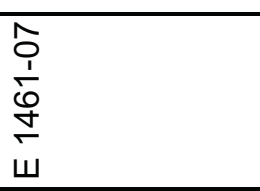 \\
\hline 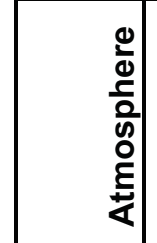 & 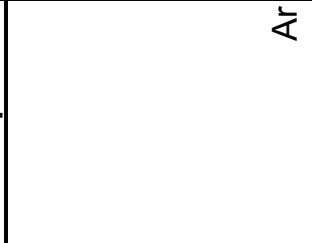 & $\frac{\grave{k}}{2}$ & 文 \\
\hline 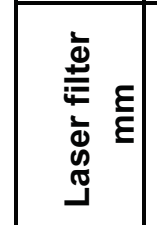 & 음 & 음 & 음 \\
\hline 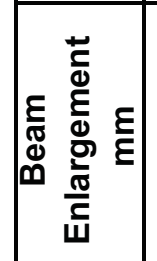 & $\stackrel{\widehat{N}}{\stackrel{N}{N}}$ & 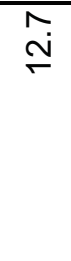 & 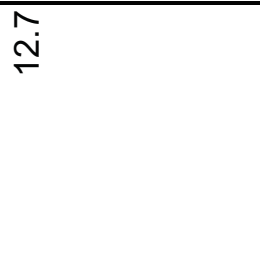 \\
\hline $\begin{array}{l}\vdots \\
\vdots \\
0 \\
⿱ 亠 凶 \\
\infty\end{array}$ & $\begin{array}{l}\text { की } \\
\text { 足 }\end{array}$ & $\begin{array}{l}\text { 足 } \\
\text { I }\end{array}$ & $\begin{array}{l}\text { की } \\
\text { की }\end{array}$ \\
\hline 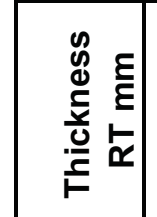 & 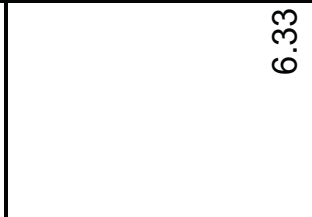 & $\begin{array}{l}m \\
\text { m. } \\
0\end{array}$ & $\begin{array}{c}\mathscr{m} \\
\oplus \\
0\end{array}$ \\
\hline 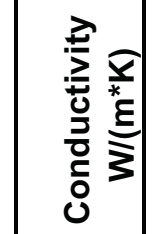 & & & \\
\hline 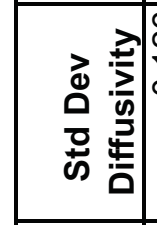 & 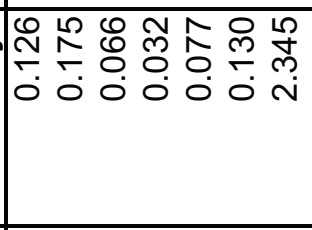 & 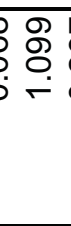 & $\begin{array}{l}\circ \\
\end{array}$ \\
\hline 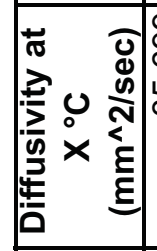 & 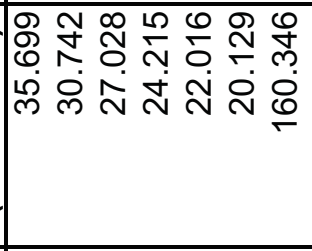 & 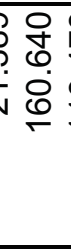 & 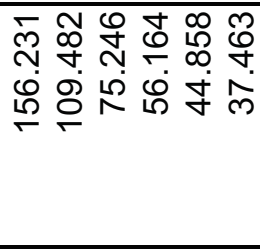 \\
\hline 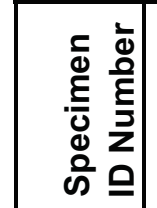 & $\begin{array}{l}\infty \\
\text { D } \\
\text { Ny}\end{array}$ & $\begin{array}{l}\text { OD } \\
\text { N }\end{array}$ & $\begin{array}{l}\stackrel{\bigcirc}{\Im} \\
\stackrel{\sim}{\sim}\end{array}$ \\
\hline
\end{tabular}




\begin{tabular}{|c|c|c|c|}
\hline$\underset{\Sigma}{\Sigma}$ & 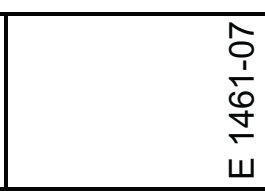 & 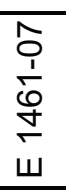 & $\begin{array}{l}\hat{O} \\
\frac{1}{0} \\
\stackrel{0}{+} \\
\dot{w}\end{array}$ \\
\hline 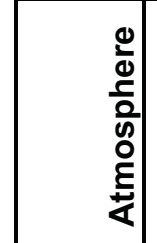 & 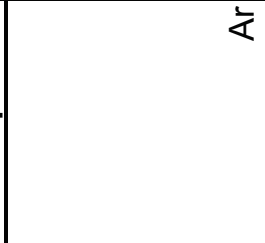 & 文 & $\grave{4}$ \\
\hline 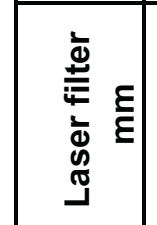 & $\stackrel{8}{\circ}$ & 음 & 음 \\
\hline 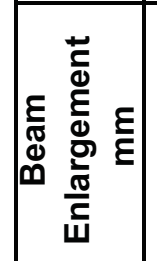 & $\stackrel{\bar{N}}{\stackrel{N}{N}}$ & 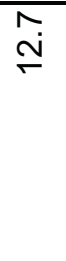 & $\stackrel{\widetilde{N}}{\underline{\mathrm{N}}}$ \\
\hline $\begin{array}{l}\vdots \\
0 \\
\stackrel{5}{\Phi} \\
\infty\end{array}$ & $\begin{array}{l}\text { की } \\
\text { की }\end{array}$ & $\begin{array}{l}\text { की } \\
\text { की }\end{array}$ & $\begin{array}{l}\text { की } \\
\text { 足 }\end{array}$ \\
\hline 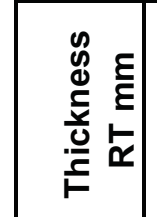 & ల్ల్ర & 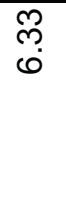 & $\begin{array}{l}\text { ల్ల } \\
0\end{array}$ \\
\hline 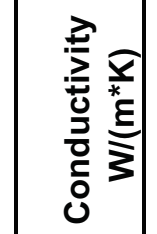 & & & \\
\hline 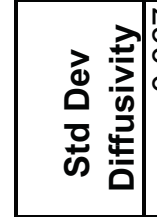 & 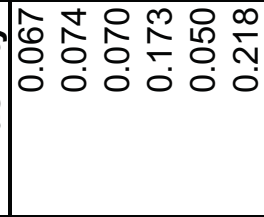 & 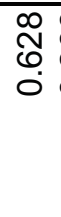 & 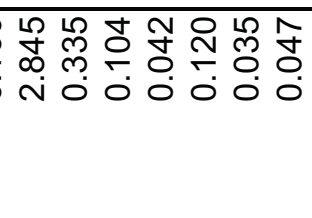 \\
\hline 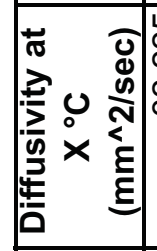 & 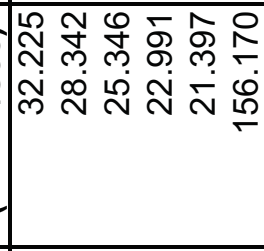 & $\stackrel{\text { L }}{\circ}$ & 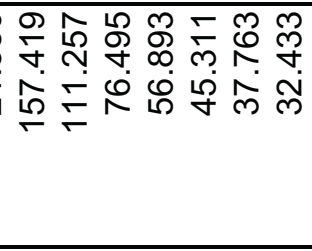 \\
\hline 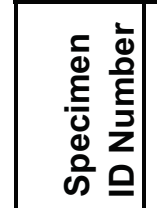 & $\stackrel{\Sigma}{\stackrel{乛}{N}}$ & $\begin{array}{l}\underset{N}{N} \\
\stackrel{N}{J}\end{array}$ & $\begin{array}{l}\frac{W}{\frac{r}{\alpha}} \\
\frac{\Delta}{0} \\
\frac{5}{5}\end{array}$ \\
\hline
\end{tabular}




\begin{tabular}{|c|c|c|c|}
\hline 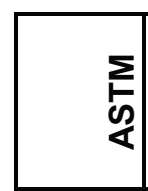 & $\begin{array}{l}\hat{o} \\
\dot{1} \\
\dot{6} \\
\dot{+} \\
\dot{\omega}\end{array}$ & 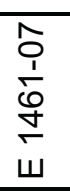 & 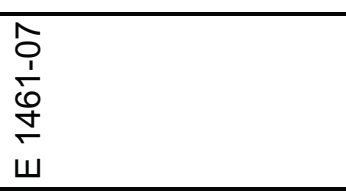 \\
\hline 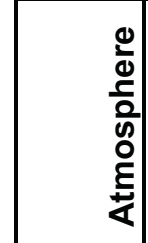 & 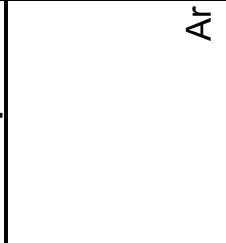 & 文 & ¿ \\
\hline 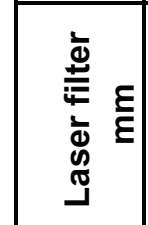 & $\stackrel{8}{\circ}$ & 8 & 음 \\
\hline 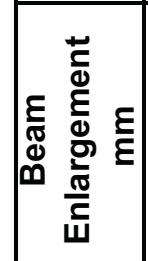 & $\stackrel{\widehat{N}}{\stackrel{N}{N}}$ & 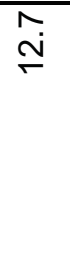 & $\widehat{\grave{I}}$ \\
\hline 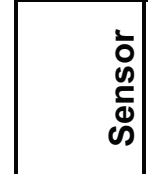 & $\begin{array}{l}\text { की } \\
\text { की }\end{array}$ & $\begin{array}{l}\text { की } \\
\text { की }\end{array}$ & की \\
\hline 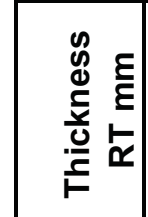 & 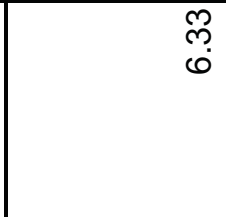 & $\begin{array}{l}\stackrel{m}{n} \\
\dot{\varphi}\end{array}$ & 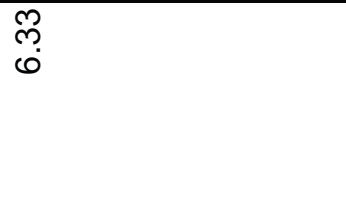 \\
\hline 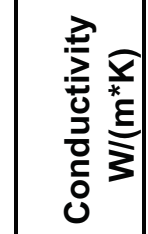 & & & \\
\hline 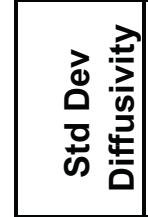 & 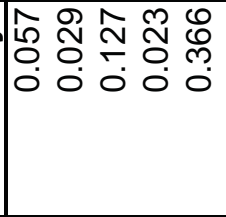 & ర & 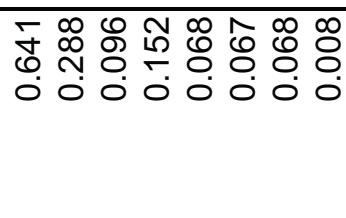 \\
\hline 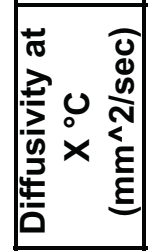 & 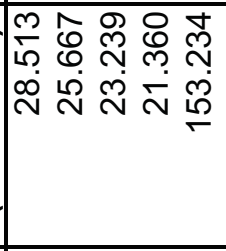 & 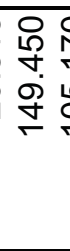 & 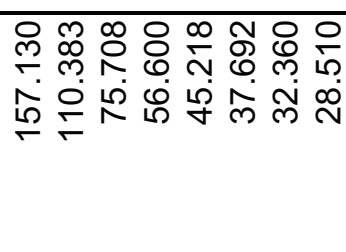 \\
\hline 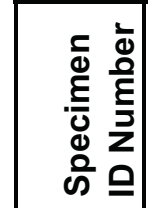 & $\begin{array}{l}\underline{w} \\
\frac{\alpha}{\alpha} \\
0 \\
\text { क } \\
\text { N }\end{array}$ & $\begin{array}{l}5 \\
5 \\
5\end{array}$ & $\begin{array}{l}\infty \\
0 \\
5\end{array}$ \\
\hline
\end{tabular}




\begin{tabular}{|c|c|}
\hline 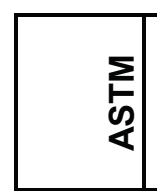 & 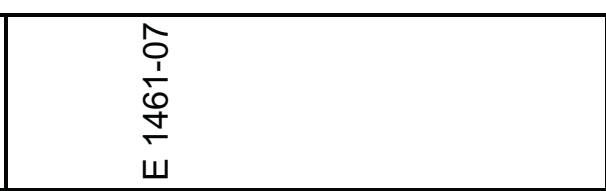 \\
\hline 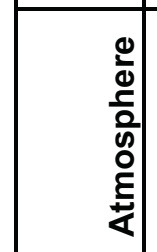 & 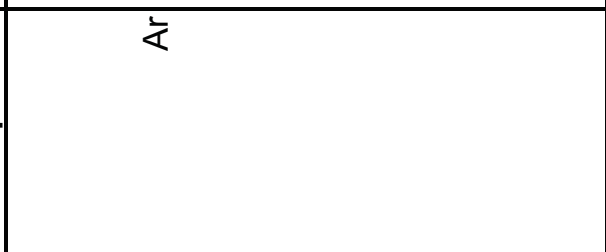 \\
\hline 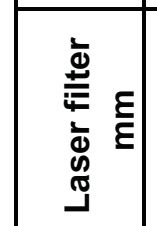 & 음 \\
\hline 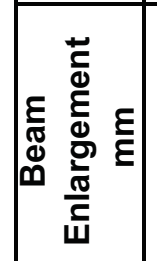 & 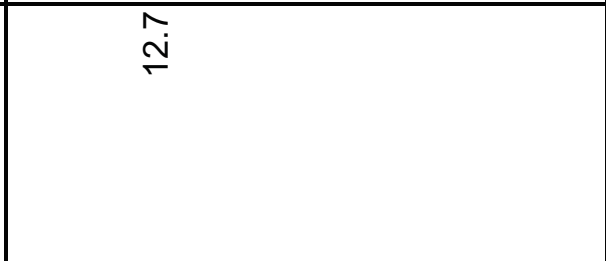 \\
\hline $\begin{array}{l}\bar{b} \\
\dot{g} \\
\bar{d} \\
\oplus\end{array}$ & की \\
\hline 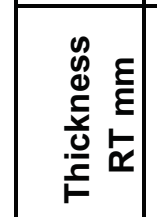 & 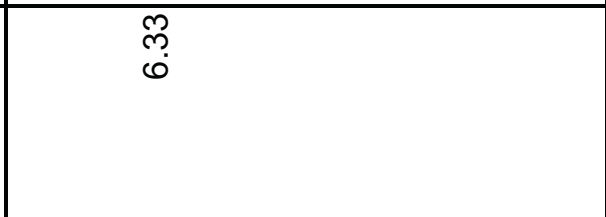 \\
\hline 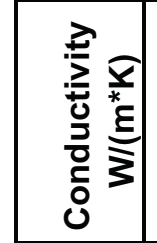 & \\
\hline 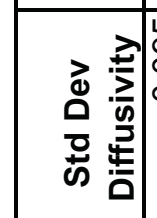 & 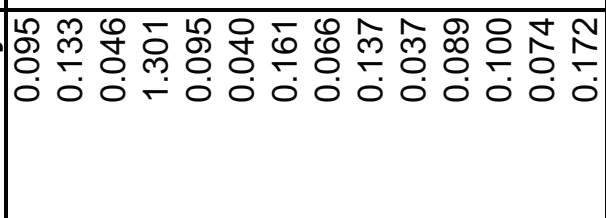 \\
\hline 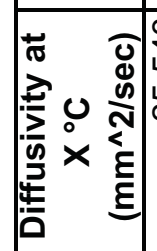 & 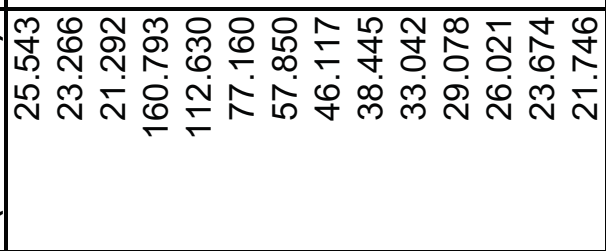 \\
\hline 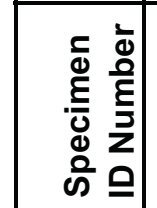 & $\begin{array}{l}8 \\
8 \\
5\end{array}$ \\
\hline
\end{tabular}




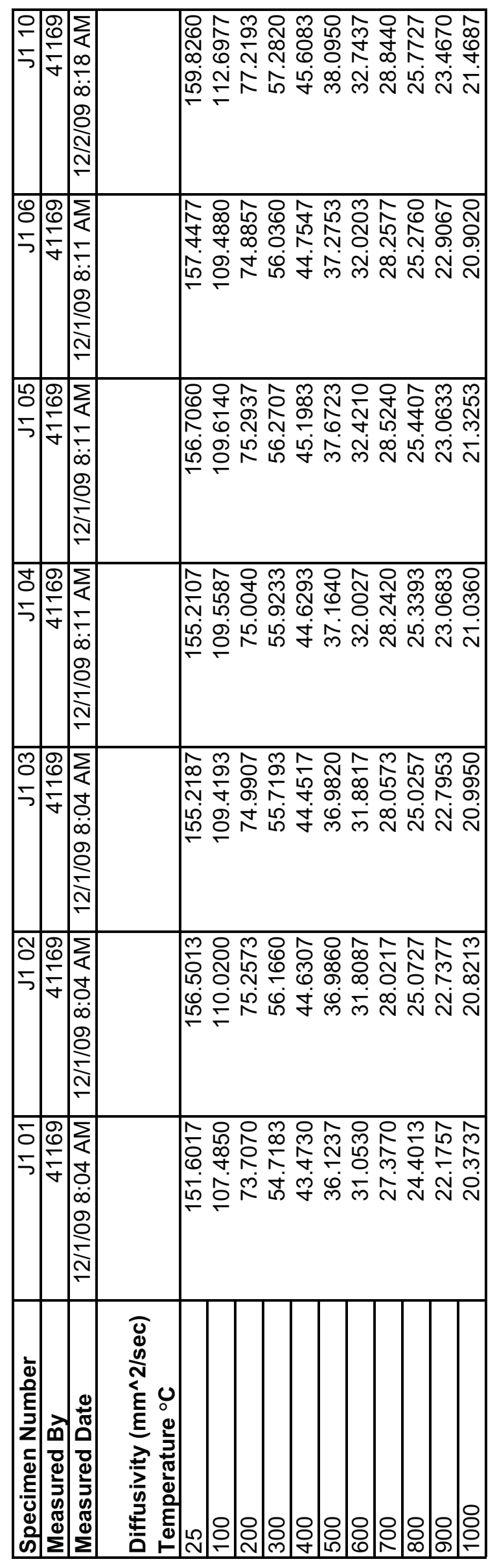




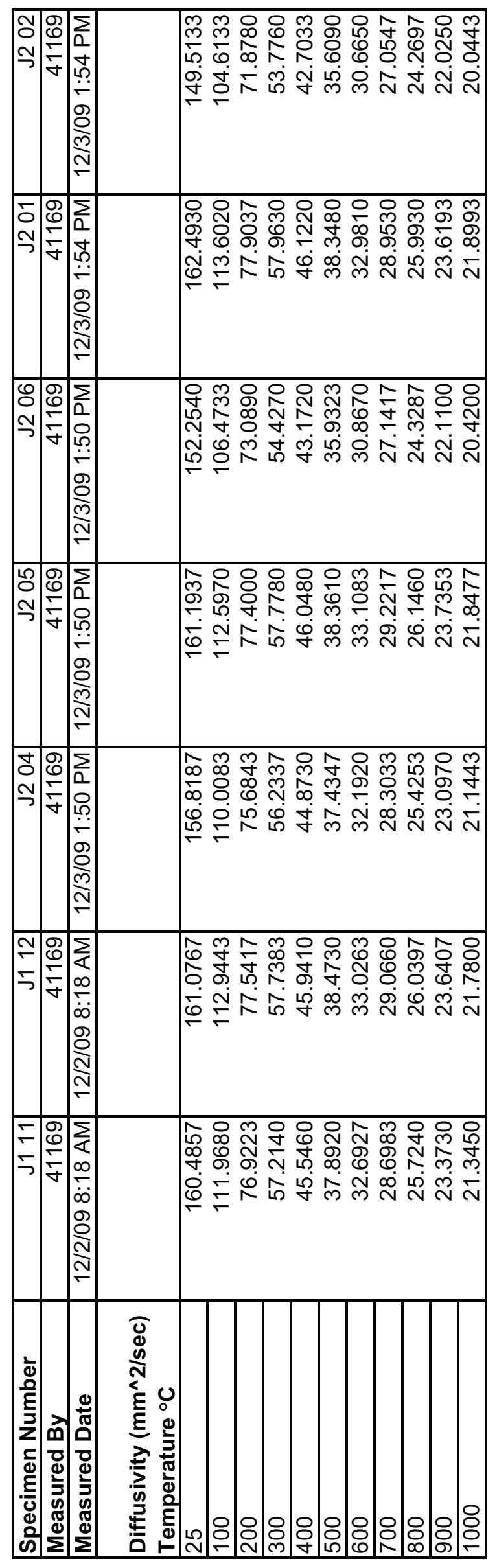




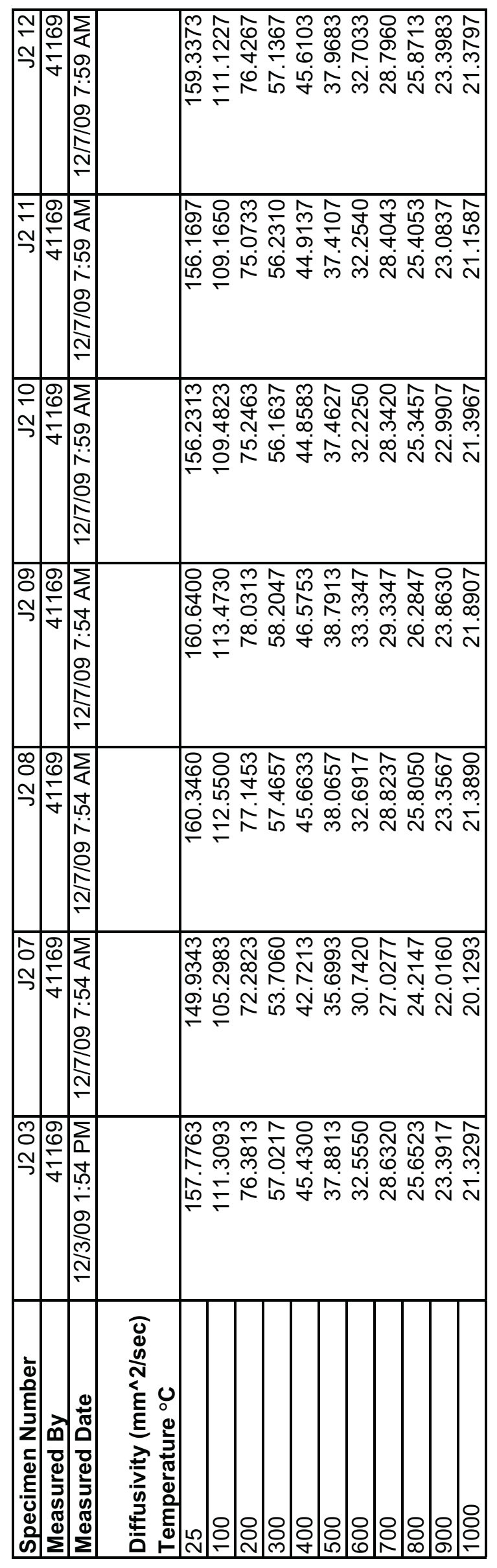




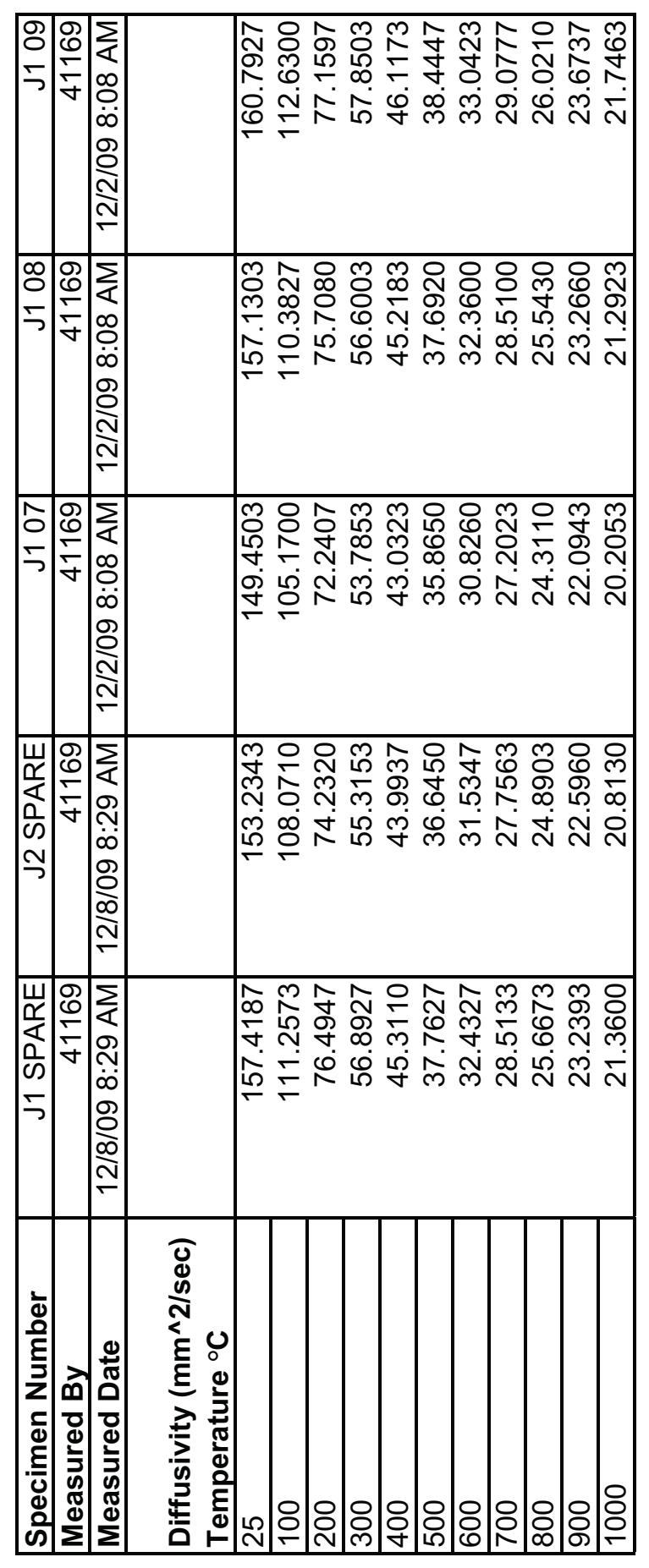




\begin{tabular}{|c|c|}
\hline 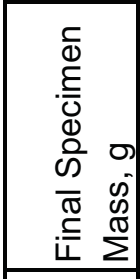 & 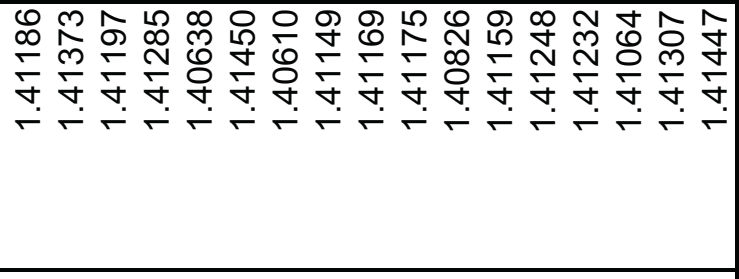 \\
\hline 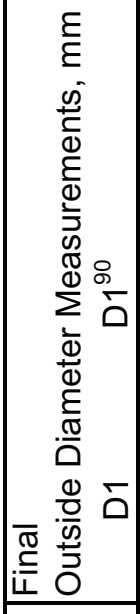 & 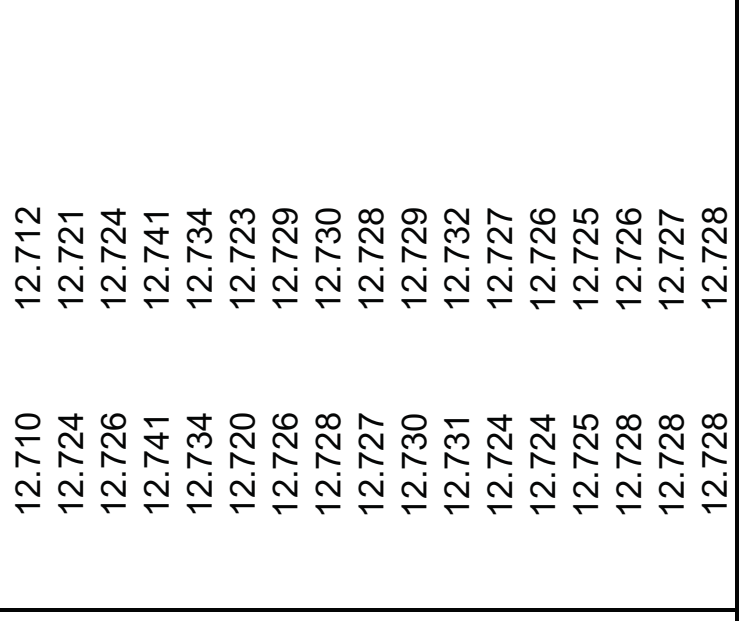 \\
\hline 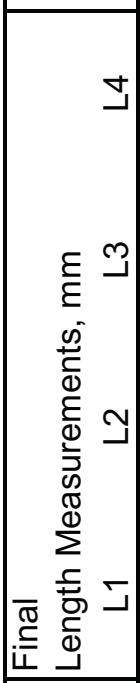 & 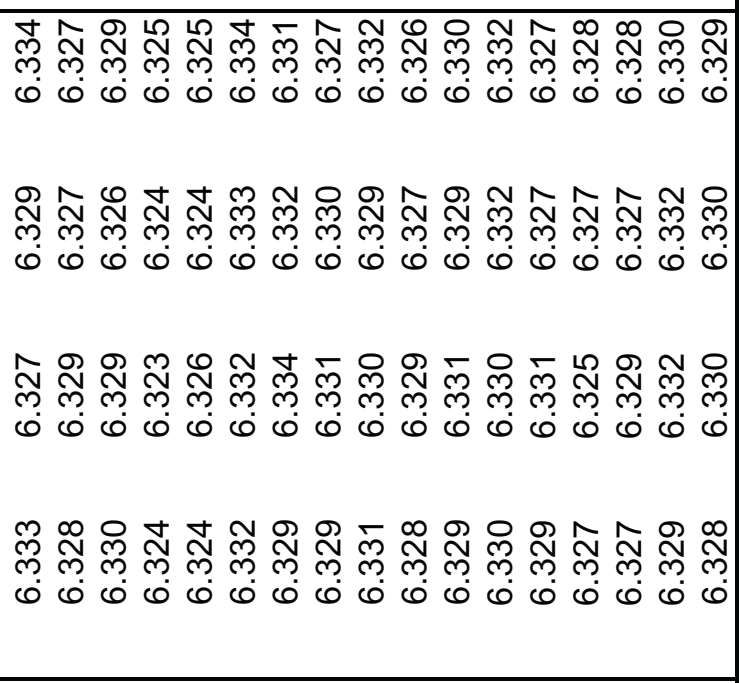 \\
\hline 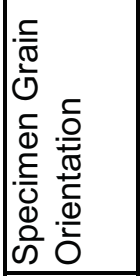 & \\
\hline 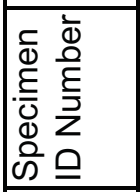 & 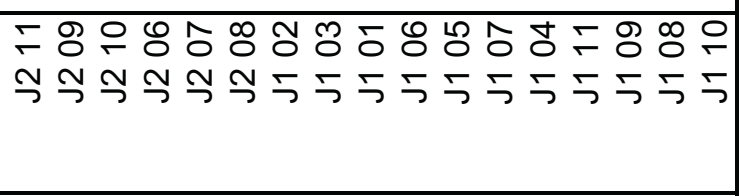 \\
\hline 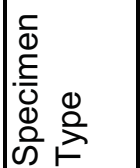 & \\
\hline
\end{tabular}




\begin{tabular}{|c|c|}
\hline 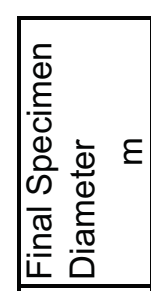 & 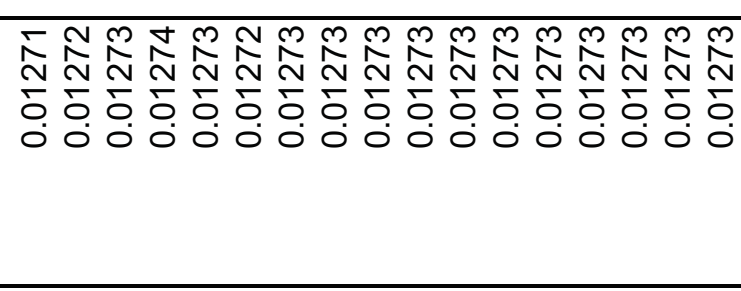 \\
\hline$\underbrace{\frac{5}{\Phi}}$ & 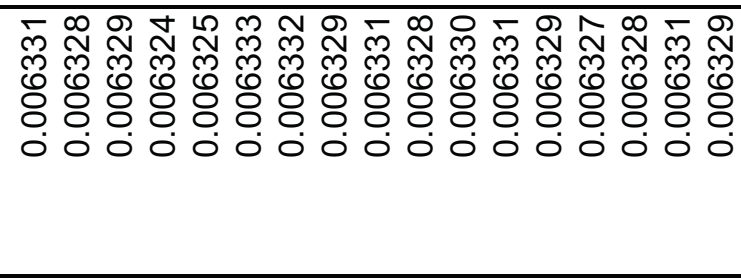 \\
\hline 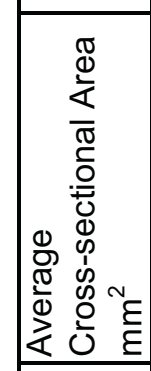 & 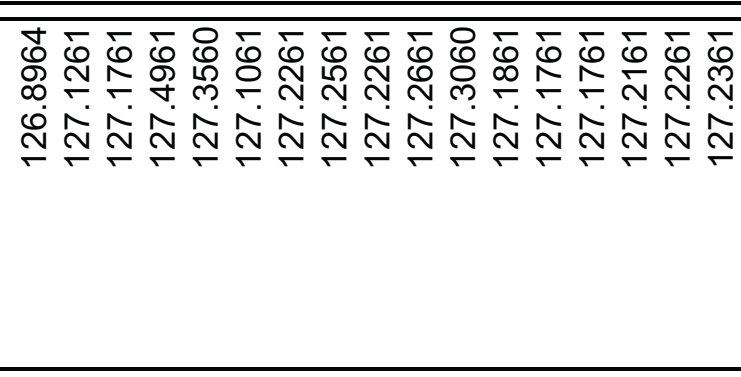 \\
\hline 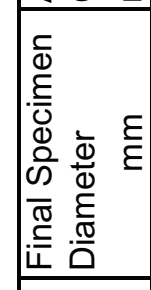 & 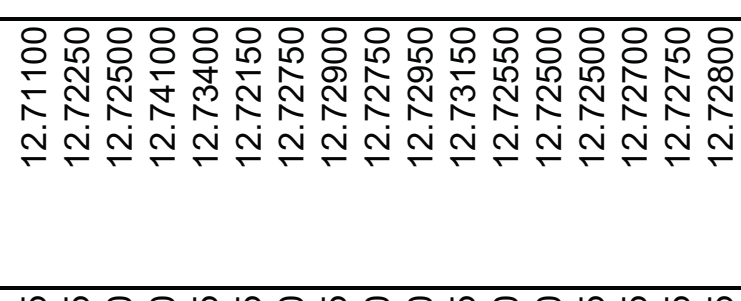 \\
\hline$=\bar{\xi}$ & 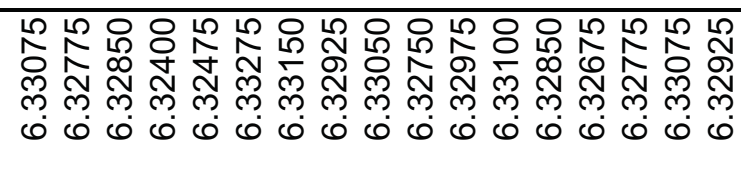 \\
\hline 总 & 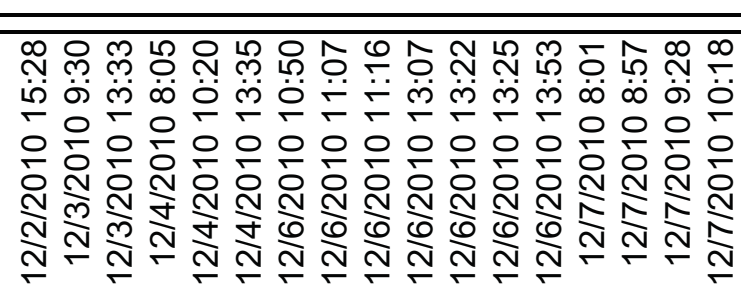 \\
\hline 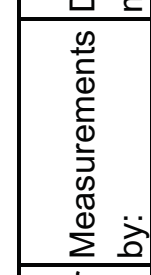 & 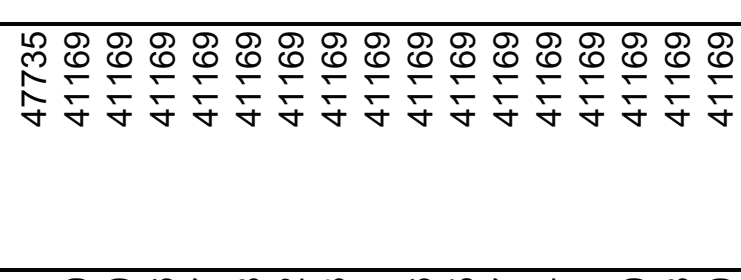 \\
\hline 这 & 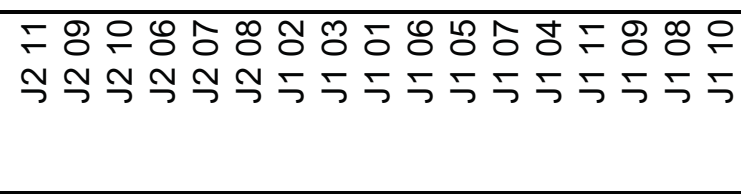 \\
\hline
\end{tabular}




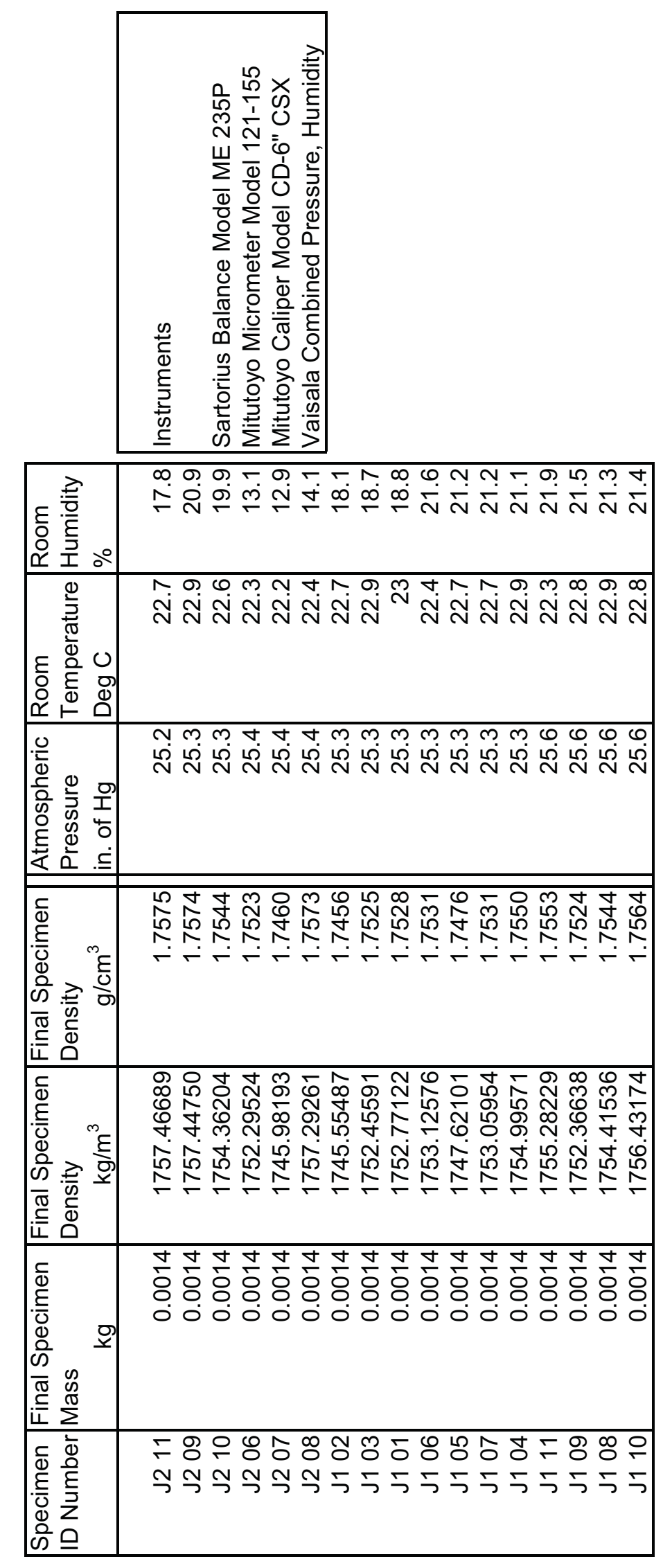




$\begin{array}{ll}\text { Graphite Grade: } & \text { IG-110 } \\ \text { Graphite Manufacturer: } & \text { Toyo Tanso } \\ \text { Forming Process: } & \text { Isostatic-molded } \\ \text { Coke Particle Size: } & \text { Fine grain } \\ \text { Coke Type: } & \text { Petroleum coke filler, pitch binder } \\ \text { ASTM Class: } & \text { INHP } \\ \text { Specimen Geometry: } & \text { Cylinder }\end{array}$

Specimen ID \#'s:

EW15 01

EW15 02

EW15 03

EW15 04

EW15 05

EW15 06

EW15 07

EW15 08

EW15 09

EW15 10

EW15 11

EW15 12

EW14 01

EW14 02

EW14 03

EW14 04

EW14 05

EW14 06

EW14 07

EW14 08

EW14 09

EW14 10

EW14 11

EW14 12 


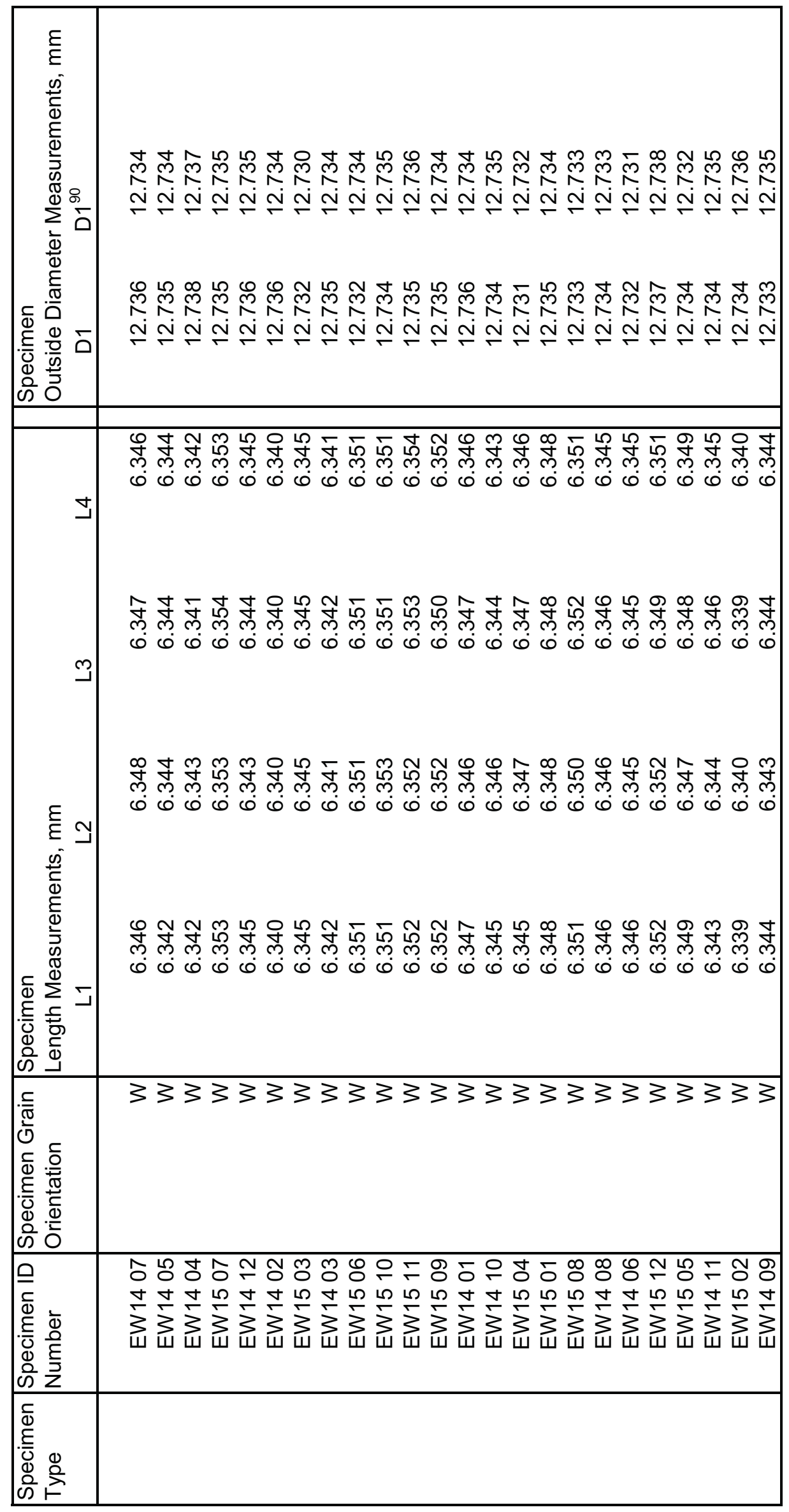




\begin{tabular}{|c|c|}
\hline 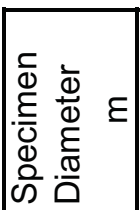 & 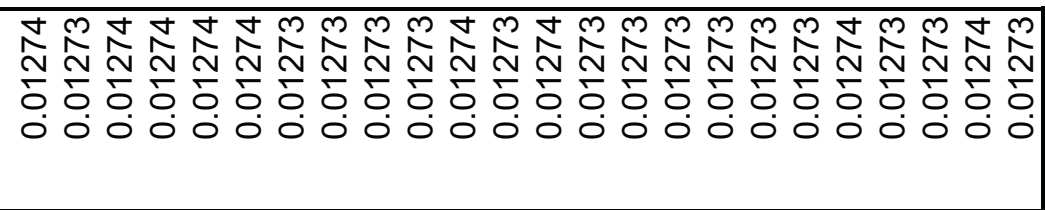 \\
\hline 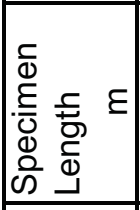 & 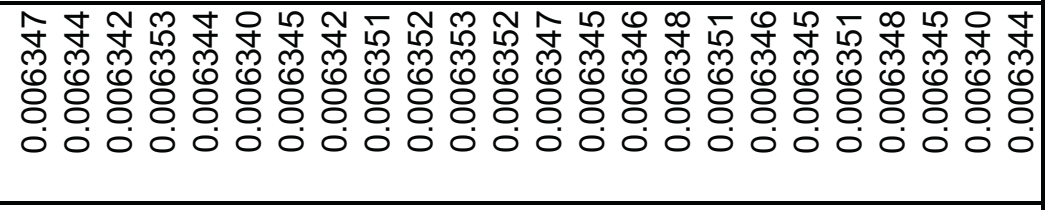 \\
\hline 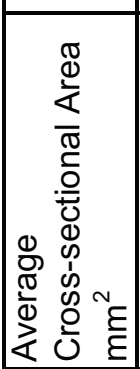 & 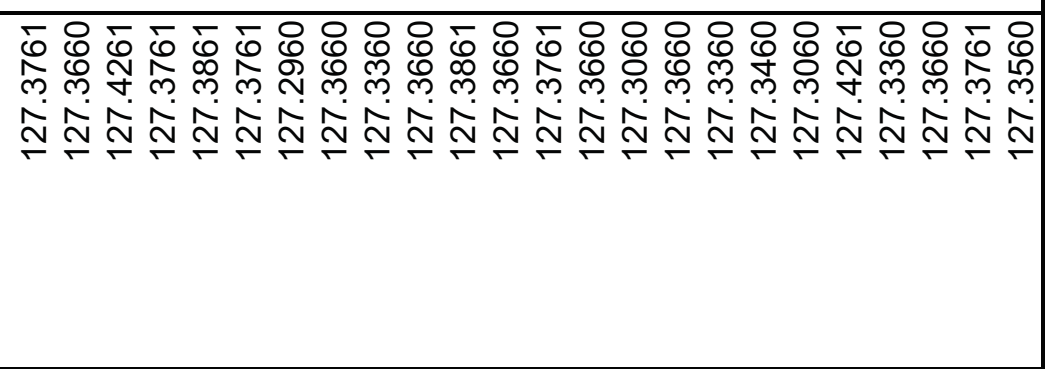 \\
\hline 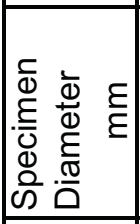 & 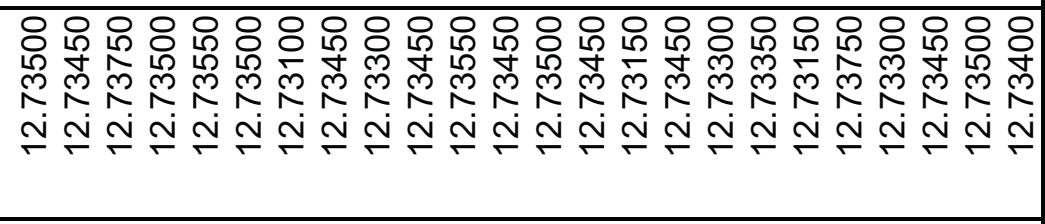 \\
\hline 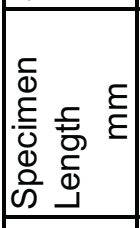 & 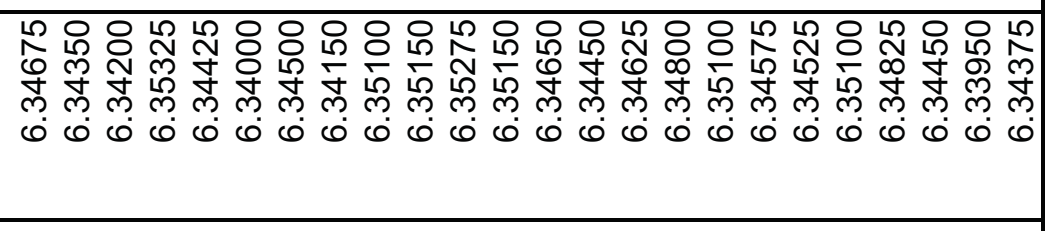 \\
\hline 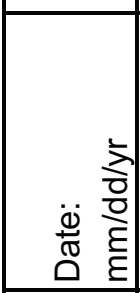 & 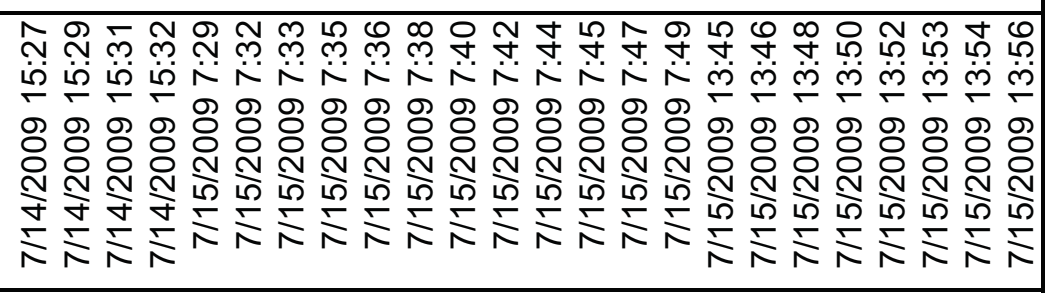 \\
\hline 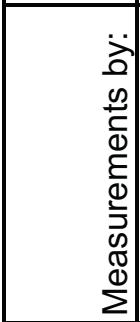 & 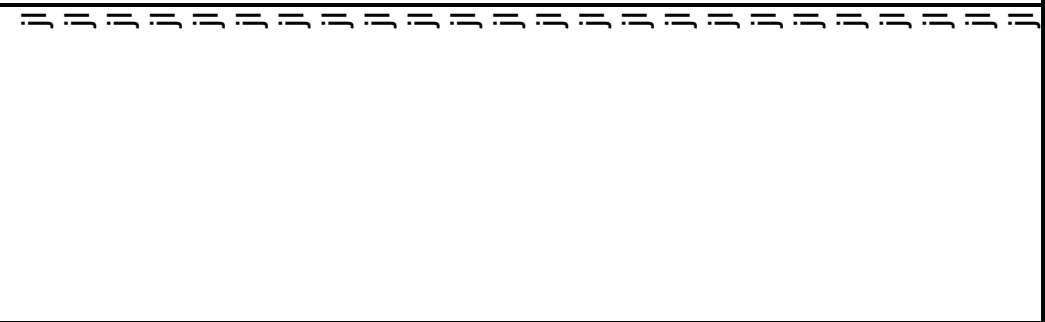 \\
\hline 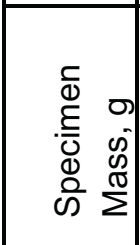 & 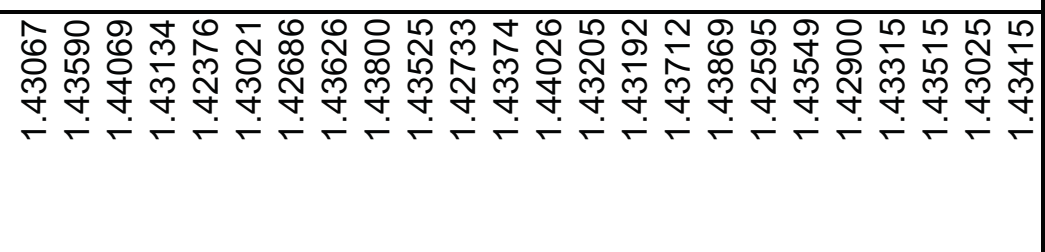 \\
\hline 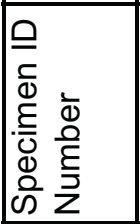 & 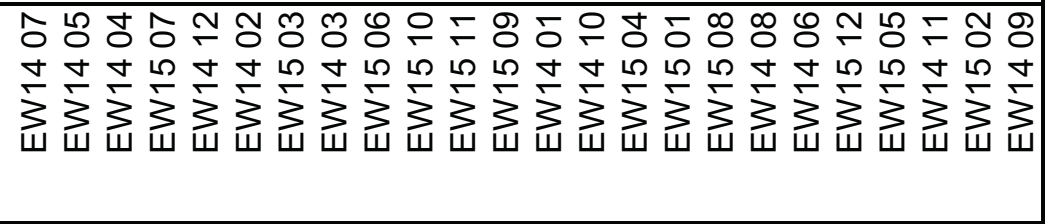 \\
\hline
\end{tabular}




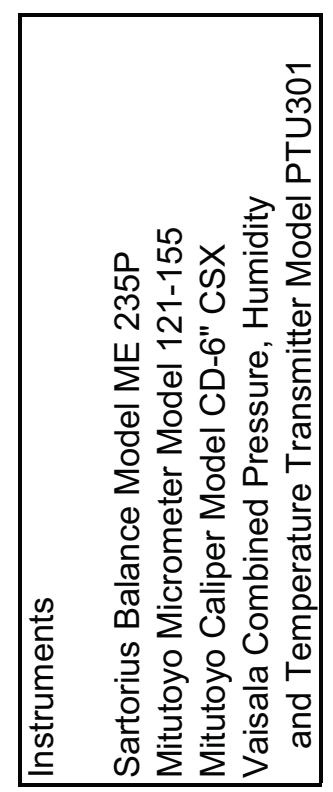

\begin{tabular}{|c|c|}
\hline \& & 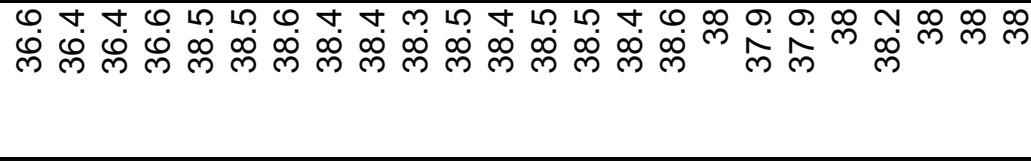 \\
\hline 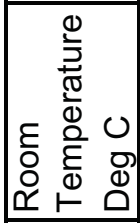 & 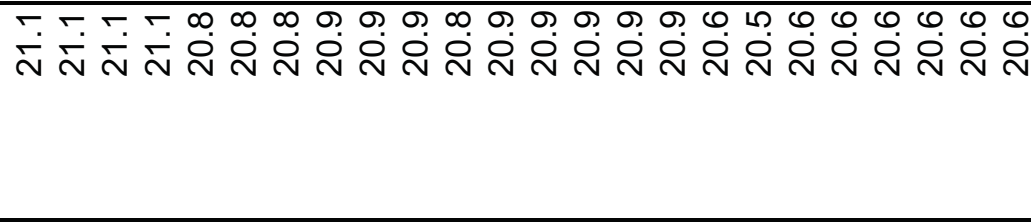 \\
\hline 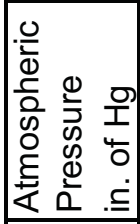 & 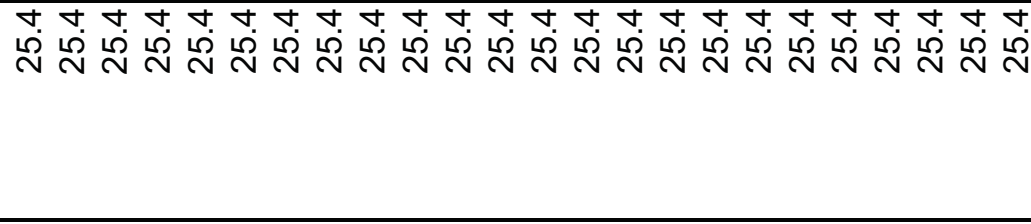 \\
\hline 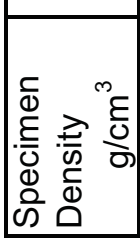 & 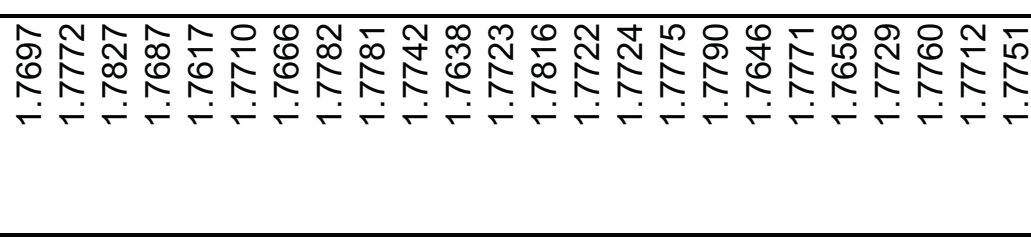 \\
\hline 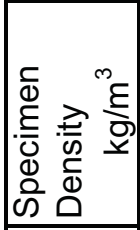 & 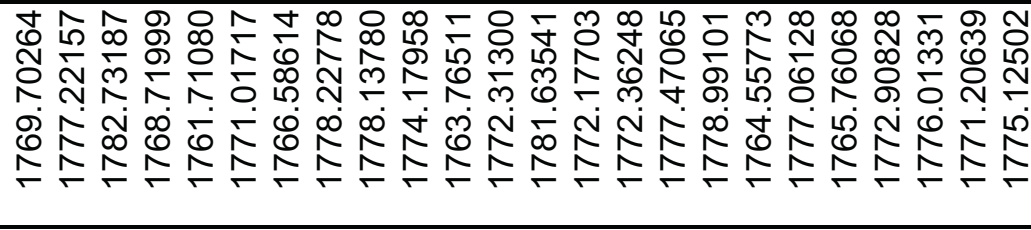 \\
\hline 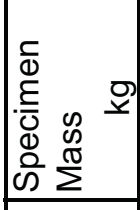 & 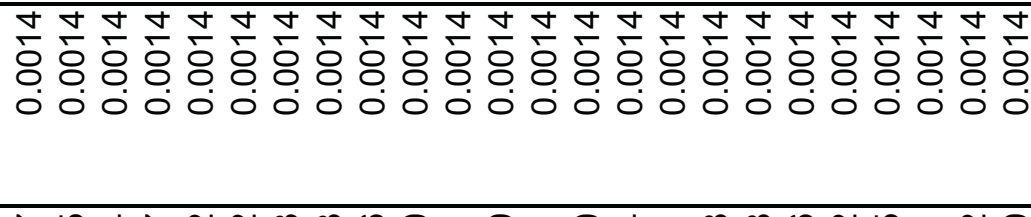 \\
\hline 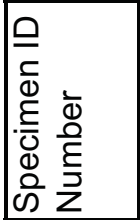 & 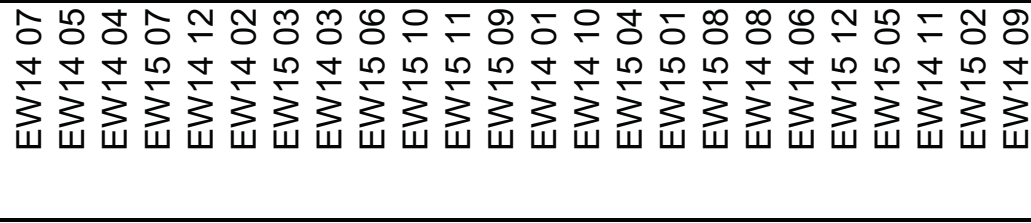 \\
\hline
\end{tabular}




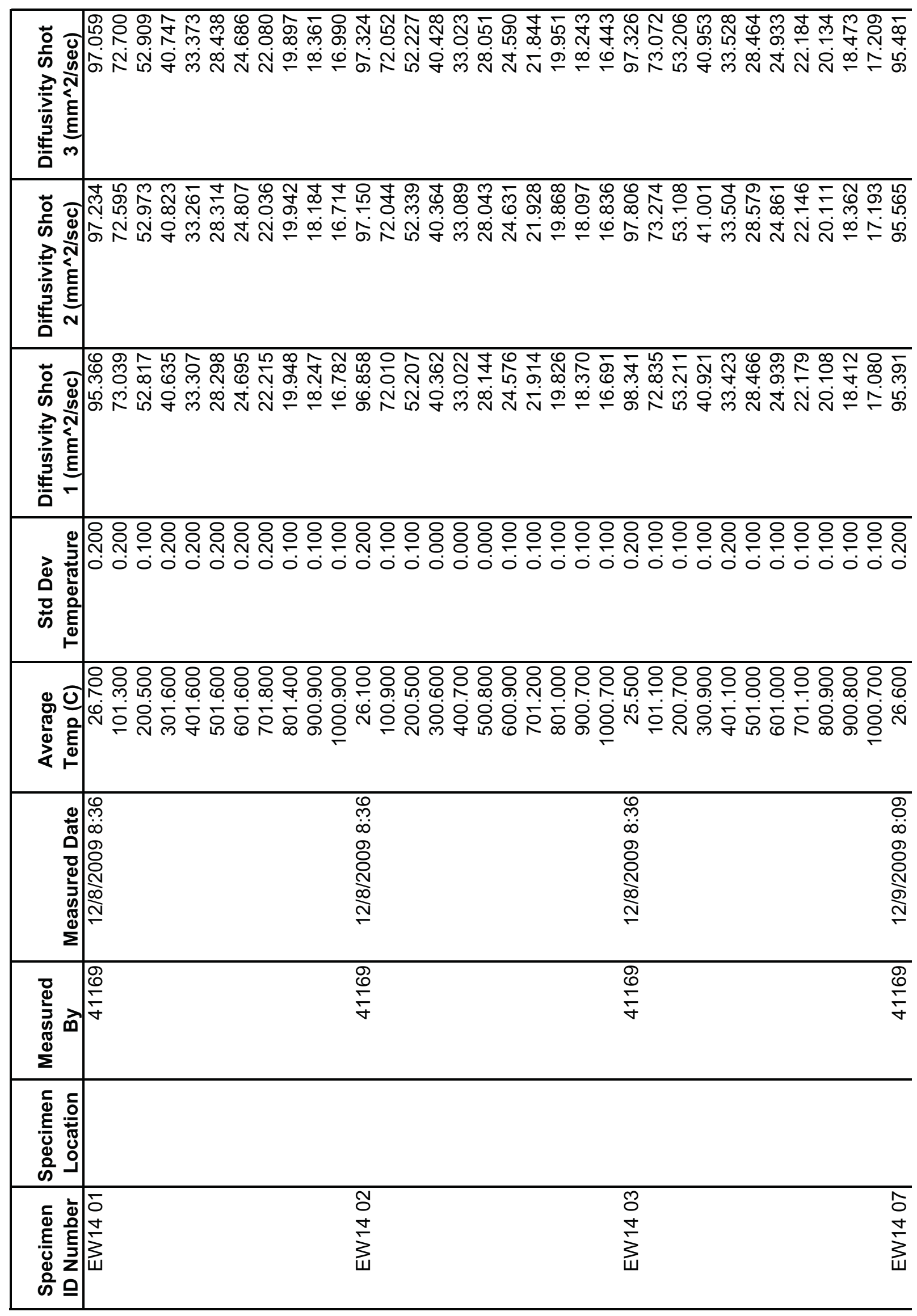




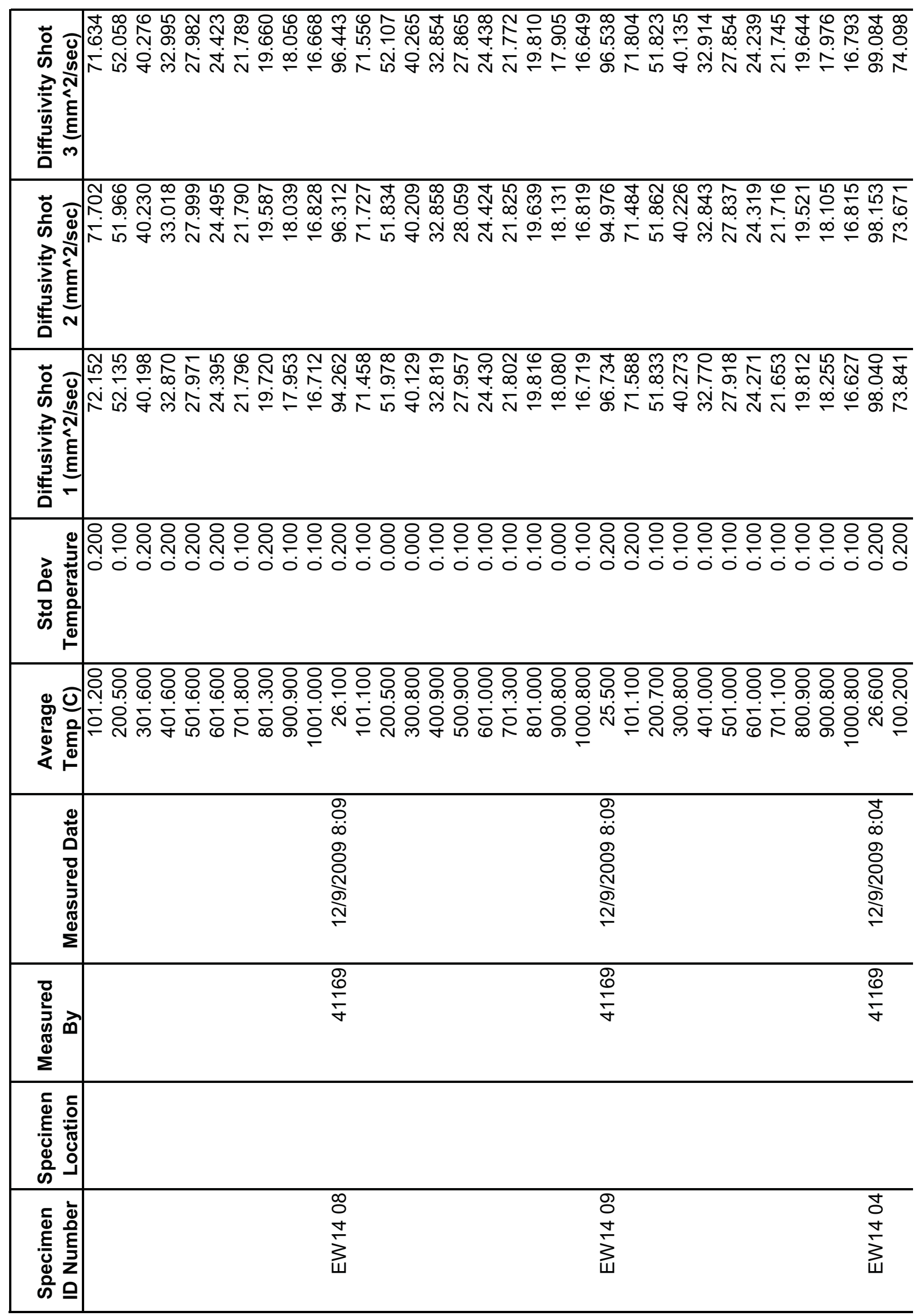




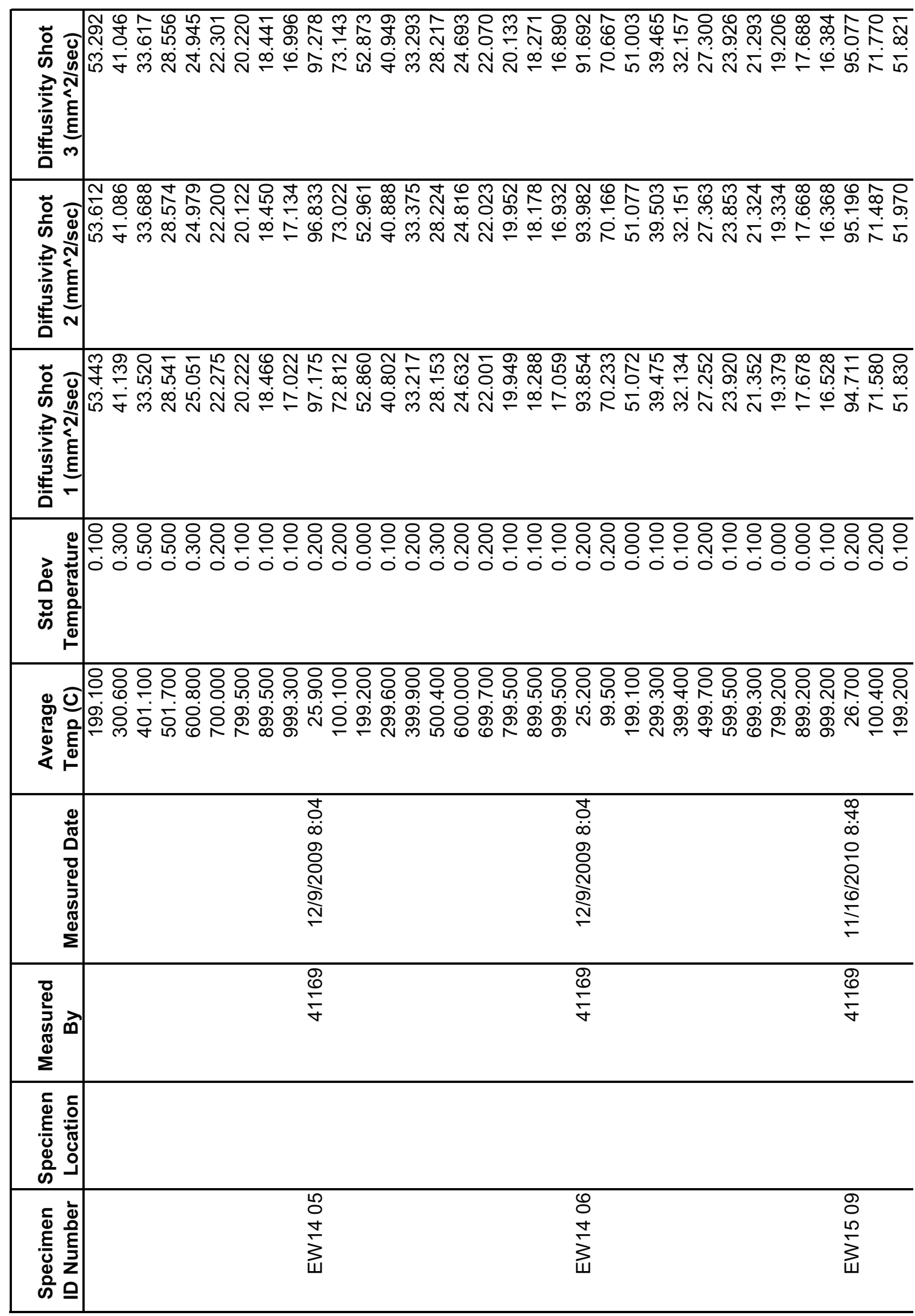




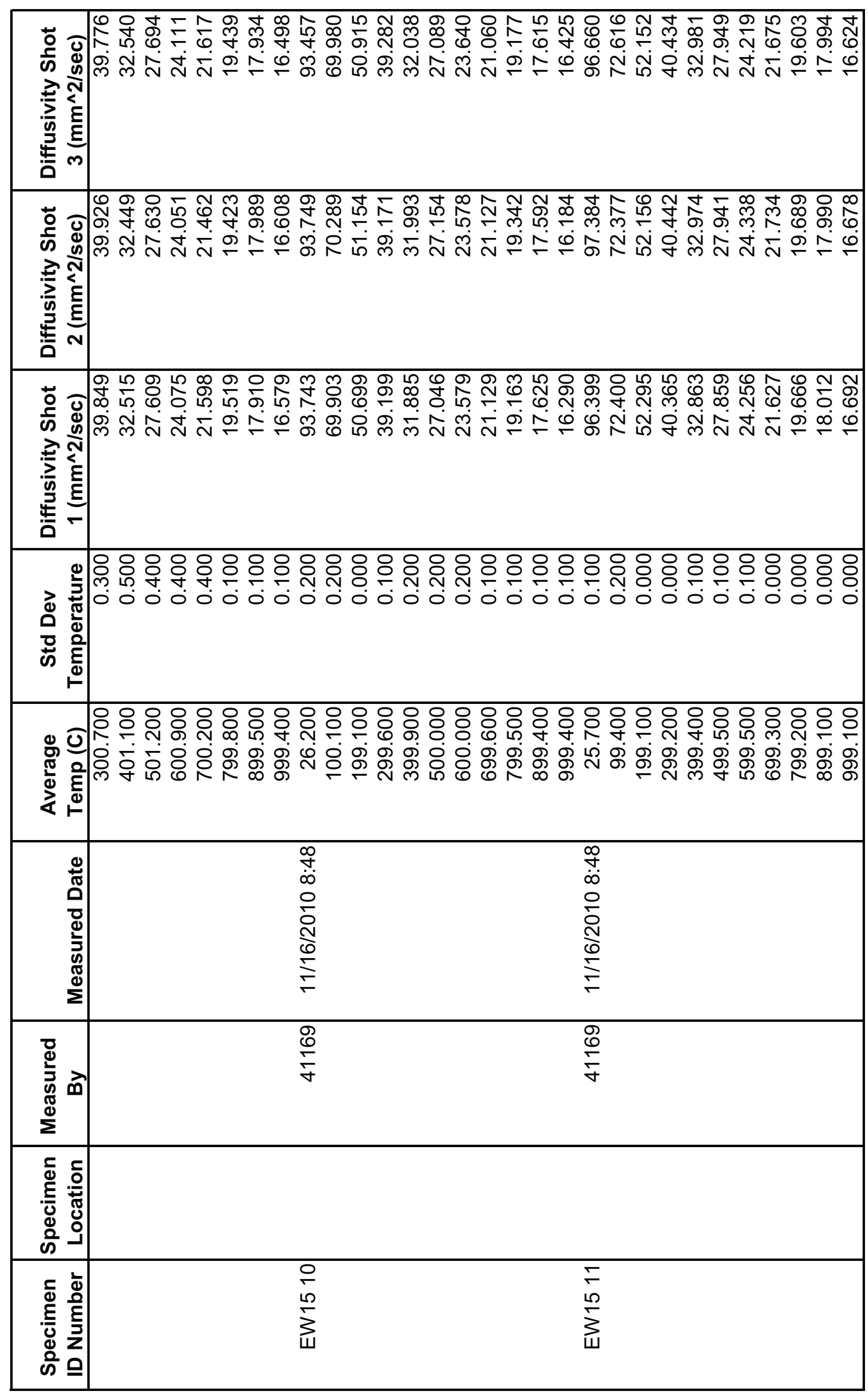




\begin{tabular}{|c|c|c|c|c|}
\hline$\underset{5}{\Sigma}$ & 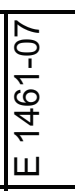 & $\begin{array}{l} \\
\\
1 \\
\dot{0} \\
\dot{+} \\
\omega \\
\end{array}$ & 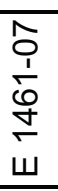 & 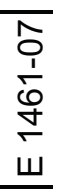 \\
\hline 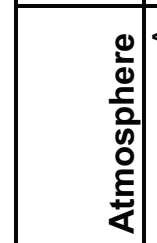 & & $\bar{z}$ & $\bar{z}$ & $\overline{\frac{\pi}{4}}$ \\
\hline 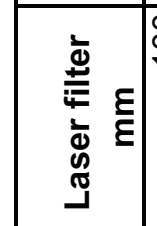 & & 음 & 음 & 음 \\
\hline 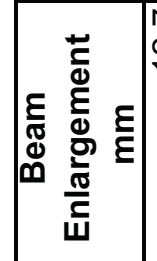 & $\stackrel{\hat{N}}{\stackrel{N}{ }}$ & $\stackrel{\stackrel{N}{\sim}}{\sim}$ & $\stackrel{\widehat{\mathrm{N}}}{\mathrm{N}}$ & $\stackrel{\stackrel{N}{\sim}}{\underline{N}}$ \\
\hline 年 & & $\begin{array}{l}\text { की } \\
\text { की }\end{array}$ & $\begin{array}{l}\text { की } \\
\text { की }\end{array}$ & $\begin{array}{l}\text { Oे } \\
\text { Dी }\end{array}$ \\
\hline 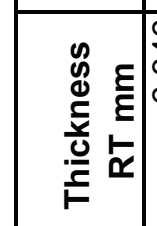 & & 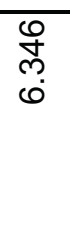 & 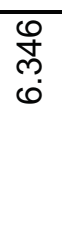 & 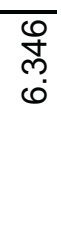 \\
\hline 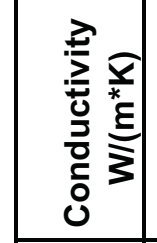 & & & & \\
\hline 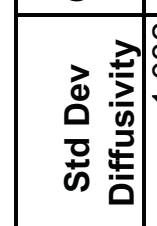 & 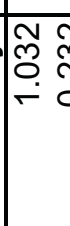 & $\stackrel{\sim}{\sim}$ & 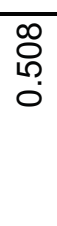 & $\begin{array}{l}\widehat{s} \\
0 \\
0 \\
0\end{array}$ \\
\hline 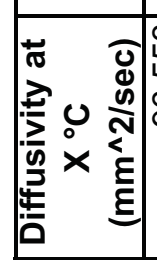 & 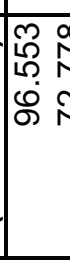 & $\begin{array}{l}\mp \mp \\
\bar{\sigma} \\
\bar{\sigma}\end{array}$ & 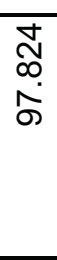 & $\begin{array}{l}\bar{g} \\
\stackrel{9}{4} \\
\stackrel{\leftrightarrow}{\circ}\end{array}$ \\
\hline 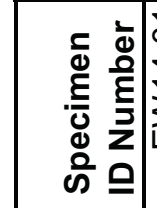 & 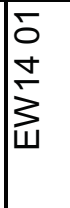 & $\begin{array}{l}\text { No } \\
\sum_{w}^{+}\end{array}$ & $\begin{array}{l}\text { O } \\
\text { o } \\
\sum_{\text {J }}^{+}\end{array}$ & $\begin{array}{l}\text { 点 } \\
\sum_{\text {J }}^{+}\end{array}$ \\
\hline
\end{tabular}




\begin{tabular}{|c|c|c|c|}
\hline 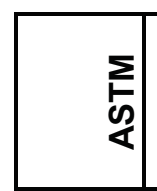 & $\begin{array}{l}\hat{o} \\
\frac{1}{0} \\
\dot{0} \\
\dot{5} \\
\end{array}$ & $\begin{array}{l}\hat{o} \\
\frac{1}{0} \\
\stackrel{0}{+} \\
\dot{\omega}\end{array}$ & 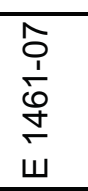 \\
\hline 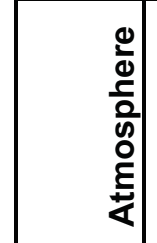 & 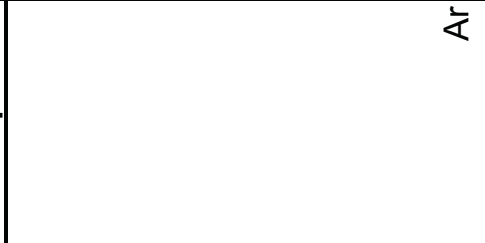 & 文 & 广 \\
\hline 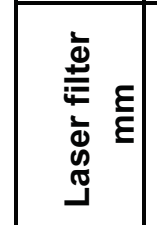 & 음 & 음 & 음 \\
\hline 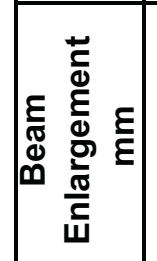 & $\stackrel{\widehat{N}}{\stackrel{N}{N}}$ & $\stackrel{\stackrel{N}{\sim}}{ }$ & 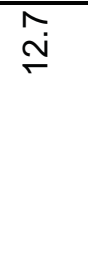 \\
\hline $\begin{array}{l}\vdots \\
\vdots \\
0 \\
\bar{d} \\
\infty\end{array}$ & $\begin{array}{l}\text { की } \\
\text { की }\end{array}$ & $\begin{array}{l}\text { 吕 } \\
\underline{\underline{n}}\end{array}$ & $\begin{array}{l}\text { की } \\
\text { की }\end{array}$ \\
\hline 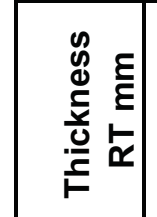 & 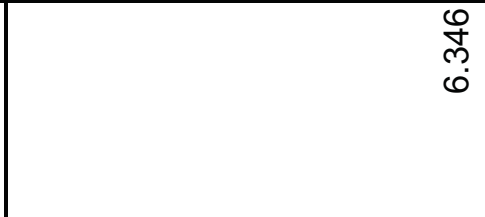 & $\begin{array}{l}0 \\
\text { @] } \\
0 \\
0\end{array}$ & $\begin{array}{l}\text { ô } \\
\text { m. } \\
0\end{array}$ \\
\hline 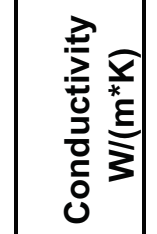 & & & \\
\hline के & 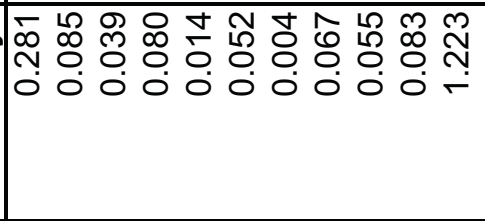 & 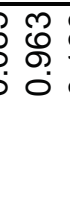 & 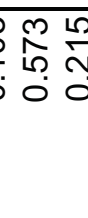 \\
\hline 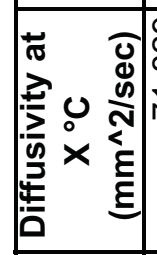 & 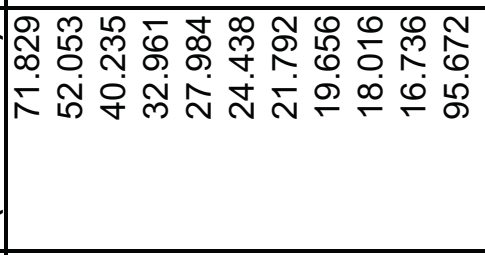 & $\begin{array}{l}3 \\
0 \\
0 \\
\dot{8} \\
\dot{8}\end{array}$ & 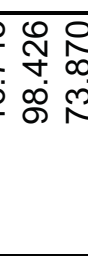 \\
\hline 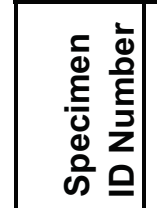 & $\begin{array}{l}\text { lo } \\
\sum^{+} \\
\sum_{4}^{+}\end{array}$ & $\begin{array}{l}8 \\
\\
\sum_{4}^{+}\end{array}$ & $\begin{array}{l} \\
0 \\
\sum_{W}^{+}\end{array}$ \\
\hline
\end{tabular}




\begin{tabular}{|c|c|c|c|}
\hline 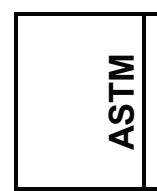 & 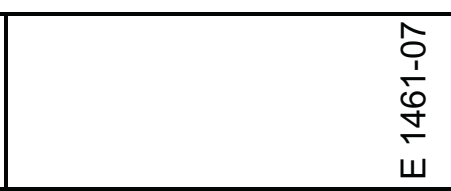 & $\begin{array}{l}\hat{o} \\
\frac{1}{0} \\
\stackrel{0}{+} \\
\dot{\omega}\end{array}$ & $\begin{array}{l}\hat{o} \\
\frac{1}{o} \\
\stackrel{+}{+} \\
\dot{\omega}\end{array}$ \\
\hline 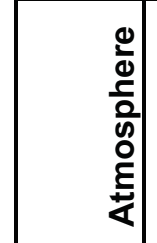 & 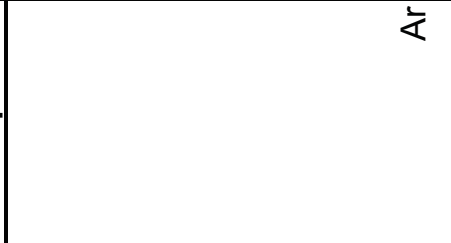 & 衣 & 交 \\
\hline 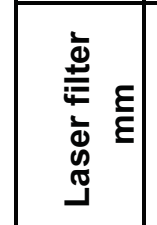 & $\stackrel{8}{\circ}$ & 음 & 음 \\
\hline 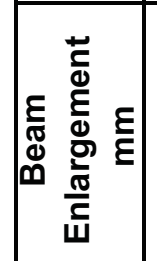 & 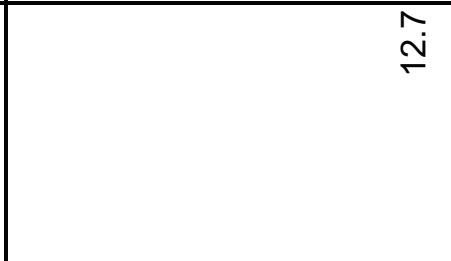 & $\stackrel{\stackrel{N}{ }}{ }$ & $\stackrel{\stackrel{\sim}{\sim}}{ }$ \\
\hline $\begin{array}{l}\bar{b} \\
\vdots \\
心 \\
\Phi \\
\infty\end{array}$ & $\begin{array}{l}\text { की } \\
\text { की }\end{array}$ & $\begin{array}{l}\text { की } \\
\text { I }\end{array}$ & $\begin{array}{l}\text { की } \\
\text { 足 }\end{array}$ \\
\hline 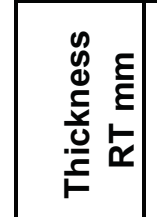 & 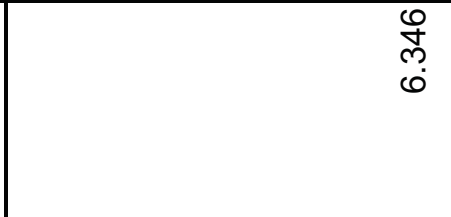 & $\begin{array}{l}0 \\
\text { @] } \\
0 \\
0\end{array}$ & 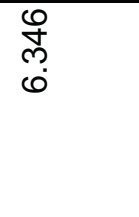 \\
\hline 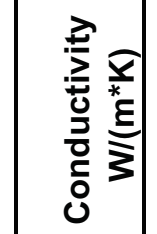 & & & \\
\hline 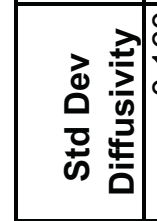 & 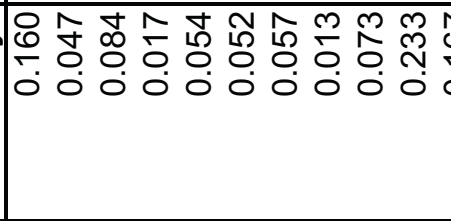 & Ș & 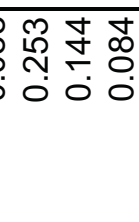 \\
\hline 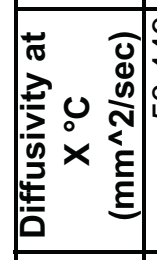 & 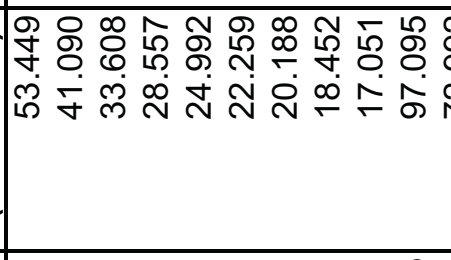 & 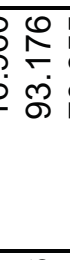 & 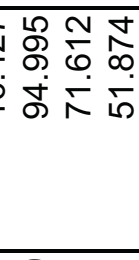 \\
\hline 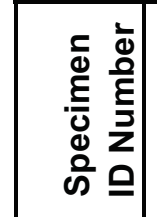 & $\begin{array}{l}\text { Lo } \\
\sum_{W}^{+}\end{array}$ & $\begin{array}{l}8 \\
\\
\sum_{4}^{+}\end{array}$ & $\begin{array}{l}8 \\
8 \\
\sum_{4}^{10} \\
\text { 10 }\end{array}$ \\
\hline
\end{tabular}




\begin{tabular}{|c|c|c|}
\hline 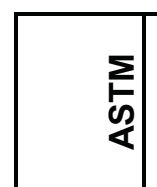 & $\begin{array}{l}\hat{o} \\
\frac{1}{1} \\
\dot{\sigma} \\
\dot{\omega} \\
\omega\end{array}$ & $\begin{array}{l}\hat{0} \\
i \\
\dot{1} \\
\dot{0} \\
\dot{\omega} \\
\end{array}$ \\
\hline 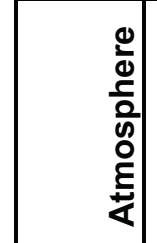 & 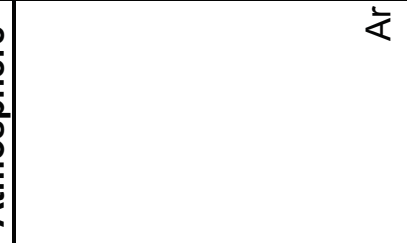 & 衣 \\
\hline 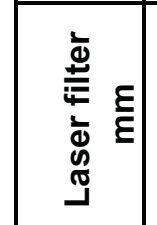 & 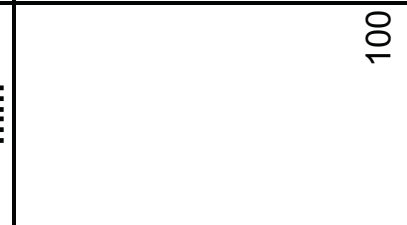 & $\stackrel{8}{\circ}$ \\
\hline 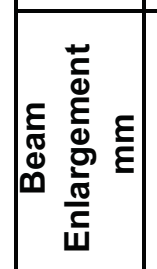 & $\stackrel{\widehat{N}}{\stackrel{N}{N}}$ & $\stackrel{\stackrel{\mathfrak{N}}{\mathrm{N}}}{ }$ \\
\hline $\begin{array}{l}\bar{b} \\
\vdots \\
\vdots \\
\Phi \\
\infty\end{array}$ & 吕 & $\begin{array}{l}\text { 吕 } \\
\underline{\underline{n}}\end{array}$ \\
\hline 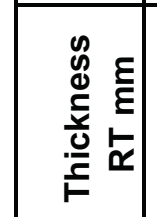 & $\begin{array}{l}\text { Oo } \\
\text { W. } \\
0\end{array}$ & $\begin{array}{l}0 \\
\text { OO } \\
0 \\
0\end{array}$ \\
\hline 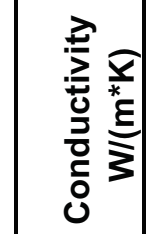 & & \\
\hline 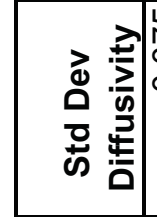 & 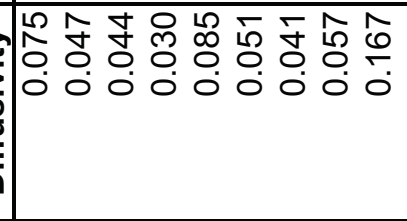 & 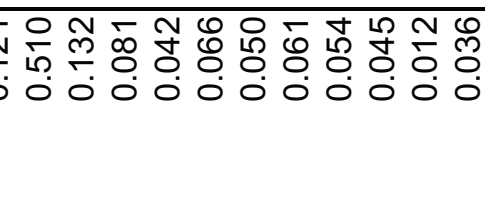 \\
\hline 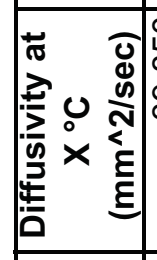 & 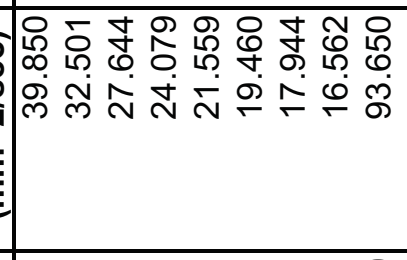 & 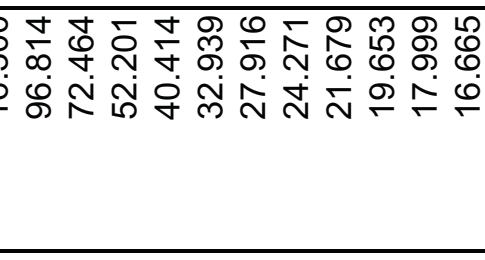 \\
\hline 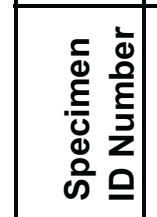 & $\sum_{\substack{\infty\\
}}^{+\infty}$ & $\sum_{i}^{\Sigma}$ \\
\hline
\end{tabular}




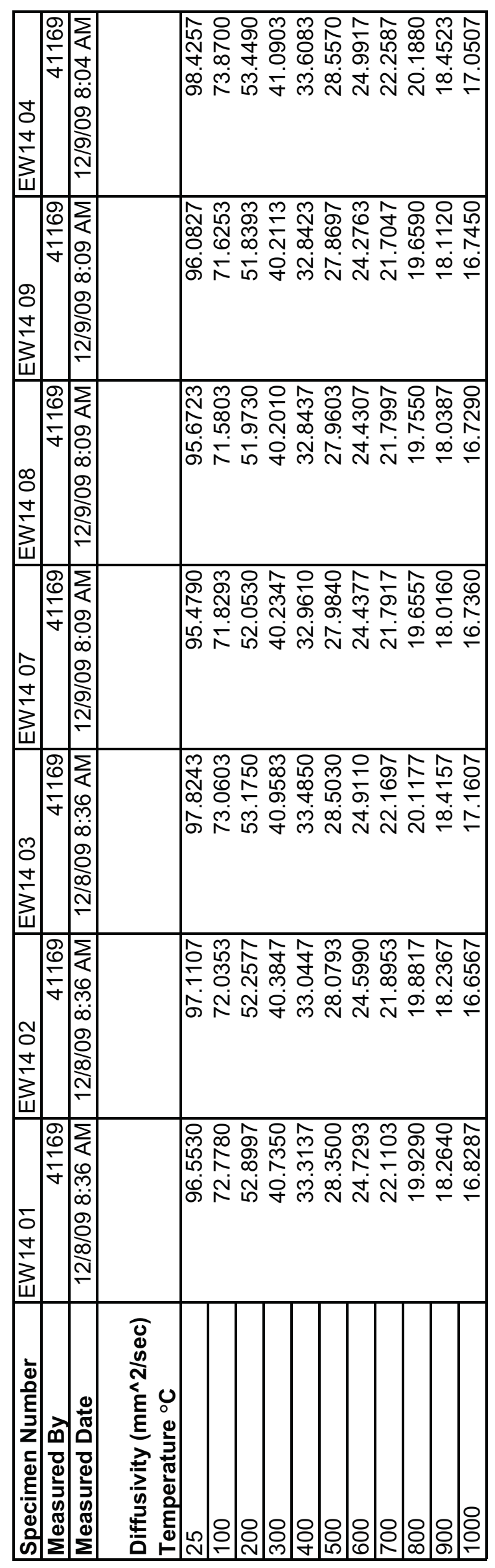




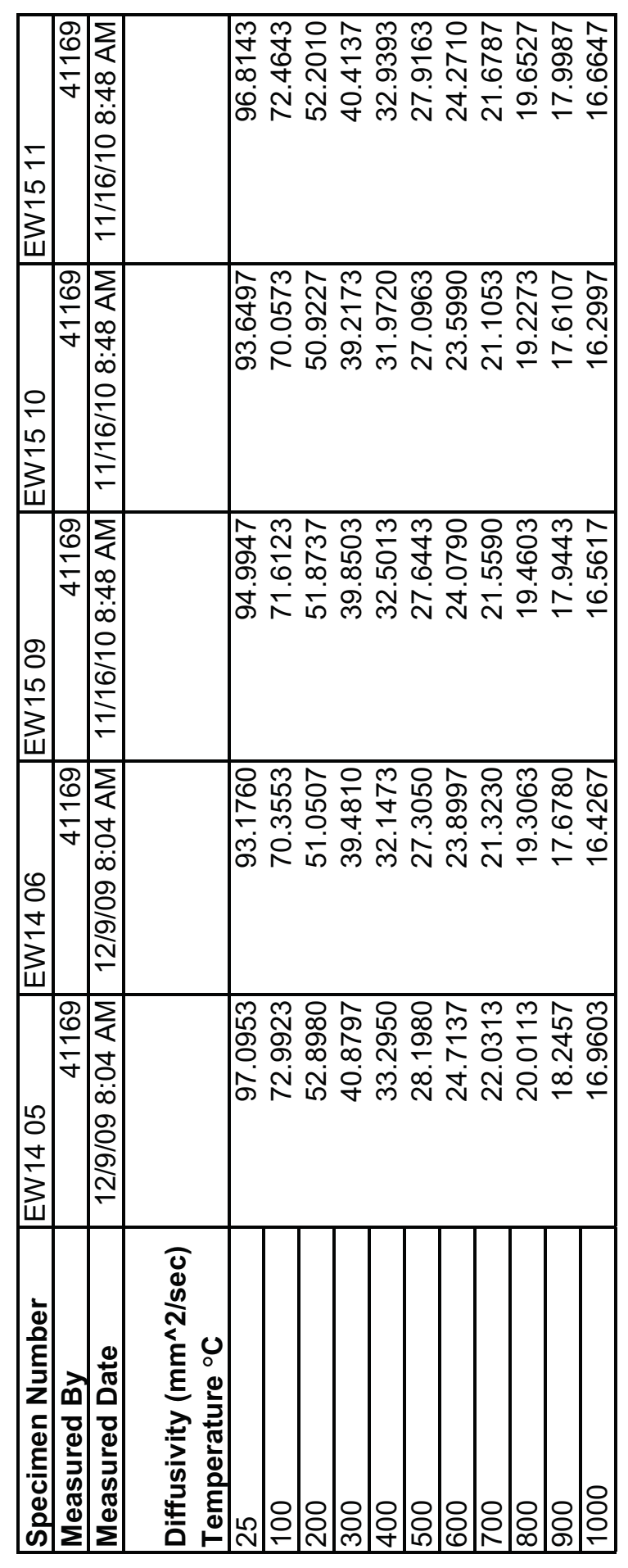




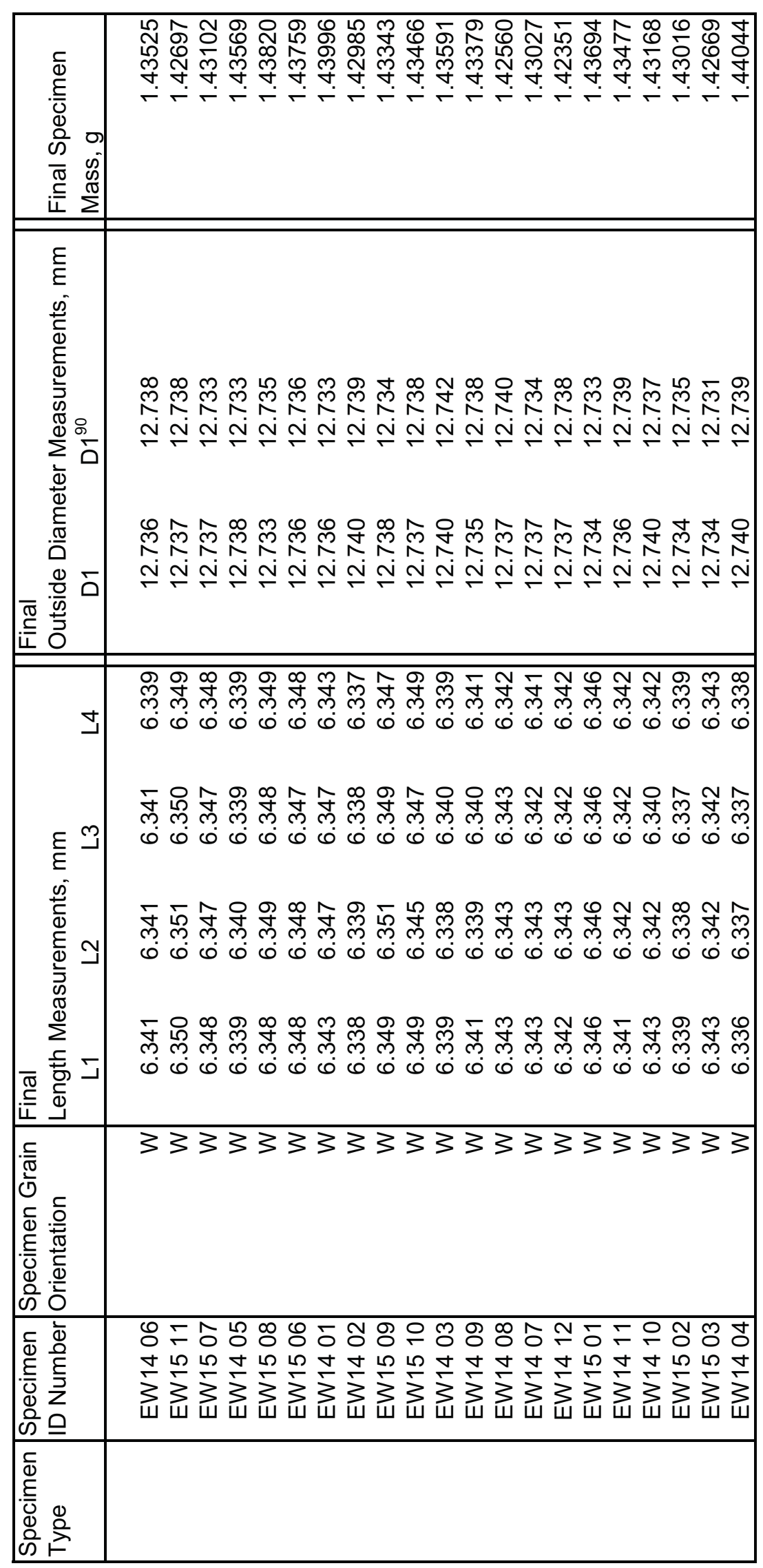




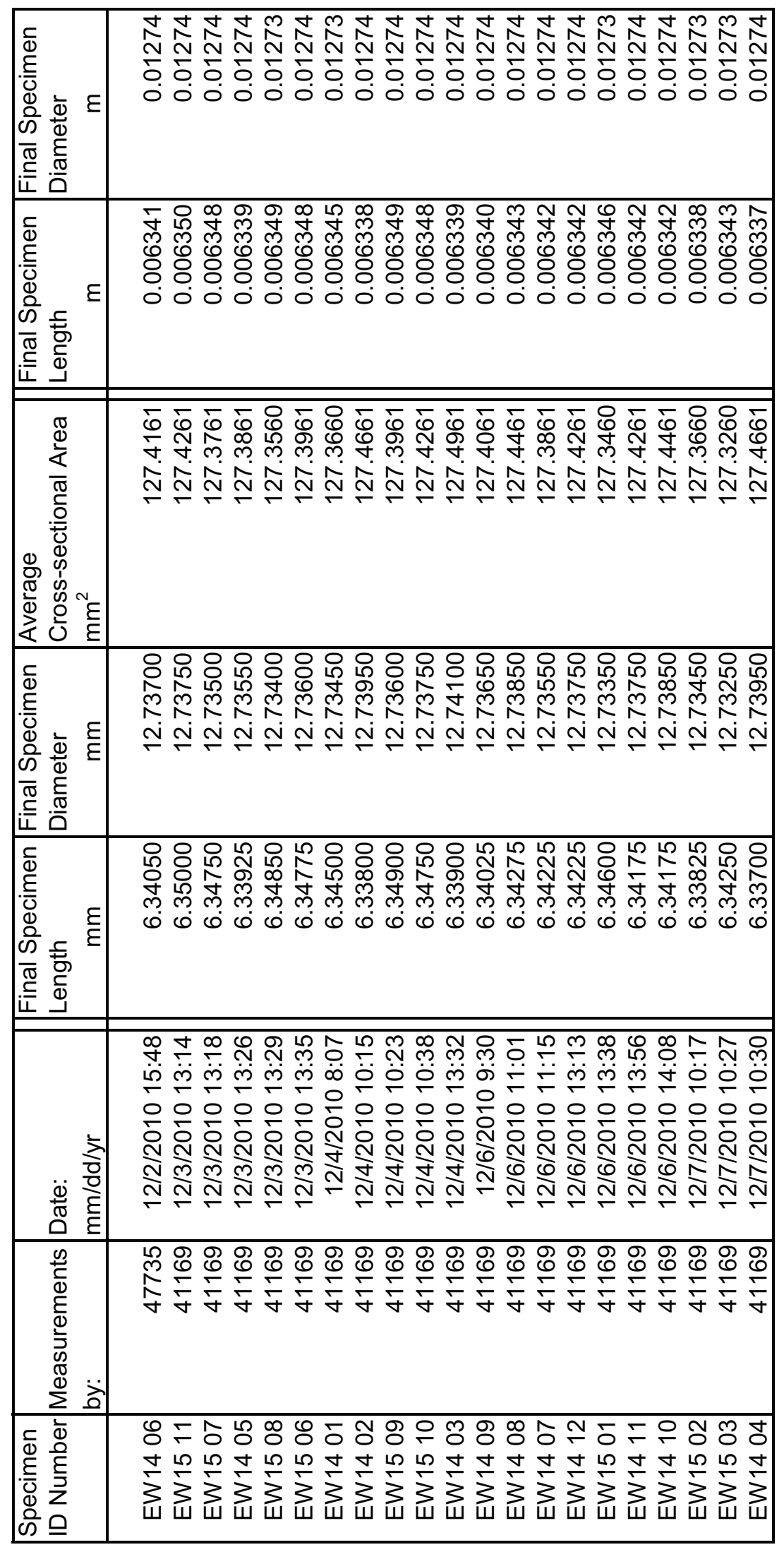




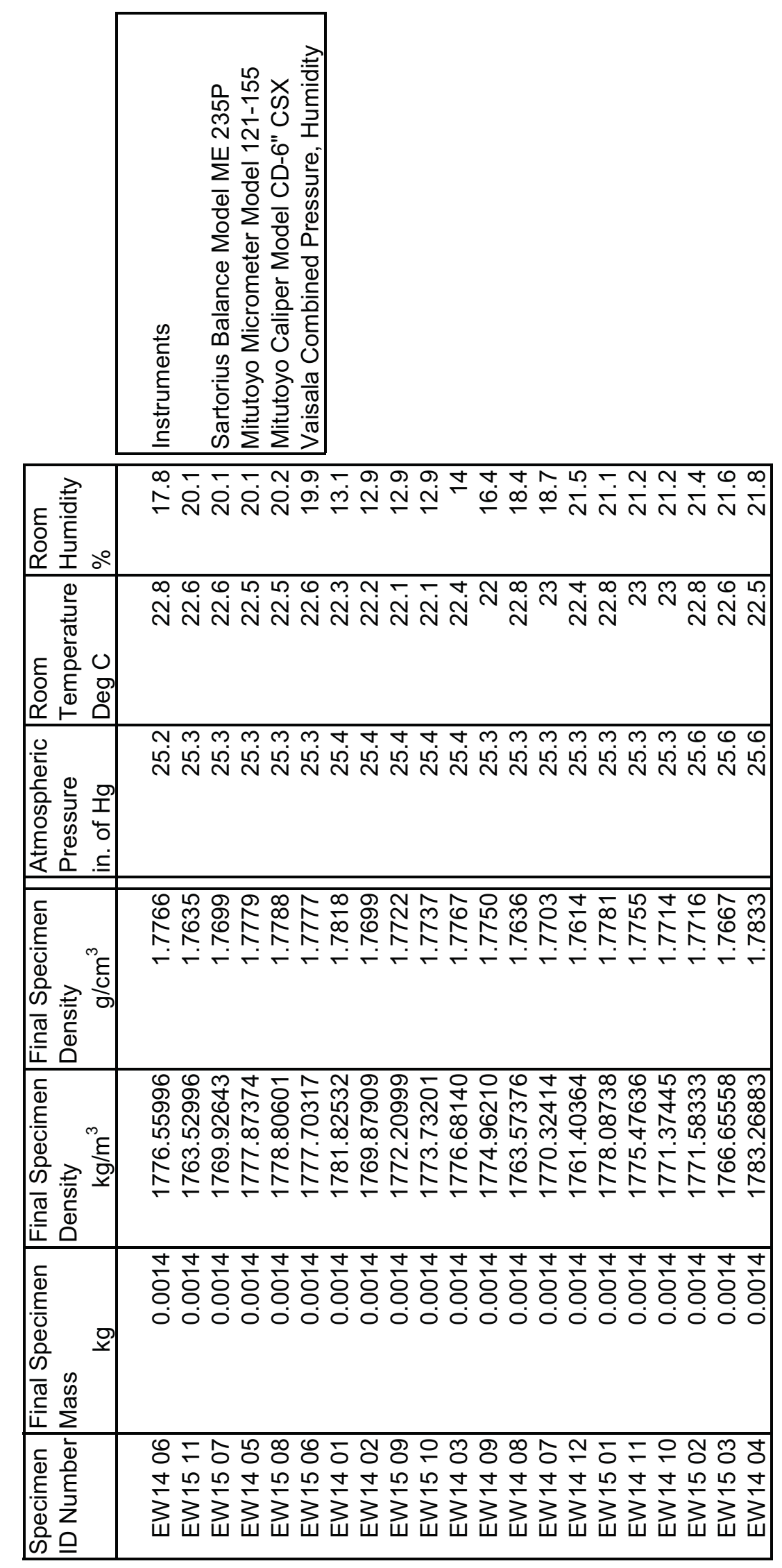




$\begin{array}{ll}\text { Graphite Grade: } & \text { IG-430 } \\ \text { Graphite Manufacturer: } & \text { Toyo Tanso } \\ \text { Forming Process: } & \text { Isostatic-molded } \\ \text { Coke Particle Size: } & \text { Fine grain } \\ \text { Coke Type: } & \text { Pitch coke } \\ \text { ASTM Class: } & \text { INHP } \\ \text { Specimen Geometry: } & \text { Cylinder }\end{array}$

Specimen ID \#'s:

FW15 01

FW15 02

FW15 03

FW15 04

FW15 05

FW15 06

FW15 07

FW15 08

FW15 09

FW15 10

FW15 11

FW15 12

FW16 01

FW16 02

FW16 03

FW16 04

FW16 05

FW16 06

FW16 07

FW16 08

FW16 09

FW16 10

FW16 11

FW16 12 


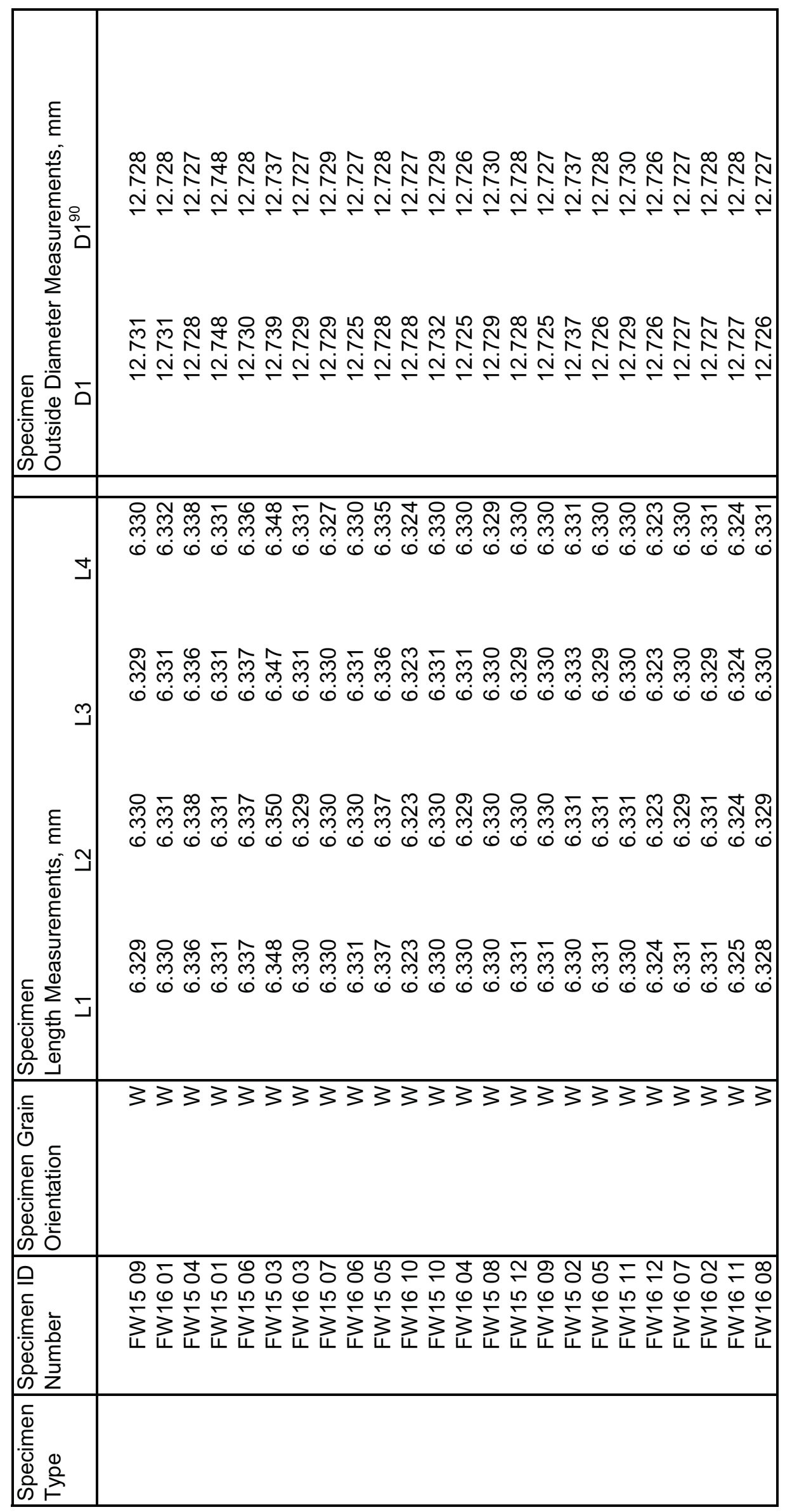




\begin{tabular}{|c|c|}
\hline 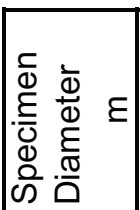 & 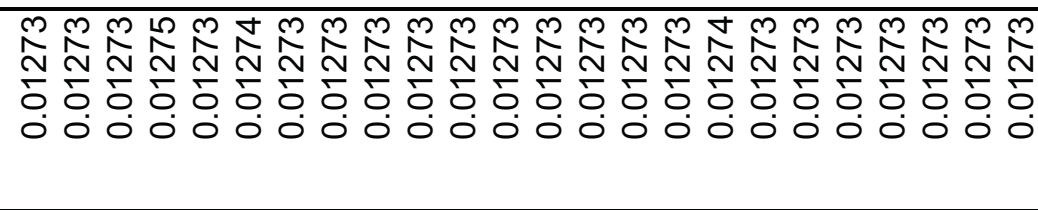 \\
\hline 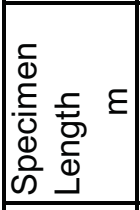 & 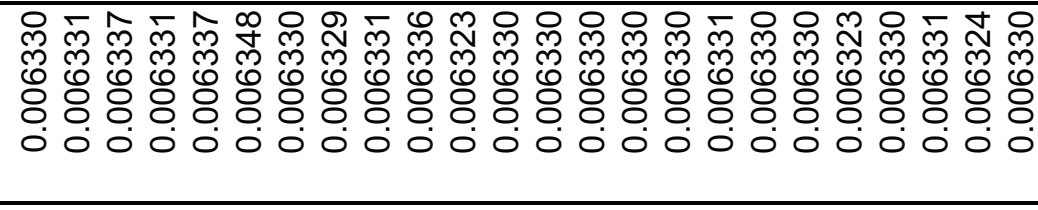 \\
\hline 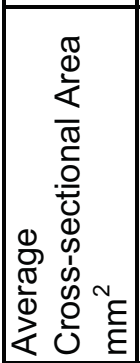 & 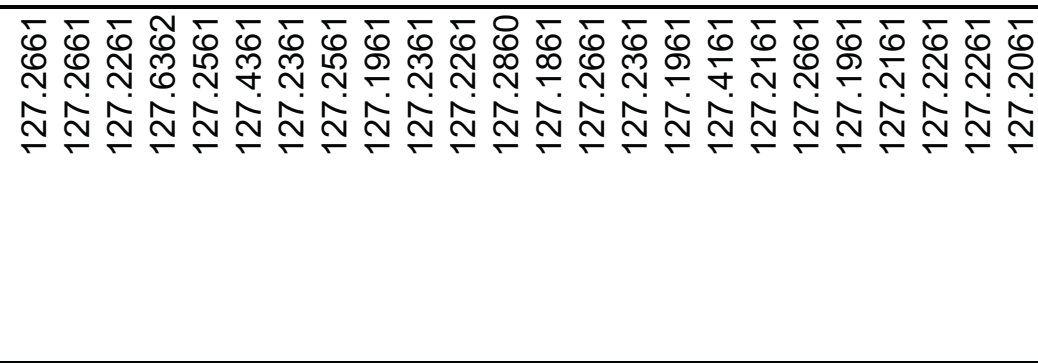 \\
\hline 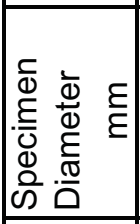 & 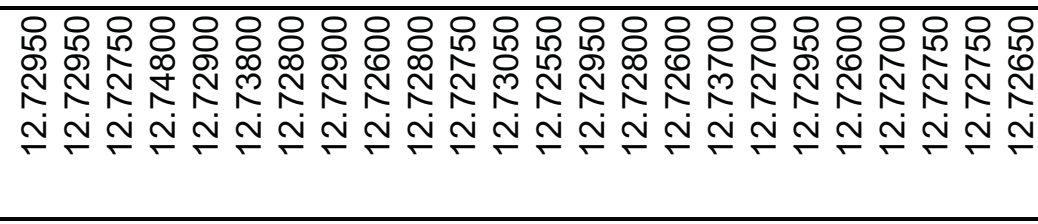 \\
\hline 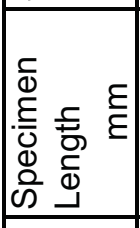 & 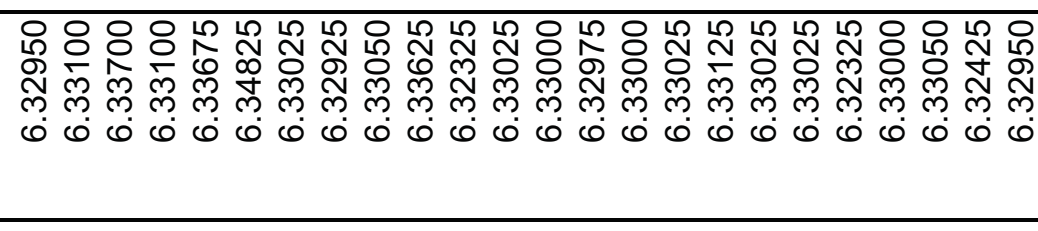 \\
\hline 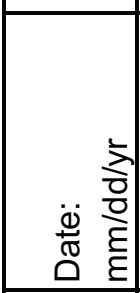 & 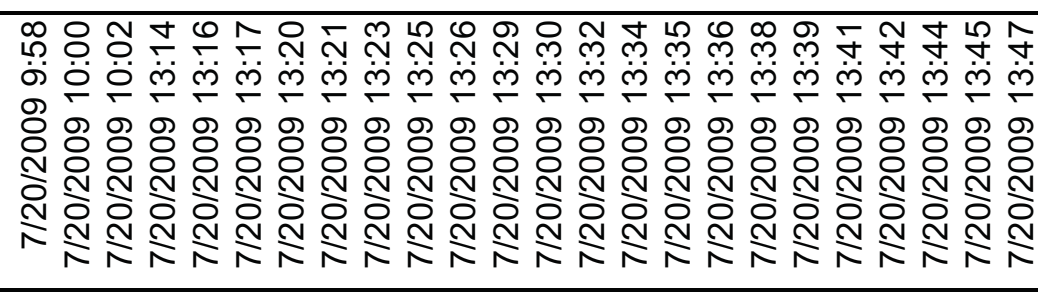 \\
\hline 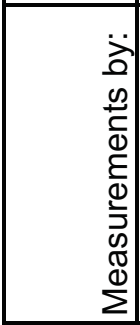 & 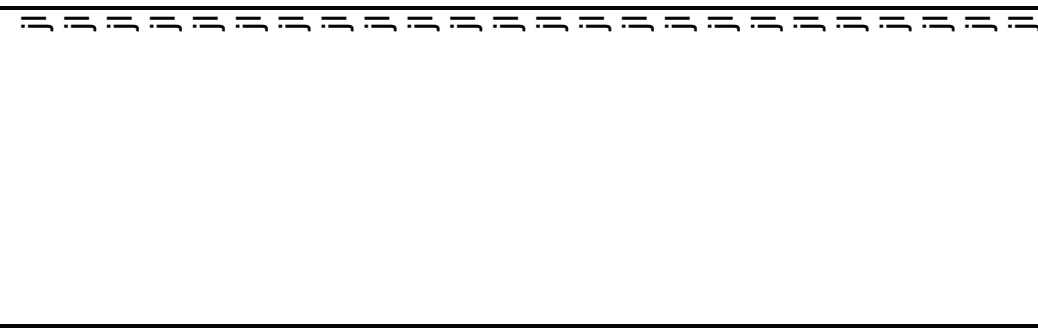 \\
\hline 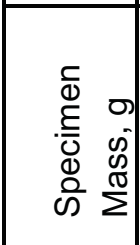 & 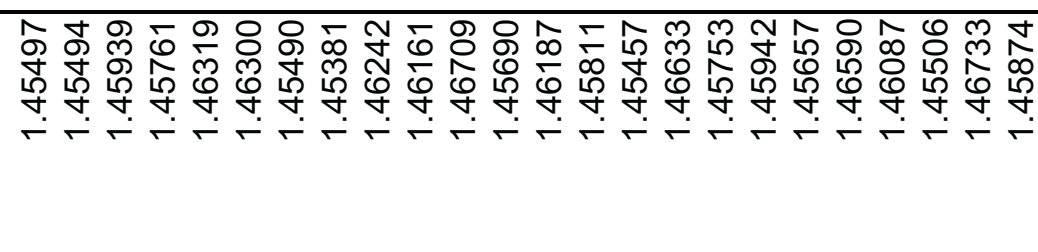 \\
\hline 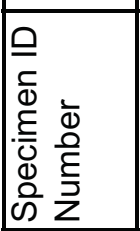 & 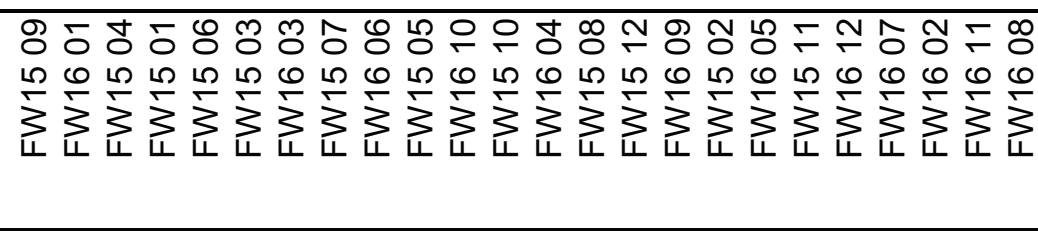 \\
\hline
\end{tabular}




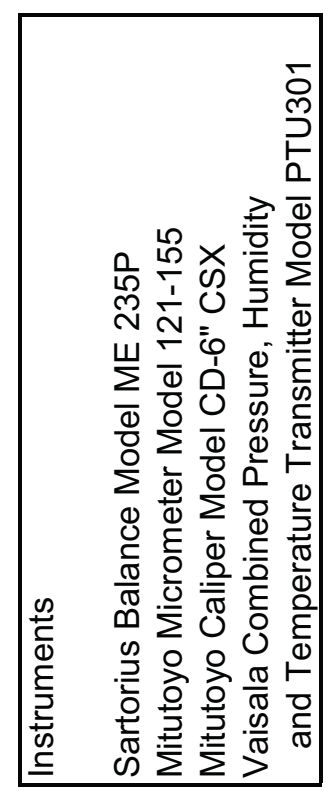

\begin{tabular}{|c|c|}
\hline \& & 岁 \\
\hline 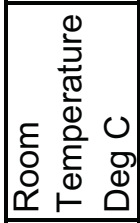 & 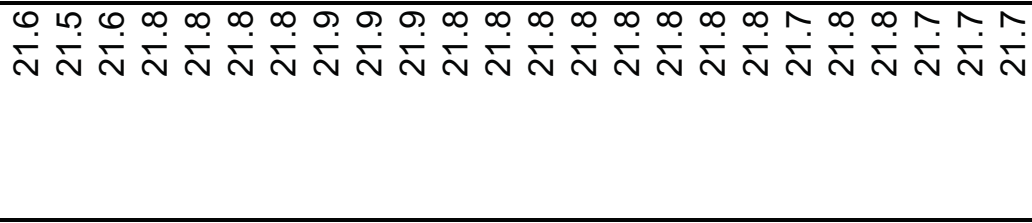 \\
\hline 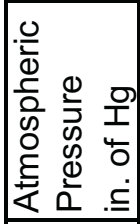 & 点 \\
\hline 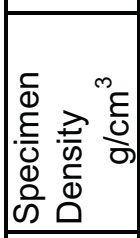 & 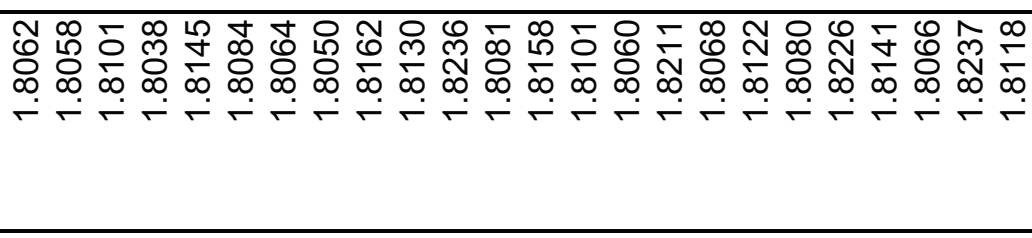 \\
\hline 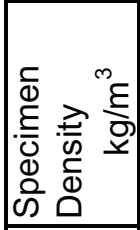 & 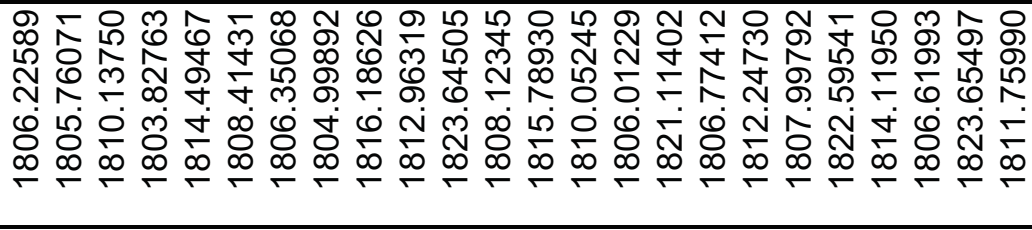 \\
\hline 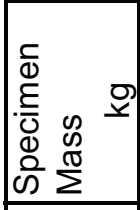 & 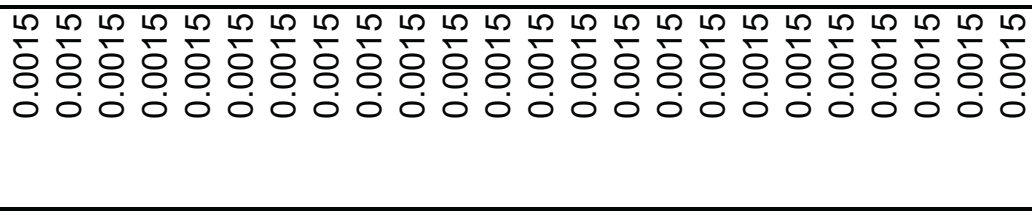 \\
\hline 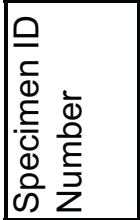 & 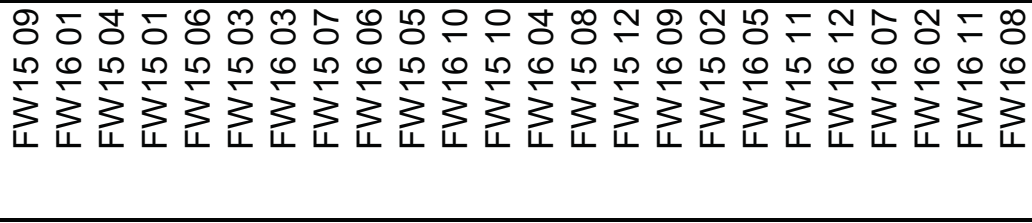 \\
\hline
\end{tabular}




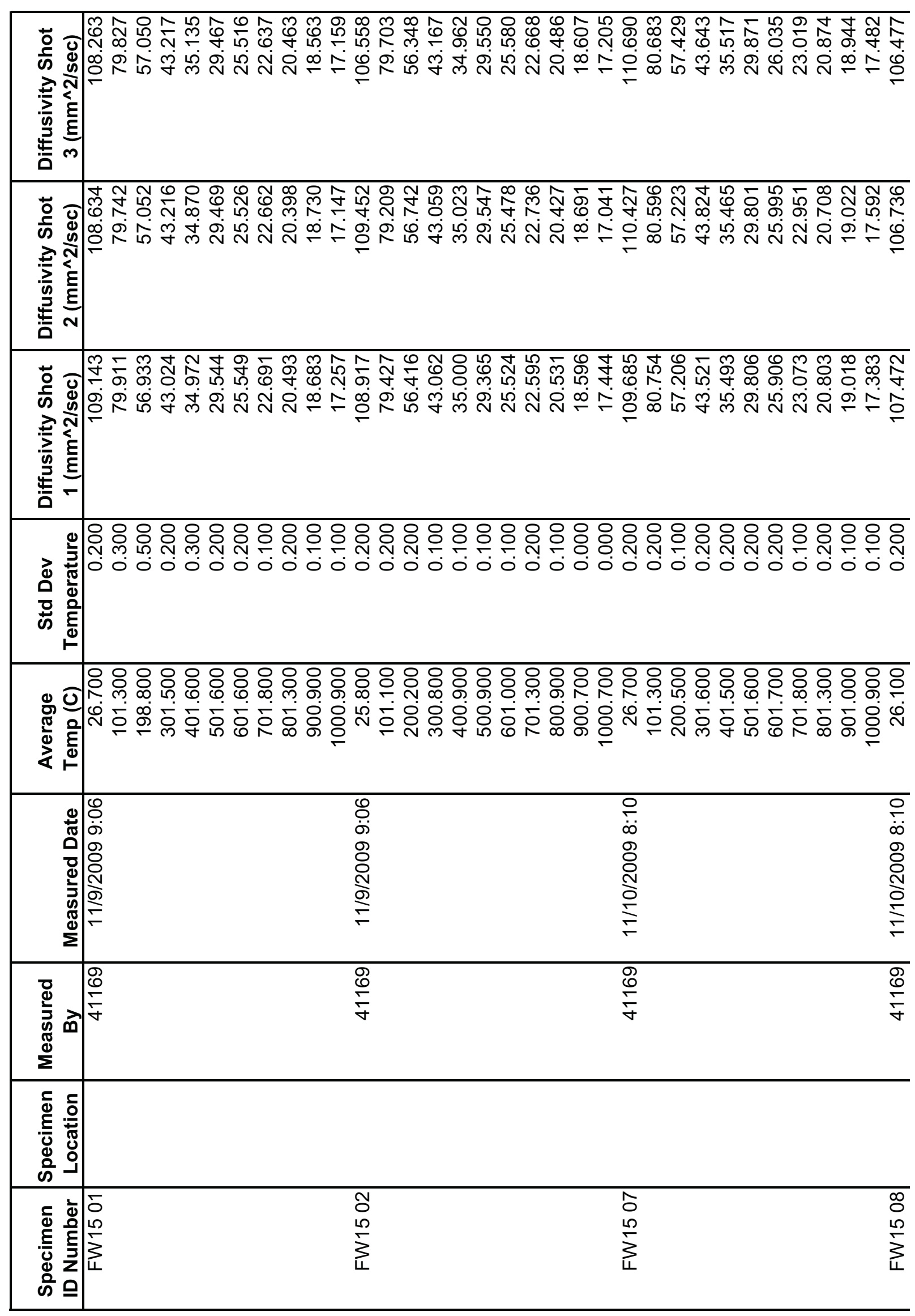




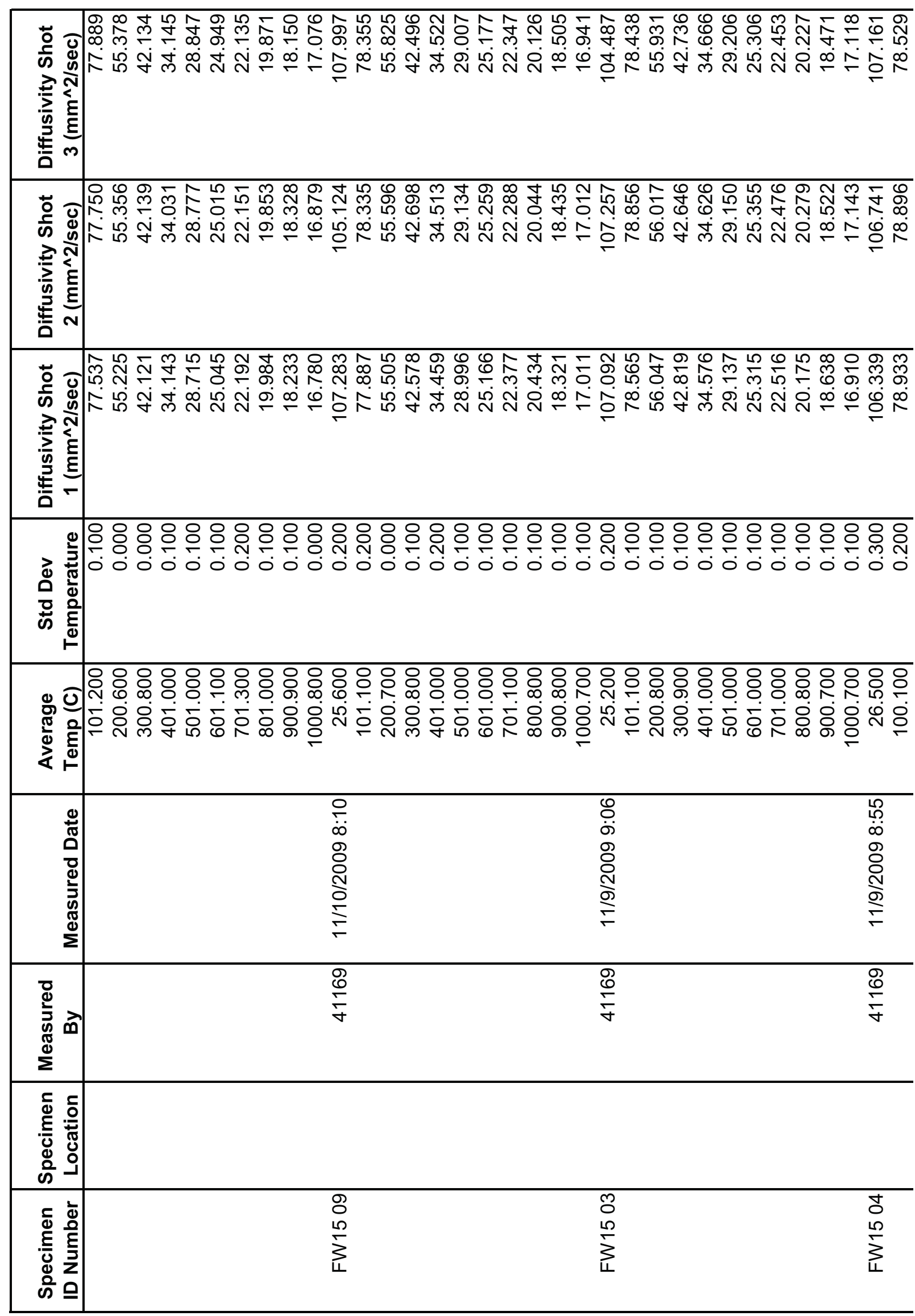




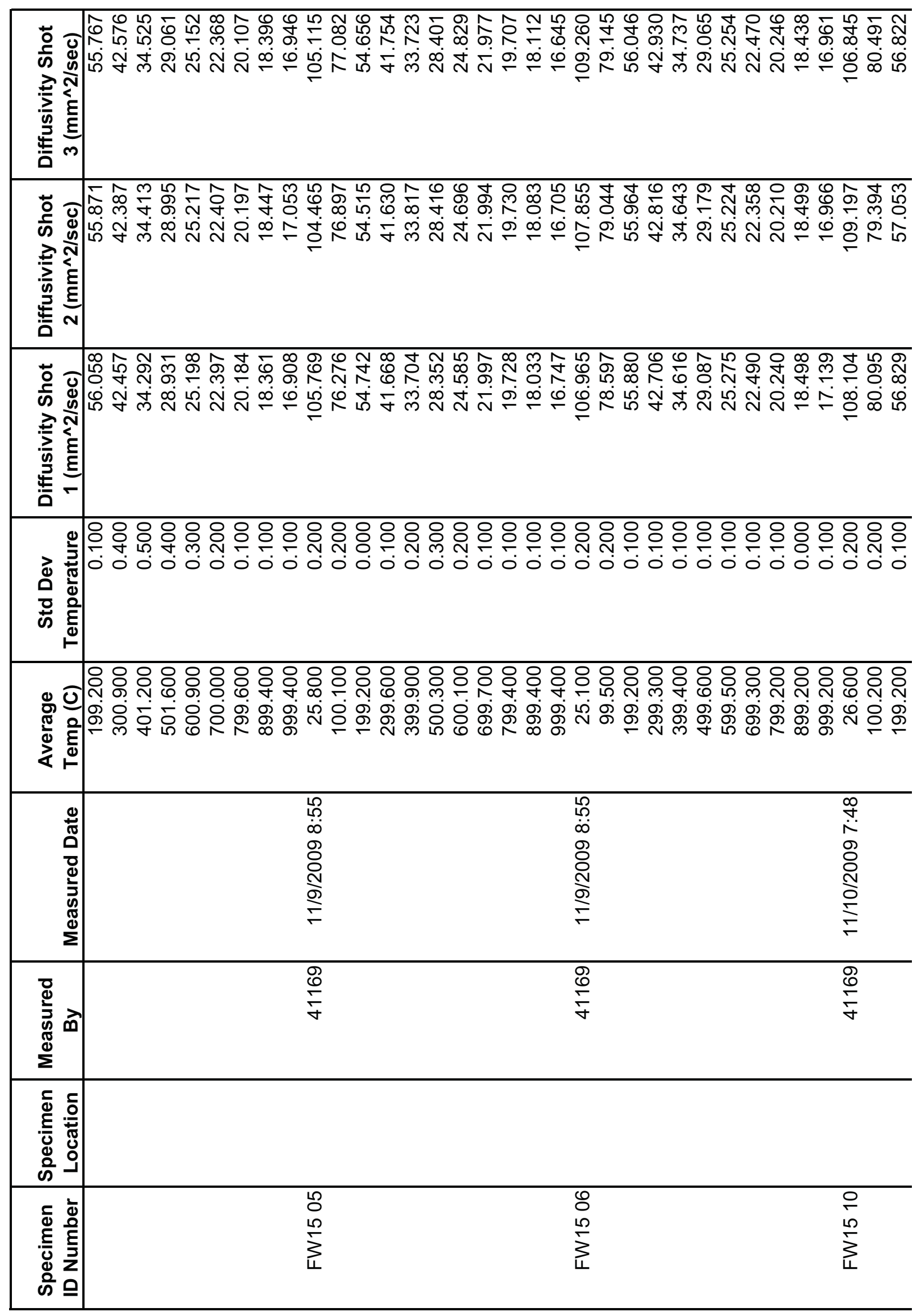




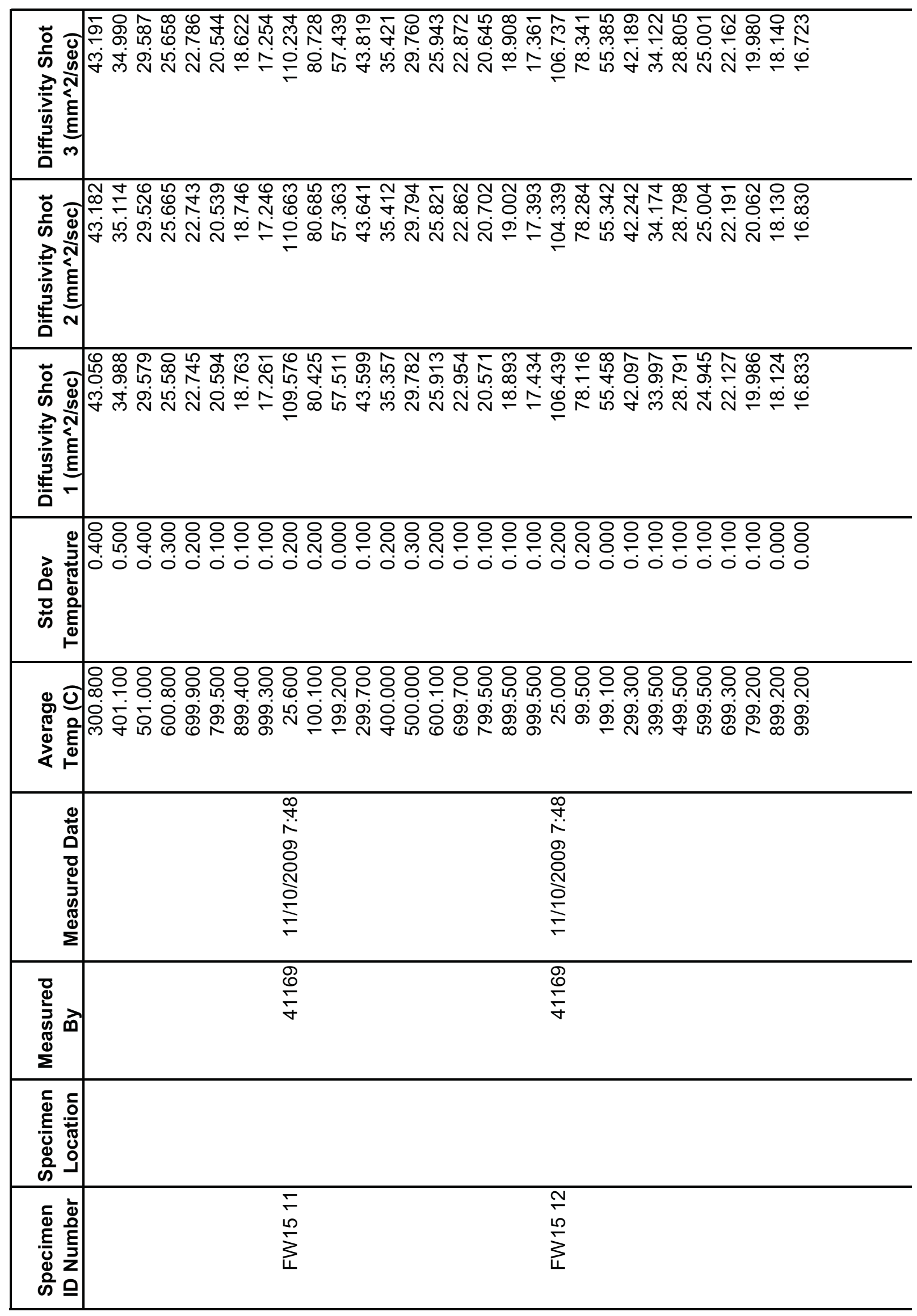




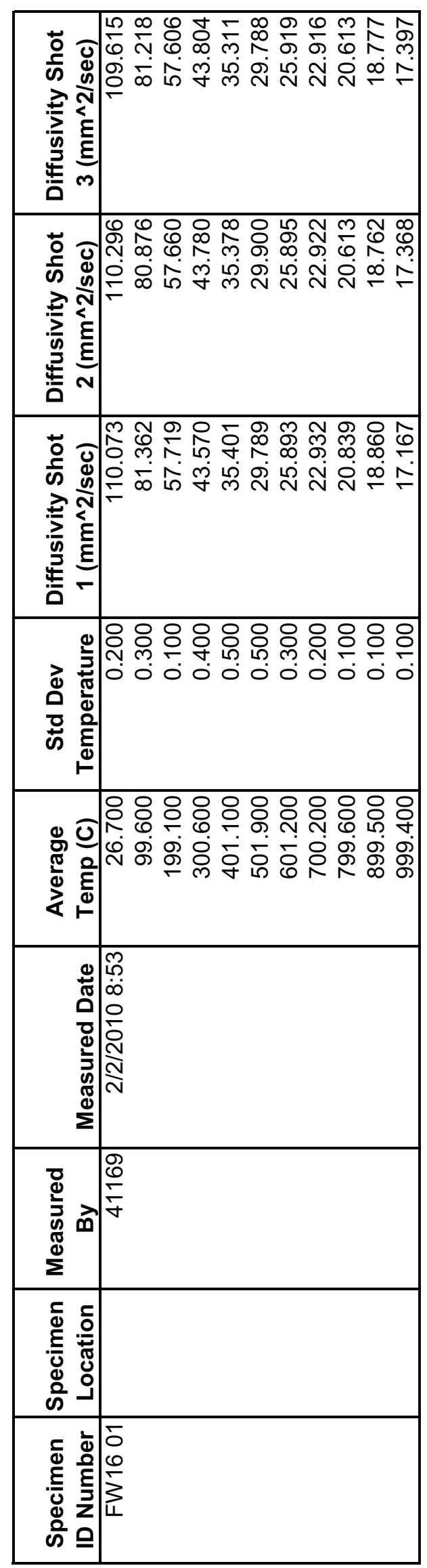




\begin{tabular}{|c|c|c|c|c|}
\hline 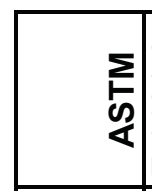 & 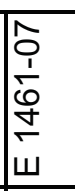 & $\begin{array}{l} \\
\\
\dot{1} \\
\dot{0} \\
\dot{+} \\
\end{array}$ & 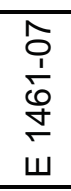 & 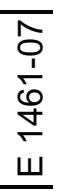 \\
\hline 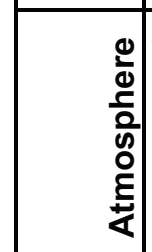 & & $\bar{z}$ & $\bar{z}$ & $\overline{\frac{1}{4}}$ \\
\hline 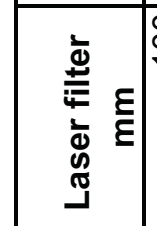 & & 음 & 음 & 8 \\
\hline 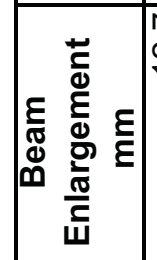 & $\stackrel{\hat{N}}{\underline{N}}$ & $\stackrel{\stackrel{N}{ }}{ }$ & $\stackrel{\stackrel{N}{N}}{ }$ & 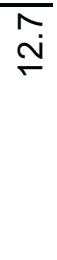 \\
\hline 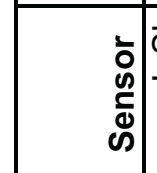 & & 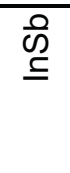 & 员 & $\begin{array}{l}\text { की } \\
\text { 足 }\end{array}$ \\
\hline 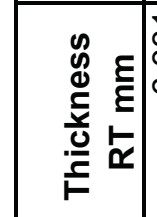 & & 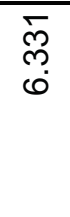 & $\begin{array}{c}\bar{m} \\
\text { } \\
0\end{array}$ & 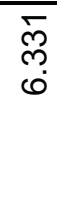 \\
\hline 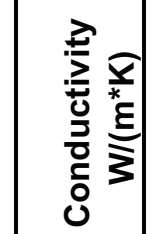 & & & & \\
\hline 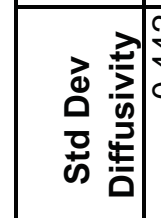 & 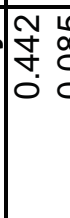 & 孚 & Sִ & \\
\hline 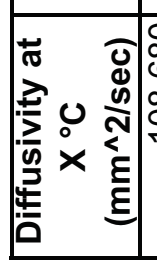 & $\mid \begin{array}{ll}8 & 1 \\
0 & 0 \\
0 & 0 \\
0 & 0 \\
0 & 0 \\
0 & 1 \\
1 & 1\end{array}$ & 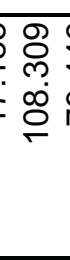 & 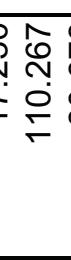 & $\begin{array}{l}\infty \\
0 \\
\infty \\
0 \\
0 \\
0\end{array}$ \\
\hline 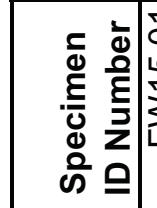 & & 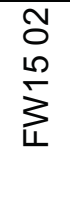 & $\begin{array}{l}\hat{0} \\
\text { L } \\
\sum_{\Downarrow L}\end{array}$ & $\begin{array}{l}\text { o } \\
\sum^{\circ} \\
\sum_{L}^{\infty}\end{array}$ \\
\hline
\end{tabular}




\begin{tabular}{|c|c|c|c|}
\hline 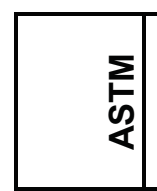 & $\begin{array}{l}\hat{o} \\
\frac{1}{0} \\
\dot{0} \\
\dot{5} \\
\end{array}$ & $\begin{array}{l}\hat{S} \\
\frac{1}{1} \\
\frac{0}{5} \\
\dot{w}\end{array}$ & 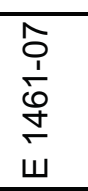 \\
\hline 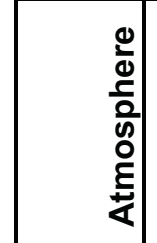 & 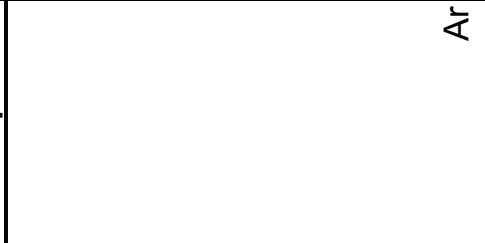 & $\frac{1}{\alpha}$ & 广 \\
\hline 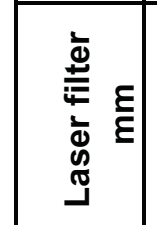 & 음 & $\stackrel{8}{\circ}$ & 음 \\
\hline 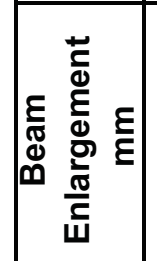 & $\stackrel{\widehat{N}}{\stackrel{N}{N}}$ & 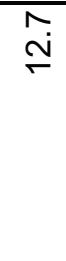 & 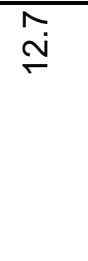 \\
\hline $\begin{array}{l}\dot{b} \\
\vdots \\
\Phi \\
\Phi \\
\infty\end{array}$ & $\begin{array}{l}\text { की } \\
\text { की }\end{array}$ & 吕 & $\begin{array}{l}\text { की } \\
\text { की }\end{array}$ \\
\hline 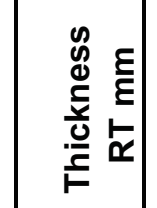 & $\begin{array}{l}\bar{m} \\
\stackrel{\emptyset}{0}\end{array}$ & $\begin{array}{c}\stackrel{m}{0} \\
\stackrel{0}{0}\end{array}$ & 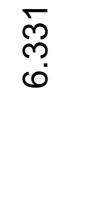 \\
\hline 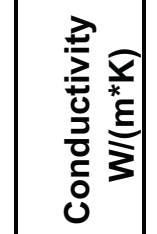 & & & \\
\hline के & 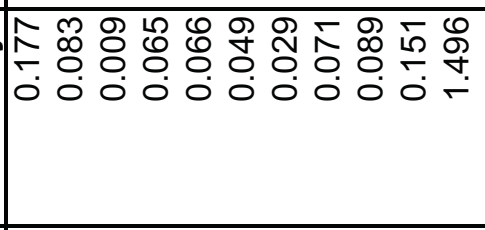 & مִ & ĩ \\
\hline 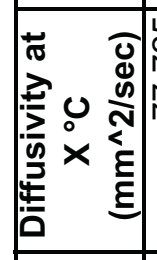 & 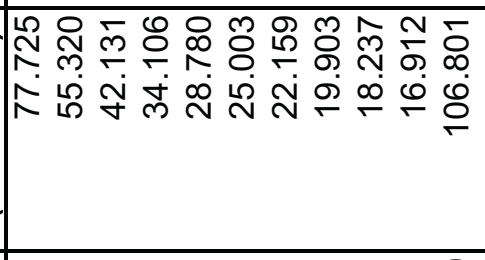 & 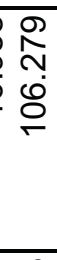 & 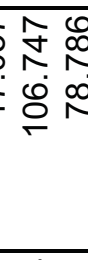 \\
\hline 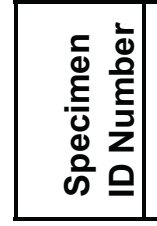 & $\begin{array}{l}8 \\
8 \\
\sum_{4}^{10} \\
4\end{array}$ & $\begin{array}{l}0 \\
0 \\
\sum^{2} \\
\sum_{4}^{2}\end{array}$ & $\begin{array}{l}0 \\
0 \\
\sum_{4}^{10} \\
4\end{array}$ \\
\hline
\end{tabular}




\begin{tabular}{|c|c|c|c|}
\hline 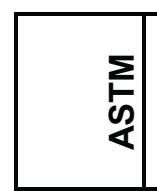 & $\begin{array}{l}\hat{o} \\
\dot{1} \\
\dot{0} \\
\dot{+} \\
\dot{w}\end{array}$ & $\begin{array}{l}\hat{o} \\
\frac{1}{0} \\
\stackrel{0}{+} \\
\dot{\omega}\end{array}$ & $\begin{array}{l}\frac{\hat{1}}{1} \\
\frac{1}{0} \\
\frac{1}{4} \\
\dot{w}\end{array}$ \\
\hline 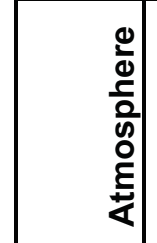 & 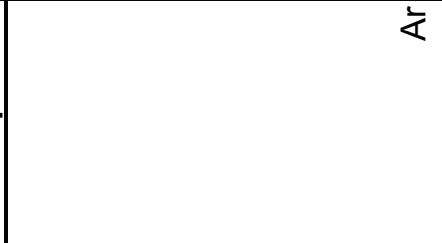 & 衣 & 交 \\
\hline 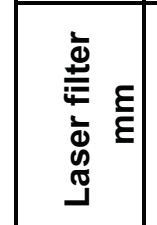 & 음 & 음 & 음 \\
\hline 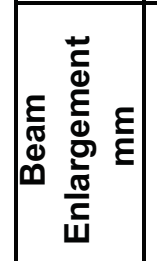 & $\stackrel{\widehat{N}}{\underline{I}}$ & $\stackrel{\stackrel{N}{ }}{ }$ & $\stackrel{\widetilde{N}}{\stackrel{N}{N}}$ \\
\hline $\begin{array}{l}\dot{b} \\
\vdots \\
\Phi \\
\Phi \\
\infty\end{array}$ & $\begin{array}{l}\text { की } \\
\text { की }\end{array}$ & 吕 & $\begin{array}{l}\text { की } \\
\text { की }\end{array}$ \\
\hline 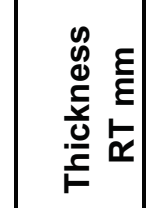 & 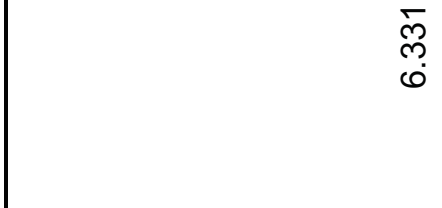 & 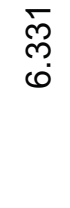 & $\begin{array}{l}\bar{\rho} \\
\stackrel{\oplus}{\oplus}\end{array}$ \\
\hline 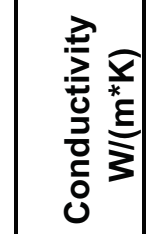 & & & \\
\hline 焉 & 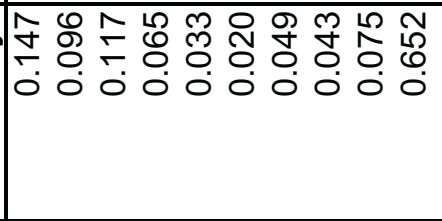 & bू & 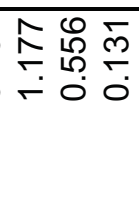 \\
\hline 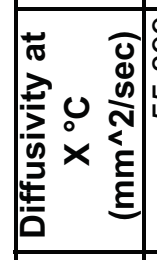 & 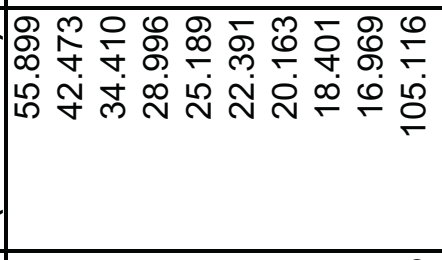 & 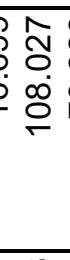 & 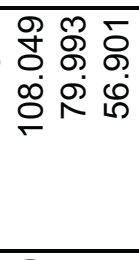 \\
\hline 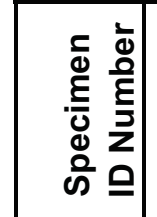 & $\begin{array}{l}20 \\
\sum_{0}^{2} \\
\sum_{4}^{2}\end{array}$ & $\begin{array}{l}8 \\
8 \\
\sum_{4}^{10} \\
4\end{array}$ & $\sum_{i}^{0}$ \\
\hline
\end{tabular}




\begin{tabular}{|c|c|c|}
\hline 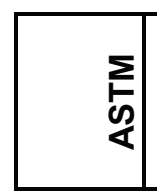 & $\begin{array}{l}\hat{o} \\
\dot{1} \\
\dot{0} \\
\dot{+} \\
\dot{W}\end{array}$ & $\begin{array}{l}\frac{\hat{1}}{1} \\
\frac{1}{6} \\
\frac{1}{\omega} \\
\end{array}$ \\
\hline $\begin{array}{c}0 \\
\frac{0}{0} \\
\frac{5}{0} \\
0 \\
0 \\
\frac{1}{4}\end{array}$ & 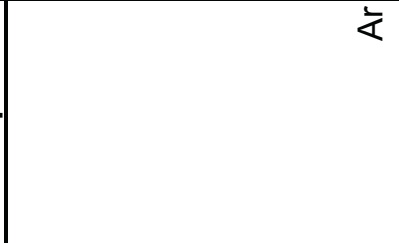 & 交 \\
\hline 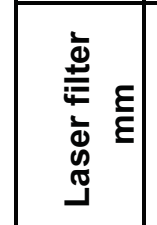 & 음 & 음 \\
\hline 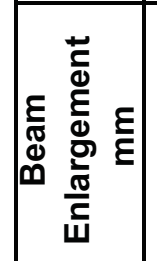 & $\stackrel{\widehat{N}}{\stackrel{N}{N}}$ & $\stackrel{\stackrel{N}{\sim}}{\stackrel{N}{*}}$ \\
\hline 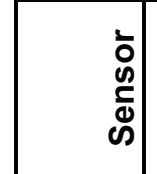 & $\begin{array}{l}\text { की } \\
\text { की }\end{array}$ & $\begin{array}{l}\text { की } \\
\text { 足 }\end{array}$ \\
\hline 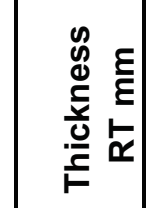 & $\begin{array}{l}\bar{m} \\
\stackrel{\emptyset}{0}\end{array}$ & 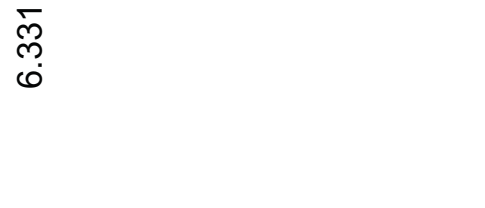 \\
\hline 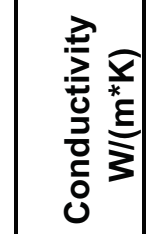 & & \\
\hline 焉 & 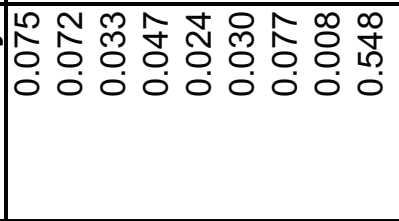 & 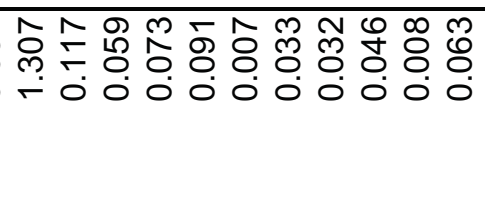 \\
\hline 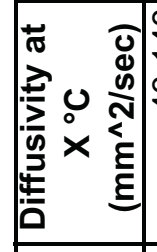 & 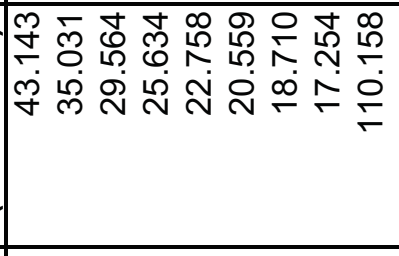 & $\begin{array}{l}\infty \\
\infty \\
\infty \\
\infty\end{array}$ \\
\hline 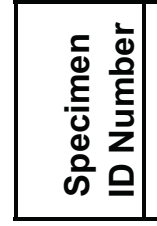 & 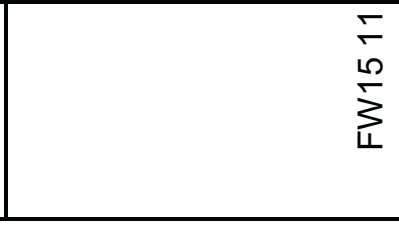 & $\sum_{\substack{n \\
L}}^{\frac{1}{20}}$ \\
\hline
\end{tabular}




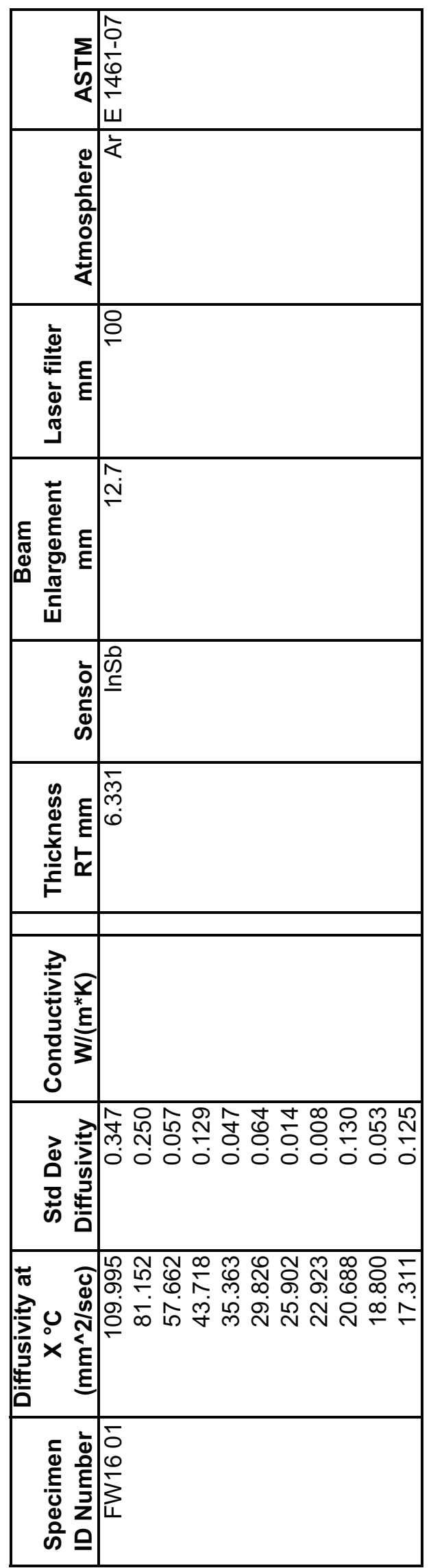




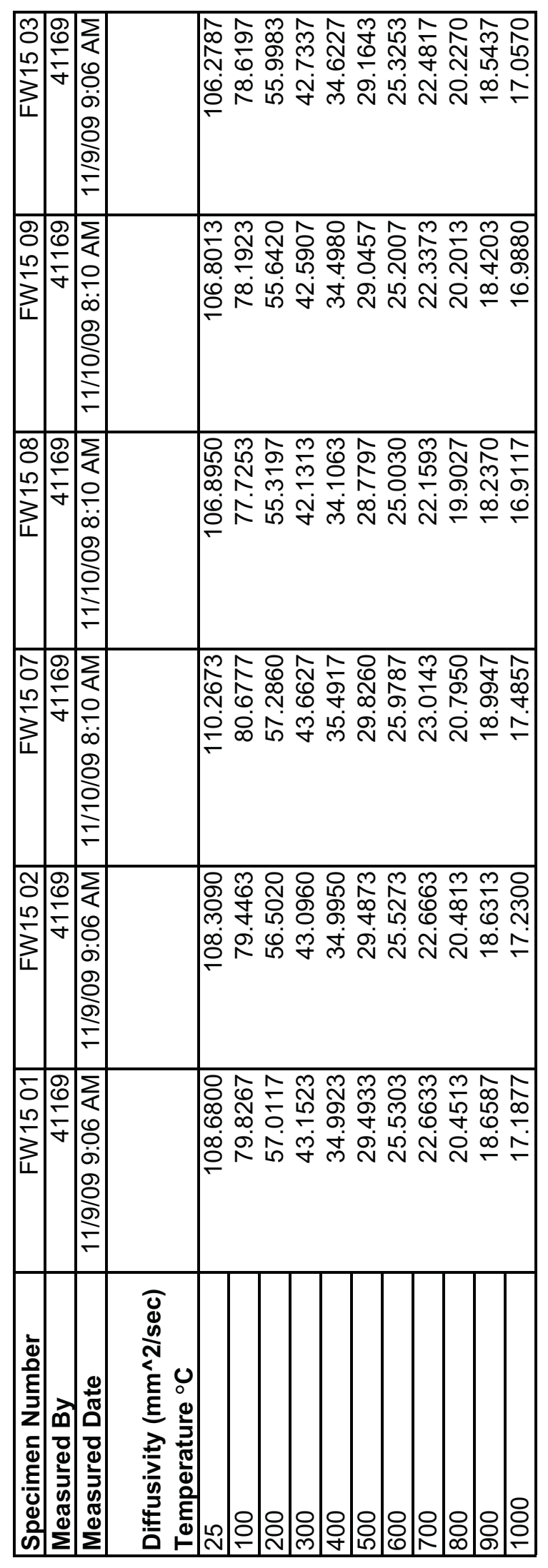




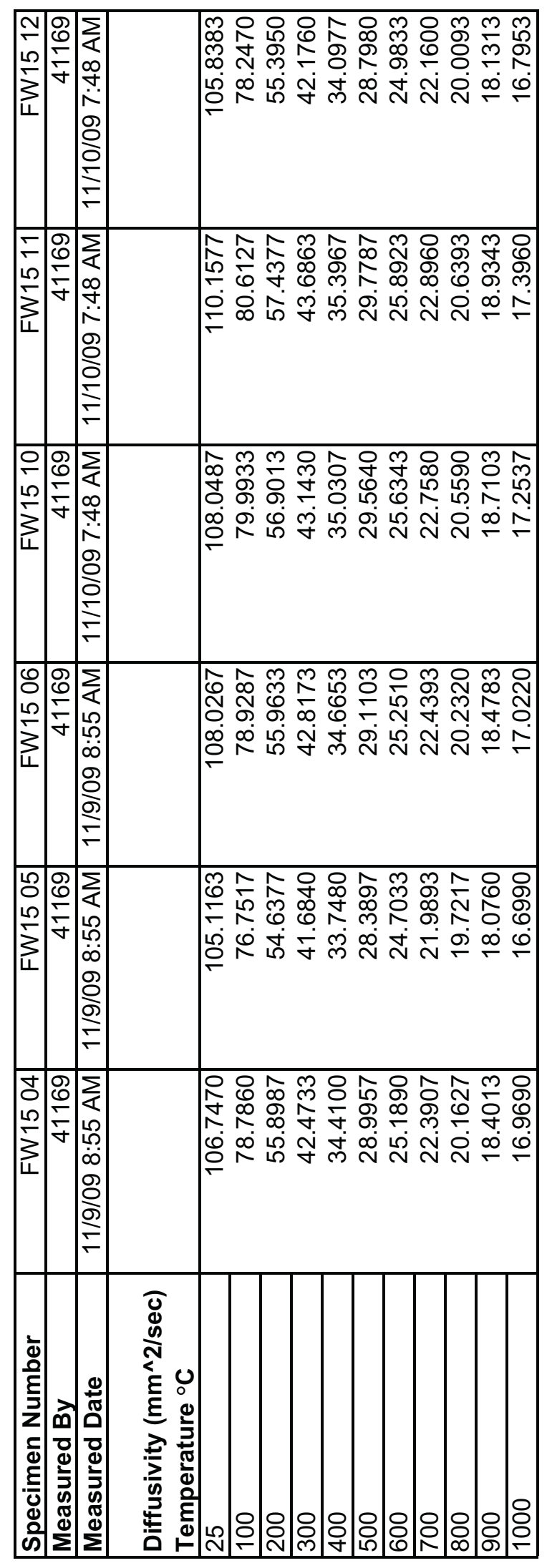




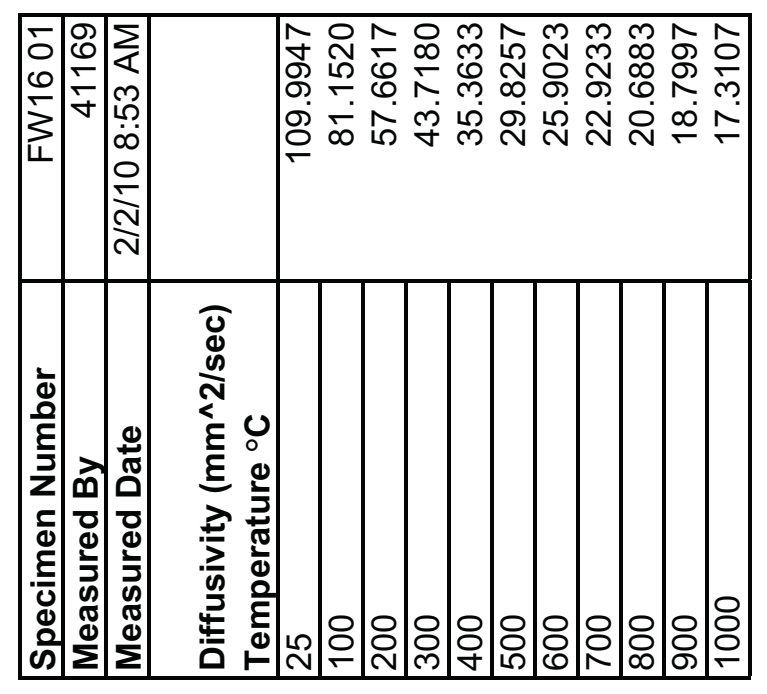

กำ 


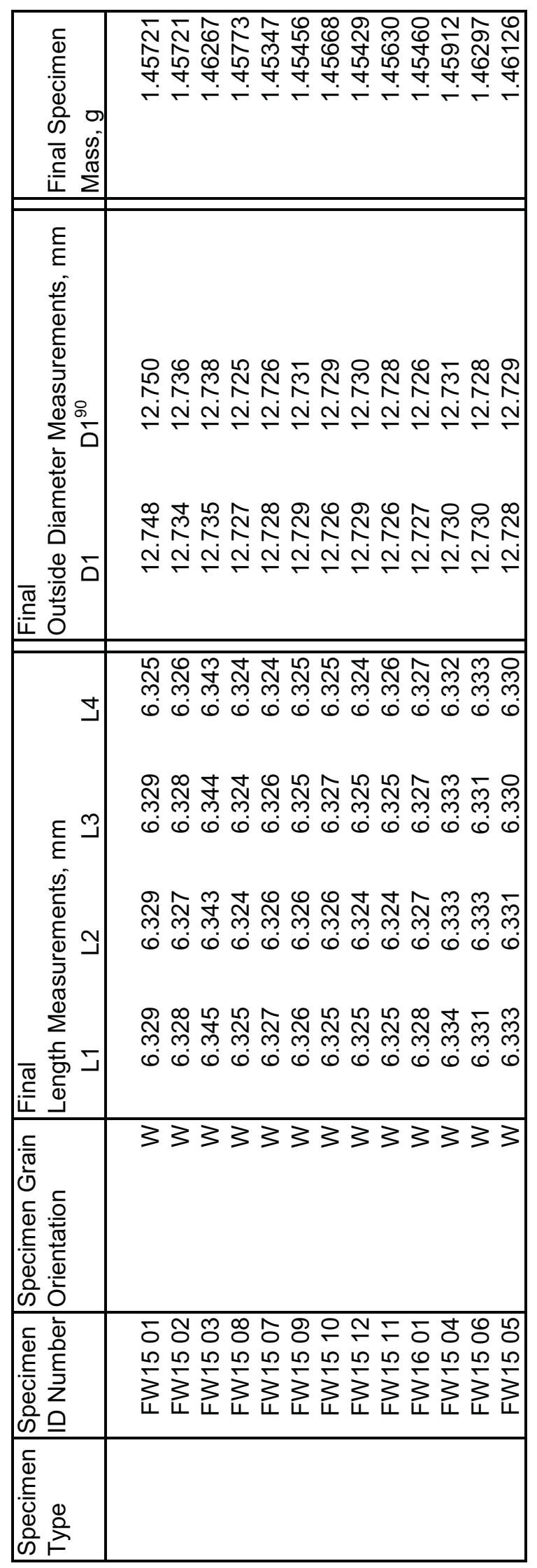




\begin{tabular}{|c|c|}
\hline 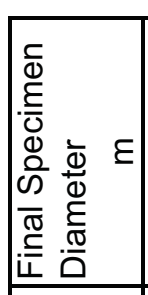 & 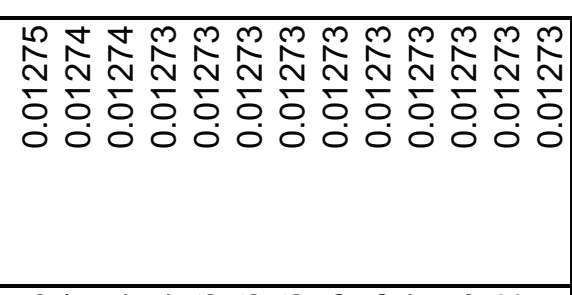 \\
\hline 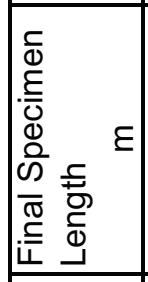 & 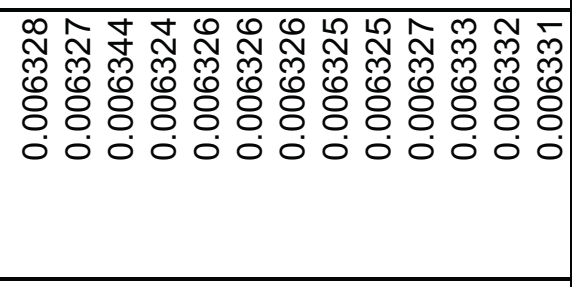 \\
\hline 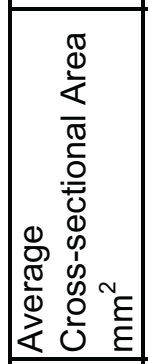 & 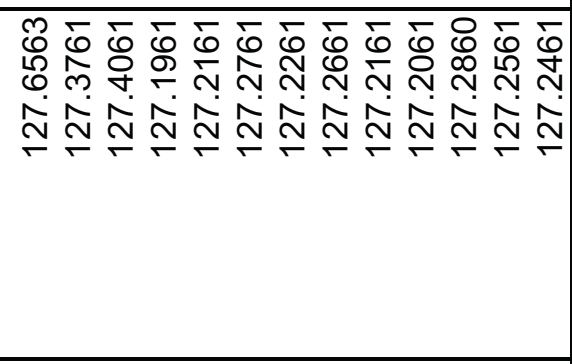 \\
\hline 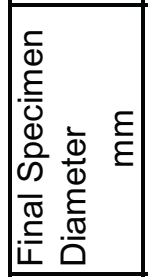 & 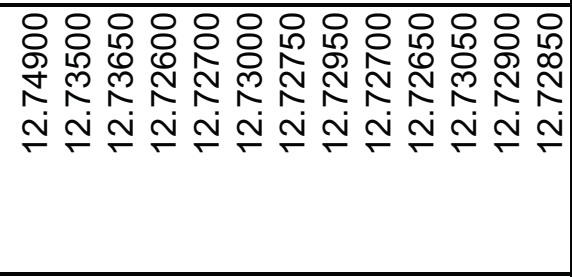 \\
\hline 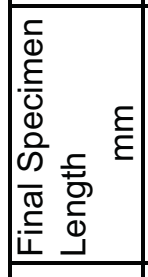 & 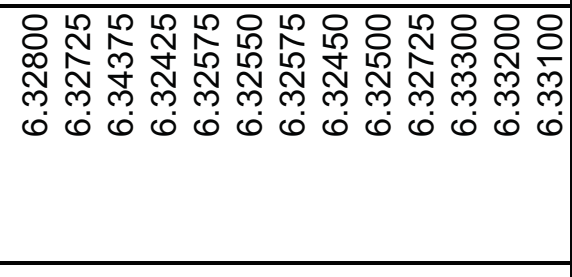 \\
\hline 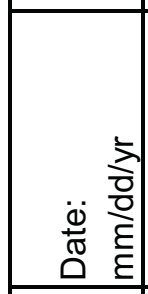 & 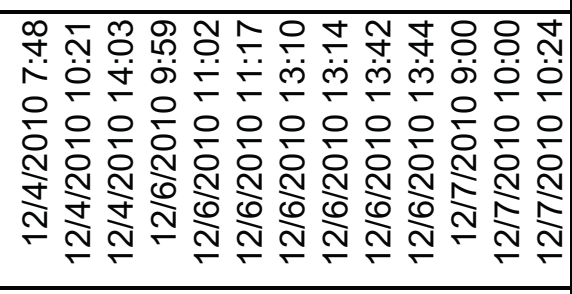 \\
\hline 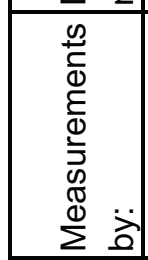 & 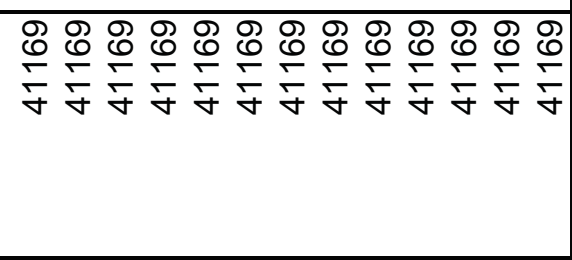 \\
\hline 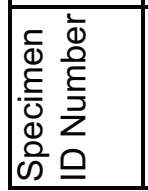 & 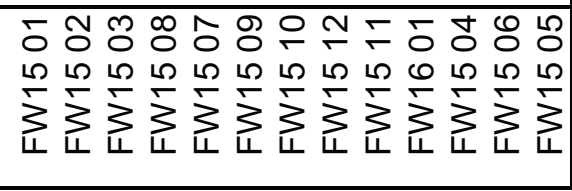 \\
\hline
\end{tabular}




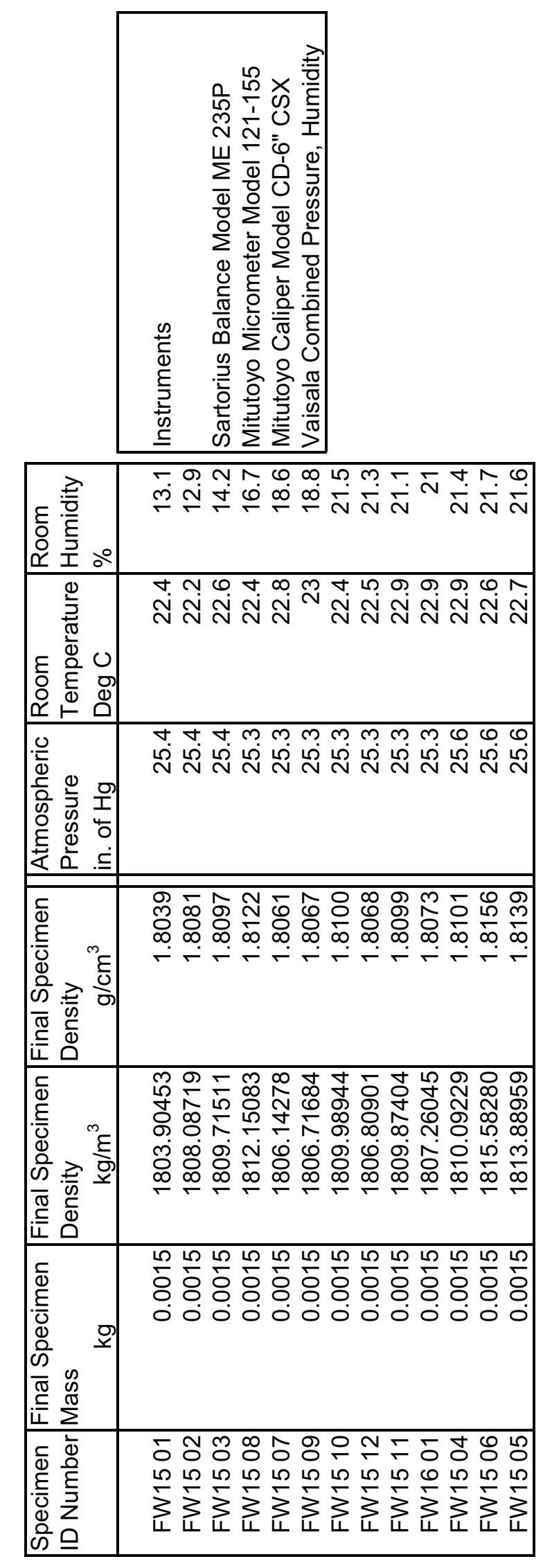


Graphite Grade:

Graphite Manufacturer:

Forming Process:

Coke Particle Size:

Coke Type:

ASTM Class:

Specimen Geometry:
NBG-10

SGL Carbon Company

Extruded

Medium grain

Pitch coke

ENHP

Cylinder

Specimen ID \#'s:

S1 01

S1 02

$\mathrm{S} 103$

S1 04

S1 05

S1 06

S1 07

S1 08

S1 09

S1 10

S1 11

S1 12

S2 01

S2 02

S2 03

S2 04

S2 05

S2 06

S2 07

S2 08

S2 09

S2 10

S2 11

S2 12 


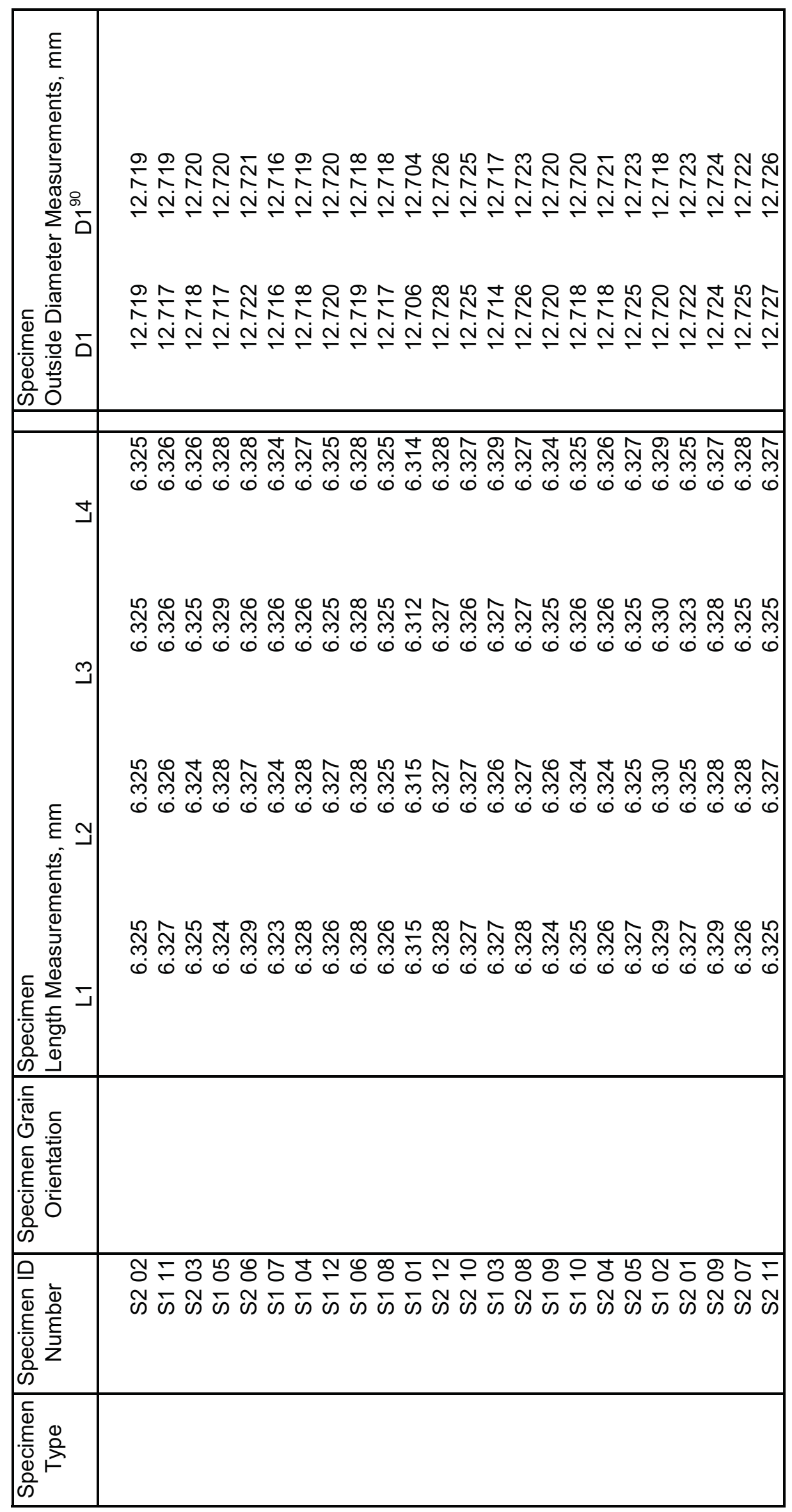




\begin{tabular}{|c|c|}
\hline 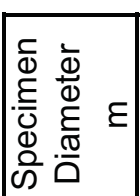 & 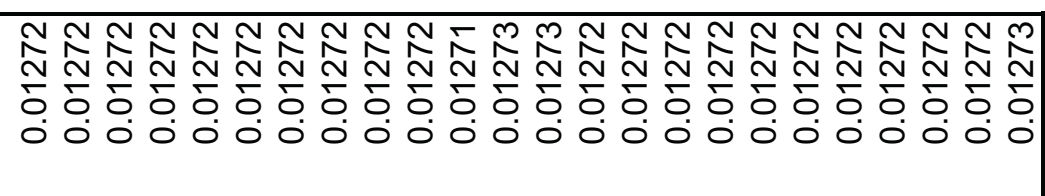 \\
\hline 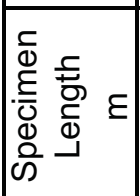 & 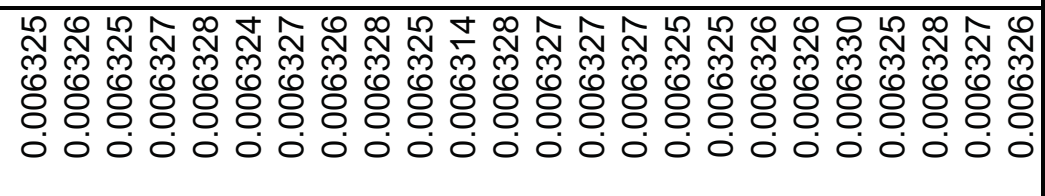 \\
\hline 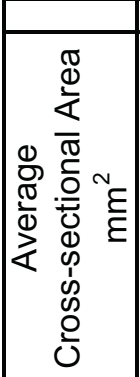 & 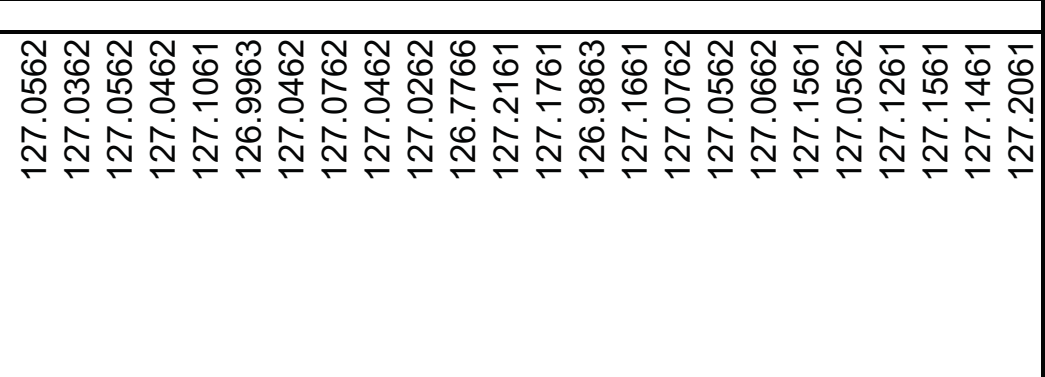 \\
\hline 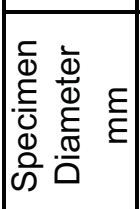 & 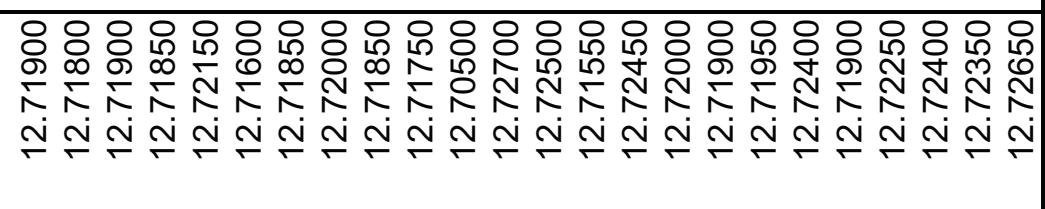 \\
\hline 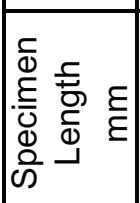 & 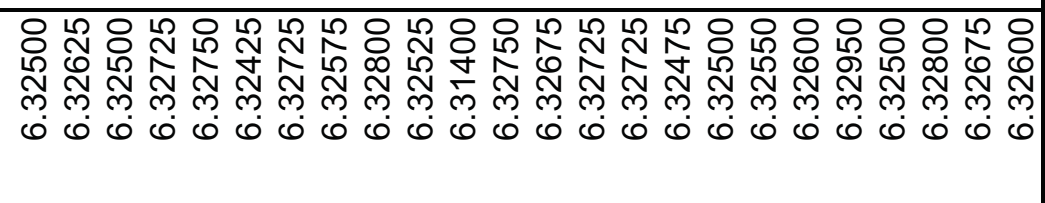 \\
\hline 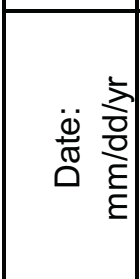 & 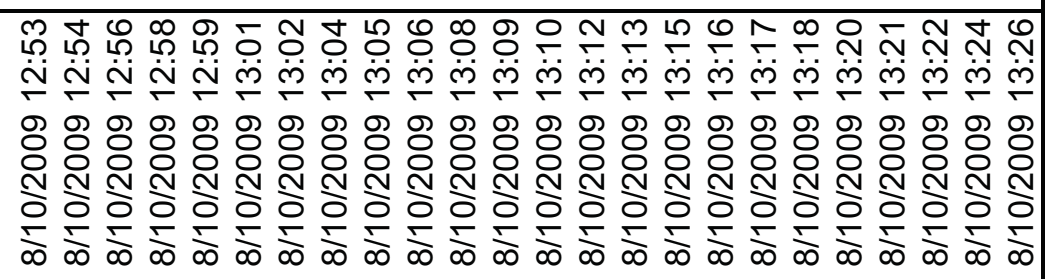 \\
\hline 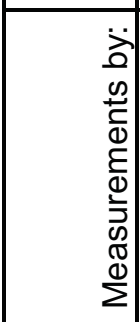 & 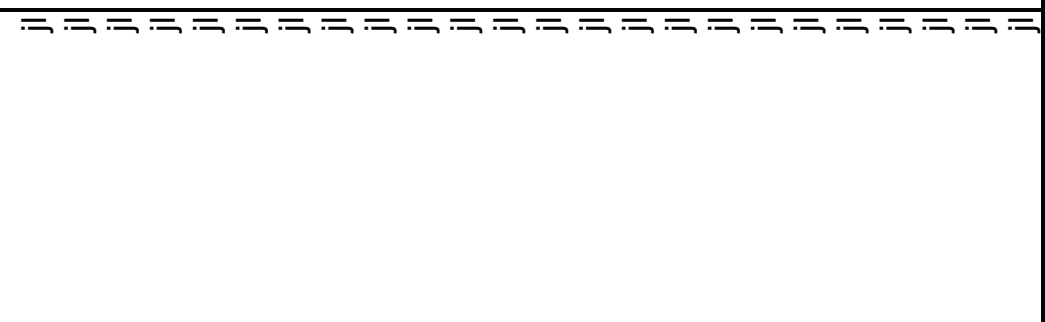 \\
\hline 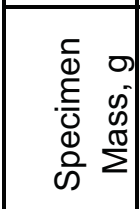 & 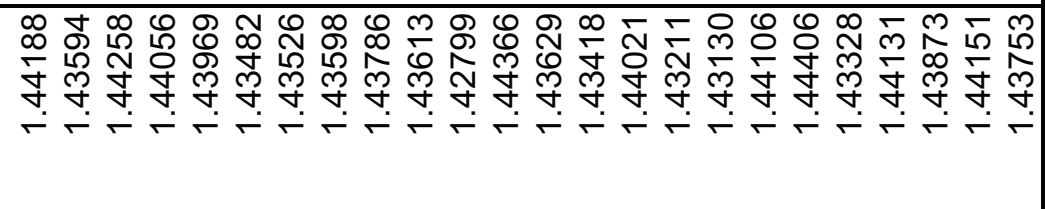 \\
\hline 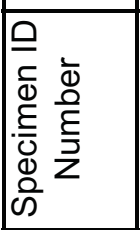 & 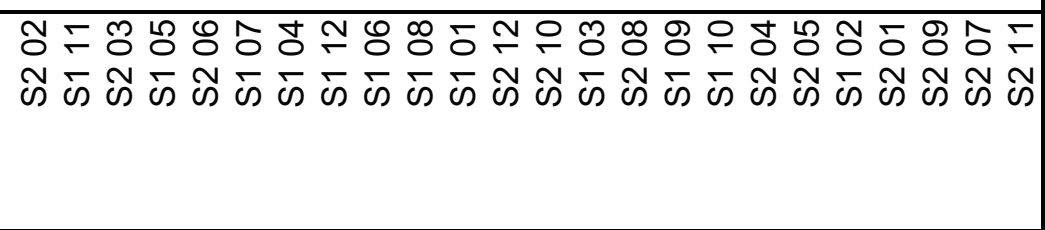 \\
\hline
\end{tabular}




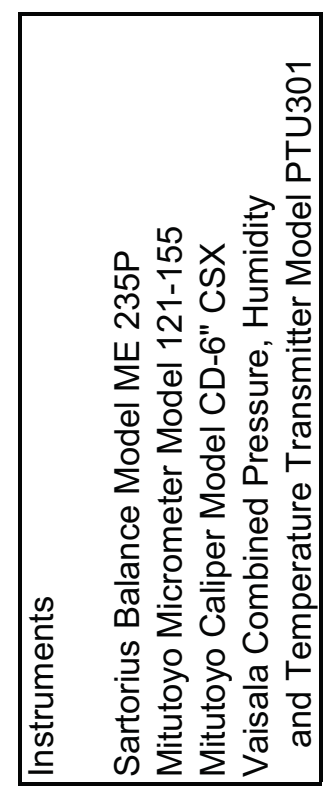

\begin{tabular}{|c|c|}
\hline 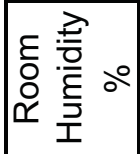 & 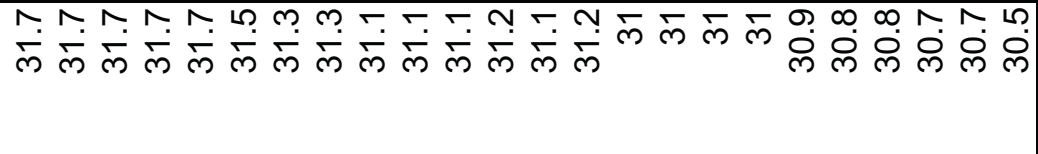 \\
\hline 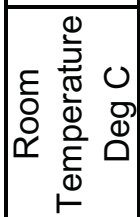 & 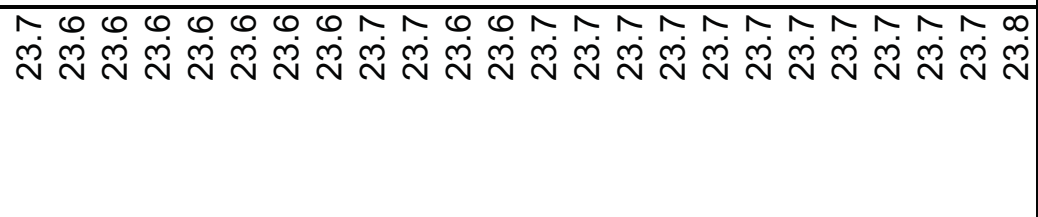 \\
\hline 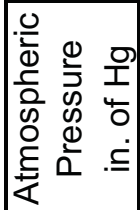 & 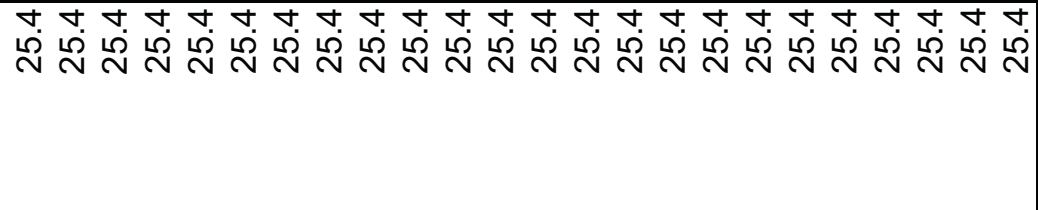 \\
\hline 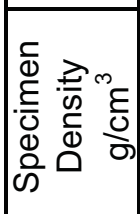 & 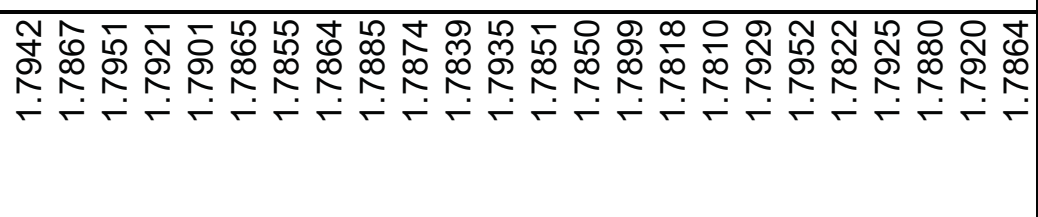 \\
\hline 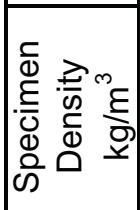 & 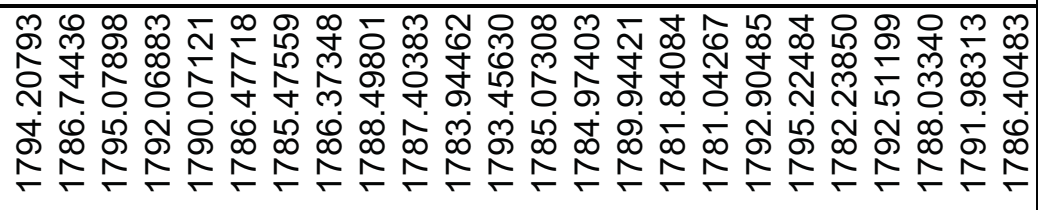 \\
\hline 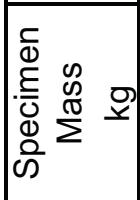 & 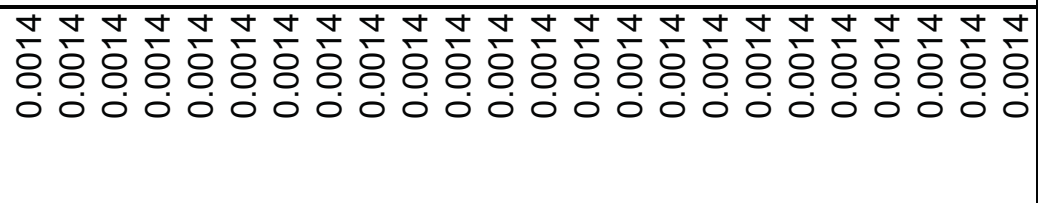 \\
\hline 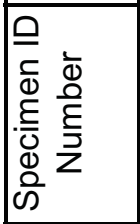 & 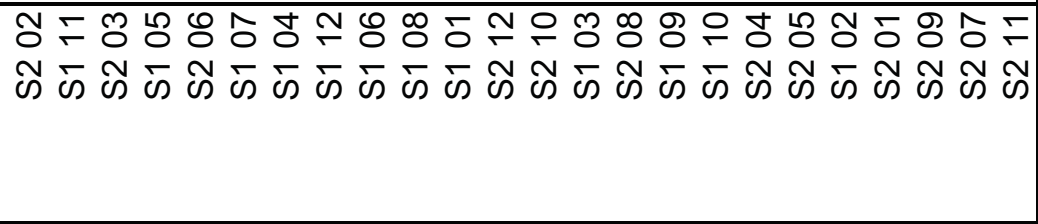 \\
\hline
\end{tabular}




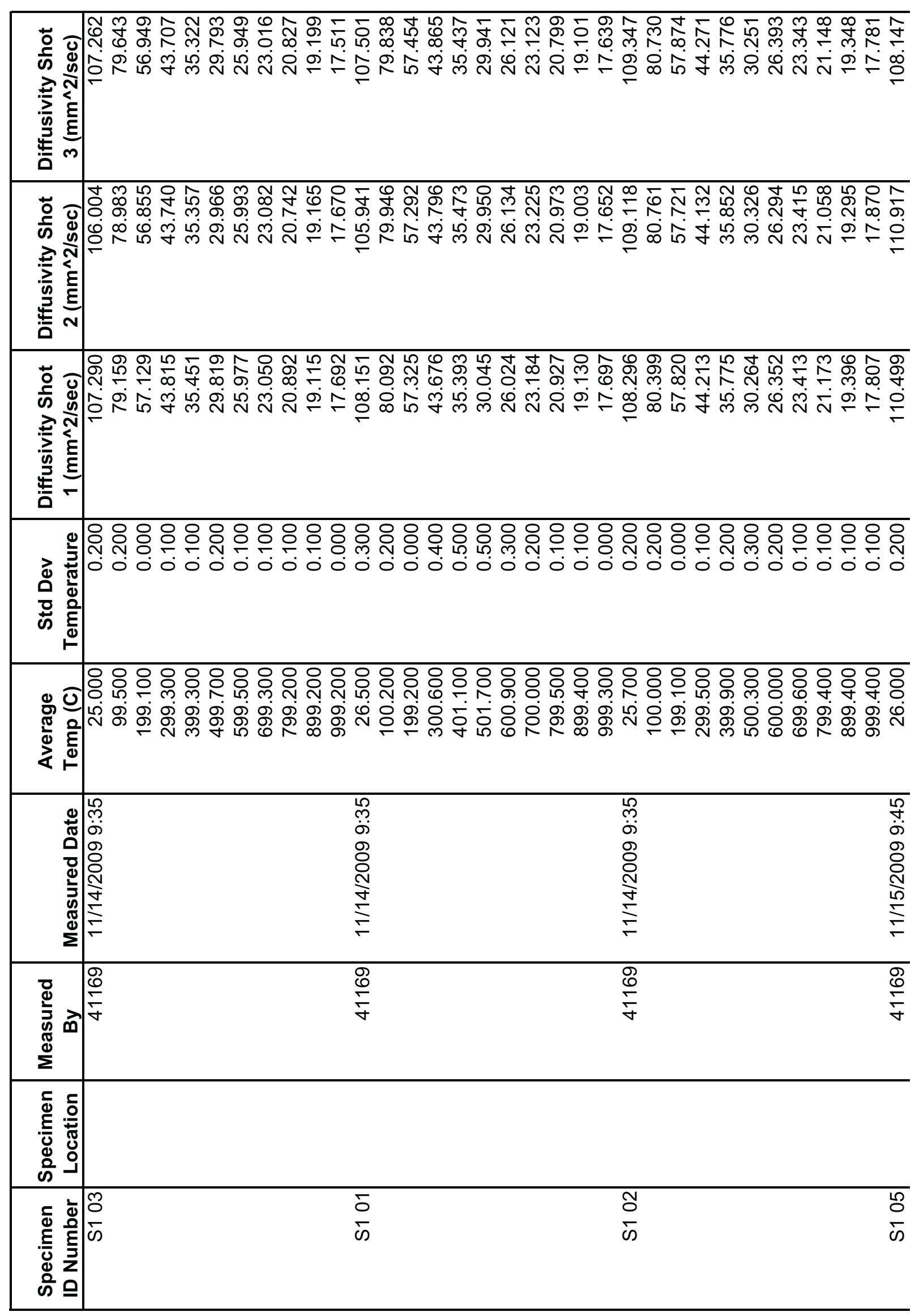




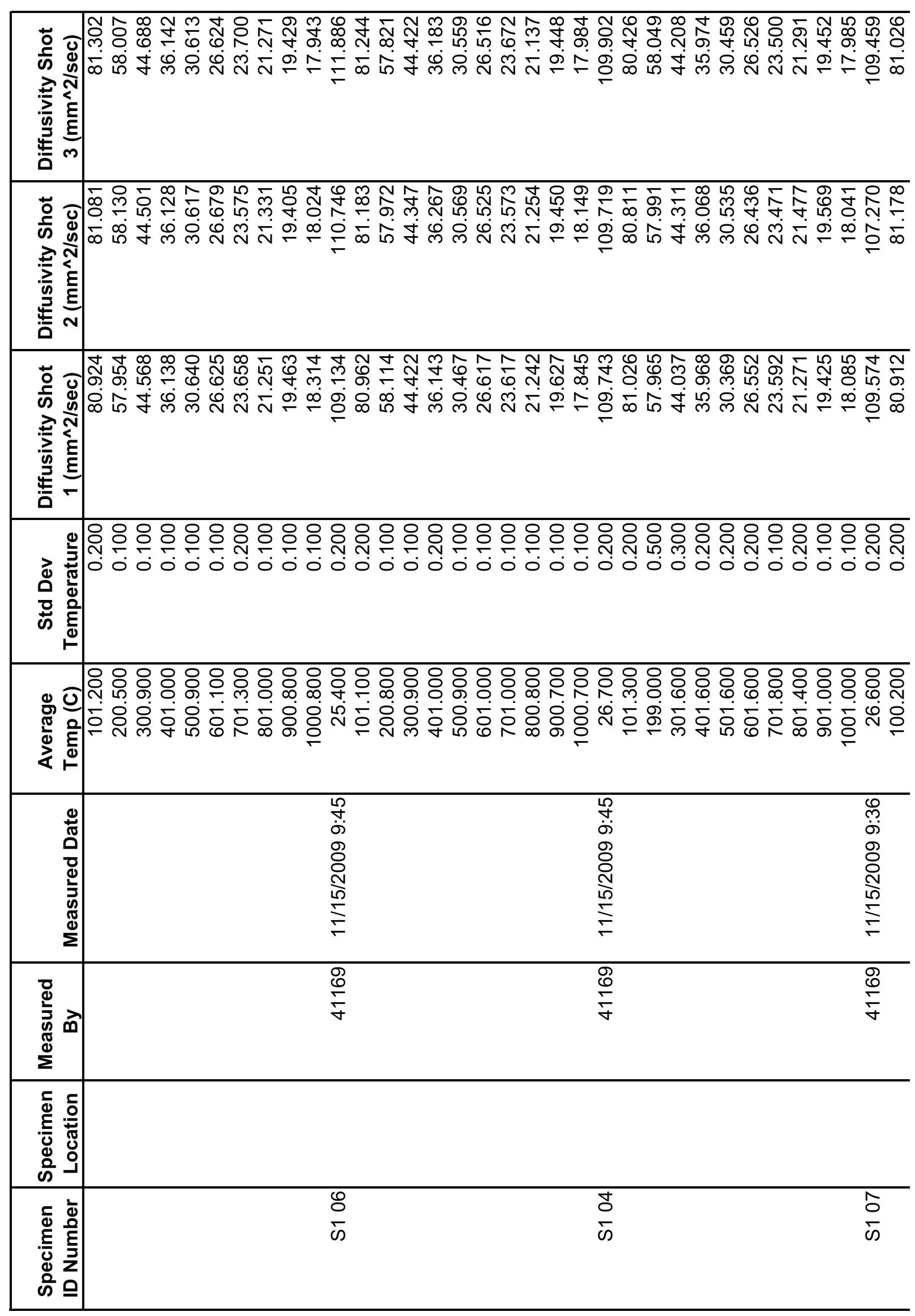




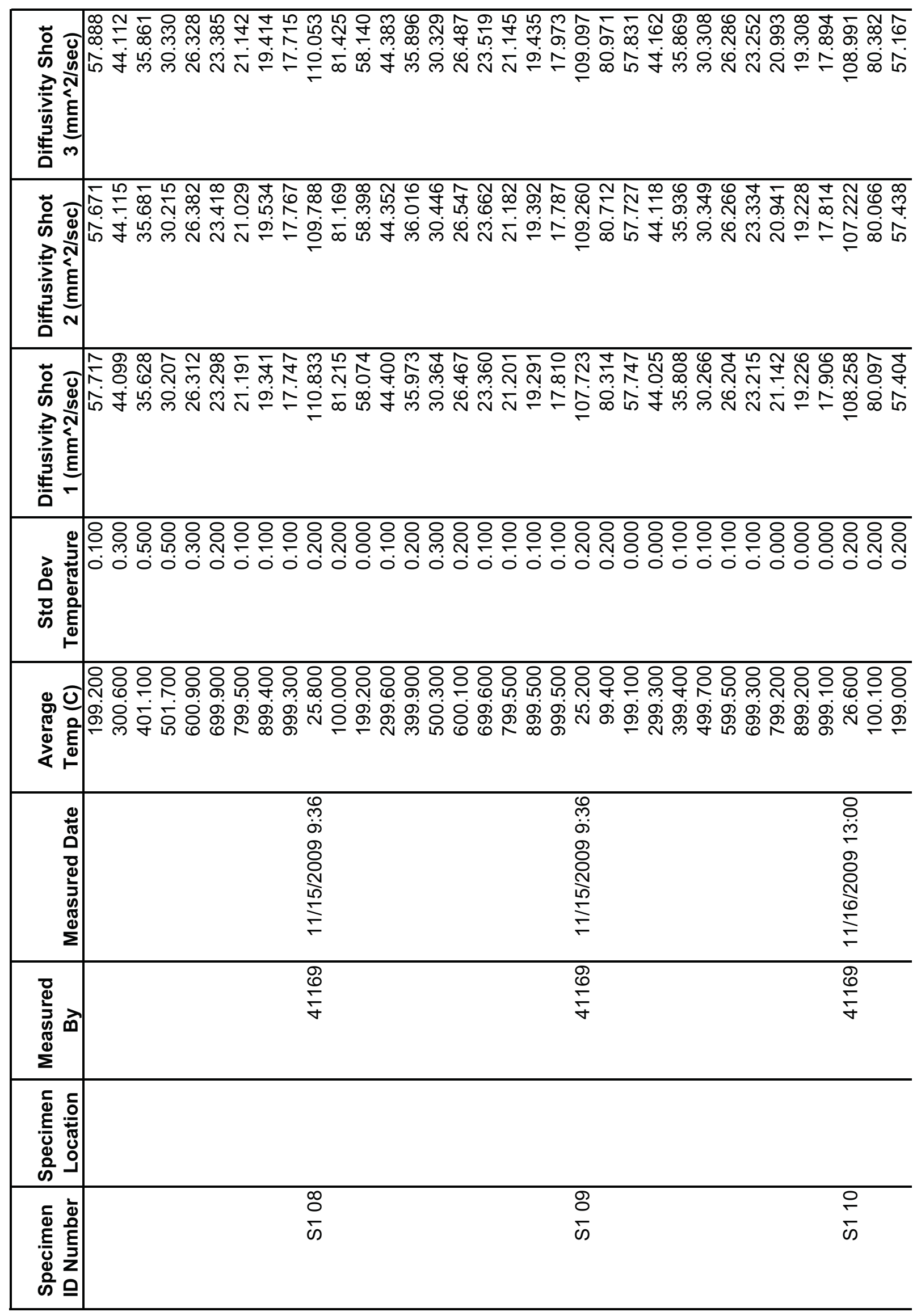




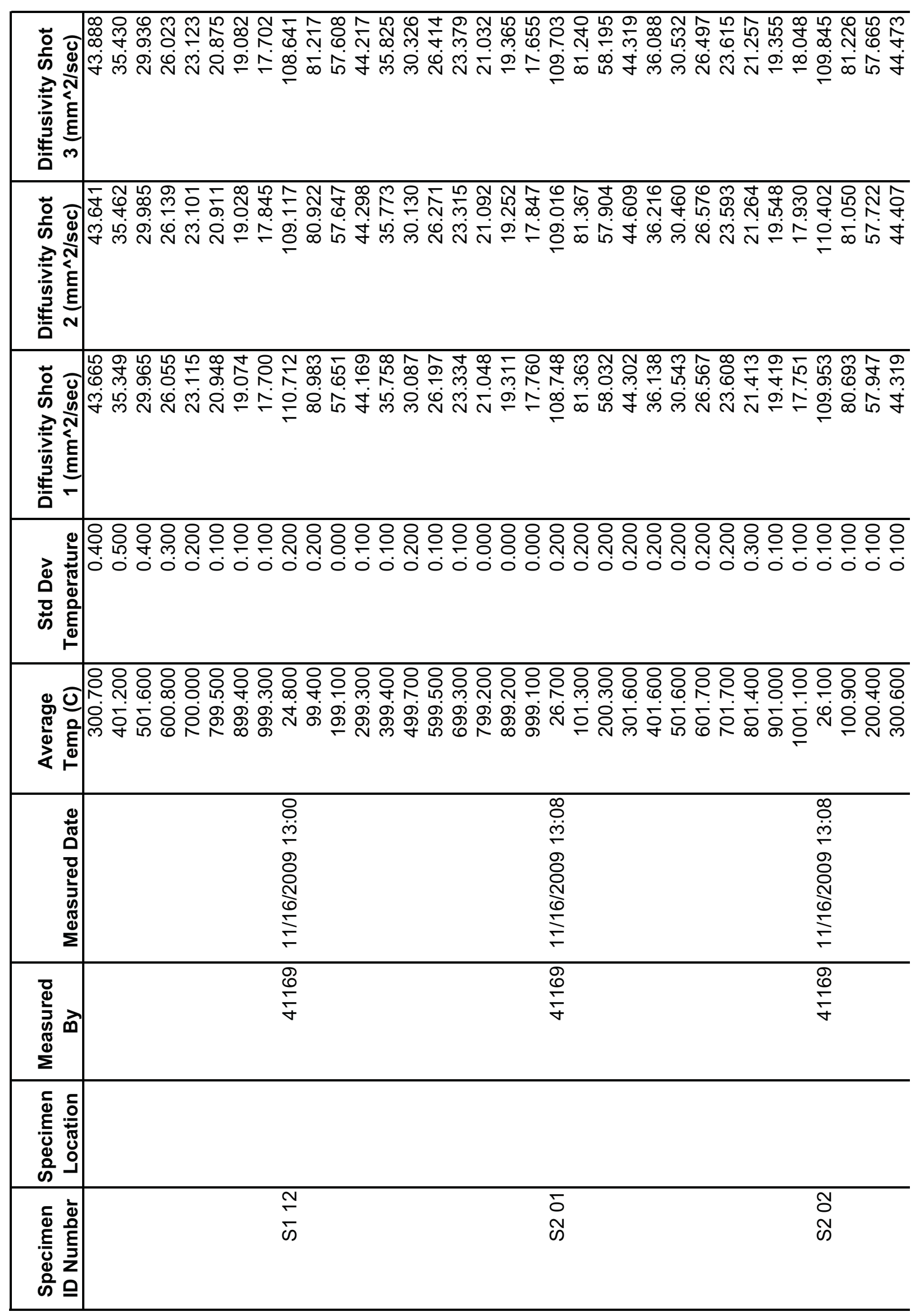




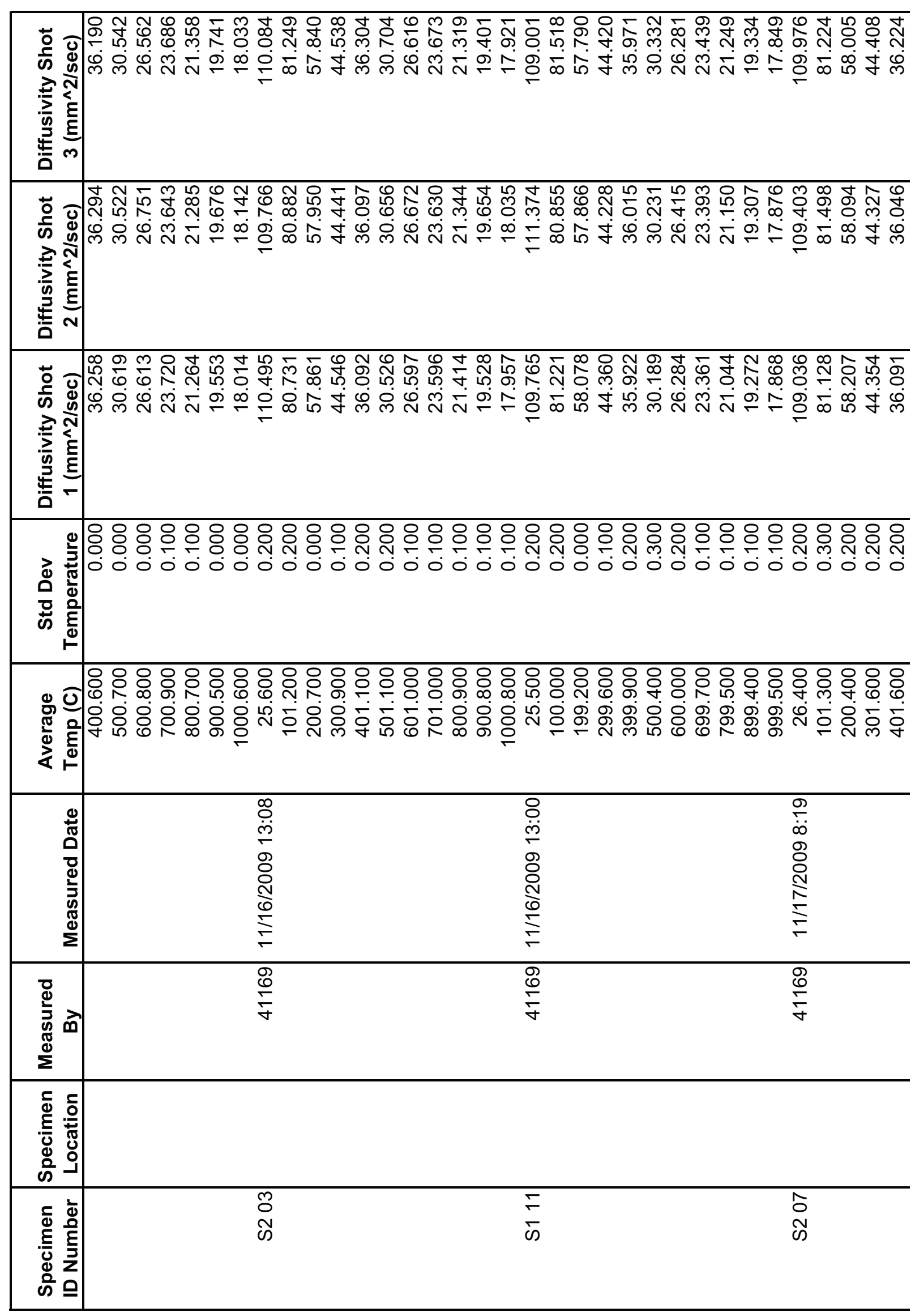




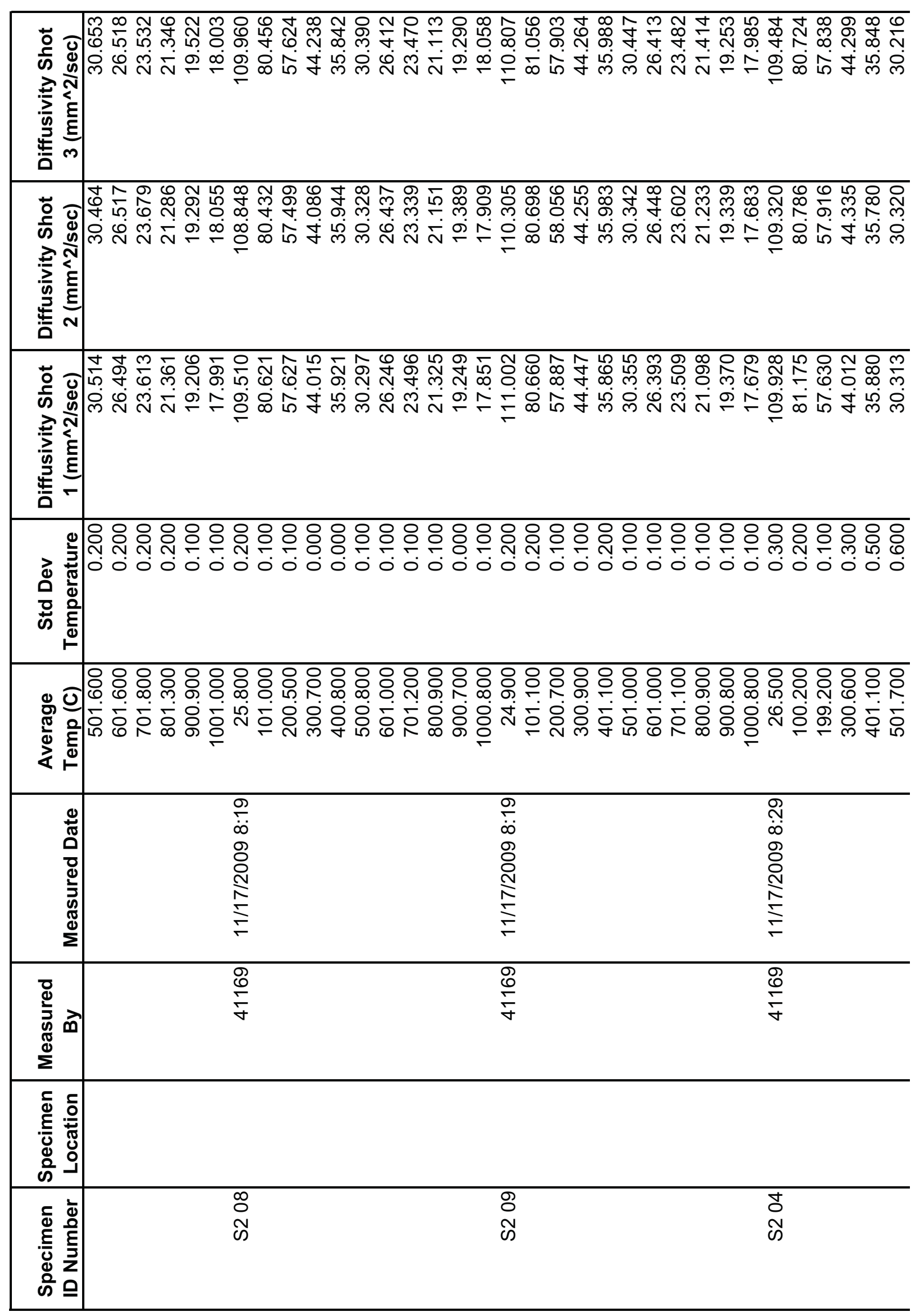




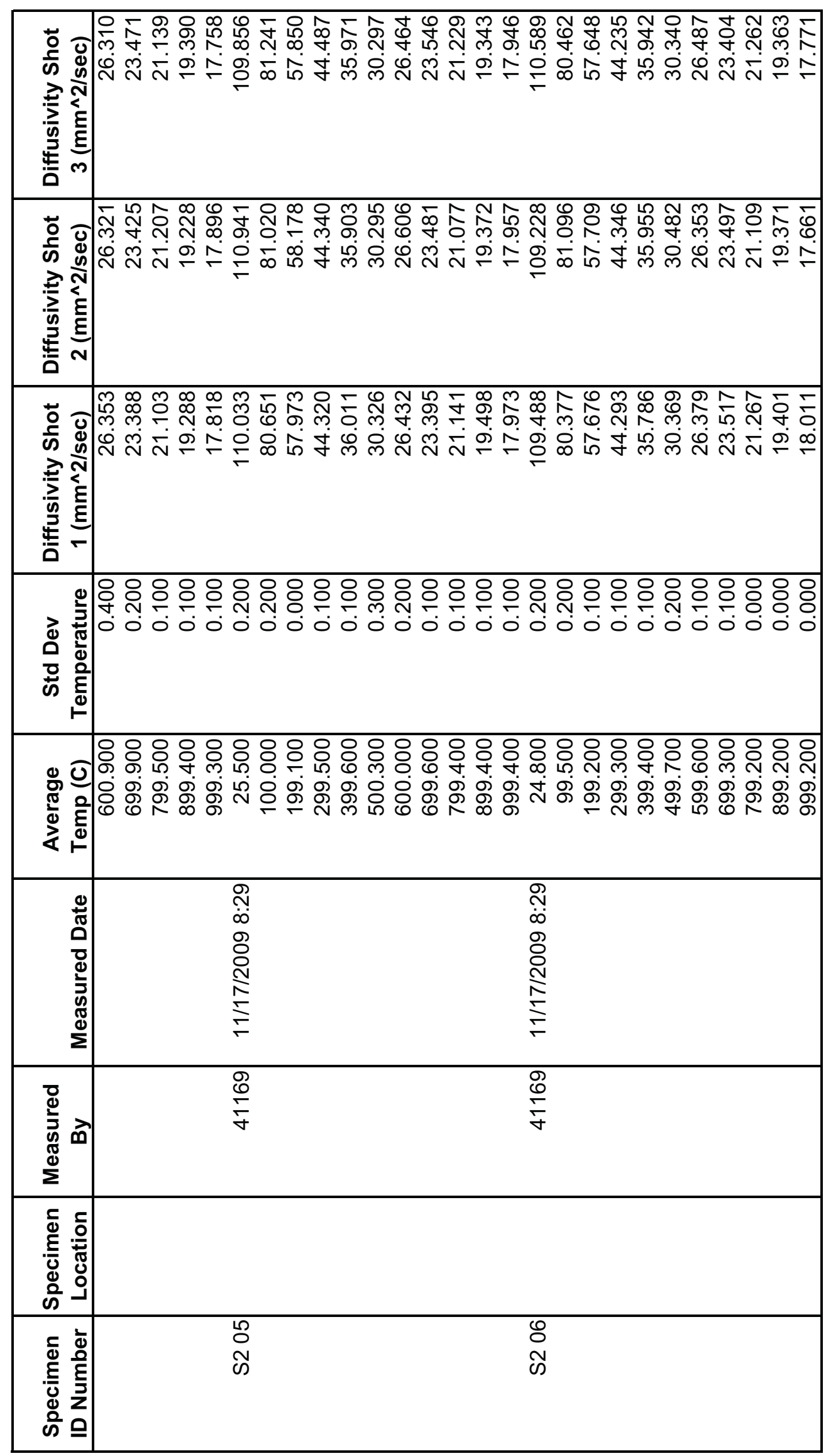




\begin{tabular}{|c|c|c|c|c|}
\hline 焉 & 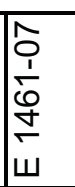 & 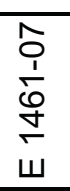 & 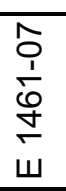 & 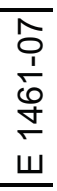 \\
\hline 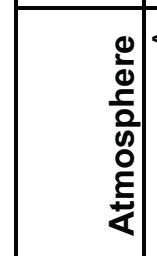 & & $\overline{\dot{z}}$ & $\bar{k}$ & $\bar{i}$ \\
\hline 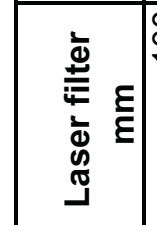 & & 음 & 음 & 음 \\
\hline 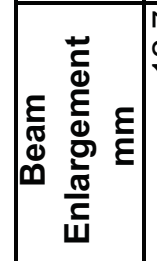 & & $\stackrel{\stackrel{N}{\sim}}{\sim}$ & $\stackrel{\widehat{N}}{\stackrel{N}{S}}$ & 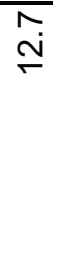 \\
\hline $\begin{array}{l}\vdots \\
\vdots \\
\grave{\omega} \\
\grave{\Phi} \\
\omega\end{array}$ & & $\begin{array}{l}\text { की } \\
\text { की }\end{array}$ & $\begin{array}{l}\text { की } \\
\text { क्ञ }\end{array}$ & की \\
\hline 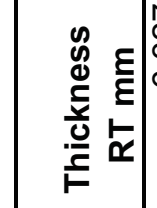 & & $\begin{array}{l}\hat{N} \\
\text { ָे } \\
\hat{0}\end{array}$ & 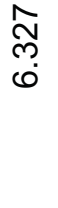 & 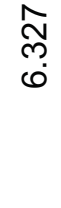 \\
\hline 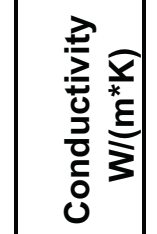 & & & & \\
\hline 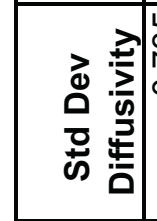 & 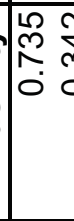 & 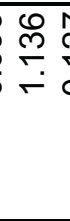 & ". & 产 \\
\hline 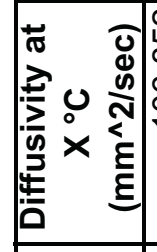 & 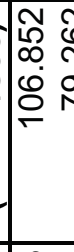 & ما & 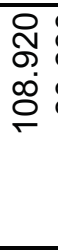 & 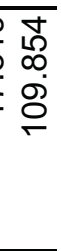 \\
\hline 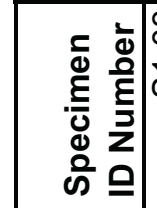 & & $\begin{array}{l}\bar{\delta} \\
\bar{\omega}\end{array}$ & $\frac{\delta}{\infty}$ & $\begin{array}{l}\frac{\mathscr{O}}{0} \\
\bar{\infty}\end{array}$ \\
\hline
\end{tabular}




\begin{tabular}{|c|c|c|c|}
\hline 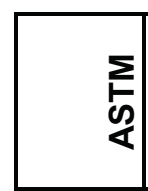 & $\begin{array}{l}\hat{o} \\
\frac{1}{0} \\
\dot{0} \\
\dot{5} \\
\end{array}$ & $\begin{array}{l}\hat{S} \\
\frac{1}{1} \\
\frac{0}{5} \\
\dot{w}\end{array}$ & 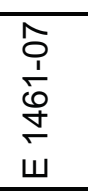 \\
\hline \begin{tabular}{|c|} 
\\
$\frac{0}{0}$ \\
$\frac{5}{0}$ \\
0 \\
0 \\
$\frac{1}{4}$
\end{tabular} & 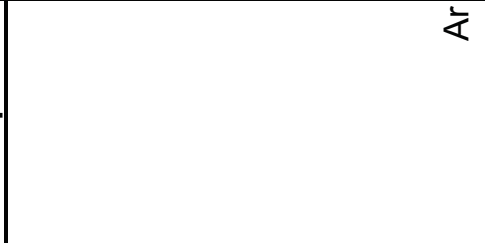 & $\frac{1}{\alpha}$ & 广 \\
\hline 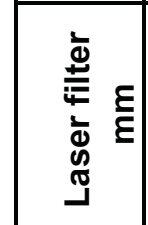 & 음 & 음 & 음 \\
\hline 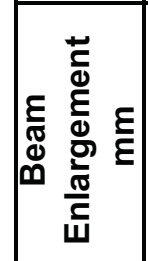 & $\stackrel{\widehat{N}}{\stackrel{N}{N}}$ & 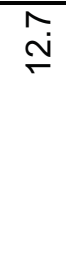 & 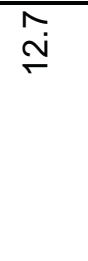 \\
\hline 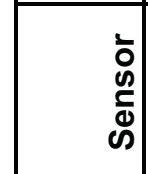 & $\begin{array}{l}\text { की } \\
\text { की }\end{array}$ & 吕 & $\begin{array}{l}\text { की } \\
\text { की }\end{array}$ \\
\hline 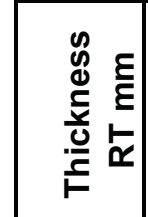 & స్ & 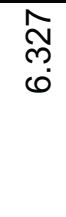 & $\begin{array}{l}\text { స్ } \\
\text { ర్ }\end{array}$ \\
\hline 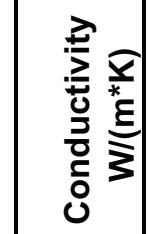 & & & \\
\hline 究 & 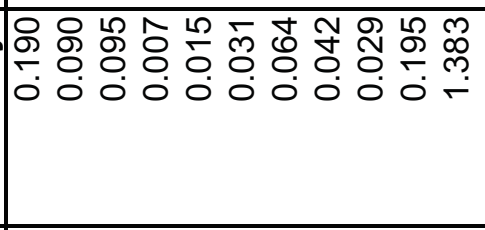 & : & 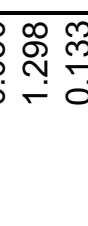 \\
\hline 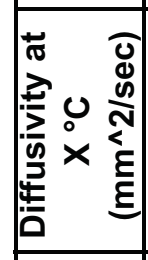 & 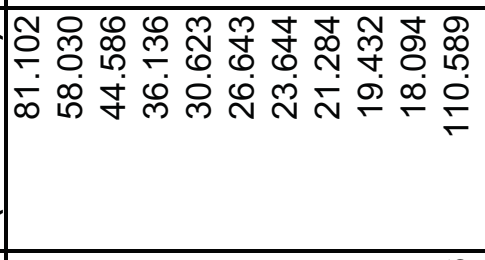 & 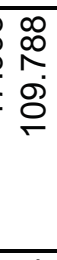 & 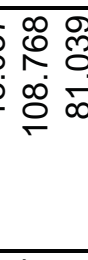 \\
\hline 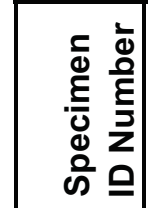 & $\begin{array}{l}8 \\
\bar{\delta} \\
\bar{n}\end{array}$ & $\begin{array}{l}\text { ठ } \\
\bar{\omega}\end{array}$ & $\begin{array}{l}\hat{0} \\
\bar{\omega}\end{array}$ \\
\hline
\end{tabular}




\begin{tabular}{|c|c|c|c|}
\hline 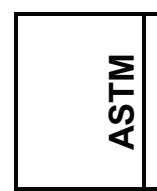 & $\begin{array}{l}\hat{o} \\
\frac{1}{0} \\
\frac{0}{+} \\
\dot{w}\end{array}$ & $\begin{array}{l}\hat{o} \\
\frac{1}{0} \\
\stackrel{0}{+} \\
\dot{\omega}\end{array}$ & $\begin{array}{l}\frac{\hat{1}}{1} \\
\frac{1}{0} \\
\frac{1}{4} \\
\dot{w}\end{array}$ \\
\hline 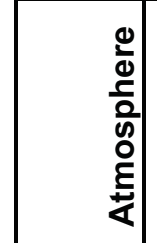 & 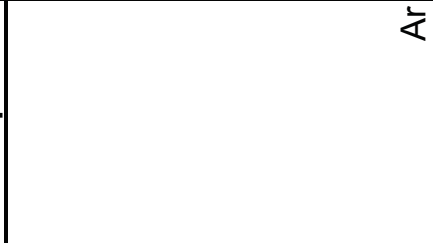 & 衣 & 交 \\
\hline 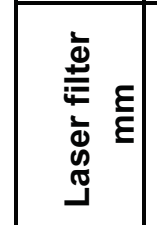 & 음 & 음 & 음 \\
\hline 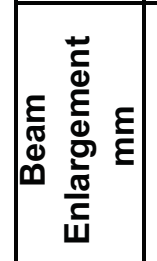 & $\stackrel{\widetilde{N}}{\stackrel{N}{N}}$ & $\stackrel{\stackrel{N}{ }}{ }$ & $\stackrel{\widetilde{N}}{\stackrel{N}{N}}$ \\
\hline $\begin{array}{l}\bar{b} \\
\vdots \\
心 \\
\Phi \\
\infty\end{array}$ & 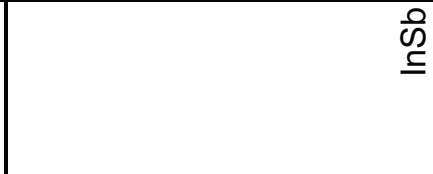 & $\begin{array}{l}\text { की } \\
\text { I }\end{array}$ & $\begin{array}{l}\text { की } \\
\text { की }\end{array}$ \\
\hline 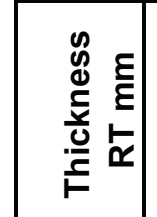 & $\begin{array}{c}\text { స్ } \\
0\end{array}$ & $\begin{array}{l}\widehat{N} \\
0 \\
0\end{array}$ & $\begin{array}{l}\widehat{N} \\
\text { ָे }\end{array}$ \\
\hline 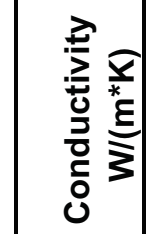 & & & \\
\hline 焉 & 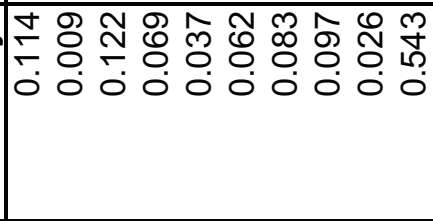 & 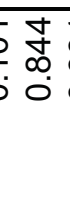 & 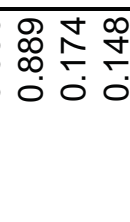 \\
\hline 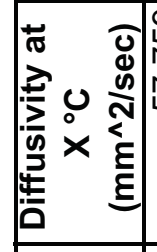 & 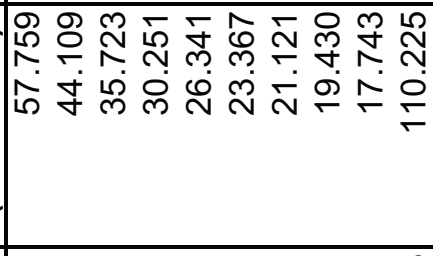 & 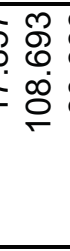 & 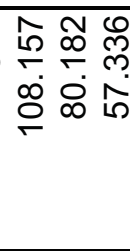 \\
\hline 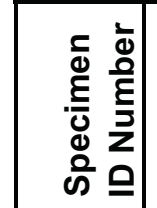 & $\begin{array}{l}\infty \\
\infty \\
\infty\end{array}$ & $\begin{array}{l}88 \\
\bar{\delta}\end{array}$ & $\begin{array}{l}\frac{\square}{\bar{\infty}} \\
\bar{\infty}\end{array}$ \\
\hline
\end{tabular}




\begin{tabular}{|c|c|c|c|}
\hline 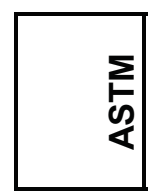 & $\begin{array}{l}\hat{o} \\
\dot{1} \\
\dot{0} \\
\dot{+} \\
\dot{w}\end{array}$ & $\begin{array}{l}\hat{o} \\
\frac{1}{0} \\
\stackrel{0}{+} \\
\dot{\omega}\end{array}$ & $\begin{array}{l}\hat{o} \\
\frac{1}{0} \\
\stackrel{0}{+} \\
\dot{w}\end{array}$ \\
\hline $\begin{array}{c}0 \\
\frac{0}{0} \\
\frac{5}{0} \\
0 \\
0 \\
\frac{1}{4}\end{array}$ & 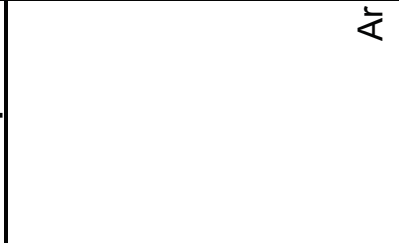 & 文 & $\frac{1}{4}$ \\
\hline 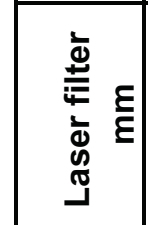 & 음 & $\stackrel{\circ}{\circ}$ & 음 \\
\hline 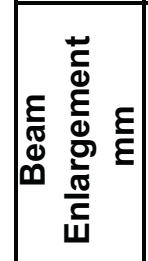 & $\stackrel{\widehat{N}}{\stackrel{N}{N}}$ & $\stackrel{\stackrel{N}{\sim}}{\mathrm{N}}$ & $\stackrel{\stackrel{\sim}{\sim}}{ }$ \\
\hline 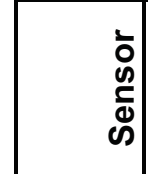 & $\begin{array}{l}\text { की } \\
\text { की }\end{array}$ & $\begin{array}{l}\text { की } \\
\text { I }\end{array}$ & $\begin{array}{l}\text { की } \\
\text { 足 }\end{array}$ \\
\hline 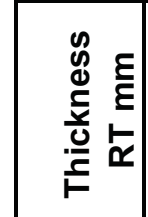 & స్ & $\begin{array}{l}\widehat{N} \\
\text { రె }\end{array}$ & 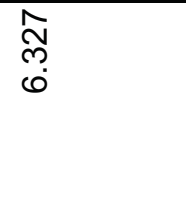 \\
\hline 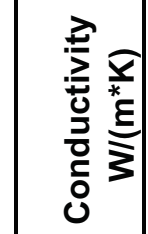 & & & \\
\hline 永辛 & 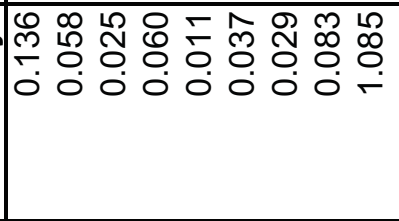 & ; & 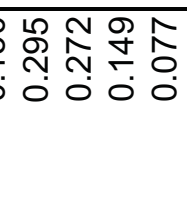 \\
\hline 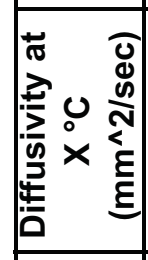 & 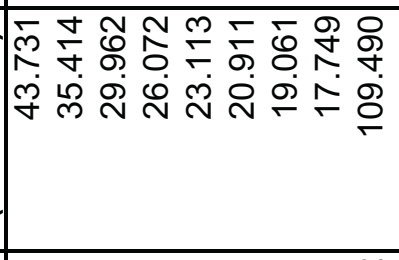 & 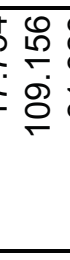 & 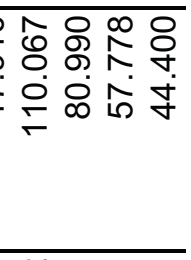 \\
\hline 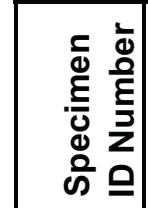 & $\frac{\mathbb{N}}{\bar{s}}$ & $\begin{array}{l}\bar{\delta} \\
\text { N }\end{array}$ & $\begin{array}{l}\text { No } \\
\text { ஸे } \\
\text { ஸे }\end{array}$ \\
\hline
\end{tabular}




\begin{tabular}{|c|c|c|c|}
\hline 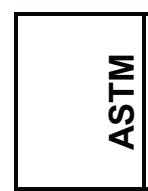 & $\begin{array}{l}\hat{O} \\
\frac{1}{0} \\
\stackrel{0}{+} \\
\dot{w}\end{array}$ & 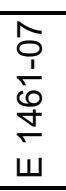 & $\begin{array}{l}\hat{O} \\
\frac{1}{0} \\
\frac{0}{+} \\
\dot{w}\end{array}$ \\
\hline 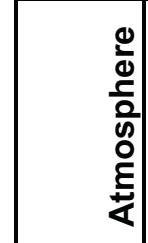 & 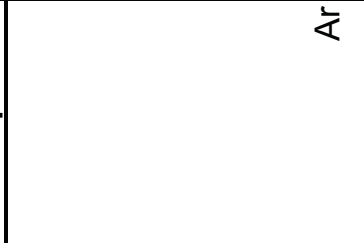 & $\frac{1}{4}$ & 交 \\
\hline 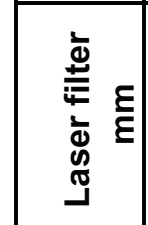 & $\stackrel{\circ}{\circ}$ & 음 & 음 \\
\hline 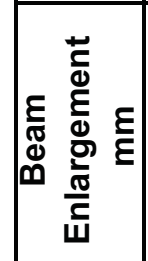 & $\stackrel{\widehat{N}}{\stackrel{N}{S}}$ & 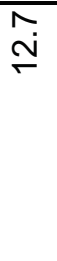 & $\stackrel{\stackrel{N}{N}}{ }$ \\
\hline 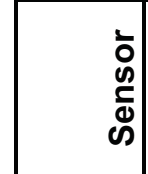 & $\begin{array}{l}\text { की } \\
\text { की }\end{array}$ & $\begin{array}{l}\text { की } \\
\text { क् }\end{array}$ & $\begin{array}{l}\text { की } \\
\text { Ф }\end{array}$ \\
\hline 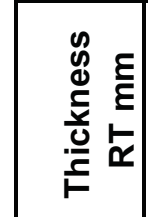 & $\begin{array}{l}\widehat{N} \\
\stackrel{N}{0}\end{array}$ & 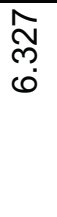 & $\begin{array}{l}\text { స్ } \\
\text { రె }\end{array}$ \\
\hline 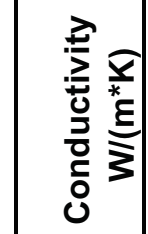 & & & \\
\hline 永辛 & 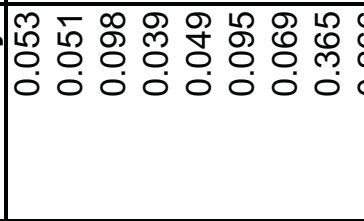 & $\stackrel{N}{\sim}$ & 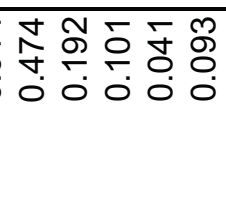 \\
\hline 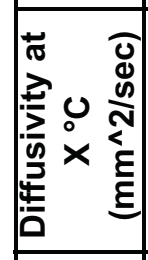 & 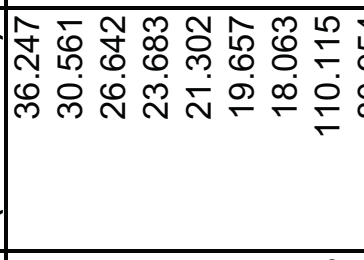 & $\begin{array}{l}\hat{y} \\
0 \\
\dot{0} \\
\end{array}$ & 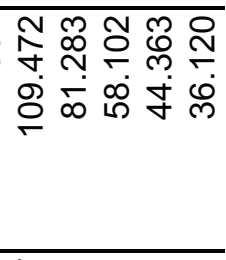 \\
\hline 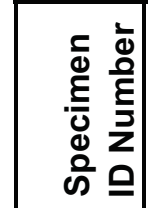 & $\begin{array}{l}\text { Oू } \\
\text { Oे }\end{array}$ & $\bar{\sigma}$ & $\begin{array}{l}\text { 今 } \\
\text { ஸे }\end{array}$ \\
\hline
\end{tabular}




\begin{tabular}{|c|c|c|c|}
\hline 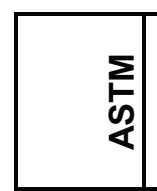 & $\begin{array}{l}\hat{o} \\
\frac{1}{0} \\
\dot{o} \\
\dot{w} \\
\end{array}$ & $\begin{array}{l}\hat{o} \\
\frac{1}{0} \\
\stackrel{0}{+} \\
\dot{\omega}\end{array}$ & $\begin{array}{l}\hat{0} \\
\frac{1}{0} \\
\stackrel{0}{+} \\
\dot{\omega}\end{array}$ \\
\hline 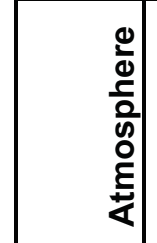 & 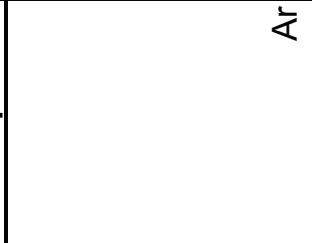 & 㐫 & $\frac{1}{4}$ \\
\hline 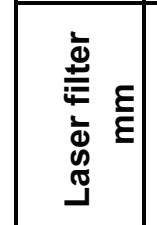 & 음 & 음 & 음 \\
\hline 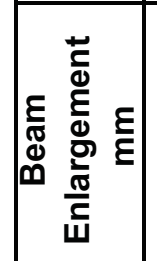 & $\stackrel{\widehat{N}}{\stackrel{N}{N}}$ & 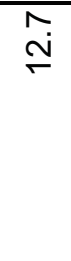 & $\stackrel{\stackrel{\sim}{\sim}}{ }$ \\
\hline $\begin{array}{l}\vdots \\
\vdots \\
0 \\
⿱ 亠 凶 \\
\infty\end{array}$ & $\begin{array}{l}\text { की } \\
\text { की }\end{array}$ & 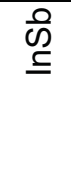 & $\begin{array}{l}\text { की } \\
\text { 足 }\end{array}$ \\
\hline 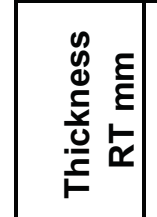 & 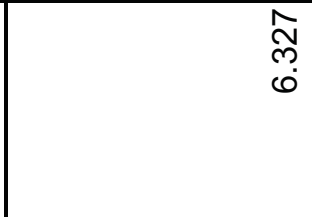 & $\begin{array}{l}\widehat{N} \\
\text { రె }\end{array}$ & $\begin{array}{l}\text { స్ } \\
\text { ర్d }\end{array}$ \\
\hline 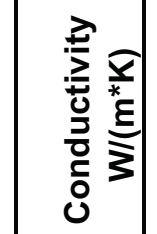 & & & \\
\hline 焉 & $\mid \begin{array}{lllll} & \end{array}$ & $\begin{array}{l}0 \\
0 \\
0 \\
0 \\
0\end{array}$ & 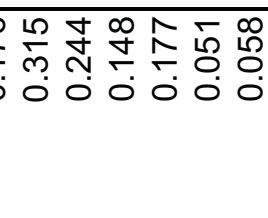 \\
\hline 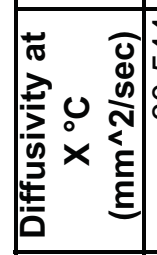 & 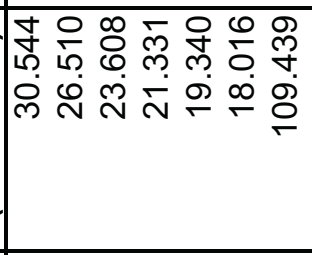 & 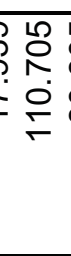 & 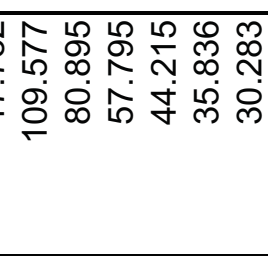 \\
\hline 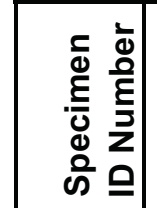 & $\begin{array}{l}\infty \\
\text { o } \\
\tilde{N}\end{array}$ & $\begin{array}{l}\text { D } \\
\text { N } \\
\text { C) }\end{array}$ & $\begin{array}{l} \pm \\
\text { Oे } \\
\text { N }\end{array}$ \\
\hline
\end{tabular}




\begin{tabular}{|c|c|c|}
\hline $\begin{array}{c}\Sigma \\
5 \\
\&\end{array}$ & $\begin{array}{l} \\
\stackrel{1}{1} \\
\frac{1}{0} \\
\frac{1}{w} \\
\end{array}$ & $\begin{array}{l}\frac{\hat{O}}{1} \\
\frac{1}{6} \\
\frac{1}{\omega} \\
\end{array}$ \\
\hline 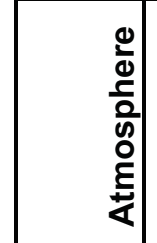 & 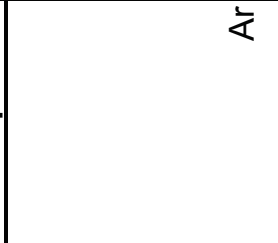 & 交 \\
\hline 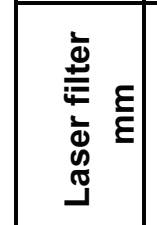 & $\stackrel{\circ}{\circ}$ & 음 \\
\hline 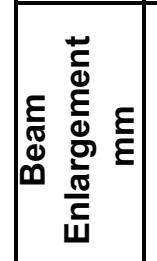 & 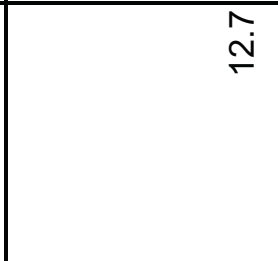 & 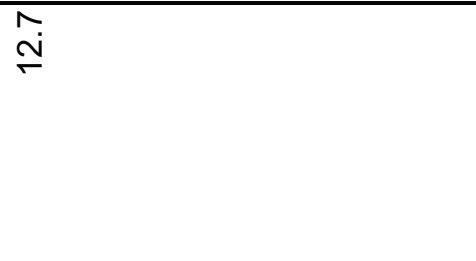 \\
\hline 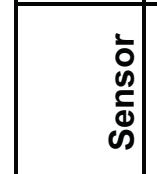 & $\begin{array}{l}\text { की } \\
\text { की }\end{array}$ & $\begin{array}{l}\text { की } \\
\text { की }\end{array}$ \\
\hline 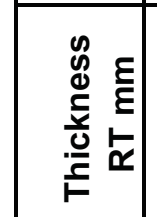 & $\begin{array}{l}\hat{N} \\
\text { ల్ }\end{array}$ & 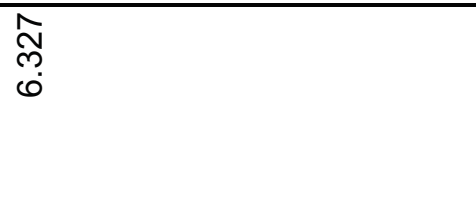 \\
\hline 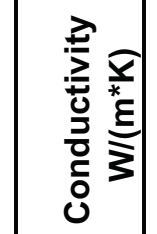 & & \\
\hline 造 & 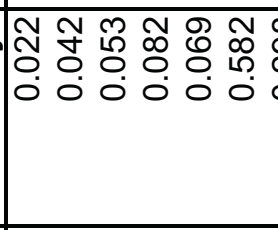 & 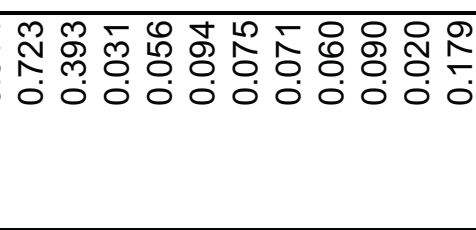 \\
\hline 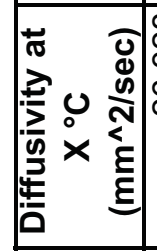 & 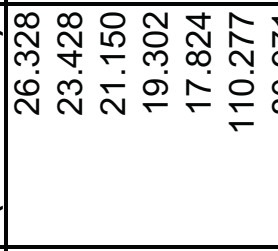 & 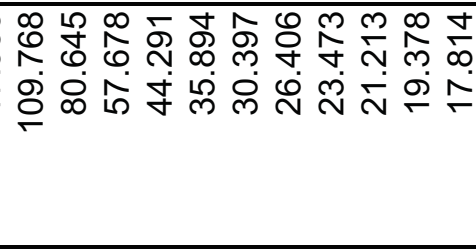 \\
\hline 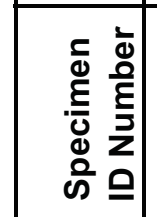 & $\begin{array}{l}\text { "ू } \\
\tilde{N}\end{array}$ & $\begin{array}{l}\dot{8} \\
\mathbb{N}\end{array}$ \\
\hline
\end{tabular}




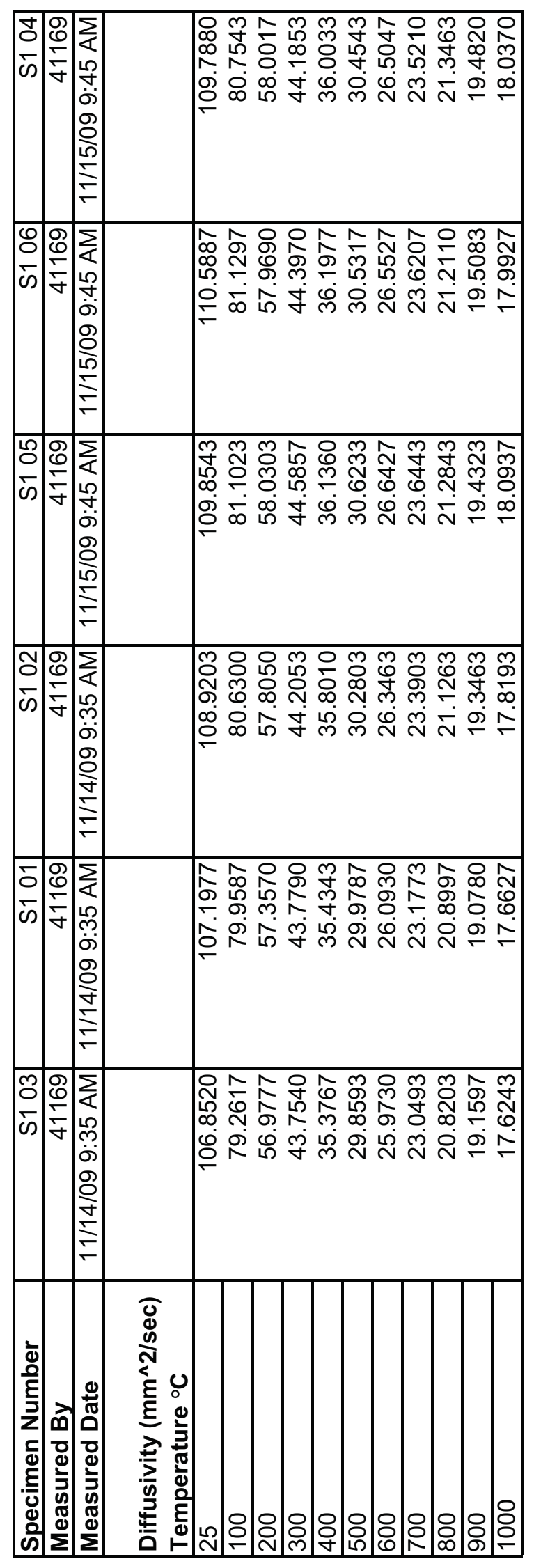




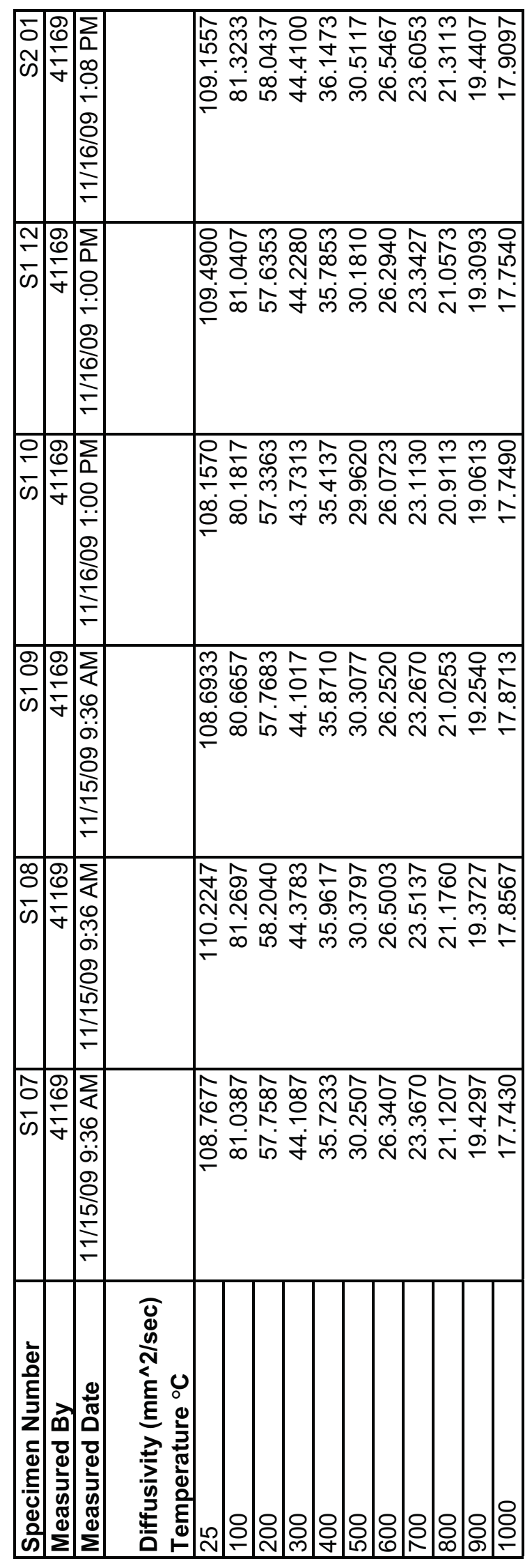




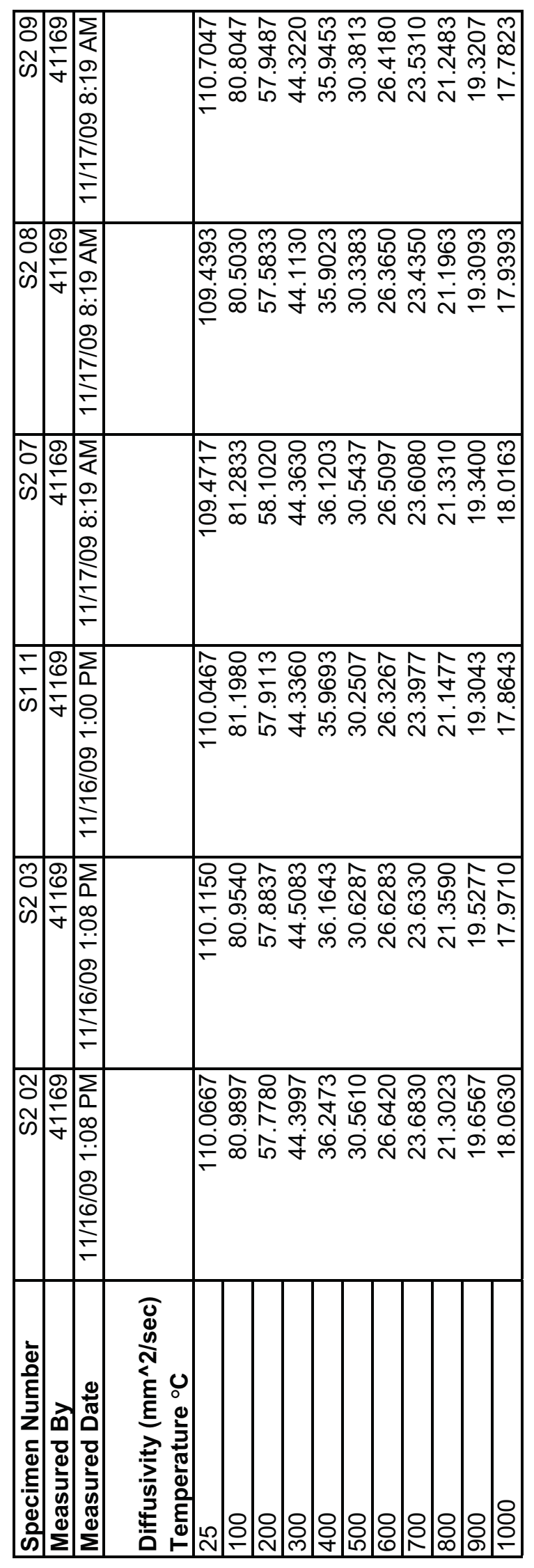




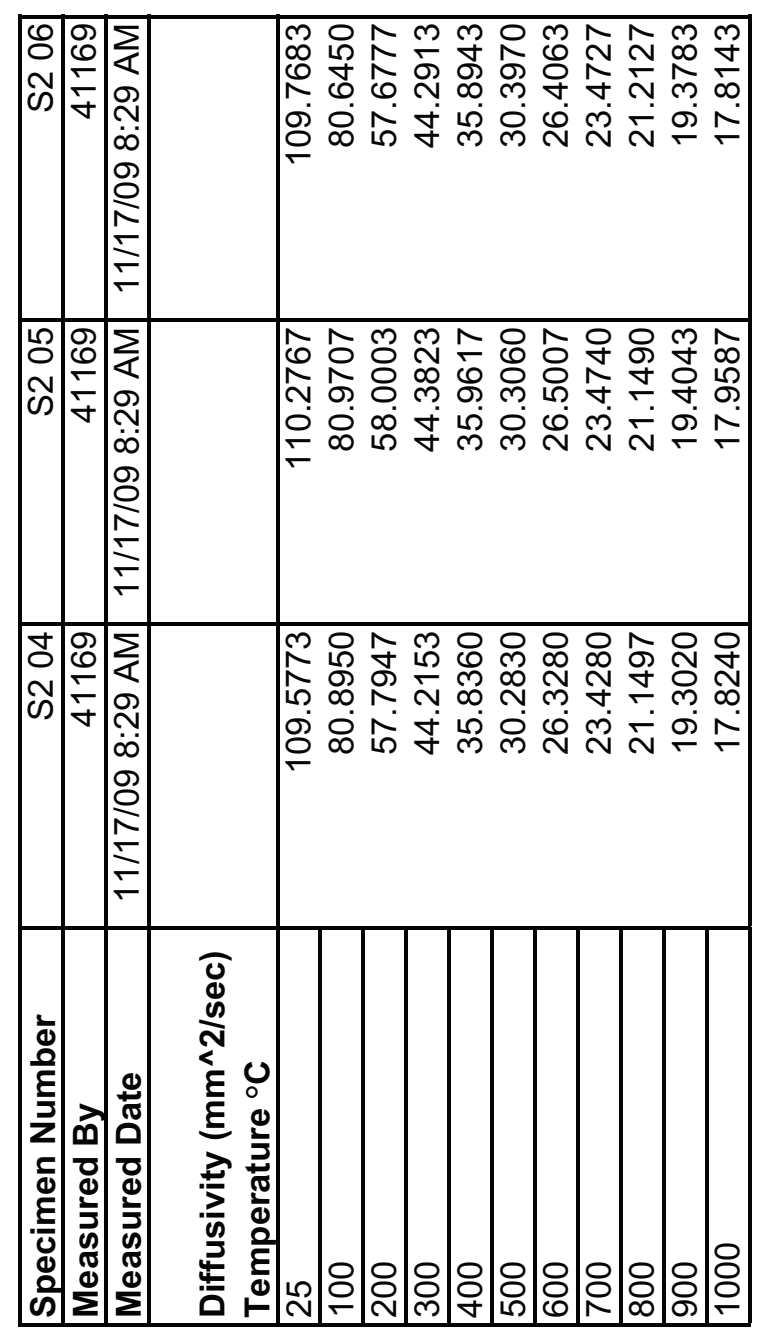




\begin{tabular}{|c|c|}
\hline 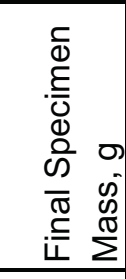 & 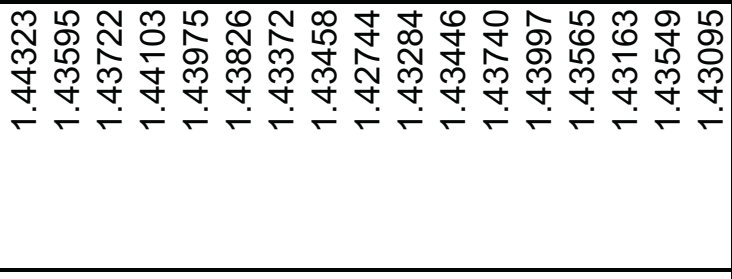 \\
\hline 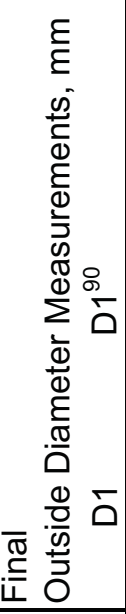 & 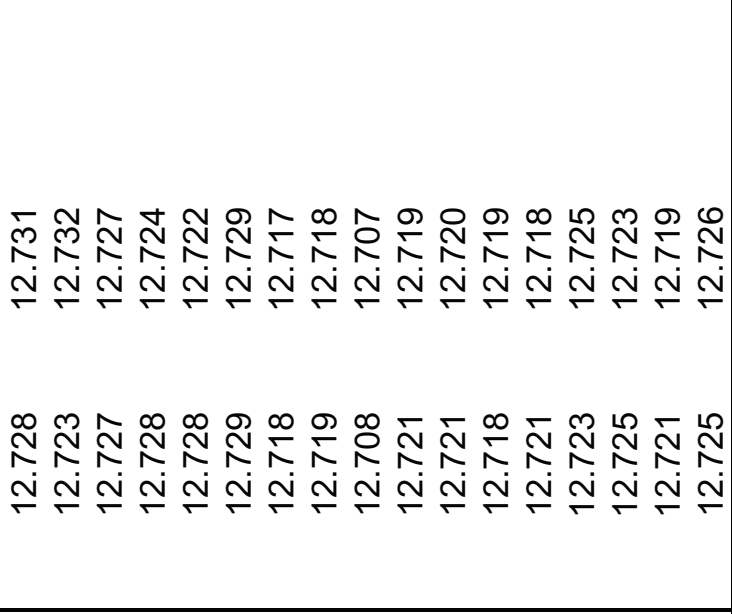 \\
\hline 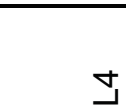 & 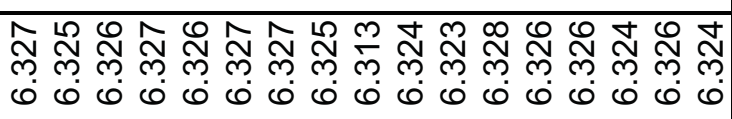 \\
\hline$\varepsilon \stackrel{m}{3}$ & 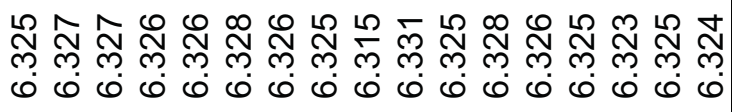 \\
\hline 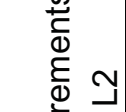 & 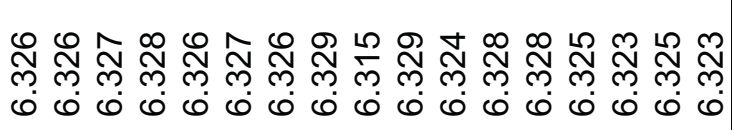 \\
\hline 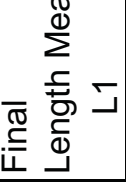 & 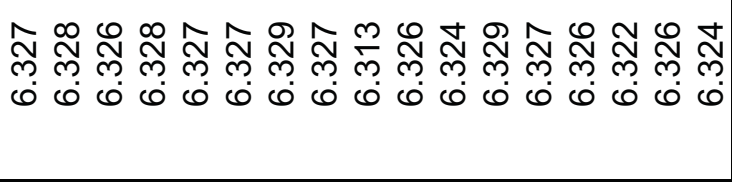 \\
\hline 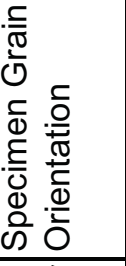 & \\
\hline 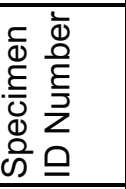 & 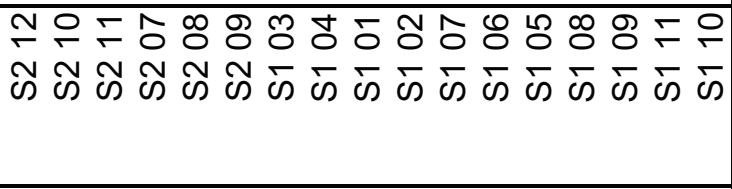 \\
\hline 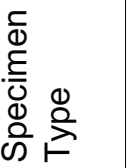 & \\
\hline
\end{tabular}




\begin{tabular}{|c|c|}
\hline 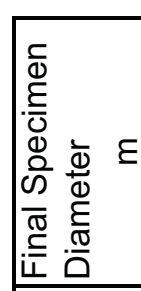 & 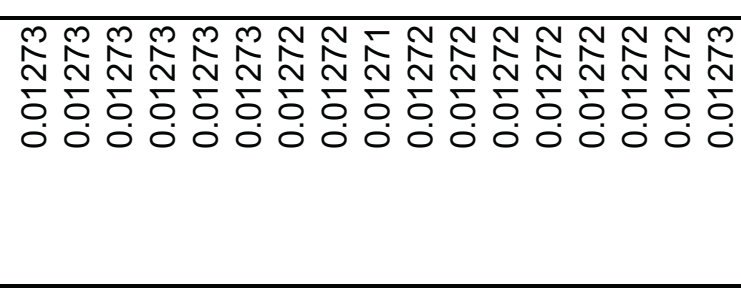 \\
\hline$\underbrace{\varepsilon}$ & 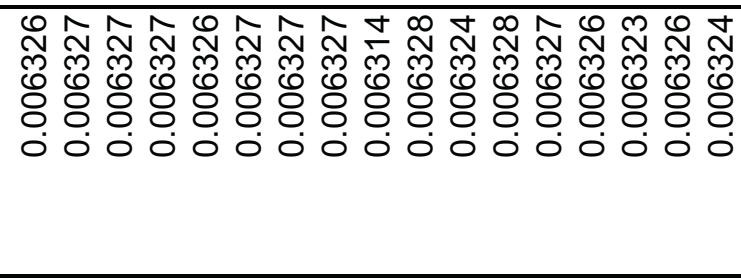 \\
\hline 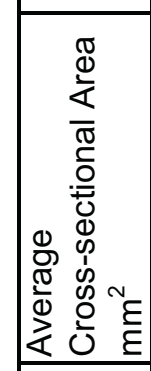 & 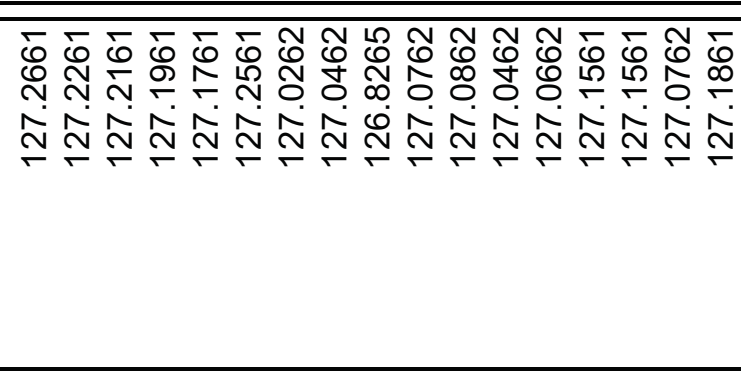 \\
\hline 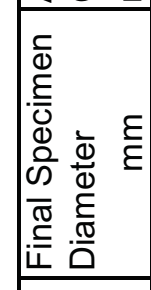 & 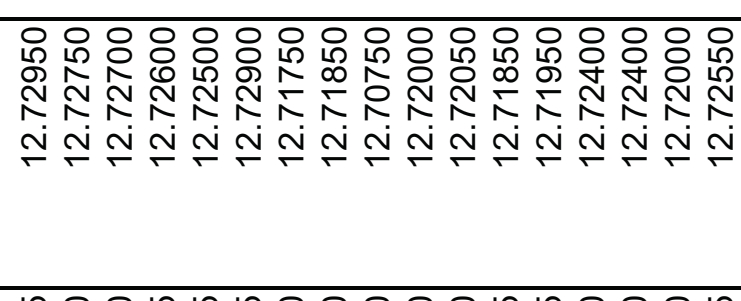 \\
\hline ह & 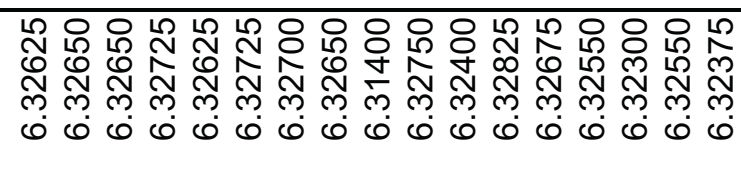 \\
\hline 总 & 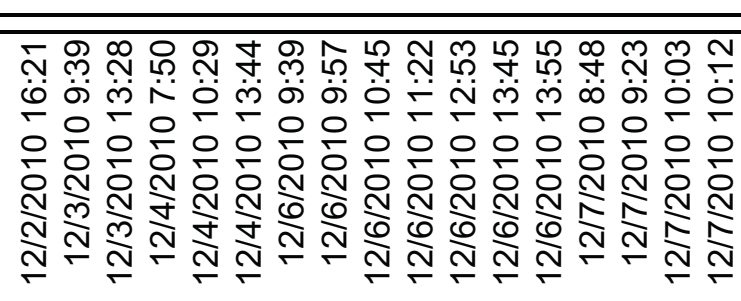 \\
\hline 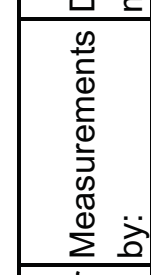 & 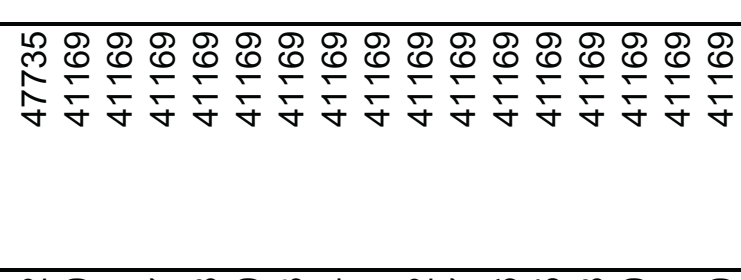 \\
\hline 焉离 & 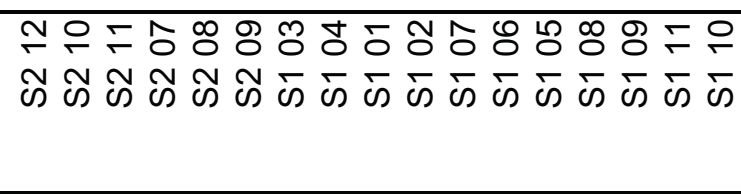 \\
\hline
\end{tabular}




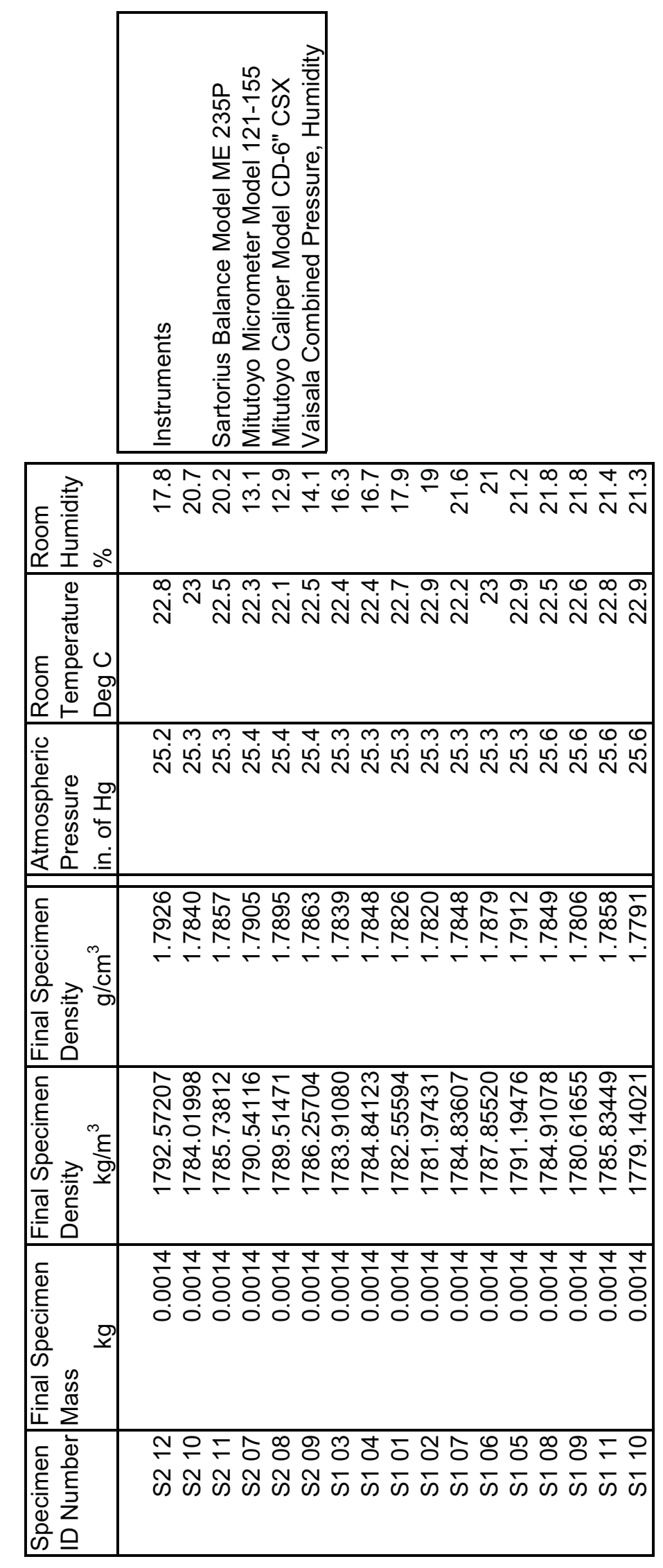




$\begin{array}{ll}\text { Graphite Grade: } & \text { NBG-17 } \\ \text { Graphite Manufacturer: } & \text { SGL Carbon Company } \\ \text { Forming Process: } & \text { Vibration molded } \\ \text { Coke Particle Size: } & \text { Medium grain } \\ \text { Coke Type: } & \text { Pitch coke filler, pitch binder } \\ \text { ASTM Class: } & \text { MNHP } \\ \text { Specimen Geometry: } & \text { Cylinder }\end{array}$

Specimen ID \#'s:

AP7 01

AP7 02

AP7 03

AP7 04

AP7 05

AP7 06

AP7 07

AP7 08

AP7 09

AP7 10

AL14 01

AL14 02

AL14 03

AL14 04

AL14 05

AL14 06

AL14 07

AL14 08

AL14 09

AL14 10

AW17 01

AW17 02

AW17 03

AW17 04

AW17 05

AW17 06

AW17 07

AW17 08

AW17 09

AW17 10 


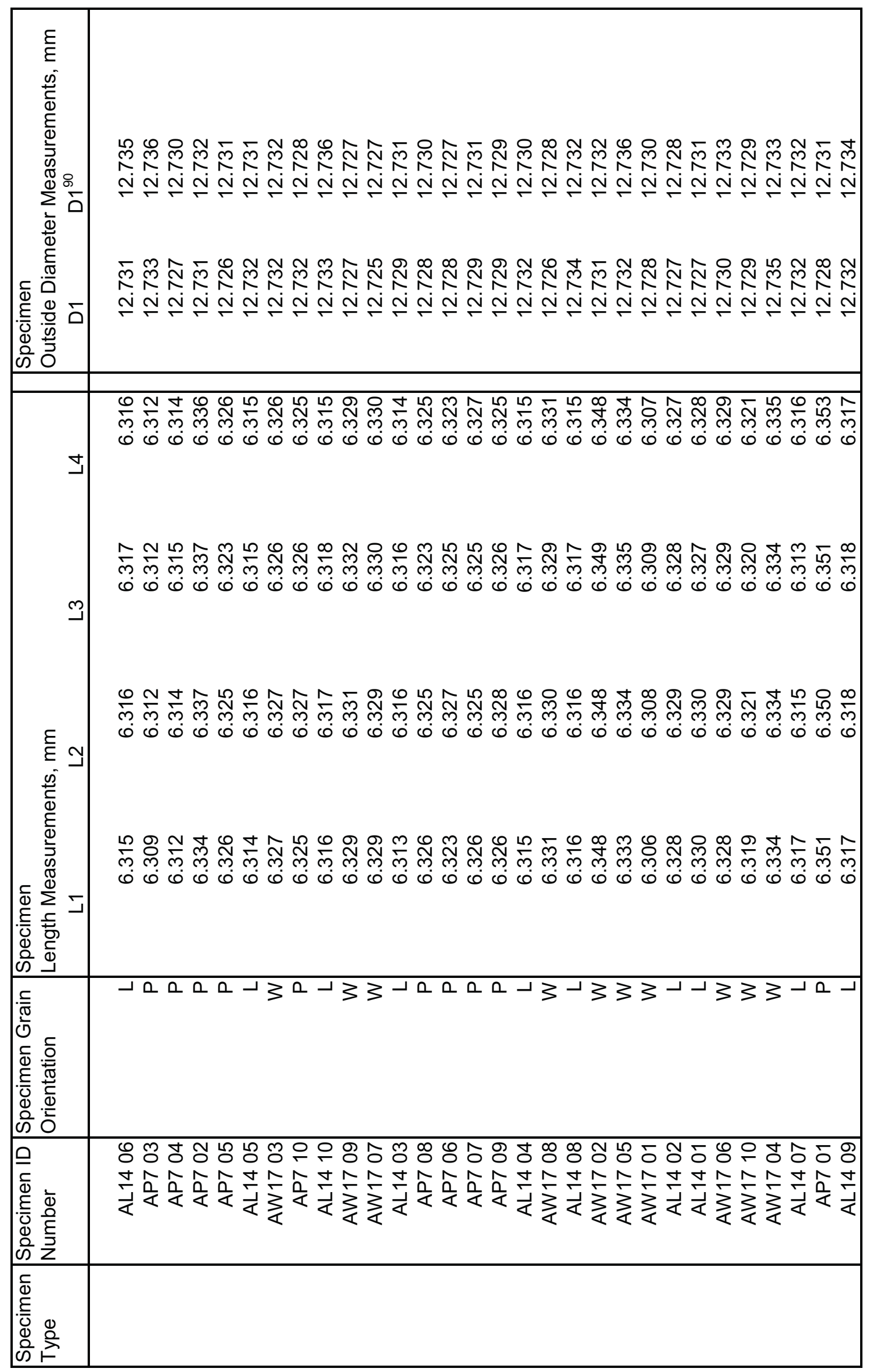




\begin{tabular}{|c|c|}
\hline 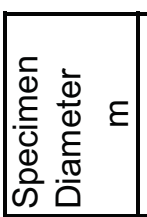 & 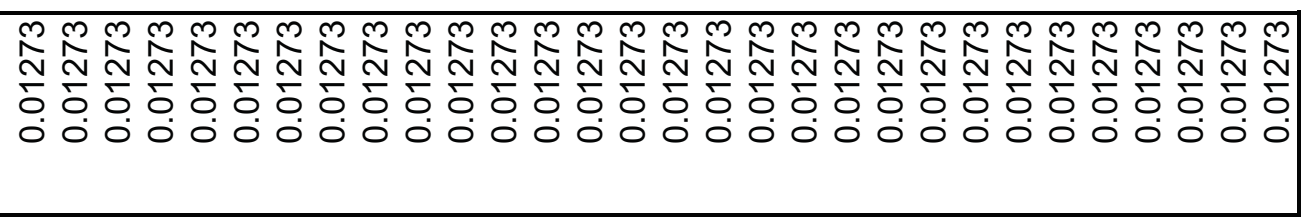 \\
\hline 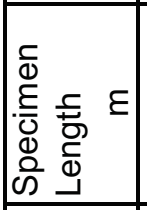 & 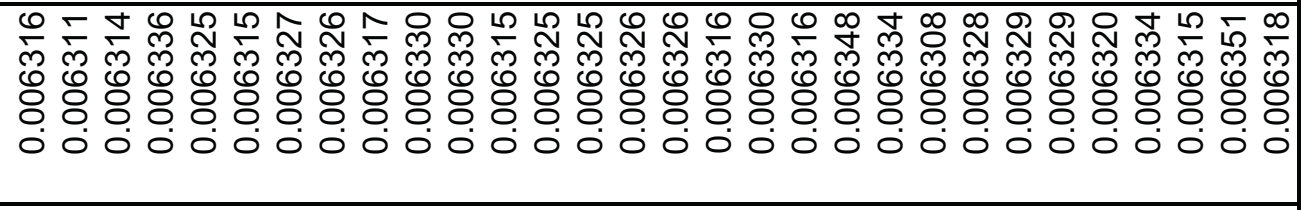 \\
\hline 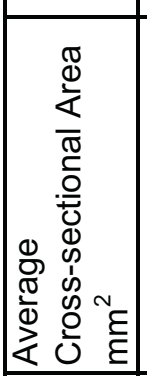 & 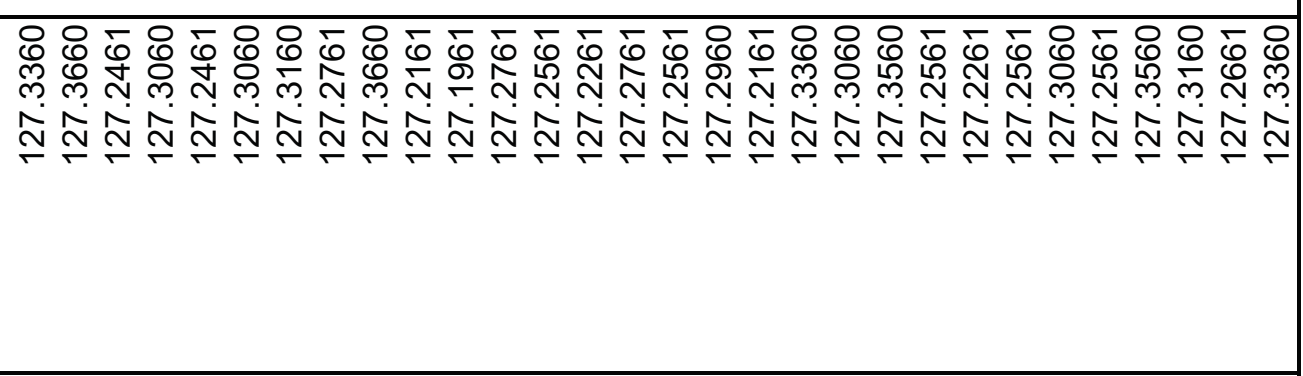 \\
\hline 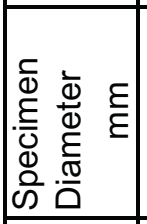 & 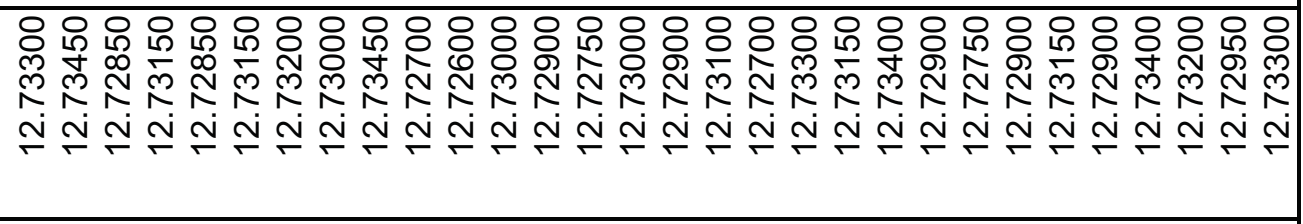 \\
\hline 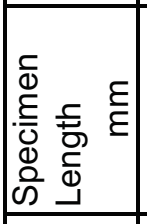 & 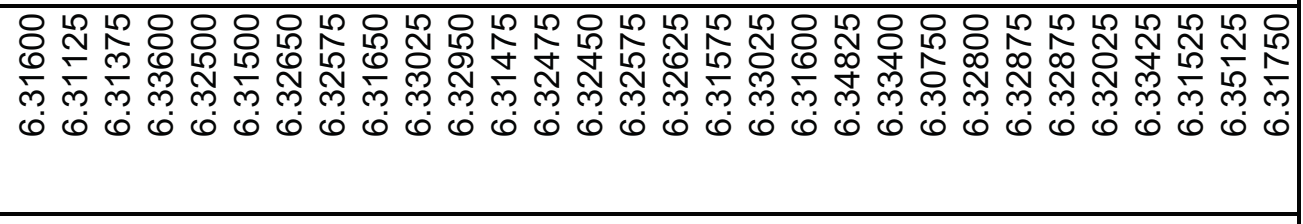 \\
\hline 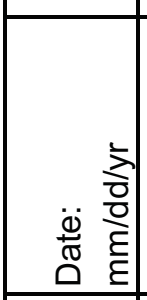 & 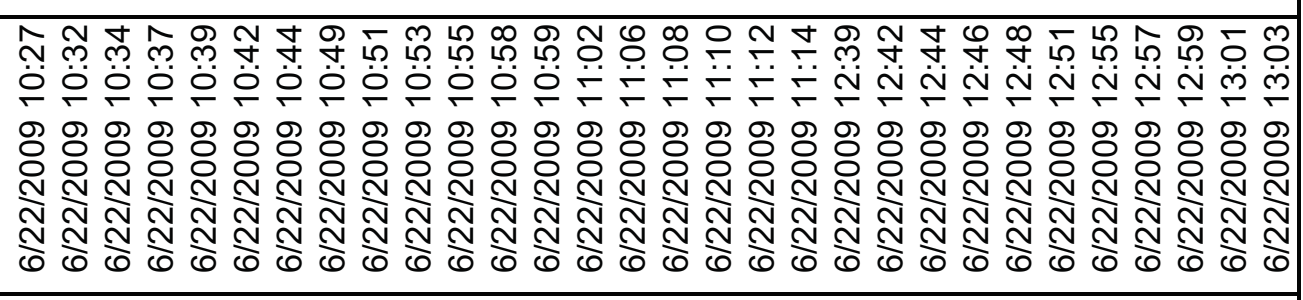 \\
\hline$\sum^{\mathbb{N}}$ & 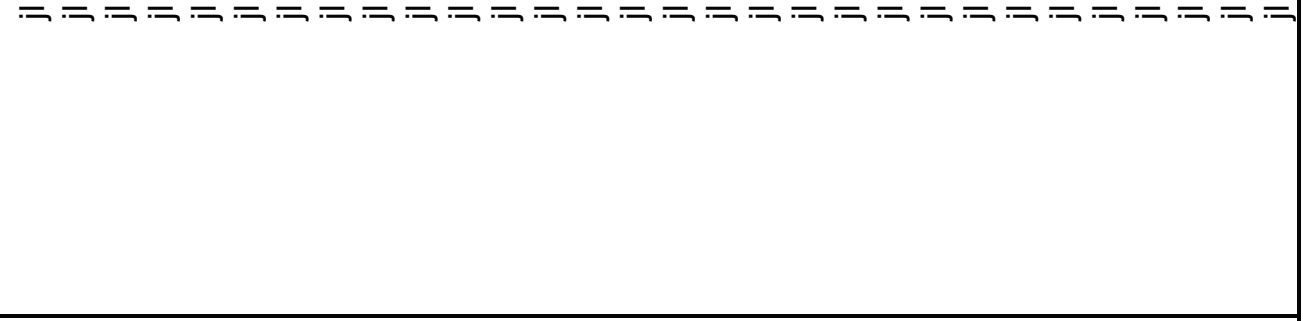 \\
\hline 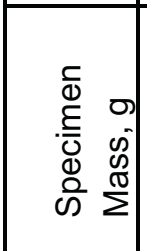 & 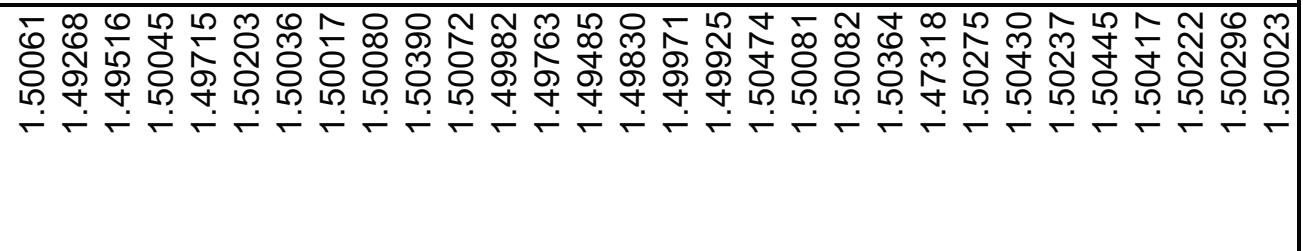 \\
\hline 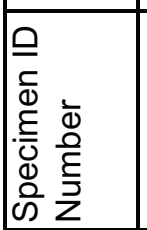 & 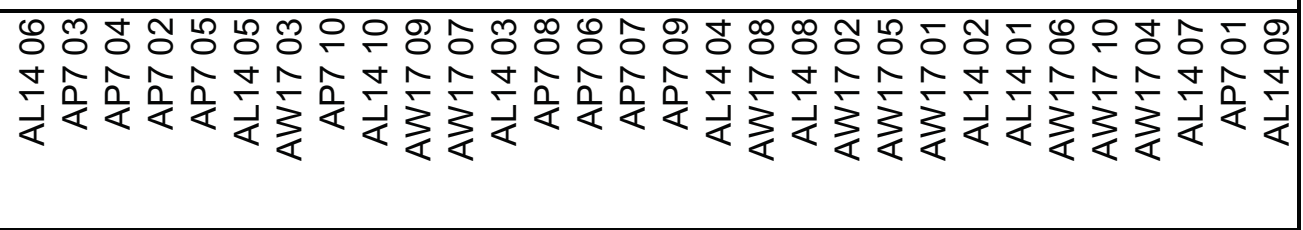 \\
\hline
\end{tabular}




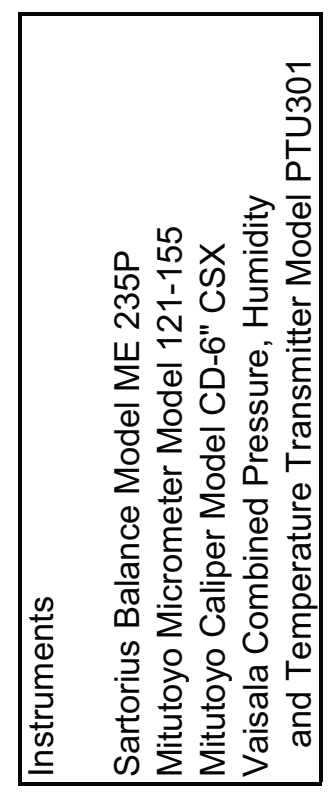

\begin{tabular}{|c|c|}
\hline 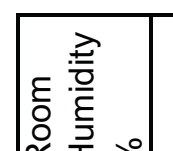 & 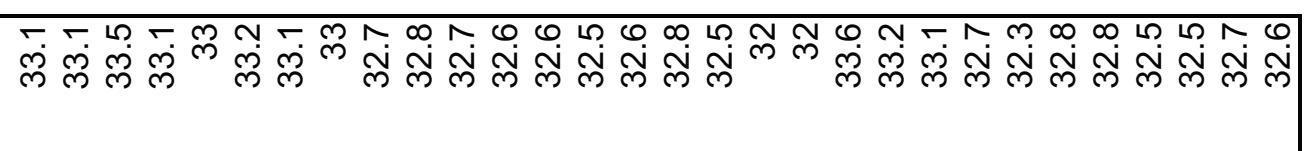 \\
\hline 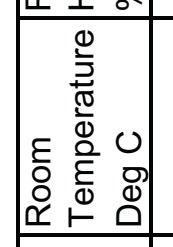 & 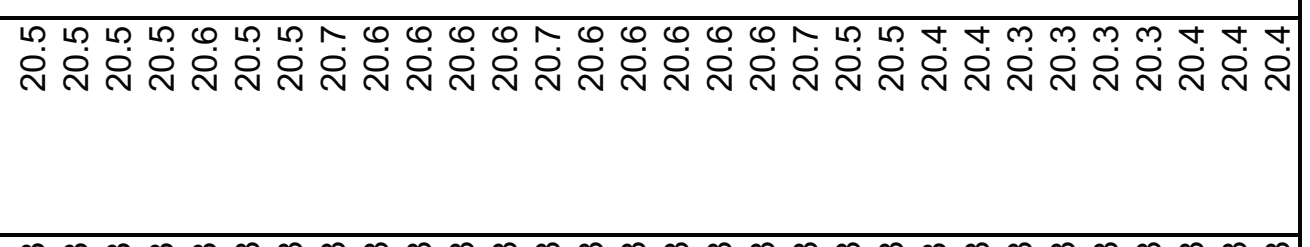 \\
\hline 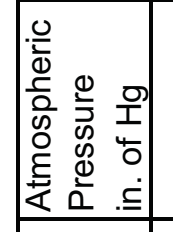 & 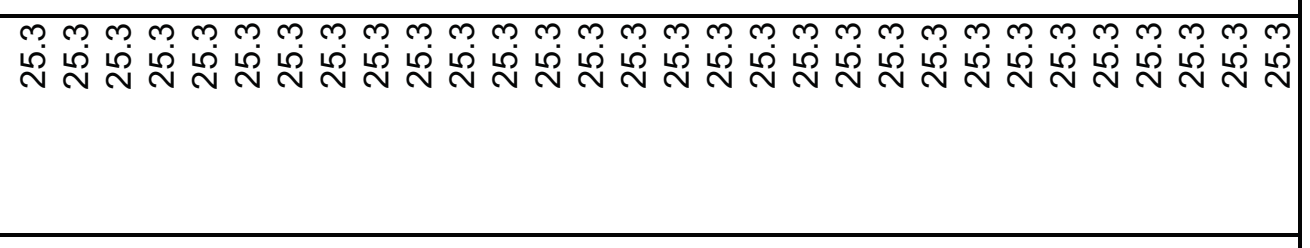 \\
\hline 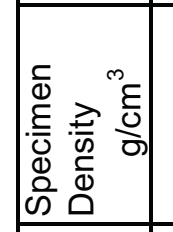 & 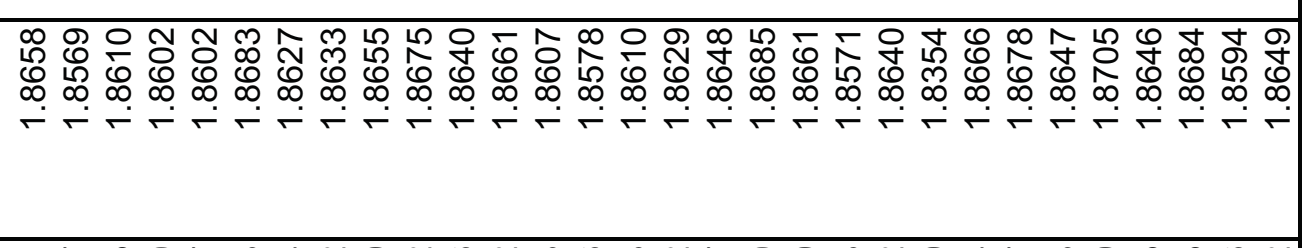 \\
\hline 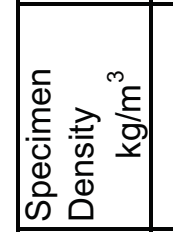 & 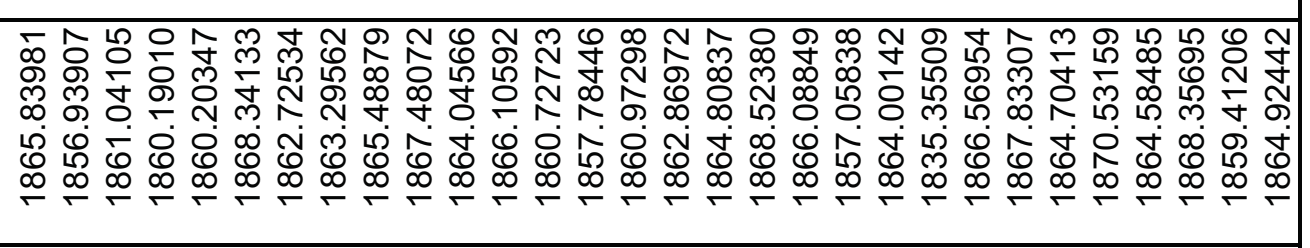 \\
\hline 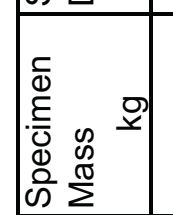 & 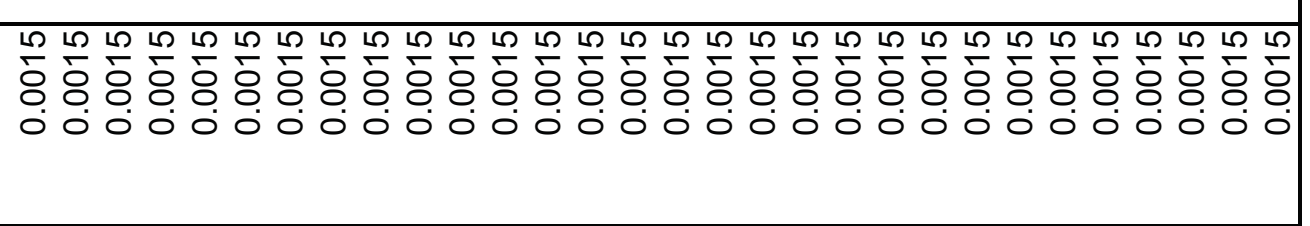 \\
\hline 焉 & 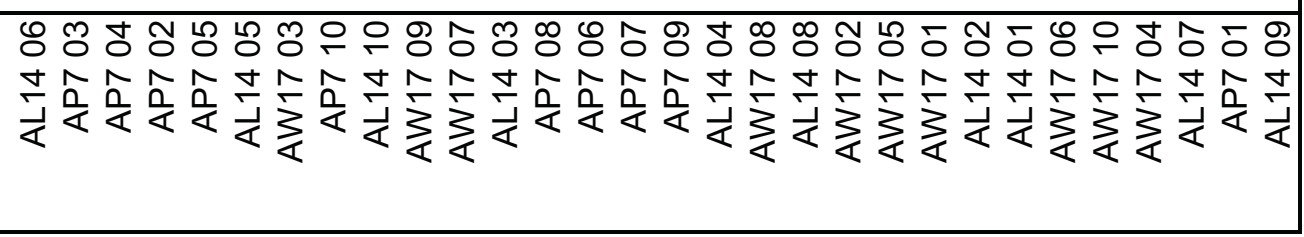 \\
\hline
\end{tabular}




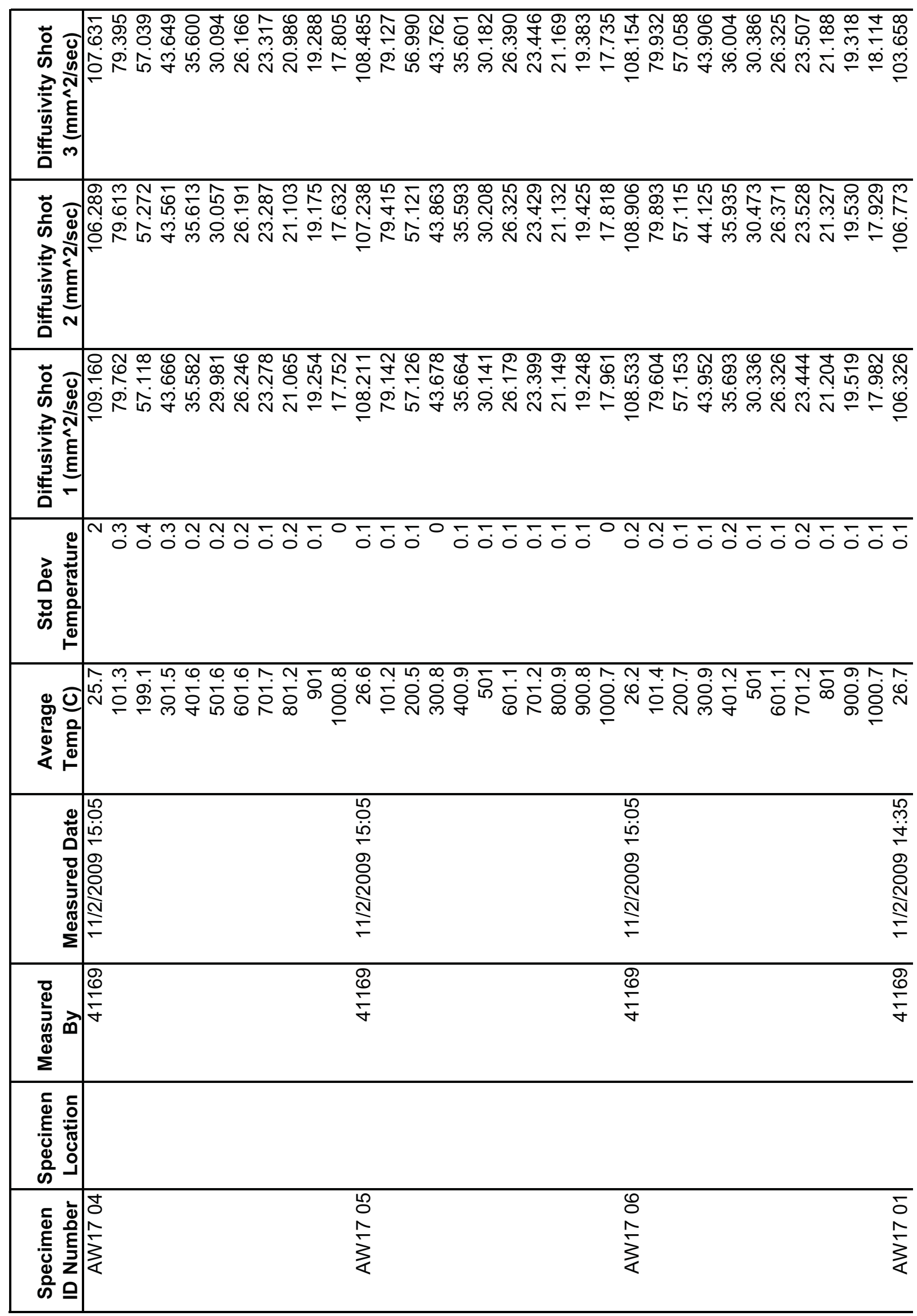




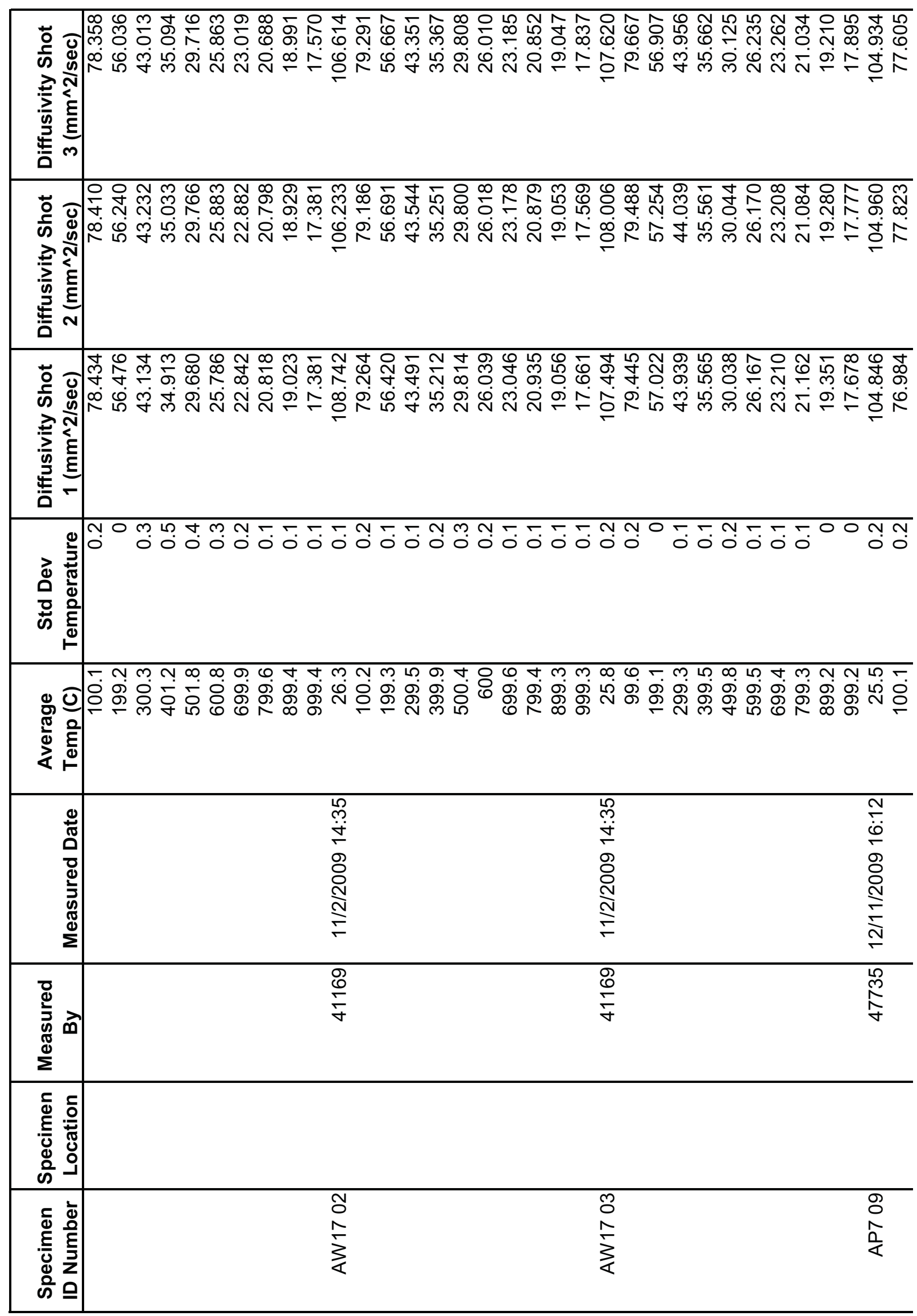




\begin{tabular}{|c|c|}
\hline 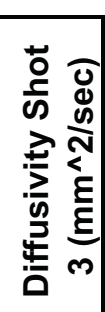 & 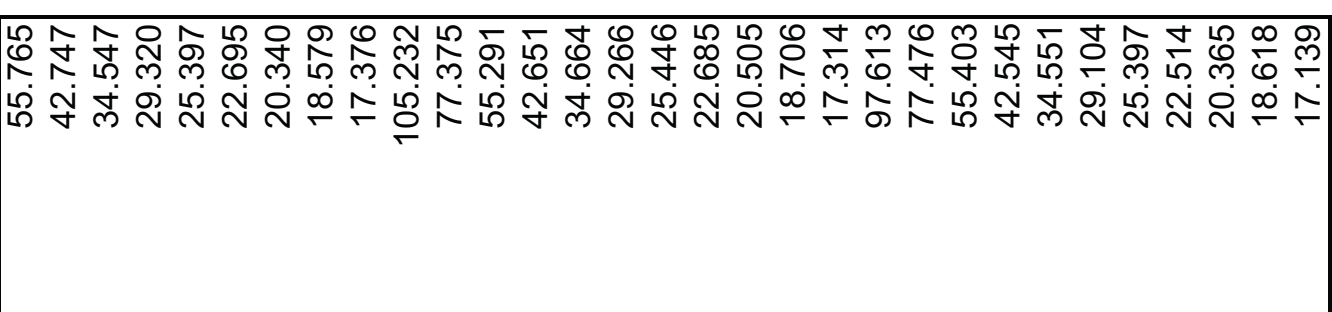 \\
\hline 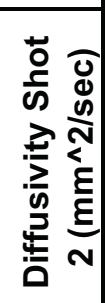 & 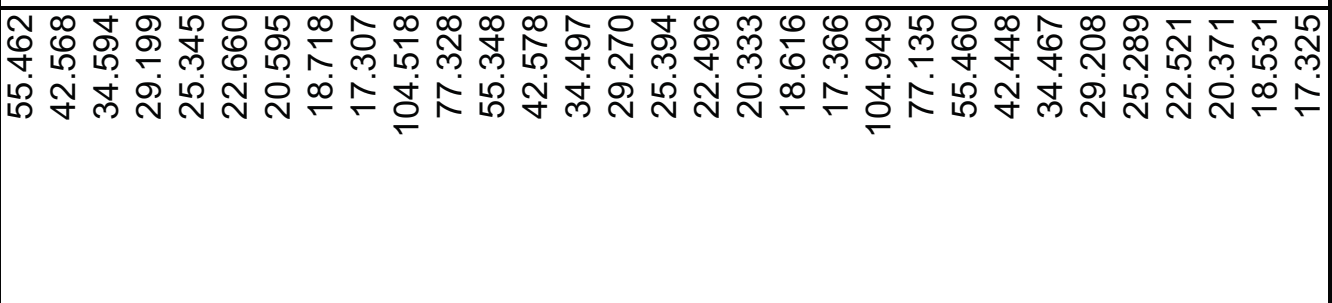 \\
\hline 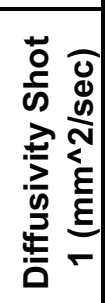 & 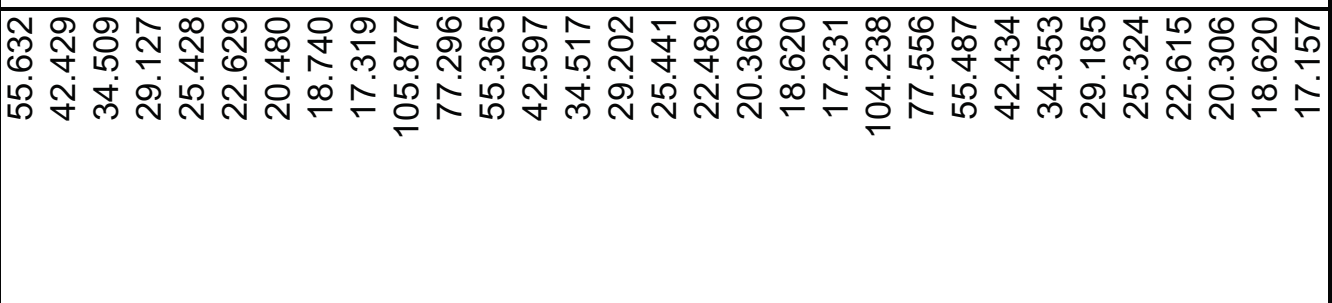 \\
\hline 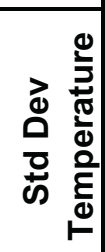 & O \\
\hline 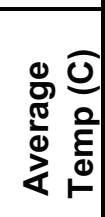 & 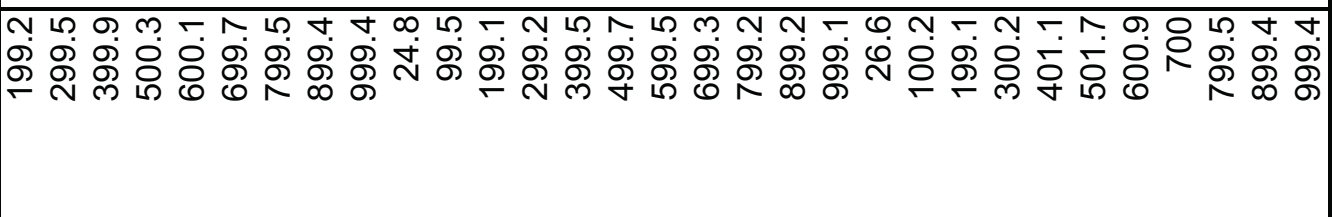 \\
\hline 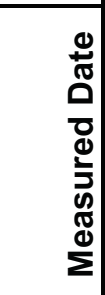 & 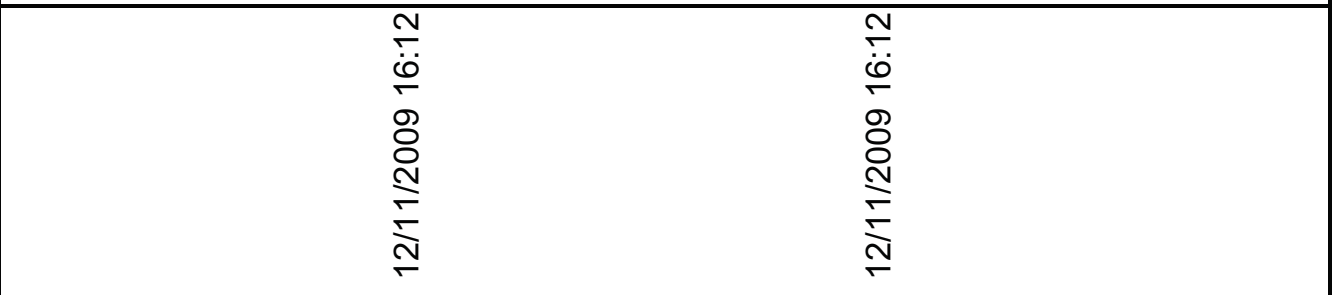 \\
\hline 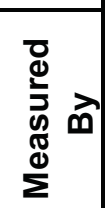 & $\begin{array}{l}\stackrel{L}{\rho} \\
\stackrel{f}{f}\end{array}$ \\
\hline 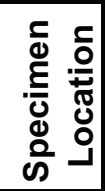 & \\
\hline 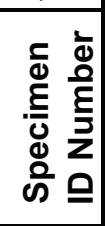 & $\begin{array}{l} \\
\\
\hat{n} \\
\frac{1}{<}\end{array}$ \\
\hline
\end{tabular}




\begin{tabular}{|c|c|c|c|c|}
\hline \begin{tabular}{|c|}
$\sum$ \\
$\infty$ \\
\\
\end{tabular} & 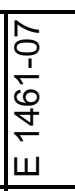 & $\begin{array}{l} \\
\\
1 \\
\dot{0} \\
\dot{+} \\
w \\
\end{array}$ & 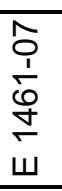 & 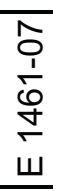 \\
\hline 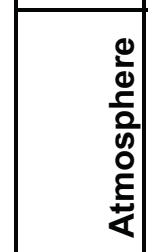 & & $\overline{⿱ 亠 乂}$ & $\overline{\dot{\alpha}}$ & $\overline{\frac{1}{4}}$ \\
\hline 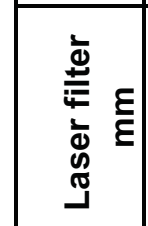 & & 음 & 음 & 음 \\
\hline 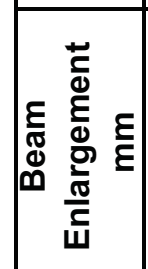 & $\stackrel{\hat{N}}{\stackrel{N}{ }}$ & 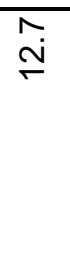 & 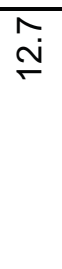 & $\overline{\stackrel{N}{N}}$ \\
\hline \begin{tabular}{l|} 
\\
$\vdots$ \\
0 \\
0 \\
$\Phi$ \\
$\infty$
\end{tabular} & & $\begin{array}{l}\text { की } \\
\text { की }\end{array}$ & $\begin{array}{l}\text { की } \\
\text { की }\end{array}$ & $\begin{array}{l}\text { Oे } \\
\text { Dी }\end{array}$ \\
\hline 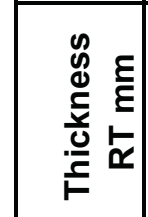 & & 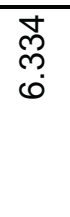 & 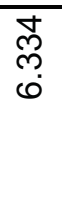 & 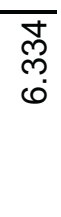 \\
\hline 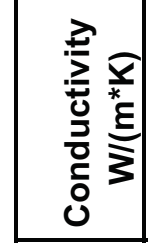 & & & & \\
\hline 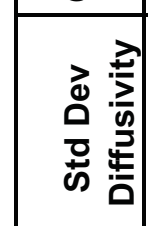 & & $\begin{array}{l}200 \\
0 \\
0 \\
0\end{array}$ & $\tilde{m}$ & b \\
\hline 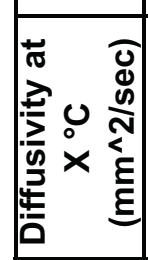 & $\begin{array}{lll}m & 0 \\
0 & 0 \\
0 & 1 \\
0 & 0 \\
0 & 0 \\
0 & 1 \\
0 & \end{array}$ & 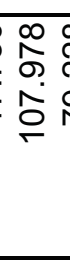 & $\begin{array}{l}\bar{\Gamma} \\
\hat{\rho} \\
0 \\
0 \\
0\end{array}$ & 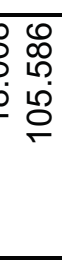 \\
\hline 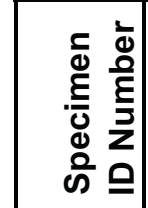 & $\mid \begin{array}{l}4 \\
0 \\
\sum \\
\sum\end{array}$ & $\begin{array}{l}\text { L } \\
\sum_{<}^{\circ}\end{array}$ & $\begin{array}{l}8 \\
8 \\
\hat{N} \\
\sum_{\varangle}\end{array}$ & \begin{tabular}{l}
5 \\
\multirow{2}{1}{} \\
$\sum_{\alpha}^{2}$
\end{tabular} \\
\hline
\end{tabular}




\begin{tabular}{|c|c|c|c|}
\hline 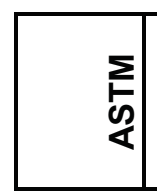 & $\begin{array}{l}\hat{o} \\
\frac{1}{0} \\
\dot{0} \\
\dot{5} \\
\end{array}$ & $\begin{array}{l}\hat{S} \\
\frac{1}{1} \\
\frac{0}{5} \\
\dot{w}\end{array}$ & 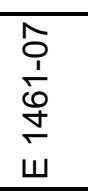 \\
\hline 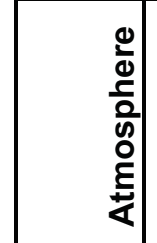 & 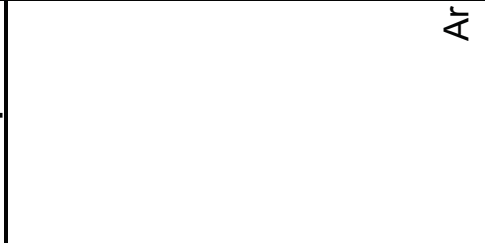 & $\frac{1}{\alpha}$ & 广 \\
\hline 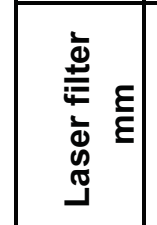 & 음 & $\stackrel{8}{\circ}$ & 음 \\
\hline 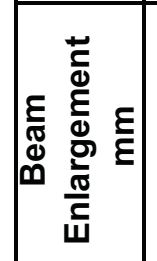 & $\stackrel{\widehat{N}}{\stackrel{N}{N}}$ & 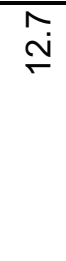 & 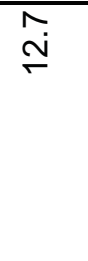 \\
\hline $\begin{array}{l}\vdots \\
\vdots \\
0 \\
\bar{d} \\
\infty\end{array}$ & $\begin{array}{l}\text { की } \\
\text { की }\end{array}$ & 吕 & $\begin{array}{l}\text { की } \\
\text { की }\end{array}$ \\
\hline 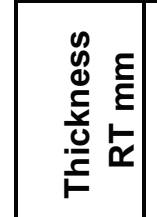 & 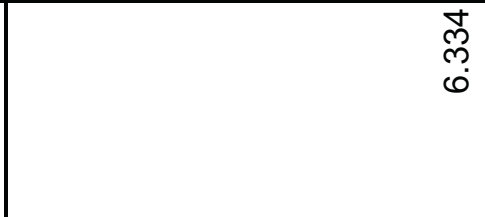 & 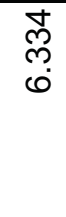 & 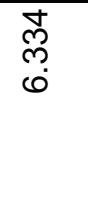 \\
\hline 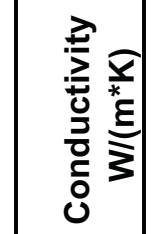 & & & \\
\hline 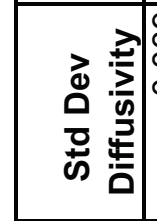 & 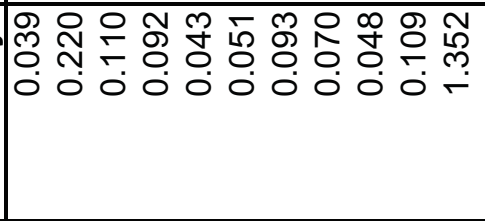 & ; & i̊ \\
\hline 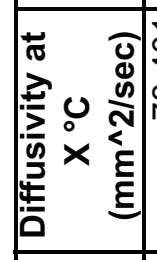 & 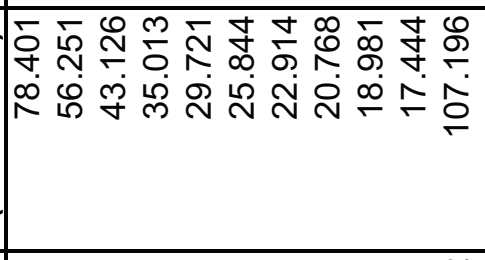 & ? & 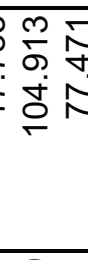 \\
\hline 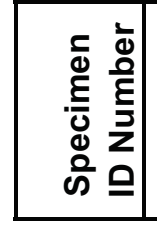 & 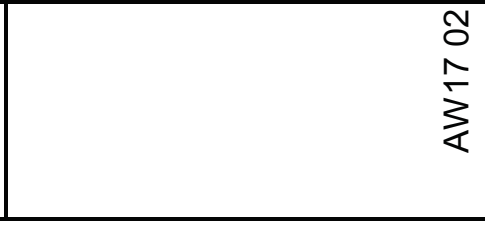 & $\begin{array}{l}0 \\
0 \\
\sum_{i}^{\circ}\end{array}$ & $\begin{array}{l}8 \\
0 \\
0 \\
\frac{1}{<}\end{array}$ \\
\hline
\end{tabular}




\begin{tabular}{|c|c|c|}
\hline $\begin{array}{l}\Sigma \\
\mathbf{E} \\
\mathbf{0}\end{array}$ & 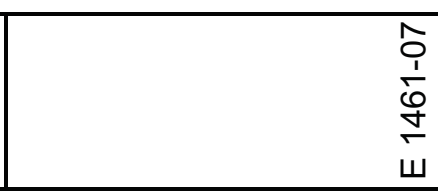 & 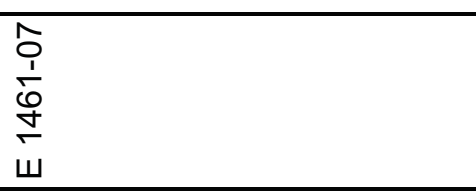 \\
\hline 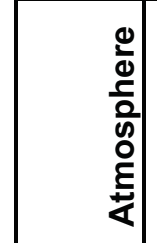 & 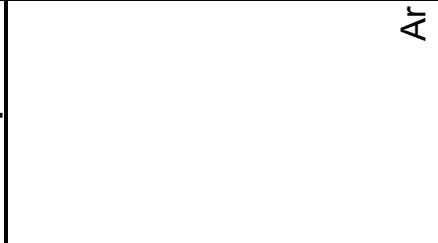 & $\frac{1}{4}$ \\
\hline 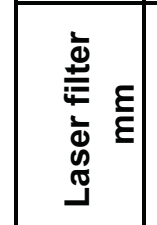 & 음 & 음 \\
\hline 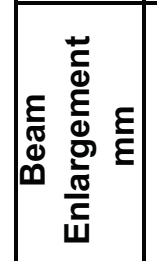 & $\stackrel{\widetilde{N}}{\stackrel{N}{N}}$ & 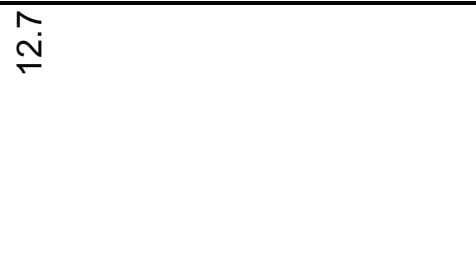 \\
\hline 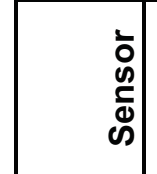 & $\begin{array}{l}\text { की } \\
\text { की }\end{array}$ & की \\
\hline 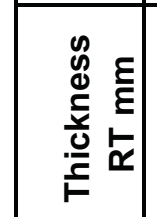 & 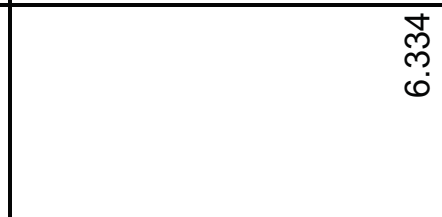 & 㥕 \\
\hline 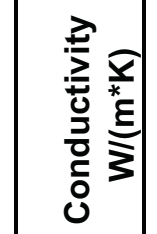 & & \\
\hline 离 & 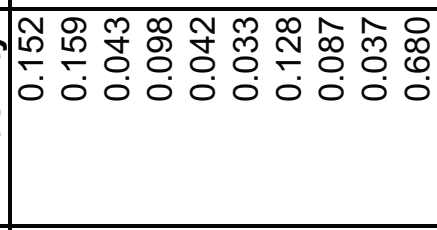 & 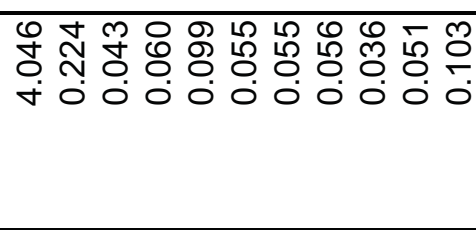 \\
\hline 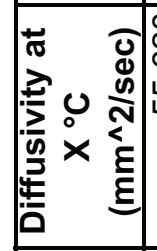 & 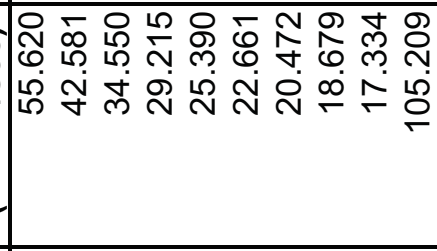 & 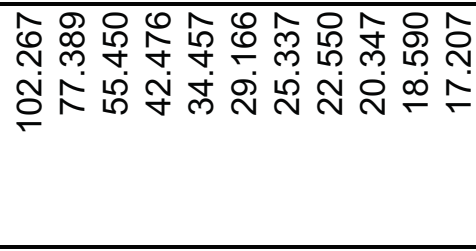 \\
\hline 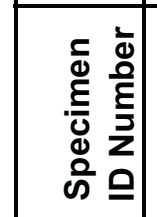 & $\begin{array}{l}\text { 운 } \\
\hat{\Lambda} \\
\frac{1}{4}\end{array}$ & $\begin{array}{l}0 \\
0 \\
\hat{\alpha} \\
\frac{0}{\alpha}\end{array}$ \\
\hline
\end{tabular}




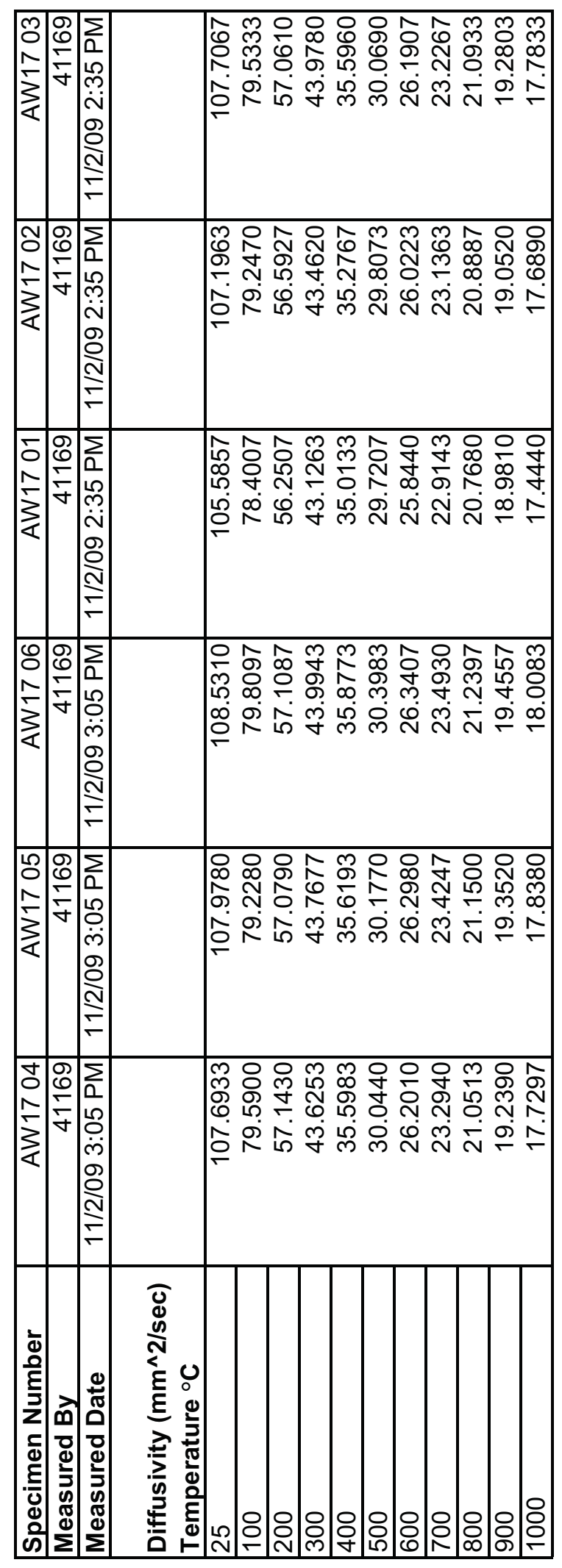




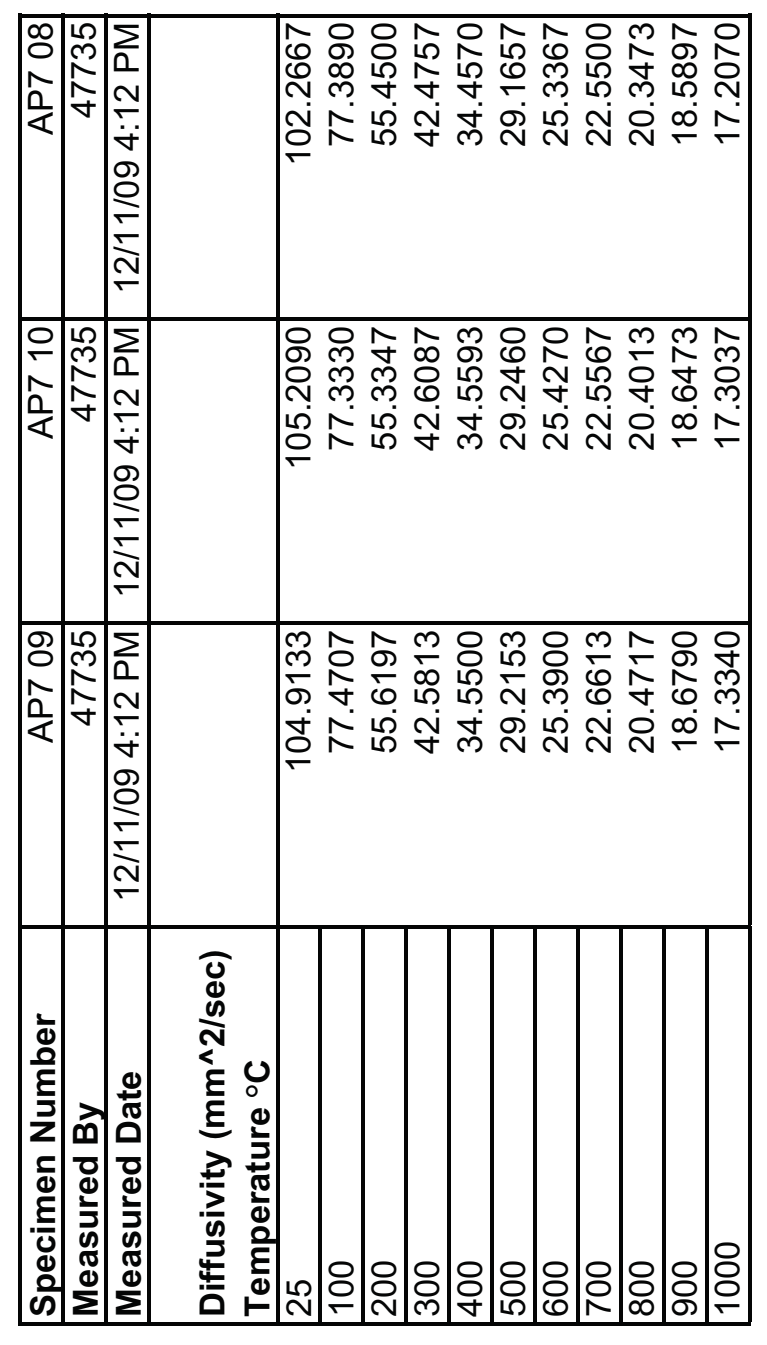

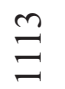




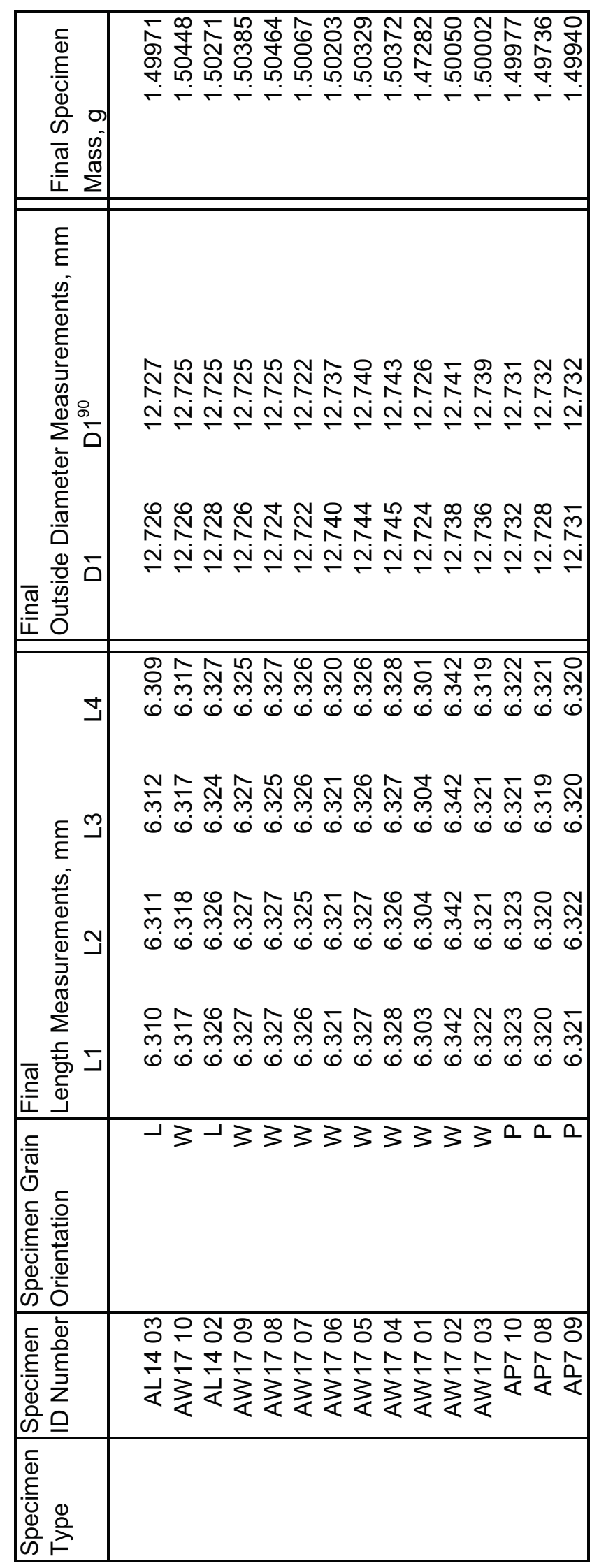




\begin{tabular}{|c|c|}
\hline 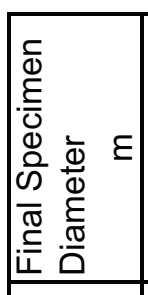 & 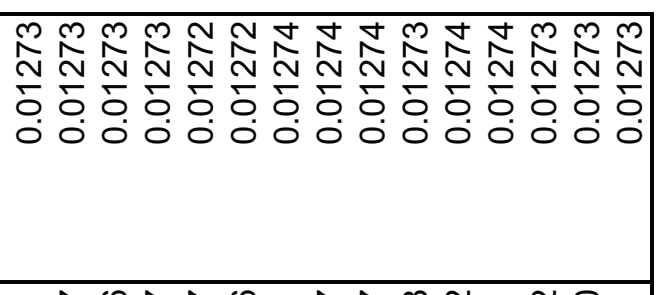 \\
\hline 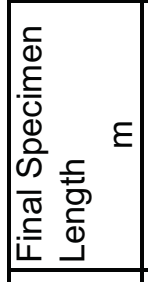 & 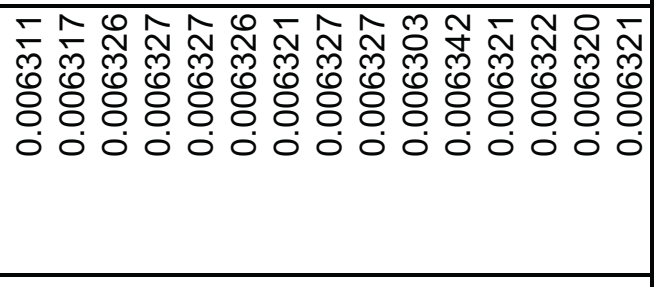 \\
\hline 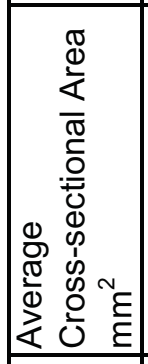 & 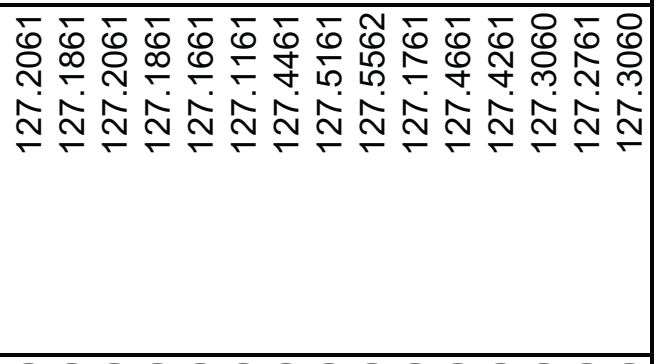 \\
\hline 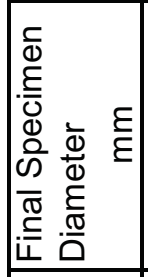 & 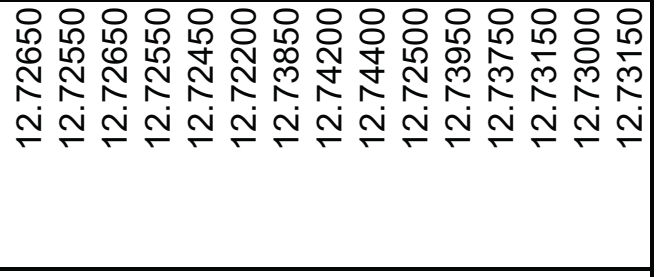 \\
\hline 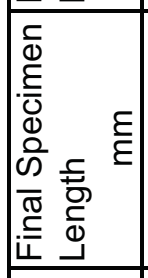 & 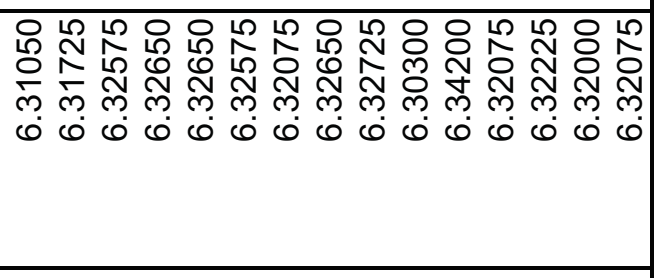 \\
\hline 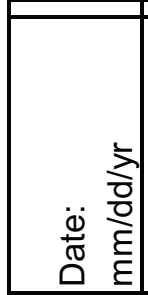 & 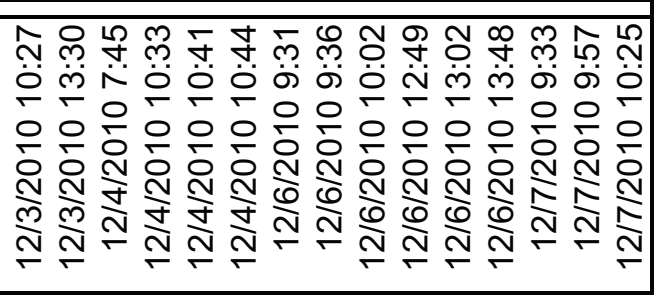 \\
\hline 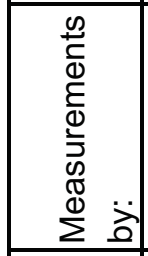 & 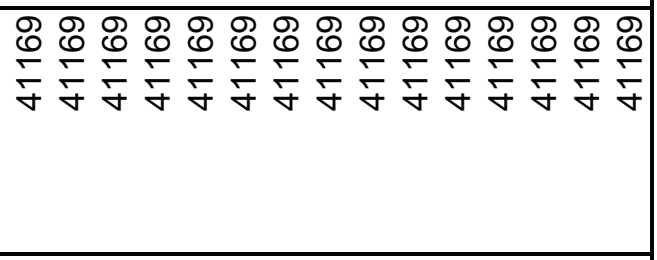 \\
\hline 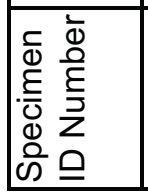 & 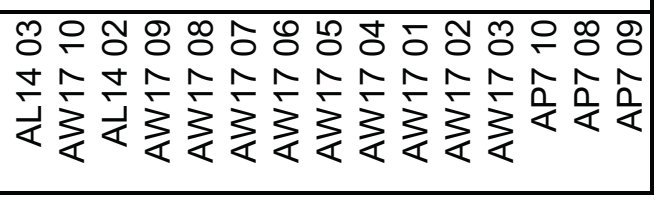 \\
\hline
\end{tabular}




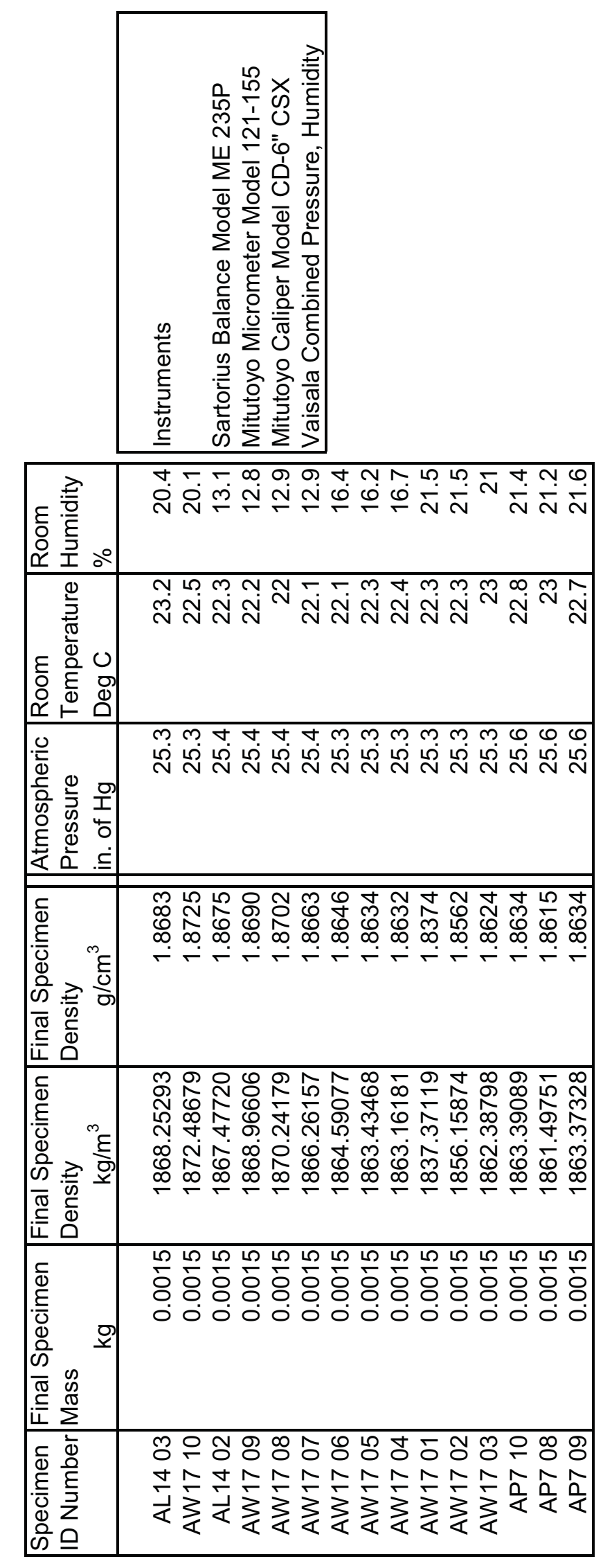




$\begin{array}{ll}\text { Graphite Grade: } & \text { NBG-18 } \\ \text { Graphite Manufacturer: } & \text { SGL Carbon Company } \\ \text { Forming Process: } & \text { Vibration molded } \\ \text { Coke Particle Size: } & \text { Medium grain } \\ \text { Coke Type: } & \text { Pitch coke filler, pitch binder } \\ \text { ASTM Class: } & \text { MNHP } \\ \text { Specimen Geometry: } & \text { Cylinder }\end{array}$

Specimen ID \#'s:

BW17 01

BW17 02

BW17 03

BW17 04

BW17 05

BW17 06

BW17 07

BW17 08

BW17 09

BW17 10

BP7 01

BP7 02

BP7 03

BP7 04

BP7 05

BP7 06

BP7 07

BP7 08

BP7 09

BP7 10

BL14-01

BL14-02

BL14-03

BL14-04

BL14-05

BL14-06

BL14-07

BL14-08

BL14-09

BL14-10

BL8-1 $\mathrm{mm}$

BL8-2mm

BL8-4mm

BL8-6mm

BL8-8mm

BL8-10mm 


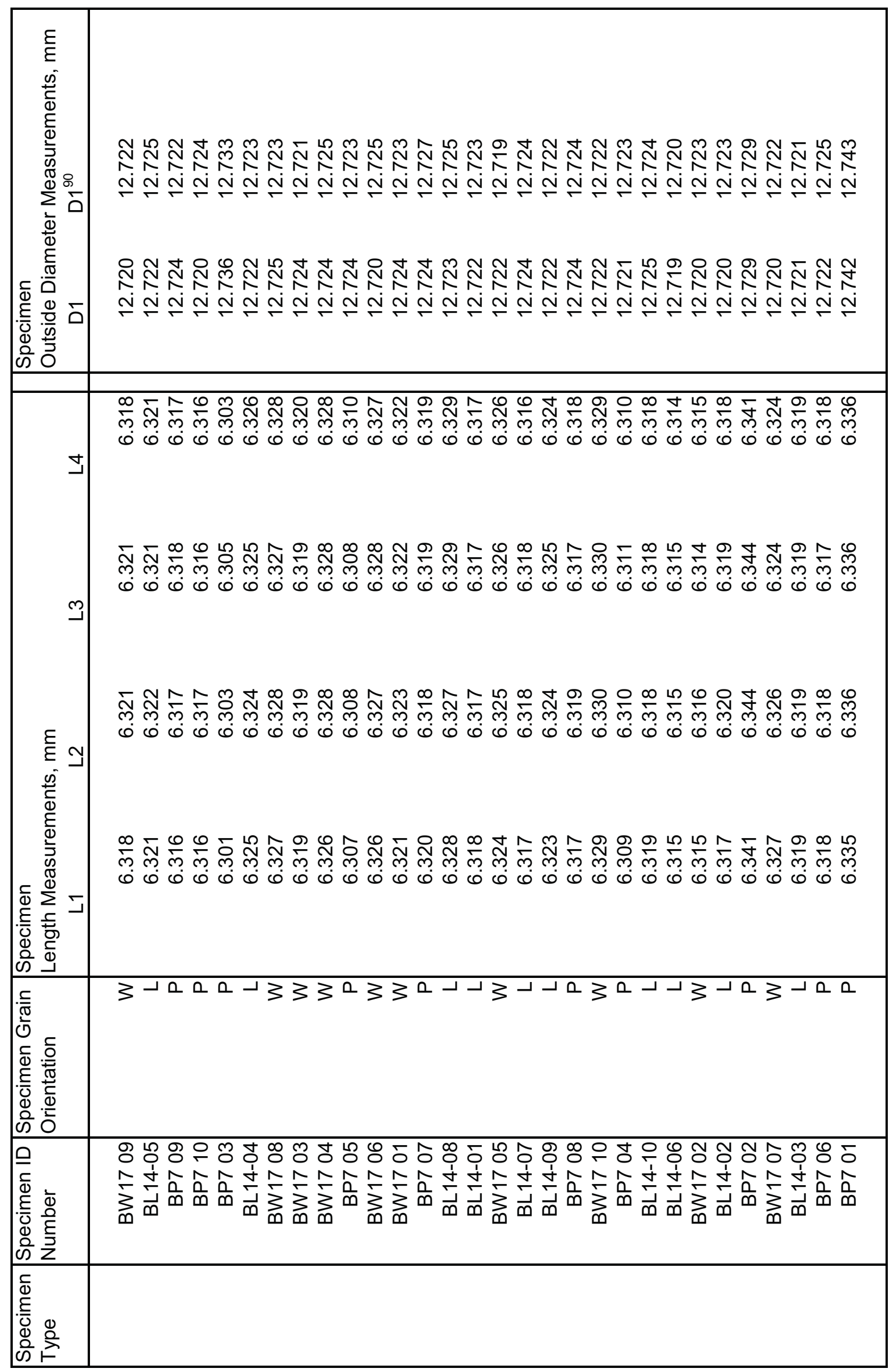




\begin{tabular}{|c|c|}
\hline 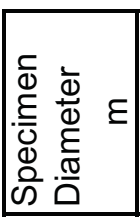 & 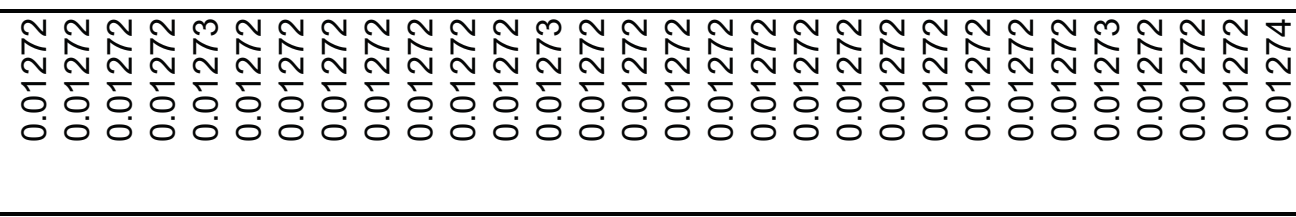 \\
\hline 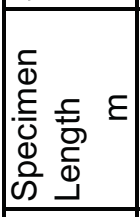 & 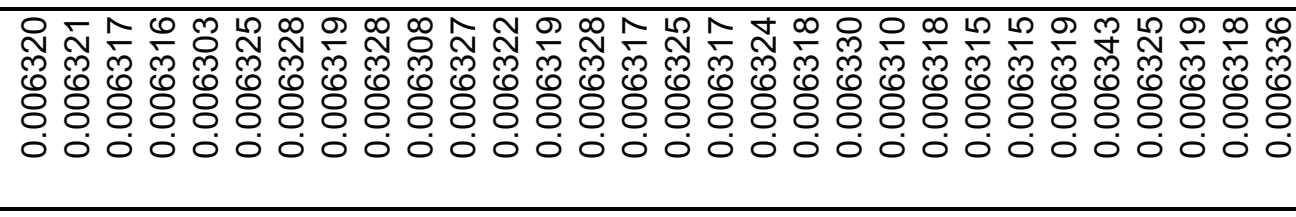 \\
\hline 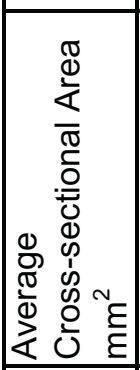 & 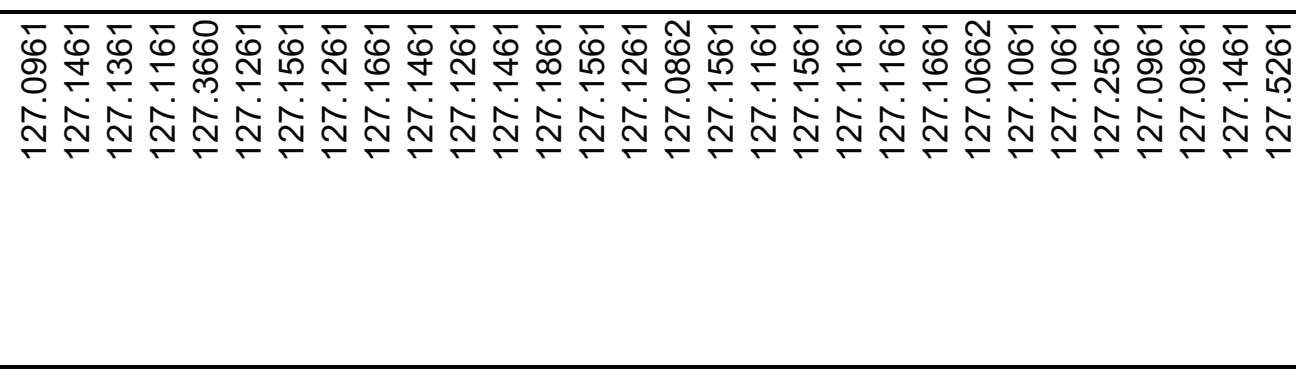 \\
\hline 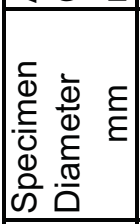 & 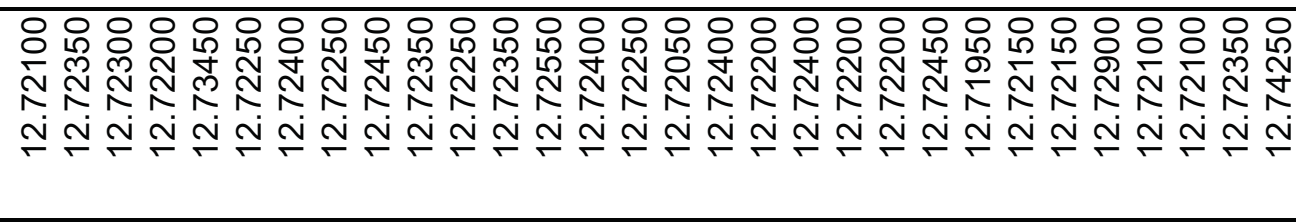 \\
\hline 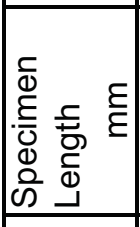 & 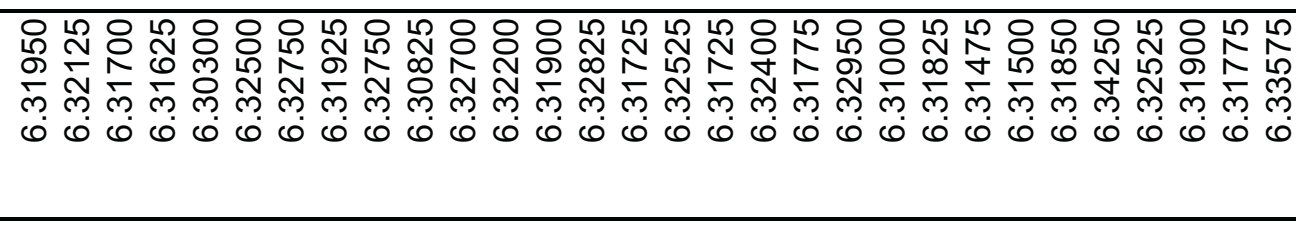 \\
\hline 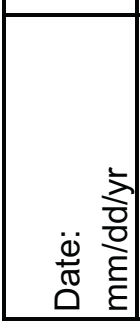 & 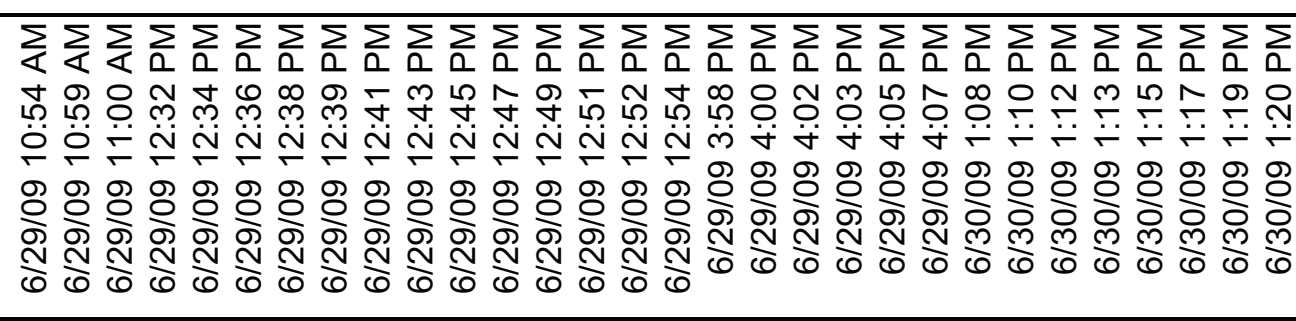 \\
\hline 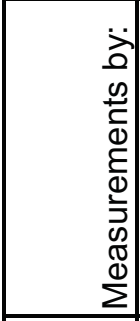 & 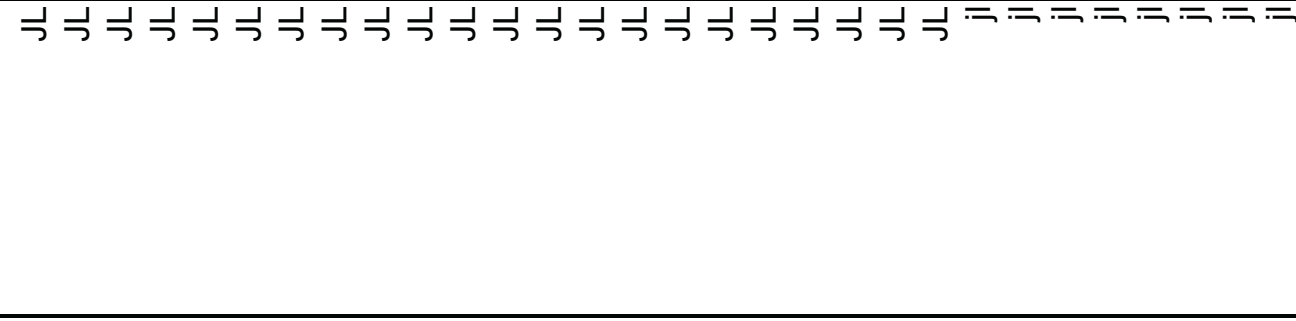 \\
\hline 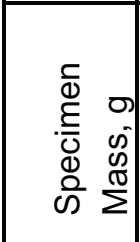 & 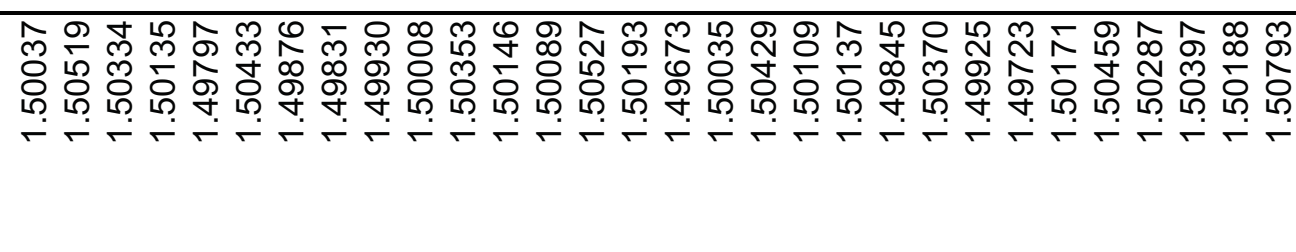 \\
\hline 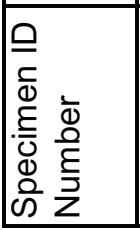 & 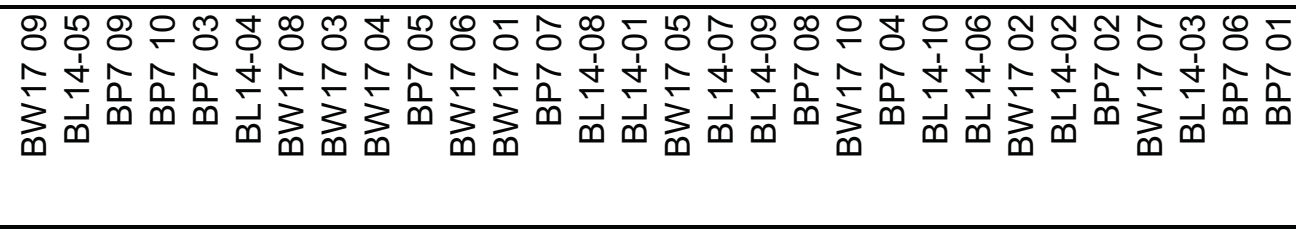 \\
\hline
\end{tabular}




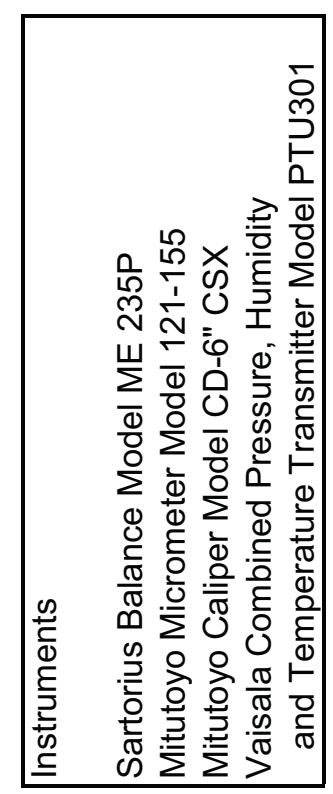

\begin{tabular}{|c|c|}
\hline 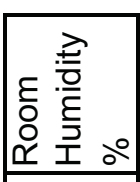 & m m の \\
\hline 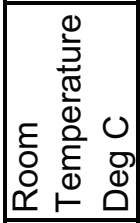 & 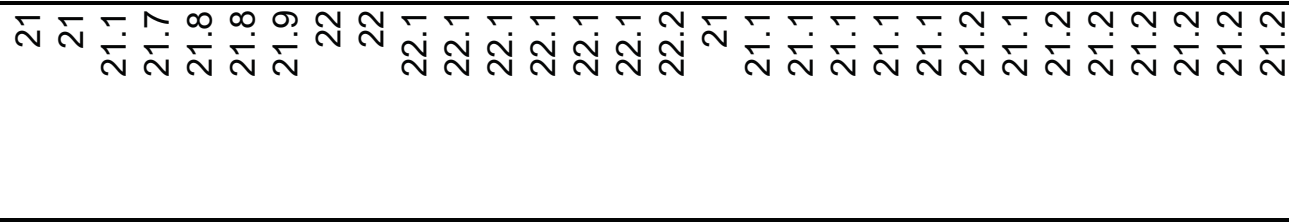 \\
\hline 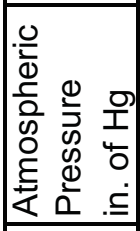 & 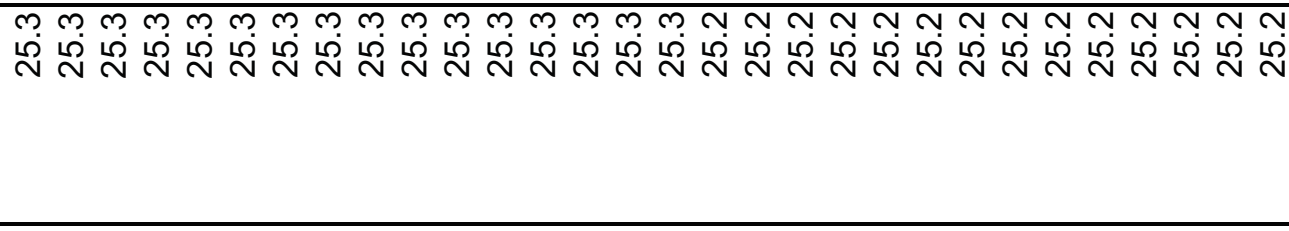 \\
\hline 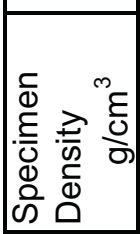 & 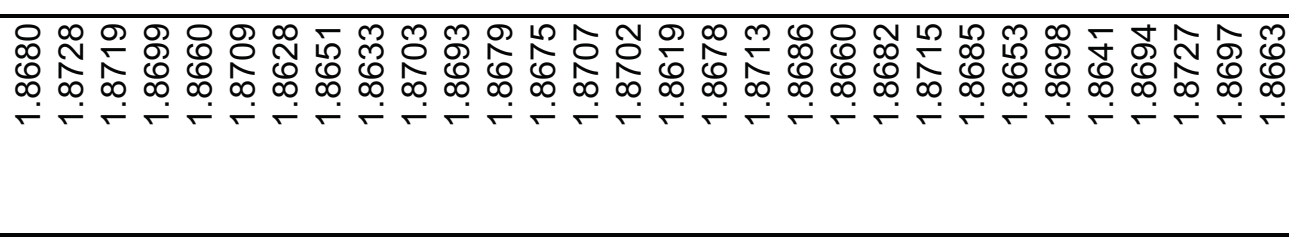 \\
\hline 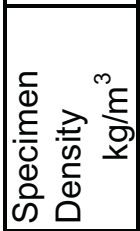 & 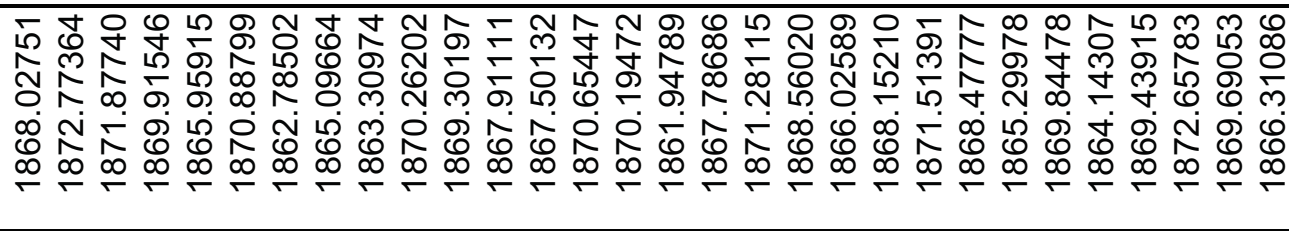 \\
\hline 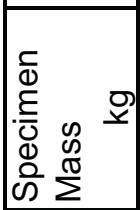 & 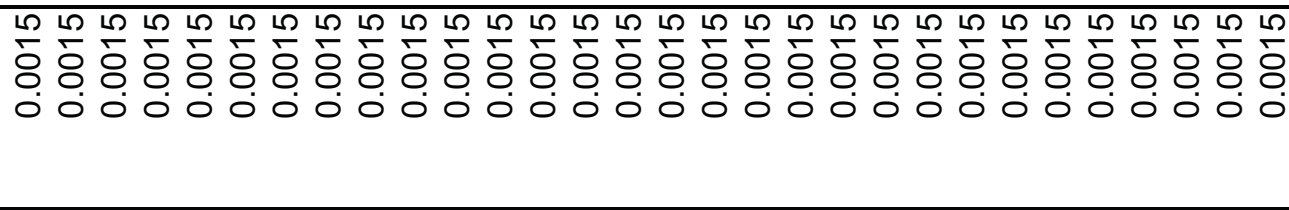 \\
\hline 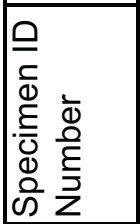 & 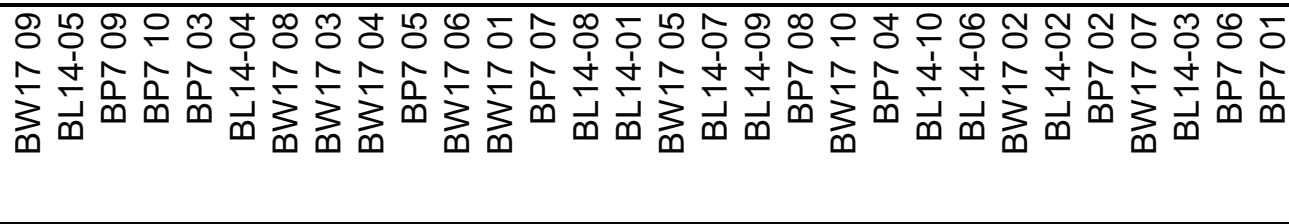 \\
\hline
\end{tabular}




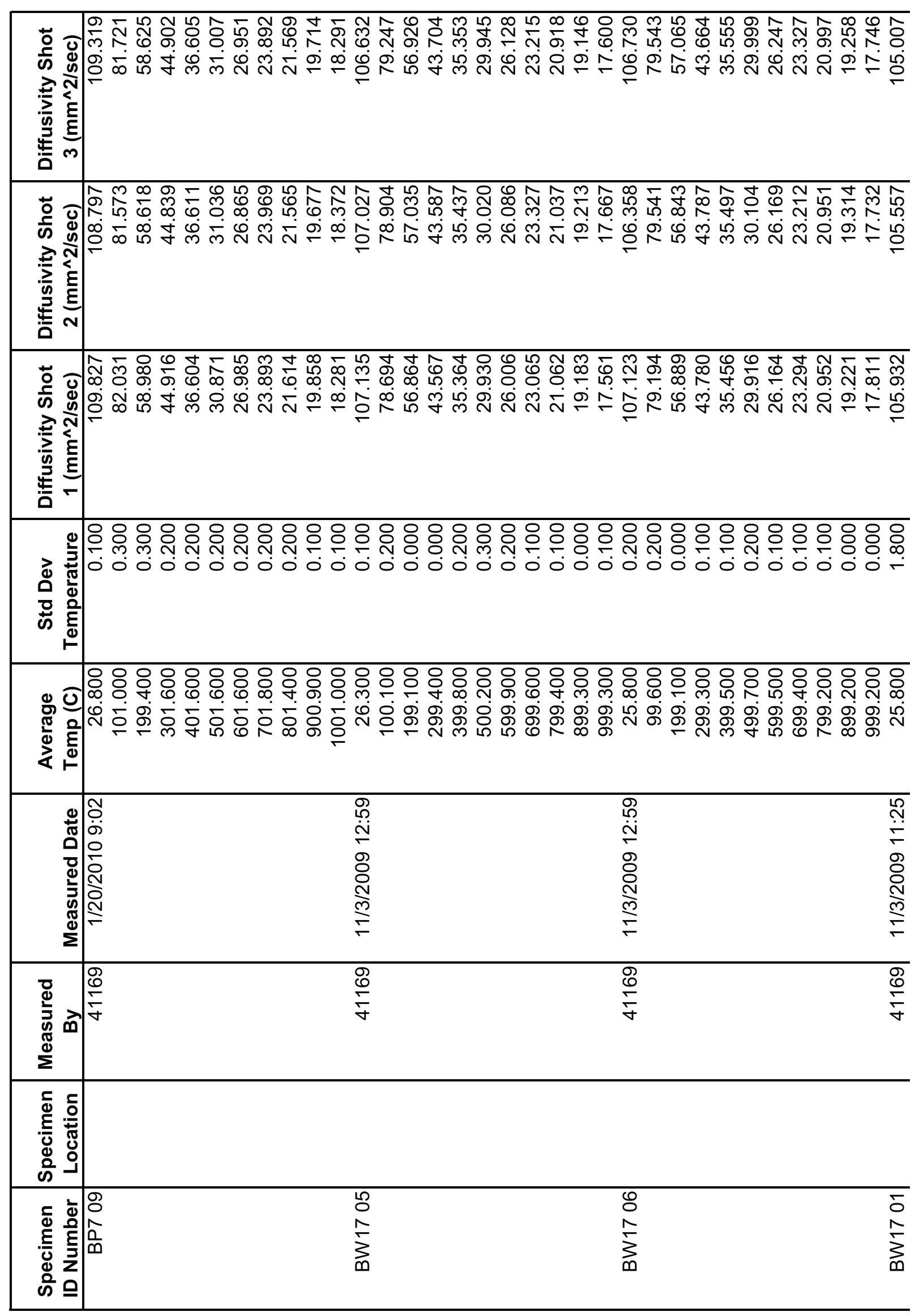




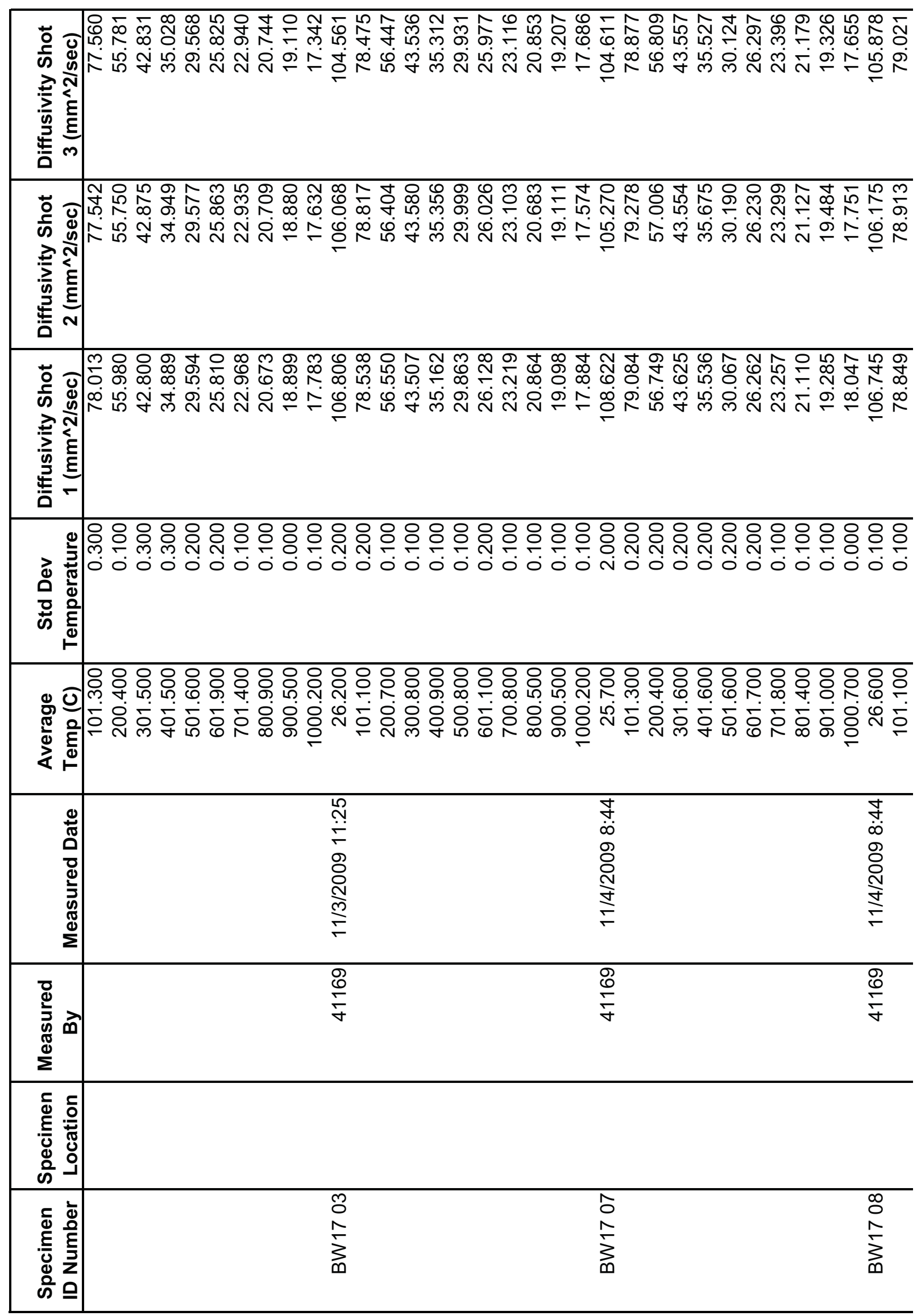




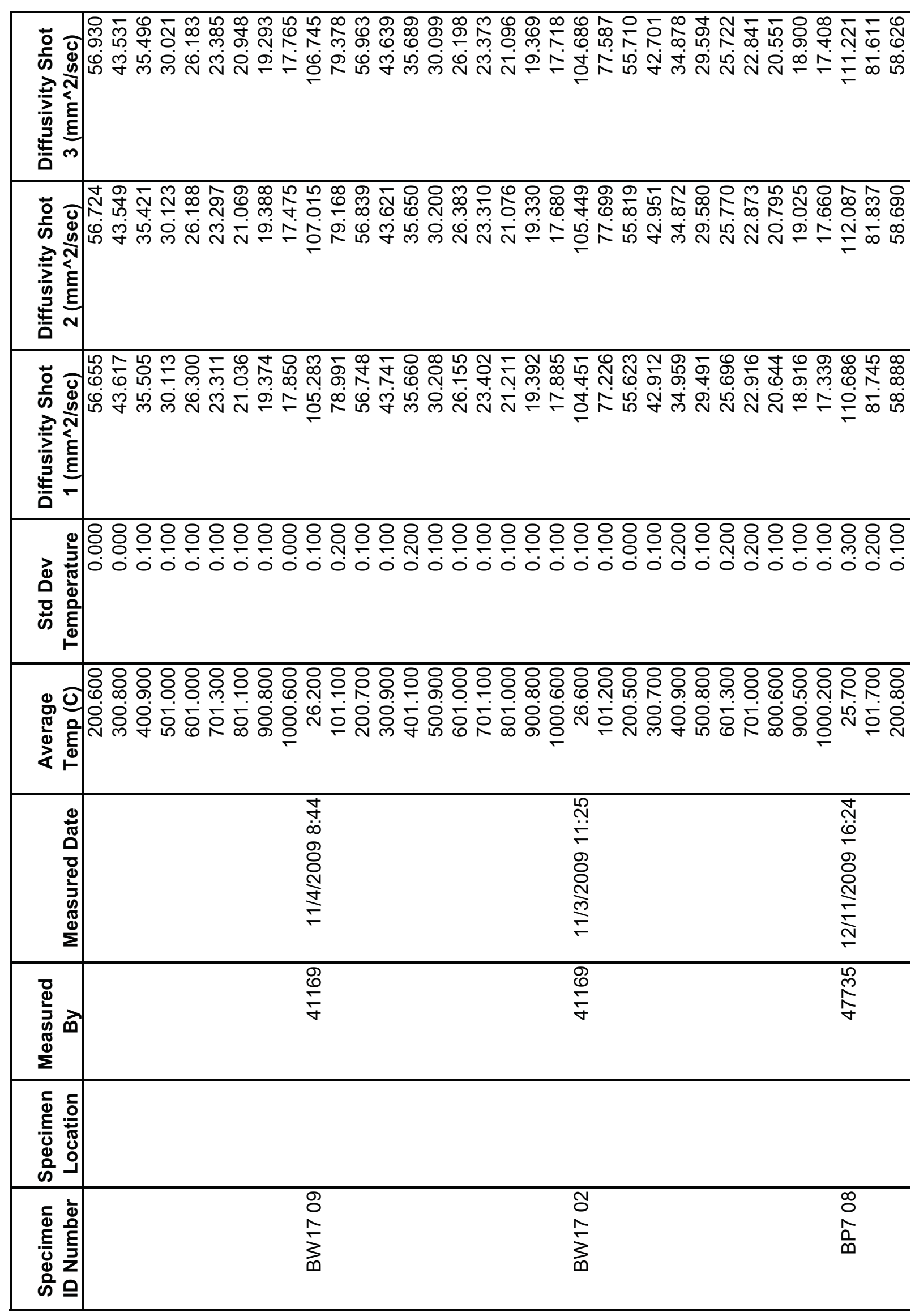




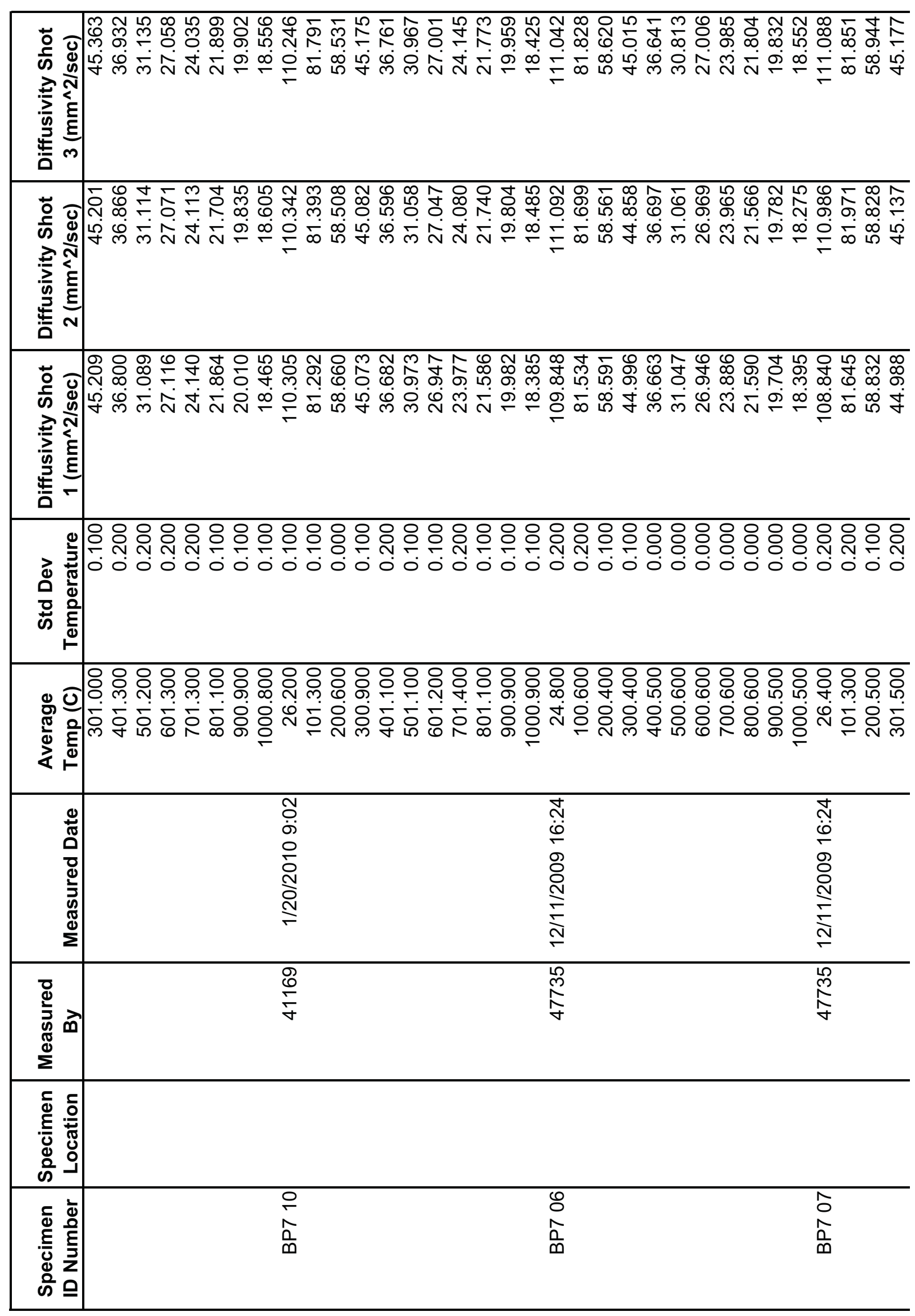




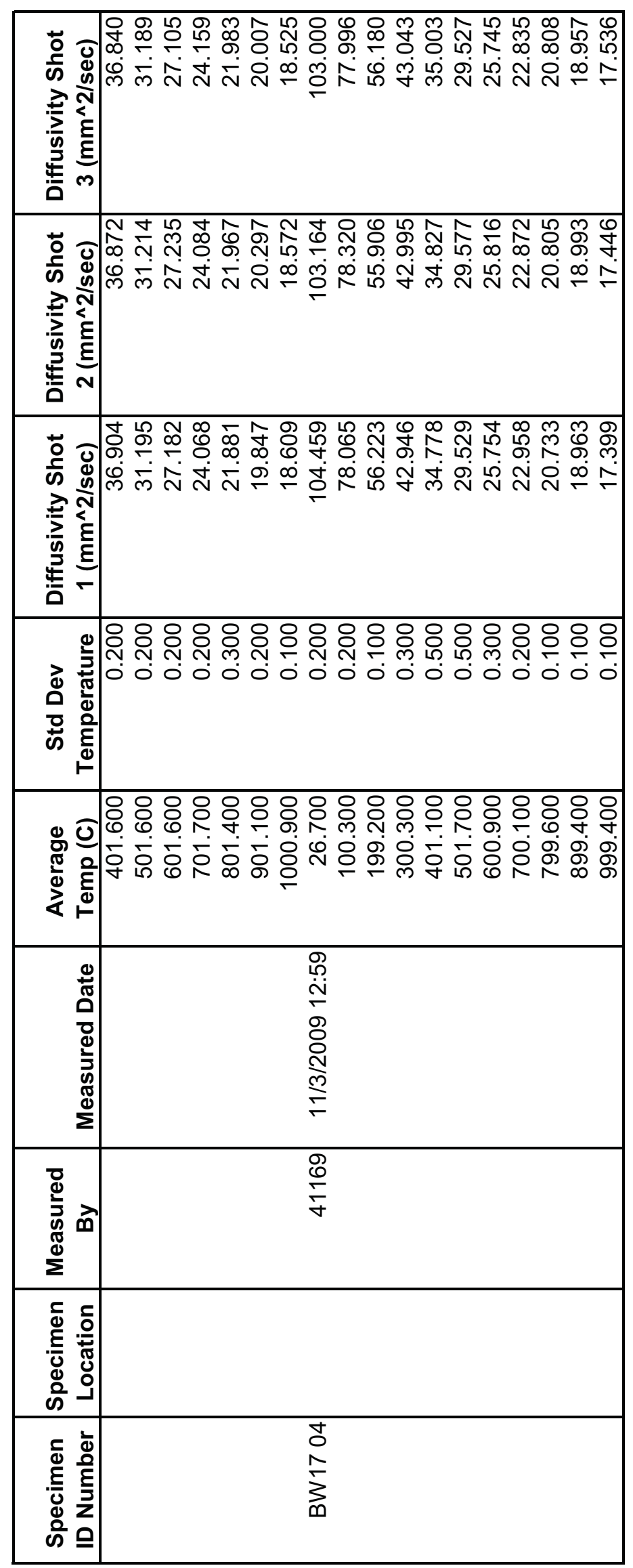




\begin{tabular}{|c|c|c|c|c|}
\hline$\underset{5}{\Sigma}$ & 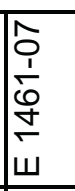 & $\begin{array}{l} \\
\\
1 \\
\dot{0} \\
\dot{+} \\
\omega \\
\end{array}$ & 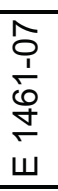 & 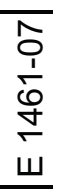 \\
\hline 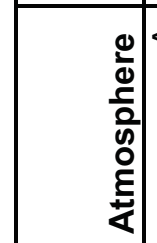 & & $\bar{z}$ & $\bar{z}$ & $\overline{\frac{\pi}{4}}$ \\
\hline 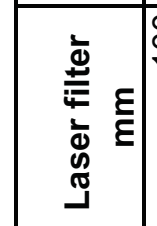 & & 음 & 음 & 음 \\
\hline 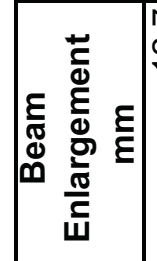 & & $\stackrel{\stackrel{N}{\sim}}{\sim}$ & $\stackrel{\widetilde{N}}{\stackrel{N}{N}}$ & 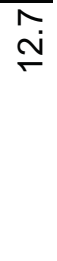 \\
\hline $\begin{array}{l}\vdots \\
\vdots \\
\vdots \\
\grave{d} \\
\omega\end{array}$ & & $\begin{array}{l}\text { की } \\
\text { की }\end{array}$ & $\begin{array}{l}\text { की } \\
\text { की }\end{array}$ & $\begin{array}{l}\text { Oे } \\
\text { Dी }\end{array}$ \\
\hline 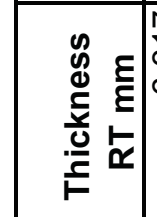 & & $\begin{array}{l}\hat{N} \\
\hat{j} \\
0\end{array}$ & $\begin{array}{c}\hat{N} \\
\overline{0}\end{array}$ & $\stackrel{\Gamma}{\hat{m}}$ \\
\hline 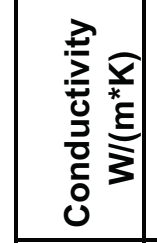 & & & & \\
\hline 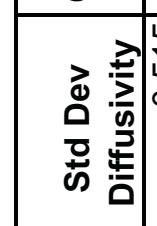 & 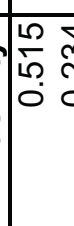 & 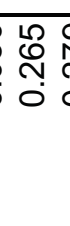 & 孞 & $\begin{array}{l}1 \\
0 \\
0 \\
0 \\
0\end{array}$ \\
\hline 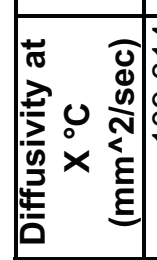 & $\mid \begin{array}{ll}0 & 5 \\
0 & 5 \\
0 & 1 \\
0 & 0 \\
0 & 0 \\
0 & 0\end{array}$ & $\begin{array}{l}\overline{\tilde{m}} \\
\circ \\
\dot{0} \\
\circ\end{array}$ & $\begin{array}{l}\widehat{0} \\
\stackrel{0}{0} \\
\dot{\theta} \\
0\end{array}$ & 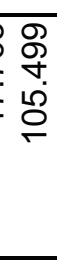 \\
\hline 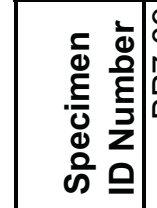 & & $\begin{array}{l}\text { Lo } \\
\sum_{0}^{\circ}\end{array}$ & $\begin{array}{l}8 \\
8 \\
\sum_{0}^{1}\end{array}$ & $\begin{array}{l}\overline{1} \\
\sum_{0}^{\circ}\end{array}$ \\
\hline
\end{tabular}




\begin{tabular}{|c|c|c|c|}
\hline 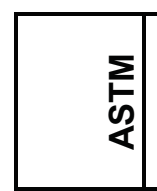 & $\begin{array}{l}\hat{o} \\
\frac{1}{1} \\
\dot{0} \\
\frac{1}{\omega} \\
\end{array}$ & $\begin{array}{l}\hat{o} \\
\frac{1}{0} \\
\stackrel{0}{+} \\
\dot{\omega}\end{array}$ & 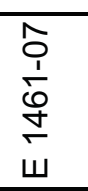 \\
\hline 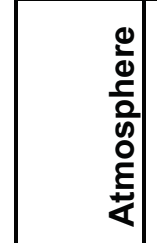 & 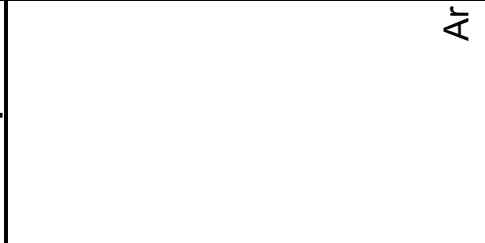 & 文 & 广 \\
\hline 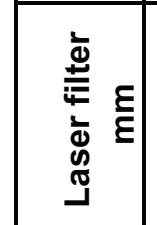 & 음 & 음 & 음 \\
\hline 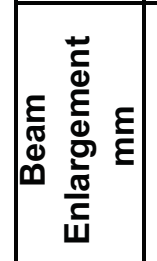 & $\stackrel{\widehat{N}}{\stackrel{N}{N}}$ & $\stackrel{\stackrel{N}{\sim}}{ }$ & 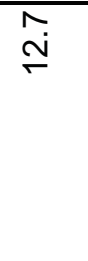 \\
\hline $\begin{array}{l}\vdots \\
\vdots \\
0 \\
\bar{d} \\
\infty\end{array}$ & $\begin{array}{l}\text { की } \\
\text { की }\end{array}$ & $\begin{array}{l}\text { 吕 } \\
\underline{\underline{n}}\end{array}$ & $\begin{array}{l}\text { की } \\
\text { की }\end{array}$ \\
\hline 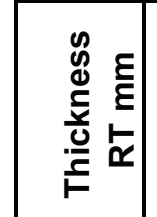 & $\underset{\substack{n \\
0}}{\hat{m}}$ & 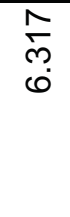 & $\begin{array}{c}\hat{n} \\
\text { o. }\end{array}$ \\
\hline 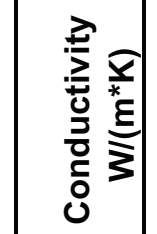 & & & \\
\hline 焉 & 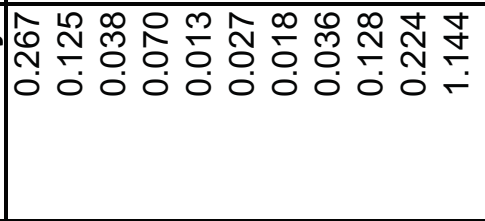 & $\frac{5}{5}$ & 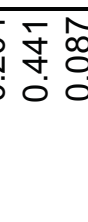 \\
\hline 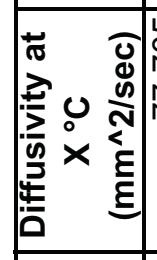 & 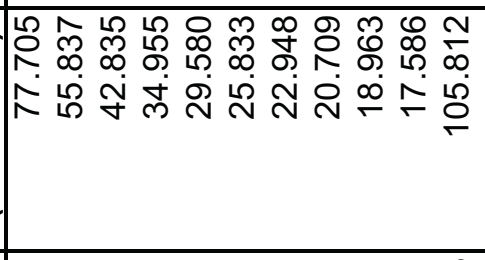 & $\begin{array}{l}2 \\
0 \\
0 \\
0 \\
0 \\
0\end{array}$ & 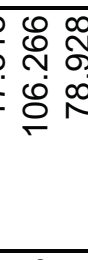 \\
\hline 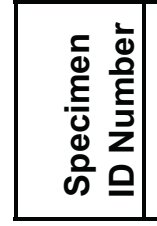 & $\begin{array}{l}\text { m } \\
\sum_{0}^{5}\end{array}$ & $\begin{array}{l}1 \\
\hat{0} \\
\sum_{0}^{5}\end{array}$ & $\begin{array}{l}\sum_{0}^{\infty} \\
\sum_{0}^{\circ}\end{array}$ \\
\hline
\end{tabular}




\begin{tabular}{|c|c|c|c|}
\hline 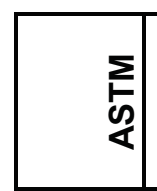 & 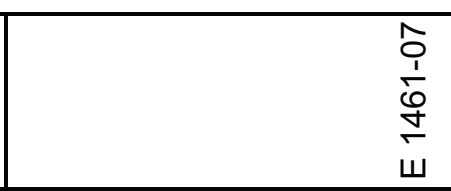 & $\begin{array}{l}\hat{o} \\
\frac{1}{0} \\
\stackrel{0}{+} \\
\dot{\omega}\end{array}$ & $\begin{array}{l}\frac{\hat{1}}{1} \\
\frac{1}{0} \\
\frac{1}{4} \\
\dot{w}\end{array}$ \\
\hline 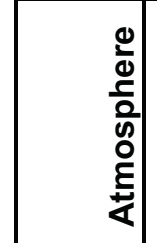 & 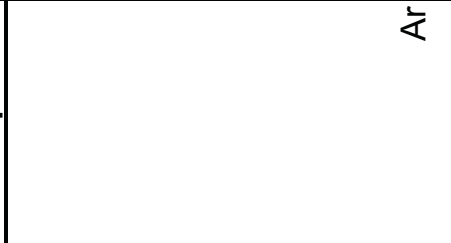 & 衣 & 交 \\
\hline 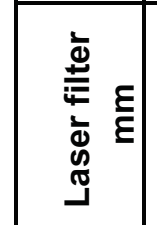 & 음 & 음 & 음 \\
\hline 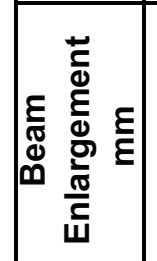 & 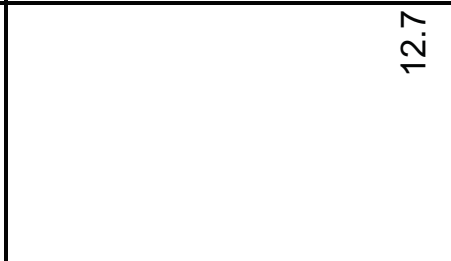 & $\stackrel{\stackrel{N}{ }}{ }$ & $\stackrel{\widetilde{N}}{\stackrel{N}{N}}$ \\
\hline $\begin{array}{l}\vdots \\
\vdots \\
0 \\
\bar{d} \\
\infty\end{array}$ & $\begin{array}{l}\text { की } \\
\text { की }\end{array}$ & 吕 & $\begin{array}{l}\text { की } \\
\text { की }\end{array}$ \\
\hline 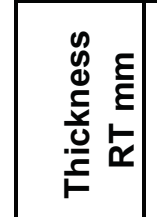 & $\stackrel{\Gamma}{\hat{m}}$ & 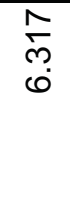 & $\stackrel{\hat{n}}{\hat{n}}$ \\
\hline 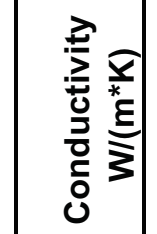 & & & \\
\hline 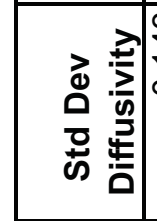 & 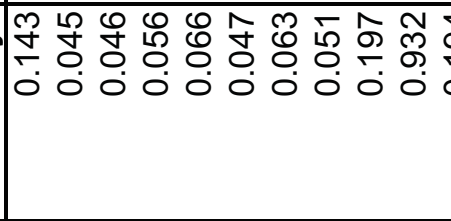 & $\begin{array}{l}3 \mathbb{N} \\
0 \\
0 \\
0 \\
0\end{array}$ & 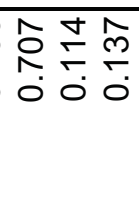 \\
\hline 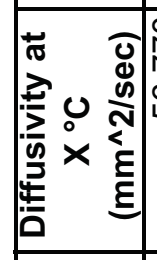 & 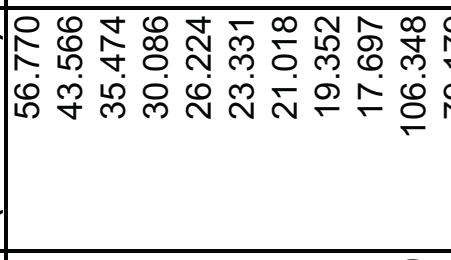 & $\begin{array}{l}50 \\
0 \\
0 \\
0 \\
0 \\
0\end{array}$ & 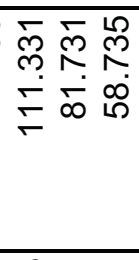 \\
\hline 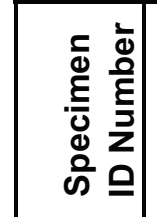 & $\begin{array}{l}8 \\
\\
\sum_{0}^{\circ}\end{array}$ & \begin{tabular}{l}
$N$ \\
\multirow{2}{N}{} \\
$\sum_{0}$
\end{tabular} & $\begin{array}{l}\text { o } \\
0 \\
\hat{0} \\
0\end{array}$ \\
\hline
\end{tabular}




\begin{tabular}{|c|c|c|c|}
\hline 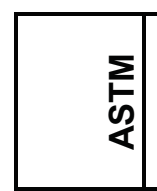 & $\begin{array}{l}\hat{o} \\
\dot{1} \\
\dot{0} \\
\dot{+} \\
\dot{w}\end{array}$ & $\begin{array}{l}\hat{o} \\
\frac{1}{0} \\
\stackrel{0}{+} \\
\dot{\omega}\end{array}$ & $\begin{array}{l}\hat{o} \\
\frac{1}{0} \\
\dot{0} \\
\dot{W} \\
\end{array}$ \\
\hline 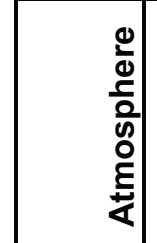 & 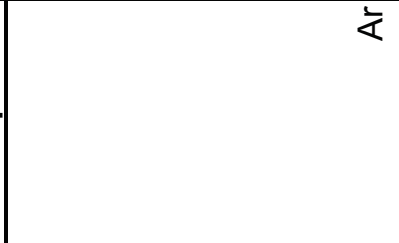 & 文 & 交 \\
\hline 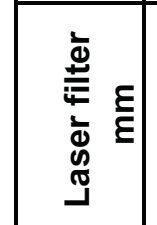 & 음 & $\stackrel{\circ}{\circ}$ & 음 \\
\hline 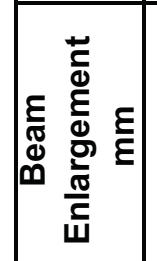 & $\stackrel{\widehat{N}}{\stackrel{N}{N}}$ & $\stackrel{\stackrel{N}{\sim}}{\mathrm{N}}$ & $\stackrel{\stackrel{\sim}{\sim}}{ }$ \\
\hline $\begin{array}{l}\bar{b} \\
0 \\
\stackrel{5}{\Phi} \\
\infty\end{array}$ & $\begin{array}{l}\text { की } \\
\text { की }\end{array}$ & $\begin{array}{l}\text { की } \\
\text { I }\end{array}$ & $\begin{array}{l}\text { की } \\
\text { 足 }\end{array}$ \\
\hline 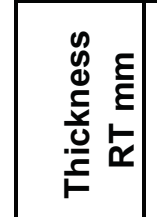 & 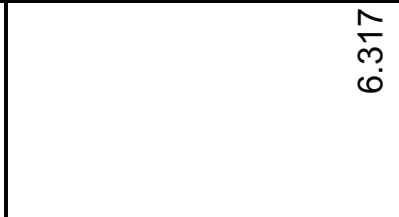 & 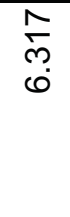 & 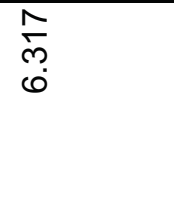 \\
\hline 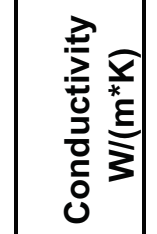 & & & \\
\hline 焉 & 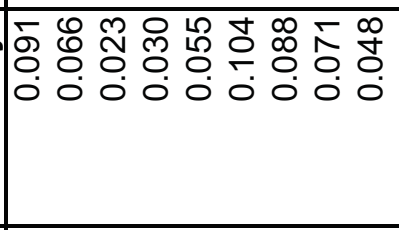 & 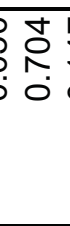 & 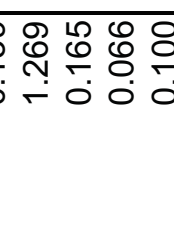 \\
\hline 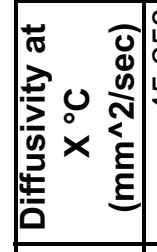 & $\begin{array}{l}\text { lo } \\
0\end{array}$ & 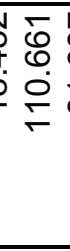 & $\begin{array}{l}l \\
0 \\
0\end{array}$ \\
\hline 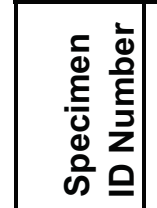 & $\begin{array}{l}\frac{0}{\alpha} \\
\frac{\hat{n}}{0}\end{array}$ & $\begin{array}{l}8 \\
0 \\
0 \\
0\end{array}$ & $\begin{array}{l}\text { o } \\
\hat{a} \\
\text { n }\end{array}$ \\
\hline
\end{tabular}




\begin{tabular}{|c|c|}
\hline$\underset{⿱ 亠 䒑}{\infty}$ & 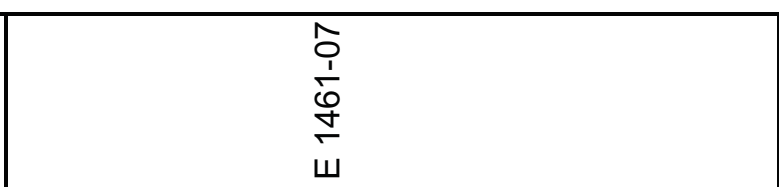 \\
\hline 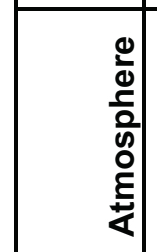 & 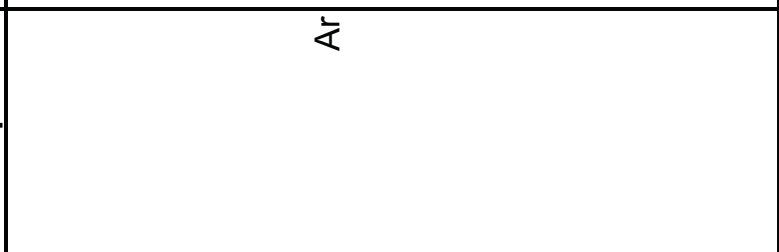 \\
\hline 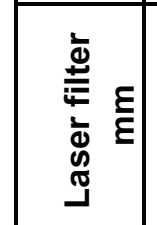 & 음 \\
\hline 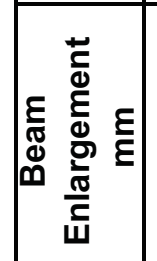 & $\stackrel{\widetilde{N}}{\stackrel{N}{N}}$ \\
\hline $\begin{array}{l}\bar{b} \\
\dot{g} \\
\bar{d} \\
\oplus\end{array}$ & $\begin{array}{l}\text { की } \\
\text { की }\end{array}$ \\
\hline 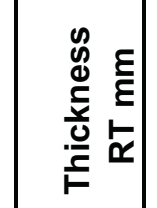 & $\begin{array}{c}\hat{m} \\
0 \\
0\end{array}$ \\
\hline 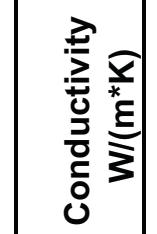 & \\
\hline 吾辛 & 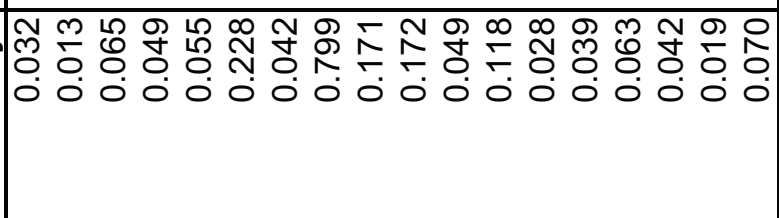 \\
\hline 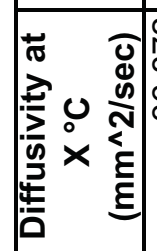 & 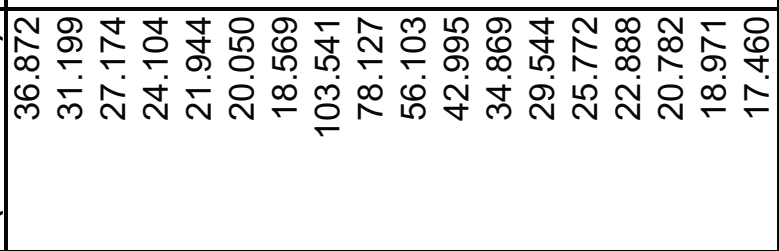 \\
\hline 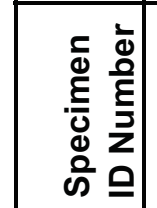 & $\begin{array}{l}\text { d } \\
\sum_{0}^{5}\end{array}$ \\
\hline
\end{tabular}




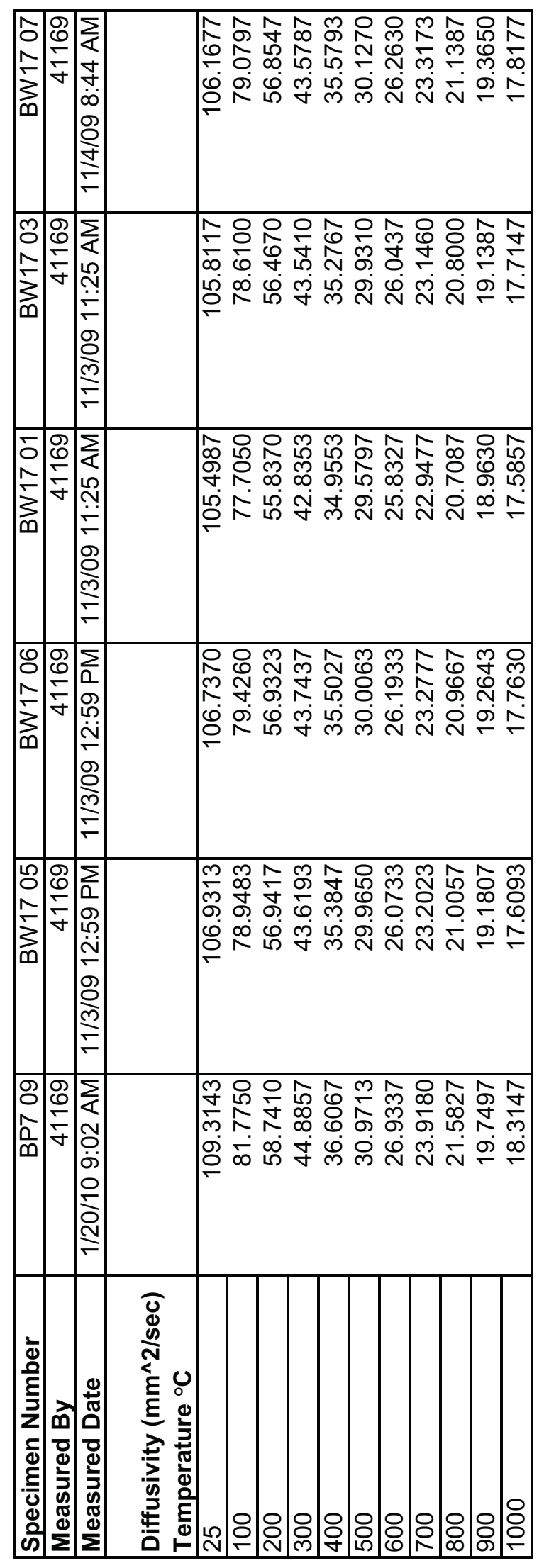




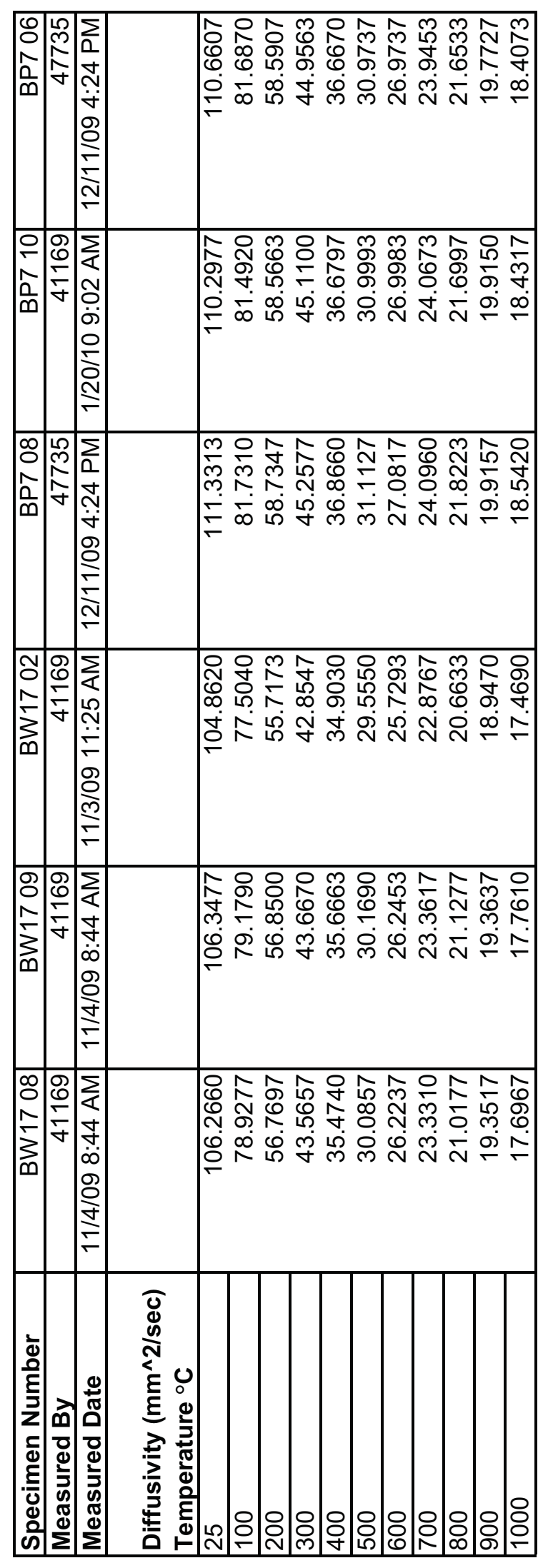

$\stackrel{N}{=}$ 


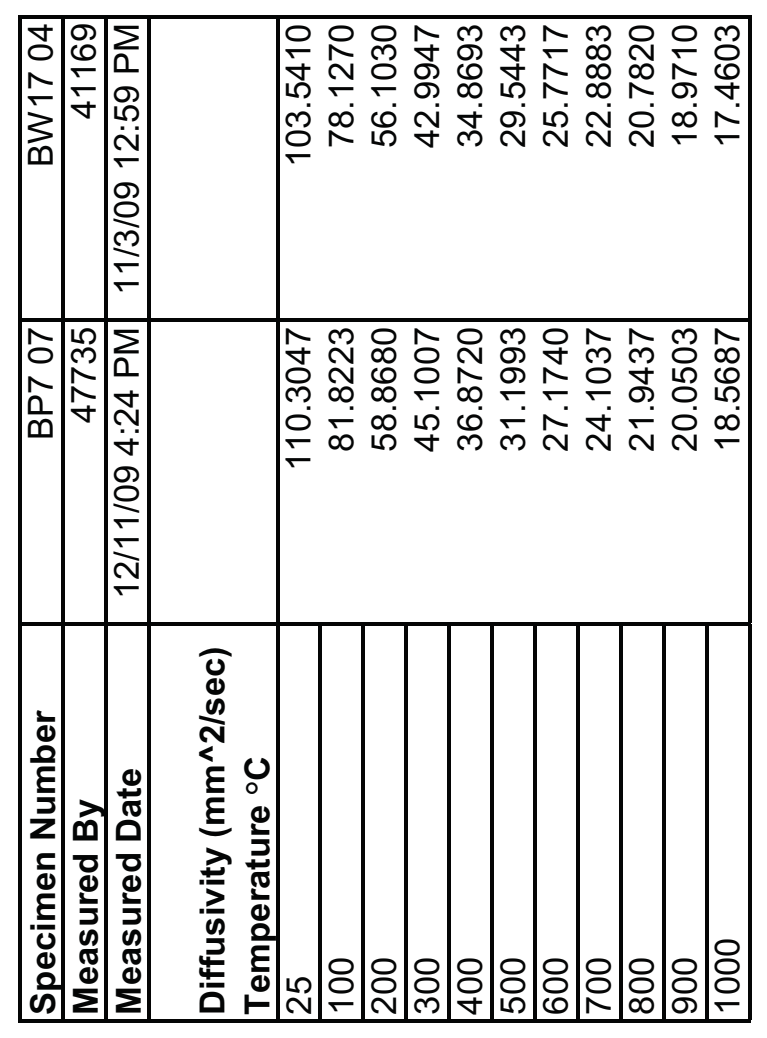

$\stackrel{m}{=}$ 


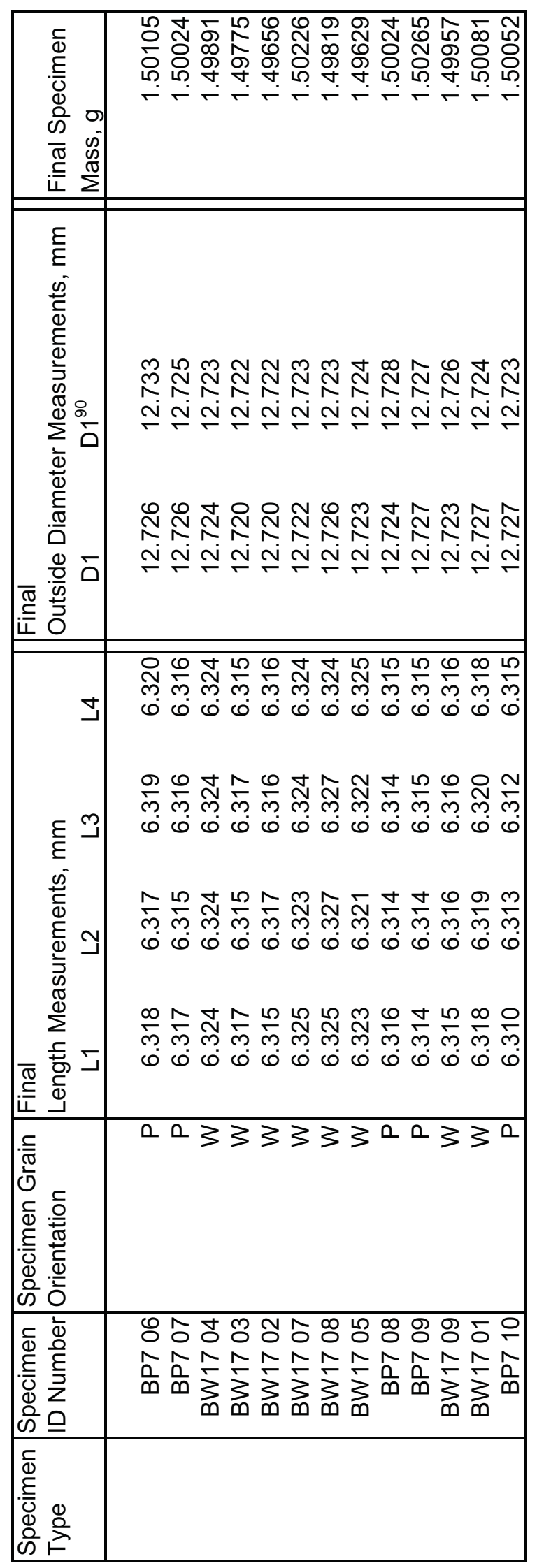




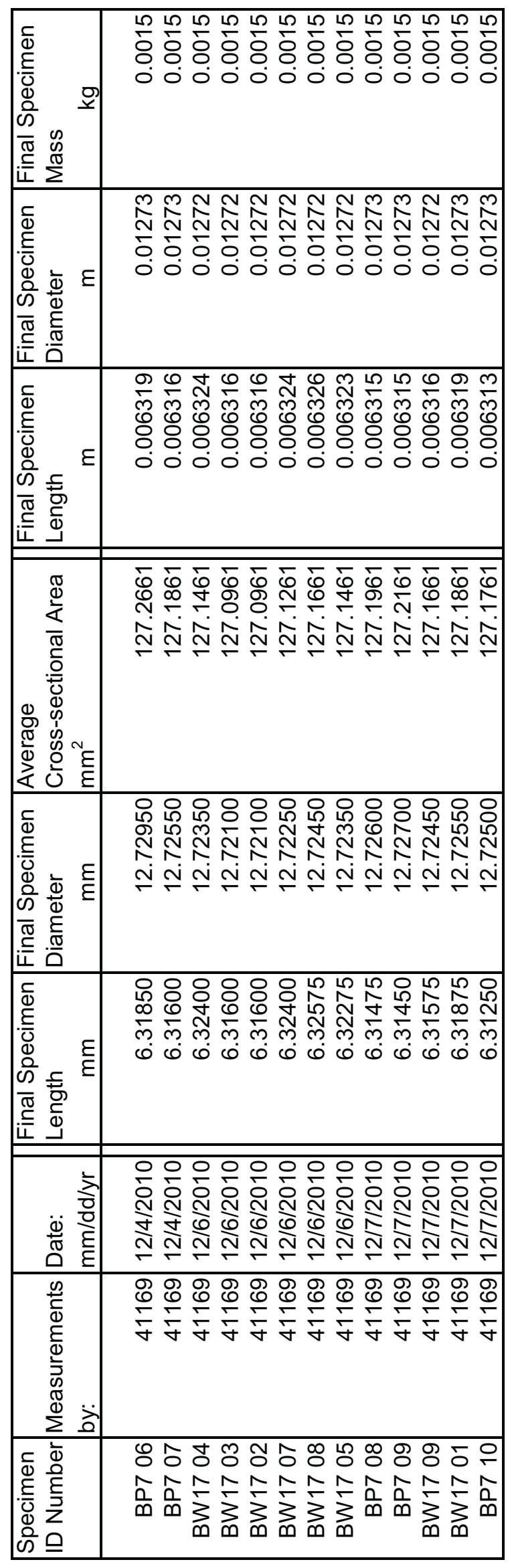




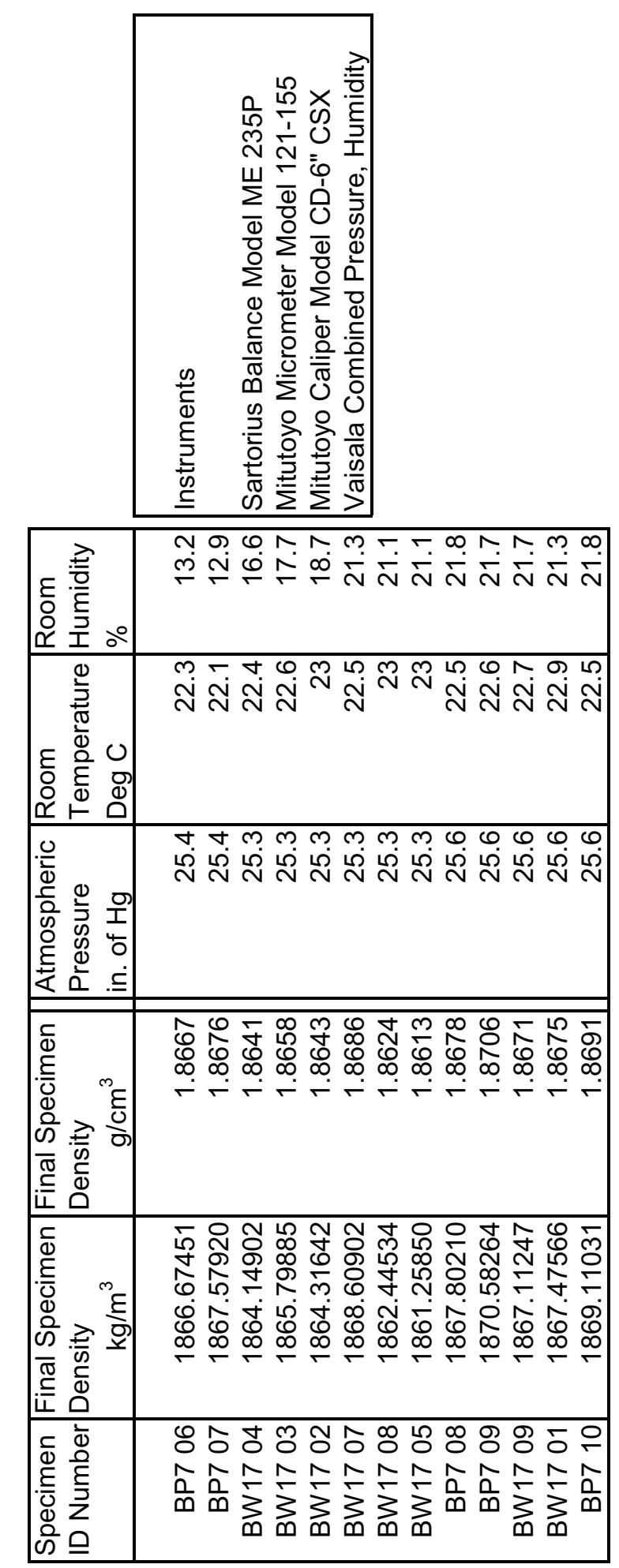

$\stackrel{0}{=}$ 
Graphite Grade:

Graphite Manufacturer:

Forming Process:

Coke Particle Size:

Coke Type:

ASTM Class:

Specimen Geometry:
NBG-25

SGL Carbon Company

Isostatic-molded

Fine grain

Petroleum coke

INHP

Cylinder

\begin{abstract}
M1-01
M1-02

M1-03

M1-04

M1-05

M1-06

M1-07

M1-08

M1-09

M1-10

M1-11

M1-12

M2-01

M2-02

M2-03

M2-04

M2-05

M2-06

M2-07

M2-08

M2-09

M2-10

M2-11

M2-12
\end{abstract}

Specimen ID \#'s: 


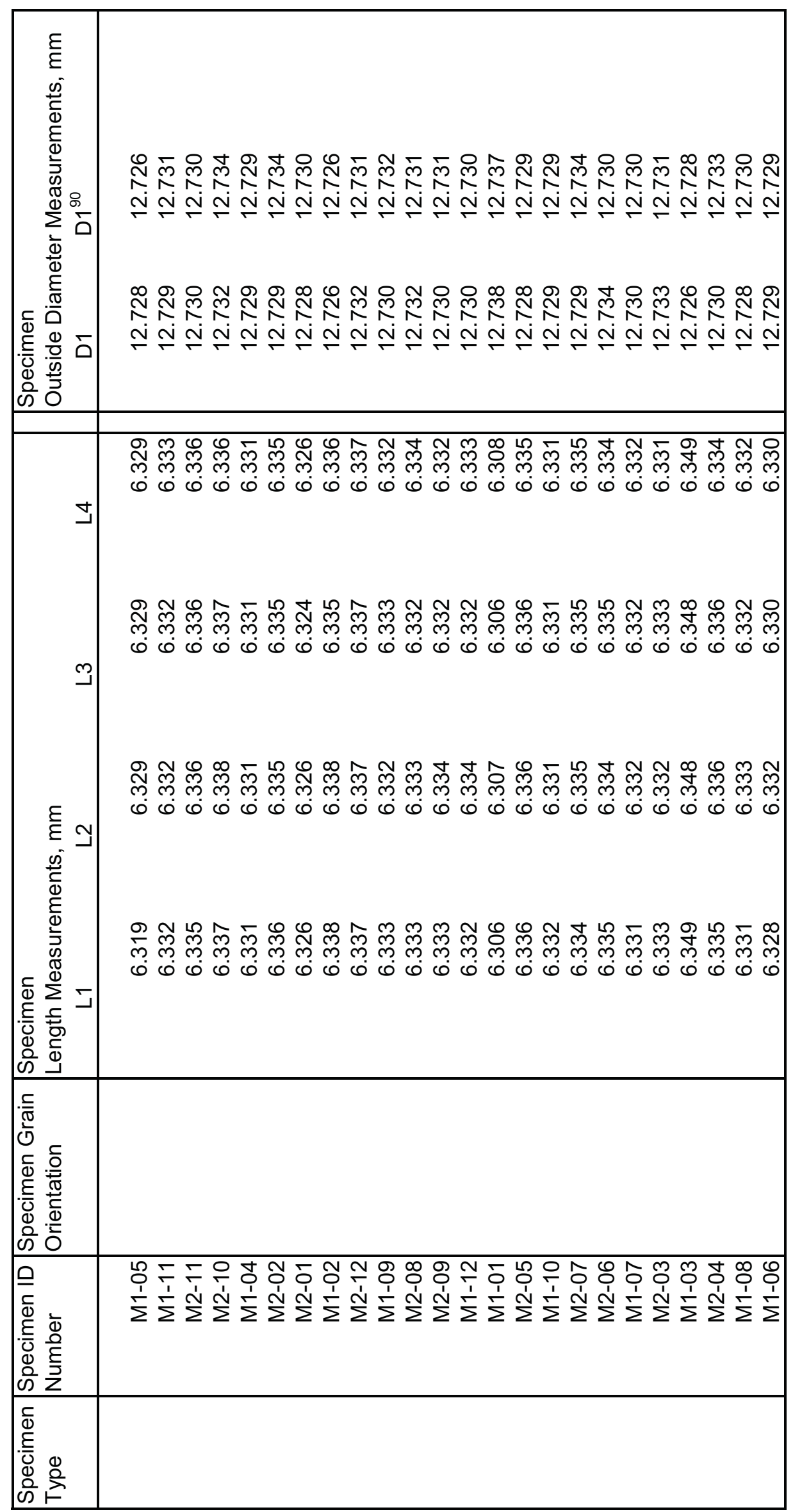




\begin{tabular}{|c|c|}
\hline 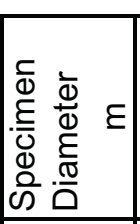 & 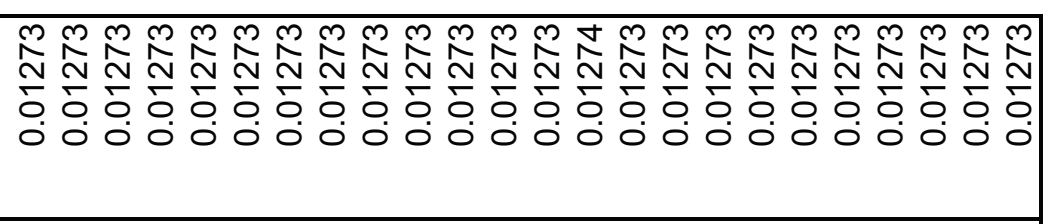 \\
\hline 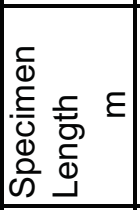 & 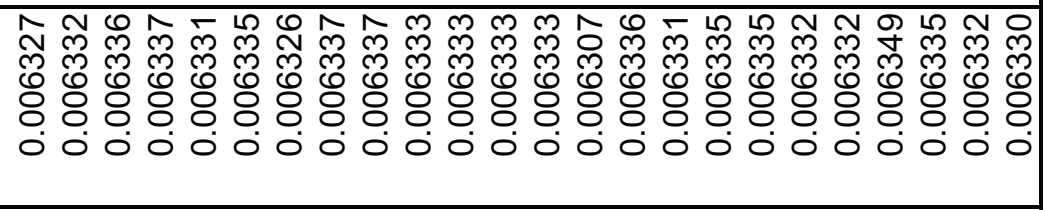 \\
\hline 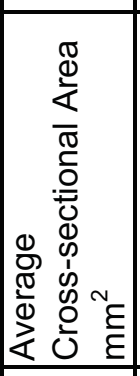 & 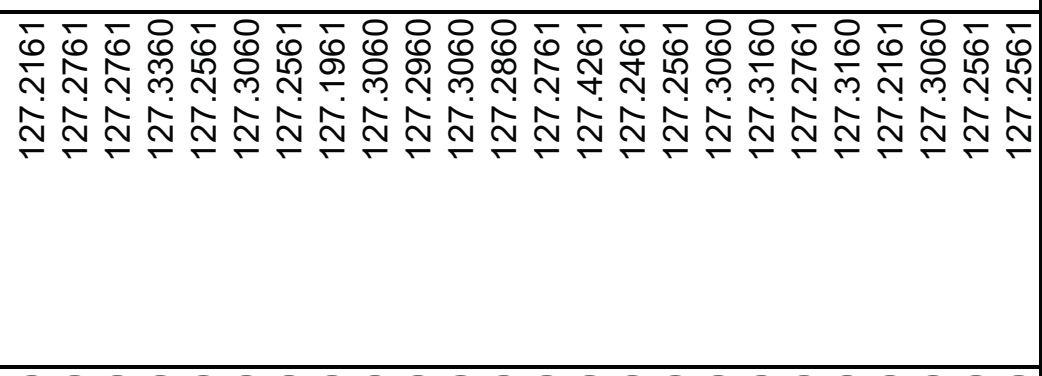 \\
\hline 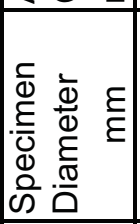 & 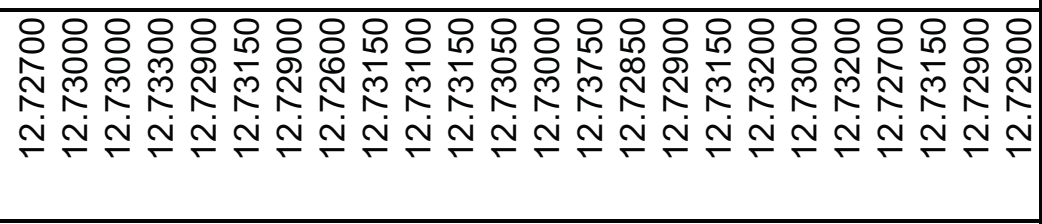 \\
\hline 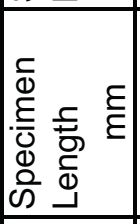 & 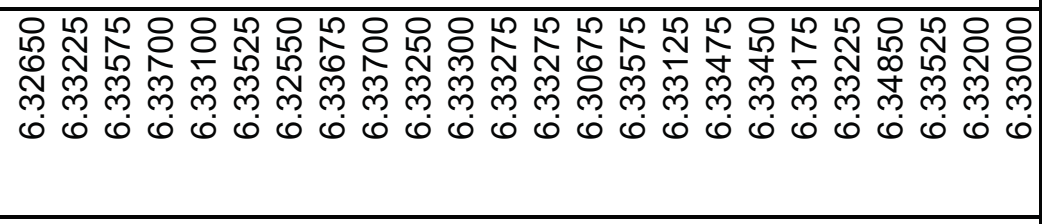 \\
\hline 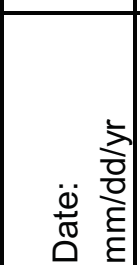 & 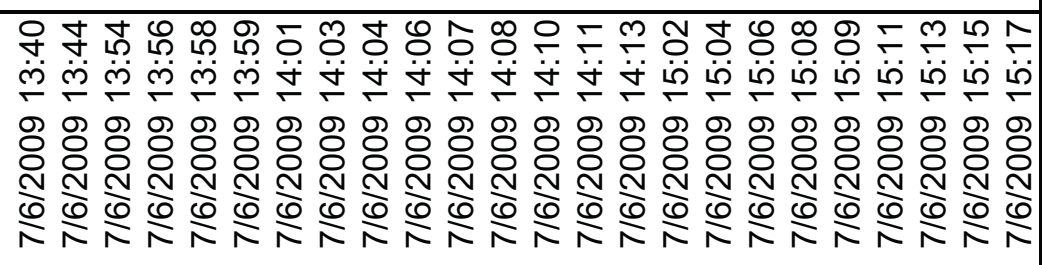 \\
\hline 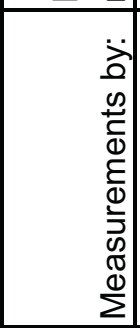 & 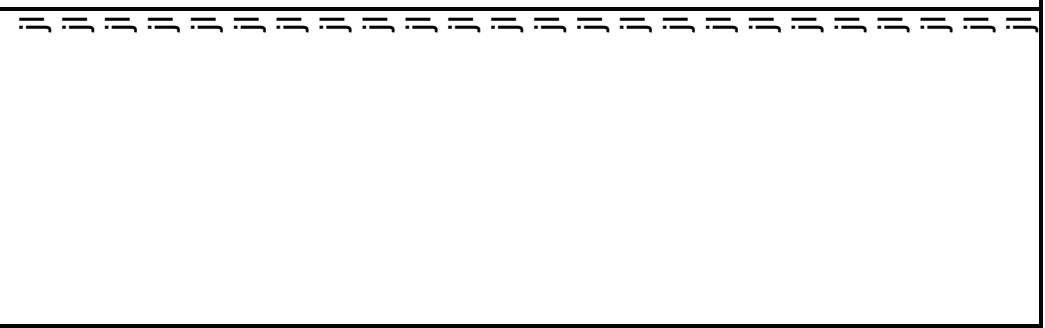 \\
\hline 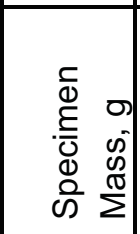 & 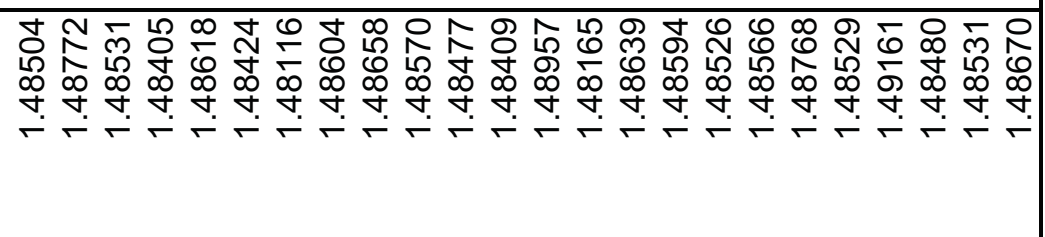 \\
\hline 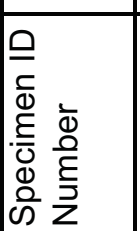 & 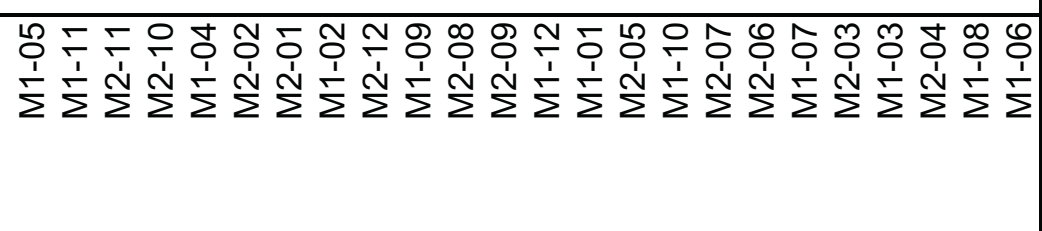 \\
\hline
\end{tabular}




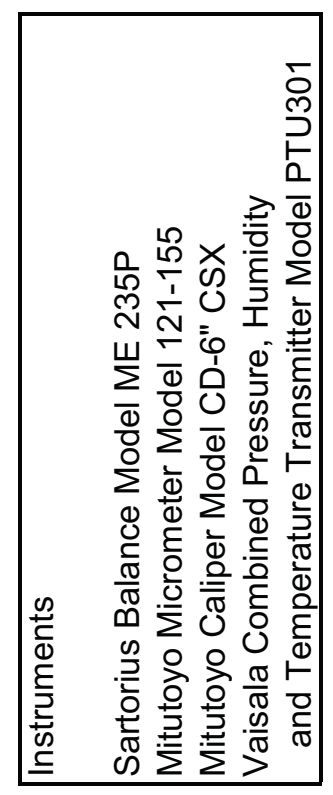

\begin{tabular}{|c|c|}
\hline \& & 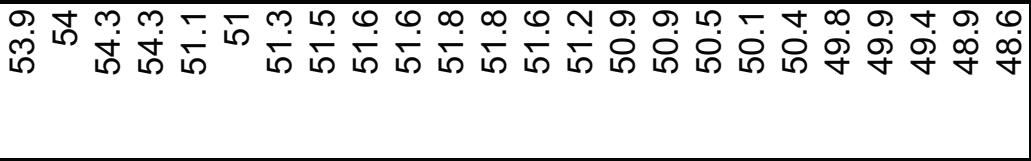 \\
\hline 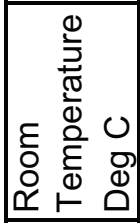 & 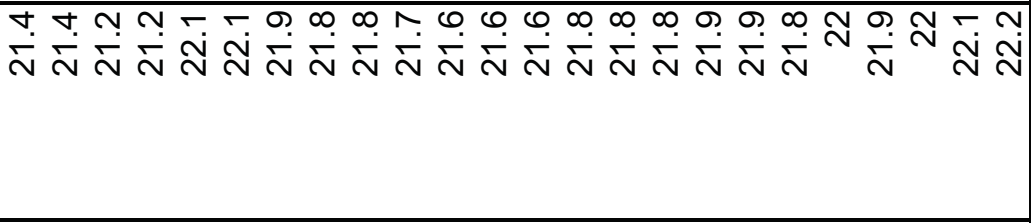 \\
\hline 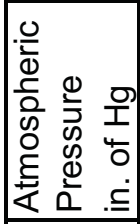 & 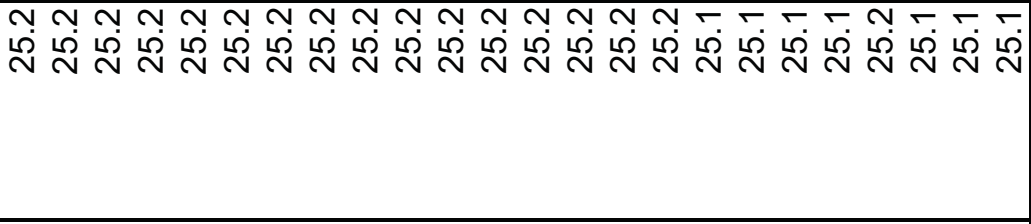 \\
\hline 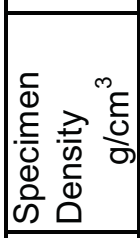 & 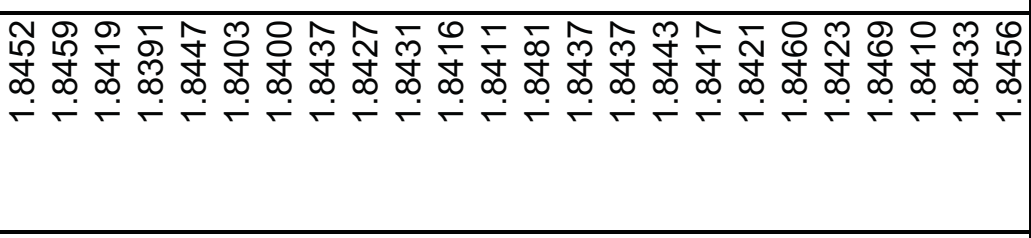 \\
\hline 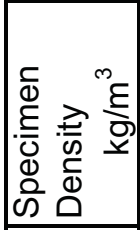 & 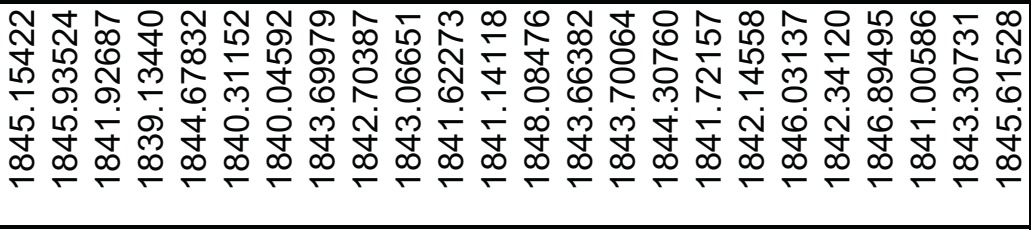 \\
\hline 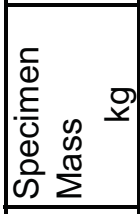 & 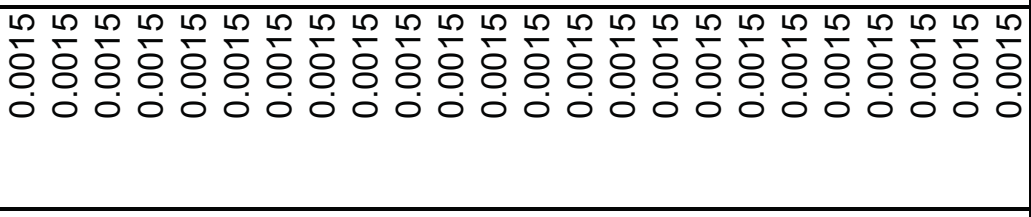 \\
\hline 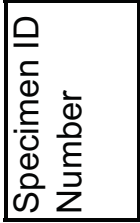 & 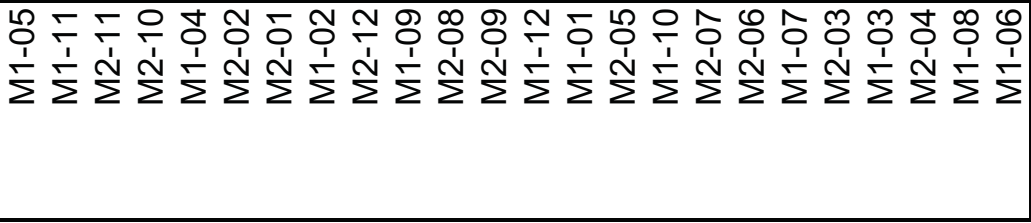 \\
\hline
\end{tabular}




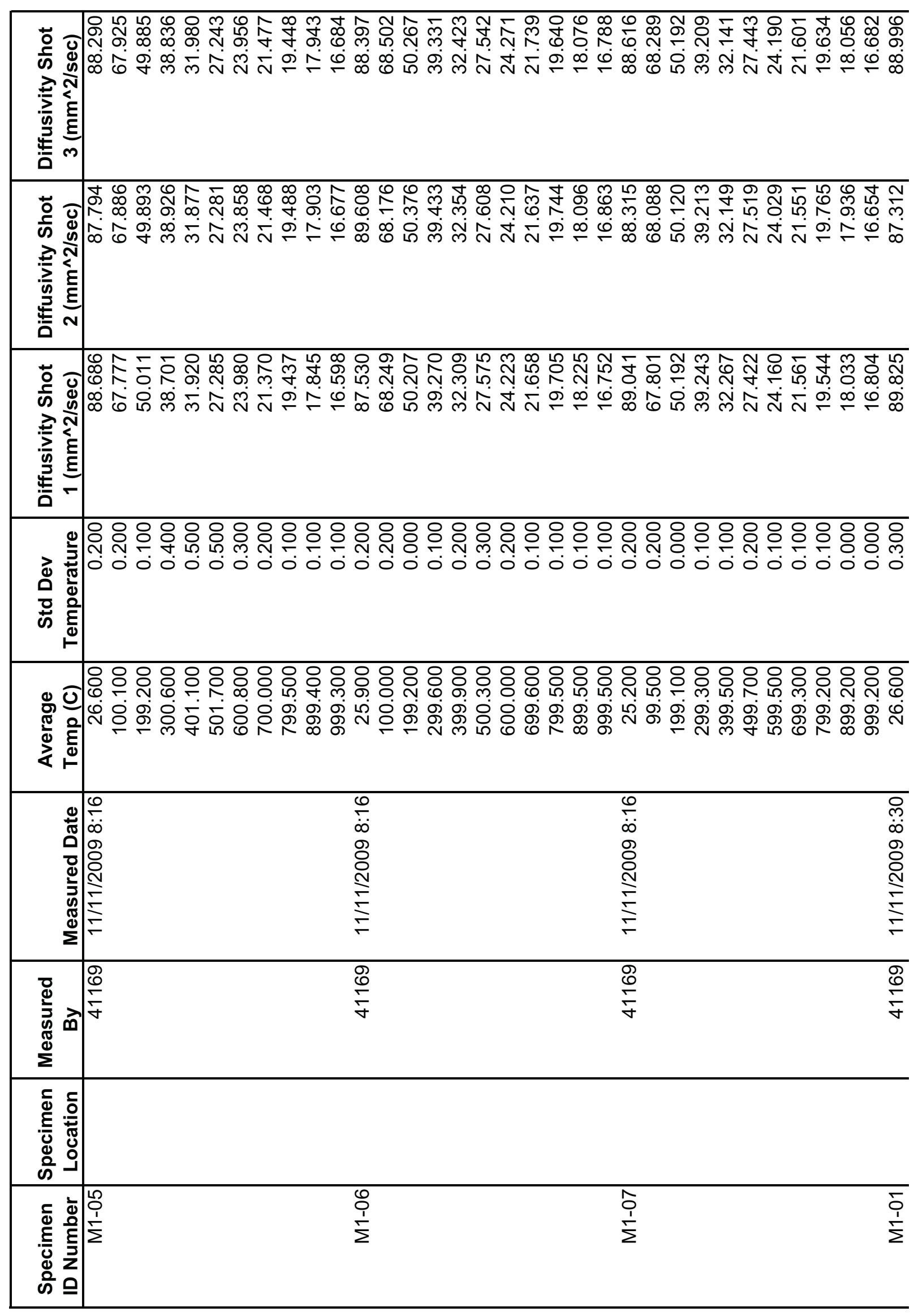




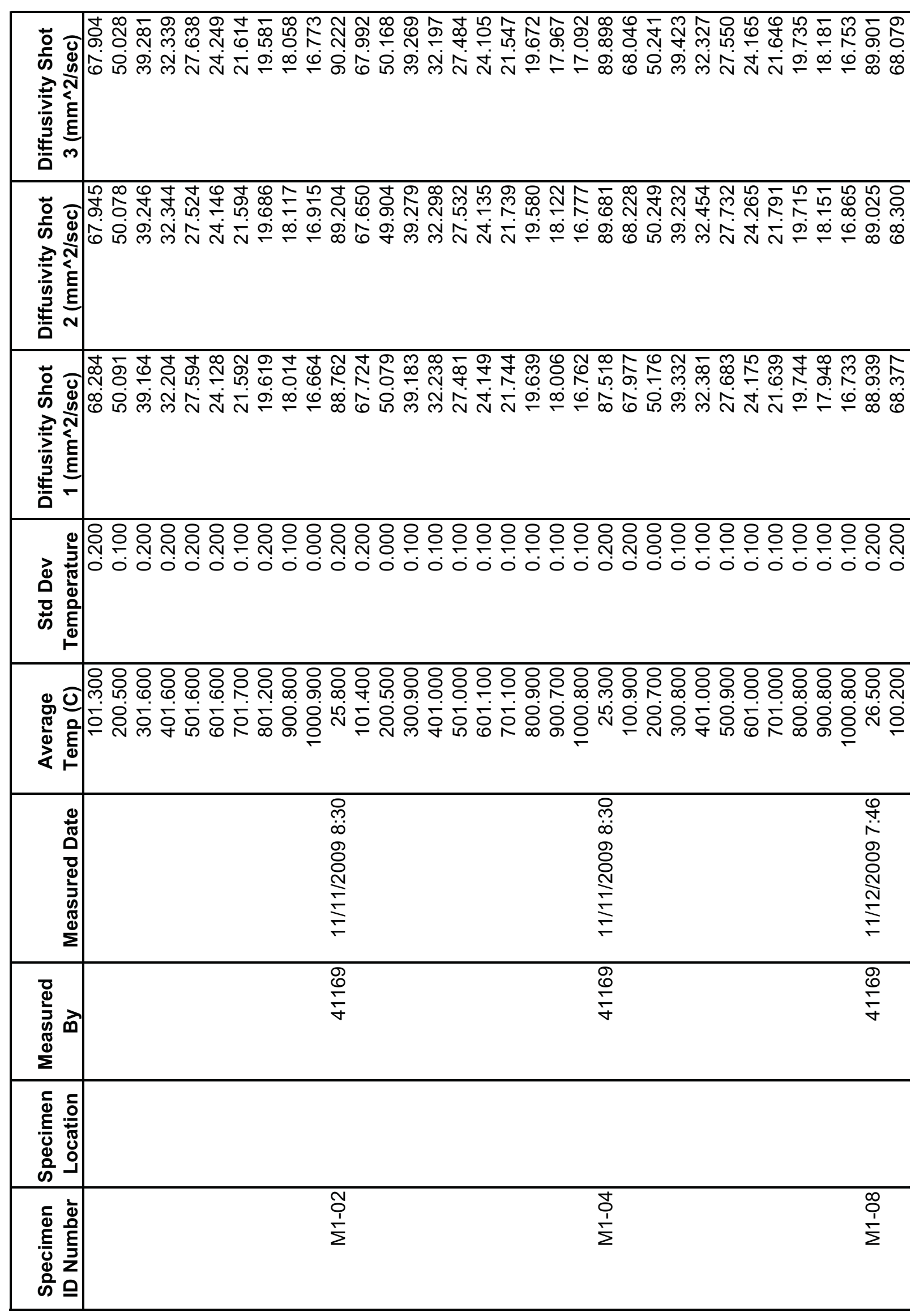




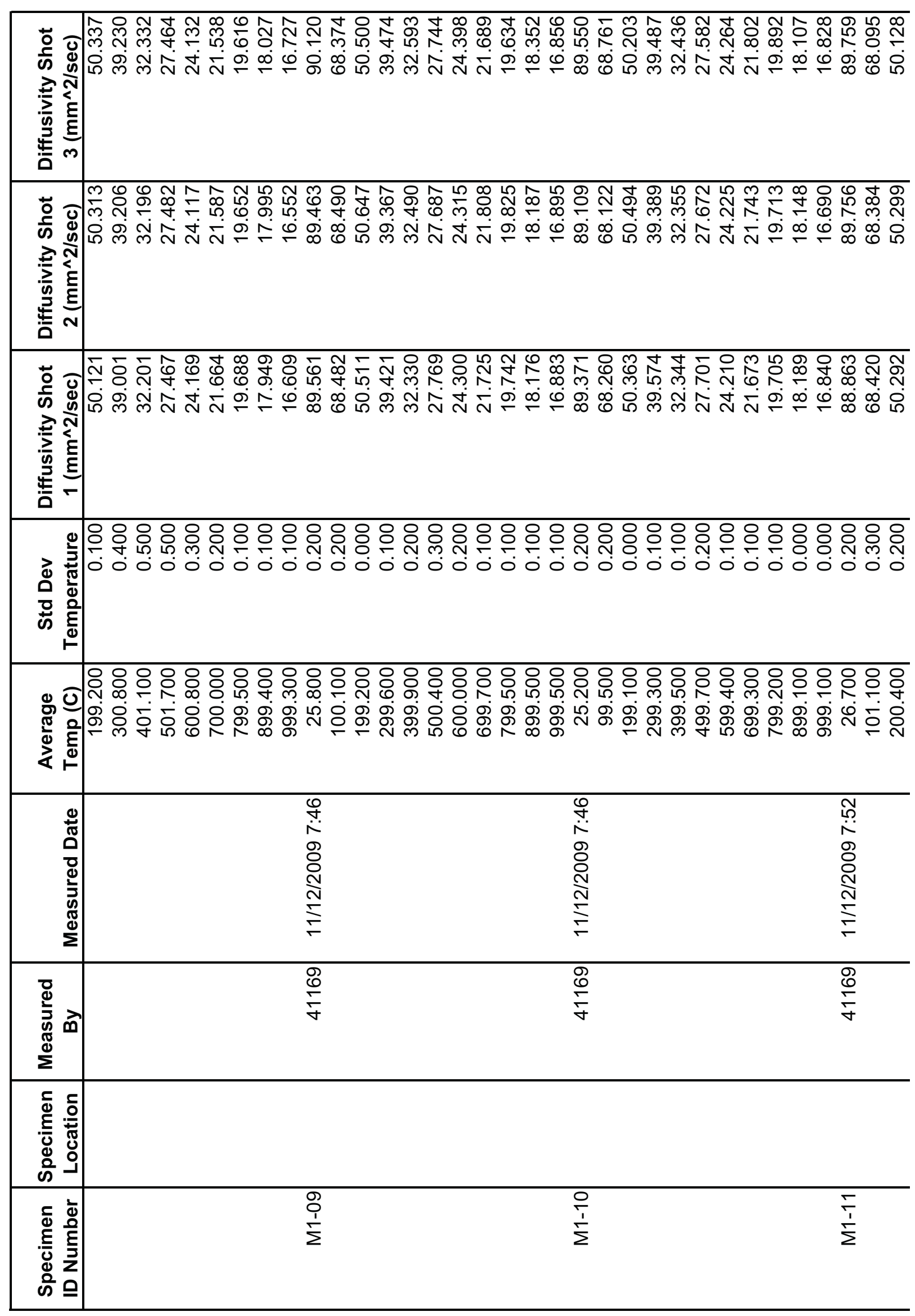




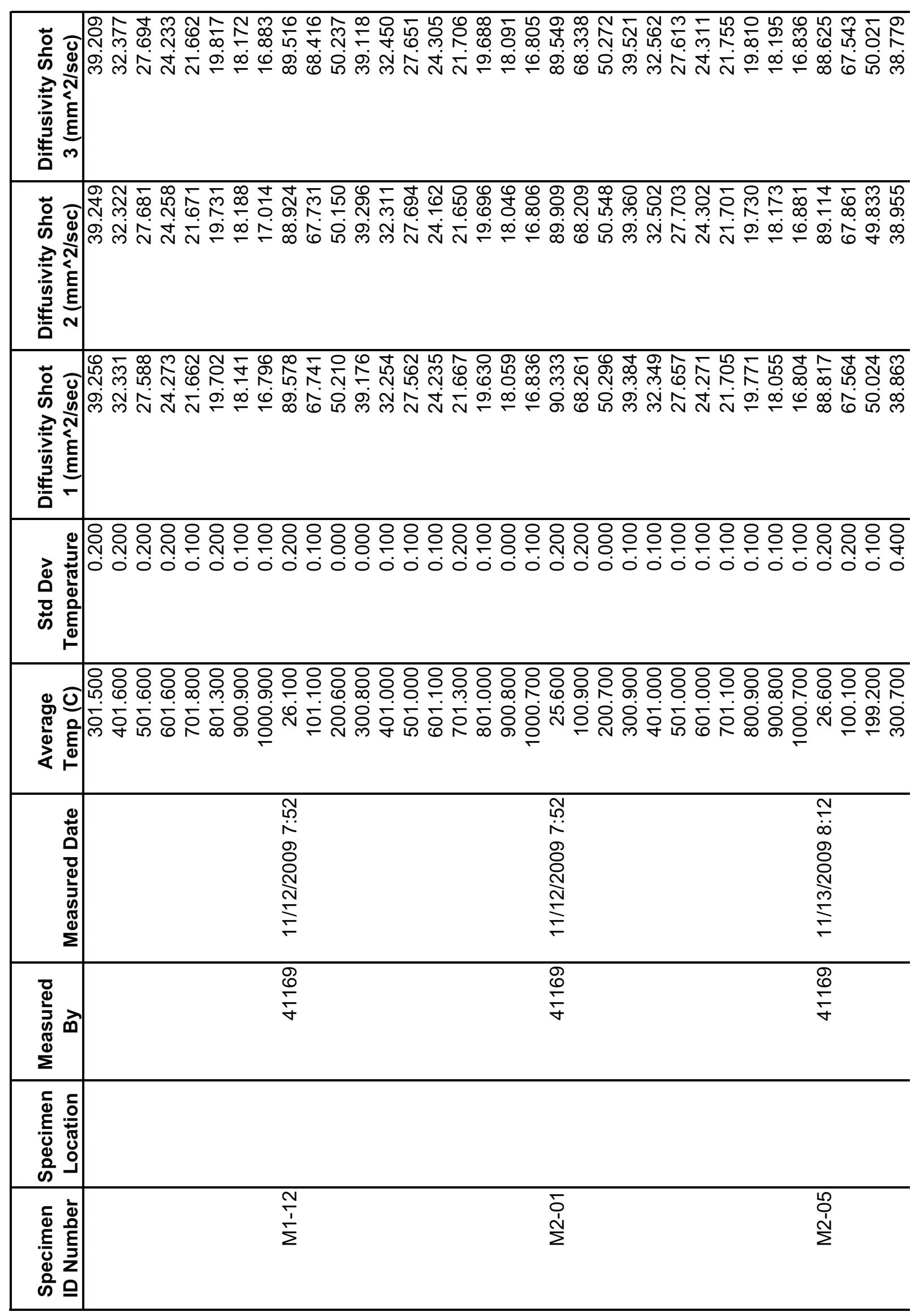




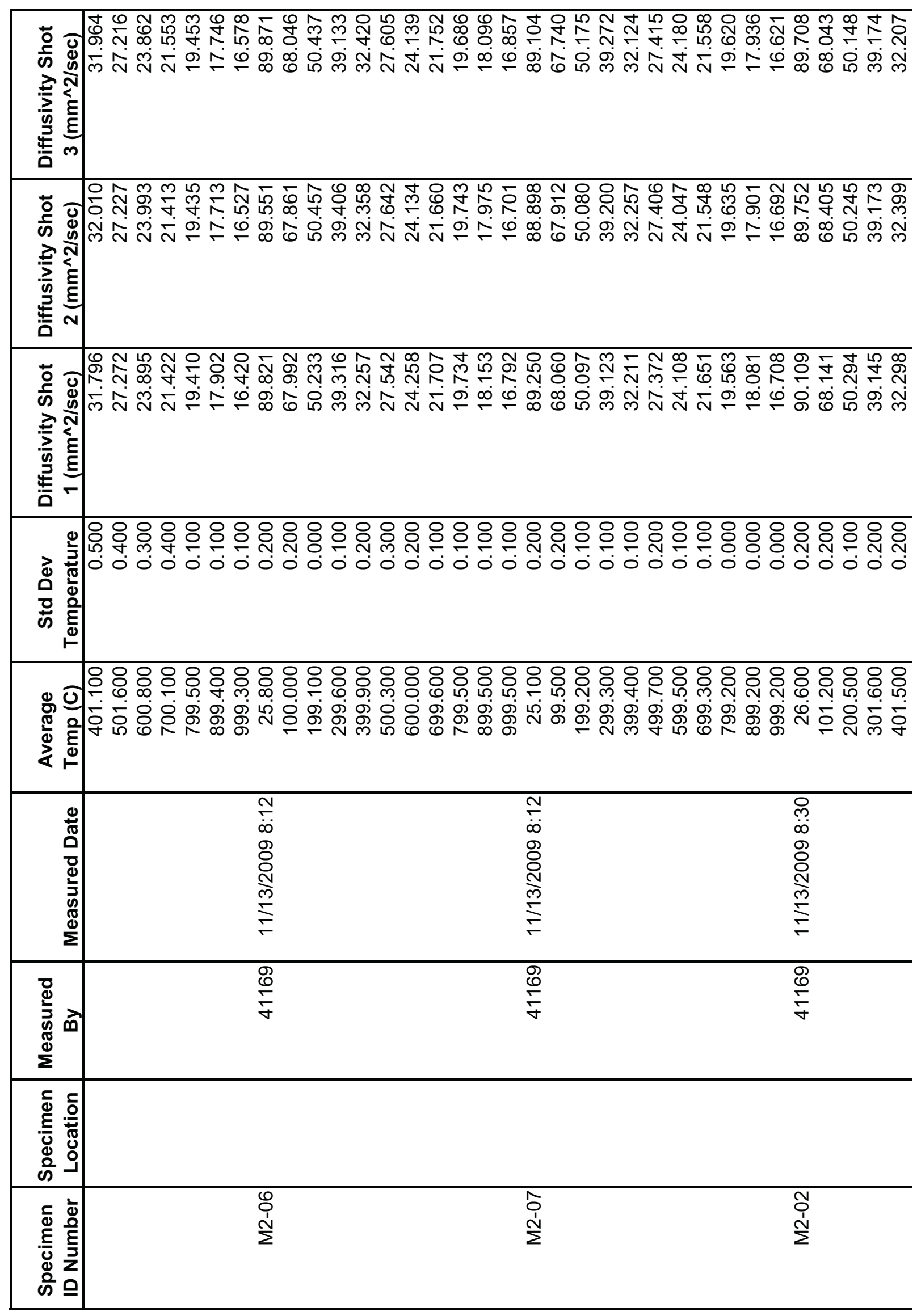




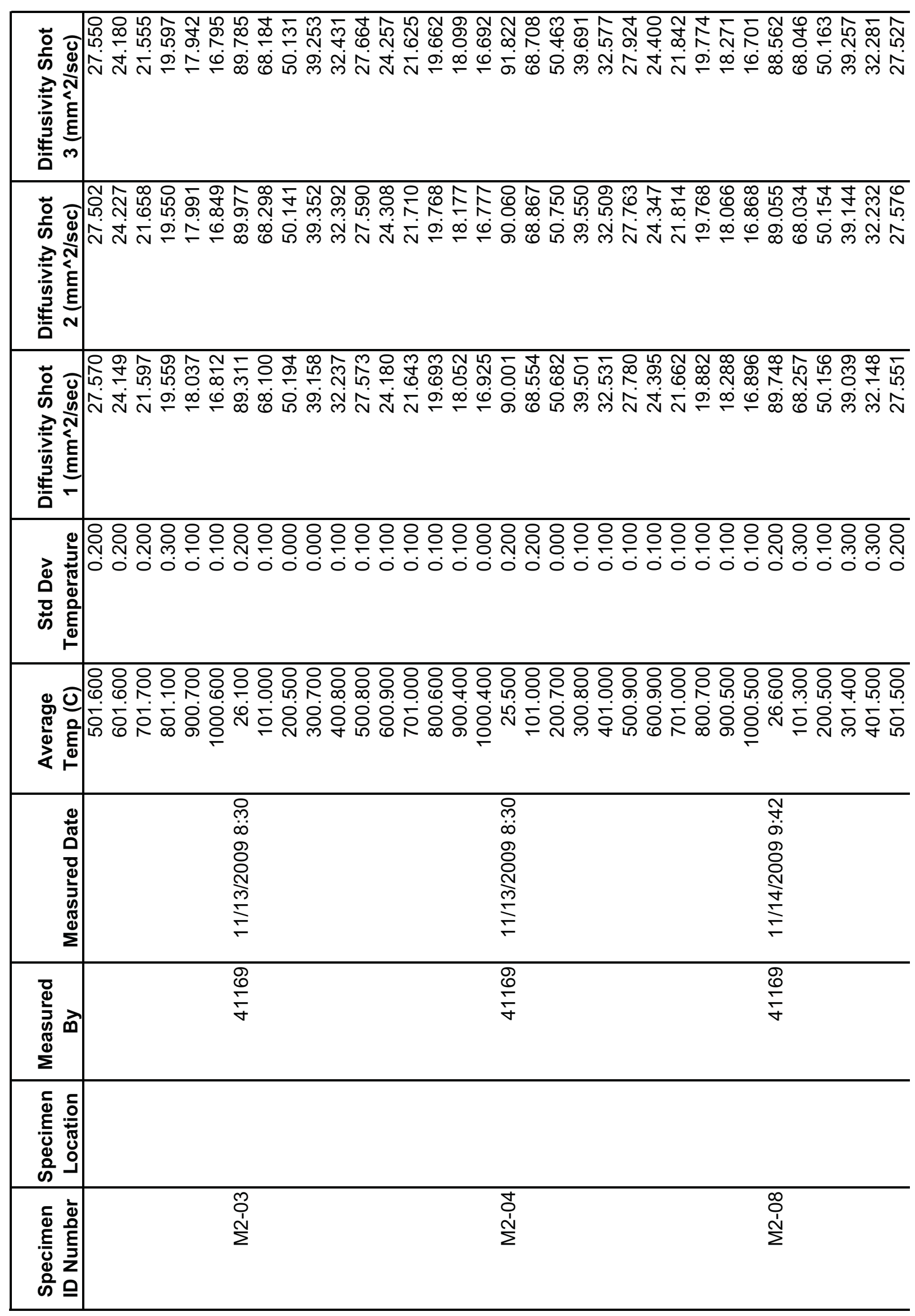




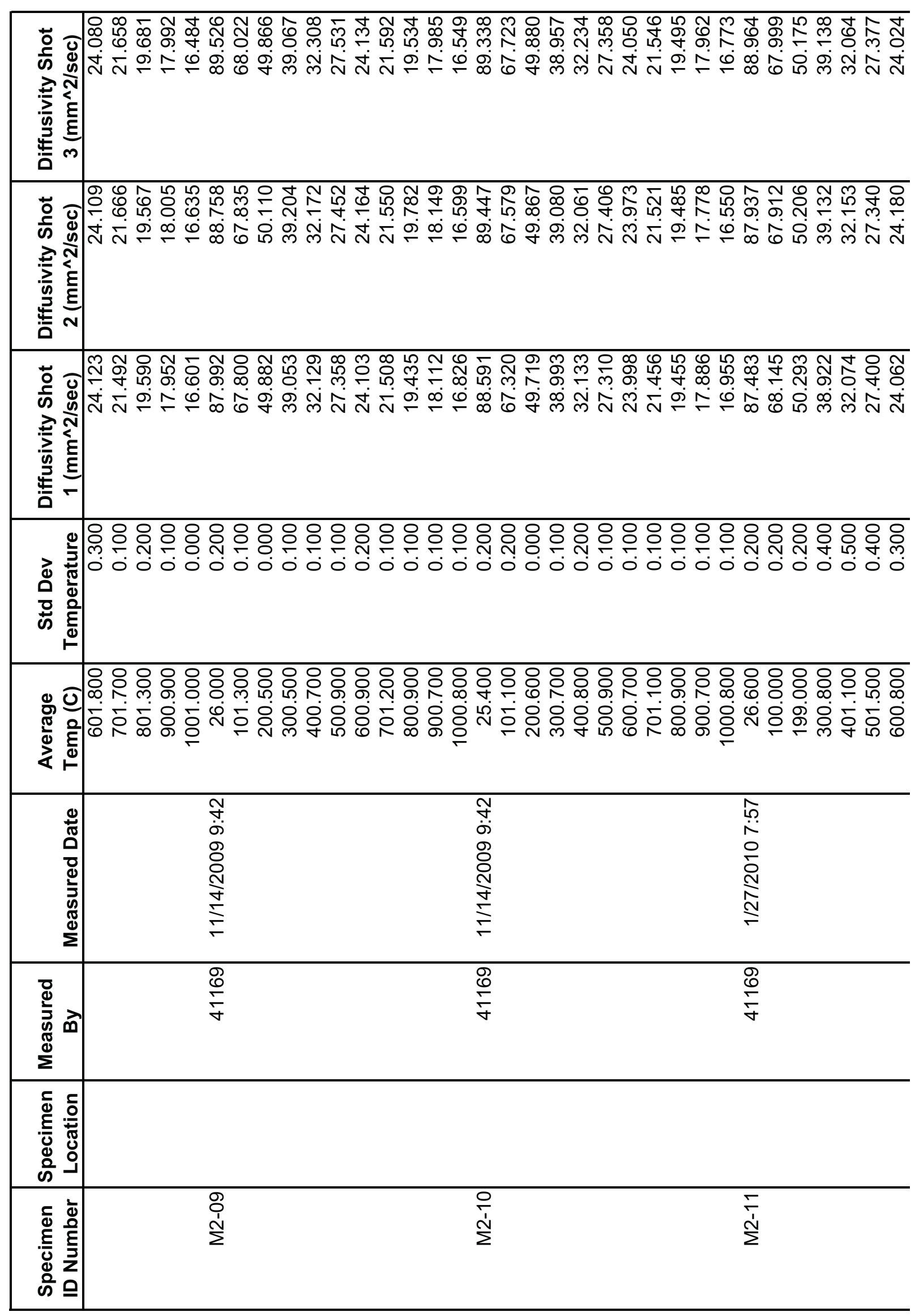




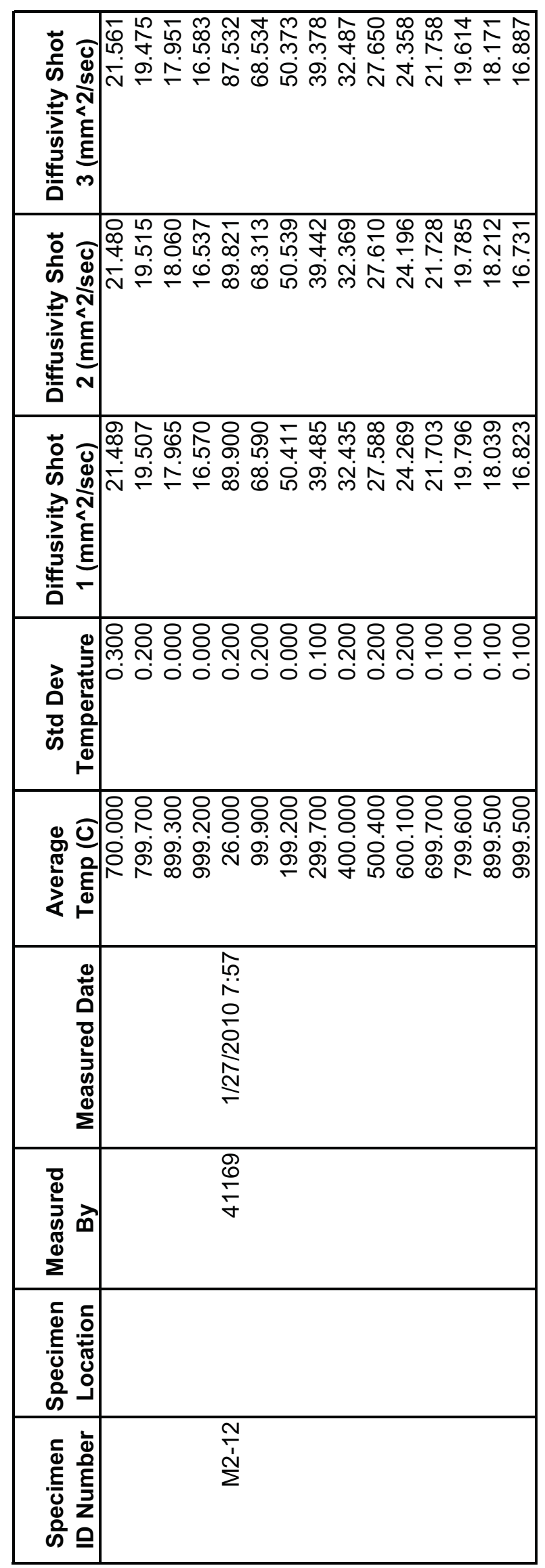




\begin{tabular}{|c|c|c|c|c|}
\hline 列 & 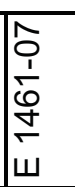 & 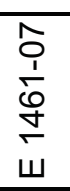 & 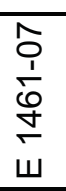 & 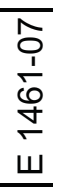 \\
\hline 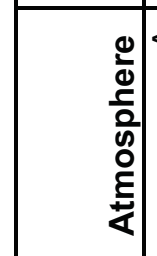 & & $\overline{\dot{z}}$ & $\bar{k}$ & $\bar{i}$ \\
\hline 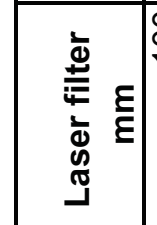 & & 음 & 음 & 음 \\
\hline 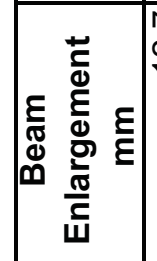 & $\stackrel{\hat{N}}{\stackrel{N}{ }}$ & $\stackrel{\stackrel{N}{\sim}}{\sim}$ & $\stackrel{\widehat{N}}{\stackrel{N}{S}}$ & 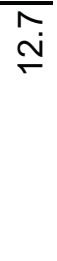 \\
\hline 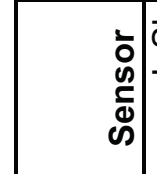 & & $\begin{array}{l}\text { की } \\
\text { की }\end{array}$ & $\begin{array}{l}\text { की } \\
\text { क्ञ }\end{array}$ & की \\
\hline 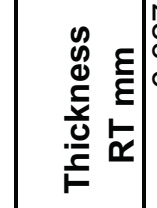 & & $\begin{array}{l}\hat{N} \\
\text { ָे } \\
\hat{0}\end{array}$ & 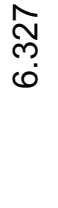 & $\begin{array}{c}\text { స్ } \\
\text { iे }\end{array}$ \\
\hline 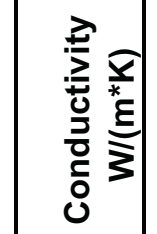 & & & & \\
\hline 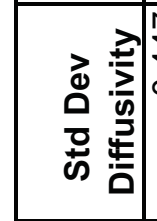 & & S & $\bar{c}$ & \\
\hline 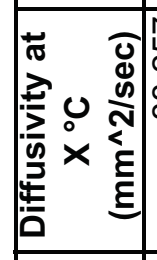 & 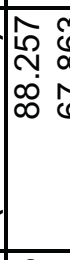 & $\begin{array}{l}N \\
\\
\infty \\
\infty \\
\infty \\
\infty\end{array}$ & 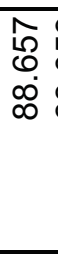 & 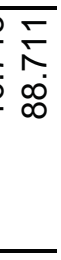 \\
\hline 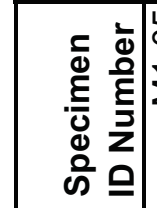 & & 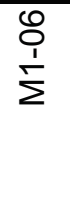 & $\begin{array}{l}\bar{N} \\
\frac{1}{\Sigma}\end{array}$ & $\begin{array}{l}\bar{\sigma} \\
\frac{1}{2}\end{array}$ \\
\hline
\end{tabular}




\begin{tabular}{|c|c|c|c|}
\hline 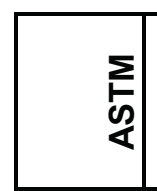 & $\begin{array}{l}\hat{o} \\
\frac{1}{0} \\
\dot{0} \\
\dot{5} \\
\end{array}$ & $\begin{array}{l}\hat{S} \\
\frac{1}{1} \\
\frac{0}{5} \\
\dot{w}\end{array}$ & 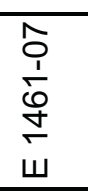 \\
\hline 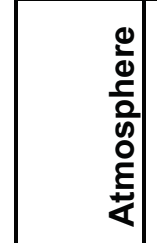 & 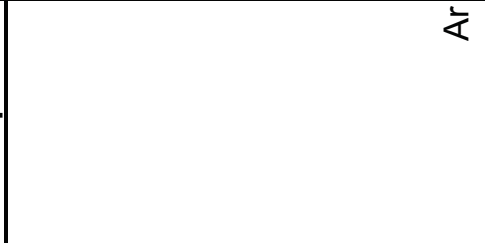 & $\frac{1}{\alpha}$ & 广 \\
\hline 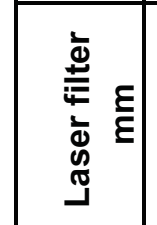 & 음 & 음 & 음 \\
\hline 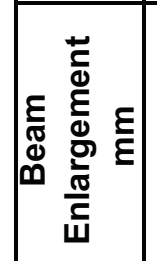 & $\stackrel{\widehat{N}}{\stackrel{N}{N}}$ & 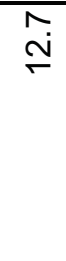 & 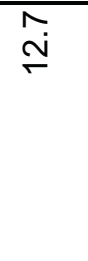 \\
\hline $\begin{array}{l}\bar{b} \\
\vdots \\
心 \\
\Phi \\
\infty\end{array}$ & $\begin{array}{l}\text { की } \\
\text { की }\end{array}$ & 吊 & $\begin{array}{l}\text { की } \\
\text { 足 }\end{array}$ \\
\hline 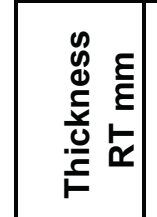 & స్ & 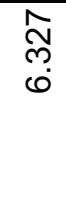 & $\begin{array}{l}\text { స్ } \\
\text { ర్ }\end{array}$ \\
\hline 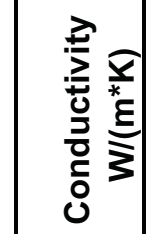 & & & \\
\hline 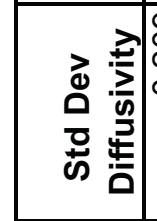 & 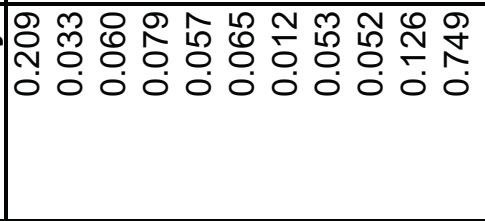 & o- & 总 \\
\hline 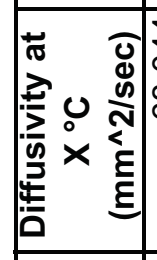 & 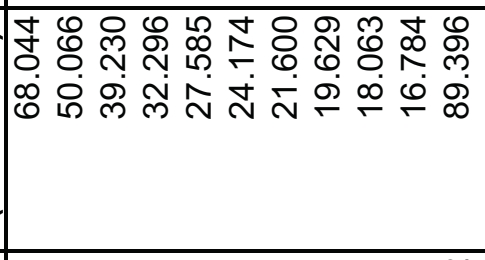 & $\begin{array}{l}\tilde{N} \\
0 \\
\infty \\
\infty \\
\infty\end{array}$ & 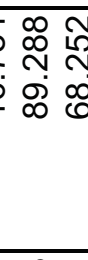 \\
\hline 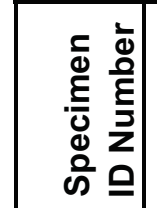 & $\begin{array}{l}\frac{N}{2} \\
\frac{1}{\Sigma}\end{array}$ & $\begin{array}{l}\text { 孛 } \\
\frac{1}{\sum}\end{array}$ & 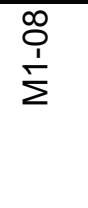 \\
\hline
\end{tabular}




\begin{tabular}{|c|c|c|c|}
\hline 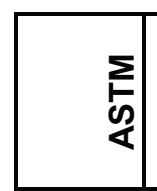 & 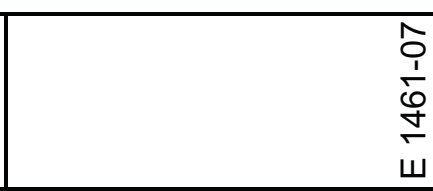 & $\begin{array}{l}\hat{o} \\
\frac{1}{0} \\
\dot{0} \\
\frac{1}{\omega} \\
\end{array}$ & 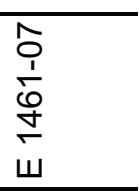 \\
\hline 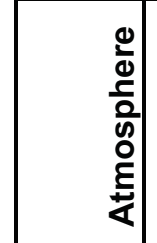 & 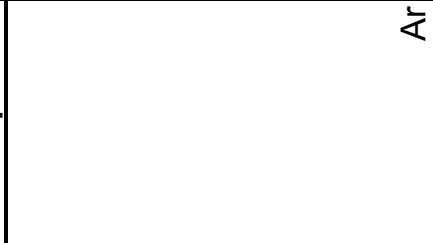 & $\frac{1}{4}$ & $\bar{z}$ \\
\hline 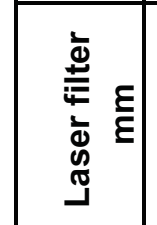 & 음 & 음 & 음 \\
\hline 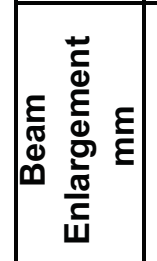 & $\stackrel{\widetilde{N}}{\stackrel{N}{N}}$ & 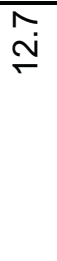 & $\stackrel{\widetilde{N}}{\stackrel{N}{N}}$ \\
\hline $\begin{array}{l}\bar{b} \\
\vdots \\
心 \\
\Phi \\
\infty\end{array}$ & 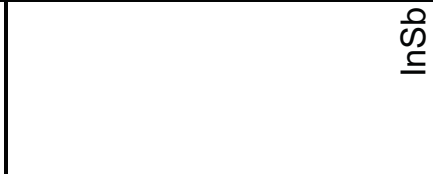 & $\begin{array}{l}\text { की } \\
\text { क् }\end{array}$ & $\begin{array}{l}\text { की } \\
\text { की }\end{array}$ \\
\hline 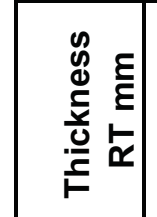 & స్ & 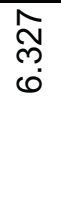 & $\begin{array}{l}\widehat{N} \\
\text { ָे }\end{array}$ \\
\hline 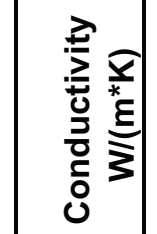 & & & \\
\hline 焉 & 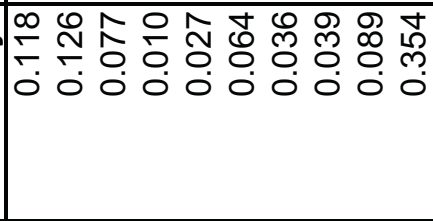 & 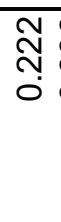 & $\begin{array}{ll}0 & \infty \\
\frac{1}{5} & \frac{1}{8} \\
0 & 0 \\
0 & 0\end{array}$ \\
\hline 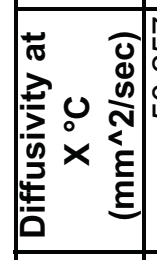 & 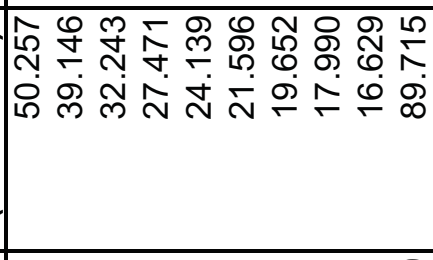 & 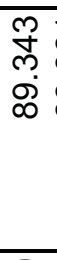 & 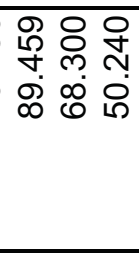 \\
\hline 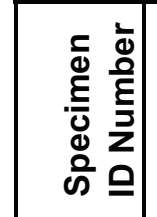 & $\begin{array}{l}8 \\
\frac{1}{2} \\
\frac{1}{2}\end{array}$ & $\frac{\frac{0}{1}}{\Sigma}$ & $\frac{\bar{T}}{\sum}$ \\
\hline
\end{tabular}




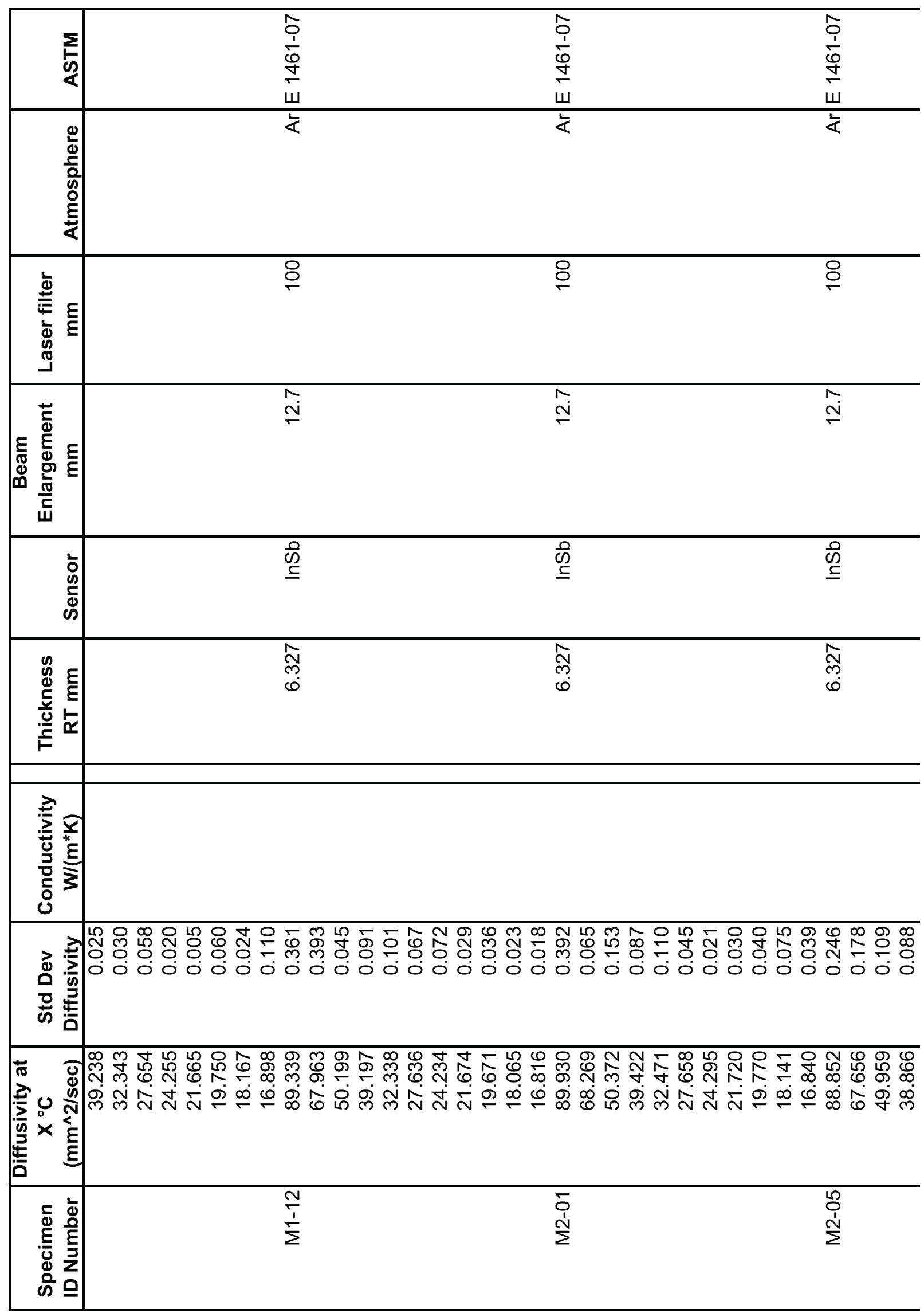




\begin{tabular}{|c|c|c|c|}
\hline 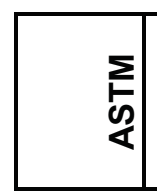 & $\begin{array}{l}\frac{\hat{O}}{1} \\
\frac{1}{6} \\
\frac{1}{+} \\
\text { W }\end{array}$ & $\begin{array}{l}\hat{o} \\
\frac{1}{0} \\
\stackrel{0}{+} \\
\dot{\omega}\end{array}$ & 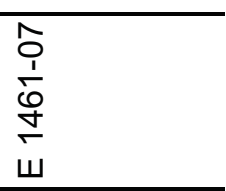 \\
\hline 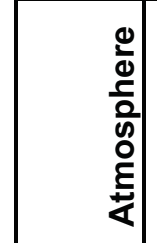 & 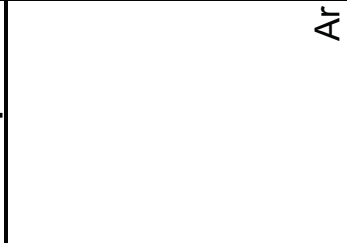 & 衣 & 交 \\
\hline 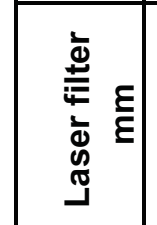 & 음 & 음 & 음 \\
\hline 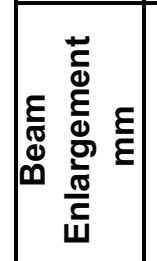 & $\stackrel{\widetilde{N}}{\stackrel{N}{N}}$ & $\stackrel{\stackrel{N}{\sim}}{ }$ & $\stackrel{\stackrel{N}{N}}{ }$ \\
\hline $\begin{array}{l}\bar{b} \\
\vdots \\
心 \\
\Phi \\
\infty\end{array}$ & $\begin{array}{l}\text { की } \\
\text { Dी }\end{array}$ & $\begin{array}{l}\text { की } \\
\text { I }\end{array}$ & $\begin{array}{l}\text { की } \\
\text { Ф }\end{array}$ \\
\hline 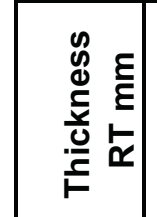 & స్ & $\begin{array}{l}\widehat{N} \\
\text { ర్d }\end{array}$ & $\begin{array}{l}\text { స్ } \\
\text { రె }\end{array}$ \\
\hline 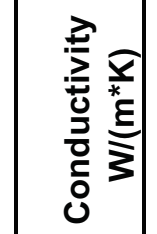 & & & \\
\hline 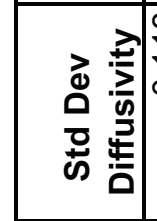 & 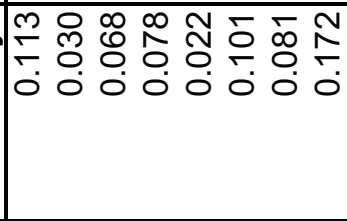 & $\frac{\hat{s}}{5}$ & 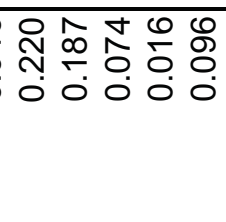 \\
\hline 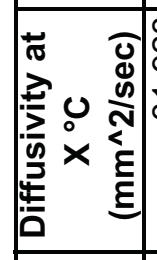 & 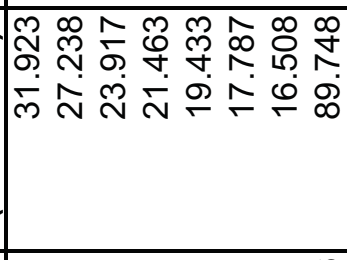 & 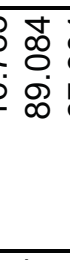 & $\begin{array}{l}0 \\
0\end{array}$ \\
\hline 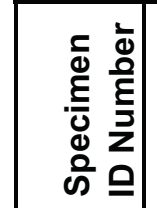 & $\begin{array}{l}\mathbb{Q} \\
\dot{1} \\
\end{array}$ & $\begin{array}{l}\text { No } \\
\stackrel{N}{\Sigma}\end{array}$ & $\begin{array}{l}\text { No } \\
\stackrel{\Lambda}{\Sigma}\end{array}$ \\
\hline
\end{tabular}




\begin{tabular}{|c|c|c|c|}
\hline 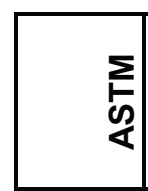 & $\begin{array}{l}\hat{o} \\
\frac{1}{0} \\
\dot{o} \\
\dot{w} \\
\end{array}$ & $\begin{array}{l}\hat{o} \\
\frac{1}{0} \\
\stackrel{0}{+} \\
\dot{\omega}\end{array}$ & $\begin{array}{l}\hat{o} \\
\frac{1}{o} \\
\dot{0} \\
\dot{W} \\
\end{array}$ \\
\hline 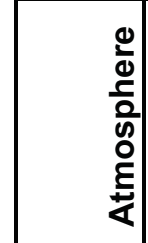 & 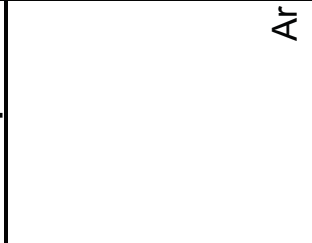 & 㐫 & 交 \\
\hline 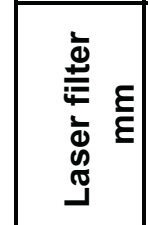 & 음 & 음 & 음 \\
\hline 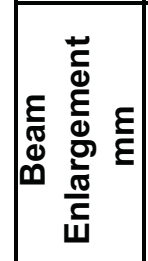 & $\stackrel{\widehat{N}}{\stackrel{N}{N}}$ & 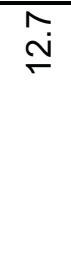 & $\stackrel{\stackrel{\sim}{\sim}}{ }$ \\
\hline 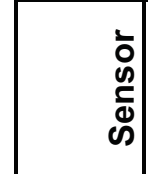 & $\begin{array}{l}\text { की } \\
\text { की }\end{array}$ & 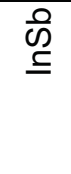 & $\begin{array}{l}\text { की } \\
\text { 足 }\end{array}$ \\
\hline 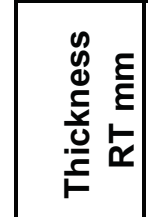 & 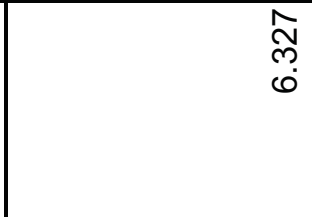 & $\begin{array}{l}\widehat{N} \\
\text { రె }\end{array}$ & $\begin{array}{l}\text { స్ } \\
\text { ర్d }\end{array}$ \\
\hline 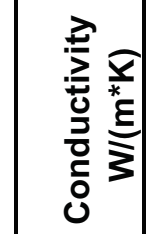 & & & \\
\hline 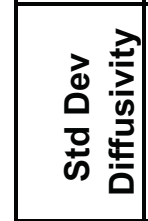 & 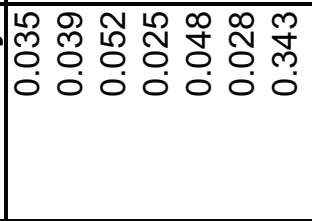 & 怘 & 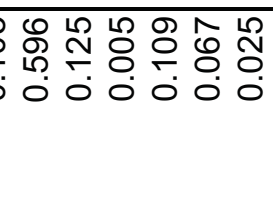 \\
\hline 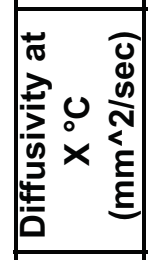 & 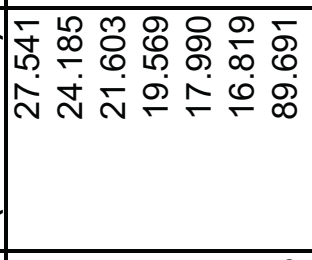 & 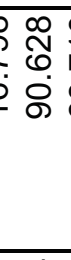 & 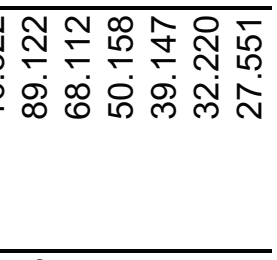 \\
\hline 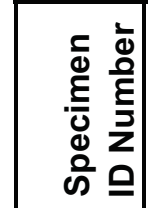 & $\begin{array}{l}\text { on } \\
\dot{N} \\
\text { Ny }\end{array}$ & $\begin{array}{l}\text { ¿े } \\
\stackrel{1}{\Sigma}\end{array}$ & 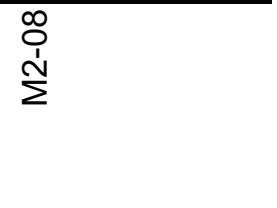 \\
\hline
\end{tabular}




\begin{tabular}{|c|c|c|c|}
\hline 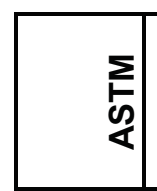 & $\begin{array}{l}\hat{o} \\
\frac{1}{0} \\
\frac{0}{+} \\
\dot{W}\end{array}$ & 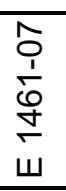 & $\begin{array}{l}\hat{O} \\
\frac{1}{0} \\
\stackrel{0}{+} \\
\dot{w}\end{array}$ \\
\hline 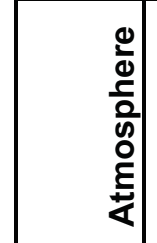 & 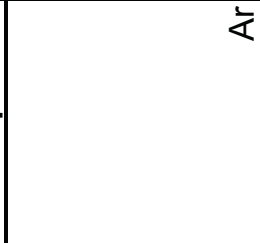 & 文 & $\bar{z}$ \\
\hline 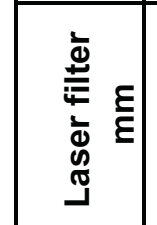 & 움 & 음 & 음 \\
\hline 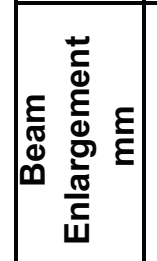 & $\stackrel{\text { }}{\stackrel{i}{\mathrm{j}}}$ & 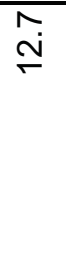 & 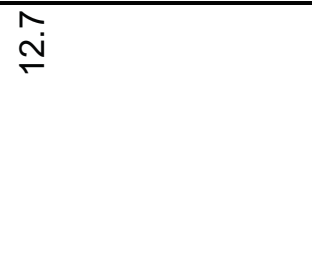 \\
\hline $\begin{array}{l}\bar{b} \\
0 \\
\stackrel{0}{\Phi} \\
心\end{array}$ & $\begin{array}{l}\text { की } \\
\text { 足 }\end{array}$ & $\begin{array}{l}\text { की } \\
\text { की }\end{array}$ & $\begin{array}{l}\text { की } \\
\text { 足 }\end{array}$ \\
\hline 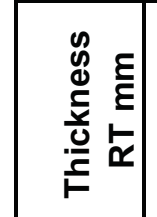 & 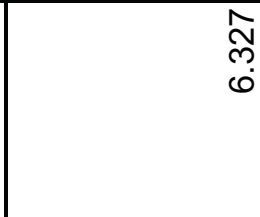 & $\begin{array}{l}\widehat{N} \\
\text { ల్ }\end{array}$ & $\begin{array}{l}\text { స్ } \\
\text { ర్ }\end{array}$ \\
\hline 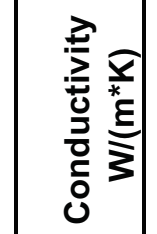 & & & \\
\hline 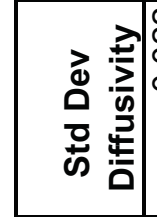 & 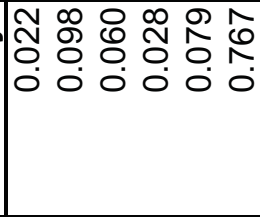 & 0 & 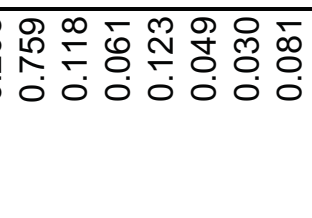 \\
\hline 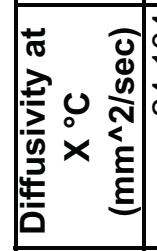 & 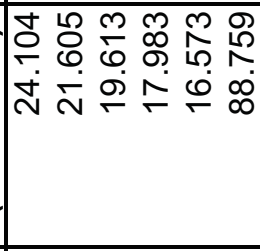 & & 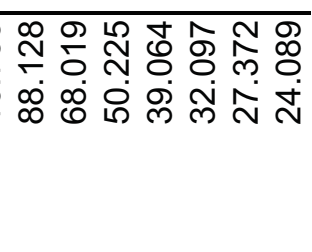 \\
\hline 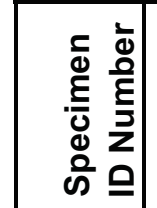 & $\begin{array}{l}\text { Oे } \\
\dot{1} \\
\text { N }\end{array}$ & 㐫 & 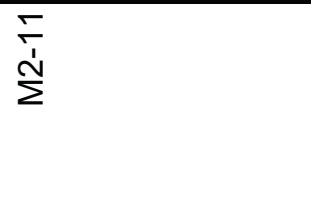 \\
\hline
\end{tabular}




\begin{tabular}{|c|c|}
\hline$\underset{\frac{2}{8}}{\frac{\Sigma}{8}}$ & 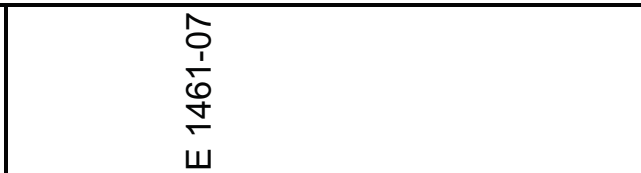 \\
\hline 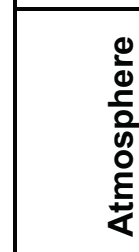 & $\bar{z}$ \\
\hline 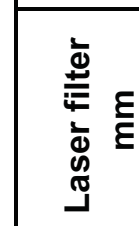 & 응 \\
\hline 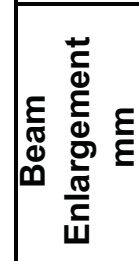 & $\stackrel{\stackrel{N}{\sim}}{\stackrel{N}{r}}$ \\
\hline 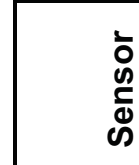 & $\begin{array}{l}\text { की } \\
\text { 号 }\end{array}$ \\
\hline 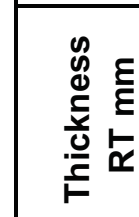 & $\begin{array}{l}\hat{\widetilde{N}} \\
0\end{array}$ \\
\hline 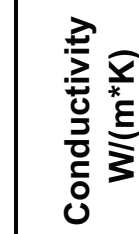 & \\
\hline 这弯 & 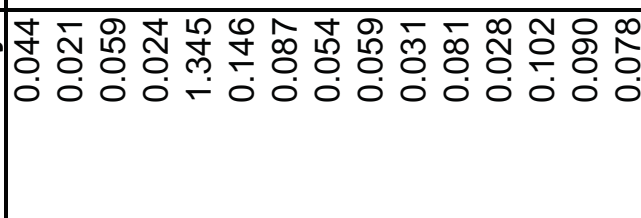 \\
\hline 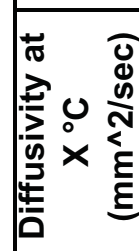 & 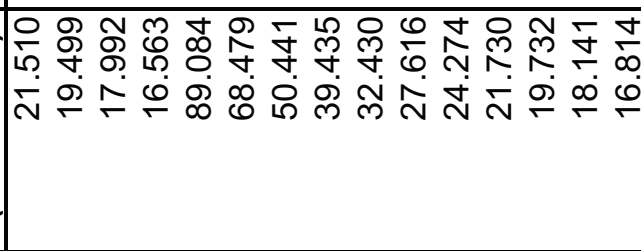 \\
\hline 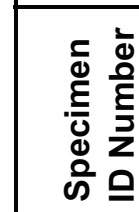 & $\begin{array}{l}\stackrel{N}{\dot{N}} \\
\stackrel{N}{\Sigma}\end{array}$ \\
\hline
\end{tabular}




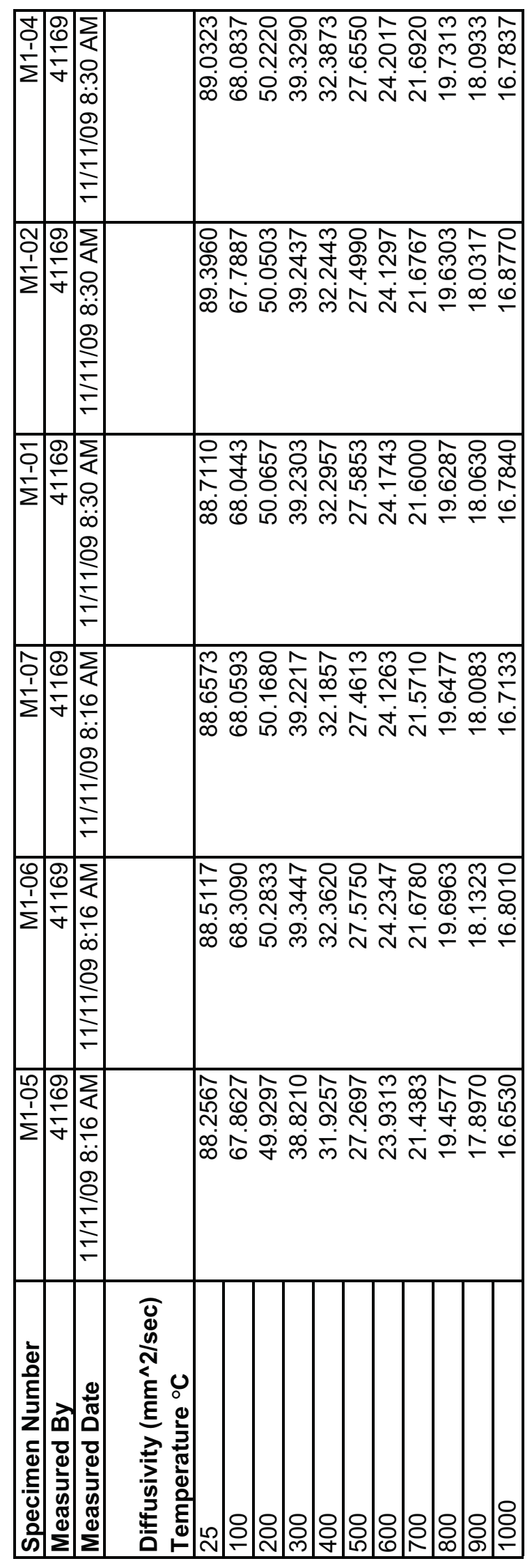




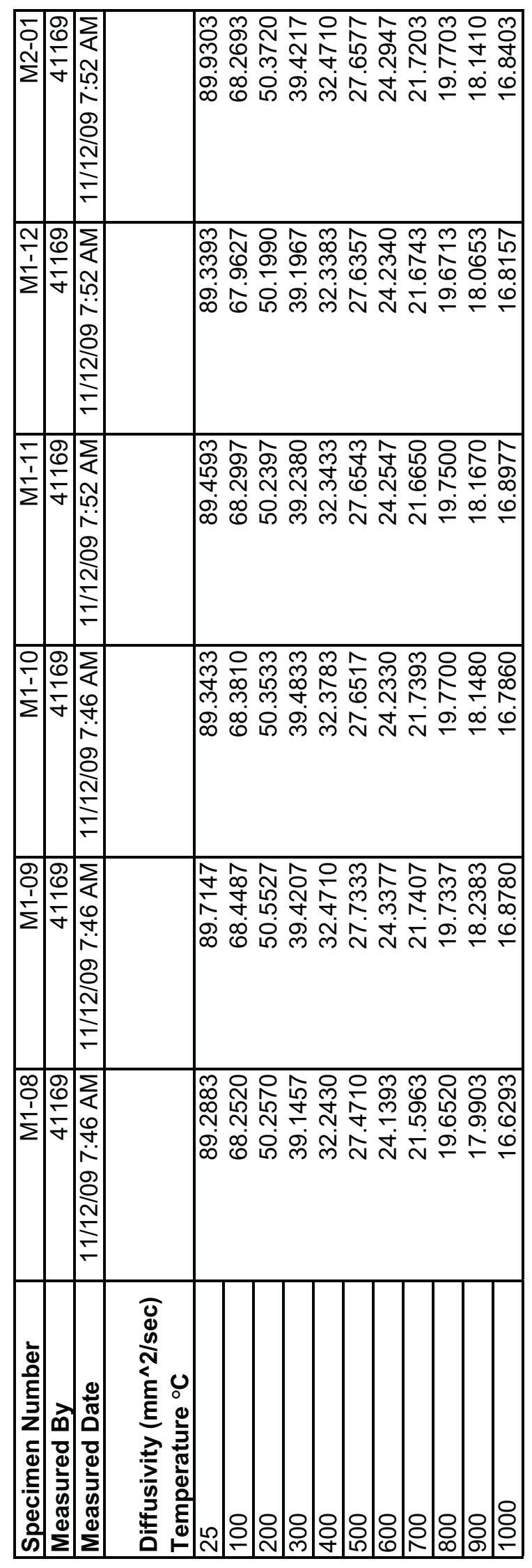




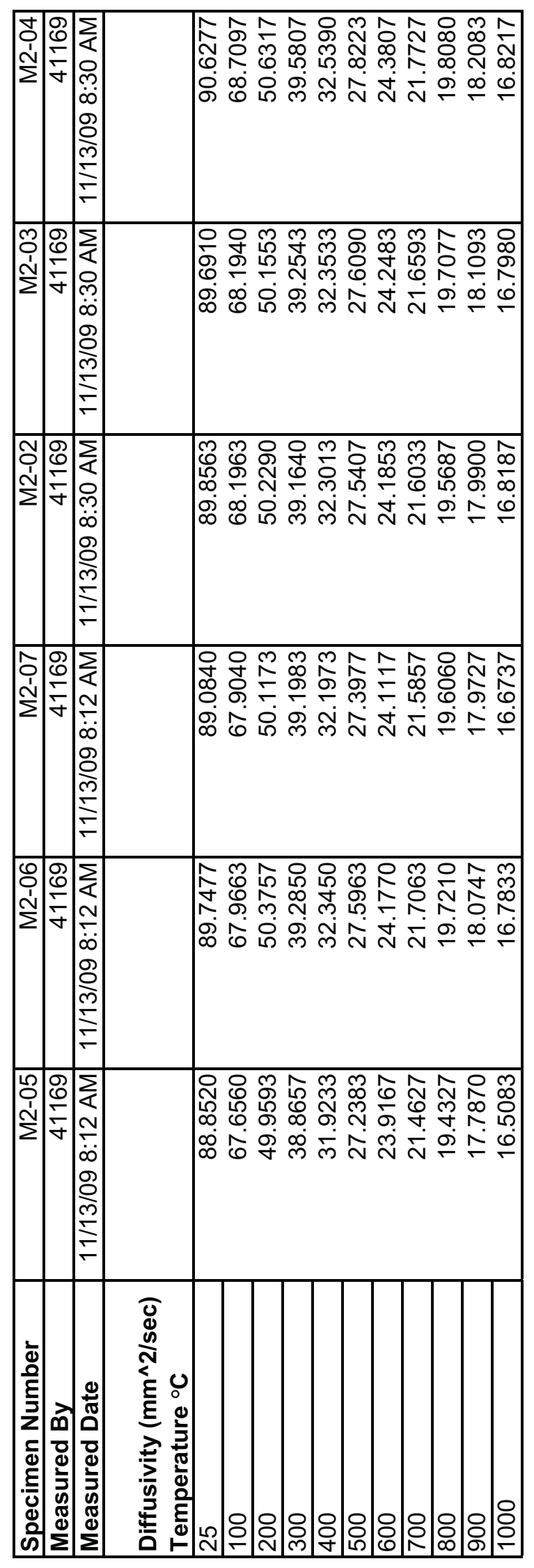




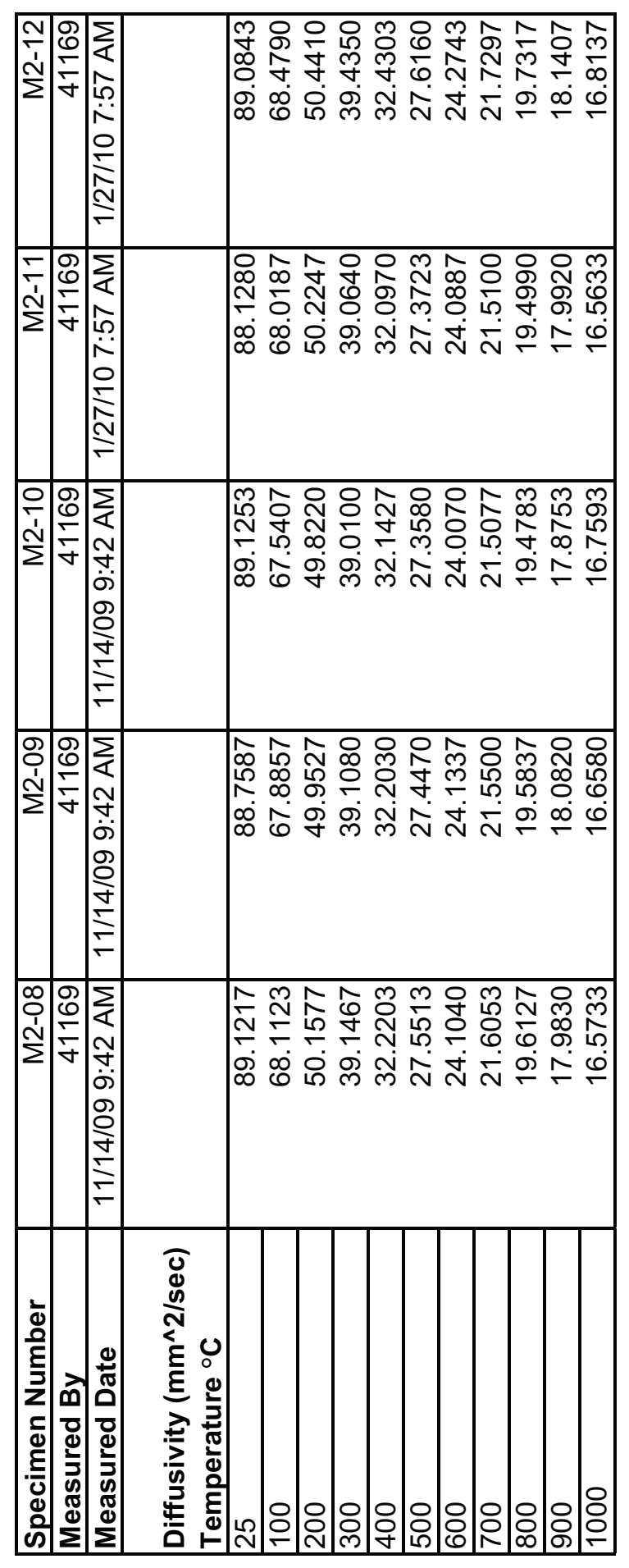

음 


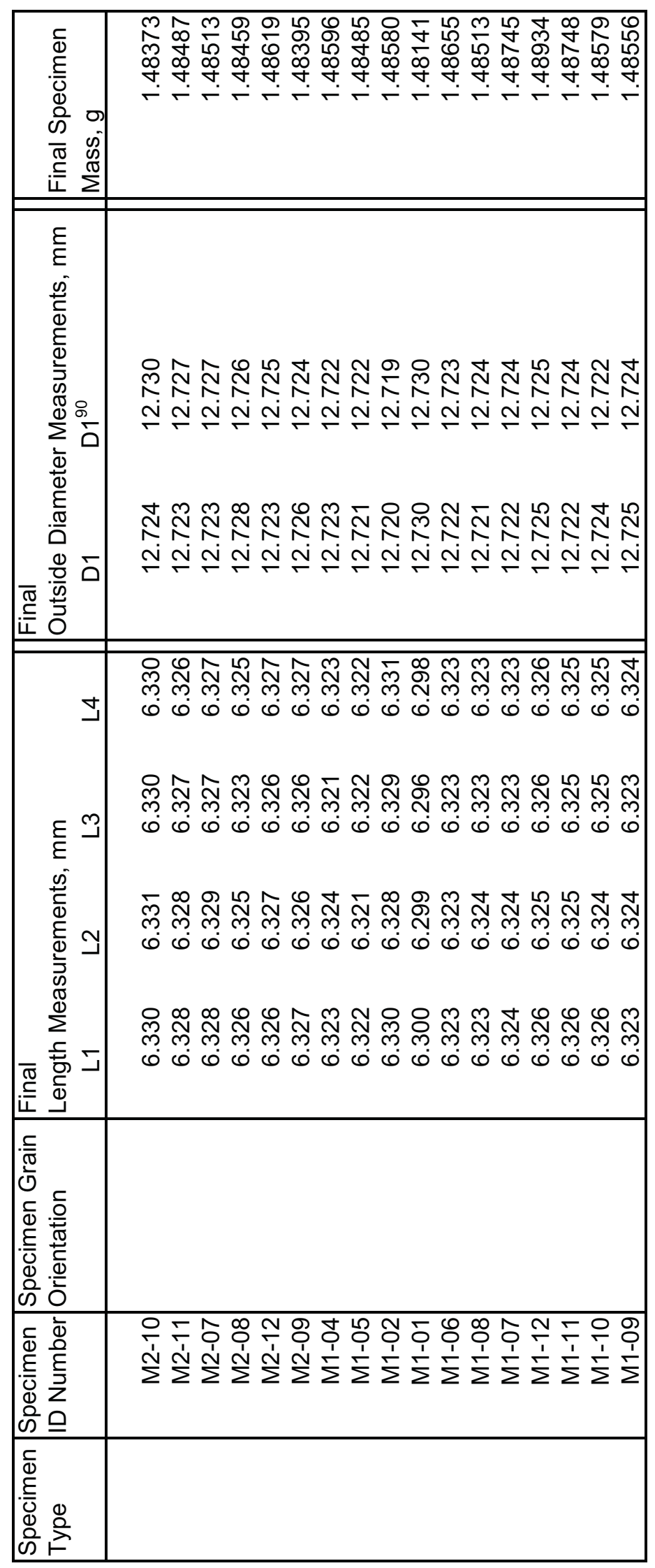




\begin{tabular}{|c|c|}
\hline 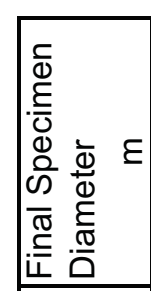 & 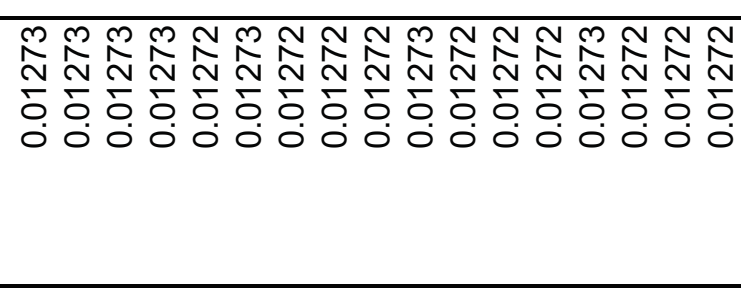 \\
\hline 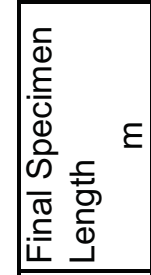 & 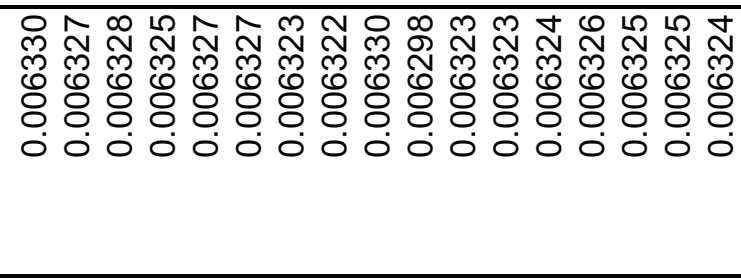 \\
\hline 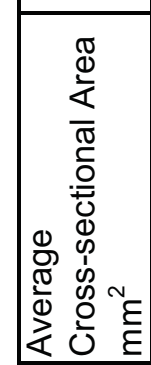 & 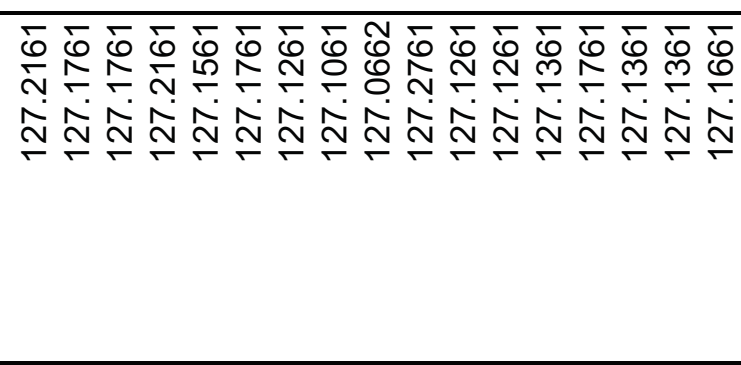 \\
\hline 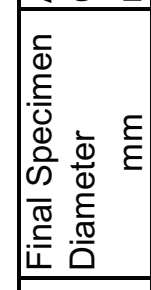 & 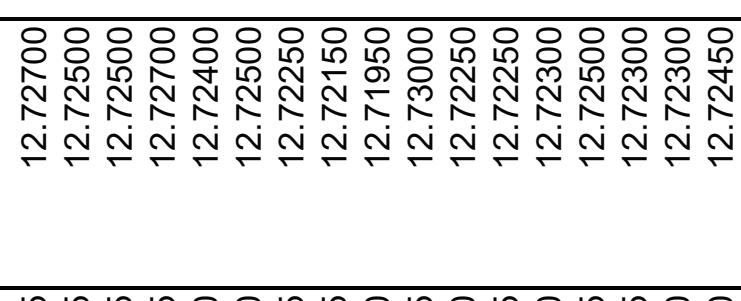 \\
\hline $\bar{\varepsilon}$ & 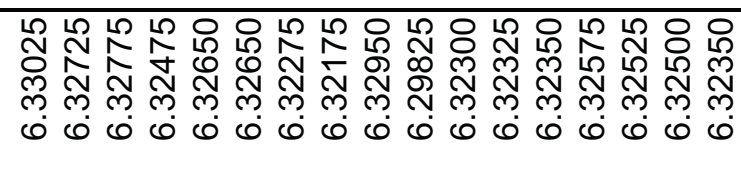 \\
\hline 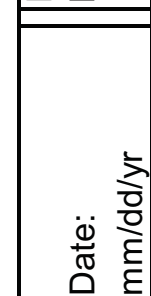 & 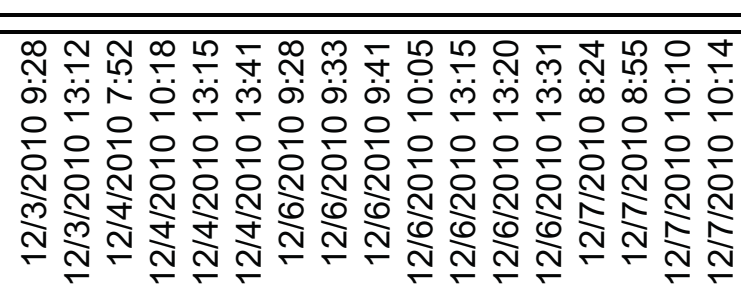 \\
\hline 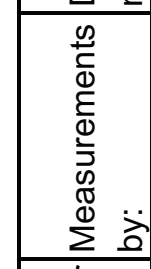 & 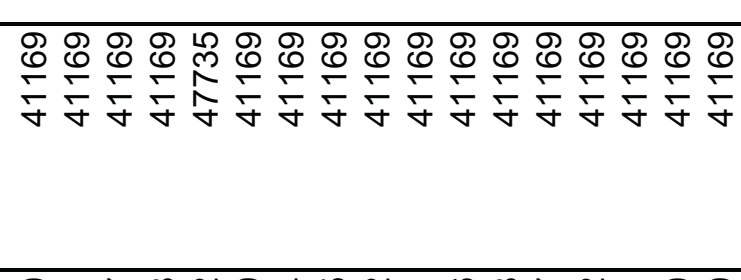 \\
\hline 这 & 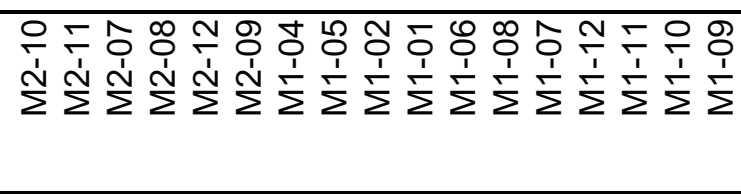 \\
\hline
\end{tabular}




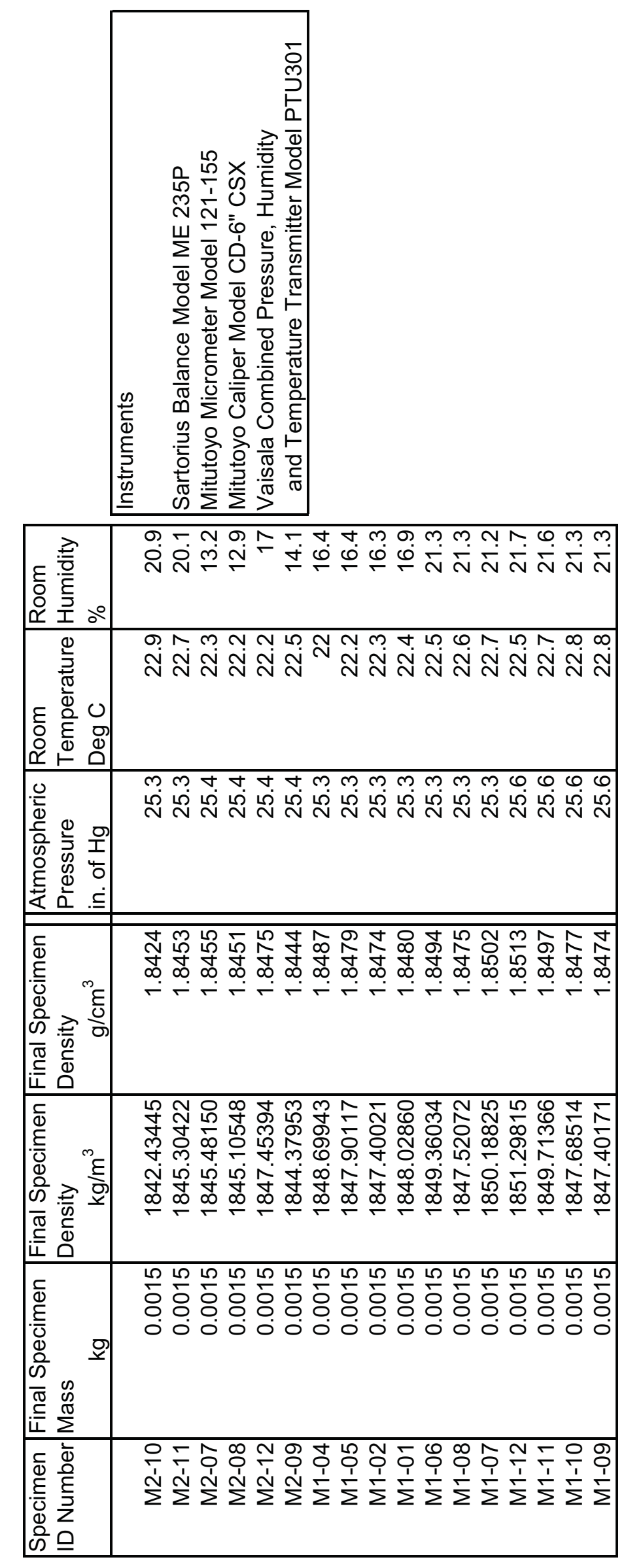


Graphite Grade:

Graphite Manufacturer:

Forming Process:

Coke Particle Size:

Coke Type:

ASTM Class:

Specimen Geometry:
A-3 New Matrix

ORNL

Unknown

Unknown

Unknown

Unknown

Cylinder

Specimen ID \#'s:

A3-H08-Z07

A3-H08-Z19

A3-H08-Z01

A3-P33-Z09

A3-P33-Z20

A3-P33-Z18

A3-P43-Z03

A3-P43-Z12

A3-P43-Z06

A3-H08-Z07p

A3-H08-Z19p

A3-H08-Z01p

A3-P33-Z09p

A3-P33-Z20p

A3-P33-Z18p

A3-P43-Z03p

A3-P43-Z12p

A3-P43-Z06p 


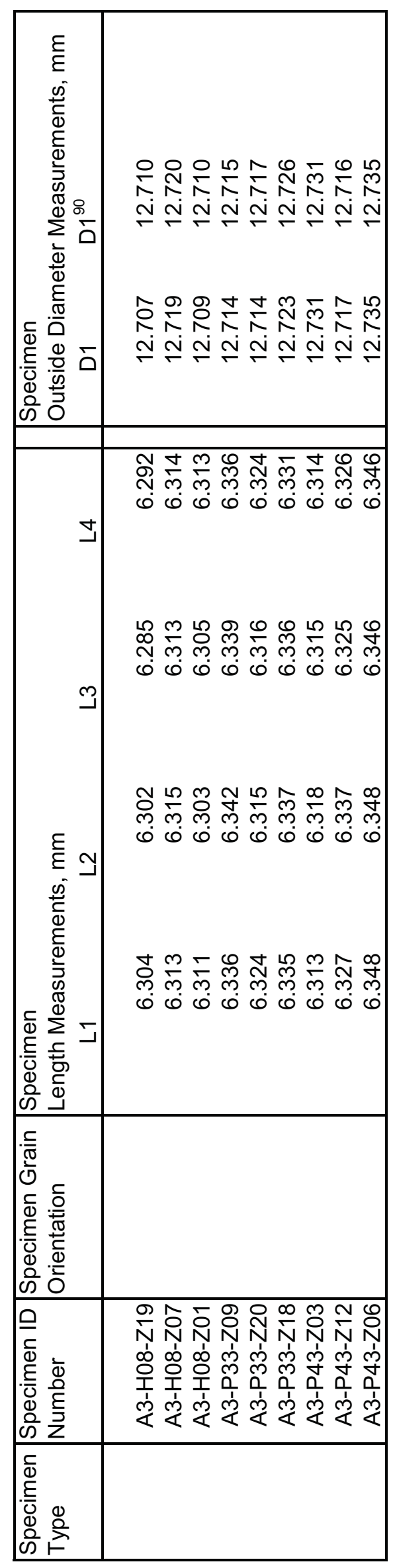

6 


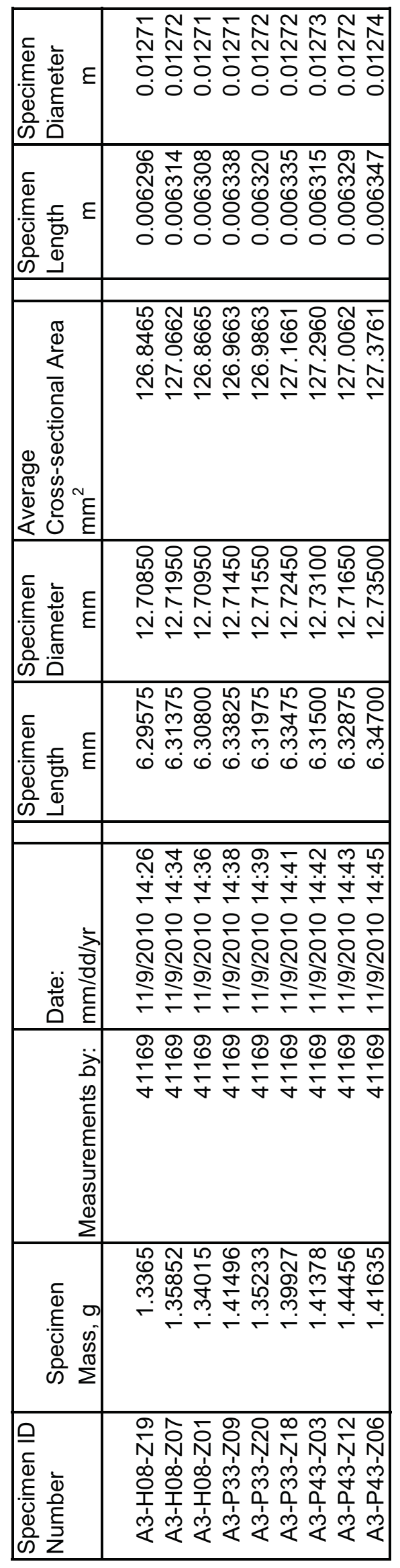




\begin{tabular}{|c|c|}
\hline 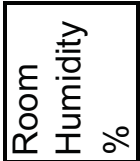 & 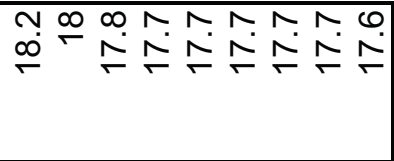 \\
\hline 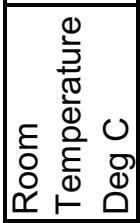 & 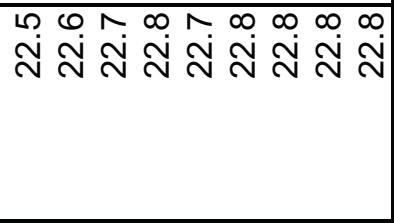 \\
\hline 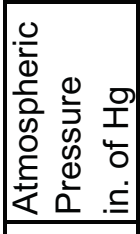 & 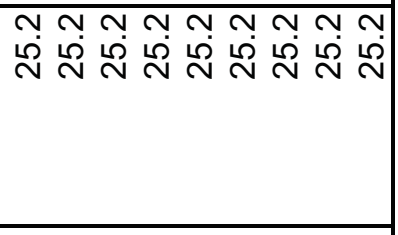 \\
\hline 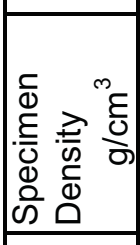 & 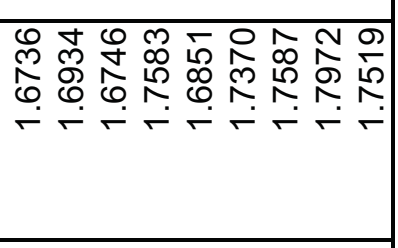 \\
\hline 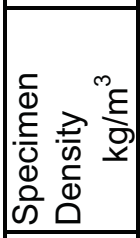 & 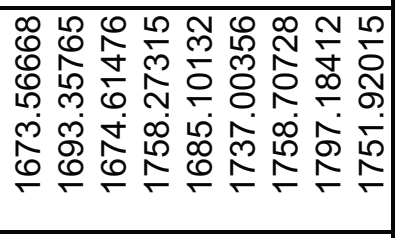 \\
\hline 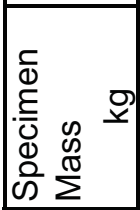 & 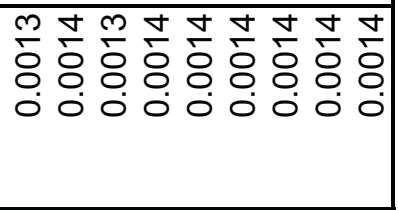 \\
\hline 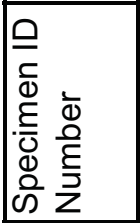 & 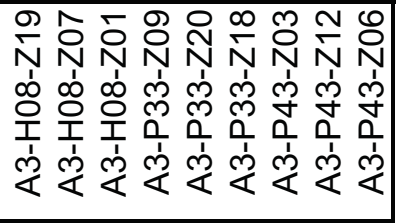 \\
\hline
\end{tabular}

$\underline{\sigma}$ 


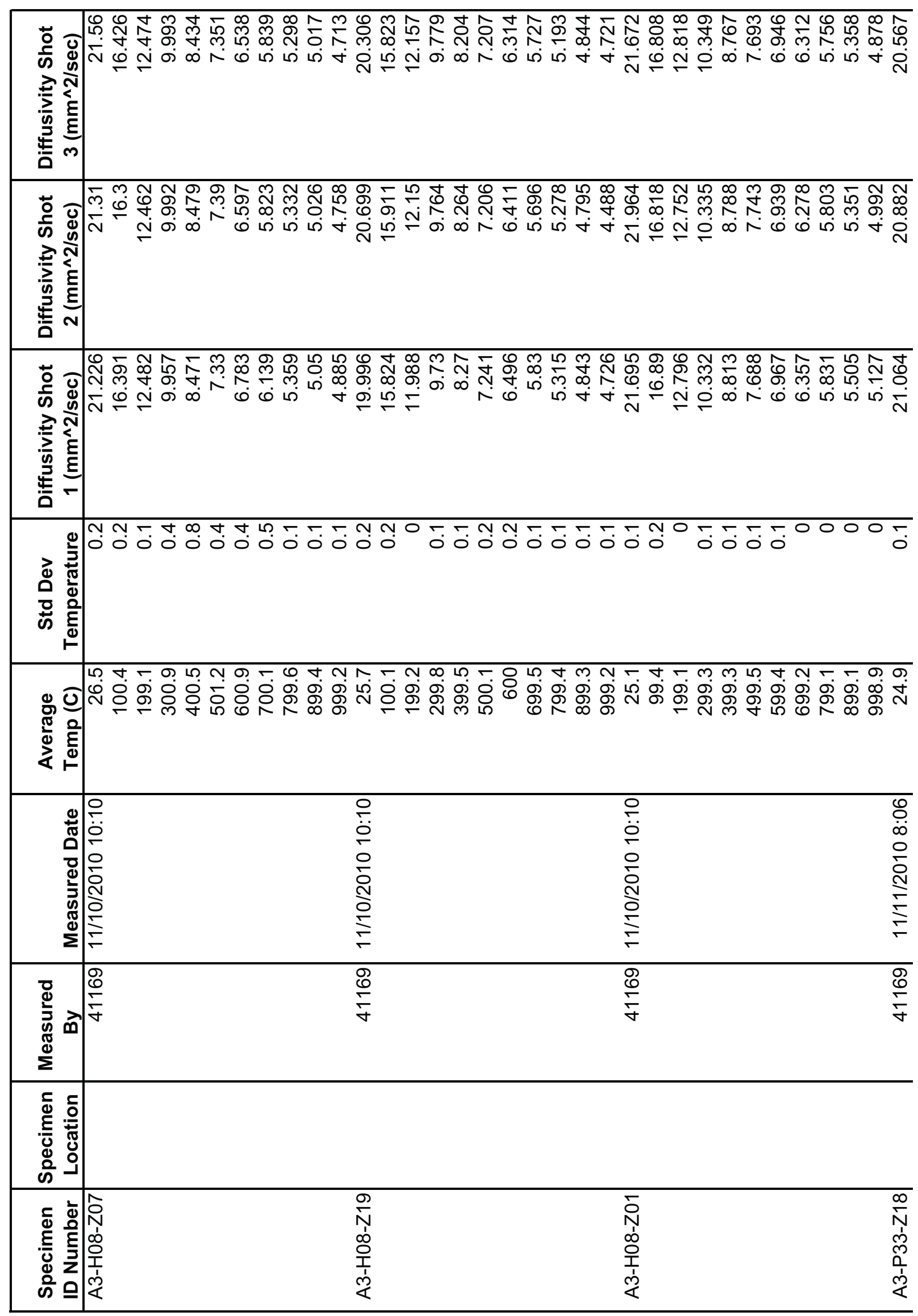




\begin{tabular}{|c|c|c|c|}
\hline 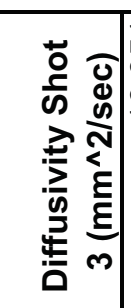 & \multicolumn{3}{|c|}{ 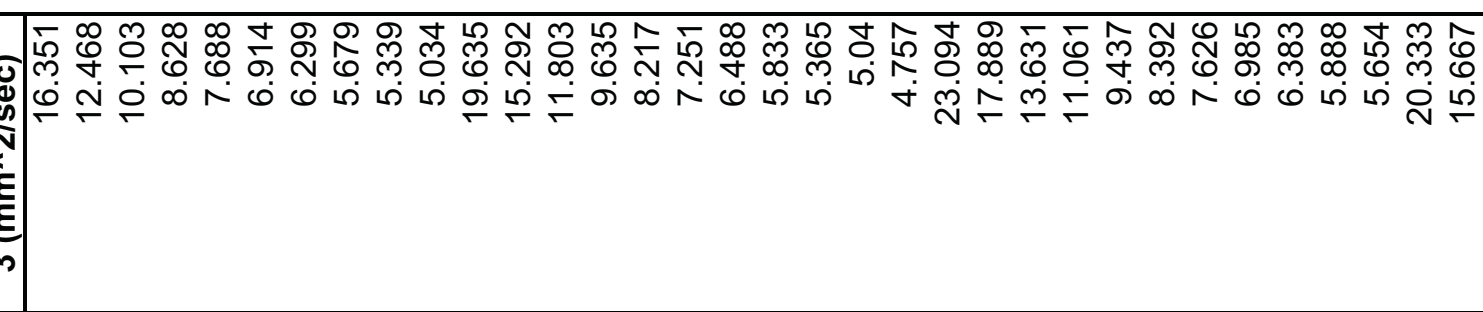 } \\
\hline 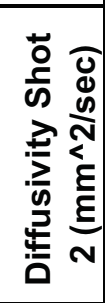 & 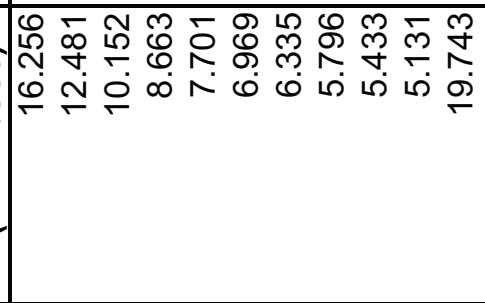 & ' & 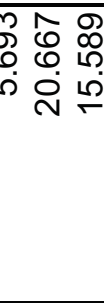 \\
\hline 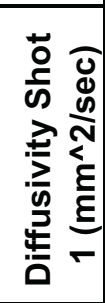 & 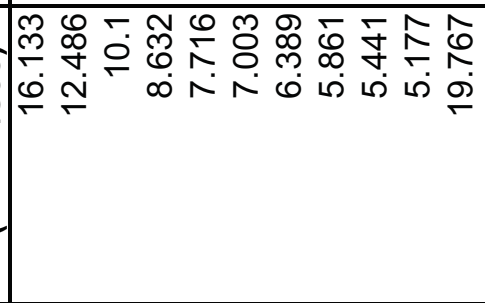 & 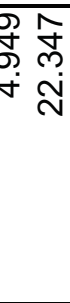 & مُ \\
\hline 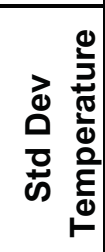 & 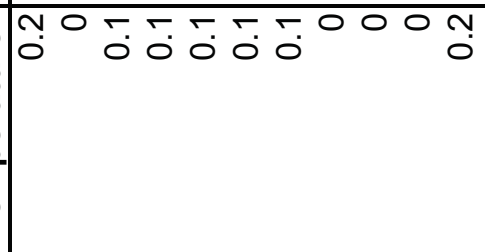 & $\overline{6}$ & $\bar{c}$ \\
\hline 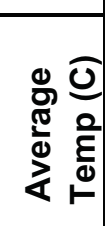 & 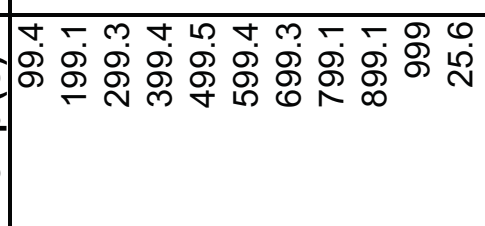 & & 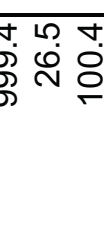 \\
\hline 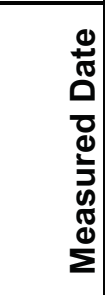 & 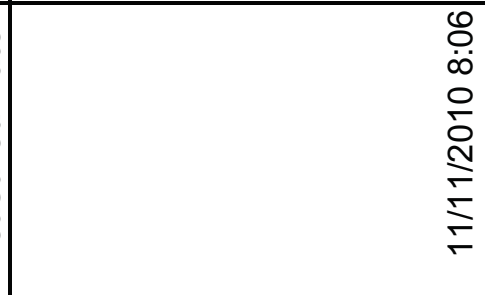 & 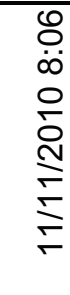 & 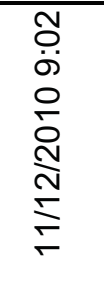 \\
\hline 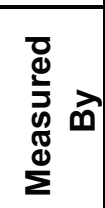 & $\begin{array}{l}\stackrel{\mathscr{O}}{0} \\
\frac{0}{q}\end{array}$ & $\frac{8}{\frac{8}{6}}$ & $\begin{array}{l}\stackrel{8}{6} \\
\frac{\sigma}{\dot{\gamma}}\end{array}$ \\
\hline 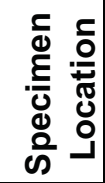 & & & \\
\hline 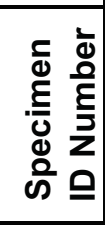 & 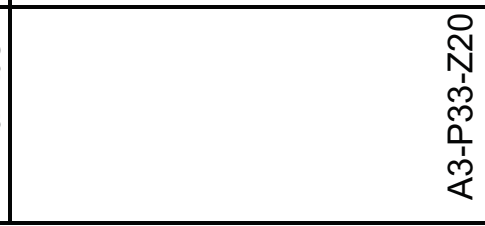 & 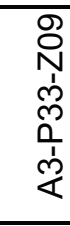 & 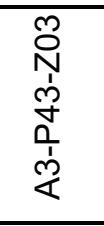 \\
\hline
\end{tabular}




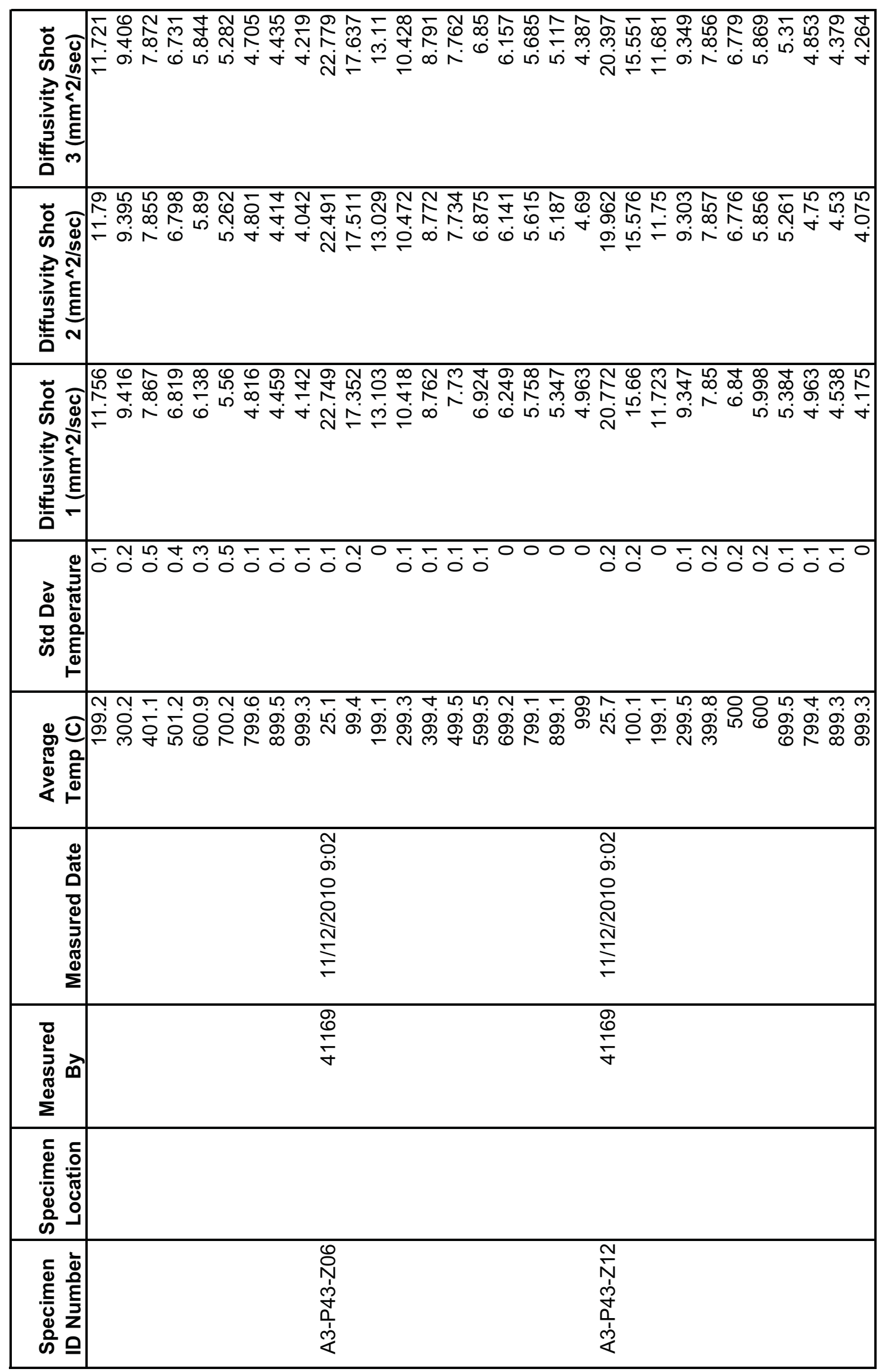




\begin{tabular}{|c|c|c|c|c|}
\hline$\underset{⿱ ㇒}{\infty} \mid$ & 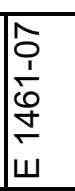 & $\begin{array}{l}\frac{0}{1} \\
\frac{1}{0} \\
\frac{1}{\square} \\
\dot{w}\end{array}$ & $\begin{array}{l}\hat{o} \\
\frac{1}{0} \\
\frac{\sigma}{+} \\
\dot{\omega}\end{array}$ & 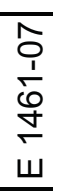 \\
\hline \begin{tabular}{l|} 
\\
$\frac{0}{0}$ \\
$\frac{0}{0}$ \\
0 \\
0 \\
$\frac{0}{\alpha}$
\end{tabular} & & 交 & $\bar{z}$ & $\dot{z}$ \\
\hline 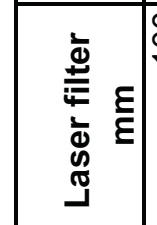 & & 음 & 음 & 음 \\
\hline 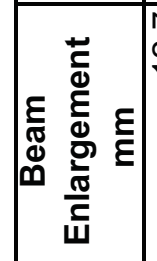 & & 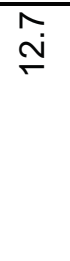 & $\stackrel{\widetilde{N}}{\widetilde{N}}$ & 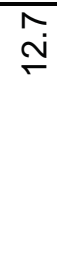 \\
\hline 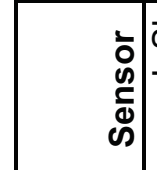 & 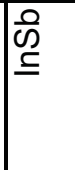 & $\begin{array}{l}\text { की } \\
\text { की }\end{array}$ & 吕 & $\begin{array}{l}\text { की } \\
\text { Dी }\end{array}$ \\
\hline 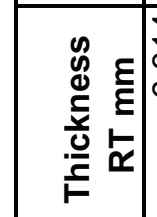 & & 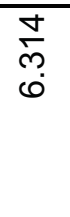 & 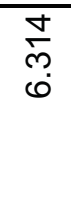 & 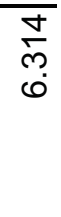 \\
\hline 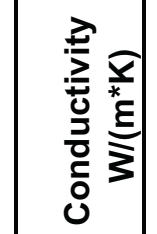 & & & & \\
\hline 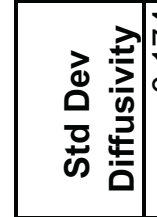 & & $\begin{array}{l}\tilde{W} \\
\text { लె } \\
0\end{array}$ & 象 & \\
\hline 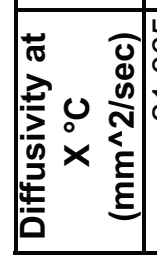 & 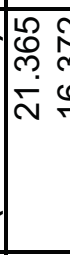 & 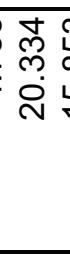 & 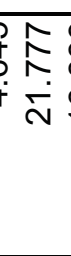 & 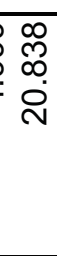 \\
\hline 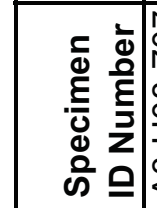 & \begin{tabular}{|l}
$\hat{D}$ \\
$N$ \\
0 \\
0 \\
0 \\
$⿱ 1$ \\
$\dot{1}$ \\
$\dot{m}<$
\end{tabular} & 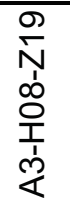 & 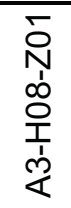 & 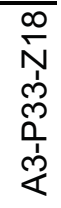 \\
\hline
\end{tabular}




\begin{tabular}{|c|c|c|c|}
\hline 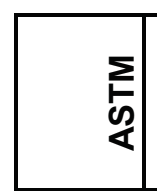 & 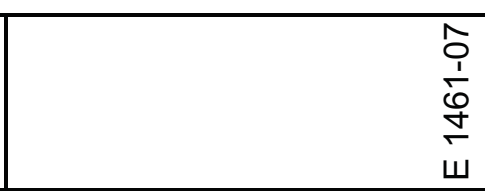 & 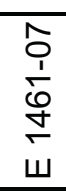 & 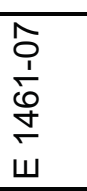 \\
\hline $\begin{array}{c}\frac{0}{0} \\
\frac{0}{0} \\
\frac{0}{0} \\
0 \\
\frac{E}{<}\end{array}$ & 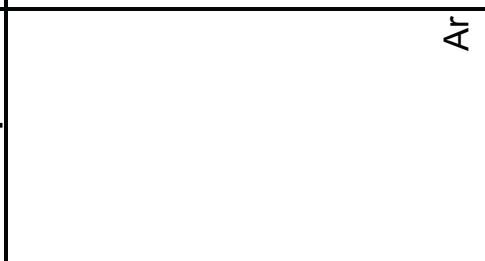 & $\frac{1}{<}$ & $\overline{\frac{1}{4}}$ \\
\hline 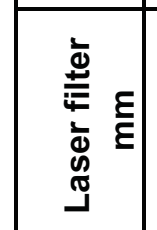 & 음 & 웅 & 음 \\
\hline 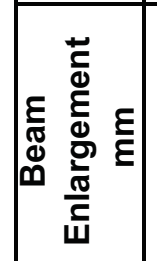 & 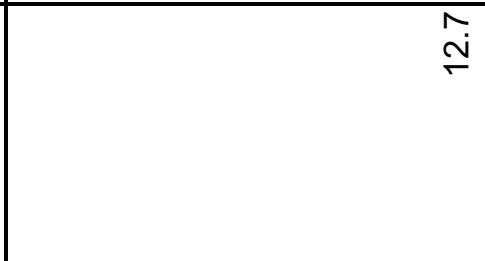 & $\stackrel{\widetilde{N}}{\stackrel{N}{N}}$ & $\widehat{\widehat{N}}$ \\
\hline 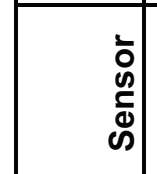 & $\begin{array}{l}\text { की } \\
\text { की }\end{array}$ & $\begin{array}{l}\text { 足 } \\
\text { I }\end{array}$ & $\begin{array}{l}\text { की } \\
\text { की }\end{array}$ \\
\hline 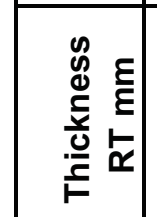 & 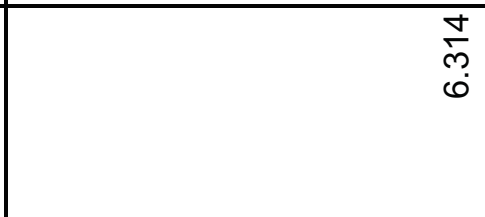 & 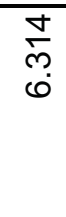 & 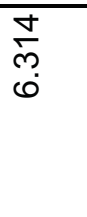 \\
\hline 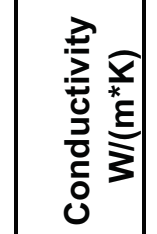 & & & \\
\hline 焉 & 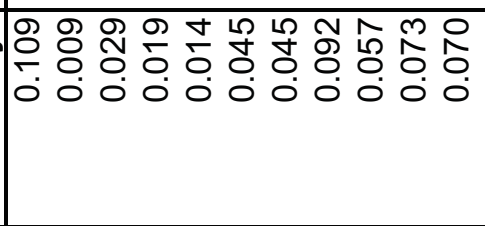 & 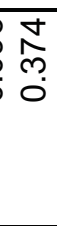 & $\begin{array}{l}0 . \\
\frac{0}{2} \\
0.0 \\
0\end{array}$ \\
\hline 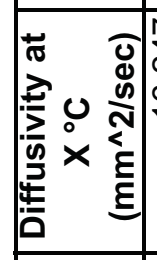 & 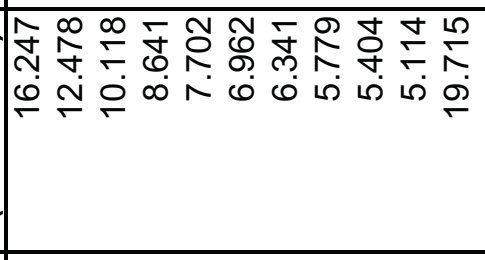 & $\begin{array}{l}\hat{N} \\
\stackrel{N}{N}\end{array}$ & 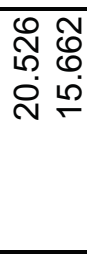 \\
\hline 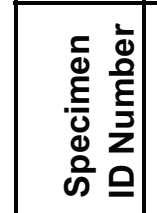 & 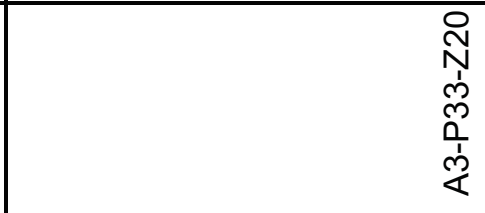 & 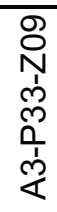 & 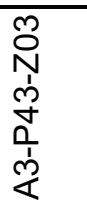 \\
\hline
\end{tabular}




\begin{tabular}{|c|c|c|}
\hline 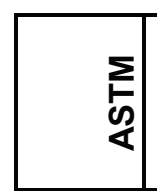 & 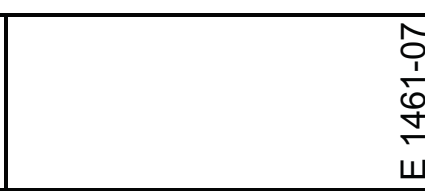 & 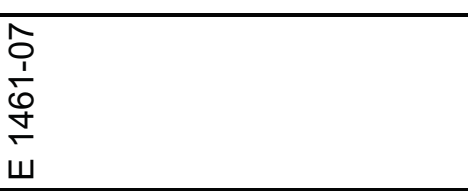 \\
\hline $\begin{array}{l}\frac{0}{0} \\
\frac{0}{0} \\
\frac{0}{0} \\
0 \\
\frac{E}{4}\end{array}$ & 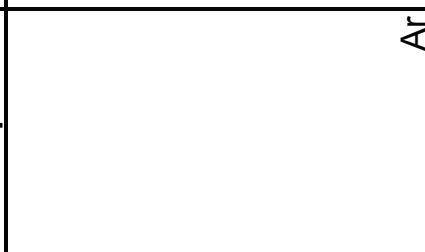 & $\frac{1}{2}$ \\
\hline 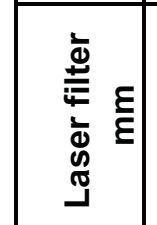 & 음 & 응 \\
\hline 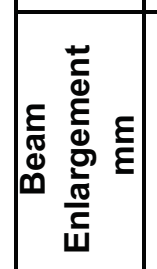 & $\stackrel{\underline{N}}{\stackrel{N}{\underline{N}}}$ & 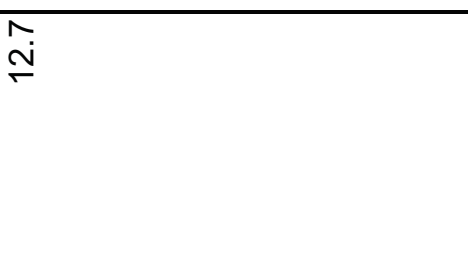 \\
\hline 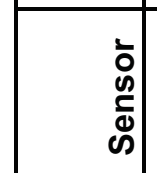 & की & 吕 \\
\hline 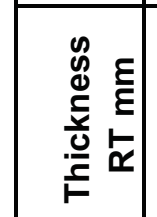 & $\stackrel{\vec{j}}{0}$ & $\begin{array}{c}\stackrel{\nabla}{m} \\
\stackrel{d}{6}\end{array}$ \\
\hline 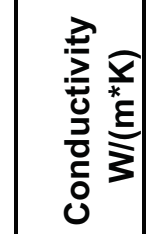 & & \\
\hline 造 & 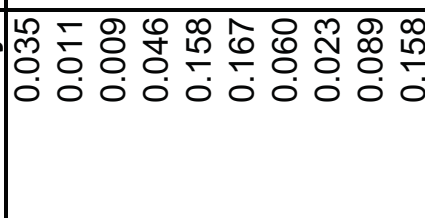 & 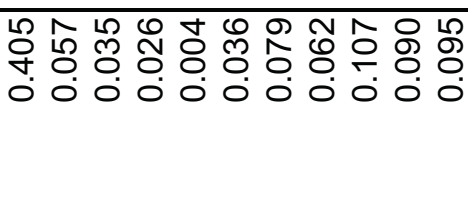 \\
\hline 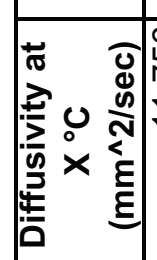 & $\begin{array}{llllll} & \\
\end{array}$ & 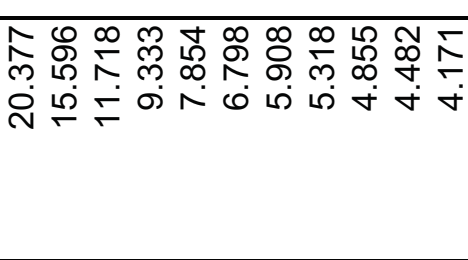 \\
\hline 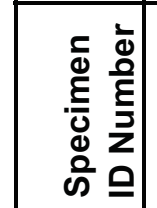 & 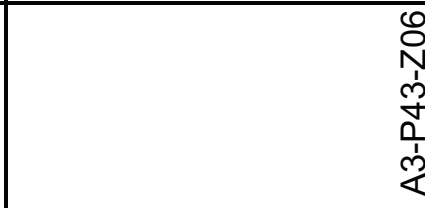 & 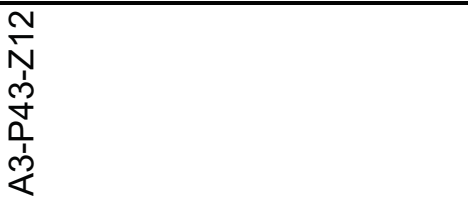 \\
\hline
\end{tabular}




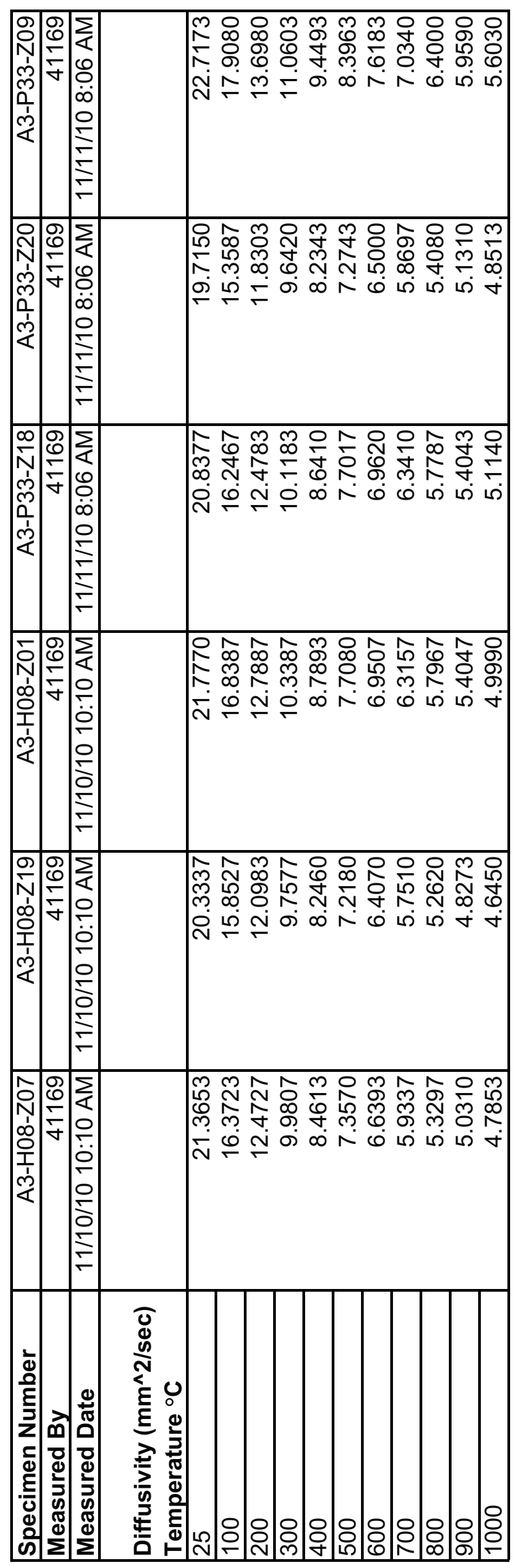




$$
\text { 㽬 }
$$




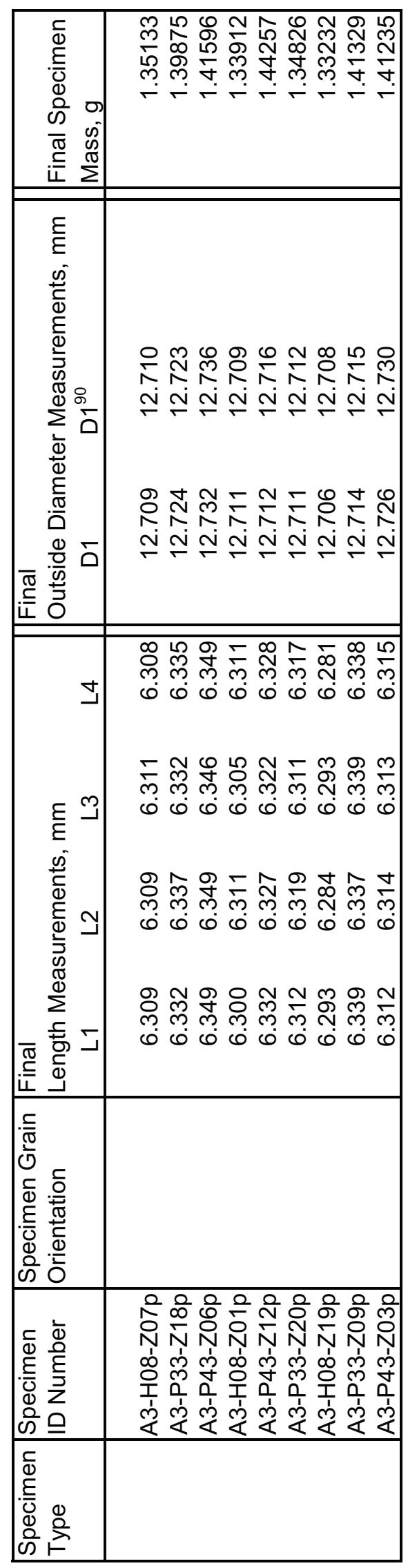

$\stackrel{2}{=}$ 


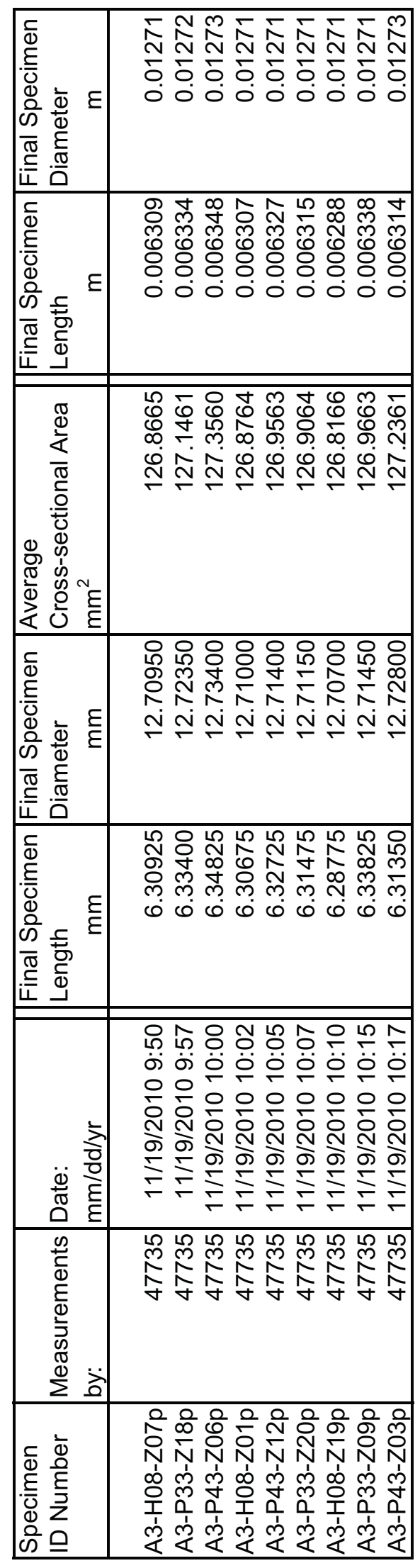

I 


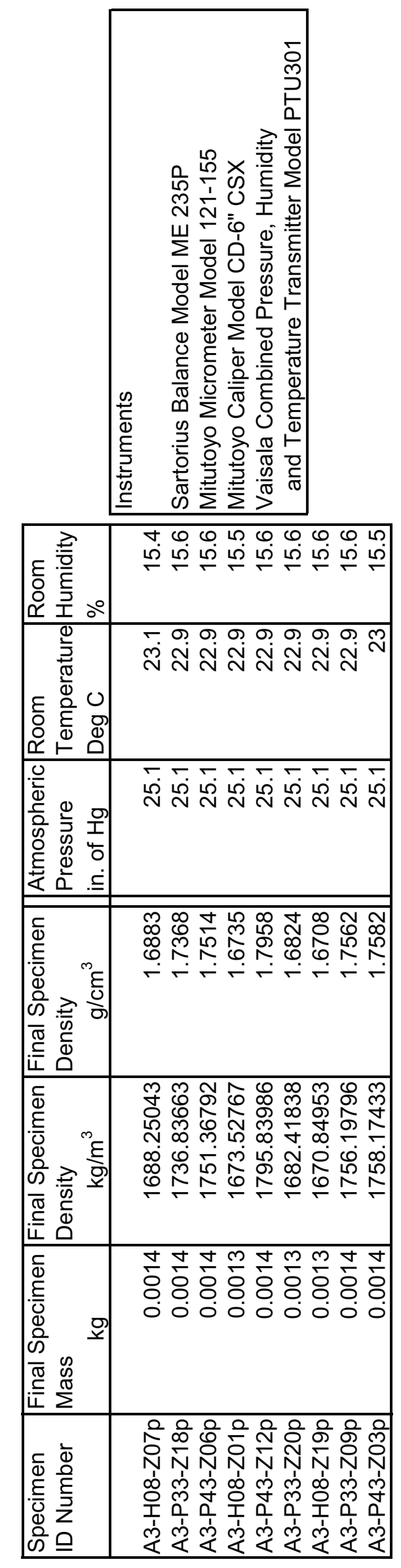

$\stackrel{\infty}{=}$ 
Graphite Grade:

PCEA

Graphite Manufacturer: Graftech International

Forming Process: Extruded

Coke Particle Size: Medium grain

Coke Type:

ASTM Class:

Pitch coke filler, pitch binder

Specimen Geometry: Cylinder

Specimen ID \#'s:

DW18 01

DW18 02

DW18 03

DW18 04

DW18 05

DW18 06

DW18 07

DW18 08

DW18 09

DW18 10

DW18 11

DW18 12

DA8 01

DA8 02

DA8 03

DA8 04

DA8 05

DA8 06

DA9 01

DA9 02

DA9 03

DA9 04

DA9 05

DA9 06 


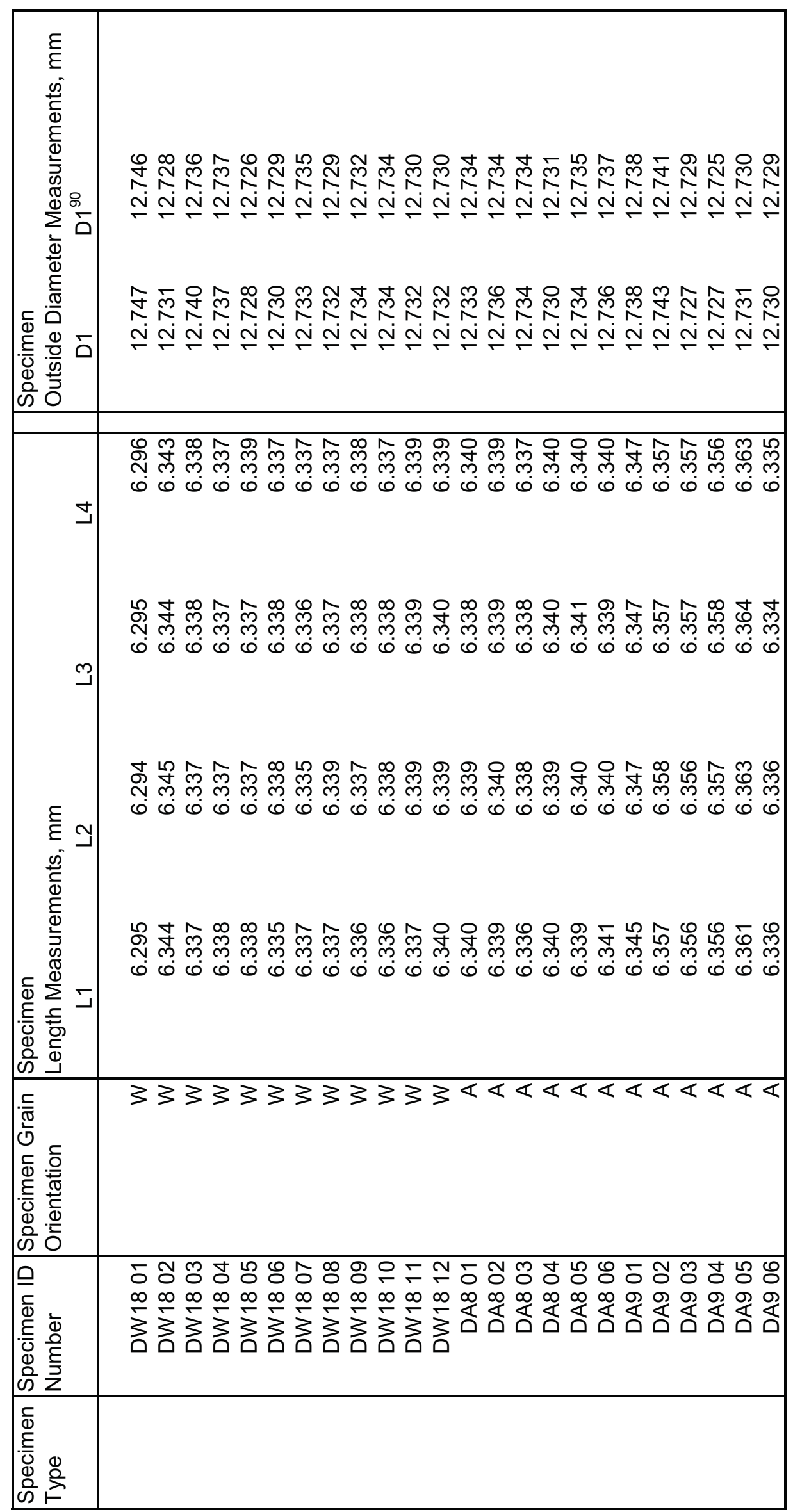




\begin{tabular}{|c|c|}
\hline 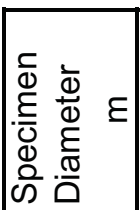 & 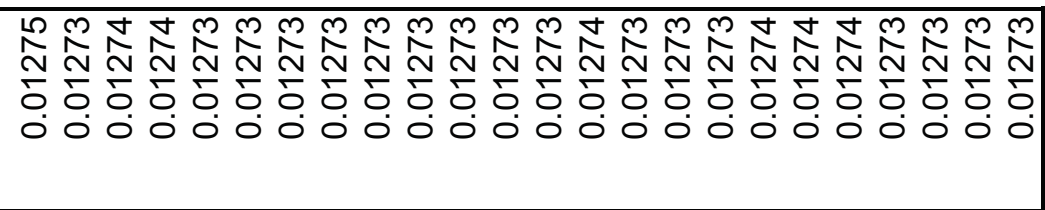 \\
\hline 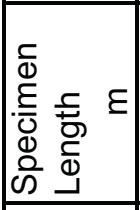 & 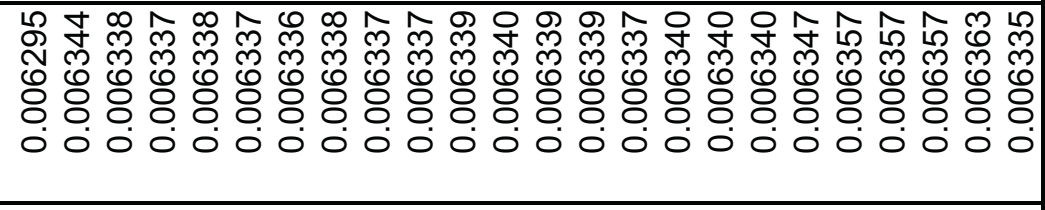 \\
\hline 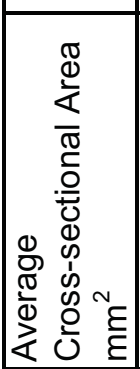 & 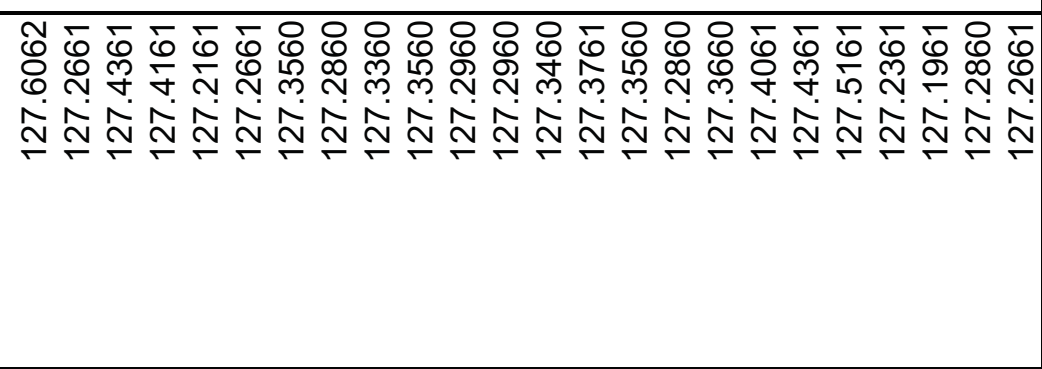 \\
\hline 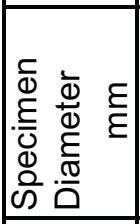 & 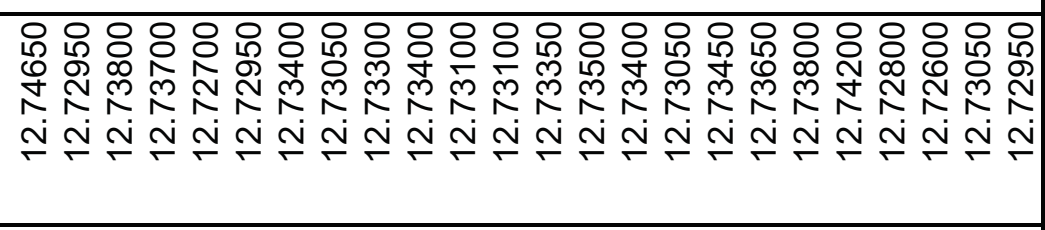 \\
\hline 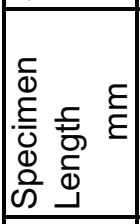 & 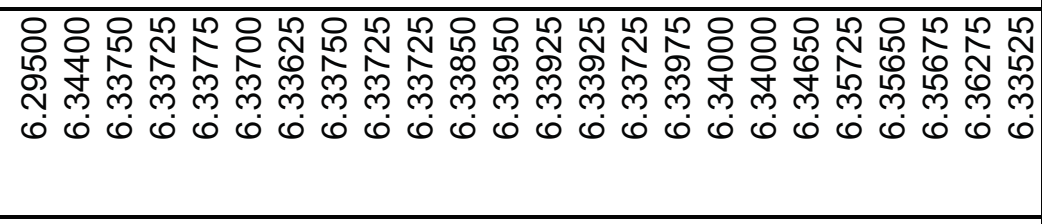 \\
\hline$\frac{\grave{\partial}}{\frac{\rho}{0}}$ & 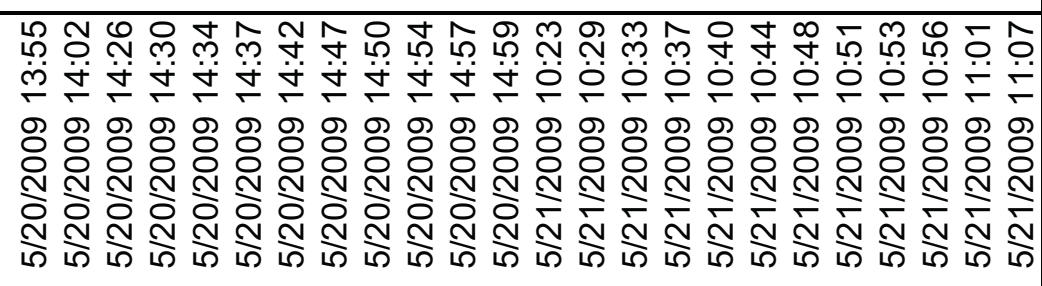 \\
\hline 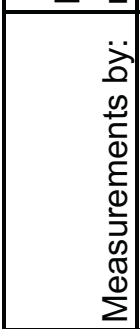 & 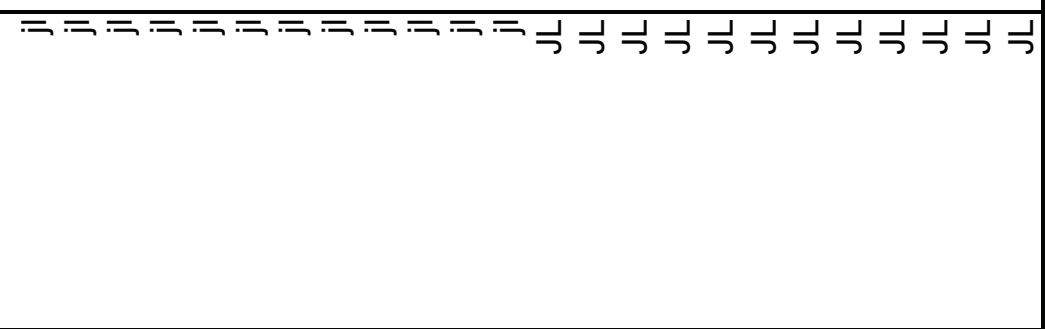 \\
\hline 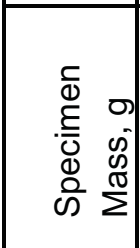 & 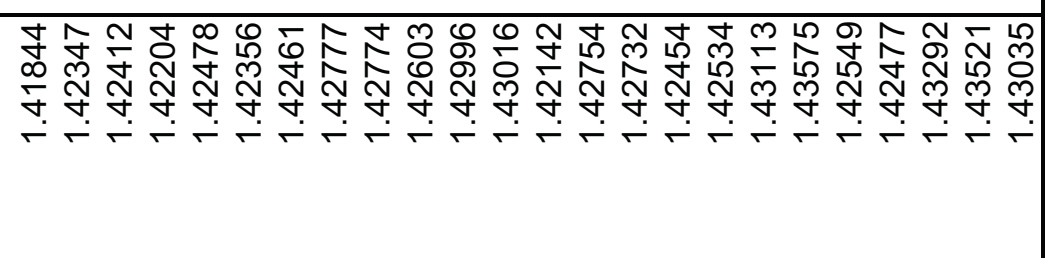 \\
\hline 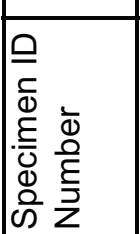 & 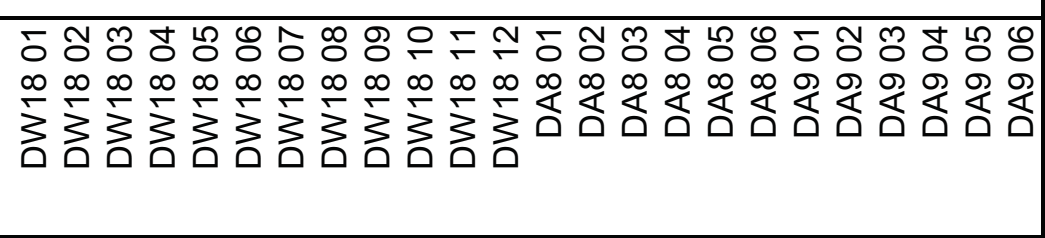 \\
\hline
\end{tabular}




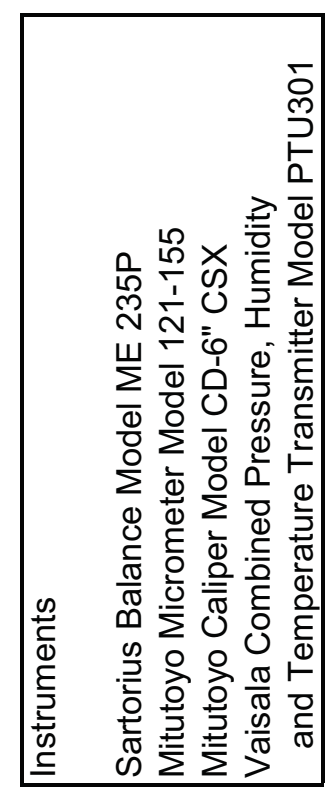

\begin{tabular}{|c|c|}
\hline \& & 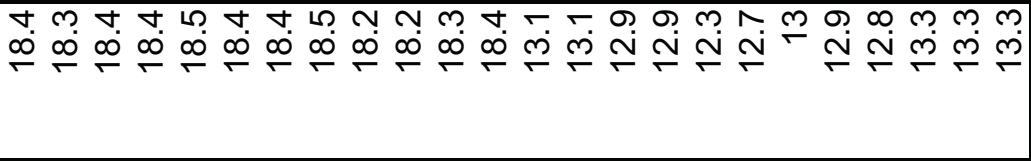 \\
\hline 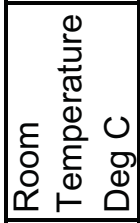 & 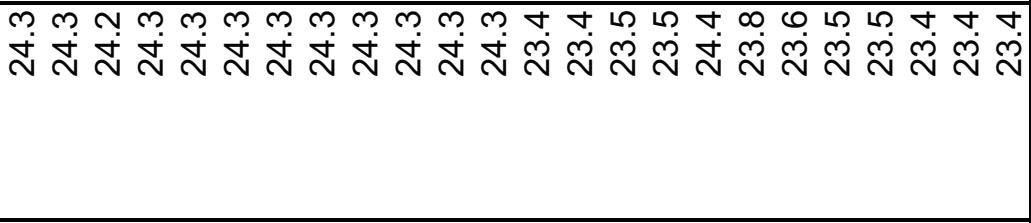 \\
\hline 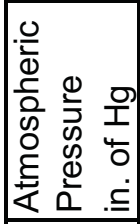 & 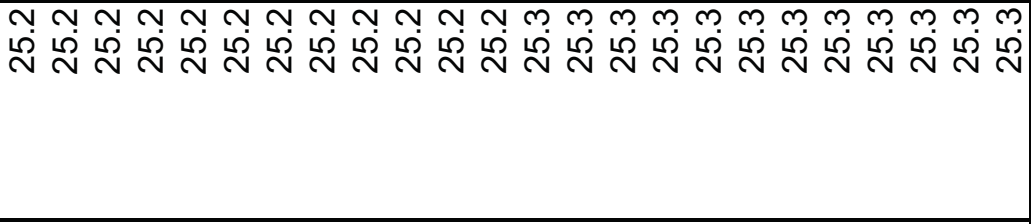 \\
\hline 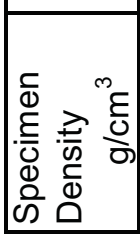 & 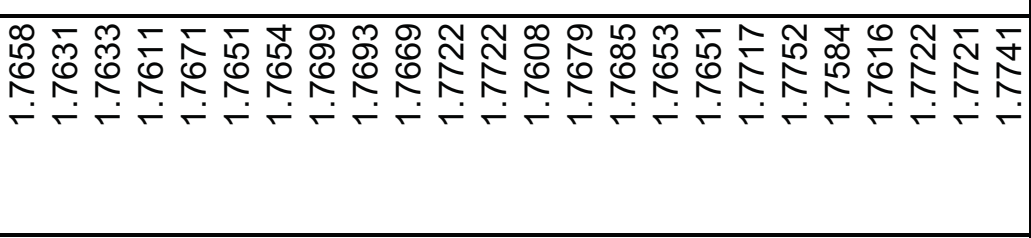 \\
\hline 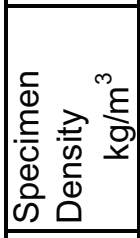 & 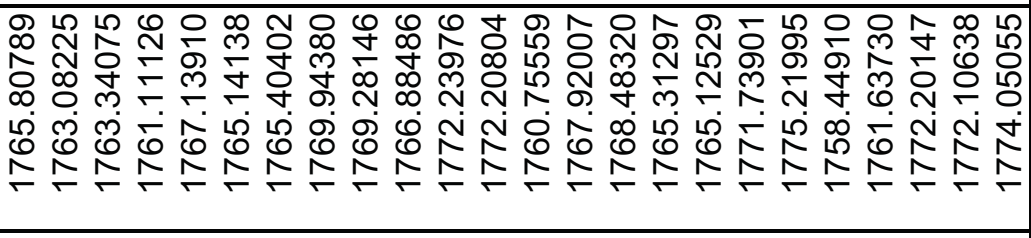 \\
\hline 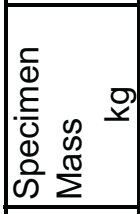 & 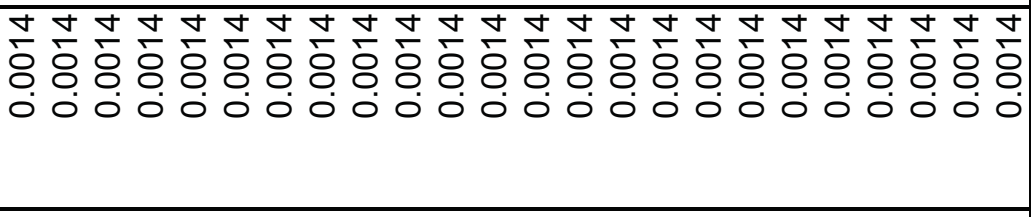 \\
\hline 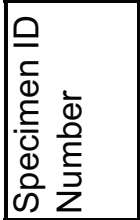 & 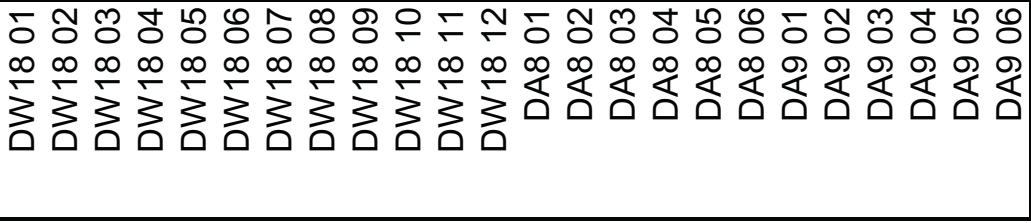 \\
\hline
\end{tabular}




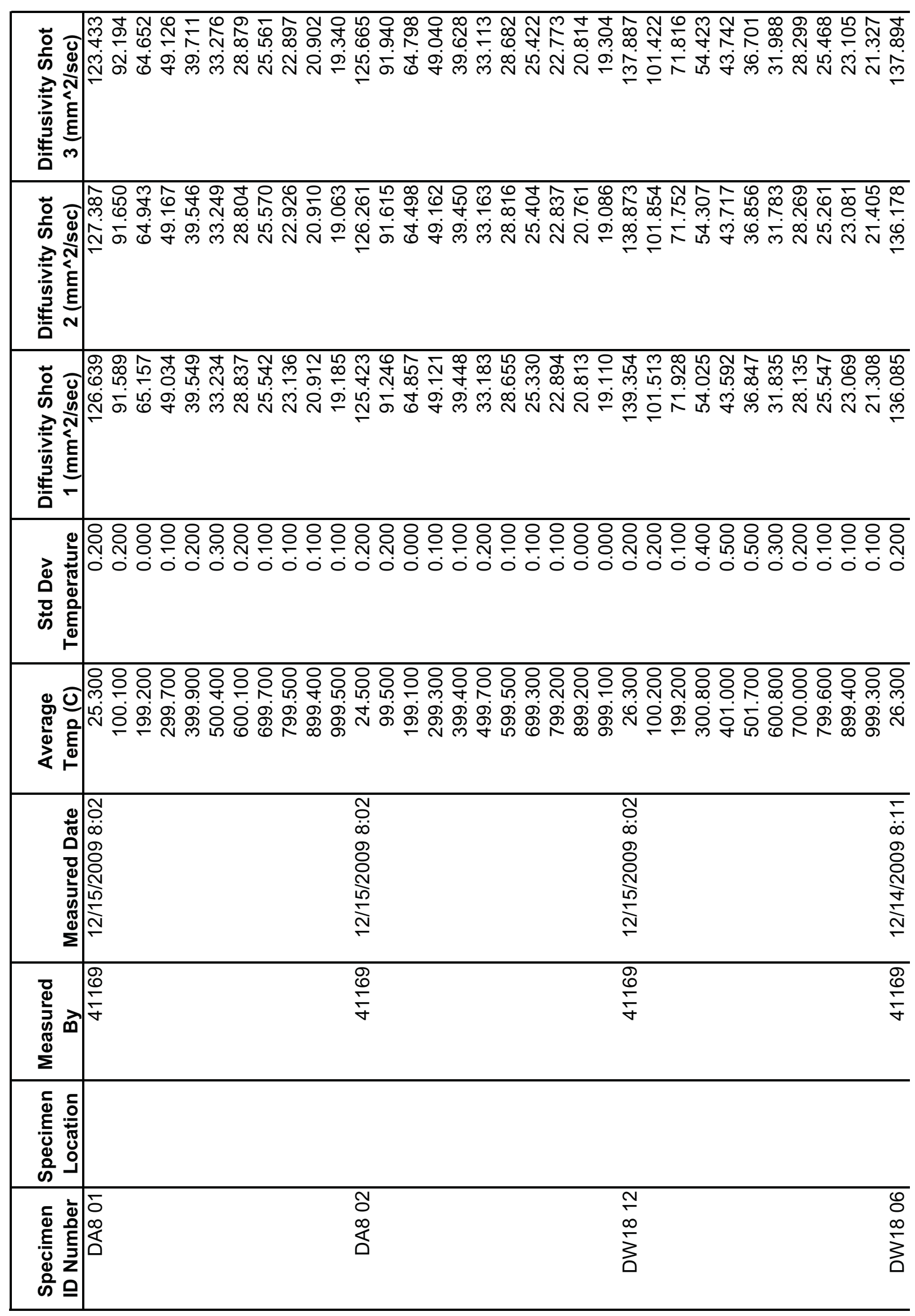




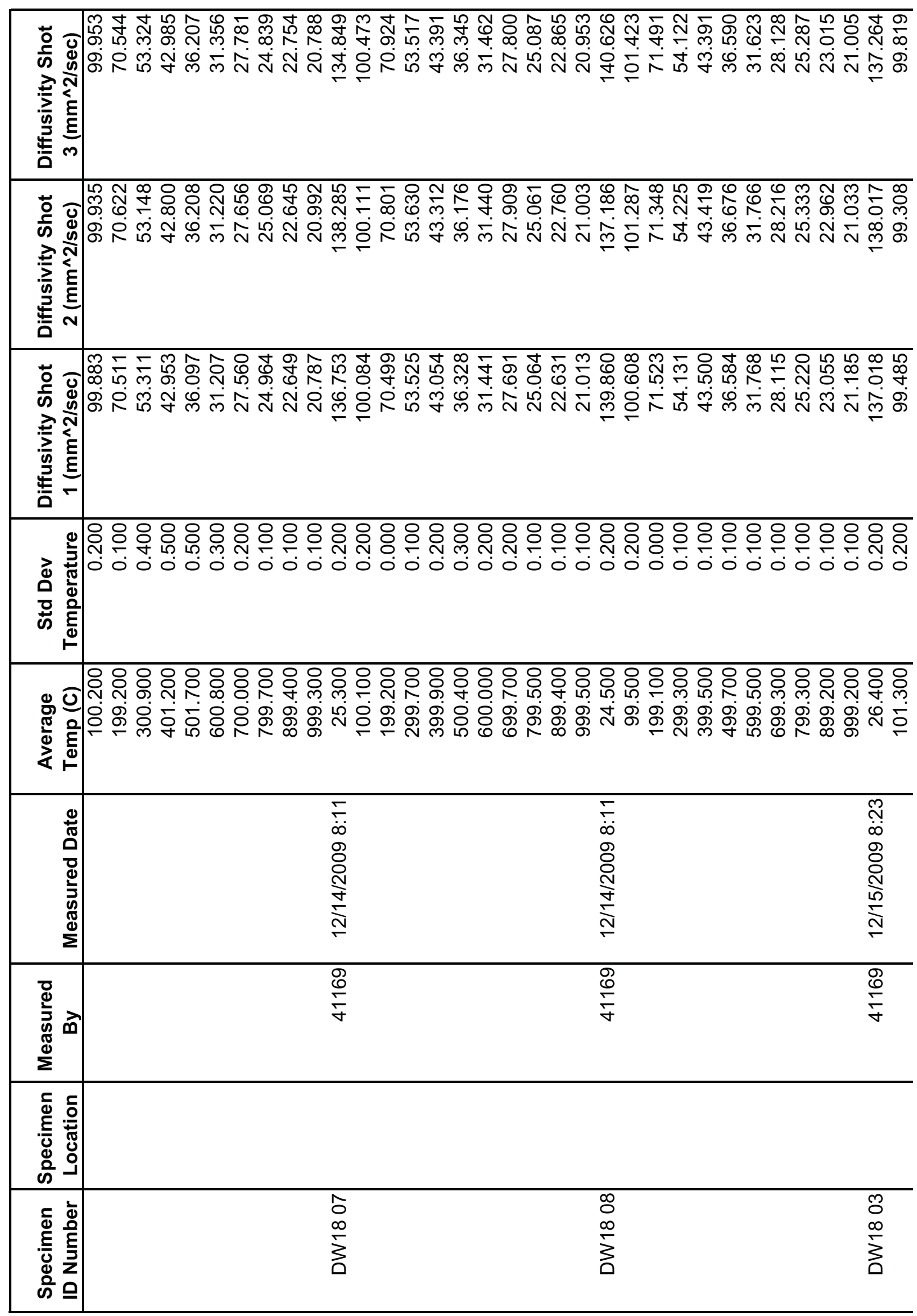




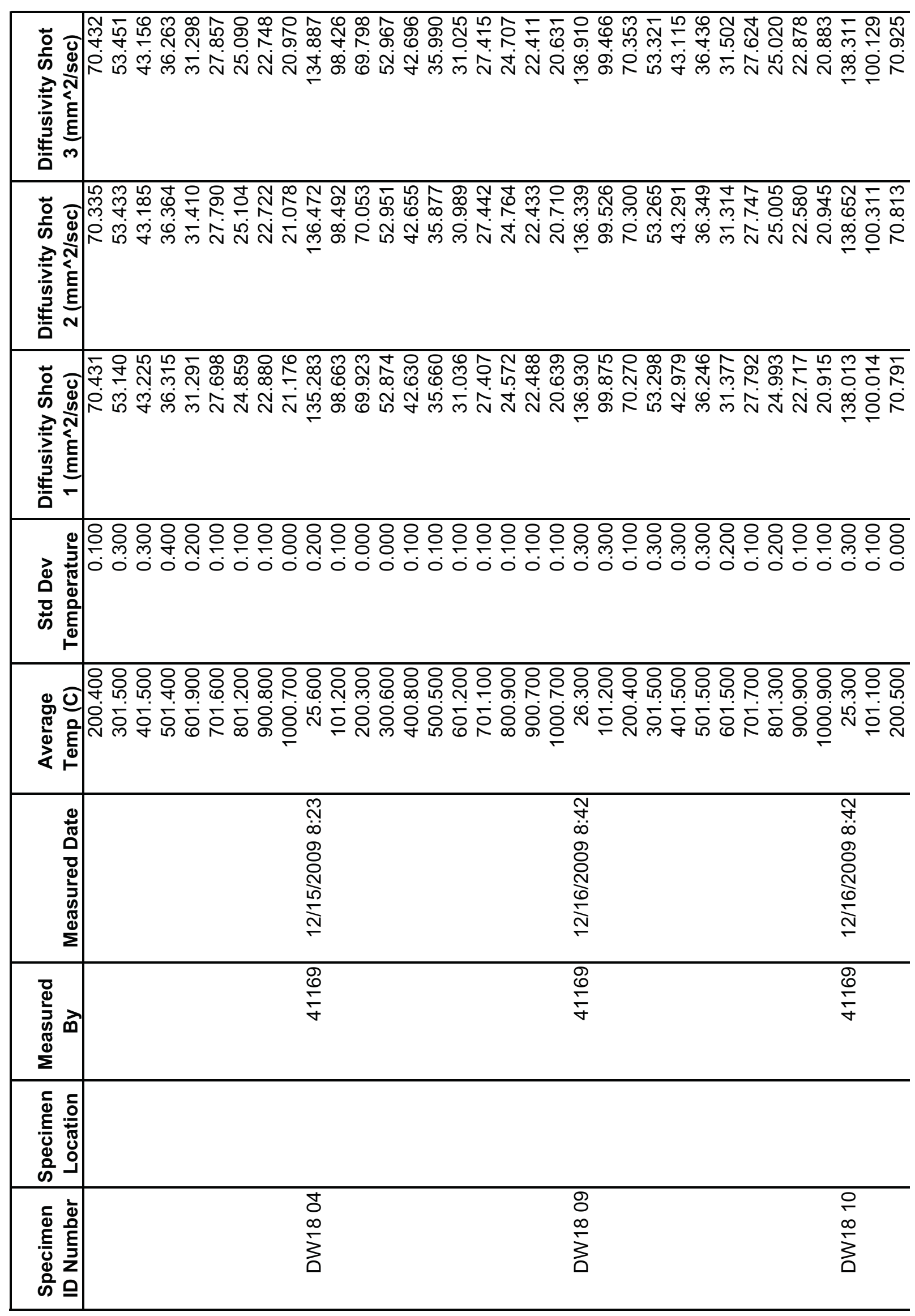




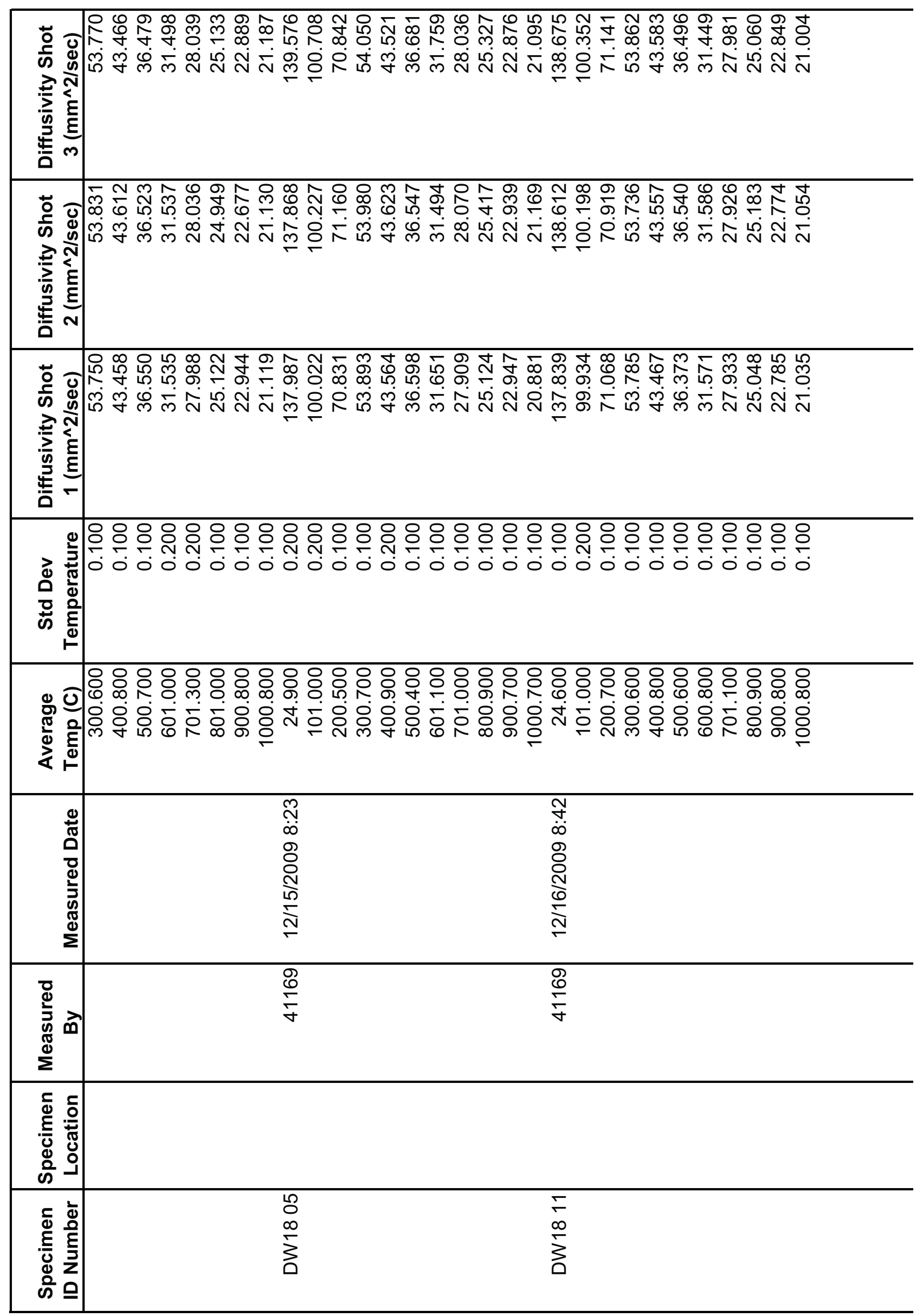




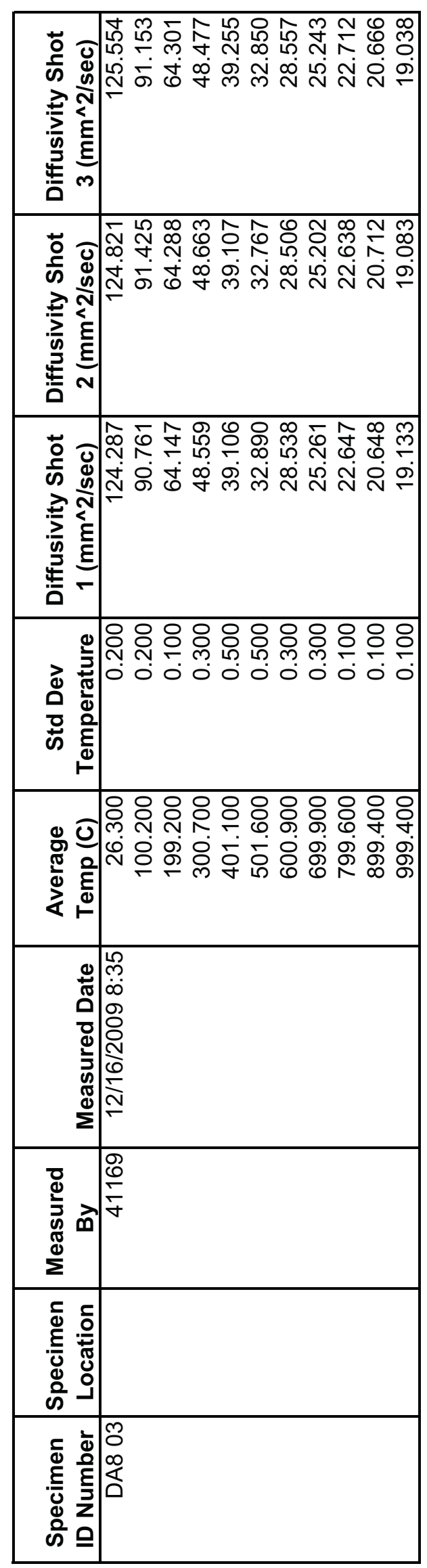

$\infty$ 


\begin{tabular}{|c|c|c|c|c|}
\hline 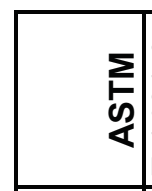 & 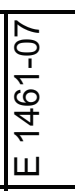 & $\begin{array}{l} \\
\\
1 \\
\dot{0} \\
\dot{+} \\
\omega \\
\end{array}$ & \begin{tabular}{l}
\multirow{1}{0}{} \\
$\frac{1}{0}$ \\
$\dot{0}$ \\
$\dot{+}$ \\
\end{tabular} & 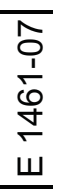 \\
\hline 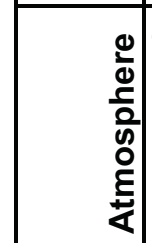 & & $\bar{z}$ & $\overline{\dot{\alpha}}$ & $\overline{\frac{\pi}{4}}$ \\
\hline 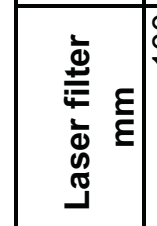 & & 음 & 음 & 음 \\
\hline 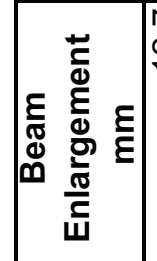 & & $\stackrel{\stackrel{N}{\sim}}{\sim}$ & $\stackrel{\widehat{\mathrm{N}}}{\mathrm{N}}$ & 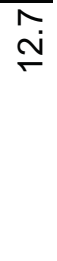 \\
\hline $\begin{array}{l}\vdots \\
\vdots \\
\vdots \\
\grave{d} \\
\omega\end{array}$ & & $\begin{array}{l}\text { की } \\
\text { की }\end{array}$ & $\begin{array}{l}\text { की } \\
\text { की }\end{array}$ & $\begin{array}{l}\text { Oे } \\
\text { Dी }\end{array}$ \\
\hline 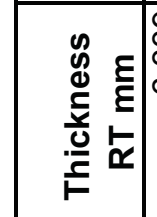 & & 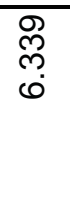 & 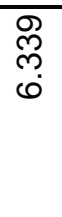 & 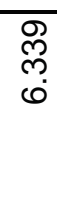 \\
\hline 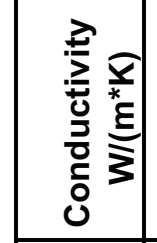 & & & & \\
\hline 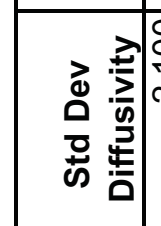 & 을 & $\overrightarrow{\tilde{m}}$ & \begin{tabular}{c}
$\infty$ \\
\multirow{2}{*}{} \\
0
\end{tabular} & 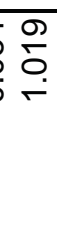 \\
\hline 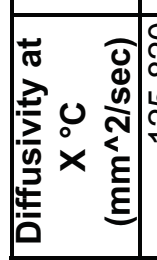 & 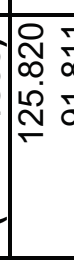 & 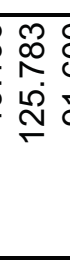 & 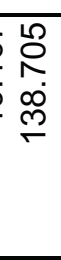 & 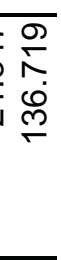 \\
\hline 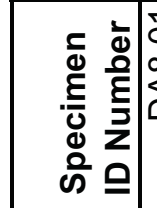 & & $\begin{array}{l}1 \\
0 \\
\infty \\
0 \\
0\end{array}$ & $\begin{array}{l}\text { No } \\
\sum_{3}^{\infty}\end{array}$ & $\begin{array}{l}8 \\
8 \\
\infty \\
\sum_{0}^{\infty}\end{array}$ \\
\hline
\end{tabular}




\begin{tabular}{|c|c|c|c|}
\hline 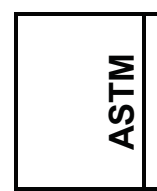 & $\begin{array}{l}\hat{o} \\
\frac{1}{0} \\
\dot{0} \\
\dot{5} \\
\end{array}$ & $\begin{array}{l}\hat{S} \\
\frac{1}{1} \\
\frac{0}{5} \\
\dot{w}\end{array}$ & 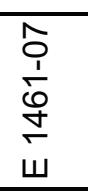 \\
\hline 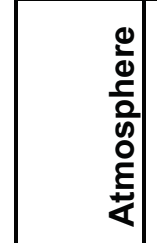 & 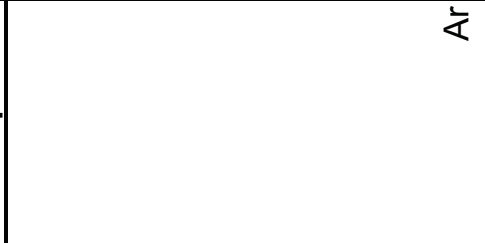 & $\frac{1}{\alpha}$ & 广 \\
\hline 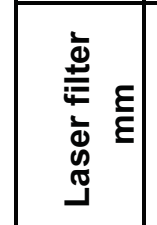 & 음 & $\stackrel{8}{\circ}$ & 음 \\
\hline 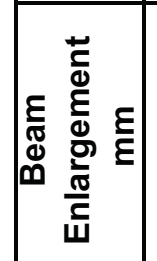 & $\stackrel{\widehat{N}}{\stackrel{N}{N}}$ & 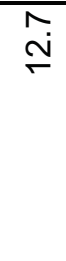 & 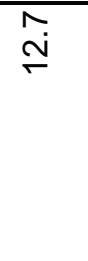 \\
\hline $\begin{array}{l}\vdots \\
\vdots \\
0 \\
\bar{d} \\
\infty\end{array}$ & $\begin{array}{l}\text { की } \\
\text { की }\end{array}$ & 吕 & $\begin{array}{l}\text { की } \\
\text { की }\end{array}$ \\
\hline 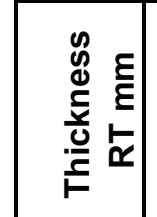 & 总 & 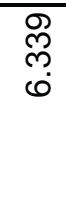 & 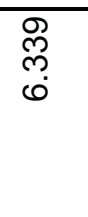 \\
\hline 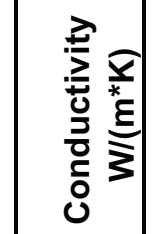 & & & \\
\hline 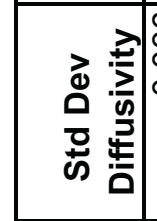 & 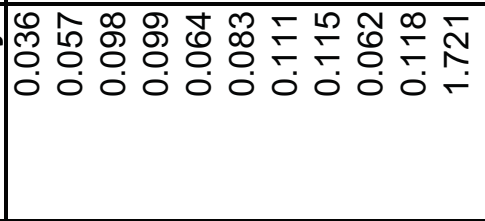 & $\stackrel{\infty}{\leftarrow}$ & 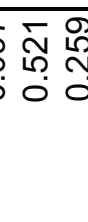 \\
\hline 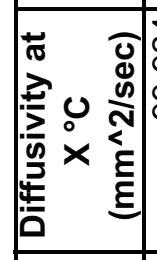 & 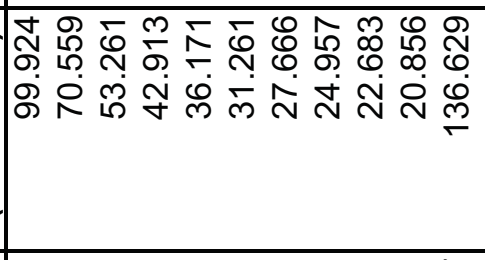 & $\begin{array}{l}\text { N̦ } \\
\text { ò } \\
\text { m. }\end{array}$ & 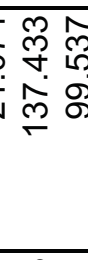 \\
\hline 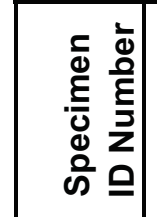 & $\begin{array}{l}1 \\
\\
\infty \\
\infty \\
\vdots \\
\vdots\end{array}$ & $\begin{array}{l}\text { o } \\
0 \\
\sum_{0}^{\infty}\end{array}$ & $\begin{array}{l}0 \\
0 \\
\infty \\
\sum_{0}^{\infty}\end{array}$ \\
\hline
\end{tabular}




\begin{tabular}{|c|c|c|c|}
\hline 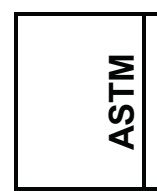 & 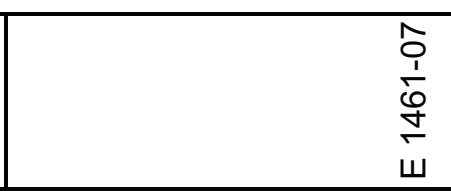 & $\begin{array}{l}\hat{o} \\
\frac{1}{0} \\
\stackrel{0}{+} \\
\dot{\omega}\end{array}$ & $\begin{array}{l}\frac{\hat{1}}{1} \\
\frac{1}{0} \\
\frac{1}{4} \\
\dot{w}\end{array}$ \\
\hline 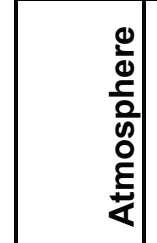 & 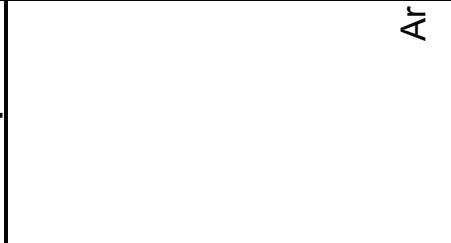 & 衣 & 交 \\
\hline 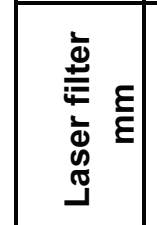 & 음 & 음 & 음 \\
\hline 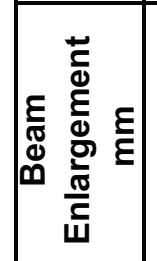 & 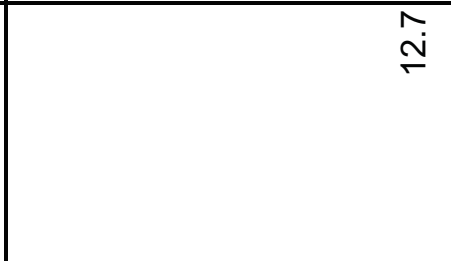 & $\stackrel{\stackrel{N}{ }}{ }$ & $\stackrel{\widetilde{N}}{\stackrel{N}{N}}$ \\
\hline $\begin{array}{l}\bar{b} \\
0 \\
\stackrel{5}{\Phi} \\
\infty\end{array}$ & $\begin{array}{l}\text { की } \\
\text { की }\end{array}$ & $\begin{array}{l}\text { की } \\
\text { I }\end{array}$ & $\begin{array}{l}\text { की } \\
\text { की }\end{array}$ \\
\hline 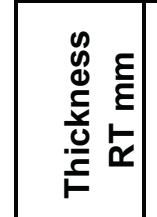 & 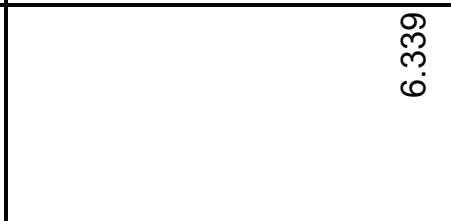 & 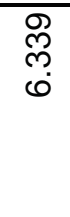 & 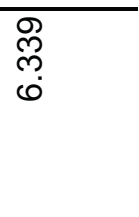 \\
\hline 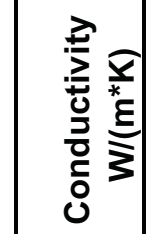 & & & \\
\hline 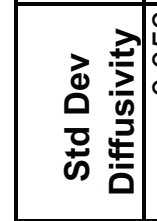 & 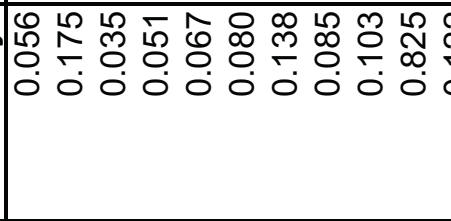 & 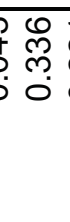 & 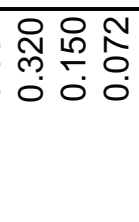 \\
\hline 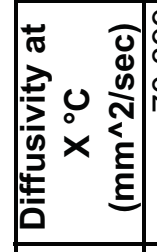 & 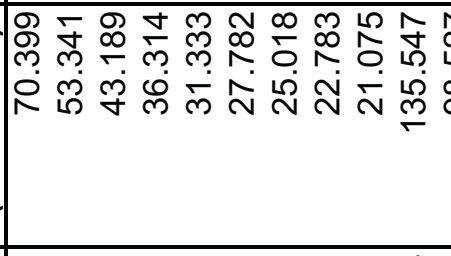 & 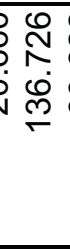 & 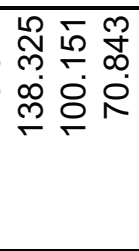 \\
\hline 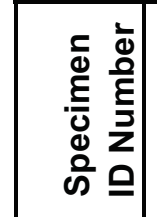 & $\begin{array}{l}\text { D } \\
\infty \\
\sum_{0}^{\infty}\end{array}$ & $\begin{array}{l}8 \\
8 \\
\infty \\
\vdots \\
\vdots\end{array}$ & $\begin{array}{l}\frac{0}{0} \\
\sum^{\infty} \\
\frac{1}{a}\end{array}$ \\
\hline
\end{tabular}




\begin{tabular}{|c|c|c|}
\hline 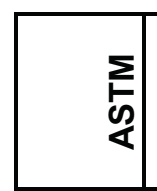 & 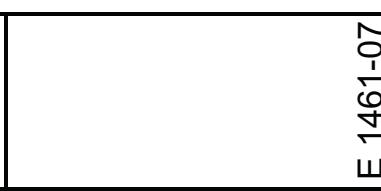 & $\begin{array}{l}\frac{\hat{1}}{1} \\
\frac{1}{6} \\
\frac{1}{\omega} \\
\end{array}$ \\
\hline $\begin{array}{c}0 \\
\frac{0}{0} \\
\frac{5}{0} \\
0 \\
0 \\
\frac{1}{4}\end{array}$ & 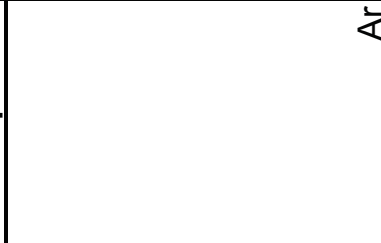 & 交 \\
\hline 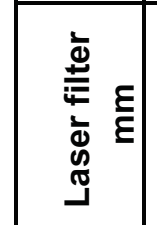 & $\stackrel{8}{\circ}$ & 음 \\
\hline 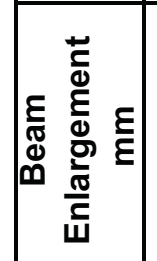 & $\stackrel{\mathrm{N}}{\mathrm{I}}$ & $\stackrel{\stackrel{N}{\sim}}{\stackrel{N}{*}}$ \\
\hline 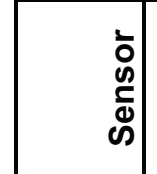 & $\begin{array}{l}\text { की } \\
\text { Dी }\end{array}$ & $\begin{array}{l}\text { की } \\
\text { 足 }\end{array}$ \\
\hline 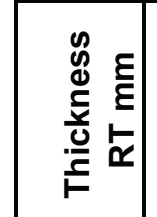 & $\begin{array}{l}\bar{D} \\
\stackrel{9}{m} \\
0 \\
0\end{array}$ & 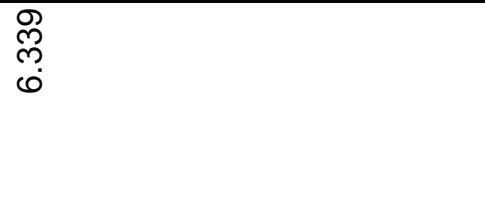 \\
\hline 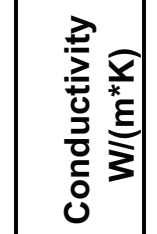 & & \\
\hline 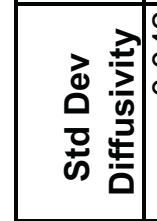 & 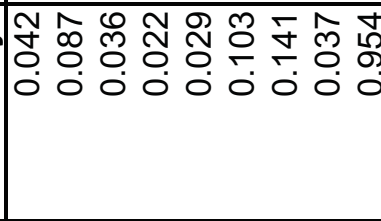 & 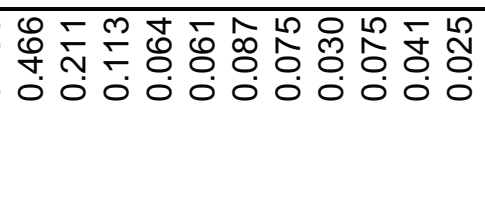 \\
\hline 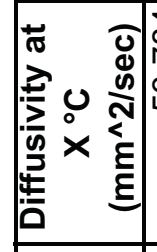 & 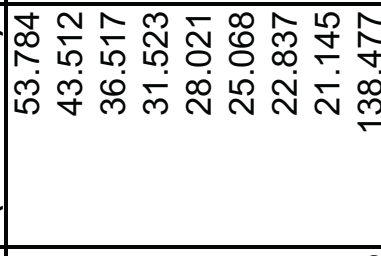 & 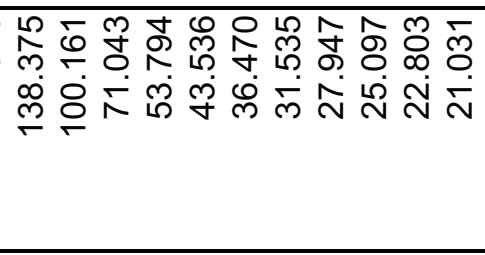 \\
\hline 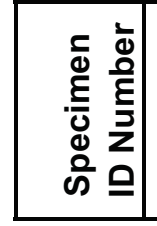 & 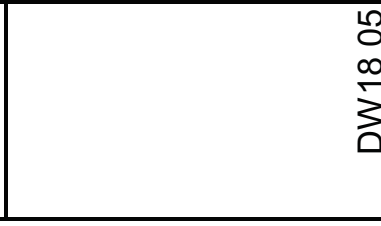 & $\frac{\Gamma}{\infty}$ \\
\hline
\end{tabular}




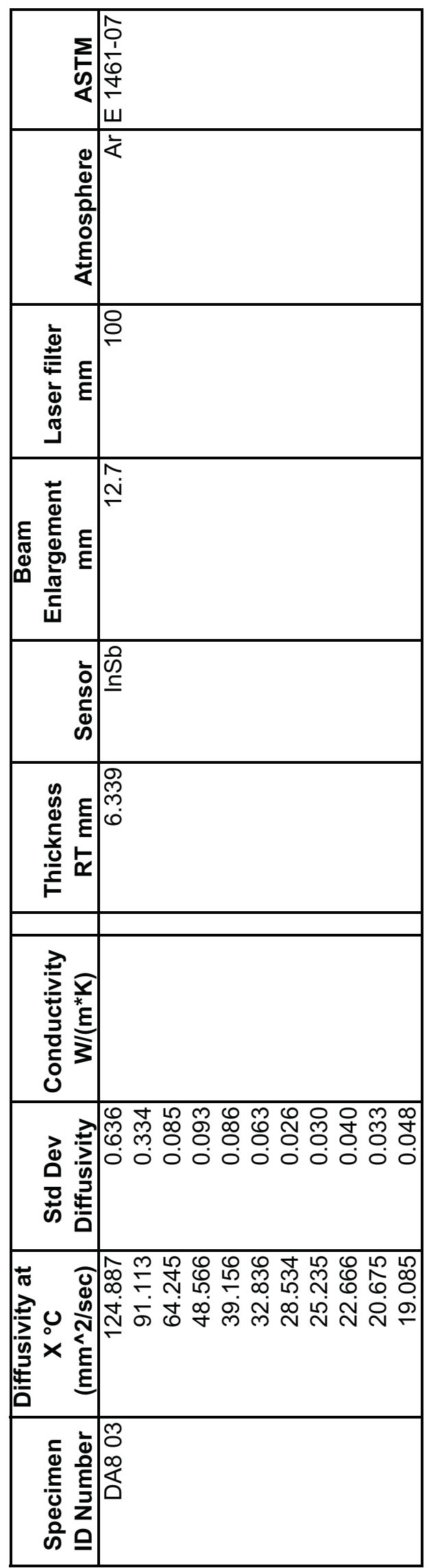

ำ 


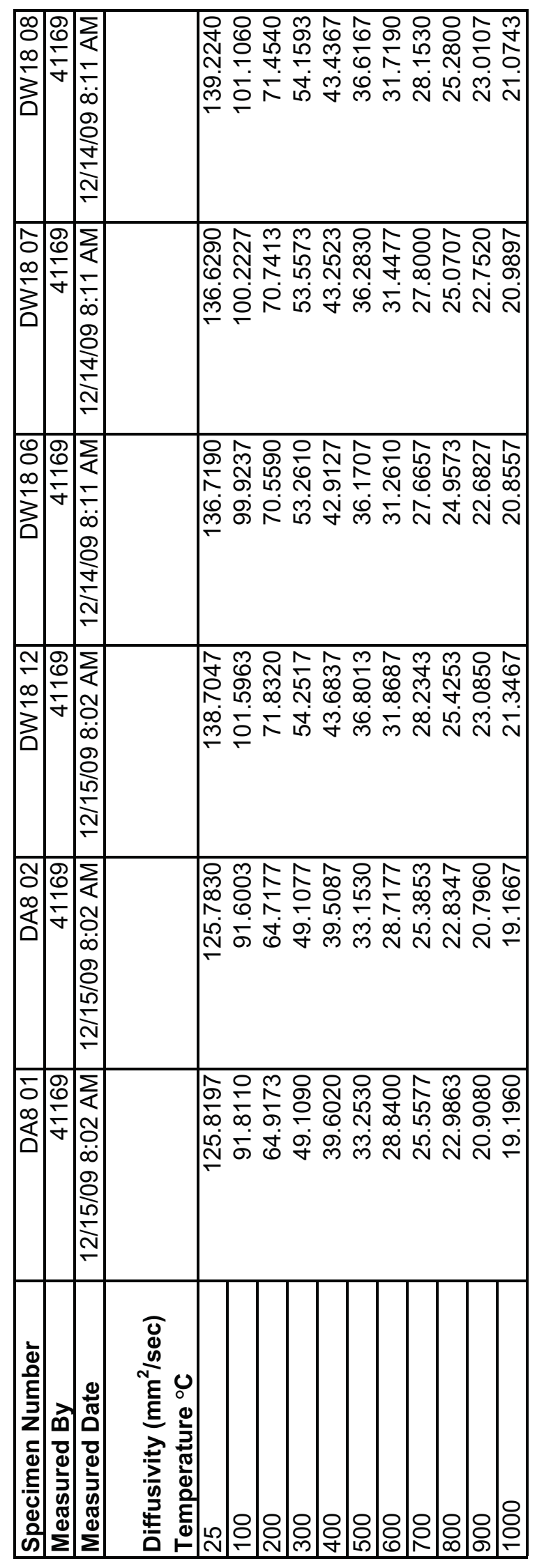




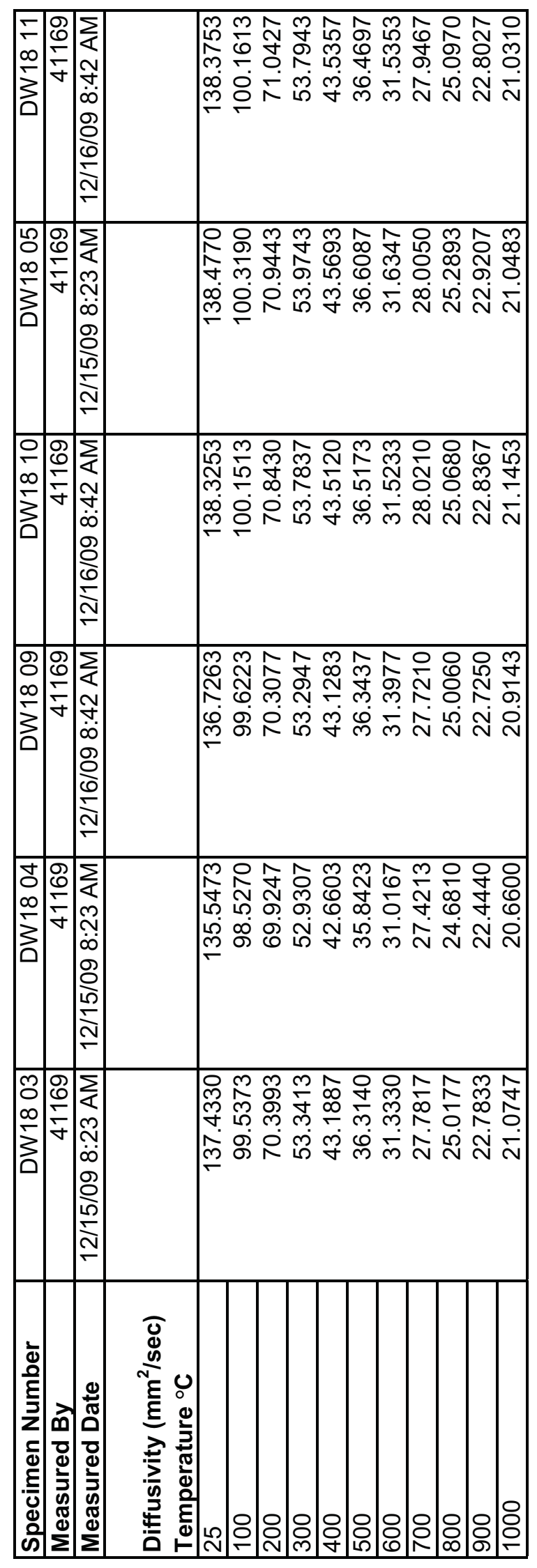




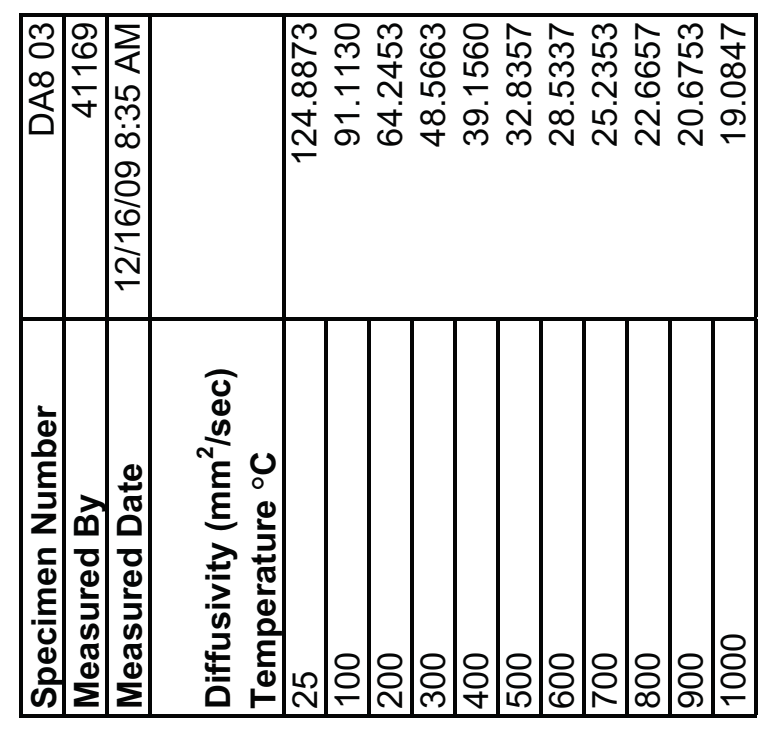

ลู 


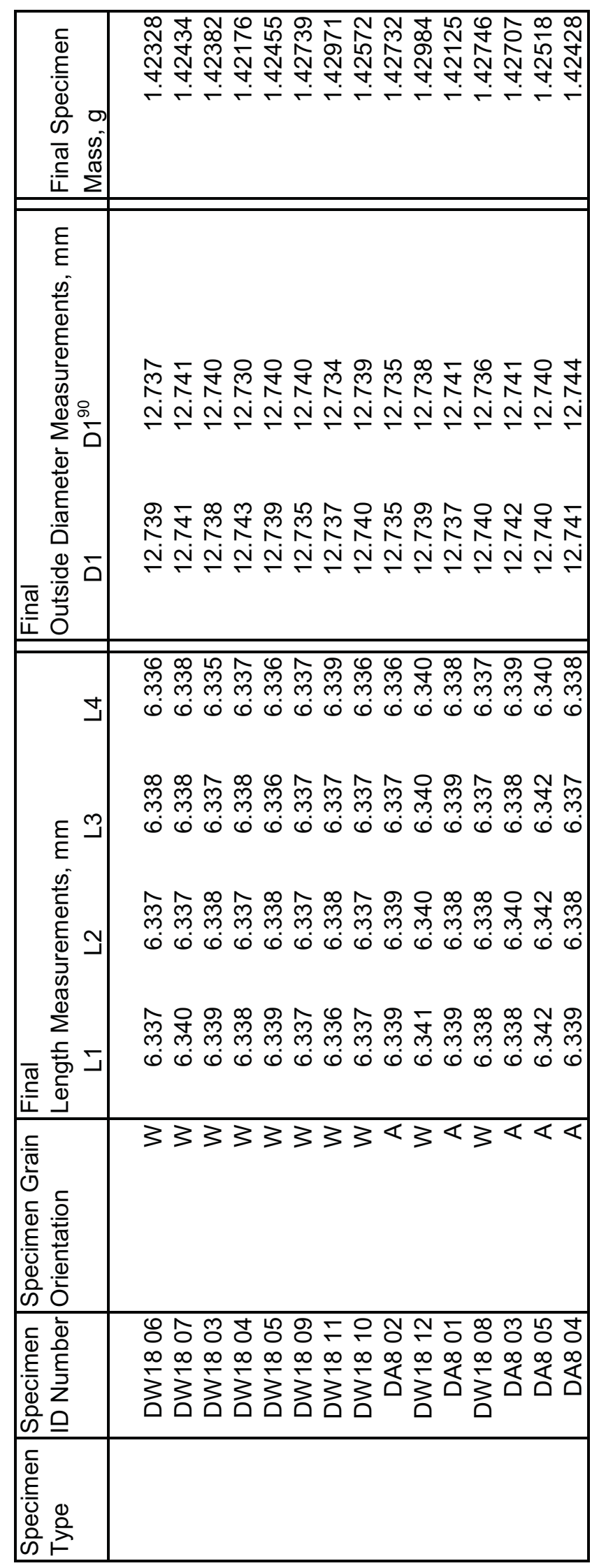




\begin{tabular}{|c|c|}
\hline 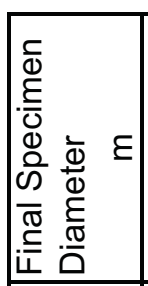 & 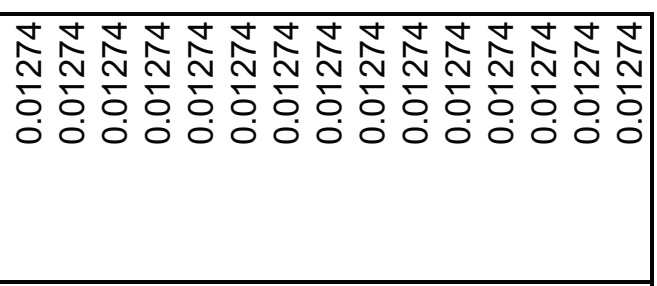 \\
\hline 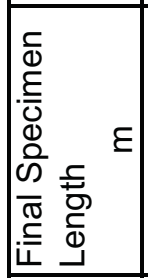 & 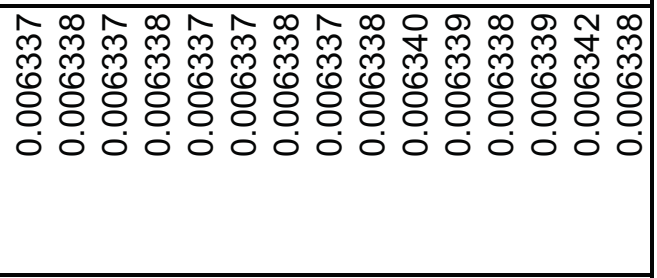 \\
\hline 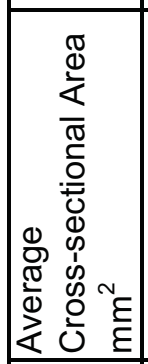 & 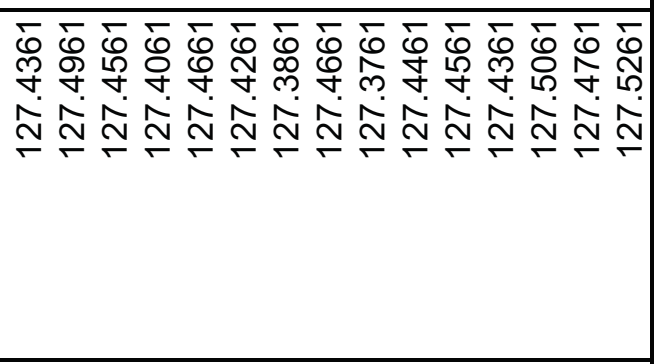 \\
\hline 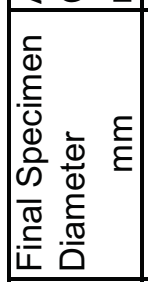 & 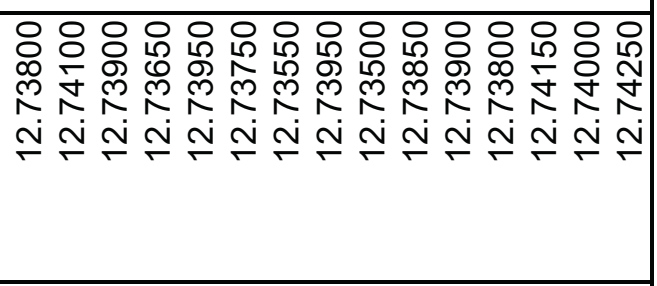 \\
\hline 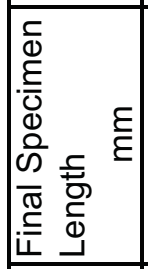 & 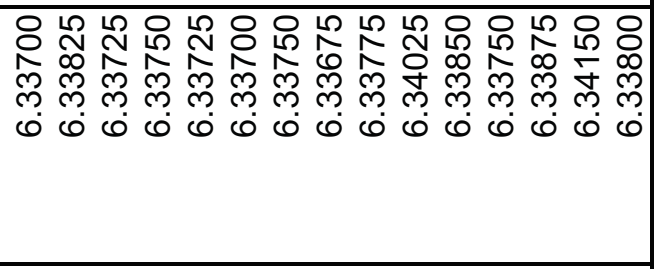 \\
\hline 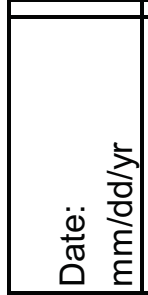 & 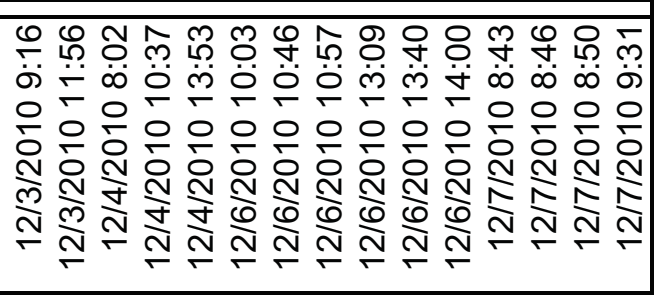 \\
\hline 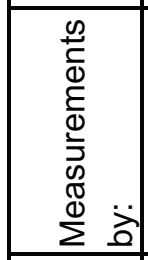 & 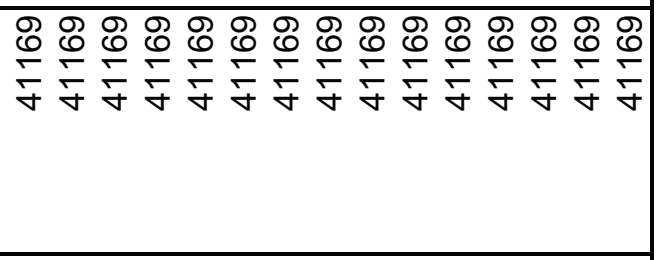 \\
\hline 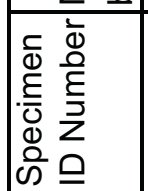 & 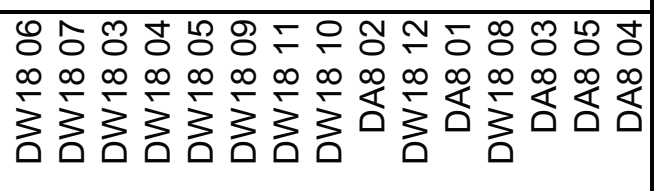 \\
\hline
\end{tabular}




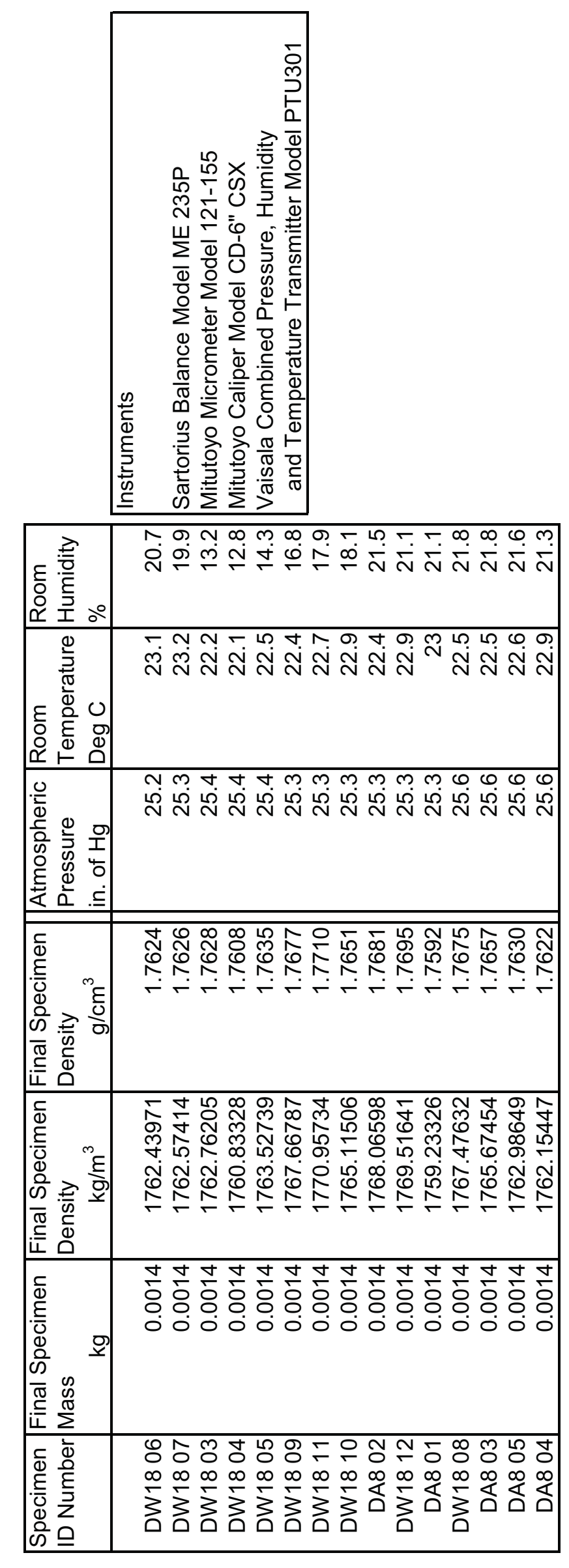


Graphite Grade:

Graphite Manufacturer:

Forming Process:

Coke Particle Size:

Coke Type:

ASTM Class:

Specimen Geometry:
PCIB

Graftech International

Isostatic-molded

Fine grain

Petroleum coke

INHP

Cylinder

Specimen ID \#'s:

P1-01

$\mathrm{P} 1-02$

$\mathrm{P} 1-03$

$\mathrm{P} 1-04$

$\mathrm{P} 1-05$

$\mathrm{P} 1-06$

$\mathrm{P} 1-07$

$\mathrm{P} 1-08$

P1-09

$\mathrm{P} 1-10$

P2-01

$\mathrm{P} 2-02$

$\mathrm{P} 2-03$

$\mathrm{P} 2-04$

$\mathrm{P} 2-05$

$\mathrm{P} 2-06$

$\mathrm{P} 2-07$

$\mathrm{P} 2-08$

P2-09

$\mathrm{P} 2-10$

P3-01

P3-02

P3-03

P3-04

P3-05

P3-06

P3-07

P3-08

P3-09

P3-10 


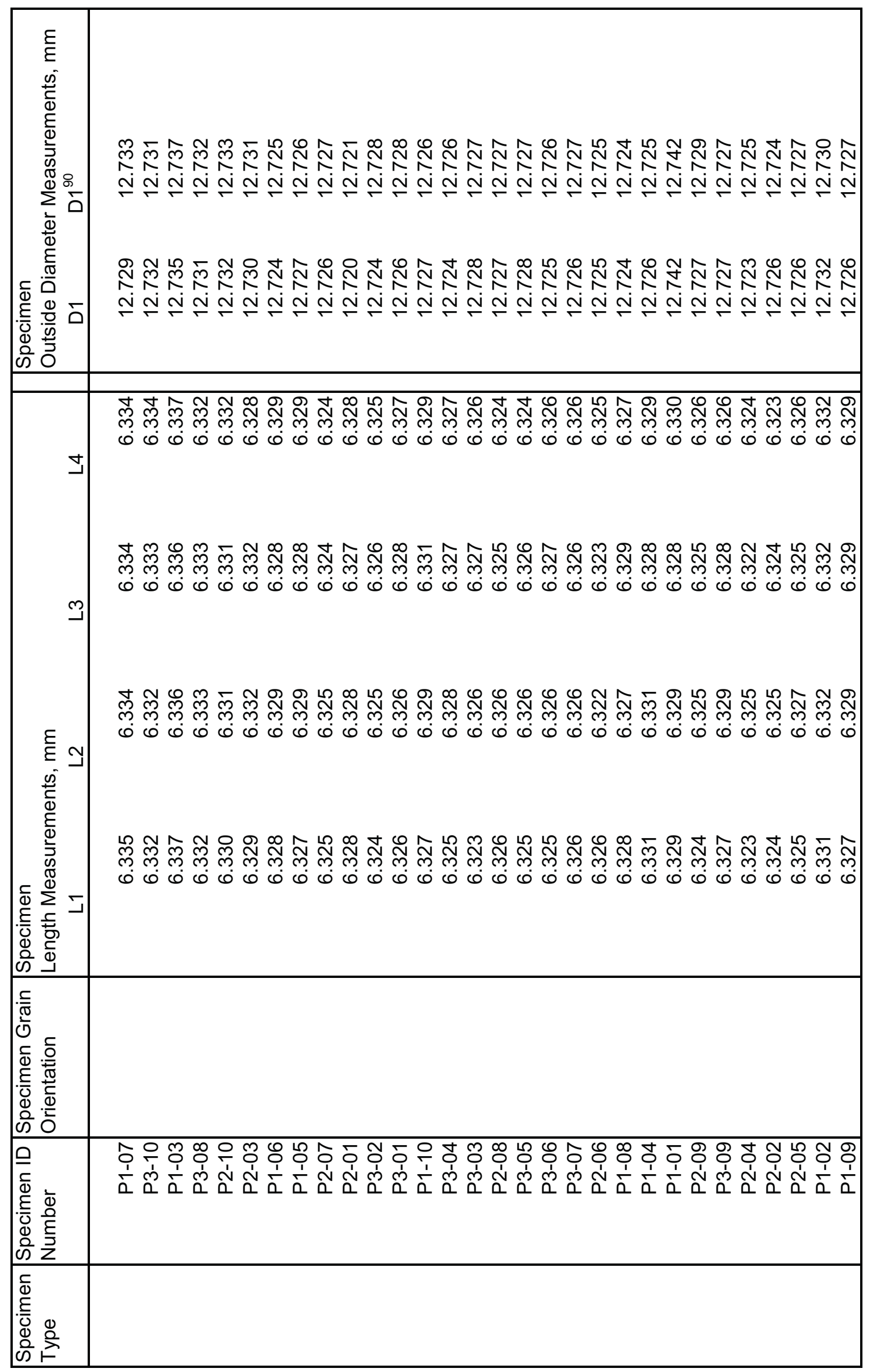




\begin{tabular}{|c|c|}
\hline 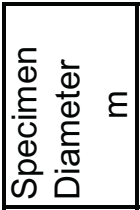 & 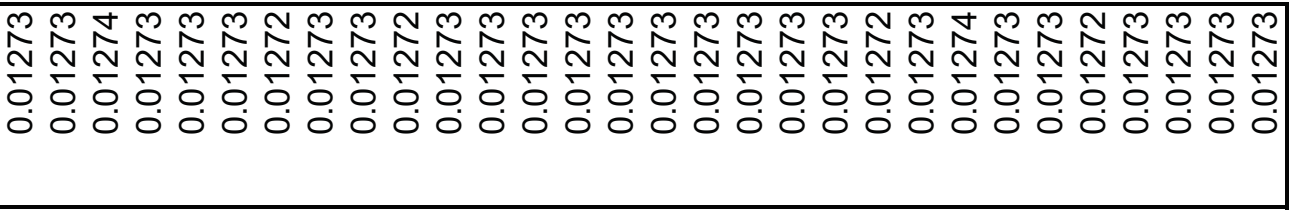 \\
\hline 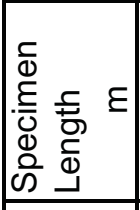 & 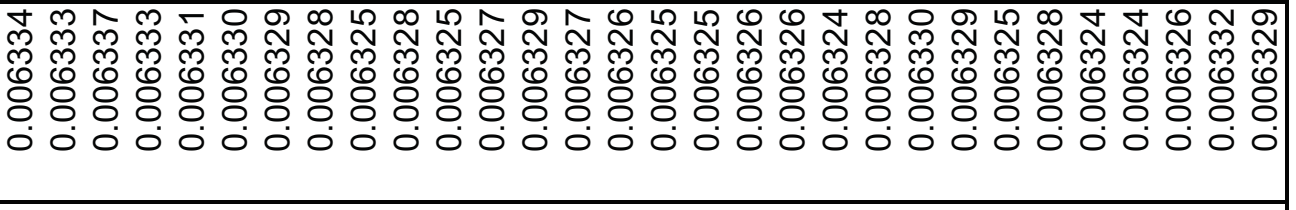 \\
\hline 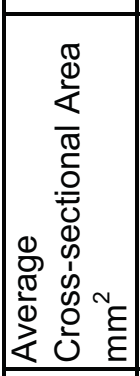 & 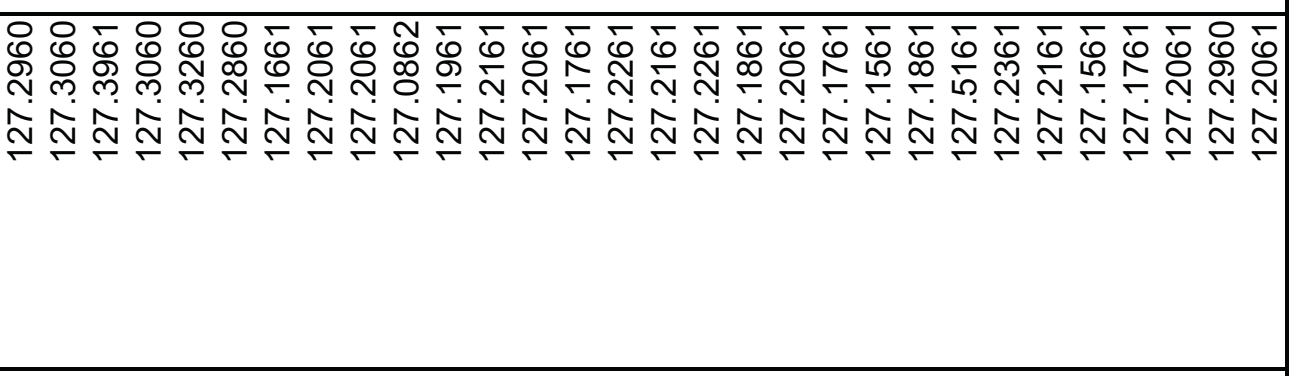 \\
\hline 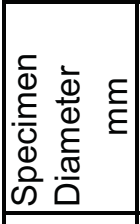 & 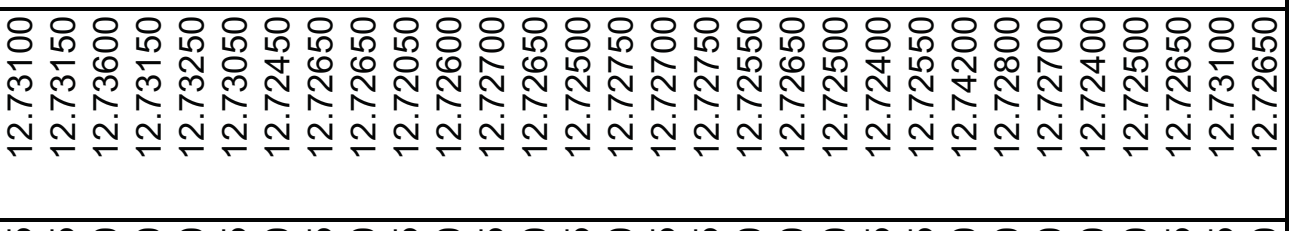 \\
\hline 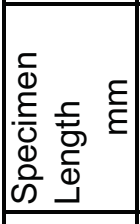 & 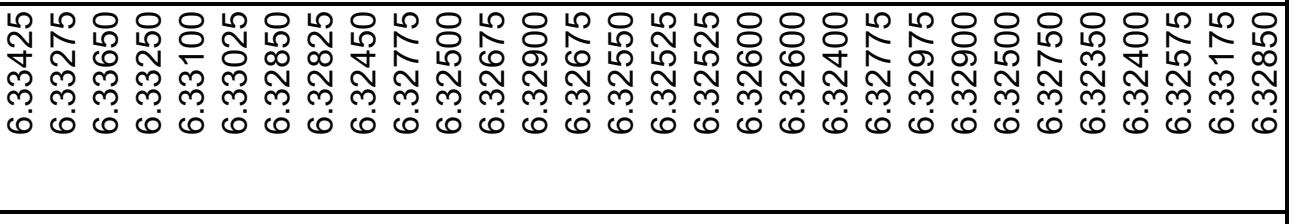 \\
\hline 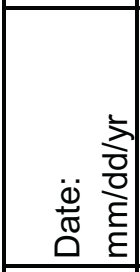 & 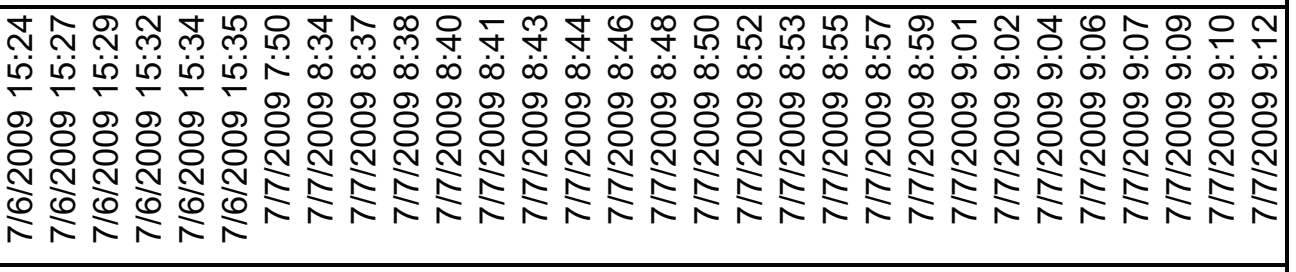 \\
\hline 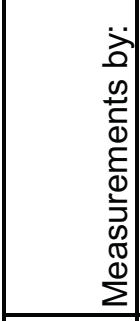 & みみ」 \\
\hline 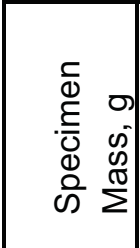 & 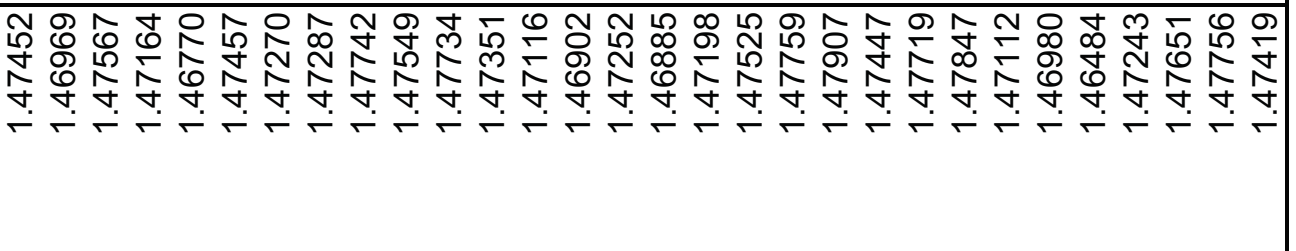 \\
\hline 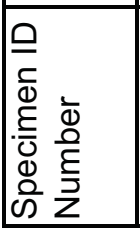 & 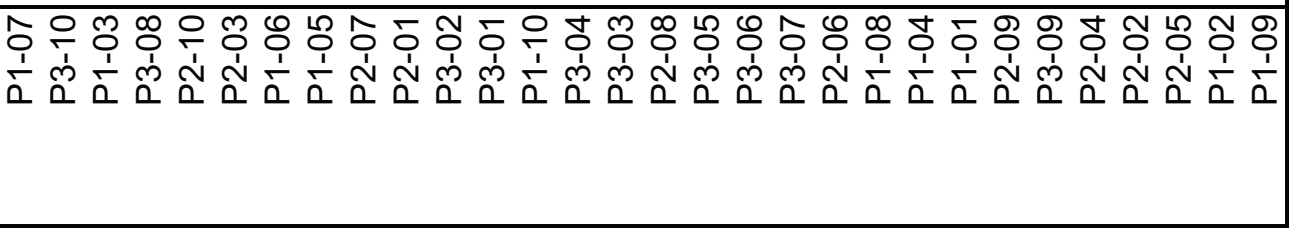 \\
\hline
\end{tabular}




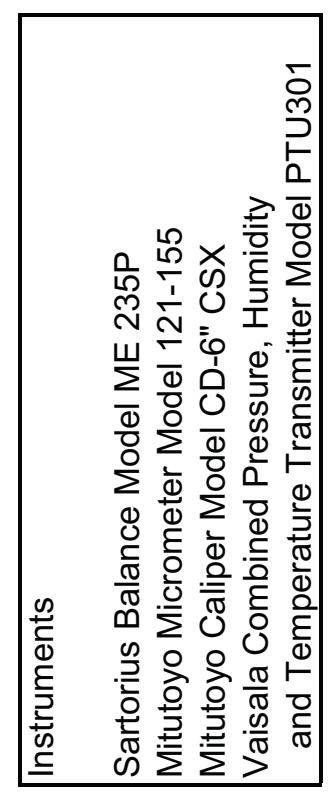

\begin{tabular}{|c|c|}
\hline 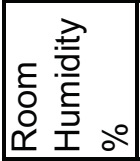 & 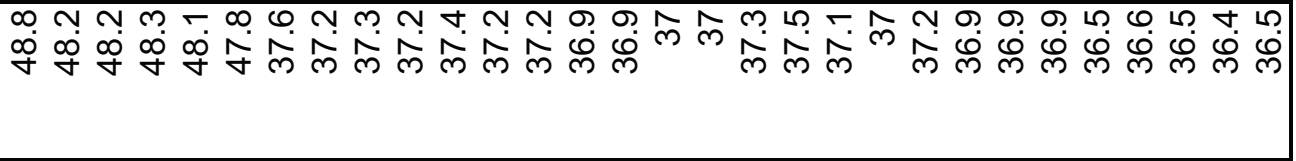 \\
\hline 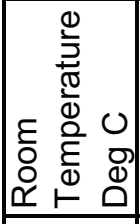 & 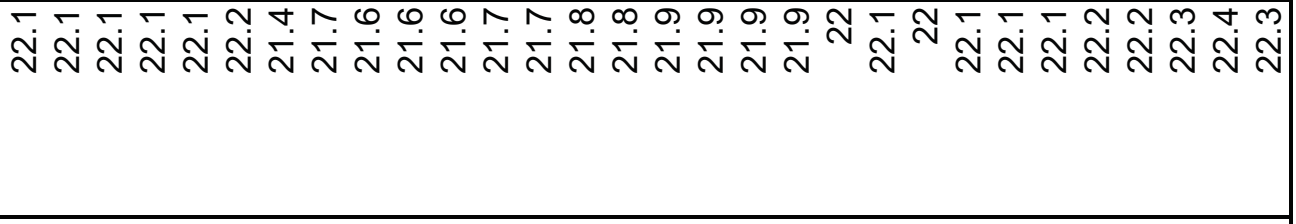 \\
\hline 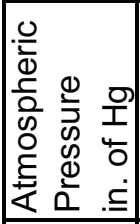 & 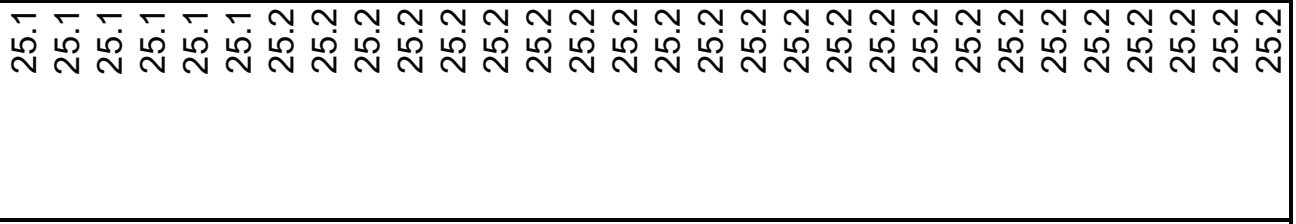 \\
\hline 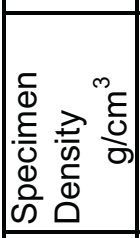 & 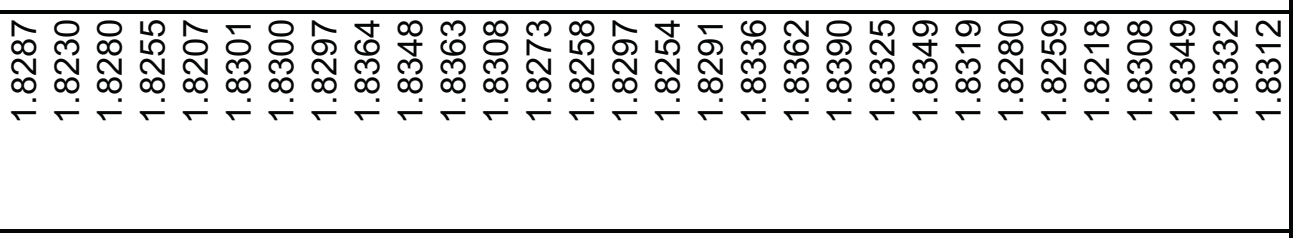 \\
\hline 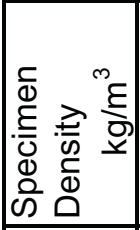 & 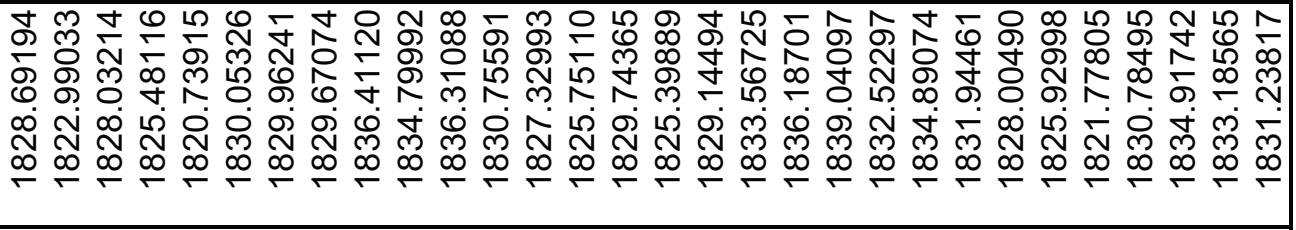 \\
\hline 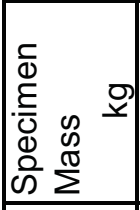 & 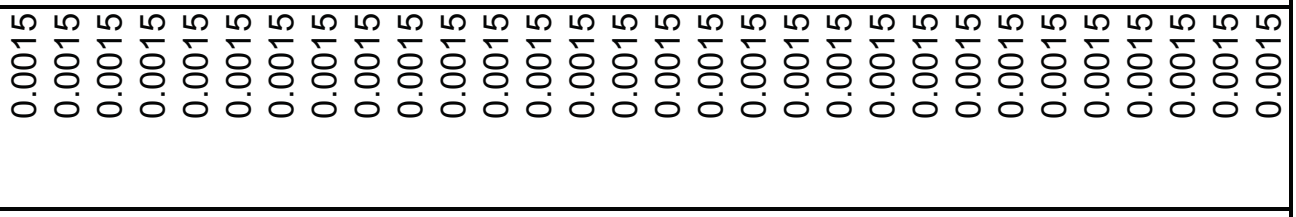 \\
\hline 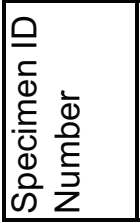 & 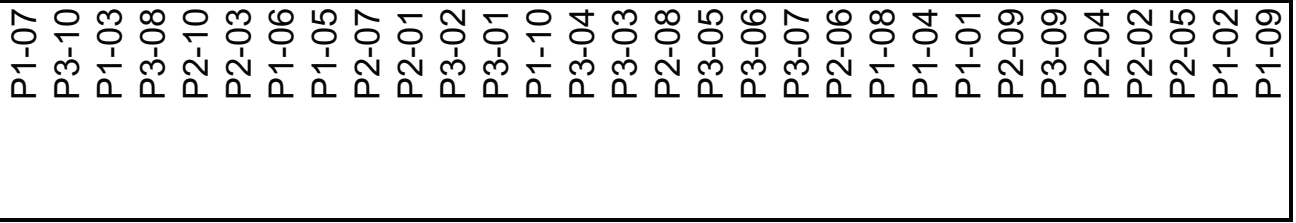 \\
\hline
\end{tabular}




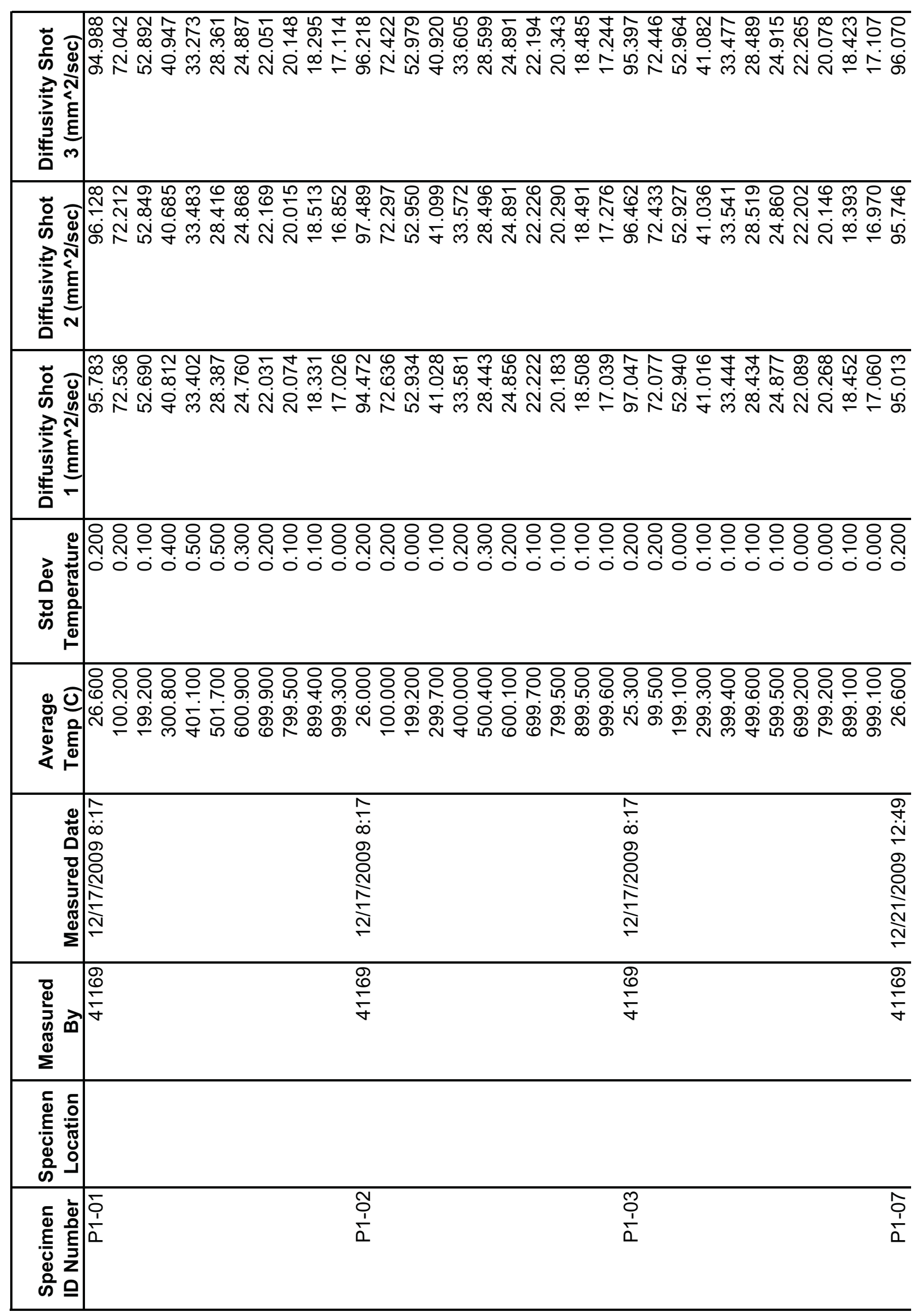




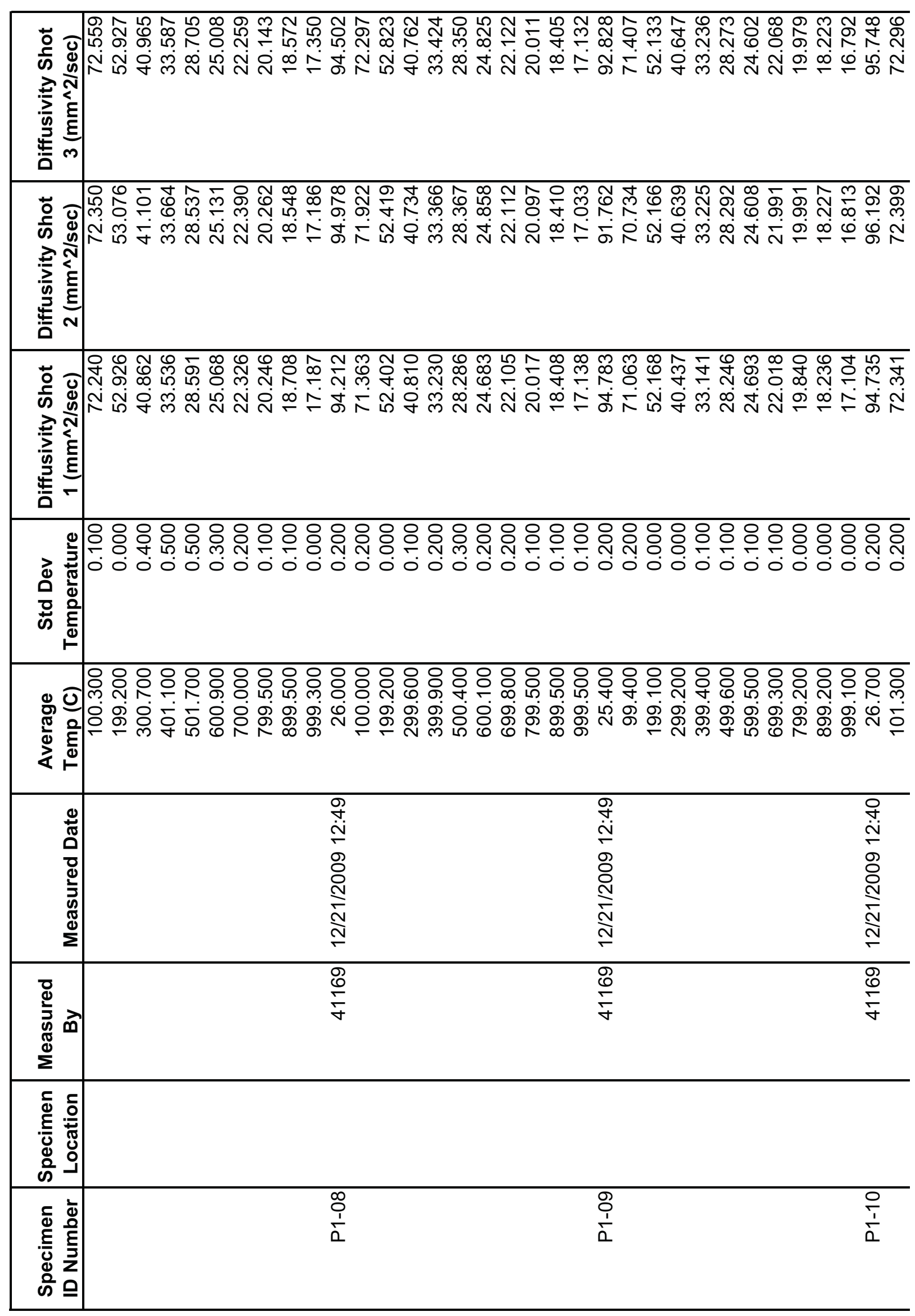




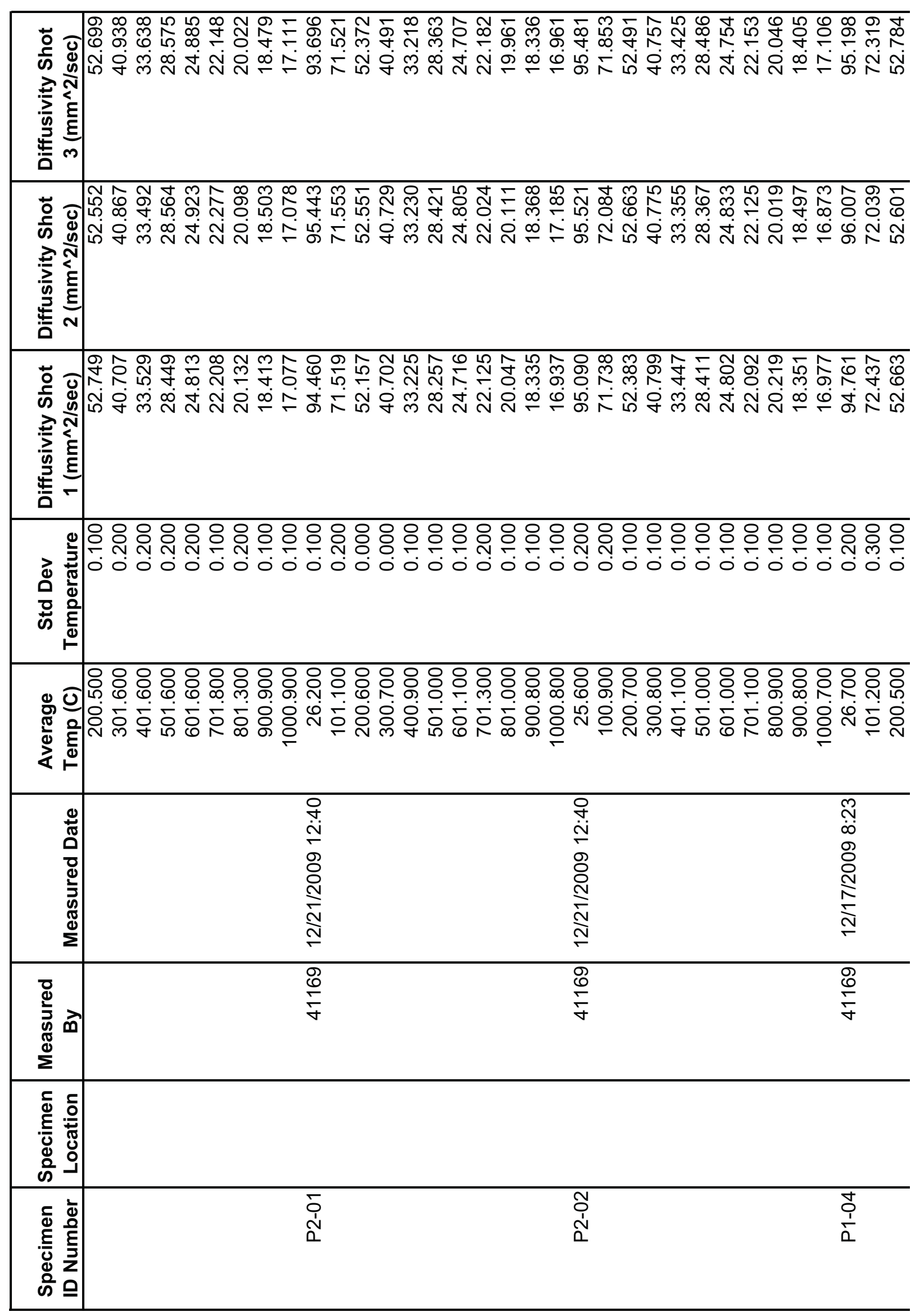




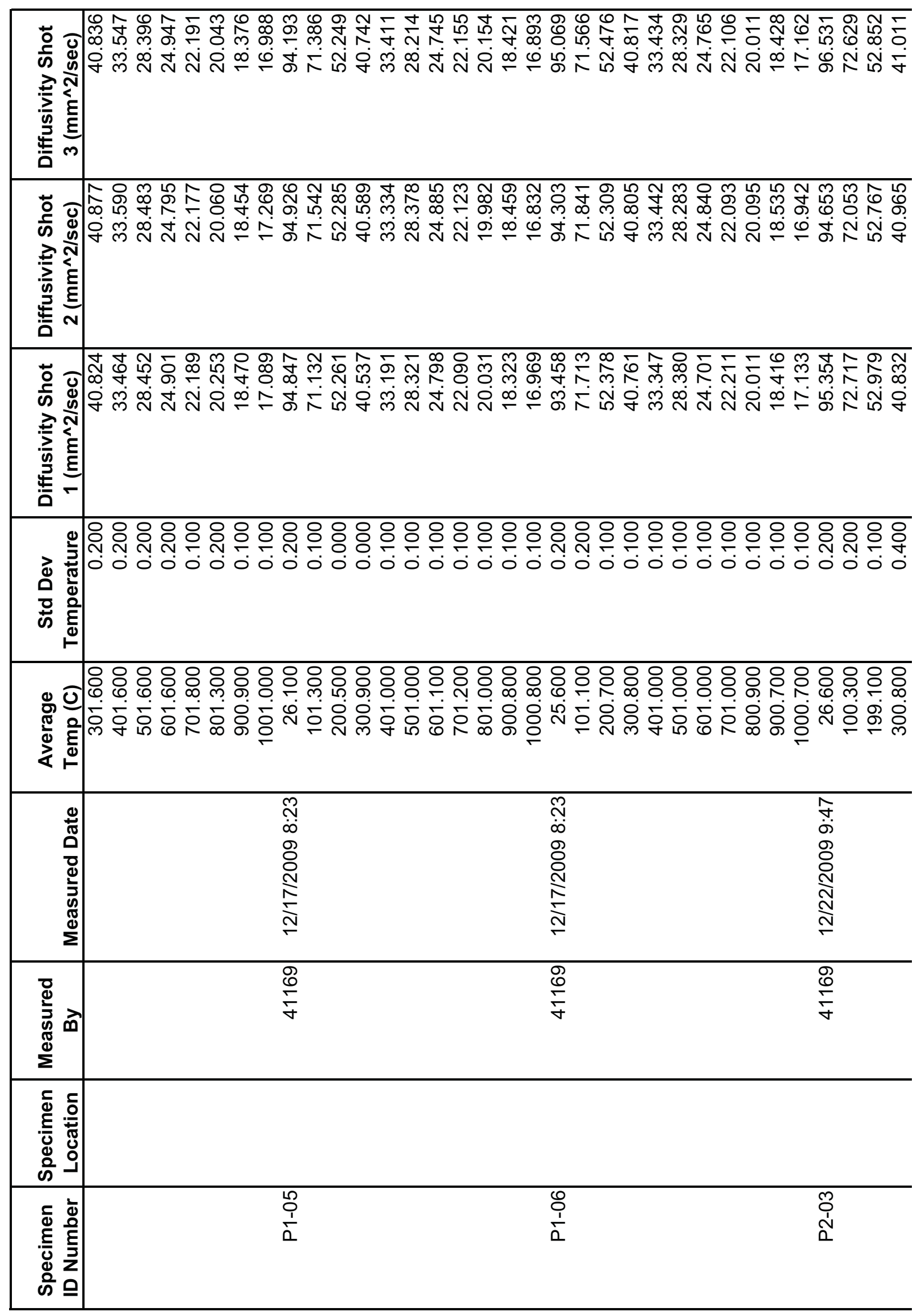




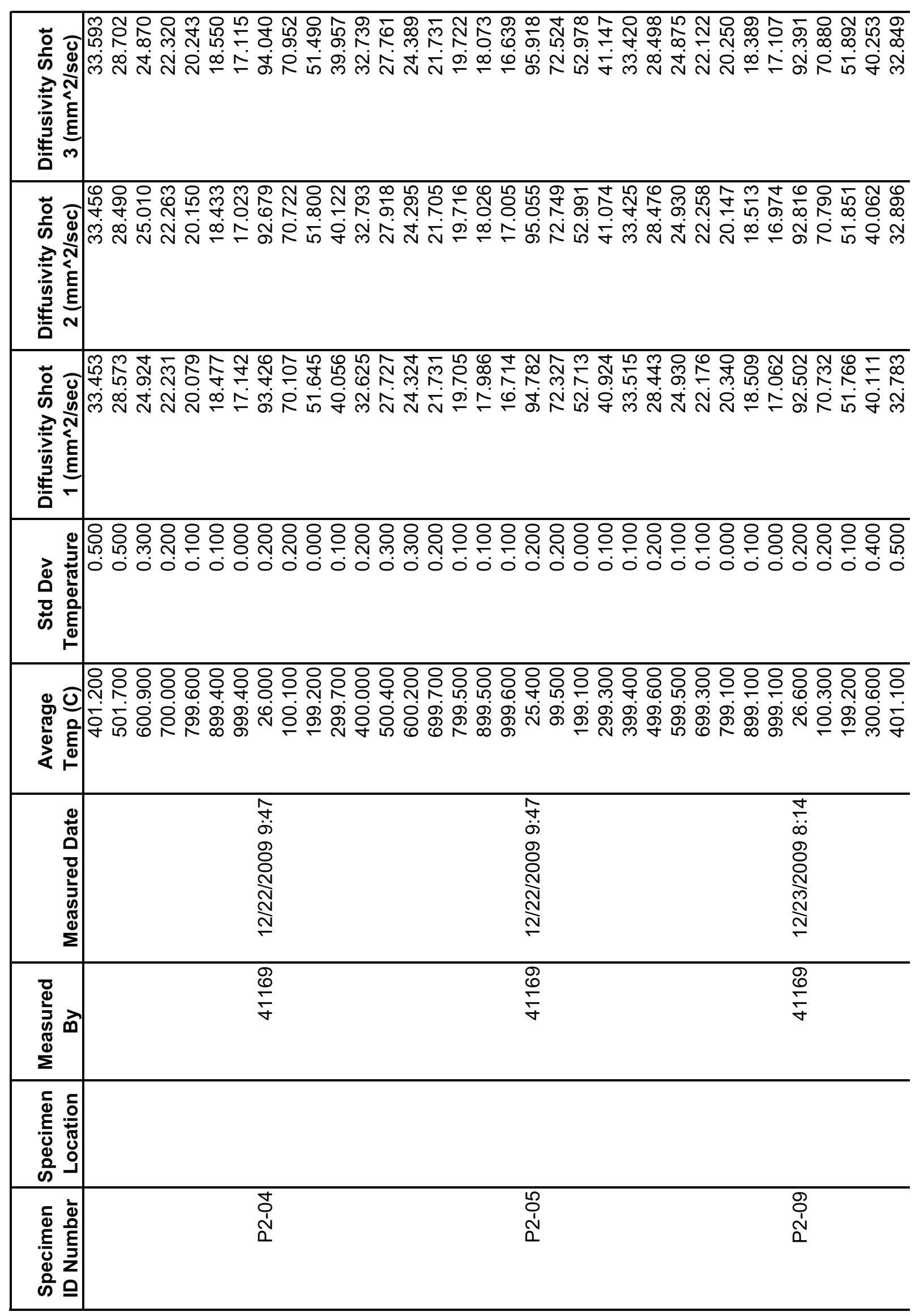




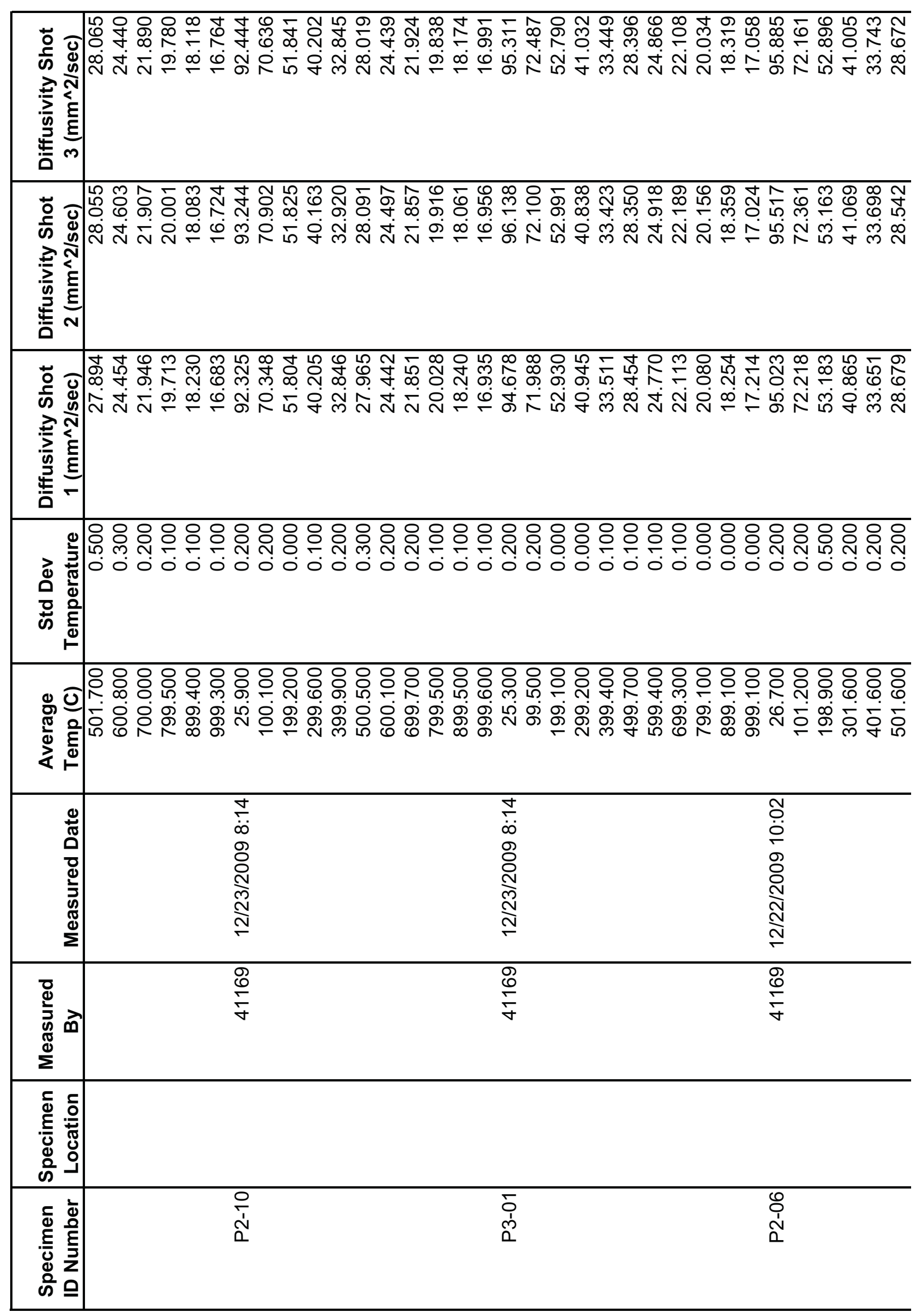




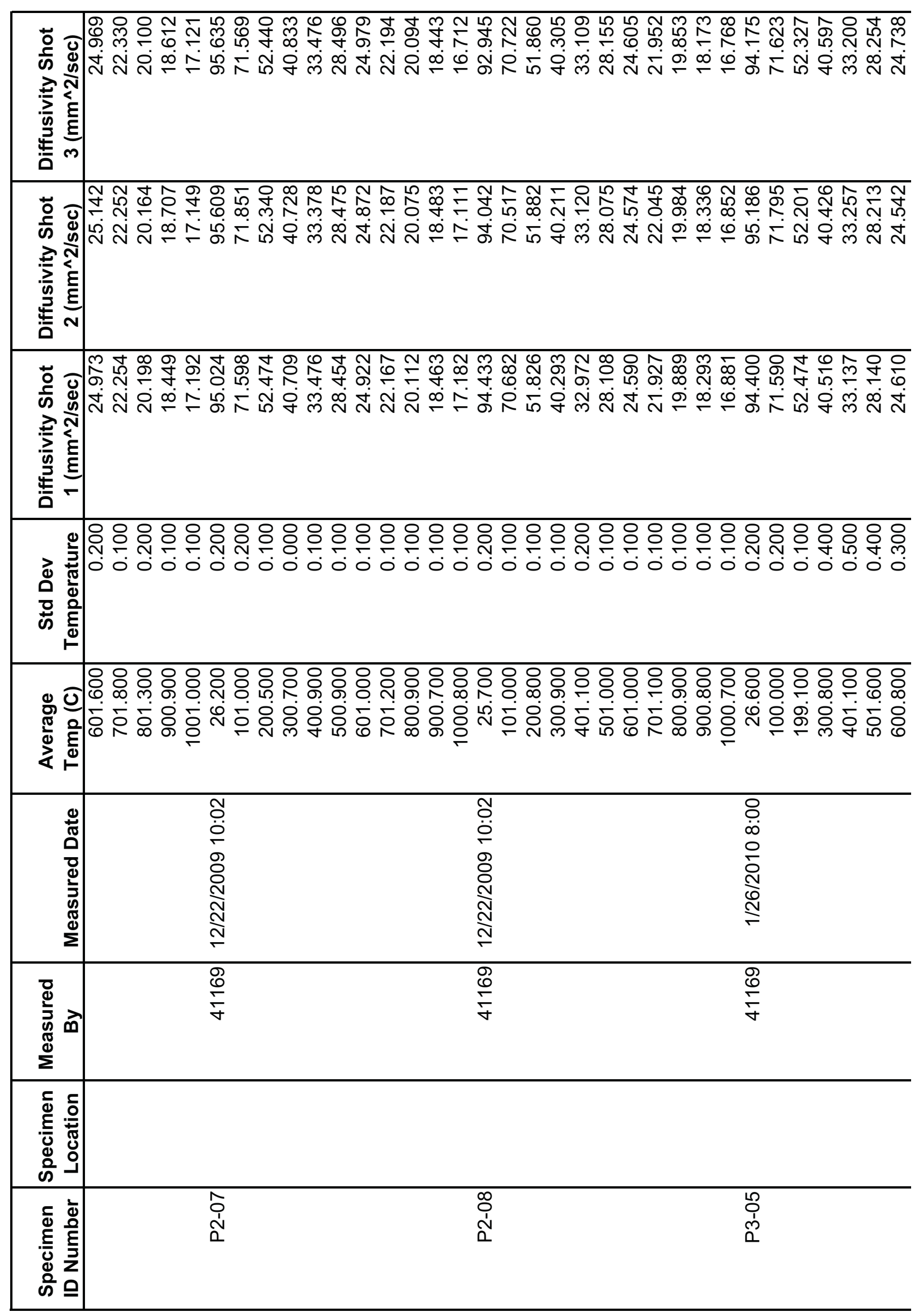




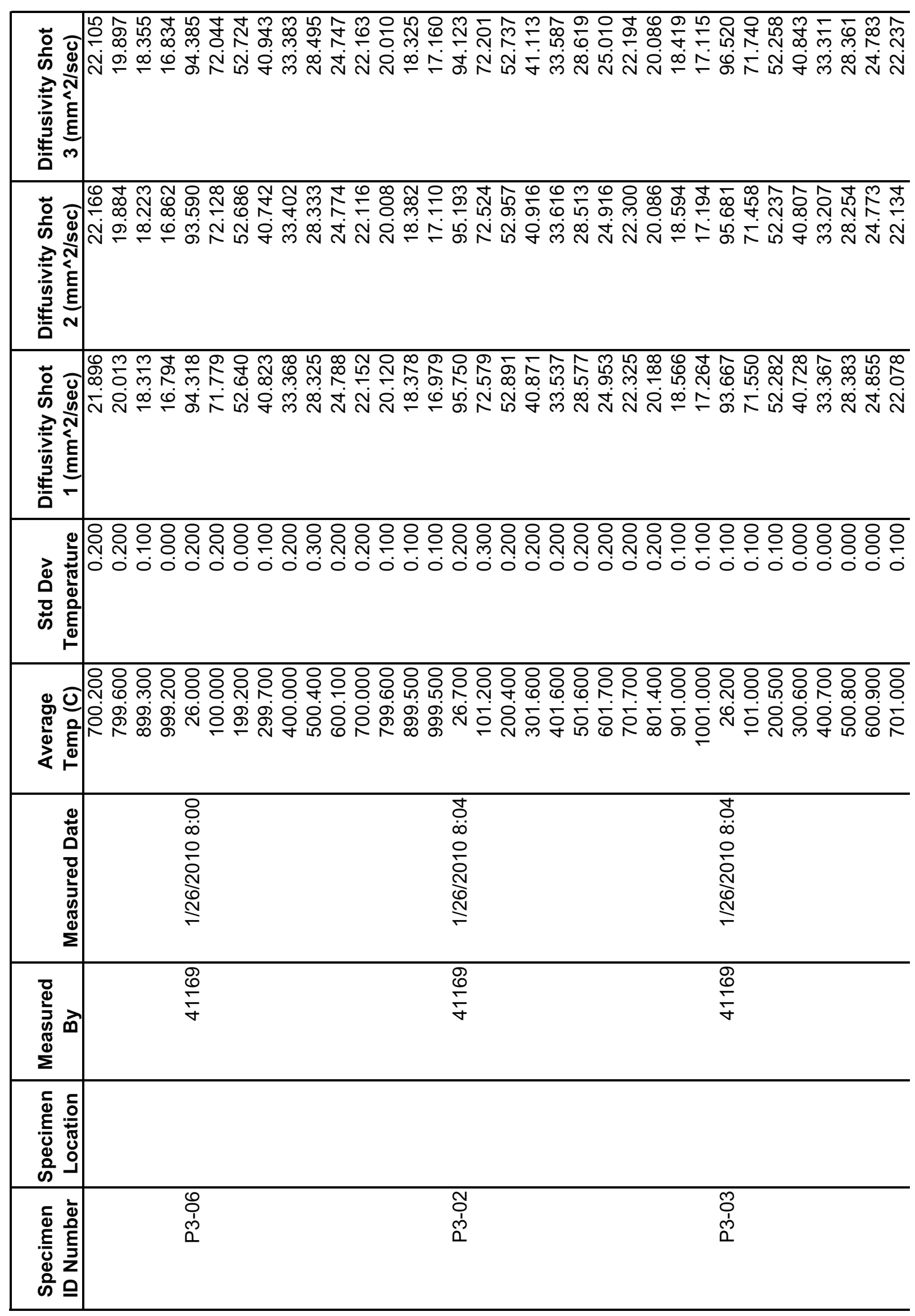




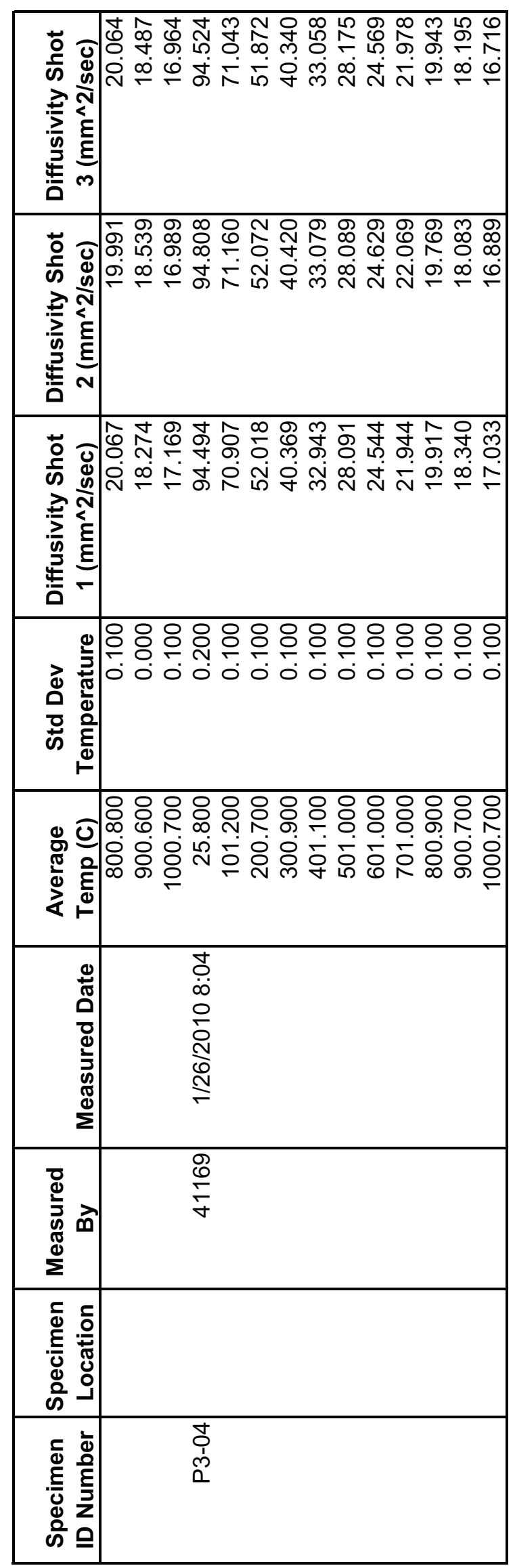




\begin{tabular}{|c|c|c|c|c|}
\hline$\underset{5}{\Sigma}$ & 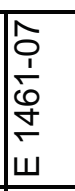 & $\begin{array}{l} \\
\\
1 \\
\dot{0} \\
\dot{+} \\
\omega \\
\end{array}$ & 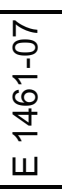 & 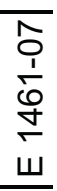 \\
\hline 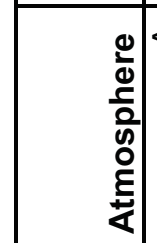 & & $\bar{z}$ & $\overline{\dot{\alpha}}$ & $\overline{\frac{\pi}{4}}$ \\
\hline 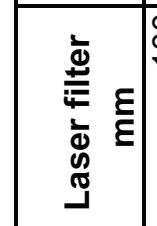 & & 음 & 음 & 음 \\
\hline 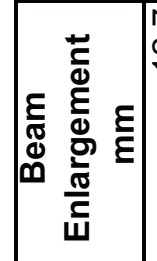 & $\stackrel{\hat{N}}{\stackrel{N}{ }}$ & $\stackrel{\stackrel{N}{\sim}}{\sim}$ & $\stackrel{\widehat{\mathrm{N}}}{\mathrm{N}}$ & 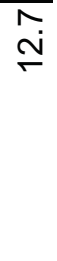 \\
\hline $\begin{array}{l}\vdots \\
\vdots \\
\vdots \\
\grave{d} \\
\omega\end{array}$ & & $\begin{array}{l}\text { की } \\
\text { की }\end{array}$ & $\begin{array}{l}\text { की } \\
\text { की }\end{array}$ & $\begin{array}{l}\text { की } \\
\text { 足 }\end{array}$ \\
\hline 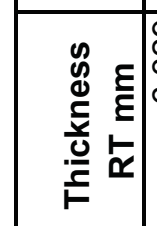 & & 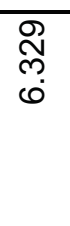 & 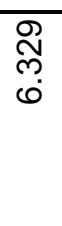 & $\begin{array}{l}\underset{\widetilde{N}}{\mathbb{N}} \\
\stackrel{0}{0}\end{array}$ \\
\hline 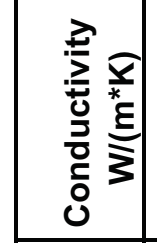 & & & & \\
\hline 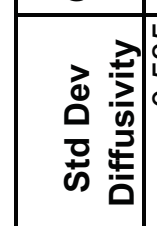 & 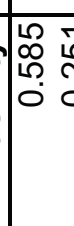 & $\stackrel{\circ}{5}$ & 0 & : \\
\hline 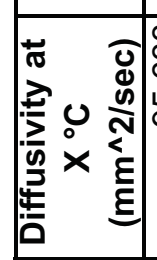 & 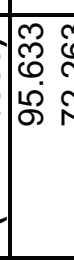 & $\begin{array}{l}8 \\
8 \\
8\end{array}$ & 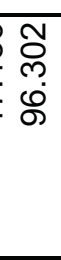 & $\begin{array}{l}0 \\
0\end{array}$ \\
\hline 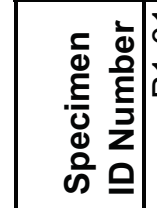 & & 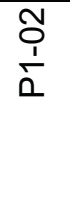 & $\begin{array}{l}\text { O̊ } \\
\frac{1}{\alpha}\end{array}$ & $\begin{array}{l}\frac{1}{1} \\
\frac{1}{2}\end{array}$ \\
\hline
\end{tabular}




\begin{tabular}{|c|c|c|c|}
\hline 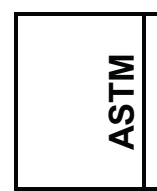 & $\begin{array}{l}\hat{o} \\
\frac{1}{0} \\
\dot{0} \\
\dot{5} \\
\end{array}$ & $\begin{array}{l}\hat{S} \\
\frac{1}{1} \\
\frac{0}{5} \\
\dot{w}\end{array}$ & 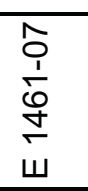 \\
\hline 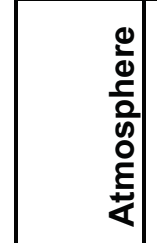 & 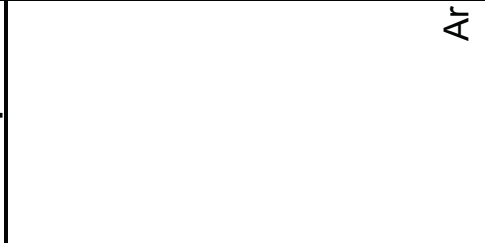 & $\frac{1}{\alpha}$ & 广 \\
\hline 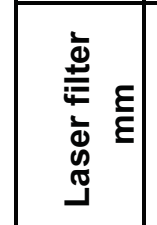 & 음 & $\stackrel{8}{\circ}$ & 음 \\
\hline 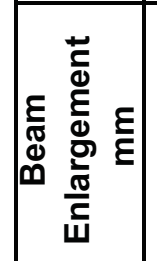 & $\stackrel{\widehat{N}}{\stackrel{N}{N}}$ & 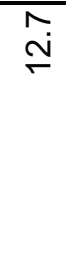 & 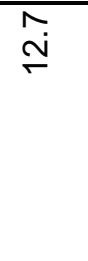 \\
\hline $\begin{array}{l}\vdots \\
\vdots \\
0 \\
\bar{d} \\
\infty\end{array}$ & $\begin{array}{l}\text { की } \\
\text { की }\end{array}$ & 吕 & $\begin{array}{l}\text { की } \\
\text { की }\end{array}$ \\
\hline 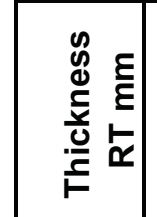 & ্ָ & 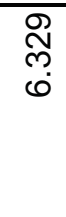 & 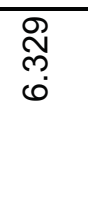 \\
\hline 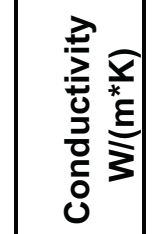 & & & \\
\hline 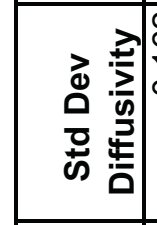 & 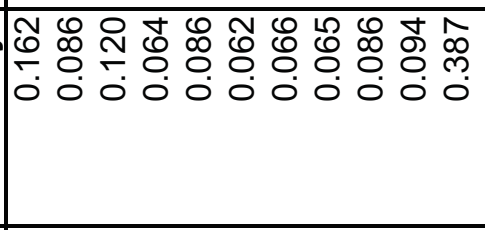 & $\stackrel{\text { ? }}{\leftarrow}$ & 年赂 \\
\hline 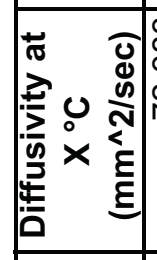 & 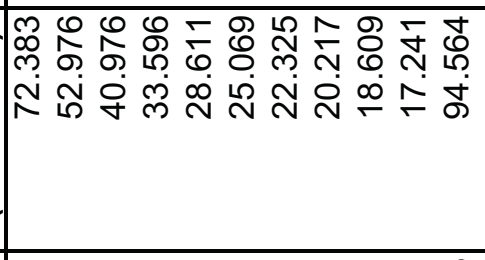 & 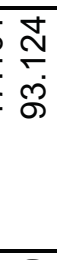 & 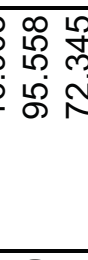 \\
\hline 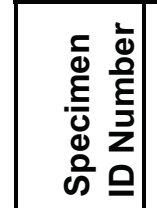 & 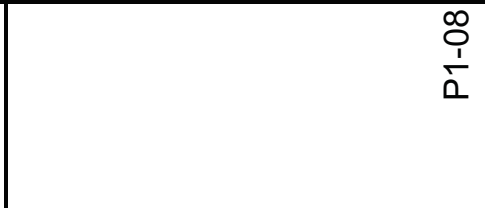 & $\begin{array}{l}0 \\
\frac{1}{1} \\
\frac{1}{0}\end{array}$ & $\frac{0}{\frac{1}{1}}$ \\
\hline
\end{tabular}




\begin{tabular}{|c|c|c|c|}
\hline 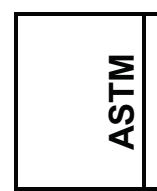 & $\begin{array}{l}\hat{o} \\
\dot{1} \\
\dot{0} \\
\dot{+} \\
\dot{w}\end{array}$ & $\begin{array}{l}\hat{o} \\
\frac{1}{0} \\
\stackrel{0}{+} \\
\dot{\omega}\end{array}$ & 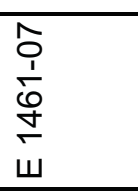 \\
\hline 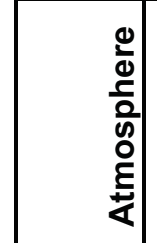 & 交 & 衣 & 交 \\
\hline 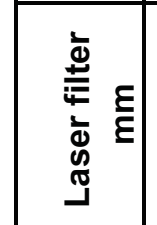 & 음 & 음 & 음 \\
\hline 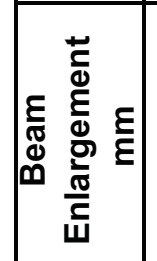 & $\stackrel{\widehat{N}}{\underline{I}}$ & $\stackrel{\stackrel{N}{ }}{ }$ & $\stackrel{\widetilde{N}}{\stackrel{N}{N}}$ \\
\hline $\begin{array}{l}\bar{b} \\
\vdots \\
心 \\
\Phi \\
\infty\end{array}$ & $\begin{array}{l}\text { की } \\
\text { की }\end{array}$ & $\begin{array}{l}\text { की } \\
\text { I }\end{array}$ & $\begin{array}{l}\text { की } \\
\text { की }\end{array}$ \\
\hline 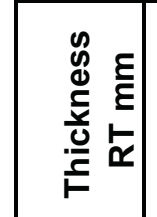 & ্ָ & $\begin{array}{l}\text { ్ָల } \\
0 \\
0\end{array}$ & 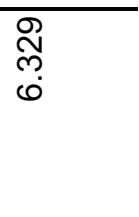 \\
\hline 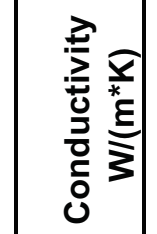 & & & \\
\hline 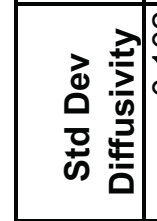 & 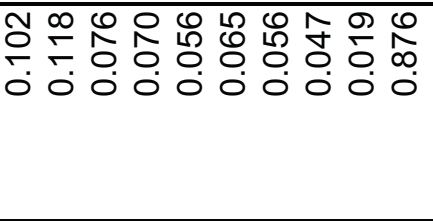 & s. & 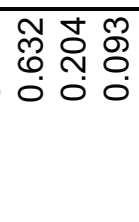 \\
\hline 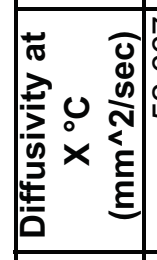 & 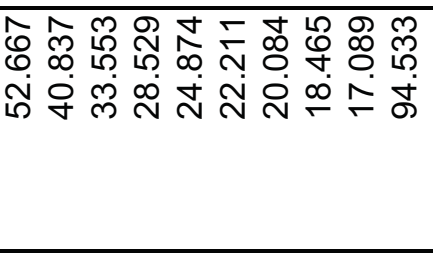 & 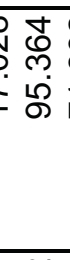 & 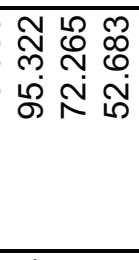 \\
\hline 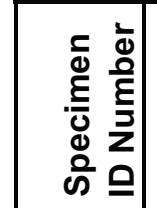 & 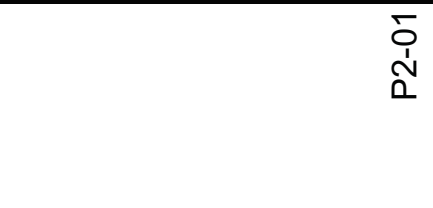 & 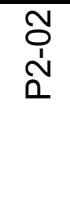 & $\begin{array}{l}\frac{1}{0} \\
\frac{1}{0}\end{array}$ \\
\hline
\end{tabular}




\begin{tabular}{|c|c|c|c|}
\hline 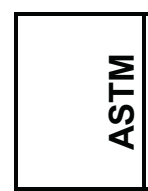 & $\begin{array}{l}\hat{o} \\
\dot{1} \\
\dot{0} \\
\dot{+} \\
\dot{w}\end{array}$ & $\begin{array}{l}\hat{D} \\
\frac{1}{1} \\
\dot{0} \\
\frac{1}{\omega} \\
\end{array}$ & $\begin{array}{l}\hat{o} \\
\frac{1}{0} \\
\stackrel{0}{+} \\
\dot{\omega}\end{array}$ \\
\hline 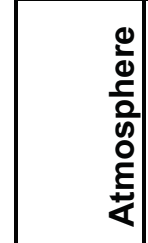 & 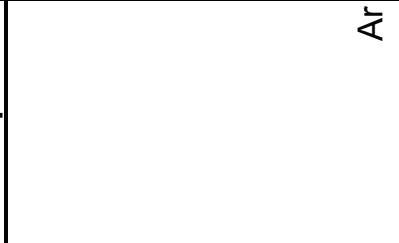 & $\frac{1}{\alpha}$ & $\frac{1}{4}$ \\
\hline 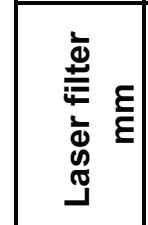 & $\stackrel{8}{\circ}$ & 음 & 음 \\
\hline 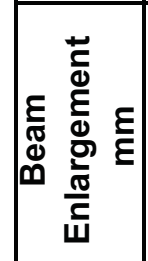 & $\stackrel{\widehat{N}}{\stackrel{N}{N}}$ & 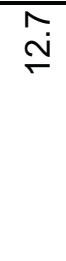 & $\stackrel{\stackrel{\sim}{\sim}}{ }$ \\
\hline 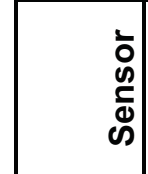 & $\begin{array}{l}\text { की } \\
\text { की }\end{array}$ & 吊 & $\begin{array}{l}\text { की } \\
\text { 足 }\end{array}$ \\
\hline 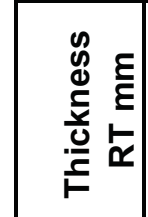 & શ్ & $\begin{array}{l}\text { స్ల } \\
0 \\
0\end{array}$ & 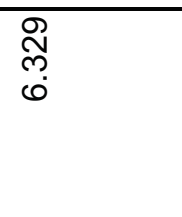 \\
\hline 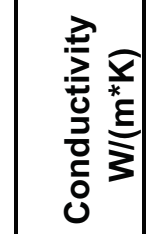 & & & \\
\hline 永辛 & 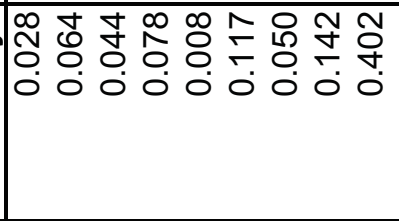 & ; & 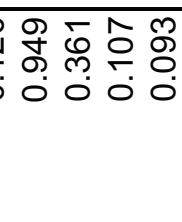 \\
\hline 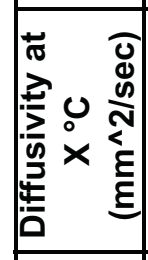 & $\mid \begin{array}{l}0 \\
0 \\
0\end{array}$ & $\stackrel{N}{\stackrel{N}{*}}$ & 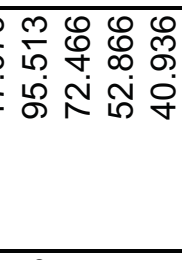 \\
\hline 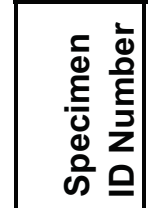 & $\begin{array}{l}\frac{n}{1} \\
\frac{1}{\Lambda}\end{array}$ & $\begin{array}{l}0 \\
\frac{1}{1} \\
\frac{1}{2}\end{array}$ & $\begin{array}{l}\text { o } \\
\grave{\Lambda} \\
\text { Qn }\end{array}$ \\
\hline
\end{tabular}




\begin{tabular}{|c|c|c|c|}
\hline 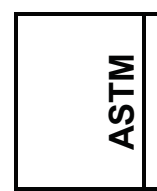 & $\begin{array}{l}\hat{O} \\
\frac{1}{0} \\
\stackrel{0}{+} \\
\dot{w}\end{array}$ & $\begin{array}{l}\hat{o} \\
\frac{1}{0} \\
\stackrel{0}{+} \\
\dot{\omega}\end{array}$ & 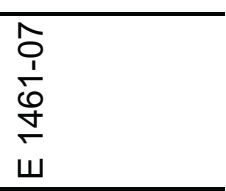 \\
\hline 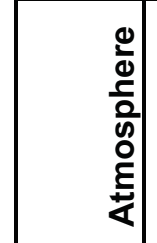 & 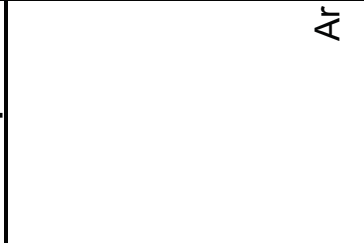 & 衣 & 交 \\
\hline 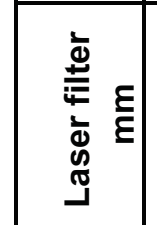 & $\stackrel{\circ}{\circ}$ & 음 & 음 \\
\hline 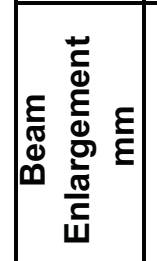 & $\stackrel{\widehat{N}}{\stackrel{N}{S}}$ & $\stackrel{\stackrel{N}{\sim}}{ }$ & $\stackrel{\stackrel{N}{N}}{ }$ \\
\hline $\begin{array}{l}\bar{b} \\
\vdots \\
心 \\
\Phi \\
\infty\end{array}$ & $\begin{array}{l}\text { की } \\
\text { की }\end{array}$ & $\begin{array}{l}\text { की } \\
\text { I }\end{array}$ & $\begin{array}{l}\text { की } \\
\text { Ф }\end{array}$ \\
\hline 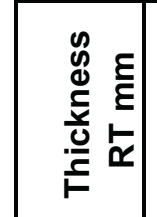 & 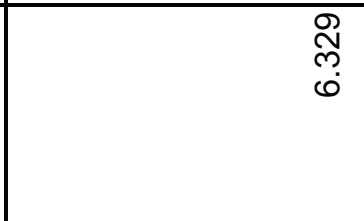 & $\begin{array}{l}\text { ్ָల } \\
0 \\
0\end{array}$ & 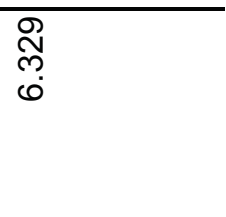 \\
\hline 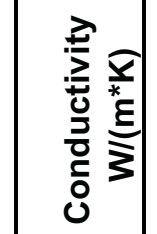 & & & \\
\hline 焉 & 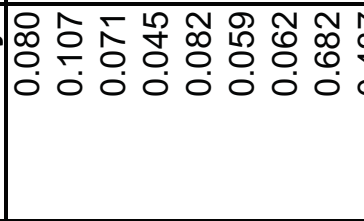 & 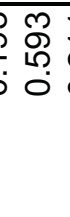 & 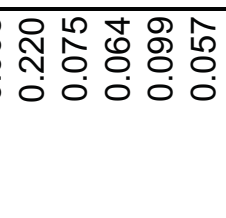 \\
\hline 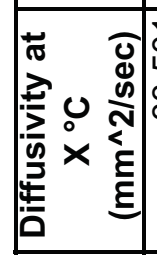 & 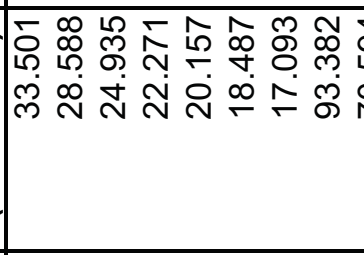 & 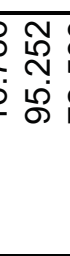 & 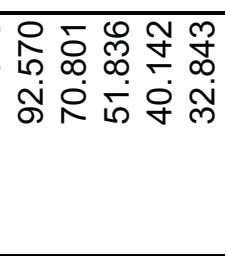 \\
\hline 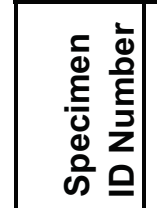 & $\begin{array}{l}\text { ¿্̀ } \\
\text { ָे }\end{array}$ & 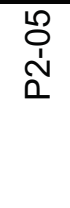 & $\begin{array}{l}\text { Oे } \\
\text { ते } \\
\text { Q }\end{array}$ \\
\hline
\end{tabular}




\begin{tabular}{|c|c|c|c|}
\hline 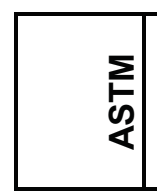 & $\begin{array}{l}\hat{o} \\
\frac{1}{0} \\
\dot{o} \\
\dot{w} \\
\end{array}$ & $\begin{array}{l}\hat{o} \\
\frac{1}{0} \\
\stackrel{0}{+} \\
\dot{\omega}\end{array}$ & $\begin{array}{l}\hat{o} \\
\frac{1}{o} \\
\dot{0} \\
\dot{W} \\
\end{array}$ \\
\hline 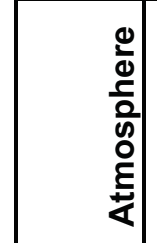 & 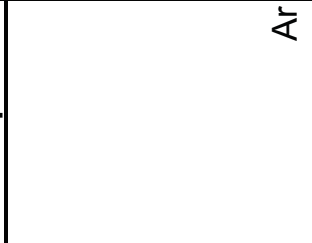 & 文 & $\frac{1}{4}$ \\
\hline 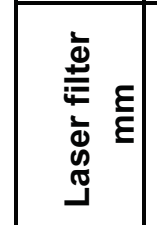 & 음 & 음 & 음 \\
\hline 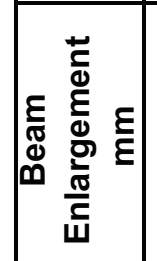 & $\stackrel{\widehat{N}}{\stackrel{N}{N}}$ & 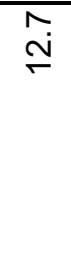 & $\stackrel{\stackrel{\sim}{\sim}}{ }$ \\
\hline $\begin{array}{l}\vdots \\
\vdots \\
0 \\
⿱ 亠 凶 \\
\infty\end{array}$ & $\begin{array}{l}\text { की } \\
\text { की }\end{array}$ & 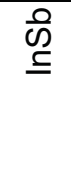 & $\begin{array}{l}\text { की } \\
\text { 足 }\end{array}$ \\
\hline 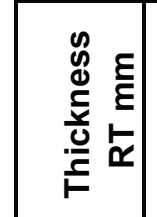 & 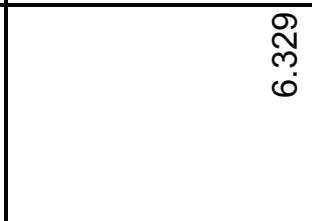 & $\begin{array}{l}\text { ్ָల } \\
0 \\
0\end{array}$ & $\begin{array}{l}\text { ్ָల్ } \\
\text { } \\
0\end{array}$ \\
\hline 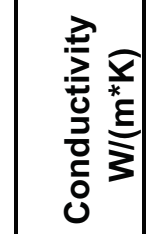 & & & \\
\hline 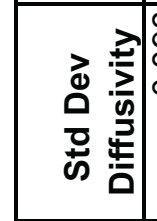 & 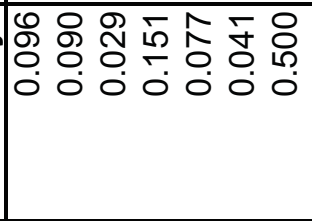 & 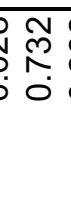 & 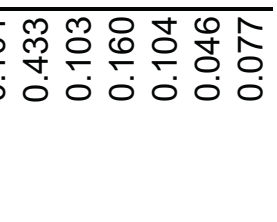 \\
\hline 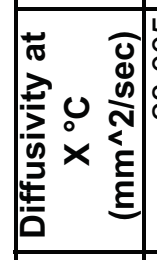 & 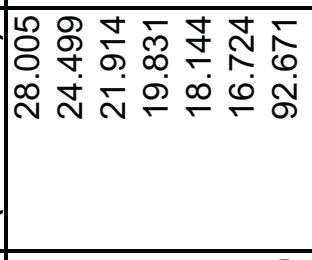 & 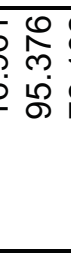 & 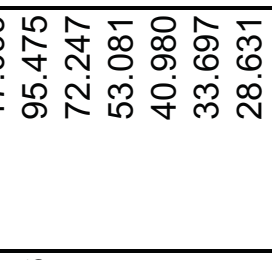 \\
\hline 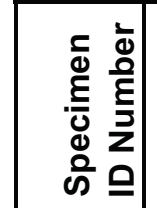 & $\begin{array}{l}\stackrel{0}{\dot{\alpha}} \\
\stackrel{\alpha}{\alpha}\end{array}$ & $\begin{array}{l}\bar{o} \\
\text { ભ }\end{array}$ & 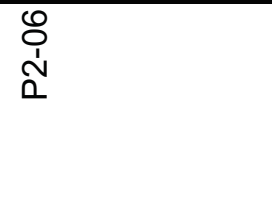 \\
\hline
\end{tabular}




\begin{tabular}{|c|c|c|c|}
\hline$\underset{\Sigma}{\Sigma}$ & $\begin{array}{l}\hat{O} \\
\frac{1}{0} \\
\stackrel{0}{0} \\
\dot{w}\end{array}$ & 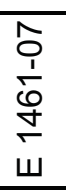 & $\begin{array}{l}\hat{O} \\
\frac{1}{0} \\
\stackrel{0}{+} \\
\dot{w}\end{array}$ \\
\hline 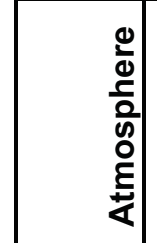 & 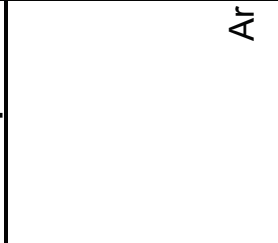 & 文 & $\bar{z}$ \\
\hline 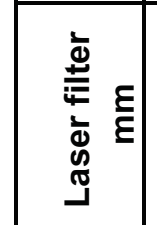 & $\stackrel{8}{\circ}$ & 음 & 음 \\
\hline 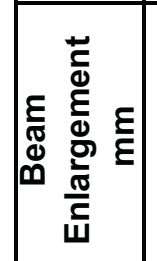 & $\stackrel{\grave{N}}{\stackrel{N}{N}}$ & 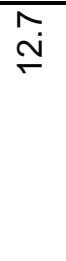 & 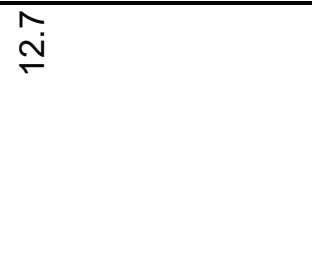 \\
\hline $\begin{array}{l}\bar{b} \\
\vdots \\
心 \\
\Phi \\
\infty\end{array}$ & $\begin{array}{l}\text { की } \\
\text { की }\end{array}$ & $\begin{array}{l}\text { की } \\
\text { की }\end{array}$ & $\begin{array}{l}\text { की } \\
\text { 足 }\end{array}$ \\
\hline 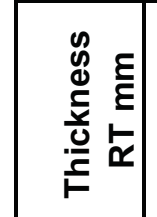 & 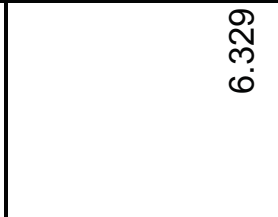 & 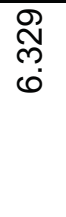 & 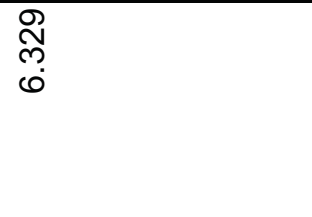 \\
\hline 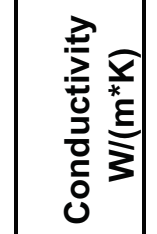 & & & \\
\hline 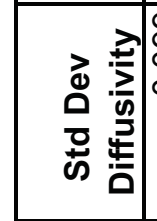 & 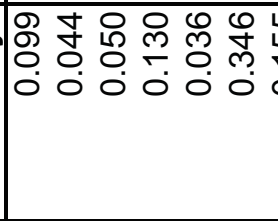 & $\hat{0}$ & 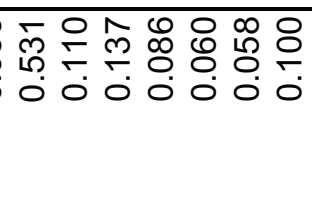 \\
\hline 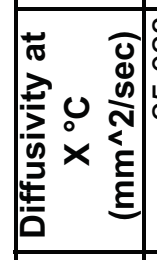 & 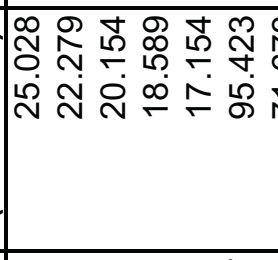 & & 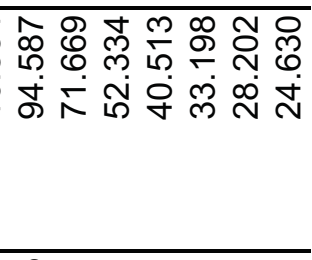 \\
\hline 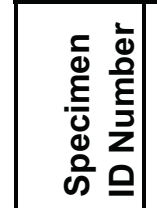 & $\begin{array}{l}\text { 文 } \\
\text { ते }\end{array}$ & $\begin{array}{l}\text { ì } \\
\text { ָे }\end{array}$ & $\begin{array}{l}\text { ஜo } \\
\text { ஸे } \\
0\end{array}$ \\
\hline
\end{tabular}




\begin{tabular}{|c|c|c|c|}
\hline$\underset{\Sigma}{\Sigma}$ & $\begin{array}{l}\hat{o} \\
\frac{1}{0} \\
\frac{0}{5} \\
\dot{w}\end{array}$ & $\begin{array}{l}\frac{0}{1} \\
\frac{1}{o} \\
\frac{1}{5} \\
\dot{w}\end{array}$ & 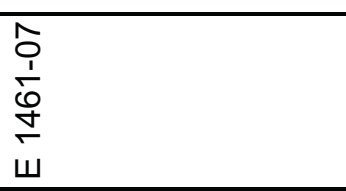 \\
\hline 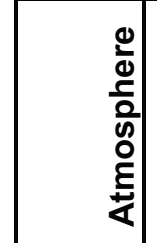 & 交 & $\frac{1}{4}$ & ¿ \\
\hline 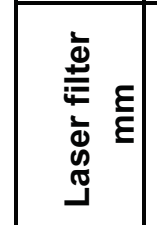 & 음 & 음 & 응 \\
\hline 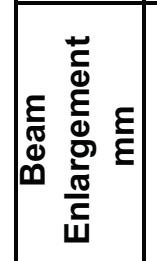 & $\hat{\stackrel{N}{N}}$ & 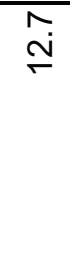 & I্ \\
\hline $\begin{array}{l}\bar{b} \\
0 \\
\stackrel{0}{\Phi} \\
心\end{array}$ & $\begin{array}{l}\text { की } \\
\text { की }\end{array}$ & $\begin{array}{l}\text { 号 } \\
\text { 足 }\end{array}$ & की \\
\hline 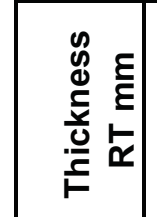 & 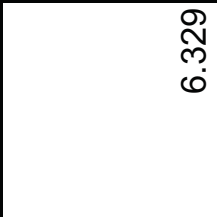 & 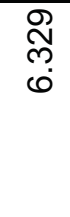 & $\begin{array}{l}\underset{\widetilde{W}}{0} \\
\text { }\end{array}$ \\
\hline 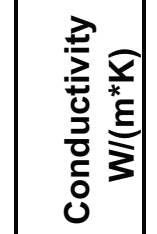 & & & \\
\hline 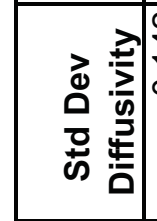 & $\begin{array}{llll} & \\
\end{array}$ & $\begin{array}{l}\widehat{N} \\
\tilde{\infty} \\
0 \\
0\end{array}$ & 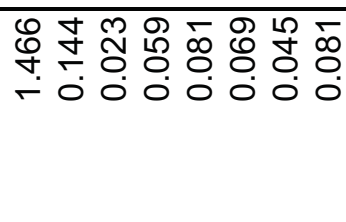 \\
\hline 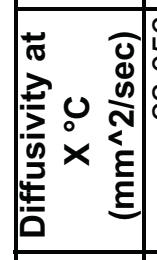 & 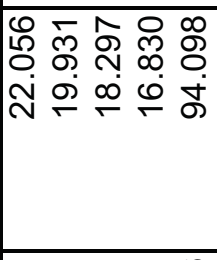 & 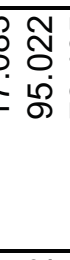 & 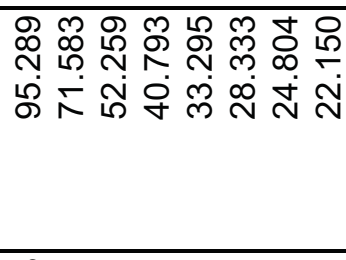 \\
\hline 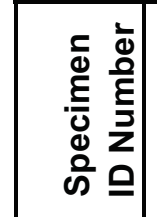 & 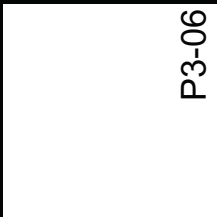 & 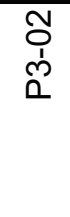 & $\begin{array}{l}\text { ôn } \\
\dot{1} \\
0\end{array}$ \\
\hline
\end{tabular}




\begin{tabular}{|c|c|}
\hline$\underset{\frac{\Sigma}{\infty}}{\frac{\Sigma}{Q}}$ & 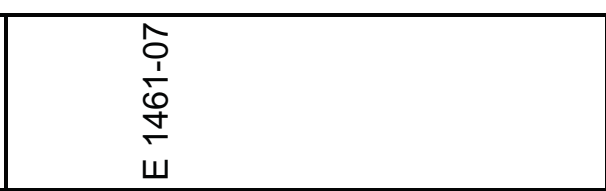 \\
\hline 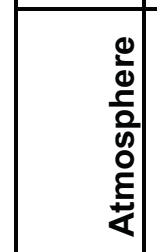 & 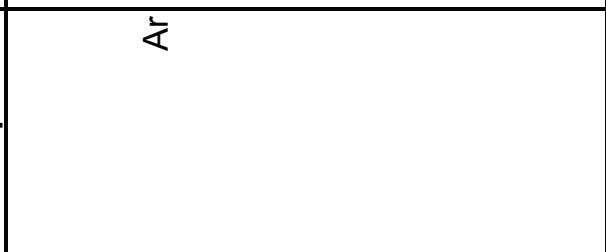 \\
\hline 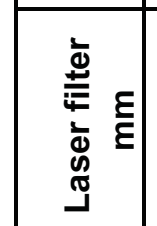 & 음 \\
\hline 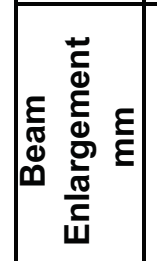 & 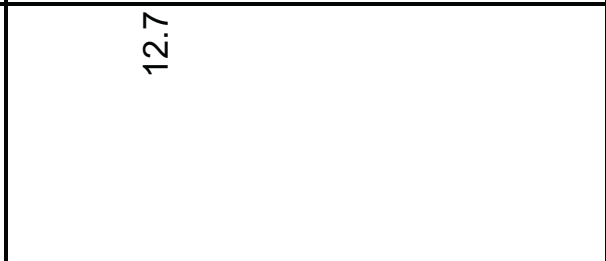 \\
\hline $\begin{array}{l}\bar{b} \\
\dot{g} \\
\bar{d} \\
\oplus\end{array}$ & की \\
\hline 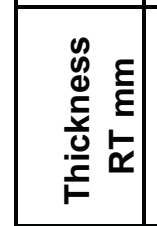 & 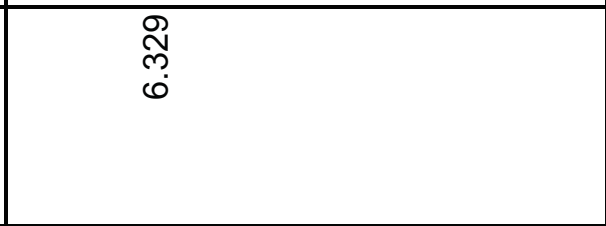 \\
\hline 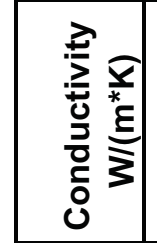 & \\
\hline 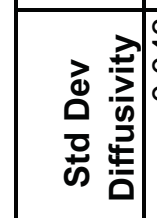 & 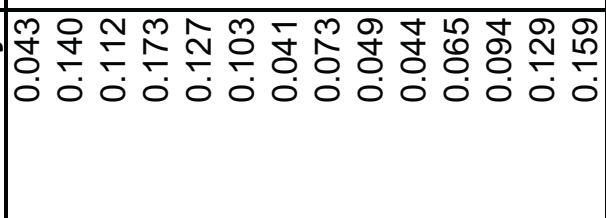 \\
\hline 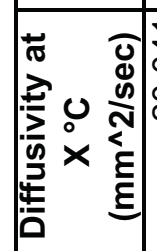 & 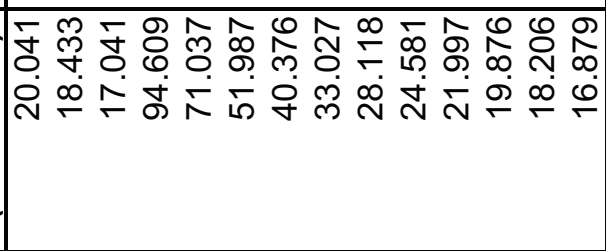 \\
\hline 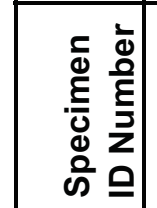 & $\begin{array}{l}\text { पे } \\
\text { ले } \\
0\end{array}$ \\
\hline
\end{tabular}




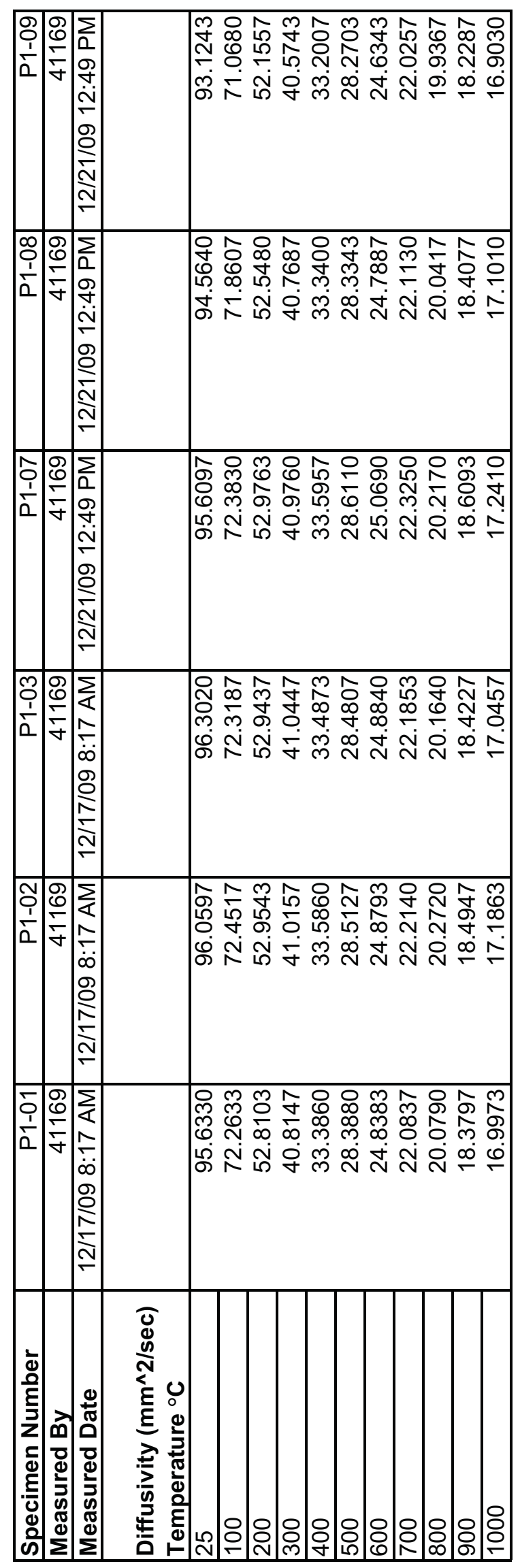




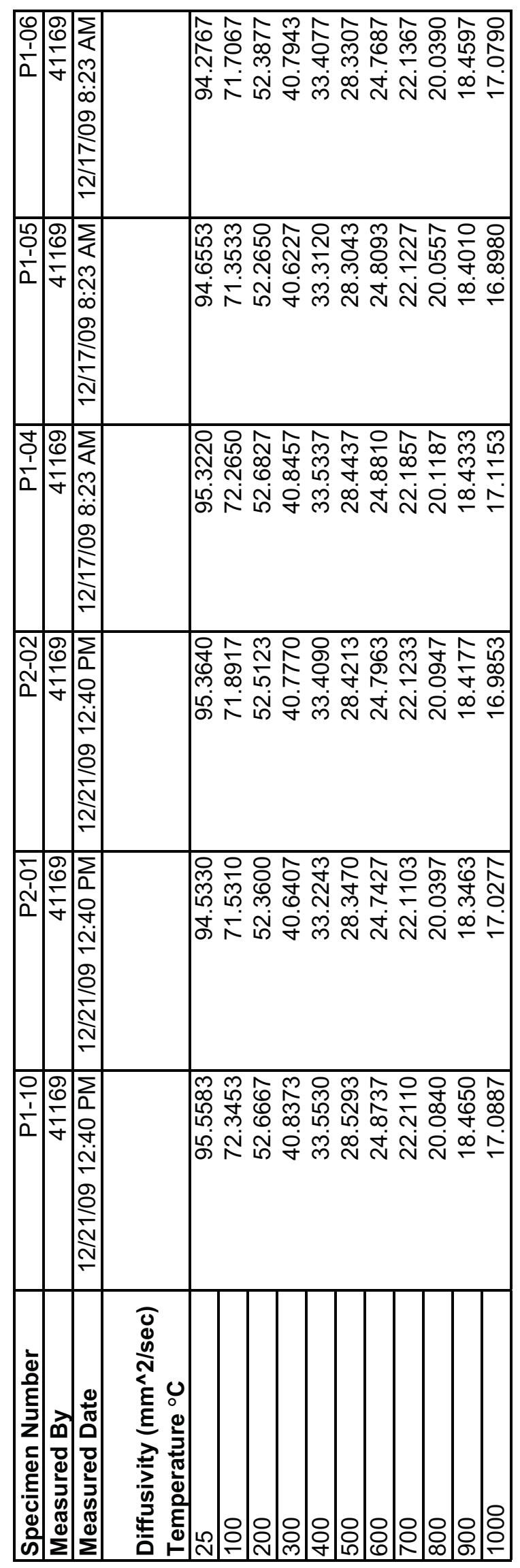




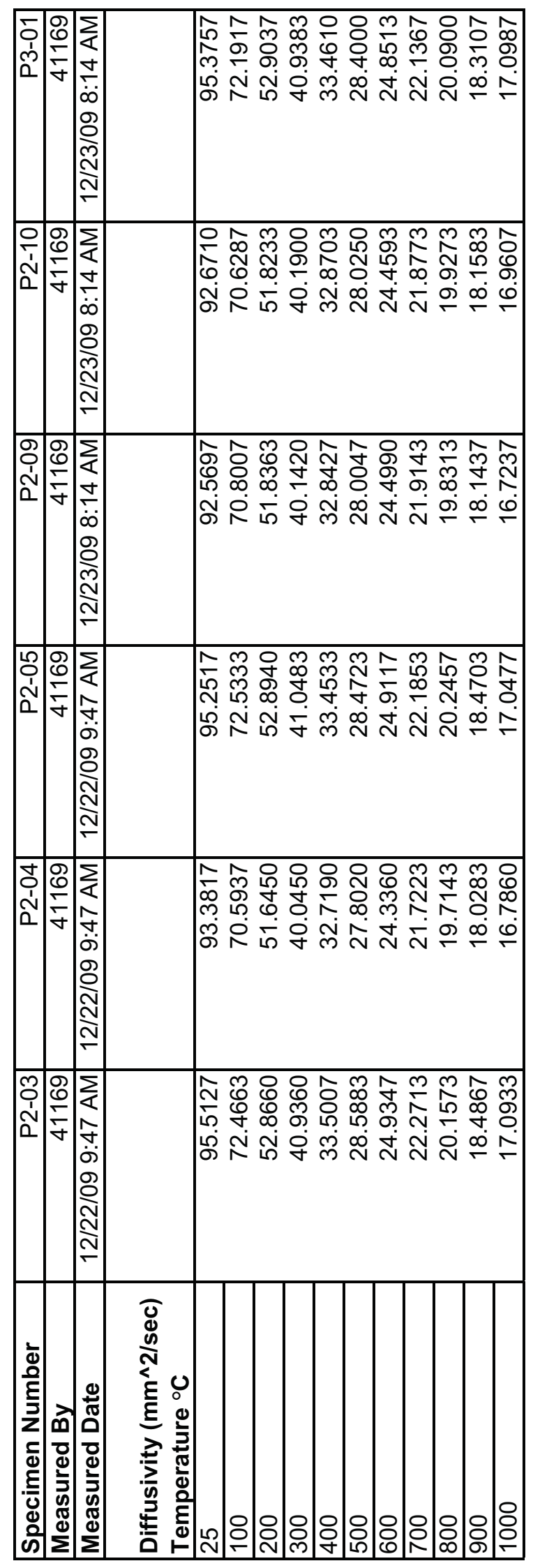




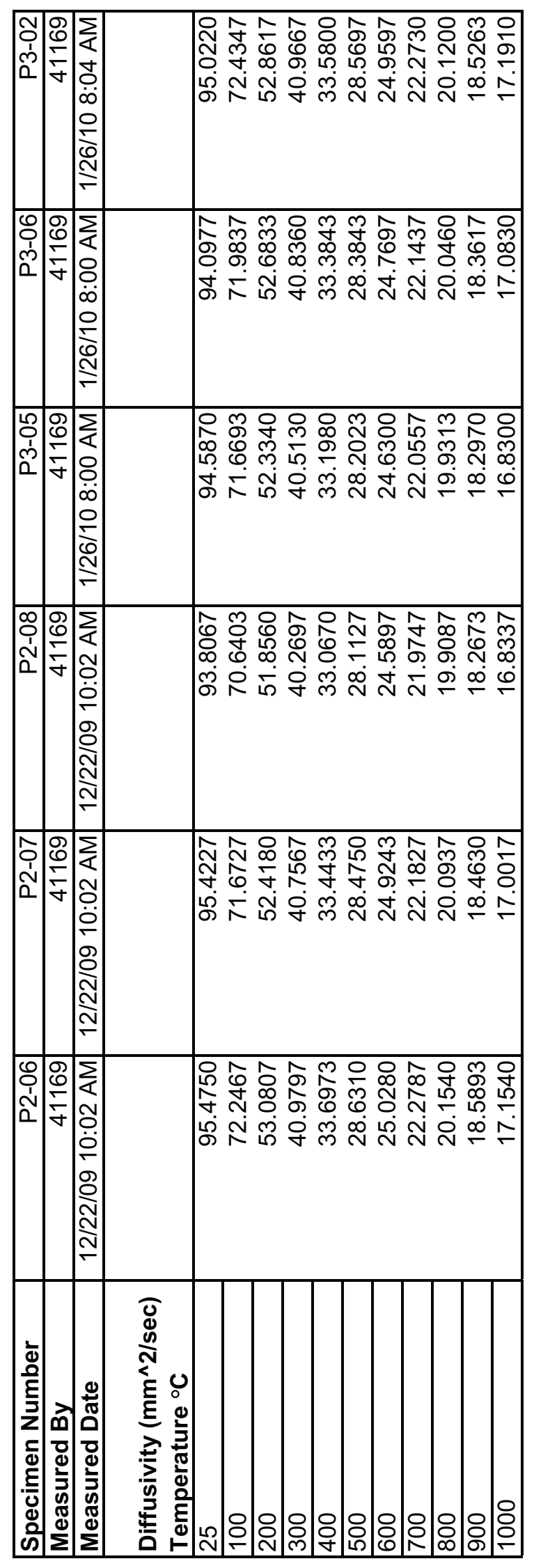




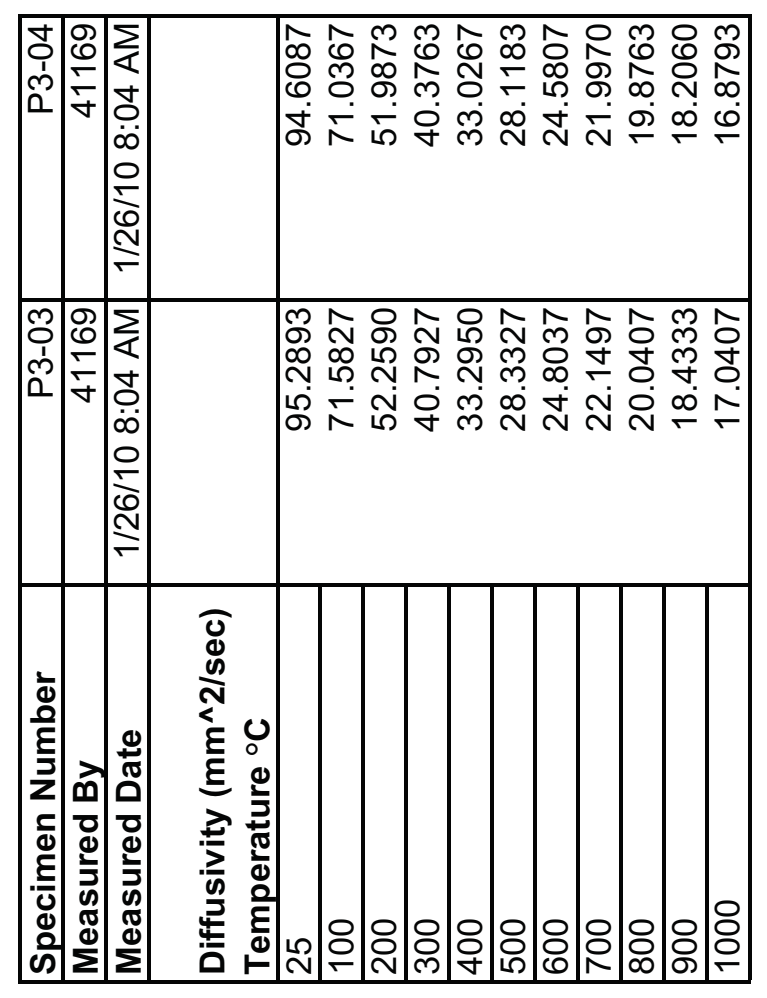

ปี 


\begin{tabular}{|c|c|}
\hline 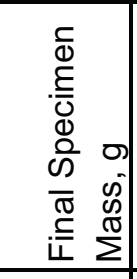 & 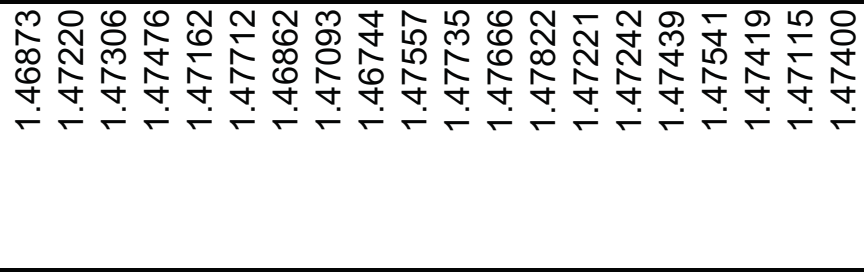 \\
\hline 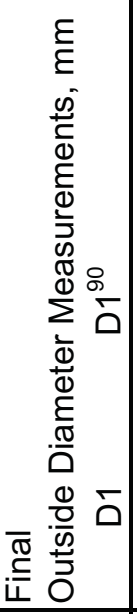 & 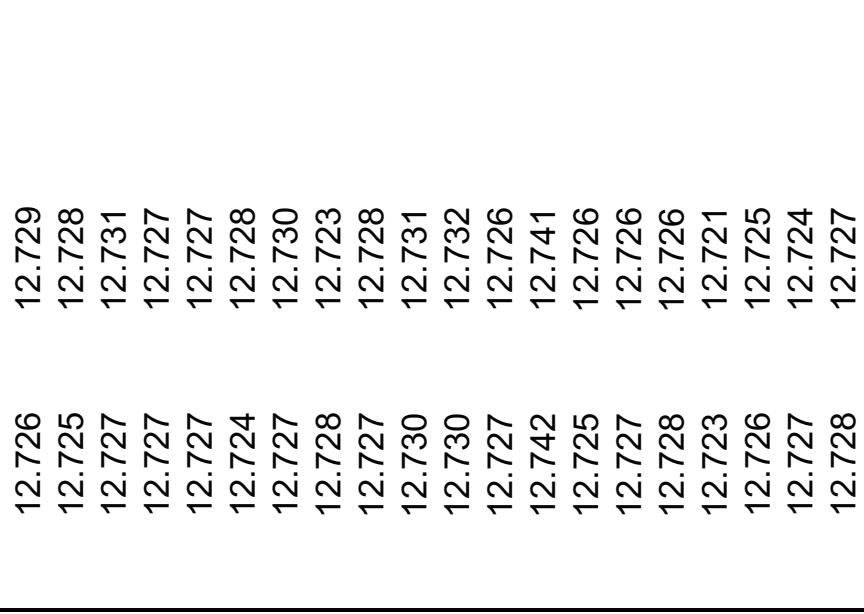 \\
\hline$\forall$ & 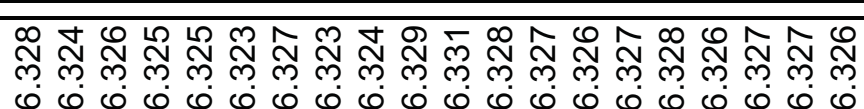 \\
\hline$\varepsilon m$ & 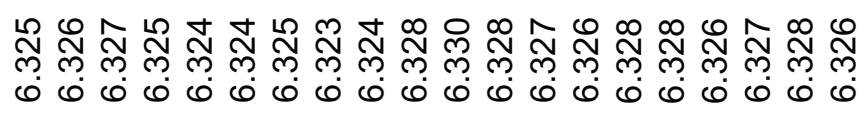 \\
\hline 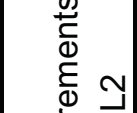 & 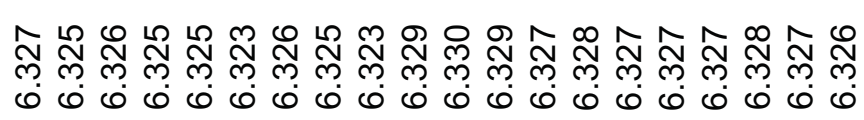 \\
\hline 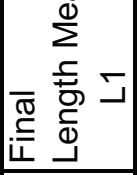 & 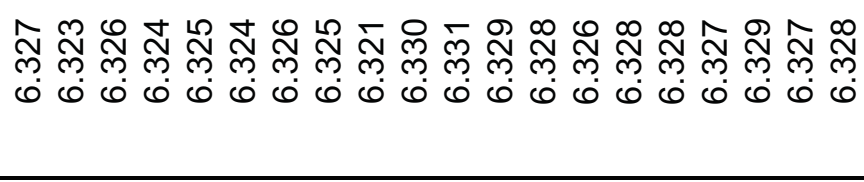 \\
\hline 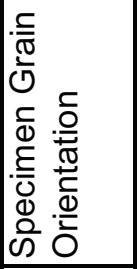 & \\
\hline 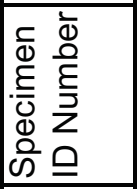 & 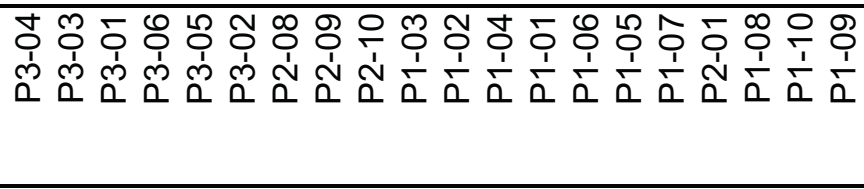 \\
\hline 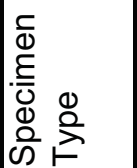 & \\
\hline
\end{tabular}




\begin{tabular}{|c|c|}
\hline 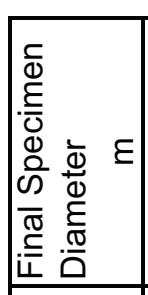 & 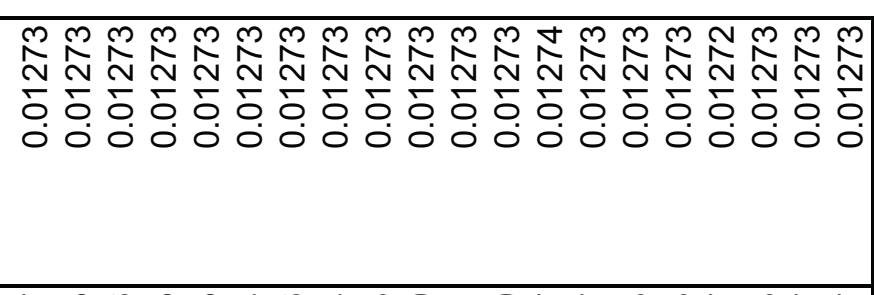 \\
\hline 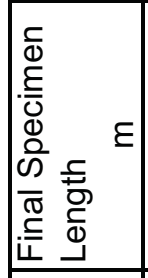 & 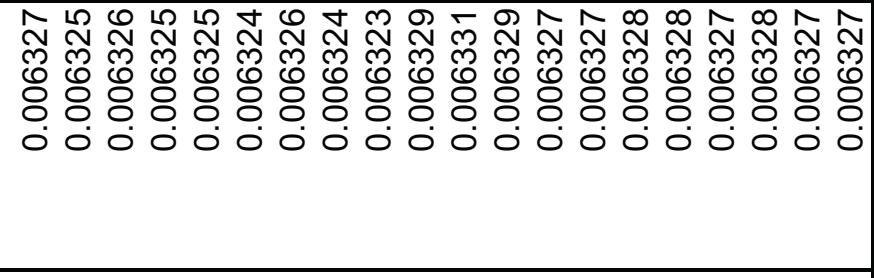 \\
\hline 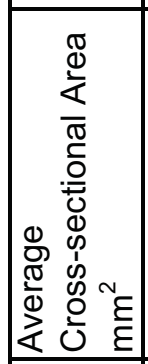 & 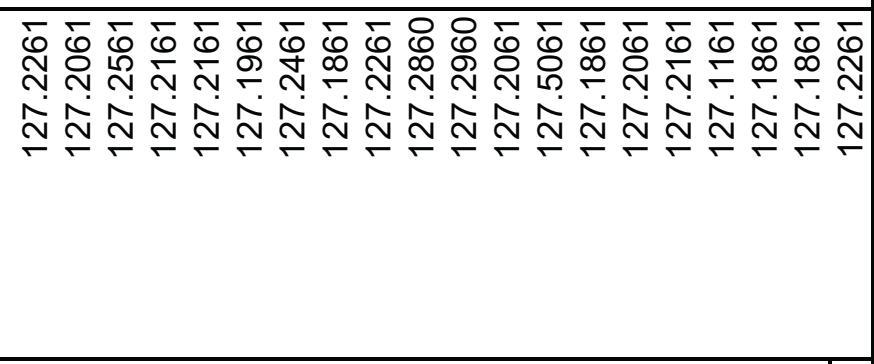 \\
\hline 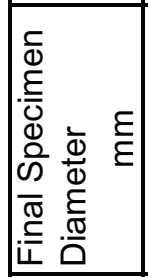 & 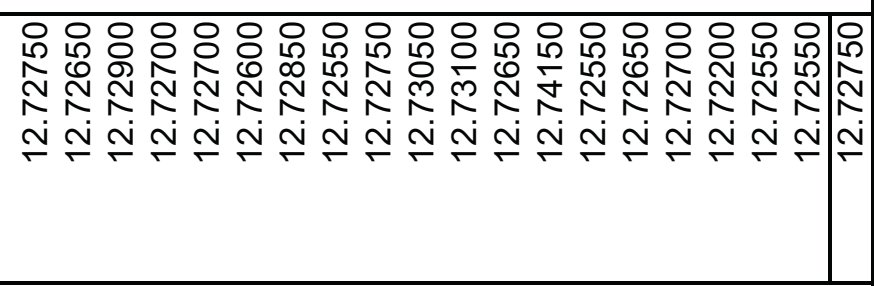 \\
\hline 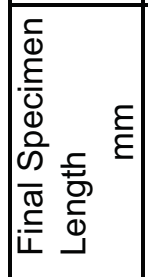 & 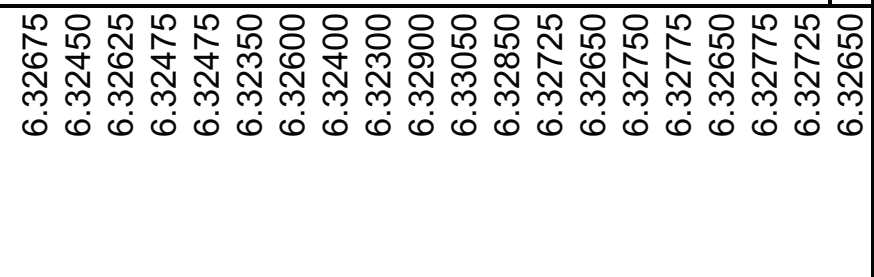 \\
\hline 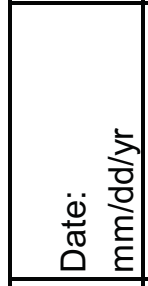 & 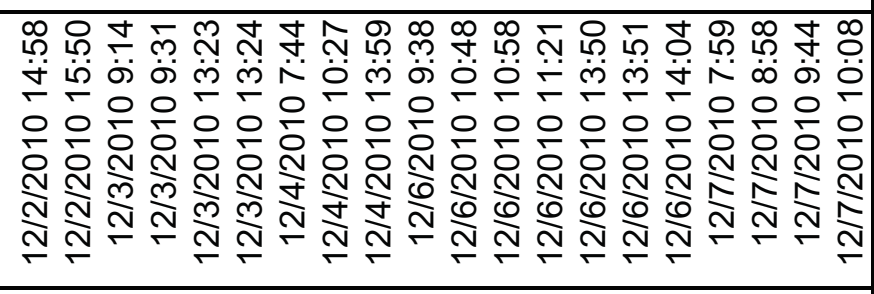 \\
\hline 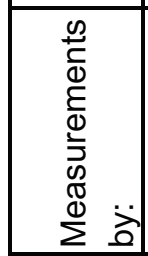 & 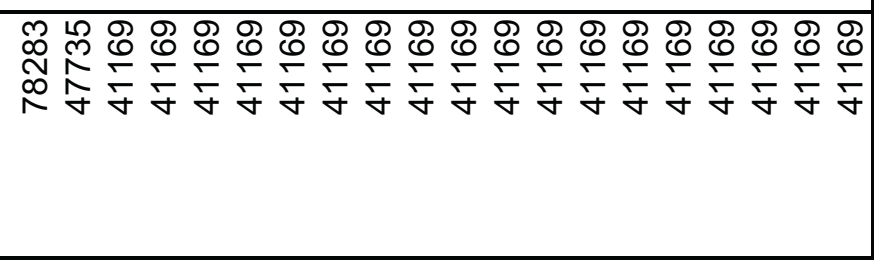 \\
\hline 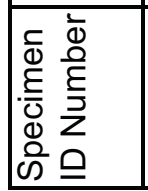 & 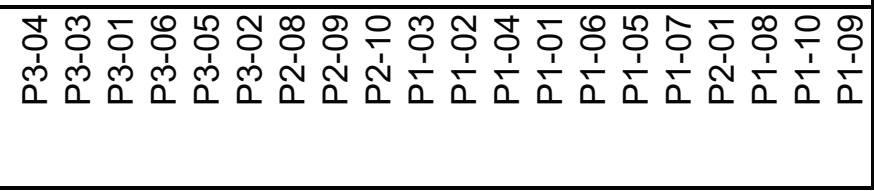 \\
\hline
\end{tabular}




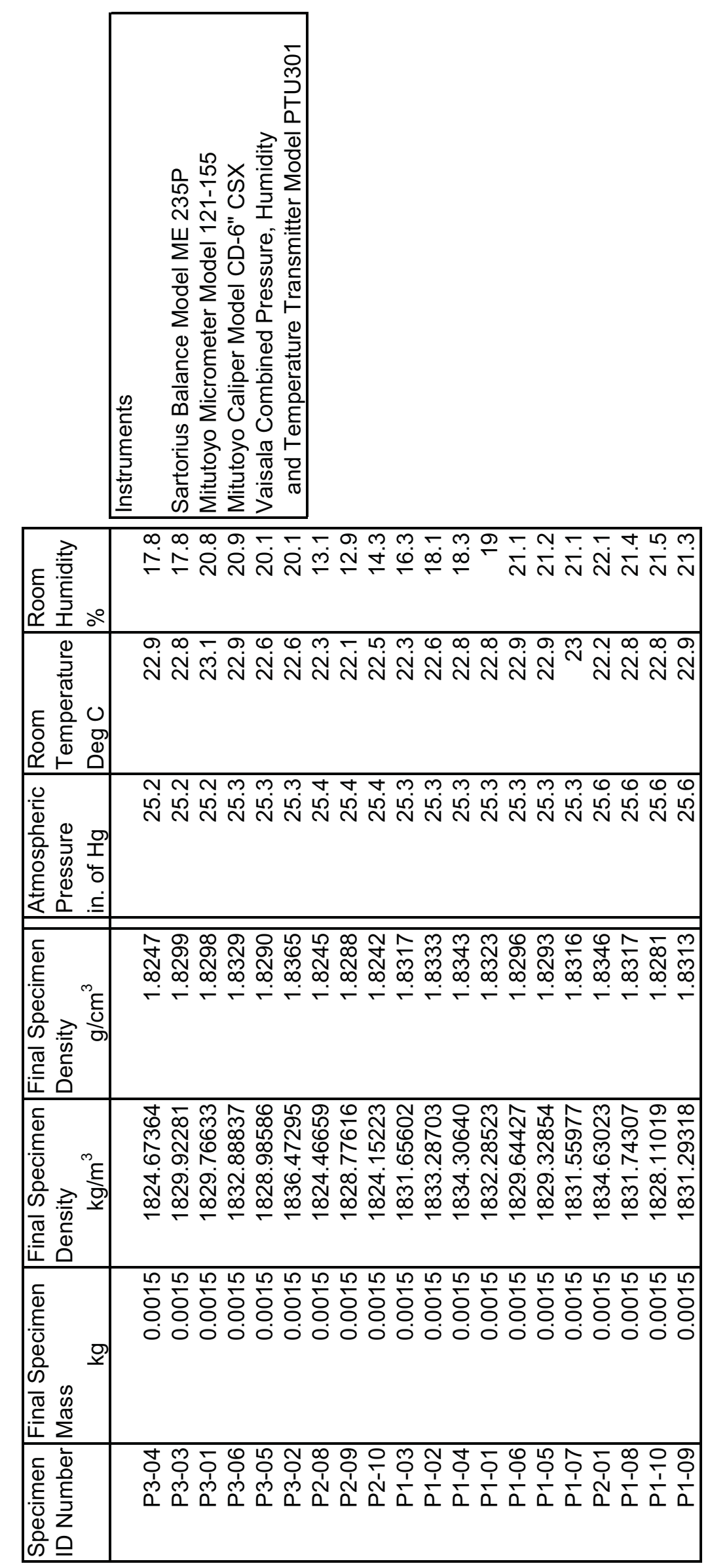


Graphite Grade:

Graphite Manufacturer:

Forming Process:

Coke Particle Size:

Coke Type:

ASTM Class:

Specimen Geometry:
PGX

Graftech International

Molded

Medium grain

Petroleum coke

MNHP

Cylinder

Specimen ID \#'s:

K1 01

$\mathrm{K} 102$

$\mathrm{K} 103$

K1 04

K1 05

K1 06

K1 07

K1 08

K1 09

K1 10

K2 01

$\mathrm{K} 202$

K2 03

K2 04

K2 05

K2 06

K2 07

K2 08

K2 09

K2 10

K3 01

K3 02

K3 03

K3 04

K3 05

K3 06

K3 07

K3 08

K3 09

K3 10 


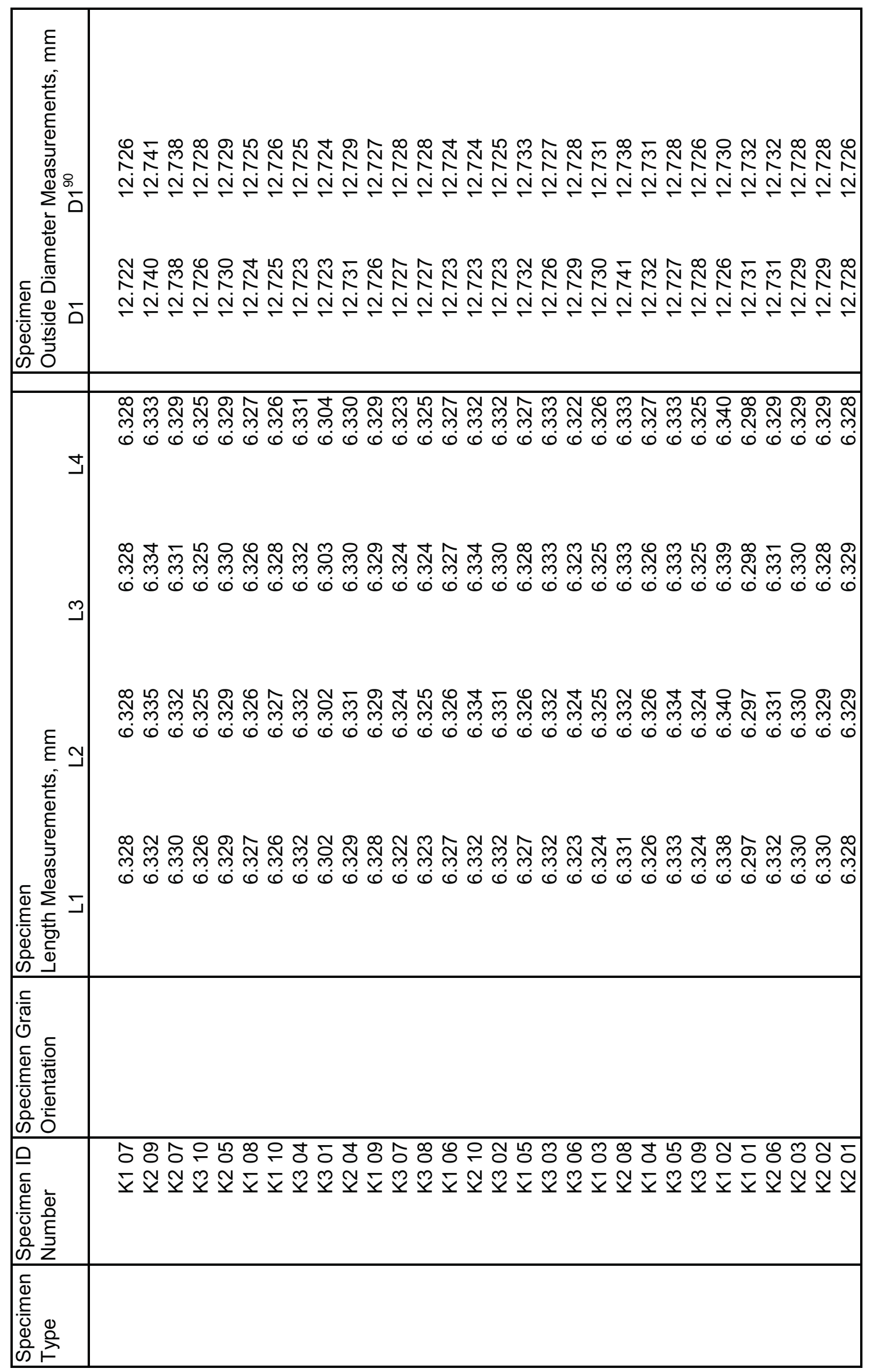




\begin{tabular}{|c|c|}
\hline 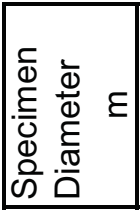 & 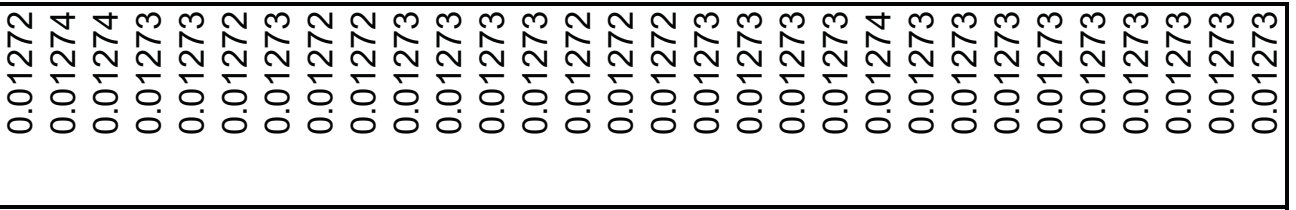 \\
\hline 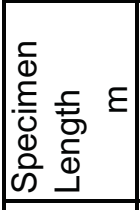 & 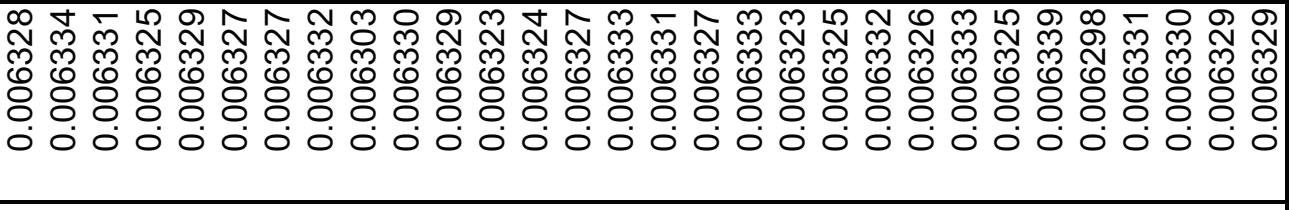 \\
\hline 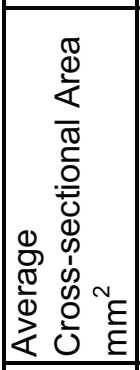 & 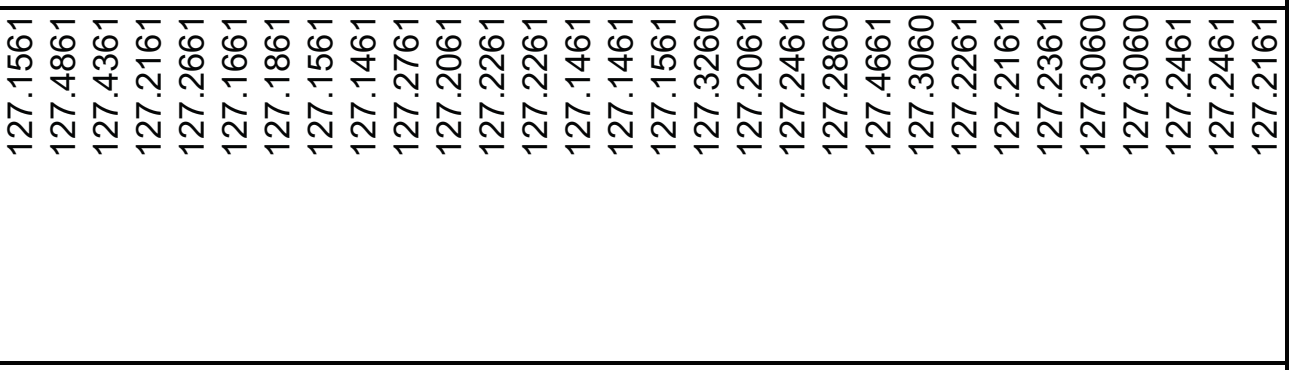 \\
\hline 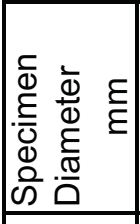 & 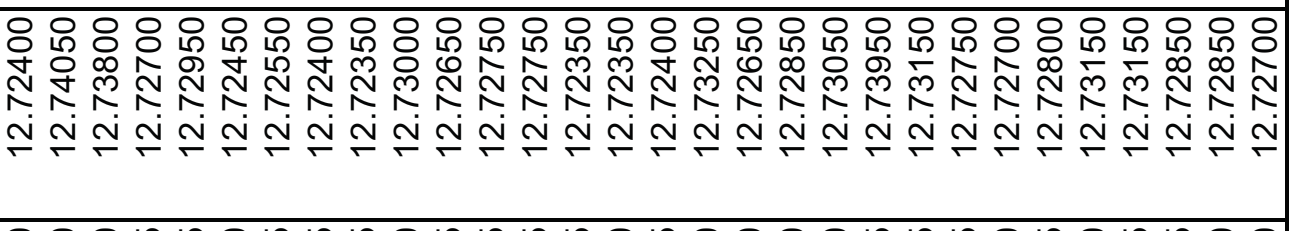 \\
\hline 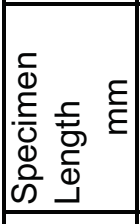 & 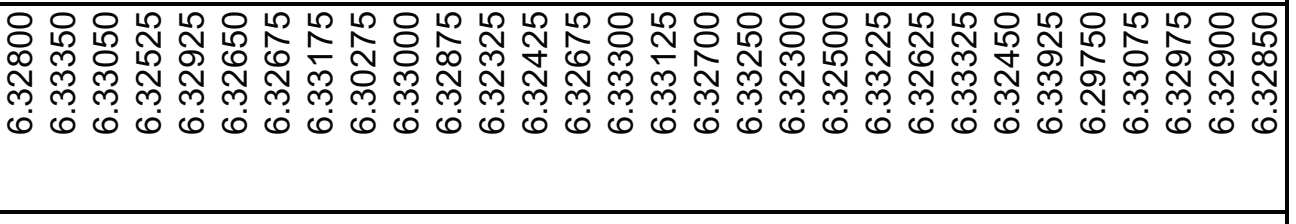 \\
\hline 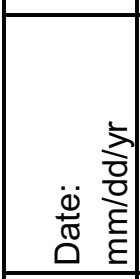 & 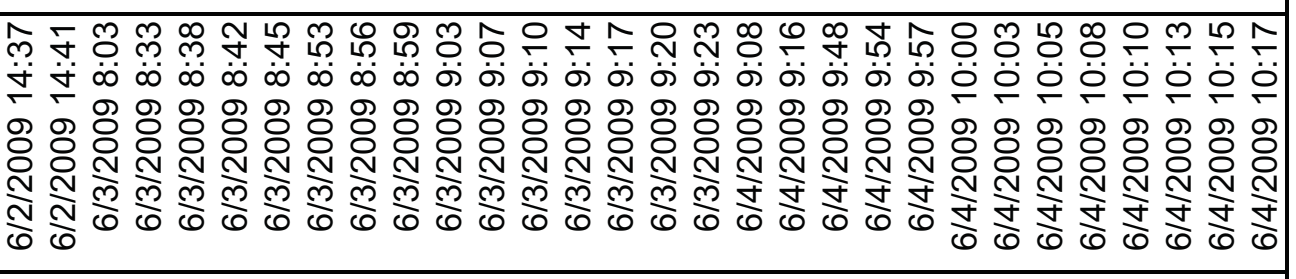 \\
\hline 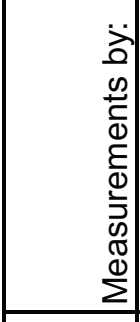 & $=====x==$ \\
\hline 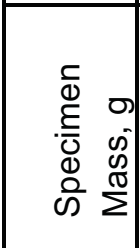 & 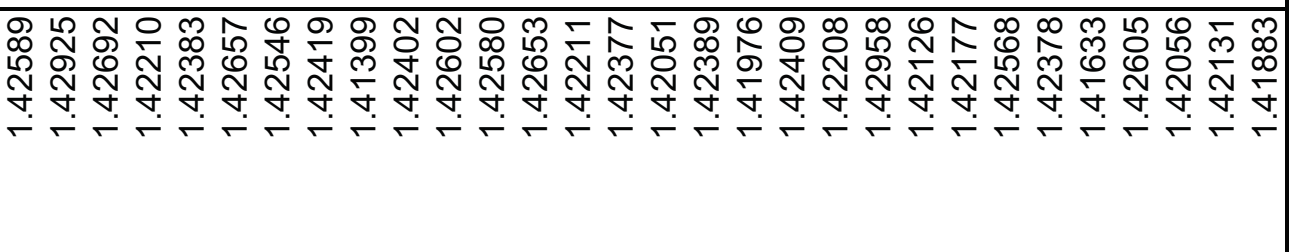 \\
\hline 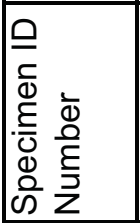 & 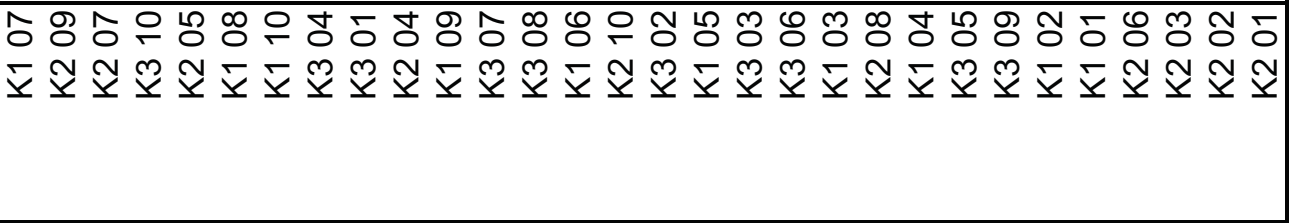 \\
\hline
\end{tabular}




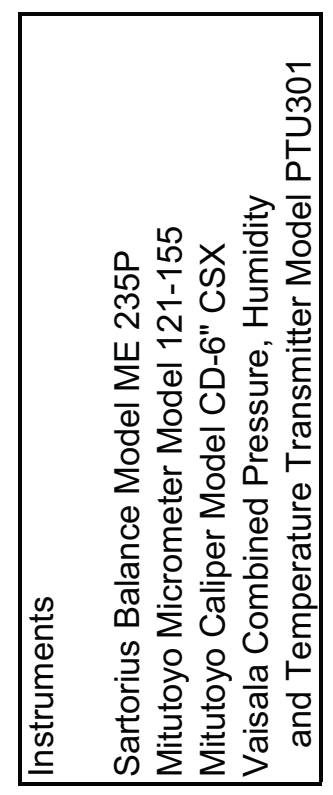

\begin{tabular}{|c|c|}
\hline 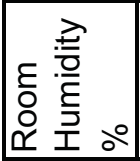 & 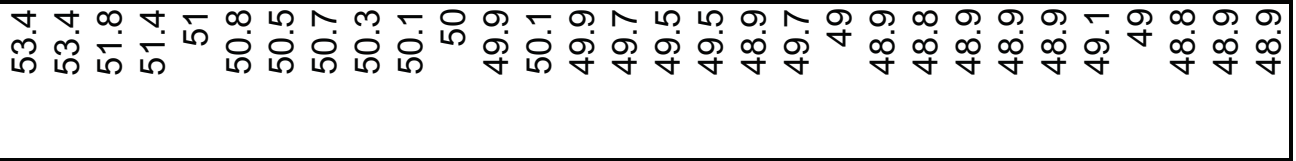 \\
\hline 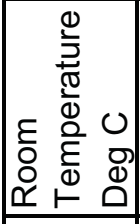 & 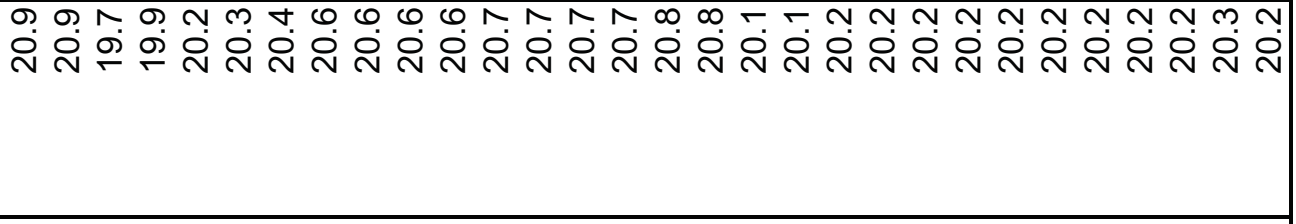 \\
\hline 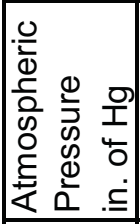 & 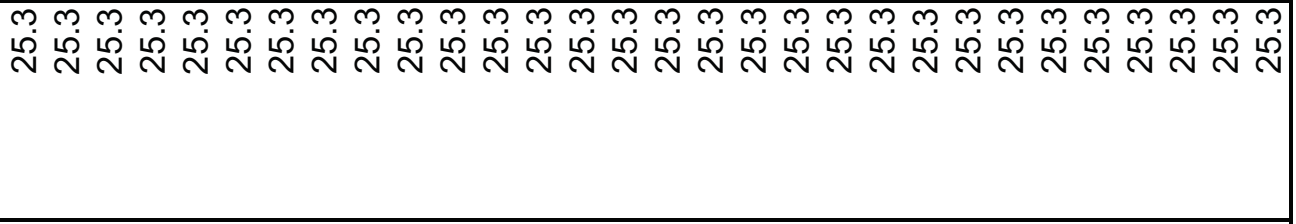 \\
\hline 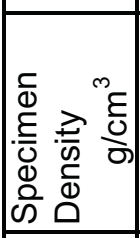 & 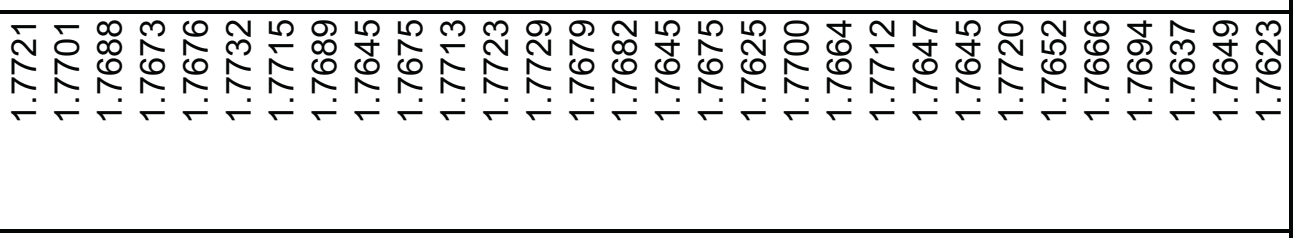 \\
\hline 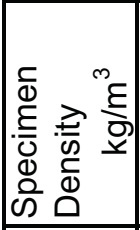 & 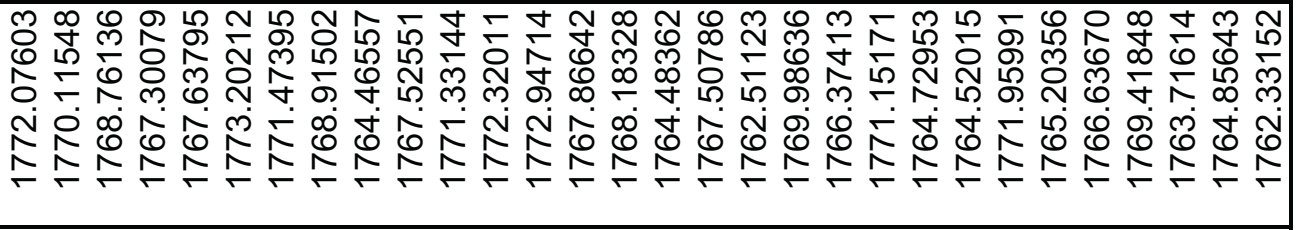 \\
\hline 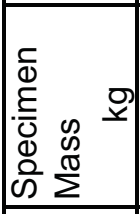 & 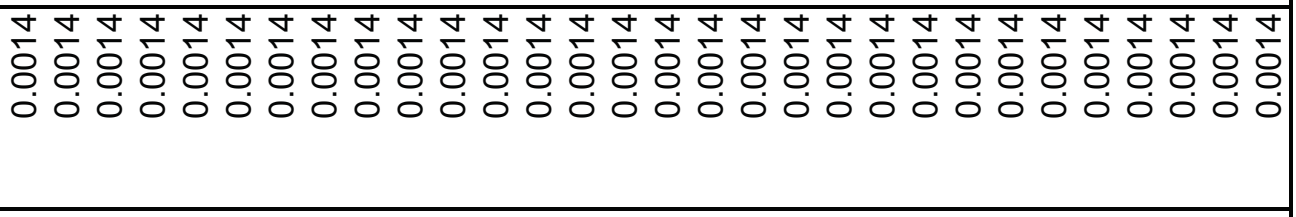 \\
\hline 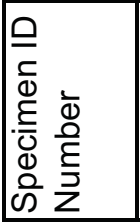 & 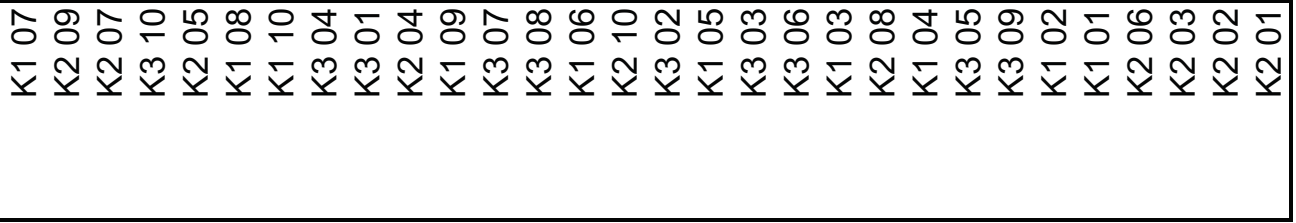 \\
\hline
\end{tabular}




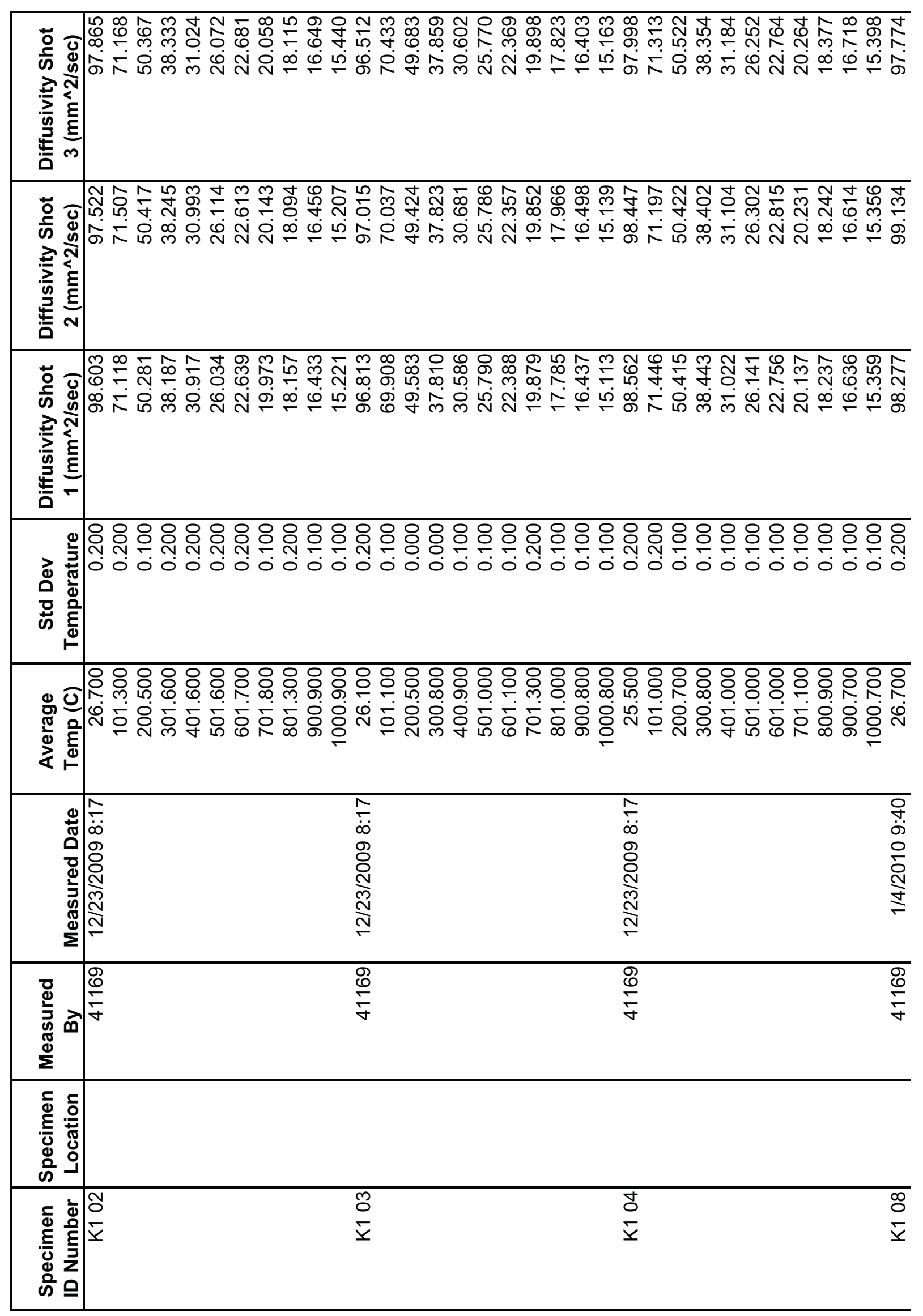




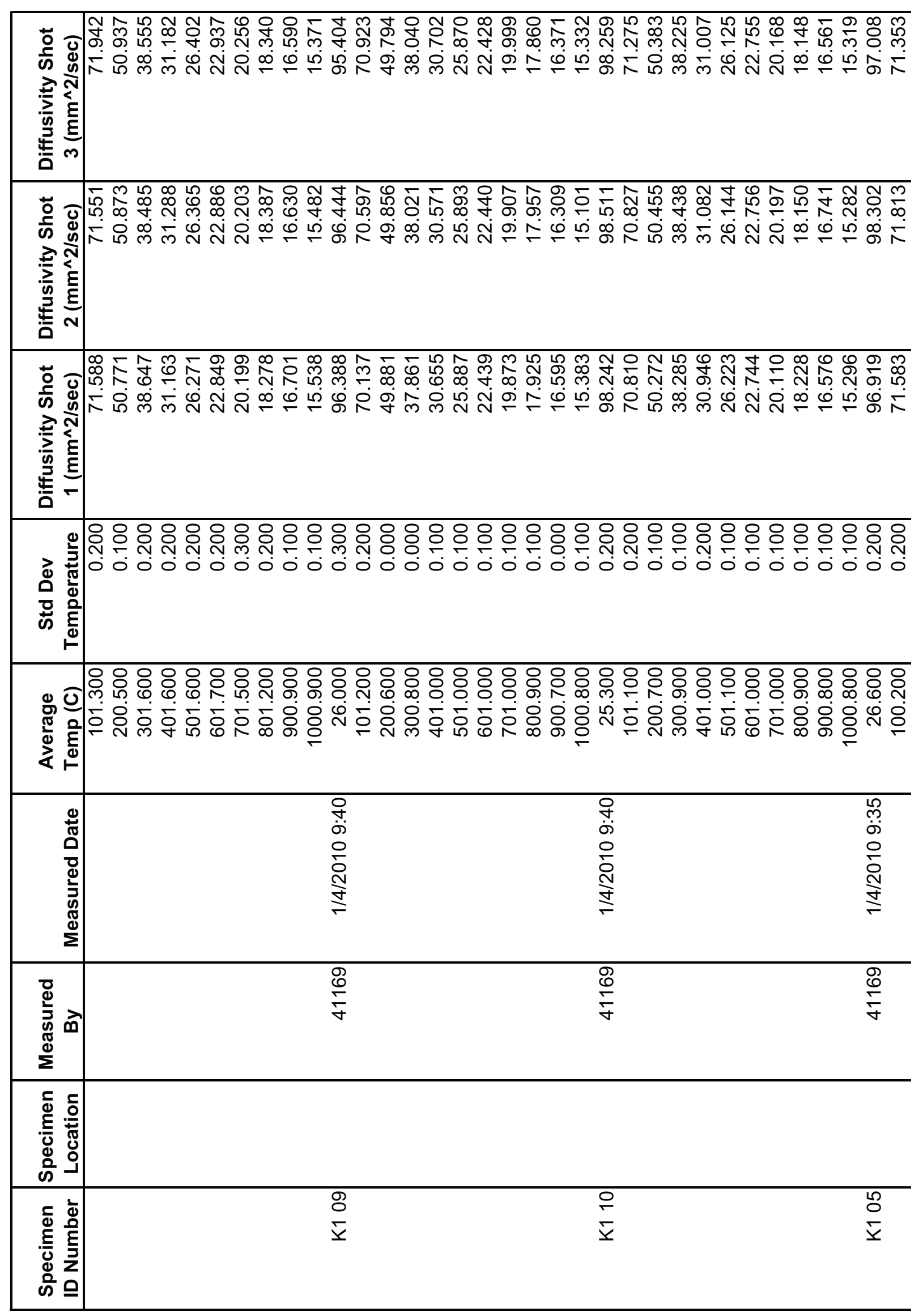




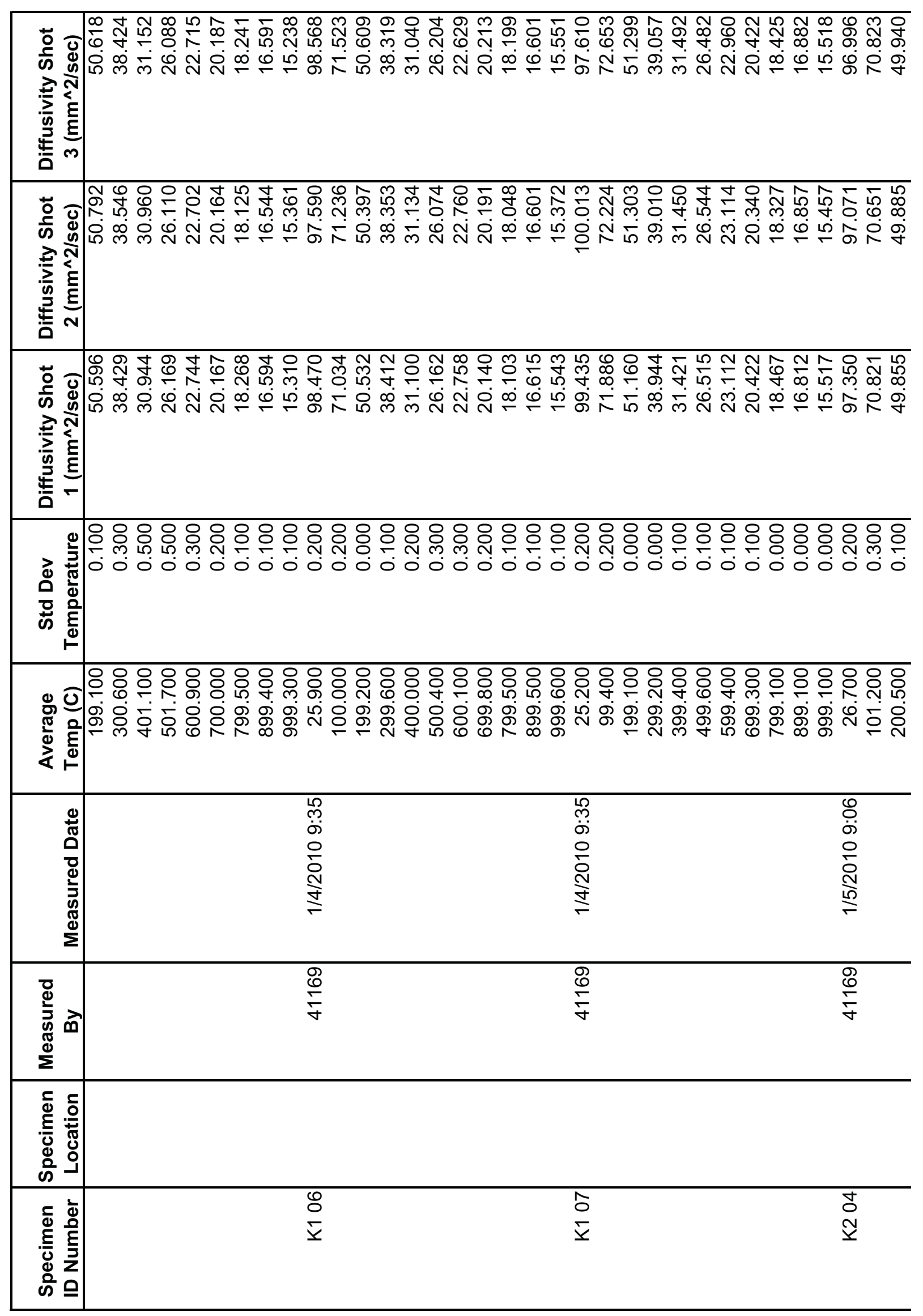




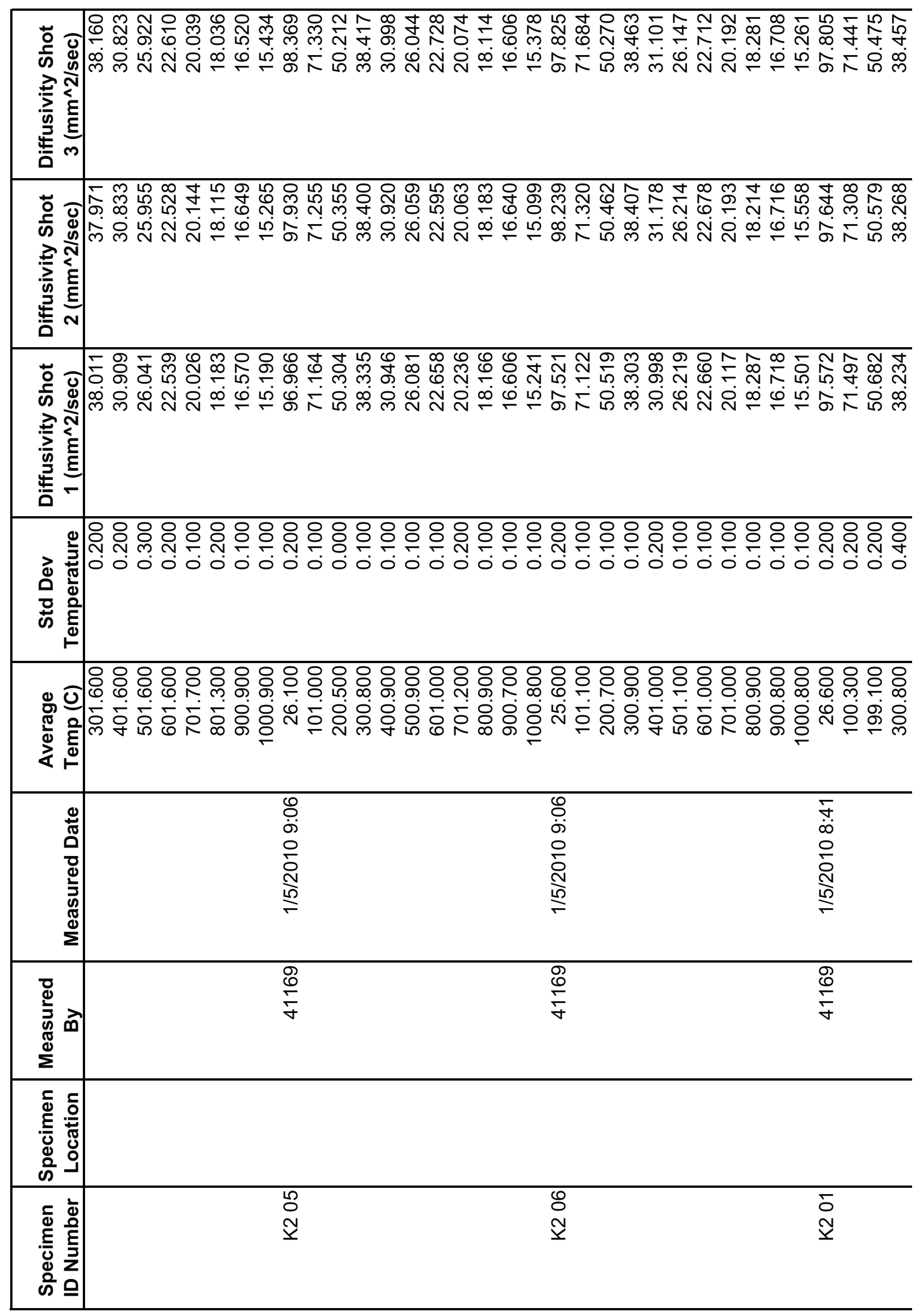




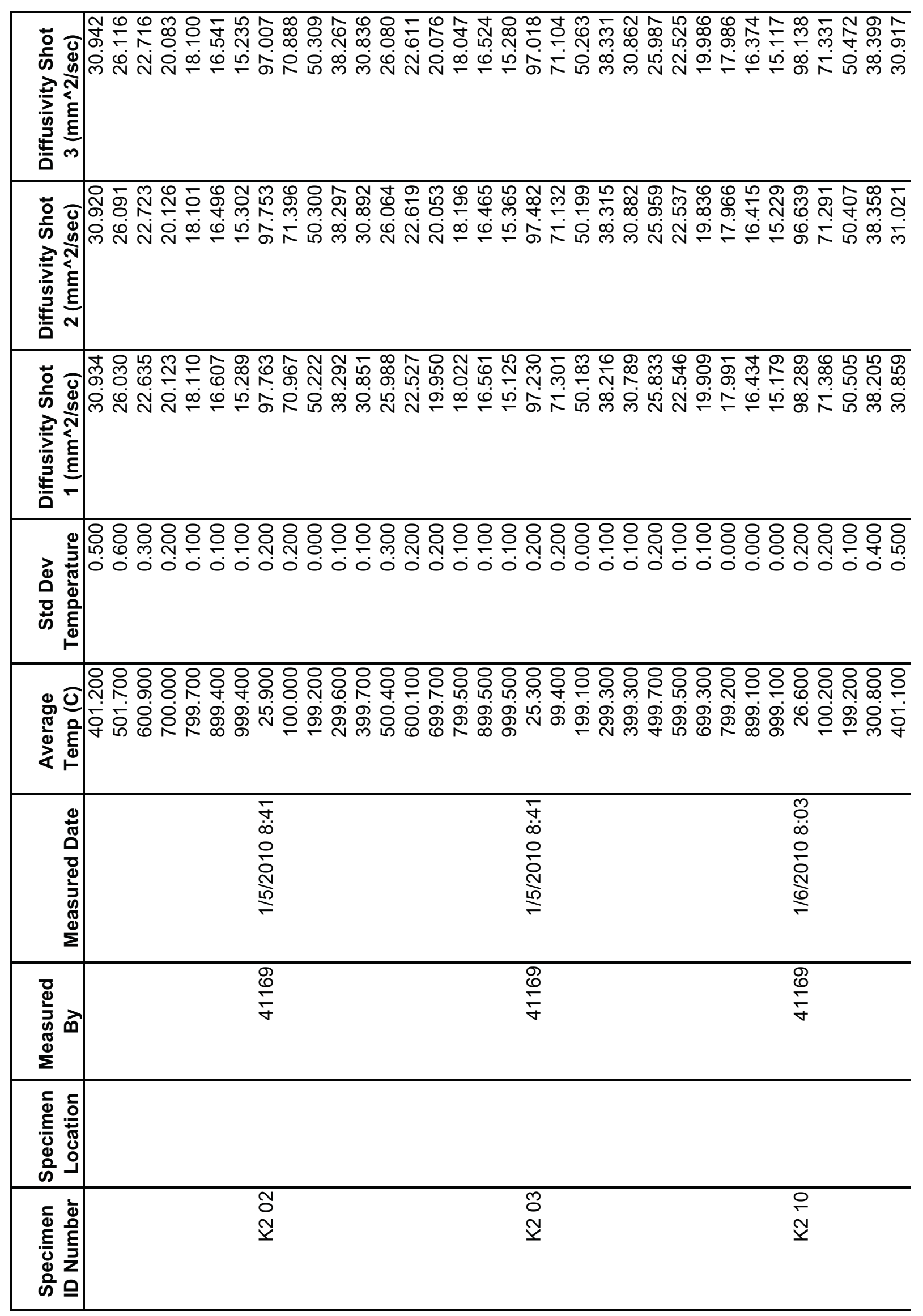




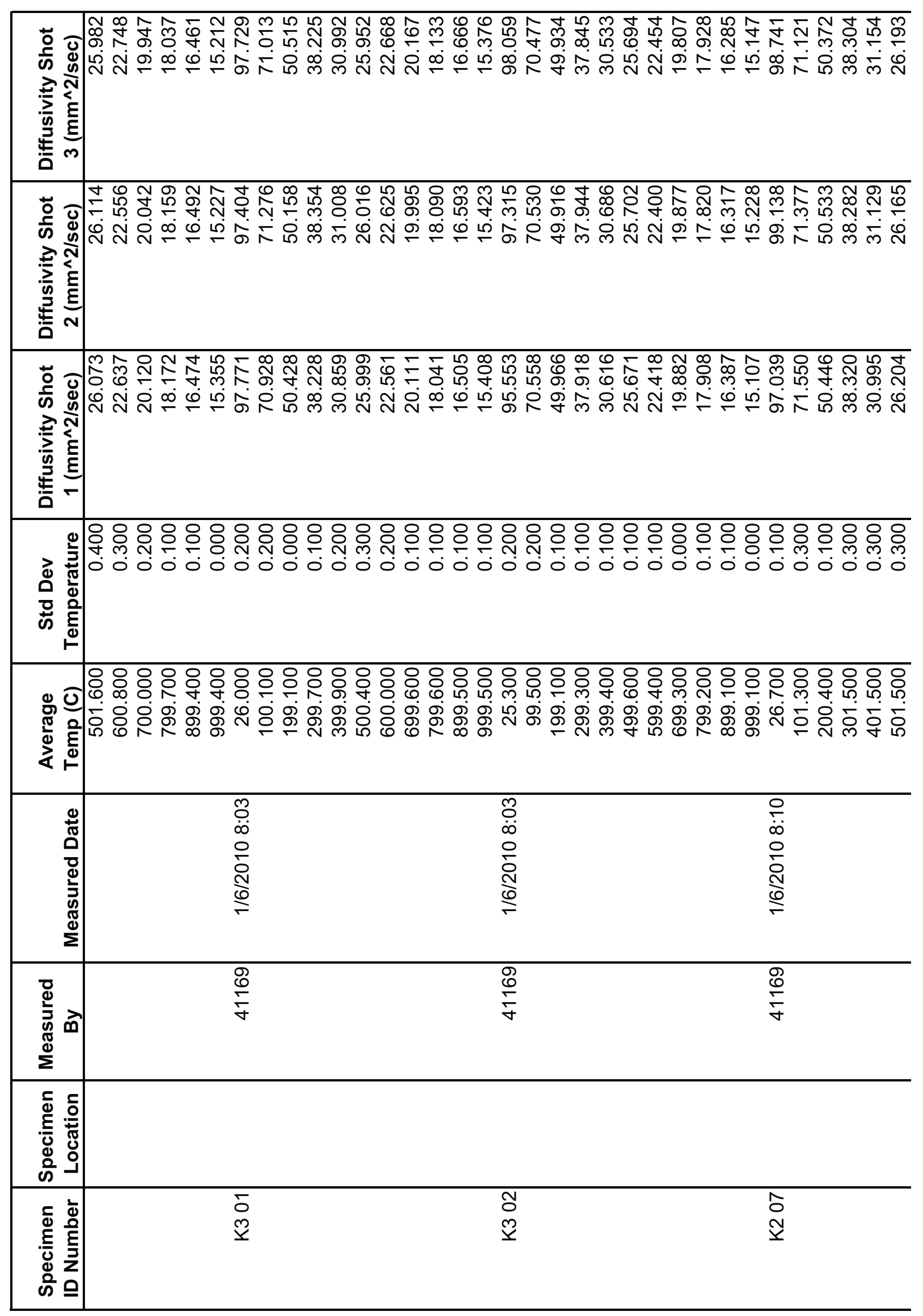




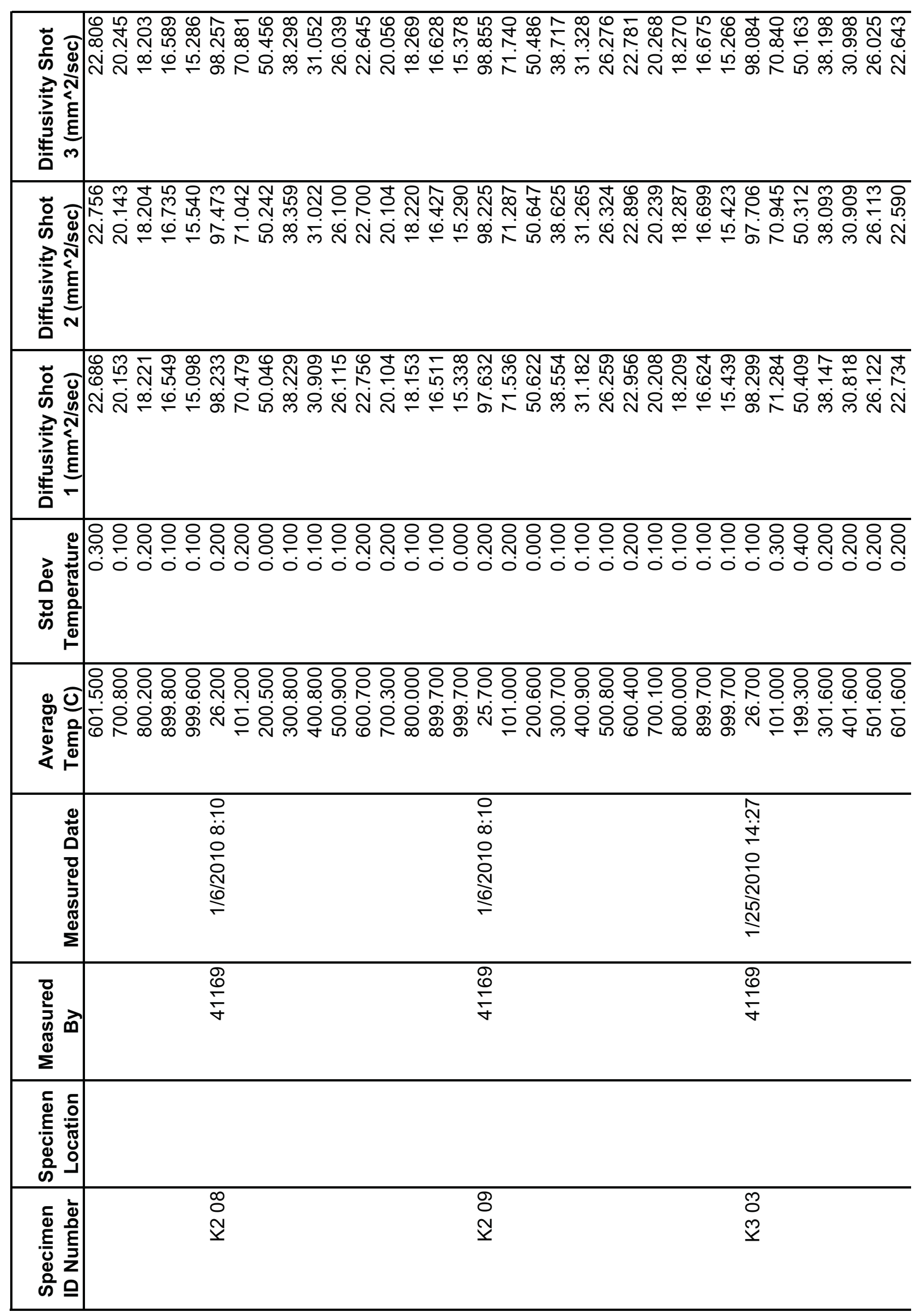




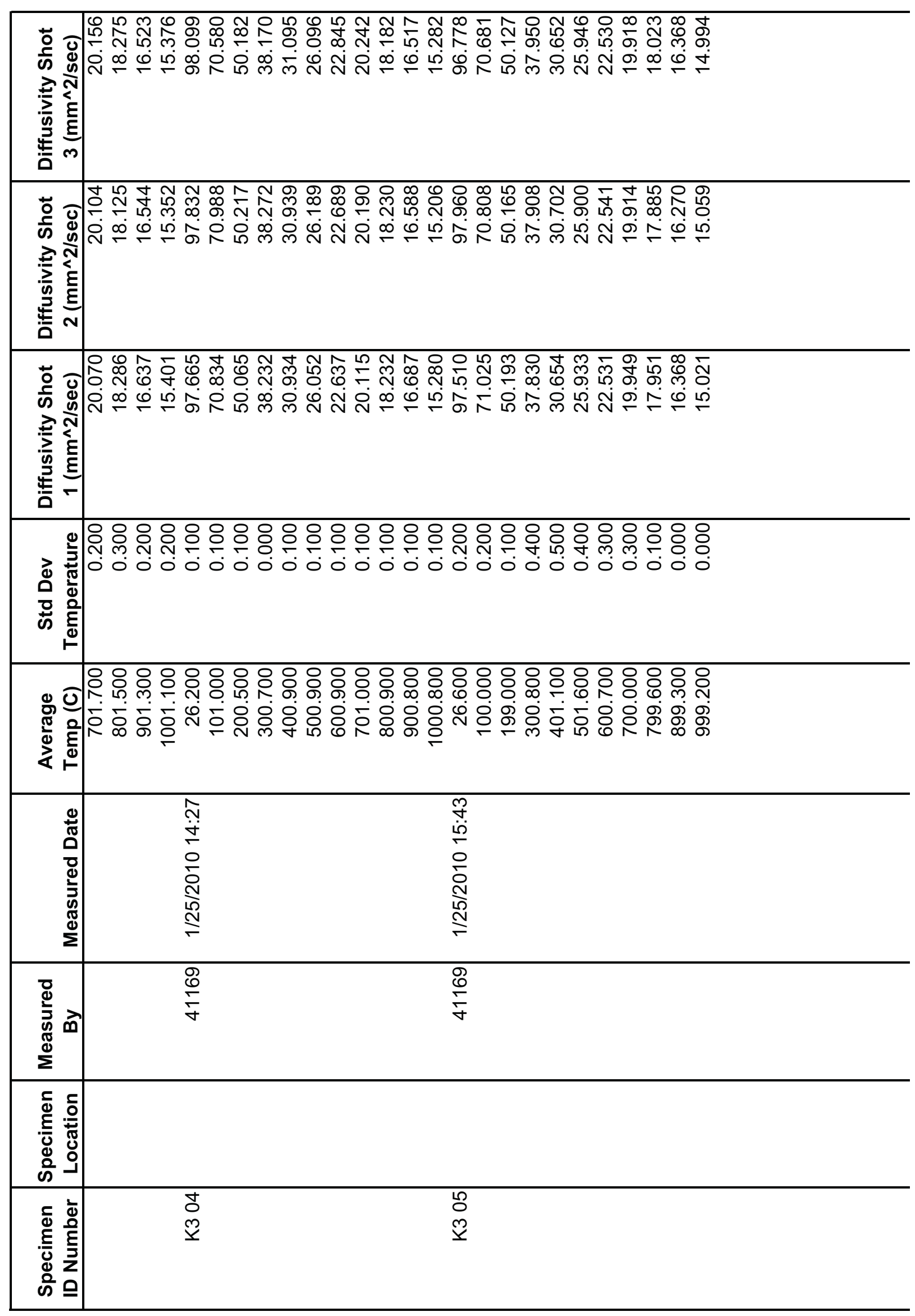




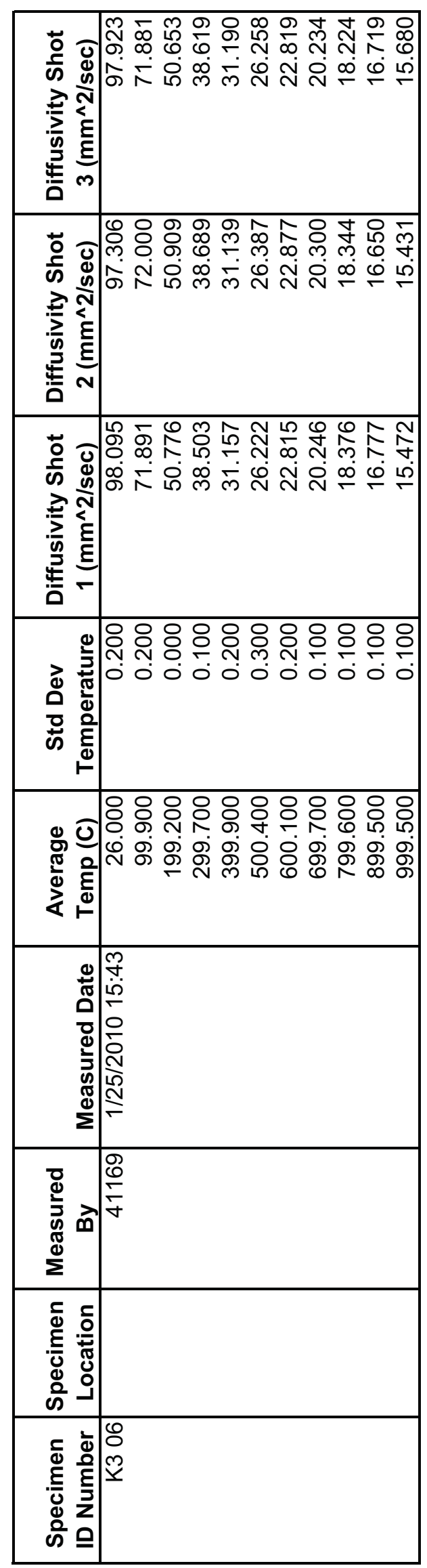

$\stackrel{\Xi}{ป}$ 


\begin{tabular}{|c|c|c|c|c|}
\hline 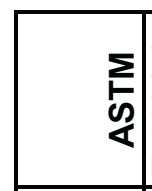 & 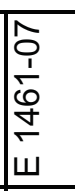 & $\begin{array}{l} \\
\\
\dot{1} \\
\dot{0} \\
\dot{+} \\
\end{array}$ & 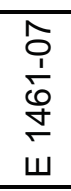 & 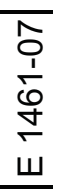 \\
\hline 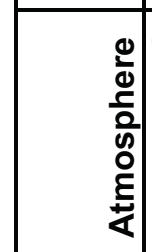 & & $\bar{z}$ & $\bar{z}$ & $\overline{\frac{1}{4}}$ \\
\hline 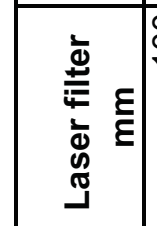 & & 음 & 음 & 8 \\
\hline 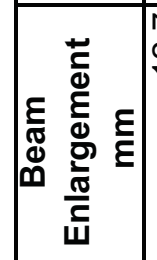 & $\stackrel{\hat{N}}{\underline{N}}$ & $\stackrel{\stackrel{N}{ }}{ }$ & $\stackrel{\stackrel{N}{N}}{ }$ & 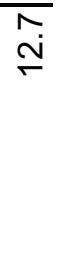 \\
\hline 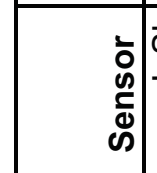 & & 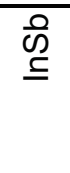 & 员 & $\begin{array}{l}\text { की } \\
\text { 足 }\end{array}$ \\
\hline 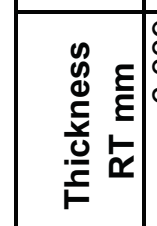 & & 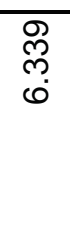 & 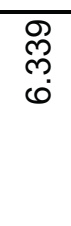 & 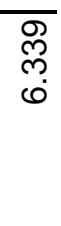 \\
\hline 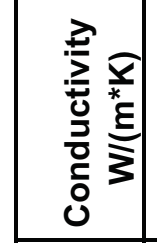 & & & & \\
\hline 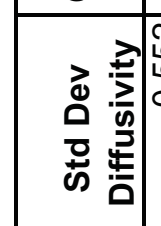 & 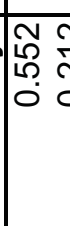 & م. & 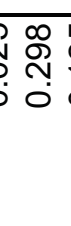 & $\begin{array}{l}\infty \\
\vdots \\
0 \\
0 \\
0\end{array}$ \\
\hline 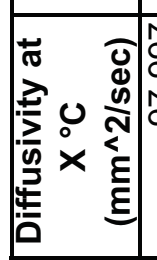 & 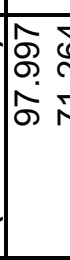 & $\begin{array}{l}8 \\
0 \\
0 \\
8 \\
8\end{array}$ & 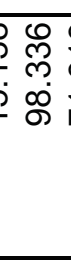 & \\
\hline 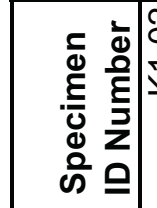 & $\begin{array}{l}\tilde{O} \\
\bar{x}\end{array}$ & $\begin{array}{l}\tilde{O} \\
\bar{y}\end{array}$ & $\begin{array}{l}\bar{J} \\
\bar{y}\end{array}$ & $\begin{array}{l}\infty \\
\stackrel{\infty}{\bar{y}}\end{array}$ \\
\hline
\end{tabular}




\begin{tabular}{|c|c|c|c|}
\hline 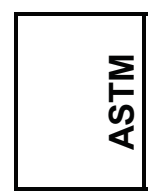 & $\begin{array}{l}\hat{o} \\
\frac{1}{0} \\
\dot{0} \\
\dot{5} \\
\end{array}$ & $\begin{array}{l}\hat{S} \\
\frac{1}{1} \\
\frac{0}{5} \\
\dot{w}\end{array}$ & 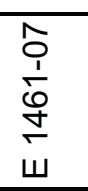 \\
\hline 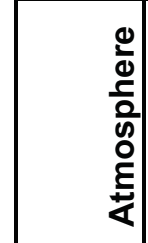 & 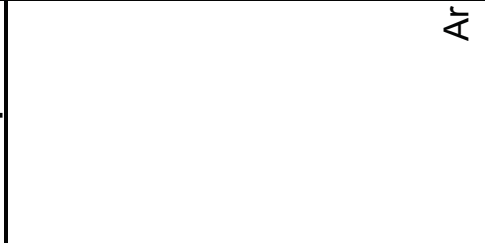 & $\frac{1}{\alpha}$ & 广 \\
\hline 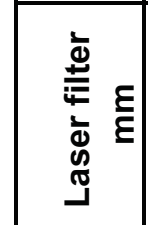 & 음 & 음 & 음 \\
\hline 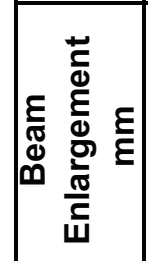 & $\stackrel{\widehat{N}}{\stackrel{N}{N}}$ & 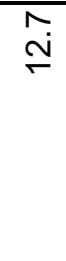 & 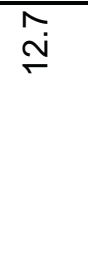 \\
\hline 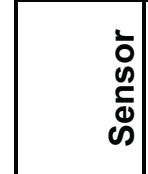 & $\begin{array}{l}\text { की } \\
\text { की }\end{array}$ & 吊 & $\begin{array}{l}\text { की } \\
\text { 足 }\end{array}$ \\
\hline 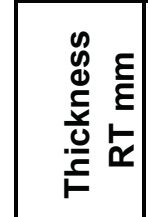 & ্ָল్ల & $\begin{array}{l}\mathscr{8} \\
\text { ల్ } \\
0\end{array}$ & 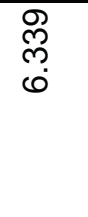 \\
\hline 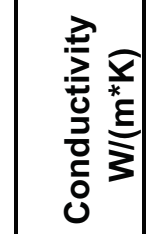 & & & \\
\hline 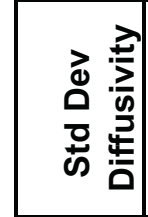 & 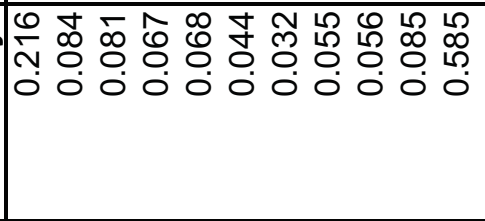 & $\check{0}$ & 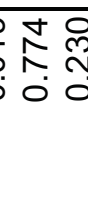 \\
\hline 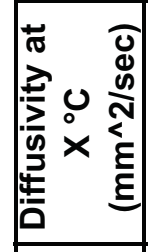 & 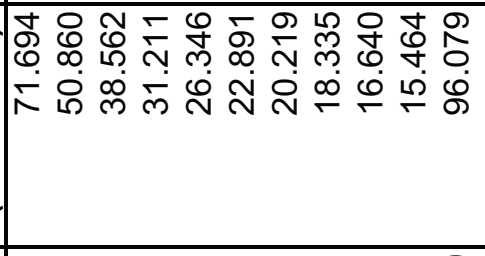 & $\begin{array}{l}\hat{m} \\
\infty \\
o \\
o\end{array}$ & 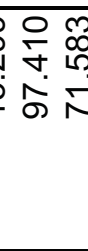 \\
\hline 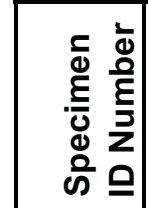 & $\begin{array}{l}\text { g } \\
\bar{y}\end{array}$ & $\frac{}{\frac{\rho}{\bar{y}}}$ & $\begin{array}{l}\mathscr{0} \\
\bar{y}\end{array}$ \\
\hline
\end{tabular}




\begin{tabular}{|c|c|c|c|}
\hline 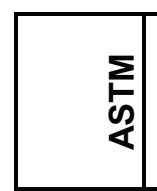 & 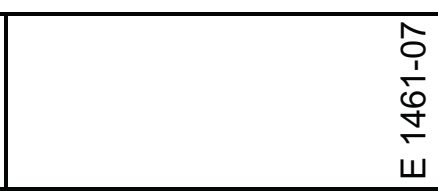 & $\begin{array}{l}\hat{o} \\
\frac{1}{0} \\
\stackrel{0}{+} \\
\dot{\omega}\end{array}$ & 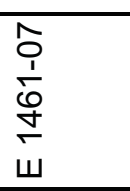 \\
\hline $\begin{array}{c}0 \\
\frac{0}{0} \\
\frac{5}{0} \\
0 \\
0 \\
\frac{1}{4}\end{array}$ & 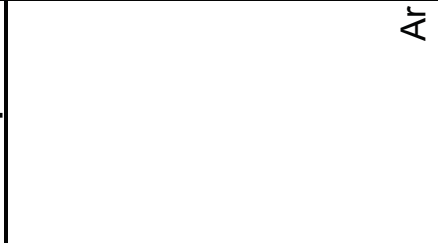 & 衣 & 交 \\
\hline 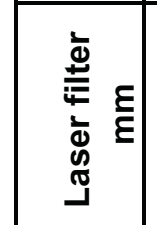 & $\stackrel{8}{\circ}$ & 음 & 음 \\
\hline 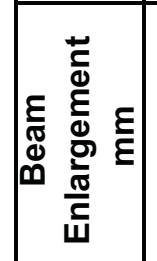 & $\stackrel{\widehat{N}}{\stackrel{N}{N}}$ & $\stackrel{\stackrel{N}{ }}{ }$ & $\stackrel{\widetilde{N}}{\stackrel{N}{N}}$ \\
\hline $\begin{array}{l}\vdots \\
\vdots \\
0 \\
\bar{d} \\
\infty\end{array}$ & $\begin{array}{l}\text { की } \\
\text { की }\end{array}$ & 吕 & $\begin{array}{l}\text { की } \\
\text { की }\end{array}$ \\
\hline 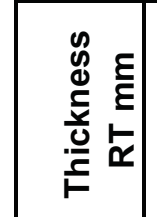 & 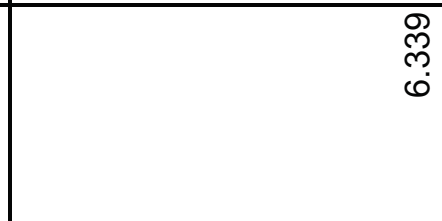 & 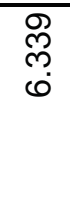 & 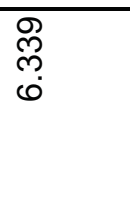 \\
\hline 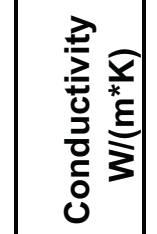 & & & \\
\hline के & 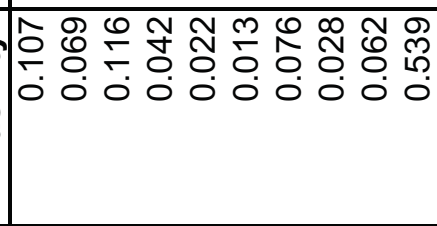 & S. & 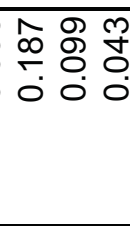 \\
\hline 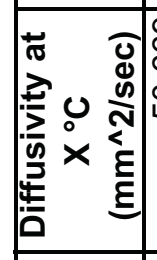 & 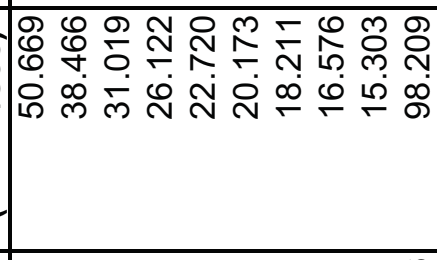 & 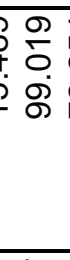 & 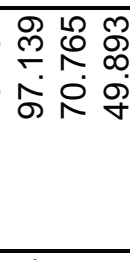 \\
\hline 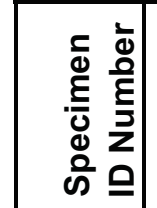 & $\begin{array}{l}8 \\
\bar{y} \\
\bar{y}\end{array}$ & $\begin{array}{l}\hat{o} \\
\bar{y}\end{array}$ & $\begin{array}{l}\tilde{J} \\
\tilde{y}\end{array}$ \\
\hline
\end{tabular}




\begin{tabular}{|c|c|c|c|}
\hline 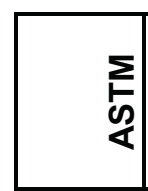 & $\begin{array}{l}\hat{o} \\
\dot{1} \\
\dot{0} \\
\dot{+} \\
\dot{w}\end{array}$ & $\begin{array}{l}\hat{o} \\
\frac{1}{0} \\
\stackrel{0}{+} \\
\dot{\omega}\end{array}$ & $\begin{array}{l}\hat{o} \\
\frac{1}{0} \\
\dot{0} \\
\dot{T} \\
\end{array}$ \\
\hline 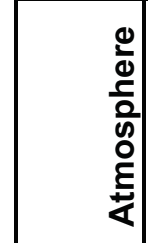 & 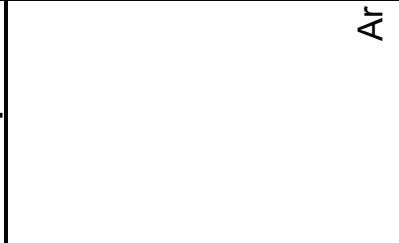 & 文 & 交 \\
\hline 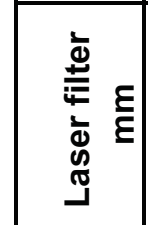 & $\stackrel{8}{\circ}$ & $\stackrel{\circ}{\circ}$ & 음 \\
\hline 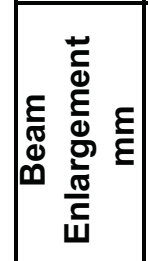 & $\stackrel{\widehat{N}}{\stackrel{N}{N}}$ & $\stackrel{\stackrel{N}{\sim}}{\mathrm{N}}$ & $\stackrel{\stackrel{\sim}{\sim}}{ }$ \\
\hline 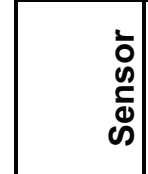 & $\begin{array}{l}\text { की } \\
\text { की }\end{array}$ & $\begin{array}{l}\text { की } \\
\text { I }\end{array}$ & $\begin{array}{l}\text { की } \\
\text { 足 }\end{array}$ \\
\hline 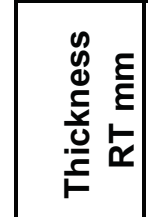 & 总 & 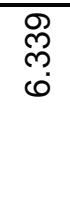 & 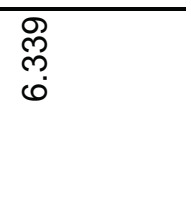 \\
\hline 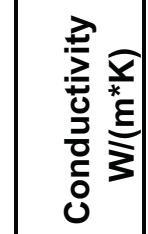 & & & \\
\hline 永辛 & $\mid \begin{array}{llllll} & \\
\end{array}$ & $\begin{array}{l}8 \\
\\
0 \\
0 \\
0\end{array}$ & : \\
\hline 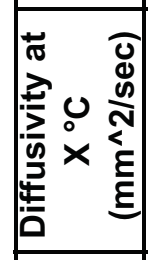 & 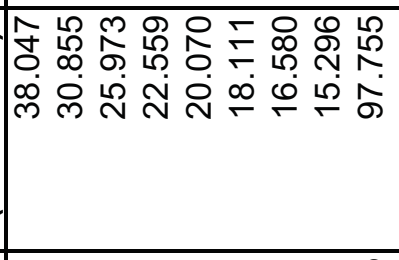 & 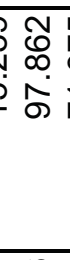 & 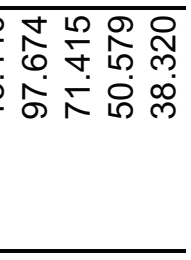 \\
\hline 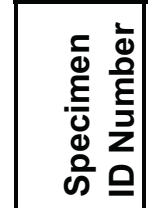 & $\begin{array}{l}\text { L̊ } \\
\tilde{\tilde{Y}}\end{array}$ & $\begin{array}{l}\mathscr{O} \\
\widetilde{\widetilde{Y}}\end{array}$ & $\begin{array}{l}\bar{\delta} \\
\tilde{\widetilde{y}}\end{array}$ \\
\hline
\end{tabular}




\begin{tabular}{|c|c|c|c|}
\hline 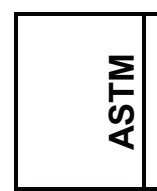 & $\begin{array}{l}\hat{O} \\
\frac{1}{0} \\
\stackrel{0}{+} \\
\dot{w}\end{array}$ & $\begin{array}{l}\hat{o} \\
\frac{1}{0} \\
\stackrel{0}{+} \\
\dot{\omega}\end{array}$ & 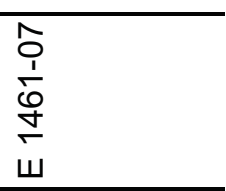 \\
\hline 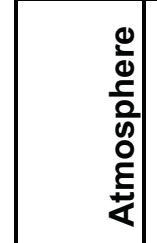 & 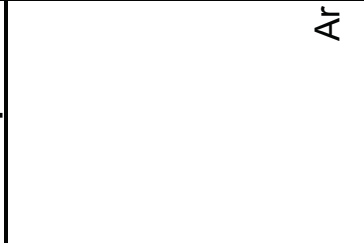 & 衣 & 交 \\
\hline 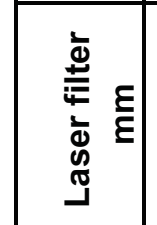 & $\stackrel{\circ}{\circ}$ & 음 & 음 \\
\hline 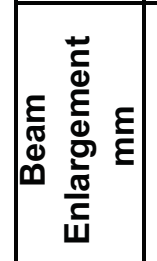 & $\stackrel{\stackrel{N}{\mathfrak{N}}}{ }$ & $\stackrel{\stackrel{N}{\sim}}{ }$ & $\stackrel{\stackrel{N}{N}}{ }$ \\
\hline $\begin{array}{l}\bar{b} \\
0 \\
\stackrel{5}{\Phi} \\
\infty\end{array}$ & $\begin{array}{l}\text { की } \\
\text { की }\end{array}$ & $\begin{array}{l}\text { की } \\
\text { I }\end{array}$ & $\begin{array}{l}\text { की } \\
\text { Ф }\end{array}$ \\
\hline 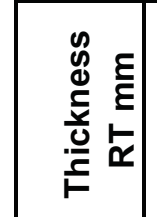 & 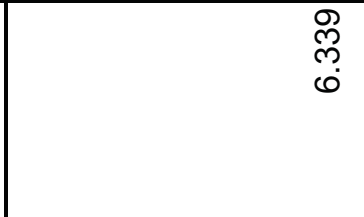 & $\begin{array}{l}\text { D్ } \\
\text { లై } \\
0\end{array}$ & 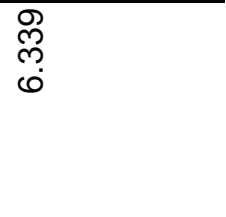 \\
\hline 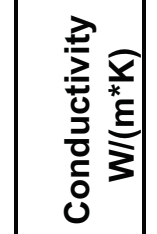 & & & \\
\hline 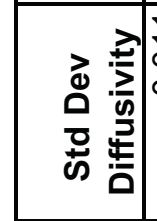 & 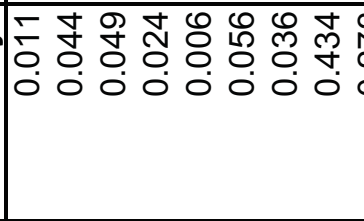 & 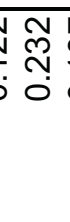 & 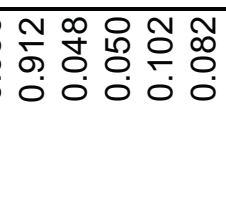 \\
\hline 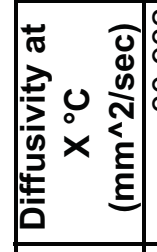 & 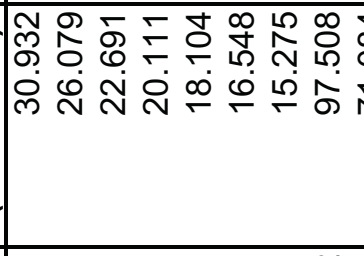 & : & 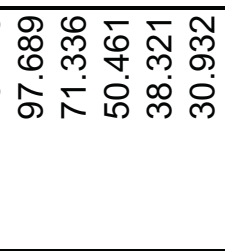 \\
\hline 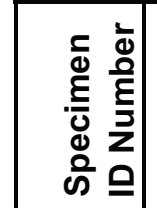 & $\begin{array}{l}\tilde{\tilde{\sigma}} \\
\widetilde{\widetilde{\Sigma}}\end{array}$ & $\begin{array}{l}\tilde{O} \\
\widetilde{\widetilde{Y}}\end{array}$ & $\begin{array}{l}\stackrel{0}{\check{Y}} \\
\underline{\underline{y}}\end{array}$ \\
\hline
\end{tabular}




\begin{tabular}{|c|c|c|c|}
\hline 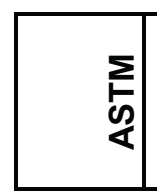 & $\begin{array}{l}\hat{o} \\
\frac{1}{0} \\
\dot{o} \\
\dot{w} \\
\end{array}$ & $\begin{array}{l}\hat{o} \\
\frac{1}{0} \\
\stackrel{0}{+} \\
\dot{\omega}\end{array}$ & $\begin{array}{l}\hat{0} \\
\frac{1}{0} \\
\stackrel{0}{+} \\
\dot{\omega}\end{array}$ \\
\hline 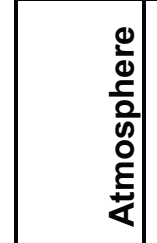 & 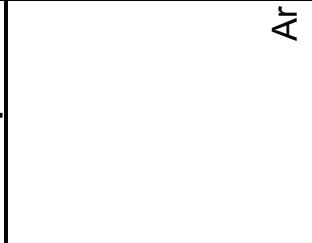 & 㐫 & 交 \\
\hline 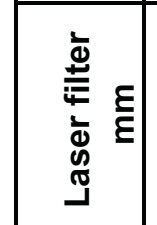 & 음 & 음 & 음 \\
\hline 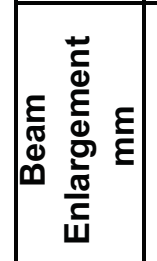 & $\stackrel{\widehat{N}}{\stackrel{N}{N}}$ & 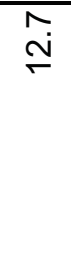 & $\stackrel{\stackrel{\sim}{\sim}}{ }$ \\
\hline $\begin{array}{l}\vdots \\
\vdots \\
0 \\
⿱ 亠 凶 \\
\infty\end{array}$ & $\begin{array}{l}\text { की } \\
\text { की }\end{array}$ & 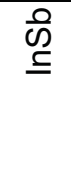 & $\begin{array}{l}\text { की } \\
\text { 足 }\end{array}$ \\
\hline 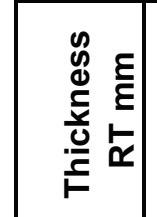 & 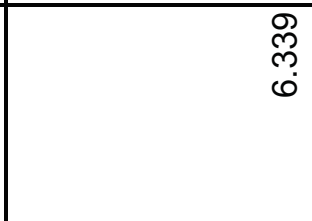 & 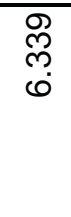 & 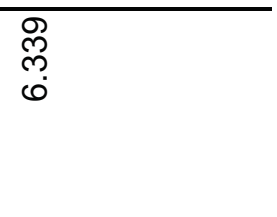 \\
\hline 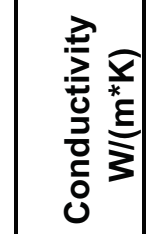 & & & \\
\hline 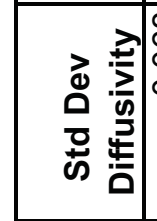 & $\mid \begin{array}{lllll} & 0 & \end{array}$ & 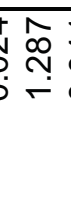 & 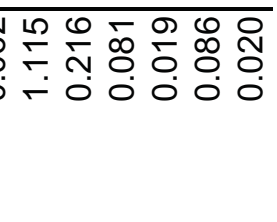 \\
\hline 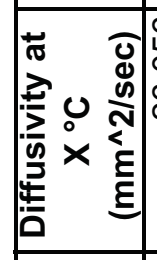 & 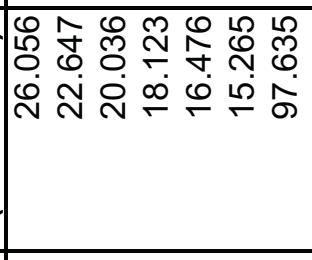 & 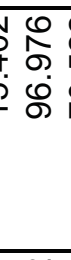 & 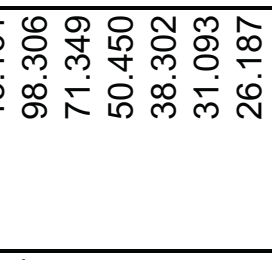 \\
\hline 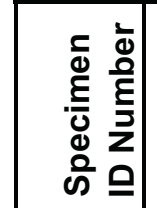 & $\begin{array}{l}\bar{\delta} \\
\underline{m}\end{array}$ & $\begin{array}{l}\tilde{N} \\
\tilde{N} \\
\underline{N}\end{array}$ & 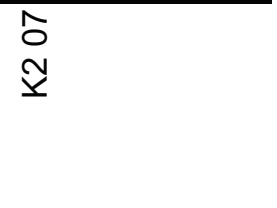 \\
\hline
\end{tabular}




\begin{tabular}{|c|c|c|c|}
\hline$\underset{\Sigma}{\Sigma}$ & $\begin{array}{l}\hat{O} \\
\frac{1}{0} \\
\stackrel{0}{0} \\
\dot{w}\end{array}$ & 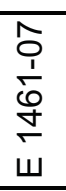 & $\begin{array}{l}\hat{O} \\
\frac{1}{0} \\
\stackrel{0}{+} \\
\dot{w}\end{array}$ \\
\hline 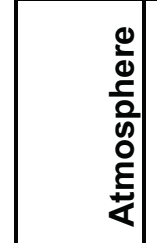 & 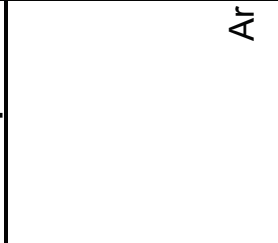 & 文 & $\bar{z}$ \\
\hline 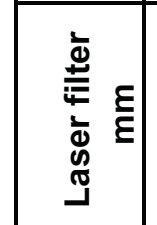 & $\stackrel{8}{\circ}$ & 음 & 음 \\
\hline 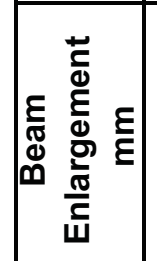 & $\stackrel{\grave{N}}{\stackrel{N}{N}}$ & 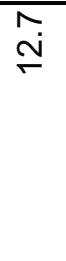 & 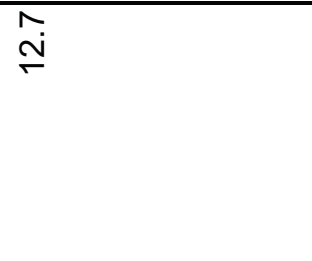 \\
\hline $\begin{array}{l}\bar{b} \\
\vdots \\
心 \\
\Phi \\
\infty\end{array}$ & $\begin{array}{l}\text { की } \\
\text { की }\end{array}$ & $\begin{array}{l}\text { की } \\
\text { की }\end{array}$ & $\begin{array}{l}\text { की } \\
\text { 足 }\end{array}$ \\
\hline 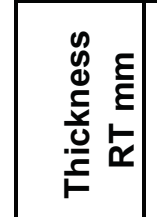 & 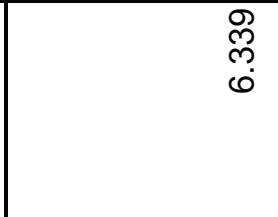 & 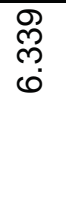 & $\begin{array}{l}\text { D్ } \\
\text { ల్ర }\end{array}$ \\
\hline 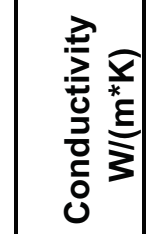 & & & \\
\hline 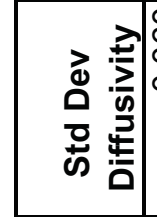 & 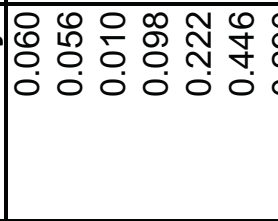 & 0 & 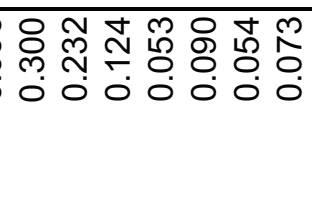 \\
\hline 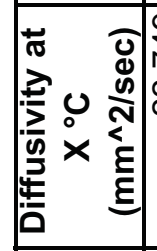 & 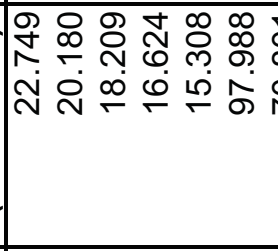 & & 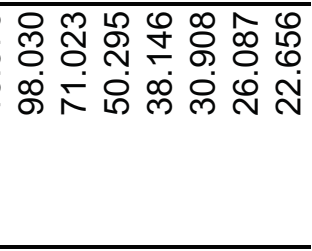 \\
\hline 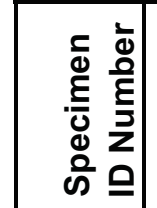 & $\begin{array}{l}\infty \\
\stackrel{\infty}{ } \\
\tilde{\Upsilon}\end{array}$ & $\begin{array}{l}\dot{\partial} \\
\tilde{\widetilde{x}}\end{array}$ & $\begin{array}{l}\tilde{m} \\
\tilde{m} \\
\underline{y}\end{array}$ \\
\hline
\end{tabular}




\begin{tabular}{|c|c|c|}
\hline 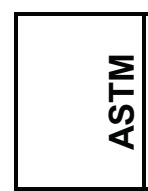 & 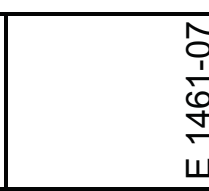 & $\begin{array}{l}\hat{S} \\
\frac{1}{5} \\
\dot{0} \\
\dot{1} \\
\end{array}$ \\
\hline $\begin{array}{c}0 \\
\frac{0}{0} \\
\frac{5}{0} \\
0 \\
0 \\
\frac{1}{4}\end{array}$ & 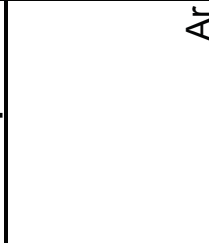 & $\frac{1}{4}$ \\
\hline 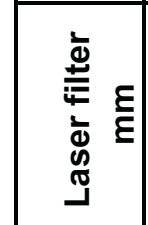 & 음 & $\stackrel{2}{\circ}$ \\
\hline 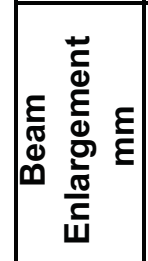 & 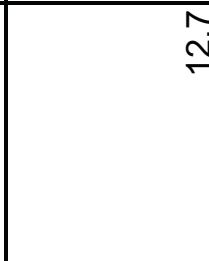 & 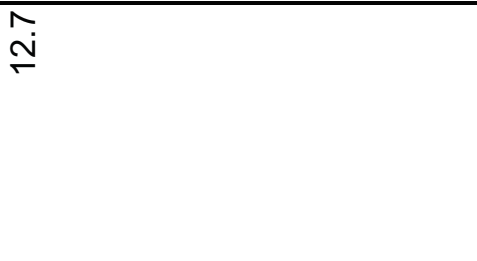 \\
\hline 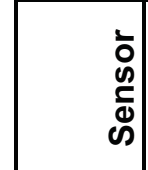 & $\begin{array}{l}\overline{0} \\
\text { c0 } \\
\end{array}$ & $\begin{array}{l}\text { की } \\
\text { की }\end{array}$ \\
\hline 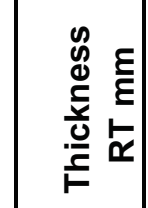 & 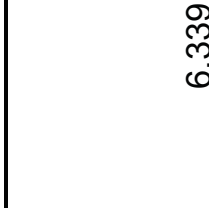 & 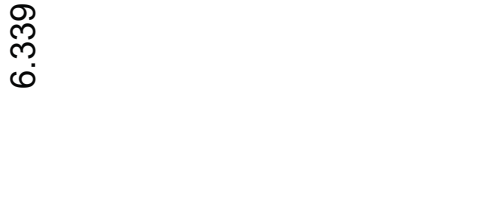 \\
\hline 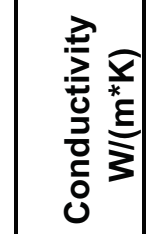 & & \\
\hline 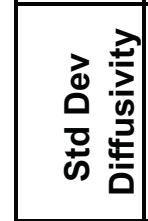 & 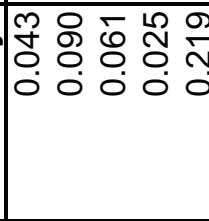 & 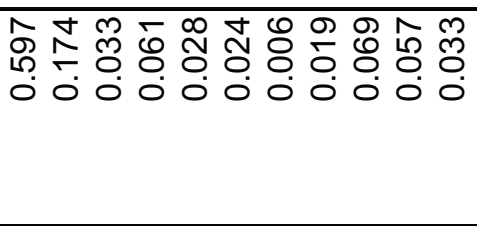 \\
\hline 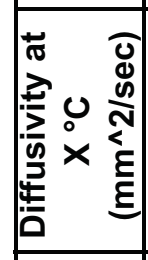 & 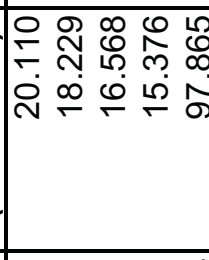 & 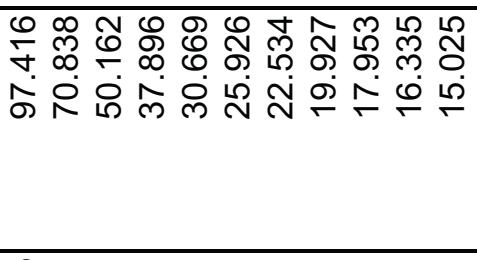 \\
\hline 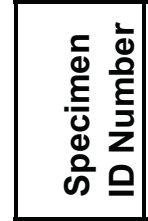 & $\begin{array}{l}\mathbf{y} \\
\text { ma }\end{array}$ & 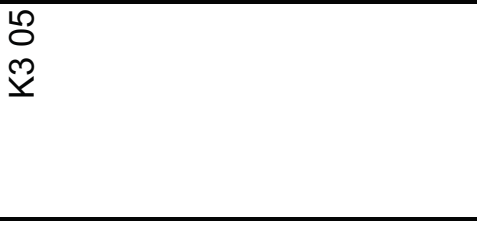 \\
\hline
\end{tabular}




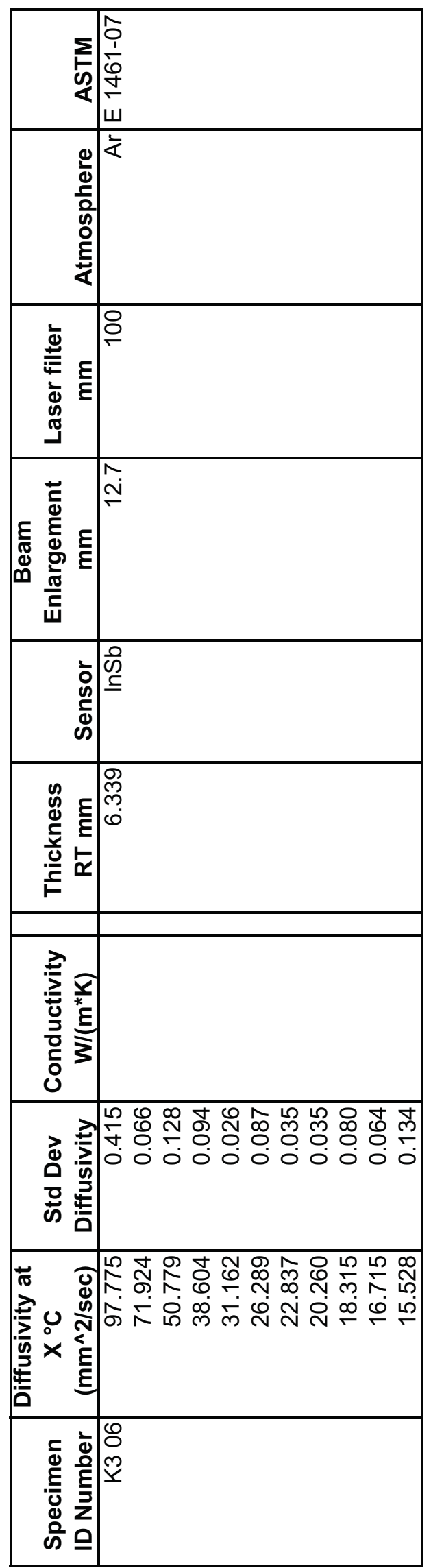




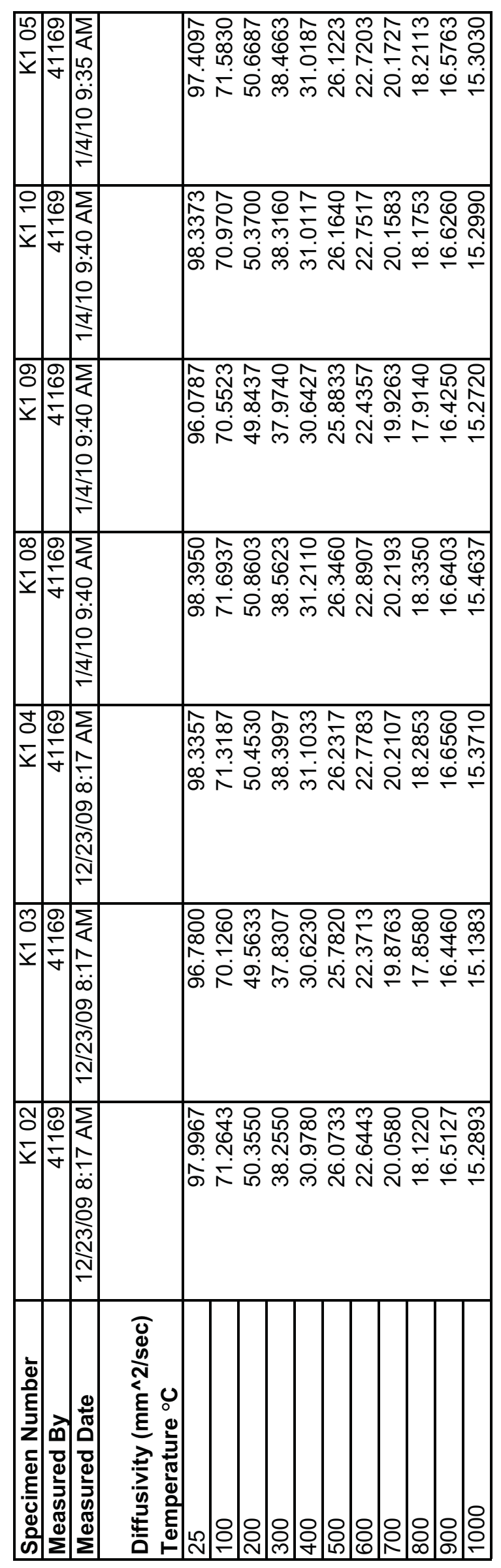




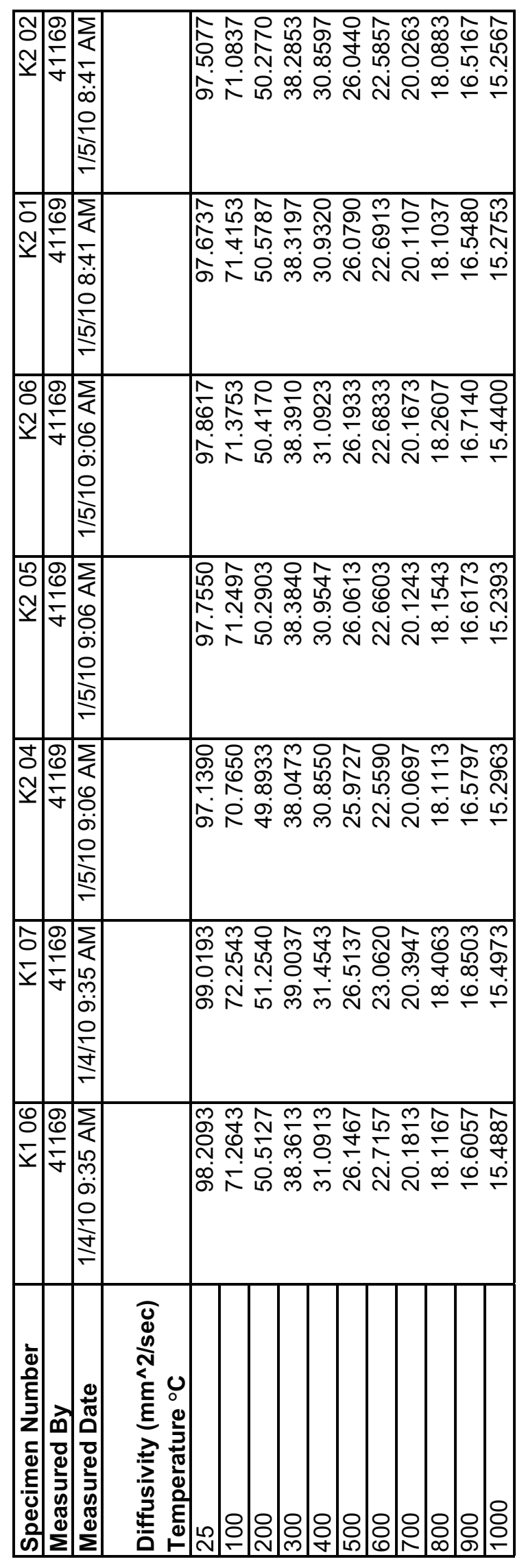




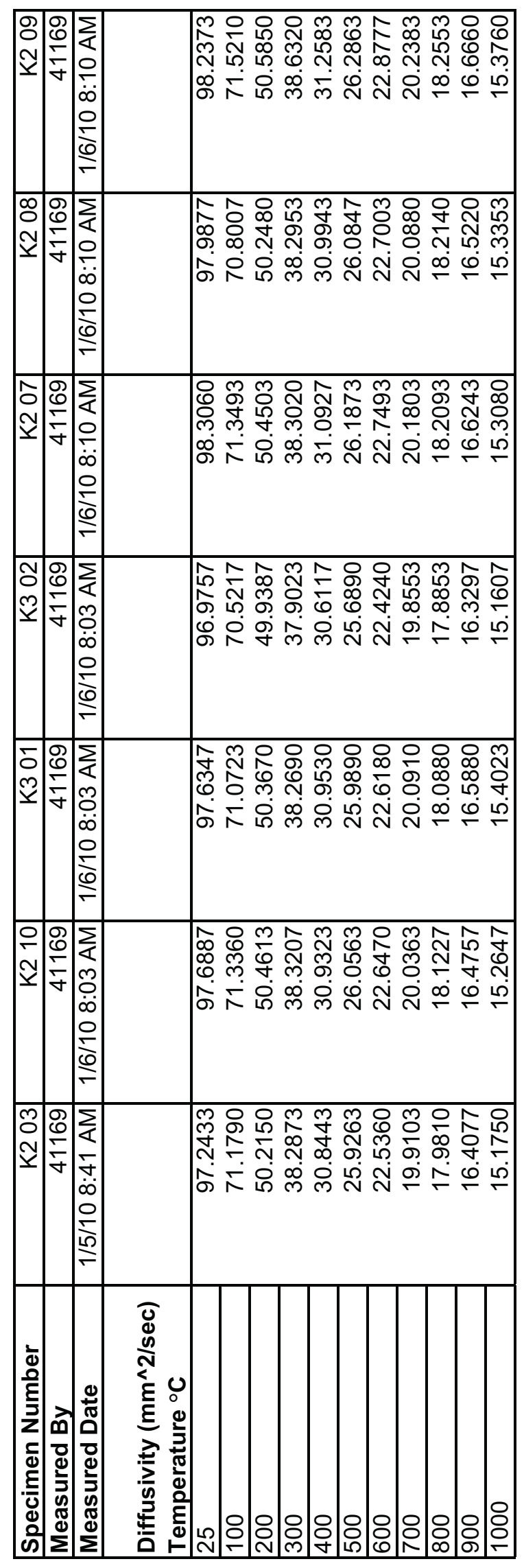




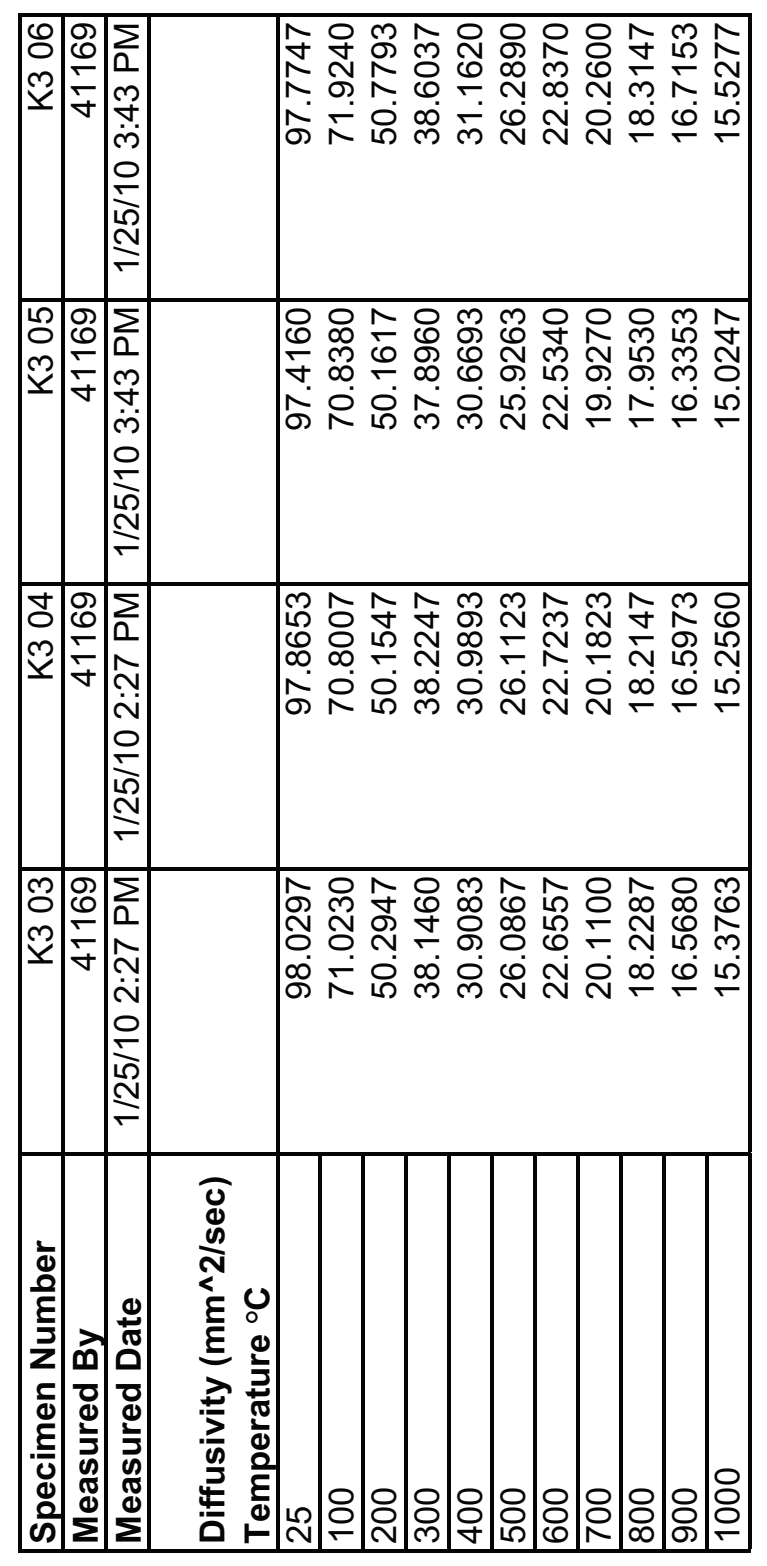




\begin{tabular}{|c|c|}
\hline 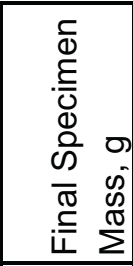 & 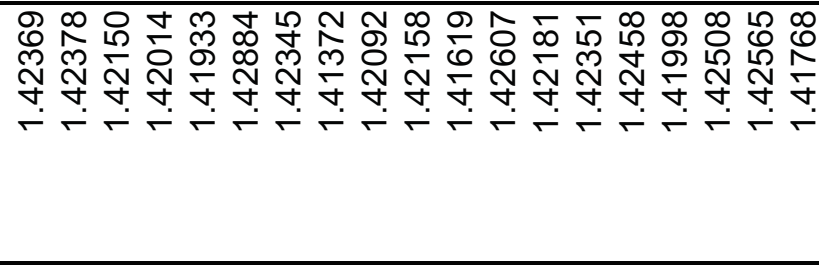 \\
\hline 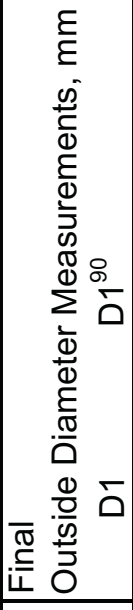 & 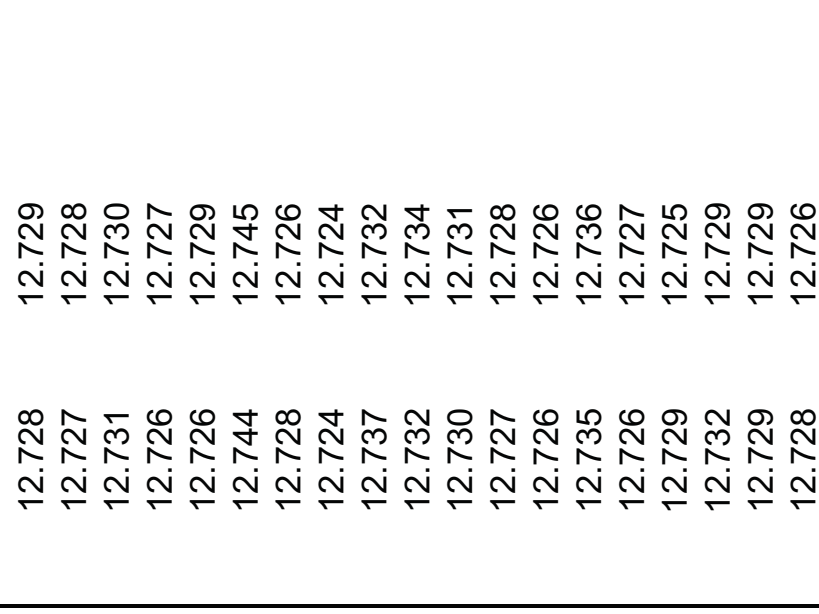 \\
\hline$\checkmark$ & 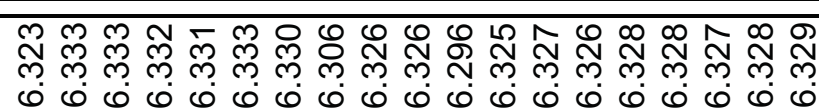 \\
\hline$\varepsilon \stackrel{3}{3}$ & 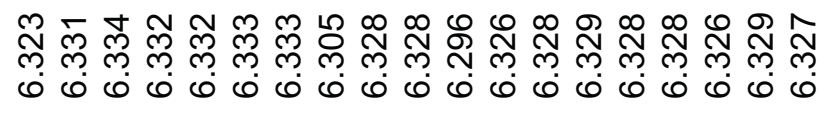 \\
\hline 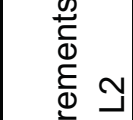 & 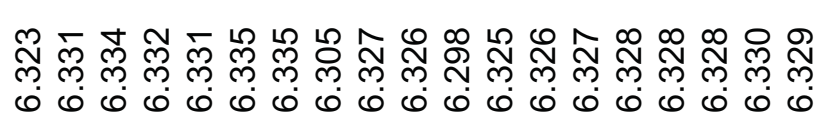 \\
\hline 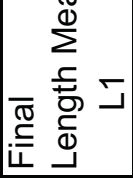 & 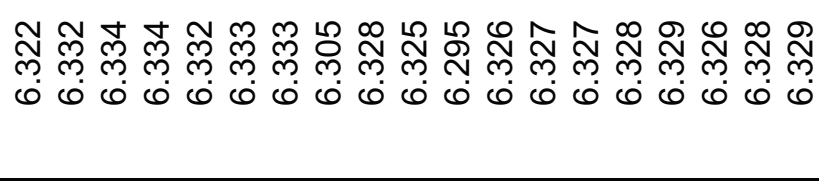 \\
\hline 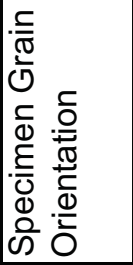 & \\
\hline 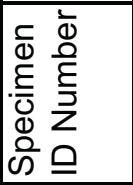 & 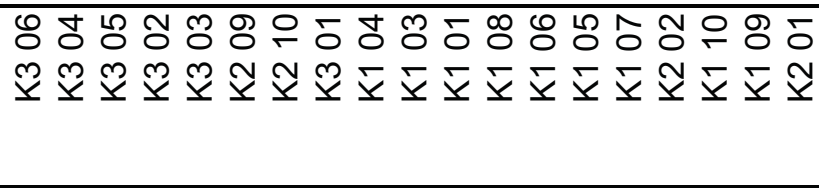 \\
\hline 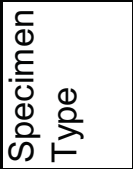 & \\
\hline
\end{tabular}




\begin{tabular}{|c|c|}
\hline 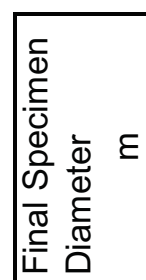 & 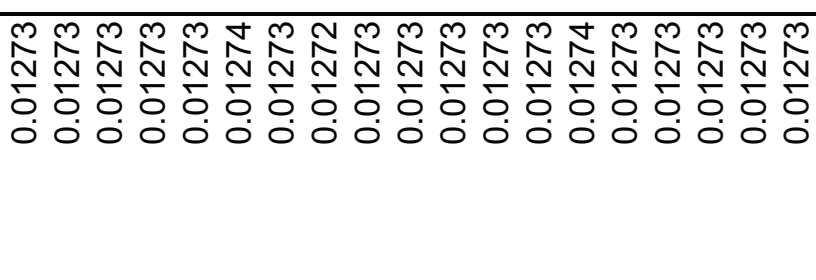 \\
\hline 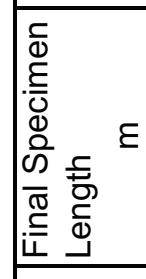 & 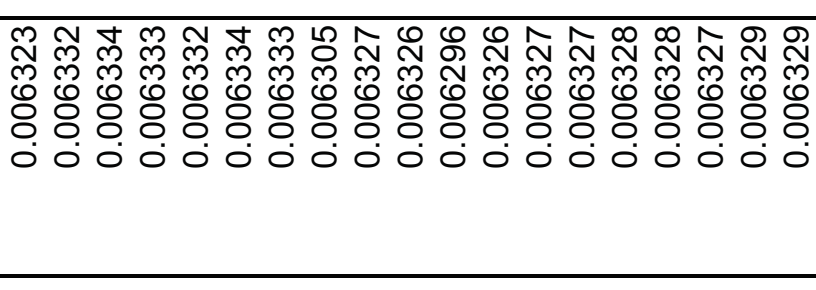 \\
\hline 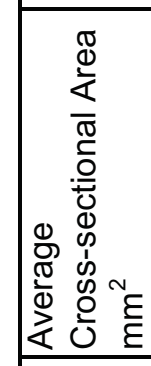 & 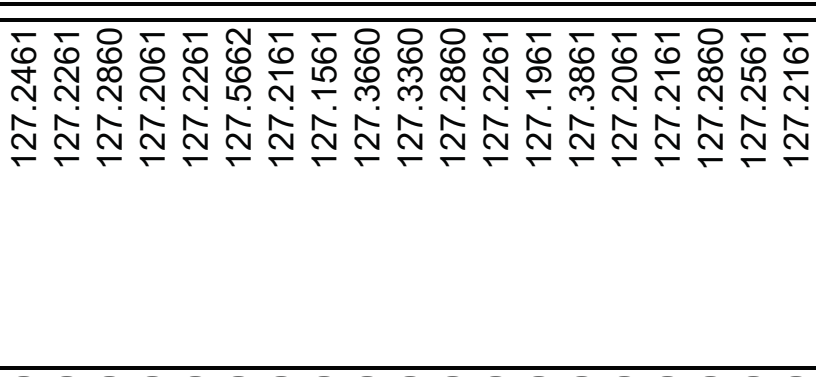 \\
\hline 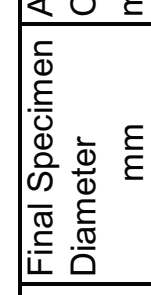 & 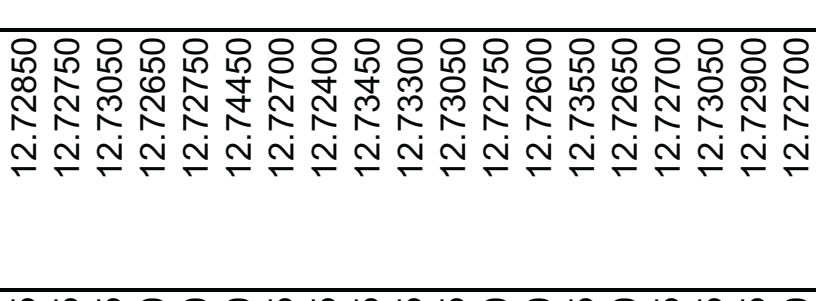 \\
\hline$E$ & 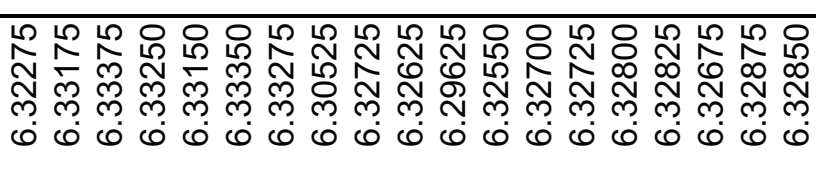 \\
\hline 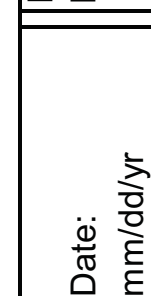 & 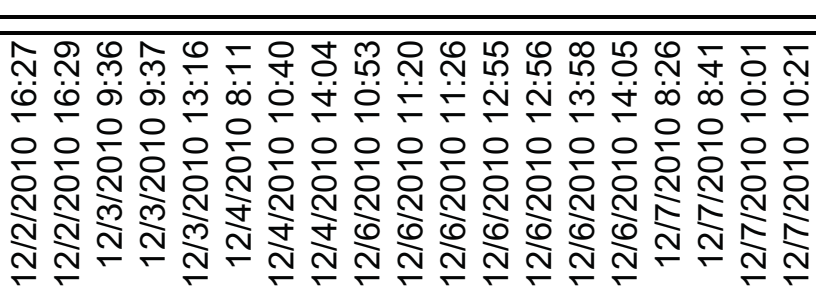 \\
\hline 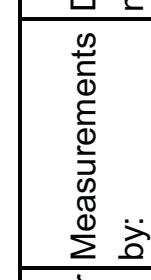 & 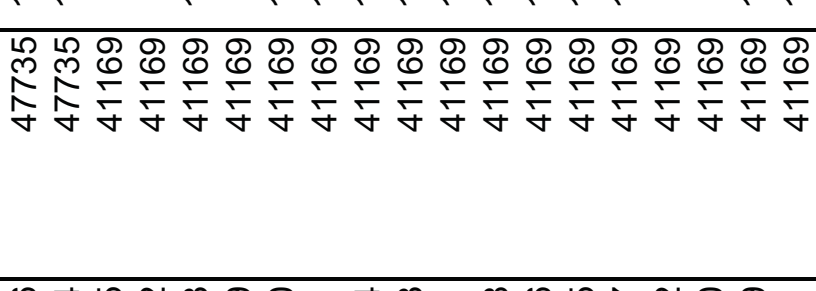 \\
\hline 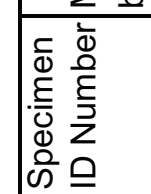 & 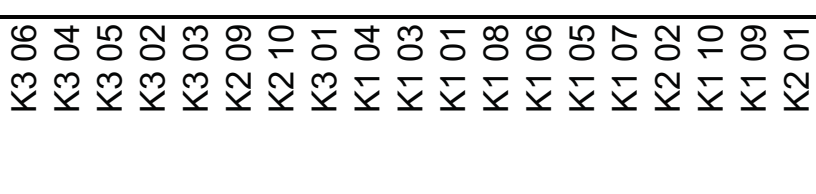 \\
\hline
\end{tabular}




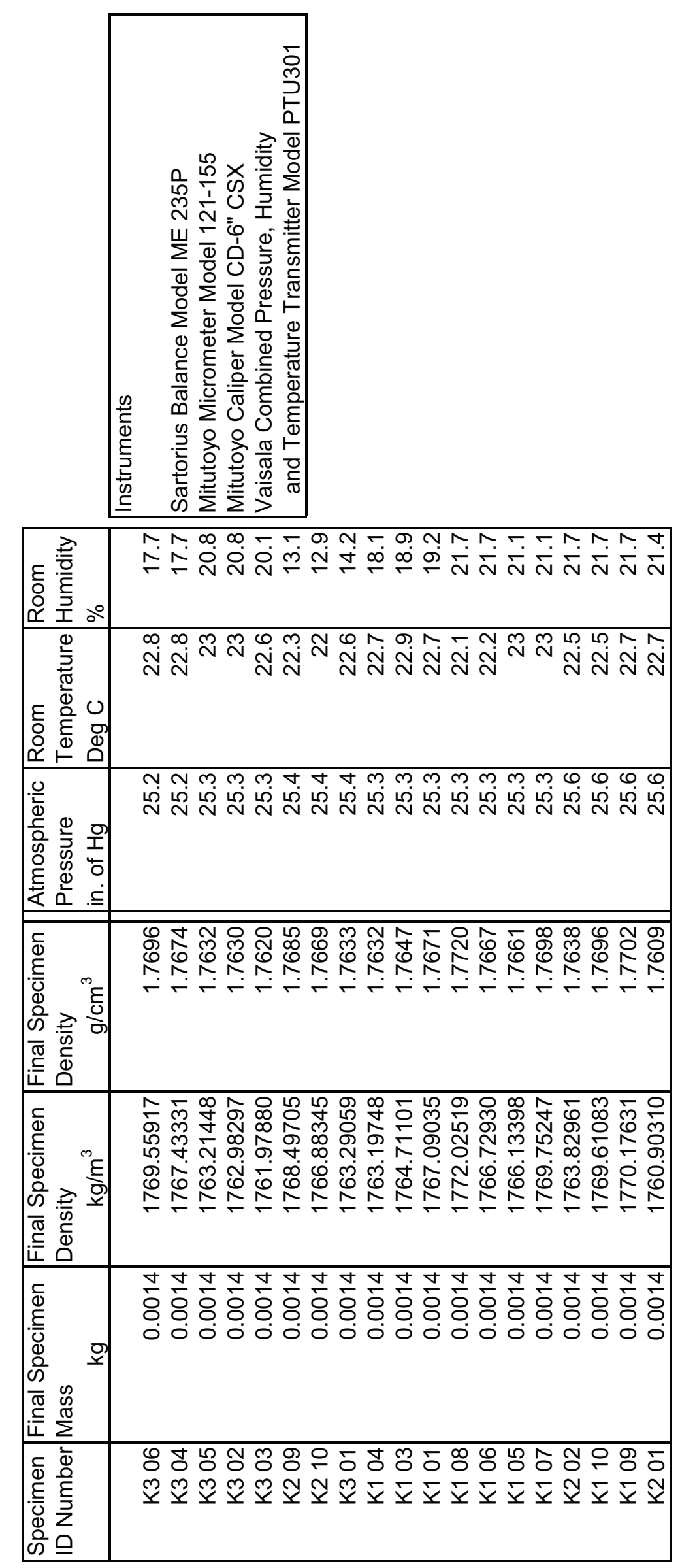


Graphite Grade:

Graphite Manufacturer: Graftech International

Forming Process:

Coke Particle Size:

Coke Type:

ASTM Class:

Specimen Geometry:
Extruded

Medium grain

Pitch coke

ENHP

Cylinder

Specimen ID \#'s:

L1 01

L1 02

L1 03

L1 04

L1 05

L1 06

L1 07

L1 08

L1 09

L1 10

L2 01

L2 02

L2 03

L2 04

L2 05

L2 06

L2 07

L2 08

L2 09

L2 10

L2 11

L3 01

L3 02

L3 03

L3 04

L3 05

L3 06

L3 07

L3 08

L3 09

L3 10 


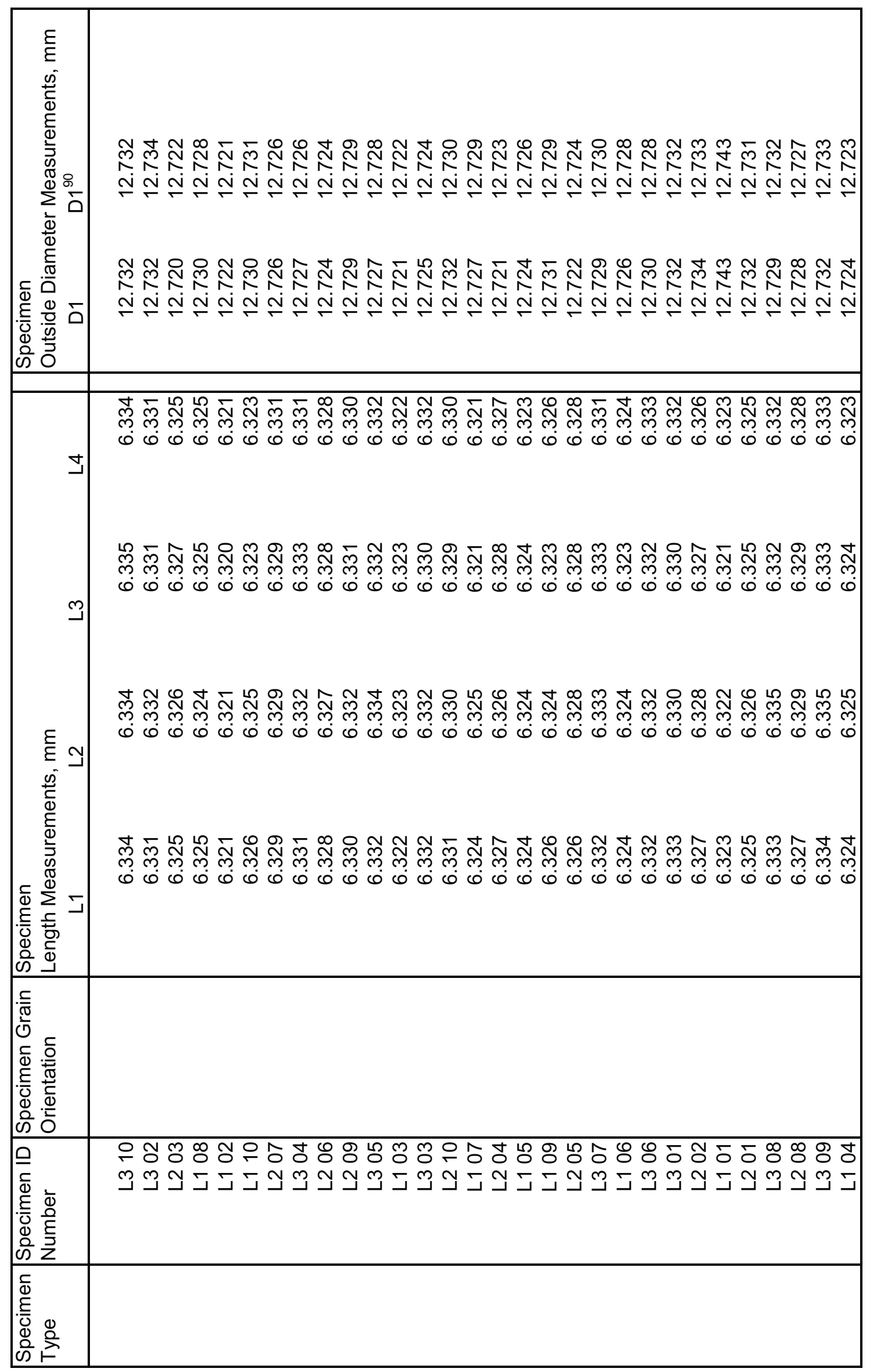




\begin{tabular}{|c|c|}
\hline 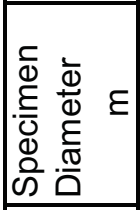 & 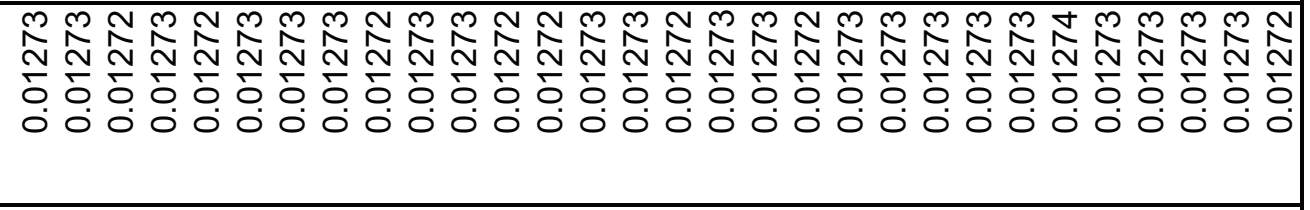 \\
\hline 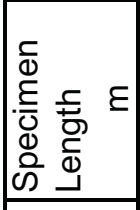 & 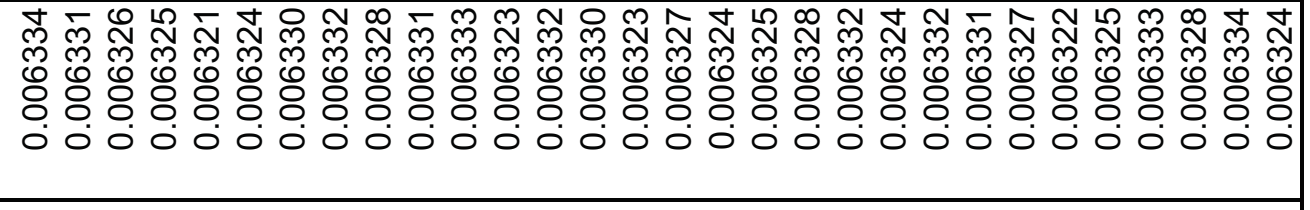 \\
\hline 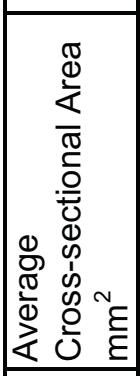 & 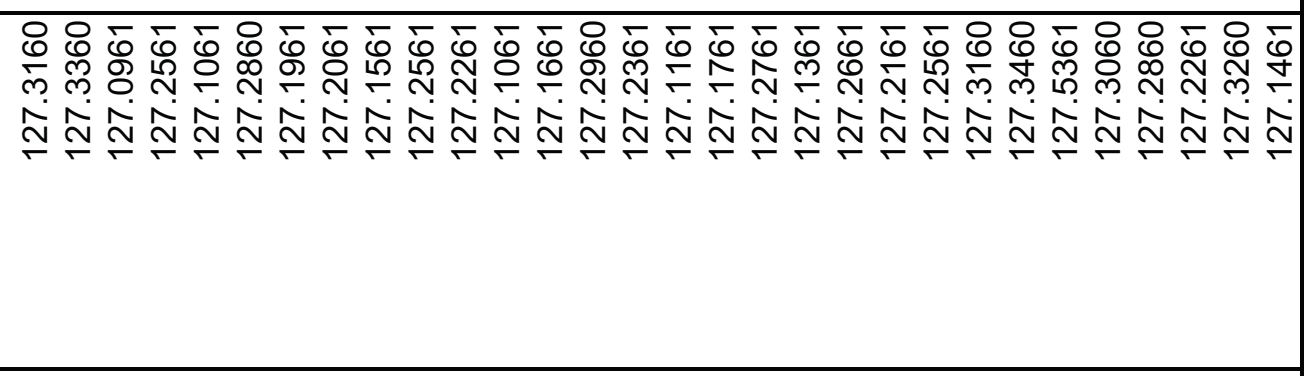 \\
\hline 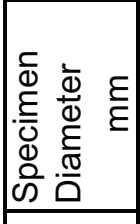 & 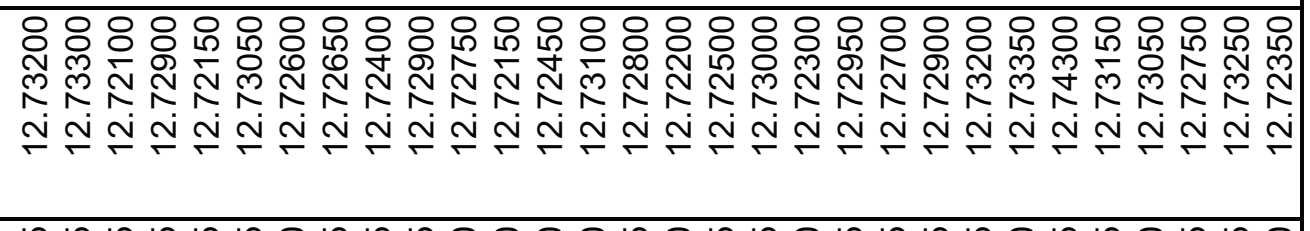 \\
\hline 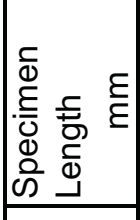 & 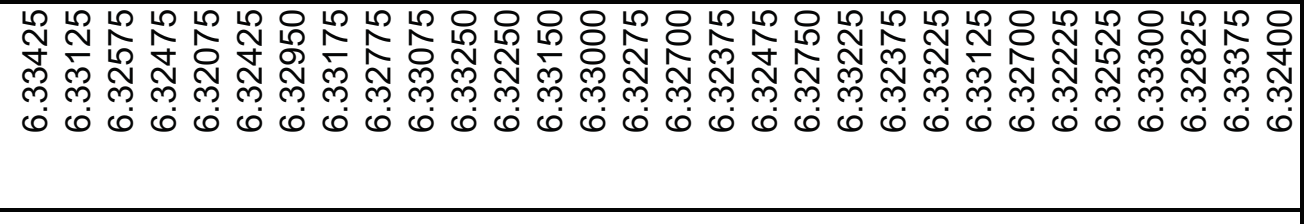 \\
\hline 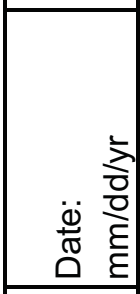 & 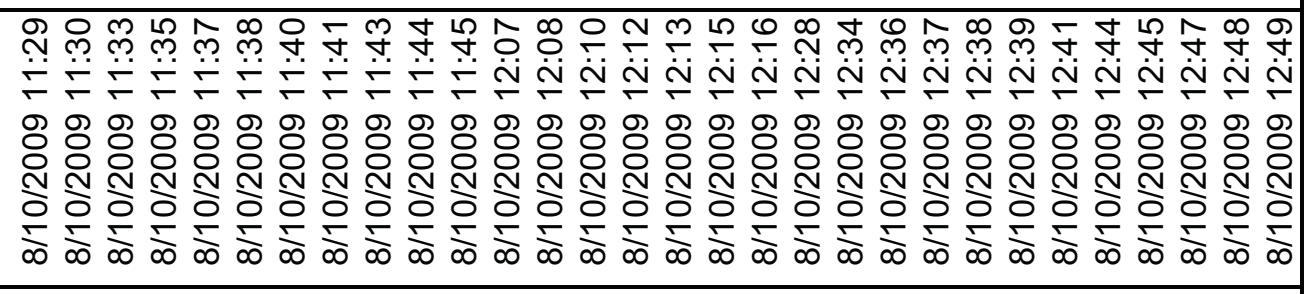 \\
\hline 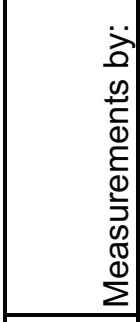 & 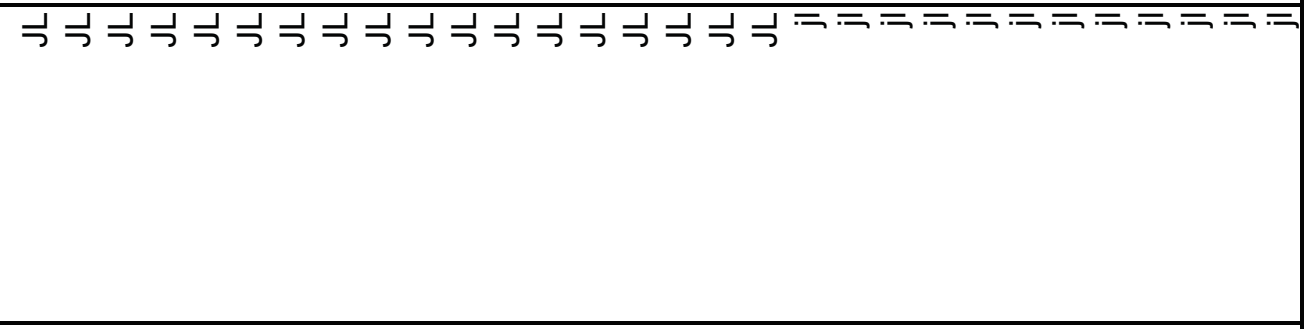 \\
\hline 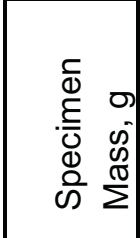 & 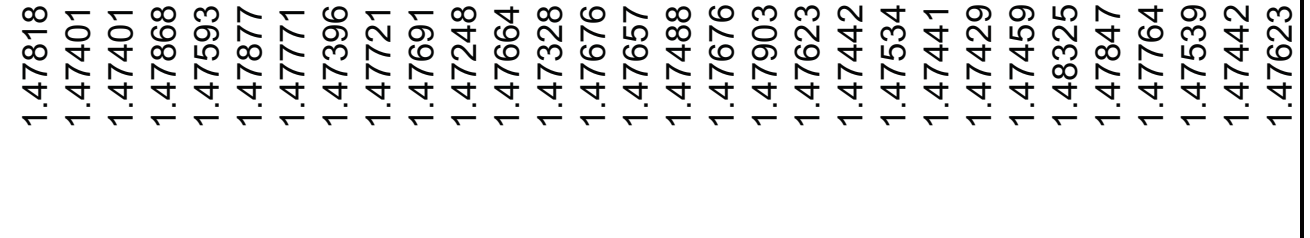 \\
\hline 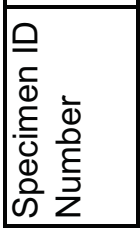 & 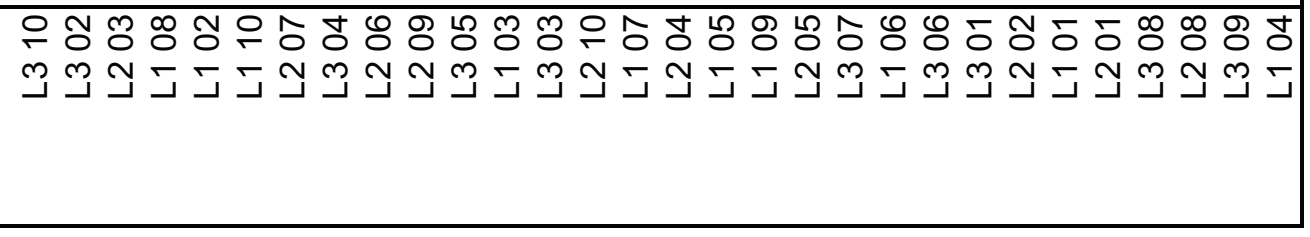 \\
\hline
\end{tabular}




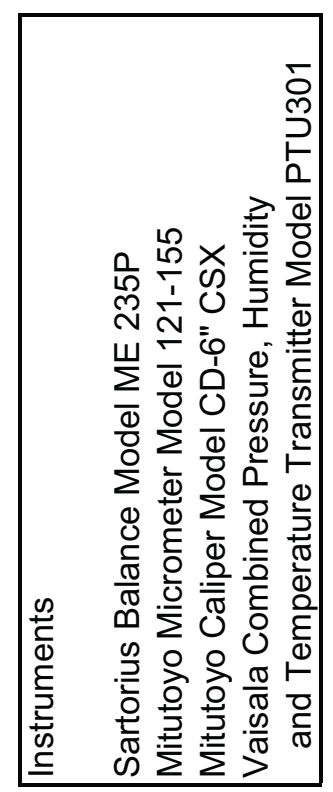

\begin{tabular}{|c|c|}
\hline 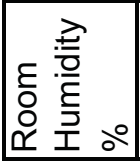 & 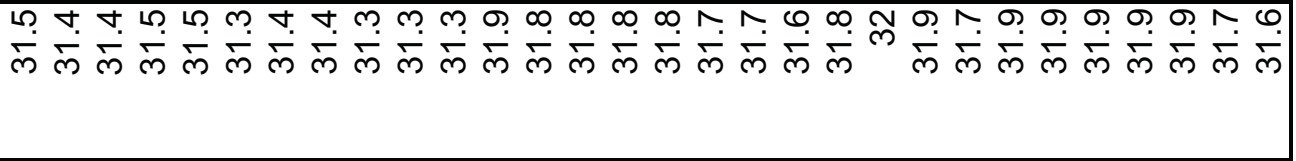 \\
\hline 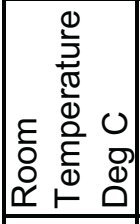 & 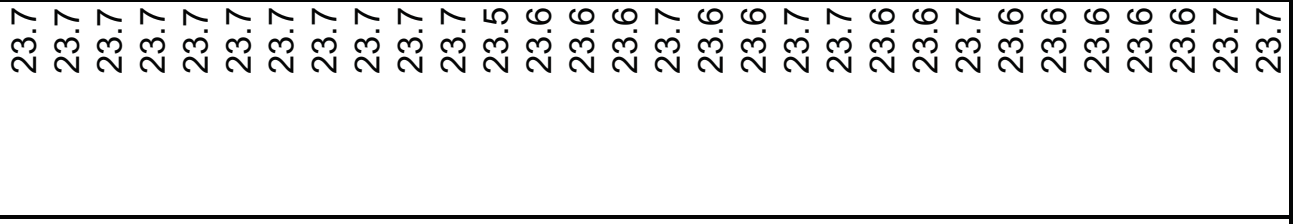 \\
\hline 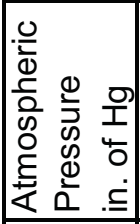 & 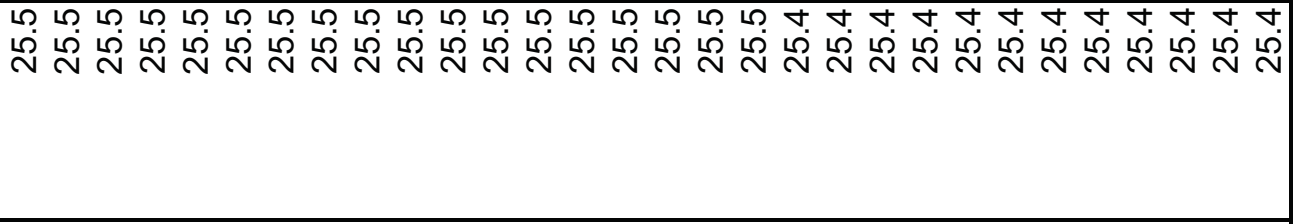 \\
\hline 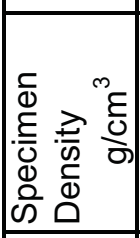 & 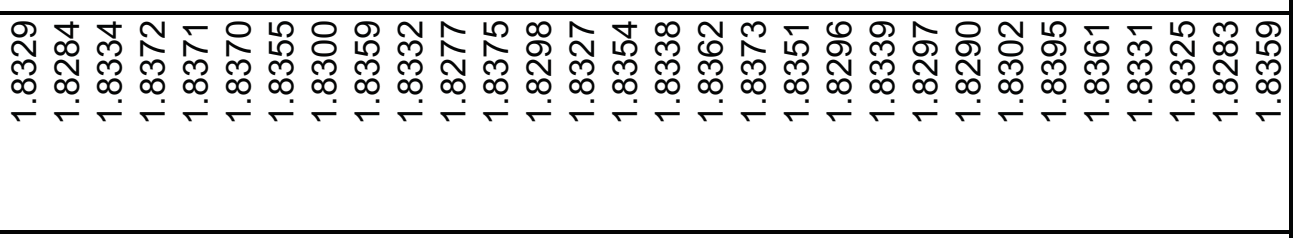 \\
\hline 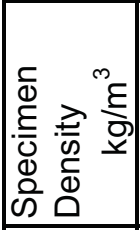 & 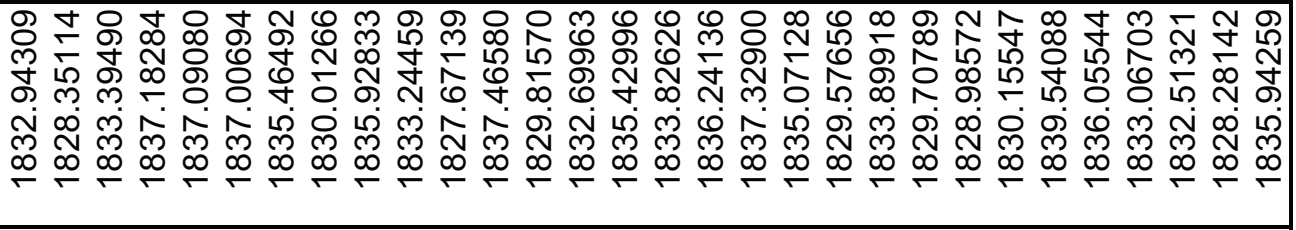 \\
\hline 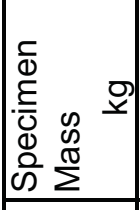 & 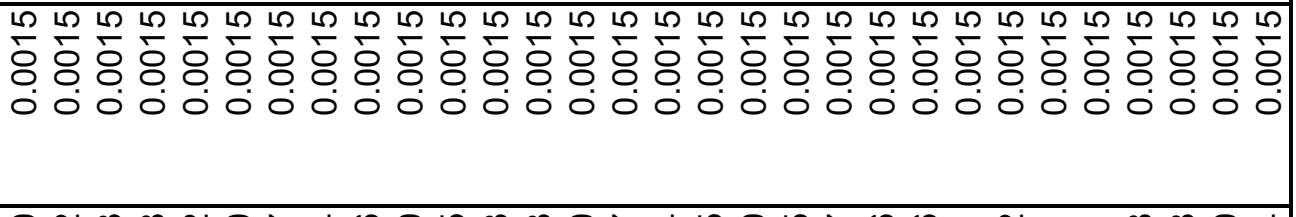 \\
\hline 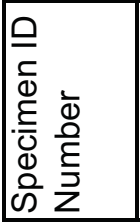 & 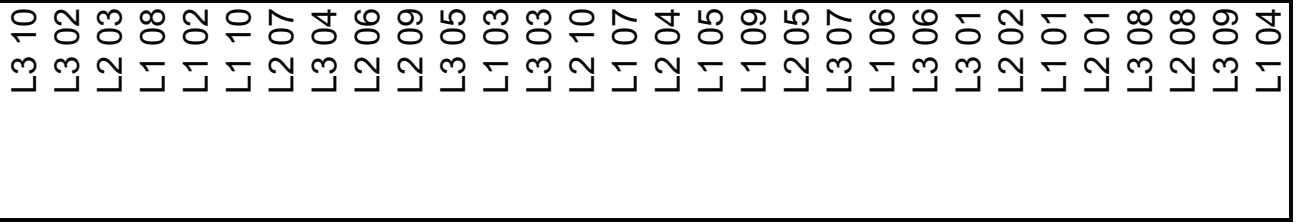 \\
\hline
\end{tabular}




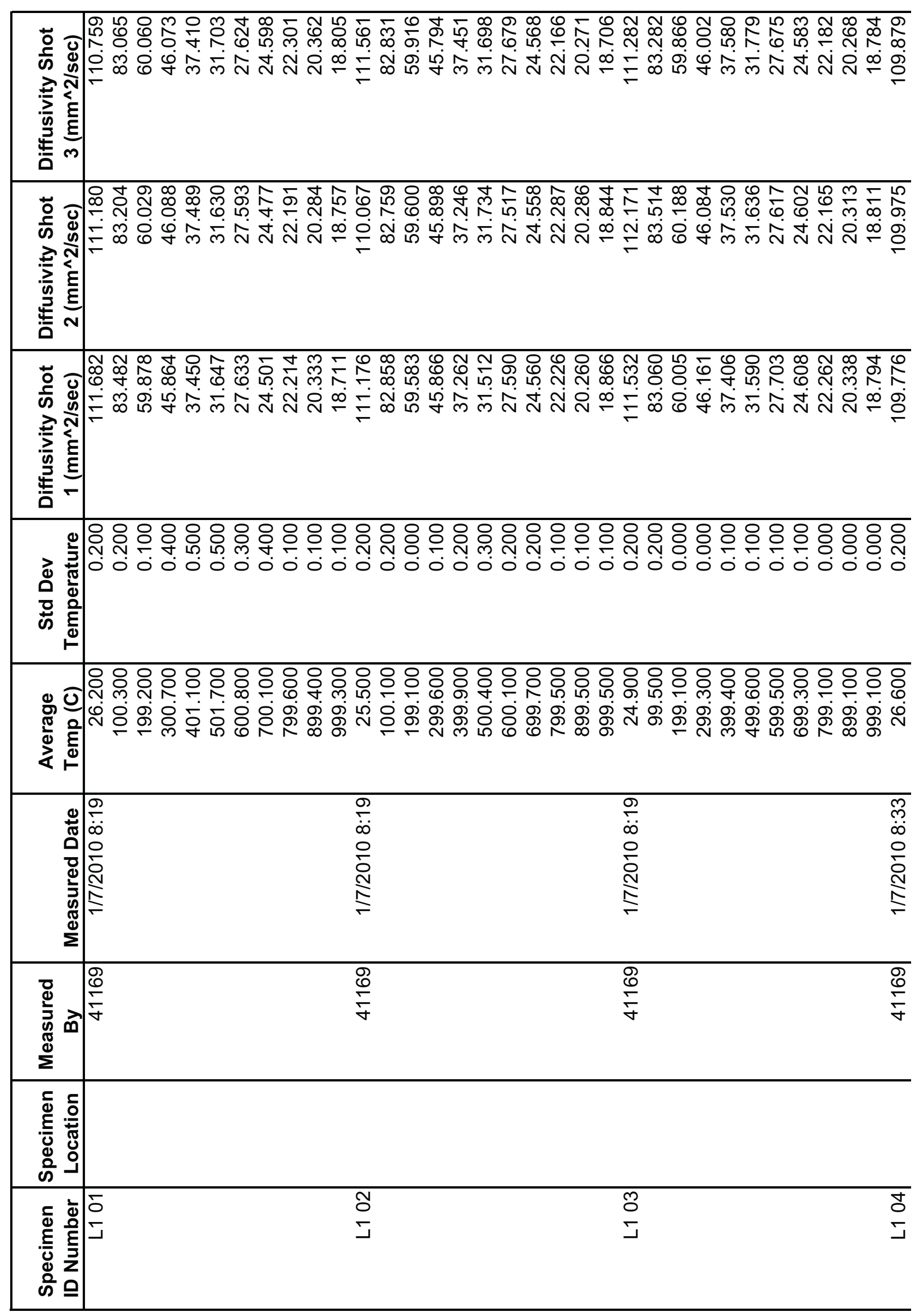




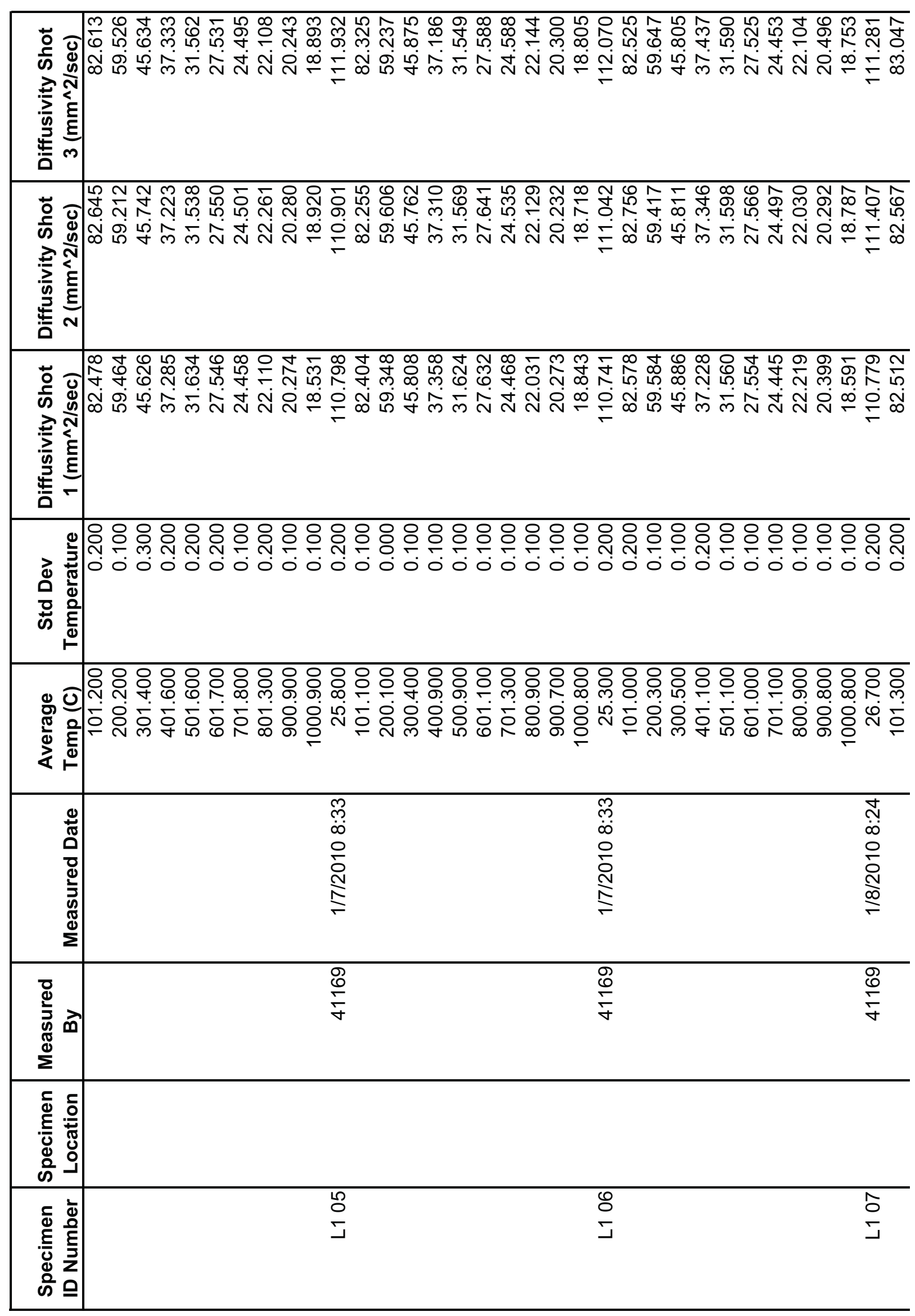




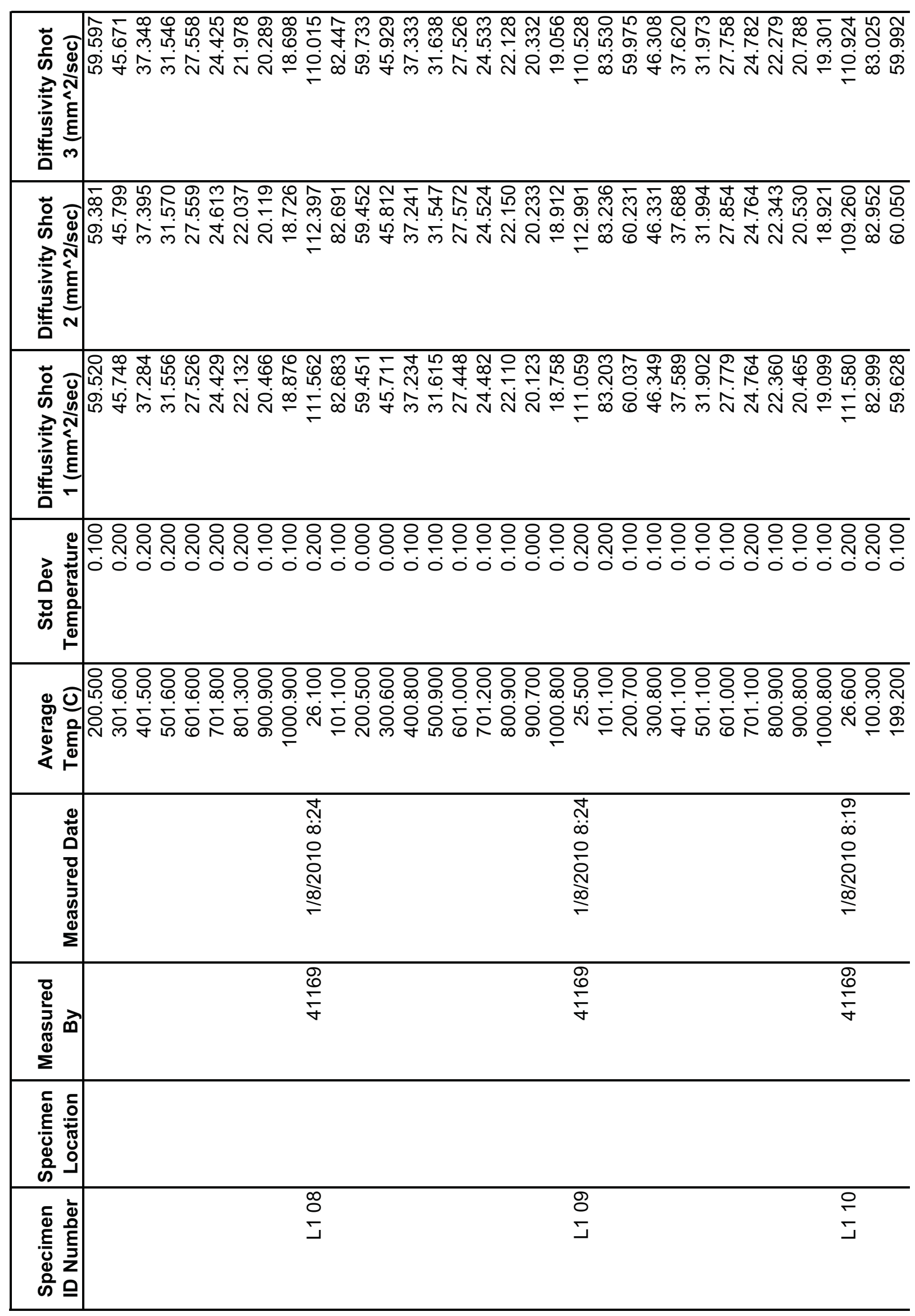




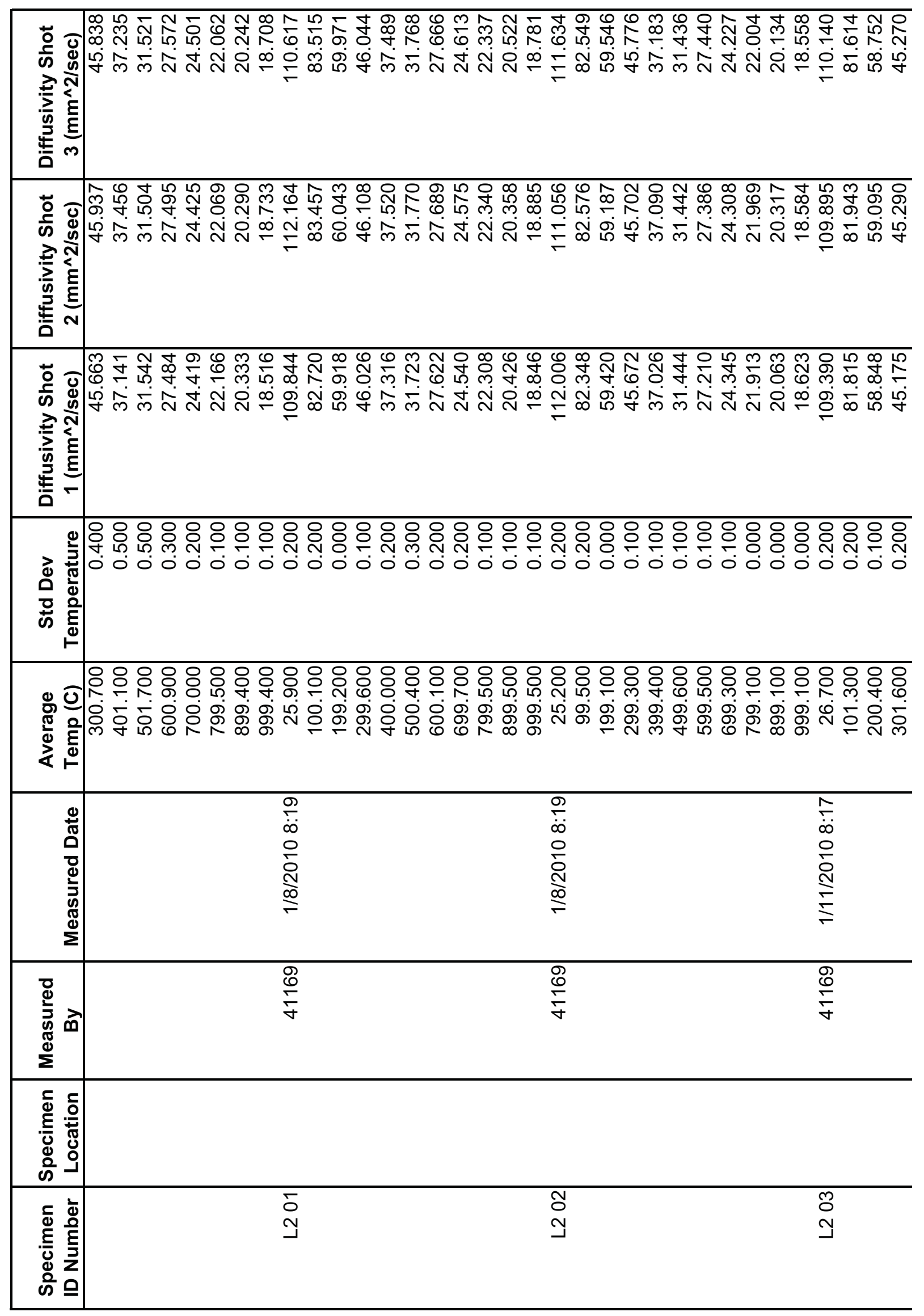




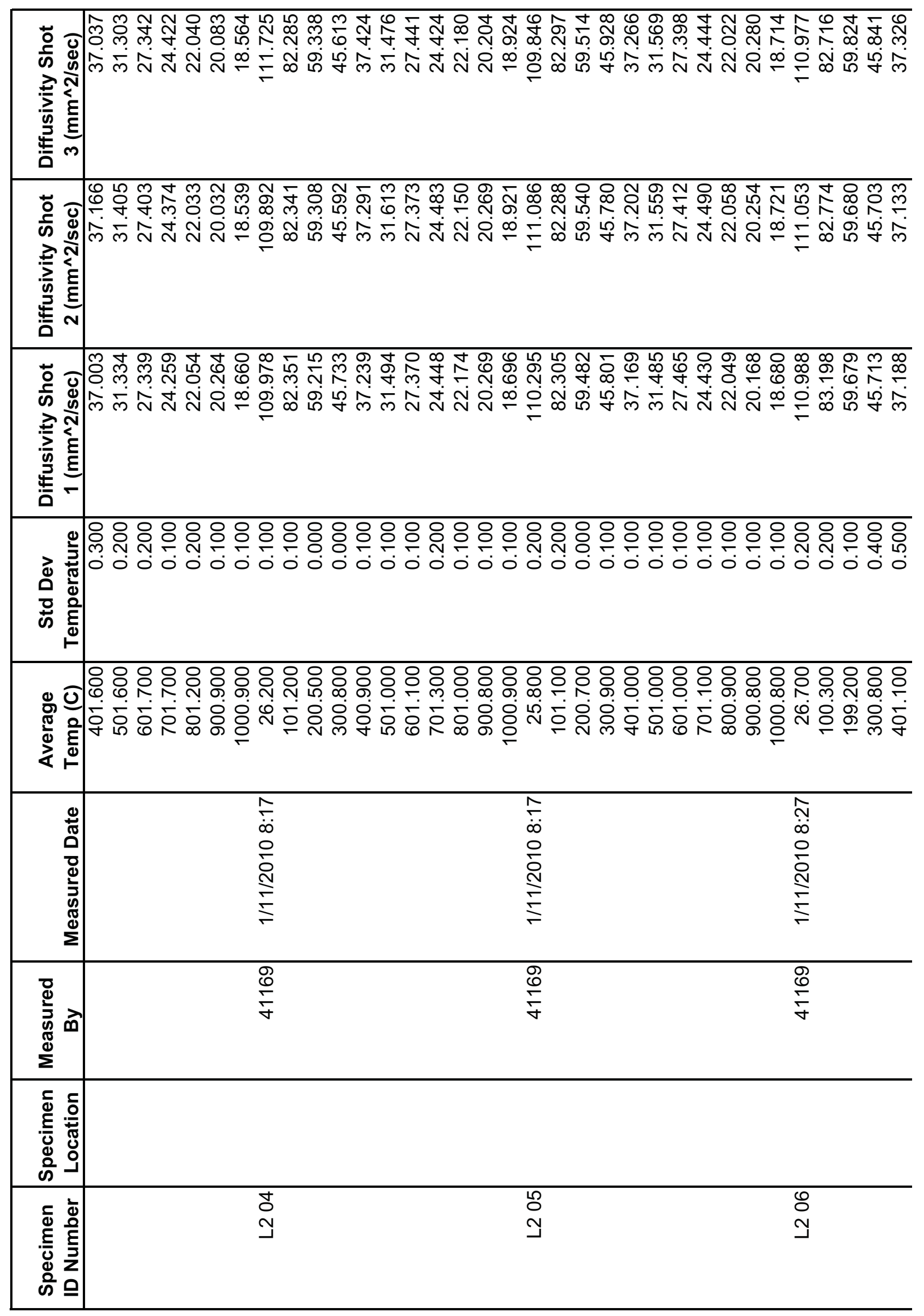




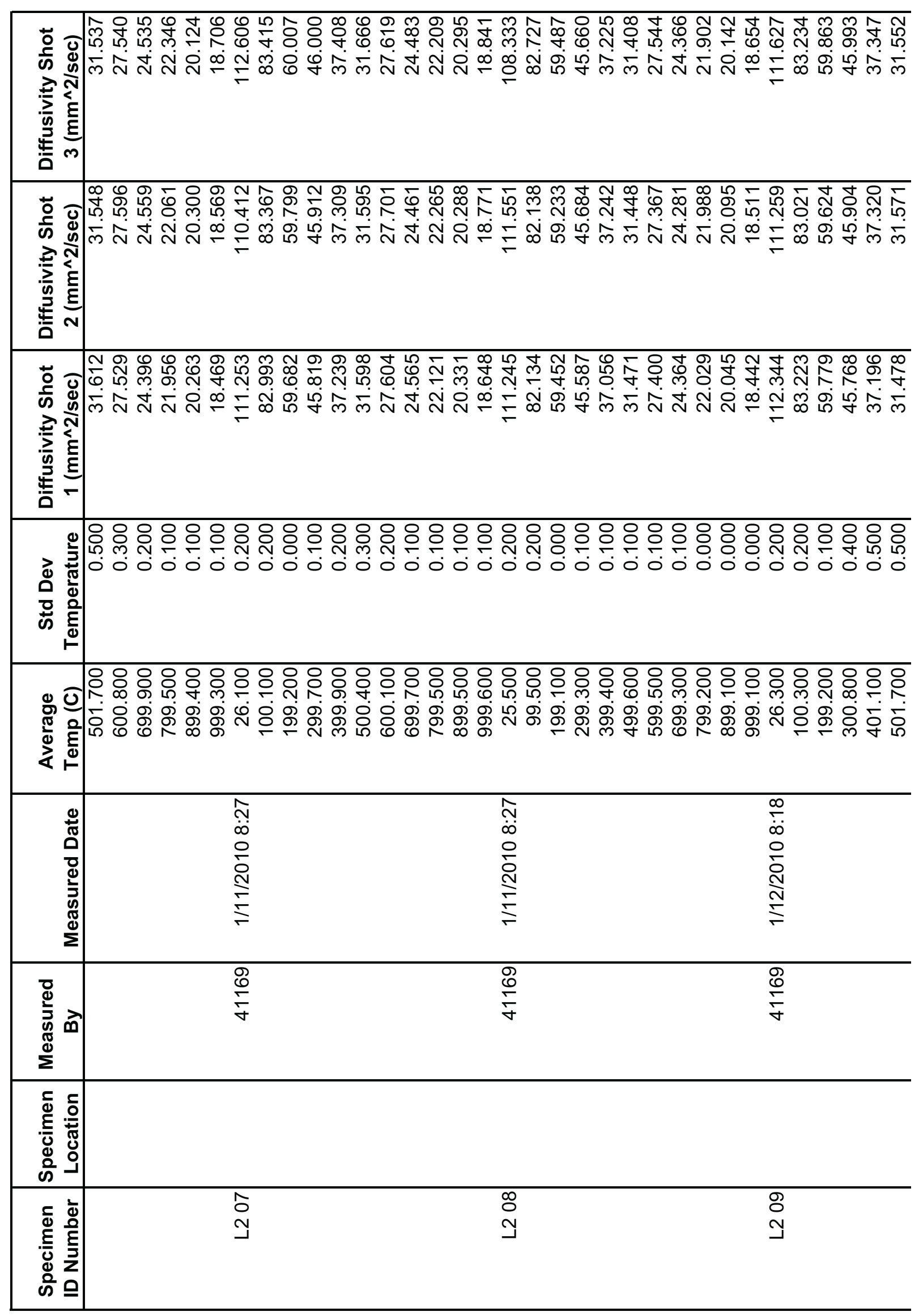




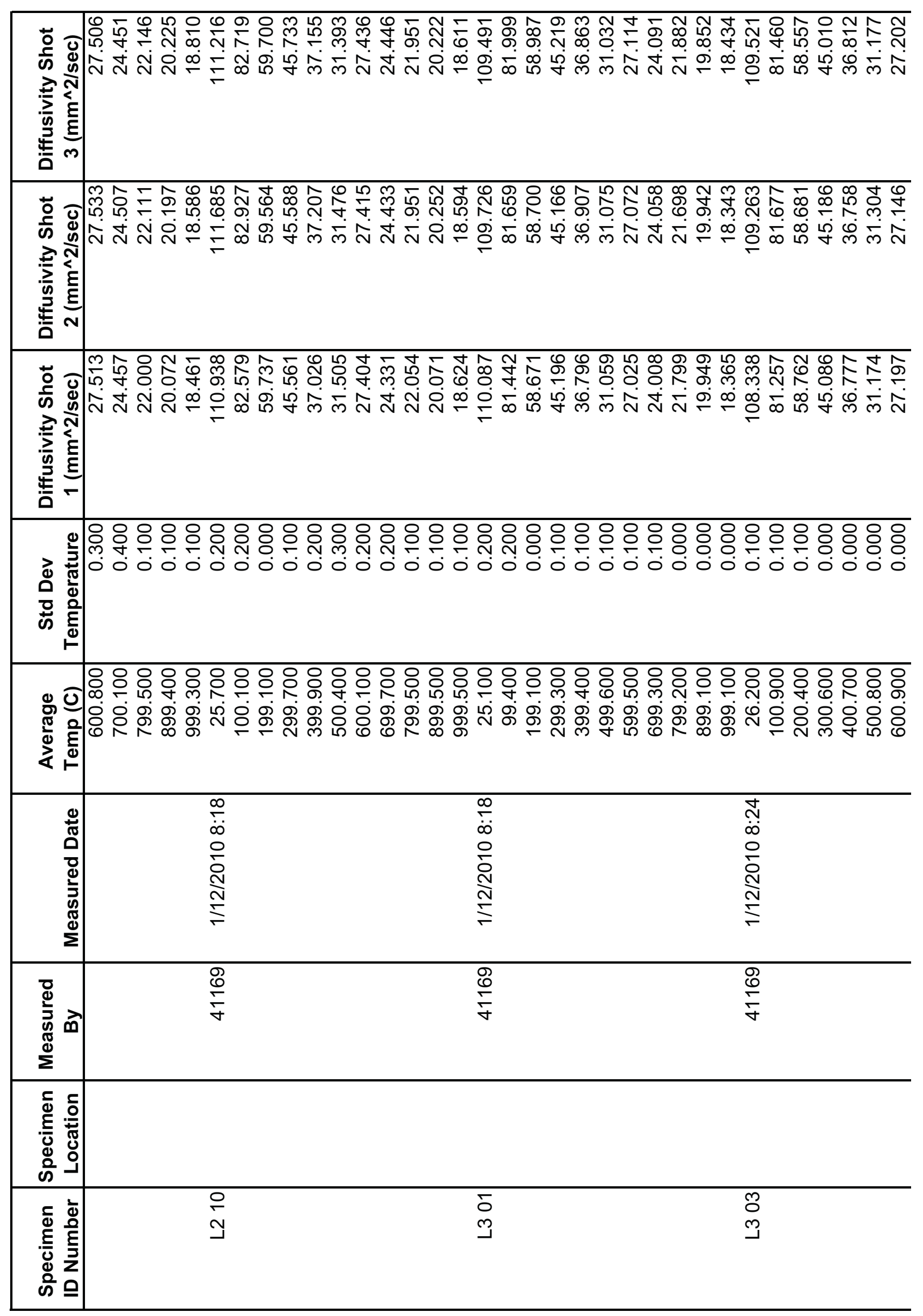




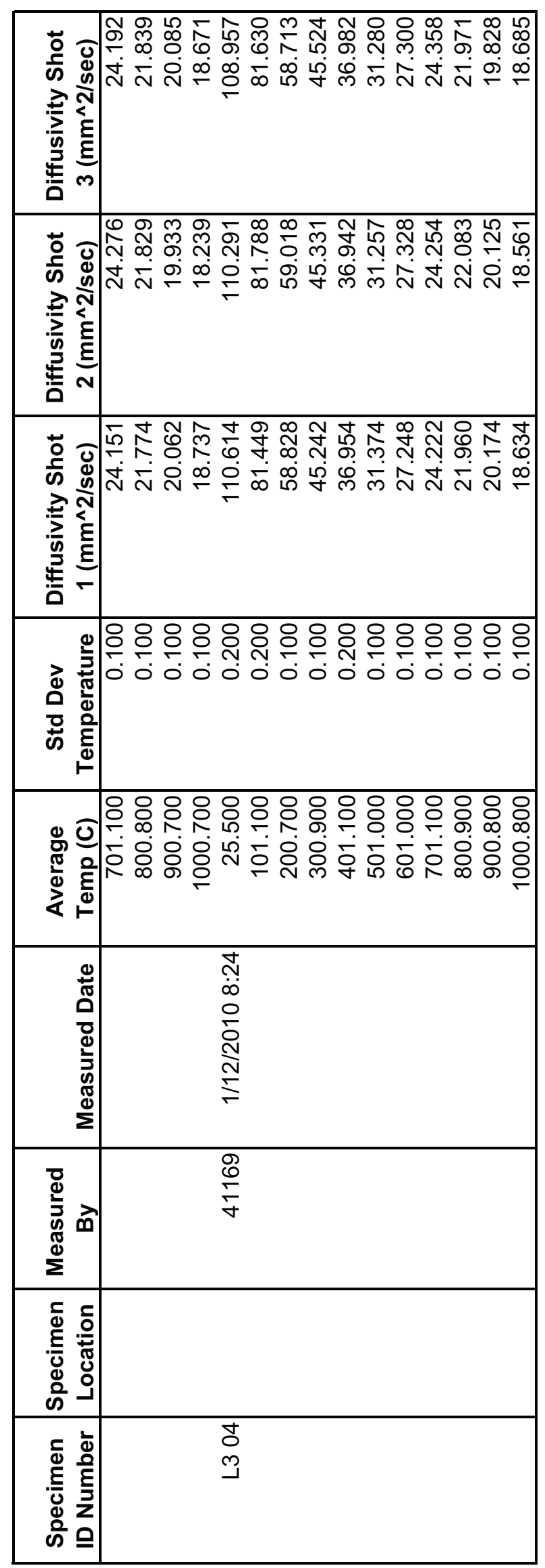




\begin{tabular}{|c|c|c|c|c|}
\hline 妾 & 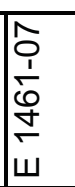 & 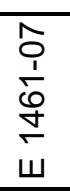 & 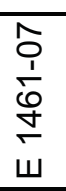 & 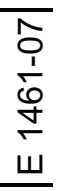 \\
\hline 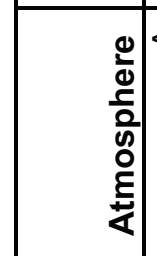 & & $\overline{\dot{z}}$ & $\bar{k}$ & $\bar{i}$ \\
\hline 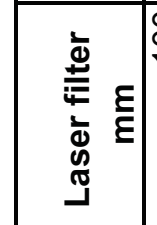 & & 음 & 음 & 음 \\
\hline 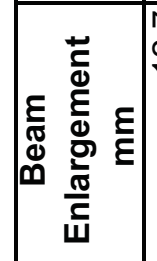 & 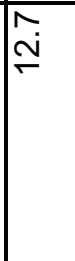 & $\stackrel{\stackrel{N}{\sim}}{\sim}$ & $\stackrel{\widehat{N}}{\stackrel{N}{S}}$ & 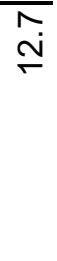 \\
\hline 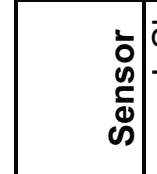 & & $\begin{array}{l}\text { की } \\
\text { की }\end{array}$ & $\begin{array}{l}\text { की } \\
\text { क्ञ }\end{array}$ & की \\
\hline 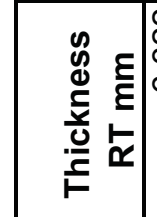 & & 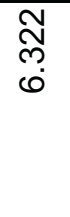 & 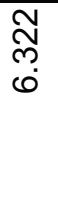 & $\begin{array}{l}\underset{\widetilde{N}}{\tilde{0}} \\
0\end{array}$ \\
\hline 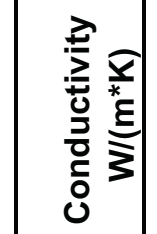 & & & & \\
\hline 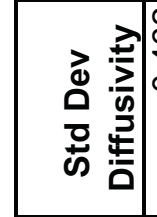 & 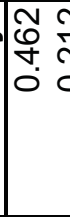 & $\begin{array}{l}\infty \\
N \\
0\end{array}$ & $\stackrel{*}{\circ}$ & $\frac{8}{0}$ \\
\hline 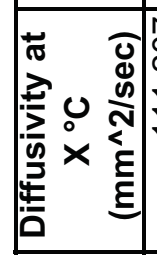 & 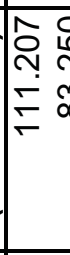 & $\begin{array}{l}n \\
\sim \\
0\end{array}$ & 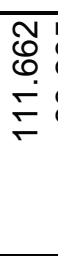 & 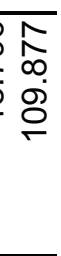 \\
\hline 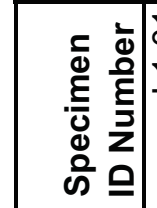 & & $\begin{array}{l}\tilde{O} \\
\tilde{J} \\
\end{array}$ & $\begin{array}{l}\text { Oू } \\
\square\end{array}$ & $\begin{array}{l}\bar{\sigma} \\
\square \\
\square\end{array}$ \\
\hline
\end{tabular}




\begin{tabular}{|c|c|c|c|}
\hline 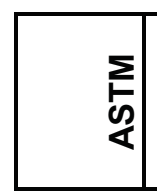 & $\begin{array}{l}\hat{o} \\
\frac{1}{0} \\
\dot{0} \\
\dot{5} \\
\end{array}$ & $\begin{array}{l}\hat{S} \\
\frac{1}{1} \\
\frac{0}{5} \\
\dot{w}\end{array}$ & 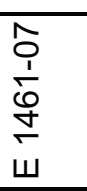 \\
\hline 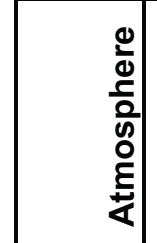 & 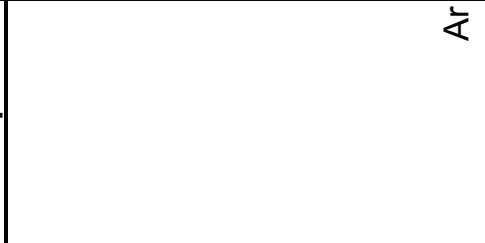 & $\frac{1}{\alpha}$ & 文 \\
\hline 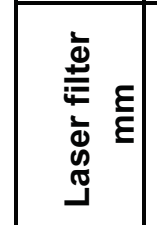 & 음 & 음 & 음 \\
\hline 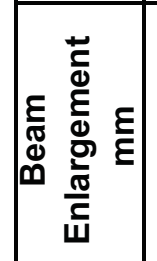 & $\stackrel{\widehat{N}}{\stackrel{N}{N}}$ & 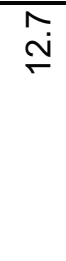 & $\stackrel{\tilde{N}}{\stackrel{N}{S}}$ \\
\hline $\begin{array}{l}\dot{b} \\
\vdots \\
\Phi \\
\Phi \\
\infty\end{array}$ & $\begin{array}{l}\text { की } \\
\text { की }\end{array}$ & 吕 & $\begin{array}{l}\text { की } \\
\text { की }\end{array}$ \\
\hline 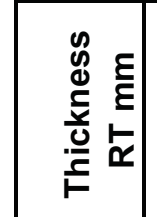 & 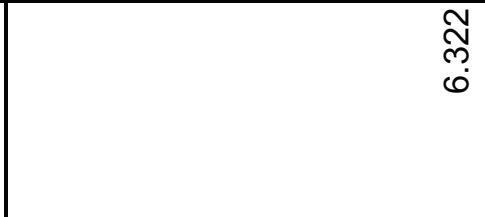 & 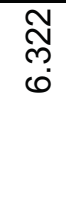 & 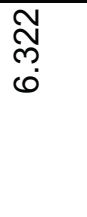 \\
\hline 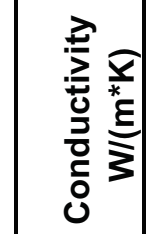 & & & \\
\hline के & 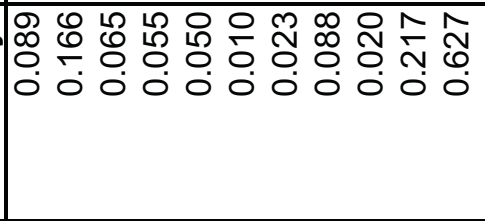 & : & $\begin{array}{l}\text { N్ల్ } \\
\text { ले } \\
0\end{array}$ \\
\hline 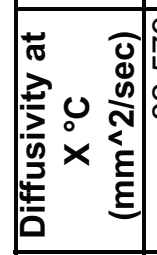 & 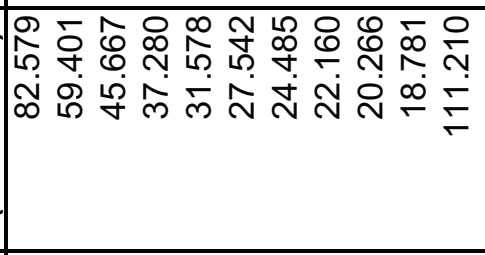 & $\stackrel{\stackrel{+}{+}}{\stackrel{\leftrightarrow}{\rightleftarrows}}$ & 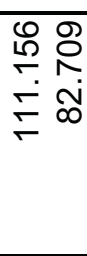 \\
\hline 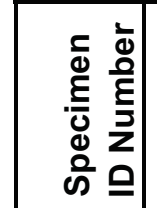 & $\begin{array}{l}\frac{1}{0} \\
\square\end{array}$ & $\begin{array}{l}8 \\
\square \\
\square\end{array}$ & $\begin{array}{l}\text { Io } \\
\text { J }\end{array}$ \\
\hline
\end{tabular}




\begin{tabular}{|c|c|c|c|}
\hline 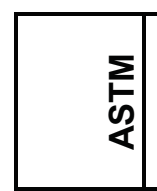 & 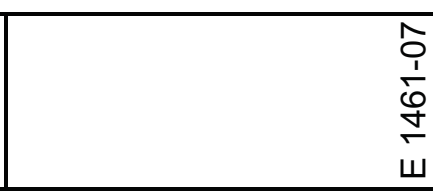 & $\begin{array}{l}\hat{o} \\
\frac{1}{0} \\
\stackrel{0}{+} \\
\dot{\omega}\end{array}$ & $\begin{array}{l}\frac{\hat{1}}{1} \\
\frac{1}{0} \\
\frac{1}{4} \\
\dot{w}\end{array}$ \\
\hline 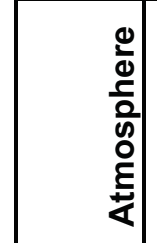 & 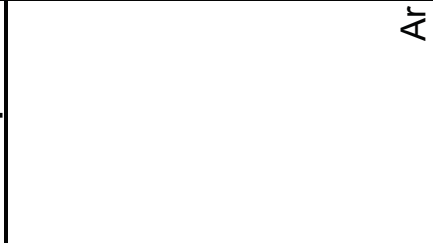 & 衣 & $\bar{z}$ \\
\hline 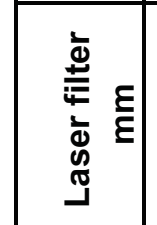 & 음 & 음 & 음 \\
\hline 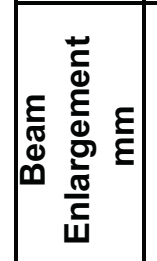 & $\stackrel{\widetilde{N}}{\stackrel{N}{N}}$ & $\stackrel{\stackrel{N}{ }}{ }$ & $\stackrel{\widetilde{N}}{\stackrel{N}{N}}$ \\
\hline $\begin{array}{l}\bar{b} \\
\vdots \\
心 \\
\Phi \\
\infty\end{array}$ & 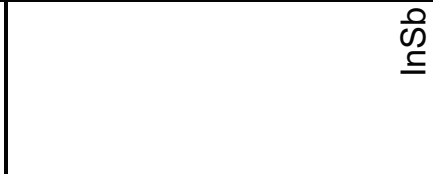 & $\begin{array}{l}\text { की } \\
\text { I }\end{array}$ & $\begin{array}{l}\text { की } \\
\text { की }\end{array}$ \\
\hline 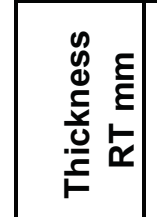 & $\begin{array}{l}\underset{N}{\mathbb{N}} \\
0\end{array}$ & $\underset{\widetilde{N}}{\tilde{N}}$ & 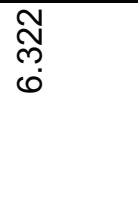 \\
\hline 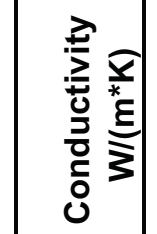 & & & \\
\hline 突 & 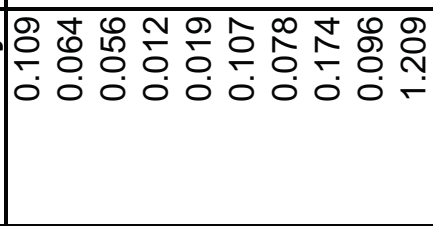 & 突 & 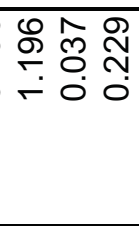 \\
\hline 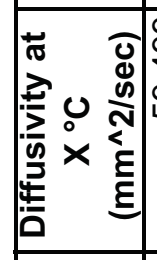 & 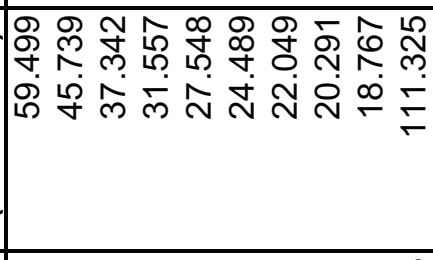 & 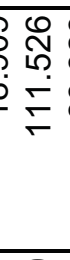 & 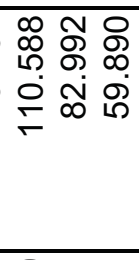 \\
\hline 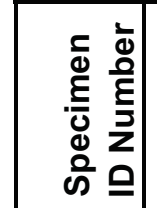 & $\begin{array}{l}\infty \\
\stackrel{0}{\circ} \\
\square\end{array}$ & $\begin{array}{l}8 \\
\\
\end{array}$ & $\begin{array}{l}\stackrel{\circ}{\frac{1}{I}} \\
\end{array}$ \\
\hline
\end{tabular}




\begin{tabular}{|c|c|c|c|}
\hline 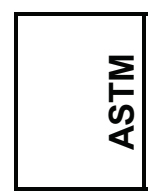 & $\begin{array}{l}\hat{o} \\
\dot{1} \\
\dot{0} \\
\dot{+} \\
\dot{w}\end{array}$ & $\begin{array}{l}\hat{D} \\
\frac{1}{1} \\
\dot{0} \\
\frac{1}{\omega} \\
\end{array}$ & $\begin{array}{l}\hat{o} \\
\frac{1}{0} \\
\stackrel{0}{+} \\
\dot{w}\end{array}$ \\
\hline $\begin{array}{c}0 \\
\frac{0}{0} \\
\frac{5}{0} \\
0 \\
0 \\
\frac{1}{4}\end{array}$ & 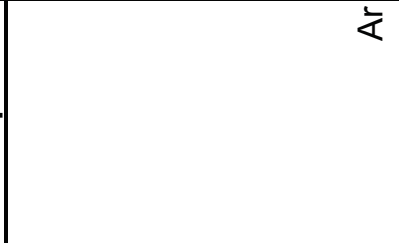 & $\frac{1}{\alpha}$ & $\frac{1}{4}$ \\
\hline 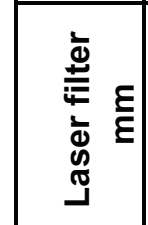 & 음 & 음 & 음 \\
\hline 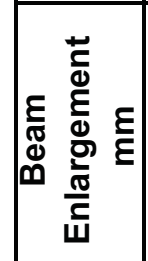 & $\stackrel{\widehat{N}}{\stackrel{N}{N}}$ & 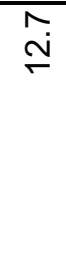 & $\stackrel{\stackrel{\sim}{\sim}}{ }$ \\
\hline 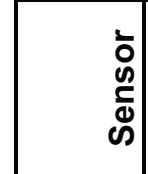 & $\begin{array}{l}\text { की } \\
\text { की }\end{array}$ & 吊 & $\begin{array}{l}\text { की } \\
\text { 足 }\end{array}$ \\
\hline 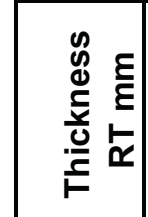 & 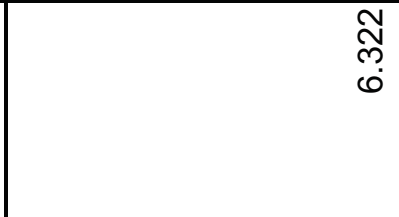 & 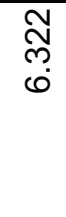 & $\begin{array}{l}\underset{N}{N} \\
\oplus \\
0\end{array}$ \\
\hline 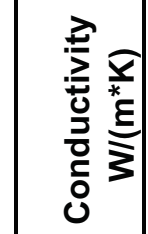 & & & \\
\hline 永辛 & 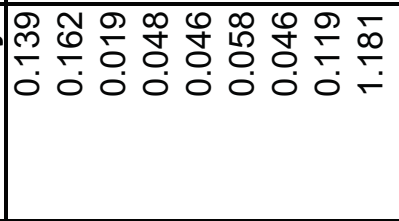 & ${ }_{0}^{\circ}$ & 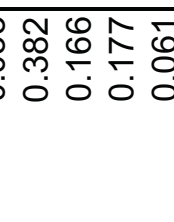 \\
\hline 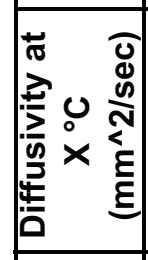 & 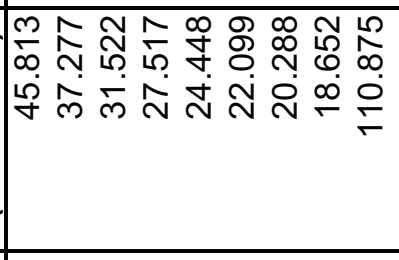 & 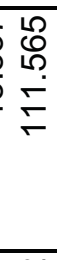 & 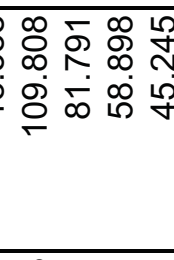 \\
\hline 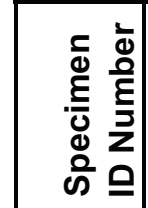 & $\begin{array}{l}\bar{\delta} \\
\mathcal{I}\end{array}$ & $\begin{array}{l}\mathcal{O} \\
\text { ב } \\
\end{array}$ & $\begin{array}{l}\text { O) } \\
\text { I }\end{array}$ \\
\hline
\end{tabular}




\begin{tabular}{|c|c|c|c|}
\hline 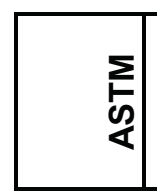 & $\begin{array}{l}\frac{\hat{o}}{1} \\
\frac{1}{0} \\
\frac{1}{+} \\
\dot{w}\end{array}$ & $\begin{array}{l}\hat{o} \\
\frac{1}{0} \\
\stackrel{0}{+} \\
\dot{\omega}\end{array}$ & $\begin{array}{l}\hat{O} \\
\frac{1}{0} \\
\frac{0}{+} \\
\dot{w}\end{array}$ \\
\hline 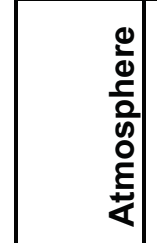 & 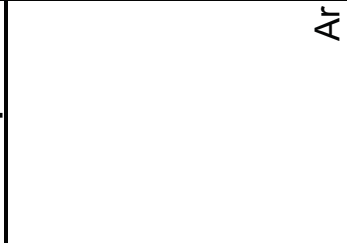 & 衣 & 交 \\
\hline 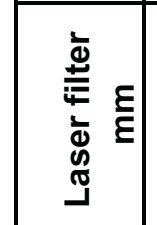 & 음 & 음 & 음 \\
\hline 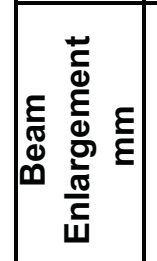 & $\stackrel{\widetilde{N}}{\stackrel{N}{N}}$ & $\stackrel{\stackrel{N}{\sim}}{ }$ & $\stackrel{\stackrel{N}{N}}{ }$ \\
\hline $\begin{array}{l}\bar{b} \\
\vdots \\
心 \\
\Phi \\
\infty\end{array}$ & $\begin{array}{l}\text { की } \\
\text { की }\end{array}$ & $\begin{array}{l}\text { की } \\
\text { I }\end{array}$ & $\begin{array}{l}\text { की } \\
\text { Ф }\end{array}$ \\
\hline 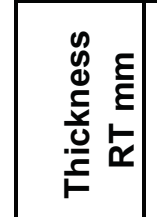 & $\begin{array}{l}\underset{N}{\mathbb{N}} \\
\text { }\end{array}$ & $\underset{\widetilde{N}}{\tilde{N}}$ & 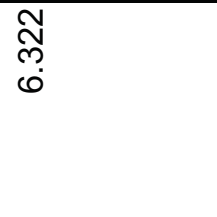 \\
\hline 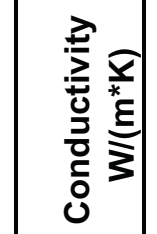 & & & \\
\hline 焉 & 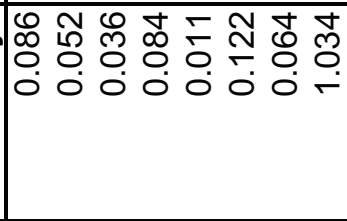 & 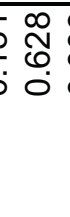 & 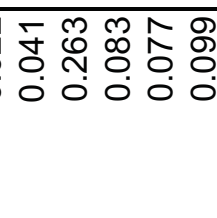 \\
\hline 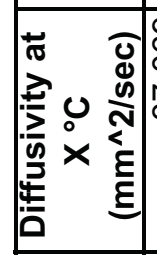 & 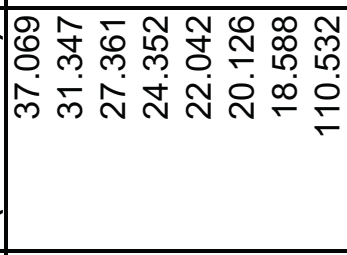 & 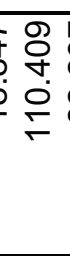 & 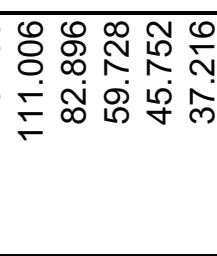 \\
\hline 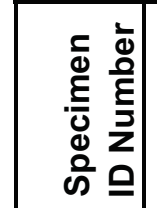 & $\begin{array}{l}\text { U } \\
\text { I }\end{array}$ & $\begin{array}{l}\text { \& } \\
\text { I }\end{array}$ & $\begin{array}{l}8 \\
\text { O }\end{array}$ \\
\hline
\end{tabular}




\begin{tabular}{|c|c|c|c|}
\hline 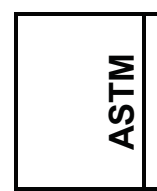 & $\begin{array}{l}\hat{o} \\
\frac{1}{0} \\
\dot{o} \\
\dot{w} \\
\end{array}$ & $\begin{array}{l}\hat{S} \\
\frac{1}{1} \\
\dot{0} \\
\dot{J} \\
\end{array}$ & 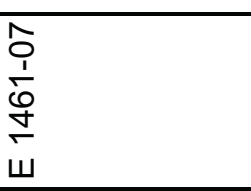 \\
\hline 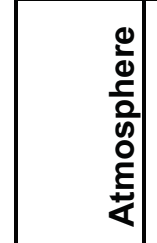 & 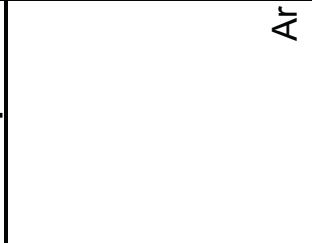 & ¿ & ¿ \\
\hline 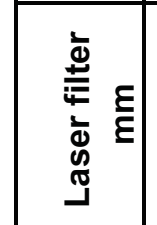 & 음 & 응 & 음 \\
\hline 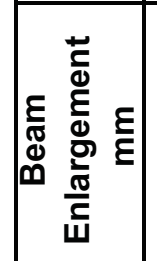 & $\stackrel{\widehat{N}}{\stackrel{N}{N}}$ & $\stackrel{\underline{N}}{\stackrel{N}{N}}$ & $\stackrel{\stackrel{N}{\mathfrak{N}}}{ }$ \\
\hline $\begin{array}{l}\vdots \\
\vdots \\
0 \\
⿱ 亠 凶 \\
\infty\end{array}$ & $\begin{array}{l}\text { की } \\
\text { की }\end{array}$ & $\begin{array}{l}\text { 号 } \\
\text { 足 }\end{array}$ & $\begin{array}{l}\text { 足 } \\
\text { 足 }\end{array}$ \\
\hline 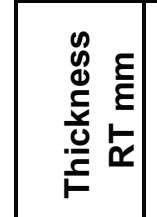 & 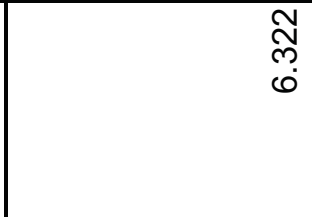 & $\underset{\widetilde{N}}{\mathbb{N}}$ & స్లై \\
\hline 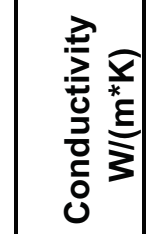 & & & \\
\hline 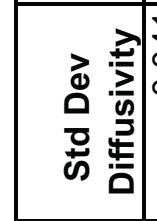 & 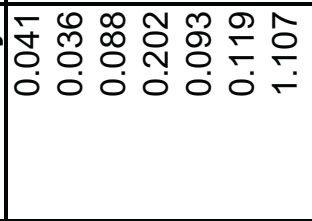 & $\tau$ & 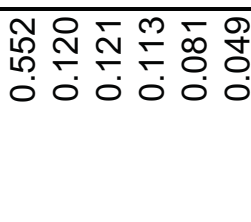 \\
\hline 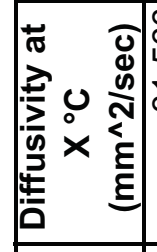 & 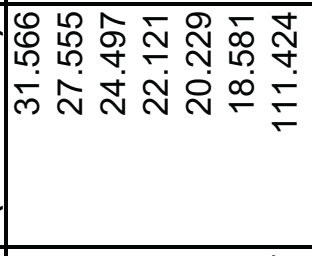 & $\begin{array}{l}\frac{0}{0} \\
\hat{0} \\
0 \\
\stackrel{0}{ }\end{array}$ & 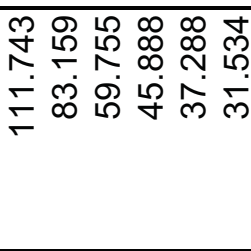 \\
\hline 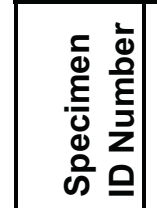 & $\begin{array}{l}\text { 今. } \\
\text { I }\end{array}$ & $\begin{array}{l}\text { 文 } \\
\text { บ }\end{array}$ & $\begin{array}{l}\text { O } \\
\text { I }\end{array}$ \\
\hline
\end{tabular}




\begin{tabular}{|c|c|c|c|}
\hline 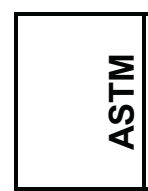 & $\begin{array}{l}\hat{o} \\
\frac{1}{0} \\
\frac{0}{+} \\
\dot{W}\end{array}$ & 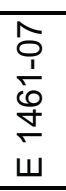 & 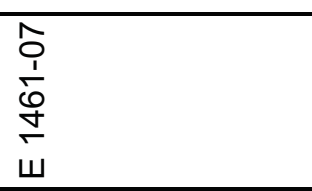 \\
\hline 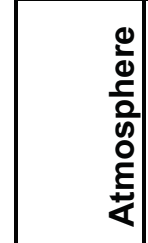 & 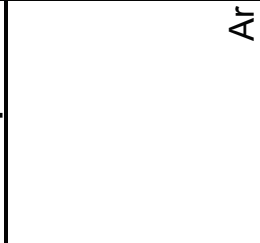 & $\frac{1}{z}$ & $\dot{z}$ \\
\hline 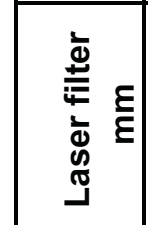 & 음 & 음 & 음 \\
\hline 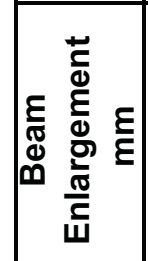 & $\stackrel{\bar{N}}{\stackrel{N}{N}}$ & $\stackrel{\tilde{N}}{\stackrel{N}{S}}$ & 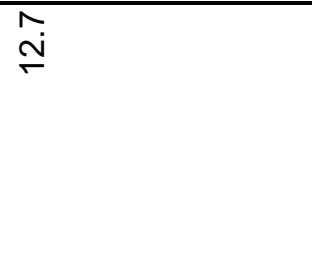 \\
\hline 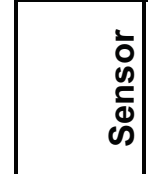 & $\begin{array}{l}\text { की } \\
\text { की }\end{array}$ & 号 & $\begin{array}{l}\text { की } \\
\text { 足 }\end{array}$ \\
\hline 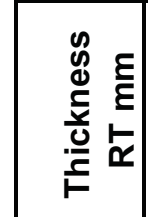 & 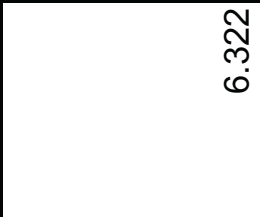 & 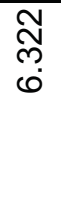 & $\begin{array}{l}\mathbb{N} \\
\text { ִָ } \\
0\end{array}$ \\
\hline 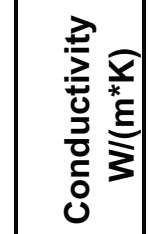 & & & \\
\hline 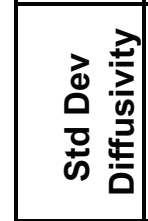 & 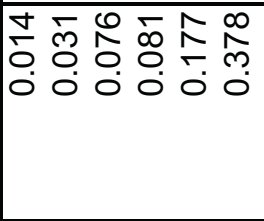 & m. & 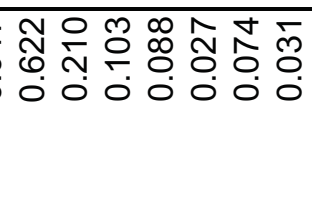 \\
\hline 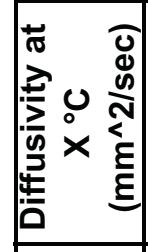 & 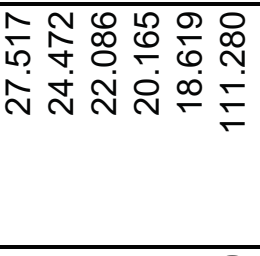 & $\begin{array}{l}0 \\
0 \\
0 \\
8 \\
0 \\
0\end{array}$ & 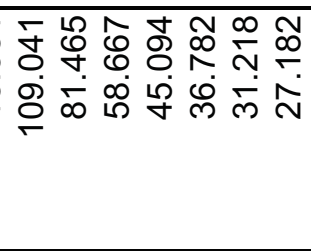 \\
\hline 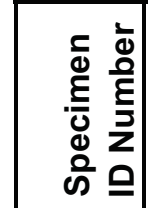 & $\begin{array}{l}\stackrel{\circ}{\simeq} \\
\text { I }\end{array}$ & ì & $\begin{array}{l}\text { o } \\
\text { m }\end{array}$ \\
\hline
\end{tabular}




\begin{tabular}{|c|c|}
\hline 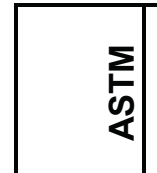 & 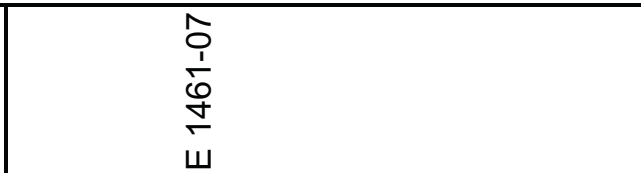 \\
\hline 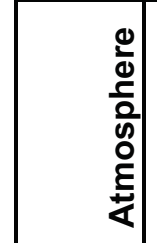 & 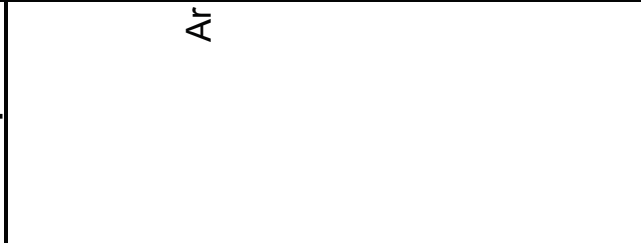 \\
\hline 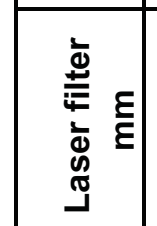 & 음 \\
\hline 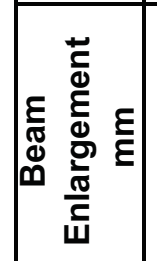 & $\stackrel{\stackrel{N}{\sim}}{\stackrel{N}{r}}$ \\
\hline $\begin{array}{l}\bar{b} \\
\dot{g} \\
\bar{d} \\
\oplus\end{array}$ & 员 \\
\hline 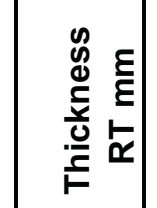 & $\begin{array}{l}\text { N } \\
\text { } \\
0\end{array}$ \\
\hline 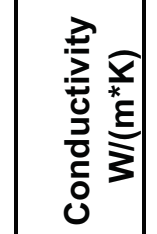 & \\
\hline 吾辛 & 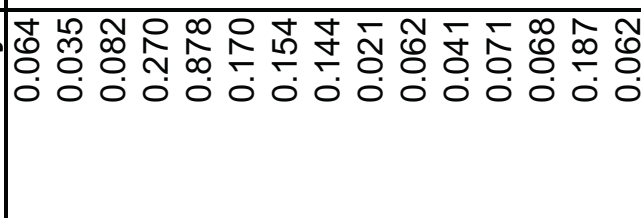 \\
\hline 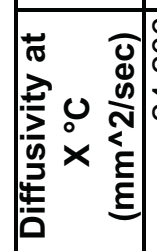 & 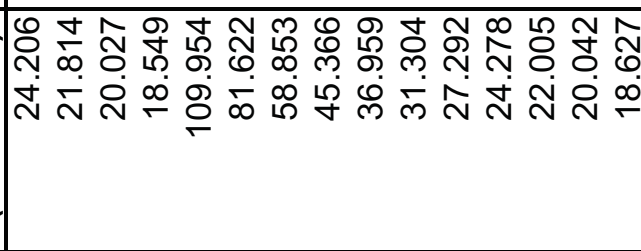 \\
\hline 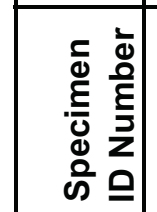 & $\begin{array}{l}\text { J } \\
\text { M }\end{array}$ \\
\hline
\end{tabular}




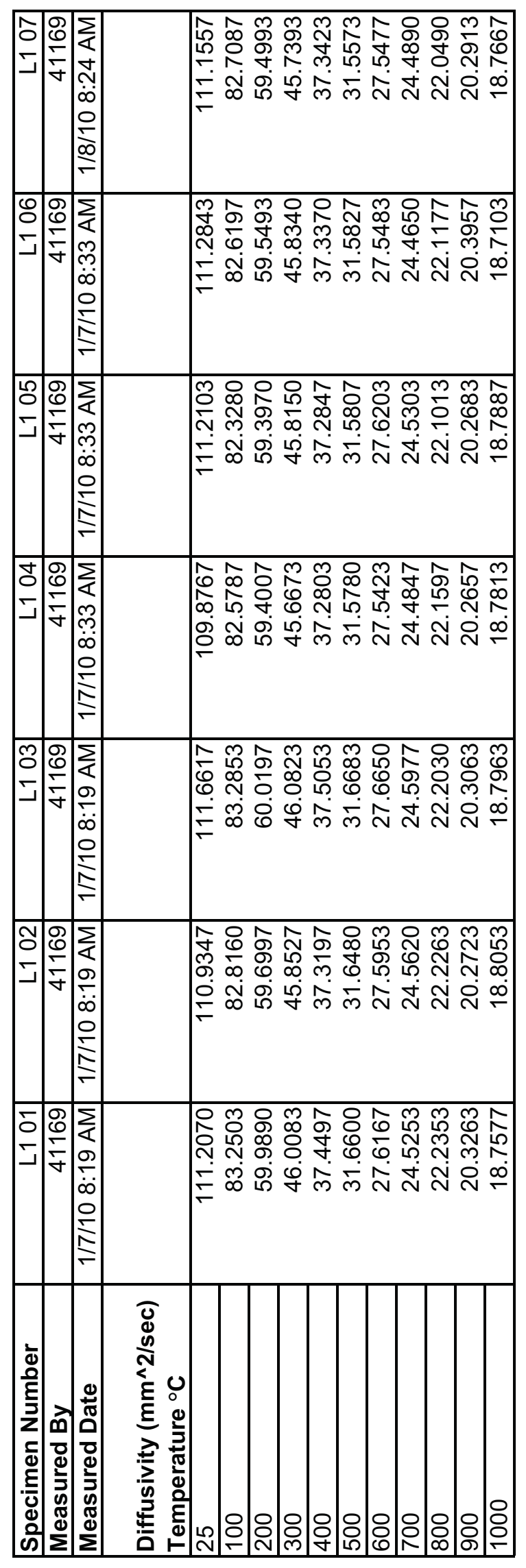




\begin{tabular}{|c|c|c|c|c|}
\hline 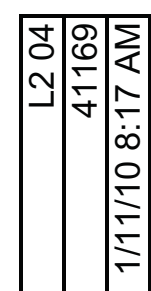 & & \multicolumn{3}{|c|}{ 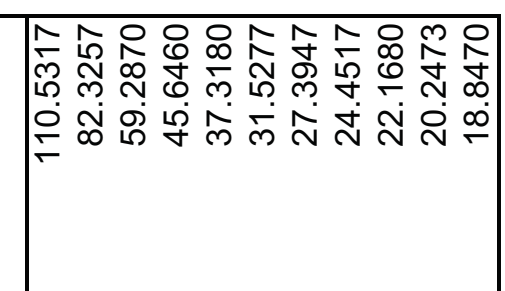 } \\
\hline 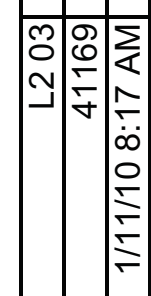 & & \multicolumn{3}{|c|}{ 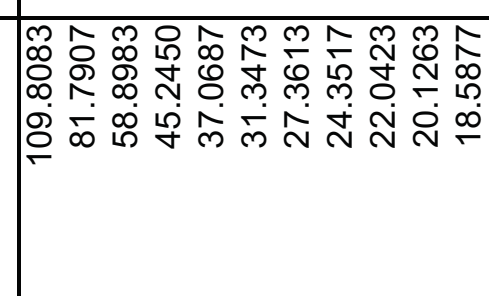 } \\
\hline 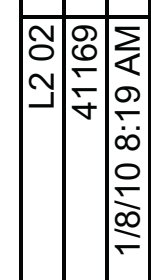 & & \multicolumn{3}{|c|}{ 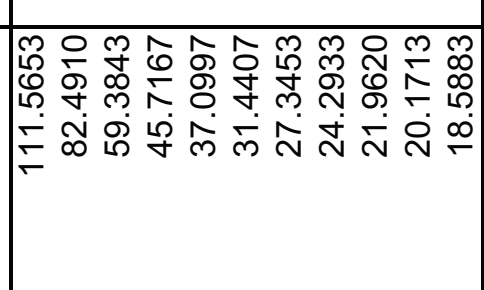 } \\
\hline 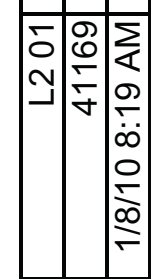 & & \multicolumn{3}{|c|}{ 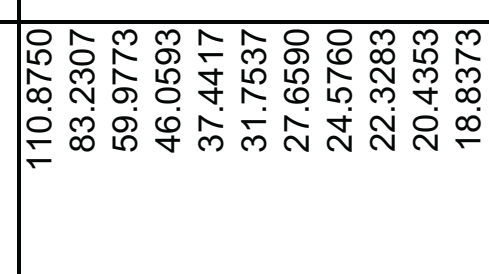 } \\
\hline 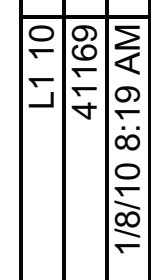 & & \multicolumn{3}{|c|}{ 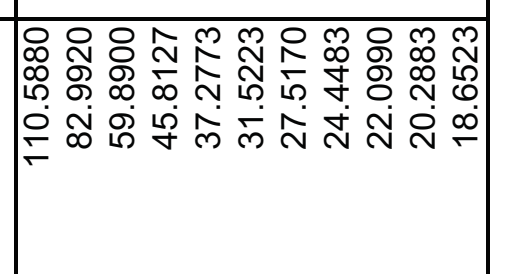 } \\
\hline$\checkmark=$ & & \multicolumn{3}{|c|}{ 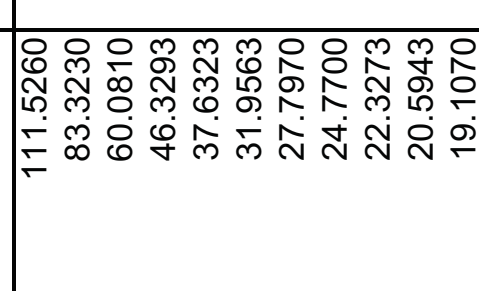 } \\
\hline 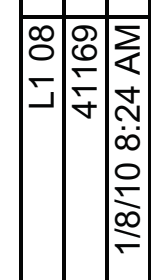 & & \multicolumn{3}{|c|}{ 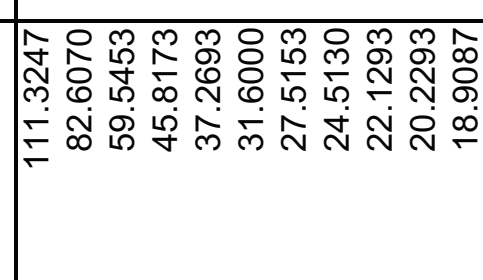 } \\
\hline 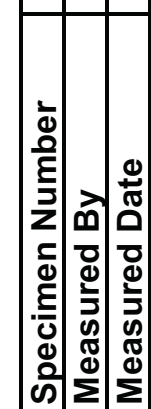 & 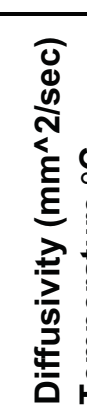 & & & 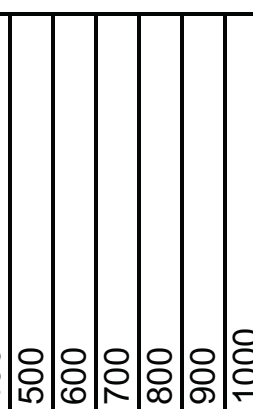 \\
\hline
\end{tabular}




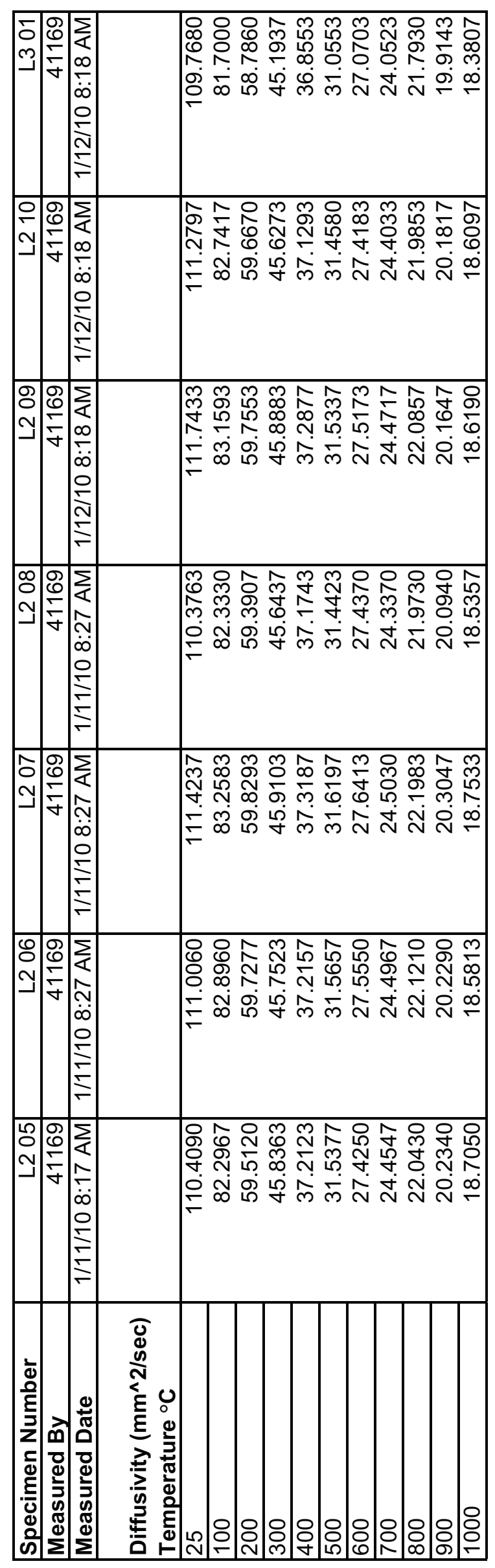




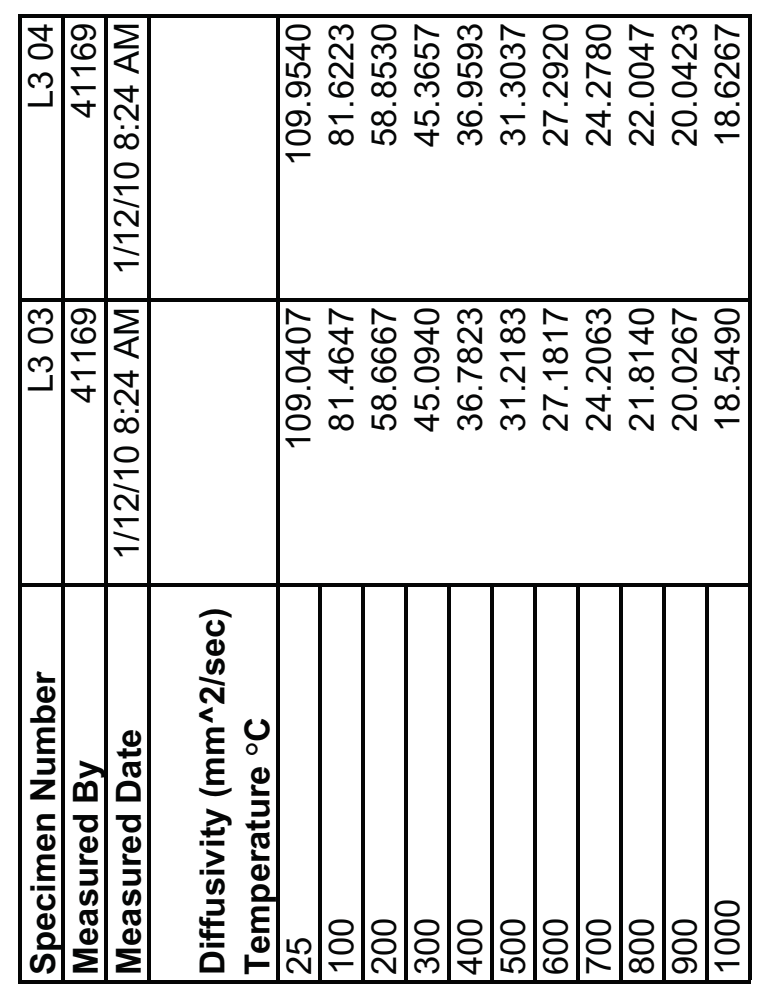

지 


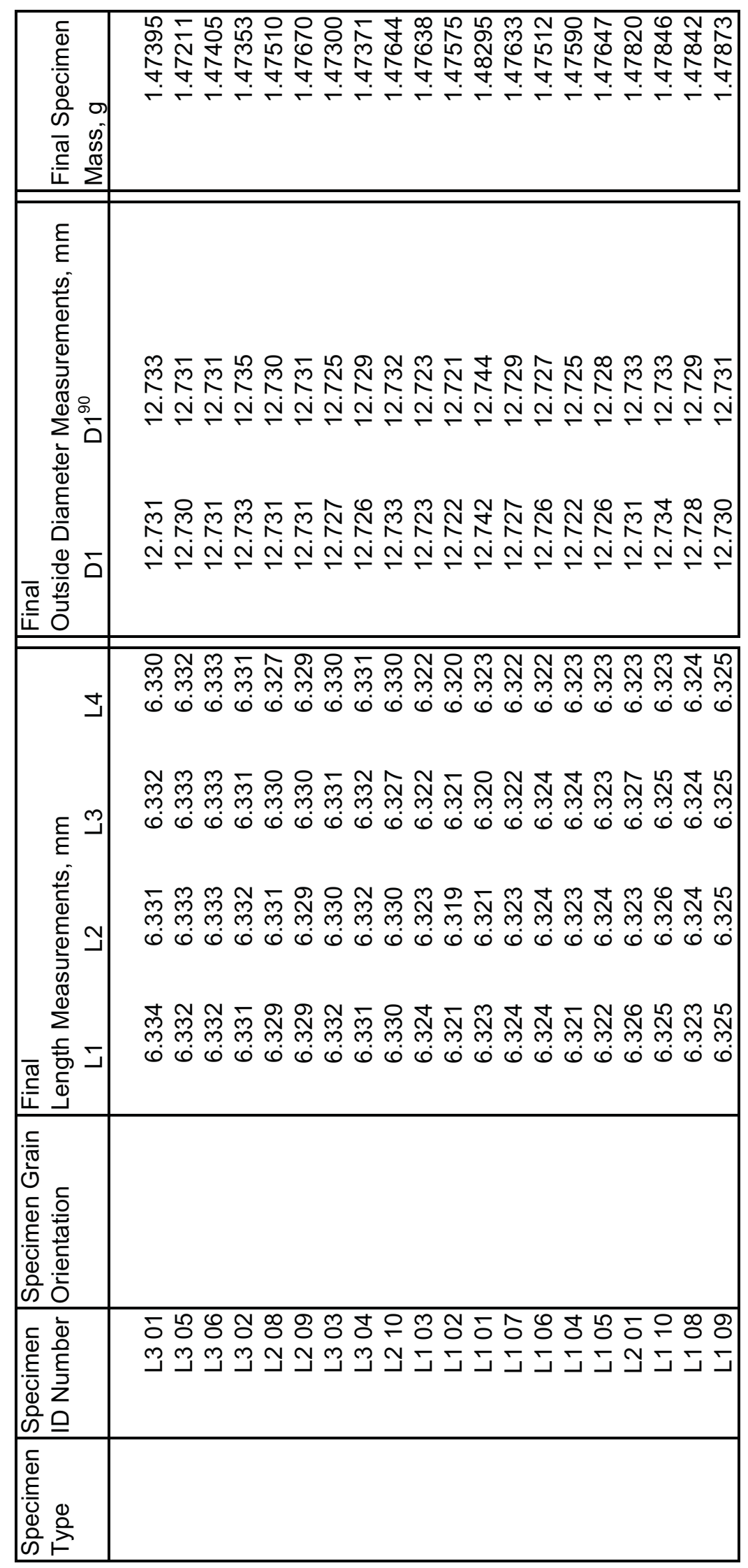




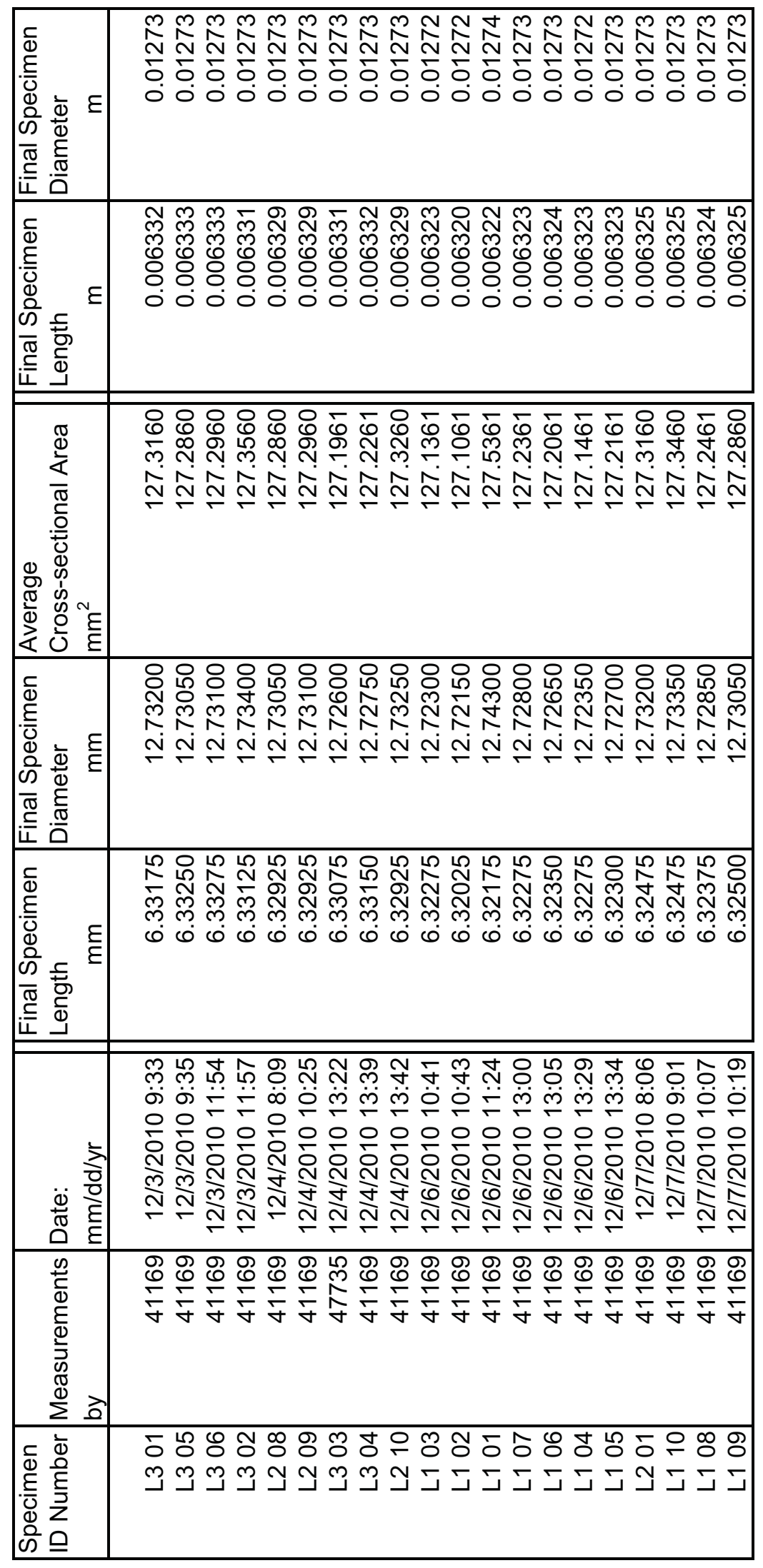




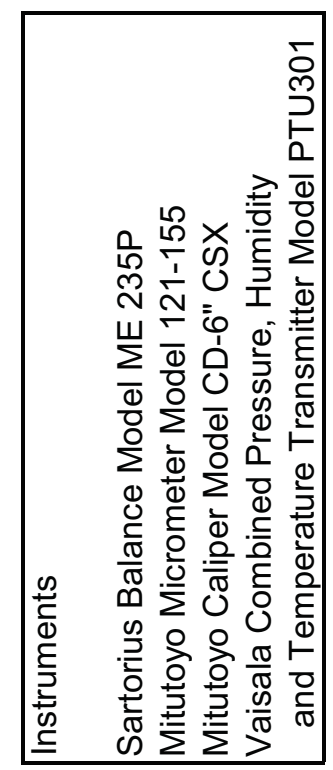

\begin{tabular}{|c|c|}
\hline 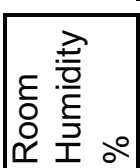 & 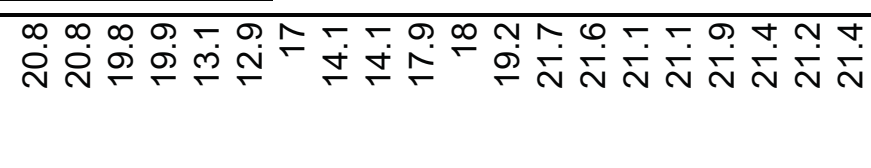 \\
\hline 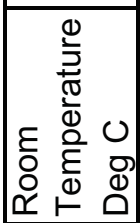 & 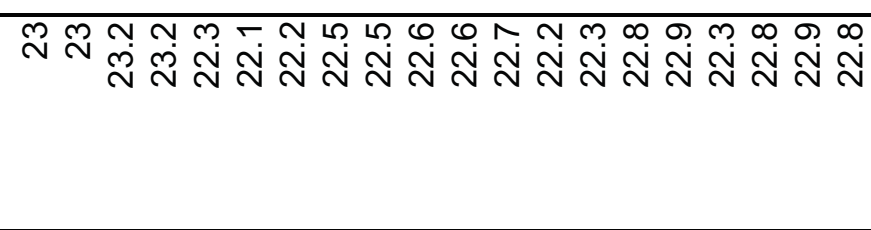 \\
\hline 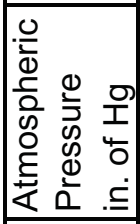 & 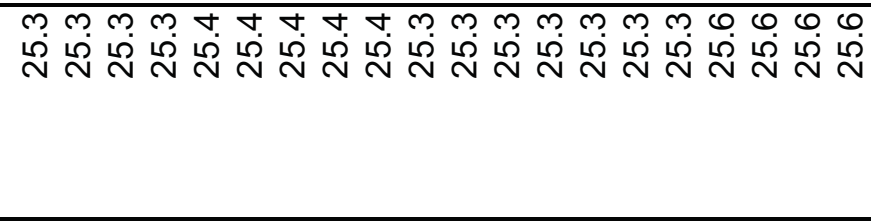 \\
\hline 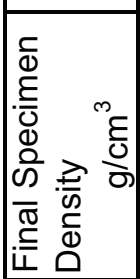 & 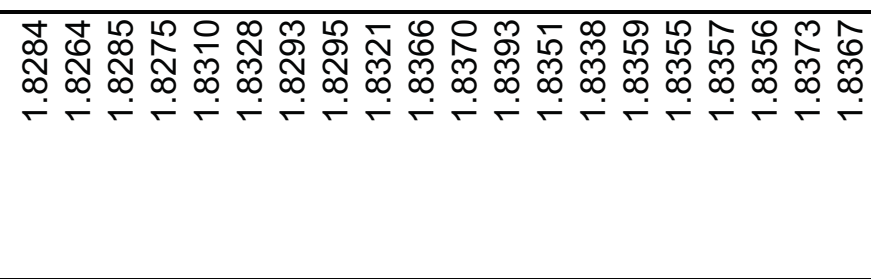 \\
\hline 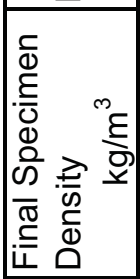 & 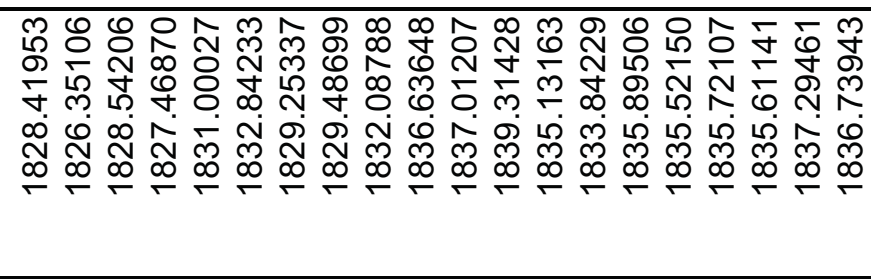 \\
\hline 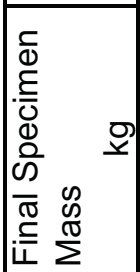 & 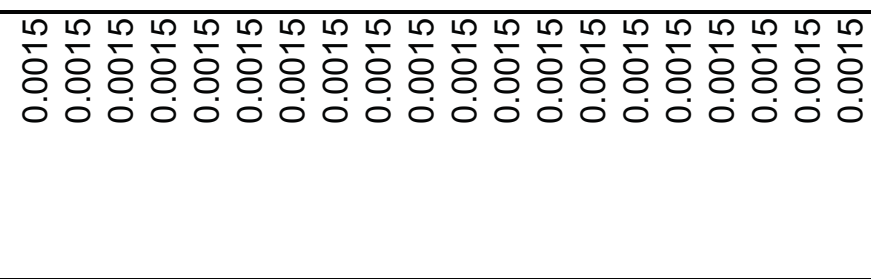 \\
\hline 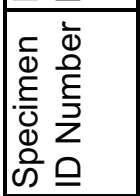 & 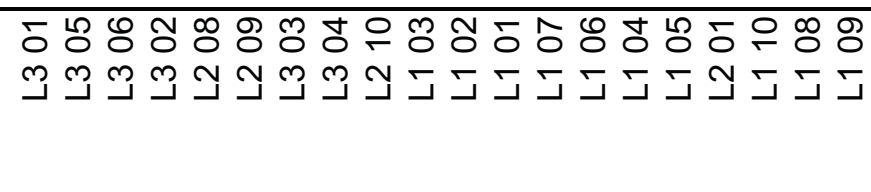 \\
\hline
\end{tabular}

